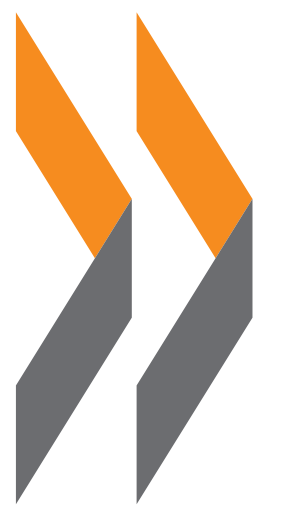

\title{
Education at a Glance 2015 OECD INDICATORS
}

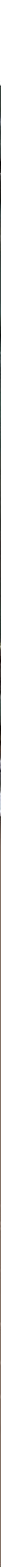

()ㅇ $\mathrm{OECD}$ 



\title{
Education at a Glance 2015
}

\author{
OECD INDICATORS
}

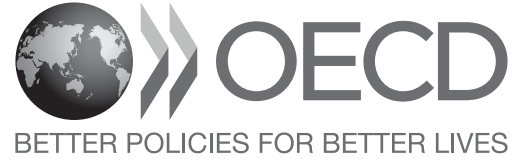


This work is published under the responsibility of the Secretary-General of the OECD. The opinions expressed and arguments employed herein do not necessarily reflect the official views of the OECD member countries.

This document and any map included herein are without prejudice to the status of or sovereignty over any territory, to the delimitation of international frontiers and boundaries and to the name of any territory, city or area.

\section{Please cite this publication as:}

OECD (2015), Education at a Glance 2015: OECD Indicators, OECD Publishing.

http://dx.doi.org/10.1787/eag-2015-en

ISBN 978-92-64-24208-1 (print)

ISBN 978-92-64-24209-8 (PDF)

The statistical data for Israel are supplied by and under the responsibility of the relevant Israeli authorities. The use of such data by the OECD is without prejudice to the status of the Golan Heights, East Jerusalem and Israeli settlements in the West Bank under the terms of international law.

Photo credits:

Stocklib Image Bank @ Cathy Yeulet

Fotolia.com @ Feng Yu

Getty Images $\odot$ blue jean images

Corrigenda to OECD publications may be found on line at: www.oecd.org/publishing/corrigenda.

(c) OECD 2015

You can copy, download or print OECD content for your own use, and you can include excerpts from OECD publications, databases and multimedia products in your own documents, presentations, blogs, websites and teaching materials, provided that suitable acknowledgement of OECD as source and copyright owner is given. All requests for public or commercial use and translation rights should be submitted to rights@oecd.org. Requests for permission to photocopy portions of this material for public or commercial use shall be addressed directly to the Copyright Clearance Center (CCC) at info@copyright.com or the Centre français d'exploitation du droit de copie (CFC) at contact@cfcopies.com. 


\section{FOREWORD}

Governments are increasingly looking to international comparisons of education opportunities and outcomes as they develop policies to enhance individuals' social and economic prospects, provide incentives for greater efficiency in schooling, and help to mobilise resources to meet rising demands. The OECD Directorate for Education and Skills contributes to these efforts by developing and analysing the quantitative, internationally comparable indicators that it publishes annually in Education at a Glance. Together with OECD country policy reviews, these indicators can be used to assist governments in building more effective and equitable education systems.

Education at a Glance addresses the needs of a range of users, from governments seeking to learn policy lessons to academics requiring data for further analysis to the general public wanting to monitor how schools are progressing in producing world-class students. The publication examines the quality of learning outcomes, the policy levers and contextual factors that shape these outcomes, and the broader private and social returns that accrue to investments in education.

Education at a Glance is the product of a long-standing, collaborative effort between OECD governments, the experts and institutions working within the framework of the OECD Indicators of Education Systems (INES) programme and the OECD Secretariat. The publication was prepared by the staff of the Innovation and Measuring Progress Division of the OECD Directorate for Education and Skills, under the responsibility of Dirk Van Damme and Corinne Heckmann and in co-operation with Étienne Albiser, Diogo Amaro de Paula, Esther Carvalhaes, Rodrigo Castañeda Valle, Éric Charbonnier, Karinne Logez, Soumaya Maghnouj, Gabriele Marconi, Ignacio Marín, Camila de Moraes, Simon Normandeau, David Potrel, Joris Ranchin, Cuauhtémoc Rebolledo Gómez, Wida Rogh, Gara Rojas González, Markus Schwabe, David Valenciano and Jean Yip. Administrative support was provided by Laetitia Dehelle, and additional advice as well as analytical support were provided by Francesco Avvisati, João Collet, Youna Lanos, William Herrera Penagos, Giannina Rech, Aurélie Rigaud and Antje Thiemann. Marilyn Achiron, Louise Binns, Marika Boiron, Célia Braga-Schich, Jennifer Cannon, Cassandra Davis, Lynda Hawe, Sophie Limoges, Camilla Lorentzen and Eric Magnusson provided valuable support in the editorial and production process. The development of the publication was steered by member countries through the INES Working Party and facilitated by the INES Networks. The members of the various bodies as well as the individual experts who have contributed to this publication and to OECD INES more generally are listed at the end of the book.

While much progress has been accomplished in recent years, member countries and the OECD continue to strive to strengthen the link between policy needs and the best available internationally comparable data. This presents various challenges and trade-offs. First, the indicators need to respond to education issues that are high on national policy agendas, and where the international comparative perspective can offer added value to what can be accomplished through national analysis and evaluation. Second, while the indicators should be as comparable as possible, they also need to be as country-specific as is necessary to allow for historical, systemic and cultural differences between countries. Third, the indicators need to be presented in as straightforward a manner as possible, while remaining sufficiently complex to reflect multi-faceted realities. Fourth, there is a general desire to keep the indicator set as small as possible, but large enough to be useful to policy makers across countries that face different challenges in education.

The OECD will continue not only to address these challenges vigorously and develop indicators in areas where it is feasible and promising to develop data, but also to advance in areas where a considerable investment still needs to be made in conceptual work. The OECD Programme for International Student Assessment (PISA) and its extension through the Survey of Adult Skills, a product of the Programme for the International Assessment of Adult Competencies (PIAAC), and the OECD Teaching and Learning International Survey (TALIS), are major efforts to this end. 



\section{TABLE OF CONTENTS}

Editorial … - 13

Introduction $\quad 15$

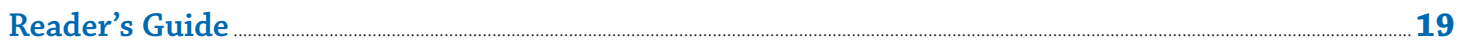

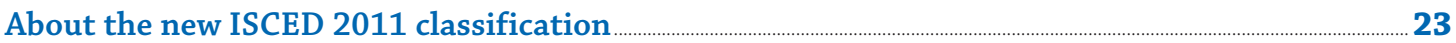

Executive Summary

CHAPTER A THE OUTPUT OF EDUCATIONAL INSTITUTIONS AND THE IMPACT

OF LEARNING 29

Indicator A1 To what level have adults studied?

Table A1.1a

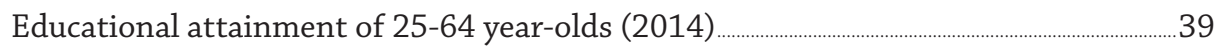

Table A1.2a Percentage of adults who have attained at least upper secondary education, by age group (2014)

Table A1.3a Percentage of adults who have attained tertiary education, by type of programme and age group (2014) … 41

Table A1.4a Trends in educational attainment, by age group (2000, 2005, 2010 and 2014)............42

Table A1.5a Adults whose highest level of education is upper secondary or post-secondary non-tertiary education, by programme orientation and gender (2014) .......................45

Table A1.6a Skills and readiness to use information and communication technologies for problem solving among 25-64 year-olds, by educational attainment (2012) _.......46

Indicator A2

How many students are expected to complete upper secondary education? _.......48

Table A2.1

Upper secondary and post-secondary non-tertiary graduation rates (2013) .............55

Table A2.2 Profile of upper secondary graduates, from general and vocational programmes (2013) ...56

Table A2.3 Profile of post-secondary non-tertiary graduates from vocational programmes (2013)....57

Table A2.4 Upper secondary and post-secondary non-tertiary graduation rates (2005 and 2013).

Indicator A3 How many young people are expected to complete tertiary education and what is their profile?

Table A3.1

First-time graduation rates, by tertiary ISCED level (2013).

Table A3.2

Profile of a first-time tertiary graduate (2013).

Table A3.3

Distribution of all tertiary graduates, by field of education (2013).

Table A3.4

Percentage of female and international first-time graduates, by tertiary ISCED level (2013).

Table A3.5 Percentage of all students and international students who graduate from sciences and engineering programmes, by tertiary ISCED level (2013)

Indicator A4 To what extent does parents' education influence their children's educational attainment? 
Table A4.2e First generation tertiary-educated adults, by part-time/full-time status, gender and age group (2012)

Table A4.3a Likelihood of being among the top 25\% in earnings, by parents' and own educational attainment, gender and age group (2012)

Indicator A5

How does educational attainment affect participation in the labour market? …......92

Table A5.1a

Employment rates, by educational attainment (2014).

Table A5.2a

Unemployment rates, by educational attainment (2014)

Table A5.3a

Trends in employment rates, by educational attainment and age group (2000, 2005, 2010 and 2014)

Table A5.4a

Trends in unemployment rates, by educational attainment and age group (2000, 2005, 2010 and 2014)

Table A5.5a

Employment, unemployment and inactivity rates of adults with upper secondary

or post-secondary non-tertiary, by programme orientation (2014)

Table A5.6a

Skills and readiness to use information and communication technologies for problem solving among 25-64 year-olds, by ICT skills required at work (2012)......114

Indicator $\mathrm{A} 6$

What are the earnings advantages from education?

Table A6.1a

Relative earnings of workers, by educational attainment, age group and gender (2013)

Table A6.2a

Differences in earnings between female and male workers, by educational attainment and age group (2013)

Table A6.3

Percentage of full-time, full-year earners, part-time earners and people without earnings, by educational attainment, age group and gender (2013)

Table A6.5a

Relative earnings of 15-24 year-old students with income from employment, by educational attainment and gender (2013).

Table A6.5b

Indicator A7 Percentage of 15-29 year-olds with income from employment among all 15-29 year-olds, by age group, gender and student status (2013)

What are the financial incentives to invest in education?

Table A7.1a

Private costs and benefits for a man attaining upper secondary or post-secondary non-tertiary education (2011)

Table A7.1b

Private costs and benefits for a woman attaining upper secondary or post-secondary non-tertiary education (2011)

Table A7.2a

Public costs and benefits for a man attaining upper secondary or post-secondary non-tertiary education (2011)

Table A7.2b

Public costs and benefits for a woman attaining upper secondary or post-secondary

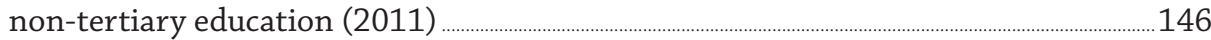

Table A7.3a

Private costs and benefits for a man attaining tertiary education (2011) 147

Table A7.3b

Private costs and benefits for a woman attaining tertiary education (2011) 148

Table A7.4a

Public costs and benefits for a man attaining tertiary education (2011) 149

Table A7.4b

Public costs and benefits for a woman attaining tertiary education (2011) 150

Indicator $\mathrm{A} 8$

How are social outcomes related to education?

Table A8.1

Likelihood of reporting to be in good health, by educational attainment (2012).......161

Table A8.2

Likelihood of reporting to volunteer at least once a month, by educational attainment (2012)

Table A8.3a

Likelihood of reporting to trust others, by educational attainment (2012)

Table A8.4

Likelihood of reporting to believe they have a say in government,

by educational attainment (2012) 
Indicator A9 What is the impact of skills on employment and earnings?

Table A9.1 (L) Likelihood of being employed, by educational attainment and literacy proficiency (2012)

Table A9.1 (N) Likelihood of being employed, by educational attainment and numeracy proficiency (2012)

Table A9.1 (P) Likelihood of being employed, by educational attainment and skills and readiness to use information and communication technologies for problem solving (2012) ....... 179

Table A9.2 (L) Difference in hourly earnings, by educational attainment and literacy proficiency (2012)

Table A9.2 (N) Difference in hourly earnings, by educational attainment and numeracy proficiency (2012)

Table A9.2 (P) Difference in hourly earnings, by educational attainment and skills and readiness to use information and communication technologies for problem solving (2012)

Indicator $\mathrm{A} 10$

Where are the gender gaps in education and employment?

Table A10.1

Percentage of low achievers in mathematics, reading and science, by gender (PISA 2012)

Table A10.2

Gender disparities at age 15 in participation in general, vocational and modular programmes.

Table A10.3

Percentage of adults, by field of study of the highest level of education attained and gender

CHAPTER B

FINANCIAL AND HUMAN RESOURCES INVESTED IN EDUCATION

Indicator B1

How much is spent per student?

Table B1.1a

Annual expenditure per student by educational institutions for all services (2012) ...... 219

Table B1.2

Annual expenditure per student by educational institutions for core services, ancillary services and R\&D (2012)

Table B1.4

Annual expenditure per student by educational institutions for all services, relative to per capita GDP (2012)

Table B1.5a

Table B1.5b

Change in expenditure per student by educational institutions for all services, relative to different factors, at the primary, secondary and post-secondary non-tertiary levels of education (2000, 2005, 2008, 2010, 2011, 2012).

Change in expenditure per student by tertiary education institutions for all services, relative to different factors (2000, 2005, 2008, 2010, 2011, 2012)

Table B1.6

Annual expenditure per student by secondary educational institutions for all services, by type of programme (2012)

Indicator B2

What proportion of national wealth is spent on education?

Table B2.1

Expenditure on educational institutions as a percentage of GDP, by level of education (2012).

Table B2.2

Trends in expenditure on educational institutions as a percentage of GDP, by level of education (2000, 2005, 2008, 2010, 2011, 2012).

Table B2.3

Expenditure on educational institutions as a percentage of GDP, by source of funding and level of education (2012)

Table B2.4

Change in public expenditure on educational institutions, as a percentage of GDP (2008, 2009, 2010, 2011, 2012) 
Indicator B3 How much public and private investment in education is there?

Table B3.1

Relative proportions of public and private expenditure on educational institutions, by level of education (2012)

Table B3.2a Trends in the proportion of public expenditure on educational institutions and index of change in public and private expenditure, at primary, secondary, post-secondary non-tertiary level (2000, 2005, 2008, 2010 to 2012)

Table B3.2b Trends in the proportion of public expenditure on tertiary education institutions and index of change in public and private expenditure (2000, 2005, 2008, 2010 to 2012) 250

Table B3.3

Annual public expenditure on educational institutions per student, by type of institution (2012)

Indicator B4

What is the total public spending on education?

Table B4.1

Total public expenditure on education (2012)

Table B4.2

(2000, 2005, 2008, 2010, 2011, and 2012) 260

Table B4.3

Indicator B5

Sources of public funds for education, for primary, secondary and post-secondary non-tertiary education (2012)

Table B5.1a

How much do tertiary students pay and what public support do they receive? .....262

Estimated annual average tuition fees charged by educational institutions

(bachelor's, master's, doctoral or equivalent level) (2013-14)

Table B5.3

Financial

Public loans to students in bachelor's, master's, doctoral or equivalent programmes (2013-14)

Table B5.5

Indicator B6

Repayment and remission of public loans to students in bachelor's, master's, doctoral or equivalent programmes (academic year 2013/14)

On what resources and services is education funding spent?

Table B6.1

Expenditure by primary and secondary educational institutions, by resource category (2012)

Table B6.2

Expenditure by educational institutions, by resource category and level of education (2012).

Indicator B7

Which factors influence the level of expenditure on education?

Table B7.1

Salary cost of teachers per student, by level of education (2013) 299

Table B7.2a

Factors used to compute the salary cost of teachers per student, in primary education (2005, 2010 and 2013)

Table B7.2b Factors used to compute the salary cost of teachers per student, in lower secondary education (2005, 2010 and 2013)

Table B7.3 Contribution of various factors to salary cost of teachers per student in primary education (2005, 2010 and 2013)

Table B7.4

Contribution of various factors to salary cost of teachers per student in lower secondary education (2005, 2010 and 2013)

Table B7.5

Contribution of various factors to salary cost of teachers per student in upper secondary education (2013) 
CHAPTER C

Indicator $\mathrm{C} 1$

Table C1.1a

Table C1.2

Table C1.3

Table C1.4a

Table C1.4b

Table C1.5

Indicator C2

Table C2.1

Table C2.2

Table C2.3

Table C2.4

Table C2.5

Indicator C3

Table C3.1

Table C3.2

Table C3.3

Table C3.4

Indicator C4

Table C4.1

Table C4.2

Table C4.3

Table C4.4

Table C4.5

Indicator C5

Table C5.2a

Table C5.3a

Table C5.4a

Table C5.4b

\section{ACCESS TO EDUCATION, PARTICIPATION AND PROGRESSION}

Percentage of students in upper secondary and post-secondary non-tertiary education, by programme orientation and age group (2013)

Percentage of students in tertiary ISCED levels and total tertiary, by intensity of study and gender (2013)

Percentage of students in primary and secondary education, by type of institution (2013)

Percentage of students in tertiary ISCED level and total tertiary, by type of institution (2013)

Expected years in education from age 5 through 39, by level of education, intensity of study and gender (2013)

How do early childhood education systems differ around the world?

Enrolment rates in early childhood education and primary education, by age (2005, 2013).

Profile of early childhood educational development programmes and pre-primary education (2013)

Expenditure on early childhood educational institutions (2012)

Profile of education-only and integrated pre-primary programmes (2013) 336

Coverage of early childhood education programmes in OECD and partner countries

How many students are expected to enter tertiary education?

First-time entry rates, by tertiary ISCED level (2013)

Profile of first-time new entrants into tertiary education (2013)

Profile of first-time new entrants into bachelor's programmes (2013) ……........................ 350

Profile of first-time new entrants, by level of education (2013)

Who studies abroad and where?

International student mobility and foreign students in tertiary education (2013)....... 363

Distribution of international and foreign students enrolled in tertiary programmes, by field of education (2013)

Distribution of international and foreign students in tertiary education, by country of origin (2013)

Students abroad in tertiary education, by country of destination (2013)

Mobility patterns of foreign and international students (2013) 369

Transition from school to work: Where are the 15-29 year-olds?

Percentage of 15-29 year-olds in education/not in education, by work status, age group and gender (2014)

Percentage of 15-29 year-olds in education/not in education, by educational attainment, work status and gender (2014)

Percentage of 15-29 year-olds in education/not in education, by number of hours worked and age group (2014)

Percentage of 15-29 year-olds in education/not in education, by number of hours worked and gender (2014)

C1
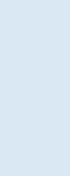

umber of

indicator

in the

2014 edition 
Indicator C6 How many adults participate in education and learning?..

Table C6.1 (P) Participation in employer-sponsored formal and/or non-formal education, by skills and readiness to use information and communication technologies for problem solving (2012)

Table C6.2a

Participation in employer-sponsored education, by educational attainment (2011, 2012)

Table C6.2c Participation in employer-sponsored education, by occupation (2011, 2012)

Table C6.2d Participation in employer-sponsored formal and/or non-formal education, by working hours and contract type (2012).

Table C6.3a

Participation in employer-sponsored formal and/or non-formal education, by use of learning at work (2012)

CHAPTER D

Indicator D1

Table D1.1

Table D1.2

Table D1.3a

Table D1.3b

Indicator D2

Table D2.1

Table D2.2

Table D2.3

Indicator D3

Table D3.1a

Table D3.2a

Table D3.3a

Table D3.4

Table D3.5a

Table D3.7c

Indicator D4

Table D4.1

Table D4.2

Indicator D5

Table D5.1

Table D5.2

Table D5.3

Table D5.4a

Table D5.4b
THE LEARNING ENVIRONMENT AND ORGANISATION OF SCHOOLS 401

How much time do students spend in the classroom?

Instruction time in compulsory general education (2015)

Organisation of compulsory general education (2015).

Instruction time per subject in primary education (2015)

Instruction time per subject in general lower secondary education (2015). 415

What is the student-teacher ratio and how big are classes?

Average class size, by type of institution and level of education (2013)

Ratio of students to teaching staff in educational institutions (2013).

Ratio of students to teaching staff, by type of institution (2013). 425

How much are teachers paid? 426

Teachers' statutory salaries, based on typical qualifications, at different points in teachers' careers (2013)

Teachers' actual salaries relative to wages of similarly educated workers (2013) ....... 442 Comparison of teachers' statutory salaries, based on typical qualifications (2013) .... 443 Average actual teachers' salaries (2013) 444

Trends in teachers' salaries, based on typical qualifications, between 2000 and 2013 445

Teachers' tasks and other criteria related to teachers' base salaries and additional payments, lower secondary level (2013) 446

How much time do teachers spend teaching? 450

Organisation of teachers' working time (2013) …….................................................................... 458

Number of teaching hours per year (2000, 2005, 2010 and 2013) 459

Who are the teachers? 460

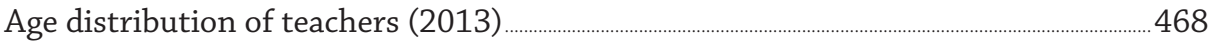

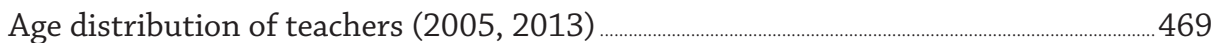

Gender distribution of teachers (2013) ... 470

Teachers' skills and readiness to use information and communication technologies for problem solving (2012)

Teachers' use of information and communication technologies at work, ICT skills required at work, and teachers' confidence in their computer skills (2012)
D1

D2

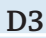

D4

D5 
Indicator D6

Table D6.1c

Table D6.5b

Table D6.9

Table D6.13

Indicator D7

Table D7.1

Table D7.3b

Table D7.4b

Table D7.8b

Table D7.9b

Indicator D8

Table D8.1

Table D8.2

Table D8.3

Table D8.4

ANNEX 1

Table X1.1a

Table X1.1b

Table X1.2a

Table X1.2b

Table X1.3

ANNEX 2

Table X2.1

Table X2.2

Table X2.3

Table X2.4a

Table X2.4b

Table X2.4c
What evaluation and assessment mechanisms are in place?

National/central examinations at the upper secondary level (2015) _..................................486

National/central assessments at the lower secondary level (2015) ........................................ 487

School inspection and school self-evaluation at the lower secondary level (2015)........488

Public school compliance-oriented reports, by domains and receiving groups (2015)....490

What teacher and school leader appraisal systems are in place?

492

Teacher appraisal and school leader appraisal at the lower secondary level (2015).......504

Teacher appraisal at the lower secondary level: Eligibility, governance

and responsibilities (2015)

Features of teacher appraisal at the lower secondary level (2015) 509

School leader appraisal at the lower secondary level: Eligibility, governance and responsibilities (2015)

Features of school leader appraisal at the lower secondary level (2015)

To what extent is information and communication technology used in teaching and learning?

Computer availability, first access to Internet and intensity of Internet use at school (PISA 2012)

Change between 2003 and 2012 in the quality of schools' educational resources

PISA score in reading for 15 -year-olds and mean score-point difference between paper-and-pencil and computer-delivered reading test, by gender (PISA 2012)

Teachers and information and communication technology

Typical graduation ages, by level of education (2013)

Typical age of entry by level of education (2013)

School year and financial year used for the calculation of indicators,

OECD countries.

School year and financial year used for the calculation of indicators, partner countries

Starting and ending age for students in compulsory education (2013)

\section{REFERENCE STATISTICS}

Overview of the economic context using basic variables

(reference period: calendar year 2012, 2012 current prices).

Basic reference statistics

(reference period: calendar year 2012, 2012 current prices)

Basic reference statistics

(reference period: calendar year 2000, 2005, 2008, 2010 and 2011)

Teachers' statutory salaries at different points in their careers, for teachers with typical qualification (2013)

Teachers' statutory salaries at different points in their careers, for teachers with minimum qualification (2013)

Trends in teachers' salaries between 2000 and 2013, for teachers

with typical qualification (2013). 
Table X2.4d Trends in teachers' salaries between 2000 and 2013, for teachers with minimum qualification (2013)

Table X2.4e Reference statistics used in calculating teachers' salaries (2000, 2005-13)

Table X2.4f

Average actual teachers' salaries in national currency (2013)

ANNEX 3

SOURCES, METHODS AND TECHNICAL NOTES

Contributors to this publication

Education Indicators in Focus

\section{Follow OECD Publications on:}

y http://twitter.com/OECD_Pubs

f $h t t p: / / w w w . f a c e b o o k . c o m / O E C D P u b l i c a t i o n s$

in. http://www.linkedin.com/groups/OECD-Publications-4645871

Vium $h$ ttp://www.youtube.com/oecdilibrary

http://www.oecd.org/oecddirect/

\section{This book has...}

StatLinks 部 SL A service that delivers Excel $^{\oplus}$ files from the printed page!

Look for the StatLinks :inst at the bottom of the tables or graphs in this book. To download the matching Excel $\AA$ spreadsheet, just type the link into your Internet browser, starting with the $h t t p: / / d x$.doi.org prefix, or click on the link from the e-book edition.

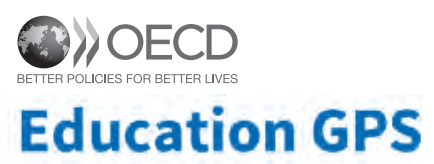

The world of education at your fingertips
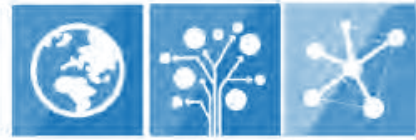

Want to keep up to date with the latest $O E C D$ data and research on education and skills?

http://gpseducation.oecd.org/ 


\section{EDITORIAL \\ Education, learning and the 2030 Agenda for Sustainable Development}

The world of education has changed enormously over the past two decades. Around the globe, more children than ever can go to school and have the opportunity to acquire the skills needed for the workplace, for their community and for life. Since 1992, the first year that Education at a Glance was published, this publication has rigorously documented such transformation as the world moves closer to achieving the goal of providing education for all.

These changes have been accompanied by an expansion of internationally comparable data on education. Only 25 years ago, many people considered education to be too local, too tied to its specific context to be measured against comparative statistical metrics. But enormous progress in statistical techniques, data collection and processing procedures have enabled policy makers, researchers and the public at large to see how education has expanded around the world, and to benchmark performance and draw lessons from other countries. The OECD has been at the forefront of this movement by pushing the measurement agenda forward. Building on the progress in ensuring universal access to, participation in and completion of education, the OECD has developed reliable metrics on student learning outcomes and equity in education, including those used in the OECD Programme for International Student Assessment (PISA) and the OECD Programme for the International Assessment of Adult Competencies (PIAAC).

This new edition of Education at a Glance is published only a few weeks after world leaders defined the global ambitions for the next 15 years by adopting 17 Sustainable Development Goals (SDGs) at the United Nations Summit in New York. Education is a cornerstone of the sustainable development agenda, and the education-related goal aims to "ensure inclusive and equitable quality education and promote lifelong learning opportunities for all" by 2030. The goal is composed of ten targets that, together, represent an ambitious commitment to develop better skills for better lives. What is new about this Education 2030 agenda is its focus on expanded access, inclusion and equity, quality and learning outcomes at all levels of education - and for people of all ages. Five of the ten targets are concerned with improving the quality of education for individual children, young people and adults, to ensure that they acquire better and more relevant knowledge and skills.

To achieve all of these targets, it is essential that every child has access to and completes a quality education of at least 12 years. Efforts to achieve universal access to education must go hand-in-hand with a renewed focus on education quality and equity. Data from PISA, the global metric used to measure the quality of learning outcomes, show why: many countries can boast that all of their children are enrolled in school, but not all of these children achieve even minimum levels of proficiency in the core subjects of reading, mathematics and science by the end of their lower secondary education. That is why the aim of achieving universal basic skills is at the heart of the SDG education agenda. This shift in focus towards quality in education for all means that the 17 SDGs and the 169 targets are universally relevant: no country, no region in the world can claim in 2015 that all of its youth have attained at least a minimum proficiency in foundation skills.

Now that the global community has defined its goal and targets for education, it needs to develop indicators on access, equity and quality that can be measured and tracked over time. These indicators will provide the basis for international accountability and for targeting policies and resources on where they can make the greatest difference. Together with other international organisations, such as UNESCO and its Institute for Statistics (UIS), UNICEF and the World Bank, the OECD stands ready to move this agenda forward. The proposed global indicators for measuring progress towards the education SDG include adaptations of existing international large-scale assessments of 
learning outcomes and skills, such as PISA and PIAAC. The indicators reported in Education at a Glance will continue to provide a strong evidence base for international comparisons of education systems. Indeed, more than two-thirds of the indicators proposed by the UN system for tracking the education SDG are already covered by existing OECD policy and data-collection instruments.

In the years to come, the education SDG targets and indicators will be fully integrated into OECD data-collection mechanisms, reporting and analyses, including Education at a Glance. By doing so, we will ensure that this flagship publication, used as a reference by many people all over the world, will continue to set the standard for measuring and monitoring global progress in education.

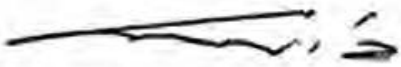

Angel Gurría

OECD Secretary-General 


\section{INTRODUCTION: THE INDICATORS AND THEIR FRAMEWORK}

\section{The organising framework}

Education at a Glance 2015: OECD Indicators offers a rich, comparable and up-to-date array of indicators that reflects a consensus among professionals on how to measure the current state of education internationally. The indicators provide information on the human and financial resources invested in education, how education and learning systems operate and evolve, and the returns to investments in education. The indicators are organised thematically, and each is accompanied by information on the policy context and an interpretation of the data. The education indicators are presented within an organising framework that:

- distinguishes between the actors in education systems: individual learners and teachers, instructional settings and learning environments, education service providers, and the education system as a whole;

- groups the indicators according to whether they address learning outcomes for individuals or countries, policy levers or circumstances that shape these outcomes, or to antecedents or constraints that put policy choices into context; and

- identifies the policy issues to which the indicators relate, with three major categories distinguishing between the quality of education outcomes and education opportunities, issues of equity in education outcomes and opportunities, and the adequacy and effectiveness of resource management.

The following matrix describes the first two dimensions:

\begin{tabular}{|c|c|c|c|c|c|c|c|}
\hline & & 1. & $\begin{array}{l}\text { Education and } \\
\text { learning outputs } \\
\text { and outcomes }\end{array}$ & 2. & $\begin{array}{l}\text { Policy levers and } \\
\text { contexts shaping } \\
\text { education outcomes }\end{array}$ & 3. & $\begin{array}{l}\text { Antecedents or } \\
\text { constraints that } \\
\text { contextualise policy }\end{array}$ \\
\hline & $\begin{array}{l}\text { Individual } \\
\text { participants } \\
\text { in education } \\
\text { and learning }\end{array}$ & 1.I. & $\begin{array}{l}\text { The quality } \\
\text { and distribution } \\
\text { of individual } \\
\text { education } \\
\text { outcomes }\end{array}$ & 2.I. & $\begin{array}{l}\text { Individual } \\
\text { attitudes towards, } \\
\text { engagement in, } \\
\text { and behaviour } \\
\text { in teaching and } \\
\text { learning }\end{array}$ & 3.I. & $\begin{array}{l}\text { Background } \\
\text { characteristics } \\
\text { of the individual } \\
\text { learners and } \\
\text { teachers }\end{array}$ \\
\hline & $\begin{array}{l}\text { Instructional } \\
\text { settings }\end{array}$ & 1.II. ' & $\begin{array}{l}\text { The quality } \\
\text { of instructional } \\
\text { delivery }\end{array}$ & 2.II. & $\begin{array}{l}\text { Pedagogy, learning } \\
\text { practices and } \\
\text { classroom climate }\end{array}$ & 3.II. & $\begin{array}{l}\text { Student learning } \\
\text { conditions and } \\
\text { teacher working } \\
\text { conditions }\end{array}$ \\
\hline & $\begin{array}{l}\text { Providers of } \\
\text { educational services }\end{array}$ & 1.III. ' & $\begin{array}{l}\text { The output of } \\
\text { educational } \\
\text { institutions } \\
\text { and institutional } \\
\text { performance }\end{array}$ & 2.III. & $\begin{array}{l}\text { School environment } \\
\text { and organisation }\end{array}$ & 3.III. & $\begin{array}{l}\text { Characteristics } \\
\text { of the service } \\
\text { providers and } \\
\text { their communities }\end{array}$ \\
\hline & $\begin{array}{l}\text { The education } \\
\text { system as a whole }\end{array}$ & 1.IV. & $\begin{array}{l}\text { The overall } \\
\text { performance of } \\
\text { the education } \\
\text { system }\end{array}$ & 2.IV. & $\begin{array}{l}\text { System-wide } \\
\text { institutional } \\
\text { settings, } \\
\text { resource allocations, } \\
\text { and policies }\end{array}$ & 3.IV. & $\begin{array}{l}\text { The national } \\
\text { educational, } \\
\text { social, economic, } \\
\text { and demographic } \\
\text { contexts }\end{array}$ \\
\hline
\end{tabular}




\section{Actors in education systems}

The OECD Indicators of Education Systems (INES) programme seeks to gauge the performance of national education systems as a whole, rather than to compare individual institutional or other sub-national entities. However, there is increasing recognition that many important features of the development, functioning and impact of education systems can only be assessed through an understanding of learning outcomes and their relationships to inputs and processes at the level of individuals and institutions. To account for this, the indicator framework distinguishes between a macro level, two meso-levels and a micro-level of education systems. These relate to:

- the education system as a whole;

- the educational institutions and providers of educational services;

- the instructional setting and the learning environment within the institutions; and

- the individual participants in education and learning.

To some extent, these levels correspond to the entities from which data are being collected, but their importance mainly centres on the fact that many features of the education system play out quite differently at different levels of the system, which needs to be taken into account when interpreting the indicators. For example, at the level of students within a classroom, the relationship between student achievement and class size may be negative, if students in small classes benefit from improved contact with teachers. At the class or school level, however, students are often intentionally grouped such that weaker or disadvantaged students are placed in smaller classes so that they receive more individual attention. At the school level, therefore, the observed relationship between class size and student achievement is often positive, suggesting that students in larger classes perform better than students in smaller classes. At higher aggregated levels of education systems, the relationship between student achievement and class size is further confounded, e.g. by the socio-economic intake of schools or by factors relating to the learning culture in different countries. Therefore, past analyses that have relied on macro-level data alone have sometimes led to misleading conclusions.

\section{Outcomes, policy levers and antecedents}

The second dimension in the organising framework further groups the indicators at each of the above levels:

- indicators on observed outputs of education systems, as well as indicators related to the impact of knowledge and skills for individuals, societies and economies, are grouped under the sub-heading output and outcomes of education and learning;

- the sub-heading policy levers and contexts groups activities seeking information on the policy levers or circumstances that shape the outputs and outcomes at each level; and

- these policy levers and contexts typically have antecedents - factors that define or constrain policy. These are represented by the sub-heading antecedents and constraints. The antecedents or constraints are usually specific for a given level of the education system; antecedents at a lower level of the system may well be policy levers at a higher level. For teachers and students in a school, for example, teacher qualifications are a given constraint while, at the level of the education system, professional development of teachers is a key policy lever.

\section{Policy issues}

Each of the resulting cells in the framework can then be used to address a variety of issues from different policy perspectives. For the purpose of this framework, policy perspectives are grouped into three classes that constitute the third dimension in the organising framework for INES:

- quality of education outcomes and education opportunities;

- equality of education outcomes and equity in education opportunities; and

- adequacy, effectiveness and efficiency of resource management.

In addition to the dimensions mentioned above, the time perspective in the framework allows for dynamic aspects of the development of education systems to be modelled as well.

The indicators that are published in Education at a Glance 2015 fit within this framework, though often they speak to more than one cell. 
Most of the indicators in Chapter A, The output of educational institutions and the impact of learning, relate to the first column of the matrix describing outputs and outcomes of education. Even so, indicators in Chapter A measuring educational attainment for different generations, for instance, not only provide a measure of the output of the education system, but also provide context for current education policies, helping to shape polices on, for example, lifelong learning.

Chapter B, Financial and human resources invested in education, provides indicators that are either policy levers or antecedents to policy, or sometimes both. For example, expenditure per student is a key policy measure that most directly affects the individual learner, as it acts as a constraint on the learning environment in schools and learning conditions in the classroom.

Chapter C, Access to education, participation and progression, provides indicators that are a mixture of outcome indicators, policy levers and context indicators. Internationalisation of education and progression rates are, for instance, outcome measures to the extent that they indicate the results of policies and practices at the classroom, school and system levels. But they can also provide contexts for establishing policy by identifying areas where policy intervention is necessary to address issues of inequity, for example.

Chapter D, The learning environment and organisation of schools, provides indicators on instruction time, teachers' working time and teachers' salaries that not only represent policy levers that can be manipulated but also provide contexts for the quality of instruction in instructional settings and for the outcomes of individual learners. It also presents data on the profile of teachers, the levels of government at which decisions about education are taken, and pathways and gateways to gain access to secondary and tertiary education.

The reader should note that this edition of Education at a Glance covers a significant amount of data from partner countries as well (please refer to the Reader's Guide for details). 



\section{READER'S GUIDE}

\section{Coverage of the statistics}

Although a lack of data still limits the scope of the indicators in many countries, the coverage extends, in principle, to the entire national education system (within the national territory), regardless of who owns or sponsors the institutions concerned and regardless of how education is delivered. With one exception (described below), all types of students and all age groups are included: children (including students with special needs), adults, nationals, foreigners, and students in open-distance learning, in special education programmes or in education programmes organised by ministries other than the ministry of education, provided that the main aim of the programme is to broaden or deepen an individual's knowledge. Vocational and technical training in the workplace, with the exception of combined school-and work-based programmes that are explicitly deemed to be part of the education system, is not included in the basic education expenditure and enrolment data.

Educational activities classified as "adult" or "non-regular" are covered, provided that the activities involve the same or similar content as "regular" education studies, or that the programmes of which they are a part lead to qualifications similar to those awarded in regular education programmes.

Courses for adults that are primarily for general interest, personal enrichment, leisure or recreation are excluded.

\section{Country coverage}

This publication features data on education from the 34 OECD countries, two partner countries that participate in the OECD Indicators of Education Systems programme (INES), namely Brazil and the Russian Federation, and other partner countries that do not participate in INES (Argentina, China, Colombia, Costa Rica, India, Indonesia, Latvia, Lithuania, Saudi Arabia and South Africa). Data sources for these latter ten countries are specified below the tables.

The statistical data for Israel are supplied by and under the responsibility of the relevant Israeli authorities. The use of such data by the OECD is without prejudice to the status of the Golan Heights, East Jerusalem and Israeli settlements in the West Bank under the terms of international law.

\section{Calculation of international means}

The main purpose of Education at a Glance is to provide an authoritative compilation of key international comparisons of education statistics. While countries attain specific values in these comparisons, readers should not assume that countries themselves are homogeneous. The country averages include significant variations among sub-national jurisdictions, much as the OECD average encompasses a variety of national experiences (see Box A1.1 in Education at a Glance 2014).

For many indicators, an OECD average is presented; for some, an OECD total is shown. The OECD average is calculated as the unweighted mean of the data values of all OECD countries for which data are available or can be estimated. The OECD average therefore refers to an average of data values at the level of the national systems and can be used to answer the question of how an indicator value for a given country compares with the value for a typical or average country. It does not take into account the absolute size of the education system in each country.

The OECD total is calculated as the weighted mean of the data values of all OECD countries for which data are available or can be estimated. It reflects the value for a given indicator when the OECD area is considered as a whole. This approach is taken for the purpose of comparing, for example, expenditure charts for individual countries with those of the entire OECD area for which valid data are available, with this area considered as a single entity. 
Both the OECD average and the OECD total can be significantly affected by missing data. Given the relatively small number of countries surveyed, no statistical methods are used to compensate for this. In the case of some countries, data may not be available for specific indicators, or specific categories may not apply. Therefore, readers should keep in mind that the term "OECD average" refers to the OECD countries included in the respective comparisons. Averages are sometimes not calculated if too many countries have missing information or have information included in other columns.

For financial tables using trend series over 1995-2012, the OECD average is also calculated for countries providing data for all reference years used. This allows for a comparison of the OECD average over time with no distortion due to the exclusion of certain countries in the different years.

For many indicators, an EU21 average is also presented. It is calculated as the unweighted mean of the data values of the 21 countries that are members of both the European Union and the OECD for which data are available or can be estimated. These 21 countries are Austria, Belgium, the Czech Republic, Denmark, Estonia, Finland, France, Germany, Greece, Hungary, Ireland, Italy, Luxembourg, the Netherlands, Poland, Portugal, Slovenia, the Slovak Republic, Spain, Sweden and the United Kingdom.

For some indicators, a G20 average is presented. The G20 average is calculated as the unweighted mean of the data values of all G20 countries for which data are available or can be estimated (Argentina, Australia, Brazil, Canada, China, France, Germany, India, Indonesia, Italy, Japan, Korea, Mexico, the Russian Federation, Saudi Arabia, South Africa, Turkey, the United Kingdom and the United States; the European Union is the 20th member of the G20 but is not included in the calculation). The G20 average is not computed if data for China or India are not available.

For some indicators, an average is presented. This average is included in tables with data from the 2012 Survey of Adult Skills, a product of the OECD Programme for the International Assessment of Adult Competencies (PIAAC). The average corresponds to the arithmetic mean of the estimates included in the table or chart from both the national and the sub-national entities (which include Flanders [Belgium] and England/Northern Ireland [UK]). Partner countries are not included in the average presented in any of the tables or charts.

\section{Standard error (S.E.)}

The statistical estimates presented in this report are based on samples of adults, rather than values that could be calculated if every person in the target population in every country had answered every question. Therefore, each estimate has a degree of uncertainty associated with sampling and measurement error, which can be expressed as a standard error. The use of confidence intervals provides a way to make inferences about the population means and proportions in a manner that reflects the uncertainty associated with the sample estimates. In this report, confidence intervals are stated at a 95\% level. In other words, the result for the corresponding population would lie within the confidence interval in 95 out of 100 replications of the measurement on different samples drawn from the same population.

In tables showing standard errors, there is one column with the heading "\%", which indicates the average percentage, and a column with the heading "S.E.", which indicates the standard error. Given the survey method, there is a sampling uncertainty in the percentages (\%) of twice the standard error (S.E.). For example, for the values: $\%=10$ and S.E. $=2.6,10 \%$ has an uncertainty zone of twice (1.96) the standard error of 2.6, assuming an error risk of $5 \%$. Thus, the true percentage would probably (error risk of $5 \%$ ) be somewhere between $5 \%$ and $15 \%$ ("confidence interval"). The confidence interval is calculated as: \% +/- 1.96 * S.E., i.e. for the previous example, $5 \%=10 \%-1.96 * 2.6$ and $15 \%=10 \%+1.96 * 2.6$.

\section{Classification of levels of education}

The classification of levels of education is based on the International Standard Classification of Education (ISCED). ISCED is an instrument for compiling statistics on education internationally. ISCED-97 was recently revised, and the new International Standard Classification of Education (ISCED 2011) was formally adopted in November 2011. This new classification is used for the first time in this edition of Education at a Glance. The major changes between ISCED 2011 and ISCED-97 are described in the section "About the new ISCED 2011 classification". 


\section{Symbols for missing data and abbreviations}

These symbols and abbreviations are used in the tables and charts:

a Data are not applicable because the category does not apply.

b There is a break in the series when data for the latest year refer to ISCED 2011 and data for previous years refer to ISCED-97.

c There are too few observations to provide reliable estimates (e.g. in PISA, there are fewer than 30 students or fewer than five schools with valid data; in the Survey of Adult Skills, there are fewer than 30 individuals).

d Includes data from another category.

m Data are not available.

0 Magnitude is either negligible or zero.

$r \quad$ Values are below a certain reliability threshold and should be interpreted with caution.

q Data have been withdrawn at the request of the country concerned.

$\mathrm{x}$ Data included in another category or column of the table (e.g. $\mathrm{x}(2)$ means that data are included in column 2 of the table).

Average is not comparable with other levels of education.

\section{Further resources}

The website www.oecd.org/education/education-at-a-glance-19991487.htm provides information on the methods used to calculate the indicators, on the interpretation of the indicators in the respective national contexts, and on the data sources involved. The website also provides access to the data underlying the indicators and to a comprehensive glossary for technical terms used in this publication.

All post-production changes to this publication are listed at www.oecd.org/publishing/corrigenda (corrections) and http://dx.doi.org/10.1787/eag-data-en (updates).

Education at a Glance uses the OECD's StatLinks service. Below each table and chart in Education at Glance 2015 is a URL that leads to a corresponding Excel file containing the underlying data for the indicator. These URLs are stable and will remain unchanged over time. In addition, readers of the Education at a Glance e-book will be able to click directly on these links and the workbook will open in a separate window.

\section{Layout of tables}

In all tables, the numbers in parentheses at the top of the columns are simply used for reference. When a consecutive number does not appear, that column is available on line only.

\section{Codes used for territorial entities}

These codes are used in certain charts. Country or territorial entity names are used in the text. Note that throughout the publication, the Flemish Community of Belgium and the French Community of Belgium may be referred to as "Belgium (Fl.)" and "Belgium (Fr.)", respectively.

$\begin{array}{lll}\text { ARG Argentina } & \text { CZE Czech Republic } & \text { ISL Iceland } \\ \text { AUS Australia } & \text { DEU Germany } & \text { ISR Israel } \\ \text { AUT Austria } & \text { DNK Denmark } & \text { ITA Italy } \\ \text { BEL Belgium } & \text { ENG England } & \text { JPN Japan } \\ \text { BFL Belgium (Flemish Community) } & \text { ESP Spain } & \text { KOR Korea } \\ \text { BFR Belgium (French Community) } & \text { EST Estonia } & \text { LUX Luxembourg } \\ \text { BRA Brazil } & \text { FIN Finland } & \text { LVA Latvia } \\ \text { CAN Canada } & \text { FRA France } & \text { LTU Lithuania } \\ \text { CHE Switzerland } & \text { GRC Greece } & \text { NZL New Zealand } \\ \text { CHL Chile } & \text { HUN Hungary } & \text { MEX Mexico } \\ \text { CHN China } & \text { IDN Indonesia } & \text { NLD Netherlands } \\ \text { COL Colombia } & \text { IND India } & \text { NOR Norway } \\ \text { CRI Costa Rica } & \text { IRL Ireland } & \text { POL Poland }\end{array}$

PRT Portugal

RUS Russian Federation

SAU Saudi Arabia

SCO Scotland

SVK Slovak Republic

SVN Slovenia

SWE Sweden

TUR Turkey

UKM United Kingdom

USA United States

ZAF South Africa 



\section{ABOUT THE NEW ISCED 2011 CLASSIFICATION}

More details can be found in the publication ISCED 2011 Operational Manual: Guidelines for Classifying National Education Programmes and Related Qualifications (OECD/Eurostat/UNESCO Institute for Statistics, 2015), http://dx.doi.org/10.1787/9789264228368-en.

\section{The need to revise ISCED}

The structure of education systems varies widely between countries. In order to produce internationally comparable education statistics and indicators, it is necessary to have a framework to collect and report data on education programmes with a similar level of educational content. UNESCO's International Standard Classification of Education (ISCED) is the reference classification for organising education programmes and related qualifications by education levels and fields. The basic concepts and definitions of ISCED are intended to be internationally valid and comprehensive of the full range of education systems.

The ISCED classification was initially developed by UNESCO in the mid-1970s, and was first revised in 1997. Due to subsequent changes in education and learning systems throughout the start of the 21st century, a further review of ISCED was undertaken between 2009 and 2011 involving extensive global consultation with countries, regional experts and international organisations. The revision took into account important shifts in the structure of higher education, such as the Bologna process in Europe, expansion of education programmes for very young children, and increasing interest in statistics on the outcomes of education, such as educational attainment. The revised ISCED 2011 classification was adopted by the UNESCO General Conference at its 36 ${ }^{\text {th }}$ session in November 2011.

\section{Major changes between ISCED 2011 and ISCED-97}

The ISCED 2011 classification is an important step forward in a long-term consultative process designed to improve the comparability of international statistics on education. The classification is used for the first time in this edition of Education at a Glance. The major changes between ISCED 2011 and ISCED-97 are the following:

- ISCED 2011 classification presents a revision of the ISCED-97 levels of education programmes (ISCED-P) and introduces for the first time a related classification of educational attainment levels (ISCED-A) based on recognised education qualifications (see Indicator A1).

- ISCED 2011 classification includes improved definitions of formal and non-formal education, educational activities and programmes.

- Compared to ISCED-97 which had seven levels of education, ISCED 2011 now has nine levels of education. In fact, higher education has been restructured taking into account changes in tertiary education, such as the Bologna structure, and now comprises four levels of education compared with two levels in ISCED-97. Programmes previously classified in level 5 of ISCED-97 will now be allocated to level 5, 6 or 7 in ISCED 2011. Moreover, while the position in the national degree structure of tertiary programmes was mentioned in ISCED-97, specific coding for this dimension has been introduced in ISCED 2011 for levels 6 and 7 (bachelor's or equivalent and master's or equivalent levels, respectively).

- ISCED level 0 has been expanded to include a new category covering early childhood educational development programmes designed for children under the age of 3 (see Indicator C2).

- Each education level within ISCED has also been more clearly delineated, which may result in some changes of classification for programmes that previously sat on the border between ISCED levels (for example, between ISCED levels 3 and 4). 
- The complementary dimensions within ISCED levels have also been revised. There are now only two categories of orientation: general and vocational. Programmes previously classified as pre-vocational (in ISCED-97) do not provide labour-market relevant qualifications and are now mainly classified as general education.

- ISCED-97 differentiated access to education at higher ISCED levels in two categories depending on the type of subsequent education, while ISCED 2011 identifies only one group of programmes that provide access to higher education levels. The ISCED 2011 sub-category "level completion with access to higher ISCED levels" corresponds to the combined destination categories A and B in ISCED-97. ISCED 2011 further sub-classifies programmes that do not provide access to higher ISCED levels into the sub-categories "no level completion", "partial level completion" and "level completion". These three sub-categories in ISCED 2011 correspond to destination category $C$ in ISCED-97.

\section{Fields of education and training}

Within ISCED, programmes and related qualifications can be classified by fields of education and training as well as by levels. The ISCED 2011 revision focused on the ISCED levels and complementary dimensions related to ISCED levels. Following the adoption of ISCED 2011, a separate review and global consultation process took place on the ISCED fields of education. The ISCED fields were revised, and the UNESCO General Conference adopted the ISCED 2013 Fields of Education and Training classification (ISCED-F 2013) in November 2013 at its 37th session. The ISCED 2013 Fields of Education and Training classification (UNESCO-UIS, 2014) is available at www.uis.unesco.org/ Education/Documents/isced-fields-of-education-training-2013.pdf and will be used for the first time in Education at a Glance 2017.

\section{Correspondence tables between ISCED versions}

The correspondence between the levels in ISCED 2011 and ISCED-97 is shown in Table 1. For more details on the correspondence between ISCED 2011 and ISCED-97 levels, see Part I of the "Isced 2011 Operational Manual: Guidelines for Classifying National Education Programmes and Related Qualifications".

Table 1. Comparison of levels of education between ISCED 2011 and ISCED-97

\begin{tabular}{c|l|c|l}
\hline \multicolumn{2}{l|}{ ISCED 2011} & \multicolumn{2}{l}{ ISCED-97 } \\
\hline 01 & Early childhood educational development & & - \\
\hline 02 & Pre-primary education & 0 & Pre-primary education \\
\hline 1 & Primary education & 1 & Primary education or first stage of basic education \\
\hline 2 & Lower secondary education & 2 & Lower secondary education or second stage of basic education \\
\hline 3 & Upper secondary education & 3 & (Upper) secondary education \\
\hline 4 & Post-secondary non-tertiary education & 4 & Post-secondary non-tertiary education \\
\hline 5 & Short-cycle tertiary education & \multirow{2}{*}{5} & $\begin{array}{l}\text { First stage of tertiary education (not leading directly to } \\
\text { an advanced research qualification) (5A, 5B) }\end{array}$ \\
\hline 6 & Bachelor's or equivalent level & \multirow{2}{*}{6} & $\begin{array}{l}\text { Second stage of tertiary education (leading to an advanced research } \\
\text { qualification) }\end{array}$ \\
\hline 7 & Master's or equivalent level & Doctoral or equivalent level &
\end{tabular}

\section{Definition of ISCED levels}

\section{Early childhood education (ISCED level 0)}

ISCED level 0 refers to early childhood programmes that have an intentional education component. ISCED level 0 programmes target children below the age of entry into primary education (ISCED level 1). These programmes aim to develop cognitive, physical and socio-emotional skills necessary for participation in school and society.

Programmes offered at ISCED level 0 are often differentiated by age. There are two categories of ISCED level 0 programmes: ISCED 010 - early childhood educational development, and ISCED 020 - pre-primary education. ISCED 010 has intentional educational content designed for younger children (typically in the age range of 0 to 2 years), while ISCED 020 is typically designed for children from the age of 3 years to the start of primary education (ISCED level 1). For international comparability purposes, the term "early childhood education" is used to label ISCED level 0 (for more details, see Indicator C2 in Education at a Glance 2015).

Programmes classified at ISCED level 0 may be referred to in many ways, for example: early childhood education and development, play school, reception, pre-primary, pre-school or educación inicial. For programmes provided 
in crèches, daycare centres, nurseries or guarderías, it is important to ensure that they meet the ISCED level 0 classification criteria specified.

\section{Primary education (ISCED level 1)}

Primary education usually begins at age 5, 6 or 7, and has a typical duration of six years. Programmes at ISCED level 1 are normally designed to give pupils a sound basic education in reading, writing and mathematics, along with an elementary understanding of other subjects, such as history, geography, natural science, social sciences, art and music. The beginning of reading activities alone is not a sufficient criterion to classify an education programme at ISCED level 1.

Programmes classified at ISCED level 1 may be referred to in many ways, for example: primary education, elementary education or basic education (stage 1 or lower grades if an education system has one programme that spans ISCED levels 1 and 2). For international comparability purposes, the term "primary education" is used to label ISCED level 1.

\section{Lower secondary education (ISCED level 2)}

Programmes at the lower secondary education level are designed to lay the foundation across a wide range of subjects and to prepare children and young people for more specialised study at upper secondary and higher levels of education. The beginning - or the end - of lower secondary education often involves a change of school for young students and also a change in the style of instruction.

In some education systems, programmes may be differentiated by orientation, although this is more common at upper secondary level. Vocational programmes, where they exist at this level, generally offer options for young people wishing to prepare for direct entry into the labour market in low- or semi-skilled jobs. They may also be the first step in vocational education, giving access to more advanced vocational programmes at the upper secondary level.

Programmes classified at ISCED level 2 may be referred to in many ways, for example: secondary school (stage one/ lower grades), junior secondary school, middle school or junior high school. If a programme spans ISCED levels 1 and 2, the terms elementary education or basic school (second stage/upper grades) are often used. For international comparability purposes, the term "lower secondary education" is used to label ISCED level 2.

\section{Upper secondary education (ISCED level 3)}

Programmes at the upper secondary education level are more specialised than those at the lower secondary level and offer students more choices and diverse pathways for completing their secondary education. The range of subjects studied by a single student tends to be narrower than at lower levels of education, but the content is more complex and the study more in-depth.

Programmes offered are differentiated by orientation and often by broad subject groups. General programmes are usually designed for students planning to continue to academic or professional studies at the tertiary level. Students will often begin to specialise in specific fields, such as the sciences, humanities or social sciences, even if they are expected to continue to take some courses in basic subjects like the national language, mathematics and, perhaps, a foreign language. There can also be general programmes at ISCED level 3 that do not provide access to tertiary education, but these are comparatively rare. Vocational programmes exist both to offer options to young people who might otherwise leave school without any qualifications from an upper secondary programme and for those wishing to prepare for skilled worker and/or technician jobs.

Second chance or re-integration programmes that either review material already covered in upper secondary programmes or provide opportunities for young people to change streams or enter an occupation requiring an upper secondary qualification that they did not earn during their previous studies, are also classified at this level.

Programmes classified at ISCED level 3 may be referred to in many ways, for example: secondary school (stage two/ upper grades), senior secondary school or (senior) high school. For international comparability purposes, the term "upper secondary education" is used to label ISCED level 3.

\section{Post-secondary non-tertiary education (ISCED level 4)}

Programmes at the post-secondary non-tertiary education level are not significantly more complex than those at the upper secondary level. They generally serve to broaden rather than deepen the knowledge, skills and competencies already gained through successful (full) level completion of upper secondary education. They may be designed to increase options for participants in the labour market, for further studies at the tertiary level, or both. 
Usually, programmes at ISCED level 4 are vocationally oriented. They may be referred to in many ways, for example: technician diploma, primary professional education or préparation aux carrières administratives. For international comparability purposes, the term "post-secondary non-tertiary education" is used to label ISCED level 4.

\section{ISCED 2011 tertiary education levels (ISCED levels 5-8)}

Tertiary education builds on secondary education, providing learning activities at a high level of complexity in specialised fields of study. Tertiary education includes what is commonly understood as academic education but also includes advanced vocational or professional education.

There is usually a clear hierarchy between qualifications granted by tertiary education programmes. It comprises ISCED levels 5 (short-cycle tertiary education), 6 (bachelor's or equivalent level), 7 (master's or equivalent level) and 8 (doctoral or equivalent level). The content of programmes at the tertiary level is more complex and advanced than in lower ISCED levels.

\section{- Short-cycle tertiary education (ISCED level 5)}

The content of ISCED level 5 programmes is noticeably more complex than in upper secondary programmes giving access to this level. ISCED level 5 programmes serve to deepen knowledge by imparting new techniques, concepts and ideas not generally covered in upper secondary education. By comparison, ISCED level 4 programmes serve to broaden knowledge and are typically not significantly more advanced than programmes at ISCED level 3.

Programmes classified at ISCED level 5 may be referred to in many ways, for example: higher technical education, community college education, technician or advanced/higher vocational training, associate degree, bac+2. For international comparability purposes, the term "short-cycle tertiary education" is used to label ISCED level 5.

\section{- Bachelor's or equivalent level (ISCED level 6)}

Programmes at ISCED level 6, or bachelor's or equivalent level, are longer and usually more theoretically oriented than ISCED level 5 programmes. They are often designed to provide participants with intermediate academic and/or professional knowledge, skills and competencies, leading to a first degree or equivalent qualification.

They typically have a duration of three to four years of full-time study at the tertiary level. They may include practical components and/or involve periods of work experience as well as theoretically based studies. They are traditionally offered by universities and equivalent tertiary educational institutions.

Programmes classified at ISCED level 6 may be referred to in many ways, for example: bachelor's programme, licence or first university cycle. For international comparability purposes, the term "bachelor's or equivalent level" is used to label ISCED level 6.

\section{- Master's or equivalent level (ISCED level 7)}

Programmes at ISCED level 7, or master's or equivalent level, have a significantly more complex content than programmes at ISCED level 6 and are usually more specialised. The content of ISCED level 7 programmes is often designed to provide participants with advanced academic and/or professional knowledge, skills and competencies, leading to a second degree or equivalent qualification. Programmes at this level may have a substantial research component but do not yet lead to the award of a doctoral qualification. The cumulative duration of studies at the tertiary level is usually five to eight years or even longer.

Programmes classified at ISCED level 7 may be referred to in many ways, for example: master's programmes or magister studies. For international comparability purposes, the term "master's or equivalent level" is used to label ISCED level 7.

\section{- Doctoral or equivalent level (ISCED level 8)}

Programmes at ISCED level 8, or doctoral or equivalent level, are designed primarily to lead to an advanced research qualification. Programmes at this ISCED level are devoted to advanced study and original research and are typically offered only by research-oriented tertiary educational institutions, such as universities. Doctoral programmes exist in both academic and professional fields.

The theoretical duration of these programmes is three years full-time in most countries, although the actual time that students take to complete the programmes is typically longer.

Programmes classified at ISCED level 8 may be referred to in many ways, for example: PhD, DPhil, D.Lit, D.Sc, LL.D, Doctorate or similar terms. For international comparability purposes the term, "doctoral or equivalent level" is used to label ISCED level 8. 


\section{EXECUTIVE SUMMARY}

\section{In the labour market and in life, education is worth the effort...}

On average, over $80 \%$ of tertiary-educated adults are employed, compared with over $70 \%$ of people with upper secondary or post-secondary non-tertiary education, and less than $60 \%$ of adults without upper secondary education. Tertiary-educated adults also earn about $60 \%$ more, on average, than adults with upper secondary as their highest level of educational attainment. In general, employment rates and earnings increase as an adult's level of education and skills increases; but the labour market still regards a diploma or degree as the primary indication of a worker's skills.

No doubt with these advantages in mind, increasing numbers of young adults in OECD countries are pursuing tertiary education. On average across the OECD countries and sub-national entities that participated in the Survey of Adult Skills, a product of the OECD Programme for the International Assessment of Adult Competencies (PIAAC), in 2012, 22\% of 25-34 year-old non-students - and in Korea, $47 \%$ of this group - have attained tertiary education even though their parents had not. These "first generation tertiary-educated adults" and the tertiary-educated adults whose parents had also completed tertiary education share similar employment rates and pursue similar fields of study. This suggests that being the first in a family to attain tertiary education is in no way a disadvantage.

Data also show that although the entry rate into bachelor's degree programmes is much higher than the entry rate into master's or doctoral programmes, there are more opportunities in the labour market - and higher earnings for adults with a master's degree than for those with only a bachelor's degree. Employed adults with a bachelor's or equivalent degree earn about $60 \%$ more than employed adults with upper secondary education, but those with a master's, doctoral or equivalent degree earn more than twice as much.

But the benefits of education are not only financial. Adults with higher educational attainment are more likely to report that they are in good health, that they participate in volunteer activities, that they trust others, and that they feel they have a say in government. In other words, more highly educated adults tend to be more engaged in the world around them.

\section{....although inequities persist}

Despite narrowing - or even inverted - gender gaps in educational attainment, women are still under-represented in certain fields of education, such as science, technology, engineering and mathematics (STEM). Young women are also less likely than young men to be employed, although the gender gap in employment is much narrower among tertiary-educated young adults than among those with lower educational attainment.

The data also show that the strongest influence on earnings is an adult's own educational attainment. Adults with tertiary education are 23 percentage points more likely to be among the $25 \%$ highest paid adults (in monthly earnings) compared with adults whose highest level of attainment is upper secondary or post-secondary nontertiary education.

\section{Between 2010 and 2012, public spending on education fell in many OECD countries}

The education sector felt a delayed reaction to the global economic crisis of 2008. Between 2010 and 2012, as GDP began to rise following the slowdown, public expenditure on educational institutions fell in more than one in three OECD countries.

The economic downturn of 2008 also had a direct impact on primary and secondary teachers' salaries. In the years immediately following the crisis, even though some countries had already begun a slow recovery, teachers' salaries were frozen or cut, such that the number of countries showing an increase in salaries, in real terms, between 2008 and 2013 shrank to about one in two OECD countries. These trends did nothing to narrow the considerable pay gap between teachers and other similarly educated workers. On average across OECD countries, pre-primary 
and primary teachers earn $78 \%$ of the salary of a similarly educated, full-time, full-year worker, lower secondary teachers are paid $80 \%$ and upper secondary teachers are paid $82 \%$ of that benchmark salary. These uncompetitive salaries will make it that much harder to attract the best candidates to the teaching profession.

Cuts in funding could also threaten professional development activities for teachers. PISA data reveal that, despite increasing investment in information and communication technologies (ICT) for schools, teachers are not using these tools systematically. Indeed, teachers who participated in the 2013 OECD Teaching and Learning International Survey (TALIS) reported that one of the areas in which they most need professional development is developing ICT skills for teaching.

\section{Other findings}

- In 2012, OECD countries spent an average of 5.3\% of their GDP on educational institutions from primary to tertiary education; 11 countries with available data spent more than $6 \%$ of their GDP.

- Education is mostly publicly funded, but tertiary institutions obtain the largest proportion of funds from private sources. Between 2000 and 2012, the average share of public funding for tertiary institutions decreased from $69 \%$ in 2000 , to $64 \%$ in 2012.

- Early childhood education is particularly beneficial for students with an immigrant background.

- In all countries and economies that participated in the OECD Programme for International Student Assessment (PISA) in 2012, the gender gap in reading performance is narrower in digital reading than in print reading. Girls outperform boys in digital reading by an average of 26 score points, compared to an average of 38 score points the equivalent of nearly one year of schooling - in print reading.

- Some $77 \%$ of adults with a vocational upper secondary or post-secondary non-tertiary qualification are employeda rate that is 7 percentage points higher than that among adults with a general upper secondary education as their highest qualification.

- One in five 20-24 year-olds is neither employed nor in education or training.

- About 57\% of employed adults with good skills in information and communication technology and in problem solving participate in employer-sponsored formal and/or non-formal education; only $9 \%$ of adults who cannot use a computer and lack problem-solving skills participate in such programmes.

- Larger classes are correlated with less time spent on teaching and learning, and more time spent on keeping order in the classroom. One additional student added to an average-size class is associated with a 0.5 percentage-point decrease in time spent on teaching and learning activities.

- The teaching force across OECD countries is ageing: in 2013,36\% of secondary school teachers were at least 50 years old. This proportion rose by 3 percentage points between 2005 and 2013, on average among countries with comparable data. 


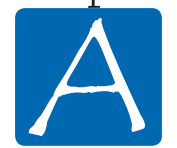

\section{THE OUTPUT OF EDUCATIONAL INSTITUTIONS AND THE IMPACT OF LEARNING}

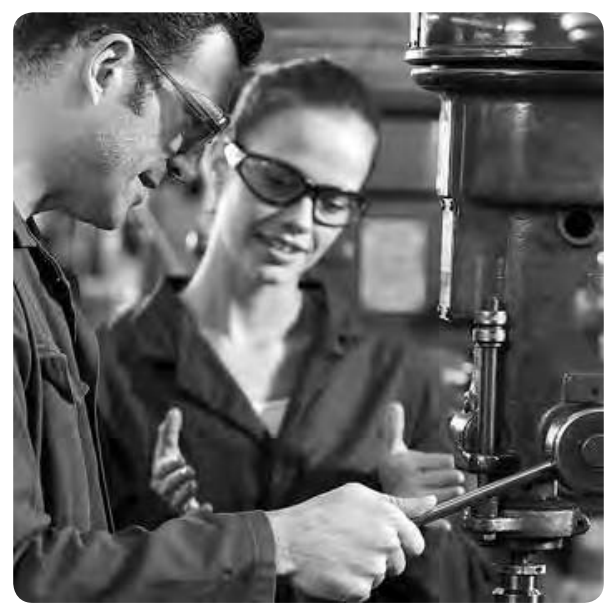

Indicator A1 To what level have adults studied?

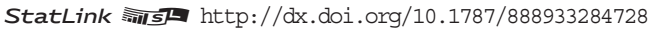

Indicator A2 How many students are expected to complete upper secondary education?

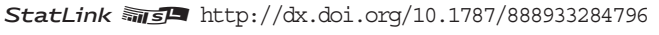

Indicator A3 How many young people are expected to complete tertiary education and what is their profile?

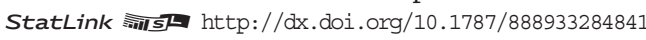

Indicator A4 To what extent does parents' education influence their children's educational attainment?

StatLink iㅔ st http://dx.doi.org/10.1787/888933284922

Indicator A5 How does educational attainment affect participation in the labour market? StatLink जiाs http://dx.doi.org/10.1787/888933284979

Indicator $\mathrm{A} 6$ What are the earnings advantages from education? StatLink 解s http://dx.doi.org/10.1787/888933285049

Indicator A7 What are the financial incentives to invest in education?

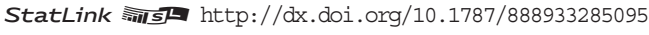

Indicator A8 How are social outcomes related to education? StatLink 部

Indicator $\mathrm{A} 9$ What is the impact of skills on employment and earnings? StatLink 刑s http://dx.doi.org/10.1787/888933285230

Indicator A10 Where are the gender gaps in education and employment?

StatLink AnIs http://dx.doi.org/10.1787/888933285306 


\section{TO WHAT LEVEL HAVE ADULTS STUDIED?}

- On average across OECD countries, about one in five younger adults (17\%) has not finished upper secondary education. Between $30 \%$ and $40 \%$ of younger adults (25-34 year-olds) in Brazil, Colombia, Portugal, Saudi Arabia and Spain, and over 50\% of younger adults in China, Costa Rica, Indonesia, Mexico and Turkey do not have an upper secondary education.

- Upper secondary education is the most commonly attained level of education in most OECD countries. On average, $43 \%$ of $25-64$ year-olds have attained upper secondary or post-secondary non-tertiary education as their highest level of education.

- In two out of five OECD countries, and Latvia, Lithuania and the Russian Federation, at least one in two younger women (25-34 year-olds) has a tertiary education, while only in Canada, Korea, Luxembourg, the Russian Federation and the United Kingdom is one in two men similarly educated.

\section{Chart A1.1. Percentage of 25-34 year-olds with attainment below upper secondary education (2014)}

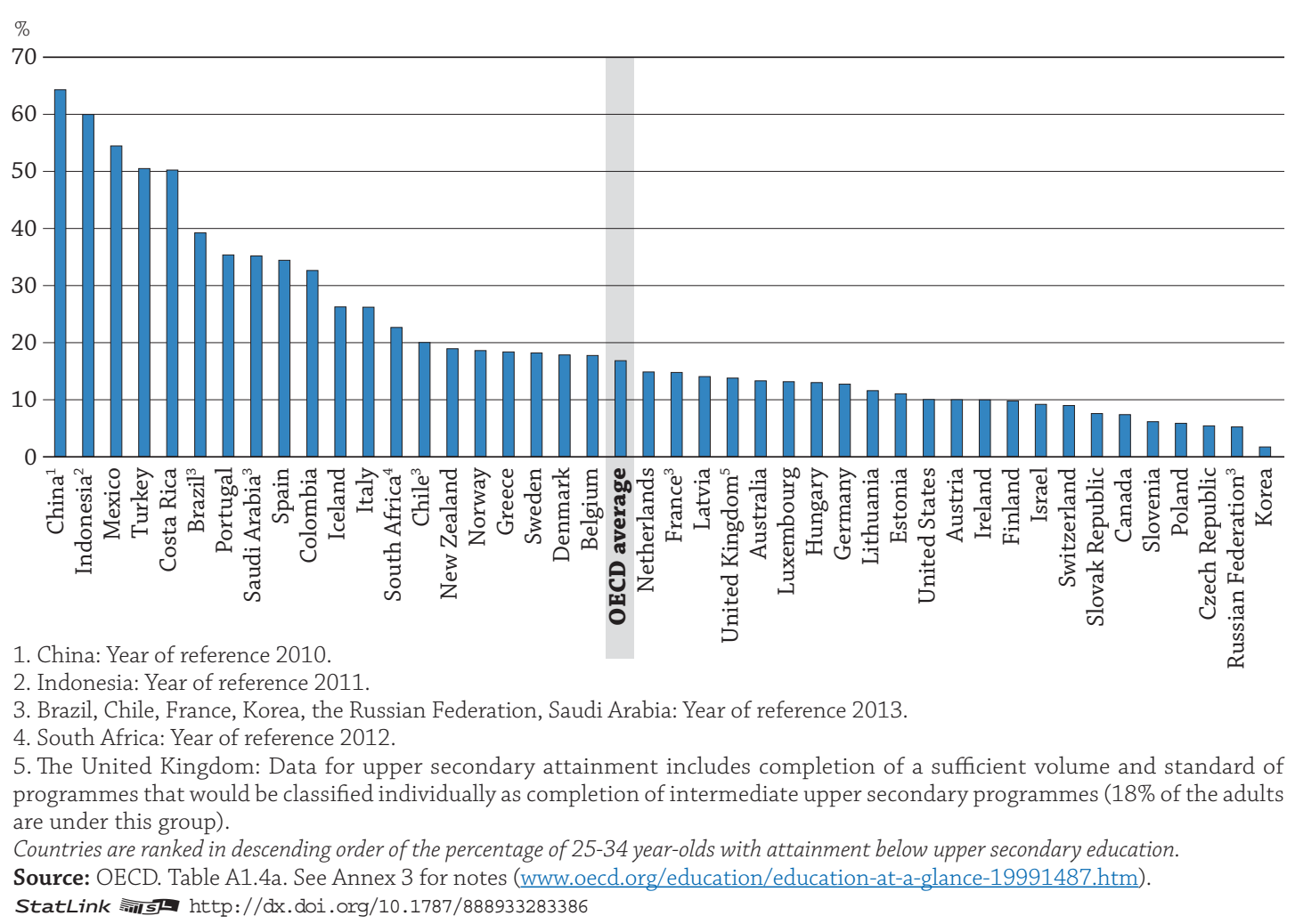

\section{Context}

The level of educational attainment shows the percentage of a population that has reached a certain level of education and holds a qualification at that level. Educational attainment is frequently used as a proxy measure of human capital and the level of an individual's skills - in other words, a measure of the skills associated with a given level of education and available in the population, and to the labour force. In this sense, qualifications certify and offer information on the type of knowledge and skills that graduates have acquired in formal schooling.

Higher levels of educational attainment are associated with several positive individual and social outcomes. Data in previous editions of Education at a Glance have shown that individuals with high educational attainment generally have better health, are more socially engaged, have higher employment rates and have higher relative earnings. Higher proficiency in skills, such as literacy and numeracy, is also strongly associated with higher levels of formal education. 
Individuals thus have incentives to pursue more education, and governments have incentives to provide appropriate infrastructure and organisation to support the expansion of higher educational attainment across the population. Over the past decades, almost all OECD countries have seen significant increases in the educational attainment of their populations, especially among the younger generations.

This indicator includes information on both educational attainment and on individuals' skills and readiness to use information and communication technologies (ICT) for problem solving.

\section{Other findings}

- Across OECD countries, 37\% of 55-64 year-old women have below upper secondary education as their highest level of attainment, but only $15 \%$ of 25-34 year-olds do.

- In China, the percentage of 25-34 year-olds with below upper secondary education as their highest level of attainment decreased by 30 percentage points in just one decade - from $94 \%$ in 2000 to $64 \%$ in 2010.

- In most OECD countries, most adults with upper secondary or post-secondary non-tertiary education as their highest level of attainment have vocational qualifications. In Canada, Chile, Costa Rica, Greece, Israel, Portugal and Spain, general qualifications are more common: more than $60 \%$ at this level of attainment of adults hold such qualifications. In Australia, Turkey and the United Kingdom, both programme orientations are equally represented.

- Skills and readiness to use ICT for problem solving increase as the level of education increases, but decrease with age. On average, $34 \%$ of men and $29 \%$ of women have good ICT and problem-solving skills.

\section{Note}

Several indicators in this publication show the level of education among individuals. Indicator A1 shows the level of attainment, i.e. the percentage of a population that has successfully completed a given level of education, and the relationship between level of attainment and the acquisition of basic skills. Graduation rates in Indicators A2 and A3 measure the estimated percentage of younger adults who are expected to graduate from a particular level of education during their lifetimes. Completion rates from upper secondary programmes in Indicator A2 estimate the proportion of students who enter a programme and complete it successfully within a certain period of time. 


\section{A1 Analysis \\ Attainment levels}

Levels of educational attainment vary greatly not only among countries, but also among generations within countries. This section examines the distribution of adults across the different aggregated levels of educational attainment: those without upper secondary education, those with upper secondary or post-secondary non-tertiary education as their highest level of attainment, and for those with a tertiary degree.

\section{Below upper secondary}

Chart A1.1 shows that there are still many young adults (25-34 year-olds) who are not benefiting from the expansion of education. Between 30\% and 40\% of younger adults (25-34 year-olds) in Brazil, Colombia, Portugal, Saudi Arabia and Spain, and over 50\% of younger adults in China, Costa Rica, Indonesia, Mexico and Turkey do not have an upper secondary education. On average across OECD countries, about one in six younger adults (17\%) has not finished upper secondary education (Table A1.4a).

The difference in attainment rates between generations is remarkable: across OECD countries, 34\% of 55-64 year-olds have not attained upper secondary education, while only $17 \%$ of 25-34 year-olds have not attained that level of education. In Brazil, China, Indonesia, Mexico, Portugal, Saudi Arabia and Turkey, more than 70\% of 55-64 year-olds have below upper secondary as their highest level of attainment, but far smaller proportions of younger adults have only this level of education (Table A1.4a).

More than one in two younger adults in China, Costa Rica, Indonesia, Mexico and Turkey have not attained upper secondary education. China is the only country where education is only just beginning to expand: in $2000,94 \%$ of 25-34 year-olds in China had not attained upper secondary education; by 2010, that proportion had shrunk 30 percentage points to $64 \%$ (Table A1.4a).

On average, the percentage of younger men without an upper secondary qualification (18\%) is higher than that of younger women (15\%). In Latvia, Portugal and Spain, this difference is about 10 or more percentage points, while in Austria, Indonesia, Mexico and Turkey, the difference is reversed (Table A1.4b, available on line).

\section{Tertiary}

As shown in Chart A1.2, the expansion in tertiary attainment over the generations has been significant. In all OECD and partner countries except Israel and South Africa, the share of younger adults with tertiary qualifications is larger than that of older adults with that level of qualification. On average, the difference between the generations (55-64 year-olds compared with 25-34 year-olds) in tertiary attainment is about 16 percentage points. The speed of the expansion varies considerably. In Brazil, Costa Rica, Estonia, Finland, Indonesia, Israel, Germany, the Russian Federation, South Africa and the United States, the difference in tertiary attainment between the two age groups is less than 10 percentage points, while in France, Ireland, Korea, Lithuania, Luxembourg, Poland, Slovenia and Spain, the difference ranges from $20 \%$ to $50 \%$. In most of the latter countries, the proportions of 55-64 year-olds with tertiary education is below the OECD average. Among the countries with the highest tertiary attainment rates, small differences between the generations, such as those observed in Israel, the Russian Federation and the United States, could reflect the fact that these countries also have the highest tertiary attainment rates among 55-64 year-olds (Table A1.4a).

On average, a larger proportion of 25-34 year-old women has attained tertiary education than men of the same age ( $46 \%$ and $35 \%$, respectively), while the opposite is true for $55-64$ year-old women and men ( $24 \%$ and $26 \%$, respectively) (Table A1.4b, available on line).

In two out of five OECD countries as well as in Latvia, Lithuania and the Russian Federation, at least one in two younger women (25-34 year-olds) has a tertiary education. Two in three younger women in Canada and the Russian Federation have a tertiary degree. In most countries, fewer than one in two men hold a tertiary degree; in Canada, Korea, Lithuania, Luxembourg, the United Kingdom and the Russian Federation roughly one in two men have attained tertiary education (Table A1.4b, available on line).

Behind the expansion of tertiary education there are large differences in the levels of tertiary education most of people have attained. For instance, in Austria and Canada, about half of all tertiary-educated adults have a qualification from a short-cycle tertiary programme, while less than $1 \%$ of tertiary-educated adults in the Czech Republic and Poland hold such a qualification. Across OECD countries, 27\% of 25-64 year-olds have at least a bachelor's degree or equivalent. In Belgium and Luxembourg, over $35 \%$ of adults hold this degree, but in Austria, Chile, France, Italy, Mexico and Turkey, less than $20 \%$ of adults do. On average across OECD countries, $16 \%$ of 25-64 year-olds have earned a bachelor's degree or equivalent, $11 \%$ have earned a master's degree, and about $1 \%$ have earned a doctoral degree or equivalent. 


\section{Chart A1.2. Percentage of younger and older tertiary-educated adults (2014)} 25-34 and 55-64 year-olds, and percentage-point difference between these two groups

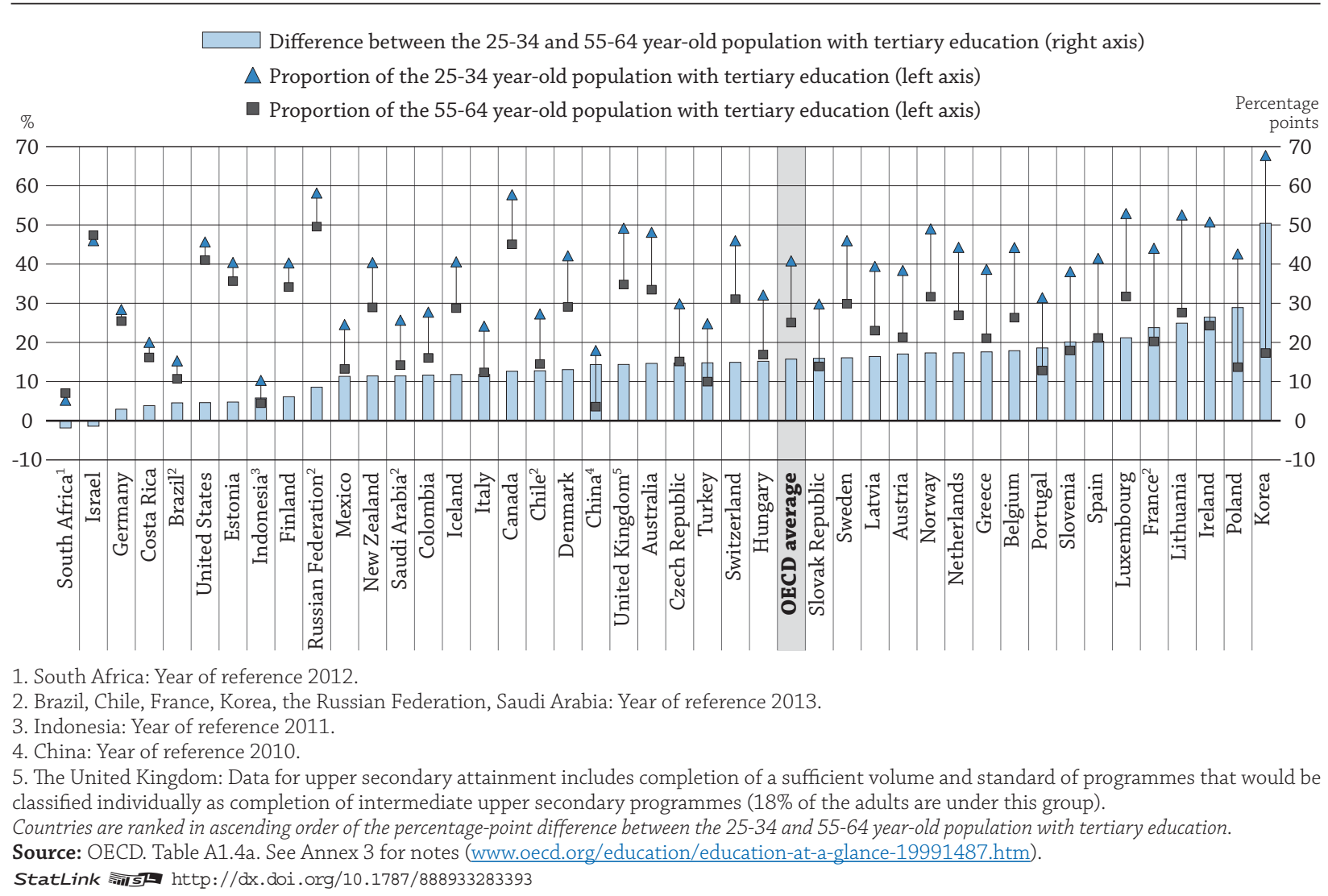

Excluding tertiary-educated adults who have completed a short-cycle programme, the majority of tertiary-educated adults in OECD countries have a bachelor's degree or equivalent as their highest level of tertiary education. But in Austria, the Czech Republic, Italy, Poland, Portugal and the Slovak Republic, about 75\% or more of tertiary-educated adults have a master's or equivalent degree as their highest tertiary qualification (Table A1.1a).

Tertiary systems have expanded at different rates across countries. For example, the proportion of people with at least a bachelor's degree is at least 20 percentages points higher among 25-34 year-olds than among 55-64 year-olds in Finland, Ireland, Korea, Lithuania, Poland and Slovenia, but is nearly the same (5 percentage points or less difference) in Brazil, Costa Rica, Germany, Israel, South Africa and the United States (Table A1.3a).

\section{Upper secondary or post-secondary non-tertiary}

Despite the expansion of tertiary education, upper secondary education is still the most commonly attained level of education in most OECD countries. More adults (25-64 year-olds) have attained upper secondary or post-secondary non-tertiary education as their highest level of education than have attained any other level of education (on average, about 43\%). Among 25-34 year-olds across OECD countries, $42 \%$ have attained upper secondary or post-secondary non-tertiary education. In the Czech Republic, the Slovak Republic and South Africa, more than $60 \%$ of younger adults have attained this level of education as their highest level of attainment (Table A1.4a).

Countries with relatively low upper secondary attainment rates can fall into one of two categories: either most individuals leave education before earning an upper secondary qualification (i.e. they have below upper secondary education), or they continue in education beyond this level until they earn a higher degree (i.e. they have attained tertiary education). The latter path is followed by most young adults in Canada, Iceland, Korea, Luxembourg and Spain, where tertiary attainment rates are higher than below upper secondary attainment rates. In China, Costa Rica, Indonesia, Mexico and Turkey, $50 \%$ and younger adults do not reach upper secondary education, thus fewer of them attain higher levels of education (Table A1.4a). 
Upper secondary or post-secondary non-tertiary vocational education and training (VET)

Upper secondary education across OECD countries is mainly divided into two types of programmes: those defined as "general", which are often designed to prepare students for further education, and those geared towards vocational education and training (VET).

There are substantial differences across OECD countries in the attainment of vocational qualifications. While at least one in two adults in the Czech Republic, Germany, Hungary, Poland and the Slovak Republic have vocational upper secondary or post-secondary non-tertiary qualifications as their highest level of attainment, in Chile, Costa Rica, Israel, Portugal, Spain and Turkey fewer than one in ten does (Table A1.5a).

Chart A1.3 shows how the shares of adults with vocational qualifications and those with general qualifications among adults with upper secondary or post-secondary non-tertiary education vary across countries. In most OECD countries, most adults with upper secondary or post-secondary non-tertiary education as their highest level of education have vocational qualifications. By contrast, in Canada, Chile, Greece, Israel, Portugal and Spain, more than $60 \%$ of adults at this level of attainment have general qualifications. In Australia, Turkey and the United Kingdom, both programme orientations are equally represented (Table A1.5a).

\section{Chart A1.3. Percentage of 25-64 year-olds whose highest level of education is upper secondary or post-secondary non-tertiary, by programme orientation (2014)}

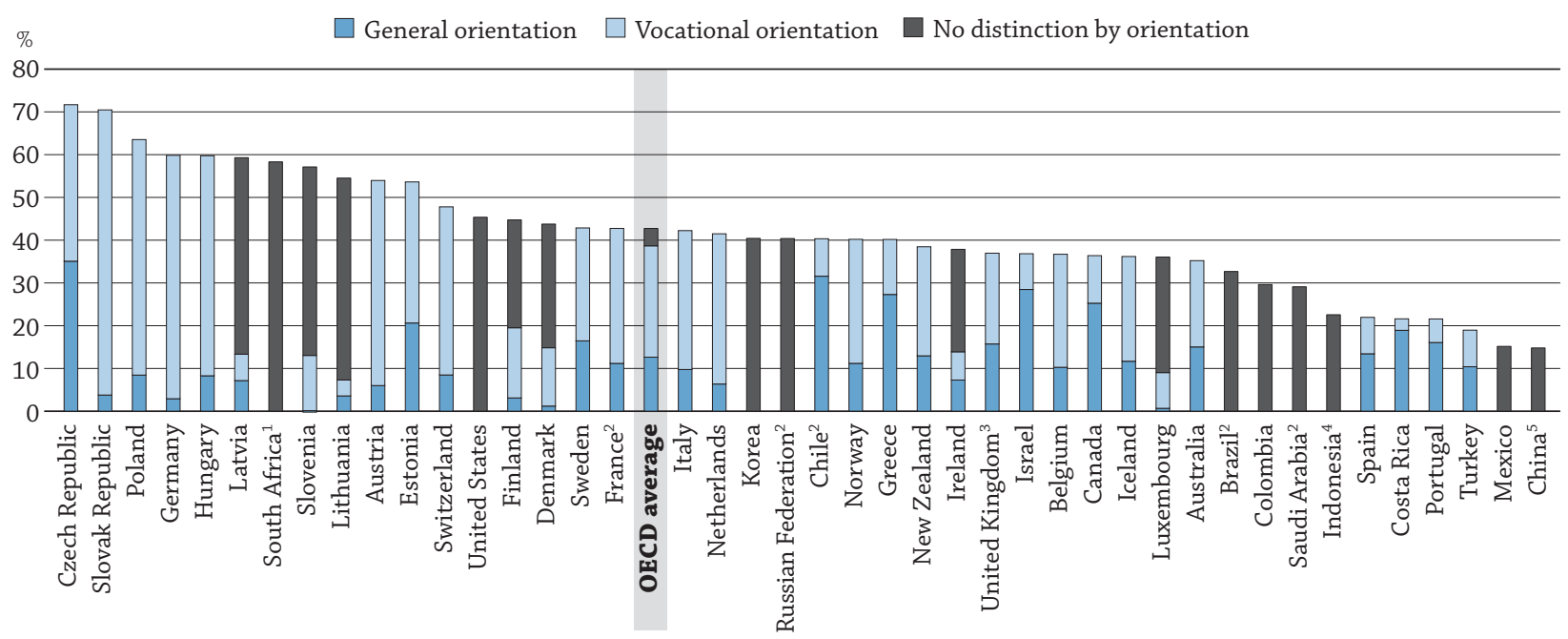

Note: Denmark, Finland, Ireland, Latvia, Lithuania, Luxembourg, Slovenia: Data for the breakdown by programme orientation are only available for 15-34 year-olds and 35-64 year-olds if those individuals had completed their highest level of education 15 years, at most, before the date of the interview.

1. South Africa: Year of reference 2012.

2. Brazil, Chile, France, the Russian Federation, Saudi Arabia: Year of reference 2013.

3. The United Kingdom: Data for upper secondary attainment includes completion of a sufficient volume and standard of programmes that would be classified individually as completion of intermediate upper secondary programmes (18\% of the adults are under this group).

4. Indonesia: Year of reference 2011.

5. China: Year of reference 2010.

Countries are ranked in descending order of the percentage of 25-64 year-olds with upper secondary or post-secondary non-tertiary education as highest level of attainment, regardless of the orientation of the programmes.

Source: OECD. Table A5.1a. See Annex 3 for notes (www.oecd.org/education/education-at-a-glance-19991487.htm).

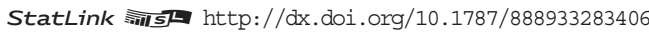

\section{Skills and readiness to use information and communication technologies for problem solving}

The 2012 Survey of Adult Skills, a product of the OECD Programme for the International Assessment of Adult Competencies (PIAAC), measured problem-solving skills in technology-rich environments and estimated the frequency with which adults use those skills at work and at home. Greater proficiency in problem solving in technology-rich environments reflects both better problem-solving skills and better skills in using digital technology, communication tools and networks to acquire and evaluate information, communicate with others and perform practical tasks (PIAAC Expert Group in Problem Solving in Technology-Rich Environments, 2009). 
The information gathered through the Survey of Adult Skills allows for the creation of an indicator that measures skills and readiness to use ICT for problem solving. This indicator combines data about performance in the problemsolving assessment and information about why some adults did not participate in the computer-based assessment and thus do not have a score in problem solving (see the Definitions section at the end of this chapter).

\section{By educational attainment}

Chart A1.4 shows that skills and readiness to use ICT for problem solving in technology-rich environments are greatly related to educational attainment. In all countries with relevant data, the results show that the proportion of the population with good ICT and problem solving-skills increases with educational attainment. On average, $7 \%$ of adults who have not attained upper secondary education have good ICT and problem solving-skills. This proportion increases to $25 \%$ among those who have attained upper secondary or post-secondary non-tertiary education, and to $52 \%$ among adults with tertiary education. The highest rates of good ICT and problem-solving skills among tertiary-educated adults are observed in the Netherlands (64\%), Sweden (62\%) and the Czech Republic (60\%); the lowest rates are observed in Poland (37\%), Estonia (35\%) and the Russian Federation (27\%). In all countries, education seems to play a central role in developing the skills that are now considered essential in modern societies (Table A1.6a).

\section{Chart A1.4. Percentage of adults with good information and communication technologies and problem-solving skills, by educational attainment (2012)}

Survey of Adult Skills, 25-64 year-olds

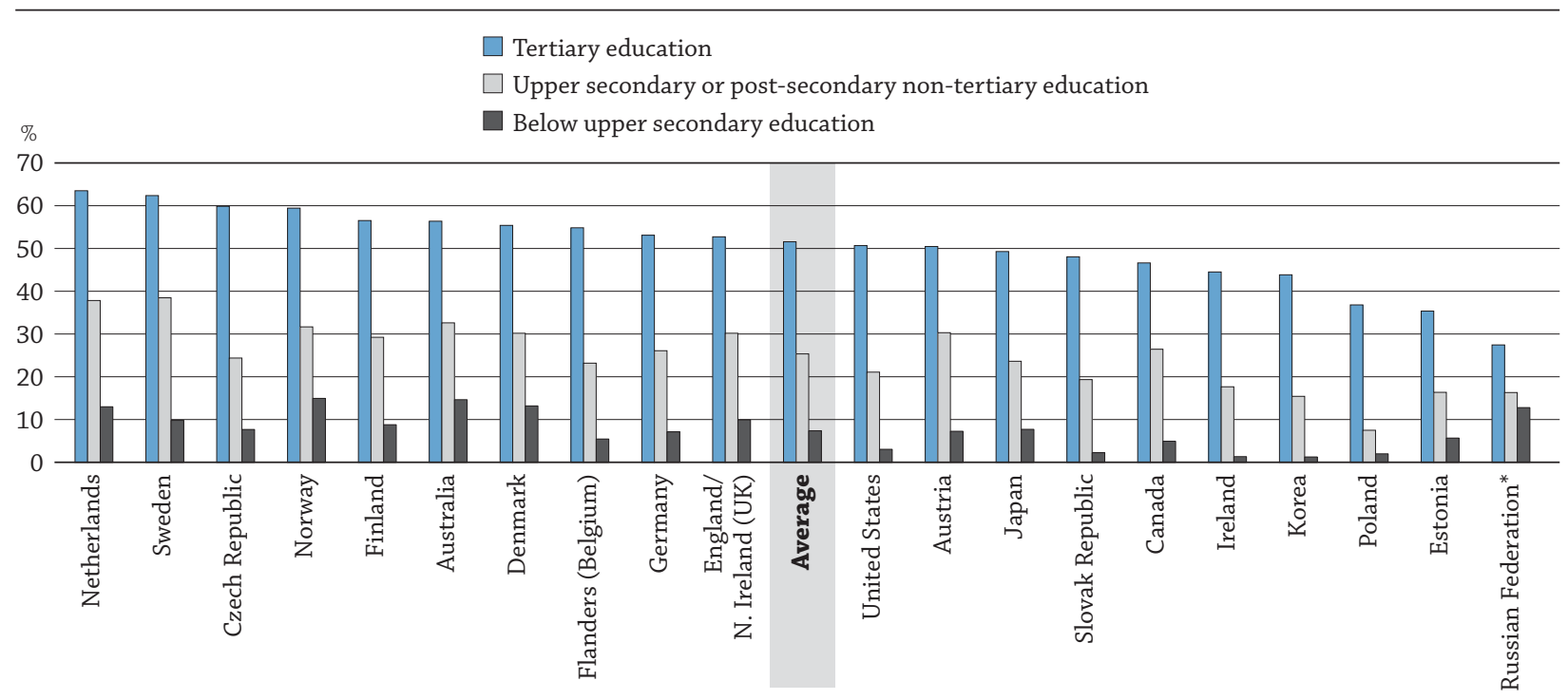

Note: Data on educational attainment are based on ISCED-97.

* See note on data for the Russian Federation in the Methodology section.

Countries are ranked in descending order of the percentage of adults with tertiary education and with good ICT and problem-solving skills (Group 4).

Source: OECD. Table 1.6a. See Annex 3 for notes (www.oecd.org/education/education-at-a-glance-19991487.htm).

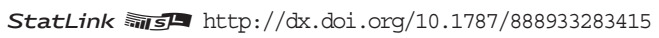

\section{By age group}

In all countries and sub-national entities that participated in the Survey of Adult Skills, the younger generation (25-34 year-olds) shows higher levels of skills and readiness to use ICT for problem solving in technologyrich environments than older generations. On average, $50 \%$ of $25-34$ year-olds, $39 \%$ of $35-44$ year-olds, $24 \%$ of 45-54 year-olds and $12 \%$ of 55-64 year-olds demonstrate good ICT and problem-solving skills. Finland has the largest proportion (67\%) of 25-34 year-olds with good ICT and problem-solving skills - much larger than the proportion of 55-64 year-olds (9\%) who demonstrate good ICT and problem-solving skills. In fact, Finland shows the widest gap between the older and the younger generations - 58 percentage points - in this area. The United States has the smallest generation gap: 20 percentage points. In the United States, more than 20\% of 55-64 year-olds have good ICT and problem-solving skills (the highest percentage among participating countries and sub-national entities) while 40\% of 25-34 year-olds do (a below-average percentage for this age group). Poland has the smallest proportion of adults, in all age groups, with good ICT and problem solving-skills (Table A1.6b, available on line). 


\section{By gender}

Among 25-64 year-olds in all countries and sub-national entities, a larger proportion of men than women have good ICT and problem-solving skills. On average, $34 \%$ of men and $29 \%$ of women have good ICT and problemsolving skills. The largest proportions of men with these skills are found in the Netherlands, Norway (both 44\%) and Sweden (43\%); the largest proportions of women with these skills are found in Sweden (39\%), Finland (38\%) and Australia (36\%). The gender gap is at least 10 percentage points wide only in Japan (40\% for men and $27 \%$ for women) (Table A1.6c, available on line).

\section{Definitions}

Age groups: adults refers to 25-64 year-olds; younger adults refers to 25-34 year-olds; older adults refers to 55-64 year-olds.

Completion of intermediate programmes for educational attainment (ISCED 2011) corresponds to recognised qualification from an ISCED 2011 level programme which is not considered as sufficient for ISCED 2011 level completion and is classified at a lower ISCED 2011 level. In addition, this recognised qualification does not give direct access to an upper ISCED 2011 level programme.

Levels of education: In this Indicator two ISCED (International Standard Classification of Education) classifications are used: ISCED 2011 and ISCED-97.

- When it is specified that ISCED 2011 is used, the levels of education are defined as: below upper secondary corresponds to ISCED 2011 Levels 0, 1 and 2, and includes recognised qualifications from ISCED 2011 Level 3 programmes, which are not considered as sufficient for ISCED 2011 Level 3 completion, and without direct access to post-secondary non-tertiary education or tertiary education; upper secondary or post-secondary non-tertiary corresponds to ISCED 2011 Levels 3 and 4; and tertiary corresponds to ISCED 2011 Levels 5, 6, 7 and 8 (UNESCO Institute for Statistics, 2012).

- When it is specified that ISCED-97 is used, the levels of education are defined as: below upper secondary corresponds to ISCED-97 Levels $0,1,2$ and $3 C$ short programmes; upper secondary or post-secondary non-tertiary corresponds to ISCED-97 Levels 3A, 3B, 3C long programmes, and Level 4; and tertiary corresponds to ISCED-97 Levels 5A, 5B and 6.

See the section About the new ISCED 2011 classification, at the beginning of this publication, for a presentation of all ISCED 2011 levels and Annex 3 for a presentation of all ISCED-97 levels.

Skill groups refer to skills and readiness to use information and communication technologies (ICT) for problem solving in technology-rich environments. Each group is described in terms of the characteristics of the types of tasks that can be successfully completed by adults, and the related scores in the assessment of problem solving in technology-rich environments in the Survey of Adult Skills.

- Group 0 (no computer experience)

- Group 1 (refused the computer-based assessment)

- Group 2 (failed ICT core stage 1 or minimal problem-solving skills - scored below Level 1 in the problem solving in technology-rich environments assessment)

- Group 3 (moderate ICT and problem-solving skills - scored at Level 1 in the problem solving in technology-rich environments assessment)

- Group 4 (good ICT and problem-solving skills - scored at Level 2 or Level 3 in the problem solving in technology-rich environments assessment)

VET: The International Standard Classification of Education (ISCED 2011) defines VET as "education programmes that are designed for learners to acquire the knowledge, skills and competencies specific to a particular occupation, trade, or class of occupations or trades. Such programmes may have work-based components (e.g. apprenticeships, dual-system education programmes). Successful completion of such programmes leads to labour market-relevant, vocational qualifications acknowledged as occupationally-oriented by the relevant national authorities and/or the labour market" (UNESCO Institute for Statistics, 2012). 


\section{Methodology}

Data on population and educational attainment for most countries are taken from OECD and Eurostat databases, which are compiled from National Labour Force Surveys by the OECD LSO (Labour Market and Social Outcomes of Learning) Network. Data on educational attainment for China, Colombia, Indonesia, Saudi Arabia and South Africa are taken from the UNESCO Institute of Statistics (UIS) database on educational attainment of the population aged 25 and older. Data on proficiency levels and mean scores are based on the Survey of Adult Skills (PIAAC) (2012). PIAAC is the OECD Programme for the International Assessment of Adult Competencies. See Annex 3 for additional information (www.oecd.org/education/education-at-a-glance-19991487.htm).

Attainment profiles are based on the percentage of the population aged 25-64 that has successfully completed a specified level of education.

In OECD statistics, recognised qualifications from ISCED 2011 Level 3 programmes that are not of sufficient duration for ISCED 2011 Level 3 completion are classified at ISCED 2011 Level 2. Where countries have been able to demonstrate equivalencies in the labour market value of attainment formally classified as "completion of intermediate upper secondary programmes" (e.g. achieving 5 good GCSEs or equivalent in the United Kingdom) and "full upper secondary attainment", attainment of these programmes are reported as ISCED 2011 Level 3 completion in the tables showing three aggregate levels of educational attainment (UNESCO Institute for Statistics, 2012).

Most OECD countries include people without education (i.e. illiterate adults or people whose educational attainment does not fit national classifications) under the international classification ISCED 0; therefore averages for the category "less than primary educational attainment" are likely to be influenced.

\section{Note regarding data from Israel}

The statistical data for Israel are supplied by and are under the responsibility of the relevant Israeli authorities. The use of such data by the OECD is without prejudice to the status of the Golan Heights, East Jerusalem and Israeli settlements in the West Bank under the terms of international law.

\section{Note regarding data from the Russian Federation in the Survey of Adult Skills (PIAAC)}

Readers should note that the sample for the Russian Federation does not include the population of the Moscow municipal area. The data published, therefore, do not represent the entire resident population aged 16-65 in Russia but rather the population of Russia excluding the population residing in the Moscow municipal area. More detailed information regarding the data from the Russian Federation as well as that of other countries can be found in the Technical Report of the Survey of Adult Skills (OECD, 2014).

\section{References}

OECD (2014), Technical Report of the Survey of Adult Skills, www.oecd.org/site/piaac/ Technical\%20Report 170CT13.pdf, pre-publication copy.

OECD (2013), OECD Skills Outlook 2013: First Results from the Survey of Adult Skills, OECD Publishing, Paris, http://dx.doi. org/10.1787/9789264204256-en.

PIAAC Expert Group in Problem Solving in Technology-Rich Environments (2009), "PIAAC Problem Solving in TechnologyRich Environments: A Conceptual Framework", OECD Education Working Papers, No. 36, OECD Publishing, Paris, http://dx.doi. org/10.1787/220262483674.

UNESCO Institute for Statistics (2012), International Standard Classification of Education: ISCED 2011, UNESCO-UIS, Montreal, Canada, www.uis.unesco.org/Education/Documents/isced-2011-en.pdf.

\section{Indicator A1 Tables}

\section{StatLink 제인 http://dx.doi.org/10.1787/888933284728}

\section{Table A1.1a Educational attainment of 25-64 year-olds (2014)}

WEB Table A1.1b Educational attainment of 25-64 year-olds, by gender (2014)

Table A1.2a Percentage of adults who have attained at least upper secondary education, by age group (2014) 
WEB Table A1.2b Percentage of adults who have attained at least upper secondary education, by age group and gender (2014)

Table A1.3a Percentage of adults who have attained tertiary education, by type of programme and age group (2014)

WEB Table A1.3b Percentage of adults who have attained tertiary education, by type of programme, age group and gender (2014)

Table A1.4a Trends in educational attainment, by age group (2000, 2005, 2010 and 2014)

WEB Table A1.4b Trends in educational attainment, by age group and gender (2000, 2005, 2010 and 2014)

Table A1.5a Adults whose highest level of education is upper secondary or post-secondary non-tertiary education, by programme orientation and gender (2014)

WEB Table A1.5b Adults whose highest level of education is upper secondary or post-secondary non-tertiary education, by programme orientation, age group and gender (2014)

Table A1.6a Skills and readiness to use information and communication technologies for problem solving among 25-64 year-olds, by educational attainment (2012)

WEB Table A1.6b

Skills and readiness to use information and communication technologies for problem solving among 25-64 year-olds, by age group (2012)

WEB Table A1.6c Skills and readiness to use information and communication technologies for problem solving among 25-64 year-olds, by gender (2012) 
Table A1.1a. Educational attainment of 25-64 year-olds (2014)

Percentage of adults with a given level of education as the highest level attained

\begin{tabular}{|c|c|c|c|c|c|c|c|c|c|c|c|c|}
\hline & \multicolumn{5}{|c|}{ Below upper secondary } & \multicolumn{2}{|c|}{$\begin{array}{l}\text { Upper secondary } \\
\text { or post-secondary } \\
\text { non-tertiary }\end{array}$} & \multicolumn{4}{|c|}{ Tertiary } & \multirow[b]{2}{*}{$\begin{array}{l}\text { All levels } \\
\text { of } \\
\text { education }\end{array}$} \\
\hline & 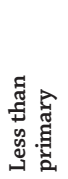 & 胥 & 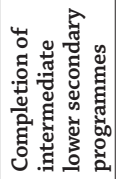 & 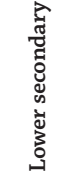 & 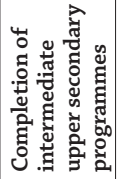 & 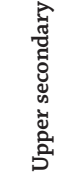 & 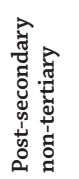 & 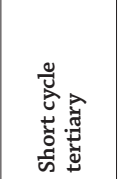 & 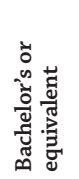 & 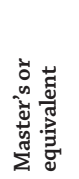 & 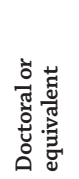 & \\
\hline & (1) & (2) & (3) & (4) & (5) & (6) & (7) & (8) & (9) & (10) & (11) & (12) \\
\hline Australia & $x(2)$ & $6^{d}$ & $a$ & 17 & a & 30 & 5 & 11 & 24 & 6 & 1 & 100 \\
\hline Austria & $x(2)$ & $1^{\mathrm{d}}$ & a & 15 & a & 52 & 2 & 15 & 2 & 11 & 1 & 100 \\
\hline Belgium & 4 & 7 & $\mathrm{a}$ & 16 & a & 35 & 1 & 0 & 20 & 16 & 1 & 100 \\
\hline Canada & $x(2)$ & $3^{\mathrm{d}}$ & a & 7 & a & 25 & 11 & 25 & 19 & $9^{d}$ & $\mathrm{x}(10)$ & 100 \\
\hline Chile $^{1}$ & 9 & 6 & a & 23 & a & 40 & $\mathrm{a}$ & 7 & 13 & $1^{\mathrm{d}}$ & $\mathrm{x}(10)$ & 100 \\
\hline Czech Republic & 0 & c & a & 7 & a & $72^{\mathrm{d}}$ & $\mathrm{x}(6)$ & 0 & 5 & 16 & 0 & 100 \\
\hline Denmark & $x(2)$ & $4^{\mathrm{d}}$ & a & 16 & a & 43 & 0 & 4 & 19 & 11 & 1 & 100 \\
\hline Estonia & 0 & 0 & $\mathrm{a}$ & 8 & a & 45 & 8 & 7 & 10 & 20 & 1 & 100 \\
\hline Finland & $\mathrm{x}(2)$ & 4 & a & 9 & a & 44 & 1 & 12 & 15 & 13 & 1 & 100 \\
\hline France $^{1}$ & 1 & 8 & a & 16 & a & 43 & 0 & 14 & 9 & 8 & 1 & 100 \\
\hline Germany & $\mathrm{x}(2)$ & $3^{\mathrm{d}}$ & a & 10 & a & 49 & 11 & 1 & 14 & 11 & 1 & 100 \\
\hline Greece & 1 & 17 & 0 & 13 & 0 & 32 & 9 & 2 & 23 & 2 & 1 & 100 \\
\hline Hungary & 0 & 1 & a & 16 & a & 51 & 8 & 1 & 13 & 9 & 1 & 100 \\
\hline Iceland & $\mathrm{x}(2)$ & $1^{\mathrm{d}}$ & a & 26 & a & 33 & 4 & 4 & 20 & 12 & 1 & 100 \\
\hline Ireland & 0 & 8 & a & 13 & a & 24 & 14 & 12 & 21 & 8 & 1 & 100 \\
\hline Israel & 2 & 5 & $\mathrm{a}$ & 7 & a & 37 & a & 14 & 22 & 11 & 1 & 100 \\
\hline Italy & 1 & 7 & a & 33 & a & 42 & 1 & c & 3 & 13 & 0 & 100 \\
\hline Japan & $\mathrm{x}(6)$ & $\mathrm{x}(6)$ & a & $\mathrm{x}(6)$ & a & $52^{\mathrm{d}}$ & $\mathrm{x}(6)$ & $21^{\mathrm{d}}$ & $28^{\mathrm{d}}$ & $\mathrm{x}(9)$ & $\mathrm{x}(9)$ & 100 \\
\hline Korea & $\mathrm{x}(2)$ & $6^{\mathrm{d}}$ & a & 9 & a & 40 & $\mathrm{~m}$ & 13 & $31^{\mathrm{d}}$ & $\mathrm{x}(9)$ & $\mathrm{x}(9)$ & 100 \\
\hline Luxembourg & c & 7 & a & 11 & a & 34 & 2 & 9 & 15 & 20 & 1 & 100 \\
\hline Mexico & 15 & 19 & 3 & 25 & 5 & 15 & a & 1 & $18^{\mathrm{d}}$ & $\mathrm{x}(9)$ & $\mathrm{x}(9)$ & 100 \\
\hline Netherlands & 1 & 6 & a & 16 & a & 41 & 0 & 2 & 20 & 12 & 1 & 100 \\
\hline New Zealand & $\mathrm{x}(2)$ & $\mathrm{x}(4)$ & a & $26^{\mathrm{d}}$ & a & 22 & 16 & 5 & 26 & 4 & 1 & 100 \\
\hline Norway & 0 & 0 & a & 17 & a & 38 & 2 & 12 & 19 & 10 & 1 & 100 \\
\hline Poland & 0 & 9 & a & 0 & a & 60 & 3 & 0 & 6 & 21 & 1 & 100 \\
\hline Portugal & 3 & 33 & a & 21 & a & 21 & 1 & $\mathrm{x}(9)$ & $5^{\mathrm{d}}$ & 17 & 1 & 100 \\
\hline Slovak Republic & 0 & 0 & 1 & 7 & 0 & 69 & 1 & 0 & 3 & 16 & 1 & 100 \\
\hline Slovenia & 0 & 1 & $\mathrm{a}$ & 13 & a & 57 & a & 7 & 6 & 13 & 2 & 100 \\
\hline Spain & 3 & 10 & a & 30 & a & 22 & 0 & 11 & 9 & 14 & 1 & 100 \\
\hline Sweden & $x(2)$ & $3^{\mathrm{d}}$ & a & 13 & 2 & 36 & 7 & 10 & 15 & 12 & 1 & 100 \\
\hline Switzerland & 0 & 2 & a & 10 & a & $48^{\mathrm{d}}$ & $\mathrm{x}(6)$ & $x(9,10,11)$ & $20^{\mathrm{d}}$ & $17^{\mathrm{d}}$ & $3^{\mathrm{d}}$ & 100 \\
\hline Turkey & 5 & 46 & a & 13 & a & 19 & a & 5 & 10 & 1 & 0 & 100 \\
\hline United Kingdom & 0 & 1 & a & 20 & 18 & 19 & a & 11 & 22 & 8 & 1 & 100 \\
\hline United States & 1 & 3 & a & 7 & $\mathrm{a}$ & $45^{\mathrm{d}}$ & $\mathrm{x}(6)$ & 11 & 22 & 10 & 2 & 100 \\
\hline OECD average & 2 & 7 & $\mathrm{~m}$ & 15 & $\mathrm{~m}$ & 39 & 5 & 8 & 16 & 11 & 1 & 100 \\
\hline EU21 average & 1 & 6 & $\mathrm{~m}$ & 14 & $\mathrm{~m}$ & 42 & 4 & 6 & 12 & 13 & 1 & 100 \\
\hline Argentina & $\mathrm{m}$ & $\mathrm{m}$ & $\mathrm{m}$ & $\mathrm{m}$ & $\mathrm{m}$ & $\mathrm{m}$ & $\mathrm{m}$ & $\mathrm{m}$ & $\mathrm{m}$ & $\mathrm{m}$ & $\mathrm{m}$ & $\mathrm{m}$ \\
\hline Brazil $^{1}$ & $\mathrm{x}(4)$ & $\mathrm{x}(4)$ & a & $54^{\mathrm{d}}$ & a & $33^{\mathrm{d}}$ & $\mathrm{x}(6)$ & $\mathrm{x}(9)$ & $14^{\mathrm{d}}$ & $\mathrm{x}(9)$ & $\mathrm{x}(9)$ & 100 \\
\hline China $^{2}$ & 3 & 25 & a & 47 & $\mathrm{a}$ & $15^{\mathrm{d}}$ & $\mathrm{x}(6)$ & 6 & 3 & $0^{\mathrm{d}}$ & $\mathrm{x}(10)$ & 100 \\
\hline Colombia & $\mathrm{x}(4)$ & $\mathrm{x}(4)$ & a & $48^{\mathrm{d}}$ & a & $30^{\mathrm{d}}$ & $\mathrm{x}(6)$ & $\mathrm{x}(9)$ & $22^{\mathrm{d}}$ & $\mathrm{x}(9)$ & $\mathrm{x}(9)$ & 100 \\
\hline Costa Rica & 2 & 37 & 8 & 7 & 2 & 16 & 5 & 1 & 15 & $2^{\mathrm{d}}$ & $\mathrm{x}(10)$ & 100 \\
\hline India & $\mathrm{m}$ & $\mathrm{m}$ & $\mathrm{m}$ & $\mathrm{m}$ & $\mathrm{m}$ & $\mathrm{m}$ & $\mathrm{m}$ & $\mathrm{m}$ & $\mathrm{m}$ & $\mathrm{m}$ & $\mathrm{m}$ & $\mathrm{m}$ \\
\hline Indonesia $^{3}$ & 22 & 31 & a & 17 & $\mathrm{a}$ & 23 & a & $\mathrm{x}(9)$ & $8^{\mathrm{d}}$ & $\mathrm{x}(9)$ & $\mathrm{x}(9)$ & 100 \\
\hline Latvia & c & 0 & $\mathrm{a}$ & 10 & a & 51 & 8 & 2 & 17 & 10 & 0 & 100 \\
\hline Lithuania & c & c & a & 6 & 2 & 34 & 21 & $\mathrm{x}(9)$ & $22^{\mathrm{d}}$ & 14 & c & 100 \\
\hline Russian Federation $^{1}$ & $x(4)$ & $\mathrm{x}(4)$ & a & $5^{\mathrm{d}}$ & a & $40^{\mathrm{d}}$ & $\mathrm{x}(6)$ & $\mathrm{x}(9)$ & $54^{\mathrm{d}}$ & $\mathrm{x}(9)$ & $x(9)$ & 100 \\
\hline Saudi Arabia ${ }^{1}$ & 16 & 15 & a & 18 & a & 24 & 5 & $\mathrm{x}(9)$ & $22^{\mathrm{d}}$ & $\mathrm{x}(9)$ & $\mathrm{x}(9)$ & 100 \\
\hline South Africa ${ }^{4}$ & 16 & 6 & a & 14 & a & 51 & 7 & $\mathrm{x}(9)$ & $7^{d}$ & $\mathrm{x}(9)$ & $\mathrm{x}(9)$ & 100 \\
\hline G20 average & 8 & 13 & $\mathrm{~m}$ & 19 & 11 & 33 & $\mathrm{~m}$ & 10 & 18 & 7 & $\mathrm{~m}$ & 100 \\
\hline
\end{tabular}

Note: In most countries, the data refer to ISCED 2011. The countries with data that refer to ISCED-97 are: Brazil, Indonesia, the Russian Federation, Saudi Arabia and South Africa. See the description of the levels of education in the Definitions section.

1. Brazil, Chile, France, the Russian Federation, Saudi Arabia: Year of reference 2013.

2. China: Year of reference 2010.

3. Indonesia: Year of reference 2011.

4. South Africa: Year of reference 2012.

Source: OECD. China, Indonesia, Saudi Arabia, South Africa: UNESCO Institute for Statistics. Colombia, Costa Rica: OECD Education Database. Latvia, Lithuania: Eurostat. See Annex 3 for notes (www.oecd.org/education/education-at-a-glance-19991487.htm).

Please refer to the Reader's Guide for information concerning symbols for missing data and abbreviations.

StatLink 겐 http://dx.doi.org/10.1787/888933284736 
Table A1.2a. Percentage of adults who have attained at least upper secondary education, by age group (2014)

\begin{tabular}{|c|c|c|c|c|c|c|}
\hline & 25-64 year-olds & $30-34$ year-olds & 25-34 year-olds & $35-44$ year-olds & 45-54 year-olds & 55-64 year-olds \\
\hline & (1) & (2) & (3) & (4) & (5) & (6) \\
\hline Australia & 77 & 86 & 87 & 82 & 71 & 65 \\
\hline Austria & 84 & 90 & 90 & 86 & 83 & 75 \\
\hline Belgium & 74 & 82 & 82 & 80 & 72 & 59 \\
\hline Canada & 90 & 93 & 93 & 93 & 89 & 85 \\
\hline Chile $^{1}$ & 61 & 77 & 80 & 66 & 53 & 42 \\
\hline Czech Republic & 93 & 95 & 95 & 96 & 94 & 88 \\
\hline Denmark & 80 & 84 & 82 & 84 & 80 & 72 \\
\hline Estonia & 91 & 89 & 89 & 89 & 94 & 92 \\
\hline Finland & 87 & 91 & 90 & 90 & 89 & 77 \\
\hline France $^{1}$ & 75 & 85 & 85 & 81 & 72 & 61 \\
\hline Germany & 87 & 87 & 87 & 87 & 87 & 86 \\
\hline Greece & 68 & 80 & 82 & 75 & 65 & 50 \\
\hline Hungary & 83 & 88 & 87 & 86 & 83 & 77 \\
\hline Iceland & 73 & 75 & 74 & 78 & 73 & 68 \\
\hline Ireland & 79 & 90 & 90 & 86 & 74 & 59 \\
\hline Israel & 85 & 90 & 91 & 87 & 82 & 78 \\
\hline Italy & 59 & 71 & 74 & 65 & 54 & 46 \\
\hline Japan & $\mathrm{m}$ & $\mathrm{m}$ & $\mathrm{m}$ & $\mathrm{m}$ & $\mathrm{m}$ & $\mathrm{m}$ \\
\hline Korea & 85 & 98 & 98 & 97 & 84 & 54 \\
\hline Luxembourg & 82 & 85 & 87 & 86 & 79 & 73 \\
\hline Mexico & 34 & 41 & 46 & 33 & 28 & 20 \\
\hline Netherlands & 76 & 85 & 85 & 80 & 74 & 65 \\
\hline New Zealand & 74 & 80 & 81 & 78 & 71 & 66 \\
\hline Norway & 82 & 82 & 81 & 86 & 80 & 80 \\
\hline Poland & 91 & 94 & 94 & 93 & 91 & 84 \\
\hline Portugal & 43 & 63 & 65 & 52 & 34 & 23 \\
\hline Slovak Republic & 91 & 93 & 92 & 94 & 92 & 84 \\
\hline Slovenia & 86 & 94 & 94 & 91 & 84 & 75 \\
\hline Spain & 57 & 67 & 66 & 65 & 53 & 39 \\
\hline Sweden & 82 & 81 & 82 & 86 & 84 & 74 \\
\hline Switzerland & 88 & 91 & 91 & 89 & 87 & 84 \\
\hline Turkey & 36 & 46 & 50 & 35 & 25 & 21 \\
\hline United Kingdom ${ }^{2}$ & 79 & 86 & 86 & 82 & 77 & 71 \\
\hline United States & 90 & 89 & 90 & 89 & 89 & 90 \\
\hline OECD average & 76 & 83 & 83 & 80 & 74 & 66 \\
\hline EU21 average & 78 & 85 & 85 & 83 & 77 & 68 \\
\hline
\end{tabular}

\begin{tabular}{|c|c|c|c|c|c|c|}
\hline $\begin{array}{l}\text { Argentina } \\
\text { Brazil }^{1}\end{array}$ & $\begin{array}{c}\mathrm{m} \\
46\end{array}$ & $\begin{array}{c}\mathrm{m} \\
59\end{array}$ & $\begin{array}{c}\mathrm{m} \\
61\end{array}$ & $\begin{array}{l}\mathrm{m} \\
48\end{array}$ & $\begin{array}{r}\mathrm{m} \\
40\end{array}$ & $\begin{array}{l}\mathrm{m} \\
28\end{array}$ \\
\hline China ${ }^{3}$ & 24 & 33 & 36 & 23 & 24 & 12 \\
\hline Colombia & 52 & 65 & 67 & 53 & 42 & 33 \\
\hline Costa Rica & 40 & 46 & 47 & 37 & 36 & 35 \\
\hline India & $\mathrm{m}$ & $\mathrm{m}$ & $\mathrm{m}$ & $\mathrm{m}$ & $\mathrm{m}$ & $\mathrm{m}$ \\
\hline Indonesia 4 & 31 & 38 & 40 & 34 & 22 & 15 \\
\hline Latvia & 90 & 85 & 86 & 88 & 95 & 90 \\
\hline Lithuania & 91 & 87 & 88 & 88 & 96 & 91 \\
\hline Russian Federation ${ }^{1}$ & 95 & 94 & 95 & 95 & 96 & 92 \\
\hline Saudi Arabia ${ }^{1}$ & 51 & 60 & 65 & 49 & 40 & 28 \\
\hline South Africa ${ }^{5}$ & 65 & 78 & 77 & 69 & 52 & 38 \\
\hline G20 average & 64 & 72 & 73 & 66 & 60 & 51 \\
\hline
\end{tabular}

Note: In most countries, the data refer to ISCED 2011. The countries with data that refer to ISCED-97 are: Brazil, Indonesia, the Russian Federation, Saudi Arabia and South Africa. See the description of the levels of education in the Definitions section.

1. Brazil, Chile, France, the Russian Federation, Saudi Arabia: Year of reference 2013.

2. The United Kingdom: Data for upper secondary attainment includes completion of a sufficient volume and standard of programmes that would be classified individually as completion of intermediate upper secondary programmes ( $18 \%$ of the adults are under this group).

3. China: Year of reference 2010.

4. Indonesia: Year of reference 2011.

5. South Africa: Year of reference 2012

Source: OECD. China, Indonesia, Saudi Arabia, South Africa: UNESCO Institute for Statistics. Colombia, Costa Rica: OECD Education Database. Latvia, Lithuania: Eurostat. See Annex 3 for notes (www.oecd.org/education/education-at-a-glance-19991487.htm).

Please refer to the Reader's Guide for information concerning symbols for missing data and abbreviations.

StatLink 젶ㄴ http://dx.doi.org/10.1787/888933284742 
Table A1.3a. Percentage of adults who have attained tertiary education, by type of programme and age group (2014)

\begin{tabular}{|c|c|c|c|c|c|c|c|c|c|c|c|c|c|c|c|c|}
\hline & \multicolumn{3}{|c|}{ Short cycle tertiary } & \multicolumn{3}{|c|}{$\begin{array}{l}\text { Bachelor's } \\
\text { or equivalent }\end{array}$} & \multicolumn{3}{|c|}{$\begin{array}{c}\text { Master's } \\
\text { or equivalent }\end{array}$} & \multicolumn{3}{|c|}{$\begin{array}{c}\text { Doctoral } \\
\text { or equivalent }\end{array}$} & \multicolumn{4}{|c|}{ Total tertiary } \\
\hline & 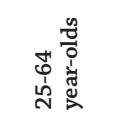 & 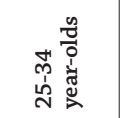 & 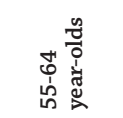 & 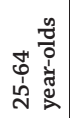 & 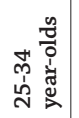 & 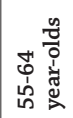 & 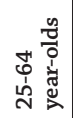 & 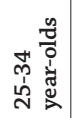 & 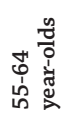 & 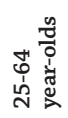 & 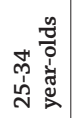 & 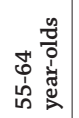 & 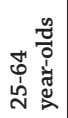 & 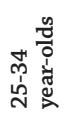 & 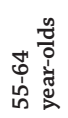 & $\begin{array}{c}25-64 \\
\text { year-olds } \\
\text { (in } \\
\text { thousands) }\end{array}$ \\
\hline & (1) & (3) & (6) & (7) & (9) & (12) & (13) & (15) & $(18)$ & (19) & (21) & (24) & $(25)$ & (27) & $(30)$ & (31) \\
\hline Australia & 11 & 10 & 11 & 24 & 30 & 18 & 6 & 7 & 4 & 1 & 1 & 1 & 42 & 48 & 33 & 5012 \\
\hline ปै Austria & 15 & 17 & 13 & 2 & 5 & 0 & 11 & 15 & 7 & 1 & 1 & 1 & 30 & 38 & 21 & 1405 \\
\hline Belgium & 0 & 0 & c & 20 & 23 & 15 & 16 & 20 & 10 & 1 & 1 & 0 & 37 & 44 & 26 & 2191 \\
\hline Canada & 25 & 24 & 23 & 19 & 24 & 14 & $9^{d}$ & $9^{d}$ & $8^{\mathrm{d}}$ & $\mathrm{x}(13)$ & $\mathrm{x}(15)$ & $\mathrm{x}(18)$ & 54 & 58 & 45 & 10293 \\
\hline Chile $^{1}$ & 7 & 9 & 4 & 13 & 18 & 9 & $1^{\mathrm{d}}$ & $1^{\mathrm{d}}$ & $1^{d}$ & $\mathrm{x}(13)$ & $\mathrm{x}(15)$ & $\mathrm{x}(18)$ & 21 & 27 & 14 & 1815 \\
\hline Czech Republic & 0 & 0 & c & 5 & 11 & 2 & 16 & 19 & 13 & 0 & 0 & 1 & 22 & 30 & 15 & 1285 \\
\hline Denmark & 4 & 4 & 4 & 19 & 22 & 18 & 11 & 15 & 7 & 1 & 1 & $0^{r}$ & 36 & 42 & 29 & 987 \\
\hline Estonia & 7 & 1 & 12 & 10 & 23 & 1 & 20 & 16 & 22 & 1 & c & c & 38 & 40 & 36 & 270 \\
\hline Finland & 12 & 0 & 17 & 15 & 26 & 7 & 13 & 14 & 9 & 1 & 0 & 1 & 42 & 40 & 34 & 1186 \\
\hline France & 14 & 18 & 9 & 9 & 12 & 6 & 8 & 14 & 5 & 1 & 1 & 1 & 32 & 44 & 20 & 10432 \\
\hline Germany & 1 & 0 & 1 & 14 & 14 & 14 & 11 & 13 & 10 & 1 & 1 & 1 & 27 & 28 & 25 & 11956 \\
\hline Greece & 2 & 1 & 2 & 23 & 34 & 17 & 2 & 3 & 1 & 1 & 0 & 1 & 28 & 39 & 21 & 1672 \\
\hline Hungary & 1 & 3 & c & 13 & 16 & 10 & 9 & 13 & 6 & 1 & 1 & 1 & 23 & 32 & 17 & 1276 \\
\hline Iceland & 4 & 3 & 4 & 20 & 26 & 15 & 12 & 12 & 9 & 1 & c & 2 & 37 & 41 & 29 & 122 \\
\hline Ireland & 12 & 12 & 8 & 21 & 29 & 11 & 8 & 9 & 4 & 1 & 1 & 0 & 41 & 51 & 24 & 982 \\
\hline Israel & 14 & 11 & 16 & 22 & 27 & 16 & 11 & 7 & 13 & 1 & 0 & 2 & 49 & 46 & 47 & 1830 \\
\hline Italy & c & c & c & 3 & 9 & 1 & 13 & 15 & 11 & 0 & 0 & 0 & 17 & 24 & 12 & 5612 \\
\hline Japan & $\mathrm{m}$ & $\mathrm{m}$ & $\mathrm{m}$ & $x(25)$ & $\mathrm{x}(27)$ & $\mathrm{x}(30)$ & $\mathrm{x}(25)$ & $\mathrm{x}(27)$ & $\mathrm{x}(30)$ & $x(25)$ & $\mathrm{x}(27)$ & $\mathrm{x}(30)$ & 28 & 37 & 21 & 17720 \\
\hline Korea & 13 & 23 & 3 & $31^{\mathrm{d}}$ & $45^{d}$ & $14^{\mathrm{d}}$ & $\mathrm{x}(7)$ & $x(9)$ & $\mathrm{x}(12)$ & $x(7)$ & $x(9)$ & $\mathrm{x}(12)$ & 45 & 68 & 17 & 13320 \\
\hline Luxembourg & 9 & 10 & 8 & 15 & 17 & 11 & 20 & 25 & 10 & 1 & 1 & 2 & 46 & 53 & 32 & 138 \\
\hline Mexico & 1 & 1 & 0 & $18^{\mathrm{d}}$ & $24^{\mathrm{d}}$ & $13^{\mathrm{d}}$ & $x(7)$ & $x(9)$ & $\mathrm{x}(12)$ & $x(7)$ & $x(9)$ & $\mathrm{x}(12)$ & 19 & 25 & 13 & 10435 \\
\hline Netherlands & 2 & 2 & 2 & 20 & 26 & 16 & 12 & 16 & 8 & 1 & 0 & 1 & 34 & 44 & 27 & 3034 \\
\hline New Zealand & 5 & 4 & 7 & 26 & 32 & 18 & 4 & 4 & 3 & 1 & 0 & 1 & 36 & 40 & 29 & 808 \\
\hline Norway & 12 & 14 & 9 & 19 & 22 & 16 & 10 & 12 & 6 & 1 & 0 & 1 & 42 & 49 & 32 & 1129 \\
\hline Poland & 0 & 0 & 0 & 6 & 11 & 2 & 21 & 31 & 11 & 1 & 0 & 0 & 27 & 43 & 14 & 5665 \\
\hline Portugal & $\mathrm{a}$ & $\mathrm{a}$ & a & 5 & 10 & 3 & 17 & 21 & 9 & 1 & c & 0 & 22 & 31 & 13 & 1236 \\
\hline Slovak Republic & 0 & $0^{x}$ & c & 3 & 6 & 1 & 16 & 23 & 12 & 1 & 1 & 0 & 20 & 30 & 14 & 646 \\
\hline Slovenia & 7 & 6 & 7 & 6 & 12 & 2 & 13 & 18 & 8 & 2 & 2 & 1 & 29 & 38 & 18 & 340 \\
\hline Spain & 11 & 13 & 5 & 9 & 11 & 7 & 14 & 17 & 8 & 1 & 0 & 1 & 35 & 41 & 21 & 9111 \\
\hline Sweden & 10 & 10 & 11 & 15 & 22 & 9 & 12 & 13 & 9 & 1 & 1 & 1 & 39 & 46 & 30 & 1905 \\
\hline Switzerland & $\mathrm{x}(7,13,19)$ & $\mathrm{x}(9,15,21)$ & $\mathrm{x}(12,18,24)$ & $20^{\mathrm{d}}$ & $24^{\mathrm{d}}$ & $15^{\mathrm{d}}$ & $17^{\mathrm{d}}$ & $19^{\mathrm{d}}$ & $13^{\mathrm{d}}$ & $3^{\mathrm{d}}$ & $2^{\mathrm{d}}$ & $3^{d}$ & 40 & 46 & 31 & 1820 \\
\hline Turkey & 5 & 7 & 4 & 10 & 15 & 5 & 1 & 2 & 1 & 0 & 0 & 0 & 17 & 25 & 10 & 5984 \\
\hline United Kingdom & 11 & 8 & 11 & 22 & 31 & 16 & 8 & 10 & 7 & 1 & 1 & 1 & 42 & 49 & 35 & 14090 \\
\hline United States & 11 & 10 & 11 & 22 & 25 & 18 & 10 & 9 & 11 & 2 & 1 & 2 & 44 & 46 & 41 & 72873 \\
\hline OECD average & 8 & 7 & 8 & 15 & 21 & 10 & 11 & 14 & 8 & 1 & 1 & 1 & 33 & 41 & 25 & 6429 \\
\hline EU21 average & 6 & 6 & 7 & 12 & 18 & 8 & 13 & 16 & 9 & 1 & 1 & 1 & 32 & 39 & 23 & 3591 \\
\hline Argentina & $\mathrm{m}$ & $\mathrm{m}$ & $\mathrm{m}$ & $\mathrm{m}$ & $\mathrm{m}$ & $\mathrm{m}$ & $\mathrm{m}$ & $\mathrm{m}$ & $\mathrm{m}$ & $\mathrm{m}$ & $\mathrm{m}$ & $\mathrm{m}$ & $\mathrm{m}$ & $\mathrm{m}$ & $\mathrm{m}$ & $\mathrm{m}$ \\
\hline Brazil $^{2}$ & $\mathrm{x}(7)$ & $\mathrm{x}(9)$ & $\mathrm{x}(12)$ & $14^{\mathrm{d}}$ & $15^{\mathrm{d}}$ & $11^{\mathrm{d}}$ & $\mathrm{x}(7)$ & $\mathrm{x}(9)$ & $\mathrm{x}(12)$ & $x(7)$ & $\mathrm{x}(9)$ & $\mathrm{x}(12)$ & 14 & 15 & 11 & 14422 \\
\hline China ${ }^{3}$ & 6 & $x(27)$ & $\mathrm{x}(30)$ & 3 & $\mathrm{x}(27)$ & $\mathrm{x}(30)$ & $0^{\mathrm{d}}$ & $\mathrm{x}(27)$ & $\mathrm{x}(30)$ & $\mathrm{x}(13)$ & $\mathrm{x}(27)$ & $\mathrm{x}(30)$ & 10 & 18 & 4 & 74086 \\
\hline Colombia & $\mathrm{x}(7)$ & $\mathrm{x}(9)$ & $\mathrm{x}(12)$ & $22^{\mathrm{d}}$ & $28^{\mathrm{d}}$ & $16^{\mathrm{d}}$ & $\mathrm{x}(7)$ & $\mathrm{x}(9)$ & $\mathrm{x}(12)$ & $x(7)$ & $\mathrm{x}(9)$ & $\mathrm{x}(12)$ & 22 & 28 & 16 & 4683 \\
\hline Costa Rica & 1 & 1 & 1 & 15 & 18 & 12 & $2^{\mathrm{d}}$ & $1^{\mathrm{d}}$ & $3^{\mathrm{d}}$ & $\mathrm{x}(13)$ & $\mathrm{x}(15)$ & $\mathrm{x}(18)$ & 18 & 20 & 16 & 441 \\
\hline India & $\mathrm{m}$ & $\mathrm{m}$ & $\mathrm{m}$ & $\mathrm{m}$ & $\mathrm{m}$ & $\mathrm{m}$ & $\mathrm{m}$ & $\mathrm{m}$ & $\mathrm{m}$ & $\mathrm{m}$ & $\mathrm{m}$ & $\mathrm{m}$ & $\mathrm{m}$ & $\mathrm{m}$ & $\mathrm{m}$ & $\mathrm{m}$ \\
\hline Indonesia $^{4}$ & $x(7)$ & $\mathrm{x}(9)$ & $\mathrm{x}(12)$ & $8^{\mathrm{d}}$ & $10^{\mathrm{d}}$ & $4^{\mathrm{d}}$ & $\mathrm{x}(7)$ & $x(9)$ & $\mathrm{x}(12)$ & $x(7)$ & $x(9)$ & $\mathrm{x}(12)$ & 8 & 10 & 4 & 10067 \\
\hline Latvia & 2 & 5 & $1^{\mathrm{r}}$ & 17 & 23 & 12 & 10 & 11 & 10 & 0 & c & c & 30 & 39 & 23 & 327 \\
\hline Lithuania & $x(7)$ & $\mathrm{x}(9)$ & $\mathrm{x}(12)$ & $22^{\mathrm{d}}$ & $38^{\mathrm{d}}$ & $12^{\mathrm{d}}$ & 14 & 14 & 15 & c & c & c & 37 & 53 & 28 & 578 \\
\hline Russian Federation ${ }^{1}$ & $\mathrm{x}(7)$ & $\mathrm{x}(9)$ & $\mathrm{x}(12)$ & $54^{\mathrm{d}}$ & $58^{\mathrm{d}}$ & $50^{\mathrm{d}}$ & $\mathrm{x}(7)$ & $x(9)$ & $\mathrm{x}(12)$ & $x(7)$ & $x(9)$ & $\mathrm{x}(12)$ & 54 & 58 & 50 & 45262 \\
\hline Saudi Arabia ${ }^{1}$ & $x(7)$ & $\mathrm{x}(9)$ & $\mathrm{x}(12)$ & $22^{\mathrm{d}}$ & $26^{\mathrm{d}}$ & $14^{\mathrm{d}}$ & $\mathrm{x}(7)$ & $x(9)$ & $\mathrm{x}(12)$ & $x(7)$ & $x(9)$ & $\mathrm{x}(12)$ & 22 & 26 & 14 & 3291 \\
\hline South Africa ${ }^{5}$ & $\mathrm{x}(7)$ & $\mathrm{x}(9)$ & $\mathrm{x}(12)$ & $7^{\mathrm{d}}$ & $5^{\mathrm{d}}$ & $7^{\mathrm{d}}$ & $\mathrm{x}(7)$ & $\mathrm{x}(9)$ & $\mathrm{x}(12)$ & $x(7)$ & $\mathrm{x}(9)$ & $\mathrm{x}(12) \mid$ & 7 & 5 & 7 & 1572 \\
\hline G20 average & 10 & $\mathrm{~m}$ & $\mathrm{~m}$ & 18 & $\mathrm{~m}$ & $\mathrm{~m}$ & 7 & $\mathrm{~m}$ & $\mathrm{~m}$ & $\mathrm{~m}$ & $\mathrm{~m}$ & $\mathrm{~m}$ & 28 & 34 & 21 & 19202 \\
\hline
\end{tabular}

Notes: In most countries, the data refer to ISCED 2011. The countries with data that refer to ISCED-97 are: Brazil, Indonesia, the Russian Federation, Saudi Arabia and South Africa. See the description of the levels of education in the Definitions section. Columns showing data for other age groups are available for consultation on line (see StatLink below)

1. Brazil, Chile, France, the Russian Federation, Saudi Arabia: Year of reference 2013

2. Japan: Data for tertiary education exclude short-cycle tertiary education.

3. China: Year of reference 2010.

4. Indonesia: Year of reference 2011

5. South Africa: Year of reference 2012.

Source: OECD. China, Indonesia, Saudi Arabia, South Africa: UNESCO Institute for Statistics. Colombia, Costa Rica: OECD Education Database. Latvia, Lithuania: Eurostat. See Annex 3 for notes (www.oecd.org/education/education-at-a-glance-19991487.htm).

Please refer to the Reader's Guide for information concerning symbols for missing data and abbreviations.

StatLink 게그 http://dx.doi.org/10.1787/888933284758 
Table A1.4a. [1/3] Trends in educational attainment, by age group (2000, 2005, 2010 and 2014)

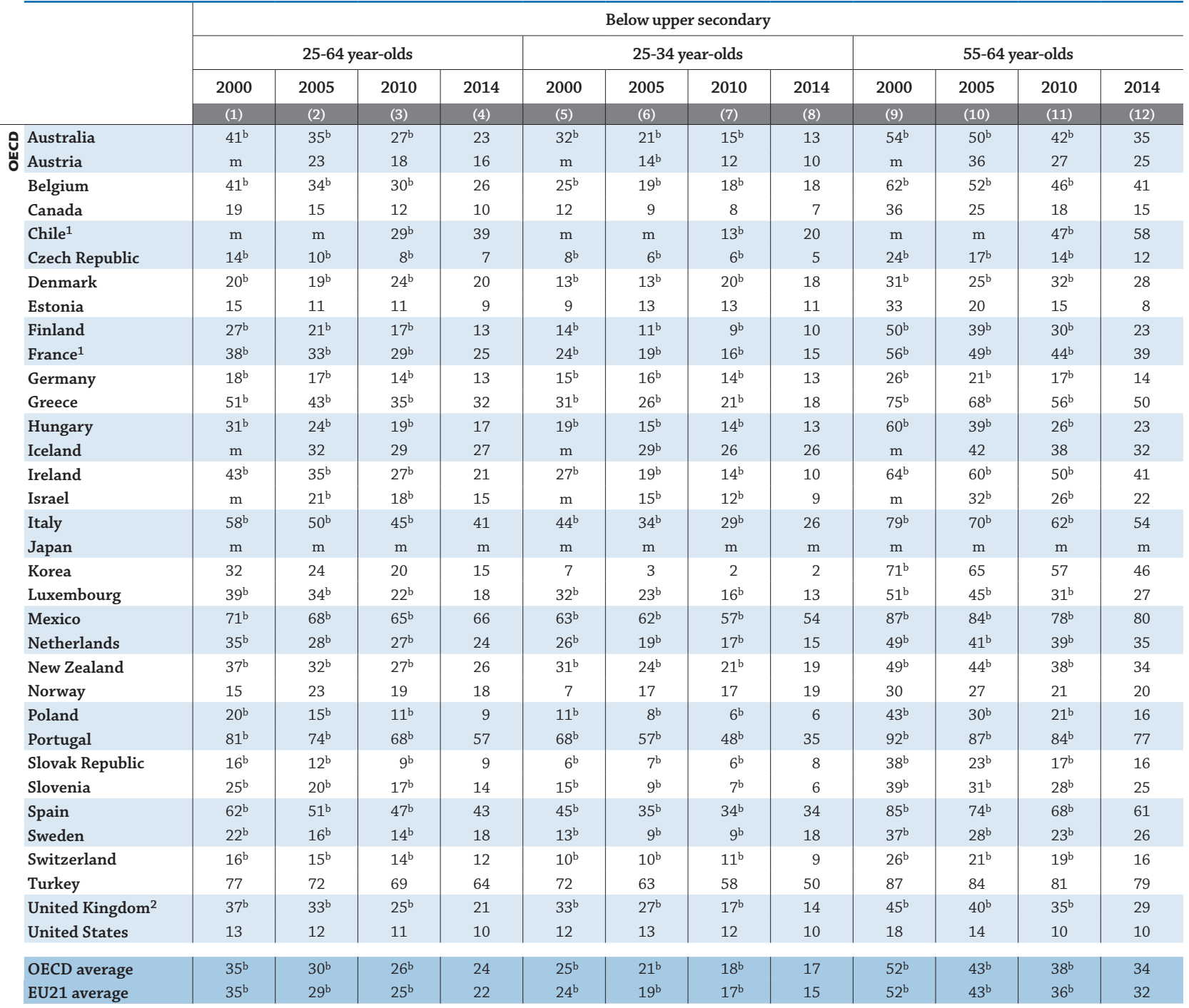

\begin{tabular}{|c|c|c|c|c|c|c|c|c|c|c|c|c|}
\hline $\begin{array}{l}\text { Argentina } \\
\text { Brazil }^{1,3}\end{array}$ & $\begin{array}{l}\mathrm{m} \\
\mathrm{m}\end{array}$ & $\begin{array}{l}\mathrm{m} \\
\mathrm{m}\end{array}$ & $\begin{array}{l}\mathrm{m} \\
59\end{array}$ & $\begin{array}{l}\mathrm{m} \\
54\end{array}$ & $\begin{array}{l}\mathrm{m} \\
\mathrm{m}\end{array}$ & $\begin{array}{l}\mathrm{m} \\
\mathrm{m}\end{array}$ & $\begin{array}{c}\mathrm{m} \\
47\end{array}$ & $\begin{array}{r}\mathrm{m} \\
39\end{array}$ & $\begin{array}{l}\mathrm{m} \\
\mathrm{m}\end{array}$ & $\begin{array}{l}\mathrm{m} \\
\mathrm{m}\end{array}$ & $\begin{array}{c}\mathrm{m} \\
75\end{array}$ & $\begin{array}{r}\mathrm{m} \\
72\end{array}$ \\
\hline $\begin{array}{l}\text { China } \\
\text { Colombia }\end{array}$ & $\begin{array}{c}95 \\
\mathrm{~m}\end{array}$ & $\begin{array}{l}\mathrm{m} \\
\mathrm{m}\end{array}$ & $\begin{array}{l}76 \\
\mathrm{~m}\end{array}$ & $\begin{array}{l}\mathrm{m} \\
48\end{array}$ & $\begin{array}{c}94 \\
\mathrm{~m}\end{array}$ & $\begin{array}{l}\mathrm{m} \\
\mathrm{m}\end{array}$ & $\begin{array}{c}64 \\
\mathrm{~m}\end{array}$ & $\begin{array}{r}\mathrm{m} \\
33\end{array}$ & $\begin{array}{r}97 \\
\mathrm{~m}\end{array}$ & $\begin{array}{l}\mathrm{m} \\
\mathrm{m}\end{array}$ & $\begin{array}{c}88 \\
\mathrm{~m}\end{array}$ & $\begin{array}{l}\mathrm{m} \\
67\end{array}$ \\
\hline $\begin{array}{l}\text { Indonesia } \\
\text { Latvia }\end{array}$ & $\begin{array}{r}\mathrm{m} \\
17\end{array}$ & $\begin{array}{c}\mathrm{m} \\
15\end{array}$ & $\begin{array}{l}74 \\
12\end{array}$ & $\begin{array}{l}69 \\
10\end{array}$ & $\begin{array}{c}\mathrm{m} \\
11\end{array}$ & $\begin{array}{c}\mathrm{m} \\
19\end{array}$ & $\begin{array}{l}63 \\
17\end{array}$ & $\begin{array}{l}60 \\
14\end{array}$ & $\begin{array}{c}\mathrm{m} \\
34\end{array}$ & $\begin{array}{l}\mathrm{m} \\
26\end{array}$ & $\begin{array}{l}92 \\
15\end{array}$ & $\begin{array}{l}85 \\
10\end{array}$ \\
\hline $\begin{array}{l}\text { Lithuania } \\
\text { Russian Federation }^{1}\end{array}$ & $\begin{array}{l}16^{\mathrm{b}} \\
\mathrm{m}\end{array}$ & $\begin{array}{c}12^{\mathrm{b}} \\
\mathrm{m}\end{array}$ & $\begin{array}{c}8^{\mathrm{b}} \\
\mathrm{m}\end{array}$ & $\begin{array}{l}9 \\
5\end{array}$ & $\begin{array}{c}8^{\mathrm{b}} \\
\mathrm{m}\end{array}$ & $\begin{array}{l}13^{b} \\
m\end{array}$ & $\begin{array}{c}12^{\mathrm{b}} \\
\mathrm{m}\end{array}$ & $\begin{array}{r}12 \\
5\end{array}$ & $\begin{array}{l}45^{b} \\
\mathrm{~m}\end{array}$ & $\begin{array}{l}29^{b} \\
m\end{array}$ & $\begin{array}{c}13^{b} \\
\mathrm{~m}\end{array}$ & $\begin{array}{l}9 \\
8\end{array}$ \\
\hline $\begin{array}{l}\text { Saudi Arabia }{ }^{1,5} \\
\text { South Africa }^{6}\end{array}$ & $\begin{array}{c}{ }^{c} \\
\mathrm{~m}\end{array}$ & $\begin{array}{l}64 \\
42\end{array}$ & $\begin{array}{l}\mathrm{m} \\
\mathrm{m}\end{array}$ & $\begin{array}{l}49 \\
35\end{array}$ & $\begin{array}{c}c \\
\mathrm{~m}\end{array}$ & $\begin{array}{l}56 \\
28\end{array}$ & $\begin{array}{l}\mathrm{m} \\
\mathrm{m}\end{array}$ & $\begin{array}{l}35 \\
23\end{array}$ & $\begin{array}{c}\mathrm{c} \\
\mathrm{m}\end{array}$ & $\begin{array}{l}85 \\
66\end{array}$ & $\begin{array}{l}\mathrm{m} \\
\mathrm{m}\end{array}$ & $\begin{array}{l}72 \\
62\end{array}$ \\
\hline
\end{tabular}

\footnotetext{
\begin{tabular}{l|l|l|l|l|l|l|l|l|l|l|l|l|l|l} 
G20 average & $45^{\mathrm{b}}$ & $\mathrm{m}$ & $40^{\mathrm{b}}$ & $\mathrm{m}$ & $37^{\mathrm{b}}$ & $\mathrm{m}$ & $31^{\mathrm{b}}$ & $\mathrm{m}$ & $60^{\mathrm{b}}$ & $\mathrm{m}$ & $54^{\mathrm{b}}$ & $\mathrm{m}$
\end{tabular}
}

Note: In most countries, there is a break in the series, represented by the code "b", as data for the latest year refer to ISCED 2011 while data for previous years refer to ISCED-97. For China and Korea data refer to ISCED-97 for all years. See the description of the levels of education in the Definitions section.

1. Brazil, Chile, France, the Russian Federation, Saudi Arabia: Data for year 2014 refer to year 2013.

2. The United Kingdom: Data for upper secondary attainment includes completion of a sufficient volume and standard of programmes that would be classified individually as completion of intermediate upper secondary programmes (18\% of the adults are under this group).

3. Brazil: Data for year 2010 refer to year 2009.

4. Indonesia: Data for year 2014 refer to year 2011, data for 2010 refer to year 2006.

5. Saudi Arabia: Data for year 2005 refer to year 2004

6. South Africa: Data for year 2014 refer to year 2012.

Source: OECD. China, Indonesia, Saudi Arabia, South Africa: UNESCO Institute for Statistics. Colombia, Costa Rica: OECD Education Database. Latvia, Lithuania: Eurostat. See Annex 3 for notes (www.oecd.org/education/education-at-a-glance-19991487.htm).

Please refer to the Reader's Guide for information concerning symbols for missing data and abbreviations.

StatLink 젶ㄴ http://dx.doi.org/10.1787/888933284763 
Table A1.4a. [2/3] Trends in educational attainment, by age group (2000, 2005, 2010 and 2014)

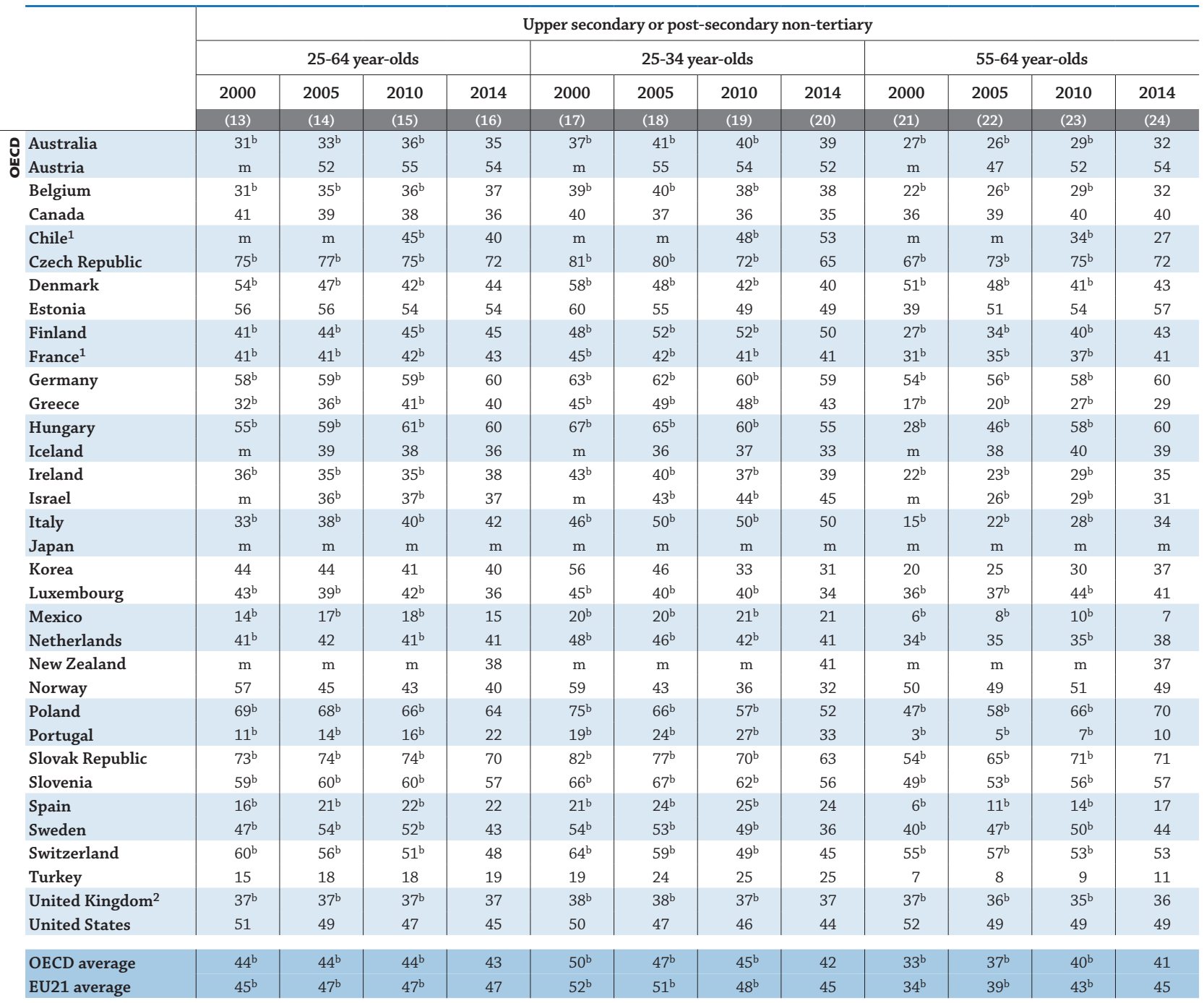

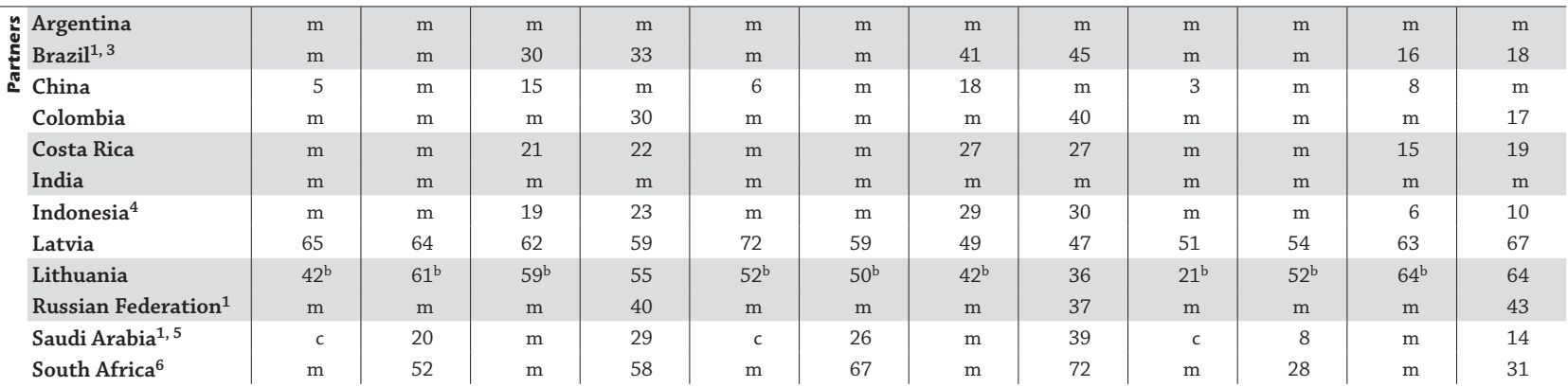

G20 average

\begin{tabular}{l|l}
$34^{\mathrm{b}}$ & $\mathrm{m}$ \\
\hline
\end{tabular}

$34^{\mathrm{b}}$

$38^{\mathrm{b}}$

Note: In most countries, there is a break in the series, represented by the code "b", as data for the latest year refer to ISCED 2011 while data for previous years refer to ISCED-97. For China and Korea data refer to ISCED-97 for all years. See the description of the levels of education in the Definitions section.

1. Brazil, Chile, France, the Russian Federation, Saudi Arabia: Data for year 2014 refer to year 2013.

2. The United Kingdom: Data for upper secondary attainment includes completion of a sufficient volume and standard of programmes that would be classified

individually as completion of intermediate upper secondary programmes ( $18 \%$ of the adults are under this group).

3. Brazil: Data for year 2010 refer to year 2009.

4. Indonesia: Data for year 2014 refer to year 2011, data for 2010 refer to year 2006.

5. Saudi Arabia: Data for year 2005 refer to year 2004.

6. South Africa: Data for year 2014 refer to year 2012

Source: OECD. China, Indonesia, Saudi Arabia, South Africa: UNESCO Institute for Statistics. Colombia, Costa Rica: OECD Education Database. Latvia, Lithuania: Eurostat. See Annex 3 for notes (www.oecd.org/education/education-at-a-glance-19991487.htm).

Please refer to the Reader's Guide for information concerning symbols for missing data and abbreviations.

StatLink त्गाड़ http://dx.doi.org/10.1787/888933284763 
Table A1.4a. [3/3] Trends in educational attainment, by age group (2000, 2005, 2010 and 2014)

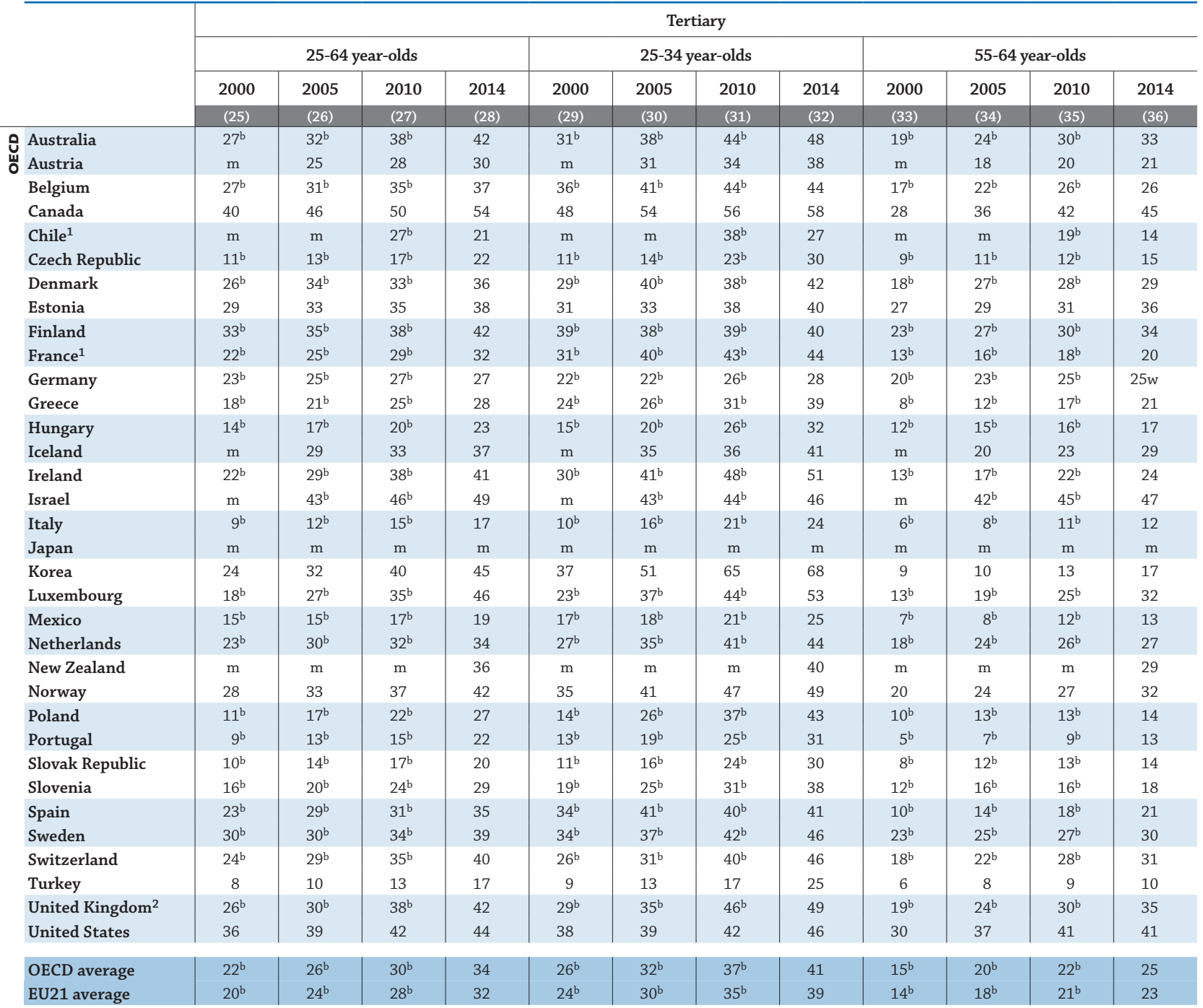

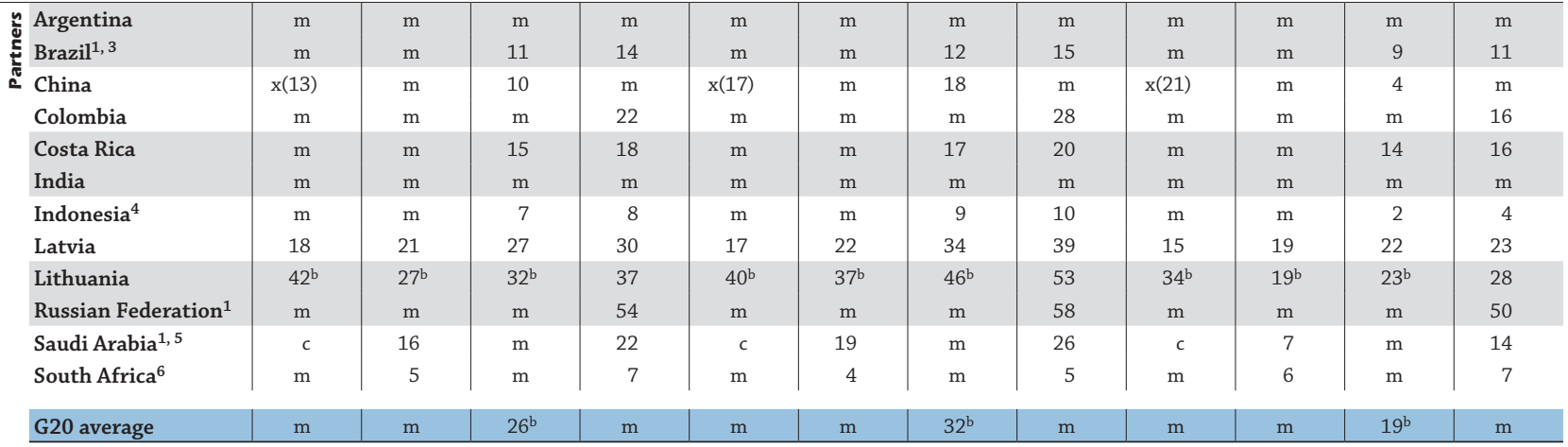

Note: In most countries, there is a break in the series, represented by the code "b", as data for the latest year refer to ISCED 2011 while data for previous years refer to ISCED-97. For China and Korea data refer to ISCED-97 for all years. See the description of the levels of education in the Definitions section.

1. Brazil, Chile, France, the Russian Federation, Saudi Arabia: Data for year 2014 refer to year 2013.

2. The United Kingdom: Data for upper secondary attainment includes completion of a sufficient volume and standard of programmes that would be classified individually as completion of intermediate upper secondary programmes (18\% of the adults are under this group).

3. Brazil: Data for year 2010 refer to year 2009.

4. Indonesia: Data for year 2014 refer to year 2011, data for 2010 refer to year 2006.

5. Saudi Arabia: Data for year 2005 refer to year 2004

6. South Africa: Data for year 2014 refer to year 2012.

Source: OECD. China, Indonesia, Saudi Arabia, South Africa: UNESCO Institute for Statistics. Colombia, Costa Rica: OECD Education Database. Latvia, Lithuania:

Eurostat. See Annex 3 for notes (www.oecd.org/education/education-at-a-glance-19991487.htm).

Please refer to the Reader's Guide for information concerning symbols for missing data and abbreviations.

StatLink 제간 http://dx.doi.org/10.1787/888933284763 
Table A1.5a. Adults whose highest level of education is upper secondary or post-secondary non-tertiary education, by programme orientation and gender (2014)

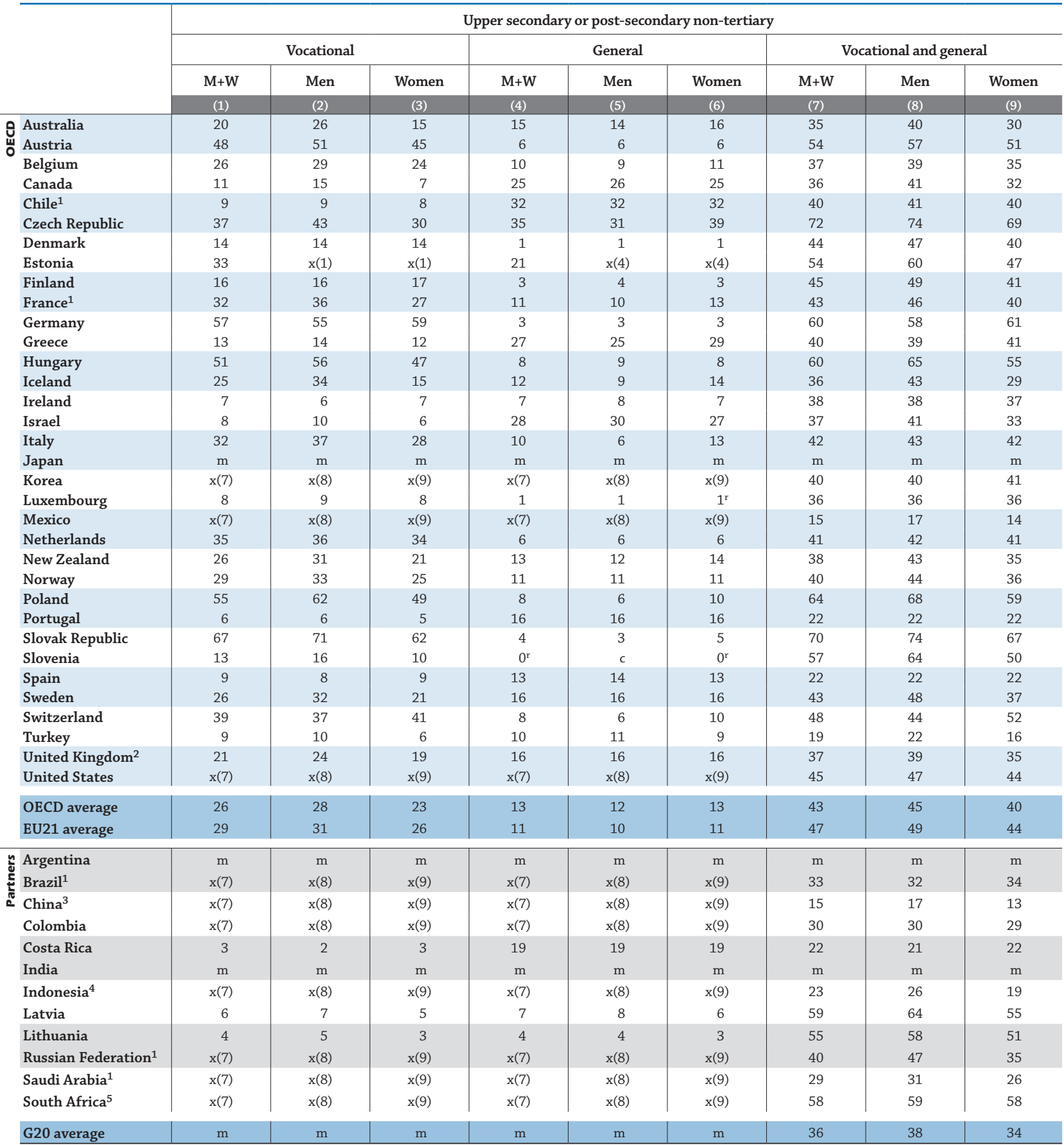

Notes: In most countries, data refer to ISCED 2011. The countries with data that refer to ISCED-97 are: Brazil, Indonesia, the Russian Federation, Saudi Arabia, South Africa. See the description of the levels of education in the Definitions section. Denmark, Finland, Ireland, Latvia, Lithuania, Luxembourg, Slovenia: Data for the breakdown by programme orientation cover only 15-34 year-olds and 35-64 year-olds if those individuals have completed their highest level of education 15 years, at most, before the data of the interview; the category "Vocational and General" covers all adults.

1. Brazil, Chile, France, the Russian Federation, Saudi Arabia: Year of reference 2013.

2. The United Kingdom: Data for upper secondary attainment includes completion of a sufficient volume and standard of programmes that would be classified individually as completion of intermediate upper secondary programmes (18\% of the adults are under this group).

3. China: Year of reference 2010.

4. Indonesia: Year of reference 2011.

5. South Africa: Year of reference 2012.

Sources: OECD. China, Indonesia, Saudi Arabia, South Africa: UNESCO Institute for Statistics. Colombia, Costa Rica: OECD Education Database. Latvia, Lithuania:

Eurostat. See Annex 3 for notes (www.oecd.org/education/education-at-a-glance-19991487.htm).

Please refer to the Reader's Guide for information concerning symbols for missing data and abbreviations.

StatLink 제그 http://dx.doi.org/10.1787/888933284773 
Table A1.6a. [1/2] Skills and readiness to use information and communication technologies for problem solving among 25-64 year-olds, by educational attainment (2012)

\begin{tabular}{|c|c|c|c|c|c|c|c|c|c|c|c|c|c|c|c|c|c|c|c|c|}
\hline & \multicolumn{10}{|c|}{ Below upper secondary } & \multicolumn{10}{|c|}{ Upper secondary or post-secondary non-tertiary } \\
\hline & \multicolumn{2}{|c|}{$\begin{array}{c}\text { Group 0 } \\
\text { (No } \\
\text { computer } \\
\text { experience) }\end{array}$} & \multicolumn{2}{|c|}{$\begin{array}{c}\text { Group 1 } \\
\text { (Refused } \\
\text { the } \\
\text { computer- } \\
\text { based } \\
\text { assessment) }\end{array}$} & \multicolumn{2}{|c|}{$\begin{array}{c}\text { Group 2 } \\
\text { (Failed ICT } \\
\text { core test } \\
\text { or minimal } \\
\text { problem- } \\
\text { solving } \\
\text { skills) }\end{array}$} & \multicolumn{2}{|c|}{$\begin{array}{l}\text { Group } 3 \\
\text { (Moderate } \\
\text { ICT } \\
\text { and } \\
\text { problem- } \\
\text { solving } \\
\text { skills) }\end{array}$} & \multicolumn{2}{|c|}{$\begin{array}{l}\text { Group 4 } \\
\text { (Good ICT } \\
\text { and } \\
\text { problem- } \\
\text { solving } \\
\text { skills) }\end{array}$} & \multicolumn{2}{|c|}{$\begin{array}{c}\text { Group 0 } \\
\text { (No } \\
\text { computer } \\
\text { experience) }\end{array}$} & \multicolumn{2}{|c|}{$\begin{array}{c}\text { Group 1 } \\
\text { (Refused } \\
\text { the } \\
\text { computer- } \\
\text { based } \\
\text { assessment) }\end{array}$} & \multicolumn{2}{|c|}{$\begin{array}{l}\text { Group 2 } \\
\text { (Failed ICT } \\
\text { core test } \\
\text { or minimal } \\
\text { problem- } \\
\text { solving } \\
\text { skills) }\end{array}$} & \multicolumn{2}{|c|}{$\begin{array}{l}\text { Group } 3 \\
\text { (Moderate } \\
\text { ICT } \\
\text { and } \\
\text { problem- } \\
\text { solving } \\
\text { skills) }\end{array}$} & \multicolumn{2}{|c|}{$\begin{array}{l}\text { Group 4 } \\
\text { (Good ICT } \\
\text { and } \\
\text { problem- } \\
\text { solving } \\
\text { skills) }\end{array}$} \\
\hline & $\%$ & S.E. & $\%$ & S.E. & $\%$ & S.E. & $\%$ & S.E. & $\%$ & S.E. & $\%$ & S.E. & $\%$ & S.E. & $\%$ & S.E. & $\%$ & S.E. & & S.E. \\
\hline & (1) & (2) & (3) & $(4)$ & (5) & $(6)$ & (7) & (8) & (9) & (10) & (11) & (12) & (13) & (14) & $(15)$ & (16) & (17) & (18) & (19) & (20) \\
\hline \multicolumn{21}{|l|}{ i National entities } \\
\hline Australia & 12 & (1.1) & 25 & (1.6) & 21 & (1.5) & 28 & (1.7) & 15 & (1.3) & 4 & (0.5) & 16 & (1.1) & 14 & (1.2) & 34 & (1.5) & 33 & (1.6) \\
\hline Austria & 33 & (1.9) & 20 & (1.7) & 22 & (1.8) & 17 & (1.9) & 7 & (1.3) & 8 & $(0.5)$ & 12 & $(0.7)$ & 15 & $(0.9)$ & 35 & (1.4) & 30 & (1.1) \\
\hline Canada & 25 & $(1.2)$ & 16 & (1.3) & 37 & (1.6) & 18 & (1.7) & 5 & (0.9) & 5 & $(0.4)$ & 9 & $(0.6)$ & 26 & (1.0) & 33 & (1.2) & 26 & (1.0) \\
\hline Czech Republic & 39 & $(4.0)$ & 22 & (2.9) & 15 & (2.5) & 16 & (3.3) & 8 & (2.1) & 10 & $(0.7)$ & 15 & (1.2) & 19 & (1.4) & 31 & (1.6) & 24 & (1.4) \\
\hline Denmark & 10 & (1.0) & 17 & (1.3) & 35 & (1.9) & 26 & (1.9) & 13 & (1.6) & 2 & $(0.3)$ & 7 & $(0.5)$ & 24 & (1.2) & 37 & (1.3) & 30 & (1.3) \\
\hline Estonia & 32 & (1.5) & 19 & (1.3) & 25 & (1.8) & 19 & (1.8) & 6 & (1.0) & 14 & $(0.7)$ & 22 & $(0.7)$ & 21 & $(0.9)$ & 27 & $(0.9)$ & 16 & $(0.9)$ \\
\hline Finland & 18 & (1.8) & 23 & (1.8) & 29 & (2.0) & 22 & (2.3) & 9 & (1.5) & 3 & $(0.4)$ & 15 & $(0.8)$ & 22 & (1.2) & 31 & (1.2) & 29 & (1.2) \\
\hline France & $\mathrm{m}$ & $\mathrm{m}$ & $\mathrm{m}$ & $\mathrm{m}$ & $\mathrm{m}$ & $\mathrm{m}$ & $\mathrm{m}$ & $\mathrm{m}$ & $\mathrm{m}$ & $\mathrm{m}$ & $\mathrm{m}$ & $\mathrm{m}$ & $\mathrm{m}$ & $\mathrm{m}$ & $\mathrm{m}$ & $\mathrm{m}$ & $\mathrm{m}$ & $\mathrm{m}$ & $\mathrm{m}$ & $\mathrm{m}$ \\
\hline Germany & 29 & (2.8) & 13 & (2.0) & 31 & (3.3) & 20 & (2.7) & 7 & (1.7) & 10 & $(0.8)$ & 8 & (0.7) & 23 & (1.2) & 34 & (1.3) & 26 & (1.0) \\
\hline Ireland & 34 & (1.5) & 29 & (1.5) & 24 & (1.7) & 11 & (1.2) & 1 & (0.5) & 6 & (0.5) & 22 & (1.3) & 20 & (1.6) & 34 & (1.5) & 18 & (1.3) \\
\hline Italy & $\mathrm{m}$ & $\mathrm{m}$ & $\mathrm{m}$ & $\mathrm{m}$ & $\mathrm{m}$ & $\mathrm{m}$ & $\mathrm{m}$ & $\mathrm{m}$ & $\mathrm{m}$ & $\mathrm{m}$ & $\mathrm{m}$ & $\mathrm{m}$ & $\mathrm{m}$ & $\mathrm{m}$ & $\mathrm{m}$ & $\mathrm{m}$ & $\mathrm{m}$ & $\mathrm{m}$ & $\mathrm{m}$ & $\mathrm{m}$ \\
\hline Japan & 44 & (2.6) & 20 & (2.0) & 18 & (2.1) & 10 & (2.0) & 8 & (1.6) & 12 & $(0.8)$ & 21 & (1.4) & 23 & (1.4) & 20 & (1.3) & 24 & $(1.2)$ \\
\hline Korea & 62 & (1.7) & 11 & (1.0) & 20 & (1.5) & 6 & (1.1) & 1 & (0.5) & 14 & $(0.8)$ & 8 & $(0.7)$ & 29 & (1.4) & 34 & (1.5) & 15 & (1.2) \\
\hline Netherlands & 11 & $(0.9)$ & 10 & $(0.9)$ & 33 & (1.7) & 34 & (1.8) & 13 & (1.2) & 1 & $(0.3)$ & 4 & $(0.6)$ & 17 & (1.1) & 40 & (1.6) & 38 & (1.6) \\
\hline Norway & 6 & $(0.9)$ & 17 & (1.4) & 30 & (2.0) & 32 & (2.0) & 15 & (1.7) & 1 & $(0.3)$ & 8 & (0.7) & 21 & (1.4) & 38 & (1.7) & 32 & (1.2) \\
\hline Poland & 65 & $(2.4)$ & 17 & (1.9) & 11 & (1.7) & 5 & (1.4) & 2 & (1.0) & 26 & $(0.9)$ & 31 & (1.0) & 21 & (1.1) & 14 & $(0.9)$ & 7 & $(0.8)$ \\
\hline Slovak Republic & 72 & (1.9) & 10 & (1.3) & 8 & (1.4) & 8 & (1.4) & 2 & $(0.7)$ & 22 & $(0.8)$ & 16 & $(0.7)$ & 13 & $(0.8)$ & 30 & (1.2) & 19 & (1.2) \\
\hline Spain & $\mathrm{m}$ & $\mathrm{m}$ & $\mathrm{m}$ & $\mathrm{m}$ & $\mathrm{m}$ & $\mathrm{m}$ & $\mathrm{m}$ & $\mathrm{m}$ & $\mathrm{m}$ & $\mathrm{m}$ & $\mathrm{m}$ & $\mathrm{m}$ & $\mathrm{m}$ & $\mathrm{m}$ & $\mathrm{m}$ & $\mathrm{m}$ & $\mathrm{m}$ & $\mathrm{m}$ & $\mathrm{m}$ & $\mathrm{m}$ \\
\hline Sweden & 6 & (1.1) & 15 & (1.7) & 42 & (2.6) & 27 & (2.6) & 10 & (1.7) & 1 & $(0.3)$ & 6 & $(0.6)$ & 18 & (1.1) & 37 & (1.3) & 38 & (1.4) \\
\hline United States & 36 & $(2.7)$ & 17 & (2.1) & 32 & (2.4) & 13 & (2.1) & 3 & (0.9) & 5 & $(0.4)$ & 9 & (1.1) & 29 & (1.6) & 36 & (1.7) & 21 & (1.5) \\
\hline \multicolumn{21}{|l|}{ Sub-national entities } \\
\hline Flanders (Belgium) & 29 & (1.6) & 10 & (1.1) & 35 & (1.9) & 20 & (1.9) & 5 & (1.2) & 8 & $(0.6)$ & 6 & $(0.5)$ & 26 & (1.1) & 36 & (1.2) & 23 & (1.3) \\
\hline England (UK) & 13 & $(1.2)$ & 9 & (1.1) & 38 & (2.0) & 30 & (2.0) & 10 & (1.2) & 3 & $(0.5)$ & 5 & $(0.8)$ & 24 & (1.7) & 37 & (2.0) & 30 & (1.7) \\
\hline Northern Ireland (UK) & 28 & (1.8) & 6 & $(0.8)$ & 36 & (2.7) & 25 & (2.5) & 6 & (1.1) & 7 & $(0.9)$ & 2 & $(0.5)$ & 27 & (2.2) & 40 & (2.3) & 24 & $(2.1)$ \\
\hline England/N. Ireland (UK) & 13 & (1.1) & 9 & (1.0) & 38 & (1.9) & 30 & (1.9) & 10 & (1.1) & 3 & $(0.5)$ & 5 & $(0.7)$ & 24 & (1.6) & 37 & (1.9) & 30 & (1.6) \\
\hline OECD average & 30 & $(0.4)$ & 17 & $(0.4)$ & 27 & $(0.5)$ & 19 & $(0.5)$ & 7 & $(0.3)$ & 8 & $(0.1)$ & 13 & $(0.2)$ & 21 & $(0.3)$ & 32 & $(0.3)$ & 25 & $(0.3)$ \\
\hline Russian Federation* & 45 & $(7.5)$ & c & c & 22 & (5.5) & 18 & (4.8) & 13 & (3.3) & 39 & (3.2) & 13 & (1.8) & 15 & (2.6) & 17 & (1.9) & 16 & (3.1) \\
\hline
\end{tabular}

Notes: Data on educational attainment are based on ISCED-97. Columns showing data for total population not broken down by level of education are available for consultation on line (see StatLink below).

* See note on data for the Russian Federation in the Methodology section.

Source: OECD. Survey of Adult Skills (PIAAC) (2012). See Annex 3 for notes (www.oecd.org/education/education-at-a-glance-19991487.htm).

Please refer to the Reader's Guide for information concerning symbols for missing data and abbreviations.

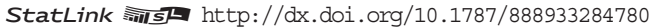


Table A1.6a. [2/2] Skills and readiness to use information and communication technologies for problem solving among 25-64 year-olds, by educational attainment (2012)

\begin{tabular}{|c|c|c|c|c|c|c|c|c|c|c|}
\hline & \multicolumn{10}{|c|}{ Tertiary } \\
\hline & \multicolumn{2}{|c|}{$\begin{array}{l}\text { Group 0 } \\
\text { (No computer } \\
\text { experience) }\end{array}$} & \multicolumn{2}{|c|}{$\begin{array}{l}\text { Group 1 } \\
\text { (Refused the } \\
\text { computer-based } \\
\text { assessment) }\end{array}$} & \multicolumn{2}{|c|}{$\begin{array}{c}\text { Group } 2 \\
\text { (Failed ICT core test } \\
\text { or minimal } \\
\text { problem-solving skills) }\end{array}$} & \multicolumn{2}{|c|}{$\begin{array}{c}\text { Group 3 } \\
\text { (Moderate ICT and } \\
\text { problem-solving skills) }\end{array}$} & \multicolumn{2}{|c|}{$\begin{array}{c}\text { Group } 4 \\
\text { (Good ICT and } \\
\text { problem-solving skills) }\end{array}$} \\
\hline & $\%$ & S.E. & $\%$ & S.E. & $\%$ & S.E. & $\%$ & S.E. & $\%$ & S.E. \\
\hline & (21) & (22) & (23) & (24) & (25) & (26) & (27) & (28) & (29) & (30) \\
\hline \multicolumn{11}{|l|}{ Q National entities } \\
\hline ప̈ Australia & 1 & $(0.2)$ & 7 & (0.7) & 10 & $(0.9)$ & 26 & (1.2) & 56 & $(1.4)$ \\
\hline Austria & 1 & $(0.4)$ & 7 & $(0.8)$ & 9 & (1.2) & 33 & (2.0) & 50 & $(2.2)$ \\
\hline Canada & 1 & $(0.1)$ & 4 & $(0.3)$ & 17 & $(0.7)$ & 31 & (1.0) & 47 & (1.0) \\
\hline Czech Republic & 0 & $(0.2)$ & 6 & (1.4) & 7 & (1.4) & 27 & (3.5) & 60 & $(3.2)$ \\
\hline Denmark & c & c & 3 & $(0.3)$ & 11 & $(0.6)$ & 31 & (1.1) & 55 & $(1.2)$ \\
\hline Estonia & 3 & $(0.3)$ & 14 & $(0.8)$ & 15 & $(0.9)$ & 33 & (1.0) & 35 & (1.3) \\
\hline Finland & c & c & 4 & $(0.4)$ & 10 & $(0.7)$ & 29 & (1.1) & 57 & (1.1) \\
\hline France & $\mathrm{m}$ & $\mathrm{m}$ & $\mathrm{m}$ & $\mathrm{m}$ & $\mathrm{m}$ & $\mathrm{m}$ & $\mathrm{m}$ & $\mathrm{m}$ & $\mathrm{m}$ & $\mathrm{m}$ \\
\hline Germany & 2 & $(0.5)$ & 4 & $(0.5)$ & 11 & (1.1) & 29 & (1.5) & 53 & (1.6) \\
\hline Ireland & 1 & $(0.2)$ & 9 & (0.6) & 11 & (1.0) & 35 & (1.6) & 45 & $(1.5)$ \\
\hline Italy & $\mathrm{m}$ & $\mathrm{m}$ & $\mathrm{m}$ & $\mathrm{m}$ & $\mathrm{m}$ & $\mathrm{m}$ & $\mathrm{m}$ & $\mathrm{m}$ & $\mathrm{m}$ & $\mathrm{m}$ \\
\hline Japan & 3 & $(0.4)$ & 12 & (0.9) & 15 & (1.1) & 22 & (1.2) & 49 & (1.3) \\
\hline Korea & 1 & $(0.2)$ & 2 & (0.3) & 14 & (1.0) & 39 & (1.6) & 44 & (1.6) \\
\hline Netherlands & 0 & $(0.2)$ & 2 & $(0.4)$ & 7 & $(0.9)$ & 27 & (1.4) & 64 & $(1.6)$ \\
\hline Norway & c & c & 2 & $(0.4)$ & 10 & $(0.8)$ & 28 & (1.5) & 59 & $(1.6)$ \\
\hline Poland & 1 & $(0.3)$ & 19 & (1.2) & 16 & (1.2) & 27 & (1.8) & 37 & (1.9) \\
\hline Slovak Republic & 1 & $(0.3)$ & 9 & (1.1) & 9 & (1.2) & 33 & (2.2) & 48 & $(2.4)$ \\
\hline Spain & $\mathrm{m}$ & $\mathrm{m}$ & $\mathrm{m}$ & $\mathrm{m}$ & $\mathrm{m}$ & $\mathrm{m}$ & $\mathrm{m}$ & $\mathrm{m}$ & $\mathrm{m}$ & $\mathrm{m}$ \\
\hline Sweden & c & c & 3 & $(0.4)$ & 9 & $(0.8)$ & 26 & (1.5) & 62 & $(1.3)$ \\
\hline United States & 1 & $(0.2)$ & 2 & (0.3) & 11 & (1.2) & 35 & (1.6) & 51 & $(1.8)$ \\
\hline \multicolumn{11}{|l|}{ Sub-national entities } \\
\hline Flanders (Belgium) & 1 & $(0.2)$ & 3 & $(0.4)$ & 10 & $(0.9)$ & 32 & (1.3) & 55 & $(1.4)$ \\
\hline England (UK) & 1 & $(0.3)$ & 3 & $(0.5)$ & 11 & (1.1) & 32 & (1.6) & 53 & $(1.7)$ \\
\hline Northern Ireland (UK) & 1 & $(0.4)$ & 1 & $(0.2)$ & 12 & (1.8) & 38 & (1.9) & 48 & $(2.4)$ \\
\hline England/N. Ireland (UK) & 1 & $(0.3)$ & 3 & $(0.5)$ & 12 & (1.0) & 32 & (1.6) & 53 & $(1.6)$ \\
\hline OECD average & 1 & $(0.1)$ & 6 & $(0.2)$ & 11 & $(0.2)$ & 30 & $(0.4)$ & 52 & $(0.4)$ \\
\hline Russian Federation* & 12 & (1.5) & 15 & (2.4) & 18 & (2.4) & 27 & (1.7) & 27 & $(2.5)$ \\
\hline
\end{tabular}

Notes: Data on educational attainment are based on ISCED-97. Columns showing data for total population not broken down by level of education are available for consultation on line (see StatLink below).

* See note on data for the Russian Federation in the Methodology section.

Source: OECD. Survey of Adult Skills (PIAAC) (2012). See Annex 3 for notes (www.oecd.org/education/education-at-a-glance-19991487.htm).

Please refer to the Reader's Guide for information concerning symbols for missing data and abbreviations.

StatLink त्ताs http://dx.doi.org/10.1787/888933284780 


\section{HOW MANY STUDENTS ARE EXPECTED TO COMPLETE UPPER SECONDARY EDUCATION?}

- Based on current patterns, it is estimated that an average of $85 \%$ of today's young people in OECD countries will complete upper secondary education over their lifetimes.

- Some $47 \%$ of men and $44 \%$ of women are expected to graduate from an upper secondary vocational programme during their lifetime, on average across OECD countries.

- Of those who graduated from an upper secondary general programme, $97 \%$ did so before they were 25 years old.

\section{Chart A2.1. Upper secondary graduation rates (2013)}

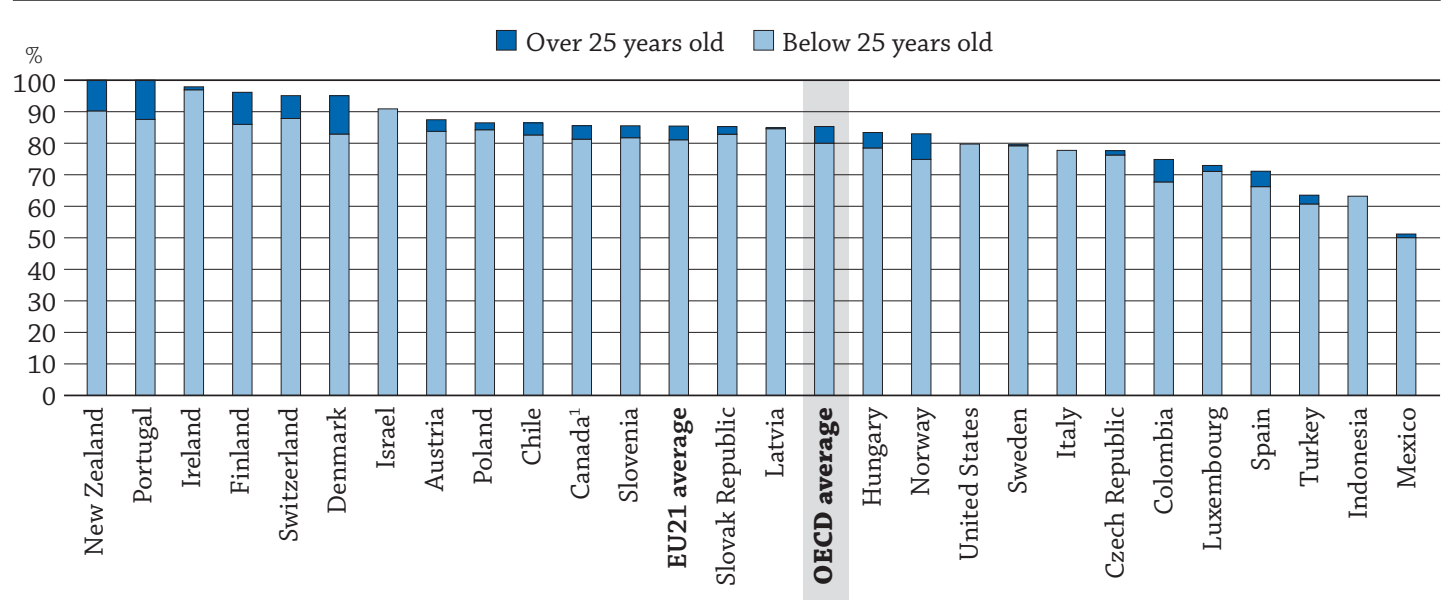

1. Year of reference 2012.

Countries are ranked in descending order of first time upper secondary graduation rates.

Source: OECD. Tables A2.1 and A2.2. See Annex 3 for notes (www.oecd.org/education/education-at-a-glance-19991487.htm).

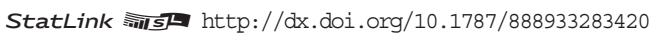

\section{Context}

Upper secondary education, which consolidates students' basic skills and knowledge through either an academic or a vocational pathway, aims to prepare students for entry into further levels of education or the labour market, and to become engaged citizens. In many countries, this level of education is not compulsory and can last from two to five years. What is crucial, however, is providing education of good quality that meets the needs of the society and the economy.

Graduating from upper secondary education has become increasingly important in all countries, as the skills needed in the labour market are becoming more knowledge-based and workers are progressively required to adapt to the uncertainties of a rapidly changing global economy. However, while graduation rates give an indication of the extent to which education systems are succeeding in preparing students to meet the labour market's minimum requirements, they do not capture the quality of education outcomes.

One of the challenges facing education systems in many OECD countries is students' disengagement and consequent dropout from the education system, meaning that they leave school without an upper secondary qualification. These young people tend to face severe difficulties entering - and remaining in - the labour market. Leaving school early is a problem, both for individuals and society. Policy makers are examining ways to reduce the number of early school-leavers, defined as those students who do not complete their upper secondary education. Internationally comparable measures of how many students successfully complete upper secondary programmes - which also imply how many students do not complete those programmes - can assist efforts to this end. 
Other findings

- In 21 of 25 OECD countries with available data, more than $75 \%$ of young people have graduated from upper secondary education. In nine countries the first-time graduation rate exceeds $90 \%$.

- On average across OECD countries, $83 \%$ of those graduating from an upper secondary vocational programme are younger than 25 and $46 \%$ are women.

- Most young men in upper secondary vocational programmes study engineering, manufacturing and construction, while young women form the majority in all other fields of study in vocational programmes.

- Some $13 \%$ of young people are expected to graduate from a post-secondary non-tertiary vocational programme; $54 \%$ of them are women.

\section{Trends}

Analysing countries for which comparable trends data are available for 2005 and 2013, the first-time graduation rate at the upper secondary level increased from $80 \%$ to $85 \%$. This increase was striking in several European countries, namely Poland (from $41 \%$ to $86 \%$ ) and Portugal (from 54\% to $100 \%$ ). By contrast, in some countries graduation rates declined during the period, including in Norway, where these rates dropped from 90\% in 2005 to 83\% in 2013.

Graduation rates from general upper secondary programmes increased slightly, on average, from $49 \%$ in 2005 to $52 \%$ in 2013, while graduation rates from vocational programmes more than compensated by increasing from $43 \%$ to $46 \%$. A few countries developed vocational education systems that grew quickly during the period. Graduation rates from vocational programmes in New Zealand and Portugal, for example, increased by more than forty percentage points.

The prevalence of post-secondary non-tertiary vocational education remained constant over the same period, with graduation rates around $10-12 \%$. In Australia, graduation rates from post-secondary nontertiary vocational education increased by 23 percentage points, so that $41 \%$ of students in Australia are now expected to graduate from one of these programmes.

\section{Note}

Graduation rates represent the estimated percentage of people from a given age cohort that is expected to graduate at some point during their lifetime. This estimate is based on the number of graduates in 2013 and the age distribution of this group. Graduation rates are based on both the population and the current pattern of graduation, and are thus sensitive to any changes in the education system, such as the introduction of new programmes, and changes in the duration of programmes. Graduation rates can be very high - even above $100 \%$ - during a period when an unexpected number of people goes back to school.

When the age breakdown is not available, the gross graduation rate is calculated instead. This refers to the total number of graduates divided by the average cohort of the population at the typical age provided by the country.

In this indicator, age refers generally to the age of students at the beginning of the calendar year. Students could be one year older than the age indicated when they graduate at the end of the school year. Twenty-five is regarded as the upper age limit for completing secondary education. Across OECD countries, more than $90 \%$ of first-time graduates from upper secondary programmes in 2013 were younger than 25. People who graduate from this level at age 25 or older are usually enrolled in second-chance programmes. 


\section{Analysis}

\section{Graduation from upper secondary programmes}

\section{A snapshot of upper secondary graduation rates}

Current estimates indicate that, on average, $85 \%$ of people across OECD countries will complete upper secondary education over their lifetime (Table A2.1). Attaining an upper secondary education is often considered to be the minimum credential for successful entry into the labour market and necessary for continuing to further education. The costs, to both individuals and society, of not completing this level of education on time can be considerable (see Indicators $\mathrm{A} 6$ and $\mathrm{A} 7$ ).

Graduation rates offer an indication of whether government initiatives have been successful in increasing the number of people who graduate from upper secondary education. The great differences in graduation rates among countries reflect the variety of systems and programmes available.

In nine countries among those with data available, more than $90 \%$ of people are expected to graduate from upper secondary school during their lifetime, but just over $50 \%$ of young people in Mexico are expected to do so (Table A2.1). In all countries, women are more likely than men to complete upper secondary education. The largest gender gap is observed in Slovenia, where 95\% of young women are expected to graduate at least once from upper secondary education, while only $76 \%$ of young men will do so.

Women are more likely than men to graduate from general programmes in all countries, while men are more likely to graduate from vocational programmes in 26 of the 35 countries with available data. Vocational education and training (VET) is an important part of upper secondary education in many OECD countries, and it can play a central role in preparing young people for work, developing adults' skills and responding to labour market needs (see Indicator A1). But in some countries, VET has been neglected and marginalised in policy discussions, often overshadowed by the increasing emphasis on general academic education. Nevertheless, an increasing number of countries are recognising that good initial vocational education and training have a major contribution to make to economic competitiveness. This is one of the explanations for the increase in graduation rates from upper secondary vocational programmes between 2005 and 2013.

\section{Chart A2.2. Trends in vocational upper secondary graduation rates (2005 and 2013)}

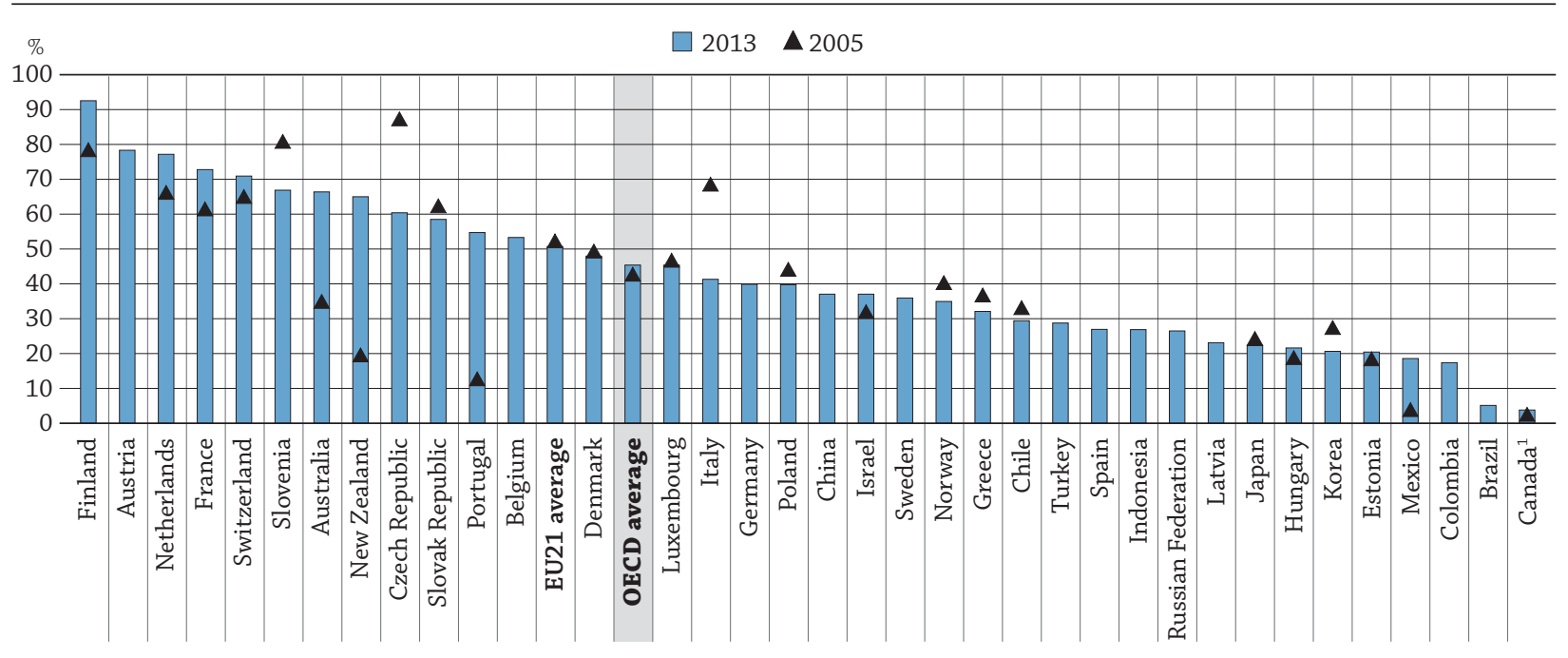

1. Year of reference 2012 intead of 2013

Countries are ranked in descending order of vocational upper secondary graduation rates in 2013.

Source: OECD. Table A2.4. See Annex 3 for notes (www.oecd.org/education/education-at-a-glance-19991487.htm).

StatLink त्गाज् http://dx.doi.org/10.1787/888933283430

On average across OECD countries, $46 \%$ of young people will graduate from an upper secondary vocational programme. In Finland, many graduates enter the labour market immediately after completing upper secondary vocational training. Some $93 \%$ of young people in Finland are expected to be awarded a vocational degree during their lifetime. By contrast, only $5 \%$ or fewer young people in Brazil and Canada will graduate from upper secondary vocational education. 
Although many countries developed extensive vocational programmes at the secondary level, in other countries, most students prefer general programmes. As shown in Chart A2.3, large proportions of students in Austria, Finland and the Netherlands are expected to graduate from an upper secondary vocational programme. But in Canada, the proportion of young people expected to graduate from a vocational programme is considerably smaller. Vocational programmes in Canada are often offered within the post-secondary system, and vocational training at the secondary level is largely a second-chance programme for older students. In fact, $65 \%$ of graduates from upper secondary vocational programmes in Canada are older than 25 (Table A2.2).

Graduation rates, however, do not imply that all graduates will pursue a tertiary degree or enter the labour force immediately. Indeed, the number of graduates who wind up neither employed nor in education or training (NEET) has been growing throughout OECD countries (see Indicator C5). For this reason, it is important to have high-quality upper secondary programmes that provide individuals with the right mix of guidance and education opportunities to ensure there are no dead ends once students have graduated.

\section{Chart A2.3. Upper secondary graduation rates, by programme orientation and gender (2013)}

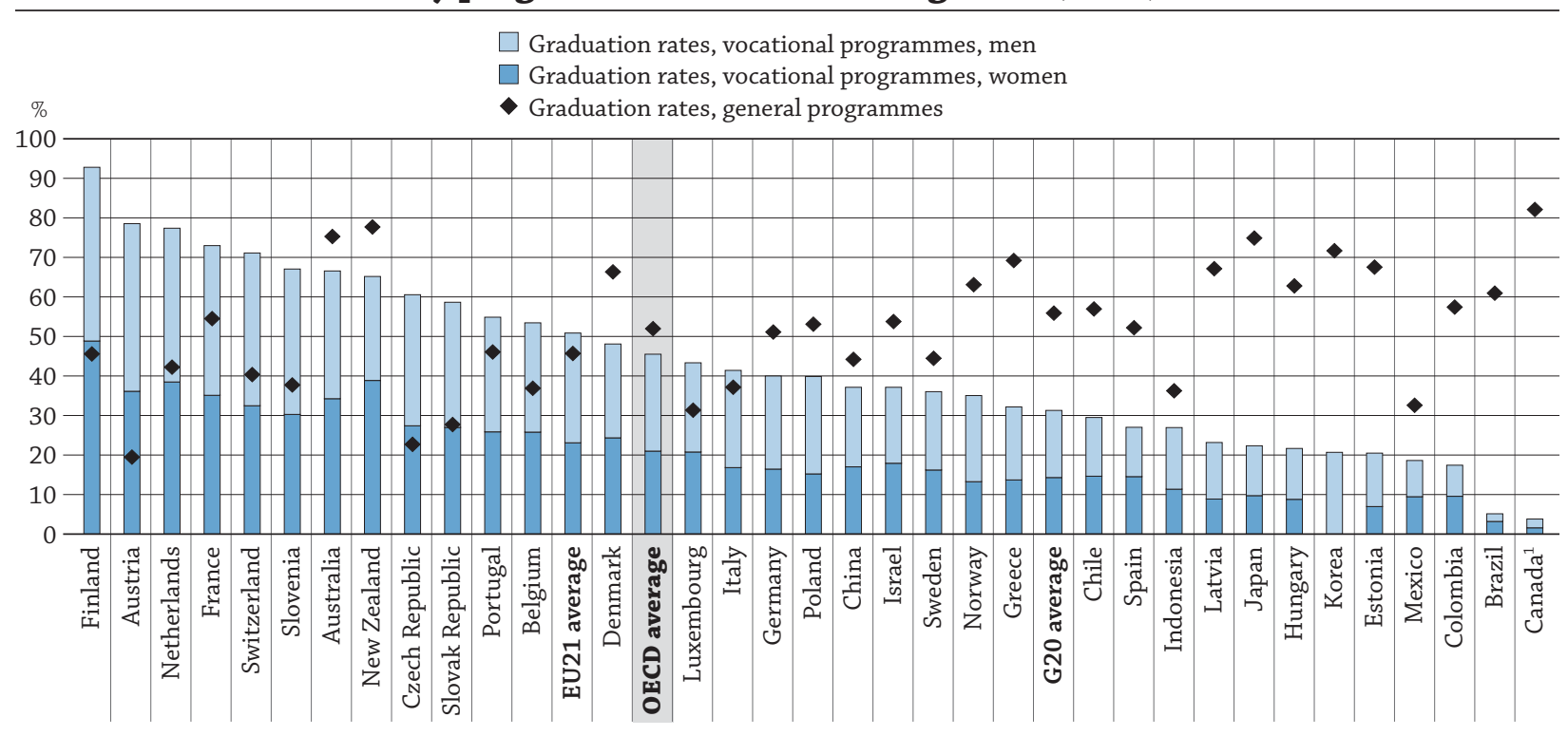

1. Year of reference 2012.

Countries are ranked in descending order of the upper secondary graduation rates in vocational programmes.

Source: OECD. Table A2.1. See Annex 3 for notes (www.oecd.org/education/education-at-a-glance-19991487.htm).

StatLink 제그 http://dx.doi.org/10.1787/888933283443

\section{Profile of an upper secondary graduate}

Graduation rates also vary according to the age of the students. Students' age at graduation can be related to changes in the education system, such as when opportunities become available to complete upper secondary education later on in life or when the duration of general and vocational programmes are altered. On average, $97 \%$ of students graduating from general upper secondary programmes are younger than 25, although in Portugal, $80 \%$ of such students are younger than 25 .

The share of older students in vocational programmes is considerably larger. On average, only $83 \%$ of graduates are younger than 25. In Australia, Canada and New Zealand, more than one in two graduates are 25 or older.

Most graduates in vocational programmes earned a degree in sciences and engineering (37\%) and education, humanities and social sciences (30\%). In two countries, Denmark (29\%) and the Netherlands (27\%), the largest proportions of graduates studied health and welfare. Gender differences are also apparent in young people's choice of field of study when pursuing vocational education. These differences can be attributed to traditional perceptions of gender roles and identities as well as the cultural values sometimes associated with particular fields of education. On average across OECD countries, $88 \%$ of the graduates from sciences and engineering programmes are men. The countries with the largest proportions of women in science and engineering at this level of education are Brazil (38\%) and Colombia (39\%). Women are, however, over-represented in all other fields of education (Table A2.2). 
As Chart A2.4 shows, across most countries, the percentage of women pursuing sciences and engineering programmes is low, averaging only $11 \%$ of all women graduating from an upper secondary vocational programme. In the fields of services, health and agriculture, there is greater gender diversity. In one-quarter of the countries, $58 \%$ or more of female graduates from upper secondary vocational training programmes choose these fields, while in another one-quarter of the countries, only $36 \%$ of women graduate from these fields of study. Conversely, across most countries, women are under-represented in sciences and engineering. In three-quarters of the countries, less than $13 \%$ of all female graduates are in these fields.

\section{Chart A2.4. Percentage of graduates from upper secondary vocational programmes in OECD countries, by field of education and gender (2013)}

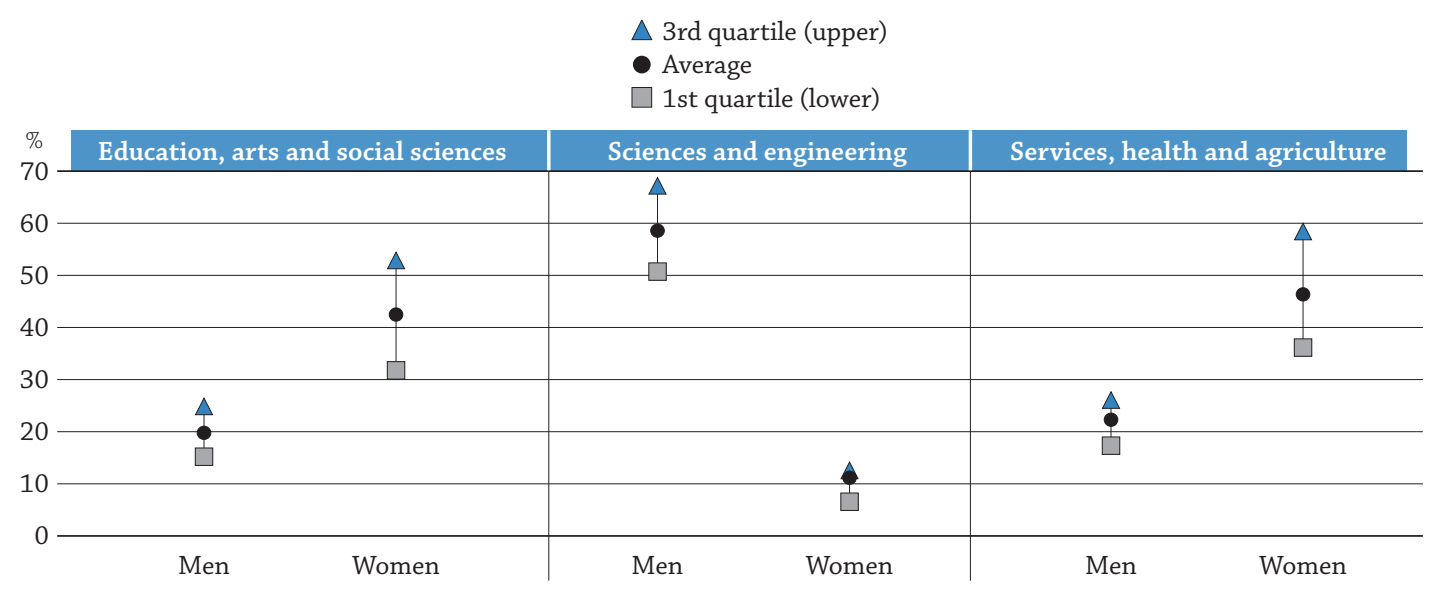

Source: OECD Database. See Annex 3 for notes (www.oecd.org/education/education-at-a-glance-19991487.htm).

StatLink 개대 http://dx.doi.org/10.1787/888933283458

\section{Vocational and educational training}

Vocational education and training (VET) is mainly designed to help participants acquire the practical skills, knowhow and understanding necessary for employment in a particular occupation or trade. Across OECD countries, $46 \%$ of students are expected to graduate from a vocational programme at the upper secondary level. However, the importance of VET systems varies widely across countries. In some, VET plays a central role in the initial education of young people, whereas in other systems, most students go into general education.

Vocational programmes can be offered in combined school- and work-based programmes, where only up to $75 \%$ of the curriculum is presented in the school environment or through distance education. These programmes include apprenticeship programmes that involve concurrent school-based and work-based training, and programmes that involve alternating periods of attendance at educational institutions and participation in work-based training. This type of "dual system" can be found in Austria, the Czech Republic, Denmark, Germany, Hungary, the Netherlands, the Slovak Republic and Switzerland (OECD, 2015). Through work-based learning, students acquire the skills that are valued in the workplace. Work-based learning is also a way to develop public-private partnerships and to allow social partners and employers to get involved in developing VET programmes, often by defining curricular frameworks.

Moreover, high-quality VET programmes tend to be effective in developing skills among those who would otherwise lack the qualifications to ensure a smooth and successful transition into the labour market. Employment rates are higher and inactivity rates are lower among people who graduated from vocational training than among those who pursued an upper secondary general programme as their highest level of educational attainment (see Indicator A5). However, it is important to ensure that graduates of upper secondary VET programmes have good employment opportunities since VET can be more expensive than other education programmes (see Indicator B1).

\section{A snapshot of post-secondary non-tertiary graduation rates}

Various kinds of post-secondary non-tertiary programmes are offered in OECD countries. These programmes straddle upper secondary and post-secondary education and may be considered either as upper secondary or postsecondary programmes, depending on the country concerned. Although the content of these programmes may not be significantly more advanced than upper secondary programmes, they broaden the knowledge of individuals who have already attained an upper secondary qualification. 
First-time graduation rates from post-secondary non-tertiary education are low compared with those from upper secondary programmes. On average, it is estimated that $13 \%$ of today's young people in OECD countries will complete vocational post-secondary non-tertiary programmes over their lifetime. The first-time graduation rate among women (15\%) is higher than that among men (10\%). In almost all countries, except Hungary, Luxembourg, Portugal and the Slovak Republic, women's first-time graduation rates at the post-secondary non-tertiary level are higher than those of men. The highest first-time graduation rates for these programmes are observed in Australia (37\%) and New Zealand (29\%) (Table A2.1). Six countries do not offer this level of education (Chile, Indonesia, Mexico, Slovenia, Turkey and the United Kingdom).

These programmes, which usually offer trade and vocational certificates, include nursery-teacher training in Austria and vocational training in dual systems for those who have attained upper secondary general qualifications in Germany. Apprenticeships designed for students who have already graduated from an upper secondary programme are also included.

\section{Profile of a post-secondary non-tertiary graduate from vocational programmes}

Post-secondary non-tertiary education vocational programmes is offered by 27 of the 34 OECD countries and by eight of the ten partner countries. Some countries that do not offer programmes at this level (ISCED 4) have high graduation rates from vocational programmes at a lower level of education (ISCED 3), such as 67\% in Slovenia and $71 \%$ in Switzerland (Table A2.1).

In comparison to upper secondary education, post-secondary non-tertiary education is fairly common among older students. On average, $41 \%$ of all graduates are older than 30 , meaning that many of them took time off education after they graduated from the previous education level. In many countries, these programmes are second-chance programmes designed to encourage adults to re-enter education. In other countries, most graduates from a postsecondary non-tertiary programme are young; for example more than $90 \%$ of all graduates are younger than 30 in Belgium (97\%) and Hungary (92\%).

The share of female graduates from post-secondary non-tertiary vocational programmes varies widely, from $73 \%$ in Austria to $24 \%$ in Luxembourg. This is partially explained by the fields of study offered at this level of education. In Austria, for instance, $62 \%$ of graduates pursued a degree in health and welfare, whereas in Luxembourg, $64 \%$ of graduates studied engineering, manufacturing and construction.

On average, most students graduate from post-secondary non-tertiary vocational programmes with degrees in social sciences, business and law (23\%) or engineering, manufacturing and construction (21\%). The least popular fields are education (5\%), sciences (4\%) and agriculture (3\%). For some countries, one single field dominates post-secondary non-tertiary education. For instance, in Denmark, $97 \%$ of students graduate with a degree in social sciences, business and law, while in the Netherlands, $66 \%$ of graduates earn a degree in engineering, manufacturing and construction.

\section{Definitions}

Graduates in the reference period can be either first-time graduates or repeat graduates. A first-time graduate is a student who has graduated for the first time at a given level of education in the reference period. Thus, if a student has graduated multiple times over the years, he or she is counted as a graduate each year, but as a first-time graduate only once.

Gross graduation rates refer to the total number of graduates (the graduates themselves may be of any age) at the specified level of education divided by the population at the typical graduation age from the specified level.

Net graduation rates represent the estimated percentage of an age group that will complete upper secondary education, based on current patterns of graduation.

Typical age is the age at the beginning of the last school/academic year of the corresponding education level and programme when the degree is obtained.

\section{Methodology}

Data refer to the academic year 2012/13 and are based on the UOE data collection on education statistics administered by the OECD in 2014 (for details, see Annex 3 at www.oecd.org/education/education-at-a-glance-19991487.htm). 
Unless otherwise indicated, graduation rates are calculated as net graduation rates (i.e. as the sum of age-specific graduation rates). Gross graduation rates are presented for countries that are unable to provide such detailed data. In order to calculate gross graduation rates, countries identify the age at which graduation typically occurs (see Annex 1). The number of graduates, regardless of their age, is divided by the population at the typical graduation age. In many countries, defining a typical age of graduation is difficult, however, because graduates are dispersed over a wide range of ages.

Graduates by programme orientation at ISCED 3 and ISCED 4 are not counted as first-time graduates given that many students graduate from more than one upper secondary or post-secondary non-tertiary programme. Therefore, graduation rates cannot be added, as some individuals would be counted twice. In addition, the typical graduation ages are not necessarily the same for the different types of programmes (see Annex 1). Vocational programmes include both school-based programmes and combined school- and work-based programmes that are recognised as part of the education system. Entirely work-based education and training programmes that are not overseen by a formal education authority are not included.

\section{Note regarding data from Israel}

The statistical data for Israel are supplied by and are under the responsibility of the relevant Israeli authorities. The use of such data by the OECD is without prejudice to the status of the Golan Heights, East Jerusalem and Israeli settlements in the West Bank under the terms of international law.

\section{References}

Falch et al. (2010), "Completion and Dropout in Upper Secondary Education in Norway: Causes and Consequences", Centre for Economic Research at NTNU, Trondheim, October 2010.

OECD (2015), "Focus on Vocational Education and Training (VET) programmes", Education Indicators in Focus, No. 33, OECD, http://dx.doi.org/10.1787/5jrxtk4cg7wg-en.

OECD (2014), PISA 2012 Results: What Students Know and Can Do (Volume I, Revised edition, February 2014): Student Performance in Mathematics, Reading and Science, PISA, OECD Publishing, Paris, http://dx.doi.org/10.1787/9789264208780-en.

OECD (2012a), Equity and Quality in Education: Supporting Disadvantaged Students and Schools, OECD Publishing, Paris, http://dx.doi.org/10.1787/9789264130852-en.

OECD (2012b), Closing the Gender Gap: Act Now, OECD Publishing, Paris, http://dx.doi.org/10.1787/9789264179370-en. OECD (2010), Learning for Jobs, OECD Reviews of Vocational Education and Training, OECD Publishing, Paris, http://dx.doi. org/10.1787/9789264087460-en.

\section{Indicator A2 Tables}

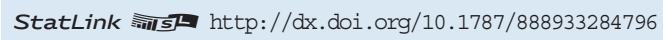

\begin{tabular}{|c|c|c|}
\hline & Table A2.1 & Upper secondary and post-secondary non-tertiary graduation rates (2013) \\
\hline & Table A2.2 & Profile of upper secondary graduates from general and vocational programmes (2013) \\
\hline & Table A2.3 & Profile of post-secondary non-tertiary graduates from vocational programmes (2013) \\
\hline & Table A2.4 & Upper secondary and post-secondary non-tertiary graduation rates (2005 and 2013) \\
\hline$W E B$ & Table A2.5 & $\begin{array}{l}\text { Share of upper secondary graduates from vocational programmes by field of education } \\
\text { and gender (2013) }\end{array}$ \\
\hline
\end{tabular}

Cut-off date for the data: 23 October 2015. Updates can be found on line at http://dx.doi.org/10.1787/eag-data-en. 
Table A2.1. Upper secondary and post-secondary non-tertiary graduation rates (2013) Sum of age-specific graduation rates, by gender and programme orientation

\begin{tabular}{|c|c|c|c|c|c|c|c|c|c|c|c|c|c|c|c|}
\hline & \multicolumn{9}{|c|}{ Upper secondary } & \multicolumn{6}{|c|}{ Post-secondary non-tertiary } \\
\hline & \multirow{2}{*}{\multicolumn{3}{|c|}{$\begin{array}{c}\begin{array}{c}\text { First-time } \\
\text { graduation rates }\end{array} \\
\text { All programmes }\end{array}$}} & \multicolumn{6}{|c|}{ Graduation rates } & \multirow{2}{*}{\multicolumn{3}{|c|}{$\begin{array}{c}\begin{array}{c}\text { First-time } \\
\text { graduation rates }\end{array} \\
\text { All programmes }\end{array}$}} & \multirow{2}{*}{\multicolumn{3}{|c|}{$\begin{array}{c}\text { Graduation rates } \\
\text { Vocational programmes }\end{array}$}} \\
\hline & & & & \multicolumn{3}{|c|}{ General programmes } & \multicolumn{3}{|c|}{ Vocational programmes } & & & & & & \\
\hline & $M+W$ & Men & Women & $M+W$ & Men & Women & $\mathrm{M}+\mathrm{W}$ & Men & Women & $M+W$ & Men & Women & $M+W$ & Men & Women \\
\hline & (1) & (2) & (3) & (4) & (5) & (6) & (7) & (8) & (9) & (10) & (11) & (12) & (13) & (14) & (15) \\
\hline Australia & $\mathrm{m}$ & $\mathrm{m}$ & $\mathrm{m}$ & 75 & 71 & 80 & 67 & 64 & 69 & 37 & 32 & 42 & 41 & 36 & 46 \\
\hline Austria & 87 & 87 & 88 & 19 & 16 & 24 & 79 & 82 & 75 & 9 & 5 & 13 & 10 & 5 & 14 \\
\hline Belgium & $\mathrm{m}$ & $\mathrm{m}$ & $\mathrm{m}$ & 37 & 32 & 43 & 53 & 54 & 53 & $\mathrm{~m}$ & $\mathrm{~m}$ & $\mathrm{~m}$ & 7 & 6 & 7 \\
\hline Canada $^{1}$ & 86 & 83 & 89 & 82 & 79 & 86 & 4 & 4 & 3 & $\mathrm{~m}$ & $\mathrm{~m}$ & $\mathrm{~m}$ & $\mathrm{~m}$ & $\mathrm{~m}$ & $\mathrm{~m}$ \\
\hline Chile & 86 & 83 & 90 & 57 & 54 & 60 & 29 & 29 & 30 & a & a & a & a & a & a \\
\hline Czech Republic & 78 & 77 & 79 & 23 & 18 & 28 & 61 & 65 & 56 & 25 & 19 & 32 & 9 & 8 & 10 \\
\hline Denmark & 95 & 90 & 100 & 66 & 60 & 73 & 48 & 47 & 49 & 1 & 1 & 1 & 1 & 1 & 1 \\
\hline Estonia & $\mathrm{m}$ & $\mathrm{m}$ & $\mathrm{m}$ & 68 & 56 & 79 & 20 & 26 & 14 & $\mathrm{~m}$ & $\mathrm{~m}$ & $\mathrm{~m}$ & 23 & 16 & 29 \\
\hline Finland & 96 & 93 & 100 & 46 & 38 & 53 & 93 & 86 & 99 & 7 & 6 & 8 & 8 & 6 & 9 \\
\hline France & $\mathrm{m}$ & $\mathrm{m}$ & $\mathrm{m}$ & 55 & 48 & 62 & 73 & 75 & 71 & $\mathrm{~m}$ & $\mathrm{~m}$ & $\mathrm{~m}$ & 0 & 0 & 0 \\
\hline Germany & $\mathrm{m}$ & $\mathrm{m}$ & $\mathrm{m}$ & 51 & 46 & 57 & 40 & 46 & 34 & 24 & 19 & 29 & 21 & 16 & 26 \\
\hline Greece & $\mathrm{m}$ & $\mathrm{m}$ & $\mathrm{m}$ & 69 & 63 & 75 & 32 & 37 & 28 & $\mathrm{~m}$ & $\mathrm{~m}$ & $\mathrm{~m}$ & 4 & 3 & 5 \\
\hline Hungary & 83 & 82 & 85 & 63 & 58 & 68 & 22 & 25 & 18 & 18 & 18 & 17 & 21 & 21 & 21 \\
\hline Iceland & $\mathrm{m}$ & $\mathrm{m}$ & $\mathrm{m}$ & $\mathrm{m}$ & $\mathrm{m}$ & $\mathrm{m}$ & $\mathrm{m}$ & $\mathrm{m}$ & $\mathrm{m}$ & $\mathrm{m}$ & $\mathrm{m}$ & $\mathrm{m}$ & $\mathrm{m}$ & $\mathrm{m}$ & $\mathrm{m}$ \\
\hline Ireland & 98 & 97 & 99 & $\mathrm{~m}$ & $\mathrm{~m}$ & $\mathrm{~m}$ & a & a & $\mathrm{a}$ & $\mathrm{m}$ & $\mathrm{m}$ & $\mathrm{m}$ & 15 & 21 & 8 \\
\hline Israel & 91 & 86 & 96 & 54 & 49 & 59 & 37 & 38 & 37 & $\mathrm{~m}$ & $\mathrm{~m}$ & $\mathrm{~m}$ & $\mathrm{a}$ & $\mathrm{a}$ & a \\
\hline Italy & 78 & 74 & 82 & 37 & 27 & 47 & 41 & 48 & 35 & 3 & 3 & 4 & 3 & 3 & 4 \\
\hline Japan & 97 & 96 & 98 & 75 & 71 & 78 & 22 & 25 & 20 & $\mathrm{~m}$ & $\mathrm{~m}$ & $\mathrm{~m}$ & $\mathrm{~m}$ & $\mathrm{~m}$ & $\mathrm{~m}$ \\
\hline Korea & 92 & 93 & 92 & 72 & 71 & 72 & 21 & 21 & 20 & $\mathrm{~m}$ & $\mathrm{~m}$ & $\mathrm{~m}$ & $\mathrm{~m}$ & $\mathrm{~m}$ & $\mathrm{~m}$ \\
\hline Luxembourg & 73 & 69 & 77 & 31 & 28 & 35 & 43 & 44 & 43 & 2 & 3 & 1 & 2 & 3 & 1 \\
\hline Mexico & 51 & 49 & 54 & 33 & 30 & 35 & 19 & 18 & 19 & a & a & $\mathrm{a}$ & a & a & a \\
\hline Netherlands & $\mathrm{m}$ & $\mathrm{m}$ & $\mathrm{m}$ & 42 & 39 & 46 & 77 & 77 & 78 & $\mathrm{~m}$ & $\mathrm{~m}$ & $\mathrm{~m}$ & 0 & 0 & 0 \\
\hline New Zealand & 100 & 96 & 100 & 78 & 74 & 82 & 65 & 52 & 78 & 29 & 23 & 34 & 33 & 26 & 40 \\
\hline Norway & 83 & 78 & 89 & 63 & 52 & 75 & 35 & 42 & 27 & 3 & 2 & 4 & 3 & 2 & 5 \\
\hline Poland & 86 & 82 & 91 & 53 & 41 & 66 & 40 & 48 & 31 & 16 & 9 & 24 & 16 & 9 & 24 \\
\hline Portugal & 100 & 98 & 100 & 46 & 40 & 52 & 55 & 58 & 52 & 5 & 6 & 4 & 5 & 6 & 4 \\
\hline Slovak Republic & 85 & 83 & 88 & 28 & 22 & 34 & 59 & 62 & 55 & 10 & 10 & 9 & 10 & 10 & 9 \\
\hline Slovenia & 86 & 76 & 95 & 38 & 29 & 47 & 67 & 72 & 61 & a & a & a & a & a & a \\
\hline Spain & 71 & 65 & 78 & 52 & 46 & 59 & 27 & 25 & 29 & $\mathrm{~m}$ & $\mathrm{~m}$ & $\mathrm{~m}$ & $\mathrm{~m}$ & $\mathrm{~m}$ & $\mathrm{~m}$ \\
\hline Sweden & 79 & 77 & 82 & 44 & 40 & 50 & 36 & 39 & 33 & 3 & 3 & 4 & 3 & 3 & 4 \\
\hline Switzerland & 95 & 94 & 97 & 40 & 34 & 47 & 71 & 76 & 66 & 1 & 1 & 1 & a & a & a \\
\hline Turkey & 64 & 61 & 66 & 35 & 32 & 38 & 29 & 29 & 28 & a & a & a & a & a & a \\
\hline United Kingdom & $\mathrm{m}$ & $\mathrm{m}$ & $\mathrm{m}$ & $\mathrm{m}$ & $\mathrm{m}$ & $\mathrm{m}$ & $\mathrm{m}$ & $\mathrm{m}$ & $\mathrm{m}$ & a & a & a & $\mathrm{a}$ & a & a \\
\hline United States & 80 & 77 & 83 & $80^{\mathrm{d}}$ & $77^{d}$ & $83^{\mathrm{d}}$ & $\mathrm{x}(4)$ & $\mathrm{x}(5)$ & $\mathrm{x}(6)$ & 21 & 16 & 27 & 21 & 16 & 27 \\
\hline OECD average & 85 & 82 & 88 & 52 & 46 & 58 & 46 & 47 & 44 & 13 & 10 & 15 & 12 & 10 & 13 \\
\hline EU21 average & 85 & 82 & 89 & 46 & 39 & 53 & 51 & 53 & 48 & 10 & 9 & 12 & 9 & 8 & 10 \\
\hline Argentina & $\mathrm{m}$ & $\mathrm{m}$ & $\mathrm{m}$ & $\mathrm{m}$ & $\mathrm{m}$ & $\mathrm{m}$ & $\mathrm{m}$ & $\mathrm{m}$ & $\mathrm{m}$ & $\mathrm{m}$ & $\mathrm{m}$ & $\mathrm{m}$ & $\mathrm{m}$ & $\mathrm{m}$ & $\mathrm{m}$ \\
\hline Brazil & $\mathrm{m}$ & $\mathrm{m}$ & $\mathrm{m}$ & 61 & 51 & 71 & 5 & 4 & 7 & $\mathrm{~m}$ & $\mathrm{~m}$ & $\mathrm{~m}$ & 7 & 6 & 8 \\
\hline China & $\mathrm{m}$ & $\mathrm{m}$ & $\mathrm{m}$ & 44 & 42 & 46 & 37 & 38 & 36 & 6 & 6 & 6 & 3 & 4 & 2 \\
\hline Colombia & 75 & 67 & 83 & 57 & 51 & 64 & 17 & 15 & 20 & 1 & 0 & 1 & a & a & a \\
\hline India & $\mathrm{m}$ & $\mathrm{m}$ & $\mathrm{m}$ & $\mathrm{m}$ & $\mathrm{m}$ & $\mathrm{m}$ & $\mathrm{m}$ & $\mathrm{m}$ & $\mathrm{m}$ & $\mathrm{m}$ & $\mathrm{m}$ & $\mathrm{m}$ & $\mathrm{m}$ & $\mathrm{m}$ & $\mathrm{m}$ \\
\hline Indonesia & 63 & 62 & 64 & 36 & 32 & 41 & 27 & 30 & 23 & a & a & a & a & a & a \\
\hline Latvia & 85 & 81 & 89 & 67 & 59 & 75 & 23 & 28 & 18 & 6 & 5 & 7 & 6 & 5 & 7 \\
\hline Russian Federation ${ }^{2}$ & $\mathrm{~m}$ & $\mathrm{~m}$ & $\mathrm{~m}$ & 51 & 44 & 58 & 27 & 39 & 14 & 6 & 6 & 6 & 6 & 6 & 6 \\
\hline Saudi Arabia & $\mathrm{m}$ & $\mathrm{m}$ & $\mathrm{m}$ & $\mathrm{m}$ & $\mathrm{m}$ & $\mathrm{m}$ & $\mathrm{m}$ & $\mathrm{m}$ & $\mathrm{m}$ & $\mathrm{m}$ & $\mathrm{m}$ & $\mathrm{m}$ & $\mathrm{m}$ & $\mathrm{m}$ & $\mathrm{m}$ \\
\hline South Africa & $\mathrm{m}$ & $\mathrm{m}$ & $\mathrm{m}$ & $\mathrm{m}$ & $\mathrm{m}$ & $\mathrm{m}$ & $\mathrm{m}$ & $\mathrm{m}$ & $\mathrm{m}$ & $\mathrm{m}$ & $\mathrm{m}$ & $\mathrm{m}$ & $\mathrm{m}$ & $\mathrm{m}$ & $\mathrm{m}$ \\
\hline G20 average & $\mathrm{m}$ & $\mathrm{m}$ & $\mathrm{m}$ & 56 & 52 & 61 & 32 & 34 & 29 & 16 & 14 & 19 & $\mathrm{~m}$ & $\mathrm{~m}$ & $\mathrm{~m}$ \\
\hline
\end{tabular}

1. Year of reference 2012.

2. Post-secondary non-tertiary includes some upper secondary graduates.

Source: OECD. Argentina, China, Colombia, India, Indonesia, Saudi Arabia, South Africa: UNESCO Institute for Statistics. Latvia: Eurostat. See Annex 3 for notes (www.oecd.org/education/education-at-a-glance-19991487.htm).

Please refer to the Reader's Guide for information concerning symbols for missing data and abbreviations.

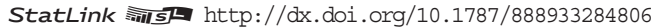


Table A2.2. Profile of upper secondary graduates from general and vocational programmes (2013)

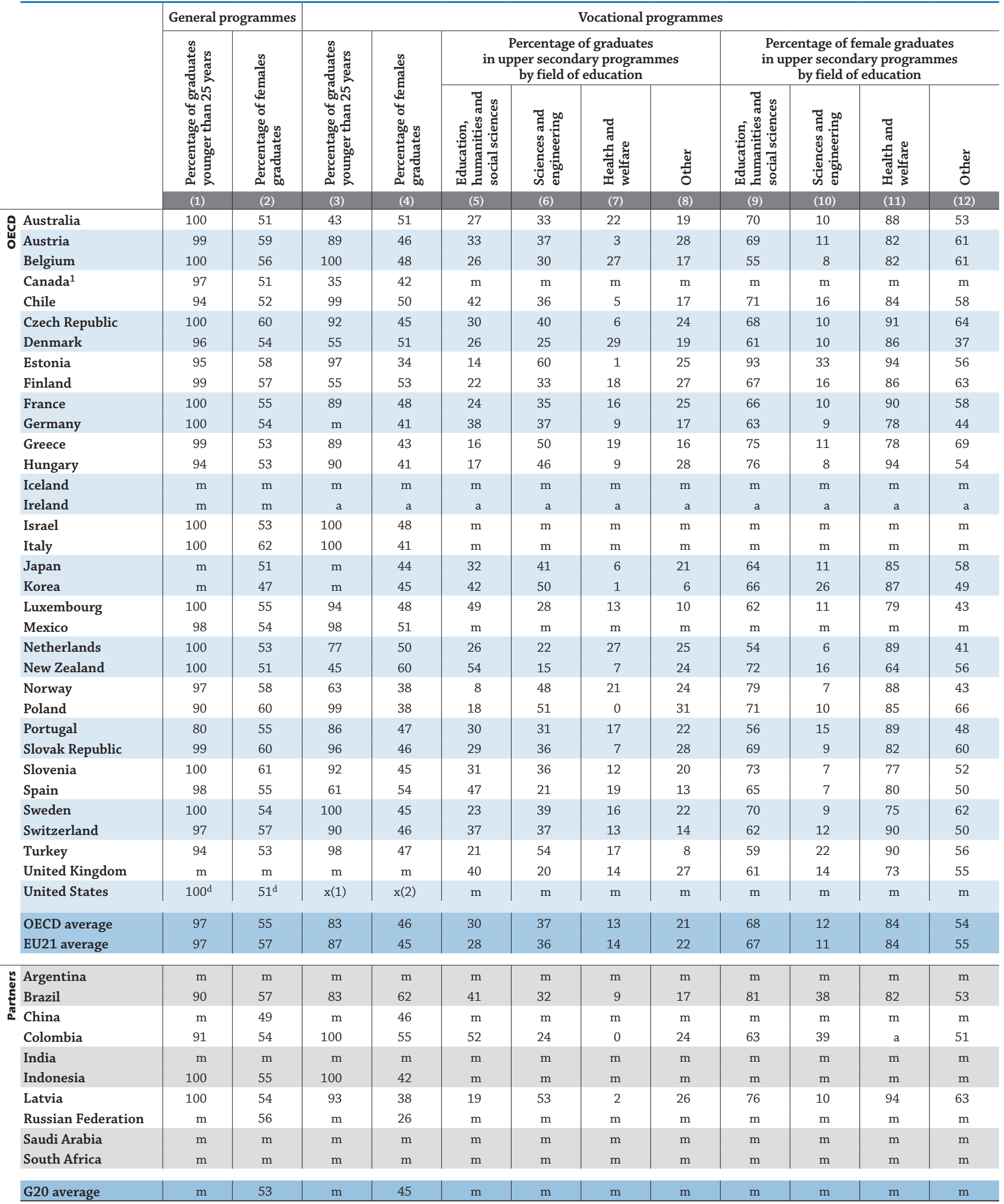

Note: The field category other includes: agriculture, services and other.

1. Year of reference 2012

Source: OECD. Argentina, China, Colombia, India, Indonesia, Saudi Arabia, South Africa: UNESCO Institute for Statistics. Latvia: Eurostat. See Annex 3 for notes (www.oecd.org/education/education-at-a-glance-19991487.htm).

Please refer to the Reader's Guide for information concerning symbols for missing data and abbreviations.

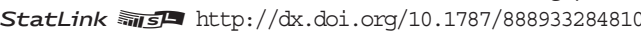


Table A2.3. Profile of post-secondary non-tertiary graduates from vocational programmes (2013)

\begin{tabular}{|c|c|c|c|c|c|c|c|c|c|c|}
\hline & \multirow[b]{2}{*}{$\begin{array}{l}\text { Percentage } \\
\text { of females } \\
\text { graduates }\end{array}$} & \multirow[b]{2}{*}{$\begin{array}{c}\text { Percentage } \\
\text { of graduates } \\
\text { younger } \\
\text { than } 30 \text { years }\end{array}$} & \multicolumn{8}{|c|}{ Percentage of graduates by field of education } \\
\hline & & & Education & $\begin{array}{c}\text { Humanities } \\
\text { and arts }\end{array}$ & $\begin{array}{c}\text { Social } \\
\text { sciences, } \\
\text { business } \\
\text { and law }\end{array}$ & Sciences & $\begin{array}{c}\text { Engineering, } \\
\text { manufacturing } \\
\text { and } \\
\text { construction }\end{array}$ & Agriculture & $\begin{array}{c}\text { Health } \\
\text { and welfare }\end{array}$ & Services \\
\hline & (1) & (2) & (3) & (4) & (5) & (6) & (7) & (8) & (9) & (10) \\
\hline Australia & 56 & 37 & 19 & 4 & 37 & 3 & 10 & 2 & 17 & 8 \\
\hline Austria & 73 & 48 & 18 & 2 & 13 & 1 & 1 & 2 & 62 & 1 \\
\hline Belgium & 53 & 97 & 0 & 7 & 12 & 1 & 22 & 2 & 34 & 22 \\
\hline Canada & $\mathrm{m}$ & $\mathrm{m}$ & $\mathrm{m}$ & $\mathrm{m}$ & $\mathrm{m}$ & $\mathrm{m}$ & $\mathrm{m}$ & $\mathrm{m}$ & $\mathrm{m}$ & $\mathrm{m}$ \\
\hline Chile & a & a & a & a & a & a & a & a & a & a \\
\hline Czech Republic & 52 & $\mathrm{~m}$ & $\mathrm{~m}$ & $\mathrm{~m}$ & $\mathrm{~m}$ & $\mathrm{~m}$ & $\mathrm{~m}$ & $\mathrm{~m}$ & $\mathrm{~m}$ & $\mathrm{~m}$ \\
\hline Denmark & 61 & 31 & 0 & 0 & 97 & 1 & 0 & 0 & 3 & 0 \\
\hline Estonia & 63 & 68 & 0 & 18 & 12 & 20 & 18 & 5 & 5 & 22 \\
\hline Finland & 58 & 10 & 2 & 2 & 51 & 0 & 25 & 2 & 5 & 13 \\
\hline France & 68 & $\mathrm{~m}$ & 0 & 55 & 13 & 11 & 1 & 0 & 0 & 20 \\
\hline Germany & 61 & $\mathrm{~m}$ & 0 & 3 & 27 & 3 & 18 & 2 & 39 & 10 \\
\hline Greece & 58 & 86 & 14 & 4 & 13 & 7 & 22 & 1 & 19 & 20 \\
\hline Hungary & 49 & 92 & 1 & 8 & 19 & 8 & 24 & 3 & 15 & 22 \\
\hline Iceland & $\mathrm{m}$ & $\mathrm{m}$ & $\mathrm{m}$ & $\mathrm{m}$ & $\mathrm{m}$ & $\mathrm{m}$ & $\mathrm{m}$ & $\mathrm{m}$ & $\mathrm{m}$ & $\mathrm{m}$ \\
\hline Ireland & 28 & 57 & 0 & 9 & 13 & 5 & 34 & 18 & 11 & 10 \\
\hline Israel & a & a & $\mathrm{a}$ & a & a & a & a & a & a & a \\
\hline Italy & 52 & $\mathrm{~m}$ & $\mathrm{~m}$ & $\mathrm{~m}$ & $\mathrm{~m}$ & $\mathrm{~m}$ & $\mathrm{~m}$ & $\mathrm{~m}$ & $\mathrm{~m}$ & $\mathrm{~m}$ \\
\hline Japan & $\mathrm{m}$ & $\mathrm{m}$ & $\mathrm{m}$ & $\mathrm{m}$ & $\mathrm{m}$ & $\mathrm{m}$ & $\mathrm{m}$ & $\mathrm{m}$ & $\mathrm{m}$ & $\mathrm{m}$ \\
\hline Korea & $\mathrm{m}$ & $\mathrm{m}$ & $\mathrm{m}$ & $\mathrm{m}$ & $\mathrm{m}$ & $\mathrm{m}$ & $\mathrm{m}$ & $\mathrm{m}$ & $\mathrm{m}$ & $\mathrm{m}$ \\
\hline Luxembourg & 24 & 65 & 4 & 8 & 0 & 0 & 64 & 2 & 5 & 18 \\
\hline Mexico & a & $\mathrm{a}$ & $\mathrm{a}$ & a & a & a & a & a & a & a \\
\hline Netherlands & 26 & 44 & 27 & 0 & 5 & 0 & 66 & 0 & 0 & 2 \\
\hline New Zealand & 60 & 58 & 2 & 27 & 24 & 6 & 10 & 3 & 13 & 14 \\
\hline Norway & 70 & 41 & 0 & 14 & 37 & 0 & 1 & 2 & 34 & 11 \\
\hline Poland & 71 & 73 & 0 & 6 & 21 & 5 & 6 & 5 & 28 & 29 \\
\hline Portugal & 36 & 82 & 0 & 5 & 13 & 8 & 33 & 5 & 5 & 30 \\
\hline Slovak Republic & 45 & 69 & 6 & 1 & 15 & 0 & 20 & 2 & 14 & 42 \\
\hline Slovenia & a & a & a & a & a & a & a & a & a & a \\
\hline Spain & $\mathrm{m}$ & $\mathrm{m}$ & $\mathrm{m}$ & $\mathrm{m}$ & $\mathrm{m}$ & $\mathrm{m}$ & $\mathrm{m}$ & $\mathrm{m}$ & $\mathrm{m}$ & $\mathrm{m}$ \\
\hline Sweden & 58 & 51 & 7 & 3 & 24 & 7 & 20 & 4 & 23 & 12 \\
\hline Switzerland & a & a & a & a & a & a & a & a & a & a \\
\hline Turkey & $\mathrm{a}$ & $\mathrm{a}$ & $\mathrm{a}$ & $\mathrm{a}$ & $\mathrm{a}$ & $\mathrm{a}$ & $\mathrm{a}$ & a & $\mathrm{a}$ & a \\
\hline United Kingdom & a & a & a & a & a & $\mathrm{a}$ & a & a & $\mathrm{a}$ & a \\
\hline United States & 61 & $\mathrm{~m}$ & 1 & 6 & 9 & 3 & 18 & 1 & 40 & 23 \\
\hline $\mathrm{OECD}$ average & 54 & 59 & 5 & 9 & 23 & 4 & 21 & 3 & 19 & 17 \\
\hline EU21 average & 52 & 62 & 5 & 8 & 22 & 5 & 23 & 3 & 17 & 17 \\
\hline
\end{tabular}

\begin{tabular}{|c|c|c|c|c|c|c|c|c|c|c|}
\hline $\begin{array}{l}\text { Argentina } \\
\text { Brazil }\end{array}$ & $\begin{array}{r}\mathrm{m} \\
57\end{array}$ & $\begin{array}{r}\mathrm{m} \\
70\end{array}$ & $\begin{array}{r}\mathrm{m} \\
0\end{array}$ & $\begin{array}{r}\mathrm{m} \\
2\end{array}$ & $\begin{array}{l}\mathrm{m} \\
20\end{array}$ & $\begin{array}{r}\mathrm{m} \\
10\end{array}$ & $\begin{array}{r}\mathrm{m} \\
21\end{array}$ & $\begin{array}{r}\mathrm{m} \\
3\end{array}$ & $\begin{array}{l}\mathrm{m} \\
29\end{array}$ & $\begin{array}{r}\mathrm{m} \\
15\end{array}$ \\
\hline$\Sigma^{\tau}$ China & 27 & $\mathrm{~m}$ & $\mathrm{~m}$ & $\mathrm{~m}$ & $\mathrm{~m}$ & $\mathrm{~m}$ & $\mathrm{~m}$ & $\mathrm{~m}$ & $\mathrm{~m}$ & $\mathrm{~m}$ \\
\hline Colombia & a & $a$ & $\mathrm{a}$ & $\mathrm{a}$ & $\mathrm{a}$ & $\mathrm{a}$ & a & a & $\mathrm{a}$ & $\mathrm{a}$ \\
\hline India & $\mathrm{m}$ & $\mathrm{m}$ & $\mathrm{m}$ & $\mathrm{m}$ & $\mathrm{m}$ & $\mathrm{m}$ & $\mathrm{m}$ & $\mathrm{m}$ & $\mathrm{m}$ & $\mathrm{m}$ \\
\hline Indonesia & a & a & $\mathrm{a}$ & $\mathrm{a}$ & $\mathrm{a}$ & $\mathrm{a}$ & a & a & $\mathrm{a}$ & $\mathrm{a}$ \\
\hline Latvia & 59 & 78 & 0 & 2 & 11 & 1 & 18 & 6 & 24 & 38 \\
\hline Russian Federation $^{1}$ & 50 & $\mathrm{~m}$ & $0^{\mathrm{d}}$ & $1^{\mathrm{d}}$ & $6^{\mathrm{d}}$ & $3^{d}$ & $47^{d}$ & $9^{d}$ & $0^{\mathrm{d}}$ & $33^{\mathrm{d}}$ \\
\hline Saudi Arabia & $\mathrm{m}$ & $\mathrm{m}$ & $\mathrm{m}$ & $\mathrm{m}$ & $\mathrm{m}$ & $\mathrm{m}$ & $\mathrm{m}$ & $\mathrm{m}$ & $\mathrm{m}$ & $\mathrm{m}$ \\
\hline South Africa & $\mathrm{m}$ & $\mathrm{m}$ & $\mathrm{m}$ & $\mathrm{m}$ & $\mathrm{m}$ & $\mathrm{m}$ & $\mathrm{m}$ & $\mathrm{m}$ & $\mathrm{m}$ & $\mathrm{m}$ \\
\hline G20 average & $\mathrm{m}$ & $\mathrm{m}$ & $\mathrm{m}$ & $\mathrm{m}$ & $\mathrm{m}$ & $\mathrm{m}$ & $\mathrm{m}$ & $\mathrm{m}$ & $\mathrm{m}$ & $\mathrm{m}$ \\
\hline
\end{tabular}

1. Data for post-secondary non-tertiary includes some upper secondary graduates.

Source: OECD. Argentina, China, Colombia, India, Indonesia, Saudi Arabia, South Africa: UNESCO Institute for Statistics. Latvia: Eurostat. See Annex 3 for notes (www.oecd.org/education/education-at-a-glance-19991487.htm).

Please refer to the Reader's Guide for information concerning symbols for missing data and abbreviations.

StatLink त्ञाI $\mathrm{http}: / / \mathrm{dx}$.doi.org/10.1787/888933284828 
Table A2.4. Upper secondary and post-secondary non-tertiary graduation rates (2005 and 2013) Sum of age-specific graduation rates, by gender and programme orientation

\begin{tabular}{|c|c|c|c|c|c|c|c|c|c|c|}
\hline & \multicolumn{6}{|c|}{ Upper secondary } & \multicolumn{4}{|c|}{ Post-secondary non-tertiary } \\
\hline & \multirow{2}{*}{\multicolumn{2}{|c|}{$\begin{array}{c}\begin{array}{c}\text { First-time } \\
\text { graduation rates }\end{array} \\
\text { All programmes }\end{array}$}} & \multicolumn{4}{|c|}{ Graduation rates } & \multirow{2}{*}{\multicolumn{2}{|c|}{$\begin{array}{c}\begin{array}{c}\text { First-time } \\
\text { graduation rates }\end{array} \\
\text { All programmes }\end{array}$}} & \multirow{2}{*}{\multicolumn{2}{|c|}{$\begin{array}{c}\text { Graduation rates } \\
\text { Vocational } \\
\text { programmes }\end{array}$}} \\
\hline & & & \multicolumn{2}{|c|}{$\begin{array}{c}\text { General } \\
\text { programmes }\end{array}$} & \multicolumn{2}{|c|}{$\begin{array}{c}\text { Vocational } \\
\text { programmes }\end{array}$} & & & & \\
\hline & 2005 & 2013 & 2005 & 2013 & 2005 & 2013 & 2005 & 2013 & 2005 & 2013 \\
\hline & (1) & (2) & $(3)$ & (4) & $(5)$ & $(6)$ & $(7)$ & $(8)$ & (9) & $(10)$ \\
\hline Australia & $\mathrm{m}$ & $\mathrm{m}$ & $\mathrm{m}$ & 75 & 35 & 67 & $\mathrm{~m}$ & 37 & 18 & 41 \\
\hline Austria & $\mathrm{m}$ & 87 & 16 & 19 & $\mathrm{~m}$ & 79 & $\mathrm{~m}$ & 9 & 28 & 10 \\
\hline Belgium & $\mathrm{m}$ & $\mathrm{m}$ & $\mathrm{m}$ & 37 & $\mathrm{~m}$ & 53 & $\mathrm{~m}$ & 5 & $\mathrm{~m}$ & 7 \\
\hline Canada $^{1}$ & $\mathrm{~m}$ & 86 & 78 & 82 & 3 & 4 & $\mathrm{~m}$ & $\mathrm{~m}$ & $\mathrm{~m}$ & $\mathrm{~m}$ \\
\hline Chile & 81 & 86 & 48 & 57 & 33 & 29 & $\mathrm{a}$ & a & $\mathrm{a}$ & a \\
\hline Czech Republic & 100 & 78 & 28 & 23 & 88 & 61 & $\mathrm{~m}$ & 25 & $\mathrm{~m}$ & 9 \\
\hline Denmark & 83 & 95 & 59 & 66 & 50 & 48 & 1 & 1 & 1 & 1 \\
\hline Estonia & $\mathrm{m}$ & $\mathrm{m}$ & 60 & 68 & 19 & 20 & $\mathrm{~m}$ & $\mathrm{~m}$ & 19 & 23 \\
\hline Finland & 94 & 96 & 52 & 46 & 79 & 93 & 6 & 7 & 6 & 8 \\
\hline France & $\mathrm{m}$ & $\mathrm{m}$ & 50 & 55 & 62 & 73 & $\mathrm{~m}$ & $\mathrm{~m}$ & 0 & 0 \\
\hline Germany & $\mathrm{m}$ & $\mathrm{m}$ & $\mathrm{m}$ & 51 & $\mathrm{~m}$ & 40 & $\mathrm{~m}$ & 24 & $\mathrm{~m}$ & 21 \\
\hline Greece & 95 & $\mathrm{~m}$ & 59 & 69 & 37 & 32 & 9 & $\mathrm{~m}$ & 9 & 4 \\
\hline Hungary & $\mathrm{m}$ & 83 & 68 & 63 & 19 & 22 & 20 & 18 & 26 & 21 \\
\hline Iceland & 79 & $\mathrm{~m}$ & 56 & $\mathrm{~m}$ & 53 & $\mathrm{~m}$ & 8 & $\mathrm{~m}$ & 8 & $\mathrm{~m}$ \\
\hline Ireland & 92 & 98 & $\mathrm{~m}$ & $\mathrm{~m}$ & a & a & 14 & $\mathrm{~m}$ & 14 & 15 \\
\hline Israel & $\mathrm{m}$ & 91 & 58 & 54 & 32 & 37 & $\mathrm{~m}$ & $\mathrm{~m}$ & $\mathrm{~m}$ & $\mathrm{a}$ \\
\hline Italy & 85 & 78 & 31 & 37 & 69 & 41 & 6 & 3 & 6 & 3 \\
\hline Japan & 95 & 97 & 71 & 75 & 24 & 22 & $\mathrm{~m}$ & $\mathrm{~m}$ & $\mathrm{~m}$ & $\mathrm{~m}$ \\
\hline Korea & 92 & 92 & 65 & 72 & 28 & 21 & a & $\mathrm{m}$ & a & $\mathrm{m}$ \\
\hline Luxembourg & 74 & 73 & 27 & 31 & 47 & 43 & $\mathrm{~m}$ & 2 & 2 & 2 \\
\hline Mexico & 40 & 51 & 36 & 33 & 4 & 19 & $\mathrm{a}$ & a & a & $\mathrm{a}$ \\
\hline Netherlands & $\mathrm{m}$ & $\mathrm{m}$ & 34 & 42 & 66 & 77 & $\mathrm{~m}$ & $\mathrm{~m}$ & 1 & 0 \\
\hline New Zealand & 88 & 100 & $\mathrm{~m}$ & 78 & 20 & 65 & 12 & 29 & 13 & 33 \\
\hline Norway & 90 & 83 & 62 & 63 & 40 & 35 & 5 & 3 & 2 & 3 \\
\hline Poland & 41 & 86 & 55 & 53 & 44 & 40 & 9 & 16 & 13 & 16 \\
\hline Portugal & 54 & 100 & 41 & 46 & 13 & 55 & $\mathrm{~m}$ & 5 & $\mathrm{~m}$ & 5 \\
\hline Slovenia & 85 & 86 & 34 & 38 & 81 & 67 & a & a & $\mathrm{a}$ & $\mathrm{a}$ \\
\hline Spain & 69 & 71 & $\mathrm{~m}$ & 52 & $\mathrm{~m}$ & 27 & a & $\mathrm{m}$ & a & $\mathrm{m}$ \\
\hline Sweden & $\mathrm{m}$ & 79 & $\mathrm{~m}$ & 44 & $\mathrm{~m}$ & 36 & $\mathrm{~m}$ & 3 & $\mathrm{~m}$ & 3 \\
\hline Switzerland & $\mathrm{m}$ & 95 & 35 & 40 & 65 & 71 & $\mathrm{~m}$ & 1 & 1 & a \\
\hline Turkey & $\mathrm{m}$ & 64 & $\mathrm{~m}$ & 35 & $\mathrm{~m}$ & 29 & a & a & a & $\mathrm{a}$ \\
\hline United Kingdom & $\mathrm{m}$ & $\mathrm{m}$ & $\mathrm{m}$ & $\mathrm{m}$ & $\mathrm{m}$ & $\mathrm{m}$ & $\mathrm{a}$ & a & a & $\mathrm{a}$ \\
\hline United States & 74 & 80 & $74^{\mathrm{d}}$ & $80^{\mathrm{d}}$ & $x(3)$ & $\mathrm{x}(4)$ & 17 & 21 & 17 & 21 \\
\hline OECD average & 80 & 85 & 49 & 52 & 43 & 46 & 10 & 12 & 10 & 12 \\
\hline EU21 average & 80 & 85 & 42 & 46 & 52 & 51 & 10 & 10 & 11 & 9 \\
\hline Argentina & $\mathrm{m}$ & $\mathrm{m}$ & $\mathrm{m}$ & $\mathrm{m}$ & $\mathrm{m}$ & $\mathrm{m}$ & $\mathrm{m}$ & $\mathrm{m}$ & $\mathrm{m}$ & $\mathrm{m}$ \\
\hline Brazil & $\mathrm{m}$ & $\mathrm{m}$ & $\mathrm{m}$ & 61 & $\mathrm{~m}$ & 5 & $\mathrm{~m}$ & $\mathrm{~m}$ & $\mathrm{~m}$ & 7 \\
\hline China & $\mathrm{m}$ & $\mathrm{m}$ & $\mathrm{m}$ & 44 & $\mathrm{~m}$ & 37 & $\mathrm{~m}$ & 6 & $\mathrm{~m}$ & 3 \\
\hline Colombia & $\mathrm{m}$ & 75 & $\mathrm{~m}$ & 57 & $\mathrm{~m}$ & 17 & $\mathrm{~m}$ & 1 & $\mathrm{~m}$ & $\mathrm{a}$ \\
\hline India & $\mathrm{m}$ & $\mathrm{m}$ & $\mathrm{m}$ & $\mathrm{m}$ & $\mathrm{m}$ & $\mathrm{m}$ & $\mathrm{m}$ & $\mathrm{m}$ & $\mathrm{m}$ & $\mathrm{m}$ \\
\hline Indonesia & $\mathrm{m}$ & 63 & $\mathrm{~m}$ & 36 & $\mathrm{~m}$ & 27 & $\mathrm{~m}$ & a & $\mathrm{m}$ & a \\
\hline Latvia & $\mathrm{m}$ & 85 & $\mathrm{~m}$ & 67 & $\mathrm{~m}$ & 23 & $\mathrm{~m}$ & 6 & $\mathrm{~m}$ & 6 \\
\hline Russian Federation ${ }^{2}$ & $\mathrm{~m}$ & $\mathrm{~m}$ & $\mathrm{~m}$ & 51 & $\mathrm{~m}$ & 27 & $\mathrm{~m}$ & 6 & $\mathrm{~m}$ & 6 \\
\hline Saudi Arabia & $\mathrm{m}$ & $\mathrm{m}$ & $\mathrm{m}$ & $\mathrm{m}$ & $\mathrm{m}$ & $\mathrm{m}$ & $\mathrm{m}$ & $\mathrm{m}$ & $\mathrm{m}$ & $\mathrm{m}$ \\
\hline South Africa & $\mathrm{m}$ & $\mathrm{m}$ & $\mathrm{m}$ & $\mathrm{m}$ & $\mathrm{m}$ & $\mathrm{m}$ & $\mathrm{m}$ & $\mathrm{m}$ & $\mathrm{m}$ & $\mathrm{m}$ \\
\hline G20 average & $\mathrm{m}$ & $\mathrm{m}$ & $\mathrm{m}$ & 56 & $\mathrm{~m}$ & 32 & $\mathrm{~m}$ & 16 & $\mathrm{~m}$ & $\mathrm{~m}$ \\
\hline
\end{tabular}

Note: Graduation rates for 2005 were calculated using typical ages of 2013 if necessary.

1. Year of reference 2012 instead of 2013.

2. Post-secondary non-tertiary graduates includes some upper secondary graduates.

Source: OECD. Argentina, China, Colombia, India, Indonesia, Saudi Arabia, South Africa: UNESCO Institute for Statistics. Latvia: Eurostat. See Annex 3 for notes (www.oecd.org/education/education-at-a-glance-19991487.htm).

Please refer to the Reader's Guide for information concerning symbols for missing data and abbreviations.

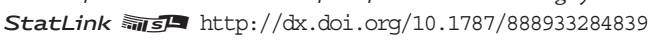





\section{HOW MANY YOUNG PEOPLE ARE EXPECTED TO COMPLETE TERTIARY EDUCATION AND WHAT IS THEIR PROFILE?}

- Based on current patterns of graduation, an average of 35\% of today's young people across OECD countries are expected to graduate from tertiary education at least once before the age of 30 .

- Women represented over half of all tertiary graduates in 2013 but are still under-represented in the fields of science and engineering.

- Graduates in fields of science and engineering combined represent less than a quarter of total tertiary graduates, but they represent $44 \%$ of graduates at the doctoral level.

\section{Chart A3.1. First-time tertiary graduation rates (2013)}

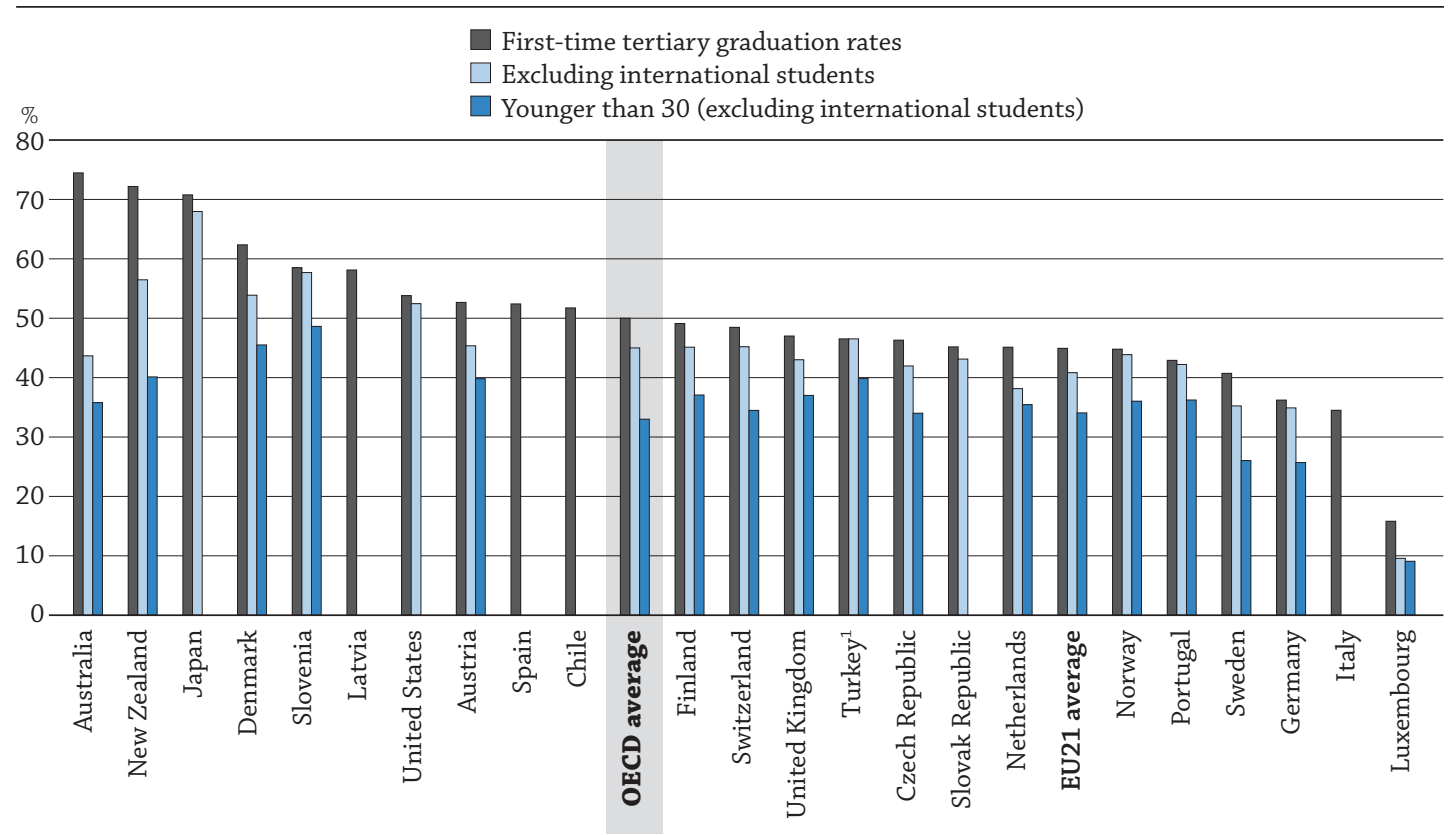

Note: Mismatches between the coverage of the population data and first-time graduates data mean that the graduation rates for those countries that are net exporters of students may be underestimated and those that are net importers may be overestimated. The first-time tertiary graduation rate excluding international students accounts for this.

1. Year of reference 2012

Countries are ranked in descending order of the first time tertiary graduation rates.

Source: OECD. Table A3.1. See Annex 3 for notes (www.oecd.org/education/education-at-a-glance-19991487.htm).

StatLink 제이 http://dx.doi.org/10.1787/888933283460

\section{Context}

Tertiary graduation rates illustrate a country's capacity to provide future workers with advanced and specialised knowledge and skills. Incentives to earn a tertiary degree, including higher salaries and better employment prospects, remain strong across OECD countries (see Indicators A5, A6 and A7 for further reading on these themes). Tertiary education varies in structure and scope among countries, and graduation rates seem to be influenced by the ease of access to and flexibility in programmes, and labour market demand for higher skills.

In recent decades, access to tertiary education has expanded remarkably, involving new types of institutions that offer more choice and new modes of delivery (OECD, 2014a). In parallel, the student population is becoming increasingly diverse in gender and in study pathways chosen. Students are also becoming more likely to seek a tertiary degree outside their country of origin.

Policy makers are exploring ways to help ease the transition from tertiary education into the labour market (OECD, 2015a). Understanding current graduation patterns would help to address the needs of recent graduates and anticipate the flow of new tertiary-educated workers into the labour force. 


\section{Other findings}

- In 2013 , a majority of first-time tertiary graduates (69\%) earned a bachelor's degree, $14 \%$ earned a master's degree and $18 \%$ earned a short-cycle tertiary diploma, on average across OECD countries.

- Advanced tertiary degrees attract more international students than bachelor's or equivalent degrees. Some $27 \%$ of students in OECD countries who graduated for the first time from a doctoral programme in 2013 were international students as were $18 \%$ of students who were awarded a master's degree or the equivalent, and 7\% of graduates who earned a bachelor's degree for the first time.

- One in three tertiary graduates in 2013 graduated with a degree in social sciences, business and law. In almost all OECD countries, the largest share of graduates pursued this field of study.

\section{Note}

Graduation rates represent the estimated percentage of an age cohort that is expected to graduate over their lifetime. This estimate is based on the total number of graduates in 2013 and the agespecific distribution of graduates. Therefore, graduation rates are based on the current pattern of graduation and thus are sensitive to any changes in education systems, such as the introduction of new programmes or any variations in a programme's duration, like those seen in many EU countries as a result of the implementation of the Bologna Process. 


\section{Analysis}

\section{Graduation rates from tertiary education}

Thanks to the new ISCED 2011 classification, statistical information on first-time graduates from tertiary education is used for the first time in this edition of Education at a Glance. First-time graduates from tertiary education are defined as students who receive a tertiary degree for the first time in their life in a given country. Based on current patterns of graduation, $50 \%$ of today's young people can be expected to graduate from tertiary education at least once during their lifetime, on average among the 22 OECD countries with comparable data for 2013. The proportion ranges from $16 \%$ in Luxembourg, where many citizens choose to study abroad, to $70 \%$ or more in Australia, Japan and New Zealand (Chart A3.1).

\section{Graduation rates, by levels of education}

More young people are expected to graduate from a bachelor's-degree programme over their lifetime than from any other level of tertiary education. Based on patterns of graduation prevailing in 2013, on average across OECD countries, $36 \%$ of young people in a given country are expected to graduate with a bachelor's degree over their lifetime, $17 \%$ are expected to earn a master's degree, $11 \%$ are expected to graduate from a short-cycle tertiary programme, and $2 \%$ are expected to graduate from a doctoral programme over their lifetime.

Although bachelor's degrees remain the most common tertiary diploma to be held by graduates in OECD countries, countries are promoting other levels of tertiary education too. In an effort to improve employability and the transition into the labour market, some countries are promoting short-cycle tertiary programmes. The probability of a person in Australia, Austria, Japan, New Zealand and the Russian Federation graduating from a short-cycle tertiary programme over his or her lifetime is $25 \%$ or more. Other ways of boosting employability and easing the transition into the labour market include promoting professional programmes at the bachelor's and master's levels of education.

Graduation rates from doctoral programmes have also increased over the past decade. In every country for which comparable data are available, the graduation rate from doctoral programmes increased between 2005 and 2013, except Austria, Finland and Slovenia, where the graduation rate decreased. Slovenia, Switzerland and the United Kingdom have the highest graduation rates at this level among all OECD countries, with $3 \%$ or more of young people - including international students - in these countries expected to graduate from doctoral programmes if 2013 patterns are maintained (Table A3.1, and Table A3.6, available on line).

\section{Graduation rates, excluding international students}

In some countries, a large proportion of graduates from tertiary education are international students. The term "international students" refers to students who have crossed borders expressly with the intent to study. For various reasons, international students have a marked impact on estimated graduation rates. By definition, they are considered first-time graduates, regardless of their previous education in other countries (i.e. an international student who graduates from a second-degree programme will be considered a first-time graduate in the country of destination). In some countries with a high proportion of international students, such as Australia and New Zealand, graduation rates are thus inflated. When international students are excluded, first-time tertiary graduation rates for Australia and New Zealand drop by 30 and 16 percentage points, respectively (Table A3.1).

\section{Graduation rates among people under the age of 30 or 35}

The first-time graduation rate from tertiary education among people under the age of 30 is an indicator of how many young people are expected to enter the labour force for the first time with a tertiary qualification. On average across the 16 countries with available data, $35 \%$ of young people (excluding international students) are expected to obtain a tertiary diploma for the first time before the age of 30. This rate varies between $49 \%$ in Slovenia and 9\% in Luxembourg.

In addition, some education systems accommodate a wider range of ages among their students than others. In Finland, Israel, New Zealand, the Slovak Republic and Switzerland, graduation rates at the bachelor's or equivalent level drops by 10 percentage points or more when restricted to young people under 30 (excluding international students). This may suggest that these education systems are more flexible in terms of access to and duration of programmes, and are more suitable for students outside the typical age of study. Finland, Israel and Switzerland also have mandatory military or civilian service that increases the length of tertiary studies (Table A3.1).

\section{Profile of graduates from tertiary education}

Over the past two decades, tertiary education in OECD countries has changed significantly: the student body is more international, more women are graduating from this level of education and, in some countries, more students are pursuing studies in science and engineering. These changes might reflect concerns about competitiveness in the global economy and the labour market. 


\section{A majority of graduates holds a bachelor's degree or the equivalent}

The new data on first-time graduates at the tertiary level allows for a more precise description of the young graduates who are entering the labour market with a tertiary diploma. They also make it easier to compare among countries by disregarding system-specific tertiary pathways.

In 2013, most first-time tertiary graduates were awarded a bachelor's degree. In fact, on average across OECD countries, $69 \%$ of first-time tertiary graduates earned a bachelor's degree, $14 \%$ earned a master's degree and $18 \%$ earned a short-cycle tertiary diploma (Table A3.2).

However, there are considerable differences across countries. In Austria, the largest share of first-time graduates (47\%) graduated from short-cycle tertiary programmes, while in Spain, the shares of first-time graduates are similar across three levels of tertiary education: short-cycle, bachelor's or the equivalent and master's or the equivalent. These differences may result from the structure of the tertiary system or because certain programmes, such as short-cycle programmes, are more vigorously promoted in some countries (Chart A3.2).

In addition, the great majority ( $82 \%$ on average across OECD countries) of first-time graduates in 2013 were 30 years old or younger, with large differences among countries. In Chile, Latvia, New Zealand, Sweden and Switzerland, at least $25 \%$ of graduates were older than 30 , whereas only $8 \%$ of first-time tertiary graduates in the Netherlands were that age (Table A3.2).

\section{Chart A3.2. Distribution of first-time graduates by level of education (2013)}

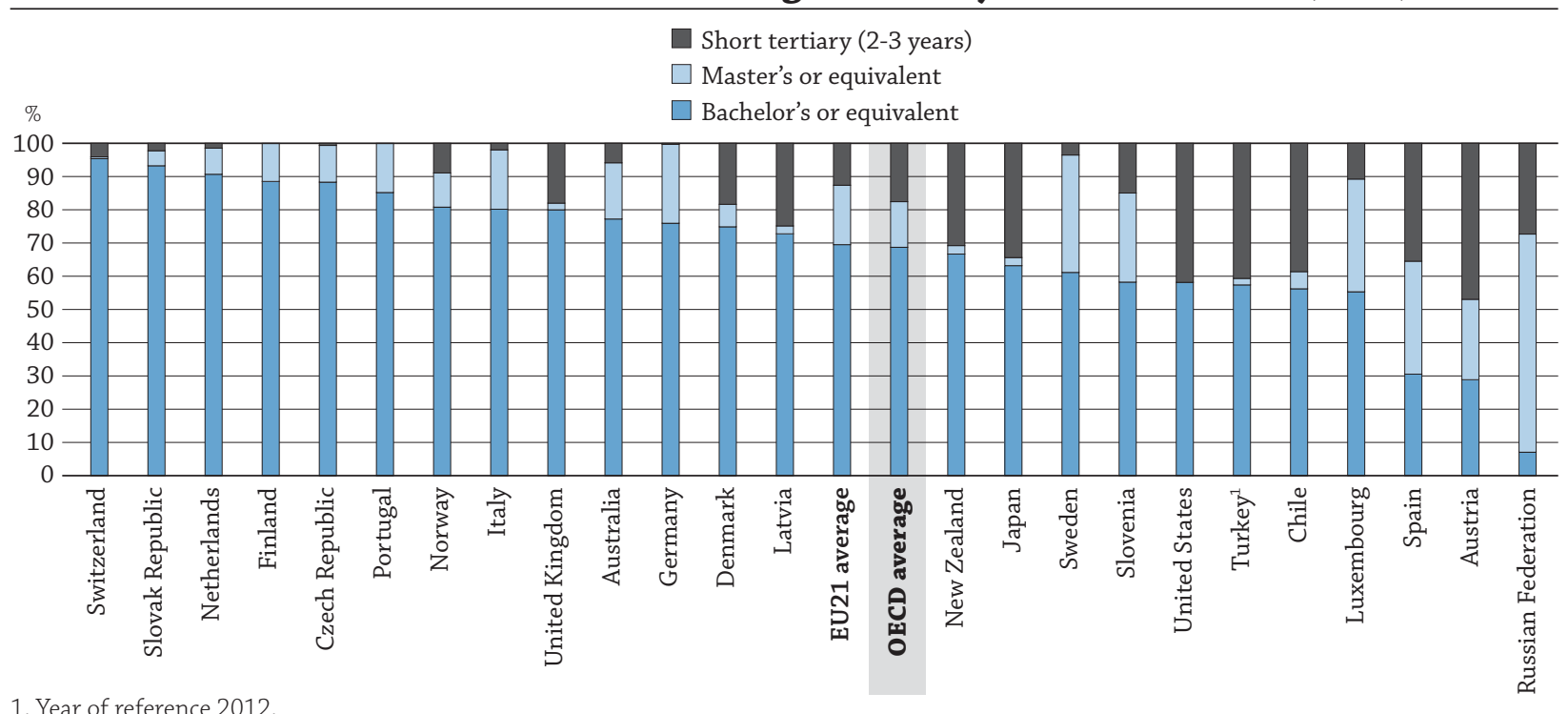

1. Year of reference 2012.

Countries are ranked in descending order of the percentage of first time graduates at bachelor's level or equivalent.

Source: OECD. Table A3.2. See Annex 3 for notes (www.oecd.org/education/education-at-a-glance-19991487.htm).

StatLink त्नाज http://dx.doi.org/10.1787/888933283475

\section{More than half of all first-time graduates are women}

Recognising the impact that education has on participation in the labour market, occupational mobility and quality of life, policy makers and educators are emphasising the importance of reducing differences in education opportunities and outcomes between men and women. In 2013, an average of 57\% of first-time graduates from tertiary education in OECD countries were women, ranging from $47 \%$ in Turkey to $69 \%$ in Latvia (Table A3.2). In addition, more than one in two first-time graduates from all levels of tertiary education - except the doctoral level - were women. On average, $58 \%$ of first-time graduates from bachelor's programmes or the equivalent were women while $47 \%$ of doctoral-level graduates were. The largest difference between the shares of women who graduated with a bachelor's degree or the equivalent and those who graduated with a doctorate (20 percentage points or more) were observed in the Czech Republic, Saudi Arabia and Sweden (Chart A3.3).

Although most tertiary graduates in 2013 were women, men still have better labour market outcomes. Earnings for tertiary-educated men are higher, on average, than those for tertiary-educated women, and tertiary-educated men tend to have higher employment rates than women with the same level of education (Indicators A5 and A6). 
In addition, even though women are over-represented among tertiary graduates, they remain under-represented in certain fields of study, such as science and engineering (see Education at a Glance online database). These results are partially explained by gender differences in young people's attitudes and aspirations. The OECD Programme for International Student Assessment (PISA) has consistently found that 15-year-old girls have higher expectations for their careers than boys, but that, on average across OECD countries, less than $5 \%$ of girls that age contemplate a career in engineering or computing (OECD, 2015b).

\section{Chart A3.3. Percentage of female graduates in tertiary levels of education (2013)}

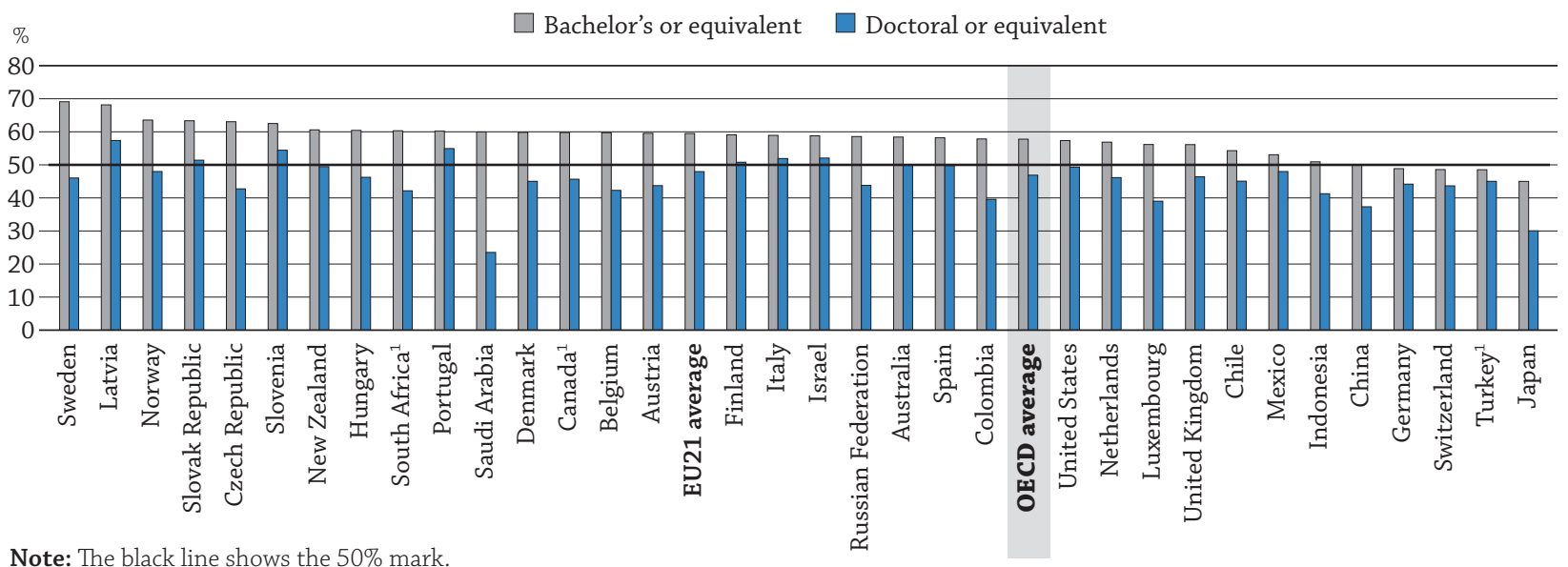

Note: The black line shows the $50 \%$ mark.

1. Year of reference 2012.

Countries are ranked in descending order of the percentage of women graduating with bachelor's or equivalent.

Source: OECD. Table A3.4. See Annex 3 for notes (www.oecd.org/education/education-at-a-glance-19991487.htm).

StatLink 젶ㄴ http://dx.doi.org/10.1787/888933283486

\section{Students choose to enrol abroad, mainly for advanced degrees}

The internationalisation of tertiary education has been more pronounced in advanced degrees, such as master's and doctoral programmes. In 2013, 27\% of doctoral graduates in OECD countries were international students as were $18 \%$ of graduates from master's programmes or the equivalent, and $7 \%$ of graduates at the bachelor's level. In Belgium, the Netherlands, New Zealand, Switzerland and the United Kingdom, 40\% or more of graduates from doctoral programmes were international students. In Luxembourg, eight out of ten doctoral graduates were international students. In Australia, master's programmes attract more international students (57\%) than doctoral programmes (36\%).

For more details on the internationalisation of tertiary education, please refer to Chapter C (Indicator C4) of this publication.

\section{Science and engineering are more popular fields of study in advanced tertiary degrees}

The distribution of graduates by field of study is related to the relative popularity of these fields among students, the relative number of positions offered in universities and equivalent institutions, and the degree structure of the various disciplines in a particular country.

One in three tertiary graduates in 2013 earned a degree from the field of social sciences, business and law. In all OECD countries but Korea, the largest share of graduates pursued this field of study. More than $45 \%$ of tertiary graduates in Colombia, Luxembourg, Turkey, Russian Federation and South Africa earned a degree in this field. The fields of science and engineering lag behind, with $14 \%$ of graduates in engineering, manufacturing and construction, and $9 \%$ of graduates in science (Table A3.3).

Many countries are pushing for a better balance in the distribution of graduates across fields of education. For instance, the United States recently took measures to increase the number of graduates with tertiary science and engineering qualifications by one million by 2022. Similarly, the European Union recently launched the Science with and for Society programme to build co-operation between science and society, recruit new talent for science, and pair scientific excellence with social awareness and responsibility. The programme aims to make science more attractive, particularly to young people, and to open further research and innovation activities across Europe. 
But the small share of graduates in science and engineering at the tertiary level hides large differences by level of tertiary education. In science, the higher the degree, the larger the share of students graduating from this field. While $5 \%$ of graduates from short-cycle tertiary programmes, $8 \%$ of graduates from bachelor's or the equivalent programmes, and $9 \%$ of graduates from master's or the equivalent programmes earned a degree in science in 2013, more than $27 \%$ of graduates from doctoral programmes did, on average across OECD countries. In Canada, Chile and France, 55\% or more of doctoral students graduated from the field of sciences or engineering in 2013 (Table A3.5).

This pattern is even clearer among international students. More than one in two international students who graduated with a doctorate earned a degree in either sciences or engineering (32\% earned a doctorate in sciences and $23 \%$ earned a doctorate in engineering), compared with one in five international students who graduated at the bachelor's level or from a short-cycle tertiary programme (Chart A3.4).

The popularity of science and engineering in doctoral programmes may be the result of policies that encourage academic research in these fields. Recent OECD work highlighted that while innovation draws on a wide set of skills, excellence in scientific research is the basis of science-based innovation, and research competence is essential for building co-operation among the scientific community, business and society. Thus, developing scientific research skills through doctoral training has become an important aim of education policy in many countries (OECD, 2014b).

\section{Chart A3.4. Percentage of students (all students and international students) who graduate from sciences and engineering at doctoral level (2013)}

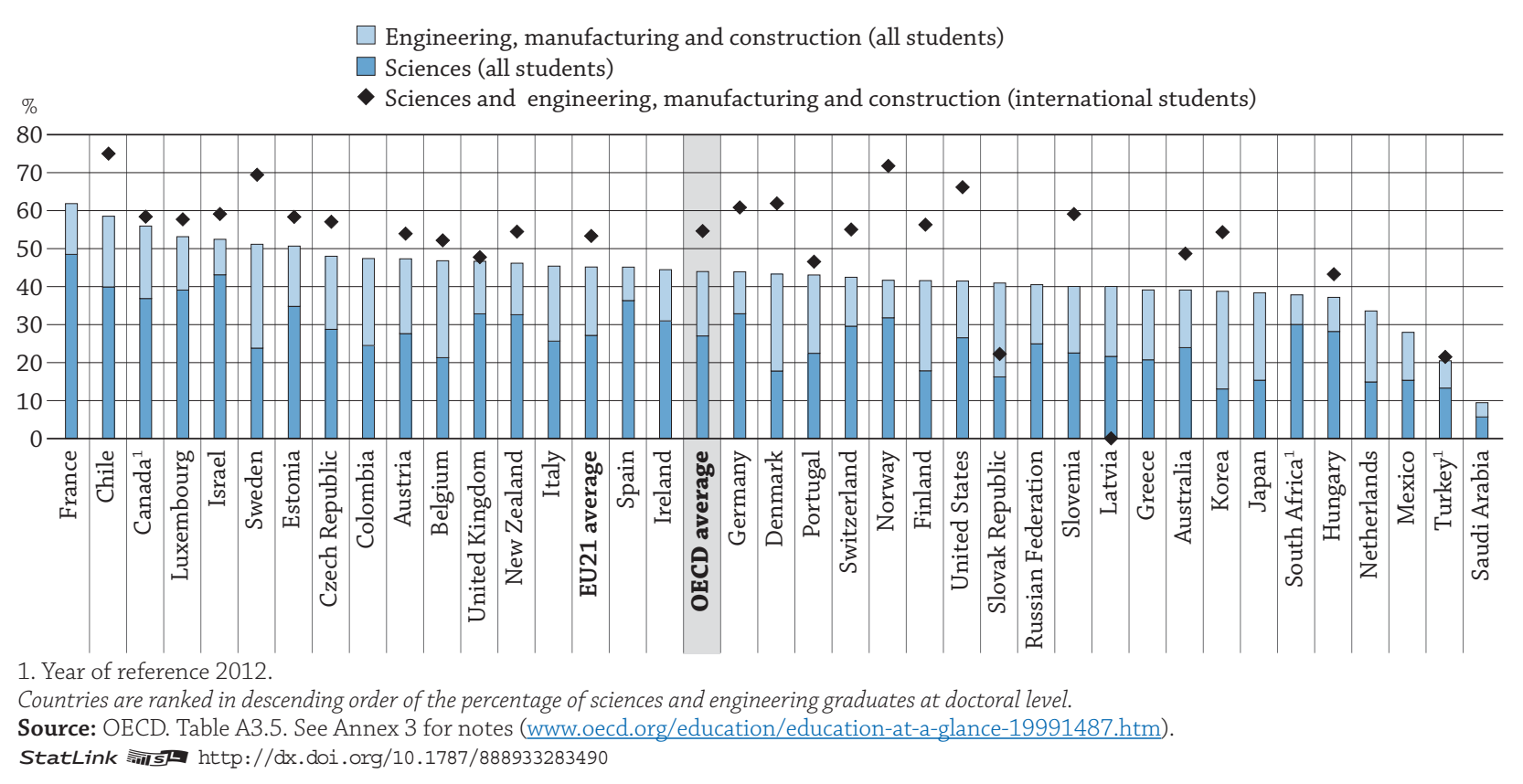

\section{Box A3.1. Do tertiary graduates perceive their workplace as innovative?}

Figuring out how to capture the value added from innovation is neither a simple nor a new problem. The OECD Innovation Strategy called for new perspectives on the measurement of innovation (OECD, 2010a, 2010b). In particular, it called for measures of innovation in the public sector, including in the education sector. The following charts are derived from Measuring Innovation in Education (OECD, 2014), responding to this call, offering new perspectives, based on REFLEX (2005) and HEGESCO (2008) surveys covering 19 European countries. In those surveys, innovation is defined as the introduction of "new or significantly improved products, processes, organisation or marketing methods". The surveys asked tertiary graduates, five years after they graduated: "How would you characterise the extent of innovation in your organisation or your workplace?" in reference to three types of innovation identified in the Oslo Manual (OECD and Eurostat, 2005): "products or services", e.g. new syllabi, textbooks or educational resources; "technology, 
tools or instruments", new processes for delivering their services, e.g. use of ICT in e-learning services, new learning-management system, new online courses, new pedagogic tools, such as maps, anatomy models, e-labs, etc.; and "knowledge or methods", e.g. new pedagogies, new administrative management systems regarding admissions or other formalities, ICT to communicate with students and parents, etc.. "High innovation" corresponds to values 4 and 5 in the scale from 1 (very low) to 5 (very high). The indicators presented below capture innovation as a significant change in key practices.

\section{Chart A3.a. Professionals in highly innovative workplaces, by sector and innovation type Percentage of graduates who perceive their workplace as highly innovative, 2005 or 2008}

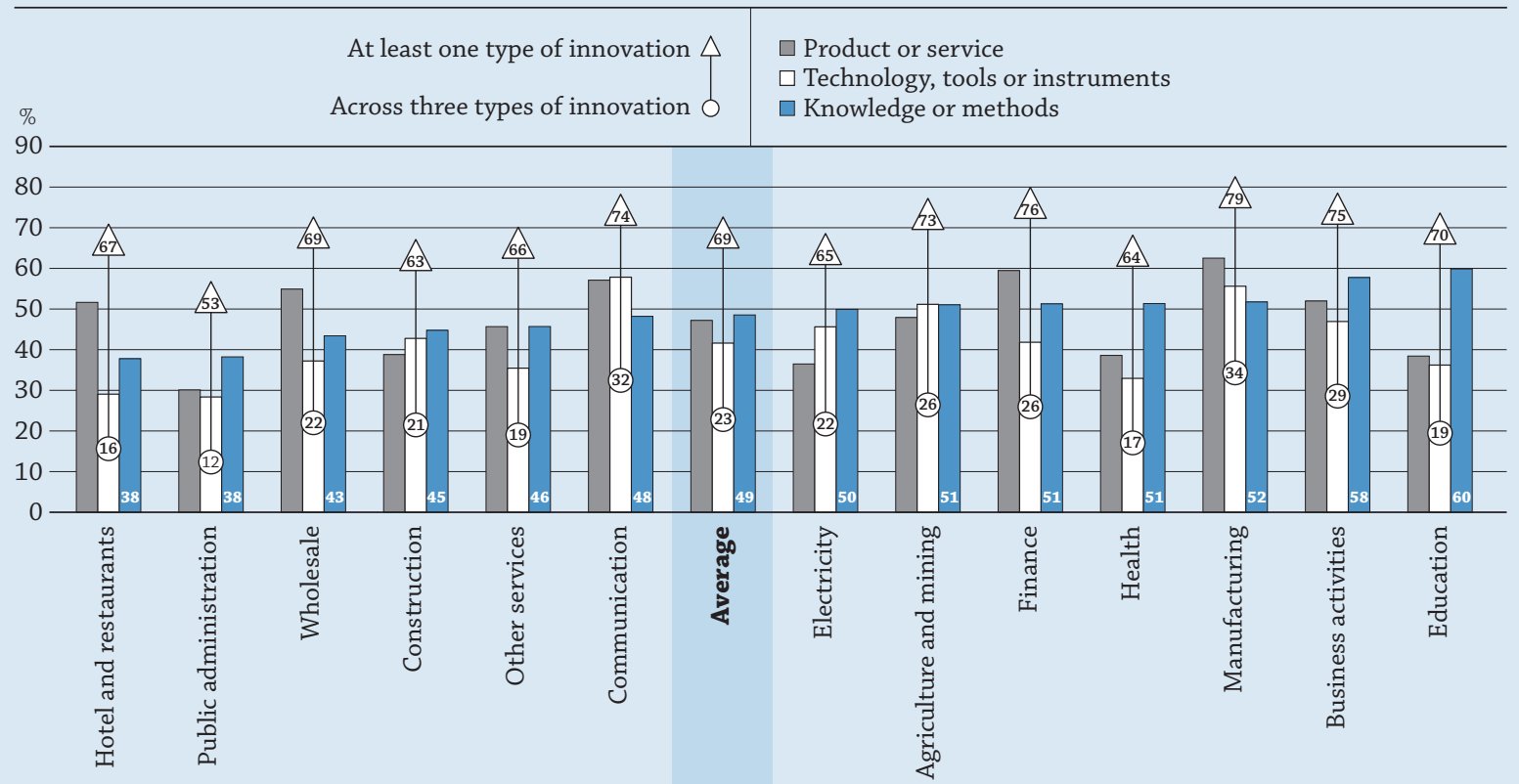

Data are ranked in ascending order of the percentage of graduates who perceive their workplace as highly innovative for knowledge or methods innovation. Source: Figures 1.4, 1.6 and 1.8 from OECD (2014), Measuring Innovation in Education: A New Perspective, Educational Research and Innovation, OECD Publishing, http://dx.doi.org/10.1787/9789264215696-en.

StatLink 제피 http://dx.doi.org/10.1787/888933283508

On average, more than two out of three tertiary graduates (69\%) across all sectors perceived their workplace as highly innovative for at least one type of innovation. Interestingly, about the same proportion (70\%) of tertiary graduates employed in the education sector, both public and private, considered their workplace as highly innovative for at least one type of innovation. Contrary to common belief, there is a fair level of innovation in the education sector, both relative to other sectors and in absolute terms. Some $60 \%$ of tertiary graduates employed in the education sector considered their workplace as highly innovative regarding knowledge or methods (compared to $49 \%$, on average, across all sectors); $38 \%$ considered their workplace as highly innovative regarding products or services (compared with $47 \%$, on average); and $36 \%$ considered their workplace as highly innovative regarding technology, tools or instruments (compared with $41 \%$, on average). Given these results, it appears that most innovation in the education sector focuses on teaching methods and knowledge (Chart A3.a).

Finland, Italy, the Netherlands, Slovenia and the United Kingdom have the largest share of graduates who considered their workplace in the education sector as highly innovative regarding at least one type of innovation. But graduates in these countries differ in how they perceive innovation in education as compared with innovation in other sectors. In Finland, graduates consider the education sector as innovative as the average across other sectors. Graduates in the Netherlands, Slovenia and the United Kingdom consider the education sector more innovative than the average across all sectors of the economy, while the opposite is observed in Italy. Interestingly, the standard deviation varies considerably across countries. Italy, the Netherlands and Slovenia show the smallest deviation across sectors. 
In contrast, the share of graduates who consider the education sector as highly innovative regarding at least one type of innovation is the smallest in the Czech Republic, France, Hungary and Portugal. Graduates working in the education sector in these four countries consider their own sector as less innovative than graduates working in other sectors of the economy. In the Czech Republic and Portugal, the shares of graduates who perceived the education sector as being highly innovative are far smaller than those who perceived other sectors of the economy as being innovative (Chart A3.b).

Interestingly, some of the countries covered by the survey reformed their education systems significantly since the release of this survey.

\section{Chart A3.b. Professionals in innovative workplaces regarding at least one type of innovation, by sector and country Percentage of graduates who perceive their workplace as highly innovative in at least one type of innovation, 2005 or 2008}

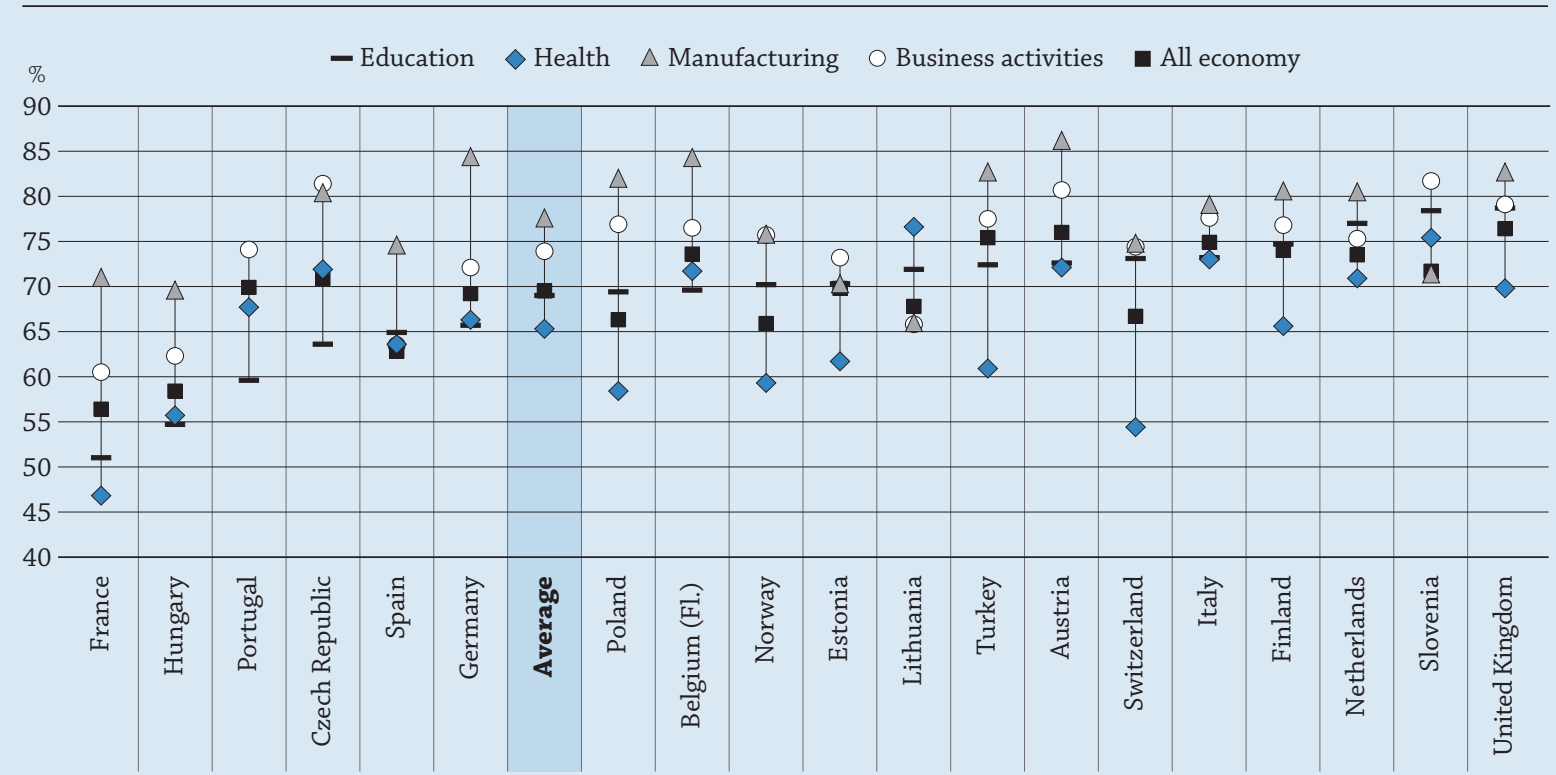

Note: Hungary, Lithuania, Poland, Slovenia and Turkey refer to HEGESCO (2008). Austria, the Belgium Flemish Community, the Czech Republic, Estonia, Finland, France, Germany, Italy, the Netherlands, Norway, Portugal, Spain, Switzerland, and the United Kingdom refer to REFLEX (2005). Data are ranked in ascending order of the percentage of graduates who perceive their workplace in education sector to be highly innovative regarding at least one type of innovation.

Source: Figure 1.5 from OECD (2014), Measuring Innovation in Education: A New Perspective, Educational Research and Innovation, OECD Publishing, http://dx.doi.org/10.1787/9789264215696-en.

StatLink 젣ㄴ http://dx.doi.org/10.1787/888933283515

In education sector more specifically, graduates in all countries reported innovations in "knowledge and methods" as the most common in 2008. On average across countries, 59\% of the graduates working in the education sector considered that their workplace is highly innovative in this respect. By contrast, $38 \%$ of graduates working in the education sector considered their workplace as highly innovative for "products or services"; and 36\% consider their workplace highly innovative for "technology, tools or instruments".

The Netherlands, Slovenia and the United Kingdom have the largest share of graduates who considered their workplace to be highly innovative regarding knowledge and methods. It is worth noting that the Netherlands and the United Kingdom are also among the countries that reported the highest level of school autonomy, as measured by the OECD Programme for International Student Assessment (PISA). Although there is no evidence of a strong correlation between school autonomy and degree of innovation in knowledge and methods across all countries, it is an interesting coincidence in some countries, suggesting that autonomous schools with control over staffing, budget, curriculum and assessments may be better equipped to introduce innovations in education. 
Turkey and the United Kingdom reported the largest share of graduates (more than 50\%) who considered their workplace in the education sector to be highly innovative considering the two other types of innovation: "products and services" and in "technology, tools or instruments". By contrast, in 2008, French and Hungarian graduates perceived the education sector as less innovative than other sectors. The share of graduates who were employed in the educator sector in highly innovative workplaces regarding "at least one type of innovation" and "all three types of innovation" was below the European average in these two countries, and each country ranked below average for all three types of innovation.

\section{Chart A3.c. Education professionals in highly innovative workplaces, by innovation type and country}

Percentage of graduates working in the education sector who perceive their workplace as highly innovative, 2005 or 2008

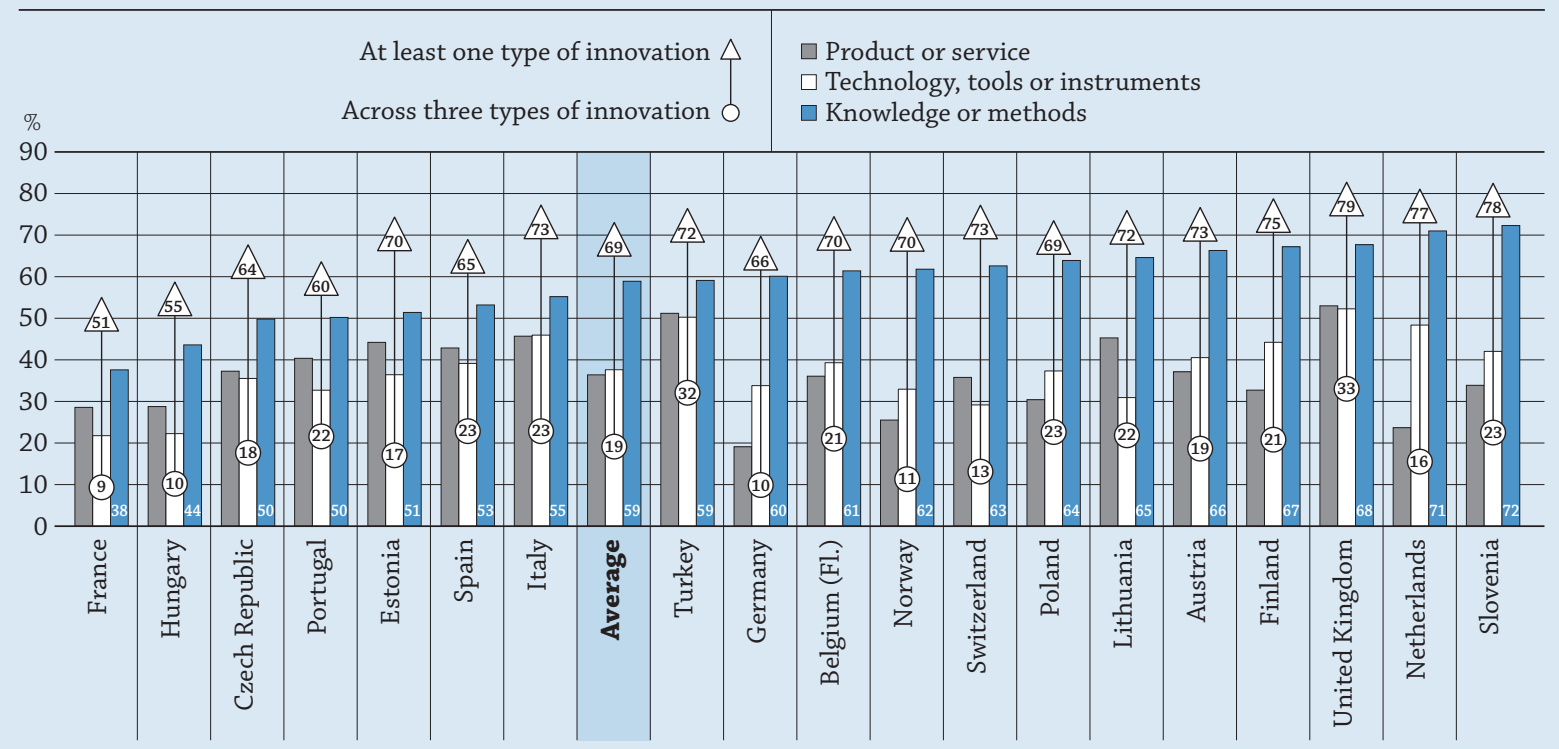

Note: Hungary, Lithuania, Poland, Slovenia and Turkey refer to HEGESCO (2008). Austria, the Belgium Flemish Community, the Czech Republic, Estonia, Finland, France, Germany, Italy, the Netherlands, Norway, Portugal, Spain, Switzerland, and the United Kingdom refer to REFLEX (2005).

Data are ranked in ascending order of the percentage of graduates who perceive their workplace in education sector to be highly innovative for knowledge or methods innovation.

Source: Figures 1.3, 1.5 and 1.7 from OECD (2014), Measuring Innovation in Education: A New Perspective, Educational Research and Innovation, OECD Publishing, http://dx.doi.org/10.1787/9789264215696-en.

StatLink 젶ㄴ http://dx.doi.org/10.1787/888933283520

How can perceived innovation, or lack thereof, be explained? Education science is relatively new so there is a comparatively small number of teaching practices that have been evaluated through research. One explanation may come from sociologists, particularly Max Weber, who have sought, since the 19th century, to explain the efforts of bureaucracies, both government and private, to rationalise and make more efficient the work and accountability of large organisations. Among the practices of public service organisations, including educational institutions that make innovation difficult are professional associations that control entry and advancement, labour agreements, expectations for transparency, and consultation outside the organisation. This may explain why France ranks low in innovation in its education and health sectors, both of which are large public service organisations with strong professional associations. In education, the traditional "decoupling" or "loose coupling" of the technical core (i.e. classroom teaching) from the formal organisation and from the policy environment hampers innovation (Dumont, Istance and Benavides [2010]).

The survey also analyses the education sector by level of education. Although no country-by-country analysis is possible, the survey found that $80 \%$ of graduates employed in tertiary education consider their workplace as highly innovative, compared to $65 \%$ of graduates employed in primary education, and $63 \%$ employed in secondary education. 


\section{Chart A3.d. Education professionals working in innovative workplace, by type of education taught}

Percentage of graduates who perceive their workplace as highly innovative regarding at least one type of innovation, 2005 or 2008

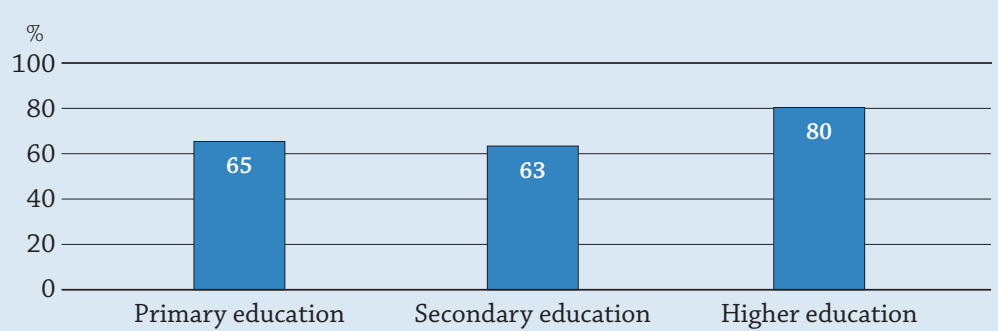

Data are ranked by level of education.

Source: Figure 1.12 from OECD (2014), Measuring Innovation in Education: A New Perspective, Educational Research and Innovation, OECD Publishing, http://dx.doi.org/10.1787/9789264215696-en.

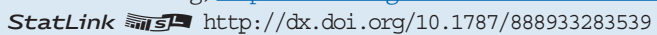

Among European countries, the increase in educational attainment over the past decades, particularly in tertiary education, may explain why that segment is considered more innovative than other segments. Despite some funding problems, tertiary education remains a hugely successful enterprise, facing rising demand worldwide as students and parents know (or believe) that life chances, and economic and social returns will be enhanced with a tertiary education. Shifting the cost of tertiary education to students/parents through rising tuition fees and, consequently, greater indebtedness among students, is unsustainable. While the more selective institutions may not be threatened, these conditions may increase the competitive advantage of for-profit providers, creating incentives to develop low-cost faculty, standardised curricula, distance-learning methods, such as MOOCs, and minimal overhead costs, etc. (Kaufmann, 2012).

\section{Methodology}

Measuring innovation and its effectiveness in the public sector, and in education in particular, is in its infancy. Measuring innovation in education is a pioneering attempt to present indicators based on existing international datasets. It aims to provide education policy makers with an estimated order of magnitude of innovation and change in education.

Measuring innovation in education presents two broad approaches to measuring innovation in education: adapting innovation surveys to the public sector (including education), and analysing organisational changes through teacher-student surveys. This indicator is based on the first approach. While such an approach asking graduates to assess the "level" of innovation - is subjective, it provides information regarding the perceived level of innovation by sector.

The REFLEX (2005) and HEGESCO (2008) surveys asked graduates of higher education five years after they graduated: "How would you characterise the extent of innovation in your organisation or your work?" regarding "products or services", "technology, tools or instruments" and "knowledge or methods". "High innovation" corresponds to values 4 and 5 in the scale from 1 (very low) to 5 (very high). The education sector includes primary, secondary and higher education as well as other non-specified education activities.

This approach is complementary to other measurement approaches. In fact, measuring innovation through several approaches would enrich the stock of knowledge, and improve the understanding of the benefits of innovation in the education sector. Measuring Innovation in Education suggests that developing an international survey on innovation in education would have several advantages, including larger country coverage (on a comparable basis) and a wider array of respondent stakeholders, targeting three levels of stakeholders: school principal/president, teachers/faculty, and students (in primary, secondary and tertiary education). Such a survey would help to identify the main areas of innovation - and even specific innovations - in the education sector and avoid the ambiguities of the "innovation" concept. 


\section{Bibliography}

Dumont, H., D. Istance and F. Benavides (eds.) (2010), The Nature of Learning: Using Research to Inspire Practice, Educational Research and Innovation, OECD Publishing, Paris, http://dx.doi.org/10.1787/9789264086487-en.

Kauffman Foundation (ed.) (2012), College 2.0: An Entrepreneurial Approach to Reforming Higher Education: Overcoming Barriers and Fostering Innovation, Papers from the Entrepreneurship in Higher Education Retreat, Ewing Marion Kauffman Foundation, www.careercollegecentral.com/pdf/entrepreneurial_approach_to_higher_ed_reform.pdf.

Looney, J. W. (2009), “Assessment and Innovation in Education”, OECD Education Working Papers, No. 24, OECD Publishing, http://dx.doi.org/10.1787/222814543073.

OECD (2014), Measuring Innovation in Education: A New Perspective, Educational Research and Innovation, OECD Publishing, http://dx.doi.org/10.1787/9789264215696-en.

OECD (2012), PISA 2012 Results: What Makes Schools Successful? (Volume IV) Resources, Policies and Practices, PISA, OECD Publishing, Paris, www.oecd.org/pisa/keyfindings/Vol4Ch4.pdf.

OECD (2010a), Measuring Innovation: A New Perspective, OECD Publishing, Paris, http://dx.doi.org/10.1787/ 9789264059474-en.

OECD (2010b), The OECD Innovation Strategy: Getting a Head Start on Tomorrow, OECD Publishing, Paris, http://dx.doi. org/10.1787/9789264083479-en.

OECD (2009), Creating Effective Teaching and Learning Environments: First Results From TALIS, OECD Publishing, Paris, http://dx.doi.org/10.1787/9789264068780-en.

OECD/Eurostat (2005), Oslo Manual: Guidelines for Collecting and Interpreting Innovation Data, 3rd Edition, The Measurement of Scientific and Technological Activities, OECD Publishing, Paris, http://dx.doi.org/10.1787/9789264013100-en.

Woessmann, L. et al. (2007), "School Accountability, Autonomy, Choice, and the Level of Student Achievement: International Evidence from PISA 2003”, OECD Education Working Papers, No. 13, OECD Publishing, Paris, http://dx.doi.org/10.1787/ 246402531617.

\section{Definitions}

First-time graduate is a student who has graduated for the first time at a given level of education during the reference period. Therefore, if a student has graduated multiple times over the years, he or she is counted as a graduate each year, but as a first-time graduate only once.

First-time tertiary graduate is a student who graduates for the first time with a tertiary diploma, regardless of the education programme in which he or she is enrolled. This definition is applied in Table A3.1 (columns 13 to 15) and Table A3.2.

First-time graduate from a given programme or level of tertiary education is a first-time graduate from the given programme, but may have a diploma from another programme. For example, a first-time graduate at the master's level has earned a master's degree for the first time, but may have graduated with a bachelor's degree previously. This definition is applied in Table A3.1 (columns 1 to 12), Table A3.4 and Table A3.5.

International students are those students who left their country of origin and moved to another country for the purpose of study. In the majority of countries, international students are considered first-time graduates, regardless of their previous education in other countries. In the calculations described here, when countries could not report the number of international students, foreign students have been used as an approximation. Foreign students are students who do not have the citizenship of the country in which they studied (for more details, please refer to Annex 3, www.oecd.org/education/education-at-a-glance-19991487.htm).

Net graduation rates represent the estimated percentage of people from a specific age cohort who will complete tertiary education over their lifetime, based on current patterns of graduation.

\section{Methodology}

Data refer to the academic year 2012/13 and are based on the UOE data collection on education statistics administered by the OECD in 2014 (for details, see Annex 3 at www.oecd.org/education/education-at-a-glance-19991487.htm).

Unless otherwise indicated, graduation rates are calculated as net graduation rates (i.e. as the sum of age-specific graduation rates). Net tertiary graduation rates represent the expected probability of graduating from tertiary education over a lifetime if current patterns are maintained. The current cohort of graduates by ages (cross-section data) is used in the calculation. 
Gross graduation rates are used when data by age are missing. In order to calculate gross graduation rates, countries identify the age at which graduation typically occurs (see Annex 1 ). The typical age of graduation for a given education level is defined in Education at a Glance as the age range comprising at least half of the graduate population. The number of graduates of which the age is unknown is divided by the population at the typical graduation age. In many countries, defining a typical age at graduation is difficult, however, because graduates are dispersed over a wide range of ages.

\section{Note regarding data from Israel}

The statistical data for Israel are supplied by and are under the responsibility of the relevant Israeli authorities. The use of such data by the OECD is without prejudice to the status of the Golan Heights, East Jerusalem and Israeli settlements in the West Bank under the terms of international law.

\section{References}

OECD (2015a), Education Policy Outlook 2015: Making Reforms Happen, OECD Publishing, Paris, http://dx.doi.org/10.1787/ 9789264225442-en.

OECD (2015b), The ABC of Gender Equality in Education: Aptitude, Behaviour, Confidence, PISA, OECD Publishing, Paris, http:// dx.doi.org/10.1787/9789264229945-en.

OECD (2014a), OECD Science, Technology and Industry Outlook 2014, OECD Publishing, Paris, http://dx.doi.org/10.1787/sti outlook-2014-en.

OECD (2014b), The State of Higher Education 2014, the OECD Higher Education Programme IMHE, OECD Publishing, Paris, www.oecd.org/edu/imhe.

\section{Indicator A3 Tables}

StatLink 施

Table A3.1 First-time graduation rates, by tertiary ISCED level (2013)

Table A3.2 Profile of a first-time tertiary graduate (2013)

Table A3.3 Distribution of all tertiary graduates, by field of education (2013)

Table A3.4 Percentage of female and international first-time graduates, by tertiary ISCED level (2013)

Table A3.5 Percentage of all students and international students who graduate from sciences and engineering programmes, by tertiary ISCED level (2013)

WEB Table A3.6 Trends in first time graduation rates, by tertiary ISCED level (2005-13)

WEB Table A3.7 Share of tertiary gradutates by field of education and gender (2013)

Cut-off date for the data: 23 October 2015. Updates can be found on line at http://dx.doi.org/10.1787/eag-data-en. 
Table A3.1. First-time graduation rates, by tertiary ISCED level (2013) Sum of age-specific graduation rates, by demographic group

\begin{tabular}{|c|c|c|c|c|c|c|c|c|c|c|c|c|c|c|c|}
\hline & \multicolumn{3}{|c|}{$\begin{array}{l}\text { Short tertiary ( } 2-3 \text { years) } \\
\text { ISCED } 5\end{array}$} & \multicolumn{3}{|c|}{$\begin{array}{l}\text { Bachelor's or equivalent } \\
\text { ISCED } 6\end{array}$} & \multicolumn{3}{|c|}{$\begin{array}{l}\text { Master's or equivalent } \\
\text { ISCED } 7\end{array}$} & \multicolumn{3}{|c|}{$\begin{array}{l}\text { Doctorate or equivalent } \\
\text { ISCED } 8\end{array}$} & \multicolumn{3}{|c|}{ First-time tertiary } \\
\hline & \multirow[b]{2}{*}{ Total } & \multicolumn{2}{|c|}{$\begin{array}{c}\text { Excluding } \\
\text { international } \\
\text { students }\end{array}$} & \multirow[b]{2}{*}{ Total } & \multicolumn{2}{|c|}{$\begin{array}{c}\text { Excluding } \\
\text { international } \\
\text { students }\end{array}$} & \multirow[b]{2}{*}{ Total } & \multicolumn{2}{|c|}{$\begin{array}{c}\text { Excluding } \\
\text { international } \\
\text { students }\end{array}$} & \multirow[b]{2}{*}{ Total } & \multicolumn{2}{|c|}{$\begin{array}{c}\text { Excluding } \\
\text { international } \\
\text { students }\end{array}$} & \multirow[b]{2}{*}{ Total } & \multicolumn{2}{|c|}{$\begin{array}{c}\text { Excluding } \\
\text { international } \\
\text { students }\end{array}$} \\
\hline & & Total & $\begin{array}{l}\text { Younger } \\
\text { than } 30\end{array}$ & & Total & $\begin{array}{l}\text { Younger } \\
\text { than } 30\end{array}$ & & Total & $\begin{array}{l}\text { Younger } \\
\text { than } 35\end{array}$ & & Total & $\begin{array}{l}\text { Younger } \\
\text { than } 35\end{array}$ & & Total & $\begin{array}{l}\text { Younger } \\
\text { than } 30\end{array}$ \\
\hline & (1) & (2) & (3) & (4) & (5) & (6) & (7) & (8) & (9) & (10) & (11) & (12) & (13) & (14) & (15) \\
\hline Australia & 28 & 26 & 12 & 61 & 43 & 34 & 18 & 8 & 5 & 2.5 & 1.6 & 0.8 & 74 & 44 & 36 \\
\hline Austria & 26 & 26 & 25 & 25 & 21 & 18 & 22 & 18 & 15 & 1.9 & 1.4 & 1.0 & 53 & 45 & 40 \\
\hline Belgium & $\mathrm{m}$ & $\mathrm{m}$ & $\mathrm{m}$ & 42 & 39 & $\mathrm{~m}$ & 11 & 8 & $\mathrm{~m}$ & 0.5 & 0.3 & $\mathrm{~m}$ & $\mathrm{~m}$ & $\mathrm{~m}$ & $\mathrm{~m}$ \\
\hline Canada $^{1}$ & 22 & 19 & 15 & 33 & 30 & 28 & 11 & 9 & 7 & 1.3 & 1.1 & 0.7 & $\mathrm{~m}$ & $\mathrm{~m}$ & $\mathrm{~m}$ \\
\hline Chile & 20 & $\mathrm{~m}$ & $\mathrm{~m}$ & 31 & $\mathrm{~m}$ & $\mathrm{~m}$ & 9 & $\mathrm{~m}$ & $\mathrm{~m}$ & 0.2 & $\mathrm{~m}$ & $\mathrm{~m}$ & 52 & $\mathrm{~m}$ & $\mathrm{~m}$ \\
\hline Czech Republic & 0 & 0 & 0 & 41 & 38 & 31 & 27 & 24 & 21 & 1.6 & 1.4 & 1.0 & 46 & 42 & 34 \\
\hline Denmark & 12 & 10 & 8 & 52 & 48 & 40 & 26 & 21 & 19 & 2.8 & 1.9 & 1.3 & 62 & 54 & 45 \\
\hline Estonia & $\mathrm{a}$ & a & a & $\mathrm{m}$ & $\mathrm{m}$ & $\mathrm{m}$ & $\mathrm{m}$ & $\mathrm{m}$ & $\mathrm{m}$ & 1.3 & $\mathrm{~m}$ & $\mathrm{~m}$ & $\mathrm{~m}$ & $\mathrm{~m}$ & $\mathrm{~m}$ \\
\hline Finland & $\mathrm{a}$ & a & $\mathrm{a}$ & 46 & 44 & 34 & 23 & 21 & 16 & 2.5 & 2.0 & 0.9 & 49 & 45 & 37 \\
\hline France & $\mathrm{m}$ & $\mathrm{m}$ & $\mathrm{m}$ & $\mathrm{m}$ & $\mathrm{m}$ & $\mathrm{m}$ & $\mathrm{m}$ & $\mathrm{m}$ & $\mathrm{m}$ & 1.7 & $\mathrm{~m}$ & $\mathrm{~m}$ & $\mathrm{~m}$ & $\mathrm{~m}$ & $\mathrm{~m}$ \\
\hline Germany & 0 & 0 & 0 & 27 & 27 & 19 & 16 & 15 & 14 & 2.7 & 2.3 & 1.9 & 36 & 35 & 26 \\
\hline Greece & a & a & a & $\mathrm{m}$ & $\mathrm{m}$ & $\mathrm{m}$ & $\mathrm{m}$ & $\mathrm{m}$ & $\mathrm{m}$ & 0.9 & $\mathrm{~m}$ & $\mathrm{~m}$ & $\mathrm{~m}$ & $\mathrm{~m}$ & $\mathrm{~m}$ \\
\hline Hungary & 7 & 7 & 7 & 22 & 21 & 15 & 15 & 14 & 12 & 0.7 & 0.7 & 0.5 & $\mathrm{~m}$ & $\mathrm{~m}$ & $\mathrm{~m}$ \\
\hline Iceland & $\mathrm{m}$ & $\mathrm{m}$ & $\mathrm{m}$ & $\mathrm{m}$ & $\mathrm{m}$ & $\mathrm{m}$ & $\mathrm{m}$ & $\mathrm{m}$ & $\mathrm{m}$ & $\mathrm{m}$ & $\mathrm{m}$ & $\mathrm{m}$ & $\mathrm{m}$ & $\mathrm{m}$ & $\mathrm{m}$ \\
\hline Ireland & $\mathrm{m}$ & $\mathrm{m}$ & $\mathrm{m}$ & $\mathrm{m}$ & $\mathrm{m}$ & $\mathrm{m}$ & $\mathrm{m}$ & $\mathrm{m}$ & $\mathrm{m}$ & 2.2 & $\mathrm{~m}$ & $\mathrm{~m}$ & $\mathrm{~m}$ & $\mathrm{~m}$ & $\mathrm{~m}$ \\
\hline Israel & $\mathrm{m}$ & $\mathrm{m}$ & $\mathrm{m}$ & 42 & 40 & 30 & 19 & 18 & 11 & 1.5 & 1.4 & 0.6 & $\mathrm{~m}$ & $\mathrm{~m}$ & $\mathrm{~m}$ \\
\hline Italy & 0 & $\mathrm{~m}$ & $\mathrm{~m}$ & 28 & $\mathrm{~m}$ & $\mathrm{~m}$ & 20 & $\mathrm{~m}$ & $\mathrm{~m}$ & 1.4 & $\mathrm{~m}$ & $\mathrm{~m}$ & 34 & $\mathrm{~m}$ & $\mathrm{~m}$ \\
\hline Japan & 25 & 24 & $\mathrm{~m}$ & 45 & 44 & $\mathrm{~m}$ & 8 & 8 & $\mathrm{~m}$ & 1.2 & 1.0 & $\mathrm{~m}$ & 71 & 68 & $\mathrm{~m}$ \\
\hline Korea & $\mathrm{m}$ & $\mathrm{m}$ & $\mathrm{m}$ & $\mathrm{m}$ & $\mathrm{m}$ & $\mathrm{m}$ & $\mathrm{m}$ & $\mathrm{m}$ & $\mathrm{m}$ & 1.6 & $\mathrm{~m}$ & $\mathrm{~m}$ & $\mathrm{~m}$ & $\mathrm{~m}$ & $\mathrm{~m}$ \\
\hline Luxembourg & 2 & 1 & 1 & 9 & 7 & 7 & 5 & 1 & 1 & 0.8 & 0.1 & 0.1 & 16 & 10 & 9 \\
\hline Mexico & 2 & $\mathrm{~m}$ & $\mathrm{~m}$ & 22 & $\mathrm{~m}$ & $\mathrm{~m}$ & 4 & $\mathrm{~m}$ & $\mathrm{~m}$ & 0.3 & $\mathrm{~m}$ & $\mathrm{~m}$ & $\mathrm{~m}$ & $\mathrm{~m}$ & $\mathrm{~m}$ \\
\hline Netherlands & 1 & 1 & 0 & 41 & 37 & 35 & $\mathrm{~m}$ & $\mathrm{~m}$ & $\mathrm{~m}$ & 2.1 & 1.3 & 1.1 & 45 & 38 & 35 \\
\hline New Zealand & 25 & 19 & 11 & 54 & 44 & 32 & 7 & 5 & 3 & 2.2 & 1.1 & 0.6 & 72 & 56 & 40 \\
\hline Norway & 4 & 4 & 3 & 37 & 36 & 30 & 17 & 15 & 12 & 1.9 & 1.3 & 0.6 & 45 & 44 & 36 \\
\hline Poland & 1 & $\mathrm{~m}$ & $\mathrm{~m}$ & $\mathrm{~m}$ & $\mathrm{~m}$ & $\mathrm{~m}$ & $\mathrm{~m}$ & $\mathrm{~m}$ & $\mathrm{~m}$ & 0.6 & $\mathrm{~m}$ & $\mathrm{~m}$ & $\mathrm{~m}$ & $\mathrm{~m}$ & $\mathrm{~m}$ \\
\hline Portugal & $\mathrm{a}$ & $\mathrm{a}$ & $\mathrm{a}$ & 36 & 36 & 30 & 20 & 19 & 16 & 1.7 & 1.5 & 0.7 & 43 & 42 & 36 \\
\hline Slovak Republic & 1 & 1 & 1 & 42 & 41 & 22 & 40 & 38 & 31 & 2.5 & 2.3 & 1.8 & 45 & 43 & $\mathrm{~m}$ \\
\hline Slovenia & 8 & 8 & 5 & 37 & 36 & 31 & 21 & 20 & 18 & 3.6 & 3.5 & 2.4 & 58 & 58 & 49 \\
\hline Spain & 20 & $\mathrm{~m}$ & $\mathrm{~m}$ & 18 & 18 & 15 & 22 & 21 & 19 & 1.5 & $\mathrm{~m}$ & $\mathrm{~m}$ & 52 & $\mathrm{~m}$ & $\mathrm{~m}$ \\
\hline Sweden & 7 & 7 & 5 & 26 & 25 & 18 & 20 & 15 & 12 & 2.7 & 1.9 & 1.0 & 41 & 35 & 26 \\
\hline Switzerland & 2 & 2 & 2 & 46 & 43 & 33 & 17 & 13 & 11 & 3.2 & 1.6 & 1.2 & 48 & 45 & 34 \\
\hline Turkey $^{1}$ & 19 & 19 & 16 & 27 & 27 & 23 & 3 & 3 & 3 & 0.7 & 0.7 & 0.5 & 47 & 47 & 40 \\
\hline United Kingdom & 8 & 8 & 5 & 45 & 38 & 33 & 27 & 15 & 10 & 3.0 & 1.7 & 1.1 & 47 & 43 & 37 \\
\hline United States & 22 & 22 & $\mathrm{~m}$ & 38 & 37 & $\mathrm{~m}$ & 20 & 18 & $\mathrm{~m}$ & 1.5 & 1.1 & $\mathrm{~m}$ & 54 & 52 & $\mathrm{~m}$ \\
\hline OECD average & 11 & 11 & 7 & 36 & 34 & 27 & 17 & 15 & 13 & 1.7 & 1.4 & 1.0 & 50 & 45 & 35 \\
\hline EU21 average & 7 & 6 & 5 & 34 & 32 & 26 & 21 & 18 & 15 & 1.8 & 1.6 & 1.1 & 45 & 41 & 34 \\
\hline
\end{tabular}

\begin{tabular}{|c|c|c|c|c|c|c|c|c|c|c|c|c|c|c|c|}
\hline $\begin{array}{l}\text { argentina } \\
\text { ș Brazil }\end{array}$ & $\begin{array}{l}\mathrm{m} \\
\mathrm{m}\end{array}$ & $\begin{array}{l}\mathrm{m} \\
\mathrm{m}\end{array}$ & $\begin{array}{l}\mathrm{m} \\
\mathrm{m}\end{array}$ & $\begin{array}{l}\mathrm{m} \\
\mathrm{m}\end{array}$ & $\begin{array}{l}\mathrm{m} \\
\mathrm{m}\end{array}$ & $\begin{array}{l}\mathrm{m} \\
\mathrm{m}\end{array}$ & $\begin{array}{l}\mathrm{m} \\
\mathrm{m}\end{array}$ & $\begin{array}{l}\mathrm{m} \\
\mathrm{m}\end{array}$ & $\begin{array}{l}\mathrm{m} \\
\mathrm{m}\end{array}$ & $\begin{array}{l}\mathrm{m} \\
\mathrm{m}\end{array}$ & $\begin{array}{l}\mathrm{m} \\
\mathrm{m}\end{array}$ & $\begin{array}{l}\mathrm{m} \\
\mathrm{m}\end{array}$ & $\begin{array}{l}\mathrm{m} \\
\mathrm{m}\end{array}$ & $\begin{array}{l}\mathrm{m} \\
\mathrm{m}\end{array}$ & $\begin{array}{l}\mathrm{m} \\
\mathrm{m}\end{array}$ \\
\hline$\varrho^{\pi}$ China & 19 & 19 & $\mathrm{~m}$ & 17 & 17 & $\mathrm{~m}$ & 2 & 2 & $\mathrm{~m}$ & 0.2 & 0.2 & $\mathrm{~m}$ & $\mathrm{~m}$ & $\mathrm{~m}$ & $\mathrm{~m}$ \\
\hline Colombia & 13 & $\mathrm{~m}$ & $\mathrm{~m}$ & 19 & $\mathrm{~m}$ & $\mathrm{~m}$ & 9 & $\mathrm{~m}$ & $\mathrm{~m}$ & 0.0 & $\mathrm{~m}$ & $\mathrm{~m}$ & $\mathrm{~m}$ & $\mathrm{~m}$ & $\mathrm{~m}$ \\
\hline India & $\mathrm{m}$ & $\mathrm{m}$ & $\mathrm{m}$ & $\mathrm{m}$ & $\mathrm{m}$ & $\mathrm{m}$ & $\mathrm{m}$ & $\mathrm{m}$ & $\mathrm{m}$ & $\mathrm{m}$ & $\mathrm{m}$ & $\mathrm{m}$ & $\mathrm{m}$ & $\mathrm{m}$ & $\mathrm{m}$ \\
\hline Indonesia & $\mathrm{m}$ & $\mathrm{m}$ & $\mathrm{m}$ & 23 & $\mathrm{~m}$ & $\mathrm{~m}$ & 1 & $\mathrm{~m}$ & $\mathrm{~m}$ & 0.1 & $\mathrm{~m}$ & $\mathrm{~m}$ & $\mathrm{~m}$ & $\mathrm{~m}$ & $\mathrm{~m}$ \\
\hline Latvia & 15 & $\mathrm{~m}$ & $\mathrm{~m}$ & 42 & $\mathrm{~m}$ & $\mathrm{~m}$ & 14 & $\mathrm{~m}$ & $\mathrm{~m}$ & 1.1 & $\mathrm{~m}$ & $\mathrm{~m}$ & 58 & $\mathrm{~m}$ & $\mathrm{~m}$ \\
\hline Russian Federation & 29 & $\mathrm{~m}$ & $\mathrm{~m}$ & 6 & $\mathrm{~m}$ & $\mathrm{~m}$ & 52 & $\mathrm{~m}$ & $\mathrm{~m}$ & 1.4 & $\mathrm{~m}$ & $\mathrm{~m}$ & $\mathrm{~m}$ & $\mathrm{~m}$ & $\mathrm{~m}$ \\
\hline South Africa ${ }^{1}$ & 6 & $\mathrm{~m}$ & $\mathrm{~m}$ & 11 & $\mathrm{~m}$ & $\mathrm{~m}$ & 1 & $\mathrm{~m}$ & $\mathrm{~m}$ & 0.2 & $\mathrm{~m}$ & $\mathrm{~m}$ & $\mathrm{~m}$ & $\mathrm{~m}$ & $\mathrm{~m}$ \\
\hline
\end{tabular}

\begin{tabular}{|c|c|c|c|c|c|c|c|c|c|c|c|c|}
\hline G2 & 14 & 17 & $\mathrm{~m}$ & 29 & 33 & $\mathrm{~m}$ & 13 & 10 & $\mathrm{~m}$ & 1.2 & 1.2 & \\
\hline
\end{tabular}

1. Year of reference 2012.

Source: OECD. Argentina, China, Colombia, India, Indonesia, Saudi Arabia, South Africa: UNESCO Institute for Statistics. Latvia: Eurostat. See Annex 3 for notes (www.oecd.org/education/education-at-a-glance-19991487.htm).

Please refer to the Reader's Guide for information concerning symbols for missing data and abbreviations.

StatLink sints http://dx.doi.org/10.1787/888933284850 
Table A3.2. Profile of a first-time tertiary graduate (2013)

\begin{tabular}{|c|c|c|c|c|c|c|}
\hline & \multirow[b]{2}{*}{$\begin{array}{c}\text { Share } \\
\text { of female graduates }\end{array}$} & \multirow{2}{*}{$\begin{array}{l}\text { Share of graduates } \\
\text { below the typical } \\
\text { age of } 30\end{array}$} & \multirow{2}{*}{$\begin{array}{c}\text { Share } \\
\text { of International } \\
\text { graduates }\end{array}$} & \multicolumn{3}{|c|}{ Share of first-time graduates by level of education } \\
\hline & & & & $\begin{array}{l}\text { Short tertiary } \\
\text { (2-3 years) }\end{array}$ & $\begin{array}{c}\text { Bachelor's } \\
\text { or equivalent }\end{array}$ & $\begin{array}{c}\text { Master's } \\
\text { or equivalent }\end{array}$ \\
\hline & (1) & (2) & (3) & (4) & (5) & (6) \\
\hline Australia & 57 & 84 & 42 & 6 & 77 & 17 \\
\hline Austria & 57 & 85 & 14 & 47 & 29 & 24 \\
\hline Belgium & $\mathrm{m}$ & $\mathrm{m}$ & $\mathrm{m}$ & $\mathrm{m}$ & $\mathrm{m}$ & $\mathrm{m}$ \\
\hline Canada & $\mathrm{m}$ & $\mathrm{m}$ & $\mathrm{m}$ & $\mathrm{m}$ & $\mathrm{m}$ & $\mathrm{m}$ \\
\hline Chile & 58 & 74 & $\mathrm{~m}$ & 39 & 56 & 5 \\
\hline Czech Republic & 63 & 81 & 9 & 1 & 88 & 11 \\
\hline Denmark & 58 & 84 & 14 & 18 & 75 & 7 \\
\hline Estonia & $\mathrm{m}$ & $\mathrm{m}$ & $\mathrm{m}$ & $\mathrm{m}$ & $\mathrm{m}$ & $\mathrm{m}$ \\
\hline Finland & 57 & 81 & 8 & $\mathrm{a}$ & 89 & 11 \\
\hline France & $\mathrm{m}$ & $\mathrm{m}$ & $\mathrm{m}$ & $\mathrm{m}$ & $\mathrm{m}$ & $\mathrm{m}$ \\
\hline Germany & 51 & 87 & 4 & 0 & 76 & 24 \\
\hline Greece & $\mathrm{m}$ & $\mathrm{m}$ & $\mathrm{m}$ & $\mathrm{m}$ & $\mathrm{m}$ & $\mathrm{m}$ \\
\hline Hungary & $\mathrm{m}$ & $\mathrm{m}$ & $\mathrm{m}$ & $\mathrm{m}$ & $\mathrm{m}$ & $\mathrm{m}$ \\
\hline Iceland & $\mathrm{m}$ & $\mathrm{m}$ & $\mathrm{m}$ & $\mathrm{m}$ & $\mathrm{m}$ & $\mathrm{m}$ \\
\hline Ireland & $\mathrm{m}$ & $\mathrm{m}$ & $\mathrm{m}$ & $\mathrm{m}$ & $\mathrm{m}$ & $\mathrm{m}$ \\
\hline Israel & $\mathrm{m}$ & $\mathrm{m}$ & $\mathrm{m}$ & $\mathrm{m}$ & $\mathrm{m}$ & $\mathrm{a}$ \\
\hline Italy & 59 & 86 & $\mathrm{~m}$ & 2 & 80 & 18 \\
\hline Japan & 51 & $\mathrm{~m}$ & 4 & 34 & 63 & 2 \\
\hline Korea & $\mathrm{m}$ & $\mathrm{m}$ & $\mathrm{m}$ & $\mathrm{m}$ & $\mathrm{m}$ & $\mathrm{m}$ \\
\hline Luxembourg & 55 & 86 & 40 & 11 & 55 & 34 \\
\hline Mexico & $\mathrm{m}$ & $\mathrm{m}$ & $\mathrm{m}$ & $\mathrm{m}$ & $\mathrm{m}$ & $\mathrm{m}$ \\
\hline Netherlands & 57 & 92 & 15 & 1 & 91 & 8 \\
\hline New Zealand & 58 & 75 & 22 & 31 & 67 & 3 \\
\hline Norway & 60 & 81 & 2 & 9 & 81 & 10 \\
\hline Poland & $\mathrm{m}$ & $\mathrm{m}$ & $\mathrm{m}$ & $\mathrm{m}$ & $\mathrm{m}$ & $\mathrm{m}$ \\
\hline Portugal & 59 & 82 & 2 & $\mathrm{a}$ & 85 & 15 \\
\hline Slovak Republic & 64 & 82 & 5 & 2 & 93 & 5 \\
\hline Slovenia & 61 & 81 & 1 & 15 & 58 & 27 \\
\hline Spain & 55 & 82 & $\mathrm{~m}$ & 35 & 31 & 34 \\
\hline Sweden & 62 & 75 & 13 & 4 & 61 & 35 \\
\hline Switzerland & 49 & 75 & 7 & 4 & 95 & 1 \\
\hline Turkey $^{1}$ & 47 & 87 & 0 & 41 & 57 & 2 \\
\hline United Kingdom & 56 & 87 & 9 & 18 & 80 & 2 \\
\hline United States & 58 & $\mathrm{~m}$ & 3 & 42 & 58 & a \\
\hline OECD average & 57 & 82 & 11 & 18 & 69 & 14 \\
\hline EU21 average & 58 & 84 & 11 & 13 & 70 & 18 \\
\hline Argentina & $\mathrm{m}$ & $\mathrm{m}$ & $\mathrm{m}$ & $\mathrm{m}$ & $\mathrm{m}$ & $\mathrm{m}$ \\
\hline Brazil & $\mathrm{m}$ & $\mathrm{m}$ & $\mathrm{m}$ & $\mathrm{m}$ & $\mathrm{m}$ & $\mathrm{m}$ \\
\hline China & $\mathrm{m}$ & $\mathrm{m}$ & $\mathrm{m}$ & $\mathrm{m}$ & $\mathrm{m}$ & $\mathrm{m}$ \\
\hline Colombia & $\mathrm{m}$ & $\mathrm{m}$ & $\mathrm{m}$ & $\mathrm{m}$ & $\mathrm{m}$ & $\mathrm{a}$ \\
\hline India & $\mathrm{m}$ & $\mathrm{m}$ & $\mathrm{m}$ & $\mathrm{m}$ & $\mathrm{m}$ & $\mathrm{m}$ \\
\hline Indonesia & $\mathrm{m}$ & $\mathrm{m}$ & $\mathrm{m}$ & $\mathrm{m}$ & $\mathrm{m}$ & $\mathrm{m}$ \\
\hline Latvia & 69 & 74 & $\mathrm{~m}$ & 25 & 73 & 2 \\
\hline Russian Federation & 59 & $\mathrm{~m}$ & $\mathrm{~m}$ & 27 & 7 & 66 \\
\hline Saudi Arabia & $\mathrm{m}$ & $\mathrm{m}$ & $\mathrm{m}$ & $\mathrm{m}$ & $\mathrm{m}$ & $\mathrm{m}$ \\
\hline South Africa & $\mathrm{m}$ & $\mathrm{m}$ & $\mathrm{m}$ & $\mathrm{m}$ & $\mathrm{m}$ & a \\
\hline G20 average & $\mathrm{m}$ & $\mathrm{m}$ & $\mathrm{m}$ & $\mathrm{m}$ & $\mathrm{m}$ & $\mathrm{m}$ \\
\hline
\end{tabular}

1. Year of reference 2012.

Source: OECD. Argentina, China, Colombia, India, Indonesia, Saudi Arabia, South Africa: UNESCO Institute for Statistics. Latvia: Eurostat. See Annex 3 for notes (www.oecd.org/education/education-at-a-glance-19991487.htm).

Please refer to the Reader's Guide for information concerning symbols for missing data and abbreviations.

StatLink 제은 http://dx.doi.org/10.1787/888933284866 
Table A3.3. Distribution of all tertiary graduates, by field of education (2013)

\begin{tabular}{|c|c|c|c|c|c|c|c|c|}
\hline & Education & $\begin{array}{l}\text { Humanities } \\
\text { and arts }\end{array}$ & $\begin{array}{c}\text { Social sciences, } \\
\text { business } \\
\text { and law }\end{array}$ & Sciences & $\begin{array}{l}\text { Engineering, } \\
\text { manufacturing } \\
\text { and } \\
\text { construction }\end{array}$ & Agriculture & $\begin{array}{c}\text { Health } \\
\text { and welfare }\end{array}$ & Services \\
\hline & (1) & (2) & (3) & (4) & $(5)$ & (6) & (7) & (8) \\
\hline Australia & 8 & 10 & 44 & 8 & 8 & 1 & 18 & 3 \\
\hline Austria & 11 & 11 & 33 & 9 & 19 & 2 & 7 & 9 \\
\hline Belgium & 10 & 11 & 32 & 5 & 12 & 2 & 25 & 2 \\
\hline Chile & 16 & 4 & 28 & 5 & 14 & 2 & 22 & 9 \\
\hline Czech Republic & 12 & 8 & 36 & 11 & 13 & 4 & 10 & 5 \\
\hline Denmark & 7 & 12 & 35 & 8 & 12 & 1 & 21 & 3 \\
\hline Estonia & 8 & 13 & 31 & 11 & 13 & 2 & 12 & 8 \\
\hline Finland & 6 & 13 & 25 & 7 & 21 & 2 & 20 & 6 \\
\hline France & 3 & 9 & 43 & 9 & 15 & 1 & 16 & 4 \\
\hline Germany & 11 & 13 & 29 & 14 & 20 & 2 & 8 & 4 \\
\hline Hungary & 14 & 9 & 43 & 6 & 11 & 2 & 8 & 8 \\
\hline Iceland & $\mathrm{m}$ & $\mathrm{m}$ & $\mathrm{m}$ & $\mathrm{m}$ & $\mathrm{m}$ & $\mathrm{m}$ & $\mathrm{m}$ & $\mathrm{m}$ \\
\hline Ireland & 9 & 13 & 31 & 11 & 12 & 1 & 16 & 6 \\
\hline Israel & $\mathrm{m}$ & $\mathrm{m}$ & $\mathrm{m}$ & $\mathrm{m}$ & $\mathrm{m}$ & $\mathrm{m}$ & $\mathrm{m}$ & $\mathrm{m}$ \\
\hline Italy & 5 & 17 & 33 & 8 & 16 & 2 & 16 & 3 \\
\hline Japan & 8 & 15 & 29 & 3 & 18 & 3 & 15 & 9 \\
\hline Korea & 7 & 18 & 22 & 7 & 24 & 1 & 14 & 7 \\
\hline Luxembourg & 24 & 8 & 48 & 10 & 6 & 0 & 4 & 0 \\
\hline Mexico & 12 & 4 & 44 & 5 & 22 & 2 & 9 & 1 \\
\hline Netherlands & 12 & 9 & 40 & 6 & 8 & 1 & 19 & 5 \\
\hline New Zealand & 12 & 14 & 33 & 12 & 7 & 1 & 15 & 5 \\
\hline Norway & 17 & 10 & 25 & 7 & 13 & 1 & 21 & 6 \\
\hline Poland & $\mathrm{m}$ & $\mathrm{m}$ & $\mathrm{m}$ & $\mathrm{m}$ & $\mathrm{m}$ & $\mathrm{m}$ & $\mathrm{m}$ & $\mathrm{m}$ \\
\hline Portugal & 9 & 9 & 31 & 8 & 18 & 1 & 17 & 6 \\
\hline Slovak Republic & 13 & 7 & 32 & 7 & 13 & 2 & 19 & 7 \\
\hline Sweden & 13 & 6 & 29 & 8 & 18 & 1 & 23 & 3 \\
\hline Switzerland & 10 & 9 & 37 & 8 & 14 & 2 & 13 & 8 \\
\hline Turkey $^{1}$ & 10 & 8 & 47 & 9 & 12 & 3 & 6 & 5 \\
\hline United Kingdom & 10 & 16 & 30 & 16 & 9 & 1 & 16 & 2 \\
\hline United States & 8 & 21 & 32 & 8 & 6 & 1 & 16 & 7 \\
\hline
\end{tabular}

\begin{tabular}{l|l|l|l|l|l|l|l|l|l|l|l|} 
OECD average & 10 & 11 & 34 & 9 & 15 & 2 \\
EU21 average & 10 & 11 & 34 & 9 & 14 & 2 \\
\hline
\end{tabular}

\begin{tabular}{|c|c|c|c|c|c|c|c|c|}
\hline $\begin{array}{l}\text { Argentina } \\
\text { Eั้ Brazil }\end{array}$ & $\begin{array}{l}\mathrm{m} \\
\mathrm{m}\end{array}$ & $\begin{array}{l}\mathrm{m} \\
\mathrm{m}\end{array}$ & $\begin{array}{l}\mathrm{m} \\
\mathrm{m}\end{array}$ & $\begin{array}{l}\mathrm{m} \\
\mathrm{m}\end{array}$ & $\begin{array}{l}\mathrm{m} \\
\mathrm{m}\end{array}$ & $\begin{array}{l}\mathrm{m} \\
\mathrm{m}\end{array}$ & $\begin{array}{l}\mathrm{m} \\
\mathrm{m}\end{array}$ & $\begin{array}{l}\mathrm{m} \\
\mathrm{m}\end{array}$ \\
\hline $\begin{array}{l}\text { Colombia } \\
\text { China }\end{array}$ & $\begin{array}{r}\mathrm{m} \\
8\end{array}$ & $\begin{array}{r}\mathrm{m} \\
3\end{array}$ & $\begin{array}{r}\mathrm{m} \\
53\end{array}$ & $\begin{array}{r}\mathrm{m} \\
4\end{array}$ & $\begin{array}{r}\mathrm{m} \\
17\end{array}$ & $\begin{array}{r}\mathrm{m} \\
2\end{array}$ & $\begin{array}{r}\mathrm{m} \\
7\end{array}$ & $\begin{array}{r}\mathrm{m} \\
4\end{array}$ \\
\hline $\begin{array}{l}\text { India } \\
\text { Indonesia }\end{array}$ & $\begin{array}{l}\mathrm{m} \\
\mathrm{m}\end{array}$ & $\begin{array}{l}\mathrm{m} \\
\mathrm{m}\end{array}$ & $\begin{array}{l}\mathrm{m} \\
\mathrm{m}\end{array}$ & $\begin{array}{l}\mathrm{m} \\
\mathrm{m}\end{array}$ & $\begin{array}{l}\mathrm{m} \\
\mathrm{m}\end{array}$ & $\begin{array}{l}\mathrm{m} \\
\mathrm{m}\end{array}$ & $\begin{array}{l}\mathrm{m} \\
\mathrm{m}\end{array}$ & $\begin{array}{l}\mathrm{m} \\
\mathrm{m}\end{array}$ \\
\hline $\begin{array}{l}\text { Latvia } \\
\text { Russian Federation }\end{array}$ & $\begin{array}{l}7 \\
8\end{array}$ & $\begin{array}{l}8 \\
4\end{array}$ & $\begin{array}{l}40 \\
50\end{array}$ & $\begin{array}{l}6 \\
6\end{array}$ & $\begin{array}{l}12 \\
21\end{array}$ & $\begin{array}{l}1 \\
1\end{array}$ & $\begin{array}{r}18 \\
5\end{array}$ & $\begin{array}{l}7 \\
5\end{array}$ \\
\hline G20 average & $\mathrm{m}$ & $\mathrm{m}$ & $\mathrm{m}$ & $\mathrm{m}$ & $\mathrm{m}$ & $\mathrm{m}$ & $\mathrm{m}$ & $\mathrm{m}$ \\
\hline
\end{tabular}

Note: Tertiary graduates include short-cycle tertiary, bachelor's or equivalent, master's or equivalent and doctorate.

1. Year of reference 2012.

Source: OECD. Argentina, China, Colombia, India, Indonesia, Saudi Arabia, South Africa: UNESCO Institute for Statistics. Latvia: Eurostat. See Annex 3 for notes (www.oecd.org/education/education-at-a-glance-19991487.htm).

Please refer to the Reader's Guide for information concerning symbols for missing data and abbreviations.

StatLink 젝ㄴ http://dx.doi.org/10.1787/888933284873 
Table A3.4. Percentage of female and international first-time graduates, by tertiary ISCED level (2013)

\begin{tabular}{|c|c|c|c|c|c|c|c|c|}
\hline & \multicolumn{4}{|c|}{ Percentage of female graduates } & \multicolumn{4}{|c|}{ Percentage of international graduates } \\
\hline & $\begin{array}{l}\text { Short tertiary } \\
(2-3 \text { years })\end{array}$ & $\begin{array}{c}\text { Bachelor's } \\
\text { or equivalent }\end{array}$ & $\begin{array}{c}\text { Master's } \\
\text { or equivalent }\end{array}$ & $\begin{array}{c}\text { Doctoral } \\
\text { or equivalent }\end{array}$ & $\begin{array}{l}\text { Short tertiary } \\
(2-3 \text { years })\end{array}$ & $\begin{array}{l}\text { Bachelor's } \\
\text { or equivalent }\end{array}$ & $\begin{array}{l}\text { Master's } \\
\text { or equivalent }\end{array}$ & $\begin{array}{c}\text { Doctoral } \\
\text { or equivalent }\end{array}$ \\
\hline & (1) & (2) & (3) & (4) & (5) & (6) & (7) & (8) \\
\hline Australia & 58 & 58 & 54 & 50 & 9 & 30 & 57 & 36 \\
\hline Austria & 54 & 60 & 55 & 44 & 1 & 14 & 18 & 29 \\
\hline Belgium & $\mathrm{m}$ & 60 & 56 & 42 & $\mathrm{~m}$ & 6 & 26 & 46 \\
\hline Canada $^{1}$ & 56 & 60 & 56 & 46 & 13 & 9 & 17 & 16 \\
\hline Chile & 61 & 54 & 55 & 45 & $\mathrm{~m}$ & $\mathrm{~m}$ & $\mathrm{~m}$ & $\mathrm{~m}$ \\
\hline Czech Republic & 66 & 63 & 61 & 43 & 3 & 7 & 10 & 13 \\
\hline Denmark & 48 & 60 & 56 & 45 & 16 & 8 & 18 & 31 \\
\hline Estonia & $\mathrm{a}$ & $\mathrm{m}$ & $\mathrm{m}$ & 60 & a & $\mathrm{m}$ & $\mathrm{m}$ & $\mathrm{m}$ \\
\hline Finland & a & 59 & 60 & 51 & a & 5 & 9 & 21 \\
\hline France & $\mathrm{m}$ & $\mathrm{m}$ & $\mathrm{m}$ & 44 & $\mathrm{~m}$ & $\mathrm{~m}$ & $\mathrm{~m}$ & $\mathrm{~m}$ \\
\hline Germany & 75 & 49 & 53 & 44 & 0 & 3 & 10 & 15 \\
\hline Greece & a & $\mathrm{m}$ & $\mathrm{m}$ & 45 & a & $\mathrm{m}$ & $\mathrm{m}$ & $\mathrm{m}$ \\
\hline Hungary & 69 & 61 & 61 & 46 & 0 & 3 & 4 & 7 \\
\hline Iceland & $\mathrm{m}$ & $\mathrm{m}$ & $\mathrm{m}$ & $\mathrm{m}$ & $\mathrm{m}$ & $\mathrm{m}$ & $\mathrm{m}$ & $\mathrm{m}$ \\
\hline Ireland & $\mathrm{m}$ & $\mathrm{m}$ & $\mathrm{m}$ & 49 & $\mathrm{~m}$ & $\mathrm{~m}$ & $\mathrm{~m}$ & $\mathrm{~m}$ \\
\hline Israel & $\mathrm{m}$ & 59 & 60 & 52 & $\mathrm{~m}$ & 3 & 3 & 3 \\
\hline Italy & 24 & 59 & 60 & 52 & $\mathrm{~m}$ & $\mathrm{~m}$ & $\mathrm{~m}$ & $\mathrm{~m}$ \\
\hline Japan & 62 & 45 & 33 & 30 & 4 & 2 & 9 & 19 \\
\hline Korea & $\mathrm{m}$ & $\mathrm{m}$ & $\mathrm{m}$ & 34 & $\mathrm{~m}$ & $\mathrm{~m}$ & $\mathrm{~m}$ & $\mathrm{~m}$ \\
\hline Luxembourg & 59 & 56 & 51 & 39 & 46 & 21 & 71 & 81 \\
\hline Mexico & 42 & 53 & 55 & 48 & $\mathrm{~m}$ & $\mathrm{~m}$ & $\mathrm{~m}$ & $\mathrm{~m}$ \\
\hline Netherlands & 51 & 57 & $\mathrm{~m}$ & 46 & a & 10 & $\mathrm{~m}$ & 40 \\
\hline New Zealand & 54 & 61 & 55 & 50 & 23 & 18 & 29 & 46 \\
\hline Norway & 24 & 64 & 58 & 48 & 0 & 2 & 11 & 30 \\
\hline Poland & 84 & $\mathrm{~m}$ & $\mathrm{~m}$ & 55 & $\mathrm{~m}$ & $\mathrm{~m}$ & $\mathrm{~m}$ & $\mathrm{~m}$ \\
\hline Portugal & $\mathrm{a}$ & 60 & 61 & 55 & a & 2 & 5 & 11 \\
\hline Slovak Republic & 70 & 63 & 64 & 51 & 1 & 4 & 4 & 6 \\
\hline Slovenia & 48 & 63 & 65 & 55 & 0 & 1 & 2 & 4 \\
\hline Spain & 52 & 58 & 56 & 50 & $\mathrm{~m}$ & 1 & 5 & $\mathrm{~m}$ \\
\hline Sweden & 55 & 69 & 55 & 46 & 0 & 3 & 25 & 32 \\
\hline Switzerland & 59 & 49 & 49 & 44 & a & 7 & 23 & 51 \\
\hline Turkey $^{1}$ & 45 & 49 & 48 & 45 & 0 & 1 & 2 & 3 \\
\hline United Kingdom & 57 & 56 & 58 & 46 & 6 & 15 & 45 & 44 \\
\hline United States & 61 & 57 & 58 & 49 & 2 & 3 & 11 & 27 \\
\hline OECD average & 56 & 58 & 56 & 47 & $\mathrm{~m}$ & 7 & 18 & 27 \\
\hline EU21 average & 58 & 60 & 58 & 48 & $\mathrm{~m}$ & 7 & 18 & 27 \\
\hline
\end{tabular}

\begin{tabular}{|c|c|c|c|c|c|c|c|c|}
\hline $\begin{array}{l}\text { Argentina } \\
\text { Brazil }\end{array}$ & $\begin{array}{l}\mathrm{m} \\
\mathrm{m}\end{array}$ & $\begin{array}{l}\mathrm{m} \\
\mathrm{m}\end{array}$ & $\begin{array}{l}\mathrm{m} \\
\mathrm{m}\end{array}$ & $\begin{array}{l}\mathrm{m} \\
\mathrm{m}\end{array}$ & $\begin{array}{l}\mathrm{m} \\
\mathrm{m}\end{array}$ & $\begin{array}{l}\mathrm{m} \\
\mathrm{m}\end{array}$ & $\begin{array}{l}\mathrm{m} \\
\mathrm{m}\end{array}$ & $\begin{array}{l}\mathrm{m} \\
\mathrm{m}\end{array}$ \\
\hline $\begin{array}{l}\text { China } \\
\text { Colombia }\end{array}$ & $\begin{array}{l}52 \\
51\end{array}$ & $\begin{array}{l}50 \\
58\end{array}$ & $\begin{array}{l}49 \\
57\end{array}$ & $\begin{array}{l}37 \\
40\end{array}$ & $\begin{array}{c}0 \\
\mathrm{~m}\end{array}$ & $\begin{array}{c}0 \\
\mathrm{~m}\end{array}$ & $\begin{array}{c}1 \\
\mathrm{~m}\end{array}$ & $\begin{array}{c}2 \\
\mathrm{~m}\end{array}$ \\
\hline $\begin{array}{l}\text { India } \\
\text { Indonesia }\end{array}$ & $\begin{array}{l}\mathrm{m} \\
\mathrm{m}\end{array}$ & $\begin{array}{c}\mathrm{m} \\
51\end{array}$ & $\begin{array}{l}\mathrm{m} \\
48\end{array}$ & $\begin{array}{r}\mathrm{m} \\
41\end{array}$ & $\begin{array}{l}\mathrm{m} \\
\mathrm{m}\end{array}$ & $\begin{array}{l}\mathrm{m} \\
\mathrm{m}\end{array}$ & $\begin{array}{l}\mathrm{m} \\
\mathrm{m}\end{array}$ & $\begin{array}{l}\mathrm{m} \\
\mathrm{m}\end{array}$ \\
\hline $\begin{array}{l}\text { Latvia } \\
\text { Russian Federation }\end{array}$ & $\begin{array}{l}71 \\
53\end{array}$ & $\begin{array}{l}68 \\
59\end{array}$ & $\begin{array}{l}69 \\
61\end{array}$ & $\begin{array}{l}57 \\
44\end{array}$ & $\begin{array}{l}\mathrm{m} \\
\mathrm{m}\end{array}$ & $\begin{array}{l}\mathrm{m} \\
\mathrm{m}\end{array}$ & $\begin{array}{l}\mathrm{m} \\
\mathrm{m}\end{array}$ & $\begin{array}{l}\mathrm{m} \\
\mathrm{m}\end{array}$ \\
\hline $\begin{array}{l}\text { Saudi Arabia } \\
\text { South Africa }^{1}\end{array}$ & $\begin{array}{l}23 \\
62\end{array}$ & $\begin{array}{l}60 \\
60\end{array}$ & $\begin{array}{l}40 \\
47\end{array}$ & $\begin{array}{l}24 \\
42\end{array}$ & $\begin{array}{l}\mathrm{m} \\
\mathrm{m}\end{array}$ & $\begin{array}{l}\mathrm{m} \\
\mathrm{m}\end{array}$ & $\begin{array}{l}\mathrm{m} \\
\mathrm{m}\end{array}$ & $\begin{array}{l}\mathrm{m} \\
\mathrm{m}\end{array}$ \\
\hline G20 average & 52 & 55 & 51 & 42 & 4 & 8 & 19 & 20 \\
\hline
\end{tabular}

1. Year of reference 2012.

Source: OECD. Argentina, China, Colombia, India, Indonesia, Saudi Arabia, South Africa: UNESCO Institute for Statistics. Latvia: Eurostat. See Annex 3 for notes (www.oecd.org/education/education-at-a-glance-19991487.htm).

Please refer to the Reader's Guide for information concerning symbols for missing data and abbreviations.

StatLink 게이 http://dx.doi.org/10.1787/888933284886 
Table A3.5. Percentage of all students and international students who graduate from sciences and engineering programmes, by tertiary ISCED level (2013)

\begin{tabular}{|c|c|c|c|c|c|c|c|c|c|c|c|c|c|c|c|c|}
\hline & \multicolumn{8}{|c|}{$\begin{array}{l}\text { Percentage of students who graduate from sciences } \\
\text { and engineering programmes }\end{array}$} & \multicolumn{8}{|c|}{$\begin{array}{l}\text { Percentage of international students who graduate from sciences } \\
\text { and engineering programmes }\end{array}$} \\
\hline & \multicolumn{4}{|c|}{ Sciences } & \multicolumn{4}{|c|}{$\begin{array}{l}\text { Engineering, manufacturing } \\
\text { and construction }\end{array}$} & \multicolumn{4}{|c|}{ Sciences } & \multicolumn{4}{|c|}{$\begin{array}{l}\text { Engineering, manufacturing } \\
\text { and construction }\end{array}$} \\
\hline & 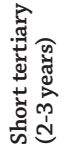 & 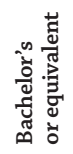 & 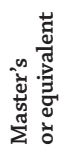 & 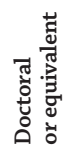 & 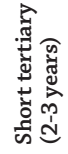 & 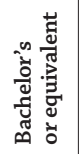 & 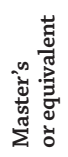 & 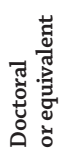 & 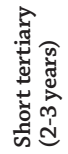 & 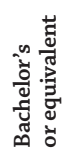 & 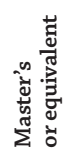 & 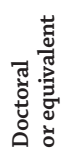 & 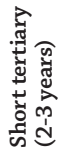 & 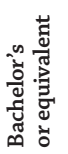 & 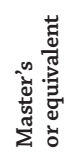 & 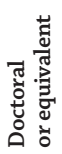 \\
\hline & (1) & (2) & (3) & (4) & (5) & (6) & (7) & (8) & (9) & $(10)$ & (11) & (12) & (13) & (14) & (15) & (16) \\
\hline Australia & 5 & 9 & 7 & 24 & 9 & 7 & 9 & 15 & 7 & 9 & 10 & 27 & 11 & 9 & 10 & 22 \\
\hline Austria & 4 & 12 & 10 & 28 & 31 & 14 & 12 & 20 & 0 & 11 & 8 & 34 & 30 & 11 & 10 & 20 \\
\hline Belgium & 0 & 4 & 7 & 21 & 0 & 11 & 14 & 26 & $\mathrm{~m}$ & 2 & 10 & 22 & $\mathrm{~m}$ & 8 & 13 & 30 \\
\hline Canada $^{1}$ & 5 & 13 & 10 & 37 & 13 & 8 & 9 & 19 & 6 & 13 & 10 & 37 & 16 & 9 & 11 & 21 \\
\hline Chile & 4 & 5 & 3 & 40 & 15 & 17 & 3 & 19 & 3 & 6 & 7 & 42 & 16 & 18 & 8 & 33 \\
\hline Czech Republic & 0 & 10 & 10 & 29 & 0 & 12 & 15 & 19 & 0 & 15 & 13 & 37 & 0 & 10 & 11 & 20 \\
\hline Denmark & 6 & 6 & 13 & 18 & 23 & 10 & 10 & 26 & 7 & 8 & 13 & 19 & 19 & 24 & 15 & 42 \\
\hline Estonia & $\mathrm{a}$ & 10 & 12 & 35 & $\mathrm{a}$ & 11 & 18 & 16 & a & 1 & 14 & 25 & a & 0 & 17 & 33 \\
\hline Finland & a & 5 & 9 & 18 & $\mathrm{a}$ & 22 & 18 & 24 & a & 4 & 15 & 23 & a & 29 & 33 & 34 \\
\hline France & 3 & 12 & 10 & 48 & 22 & 8 & 17 & 13 & $\mathrm{~m}$ & $\mathrm{~m}$ & $\mathrm{~m}$ & $\mathrm{~m}$ & $\mathrm{~m}$ & $\mathrm{~m}$ & $\mathrm{~m}$ & $\mathrm{~m}$ \\
\hline Germany & 0 & 11 & 17 & 33 & 31 & 24 & 15 & 11 & a & 12 & 13 & 46 & a & 28 & 25 & 15 \\
\hline Greece & $\mathrm{a}$ & 11 & 17 & 21 & $\mathrm{a}$ & 19 & 15 & 18 & a & $\mathrm{m}$ & $\mathrm{m}$ & $\mathrm{m}$ & a & $\mathrm{m}$ & $\mathrm{m}$ & $\mathrm{m}$ \\
\hline Hungary & 9 & 5 & 6 & 28 & 3 & 12 & 12 & 9 & 3 & 6 & 3 & 35 & 13 & 9 & 4 & 8 \\
\hline Iceland & $\mathrm{m}$ & $\mathrm{m}$ & $\mathrm{m}$ & $\mathrm{m}$ & $\mathrm{m}$ & $\mathrm{m}$ & $\mathrm{m}$ & $\mathrm{m}$ & $\mathrm{m}$ & $\mathrm{m}$ & $\mathrm{m}$ & $\mathrm{m}$ & $\mathrm{m}$ & $\mathrm{m}$ & $\mathrm{m}$ & $\mathrm{m}$ \\
\hline Ireland & 12 & 11 & 9 & 31 & 20 & 12 & 4 & 13 & $\mathrm{~m}$ & $\mathrm{~m}$ & $\mathrm{~m}$ & $\mathrm{~m}$ & $\mathrm{~m}$ & $\mathrm{~m}$ & $\mathrm{~m}$ & $\mathrm{~m}$ \\
\hline Israel & $\mathrm{m}$ & 8 & 7 & 43 & $\mathrm{~m}$ & 13 & 5 & 9 & $\mathrm{~m}$ & 8 & 8 & 41 & $\mathrm{~m}$ & 11 & 4 & 18 \\
\hline Italy & 10 & 8 & 7 & 26 & 69 & 15 & 18 & 20 & $\mathrm{~m}$ & $\mathrm{~m}$ & $\mathrm{~m}$ & $\mathrm{~m}$ & $\mathrm{~m}$ & $\mathrm{~m}$ & $\mathrm{~m}$ & $\mathrm{~m}$ \\
\hline Japan & 0 & 4 & 10 & 15 & 15 & 17 & 33 & 23 & $\mathrm{~m}$ & $\mathrm{~m}$ & $\mathrm{~m}$ & $\mathrm{~m}$ & $\mathrm{~m}$ & $\mathrm{~m}$ & $\mathrm{~m}$ & $\mathrm{~m}$ \\
\hline Korea & 2 & 10 & 6 & 13 & 28 & 23 & 17 & 26 & 1 & 4 & 5 & 25 & 37 & 12 & 16 & 30 \\
\hline Luxembourg & 1 & 6 & 12 & 39 & 10 & 7 & 4 & 14 & 0 & 5 & 15 & 44 & 0 & 2 & 4 & 13 \\
\hline Mexico & 1 & 6 & 4 & 15 & 52 & 22 & 7 & 13 & $\mathrm{~m}$ & $\mathrm{~m}$ & $\mathrm{~m}$ & $\mathrm{~m}$ & $\mathrm{~m}$ & $\mathrm{~m}$ & $\mathrm{~m}$ & $\mathrm{~m}$ \\
\hline Netherlands & 2 & 6 & 6 & 15 & 6 & 8 & 8 & 19 & a & 3 & 9 & $\mathrm{~m}$ & a & 5 & 11 & $\mathrm{~m}$ \\
\hline New Zealand & 12 & 12 & 13 & 33 & 6 & 6 & 15 & 14 & 15 & 16 & 16 & 37 & 6 & 7 & 19 & 17 \\
\hline Norway & 3 & 5 & 10 & 32 & 55 & 8 & 14 & 10 & 0 & 6 & 18 & 52 & 50 & 5 & 19 & 20 \\
\hline Poland & 0 & 7 & 6 & $\mathrm{~m}$ & 0 & 11 & 12 & $\mathrm{~m}$ & $\mathrm{a}$ & $4^{\mathrm{d}}$ & $\mathrm{x}(10)$ & $\mathrm{m}$ & a & $6^{\mathrm{d}}$ & $\mathrm{x}(14)$ & $\mathrm{m}$ \\
\hline Portugal & a & 6 & 8 & 22 & a & 18 & 18 & 21 & $\mathrm{a}$ & 6 & 8 & 26 & a & 21 & 16 & 20 \\
\hline Slovak Republic & 2 & 8 & 7 & 16 & 3 & 13 & 13 & 25 & 0 & 2 & 2 & 7 & 0 & 9 & 3 & 15 \\
\hline Slovenia & 6 & 10 & 8 & 22 & 24 & 15 & 15 & 18 & 0 & 10 & 8 & 41 & 17 & 16 & 14 & 18 \\
\hline Spain & 7 & 7 & 10 & 36 & 19 & 20 & 12 & 9 & $\mathrm{~m}$ & 5 & 7 & $\mathrm{~m}$ & $\mathrm{~m}$ & 9 & 9 & $\mathrm{~m}$ \\
\hline Sweden & 9 & 6 & 8 & 24 & 28 & 10 & 24 & 27 & 14 & 12 & 20 & 32 & 21 & 15 & 37 & 38 \\
\hline Switzerland & 1 & 6 & 10 & 30 & 2 & 16 & 12 & 13 & a & 10 & 12 & 37 & a & 17 & 14 & 18 \\
\hline Turkey $^{1}$ & 7 & 9 & 10 & 13 & 19 & 8 & 9 & 7 & 2 & 10 & 12 & 16 & 9 & 16 & 17 & 5 \\
\hline United Kingdom & 12 & 20 & 11 & 33 & 8 & 9 & 10 & 14 & 9 & 14 & 11 & 29 & 10 & 15 & 13 & 19 \\
\hline United States & 5 & 11 & 6 & 26 & 7 & 6 & 6 & 15 & 6 & 13 & 18 & 35 & 4 & 12 & 21 & 31 \\
\hline OECD average & 5 & 8 & 9 & 27 & 19 & 13 & 13 & 17 & 4 & 8 & 11 & 32 & 15 & 12 & 14 & 23 \\
\hline EU21 average & 5 & 9 & 10 & 27 & 19 & 13 & 13 & 18 & 4 & 7 & 10 & 30 & 12 & 13 & 15 & 23 \\
\hline
\end{tabular}

\begin{tabular}{|c|c|c|c|c|c|c|c|c|c|c|c|c|c|c|c|c|}
\hline $\begin{array}{l}\text { Argentina } \\
\text { Brazil }\end{array}$ & $\begin{array}{c}\mathrm{m} \\
2\end{array}$ & $\begin{array}{c}\mathrm{m} \\
6\end{array}$ & $\begin{array}{l}\mathrm{m} \\
\mathrm{m}\end{array}$ & $\begin{array}{l}\mathrm{m} \\
\mathrm{m}\end{array}$ & $\begin{array}{r}\mathrm{m} \\
0\end{array}$ & $\begin{array}{r}\mathrm{m} \\
8\end{array}$ & $\begin{array}{l}\mathrm{m} \\
\mathrm{m}\end{array}$ & $\begin{array}{l}\mathrm{m} \\
\mathrm{m}\end{array}$ & $\begin{array}{r}\mathrm{m} \\
0\end{array}$ & $\begin{array}{r}\mathrm{m} \\
6\end{array}$ & $\begin{array}{l}\mathrm{m} \\
\mathrm{m}\end{array}$ & $\begin{array}{l}\mathrm{m} \\
\mathrm{m}\end{array}$ & $\begin{array}{c}\mathrm{m} \\
0\end{array}$ & $\begin{array}{c}\mathrm{m} \\
14\end{array}$ & $\begin{array}{l}\mathrm{m} \\
\mathrm{m}\end{array}$ & $\begin{array}{l}\mathrm{m} \\
\mathrm{m}\end{array}$ \\
\hline China & $\mathrm{m}$ & $\mathrm{m}$ & $\mathrm{m}$ & $\mathrm{m}$ & $\mathrm{m}$ & $\mathrm{m}$ & $\mathrm{m}$ & $\mathrm{m}$ & $\mathrm{m}$ & $\mathrm{m}$ & $\mathrm{m}$ & $\mathrm{m}$ & $\mathrm{m}$ & $\mathrm{m}$ & $\mathrm{m}$ & $\mathrm{m}$ \\
\hline Colombia & 8 & 2 & 2 & 24 & 18 & 22 & 6 & 23 & $\mathrm{~m}$ & $\mathrm{~m}$ & $\mathrm{~m}$ & $\mathrm{~m}$ & $\mathrm{~m}$ & $\mathrm{~m}$ & $\mathrm{~m}$ & $\mathrm{~m}$ \\
\hline India & $\mathrm{m}$ & $\mathrm{m}$ & $\mathrm{m}$ & $\mathrm{m}$ & $\mathrm{m}$ & $\mathrm{m}$ & $\mathrm{m}$ & $\mathrm{m}$ & $\mathrm{m}$ & $\mathrm{m}$ & $\mathrm{m}$ & $\mathrm{m}$ & $\mathrm{m}$ & $\mathrm{m}$ & $\mathrm{m}$ & $\mathrm{m}$ \\
\hline Indonesia & $\mathrm{m}$ & $\mathrm{m}$ & $\mathrm{m}$ & $\mathrm{m}$ & $\mathrm{m}$ & $\mathrm{m}$ & $\mathrm{m}$ & $\mathrm{m}$ & $\mathrm{m}$ & $\mathrm{m}$ & $\mathrm{m}$ & $\mathrm{m}$ & $\mathrm{m}$ & $\mathrm{m}$ & $\mathrm{m}$ & $\mathrm{m}$ \\
\hline Latvia & 4 & 5 & 9 & 22 & 9 & 12 & 14 & 18 & 0 & 2 & 2 & 0 & 14 & 3 & 4 & 0 \\
\hline Russian Federation & 6 & 10 & 5 & 25 & 32 & 14 & 17 & 16 & $\mathrm{~m}$ & $\mathrm{~m}$ & $\mathrm{~m}$ & $\mathrm{~m}$ & $\mathrm{~m}$ & $\mathrm{~m}$ & $\mathrm{~m}$ & $\mathrm{~m}$ \\
\hline Saudi Arabia & 21 & 18 & 6 & 6 & 26 & 5 & 2 & 4 & $\mathrm{~m}$ & $\mathrm{~m}$ & $\mathrm{~m}$ & $\mathrm{~m}$ & $\mathrm{~m}$ & $\mathrm{~m}$ & $\mathrm{~m}$ & $\mathrm{~m}$ \\
\hline South Africa ${ }^{1}$ & 10 & 11 & 13 & 30 & 9 & 7 & 11 & 8 & $\mathrm{~m}$ & $\mathrm{~m}$ & $\mathrm{~m}$ & $\mathrm{~m}$ & $\mathrm{~m}$ & $\mathrm{~m}$ & $\mathrm{~m}$ & $\mathrm{~m}$ \\
\hline
\end{tabular}

\begin{tabular}{l|c|c|c|c|c|c|c|c|c|c|c|c|c|c|c|c|} 
G20 average & $\mathrm{m}$ & $\mathrm{m}$ & $\mathrm{m}$ & $\mathrm{m}$ & $\mathrm{m}$ & $\mathrm{m}$ & $\mathrm{m}$ & $\mathrm{m}$ & $\mathrm{m}$ & $\mathrm{m}$ & $\mathrm{m}$ & $\mathrm{m}$ & $\mathrm{m}$ & $\mathrm{m}$ & $\mathrm{m}$ & $\mathrm{m}$ \\
\hline
\end{tabular}

1. Year of reference 2012.

Source: OECD. Argentina, China, Colombia, India, Indonesia, Saudi Arabia, South Africa: UNESCO Institute for Statistics. Latvia: Eurostat. See Annex 3 for notes (www.oecd.org/education/education-at-a-glance-19991487.htm).

Please refer to the Reader's Guide for information concerning symbols for missing data and abbreviations.

StatLink Nints http://dx.doi.org/10.1787/888933284899 



\section{TO WHAT EXTENT DOES PARENTS' EDUCATION INFLUENCE THEIR CHILDREN'S EDUCATIONAL ATTAINMENT?}

- On average across OECD countries and sub-national entities that participated in the Survey of Adult Skills (PIAAC) in 2012, 22\% of 25-34 year-old non-students - and in Korea, $47 \%$ of this group - have attained tertiary education even though their parents have not (upward mobility).

- First generation tertiary-educated adults and tertiary-educated adults whose parents also hold a tertiary degree share similar employment rates and pursue similar fields of study.

- When parents' education is taken into account, adults with tertiary education are 23 percentage points more likely than those with upper secondary or post-secondary non-tertiary education as their highest level of education to be among the top $25 \%$ in monthly earnings, on average.

\section{Chart A4.1. Intergenerational mobility in education (2012)}

Survey of Adult Skills, educational attainment of 25-34 year-old non-students

compared with their parents

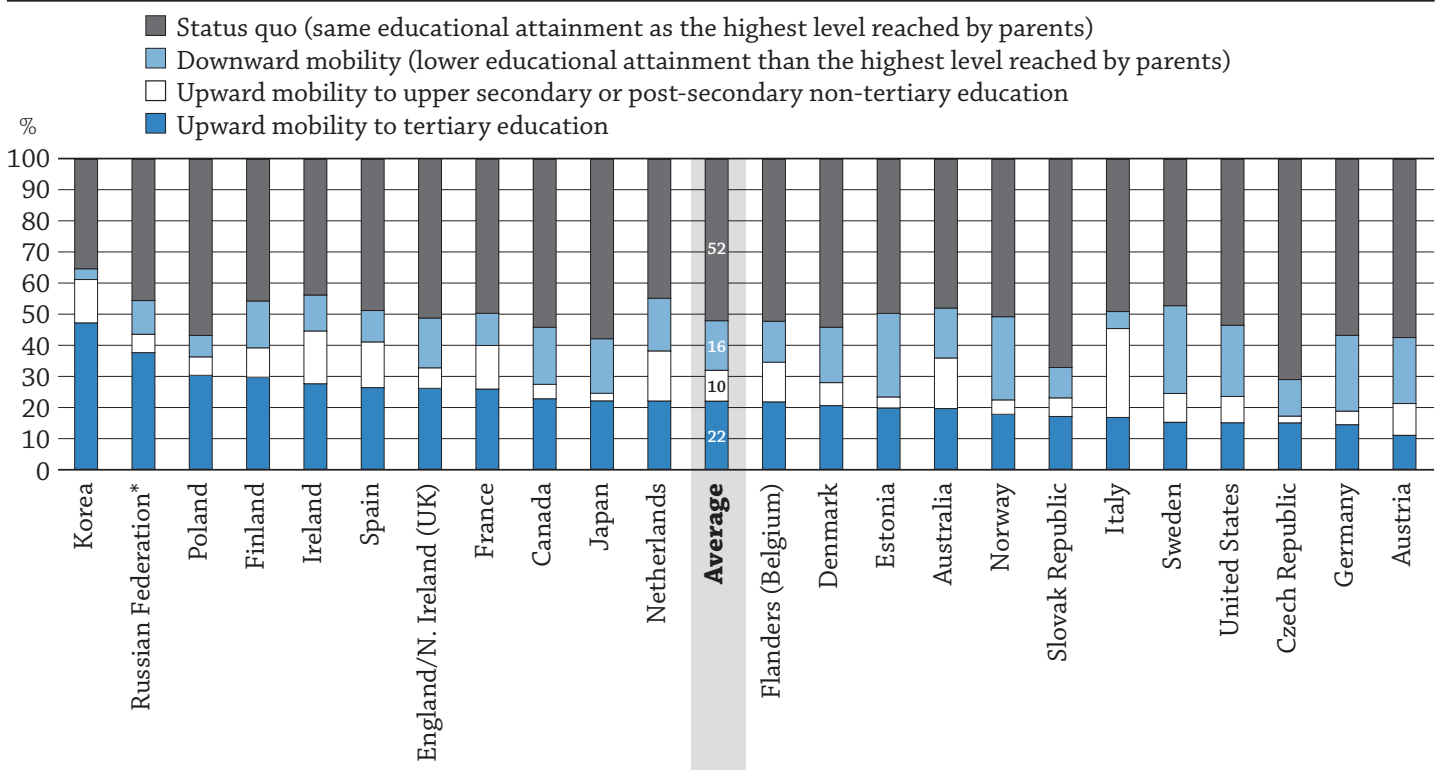

* See note on data for the Russian Federation in the Methodology section.

Countries are ranked in descending order of upward mobility to tertiary education among tertiary-educated 25-34 year-old non-students. Source: OECD. Table A4.1a. See Annex 3 for notes (www.oecd.org/education/education-at-a-glance- 19991487.htm).

StatLink 제이 http://dx.doi.org/10.1787/888933283540

\section{Context}

Because of its strong links to employment, earnings, overall wealth and the well-being of individuals, education can reduce inequalities in societies - but it can also perpetuate them. Giving all young people a fair chance to obtain a quality education is a fundamental part of the social contract. It is critically important to address inequalities in education opportunities in order to maintain social mobility and broaden the pool of candidates for higher education and high-skilled jobs. This indicator draws from the Survey of Adult Skills, a product of the OECD Programme for the International Assessment of Adult Competencies (PIAAC) (2012), to analyse the incidence of tertiary education among adults whose parents had not attained that level of education (first generation tertiary-educated adults) and intergenerational social mobility.

In today's fast-changing labour markets, the gap in returns to low- and high-qualified workers is growing. On average, less-educated adults have the highest unemployment and inactivity rates and have the lowest wages over their working lives (see Indicators A5 and A6). Having a large population of low-qualified workers may thus lead to a heavier social burden and deepening inequalities that are both difficult and costly to address once people have left initial education. 
PISA 2012 results show that in several countries that have designed and implemented policies with a stronger focus on equity, students from disadvantaged backgrounds have improved their performance at school. A significant number of countries that underperformed in 2003 improved their PISA scores markedly by 2012. In several of these countries, the improvement was mainly due to giving more students higher-quality education (OECD, 2013). Various policy options, such as maintaining reasonable costs for higher education and funding student support systems can help disadvantaged students. Ensuring access to and success in tertiary education for all is important, but so is addressing inequalities at the earliest stages of schooling.

\section{Other findings}

- The opportunity for individuals to attain tertiary education and surpass their parents' education has stayed the same or increased over time in most countries. Among individuals whose parents' highest level of education was upper secondary or post-secondary non-tertiary, 38\% of 25-34 year-olds attained tertiary education, on average, as did $37 \%$ of $35-44$ year-olds, $36 \%$ of $45-54$ year-olds, and $34 \%$ of $55-64$ year-olds.

- In some countries, it is natural that a large proportion of younger adults appears unlikely to surpass their parents in educational attainment because many of those parents have already attained tertiary education, leaving no room for upward mobility for their children. This kind of status quo in educational attainment is a positive outcome.

- On average, $88 \%$ of first generation tertiary-educated 25-34 year-old non-students are employed. In Flanders (Belgium), $98 \%$ of this group are employed. 


\section{Analysis}

\section{First generation tertiary-educated adults}

Intergenerational mobility in education, as measured by the OECD Programme for the International Assessment of Adult Competencies (PIAAC) (2012), refers to the proportion of individuals whose highest level of qualification is different from that of their parents: higher in the case of upward mobility, and lower in the case of downward mobility across generations. Status quo in education is when children attain the same level of education as their parents.

Chart A4.1. summarises the findings of educational mobility among 25-34 year-old non-students presented in Indicator A4 in Education at a Glance 2014, with an additional breakdown by level of upward mobility (Table A4.4 in OECD, 2014a). It shows that, across the countries and sub-national entities that participated in the Survey of Adult Skills, the majority of younger adults attained the same level of education as their parents (52\%). Some $16 \%$ of younger adults attained a lower level of education than their parents (downward mobility) while the remaining $32 \%$ were upwardly mobile compared to their parents, either attaining upper secondary or post-secondary non-tertiary education (10\%) or tertiary education (22\%). The latter group is referred to as first generation tertiary-educated adults (Table A4.1a).

In Korea, $47 \%$ of young adults surpassed their parents' educational attainment when they themselves attained tertiary education. This contrasts with Austria, the Czech Republic, Germany, Sweden and the United States, where $15 \%$ or less of younger adults are first generation tertiary-educated. In Austria, the Czech Republic and Germany, a large share of students choose vocational education, which may explain the low percentage of young adults who are first generation tertiary educated. In the United States, the proportion of tertiary-educated older adults is one of the highest among OECD countries, which leaves less room for upward mobility to tertiary education (Table A4.1a and see Table A1.3a).

As Indicator A1 shows, the level of education rose significantly in recent years, especially among younger adults. Over the past 30 years, almost all OECD countries have seen significant increases in the educational attainment of their populations, this can be observed by comparing the educational attainment of younger and older adults. By 2014, about one in three adults in OECD countries held a tertiary qualification, including qualifications from more technical tertiary programmes as well as from universities (see Table A1.4a).

As the share of adults whose parents have a tertiary education increases, fewer younger adults show upward educational mobility. This is why, on average across the countries and sub-national entities that participated in the Survey of Adult Skills, the proportion of younger adults with a higher level of education than their parents is shrinking. At the same time, when looking at the data in light of parents' educational attainment, opportunities to attain higher levels of education are growing (see Table A4.2 in OECD, 2014a).

As Chart A4.2 shows, there is a clear progression of educational attainment and a continuous increase in opportunities to attain tertiary education. The proportion of younger adults who completed tertiary education is larger than the proportion of older adults who did so, regardless of their parents' level of education (Table A4.2 in OECD, 2014a).

The results also show an increasing proportion of children of tertiary-educated parents who also attained tertiary education. On average, $60 \%$ of older adults attained tertiary education as their parents did, as did $65 \%$ of younger adults (see Table A4.2 in OECD, 2014a).

Chart A4.2 not only shows an increase in tertiary attainment across younger age groups, it also confirms the impact of parents' education on children's own attainment. The share of younger adults who complete tertiary education and whose parents completed below upper secondary as their highest level of education is increasing among younger adults, but remains small - 23\% among younger adults - compared to the proportion of younger adults of the same age who complete tertiary education and whose parents also completed that level of education (65\%) (see Table A4.2 in OECD, 2014a).

The differences, related to parents' educational attainment, in the proportions of individuals who completed tertiary education is stable across the generations. Some $23 \%$ of younger adults whose parents did not attain upper secondary education attained tertiary education themselves, compared with $65 \%$ of adults of the same age whose parents also attained tertiary education (a difference of 42 percentage points). Among 35-44 year-olds, this difference is 44 percentage points ( $24 \%$ and $68 \%$, respectively), it is 43 percentage points among $45-54$ year-olds ( $19 \%$ and $63 \%$, respectively) and 43 percentage points among older adults ( $17 \%$ and $60 \%$, respectively). These trends show that there is room for reducing inequities to allow a greater proportion of individuals whose parents have low educational attainment to complete tertiary education (see Table A4.2 in OECD, 2014a). 


\section{Chart A4.2. Percentage of non-students who completed tertiary education, by age group and parents' educational attainment (2012)}

Survey of Adult Skills, average

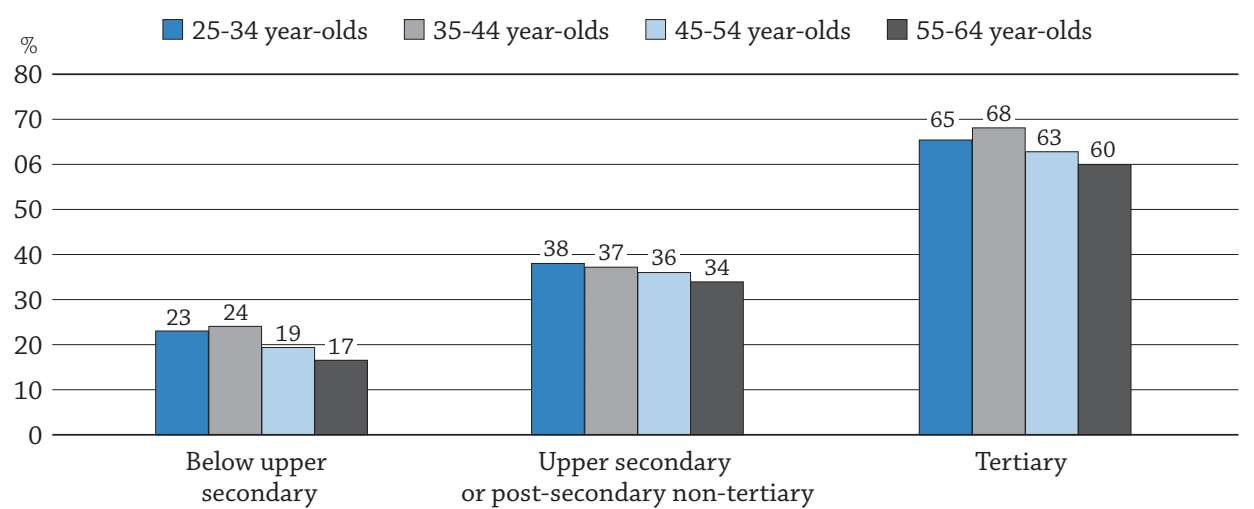

Source: OECD. Education at a Glance 2014: OECD Indicators. Table A4.2. See Annex 3 for notes (www.oecd.org/education/education-at-a-glance19991487.htm).

StatLink 需5 http://dx.doi.org/10.1787/888933283558

\section{Gender}

Chart A4.3 shows that in most countries and sub-national entities that participated in the Survey of Adult Skills in 2012, a larger proportion of women than men are first generation tertiary-educated, both among younger adults and among 35-44 year-olds. But the gap in favour of women is larger among 25-34 year-olds than among 35-44 year-oldsconfirming conclusions drawn from Indicator A1 on educational attainment that find a larger proportion of adult women than men complete tertiary education, with a particularly wide gender gap among younger adults. The Czech Republic is the only country with a difference of more than 15 percentage points in the proportion of first generation tertiary-educated women among younger adults (68\%) compared to that among 35-44 year-olds (37\%). The largest proportion of first generation tertiary-educated 25-34 year-old women is also found in the Czech Republic: a proportion that is 10 percentage points larger than the average (Table A4.2b, available on line).

Chart A4.3. First generation tertiary-educated women, by age group (2012) Survey of Adult Skills, 25-34 and 35-44 year-old first generation tertiary-educated non-student women

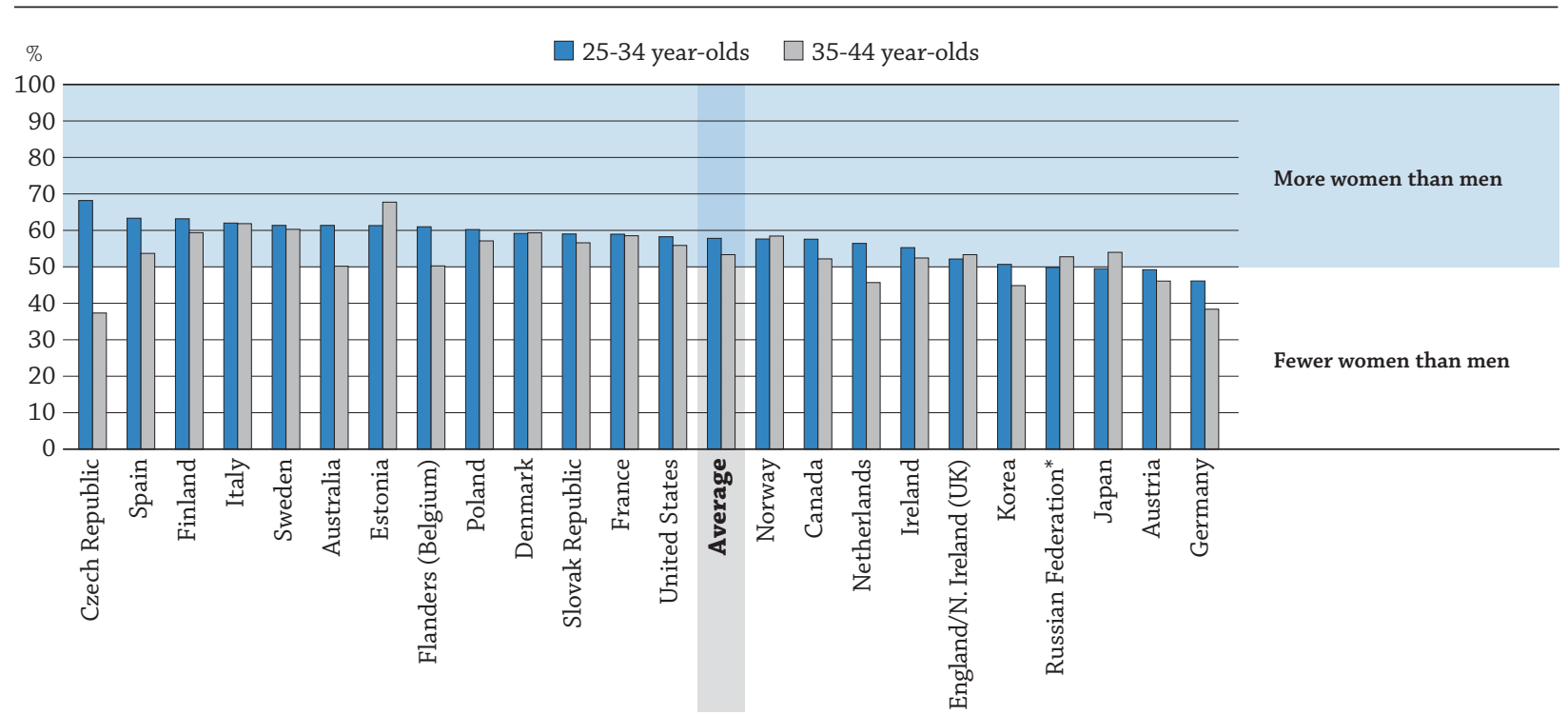

* See note on data for the Russian Federation in the Methodology section.

Countries are ranked in descending order of the proportion of first generation tertiary-educated 25-34 year-old non-student women.

Source: OECD. Table A4.2b, available on line. See Annex 3 for notes (www.oecd.org/education/education-at-a-glance- 19991487.htm).

StatLink 제그 http://dx.doi.org/10.1787/888933283569 


\section{Field of education}

Chart A4.4 shows that in most countries, first generation tertiary-educated individuals pursued fields of education similar to those chosen by individuals who were not first generation tertiary-educated. On average, the largest proportion of all tertiary-educated younger adults holds a degree in social sciences, business and law (28\%, on average). The second most popular field of education is engineering, manufacturing and construction (17\%). In the Czech Republic, $41 \%$ of first generation tertiary-educated younger adults completed their degree in social sciences, business and law, the largest proportion among participating countries and sub-national entities. By contrast, in the Russian Federation, 18\% of first generation tertiary-educated younger adults completed their degree in social sciences, business and law, and 34\% completed their degree in engineering, manufacturing and construction. In England/Northern Ireland (UK), the Netherlands and the United States, among both first generation tertiary-educated adults and tertiary-educated adults whose parents also hold a tertiary degree, there is a difference of more than 20 percentage points between the proportions of adults who studied social sciences, business and law and those who studied engineering, manufacturing and construction (Table A4.2c, available on line).

\section{Chart A4.4. First generation/not first generation tertiary-educated adults, by selected field of education (2012)}

Survey of Adult Skills, 25-34 year-old non-students

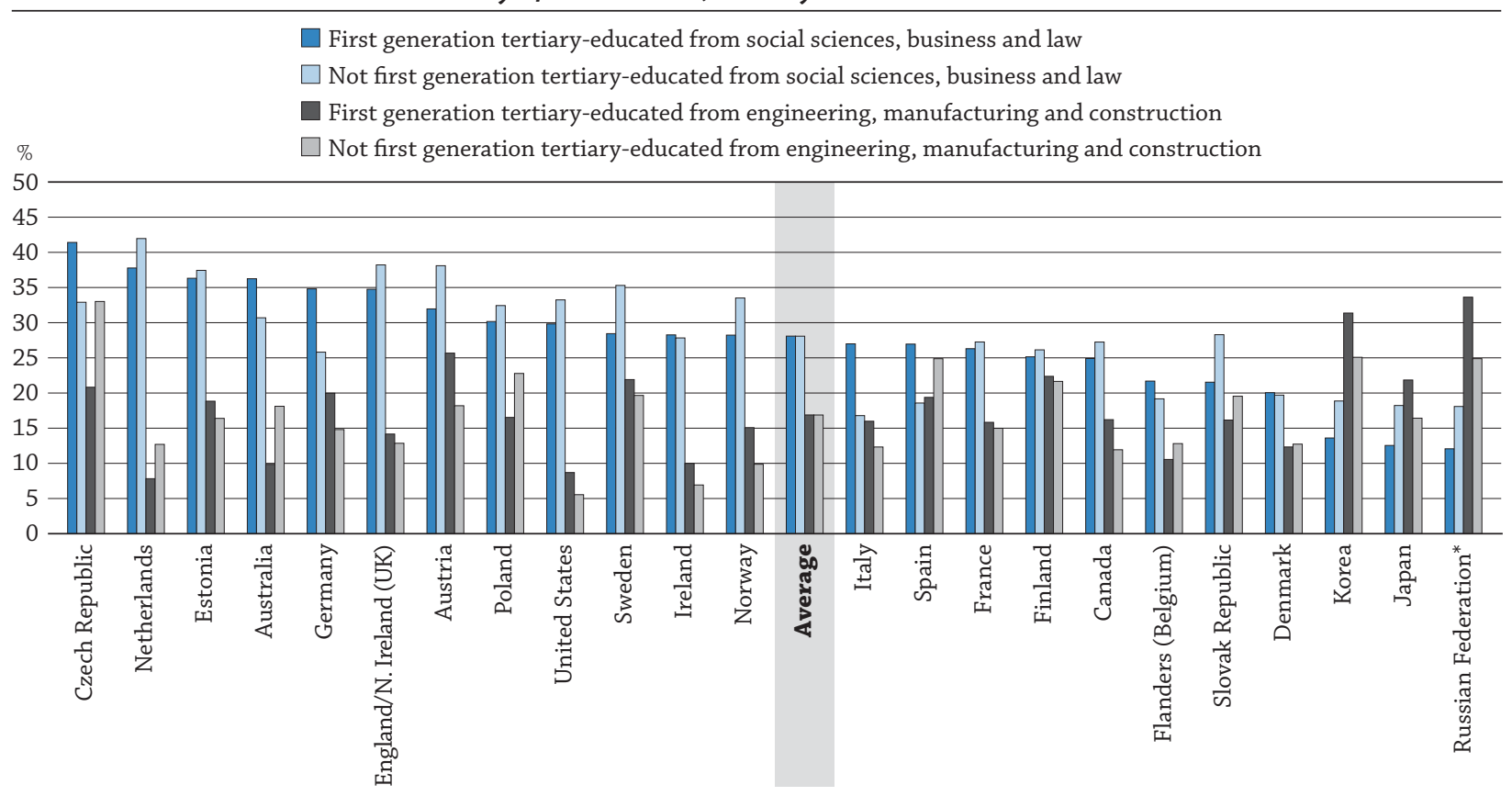

* See note on data for the Russian Federation in the Methodology section.

Countries are ranked in descending order of first generation tertiary-educated 25-34 year-old non-student from social sciences, business and law. Source: OECD. Table A4.2c, available on line. See Annex 3 for notes (www.oecd.org/education/education-at-a-glance- 19991487.htm). StatLink 츔ㄴ http://dx.doi.org/10.1787/888933283575

\section{Labour status of first generation tertiary-educated adults}

Indicator A5 shows that employment rates are the highest among tertiary-educated adults. Similar conclusions can be drawn from Chart A4.5, which shows that, on average, 88\% of first generation tertiary-educated 25-34 year-old non-students are employed, as are $90 \%$ of those who attained tertiary education and whose parents hold a tertiary degree. The difference in employment rates between those who are first generation tertiary-educated and those who are not is not statistically significant in most countries. Thus, in general, first generation tertiary-educated adults do not have a better or worse chance of being employed compared to tertiary-educated adults whose parents had also attained that level of education (Table A4.2d and see Table A5.3a).

When comparing age groups among first generation tertiary-educated adults, the difference in the proportion of employed first generation tertiary-educated adults is less than 3 percentage points between 25-34 year-olds and 35-44 year-olds in 16 of the 24 countries and sub-national entities that participated in the Survey of Adult Skills. 
In Austria, Flanders (Belgium), Ireland, Japan, Northern Ireland (UK) and the Russian Federation, the proportion of first generation tertiary-educated 25-34 year-olds who are employed is greater than that among 35-44 year-olds. In these countries and sub-national entities, younger cohorts of first generation tertiary-educated adults have better employment prospects than older groups (Table A4.2d).

\section{Chart A4.5. Employment rates among first generation/not first generation tertiary-educated adults (2012)}

Survey of Adult Skills, 25-34 year-old non-students

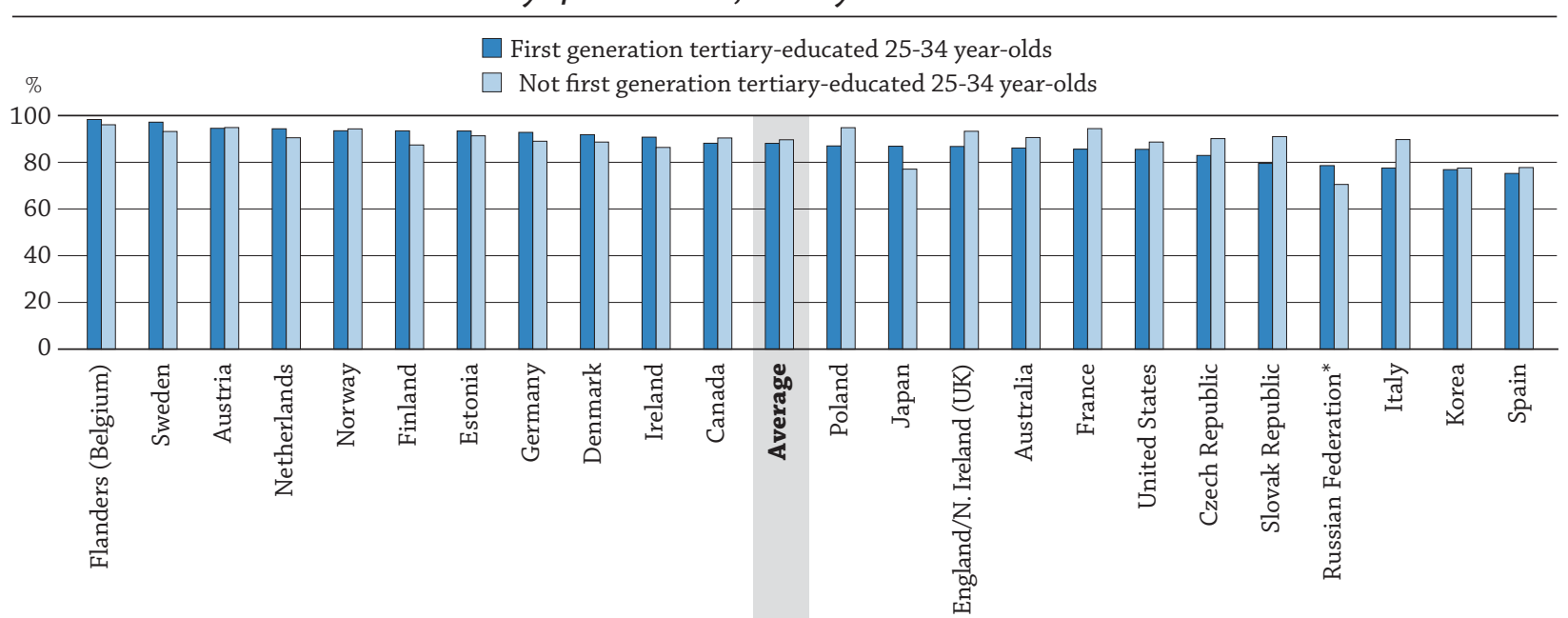

* See note on data for the Russian Federation in the Methodology section.

Countries are ranked in descending order of the employment rate of first generation tertiary-educated 25-34 year-olds.

Source: OECD. Table A4.2d. See Annex 3 for notes (www.oecd.org/education/education-at-a-glance- 19991487.htm).

StatLink 제그 http://dx.doi.org/10.1787/888933283588

\section{Part-time/full-time work}

On average across the countries and sub-national entities that participated in the survey, $88 \%$ of employed first generation tertiary-educated 25-34 year-old non-students work 30 hours per week or more (paid or unpaid). This means that a large majority of first generation tertiary-educated adults work full time after they finish tertiary education. However, there are significant variations among countries. For example, in the Netherlands, $72 \%$ of first generation tertiary-educated 25-34 year-olds work full time, but only 57\% of Dutch women who are of the same age and are first generation tertiary-educated do. By contrast, in Denmark, $96 \%$ of first generation tertiary-educated 25-34 year-olds - $98 \%$ of men and $94 \%$ of women - work full time (Table A4.2e).

\section{Intergenerational social mobility}

The findings shown in Chart A4.6 confirm the general conclusions of Indicator A6: higher educational attainment is positively associated with earnings. But they also show that parents' education has less impact on individuals' earnings compared to the effect of one's own level of education. In fact, when controlling for adults' own education, parents' education is significant in only 8 of the 22 countries and sub-national entities shown in the chart (Table A4.3a).

On average, there is a 4 percentage-point increase in the probability of having among the highest $25 \%$ in monthly earnings when parents' highest level of education is tertiary compared with those whose parents' highest level of education is upper secondary or post-secondary non-tertiary. This means that, after accounting for an adult's own educational attainment, having tertiary-educated parents is a positive influence on children's earnings meaning also that it still has a somewhat enduring (additional) effect on children's economic situation, even if the strongest influence on earnings is through an adult's own educational attainment. Adults with tertiary education are 23 percentage points more likely to have among the highest $25 \%$ in monthly earnings compared with adults whose highest level of attainment is upper secondary or post-secondary non-tertiary education (Table A4.3a).

A similar situation, with a significant role of parents' tertiary education, is present in eight countries, highest in the Czech Republic and the Slovak Republic, then in Estonia and England/Northern Ireland (UK). This emphasises the importance of bringing up the relationship between parents' education and own education in an examination of intergenerational social mobility (Table A4.3a). 
Other analyses on the likelihood of being in a skilled occupation or being a top performer in literacy and numeracy based on educational attainment of parents and own educational attainment are available on line (Tables A4.3b, c and d, available on line).

\section{Chart A4.6. Likelihood of being among the top $25 \%$ in earnings, by parents' and own educational attainment (2012)}

Survey of Adult Skills, 25-64 year-olds, upper secondary or post-secondary non-tertiary education as reference category

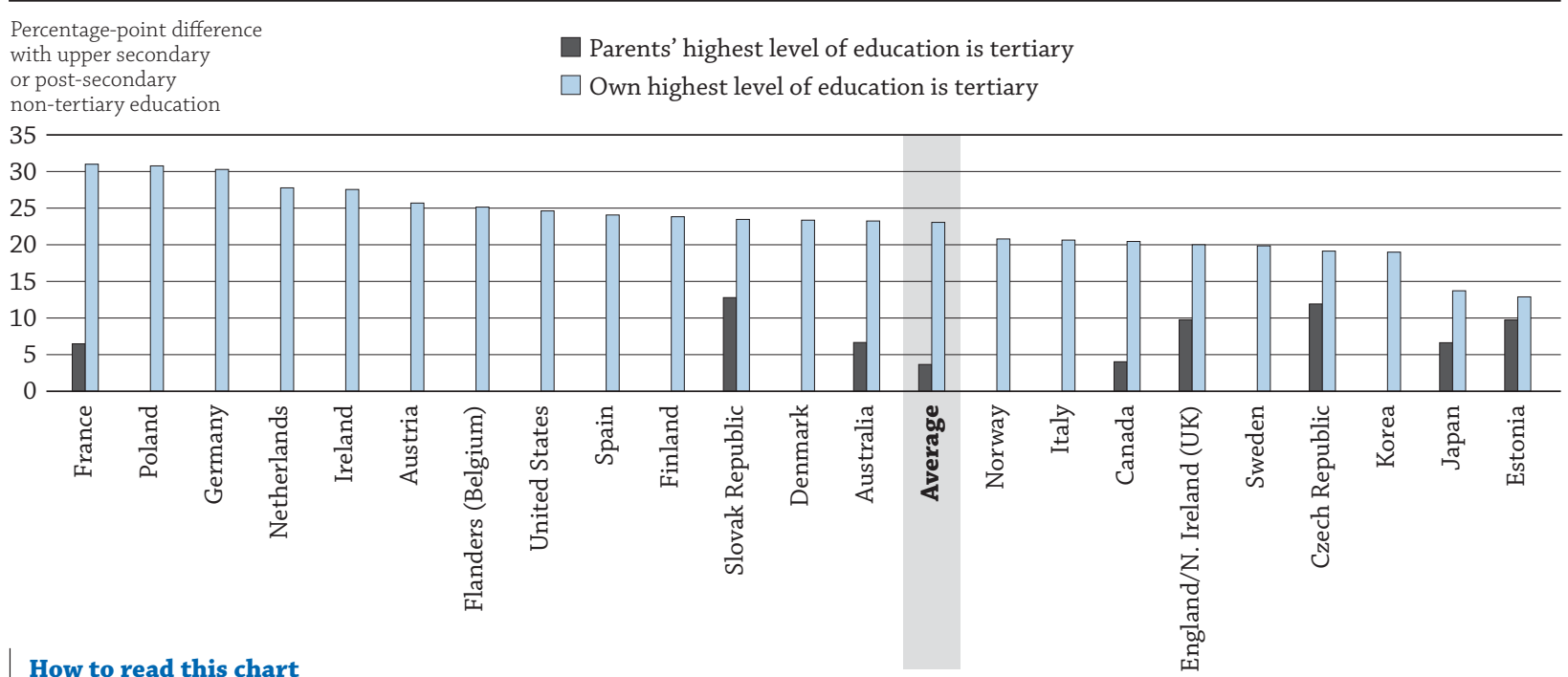

On average, the percentage of individuals with monthly earnings in the highest $25 \%$ of the monthly earnings distribution and whose parents reached tertiary education increases by 4 percentage points compared with those whose parents have upper secondary or post-secondary nontertiary education. However, when the individual's own highest level of education is tertiary, the increase is 23 percentage points compared with one who attained upper secondary or post-secondary non-tertiary education.

Note: Differences between the groups that are not statistically significant at $95 \%$ are not presented.

Countries are ranked in descending order of the percentage-point difference in likelihood of being in the highest $25 \%$ of earnings distribution when own highest level of education is tertiary (upper secondary or post-secondary non-tertiary education is the reference category).

Source: OECD. Table A4.3a. See Annex 3 for notes (www.oecd.org/education/education-at-a-glance-19991487.htm).

StatLink 形 1 http://dx.doi.org/10.1787/888933283590

\section{Definitions}

Age groups: Adults refers to 25-64 year-olds; younger adults refers to 25-34 year-olds.

Earnings refer to monthly wages, including bonuses for wage and salary earners and self-employed adults.

First generation tertiary-educated adults are indivuals who have attained tertiary education but whose parents level of education is lower. The comparison is made only with the adult's parents, not with earlier generations.

Levels of education: Below upper secondary corresponds to ISCED-97 Levels 0, 1, 2 and 3C short programmes; upper secondary or post-secondary non-tertiary corresponds to ISCED-97 Levels 3A, 3B, 3C long programmes, and Level 4; and tertiary corresponds to ISCED-97 Levels 5A, 5B and 6.

Non-student refers to an individual who was not enrolled as a student at the time of the survey. For example, "nonstudents who completed tertiary education" refers to individuals who had completed tertiary education and were not students when the survey was conducted.

Parents' educational attainment: Below upper secondary means that both parents have attained ISCED-97 Levels $0,1,2$ or $3 C$ short programmes; upper secondary or post-secondary non-tertiary means that at least one parent (whether mother or father) has attained ISCED-97 Levels 3A, 3B, 3C long programmes, or Level 4; and tertiary means that at least one parent (whether mother or father) has attained ISCED-97 Levels 5A, 5B or 6.

Working hours: Full time refers to individuals who work 30 hours per week or more (paid or unpaid); part time refers to individuals who work less than 30 hours per week (paid or unpaid). 


\section{Methodology}

All data are based on the Survey of Adult Skills (PIAAC) (2012). PIAAC is the OECD Programme for the International Assessment of Adult Competencies. See Annex 3 (www.oecd.org/education/education-at-a-glance-19991487.htm) for additional information.

Data on first generation tertiary-educated adults are also based on data from the Survey of Adult Skills, which was not specifically designed for this population. The sample is smaller than in other indicators that use the whole population, explaining why standard errors are slightly higher than usual. Data should, therefore, be interpreted with caution.

Note regarding data from the Russian Federation in the Survey of Adult Skills (PIAAC)

Readers should note that the sample for the Russian Federation does not include the population of the Moscow municipal area. The data published, therefore, do not represent the entire resident population aged 16-65 in Russia but rather the population of Russia excluding the population residing in the Moscow municipal area. More detailed information regarding the data from the Russian Federation as well as that of other countries can be found in the Technical Report of the Survey of Adult Skills (OECD, 2014b).

\section{References}

OECD (2014a), Education at a Glance 2014: OECD Indicators, OECD Publishing, Paris, http://dx.doi.org/10.1787/eag-2014-en. OECD (2014b), Technical Report of the Survey of Adult Skills, www.oecd.org/site/piaac/ Technical\%20Report 17OCT13.pdf, pre-publication copy.

OECD (2013), PISA 2012 Results: Excellence through Equity (Volume II): Giving Every Student the Chance to Succeed, PISA, OECD Publishing, Paris, http://dx.doi.org/10.1787/9789264201132-en.

\section{Indicator A4 Tables}

StatLink त्ञाज http://dx.doi.org/10.1787/888933284922

Table A4.1a Intergenerational mobility in education, by age group (2012)

WEB Table A4.1b Men's intergenerational mobility in education, by age group (2012)

WEB Table A4.1c Women's intergenerational mobility in education, by age group (2012)

WEB Table A4.2a First generation tertiary-educated adults, by parents' education level, gender and age group (2012)

WEB Table A4.2b First generation tertiary-educated adults, by gender and age group (2012)

WEB Table A4.2c First generation/not first generation tertiary-educated adults, by field of education, gender and age group (2012)

Table A4.2d First generation/not first generation tertiary-educated adults, by labour force status, gender and age group (2012)

Table A4.2e First generation tertiary-educated adults, by part-time/full-time status, gender and age group (2012)

WEB Table A4.2f First generation tertiary-educated adults, by monthly earnings, gender and age group (2012)

WEB Table A4.2g

First generation tertiary-educated adults, by time off/no time off from education, gender and age group (2012)

Table A4.3a Likelihood of being among the top $25 \%$ in earnings, by parents' and own educational attainment, gender and age group (2012)

WEB Table A4.3b Likelihood of being in a skilled occupation, by parents' and own educational attainment, gender and age group (2012)

WEB Table A4.3c Likelihood of being a top performer in literacy, by parents' and own educational attainment, gender and age group (2012)

WEB Table A4.3d Likelihood of being a top performer in numeracy, by parents' and own educational attainment, gender and age group (2012) 
Table A4.1a. Intergenerational mobility in education, by age group (2012) 25-34 and 35-44 year-old non-students whose educational attainment is lower than (downward mobility), higher than (upward mobility), or the same as (status quo) that of their parents

\begin{abstract}
How to read this table: In Australia, among 25-34 year-olds, $6 \%$ completed below upper secondary (a level lower than that attained by their parents), $10 \%$ completed upper secondary or post-secondary education (a level lower than that attained by their parents), $16 \%$ completed upper secondary or post-secondary education (a level higher than that attained by their parents), 20\% completed tertiary education (a level higher than that attained by their parents), $8 \%$ completed below upper secondary (a level equivalent to that attained by their parents), $13 \%$ completed upper secondary or post-secondary education (a level equivalent to that attained by their parents) and $27 \%$ completed tertiary education (a level equivalent to that attained by their parents).
\end{abstract}

\begin{tabular}{|c|c|c|c|c|c|c|c|c|c|c|c|c|c|c|c|c|c|c|c|c|}
\hline & \multicolumn{20}{|c|}{ 25-34 year-olds } \\
\hline & \multicolumn{6}{|c|}{ Downward mobility } & \multicolumn{6}{|c|}{ Upward mobility } & \multicolumn{8}{|c|}{ Status quo } \\
\hline & \multicolumn{2}{|c|}{$\begin{array}{c}\text { Own } \\
\text { education: } \\
\text { below upper } \\
\text { secondary }\end{array}$} & \multicolumn{2}{|c|}{$\begin{array}{c}\text { Own } \\
\text { education: } \\
\text { upper } \\
\text { secondary } \\
\text { or post- } \\
\text { secondary } \\
\text { non-tertiary }\end{array}$} & \multicolumn{2}{|c|}{$\begin{array}{c}\text { Own } \\
\text { education: } \\
\text { all levels }\end{array}$} & \multicolumn{2}{|c|}{\begin{tabular}{|c|} 
Own \\
education: \\
upper \\
secondary \\
or post- \\
secondary \\
non-tertiary
\end{tabular}} & \multicolumn{2}{|c|}{$\begin{array}{c}\text { Own } \\
\text { education: } \\
\text { tertiary }\end{array}$} & \multicolumn{2}{|c|}{$\begin{array}{c}\text { Own } \\
\text { education: } \\
\text { all levels }\end{array}$} & \multicolumn{2}{|c|}{$\begin{array}{c}\text { Own } \\
\text { education: } \\
\text { below upper } \\
\text { secondary }\end{array}$} & \multicolumn{2}{|c|}{$\begin{array}{c}\text { Own } \\
\text { education: } \\
\text { upper } \\
\text { secondary } \\
\text { or post- } \\
\text { secondary } \\
\text { non-tertiary }\end{array}$} & \multicolumn{2}{|c|}{$\begin{array}{c}\text { Own } \\
\text { education: } \\
\text { tertiary }\end{array}$} & \multicolumn{2}{|c|}{$\begin{array}{c}\text { Own } \\
\text { education: } \\
\text { all levels }\end{array}$} \\
\hline & $\%$ & S.E. & $\%$ & S.E. & $\%$ & S.E. & $\%$ & S.E. & $\%$ & S.E. & $\%$ & S.E. & $\%$ & S.E. & $\%$ & S.E. & $\%$ & S.E. & $\%$ & S.E. \\
\hline & & (2) & (3) & (4) & (5) & (6) & (7) & (8) & (9) & $(10)$ & $(11)$ & (12) & (13) & (14) & $(15)$ & (16) & $(17)$ & (18) & (19) & (20) \\
\hline \multicolumn{21}{|l|}{ Q National entities } \\
\hline Australia & 6 & (1.0) & 10 & $(1.0)$ & 16 & (1.4) & 16 & (1.5) & 20 & (1.5) & 36 & (1.9) & 8 & (1.0) & 13 & $(1.2)$ & 27 & (1.5) & 48 & (2.0) \\
\hline Austria & 7 & (0.9) & 14 & $(1.2)$ & 21 & (1.4) & 10 & (1.2) & 11 & $(0.9)$ & 21 & (1.4) & 6 & $(0.6)$ & 44 & (1.6) & 8 & $(0.7)$ & 57 & (1.8) \\
\hline Canada & 5 & $(0.6)$ & 14 & (1.1) & 18 & (1.1) & 5 & $(0.5)$ & 23 & $(1.2)$ & 27 & (1.4) & 3 & $(0.5)$ & 16 & (1.1) & 35 & (1.3) & 54 & (1.5) \\
\hline Czech Republic & 6 & (1.0) & 6 & (1.1) & 12 & (1.5) & 2 & $(0.5)$ & 15 & (1.1) & 17 & $(1.2)$ & 1 & $(0.3)$ & 57 & (2.0) & 13 & (1.3) & 71 & (1.8) \\
\hline Denmark & 8 & (1.3) & 9 & $(1.2)$ & 18 & (1.6) & 7 & (1.1) & 21 & (1.3) & 28 & (1.5) & 6 & (1.0) & 19 & (1.6) & 29 & (1.5) & 54 & (1.8) \\
\hline Estonia & 11 & $(0.9)$ & 16 & (1.2) & 27 & (1.4) & 4 & $(0.6)$ & 20 & (1.1) & 23 & (1.3) & 3 & (0.6) & 22 & (1.3) & 25 & (1.3) & 50 & (1.5) \\
\hline Finland & 6 & (1.0) & 9 & (1.0) & 15 & (1.4) & 9 & (1.2) & 30 & (1.7) & 39 & (1.9) & 1 & $(0.5)$ & 26 & (1.3) & 18 & (1.2) & 46 & (1.7) \\
\hline France & 5 & $(0.7)$ & 5 & $(0.7)$ & 10 & (1.0) & 14 & (1.1) & 26 & (1.3) & 40 & (1.4) & 8 & $(0.8)$ & 23 & (1.4) & 19 & (1.1) & 50 & (1.5) \\
\hline Germany & 8 & (1.2) & 17 & (1.5) & 24 & (1.9) & 4 & (0.9) & 14 & (1.5) & 19 & (1.7) & 2 & $(0.7)$ & 34 & (2.1) & 21 & (1.5) & 57 & (2.0) \\
\hline Ireland & 4 & (0.6) & 8 & $(0.9)$ & 12 & (1.0) & 17 & (1.1) & 28 & (1.2) & 45 & (1.5) & 9 & $(0.8)$ & 16 & (1.3) & 19 & (1.1) & 44 & (1.6) \\
\hline Italy & 3 & $(0.9)$ & 2 & (0.7) & 5 & (1.2) & 29 & (1.6) & 17 & (1.2) & 45 & (1.9) & 28 & (2.1) & 16 & (1.4) & 5 & (1.0) & 49 & (2.0) \\
\hline Japan & 6 & $(0.9)$ & 11 & (1.3) & 18 & (1.2) & 2 & (0.6) & 22 & (1.5) & 24 & (1.6) & 2 & (0.6) & 21 & (1.4) & 35 & (1.7) & 58 & (1.7) \\
\hline Korea & 1 & $(0.2)$ & 3 & $(0.6)$ & 3 & $(0.6)$ & 14 & (1.0) & 47 & (1.2) & 61 & (1.5) & 2 & $(0.4)$ & 16 & (1.1) & 17 & (1.2) & 35 & (1.5) \\
\hline Netherlands & 8 & (1.0) & 9 & (1.2) & 17 & (1.4) & 16 & (1.5) & 22 & (1.6) & 38 & $(2.2)$ & 10 & (1.3) & 15 & (1.5) & 20 & (1.5) & 45 & (2.1) \\
\hline Norway & 13 & (1.4) & 14 & (1.6) & 27 & (1.9) & 5 & (0.7) & 18 & (1.2) & 22 & (1.3) & 4 & $(0.9)$ & 18 & (1.5) & 28 & (1.5) & 51 & (2.3) \\
\hline Poland & 4 & (0.7) & 3 & $(0.7)$ & 7 & (1.0) & 6 & $(0.8)$ & 30 & (1.6) & 36 & (1.7) & 2 & $(0.4)$ & 41 & (1.6) & 14 & (1.2) & 57 & (1.8) \\
\hline Slovak Republic & 4 & $(0.5)$ & 6 & $(0.7)$ & 10 & $(0.8)$ & 6 & $(0.8)$ & 17 & (1.5) & 23 & (1.6) & 9 & (1.0) & 49 & (1.5) & 10 & (1.0) & 67 & (1.8) \\
\hline Spain & 7 & $(0.9)$ & 3 & $(0.6)$ & 10 & (1.0) & 15 & (1.2) & 26 & (1.3) & 41 & (1.6) & 31 & (1.5) & 8 & (1.1) & 10 & $(0.9)$ & 49 & (1.6) \\
\hline Sweden & 9 & (1.2) & 20 & (1.6) & 28 & (1.7) & 9 & (1.1) & 15 & (1.3) & 24 & (1.8) & 5 & (1.0) & 18 & (1.6) & 25 & (1.5) & 47 & (2.1) \\
\hline United States & 5 & $(0.9)$ & 18 & (1.7) & 23 & (1.9) & 8 & $(0.9)$ & 15 & (1.4) & 24 & (1.7) & 5 & $(0.8)$ & 22 & (1.4) & 27 & (1.6) & 54 & (2.1) \\
\hline \multicolumn{21}{|l|}{ Sub-national entities } \\
\hline Flanders (Belgium) & 4 & (0.7) & 9 & (1.1) & 13 & (1.3) & 13 & (1.2) & 22 & (1.5) & 35 & (1.6) & 3 & (0.7) & 23 & (1.3) & 26 & (1.5) & 52 & (1.6) \\
\hline England (UK) & 8 & $(0.9)$ & 7 & (1.0) & 16 & (1.2) & 7 & $(0.9)$ & 26 & $(1.8)$ & 32 & (1.9) & 6 & $(0.9)$ & 19 & (1.7) & 27 & (1.9) & 51 & $(2.2)$ \\
\hline Northern Ireland (UK) & 8 & (1.5) & 5 & (1.3) & 13 & (1.7) & 9 & (1.3) & 27 & (1.8) & 36 & (1.9) & 11 & (1.4) & 23 & (1.7) & 17 & (1.8) & 51 & (2.2) \\
\hline England/N. Ireland (UK) & 8 & $(0.9)$ & 7 & $(0.9)$ & 16 & (1.1) & 7 & $(0.9)$ & 26 & (1.7) & 33 & (1.9) & 6 & $(0.9)$ & 19 & (1.6) & 26 & (1.8) & 51 & (2.1) \\
\hline Average & 6 & $(0.2)$ & 10 & $(0.2)$ & 16 & $(0.3)$ & 10 & $(0.2)$ & 22 & $(0.3)$ & 32 & $(0.3)$ & 7 & $(0.2)$ & 24 & $(0.3)$ & 21 & $(0.3)$ & 52 & $(0.4)$ \\
\hline Russian Federation* & 5 & $(0.7)$ & 6 & (1.6) & 11 & $(2.2)$ & 6 & (1.5) & 38 & (3.0) & 44 & (4.3) & 2 & $(0.6)$ & 13 & (1.3) & 31 & (3.0) & 46 & $(4.0)$ \\
\hline
\end{tabular}

Note: Columns showing data for 35-44 year-olds are available for consultation on line (see StatLink below).

* See note on data for the Russian Federation in the Methodology section.

Source: OECD. Survey of Adult Skills (PIAAC) (2012). See Annex 3 for notes (www.oecd.org/education/education-at-a-glance-19991487.htm).

Please refer to the Reader's Guide for information concerning symbols for missing data and abbreviations.

StatLink 제이 http://dx.doi.org/10.1787/888933284933 
Table A4.2d. First generation/not first generation tertiary-educated adults, by labour force status, gender and age group (2012)

25-34 and 35-44 year-old first generation/not first generation tertiary-educated non-students

\begin{tabular}{|c|c|c|c|c|c|c|c|c|c|c|c|c|c|c|c|c|c|c|}
\hline & \multicolumn{18}{|c|}{ First generation tertiary-educated 25-34 year-olds } \\
\hline & \multicolumn{6}{|c|}{ Employed } & \multicolumn{6}{|c|}{ Unemployed } & \multicolumn{6}{|c|}{ Inactive } \\
\hline & \multicolumn{2}{|c|}{ Men } & \multicolumn{2}{|c|}{ Women } & \multicolumn{2}{|c|}{$\mathrm{M}+\mathrm{W}$} & \multicolumn{2}{|c|}{ Men } & \multicolumn{2}{|c|}{ Women } & \multicolumn{2}{|c|}{$\mathrm{M}+\mathrm{W}$} & \multicolumn{2}{|c|}{ Men } & \multicolumn{2}{|c|}{ Women } & \multicolumn{2}{|c|}{$\mathrm{M}+\mathrm{W}$} \\
\hline & $\%$ & S.E. & $\%$ & S.E. & $\%$ & S.E. & $\%$ & S.E. & $\%$ & S.E. & $\%$ & S.E. & $\%$ & S.E. & $\%$ & S.E. & $\%$ & S.E. \\
\hline \multirow{2}{*}{\multicolumn{19}{|c|}{ National entities }} \\
\hline & & & & & & & & & & & & & & & & & & \\
\hline Australia & 93 & $(4.2)$ & 82 & $(4.1)$ & 86 & (3.0) & 5 & (3.0) & 4 & (2.1) & 4 & (1.6) & c & c & 14 & (3.9) & 10 & (2.5) \\
\hline Austria & 98 & (1.8) & 91 & (4.9) & 95 & (2.5) & c & c & c & c & c & c & c & c & 6 & (3.8) & 3 & (1.9) \\
\hline Canada & 94 & (2.5) & 84 & (3.1) & 88 & (2.2) & 5 & (2.3) & 4 & (1.7) & 5 & (1.4) & 2 & (0.9) & 11 & (2.3) & 7 & (1.4) \\
\hline Czech Republic & 98 & (1.5) & 76 & (5.1) & 83 & (3.9) & c & c & 3 & (3.3) & 3 & (2.1) & c & c & 21 & (4.5) & 14 & (3.2) \\
\hline Denmark & 94 & (2.9) & 90 & (3.1) & 92 & (2.1) & 5 & (2.8) & 5 & (2.2) & 5 & (1.7) & c & c & 4 & (2.1) & 3 & (1.3) \\
\hline Estonia & 96 & (2.4) & 92 & (2.2) & 93 & (1.6) & c & c & c & c & 2 & (1.0) & c & c & 7 & (2.3) & 5 & (1.4) \\
\hline Finland & 93 & (2.8) & 94 & $(2.0)$ & 93 & (1.7) & c & c & 2 & (1.1) & 2 & (1.0) & 4 & (2.3) & 4 & (1.6) & 4 & (1.4) \\
\hline France & 86 & (2.9) & 85 & (2.6) & 86 & (1.9) & 9 & (2.5) & 7 & (2.3) & 8 & (1.7) & 5 & (2.2) & 8 & (1.5) & 7 & (1.4) \\
\hline Germany & 98 & (2.5) & 87 & (5.1) & 93 & (2.5) & c & c & c & c & c & c & c & c & 11 & (4.8) & 5 & (2.1) \\
\hline Ireland & 91 & (2.9) & 91 & (2.3) & 91 & (1.7) & 7 & (2.4) & 4 & (1.6) & 5 & (1.5) & 2 & (1.7) & 5 & (1.5) & 4 & (1.1) \\
\hline Italy & 87 & (6.0) & 72 & (7.4) & 78 & (5.7) & c & c & 21 & (7.2) & 15 & (5.2) & 8 & $(4.8)$ & 7 & (3.1) & 7 & (2.6) \\
\hline Japan & 96 & (2.1) & 78 & (3.7) & 87 & (2.3) & c & c & c & c & c & c & 3 & (1.8) & 22 & (3.7) & 13 & (2.2) \\
\hline Korea & 89 & (2.1) & 65 & (2.8) & 77 & (1.7) & 3 & (1.1) & 4 & (1.2) & 4 & $(0.8)$ & 8 & (2.0) & 31 & (2.7) & 20 & (1.6) \\
\hline Netherlands & 98 & (2.3) & 92 & (3.6) & 94 & (2.4) & c & c & c & c & 3 & (1.9) & c & c & 4 & (2.4) & 2 & (1.4) \\
\hline Norway & 97 & (2.2) & 91 & (3.0) & 93 & (2.0) & c & c & 4 & (2.1) & 3 & (1.3) & c & c & 5 & (2.2) & 4 & (1.5) \\
\hline Poland & 95 & (1.6) & 81 & (3.0) & 87 & (1.8) & 4 & (1.6) & 7 & (1.5) & 6 & (1.0) & 0 & (0.3) & 12 & (2.6) & 7 & (1.5) \\
\hline Slovak Republic & 89 & (3.5) & 73 & (4.3) & 80 & (2.9) & 6 & (2.6) & 4 & (1.6) & 5 & (1.5) & 5 & (3.0) & 23 & $(4.1)$ & 16 & (2.8) \\
\hline Spain & 76 & (5.1) & 75 & $(4.2)$ & 75 & (3.2) & 16 & (4.5) & 15 & (3.2) & 16 & (2.7) & 8 & (3.3) & 10 & (2.7) & 9 & (2.3) \\
\hline Sweden & 98 & (1.8) & 96 & (1.8) & 97 & (1.3) & c & c & 3 & (1.6) & 2 & (1.2) & c & c & c & c & c & c \\
\hline United States & 90 & (5.2) & 83 & $(4.2)$ & 86 & (3.3) & c & c & 8 & (3.1) & 6 & (2.2) & c & c & 10 & (2.9) & 9 & (2.6) \\
\hline \multicolumn{19}{|l|}{ Sub-national entities } \\
\hline Flanders (Belgium) & 98 & (1.6) & 98 & (1.2) & 98 & (1.0) & c & c & c & c & c & c & c & c & c & c & c & c \\
\hline England (UK) & 92 & (3.5) & 82 & (3.5) & 87 & (2.4) & 8 & (3.5) & 3 & (1.6) & 6 & (2.0) & c & c & 15 & (3.2) & 8 & (1.7) \\
\hline Northern Ireland (UK) & 89 & (7.1) & 92 & (2.9) & 91 & (3.5) & 9 & (7.0) & c & c & 6 & (3.3) & c & c & 6 & (2.3) & 4 & (1.4) \\
\hline England/N. Ireland (UK) & 92 & (3.4) & 82 & (3.4) & 87 & (2.3) & 8 & (3.4) & 3 & (1.5) & 6 & (1.9) & c & c & 14 & (3.0) & 7 & (1.6) \\
\hline Average & 93 & (0.7) & 84 & $(0.8)$ & 88 & (0.6) & 7 & $(0.9)$ & 6 & $(0.7)$ & 6 & $(0.5)$ & 5 & $(0.8)$ & 11 & $(0.7)$ & 8 & $(0.4)$ \\
\hline Russian Federation* & 90 & (2.4) & 67 & (3.9) & 79 & (2.4) & 2 & $(0.9)$ & c & c & 1 & (0.5) & 8 & (2.2) & 32 & (3.9) & 20 & (2.3) \\
\hline
\end{tabular}

Notes: First generation refers to the comparison with an adult's parents only, not with earlier generations. Columns showing data for $35-44$ year-olds and data for not first generation tertiary-educated adults are available for consultation on line (see StatLink below).

* See note on data for the Russian Federation in the Methodology section.

Source: OECD. Survey of Adult Skills (PIAAC) (2012). See Annex 3 for notes (www.oecd.org/education/education-at-a-glance-19991487.htm).

Please refer to the Reader's Guide for information concerning symbols for missing data and abbreviations.

StatLink 部实 http://dx.doi.org/10.1787/888933284941 
Table A4.2e. First generation tertiary-educated adults, by part-time/full-time status, gender and age group (2012)

25-34 and 35-44 year-old first generation tertiary-educated non-students

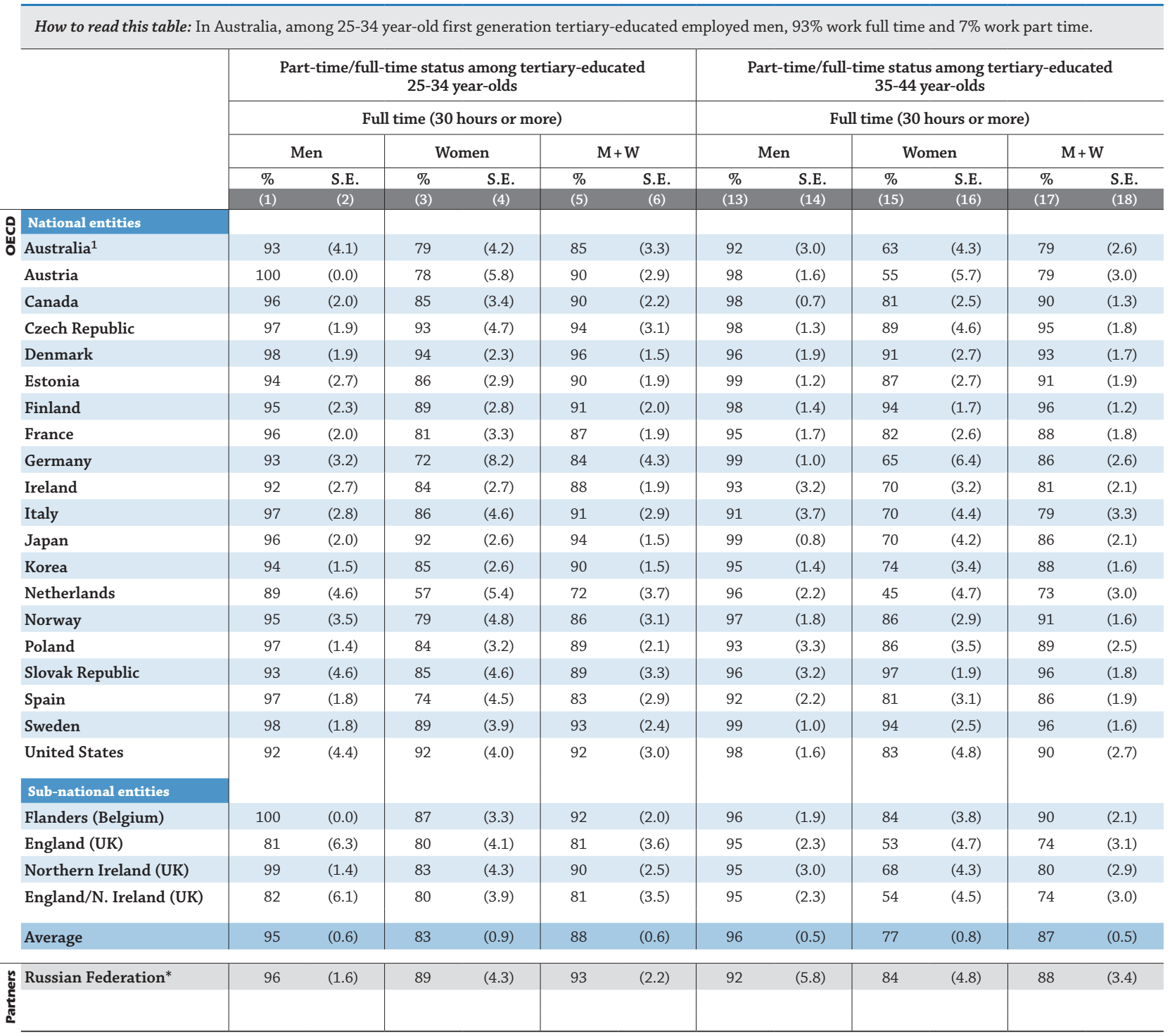

Notes: First generation refers to the comparison with an adult's parents only, not with earlier generations. Columns showing data for people working part time are available for consultation on line (see StatLink below).

1. For Australia, data based on full-time/part-time status use a variable that is capped at 60 hours per week; there is no upper limit for other countries.

* See note on data for the Russian Federation in the Methodology section.

Source: OECD. Survey of Adult Skills (PIAAC) (2012). See Annex 3 for notes (www.oecd.org/education/education-at-a-glance-19991487.htm).

Please refer to the Reader's Guide for information concerning symbols for missing data and abbreviations.

StatLink 제대 http://dx.doi.org/10.1787/888933284955 


\title{
Table A4.3a. [1/3] Likelihood of being among the top $25 \%$ in earnings, by parents' and own educational attainment, gender and age group (2012) 25-64 year-olds, percentage-point difference
}

\begin{abstract}
The percentages presented in the first eight columns are not related to the regression. They should be used as a reference to better understand the percentagepoint differences presented in the other columns.

How to read this table: In Model 1, the independent variables used are: parents' educational attainment, gender and age group. In Model 2, the individual's own educational attainment is included. This approach allows for a comparison of the effect of the individual's own educational attainment on the percentage-point differences for the variables included in the first model. For example, in Australia, the percentage of individuals with monthly earnings in the highest $25 \%$ of the monthly earnings distribution, and whose parents reached below upper secondary education, decreases by 4 percentage points compared with those whose parents have upper secondary or post-secondary non-tertiary education. However, when controlling for own educational attainment, the difference between an individual whose parents reached below upper secondary education and one whose parents have upper secondary or post-secondary non-tertiary education becomes nul and not significant.
\end{abstract}

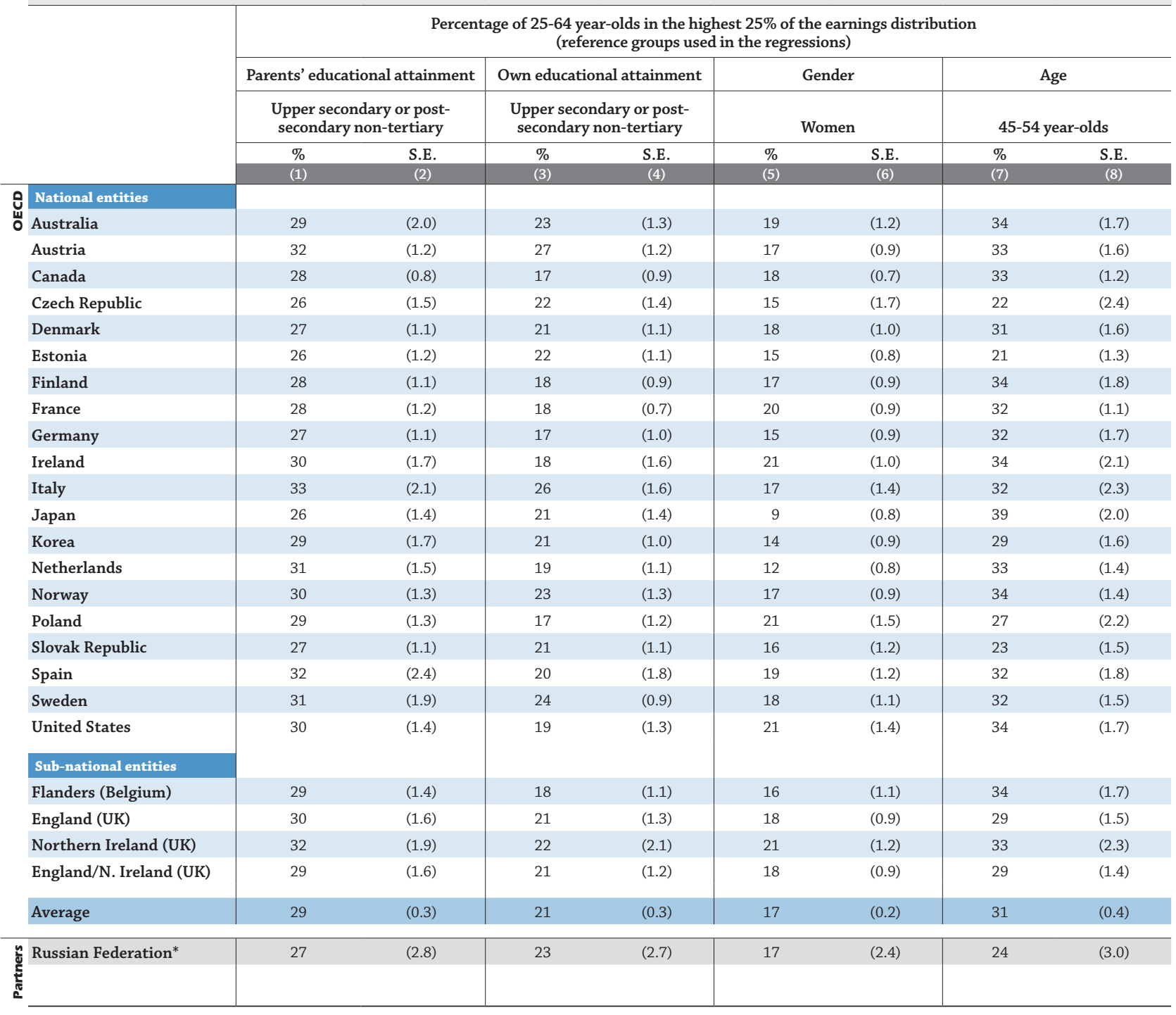

1. Model 1 is a linear regression where the dependent variable is "monthly earnings in the highest $25 \%$ of monthly earnings distribution" and where the independent variables are parents' educational attainment, gender and age group. The difference with Model 2 is that it doesn't include "own educational attainment".

2. Model 2 is a linear regression where the dependent variable is "monthly earnings in the highest $25 \%$ of monthly earnings distribution" and where the independent variables are parents' educational attainment, own educational attainment, gender and age group.

3. The reference category is upper secondary or post-secondary non-tertiary education.

4. The reference category is women.

5. The reference category is $45-54$ year-olds.

* See note on data for the Russian Federation in the Methodology section.

Source: OECD. Survey of Adult Skills (PIAAC) (2012). See Annex 3 for notes (www.oecd.org/education/education-at-a-glance-19991487.htm).

Please refer to the Reader's Guide for information concerning symbols for missing data and abbreviations.

StatLink 게 St http://dx.doi.org/10.1787/888933284966 
Table A4.3a. [2/3] Likelihood of being among the top $25 \%$ in earnings, by parents' and own educational attainment, gender and age group (2012) 25-64 year-olds, percentage-point difference

The percentages presented in the first eight columns are not related to the regression. They should be used as a reference to better understand the percentagepoint differences presented in the other columns.

How to read this table: In Model 1, the independent variables used are: parents' educational attainment, gender and age group. In Model 2, the individual's own educational attainment is included. This approach allows for a comparison of the effect of the individual's own educational attainment on the percentage-point differences for the variables included in the first model. For example, in Australia, the percentage of individuals with monthly earnings in the highest $25 \%$ of the monthly earnings distribution, and whose parents reached below upper secondary education, decreases by 4 percentage points compared with those whose parents have upper secondary or post-secondary non-tertiary education. However, when controlling for own educational attainment, the difference between an individual whose parents reached below upper secondary education and one whose parents have upper secondary or post-secondary non-tertiary education becomes nul and not significant.

\begin{tabular}{|c|c|c|c|c|c|c|c|c|c|c|c|c|}
\hline & \multicolumn{12}{|c|}{ Monthly earnings in the highest $25 \%$ of monthly earnings distribution, dependent on: } \\
\hline & \multicolumn{12}{|c|}{ Model $1^{1}$} \\
\hline & \multicolumn{4}{|c|}{ Parents' educational attainment ${ }^{3}$} & \multirow{2}{*}{\multicolumn{2}{|c|}{$\begin{array}{c}\text { Gender }^{4} \\
\text { Men }\end{array}$}} & \multicolumn{6}{|c|}{ Age group $^{5}$} \\
\hline & \multicolumn{2}{|c|}{$\begin{array}{l}\text { Below upper } \\
\text { secondary }\end{array}$} & \multicolumn{2}{|c|}{ Tertiary } & & & \multicolumn{2}{|c|}{$\begin{array}{c}25-34 \\
\text { year-olds }\end{array}$} & \multicolumn{2}{|c|}{$\begin{array}{c}35-44 \\
\text { year-olds }\end{array}$} & \multicolumn{2}{|c|}{$\begin{array}{c}55-64 \\
\text { year-olds }\end{array}$} \\
\hline & pp & S.E. & pp & S.E. & pp & S.E. & $\mathrm{pp}$ & S.E. & $\mathrm{pp}$ & S.E. & $\mathrm{pp}$ & S.E. \\
\hline & (9) & (10) & (11) & (12) & (13) & (14) & (15) & (16) & (17) & (18) & (19) & (20) \\
\hline \multicolumn{13}{|l|}{ Q National entities } \\
\hline ڤ̆ Australia & -4 & $(0.02)$ & 13 & $(0.03)$ & 20 & $(0.02)$ & -14 & $(0.02)$ & -2 & $(0.02)$ & -9 & $(0.03)$ \\
\hline Austria & -14 & $(0.02)$ & 3 & $(0.02)$ & 25 & $(0.02)$ & -14 & $(0.02)$ & -4 & $(0.02)$ & 2 & $(0.04)$ \\
\hline Canada & -6 & $(0.01)$ & 8 & $(0.01)$ & 21 & $(0.01)$ & -17 & $(0.02)$ & -1 & $(0.02)$ & -4 & $(0.02)$ \\
\hline Czech Republic & -13 & $(0.03)$ & 20 & $(0.04)$ & 21 & $(0.03)$ & 4 & $(0.04)$ & 6 & $(0.04)$ & -3 & $(0.04)$ \\
\hline Denmark & -4 & $(0.02)$ & 10 & $(0.02)$ & 21 & $(0.01)$ & -15 & $(0.02)$ & 0 & $(0.02)$ & -4 & $(0.02)$ \\
\hline Estonia & -7 & $(0.02)$ & 12 & $(0.02)$ & 24 & $(0.01)$ & 5 & $(0.02)$ & 5 & $(0.02)$ & -5 & $(0.02)$ \\
\hline Finland & -6 & $(0.02)$ & 7 & $(0.02)$ & 22 & $(0.02)$ & -21 & $(0.02)$ & -4 & $(0.03)$ & -9 & $(0.02)$ \\
\hline France & -8 & $(0.02)$ & 17 & $(0.02)$ & 14 & $(0.01)$ & -20 & $(0.02)$ & -6 & $(0.02)$ & -4 & $(0.02)$ \\
\hline Germany & -12 & $(0.03)$ & 11 & $(0.02)$ & 25 & $(0.01)$ & -15 & $(0.02)$ & -4 & $(0.02)$ & -4 & $(0.03)$ \\
\hline Ireland & -10 & $(0.02)$ & 8 & $(0.03)$ & 13 & $(0.02)$ & -20 & $(0.03)$ & -2 & $(0.02)$ & -10 & $(0.03)$ \\
\hline Italy & -16 & $(0.02)$ & 10 & $(0.05)$ & 17 & $(0.02)$ & -22 & $(0.03)$ & -8 & $(0.03)$ & 6 & $(0.05)$ \\
\hline Japan & -6 & $(0.02)$ & 11 & $(0.02)$ & 33 & $(0.01)$ & -31 & $(0.02)$ & -11 & $(0.02)$ & -12 & $(0.03)$ \\
\hline Korea & -10 & $(0.02)$ & 7 & $(0.03)$ & 23 & $(0.01)$ & -17 & $(0.02)$ & 4 & $(0.02)$ & -9 & $(0.02)$ \\
\hline Netherlands & -8 & $(0.02)$ & 8 & $(0.02)$ & 32 & $(0.01)$ & -20 & $(0.02)$ & 0 & $(0.02)$ & -2 & $(0.02)$ \\
\hline Norway & -9 & $(0.02)$ & 7 & $(0.02)$ & 25 & $(0.02)$ & -18 & $(0.02)$ & -1 & $(0.02)$ & -5 & $(0.02)$ \\
\hline Poland & -15 & $(0.02)$ & 12 & $(0.03)$ & 12 & $(0.02)$ & -5 & $(0.03)$ & -2 & $(0.03)$ & -2 & $(0.04)$ \\
\hline Slovak Republic & -16 & $(0.02)$ & 23 & $(0.04)$ & 19 & $(0.02)$ & -3 & $(0.02)$ & 0 & $(0.02)$ & -1 & $(0.03)$ \\
\hline Spain & -15 & $(0.03)$ & 3 & $(0.04)$ & 14 & $(0.02)$ & -20 & $(0.02)$ & -6 & $(0.02)$ & 2 & $(0.04)$ \\
\hline Sweden & -11 & $(0.02)$ & 3 & $(0.03)$ & 19 & $(0.02)$ & -18 & $(0.02)$ & -3 & $(0.02)$ & -3 & $(0.03)$ \\
\hline United States & -19 & $(0.02)$ & 11 & $(0.02)$ & 16 & $(0.02)$ & -20 & $(0.02)$ & -3 & $(0.03)$ & 0 & $(0.02)$ \\
\hline \multicolumn{13}{|l|}{ Sub-national entities } \\
\hline Flanders (Belgium) & -12 & $(0.02)$ & 7 & $(0.02)$ & 19 & $(0.02)$ & -24 & $(0.02)$ & -10 & $(0.02)$ & -2 & $(0.03)$ \\
\hline England (UK) & -9 & $(0.03)$ & 15 & $(0.03)$ & 22 & $(0.02)$ & -16 & $(0.03)$ & 2 & $(0.03)$ & -9 & $(0.02)$ \\
\hline Northern Ireland (UK) & -12 & $(0.03)$ & 15 & $(0.04)$ & 18 & $(0.02)$ & -15 & $(0.03)$ & -3 & $(0.03)$ & -6 & $(0.05)$ \\
\hline England/N. Ireland (UK) & -9 & $(0.02)$ & 15 & $(0.03)$ & 21 & $(0.02)$ & -16 & $(0.03)$ & 1 & $(0.03)$ & -9 & $(0.02)$ \\
\hline Average & -10 & $(0.00)$ & 10 & $(0.01)$ & 21 & $(0.00)$ & -15 & $(0.01)$ & -2 & $(0.01)$ & -4 & $(0.01)$ \\
\hline Russian Federation* & -13 & $(0.03)$ & 5 & $(0.04)$ & 14 & $(0.04)$ & 0 & $(0.04)$ & -5 & $(0.06)$ & -9 & $(0.04)$ \\
\hline
\end{tabular}

1. Model 1 is a linear regression where the dependent variable is "monthly earnings in the highest $25 \%$ of monthly earnings distribution" and where the independent variables are parents' educational attainment, gender and age group. The difference with Model 2 is that it doesn't include "own educational attainment".

2. Model 2 is a linear regression where the dependent variable is "monthly earnings in the highest $25 \%$ of monthly earnings distribution" and where the independent variables are parents' educational attainment, own educational attainment, gender and age group.

3. The reference category is upper secondary or post-secondary non-tertiary education.

4. The reference category is women.

5. The reference category is $45-54$ year-olds.

* See note on data for the Russian Federation in the Methodology section.

Source: OECD. Survey of Adult Skills (PIAAC) (2012). See Annex 3 for notes (www.oecd.org/education/education-at-a-glance-19991487.htm).

Please refer to the Reader's Guide for information concerning symbols for missing data and abbreviations.

StatLink 제개 http://dx.doi.org/10.1787/888933284966 


\title{
Table A4.3a. [3/3] Likelihood of being among the top $25 \%$ in earnings, by parents' and own educational attainment, gender and age group (2012) 25-64 year-olds, percentage-point difference
}

\begin{abstract}
The percentages presented in the first eight columns are not related to the regression. They should be used as a reference to better understand the percentagepoint differences presented in the other columns.

How to read this table: In Model 1, the independent variables used are: parents' educational attainment, gender and age group. In Model 2, the individual's own educational attainment is included. This approach allows for a comparison of the effect of the individual's own educational attainment on the percentage-point differences for the variables included in the first model. For example, in Australia, the percentage of individuals with monthly earnings in the highest $25 \%$ of the monthly earnings distribution, and whose parents reached below upper secondary education, decreases by 4 percentage points compared with those whose parents have upper secondary or post-secondary non-tertiary education. However, when controlling for own educational attainment, the difference between an individual whose parents reached below upper secondary education and one whose parents have upper secondary or post-secondary non-tertiary education becomes nul and not significant.
\end{abstract}

\begin{tabular}{|c|c|c|c|c|c|c|c|c|c|c|c|c|c|c|c|c|c|}
\hline & & \multicolumn{16}{|c|}{ Monthly earnings in the highest $25 \%$ of monthly earnings distribution, dependent on: } \\
\hline & & \multicolumn{16}{|c|}{ Model $2^{2}$} \\
\hline & & \multicolumn{4}{|c|}{$\begin{array}{c}\text { Parents' educational } \\
\text { attainment }^{3}\end{array}$} & \multicolumn{4}{|c|}{ Own educational attainment ${ }^{3}$} & \multicolumn{2}{|c|}{ Gender 4} & \multicolumn{6}{|c|}{ Age group 5} \\
\hline & & \multicolumn{2}{|c|}{$\begin{array}{l}\text { Below upper } \\
\text { secondary }\end{array}$} & \multicolumn{2}{|c|}{ Tertiary } & \multicolumn{2}{|c|}{$\begin{array}{l}\text { Below upper } \\
\text { secondary }\end{array}$} & \multicolumn{2}{|c|}{ Tertiary } & \multicolumn{2}{|c|}{ Men } & \multicolumn{2}{|c|}{$\begin{array}{c}25-34 \\
\text { year-olds }\end{array}$} & \multicolumn{2}{|c|}{$\begin{array}{c}35-44 \\
\text { year-olds }\end{array}$} & \multicolumn{2}{|c|}{$\begin{array}{c}55-64 \\
\text { year-olds }\end{array}$} \\
\hline & & $\mathrm{pp}$ & S.E. & $\mathrm{pp}$ & S.E. & pp & S.E. & pp & S.E. & $\mathrm{pp}$ & S.E. & $\mathrm{pp}$ & S.E. & $\mathrm{pp}$ & S.E. & pp & S.E. \\
\hline & National entities & & & & & & & & & & & & & & & & \\
\hline & Australia & 0 & $(0.02)$ & 7 & $(0.03)$ & -6 & $(0.02)$ & 23 & $(0.02)$ & 23 & $(0.02)$ & -16 & $(0.02)$ & -4 & $(0.02)$ & -9 & $(0.02)$ \\
\hline & Austria & -9 & $(0.02)$ & -4 & $(0.02)$ & -18 & $(0.02)$ & 26 & $(0.02)$ & 23 & $(0.02)$ & -14 & $(0.02)$ & -4 & $(0.02)$ & 2 & $(0.03)$ \\
\hline & Canada & -3 & $(0.01)$ & 4 & $(0.01)$ & -6 & $(0.02)$ & 20 & $(0.01)$ & 22 & $(0.01)$ & -17 & $(0.02)$ & -2 & $(0.02)$ & -3 & $(0.02)$ \\
\hline & Czech Republic & -9 & $(0.03)$ & 12 & $(0.04)$ & -6 & $(0.03)$ & 19 & $(0.04)$ & 21 & $(0.03)$ & 2 & $(0.04)$ & 5 & $(0.04)$ & -4 & $(0.04)$ \\
\hline & Denmark & -2 & $(0.02)$ & 3 & $(0.02)$ & -6 & $(0.02)$ & 23 & $(0.01)$ & 24 & $(0.01)$ & -17 & $(0.02)$ & -2 & $(0.02)$ & -4 & $(0.02)$ \\
\hline & Finland & -3 & $(0.02)$ & 3 & $(0.02)$ & -5 & $(0.03)$ & 24 & $(0.01)$ & 27 & $(0.02)$ & -20 & $(0.02)$ & -5 & $(0.02)$ & -7 & $(0.02)$ \\
\hline & France & -2 & $(0.02)$ & 6 & $(0.02)$ & -10 & $(0.02)$ & 31 & $(0.01)$ & 18 & $(0.01)$ & -25 & $(0.02)$ & -10 & $(0.02)$ & -2 & $(0.02)$ \\
\hline & Germany & -6 & $(0.02)$ & 2 & $(0.02)$ & -11 & $(0.02)$ & 30 & $(0.02)$ & 24 & $(0.01)$ & -15 & $(0.02)$ & -4 & $(0.02)$ & -7 & $(0.03)$ \\
\hline & Ireland & -2 & $(0.02)$ & 1 & $(0.03)$ & -11 & $(0.02)$ & 27 & $(0.02)$ & 17 & $(0.02)$ & -24 & $(0.03)$ & -6 & $(0.02)$ & -5 & $(0.03)$ \\
\hline & Italy & -8 & $(0.02)$ & 2 & $(0.05)$ & -10 & $(0.02)$ & 21 & $(0.03)$ & 21 & $(0.02)$ & -24 & $(0.03)$ & -9 & $(0.03)$ & 5 & $(0.05)$ \\
\hline & Japan & -3 & $(0.02)$ & 7 & $(0.02)$ & -5 & $(0.03)$ & 14 & $(0.02)$ & 33 & $(0.01)$ & -31 & $(0.02)$ & -10 & $(0.02)$ & -9 & $(0.03)$ \\
\hline & Korea & -5 & $(0.02)$ & 3 & $(0.03)$ & -10 & $(0.02)$ & 19 & $(0.02)$ & 21 & $(0.01)$ & -23 & $(0.03)$ & 0 & $(0.02)$ & -4 & $(0.02)$ \\
\hline & Netherlands & -3 & $(0.02)$ & 1 & $(0.02)$ & -7 & $(0.02)$ & 28 & $(0.02)$ & 32 & $(0.01)$ & -20 & $(0.02)$ & -1 & $(0.02)$ & -1 & $(0.02)$ \\
\hline & Norway & -5 & $(0.02)$ & 2 & $(0.02)$ & -8 & $(0.02)$ & 21 & $(0.02)$ & 28 & $(0.01)$ & -17 & $(0.02)$ & -3 & $(0.02)$ & -6 & $(0.02)$ \\
\hline & Poland & -9 & $(0.02)$ & 0 & $(0.03)$ & -8 & $(0.03)$ & 31 & $(0.02)$ & 17 & $(0.02)$ & -9 & $(0.03)$ & -3 & $(0.03)$ & -1 & $(0.03)$ \\
\hline & Slovak Republic & -11 & $(0.02)$ & 13 & $(0.04)$ & -9 & $(0.02)$ & 23 & $(0.03)$ & 21 & $(0.02)$ & -3 & $(0.02)$ & 0 & $(0.02)$ & -1 & $(0.03)$ \\
\hline & Spain & -8 & $(0.03)$ & -3 & $(0.03)$ & -12 & $(0.02)$ & 24 & $(0.02)$ & 17 & $(0.02)$ & -20 & $(0.02)$ & -8 & $(0.02)$ & 5 & $(0.03)$ \\
\hline & Sweden & -8 & $(0.02)$ & -1 & $(0.03)$ & -13 & $(0.03)$ & 20 & $(0.02)$ & 23 & $(0.02)$ & -19 & $(0.02)$ & -5 & $(0.02)$ & -2 & $(0.02)$ \\
\hline & United States & -10 & $(0.02)$ & 4 & $(0.02)$ & -13 & $(0.02)$ & 25 & $(0.02)$ & 18 & $(0.02)$ & -19 & $(0.02)$ & -3 & $(0.03)$ & -1 & $(0.02)$ \\
\hline & Flanders (Belgium) & -6 & $(0.02)$ & 1 & $(0.02)$ & -8 & $(0.02)$ & 25 & $(0.02)$ & 22 & $(0.02)$ & -23 & $(0.02)$ & -9 & $(0.02)$ & 0 & $(0.03)$ \\
\hline & England (UK) & -5 & $(0.02)$ & 10 & $(0.03)$ & -4 & $(0.03)$ & 20 & $(0.02)$ & 23 & $(0.02)$ & -17 & $(0.03)$ & 1 & $(0.03)$ & -7 & $(0.02)$ \\
\hline & Northern Ireland (UK) & -4 & $(0.03)$ & 6 & $(0.04)$ & -15 & $(0.03)$ & 27 & $(0.03)$ & 20 & $(0.02)$ & -18 & $(0.03)$ & -4 & $(0.03)$ & -2 & $(0.04)$ \\
\hline & England/N. Ireland (UK) & -5 & $(0.02)$ & 10 & $(0.03)$ & -5 & $(0.03)$ & 20 & $(0.02)$ & 22 & $(0.02)$ & -17 & $(0.03)$ & 1 & $(0.03)$ & -7 & $(0.02)$ \\
\hline & Average & -5 & $(0.00)$ & 4 & $(0.01)$ & -9 & $(0.01)$ & 23 & $(0.00)$ & 23 & $(0.00)$ & -17 & $(0.01)$ & -3 & $(0.01)$ & -3 & $(0.01)$ \\
\hline & Russian Federation* & -13 & $(0.03)$ & 5 & $(0.04)$ & -2 & $(0.08)$ & 0 & $(0.03)$ & 14 & $(0.04)$ & 0 & $(0.04)$ & -5 & $(0.06)$ & -9 & $(0.04)$ \\
\hline
\end{tabular}

1. Model 1 is a linear regression where the dependent variable is "monthly earnings in the highest $25 \%$ of monthly earnings distribution" and where the independent variables are parents' educational attainment, gender and age group. The difference with Model 2 is that it doesn't include "own educational attainment".

2. Model 2 is a linear regression where the dependent variable is "monthly earnings in the highest $25 \%$ of monthly earnings distribution" and where the independent variables are parents' educational attainment, own educational attainment, gender and age group.

3 . The reference category is upper secondary or post-secondary non-tertiary education.

4. The reference category is women.

5. The reference category is $45-54$ year-olds.

* See note on data for the Russian Federation in the Methodology section.

Source: OECD. Survey of Adult Skills (PIAAC) (2012). See Annex 3 for notes (www.oecd.org/education/education-at-a-glance-19991487.htm).

Please refer to the Reader's Guide for information concerning symbols for missing data and abbreviations.

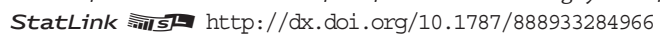




\section{INDICATOR A5 HOW DOES EDUCATIONAL ATTAINMENT AFFECT PARTICIPATION IN THE LABOUR MARKET?}

- On average, over $80 \%$ of tertiary-educated people are employed compared with over $70 \%$ of people with an upper secondary or post-secondary non-tertiary education and less than $60 \%$ of people with below upper secondary education.

- Unemployment rates are higher among younger adults (25-34 year-olds) than among older adults (55-64 year-olds), for all levels of education.

- Despite their higher educational attainment, young women still have lower employment rates than young men, although the gender gap is much narrower among tertiary-educated young adults than among those with lower educational attainment.

\section{Chart A5.1. Unemployment rates, by educational attainment (2014)} 25-64 year-olds

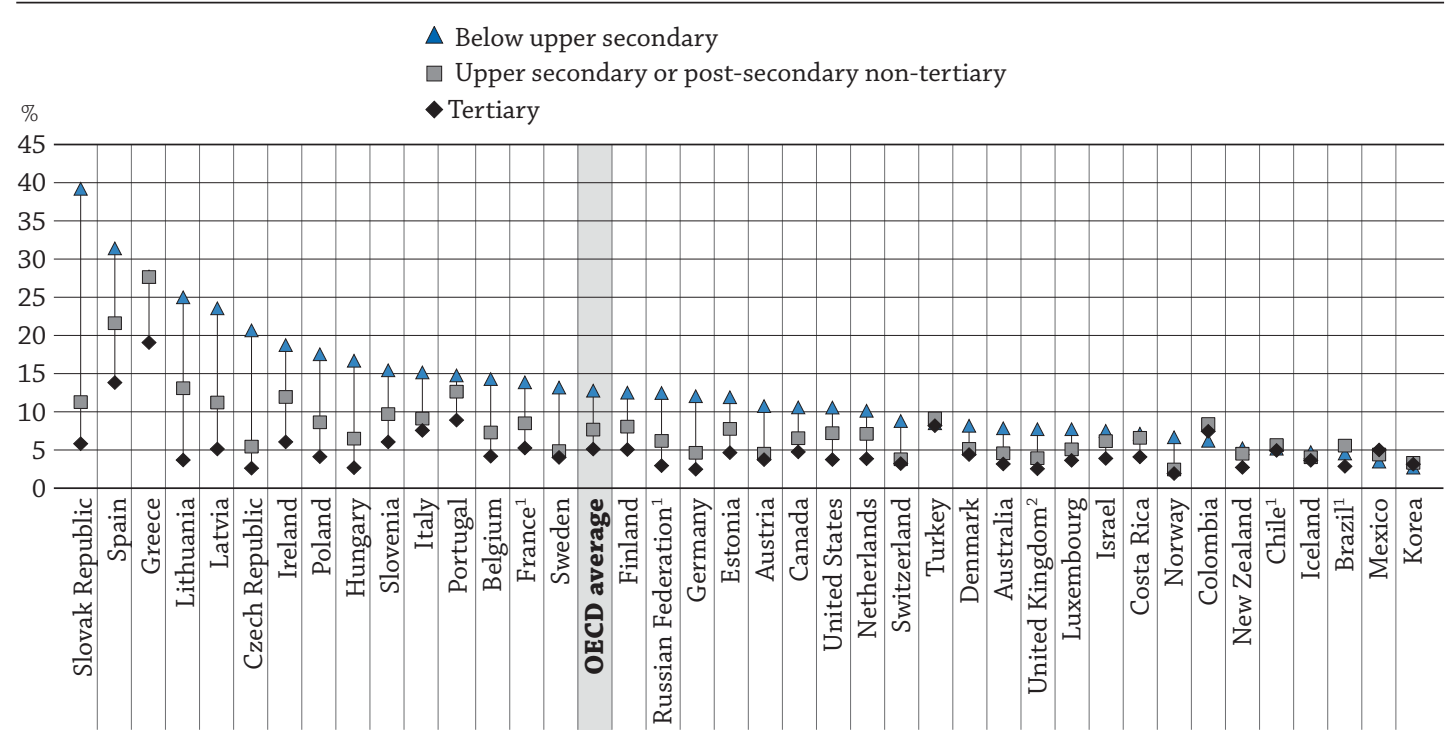

1. Brazil, Chile, France, the Russian Federation: Data for year 2014 refer to year 2013.

2. The United Kingdom: Data for upper secondary attainment includes completion of a sufficient volume and standard of programmes that would be classified individually as completion of intermediate upper secondary programmes (18\% of the adults are under this group).

Countries are ranked in descending order of the unemployment rate of adults with below upper secondary education.

Source: OECD. Table A5.4a. See Annex 3 for notes (www.oecd.org/education/education-at-a-glance- 19991487.htm).

StatLink 게에 $\mathrm{http}: / / \mathrm{dx}$.doi.org/10.1787/888933283600

\section{Context}

Educational qualifications are frequently used as a proxy measure of the skills available in the population and the labour force. The economies of OECD countries depend upon a sufficient supply of high-skilled workers. In most OECD countries, people with high qualifications are most likely to be employed. At the same time, people with the lowest educational qualifications are at greater risk of being unemployed. Given the technological advances that have been transforming the needs of the global labour market, people with higher or specific skills are in strong demand. Favourable employment prospects confirm the value of attaining high levels of education: on average, $12.8 \%$ of adults with low qualifications are unemployed, while among those with tertiary qualifications only $5.1 \%$ are unemployed. 


\section{Other findings}

- Unemployment rates are slightly lower among individuals with vocational upper secondary or postsecondary non-tertiary education (8.5\%), on average, than among individuals with a general upper secondary education (8.9\%).

- In Colombia and Mexico, unemployment rates are higher among tertiary-educated adults $(7.4 \%$ and $5.0 \%$, respectively) than among those with below upper secondary education $(6.2 \%$ and $3.5 \%$, respectively).

- Employment rates among adults without an upper secondary qualification are below $40 \%$ in the Slovak Republic (33\%) and Poland (39\%).

\section{INDICATOR A5}




\section{Analysis}

\section{Labour market outcomes}

Chart A5.1 shows that, across all countries for which data are available, having a tertiary education reduces the risks of being unemployed. Across OECD countries, $5.1 \%$ of adults with tertiary education are unemployed compared with $7.7 \%$ of adults with upper secondary or post-secondary non-tertiary education, and $12.8 \%$ of adults with below upper secondary education (Table A5.4a).

The difference in the unemployment rates between high- and low-qualified adults is largest in the Slovak Republic: $5.8 \%$ of tertiary-educated adults are unemployed compared with $39.2 \%$ of adults with below upper secondary education. More than 20\% of adults with below upper secondary education in the Czech Republic and Latvia are unemployed, as are $31.4 \%$ of adults with that level of education in Spain. In all three countries unemployment rates among tertiary-educated adults are around 18 percentage points lower than unemployment rates among adults with below upper secondary education. Some other countries show relatively low unemployment rates across all levels of education. For instance, in Chile, the unemployment rate for adults with tertiary education (4.9\%) is similar to that of adults with upper secondary or post-secondary non-tertiary education (5.6\%) or with below upper secondary education (5.2\%) (Table A5.4a).

On average across OECD countries, over $80 \%$ of tertiary-educated adults are employed compared with over $70 \%$ of adults with upper secondary or post-secondary non-tertiary education as their highest level of attainment, and less than $60 \%$ of people with below upper secondary as their highest level of attainment. In some countries, the difference in employment rates between people who hold a tertiary qualification and those whose highest qualification is below upper secondary education is large. In Austria, Belgium, the Czech Republic, Finland, France, Germany, Hungary, Ireland, Israel, Latvia, Lithuania, Poland, the Russian Federation, the Slovak Republic and Slovenia, for example, the difference in employment rates between these two groups is at least 30 percentage points (Table A5.3a).

\section{Chart A5.2. Employment rates for younger and older tertiary-educated adults (2014)} 25-34 and 55-64 year-olds, and percentage-point difference between these two groups

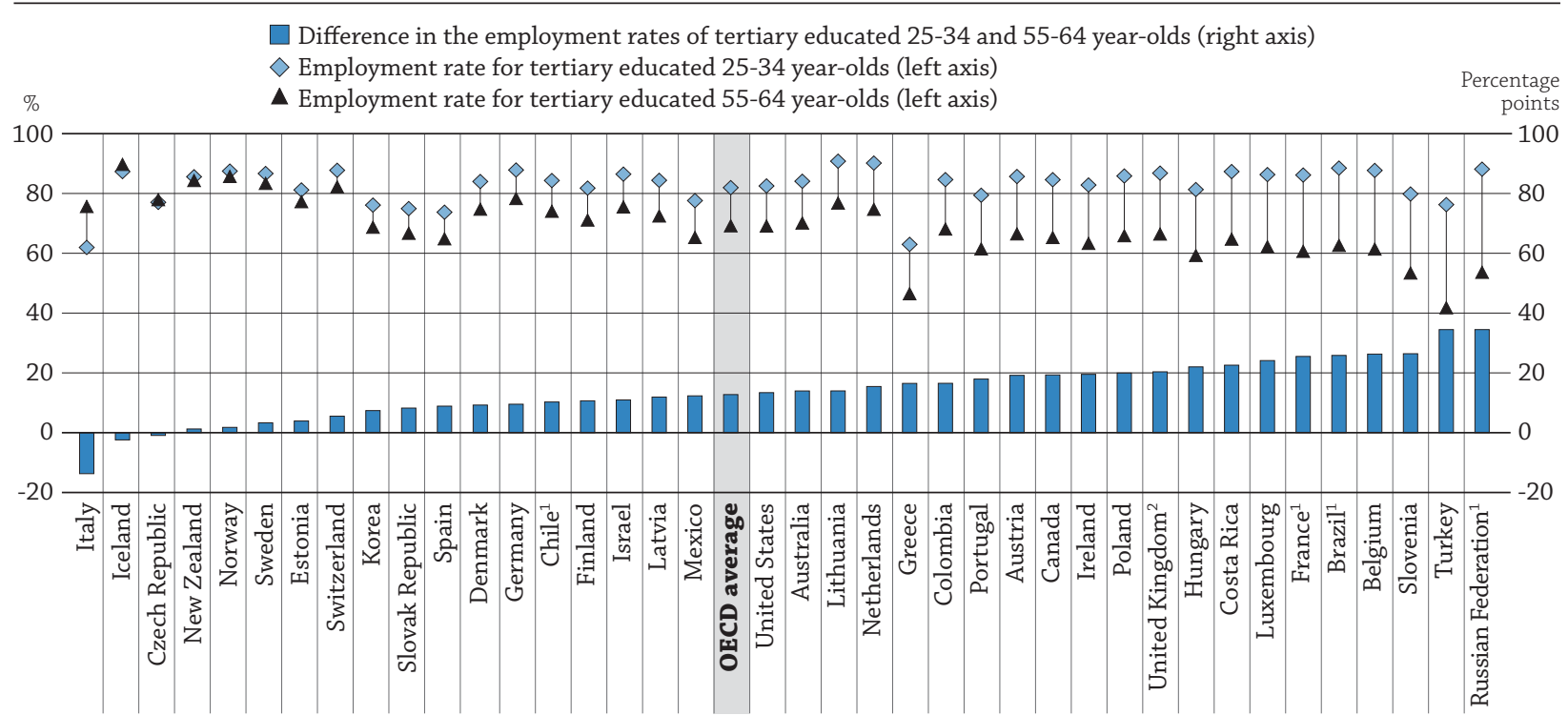

1. Brazil, Chile, France, the Russian Federation: Data for year 2014 refer to year 2013.

2. The United Kingdom: Data for upper secondary attainment includes completion of a sufficient volume and standard of programmes that would be classified individually as completion of intermediate upper secondary programmes (18\% of the adults are under this group).

Countries are ranked in ascending order of the percentage-point difference between the employment rate of the tertiary-educated 25-34 and 55-64 year-olds. Source: OECD. Table A5.3a. See Annex 3 for notes (www.oecd.org/education/education-at-a-glance- 19991487.htm).

StatLink 初西 http://dx.doi.org/10.1787/888933283613

\section{By age group}

A larger proportion of older adults (55-64 year-olds) than younger adults (25-34 year-olds) are out of the labour force, largely because of retirement. Chart A5.2 shows that employment rates are consistently higher for younger tertiary-educated adults. The proportion of 25-34 year-olds with tertiary education who are employed is, 
on average, about 13 percentage points larger than that of 55-64 year-olds who have attained the same level of education ( $82 \%$ and $69 \%$, respectively). The Russian Federation and Turkey show the largest difference in employment rates between younger and older adults (34 percentage points). In some countries, such as the Russian Federation, the large difference in employment rates is due to lower retirement ages (60 years or younger). In both countries, employment rates of tertiary-educated older adults ( $54 \%$ and $42 \%$, respectively) are below the OECD average (69\%), but are close to or above the OECD average among younger adults ( $88 \%$ and $76 \%$, respectively) (Table A5.3a).

The largest gaps in employment rates between age groups and educational attainment are seen in Austria, Belgium, Mexico, the Russian Federation, Slovenia and Turkey among adults with upper secondary or post-secondary non-tertiary education. In those countries, the employment rates between younger and older adults with this level of attainment differ by more than 35 percentages points (Table A5.3a).

Unemployment hits younger people the hardest, and unemployment rates are higher among younger adults (25-34 year-olds) than among older adults (55-64 year-olds), for all levels of education. On average across OECD countries, about $9 \%$ of older adults who have not attained upper secondary education are unemployed compared with about $19 \%$ of younger adults with the same level of education. Similarly, $10.2 \%$ of younger adults with an upper secondary or post-secondary non-tertiary education are unemployed, compared to $6.5 \%$ of older adults with the same level of education. The gap between the two age groups is the smallest among tertiaryeducated adults: about $7 \%$ of younger adults in this group are unemployed compared to about $4 \%$ of older adults (Table A5.4a).

\section{By gender}

Across all OECD countries and education levels, only $66 \%$ of women are employed compared with $80 \%$ of men despite women's higher educational attainment, in general. On average, employment rates for those with the lowest qualifications (below upper secondary education) are significantly higher among younger men than among younger women. The gender gap in employment rates is the largest among adults with the least education (Tables A5.1b, A5.3b and c, available on line).

On average across OECD countries, the gender difference in employment rates among 25-64 year-olds with below upper secondary education as their highest level of attainment is 20 percentage points (66\% for men and $46 \%$ for women). This difference shrinks to 15 percentage points among individuals with upper secondary or post-secondary non-tertiary education ( $81 \%$ for men and $66 \%$ for women), and to just 9 percentage points among tertiary-educated men and women ( $88 \%$ for men and $79 \%$ for women) (Tables A5.3b and c, available on line).

Although the gap between men's and women's employment rates narrows as educational attainment increases, the employment rate among tertiary-educated women across OECD countries is still considerably lower than that of men - despite the fact that a larger proportion of women (36\%) than men (31\%) in OECD countries now has a tertiary education (Tables A1.4b, A5.3b and c, available on line).

In all OECD countries except the Slovak Republic, the gender gap in employment is smaller among 25-64 year-olds with tertiary education than among those who have not attained upper secondary education. The difference is particularly large in Chile, Mexico and Turkey, where it exceeds 25 percentage points (Tables A5.3b and c, available on line).

Gender differences in unemployment rates are, on average, less pronounced than they are in employment rates. Among adults with below upper secondary education, unemployment rates are similar for women and men (12.6\% and $12.9 \%$, respectively). Among adults who have an upper secondary or post-secondary non-tertiary education, unemployment rates are higher among women (8.6\%) than among men (7.1\%). This also observed among tertiaryeducated adults, where the unemployment rate is about $6 \%$ for women and $5 \%$ for men (Tables A5.4b and c, available on line).

Gender differences in unemployment rates are particularly large in Greece and Turkey. In Turkey, 12.0\% of tertiaryeducated women were unemployed in 2014 compared to only 5.9\% of tertiary-educated men; in Greece, $21.4 \%$ of tertiary-educated women and $16.8 \%$ of tertiary-educated men were unemployed that year. These differences were even more pronounced among adults with upper secondary education as their highest level of attainment: in Turkey, $16.7 \%$ of women were unemployed compared with $7.0 \%$ of men; while in Greece, $33.7 \%$ of women and $22.8 \%$ of men with this level of education were unemployed in 2014 (Tables A5.4b and c, available on line). 


\section{Among those with below upper secondary education}

While there is still work for adults with low levels of education across OECD labour markets, unemployment among these individuals increased in many countries and unemployment rates are higher compared to those seen among better better-qualified people. Some $13 \%$ of adults with below upper secondary education are unemployed, on average. Among this group of adults, unemployment rates exceed the OECD average, and are around 20\% or more, in the Czech Republic, Greece, Latvia, Lithuania, the Slovak Republic and Spain. By contrast, less than 5\% of people with below upper secondary education in Brazil, Iceland, Korea and Mexico are unemployed (Table A5.4a).

Across OECD countries, only about one in two adults with below upper secondary education is employed (56\%) compared with $74 \%$ of adults with upper secondary or post-secondary non-tertiary and $83 \%$ of adults with tertiary qualifications. The employment rates among adults without an upper secondary qualification drop below $40 \%$ in the Slovak Republic (33\%) and Poland (39\%). But in some countries, the employment rates for adults without an upper secondary qualification are high: in Brazil, Colombia, Iceland, Korea, New Zealand, Sweden and Switzerland at least two in three adults with low educational attainment are employed (Table 5.3a).

\section{Among those with upper secondary education (vocational or general)}

Higher levels of attainment come with a change in labour market outcomes. People with upper secondary education have lower unemployment rates $(7.5 \%)$ and higher employment rates $(74 \%)$ than people with a lower level of education ( $12.8 \%$ and $56 \%$, respectively). In some countries that were hit hard by the economic crisis, like Greece, Lithuania and Spain, unemployment rates even among adults with upper secondary or post-secondary non-tertiary education were $20 \%$ or higher in 2014 , and unemployment rates among those with below upper secondary education were even higher (Tables A5.3a and A5.4a).

\section{Chart A5.3. Employment rates among adults whose highest level of education is upper secondary or post-secondary non-tertiary, by programme orientation (2014)

\author{
25-64 year-olds
}

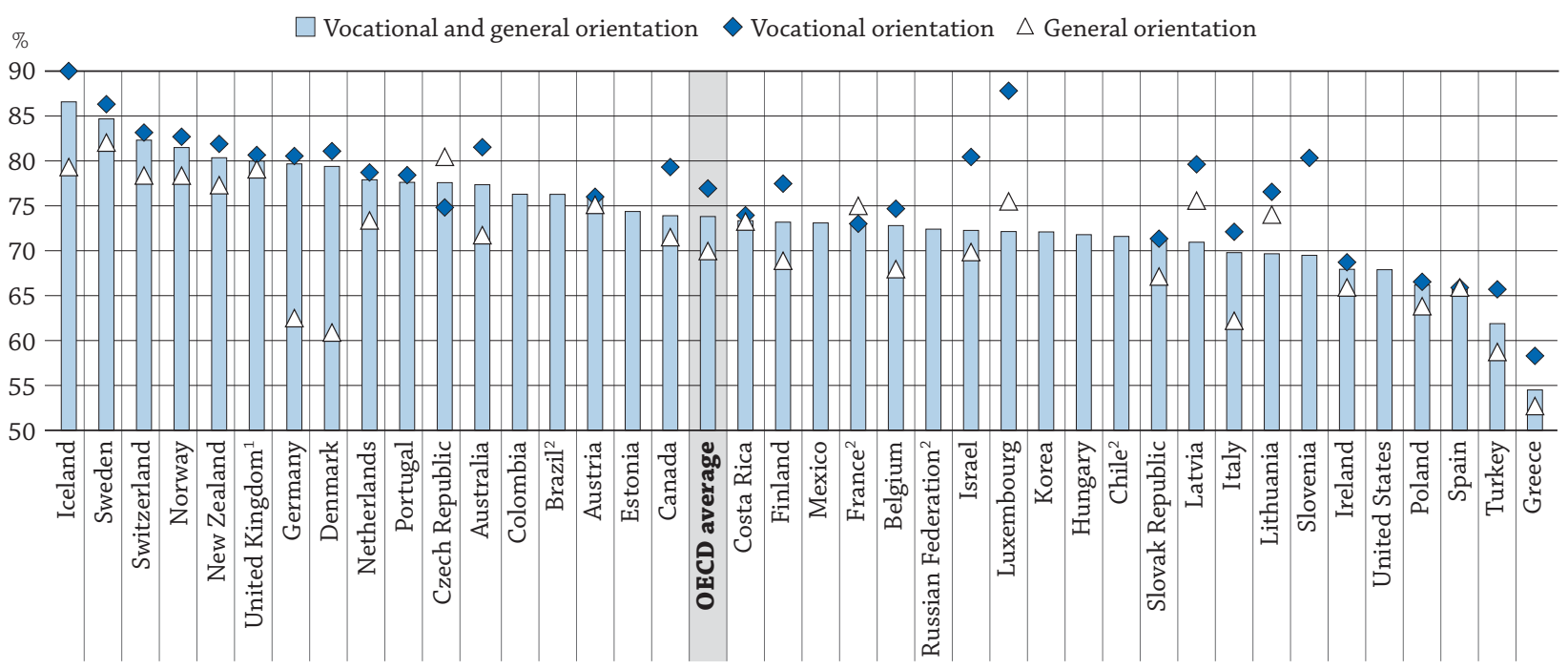

Note: Denmark, Finland, Ireland, Latvia, Lithuania, Luxembourg, Slovenia: Data for the breakdown by programme orientation are only available for 15-34 year-olds and 35-64 year-olds if those individuals had completed their highest level of education 15 years, at most, before the date of the interview; the category "Vocational and general" covers all adults.

1. The United Kingdom: Data for upper secondary attainment includes completion of a sufficient volume and standard of programmes that would be classified individually as completion of intermediate upper secondary programmes ( $18 \%$ of the adults are under this group).

2. Brazil, Chile, France, the Russian Federation: Year of reference 2013.

Countries are ranked in descending order of the employment rate of 25-64 year-olds with upper secondary or post-secondary non-tertiary education as highest level of attainment, regardless of the orientation of the programmes.

Source: OECD. Table A5.5a. See Annex 3 for notes (www.oecd.org/education/education-at-a-glance-19991487.htm).

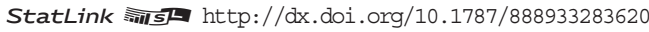

The labour market outcomes of the population with upper secondary or post-secondary non-tertiary education vary according to the type of programme pursued. Across OECD countries, three out of five adults with an upper secondary or post-secondary non-tertiary education have a vocational qualification, while one in three has a general qualification. As shown in Chart A5.3, across OECD countries, $77 \%$ of individuals with a vocational upper secondary 
or post-secondary non-tertiary qualification are employed - a rate that is 7 percentage points higher than that among individuals with a general upper secondary education as their highest qualification. In Denmark, Germany and Slovenia, employment rates are 18 or more percentage points higher among adults with a vocational qualification than among those with a general qualification, whereas in the Czech Republic and France the employment rates among adults with a vocational qualification are slightly lower than among those with a general qualification (Table A5.5a and see Table A1.1a).

The difference may be explained by the high-quality vocational education and training (VET) programmes available in many countries. In some systems, school-based learning is widely combined with workplace learning. Examples of this type of "dual system" can be found in Austria, Germany, the Netherlands and Switzerland. One of the strengths of this practice is that it forms a series of public-private partnerships, allowing social partners and employers to get involved in the development of VET programmes, often including the definition of curricular frameworks. In many of these systems, employers invest significantly in VET programmes by financing apprenticeships, assuming the costs of instructors, materials and/or equipment (CEDEFOP, 2011).

Among other positive effects, combining school-based and workplace learning in an integrated formal education supports the incorporation of VET students into the labour market. Research has shown that VET can yield good economic returns on public investment, and some countries with strong VET systems, like Germany, have been relatively successful in tackling the problem of youth unemployment (CEDEFOP, 2011).

Unemployment rates are generally slightly lower among individuals with vocational upper secondary or postsecondary non-tertiary education ( $8.5 \%$, on average) compared with adults with a general upper secondary education (8.9\%, on average). In Denmark the unemployment rate among individuals with vocational upper secondary or post-secondary non-tertiary education is about 5 percentage points lower than the unemployment rate among individuals with a general upper secondary or post-secondary non-tertiary education. The opposite pattern is observed in the Czech Republic, Greece and Portugal (Table A5.5a).

The lower employment rates and higher unemployment rates for people with an upper secondary or postsecondary non-tertiary general qualification may be also be explained by the fact that adults with a diploma in general programmes are more likely to continue education after they graduate from secondary school, while those who do not pursue further education seem to lack qualifications to ensure a successful integration into the labour market.

\section{Among those with tertiary qualifications}

Across OECD countries, tertiary-educated adults have the best outcomes in the labour market. On average, $83 \%$ of all tertiary-educated adults were employed in 2014 and 5\% were unemployed. Employment rates among tertiaryeducated adults are also higher than among adults with upper secondary vocational qualifications in all countries for which information is available. Some $77 \%$ of adults with a vocational upper secondary qualification were employed in 2014 compared with $83 \%$ of adults with a tertiary qualification. Only in a couple of countries are the differences in rates between the two levels of qualifications small and comparable. Employment rates among adults with upper secondary VET qualifications are similar to those among adults with tertiary education by less than three percentage points in Australia, Canada and Iceland (Tables A5.3a and A5.5a).

Unemployment rates are generally lower for adults with tertiary education than for adults with less education. Some 13\% of adults without upper secondary education were unemployed in 2014 compared with $5.1 \%$ of adults with tertiary education. However, unemployment rates are still high among younger adults (25-34 year-olds) with a tertiary qualification in some countries, namely Greece (32.5\%), Italy (17.7\%), Portugal (14.0\%), Slovenia (11.9\%), Spain (19.4\%) and Turkey (11.4\%). In addition, in general, younger adults with tertiary education have higher unemployment rates than older adults with comparable qualifications: the OECD average is $7.5 \%$ among 25-34 year-olds and 3.8\% among 55-64 year-olds (Table A5.4a).

In Colombia and Mexico, unemployment rates are higher among tertiary-educated adults than among those with below upper secondary as their highest level of attainment. For example, in Mexico, unemployment rates increase as education levels increase. The unemployment rate is higher among tertiary-educated adults than among those who have not attained upper secondary education. This is the case among all adults ( $5.0 \%$ and $3.5 \%$, respectively) as among older (3.5\% and $2.5 \%$, respectively) and younger ( $7.3 \%$ and $4.7 \%$, respectively) adults. In Mexico, the highest unemployment rates across all levels of education are those for the tertiary-educated, 25-34 year-old men (7.9\%) (Table A5.4a). 
Chart A5.4. Unemployment rates for 25-34 year-olds with tertiary education, by gender (2014)

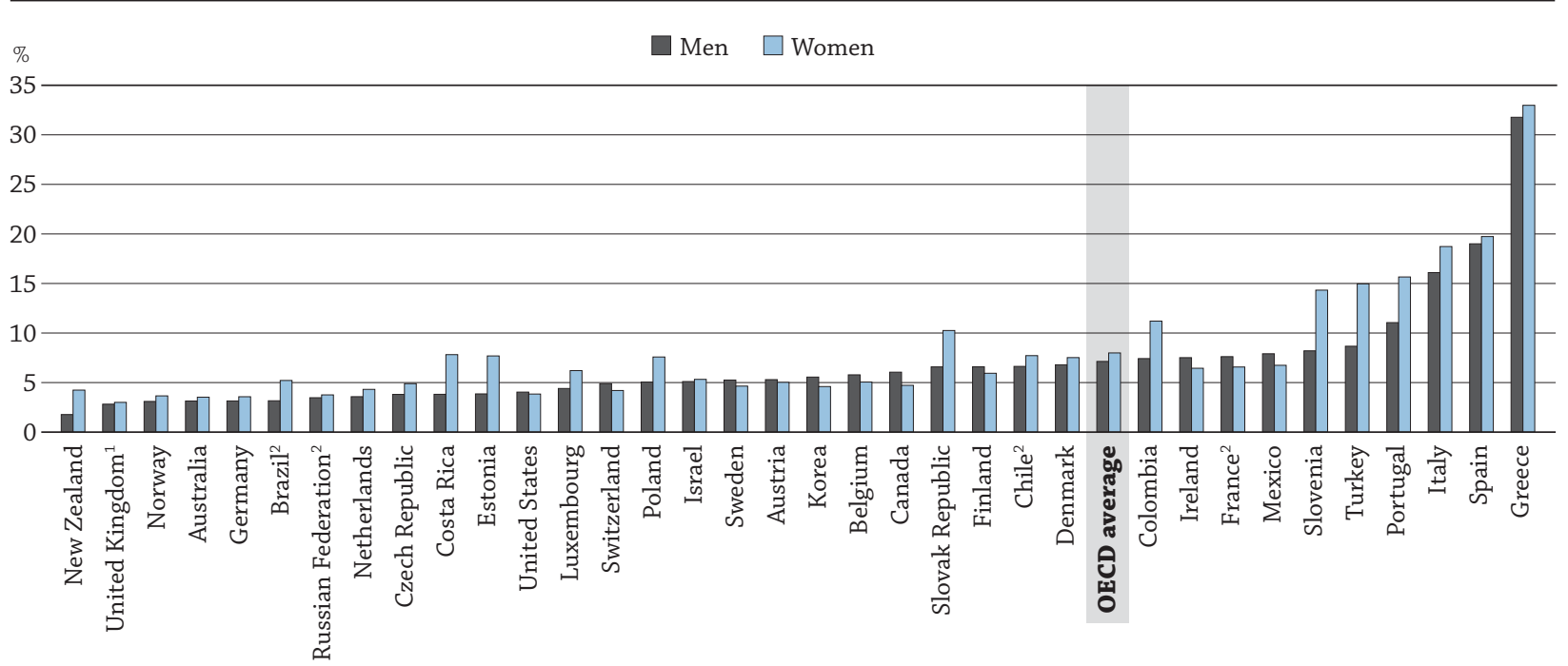

1. The United Kingdom: Data for upper secondary attainment includes completion of a sufficient volume and standard of programmes that would be classified individually as completion of intermediate upper secondary programmes (18\% of the adults are under this group).

2. Brazil, Chile, France, the Russian Federation: Data for year 2014 refer to year 2013.

Countries are ranked in ascending order of the unemployment rate of tertiary-educated 25-34 year-old men.

Source: OECD. Tables A5.4b and c, available on line. See Annex 3 for notes (www.oecd.org/education/education-at-a-glance- 19991487.htm).

StatLink 제이 http://dx.doi.org/10.1787/888933283632

In general, the higher the level of educational attainment, the higher the employment rate and the lower the unemployment rate. This holds true for the various levels within tertiary education, as well. In OECD countries, the employment rate among adults with a short-cycle tertiary qualification or bachelor's or equivalent degree is about 10 percentage points lower than the employment rate among adults with a doctoral or equivalent degree $(79 \%, 82 \%$ and $91 \%$, respectively), while the respective unemployment rates are about two percentage points higher (5.1\%, $5.6 \%$ and $3.4 \%$, respectively) (Tables A5.1a and A5.2a).

In most OECD and partner countries, labour market opportunities are better for adults with a master's degree or equivalent than for adults with a bachelor's degree. For instance, in Austria, Chile, Costa Rica, Portugal and Turkey, the unemployment rates among adults with a master's degree are half or less of those among adults with a bachelor's degree. In these countries, except for Costa Rica, the employment rates are about 10 percentage points higher for those with a master's degree (Table A5.1a).

\section{Skills and readiness to use information and communication technologies for problem solving}

The 2012 Survey of Adult Skills, a product of the OECD Programme for the International Assessment of Adult Competencies (PIAAC), measured problem-solving skills in technology-rich environments and estimated the frequency with which adults use those skills at work and at home. Greater proficiency in problem solving in technology-rich environments reflects both better problem-solving skills and better skills in using digital technology, communication tools and networks to acquire and evaluate information, communicate with others and perform practical tasks (PIAAC Expert Group in Problem Solving in Technology-Rich Environments, 2009).

The information gathered through the Survey of Adult Skills allows for the creation of an indicator that measures skills and readiness to use ICT for problem solving. This indicator combines data about performance in the problemsolving assessment and information about why some adults did not participate in the computer-based assessment and thus do not have a score in problem solving (see the Definitions section at the end of this chapter).

For most of today's workers, ICT skills are key to getting a job and/or a better salary. For economies, they are crucial for remaining competitive in the global market. OECD countries anticipate that technology will continue to be a key driver of job creation, and have placed the development of ICT skills as the most important policy strategy for economic recovery (Chinien and Boutin, 2011; OECD, 2010). 
Across all countries, skills and readiness to use ICT for problem solving increase with the complexity of ICT skills required at work. On average, $66 \%$ of adults who reported they are required to complete complex ICT tasks at work have good ICT and problem-solving skills. This proportion is the largest in Sweden (77\%) and Germany (75\%) and the smallest in Ireland (60\%), Poland (53\%) and the Russian Federation (42\%). On average, a smaller proportion of people who are required to complete moderate-level ICT tasks at work has good ICT and problem-solving skills compared to those who are required to complete complex ICT tasks at work. For example, in Estonia $66 \%$ of the people who reported that they are required to complete complex ICT tasks at work have good ICT and problemsolving skills, but this proportion decreases to $39 \%$ among workers who are required to complete moderate-level ICT tasks at work. Across all participating countries and sub-national entities, there is a positive relationship between the complexity of ICT skills required at work and the skills and readiness to use ICT for problem solving (Table A5.6a).

\section{Chart A5.5. Percentage of adults with good information and communication technologies and problem-solving skills, by selected industry (2012)}

Survey of Adult Skills, 25-64 year-olds

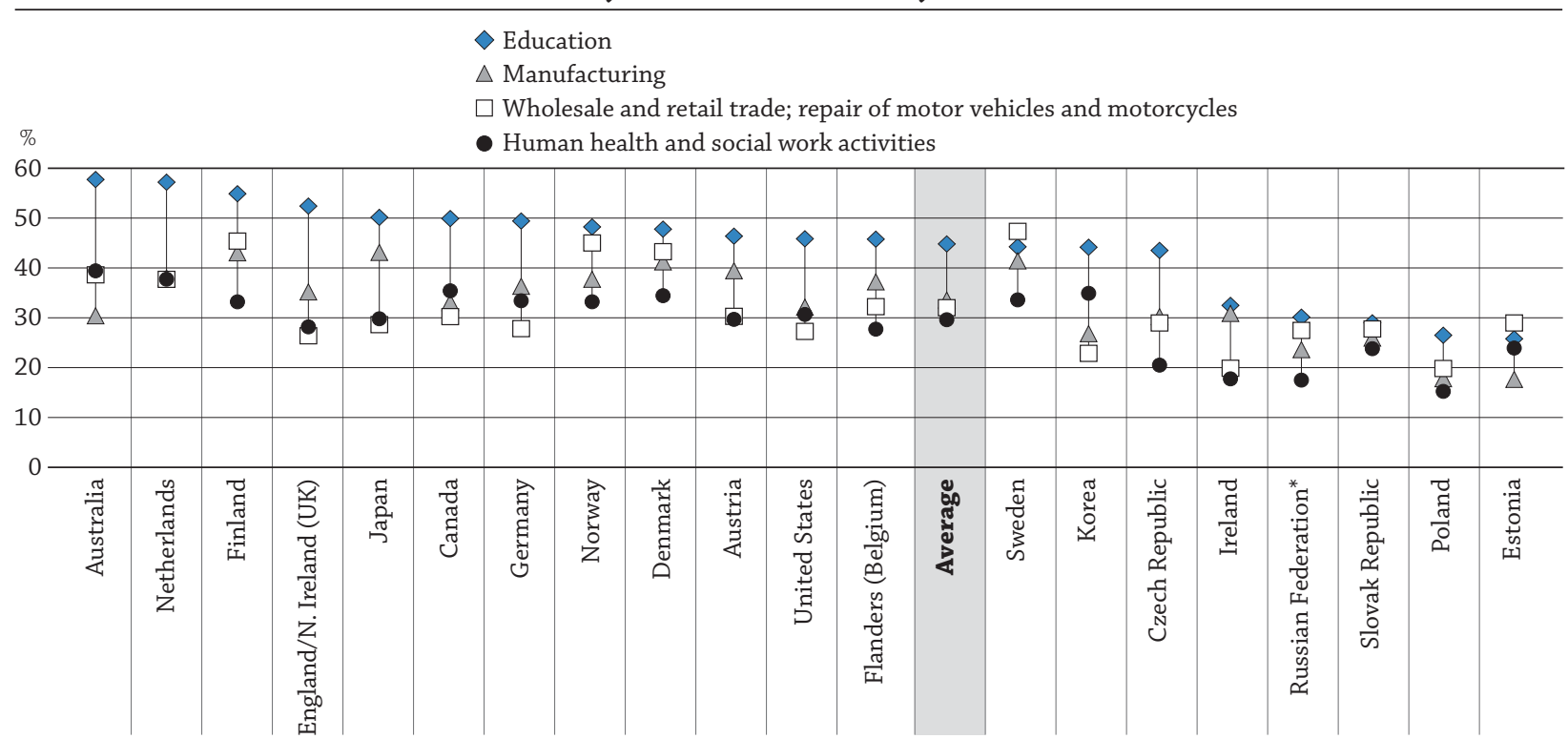

* See note on data for the Russian Federation in the Methodology section.

Countries are ranked in descending order of the percentage of adults working in education with good ICT and problem-solving skills.

Source: OECD. Table A5.6d, available on line. See Annex 3 for notes (www.oecd.org/education/education-at-a-glance- 19991487.htm).

StatLink 제그 http://dx.doi.org/10.1787/888933283646

Chart A5.5 shows the proportion of the survey respondents with the highest skills and readiness to use ICT for problem solving among workers in education, manufacturing, wholesale and retail trade, and human health and social work activities. Across these industries, the largest proportion of respondents with good ICT and problemsolving skills was composed of people who work in education. This is especially true in Australia, Canada, England/ Northern Ireland (UK), Finland, Japan and the Netherlands, where at least one in two respondents who work in education have good ICT and problem-solving skills. On average, $45 \%$ of workers in education have good ICT and problem-solving skills, as do $34 \%$ of workers in manufacturing, $32 \%$ of workers in wholesale and retail trade, and $30 \%$ of workers in human health and social work activities (Table A5.6d, available on line).

\section{Box A5.1. Labour market outcomes for recent graduates}

The difficulties young adults face in entering the labour force are apparent from both international and national indicators. For example, unemployment rates among tertiary-educated 25-34 year-olds increased 2.0 percentage points between 2005 and 2012 to an average of 7.4\% across OECD countries. During the same period, unemployment rates among similarly educated 55-64 year-olds rose by 0.4 percentage points to $3.9 \%$ (Table A5.4a in OECD, 2014a). However, the unemployment rates among graduates who had completed their degrees in the previous year were appreciably higher than these rates, and graduates in some countries could not find work. 
In $2013,31 \%$ of $15-34$ year-olds who had completed an upper secondary or post-secondary non-tertiary programme in the previous year, and who were not enrolled in further education, were unemployed. This unemployment rate ranged from $9 \%$ in Germany and 10\% in Austria and the Netherlands, to 59\% in Italy, 62\% in Spain, and $76 \%$ in Greece. The majority of 15-34 year-old-non-students with an upper secondary or postsecondary non-tertiary education were able to find employment in the year following their graduation. Some $61 \%$ of these non-students across the 26 OECD countries with available data were employed in 2013 . There was a wide range in employment rates among recent graduates from this level of education, ranging from $16 \%$ in Greece, 26\% in Italy and 31\% in Spain to 84\% in Austria and Iceland, and 85\% in Germany.

\section{Chart A5.a. Employment rates of 15-34 year-olds with upper secondary or post-secondary non-tertiary education not in education or training, by years since graduation (2013)}

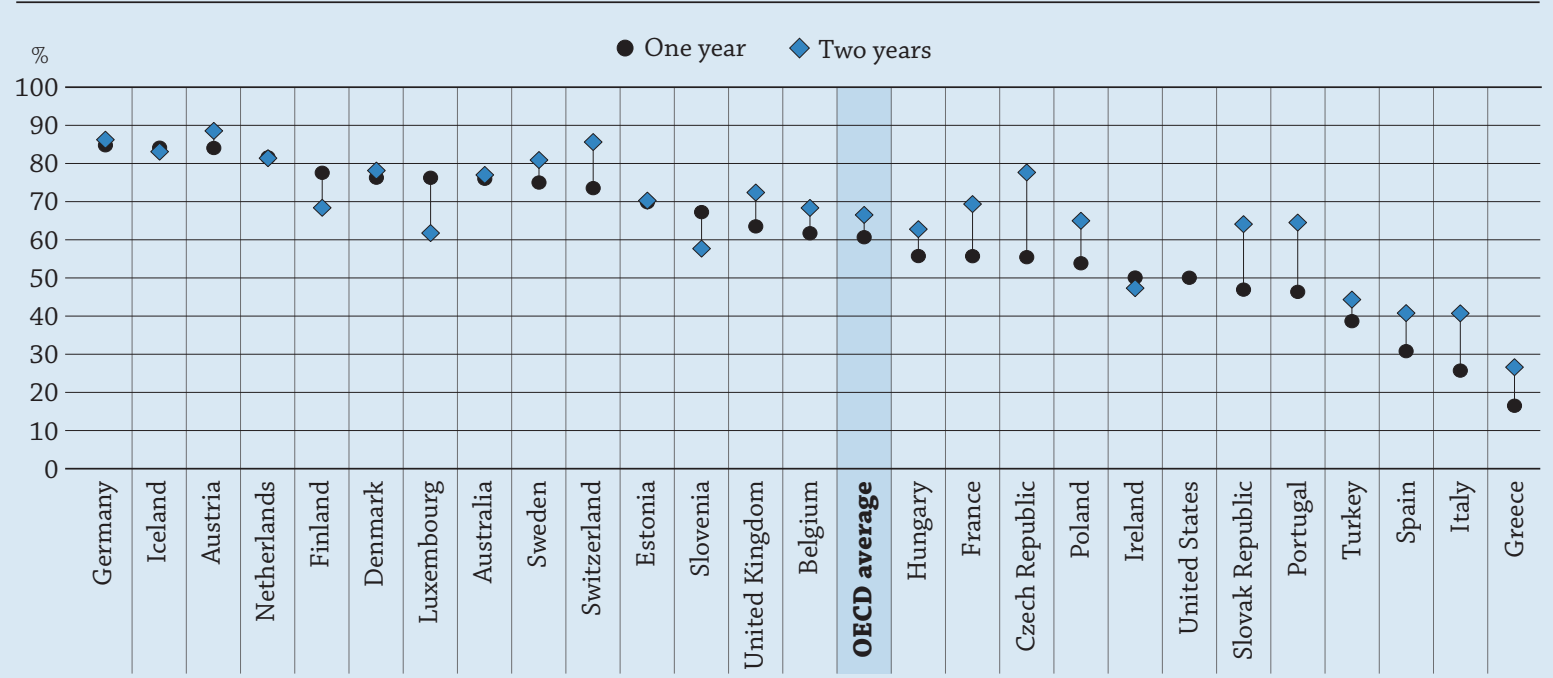

Countries are ranked in descending order of the employment rate after one year since graduation.

Source: OECD. Table A5.a, available on line. See Annex 3 for notes (www.oecd.org/education/education-at-a-glance-19991487.htm).

StatLink त्नाजा http://dx.doi.org/10.1787/888933283658

\section{Chart A5.b. Employment rates among 20-34 year-olds with tertiary education and not in education or training, by years since graduation (2013)}

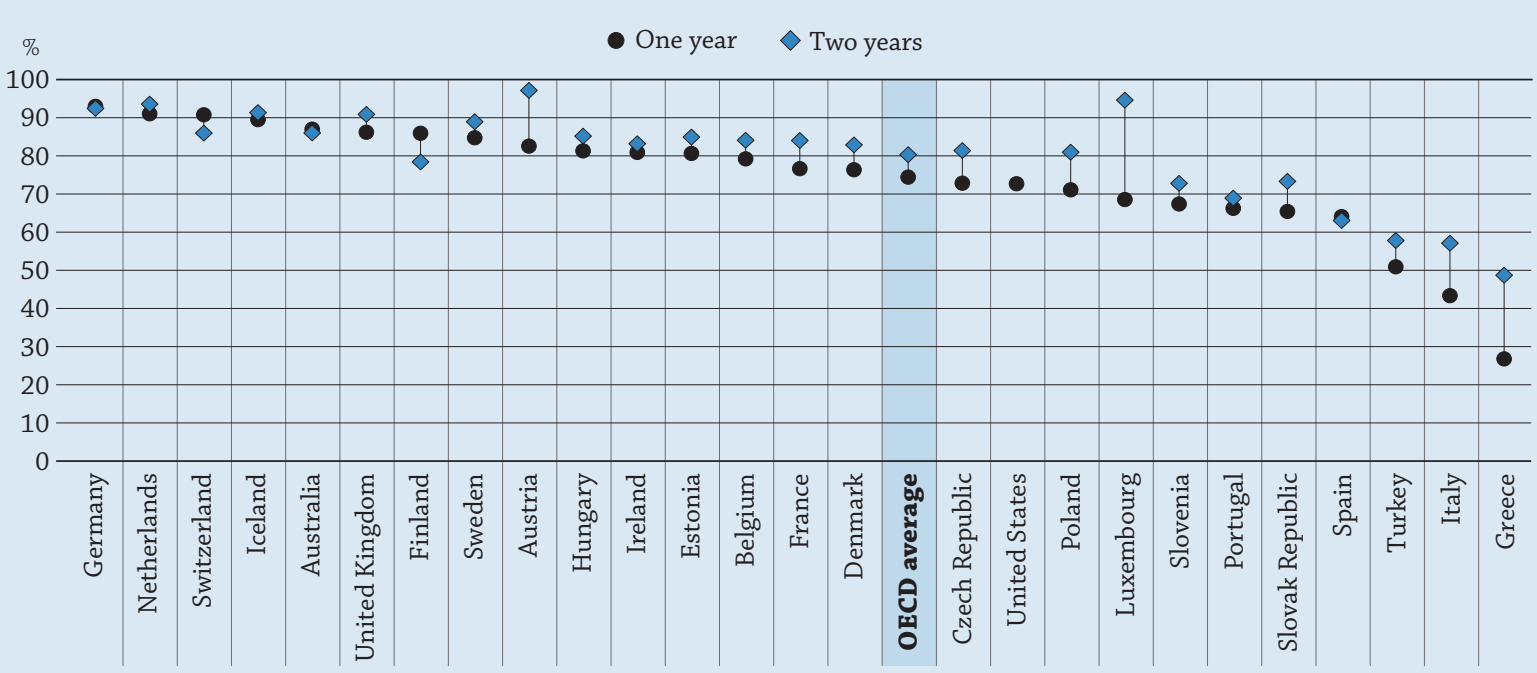

Countries are ranked in descending order of the employment rate after one year since graduation.

Source: OECD. Table A5.b, available on line. See Annex 3 for notes (www.oecd.org/education/education-at-a-glance-19991487.htm).

StatLink 제매 http://dx.doi.org/10.1787/888933283660 
Most countries show higher employment rates among adults with tertiary education compared to adults with upper secondary or post-secondary non-tertiary education. For example, the average employment rate one year after completing education among tertiary-educated 20-34 year-olds who were no longer in education or training was $74 \%$ in 2013 , compared to $61 \%$ among $15-34$ year-olds who had completed only upper secondary or post-secondary non-tertiary education. Some countries, such as Austria and Denmark, showed similar employment rates for these two groups of graduates. However, many countries showed wide differences, including Spain (64\% among those with tertiary education compared to $31 \%$ among those with an upper secondary or post-secondary non-tertiary education) and the United States (73\% and 50\%, respectively). Employment rates among 20-34 year-old non-students with a tertiary education ranged from $27 \%$ in Greece, $43 \%$ in Italy and 51\% in Turkey to $91 \%$ in the Netherlands and Switzerland, and $93 \%$ in Germany.

In $2013,19 \%$ of $20-34$ year-old non-students who had completed a tertiary programme in the past year were unemployed compared to $31 \%$ of 15 -34 year-old non-students who had completed an upper secondary or postsecondary non-tertiary programme. Consistent with other findings, unemployment rates among young adults with tertiary education fell in the years following their graduation. Some $19 \%$ of tertiary-educated young adults were unemployed in the year following graduation, compared to $14 \%$ two years after graduation and $13 \%$ three years after graduation. As observed in the unemployment rates among young non-students with an upper secondary or post-secondary non-tertiary education, there was also a wide range in unemployment rates among young tertiary-educated non-students. Unemployment rates among those who had completed a tertiary programme in the previous year ranged from $4 \%$ in Germany, $7 \%$ in Australia, $8 \%$ in the Netherlands and $9 \%$ in Sweden, to 34\% in Spain, 37\% in Italy and 62\% in Greece. While the unemployment rates were typically lower two or three years after graduation, the high rates for young non-students in some countries underscore the challenges in securing stable employment.

\section{Box A5.1 Tables}

WEB Table A5.a. Employment and unemployment rates among 15-34 year-olds with upper secondary or post-secondary non-tertiary education and not in education or training, by years since graduation $(2011,2012,2013)$

WEB Table A5.b. Employment and unemployment rates among 20-34 year-olds with tertiary education and not in education or training, by years since graduation (2011, 2012, 2013)

\section{Definitions}

Active population (labour force) is the total number of employed and unemployed persons, in accordance with the definition in the Labour Force Survey.

Age groups: Adults refers to 25-64 year-olds; younger adults refers to 25-34 year-olds; and older adults refers to 55-64 year-olds. The working-age population is the total population aged 25-64.

Employed individuals are those who, during the survey reference week: $i$ ) work for pay (employees) or profit (self-employed and unpaid family workers) for at least one hour; or ii) have a job but are temporarily not at work (through injury, illness, holiday, strike or lock-out, educational or training leave, maternity or parental leave, etc.).

The employment rate refers to the number of persons in employment as a percentage of the working-age population (the number of employed people is divided by the number of all working-age people). Employment rates by gender, age, educational attainment, programme orientation and age groups are calculated within each of these categories; for example the employment rate among women is calculated by dividing the number of employed women by the total number of working-age women.

Inactive individuals are those who are, during the survey reference week, neither employed nor unemployed, i.e. individuals who are not looking for a job. The number of inactive individuals is calculated by subtracting the number of active people (labour force) from the number of all working-age people.

The inactive rate refers to inactive persons as a percentage of the population (i.e. the number of inactive people is divided by the number of all working-age people). Inactive rates by gender, age, educational attainment, programme 
orientation and age groups are calculated within each of these categories; for example, the inactive rate among individuals with a tertiary education degree is calculated by dividing the number of inactive individuals with tertiary education by the total number of working-age people with tertiary education.

Completion of intermediate programmes for educational attainment (ISCED 2011) corresponds to recognised qualification from an ISCED 2011 level programme which is not considered as sufficient for ISCED 2011 level completion and is classified at a lower ISCED 2011 level. In addition, this recognised qualification does not give direct access to an upper ISCED 2011 level programme.

Levels of education: In this Indicator two ISCED (International Standard Classification of Education) classifications are used: ISCED 2011 and ISCED-97.

- When it is specified that ISCED 2011 is used, the levels of education are defined as: below upper secondary corresponds to ISCED 2011 Levels 0, 1 and 2, and includes recognised qualifications from ISCED 2011 Level 3 programmes, which are not considered as sufficient for ISCED 2011 Level 3 completion, and without direct access to post-secondary non-tertiary education or tertiary education; upper secondary or post-secondary nontertiary corresponds to ISCED 2011 Levels 3 and 4; and tertiary corresponds to ISCED 2011 Levels 5, 6, 7 and 8 (UNESCO Institute for Statistics, 2012).

- When it is specified that ISCED-97 is used, the levels of education are defined as: below upper secondary corresponds to ISCED-97 Levels 0, 1, 2 and 3C short programmes; upper secondary or post-secondary nontertiary corresponds to ISCED-97 Levels 3A, 3B, 3C long programmes, and Level 4; and tertiary corresponds to ISCED-97 Levels 5A, 5B and 6.

See the section About the new ISCED 2011 classification, at the beginning of this publication, for a presentation of all ISCED 2011 levels, and Annex 3 for a presentation of all ISCED-97 levels.

Skill groups refer to skills and readiness to use information and communication technologies (ICT) for problem solving in technology-rich environments. Each group is described in terms of the characteristics of the types of tasks that can be successfully completed by adults and the related scores in the assessment of problem solving in technology-rich environments in the Survey of Adult Skills.

- Group 0 (no computer experience)

- Group 1 (refused the computer-based assessment)

- Group 2 (failed ICT core stage 1 or minimal problem-solving skills - scored below Level 1 in the problem solving in technology-rich environments assessment)

- Group 3 (moderate ICT and problem-solving skills - scored at Level 1 in the problem solving in technology-rich environments assessment)

- Group 4 (good ICT and problem-solving skills - scored at Level 2 or Level 3 in the problem solving in technologyrich environments assessment)

Skills required at work refers to the use of computers needed at work. Four levels of use are identified: "ICT skills not required at work" corresponds to individuals who reported they do not use a computer in their job; "Straightforward" indicates using a computer for routine tasks, such as data entry or sending and receiving e-mails; "Moderate" indicates using a computer for word-processing, spreadsheets or database management; and "Complex" indicates developing software or modifying computer games, programming using languages like java, sql, php or perl, or maintaining a computer network.

The unemployment rate refers to unemployed persons as a percentage of the labour force (i.e. the number of unemployed people is divided by the sum of employed and unemployed people). Unemployment rates by gender, age, educational attainment, programme orientation and age groups are calculated within each of these categories; for example, the unemployment rate among women is calculated by dividing the number of unemployed women by the total number of women who are active in the labour force.

Unemployed individuals are those who are, during the survey reference week, without work (i.e. neither had a job nor were at work for one hour or more in paid employment or self-employment), actively seeking employment (i.e. had taken specific steps during the four weeks prior to the reference week to seek paid employment or selfemployment), and currently available to start work (i.e. were available for paid employment or self-employment before the end of the two weeks following the reference week). 


\section{Methodology}

Data on population and educational attainment for most countries are taken from OECD and Eurostat databases, which are compiled from National Labour Force Surveys by the OECD LSO (Labour Market and Social Outcomes of Learning) Network. Data on educational attainment for China, Colombia, Indonesia, Saudi Arabia and South Africa are taken from the UNESCO Institute of Statistics (UIS) database on educational attainment of the population aged 25 and older. Data on proficiency levels and mean scores are based on the Survey of Adult Skills (PIAAC) (2012). PIAAC is the OECD Programme for the International Assessment of Adult Competencies. See Annex 3 for additional information (www.oecd.org/education/education-at-a-glance-19991487.htm).

\section{Note regarding data from Israel}

The statistical data for Israel are supplied by and are under the responsibility of the relevant Israeli authorities. The use of such data by the OECD is without prejudice to the status of the Golan Heights, East Jerusalem and Israeli settlements in the West Bank under the terms of international law.

\section{Note regarding data from the Russian Federation in the Survey of Adult Skills (PIAAC)}

Readers should note that the sample for the Russian Federation does not include the population of the Moscow municipal area. The data published, therefore, do not represent the entire resident population aged 16-65 in Russia but rather the population of Russia excluding the population residing in the Moscow municipal area. More detailed information regarding the data from the Russian Federation as well as that of other countries can be found in the Technical Report of the Survey of Adult Skills (OECD, 2014b).

\section{References}

Chinien, C. and F. Boutin (2011), "Defining Essential Digital Skills in the Canadian Workplace: Final Report”, WDM-Consultants. European Centre for the Development of Vocational Training (CEDEFOP) (2011), The Benefits of Vocational Education and Training, Publications Office of the European Union, Luxembourg.

OECD (2014a), Education at a Glance 2014: OECD Indicators, OECD Publishing, Paris, http://dx.doi.org/10.1787/eag-2014-en. OECD (2014b), Technical Report of the Survey of Adult Skills, www.oecd.org/site/piaac/_Technical\%20Report_17OCT13.pdf, pre-publication copy.

OECD (2013), OECD Skills Outlook 2013: First Results from the Survey of Adult Skills, OECD Publishing, Paris, http://dx.doi. org/10.1787/9789264204256-en.

OECD (2010), "Highlights", in OECD Information Technology Outlook 2010, OECD Publishing, Paris, http://dx.doi.org/10.1787/ it outlook-2010-2-en.

PIAAC Expert Group in Problem Solving in Technology-Rich Environments (2009), "PIAAC Problem Solving in TechnologyRich Environments: A Conceptual Framework”, OECD Education Working Papers, No. 36, OECD Publishing, Paris, http://dx.doi. org/10.1787/220262483674.

UNESCO Institute for Statistics (2012), International Standard Classification of Education: ISCED 2011, UNESCO-UIS, Montreal, Canada, www.uis.unesco.org/Education/Documents/isced-2011-en.pdf.

\section{Indicator A5 Tables}

\begin{tabular}{|c|c|c|}
\hline & Table A5.1a & Employment rates, by educational attainment (2014) \\
\hline WEB & Table A5.1b & Employment rates, by educational attainment and gender (2014) \\
\hline & Table A5.2a & Unemployment rates, by educational attainment (2014) \\
\hline WEB & Table A5.2b & Unemployment rates, by educational attainment and gender (2014) \\
\hline & Table A5.3a & Trends in employment rates, by educational attainment and age group $(2000,2005,2010$ and 2014) \\
\hline WEB & Table A5.3b & $\begin{array}{l}\text { Trends in employment rates among men, by educational attainment and age group } \\
(2000,2005,2010 \text { and 2014) }\end{array}$ \\
\hline$W E B$ & Table A5.3c & $\begin{array}{l}\text { Trends in employment rates among women, by educational attainment and age group } \\
(2000,2005,2010 \text { and } 2014)\end{array}$ \\
\hline
\end{tabular}


Table A5.4a Trends in unemployment rates, by educational attainment and age group (2000, 2005, 2010 and 2014)

WEB Table A5.4b Trends in unemployment rates among men, by educational attainment and age group (2000, 2005, 2010 and 2014)

WEB Table A5.4c Trends in unemployment rates among women, by educational attainment and age group $(2000,2005,2010$ and 2014)

Table A5.5a Employment, unemployment and inactivity rates of adults with upper secondary or post-secondary non-tertiary, by programme orientation (2014)

WEB Table A5.5b Employment, unemployment and inactivity rates of adults with upper secondary or post-secondary non-tertiary, by programme orientation and gender (2014)

WEB Table A5.5c Employment, unemployment and inactivity rates of adults with upper secondary or post-secondary non-tertiary, by programme orientation and age group (2014)

Table A5.6a Skills and readiness to use information and communication technologies for problem solving among 25-64 year-olds, by ICT skills required at work (2012)

WEB Table A5.6b Skills and readiness to use information and communication technologies for problem solving among 25-64 year-olds, by confidence in using computers at work (2012)

WEB Table A5.6c Skills and readiness to use information and communication technologies for problem solving among 25-64 year-olds, by impact of ICT skills on career opportunities (2012)

WEB Table A5.6d Skills and readiness to use information and communication technologies for problem solving among 25-64 year-olds, by selected industry (2012) 
Table A5.1a. Employment rates, by educational attainment (2014) Percentage of employed 25-64 year-olds among all 25-64 year-olds

\begin{tabular}{|c|c|c|c|c|c|c|c|c|c|c|c|c|}
\hline & \multicolumn{5}{|c|}{ Below upper secondary } & \multicolumn{2}{|c|}{$\begin{array}{l}\text { Upper secondary } \\
\text { or post-secondary } \\
\text { non-tertiary }\end{array}$} & \multicolumn{4}{|c|}{ Tertiary } & \multirow[b]{2}{*}{$\begin{array}{l}\text { All levels } \\
\text { of } \\
\text { education }\end{array}$} \\
\hline & 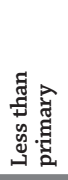 & 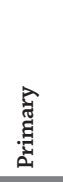 & 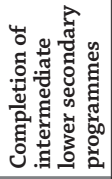 & 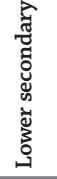 & 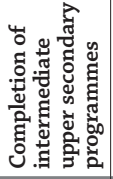 & 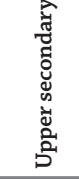 & 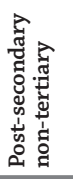 & 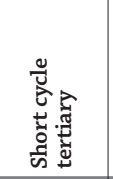 & 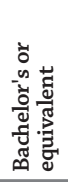 & 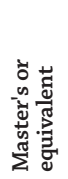 & 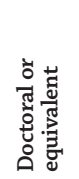 & \\
\hline & (1) & (2) & (3) & (4) & (5) & (6) & (7) & (8) & (9) & (10) & (11) & (12) \\
\hline Australia & $\mathrm{x}(2)$ & $42^{\mathrm{d}}$ & a & 66 & a & 77 & 81 & 80 & 83 & 85 & 92 & 76 \\
\hline Austria & $\mathrm{x}(2)$ & $30^{\mathrm{d}}$ & a & 55 & a & 76 & 80 & 84 & 77 & 89 & 88 & 75 \\
\hline Belgium & 29 & 41 & a & 55 & a & 72 & 82 & 76 & 84 & 86 & 90 & 71 \\
\hline Canada & $x(2)$ & $45^{\mathrm{d}}$ & a & 60 & a & 72 & 79 & 81 & 82 & $83^{\mathrm{d}}$ & $\mathrm{x}(10)$ & 76 \\
\hline Chile $^{1}$ & 53 & 55 & a & 66 & a & 72 & a & 81 & 85 & $94^{\mathrm{d}}$ & $\mathrm{x}(10)$ & 70 \\
\hline Czech Republic & $\mathrm{m}$ & c & a & 44 & a & $78^{\mathrm{d}}$ & $\mathrm{x}(6)$ & 89 & 79 & 86 & 94 & 77 \\
\hline Denmark & $\mathrm{m}$ & 42 & $\mathrm{a}$ & 67 & $\mathrm{a}$ & 79 & 93 & 85 & 84 & 90 & 96 & 78 \\
\hline Estonia & c & 40 & $\mathrm{~m}$ & 63 & $\mathrm{~m}$ & 74 & 75 & 78 & 83 & 86 & 95 & 77 \\
\hline Finland & $\mathrm{x}(2)$ & $38^{\mathrm{d}}$ & a & 60 & a & 73 & 92 & 81 & 82 & 86 & 91 & 75 \\
\hline France $^{1}$ & 40 & 43 & a & 61 & a & 73 & 68 & 84 & 82 & 87 & 87 & 72 \\
\hline Germany & $x(2)$ & $47^{\mathrm{d}}$ & a & 61 & a & 78 & 85 & 90 & 88 & 87 & 93 & 79 \\
\hline Greece & 27 & 43 & 48 & 53 & $53^{r}$ & 54 & 57 & 64 & 67 & 79 & 87 & 56 \\
\hline Hungary & c & 25 & a & 47 & a & 71 & 78 & 82 & 80 & 85 & 88 & 70 \\
\hline Iceland & $x(2)$ & $66^{\mathrm{d}}$ & a & 77 & a & 86 & 95 & 88 & 89 & 94 & 99 & 86 \\
\hline Ireland & c & 35 & a & 55 & a & 67 & 69 & 77 & 81 & 86 & 92 & 69 \\
\hline Israel & 34 & 42 & a & 58 & a & 72 & a & 82 & 86 & 90 & 92 & 76 \\
\hline Italy & 31 & 28 & a & 55 & a & 70 & 73 & c & 69 & 80 & 90 & 63 \\
\hline Japan & $\mathrm{x}(6)$ & $\mathrm{x}(6)$ & a & $\mathrm{x}(6)$ & a & $76^{d}$ & $\mathrm{x}(8)$ & $76^{d}$ & $86^{d}$ & $\mathrm{x}(9)$ & $x(9)$ & 79 \\
\hline Korea & $\mathrm{x}(2)$ & 63 & a & 69 & a & 72 & $\mathrm{~m}$ & 76 & $78^{\mathrm{d}}$ & $\mathrm{x}(9)$ & $x(9)$ & 74 \\
\hline Luxembourg & c & 59 & a & 62 & a & 72 & 75 & 79 & 82 & 89 & 85 & 76 \\
\hline Mexico & 58 & 62 & 68 & 67 & 64 & 73 & a & 71 & $79^{d}$ & $\mathrm{x}(9)$ & $\mathrm{x}(9)$ & 68 \\
\hline Netherlands & 35 & 51 & a & 64 & a & 78 & 87 & 84 & 87 & 89 & 94 & 77 \\
\hline New Zealand & $\mathrm{x}(4)$ & $\mathrm{x}(4)$ & $\mathrm{a}$ & $71^{\mathrm{d}}$ & $\mathrm{a}$ & 77 & 85 & 86 & 87 & 87 & 91 & 80 \\
\hline Norway & 35 & 50 & $\mathrm{a}$ & 62 & $\mathrm{a}$ & 82 & 79 & 84 & 91 & 93 & 97 & 81 \\
\hline Poland & 6 & 40 & a & 44 & a & 66 & 70 & 50 & 82 & 87 & 93 & 69 \\
\hline Portugal & 32 & 60 & a & 72 & a & 78 & 77 & $\mathrm{x}(9)$ & $72^{\mathrm{d}}$ & 85 & 91 & 70 \\
\hline Slovak Republic & c & c & 53 & 31 & c & 71 & 75 & 73 & 74 & 81 & 81 & 69 \\
\hline Slovenia & c & 33 & a & 50 & $\mathrm{a}$ & 69 & $\mathrm{a}$ & 76 & 81 & 86 & 94 & 70 \\
\hline Spain & 25 & 39 & a & 55 & a & 66 & 64 & 74 & 77 & 79 & 87 & 63 \\
\hline Sweden & $\mathrm{x}(2)$ & $44^{\mathrm{d}}$ & $\mathrm{a}$ & 68 & 82 & 85 & 84 & 84 & 89 & 92 & 94 & 83 \\
\hline Switzerland & 53 & 67 & $\mathrm{a}$ & 70 & $\mathrm{a}$ & 82 & a & $x(9,10,11)$ & $89^{d}$ & $88^{\mathrm{d}}$ & $93^{\mathrm{d}}$ & 83 \\
\hline Turkey & 35 & 50 & a & 60 & a & 62 & $\mathrm{a}$ & 68 & 78 & 87 & 95 & 57 \\
\hline United Kingdom & 41 & 61 & a & 60 & 77 & 83 & $\mathrm{a}$ & 83 & 85 & 86 & 91 & 78 \\
\hline United States & 57 & 58 & a & 53 & a & $68^{\mathrm{d}}$ & $\mathrm{x}(6)$ & 76 & 80 & 84 & 86 & 72 \\
\hline OECD average & 37 & 47 & $\mathrm{~m}$ & 59 & $\mathrm{~m}$ & 74 & 79 & 79 & 82 & 87 & 91 & 73 \\
\hline EU21 average & 30 & 42 & $\mathrm{~m}$ & 56 & $\mathrm{~m}$ & 73 & 77 & 79 & 80 & 86 & 90 & 72 \\
\hline Argentina & $\mathrm{m}$ & $\mathrm{m}$ & $\mathrm{m}$ & $\mathrm{m}$ & $\mathrm{m}$ & $\mathrm{m}$ & $\mathrm{m}$ & $\mathrm{m}$ & $\mathrm{m}$ & $\mathrm{m}$ & $\mathrm{m}$ & $\mathrm{m}$ \\
\hline Brazil $^{1}$ & $\mathrm{x}(4)$ & $\mathrm{x}(4)$ & $\mathrm{x}(4)$ & $67^{\mathrm{d}}$ & a & $76^{\mathrm{d}}$ & $\mathrm{x}(6)$ & $\mathrm{x}(9)$ & $85^{\mathrm{d}}$ & $\mathrm{x}(9)$ & $\mathrm{x}(9)$ & 72 \\
\hline China & $\mathrm{m}$ & $\mathrm{m}$ & $\mathrm{m}$ & $\mathrm{m}$ & $\mathrm{m}$ & $\mathrm{m}$ & $\mathrm{m}$ & $\mathrm{m}$ & $\mathrm{m}$ & $\mathrm{m}$ & $\mathrm{m}$ & $\mathrm{m}$ \\
\hline Colombia & $\mathrm{x}(4)$ & $\mathrm{x}(4)$ & a & $72^{\mathrm{d}}$ & a & 76 & a & $\mathrm{x}(9)$ & $84^{\mathrm{d}}$ & $\mathrm{x}(9)$ & $x(9)$ & 76 \\
\hline Costa Rica & 51 & 64 & 70 & 71 & 69 & 73 & 74 & 75 & 84 & $91^{\mathrm{d}}$ & $\mathrm{x}(10)$ & 71 \\
\hline India & $\mathrm{m}$ & $\mathrm{m}$ & $\mathrm{m}$ & $\mathrm{m}$ & $\mathrm{m}$ & $\mathrm{m}$ & $\mathrm{m}$ & $\mathrm{m}$ & $\mathrm{m}$ & $\mathrm{m}$ & $\mathrm{m}$ & $\mathrm{m}$ \\
\hline Indonesia & $\mathrm{m}$ & $\mathrm{m}$ & $\mathrm{m}$ & $\mathrm{m}$ & $\mathrm{m}$ & $\mathrm{m}$ & $\mathrm{m}$ & $\mathrm{m}$ & $\mathrm{m}$ & $\mathrm{m}$ & $\mathrm{m}$ & $\mathrm{m}$ \\
\hline Latvia & c & c & a & 53 & a & 70 & 74 & 84 & 83 & 86 & 97 & 73 \\
\hline Lithuania & c & c & $\mathrm{a}$ & 46 & 64 & 67 & 73 & $\mathrm{x}(9)$ & 89 & 90 & c & 75 \\
\hline Russian Federation ${ }^{1}$ & $\mathrm{x}(4)$ & $\mathrm{x}(4)$ & a & $49^{d}$ & a & $72^{\mathrm{d}}$ & $\mathrm{x}(6)$ & $x(9)$ & $83^{d}$ & $\mathrm{x}(9)$ & $\mathrm{x}(9)$ & 77 \\
\hline Saudi Arabia & $\mathrm{m}$ & $\mathrm{m}$ & $\mathrm{m}$ & $\mathrm{m}$ & $\mathrm{m}$ & $\mathrm{m}$ & $\mathrm{m}$ & $\mathrm{m}$ & $\mathrm{m}$ & $\mathrm{m}$ & $\mathrm{m}$ & $\mathrm{m}$ \\
\hline South Africa & $\mathrm{m}$ & $\mathrm{m}$ & $\mathrm{m}$ & $\mathrm{m}$ & $\mathrm{m}$ & $\mathrm{m}$ & $\mathrm{m}$ & $\mathrm{m}$ & $\mathrm{m}$ & $\mathrm{m}$ & $\mathrm{m}$ & $\mathrm{m}$ \\
\hline G20 average & $\mathrm{m}$ & $\mathrm{m}$ & $\mathrm{m}$ & $\mathrm{m}$ & $\mathrm{m}$ & $\mathrm{m}$ & $\mathrm{m}$ & $\mathrm{m}$ & $\mathrm{m}$ & $\mathrm{m}$ & $\mathrm{m}$ & $\mathrm{m}$ \\
\hline
\end{tabular}

Note: In most countries data refer to ISCED 2011. The countries with data that refer to ISCED-97 are: Brazil, Indonesia, the Russian Federation, Saudi Arabia and South Africa. See the description of the levels of education in the Definitions section.

1. Brazil, Chile, France, the Russian Federation: Year of reference 2013.

Source: OECD. Colombia, Costa Rica: OECD Education Database. Latvia, Lithuania: Eurostat. See Annex 3 for notes (www.oecd.org/education/education-at-aglance-19991487.htm)

Please refer to the Reader's Guide for information concerning symbols for missing data and abbreviations.

StatLink 게대 http://dx.doi.org/10.1787/888933284983 
Table A5.2a. Unemployment rates, by educational attainment (2014)

Percentage of unemployed 25-64 year-olds among 25-64 year-olds in the labour force

\begin{tabular}{|c|c|c|c|c|c|c|c|c|c|c|c|c|}
\hline & \multicolumn{5}{|c|}{ Below upper secondary } & \multicolumn{2}{|c|}{$\begin{array}{l}\text { Upper secondary } \\
\text { or post-secondary } \\
\text { non-tertiary }\end{array}$} & \multicolumn{4}{|c|}{ Tertiary } & \multirow[b]{2}{*}{$\begin{array}{l}\text { All levels } \\
\text { of } \\
\text { education }\end{array}$} \\
\hline & 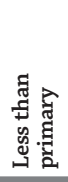 & 跑 & 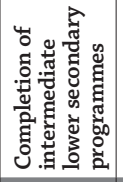 & 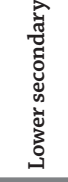 & 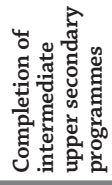 & 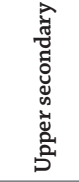 & 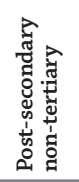 & 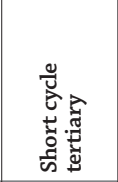 & 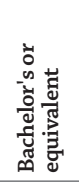 & 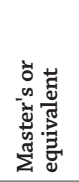 & 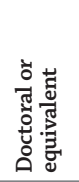 & \\
\hline & (1) & (2) & (3) & (4) & (5) & (6) & (7) & (8) & (9) & (10) & (11) & (12) \\
\hline Australia & $\mathrm{x}(2)$ & $11.4^{\mathrm{d}}$ & $\mathrm{a}$ & 7.0 & a & 4.6 & 4.2 & 4.0 & 2.7 & 3.9 & c & 4.5 \\
\hline Austria & $x(2)$ & $23.1^{\mathrm{d}}$ & a & 10.2 & $\mathrm{a}$ & 4.6 & c & 3.5 & 6.5 & 3.4 & 5.7 & 5.0 \\
\hline Belgium & 22.6 & 14.9 & a & 12.9 & a & 7.4 & c & c & 4.0 & 4.3 & c & 7.3 \\
\hline Canada & $\mathrm{x}(2)$ & $11.7^{\mathrm{d}}$ & $\mathrm{a}$ & 10.3 & $\mathrm{a}$ & 6.7 & 6.2 & 4.8 & 4.7 & $4.8^{\mathrm{d}}$ & $\mathrm{x}(10)$ & 5.8 \\
\hline Chile $^{1}$ & 4.6 & 5.1 & a & 5.4 & a & 5.6 & a & 5.7 & 4.9 & $1.3^{\mathrm{d}}$ & $\mathrm{x}(10)$ & 5.3 \\
\hline Czech Republic & $\mathrm{m}$ & c & a & 20.7 & a & $5.4^{\mathrm{d}}$ & $\mathrm{x}(6)$ & c & 3.8 & 2.3 & c & 5.5 \\
\hline Denmark & $\mathrm{x}(2)$ & $9.9^{\mathrm{d}}$ & a & 7.9 & a & 5.2 & c & 4.8 & 4.0 & 5.0 & c & 5.4 \\
\hline Estonia & c & c & $\mathrm{m}$ & 11.5 & $\mathrm{~m}$ & 7.8 & 7.7 & 5.7 & 5.0 & 4.3 & 0.0 & 6.8 \\
\hline Finland & $x(2)$ & $13.9^{\mathrm{d}}$ & a & 12.1 & a & 8.2 & c & 4.5 & 5.9 & 4.8 & c & 7.1 \\
\hline France $^{1}$ & 14.1 & 13.2 & a & 14.1 & a & 8.5 & c & 4.8 & 6.0 & 5.3 & 5.1 & 8.4 \\
\hline Germany & $\mathrm{x}(2)$ & $16.0^{\mathrm{d}}$ & a & 11.0 & a & 5.0 & 2.9 & c & 2.4 & 2.8 & 1.9 & 4.8 \\
\hline Greece & 40.5 & 25.7 & $24.5^{x}$ & 29.0 & $44.0^{x}$ & 26.9 & 30.1 & $2.8^{\mathrm{r}}$ & 20.8 & 15.4 & $6.7^{\mathrm{x}}$ & 24.9 \\
\hline Hungary & c & 29.2 & a & 16.2 & a & 6.7 & 5.2 & c & 3.1 & 2.0 & c & 6.7 \\
\hline Iceland & $x(2)$ & $c^{d}$ & a & 4.8 & a & 4.3 & c & c & 3.5 & 3.8 & c & 4.1 \\
\hline Ireland & c & 21.5 & a & 17.4 & a & 10.8 & 13.8 & 7.1 & 6.0 & 5.0 & c & 10.3 \\
\hline Israel & 7.0 & 7.8 & a & 7.5 & a & 6.2 & a & 4.7 & 4.1 & 2.8 & 2.4 & 5.1 \\
\hline Italy & 21.7 & 19.9 & a & 14.5 & a & 9.1 & 11.7 & c & 11.6 & 6.8 & 3.9 & 10.8 \\
\hline Japan & $\mathrm{x}(6)$ & $\mathrm{x}(6)$ & a & $\mathrm{x}(6)$ & a & $4.1^{\mathrm{d}}$ & $\mathrm{x}(8)$ & $3.4^{\mathrm{d}}$ & $2.5^{\mathrm{d}}$ & $\mathrm{x}(9)$ & $\mathrm{x}(9)$ & 3.5 \\
\hline Korea & $x(2)$ & 2.8 & $\mathrm{a}$ & 2.6 & $\mathrm{a}$ & 3.3 & $\mathrm{~m}$ & 3.3 & $3.1^{\mathrm{d}}$ & $\mathrm{x}(9)$ & $\mathrm{x}(9)$ & 3.1 \\
\hline Luxembourg & c & 9.7 & a & 6.4 & a & 4.9 & c & 4.9 & 3.5 & 3.2 & c & 4.7 \\
\hline Mexico & 2.3 & 2.9 & 5.3 & 4.2 & 4.4 & 4.4 & a & 5.9 & $5.0^{\mathrm{d}}$ & $\mathrm{x}(9)$ & $\mathrm{x}(9)$ & 4.0 \\
\hline Netherlands & 18.2 & 11.1 & a & 9.4 & a & 7.1 & c & 4.6 & 4.0 & 3.6 & c & 6.4 \\
\hline New Zealand & $\mathrm{x}(4)$ & $\mathrm{x}(4)$ & a & $5.2^{\mathrm{d}}$ & a & 5.3 & 3.5 & 3.7 & 2.4 & 3.0 & c & 4.0 \\
\hline Norway & 0.0 & 7.1 & & 6.7 & $\mathrm{~m}$ & 2.4 & 3.0 & 3.3 & 1.2 & 1.8 & 0.9 & 2.8 \\
\hline Poland & 0.0 & 16.9 & a & 27.6 & a & 8.7 & 7.8 & 0.0 & 6.0 & 3.7 & 0.9 & 7.7 \\
\hline Portugal & 23.8 & 14.5 & a & 14.5 & a & 12.5 & 16.8 & $\mathrm{x}(9)$ & $14.1^{\mathrm{d}}$ & 7.7 & c & 12.8 \\
\hline Slovak Republic & c & c & 16.8 & 42.7 & c & 11.4 & c & c & 7.5 & 5.5 & c & 11.8 \\
\hline Slovenia & c & $22.4^{r}$ & a & 15.1 & a & 9.7 & a & $4.9^{r}$ & 9.4 & 5.6 & $2.6^{x}$ & 9.1 \\
\hline Spain & 47.7 & 36.6 & a & 29.0 & a & 21.6 & c & 17.0 & 12.9 & 12.4 & 8.2 & 22.4 \\
\hline Sweden & $\mathrm{x}(2)$ & $26.2^{\mathrm{d}}$ & a & 11.7 & 8.8 & 4.6 & 6.1 & 5.7 & 3.8 & 3.3 & c & 5.8 \\
\hline Switzerland & c & c & a & 9.4 & $\mathrm{a}$ & $3.8^{\mathrm{d}}$ & $\mathrm{x}(6)$ & $x(9,10,11)$ & $2.9^{\mathrm{d}}$ & $3.8^{\mathrm{d}}$ & $c^{\mathrm{d}}$ & 4.1 \\
\hline Turkey & 11.1 & 8.0 & a & 9.5 & a & 9.1 & a & 10.0 & 8.1 & 4.6 & c & 8.6 \\
\hline United Kingdom & c & 9.8 & a & 7.7 & 4.8 & 3.2 & a & 2.6 & 2.7 & 2.1 & c & 3.9 \\
\hline United States & 7.3 & 7.5 & a & 12.5 & a & $7.2^{\mathrm{d}}$ & $\mathrm{x}(6)$ & 4.9 & 3.9 & 2.6 & 2.3 & 5.8 \\
\hline OECD average & 15.8 & 14.8 & $\mathrm{~m}$ & 12.9 & $\mathrm{~m}$ & 7.5 & 9.1 & 5.1 & 5.6 & 4.5 & 3.4 & 7.3 \\
\hline EU21 average & 23.6 & 18.6 & $\mathrm{~m}$ & 16.3 & $\mathrm{~m}$ & 9.0 & 11.3 & 5.2 & 6.8 & 5.2 & 3.9 & 9.0 \\
\hline Argentina & $\mathrm{m}$ & $\mathrm{m}$ & $\mathrm{m}$ & $\mathrm{m}$ & $\mathrm{m}$ & $\mathrm{m}$ & $\mathrm{m}$ & $\mathrm{m}$ & $\mathrm{m}$ & $\mathrm{m}$ & $\mathrm{m}$ & $\mathrm{m}$ \\
\hline Brazil $^{1}$ & $\mathrm{x}(4)$ & $\mathrm{x}(4)$ & a & $4.5^{\mathrm{d}}$ & a & $5.6^{\mathrm{d}}$ & $x(6)$ & $\mathrm{x}(9)$ & $2.9^{\mathrm{d}}$ & $x(9)$ & $x(9)$ & 4.6 \\
\hline China & $\mathrm{m}$ & $\mathrm{m}$ & $\mathrm{m}$ & $\mathrm{m}$ & $\mathrm{m}$ & $\mathrm{m}$ & $\mathrm{m}$ & $\mathrm{m}$ & $\mathrm{m}$ & $\mathrm{m}$ & $\mathrm{m}$ & $\mathrm{m}$ \\
\hline Colombia & $\mathrm{x}(4)$ & $\mathrm{x}(4)$ & a & $6.2^{\mathrm{d}}$ & a & $8.4^{\mathrm{d}}$ & $\mathrm{x}(6)$ & $\mathrm{x}(9)$ & $7.4^{\mathrm{d}}$ & $\mathrm{x}(9)$ & $\mathrm{x}(9)$ & 7.2 \\
\hline Costa Rica & 7.7 & 7.0 & 7.4 & 7.5 & 6.1 & 6.8 & 6.0 & 5.3 & 4.4 & $1.4^{\mathrm{d}}$ & $\mathrm{x}(10)$ & 6.4 \\
\hline India & $\mathrm{m}$ & $\mathrm{m}$ & $\mathrm{m}$ & $\mathrm{m}$ & $\mathrm{m}$ & $\mathrm{m}$ & $\mathrm{m}$ & $\mathrm{m}$ & $\mathrm{m}$ & $\mathrm{m}$ & $\mathrm{m}$ & $\mathrm{m}$ \\
\hline Indonesia & $\mathrm{m}$ & $\mathrm{m}$ & $\mathrm{m}$ & $\mathrm{m}$ & $\mathrm{m}$ & $\mathrm{m}$ & $\mathrm{m}$ & $\mathrm{m}$ & $\mathrm{m}$ & $\mathrm{m}$ & $\mathrm{m}$ & $\mathrm{m}$ \\
\hline Latvia & c & c & a & 22.7 & a & 11.6 & 9.2 & c & 6.2 & 3.5 & c & 10.3 \\
\hline Lithuania & c & c & a & 27 & c & 15 & 10 & $\mathrm{x}(9)$ & $5^{d}$ & c & c & 10 \\
\hline Russian Federation $^{1}$ & $\mathrm{x}(4)$ & $\mathrm{x}(4)$ & a & $12.5^{\mathrm{d}}$ & a & $6.2^{\mathrm{d}}$ & $x(6)$ & $x(9)$ & $2.9^{\mathrm{d}}$ & $\mathrm{x}(9)$ & $x(9)$ & 4.6 \\
\hline Saudi Arabia & $\mathrm{m}$ & $\mathrm{m}$ & $\mathrm{m}$ & $\mathrm{m}$ & $\mathrm{m}$ & $\mathrm{m}$ & $\mathrm{m}$ & $\mathrm{m}$ & $\mathrm{m}$ & $\mathrm{m}$ & $\mathrm{m}$ & $\mathrm{m}$ \\
\hline South Africa & $\mathrm{m}$ & $\mathrm{m}$ & $\mathrm{m}$ & $\mathrm{m}$ & $\mathrm{m}$ & $\mathrm{m}$ & $\mathrm{m}$ & $\mathrm{m}$ & $\mathrm{m}$ & $\mathrm{m}$ & $\mathrm{m}$ & $\mathrm{m}$ \\
\hline G20 average & $\mathrm{m}$ & $\mathrm{m}$ & $\mathrm{m}$ & $\mathrm{m}$ & $\mathrm{m}$ & $\mathrm{m}$ & $\mathrm{m}$ & $\mathrm{m}$ & $\mathrm{m}$ & $\mathrm{m}$ & $\mathrm{m}$ & $\mathrm{m}$ \\
\hline
\end{tabular}

Note: In most countries data refer to ISCED 2011. The countries with data that refer to ISCED-97 are: Brazil, Indonesia, the Russian Federation, Saudi Arabia and South Africa. See the description of the levels of education in the Definitions section.

1. Brazil, Chile, France, the Russian Federation: Year of reference 2013.

Source: OECD. Colombia, Costa Rica: OECD Education Database. Latvia, Lithuania: Eurostat. See Annex 3 for notes (www.oecd.org/education/education-at-aglance-19991487.htm)

Please refer to the Reader's Guide for information concerning symbols for missing data and abbreviations.

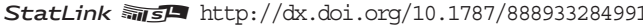


Table A5.3a. [1/3] Trends in employment rates, by educational attainment and age group (2000, 2005, 2010 and 2014)

Percentage of employed adults, by age group among all adults in the same age group

\begin{tabular}{|c|c|c|c|c|c|c|c|c|c|c|c|c|}
\hline & \multicolumn{12}{|c|}{ Below upper secondary } \\
\hline & \multicolumn{4}{|c|}{ Employment rates of 25-64 year-olds } & \multicolumn{4}{|c|}{ Employment rates of $25-34$ year-olds } & \multicolumn{4}{|c|}{ Employment rates of 55-64 year-olds } \\
\hline & 2000 & 2005 & 2010 & 2014 & 2000 & 2005 & 2010 & 2014 & 2000 & 2005 & 2010 & 2014 \\
\hline & (1) & (2) & (3) & (4) & (5) & (6) & (7) & (8) & (17) & (18) & (19) & (20) \\
\hline Australia & $61^{b}$ & $63^{b}$ & $65^{\mathrm{b}}$ & 60 & $64^{\mathrm{b}}$ & $64^{b}$ & $61^{b}$ & 59 & $39^{b}$ & $46^{b}$ & $53^{b}$ & 50 \\
\hline Austria & $\mathrm{m}$ & 53 & 55 & 53 & $\mathrm{~m}$ & 61 & 59 & 58 & $\mathrm{~m}$ & 23 & 30 & 30 \\
\hline Belgium & $51^{\mathrm{b}}$ & $49^{b}$ & $49^{b}$ & 47 & $64^{\mathrm{b}}$ & $57^{b}$ & $56^{\mathrm{b}}$ & 52 & $19^{\mathrm{b}}$ & $21^{\mathrm{b}}$ & $26^{\mathrm{b}}$ & 30 \\
\hline Canada & 55 & 56 & 55 & 56 & 60 & 62 & 58 & 57 & 37 & 40 & 43 & 48 \\
\hline Chile $^{1}$ & $\mathrm{~m}$ & $\mathrm{~m}$ & $62^{\mathrm{b}}$ & 61 & $\mathrm{~m}$ & $\mathrm{~m}$ & $59^{b}$ & 61 & $\mathrm{~m}$ & $\mathrm{~m}$ & $55^{b}$ & 54 \\
\hline Czech Republic & $47^{b}$ & $41^{\mathrm{b}}$ & $43^{b}$ & 43 & $51^{\mathrm{b}}$ & $43^{b}$ & $47^{b}$ & 46 & $17^{\mathrm{b}}$ & $20^{\mathrm{b}}$ & $26^{\mathrm{b}}$ & 29 \\
\hline Denmark & $62^{b}$ & $62^{\mathrm{b}}$ & $63^{b}$ & 62 & $70^{\mathrm{b}}$ & $64^{\mathrm{b}}$ & $65^{\mathrm{b}}$ & 57 & $41^{b}$ & $42^{b}$ & $46^{b}$ & 53 \\
\hline Estonia & 42 & 50 & 45 & 60 & 53 & 60 & 51 & 66 & 24 & 36 & 30 & 44 \\
\hline Finland & $60^{\mathrm{b}}$ & $58^{\mathrm{b}}$ & $55^{\mathrm{b}}$ & 54 & $69^{b}$ & $63^{b}$ & $59^{b}$ & 52 & $33^{b}$ & $43^{b}$ & $44^{\mathrm{b}}$ & 45 \\
\hline France $^{1}$ & 56 & 59 & 55 & 54 & 61 & 63 & 57 & 55 & 24 & 32 & 32 & 37 \\
\hline Germany & $51^{b}$ & $52^{\mathrm{b}}$ & $55^{\mathrm{b}}$ & 58 & $60^{\mathrm{b}}$ & $52^{b}$ & $55^{b}$ & 55 & $26^{\mathrm{b}}$ & $32^{b}$ & $40^{\mathrm{b}}$ & 48 \\
\hline Greece & $58^{\mathrm{b}}$ & $59^{b}$ & $57^{\mathrm{b}}$ & 47 & $67^{\mathrm{b}}$ & $72^{b}$ & $64^{\mathrm{b}}$ & 51 & $39^{\mathrm{b}}$ & $39^{b}$ & $40^{\mathrm{b}}$ & 33 \\
\hline Hungary & $36^{b}$ & $38^{\mathrm{b}}$ & $38^{\mathrm{b}}$ & 45 & $50^{\mathrm{b}}$ & $49^{b}$ & $40^{\mathrm{b}}$ & 49 & $12^{\mathrm{b}}$ & $16^{\mathrm{b}}$ & $20^{\mathrm{b}}$ & 25 \\
\hline Iceland & $\mathrm{m}$ & 82 & 75 & 77 & $\mathrm{~m}$ & 81 & 67 & 74 & $\mathrm{~m}$ & 81 & 74 & 76 \\
\hline Ireland & $56^{\mathrm{b}}$ & $58^{\mathrm{b}}$ & $48^{\mathrm{b}}$ & 47 & $68^{\mathrm{b}}$ & $64^{\mathrm{b}}$ & $44^{\mathrm{b}}$ & 38 & $39^{b}$ & $45^{b}$ & $41^{b}$ & 42 \\
\hline Israel & $\mathrm{m}$ & $41^{\mathrm{b}}$ & $45^{b}$ & 49 & $\mathrm{~m}$ & $43^{b}$ & $45^{b}$ & 56 & $\mathrm{~m}$ & $32^{\mathrm{b}}$ & $38^{b}$ & 43 \\
\hline Italy & $49^{b}$ & $52^{\mathrm{b}}$ & $50^{\mathrm{b}}$ & 50 & $60^{\mathrm{b}}$ & $65^{b}$ & $57^{b}$ & 50 & $23^{b}$ & $24^{\mathrm{b}}$ & $26^{\mathrm{b}}$ & 33 \\
\hline Japan & $\mathrm{m}$ & $\mathrm{m}$ & $\mathrm{m}$ & $\mathrm{m}$ & $\mathrm{m}$ & $\mathrm{m}$ & $\mathrm{m}$ & $\mathrm{m}$ & $\mathrm{m}$ & $\mathrm{m}$ & $\mathrm{m}$ & $\mathrm{m}$ \\
\hline Korea & 68 & 66 & 65 & 66 & 65 & 62 & 57 & 59 & 59 & 58 & 59 & 64 \\
\hline Luxembourg & $58^{\mathrm{b}}$ & $62^{\mathrm{b}}$ & $62^{\mathrm{b}}$ & 61 & $78^{\mathrm{b}}$ & $79 \mathrm{~b}$ & $78^{b}$ & 73 & $15^{b}$ & $22^{b}$ & $25^{b}$ & 26 \\
\hline Mexico & $61^{b}$ & $62^{\mathrm{b}}$ & $63^{b}$ & 63 & $63^{b}$ & $63^{b}$ & $63^{b}$ & 65 & $51^{b}$ & $52^{b}$ & $53^{b}$ & 53 \\
\hline Netherlands & $58^{\mathrm{b}}$ & $60^{\mathrm{b}}$ & $61^{\mathrm{b}}$ & 59 & $72^{\mathrm{b}}$ & $70^{\mathrm{b}}$ & $70^{\mathrm{b}}$ & 63 & $28^{\mathrm{b}}$ & $35^{b}$ & $42^{b}$ & 46 \\
\hline New Zealand & $65^{b}$ & $70^{\mathrm{b}}$ & $68^{\mathrm{b}}$ & 71 & $63^{b}$ & $68^{b}$ & $64^{b}$ & 65 & $49^{b}$ & $61^{b}$ & $64^{b}$ & 66 \\
\hline Norway & $65^{b}$ & $64^{\mathrm{b}}$ & $64^{\mathrm{b}}$ & 62 & $67^{\mathrm{b}}$ & $66^{\mathrm{b}}$ & $64^{\mathrm{b}}$ & 61 & $53^{b}$ & $48^{b}$ & $51^{b}$ & 53 \\
\hline Poland & $43^{b}$ & $38^{\mathrm{b}}$ & $40^{\mathrm{b}}$ & 39 & $50^{\mathrm{b}}$ & $45^{b}$ & $49^{b}$ & 45 & $24^{\mathrm{b}}$ & $21^{b}$ & $22^{b}$ & 25 \\
\hline Portugal & $73^{b}$ & $71^{\mathrm{b}}$ & $68^{\mathrm{b}}$ & 63 & $83^{b}$ & $81^{\mathrm{b}}$ & $75^{b}$ & 71 & $50^{\mathrm{b}}$ & $50^{\mathrm{b}}$ & $48^{b}$ & 45 \\
\hline Slovak Republic & $31^{b}$ & $26^{\mathrm{b}}$ & $30^{\mathrm{b}}$ & 33 & $29^{b}$ & $16^{\mathrm{b}}$ & $21^{\mathrm{b}}$ & 28 & $7^{\mathrm{b}}$ & $9^{b}$ & $21^{b}$ & 24 \\
\hline Slovenia & $53^{\mathrm{b}}$ & $56^{\mathrm{b}}$ & $51^{b}$ & 49 & $75^{\mathrm{b}}$ & $70^{\mathrm{b}}$ & $60^{\mathrm{b}}$ & 53 & $20^{\mathrm{b}}$ & $27^{\mathrm{b}}$ & $28^{\mathrm{b}}$ & 29 \\
\hline Spain & $54^{\mathrm{b}}$ & $59^{b}$ & $53^{b}$ & 49 & $65^{\mathrm{b}}$ & $72^{b}$ & $59^{b}$ & 55 & $33^{b}$ & $38^{b}$ & $36^{b}$ & 35 \\
\hline Sweden & $68^{b}$ & $66^{\mathrm{b}}$ & $63^{\mathrm{b}}$ & 66 & $67^{\mathrm{b}}$ & $65^{b}$ & $60^{\mathrm{b}}$ & 65 & $56^{\mathrm{b}}$ & $59^{b}$ & $60^{\mathrm{b}}$ & 63 \\
\hline Switzerland & $64^{\mathrm{b}}$ & $65^{b}$ & $69^{b}$ & 69 & $68^{\mathrm{b}}$ & $68^{b}$ & $70^{\mathrm{b}}$ & 67 & $47^{\mathrm{b}}$ & $51^{b}$ & $54^{\mathrm{b}}$ & 56 \\
\hline Turkey & 53 & 47 & 49 & 51 & 55 & 49 & 51 & 54 & 38 & 30 & 31 & 33 \\
\hline United Kingdom² & $65^{b}$ & $65^{b}$ & $56^{\mathrm{b}}$ & 60 & $66^{\mathrm{b}}$ & $64^{\mathrm{b}}$ & $56^{\mathrm{b}}$ & 57 & $51^{\mathrm{b}}$ & $56^{b}$ & $44^{\mathrm{b}}$ & 48 \\
\hline United States & 58 & 57 & 52 & 55 & 64 & 62 & 55 & 58 & 40 & 39 & 40 & 40 \\
\hline OECD average & $56^{b}$ & $56^{\mathrm{b}}$ & $55^{\mathrm{b}}$ & 56 & $63^{b}$ & $61^{b}$ & $57^{\mathrm{b}}$ & 57 & $34^{\mathrm{b}}$ & $38^{b}$ & $41^{b}$ & 43 \\
\hline EU21 average & $53^{b}$ & $54^{\mathrm{b}}$ & $52^{\mathrm{b}}$ & 52 & $63^{b}$ & $61^{\mathrm{b}}$ & $56^{b}$ & 54 & $29^{b}$ & $33^{b}$ & $35^{\mathrm{b}}$ & 38 \\
\hline
\end{tabular}

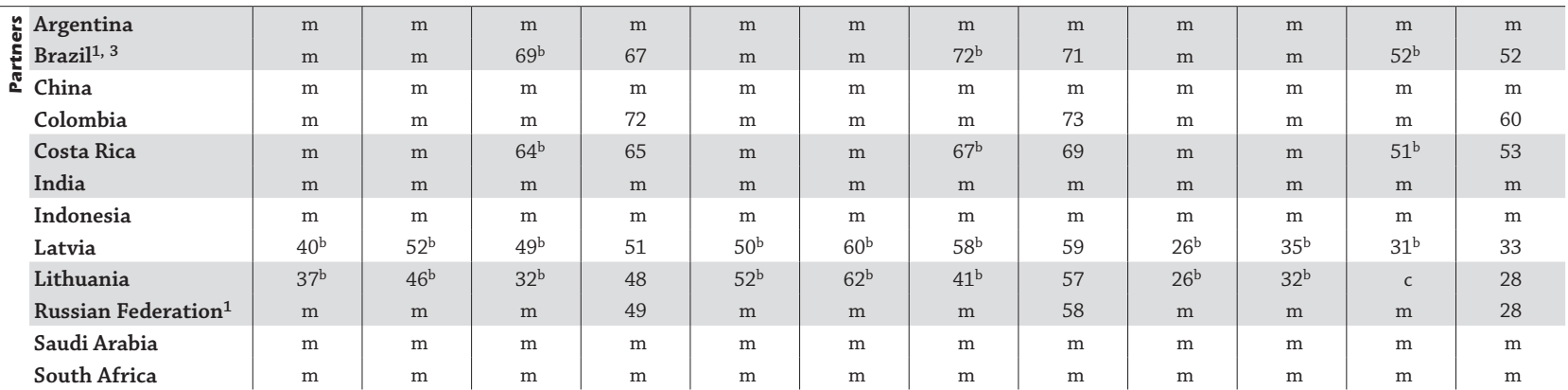

\section{G20 average}

Notes: In most countries, there is a break in the series, represented by the code " $b$ ", as data for the latest year refer to ISCED 2011 while data for previous years refer to ISCED-97. For Korea data refer to ISCED-97 for all years. See the description of the levels of education in the Definitions section. Columns showing data for other age groups are available for consultation on line (see StatLink below).

1. Brazil, Chile, France, the Russian Federation: Data for year 2014 refer to year 2013.

2. The United Kingdom: Data for upper secondary attainment includes completion of a sufficient volume and standard of programmes that would be classified individually as completion of intermediate upper secondary programmes ( $18 \%$ of the adults are under this group).

3. Brazil: Data for year 2010 refer to year 2009.

Source: OECD. Colombia, Costa Rica: OECD Education Database. Latvia, Lithuania: Eurostat. See Annex 3 for notes (www.oecd.org/education/education-at-aglance-19991487.htm).

Please refer to the Reader's Guide for information concerning symbols for missing data and abbreviations.

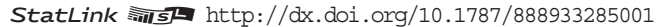


Table A5.3a. [2/3] Trends in employment rates, by educational attainment and age group (2000, 2005, 2010 and 2014)

Percentage of employed adults, by age group among all adults in the same age group

\begin{tabular}{|c|c|c|c|c|c|c|c|c|c|c|c|c|}
\hline & \multicolumn{12}{|c|}{ Upper secondary or post-secondary non-tertiary } \\
\hline & \multicolumn{4}{|c|}{ Employment rates of 25-64 year-olds } & \multicolumn{4}{|c|}{ Employment rates of $25-34$ year-olds } & \multicolumn{4}{|c|}{ Employment rates of 55-64 year-olds } \\
\hline & 2000 & 2005 & 2010 & 2014 & 2000 & 2005 & 2010 & 2014 & 2000 & 2005 & 2010 & 2014 \\
\hline & (21) & (22) & (23) & (24) & (25) & (26) & (27) & (28) & (37) & (38) & (39) & (40) \\
\hline Australia & $77^{\mathrm{b}}$ & $80^{\mathrm{b}}$ & $80^{\mathrm{b}}$ & 77 & $80^{\mathrm{b}}$ & $81^{\mathrm{b}}$ & $78^{\mathrm{b}}$ & 78 & $53^{b}$ & $62^{\mathrm{b}}$ & $71^{\mathrm{b}}$ & 67 \\
\hline Austria & $\mathrm{m}$ & 73 & 76 & 76 & $\mathrm{~m}$ & 83 & 83 & 83 & $\mathrm{~m}$ & 28 & 39 & 44 \\
\hline Belgium & $75^{\mathrm{b}}$ & $74^{\mathrm{b}}$ & $74^{b}$ & 73 & $84^{b}$ & $81^{b}$ & $80^{\mathrm{b}}$ & 79 & $31^{\mathrm{b}}$ & $38^{b}$ & $41^{b}$ & 44 \\
\hline Canada & 76 & 76 & 74 & 74 & 79 & 80 & 77 & 77 & 52 & 57 & 58 & 59 \\
\hline Chile $^{1}$ & $\mathrm{~m}$ & $\mathrm{~m}$ & $72^{b}$ & 72 & $\mathrm{~m}$ & $\mathrm{~m}$ & $74^{\mathrm{b}}$ & 70 & $\mathrm{~m}$ & $\mathrm{~m}$ & $59^{b}$ & 62 \\
\hline Czech Republic & $76^{\mathrm{b}}$ & $75^{\mathrm{b}}$ & $74^{\mathrm{b}}$ & 78 & $77^{\mathrm{b}}$ & $78^{\mathrm{b}}$ & $76^{\mathrm{b}}$ & 78 & $39^{b}$ & $47^{b}$ & $46^{\mathrm{b}}$ & 53 \\
\hline Denmark & $81^{b}$ & $80^{\mathrm{b}}$ & $79^{\mathrm{b}}$ & 79 & $85^{b}$ & $83^{b}$ & $82^{\mathrm{b}}$ & 79 & $57^{\mathrm{b}}$ & $61^{\mathrm{b}}$ & $59^{b}$ & 63 \\
\hline Estonia & 70 & 74 & 69 & 74 & 74 & 77 & 70 & 76 & 46 & 53 & 54 & 58 \\
\hline Finland & $75^{\mathrm{b}}$ & $75^{\mathrm{b}}$ & $74^{\mathrm{b}}$ & 73 & $76^{\mathrm{b}}$ & $77^{\mathrm{b}}$ & $76^{\mathrm{b}}$ & 74 & $42^{b}$ & $53^{b}$ & $55^{\mathrm{b}}$ & 57 \\
\hline France $^{1}$ & 75 & 76 & 74 & 73 & 80 & 80 & 79 & 76 & 31 & 40 & 41 & 46 \\
\hline Germany & $70^{\mathrm{b}}$ & $71^{\mathrm{b}}$ & $76^{\mathrm{b}}$ & 80 & $79^{\mathrm{b}}$ & $74^{\mathrm{b}}$ & $78^{\mathrm{b}}$ & 82 & $37^{\mathrm{b}}$ & $43^{b}$ & $56^{\mathrm{b}}$ & 64 \\
\hline Greece & $65^{\mathrm{b}}$ & $69^{b}$ & $67^{\mathrm{b}}$ & 54 & $69^{\mathrm{b}}$ & $73^{b}$ & $71^{\mathrm{b}}$ & 54 & $31^{\mathrm{b}}$ & $38^{\mathrm{b}}$ & $37^{\mathrm{b}}$ & 27 \\
\hline Hungary & $72^{b}$ & $70^{\mathrm{b}}$ & $66^{\mathrm{b}}$ & 72 & $75^{b}$ & $75^{\mathrm{b}}$ & $71^{\mathrm{b}}$ & 77 & $29^{b}$ & $39^{b}$ & $35^{b}$ & 43 \\
\hline Iceland & $\mathrm{m}$ & 89 & 83 & 87 & $\mathrm{~m}$ & 82 & 73 & 78 & $\mathrm{~m}$ & 87 & 82 & 87 \\
\hline Ireland & $77^{\mathrm{b}}$ & $77^{\mathrm{b}}$ & $66^{\mathrm{b}}$ & 68 & $85^{b}$ & $83^{b}$ & $67^{\mathrm{b}}$ & 67 & $48^{b}$ & $56^{\mathrm{b}}$ & $55^{\mathrm{b}}$ & 59 \\
\hline Israel & $\mathrm{m}$ & $67^{\mathrm{b}}$ & $70^{\mathrm{b}}$ & 72 & $\mathrm{~m}$ & $65^{\mathrm{b}}$ & $68^{\mathrm{b}}$ & 71 & $\mathrm{~m}$ & $53^{b}$ & $62^{\mathrm{b}}$ & 65 \\
\hline Italy & $71^{\mathrm{b}}$ & $74^{\mathrm{b}}$ & $73^{b}$ & 70 & $67^{\mathrm{b}}$ & $72^{\mathrm{b}}$ & $69^{b}$ & 63 & $41^{b}$ & $44^{\mathrm{b}}$ & $48^{b}$ & 57 \\
\hline Japan & $\mathrm{m}$ & $\mathrm{m}$ & $\mathrm{m}$ & $\mathrm{m}$ & $\mathrm{m}$ & $\mathrm{m}$ & $\mathrm{m}$ & $\mathrm{m}$ & $\mathrm{m}$ & $\mathrm{m}$ & $\mathrm{m}$ & $\mathrm{m}$ \\
\hline Korea & 69 & 70 & 71 & 72 & 64 & 64 & 64 & 63 & 53 & 59 & 62 & 67 \\
\hline Luxembourg & $73^{b}$ & $72^{\mathrm{b}}$ & $72^{b}$ & 72 & $85^{\mathrm{b}}$ & $82^{\mathrm{b}}$ & $83^{b}$ & 84 & $32^{\mathrm{b}}$ & $30^{\mathrm{b}}$ & $35^{\mathrm{b}}$ & 38 \\
\hline Mexico & $71^{\mathrm{b}}$ & $71^{\mathrm{b}}$ & $72^{\mathrm{b}}$ & 73 & $71^{\mathrm{b}}$ & $71^{\mathrm{b}}$ & $72^{\mathrm{b}}$ & 71 & $48^{\mathrm{b}}$ & $46^{\mathrm{b}}$ & $50^{b}$ & 60 \\
\hline Netherlands & $79^{\mathrm{b}}$ & $78^{\mathrm{b}}$ & $80^{\mathrm{b}}$ & 78 & $88^{\mathrm{b}}$ & $86^{\mathrm{b}}$ & $87^{b}$ & 82 & $43^{b}$ & $49^{b}$ & $57^{\mathrm{b}}$ & 63 \\
\hline New Zealand & $80^{\mathrm{b}}$ & $84^{\mathrm{b}}$ & $82^{\mathrm{b}}$ & 80 & $78^{\mathrm{b}}$ & $82^{\mathrm{b}}$ & $77^{\mathrm{b}}$ & 76 & $65^{\mathrm{b}}$ & $75^{b}$ & $78^{\mathrm{b}}$ & 78 \\
\hline Norway & $83^{b}$ & $82^{\mathrm{b}}$ & $82^{\mathrm{b}}$ & 81 & $84^{\mathrm{b}}$ & $84^{\mathrm{b}}$ & $85^{b}$ & 84 & $68^{\mathrm{b}}$ & $70^{\mathrm{b}}$ & $68^{\mathrm{b}}$ & 71 \\
\hline Poland & $67^{b}$ & $62^{\mathrm{b}}$ & $65^{\mathrm{b}}$ & 66 & $71^{b}$ & $68^{\mathrm{b}}$ & $74^{\mathrm{b}}$ & 74 & $28^{\mathrm{b}}$ & $28^{\mathrm{b}}$ & $34^{\mathrm{b}}$ & 42 \\
\hline Portugal & $83^{b}$ & $79^{\mathrm{b}}$ & $80^{\mathrm{b}}$ & 78 & $83^{b}$ & $78^{\mathrm{b}}$ & $80^{\mathrm{b}}$ & 78 & $51^{\mathrm{b}}$ & $48^{b}$ & $51^{b}$ & 55 \\
\hline Slovak Republic & $71^{\mathrm{b}}$ & $71^{\mathrm{b}}$ & $70^{\mathrm{b}}$ & 71 & $72^{b}$ & $73^{\mathrm{b}}$ & $72^{b}$ & 71 & $27^{b}$ & $34^{b}$ & $41^{\mathrm{b}}$ & 45 \\
\hline Slovenia & $74^{\mathrm{b}}$ & $75^{b}$ & $73^{b}$ & 69 & $86^{\mathrm{b}}$ & $84^{\mathrm{b}}$ & $81^{\mathrm{b}}$ & 75 & $18^{\mathrm{b}}$ & $27^{b}$ & $32^{b}$ & 33 \\
\hline Spain & $72^{\mathrm{b}}$ & $75^{\mathrm{b}}$ & $69^{\mathrm{b}}$ & 66 & $73^{b}$ & $78^{\mathrm{b}}$ & $69^{\mathrm{b}}$ & 65 & $51^{\mathrm{b}}$ & $51^{b}$ & $53^{b}$ & 53 \\
\hline Sweden & $82^{b}$ & $81^{\mathrm{b}}$ & $81^{b}$ & 85 & $83^{b}$ & $81^{b}$ & $80^{\mathrm{b}}$ & 84 & $66^{\mathrm{b}}$ & $69^{b}$ & $70^{\mathrm{b}}$ & 75 \\
\hline Switzerland & $81^{\mathrm{b}}$ & $80^{\mathrm{b}}$ & $81^{\mathrm{b}}$ & 82 & $84^{\mathrm{b}}$ & $83^{b}$ & $84^{\mathrm{b}}$ & 85 & $66^{\mathrm{b}}$ & $65^{\mathrm{b}}$ & $67^{\mathrm{b}}$ & 70 \\
\hline Turkey & 64 & 62 & 60 & 62 & 67 & 64 & 64 & 65 & 20 & 24 & 24 & 29 \\
\hline United Kingdom ${ }^{2}$ & $81^{\mathrm{b}}$ & $82^{\mathrm{b}}$ & $78^{\mathrm{b}}$ & 80 & $83^{b}$ & $81^{b}$ & $79^{\mathrm{b}}$ & 81 & $65^{\mathrm{b}}$ & $69^{b}$ & $63^{b}$ & 67 \\
\hline United States & 77 & 73 & 68 & 68 & 80 & 74 & 68 & 68 & 58 & 58 & 57 & 58 \\
\hline OECD average & $75^{\mathrm{b}}$ & $75^{\mathrm{b}}$ & $74^{\mathrm{b}}$ & 74 & $78^{\mathrm{b}}$ & $77^{\mathrm{b}}$ & $76^{\mathrm{b}}$ & 75 & $45^{\mathrm{b}}$ & $50^{\mathrm{b}}$ & $53^{b}$ & 56 \\
\hline EU21 average & $74^{\mathrm{b}}$ & $74^{\mathrm{b}}$ & $73^{b}$ & 73 & $79^{b}$ & $78^{\mathrm{b}}$ & $77^{\mathrm{b}}$ & 75 & $41^{\mathrm{b}}$ & $45^{\mathrm{b}}$ & $48^{\mathrm{b}}$ & 52 \\
\hline
\end{tabular}

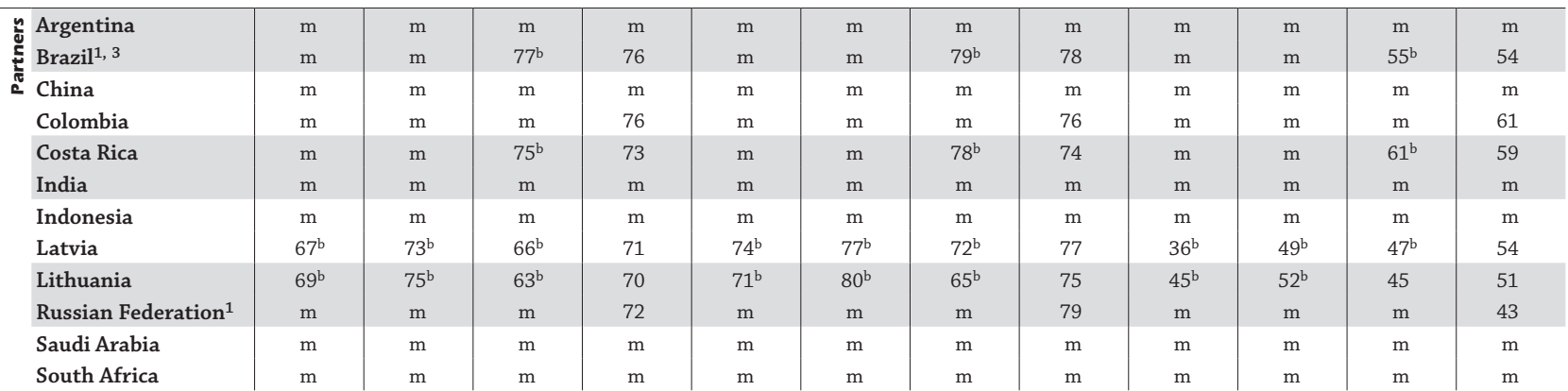

\begin{tabular}{l|l|l|l|l|l|l|l|l|l|l|l|l|l} 
G20 average & $\mathrm{m}$ & $\mathrm{m}$ & $\mathrm{m}$ & $\mathrm{m}$ & $\mathrm{m}$ & $\mathrm{m}$ & $\mathrm{m}$ & $\mathrm{m}$ & $\mathrm{m}$ & $\mathrm{m}$ & $\mathrm{m}$ & $\mathrm{m}$ \\
\hline
\end{tabular}

Notes: In most countries, there is a break in the series, represented by the code "b", as data for the latest year refer to ISCED 2011 while data for previous years refer to ISCED-97. For Korea data refer to ISCED-97 for all years. See the description of the levels of education in the Definitions section. Columns showing data for other age groups are available for consultation on line (see StatLink below).

1. Brazil, Chile, France, the Russian Federation: Data for year 2014 refer to year 2013.

2. The United Kingdom: Data for upper secondary attainment includes completion of a sufficient volume and standard of programmes that would be classified individually as completion of intermediate upper secondary programmes ( $18 \%$ of the adults are under this group).

3. Brazil: Data for year 2010 refer to year 2009.

Source: OECD. Colombia, Costa Rica: OECD Education Database. Latvia, Lithuania: Eurostat. See Annex 3 for notes (www.oecd.org/education/education-at-aglance-19991487.htm).

Please refer to the Reader's Guide for information concerning symbols for missing data and abbreviations.

StatLink 제느 http://dx.doi.org/10.1787/888933285001 
Table A5.3a. [3/3] Trends in employment rates, by educational attainment and age group (2000, 2005, 2010 and 2014)

Percentage of employed adults, by age group among all adults in the same age group

\begin{tabular}{|c|c|c|c|c|c|c|c|c|c|c|c|c|}
\hline & \multicolumn{12}{|c|}{ Tertiary } \\
\hline & \multicolumn{4}{|c|}{ Employment rates of 25-64 year-olds } & \multicolumn{4}{|c|}{ Employment rates of 25-34 year-olds } & \multicolumn{4}{|c|}{ Employment rates of 55-64 year-olds } \\
\hline & 2000 & 2005 & 2010 & 2014 & 2000 & 2005 & 2010 & 2014 & 2000 & 2005 & 2010 & 2014 \\
\hline & $(41)$ & $(42)$ & $(43)$ & $(44)$ & $(45)$ & $(46)$ & $(47)$ & $(48)$ & (57) & $(58)$ & (59) & $(60)$ \\
\hline Australia & $83^{\mathrm{b}}$ & $84^{\mathrm{b}}$ & $84^{\mathrm{b}}$ & 83 & $84^{\mathrm{b}}$ & $85^{\mathrm{b}}$ & $85^{\mathrm{b}}$ & 84 & $65^{\mathrm{b}}$ & $69^{\mathrm{b}}$ & $75^{b}$ & 70 \\
\hline Austria & $\mathrm{m}$ & 83 & 85 & 85 & $\mathrm{~m}$ & 86 & 86 & 86 & $\mathrm{~m}$ & 48 & 61 & 66 \\
\hline Belgium & $85^{b}$ & $84^{b}$ & $84^{b}$ & 85 & $92^{\mathrm{b}}$ & $90^{\mathrm{b}}$ & $89^{b}$ & 88 & $46^{\mathrm{b}}$ & $49^{b}$ & $53^{b}$ & 61 \\
\hline Canada & 83 & 82 & 81 & 82 & 86 & 85 & 84 & 85 & 58 & 62 & 65 & 65 \\
\hline Chile $^{1}$ & $\mathrm{~m}$ & $\mathrm{~m}$ & $79^{b}$ & 84 & $\mathrm{~m}$ & $\mathrm{~m}$ & $75^{b}$ & 84 & $\mathrm{~m}$ & $\mathrm{~m}$ & $74^{\mathrm{b}}$ & 74 \\
\hline Czech Republic & $87^{b}$ & $86^{b}$ & $83^{b}$ & 84 & $83^{b}$ & $81^{\mathrm{b}}$ & $77^{b}$ & 77 & $66^{\mathrm{b}}$ & $69^{\mathrm{b}}$ & $71^{b}$ & 78 \\
\hline Denmark & $88^{b}$ & $86^{b}$ & $86^{b}$ & 86 & $88^{\mathrm{b}}$ & $87^{b}$ & $86^{b}$ & 84 & $73^{b}$ & $73^{b}$ & $71^{\mathrm{b}}$ & 75 \\
\hline Estonia & 83 & 84 & 80 & 84 & 85 & 84 & 81 & 81 & 62 & 74 & 66 & 77 \\
\hline Finland & $84^{\mathrm{b}}$ & $84^{\mathrm{b}}$ & $84^{\mathrm{b}}$ & 83 & $84^{\mathrm{b}}$ & $86^{b}$ & $84^{\mathrm{b}}$ & 82 & $60^{\mathrm{b}}$ & $66^{\mathrm{b}}$ & $70^{\mathrm{b}}$ & 71 \\
\hline France $^{1}$ & 83 & 83 & 84 & 84 & 85 & 86 & 87 & 86 & 50 & 56 & 55 & 61 \\
\hline Germany & $83^{b}$ & $83^{b}$ & $87^{b}$ & 88 & $89^{b}$ & $85^{b}$ & $88^{b}$ & 88 & $58^{\mathrm{b}}$ & $63^{b}$ & $73^{\mathrm{b}}$ & 78 \\
\hline Greece & $81^{\mathrm{b}}$ & $82^{b}$ & $80^{\mathrm{b}}$ & 69 & $79^{\mathrm{b}}$ & $79^{b}$ & $77^{\mathrm{b}}$ & 63 & $50^{\mathrm{b}}$ & $59^{b}$ & $57^{\mathrm{b}}$ & 46 \\
\hline Hungary & $82^{b}$ & $83^{b}$ & $79^{b}$ & 82 & $83^{b}$ & $83^{b}$ & $79^{b}$ & 81 & $52^{\mathrm{b}}$ & $60^{\mathrm{b}}$ & $54^{\mathrm{b}}$ & 59 \\
\hline Iceland & $\mathrm{m}$ & 94 & 90 & 91 & $\mathrm{~m}$ & 94 & 88 & 87 & $\mathrm{~m}$ & 90 & 89 & 90 \\
\hline Ireland & $88^{b}$ & $87^{b}$ & $81^{b}$ & 81 & $91^{\mathrm{b}}$ & $89^{b}$ & $83^{b}$ & 83 & $67^{\mathrm{b}}$ & $70^{\mathrm{b}}$ & $66^{\mathrm{b}}$ & 63 \\
\hline Israel & $\mathrm{m}$ & $81^{b}$ & $82^{b}$ & 86 & $\mathrm{~m}$ & $82^{b}$ & $82^{b}$ & 86 & $\mathrm{~m}$ & $68^{\mathrm{b}}$ & $71^{\mathrm{b}}$ & 75 \\
\hline Italy & $81^{b}$ & $80^{\mathrm{b}}$ & $78^{b}$ & 78 & $73^{b}$ & $69^{b}$ & $67^{b}$ & 62 & $58^{b}$ & $67^{b}$ & $67^{b}$ & 76 \\
\hline Japan & $\mathrm{m}$ & $\mathrm{m}$ & $\mathrm{m}$ & $\mathrm{m}$ & $\mathrm{m}$ & $\mathrm{m}$ & $\mathrm{m}$ & $\mathrm{m}$ & $\mathrm{m}$ & $\mathrm{m}$ & $\mathrm{m}$ & $\mathrm{m}$ \\
\hline Korea & 75 & 77 & 76 & 77 & 74 & 74 & 74 & 76 & 57 & 61 & 64 & 69 \\
\hline Luxembourg & $84^{\mathrm{b}}$ & $84^{\mathrm{b}}$ & $85^{b}$ & 85 & $83^{b}$ & $87^{b}$ & $87^{b}$ & 86 & $65^{\mathrm{b}}$ & $60^{\mathrm{b}}$ & $67^{\mathrm{b}}$ & 62 \\
\hline Mexico & $82^{b}$ & $82^{b}$ & $81^{b}$ & 79 & $80^{\mathrm{b}}$ & $79^{b}$ & $80^{\mathrm{b}}$ & 78 & $69^{\mathrm{b}}$ & $68^{\mathrm{b}}$ & $66^{\mathrm{b}}$ & 65 \\
\hline Netherlands & $86^{\mathrm{b}}$ & $86^{\mathrm{b}}$ & $88^{b}$ & 88 & $93^{b}$ & $92^{\mathrm{b}}$ & $93^{b}$ & 90 & $56^{\mathrm{b}}$ & $62^{\mathrm{b}}$ & $69^{b}$ & 75 \\
\hline New Zealand & $82^{\mathrm{b}}$ & $84^{\mathrm{b}}$ & $84^{\mathrm{b}}$ & 87 & $82^{\mathrm{b}}$ & $81^{b}$ & $81^{b}$ & 86 & $67^{\mathrm{b}}$ & $78^{\mathrm{b}}$ & $82^{b}$ & 84 \\
\hline Norway & $90^{\mathrm{b}}$ & $89^{b}$ & $90^{\mathrm{b}}$ & 90 & $87^{b}$ & $86^{b}$ & $89^{b}$ & 87 & $86^{\mathrm{b}}$ & $85^{b}$ & $84^{\mathrm{b}}$ & 86 \\
\hline Poland & $85^{\mathrm{b}}$ & $83^{b}$ & $85^{b}$ & 86 & $87^{b}$ & $83^{b}$ & $86^{b}$ & 86 & $51^{\mathrm{b}}$ & $55^{b}$ & $56^{b}$ & 66 \\
\hline Portugal & $91^{\mathrm{b}}$ & $87^{b}$ & $85^{b}$ & 83 & $91^{\mathrm{b}}$ & $87^{b}$ & $85^{b}$ & 79 & $69^{b}$ & $61^{\mathrm{b}}$ & $58^{b}$ & 61 \\
\hline Slovak Republic & $86^{\mathrm{b}}$ & $84^{b}$ & $82^{b}$ & 80 & $83^{\mathrm{b}}$ & $84^{b}$ & $78^{\mathrm{b}}$ & 75 & $54^{\mathrm{b}}$ & $54^{\mathrm{b}}$ & $66^{\mathrm{b}}$ & 67 \\
\hline Slovenia & $86^{\mathrm{b}}$ & $87^{b}$ & $87^{b}$ & 83 & $92^{\mathrm{b}}$ & $91^{\mathrm{b}}$ & $88^{b}$ & 80 & $48^{\mathrm{b}}$ & $51^{\mathrm{b}}$ & $57^{b}$ & 53 \\
\hline Spain & $80^{\mathrm{b}}$ & $83^{b}$ & $80^{b}$ & 77 & $75^{b}$ & $82^{b}$ & $79^{b}$ & 74 & $64^{\mathrm{b}}$ & $65^{b}$ & $64^{b}$ & 65 \\
\hline Sweden & $87^{\mathrm{b}}$ & $87^{b}$ & $88^{b}$ & 89 & $82^{\mathrm{b}}$ & $84^{\mathrm{b}}$ & $85^{b}$ & 87 & $79^{\mathrm{b}}$ & $83^{b}$ & $81^{\mathrm{b}}$ & 83 \\
\hline Switzerland & $90^{\mathrm{b}}$ & $90^{\mathrm{b}}$ & $88^{b}$ & 89 & $91^{\mathrm{b}}$ & $91^{\mathrm{b}}$ & $87^{b}$ & 88 & $78^{\mathrm{b}}$ & $79^{\mathrm{b}}$ & $79^{b}$ & 82 \\
\hline Turkey & 78 & 75 & 76 & 76 & 83 & 79 & 77 & 76 & 37 & 34 & 38 & 42 \\
\hline United Kingdom ${ }^{2}$ & $88^{\mathrm{b}}$ & $88^{b}$ & $84^{b}$ & 85 & $91^{\mathrm{b}}$ & $90^{\mathrm{b}}$ & $87^{b}$ & 87 & $66^{\mathrm{b}}$ & $72^{b}$ & $65^{\mathrm{b}}$ & 66 \\
\hline United States & 85 & 82 & 80 & 80 & 87 & 83 & 82 & 82 & 70 & 72 & 70 & 69 \\
\hline OECD average & $84^{\mathrm{b}}$ & $84^{b}$ & $83^{b}$ & 83 & $85^{b}$ & $85^{b}$ & $83^{b}$ & 82 & $61^{\mathrm{b}}$ & $65^{b}$ & $67^{b}$ & 69 \\
\hline EU21 average & $85^{\mathrm{b}}$ & $85^{b}$ & $84^{b}$ & 83 & $85^{\mathrm{b}}$ & $85^{b}$ & $83^{b}$ & 81 & $60^{\mathrm{b}}$ & $63^{b}$ & $64^{\mathrm{b}}$ & 67 \\
\hline
\end{tabular}

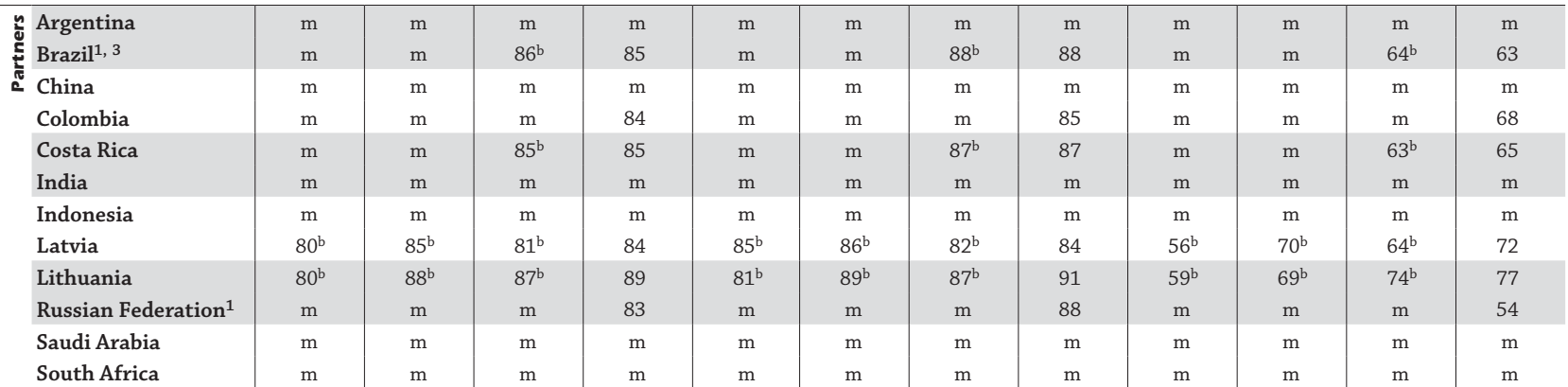

\begin{tabular}{l|l|l|l|l|l|l|l|l|l|l|l|l|l} 
G20 average & $\mathrm{m}$ & $\mathrm{m}$ & $\mathrm{m}$ & $\mathrm{m}$ & $\mathrm{m}$ & $\mathrm{m}$ & $\mathrm{m}$ & $\mathrm{m}$ & $\mathrm{m}$ & $\mathrm{m}$ & $\mathrm{m}$ & $\mathrm{m}$
\end{tabular}

Notes: In most countries, there is a break in the series, represented by the code "b", as data for the latest year refer to ISCED 2011 while data for previous years refer to ISCED-97. For Korea data refer to ISCED-97 for all years. See the description of the levels of education in the Definitions section. Columns showing data for other age groups are available for consultation on line (see StatLink below).

1. Brazil, Chile, France, the Russian Federation: Data for year 2014 refer to year 2013.

2. The United Kingdom: Data for upper secondary attainment includes completion of a sufficient volume and standard of programmes that would be classified individually as completion of intermediate upper secondary programmes (18\% of the adults are under this group).

3. Brazil: Data for year 2010 refer to year 2009.

Source: OECD. Colombia, Costa Rica: OECD Education Database. Latvia, Lithuania: Eurostat. See Annex 3 for notes (www.oecd.org/education/education-at-aglance-19991487.htm).

Please refer to the Reader's Guide for information concerning symbols for missing data and abbreviations.

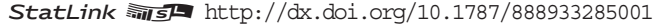


Table A5.4a. [1/3] Trends in unemployment rates, by educational attainment and age group (2000, 2005, 2010 and 2014)

Percentage of unemployed adults, by age group among adults in the same age group who are in the labour force

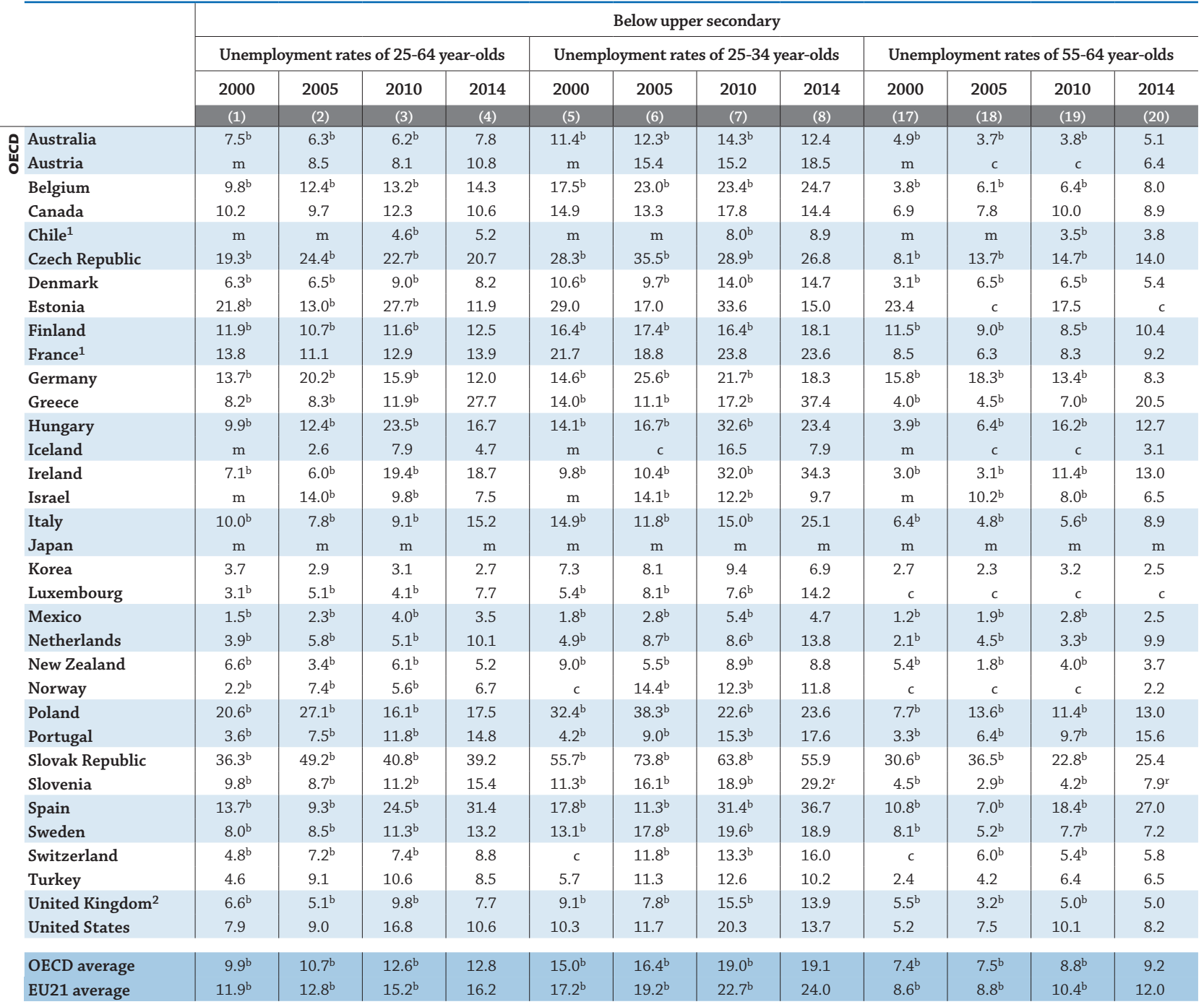

\begin{tabular}{|c|c|c|c|c|c|c|c|c|c|c|c|c|}
\hline $\begin{array}{l}\text { Argentina } \\
\text { Brazil } \\
\text { 1, } 3\end{array}$ & $\begin{array}{l}\mathrm{m} \\
\mathrm{m}\end{array}$ & $\begin{array}{l}\mathrm{m} \\
\mathrm{m}\end{array}$ & $\begin{array}{c}\mathrm{m} \\
5.7^{\mathrm{b}}\end{array}$ & $\begin{array}{r}\mathrm{m} \\
4.5\end{array}$ & $\begin{array}{l}\mathrm{m} \\
\mathrm{m}\end{array}$ & $\begin{array}{l}\mathrm{m} \\
\mathrm{m}\end{array}$ & $\begin{array}{c}\mathrm{m} \\
8.5^{\mathrm{b}}\end{array}$ & $\begin{array}{r}\mathrm{m} \\
7.4\end{array}$ & $\begin{array}{l}\mathrm{m} \\
\mathrm{m}\end{array}$ & $\begin{array}{l}\mathrm{m} \\
\mathrm{m}\end{array}$ & $\begin{array}{c}\mathrm{m} \\
2.8^{\mathrm{b}}\end{array}$ & $\begin{array}{r}\mathrm{m} \\
2.4\end{array}$ \\
\hline $\begin{array}{l}\text { ¿ China } \\
\text { Colombia }\end{array}$ & $\begin{array}{l}\mathrm{m} \\
\mathrm{m}\end{array}$ & $\begin{array}{l}\mathrm{m} \\
\mathrm{m}\end{array}$ & $\begin{array}{l}\mathrm{m} \\
\mathrm{m}\end{array}$ & $\begin{array}{r}\mathrm{m} \\
6.2\end{array}$ & $\begin{array}{l}\mathrm{m} \\
\mathrm{m}\end{array}$ & $\begin{array}{l}\mathrm{m} \\
\mathrm{m}\end{array}$ & $\begin{array}{l}\mathrm{m} \\
\mathrm{m}\end{array}$ & $\begin{array}{r}\mathrm{m} \\
8.0\end{array}$ & $\begin{array}{l}\mathrm{m} \\
\mathrm{m}\end{array}$ & $\begin{array}{l}\mathrm{m} \\
\mathrm{m}\end{array}$ & $\begin{array}{l}\mathrm{m} \\
\mathrm{m}\end{array}$ & $\begin{array}{r}\mathrm{m} \\
5.6\end{array}$ \\
\hline $\begin{array}{l}\text { Costa Rica } \\
\text { India }\end{array}$ & $\begin{array}{l}\mathrm{m} \\
\mathrm{m}\end{array}$ & $\begin{array}{l}\mathrm{m} \\
\mathrm{m}\end{array}$ & $\begin{array}{c}7.5^{b} \\
\mathrm{~m}\end{array}$ & $\begin{array}{r}7.1 \\
\mathrm{~m}\end{array}$ & $\begin{array}{l}\mathrm{m} \\
\mathrm{m}\end{array}$ & $\begin{array}{l}\mathrm{m} \\
\mathrm{m}\end{array}$ & $\begin{array}{c}9.4^{\mathrm{b}} \\
\mathrm{m}\end{array}$ & $\begin{array}{r}9.9 \\
\mathrm{~m}\end{array}$ & $\begin{array}{l}\mathrm{m} \\
\mathrm{m}\end{array}$ & $\begin{array}{l}\mathrm{m} \\
\mathrm{m}\end{array}$ & $\begin{array}{c}5.6^{\mathrm{b}} \\
\mathrm{m}\end{array}$ & $\begin{array}{r}4.9 \\
\mathrm{~m}\end{array}$ \\
\hline $\begin{array}{l}\text { Indonesia } \\
\text { Latvia }\end{array}$ & $\begin{array}{c}\mathrm{m} \\
19.0^{\mathrm{b}}\end{array}$ & $\begin{array}{c}\mathrm{m} \\
12.9^{\mathrm{b}}\end{array}$ & $\begin{array}{c}\mathrm{m} \\
27.6^{\mathrm{b}}\end{array}$ & $\begin{array}{r}\mathrm{m} \\
23.6\end{array}$ & $\begin{array}{c}\mathrm{m} \\
25.7^{\mathrm{b}}\end{array}$ & $\begin{array}{c}\mathrm{m} \\
16.4^{\mathrm{b}}\end{array}$ & $\begin{array}{c}\mathrm{m} \\
26.7^{\mathrm{b}}\end{array}$ & $\begin{array}{r}\mathrm{m} \\
24.3\end{array}$ & $\begin{array}{c}\mathrm{m} \\
8.0^{\mathrm{b}}\end{array}$ & $\begin{array}{c}\mathrm{m} \\
7.6^{\mathrm{b}}\end{array}$ & $\begin{array}{c}\mathrm{m} \\
24.0^{\mathrm{b}}\end{array}$ & $\begin{array}{c}\mathrm{m} \\
22.9^{\mathrm{r}}\end{array}$ \\
\hline $\begin{array}{l}\text { Saudi Arabia } \\
\text { South Africa }\end{array}$ & $\begin{array}{l}\mathrm{m} \\
\mathrm{m}\end{array}$ & $\begin{array}{l}\mathrm{m} \\
\mathrm{m}\end{array}$ & $\begin{array}{l}\mathrm{m} \\
\mathrm{m}\end{array}$ & $\begin{array}{l}\mathrm{m} \\
\mathrm{m}\end{array}$ & $\begin{array}{l}\mathrm{m} \\
\mathrm{m}\end{array}$ & $\begin{array}{l}\mathrm{m} \\
\mathrm{m}\end{array}$ & $\begin{array}{l}\mathrm{m} \\
\mathrm{m}\end{array}$ & $\begin{array}{l}\mathrm{m} \\
\mathrm{m}\end{array}$ & $\begin{array}{l}\mathrm{m} \\
\mathrm{m}\end{array}$ & $\begin{array}{l}\mathrm{m} \\
\mathrm{m}\end{array}$ & $\begin{array}{l}\mathrm{m} \\
\mathrm{m}\end{array}$ & $\begin{array}{l}\mathrm{m} \\
\mathrm{m}\end{array}$ \\
\hline G20 average & $\mathrm{m}$ & $\mathrm{m}$ & $\mathrm{m}$ & $\mathrm{m}$ & $\mathrm{m}$ & $\mathrm{m}$ & $\mathrm{m}$ & $\mathrm{m}$ & $\mathrm{m}$ & $\mathrm{m}$ & $\mathrm{m}$ & $\mathrm{m}$ \\
\hline
\end{tabular}

Notes: In most countries, there is a break in the series, represented by the code "b", as data for the latest year refer to ISCED 2011 while data for previous years refer to ISCED-97. For Korea data refer to ISCED-97 for all years. See the description of the levels of education in the Definitions section. Columns showing data for other age groups are available for consultation on line (see StatLink below).

1. Brazil, Chile, France, the Russian Federation: Data for year 2014 refer to year 2013.

2. The United Kingdom: Data for upper secondary attainment includes completion of a sufficient volume and standard of programmes that would be classified individually as completion of intermediate upper secondary programmes ( $18 \%$ of the adults are under this group).

3. Brazil: Data for year 2010 refer to year 2009.

Source: OECD. Colombia, Costa Rica: OECD Education Database. Latvia, Lithuania: Eurostat. See Annex 3 for notes (www.oecd.org/education/education-at-aglance-19991487.htm).

Please refer to the Reader's Guide for information concerning symbols for missing data and abbreviations.

StatLink 제개 http://dx.doi.org/10.1787/888933285011 
Table A5.4a. [2/3] Trends in unemployment rates, by educational attainment and age group (2000, 2005, 2010 and 2014)

Percentage of unemployed adults, by age group among adults in the same age group who are in the labour force

\begin{tabular}{|c|c|c|c|c|c|c|c|c|c|c|c|c|}
\hline & \multicolumn{12}{|c|}{ Upper secondary or post-secondary non-tertiary } \\
\hline & \multicolumn{4}{|c|}{ Unemployment rates of 25-64 year-olds } & \multicolumn{4}{|c|}{ Unemployment rates of 25-34 year-olds } & \multicolumn{4}{|c|}{ Unemployment rates of 55-64 year-olds } \\
\hline & 2000 & 2005 & 2010 & 2014 & 2000 & 2005 & 2010 & 2014 & 2000 & 2005 & 2010 & 2014 \\
\hline & (21) & (22) & (23) & (24) & (25) & $(26)$ & (27) & $(28)$ & (37) & (38) & (39) & (40) \\
\hline Australia & $4.5^{\mathrm{b}}$ & $3.4^{\mathrm{b}}$ & $3.6^{b}$ & 4.6 & $5.3^{b}$ & $4.0^{\mathrm{b}}$ & $5.0^{\mathrm{b}}$ & 5.9 & $4.1^{b}$ & $3.4^{b}$ & $2.5^{\mathrm{b}}$ & 2.9 \\
\hline Austria & $\mathrm{m}$ & 4.5 & 4.0 & 4.5 & $\mathrm{~m}$ & 5.3 & 5.7 & 6.3 & $\mathrm{~m}$ & c & 2.8 & 3.6 \\
\hline Belgium & $5.3^{\mathrm{b}}$ & $6.9^{b}$ & $6.6^{b}$ & 7.3 & $6.7^{b}$ & $9.4^{\mathrm{b}}$ & $10.2^{\mathrm{b}}$ & 10.8 & $3.5^{b}$ & $4.1^{b}$ & $4.1^{b}$ & 5.4 \\
\hline Chile $^{1}$ & $\mathrm{~m}$ & $\mathrm{~m}$ & $6.2^{\mathrm{b}}$ & 5.6 & $\mathrm{~m}$ & $\mathrm{~m}$ & $8.1^{b}$ & 7.5 & $\mathrm{~m}$ & $\mathrm{~m}$ & $4.3^{b}$ & 3.8 \\
\hline Czech Republic & $6.7^{\mathrm{b}}$ & $6.2^{\mathrm{b}}$ & $6.2^{\mathrm{b}}$ & 5.4 & $8.7^{b}$ & $7.0^{\mathrm{b}}$ & $7.4^{\mathrm{b}}$ & 7.0 & $5.3^{b}$ & $4.9^{b}$ & $6.5^{b}$ & 5.0 \\
\hline Denmark & $3.9^{b}$ & $4.0^{\mathrm{b}}$ & $6.1^{\mathrm{b}}$ & 5.1 & $3.9^{b}$ & $4.3^{\mathrm{b}}$ & $7.6^{\mathrm{b}}$ & 6.9 & $4.9^{\mathrm{b}}$ & $5.7^{\mathrm{b}}$ & $6.3^{b}$ & 5.2 \\
\hline Estonia & 14.5 & 8.4 & 18.0 & 7.8 & 15.4 & 7.2 & 19.4 & 9.2 & 3.9 & 5.9 & 17.3 & 7.4 \\
\hline Finland & $8.8^{b}$ & $7.4^{\mathrm{b}}$ & $7.5^{b}$ & 8.1 & $10.4^{\mathrm{b}}$ & $8.0^{\mathrm{b}}$ & $8.1^{b}$ & 9.3 & $9.7^{b}$ & $7.0^{\mathrm{b}}$ & $7.5^{b}$ & 7.9 \\
\hline France $^{1}$ & 8.0 & 6.6 & 7.2 & 8.5 & 10.3 & 9.3 & 10.8 & 13.5 & 7.7 & 4.6 & 6.4 & 6.6 \\
\hline Germany & $7.8^{\mathrm{b}}$ & $11.0^{\mathrm{b}}$ & $6.9^{b}$ & 4.6 & $6.2^{\mathrm{b}}$ & $10.9^{\mathrm{b}}$ & $7.4^{\mathrm{b}}$ & 4.9 & $13.7^{\mathrm{b}}$ & $13.9^{\mathrm{b}}$ & $8.4^{b}$ & 5.6 \\
\hline Hungary & $5.3^{b}$ & $6.0^{\mathrm{b}}$ & $9.5^{b}$ & 6.5 & $6.8^{b}$ & $7.3^{b}$ & $11.4^{\mathrm{b}}$ & 8.5 & $3.6^{b}$ & $4.0^{\mathrm{b}}$ & $7.9^{b}$ & 6.5 \\
\hline Iceland & $\mathrm{m}$ & c & 6.8 & 4.1 & $\mathrm{~m}$ & c & 11.8 & 7.1 & $\mathrm{~m}$ & c & c & 3.1 \\
\hline Ireland & $2.6^{b}$ & $3.1^{b}$ & $13.8^{\mathrm{b}}$ & 11.9 & $2.7^{\mathrm{b}}$ & $3.7^{\mathrm{b}}$ & $18.7^{\mathrm{b}}$ & 16.5 & c & c & $8.6^{b}$ & 8.3 \\
\hline Israel & $\mathrm{m}$ & $9.4^{b}$ & $6.8^{\mathrm{b}}$ & 6.2 & $\mathrm{~m}$ & $10.4^{\mathrm{b}}$ & $8.0^{\mathrm{b}}$ & 7.8 & $\mathrm{~m}$ & $9.9^{b}$ & $5.2^{\mathrm{b}}$ & 4.8 \\
\hline Italy & $7.2^{\mathrm{b}}$ & $5.2^{b}$ & $6.1^{b}$ & 9.1 & $12.2^{\mathrm{b}}$ & $8.1^{b}$ & $10.1^{\mathrm{b}}$ & 15.9 & $1.6^{b}$ & $2.4^{\mathrm{b}}$ & $2.5^{b}$ & 4.2 \\
\hline Japan & $\mathrm{m}$ & $\mathrm{m}$ & $\mathrm{m}$ & $\mathrm{m}$ & $\mathrm{m}$ & $\mathrm{m}$ & $\mathrm{m}$ & $\mathrm{m}$ & $\mathrm{m}$ & $\mathrm{m}$ & $\mathrm{m}$ & $\mathrm{m}$ \\
\hline Korea & 4.1 & 3.8 & 3.5 & 3.3 & 5.0 & 5.7 & 6.2 & 7.0 & 3.7 & 3.3 & 2.7 & 2.5 \\
\hline Luxembourg & $1.6^{\mathrm{b}}$ & $3.2^{\mathrm{b}}$ & $3.6^{\mathrm{b}}$ & 5.1 & $2.2^{\mathrm{b}}$ & $4.0^{\mathrm{b}}$ & $4.8^{\mathrm{b}}$ & $4.2^{\mathrm{r}}$ & c & c & c & $5.3^{r}$ \\
\hline Mexico & $2.2^{\mathrm{b}}$ & $3.1^{b}$ & $4.5^{\mathrm{b}}$ & 4.4 & $2.5^{\mathrm{b}}$ & $4.1^{b}$ & $5.8^{b}$ & 5.7 & $2.6^{b}$ & $2.4^{b}$ & $3.9^{b}$ & 4.1 \\
\hline Netherlands & $2.3^{\mathrm{b}}$ & $4.1^{b}$ & $3.1^{\mathrm{b}}$ & 7.1 & $2.2^{\mathrm{b}}$ & $3.9^{\mathrm{b}}$ & $3.4^{\mathrm{b}}$ & 7.1 & $2.5^{b}$ & $4.6^{b}$ & $2.5^{\mathrm{b}}$ & 8.4 \\
\hline New Zealand & $3.9^{b}$ & $2.3^{b}$ & $4.5^{b}$ & 4.5 & $4.7^{\mathrm{b}}$ & $3.0^{\mathrm{b}}$ & $7.2^{\mathrm{b}}$ & 6.7 & $3.8^{\mathrm{b}}$ & $1.7^{\mathrm{b}}$ & $3.4^{\mathrm{b}}$ & 3.1 \\
\hline Norway & $2.6^{b}$ & $2.6^{b}$ & $2.2^{\mathrm{b}}$ & 2.4 & $3.7^{b}$ & $4.1^{\mathrm{b}}$ & $3.8^{b}$ & 3.7 & c & c & c & 1.4 \\
\hline Poland & $13.9^{\mathrm{b}}$ & $16.6^{\mathrm{b}}$ & $8.9^{b}$ & 8.6 & $16.8^{\mathrm{b}}$ & $19.9^{b}$ & $11.5^{\mathrm{b}}$ & 11.7 & $11.6^{\mathrm{b}}$ & $13.0^{\mathrm{b}}$ & $7.8^{b}$ & 7.1 \\
\hline Portugal & $3.5^{b}$ & $6.7^{\mathrm{b}}$ & $9.7^{\mathrm{b}}$ & 12.6 & $3.5^{b}$ & $8.3^{b}$ & $11.5^{\mathrm{b}}$ & 14.9 & c & c & c & 12.9 \\
\hline Slovak Republic & $14.3^{\mathrm{b}}$ & $12.7^{\mathrm{b}}$ & $12.3^{\mathrm{b}}$ & 11.3 & $17.7^{\mathrm{b}}$ & $13.8^{\mathrm{b}}$ & $14.6^{\mathrm{b}}$ & 15.4 & $10.1^{\mathrm{b}}$ & $11.6^{\mathrm{b}}$ & $9.9^{b}$ & 10.3 \\
\hline Slovenia & $5.7^{\mathrm{b}}$ & $5.7^{b}$ & $6.9^{b}$ & 9.7 & $5.8^{\mathrm{b}}$ & $6.7^{\mathrm{b}}$ & $10.2^{\mathrm{b}}$ & 14.5 & $10.9^{\mathrm{b}}$ & $6.3^{b}$ & $5.0^{\mathrm{b}}$ & 10.0 \\
\hline Switzerland & $2.2^{\mathrm{b}}$ & $3.7^{\mathrm{b}}$ & $4.1^{\mathrm{b}}$ & 3.8 & $2.8^{b}$ & $4.7^{\mathrm{b}}$ & $5.4^{\mathrm{b}}$ & 5.1 & c & $3.7^{b}$ & $3.6^{b}$ & 3.1 \\
\hline Turkey & 5.5 & 9.1 & 11.3 & 9.1 & 7.1 & 11.9 & 13.3 & 10.9 & 0.0 & 4.5 & 10.7 & 7.7 \\
\hline United Kingdom ${ }^{2}$ & $4.0^{\mathrm{b}}$ & $3.1^{\mathrm{b}}$ & $5.9^{\mathrm{b}}$ & 3.9 & $4.7^{\mathrm{b}}$ & $4.1^{\mathrm{b}}$ & $8.1^{b}$ & 5.4 & $4.0^{\mathrm{b}}$ & $2.4^{\mathrm{b}}$ & $5.0^{\mathrm{b}}$ & 3.3 \\
\hline United States & 3.6 & 5.1 & 11.2 & 7.2 & 4.4 & 6.9 & 14.3 & 10.1 & 3.1 & 4.2 & 8.8 & 5.4 \\
\hline OECD average & $6.1^{\mathrm{b}}$ & $6.2^{\mathrm{b}}$ & $7.6^{\mathrm{b}}$ & 7.7 & $7.3^{\mathrm{b}}$ & $7.5^{b}$ & $9.9^{b}$ & 10.2 & $5.6^{b}$ & $5.7^{b}$ & $6.4^{\mathrm{b}}$ & 6.5 \\
\hline EU21 average & $7.1^{\mathrm{b}}$ & $6.9^{\mathrm{b}}$ & $8.5^{b}$ & 9.1 & $8.5^{b}$ & $8.2^{b}$ & $10.8^{\mathrm{b}}$ & 11.9 & $6.6^{\mathrm{b}}$ & $6.4^{\mathrm{b}}$ & $7.1^{\mathrm{b}}$ & 7.9 \\
\hline
\end{tabular}

\begin{tabular}{|c|c|c|c|c|c|c|c|c|c|c|c|c|}
\hline 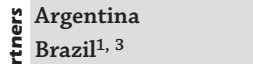 & $\begin{array}{l}\mathrm{m} \\
\mathrm{m}\end{array}$ & $\begin{array}{l}\mathrm{m} \\
\mathrm{m}\end{array}$ & $\begin{array}{c}\mathrm{m} \\
7.2^{\mathrm{b}}\end{array}$ & $\begin{array}{r}\mathrm{m} \\
5.6\end{array}$ & $\begin{array}{l}\mathrm{m} \\
\mathrm{m}\end{array}$ & $\begin{array}{l}\mathrm{m} \\
\mathrm{m}\end{array}$ & $\begin{array}{c}\mathrm{m} \\
9.8^{\mathrm{b}}\end{array}$ & $\begin{array}{r}\mathrm{m} \\
7.5\end{array}$ & $\begin{array}{l}\mathrm{m} \\
\mathrm{m}\end{array}$ & $\begin{array}{l}\mathrm{m} \\
\mathrm{m}\end{array}$ & $\begin{array}{c}\mathrm{m} \\
4.2^{\mathrm{b}}\end{array}$ & $\begin{array}{r}\mathrm{m} \\
2.6\end{array}$ \\
\hline e China & $\mathrm{m}$ & $\mathrm{m}$ & $\mathrm{m}$ & $\mathrm{m}$ & $\mathrm{m}$ & $\mathrm{m}$ & $\mathrm{m}$ & $\mathrm{m}$ & $\mathrm{m}$ & $\mathrm{m}$ & $\mathrm{m}$ & $\mathrm{m}$ \\
\hline Colombia & $\mathrm{m}$ & & $\mathrm{m}$ & 8.4 & $\mathrm{~m}$ & $\mathrm{~m}$ & & 10.3 & & & & 5.6 \\
\hline $\begin{array}{l}\text { Costa Rica } \\
\text { India }\end{array}$ & $\begin{array}{l}\mathrm{m} \\
\mathrm{m}\end{array}$ & $\begin{array}{l}\mathrm{m} \\
\mathrm{m}\end{array}$ & $\begin{array}{c}4.6^{b} \\
\mathrm{~m}\end{array}$ & $\begin{array}{r}6.6 \\
\mathrm{~m}\end{array}$ & $\begin{array}{l}\mathrm{m} \\
\mathrm{m}\end{array}$ & $\begin{array}{l}\mathrm{m} \\
\mathrm{m}\end{array}$ & $\begin{array}{c}6.4^{\mathrm{b}} \\
\mathrm{m}\end{array}$ & $\begin{array}{r}11.0 \\
\mathrm{~m}\end{array}$ & $\begin{array}{l}\mathrm{m} \\
\mathrm{m}\end{array}$ & $\begin{array}{l}\mathrm{m} \\
\mathrm{m}\end{array}$ & $\begin{array}{c}0.3^{b} \\
\mathrm{~m}\end{array}$ & $\begin{array}{r}1.5 \\
\mathrm{~m}\end{array}$ \\
\hline Indonesia & $\mathrm{m}$ & $\mathrm{m}$ & $\mathrm{m}$ & $\mathrm{m}$ & $\mathrm{m}$ & $\mathrm{m}$ & $\mathrm{m}$ & $\mathrm{m}$ & $\mathrm{m}$ & $\mathrm{m}$ & $\mathrm{m}$ & $\mathrm{m}$ \\
\hline Latvia & $14.5^{\mathrm{b}}$ & $9.0^{\mathrm{b}}$ & $18.7^{\mathrm{b}}$ & 11.2 & $14.1^{\mathrm{b}}$ & $9.4^{\mathrm{b}}$ & $18.1^{\mathrm{b}}$ & 11.3 & $11.1^{\mathrm{b}}$ & $10.1^{\mathrm{b}}$ & $17.3^{\mathrm{b}}$ & 10.9 \\
\hline Saudi Arabia & $\mathrm{m}$ & $\mathrm{m}$ & $\mathrm{m}$ & $\mathrm{m}$ & $\mathrm{m}$ & $\mathrm{m}$ & $\mathrm{m}$ & $\mathrm{m}$ & $\mathrm{m}$ & $\mathrm{m}$ & $\mathrm{m}$ & $\mathrm{m}$ \\
\hline South Africa & $\mathrm{m}$ & $\mathrm{m}$ & $\mathrm{m}$ & $\mathrm{m}$ & $\mathrm{m}$ & $\mathrm{m}$ & $\mathrm{m}$ & $\mathrm{m}$ & $\mathrm{m}$ & $\mathrm{m}$ & $\mathrm{m}$ & $\mathrm{m}$ \\
\hline
\end{tabular}

\begin{tabular}{l|l|l|l|l|l|l|l|l|l|l|l|l|l|l} 
G20 average & $\mathrm{m}$ & $\mathrm{m}$ & $\mathrm{m}$ & $\mathrm{m}$ & $\mathrm{m}$ & $\mathrm{m}$ & $\mathrm{m}$ & $\mathrm{m}$ & $\mathrm{m}$ & $\mathrm{m}$ & $\mathrm{m}$ & $\mathrm{m}$ \\
\hline
\end{tabular}

Notes: In most countries, there is a break in the series, represented by the code "b", as data for the latest year refer to ISCED 2011 while data for previous years refer to ISCED-97. For Korea data refer to ISCED-97 for all years. See the description of the levels of education in the Definitions section. Columns showing data for other age groups are available for consultation on line (see StatLink below).

1. Brazil, Chile, France, the Russian Federation: Data for year 2014 refer to year 2013.

2. The United Kingdom: Data for upper secondary attainment includes completion of a sufficient volume and standard of programmes that would be classified individually as completion of intermediate upper secondary programmes ( $18 \%$ of the adults are under this group).

3. Brazil: Data for year 2010 refer to year 2009.

Source: OECD. Colombia, Costa Rica: OECD Education Database. Latvia, Lithuania: Eurostat. See Annex 3 for notes (www.oecd.org/education/education-at-aglance-19991487.htm).

Please refer to the Reader's Guide for information concerning symbols for missing data and abbreviations.

StatLink 제개 http://dx.doi.org/10.1787/888933285011 
Table A5.4a. [3/3] Trends in unemployment rates, by educational attainment and age group (2000, 2005, 2010 and 2014)

Percentage of unemployed adults, by age group among adults in the same age group who are in the labour force

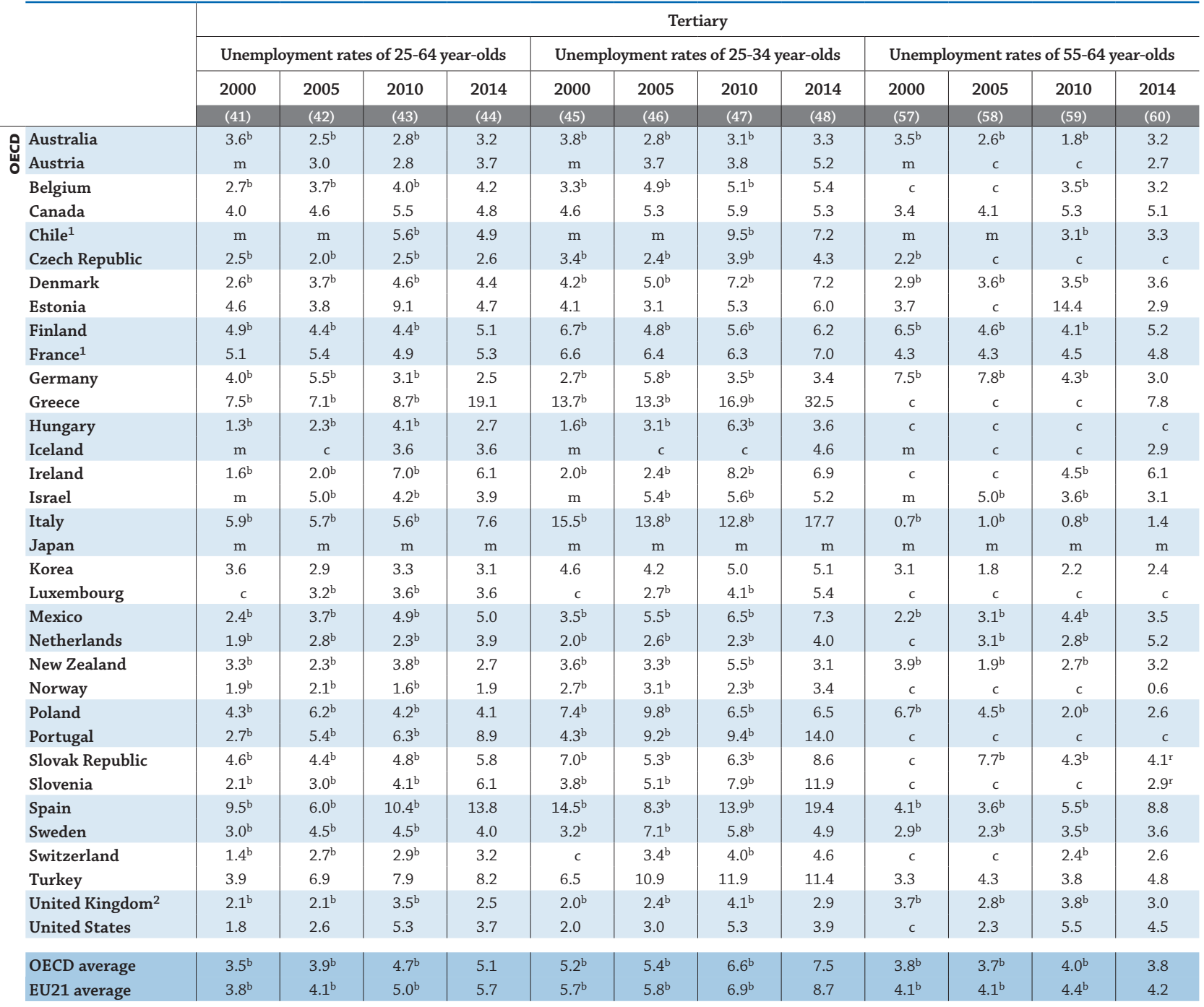

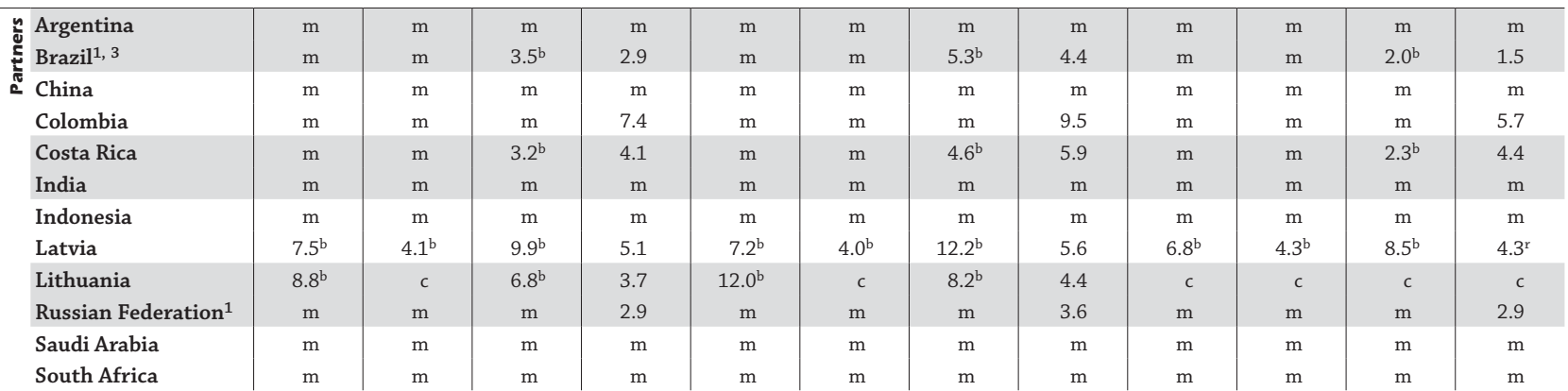

G20 average

\begin{tabular}{l|l|l|l|l}
$\mathrm{m}$ & $\mathrm{m}$ & $\mathrm{m}$ & $\mathrm{m}$ & $\mathrm{m}$
\end{tabular}

\begin{tabular}{l|l}
$\mathrm{m}$ & $\mathrm{m}$
\end{tabular}

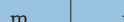

\begin{tabular}{l|l|l}
$\mathrm{m}$ & $\mathrm{m}$ & $\mathrm{m}$
\end{tabular}

Notes: In most countries, there is a break in the series, represented by the code "b", as data for the latest year refer to ISCED 2011 while data for previous years refer to ISCED-97. For Korea data refer to ISCED-97 for all years. See the description of the levels of education in the Definitions section. Columns showing data for other age groups are available for consultation on line (see StatLink below).

1. Brazil, Chile, France, the Russian Federation: Data for year 2014 refer to year 2013.

2. The United Kingdom: Data for upper secondary attainment includes completion of a sufficient volume and standard of programmes that would be classified individually as completion of intermediate upper secondary programmes ( $18 \%$ of the adults are under this group).

3. Brazil: Data for year 2010 refer to year 2009.

Source: OECD. Colombia, Costa Rica: OECD Education Database. Latvia, Lithuania: Eurostat. See Annex 3 for notes (www.oecd.org/education/education-at-aglance-19991487.htm).

Please refer to the Reader's Guide for information concerning symbols for missing data and abbreviations.

StatLink 제기 http://dx.doi.org/10.1787/888933285011 
Table A5.5a. Employment, unemployment and inactivity rates of adults with upper secondary or post-secondary non-tertiary education, by programme orientation (2014)

25-64 year-olds with upper secondary or post-secondary non-tertiary education as the highest level of attainment

\begin{tabular}{|c|c|c|c|c|c|c|c|c|c|}
\hline & \multicolumn{3}{|c|}{ Employment rate } & \multicolumn{3}{|c|}{ Unemployment rate } & \multicolumn{3}{|c|}{ Inactivity rate } \\
\hline & Vocational & General & $\begin{array}{l}\text { Vocational } \\
\text { and general }\end{array}$ & Vocational & General & $\begin{array}{l}\text { Vocational } \\
\text { and general }\end{array}$ & Vocational & General & $\begin{array}{l}\text { Vocational } \\
\text { and general }\end{array}$ \\
\hline & (1) & (2) & (3) & $(4)$ & $(5)$ & (6) & (7) & $(8)$ & (9) \\
\hline Australia & 82 & 72 & 77 & 4.6 & 4.5 & 4.6 & 15 & 25 & 19 \\
\hline Austria & 76 & 75 & 76 & 4.3 & 6.0 & 4.5 & 21 & 20 & 21 \\
\hline Belgium & 75 & 68 & 73 & 6.6 & 9.1 & 7.3 & 20 & 25 & 21 \\
\hline Canada & 79 & 72 & 74 & 6.2 & 6.7 & 6.5 & 15 & 23 & 21 \\
\hline Chile $^{1}$ & 77 & 70 & 72 & 4.6 & 6.0 & 5.6 & 20 & 25 & 24 \\
\hline Czech Republic & 75 & 80 & 78 & 6.9 & 4.0 & 5.4 & 20 & 16 & 18 \\
\hline Denmark & 81 & 61 & 79 & 6.6 & $12.1 \mathrm{r}$ & 5.1 & 13 & 31 & 16 \\
\hline Estonia & $\mathrm{m}$ & $\mathrm{m}$ & 74 & $\mathrm{~m}$ & $\mathrm{~m}$ & 7.8 & $\mathrm{~m}$ & $\mathrm{~m}$ & 19 \\
\hline Finland & 77 & 69 & 73 & 9.0 & 8.3 & 8.1 & 15 & 25 & 20 \\
\hline France $^{1}$ & 73 & 75 & 73 & 9.0 & 8.0 & 8.0 & 20 & 19 & 20 \\
\hline Germany & 81 & 62 & 80 & 4.6 & 6.2 & 4.6 & 16 & 33 & 16 \\
\hline Greece & 58 & 53 & 54 & 30.4 & 26.1 & 27.6 & 16 & 29 & 25 \\
\hline Hungary & $\mathrm{m}$ & $\mathrm{m}$ & 72 & $\mathrm{~m}$ & $\mathrm{~m}$ & 6.5 & $\mathrm{~m}$ & $\mathrm{~m}$ & 23 \\
\hline Iceland & 90 & 79 & 87 & 3.5 & 5.3 & 4.1 & 7 & 16 & 10 \\
\hline Ireland & 69 & 66 & 68 & 16.4 & 15.8 & 11.9 & 18 & 22 & 23 \\
\hline Israel & 80 & 70 & 72 & 5.3 & 6.5 & 6.2 & 15 & 25 & 23 \\
\hline Italy & 72 & 62 & 70 & 8.9 & 9.9 & 9.1 & 21 & 31 & 23 \\
\hline Japan & $\mathrm{m}$ & $\mathrm{m}$ & $\mathrm{m}$ & $\mathrm{m}$ & $\mathrm{m}$ & $\mathrm{m}$ & $\mathrm{m}$ & $\mathrm{m}$ & $\mathrm{m}$ \\
\hline Korea & $\mathrm{m}$ & $\mathrm{m}$ & 72 & $\mathrm{~m}$ & $\mathrm{~m}$ & 3.3 & $\mathrm{~m}$ & $\mathrm{~m}$ & 25 \\
\hline Luxembourg & 88 & 75 & 72 & 5.2 & c & 5.1 & 7 & c & 24 \\
\hline Mexico & $\mathrm{m}$ & $\mathrm{m}$ & 73 & $\mathrm{~m}$ & $\mathrm{~m}$ & 4.4 & $\mathrm{~m}$ & $\mathrm{~m}$ & 24 \\
\hline Netherlands & 79 & 73 & 78 & 6.9 & 8.6 & 7.1 & 16 & 20 & 16 \\
\hline New Zealand & 82 & 77 & 80 & 4.6 & 4.3 & 4.5 & 14 & 19 & 16 \\
\hline Norway & 83 & 78 & 81 & 2.1 & 3.3 & 2.4 & 16 & 19 & 16 \\
\hline Poland & 67 & 64 & 66 & 8.4 & 10.4 & 8.6 & 27 & 29 & 28 \\
\hline Portugal & 78 & 77 & 78 & 14.9 & 11.8 & 12.6 & 8 & 12 & 11 \\
\hline Slovak Republic & 71 & 67 & 71 & 11.2 & 12.2 & 11.3 & 20 & 24 & 20 \\
\hline Slovenia & 80 & $48 \mathrm{r}$ & 69 & 13.7 & c & 9.7 & 7 & $42^{r}$ & 23 \\
\hline Spain & 66 & 66 & 66 & 22.9 & 20.7 & 21.6 & 15 & 17 & 16 \\
\hline Sweden & 86 & 82 & 85 & 4.3 & 5.8 & 4.9 & 10 & 13 & 11 \\
\hline Switzerland & 83 & 78 & 82 & 3.5 & 5.2 & 3.8 & 14 & 17 & 14 \\
\hline Turkey & 66 & 59 & 62 & 8.1 & 10.1 & 9.1 & 29 & 35 & 32 \\
\hline United Kingdom ${ }^{2}$ & 81 & 79 & 80 & 4.1 & 3.6 & 3.9 & 16 & 18 & 17 \\
\hline United States & $\mathrm{m}$ & $\mathrm{m}$ & 68 & $\mathrm{~m}$ & $\mathrm{~m}$ & 7.2 & $\mathrm{~m}$ & $\mathrm{~m}$ & 27 \\
\hline OECD average & 77 & 70 & 74 & 8.5 & 8.9 & 7.7 & 16 & 23 & 20 \\
\hline EU21 average & 75 & 69 & 73 & 10.2 & 10.5 & 9.1 & 16 & 24 & 20 \\
\hline Argentina & $\mathrm{m}$ & $\mathrm{m}$ & $\mathrm{m}$ & $\mathrm{m}$ & $\mathrm{m}$ & $\mathrm{m}$ & $\mathrm{m}$ & $\mathrm{m}$ & $\mathrm{m}$ \\
\hline Brazil $^{1}$ & $\mathrm{~m}$ & $\mathrm{~m}$ & 76 & $\mathrm{~m}$ & $\mathrm{~m}$ & 5.6 & $\mathrm{~m}$ & $\mathrm{~m}$ & 19 \\
\hline China & $\mathrm{m}$ & $\mathrm{m}$ & $\mathrm{m}$ & $\mathrm{m}$ & $\mathrm{m}$ & $\mathrm{m}$ & $\mathrm{m}$ & $\mathrm{m}$ & $\mathrm{m}$ \\
\hline Colombia & $\mathrm{m}$ & $\mathrm{m}$ & 76 & $\mathrm{~m}$ & $\mathrm{~m}$ & 8.4 & $\mathrm{~m}$ & $\mathrm{~m}$ & 17 \\
\hline Costa Rica & 74 & 73 & 73 & 4.2 & 6.9 & 6.6 & 23 & 21 & 21 \\
\hline India & $\mathrm{m}$ & $\mathrm{m}$ & $\mathrm{m}$ & $\mathrm{m}$ & $\mathrm{m}$ & $\mathrm{m}$ & $\mathrm{m}$ & $\mathrm{m}$ & $\mathrm{m}$ \\
\hline Indonesia & $\mathrm{m}$ & $\mathrm{m}$ & $\mathrm{m}$ & $\mathrm{m}$ & $\mathrm{m}$ & $\mathrm{m}$ & $\mathrm{m}$ & $\mathrm{m}$ & $\mathrm{m}$ \\
\hline Latvia & 80 & 76 & 71 & 10.6 & 11.0 & 11.2 & 11 & 15 & 20 \\
\hline Lithuania & 77 & 74 & 70 & 14.8 & 15.1 & 13.1 & c & 13 & 20 \\
\hline Russian Federation ${ }^{1}$ & $\mathrm{~m}$ & $\mathrm{~m}$ & 72 & $\mathrm{~m}$ & m & 6.2 & $\mathrm{~m}$ & $\mathrm{~m}$ & 23 \\
\hline Saudi Arabia & $\mathrm{m}$ & $\mathrm{m}$ & $\mathrm{m}$ & $\mathrm{m}$ & $\mathrm{m}$ & $\mathrm{m}$ & $\mathrm{m}$ & $\mathrm{m}$ & $\mathrm{m}$ \\
\hline South Africa & $\mathrm{m}$ & $\mathrm{m}$ & $\mathrm{m}$ & $\mathrm{m}$ & $\mathrm{m}$ & $\mathrm{m}$ & $\mathrm{m}$ & $\mathrm{m}$ & $\mathrm{m}$ \\
\hline G20 average & $\mathrm{m}$ & $\mathrm{m}$ & $\mathrm{m}$ & $\mathrm{m}$ & $\mathrm{m}$ & $\mathrm{m}$ & $\mathrm{m}$ & $\mathrm{m}$ & $\mathrm{m}$ \\
\hline
\end{tabular}

Notes: "Vocational and general" also includes people in programmes for which no orientation is specified. Denmark, Finland, Ireland, Latvia, Lithuania, Luxembourg, Slovenia: Data for the breakdown by programme orientation cover only 15-34 year-olds and 35-64 year-olds if those individuals had completed their highest level of education 15 years, at most, before the date of the interview. In most countries, data refer to ISCED 2011. The countries with data that refer to ISCED-97 are: Brazil, Indonesia, the Russian Federation, Saudi Arabia and South Africa. See the description of the levels of education in the Definitions section.

1. Brazil, Chile, France, the Russian Federation: Year of reference 2013.

2. The United Kingdom: Data for upper secondary attainment includes completion of a sufficient volume and standard of programmes that would be classified individually as completion of intermediate upper secondary programmes (18\% of the adults are under this group).

Source: OECD. Colombia, Costa Rica: OECD Education Database. Latvia, Lithuania: Eurostat. See Annex 3 for notes (www.oecd.org/education/education-at-aglance-19991487.htm).

Please refer to the Reader's Guide for information concerning symbols for missing data and abbreviations.

StatLink 제느 http://dx.doi.org/10.1787/888933285023 
Table A5.6a. [1/2] Skills and readiness to use information and communication technologies for problem solving among 25-64 year-olds, by ICT skills required at work (2012)

\begin{tabular}{|c|c|c|c|c|c|c|c|c|c|c|c|c|c|c|c|c|}
\hline & \multicolumn{8}{|c|}{ Complex ICT skills required at work } & \multicolumn{8}{|c|}{ Moderate ICT skills required at work } \\
\hline & \multicolumn{2}{|c|}{\begin{tabular}{|c|} 
Group 1 \\
(Refused \\
the computer- \\
based \\
assessment)
\end{tabular}} & \multicolumn{2}{|c|}{\begin{tabular}{|c|} 
Group 2 \\
(Failed ICT core \\
test or minimal \\
problem- \\
solving skills) \\
\end{tabular}} & \multicolumn{2}{|c|}{$\begin{array}{l}\text { Group } 3 \\
\text { (Moderate ICT } \\
\text { and problem- } \\
\text { solving skills) }\end{array}$} & \multicolumn{2}{|c|}{$\begin{array}{c}\text { Group } 4 \\
\text { (Good ICT } \\
\text { and problem- } \\
\text { solving skills) }\end{array}$} & \multicolumn{2}{|c|}{$\begin{array}{c}\text { Group 1 } \\
\text { (Refused } \\
\text { the computer- } \\
\text { based } \\
\text { assessment) }\end{array}$} & \multicolumn{2}{|c|}{\begin{tabular}{|c|} 
Group 2 \\
(Failed ICT core \\
test or minimal \\
problem- \\
solving skills) \\
\end{tabular}} & \multicolumn{2}{|c|}{$\begin{array}{l}\text { Group } 3 \\
\text { (Moderate ICT } \\
\text { and problem- } \\
\text { solving skills) }\end{array}$} & \multicolumn{2}{|c|}{$\begin{array}{c}\text { Group } 4 \\
\text { (Good ICT } \\
\text { and problem- } \\
\text { solving skills) }\end{array}$} \\
\hline & $\%$ & S.E. & $\%$ & S.E. & $\%$ & S.E. & $\%$ & S.E. & $\%$ & S.E. & $\%$ & S.E. & $\%$ & S.E. & $\%$ & S.E. \\
\hline & (1) & (2) & (3) & $(4)$ & (5) & $(6)$ & (7) & $(8)$ & (9) & (10) & (11) & (12) & (13) & (14) & (15) & (16) \\
\hline \multicolumn{17}{|l|}{ National entities } \\
\hline Australia & 3 & $(1.0)$ & 4 & (1.4) & 26 & $(3.1)$ & 67 & (3.3) & 6 & $(0.7)$ & 8 & $(0.9)$ & 30 & $(1.4)$ & 56 & $(1.7)$ \\
\hline Austria & c & c & 7 & (2.7) & 25 & $(5.0)$ & 68 & $(5.1)$ & 4 & $(0.6)$ & 9 & (1.0) & 36 & $(2.1)$ & 51 & (2.1) \\
\hline Canada & 1 & $(0.4)$ & 9 & (1.5) & 24 & $(2.5)$ & 66 & $(2.7)$ & 2 & $(0.3)$ & 13 & $(0.7)$ & 32 & $(1.1)$ & 53 & (1.0) \\
\hline Czech Republic & c & $c$ & 3 & (3.4) & 30 & $(7.6)$ & 66 & $(7.6)$ & 6 & $(1.0)$ & 9 & $(1.4)$ & 31 & $(2.7)$ & 54 & $(2.6)$ \\
\hline Denmark & 1 & $(0.4)$ & 6 & (1.3) & 24 & $(3.5)$ & 69 & (3.4) & 2 & $(0.3)$ & 11 & $(0.8)$ & 36 & $(1.1)$ & 51 & $(1.3)$ \\
\hline Estonia & 4 & (1.4) & 8 & (2.3) & 23 & $(3.8)$ & 66 & $(4.1)$ & 10 & $(0.6)$ & 14 & $(0.9)$ & 37 & $(1.2)$ & 39 & (1.4) \\
\hline Finland & 2 & $(0.8)$ & 10 & (2.4) & 20 & $(3.8)$ & 69 & $(4.4)$ & 3 & $(0.4)$ & 9 & $(0.9)$ & 31 & $(1.2)$ & 57 & (1.1) \\
\hline Germany & c & c & 5 & (1.8) & 19 & $(4.6)$ & 75 & $(4.6)$ & 2 & $(0.4)$ & 9 & (1.1) & 34 & (1.6) & 56 & (1.8) \\
\hline Ireland & c & c & 8 & (2.3) & 31 & $(4.0)$ & 60 & $(4.5)$ & 5 & $(0.7)$ & 10 & (1.1) & 37 & $(2.0)$ & 48 & (1.8) \\
\hline Italy & $\mathrm{m}$ & $\mathrm{m}$ & $\mathrm{m}$ & $\mathrm{m}$ & $\mathrm{m}$ & $\mathrm{m}$ & $\mathrm{m}$ & $\mathrm{m}$ & $\mathrm{m}$ & $\mathrm{m}$ & $\mathrm{m}$ & $\mathrm{m}$ & $\mathrm{m}$ & $\mathrm{m}$ & $\mathrm{m}$ & $\mathrm{m}$ \\
\hline Japan & c & c & 11 & (3.8) & 14 & $(4.3)$ & 73 & (5.5) & 6 & $(0.7)$ & 15 & (1.1) & 23 & (1.4) & 57 & (1.5) \\
\hline Korea & c & c & 7 & (2.6) & 31 & $(5.7)$ & 61 & $(5.8)$ & 1 & $(0.3)$ & 14 & (1.2) & 37 & $(1.8)$ & 48 & (2.1) \\
\hline Netherlands & c & c & 6 & (1.9) & 24 & $(3.3)$ & 69 & $(3.4)$ & 1 & $(0.3)$ & 8 & $(0.8)$ & 33 & $(1.5)$ & 58 & $(1.6)$ \\
\hline Norway & c & c & 8 & (2.0) & 25 & $(3.2)$ & 66 & $(3.3)$ & 1 & $(0.2)$ & 9 & $(0.8)$ & 34 & $(1.3)$ & 56 & (1.4) \\
\hline Poland & 8 & (3.1) & 17 & (3.6) & 23 & $(5.8)$ & 53 & $(6.2)$ & 18 & $(1.5)$ & 17 & (1.6) & 29 & $(2.3)$ & 36 & (2.0) \\
\hline Slovak Republic & c & c & 6 & (2.3) & 28 & $(5.5)$ & 64 & $(5.7)$ & 7 & $(0.8)$ & 11 & $(1.1)$ & 39 & (1.9) & 42 & (1.9) \\
\hline Spain & $\mathrm{m}$ & $\mathrm{m}$ & $\mathrm{m}$ & $\mathrm{m}$ & $\mathrm{m}$ & $\mathrm{m}$ & $\mathrm{m}$ & $\mathrm{m}$ & $\mathrm{m}$ & $\mathrm{m}$ & $\mathrm{m}$ & $\mathrm{m}$ & $\mathrm{m}$ & $\mathrm{m}$ & $\mathrm{m}$ & $\mathrm{m}$ \\
\hline Sweden & c & c & 5 & (1.5) & 16 & $(2.8)$ & 77 & (3.1) & 2 & $(0.3)$ & 9 & $(0.8)$ & 31 & (1.4) & 58 & (1.4) \\
\hline United States & c & c & 8 & (2.6) & 30 & $(4.1)$ & 61 & $(4.6)$ & 2 & $(0.5)$ & 12 & $(1.2)$ & 35 & $(2.1)$ & 52 & $(2.2)$ \\
\hline \multicolumn{17}{|l|}{ Sub-national entities } \\
\hline Northern Ireland (UK) & c & c & c & c & 26 & $(6.0)$ & 71 & $(6.8)$ & 0 & $(0.2)$ & 10 & (1.8) & 40 & $(2.1)$ & 49 & (2.1) \\
\hline England/N. Ireland (UK) & c & c & 4 & (1.6) & 21 & $(3.3)$ & 73 & $(3.7)$ & 2 & $(0.5)$ & 9 & (1.0) & 34 & $(1.7)$ & 55 & (1.6) \\
\hline Average & $\mathrm{m}$ & $\mathrm{m}$ & 7 & $(0.5)$ & 24 & $(1.0)$ & 66 & $(1.1)$ & 4 & $(0.1)$ & 11 & $(0.2)$ & 33 & $(0.4)$ & 52 & $(0.4)$ \\
\hline Russian Federation* & 8 & $(3.2)$ & 22 & $(10.1)$ & 28 & $(9.3)$ & 42 & $(8.7)$ & 13 & $(4.4)$ & 16 & $(3.2)$ & 32 & $(4.2)$ & 39 & (3.9) \\
\hline
\end{tabular}

* See note on data for the Russian Federation in the Methodology section.

Source: OECD. Survey of Adult Skills (PIAAC) (2012). See Annex 3 for notes (www.oecd.org/education/education-at-a-glance-19991487.htm).

Please refer to the Reader's Guide for information concerning symbols for missing data and abbreviations.

StatLink 켚ㄴ http://dx.doi.org/10.1787/888933285038 
Table A5.6a. [2/2] Skills and readiness to use information and communication technologies for problem solving among 25-64 year-olds, by ICT skills required at work (2012)

\begin{tabular}{|c|c|c|c|c|c|c|c|c|c|c|c|c|c|c|c|c|c|c|}
\hline & \multicolumn{8}{|c|}{ Straightforward ICT skills required at work } & \multicolumn{10}{|c|}{ ICT skills not required at work } \\
\hline & \multicolumn{2}{|c|}{$\begin{array}{l}\text { Group 1 } \\
\text { (Refused } \\
\text { the } \\
\text { computer- } \\
\text { based } \\
\text { assessment) }\end{array}$} & \multicolumn{2}{|c|}{$\begin{array}{c}\text { Group 2 } \\
\text { (Failed ICT } \\
\text { core test } \\
\text { or minimal } \\
\text { problem- } \\
\text { solving skills) }\end{array}$} & \multicolumn{2}{|c|}{$\begin{array}{l}\text { Group } 3 \\
\text { (Moderate } \\
\text { ICT and } \\
\text { problem- } \\
\text { solving skills) }\end{array}$} & \multicolumn{2}{|c|}{$\begin{array}{c}\text { Group 4 } \\
\text { (Good ICT } \\
\text { and problem- } \\
\text { solving skills) }\end{array}$} & \multicolumn{2}{|c|}{$\begin{array}{c}\text { Group } 0 \\
\text { (No computer } \\
\text { experience) }\end{array}$} & \multicolumn{2}{|c|}{$\begin{array}{c}\text { Group 1 } \\
\text { (Refused } \\
\text { the } \\
\text { computer- } \\
\text { based } \\
\text { assessment) }\end{array}$} & \multicolumn{2}{|c|}{$\begin{array}{c}\text { Group 2 } \\
\text { (Failed ICT } \\
\text { core test } \\
\text { or minimal } \\
\text { problem- } \\
\text { solving skills) }\end{array}$} & \multicolumn{2}{|c|}{$\begin{array}{l}\text { Group } 3 \\
\text { (Moderate } \\
\text { ICT and } \\
\text { problem- } \\
\text { solving skills) }\end{array}$} & \multicolumn{2}{|c|}{$\begin{array}{c}\text { Group 4 } \\
\text { (Good ICT } \\
\text { and problem- } \\
\text { solving skills) }\end{array}$} \\
\hline & $\%$ & S.E. & $\%$ & S.E. & $\%$ & S.E. & $\%$ & S.E. & $\%$ & S.E. & $\%$ & S.E. & $\%$ & S.E. & $\%$ & S.E. & $\%$ & S.E. \\
\hline & (17) & (18) & (19) & (20) & (21) & (22) & (23) & (24) & (25) & (26) & (27) & $(28)$ & (29) & $(30)$ & (31) & (32) & (33) & $(34)$ \\
\hline \multicolumn{19}{|l|}{ Q National entities } \\
\hline Australia & 17 & (1.4) & 18 & $(1.8)$ & 37 & (1.8) & 28 & $(2.0)$ & 11 & (1.0) & 31 & (1.8) & 18 & (1.6) & 25 & $(2.2)$ & 14 & (1.9) \\
\hline Austria & 15 & (1.4) & 19 & (1.9) & 40 & (2.6) & 27 & (1.9) & 27 & (1.6) & 22 & (1.4) & 20 & (1.7) & 22 & (2.0) & 10 & (1.4) \\
\hline Canada & 8 & $(0.7)$ & 30 & $(1.2)$ & 36 & (1.6) & 27 & (1.2) & 16 & $(0.8)$ & 14 & $(0.9)$ & 32 & (1.4) & 24 & $(1.2)$ & 13 & (1.1) \\
\hline Czech Republic & 20 & (2.3) & 21 & (2.9) & 38 & (3.4) & 21 & $(2.7)$ & 20 & (1.7) & 19 & $(2.1)$ & 25 & $(2.6)$ & 24 & (2.5) & 12 & (1.9) \\
\hline Denmark & 7 & $(0.7)$ & 28 & (1.9) & 39 & (1.9) & 26 & (1.8) & 8 & (1.0) & 16 & (1.5) & 36 & $(2.3)$ & 26 & $(2.4)$ & 13 & (2.1) \\
\hline Estonia & 22 & $(1.2)$ & 28 & $(1.8)$ & 34 & $(1.8)$ & 16 & (1.6) & 19 & $(0.9)$ & 26 & (1.0) & 24 & (1.3) & 22 & (1.2) & 8 & $(0.9)$ \\
\hline Finland & 11 & (1.0) & 25 & (1.8) & 37 & (1.9) & 28 & (1.8) & 12 & (1.4) & 24 & (1.6) & 26 & $(1.8)$ & 25 & $(2.0)$ & 14 & (1.6) \\
\hline France & $\mathrm{m}$ & $\mathrm{m}$ & $\mathrm{m}$ & $\mathrm{m}$ & $\mathrm{m}$ & $\mathrm{m}$ & $\mathrm{m}$ & $\mathrm{m}$ & $\mathrm{m}$ & $\mathrm{m}$ & $\mathrm{m}$ & $\mathrm{m}$ & $\mathrm{m}$ & $\mathrm{m}$ & $\mathrm{m}$ & $\mathrm{m}$ & $\mathrm{m}$ & $\mathrm{m}$ \\
\hline Germany & 6 & $(0.9)$ & 27 & (2.5) & 40 & $(2.2)$ & 27 & (1.8) & 23 & (1.9) & 13 & (1.4) & 29 & $(2.0)$ & 24 & (1.7) & 11 & (1.2) \\
\hline Ireland & 22 & (1.6) & 25 & $(2.2)$ & 35 & $(2.2)$ & 18 & (1.5) & 23 & (1.3) & 28 & (1.7) & 21 & (1.7) & 19 & $(1.8)$ & 8 & (1.1) \\
\hline Italy & $\mathrm{m}$ & $\mathrm{m}$ & $\mathrm{m}$ & $\mathrm{m}$ & $\mathrm{m}$ & $\mathrm{m}$ & $\mathrm{m}$ & $\mathrm{m}$ & $\mathrm{m}$ & $\mathrm{m}$ & $\mathrm{m}$ & $\mathrm{m}$ & $\mathrm{m}$ & $\mathrm{m}$ & $\mathrm{m}$ & $\mathrm{m}$ & $\mathrm{m}$ & $\mathrm{m}$ \\
\hline Japan & 21 & (1.7) & 25 & (1.9) & 24 & (1.6) & 30 & (1.7) & 34 & (1.8) & 25 & (1.9) & 19 & (1.5) & 13 & (1.3) & 9 & (1.4) \\
\hline Korea & 9 & (1.0) & 31 & (1.7) & 41 & $(2.2)$ & 19 & (1.6) & 42 & (1.5) & 9 & $(0.8)$ & 24 & (1.3) & 19 & (1.3) & 6 & $(0.8)$ \\
\hline Netherlands & 5 & $(0.9)$ & 25 & (2.3) & 44 & $(2.6)$ & 26 & $(2.2)$ & 12 & (1.4) & 12 & (1.4) & 31 & $(2.1)$ & 32 & (2.3) & 13 & (1.8) \\
\hline Norway & 10 & (1.1) & 25 & (1.9) & 39 & $(2.1)$ & 26 & (1.7) & 6 & $(0.9)$ & 21 & (1.8) & 35 & $(2.7)$ & 28 & $(2.8)$ & 12 & (1.7) \\
\hline Poland & 29 & $(2.0)$ & 28 & $(2.2)$ & 25 & (2.3) & 18 & $(2.0)$ & 34 & (1.2) & 32 & (1.3) & 18 & (1.4) & 10 & (1.1) & 5 & $(0.8)$ \\
\hline Slovak Republic & 22 & $(2.1)$ & 18 & (2.5) & 39 & (3.0) & 21 & (2.2) & 40 & (1.4) & 17 & (1.1) & 12 & (1.1) & 21 & (1.3) & 11 & (1.2) \\
\hline Spain & $\mathrm{m}$ & $\mathrm{m}$ & $\mathrm{m}$ & $\mathrm{m}$ & $\mathrm{m}$ & $\mathrm{m}$ & $\mathrm{m}$ & $\mathrm{m}$ & $\mathrm{m}$ & $\mathrm{m}$ & $\mathrm{m}$ & $\mathrm{m}$ & $\mathrm{m}$ & $\mathrm{m}$ & $\mathrm{m}$ & $\mathrm{m}$ & $\mathrm{m}$ & $\mathrm{m}$ \\
\hline Sweden & 5 & $(0.7)$ & 25 & (1.7) & 40 & $(2.0)$ & 30 & (1.9) & 6 & (1.5) & 17 & (1.9) & 32 & $(2.5)$ & 26 & $(2.6)$ & 19 & (2.1) \\
\hline United States & 7 & (1.0) & 28 & $(2.0)$ & 44 & $(2.4)$ & 22 & (2.1) & 20 & $(2.0)$ & 14 & (1.5) & 34 & $(2.8)$ & 23 & (2.3) & 8 & (1.2) \\
\hline \multicolumn{19}{|l|}{ Sub-national entities } \\
\hline Flanders (Belgium) & 6 & (1.0) & 30 & $(2.0)$ & 41 & (2.4) & 23 & (2.1) & 21 & (1.4) & 11 & (1.1) & 33 & $(2.2)$ & 26 & (1.9) & 10 & (1.1) \\
\hline England (UK) & 5 & $(1.0)$ & 32 & $(2.4)$ & 41 & $(2.7)$ & 23 & $(2.0)$ & 10 & (1.4) & 10 & (1.4) & 35 & $(2.9)$ & 32 & (2.7) & 12 & (1.7) \\
\hline Northern Ireland (UK) & 3 & $(0.7)$ & 32 & (3.2) & 42 & (3.0) & 23 & (2.8) & 29 & (2.4) & 4 & (1.0) & 35 & (3.1) & 26 & $(2.7)$ & 6 & (1.5) \\
\hline England/N. Ireland (UK) & 5 & $(0.9)$ & 32 & $(2.3)$ & 41 & (2.6) & 23 & (1.9) & 11 & (1.4) & 10 & (1.4) & 35 & $(2.9)$ & 32 & (2.6) & 11 & (1.6) \\
\hline Average & 13 & $(0.3)$ & 26 & $(0.5)$ & 38 & $(0.5)$ & 24 & $(0.4)$ & 20 & $(0.3)$ & 19 & $(0.3)$ & 27 & $(0.5)$ & 23 & $(0.5)$ & 11 & $(0.3)$ \\
\hline Russian Federation* & 17 & $(2.4)$ & 19 & (3.6) & 32 & (3.7) & 32 & (3.9) & 31 & (3.3) & 14 & (1.7) & 17 & $(2.7)$ & 21 & $(2.0)$ & 17 & (1.7) \\
\hline
\end{tabular}

* See note on data for the Russian Federation in the Methodology section.

Source: OECD. Survey of Adult Skills (PIAAC) (2012). See Annex 3 for notes (www.oecd.org/education/education-at-a-glance-19991487.htm).

Please refer to the Reader's Guide for information concerning symbols for missing data and abbreviations.

StatLink 겐 http://dx.doi.org/10.1787/888933285038 


\section{WHAT ARE THE EARNINGS ADVANTAGES FROM EDUCATION?}

- In all OECD countries, adults with tertiary education earn more than adults with upper secondary education who, in turn, earn more than adults with below upper secondary education.

- Across OECD countries, compared with adults with upper secondary education with income from employment, those without that level of education earn about $20 \%$ less, those with post-secondary non-tertiary education earn about $10 \%$ more, and those with a tertiary degree earn about $60 \%$ more.

- Across OECD countries, people with a master's, doctoral or equivalent degree have the highest earnings advantages. In Brazil and Chile, these people are the most highly rewarded, relative to people with less education, as they earn more than four times the income of a person with upper secondary education.

\section{Chart A6.1. Relative earnings of tertiary-educated workers, by level of tertiary education (2013)}

25-64 year-olds with income from employment; upper secondary education $=100$

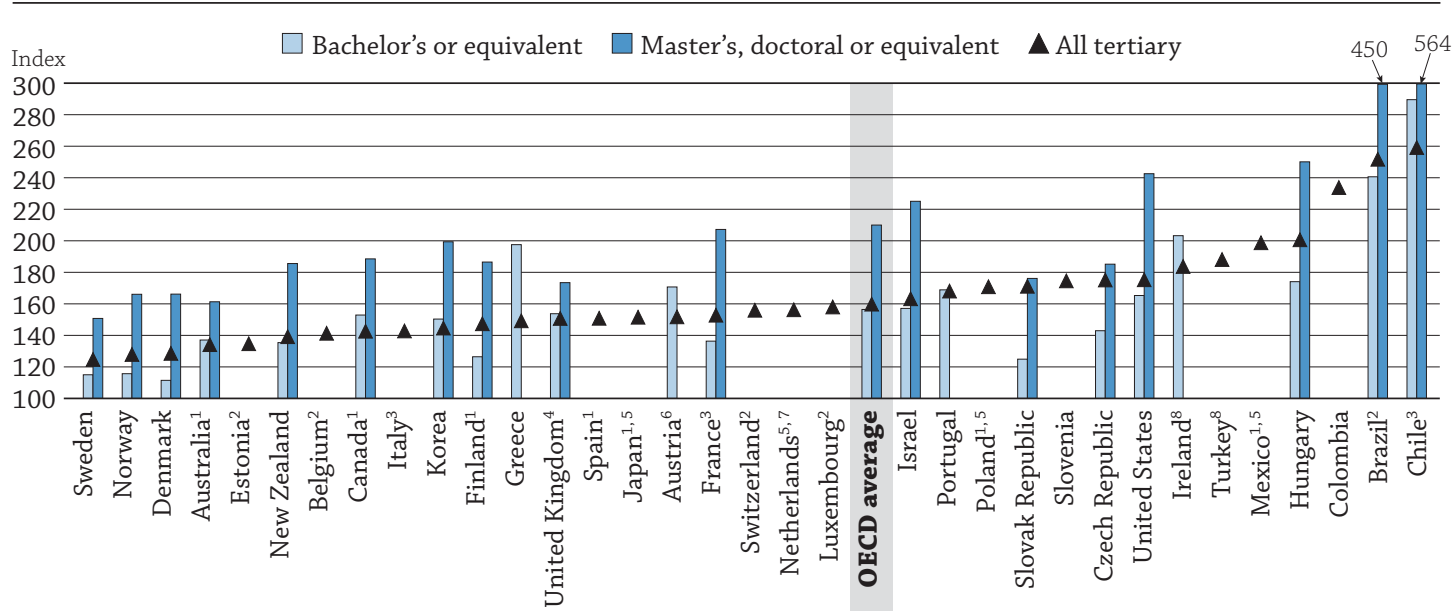

Note: Tertiary education includes short cycle tertiary, bachelor's, master's, doctoral or equivalent degrees.

1. Australia, Canada, Finland, Japan, Mexico, Poland, Spain: Year of reference 2012.

2. Belgium, Brazil, Estonia, Luxembourg, Switzerland: Index 100 refers to the combined ISCED levels 3 and 4 of the educational attainment levels in the ISCED 2011 classification.

3. Chile, France, Italy: Year of reference 2011.

4. The United Kingdom: Data for upper secondary attainment includes completion of a sufficient volume and standard of programmes that would be classified individually as completion of intermediate upper secondary programmes ( $18 \%$ of the adults are under this group).

5. Japan, Mexico, the Netherlands, Poland: Index 100 refers to the combined ISCED levels 3 and 4 of the educational attainment levels in the ISCED-97 classification.

6. Austria: Master's, doctoral or equivalent are included in bachelor's or equivalent.

7. The Netherlands: Year of reference 2010.

8. Ireland, Turkey: Earnings net of income tax.

Countries are ranked in ascending order of the relative earnings of 25-64 year-olds with tertiary education.

Source: OECD. Table A6.1a. See Annex 3 for notes (www.oecd.org/education/education-at-a-glance-19991487.htm).

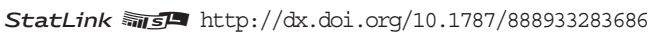

\section{Context}

Even if having a better job is only one among many of the positive social and individual outcomes of attaining higher qualifications, data show that higher levels of education usually translate into better chances of employment (see Indicator A5) and higher earnings. In fact, in all OECD countries for which information is available, the higher the level of education, the greater the relative earnings. This also seems to hold true for skills: individuals with high literacy proficiency, as measured in the Survey of Adult Skills, a product of the OECD Programme for the International Assessment of Adult Competencies (PIAAC), were observed to earn the highest wages, while those with low skills proficiency generally earned the lowest income (see Indicator A6 in Education at a Glance 2014) (OECD, 2014). 
The potential to earn more and see those earnings increase over time, along with other social benefits, is an incentive for individuals to pursue education and training; this is true even though the economic rewards vary, according to the chosen field of education (See Box A6.1 in Education at a Glance 2013, OECD [2013]). While relative earnings for individuals with higher educational attainment tend to increase with age, relative earnings for people with below upper secondary education tend to decrease. "Relative earnings" are percentages of the earnings of adults with levels of education other than upper secondary relative to the earnings of those with upper secondary education.

Variations in relative earnings among countries reflect a number of factors, including the demand for skills in the labour market, the supply of workers at various levels of educational attainment, minimum wage laws, the strength of labour unions, the coverage of collective-bargaining agreements, relative incidence of part-time and seasonal work and/or the age composition of the labour force. Variations in relative earnings among countries can also be due to more homogeneous earnings independent of education levels or to particularly high or low earnings for the reference group.

The data in this indicator show that earnings advantages vary according to education, age and gender. Each of these factors seems to play a role in individuals' earnings advantages to different extents. The higher the qualification attained, the better-placed individuals are to earn higher wages and to see increases in those wages over time. However, in many countries, gender gaps in earnings persist, regardless of the level of education.

\section{Other findings}

- About $25 \%$ of tertiary-educated individuals earn more than twice the median. They are substantially less likely to be in the low-earnings category than those with below upper secondary education. About $10 \%$ of tertiary-educated workers earn at or below half the median, compared with about $25 \%$ of workers with below upper secondary education. Only $3 \%$ of those workers earn more than twice the median.

- About $65 \%$ of $15-24$ year-old non-students have earnings from employment, while fewer than half of students (around 40\%) do. In OECD countries, about 50\% of 15-24 year-olds have income from employment. 


\section{Analysis}

\section{Educational attainment and relative earnings}

The higher the level of education, the higher the relative earnings. "Relative earnings" refers to the earnings of adults with income from employment who have an educational attainment other than upper secondary, relative to the earnings of those with upper secondary education.

In all OECD countries, adults with tertiary education earn more than adults with upper secondary education who, in turn, earn more than adults with below upper secondary education. In many countries, upper secondary education is the level beyond which further education and training implies high relative earnings. As such, upper secondary education can be considered the benchmark against which earnings related to educational attainment can be measured. Since private investment costs beyond upper secondary education rise considerably in most countries, a high earnings advantage is an important incentive for individuals to invest time and money in further education (Table A6.1a).

Earnings differentials between adults with tertiary education and those with upper secondary education are generally more pronounced than the differentials between upper secondary and below upper secondary education. Across OECD countries, compared with adults with upper secondary education with income from employment, those without this qualification earn about $20 \%$ less, those with post-secondary non-tertiary education about $10 \%$ more, those with a tertiary degree earn about $60 \%$ more. The $60 \%$ earnings advantage accrues only to those with a bachelor's or equivalent degree. Those with a short-cycle tertiary degree earn only about $25 \%$ more, but those with a master's, doctoral or equivalent degree earn more than twice as much as those with upper secondary education as their highest level of attainment. The results show that continuing tertiary education after a bachelor's degree pays off (Table A6.1a).

Chart A6.1 shows that the relative earnings advantages of tertiary-educated workers are largest in Brazil, Chile and Colombia and are smallest in Denmark, Norway and Sweden. This is even more pronounced when looking at people with a master's, doctoral or equivalent degree. In Brazil and Chile, people with those degrees are the most highly rewarded, relative to persons with less education, as they earn more than four times the income of a person with upper secondary education as their highest level of attainment (Table A6.1a).

\section{Relative earnings, by gender}

Across OECD countries, relative earnings are affected by educational attainment to various degrees. On average, there are no large gender differences in the relative earnings of tertiary-educated adults with income from employment. A man or a woman with tertiary education earns about $65 \%$ more than a person of the same gender with upper secondary education as his or her highest level of attainment (Table A6.1a.).

However, gender differences in relative earnings among tertiary-educated adults do vary across countries. In all cases, the differences are relative to the earnings of adults of the same gender with upper secondary education as their highest level of attainment and who have income from employment. In Australia, Belgium, Colombia, Estonia, Japan, Korea, Spain, Turkey and the United Kingdom, women's relative earnings are more than 10 percentage points higher than men's, while in Austria, the Czech Republic, Denmark, Finland, France, Hungary, Israel, Italy, Mexico, Poland, the Slovak Republic, Slovenia and the United States, men's relative earnings are more than 10 percentage points higher than women's. The gender differences in relative earnings tend to be larger among adults with a master's, doctoral or equivalent degree than with any other type of tertiary degree. When comparing the genders, it should be borne in mind that there may be large differences between the two in the proportion of people with income from employment (Table A6.1a).

\section{Relative earnings, by age}

Higher educational attainment is associated with higher earnings during a person's working life. On average across OECD countries, earnings increase with the level of educational attainment, but this increase is particularly large for older workers. People with higher levels of education are more likely to be employed, and remain employed, and have more opportunities to gain experience on the job.

Taking the OECD average as an example, young adults with tertiary education earn about $41 \%$ more of what young adults with upper secondary education earn. Older adults earn about $77 \%$ more. Chart A6.2 shows the difference between these two age groups. 


\section{Chart A6.2. Difference in relative earnings between older and younger tertiary-educated workers, by level of tertiary education (2013)}

Relative earnings of 55-64 year-olds minus that of 25-34 year-olds with income from employment; compared to earnings of workers with only upper secondary education

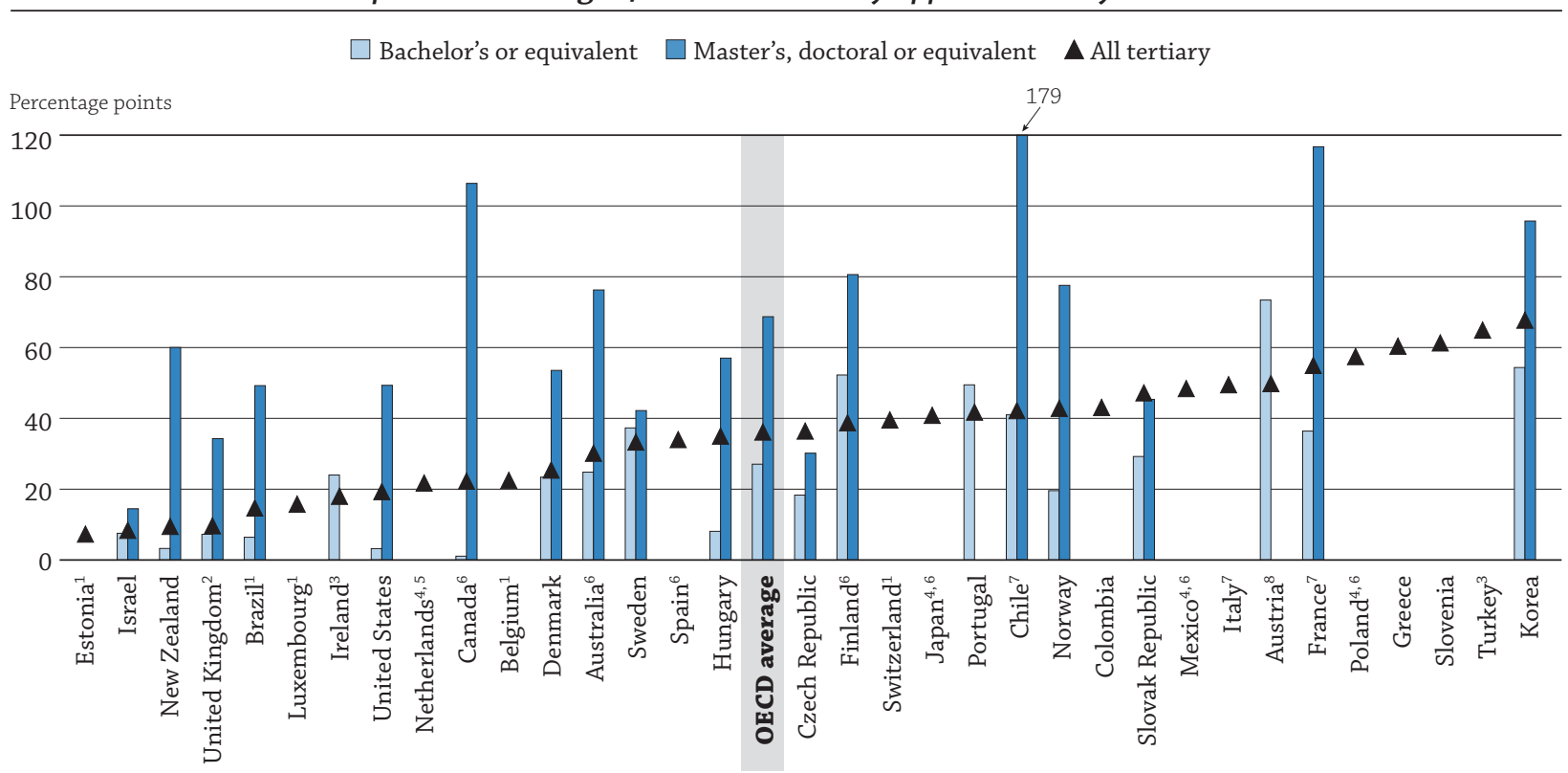

Note: Tertiary education includes short cycle tertiary, bachelor's, master's, doctoral or equivalent degrees.

1. Belgium, Brazil, Estonia, Luxembourg, Switzerland: Index 100 refers to the combined ISCED levels 3 and 4 of the educational attainment levels in the ISCED 2011 classification.

2. The United Kingdom: Data for upper secondary attainment includes completion of a sufficient volume and standard of programmes that would be classified individually as completion of intermediate upper secondary programmes (18\% of the adults are under this group).

3. Ireland, Turkey: Earnings net of income tax.

4. Japan, Mexico, the Netherlands, Poland: Index 100 refers to the combined ISCED levels 3 and 4 of the educational attainment levels in the ISCED-97 classification.

5. The Netherlands: Year of reference 2010.

6. Australia, Canada, Finland, Japan, Mexico, Poland, Spain: Year of reference 2012.

7. Chile, France, Italy: Year of reference 2011.

8. Austria: Master's, doctoral or equivalent are included in bachelor's or equivalent.

Countries are ranked in ascending order of the percentage-point difference in relative earnings between 55-64 year-old and 25-34 year-old tertiary educated workers.

Source: OECD. Table A6.1a. See Annex 3 for notes (www.oecd.org/education/education-at-a-glance-19991487.htm).

StatLink ints http://dx.doi.org/10.1787/888933283698

The relative earnings for tertiary-educated older adults are higher than those for younger adults in all OECD countries. On average, the differential between the two groups is about 35 percentage points and is higher than 50 percentage points in France, Greece, Korea, Poland, Slovenia and Turkey. The earnings differentials also increase with the different levels of education within the category of tertiary education. Across OECD countries, the difference is 27 percentage points for younger and older adults with a bachelor's or equivalent qualification, and increases to 69 percentage points for the two groups who hold a master's, doctoral or equivalent qualification (Table A6.1a).

\section{Distribution of earnings within levels of educational attainment}

Data on the distribution of earnings within groups with different levels of education can show how tightly earnings centre around the country median. In addition to providing information on equity in earnings, these data indicate the risks associated with investing in education, as risk is typically measured by the variation in outcomes. Data on the distribution of earnings (Table A6.4, available on line) include earnings from all employed individuals. This limits the analysis as the hours worked influence earnings, in general, and the distribution of earnings, in particular (see the Methodology section for further information).

For people with income from employment, the five earnings categories reported range from "At or below half the median" income to "More than twice the median" income. As expected, there is a large difference between the earnings of people with below upper secondary education and those with tertiary education. On average, tertiaryeducated individuals are substantially more likely to earn twice as much as the median worker (about $25 \%$ of these 
people do) and are substantially less likely to be in the low-earnings category (about 10\% are) than people with below upper secondary education as their highest level of attainment (3\% earn more than twice the median and about $25 \%$ earn at or below half the median) (Table A6.4, available on line).

Chart A6.3 shows the results for tertiary-educated workers (excluding short-cycle tertiary education) by comparing the proportion of wage-earners at or below the median to those earning more than the median. On average, about $70 \%$ of tertiary-educated workers earn more than the median and $30 \%$ less. There are some notable differences in how well tertiary-educated individuals fare in different countries. In Brazil, Chile and Greece, people with a tertiary degree can expect to earn more than the median (about $90 \%$ do) whereas in Norway tertiary-educated adults have a 50/50 chance of earning more or less than the median. In most of other countries, about $70 \%$ of tertiary-educated workers can expect to earn more than the median. In Brazil and Chile, $60 \%$ or more of tertiary-educated adults earn twice as much as the median worker, while less than $5 \%$ of tertiaryeducated workers in Brazil, Chile, the Czech Republic, Greece, Hungary and Portugal earn less than half the median (Table A6.4, available on line).

In all countries, individuals who remain with below upper secondary qualifications through their working life usually face large earnings disadvantages. On average across OECD countries, less than 5\% of those with below upper secondary education earn twice the national median. Only in Brazil, Canada and Mexico is this proportion larger than 5\%. On average, over $25 \%$ of workers with below upper secondary education as their highest level of attainment earn less than half the national median; in the United States, about $45 \%$ of this group do (Table A6.4, available on line).

\section{Chart A6.3. Earnings of adults with a bachelor's, master's, doctoral or equivalent degree relative to the median earnings of all workers (2013) 25-64 year-olds with income from employment}

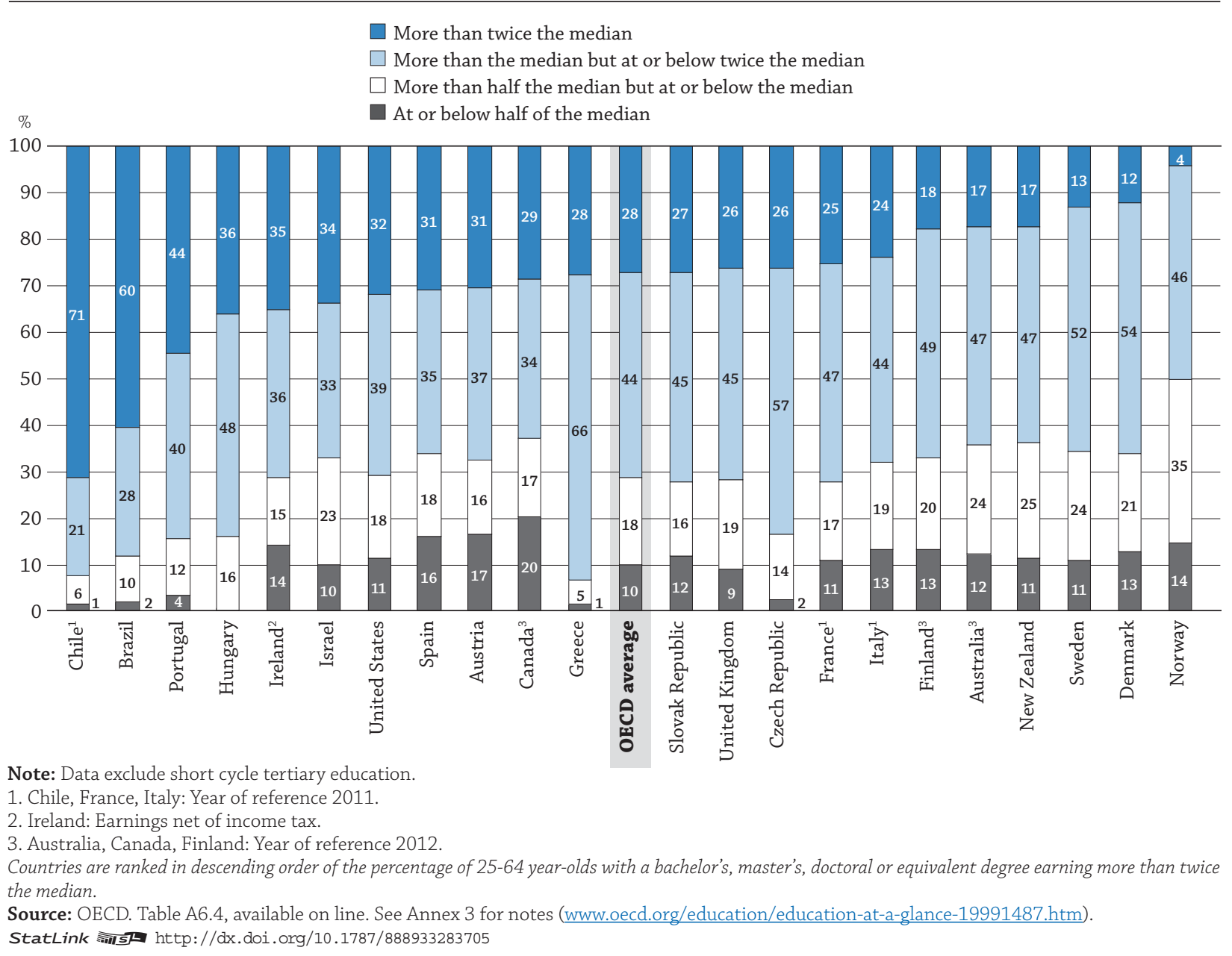




\section{Relative earnings of students}

In OECD countries, about $50 \%$ of $15-24$ year-olds have income from employment. In this age group, a majority of non-students (about 65\%) has earnings from employment, while less than half of students do (about 40\%). In Belgium, Chile and Greece, about $10 \%$ or less of $15-24$ year-old students have earnings from employment. In some countries, such as Switzerland, a proportion of students enrolled in upper secondary education has earnings based on apprenticeship contracts, but these students are not included in these calculations (Table A6.5b).

Students with income from employment have fewer earnings than non-students. The relative earnings increase from $43 \%$ for those with below upper secondary education to $63 \%$ for those with tertiary education (Table A6.5a).

These findings support the widespread notion that schooling beyond compulsory education implies a temporary loss of income, even when combining studies and work. This loss of income, together with tuition fees and the need to repay loans, may discourage some individuals from studying while being active in the labour market.

\section{Relative earnings and skills supply}

\section{Chart A6.4. Relative earnings of tertiary-educated workers and their share in the population (2013)}

25-64 year-olds with income from employment; upper secondary education $=100$

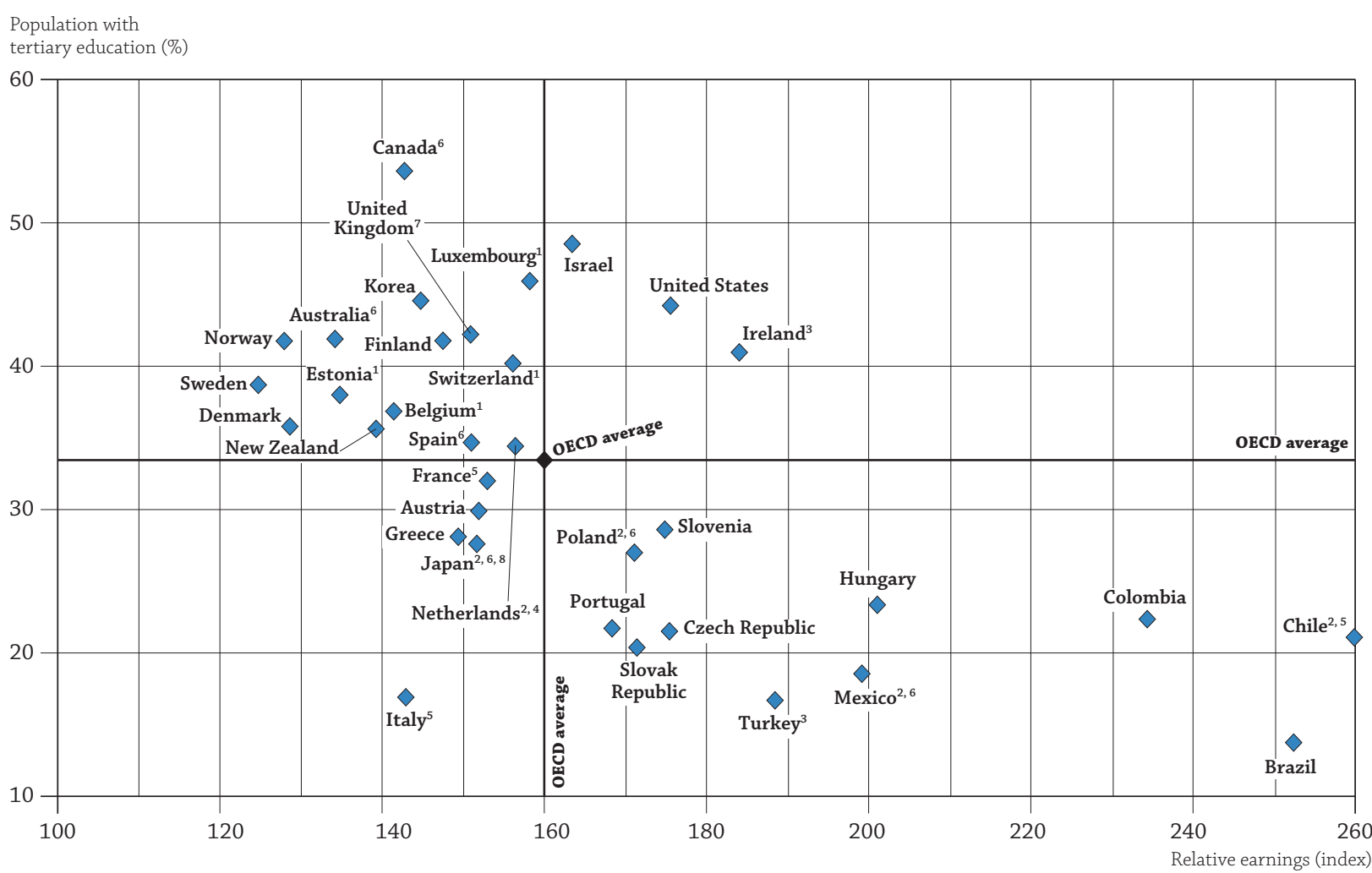

Note: All tertiary includes short cycle tertiary, bachelor's, master's, doctoral or equivalent degrees. Data on educational attainment refers to year 2014 or latest available year.

1. Belgium, Estonia, Luxembourg, Switzerland: Index 100 refers to the combined ISCED levels 3 and 4 of the educational attainment levels in the ISCED 2011 classification.

2. Chile, Japan, Mexico, the Netherlands, Poland: Index 100 refers to the combined ISCED levels 3 and 4 of the educational attainment levels in the ISCED-97 classification.

3. Ireland, Turkey: Earnings net of income tax.

4. The Netherlands: Year of reference 2010.

5. Chile, France, Italy: Year of reference 2011.

6. Australia, Canada, Finland, Japan, Mexico, Poland, Spain: Year of reference 2012.

7. The United Kingdom: Data for upper secondary attainment includes completion of a sufficient volume and standard of programmes that would be classified individually as partial level completion of upper secondary education.

8. Japan: Data on educational attainment exclude short-cycle tertiary education at the tertiary level.

Source: OECD. Tables A1.3a and A6.1a. See Annex 3 for notes (www.oecd.org/education/education-at-a-glance-19991487.htm).

StatLink त्ता St http://dx.doi.org/10.1787/888933283719 
There are various factors explaining the variation in earnings. Chart A6.4 shows countries by the relative earnings of tertiary-educated workers and the share of these workers in the population. The chart also provides some evidence of the influence of the supply-and-demand of tertiary-educated workers on relative earnings. The earnings advantages are largest in countries with a small share of tertiary-educated people, such as Brazil, Chile, Colombia, Hungary and Mexico, whereas earnings advantages are smallest in countries with a large share of tertiary-educated people, such as Norway and Sweden. But other factors, not shown in Chart A6.4, may explain deviations from this pattern. For instance, the share of people with a tertiary degree is above the OECD average in Canada (54\%) and below the average in Italy (17\%), but relative earnings for these workers are below the OECD average in both countries (43\% higher earnings for a person with a tertiary degree compared with a person with upper secondary education as the highest level of education) (Table A6.1a).

\section{Definitions}

Age groups: adults refers to 25-64 year-olds; younger adults refers to 25-34 year-olds; older adults refers to 55-64 year-olds. The working-age population is the total population aged 25-64.

Completion of intermediate programmes for educational attainment (ISCED 2011) corresponds to recognised qualification from an ISCED 2011 level programme which is not considered as sufficient for ISCED 2011 level completion and is classified at a lower ISCED 2011 level. In addition, this recognised qualification does not give direct access to an upper ISCED 2011 level programme.

Levels of education: In this Indicator two ISCED (International Standard Classification of Education) classifications are used: ISCED 2011 and ISCED-97.

- When it is specified that ISCED 2011 is used, the levels of education are defined as: below upper secondary corresponds to ISCED 2011 Levels 0, 1 and 2, and includes recognised qualifications from ISCED 2011 Level 3 programmes, which are not considered as sufficient for ISCED 2011 Level 3 completion, and without direct access to post-secondary non-tertiary education or tertiary education; upper secondary or post-secondary non-tertiary corresponds to ISCED 2011 Levels 3 and 4; and tertiary corresponds to ISCED 2011 Levels 5, 6, 7 and 8 (UNESCO Institute for Statistics, 2012).

- When it is specified that ISCED-97 is used, the levels of education are defined as: below upper secondary corresponds to ISCED-97 Levels $0,1,2$ and $3 C$ short programmes; upper secondary or post-secondary non-tertiary corresponds to ISCED-97 Levels 3A, 3B, 3C long programmes, and Level 4; and tertiary corresponds to ISCED-97 Levels 5A, 5B and 6.

See the section About the new ISCED 2011 classification, at the beginning of this publication, for a presentation of all ISCED 2011 levels and Annex 3 for a presentation of all ISCED-97 levels.

\section{Methodology}

The indicator is based on the regular data collection by the OECD LSO (Labour Market and Social Outcomes of Learning) Network that takes account of earnings from work for all individuals during the reference period, even if the individual has worked part time or part year. This database contains data on student versus non-student earnings. It also gathers information on the earnings of those working full time and full year, for Tables A6.2a and A6.3a, and Table A6.2b, available on line. See Annex 3 (www.oecd.org/education/education-at-a-glance-19991487.htm) for additional information.

\section{Regular earnings data collection}

Regular earnings data collection (used in all tables) provides information based on an annual, monthly or weekly reference period, depending on the country. The length of the reference period for earnings also differs. Australia, New Zealand and the United Kingdom reported data on weekly earnings; Belgium, Brazil, Chile, Colombia, Estonia, Israel (three months), Korea and Portugal reported monthly data; and all other countries reported annual data. Data on earnings are before income tax, except for Ireland and Turkey, where earnings reported are net of income tax. For Belgium data on dispersion of earnings from work and earnings of students and non-students are net of income tax. Data on earnings for individuals in part-time work are excluded in the regular data collection for Slovenia; and data on part-year earnings are excluded for Slovenia. Earnings of self-employed people are excluded for many countries and, in general, there is no simple and comparable method to separate earnings from employment and returns to capital invested in the business. 
Since earnings data differ across countries in a number of ways, the results should be interpreted with caution. For example:

- in countries reporting annual earnings, differences in the incidence of seasonal work among individuals with different levels of educational attainment will have an effect on relative earnings that is not similarly reflected in the data for countries reporting weekly or monthly earnings;

- migrants are not separated from non-migrants, and in some countries this could have an effect on earnings, as there could be a migrant earning penalty that is independent of qualification return;

- countries may include earnings for self-employed or part-time work; and

- countries may differ in the extent to which there are employer contributions to pensions, health insurance, etc. on top of the salary.

In addition, data available in Tables A6.1a and $\mathrm{b}$ concern relative earnings and should therefore be used with caution in assessing the evolution of relative earnings for different levels of education. For Tables A6.5a and b, differences between countries could be the result of differences in data sources and in the length of the reference period. For further details, see Annex 3 (www.oecd.org/education/education-at-a-glance-19991487.htm).

This indicator does not take into consideration the impact of effective income from free government services. In some countries, incomes could be high but they may have to cover, for instance, health insurance and schooling/ tertiary education for children/students, while in other countries incomes could be lower but the state provides both free health care and schooling.

The total (men plus women, i.e. $\mathrm{M}+\mathrm{W}$ ) average for earnings is not the simple average of the earnings figures for men and women, but the average based on earnings of the total population. This overall average weights the average earnings figure separately for men and women by the share of men and women at different levels of attainment.

\section{Full-time and full-year data collection}

Full-time and full-year data collection supplies the data for Table A6.2a and b (gender differences in full-time earnings) and Table A6.3 (differences in full-time earnings by educational attainment).

For the definition of full-time earnings (in Tables A6.2a and b and A6.3), countries were asked whether they had applied a self-designated full-time status or a threshold value of the typical number of hours worked per week. Belgium, France, Germany, Italy, Luxembourg, Portugal, Spain, Sweden, Switzerland and the United Kingdom reported self-designated full-time status; the other countries defined the full-time status by the number of working hours per week. The threshold was 44/45 hours per week in Chile, 36 hours per week in Hungary, the Slovak Republic and Slovenia, 35 hours in Australia, Brazil, Canada, Colombia, Estonia, Israel, Korea, Norway and the United States, and 30 hours in the Czech Republic, Greece, Ireland, New Zealand and Turkey. Other participating countries did not report a minimum normal number of working hours for full-time work. For some countries, data on full-time, full-year earnings are based on the European Survey on Income and Living Conditions (EU-SILC), which uses a self-designated approach in establishing full-time status.

\section{Note regarding data from Israel}

The statistical data for Israel are supplied by and are under the responsibility of the relevant Israeli authorities. The use of such data by the OECD is without prejudice to the status of the Golan Heights, East Jerusalem and Israeli settlements in the West Bank under the terms of international law.

\section{References}

OECD (2014), Education at a Glance 2014: OECD Indicators, OECD Publishing, Paris, http://dx.doi.org/10.1787/eag-2014-en. OECD (2013), Education at a Glance 2013: OECD Indicators, OECD Publishing, Paris, http://dx.doi.org/10.1787/eag-2013-en. UNESCO Institute for Statistics (2012), International Standard Classification of Education: ISCED 2011, UNESCO-UIS, Montreal, Canada, www.uis.unesco.org/Education/Documents/isced-2011-en.pdf.

\section{Indicator A6 Tables}

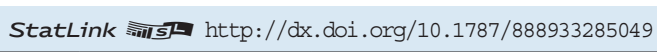

Table A6.1a Relative earnings of workers, by educational attainment, age group and gender (2013)

WEB Table A6.1b Trends in relative earnings of workers, by educational attainment and gender $(2005,2010-13)$ 
Table A6.2a

WEB Table A6.2b
Differences in earnings between female and male workers, by educational attainment and age group (2013)

Trends in the differences in earnings between female and male workers, by educational attainment (2005, 2010-13)

Table A6.3 Percentage of full-time, full-year earners, part-time earners and people without earnings, by educational attainment, age group and gender (2013)

WEB Table A6.4 Level of earnings relative to median earnings, by gender and educational attainment (2013)

Table A6.5a Relative earnings of 15-24 year-old students with income from employment, by educational attainment and gender (2013)

Table A6.5b Percentage of 15-29 year-olds with income from employment among all 15-29 year-olds, by age group, gender and student status (2013) 
Table A6.1a. Relative earnings of workers, by educational attainment, age group and gender (2013) Adults with income from employment; upper secondary education $=100$

\begin{tabular}{|c|c|c|c|c|c|c|c|}
\hline & \multirow[b]{4}{*}{ Year } & \multicolumn{6}{|c|}{ Men and women } \\
\hline & & $\begin{array}{l}\text { Below upper } \\
\text { secondary }\end{array}$ & $\begin{array}{c}\text { Post-secondary } \\
\text { non-tertiary }\end{array}$ & $\begin{array}{c}\text { Short cycle } \\
\text { tertiary }\end{array}$ & $\begin{array}{c}\text { Bachelor's } \\
\text { or equivalent }\end{array}$ & $\begin{array}{c}\text { Master's, doctoral } \\
\text { or equivalent }\end{array}$ & $\begin{array}{c}\text { All tertiary } \\
\text { education }\end{array}$ \\
\hline & & $25-64$ & $25-64$ & $25-64$ & $25-64$ & $25-64$ & $25-64$ \\
\hline & & (1) & (4) & (7) & (10) & (13) & (16) \\
\hline Australia & 2012 & 83 & 99 & 114 & 137 & 161 & 134 \\
\hline Ј Austria & 2013 & 71 & $\mathrm{~m}$ & 136 & $171^{\mathrm{d}}$ & $\mathrm{x}(10)$ & 152 \\
\hline Belgium $^{1}$ & 2013 & 87 & $\mathrm{~m}$ & $\mathrm{~m}$ & $\mathrm{~m}$ & $\mathrm{~m}$ & 141 \\
\hline Canada & 2012 & 84 & 119 & 118 & 153 & 189 & 143 \\
\hline Chile $^{2}$ & 2011 & 66 & $\mathrm{a}$ & 151 & 290 & 564 & 260 \\
\hline Czech Republic & 2013 & 74 & $\mathrm{~m}$ & 117 & 143 & 185 & 175 \\
\hline Denmark & 2013 & 82 & 134 & 114 & 111 & 166 & 129 \\
\hline Estonia $^{1}$ & 2013 & 91 & $\mathrm{~m}$ & $\mathrm{~m}$ & $\mathrm{~m}$ & $\mathrm{~m}$ & 135 \\
\hline Finland & 2012 & 93 & 128 & 129 & 127 & 187 & 148 \\
\hline France & 2011 & 82 & $\mathrm{~m}$ & 127 & 136 & 207 & 153 \\
\hline Germany & & $\mathrm{m}$ & $\mathrm{m}$ & $\mathrm{m}$ & $\mathrm{m}$ & $\mathrm{m}$ & $\mathrm{m}$ \\
\hline Greece & 2013 & 79 & 109 & 148 & 198 & $\mathrm{~m}$ & 149 \\
\hline Hungary & 2013 & 75 & 99 & 102 & 174 & 250 & 201 \\
\hline Iceland & & $\mathrm{m}$ & $\mathrm{m}$ & $\mathrm{m}$ & $\mathrm{m}$ & $\mathrm{m}$ & $\mathrm{m}$ \\
\hline Ireland $^{3}$ & 2013 & 83 & 92 & 144 & 204 & $\mathrm{~m}$ & 184 \\
\hline Israel & 2013 & 84 & a & 117 & 157 & 225 & 163 \\
\hline Italy & 2011 & 78 & $\mathrm{~m}$ & $\mathrm{~m}$ & $\mathrm{~m}$ & $\mathrm{~m}$ & 143 \\
\hline Japan $^{2}$ & 2012 & 78 & $\mathrm{~m}$ & $\mathrm{~m}$ & $\mathrm{~m}$ & $\mathrm{~m}$ & 152 \\
\hline Korea & 2013 & 71 & $\mathrm{~m}$ & 115 & 150 & 200 & 145 \\
\hline Luxembourg ${ }^{1}$ & 2013 & 64 & $\mathrm{~m}$ & $\mathrm{~m}$ & $\mathrm{~m}$ & $\mathrm{~m}$ & 158 \\
\hline Mexico $^{2}$ & 2012 & 54 & $\mathrm{a}$ & $\mathrm{m}$ & $\mathrm{m}$ & $\mathrm{m}$ & 199 \\
\hline Netherlands ${ }^{2}$ & 2010 & 83 & $\mathrm{~m}$ & $\mathrm{~m}$ & $\mathrm{~m}$ & $\mathrm{~m}$ & 156 \\
\hline New Zealand & 2013 & 92 & 116 & 114 & 135 & 186 & 139 \\
\hline Norway & 2013 & 77 & 101 & 120 & 116 & 166 & 128 \\
\hline Poland $^{2}$ & 2012 & 84 & $\mathrm{~m}$ & $\mathrm{~m}$ & $\mathrm{~m}$ & $\mathrm{~m}$ & 171 \\
\hline Portugal & 2013 & 71 & 102 & 162 & 169 & $\mathrm{~m}$ & 168 \\
\hline Slovak Republic & 2013 & 68 & $\mathrm{~m}$ & 125 & 125 & 176 & 171 \\
\hline Slovenia & 2013 & 79 & $\mathrm{a}$ & $\mathrm{m}$ & $\mathrm{m}$ & $\mathrm{m}$ & 175 \\
\hline Spain & 2012 & 75 & 124 & $\mathrm{~m}$ & $\mathrm{~m}$ & $\mathrm{~m}$ & 151 \\
\hline Sweden & 2013 & 78 & 117 & 106 & 115 & 151 & 125 \\
\hline Switzerland ${ }^{1}$ & 2013 & 78 & $\mathrm{~m}$ & $\mathrm{~m}$ & $\mathrm{~m}$ & $\mathrm{~m}$ & 156 \\
\hline Turkey $^{3}$ & 2013 & 65 & $\mathrm{a}$ & $\mathrm{m}$ & $\mathrm{m}$ & $\mathrm{m}$ & 188 \\
\hline United Kingdom 4 & 2013 & 74 & $\mathrm{a}$ & 123 & 154 & 174 & 151 \\
\hline United States & 2013 & 70 & $\mathrm{~m}$ & 116 & 165 & 243 & 176 \\
\hline $\mathrm{OECD}$ average & & 77 & 112 & 125 & 157 & 214 & 160 \\
\hline EU21 average & & 79 & 113 & 128 & 152 & 187 & 157 \\
\hline
\end{tabular}

\begin{tabular}{|c|c|c|c|c|c|c|c|}
\hline $\begin{array}{l}\text { Argentina } \\
\text { Brazil }\end{array}$ & 2013 & $\begin{array}{l}\mathrm{m} \\
60\end{array}$ & $\begin{array}{l}\mathrm{m} \\
\mathrm{m}\end{array}$ & $\begin{array}{l}\mathrm{m} \\
\mathrm{m}\end{array}$ & $\begin{array}{r}\mathrm{m} \\
241\end{array}$ & $\begin{array}{r}\mathrm{m} \\
450\end{array}$ & $\begin{array}{r}\mathrm{m} \\
252\end{array}$ \\
\hline $\begin{array}{l}\text { China } \\
\text { Colombia }\end{array}$ & 2013 & $\begin{array}{l}\mathrm{m} \\
65\end{array}$ & $\begin{array}{l}\mathrm{m} \\
\mathrm{m}\end{array}$ & $\begin{array}{l}\mathrm{m} \\
\mathrm{m}\end{array}$ & $\begin{array}{l}\mathrm{m} \\
\mathrm{m}\end{array}$ & $\begin{array}{l}\mathrm{m} \\
\mathrm{m}\end{array}$ & $\begin{array}{r}m \\
234\end{array}$ \\
\hline $\begin{array}{l}\text { India } \\
\text { Indonesia }\end{array}$ & & $\begin{array}{l}\mathrm{m} \\
\mathrm{m}\end{array}$ & $\begin{array}{l}\mathrm{m} \\
\mathrm{m}\end{array}$ & $\begin{array}{l}\mathrm{m} \\
\mathrm{m}\end{array}$ & $\begin{array}{l}\mathrm{m} \\
\mathrm{m}\end{array}$ & $\begin{array}{l}\mathrm{m} \\
\mathrm{m}\end{array}$ & $\begin{array}{l}\mathrm{m} \\
\mathrm{m}\end{array}$ \\
\hline $\begin{array}{l}\text { Latvia } \\
\text { Russian Federation }\end{array}$ & & $\begin{array}{l}\mathrm{m} \\
\mathrm{m}\end{array}$ & $\begin{array}{l}\mathrm{m} \\
\mathrm{m}\end{array}$ & $\begin{array}{l}\mathrm{m} \\
\mathrm{m}\end{array}$ & $\begin{array}{l}\mathrm{m} \\
\mathrm{m}\end{array}$ & $\begin{array}{l}\mathrm{m} \\
\mathrm{m}\end{array}$ & $\begin{array}{l}\mathrm{m} \\
\mathrm{m}\end{array}$ \\
\hline $\begin{array}{l}\text { Saudi Arabia } \\
\text { South Africa }\end{array}$ & & $\begin{array}{l}\mathrm{m} \\
\mathrm{m}\end{array}$ & $\begin{array}{l}\mathrm{m} \\
\mathrm{m}\end{array}$ & $\begin{array}{l}\mathrm{m} \\
\mathrm{m}\end{array}$ & $\begin{array}{l}\mathrm{m} \\
\mathrm{m}\end{array}$ & $\begin{array}{l}\mathrm{m} \\
\mathrm{m}\end{array}$ & $\begin{array}{l}\mathrm{m} \\
\mathrm{m}\end{array}$ \\
\hline
\end{tabular}

1. Belgium, Estonia, Luxembourg, Switzerland: Index 100 refers to the combined ISCED levels 3 and 4 of the educational attainment levels in the ISCED 2011 classification.

2. Chile, Japan, Mexico, the Netherlands, Poland: Index 100 refers to the combined ISCED levels 3 and 4 of the educational attainment levels in the ISCED-97 classification.

3. Ireland, Turkey: Earnings net of income tax.

4. The United Kingdom: Data for upper secondary attainment includes completion of a sufficient volume and standard of programmes that would be classified individually as completion of intermediate upper secondary programmes (18\% of the adults are under this group).

Source: OECD. See Annex 3 for notes (www.oecd.org/education/education-at-a-glance-19991487.htm).

Please refer to the Reader's Guide for information concerning symbols for missing data and abbreviations.

StatLink 젝ㄴ http://dx.doi.org/10.1787/888933285052 
Table A6.2a. Differences in earnings between female and male workers, by educational attainment and age group (2013)

Adults with income from employment; average annual full-time, full-year earnings of women as a percentage of men's earnings

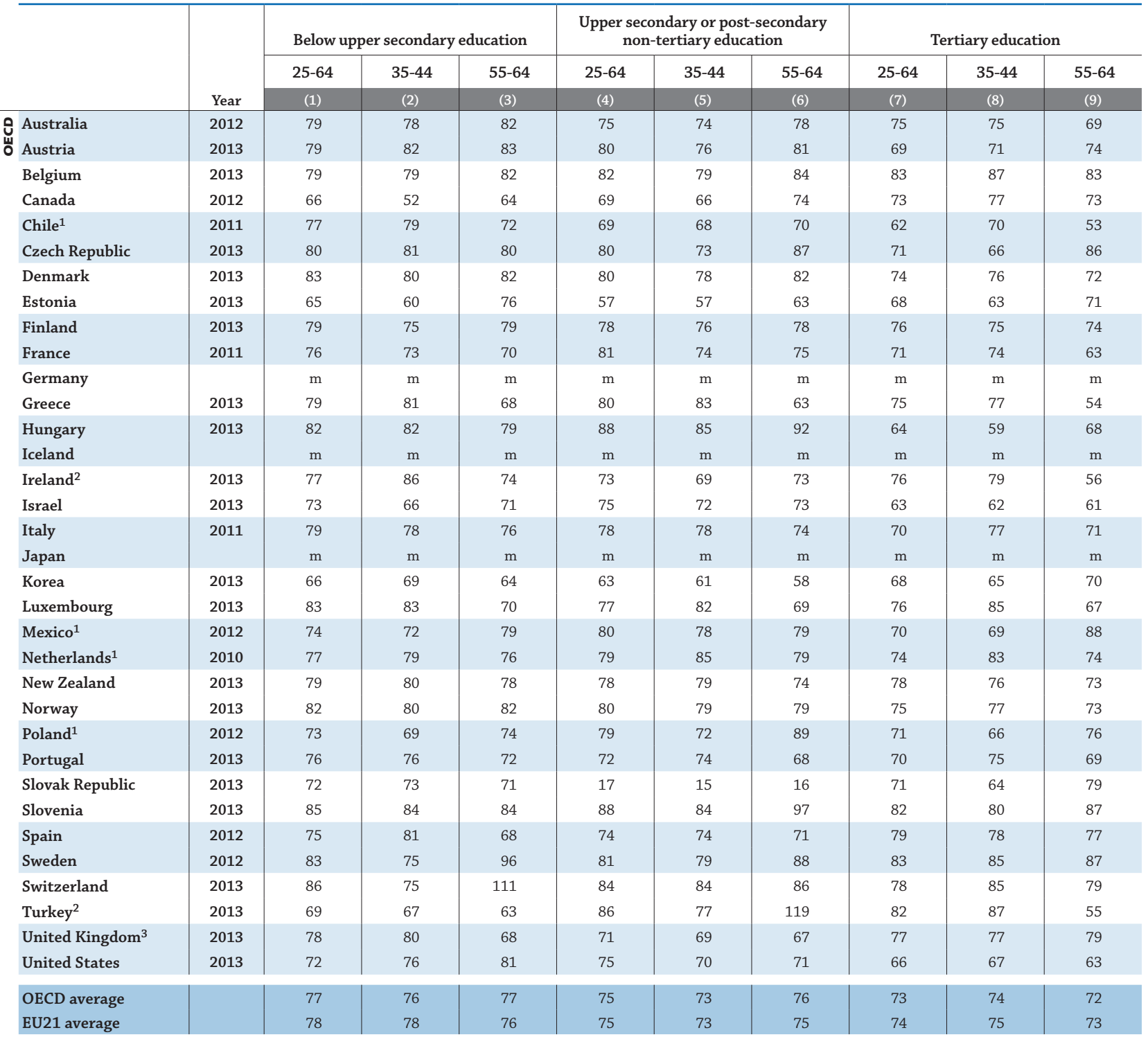

\begin{tabular}{|c|c|c|c|c|c|c|c|c|c|c|}
\hline $\begin{array}{l}\text { Argentina } \\
\text { Brazil }\end{array}$ & 2013 & $\begin{array}{l}\mathrm{m} \\
67\end{array}$ & $\begin{array}{l}\mathrm{m} \\
68\end{array}$ & $\begin{array}{r}\mathrm{m} \\
62\end{array}$ & $\begin{array}{l}\mathrm{m} \\
62\end{array}$ & $\begin{array}{l}\mathrm{m} \\
63\end{array}$ & $\begin{array}{l}\mathrm{m} \\
54\end{array}$ & $\begin{array}{l}\mathrm{m} \\
62\end{array}$ & $\begin{array}{l}\mathrm{m} \\
66\end{array}$ & $\begin{array}{l}\mathrm{m} \\
60\end{array}$ \\
\hline $\begin{array}{l}\text { China } \\
\text { Colombia }\end{array}$ & 2013 & $\begin{array}{l}\mathrm{m} \\
77\end{array}$ & $\begin{array}{c}\mathrm{m} \\
78\end{array}$ & $\begin{array}{r}\mathrm{m} \\
77\end{array}$ & $\begin{array}{l}\mathrm{m} \\
74\end{array}$ & $\begin{array}{l}\mathrm{m} \\
70\end{array}$ & $\begin{array}{l}\mathrm{m} \\
67\end{array}$ & $\begin{array}{r}\mathrm{m} \\
75\end{array}$ & $\begin{array}{l}\mathrm{m} \\
78\end{array}$ & $\begin{array}{r}\mathrm{m} \\
73\end{array}$ \\
\hline $\begin{array}{l}\text { India } \\
\text { Indonesia }\end{array}$ & & $\begin{array}{l}\mathrm{m} \\
\mathrm{m}\end{array}$ & $\begin{array}{l}\mathrm{m} \\
\mathrm{m}\end{array}$ & $\begin{array}{l}\mathrm{m} \\
\mathrm{m}\end{array}$ & $\begin{array}{l}\mathrm{m} \\
\mathrm{m}\end{array}$ & $\begin{array}{l}\mathrm{m} \\
\mathrm{m}\end{array}$ & $\begin{array}{l}\mathrm{m} \\
\mathrm{m}\end{array}$ & $\begin{array}{l}\mathrm{m} \\
\mathrm{m}\end{array}$ & $\begin{array}{l}\mathrm{m} \\
\mathrm{m}\end{array}$ & $\begin{array}{l}\mathrm{m} \\
\mathrm{m}\end{array}$ \\
\hline $\begin{array}{l}\text { Latvia } \\
\text { Russian Federation }\end{array}$ & & $\begin{array}{l}\mathrm{m} \\
\mathrm{m}\end{array}$ & $\begin{array}{l}\mathrm{m} \\
\mathrm{m}\end{array}$ & $\begin{array}{l}\mathrm{m} \\
\mathrm{m}\end{array}$ & $\begin{array}{l}\mathrm{m} \\
\mathrm{m}\end{array}$ & $\begin{array}{l}\mathrm{m} \\
\mathrm{m}\end{array}$ & $\begin{array}{l}\mathrm{m} \\
\mathrm{m}\end{array}$ & $\begin{array}{l}\mathrm{m} \\
\mathrm{m}\end{array}$ & $\begin{array}{l}\mathrm{m} \\
\mathrm{m}\end{array}$ & $\begin{array}{l}\mathrm{m} \\
\mathrm{m}\end{array}$ \\
\hline $\begin{array}{l}\text { Saudi Arabia } \\
\text { South Africa }\end{array}$ & & $\begin{array}{l}\mathrm{m} \\
\mathrm{m}\end{array}$ & $\begin{array}{l}\mathrm{m} \\
\mathrm{m}\end{array}$ & $\begin{array}{l}\mathrm{m} \\
\mathrm{m}\end{array}$ & $\begin{array}{l}\mathrm{m} \\
\mathrm{m}\end{array}$ & $\begin{array}{l}\mathrm{m} \\
\mathrm{m}\end{array}$ & $\begin{array}{l}\mathrm{m} \\
\mathrm{m}\end{array}$ & $\begin{array}{l}\mathrm{m} \\
\mathrm{m}\end{array}$ & $\begin{array}{l}\mathrm{m} \\
\mathrm{m}\end{array}$ & $\begin{array}{l}\mathrm{m} \\
\mathrm{m}\end{array}$ \\
\hline G20 average & & $\mathrm{m}$ & $\mathrm{m}$ & $\mathrm{m}$ & $\mathrm{m}$ & $\mathrm{m}$ & $\mathrm{m}$ & $\mathrm{m}$ & $\mathrm{m}$ & $\mathrm{m}$ \\
\hline
\end{tabular}

Note: Columns showing the relative earnings for all levels of education combined are available for consultation on line (see StatLink below).

1. Chile, Mexico, the Netherlands, Poland: Educational attainment levels are based in the ISCED-97 classification.

2. Ireland, Turkey: Earnings net of income tax.

3. The United Kingdom: Data for upper secondary attainment includes completion of a sufficient volume and standard of programmes that would be classified individually as completion of intermediate upper secondary programmes (18\% of the adults are under this group).

Source: OECD. See Annex 3 for notes (www.oecd.org/education/education-at-a-glance-19991487.htm).

Please refer to the Reader's Guide for information concerning symbols for missing data and abbreviations.

StatLink 제에 $\mathrm{http}: / / \mathrm{dx}$.doi.org/10.1787/888933285069 
Table A6.3. [1/3] Percentage of full-time, full-year earners, part-time earners and people without earnings, by educational attainment, age group and gender (2013)

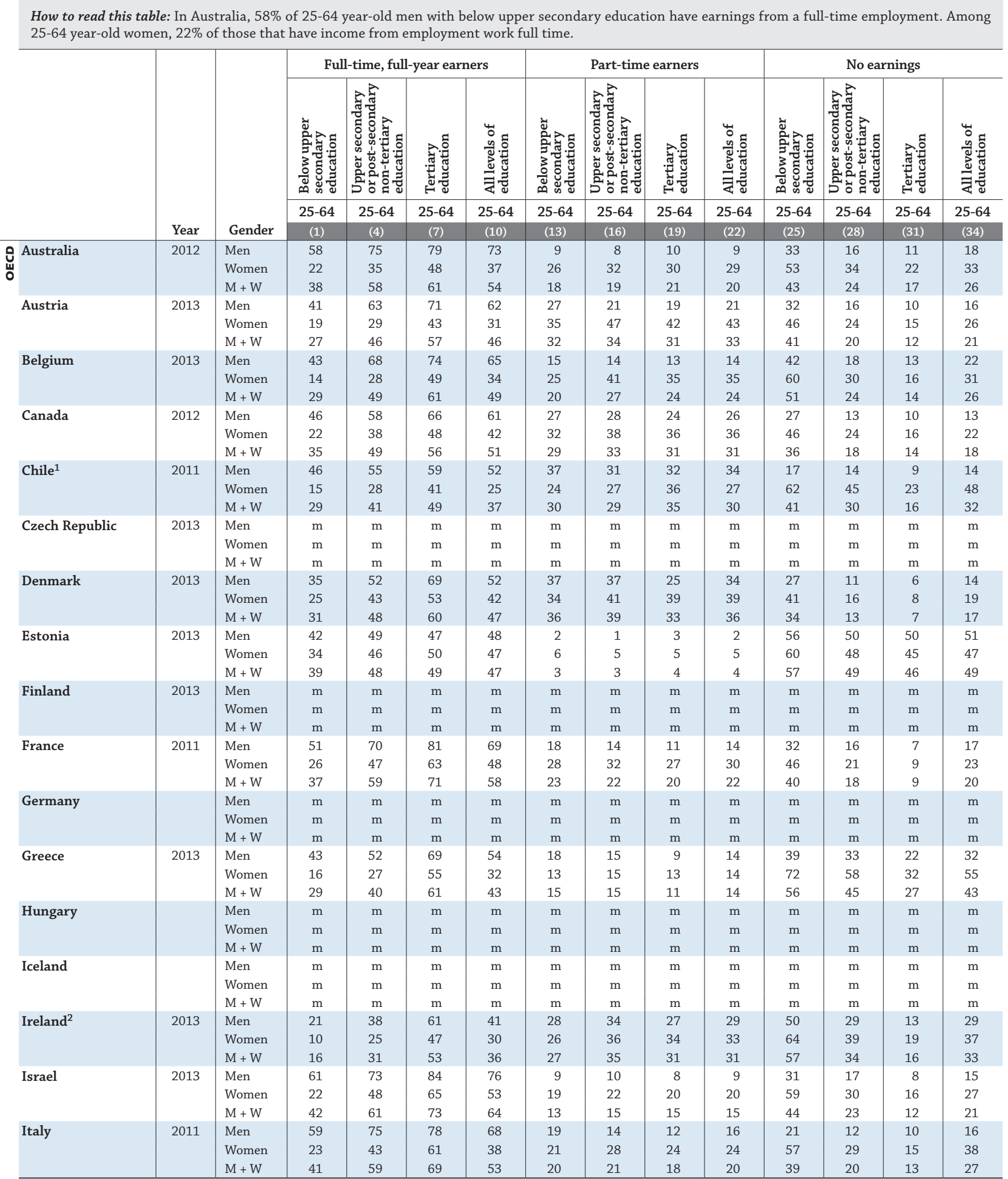

Notes: The length of the reference period varies from one week to one year. Self-employed individuals are excluded in some countries. See the Methodology section and Annex 3 for further information. Columns showing data for other age groups are available for consultation on line (see StatLink below).

1. Chile, Mexico: Educational attainment levels are based in the ISCED-97 classification.

2. Ireland, Turkey: Earnings net of income tax.

3. The United Kingdom: Data for upper secondary attainment includes completion of a sufficient volume and standard of programmes that would be classified individually as completion of intermediate upper secondary programmes ( $18 \%$ of the adults are under this group).

Source: OECD. See Annex 3 for notes (www.oecd.org/education/education-at-a-glance-19991487.htm).

Please refer to the Reader's Guide for information concerning symbols for missing data and abbreviations.

StatLink 제그 http://dx.doi.org/10.1787/888933285075 
Table A6.3. [2/3] Percentage of full-time, full-year earners, part-time earners and people without earnings, by educational attainment, age group and gender (2013)

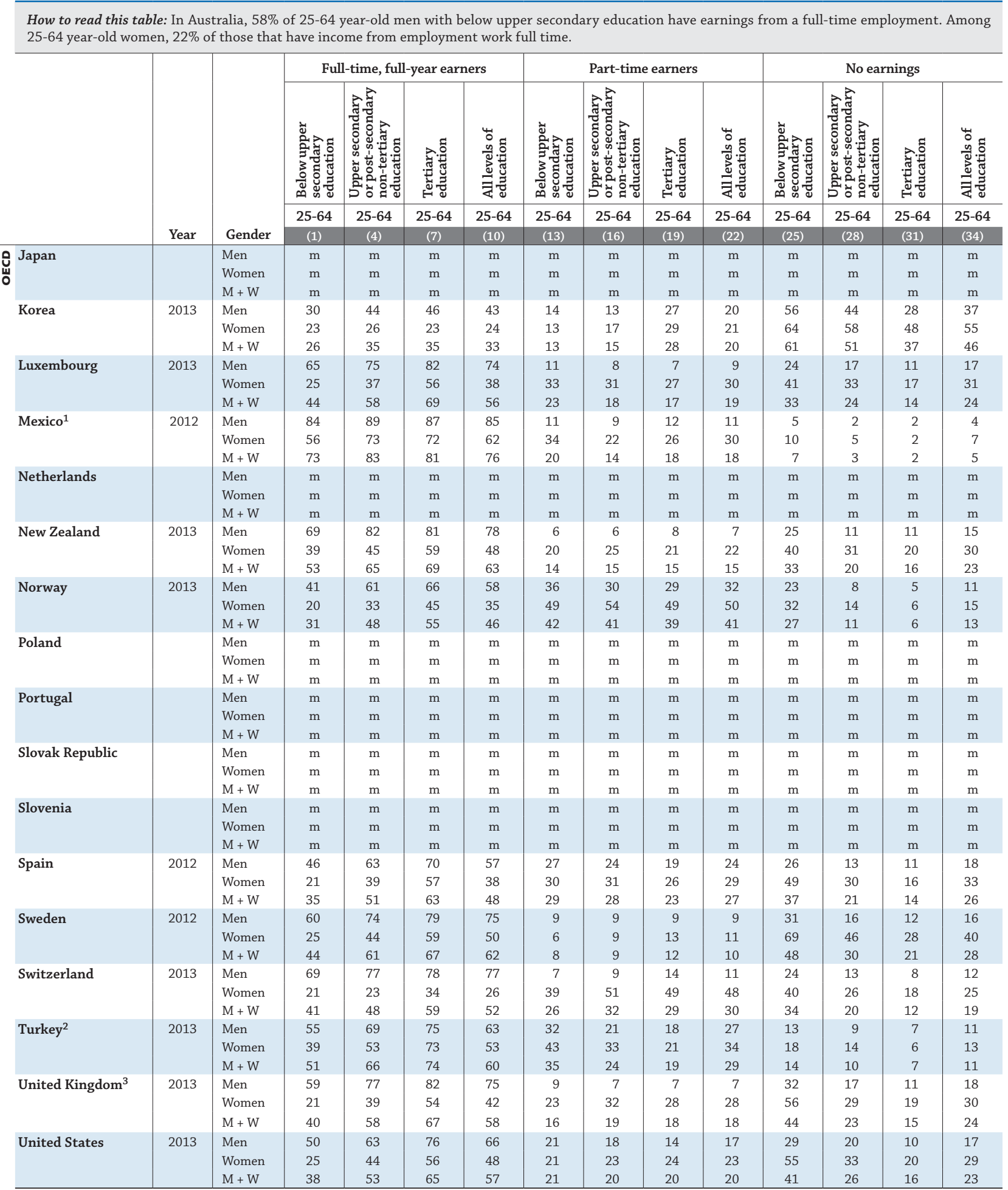

Notes: The length of the reference period varies from one week to one year. Self-employed individuals are excluded in some countries. See the Methodology section and Annex 3 for further information. Columns showing data for other age groups are available for consultation on line (see StatLink below).

1. Chile, Mexico: Educational attainment levels are based in the ISCED-97 classification.

2. Ireland, Turkey: Earnings net of income tax.

3. The United Kingdom: Data for upper secondary attainment includes completion of a sufficient volume and standard of programmes that would be classified individually as completion of intermediate upper secondary programmes (18\% of the adults are under this group).

Source: OECD. See Annex 3 for notes (www.oecd.org/education/education-at-a-glance-19991487.htm).

Please refer to the Reader's Guide for information concerning symbols for missing data and abbreviations.

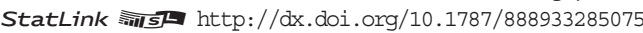


Table A6.3. [3/3] Percentage of full-time, full-year earners, part-time earners and people without earnings, by educational attainment, age group and gender (2013)

\begin{tabular}{|c|c|c|c|c|c|c|c|c|c|c|c|c|c|c|}
\hline & \multirow[b]{4}{*}{ Year } & \multirow[b]{4}{*}{ Gender } & \multicolumn{4}{|c|}{ Full-time, full-year earners } & \multicolumn{4}{|c|}{ Part-time earners } & \multicolumn{4}{|c|}{ No earnings } \\
\hline & & & 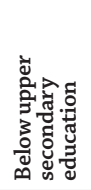 & 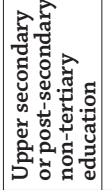 & 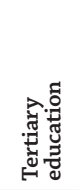 & 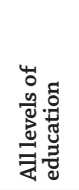 & 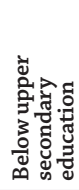 & 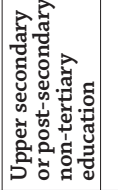 & 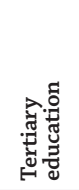 & 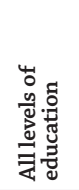 & 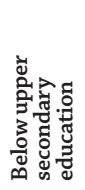 & 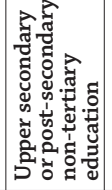 & 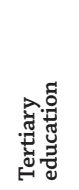 & 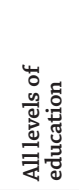 \\
\hline & & & $25-64$ & $25-64$ & $25-64$ & $25-64$ & $25-64$ & $25-64$ & $25-64$ & $25-64$ & $25-64$ & $25-64$ & $25-64$ & $25-64$ \\
\hline & & & (1) & (4) & (7) & (10) & (13) & (16) & (19) & (22) & (25) & (28) & $(31)$ & (34) \\
\hline of OECD average & & Men & 51 & 65 & 72 & 64 & 19 & 17 & 16 & 17 & 30 & 18 & 12 & 19 \\
\hline 品 & & Women & 24 & 39 & 59 & 40 & 26 & 30 & 29 & 29 & 50 & 31 & 19 & 31 \\
\hline & & $M+W$ & 38 & 53 & 62 & 52 & 22 & 23 & 22 & 23 & 40 & 24 & 16 & 25 \\
\hline EU21 average & & Men & 47 & 63 & 72 & 62 & 18 & 17 & 13 & 16 & 34 & 21 & 15 & 22 \\
\hline & & Women & 22 & 37 & 54 & 39 & 23 & 29 & 26 & 27 & 55 & 34 & 20 & 34 \\
\hline & & $M+W$ & 34 & 51 & 62 & 50 & 21 & 23 & 20 & 21 & 45 & 27 & 17 & 28 \\
\hline \multirow[t]{3}{*}{ Argentina } & & Men & $\mathrm{m}$ & $\mathrm{m}$ & $\mathrm{m}$ & $\mathrm{m}$ & $\mathrm{m}$ & $\mathrm{m}$ & $\mathrm{m}$ & $\mathrm{m}$ & $\mathrm{m}$ & $\mathrm{m}$ & $\mathrm{m}$ & $\mathrm{m}$ \\
\hline & & Women & $\mathrm{m}$ & $\mathrm{m}$ & $\mathrm{m}$ & $\mathrm{m}$ & $\mathrm{m}$ & $\mathrm{m}$ & $\mathrm{m}$ & $\mathrm{m}$ & $\mathrm{m}$ & $\mathrm{m}$ & $\mathrm{m}$ & $\mathrm{m}$ \\
\hline & & $M+W$ & $\mathrm{~m}$ & $\mathrm{~m}$ & $\mathrm{~m}$ & $\mathrm{~m}$ & $\mathrm{~m}$ & $\mathrm{~m}$ & $\mathrm{~m}$ & $\mathrm{~m}$ & $\mathrm{~m}$ & $\mathrm{~m}$ & $\mathrm{~m}$ & $\mathrm{~m}$ \\
\hline \multirow[t]{3}{*}{ Brazil } & 2013 & Men & 71 & 75 & 72 & 72 & 26 & 21 & 20 & 24 & 3 & 4 & 8 & 4 \\
\hline & & Women & 47 & 62 & 60 & 55 & 51 & 35 & 34 & 42 & 2 & 3 & 6 & 3 \\
\hline & & $M+W$ & 62 & 69 & 65 & 65 & 36 & 28 & 28 & 32 & 3 & 4 & 7 & 4 \\
\hline \multirow[t]{3}{*}{ China } & & Men & $\mathrm{m}$ & $\mathrm{m}$ & $\mathrm{m}$ & $\mathrm{m}$ & $\mathrm{m}$ & $\mathrm{m}$ & $\mathrm{m}$ & $\mathrm{m}$ & $\mathrm{m}$ & $\mathrm{m}$ & $\mathrm{m}$ & $\mathrm{m}$ \\
\hline & & Women & $\mathrm{m}$ & $\mathrm{m}$ & $\mathrm{m}$ & $\mathrm{m}$ & $\mathrm{m}$ & $\mathrm{m}$ & $\mathrm{m}$ & $\mathrm{m}$ & $\mathrm{m}$ & $\mathrm{m}$ & $\mathrm{m}$ & $\mathrm{m}$ \\
\hline & & $M+W$ & $\mathrm{~m}$ & $\mathrm{~m}$ & $\mathrm{~m}$ & $\mathrm{~m}$ & $\mathrm{~m}$ & $\mathrm{~m}$ & $\mathrm{~m}$ & $\mathrm{~m}$ & $\mathrm{~m}$ & $\mathrm{~m}$ & $\mathrm{~m}$ & $\mathrm{~m}$ \\
\hline \multirow[t]{3}{*}{ Colombia } & 2013 & Men & 83 & 85 & 81 & 83 & 15 & 13 & 18 & 15 & 2 & 2 & 1 & 2 \\
\hline & & Women & 52 & 64 & 70 & 60 & 39 & 30 & 28 & 33 & 9 & 6 & 2 & 6 \\
\hline & & $\mathrm{M}+\mathrm{W}$ & 71 & 76 & 75 & 73 & 25 & 21 & 23 & 23 & 5 & 4 & 2 & 4 \\
\hline \multirow{3}{*}{ India } & & Men & $\mathrm{m}$ & $\mathrm{m}$ & $\mathrm{m}$ & $\mathrm{m}$ & $\mathrm{m}$ & $\mathrm{m}$ & $\mathrm{m}$ & $\mathrm{m}$ & $\mathrm{m}$ & $\mathrm{m}$ & $\mathrm{m}$ & $\mathrm{m}$ \\
\hline & & Women & $\mathrm{m}$ & $\mathrm{m}$ & $\mathrm{m}$ & $\mathrm{m}$ & $\mathrm{m}$ & $\mathrm{m}$ & $\mathrm{m}$ & $\mathrm{m}$ & $\mathrm{m}$ & $\mathrm{m}$ & $\mathrm{m}$ & $\mathrm{m}$ \\
\hline & & $M+W$ & $\mathrm{~m}$ & $\mathrm{~m}$ & $\mathrm{~m}$ & $\mathrm{~m}$ & $\mathrm{~m}$ & $\mathrm{~m}$ & $\mathrm{~m}$ & $\mathrm{~m}$ & $\mathrm{~m}$ & $\mathrm{~m}$ & $\mathrm{~m}$ & $\mathrm{~m}$ \\
\hline \multirow[t]{3}{*}{ Indonesia } & & Men & $\mathrm{m}$ & $\mathrm{m}$ & $\mathrm{m}$ & $\mathrm{m}$ & $\mathrm{m}$ & $\mathrm{m}$ & $\mathrm{m}$ & $\mathrm{m}$ & $\mathrm{m}$ & $\mathrm{m}$ & $\mathrm{m}$ & $\mathrm{m}$ \\
\hline & & Women & $\mathrm{m}$ & $\mathrm{m}$ & $\mathrm{m}$ & $\mathrm{m}$ & $\mathrm{m}$ & $\mathrm{m}$ & $\mathrm{m}$ & $\mathrm{m}$ & $\mathrm{m}$ & $\mathrm{m}$ & $\mathrm{m}$ & $\mathrm{m}$ \\
\hline & & $M+W$ & $\mathrm{~m}$ & $\mathrm{~m}$ & $\mathrm{~m}$ & $\mathrm{~m}$ & $\mathrm{~m}$ & $\mathrm{~m}$ & $\mathrm{~m}$ & $\mathrm{~m}$ & $\mathrm{~m}$ & $\mathrm{~m}$ & $\mathrm{~m}$ & $\mathrm{~m}$ \\
\hline \multirow[t]{3}{*}{ Latvia } & & Men & $\mathrm{m}$ & $\mathrm{m}$ & $\mathrm{m}$ & $\mathrm{m}$ & $\mathrm{m}$ & $\mathrm{m}$ & $\mathrm{m}$ & $\mathrm{m}$ & $\mathrm{m}$ & $\mathrm{m}$ & $\mathrm{m}$ & $\mathrm{m}$ \\
\hline & & Women & $\mathrm{m}$ & $\mathrm{m}$ & $\mathrm{m}$ & $\mathrm{m}$ & $\mathrm{m}$ & $\mathrm{m}$ & $\mathrm{m}$ & $\mathrm{m}$ & $\mathrm{m}$ & $\mathrm{m}$ & $\mathrm{m}$ & $\mathrm{m}$ \\
\hline & & $M+W$ & $\mathrm{~m}$ & $\mathrm{~m}$ & $\mathrm{~m}$ & $\mathrm{~m}$ & $\mathrm{~m}$ & $\mathrm{~m}$ & $\mathrm{~m}$ & $\mathrm{~m}$ & $\mathrm{~m}$ & $\mathrm{~m}$ & $\mathrm{~m}$ & $\mathrm{~m}$ \\
\hline \multirow[t]{3}{*}{ Russian Federation } & & Men & $\mathrm{m}$ & $\mathrm{m}$ & $\mathrm{m}$ & $\mathrm{m}$ & $\mathrm{m}$ & $\mathrm{m}$ & $\mathrm{m}$ & $\mathrm{m}$ & $\mathrm{m}$ & $\mathrm{m}$ & $\mathrm{m}$ & $\mathrm{m}$ \\
\hline & & Women & $\mathrm{m}$ & $\mathrm{m}$ & $\mathrm{m}$ & $\mathrm{m}$ & $\mathrm{m}$ & $\mathrm{m}$ & $\mathrm{m}$ & $\mathrm{m}$ & $\mathrm{m}$ & $\mathrm{m}$ & $\mathrm{m}$ & $\mathrm{m}$ \\
\hline & & $M+W$ & $\mathrm{~m}$ & $\mathrm{~m}$ & $\mathrm{~m}$ & $\mathrm{~m}$ & $\mathrm{~m}$ & $\mathrm{~m}$ & $\mathrm{~m}$ & $\mathrm{~m}$ & $\mathrm{~m}$ & $\mathrm{~m}$ & $\mathrm{~m}$ & $\mathrm{~m}$ \\
\hline \multirow[t]{3}{*}{ Saudi Arabia } & & Men & $\mathrm{m}$ & $\mathrm{m}$ & $\mathrm{m}$ & $\mathrm{m}$ & $\mathrm{m}$ & $\mathrm{m}$ & $\mathrm{m}$ & $\mathrm{m}$ & $\mathrm{m}$ & $\mathrm{m}$ & $\mathrm{m}$ & $\mathrm{m}$ \\
\hline & & Women & $\mathrm{m}$ & $\mathrm{m}$ & $\mathrm{m}$ & $\mathrm{m}$ & $\mathrm{m}$ & $\mathrm{m}$ & $\mathrm{m}$ & $\mathrm{m}$ & $\mathrm{m}$ & $\mathrm{m}$ & $\mathrm{m}$ & $\mathrm{m}$ \\
\hline & & $M+W$ & $\mathrm{~m}$ & $\mathrm{~m}$ & $\mathrm{~m}$ & $\mathrm{~m}$ & $\mathrm{~m}$ & $\mathrm{~m}$ & $\mathrm{~m}$ & $\mathrm{~m}$ & $\mathrm{~m}$ & $\mathrm{~m}$ & $\mathrm{~m}$ & $\mathrm{~m}$ \\
\hline \multirow[t]{3}{*}{ South Africa } & & Men & $\mathrm{m}$ & $\mathrm{m}$ & $\mathrm{m}$ & $\mathrm{m}$ & $\mathrm{m}$ & $\mathrm{m}$ & $\mathrm{m}$ & $\mathrm{m}$ & $\mathrm{m}$ & $\mathrm{m}$ & $\mathrm{m}$ & $\mathrm{m}$ \\
\hline & & Women & $\mathrm{m}$ & $\mathrm{m}$ & $\mathrm{m}$ & $\mathrm{m}$ & $\mathrm{m}$ & $\mathrm{m}$ & $\mathrm{m}$ & $\mathrm{m}$ & $\mathrm{m}$ & $\mathrm{m}$ & $\mathrm{m}$ & $\mathrm{m}$ \\
\hline & & $M+W$ & $\mathrm{~m}$ & $\mathrm{~m}$ & $\mathrm{~m}$ & $\mathrm{~m}$ & $\mathrm{~m}$ & $\mathrm{~m}$ & $\mathrm{~m}$ & $\mathrm{~m}$ & $\mathrm{~m}$ & $\mathrm{~m}$ & $\mathrm{~m}$ & $\mathrm{~m}$ \\
\hline \multirow[t]{3}{*}{ G20 average } & & Men & $\mathrm{m}$ & $\mathrm{m}$ & $\mathrm{m}$ & $\mathrm{m}$ & $\mathrm{m}$ & $\mathrm{m}$ & $\mathrm{m}$ & $\mathrm{m}$ & $\mathrm{m}$ & $\mathrm{m}$ & $\mathrm{m}$ & $\mathrm{m}$ \\
\hline & & Women & $\mathrm{m}$ & $\mathrm{m}$ & $\mathrm{m}$ & $\mathrm{m}$ & $\mathrm{m}$ & $\mathrm{m}$ & $\mathrm{m}$ & $\mathrm{m}$ & $\mathrm{m}$ & $\mathrm{m}$ & $\mathrm{m}$ & $\mathrm{m}$ \\
\hline & & $M+W$ & $\mathrm{~m}$ & $\mathrm{~m}$ & $\mathrm{~m}$ & $\mathrm{~m}$ & $\mathrm{~m}$ & $\mathrm{~m}$ & $\mathrm{~m}$ & $\mathrm{~m}$ & $\mathrm{~m}$ & $\mathrm{~m}$ & $\mathrm{~m}$ & $\mathrm{~m}$ \\
\hline
\end{tabular}

Notes: The length of the reference period varies from one week to one year. Self-employed individuals are excluded in some countries. See the Methodology section and Annex 3 for further information. Columns showing data for other age groups are available for consultation on line (see StatLink below).

1. Chile, Mexico: Educational attainment levels are based in the ISCED-97 classification.

2. Ireland, Turkey: Earnings net of income tax.

3. The United Kingdom: Data for upper secondary attainment includes completion of a sufficient volume and standard of programmes that would be classified individually as completion of intermediate upper secondary programmes ( $18 \%$ of the adults are under this group).

Source: OECD. See Annex 3 for notes (www.oecd.org/education/education-at-a-glance-19991487.htm).

Please refer to the Reader's Guide for information concerning symbols for missing data and abbreviations.

StatLink 게그 http://dx.doi.org/10.1787/888933285075 
Table A6.5a. Relative earnings of 15-24 year-old students with income from employment, by educational attainment and gender (2013)

Earnings of 15-24 year-old students with income from employment compared with earnings of 15-24 year-old non-students with income from employment; non-students with income from employment $=100$

\begin{tabular}{|c|c|c|c|c|c|c|c|c|c|c|}
\hline & \multirow[b]{3}{*}{ Year } & \multicolumn{3}{|c|}{ Below upper secondary education } & \multicolumn{3}{|c|}{$\begin{array}{l}\text { Upper secondary or post-secondary } \\
\text { non-tertiary education }\end{array}$} & \multicolumn{3}{|c|}{ Tertiary education } \\
\hline & & $M+W$ & Men & Women & $M+W$ & Men & Women & $M+W$ & Men & Women \\
\hline & & (1) & (2) & (3) & (4) & (5) & (6) & (7) & (8) & (9) \\
\hline Australia & 2012 & 29 & c & c & 52 & 51 & 57 & 68 & c & c \\
\hline Austria & 2013 & 62 & 64 & 56 & 32 & 37 & 30 & 39 & 47 & 33 \\
\hline Belgium $^{1}$ & 2013 & 47 & 55 & 41 & 64 & 65 & 65 & 65 & 75 & 81 \\
\hline Canada & 2012 & 34 & 31 & 45 & 42 & 42 & 49 & 49 & 40 & 55 \\
\hline Chile $^{2}$ & 2011 & 68 & 66 & 77 & 113 & 124 & 90 & $\mathrm{~m}$ & $\mathrm{~m}$ & $\mathrm{~m}$ \\
\hline Czech Republic & & $\mathrm{m}$ & $\mathrm{m}$ & $\mathrm{m}$ & $\mathrm{m}$ & $\mathrm{m}$ & $\mathrm{m}$ & $\mathrm{m}$ & $\mathrm{m}$ & $\mathrm{m}$ \\
\hline Denmark & 2012 & 44 & 45 & 46 & 47 & 42 & 55 & 43 & 43 & 43 \\
\hline Estonia & 2013 & 83 & 63 & 116 & 82 & 87 & 92 & 88 & $\mathrm{~m}$ & 106 \\
\hline Finland & 2012 & 39 & 38 & 45 & 56 & 56 & 58 & 53 & 61 & 51 \\
\hline France & 2011 & c & c & c & 55 & 69 & 44 & 41 & 52 & 32 \\
\hline Germany & & $\mathrm{m}$ & $\mathrm{m}$ & $\mathrm{m}$ & $\mathrm{m}$ & $\mathrm{m}$ & $\mathrm{m}$ & $\mathrm{m}$ & $\mathrm{m}$ & $\mathrm{m}$ \\
\hline Greece & 2013 & $\mathrm{~m}$ & $\mathrm{~m}$ & $\mathrm{~m}$ & 48 & 54 & 44 & $\mathrm{~m}$ & $\mathrm{~m}$ & $\mathrm{~m}$ \\
\hline Hungary & & $\mathrm{m}$ & $\mathrm{m}$ & $\mathrm{m}$ & $\mathrm{m}$ & $\mathrm{m}$ & $\mathrm{m}$ & $\mathrm{m}$ & $\mathrm{m}$ & $\mathrm{m}$ \\
\hline Iceland & & $\mathrm{m}$ & $\mathrm{m}$ & $\mathrm{m}$ & $\mathrm{m}$ & $\mathrm{m}$ & $\mathrm{m}$ & $\mathrm{m}$ & $\mathrm{m}$ & $\mathrm{m}$ \\
\hline Ireland $^{1}$ & 2013 & 19 & $\mathrm{~m}$ & $\mathrm{~m}$ & 45 & 41 & 51 & 57 & $\mathrm{~m}$ & 63 \\
\hline Israel & 2013 & 27 & 21 & 45 & 97 & 97 & 102 & 73 & $\mathrm{~m}$ & 59 \\
\hline Italy & 2011 & 54 & 83 & 31 & 63 & 62 & 69 & 110 & 119 & 106 \\
\hline Japan & & $\mathrm{m}$ & $\mathrm{m}$ & $\mathrm{m}$ & $\mathrm{m}$ & $\mathrm{m}$ & $\mathrm{m}$ & $\mathrm{m}$ & $\mathrm{m}$ & $\mathrm{m}$ \\
\hline Korea & 2013 & 44 & 61 & 34 & 51 & 57 & 46 & $\mathrm{~m}$ & $\mathrm{~m}$ & $\mathrm{~m}$ \\
\hline Luxembourg & & $\mathrm{m}$ & $\mathrm{m}$ & $\mathrm{m}$ & $\mathrm{m}$ & $\mathrm{m}$ & $\mathrm{m}$ & $\mathrm{m}$ & $\mathrm{m}$ & $\mathrm{m}$ \\
\hline Mexico $^{2}$ & 2012 & 57 & 55 & 63 & 75 & 72 & 79 & 80 & 65 & 96 \\
\hline Netherlands & & $\mathrm{m}$ & $\mathrm{m}$ & $\mathrm{m}$ & $\mathrm{m}$ & $\mathrm{m}$ & $\mathrm{m}$ & $\mathrm{m}$ & $\mathrm{m}$ & $\mathrm{m}$ \\
\hline New Zealand & 2013 & 42 & 50 & 34 & 50 & 58 & 44 & 62 & 54 & 67 \\
\hline Norway & 2013 & 37 & 40 & 34 & 40 & 38 & 46 & 38 & 36 & 40 \\
\hline Poland & & $\mathrm{m}$ & $\mathrm{m}$ & $\mathrm{m}$ & $\mathrm{m}$ & $\mathrm{m}$ & $\mathrm{m}$ & $\mathrm{m}$ & $\mathrm{m}$ & $\mathrm{m}$ \\
\hline Portugal & & $\mathrm{m}$ & $\mathrm{m}$ & $\mathrm{m}$ & $\mathrm{m}$ & $\mathrm{m}$ & $\mathrm{m}$ & $\mathrm{m}$ & $\mathrm{m}$ & $\mathrm{m}$ \\
\hline Slovak Republic & & $\mathrm{m}$ & $\mathrm{m}$ & $\mathrm{m}$ & $\mathrm{m}$ & $\mathrm{m}$ & $\mathrm{m}$ & $\mathrm{m}$ & $\mathrm{m}$ & $\mathrm{m}$ \\
\hline Slovenia & & $\mathrm{m}$ & $\mathrm{m}$ & $\mathrm{m}$ & $\mathrm{m}$ & $\mathrm{m}$ & $\mathrm{m}$ & $\mathrm{m}$ & $\mathrm{m}$ & $\mathrm{m}$ \\
\hline Spain & 2012 & 52 & 66 & 34 & 36 & 31 & 45 & 41 & 49 & 33 \\
\hline Sweden & & $\mathrm{m}$ & $\mathrm{m}$ & $\mathrm{m}$ & $\mathrm{m}$ & $\mathrm{m}$ & $\mathrm{m}$ & $\mathrm{m}$ & $\mathrm{m}$ & $\mathrm{m}$ \\
\hline Switzerland & 2013 & 10 & 6 & 35 & 43 & 47 & 41 & 53 & 55 & 50 \\
\hline Turkey $^{1}$ & 2013 & 62 & 64 & 60 & 99 & 103 & 91 & 113 & 107 & 115 \\
\hline United Kingdom $^{3}$ & 2013 & 28 & 32 & 23 & 28 & 33 & 25 & 61 & 73 & 49 \\
\hline United States & 2013 & 27 & 26 & 30 & 57 & 56 & 61 & 66 & 60 & 74 \\
\hline OECD average & & 43 & 48 & 47 & 58 & 60 & 58 & 63 & 62 & 64 \\
\hline EU21 average & & 47 & 56 & 49 & 51 & 52 & 53 & 60 & 65 & 60 \\
\hline
\end{tabular}

\begin{tabular}{|c|c|c|c|c|c|c|c|c|c|c|}
\hline $\begin{array}{l}\text { Argentina } \\
\text { Brazil }\end{array}$ & 2013 & $\begin{array}{l}\mathrm{m} \\
60\end{array}$ & $\begin{array}{l}\mathrm{m} \\
58\end{array}$ & $\begin{array}{l}\mathrm{m} \\
71\end{array}$ & $\begin{array}{r}\mathrm{m} \\
111\end{array}$ & $\begin{array}{r}\mathrm{m} \\
112\end{array}$ & $\begin{array}{r}\mathrm{m} \\
116\end{array}$ & $\begin{array}{r}\mathrm{m} \\
102\end{array}$ & $\begin{array}{l}\mathrm{m} \\
86\end{array}$ & $\begin{array}{r}\mathrm{m} \\
116\end{array}$ \\
\hline $\begin{array}{l}\text { China } \\
\text { Colombia }\end{array}$ & 2013 & $\begin{array}{r}\mathrm{m} \\
55\end{array}$ & $\begin{array}{l}\mathrm{m} \\
51\end{array}$ & $\begin{array}{l}\mathrm{m} \\
73\end{array}$ & $\begin{array}{r}\mathrm{m} \\
97\end{array}$ & $\begin{array}{r}\mathrm{m} \\
94\end{array}$ & $\begin{array}{r}\mathrm{m} \\
106\end{array}$ & $\begin{array}{l}\mathrm{m} \\
99\end{array}$ & $\begin{array}{r}\mathrm{m} \\
98\end{array}$ & $\begin{array}{r}\mathrm{m} \\
101\end{array}$ \\
\hline $\begin{array}{l}\text { India } \\
\text { Indonesia }\end{array}$ & & $\begin{array}{l}\mathrm{m} \\
\mathrm{m}\end{array}$ & $\begin{array}{l}\mathrm{m} \\
\mathrm{m}\end{array}$ & $\begin{array}{l}\mathrm{m} \\
\mathrm{m}\end{array}$ & $\begin{array}{l}\mathrm{m} \\
\mathrm{m}\end{array}$ & $\begin{array}{l}\mathrm{m} \\
\mathrm{m}\end{array}$ & $\begin{array}{l}\mathrm{m} \\
\mathrm{m}\end{array}$ & $\begin{array}{l}\mathrm{m} \\
\mathrm{m}\end{array}$ & $\begin{array}{l}\mathrm{m} \\
\mathrm{m}\end{array}$ & $\begin{array}{l}\mathrm{m} \\
\mathrm{m}\end{array}$ \\
\hline $\begin{array}{l}\text { Latvia } \\
\text { Russian Federation }\end{array}$ & & $\begin{array}{l}\mathrm{m} \\
\mathrm{m}\end{array}$ & $\begin{array}{l}\mathrm{m} \\
\mathrm{m}\end{array}$ & $\begin{array}{l}\mathrm{m} \\
\mathrm{m}\end{array}$ & $\begin{array}{l}\mathrm{m} \\
\mathrm{m}\end{array}$ & $\begin{array}{l}\mathrm{m} \\
\mathrm{m}\end{array}$ & $\begin{array}{l}\mathrm{m} \\
\mathrm{m}\end{array}$ & $\begin{array}{l}\mathrm{m} \\
\mathrm{m}\end{array}$ & $\begin{array}{l}\mathrm{m} \\
\mathrm{m}\end{array}$ & $\begin{array}{l}\mathrm{m} \\
\mathrm{m}\end{array}$ \\
\hline $\begin{array}{l}\text { Saudi Arabia } \\
\text { South Africa }\end{array}$ & & $\begin{array}{l}\mathrm{m} \\
\mathrm{m}\end{array}$ & $\begin{array}{l}\mathrm{m} \\
\mathrm{m}\end{array}$ & $\begin{array}{l}\mathrm{m} \\
\mathrm{m}\end{array}$ & $\begin{array}{l}\mathrm{m} \\
\mathrm{m}\end{array}$ & $\begin{array}{l}\mathrm{m} \\
\mathrm{m}\end{array}$ & $\begin{array}{l}\mathrm{m} \\
\mathrm{m}\end{array}$ & $\begin{array}{l}\mathrm{m} \\
\mathrm{m}\end{array}$ & $\begin{array}{l}\mathrm{m} \\
\mathrm{m}\end{array}$ & $\begin{array}{l}\mathrm{m} \\
\mathrm{m}\end{array}$ \\
\hline
\end{tabular}

Notes: For some countries in this table the age breakdown is $16-24$ year-olds. Columns showing the relative earnings for all levels of education combined are available for consultation on line (see StatLink below).

1. Belgium, Ireland, Turkey: Earnings net of income tax.

2. Chile, Mexico: Data refer to broad ISCED-97 attainment categories.

3. The United Kingdom: Data for upper secondary attainment includes completion of a sufficient volume and standard of programmes that would be classified individually as completion of intermediate upper secondary programmes (18\% of the adults are under this group).

Source: OECD. See Annex 3 for notes (www.oecd.org/education/education-at-a-glance-19991487.htm).

Please refer to the Reader's Guide for information concerning symbols for missing data and abbreviations.

StatLink 제개 http://dx.doi.org/10.1787/888933285081 
Table A6.5b. Percentage of 15-29 year-olds with income from employment among all 15-29 year-olds, by age group, gender and student status (2013)

\begin{tabular}{|c|c|c|c|c|c|c|c|}
\hline & \multirow[b]{4}{*}{ Year } & \multicolumn{6}{|c|}{ Men and women } \\
\hline & & \multicolumn{3}{|c|}{$15-24$ year-olds } & \multicolumn{3}{|c|}{$25-29$ year-olds } \\
\hline & & Non-students & Students & Total & Non-students & Students & Total \\
\hline & & (1) & (2) & (3) & (4) & (5) & (6) \\
\hline Australia & 2012 & 70 & 47 & 56 & 79 & 71 & 77 \\
\hline Austria & 2013 & 87 & 61 & 72 & 90 & 78 & 88 \\
\hline Belgium $^{1}$ & 2013 & 28 & 2 & 11 & 35 & 18 & 34 \\
\hline Canada & 2012 & 89 & 74 & 78 & 90 & 84 & 88 \\
\hline Chile & 2011 & 50 & 10 & 28 & 70 & 45 & 66 \\
\hline Czech Republic & & $\mathrm{m}$ & $\mathrm{m}$ & $\mathrm{m}$ & $\mathrm{m}$ & $\mathrm{m}$ & $\mathrm{m}$ \\
\hline Denmark & 2012 & 71 & 71 & 71 & 81 & 82 & 82 \\
\hline Estonia & 2013 & 41 & 13 & 22 & 54 & 45 & 52 \\
\hline Finland & 2012 & 77 & 77 & 77 & 84 & 85 & 84 \\
\hline France & 2011 & 76 & 37 & 55 & 92 & 73 & 91 \\
\hline Germany & 2012 & 66 & 37 & 46 & 70 & 62 & 68 \\
\hline Greece & 2013 & 27 & 4 & 12 & 54 & 27 & 51 \\
\hline Hungary & & $\mathrm{m}$ & $\mathrm{m}$ & $\mathrm{m}$ & $\mathrm{m}$ & $\mathrm{m}$ & $\mathrm{m}$ \\
\hline Iceland & & $\mathrm{m}$ & $\mathrm{m}$ & $\mathrm{m}$ & $\mathrm{m}$ & $\mathrm{m}$ & $\mathrm{m}$ \\
\hline Ireland $^{1}$ & 2013 & 36 & 23 & 28 & 67 & 51 & 65 \\
\hline Israel & 2013 & 68 & 18 & 45 & 80 & 74 & 79 \\
\hline Italy & 2011 & 56 & 13 & 32 & 79 & 44 & 72 \\
\hline Japan & & $\mathrm{m}$ & $\mathrm{m}$ & $\mathrm{m}$ & $\mathrm{m}$ & $\mathrm{m}$ & $\mathrm{m}$ \\
\hline Korea & 2013 & 52 & 11 & 23 & 67 & 29 & 64 \\
\hline Luxembourg & & $\mathrm{m}$ & $\mathrm{m}$ & $\mathrm{m}$ & $\mathrm{m}$ & $\mathrm{m}$ & $\mathrm{m}$ \\
\hline Mexico & 2012 & 89 & 81 & 87 & 94 & 96 & 94 \\
\hline Netherlands & & $\mathrm{m}$ & $\mathrm{m}$ & $\mathrm{m}$ & $\mathrm{m}$ & $\mathrm{m}$ & $\mathrm{m}$ \\
\hline New Zealand & 2013 & 69 & 33 & 47 & 76 & 60 & 74 \\
\hline Norway & 2013 & 82 & 67 & 72 & 89 & 90 & 89 \\
\hline Poland & & $\mathrm{m}$ & $\mathrm{m}$ & $\mathrm{m}$ & $\mathrm{m}$ & $\mathrm{m}$ & $\mathrm{m}$ \\
\hline Portugal & & $\mathrm{m}$ & $\mathrm{m}$ & $\mathrm{m}$ & $\mathrm{m}$ & $\mathrm{m}$ & $\mathrm{m}$ \\
\hline Slovak Republic & & $\mathrm{m}$ & $\mathrm{m}$ & $\mathrm{m}$ & $\mathrm{m}$ & $\mathrm{m}$ & $\mathrm{m}$ \\
\hline Slovenia & & $\mathrm{m}$ & $\mathrm{m}$ & $\mathrm{m}$ & $\mathrm{m}$ & $\mathrm{m}$ & $\mathrm{m}$ \\
\hline Spain & 2012 & 60 & 20 & 34 & 82 & 71 & 80 \\
\hline Sweden & & $\mathrm{m}$ & $\mathrm{m}$ & $\mathrm{m}$ & $\mathrm{m}$ & $\mathrm{m}$ & $\mathrm{m}$ \\
\hline Switzerland & 2013 & 72 & 53 & 60 & 85 & 61 & 81 \\
\hline Turkey $^{1}$ & 2013 & 76 & 76 & 76 & 86 & 87 & 86 \\
\hline United Kingdom ${ }^{2}$ & 2013 & 56 & 32 & 46 & 80 & 63 & 78 \\
\hline United States & 2013 & 73 & 41 & 54 & 82 & 62 & 79 \\
\hline OECD average & & 64 & 39 & 49 & 77 & 63 & 75 \\
\hline EU21 average & & 57 & 32 & 42 & 72 & 58 & 71 \\
\hline Argentina & & $\mathrm{m}$ & $\mathrm{m}$ & $\mathrm{m}$ & $\mathrm{m}$ & $\mathrm{m}$ & $\mathrm{m}$ \\
\hline Brazil & 2013 & 62 & 32 & 48 & 75 & 70 & 74 \\
\hline China & & $\mathrm{m}$ & $\mathrm{m}$ & $\mathrm{m}$ & $\mathrm{m}$ & $\mathrm{m}$ & $\mathrm{m}$ \\
\hline Colombia & 2013 & 91 & 79 & 88 & 96 & 97 & 96 \\
\hline India & & $\mathrm{m}$ & $\mathrm{m}$ & $\mathrm{m}$ & $\mathrm{m}$ & $\mathrm{m}$ & $\mathrm{m}$ \\
\hline Indonesia & & $\mathrm{m}$ & $\mathrm{m}$ & $\mathrm{m}$ & $\mathrm{m}$ & $\mathrm{m}$ & $\mathrm{m}$ \\
\hline Latvia & & $\mathrm{m}$ & $\mathrm{m}$ & $\mathrm{m}$ & $\mathrm{m}$ & $\mathrm{m}$ & $\mathrm{m}$ \\
\hline Russian Federation & & $\mathrm{m}$ & $\mathrm{m}$ & $\mathrm{m}$ & $\mathrm{m}$ & $\mathrm{m}$ & $\mathrm{m}$ \\
\hline Saudi Arabia & & $\mathrm{m}$ & $\mathrm{m}$ & $\mathrm{m}$ & $\mathrm{m}$ & $\mathrm{m}$ & $\mathrm{m}$ \\
\hline South Africa & & $\mathrm{m}$ & $\mathrm{m}$ & $\mathrm{m}$ & $\mathrm{m}$ & $\mathrm{m}$ & $\mathrm{m}$ \\
\hline G20 average & & $\mathrm{m}$ & $\mathrm{m}$ & $\mathrm{m}$ & $\mathrm{m}$ & $\mathrm{m}$ & $\mathrm{m}$ \\
\hline
\end{tabular}

Notes: For some countries in this table the age breakdown is 16-24 year-olds instead of 15-24 year-olds. Columns showing data for men and women separately are available for consultation on line (see StatLink below).

1. Belgium, Ireland, Turkey: Earnings net of income tax.

2. The United Kingdom: Data for upper secondary attainment includes completion of a sufficient volume and standard of programmes that would be classified individually as completion of intermediate upper secondary programmes (18\% of the adults are under this group).

Source: OECD. See Annex 3 for notes (www.oecd.org/education/education-at-a-glance-19991487.htm).

Please refer to the Reader's Guide for information concerning symbols for missing data and abbreviations.

StatLink 게대 http://dx.doi.org/10.1787/888933283672 


\section{WHAT ARE THE FINANCIAL INCENTIVES TO INVEST IN EDUCATION?}

- Adults completing tertiary education benefit from substantial returns on investment: they are more likely to be employed and earn more than adults without tertiary education do.

- Not only does education pay off for individuals, but the public benefits of education, in greater tax revenues and social contributions from a larger proportion of tertiary-educated adults, also outweigh the cost.

- Across OECD countries, the net public return on investment for a woman with tertiary education is USD 65500 over her lifetime - 1.2 times the public cost of investment in her education. For a man, the net public return is over USD 127400 , which is almost 2.5 times the public cost of investment in his education.

\section{Chart A7.1. Private net financial returns for a woman attaining tertiary education (2011)}

\section{As compared with returns to upper secondary or post-secondary non-tertiary education, in equivalent USD converted using PPPs for GDP}

USD converted

using PPPs for GDP

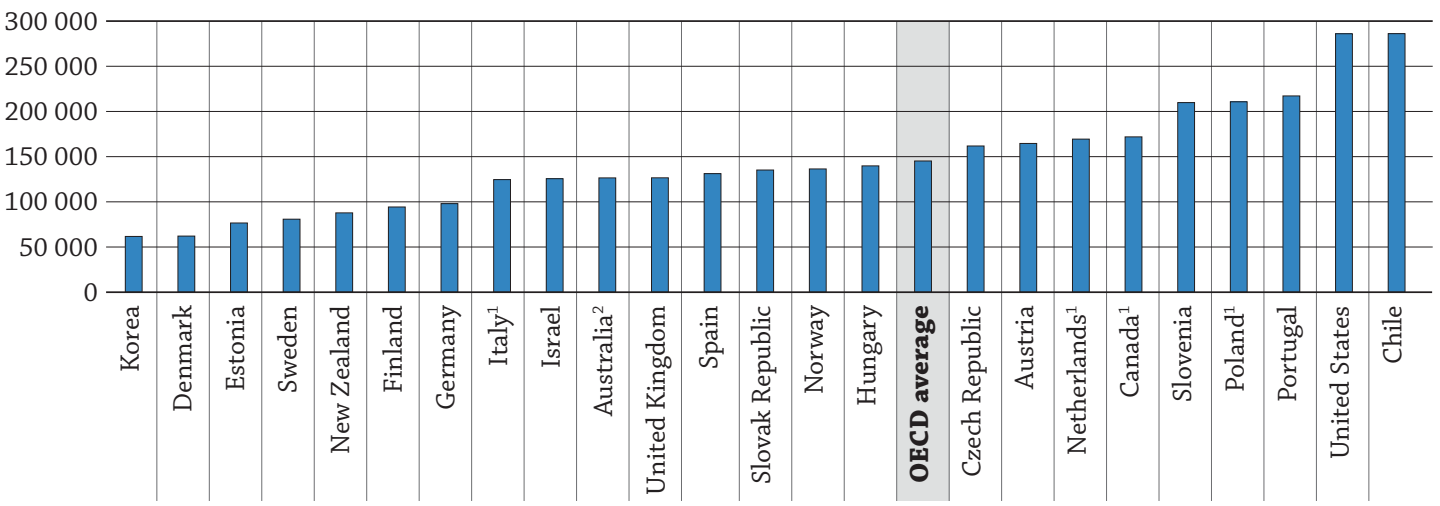

1. Canada, Italy, the Netherlands, Poland: Year of reference 2010.

2. Australia: Year of reference 2009.

Countries are ranked in ascending order of private net financial returns.

Source: OECD. Tables A7.3b and A7.4b. See Annex 3 for notes (www.oecd.org/education/education-at-a-glance- 19991487.htm). StatLink 제내 http://dx.doi.org/10.1787/888933283725

\section{Context}

Devoting time and money in education is an investment in human capital. For adults, having higher educational attainment improves chances for employment and reduces the risk of unemployment. Better opportunities in the labour market (see Indicator A5) and higher earnings (see Indicator A6) are strong incentives for adults to invest in education and to postpone consumption and earnings for future rewards. Countries, in turn, benefit through reduced public expenditure on social welfare programmes and revenues earned through taxes paid once individuals enter the labour market.

It is crucial for policy makers to understand the economic incentives for individuals to invest in education. For instance, large increases in labour market demand for more highly educated workers can drive up earnings and returns before supply catches up. That signals a need for additional investment in education. In countries with rigid labour laws and structures that tend to limit differences in wages across the board, this signal will be weaker.

An understanding of the returns from education is also relevant for policies that address access to education, taxes and the costs of further education for the individual. It is important, then, to consider the balance between private and public returns together with the information from other indicators in this publication. It is not sufficient to consider only the public rate of return to determine the optimal amount governments should invest in education (see Box A7.1 in Education at a Glance 2013 [OECD, 2013]). 
In countries with lengthy tertiary programmes and relatively high incomes after upper secondary or post-secondary non-tertiary education, the effect of foregone earnings is considerable. The magnitude of this effect also depends on expected wage levels and the probability of finding a job with or without having tertiary qualifications. As the labour market for young adults worsens (see Indicator C5), the effect of foregone earnings is reduced, making tertiary education a less costly investment. Since more highly educated people tend to fare better in the labour market in times of economic hardship (see Indicator A5), larger earnings differentials add to the benefit to both the individual and society. Data from 2011 (used in this volume), show that both private and public returns to tertiary education were higher than returns to upper secondary and post-secondary non-tertiary education.

It should be kept in mind that a host of education-related and contextual factors not reflected in this indicator affect the financial returns to education. These include the field of study, countries' specific economic situation, labour market context and institutional setting, as well as social and cultural factors.

\section{Other findings}

- On average across OECD countries, the calculated financial return to tertiary education for a single worker with no children is around twice as large as returns to such a person with upper secondary or post-secondary non-tertiary education as his or her highest level of attainment.

- Gross earnings benefits from tertiary education over the course of a lifetime are USD 477400 for men and USD 332600 for women across OECD countries.

- Gross earnings benefits for an adult with upper secondary or post-secondary non-tertiary degree, compared to benefits for an adult who has not attained this level of education, are particularly large in Austria, Luxembourg and the United States. In these countries, gross earnings benefits amount to more than USD 400000 for a man and USD 250000 for a woman over their lifetime.

- On average across the 26 OECD countries with available data, the net public return for a woman who completed upper secondary or post-secondary non-tertiary education is about USD 48000 compared with a woman who did not complete that level of education. For a man, the net public return is USD 70300.

- Across OECD countries, people invest around USD 55000 to earn a tertiary degree. In the Netherlands and the United States, average investment exceeds USD 100000 when direct and indirect costs are taken into account. 


\section{Analysis}

This indicator provides information on the incentives to invest in further education by considering its costs and benefits, including net financial returns and internal rate of return. It examines the choice between pursuing higher levels of education and entering the labour market. The indicator focuses on two scenarios:

- Investing in tertiary education compared to entering the labour market with an upper secondary or postsecondary non-tertiary degree; and

- Investing in upper secondary or post-secondary non-tertiary education compared to entering the labour market without an upper secondary or post-secondary non-tertiary degree.

Two types of investors are considered:

- The person (referred to here as "Private") who chooses to pursue higher levels of education based on the additional net earnings and costs he or she can expect; and

- The government (referred to here as "Public") that decides to invest in education based on the additional revenue it would receive (tax receipts) and the costs involved.

Values are presented separately for men and women to account for gender-specific differences in earnings and unemployment rates.

\section{Financial incentives for people to invest in education (private financial returns on investment)}

Attracted by higher earnings and employment prospects, more people than ever before are attaining upper secondary or post-secondary education and tertiary education (see Indicators A1, A5 and A6). Different financial components, such as level of household out-of-pocket spending on education, the additional earnings over the lifecourse, and the tax and benefits systems can influence someone's decision to pursue further formal education.

\section{Net private financial returns on investment}

In almost all countries with available data for upper secondary and post-secondary non-tertiary and tertiary education, financial private returns to education are higher for tertiary education than for upper secondary education. A woman can expect a net financial return on investment in upper secondary or post-secondary nontertiary education of USD 62 000; her expected financial return on investment in tertiary education is USD 145200 (Tables A7.1b and A7.3b).

Men can expect a higher return to investment in education than women at both the upper secondary or postsecondary non-tertiary (USD 107 100) and tertiary (USD 229 000) levels of education (Tables A7.1a and A7.3a). These results are consistent with the higher earnings and lower uemployment rates enjoyed by men (see Indicators A5 and A6).

\section{The total private cost of education}

Direct costs and foregone earnings are the two components of the total cost of education considered in the computations of this Indicator. Accounting for both the direct cost and forgone earnings, a woman invests USD 31200 in upper secondary or post-secondary non tertiary and USD 57200 in tertiary education (Tables A7.1b and $\mathrm{A7.3b).}$

Most adults consider the direct private costs (household expenditure) of education as they decide whether or not to invest in further education. The direct cost for a person is, on average, lower for upper secondary education (USD 2 800) than for tertiary education (USD 13 200). In addition, direct private costs for tertiary education vary more across countries than costs for upper secondary non-tertiary education, ranging from less than USD 2500 in Austria, Norway and Sweden, to more than USD 25000 in Australia, Chile, the United Kingdom and the United States. In fact, the direct costs for tertiary education in the United States - USD 55000 - are the highest among all OECD countries (Tables A7.1b and A7.3b).

While they are the most visible part of the total cost of education, direct costs of education represent only a small share of this cost ( $10 \%$ of the total cost, on average, for upper secondary non-tertiary education and $20 \%$ for tertiary education). The main costs are the foregone earnings - what a student could potentially earn if not in school. Foregone earnings vary substantially across countries, depending on the length of education, earnings levels and the difference in earnings across levels of educational attainment. In Estonia and Spain, foregone earnings from investing in upper secondary or post-secondary non-tertiary education are estimated at less than USD 15000 for both women and men, while in Austria, Luxembourg, the Netherlands and Norway, they exceed USD 45000 (Tables A7.1a and b). 


\section{Chart A7.2. Private costs and benefits of education for a woman, by educational attainment (2011) \\ In equivalent USD converted using PPPs for GDP}

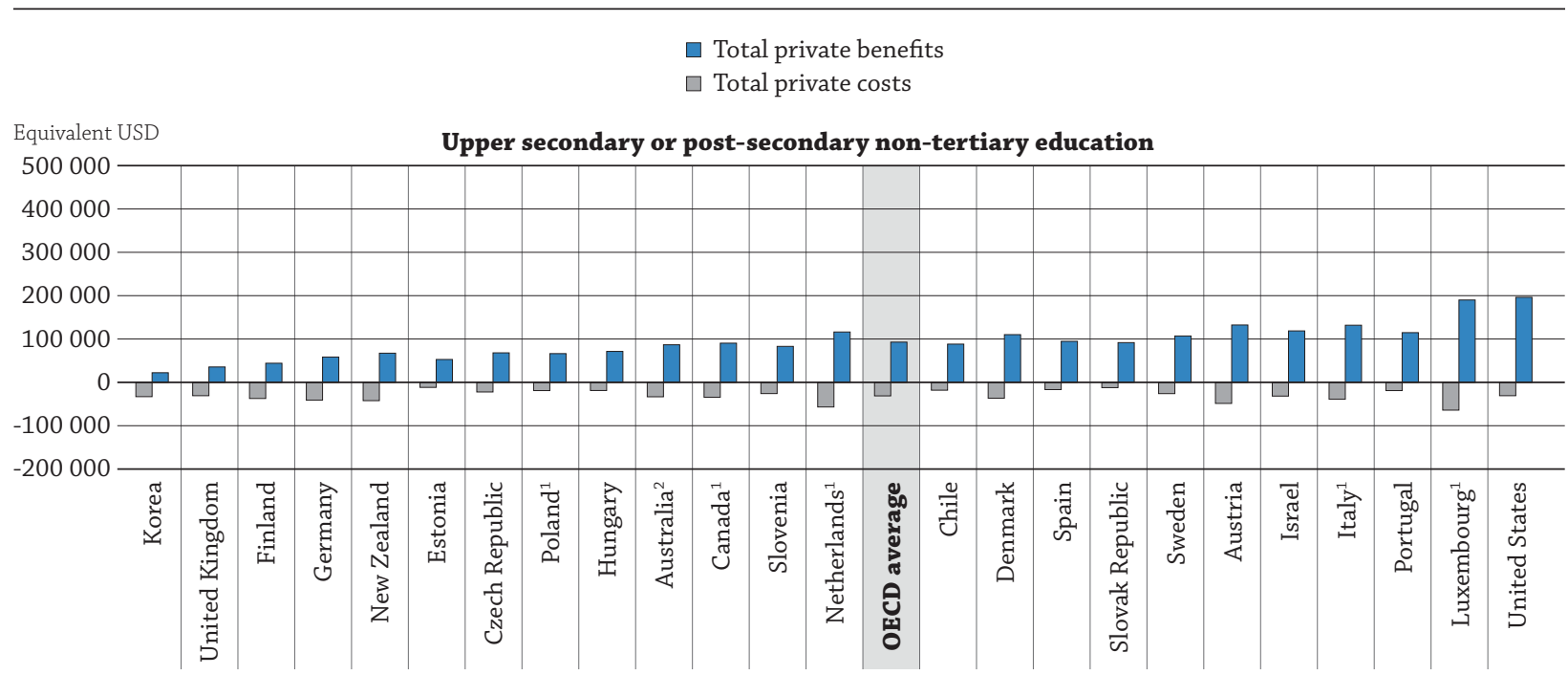

Equivalent USD

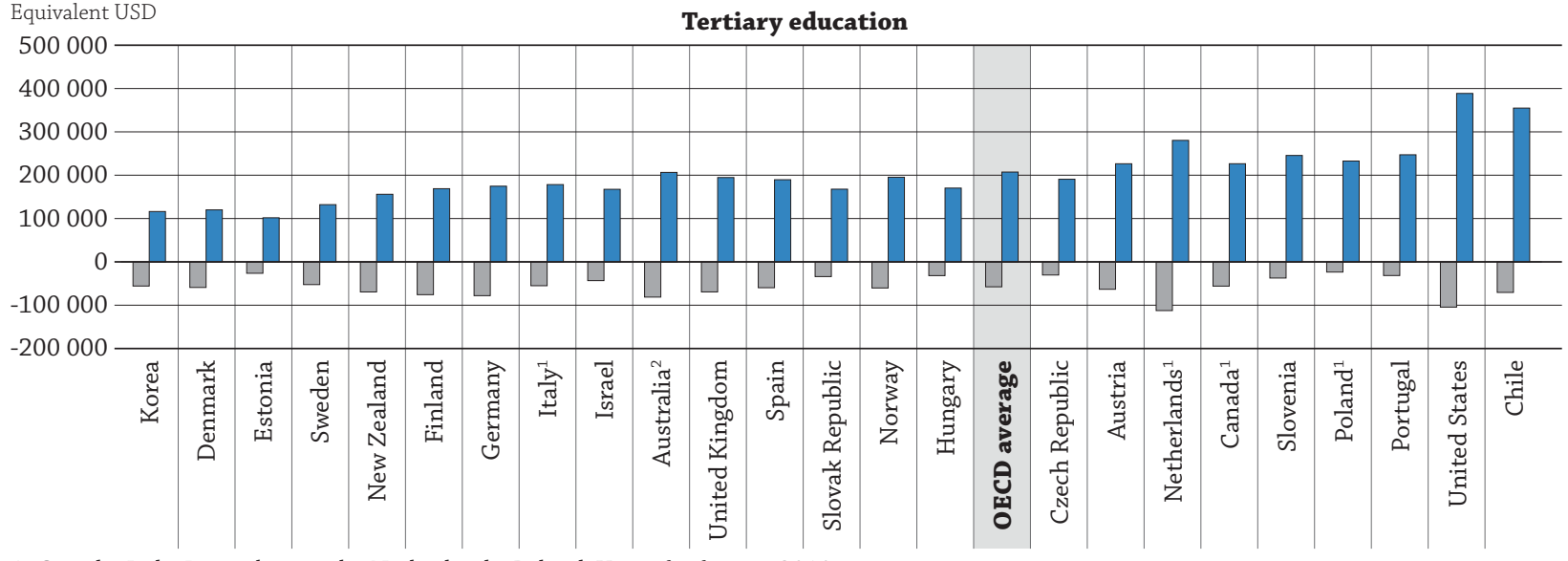

1. Canada, Italy, Luxembourg, the Netherlands, Poland: Year of reference 2010.

2. Australia: Year of reference 2009.

Countries are ranked in ascending order of net financial private returns (benefits-costs).

Source: OECD. Tables A7.1b and A7.3b. See Annex 3 for notes (www.oecd.org/education/education-at-a-glance- 19991487.htm).

StatLink 部西 http://dx.doi.org/10.1787/888933283733

\section{The total private benefits of education}

Chart A7.2 shows that while total costs of investing in tertiary education (USD 57200 for a woman) are higher, on average, than investing in upper secondary or post-secondary non-tertiary education (USD 31200 for a woman), even greater total benefits (USD 208300 for a woman) accrue to tertiary-educated adults than to adults with upper secondary or post-secondary non-tertiary education as their highest level of attainment (USD 92800 for a woman) (Tables A7.1b and A7.3b).

In general, further education yields higher earnings over a lifetime. A woman with upper secondary or postsecondary non-tertiary education as her highest level of attainment can expect to earn USD 151800 more in gross earnings than a woman with a lower level of attainment over the course of her life (Table A7.1b). A tertiaryeducated woman can expect to earn USD 332600 more in gross earnings than a woman with upper secondary or post-secondary non-tertiary education. On average, the gross earning benefits from tertiary education are double the gross earning benefits from upper secondary or post-secondary non-tertiary education for both men and women. While gross earnings benefits from tertiary education for a woman are only about USD 130000 in Estonia and Korea, they are more than USD 460000 in Chile, Luxembourg, Slovenia, the Netherlands and the United States (Table A7.3b). 
As shown in Indicator A6, earnings premiums from higher educational attainment are greater for men than for women. Gross earnings benefits for men are $40 \%$ higher than for women from both upper secondary or postsecondary non-tertiary and tertiary education (Tables A7.1a and b, and Tables A7.3a and b).

Countries' tax and social benefits systems also have an impact on people's decisions to pursue - or not - further education. Higher income taxes and social contributions and lower social transfers related to higher earnings can act as disincentives to invest in further education by creating a wedge between the level of gross earnings needed to recover the cost of education and the final net earnings perceived by the individual (Brys and Torres, 2013). For instance, a man who chose to invest in tertiary education will pay, on average, $40 \%$ of his additional income associated with tertiary education in taxes and social contributions. In Canada, Chile, the Czech Republic, Estonia, Greece, Korea, New Zealand, Poland, the Slovak Republic and Spain, income taxes and social contributions amount to less than a third of the gross earning benefits, while in Denmark, Germany, Italy and Slovenia they add up to about half of the gross earning benefits (Table A7.3a).

\section{Financial incentives for governments to invest in education (public financial returns on investment)}

In an era of budget constraints, government investment in education attracts even greater scrutiny. Governments are major investors in education and, from a budget point of view, are interested to know if they will recover their investment.

Higher levels of educational attainment tends to translate into higher income, on average (see Indicator A6). In this sense, investments in education generate public returns as tertiary-educated adults pay higher income taxes and social insurance payments and require fewer social transfers.

The discussion of the public returns to education in this chapter is limited to budget considerations and does not take into account other sources of returns to education enjoyed by society as a whole, such as higher productivity, better health and life expectancy, and other social outcomes.

\section{Net financial returns on investment for governments}

On average across OECD countries, the net public return for a woman attaining tertiary education is USD 65500 and USD 48000 for a woman attaining upper secondary or post-secondary non-tertiary education (Tables A7.2b and $\mathrm{A} 7.4 \mathrm{~b})$.

In Estonia, New Zealand, Spain, Sweden, Switzerland and the United Kingdom, public net financial returns on education are higher for a man with upper secondary or post-secondary non-tertiary education than for a tertiaryeducated man. This could be either because of the relatively higher public direct cost of education at the tertiary level in some of these countries or because of relatively less progressive tax systems in others (Table A7.1a and Table A7.3a).

\section{The total public cost of education}

The total public cost of investment in an individual's education include direct government expenditure on education per student (direct public cost), as well as the foregone tax receipts the government would have received if the individual had entered the labour market instead of pursuing further education. Chart A7.3 shows that on average, upper secondary or post-secondary non-tertiary education for a woman costs about USD 31700 for OECD countries and ranges from below USD 13000 in Chile, Hungary and the United Kingdom to over USD 55000 in Austria, Denmark, Luxembourg, Norway and Switzerland. Tertiary education for a woman in OECD countries costs an average of USD 53900 (Tables A7.2b and A7.4b).

In Denmark, Finland, Germany, Sweden and Switzerland, the direct cost to governments per tertiary student is higher than USD 85 000, while this direct public cost in Chile, Israel, Korea and Poland is below USD 20 000. Since these calculations do not take into account public loans, direct public costs in countries that widely offer public loans, such as Australia, the United Kingdom and the United States, might be underestimated (see Indicator B5).

\section{The total public benefits of education}

Governments offset the costs of direct investment and foregone tax receipts by receiving additional tax receipts and social contributions from higher-educated adults. Overall, taking into account unemployment rate differences and benefits, total public benefits accruing over the lifetime of a woman whose highest level of attainment is upper secondary or post-secondary non-tertiary education are USD 77 300, and USD 123600 for a tertiary-educated woman (Tables A7.2b and A7.4b). 
Chart A7.3. Public costs and benefits of education for a woman, by educational attainment (2011)

In equivalent USD converted using PPPs for GDP

Total public benefits

$\square$ Total public costs

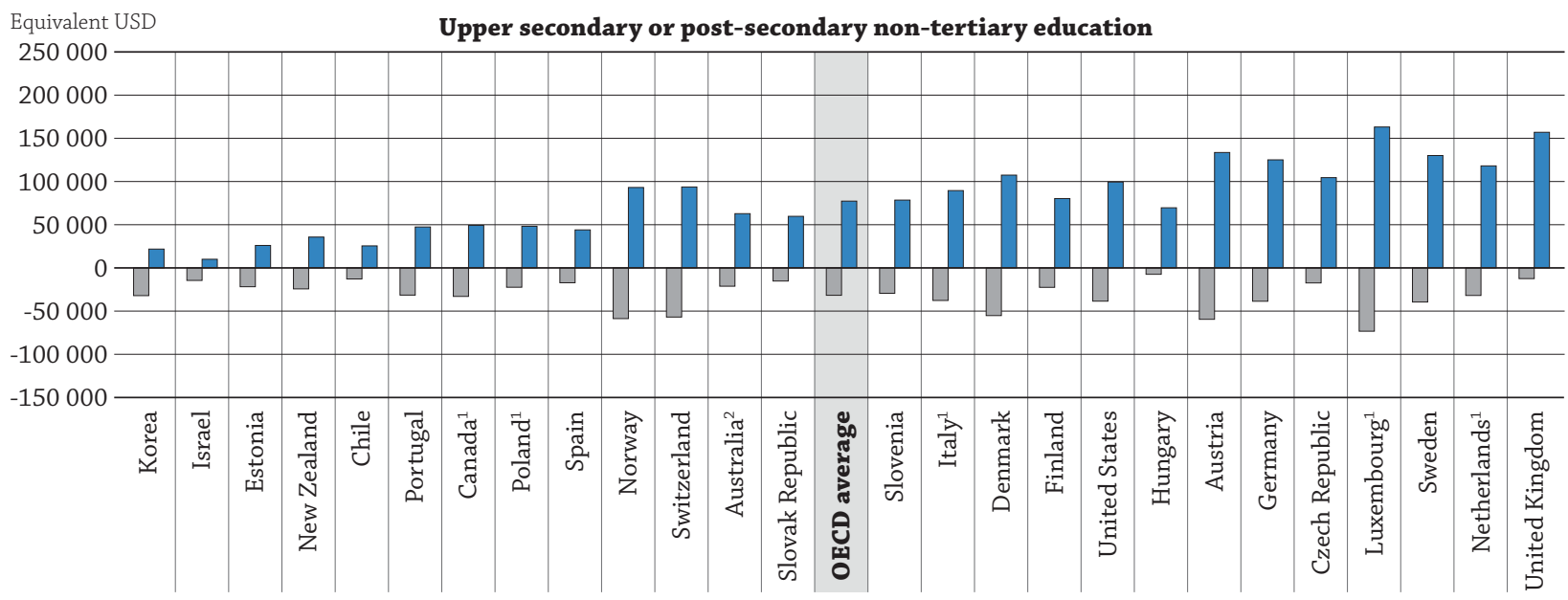

Equivalent USD

Tertiary education

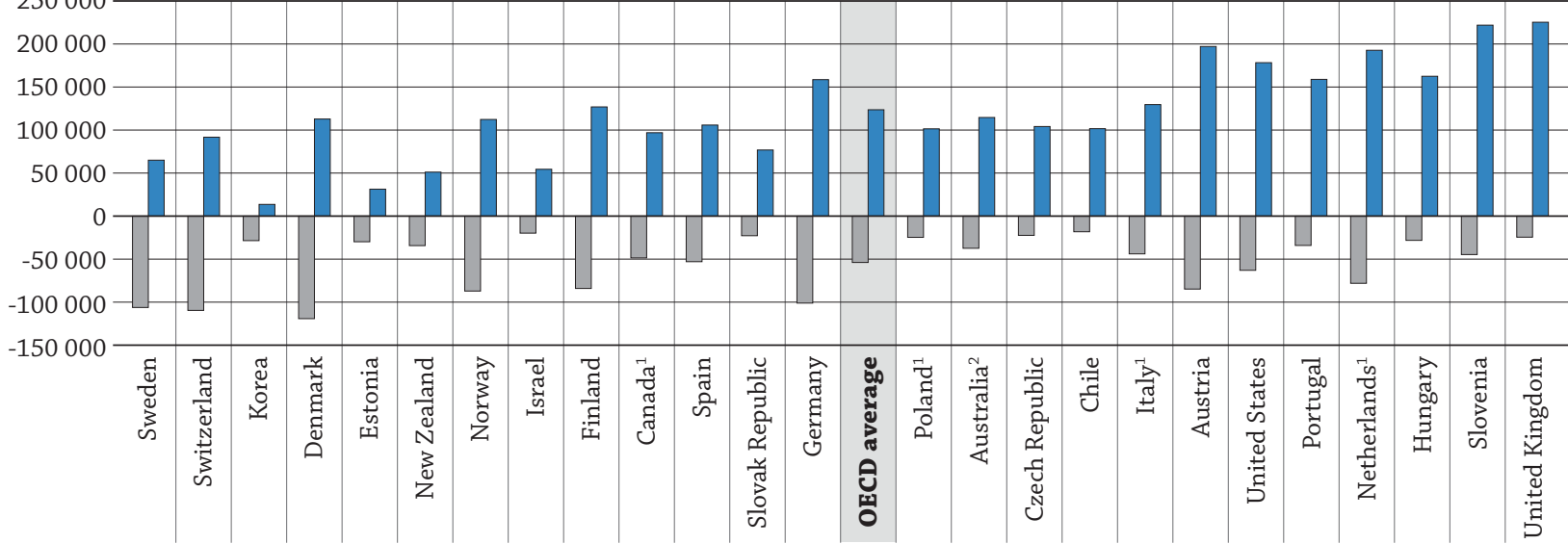

1. Canada, Italy, Luxembourg, the Netherlands, Poland: Year of reference 2010.

2. Australia: Year of reference 2009.

Countries are ranked in ascending order of net financial public returns (benefits-costs).

Source: OECD. Tables A7.2b and A7.4b. See Annex 3 for notes (www.oecd.org/education/education-at-a-glance- 19991487.htm).

StatLink त्ञात http://dx.doi.org/10.1787/888933283748

Given that gross earnings benefits vary substantially among OECD countries, tax payments and benefits to the public sector also vary in ways that are somewhat counterintuitive. The largest public gains in tax and social security benefits from higher education are most often found in countries where earnings differentials are large, or where average earnings reach high income-tax brackets. In Luxembourg, the Netherlands, Slovenia and the United Kingdom, tertiary-educated adults pay more in taxes and social contributions, resulting in more than USD 200000 in total public benefits from tertiary-educated women. Conversely, because gross earnings benefits from tertiary education are relatively low in Estonia, Israel, Korea, and New Zealand, the public benefits from education are relatively low (Table A7.4b).

Since higher taxes can sometimes deter private investment in education, a number of countries have tax policies that effectively lower the actual tax paid by adults, particularly by those in high-income brackets. Tax relief for interest payments on mortgage debt has been introduced in many OECD countries to encourage homeownership. These benefits favour those with higher education and high marginal tax rates. The tax incentives for housing are particularly large in the Czech Republic, Denmark, Finland, Greece, the Netherlands, Norway, Sweden and the United States (Andrews et al., 2011). 


\section{Definitions}

Adults refers to 15-64 year-olds.

Direct costs are the direct expenditure on education per student during the time spent in school.

Private direct cost is households' total expenditure on education and includes net payments to educational institutions as well as payments for educational goods and services outside of educational institutions (school supply, tutoring, etc.).

Public direct cost is the government's spending on a student's education. It includes the direct public expenditure on educational institutions, government scholarships and other grants to students and households and transfers and payments to other private entities for educational purposes.

Foregone earnings are the (unobserved) net earnings an individual would have had if he or she entered the labour force and successfully found a job instead of choosing to do further studies.

Foregone taxes on earnings are the (unobserved) tax receipts the government would have received if the individual chose to enter the labour force and successfully found a job instead of choosing to pursue further studies.

Gross earnings benefits are the discounted sum of earnings premiums over the course of a lifetime associated with a higher level of education provided that the individual successfully enters the labour market.

The income tax effect is the discounted sum of additional level of income tax paid by the private individual or earned by the government over the course of a lifetime and associated with a higher level of education.

The internal rate of return is the (hypothetical) real interest rate equalising the costs and the benefits related to the educational investment.

Levels of education: Below upper secondary corresponds to ISCED-97 Levels 0, 1, 2 and $3 C$ short programmes; upper secondary or post-secondary non-tertiary corresponds to ISCED-97 Levels 3A, 3B, 3C long programmes, and Level 4; and tertiary corresponds to ISCED-97 Levels 5A, 5B and 6.

The net financial returns are the net present value of the financial investment in education. The net financial returns are the difference between the discounted financial benefits and the discounted financial cost of education, and represent the additional value that education produces over and above the $2 \%$ real interest that is charged on these cash flows.

The social contribution effect is the discounted sum of additional employee social contribution paid by the private individual or received by the government over the course of a lifetime and associated with a higher level of education.

The transfers effect is the discounted sum of additional social transfers from the government to the private individual associated with a higher education level over the course of a lifetime. Social transfers include two types of benefits: housing benefits and social assistance.

\section{Methodology}

\section{The general approach}

This indicator estimates the financial returns on investment in education from the age of entry into further education to a theoretical age of retirement (64 years old). Returns to education are studied purely from the perspective of financial investment that weighs the cost and benefits of the investment.

Two periods are considered (Figure 1):

- Time spent in school during which the private person and the government pay the cost of education.

- Time spent in the labour market during which the private person and the government receive the added payments associated with further education.

In calculating the financial returns to education, the approach taken here is the net present value (NPV) of the investment. The NPV expresses cash transfers happening at different time in present value which allow for direct comparability of the cost and benefit. In this framework, lifetime costs and benefits are transferred back to the start of the investment. This is done by discounting all cash flows back to the beginning of the investment (Y1 in Figure 1) with a set rate of interest (discount rate). 
Figure 1. Financial returns on investment in education over a life-time for a representative individual

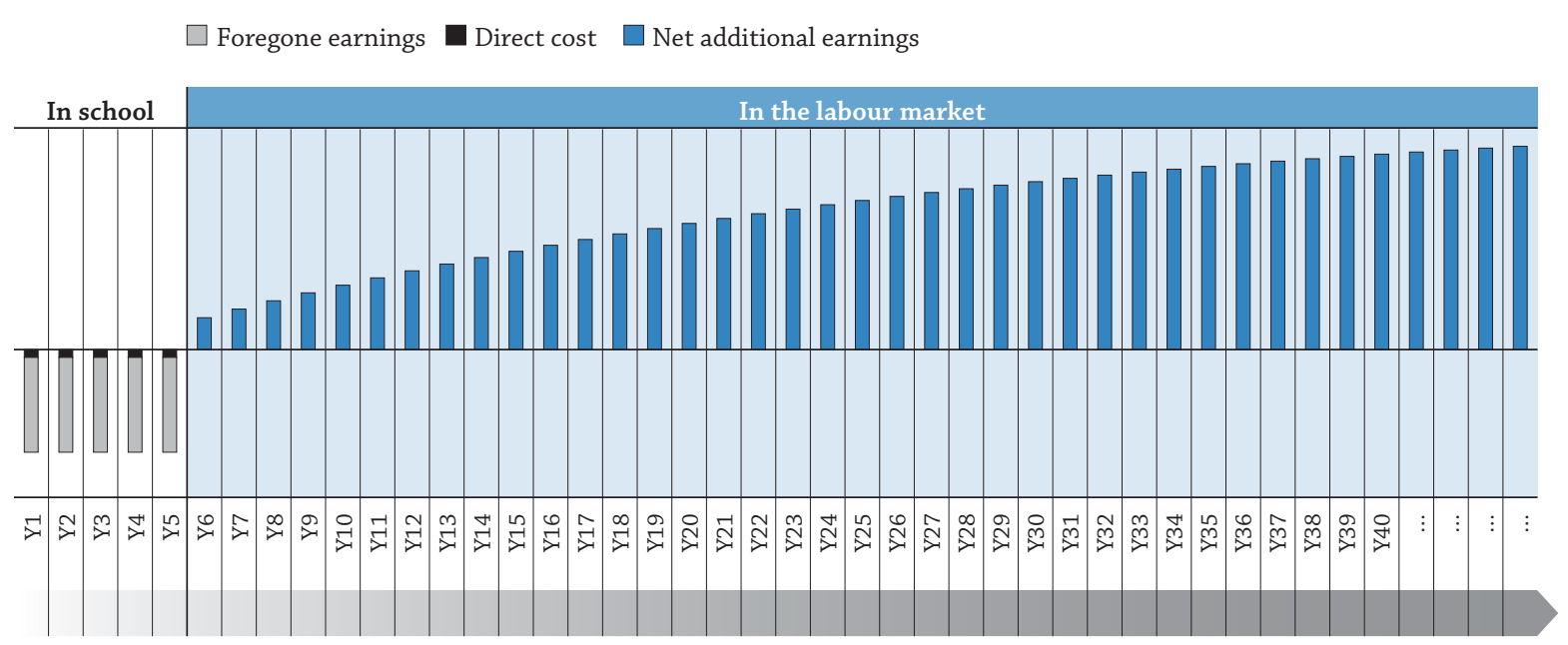

To set a value for the discount rate, long-term government bonds have been used as a benchmark. The average longterm interest rate across OECD countries was approximately 4.9\% in 2011 which leads to an average real interest on government bonds across OECD countries of approximately $2 \%$. The $2 \%$ real discount rate used in this indicator reflects the fact that calculations are made in constant prices (OECD, 2015a; 2015b).

The choice of discount rate is difficult, as it should reflect not only the overall time horizon of the investment, but also the cost of borrowing or the perceived risk of the investment. To allow for comparability, and to make the interpretation of results easier, the same discount rate (2\%) is applied across all OECD countries. All values presented in the tables of this indicator are in net present value equivalent USD using purchasing power parities (PPP).

\section{The costs}

\section{Total cost}

Investing in a higher level of education has direct and indirect costs. The direct cost is the upfront expenditure paid during the years of additional studies. The indirect costs for a private person are the foregone earnings that the individual would have received if he or she had decided to work instead of pursue an additional degree of education. Similarly, the indirect costs for the public are the foregone tax receipts not received because the person chose to pursue further education instead of entering the labour market.

Private cost $=$ Direct cost + Foregone earnings

Public cost $=$ Direct cost + Foregone tax receipts

\section{Direct cost of education}

The source of direct costs of education is the UOE data collection on finance (year of reference 2011 unless otherwise specified in the tables). The direct cost includes all expenditures on education for all levels of government combined (public direct cost) and all education-related household expenditure (private direct cost).

Private direct cost is net of loans and grants; public loans are not included in public direct costs. The exclusion of loans from the public cost may lead to an underestimation of public costs for some countries, particularly at the tertiary level. Further details on student loans can be found in Indicator B5.

Please note that, because of significant differences in methodology, direct costs are not comparable between this edition of Education at a Glance and previous editions. For further details, please refer to Annex 3 (www.oecd.org/ education/education-at-a-glance-19991487.htm).

\section{Shadow cost of education (foregone earnings and tax receipts)}

Investing in further education also has a shadow cost, or opportunity cost, which is the income the private individual or the government does not receive while the student is in school. 
The shadow cost for a person is the foregone earnings an adult would have made if he or she had entered the labour force instead of pursuing a higher level of education. From the government's perspective, the shadow cost is the foregone taxes the government is not collecting while the person is studying instead of working. To simplify, the indicator assumes that students do not have earnings or pay taxes while they are studying. To compute foregone earnings and foregone tax receipts, the indicator assumes that the earnings lost are equal to the minimum wage. This simplification is used to allow for better comparability of the data across countries. The price for this assumption is an upward bias in the calculated net present value, as the potential earnings of many young people is likely to be higher than the minimum wage.

\section{The benefits}

\section{Total benefits}

The benefits of investing in education are the added income associated with a higher level of education given the probability of successfully finding a job. For the private individual, this additional income is the added net earnings expected from an additional level of education, given that the person successfully enters the labour market. Public benefits are constructed to mirror private benefits. Public benefits are the sum of added tax receipts that accrue to the government from an adult with a higher level of education, provided that the person successfully enters the labour market.

For $j$, the highest level of attainment, and $j-1$, a lower level of attainment, total public and private benefits can be written as:

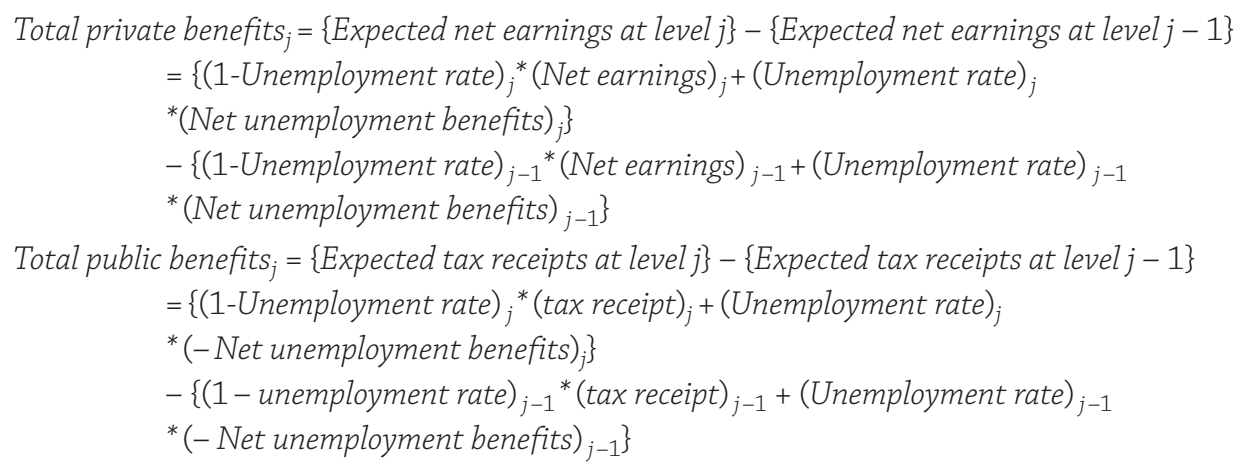

Please note that, because of significant differences in methodology, direct costs are not comparable between this edition of Education at a Glance and previous editions. For further details, please refer to Annex 3 (www.oecd.org/ education/education-at-a-glance-19991487.htm).

\section{Decomposition of net earning and tax receipt effects}

The indicator also presents the decomposition of net earnings and tax receipts effects, defined as the difference in income associated with a higher level of attainment. These elements help explain the differences between countries in total benefits, as tax and benefits levels can create a wedge between the added gross earnings associated with a higher level of education and the equivalent net earnings.

Gross earnings effect is the discounted sum of additional gross earnings level associated with a higher level of educational attainment. The data on earnings come from the earnings data collection of the OECD Network on Labour Market, Economic and Social Outcomes of Learning (LSO Network). Earnings are age-, gender- and attainment level-specific.

The income tax effect is the discounted sum of added level of income tax paid by the individual and received by the government for an additional level of education. Income tax data are computed using the OECD Taxing Wages model. The Taxing Wages model determines the level of taxes due on a given level of income. It is assumed that annual income from employment is equal to a given ratio of the average full-time gross wage earnings for an adult for each OECD economy. The Taxing Wages model computes the level of the tax wedge on income for several household composition scenarios. The scenario used for this indicator is of a single worker with no children. For country-specific details on the income tax in the Taxing Wages model, see OECD Taxing Wages 2014 (OECD, 2014).

The social contribution effect is the discounted sum of added level of employee social contributions associated with a higher level of attainment, paid by the individual and received by the state. Employee social contributions are computed using the OECD Taxing Wages model's scenario of a single worker with no children. For country-specific details on employee social contributions in the Taxing Wages model, see OECD Taxing Wages 2015 (OECD, 2015c). 
The social transfers effect is the discounted sum of added level of social transfers associated with a higher level of attainment. Social transfers correspond to the sum of social assistance and housing benefits paid by the government to individuals. Social transfers are computed using the OECD Tax and Benefits model under the assumption of a single worker with no children aged 40. For country-specific details on social transfers in the Tax and Benefits model, see OECD Benefits and Wages country-specific information, available on line (see www.oecd.org/els/soc/ benefits-and-wages-country-specific-information.htm).

\section{Net financial returns}

The net financial return to education is the difference between the costs and benefits of an added level of education and is calculated as follow:

Net financial returns $=$ total benefit+total cost

\section{Methodological caveats}

To allow for better comparability across countries, the model relies on some assumptions and simplifications. A list of the main assumptions and model limitation is available on line in Annex 3 (see www.oecd.org/education/ education-at-a-glance-19991487.htm).

In addition, the data reported are accounting-based values only. The results probably differ from econometric estimates that would use the same data on the micro level (i.e. data from household or individual surveys) rather than a lifetime stream of earnings derived from average earnings.

The approach used here estimates future earnings for adults with different levels of education, based on knowledge of how average present gross earnings vary by level of attainment and age. However, the relationship between different levels of educational attainment and earnings may differ in the future, as technological, economic and social changes may all alter how wage levels relate to education levels.

In estimating benefits, the effect of education on the likelihood of finding employment when an individual wants to work is taken into account. However, this also makes the estimate sensitive to the stage in the economic cycle at which the data are collected. As more highly educated adults typically have a stronger attachment to the labour market, the value of education generally increases in times of slow economic growth.

Given these factors, the returns on education in different countries should be interpreted with caution.

For further information on methodology, see OECD, 2011, and Annex 3 at www.oecd.org/education/education-ata-glance-19991487.htm.

\section{Note regarding data from Israel}

The statistical data for Israel are supplied by and are under the responsibility of the relevant Israeli authorities. The use of such data by the OECD is without prejudice to the status of the Golan Heights, East Jerusalem and Israeli settlements in the West Bank under the terms of international law.

\section{References}

Andrews, D., A. Caldera Sánchez and Å. Johansson (2011), "Housing Markets and Structural Policies in OECD Countries", OECD Economics Department Working Papers, No. 836, OECD Publishing, Paris, http://dx.doi.org/10.1787/5kgk8t2k9vf3-en.

Brys, B. and C. Torres (2013), "Effective Personal Tax Rates on Marginal Skills Investments in OECD Countries: A New Methodology", OECD Taxation Working Papers, No. 16, OECD Publishing, Paris, http://dx.doi.org/10.1787/5k425747xbr6-en.

OECD (2015a), "Exchange rates (USD monthly averages)", Monthly Monetary and Financial Statistics (MEI) (database), http://stats. oecd.org/Index.aspx?QueryId=169.

OECD (2015b), “Consumer prices: Annual inflation”, Monthly Monetary and Financial Statistics (MEI) (database), http://stats. oecd.org/Index.aspx?QueryId=169.

OECD (2015c), Taxing Wages 2015, OECD Publishing, Paris, http://dx.doi.org/10.1787/tax wages-2015-en.

OECD (2014), Taxing Wages 2014, OECD Publishing, Paris, http://dx.doi.org/10.1787/tax wages-2014-en.

OECD (2013), Education at a Glance 2013: OECD Indicators, OECD Publishing, Paris, http://dx.doi.org/10.1787/eag-2013-en.

OECD (2011), "A User's Guide to Indicator A9 - Incentives to Invest in Education", in Education at a Glance 2011: OECD Indicators, OECD Publishing, Paris, http://dx.doi.org/10.1787/eag-2011-en. 


\section{A7 Indicator A7 Tables}

StatLink त्माज्ञा http://dx.doi.org/10.1787/888933285095

Table A7.1a Private costs and benefits for a man attaining upper secondary or post-secondary non-tertiary education (2011)

Table A7.1b Private costs and benefits for a woman attaining upper secondary or post-secondary non-tertiary education (2011)

Table A7.2a Public costs and benefits for a man attaining upper secondary or post-secondary non-tertiary education (2011)

Table A7.2b Public costs and benefits for a woman attaining upper secondary or post-secondary non-tertiary education (2011)

Table A7.3a Private costs and benefits for a man attaining tertiary education (2011)

Table A7.3b Private costs and benefits for a woman attaining tertiary education (2011)

Table A7.4a Public costs and benefits for a man attaining tertiary education (2011)

Table A7.4b Public costs and benefits for a woman attaining tertiary education (2011) 
Table A7.1a. Private costs and benefits for a man attaining upper secondary or post-secondary non-tertiary education (2011)

As compared with a man with below upper secondary education, in equivalent USD converted using PPPs for GDP

\begin{tabular}{|c|c|c|c|c|c|c|c|c|c|c|}
\hline & \multirow[b]{2}{*}{ Direct costs } & \multirow[b]{2}{*}{$\begin{array}{l}\text { Foregone } \\
\text { earnings }\end{array}$} & \multirow[b]{2}{*}{ Total costs } & \multicolumn{4}{|c|}{ Earnings benefits decomposition } & \multirow[b]{2}{*}{$\begin{array}{c}\text { Total } \\
\text { benefits }\end{array}$} & \multirow[b]{2}{*}{$\begin{array}{c}\text { Net } \\
\text { financial } \\
\text { returns }\end{array}$} & \multirow[b]{2}{*}{$\begin{array}{l}\text { Internal } \\
\text { rate } \\
\text { of return }\end{array}$} \\
\hline & & & & $\begin{array}{c}\text { Gross } \\
\text { earnings } \\
\text { benefits }\end{array}$ & $\begin{array}{c}\text { Income tax } \\
\text { effect }\end{array}$ & $\begin{array}{c}\text { Social } \\
\text { contribution } \\
\text { effect }\end{array}$ & $\begin{array}{c}\text { Transfers } \\
\text { effect }\end{array}$ & & & \\
\hline & (1) & (2) & $(3)=(1)+(2)$ & (4) & (5) & (6) & (7) & (8) & $(9)=(8)+(3)$ & $(10)$ \\
\hline Australia $^{2}$ & -4600 & -27700 & -32300 & 213400 & -70300 & 0 & -2600 & 155100 & 122800 & $21.4 \%$ \\
\hline Austria & -1600 & -48800 & -50500 & 430400 & -113500 & -81100 & -1900 & 238300 & 187800 & $12.5 \%$ \\
\hline Belgium $^{3}$ & a & a & $\mathbf{a}$ & a & a & a & a & $\mathbf{a}$ & $\mathbf{a}$ & a \\
\hline Canada ${ }^{4}$ & -1300 & -31100 & -32400 & 220000 & -57600 & -14500 & 0 & 152000 & 119600 & $14.7 \%$ \\
\hline Chile & -3700 & -19000 & -22700 & 188000 & -6400 & -31600 & -1800 & 141500 & 118800 & $15.6 \%$ \\
\hline Czech Republic & -2600 & -17800 & -20400 & 132900 & -26700 & -14600 & -10400 & 97500 & 77100 & $16.7 \%$ \\
\hline Denmark & -200 & -35400 & -35500 & 286800 & -119100 & 0 & -16900 & 143200 & 107700 & $16.3 \%$ \\
\hline Estonia & -200 & -10800 & - 10900 & 96800 & -19800 & -2700 & 0 & 94300 & 83300 & $22.6 \%$ \\
\hline Finland & -300 & -35800 & -36100 & 110600 & -35300 & -8200 & -5500 & 69600 & 33400 & $8.3 \%$ \\
\hline France & $q$ & $q$ & $\mathbf{q}$ & $q$ & $\mathrm{q}$ & $q$ & $q$ & $\mathbf{q}$ & $\mathbf{q}$ & $\mathrm{q}$ \\
\hline Greece & $\mathrm{m}$ & -18300 & $\mathbf{m}$ & 112200 & -8900 & -18200 & 0 & 75400 & $\mathbf{m}$ & $\mathrm{m}$ \\
\hline Hungary & -400 & -17600 & -18100 & 107500 & -22100 & -18800 & 0 & 72400 & 54300 & $15.1 \%$ \\
\hline Iceland & $\mathrm{m}$ & $\mathrm{m}$ & $\mathbf{m}$ & $\mathrm{m}$ & $\mathrm{m}$ & $\mathrm{m}$ & $\mathrm{m}$ & $\mathbf{m}$ & $\mathbf{m}$ & $\mathrm{m}$ \\
\hline Ireland & $\mathrm{m}$ & $\mathrm{m}$ & $\mathbf{m}$ & $\mathrm{m}$ & $\mathrm{m}$ & $\mathrm{m}$ & $\mathrm{m}$ & $\mathbf{m}$ & $\mathbf{m}$ & $\mathrm{m}$ \\
\hline Israel & -2700 & -29600 & -32300 & 204900 & -28900 & -23200 & 0 & 154700 & 122400 & $12.4 \%$ \\
\hline Italy $^{4}$ & -7700 & -34000 & -41700 & 217500 & -69100 & -20600 & 0 & 129800 & 88100 & $7.1 \%$ \\
\hline Japan & $\mathrm{m}$ & $\mathrm{m}$ & $\mathbf{m}$ & $\mathrm{m}$ & & $\mathrm{m}$ & $\mathrm{m}$ & $\mathbf{m}$ & $\mathbf{m}$ & $\mathrm{m}$ \\
\hline Korea & -9100 & -24400 & -33500 & 91400 & -800 & -7400 & -2800 & 76600 & 43100 & $6.7 \%$ \\
\hline Luxembourg $^{4}$ & -2100 & -53100 & -55200 & 414200 & -117100 & -51200 & -2800 & 240800 & 185500 & $13.5 \%$ \\
\hline Mexico & $\mathrm{m}$ & $\mathrm{m}$ & $\mathbf{m}$ & $\mathrm{m}$ & $\mathrm{m}$ & $\mathrm{m}$ & & $\mathbf{m}$ & $\mathbf{m}$ & $\mathrm{m}$ \\
\hline Netherlands ${ }^{4}$ & -4900 & -52100 & -57000 & 205900 & -71400 & -13000 & 0 & 125200 & 68200 & $5.7 \%$ \\
\hline New Zealand & -5900 & -37100 & -43000 & 200000 & -55500 & 0 & -1600 & 155400 & 112400 & $11.4 \%$ \\
\hline Norway & $\mathrm{m}$ & -46700 & $\mathbf{m}$ & 337000 & -96800 & -26300 & -300 & 218000 & $\mathbf{m}$ & $\mathrm{m}$ \\
\hline Slovak Republic & -3800 & -15600 & -19400 & 149000 & -24500 & -20000 & 0 & 133700 & 114300 & $27.6 \%$ \\
\hline Slovenia & -2600 & -24200 & -26700 & 154200 & -29000 & -34100 & 0 & 91800 & 65100 & $11.4 \%$ \\
\hline Spain & -1600 & -14500 & -16100 & 136500 & -34000 & -8700 & 0 & 96500 & 80300 & $14.5 \%$ \\
\hline Sweden & 0 & -24200 & -24200 & 242700 & -54400 & -17000 & -13300 & 156400 & 132200 & $24.3 \%$ \\
\hline Switzerland & $\mathrm{m}$ & $\mathrm{m}$ & $\mathbf{m}$ & $\mathrm{m}$ & $\mathrm{m}$ & $\mathrm{m}$ & $\mathrm{m}$ & $\mathbf{m}$ & $\mathbf{m}$ & $\mathrm{m}$ \\
\hline Turkey & $\mathrm{m}$ & $\mathrm{m}$ & $\mathbf{m}$ & $\mathrm{m}$ & $\mathrm{m}$ & $\mathrm{m}$ & $\mathrm{m}$ & $\mathbf{m}$ & $\mathbf{m}$ & $\mathrm{m}$ \\
\hline United Kingdom & -3700 & -24000 & -27700 & 342400 & -70400 & -41100 & -23100 & 216700 & 189000 & $18.2 \%$ \\
\hline United States & -3700 & -26300 & -30000 & 402700 & -92700 & -22800 & -6200 & 270600 & 240600 & $23.5 \%$ \\
\hline OECD average & -2800 & -28600 & -31100 & 210300 & -51300 & -21000 & -3800 & 138900 & 107100 & $14.7 \%$ \\
\hline EU21 average & -2200 & -27900 & -30600 & 200600 & -51300 & -23400 & -4700 & 127100 & 99500 & $14.5 \%$ \\
\hline
\end{tabular}

Notes: Values are based on the difference between men who attained an upper secondary or post-secondary non-tertiary education compared with those who have not attained that level of education. Values have been rounded up to the nearest hundred.

1. Total benefit is a weighted sum of gross earnings benefits (4), income tax effect (5), social contribution effect (6) and transfer effects (7), taking into account the probability of employment and the unemployment benefits in case of unemployment. For further details, please refer to the Methodology section.

2. Australia: Year of reference 2009.

3. Belgium: Data are not included in the table because upper secondary education is compulsory.

4. Canada, Italy, Luxembourg, the Netherlands, Poland: Year of reference 2010.

Source: OECD. See Annex 3 for notes (www.oecd.org/education/education-at-a-glance-19991487.htm).

Please refer to the Reader's Guide for information concerning symbols for missing data and abbreviations.

StatLink 젝ㄴ http://dx.doi.org/10.1787/888933285101 
Table A7.1b. Private costs and benefits for a woman attaining upper secondary or post-secondary non-tertiary education (2011)

As compared with a woman with below upper secondary education, in equivalent USD converted using PPPs for GDP

\begin{tabular}{|c|c|c|c|c|c|c|c|c|c|c|}
\hline & \multirow[b]{2}{*}{ Direct costs } & \multirow[b]{2}{*}{$\begin{array}{l}\text { Foregone } \\
\text { earnings }\end{array}$} & \multirow[b]{2}{*}{ Total costs } & \multicolumn{4}{|c|}{ Earnings benefits decomposition } & \multirow[b]{2}{*}{$\begin{array}{c}\text { Total } \\
\text { benefits }\end{array}$} & \multirow[b]{2}{*}{$\begin{array}{c}\text { Net } \\
\text { financial } \\
\text { returns }\end{array}$} & \multirow[b]{2}{*}{$\begin{array}{c}\text { Internal } \\
\text { rate } \\
\text { of return }\end{array}$} \\
\hline & & & & $\begin{array}{l}\text { Gross } \\
\text { earnings } \\
\text { benefits }\end{array}$ & $\begin{array}{l}\text { Income tax } \\
\text { effect }\end{array}$ & $\begin{array}{c}\text { Social } \\
\text { contribution } \\
\text { effect }\end{array}$ & $\begin{array}{c}\text { Transfers } \\
\text { effect }\end{array}$ & & & \\
\hline & (1) & (2) & $(3)=(1)+(2)$ & (4) & (5) & (6) & (7) & (8) & $(9)=(8)+(3)$ & (10) \\
\hline \multirow{2}{*}{$\begin{array}{l}\text { Australia }{ }^{2} \\
\text { ơ Austria }\end{array}$} & -4600 & -28800 & $-\mathbf{3 3} 300$ & 141000 & -31600 & 0 & -25000 & 86700 & 53400 & $10.5 \%$ \\
\hline & -1600 & -47000 & -48600 & 254800 & -42500 & -51000 & -26500 & 132300 & 83600 & $8.2 \%$ \\
\hline Belgium $^{3}$ & a & a & $\mathbf{a}$ & a & a & a & a & $\mathbf{a}$ & $\mathbf{a}$ & a \\
\hline Canada $^{4}$ & -1300 & -33100 & -34400 & 117000 & -22700 & -9400 & 0 & 90400 & 55900 & $7.6 \%$ \\
\hline Chile & -3700 & -14400 & -18100 & 115000 & -2000 & -23200 & -1400 & 88200 & 70200 & $\mathrm{~m}$ \\
\hline Czech Republic & -2600 & -19600 & -22200 & 115400 & -23200 & -12700 & -23200 & 68100 & 45800 & $11.7 \%$ \\
\hline Denmark & -200 & -36400 & -36600 & 200500 & -81800 & 0 & 0 & 110100 & 73500 & $12.4 \%$ \\
\hline Estonia & -200 & -11400 & -11600 & 59200 & -12100 & -1700 & 0 & 52800 & 41200 & $21.1 \%$ \\
\hline Finland & -300 & -36900 & -37200 & 83800 & -18600 & -6300 & -21600 & 44100 & 6900 & $3.9 \%$ \\
\hline France & $q$ & $q$ & $\mathbf{q}$ & $q$ & $q$ & $\mathrm{q}$ & $\mathrm{q}$ & $\mathbf{q}$ & $\mathbf{q}$ & $\mathrm{q}$ \\
\hline Greece & $\mathrm{m}$ & -12900 & $\mathbf{m}$ & 82500 & 0 & -13400 & 0 & 49100 & & $\mathrm{~m}$ \\
\hline Hungary & -400 & -18300 & -18800 & 102700 & -20900 & -18000 & 0 & 71400 & 52600 & $13.2 \%$ \\
\hline Iceland & $\mathrm{m}$ & $\mathrm{m}$ & $\mathbf{m}$ & $\mathrm{m}$ & $\mathrm{m}$ & $\mathrm{m}$ & $\mathrm{m}$ & $\mathbf{m}$ & $\mathbf{m}$ & $\mathrm{m}$ \\
\hline Ireland & $\mathrm{m}$ & $\mathrm{m}$ & $\mathbf{m}$ & $\mathrm{m}$ & $\mathrm{m}$ & $\mathrm{m}$ & $\mathrm{m}$ & $\mathbf{m}$ & $\mathbf{m}$ & $\mathrm{m}$ \\
\hline Israel & -2700 & -28900 & -31700 & 131600 & -5600 & -7100 & 0 & 118700 & 87100 & $9.7 \%$ \\
\hline Italy ${ }^{4}$ & -7700 & -31200 & -38900 & 212200 & -62300 & -20100 & 0 & 131800 & 93000 & $9.0 \%$ \\
\hline Japan & $\mathrm{m}$ & $\mathrm{m}$ & $\mathbf{m}$ & $\mathrm{m}$ & $\mathrm{m}$ & $\mathrm{m}$ & $\mathrm{m}$ & $\mathbf{m}$ & $\mathbf{m}$ & $\mathrm{m}$ \\
\hline Korea & -9100 & -23900 & -33000 & 45300 & 0 & -3600 & -17800 & 22200 & -10800 & $0.6 \%$ \\
\hline Luxembourg $^{4}$ & -2100 & -62000 & -64100 & 358300 & -60100 & -44300 & -59200 & 190000 & 125800 & $6.9 \%$ \\
\hline Mexico & $\mathrm{m}$ & $\mathrm{m}$ & $\mathbf{m}$ & $\mathrm{m}$ & $\mathrm{m}$ & $\mathrm{m}$ & $\mathrm{m}$ & $\mathbf{m}$ & $\mathbf{m}$ & $\mathrm{m}$ \\
\hline Netherlands 4 & -4900 & -51900 & -56800 & 213000 & -48400 & -41600 & -8000 & 116200 & 59400 & $5.2 \%$ \\
\hline New Zealand & -5900 & -36300 & -42200 & 80000 & -13400 & 0 & -7200 & 67300 & 25100 & $5.2 \%$ \\
\hline Norway & $\mathrm{m}$ & -46500 & $\mathbf{m}$ & 206500 & -53700 & -16100 & -12300 & 125000 & $\mathbf{m}$ & $\mathrm{m}$ \\
\hline Slovak Republic & -3800 & -8500 & -12200 & 101100 & -16000 & -13500 & 0 & 91700 & 79500 & $31.4 \%$ \\
\hline Slovenia & -2600 & -23400 & - 25900 & 148500 & -35200 & -32800 & 0 & 83000 & 57100 & $9.1 \%$ \\
\hline Spain & -1600 & -15100 & - 16700 & 129800 & -33700 & -8200 & 0 & 94700 & 78000 & $11.3 \%$ \\
\hline Sweden & 0 & -26000 & -26000 & 198200 & -41200 & -13800 & -35500 & 107000 & 81000 & $12.3 \%$ \\
\hline Switzerland & $\mathrm{m}$ & $\mathrm{m}$ & $\mathbf{m}$ & $\mathrm{m}$ & $\mathrm{m}$ & $\mathrm{m}$ & $\mathrm{m}$ & $\mathbf{m}$ & $\mathbf{m}$ & $\mathrm{m}$ \\
\hline Turkey & $\mathrm{m}$ & $\mathrm{m}$ & $\mathbf{m}$ & $\mathrm{m}$ & $\mathrm{m}$ & $\mathrm{m}$ & $\mathrm{m}$ & $\mathbf{m}$ & $\mathbf{m}$ & $\mathrm{m}$ \\
\hline United Kingdom & -3700 & -27100 & -30800 & 147500 & -79900 & -17700 & -32300 & 35500 & 4700 & $3.8 \%$ \\
\hline United States & -3700 & -27100 & -30800 & 296200 & -61900 & -16700 & -17900 & 196200 & 165300 & $17.4 \%$ \\
\hline OECD average & -2800 & -28500 & -31200 & 151800 & -32200 & -16800 & -12400 & 92800 & 62000 & $10.3 \%$ \\
\hline EU21 average & -2200 & -27900 & -30900 & 156400 & -36000 & -20100 & -13400 & 89800 & 61300 & $11.0 \%$ \\
\hline
\end{tabular}

Notes: Values are based on the difference between women who attained an upper secondary or post-secondary non-tertiary education compared with those who have not attained that level of education. Values have been rounded up to the nearest hundred.

1. Total benefit is a weighted sum of gross earnings benefits (4), income tax effect (5), social contribution effect (6) and transfer effects (7), taking into account the probability of employment and the unemployment benefits in case of unemployment. For further details, please refer to the Methodology section.

2. Australia: Year of reference 2009.

3. Belgium: Data are not included in the table because upper secondary education is compulsory.

4. Canada, Italy, Luxembourg, the Netherlands, Poland: Year of reference 2010.

Source: OECD. See Annex 3 for notes (www.oecd.org/education/education-at-a-glance-19991487.htm).

Please refer to the Reader's Guide for information concerning symbols for missing data and abbreviations.

StatLink 젬ㄴ http://dx.doi.org/10.1787/888933285119 
Table A7.2a. Public costs and benefits for a man attaining upper secondary or post-secondary non-tertiary education (2011)

As compared with a man with below upper secondary education, in equivalent USD converted using PPPs for GDP

\begin{tabular}{|c|c|c|c|c|c|c|c|c|c|}
\hline & \multirow[b]{2}{*}{ Direct costs } & \multirow[b]{2}{*}{$\begin{array}{l}\text { Foregone } \\
\text { taxes on } \\
\text { earnings }\end{array}$} & \multirow[b]{2}{*}{ Total costs } & \multicolumn{3}{|c|}{ Earnings benefits decomposition } & \multirow[b]{2}{*}{$\begin{array}{c}\text { Total } \\
\text { benefits }{ }^{1}\end{array}$} & \multirow[b]{2}{*}{$\begin{array}{l}\text { Net } \\
\text { financial } \\
\text { returns }\end{array}$} & \multirow[b]{2}{*}{$\begin{array}{c}\text { Internal } \\
\text { rate } \\
\text { of return }\end{array}$} \\
\hline & & & & $\begin{array}{c}\text { Income tax } \\
\text { effect }\end{array}$ & $\begin{array}{c}\text { Social } \\
\text { contribution } \\
\text { effect }\end{array}$ & $\begin{array}{c}\text { Transfers } \\
\text { effect }\end{array}$ & & & \\
\hline & (1) & (2) & $(3)=(1)+(2)$ & (4) & (5) & (6) & (7) & $(8)=(7)+(3)$ & (9) \\
\hline Australia $^{2}$ & -18000 & -3100 & -21100 & 70300 & 0 & 2600 & 91900 & 70800 & $20.0 \%$ \\
\hline Austria & -51200 & -8600 & -59800 & 113500 & 81100 & 1900 & 211900 & 152100 & $9.6 \%$ \\
\hline Belgium $^{3}$ & a & a & $\mathbf{a}$ & a & a & a & $\mathbf{a}$ & $\mathbf{a}$ & a \\
\hline Canada ${ }^{4}$ & -29800 & -3000 & -32800 & 57600 & 14500 & 0 & 92400 & 59600 & $9.0 \%$ \\
\hline Chile & -12800 & -100 & -12800 & 6400 & 31600 & 1800 & 37800 & 25000 & $8.8 \%$ \\
\hline Czech Republic & -21200 & 3400 & -17800 & 26700 & 14600 & 10400 & 122300 & 104500 & $24.6 \%$ \\
\hline Denmark & -41300 & -13500 & -54800 & 119100 & 0 & 16900 & 151400 & 96600 & $9.6 \%$ \\
\hline Estonia & -20100 & -1600 & -21700 & 19800 & 2700 & 0 & 56000 & 34300 & $8.7 \%$ \\
\hline Finland & -26200 & 3600 & -22600 & 35300 & 8200 & 5500 & 80700 & 58100 & $18.4 \%$ \\
\hline France & $\mathrm{q}$ & $\mathrm{q}$ & $\mathbf{q}$ & $q$ & $q$ & $q$ & $\mathbf{q}$ & $\mathbf{q}$ & $q$ \\
\hline Germany & -31200 & -7400 & -38600 & 38500 & 31800 & 10800 & 136600 & 98000 & $15.0 \%$ \\
\hline Greece & $\mathrm{m}$ & -4400 & $\mathbf{m}$ & 8900 & 18200 & 0 & 30800 & $\mathbf{m}$ & $\mathrm{m}$ \\
\hline Hungary & -8600 & 1100 & -7400 & 22100 & 18800 & 0 & 72600 & 65200 & $27.9 \%$ \\
\hline Iceland & $\mathrm{m}$ & $\mathrm{m}$ & $\mathbf{m}$ & $\mathrm{m}$ & $\mathrm{m}$ & $\mathrm{m}$ & $\mathbf{m}$ & $\mathbf{m}$ & $\mathrm{m}$ \\
\hline Ireland & $\mathrm{m}$ & $\mathrm{m}$ & $\mathbf{m}$ & $\mathrm{m}$ & $\mathrm{m}$ & $\mathrm{m}$ & $\mathbf{m}$ & $\mathbf{m}$ & $\mathrm{m}$ \\
\hline Israel & -14600 & 100 & -14500 & 28900 & 23200 & 0 & 54100 & 39500 & $9.2 \%$ \\
\hline Italy ${ }^{4}$ & -31300 & -7000 & -38300 & 69100 & 20600 & 0 & 106200 & 67800 & $6.5 \%$ \\
\hline Japan & $\mathrm{m}$ & $\mathrm{m}$ & $\mathbf{m}$ & $\mathrm{m}$ & $\mathrm{m}$ & $\mathrm{m}$ & $\mathbf{m}$ & $\mathbf{m}$ & $\mathrm{m}$ \\
\hline Korea & -21500 & -10800 & -32300 & 800 & 7400 & 2800 & 11900 & -20500 & $-1.9 \%$ \\
\hline Luxembourg ${ }^{4}$ & -68000 & -4500 & -72500 & 117100 & 51200 & 2800 & 185500 & 112900 & $8.7 \%$ \\
\hline Mexico & $\mathrm{m}$ & $\mathrm{m}$ & $\mathbf{m}$ & $\mathrm{m}$ & $\mathrm{m}$ & $\mathrm{m}$ & $\mathbf{m}$ & $\mathbf{m}$ & $\mathrm{m}$ \\
\hline Netherlands ${ }^{4}$ & -29100 & -2800 & -31900 & 71400 & 13000 & 0 & 106500 & 74600 & $9.6 \%$ \\
\hline New Zealand & -23200 & -1100 & -24300 & 55500 & 0 & 1600 & 73300 & 49000 & $9.7 \%$ \\
\hline Norway & -49200 & -9600 & -58700 & 96800 & 26300 & 300 & 142600 & 83900 & $8.4 \%$ \\
\hline Poland 4 & -17000 & -5900 & -22900 & 6200 & 12500 & 0 & 41900 & 19000 & $6.3 \%$ \\
\hline Portugal & -29300 & -2300 & -31600 & 64000 & 27400 & 0 & 81300 & 49600 & $6.9 \%$ \\
\hline Slovak Republic & -17100 & 3400 & -13700 & 24500 & 20000 & 0 & 98900 & 85100 & $21.8 \%$ \\
\hline Slovenia & -22500 & -7200 & -29600 & 29000 & 34100 & 0 & 88200 & 58600 & $10.5 \%$ \\
\hline Spain & -19200 & 1900 & -17300 & 34000 & 8700 & 0 & 67700 & 50400 & $11.4 \%$ \\
\hline Sweden & -35000 & -4200 & - 39200 & 54400 & 17000 & 13300 & 116900 & 77700 & $19.5 \%$ \\
\hline Switzerland & -41600 & -14000 & -55600 & 52000 & 18900 & 0 & 114200 & 58600 & $7.6 \%$ \\
\hline Turkey & $\mathrm{m}$ & $\mathrm{m}$ & $\mathbf{m}$ & $\mathrm{m}$ & $\mathrm{m}$ & $\mathrm{m}$ & $\mathbf{m}$ & $\mathbf{m}$ & $\mathrm{m}$ \\
\hline United Kingdom & -14500 & 1800 & -12700 & 70400 & 41100 & 23100 & 184900 & 172200 & $53.4 \%$ \\
\hline United States & -34500 & -3800 & -38300 & 92700 & 22800 & 6200 & 123800 & 85500 & $11.8 \%$ \\
\hline OECD average & -28000 & -3700 & -31600 & 51300 & 21000 & 3700 & 99300 & 70300 & $13.5 \%$ \\
\hline EU21 average & -28400 & -3000 & -31300 & 51300 & 23400 & 4700 & 107800 & 81000 & $15.8 \%$ \\
\hline
\end{tabular}

Notes: Values are based on the difference between men who attained an upper secondary or post-secondary non-tertiary education compared with those who have not attained that level of education. Values have been rounded up to the nearest hundred.

1. Total benefit is a weighted sum of gross earnings benefits (4), income tax effect (5), social contribution effect (6) and transfer effects (7), taking into account the probability of employment and the unemployment benefits in case of unemployment. For further details, please refer to the Methodology section.

2. Australia: Year of reference 2009.

3. Belgium: Data are not included in the table because upper secondary education is compulsory.

4. Canada, Italy, Luxembourg, the Netherlands, Poland: Year of reference 2010.

Source: OECD. See Annex 3 for notes (www.oecd.org/education/education-at-a-glance-19991487.htm).

Please refer to the Reader's Guide for information concerning symbols for missing data and abbreviations.

StatLink 젝ㄴ http://dx.doi.org/10.1787/888933285127 
Table A7.2b. Public costs and benefits for a woman attaining upper secondary or post-secondary non-tertiary education (2011)

As compared with a woman with below upper secondary education, in equivalent USD converted using PPPs for GDP

\begin{tabular}{|c|c|c|c|c|c|c|c|c|c|}
\hline & \multirow[b]{2}{*}{ Direct costs } & \multirow[b]{2}{*}{$\begin{array}{l}\text { Foregone } \\
\text { taxes on } \\
\text { earnings }\end{array}$} & \multirow[b]{2}{*}{ Total costs } & \multicolumn{3}{|c|}{ Earnings benefits decomposition } & \multirow[b]{2}{*}{$\begin{array}{c}\text { Total } \\
\text { benefits }\end{array}$} & \multirow[b]{2}{*}{$\begin{array}{c}\text { Net } \\
\text { financial } \\
\text { returns }\end{array}$} & \multirow[b]{2}{*}{$\begin{array}{c}\text { Internal } \\
\text { rate } \\
\text { of return }\end{array}$} \\
\hline & & & & $\begin{array}{c}\text { Income tax } \\
\text { effect }\end{array}$ & $\begin{array}{c}\text { Social } \\
\text { contribution } \\
\text { effect }\end{array}$ & $\begin{array}{c}\text { Transfers } \\
\text { effect }\end{array}$ & & & \\
\hline & $(1)$ & $(2)$ & $(3)=(1)+(2)$ & $(4)$ & $(5)$ & (6) & $(7)$ & $(8)=(7)+(3)$ & (9) \\
\hline Australia $^{2}$ & -18000 & -3200 & -21200 & 31600 & 0 & 25000 & 62800 & 41600 & $22.4 \%$ \\
\hline Austria & -51200 & -8200 & -59500 & 42500 & 51000 & 26500 & 133600 & 74200 & $8.0 \%$ \\
\hline Belgium $^{3}$ & a & a & $\mathbf{a}$ & a & a & a & $\mathbf{a}$ & $\mathbf{a}$ & a \\
\hline Canada $^{4}$ & -29800 & -3200 & -33000 & 22700 & 9400 & 0 & 49200 & 16200 & $4.7 \%$ \\
\hline Chile & -12800 & -100 & -12800 & 2000 & 23200 & 1400 & 25500 & 12700 & $7.2 \%$ \\
\hline Czech Republic & -21200 & 3800 & -17400 & 23200 & 12700 & 23200 & 104500 & 87100 & $20.6 \%$ \\
\hline Denmark & -41300 & -13900 & -55200 & 81800 & 0 & 0 & 107400 & 52100 & $7.7 \%$ \\
\hline Estonia & -20100 & -1700 & -21800 & 12100 & 1700 & 0 & 26000 & 4200 & $4.6 \%$ \\
\hline Finland & -26200 & 3700 & -22500 & 18600 & 6300 & 21600 & 80300 & 57800 & $22.8 \%$ \\
\hline France & $\mathrm{q}$ & $\mathrm{q}$ & $\mathbf{q}$ & $q$ & $q$ & $q$ & $\mathbf{q}$ & $\mathbf{q}$ & $q$ \\
\hline Greece & $\mathrm{m}$ & -3100 & $\mathbf{m}$ & 0 & 13400 & 0 & 13000 & $\mathbf{m}$ & $\mathrm{m}$ \\
\hline Hungary & -8600 & 1200 & -7400 & 20900 & 18000 & 0 & 69600 & 62300 & $25.2 \%$ \\
\hline Iceland & $\mathrm{m}$ & $\mathrm{m}$ & $\mathbf{m}$ & $\mathrm{m}$ & $\mathrm{m}$ & $\mathrm{m}$ & $\mathbf{m}$ & $\mathbf{m}$ & $\mathrm{m}$ \\
\hline Ireland & $\mathrm{m}$ & $\mathrm{m}$ & $\mathbf{m}$ & $\mathrm{m}$ & $\mathrm{m}$ & $\mathrm{m}$ & $\mathbf{m}$ & $\mathbf{m}$ & $\mathrm{m}$ \\
\hline Israel & -14600 & 100 & - 14500 & 5600 & 7100 & 0 & 10000 & -4600 & $1.1 \%$ \\
\hline Italy ${ }^{4}$ & -31300 & -6500 & -37700 & 62300 & 20100 & 0 & 89500 & 51800 & $6.6 \%$ \\
\hline Japan & $\mathrm{m}$ & $\mathrm{m}$ & $\mathbf{m}$ & $\mathrm{m}$ & $\mathrm{m}$ & $\mathrm{m}$ & $\mathbf{m}$ & $\mathbf{m}$ & $\mathrm{m}$ \\
\hline Korea & -21500 & -10600 & -32100 & 0 & 3600 & 17800 & 21800 & -10300 & $0.5 \%$ \\
\hline Luxembourg $^{4}$ & -68000 & -5300 & -73300 & 60100 & 44300 & 59200 & 163100 & 89800 & $11.5 \%$ \\
\hline Mexico & $\mathrm{m}$ & $\mathrm{m}$ & $\mathbf{m}$ & $\mathrm{m}$ & $\mathrm{m}$ & $\mathrm{m}$ & $\mathbf{m}$ & $\mathbf{m}$ & $\mathrm{m}$ \\
\hline Netherlands 4 & -29100 & -2800 & -31900 & 48400 & 41600 & 8000 & 118100 & 86200 & $12.0 \%$ \\
\hline New Zealand & -23200 & -1100 & -24300 & 13400 & 0 & 7200 & 35700 & 11400 & $5.6 \%$ \\
\hline Norway & -49200 & -9500 & -58700 & 53700 & 16100 & 12300 & 93100 & 34400 & $5.8 \%$ \\
\hline Slovak Republic & -17100 & 1800 & -15200 & 16000 & 13500 & 0 & 59700 & 44500 & $16.0 \%$ \\
\hline Slovenia & -22500 & -6900 & -29400 & 35200 & 32800 & 0 & 78500 & 49100 & $9.6 \%$ \\
\hline Spain & -19200 & 1900 & -17200 & 33700 & 8200 & 0 & 44000 & 26700 & $7.8 \%$ \\
\hline Sweden & -35000 & -4500 & -39500 & 41200 & 13800 & 35500 & 130100 & 90500 & $22.4 \%$ \\
\hline Switzerland & -41600 & -15300 & -56900 & 26700 & 14600 & 10700 & 93700 & 36800 & $7.5 \%$ \\
\hline Turkey & $\mathrm{m}$ & $\mathrm{m}$ & $\mathbf{m}$ & $\mathrm{m}$ & $\mathrm{m}$ & $\mathrm{m}$ & $\mathbf{m}$ & $\mathbf{m}$ & $\mathrm{m}$ \\
\hline United Kingdom & -14500 & 2000 & -12400 & 79900 & 17700 & 32300 & 157000 & 144600 & $37.8 \%$ \\
\hline United States & -34500 & -3900 & -38500 & 61900 & 16700 & 17900 & 99400 & 60900 & $12.0 \%$ \\
\hline OECD average & -28000 & -3700 & -31700 & 32000 & 16700 & 12400 & 77300 & 48000 & $11.8 \%$ \\
\hline EU21 average & -28400 & -3000 & -31300 & 36000 & 20100 & 13400 & 88600 & 61700 & $14.2 \%$ \\
\hline
\end{tabular}

Notes: Values are based on the difference between women who attained an upper secondary or post-secondary non-tertiary education compared with those who have not attained that level of education. Values have been rounded up to the nearest hundred.

1. Total benefit is a weighted sum of gross earnings benefits (4), income tax effect (5), social contribution effect (6) and transfer effects (7), taking into account the probability of employment and the unemployment benefits in case of unemployment. For further details, please refer to the Methodology section.

2. Australia: Year of reference 2009.

3. Belgium: Data are not included in the table because upper secondary education is compulsory.

4. Canada, Italy, Luxembourg, the Netherlands, Poland: Year of reference 2010.

Source: OECD. See Annex 3 for notes (www.oecd.org/education/education-at-a-glance-19991487.htm).

Please refer to the Reader's Guide for information concerning symbols for missing data and abbreviations.

StatLink 젬ㄴ http://dx.doi.org/10.1787/888933285133 
Table A7.3a. Private costs and benefits for a man attaining tertiary education (2011)

As compared with a man attaining upper secondary or post-secondary non-tertiary education, in equivalent USD converted using PPPs for GDP

\begin{tabular}{|c|c|c|c|c|c|c|c|c|c|c|}
\hline & \multirow[b]{2}{*}{ Direct costs } & \multirow[b]{2}{*}{$\begin{array}{l}\text { Foregone } \\
\text { earnings }\end{array}$} & \multirow[b]{2}{*}{ Total costs } & \multicolumn{4}{|c|}{ Earnings benefits decomposition } & \multirow[b]{2}{*}{$\begin{array}{c}\text { Total } \\
\text { benefits }{ }^{1}\end{array}$} & \multirow[b]{2}{*}{$\begin{array}{c}\text { Net } \\
\text { financial } \\
\text { returns }\end{array}$} & \multirow[b]{2}{*}{$\begin{array}{c}\text { Internal } \\
\text { rate } \\
\text { of return }\end{array}$} \\
\hline & & & & $\begin{array}{c}\text { Gross } \\
\text { earnings } \\
\text { benefits }\end{array}$ & $\begin{array}{c}\text { Income tax } \\
\text { effect }\end{array}$ & \begin{tabular}{|c|} 
Social \\
contribution \\
effect
\end{tabular} & $\begin{array}{c}\text { Transfers } \\
\text { effect }\end{array}$ & & & \\
\hline & (1) & (2) & $(3)=(1)+(2)$ & (4) & (5) & (6) & (7) & (8) & $(9)=(8)+(3)$ & (10) \\
\hline Australia $^{2}$ & -27400 & -52200 & -79600 & 483700 & -172400 & 0 & 0 & 302800 & 223200 & $10.4 \%$ \\
\hline Austria & -1900 & -61000 & -62900 & 559500 & -181100 & -73200 & 0 & 306500 & 243600 & $11.0 \%$ \\
\hline Belgium & $\mathrm{m}$ & $\mathrm{m}$ & $\mathbf{m}$ & $\mathrm{m}$ & $\mathrm{m}$ & $\mathrm{m}$ & $\mathrm{m}$ & $\mathbf{m}$ & $\mathbf{m}$ & $\mathrm{m}$ \\
\hline Canada $^{3}$ & -17400 & -36800 & -54200 & 395000 & -121300 & -6400 & 0 & 260600 & 206400 & $12.2 \%$ \\
\hline Chile & -38100 & -33900 & -71900 & 766000 & -70400 & -83600 & -1300 & 587100 & 515100 & $15.9 \%$ \\
\hline Czech Republic & -3300 & -27100 & -30400 & 488800 & -98300 & -53800 & 0 & 331900 & 301500 & $23.5 \%$ \\
\hline Denmark & -4300 & -52400 & -56700 & 421500 & -214700 & 0 & -10800 & 189900 & 133200 & $8.9 \%$ \\
\hline Estonia & -4900 & -20100 & -25000 & 220400 & -45000 & -6200 & 0 & 172200 & 147200 & $20.3 \%$ \\
\hline Finland & -3400 & -69200 & -72600 & 466100 & -177700 & -34000 & 0 & 252800 & 180200 & $9.6 \%$ \\
\hline France & $q$ & $\mathrm{q}$ & $\mathbf{q}$ & $\mathrm{q}$ & $\mathrm{q}$ & $q$ & $q$ & $\mathbf{q}$ & $\mathbf{q}$ & $\mathrm{q}$ \\
\hline Greece & $\mathrm{m}$ & -26800 & $\mathbf{m}$ & 234100 & -35900 & -37900 & 0 & 151400 & $\mathbf{m}$ & $\mathrm{m}$ \\
\hline Hungary & -9100 & -22200 & -31300 & 620900 & -156300 & -108700 & 0 & 346900 & 315600 & $25.4 \%$ \\
\hline Iceland & $\mathrm{m}$ & $\mathrm{m}$ & $\mathbf{m}$ & $\mathrm{m}$ & $\mathrm{m}$ & $\mathrm{m}$ & $\mathrm{m}$ & $\mathbf{m}$ & $\mathbf{m}$ & $\mathrm{m}$ \\
\hline Ireland & $\mathrm{m}$ & $\mathrm{m}$ & $\mathbf{m}$ & $\mathrm{m}$ & $\mathrm{m}$ & $\mathrm{m}$ & $\mathrm{m}$ & $\mathbf{m}$ & & $\mathrm{m}$ \\
\hline Israel & -11300 & -31800 & -43100 & 371300 & -82900 & -44600 & 0 & 239300 & 196300 & $13.2 \%$ \\
\hline Italy $^{3}$ & -15800 & -40200 & -56000 & 487500 & -184400 & -48600 & 0 & 248800 & 192800 & $9.5 \%$ \\
\hline Japan & $\mathrm{m}$ & $\mathrm{m}$ & $\mathbf{m}$ & $\mathrm{m}$ & $\mathrm{m}$ & $\mathrm{m}$ & $\mathrm{m}$ & $\mathbf{m}$ & $\mathbf{m}$ & $\mathrm{m}$ \\
\hline Korea & -20300 & -33700 & -54000 & 154200 & -2800 & -12400 & 0 & 137200 & 83200 & $6.2 \%$ \\
\hline Luxembourg $^{3}$ & $\mathrm{~m}$ & -61900 & $\mathbf{m}$ & 946300 & -327000 & -110800 & 0 & 496700 & $\mathbf{m}$ & $\mathrm{m}$ \\
\hline Mexico & $\mathrm{m}$ & $\mathrm{m}$ & $\mathbf{m}$ & $\mathrm{m}$ & $\mathrm{m}$ & $\mathrm{m}$ & $\mathrm{m}$ & $\mathbf{m}$ & $\mathbf{m}$ & $\mathrm{m}$ \\
\hline Netherlands ${ }^{3}$ & -16900 & -95000 & - 111900 & 615300 & -273200 & -1300 & 0 & 336400 & 224500 & $9.5 \%$ \\
\hline New Zealand & -14000 & -54400 & -68400 & 240500 & -73500 & 0 & 0 & 165500 & 97100 & $7.1 \%$ \\
\hline Norway & -2300 & -55900 & -58200 & 419100 & -152700 & -32700 & 0 & 234700 & 176500 & $8.4 \%$ \\
\hline Poland $^{3}$ & -6100 & -18000 & -24100 & 495800 & -43900 & -88400 & 0 & 362200 & 338200 & $29.2 \%$ \\
\hline Slovak Republic & -9100 & -24500 & -33600 & 390700 & -64700 & -49900 & 0 & 280900 & 247300 & $20.6 \%$ \\
\hline Slovenia & -4100 & -33600 & -37700 & 593000 & -155900 & -131100 & 0 & 291900 & 254200 & $17.4 \%$ \\
\hline Spain & -12900 & -45900 & -58800 & 242500 & -61700 & -15400 & 0 & 161500 & 102700 & $9.1 \%$ \\
\hline Sweden & -200 & -51900 & -51900 & 303600 & -117000 & -12400 & 0 & 169600 & 117700 & $8.3 \%$ \\
\hline Switzerland & $\mathrm{m}$ & $\mathrm{m}$ & $\mathbf{m}$ & $\mathrm{m}$ & $\mathrm{m}$ & $\mathrm{m}$ & $\mathrm{m}$ & $\mathbf{m}$ & $\mathbf{m}$ & $\mathrm{m}$ \\
\hline Turkey & $\mathrm{m}$ & $\mathrm{m}$ & $\mathbf{m}$ & $\mathrm{m}$ & $\mathrm{m}$ & $\mathrm{m}$ & $\mathrm{m}$ & $\mathbf{m}$ & $\mathbf{m}$ & $\mathrm{m}$ \\
\hline United Kingdom & -25900 & -40700 & -66600 & 538400 & -121000 & -58100 & -1800 & 353600 & 287000 & $15.7 \%$ \\
\hline United States & -55000 & -46200 & -101300 & 861000 & -261800 & -48600 & 0 & 547600 & 446300 & $15.7 \%$ \\
\hline OECD average & -13200 & -43500 & -56700 & 477400 & -137000 & -46700 & -500 & 288600 & 229000 & $14.0 \%$ \\
\hline EU21 average & -8200 & -43700 & -51800 & 484600 & -145800 & -54700 & -700 & 279400 & 222000 & $15.5 \%$ \\
\hline
\end{tabular}

Notes: Values are based on the difference between men who attained a tertiary education compared with those who have attained an upper secondary or post-secondary non-tertiary education. Values have been rounded up to the nearest hundred.

1. Total benefit is a weighted sum of gross earnings benefits (4), income tax effect (5), social contribution effect (6) and transfer effects (7), taking into account the probability of employment and the unemployment benefits in case of unemployment. For further details, please refer to the Methodology section.

2. Australia: Year of reference 2009.

3. Canada, Italy, Luxembourg, the Netherlands, Poland: Year of reference 2010.

Source: OECD. See Annex 3 for notes (www.oecd.org/education/education-at-a-glance-19991487.htm).

Please refer to the Reader's Guide for information concerning symbols for missing data and abbreviations.

StatLink 部 ST http://dx.doi.org/10.1787/888933285143 
Table A7.3b. Private costs and benefits for a woman attaining tertiary education (2011)

As compared with a woman attaining upper secondary or post-secondary non-tertiary education, in equivalent USD converted using PPPs for GDP

\begin{tabular}{|c|c|c|c|c|c|c|c|c|c|c|}
\hline & \multirow[b]{2}{*}{ Direct costs } & \multirow[b]{2}{*}{$\begin{array}{l}\text { Foregone } \\
\text { earnings }\end{array}$} & \multirow[b]{2}{*}{ Total costs } & \multicolumn{4}{|c|}{ Earnings benefits decomposition } & \multirow[b]{2}{*}{$\begin{array}{c}\text { Total } \\
\text { benefits }\end{array}$} & \multirow[b]{2}{*}{$\begin{array}{c}\text { Net } \\
\text { financial } \\
\text { returns }\end{array}$} & \multirow[b]{2}{*}{$\begin{array}{l}\text { Internal } \\
\text { rate } \\
\text { of return }\end{array}$} \\
\hline & & & & $\begin{array}{c}\text { Gross } \\
\text { earnings } \\
\text { benefits }\end{array}$ & $\begin{array}{c}\text { Income tax } \\
\text { effect }\end{array}$ & $\begin{array}{c}\text { Social } \\
\text { contribution } \\
\text { effect }\end{array}$ & $\begin{array}{c}\text { Transfers } \\
\text { effect }\end{array}$ & & & \\
\hline & (1) & (2) & $(3)=(1)+(2)$ & (4) & (5) & (6) & (7) & (8) & $(9)=(8)+(3)$ & (10) \\
\hline Australia $^{2}$ & -27400 & -53500 & -81000 & 321200 & -112300 & 0 & 0 & 207500 & 126500 & $8.5 \%$ \\
\hline Austria & -1900 & -61000 & -62900 & 432400 & -120400 & -81600 & 0 & 227500 & 164600 & $8.8 \%$ \\
\hline Belgium & $\mathrm{m}$ & $\mathrm{m}$ & $\mathbf{m}$ & $\mathrm{m}$ & $\mathrm{m}$ & $\mathrm{m}$ & $\mathrm{m}$ & $\mathbf{m}$ & $\mathbf{m}$ & $\mathrm{m}$ \\
\hline Canada $^{3}$ & -17400 & -38200 & -55600 & 328800 & -73600 & -25100 & 0 & 227600 & 171900 & $13.5 \%$ \\
\hline Chile & -38100 & -32100 & -70200 & 463000 & -25900 & -75800 & -1300 & 356300 & 286100 & $13.7 \%$ \\
\hline Czech Republic & -3300 & -26600 & -29900 & 282400 & -56800 & -31100 & -3700 & 191700 & 161800 & $16.3 \%$ \\
\hline Denmark & -4300 & -54400 & -58700 & 236600 & -98300 & 0 & -13000 & 120800 & 62100 & $6.9 \%$ \\
\hline Estonia & -4900 & -21000 & -25900 & 133200 & -27200 & -3700 & 0 & 102500 & 76600 & $13.8 \%$ \\
\hline Finland & -3400 & -72100 & -75400 & 290100 & -95500 & -21700 & -2600 & 169800 & 94300 & $7.1 \%$ \\
\hline France & $\mathrm{q}$ & q & q & q & $\mathrm{q}$ & $\mathrm{q}$ & $\mathrm{q}$ & q & q & q \\
\hline Germany & -5200 & -72500 & -77700 & 326000 & -83300 & -68000 & 0 & 175600 & 98000 & $6.4 \%$ \\
\hline Greece & $\mathrm{m}$ & -21900 & $\mathbf{m}$ & 235300 & -16700 & -38100 & 0 & 152900 & $\mathbf{m}$ & $\mathrm{m}$ \\
\hline Hungary & -9100 & -22200 & -31300 & 323200 & -93800 & -56600 & 0 & 171200 & 139800 & $16.2 \%$ \\
\hline Iceland & $\mathrm{m}$ & $\mathrm{m}$ & $\mathbf{m}$ & $\mathrm{m}$ & $\mathrm{m}$ & $\mathrm{m}$ & $\mathrm{m}$ & $\mathbf{m}$ & $\mathbf{m}$ & $\mathrm{m}$ \\
\hline Ireland & $\mathrm{m}$ & $\mathrm{m}$ & $\mathbf{m}$ & $\mathrm{m}$ & $\mathrm{m}$ & $\mathrm{m}$ & $\mathrm{m}$ & $\mathbf{m}$ & $\mathbf{m}$ & $\mathrm{m}$ \\
\hline Israel & -11300 & -31600 & -42900 & 225300 & -31700 & -25800 & 0 & 168400 & 125600 & $11.0 \%$ \\
\hline Italy $^{3}$ & -15800 & -38900 & -54700 & 316800 & -102900 & -30100 & 0 & 179300 & 124600 & $9.5 \%$ \\
\hline Japan & $\mathrm{m}$ & $\mathrm{m}$ & $\mathbf{m}$ & $\mathrm{m}$ & $\mathrm{m}$ & $\mathrm{m}$ & $\mathrm{m}$ & $\mathbf{m}$ & $\mathbf{m}$ & $\mathrm{m}$ \\
\hline Korea & -20300 & -35000 & -55400 & 131600 & -900 & -10600 & 0 & 117000 & 61700 & $5.5 \%$ \\
\hline Luxembourg ${ }^{3}$ & $\mathrm{~m}$ & -65200 & $\mathbf{m}$ & 721500 & -223400 & -89100 & 0 & 407200 & $\mathbf{m}$ & $\mathrm{m}$ \\
\hline Mexico & $\mathrm{m}$ & $\mathrm{m}$ & $\mathbf{m}$ & $\mathrm{m}$ & $\mathrm{m}$ & $\mathrm{m}$ & $\mathrm{m}$ & $\mathbf{m}$ & $\mathbf{m}$ & $\mathrm{m}$ \\
\hline Netherlands ${ }^{3}$ & -16900 & -95300 & -112200 & 479300 & -189100 & -6900 & 0 & 281600 & 169400 & $8.6 \%$ \\
\hline New Zealand & -14000 & -55100 & -69100 & 206300 & -44100 & 0 & -3300 & 156900 & 87800 & $8.1 \%$ \\
\hline Norway & -2300 & -57600 & -59900 & 304100 & -85100 & -23700 & 0 & 196300 & 136400 & $8.9 \%$ \\
\hline Poland $^{3}$ & -6100 & -17000 & -23100 & 316400 & -28000 & -56400 & 0 & 233800 & 210700 & $24.0 \%$ \\
\hline Portugal & -8600 & -22500 & -31100 & 413600 & -119700 & -45500 & 0 & 248300 & 217200 & $20.5 \%$ \\
\hline Slovak Republic & -9100 & -24400 & -33500 & 233600 & -38400 & -31300 & 0 & 168700 & 135200 & $14.8 \%$ \\
\hline Slovenia & -4100 & -32800 & -36900 & 463800 & -110500 & -102500 & 0 & 246700 & 209800 & $16.1 \%$ \\
\hline Spain & -12900 & -46400 & -59300 & 284200 & -73100 & -18000 & 0 & 190600 & 131200 & $10.5 \%$ \\
\hline Sweden & -200 & -52100 & -52100 & 190400 & -43300 & -13300 & 0 & 132900 & 80800 & $7.3 \%$ \\
\hline Switzerland & $\mathrm{m}$ & $\mathrm{m}$ & $\mathbf{m}$ & $\mathrm{m}$ & $\mathrm{m}$ & $\mathrm{m}$ & $\mathrm{m}$ & $\mathbf{m}$ & m & $\mathrm{m}$ \\
\hline Turkey & $\mathrm{m}$ & $\mathrm{m}$ & $\mathbf{m}$ & $\mathrm{m}$ & $\mathrm{m}$ & $\mathrm{m}$ & $\mathrm{m}$ & $\mathbf{m}$ & $\mathbf{m}$ & $\mathrm{m}$ \\
\hline United Kingdom & -25900 & -43100 & -69000 & 422200 & -93000 & -50700 & -80300 & 195600 & 126600 & $8.7 \%$ \\
\hline United States & -55000 & -49200 & -104200 & 566600 & -139100 & -32000 & 0 & 390200 & 286000 & $12.2 \%$ \\
\hline OECD average & -13200 & -43900 & -57200 & 332600 & -81800 & -36100 & -4000 & 208300 & 145200 & $11.5 \%$ \\
\hline EU21 average & -8200 & -43900 & -52100 & 338900 & -89600 & -41400 & -5500 & 199800 & 137700 & $12.2 \%$ \\
\hline
\end{tabular}

Notes: Values are based on the difference between women who attained a tertiary education compared with those who have attained an upper secondary or postsecondary non-tertiary education. Values have been rounded up to the nearest hundred.

1. Total benefit is a weighted sum of gross earnings benefits (4), income tax effect (5), social contribution effect (6) and transfer effects (7), taking into account the probability of employment and the unemployment benefits in case of unemployment. For further details, please refer to the Methodology section.

2. Australia: Year of reference 2009.

3. Canada, Italy, Luxembourg, the Netherlands, Poland: Year of reference 2010.

Source: OECD. See Annex 3 for notes (www.oecd.org/education/education-at-a-glance-19991487.htm).

Please refer to the Reader's Guide for information concerning symbols for missing data and abbreviations.

StatLink त्ताजा http://dx.doi.org/10.1787/888933285155 
Table A7.4a. Public costs and benefits for a man attaining tertiary education (2011)

As compared with a man attaining upper secondary or post-secondary non-tertiary education, in equivalent USD converted using PPPs for GDP

\begin{tabular}{|c|c|c|c|c|c|c|c|c|c|}
\hline & \multirow[b]{2}{*}{ Direct costs } & \multirow[b]{2}{*}{$\begin{array}{c}\text { Foregone } \\
\text { taxes on } \\
\text { earnings }\end{array}$} & \multirow[b]{2}{*}{ Total costs } & \multicolumn{3}{|c|}{ Earnings benefits decomposition } & \multirow[b]{2}{*}{$\begin{array}{c}\text { Total } \\
\text { benefits }\end{array}$} & \multirow[b]{2}{*}{$\begin{array}{c}\text { Net } \\
\text { financial } \\
\text { returns }\end{array}$} & \multirow[b]{2}{*}{$\begin{array}{l}\text { Internal } \\
\text { rate } \\
\text { of return }\end{array}$} \\
\hline & & & & $\begin{array}{c}\text { Income tax } \\
\text { effect }\end{array}$ & $\begin{array}{c}\text { Social } \\
\text { contribution } \\
\text { effect }\end{array}$ & $\begin{array}{c}\text { Transfers } \\
\text { effect }\end{array}$ & & & \\
\hline & (1) & (2) & $(3)=(1)+(2)$ & (4) & (5) & (6) & (7) & $(8)=(7)+(3)$ & (9) \\
\hline Australia $^{2}$ & -31400 & -5800 & -37200 & 172400 & 0 & 0 & 168800 & 131500 & $11.6 \%$ \\
\hline Austria & -74100 & -10700 & -84800 & 181100 & 73200 & 0 & 260100 & 175300 & $8.0 \%$ \\
\hline Belgium & $\mathrm{m}$ & $\mathrm{m}$ & $\mathbf{m}$ & $\mathrm{m}$ & $\mathrm{m}$ & $\mathrm{m}$ & $\mathbf{m}$ & $\mathbf{m}$ & $\mathrm{m}$ \\
\hline Canada $^{3}$ & -44900 & -3600 & -48400 & 121300 & 6400 & 0 & 136100 & 87700 & $8.0 \%$ \\
\hline Chile & -18100 & -100 & -18200 & 70400 & 83600 & 1300 & 149200 & 131000 & $16.4 \%$ \\
\hline Czech Republic & -27600 & 5200 & -22300 & 98300 & 53800 & 0 & 156600 & 134200 & $17.0 \%$ \\
\hline Denmark & -98400 & -20000 & -118400 & 214700 & 0 & 10800 & 226200 & 107800 & $5.5 \%$ \\
\hline Estonia & -26600 & -3000 & -29600 & 45000 & 6200 & 0 & 56100 & 26500 & $7.5 \%$ \\
\hline Finland & -91300 & 6900 & -84400 & 177700 & 34000 & 0 & 217300 & 133000 & $7.5 \%$ \\
\hline France & $\mathrm{q}$ & $\mathrm{q}$ & $\mathbf{q}$ & $\mathrm{q}$ & $q$ & q & $\mathbf{q}$ & q & $q$ \\
\hline Germany & -87500 & -13400 & -100900 & 189800 & 97700 & 0 & 306500 & 205600 & $8.7 \%$ \\
\hline Greece & $\mathrm{m}$ & -6400 & $\mathbf{m}$ & 35900 & 37900 & 0 & 76300 & $\mathbf{m}$ & $\mathrm{m}$ \\
\hline Hungary & -29600 & 1400 & -28200 & 156300 & 108700 & 0 & 271200 & 243000 & $24.1 \%$ \\
\hline Iceland & $\mathrm{m}$ & $\mathrm{m}$ & $\mathbf{m}$ & $\mathrm{m}$ & $\mathrm{m}$ & & $\mathbf{m}$ & $\mathbf{m}$ & $\mathrm{m}$ \\
\hline Ireland & $\mathrm{m}$ & $\mathrm{m}$ & $\mathbf{m}$ & $\mathrm{m}$ & $\mathrm{m}$ & $\mathrm{m}$ & $\mathbf{m}$ & $\mathbf{m}$ & $\mathrm{m}$ \\
\hline Israel & -19900 & 100 & -19800 & 82900 & 44600 & 0 & 121300 & 101500 & $11.6 \%$ \\
\hline Italy ${ }^{3}$ & -35900 & -8300 & -44200 & 184400 & 48600 & 0 & 226900 & 182700 & $9.4 \%$ \\
\hline Japan & $\mathrm{m}$ & $\mathrm{m}$ & $\mathbf{m}$ & $\mathrm{m}$ & $\mathrm{m}$ & $\mathrm{m}$ & $\mathbf{m}$ & m & $\mathrm{m}$ \\
\hline Korea & -13100 & -14900 & -27900 & 2800 & 12400 & 0 & 17200 & -10800 & $0.5 \%$ \\
\hline Luxembourg $^{3}$ & $\mathrm{~m}$ & -5200 & $\mathbf{m}$ & 327000 & 110800 & 0 & 408000 & $\mathbf{m}$ & $\mathrm{m}$ \\
\hline Mexico & $\mathrm{m}$ & $\mathrm{m}$ & $\mathbf{m}$ & $\mathrm{m}$ & & & $\mathbf{m}$ & $\mathbf{m}$ & $\mathrm{m}$ \\
\hline Netherlands ${ }^{3}$ & -73000 & -5100 & -78100 & 273200 & 1300 & 0 & 272600 & 194600 & $\mathrm{~m}$ \\
\hline New Zealand & -32600 & -1700 & -34300 & 73500 & 0 & 0 & 74300 & 40000 & $6.3 \%$ \\
\hline Norway & -75300 & -11400 & -86800 & 152700 & 32700 & 0 & 192600 & 105800 & $5.5 \%$ \\
\hline Poland $^{3}$ & -19100 & -6000 & -25000 & 43900 & 88400 & 0 & 143100 & 118100 & $15.1 \%$ \\
\hline Portugal & -31400 & -3000 & -34300 & 177300 & 57400 & 0 & 211800 & 177500 & $11.5 \%$ \\
\hline Slovak Republic & -28100 & 5300 & -22800 & 64700 & 49900 & 0 & 123100 & 100400 & $14.6 \%$ \\
\hline Slovenia & -34900 & -9900 & -44900 & 155900 & 131100 & 0 & 284300 & 239400 & $14.0 \%$ \\
\hline Spain & -59000 & 5900 & -53100 & 61700 & 15400 & 0 & 100700 & 47600 & $6.2 \%$ \\
\hline Sweden & -97200 & -9000 & -106100 & 117000 & 12400 & 0 & 128800 & 22700 & $3.1 \%$ \\
\hline Switzerland & -91300 & -18500 & -109700 & 125200 & 36200 & 0 & 161900 & 52200 & $4.0 \%$ \\
\hline Turkey & $\mathrm{m}$ & $\mathrm{m}$ & $\mathbf{m}$ & $\mathrm{m}$ & $\mathrm{m}$ & $\mathrm{m}$ & $\mathbf{m}$ & $\mathbf{m}$ & $\mathrm{m}$ \\
\hline United Kingdom & -27700 & 3100 & -24700 & 121000 & 58100 & 1800 & 191800 & 167100 & $23.4 \%$ \\
\hline United States & -55900 & -6700 & -62600 & 261800 & 48600 & 0 & 334200 & 271700 & $14.5 \%$ \\
\hline OECD average & -49000 & -5000 & -53900 & 136600 & 46300 & 500 & 185800 & 127400 & $10.6 \%$ \\
\hline EU21 average & -52600 & -4000 & -56400 & 145800 & 54700 & 700 & 203400 & 142200 & $11.7 \%$ \\
\hline
\end{tabular}

Notes: Values are based on the difference between men who attained a tertiary education compared with those who have attained an upper secondary or post-secondary non-tertiary education. Values have been rounded up to the nearest hundred.

1. Total benefit is a weighted sum of gross earnings benefits (4), income tax effect (5), social contribution effect (6) and transfer effects (7), taking into account the probability of employment and the unemployment benefits in case of unemployment. For further details, please refer to the Methodology section.

2. Australia: Year of reference 2009.

3. Canada, Italy, Luxembourg, the Netherlands, Poland: Year of reference 2010.

Source: OECD. See Annex 3 for notes (www.oecd.org/education/education-at-a-glance-19991487.htm).

Please refer to the Reader's Guide for information concerning symbols for missing data and abbreviations.

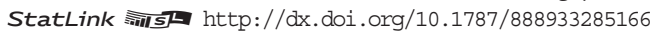


Table A7.4b. Public costs and benefits for a woman attaining tertiary education (2011)

As compared with a woman attaining upper secondary or post-secondary non-tertiary education, in equivalent USD converted using PPPs for GDP

\begin{tabular}{|c|c|c|c|c|c|c|c|c|c|}
\hline & \multirow[b]{2}{*}{ Direct costs } & \multirow[b]{2}{*}{$\begin{array}{l}\text { Foregone } \\
\text { taxes on } \\
\text { earnings }\end{array}$} & \multirow[b]{2}{*}{ Total costs } & \multicolumn{3}{|c|}{ Earnings benefits decomposition } & \multirow[b]{2}{*}{$\begin{array}{c}\text { Total } \\
\text { benefits }{ }^{1}\end{array}$} & \multirow[b]{2}{*}{$\begin{array}{c}\text { Net } \\
\text { financial } \\
\text { returns }\end{array}$} & \multirow[b]{2}{*}{$\begin{array}{l}\text { Internal } \\
\text { rate } \\
\text { of return }\end{array}$} \\
\hline & & & & $\begin{array}{c}\text { Income tax } \\
\text { effect }\end{array}$ & \begin{tabular}{|c|} 
Social \\
contribution \\
effect
\end{tabular} & $\begin{array}{c}\text { Transfers } \\
\text { effect }\end{array}$ & & & \\
\hline & (1) & (2) & $(3)=(1)+(2)$ & (4) & (5) & (6) & (7) & $(8)=(7)+(3)$ & (9) \\
\hline Australia $^{2}$ & -31400 & -6000 & -37400 & 112300 & 0 & 0 & 114600 & 77200 & $9.7 \%$ \\
\hline Austria & -74100 & -10700 & -84800 & 120400 & 81600 & 0 & 197100 & 112400 & $6.2 \%$ \\
\hline Belgium & $\mathrm{m}$ & $\mathrm{m}$ & $\mathbf{m}$ & $\mathrm{m}$ & $\mathrm{m}$ & $\mathrm{m}$ & $\mathbf{m}$ & $\mathbf{m}$ & $\mathrm{m}$ \\
\hline Canada $^{3}$ & -44900 & -3700 & -48600 & 73600 & 25100 & 0 & 96900 & 48300 & $6.7 \%$ \\
\hline Chile & -18100 & -100 & -18200 & 25900 & 75800 & 1300 & 101600 & 83400 & $14.6 \%$ \\
\hline Czech Republic & -27600 & 5100 & -22400 & 56800 & 31100 & 3700 & 104100 & 81700 & $13.5 \%$ \\
\hline Denmark & -98400 & -20800 & - 119200 & 98300 & 0 & 13000 & 112800 & -6400 & $2.2 \%$ \\
\hline Estonia & -26600 & -3200 & -29800 & 27200 & 3700 & 0 & 31300 & 1600 & $3.6 \%$ \\
\hline Finland & -91300 & 7200 & -84100 & 95500 & 21700 & 2600 & 126900 & 42800 & $4.6 \%$ \\
\hline France & $\mathrm{q}$ & q & $\mathbf{q}$ & $\mathrm{q}$ & $\mathrm{q}$ & $\mathrm{q}$ & q & q & $\mathrm{q}$ \\
\hline Germany & -87500 & -13600 & -101100 & 83300 & 68000 & 0 & 158600 & 57500 & $4.5 \%$ \\
\hline Greece & $\mathrm{m}$ & -5200 & m & 16700 & 38100 & 0 & 65900 & $\mathbf{m}$ & $\mathrm{m}$ \\
\hline Hungary & -29600 & 1400 & -28200 & 93800 & 56600 & 0 & 162500 & 134300 & $16.9 \%$ \\
\hline Iceland & $\mathrm{m}$ & $\mathrm{m}$ & $\mathbf{m}$ & $\mathrm{m}$ & $\mathrm{m}$ & $\mathrm{m}$ & $\mathbf{m}$ & $\mathbf{m}$ & $\mathrm{m}$ \\
\hline Ireland & $\mathrm{m}$ & $\mathrm{m}$ & $\mathbf{m}$ & $\mathrm{m}$ & $\mathrm{m}$ & $\mathrm{m}$ & $\mathbf{m}$ & $\mathbf{m}$ & $\mathrm{m}$ \\
\hline Israel & -19900 & 100 & -19800 & 31700 & 25800 & 0 & 54400 & 34600 & $7.3 \%$ \\
\hline Italy $^{3}$ & -35900 & -8000 & -43900 & 102900 & 30100 & 0 & 129600 & 85700 & $7.8 \%$ \\
\hline Japan & $\mathrm{m}$ & $\mathrm{m}$ & $\mathbf{m}$ & $\mathrm{m}$ & $\mathrm{m}$ & $\mathrm{m}$ & $\mathbf{m}$ & $\mathbf{m}$ & $\mathrm{m}$ \\
\hline Korea & -13100 & -15500 & -28500 & 900 & 10600 & 0 & 13600 & -14900 & $-0.6 \%$ \\
\hline Luxembourg $^{3}$ & $\mathrm{~m}$ & -5500 & $\mathbf{m}$ & 223400 & 89100 & 0 & 287300 & $\mathbf{m}$ & $\mathrm{m}$ \\
\hline Mexico & $\mathrm{m}$ & $\mathrm{m}$ & $\mathbf{m}$ & $\mathrm{m}$ & $\mathrm{m}$ & $\mathrm{m}$ & $\mathbf{m}$ & $\mathbf{m}$ & $\mathrm{m}$ \\
\hline Netherlands ${ }^{3}$ & -73000 & -5100 & -78100 & 189100 & 6900 & 0 & 192700 & 114600 & $\mathrm{~m}$ \\
\hline New Zealand & -32600 & -1700 & -34300 & 44100 & 0 & 3300 & 51100 & 16800 & $5.1 \%$ \\
\hline Norway & -75300 & -11800 & -87100 & 85100 & 23700 & 0 & 112300 & 25200 & $3.4 \%$ \\
\hline Poland $^{3}$ & -19100 & -5600 & -24700 & 28000 & 56400 & 0 & 101400 & 76600 & $12.6 \%$ \\
\hline Portugal & -31400 & -2700 & -34100 & 119700 & 45500 & 0 & 158900 & 124800 & $11.0 \%$ \\
\hline Slovak Republic & -28100 & 5300 & -22800 & 38400 & 31300 & 0 & 76800 & 54000 & $10.5 \%$ \\
\hline Slovenia & -34900 & -9700 & -44700 & 110500 & 102500 & 0 & 221900 & 177200 & $11.3 \%$ \\
\hline Spain & -59000 & 6000 & -53000 & 73100 & 18000 & 0 & 105800 & 52800 & $6.8 \%$ \\
\hline Sweden & -97200 & -9000 & -106200 & 43300 & 13300 & 0 & 65000 & -41200 & $0.3 \%$ \\
\hline Switzerland & -91300 & -18300 & -109600 & 73300 & 28900 & 0 & 91700 & -17900 & $1.5 \%$ \\
\hline Turkey & $\mathrm{m}$ & $\mathrm{m}$ & $\mathbf{m}$ & $\mathrm{m}$ & $\mathrm{m}$ & $\mathrm{m}$ & $\mathbf{m}$ & $\mathbf{m}$ & $\mathrm{m}$ \\
\hline United Kingdom & -27700 & 3200 & -24500 & 93000 & 50700 & 80300 & 225300 & 200800 & $37.2 \%$ \\
\hline United States & -55900 & -7100 & -63000 & 139100 & 32000 & 0 & 178300 & 115300 & $9.2 \%$ \\
\hline OECD average & -49000 & -5000 & -53900 & 81500 & 35800 & 3900 & 123600 & 65500 & $8.6 \%$ \\
\hline EU21 average & -52600 & -3900 & -56400 & 89600 & 41400 & 5500 & 140200 & 79300 & $9.9 \%$ \\
\hline
\end{tabular}

Notes: Values are based on the difference between women who attained a tertiary education compared with those who have attained an upper secondary or postsecondary non-tertiary education. Values have been rounded up to the nearest hundred.

1. Total benefit is a weighted sum of gross earnings benefits (4), income tax effect (5), social contribution effect (6) and transfer effects (7), taking into account the probability of employment and the unemployment benefits in case of unemployment. For further details, please refer to the Methodology section.

2. Australia: Year of reference 2009.

3. Canada, Italy, Luxembourg, the Netherlands, Poland: Year of reference 2010.

Source: OECD. See Annex 3 for notes (www.oecd.org/education/education-at-a-glance-19991487.htm).

Please refer to the Reader's Guide for information concerning symbols for missing data and abbreviations.

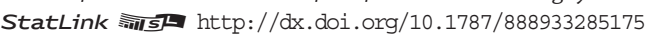





\section{HOW ARE SOCIAL OUTCOMES RELATED TO EDUCATION?}

- On average across countries and sub-national entities that participated in the Survey of Adult Skills (PIAAC) (2012), adults with higher qualifications were more likely to report desirable social outcomes, including good or excellent health, participation in volunteer activities, interpersonal trust, and political efficacy (i.e. having a say in government).

- The proportion of adults who reported that they have a say in government (political efficacy) grows with each additional level of education; and the difference in these proportions is larger between adults with upper secondary or post-secondary non-tertiary education and those with tertiary education than between adults who have below upper secondary education and those with upper secondary or post-secondary non-tertiary education.

- The proportion of adults who reported that they volunteer and enjoy good-to-excellent health grows with each additional level of education; and the difference in these proportions is larger between adults with below upper secondary education and those with upper secondary or postsecondary non-tertiary education than between adults with upper secondary or post-secondary non-tertiary and those with tertiary education.

\section{Chart A8.1. Social outcomes related to education (2012)}

Survey of Adult Skills, average, 25-64 year-olds, upper secondary or post-secondary non-tertiary education as reference category

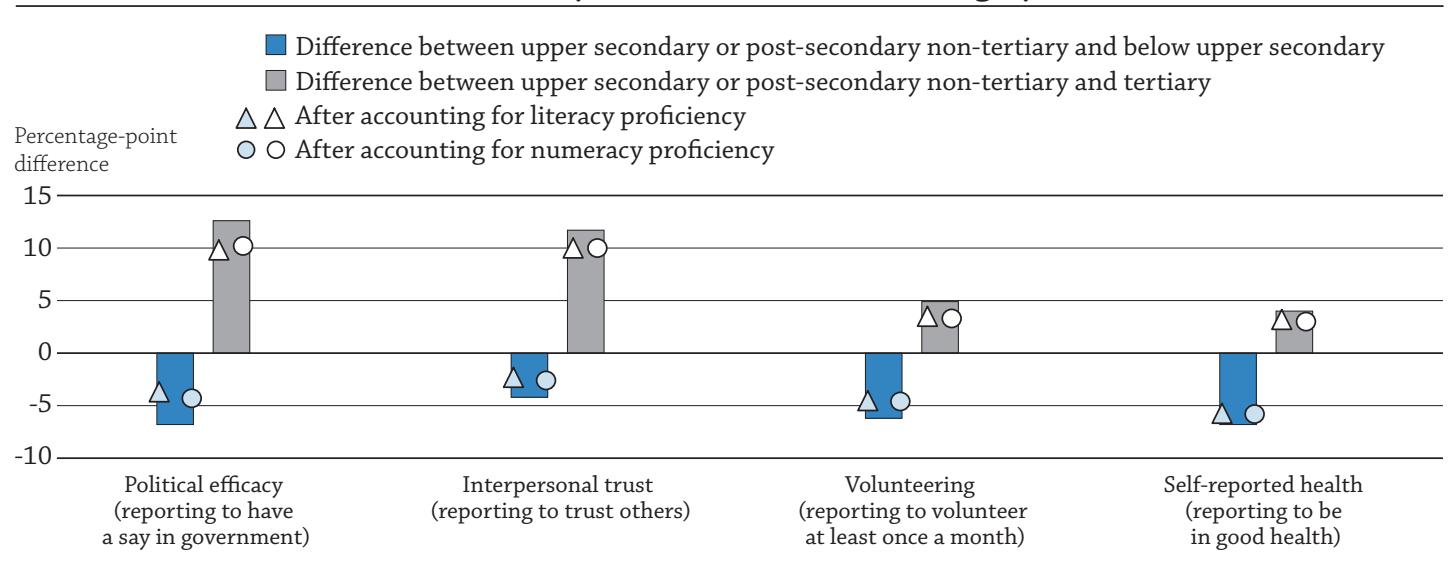

\section{How to read this chart}

Percentage-point difference reflects the relative change of social outcomes compared to the reference category. For example, on average the percentage of individuals with tertiary education reporting that they have a say in government increases by 13 percentage points compared to someone who has upper secondary or post-secondary non-tertiary education. After accounting for literacy proficiency or numeracy proficiency the increase drops to 10 percentage points. On the other hand, on average the percentage of individuals with below upper secondary education reporting that they have a say in government decreases by 7 percentage points compared to someone who has upper secondary or post-secondary non-tertiary education. After accounting for literacy proficiency or numeracy proficiency the decrease rises to 4 percentage points.

Note: Calculations are based on a linear regression after accounting for gender, age and monthly earnings.

Social outcomes are ranked in descending order of percentage-point difference between upper secondary or post-secondary non-tertiary and tertiary education.

Source: OECD. Tables A8.1, A8.2, A8.3a, A8.4, and Tables A8.1 (L), A8.1 (N), A8.2 (L), A8.2 (N), A8.3a (L), A8.3a (N), A8.4 (L) and A8.4 (N), available on line. See annex 3 for notes (www.oecd.org/education/education-at-a-glance-19991487.htm).

StatLink 케대 http://dx.doi.org/10.1787/888933283755 
Health is not the sole social outcome related to education. Interpersonal trust, volunteering and political engagement are also positively associated with education. Without trust in others and the rule of law, all relationships, whether business, political or social, function less efficiently. When people feel they have something to offer, when they are aware of others around them, they are more apt to participate in social change through volunteering. And when people feel they understand the political issues facing their country and could make a difference in how their country is run, they are more likely to be politically engaged (OECD, 2013b).

\section{Other findings}

- The differences in the shares of the population reporting positive social outcomes observed among adults with different levels of educational attainment partly reflect differences in age, gender and earnings. In most countries, accounting for these factors reduces, but does not eliminate, the differences observed in social outcomes across levels of educational attainment.

- The outcome that is most strongly influenced by individuals' age, gender and earnings is health, where consideration of these factors reduces, by about half, differences in the share of adults reporting good or excellent health across levels of educational attainment. The differences across levels of educational attainment are generally not strongly related to these factors for volunteering, interpersonal trust and political efficacy.

- Both literacy and numeracy skills are associated with positive social outcomes, although educational attainment itself is the primary factor associated with differences in social outcomes. 


\section{Analysis}

This year's indicator on social outcomes related to education (and skills) includes measures of self-reported health, volunteering, interpersonal trust and political efficacy, as assessed in the Survey of Adult Skills, a product of the OECD Programme for the International Assessment of Adult Competencies (PIAAC). These four social outcome measures are considered to be key indicators of individual and national well-being (OECD, 2013a).

Results show that educational attainment is positively associated with these measures of social outcomes even after accounting for gender, age, monthly earnings and proficiency in literacy or numeracy. Although country-specific patterns vary, the overall results show that proficiency in literacy and numeracy are related to all measured social outcomes, whereas gender, age and monthly earnings are related to self-reported health only.

As Chart A8.1 shows, the proportion of adults who believe they have a say in government is 13 percentage points larger among adults with tertiary education than among adults with upper secondary or post-secondary nontertiary education. There is a 12 percentage-point difference between these two groups when considering adults who reported that they trust others, a 5 percentage-point difference when considering adults who reported that they volunteer at least once a month, and a 4 percentage-point difference between the two groups of adults when considering adults who reported that they are in good health. By contrast, the proportions of adults reporting each of these positive social outcomes decrease by a range of 4 to 7 percentage points among adults whose highest level of education is below upper secondary than among adults with upper secondary or post-secondary non-tertiary education (Tables A8.1, A8.2, A8.3a and A8.4).

\section{Self-reported health}

On average across countries and sub-national entities that participated in the Survey of Adult Skills, $79 \%$ of adults with upper secondary or post-secondary non-tertiary education reported that they are "in good health". In all participating countries and sub-national entities, there is a positive relationship between educational attainment and self-reported health. On average, the proportion of adults who reported that they are in good health is 15 percentage points smaller among adults with below upper secondary as their highest attainment level compared with adults with upper secondary or post-secondary non-tertiary education. This proportion is 9 percentage points larger among tertiaryeducated adults compared with adults with upper secondary or post-secondary non-tertiary education (Table A8.1).

After accounting for gender, age and monthly earnings, educational attainment loses some of its impact on selfreported health. Proficiency in literacy and numeracy also moderate the impact of educational attainment on self-reported health. For example, on average, after accounting for gender and age, the proportion of adults who reported that they are in good health is 12 percentage points smaller among adults with below upper secondary as their highest attainment level compared with adults with upper secondary or post-secondary non-tertiary education. It is 7 percentage points smaller when also accounting for monthly earnings, and 6 percentage points smaller when literacy or numeracy proficiency are also taken into account (Tables A8.1 [L] and A8.1 [N], available on line).

Chart A8.2 shows the percentage-point difference in self-reported health between levels of educational attainment after accounting for gender, age, monthly earnings and proficiency in literacy or numeracy. Results show that after accounting for all of these variables, educational attainment continues to play a determinant role in self-reported health. On average, after accounting for gender, age and monthly earnings, the difference is larger when comparing upper secondary or post-secondary non-tertiary education with below upper secondary education ( 7 percentage points) than when comparing upper secondary or post-secondary non-tertiary education with tertiary education (4 percentage points). Large negative differences are observed in the Czech Republic, Estonia, Korea, the Slovak Republic and the United States, where the proportion of adults reporting to be in good health is about 10 percentage points smaller among those with below upper secondary education compared to adults with upper secondary or post-secondary non-tertiary education. By contrast, only in Estonia is the proportion of adults who reported to be in good health at least 10 percentage points larger among tertiary-educated adults than among adults with upper secondary or post-secondary non-tertiary education (Table A8.1, and Tables A8.1 [L] and A8.1 [N], available on line).

\section{Volunteering}

On average across participating countries and sub-national entities, $18 \%$ of adults with upper secondary or postsecondary non-tertiary education reported that they volunteer at least once a month. All participating countries and sub-national entities show a positive relationship between educational attainment and volunteering. On average, the proportion of adults who reported that they volunteer once a month is 5 percentage points smaller among adults with below upper secondary education than among adults who have attained upper secondary or post-secondary non-tertiary education. This proportion is 5 percentage points larger among tertiary-educated adults than among adults with upper secondary or post-secondary non-tertiary education (Table A8.2). 


\section{Chart A8.2. Likelihood of reporting to be in good health, by educational attainment (2012)}

Survey of Adult Skills, 25-64 year-olds, upper secondary or post-secondary non-tertiary education as reference category

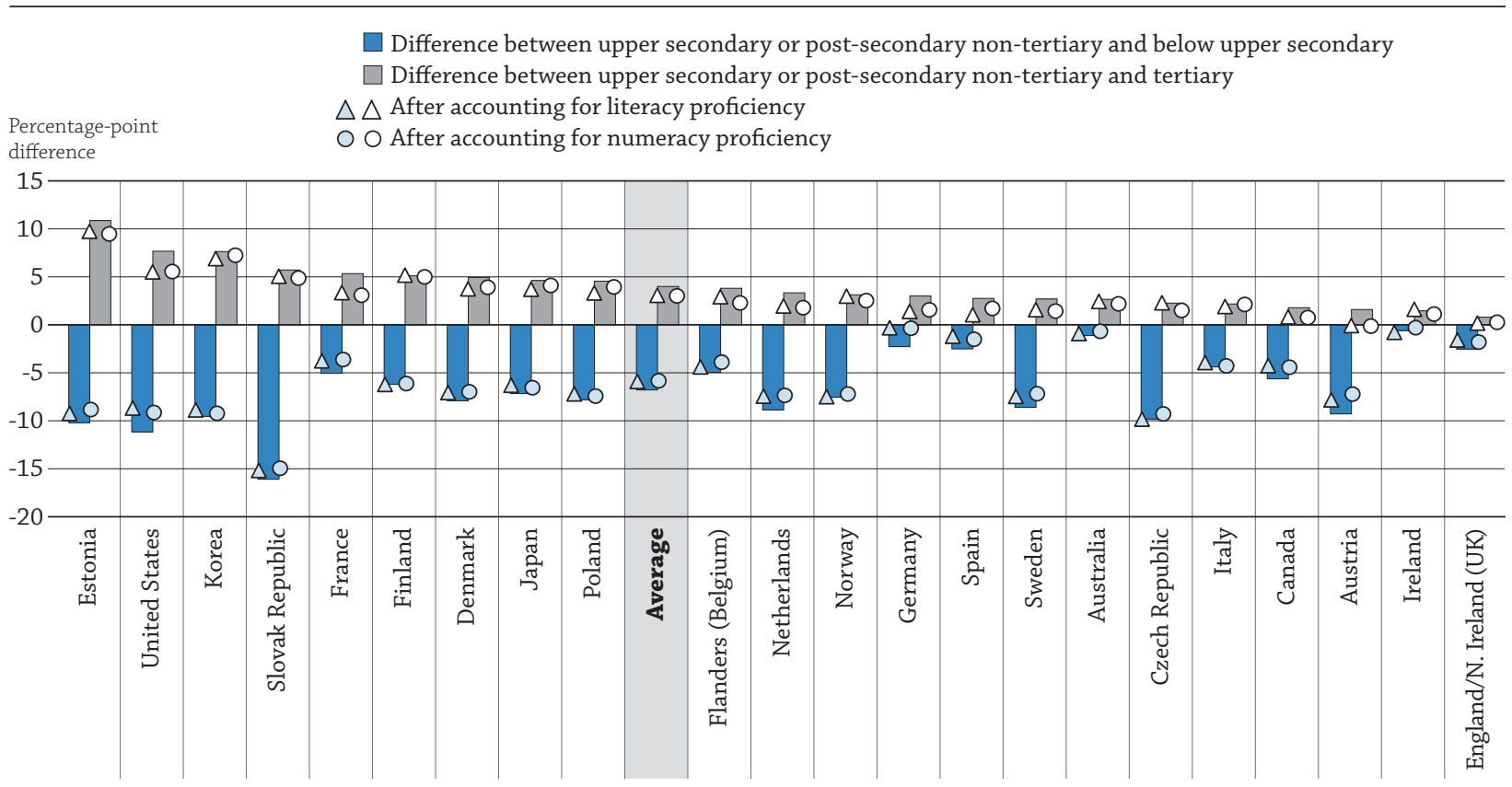

How to read this chart

Percentage-point difference reflects the relative change of reporting to be in good health compared to the reference category. For example, in Estonia, the percentage of individuals with tertiary education reporting to be in good health increases by 11 percentage points compared to someone who has upper secondary or post-secondary non-tertiary education. Similarly, after accounting for literacy proficiency, the percentage of individuals with tertiary education increases by 10 percentage points compared to someone who has upper secondary or post-secondary non-tertiary education.

Note: Calculations are based on a linear regression after accounting for gender, age and monthly earnings.

Countries are ranked in descending order of percentage-point difference between upper secondary or post-secondary non-tertiary and tertiary education.

Source: OECD. Table A8.1, and Tables A8.1 (L) and A8.1 (N), available on line. See Annex 3 for notes (www.oecd.org/education/education-at-aglance-19991487.htm).

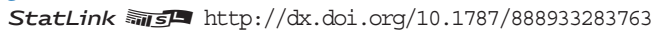

When comparing adults with upper secondary or post-secondary non-tertiary education and those with tertiary education, these proportions do not change after accounting for gender and age. When accounting for proficiency in literacy or numeracy, the percentage-point increase shrinks slightly from 5 to 3 percentage points (Tables A8.2 [L] and A8.2 [N], available on line).

Chart A8.3 shows the percentage-point difference, between levels of educational attainment, in the proportion of adults who reported that they volunteer at least once a month, after accounting for gender, age and monthly earnings. It also shows the impact of accounting for literacy or numeracy proficiency. Results show that after accounting for all of these variables, educational attainment continues to play a determinant role in whether adults report that they volunteer at least once a month. On average, after accounting for gender, age and monthly earnings, the difference is larger when comparing upper secondary or post-secondary non-tertiary education with below upper secondary education (-6 percentage points) than when comparing upper secondary or post-secondary non-tertiary education with tertiary education (5 percentage points) (Table A8.2, and Tables A8.2 [L] and A8.2 [N], available on line).

Large negative differences are observed in Austria, Germany and the United States, where the proportion of adults who reported that they volunteer is about 10 percentage points smaller among adults with below upper secondary as their highest level of attainment than among adults with upper secondary or post-secondary non-tertiary education. By contrast, only in the United States is the proportion of adults who reported that they volunteer more than 10 percentage points larger among tertiary-educated adults than among adults with upper secondary or post-secondary non-tertiary education (Table A8.2, and Tables A8.2 [L] and A8.2 [N], available on line). 


\section{Chart A8.3. Likelihood of reporting to volunteer at least once a month, by educational attainment (2012)}

Survey of Adult Skills, 25-64 year-olds, upper secondary or post-secondary non-tertiary education as reference category

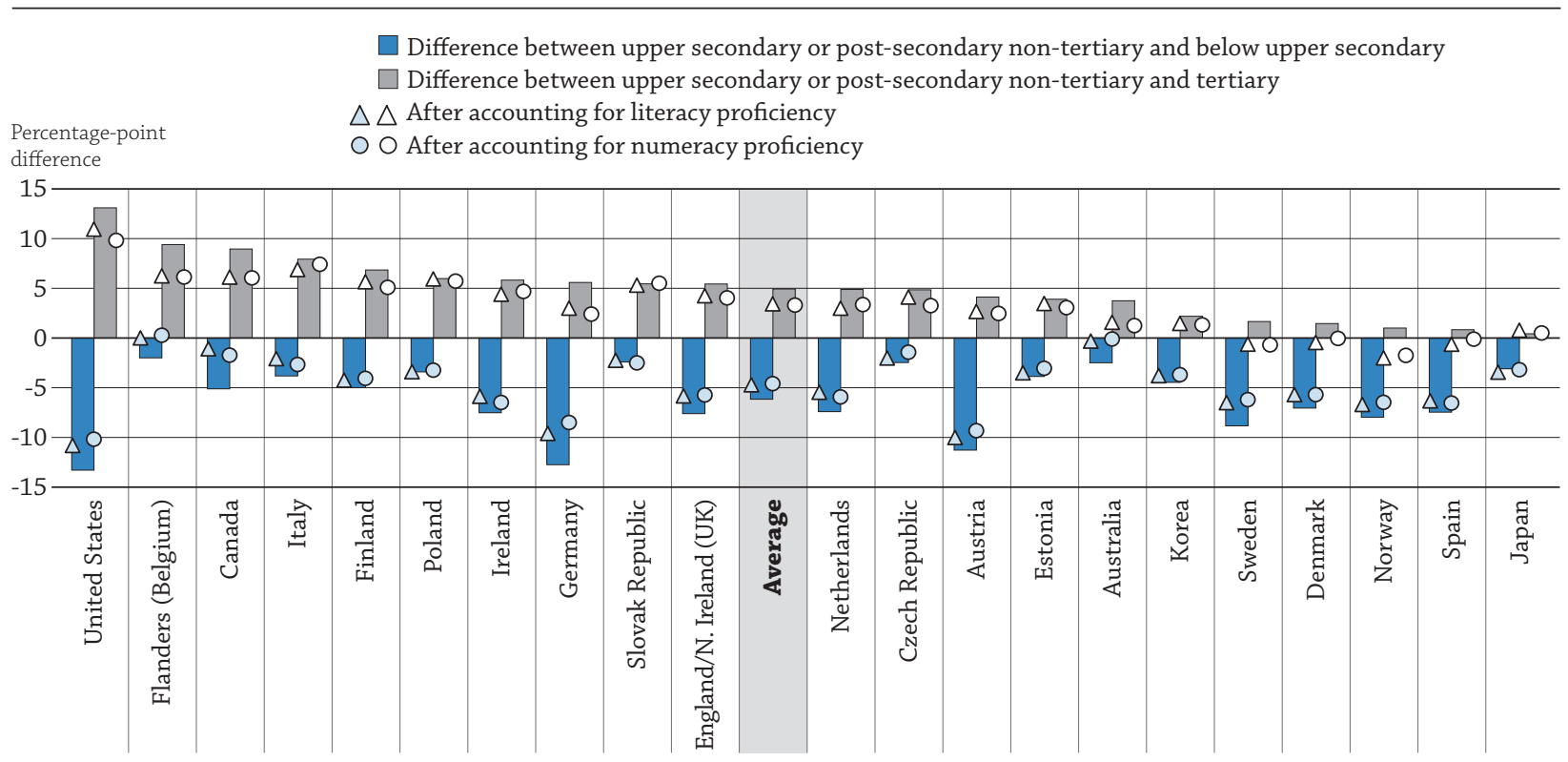

How to read this chart

Percentage-point difference reflects the relative change of reporting to volunteer at least once a month compared to the reference category. For example, in the United States, the percentage of individuals with tertiary education reporting to volunteer at least once a month increases by 13 percentage points compared to someone who has upper secondary or post-secondary non-tertiary education. Similarly, after accounting for literacy proficiency, the percentage of individuals with tertiary education increases by 11 percentage points compared to someone who has upper secondary or post-secondary non-tertiary education.

Note: Calculations are based on a linear regression after accounting for gender, age and monthly earnings.

Countries are ranked in descending order of percentage-point difference between upper secondary or post-secondary non-tertiary and tertiary education.

Source: OECD. Table A8.2, and Tables A8.2 (L) and A8.2 (N), available on line. See Annex 3 for notes (www.oecd.org/education/education-at-aglance-19991487.htm).

StatLink 제매 http://dx.doi.org/10.1787/888933283778

\section{Interpersonal trust}

On average across participating countries and sub-national entities, $18 \%$ of adults with upper secondary or postsecondary non-tertiary education reported that they trust others. In all participating countries and sub-national entities, there is a positive relationship between educational attainment and interpersonal trust. On average, the proportion of adults who reported that they trust others is 4 percentage points smaller among adults with below upper secondary education than among adults with upper secondary or post-secondary non-tertiary education, and is 12 percentage points larger among tertiary-educated adults than among adults whose highest attainment is upper secondary or post-secondary non-tertiary education (Table A8.3a).

After accounting for gender, age and monthly earnings, there is no change in these differences between levels of educational attainment. But when also accounting for proficiency in literacy or numeracy, the differences between levels of educational attainment shrink slightly. The proportion of tertiary-educated adults who reported that they trust others is 10 percentage points larger than the proportion of adults with upper secondary or post-secondary non-tertiary education who so reported (Tables A8.3a [L] and A8.3a [N], available on line).

Chart A8.4 shows the differences in the proportion of adults who reported that they trust others related to the level of educational attainment, after accounting for gender, age and monthly earnings. Particularly larges differences between tertiary-educated adults and those with upper secondary or post-secondary non-tertiary education are observed in Norway (20 percentage points) and Denmark (19 percentage points). The largest differences between adults with below upper secondary education and those with upper secondary or post-secondary non-tertiary education are seen in Denmark (-10 percentage points) and the Netherlands ( -9 percentage points) (Table A8.3a). 
After accounting for proficiency in literacy or numeracy, the proportion of adults who reported that they trust others is 10 percentage points larger, on average, among tertiary-educated adults than among adults with upper secondary or post-secondary non-tertiary education. The largest differences are observed in Flanders (Belgium) and Norway (16 percentage points or more). Conversely, the proportion of adults who so reported is 3 percentage points smaller, on average, among adults with below upper secondary education than among adults with upper secondary or post-secondary non-tertiary education. A notable difference between these two groups is observed in Flanders (Belgium) (-9 percentage points) (Tables A8.3a [L] and A8.3a [N], available on line).

\section{Chart A8.4. Likelihood of reporting to trust others, by educational attainment (2012) Survey of Adult Skills, 25-64 year-olds, upper secondary or post-secondary non-tertiary education as reference category}

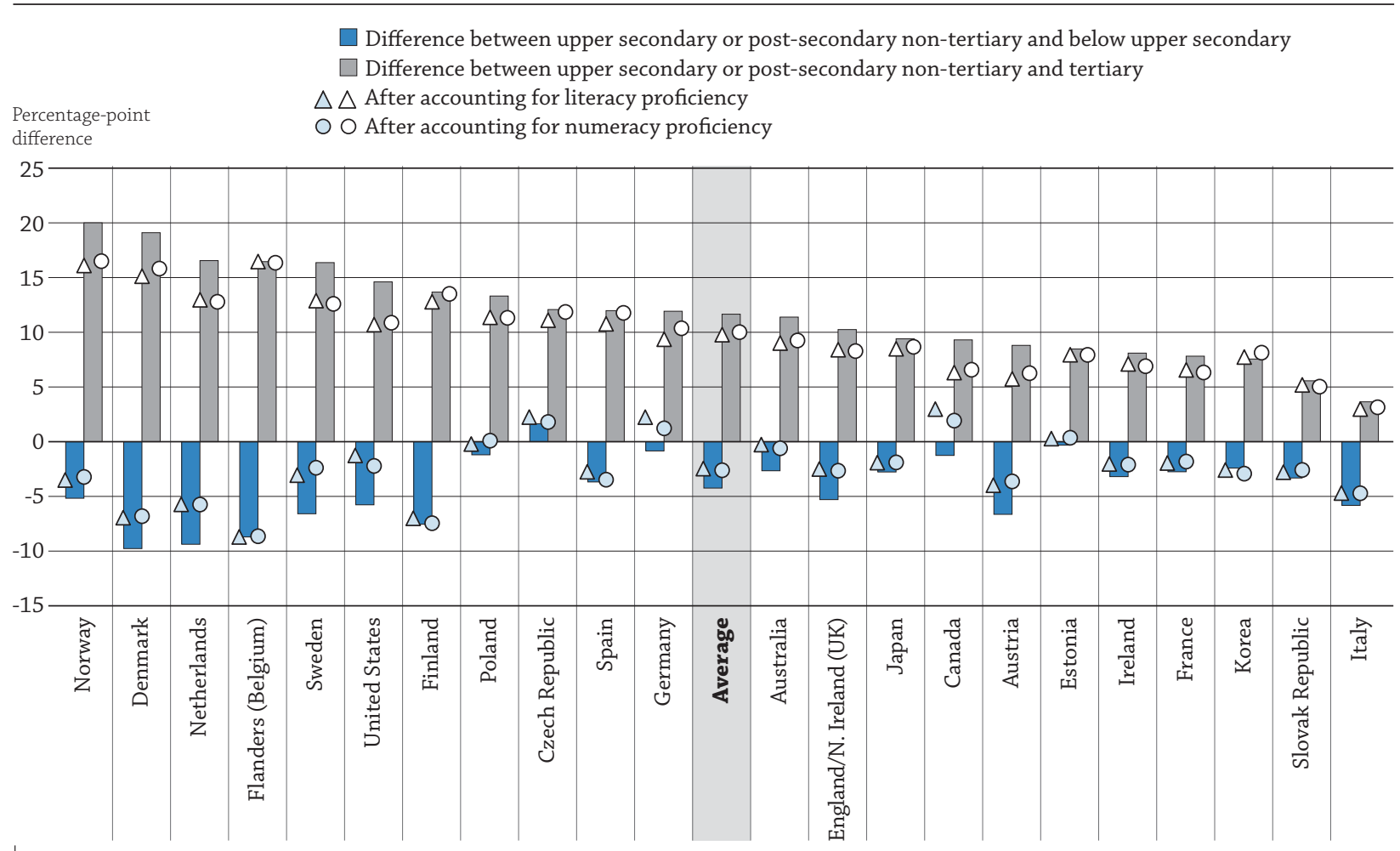

How to read this chart

Percentage-point difference reflects the relative change of reporting to trust others compared to the reference category. For example, in Norway, the percentage of individuals with tertiary education reporting to trust others increases by 20 percentage points compared to someone who has upper secondary or post-secondary non-tertiary education. Similarly, after accounting for literacy proficiency, the percentage of individuals with tertiary education increases by 16 percentage points compared to someone who has upper secondary or post-secondary non-tertiary education.

Note: Calculations are based on a linear regression after accounting for gender, age and monthly earnings.

Countries are ranked in descending order of percentage-point difference between upper secondary or post-secondary non-tertiary and tertiary education.

Source: OECD. Table A8.3a, and Tables A8.3a (L) and A8.3a (N), available on line. See Annex 3 for notes (www.oecd.org/education/education-at-aglance-19991487.htm).

StatLink त्राजा http://dx.doi.org/10.1787/888933283784

\section{Political efficacy}

On average across participating countries and sub-national entities, $30 \%$ of adults with upper secondary or postsecondary non-tertiary education reported that they have a say in government. In all participating countries and sub-national entities, there is a positive relationship between educational attainment and political efficacy. On average, the proportion of adults who reported that they have a say in government is 7 percentage points smaller among adults with below upper secondary education than among adults with upper secondary or post-secondary non-tertiary education, and is 13 percentage points larger among tertiary-educated adults than among adults with upper secondary or post-secondary education (Table A8.4). 
After accounting for gender, age and monthly earnings, there is no change in these differences between levels of educational attainment. When accounting for proficiency in literacy or numeracy, the proportion of adults who reported that they have a say in government is 4 percentage points smaller among adults with below upper secondary education than among adults with upper secondary or post-secondary non-tertiary education. This proportion is 10 percentage points larger among tertiary-educated adults than among adults with upper secondary or post-secondary non-tertiary education (Tables A8.4 [L] and A8.4 [N], available on line).

As shown in Chart A8.5, the proportion of adults who reported that they believe they have a say in government is 13 percentage points larger among tertiary-educated adults than among adults with upper secondary or postsecondary non-tertiary education, after accounting for gender, age and monthly earnings. Although literacy and numeracy skills moderate the effect of educational attainment, it continues to play a determinant role in this social outcome. Particularly large differences in these proportions between adults with upper secondary or postsecondary non-tertiary education and tertiary-educated adults are seen in the Netherlands and Norway. By contrast, in the United States, the proportion of adults who reported that they believe they have a say in government is strikingly smaller among adults with below upper secondary education than among adults with upper secondary or post-secondary non-tertiary education (Table A8.4, and Tables A8.4 [L] and A8.4 [N], available on line).

\section{Chart A8.5. Likelihood of reporting to believe that they have a say in government, by educational attainment (2012)}

\section{Survey of Adult Skills, 25-64 year-olds, upper secondary or post-secondary non-tertiary education}

\section{as reference category}

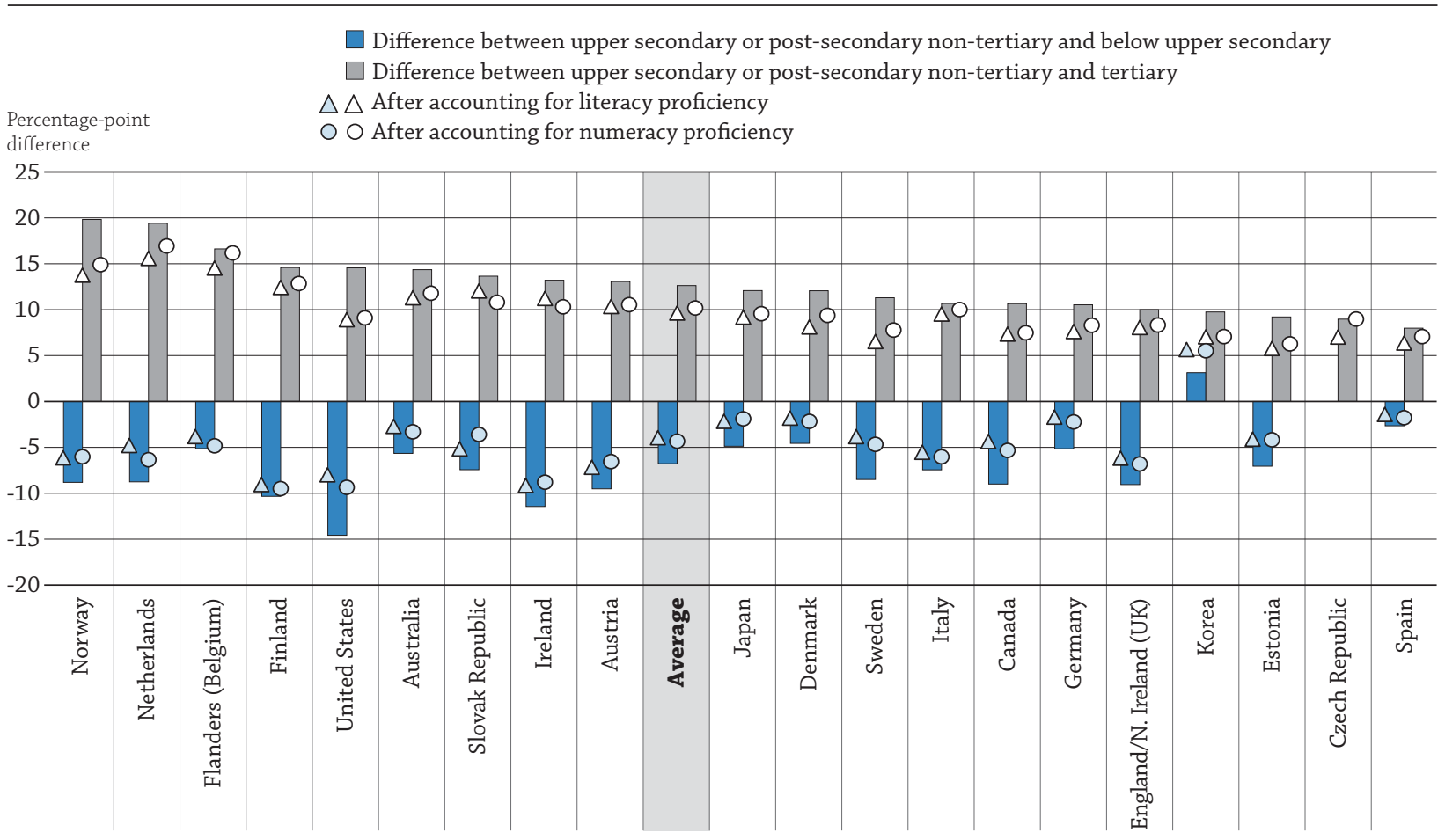

\footnotetext{
How to read this chart

Percentage-point difference reflects the relative change of reporting that they have a say in government compared to the reference category. For example, in Norway, the percentage of individuals with tertiary education reporting that they have a say in government increases by 20 percentage points compared to someone who has upper secondary or post-secondary non-tertiary education. Similarly, after accounting for literacy proficiency, the percentage of individuals with tertiary education increases by 14 percentage points compared to someone who has
} upper secondary or post-secondary non-tertiary education.

Note: Calculations are based on a linear regression after accounting for gender, age and monthly earnings. Differences between the groups that are not statistically significant at $95 \%$ are not presented.

Countries are ranked in descending order of percentage-point difference between upper secondary or post-secondary non-tertiary and tertiary education.

Source: OECD. Table A8.4, and Tables A8.4 (L) and A8.4 (N), available on line. See Annex 3 for notes (www.oecd.org/education/education-at-aglance-19991487.htm).

StatLink त्माज् http://dx.doi.org/10.1787/888933291269 


\section{Definitions}

Adults refer to 25-64 year-olds.

Levels of education: Below upper secondary corresponds to ISCED-97 Levels 0, 1, 2 and 3C short programmes; upper secondary or post-secondary non-tertiary corresponds to ISCED-97 Levels 3A, 3B, 3C long programmes, and Level 4; and tertiary corresponds to ISCED-97 Levels 5A, 5B and 6.

Literacy is the ability to understand, evaluate, use and engage with written texts to participate in society, to achieve one's goals, and to develop one's knowledge and potential. Literacy encompasses a range of skills from the decoding of written words and sentences to the comprehension, interpretation, and evaluation of complex texts. It does not, however, involve the production of text (writing). Information on the skills of adults with low levels of proficiency is provided by an assessment of reading components that covers text vocabulary, sentence comprehension and passage fluency.

Numeracy is the ability to access, use, interpret and communicate mathematical information and ideas in order to engage in and manage the mathematical demands of a range of situations in adult life. To this end, numeracy involves managing a situation or solving a problem in a real context, by responding to mathematical content/ information/ideas represented in multiple ways.

Reporting to be in good health includes adults who reported that they are in excellent, very good or good health.

Reporting to believe they have a say in government includes adults who strongly disagreed or disagreed with the statement: "People like me don't have any say about what the government does".

Reporting to trust others includes adults who strongly disagreed or disagreed that there are only a few people you can trust completely.

Reporting to volunteer includes adults who reported that they volunteer at least once a month.

\section{Methodology}

All data are based on the Survey of Adult Skills (PIAAC) (2012). PIAAC is the OECD Programme for the International Assessment of Adult Competencies. See Annex 3 (www.oecd.org/education/education-at-a-glance-19991487.htm) for additional information.

Ordinary least-square regressions using level of education, gender, age and monthly earnings as independent variables were run to determine the impact of education on self-reported health, volunteering, interpersonal trust and political efficacy. Regressions were run in a stepwise fashion, including educational attainment first, then gender and age, and monthly earnings in a final step. Separate analyses were also conducted to control for literacy or numeracy in each of these steps.

Note regarding data from the Russian Federation in the Survey of Adult Skills (PIAAC)

Readers should note that the sample for the Russian Federation does not include the population of the Moscow municipal area. The data published, therefore, do not represent the entire resident population aged 16-65 in Russia but rather the population of Russia excluding the population residing in the Moscow municipal area. More detailed information regarding the data from the Russian Federation as well as that of other countries can be found in the Technical Report of the Survey of Adult Skills (OECD, 2014).

\section{References}

Cutler, D.M. and A. Lleras-Muney (2006), "Education and Health: Evaluating Theories and Evidence”, NBER Working Paper, No. 12352.

OECD (2014), Technical Report of the Survey of Adult Skills, www.oecd.org/site/piaac/_Technical\%20Report_170CT13.pdf, pre-publication copy.

OECD (2013a), How's Life? 2013: Measuring Well-being, OECD Publishing, Paris, http://dx.doi.org/10.1787/9789264201392-en. OECD (2013b), OECD Skills Outlook 2013: First Results from the Survey of Adult Skills, OECD Publishing, Paris, http://dx.doi. org/10.1787/9789264204256-en. 


\section{Indicator A8 Tables}

StatLink 部

Table A8.1 Likelihood of reporting to be in good health, by educational attainment (2012)

WEB Table A8.1 (L) Likelihood of reporting to be in good health, by educational attainment, after accounting for literacy proficiency (2012)

WEB Table A8.1 (N) Likelihood of reporting to be in good health, by educational attainment, after accounting for numeracy proficiency (2012)

Table A8.2 Likelihood of reporting to volunteer at least once a month, by educational attainment (2012)

WEB Table A8.2 (L) Likelihood of reporting to volunteer at least once a month, by educational attainment, after accounting for literacy proficiency (2012)

WEB Table A8.2 (N) Likelihood of reporting to volunteer at least once a month, by educational attainment, after accounting for numeracy proficiency (2012)

Table A8.3a Likelihood of reporting to trust others, by educational attainment (2012)

WEB Table A8.3a (L) Likelihood of reporting to trust others, by educational attainment, after accounting for literacy proficiency (2012)

Table A8.3a (N) Likelihood of reporting to trust others, by educational attainment, after accounting for numeracy proficiency (2012)

Table A8.3b Likelihood of reporting that others do not take advantage of them, by educational attainment (2012)

Table A8.3b (L) Likelihood of reporting that others do not take advantage of them, by educational attainment, after accounting for literacy proficiency (2012)

Table A8.3b (N) Likelihood of reporting that others do not take advantage of them, by educational attainment, after accounting for numeracy proficiency (2012)

Table A8.4 Likelihood of reporting to believe they have a say in government, by educational attainment (2012)

WEB Table A8.4 (L) Likelihood of reporting to believe they have a say in government, by educational attainment, after accounting for literacy proficiency (2012)

WEB Table A8.4 (N) Likelihood of reporting to believe they have a say in government, by educational attainment, after accounting for numeracy proficiency (2012) 
Table A8.1. Likelihood of reporting to be in good health, by educational attainment (2012) 25-64 year-olds, upper secondary or post-secondary non-tertiary education as reference category, percentage-point difference

The percentages presented in the first column are not related to the regression. They should be used as a reference to better understand the percentage-point difference presented in the other columns.

How to read this table: In Australia, the percentage of individuals with below upper secondary education reporting to be in good health decreases by 8 percentage points compared to someone who has upper secondary or post-secondary non-tertiary education. After accounting for gender and age, the percentage of individuals with below upper secondary education reporting to be in good health decreases by 6 percentage points compared to someone who has upper secondary or post-secondary non-tertiary education. And after accounting for gender, age and monthly earnings, the percentage of individuals with below upper secondary education reporting to be in good health decreases by 1 percentage point compared to someone who has upper secondary or post-secondary nontertiary education.

\begin{tabular}{|c|c|c|c|c|c|c|c|c|c|c|c|c|c|c|c|}
\hline & & \multirow{2}{*}{\multicolumn{2}{|c|}{$\begin{array}{l}\text { Percentage } \\
\text { of adults reporting } \\
\text { that they are in } \\
\text { good health among } \\
\text { those who have } \\
\text { upper secondary } \\
\text { or post-secondary } \\
\text { non-tertiary } \\
\text { education }\end{array}$}} & \multicolumn{6}{|c|}{$\begin{array}{c}\text { Difference between upper secondary or post-secondary } \\
\text { non-tertiary and below upper secondary }\end{array}$} & \multicolumn{6}{|c|}{$\begin{array}{l}\text { Difference between upper secondary } \\
\text { or post-secondary non-tertiary and tertiary }\end{array}$} \\
\hline & & & & \multicolumn{2}{|c|}{$\begin{array}{l}\text { No control } \\
\text { variable }\end{array}$} & \multicolumn{2}{|c|}{$\begin{array}{l}\text { Accounting } \\
\text { for gender } \\
\text { and age }\end{array}$} & \multicolumn{2}{|c|}{$\begin{array}{c}\text { Accounting } \\
\text { for gender, age and } \\
\text { monthly earnings }\end{array}$} & \multicolumn{2}{|c|}{$\begin{array}{l}\text { No control } \\
\text { variable }\end{array}$} & \multicolumn{2}{|c|}{$\begin{array}{l}\text { Accounting } \\
\text { for gender } \\
\text { and age }\end{array}$} & \multicolumn{2}{|c|}{$\begin{array}{l}\text { Accounting } \\
\text { for gender, age } \\
\text { and monthly } \\
\text { earnings }\end{array}$} \\
\hline & & $\%$ & S.E. & $\mathrm{pp}$ & S.E. & $\mathrm{pp}$ & S.E. & pp & S.E. & pp & S.E. & pp & S.E. & pp & S.E. \\
\hline & & (1) & (2) & (3) & $(4)$ & (5) & (6) & (7) & $(8)$ & (9) & (10) & (11) & (12) & (13) & (14) \\
\hline \multirow{2}{*}{ 品 } & National entities & & & & & & & & & & & & & & \\
\hline & Australia & 84 & (1.1) & -8 & $(0.02)$ & -6 & $(0.02)$ & -1 & $(0.02)$ & 6 & $(0.01)$ & 6 & $(0.01)$ & 3 & $(0.01)$ \\
\hline & Austria & 83 & $(0.6)$ & -16 & $(0.02)$ & -13 & $(0.02)$ & -9 & $(0.02)$ & 7 & $(0.01)$ & 7 & $(0.01)$ & 2 & $(0.01)$ \\
\hline & Canada & 87 & $(0.6)$ & -13 & $(0.02)$ & -12 & $(0.02)$ & -6 & $(0.02)$ & 5 & $(0.01)$ & 5 & $(0.01)$ & 2 & $(0.01)$ \\
\hline & Czech Republic & 88 & $(0.9)$ & -20 & $(0.04)$ & -17 & $(0.03)$ & -10 & $(0.03)$ & 9 & $(0.01)$ & 6 & $(0.01)$ & 2 & $(0.01)$ \\
\hline & Denmark & 81 & $(0.9)$ & -16 & $(0.02)$ & -15 & $(0.02)$ & -8 & $(0.02)$ & 9 & $(0.01)$ & 8 & $(0.01)$ & 5 & $(0.01)$ \\
\hline & Finland & 77 & (1.0) & -15 & $(0.02)$ & -9 & $(0.02)$ & -6 & $(0.03)$ & 12 & $(0.01)$ & 11 & $(0.01)$ & 5 & $(0.01)$ \\
\hline & France & 80 & $(0.8)$ & -12 & $(0.01)$ & -9 & $(0.01)$ & -5 & $(0.02)$ & 10 & $(0.01)$ & 8 & $(0.01)$ & 5 & $(0.01)$ \\
\hline & Germany & 86 & $(0.8)$ & -11 & $(0.03)$ & -11 & $(0.02)$ & -2 & $(0.03)$ & 6 & $(0.01)$ & 5 & $(0.01)$ & 3 & $(0.01)$ \\
\hline & Ireland & 89 & $(0.8)$ & -11 & $(0.02)$ & -9 & $(0.02)$ & -1 & $(0.02)$ & 5 & $(0.01)$ & 4 & $(0.01)$ & 1 & $(0.01)$ \\
\hline & Italy & 87 & (1.1) & -15 & $(0.02)$ & -9 & $(0.01)$ & -4 & $(0.02)$ & 3 & $(0.02)$ & 2 & $(0.02)$ & 2 & $(0.02)$ \\
\hline & Japan & 70 & (1.3) & -10 & $(0.03)$ & -8 & $(0.03)$ & -7 & $(0.04)$ & 8 & $(0.02)$ & 6 & $(0.02)$ & 5 & $(0.02)$ \\
\hline & Korea & 46 & $(1.3)$ & -20 & $(0.02)$ & -13 & $(0.02)$ & -10 & $(0.03)$ & 11 & $(0.02)$ & 9 & $(0.02)$ & 8 & $(0.02)$ \\
\hline & Netherlands & 81 & $(1.0)$ & -12 & $(0.02)$ & -9 & $(0.02)$ & -9 & $(0.02)$ & 7 & $(0.01)$ & 7 & $(0.01)$ & 3 & $(0.01)$ \\
\hline & Norway & 81 & $(1.0)$ & -14 & $(0.02)$ & -12 & $(0.02)$ & -8 & $(0.02)$ & 8 & $(0.01)$ & 8 & $(0.01)$ & 3 & $(0.01)$ \\
\hline & Poland & 76 & $(0.9)$ & -23 & $(0.02)$ & -18 & $(0.02)$ & -8 & $(0.04)$ & 17 & $(0.01)$ & 10 & $(0.01)$ & 5 & $(0.01)$ \\
\hline & Slovak Republic & 79 & $(0.7)$ & -25 & $(0.02)$ & -21 & $(0.02)$ & -16 & $(0.04)$ & 12 & $(0.01)$ & 9 & $(0.01)$ & 6 & $(0.01)$ \\
\hline & Spain & 80 & (1.4) & -11 & $(0.02)$ & -7 & $(0.02)$ & -3 & $(0.02)$ & 6 & $(0.02)$ & 5 & $(0.02)$ & 3 & $(0.02)$ \\
\hline & Sweden & 84 & $(0.9)$ & -16 & $(0.02)$ & -15 & $(0.02)$ & -9 & $(0.03)$ & 6 & $(0.01)$ & 6 & $(0.01)$ & 3 & $(0.01)$ \\
\hline \multicolumn{16}{|c|}{ Sub-national entities } \\
\hline & Flanders (Belgium) & 84 & $(0.8)$ & -11 & $(0.02)$ & -9 & $(0.02)$ & -5 & $(0.02)$ & 6 & $(0.01)$ & 6 & $(0.01)$ & 4 & $(0.01)$ \\
\hline & England (UK) & 85 & $(1.0)$ & -13 & $(0.02)$ & -12 & $(0.02)$ & -2 & $(0.02)$ & 4 & $(0.01)$ & 4 & $(0.01)$ & 1 & $(0.01)$ \\
\hline & Northern Ireland (UK) & 83 & (1.4) & -15 & $(0.02)$ & -12 & $(0.02)$ & -5 & $(0.02)$ & 7 & $(0.02)$ & 7 & $(0.02)$ & 2 & $(0.01)$ \\
\hline & England/N. Ireland (UK) & 85 & $(1.0)$ & -13 & $(0.02)$ & -12 & $(0.02)$ & -3 & $(0.02)$ & 4 & $(0.01)$ & 4 & $(0.01)$ & 1 & $(0.01)$ \\
\hline & Average & 79 & $(0.2)$ & -15 & $(0.00)$ & -12 & $(0.00)$ & -7 & $(0.01)$ & 9 & $(0.00)$ & 7 & $(0.00)$ & 4 & $(0.00)$ \\
\hline$\frac{n}{2}$ & Russian Federation* & $q$ & $q$ & q & $q$ & $q$ & $q$ & $q$ & $\mathrm{q}$ & $q$ & $\mathrm{q}$ & $q$ & q & $q$ & q \\
\hline
\end{tabular}

Note: Calculations for percentage-point difference are based on linear regressions where the dependent variable is reporting to be in good health and where the independent variables vary according to the model. In the first regression (columns labelled "No control variable") only educational attainment is used as an independent variable. In the second regression (columns labelled "Accounting for gender and age"), gender and age are added as independent variables. In the third regression (columns labelled "Accounting for gender, age and monthly earnings"), educational attainment, gender, age and monthly earnings are included as independent variables.

* See note on data for the Russian Federation in the Methodology section.

Source: OECD. Survey of Adult Skills (PIAAC) (2012). See Annex 3 for notes (www.oecd.org/education/education-at-a-glance-19991487.htm).

Please refer to the Reader's Guide for information concerning symbols for missing data and abbreviations.

StatLink 제므 http://dx.doi.org/10.1787/888933285195 
Table A8.2. Likelihood of reporting to volunteer at least once a month, by educational attainment (2012)

25-64 year-olds, upper secondary or post-secondary non-tertiary education as reference category, percentage-point difference

\begin{abstract}
The percentages presented in the first column are not related to the regression. They should be used as a reference to better understand the percentage-point difference presented in the other columns.

How to read this table: In Australia, the percentage of individuals with below upper secondary education reporting to volunteer at least once a month decreases by 2 percentage points compared to someone who has upper secondary or post-secondary non-tertiary education. After accounting for gender and age, the percentage of individuals with below upper secondary education reporting to volunteer at least once a month decreases by 5 percentage points compared to someone who has upper secondary or post-secondary non-tertiary education. And after accounting for gender, age and monthly earnings, the percentage of individuals with below upper secondary education reporting to volunteer at least once a month decreases by 2 percentage points compared to someone who has upper secondary or post-secondary non-tertiary education.
\end{abstract}

\begin{tabular}{|c|c|c|c|c|c|c|c|c|c|c|c|c|c|c|c|}
\hline & & \multirow{2}{*}{\multicolumn{2}{|c|}{$\begin{array}{c}\text { Percentage } \\
\text { of adults reporting } \\
\text { that they volunteer } \\
\text { at least once a } \\
\text { month among } \\
\text { those who have } \\
\text { upper secondary } \\
\text { or post-secondary } \\
\text { non-tertiary } \\
\text { education }\end{array}$}} & \multicolumn{6}{|c|}{$\begin{array}{l}\text { Difference between upper secondary or post-secondary } \\
\text { non-tertiary and below upper secondary }\end{array}$} & \multicolumn{6}{|c|}{$\begin{array}{l}\text { Difference between upper secondary } \\
\text { or post-secondary non-tertiary and tertiary }\end{array}$} \\
\hline & & & & \multicolumn{2}{|c|}{$\begin{array}{l}\text { No control } \\
\text { variable }\end{array}$} & \multicolumn{2}{|c|}{$\begin{array}{l}\text { Accounting } \\
\text { for gender } \\
\text { and age }\end{array}$} & \multicolumn{2}{|c|}{$\begin{array}{l}\text { Accounting } \\
\text { for gender, } \\
\text { age and monthly } \\
\text { earnings }\end{array}$} & \multicolumn{2}{|c|}{$\begin{array}{l}\text { No control } \\
\text { variable }\end{array}$} & \multicolumn{2}{|c|}{$\begin{array}{l}\text { Accounting } \\
\text { for gender } \\
\text { and age }\end{array}$} & \multicolumn{2}{|c|}{$\begin{array}{l}\text { Accounting } \\
\text { for gender, } \\
\text { age and } \\
\text { monthly } \\
\text { earnings }\end{array}$} \\
\hline & & (1) & (2) & (3) & (4) & (5) & (6) & (7) & (8) & (9) & (10) & (11) & (12) & (13) & (14) \\
\hline \multirow{2}{*}{$\underset{\mathrm{u}}{\mathrm{O}}$} & National entities & & & & & & & & & & & & & & \\
\hline & Australia & 20 & $(1.1)$ & -2 & $(0.02)$ & -5 & $(0.02)$ & -2 & $(0.02)$ & 4 & $(0.02)$ & 3 & $(0.02)$ & 4 & $(0.02)$ \\
\hline & Austria & 23 & $(0.8)$ & -8 & $(0.01)$ & -8 & $(0.01)$ & -11 & $(0.02)$ & 6 & $(0.02)$ & 6 & $(0.02)$ & 4 & $(0.02)$ \\
\hline & Canada & 20 & $(0.8)$ & -4 & $(0.01)$ & -4 & $(0.01)$ & -5 & $(0.02)$ & 9 & $(0.01)$ & 9 & $(0.01)$ & 9 & $(0.01)$ \\
\hline & Czech Republic & 9 & $(1.0)$ & -4 & $(0.02)$ & -3 & $(0.02)$ & -2 & $(0.04)$ & 3 & $(0.02)$ & 3 & $(0.02)$ & 5 & $(0.03)$ \\
\hline & Denmark & 27 & $(1.2)$ & -8 & $(0.02)$ & -8 & $(0.02)$ & -7 & $(0.02)$ & 0 & $(0.01)$ & 2 & $(0.01)$ & 1 & $(0.02)$ \\
\hline & Finland & 19 & $(1.0)$ & -2 & $(0.02)$ & -3 & $(0.02)$ & -5 & $(0.02)$ & 6 & $(0.01)$ & 7 & $(0.01)$ & 7 & $(0.02)$ \\
\hline & France & $\mathrm{q}$ & $\mathrm{q}$ & $\mathrm{q}$ & $\mathrm{q}$ & $\mathrm{q}$ & $\mathrm{q}$ & $\mathrm{q}$ & $\mathrm{q}$ & $q$ & $\mathrm{q}$ & $q$ & $\mathrm{q}$ & $q$ & $\mathrm{q}$ \\
\hline & Germany & 22 & $(1.0)$ & -11 & $(0.02)$ & -11 & $(0.02)$ & -13 & $(0.03)$ & 6 & $(0.01)$ & 6 & $(0.01)$ & 6 & $(0.02)$ \\
\hline & Ireland & 20 & $(0.9)$ & -5 & $(0.01)$ & -9 & $(0.01)$ & -8 & $(0.03)$ & 4 & $(0.01)$ & 6 & $(0.01)$ & 6 & $(0.02)$ \\
\hline & Italy & 14 & $(1.0)$ & -4 & $(0.01)$ & -5 & $(0.01)$ & -4 & $(0.02)$ & 7 & $(0.02)$ & 7 & $(0.02)$ & 8 & $(0.03)$ \\
\hline & Japan & 12 & $(0.9)$ & -3 & $(0.01)$ & -5 & $(0.01)$ & -3 & $(0.02)$ & 0 & $(0.01)$ & 2 & $(0.01)$ & 0 & $(0.01)$ \\
\hline & Korea & 12 & $(0.7)$ & -1 & $(0.01)$ & -5 & $(0.01)$ & -4 & $(0.02)$ & 1 & $(0.01)$ & 2 & $(0.01)$ & 2 & $(0.01)$ \\
\hline & Netherlands & 28 & $(1.1)$ & -3 & $(0.02)$ & -7 & $(0.02)$ & -7 & $(0.02)$ & 4 & $(0.02)$ & 4 & $(0.02)$ & 5 & $(0.02)$ \\
\hline & Norway & 32 & $(1.3)$ & -9 & $(0.02)$ & -9 & $(0.02)$ & -8 & $(0.02)$ & 1 & $(0.02)$ & 2 & $(0.02)$ & 1 & $(0.02)$ \\
\hline & Poland & 6 & $(0.5)$ & -3 & $(0.01)$ & -3 & $(0.01)$ & -3 & $(0.02)$ & 7 & $(0.01)$ & 7 & $(0.01)$ & 6 & $(0.01)$ \\
\hline & Slovak Republic & 8 & $(0.5)$ & -3 & $(0.01)$ & -3 & $(0.01)$ & -2 & $(0.02)$ & 5 & $(0.01)$ & 5 & $(0.01)$ & 5 & $(0.01)$ \\
\hline & Spain & 13 & $(1.2)$ & -6 & $(0.01)$ & -7 & $(0.01)$ & -7 & $(0.02)$ & 2 & $(0.02)$ & 2 & $(0.02)$ & 1 & $(0.02)$ \\
\hline & Sweden & 21 & $(1.1)$ & -8 & $(0.02)$ & -9 & $(0.02)$ & -9 & $(0.02)$ & 1 & $(0.02)$ & 2 & $(0.02)$ & 2 & $(0.02)$ \\
\hline \multicolumn{16}{|c|}{ Sub-national entities } \\
\hline & Flanders (Belgium) & 18 & $(1.0)$ & -4 & $(0.02)$ & -5 & $(0.02)$ & -2 & $(0.02)$ & 6 & $(0.01)$ & 7 & $(0.01)$ & 9 & $(0.01)$ \\
\hline & England (UK) & 17 & (1.1) & -6 & $(0.02)$ & -7 & $(0.02)$ & -8 & $(0.02)$ & 6 & $(0.02)$ & 7 & $(0.01)$ & 5 & $(0.02)$ \\
\hline & Northern Ireland (UK) & 17 & $(1.5)$ & -8 & $(0.02)$ & -10 & $(0.02)$ & -12 & $(0.03)$ & 9 & $(0.02)$ & 10 & $(0.02)$ & 8 & $(0.02)$ \\
\hline & England/N. Ireland (UK) & 17 & $(1.1)$ & -6 & $(0.02)$ & -7 & $(0.02)$ & -8 & $(0.02)$ & 6 & $(0.01)$ & 7 & $(0.01)$ & 5 & $(0.02)$ \\
\hline & Average & 18 & $(0.2)$ & -5 & $(0.00)$ & -6 & $(0.00)$ & -6 & $(0.00)$ & 5 & $(0.00)$ & 5 & $(0.00)$ & 5 & $(0.00)$ \\
\hline 产 & Russian Federation* & $q$ & $q$ & q & $\mathrm{q}$ & $\mathrm{q}$ & $\mathrm{q}$ & q & $q$ & $\mathrm{q}$ & q & $\mathrm{q}$ & $\mathrm{q}$ & $q$ & $\mathrm{q}$ \\
\hline
\end{tabular}

Note: Calculations for percentage-point difference are based on linear regressions where the dependent variable is reporting to volunteer at least once a month and where the independent variables vary according to the model. In the first regression (columns labelled "No control variable") only educational attainment is used as an independent variable. In the second regression (columns labelled "Accounting for gender and age"), gender and age are added as independent variables. In the third regression (columns labelled "Accounting for gender, age and monthly earnings"), educational attainment, gender, age and monthly earnings are included as independent variables.

* See note on data for the Russian Federation in the Methodology section.

Source: OECD. Survey of Adult Skills (PIAAC) (2012). See Annex 3 for notes (www.oecd.org/education/education-at-a-glance-19991487.htm).

Please refer to the Reader's Guide for information concerning symbols for missing data and abbreviations.

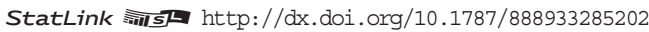


Table A8.3a. Likelihood of reporting to trust others, by educational attainment (2012) 25-64 year-olds, upper secondary or post-secondary non-tertiary education as reference category, percentage-point difference

\begin{abstract}
The percentages presented in the first column are not related to the regression. They should be used as a reference to better understand the percentage-point difference presented in the other columns.

How to read this table: In Australia, the percentage of individuals with below upper secondary education reporting to trust others decreases by 4 percentage points compared to someone who has upper secondary or post-secondary non-tertiary education. After accounting for gender and age, the percentage of individuals with below upper secondary education reporting to trust others decreases by 5 percentage points compared to someone who has upper secondary or post-secondary non-tertiary education. And after accounting for gender, age and monthly earnings, the percentage of individuals with below upper secondary education reporting to trust others decreases by 3 percentage points compared to someone who has upper secondary or post-secondary non-tertiary education.
\end{abstract}

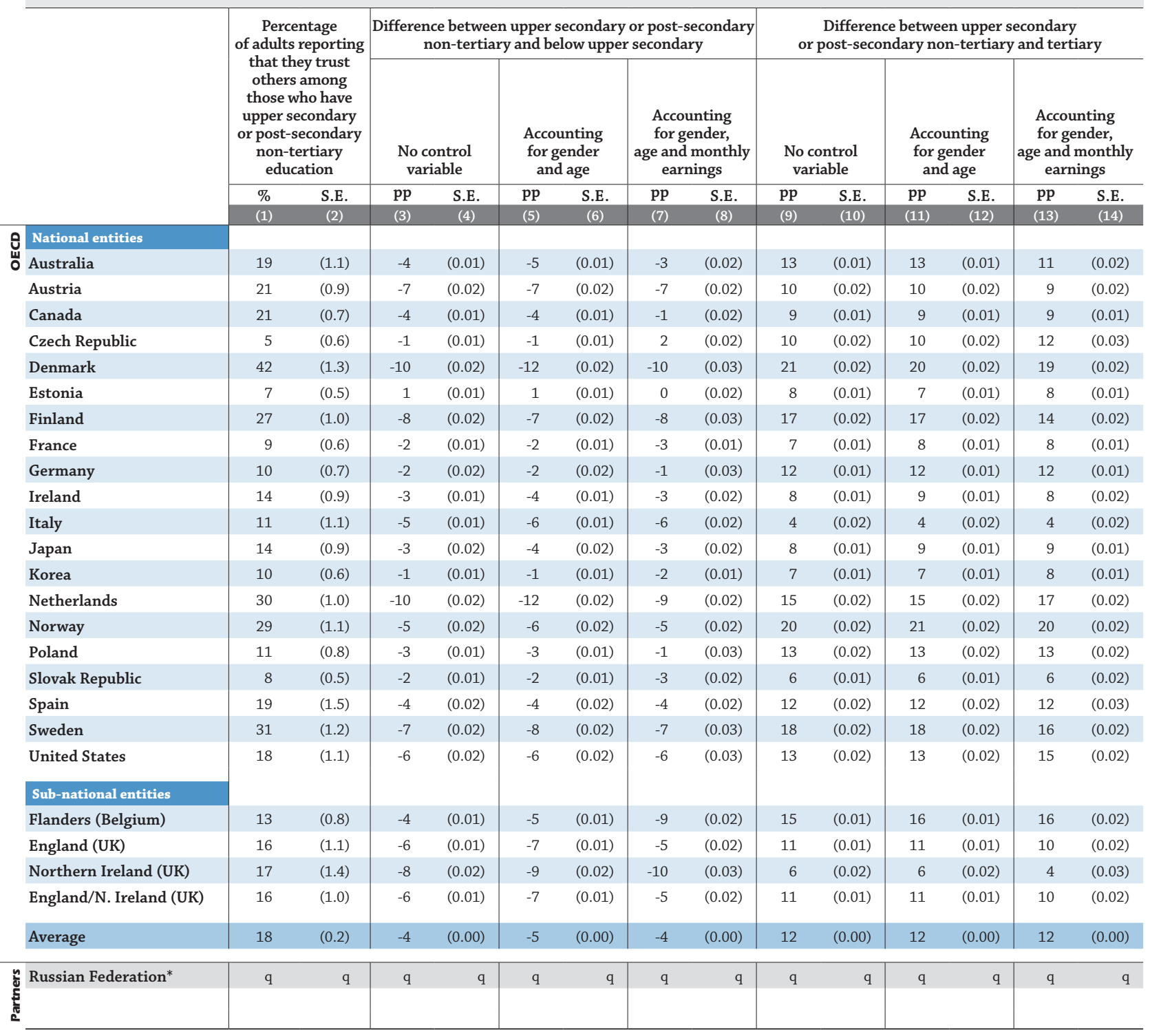

Note: Calculations for percentage-point difference are based on linear regressions where the dependent variable is reporting to trust others and where the independent variables vary according to the model. In the first regression (columns labelled "No control variable") only educational attainment is used as an independent variable. In the second regression (columns labelled "Accounting for gender and age"), gender and age are added as independent variables. In the third regression (columns labelled "Accounting for gender, age and monthly earnings"), educational attainment, gender, age and monthly earnings are included as independent variables.

* See note on data for the Russian Federation in the Methodology section.

Source: OECD. Survey of Adult Skills (PIAAC) (2012). See Annex 3 for notes (www.oecd.org/education/education-at-a-glance-19991487.htm).

Please refer to the Reader's Guide for information concerning symbols for missing data and abbreviations.

StatLink 게그 http://dx.doi.org/10.1787/888933285214 


\section{Table A8.4. Likelihood of reporting to believe that they have a say in government, by educational attainment (2012)}

\section{5-64 year-olds, upper secondary or post-secondary non-tertiary education as reference category,} percentage-point difference

The percentages presented in the first column are not related to the regression. They should be used as a reference to better understand the percentage-point difference presented in the other columns.

How to read this table: In Australia, the percentage of individuals with below upper secondary education reporting to believe that they have a say in goverment decreases by 6 percentage points compared to someone who has upper secondary or post-secondary non-tertiary education. After accounting for gender and age, the percentage of individuals with below upper secondary education reporting to believe that they have a say in goverment decreases by 8 percentage points compared to someone who has upper secondary or post-secondary non-tertiary education. And after accounting for gender, age and monthly earnings, the percentage of individuals with below upper secondary education reporting to believe that they have a say in goverment decreases by 6 percentage points compared to someone who has upper secondary or post-secondary non-tertiary education.

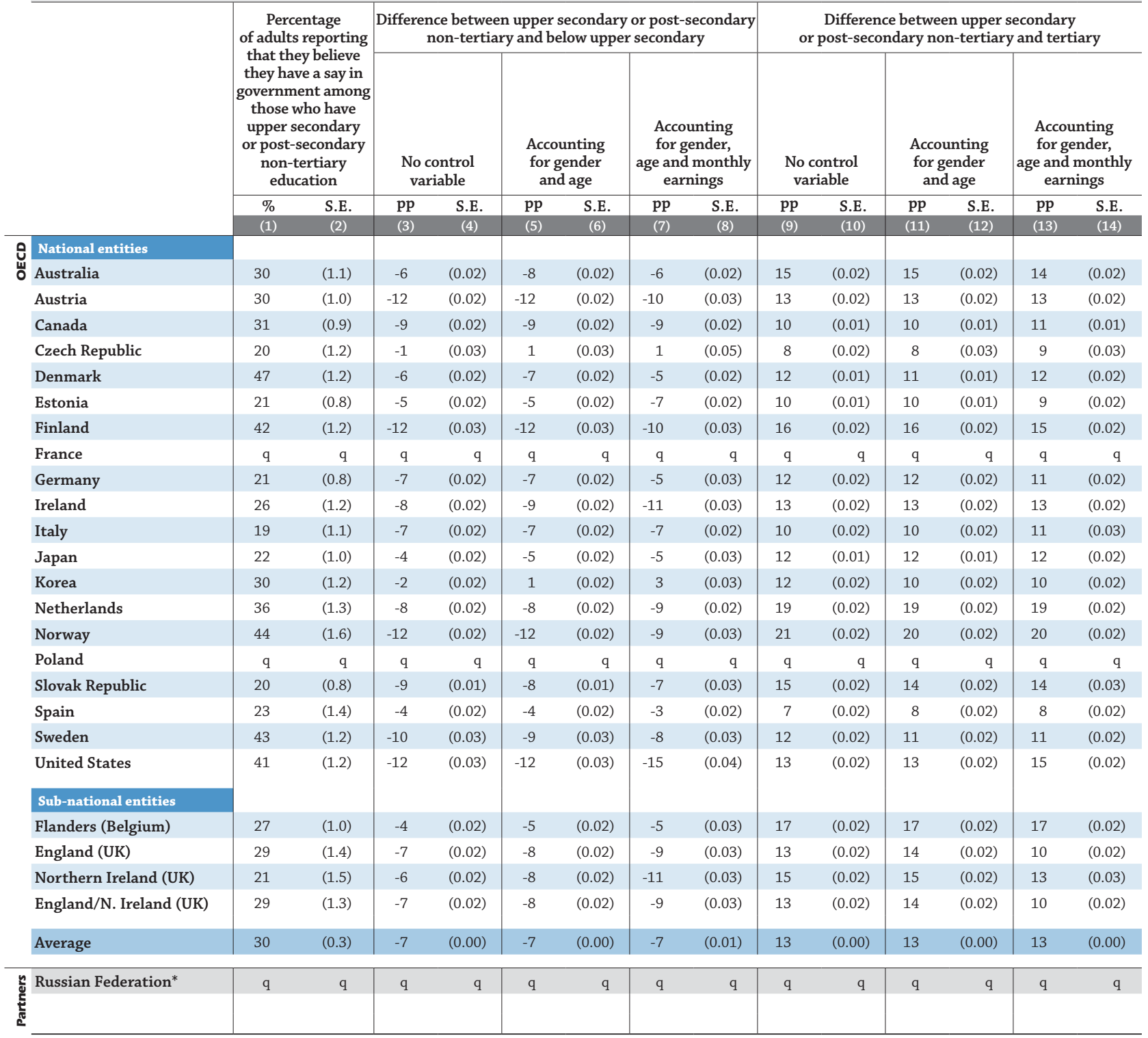

Note: Calculations for percentage-point difference are based on linear regressions where the dependent variable is reporting to believe that they have a say in government and where the independent variables vary according to the model. In the first regression (columns labelled "No control variable") only educational attainment is used as an independent variable. In the second regression (columns labelled "Accounting for gender and age"), gender and age are added as independent variables. In the third regression (columns labelled "Accounting for gender, age and monthly earnings"), educational attainment, gender, age and monthly earnings are included as independent variables.

* See note on data for the Russian Federation in the Methodology section.

Source: OECD. Survey of Adult Skills (PIAAC) (2012). See Annex 3 for notes (www.oecd.org/education/education-at-a-glance-19991487.htm).

Please refer to the Reader's Guide for information concerning symbols for missing data and abbreviations.

StatLink 제이 http://dx.doi.org/10.1787/888933285229 



\section{WHAT IS THE IMPACT OF SIKILLS ON EMPLOYMENT AND EARNINGS?}

- On average across countries and sub-national entities that participated in the Survey of Adult Skills (PIAAC) (2012), employment rates and earnings increase with educational attainment and, to a lesser extent, with higher skills.

- The highest returns to greater skills proficiency accrue to individuals who have attained tertiary education.

- Among adults with tertiary education and those with upper secondary or post-secondary nontertiary education, skills in using ICT for problem solving are associated with higher earnings compared to adults who are equally proficient in numeracy, and proficiency in numeracy yields higher returns than equivalent proficiency in literacy.

\section{Chart A9.1. Difference in hourly earnings, by educational attainment and skills (2012)}

Survey of Adult Skills, 25-64 year-old non-students, average across OECD countries, reference category is below upper secondary education and proficiency Level 1 or below, or skills Group 0 or 1
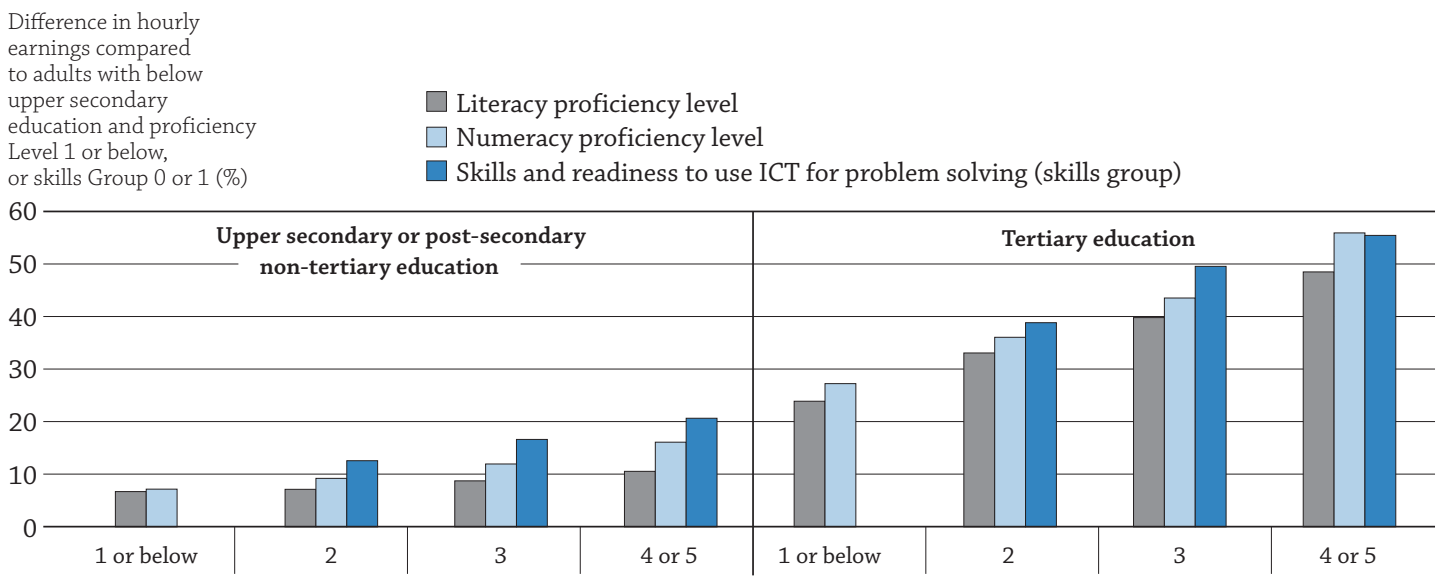

How to read this chart

On average, tertiary-educated adults with literacy proficiency of Level 4 or 5 earn $48 \%$ more compared with adults with below upper secondary education and literacy proficiency of Level 1 or below.

The percentages represent the earnings outcomes compared to the reference category (reference category is below upper secondary education and proficiency Level 1 or below, or skills Group 0 or 1 ).

Notes: Literacy and numeracy are based on proficiency levels whereas skills and readiness to use ICT for problem solving is based on skill groups which follow a different approach. For skills and readiness to use ICT for problem solving " 4 or 5 " should be interpreted as Group 4. Values are not shown when there are too few observations to provide reliable estimates.

Source: OECD. Tables A9.2 (L), A9.2 (N) and A9.2 (P). See Annex 3 for notes (www.oecd.org/education/education-at-a-glance19991487.htm).

StatLink 제에 $h$ htp://dx.doi.org/10.1787/888933283798

\section{Context}

Basic literacy, numeracy and problem-solving skills are usually acquired in formal schooling (Green and Riddell, 2012). But adults who have attained the same level of education can have different levels of proficiency in literacy and numeracy skills, and in skills related to using information and communication technology (ICT) to solve problems. To the extent that workers' productivity is related to the knowledge and skills they possess, and that wages reflect such productivity, albeit imperfectly, individuals with more skills should expect higher returns from labour market participation, and would thus be more likely to participate in it. Thus, improving the teaching of literacy and numeracy in schools and in programmes for adults with poor skills and limited familiarity with ICT may provide considerable economic and social returns for individuals and society as a whole (OECD, 2013). 


\section{Other findings}

- Adults with upper secondary or post-secondary non-tertiary education and numeracy proficiency of Level 1 or below earn $7 \%$ more per hour than adults with below upper secondary education and numeracy proficiency of Level 1 or below, while adults with upper secondary or post-secondary non-tertiary education and numeracy proficiency of Level 4 or 5 earn $16 \%$ more per hour than adults with below upper secondary education and numeracy proficiency of Level 1 or below. High skills, combined with a tertiary education, are even more highly rewarded. Tertiary-educated adults with numeracy proficiency of Level 4 or 5 earn $56 \%$ more than adults with below upper secondary education and numeracy proficiency of Level 1 or below - a difference of 40 percentage points.

- The odds of being employed do not necessarily increase as literacy skills improve. For example, in Poland, the odds ratio of being employed for an adult with tertiary education and literacy proficiency of Level 1 or below is the highest (11.7), whereas the odds ratio for a tertiary-educated adult with literacy proficiency of Level 4 or 5 is 9.0.

- The greatest returns for individuals with tertiary education and numeracy proficiency of Level 4 or 5 are observed in the Slovak Republic. The hourly earnings of adults with those levels of education and skills are 108\% higher than those of adults with below upper secondary education and Level 1 or below proficiency in numeracy - a much larger difference than the average (56\%).

\section{INDICATOR A9}




\section{Analysis}

This indicator deepens the analyses discussed in Indicators A5 and A6 that show that employment rates and earnings increase as the level of education increases. More specifically, it evaluates the relative impact on employment rates and hourly earnings of higher levels of educational attainment and greater proficiency in literacy, numeracy and using ICT for problem solving. Findings are based on the Survey of Adult Skills, a product of the OECD Programme for the International Assessment of Adult Competencies (PIAAC) (2012).

Results show that employment rates and earnings increase with educational attainment and, to a lesser extent, with higher skills. This means that the labour market rewards educational attainment more highly than the skills measured by the Survey of Adult Skills.

\section{The impact of education and skills on employment}

\section{Impact of education and literacy skills on employment}

Higher educational attainment and higher levels of skills have a positive impact on employment. On average across the OECD countries and sub-national entities that participated in the Survey of Adult Skills in 2012, $48 \%$ of adults with below upper secondary qualifications and literacy proficiency of Level 1 or below are employed (reference group). Individuals with upper secondary or post-secondary non-tertiary education as their highest level of attainment and with literacy proficiency of Level 4 or 5 are more likely to be employed compared with the reference group (odds ratio of 2.4 - see Box A9.1. for how to interpret odds ratios). The likelihood of being employed increases for adults with tertiary qualifications and literacy proficiency of Level 4 or 5 (odds ratio of 4.2) (Table A9.1 [L]).

The returns associated with greater literacy proficiency within education levels appear to be more limited. For individuals with below upper secondary education, there is a small difference between those with Level 2 or Level 3 in literacy (odds ratio of 1.3 and 1.2, respectively). For those with an upper secondary or post-secondary non-tertiary education, the odds ratio remains the same (2.0), regardless of whether an adult is proficient to Level 1 or below, Level 2 or Level 3 in literacy. When an adult at that level of education is proficient to Level 4 or 5 , the odds of being employed are 2.4. For tertiary-educated adults, the odds of being employed increase as proficiency in literacy increases: odds ratio of 2.9 for Level 1 or below, 3.7 for Levels 2 and 3, and 4.2 for Level 4 or 5 (Table A9.1 [L]).

In all participating countries and sub-national entities, the odds of being employed are greater for individuals with tertiary education, regardless of their proficiency in literacy. Data also show that the odds of being employed do not necessarily increase as an individual improves in literacy. For example, in Poland, the odds ratio of being employed for an adult with tertiary education and literacy proficiency of Level 1 or below is the highest (11.7), whereas the odds ratio for an adult with similar educational attainment, but whose literacy proficiency is Level 4 or 5 is 9.0. In the Slovak Republic, tertiary-educated adults with literacy proficiency of Level 2 or 3 have an odds ratio of more than 5.0 of being employed while the odds ratio of those with similar educational attainment and with literacy proficiency of Level 4 or 5 is 3.4, another example where higher literacy skills are not necessarily associated with higher employment (Table A9.1 [L]).

\section{Impact of education and numeracy skills on employment}

Compared to literacy skills, numeracy skills have a more significant impact on employment outcomes. On average across participating OECD countries and sub-national entities, $47 \%$ of individuals with below upper secondary education as their highest level of attainment and with numeracy proficiency of Level 1 or below are employed (reference group). For those individuals with below upper secondary education, an increase in numeracy proficiency from Level 1 or below to Level 2 improves the probability of being employed (odds ratio of 1.5). When such an individual improves in numeracy from Level 1 or below to Level 3, the odds of being employed increases to 1.8 (Table A9.1 [N]).

An adult with upper secondary or post-secondary non-tertiary education as his or her highest level of attainment and numeracy proficiency at or below Level 1 has an odds ratio of being employed of 1.9. If that person were to improve his or her numeracy skills to Level 2, the odds ratio would improve to 2.5; Level 3 proficiency would result in an odds ratio of 3.0, and a proficiency of Level 4 or 5 would yield a ratio of 3.8.

Similarly, improvements in numeracy proficiency for tertiary-educated adults increase the probability of being employed: the odds ratio is 2.8 for Level 1 or below; 4.5 for Level 2; 5.5 for Level 3; and 7.6 for Level 4 or 5 (Table A9.1 [N]). 


\section{Chart A9.2. Likelihood of being employed, by educational attainment and numeracy proficiency (2012)}

Survey of Adult Skills, 25-64 year-old non-students, reference category is below upper secondary education and numeracy proficiency of Level 1 or below

\begin{tabular}{|c|c|c|c|c|c|c|c|c|c|c|c|c|c|c|c|c|c|c|c|c|c|}
\hline \multirow{3}{*}{$\begin{array}{l}\text { Odds } \\
\text { ratio } \\
20+1 \\
\end{array}$} & \multirow{2}{*}{\multicolumn{4}{|c|}{$\begin{array}{l}\text { Level 3: } 27 \\
\text { Level } 4 \text { or 5: } 34 \\
\text { Level } 4 \text { or 5: } 22\end{array}$}} & \multicolumn{11}{|c|}{ Tertiary education } & & & & & & \\
\hline & & & & & \multicolumn{4}{|c|}{ Level 1 or below } & \multicolumn{2}{|c|}{$\square$ Level 2} & \multicolumn{2}{|c|}{ ○ Level } & \multicolumn{3}{|c|}{$\Delta$ Level 4 or 5} & & & & & & \\
\hline & & & & & & & & & & & & & & & & & & & & & \\
\hline 16 & & & & & & & & & & & & & & & & & & & & & \\
\hline 14 & & & & & & & & & & & & & & & & & & & & & \\
\hline 12 & & $\Delta$ & & & & & & & & & & & & & & & & & & & \\
\hline 10 & 0 & & $\Delta$ & & & & & & & & & & & & & & & & & & \\
\hline 8 & 4 & & & 4 & $\Delta$ & $\theta$ & $\Delta$ & & & & & & & & & & & & & & \\
\hline & & & & 1 & & & 0 & 4 & 4 & & & & & & & & & & & $10=$ & \\
\hline 4 & & 0 & - & 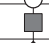 & 9 & 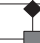 & I & & 8 & 5 & $\theta$ & 4 & 4 & A & & & a & & & & \\
\hline & & 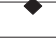 & & 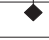 & ப & 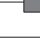 & & $\bullet$ & 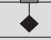 & & 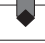 & $\checkmark$ & $\rho$ & 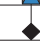 & $\Delta$ & 1 & 8 & A & A & a & $\mathrm{n}$ \\
\hline $0 \ldots \ldots$ & $\cdots \cdot$ & $\cdots .$. & ..... & $\cdots$ & & .... & ..... & $\ldots$ & (i) & ......... & ........ & 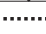 & & & ....... & $\cdots$ & $\cdots$ & 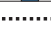 & & $\ldots$ & ..... \\
\hline 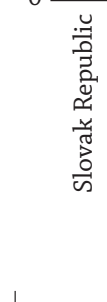 & 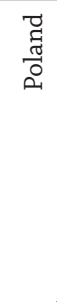 & 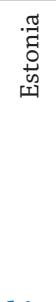 & 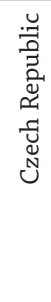 & 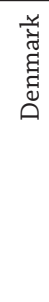 & 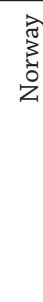 & 疍 & 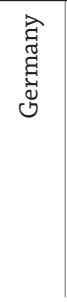 & 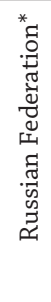 & 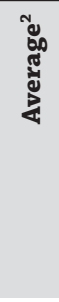 & 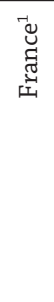 & 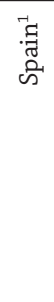 & $\begin{array}{l}\text { శ్ } \\
\text { శ్ } \\
\widetilde{J}\end{array}$ & 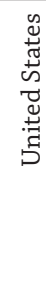 & 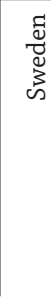 & 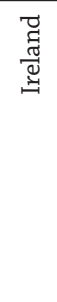 & 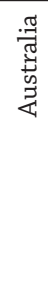 & 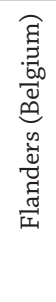 & 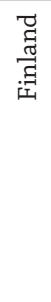 & 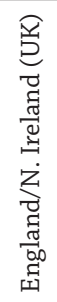 & 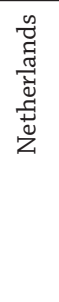 & 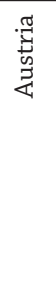 \\
\hline
\end{tabular}

How to read this chart

In the Slovak Republic, a person with tertiary education and a numeracy proficiency of Level 4 or 5 is 33.8 times as likely (in terms of odds ratio) of being employed as someone with below upper secondary education and a numeracy proficiency of Level 1 or below.

The "odds ratio" reflects the relative likelihood of being employed. The reference category is below upper secondary education and a numeracy proficiency of Level 1 or below and their odds ratio are set to equal 1 (thicker line).

Notes: The odds ratio are based on a logistic regression, after accounting for: age, gender, parents' educational attainment, immigration background, parental status (have a child or not), cohabitation status (living with spouse/partner or not), literacy proficiency, skills and readiness to use ICT for problem solving. Differences between the groups are not shown when they are not statistically significant at $95 \%$.

1. The coefficients for France, Italy and Spain have been estimated without accounting for skills and readiness to use ICT for problem solving since it was not tested in these countries. Since there is positive correlation between skills and readiness to use ICT for problem solving and numeracy, literacy and education, the effect of excluding skills and readiness to use ICT for problem solving is likely to be that the coefficients on the proficiency by education level are overestimated, relative to the results for other countries.

2. Average for the regression excludes France, Italy and Spain as a different model specification was used for these countries.

* See note on data for the Russian Federation in the Methodology section.

Countries are ranked in descending order of the odds ratio of being employed for individuals with tertiary education and a numeracy proficiency of Level 4 or 5.

Source: OECD. Table A9.1 (N). See Annex 3 for notes (www.oecd.org/education/education-at-a-glance-19991487.htm).

StatLink 젠 http://dx.doi.org/10.1787/888933283802

Chart A9.2 shows the odds of being employed among adults with tertiary education and different levels of numeracy proficiency relative to the odds of being employed among adults with below upper secondary education with numeracy proficiency of Level 1 or below. As observed with literacy proficiency, adults with tertiary education have greater odds of being employed, regardless of their proficiency in numeracy. Results show that numeracy has a stronger impact on employment compared to literacy. For example, in Poland, the odds ratio of being employed for those with numeracy proficiency of Level 3 is 11.0 whereas it is 22.4 for adults with similar educational attainment and with numeracy proficiency of Level 4 or 5 , the largest difference among participating countries and sub-national entities. On average, the odds ratio of being employed for individuals with numeracy proficiency of Level 4 or 5 (7.6) is higher than that of adults with similar educational attainment and with literacy proficiency of Level 4 or 5 (4.2) (Tables A9.1 [L] and A9.1 [N]).

\section{Impact of education and skills and readiness to use ICT for problem solving on employment}

On average, $38 \%$ of adults with ICT and problem-solving skills of Group 0 or 1 (see the Definitions section below for a description of skill groups) and below upper secondary education are employed (reference group). Adults with upper secondary or post-secondary non-tertiary education as their highest level of attainment and good ICT and problemsolving skills are more likely to be employed compared with the reference group (odds ratio of 2.8). The likelihood of being employed increases for individuals with tertiary qualifications and good ICT and problem-solving skills (odds ratio of 5.1) (Table A9.1 [P]). 
As with literacy and numeracy, tertiary-educated adults have greater odds of being employed, regardless of their skills and readiness to use ICT for problem solving. The odds ratio of being employed for adults with tertiary education and good ICT and problem-solving skills are the highest in Norway and Poland (odds ratio of 9.8 and 11.7, respectively). In these two countries, the odds ratio of being employed increase as skills improve. This is not the case in Estonia and the Slovak Republic, where the odds of being employed are the fourth and third highest, respectively, among adults with a similar profile (odds ratio of 8.4 and 8.9, respectively). In these two countries, adults with lower skills have greater odds of being employed (Table A9.1 [P]).

\section{Employment returns to education and skills proficiency}

In most countries, improvements in skills proficiency for adults with below upper secondary as their highest level of education do not have a statistically significant effect on employment. At the upper secondary or post-secondary nontertiary level, the strongest impact on employment related to changes in skills proficiency tend to be associated with numeracy proficiency, especially when moving from Level 3 to Level 4 or 5. For example, in Italy and the Slovak Republic, there is an increase of more than 4 points in the odds ratio between these two proficiency levels (Table A9.1 [N]).

Among tertiary-educated adults, the strongest impact on employment related to changes in skills proficiency also tend to be associated with numeracy proficiency, especially when moving from Level 3 to Level 4 or 5 . In the Czech Republic, Estonia, Poland and the Slovak Republic, the odds ratio increases by more than 5 points between these two proficiency levels (Table A9.1 [N]).

\section{The impact of education and skills on earnings}

Chart A9.1 shows the impact of educational attainment and skills on hourly earnings. Data for adults with below upper secondary as their highest level of education are not presented in this chart because differentials in hourly earnings are not statistically significant across proficiency levels and skills groups among adults with this level of education. However, pattern starts to take shape when comparing adults with upper secondary or post-secondary non-tertiary education with various levels of skills, and adults with below upper secondary education and the lowest level of skills (the reference category) (Tables A9.2 [L], A9.2 [N] and A9.2 [P]).

Results show that among adults with literacy or numeracy proficiency at Level 1 or below, adults with upper secondary or post-secondary non-tertiary education earn $7 \%$ more per hour than adults with below upper secondary education. For skills and readiness to use ICT for problem solving, this difference is not significant and thus not displayed in the chart (Tables A9.2 [L], A9.2 [N] and A9.2 [P]).

When moving to higher skills levels, results become significant for all skills measured, and show that literacy proficiency has less of an effect on earnings than numeracy proficiency, which, in turn, has less of an effect than ICT skills and the readiness to use ICT for problem solving. The differences in the effect on hourly earnings between these three skills tend to increase with proficiency, meaning that an adult with upper secondary or post-secondary non-tertiary education and with good ICT and problem-solving skills can expect greater returns compared with top performers in literacy and numeracy (Tables A9.2 [L], A9.2 [N] and A9.2 [P]).

The chart shows larger returns to adults with higher skills, but it also shows that attaining higher levels of education yields greater returns. For example, adults with upper secondary or post-secondary non-tertiary education and numeracy proficiency of Level 1 or below earn $7 \%$ more per hour than adults with below upper secondary education and numeracy proficiency of Level 1 or below, while adults with upper secondary or post-secondary non-tertiary education and numeracy proficiency of Level 4 or 5 earn $16 \%$ more per hour than adults with below upper secondary education and numeracy proficiency of Level 1 or below. High skills, combined with a tertiary education, are even more highly rewarded. Tertiary-educated adults with numeracy proficiency of Level 4 or 5 earn $56 \%$ more than adults with below upper secondary education and numeracy proficiency of Level 1 or below - a difference of 40 percentage points (Tables A9.2 [L], A9.2 [N] and A9.2 [P]).

\section{Impact of education and literacy skills on earnings}

In general, within each education level, there are positive returns to greater literacy proficiency; but the returns are even greater to higher educational attainment. In many countries, adults with upper secondary or post-secondary non-tertiary education and the highest skills in literacy earn less than adults with tertiary education and the lowest literacy skills (Table A9.2 [L]).

Individuals with upper secondary or post-secondary non-tertiary education and literacy proficiency of Level 1 or below earn 7\% more than adults with below upper secondary education and literacy proficiency of Level 1 or below (reference category), while adults with upper secondary or post-secondary non-tertiary education and literacy 
proficiency Level 4 or 5 earn 11\% more per hour than adults in the reference category. A similar analysis among tertiary-educated adults reveals a 24\% increase in hourly earnings for adults with literacy proficiency of Level 1 or below and a $48 \%$ increase for individual with literacy proficiency of Level 4 or 5 compared with adults in the reference category. (Table A9.2 [L]).

These estimates are averages; there is significant variation across national and sub-national entities. For instance, increases in hourly earnings are largest in the Slovak Republic for adults with tertiary education and literacy proficiency of Level 4 or 5 compared to the reference category. The hourly earnings of these adults are $87 \%$ higher than the earnings of adults with below upper secondary education and literacy proficiency of Level 1 or below much larger than the average difference (48\%) between these two groups. By contrast, this difference is less than $30 \%$ in Finland, Japan, Norway and Sweden (Table A9.2 [L]).

Korea shows the largest difference in returns, related to skills proficiency, among tertiary-educated adults. The hourly earnings of those with tertiary education and literacy proficiency of Level 1 or below are $42 \%$ higher than the earnings of those with below upper secondary education and literacy proficiency of Level 1 or below. The earnings of those with tertiary education and literacy proficiency of Level 4 or 5 are 83\% higher (Table A9.2 [L]).

\section{Impact of education and numeracy skills on earnings}

On average across OECD countries and sub-national entities that participated in the Survey of Adult Skills, compared to an adult with below upper secondary education and with numeracy proficiency at Level 1 or below (reference group), an adult with upper secondary or post-secondary non-tertiary education with similar proficiency in numeracy earns $7 \%$ more per hour. Those with similar educational attainment but with numeracy proficiency at Level 4 or 5 see a $16 \%$ increase in their hourly earnings. For tertiary-educated adults, the earnings outcomes, compared to the reference group, range from an increase of $27 \%$ among those with proficiency Level 1 or below in numeracy to an increase of $56 \%$ among those with proficiency Level 4 or 5 in numeracy (Table A9.2 [N]).

As shown in Chart A9.3, the greatest returns for individuals with tertiary education and numeracy proficiency of Level 4 or 5, compared to the reference category, are observed in the Slovak Republic. These adults earn 108\% more per hour than adults with below upper secondary education and numeracy proficiency of Level 1 or below - a much larger difference between the two groups than the average (56\%). Estonia shows the largest difference in returns related to numeracy proficiency for tertiary-educated adults. Adults at this level of education who are proficient at Level 1 or below in numeracy earn 31\% more than the reference group, while those with the same level of education but with numeracy proficiency at Level 4 or 5 earn $76 \%$ more (Table A9.2 [N]).

\section{Impact of education and skills and readiness to use ICT for problem solving on earnings}

As shown in Chart A9.1, the advantage in having higher ICT and problem-solving skills is greatest among tertiaryeducated adults. In Austria, the Czech Republic, England/Northern Ireland (UK), Korea and the United States, the relative hourly earnings advantage for tertiary-educated adults who have moderate ICT and problem-solving skills (Group 3) is at least 15 percentage points greater than for adults with minimal problem-solving skills in technology-rich environments and adults who failed the first stage of the computer-based assessment (Group 2). In England/Northern Ireland (UK) and Korea, adults with good ICT and problem-solving skills (Group 4) add 18 and 12 percentage points, respectively, to the relative hourly earnings of adults in Group 3. More generally, across OECD countries and sub-national entities, the relative hourly earnings advantage for tertiary-educated adults with moderate ICT and problem-solving skills is 11 percentage points greater than for tertiary-educated adults with minimal skills in problem solving using ICT and adults who failed the first stage of the computer-based assessment. The relative earnings is 5 percentage points greater for adults with good ICT and problem-solving skills compared to those with moderate ICT and problem-solving skills (Table A9.2 [P]).

\section{Earnings returns to education and skills proficiency}

The analysis shows the relative importance of each set of skills at different levels of educational attainment. For example, across countries and sub-national entities that participated in the survey, adults with upper secondary or post-secondary non-tertiary education, proficiency in using ICT for problem solving is associated with higher earnings compared to equivalent numeracy skills, which, in turn, yield larger returns than equivalent literacy skills. On average, adults with upper secondary or post-secondary non-tertiary education and good ICT and problem-solving skills (Group 4) earn 21\% more per hour than adults with below upper secondary education and no computer experience or who refused the computer-based assessment (Group 0 or 1). Those at Level 4 or 5 in numeracy proficiency earn 16\% more per hour and those at Level 4 or 5 in literacy earn $11 \%$ more compared to adults with below upper secondary education and proficiency Level 1 or below in these skills (Tables A9.2 [L], A9.2 [N] and A9.2 [P]). 


\section{Chart A9.3. Difference in hourly earnings, by educational attainment and numeracy proficiency (2012)}

Survey of Adult Skills, 25-64 year-old non-students, reference category is below upper secondary education and numeracy proficiency of Level 1 or below

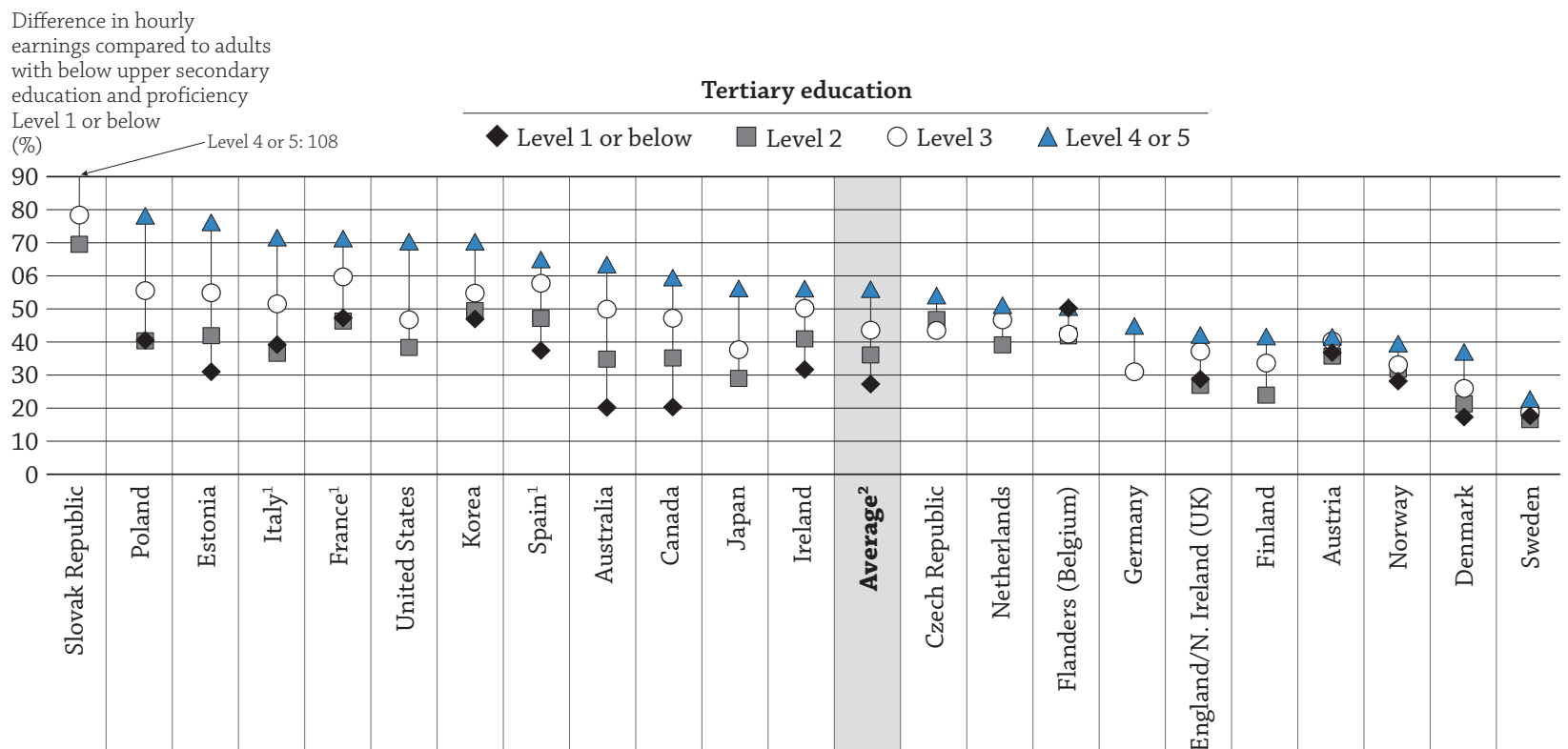

How to read this chart

In the Slovak Republic, tertiary-educated adults with numeracy proficiency of Level 4 or 5 earn $108 \%$ more compared with adults with below upper secondary education and numeracy proficiency of Level 1 or below.

The percentages represent the earnings outcomes compared to the reference category (below upper secondary education and a numeracy proficiency of Level 1 or below).

Notes: The values are based on a linear regression, after accounting for: age, gender, parents' educational attainment, immigration background, parental status (have a child or not), cohabitation status (living with spouse/partner or not), literacy proficiency, skills and readiness to use ICT for problem solving. Differences between the groups are not shown when they are not statistically significant at $95 \%$.

1. The coefficients for France, Italy and Spain have been estimated without accounting for skills and readiness to use ICT for problem solving since it was not tested in these countries. Since there is positive correlation between skills and readiness to use ICT for problem solving and numeracy, literacy and education, the effect of excluding skills and readiness to use ICT for problem solving is likely to be that the coefficients on the proficiency by education level are overestimated, relative to the results for other countries.

2. Average for the regression excludes France, Italy and Spain as a different model specification was used for these countries.

Countries are ranked in descending order of the percentage increase in earnings for individuals with tertiary education and a numeracy proficiency of Level 4 or 5. Source: OECD. Table A9.2 (N). See Annex 3 for notes (www.oecd.org/education/education-at-a-glance-19991487.htm).

StatLink 젠 http://dx.doi.org/10.1787/888933283811

\section{Definitions}

Adults refer to 25-64 year-olds.

Earnings refer to hourly earnings excluding bonuses for wage and salary earners.

The employment rate refers to the number of persons in employment as a percentage of the working-age population (the number of employed people is divided by the number of all working-age people). Employment rates by gender, age, educational attainment, programme orientation and age groups are calculated within each of these categories; for example the employment rate among women is calculated by dividing the number of employed women by the total number of working-age women.

Levels of education: Below upper secondary corresponds to ISCED-97 Levels 0, 1, 2 and 3C short programmes; upper secondary or post-secondary non-tertiary corresponds to ISCED-97 Levels 3A, 3B, 3C long programmes, and Level 4; and tertiary corresponds to ISCED-97 Levels 5A, 5B and 6.

Literacy is the ability to understand, evaluate, use and engage with written texts to participate in society, to achieve one's goals, and to develop one's knowledge and potential. Literacy encompasses a range of skills from the decoding of written words and sentences to the comprehension, interpretation, and evaluation of complex texts. It does not, however, involve the production of text (writing). Information on the skills of adults with 
low levels of proficiency is provided by an assessment of reading components that covers text vocabulary, sentence comprehension and passage fluency.

Numeracy is the ability to access, use, interpret and communicate mathematical information and ideas in order to engage in and manage the mathematical demands of a range of situations in adult life. To this end, numeracy involves managing a situation or solving a problem in a real context, by responding to mathematical content/ information/ideas represented in multiple ways.

Problem solving in technology-rich environments is the ability to use digital technology, communication tools and networks to acquire and evaluate information, communicate with others and perform practical tasks. The assessment focuses on the abilities to solve problems for personal, work and civic purposes by setting up appropriate goals and plans, and accessing and making use of information through computers and computer networks.

Proficiency levels for literacy and numeracy are based on a 500-point scale. Each level has been defined by particular score-point ranges. Six levels are defined for literacy and numeracy (Below Level 1 and Levels 1 through 5) which are grouped in four proficiency levels in Education at a Glance: Level 1 or below - all scores below 226 points; Level 2 scores from 226 points to less than 276 points; Level 3 - scores from 276 points to less than 326 points; Level 4 or 5 - scores from 326 points and higher.

Skill groups refer to skills and readiness to use information and communication technologies (ICT) for problem solving in technology-rich environments. Each group is described in terms of the characteristics of the types of tasks that can be successfully completed by adults and the related scores in the assessment of problem solving in technology-rich environments in the Survey of Adult Skills.

- Group 0 or 1 (no computer experience or refused the computer-based assessment)

- Group 2 (failed ICT core test or minimal problem-solving skills - scored below Level 1 in the problem solving in technology-rich environments assessment)

- Group 3 (moderate ICT and problem-solving skills - scored at Level 1 in the problem solving in technology-rich environments assessment)

- Group 4 (good ICT and problem-solving skills - scored at Level 2 or Level 3 in the problem solving in technologyrich environments assessment)

\section{Methodology}

All data are based on the Survey of Adult Skills (PIAAC) (2012). PIAAC is the OECD Programme for the International Assessment of Adult Competencies. See Annex 3 (www.oecd.org/education/education-at-a-glance-19991487.htm) for additional information.

The sample under consideration is restricted to non-students, as including the employment status and reported earnings for students would likely obscure the impact of skills on labour market outcomes. There is no restriction based on age implying that the sample includes those aged between 25 and 64 . When the impact of skills and education on earnings is undertaken, the self-employed are excluded.

\section{Box A9.1. Description of logistic regression analysis and interpretation of odds ratio}

Logistic regression analysis enables an estimation of the relationship between one or more independent variables (predictors) on categorical dependent (predicted) variables with two categories (binary logistic regression) or more than two categories (multinomial logistic regression). Multinomial logistic regression compares multiple groups through a combination of binary logistic regressions. Logistic regression analyses were carried out to evaluate the likelihood of being employed for different levels of skills and education. When a logistic regression is calculated, the statistical software output generates the regression coefficient ( $ß$ ), which is the estimated increase in the log odds of the outcome per unit increase in the value of the predictor variable. Odds ratio (OR) is obtained with the exponential function of the regression coefficient (exp (B)). The transformation of log odds $(ß)$ into odds ratios $(O R=\exp (ß))$ makes the data more interpretable in terms of probability. Three types of outcomes are possible for the odds ratios:

- $\mathrm{OR}=1$ Predictor variable does not affect odds of outcome

- $\mathrm{OR}>1$ Predictor variable associated with higher odds of outcome

- OR <1 Predictor variable associated with lower odds of outcome 
In odds ratios, categories are compared with a predetermined reference category. For example, in Table A9.1 (L) the reference category is 25-64 year-olds with literacy proficiency of Level 1 or below and educational attainment of below upper secondary education. Odds ratios can be interpreted in such a way that for a unit change in the predictor variable (e.g. level of education changing from below upper secondary education to upper secondary or post-secondary non-tertiary education), the odds ratio of the outcome variable relative to the reference category is expected to change by a factor of the respective parameter estimate, given that the other variables in the model are held constant.

It is also important to note that the odds of being employed are not the same as the probability of employment although there is a correspondence between the measures:

$$
\text { Odds = Probability } /(1-\text { Probability }) \text { and conversely, Probability }=\text { Odds } /(1+O d d s)
$$

The odds of being employed can be defined as the probability of employment over the probability of nonemployment so, for example, a probability of $50 \%$ corresponds to odds of 1 . As a further example, on average the probability of being employed stands at $48 \%$ for the reference category (i.e. below upper secondary education and literacy proficiency of Level 1 or below) corresponding to employment odds of $0.92(=0.48 /(1.0-0.48)$ ). To compare the employment outcomes of different groups of individuals, we estimate the odds ratio, which is the employment odds of the selected group divided by the employment odds of the reference category. Therefore, taking the previous example, if the employment odds of the reference category stands at 0.92 and the odds ratio is 4.2 for individuals with tertiary education and literacy proficiency of Level 4 or 5 , then the odds of being employed for this selected group are 4.2 times the odds of being employed for the baseline group (i.e. $3.86=4.2 * 0.92$ ). Using this figure, we could convert back to probabilities and say that the employment rate for individuals with tertiary education and literacy proficiency of Level 4 or 5 is approximately $79 \%$ $(=3.86 /(1+3.86))$.

\section{Note regarding data from the Russian Federation in the Survey of Adult Skills (PIAAC)}

Readers should note that the sample for the Russian Federation does not include the population of the Moscow municipal area. The data published, therefore, do not represent the entire resident population aged 16-65 in Russia but rather the population of Russia excluding the population residing in the Moscow municipal area. More detailed information regarding the data from the Russian Federation as well as that of other countries can be found in the Technical Report of the Survey of Adult Skills (OECD, 2014).

\section{References}

Green, D.A., W.C. Riddell (2012), Understanding Educational Impacts: The Role of Literacy and Numeracy Skills, Department of Economics, University of British Columbia.

OECD (2014), Technical Report of the Survey of Adult Skills, www.oecd.org/site/piaac/ Technical\%20Report 170CT13.pdf, pre-publication copy.

OECD (2013), OECD Skills Outlook 2013: First Results from the Survey of Adult Skills, OECD Publishing, Paris, http://dx.doi. org/10.1787/9789264204256-en.

\section{Indicator A9 Tables}

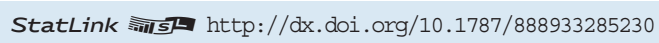

Table A9.1 (L) Likelihood of being employed, by educational attainment and literacy proficiency (2012)

Table A9.1 (N) Likelihood of being employed, by educational attainment and numeracy proficiency (2012)

Table A9.1 (P) Likelihood of being employed, by educational attainment and skills and readiness to use information and communication technologies for problem solving (2012)

Table A9.2 (L) Difference in hourly earnings, by educational attainment and literacy proficiency (2012)

Table A9.2 (N) Difference in hourly earnings, by educational attainment and numeracy proficiency (2012)

Table A9.2 (P) Difference in hourly earnings, by educational attainment and skills and readiness to use information and communication technologies for problem solving (2012) 
Table A9.1 (L). [1/2] Likelihood of being employed, by educational attainment and literacy proficiency (2012)

25-64 year-old non-students, below upper secondary education and proficiency Level 1 or below as reference category, odds ratio Logistic regression is used to estimate the odds ratios and p-values; an odds ratio reflects the relative likelihood of being employed compared to someone with
an education level of below upper secondary education and a literacy proficiency of Level 1 or below. The latter group is taken as the reference category for the
interpretation of the relative likelihood and therefore their odds ratio are set to equal 1 . Differences between the groups are statistically significant at $95 \%$ if
the "p-value" associated with the odds ratio is below 0.05 .
How to read this table: In Australia, a person with tertiary education and a literacy proficiency of Level 4 or 5 is 2.6 times as likely (in terms of odds ratio) of being
employed as someone with below upper secondary education and a literacy proficiency of Level 1 or below.

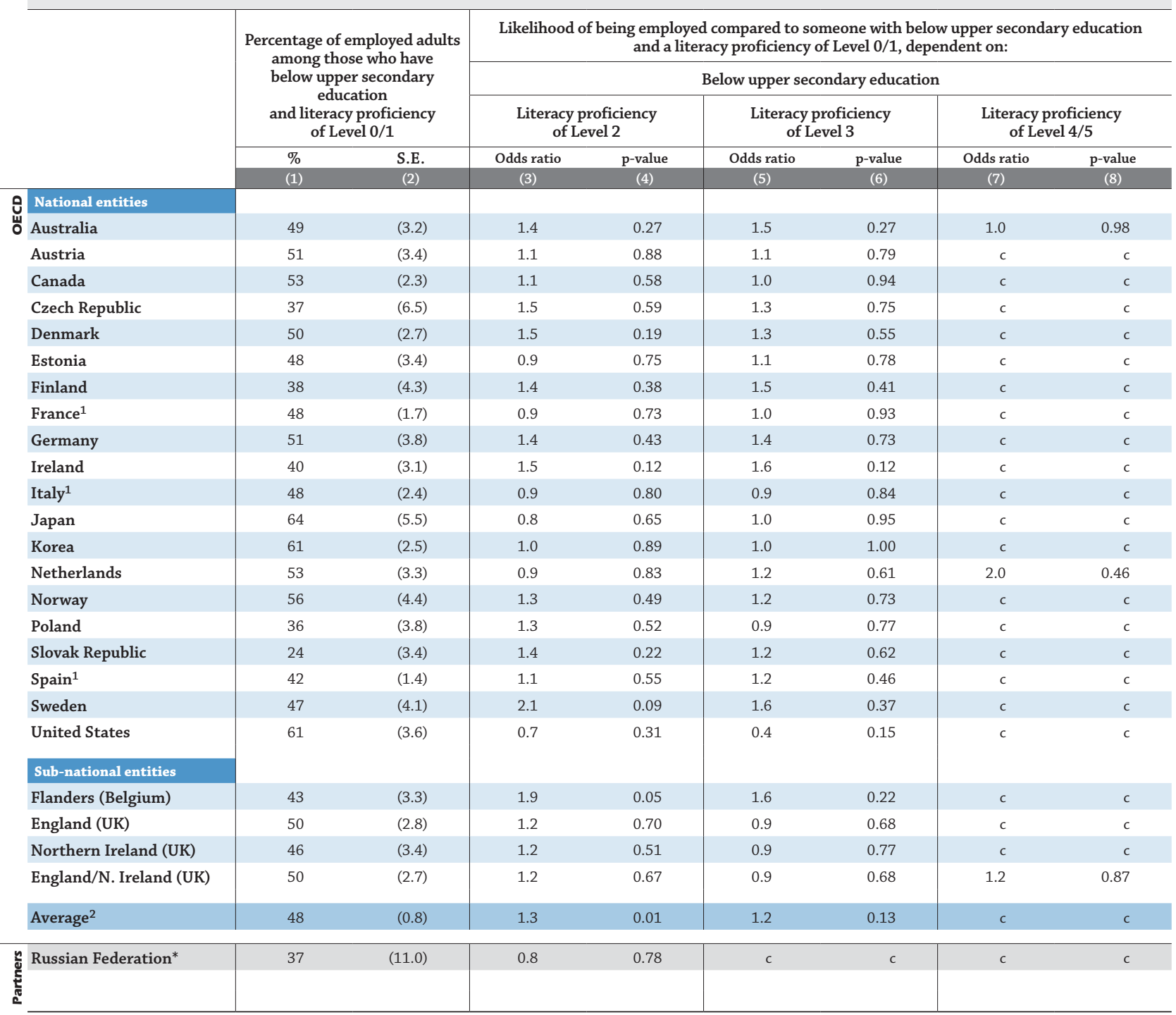

Note: Calculations for odds ratio are based on logistic regressions where the dependent variable is the likelihood of being employed and where the independent variables are educational attainment and proficiency levels, after accounting for: age, gender, parents' educational attainment, immigration background, parental status (have a child or not), cohabitation status (living with spouse/partner or not), numeracy proficiency, skills and readiness to use ICT for problem solving. The reference category is below upper secondary education and proficiency Level 1 or below.

1. The coefficients for France, Italy and Spain have been estimated without accounting for skills and readiness to use ICT for problem solving since it was not tested in these countries. Since there is positive correlation between skills and readiness to use ICT for problem solving and numeracy, literacy and education, the effect of excluding skills and readiness to use ICT for problem solving is likely to be that the coefficients on the proficiency by education level are overestimated, relative to the results for other countries.

2. Average for the regression excludes France, Italy and Spain as a different model specification was used for these countries.

* See note on data for the Russian Federation in the Methodology section.

Source: OECD. Survey of Adult Skills (PIAAC) (2012). See Annex 3 for notes (www.oecd.org/education/education-at-a-glance-19991487.htm).

Please refer to the Reader's Guide for information concerning symbols for missing data and abbreviations.

StatLink 젝ㄴ http://dx.doi.org/10.1787/888933285244 


\section{Table A9.1 (L). [2/2] Likelihood of being employed, by educational attainment and literacy proficiency (2012)}

\section{5-64 year-old non-students, below upper secondary education and proficiency Level 1 or below} as reference category, odds ratio

Logistic regression is used to estimate the odds ratios and p-values; an odds ratio reflects the relative likelihood of being employed compared to someone with an education level of below upper secondary education and a literacy proficiency of Level 1 or below. The latter group is taken as the reference category for the interpretation of the relative likelihood and therefore their odds ratio are set to equal 1 . Differences between the groups are statistically significant at $95 \%$ if the "p-value" associated with the odds ratio is below 0.05 .

How to read this table: In Australia, a person with tertiary education and a literacy proficiency of Level 4 or 5 is 2.6 times as likely (in terms of odds ratio) of being employed as someone with below upper secondary education and a literacy proficiency of Level 1 or below.

\begin{tabular}{|c|c|c|c|c|c|c|c|c|c|c|c|c|c|c|c|c|}
\hline & \multicolumn{16}{|c|}{$\begin{array}{l}\text { Likelihood of being employed compared to someone with below upper secondary education } \\
\text { and a literacy proficiency of Level 0/1, dependent on: }\end{array}$} \\
\hline & \multicolumn{8}{|c|}{ Upper secondary or post-secondary non-tertiary education } & \multicolumn{8}{|c|}{ Tertiary education } \\
\hline & \multicolumn{2}{|c|}{$\begin{array}{c}\text { Literacy } \\
\text { proficiency } \\
\text { of Level 0/1 }\end{array}$} & \multicolumn{2}{|c|}{$\begin{array}{c}\text { Literacy } \\
\text { proficiency } \\
\text { of Level } 2\end{array}$} & \multicolumn{2}{|c|}{$\begin{array}{c}\text { Literacy } \\
\text { proficiency } \\
\text { of Level } 3\end{array}$} & \multicolumn{2}{|c|}{$\begin{array}{c}\text { Literacy } \\
\text { proficiency } \\
\text { of Level } 4 / 5\end{array}$} & \multicolumn{2}{|c|}{$\begin{array}{c}\text { Literacy } \\
\text { proficiency } \\
\text { of Level } 0 / 1\end{array}$} & \multicolumn{2}{|c|}{$\begin{array}{c}\text { Literacy } \\
\text { proficiency } \\
\text { of Level } 2\end{array}$} & \multicolumn{2}{|c|}{$\begin{array}{c}\text { Literacy } \\
\text { proficiency } \\
\text { of Level } 3\end{array}$} & \multicolumn{2}{|c|}{$\begin{array}{c}\text { Literacy } \\
\text { proficiency } \\
\text { of Level } 4 / 5\end{array}$} \\
\hline & $\begin{array}{l}\text { Odds } \\
\text { ratio }\end{array}$ & $\mathrm{p}$-value & $\begin{array}{l}\text { Odds } \\
\text { ratio }\end{array}$ & $\mathrm{p}$-value & $\begin{array}{l}\text { Odds } \\
\text { ratio }\end{array}$ & $\mathrm{p}$-value & $\begin{array}{l}\text { Odds } \\
\text { ratio }\end{array}$ & $\mathrm{p}$-value & $\begin{array}{l}\text { Odds } \\
\text { ratio }\end{array}$ & $\mathrm{p}$-value & $\begin{array}{l}\text { Odds } \\
\text { ratio }\end{array}$ & $\mathrm{p}$-value & $\begin{array}{l}\text { Odds } \\
\text { ratio }\end{array}$ & $\mathrm{p}$-value & $\begin{array}{l}\text { Odds } \\
\text { ratio }\end{array}$ & $\mathrm{p}$-value \\
\hline & (9) & (10) & (11) & (12) & (13) & (14) & (15) & (16) & (17) & (18) & (19) & (20) & (21) & (22) & (23) & $(24)$ \\
\hline \multicolumn{17}{|l|}{ Q National entities } \\
\hline ठै Australia & 1.5 & 0.25 & 1.6 & 0.13 & 1.4 & 0.37 & 1.5 & 0.42 & 2.4 & 0.07 & 2.6 & 0.00 & 2.7 & 0.00 & 2.6 & 0.03 \\
\hline Austria & 1.6 & 0.13 & 1.8 & 0.04 & 2.2 & 0.02 & 2.7 & 0.23 & 1.1 & 0.85 & 2.7 & 0.01 & 3.0 & 0.01 & 2.4 & 0.13 \\
\hline Canada & 2.0 & 0.00 & 2.0 & 0.00 & 1.9 & 0.00 & 1.6 & 0.24 & 2.2 & 0.00 & 2.7 & 0.00 & 2.7 & 0.00 & 2.4 & 0.00 \\
\hline Czech Republic & 3.9 & 0.02 & 3.4 & 0.04 & 3.5 & 0.05 & 5.5 & 0.09 & c & c & 7.8 & 0.00 & 4.9 & 0.03 & 6.5 & 0.04 \\
\hline Denmark & 1.7 & 0.02 & 1.9 & 0.01 & 1.5 & 0.18 & 1.9 & 0.40 & 2.7 & 0.00 & 3.8 & 0.00 & 3.5 & 0.00 & 3.9 & 0.02 \\
\hline Estonia & 2.3 & 0.00 & 2.0 & 0.00 & 1.9 & 0.02 & 2.3 & 0.09 & 3.7 & 0.00 & 3.9 & 0.00 & 3.1 & 0.00 & 5.2 & 0.00 \\
\hline Finland & 1.3 & 0.50 & 1.7 & 0.07 & 2.1 & 0.04 & 3.6 & 0.01 & 1.4 & 0.48 & 2.9 & 0.00 & 3.4 & 0.00 & 3.0 & 0.01 \\
\hline France $^{1}$ & 1.5 & 0.04 & 1.7 & 0.00 & 1.3 & 0.30 & 1.1 & 0.84 & 1.3 & 0.46 & 2.6 & 0.00 & 2.9 & 0.00 & 2.6 & 0.02 \\
\hline Germany & 2.6 & 0.00 & 2.3 & 0.00 & 2.2 & 0.03 & 2.3 & 0.17 & 2.7 & 0.04 & 3.4 & 0.00 & 4.1 & 0.00 & 3.6 & 0.03 \\
\hline Ireland & 2.1 & 0.01 & 1.9 & 0.00 & 2.4 & 0.00 & 3.2 & 0.06 & 3.6 & 0.00 & 4.3 & 0.00 & 4.5 & 0.00 & 6.0 & 0.00 \\
\hline Italy $^{1}$ & 2.2 & 0.01 & 1.3 & 0.34 & 1.7 & 0.15 & 3.3 & 0.19 & 3.8 & 0.00 & 2.6 & 0.00 & 3.6 & 0.00 & 3.2 & 0.18 \\
\hline Japan & 1.1 & 0.93 & 0.8 & 0.62 & 0.7 & 0.48 & 0.6 & 0.46 & c & c & 1.0 & 0.96 & 0.7 & 0.51 & 0.5 & 0.22 \\
\hline Korea & 1.1 & 0.71 & 1.0 & 0.99 & 0.9 & 0.85 & 0.7 & 0.62 & 2.0 & 0.22 & 0.9 & 0.68 & 0.8 & 0.64 & 0.7 & 0.53 \\
\hline Netherlands & 1.9 & 0.08 & 1.7 & 0.04 & 1.5 & 0.19 & 1.2 & 0.70 & 2.7 & 0.22 & 2.4 & 0.02 & 2.7 & 0.01 & 2.1 & 0.08 \\
\hline Norway & 1.9 & 0.04 & 1.9 & 0.07 & 1.4 & 0.51 & 1.5 & 0.63 & 1.8 & 0.16 & 2.4 & 0.03 & 3.5 & 0.01 & 2.9 & 0.10 \\
\hline Poland & 2.2 & 0.00 & 2.3 & 0.00 & 2.0 & 0.03 & 1.8 & 0.24 & 11.7 & 0.00 & 6.9 & 0.00 & 6.4 & 0.00 & 9.0 & 0.00 \\
\hline Slovak Republic & 4.4 & 0.00 & 3.8 & 0.00 & 2.7 & 0.00 & 1.6 & 0.32 & c & c & 7.6 & 0.00 & 5.5 & 0.00 & 3.4 & 0.04 \\
\hline Spain $^{1}$ & 2.4 & 0.00 & 1.9 & 0.00 & 1.8 & 0.02 & 1.8 & 0.50 & 3.3 & 0.00 & 3.0 & 0.00 & 2.9 & 0.00 & 3.2 & 0.01 \\
\hline Sweden & 2.1 & 0.06 & 3.6 & 0.00 & 4.1 & 0.00 & 7.8 & 0.03 & 2.3 & 0.07 & 6.6 & 0.00 & 9.9 & 0.00 & 15.0 & 0.00 \\
\hline United States & 1.0 & 0.94 & 0.9 & 0.68 & 1.1 & 0.83 & 1.2 & 0.74 & 1.4 & 0.47 & 1.4 & 0.36 & 1.3 & 0.44 & 1.2 & 0.73 \\
\hline \multicolumn{17}{|l|}{ Sub-national entities } \\
\hline Flanders (Belgium) & 1.8 & 0.03 & 2.6 & 0.00 & 2.5 & 0.01 & 3.4 & 0.04 & 3.2 & 0.04 & 4.6 & 0.00 & 5.4 & 0.00 & 7.0 & 0.00 \\
\hline England (UK) & 1.9 & 0.07 & 1.4 & 0.30 & 1.8 & 0.08 & 1.6 & 0.41 & 1.7 & 0.31 & 1.8 & 0.07 & 1.9 & 0.04 & 1.6 & 0.27 \\
\hline Northern Ireland (UK) & 1.8 & 0.09 & 1.7 & 0.09 & 1.6 & 0.22 & 1.4 & 0.48 & 1.3 & 0.62 & 2.4 & 0.01 & 2.1 & 0.09 & 2.0 & 0.28 \\
\hline England/N. Ireland (UK) & 1.9 & 0.06 & 1.4 & 0.26 & 1.8 & 0.07 & 1.6 & 0.39 & 1.7 & 0.30 & 1.9 & 0.05 & 1.9 & 0.03 & 1.6 & 0.24 \\
\hline Average $^{2}$ & 2.0 & 0.00 & 2.0 & 0.00 & 2.0 & 0.00 & 2.4 & 0.00 & 2.9 & 0.00 & 3.7 & 0.00 & 3.7 & 0.00 & 4.2 & 0.00 \\
\hline Russian Federation* & 6.3 & 0.01 & 4.8 & 0.01 & 3.7 & 0.10 & 1.7 & 0.56 & 2.2 & 0.20 & 4.0 & 0.03 & 5.0 & 0.02 & 4.8 & 0.03 \\
\hline
\end{tabular}

Note: Calculations for odds ratio are based on logistic regressions where the dependent variable is the likelihood of being employed and where the independent variables are educational attainment and proficiency levels, after accounting for: age, gender, parents' educational attainment, immigration background, parental status (have a child or not), cohabitation status (living with spouse/partner or not), numeracy proficiency, skills and readiness to use ICT for problem solving. The reference category is below upper secondary education and proficiency Level 1 or below.

1. The coefficients for France, Italy and Spain have been estimated without accounting for skills and readiness to use ICT for problem solving since it was not tested in these countries. Since there is positive correlation between skills and readiness to use ICT for problem solving and numeracy, literacy and education, the effect of excluding skills and readiness to use ICT for problem solving is likely to be that the coefficients on the proficiency by education level are overestimated, relative to the results for other countries.

2. Average for the regression excludes France, Italy and Spain as a different model specification was used for these countries.

* See note on data for the Russian Federation in the Methodology section.

Source: OECD. Survey of Adult Skills (PIAAC) (2012). See Annex 3 for notes (www.oecd.org/education/education-at-a-glance-19991487.htm).

Please refer to the Reader's Guide for information concerning symbols for missing data and abbreviations.

StatLink 제내 http://dx.doi.org/10.1787/888933285244 


\title{
Table A9.1 (N). [1/2] Likelihood of being employed, by educational attainment and numeracy proficiency (2012)
}

25-64 year-old non-students, below upper secondary education and proficiency Level 1 or below as reference category, odds ratio

\begin{abstract}
Logistic regression is used to estimate the odds ratios and p-values; an odds ratio reflects the relative likelihood of being employed compared to someone with an education level of below upper secondary education and a numeracy proficiency of Level 1 or below. The latter group is taken as the reference category for the interpretation of the relative likelihood and therefore their odds ratio are set to equal 1 . Differences between the groups are statistically significant at $95 \%$ if the "p-value" associated with the odds ratio is below 0.05 .

How to read this table: In Australia, a person with tertiary education and a numeracy proficiency of Level 4 or 5 is 3.5 times as likely (in terms of odds ratio) of being employed as someone with below upper secondary education and a numeracy proficiency of Level 1 or below.
\end{abstract}

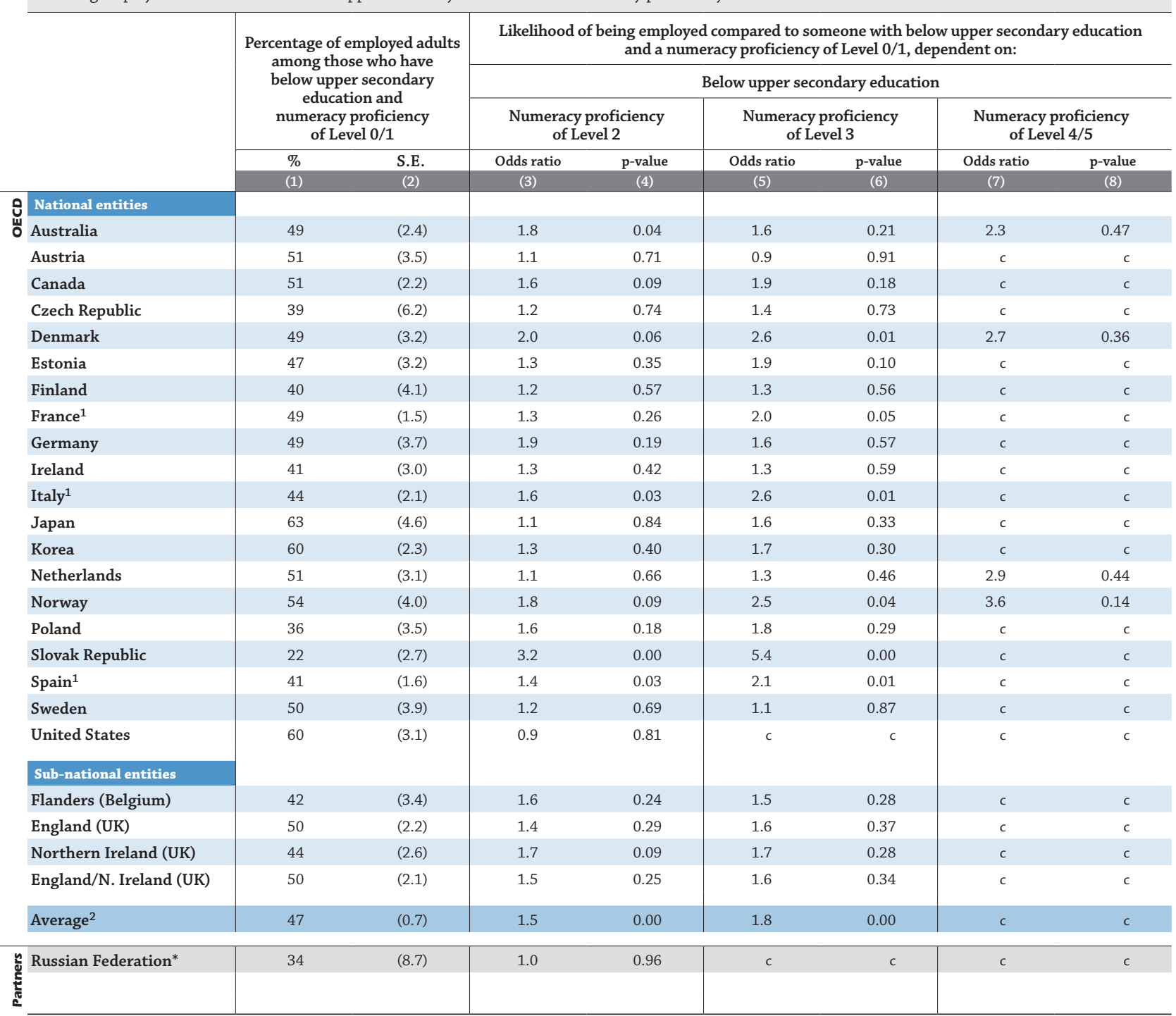

Note: Calculations for odds ratio are based on logistic regressions where the dependent variable is the likelihood of being employed and where the independent variables are educational attainment and proficiency levels, after accounting for: age, gender, parents' educational attainment, immigration background, parental status (have a child or not), cohabitation status (living with spouse/partner or not), literacy proficiency, skills and readiness to use ICT for problem solving. The reference category is below upper secondary education and proficiency Level 1 or below.

1. The coefficients for France, Italy and Spain have been estimated without accounting for skills and readiness to use ICT for problem solving since it was not tested in these countries. Since there is positive correlation between skills and readiness to use ICT for problem solving and numeracy, literacy and education, the effect of excluding skills and readiness to use ICT for problem solving is likely to be that the coefficients on the proficiency by education level are overestimated, relative to the results for other countries.

2. Average for the regression excludes France, Italy and Spain as a different model specification was used for these countries.

* See note on data for the Russian Federation in the Methodology section.

Source: OECD. Survey of Adult Skills (PIAAC) (2012). See Annex 3 for notes (www.oecd.org/education/education-at-a-glance-19991487.htm).

Please refer to the Reader's Guide for information concerning symbols for missing data and abbreviations.

StatLink 겜 http://dx.doi.org/10.1787/888933285257 


\section{Table A9.1 (N). [2/2] Likelihood of being employed, by educational attainment and numeracy proficiency (2012)}

\section{5-64 year-old non-students, below upper secondary education and proficiency Level 1 or below} as reference category, odds ratio

Logistic regression is used to estimate the odds ratios and p-values; an odds ratio reflects the relative likelihood of being employed compared to someone with an education level of below upper secondary education and a numeracy proficiency of Level 1 or below. The latter group is taken as the reference category for the interpretation of the relative likelihood and therefore their odds ratio are set to equal 1 . Differences between the groups are statistically significant at $95 \%$ if the "p-value" associated with the odds ratio is below 0.05 .

How to read this table: In Australia, a person with tertiary education and a numeracy proficiency of Level 4 or 5 is 3.5 times as likely (in terms of odds ratio) of being employed as someone with below upper secondary education and a numeracy proficiency of Level 1 or below.

\begin{tabular}{|c|c|c|c|c|c|c|c|c|c|c|c|c|c|c|c|c|}
\hline & \multicolumn{16}{|c|}{$\begin{array}{l}\text { Likelihood of being employed compared to someone with below upper secondary education } \\
\text { and a numeracy proficiency of Level } 0 / 1 \text {, dependent on: }\end{array}$} \\
\hline & \multicolumn{8}{|c|}{ Upper secondary or post-secondary non-tertiary education } & \multicolumn{8}{|c|}{ Tertiary education } \\
\hline & \multicolumn{2}{|c|}{$\begin{array}{l}\text { Numeracy } \\
\text { proficiency } \\
\text { of Level } 0 / 1\end{array}$} & \multicolumn{2}{|c|}{$\begin{array}{l}\text { Numeracy } \\
\text { proficiency } \\
\text { of Level } 2\end{array}$} & \multicolumn{2}{|c|}{$\begin{array}{l}\text { Numeracy } \\
\text { proficiency } \\
\text { of Level } 3\end{array}$} & \multicolumn{2}{|c|}{$\begin{array}{c}\text { Numeracy } \\
\text { proficiency } \\
\text { of Level } 4 / 5\end{array}$} & \multicolumn{2}{|c|}{$\begin{array}{c}\text { Numeracy } \\
\text { proficiency } \\
\text { of Level 0/1 }\end{array}$} & \multicolumn{2}{|c|}{$\begin{array}{c}\text { Numeracy } \\
\text { proficiency } \\
\text { of Level } 2\end{array}$} & \multicolumn{2}{|c|}{$\begin{array}{l}\text { Numeracy } \\
\text { proficiency } \\
\text { of Level } 3\end{array}$} & \multicolumn{2}{|c|}{$\begin{array}{l}\text { Numeracy } \\
\text { proficiency } \\
\text { of Level } 4 / 5\end{array}$} \\
\hline & $\begin{array}{l}\text { Odds } \\
\text { ratio }\end{array}$ & $\mathrm{p}$-value & $\begin{array}{l}\text { Odds } \\
\text { ratio }\end{array}$ & $\mathrm{p}$-value & $\begin{array}{l}\text { Odds } \\
\text { ratio }\end{array}$ & p-value & $\begin{array}{l}\text { Odds } \\
\text { ratio }\end{array}$ & $\mathrm{p}$-value & $\begin{array}{l}\text { Odds } \\
\text { ratio }\end{array}$ & $\mathrm{p}$-value & $\begin{array}{l}\text { Odds } \\
\text { ratio }\end{array}$ & $\mathrm{p}$-value & $\begin{array}{l}\text { Odds } \\
\text { ratio }\end{array}$ & $\mathrm{p}$-value & $\begin{array}{l}\text { Odds } \\
\text { ratio }\end{array}$ & $\mathrm{p}$-value \\
\hline & (9) & (10) & (11) & (12) & (13) & (14) & (15) & (16) & (17) & (18) & (19) & (20) & (21) & (22) & (23) & (24) \\
\hline \multicolumn{17}{|l|}{8 National entities } \\
\hline ○ Australia & 1.4 & 0.17 & 1.6 & 0.06 & 1.9 & 0.07 & 1.6 & 0.47 & 2.8 & 0.01 & 2.9 & 0.00 & 3.4 & 0.00 & 3.5 & 0.01 \\
\hline Austria & 1.6 & 0.17 & 1.8 & 0.04 & 1.9 & 0.05 & 3.1 & 0.06 & 1.2 & 0.82 & 2.7 & 0.01 & 3.0 & 0.01 & 2.1 & 0.15 \\
\hline Canada & 2.1 & 0.00 & 2.7 & 0.00 & 2.8 & 0.00 & 3.8 & 0.01 & 2.5 & 0.00 & 3.7 & 0.00 & 4.0 & 0.00 & 5.7 & 0.00 \\
\hline Czech Republic & 2.5 & 0.07 & 3.5 & 0.01 & 4.0 & 0.01 & 5.9 & 0.04 & c & c & 5.7 & 0.01 & 5.9 & 0.00 & 10.9 & 0.00 \\
\hline Denmark & 1.8 & 0.05 & 2.6 & 0.00 & 2.9 & 0.00 & 3.5 & 0.02 & 3.4 & 0.00 & 4.9 & 0.00 & 6.3 & 0.00 & 9.8 & 0.00 \\
\hline Estonia & 2.2 & 0.00 & 2.7 & 0.00 & 3.2 & 0.00 & 4.3 & 0.01 & 3.8 & 0.00 & 5.2 & 0.00 & 5.3 & 0.00 & 12.7 & 0.00 \\
\hline Finland & 1.1 & 0.78 & 1.7 & 0.07 & 1.7 & 0.15 & 2.6 & 0.05 & 1.3 & 0.53 & 3.1 & 0.00 & 2.6 & 0.01 & 2.5 & 0.04 \\
\hline France $^{1}$ & 1.6 & 0.00 & 2.0 & 0.00 & 2.2 & 0.00 & 3.1 & 0.03 & 1.5 & 0.15 & 3.8 & 0.00 & 4.7 & 0.00 & 6.1 & 0.00 \\
\hline Germany & 2.4 & 0.00 & 2.8 & 0.00 & 4.4 & 0.00 & 5.0 & 0.00 & 2.4 & 0.05 & 4.9 & 0.00 & 6.7 & 0.00 & 8.4 & 0.00 \\
\hline Ireland & 1.7 & 0.01 & 1.7 & 0.04 & 1.9 & 0.06 & 1.9 & 0.28 & 3.8 & 0.00 & 3.5 & 0.00 & 3.6 & 0.00 & 3.6 & 0.01 \\
\hline Italy $^{1}$ & 2.6 & 0.00 & 2.2 & 0.00 & 3.3 & 0.00 & 8.2 & 0.00 & 5.7 & 0.00 & 3.9 & 0.00 & 8.5 & 0.00 & 8.9 & 0.01 \\
\hline Japan & 1.0 & 0.92 & 1.1 & 0.88 & 1.3 & 0.43 & 1.8 & 0.26 & 1.6 & 0.46 & 1.1 & 0.72 & 1.3 & 0.53 & 1.6 & 0.32 \\
\hline Korea & 1.1 & 0.75 & 1.3 & 0.36 & 1.5 & 0.29 & 1.7 & 0.45 & 1.7 & 0.25 & 1.2 & 0.46 & 1.2 & 0.58 & 1.4 & 0.55 \\
\hline Netherlands & 1.6 & 0.18 & 2.3 & 0.00 & 1.5 & 0.28 & 1.4 & 0.56 & 2.4 & 0.26 & 3.1 & 0.00 & 2.9 & 0.00 & 2.1 & 0.14 \\
\hline Norway & 2.0 & 0.03 & 2.4 & 0.00 & 2.6 & 0.04 & 4.1 & 0.04 & 1.7 & 0.14 & 3.9 & 0.00 & 5.8 & 0.00 & 9.3 & 0.00 \\
\hline Poland & 2.2 & 0.00 & 3.1 & 0.00 & 3.4 & 0.00 & 4.0 & 0.02 & 9.2 & 0.00 & 9.5 & 0.00 & 11.0 & 0.00 & 22.4 & 0.00 \\
\hline Slovak Republic & 4.1 & 0.00 & 8.3 & 0.00 & 12.1 & 0.00 & 16.6 & 0.00 & c & c & 15.9 & 0.00 & 27.3 & 0.00 & 33.8 & 0.00 \\
\hline Spain $^{1}$ & 2.7 & 0.00 & 2.3 & 0.00 & 3.0 & 0.00 & 5.3 & 0.07 & 3.3 & 0.00 & 3.9 & 0.00 & 5.1 & 0.00 & 5.8 & 0.00 \\
\hline Sweden & 2.1 & 0.03 & 2.1 & 0.05 & 2.4 & 0.07 & 2.7 & 0.13 & 2.5 & 0.04 & 4.6 & 0.00 & 4.9 & 0.00 & 4.5 & 0.02 \\
\hline United States & 1.1 & 0.72 & 1.5 & 0.15 & 2.5 & 0.01 & 3.4 & 0.07 & 1.5 & 0.20 & 2.1 & 0.01 & 2.8 & 0.01 & 5.6 & 0.00 \\
\hline \multicolumn{17}{|l|}{ Sub-national entities } \\
\hline Flanders (Belgium) & 1.9 & 0.02 & 2.2 & 0.00 & 1.8 & 0.09 & 1.7 & 0.25 & 3.1 & 0.08 & 4.6 & 0.00 & 3.8 & 0.00 & 3.0 & 0.01 \\
\hline England (UK) & 1.7 & 0.05 & 2.0 & 0.01 & 2.9 & 0.02 & 2.4 & 0.15 & 2.1 & 0.02 & 2.4 & 0.00 & 3.0 & 0.00 & 2.4 & 0.06 \\
\hline Northern Ireland (UK) & 2.1 & 0.04 & 2.0 & 0.02 & 3.3 & 0.00 & 2.5 & 0.18 & 1.5 & 0.34 & 3.7 & 0.00 & 3.5 & 0.00 & 3.7 & 0.06 \\
\hline England/N. Ireland (UK) & 1.7 & 0.04 & 2.0 & 0.01 & 2.9 & 0.01 & 2.5 & 0.13 & 2.1 & 0.01 & 2.4 & 0.00 & 3.0 & 0.00 & 2.5 & 0.05 \\
\hline Average $^{2}$ & 1.9 & 0.00 & 2.5 & 0.00 & 3.0 & 0.00 & 3.8 & 0.00 & 2.8 & 0.00 & 4.5 & 0.00 & 5.5 & 0.00 & 7.6 & 0.00 \\
\hline Russian Federation* & 6.4 & 0.00 & 6.6 & 0.00 & 5.0 & 0.02 & 2.0 & 0.50 & 3.1 & 0.05 & 6.2 & 0.00 & 6.5 & 0.00 & 7.7 & 0.00 \\
\hline
\end{tabular}

Note: Calculations for odds ratio are based on logistic regressions where the dependent variable is the likelihood of being employed and where the independent variables are educational attainment and proficiency levels, after accounting for: age, gender, parents' educational attainment, immigration background, parental status (have a child or not), cohabitation status (living with spouse/partner or not), literacy proficiency, skills and readiness to use ICT for problem solving. The reference category is below upper secondary education and proficiency Level 1 or below.

1. The coefficients for France, Italy and Spain have been estimated without accounting for skills and readiness to use ICT for problem solving since it was not tested in these countries. Since there is positive correlation between skills and readiness to use ICT for problem solving and numeracy, literacy and education, the effect of excluding skills and readiness to use ICT for problem solving is likely to be that the coefficients on the proficiency by education level are overestimated, relative to the results for other countries.

2. Average for the regression excludes France, Italy and Spain as a different model specification was used for these countries.

* See note on data for the Russian Federation in the Methodology section.

Source: OECD. Survey of Adult Skills (PIAAC) (2012). See Annex 3 for notes (www.oecd.org/education/education-at-a-glance-19991487.htm).

Please refer to the Reader's Guide for information concerning symbols for missing data and abbreviations.

StatLink 제내 http://dx.doi.org/10.1787/888933285257 
Table A9.1 (P). [1/2] Likelihood of being employed, by educational attainment and skills and readiness to use information and communication technologies for problem solving (2012)

25-64 year-olds, reference category, below upper secondary education and skills Group 0 or 1 as reference category, odds ratio

Logistic regression is used to estimate the odds ratios and p-values; an odds ratio reflects the relative likelihood of being employed compared to someone with
a level of skills and readiness to use ICT for problem solving of Group 0 or 1 . The latter group is taken as the reference category for the interpretation of the
relative likelihood and therefore their odds ratio are set to equal 1 . Differences between the groups are statistically significant at $95 \%$ if the "p-value" associated
with the odds ratio is below 0.05 .
How to read this table: In Australia, a person with tertiary education and among Group 4 of skills and readiness to use ICT for problem solving is 5.2 times as
likely (in terms of odds ratio) of being employed as someone with below upper secondary education and among Group 0 or 1 of skills and readiness to use ICT
for problem solving.

\begin{tabular}{|c|c|c|c|c|c|c|c|c|}
\hline & \multirow{2}{*}{\multicolumn{2}{|c|}{$\begin{array}{l}\text { Percentage of employed adults } \\
\text { among those who have } \\
\text { below upper secondary } \\
\text { education and skills } \\
\text { and readiness to use ICT for } \\
\text { problem solving of Group } 0 / 1\end{array}$}} & \multicolumn{6}{|c|}{$\begin{array}{l}\text { Likelihood of being employed compared to someone with below upper secondary education } \\
\text { and a level of skills and readiness to use ICT for problem solving of Group } 0 / 1 \text {, dependent on: }\end{array}$} \\
\hline & & & \multicolumn{6}{|c|}{ Below upper secondary education } \\
\hline & $\%$ & S.E. & Odds ratio & p-value & Odds ratio & p-value & Odds ratio & p-value \\
\hline & (1) & (2) & (3) & (4) & (5) & (6) & (7) & (8) \\
\hline \multicolumn{9}{|l|}{ (ิ) National entities } \\
\hline ○ Australia & 31 & (3.5) & 1.5 & 0.15 & 2.5 & 0.00 & 2.7 & 0.01 \\
\hline Austria & 40 & $(2.6)$ & 0.9 & 0.82 & 1.0 & 0.98 & 0.7 & 0.67 \\
\hline Canada & 41 & $(3.0)$ & 1.0 & 0.84 & 1.2 & 0.43 & 1.0 & 0.94 \\
\hline Czech Republic & 28 & $(5.2)$ & 2.1 & 0.27 & 0.5 & 0.32 & 0.5 & 0.52 \\
\hline Denmark & 31 & $(5.1)$ & 1.8 & 0.01 & 2.0 & 0.02 & 1.1 & 0.87 \\
\hline Estonia & 32 & $(2.7)$ & 2.2 & 0.00 & 2.3 & 0.02 & 3.2 & 0.09 \\
\hline France & $\mathrm{m}$ & $\mathrm{m}$ & $\mathrm{m}$ & $\mathrm{m}$ & $\mathrm{m}$ & $\mathrm{m}$ & $\mathrm{m}$ & $\mathrm{m}$ \\
\hline Germany & 43 & $(5.5)$ & 1.1 & 0.82 & 0.6 & 0.34 & 0.7 & 0.63 \\
\hline Ireland & 40 & $(2.9)$ & 1.4 & 0.17 & 1.7 & 0.07 & 2.2 & 0.45 \\
\hline Italy & $\mathrm{m}$ & $\mathrm{m}$ & $\mathrm{m}$ & $\mathrm{m}$ & $\mathrm{m}$ & $\mathrm{m}$ & $\mathrm{m}$ & $\mathrm{m}$ \\
\hline Japan & 62 & $(4.0)$ & 0.6 & 0.16 & 1.0 & 0.99 & 1.3 & 0.75 \\
\hline Korea & 60 & $(1.7)$ & 1.1 & 0.72 & 2.2 & 0.23 & 0.6 & 0.67 \\
\hline Netherlands & 39 & $(4.7)$ & 1.2 & 0.47 & 2.0 & 0.01 & 3.9 & 0.00 \\
\hline Norway & 22 & $(6.9)$ & 2.5 & 0.01 & 2.7 & 0.00 & 3.3 & 0.04 \\
\hline Poland & 31 & $(2.7)$ & 1.4 & 0.49 & 0.6 & 0.46 & c & c \\
\hline Slovak Republic & 27 & $(2.1)$ & 1.1 & 0.75 & 2.1 & 0.06 & 2.9 & 0.08 \\
\hline Spain & $\mathrm{m}$ & $\mathrm{m}$ & $\mathrm{m}$ & $\mathrm{m}$ & $\mathrm{m}$ & $\mathrm{m}$ & $\mathrm{m}$ & $\mathrm{m}$ \\
\hline Sweden & 35 & $(9.1)$ & 1.6 & 0.20 & 1.1 & 0.80 & 0.8 & 0.78 \\
\hline United States & 61 & $(3.8)$ & 0.9 & 0.85 & 0.7 & 0.55 & 0.3 & 0.13 \\
\hline \multicolumn{9}{|l|}{ Sub-national entities } \\
\hline England (UK) & 32 & $(5.0)$ & 2.0 & 0.05 & 2.2 & 0.03 & 2.1 & 0.19 \\
\hline Northern Ireland (UK) & 40 & $(3.0)$ & 1.7 & 0.04 & 1.9 & 0.07 & 2.2 & 0.22 \\
\hline England/N. Ireland (UK) & 33 & $(4.6)$ & 2.0 & 0.04 & 2.2 & 0.02 & 2.1 & 0.17 \\
\hline Average & 38 & $(1.0)$ & 1.5 & 0.00 & 1.5 & 0.00 & 1.7 & 0.00 \\
\hline nussian Federation* & 21 & $(5.6)$ & c & c & c & c & c & c \\
\hline
\end{tabular}

Note: Calculations for odds ratio are based on logistic regressions where the dependent variable is the likelihood of being employed and where the independent variables are educational attainment and skill groups, after accounting for: age, gender, parents' educational attainment, immigration background, parental status (have a child or not), cohabitation status (living with spouse/partner or not), literacy proficiency and numeracy proficiency. The reference category is below upper secondary education and Group 0 or 1.

* See note on data for the Russian Federation in the Methodology section.

Source: OECD. Survey of Adult Skills (PIAAC) (2012). See Annex 3 for notes (www.oecd.org/education/education-at-a-glance-19991487.htm).

Please refer to the Reader's Guide for information concerning symbols for missing data and abbreviations.

StatLink 젝ㄴ http://dx.doi.org/10.1787/888933285268 
Table A9.1 (P). [2/2] Likelihood of being employed, by educational attainment and skills and readiness to use information and communication technologies for problem solving (2012)

25-64 year-olds, reference category, below upper secondary education and skills Group 0 or 1 as reference category, odds ratio

Logistic regression is used to estimate the odds ratios and p-values; an odds ratio reflects the relative likelihood of being employed compared to someone with a level of skills and readiness to use ICT for problem solving of Group 0 or 1 . The latter group is taken as the reference category for the interpretation of the relative likelihood and therefore their odds ratio are set to equal 1. Differences between the groups are statistically significant at $95 \%$ if the "p-value" associated with the odds ratio is below 0.05 .

How to read this table: In Australia, a person with tertiary education and among Group 4 of skills and readiness to use ICT for problem solving is 5.2 times as likely (in terms of odds ratio) of being employed as someone with below upper secondary education and among Group 0 or 1 of skills and readiness to use ICT for problem solving.

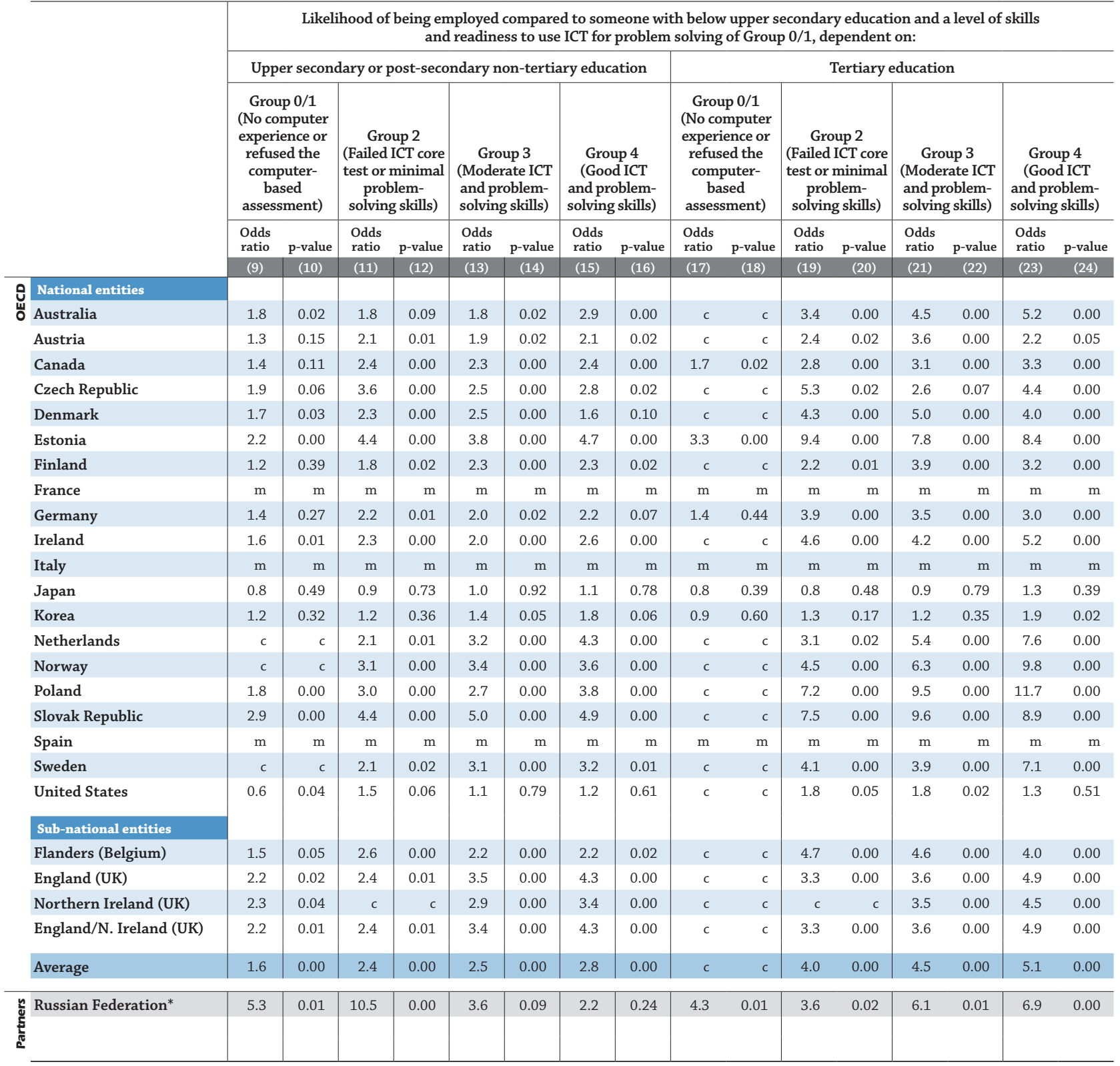

Note: Calculations for odds ratio are based on logistic regressions where the dependent variable is the likelihood of being employed and where the independent variables are educational attainment and skill groups, after accounting for: age, gender, parents' educational attainment, immigration background, parental status (have a child or not), cohabitation status (living with spouse/partner or not), literacy proficiency and numeracy proficiency. The reference category is below upper secondary education and Group 0 or 1.

* See note on data for the Russian Federation in the Methodology section.

Source: OECD. Survey of Adult Skills (PIAAC) (2012). See Annex 3 for notes (www.oecd.org/education/education-at-a-glance-19991487.htm).

Please refer to the Reader's Guide for information concerning symbols for missing data and abbreviations.

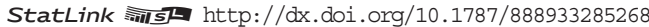




\section{Table A9.2 (L). [1/2] Difference in hourly earnings, by educational attainment and literacy proficiency (2012) 25-64 year-old non-students, below upper secondary and proficiency Level 1 or below as reference category}

Ordinary least square regression, used to estimate percentage, reflects the change in hourly earnings compared to someone with below upper secondary education and a literacy proficiency of Level 1 or below. The latter group is taken as the reference category for the interpretation of the percentage difference. How to read this table: In Australia, a person with tertiary education and a literacy proficiency of Level 4 or 5 is earning $37 \%$ more, compared with someone with below upper secondary education and a literacy proficiency of Level 1 or below.

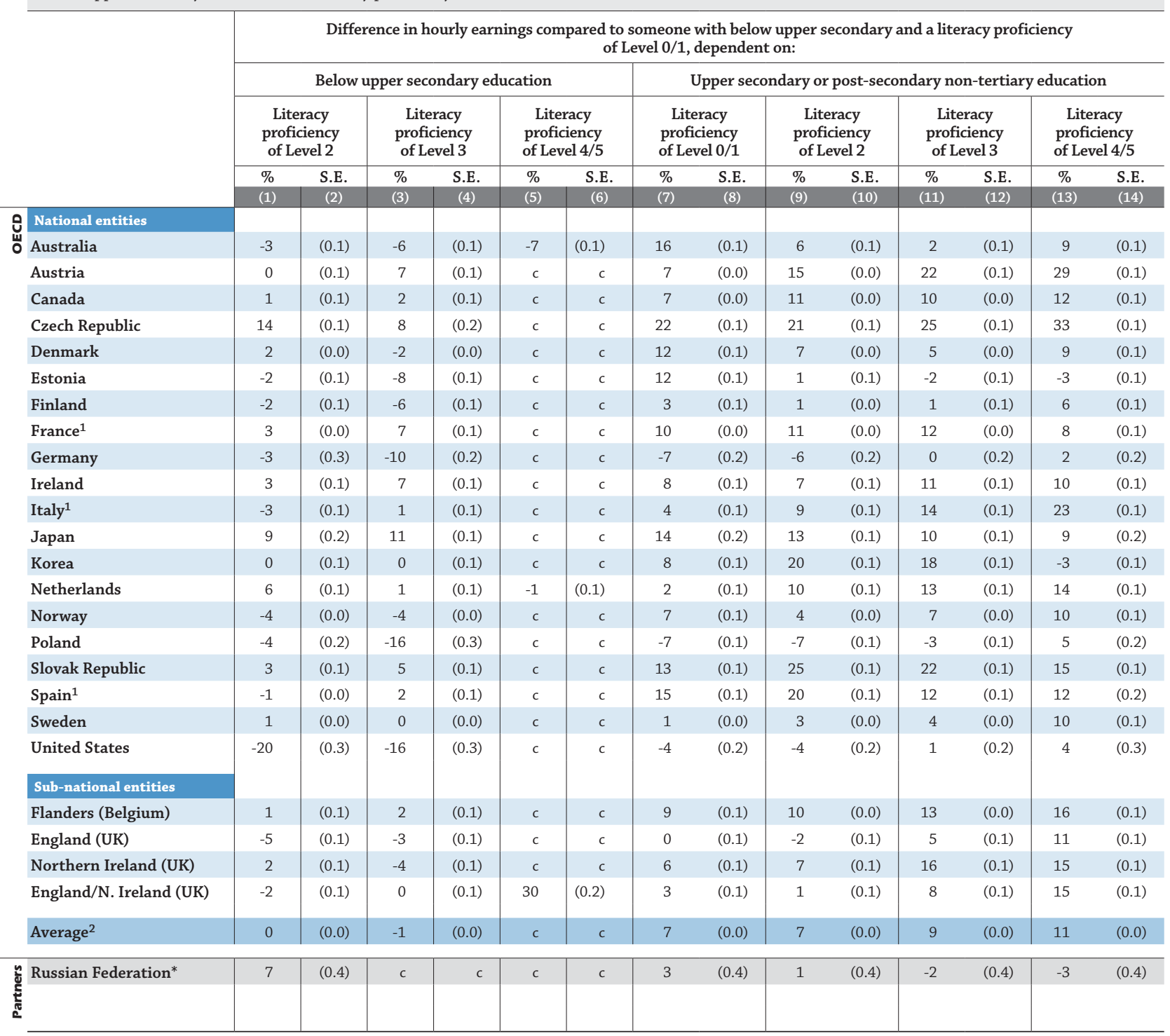

Note: Calculations are based on ordinary least square regressions where the dependent variable is hourly earnings and where the independent variables are educational attainment and proficiency levels, after accounting for: age, gender, parents' educational attainment, immigration background, parental status (have a child or not), cohabitation status (living with spouse/partner or not), numeracy proficiency, skills and readiness to use ICT for problem solving. The reference category is below upper secondary education and proficiency Level 1 or below.

1. The coefficients for France, Italy and Spain have been estimated without accounting for skills and readiness to use ICT for problem solving since it was not tested in these countries. Since there is positive correlation between skills and readiness to use ICT for problem solving and numeracy, literacy and education, the effect of excluding skills and readiness to use ICT for problem solving is likely to be that the coefficients on the proficiency by education level are overestimated, relative to the results for other countries.

2. Average excludes France, Italy and Spain as a different model specification was used for these countries.

* See note on data for the Russian Federation in the Methodology section.

Source: OECD. Survey of Adult Skills (PIAAC) (2012). See Annex 3 for notes (www.oecd.org/education/education-at-a-glance-19991487.htm).

Please refer to the Reader's Guide for information concerning symbols for missing data and abbreviations.

StatLink 제그 http://dx.doi.org/10.1787/888933285270 
Table A9.2 (L). [2/2] Difference in hourly earnings, by educational attainment and literacy proficiency (2012)

25-64 year-old non-students, below upper secondary and proficiency Level 1 or below as reference category

Ordinary least square regression, used to estimate percentage, reflects the change in hourly earnings compared to someone with below upper secondary education and a literacy proficiency of Level 1 or below. The latter group is taken as the reference category for the interpretation of the percentage difference.

How to read this table: In Australia, a person with tertiary education and a literacy proficiency of Level 4 or 5 is earning $37 \%$ more, compared with someone with below upper secondary education and a literacy proficiency of Level 1 or below.

\begin{tabular}{|c|c|c|c|c|c|c|c|c|}
\hline & \multicolumn{8}{|c|}{$\begin{array}{l}\text { Difference in hourly earnings compared to someone with below upper secondary and a literacy proficiency } \\
\text { of Level } 0 / 1 \text {, dependent on: }\end{array}$} \\
\hline & \multicolumn{8}{|c|}{ Tertiary education } \\
\hline & \multicolumn{2}{|c|}{$\begin{array}{l}\text { Literacy proficiency } \\
\text { of Level } 0 / 1\end{array}$} & \multicolumn{2}{|c|}{$\begin{array}{l}\text { Literacy proficiency } \\
\text { of Level } 2\end{array}$} & \multicolumn{2}{|c|}{$\begin{array}{l}\text { Literacy proficiency } \\
\text { of Level } 3\end{array}$} & \multicolumn{2}{|c|}{$\begin{array}{l}\text { Literacy proficiency } \\
\text { of Level } 4 / 5\end{array}$} \\
\hline & $\%$ & S.E. & $\%$ & S.E. & $\%$ & S.E. & $\%$ & S.E. \\
\hline & (15) & (16) & (17) & (18) & (19) & (20) & (21) & (22) \\
\hline \multicolumn{9}{|l|}{ Q National entities } \\
\hline む̈ Australia & 17 & $(0.1)$ & 25 & $(0.1)$ & 32 & $(0.1)$ & 37 & $(0.1)$ \\
\hline Austria & 31 & $(0.1)$ & 39 & $(0.1)$ & 51 & $(0.1)$ & 63 & $(0.1)$ \\
\hline Canada & 19 & $(0.1)$ & 28 & $(0.0)$ & 39 & $(0.0)$ & 42 & $(0.1)$ \\
\hline Czech Republic & c & c & 46 & $(0.1)$ & 58 & $(0.1)$ & 75 & $(0.1)$ \\
\hline Denmark & 22 & $(0.1)$ & 22 & $(0.0)$ & 29 & $(0.0)$ & 36 & $(0.1)$ \\
\hline Estonia & 17 & $(0.1)$ & 22 & $(0.1)$ & 24 & $(0.1)$ & 37 & $(0.1)$ \\
\hline Finland & 22 & $(0.1)$ & 22 & $(0.0)$ & 26 & $(0.1)$ & 28 & $(0.1)$ \\
\hline France $^{1}$ & 42 & $(0.1)$ & 44 & $(0.0)$ & 44 & $(0.0)$ & 51 & $(0.1)$ \\
\hline Germany & 15 & $(0.2)$ & 23 & $(0.2)$ & 32 & $(0.2)$ & 48 & $(0.2)$ \\
\hline Ireland & 32 & $(0.1)$ & 38 & $(0.1)$ & 44 & $(0.1)$ & 43 & $(0.1)$ \\
\hline Italy $^{1}$ & 38 & $(0.1)$ & 42 & $(0.1)$ & 53 & $(0.1)$ & 60 & $(0.1)$ \\
\hline Japan & c & c & 27 & $(0.1)$ & 27 & $(0.1)$ & 29 & $(0.2)$ \\
\hline Korea & 42 & $(0.1)$ & 50 & $(0.1)$ & 65 & $(0.1)$ & 83 & $(0.1)$ \\
\hline Netherlands & 17 & $(0.1)$ & 39 & $(0.1)$ & 48 & $(0.1)$ & 49 & $(0.1)$ \\
\hline Norway & 21 & $(0.1)$ & 28 & $(0.1)$ & 28 & $(0.0)$ & 28 & $(0.1)$ \\
\hline Poland & 37 & $(0.2)$ & 33 & $(0.1)$ & 40 & $(0.1)$ & 61 & $(0.2)$ \\
\hline Slovak Republic & c & c & 68 & $(0.1)$ & 75 & $(0.1)$ & 87 & $(0.1)$ \\
\hline Spain $^{1}$ & 35 & $(0.1)$ & 40 & $(0.1)$ & 43 & $(0.1)$ & 51 & $(0.1)$ \\
\hline Sweden & 12 & $(0.1)$ & 19 & $(0.0)$ & 23 & $(0.0)$ & 27 & $(0.1)$ \\
\hline United States & 10 & $(0.3)$ & 28 & $(0.2)$ & 34 & $(0.3)$ & 51 & $(0.2)$ \\
\hline \multicolumn{9}{|l|}{ Sub-national entities } \\
\hline Flanders (Belgium) & 52 & $(0.1)$ & 39 & $(0.1)$ & 39 & $(0.0)$ & 43 & $(0.1)$ \\
\hline England (UK) & 12 & $(0.1)$ & 31 & $(0.1)$ & 38 & $(0.1)$ & 49 & $(0.1)$ \\
\hline Northern Ireland (UK) & 31 & $(0.2)$ & 35 & $(0.1)$ & 38 & $(0.1)$ & 41 & $(0.1)$ \\
\hline England/N. Ireland (UK) & 16 & $(0.1)$ & 35 & $(0.1)$ & 42 & $(0.1)$ & 54 & $(0.1)$ \\
\hline Average $^{2}$ & 24 & $(0.0)$ & 33 & $(0.0)$ & 40 & $(0.0)$ & 48 & $(0.0)$ \\
\hline Russian Federation* & 0 & $(0.4)$ & 4 & $(0.4)$ & 5 & (0.4) & 19 & $(0.4)$ \\
\hline
\end{tabular}

Note: Calculations are based on ordinary least square regressions where the dependent variable is hourly earnings and where the independent variables are educational attainment and proficiency levels, after accounting for: age, gender, parents' educational attainment, immigration background, parental status (have a child or not), cohabitation status (living with spouse/partner or not), numeracy proficiency, skills and readiness to use ICT for problem solving. The reference category is below upper secondary education and proficiency Level 1 or below.

1. The coefficients for France, Italy and Spain have been estimated without accounting for skills and readiness to use ICT for problem solving since it was not tested in these countries. Since there is positive correlation between skills and readiness to use ICT for problem solving and numeracy, literacy and education, the effect of excluding skills and readiness to use ICT for problem solving is likely to be that the coefficients on the proficiency by education level are overestimated, relative to the results for other countries.

2. Average excludes France, Italy and Spain as a different model specification was used for these countries.

* See note on data for the Russian Federation in the Methodology section.

Source: OECD. Survey of Adult Skills (PIAAC) (2012). See Annex 3 for notes (www.oecd.org/education/education-at-a-glance-19991487.htm).

Please refer to the Reader's Guide for information concerning symbols for missing data and abbreviations.

StatLink ज्ञाs http://dx.doi.org/10.1787/888933285270 
Table A9.2 (N). [1/2] Difference in hourly earnings, by educational attainment and numeracy proficiency (2012) 25-64 year-old non-students, below upper secondary and proficiency Level 1 or below as reference category

Ordinary least square regression, used to estimate percentage, reflects the change in hourly earnings compared to someone with below upper secondary education and a numeracy proficiency of Level 1 or below. The latter group is taken as the reference category for the interpretation of the percentage difference. How to read this table: In Australia, a person with tertiary education and a numeracy proficiency of Level 4 or 5 is earning $63 \%$ more, compared with someone with below upper secondary education and a numeracy proficiency of Level 1 or below.

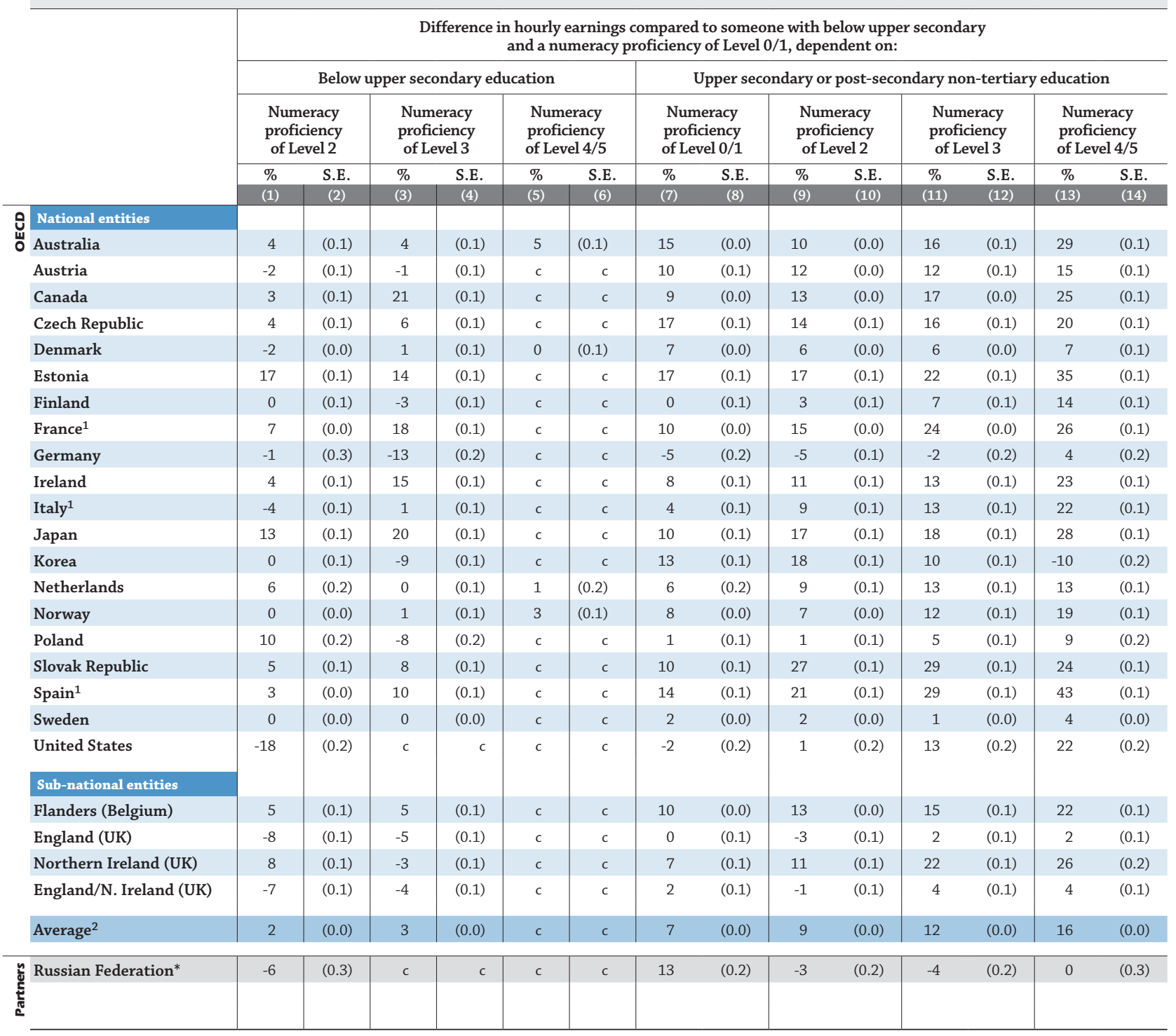

Note: Calculations are based on ordinary least square regressions where the dependent variable is hourly earnings and where the independent variables are educational attainment and proficiency levels, after accounting for: age, gender, parents' educational attainment, immigration background, parental status (have a child or not), cohabitation status (living with spouse/partner or not), literacy proficiency, skills and readiness to use ICT for problem solving. The reference category is below upper secondary education and proficiency Level 1 or below.

1. The coefficients for France, Italy and Spain have been estimated without accounting for skills and readiness to use ICT for problem solving since it was not tested in these countries. Since there is positive correlation between skills and readiness to use ICT for problem solving and numeracy, literacy and education, the effect of excluding skills and readiness to use ICT for problem solving is likely to be that the coefficients on the proficiency by education level are overestimated, relative to the results for other countries.

2. Average excludes France, Italy and Spain as a different model specification was used for these countries.

* See note on data for the Russian Federation in the Methodology section.

Source: OECD. Survey of Adult Skills (PIAAC) (2012). See Annex 3 for notes (www.oecd.org/education/education-at-a-glance-19991487.htm).

Please refer to the Reader's Guide for information concerning symbols for missing data and abbreviations.

StatLink त्गाजम http://dx.doi.org/10.1787/888933285280 
Table A9.2 (N). [2/2] Difference in hourly earnings,

by educational attainment and numeracy proficiency (2012)

25-64 year-old non-students, below upper secondary and proficiency Level 1 or below as reference category

Ordinary least square regression, used to estimate percentage, reflects the change in hourly earnings compared to someone with below upper secondary education and a numeracy proficiency of Level 1 or below. The latter group is taken as the reference category for the interpretation of the percentage difference.

How to read this table: In Australia, a person with tertiary education and a numeracy proficiency of Level 4 or 5 is earning $63 \%$ more, compared with someone with below upper secondary education and a numeracy proficiency of Level 1 or below.

\begin{tabular}{|c|c|c|c|c|c|c|c|c|c|}
\hline & & \multicolumn{8}{|c|}{$\begin{array}{l}\text { Difference in hourly earnings compared to someone with below upper secondary } \\
\text { and a numeracy proficiency of Level } 0 / 1 \text {, dependent on: }\end{array}$} \\
\hline & & \multicolumn{8}{|c|}{ Tertiary education } \\
\hline & & \multicolumn{2}{|c|}{$\begin{array}{l}\text { Numeracy proficiency } \\
\text { of Level } 0 / 1\end{array}$} & \multicolumn{2}{|c|}{$\begin{array}{l}\text { Numeracy proficiency } \\
\text { of Level } 2\end{array}$} & \multicolumn{2}{|c|}{$\begin{array}{l}\text { Numeracy proficiency } \\
\text { of Level } 3\end{array}$} & \multicolumn{2}{|c|}{$\begin{array}{l}\text { Numeracy proficiency } \\
\text { of Level } 4 / 5\end{array}$} \\
\hline & & $\%$ & S.E. & $\%$ & S.E. & $\%$ & S.E. & $\%$ & S.E. \\
\hline & & (15) & $(16)$ & $(17)$ & $(18)$ & (19) & $(20)$ & $(21)$ & $(22)$ \\
\hline 岂 & Australia & 20 & $(0.1)$ & 35 & $(0.1)$ & 50 & $(0.1)$ & 63 & $(0.1)$ \\
\hline & Austria & 37 & $(0.1)$ & 36 & $(0.1)$ & 40 & $(0.1)$ & 42 & $(0.1)$ \\
\hline & Canada & 20 & $(0.0)$ & 35 & $(0.0)$ & 47 & $(0.0)$ & 59 & $(0.1)$ \\
\hline & Czech Republic & c & c & 47 & $(0.2)$ & 43 & $(0.1)$ & 54 & $(0.1)$ \\
\hline & Denmark & 17 & $(0.1)$ & 21 & $(0.0)$ & 26 & $(0.0)$ & 37 & $(0.1)$ \\
\hline & Estonia & 31 & $(0.1)$ & 42 & $(0.1)$ & 55 & $(0.1)$ & 76 & $(0.1)$ \\
\hline & Finland & 15 & $(0.1)$ & 24 & $(0.1)$ & 34 & $(0.1)$ & 42 & $(0.1)$ \\
\hline & Germany & 24 & $(0.2)$ & 22 & $(0.2)$ & 31 & $(0.2)$ & 45 & $(0.2)$ \\
\hline & Ireland & 32 & $(0.1)$ & 41 & $(0.1)$ & 50 & $(0.1)$ & 56 & $(0.1)$ \\
\hline & Italy $^{1}$ & 39 & $(0.1)$ & 37 & $(0.1)$ & 51 & $(0.1)$ & 71 & $(0.1)$ \\
\hline & Japan & 11 & $(0.1)$ & 29 & $(0.1)$ & 38 & $(0.1)$ & 56 & $(0.1)$ \\
\hline & Korea & 47 & $(0.1)$ & 49 & $(0.1)$ & 55 & $(0.1)$ & 70 & $(0.1)$ \\
\hline & Netherlands & 18 & $(0.2)$ & 39 & $(0.1)$ & 47 & $(0.1)$ & 51 & $(0.2)$ \\
\hline & Norway & 28 & $(0.1)$ & 32 & $(0.1)$ & 33 & $(0.1)$ & 39 & $(0.1)$ \\
\hline & Poland & 40 & $(0.1)$ & 40 & $(0.1)$ & 55 & $(0.1)$ & 78 & $(0.1)$ \\
\hline & Slovak Republic & c & c & 69 & $(0.1)$ & 78 & $(0.1)$ & 108 & $(0.1)$ \\
\hline & Spain $^{1}$ & 37 & $(0.1)$ & 47 & $(0.1)$ & 58 & $(0.1)$ & 65 & $(0.1)$ \\
\hline & Sweden & 18 & $(0.1)$ & 17 & $(0.0)$ & 19 & $(0.0)$ & 23 & $(0.0)$ \\
\hline & United States & 26 & $(0.2)$ & 38 & $(0.2)$ & 47 & $(0.2)$ & 70 & $(0.2)$ \\
\hline \multicolumn{10}{|c|}{ Sub-national entities } \\
\hline & England (UK) & 26 & $(0.1)$ & 24 & $(0.1)$ & 35 & $(0.1)$ & 40 & $(0.1)$ \\
\hline & Northern Ireland (UK) & 35 & $(0.1)$ & 39 & $(0.1)$ & 44 & $(0.1)$ & 54 & $(0.1)$ \\
\hline & England/N. Ireland (UK) & 29 & $(0.1)$ & 27 & $(0.1)$ & 37 & $(0.1)$ & 42 & $(0.1)$ \\
\hline & Average $^{2}$ & 27 & $(0.0)$ & 36 & $(0.0)$ & 44 & $(0.0)$ & 56 & $(0.0)$ \\
\hline 产 & Russian Federation* & -3 & $(0.3)$ & 3 & $(0.2)$ & 7 & $(0.2)$ & 13 & $(0.3)$ \\
\hline
\end{tabular}

Note: Calculations are based on ordinary least square regressions where the dependent variable is hourly earnings and where the independent variables are educational attainment and proficiency levels, after accounting for: age, gender, parents' educational attainment, immigration background, parental status (have a child or not), cohabitation status (living with spouse/partner or not), literacy proficiency, skills and readiness to use ICT for problem solving. The reference category is below upper secondary education and proficiency Level 1 or below.

1. The coefficients for France, Italy and Spain have been estimated without accounting for skills and readiness to use ICT for problem solving since it was not tested in these countries. Since there is positive correlation between skills and readiness to use ICT for problem solving and numeracy, literacy and education, the effect of excluding skills and readiness to use ICT for problem solving is likely to be that the coefficients on the proficiency by education level are overestimated, relative to the results for other countries.

2. Average excludes France, Italy and Spain as a different model specification was used for these countries.

* See note on data for the Russian Federation in the Methodology section.

Source: OECD. Survey of Adult Skills (PIAAC) (2012). See Annex 3 for notes (www.oecd.org/education/education-at-a-glance-19991487.htm).

Please refer to the Reader's Guide for information concerning symbols for missing data and abbreviations.

StatLink 제그 http://dx.doi.org/10.1787/888933285280 
Table A9.2 (P). [1/2] Difference in hourly earings, by educational attainment and skills and readiness to use information and communication technologies for problem solving (2012) 25-64 year-old non-students, below upper secondary and a level of skills and readiness to use ICT for problem solving of Group 0 or 1 as reference category

Ordinary least square regression, used to estimate the percentage, reflects the change in hourly earnings compared to someone with below upper secondary education and a level of skills and readiness to use ICT for problem solving of Group 0 or 1 . The latter group is taken as the reference category for the interpretation of the percentage difference.

How to read this table: In Australia, a person with tertiary education and among Group 4 of skills and readiness to use ICT for problem solving is earning $48 \%$ more, compared with someone with below upper secondary education and among Group 0 or 1 of skills and readiness to use ICT for problem solving.

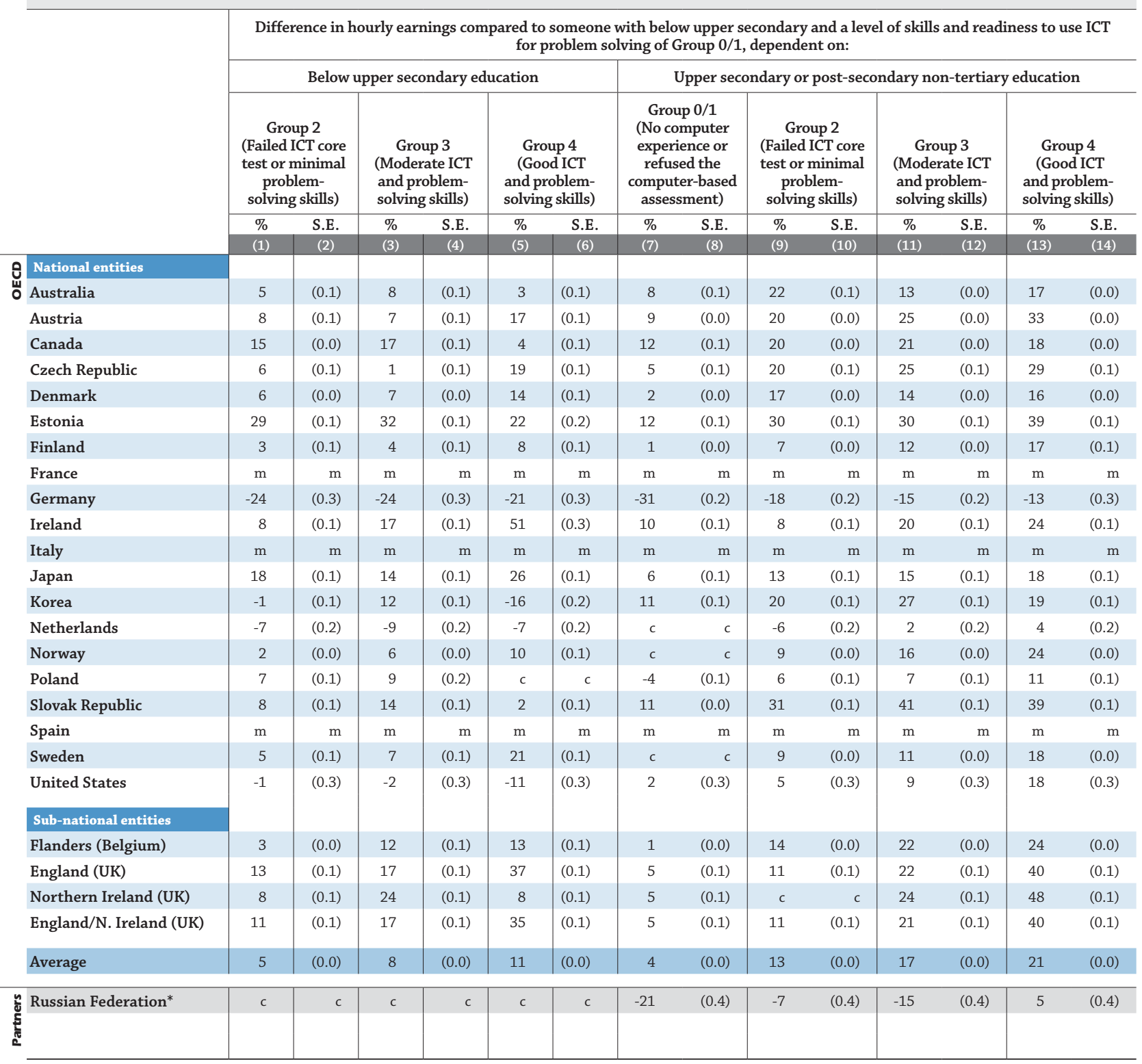

Note: Calculations are based on ordinary least square regressions where the dependent variable is hourly earnings and where the independent variables are educational attainment and skill groups, after accounting for: age, gender, parents' educational attainment, immigration background, parental status (have a child or not), cohabitation status (living with spouse/partner or not), literacy proficiency and numeracy proficiency. The reference category is below upper secondary education and Group 0/1.

* See note on data for the Russian Federation in the Methodology section.

Source: OECD. Survey of Adult Skills (PIAAC) (2012). See Annex 3 for notes (www.oecd.org/education/education-at-a-glance-19991487.htm).

Please refer to the Reader's Guide for information concerning symbols for missing data and abbreviations.

StatLink 겍 http://dx.doi.org/10.1787/888933285291 
Table A9.2 (P). [2/2] Difference in hourly earings, by educational attainment and skills and readiness to use information and communication technologies for problem solving (2012) 25-64 year-old non-students, below upper secondary and a level of skills and readiness to use ICT for problem solving of Group 0 or 1 as reference category

Ordinary least square regression, used to estimate the percentage, reflects the change in hourly earnings compared to someone with below upper secondary education and a level of skills and readiness to use ICT for problem solving of Group 0 or 1 . The latter group is taken as the reference category for the interpretation of the percentage difference.

How to read this table: In Australia, a person with tertiary education and among Group 4 of skills and readiness to use ICT for problem solving is earning $48 \%$ more, compared with someone with below upper secondary education and among Group 0 or 1 of skills and readiness to use ICT for problem solving.

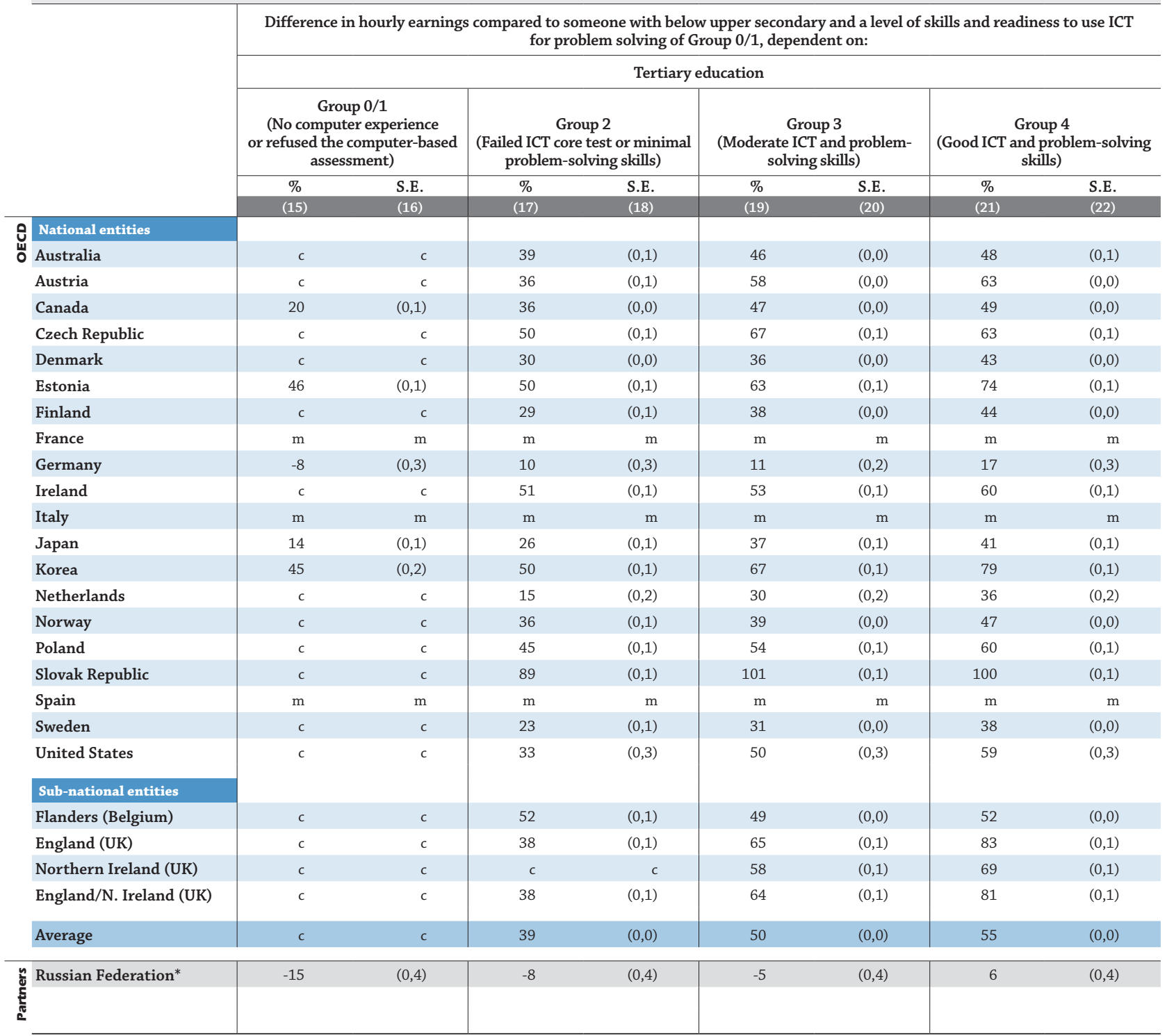

Note: Calculations are based on ordinary least square regressions where the dependent variable is hourly earnings and where the independent variables are educational attainment and skill groups, after accounting for: age, gender, parents' educational attainment, immigration background, parental status (have a child or not), cohabitation status (living with spouse/partner or not), literacy proficiency and numeracy proficiency. The reference category is below upper secondary education and Group 0/1.

* See note on data for the Russian Federation in the Methodology section.

Source: OECD. Survey of Adult Skills (PIAAC) (2012). See Annex 3 for notes (www.oecd.org/education/education-at-a-glance-19991487.htm).

Please refer to the Reader's Guide for information concerning symbols for missing data and abbreviations.

StatLink 제개 http://dx.doi.org/10.1787/888933285291 



\section{INDICATOR A10}

\section{WHERE ARE THE GENDER GAPS IN EDUCATION AND EMPLOYMENT?}

- Over the three past decades, OECD countries have made significant progress in narrowing or closing long-standing gender gaps in many areas of education and employment, including educational attainment, pay and labour market participation.

- In 2014, a larger proportion of 25-34 year-old women than men of the same age had attained tertiary education ( $46 \%$ and $35 \%$, respectively). This pattern is observed in 40 out of 42 countries with available data.

- New gender gaps in education are opening: young men are significantly more likely than young women to have low skills and poor academic achievement; while in tertiary education and beyond, young women are still under-represented in the fields of mathematics, physical science and computing.

\section{Chart A10.1. Percentage of 25-34 year-olds who have attained tertiary education, by gender (2014)}

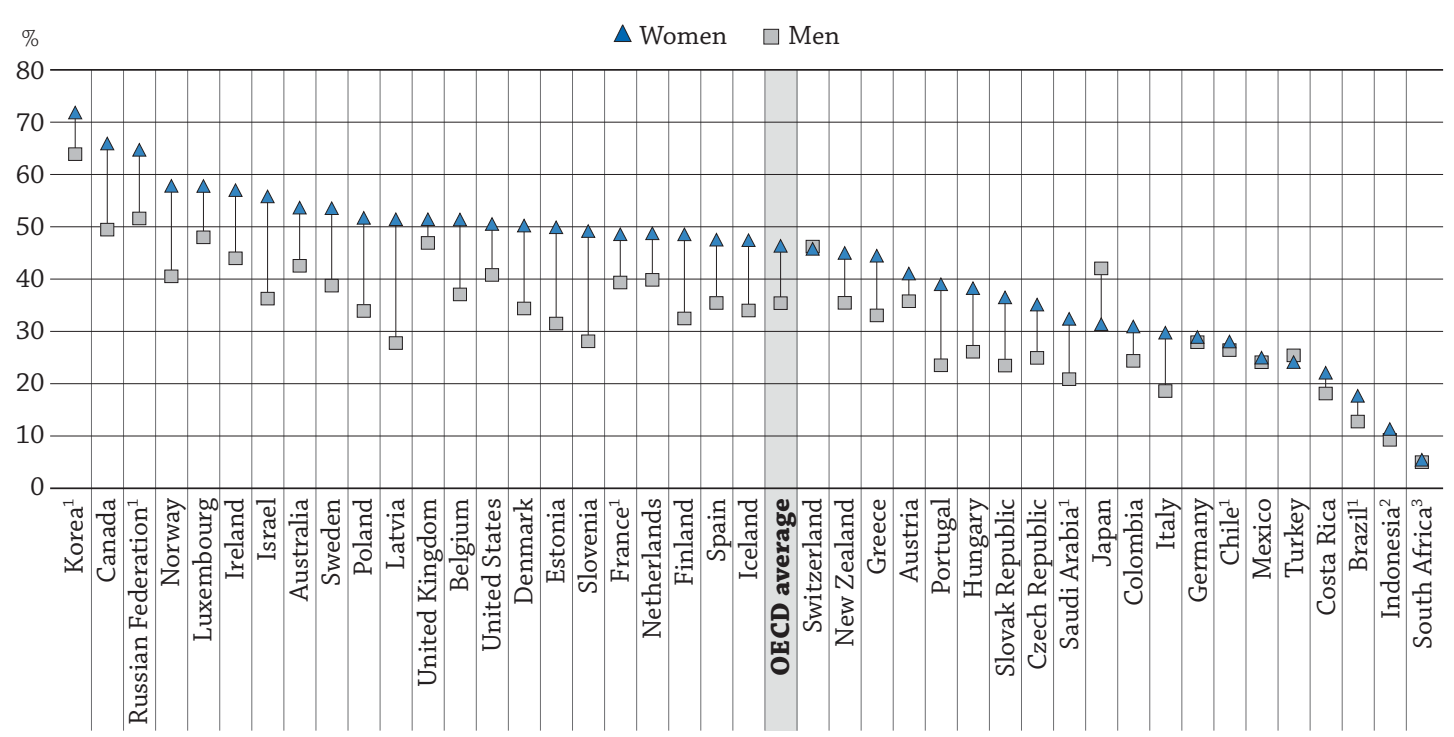

1. Brazil, Chile, France, Korea, Russian Federation, Saudi Arabia: Year of reference 2013.

2. Indonesia: Year of reference 2011.

3. South Africa: Year of reference 2012

Countries are ranked in descending order of the percentage of women who attained tertiary education.

Source: OECD. Table A1.4b. See Annex 3 for notes (www.oecd.org/education/education-at-a-glance-19991487.htm).

StatLink त्ञाजा http://dx.doi.org/10.1787/888933283820

\section{Context}

To compete successfully in today's global economy, countries need to develop the potential of all of their citizens. They need to ensure that men and women develop the right skills and find opportunities to use them productively. Recognising the impact that the gender gap has on participation in labour markets, occupational mobility and quality of life, policy makers and educators need to help to eliminate gender differences in education, at the workplace and in access to jobs.

In education, many countries have managed to close gender gaps in learning outcomes. In fact, as women now surpass men in many aspects of education in OECD countries, there is growing concern about the underachievement of young men in certain areas, such as reading. Gender differences in student performance, as well as perceptions that some fields of education are more "suitable" for either women or men, need to be addressed if greater gender equity in education outcomes is to be achieved. Gender equality is not only a goal in itself, it is also economically beneficial. Education programmes that attract candidates of mostly one gender are in danger of excluding many capable students. 
Other findings

- PISA (the OECD Programme for International Student Assessment) finds that 15-year-old boys are more likely than girls of the same age to be low achievers. In 2012, 14\% of boys and 9\% of girls did not attain the PISA baseline level of proficiency in any of the three core subjects measured in PISA - reading, mathematics and science (OECD, 2014a).

- Across OECD countries, boys are still marginally more represented than girls in upper secondary vocational education.

- Men are five times more likely than women to have studied engineering, manufacturing and construction, on average across OECD countries. By contrast, women are three times more likely than men to have studied education science.

- In all countries and economies that distributed the PISA parent questionnaire, parents were more likely to expect their sons, rather than their daughters, to work in science, technology, engineering or mathematics, even when boys and girls perform at the same level in mathematics.

- Young women have higher educational attainment, but lower employment rates, than young men. The gender gap is much wider at the lower levels of educational attainment than at the highest levels.

- Across OECD countries, a 35-44 year-old tertiary-educated woman earns about 74\% of what a similarly educated man earns. But this result also reflects the under-representation of women at the highest levels of tertiary education and in some fields of education that are highly rewarded by the labour market.

\section{Trends}

Over the three past decades, the gender gap in educational attainment has reversed. Among 55-64 year-olds, more men than women hold tertiary qualifications; but the opposite is true among 25-34 year-olds.

\section{INDICATOR A10}




\section{Analysis}

\section{The gender gap in attainment rates has reversed}

As most OECD countries have made secondary education compulsory, attaining that level of education has become the norm for men and women. Not only are more young women than ever before participating in formal education and enrolling in tertiary education, over the past few decades, the gender hierarchy in educational attainment has been inverted (see Tables A1.2b and A1.4b, available on line).

In 2014, a larger proportion of 25-34 year-old women than men of the same age had attained tertiary education ( $46 \%$ and $35 \%$, respectively), while the opposite was true for 55-64 year-old women and men ( $24 \%$ and $26 \%$, respectively). Israel, Latvia and Slovenia show the widest gender gap among 25-34 year-olds: 20 percentage points or more, in favour of women, among tertiary-educated younger adults (see Table A1.4b, available on line). That same year, some $85 \%$ of younger women had attained at least upper secondary education while $82 \%$ of younger men did, on average across OECD countries (see Tables A1.2b and A1.4b, available on line).

This trend is also observed among students younger than 25. In 2013, 58\% of graduates from upper secondary general programmes were women and $46 \%$ were men. However, women are still under-represented at the very highest levels of education. In most OECD countries in 2013, around 45\% of advanced research degrees were awarded to women (see Indicators A2 and A3).

\section{Low-performing boys}

In all 65 countries and economies that participated in PISA 2012, girls outperformed boys in reading by an average of 38 score points (across OECD countries) - the equivalent of one year of school - as they have done consistently throughout all the PISA cycles since 2000. Boys, however, continued to outperform girls in mathematics in 38 participating countries and economies by an average of 11 score points (across OECD countries) - equivalent to around three months of school. PISA also reveals that there is very little difference in science performance between boys and girls (PISA 2012 database).

Stark gender differences are observed among the lowest-performing students - those who score below PISA proficiency Level 2, which is considered to be the baseline level of proficiency, in all subjects assessed in PISA. While the proportion of girls is marginally larger than that of boys among poor performers in mathematics, in all but six participating countries and economies, a larger proportion of boys than girls does not even achieve the baseline level of proficiency in any of the three core PISA subjects - reading, mathematics and science. Across OECD countries, boys are 5 percentage points more likely than girls to be low achievers in reading, science and mathematics. In 2012, 14\% of boys and 9\% of girls did not attain the PISA baseline level of proficiency in any of the three core subjects (Table A10.1 and Chart A10.2).

The percentage of boys who failed to reach the baseline level of proficiency in any subject is worryingly high in many countries. More than one in five boys in Chile, Greece, Israel, Mexico, the Slovak Republic and Turkey failed to make the grade in any of the three core PISA subjects. Among partner countries and economies, the proportions are even larger. In Indonesia, Jordan, Peru and Qatar more than one in two students failed to make the grade.

The proportion of girls who failed to make the grade is much smaller. Peru is the only country or economy that participated in PISA 2012 where more than one in two girls did not reach the baseline level of proficiency in any of the three subjects. In Chile and Mexico, more than one in five girls failed to make the grade in all three subjects, and in eight partner countries and economies, more than one in three girls failed to make the grade (Table A10.1 and Chart A10.2).

The sizeable number of boys who fail to attain the baseline level of proficiency in all three core PISA subjects is a major challenge for education systems. Students who perform poorly in all subjects are hard to motivate and keep in school. Because of their low levels of skills, these students may also feel disconnected from and disengaged with school. It may then become easier for these students to build an identity based on rebellion against school and formal education than to engage and invest the effort needed to break the vicious cycle of low performance and low motivation.

\section{Gender differences in participation in pre-vocational and vocational programmes}

Students who participated in PISA 2012 were asked to report on the kind of programme in which they were enrolled. On average across OECD countries, $82 \%$ of 15 -year-old students were enrolled in a programme with a general curriculum, $14 \%$ were enrolled in a programme with a pre-vocational or vocational curriculum, and $4 \%$ were in modular programmes that combine any or all of these curricula (Table A10.2 and Chart A10.3). 

Chart A10.2. Gender differences in the percentage of 15-year-old students who are
low achievers in all subjects (e.g. mathematics, reading, science) (PISA 2012)

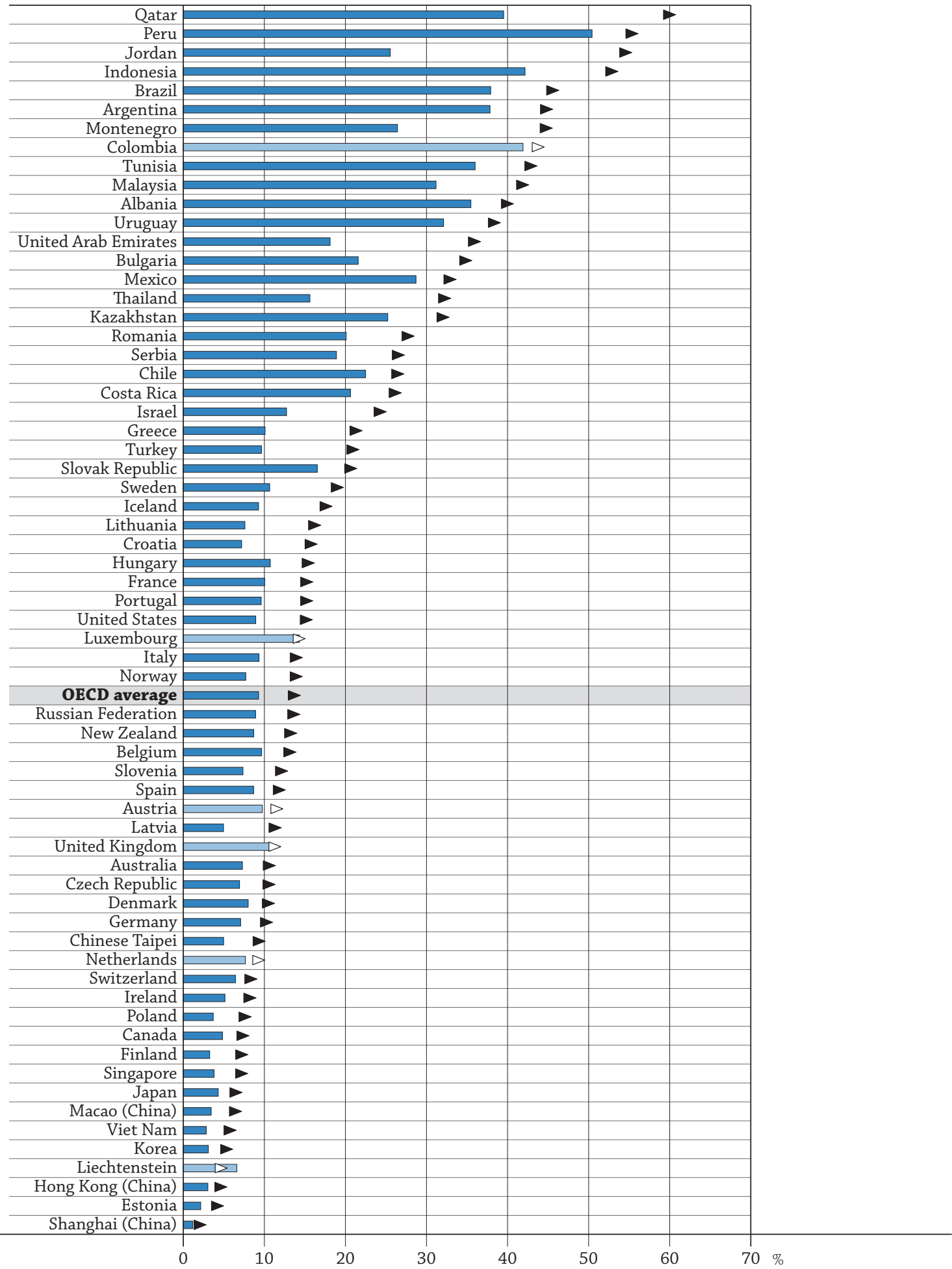

Note: Gender differences that are statistically significant are marked in a darker tone.

Countries and economies are ranked in descending order of the percentage of boys who are low performers (below PISA proficiency Level 2) in reading, mathematics and science.

Source: OECD. Table A10.1. See Annex 3 for notes (www.oecd.org/education/education-at-a-glance-19991487.htm).

StatLink 部 S1 http://dx.doi.org/10.1787/888933283830 

Chart A10.3. Gender gap in favour of boys in the percentage of 15-year-old students who are
enrolled in a vocational programme (PISA 2012)

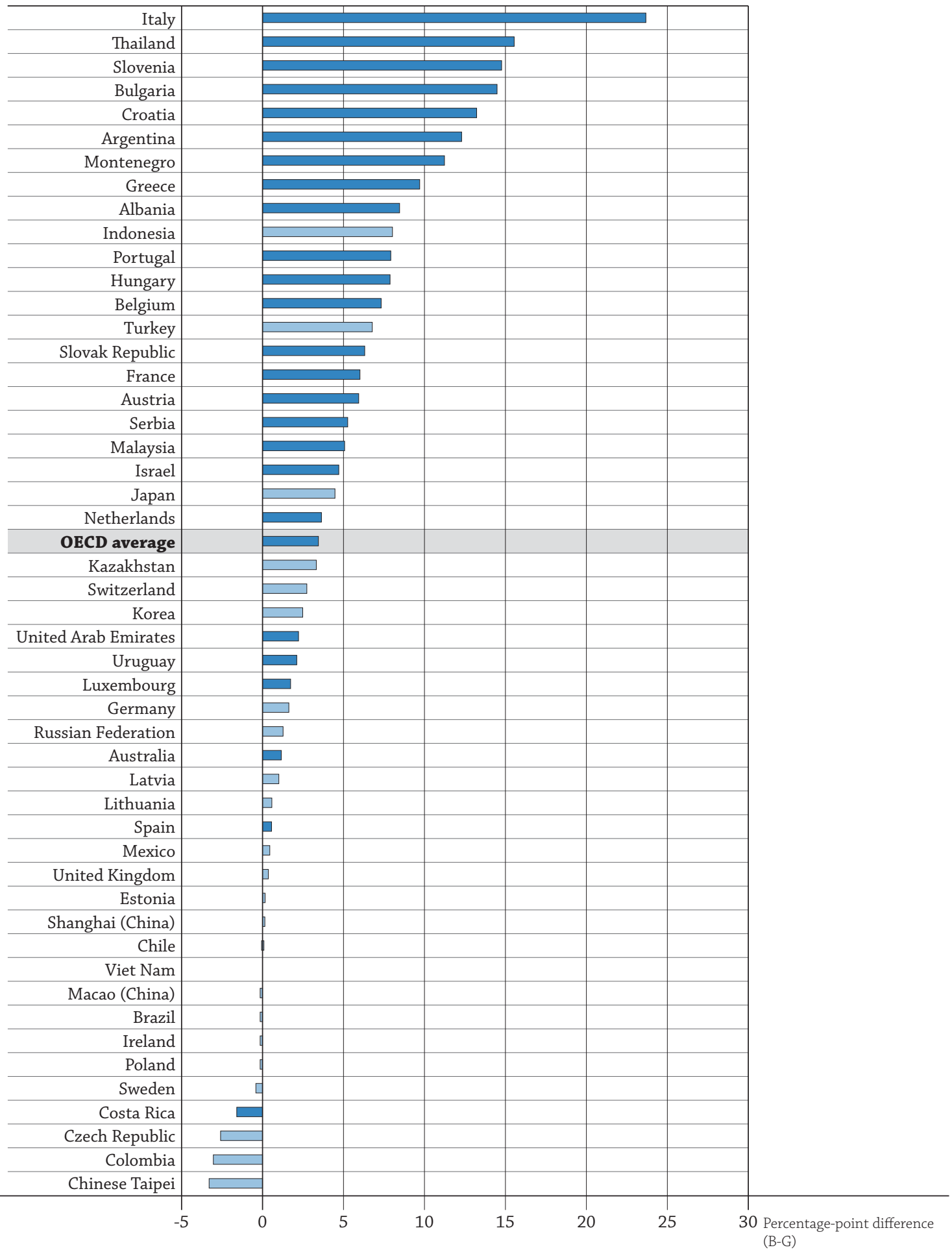

Note: Differences that are statistically significant are marked in a darker tone .

The figure only shows countries and economies where 15-year-old students have the option of enrolling in vocational programmes.

Data for the Slovak Republic do not consider gender differences in participation in modular programmes.

Countries and economies are ranked in descending order of the percentage-point difference between boys and girls who are enrolled in vocational programmes rather than general programmes.

Source: OECD. Table A10.2. See Annex 3 for notes (www.oecd.org/education/education-at-a-glance-19991487.htm).

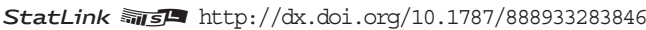


On average across OECD countries, $16 \%$ of 15 -year-old boys and $13 \%$ of 15 -year-old girls attend pre-vocational and vocational schools. However, in many of the countries where large proportions of students are enrolled in prevocational and vocational programmes, boys are heavily over-represented in these programmes. For example, in Italy $50 \%$ of students are enrolled in pre-vocational and vocational programmes. However, while $61 \%$ of boys attend such programmes, only $37 \%$ of girls do (Chart A10.3).

In part, this disparity might reflect the fact that boys are more likely to be low achievers than girls, and low achievers are over-represented among technical and vocational school students. But boys' over-representation in these tracks might also reflect a greater awareness among boys for the need to be prepared for the labour market, the need to acquire more practical skills, or simply the fact that boys might enjoy the content and ways of learning in vocational programmes more than girls.

\section{Gender differences in field of study}

Across OECD countries, boys are still marginally more represented than girls in upper secondary vocational education. Similarly, 15-year-old boys and girls hold different expectations for the field in which they expect to be working as young adults. Boys are significantly more likely to expect to work in science, technology, engineering and mathematics (STEM) occupations; and parents are more likely to expect their sons, rather than their daughters, to work in a STEM field, even when boys and girls perform at the same level in mathematics (see more details in Box A10.1).

On average across OECD countries, 16-65 year-old men surveyed in the 2012 Survey of Adult Skills (OECD, 2013), a product of the OECD Programme for the International Assessment of Adult Competencies (PIAAC) are, on average, five times more likely than women of the same age to have studied engineering, manufacturing and construction (38\% of men reported that they had studied these subjects while only $7 \%$ of women so reported) and are 3 percentage points more likely to have studied science, mathematics and computing ( $10 \%$ of men reported that they had studied these subjects while 7\% of women so reported). By contrast, 16-65 year-old women are around four times more likely than men to have studied health and welfare ( $15 \%$ of women and $4 \%$ of men so reported), around three times more likely to have studied education science and to have enrolled in teacher training ( $9 \%$ of women and $3 \%$ of men so reported), and around twice as likely as men to have studied humanities, languages and arts (10\% of women and $5 \%$ of men so reported). (Table A10.3 and Chart A10.4).

Differences in the percentage of 16-65 year-old men and women who reported that they had studied engineering, manufacturing and construction are larger than 19 percentage points in all countries and economies examined. These differences are particularly wide in Austria, the Czech Republic, Finland, Germany and the Slovak Republic, where men are over 40 percentage points more likely than women to have studied these subjects, according to their reports. Differences are smallest in Canada, Estonia, Italy, Korea and the United Kingdom (Table A10.3 and Chart A10.4).

\section{Box A10.1. Parents' expectations for their children}

Results presented in Chart A10.a suggest that parents still hold different expectations for their sons and daughters. This could be because parents still harbour stereotypical notions of what women and men excel at and the career they can pursue when they enter the labour market - which is, in turn, related to occupational segregation in the labour market.

In Chile, Croatia, Germany, Hong Kong (China), Hungary, Italy, Korea, Macao (China), Mexico and Portugal, students who participated in PISA 2012 were asked to take home a questionnaire for their parents to complete. The responses collected allow for more in-depth analyses of parents' attitudes and perceptions. Among other things, parents were asked what occupation they expected their 15-year-old child to work in when he or she is 30 years old.

Chart A10.a shows that, in all countries and economies that distributed the parent questionnaire, parents were more likely to expect their sons, rather than their daughters, to work in a STEM field. For example, in Chile, $50 \%$ of 15 -year-old boys' parents expected that they would work in STEM occupations; only $16 \%$ of girls' parents reported so.

The gender gap in the percentage of 15-year-old boys and girls whose parents expected them to work in STEM occupations is larger than 30 percentage points in Chile, Hungary and Portugal. In Korea, relatively few students have parents who expected them to work in STEM occupations - $17 \%$ of boys and $9 \%$ of girls; but even so, the gender gap is a substantial 7 percentage points. 
The gender differences in academic performance do not explain the observed differences in parents' expectations for their sons and daughters to work in STEM fields. The gender difference remains large and significant in all participating countries and economies, even when accounting for students' performance in reading, mathematics and science. As expected, results indicate that parents are more likely to expect that their children will work in STEM fields if they perform better in mathematics. In other words, the better the student's mathematic performance, the greater the likelihood that his or her parents will expect that student to work in a STEM occupation. In Croatia and Italy, parents are less likely to expect their children to work in STEM occupations if they perform better in reading.

\section{Chart A10.a. Parents' expectations for their children's careers (PISA 2012)}

\section{Percentage of students whose parents expect that they will work in STEM occupations}

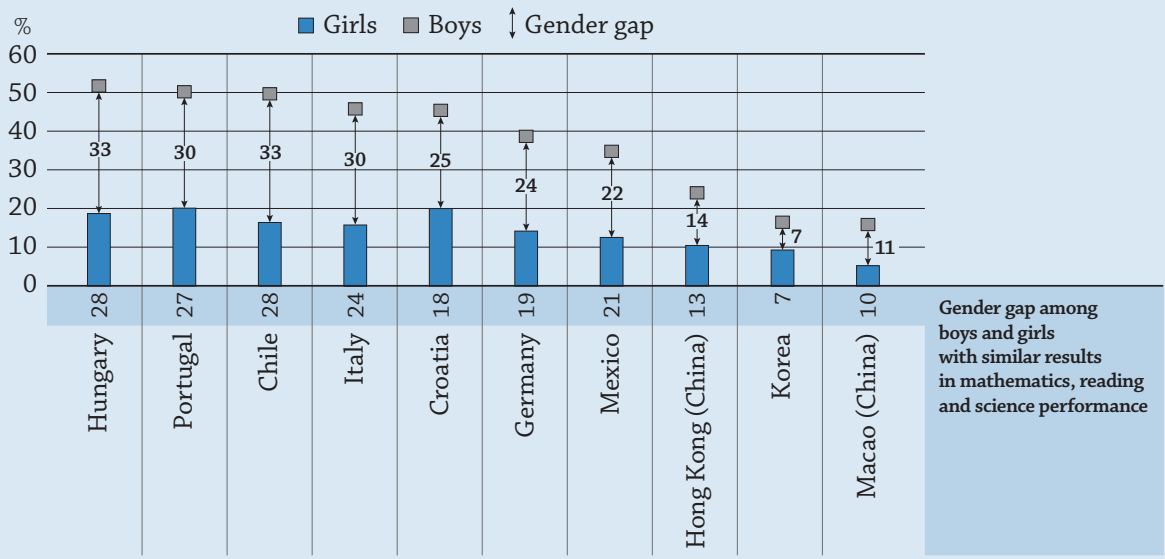

Note: All gender differences are statistically significant. STEM stands for science, technology, engineering, and mathematics.

Countries and economies are ranked in descending order of the percentage of boys whose parents expect that they will work in STEM occupations when they are 30 years old.

Source: OECD (2015), The ABC of Gender Equality in Education: Aptitude, Behaviour, Confidence (Table 5.4). PISA 2012 Database. See Annex 3 for notes (www.oecd.org/education/education-at-a-glance-19991487.htm).

StatLink त्राज्ञा http://dx.doi.org/10.1787/888933283880

PISA results also suggest that in Hong Kong (China), Korea, Macao (China), Mexico and Portugal, when comparing students of similar performance in reading, mathematics and science, students from socio-economically advantaged households are more likely than students from disadvantaged households to have parents who expect them to work in STEM occupations. Italy is the only country where advantaged students are less likely to have parents who expect them to work in STEM occupations.

The literature often suggests that girls' lack of confidence in their abilities in mathematics and science may be due to an absence of role models. The paucity of women scientists means that young girls have little in the way of tangible evidence to disprove the stereotypical notion that mathematics and science are somehow more "masculine" disciplines. PISA results show that few mothers of 15-year-olds, worldwide, work in STEM occupations; indeed, in all countries and economies there are far fewer women than men employed in these sectors. But PISA does not provide strong evidence that the gender gap in mathematics performance is narrower in households where the mother does work in a STEM occupation. In fact, in Belgium, Bulgaria, Canada, France, Greece, the Netherlands, Qatar, the Slovak Republic, Turkey and Uruguay, the gender gap in mathematics performance, in favour of boys, appears to be much wider among students whose mother works in a STEM field.

What these results suggest is that many parents still expect their sons and daughters to pursue different occupations, even when they perform similarly in mathematics. While having positive role models is important for girls, many girls who have parents, and mothers in particular, who work in science- and mathematicsrelated fields often underperform in mathematics compared to boys from similar households. One reason may be the much higher level of anxiety towards mathematics that girls report, and the fact that they are often more driven to perform well in school and achieve at a high level. High anxiety coupled with high expectations often lead to choking under pressure. 
Chart A10.4. Proportion of the population surveyed in the 2012 Survey of Adult Skills, by field of study

Percentage of 16-65 year-old men and women with each field of study.

$\square \square$ Men $\triangle \boldsymbol{\Delta}$ Women
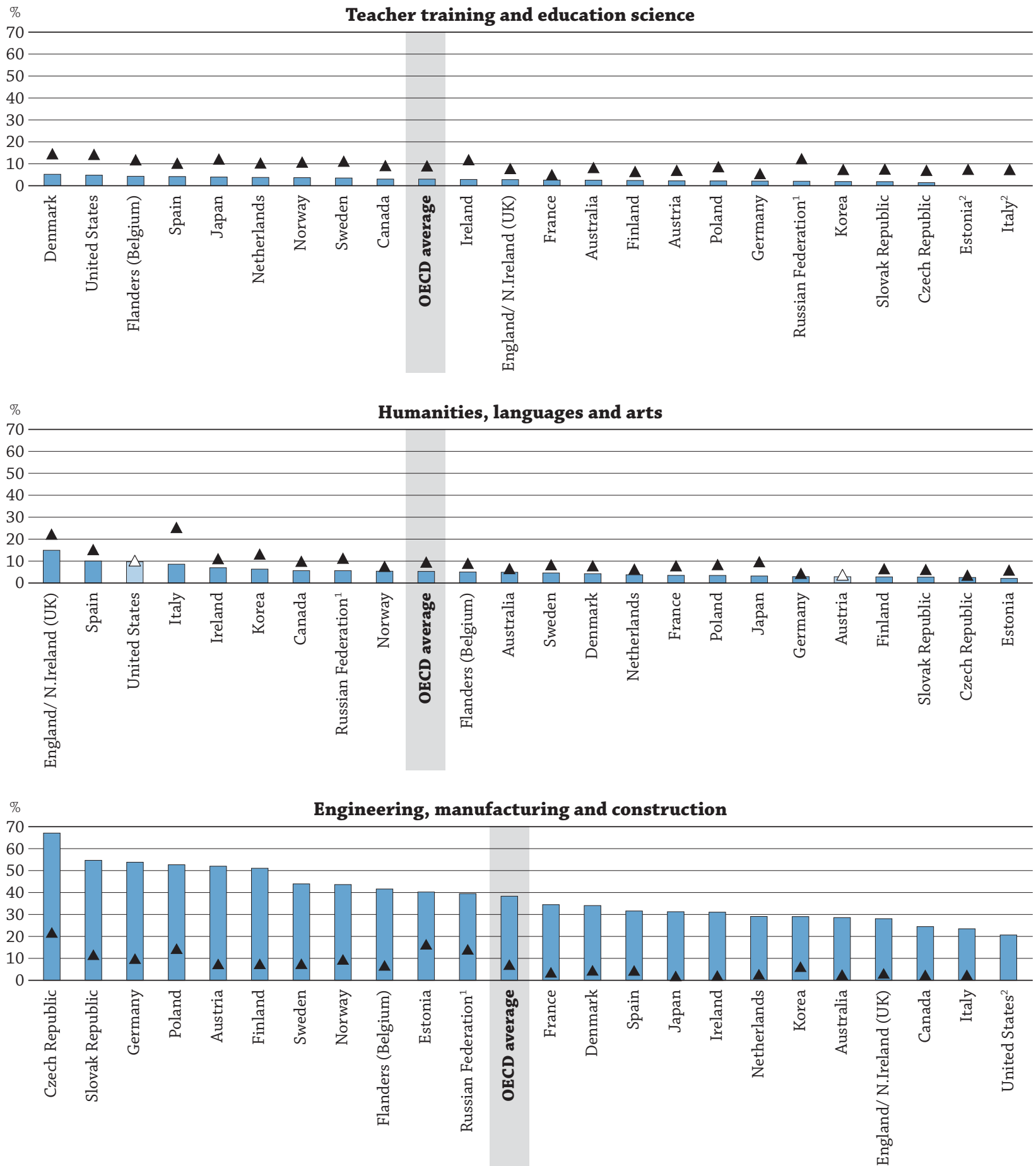

Note: Gender differences that are statistically significant are marked in a darker tone.

1. See note on data for the Russian Federation in the Mehtodology section.

2. Sample size too small.

Countries and economies are ranked in descending order of the percentage of men in each field of study.

Source: OECD. Survey of Adult Skills (PIAAC) (2012), Table A10.3. See Annex 3 for notes (www.oecd.org/education/education-at-a-glance-19991487.htm).

StatLink 제그 http://dx.doi.org/10.1787/888933283850 
In all of these countries except Estonia, the gender difference (in percentage points) is smaller because fewer individuals have studied these fields, not because there is greater gender equity in enrolment in these fields. Similarly, countries showing a small difference in the percentage of men and women who reported that they had studied health and welfare tend to be those where these programmes attract comparatively fewer candidates. For example, Italy, Korea, Poland and the Russian Federation show a small or no gender gap in these fields - but also relatively few adults reported that they had studied these subjects (Chart A10.4).

\section{Employment rates, by gender}

In 2014, women were still less likely than men to participate in the labour market - even when they have a tertiary qualification - and were also more likely to work part-time. Across all OECD countries and education levels, only $66 \%$ of women were employed compared with $80 \%$ of men - despite women's higher educational attainment, in general. On average, employment rates for those with the lowest qualifications (below upper secondary education) are significantly higher among younger men than among younger women. The gender gap in employment rates is the largest among adults with the least education (see Tables A5.1b, A5.3b and c, available on line).

On average across OECD countries, the gender difference in employment rates among 25-64 year-olds with below upper secondary education as their highest level of attainment is 20 percentage points ( $66 \%$ for men and $47 \%$ for women). This difference shrinks to 15 percentage points among individuals with upper secondary or post-secondary non-tertiary education ( $81 \%$ for men and $66 \%$ for women), and to 8 percentage points among tertiary-educated men and women (88\% for men and 79\% for women) (Chart A10.5, and see Tables A5.3b and c, available on line).

\section{Chart A10.5. Employment rates of 25-64 year-old men and women with below upper secondary and tertiary education (2014)}

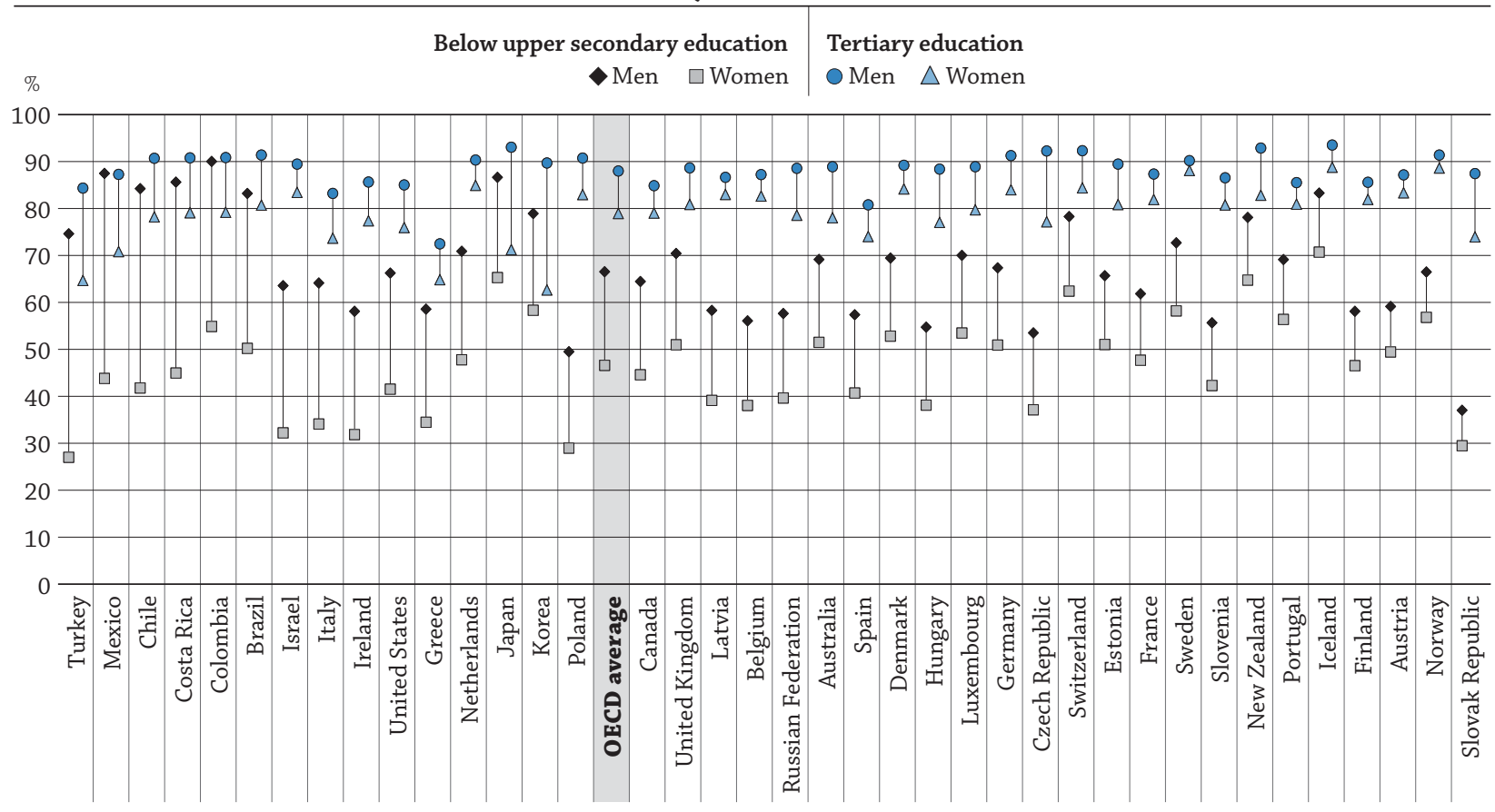

Countries are ranked in descending order of the difference between employment rate of 25-64 year-old men and women with below upper secondary education. Source: OECD. Tables A5.3b and c. See Annex 3 for notes (www.oecd.org/education/education-at-a-glance-19991487.htm).

StatLink s-ins http://dx.doi.org/10.1787/888933283861

Although the gap between men's and women's employment rates narrows as educational attainment increases, the employment rate among tertiary-educated women across OECD countries is still considerably lower than that of men - despite the fact that a larger proportion of 25-64 year-old women (35\%) than men of the same age (32\%) in OECD countries now has a tertiary education (see Tables A1.3b, A5.3b and c, available on line).

In all OECD countries except the Slovak Republic, the gender gap in employment is smaller among 25-64 year-olds with tertiary education than among those who have not attained upper secondary education. The difference is particularly large in Chile, Mexico and Turkey, where it exceeds 25 percentage points (see Tables A5.3b and c, available on line). 
Gender differences in unemployment rates are less pronounced than they are in employment rates, on average. This can be partly explained by differences in labour market participation between women and men. Among adults with below upper secondary education, unemployment rates are similar for women and men (about 12.5\% for both). Among adults who have an upper secondary or post-secondary non-tertiary education, unemployment rates are higher among women (8\%) than among men (7\%). This also observed among tertiary-educated adults, where the unemployment rate is about $6 \%$ for women and $5 \%$ men (see Tables A5.4b and c, available on line).

Gender differences in unemployment rates are particularly large in Greece and Turkey. In Turkey, $12 \%$ of tertiaryeducated women were unemployed in 2014 compared to only 6\% of tertiary-educated men; in Greece, $21 \%$ of tertiary-educated women and $17 \%$ of tertiary-educated men were unemployed that year. These differences were even more pronounced among adults with upper secondary education as their highest level of attainment. In Turkey, $17 \%$ of women were unemployed in 2014 compared with $7 \%$ of men; while in Greece, 34\% of women and $23 \%$ of men with this level of education were unemployed that year (see Tables A5.4b and c, available on line).

\section{Differences in earnings between employed men and women, by educational attainment}

Not only are there fewer women in the labour market, when they do work they tend to earn less than men. Regardless of the level of education, the gender gap in earnings persists. Even among tertiary-educated adults, women earn less than men. The available data on full-time, full-year earners show that the largest gender gap in earnings is among workers with tertiary education. Across OECD countries, a 35-44 year-old tertiary-educated woman earns about $74 \%$ of what a similarly educated man earns. Only in Belgium, Luxembourg, Sweden, Switzerland and Turkey do the earnings of tertiary-educated women amount to $85 \%$ or more of men's earnings. In Estonia, Hungary, Israel, Korea and the Slovak Republic, women with a tertiary degree earn $65 \%$ or less of what tertiary-educated men earn (Chart A10.6 and see Table A6.2a).

However, these findings should be interpreted with some caution. In fact, the results are partly explained by the under-representation of women at the highest levels of tertiary education and in some fields of education, such as engineering, manufacturing and construction, which are highly rewarded by the labour market.

\section{Chart A10.6. Earnings of 35-44 year-old tertiary-educated women as a percentage of the earnings of men of the same age and educational attainment (2013 or most recent year available)} Adults with income from employment; average annual full-time, full-year earnings

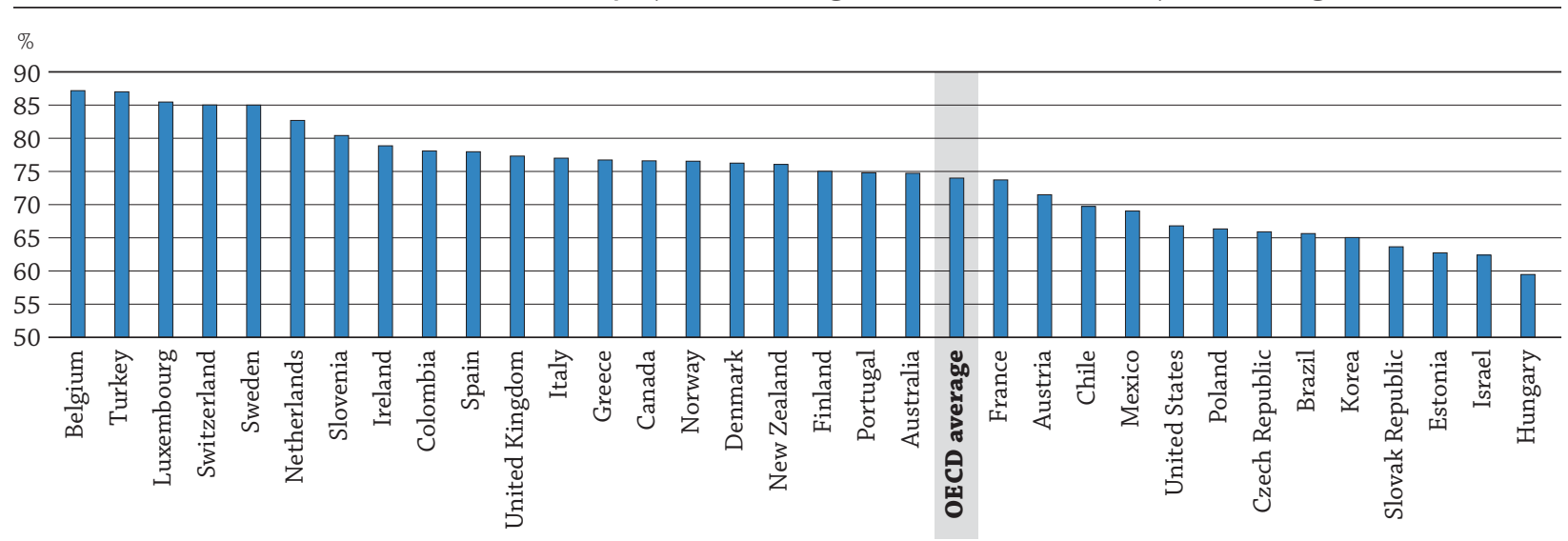

Countries are ranked in descending order of the earnings of 35-44 year-old women with tertiary qualification as a percentage of the earnings of men with the same characteristics.

Source: OECD. Table A6.2a. See Annex 3 for notes (www.oecd.org/education/education-at-a-glance-19991487.htm).

StatLink 젝ㄴ http://dx.doi.org/10.1787/888933283875

\section{Methodology}

Data on population and educational attainment for most countries are taken from OECD and Eurostat databases, which are compiled from National Labour Force Surveys by the OECD LSO (Labour Market and Social Outcomes of Learning) Network. Data on educational attainment for Argentina, China, Colombia, Indonesia, Saudi Arabia and South Africa are taken from the UNESCO Institute of Statistics (UIS) database on educational attainment of the population aged 25 and older. 
The PISA target population is 15-year-old students. Operationally, these are students who were from 15 years and 3 (completed) months to 16 years and 2 (completed) months at the beginning of the testing period, and who were enrolled in an educational institution, regardless of the grade level or type of institution and of whether they participated in school full-time or part-time.

Data on field of studies are based on the Survey of Adult Skills (PIAAC) (2012). PIAAC is the OECD Programme for the International Assessment of Adult Competencies. See Annex 3 for additional information (www.oecd.org/ education/education-at-a-glance-19991487.htm).

The indicator is based on the regular data collection by the OECD LSO (Labour Market and Social Outcomes of Learning) Network that takes account of earnings from work for all individuals during the reference period, even if the individual has worked part time or part year. This indicator is based on the earnings of those working full time and full year. See Annex 3 (www.oecd.org/education/education-at-a-glance-19991487.htm) for additional information.

\section{Note regarding data from Israel}

The statistical data for Israel are supplied by and are under the responsibility of the relevant Israeli authorities. The use of such data by the OECD is without prejudice to the status of the Golan Heights, East Jerusalem and Israeli settlements in the West Bank under the terms of international law.

\section{Note regarding data from the Russian Federation in the Survey of Adult Skills (PIAAC)}

Readers should note that the sample for the Russian Federation does not include the population of the Moscow municipal area. The data published, therefore, do not represent the entire resident population aged 16-65 in Russia but rather the population of Russia excluding the population residing in the Moscow municipal area. More detailed information regarding the data from the Russian Federation as well as that of other countries can be found in the Technical Report of the Survey of Adult Skills (OECD, 2014b).

\section{References}

OECD (2015), The ABC of Gender Equality in Education: Aptitude, Behaviour, Confidence, PISA, OECD Publishing, Paris, http://dx.doi. org/10.1787/9789264229945-en.

OECD (2014a), PISA 2012 Results: What Students Know and Can Do (Volume I, Revised edition, February 2014): Student Performance in Mathematics, Reading and Science, PISA, OECD Publishing, Paris, http://dx.doi.org/10.1787/9789264208780-en.

OECD (2014b), Technical Report of the Survey of Adult Skills, www.oecd.org/site/piaac/ Technical\%20Report 170CT13.pdf, pre-publication copy.

OECD (2013), OECD Skills Outlook 2013: First Results from the Survey of Adult Skills, OECD Publishing, Paris, http://dx.doi. org/10.1787/9789264204256-en.

\section{Indicator A10 Tables}

StatLink त्तारा http://dx.doi.org/10.1787/888933285306

Table A10.1 Percentage of low achievers in mathematics, reading and science, by gender (PISA 2012)

Table A10.2 Gender disparities at age 15 in participation in general, vocational and modular programmes

Table A10.3 Percentage of adults, by field of study of the highest level of education attained and gender 
Table A10.1. [1/3] Percentage of low achievers in mathematics, reading and science, by gender (PISA 2012) Percentage of 15-year-old students who do not attain the PISA baseline proficiency Level 2

\begin{tabular}{|c|c|c|c|c|c|c|c|c|c|c|c|}
\hline & & & & & & & are: & & & & \\
\hline & & $\begin{array}{r}\text { Not lo } \\
\text { any of th }\end{array}$ & $\begin{array}{l}\text { vers in } \\
\text { domains }\end{array}$ & $\begin{array}{l}\text { Low } \\
\text { in }\end{array}$ & $\begin{array}{l}\text { s only } \\
\text { atics }\end{array}$ & $\begin{array}{r}\text { Low a } \\
\text { ir }\end{array}$ & $\begin{array}{l}\text { s only } \\
\text { ig }\end{array}$ & $\begin{array}{r}\text { Low } \\
\mathrm{i}\end{array}$ & $\begin{array}{l}\text { s only } \\
\text { e }\end{array}$ & $\begin{array}{l}\text { Low } \\
\text { all tr }\end{array}$ & $\begin{array}{l}\text { ers in } \\
\text { nains }\end{array}$ \\
\hline & & $\%$ & S.E. & $\%$ & S.E. & $\%$ & S.E. & $\%$ & S.E. & $\%$ & S.E. \\
\hline & & (1) & (2) & (3) & (4) & (5) & (6) & (7) & (8) & (9) & (10) \\
\hline 웁 & Australia & 76 & $(0.8)$ & 18 & $(0.7)$ & 19 & $(0.6)$ & 14 & $(0.6)$ & 11 & $(0.6)$ \\
\hline 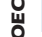 & Austria & 72 & (1.7) & 16 & (1.4) & 26 & (1.7) & 16 & (1.4) & 12 & (1.2) \\
\hline & Belgium & 75 & $(1.2)$ & 19 & (1.3) & 20 & $(1.2)$ & 18 & $(1.2)$ & 13 & (1.1) \\
\hline & Chile & 48 & $(2.1)$ & 45 & (2.1) & 39 & $(2.2)$ & 33 & $(1.9)$ & 26 & (1.8) \\
\hline & Czech Republic & 72 & (1.7) & 19 & (1.4) & 23 & (1.7) & 14 & (1.4) & 11 & (1.2) \\
\hline & Denmark & 76 & (1.5) & 15 & (1.3) & 19 & (1.5) & 16 & (1.3) & 11 & (1.1) \\
\hline & Estonia & 83 & $(1.1)$ & 11 & $(0.9)$ & 14 & (1.1) & 6 & $(0.6)$ & 4 & $(0.6)$ \\
\hline & Finland & 79 & (1.1) & 14 & $(0.9)$ & 18 & $(1.1)$ & 10 & (0.9) & 7 & (0.6) \\
\hline & France & 69 & $(1.4)$ & 22 & (1.1) & 25 & (1.3) & 20 & (1.4) & 15 & (1.0) \\
\hline & Germany & 77 & (1.1) & 17 & (1.1) & 20 & $(1.2)$ & 13 & (1.0) & 10 & (1.0) \\
\hline & Greece & 57 & (1.9) & 34 & (1.7) & 32 & (1.7) & 30 & (1.8) & 21 & (1.5) \\
\hline & Ireland & 81 & $(1.5)$ & 15 & (1.4) & 13 & (1.3) & 11 & (1.2) & 8 & (1.1) \\
\hline & Israel & 60 & $(2.8)$ & 34 & (2.6) & 32 & $(2.6)$ & 31 & $(2.6)$ & 24 & (2.2) \\
\hline & Italy & 68 & (1.0) & 23 & $(0.9)$ & 26 & $(0.9)$ & 19 & $(0.7)$ & 14 & $(0.6)$ \\
\hline & Japan & 84 & $(1.5)$ & 11 & (1.2) & 13 & (1.3) & 9 & (1.1) & 7 & $(0.9)$ \\
\hline & Korea & 87 & (1.5) & 9 & (1.2) & 10 & (1.4) & 7 & (1.1) & 5 & (1.0) \\
\hline & Luxembourg & 69 & $(1.0)$ & 20 & $(0.8)$ & 26 & $(0.9)$ & 20 & $(0.8)$ & 14 & $(0.7)$ \\
\hline & Mexico & 38 & $(0.9)$ & 51 & (1.0) & 47 & (1.1) & 45 & (1.0) & 33 & (1.0) \\
\hline & Netherlands & 80 & (1.5) & 14 & (1.3) & 17 & (1.4) & 13 & (1.3) & 9 & (1.1) \\
\hline & New Zealand & 73 & $(1.3)$ & 22 & (1.2) & 21 & $(1.2)$ & 17 & (1.1) & 13 & $(0.9)$ \\
\hline & Norway & 70 & (1.4) & 23 & (1.2) & 22 & (1.4) & 21 & (1.3) & 14 & (1.0) \\
\hline & Poland & 79 & (1.3) & 15 & (1.2) & 16 & $(1.2)$ & 10 & $(0.9)$ & 8 & $(0.8)$ \\
\hline & Portugal & 69 & (1.8) & 24 & (1.5) & 25 & (1.8) & 20 & (1.6) & 15 & (1.4) \\
\hline & Slovak Republic & 60 & $(1.9)$ & 28 & (1.6) & 35 & $(2.0)$ & 27 & $(1.8)$ & 21 & (1.7) \\
\hline & Slovenia & 66 & $(1.2)$ & 20 & (1.0) & 30 & (1.1) & 15 & $(0.8)$ & 12 & $(0.7)$ \\
\hline & Spain & 70 & (1.1) & 22 & (1.0) & 23 & $(0.9)$ & 16 & $(0.8)$ & 12 & $(0.7)$ \\
\hline & Sweden & 63 & (1.6) & 28 & (1.4) & 31 & (1.8) & 25 & (1.5) & 19 & (1.5) \\
\hline & Switzerland & 79 & $(1.2)$ & 12 & $(0.8)$ & 18 & (1.1) & 13 & (1.0) & 8 & $(0.7)$ \\
\hline & OECD average & 70 & $(0.3)$ & 22 & $(0.2)$ & 24 & $(0.3)$ & 18 & $(0.2)$ & 14 & $(0.2)$ \\
\hline$n$ & Albania & 27 & (1.3) & 61 & (1.5) & 55 & (1.9) & 55 & (1.5) & 40 & (1.2) \\
\hline छ & Argentina & 28 & $(2.0)$ & 63 & (2.4) & 61 & $(2.0)$ & 52 & $(2.5)$ & 45 & (2.3) \\
\hline$\frac{\pi}{2}$ & Brazil & 28 & (1.1) & 64 & (1.1) & 59 & $(1.2)$ & 55 & (1.3) & 46 & (1.2) \\
\hline & Bulgaria & 43 & (2.3) & 45 & (2.2) & 51 & (2.4) & 42 & (2.4) & 35 & (2.2) \\
\hline & Colombia & 28 & $(1.8)$ & 67 & (1.9) & 57 & $(1.8)$ & 51 & $(1.8)$ & 44 & (1.8) \\
\hline & Costa Rica & 41 & $(2.3)$ & 52 & (2.3) & 39 & $(2.1)$ & 36 & $(2.0)$ & 26 & (1.8) \\
\hline & Croatia & 65 & (1.9) & 29 & (1.7) & 27 & (1.9) & 19 & (1.4) & 16 & (1.3) \\
\hline & Hong Kong (China) & 89 & $(1.2)$ & 9 & (1.0) & 9 & $(1.0)$ & 6 & $(0.7)$ & 5 & $(0.6)$ \\
\hline & Indonesia & 19 & $(2.4)$ & 74 & $(2.4)$ & 62 & $(2.6)$ & 67 & $(2.4)$ & 53 & (2.5) \\
\hline & Jordan & 21 & (2.1) & 72 & (2.4) & 69 & (2.3) & 61 & $(2.7)$ & 55 & (2.7) \\
\hline & Kazakhstan & 29 & (2.1) & 45 & (2.0) & 67 & (2.0) & 44 & (2.2) & 32 & (1.8) \\
\hline & Latvia & 69 & $(1.7)$ & 22 & (1.5) & 25 & (1.9) & 15 & (1.4) & 11 & (1.3) \\
\hline & Liechtenstein & 82 & (3.1) & 11 & (2.8) & 14 & (3.0) & 8 & $(2.2)$ & 5 & (2.3) \\
\hline & Lithuania & 63 & (1.6) & 28 & (1.3) & 32 & (1.7) & 19 & (1.3) & 16 & (1.1) \\
\hline & Macao (China) & 80 & $(0.8)$ & 12 & $(0.7)$ & 16 & $(0.7)$ & 10 & $(0.6)$ & 6 & $(0.5)$ \\
\hline & Malaysia & 31 & (1.9) & 54 & (2.0) & 63 & (2.0) & 48 & (1.9) & 42 & (1.8) \\
\hline & Montenegro & 33 & $(0.9)$ & 57 & (1.1) & 57 & (1.1) & 54 & (1.0) & 45 & (1.1) \\
\hline & Peru & 21 & $(1.7)$ & 71 & (1.9) & 65 & (1.9) & 67 & (2.1) & 55 & (2.0) \\
\hline & Qatar & 23 & $(0.6)$ & 71 & $(0.6)$ & 68 & $(0.5)$ & 68 & $(0.6)$ & 60 & $(0.6)$ \\
\hline & Romania & 44 & $(2.2)$ & 40 & (2.2) & 47 & (2.2) & 39 & (2.0) & 28 & (1.9) \\
\hline & Russian Federation & 63 & $(1.7)$ & 25 & (1.4) & 29 & $(1.8)$ & 20 & $(1.5)$ & 14 & (1.1) \\
\hline & Serbia & 48 & $(2.1)$ & 37 & (2.0) & 43 & $(1.9)$ & 37 & $(2.3)$ & 27 & (1.9) \\
\hline & Shanghai (China) & 94 & $(0.8)$ & 4 & $(0.7)$ & 4 & $(0.6)$ & 3 & $(0.5)$ & 2 & $(0.4)$ \\
\hline & Singapore & 84 & $(0.7)$ & 10 & $(0.7)$ & 13 & $(0.6)$ & 11 & $(0.7)$ & 7 & $(0.7)$ \\
\hline & Chinese Taipei & 81 & $(1.4)$ & 14 & (1.3) & 16 & $(1.3)$ & 11 & $(1.1)$ & 9 & (1.1) \\
\hline & Thailand & 37 & $(1.8)$ & 54 & (1.8) & 48 & (1.8) & 39 & (1.8) & 32 & (1.8) \\
\hline & Tunisia & 26 & $(2.1)$ & 64 & $(2.2)$ & 57 & $(2.4)$ & 55 & $(2.2)$ & 43 & $(2.4)$ \\
\hline & United Arab Emirates & 43 & (1.8) & 48 & (1.7) & 48 & (1.7) & 42 & $(1.9)$ & 36 & (1.6) \\
\hline & Uruguay & 35 & $(1.6)$ & 53 & $(1.8)$ & 55 & $(1.8)$ & 47 & $(1.7)$ & 38 & $(1.7)$ \\
\hline & Viet Nam & 81 & $(2.4)$ & 14 & $(2.1)$ & 14 & $(2.0)$ & 8 & $(1.4)$ & 6 & (1.3) \\
\hline
\end{tabular}

Note: Values that are statistically significant are indicated in bold.

Source: OECD. PISA 2012 Database. See Annex 3 for notes (www.oecd.org/education/education-at-a-glance-19991487.htm).

Please refer to the Reader's Guide for information concerning symbols for missing data and abbreviations.

StatLink 제 SL http://dx.doi.org/10.1787/888933285319 
Table A10.1. [2/3] Percentage of low achievers in mathematics, reading and science, by gender (PISA 2012) Percentage of 15-year-old students who do not attain the PISA baseline proficiency Level 2

\begin{tabular}{|c|c|c|c|c|c|c|c|c|c|c|c|}
\hline & \multicolumn{10}{|c|}{ Girls who are: } \\
\hline & & \multicolumn{2}{|c|}{$\begin{array}{c}\text { Not low achievers in } \\
\text { any of the three domains }\end{array}$} & \multicolumn{2}{|c|}{$\begin{array}{l}\text { Low achievers only } \\
\text { in mathematics }\end{array}$} & \multicolumn{2}{|c|}{$\begin{array}{l}\text { Low achievers only } \\
\text { in reading }\end{array}$} & \multicolumn{2}{|c|}{$\begin{array}{l}\text { Low achievers only } \\
\text { in science }\end{array}$} & \multicolumn{2}{|c|}{$\begin{array}{l}\text { Low achievers in } \\
\text { all three domain }\end{array}$} \\
\hline & & $\%$ & S.E. & $\%$ & S.E. & $\%$ & S.E. & $\%$ & S.E. & $\%$ & S.E. \\
\hline & & (11) & (12) & (13) & (14) & (15) & (16) & (17) & (18) & (19) & (20) \\
\hline \multirow{30}{*}{$\begin{array}{l}\mathbf{u} \\
\text { ö } \\
\text {. }\end{array}$} & Australia & 77 & $(0.8)$ & 21 & $(0.8)$ & 9 & $(0.6)$ & 13 & $(0.7)$ & 7 & $(0.5)$ \\
\hline & Austria & 76 & (1.5) & 21 & (1.4) & 13 & (1.1) & 15 & $(1.4)$ & 10 & (1.0) \\
\hline & Belgium & 77 & (1.1) & 19 & (1.1) & 12 & $(0.9)$ & 17 & $(1.0)$ & 10 & $(0.8)$ \\
\hline & Chile & 41 & (1.8) & 57 & (1.9) & 27 & (1.7) & 35 & (1.7) & 22 & (1.7) \\
\hline & Czech Republic & 75 & (1.6) & 23 & $(1.7)$ & 11 & (1.2) & 13 & (1.4) & 7 & (1.0) \\
\hline & Denmark & 77 & (1.2) & 19 & (1.1) & 10 & (0.9) & 17 & $(1.0)$ & 8 & (0.9) \\
\hline & Estonia & 88 & $(0.8)$ & 10 & $(0.8)$ & 4 & $(0.7)$ & 4 & $(0.5)$ & 2 & $(0.4)$ \\
\hline & Finland & 89 & $(0.7)$ & 10 & $(0.7)$ & 5 & $(0.6)$ & 6 & $(0.5)$ & 3 & $(0.5)$ \\
\hline & France & 75 & (1.1) & 22 & (1.1) & 13 & (1.1) & 17 & $(1.0)$ & 10 & $(0.9)$ \\
\hline & Germany & 80 & (1.4) & 19 & $(1.3)$ & 9 & $(0.9)$ & 11 & $(1.0)$ & 7 & (0.9) \\
\hline & Greece & 60 & (1.7) & 37 & (1.7) & 13 & (1.1) & 21 & $(1.5)$ & 10 & (1.1) \\
\hline & Hungary & 70 & (1.6) & 28 & $(1.7)$ & 13 & (1.2) & 17 & (1.4) & 11 & (1.1) \\
\hline & Ireland & 80 & (1.4) & 19 & (1.2) & 6 & $(0.9)$ & 10 & (1.1) & 5 & $(0.7)$ \\
\hline & Israel & 63 & (1.6) & 33 & (1.6) & 15 & (1.3) & 26 & $(1.6)$ & 13 & (1.3) \\
\hline & Italy & 70 & (1.1) & 27 & (1.0) & 13 & $(0.7)$ & 18 & $(1.0)$ & 9 & $(0.6)$ \\
\hline & Japan & 87 & (1.2) & 11 & (1.1) & 6 & $(0.8)$ & 8 & $(1.0)$ & 4 & $(0.7)$ \\
\hline & Korea & 90 & $(1.2)$ & 9 & (1.1) & 4 & $(0.7)$ & 5 & $(0.8)$ & 3 & $(0.6)$ \\
\hline & Luxembourg & 67 & $(0.9)$ & 29 & $(0.7)$ & 17 & $(0.9)$ & 24 & $(0.9)$ & 14 & $(0.9)$ \\
\hline & Mexico & 35 & (1.0) & 58 & $(0.9)$ & 35 & (1.0) & 48 & $(0.9)$ & 29 & $(0.8)$ \\
\hline & Netherlands & 81 & (1.6) & 16 & (1.5) & 11 & (1.3) & 13 & $(1.2)$ & 8 & (1.0) \\
\hline & New Zealand & 74 & (1.2) & 24 & (1.1) & 11 & (0.9) & 15 & (1.1) & 9 & $(0.8)$ \\
\hline & Norway & 74 & (1.5) & 22 & (1.4) & 10 & $(0.9)$ & 18 & $(1.3)$ & 8 & $(0.9)$ \\
\hline & Poland & 84 & $(1.2)$ & 14 & $(1.0)$ & 5 & $(0.7)$ & 8 & $(0.9)$ & 4 & $(0.5)$ \\
\hline & Portugal & 71 & $(1.9)$ & 26 & $(1.8)$ & 12 & $(1.5)$ & 17 & $(1.6)$ & 10 & $(1.2)$ \\
\hline & Slovak Republic & 67 & $(2.1)$ & 27 & (1.7) & 20 & $(2.2)$ & 27 & $(2.1)$ & 17 & (1.6) \\
\hline & Slovenia & 78 & (1.1) & 20 & (1.0) & 11 & (0.9) & 11 & $(0.8)$ & 7 & $(0.6)$ \\
\hline & Spain & 72 & $(1.0)$ & 25 & $(1.0)$ & 13 & $(0.8)$ & 15 & $(0.8)$ & 9 & $(0.6)$ \\
\hline & Sweden & 70 & (1.5) & 26 & $(1.3)$ & 14 & $(0.9)$ & 19 & $(1.1)$ & 11 & $(0.7)$ \\
\hline & Switzerland & 83 & $(1.0)$ & 13 & $(0.9)$ & 9 & $(0.8)$ & 12 & $(0.8)$ & 6 & $(0.6)$ \\
\hline & OECD average & 73 & $(0.2)$ & 24 & $(0.2)$ & 12 & $(0.2)$ & 17 & $(0.2)$ & 9 & $(0.2)$ \\
\hline \multirow{30}{*}{ 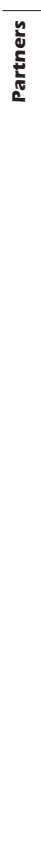 } & Albania & 29 & (1.3) & 60 & $(1.6)$ & 49 & (1.5) & 50 & $(1.3)$ & 35 & $(1.2)$ \\
\hline & Argentina & 28 & $(2.0)$ & 70 & $(2.1)$ & 46 & (1.9) & 49 & $(2.3)$ & 38 & (1.9) \\
\hline & Brazil & 25 & (1.1) & 72 & (1.1) & 43 & (1.3) & 55 & $(1.2)$ & 38 & $(1.2)$ \\
\hline & Bulgaria & 53 & $(2.1)$ & 42 & $(2.0)$ & 27 & (2.1) & 31 & $(2.0)$ & 22 & (1.9) \\
\hline & Colombia & 19 & $(1.5)$ & 80 & $(1.5)$ & 47 & $(2.2)$ & 60 & $(2.1)$ & 42 & (2.1) \\
\hline & Costa Rica & 31 & $(2.0)$ & 66 & (1.9) & 26 & (1.8) & 42 & $(2.2)$ & 21 & (1.6) \\
\hline & Croatia & 68 & $(1.8)$ & 31 & $(1.8)$ & 9 & (1.0) & 15 & $(1.0)$ & 7 & $(0.8)$ \\
\hline & Hong Kong (China) & 91 & $(1.0)$ & 8 & $(0.9)$ & 4 & $(0.7)$ & 5 & $(0.7)$ & 3 & $(0.6)$ \\
\hline & Indonesia & 18 & $(2.1)$ & 77 & $(2.2)$ & 48 & (2.4) & 66 & $(2.5)$ & 42 & $(2.4)$ \\
\hline & Jordan & 33 & $(1.8)$ & 65 & $(1.9)$ & 33 & (1.5) & 38 & $(1.6)$ & 26 & (1.4) \\
\hline & Kazakhstan & 38 & $(1.9)$ & 45 & $(1.9)$ & 47 & $(1.7)$ & 39 & $(2.1)$ & 25 & $(1.4)$ \\
\hline & Latvia & 80 & (1.4) & 18 & (1.4) & 8 & (1.1) & 9 & $(1.0)$ & 5 & (1.0) \\
\hline & Liechtenstein & 79 & (3.6) & 17 & $(3.5)$ & 10 & (2.8) & 13 & $(3.3)$ & 7 & (2.6) \\
\hline & Lithuania & 74 & $(1.5)$ & 24 & $(1.4)$ & 10 & $(0.9)$ & 12 & (1.1) & 8 & $(0.8)$ \\
\hline & Macao (China) & 87 & $(0.6)$ & 10 & $(0.7)$ & 6 & $(0.5)$ & 7 & $(0.6)$ & 3 & $(0.4)$ \\
\hline & Malaysia & 41 & (1.9) & 49 & $(2.0)$ & 43 & (1.8) & 42 & $(1.7)$ & 31 & (1.6) \\
\hline & Montenegro & 40 & (1.3) & 56 & $(1.4)$ & 29 & (1.2) & 46 & $(1.0)$ & 26 & (1.0) \\
\hline & Peru & 19 & $(2.1)$ & 77 & $(2.2)$ & 55 & (2.4) & 69 & $(2.4)$ & 50 & $(2.4)$ \\
\hline & Qatar & 28 & $(0.6)$ & 68 & $(0.7)$ & 45 & $(0.6)$ & 57 & $(0.7)$ & 40 & $(0.6)$ \\
\hline & Romania & 50 & $(2.1)$ & 41 & $(2.2)$ & 28 & (1.9) & 35 & $(1.8)$ & 20 & (1.7) \\
\hline & Russian Federation & 71 & (1.4) & 23 & $(1.2)$ & 15 & (1.2) & 17 & $(1.1)$ & 9 & $(0.8)$ \\
\hline & Serbia & 54 & $(2.0)$ & 40 & $(1.8)$ & 23 & (1.7) & 33 & $(1.9)$ & 19 & (1.5) \\
\hline & Shanghai (China) & 96 & $(0.6)$ & 4 & $(0.6)$ & 2 & $(0.3)$ & 2 & $(0.4)$ & 1 & $(0.3)$ \\
\hline & Singapore & 89 & $(0.8)$ & 7 & $(0.6)$ & 6 & $(0.6)$ & 8 & $(0.6)$ & 4 & $(0.4)$ \\
\hline & Chinese Taipei & 87 & (1.0) & 11 & $(0.9)$ & 7 & $(0.8)$ & 8 & $(0.8)$ & 5 & $(0.7)$ \\
\hline & Thailand & 50 & $(2.0)$ & 46 & $(2.1)$ & 21 & (1.4) & 29 & $(1.7)$ & 16 & $(1.2)$ \\
\hline & Tunisia & 24 & $(1.8)$ & 71 & $(1.9)$ & 42 & $(2.4)$ & 55 & $(2.0)$ & 36 & $(2.4)$ \\
\hline & United Arab Emirates & 53 & $(1.8)$ & 44 & $(1.7)$ & 23 & (1.5) & 28 & $(1.8)$ & 18 & (1.4) \\
\hline & Uruguay & 36 & $(1.4)$ & 58 & $(1.6)$ & 40 & (1.5) & 46 & $(1.5)$ & 32 & (1.4) \\
\hline & Viet Nam & 84 & $(1.7)$ & 14 & $(1.6)$ & 5 & (1.0) & 6 & $(1.0)$ & 3 & $(0.7)$ \\
\hline
\end{tabular}

Note: Values that are statistically significant are indicated in bold.

Source: OECD, PISA 2012 Database. See Annex 3 for notes (www.oecd.org/education/education-at-a-glance-19991487.htm).

Please refer to the Reader's Guide for information concerning symbols for missing data and abbreviations.

StatLink Niाs h http://dx.doi.org/10.1787/888933285319 
Table A10.1. [3/3] Percentage of low achievers in mathematics, reading and science, by gender (PISA 2012) Percentage of 15-year-old students who do not attain the PISA baseline proficiency Level 2

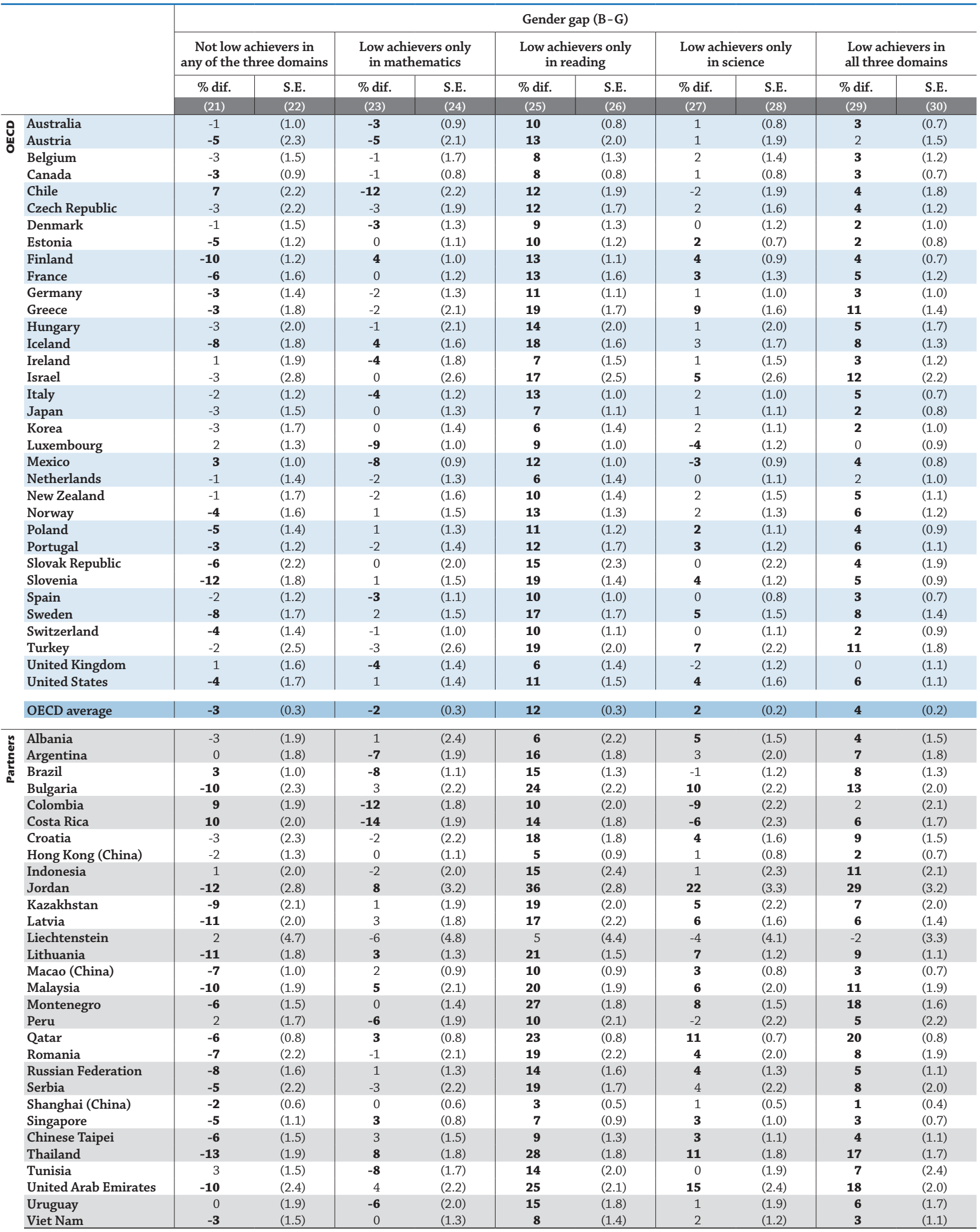

Note: Values that are statistically significant are indicated in bold.

Source: OECD, PISA 2012 Database. See Annex 3 for notes (www.oecd.org/education/education-at-a-glance-19991487.htm).

Please refer to the Reader's Guide for information concerning symbols for missing data and abbreviations.

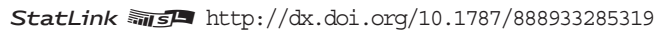


Table A10.2 Gender disparities at age 15 in participation in general, vocational and modular programmes Results based on students' self-reports (PISA 2012)

\begin{tabular}{|c|c|c|c|c|c|c|c|c|c|c|c|c|c|c|c|c|c|c|}
\hline & \multicolumn{6}{|c|}{$\begin{array}{l}\text { Percentage of boys who are enrolled in a } \\
\text { programme whose curriculum is: }\end{array}$} & \multicolumn{6}{|c|}{$\begin{array}{l}\text { Percentage of girls who are enrolled } \\
\text { in a programme whose curriculum is: }\end{array}$} & \multicolumn{6}{|c|}{$\begin{array}{l}\text { Gender gap (B-G) in the percentage } \\
\text { of students who are enrolled in a programme } \\
\text { whose curriculum is: }\end{array}$} \\
\hline & \multicolumn{2}{|c|}{ General } & \multicolumn{2}{|c|}{ Vocational } & \multicolumn{2}{|c|}{ Modular } & \multicolumn{2}{|c|}{ General } & \multicolumn{2}{|c|}{ Vocational } & \multicolumn{2}{|c|}{ Modular } & \multicolumn{2}{|c|}{ General } & \multicolumn{2}{|c|}{ Vocational } & \multicolumn{2}{|c|}{ Modular } \\
\hline & $\%$ & S.E. & $\%$ & S.E. & $\%$ & S.E. & $\%$ & S.E. & $\%$ & S.E. & $\%$ & S.E. & $\begin{array}{c}\text { \%-pt } \\
\text { dif. }\end{array}$ & S.E. & $\begin{array}{c}\text { \%-pt } \\
\text { dif. }\end{array}$ & S.E. & $\begin{array}{c}\text { \%-pt } \\
\text { dif. }\end{array}$ & S.E. \\
\hline & (1) & (2) & $(3)$ & (4) & (5) & (6) & (7) & (8) & (9) & $(10)$ & (11) & (12) & (13) & (14) & (15) & $(16)$ & (17) & (18) \\
\hline Australia & 89 & $(0.6)$ & 11 & (0.6) & 0 & c & 90 & $(0.6)$ & 10 & $(0.6)$ & 0 & c & -1 & $(0.6)$ & 1 & $(0.6)$ & 0 & c \\
\hline Austria & 28 & (1.6) & 72 & (1.6) & 0 & c & 34 & (1.6) & 66 & (1.6) & 0 & c & -6 & $(2.6)$ & 6 & $(2.6)$ & 0 & c \\
\hline Belgium & 52 & (1.4) & 48 & (1.4) & 0 & c & 60 & (1.5) & 40 & (1.5) & 0 & c & -7 & (2.0) & 7 & (2.0) & 0 & c \\
\hline Canada & 0 & c & 0 & c & 100 & c & 0 & c & 0 & c & 100 & c & 0 & c & 0 & c & 0 & c \\
\hline Chile & 97 & $(0.3)$ & 3 & (0.3) & 0 & c & 97 & $(0.3)$ & 3 & $(0.3)$ & 0 & c & 0 & $(0.5)$ & 0 & $(0.5)$ & 0 & c \\
\hline Czech Republic & 70 & $(2.2)$ & 30 & (2.2) & 0 & c & 68 & $(2.4)$ & 32 & $(2.4)$ & 0 & c & 3 & (3.9) & -3 & (3.9) & 0 & c \\
\hline Denmark & 100 & c & 0 & c & 0 & c & 100 & c & 0 & $c$ & 0 & c & 0 & c & 0 & c & 0 & c \\
\hline Estonia & 100 & $(0.3)$ & 0 & (0.3) & 0 & c & 100 & $(0.2)$ & 0 & $(0.2)$ & 0 & c & 0 & $(0.4)$ & 0 & $(0.4)$ & 0 & c \\
\hline Finland & 100 & c & 0 & c & 0 & c & 100 & c & 0 & c & 0 & c & 0 & c & 0 & c & 0 & c \\
\hline France & 82 & $(1.6)$ & 18 & (1.6) & 0 & c & 88 & $(1.5)$ & 12 & $(1.5)$ & 0 & c & -6 & $(2.0)$ & 6 & $(2.0)$ & 0 & c \\
\hline Germany & 97 & (1.3) & 3 & (1.3) & 0 & c & 99 & $(0.6)$ & 1 & $(0.6)$ & 0 & c & -2 & $(1.2)$ & 2 & $(1.2)$ & 0 & c \\
\hline Greece & 82 & $(2.6)$ & 18 & (2.6) & 0 & c & 91 & (2.1) & 9 & $(2.1)$ & 0 & c & -10 & (1.2) & 10 & (1.2) & 0 & c \\
\hline Hungary & 82 & (1.8) & 18 & (1.8) & 0 & c & 90 & (1.1) & 10 & (1.1) & 0 & c & -8 & (2.1) & 8 & (2.1) & 0 & c \\
\hline Iceland & 100 & c & 0 & c & 0 & c & 100 & $c$ & 0 & c & 0 & c & 0 & $c$ & 0 & $c$ & 0 & c \\
\hline Ireland & 99 & $(0.3)$ & 1 & (0.3) & 0 & c & 99 & $(0.2)$ & 1 & $(0.2)$ & 0 & c & 0 & $(0.3)$ & 0 & $(0.3)$ & 0 & c \\
\hline Israel & 95 & $(0.6)$ & 5 & (0.6) & 0 & c & 99 & $(0.3)$ & 1 & $(0.3)$ & 0 & c & -5 & $(0.9)$ & 5 & $(0.9)$ & 0 & c \\
\hline Italy & 39 & (1.1) & 61 & (1.1) & 0 & c & 63 & (1.6) & 37 & (1.6) & 0 & c & -24 & (2.1) & 24 & (2.1) & 0 & c \\
\hline Japan & 74 & (1.7) & 26 & (1.7) & 0 & c & 78 & (1.6) & 22 & (1.6) & 0 & c & -4 & (3.0) & 4 & (3.0) & 0 & c \\
\hline Korea & 79 & (2.6) & 21 & (2.6) & 0 & c & 81 & $(2.4)$ & 19 & (2.4) & 0 & c & -2 & (4.1) & 2 & (4.1) & 0 & c \\
\hline Luxembourg & 77 & $(0.3)$ & 15 & $(0.2)$ & 8 & $(0.3)$ & 81 & $(0.3)$ & 14 & $(0.2)$ & 6 & $(0.2)$ & -4 & $(0.4)$ & 2 & $(0.3)$ & 2 & $(0.3)$ \\
\hline Mexico & 75 & $(1.0)$ & 25 & (1.0) & 0 & c & 75 & (1.1) & 25 & (1.1) & 0 & c & 0 & $(0.7)$ & 0 & $(0.7)$ & 0 & c \\
\hline Netherlands & 76 & (1.9) & 24 & (1.9) & 0 & c & 80 & (1.9) & 20 & (1.9) & 0 & c & -4 & (1.5) & 4 & (1.5) & 0 & c \\
\hline New Zealand & 100 & c & 0 & c & 0 & c & 100 & c & 0 & c & 0 & c & 0 & c & 0 & c & 0 & c \\
\hline Norway & 100 & c & 0 & c & 0 & c & 100 & c & 0 & c & 0 & c & 0 & c & 0 & c & 0 & c \\
\hline Poland & 100 & c & 0 & c & 0 & c & 100 & $(0.1)$ & 0 & $(0.1)$ & 0 & c & 0 & $(0.1)$ & 0 & $(0.1)$ & 0 & c \\
\hline Portugal & 79 & $(2.4)$ & 21 & (2.4) & 0 & c & 87 & (1.8) & 13 & (1.8) & 0 & c & -8 & $(1.3)$ & 8 & (1.3) & 0 & c \\
\hline Slovak Republic & 63 & $(2.3)$ & 11 & $(2.2)$ & 26 & (1.8) & 69 & $(2.3)$ & 5 & $(1.2)$ & 26 & (2.1) & -7 & (3.7) & 6 & (2.1) & 0 & $(2.8)$ \\
\hline Slovenia & 40 & $(0.8)$ & 60 & $(0.8)$ & 0 & c & 54 & $(0.6)$ & 46 & $(0.6)$ & 0 & c & -15 & $(1.0)$ & 15 & $(1.0)$ & 0 & c \\
\hline Spain & 99 & $(0.2)$ & 1 & $(0.2)$ & 0 & c & 100 & $(0.1)$ & 0 & $(0.1)$ & 0 & c & -1 & $(0.2)$ & $\mathbf{1}$ & $(0.2)$ & 0 & c \\
\hline Sweden & 100 & $(0.1)$ & 0 & (0.1) & 0 & c & 99 & $(0.2)$ & 1 & $(0.2)$ & 0 & c & 0 & $(0.2)$ & 0 & $(0.2)$ & 0 & c \\
\hline Switzerland & 88 & $(2.2)$ & 12 & (2.2) & 0 & c & 91 & (2.0) & 9 & $(2.0)$ & 0 & c & -3 & (3.7) & 3 & (3.7) & 0 & c \\
\hline Turkey & 59 & $(2.1)$ & 41 & (2.1) & 0 & c & 65 & $(2.5)$ & 35 & $(2.5)$ & 0 & c & -7 & $(4.5)$ & 7 & $(4.5)$ & 0 & c \\
\hline United Kingdom & 99 & $(0.2)$ & 1 & $(0.2)$ & 0 & a & 99 & $(0.2)$ & 1 & $(0.2)$ & 0 & a & 0 & $(0.3)$ & 0 & $(0.3)$ & 0 & a \\
\hline United States & 100 & c & 0 & c & 0 & c & 100 & c & 0 & $c$ & 0 & c & 0 & c & 0 & c & 0 & c \\
\hline
\end{tabular}

\begin{tabular}{l|ll|ll|ll|ll|ll|ll|ll|ll|ll|} 
OECD average & 80 & $(0.2)$ & 16 & $(0.2)$ & 4 & $(0.1)$ & 83 & $(0.2)$ & 13 & $(0.2)$ & 4 & $(0.1)$ & $\mathbf{- 4}$ & $(0.3)$ & $\mathbf{3}$ & $(0.3)$ & 0 & $(0.1)$ \\
\hline
\end{tabular}

\begin{tabular}{|c|c|c|c|c|c|c|c|c|c|c|c|c|c|c|c|c|c|c|}
\hline Albania & 88 & $(2.9)$ & 12 & (2.9) & 0 & c & 96 & (1.3) & 4 & (1.3) & 0 & c & -8 & $(2.6)$ & 8 & (2.6) & 0 & $\mathrm{c}$ \\
\hline Argentina & 79 & (3.7) & 21 & (3.7) & 0 & $c$ & 91 & (1.7) & 9 & (1.7) & 0 & c & -12 & $(2.4)$ & 12 & (2.4) & 0 & $c$ \\
\hline Brazil & 100 & $(0.0)$ & 0 & $(0.0)$ & 0 & c & 100 & $(0.0)$ & 0 & $(0.0)$ & 0 & c & 0 & $(0.0)$ & 0 & $(0.0)$ & 0 & c \\
\hline Bulgaria & 52 & $(2.4)$ & 48 & (2.4) & 0 & c & 67 & $(2.8)$ & 33 & $(2.8)$ & 0 & c & -14 & $(4.0)$ & 14 & (4.0) & 0 & c \\
\hline Colombia & 76 & (2.3) & 24 & (2.3) & 0 & c & 73 & (2.6) & 27 & (2.6) & 0 & c & 3 & $(1.8)$ & -3 & (1.8) & 0 & c \\
\hline Costa Rica & 92 & (1.8) & 8 & (1.8) & 0 & c & 90 & (1.6) & 10 & $(1.6)$ & 0 & c & 2 & $(0.5)$ & -2 & $(0.5)$ & 0 & c \\
\hline Croatia & 23 & (1.5) & 77 & (1.5) & 0 & c & 37 & (1.5) & 63 & (1.5) & 0 & c & -13 & (1.8) & 13 & (1.8) & 0 & c \\
\hline Hong Kong (China) & 100 & c & 0 & c & 0 & c & 100 & c & 0 & c & 0 & c & 0 & c & 0 & c & 0 & c \\
\hline Indonesia & 76 & $(4.2)$ & 24 & $(4.2)$ & 0 & c & 84 & (3.1) & 16 & (3.1) & 0 & c & -8 & $(4.2)$ & 8 & $(4.2)$ & 0 & c \\
\hline Jordan & 100 & c & 0 & c & 0 & c & 100 & c & 0 & c & 0 & c & 0 & c & 0 & c & 0 & c \\
\hline Latvia & 99 & $(0.9)$ & 1 & $(0.9)$ & 0 & c & 100 & $(0.4)$ & 0 & $(0.4)$ & 0 & c & -1 & $(1.0)$ & 1 & (1.0) & 0 & c \\
\hline Liechtenstein & 100 & c & 0 & c & 0 & c & 100 & c & 0 & c & 0 & c & 0 & c & 0 & c & 0 & c \\
\hline Lithuania & 99 & $(0.4)$ & 1 & $(0.4)$ & 0 & c & 100 & $(0.1)$ & 0 & $(0.1)$ & 0 & c & -1 & $(0.4)$ & 1 & $(0.4)$ & 0 & c \\
\hline Macao (China) & 98 & $(0.0)$ & 2 & $(0.0)$ & 0 & c & 98 & $(0.1)$ & 2 & $(0.1)$ & 0 & c & 0 & $(0.0)$ & 0 & $(0.0)$ & c & c \\
\hline Malaysia & 84 & (1.5) & 16 & (1.5) & 0 & c & 89 & $(1.2)$ & 11 & (1.2) & 0 & c & -5 & (1.5) & 5 & (1.5) & 0 & c \\
\hline Montenegro & 28 & $(0.4)$ & 72 & $(0.4)$ & 0 & c & 40 & $(0.2)$ & 60 & $(0.2)$ & 0 & c & -11 & $(0.5)$ & 11 & $(0.5)$ & 0 & c \\
\hline Peru & 100 & c & 0 & c & 0 & c & 100 & c & 0 & c & 0 & c & 0 & c & 0 & c & 0 & c \\
\hline Qatar & 100 & c & 0 & c & 0 & c & 100 & c & 0 & c & 0 & c & 0 & c & 0 & c & 0 & c \\
\hline Romania & 100 & c & 0 & c & 0 & c & 100 & c & 0 & c & 0 & c & 0 & c & 0 & $c$ & 0 & c \\
\hline Serbia & 23 & (1.3) & 77 & (1.3) & 0 & c & 28 & $(1.2)$ & 72 & $(1.2)$ & 0 & c & -5 & $(1.6)$ & 5 & (1.6) & 0 & c \\
\hline Shanghai (China) & 79 & $(1.0)$ & 21 & (1.0) & 0 & c & 79 & (1.2) & 21 & $(1.2)$ & 0 & c & 0 & (1.9) & 0 & (1.9) & 0 & c \\
\hline Singapore & 100 & c & 0 & c & 0 & c & 100 & c & 0 & c & 0 & c & 0 & c & 0 & $c$ & 0 & c \\
\hline Chinese Taipei & 67 & $(2.1)$ & 33 & (2.1) & 0 & c & 64 & $(2.0)$ & 36 & $(2.0)$ & 0 & c & 3 & $(3.0)$ & -3 & (3.0) & 0 & c \\
\hline Thailand & 72 & (1.4) & 28 & (1.4) & 0 & $c$ & 87 & $(1.2)$ & 13 & $(1.2)$ & 0 & c & -16 & $(2.2)$ & 16 & (2.2) & 0 & c \\
\hline Tunisia & 100 & c & 0 & c & 0 & c & 100 & c & 0 & $c$ & 0 & c & 0 & c & 0 & $c$ & 0 & c \\
\hline United Arab Emirates & 96 & $(0.2)$ & 4 & $(0.2)$ & 0 & $c$ & 98 & $(0.1)$ & 2 & $(0.1)$ & 0 & c & -2 & $(0.2)$ & 2 & $(0.2)$ & 0 & $c$ \\
\hline Uruguay & 95 & $(0.8)$ & 3 & $(0.8)$ & 2 & $(0.5)$ & 99 & $(0.2)$ & 0 & $(0.1)$ & 1 & $(0.2)$ & -4 & $(0.8)$ & 2 & (0.8) & 2 & (0.5) \\
\hline Viet Nam & 99 & (1.1) & 0 & $(0.0)$ & 1 & (1.1) & 100 & $(0.3)$ & 0 & $(0.0)$ & 0 & (0.3) & -1 & $(0.7)$ & 0 & $(0.0)$ & 1 & (0.7) \\
\hline
\end{tabular}

Note: Values that are statistically significant are indicated in bold.

Source: OECD, PISA 2012 Database. See Annex 3 for notes (www.oecd.org/education/education-at-a-glance-19991487.htm).

Please refer to the Reader's Guide for information concerning symbols for missing data and abbreviations.

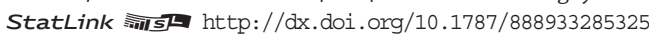


Table A10.3. [1/2] Percentage of adults, by field of study of the highest level of education attained and gender 16-65 year-olds surveyed in the 2012 Survey of Adult Skills

\begin{tabular}{|c|c|c|c|c|c|c|c|c|c|c|c|c|c|c|c|c|c|c|}
\hline & \multicolumn{6}{|c|}{ General programmes } & \multicolumn{6}{|c|}{ Teacher training and education science } & \multicolumn{6}{|c|}{ Humanities, languages and arts } \\
\hline & \multicolumn{2}{|c|}{ Men } & \multicolumn{2}{|c|}{ Women } & \multicolumn{2}{|c|}{$\begin{array}{c}\text { \%-point } \\
\text { difference } \\
(M-W)\end{array}$} & \multicolumn{2}{|c|}{ Men } & \multicolumn{2}{|c|}{ Women } & \multicolumn{2}{|c|}{$\begin{array}{c}\text { \%-point } \\
\text { difference } \\
(\mathrm{M}-\mathrm{W})\end{array}$} & \multicolumn{2}{|c|}{ Men } & \multicolumn{2}{|c|}{ Women } & \multicolumn{2}{|c|}{$\begin{array}{c}\text { \%-point } \\
\text { difference } \\
(\mathrm{M}-\mathrm{W})\end{array}$} \\
\hline & $\%$ & S.E. & $\%$ & S.E. & $\%$ & S.E. & $\%$ & S.E. & $\%$ & S.E. & $\%$ & S.E. & $\%$ & S.E. & $\%$ & S.E. & $\%$ & S.E. \\
\hline & (1) & (2) & (3) & $(4)$ & (5) & (6) & (7) & $(8)$ & (9) & (10) & (11) & (12) & (13) & (14) & (15) & (16) & (17) & (18) \\
\hline \multicolumn{19}{|l|}{ National entities } \\
\hline Australia & 31 & $(0.9)$ & 28 & (1.1) & 3 & (1.7) & 3 & $(0.3)$ & 8 & $(0.5)$ & -6 & $(0.6)$ & 5 & $(0.4)$ & 7 & $(0.6)$ & -2 & $(0.7)$ \\
\hline Щّ Austria & 9 & $(0.7)$ & 10 & $(0.7)$ & -1 & (1.0) & 2 & $(0.3)$ & 7 & $(0.5)$ & -5 & $(0.5)$ & 3 & $(0.4)$ & 4 & $(0.5)$ & -1 & $(0.6)$ \\
\hline - Canada & 29 & $(0.6)$ & 28 & $(0.6)$ & 0 & (1.1) & 3 & $(0.3)$ & 9 & $(0.4)$ & -6 & $(0.5)$ & 6 & $(0.4)$ & 10 & $(0.4)$ & -4 & $(0.5)$ \\
\hline Czech Republic & 4 & $(0.0)$ & 8 & $(0.0)$ & -4 & $(0.1)$ & 1 & $(0.0)$ & 7 & $(0.0)$ & -6 & $(0.0)$ & 3 & $(0.0)$ & 4 & $(0.2)$ & -1 & $(0.2)$ \\
\hline Denmark & 11 & $(0.7)$ & 15 & $(0.7)$ & -4 & (1.0) & 5 & $(0.4)$ & 15 & $(0.7)$ & -9 & $(0.9)$ & 4 & $(0.4)$ & 8 & $(0.5)$ & -4 & $(0.6)$ \\
\hline Estonia & 29 & $(0.8)$ & 23 & $(0.7)$ & 6 & (1.1) & c & c & 7 & $(0.5)$ & c & c & 2 & $(0.3)$ & 6 & $(0.4)$ & -4 & $(0.5)$ \\
\hline Finland & 14 & $(0.8)$ & 14 & $(0.7)$ & 0 & (1.2) & 2 & $(0.3)$ & 6 & $(0.5)$ & -4 & (0.6) & 3 & (0.3) & 6 & $(0.6)$ & -4 & $(0.7)$ \\
\hline France & 11 & $(0.8)$ & 16 & (1.0) & -5 & $(0.8)$ & 3 & $(0.3)$ & 5 & $(0.3)$ & -2 & (0.5) & 3 & $(0.3)$ & 8 & $(0.5)$ & -4 & $(0.6)$ \\
\hline Germany & 6 & $(0.4)$ & 7 & $(0.4)$ & -1 & $(0.6)$ & 2 & $(0.3)$ & 6 & $(0.4)$ & -3 & $(0.5)$ & 3 & $(0.4)$ & 4 & $(0.5)$ & -1 & $(0.6)$ \\
\hline Ireland & 5 & $(0.7)$ & 10 & $(0.8)$ & -5 & (1.1) & 3 & $(0.5)$ & 12 & $(0.7)$ & -9 & $(0.9)$ & 7 & $(0.8)$ & 11 & $(0.9)$ & -4 & (1.2) \\
\hline Italy & 11 & (1.2) & 9 & (0.9) & 1 & (1.3) & c & c & 7 & (0.9) & c & c & 9 & (0.8) & 25 & (1.2) & -17 & (1.3) \\
\hline Japan & 34 & (1.1) & 46 & (1.3) & -12 & (1.5) & 4 & $(0.4)$ & 12 & (1.0) & -8 & (1.0) & 3 & $(0.4)$ & 10 & $(0.6)$ & -7 & $(0.6)$ \\
\hline Korea & 33 & (1.0) & 41 & (1.1) & -8 & (1.5) & 2 & $(0.3)$ & 7 & $(0.6)$ & -5 & $(0.6)$ & 6 & $(0.5)$ & 13 & $(0.6)$ & -7 & $(0.7)$ \\
\hline Netherlands & 9 & $(0.7)$ & 13 & (0.7) & -4 & (1.0) & 4 & $(0.4)$ & 10 & $(0.8)$ & -7 & (0.9) & 4 & $(0.5)$ & 6 & $(0.6)$ & -3 & $(0.7)$ \\
\hline Norway & 8 & $(0.6)$ & 10 & $(0.8)$ & -2 & (1.1) & 4 & $(0.4)$ & 11 & $(0.6)$ & -7 & $(0.8)$ & 5 & $(0.5)$ & 8 & $(0.5)$ & -2 & $(0.6)$ \\
\hline Poland & 10 & $(0.5)$ & 13 & (0.7) & -4 & $(0.9)$ & 2 & $(0.3)$ & 9 & $(0.7)$ & -6 & $(0.7)$ & 3 & (0.4) & 8 & $(0.5)$ & -5 & $(0.6)$ \\
\hline Slovak Republic & 7 & (0.6) & 11 & (0.7) & -4 & $(0.9)$ & 2 & $(0.3)$ & 8 & $(0.6)$ & -6 & $(0.6)$ & 3 & $(0.4)$ & 6 & $(0.5)$ & -3 & $(0.7)$ \\
\hline Spain & 12 & (1.0) & 12 & $(0.9)$ & 0 & (1.6) & 4 & $(0.6)$ & 10 & $(0.8)$ & -6 & (1.0) & 10 & $(0.8)$ & 15 & (1.0) & -5 & (1.3) \\
\hline Sweden & 11 & $(0.7)$ & 14 & (0.9) & -4 & (1.1) & 4 & $(0.3)$ & 11 & $(0.7)$ & -8 & $(0.8)$ & 5 & $(0.5)$ & 8 & $(0.7)$ & -4 & $(0.9)$ \\
\hline United States & 8 & $(0.9)$ & 9 & $(0.7)$ & -1 & (1.1) & 5 & $(0.8)$ & 14 & (1.1) & -9 & (1.2) & 10 & $(0.8)$ & 10 & $(0.8)$ & -1 & (1.1) \\
\hline \multicolumn{19}{|l|}{ Sub-national entities } \\
\hline Flanders (Belgium) & 13 & $(0.7)$ & 20 & (0.9) & -7 & (1.1) & 4 & $(0.5)$ & 12 & $(0.7)$ & -7 & $(0.8)$ & 5 & (0.5) & 9 & $(0.7)$ & -4 & $(0.8)$ \\
\hline England/N.Ireland (UK) & 19 & (1.2) & 21 & (1.0) & -2 & (1.5) & 3 & $(0.4)$ & 8 & $(0.6)$ & -5 & $(0.7)$ & 15 & (1.0) & 22 & (1.1) & -7 & (1.6) \\
\hline Average & 15 & $(0.2)$ & 17 & $(0.2)$ & -2 & $(0.3)$ & 3 & $(0.1)$ & 9 & $(0.1)$ & -6 & $(0.2)$ & 5 & $(0.1)$ & 10 & $(0.1)$ & -4 & $(0.2)$ \\
\hline Russian Federation* & 21 & $(0.7)$ & 17 & $(1.0)$ & 4 & (1.1) & 2 & $(0.4)$ & 12 & $(1.2)$ & -10 & $(1.2)$ & 6 & $(0.9)$ & 11 & (1.0) & -6 & (1.3) \\
\hline & & & & & & & & & & & & & & & & & & \\
\hline
\end{tabular}

\begin{tabular}{|c|c|c|c|c|c|c|c|c|c|c|c|c|c|c|c|c|c|c|}
\hline & \multicolumn{6}{|c|}{ Social sciences, business and law } & \multicolumn{6}{|c|}{ Science, mathematics and computing } & \multicolumn{6}{|c|}{$\begin{array}{l}\text { Engineering, manufacturing } \\
\text { and construction }\end{array}$} \\
\hline & \multicolumn{2}{|c|}{ Men } & \multicolumn{2}{|c|}{ Women } & \multicolumn{2}{|c|}{$\begin{array}{c}\text { \%-point } \\
\text { difference } \\
(\mathrm{M}-\mathrm{W})\end{array}$} & \multicolumn{2}{|c|}{ Men } & \multicolumn{2}{|c|}{ Women } & \multicolumn{2}{|c|}{$\begin{array}{c}\text { \%-point } \\
\text { difference } \\
(\mathrm{M}-\mathrm{W})\end{array}$} & \multicolumn{2}{|c|}{ Men } & \multicolumn{2}{|c|}{ Women } & \multicolumn{2}{|c|}{$\begin{array}{c}\text { \%-point } \\
\text { difference } \\
(\mathrm{M}-\mathrm{W})\end{array}$} \\
\hline & $\%$ & S.E. & $\%$ & S.E. & $\%$ & S.E. & $\%$ & S.E. & $\%$ & S.E. & $\%$ & S.E. & $\%$ & S.E. & $\%$ & S.E. & $\%$ & S.E. \\
\hline & (19) & $(20)$ & (21) & $(22)$ & (23) & (24) & (25) & (26) & (27) & (28) & (29) & (30) & (31) & (32) & (33) & (34) & (35) & $(36)$ \\
\hline \multicolumn{19}{|l|}{ National entities } \\
\hline Australia & 17 & $(0.8)$ & 25 & $(0.8)$ & -8 & (1.2) & 6 & $(0.5)$ & 4 & $(0.4)$ & 2 & $(0.6)$ & 29 & (1.1) & 3 & $(0.4)$ & 26 & (1.3) \\
\hline Austria & 18 & $(0.8)$ & 40 & $(1.2)$ & -22 & (1.3) & 3 & $(0.3)$ & 2 & $(0.3)$ & 1 & $(0.4)$ & 52 & (1.1) & 7 & $(0.7)$ & 45 & (1.2) \\
\hline Canada & 15 & $(0.6)$ & 19 & $(0.6)$ & -5 & $(0.8)$ & 13 & (0.5) & 8 & $(0.4)$ & 5 & (0.6) & 24 & $(0.7)$ & 2 & $(0.2)$ & 22 & $(0.7)$ \\
\hline Czech Republic & 8 & $(0.0)$ & 32 & $(0.2)$ & -23 & $(0.2)$ & 3 & $(0.0)$ & 2 & $(0.0)$ & 1 & $(0.0)$ & 67 & $(0.2)$ & 22 & $(0.1)$ & 45 & $(0.2)$ \\
\hline Denmark & 14 & $(0.7)$ & 16 & $(0.7)$ & -2 & $(0.9)$ & 12 & $(0.7)$ & 7 & $(0.5)$ & 5 & $(0.9)$ & 34 & $(0.9)$ & 4 & $(0.4)$ & 30 & (1.1) \\
\hline Estonia & 8 & $(0.5)$ & 24 & $(0.6)$ & -16 & $(0.9)$ & 4 & $(0.3)$ & 3 & $(0.3)$ & 2 & (0.5) & 40 & (1.0) & 16 & $(0.6)$ & 24 & (1.2) \\
\hline Finland & 12 & $(0.6)$ & 23 & $(0.8)$ & -11 & (1.0) & 2 & (0.3) & 3 & $(0.3)$ & 0 & $(0.4)$ & 51 & (1.2) & 7 & (0.5) & 44 & (1.3) \\
\hline France & 12 & $(0.6)$ & 20 & $(0.8)$ & -8 & (1.0) & 12 & $(0.5)$ & 10 & $(0.5)$ & 2 & $(0.7)$ & 34 & $(0.9)$ & 4 & $(0.3)$ & 31 & $(0.9)$ \\
\hline Germany & 17 & $(0.8)$ & 39 & (1.3) & -22 & (1.5) & 5 & (0.5) & 3 & $(0.4)$ & 1 & (0.6) & 54 & (1.1) & 10 & $(0.7)$ & 44 & (1.3) \\
\hline Ireland & 18 & (1.2) & 25 & (1.1) & -7 & (1.7) & 18 & (1.2) & 11 & $(0.8)$ & 7 & (1.3) & 31 & (1.5) & 2 & $(0.4)$ & 29 & (1.6) \\
\hline Italy & 16 & (1.2) & 21 & (1.2) & -5 & (1.6) & 23 & (1.3) & 19 & (1.2) & 4 & (1.7) & 23 & (1.4) & 2 & $(0.4)$ & 21 & (1.4) \\
\hline Japan & 16 & $(0.8)$ & 10 & $(0.8)$ & 5 & (1.0) & 3 & $(0.4)$ & 2 & $(0.3)$ & 1 & $(0.5)$ & 31 & (1.3) & 2 & $(0.3)$ & 29 & (1.3) \\
\hline Korea & 11 & $(0.7)$ & 12 & $(0.8)$ & 0 & $(0.9)$ & 9 & (0.6) & 10 & $(0.6)$ & -1 & $(0.9)$ & 29 & (1.0) & 6 & $(0.5)$ & 23 & (1.2) \\
\hline Netherlands & 30 & (1.0) & 28 & (1.1) & 2 & (1.6) & 11 & $(0.7)$ & 3 & $(0.4)$ & 8 & $(0.8)$ & 29 & (1.1) & 3 & $(0.4)$ & 26 & (1.1) \\
\hline Norway & 18 & $(0.8)$ & 23 & $(0.9)$ & -5 & (1.1) & 9 & (0.5) & 6 & $(0.6)$ & 3 & (0.6) & 44 & (1.0) & 9 & $(0.7)$ & 34 & (1.2) \\
\hline Poland & 9 & $(0.6)$ & 15 & $(0.7)$ & -7 & (1.1) & 6 & $(0.5)$ & 8 & $(0.6)$ & -2 & $(0.8)$ & 53 & (1.1) & 14 & $(0.8)$ & 38 & (1.3) \\
\hline Slovak Republic & 6 & $(0.5)$ & 19 & $(0.8)$ & -13 & (1.0) & 9 & $(0.6)$ & 5 & $(0.6)$ & 4 & $(0.9)$ & 55 & (1.1) & 12 & $(0.8)$ & 43 & (1.4) \\
\hline Spain & 19 & (1.1) & 26 & (1.2) & -7 & (1.6) & 14 & (1.0) & 10 & $(0.8)$ & 3 & (1.3) & 32 & (1.3) & 4 & $(0.5)$ & 27 & (1.5) \\
\hline Sweden & 17 & (1.1) & 24 & (1.1) & -7 & (1.6) & 7 & $(0.5)$ & 4 & $(0.4)$ & 3 & $(0.7)$ & 44 & (1.0) & 8 & $(0.6)$ & 36 & $(1.2)$ \\
\hline United States & 24 & (1.4) & 21 & (1.2) & 3 & (1.4) & 18 & (1.4) & 11 & $(0.8)$ & 7 & (1.6) & 21 & (1.4) & c & c & c & c \\
\hline \multicolumn{19}{|l|}{ Sub-national entities } \\
\hline Flanders (Belgium) & 14 & $(0.8)$ & 19 & $(0.9)$ & -5 & (1.3) & 13 & $(0.8)$ & 8 & $(0.5)$ & 5 & (0.9) & 42 & (1.0) & 7 & $(0.6)$ & 35 & $(1.2)$ \\
\hline England/N.Ireland (UK) & 16 & (1.0) & 23 & (1.1) & -7 & (1.5) & 15 & (1.0) & 9 & $(0.6)$ & 6 & (1.1) & 28 & (1.1) & 3 & $(0.4)$ & 25 & $(1.2)$ \\
\hline Average & 15 & $(0.2)$ & 23 & $(0.2)$ & -8 & $(0.3)$ & 10 & $(0.2)$ & 7 & $(0.1)$ & 3 & $(0.2)$ & 38 & $(0.2)$ & 7 & $(0.1)$ & 32 & $(0.3)$ \\
\hline Russian Federation* & 7 & $(0.8)$ & 11 & $(0.7)$ & -5 & (1.3) & 9 & $(0.7)$ & 10 & $(0.8)$ & -1 & $(0.9)$ & 40 & (2.0) & 14 & (1.5) & 25 & (1.4) \\
\hline & & & & & & & & & & & & & & & & & & \\
\hline
\end{tabular}

Note: Values that are statistically significant are indicated in bold.

* See note on data for the Russian Federation in the Methodology section.

Source: OECD. Survey of Adult Skills (PIAAC) (2012). See Annex 3 for notes (www.oecd.org/education/education-at-a-glance-19991487.htm).

Please refer to the Reader's Guide for information concerning symbols for missing data and abbreviations.

StatLink 需可 http://dx.doi.org/10.1787/888933285332 
Table A10.3. [2/2] Percentage of adults, by field of study of the highest level of education attained and gender 16-65 year-olds surveyed in the 2012 Survey of Adult Skills

\begin{tabular}{|c|c|c|c|c|c|c|c|c|c|c|c|c|c|c|c|c|c|c|c|}
\hline & & \multicolumn{6}{|c|}{ Agriculture and veterinary medicine } & \multicolumn{6}{|c|}{ Health and welfare } & \multicolumn{6}{|c|}{ Services } \\
\hline & & \multicolumn{2}{|c|}{ Men } & \multicolumn{2}{|c|}{ Women } & \multicolumn{2}{|c|}{$\begin{array}{c}\text { \%-point } \\
\text { difference } \\
(\mathrm{M}-\mathrm{W})\end{array}$} & \multicolumn{2}{|c|}{ Men } & \multicolumn{2}{|c|}{ Women } & \multicolumn{2}{|c|}{$\begin{array}{c}\text { \%-point } \\
\text { difference } \\
(\mathrm{M}-\mathrm{W})\end{array}$} & \multicolumn{2}{|c|}{ Men } & \multicolumn{2}{|c|}{ Women } & \multicolumn{2}{|c|}{$\begin{array}{c}\text { \%-point } \\
\text { difference } \\
(\mathrm{M}-\mathrm{W})\end{array}$} \\
\hline & & $\%$ & S.E. & $\%$ & S.E. & $\%$ & S.E. & $\%$ & S.E. & $\%$ & S.E. & $\%$ & S.E. & $\%$ & S.E. & $\%$ & S.E. & $\%$ & S.E. \\
\hline & & $(37)$ & $(38)$ & $(39)$ & $(40)$ & $(41)$ & $(42)$ & $(43)$ & $(44)$ & $(45)$ & (46) & (47) & $(48)$ & $(49)$ & $(50)$ & $(51)$ & $(52)$ & $(53)$ & $(54)$ \\
\hline \multicolumn{20}{|c|}{ National entities } \\
\hline 峈 & Austria & 5 & $(0.4)$ & 3 & $(0.4)$ & 2 & $(0.6)$ & 2 & $(0.3)$ & 10 & $(0.6)$ & -7 & $(0.7)$ & 6 & $(0.6)$ & 18 & $(0.9)$ & -12 & (1.0) \\
\hline & Canada & 2 & $(0.2)$ & 1 & $(0.2)$ & 0 & $(0.3)$ & 3 & $(0.3)$ & 15 & $(0.6)$ & -12 & $(0.7)$ & 6 & $(0.4)$ & 6 & $(0.3)$ & 0 & $(0.5)$ \\
\hline & Czech Republic & 5 & $(0.0)$ & 5 & $(0.0)$ & 0 & $(0.0)$ & c & c & 4 & $(0.5)$ & c & c & 8 & $(0.2)$ & 16 & $(0.5)$ & -8 & $(0.5)$ \\
\hline & Denmark & 5 & (0.3) & 2 & $(0.3)$ & 3 & $(0.4)$ & 3 & $(0.3)$ & 21 & $(0.7)$ & -18 & $(0.8)$ & 12 & $(0.7)$ & 13 & $(0.7)$ & -1 & (0.9) \\
\hline & Estonia & 4 & $(0.4)$ & 4 & $(0.3)$ & 0 & (0.5) & c & c & 7 & $(0.4)$ & c & c & 11 & $(0.6)$ & 9 & $(0.4)$ & 2 & $(0.7)$ \\
\hline & Finland & 6 & $(0.5)$ & 2 & $(0.3)$ & 4 & $(0.6)$ & 4 & $(0.4)$ & 23 & $(0.7)$ & -19 & $(0.9)$ & 6 & $(0.5)$ & 15 & $(0.7)$ & -9 & (1.0) \\
\hline & France & 6 & $(0.5)$ & 2 & $(0.3)$ & 4 & (0.6) & 3 & $(0.3)$ & 16 & $(0.7)$ & -13 & $(0.8)$ & 16 & $(0.9)$ & 19 & $(0.8)$ & -3 & (1.1) \\
\hline & Germany & 3 & $(0.5)$ & 2 & $(0.3)$ & 2 & $(0.5)$ & 4 & $(0.5)$ & 19 & $(0.8)$ & -15 & $(0.9)$ & 6 & $(0.6)$ & 10 & $(0.7)$ & -4 & (0.9) \\
\hline & Ireland & 4 & $(0.6)$ & c & c & c & c & 5 & $(0.7)$ & 16 & (1.0) & -11 & (1.2) & 8 & (0.9) & 11 & $(0.9)$ & -3 & (1.3) \\
\hline & Japan & 5 & (0.6) & 1 & $(0.3)$ & 4 & $(0.6)$ & 2 & $(0.3)$ & 11 & $(0.6)$ & -9 & $(0.7)$ & 2 & $(0.3)$ & 7 & $(0.6)$ & -5 & $(0.7)$ \\
\hline & Korea & 4 & $(0.3)$ & c & c & c & c & 3 & $(0.4)$ & 6 & $(0.4)$ & -4 & $(0.6)$ & 3 & $(0.3)$ & 3 & $(0.4)$ & -1 & $(0.6)$ \\
\hline & Netherlands & 5 & (0.5) & 2 & $(0.4)$ & 2 & $(0.6)$ & 5 & $(0.5)$ & 31 & (1.0) & -26 & $(1.2)$ & 4 & $(0.5)$ & 4 & $(0.5)$ & 1 & $(0.7)$ \\
\hline & Norway & 3 & $(0.4)$ & 2 & $(0.3)$ & 2 & $(0.5)$ & 5 & $(0.4)$ & 27 & (1.1) & -23 & (1.1) & 4 & $(0.4)$ & 4 & $(0.4)$ & 0 & $(0.6)$ \\
\hline & Poland & 6 & (0.6) & 7 & $(0.6)$ & -1 & $(0.8)$ & 1 & $(0.3)$ & 6 & $(0.5)$ & -5 & $(0.6)$ & 10 & (0.6) & 18 & $(0.8)$ & -8 & (1.0) \\
\hline & Slovak Republic & 8 & $(0.7)$ & 6 & $(0.5)$ & 1 & (0.9) & c & $c$ & 9 & $(0.7)$ & c & c & 10 & $(0.7)$ & 24 & $(1.0)$ & -14 & (1.3) \\
\hline & Spain & 3 & $(0.5)$ & c & c & c & c & 4 & $(0.6)$ & 16 & $(0.9)$ & -11 & (1.1) & 3 & $(0.5)$ & 5 & $(0.6)$ & -2 & $(0.8)$ \\
\hline & Sweden & 4 & $(0.5)$ & 2 & $(0.3)$ & 2 & $(0.6)$ & 5 & $(0.5)$ & 24 & $(1.0)$ & -19 & (1.1) & 5 & $(0.5)$ & 5 & $(0.5)$ & 0 & $(0.7)$ \\
\hline & United States & c & c & c & c & c & c & 6 & $(0.7)$ & 25 & (1.1) & -19 & (1.5) & 8 & $(0.6)$ & 7 & $(0.7)$ & 1 & (0.9) \\
\hline \multicolumn{20}{|c|}{ Sub-national entities } \\
\hline & Flanders (Belgium) & 3 & $(0.4)$ & c & c & c & c & 4 & $(0.4)$ & 20 & $(1.0)$ & -16 & (1.0) & 2 & $(0.3)$ & 5 & $(0.6)$ & -3 & $(0.6)$ \\
\hline & England/N.Ireland (UK) & 1 & $(0.2)$ & c & c & c & c & 3 & $(0.4)$ & 12 & $(0.8)$ & -9 & $(0.8)$ & c & c & c & c & c & c \\
\hline & Average & 4 & $(0.1)$ & 3 & $(0.1)$ & 2 & $(0.2)$ & 4 & $(0.1)$ & 15 & $(0.2)$ & -13 & $(0.2)$ & 7 & $(0.1)$ & 10 & $(0.1)$ & -3 & $(0.2)$ \\
\hline$\tilde{n}$ & Russian Federation* & 8 & (1.3) & 4 & $(0.7)$ & 4 & (1.0) & 3 & $(0.8)$ & 8 & $(0.4)$ & -5 & $(1.0)$ & 5 & $(0.9)$ & 12 & $(1.0)$ & -7 & $(1.0)$ \\
\hline$\frac{5}{\pi}$ & & & & & & & & & & & & & & & & & & & \\
\hline
\end{tabular}

Note: Values that are statistically significant are indicated in bold.

* See note on data for the Russian Federation in the Methodology section.

Source: OECD. Survey of Adult Skills (PIAAC) (2012). See Annex 3 for notes (www.oecd.org/education/education-at-a-glance-19991487.htm).

Please refer to the Reader's Guide for information concerning symbols for missing data and abbreviations.

StatLink 제느 http://dx.doi.org/10.1787/888933285332 


\section{Chapter}

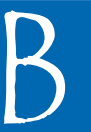

\section{FINANCIAL AND HUMAN RESOURCES INVESTED IN EDUCATION}

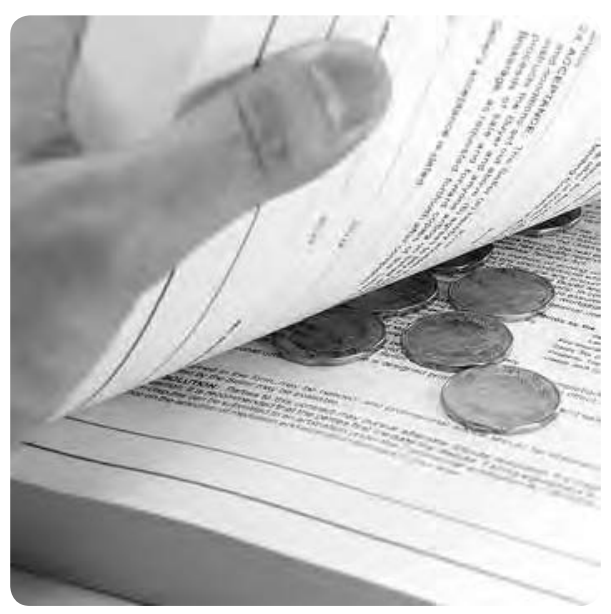

Indicator B1 How much is spent per student?

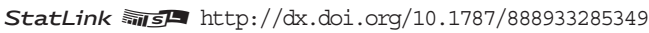

Indicator B2 What proportion of national wealth is spent on education? StatLink Ants http://dx.doi.org/10.1787/888933285413

Indicator B3 How much public and private investment in education is there? StatLink ज्ञाई

Indicator B4 What is the total public spending on education? StatLink ज्ञाइ http://dx.doi.org/10.1787/888933285519

Indicator B5 How much do tertiary students pay and what public support do they receive? StatLink 게제 $h t t p: / / d x . d o i . o r g / 10.1787 / 888933285558$

Indicator B6 On what resources and services is education funding spent?

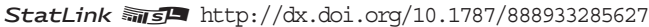

Indicator B7 Which factors influence the level of expenditure on education?

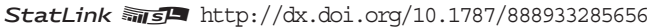




\section{Classification of educational expenditure}

Educational expenditure in this chapter is classified through three dimensions:

- The first dimension - represented by the horizontal axis in the diagram below - relates to the location where spending occurs. Spending on schools and universities, education ministries and other agencies directly involved in providing and supporting education is one component of this dimension. Spending on education outside these institutions is another.

- The second dimension - represented by the vertical axis in the diagram below - classifies the goods and services that are purchased. Not all expenditure on educational institutions can be classified as direct educational or instructional expenditure. Educational institutions in many OECD countries offer various ancillary services - such as meals, transport, housing, etc. - in addition to teaching services to support students and their families. At the tertiary level, spending on research and development can be significant. Not all spending on educational goods and services occurs within educational institutions. For example, families may purchase textbooks and materials themselves or seek private tutoring for their children.

- The third dimension - represented by the colours in the diagram below - distinguishes among the sources from which funding originates. These include the public sector and international agencies (indicated by light blue), and households and other private entities (indicated by medium-blue). Where private expenditure on education is subsidised by public funds, this is indicated by cells in the grey colour.

\begin{tabular}{|c|c|c|}
\hline & Public sources of funds & Private funds publicly subsidised \\
\hline & $\begin{array}{l}\text { Spending on educational institutions } \\
\text { (e.g. schools, universities, } \\
\text { educational administration } \\
\text { and student welfare services) }\end{array}$ & $\begin{array}{l}\text { Spending on education outside educational } \\
\text { institutions } \\
\text { (e.g. private purchases of educational goods } \\
\text { and services, including private tutoring) }\end{array}$ \\
\hline \multirow{3}{*}{$\begin{array}{r}\text { Spending on } \\
\text { core educational } \\
\text { services }\end{array}$} & $\begin{array}{l}\text { e.g. public spending on instructional } \\
\text { services in educational institutions }\end{array}$ & e.g. subsidised private spending on books \\
\hline & $\begin{array}{l}\text { e.g. subsidised private spending on } \\
\text { instructional services in educational } \\
\text { institutions }\end{array}$ & \multirow[t]{2}{*}{$\begin{array}{l}\text { e.g. private spending on books and other } \\
\text { school materials or private tutoring }\end{array}$} \\
\hline & e.g. private spending on tuition fees & \\
\hline \multirow{2}{*}{$\begin{array}{l}\text { Spending on } \\
\text { research and } \\
\text { development }\end{array}$} & e.g. public spending on university research & \\
\hline & $\begin{array}{l}\text { e.g. funds from private industry for } \\
\text { research and development in educational } \\
\text { institutions }\end{array}$ & \\
\hline \multirow{2}{*}{$\begin{array}{r}\text { Spending } \\
\text { on educational } \\
\text { services other } \\
\text { than instruction }\end{array}$} & $\begin{array}{l}\text { e.g. public spending on ancillary services } \\
\text { such as meals, transport to schools, or } \\
\text { housing on the campus }\end{array}$ & $\begin{array}{l}\text { e.g. subsidised private spending on student } \\
\text { living costs or reduced prices for transport }\end{array}$ \\
\hline & $\begin{array}{l}\text { e.g. private spending on fees for ancillary } \\
\text { services }\end{array}$ & $\begin{array}{l}\text { e.g. private spending on student living } \\
\text { costs or transport }\end{array}$ \\
\hline
\end{tabular}




\section{Coverage diagrams}

For Indicators B1, B2, B3 and B6

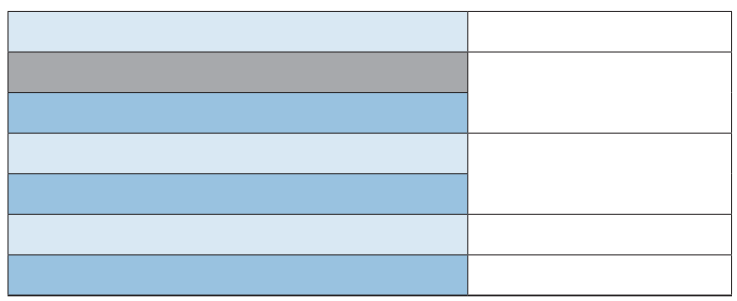

\section{CHAPTER B}

For Indicator B4

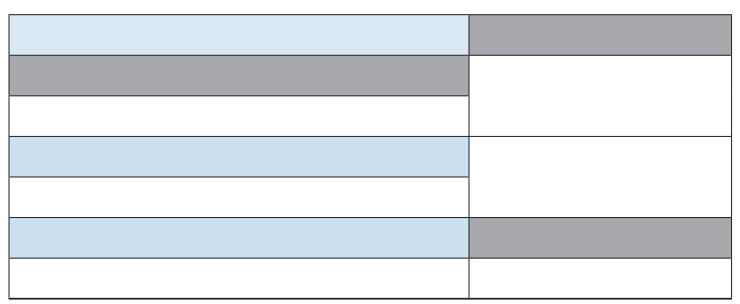




\section{HOW MUCH IS SPENT PER STUDENT?}

INDICATOR B1

- On average, OECD countries spend USD 10220 per student per year from primary through tertiary education: USD 8247 per primary student, USD 9518 per secondary student, and USD 15028 per tertiary student.

- In primary, secondary and post-secondary non-tertiary education, $90 \%$ of total expenditure per student is devoted to core educational services. Greater differences are seen at the tertiary level, partly because expenditure on research and development (R\&D) represents an average of $32 \%$ of total expenditure per student.

- From 2005 to 2012, expenditure per student in primary, secondary and post-secondary non-tertiary educational institutions increased by 21\%, on average across OECD countries; but between 2008 and 2012, investment in education fell in nearly one-quarter of countries as a result of the economic crisis, and resulted in a decrease of expenditure per student in a few countries.

\section{Chart B1.1. Annual expenditure by educational institutions per student, by types of service, from primary to tertiary education (2012) \\ In equivalent USD converted using PPPs, based on full-time equivalents, for primary through tertiary education}
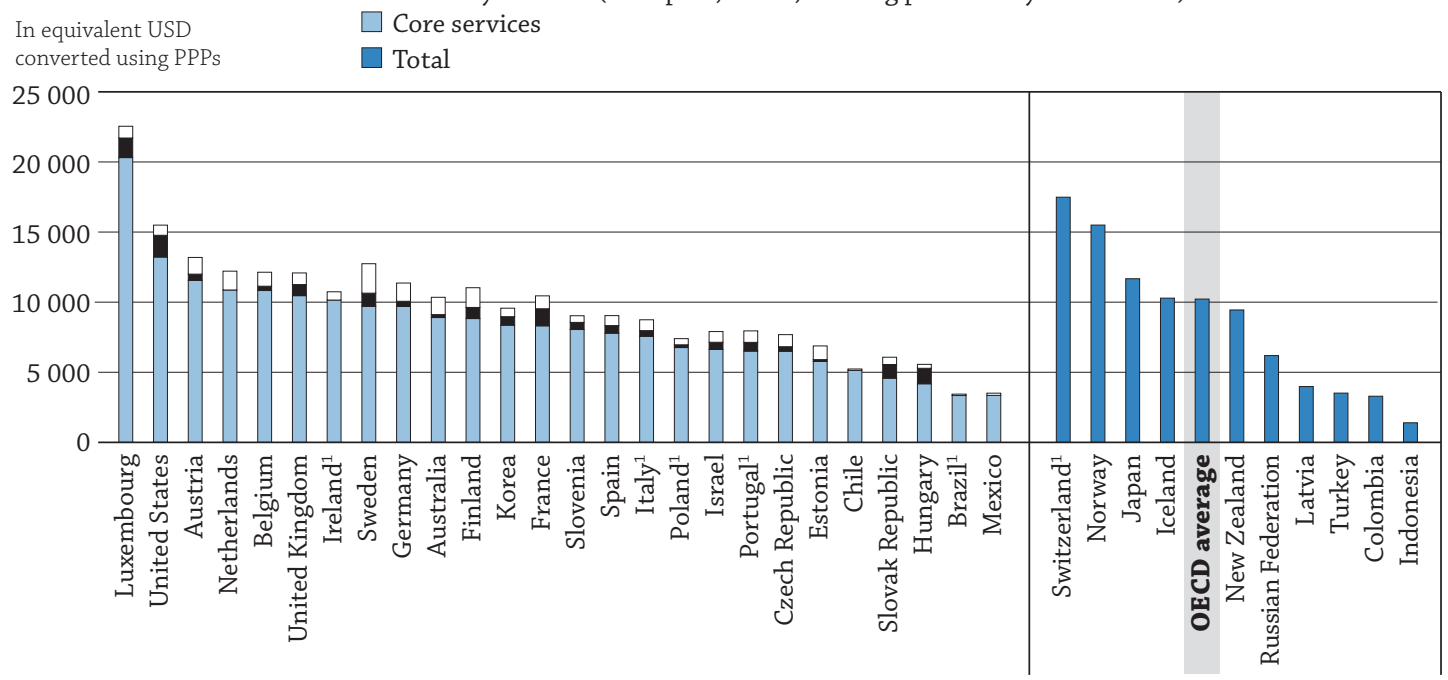

1. Public institutions only.

Countries are ranked in descending order of expenditure per student by educational institutions for core services.

Source: OECD. Table B1.2. See Annex 3 for notes (www.oecd.org/education/education-at-a-glance-19991487.htm).

StatLink त्नाज्ञ http://dx.doi.org/10.1787/888933283897

\section{Context}

The demand for high-quality education, which can translate into higher costs per student, must be balanced against other demands on public expenditure and the overall tax burden. Policy makers must also balance the importance of improving the quality of education services with the desirability of expanding access to education opportunities, notably at the tertiary level. A comparative review of trends in expenditure per student by educational institutions shows that, in many OECD countries, expenditure has not kept up with expanding enrolments. In addition, some OECD countries emphasise broad access to higher education, while others invest in near-universal education for children as young as three or four. Both the extent of investment in education and the number of students enrolled can be affected by financial crises. Consequently, the recent global economic crisis is likely to have resulted in changes in the level of expenditure per student. However, because the crisis began in late 2008, available data until 2012 cannot yet show the full extent of this impact. 
Expenditure per student by educational institutions is largely influenced by teachers' salaries (see Indicators B7 and D3), pension systems, instructional and teaching hours (see Indicator B7), the cost of teaching materials and facilities, the programme provided (e.g. general or vocational), and the number of students enrolled in the education system (see Indicator C1). Policies to attract new teachers or to reduce average class size or change staffing patterns (see Indicator D2) have also contributed to changes in expenditure per student by educational institutions over time. Ancillary and R\&D services can also influence the level of expenditure per student.

\section{Other findings}

- Among the ten countries with the highest expenditure per student by secondary educational institutions, high teachers' salaries and low student-teacher ratios are often the main factors explaining the level of expenditure.

- At the primary and secondary levels there is a strong positive relationship between spending per student by educational institutions and per capita GDP. The relationship is weaker at the tertiary level, mainly because financing mechanisms and enrolment patterns differ more at this level.

- Excluding activities peripheral to instruction (R\&D and ancillary services, such as welfare services to students), OECD countries annually spend USD 8561 per student from primary through tertiary education, on average. Compared with average total expenditure, this lower amount is mainly the result of much lower expenditure per student at the tertiary level when peripheral activities are excluded.

- On average, OECD countries spend around two-thirds more per student at the tertiary level than at the primary, secondary and post-secondary non-tertiary levels. However, R\&D activities or ancillary services can account for a significant proportion of expenditure at the tertiary level. When these are excluded, expenditure per student on core educational services at the tertiary level is still, on average, $21 \%$ higher than at the primary, secondary and post-secondary non-tertiary levels.

- The orientation of secondary school programmes influences the level of expenditure per student in most countries. Among the 23 OECD countries with separate data on expenditure for general and vocational programmes at the upper secondary and post-secondary non-tertiary levels, an average of USD 328 more was spent per student in a vocational programme than in a general programme in 2012.

\section{Trends}

At primary, secondary and post-secondary non-tertiary levels, the period 2000 to 2012 was one of relative stability in student enrolments in most countries. During that time, expenditure per student by educational institutions increased in every country with available data, except Italy, and by an average of $43 \%$ (among countries with available data for all years). On average across OECD countries, the increase was relatively smaller over the period 2000-05 than over the period 2005-12. The largest increases in expenditure per student between 2005 and 2012 were seen in countries that were still among those with the lowest expenditure per student in 2012. Since the beginning of the economic crisis in 2008, expenditure per primary, secondary and post-secondary non-tertiary student continued to increase, except in Estonia, Hungary, Iceland, Italy, Slovenia and Spain.

At the tertiary level, spending per student increased between 2000 and 2012 in most countries, except Brazil, Chile, Hungary, Iceland, Ireland, Israel and Switzerland. On average across OECD countries, expenditure per tertiary student increased by about $4 \%$ between 2000 and 2005 and by $11 \%$ between 2005 and 2012. Since the beginning of the economic crisis in 2008, expenditure per tertiary student decreased in more than a third of countries, mainly because enrolment increased faster than expenditure. In Hungary, Iceland, Ireland, Italy, Portugal, the Russian Federation and Spain, however, there was an actual decrease in expenditure by tertiary educational institutions. This led to a decrease in expenditure per student in all of these countries except Italy and the Russian Federation, where tertiary enrolment fell even faster. 


\section{Analysis}

\section{Expenditure per student by educational institutions}

Annual spending per student from primary through tertiary education in 2012 ranged from USD 4000 or less per student in Brazil, Colombia, Indonesia, Latvia, Mexico and Turkey to more than USD 10000 per student in Australia, Austria, Belgium, Finland, France, Germany, Iceland, Ireland, Japan, the Netherlands, Sweden and the United Kingdom, and by over USD 15000 in Luxembourg, Norway, Switzerland and the United States. In more than one-quarter of countries (11 of 37 countries with available data), spending per student ranged from USD 10000 to less than USD 13000 from primary through tertiary education (Chart B1.1 and Table B1.1a).

Countries have different priorities for allocating their resources (see Indicator B7). For example, among the ten OECD countries with the largest expenditure per student by educational institutions at the lower secondary level (Table B1.1a), Denmark, Ireland, Luxembourg, Switzerland and the United States have among the highest teachers' salaries after 15 years of experience at the lower secondary level, and Austria, Finland, Luxembourg and Norway have some of the lowest student-teacher ratios at that level (see Table B7.2b).

Even if spending per student from primary through tertiary education is similar among some OECD countries, the ways in which resources are allocated to the different levels of education vary widely. Spending per student by educational institutions in a typical OECD country (as represented by the simple mean among all OECD countries) amounts to USD 8247 at the primary level, USD 9518 at the secondary level, and USD 15028 at the tertiary level (Table B1.1a and Chart B1.2). The average spending per tertiary student is affected by high expenditure - more than USD 20000 - in a few OECD countries, notably Canada, Luxembourg, Norway, Sweden, Switzerland, the United Kingdom and the United States.

These averages mask a broad range of expenditure per student by educational institutions across OECD countries, varying by a factor of less than 8 at the primary level and by a factor of 7 at the secondary level. At the primary level, expenditures range from USD 2700 or less per student in Colombia, Indonesia, Mexico, South Africa and Turkey, to more than USD 20000 in Luxembourg. At the secondary level, expenditure ranges from USD 3100 or less per student in Brazil, Colombia, Indonesia, Mexico, South Africa and Turkey to more than USD 20000 in Luxembourg (Table B1.1a and Chart B1.2). These differences in annual expenditure per student at each level of education can also lead to large differences in the cumulative expenditure per student over the duration of studies (see Table B1.3 and Chart B1.6, available on line, for differences in cumulative expenditure per student over the duration of primary and secondary education).

These comparisons are based on purchasing power parities (PPPs) for GDP, not on market exchange rates. Therefore, they reflect the amount of a national currency required to produce the same basket of goods and services in a given country as produced by the United States in USD.

\section{Expenditure per student on core education services}

On average across OECD countries, expenditure on core education services represents $84 \%$ of total expenditure per student from primary through tertiary education, and exceeds $90 \%$ in Ireland, Luxembourg, Mexico and Poland. In 4 of the 20 countries for which data are available - France, Hungary, the Slovak Republic and Sweden core educational services account for less than $80 \%$ of expenditure per student. Annual expenditure on R\&D and ancillary services influence the ranking of countries for all services combined. However, this overall picture masks large variations among the levels of education (Table B1.2).

At the primary, secondary and post-secondary non-tertiary levels, expenditure is dominated by spending on core education services. On average, OECD countries for which data are available spend $90 \%$ of the total expenditure per student (or USD 8 080) on core educational services. In 11 of the 26 countries for which data are available, ancillary services provided by these institutions account for less than $5 \%$ of the total expenditure per student. The proportion of total expenditure per student devoted to ancillary services exceeds $10 \%$ in Finland, France, Hungary, Korea, the Slovak Republic and Sweden (Table B1.2).

Greater differences are seen at the tertiary level, partly because R\&D expenditure can account for a significant proportion of spending on education. The OECD countries in which most R\&D is performed in tertiary educational institutions (e.g. Portugal and Switzerland, and Sweden for publicly funded R\&D) may report higher expenditure per student on educational institutions than those in which a large proportion of R\&D is performed in other public institutions or in industry. 


\section{Chart B1.2. Annual expenditure per student by educational institutions for all services, by level of education (2012)}

Expenditure on core, ancillary services and R\&D, in equivalent USD converted using PPPs, based on full-time equivalents
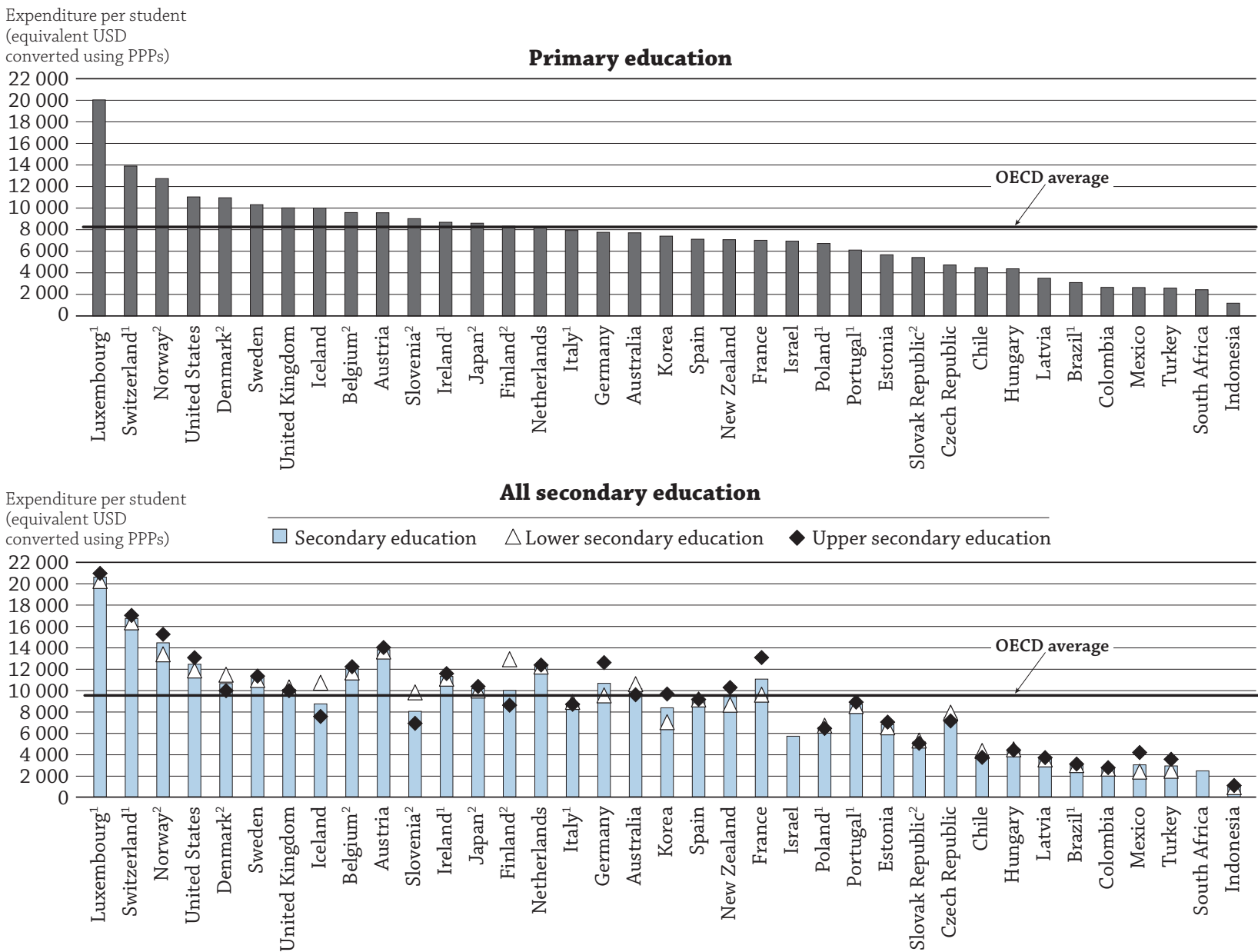

Expenditure per student

(equivalent USD

converted using PPPs) Tertiary education

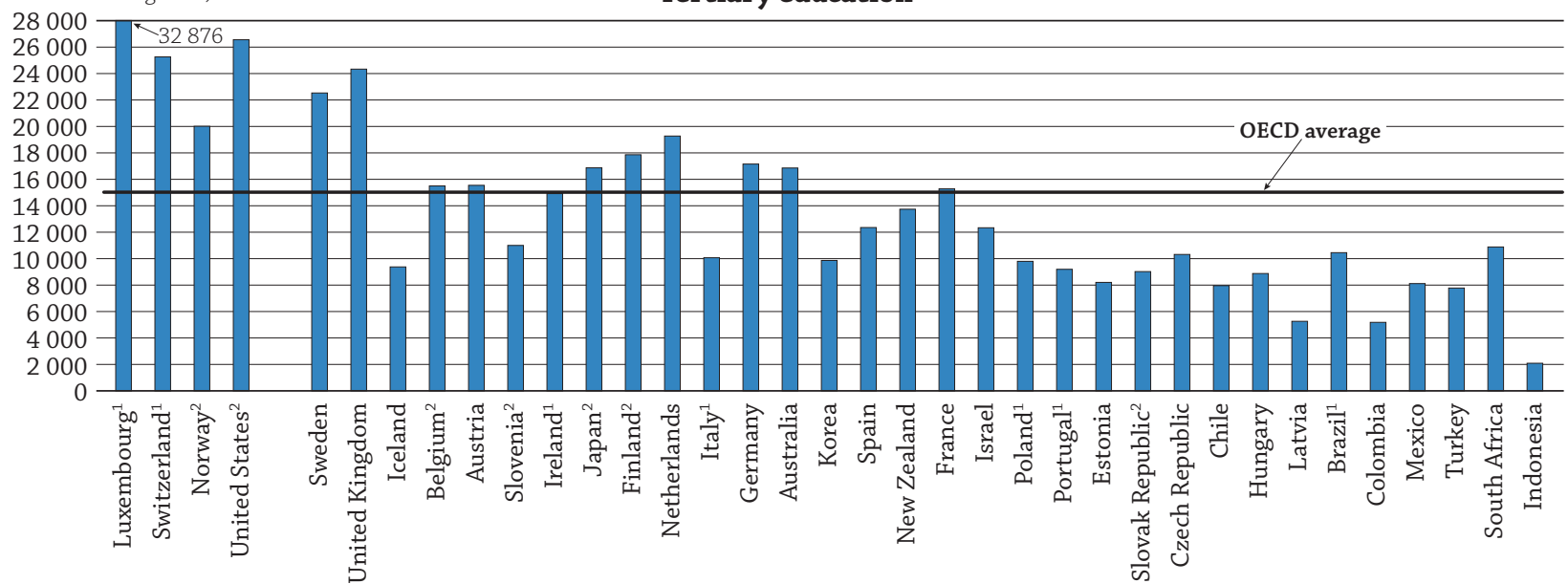

1. Public institutions only (for Italy, except in tertiary education; for Luxembourg, at tertiary level only).

2. Some levels of education are included with others. Refer to "x" code in Table B1.1a for details.

Countries are ranked in descending order of expenditure on educational institutions per student in primary education.

Source: OECD. Table B1.1a. See Annex 3 for notes (www.oecd.org/education/education-at-a-glance-19991487.htm).

StatLink 세대 http://dx.doi.org/10.1787/888933283903 
Excluding R\&D activities and ancillary services (peripheral services, such as student welfare services), expenditure on core education services in tertiary institutions is, on average across OECD countries, USD 9782 per student. It ranges from USD 5000 or less in Estonia, Latvia, Portugal and the Slovak Republic to more than USD 10000 in Austria, Canada, Finland, Ireland, the Netherlands, Norway, Sweden and the United Kingdom, and more than USD 20000 in the United States (Table B1.2).

On average across OECD countries, expenditure on R\&D and ancillary services at the tertiary level represents $32 \%$ and $5 \%$, respectively, of all expenditure per student by tertiary institutions. In 10 of the 23 OECD countries for which data on R\&D and ancillary services are available separately from total expenditure - Australia, Estonia, Germany, Israel, Italy, Norway, Portugal, the Slovak Republic, Sweden and Switzerland - expenditure on R\&D and ancillary services represents at least $40 \%$ of total tertiary expenditure per student by educational institutions. This can translate into significant amounts: in Australia, Germany, Norway, Sweden and Switzerland, expenditure for $\mathrm{R} \& \mathrm{D}$ and ancillary services amounts to more than USD 6000 per student. This is also the case for Canada, Finland, the Netherlands, the United Kingdom and the United States, where expenditure for R\&D and ancillary services represent a smaller share of expenditure (Table B1.2).

\section{Expenditure per student by educational institutions at different levels of education}

Expenditure per student by educational institutions rises with the level of education in almost all countries, but the size of the differentials varies markedly (Table B1.1a and Chart B1.3). Expenditure on secondary education is 1.2 times greater than expenditure on primary education, on average. This ratio reaches or exceeds 1.5 in the Czech Republic, France and the Netherlands largely because of the concurrent increase in the number of instructional hours for students and significant decrease in the number of teachers' teaching hours between primary and secondary education, as compared to the OECD average. The ratio may also be greater due to large enrolments in vocational programmes, as is observed in the Netherlands (see below). In these countries (except the Czech Republic), teachers' salaries are also lower in primary education compared to lower secondary education (see Indicators B7, D1 and D4).

\section{Chart B1.3. Expenditure per student by educational institutions for all services, at secondary and tertiary levels of education relative to primary education (2012)}

Primary education $=100$

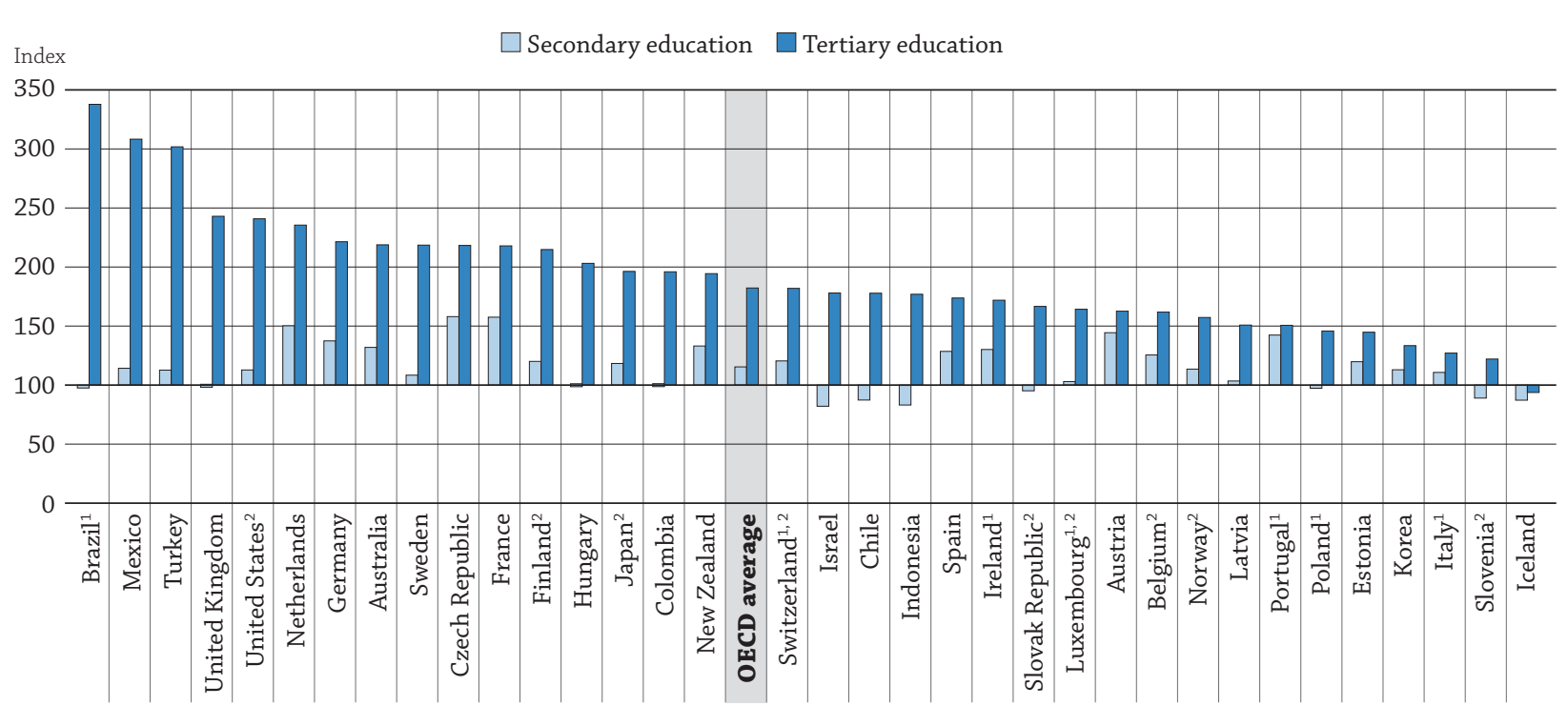

Notes: A ratio of 300 for tertiary education means that expenditure per tertiary student by educational institutions is three times the expenditure per primary student by educational institutions.

A ratio of 50 for secondary education means that expenditure per secondary student by educational institutions is half the expenditure per primary student by educational institutions.

1. Public institutions only.

2. Some levels of education are included with others. Refer to " $\mathrm{x}$ " code in Table B1.1a for details.

Countries are ranked in descending order of expenditure per student by educational institutions in tertiary education relative to primary education.

Source: OECD. Table B1.1a. See Annex 3 for notes (www.oecd.org/education/education-at-a-glance-19991487.htm).

StatLink 헤프 http://dx.doi.org/10.1787/888933283917 
Educational institutions in OECD countries spend an average of 1.8 times more per tertiary student than per primary student, but spending patterns vary widely, mainly because education policies vary more at the tertiary level (see Indicator B5). For example, Estonia, Iceland, Italy, Korea, Poland and Slovenia spend less than 1.5 times more on a tertiary student than on a primary student, but Brazil, Mexico and Turkey spend three times as much, and South Africa four times as much (Table B1.1a and Chart B1.3).

\section{Differences in expenditure per student between general and vocational programmes}

On average across the 23 OECD countries for which data are available, only USD 328 more is spent per student in vocational than in general programmes at upper secondary and post-secondary non-tertiary education, but this masks large differences in expenditure per student within countries. The difference is less than USD 100 in Chile and Latvia, but exceeds USD 6000 in three countries, with higher expenditure per student in vocational than in general programmes in Israel, and higher expenditure per student in general than in vocational programmes in Slovenia and Switzerland. Countries with large enrolments in dual-system apprenticeship programmes at the upper secondary level (e.g. Austria, Finland, Germany, Luxembourg and the Netherlands) tend to have higher expenditure per student in vocational programmes than in general programmes. The difference is USD 627 in Austria, USD 1350 in Finland, USD 2640 in Germany, USD 2439 in Luxembourg and USD 3146 in the Netherlands. Exceptions to this pattern are Australia, Belgium, Chile, Hungary, Slovenia, Switzerland and the United Kingdom, where expenditure per student enrolled in a general programme is higher than expenditure per student in a vocational programme. The underestimation of the expenditure made by private enterprises on dual vocational programmes can partly explain these differences (Table B1.6, and see Table C1.3 and Box B3.1). However, other countries with smaller proportions of students in vocational programmes also have much higher expenditure per student in vocational than in general programmes (for example, Israel spends USD 6167 more per vocational than per general student, and Spain spends USD 2106 more) (Table B1.6).

\section{Expenditure per student by educational institutions relative to per capita GDP}

Since access to education is universal (and usually compulsory) at the lower levels of schooling in most OECD countries, spending per student by educational institutions at those levels can be interpreted as the resources spent on the school-age population relative to a country's ability to pay. At higher levels of education, this measure is more difficult to interpret because student enrolments vary sharply among countries. At the tertiary level, for example, OECD countries may rank relatively high on this measure if a large proportion of their wealth is spent on educating a relatively small number of students.

In OECD countries, expenditure per student by educational institutions averages $22 \%$ of per capita GDP at the primary level, $25 \%$ at the secondary level, and $40 \%$ at the tertiary level. Overall, from primary to tertiary levels of education, expenditure per student averages $27 \%$ of per capita GDP in OECD countries (Table B1.4).

Countries with low levels of expenditure per student may nonetheless show distributions of investment relative to per capita GDP that are similar to those of countries with a high level of spending per student. For example, Korea and Portugal - countries with below-OECD-average expenditure per student by educational institutions at the secondary level and below-OECD-average per capita GDP - spend more per student relative to per capita GDP than the OECD average.

The relationship between per capita GDP and expenditure per student by educational institutions is difficult to interpret. However, there is a clear positive relationship between the two at both the primary and secondary levels of education - in other words, poorer countries tend to spend less per student than richer ones. Although the relationship is generally positive at these levels, there are variations, even among countries with similar levels of per capita GDP, and especially those in which per capita GDP exceeds USD 30 000. Ireland and Austria, for example, have similar levels of per capita GDP (see Table X2.1 in Annex 2) but spend very different proportions of it on primary and secondary education. In Ireland, the proportions are 19\% at the primary level and 25\% at the secondary level (below or at the OECD averages of $22 \%$ and $25 \%$, respectively), while in Austria, the proportions are $21 \%$ and $31 \%$, respectively, and are among the largest at the secondary level (Table B1.4 and Chart B1.7, available on line).

There is more variation in spending at the tertiary level, and the relationship between countries' relative wealth and their expenditure levels varies as well. Canada, Sweden, the United Kingdom and the United States spend more than $50 \%$ of per capita GDP on each tertiary student - among the largest proportions after Brazil and South Africa (Table B1.4 and Chart B1.7, available on line). Brazil spends the equivalent of $83 \%$ of per capita GDP on each tertiary student; however, tertiary students represent only $5 \%$ of students enrolled in all levels of education combined (Table B1.7, available on line). 


\section{Chart B1.4. Annual expenditure per student by educational institutions in 2012 related to change since 2005 , by level of education

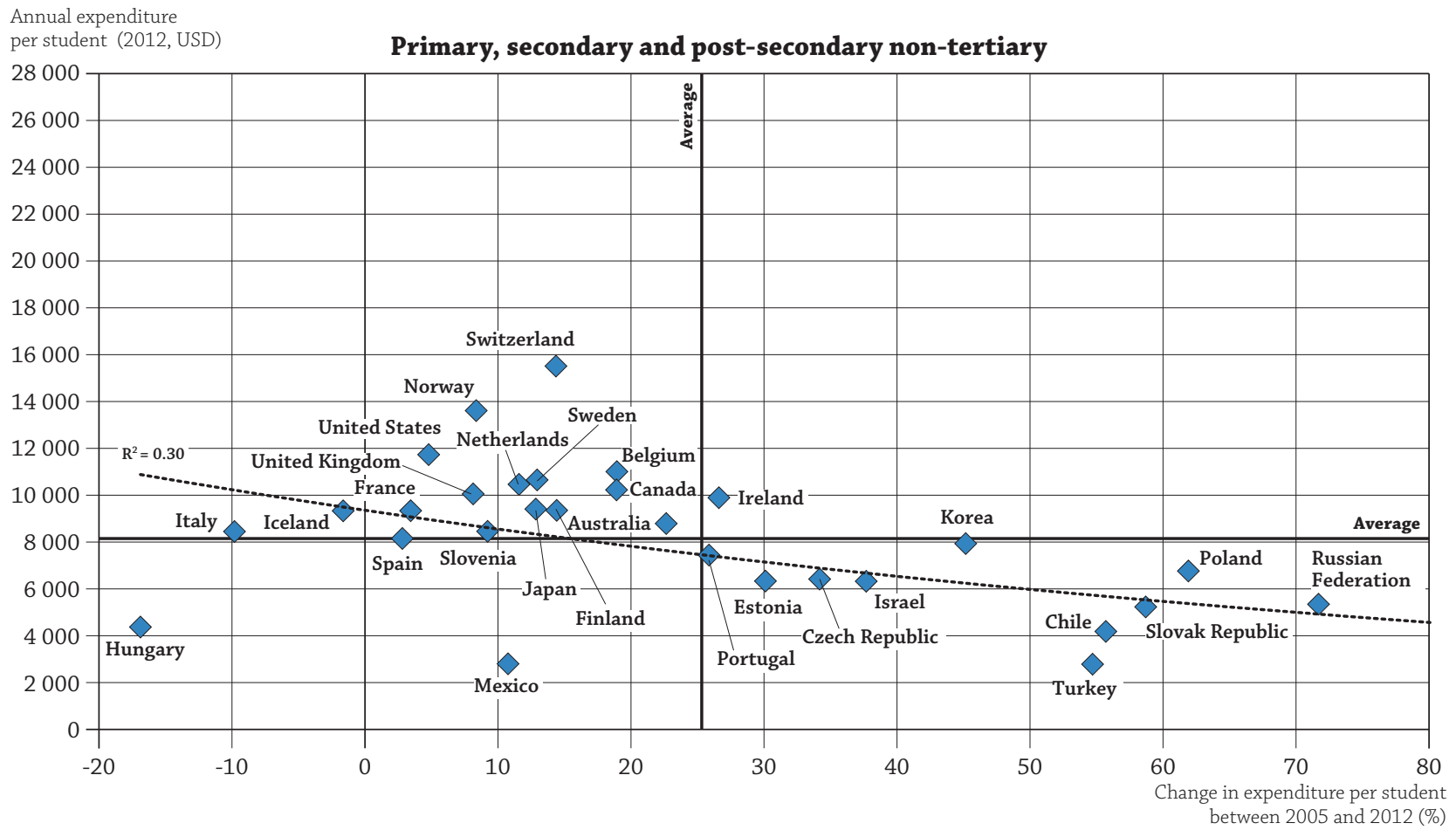

Annual expenditure

per student (2012, USD)

Tertiary education

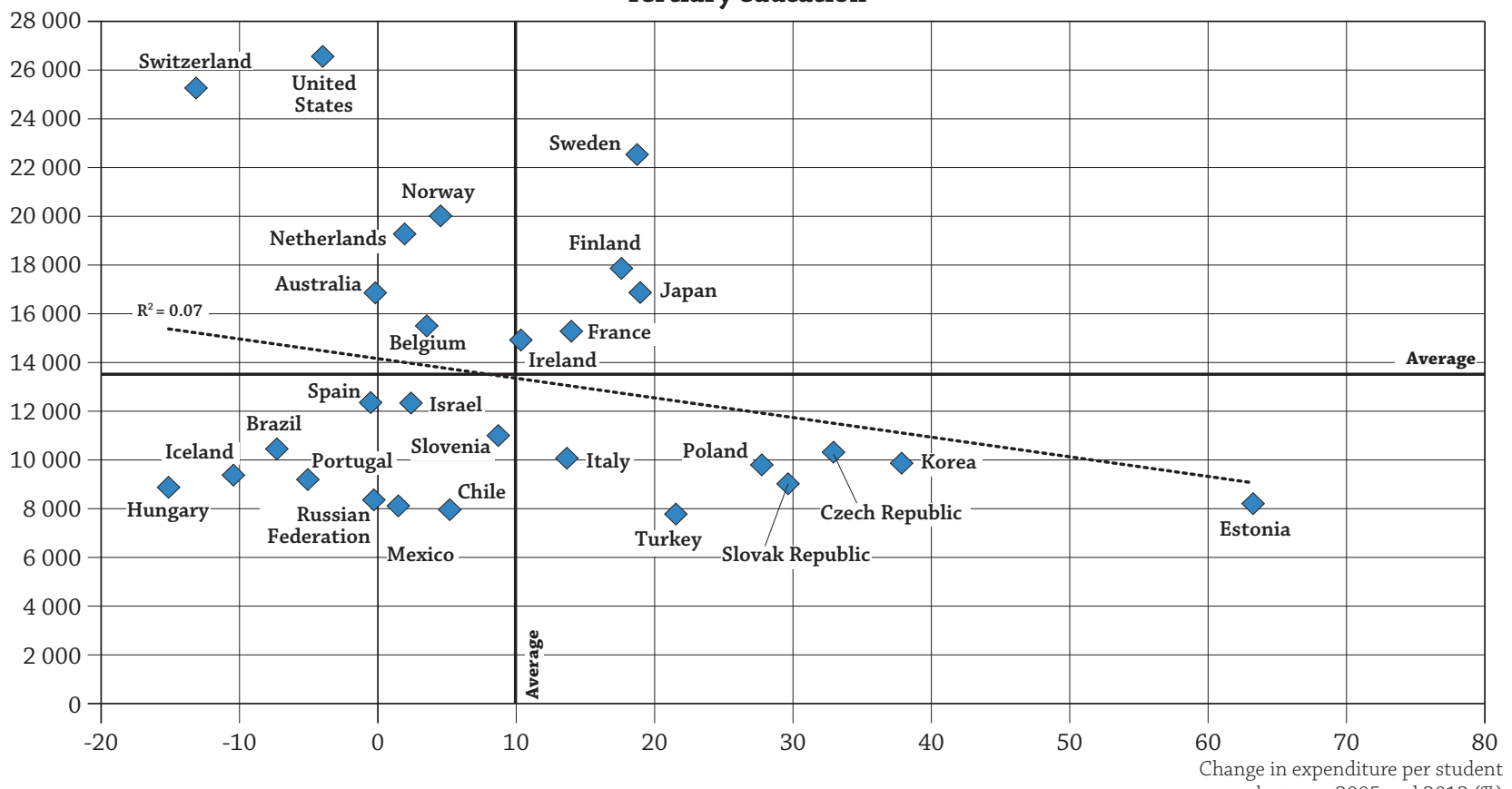

between 2005 and 2012 (\%)

Notes: Average values refer to countries with available data for both 2005 and 2012. At the primary, secondary, and post-secondary non-tertiary levels, Brazil does not appear in the chart as expenditure per student increased by more than $110 \%$ between 2005 and 2012.

Source: OECD. Tables B1.2, B1.5a and B1.5b. See Annex 3 for notes (www.oecd.org/education/education-at-a-glance-19991487.htm).

StatLink 제개 http://dx.doi.org/10.1787/888933283927 


\section{Change in expenditure per student by educational institutions between 2000 and 2012}

Changes in expenditure by educational institutions largely reflect changes in the size of the school-age population and in teachers' salaries. These tend to rise over time in real terms: teachers' salaries, the main component of costs, have increased in the majority of countries during the past decade (see Indicator D3). The size of the schoolage population influences both enrolment levels and the amount of resources and organisational effort a country must invest in its education system. The larger this population, the greater the potential demand for education services. Change in expenditure per student over years may also vary between levels of education within countries, as both enrolment and expenditure may follow different trends at different levels of education. At the tertiary level, compared to other levels of education, expenditure per student decreased in more countries between 2005 and 2012 (Tables B1.5a and b, and Chart B1.4).

Expenditure per primary, secondary and post-secondary non-tertiary student by educational institutions increased in every country except Italy, and by an average of more than 43\% between 2000 and 2012, a time during which student enrolment at these levels was relatively stable. In most countries, the increase was slightly smaller over the period 2000-05 than over the period 2005-12, as a result of the combination of a larger increase in expenditure with a smaller decrease in student numbers in the former than in the latter period.

Between 2005 and 2012, in 23 of the 30 countries for which data are available, expenditure per primary, secondary and post-secondary non-tertiary student by educational institutions increased by at least $10 \%$. The increase exceeded 50\% in Brazil, Chile, Poland, the Russian Federation, the Slovak Republic and Turkey. By contrast, in France, Spain and the United States this expenditure increased by only $5 \%$ or less. Only Hungary, Iceland and Italy showed a decrease in expenditure per primary, secondary and post-secondary non-tertiary student during this period (Table B1.5a).

In Brazil, Chile, Poland, the Russian Federation, the Slovak Republic and Turkey, increases in expenditure per student over 2005-12 are among the largest, but these countries were still among those with the lowest expenditure per student in 2012. The correlation between the level of expenditure per student and its variation over time is weak. For example, Chile and Hungary, with similar levels of expenditure per student in 2012, did not increase expenditure per student in similar ways. Expenditure per student decreased in Hungary over the period as a result of a drop in both expenditure and enrolment (with a larger decrease in expenditure than in student numbers). In Chile, enrolments decreased as much as in Hungary, but expenditure increased significantly over the period (Table B1.5a and Chart B1.4).

Since the beginning of the economic crisis in 2008, however, expenditure per primary, secondary and post-secondary non-tertiary student decreased in a few countries, namely Estonia, Hungary, Iceland, Italy, Slovenia and Spain. In all these countries, this trend resulted from a decrease in expenditure (combined with a smaller decrease in enrolment or, in Spain, with an increase in enrolment). In other countries, expenditure per student increased as in most of them, expenditure continued to increase even as enrolments dropped (except in Australia, Ireland, Israel, Mexico, Norway, Turkey and the United Kingdom, where enrolments increased). This demonstrates that, in most countries, the global economic crisis had not yet affected the overall investment in education (Chart B1.5).

The pattern is different at the tertiary level. Spending per student increased between 2000 and 2012 in most countries, except in Brazil, Chile, Hungary, Iceland, Ireland, Israel and Switzerland, where expenditure did not keep up with expanding enrolments. On average across OECD countries, expenditure per tertiary student by educational institutions increased by nearly 4\% between 2000 and 2005 and by 11\% between 2005 and 2012.

Between 2005 and 2012, expenditure per tertiary student increased in most countries. The increase reached 38\% in Korea and 63\% in Estonia as a result of a large increase in expenditure combined with constant enrolment. By contrast, over this period, expenditure per student decreased in a quarter of countries (7 of 28 countries with available data), particularly in Hungary, Iceland and Switzerland (by more than 10\%) and to a lesser extent in Brazil, Portugal, Spain and the United States. In Hungary, this resulted from a larger decrease in expenditure than in student numbers; whereas in the other countries, the decline was the result of a rapid increase in the number of tertiary students (Table B1.5b and Chart B1.4).

Since the beginning of the economic crisis in 2008, expenditure on tertiary institutions has decreased in 7 of the 32 countries with available data: Hungary, Iceland, Ireland, Italy, Portugal, the Russian Federation and Spain. This led to a drop in expenditure per student in all of these countries except Italy and the Russian Federation, where tertiary enrolment fell even faster. Globally, expenditure per student decreased in a third of countries between 2008 and 2012, mainly as enrolment increased faster than expenditure (Chart B1.5). 


\section{Chart B1.5. Changes in the number of students, expenditure on educational institutions and expenditure per student, by level of education $(2008,2012)$}

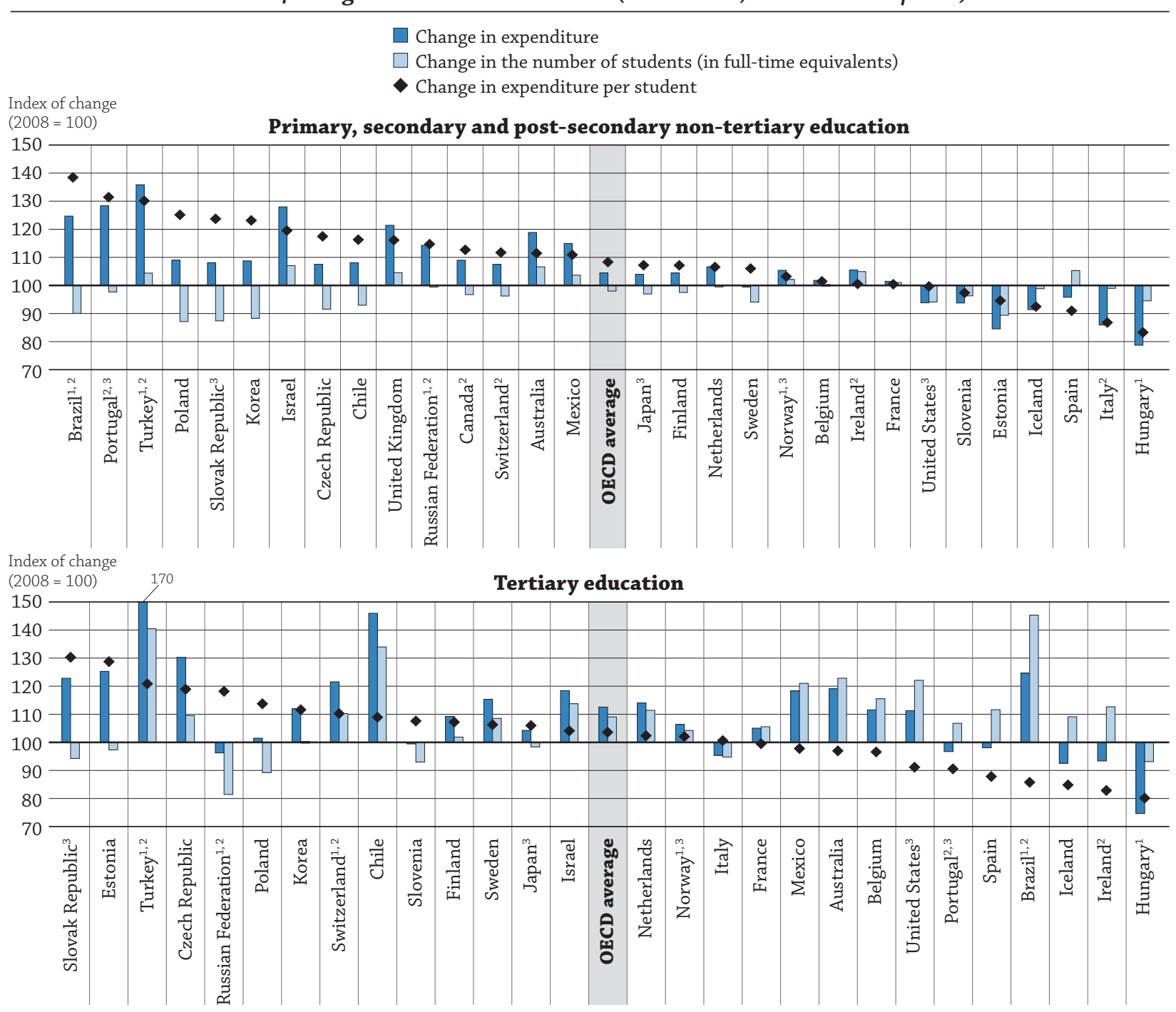

1. Public expenditure only.

2. Public institutions only.

3. Some levels of education are included with others. Refer to " $x$ " code in Table B1.1a for details.

Countries are ranked in descending order of change in expenditure per student by educational institutions.

Source: OECD. Tables B1.5a and B1.5b. See Annex 3 for notes (www.oecd.org/education/education-at-a-glance-19991487.htm).

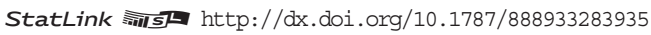

\section{Definitions}

Ancillary services are services provided by educational institutions that are peripheral to the main educational mission. The main component of ancillary services is student welfare services. In primary, secondary and postsecondary non-tertiary education, student welfare services include meals, school health services and transportation to and from school. At the tertiary level, they include residence halls (dormitories), dining halls and health care.

Core educational services are directly related to instruction in educational institutions, including teachers' salaries, construction and maintenance of school buildings, teaching materials, books and administration of schools.

Research and development (R\&D) includes research performed at universities and other tertiary educational institutions, regardless of whether the research is financed from general institutional funds or through separate grants or contracts from public or private sponsors. 


\section{Methodology}

Data refer to the financial year 2012 and are based on the UOE data collection on education statistics administered by the OECD in 2014 (for details see Annex 3 at www.oecd.org/education/education-at-a-glance-19991487.htm).

Tables B1.5a and $\mathrm{b}$ show the changes in expenditure per student by educational institutions between the financial years 2000, 2005, 2008, 2010, 2011 and 2012. OECD countries were asked to collect 2000, 2005, 2008, 2010 and 2011 data according to the definitions and coverage of UOE 2014 data collection. All expenditure data and GDP information for 2000, 2005, 2008, 2010 and 2011 are adjusted to 2012 prices using the GDP price deflator.

The indicator shows direct public and private expenditure by educational institutions in relation to the number of full-time equivalent students enrolled. Public subsidies for students' living expenses outside educational institutions have been excluded to ensure international comparability.

Core educational services are estimated as the residual of all expenditure, that is, total expenditure on educational institutions net of expenditure on R\&D and ancillary services. The classification of R\&D expenditure is based on data collected from the institutions carrying out R\&D, rather than on the sources of funds.

Expenditure per student by educational institutions at a particular level of education is calculated by dividing total expenditure by educational institutions at that level by the corresponding full-time equivalent enrolment. Only educational institutions and programmes for which both enrolment and expenditure data are available are taken into account. Expenditure in national currency is converted into equivalent USD by dividing the national currency figure by the purchasing power parity (PPP) index for GDP. The PPP exchange rate is used because the market exchange rate is affected by many factors (interest rates, trade policies, expectations of economic growth, etc.) that have little to do with current relative domestic purchasing power in different OECD countries (see Annex 2 for further details).

Expenditure data for students in private educational institutions are not available for certain countries, and some other countries provide incomplete data on independent private institutions. Where this is the case, only expenditure on public and government-dependent private institutions has been taken into account.

Expenditure per student by educational institutions relative to per capita GDP is calculated by expressing expenditure per student by educational institutions in units of national currency as a percentage of per capita GDP, also in national currency. In cases where the educational expenditure data and the GDP data pertain to different reference periods, the expenditure data are adjusted to the same reference period as the GDP data, using inflation rates for the $\mathrm{OECD}$ country in question (see Annex 2).

Full-time equivalent student: The ranking of OECD countries by annual expenditure on educational services per student is affected by differences in how countries define full-time, part-time and full-time equivalent enrolment. Some OECD countries count every participant at the tertiary level as a full-time student, while others determine a student's intensity of participation by the credits that he/she obtains for successful completion of specific course units during a specified reference period. OECD countries that can accurately account for part-time enrolment have higher apparent expenditure per full-time equivalent student by educational institutions than OECD countries that cannot differentiate among the different types of student attendance.

\section{Note regarding data from Israel}

The statistical data for Israel are supplied by and are under the responsibility of the relevant Israeli authorities. The use of such data by the OECD is without prejudice to the status of the Golan Heights, East Jerusalem and Israeli settlements in the West Bank under the terms of international law.

\section{Indicator B1 Tables}

\section{StatLink त्ञाI $\mathrm{ht}$ htp://dx.doi.org/10.1787/888933285349}

\section{Table B1.1a Annual expenditure per student by educational institutions for all services (2012)}

WEB Table B1.1b Annual expenditure per student by educational institutions for core services (2012)

Table B1.2 Annual expenditure per student by educational institutions for core services, ancillary services and R\&D (2012) 
WEB Table B1.3 Cumulative expenditure per student by educational institutions for all services over the theoretical duration of primary and secondary studies (2012)

Table B1.4 Annual expenditure per student by educational institutions for all services, relative to per capita GDP (2012)

Table B1.5a Change in expenditure per student by educational institutions for all services, relative to different factors, at the primary, secondary and post-secondary non-tertiary levels of education (2000, 2005, 2008, 2010, 2011, 2012)

Table B1.5b Change in expenditure per student by tertiary education institutions for all services, relative to different factors $(2000,2005,2008,2010,2011,2012)$

Table B1.6 Annual expenditure per student by secondary educational institutions for all services, by type of programme (2012)

WEB Table B1.7 Percentage of expenditure by educational institutions compared to the proportion of students enrolled at each level of education (2012)

\section{Additional material available on line only}

StatLink 젬ㄴ http://dx.doi.org/10.1787/888933285349

WEB Chart B1.6 Cumulative expenditure per student by educational institutions over the theoretical duration of primary and secondary studies (2012)

WEB Chart B1.7 Annual expenditure per student by educational institutions relative to per capita GDP (2012)

Cut-off date for the data: 23 October 2015. Updates can be found on line at http://dx.doi.org/10.1787/eag-data-en. 
Table B1.1a. Annual expenditure per student by educational institutions for all services (2012) In equivalent USD converted using PPPs for GDP, by level of education, based on full-time equivalents

\begin{tabular}{|c|c|c|c|c|c|c|c|c|c|c|}
\hline & \multirow[b]{2}{*}{ Primary } & \multicolumn{3}{|c|}{ Secondary } & \multirow[b]{2}{*}{$\begin{array}{c}\text { Post- } \\
\text { secondary } \\
\text { non-tertiary }\end{array}$} & \multicolumn{3}{|c|}{ Tertiary (including R\&D activities) } & \multirow[b]{2}{*}{$\begin{array}{l}\text { All tertiary } \\
\text { excluding } \\
\text { R\&D } \\
\text { activities }\end{array}$} & \multirow[b]{2}{*}{$\begin{array}{c}\text { Primary } \\
\text { to tertiary } \\
\text { (including R\&D } \\
\text { activities and } \\
\text { undistributed } \\
\text { programmes) }\end{array}$} \\
\hline & & $\begin{array}{c}\text { Lower } \\
\text { secondary }\end{array}$ & $\begin{array}{c}\text { Upper } \\
\text { secondary }\end{array}$ & $\begin{array}{c}\text { All } \\
\text { secondary }\end{array}$ & & $\begin{array}{c}\text { Short-cycle } \\
\text { tertiary }\end{array}$ & $\begin{array}{c}\text { Bachelor's, } \\
\text { master's, } \\
\text { doctoral or } \\
\text { equivalent } \\
\text { level }\end{array}$ & $\begin{array}{c}\text { All } \\
\text { tertiary }\end{array}$ & & \\
\hline & (1) & (2) & (3) & (4) & (5) & (6) & (7) & $(8)$ & (9) & (10) \\
\hline Austria & 9563 & 13632 & 14013 & 13806 & 5212 & 15071 & 15641 & 15549 & 11616 & 13189 \\
\hline Belgium & 9581 & 11670 & $12210^{\mathrm{d}}$ & $12025^{d}$ & $x(3)$ & 8212 & 15785 & 15503 & 10156 & 12135 \\
\hline Canada $^{1,2}$ & $9680^{\mathrm{d}}$ & $\mathrm{x}(1)$ & 11695 & $\mathrm{~m}$ & $\mathrm{~m}$ & 15348 & 25525 & 22006 & 15788 & $\mathbf{m}$ \\
\hline Chile $^{3}$ & 4476 & 4312 & 3706 & 3909 & a & 4186 & 9409 & 7960 & 7600 & 5235 \\
\hline Czech Republic & 4728 & 7902 & 7119 & 7469 & 2445 & 16645 & 10304 & 10319 & 6807 & 7684 \\
\hline Denmark & 10953 & 11460 & 9959 & 10632 & a & $\mathrm{m}$ & $\mathrm{m}$ & $\mathrm{m}$ & $\mathrm{m}$ & $\mathbf{m}$ \\
\hline Estonia & 5668 & 6524 & 7013 & 6791 & 7478 & a & 8206 & 8206 & 4690 & 6878 \\
\hline France & 7013 & 9588 & 13070 & 11046 & $\mathrm{~m}$ & 12346 & 16279 & 15281 & 10361 & 10450 \\
\hline Germany & 7749 & 9521 & 12599 & 10650 & 10041 & 8265 & 17159 & 17157 & 10025 & 11363 \\
\hline Greece & $\mathrm{m}$ & $\mathrm{m}$ & $\mathrm{m}$ & $\mathrm{m}$ & $\mathrm{m}$ & $\mathrm{m}$ & $\mathrm{m}$ & $\mathrm{m}$ & $\mathrm{m}$ & $\mathbf{m}$ \\
\hline Hungary & 4370 & 4459 & 4386 & 4419 & 3698 & 2897 & 9658 & 8876 & 7405 & 5564 \\
\hline Iceland & 10003 & 10706 & 7541 & 8724 & 11140 & 9665 & 9373 & 9377 & $\mathrm{~m}$ & 10287 \\
\hline Ireland $^{2}$ & 8681 & 11087 & 11564 & 11298 & 12856 & $\mathrm{x}(8)$ & $x(8)$ & 14922 & 11418 & 10740 \\
\hline Israel & 6931 & $\mathrm{x}(4)$ & $\mathrm{x}(4)$ & 5689 & 2326 & 6366 & 13777 & 12338 & 7710 & 7903 \\
\hline Italy $^{2}$ & 7924 & 8905 & 8684 & 8774 & $\mathrm{~m}$ & $\mathrm{~m}$ & 10071 & 10071 & 6369 & 8744 \\
\hline Japan & 8595 & 9976 & $10360^{\mathrm{d}}$ & $10170^{\mathrm{d}}$ & $x(3,6,7)$ & $10532^{\mathrm{d}}$ & $18557^{\mathrm{d}}$ & $16872^{\mathrm{d}}$ & $\mathrm{m}$ & 11671 \\
\hline Korea & 7395 & 7008 & 9651 & 8355 & $\mathrm{~m}$ & 5540 & 11173 & 9866 & 8026 & 9569 \\
\hline Luxembourg ${ }^{2,4}$ & 20020 & 20247 & 20962 & 20617 & 1257 & 3749 & 34739 & 32876 & 21358 & 22545 \\
\hline Mexico & 2632 & 2367 & 4160 & 3007 & $\mathrm{a}$ & $\mathrm{x}(8)$ & $x(8)$ & 8115 & 6647 & 3509 \\
\hline Netherlands & 8185 & 12227 & 12368 & 12296 & 11554 & 11580 & 19305 & 19276 & 12505 & 12211 \\
\hline New Zealand & 7069 & 8644 & 10262 & 9409 & 9542 & 10289 & 14543 & 13740 & 10841 & 9443 \\
\hline Portugal $^{2}$ & 6105 & 8524 & $8888^{d}$ & $8691^{d}$ & $x(3,7)$ & a & $9196^{\mathrm{d}}$ & $9196^{\mathrm{d}}$ & 4917 & 7952 \\
\hline Slovak Republic & 5415 & 5283 & $5027^{d}$ & $5152^{d}$ & $x(3)$ & $\mathrm{x}(3)$ & 9022 & 9022 & 6191 & 6072 \\
\hline Slovenia & 9015 & 9802 & $6898^{\mathrm{d}}$ & $8022^{\mathrm{d}}$ & $x(3)$ & 6874 & 11615 & 11002 & 8888 & 9031 \\
\hline Spain & 7111 & 9137 & $9145^{d}$ & $9141^{\mathrm{d}}$ & $\mathrm{x}(3)$ & 9394 & 13040 & 12356 & 8983 & 9040 \\
\hline Sweden & 10312 & 10966 & 11329 & 11177 & 3610 & 5897 & 24025 & 22534 & 10589 & 12742 \\
\hline Switzerland ${ }^{2}$ & 13889 & 16370 & $17024^{\mathrm{d}}$ & $16731^{\mathrm{d}}$ & $x(3)$ & $\mathrm{x}(8)$ & $\mathrm{x}(8)$ & 25264 & 11632 & 17485 \\
\hline Turkey & 2577 & 2448 & 3524 & 2904 & $\mathrm{a}$ & $x(8)$ & $x(8)$ & 7779 & 5557 & 3514 \\
\hline United Kingdom & 10017 & 10271 & 9963 & 10085 & a & $x(8)$ & $x(8)$ & 24338 & 18593 & 12084 \\
\hline United States & 11030 & 11856 & 13059 & 12442 & $\mathrm{x}(8)$ & $x(8)$ & $x(8)$ & $26562^{d}$ & 23706 & 15494 \\
\hline OECD average & 8247 & 9627 & 9876 & 9518 & 6734 & 8968 & 15111 & 15028 & 10309 & 10220 \\
\hline EU21 average & 8372 & 10040 & 10011 & 9931 & 6461 & 9097 & 14807 & 14955 & 9963 & 10361 \\
\hline Argentina & $\mathrm{m}$ & $\mathrm{m}$ & $\mathrm{m}$ & $\mathrm{m}$ & a & $\mathrm{m}$ & $\mathrm{m}$ & $\mathrm{m}$ & $\mathrm{m}$ & $\mathbf{m}$ \\
\hline Brazil $^{2}$ & 3095 & 2981 & 3078 & 3020 & a & $x(8)$ & $x(8)$ & 10455 & 9595 & 3441 \\
\hline China & $\mathrm{m}$ & $\mathrm{m}$ & $\mathrm{m}$ & $\mathrm{m}$ & $\mathrm{m}$ & $\mathrm{m}$ & $\mathrm{m}$ & $\mathrm{m}$ & $\mathrm{m}$ & $\mathbf{m}$ \\
\hline Colombia $^{3}$ & 2645 & 2651 & $2742^{\mathrm{d}}$ & $2677^{d}$ & $\mathrm{x}(3)$ & $\mathrm{x}(8)$ & $x(8)$ & 5183 & $\mathrm{~m}$ & 3291 \\
\hline India & $\mathrm{m}$ & $\mathrm{m}$ & $\mathrm{m}$ & $\mathrm{m}$ & $\mathrm{m}$ & $\mathrm{m}$ & $\mathrm{m}$ & $\mathrm{m}$ & $\mathrm{m}$ & $\mathbf{m}$ \\
\hline Indonesia $^{3}$ & 1180 & 915 & 1067 & 981 & a & $x(8)$ & $x(8)$ & 2089 & $\mathrm{~m}$ & 1397 \\
\hline Latvia & 3489 & 3515 & 3685 & 3610 & 4153 & 5091 & 5290 & 5262 & 4303 & 3983 \\
\hline Russian Federation & $\mathrm{x}(4)$ & $\mathrm{x}(4)$ & $\mathrm{x}(4)$ & $5345^{d}$ & $\mathrm{x}(4)$ & 5183 & 9115 & 8363 & 7641 & 6190 \\
\hline Saudi Arabia & $\mathrm{m}$ & $\mathrm{m}$ & $\mathrm{m}$ & $\mathrm{m}$ & $\mathrm{m}$ & $\mathrm{m}$ & $\mathrm{m}$ & $\mathrm{m}$ & $\mathrm{m}$ & $\mathbf{m}$ \\
\hline South Africa ${ }^{2}$ & 2431 & $\mathrm{x}(4)$ & $x(4)$ & 2440 & 5188 & $x(8)$ & $x(8)$ & 10885 & $\mathrm{~m}$ & 3633 \\
\hline G20 average & $\mathrm{m}$ & $\mathrm{m}$ & $\mathrm{m}$ & $\mathrm{m}$ & $\mathrm{m}$ & $\mathrm{m}$ & $\mathrm{m}$ & $\mathrm{m}$ & $\mathrm{m}$ & $\mathbf{m}$ \\
\hline
\end{tabular}

Note: Data on early childhood education is available in Indicator $C 2$.

1. Year of reference 2011.

2. Public institutions only (for Canada and Luxembourg, in tertiary education only; for Italy, except in tertiary education).

3. Year of reference 2013

4. Pre-primary and primary education include reimbursements from local authorities for previous years.

Source: OECD. Argentina, China, Colombia, India, Indonesia, Saudi Arabia, South Africa: UNESCO Institute for Statistics. Latvia: Eurostat. See Annex 3 for notes (www.oecd.org/education/education-at-a-glance-19991487.htm).

Please refer to the Reader's Guide for information concerning symbols for missing data and abbreviations.

StatLink 제그 http://dx.doi.org/10.1787/888933285351 
Table B1.2. Annual expenditure per student by educational institutions for core services, ancillary services and R\&D (2012)

In equivalent USD converted using PPPs for GDP, by level of education and type of service, based on full-time equivalents



1. Some levels of education are included with others. Refer to " $\mathrm{x}$ " code in Table B1.1a for details.

2. Year of reference 2011.

3. Public institutions only (for Canada and Luxembourg, in tertiary education only; for Italy, except in tertiary education).

4. Year of reference 2013

5. Excludes post-secondary non-tertiary education and short-cycle tertiary programmes.

Source: OECD. Argentina, China, Colombia, India, Indonesia, Saudi Arabia, South Africa: UNESCO Institute for Statistics. Latvia: Eurostat. See Annex 3 for notes (www.oecd.org/education/education-at-a-glance-19991487.htm).

Please refer to the Reader's Guide for information concerning symbols for missing data and abbreviations.

StatLink 젶ㄴ http://dx.doi.org/10.1787/888933285363 
Table B1.4. Annual expenditure per student by educational institutions for all services, relative to per capita GDP (2012)

In percentage of GDP per capita, by level of education, based on full-time equivalents

\begin{tabular}{|c|c|c|c|c|c|c|c|c|c|c|}
\hline & \multirow[b]{2}{*}{ Primary } & \multicolumn{3}{|c|}{ Secondary } & \multirow[b]{2}{*}{$\begin{array}{c}\text { Post- } \\
\text { secondary } \\
\text { non-tertiary }\end{array}$} & \multicolumn{3}{|c|}{ Tertiary (including R\&D activities) } & \multirow[b]{2}{*}{$\begin{array}{l}\text { All tertiary } \\
\text { excluding } \\
\text { R\&D } \\
\text { activities }\end{array}$} & \multirow[b]{2}{*}{$\begin{array}{c}\text { Primary } \\
\text { to tertiary } \\
\text { (including R\&D } \\
\text { activities and } \\
\text { undistributed } \\
\text { programmes) }\end{array}$} \\
\hline & & $\begin{array}{c}\text { Lower } \\
\text { secondary }\end{array}$ & $\begin{array}{c}\text { Upper } \\
\text { secondary }\end{array}$ & $\begin{array}{c}\text { All } \\
\text { secondary }\end{array}$ & & $\begin{array}{c}\text { Short-cycle } \\
\text { tertiary }\end{array}$ & $\begin{array}{c}\text { Bachelor's, } \\
\text { master's, } \\
\text { doctoral or } \\
\text { equivalent } \\
\text { level }\end{array}$ & $\begin{array}{c}\text { All } \\
\text { tertiary }\end{array}$ & & \\
\hline & (1) & (2) & (3) & (4) & (5) & (6) & (7) & (8) & (9) & (10) \\
\hline Australia & 18 & 25 & 22 & 24 & 15 & 19 & 44 & 39 & 24 & 24 \\
\hline Austria & 21 & 30 & 31 & 31 & 12 & 34 & 35 & 35 & 26 & 29 \\
\hline Belgium & 23 & 28 & $29^{\mathrm{d}}$ & $29^{\mathrm{d}}$ & $x(3)$ & 20 & 38 & 37 & 24 & 29 \\
\hline Canada ${ }^{1,2}$ & $23^{\mathrm{d}}$ & $\mathrm{x}(1)$ & 27 & $\mathrm{~m}$ & $\mathrm{~m}$ & 36 & 60 & 52 & 37 & $\mathbf{m}$ \\
\hline Chile $^{3}$ & 21 & 20 & 17 & 18 & a & 20 & 44 & 37 & 36 & 25 \\
\hline Czech Republic & 16 & 28 & 25 & 26 & 9 & 58 & 36 & 36 & 24 & 27 \\
\hline Denmark & 25 & 26 & 23 & 24 & a & $\mathrm{m}$ & $\mathrm{m}$ & $\mathrm{m}$ & $\mathrm{m}$ & $\mathbf{m}$ \\
\hline Estonia & 23 & 26 & 28 & 28 & 30 & a & 33 & 33 & 19 & 28 \\
\hline Finland & 21 & 32 & $21^{\mathrm{d}}$ & $25^{\mathrm{d}}$ & $x(3)$ & a & 44 & 44 & 27 & 27 \\
\hline France & 19 & 26 & 35 & 30 & 0 & 33 & 44 & 41 & 28 & 28 \\
\hline Germany & 18 & 22 & 29 & 25 & 23 & 19 & 40 & 40 & 23 & 27 \\
\hline Greece & $\mathrm{m}$ & $\mathrm{m}$ & $\mathrm{m}$ & $\mathrm{m}$ & $\mathrm{m}$ & $\mathrm{m}$ & $\mathrm{m}$ & $\mathrm{m}$ & $\mathrm{m}$ & $\mathbf{m}$ \\
\hline Hungary & 19 & 20 & 20 & 20 & 16 & 13 & 43 & 39 & 33 & 25 \\
\hline Iceland & 25 & 26 & 19 & 22 & 28 & 24 & 23 & 23 & $\mathrm{~m}$ & 25 \\
\hline Ireland $^{2}$ & 19 & 25 & 26 & 25 & 28 & $\mathrm{x}(8)$ & $\mathrm{x}(8)$ & 33 & 25 & 24 \\
\hline Israel & 22 & $\mathrm{x}(4)$ & $\mathrm{x}(4)$ & 18 & 7 & 20 & 44 & 39 & 25 & 25 \\
\hline Italy ${ }^{2}$ & 22 & 25 & 25 & 25 & $\mathrm{~m}$ & $\mathrm{~m}$ & 29 & 29 & 18 & 25 \\
\hline Japan & 24 & 28 & $29^{\mathrm{d}}$ & $28^{\mathrm{d}}$ & $x(3,6,7)$ & $30^{\mathrm{d}}$ & $52^{\mathrm{d}}$ & $47^{\mathrm{d}}$ & $\mathrm{m}$ & 33 \\
\hline Korea & 23 & 22 & 30 & 26 & $\mathrm{~m}$ & 17 & 35 & 31 & 25 & 30 \\
\hline Luxembourg & 22 & 22 & 23 & 22 & 1 & 4 & 38 & 36 & 23 & 25 \\
\hline Mexico & 16 & 14 & 25 & 18 & a & $\mathrm{x}(8)$ & $\mathrm{x}(8)$ & 48 & 40 & 21 \\
\hline Netherlands & 18 & 27 & 27 & 27 & 25 & 25 & 42 & 42 & 27 & 27 \\
\hline New Zealand & 22 & 27 & 32 & 29 & 30 & 32 & 45 & 43 & 34 & 29 \\
\hline Norway & 25 & 26 & $30^{\mathrm{d}}$ & $28^{\mathrm{d}}$ & $\mathrm{x}(3)$ & $\mathrm{x}(3)$ & 39 & 39 & 23 & 30 \\
\hline Poland $^{2}$ & 29 & 29 & 28 & 29 & $\mathrm{~m}$ & 36 & 43 & 43 & 34 & 32 \\
\hline Portugal $^{2}$ & 22 & 31 & $33^{\mathrm{d}}$ & $32^{\mathrm{d}}$ & $\mathrm{x}(3,7)$ & a & $34^{\mathrm{d}}$ & $34^{\mathrm{d}}$ & 18 & 29 \\
\hline Slovak Republic & 21 & 21 & $20^{\mathrm{d}}$ & $20^{\mathrm{d}}$ & $\mathrm{x}(3)$ & $\mathrm{x}(3)$ & 35 & 35 & 24 & 24 \\
\hline Slovenia & 32 & 34 & $24^{\mathrm{d}}$ & $28^{\mathrm{d}}$ & $\mathrm{x}(3)$ & 24 & 41 & 39 & 31 & 32 \\
\hline Spain & 22 & 28 & $28^{\mathrm{d}}$ & $28^{\mathrm{d}}$ & $\mathrm{x}(3)$ & 29 & 40 & 38 & 27 & 28 \\
\hline Sweden & 24 & 25 & 26 & 25 & 8 & 13 & 55 & 51 & 24 & 29 \\
\hline Switzerland $^{2}$ & 25 & 29 & $31^{\mathrm{d}}$ & $30^{\mathrm{d}}$ & $x(3)$ & $\mathrm{x}(8)$ & $\mathrm{x}(8)$ & 45 & 21 & 31 \\
\hline Turkey & 14 & 14 & 20 & 16 & a & $\mathrm{x}(8)$ & $\mathrm{x}(8)$ & 43 & $\mathrm{~m}$ & 20 \\
\hline United Kingdom & 27 & 28 & 27 & 27 & a & $\mathrm{x}(8)$ & $\mathrm{x}(8)$ & 65 & 50 & 33 \\
\hline United States & 22 & 24 & 26 & 25 & $\mathrm{x}(8)$ & $\mathrm{x}(8)$ & $\mathrm{x}(8)$ & $53^{\mathrm{d}}$ & 48 & 31 \\
\hline OECD average & 22 & 25 & 26 & 25 & 17 & 25 & 41 & 40 & 28 & 27 \\
\hline EU21 average & 22 & 27 & 26 & 26 & 15 & 26 & 39 & 39 & 27 & 28 \\
\hline Argentina & $\mathrm{m}$ & $\mathrm{m}$ & $\mathrm{m}$ & $\mathrm{m}$ & a & $\mathrm{m}$ & $\mathrm{m}$ & $\mathrm{m}$ & $\mathrm{m}$ & $\mathbf{m}$ \\
\hline Brazil $^{2}$ & 25 & 24 & 24 & 24 & a & $\mathrm{x}(8)$ & $\mathrm{x}(8)$ & 83 & 76 & 27 \\
\hline China & $\mathrm{m}$ & $\mathrm{m}$ & $\mathrm{m}$ & $\mathrm{m}$ & $\mathrm{m}$ & $\mathrm{m}$ & $\mathrm{m}$ & $\mathrm{m}$ & $\mathrm{m}$ & $\mathbf{m}$ \\
\hline Colombia $^{3}$ & 22 & 22 & $23^{\mathrm{d}}$ & $22^{\mathrm{d}}$ & $\mathrm{x}(3)$ & $\mathrm{x}(8)$ & $\mathrm{x}(8)$ & 43 & $\mathrm{~m}$ & 27 \\
\hline India & $\mathrm{m}$ & $\mathrm{m}$ & $\mathrm{m}$ & $\mathrm{m}$ & $\mathrm{m}$ & $\mathrm{m}$ & $\mathrm{m}$ & $\mathrm{m}$ & $\mathrm{m}$ & $\mathbf{m}$ \\
\hline Indonesia $^{3}$ & 12 & 9 & 11 & 10 & a & $\mathrm{x}(8)$ & $\mathrm{x}(8)$ & 21 & $\mathrm{~m}$ & 14 \\
\hline Latvia & 23 & 23 & 25 & 24 & 28 & 34 & 35 & 35 & 29 & 27 \\
\hline Russian Federation & $\mathrm{x}(4)$ & $\mathrm{x}(4)$ & $\mathrm{x}(4)$ & $22^{\mathrm{d}}$ & $\mathrm{x}(4)$ & 22 & 38 & 35 & 32 & 26 \\
\hline Saudi Arabia & $\mathrm{m}$ & $\mathrm{m}$ & $\mathrm{m}$ & $\mathrm{m}$ & $\mathrm{m}$ & $\mathrm{m}$ & $\mathrm{m}$ & $\mathrm{m}$ & $\mathrm{m}$ & $\mathbf{m}$ \\
\hline South Africa ${ }^{2}$ & 19 & $\mathrm{x}(4)$ & $\mathrm{x}(4)$ & 19 & 41 & $\mathrm{x}(8)$ & $\mathrm{x}(8)$ & 87 & $\mathrm{~m}$ & 29 \\
\hline
\end{tabular}

\begin{tabular}{l|l|l|l|l|l|l|l|l|l|l|} 
G20 average & $\mathrm{m}$ & $\mathrm{m}$ & $\mathrm{m}$ & $\mathrm{m}$ & $\mathrm{m}$ & $\mathrm{m}$ & $\mathrm{m}$ & $\mathrm{m}$ & $\mathrm{m}$ & $\mathbf{m}$
\end{tabular}

1. Year of reference 2011.

2. Public institutions only (for Canada and Luxembourg, in tertiary education only; for Italy, except in tertiary education).

3. Year of reference 2013.

Sources: OECD. Argentina, China, Colombia, India, Indonesia, Saudi Arabia, South Africa: UNESCO Institute for Statistics. Latvia: Eurostat. See Annex 3 for notes (www.oecd.org/education/education-at-a-glance-19991487.htm).

Please refer to the Reader's Guide for information concerning symbols for missing data and abbreviations.

StatLink 첵ㄴ http://dx.doi.org/10.1787/888933285378 
Table B1.5a. Change in expenditure per student by educational institutions for all services, relative to different factors, at the primary, secondary and post-secondary non-tertiary levels of education $(2000,2005,2008,2010,2011,2012)$

Index of change (GDP deflator $2005=100$, constant prices )

\begin{tabular}{|c|c|c|c|c|c|c|c|c|c|c|c|c|c|c|c|}
\hline & \multicolumn{15}{|c|}{ Primary, secondary and post-secondary non-tertiary } \\
\hline & \multicolumn{5}{|c|}{$\begin{array}{l}\text { Change in expenditure } \\
(2005=100)\end{array}$} & \multicolumn{5}{|c|}{$\begin{array}{l}\text { Change in the number of students } \\
\qquad(2005=100)\end{array}$} & \multicolumn{5}{|c|}{$\begin{array}{l}\text { Change in expenditure per student } \\
(2005=100)\end{array}$} \\
\hline & 2000 & 2008 & 2010 & 2011 & 2012 & 2000 & 2008 & 2010 & 2011 & 2012 & 2000 & 2008 & 2010 & 2011 & 2012 \\
\hline & (1) & (2) & (3) & (4) & (5) & (6) & (7) & (8) & (9) & (10) & (11) & (12) & (13) & (14) & (15) \\
\hline $\begin{array}{l}\text { Australia } \\
\text { Austria }\end{array}$ & $\begin{array}{c}83 \\
\mathrm{~m}\end{array}$ & $\begin{array}{r}110 \\
\mathrm{~m}\end{array}$ & $\begin{array}{r}133 \\
\mathrm{~m}\end{array}$ & $\begin{array}{r}130 \\
\mathrm{~m}\end{array}$ & $\begin{array}{r}130 \\
\mathrm{~m}\end{array}$ & $\begin{array}{c}93 \\
\mathrm{~m}\end{array}$ & $\begin{array}{r}100 \\
\mathrm{~m}\end{array}$ & $\begin{array}{r}101 \\
\mathrm{~m}\end{array}$ & $\begin{array}{r}103 \\
\mathrm{~m}\end{array}$ & $\begin{array}{r}106 \\
\mathrm{~m}\end{array}$ & $\begin{array}{c}\mathbf{8 9} \\
\mathbf{m}\end{array}$ & $\begin{array}{r}110 \\
\mathbf{m}\end{array}$ & $\begin{array}{r}131 \\
\mathbf{m}\end{array}$ & $\begin{array}{r}126 \\
\mathrm{~m}\end{array}$ & $\begin{array}{r}123 \\
\mathrm{~m}\end{array}$ \\
\hline Belgium & $\mathrm{m}$ & 113 & 112 & 113 & 115 & 91 & 96 & 95 & 96 & 96 & $\mathbf{m}$ & 117 & 117 & 118 & 119 \\
\hline Canada ${ }^{1,2,4}$ & 84 & 105 & 117 & 118 & 115 & 99 & 100 & 98 & 97 & 96 & 84 & 106 & 119 & 121 & 119 \\
\hline Chile $^{3}$ & 96 & 129 & 126 & 147 & 140 & 99 & 96 & 93 & 91 & 90 & 97 & 134 & 135 & 162 & 156 \\
\hline Czech Republic & 77 & 106 & 110 & 113 & 114 & 107 & 93 & 89 & 87 & 85 & 72 & 114 & 124 & 130 & 134 \\
\hline Denmark & 86 & 99 & 108 & 100 & 81 & 95 & $\mathrm{~m}$ & 105 & 111 & $\mathrm{~m}$ & 90 & $\mathbf{m}$ & 103 & 90 & $\mathbf{m}$ \\
\hline Estonia & $\mathrm{m}$ & 124 & 109 & 104 & 105 & 121 & 90 & 85 & 83 & 81 & $\mathbf{m}$ & 138 & 129 & 125 & 130 \\
\hline Finland & 83 & 107 & 112 & 113 & 112 & 95 & 101 & 100 & 99 & 98 & 87 & 107 & 112 & 114 & 114 \\
\hline France & 99 & 103 & 106 & 105 & 104 & 102 & 100 & 100 & 100 & 101 & 98 & 103 & 106 & 105 & 103 \\
\hline Germany & $\mathrm{m}$ & $\mathrm{m}$ & $\mathrm{m}$ & $\mathrm{m}$ & $\mathrm{m}$ & $\mathrm{m}$ & $\mathrm{m}$ & $\mathrm{m}$ & $\mathrm{m}$ & $\mathrm{m}$ & $\mathbf{m}$ & $\mathbf{m}$ & $\mathbf{m}$ & $\mathbf{m}$ & $\mathbf{m}$ \\
\hline Greece $^{1}$ & 77 & $\mathrm{~m}$ & $\mathrm{~m}$ & $\mathrm{~m}$ & $\mathrm{~m}$ & 101 & $\mathrm{~m}$ & $\mathrm{~m}$ & $\mathrm{~m}$ & $\mathrm{~m}$ & 77 & $\mathbf{m}$ & $\mathbf{m}$ & m & $\mathbf{m}$ \\
\hline Hungary $^{5}$ & 68 & 96 & 85 & 79 & 75 & 104 & 96 & 94 & 92 & 91 & 66 & 100 & 90 & 86 & 83 \\
\hline Iceland & 73 & 108 & 96 & 99 & 98 & 94 & 101 & 101 & 101 & 100 & 77 & 106 & 95 & 98 & 98 \\
\hline Ireland $^{4}$ & 69 & 131 & 140 & 138 & 139 & 97 & 104 & 108 & 109 & 109 & 71 & 126 & 129 & 127 & 127 \\
\hline Israel & 99 & 120 & 130 & 144 & 154 & 94 & 104 & 108 & 111 & 112 & 106 & 115 & 120 & 130 & 138 \\
\hline Italy 4,6 & 96 & 104 & 97 & 93 & 90 & 99 & 100 & 100 & 101 & 99 & 98 & 104 & 97 & 92 & 90 \\
\hline Japan $^{1}$ & 99 & 102 & 105 & 105 & 106 & 109 & 97 & 96 & 95 & 94 & 90 & 105 & 109 & 110 & 113 \\
\hline Korea & 69 & 115 & 126 & 127 & 125 & 102 & 98 & 93 & 90 & 86 & 67 & 118 & 135 & 142 & 145 \\
\hline Luxembourg ${ }^{4,5}$ & $\mathrm{~m}$ & $\mathrm{~m}$ & 105 & 101 & 96 & $\mathrm{~m}$ & $\mathrm{~m}$ & $\mathrm{~m}$ & $\mathrm{~m}$ & $\mathrm{~m}$ & $\mathbf{m}$ & $\mathbf{m}$ & $\mathbf{m}$ & $\mathbf{m}$ & $\mathbf{m}$ \\
\hline Mexico & 80 & 103 & 111 & 116 & 119 & 95 & 103 & 105 & 106 & 107 & 85 & 100 & 106 & 109 & 111 \\
\hline Netherlands & 82 & 106 & 114 & 113 & 113 & 97 & 101 & 102 & 102 & 101 & 85 & 105 & 112 & 111 & 112 \\
\hline New Zealand & $\mathrm{m}$ & $\mathrm{m}$ & $\mathrm{m}$ & $\mathrm{m}$ & $\mathrm{m}$ & $\mathrm{m}$ & $\mathrm{m}$ & $\mathrm{m}$ & $\mathrm{m}$ & $\mathrm{m}$ & $\mathbf{m}$ & $\mathbf{m}$ & $\mathbf{m}$ & $\mathbf{m}$ & $\mathbf{m}$ \\
\hline Norway ${ }^{1,5}$ & 87 & 107 & 113 & 112 & 112 & 95 & 102 & 102 & 102 & 104 & 92 & 105 & 111 & 109 & 108 \\
\hline Poland & 90 & 114 & 122 & 120 & 125 & 110 & 88 & 83 & 80 & 77 & 82 & 129 & 147 & 149 & 162 \\
\hline Portugal $^{1,4}$ & 100 & 95 & 108 & 101 & 123 & 111 & 100 & 99 & 97 & 97 & 90 & 96 & 109 & 104 & 126 \\
\hline Slovak Republic ${ }^{1}$ & 74 & 115 & 135 & 125 & 125 & 108 & 90 & 84 & 82 & 79 & 68 & 128 & 159 & 154 & 159 \\
\hline Slovenia & $\mathrm{m}$ & 104 & 103 & 101 & 98 & $\mathrm{~m}$ & 93 & 90 & 90 & 89 & $\mathbf{m}$ & 112 & 114 & 113 & 109 \\
\hline Spain & 92 & 115 & 119 & 116 & 110 & 107 & 102 & 105 & 107 & 107 & 86 & 113 & 113 & 109 & 103 \\
\hline Sweden & 88 & 103 & 102 & 102 & 103 & 98 & 97 & 91 & 91 & 91 & 90 & 107 & 112 & 113 & 113 \\
\hline Switzerland ${ }^{4}$ & 87 & 103 & 107 & 109 & 110 & 100 & 100 & 98 & 97 & 97 & 86 & 102 & 109 & 112 & 114 \\
\hline Turkey ${ }^{4,5}$ & 71 & 121 & 147 & 149 & 165 & 92 & 102 & 106 & 105 & 107 & 77 & 119 & 138 & 141 & 155 \\
\hline United Kingdom & $\mathrm{m}$ & 99 & 106 & 109 & 112 & 113 & 99 & 100 & 102 & 104 & $\mathbf{m}$ & 100 & 106 & 107 & 108 \\
\hline United States $^{1}$ & 86 & 111 & 109 & 107 & 104 & 98 & 106 & 100 & 100 & 99 & 88 & 105 & 110 & 107 & 105 \\
\hline OECD average & 84 & 109 & 114 & 114 & 114 & 101 & 99 & 98 & 97 & 97 & 85 & 112 & 117 & 118 & 121 \\
\hline EU21 average & 84 & 108 & 111 & 108 & 108 & 103 & 97 & 96 & 96 & 94 & 83 & 112 & 117 & 115 & 118 \\
\hline Argentina & $\mathrm{m}$ & $\mathrm{m}$ & $\mathrm{m}$ & $\mathrm{m}$ & $\mathrm{m}$ & $\mathrm{m}$ & $\mathrm{m}$ & $\mathrm{m}$ & $\mathrm{m}$ & $\mathrm{m}$ & $\mathbf{m}$ & $\mathbf{m}$ & $\mathbf{m}$ & $\mathbf{m}$ & $\mathbf{m}$ \\
\hline Brazil $1^{4,5}$ & 66 & 146 & 170 & 175 & 182 & 98 & 96 & 91 & 89 & 87 & 67 & 152 & 187 & 197 & 210 \\
\hline China & $\mathrm{m}$ & $\mathrm{m}$ & $\mathrm{m}$ & $\mathrm{m}$ & $\mathrm{m}$ & $\mathrm{m}$ & $\mathrm{m}$ & $\mathrm{m}$ & $\mathrm{m}$ & $\mathrm{m}$ & $\mathbf{m}$ & $\mathbf{m}$ & $\mathbf{m}$ & $\mathbf{m}$ & $\mathbf{m}$ \\
\hline Colombia & $\mathrm{m}$ & $\mathrm{m}$ & $\mathrm{m}$ & $\mathrm{m}$ & $\mathrm{m}$ & $\mathrm{m}$ & $\mathrm{m}$ & $\mathrm{m}$ & $\mathrm{m}$ & $\mathrm{m}$ & $\mathbf{m}$ & $\mathbf{m}$ & $\mathbf{m}$ & $\mathbf{m}$ & $\mathbf{m}$ \\
\hline India & $\mathrm{m}$ & $\mathrm{m}$ & $\mathrm{m}$ & $\mathrm{m}$ & $\mathrm{m}$ & $\mathrm{m}$ & $\mathrm{m}$ & $\mathrm{m}$ & $\mathrm{m}$ & $\mathrm{m}$ & $\mathbf{m}$ & $\mathbf{m}$ & $\mathbf{m}$ & m & $\mathbf{m}$ \\
\hline Indonesia & $\mathrm{m}$ & $\mathrm{m}$ & $\mathrm{m}$ & $\mathrm{m}$ & $\mathrm{m}$ & $\mathrm{m}$ & $\mathrm{m}$ & $\mathrm{m}$ & $\mathrm{m}$ & $\mathrm{m}$ & $\mathbf{m}$ & $\mathbf{m}$ & $\mathbf{m}$ & m & $\mathbf{m}$ \\
\hline Latvia & $\mathrm{m}$ & $\mathrm{m}$ & $\mathrm{m}$ & $\mathrm{m}$ & $\mathrm{m}$ & $\mathrm{m}$ & $\mathrm{m}$ & $\mathrm{m}$ & $\mathrm{m}$ & $\mathrm{m}$ & $\mathbf{m}$ & $\mathbf{m}$ & $\mathbf{m}$ & $\mathbf{m}$ & $\mathbf{m}$ \\
\hline Russian Federation ${ }^{4,5}$ & 66 & 132 & 126 & 129 & 151 & $\mathrm{~m}$ & 88 & 87 & 88 & 88 & $\mathbf{m}$ & 150 & 144 & 147 & 172 \\
\hline Saudi Arabia & $\mathrm{m}$ & $\mathrm{m}$ & $\mathrm{m}$ & $\mathrm{m}$ & $\mathrm{m}$ & $\mathrm{m}$ & $\mathrm{m}$ & $\mathrm{m}$ & $\mathrm{m}$ & $\mathrm{m}$ & $\mathbf{m}$ & $\mathbf{m}$ & $\mathbf{m}$ & $\mathbf{m}$ & $\mathbf{m}$ \\
\hline South Africa & $\mathrm{m}$ & $\mathrm{m}$ & $\mathrm{m}$ & $\mathrm{m}$ & $\mathrm{m}$ & $\mathrm{m}$ & $\mathrm{m}$ & $\mathrm{m}$ & $\mathrm{m}$ & $\mathrm{m}$ & $\mathbf{m}$ & $\mathbf{m}$ & $\mathbf{m}$ & $\mathbf{m}$ & m \\
\hline
\end{tabular}

\begin{tabular}{l|c|c|c|c|c|c|c|c|c|c|c|c|c|c|c} 
G20 average & $\mathrm{m}$ & $\mathrm{m}$ & $\mathrm{m}$ & $\mathrm{m}$ & $\mathrm{m}$ & $\mathrm{m}$ & $\mathrm{m}$ & $\mathrm{m}$ & $\mathrm{m}$ & $\mathrm{m}$ & $\mathbf{m}$ & $\mathbf{m}$ & $\mathbf{m}$ & $\mathbf{m}$ & $\mathbf{m}$ \\
\hline
\end{tabular}

1. Some levels of education are included with others. Refer to "x" code in Table B1.1a for details.

2. Year of reference 2011 instead of 2012. Year of reference 2004 instead of 2005.

3. Year of reference 2013 instead of 2012. Year of reference 2006 instead of 2005.

4. Public institutions only.

5. Public expenditure only.

6. Excluding post-secondary non-tertiary education.

Source: OECD. See Annex 3 for notes (www.oecd.org/education/education-at-a-glance-19991487.htm)

Please refer to the Reader's Guide for information concerning symbols for missing data and abbreviations.

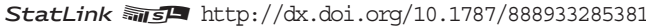


Table B1.5b. Change in expenditure per student by tertiary educational institutions for all services, relative to different factors $(2000,2005,2008,2010,2011,2012)$

Index of change (GDP deflator $2005=100$, constant prices)

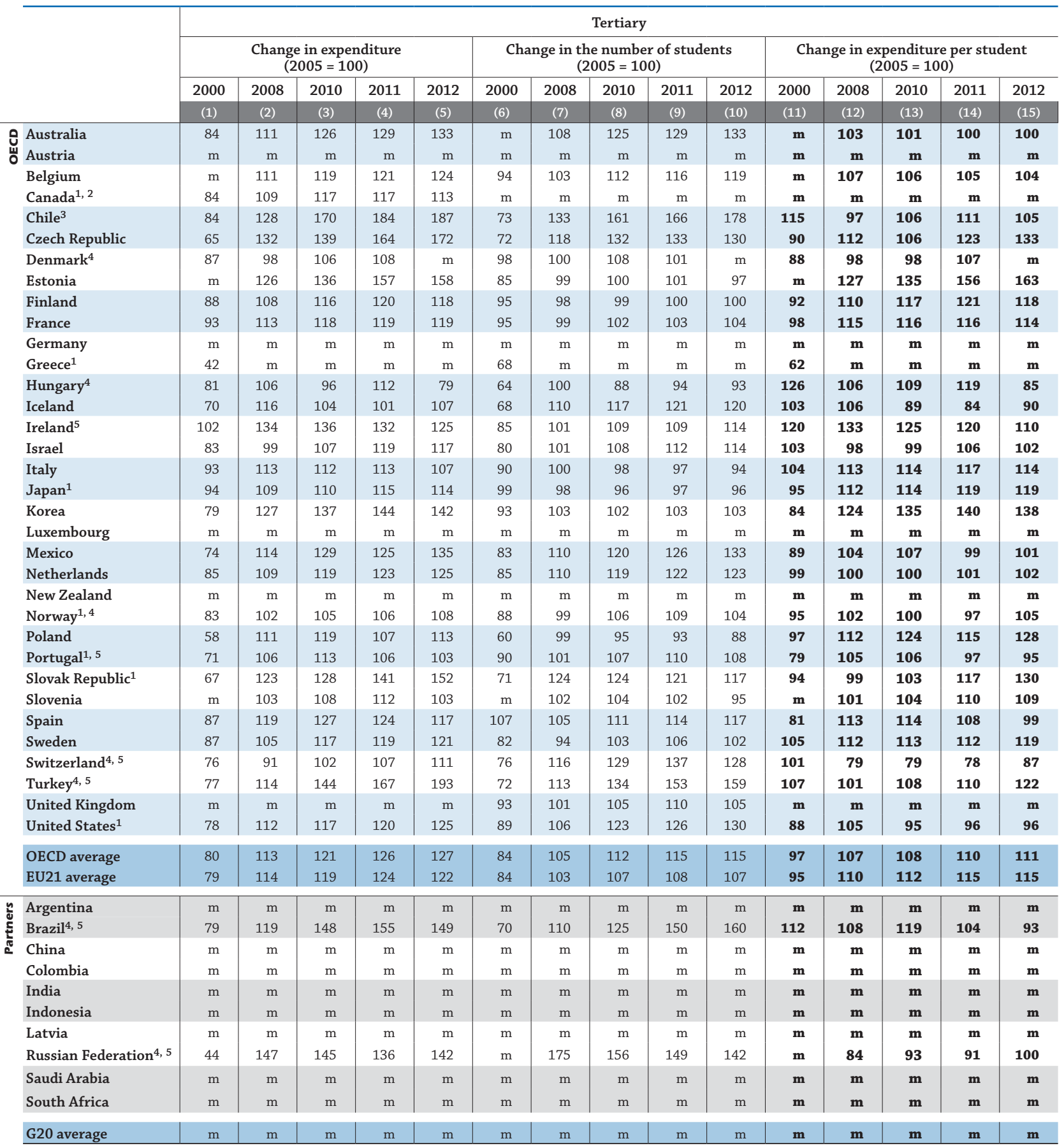

1. Some levels of education are included with others. Refer to " $\mathrm{x}$ " code in Table B1.1a for details.

2. Year of reference 2011 instead of 2012. Year of reference 2004 instead of 2005.

3. Year of reference 2013 instead of 2012. Year of reference 2006 instead of 2005.

4. Public expenditure only.

5. Public institutions only.

Source: OECD. See Annex 3 for notes (www.oecd.org/education/education-at-a-glance-19991487.htm).

Please refer to the Reader's Guide for information concerning symbols for missing data and abbreviations.

StatLink 제개 http://dx.doi.org/10.1787/888933285399 
Table B1.6. Annual expenditure per student by secondary educational institutions for all services, by type of programme (2012)

In equivalent USD converted using PPPs for GDP, by level of education, based on full-time equivalents

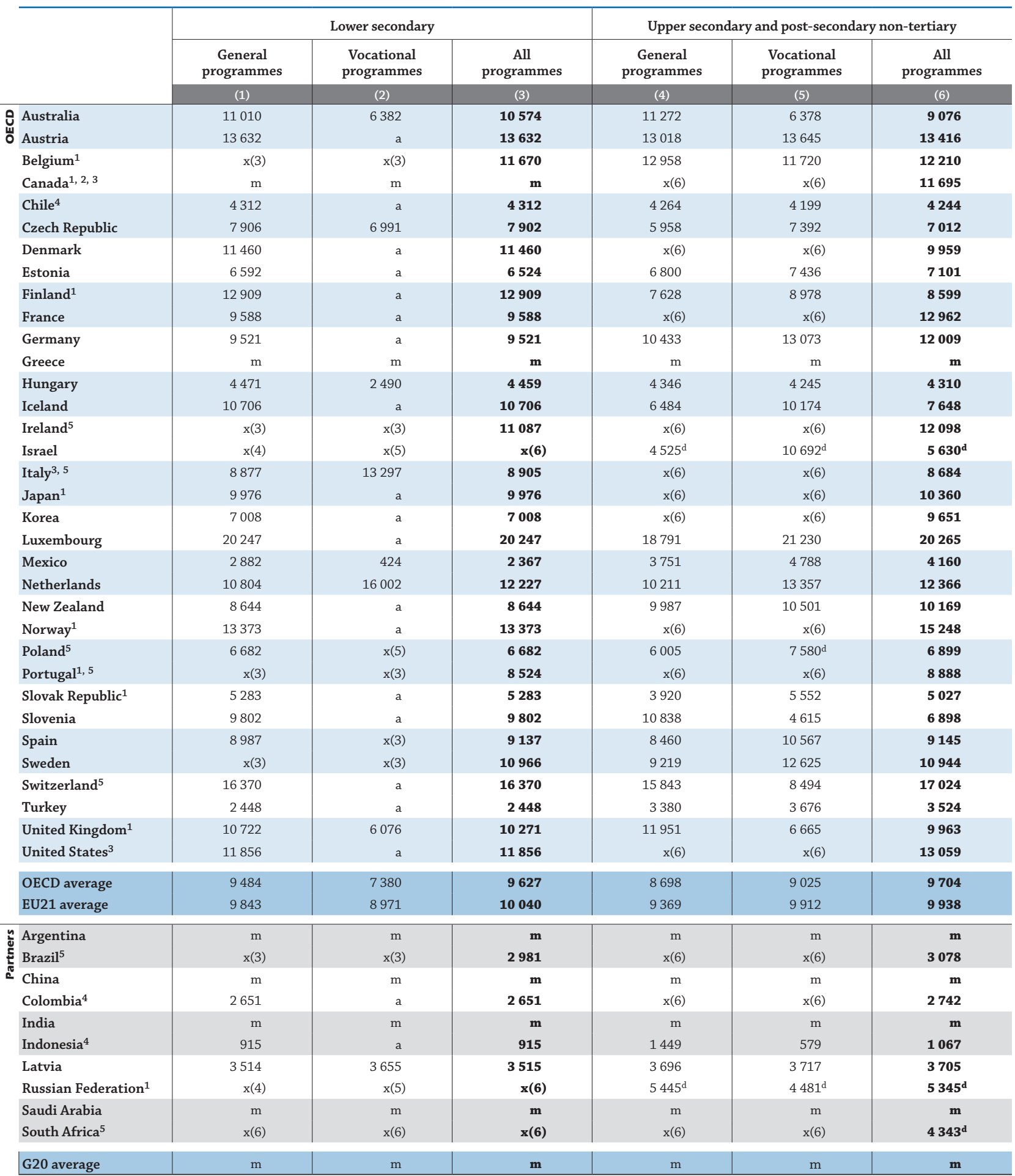

1. Some levels of education are included with others. Refer to "x" code in Table B1.1a for details.

2. Year of reference 2011.

3. Excludes post-secondary non-tertiary education.

4. Year of reference 2013.

5. Public institutions only.

Source: OECD. Argentina, China, Colombia, India, Indonesia, Saudi Arabia, South Africa: UNESCO Institute for Statistics. Latvia: Eurostat. See Annex 3 for notes (www.oecd.org/education/education-at-a-glance-19991487.htm).

Please refer to the Reader's Guide for information concerning symbols for missing data and abbreviations.

StatLink 젝ㄴ http://dx.doi.org/10.1787/888933285401 



\section{WHAT PROPORTION OF NATIONAL WEALTH IS SPENT ON EDUCATION?}

- In 2012, OECD countries spent an average of 5.3\% of their GDP on educational institutions from primary to tertiary education; 11 countries with available data (Canada, Chile, Colombia, Iceland, Israel, Korea, New Zealand, Norway, South Africa, the United Kingdom and the United States) spent $6 \%$ or more.

- Between 2000 and 2012, expenditure on primary to tertiary education increased at a faster rate than GDP growth in more than two out of three countries for which data are available. In the other countries, the share of GDP devoted to education decreased by less than 0.5 percentage point.

- Since the beginning of the economic crisis in 2008 and up to 2010, GDP decreased, in real terms, in 20 of 36 countries with available data, while public expenditure on educational institutions fell in only six countries. As a result, public expenditure as a percentage of GDP decreased in five countries during this period. Still, GDP rose, in real terms, in most countries between 2010 and 2012, and public expenditure on educational institutions fell in more than one out of three OECD countries as a result of fiscal-consolidation policies.

\section{Chart B2.1. Expenditure on primary to tertiary education institutions as a percentage of GDP (2012) \\ From public and private sources, including undistributed programmes}

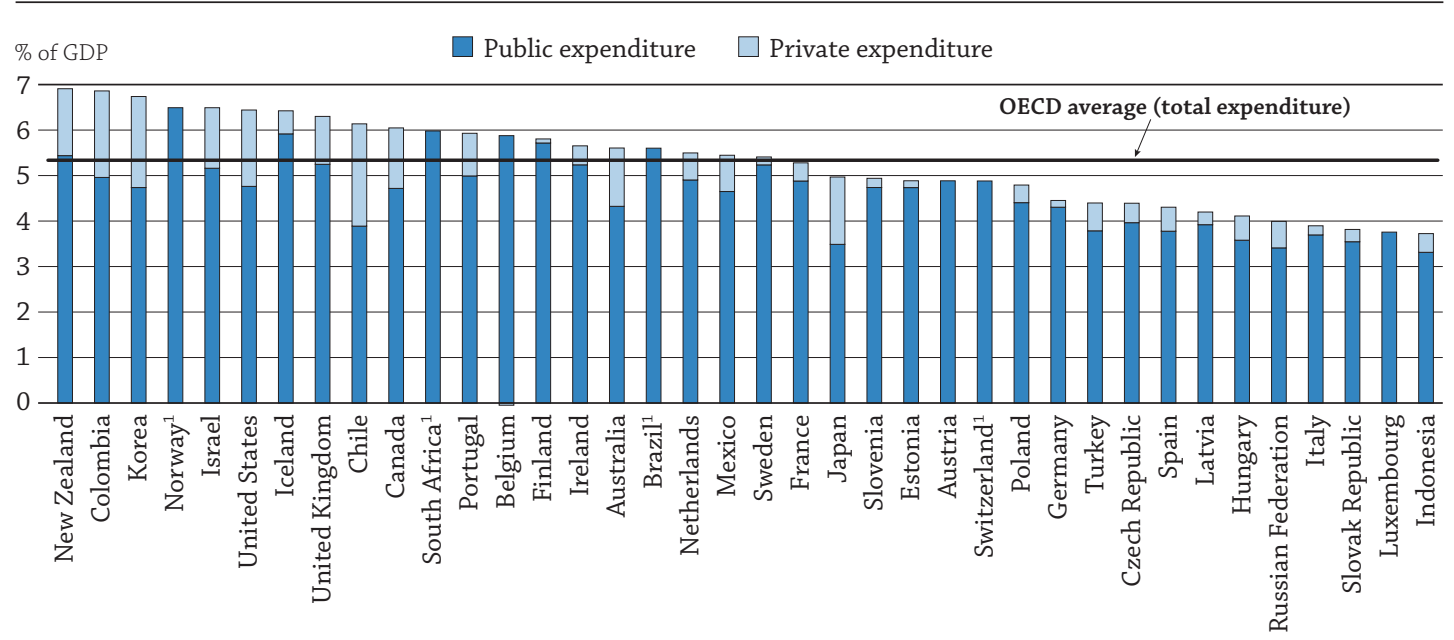

1. Public expenditure only (for Switzerland, in tertiary education only; for Norway, in primary, secondary and post-secondary non-tertiary education only).

Countries are ranked in descending order of expenditure from both public and private sources on educational institutions

Source: OECD. Table B2.3. See Annex 3 for notes (www.oecd.org/education/education-at-a-glance-19991487.htm).

StatLink 젬ㄴ http://dx.doi.org/10.1787/888933283940

\section{Context}

Countries invest in educational institutions to help foster economic growth, enhance productivity, contribute to personal and social development, and reduce social inequality, among other reasons. The proportion of education expenditure relative to GDP depends on the different preferences of various public and private actors. Nevertheless, expenditure on education largely comes from public budgets and is closely scrutinised by governments. During economic downturns, even core sectors like education can be subject to budget cuts.

The level of expenditure on educational institutions is affected by the size of a country's school-age population, enrolment rates, level of teachers' salaries, and the organisation and delivery of instruction. At the primary and lower secondary levels of education (corresponding broadly to the 5-14 year-old population), enrolment rates are close to $100 \%$ in OECD countries, and changes in the number of students are closely related to demographic changes. This is not as much the case in upper secondary and tertiary education, because part of the concerned population has left the education system (see Indicator $\mathrm{C} 1$ ). 
This indicator presents a measure of expenditure on educational institutions relative to a nation's wealth. National wealth is estimated based on GDP, and expenditure on education includes spending by governments, enterprises and individual students and their families.

\section{Other findings}

- Primary, secondary and post-secondary non-tertiary education accounts for two-thirds of expenditure on primary to tertiary educational institutions, or 3.7\% of GDP, on average across OECD countries. New Zealand spends the most among OECD and partner countries, with $5.0 \%$ of its GDP devoted to these levels of education, while the Czech Republic, Hungary, Indonesia, Japan, Latvia, the Russian Federation and the Slovak Republic spend less than 3\% of their GDP on these levels of education.

- Tertiary education accounts for more than one-quarter of expenditure on educational institutions, or $1.5 \%$ of GDP, on average across OECD countries. Canada, Chile, Korea and the United States spend between $2.3 \%$ and $2.8 \%$ of their GDP on tertiary institutions.

- Private expenditure on educational institutions as a percentage of GDP is highest at the tertiary level, on average across OECD countries. This share is highest in Chile, Korea and the United States, where it ranges from $1.4 \%$ to $1.5 \%$ of GDP.

\section{Trends}

Between 2008 and 2010, public investment in primary to tertiary education increased by an average of $5 \%$ among OECD countries. However, the growth of public expenditure on educational institutions slowed afterwards, and remained stable between 2010 and 2012, on average across OECD countries. Between 2008 and 2012, the average annual growth rate across OECD countries decreased continuously, from 3\% in 2008-09 to 0\% in 2011-12.

Over the period 2008-10, Estonia, Hungary, Iceland, Italy, the Russian Federation and the United States cut (in real terms) public expenditure on educational institutions, from about $1 \%$ in the United States to $11 \%$ in Hungary and Iceland. Over the period 2010-12, public expenditure continued to decrease in four of these six countries (the exceptions were Iceland and the Russian Federation) and also decreased in seven other countries. Among these 11 countries, the decrease during this period reached 5\% or more in Hungary, Italy, Portugal, Slovenia and Spain. 


\section{Analysis}

\section{Overall investment relative to GDP}

The share of national wealth devoted to educational institutions is substantial in all OECD and partner countries with available data. In 2012, OECD countries spent an average of 5.3\% of their GDP on educational institutions from primary to tertiary education (see Table C2.3 for the share of GDP devoted to early childhood education).

In 2012, expenditure on primary to tertiary educational institutions relative to GDP reached $6 \%$ or more in 11 countries with available data, and exceeded $6.7 \%$ in Colombia, Korea and New Zealand. At the other end of the spectrum, Indonesia, Italy, Luxembourg, the Russian Federation and the Slovak Republic spent less than 4\% of their GDP on education (Chart B2.1 and Table B2.1).

\section{Chart B2.2. Expenditure on educational institutions as a percentage of GDP (2012)} From public and private sources, by level of education and source of funds

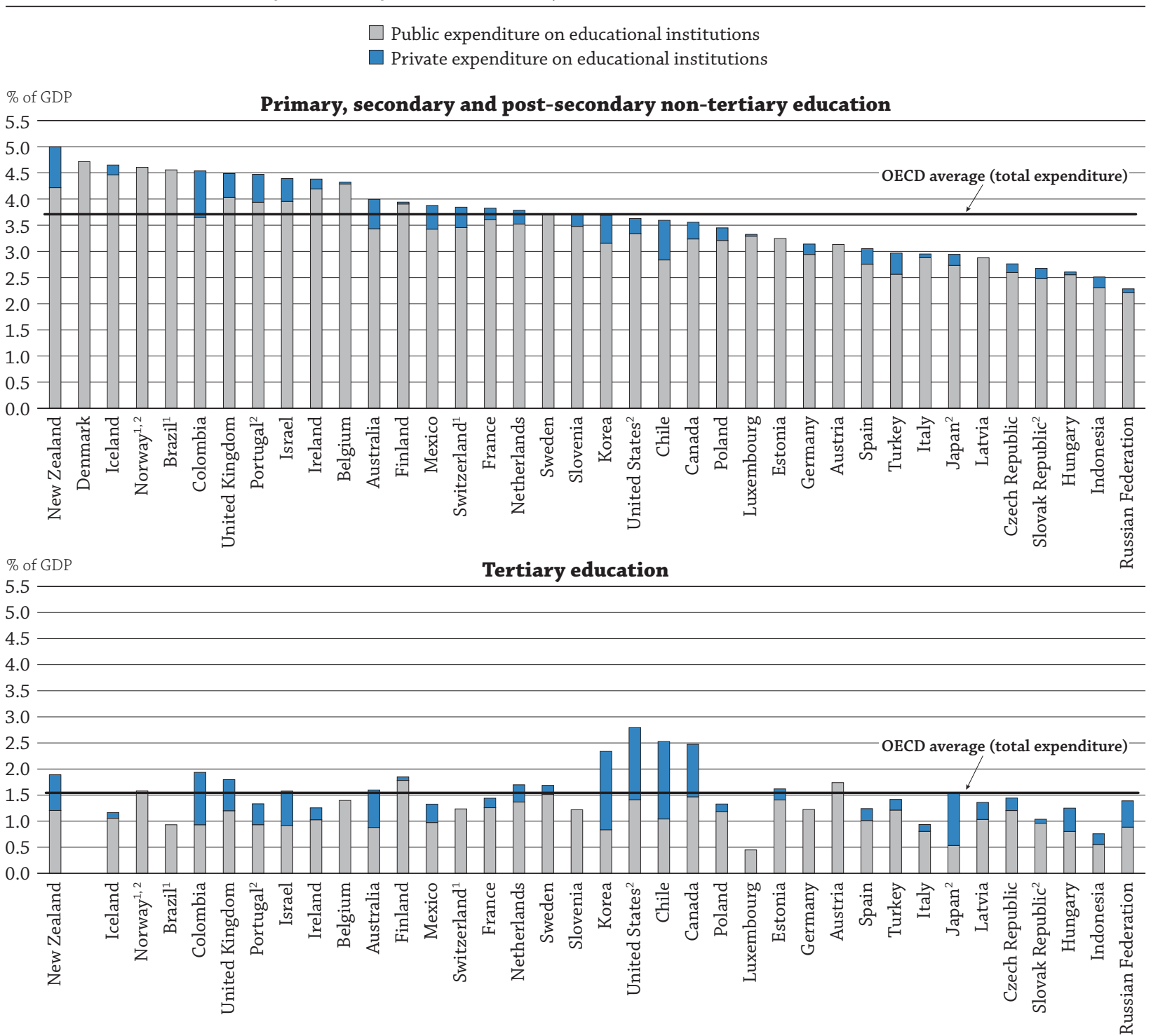

1. Public expenditure only (for Switzerland, in tertiary education only; for Norway, in primary, secondary and post-secondary non-tertiary education only).

2. Some levels of education are included with others. Refer to "x" code in Table B1.1a for details.

Countries are ranked in descending order of expenditure from both public and private sources on educational institutions in primary, secondary and post-secondary non-tertiary education.

Source: OECD. Table B2.3. See Annex 3 for notes (www.oecd.org/education/education-at-a-glance-19991487.htm).

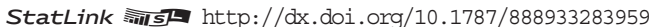




\section{Expenditure on educational institutions, by level of education}

An average of two-thirds of the expenditure on education (excluding early childhood education) in all OECD countries is devoted to primary, secondary and post-secondary non-tertiary education, while a more than a quarter goes to tertiary education and $0.1 \%$ is not distributed to specific levels of education. Primary and lower secondary levels receive about $47 \%$ of educational expenditure in OECD countries, on average. Expenditure on educational institutions depends on the age of the population. In most cases, countries with above-average expenditure on educational institutions relative to GDP are usually those with an above-average proportion of people whose age corresponds to primary and lower secondary education (Table B2.2 and see Indicator C1).

In all OECD and partner countries with available data, the level of national resources devoted to primary, secondary and post-secondary non-tertiary education combined is much larger than the share devoted to tertiary education. This share exceeds $50 \%$ of educational expenditure (excluding early childhood education) in all countries. For primary, secondary and post-secondary non-tertiary education, expenditure as a percentage of GDP ranges from less than 3\% in the Czech Republic, Hungary, Indonesia, Japan, Latvia, the Russian Federation and the Slovak Republic to $5 \%$ in New Zealand.

Expenditure on primary and lower secondary education amounts to $1.5 \%$ or more of GDP in all countries, and reaches 3\% or more in Australia, Brazil, Colombia, Denmark, Iceland, Ireland, Mexico, New Zealand, Norway and the United Kingdom (Table B2.1).

Expenditure on tertiary education amounts to at least 1\% of GDP in nearly all countries except Brazil, Indonesia, Italy (expenditure on short-cycle tertiary programmes is not included), Luxembourg and South Africa, and exceeds 2.3\% in Canada, Chile, Korea and the United States (Table B2.3 and Chart B2.2).

\section{Changes in overall spending on educational institutions between 2000 and 2012}

The increase in the number of students enrolled in upper secondary and tertiary education between 2000 and 2012 was accompanied in most countries by an increase in financial investments at these levels.

Over the period 2000-12, in all countries with comparable data, both expenditure on primary to tertiary educational institutions and GDP increased (see Table X2.3). In Chile, France, Italy, Norway, Poland and Sweden, expenditure on education increased less than GDP, leading to a decrease in expenditure as a proportion of GDP of up to 0.5 percentage point. In all other countries with comparable data, expenditure on educational institutions (primary to tertiary) increased at a faster rate than GDP, resulting in an increase in expenditure on educational institutions as a percentage of GDP (Chart B2.3). The increase was one percentage point or more in Brazil (from $3.1 \%$ to $5.6 \%$ ), Ireland (from $4.3 \%$ to $5.6 \%$ ), the Netherlands (from $4.5 \%$ to $5.5 \%$ ), Portugal (from $4.7 \%$ to $5.8 \%$ ), the Russian Federation (from 2.1\% to $3.7 \%$ ) and Turkey (from $2.5 \%$ to $3.8 \%$ ) (Table B2.2).

\section{Chart B2.3. Expenditure on primary to tertiary education institutions as a percentage of GDP (2000, 2008 and 2012) \\ From public and private sources, excluding undistributed programmes}

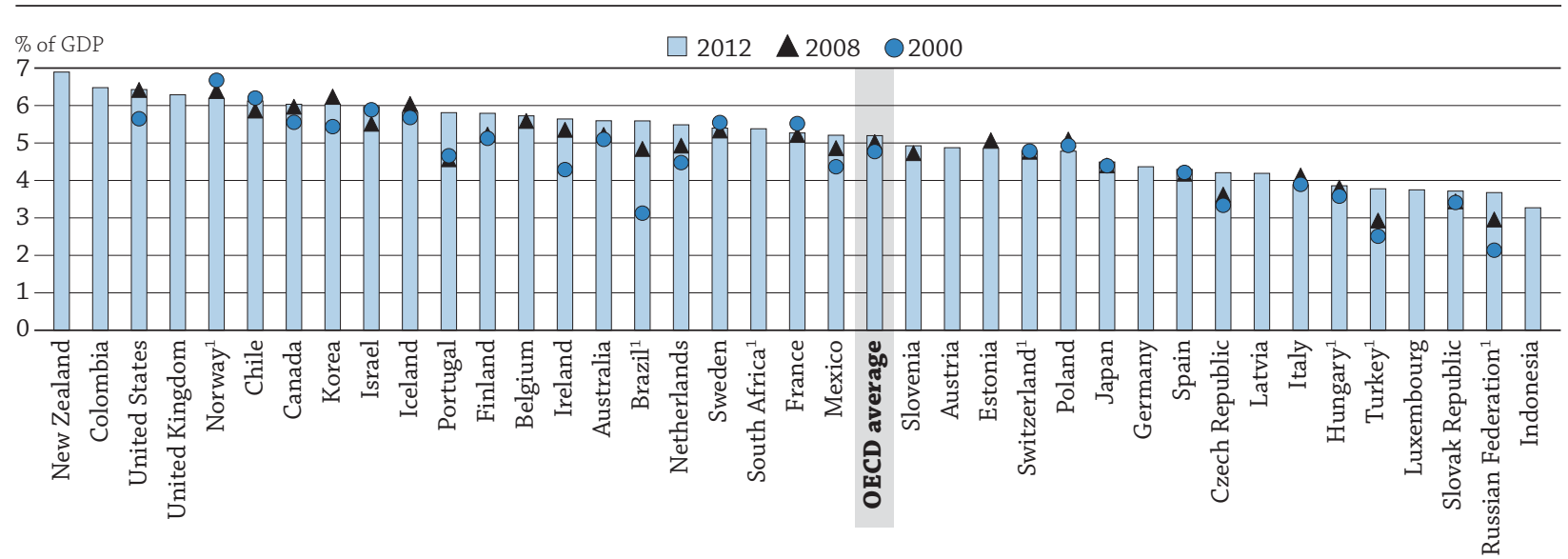

1. Public expenditure only (for Switzerland, in tertiary education only; for Norway, in primary, secondary and post-secondary non-tertiary education only).

Countries are ranked in descending order of expenditure from both public and private sources on educational institutions in 2012

Source: OECD. Table B2.2. See Annex 3 for notes (www.oecd.org/education/education-at-a-glance-19991487.htm).

StatLink 제에 $\mathrm{http}: / / \mathrm{dx}$.doi.org/10.1787/888933283964 
Chart B2.4. Impact of the economic crisis on public expenditure on education and index of change in public expenditure on educational institutions and in GDP (2010 to 2012)

Index of change between 2010 and 2012 in expenditure on educational institutions as a percentage of GDP, primary to tertiary levels of education $(2010=100,2012$ constant prices)

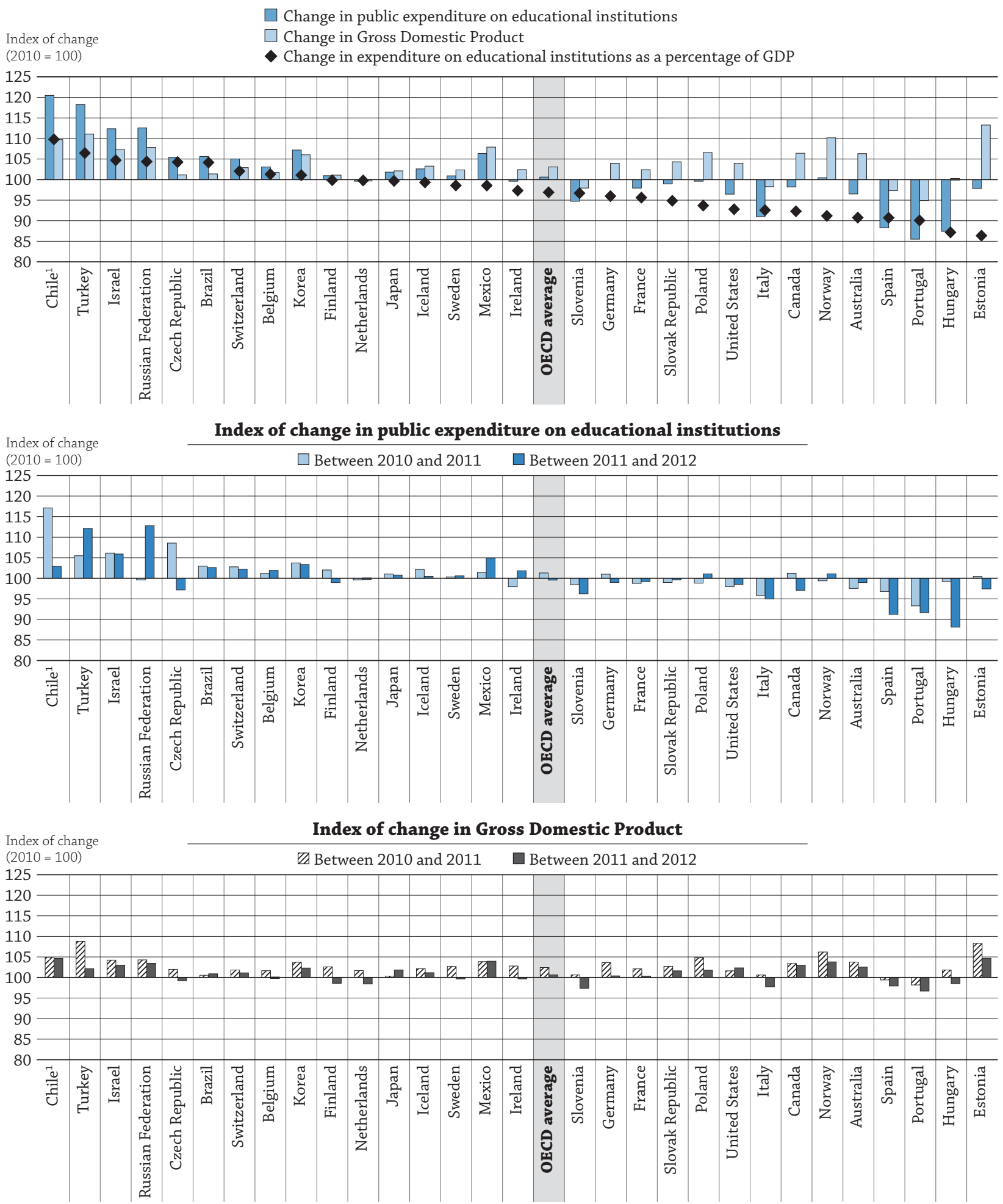

1. Data refer to 2011-2013 instead of 2010-2012.

Countries are ranked in descending order of the change in expenditure on educational institutions as a percentage of GDP.

Source: OECD. Table B2.4. See Annex 3 for notes (www.oecd.org/education/education-at-a-glance-19991487.htm).

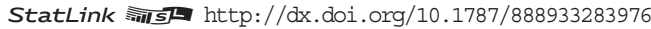


There were similar changes in expenditure on primary, secondary and post-secondary non-tertiary education combined, as well as on tertiary education.

\section{Effect of the economic crisis on public expenditure on educational institutions between 2008 and 2012}

The global economic crisis that began in 2008 had - and is still having - major adverse effects on different sectors of the economy. Data from 2008 to 2012 show clearly the impact of the crisis on the funding of educational institutions, especially when comparing the periods 2008-10 and 2010-12.

Between 2008 and 2010, GDP (expressed in constant prices) fell in the majority of the countries (20 out of 36 countries with available data), and by $5 \%$ or more in Estonia, Finland, Greece, Hungary, Iceland, Ireland and Slovenia. As more than three-quarters of education expenditure in most countries comes from public sources, how did the downturn in GDP growth affect public spending on education? Available figures show that the education sector was still relatively untouched by early budget cuts.

Since public budgets in most countries are approved many months before the funds are actually spent, there are certain built-in rigidities to the funding of education. Moreover, most governments try to protect education from dramatic reductions in public investment.

Among the 36 countries with available data for the 2008-10 period, only six countries cut (in real terms) public expenditure on educational institutions: Estonia (by 10\%), Hungary (by 11\%), Iceland (by 11\%), Italy (by 6\%), the Russian Federation (by 4\%) and the United States (by 1\%). In Hungary, Iceland and Italy, this translated into a decrease in expenditure on educational institutions as a percentage of GDP (as the reduction in expenditure was larger than the decrease in GDP, or as GDP increased at the same time). In the three other countries, the share of GDP devoted to education did not change or increased as the decrease in expenditure was balanced with similar or larger decreases in GDP.

In all other countries, public expenditure on educational institutions increased or was stable, while GDP decreased in some of them. As a result, the share of GDP devoted to education continued to rise between 2008 and 2010 (by $6 \%$, on average across OECD countries), except in Chile and Poland. In Chile and Poland, GDP increased at a faster rate than public expenditure on educational institutions, resulting in a slight decrease of public expenditure on educational institution as a percentage of GDP (Table B2.4).

During 2010-12, the crisis had a stronger impact on public expenditure on education. While GDP decreased between 2008 and 2010 in two-thirds of the countries with available data, it stayed constant or increased between 2010 and 2012 in all countries except five. The GDP decreased in Greece (by 15\%), Italy (by 2\%), Portugal (by 5\%), Slovenia (by 2\%) and Spain (by 3\%) (Chart B2.4).

Public expenditure on educational institutions started to fall between 2010 and 2012 - later than decreases in GDP, as a result of the necessary time gap to adjust public budgets. Public expenditure decreased between 2010 and 2011, or between 2011 and 2012, or continuously over the two-year period in a larger number of countries than between 2008 and 2010. Over the whole period 2010-12, public expenditure on educational institutions decreased in 11 countries, and by $5 \%$ or more in Hungary, Italy, Portugal, Slovenia and Spain. Combined with increases in GDP, this led to decreases of public expenditure on education as a percentage of GDP in all of these 11 countries, most significantly in Estonia (by 14\%) and Hungary (by 13\%).

\section{Methodology}

Data refer to the financial year 2012 and are based on the UOE data collection on education statistics administered by the OECD in 2014 (for details see Annex 3 at www.oecd.org/education/education-at-a-glance-19991487.htm).

Note regarding data from Israel

The statistical data for Israel are supplied by and are under the responsibility of the relevant Israeli authorities. The use of such data by the OECD is without prejudice to the status of the Golan Heights, East Jerusalem and Israeli settlements in the West Bank under the terms of international law. 


\section{Indicator B2 Tables}

StatLink 齐可 http://dx.doi.org/10.1787/888933285413

Table B2.1 Expenditure on educational institutions as a percentage of GDP, by level of education (2012)

Table B2.2 Trends in expenditure on educational institutions as a percentage of GDP, by level of education (2000, 2005, 2008, 2010, 2011, 2012)

Table B2.3 Expenditure on educational institutions as a percentage of GDP, by source of funding and level of education (2012)

Table B2.4 Change in public expenditure on educational institutions, as a percentage of GDP $(2008,2009,2010,2011,2012)$

Cut-off date for the data: 23 October 2015. Updates can be found on line at http://dx.doi.org/10.1787/eag-data-en. 
Table B2.1. Expenditure on educational institutions as a percentage of GDP, by level of education (2012) From public and private sources of funds ${ }^{1}$

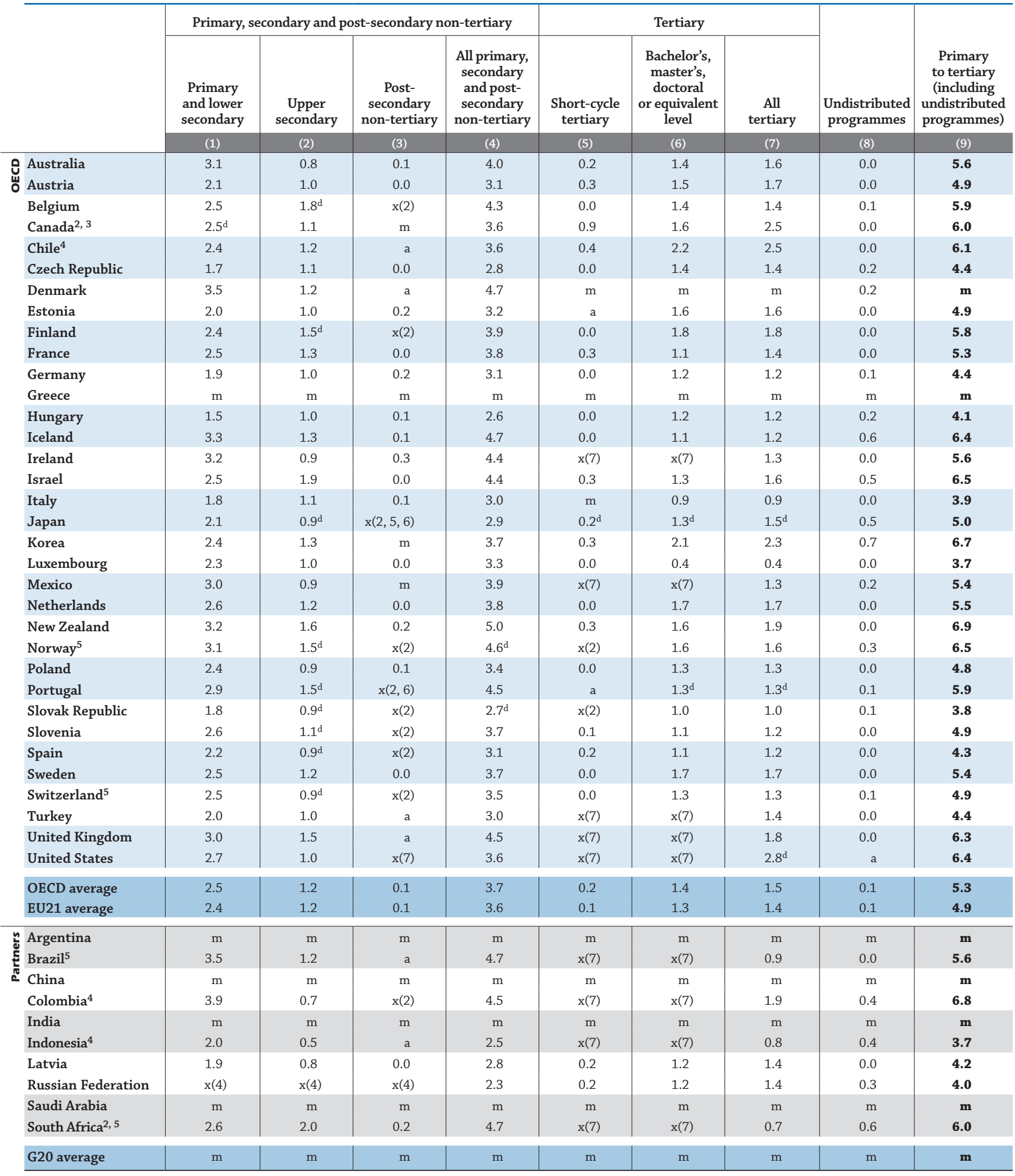

Note: Data on early childhood education is available in Indicator C2.

1. Including international sources.

2. Column 1 only refers to primary education and column 2 refers to all secondary education.

3. Year of reference 2011.

4. Year of reference 2013

5. Public expenditure only (for Switzerland, in tertiary education only; for Norway, in primary, secondary and post-secondary non-tertiary education only).

Source: OECD. Argentina, China, Colombia, India, Indonesia, Saudi Arabia, South Africa: UNESCO Institute for Statistics. Latvia: Eurostat. See Annex 3 for notes

(www.oecd.org/education/education-at-a-glance-19991487.htm).

Please refer to the Reader's Guide for information concerning symbols for missing data and abbreviations.

StatLink 젝ㄴ http://dx.doi.org/10.1787/888933285427 
Table B2.2. Trends in expenditure on educational institutions as a percentage of GDP, by level of education (2000, 2005, 2008, 2010, 2011, 2012)

From public and private sources, by year

\begin{tabular}{|c|c|c|c|c|c|c|c|c|c|c|c|c|c|c|c|c|c|c|}
\hline & \multicolumn{6}{|c|}{$\begin{array}{c}\text { Primary, secondary } \\
\text { and post-secondary non-tertiary }\end{array}$} & \multicolumn{6}{|c|}{ Tertiary } & \multicolumn{6}{|c|}{$\begin{array}{c}\text { Primary to tertiary } \\
\text { (excluding undistributed programmes) }\end{array}$} \\
\hline & 2000 & 2005 & 2008 & 2010 & 2011 & 2012 & 2000 & 2005 & 2008 & 2010 & 2011 & 2012 & 2000 & 2005 & 2008 & 2010 & 2011 & 2012 \\
\hline & (1) & (2) & (3) & (4) & (5) & (6) & (7) & (8) & (9) & $(10)$ & (11) & (12) & (13) & (14) & (15) & (16) & (17) & (18) \\
\hline Australia & 3.6 & 3.7 & 3.7 & 4.3 & 4.1 & 4.0 & 1.4 & 1.5 & 1.5 & 1.6 & 1.6 & 1.6 & 5.1 & 5.2 & 5.2 & 5.9 & 5.7 & 5.6 \\
\hline Austria & $\mathrm{m}$ & $\mathrm{m}$ & $\mathrm{m}$ & $\mathrm{m}$ & $\mathrm{m}$ & 3.1 & $\mathrm{~m}$ & $\mathrm{~m}$ & $\mathrm{~m}$ & $\mathrm{~m}$ & $\mathrm{~m}$ & 1.7 & $\mathbf{m}$ & $\mathbf{m}$ & $\mathbf{m}$ & $\mathbf{m}$ & m & 4.9 \\
\hline Belgium & $\mathrm{m}$ & 4.1 & 4.3 & 4.3 & 4.2 & 4.3 & $\mathrm{~m}$ & 1.2 & 1.3 & 1.4 & 1.4 & 1.4 & $\mathbf{m}$ & 5.3 & 5.6 & 5.7 & 5.6 & 5.7 \\
\hline Canada $^{1,2}$ & 3.3 & 3.5 & 3.4 & 3.9 & 3.8 & 3.6 & 2.3 & 2.5 & 2.5 & 2.7 & 2.6 & 2.5 & 5.5 & 6.0 & 6.0 & 6.6 & 6.4 & 6.0 \\
\hline Chile $^{3}$ & 4.2 & 3.2 & 3.9 & 3.4 & 3.7 & 3.6 & 2.0 & 1.7 & 2.0 & 2.4 & 2.5 & 2.5 & 6.2 & 4.9 & 5.9 & 5.7 & 6.2 & 6.1 \\
\hline Czech Republic & 2.6 & 2.8 & 2.5 & 2.7 & 2.7 & 2.8 & 0.8 & 1.0 & 1.1 & 1.2 & 1.4 & 1.4 & 3.3 & 3.7 & 3.6 & 3.9 & 4.1 & 4.2 \\
\hline Denmark & 4.0 & 4.4 & 4.1 & 4.7 & 4.3 & 4.7 & 1.5 & 1.7 & 1.6 & 1.8 & 1.9 & $\mathrm{~m}$ & 5.5 & 6.0 & 5.8 & 6.5 & 6.1 & $\mathbf{m}$ \\
\hline Estonia & $\mathrm{m}$ & 3.4 & 3.8 & 3.8 & 3.3 & 3.2 & $\mathrm{~m}$ & 1.1 & 1.3 & 1.6 & 1.7 & 1.6 & $\mathbf{m}$ & 4.6 & 5.1 & 5.4 & 5.0 & 4.9 \\
\hline Finland & 3.5 & 3.7 & 3.6 & 4.0 & 3.9 & 3.9 & 1.6 & 1.7 & 1.6 & 1.8 & 1.9 & 1.8 & 5.1 & 5.4 & 5.2 & 5.8 & 5.8 & 5.8 \\
\hline France & 4.2 & 3.9 & 3.8 & 4.0 & 3.9 & 3.8 & 1.3 & 1.3 & 1.4 & 1.5 & 1.5 & 1.4 & 5.5 & 5.2 & 5.2 & 5.4 & 5.3 & 5.3 \\
\hline Germany & $\mathrm{m}$ & $\mathrm{m}$ & $\mathrm{m}$ & 3.3 & 3.2 & 3.1 & $\mathrm{~m}$ & $\mathrm{~m}$ & $\mathrm{~m}$ & 1.2 & 1.2 & 1.2 & $\mathbf{m}$ & $\mathbf{m}$ & $\mathbf{m}$ & 4.5 & 4.4 & 4.4 \\
\hline Greece $^{2}$ & 2.6 & 2.7 & $\mathrm{~m}$ & $\mathrm{~m}$ & $\mathrm{~m}$ & $\mathrm{~m}$ & 0.7 & 1.5 & $\mathrm{~m}$ & $\mathrm{~m}$ & $\mathrm{~m}$ & $\mathrm{~m}$ & 3.3 & 4.2 & $\mathbf{m}$ & $\mathbf{m}$ & m & $\mathbf{m}$ \\
\hline Hungary ${ }^{4}$ & 2.7 & 3.2 & 2.9 & 2.8 & 2.6 & 2.6 & 0.8 & 0.9 & 0.9 & 0.8 & 1.0 & 1.2 & 3.6 & 4.1 & 3.8 & 3.6 & 3.5 & 3.9 \\
\hline Iceland & 4.6 & 5.2 & 4.8 & 4.7 & 4.7 & 4.7 & 1.0 & 1.2 & 1.2 & 1.2 & 1.1 & 1.2 & 5.7 & 6.4 & 6.0 & 5.8 & 5.8 & 5.8 \\
\hline Ireland & 2.9 & 3.3 & 4.0 & 4.5 & 4.3 & 4.4 & 1.4 & 1.1 & 1.4 & 1.5 & 1.4 & 1.3 & 4.3 & 4.4 & 5.3 & 6.0 & 5.7 & 5.6 \\
\hline Israel & 4.2 & 3.8 & 4.0 & 4.0 & 4.2 & 4.4 & 1.7 & 1.8 & 1.5 & 1.6 & 1.7 & 1.6 & 5.9 & 5.6 & 5.5 & 5.5 & 5.9 & 6.0 \\
\hline Italy & 3.1 & 3.1 & 3.2 & 3.1 & 3.0 & 3.0 & 0.8 & 0.8 & 0.9 & 1.0 & 1.0 & 0.9 & 3.9 & 4.0 & 4.1 & 4.1 & 4.0 & 3.9 \\
\hline $\mathrm{Japan}^{2}$ & 3.0 & 2.9 & 2.9 & 3.0 & 3.0 & 2.9 & 1.4 & 1.4 & 1.5 & 1.5 & 1.6 & 1.5 & 4.4 & 4.3 & 4.4 & 4.5 & 4.5 & 4.5 \\
\hline Korea & 3.3 & 3.8 & 3.9 & 3.9 & 3.8 & 3.7 & 2.1 & 2.1 & 2.4 & 2.4 & 2.4 & 2.3 & 5.4 & 6.0 & 6.2 & 6.3 & 6.2 & 6.0 \\
\hline Luxembourg & $\mathrm{m}$ & $\mathrm{m}$ & 3.1 & 3.5 & 3.3 & 3.3 & $\mathrm{~m}$ & $\mathrm{~m}$ & $\mathrm{~m}$ & $\mathrm{~m}$ & $\mathrm{~m}$ & 0.4 & $\mathbf{m}$ & $\mathbf{m}$ & $\mathbf{m}$ & $\mathbf{m}$ & $\mathbf{m}$ & 3.7 \\
\hline Mexico & 3.4 & 3.9 & 3.6 & 3.9 & 3.9 & 3.9 & 0.9 & 1.2 & 1.2 & 1.4 & 1.3 & 1.3 & 4.4 & 5.0 & 4.9 & 5.3 & 5.2 & 5.2 \\
\hline Netherlands & 3.2 & 3.6 & 3.5 & 3.8 & 3.7 & 3.8 & 1.3 & 1.5 & 1.5 & 1.6 & 1.6 & 1.7 & 4.5 & 5.1 & 4.9 & 5.4 & 5.4 & 5.5 \\
\hline New Zealand & $\mathrm{m}$ & $\mathrm{m}$ & $\mathrm{m}$ & $\mathrm{m}$ & $\mathrm{m}$ & 5.0 & $\mathrm{~m}$ & $\mathrm{~m}$ & $\mathrm{~m}$ & $\mathrm{~m}$ & $\mathrm{~m}$ & 1.9 & $\mathbf{m}$ & $\mathbf{m}$ & $\mathbf{m}$ & $\mathbf{m}$ & $\mathbf{m}$ & 6.9 \\
\hline Norway ${ }^{2,4}$ & 5.0 & 5.1 & 4.8 & 5.1 & 4.7 & 4.6 & 1.6 & 1.7 & 1.6 & 1.6 & 1.5 & 1.6 & 6.7 & 6.8 & 6.4 & 6.7 & 6.3 & 6.2 \\
\hline Poland & 3.9 & 3.7 & 3.6 & 3.6 & 3.4 & 3.4 & 1.1 & 1.6 & 1.5 & 1.5 & 1.3 & 1.3 & 4.9 & 5.3 & 5.1 & 5.1 & 4.7 & 4.8 \\
\hline Portugal $^{2}$ & 3.7 & 3.6 & 3.3 & 3.7 & 3.6 & 4.5 & 0.9 & 1.3 & 1.3 & 1.4 & 1.3 & 1.3 & 4.7 & 4.8 & 4.6 & 5.1 & 4.9 & 5.8 \\
\hline Slovak Republic ${ }^{2}$ & 2.6 & 2.8 & 2.6 & 3.0 & 2.7 & 2.7 & 0.8 & 0.9 & 0.9 & 0.9 & 1.0 & 1.0 & 3.4 & 3.7 & 3.4 & 3.9 & 3.7 & 3.7 \\
\hline Slovenia & $\mathrm{m}$ & 4.1 & 3.6 & 3.8 & 3.7 & 3.7 & $\mathrm{~m}$ & 1.3 & 1.1 & 1.2 & 1.3 & 1.2 & $\mathbf{m}$ & 5.3 & 4.7 & 5.1 & 5.0 & 4.9 \\
\hline Spain & 3.1 & 2.8 & 3.0 & 3.2 & 3.1 & 3.1 & 1.1 & 1.1 & 1.2 & 1.3 & 1.3 & 1.2 & 4.2 & 3.9 & 4.2 & 4.5 & 4.4 & 4.3 \\
\hline Sweden & 4.0 & 4.0 & 3.8 & 3.8 & 3.7 & 3.7 & 1.5 & 1.5 & 1.5 & 1.7 & 1.7 & 1.7 & 5.5 & 5.5 & 5.3 & 5.4 & 5.3 & 5.4 \\
\hline Switzerland ${ }^{4}$ & 3.7 & 4.0 & 3.7 & 3.8 & 3.8 & 3.5 & 1.1 & 1.3 & 1.1 & 1.2 & 1.2 & 1.3 & 4.8 & 5.3 & 4.8 & 5.0 & 5.0 & 4.8 \\
\hline Turkey $^{4}$ & 1.8 & 2.0 & 2.2 & 2.5 & 2.3 & 2.6 & 0.7 & 0.8 & 0.8 & 0.9 & 1.0 & 1.2 & 2.5 & 2.8 & 2.9 & 3.5 & 3.3 & 3.8 \\
\hline United Kingdom ${ }^{4}$ & $\mathrm{~m}$ & 4.2 & 3.9 & 4.3 & 4.4 & 4.5 & $\mathrm{~m}$ & $\mathrm{~m}$ & $\mathrm{~m}$ & $\mathrm{~m}$ & $\mathrm{~m}$ & 1.8 & m & $\mathbf{m}$ & $\mathbf{m}$ & $\mathbf{m}$ & $\mathbf{m}$ & 6.3 \\
\hline United States $^{2}$ & 3.6 & 3.6 & 3.9 & 3.8 & 3.7 & 3.6 & 2.1 & 2.3 & 2.5 & 2.6 & 2.7 & 2.8 & 5.6 & 6.0 & 6.4 & 6.5 & 6.4 & 6.4 \\
\hline OECD average & 3.5 & 3.6 & 3.6 & 3.8 & 3.6 & 3.7 & 1.3 & 1.4 & 1.4 & 1.5 & 1.5 & 1.5 & 4.8 & 5.0 & 5.0 & 5.3 & 5.2 & 5.2 \\
\hline EU21 average & 3.3 & 3.5 & 3.5 & 3.7 & 3.5 & 3.6 & 1.1 & 1.3 & 1.3 & 1.4 & 1.4 & 1.4 & 4.4 & 4.7 & 4.8 & 5.0 & 4.9 & 4.9 \\
\hline $\begin{array}{l}\text { OECD mean for } \\
\text { countries with data } \\
\text { for all reference years }\end{array}$ & 3.5 & 3.6 & 3.5 & 3.7 & 3.6 & 3.6 & 1.3 & 1.4 & 1.5 & 1.6 & 1.6 & 1.6 & 4.8 & 5.0 & 5.0 & 5.2 & 5.2 & 5.2 \\
\hline Argentina & $\mathrm{m}$ & $\mathrm{m}$ & $\mathrm{m}$ & $\mathrm{m}$ & $\mathrm{m}$ & $\mathrm{m}$ & $\mathrm{m}$ & $\mathrm{m}$ & $\mathrm{m}$ & $\mathrm{m}$ & $\mathrm{m}$ & $\mathrm{m}$ & $\mathbf{m}$ & $\mathbf{m}$ & $\mathbf{m}$ & $\mathbf{m}$ & $\mathbf{m}$ & $\mathbf{m}$ \\
\hline Brazil $^{4}$ & 2.4 & 3.2 & 4.1 & 4.3 & 4.4 & 4.7 & 0.7 & 0.8 & 0.8 & 0.9 & 0.9 & 0.9 & 3.1 & 4.0 & 4.8 & 5.2 & 5.3 & 5.6 \\
\hline China & $\mathrm{m}$ & $\mathrm{m}$ & $\mathrm{m}$ & $\mathrm{m}$ & $\mathrm{m}$ & $\mathrm{m}$ & $\mathrm{m}$ & $\mathrm{m}$ & $\mathrm{m}$ & $\mathrm{m}$ & $\mathrm{m}$ & $\mathrm{m}$ & $\mathbf{m}$ & $\mathbf{m}$ & $\mathbf{m}$ & $\mathbf{m}$ & $\mathbf{m}$ & $\mathbf{m}$ \\
\hline Colombia $^{3}$ & $\mathrm{~m}$ & $\mathrm{~m}$ & $\mathrm{~m}$ & $\mathrm{~m}$ & $\mathrm{~m}$ & 4.5 & $\mathrm{~m}$ & $\mathrm{~m}$ & $\mathrm{~m}$ & $\mathrm{~m}$ & $\mathrm{~m}$ & 1.9 & $\mathbf{m}$ & m & $\mathbf{m}$ & $\mathbf{m}$ & $\mathbf{m}$ & 6.5 \\
\hline India & $\mathrm{m}$ & $\mathrm{m}$ & $\mathrm{m}$ & $\mathrm{m}$ & $\mathrm{m}$ & $\mathrm{m}$ & $\mathrm{m}$ & $\mathrm{m}$ & $\mathrm{m}$ & $\mathrm{m}$ & $\mathrm{m}$ & $\mathrm{m}$ & $\mathbf{m}$ & $\mathbf{m}$ & $\mathbf{m}$ & $\mathbf{m}$ & $\mathbf{m}$ & $\mathbf{m}$ \\
\hline Indonesia $^{3}$ & $\mathrm{~m}$ & $\mathrm{~m}$ & $\mathrm{~m}$ & $\mathrm{~m}$ & $\mathrm{~m}$ & 2.5 & $\mathrm{~m}$ & $\mathrm{~m}$ & $\mathrm{~m}$ & $\mathrm{~m}$ & $\mathrm{~m}$ & 0.8 & m & m & $\mathbf{m}$ & $\mathbf{m}$ & $\mathbf{m}$ & 3.3 \\
\hline Latvia & $\mathrm{m}$ & $\mathrm{m}$ & $\mathrm{m}$ & $\mathrm{m}$ & $\mathrm{m}$ & 2.8 & $\mathrm{~m}$ & $\mathrm{~m}$ & $\mathrm{~m}$ & $\mathrm{~m}$ & $\mathrm{~m}$ & 1.4 & m & $\mathbf{m}$ & $\mathbf{m}$ & $\mathbf{m}$ & $\mathbf{m}$ & 4.2 \\
\hline Russian Federation ${ }^{4}$ & 1.7 & 1.9 & 2.0 & 2.0 & 2.0 & 2.3 & 0.5 & 0.8 & 0.9 & 1.0 & 0.9 & 1.4 & 2.1 & 2.7 & 3.0 & 3.0 & 2.8 & 3.7 \\
\hline Saudi Arabia & $\mathrm{m}$ & $\mathrm{m}$ & $\mathrm{m}$ & $\mathrm{m}$ & $\mathrm{m}$ & $\mathrm{m}$ & $\mathrm{m}$ & $\mathrm{m}$ & $\mathrm{m}$ & $\mathrm{m}$ & $\mathrm{m}$ & $\mathrm{m}$ & $\mathbf{m}$ & $\mathbf{m}$ & $\mathbf{m}$ & $\mathbf{m}$ & $\mathbf{m}$ & $\mathbf{m}$ \\
\hline South Africa $^{4}$ & $\mathrm{~m}$ & $\mathrm{~m}$ & $\mathrm{~m}$ & $\mathrm{~m}$ & $\mathrm{~m}$ & 4.7 & $\mathrm{~m}$ & $\mathrm{~m}$ & $\mathrm{~m}$ & $\mathrm{~m}$ & $\mathrm{~m}$ & 0.7 & m & $\mathbf{m}$ & $\mathbf{m}$ & $\mathbf{m}$ & $\mathbf{m}$ & 5.4 \\
\hline G20 average & $\mathrm{m}$ & $\mathrm{m}$ & $\mathrm{m}$ & $\mathrm{m}$ & $\mathrm{m}$ & $\mathrm{m}$ & $\mathrm{m}$ & $\mathrm{m}$ & $\mathrm{m}$ & $\mathrm{m}$ & $\mathrm{m}$ & $\mathrm{m}$ & m & $\mathbf{m}$ & $\mathbf{m}$ & $\mathbf{m}$ & $\mathbf{m}$ & m \\
\hline
\end{tabular}

1. Year of reference 2011 instead of 2012. Year of reference 2004 instead of 2005.

2. Some levels of education are included with others. Refer to " $\mathrm{x}$ " code in Table B1.1a for details.

3. Year of reference 2013 instead of 2012. Year of reference 2006 instead of 2005.

4. Public expenditure only (for Switzerland, in tertiary education only; for Norway, in primary, secondary and post-secondary non-tertiary education only; the Russian Federation, data available for 1995 and 2000 only).

Source: OECD. Argentina, China, Colombia, India, Indonesia, Saudi Arabia, South Africa: UNESCO Institute for Statistics. Latvia: Eurostat. See Annex 3 for notes (www.oecd.org/education/education-at-a-glance-19991487.htm).

Please refer to the Reader's Guide for information concerning symbols for missing data and abbreviations.

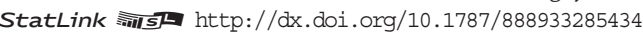


Table B2.3. Expenditure on educational institutions as a percentage of GDP, by source of funding and level of education (2012)

From public and private sources of funds

\begin{tabular}{|c|c|c|c|c|c|c|c|c|c|}
\hline & \multicolumn{3}{|c|}{$\begin{array}{l}\text { Primary, secondary } \\
\text { and post-secondary non-tertiary }\end{array}$} & \multicolumn{3}{|c|}{ Tertiary } & \multicolumn{3}{|c|}{$\begin{array}{l}\text { Primary to tertiary } \\
\text { (including undistributed programmes) }\end{array}$} \\
\hline & Public ${ }^{1}$ & Private $^{2}$ & Total & Public $^{1}$ & Private $^{2}$ & Total & Public $^{1}$ & Private $^{2}$ & Total \\
\hline & (1) & (2) & (3) & (4) & (5) & (6) & (7) & (8) & (9) \\
\hline Australia & 3.4 & 0.6 & 4.0 & 0.9 & 0.7 & 1.6 & 4.3 & 1.3 & 5.6 \\
\hline Austria & 3.1 & 0.0 & 3.1 & 1.7 & 0.0 & 1.7 & 4.9 & 0.0 & 4.9 \\
\hline Belgium & 4.3 & 0.0 & 4.3 & 1.4 & 0.0 & 1.4 & 5.9 & 0.0 & 5.9 \\
\hline Canada $^{3,4}$ & $3.2^{\mathrm{d}}$ & $0.3^{\mathrm{d}}$ & $3.6^{\mathrm{d}}$ & 1.5 & 1.0 & 2.5 & 4.7 & 1.3 & 6.0 \\
\hline Chile $^{5}$ & 2.8 & 0.8 & 3.6 & 1.0 & 1.5 & 2.5 & 3.9 & 2.2 & 6.1 \\
\hline Czech Republic & 2.6 & 0.2 & 2.8 & 1.2 & 0.2 & 1.4 & 4.0 & 0.4 & 4.4 \\
\hline Denmark & 4.7 & 0.0 & 4.7 & $\mathrm{~m}$ & $\mathrm{~m}$ & $\mathbf{m}$ & $\mathrm{m}$ & $\mathrm{m}$ & $\mathbf{m}$ \\
\hline Estonia & 3.2 & 0.0 & 3.2 & 1.4 & 0.2 & 1.6 & 4.7 & 0.1 & 4.9 \\
\hline Finland & 3.9 & 0.0 & 3.9 & 1.8 & 0.1 & 1.8 & 5.7 & 0.1 & 5.8 \\
\hline France & 3.6 & 0.2 & 3.8 & 1.3 & 0.2 & 1.4 & 4.9 & 0.4 & 5.3 \\
\hline Germany & 2.9 & 0.2 & 3.1 & 1.2 & 0.0 & 1.2 & 4.3 & 0.1 & 4.4 \\
\hline Greece & $\mathrm{m}$ & $\mathrm{m}$ & $\mathbf{m}$ & $\mathrm{m}$ & $\mathrm{m}$ & $\mathbf{m}$ & $\mathrm{m}$ & $\mathrm{m}$ & $\mathbf{m}$ \\
\hline Hungary & 2.6 & 0.1 & 2.6 & 0.8 & 0.4 & 1.2 & 3.6 & 0.5 & 4.1 \\
\hline Iceland & 4.5 & 0.2 & 4.7 & 1.1 & 0.1 & 1.2 & 5.9 & 0.5 & 6.4 \\
\hline Ireland & 4.2 & 0.2 & 4.4 & 1.0 & 0.2 & 1.3 & 5.2 & 0.4 & 5.6 \\
\hline Israel & 4.0 & 0.4 & 4.4 & 0.9 & 0.7 & 1.6 & 5.1 & 1.3 & 6.5 \\
\hline Italy ${ }^{6}$ & 2.9 & 0.1 & 3.0 & 0.8 & 0.1 & 0.9 & 3.7 & 0.2 & 3.9 \\
\hline $\mathrm{Japan}^{4}$ & 2.7 & 0.2 & 2.9 & 0.5 & 1.0 & 1.5 & 3.5 & 1.5 & 5.0 \\
\hline Korea & 3.2 & 0.5 & 3.7 & 0.8 & 1.5 & 2.3 & 4.7 & 2.0 & 6.7 \\
\hline Luxembourg & 3.3 & 0.0 & 3.3 & 0.4 & 0.0 & 0.4 & 3.7 & 0.0 & 3.7 \\
\hline Mexico & 3.4 & 0.5 & 3.9 & 1.0 & 0.4 & 1.3 & 4.6 & 0.8 & 5.4 \\
\hline Netherlands & 3.5 & 0.3 & 3.8 & 1.4 & 0.3 & 1.7 & 4.9 & 0.6 & 5.5 \\
\hline New Zealand & 4.2 & 0.8 & 5.0 & 1.2 & 0.7 & 1.9 & 5.4 & 1.5 & 6.9 \\
\hline Norway ${ }^{4}$ & 4.6 & 0.0 & 4.6 & 1.6 & 0.0 & 1.6 & 6.5 & 0.0 & 6.5 \\
\hline Poland & 3.2 & 0.2 & 3.4 & 1.2 & 0.1 & 1.3 & 4.4 & 0.4 & 4.8 \\
\hline Portugal $^{4}$ & 3.9 & 0.5 & 4.5 & 0.9 & 0.4 & 1.3 & 5.0 & 0.9 & 5.9 \\
\hline Slovak Republic ${ }^{4}$ & 2.5 & 0.2 & 2.7 & 1.0 & 0.1 & 1.0 & 3.5 & 0.3 & 3.8 \\
\hline Slovenia & 3.5 & 0.2 & 3.7 & 1.2 & 0.0 & 1.2 & 4.7 & 0.2 & 4.9 \\
\hline Spain & 2.8 & 0.3 & 3.1 & 1.0 & 0.2 & 1.2 & 3.8 & 0.5 & 4.3 \\
\hline Sweden & 3.7 & 0.0 & 3.7 & 1.5 & 0.2 & 1.7 & 5.2 & 0.2 & 5.4 \\
\hline Switzerland & 3.5 & 0.0 & 3.5 & 1.2 & 0.0 & 1.2 & 4.9 & 0.0 & 4.9 \\
\hline Turkey & 2.6 & 0.4 & 3.0 & 1.2 & 0.2 & 1.4 & 3.8 & 0.6 & 4.4 \\
\hline United Kingdom & 4.0 & 0.5 & 4.5 & 1.2 & 0.6 & 1.8 & 5.2 & 1.0 & 6.3 \\
\hline United States ${ }^{4}$ & 3.3 & 0.3 & 3.6 & 1.4 & 1.4 & 2.8 & 4.7 & 1.7 & 6.4 \\
\hline $\mathrm{OECD}$ average & 3.5 & 0.2 & 3.7 & 1.2 & 0.4 & 1.5 & 4.7 & 0.7 & 5.3 \\
\hline EU21 average & 3.4 & 0.2 & 3.6 & 1.2 & 0.2 & 1.4 & 4.6 & 0.3 & 4.9 \\
\hline Argentina & $\mathrm{m}$ & $\mathrm{m}$ & $\mathbf{m}$ & $\mathrm{m}$ & $\mathrm{m}$ & m & $\mathrm{m}$ & $\mathrm{m}$ & m \\
\hline Brazil & 4.6 & 0.0 & 4.6 & 0.9 & 0.0 & 0.9 & 5.6 & 0.0 & 5.6 \\
\hline China & $\mathrm{m}$ & $\mathrm{m}$ & $\mathbf{m}$ & $\mathrm{m}$ & $\mathrm{m}$ & $\mathbf{m}$ & $\mathrm{m}$ & $\mathrm{m}$ & $\mathbf{m}$ \\
\hline Colombia $^{5}$ & 3.6 & 0.9 & 4.5 & 0.9 & 1.0 & 1.9 & 4.9 & 1.9 & 6.8 \\
\hline India & $\mathrm{m}$ & $\mathrm{m}$ & $\mathbf{m}$ & $\mathrm{m}$ & $\mathrm{m}$ & $\mathbf{m}$ & $\mathrm{m}$ & $\mathrm{m}$ & m \\
\hline Indonesia $^{5}$ & 2.3 & 0.2 & 2.5 & 0.6 & 0.2 & 0.8 & 3.3 & 0.4 & 3.7 \\
\hline Latvia & 2.9 & 0.0 & 2.8 & 1.0 & 0.3 & 1.4 & 3.9 & 0.3 & 4.2 \\
\hline Russian Federation & 2.2 & 0.1 & 2.3 & 0.9 & 0.5 & 1.4 & 3.4 & 0.6 & 4.0 \\
\hline Saudi Arabia & $\mathrm{m}$ & $\mathrm{m}$ & $\mathbf{m}$ & $\mathrm{m}$ & $\mathrm{m}$ & $\mathbf{m}$ & $\mathrm{m}$ & $\mathrm{m}$ & $\mathbf{m}$ \\
\hline South Africa & 4.7 & $\mathrm{~m}$ & m & 0.7 & $\mathrm{~m}$ & $\mathbf{m}$ & 6.0 & $\mathrm{~m}$ & m \\
\hline G20 average & $\mathrm{m}$ & $\mathrm{m}$ & m & $\mathrm{m}$ & $\mathrm{m}$ & $\mathbf{m}$ & $\mathrm{m}$ & $\mathrm{m}$ & $\mathbf{m}$ \\
\hline
\end{tabular}

1. Including public subsidies to households attributable for educational institutions, and direct expenditure on educational institutions from international sources.

2. Net of public subsidies attributable for educational institutions.

3. Year of reference 2011.

4. Some levels of education are included with others. Refer to " $\mathrm{x}$ " code in Table B1.1a for details.

5. Year of reference 2013.

6. Excludes short-cycle tertiary programmes.

Source: OECD. Argentina, China, Colombia, India, Indonesia, Saudi Arabia, South Africa: UNESCO Institute for Statistics. Latvia: Eurostat. See Annex 3 for notes (www.oecd.org/education/education-at-a-glance-19991487.htm).

Please refer to the Reader's Guide for information concerning symbols for missing data and abbreviations.

StatLink 세개 http://dx.doi.org/10.1787/888933285443 
Table B2.4. Change in public expenditure on educational institutions as a percentage of GDP (2008, 2009, 2010, 2011, 2012)

Index of change between 2008 and 2012 in public expenditure on educational institutions as a percentage of GDP, primary to tertiary education (2012 constant prices)

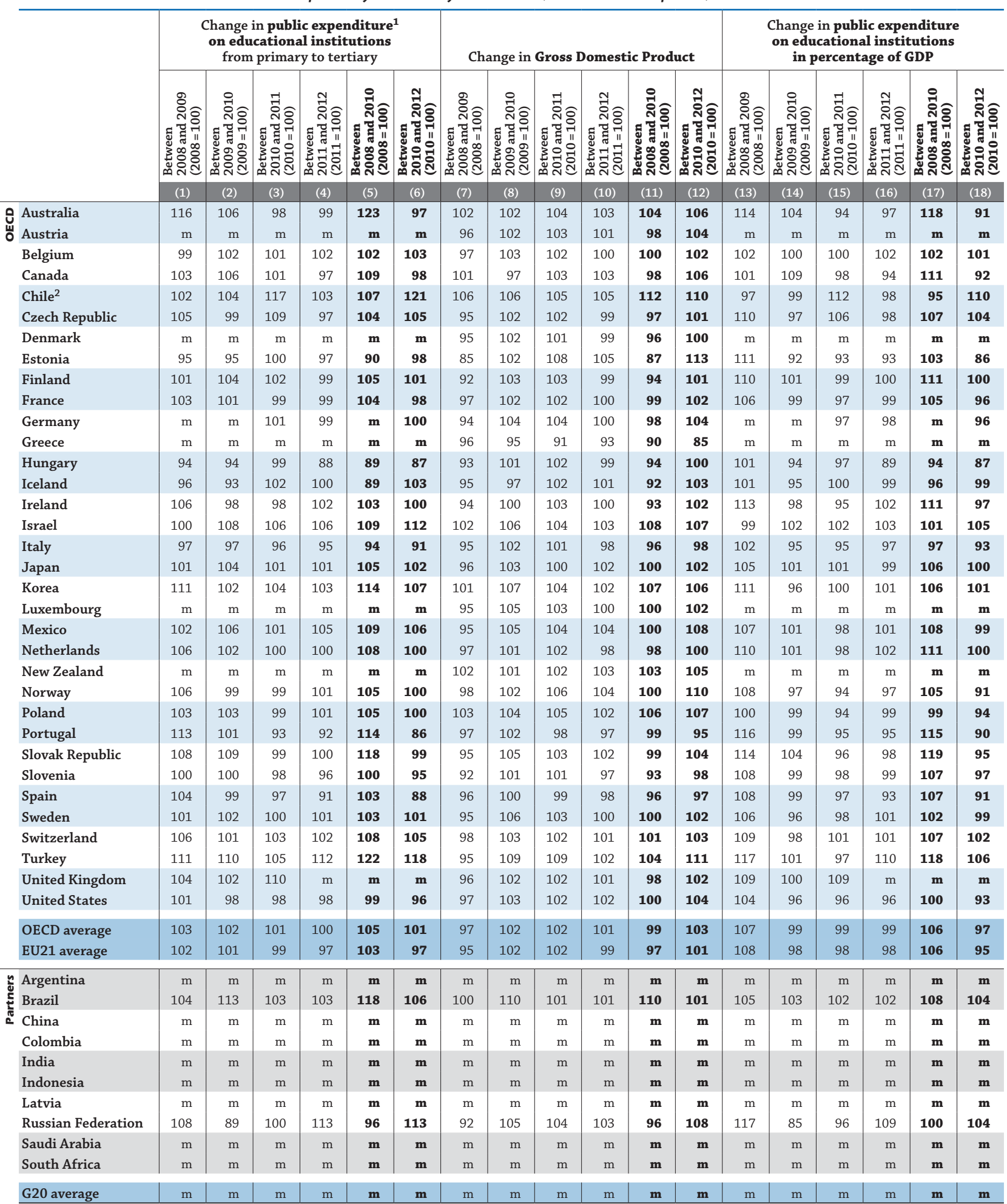

1. Excluding subsidies attributable to payments to educational institutions received from public sources.

2. Data refer to 2009-2013 instead of 2008-2012.

Source: OECD. Argentina, China, Colombia, India, Indonesia, Saudi Arabia, South Africa: UNESCO Institute for Statistics. Latvia: Eurostat. See Annex 3 for notes (www.oecd.org/education/education-at-a-glance-19991487.htm).

Please refer to the Reader's Guide for information concerning symbols for missing data and abbreviations.

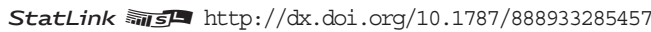





\section{HOW MUCH PUBLIC AND PRIVATE INVESTMENT IN EDUCATION IS THERE?}

- Public funding accounts for $83 \%$ of all funds for educational institutions from primary to tertiary education, on average across OECD countries.

- Nearly $91 \%$ of the funds for primary, secondary and post-secondary non-tertiary educational institutions come from public sources, on average across OECD countries; only in Chile and Colombia is this share less than $80 \%$.

- Of primary to tertiary levels of education, tertiary institutions obtain the largest proportion of funds $-30 \%$ - from private sources.

\section{Chart B3.1. Share of private expenditure on educational institutions (2012)}

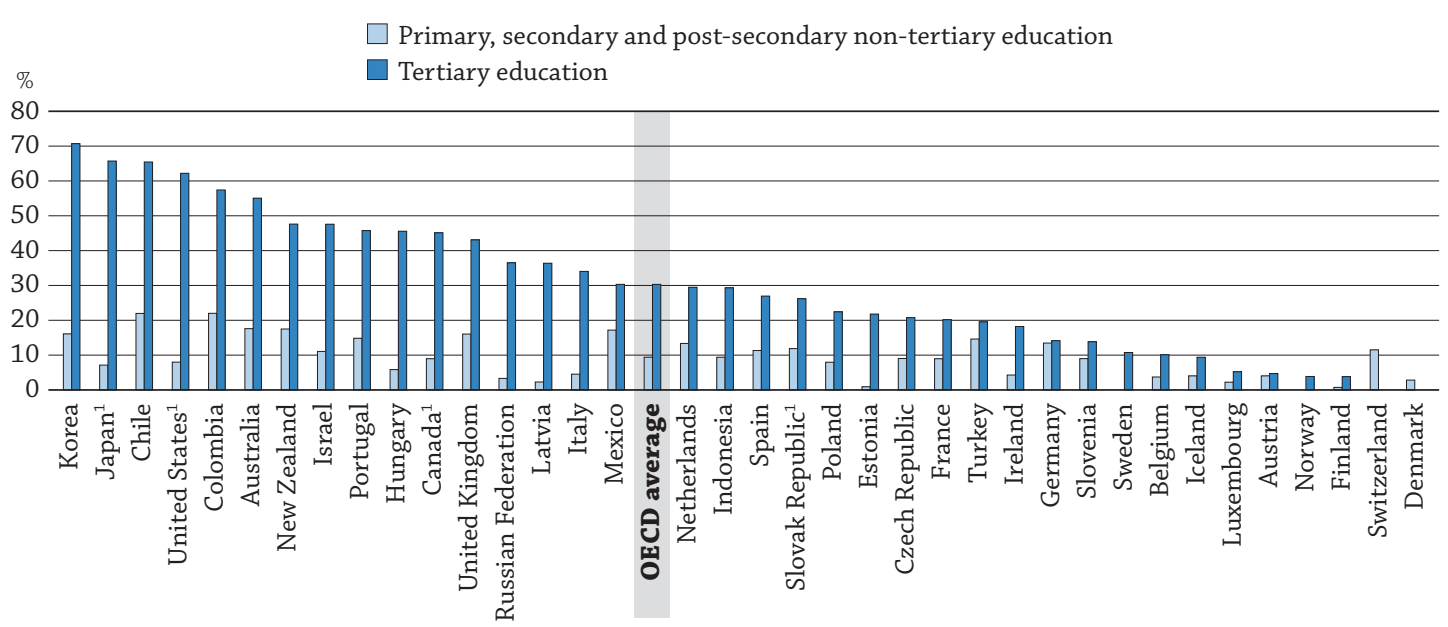

\section{How to read this chart}

The chart shows private spending on educational institutions as a percentage of total spending on educational institutions. This includes all money transferred to educational institutions from private sources, including public funding via subsidies to households, private fees for educational services or other private spending (e.g. on accommodation) which goes through the institution.

1. Some levels of education are included with others. Refer to " $\mathrm{x}$ " code in Table B1.1a for details.

Countries are ranked in descending order of the share of private expenditure on educational institutions for tertiary education.

Source: OECD. Table B3.1. See Annex 3 for notes (www.oecd.org/education/education-at-a-glance-19991487.htm).

StatLink 제개 http://dx.doi.org/10.1787/888933283989

\section{Context}

More people are participating in a wider range of educational programmes offered by increasing numbers of providers than ever before. As a result, the question of who should support an individual's efforts to acquire more education - governments or the individuals themselves - is becoming increasingly important. In the current economic environment, many governments are finding it difficult to provide the necessary resources to support the increased demand for education through public funds alone. In addition, some policy makers assert that those who benefit the most from education - the individuals who receive it - should bear at least some of the costs. While public funding still represents a large part of countries' investment in education, the role of private sources of funding is becoming increasingly prominent.

The balance between public and private financing of education is an important policy issue in many OECD countries, especially at the pre-primary and tertiary levels of education, for which full or nearly full public funding is less common. At these levels, private funding comes mainly from households, raising concerns about equity of access to education. The debate is particularly intense with respect to funding for tertiary education. Some stakeholders are concerned that the balance between public and private funding should not become so tilted as to discourage potential students from entering tertiary education. Others believe that countries should significantly increase public 
support to students, while still others support efforts to increase the amount of funding to tertiary education provided by private enterprises. By contrast, primary, secondary and post-secondary non-tertiary education, which is mainly compulsory, is usually conceived as a public good and is thus largely financed by public funds.

\section{INDICATOR B3}

\section{Other findings}

- Public funding for educational institutions, including primary, secondary and post-secondary non-tertiary education combined, and for tertiary education, increased between 2000 and 2012 in nearly all countries for which comparable data are available. However, at the tertiary level, more households are sharing the cost of education, thus private funding increased at an even greater rate in more than three-quarters of countries.

- Public funds are mainly allocated to public institutions, but also to private institutions to varying degrees. For primary to tertiary levels of education combined, public expenditure on public institutions, per student, is $91 \%$ higher than the level of public expenditure on private institutions, on average across OECD countries. However, the proportion varies from $64 \%$ higher for primary, secondary and post-secondary non-tertiary education combined to more than double that proportion (151\% higher) at the tertiary level.

- Five of the six countries with the lowest amounts of public expenditure per student, in public and private tertiary institutions, are also those (except one) with the fewest students enrolled in public tertiary institutions.

- In most countries for which data are available, individual households account for most of the private expenditure on tertiary education. Austria, Belgium, the Czech Republic, Sweden and the United Kingdom are the exceptions, where private expenditure from entities other than households (e.g. private businesses and non-profit organisations) is more significant than private expenditure from households, mainly because tuition fees charged by tertiary institutions are low or negligible in these countries (except the United Kingdom).

\section{Trends}

Between 2000 and 2012, the average share of public funding for tertiary institutions decreased from $68.8 \%$ in 2000, to $64.9 \%$ in 2005 and decreased slightly again to $64.5 \%$ in 2012 (on average across the 20 OECD countries for which trend data are available for all years) (Table B3.2b). This trend is mainly influenced by some European countries, where there were significant changes in tuition fees and where enterprises participate more actively in providing grants to finance tertiary institutions.

Between 2000 and 2012, the share of private funding for tertiary education increased in more than three-quarters of the countries ( 17 of 22 countries for which comparable data are available). This share increased by more than four percentage points, on average across OECD countries, and by more than nine percentage points in Hungary, Italy, Mexico, Portugal and the Slovak Republic (Table B3.2b). In these countries, the share of private funding increased the most between 2000 and 2008 as a result of a much larger increase in private funding than in public funding.

Between 2000 and 2012, the share of private funding did not change much at the primary, secondary, post-secondary non-tertiary levels, on average across OECD countries for which comparable data are available, but it increased in some countries - most significantly (by nine percentage points or more) in Portugal and the Slovak Republic. Chile is the only country with a significant increase (more than nine percentage points) in the share of public funding during this period (Tables B3.2a). 


\section{Analysis}

\section{Public and private expenditure on educational institutions}

Educational institutions in OECD countries are mainly publicly funded, although there is a substantial - and growing - level of private funding at the tertiary level. On average across OECD countries, $83 \%$ of all funds for primary to tertiary educational institutions come directly from public sources; $17 \%$ come from private sources (Chart B3.1 and Table B3.1).

However, the share of public and private funding varies widely among countries. Comparing expenditure on primary to tertiary levels of education combined, the share of private funds exceeds $20 \%$ in Canada, Israel, Portugal and the United Kingdom, 25\% in Australia, Japan and New Zealand, 30\% in Colombia, Korea and the United States, and reaches nearly $40 \%$ in Chile. By contrast, in Finland, Luxembourg and Sweden, less than $4 \%$ of expenditure on education comes from private sources (Table B3.1).

\section{Public and private expenditure on primary, secondary and post-secondary non-tertiary educational institutions}

Public funding dominates primary, secondary and post-secondary non-tertiary education in all countries. In half of the countries, less than $10 \%$ of funding for these levels of education comes from private sources, and the share of private funding exceeds $20 \%$ only in Chile and Colombia (among countries with available data) (Table B3.1 and Chart B3.1). In most countries, the largest share of private expenditure at these levels comes from households and goes mainly towards tuition fees (Chart B3.2). However, in the Netherlands and Switzerland, most private expenditure takes the form of contributions from the business sector to the dual system of apprenticeship in upper secondary and post-secondary non-tertiary education (see Box B3.1). In Canada, too, the contribution from other private entities is larger than that from households.

Between 2000 and 2012, the share of public funding for primary, secondary and post-secondary non-tertiary education decreased slightly among the 19 countries with available data for all years (from $90.6 \%$ in 2000 to $89.7 \%$ in 2012, on average). This share shrank by two percentage points or more in Israel, Italy, Mexico, Poland and Spain, and by nine percentage points or more in Portugal and the Slovak Republic. In the other countries, shifts in the opposite direction, i.e. towards public funding, between 2000 and 2012 exceeded three percentage points in Japan and Korea and nine percentage points in Chile (Table B3.2a).

\section{Box B3.1. Private expenditure on the work-based component of educational programmes}

Many countries have some form of combined school- and work-based educational programmes (e.g. apprenticeship programmes, dual systems). The impact of reporting these programmes in the financial indicators is strong in a few countries, even if it is not significant in most countries (see Table at the end of this box). Expenditure by private employers on training apprentices (e.g. compensation of instructors and cost of instructional materials and equipment) and other participants in these programmes is included in the financial indicators published in Education at a Glance. Expenditure to train company instructors is also included.

Among countries with some form of dual education systems, only Germany, Switzerland and, to some extent, the Netherlands, conduct surveys about private expenditure by employers. In a number of countries, such as the Czech Republic, Finland, Norway and the Slovak Republic, workplace training is directly financed by the government, or firms are reimbursed for their expenses; thus private expenditures are implicitly included in public expenditures reported in the indicators for most of these countries.

However, 10 of 17 countries with medium to large dual systems - Australia, Austria, Denmark, Estonia, France, Hungary, Iceland, Luxembourg, the Russian Federation and the United Kingdom - do not include private expenditure by enterprises that relate to these programmes in the financial indicators published in Education at a Glance. This is mainly because of a lack of such data.

The size of the work-based component varies widely among these countries and can have a significant impact on total expenditure in some. Among countries with available data on upper secondary education, Germany, the Netherlands and Switzerland have a significant proportion of all students enrolled in vocational education and training programmes (VET) with a work-based component (a minimum of $20 \%$ in the Netherlands, varying to a maximum of $80 \%$ dependending on the VET track of school or work based, $50 \%$ in Germany and $60 \%$ in Switzerland). The corresponding expenditure on these programmes represents between $0.3 \%$ and $0.5 \%$ of GDP (see Indicator B2). 
Further research has shown that $6 \%$ to $30 \%$ of upper secondary students (a "medium" share) are enrolled in VET programmes with a work-based component in Australia, Finland, France, Hungary, Iceland, Luxembourg, Norway, the Russian Federation, the Slovak Republic and the United Kingdom, while more than $30 \%$ of upper secondary students (a "large" proportion) in Austria, the Czech Republic, Denmark and Estonia are enrolled in such programmes. Among the group of countries with missing data on training expenditures, the impact of not reporting such expenditures is expected to be small for Australia, Denmark, Estonia, Iceland, Norway and the Slovak Republic, but is potentially large for Austria, France, Hungary, Luxembourg, the Russian Federation and the United Kingdom (see Table below).

In the financial indicators published in Education at a Glance, the cost of apprentices' salaries, social security contributions, and other compensation paid to students or apprentices in combined school- and workbased educational programmes is not included. Private investment in upper secondary VET programmes with a work-based component is considered to be moderate in Austria, France, Hungary, Luxembourg, the Netherlands, the Russian Federation and the United Kingdom, and large in Germany and Switzerland, where apprentices spend a substantial portion of their time in the workplace and where training is intensive (see Table below).

\begin{tabular}{|c|c|c|c|}
\hline \multirow[b]{2}{*}{$\begin{array}{l}\text { Share of dual/part-time } \\
\text { VET to all students }\end{array}$} & \multicolumn{3}{|c|}{ Importance of investment by firms } \\
\hline & Low & Medium & High \\
\hline Large (> 30\%) & $\begin{array}{l}\text { The Czech Republic, Denmark, } \\
\text { Estonia }\end{array}$ & Austria & Germany, Switzerland \\
\hline Medium (6-30\%) & $\begin{array}{l}\text { Australia, Finland, } \\
\text { Iceland, Norway, } \\
\text { the Slovak Republic }\end{array}$ & $\begin{array}{l}\text { France, Hungary } \\
\text { Luxembourg, } \\
\text { the Netherlands, } \\
\text { the Russian Federation, } \\
\text { the United Kingdom }\end{array}$ & \\
\hline Low $(<6 \%)$ & $\begin{array}{l}\text { Belgium, Brazil, Canada, Chile, } \\
\text { Greece, Ireland, Israel, Italy, } \\
\text { Japan, Korea, Mexico, } \\
\text { New Zealand, Poland, } \\
\text { Portugal, Slovenia, } \\
\text { Spain, Sweden, Turkey, } \\
\text { the United States }\end{array}$ & & \\
\hline
\end{tabular}

* The importance of investment by firms is an index that reflects the time that trainees spend in the workplace, the intensity of training (weekly instruction time) at the workplace, and controls for public reimbursement of such expenditure.

\section{Public and private expenditure on tertiary educational institutions}

High private returns to tertiary education (see Indicator A7) suggest that a greater contribution to the costs of education by individuals and other private entities may be justified, as long as there are ways to ensure that funding is available to students regardless of their economic backgrounds (see Indicator B5). In all countries, the proportion of private expenditure on education is far higher for tertiary education - an average of nearly $30 \%$ of total expenditure at this level - than it is for primary, secondary and post-secondary non-tertiary education (Chart B3.1 and Table B3.1).

The proportion of expenditure on tertiary institutions covered by individuals, businesses and other private sources, including subsidised private payments, ranges from less than 5\% in Austria, Finland and Norway (tuition fees charged by tertiary institutions are low or negligible in these countries) to more than $40 \%$ in a third of countries with available data. The proportion is more than $40 \%$ in Australia, Canada, Colombia, Hungary, Israel, New Zealand, Portugal and the United Kindgom, and exceeds 60\% in Chile, Japan, Korea and the United States. These proportions may be related to the level of tuition fees charged by tertiary institutions (Chart B3.2 and Table B3.1, see Indicator B5). 


\section{Chart B3.2. Distribution of public and private expenditure on educational institutions (2012)}

\section{By level of education}
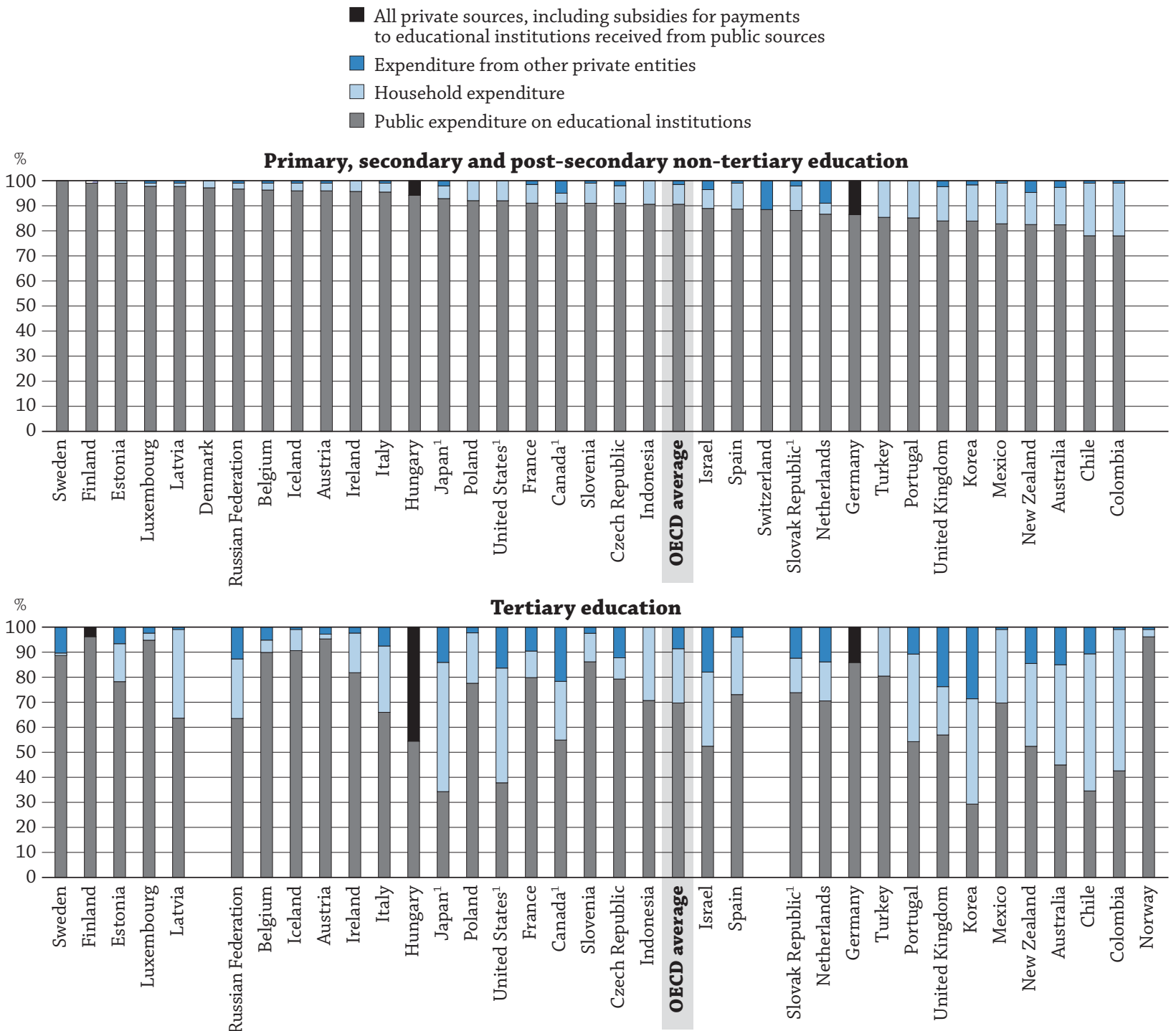

1. Some levels of education are included with others. Refer to " $\mathrm{x}$ " code in Table B1.1a for details.

Countries are ranked in descending order of the proportion of public expenditure on educational institutions in primary, secondary and post-secondary non-tertiary education.

Source: OECD. Table B3.1. See Annex 3 for notes (www.oecd.org/education/education-at-a-glance-19991487.htm).

StatLink 젬 http://dx.doi.org/10.1787/888933283996

Of these countries, in Korea and the United Kingdom, most students are enrolled in private institutions (around $80 \%$ in private universities in Korea; $100 \%$ in government-dependent private institutions in the United Kingdom). In Korea, more than $40 \%$ of the education budget comes from tuition fees. In the United Kingdom (England, Northern Ireland, Wales), tertiary education is funded through a combination of tuition fees (paid by students directly to the institution), and central government grants paid indirectly from a higher education-funding agency (see Indicators B5, and Indicator C7 in Education at a Glance 2014).

The contribution from private entities other than households to financing educational institutions is larger for tertiary education than for other levels of education, on average across OECD countries. The share of expenditure on tertiary institutions covered by private entities other than households is above $10 \%$ in more than one-third of countries with available data. In Sweden, these contributions are largely devoted to sponsoring research and development. This share reaches $15 \%$ or more in Australia, Israel and the United States, and exceeds $20 \%$ in Canada, Korea and the United Kingdom (see Box B3.2). 


\section{Box B3.2. Expenditure by private entities other than households on tertiary education}

Expenditure by other private entities is reported in the financial indicators published in Education at a Glance. The share of private expenditure on educational institutions varies between countries due to differences in the extent of the contribution by these private entities and in whether these expenditures are covered in the data reported.

The OECD launched a survey in 2015 to better analyse how these expenditures are covered in financial indicators. The replies from the 14 participating countries (excluding Brazil, which reports only public expenditure in financial indicators; see the list of participating countries below) do not allow for drawing definitive conclusions; nonetheless, they show that, in most of these countries, both private enterprises and non-profit organisations fund educational institutions. However, tertiary institutions in the Netherlands do not receive funds from non-profit organisations, nor do short-cycle tertiary programmes in the Slovak Republic and Sweden.

For most of these countries, expenditure from private enterprises and non-profit organisations are at least partially covered in the financial indicators. Expenditure from private enterprises are fully covered only in Canada, Finland, the Netherlands, Sweden and the United Kingdom (at bachelor's, master's, doctoral or equivalent levels), and expenditure from non-profit organisations are also fully covered in Canada, Israel, Slovenia and Sweden. Some of these countries, such as Canada and Israel, are among those with the largest proportions of funds from other private entities in OECD countries (see Table B3.1).

These types of expenditure are difficult to cover because of a lack of available sources of data. Usually only expenditure related to public educational institutions can be accounted for; only a few countries estimate the extent of the partial coverage. For example, the Slovak Republic estimated that up to $10 \%$ of expenditure from private enterprises is not covered, which could have an impact of one to two percentage points on the share of private expenditure on educational institutions.

Payments to educational institutions from both private enterprises and non-profit organisations are more widely covered than support to households and students. In the 12 countries providing information on the coverage of various types of expenditure from these private entities (excluding the Slovak Republic and Slovenia), half of the countries cover support, at least partially, while all countries cover payments to educational institutions, at least partially.

Expenditure to educational institutions for research and development is the most widely covered (it is fully covered in the Flemish Community of Belgium, Canada, Finland, Hungary, the Netherlands, Sweden, Switzerland, the United Kingdom and the United States, and partially covered in Australia, New Zealand and Portugal). Payments for specific educational activities and fees paid to institutions for ancillary services are less widely covered. Australia, the Flemish Community of Belgium, Sweden and the United Kingdom do not have data on one or both of these types of expenditure.

Support to households and students is not as well covered, but is often funded from public sources only. These data are also more difficult to collect: Australia, Canada, New Zealand, Portugal and Sweden do not cover scholarships and other grants to households and students, for example.

\begin{tabular}{l|c|c|c|c}
\cline { 2 - 5 } & $\begin{array}{c}\text { Fully } \\
\text { covered }\end{array}$ & $\begin{array}{c}\text { Partially } \\
\text { covered }\end{array}$ & $\begin{array}{c}\text { Not } \\
\text { covered }\end{array}$ & $\begin{array}{c}\text { Not } \\
\text { applicable }\end{array}$ \\
\hline Payments to educational institutions & $\mathbf{3 1 \%}$ & $\mathbf{6 9 \%}$ & $\mathbf{0 \%}$ & $\mathbf{0 \%}$ \\
\hline of which: Payments for specif. educational activities & $38 \%$ & $23 \%$ & $31 \%$ & $8 \%$ \\
\hline of which: Fees paid to institutions for ancillary services & $31 \%$ & $23 \%$ & $15 \%$ & $31 \%$ \\
\hline of which: Payments for R\&D expenditure & $69 \%$ & $23 \%$ & $0 \%$ & $8 \%$ \\
\hline Support to households and students & $\mathbf{3 8 \%}$ & $\mathbf{1 5 \%}$ & $\mathbf{3 1 \%}$ & $\mathbf{1 5 \%}$ \\
\hline Scholarships and other grants to households and students & $31 \%$ & $8 \%$ & $38 \%$ & $23 \%$ \\
\hline Student loans & $31 \%$ & $8 \%$ & $31 \%$ & $31 \%$ \\
\hline
\end{tabular}

List of respondent countries: Australia, Belgium (Flemish Community), Brazil, Canada, Finland, Hungary, Israel, the Netherlands, New Zealand, Portugal, the Slovak Republic, Sweden, Switzerland, the United Kingdom and the United States.

For more details on countries' responses, see Annex 3 (www.oecd.org/education/education-at-a-glance-19991487.htm). 
In many OECD countries, greater participation in tertiary education (see Indicator $\mathrm{C} 1$ ) reflects strong individual and social demand. The increases in enrolment have been accompanied by increases in investment from both public and private sources, and changes in the proportions of public and private expenditure. On average across the 20 OECD countries for which trend data are available for all reference years, the share of public funding for tertiary institutions decreased by more than four percentage points between 2000 and 2012, from $68.8 \%$ in 2000 to $64.9 \%$ in 2005 , and continued to decrease slightly over the following years to $64.5 \%$ in 2012 . The decrease in this share is particularly large in some, mostly European, countries where there had been large increases in private funding, from tuition fees and/or as enterprises participated more actively, largely through grants to tertiary institutions.

In most countries with available data for the different years, the change in the share of public/private funding since 2005 was small; between 2005 and 2012, changes were smallest since 2008 (Table B3.2b, Chart B3.3 and see Indicator B5).

Seventeen of the 22 countries for which comparable data are available for 2000 and 2012 showed an increase in the share of private funding for tertiary education. This increase exceeded 9 percentage points in Hungary, Italy, Mexico, Portugal and the Slovak Republic. Some countries reported particularly large increases before 2000 (see Education at a Glance 2014). For example, in Australia, the increase between 1995 and 2000 was notable and associated with changes to the Higher Education Contribution Scheme/Higher Education Loan Programme implemented in 1997. By contrast, Chile, Korea and Poland show significant decreases (by 6 percentage points or more) in the share of private expenditure on tertiary educational institutions between 2000 and 2012. In Chile and Korea, where tuition fees are particularly high (see Indicator B5), the decrease resulted from a larger increase in public expenditure than in private expenditure.

In some countries, trends in the changes in the share of public/private funding move in opposite directions before and after 2008. This is most significant in Chile, Korea and Poland, where the share of private funds increased between 2005 and 2008, and then decreased between 2008 and 2012, resulting in an overall decrease in the share of private funds between 2005 and 2012. In these countries, contrary to what was observed in most other countries, the change in the share of private expenditure was larger between 2008 and 2012 than between 2005 and 2008 (Chart B3.3).

Between 2000 and 2012, private expenditure on educational institutions generally increased faster than public expenditure. Nevertheless, public investment in tertiary education also increased in most countries for which 2000 and 2012 data are available, regardless of the changes in private spending. Five of the ten countries with the largest increases in private expenditure during this period (Chile, the Czech Republic, Iceland, Mexico and the Slovak Republic) are also among the ten countries with the largest increases in public expenditure (Table B3.2b).

\section{Public expenditure on educational institutions per student, by type of institution}

The level of public expenditure partly shows the degree to which governments value education (see Indicators B2 and B4). Naturally, public funds go to public institutions; but in some cases a significant part of the public budget may be devoted to private educational institutions (government-dependent private institutions and independent private institutions).

Table B3.3 shows public investment in educational institutions relative to the size of the education system. The data focus on public expenditure, per student, on public and private educational institutions (private funds are excluded from Table B3.3, although in some countries they represent a significant share of the resources of educational institutions, especially at the tertiary level). This measure complements data on public expenditure relative to national income (see Indicator B2).

On average across OECD countries, at primary to tertiary levels of education combined, public expenditure, per student, on public institutions is $91 \%$ higher than public expenditure, per student, on private institutions (USD 9317 and USD 4 889, respectively). However, the difference varies according to the level of education. At the primary, secondary and post-secondary non-tertiary levels of education, public expenditure, per student, on public institutions is around 1.6 times that on private institutions (USD 8683 and USD 5 284, respectively), whereas at the tertiary level, public expenditure, per student, on public institutions is 2.5 times that on private institutions (USD 11913 and USD 4 751, respectively).

At the primary, secondary and post-secondary non-tertiary levels of education (the levels with the largest proportion of public funds, Table B3.2b), public expenditure per student on both public and private institutions averages USD 8039 in OECD countries, but varies from less than USD 2350 in Mexico and partner countries Colombia 
and Indonesia, to more than USD 10000 in Austria, Belgium, Denmark, Norway, Sweden and the United States, and exceeds USD 19000 in Luxembourg. At this level, most students are enrolled in public institutions, and public expenditure per student is usually higher on public than on private institutions, except in Denmark, Israel and, to a lesser extent, Hungary and the Slovak Republic (Table B3.3). In these four OECD countries, between $9 \%$ and $20 \%$ of students are enrolled in private institutions. In Mexico and the Netherlands, the amount of public expenditure, per student, on private institutions is small or negligible, as the private sector is marginal and receives little or no public funds (see Table C1.4).

\section{Chart B3.3. Share of private expenditure on tertiary education institutions (2005 and 2012) and change, in percentage points, in the share of private expenditure between 2005 and 2012}
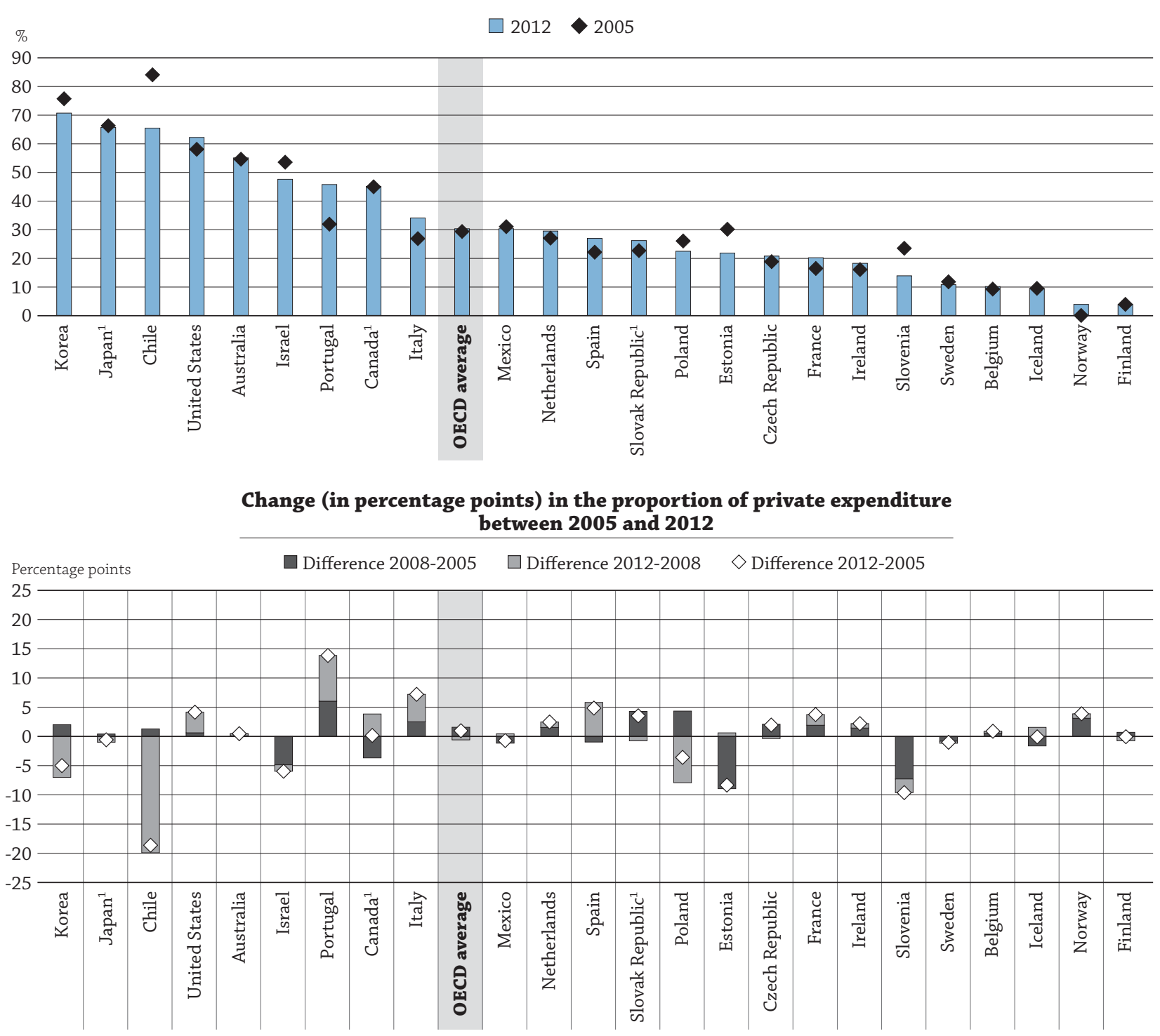

1. Some levels of education are included with others. Refer to "x" code in Table B1.1a for details.

Countries are ranked in descending order of the share of private expenditure on educational institutions in 2012.

Source: OECD. Table B3.2b. See Annex 3 for notes (www.oecd.org/education/education-at-a-glance-19991487.htm).

StatLink त्राजा http://dx.doi.org/10.1787/888933284007

At the tertiary level, public expenditure, per student, on both public and private institutions averages USD 9223 in OECD countries, but varies from about USD 2800 or less in Chile, Colombia and Indonesia, to more than USD 17000 in Finland, Norway and Sweden - three countries where the share of private expenditure is small or negligible. In all countries with available data except Israel and Latvia, public expenditure, per student, is higher on public than on private institutions (Table B3.3 and Chart B3.4). 
At this level, patterns in the allocation of public funds to public and private institutions differ. In the Netherlands, at least $90 \%$ of students are enrolled in public institutions, and most public expenditure goes to these institutions. Public expenditure, per student, on public institutions is higher than the OECD average, and public expenditure, per student, on private institutions is negligible. About 30\% of total expenditure on tertiary institutions in the Netherlands come from private sources (Chart B3.4 and Table B3.1).

In Belgium, Estonia, Hungary, Iceland, Israel, Latvia and Sweden, public expenditure goes to both public and private institutions, and public expenditure, per student, on private institutions represents at least $50 \%$ - and up to more than $100 \%$ - of the level of public expenditure, per student, on public tertiary institutions (Table B3.3). However, these countries show different participation patterns. In Hungary and Sweden, at least $80 \%$ of students are enrolled in public institutions, whereas in Belgium, Estonia, Israel and Latvia, tertiary students are mainly enrolled in private institutions. In the remaining countries, public expenditure goes mainly to public institutions (Chart B3.4 and Table B3.3).

\section{Chart B3.4. Annual public expenditure on educational institutions per student in tertiary education, by type of institution (2012)}

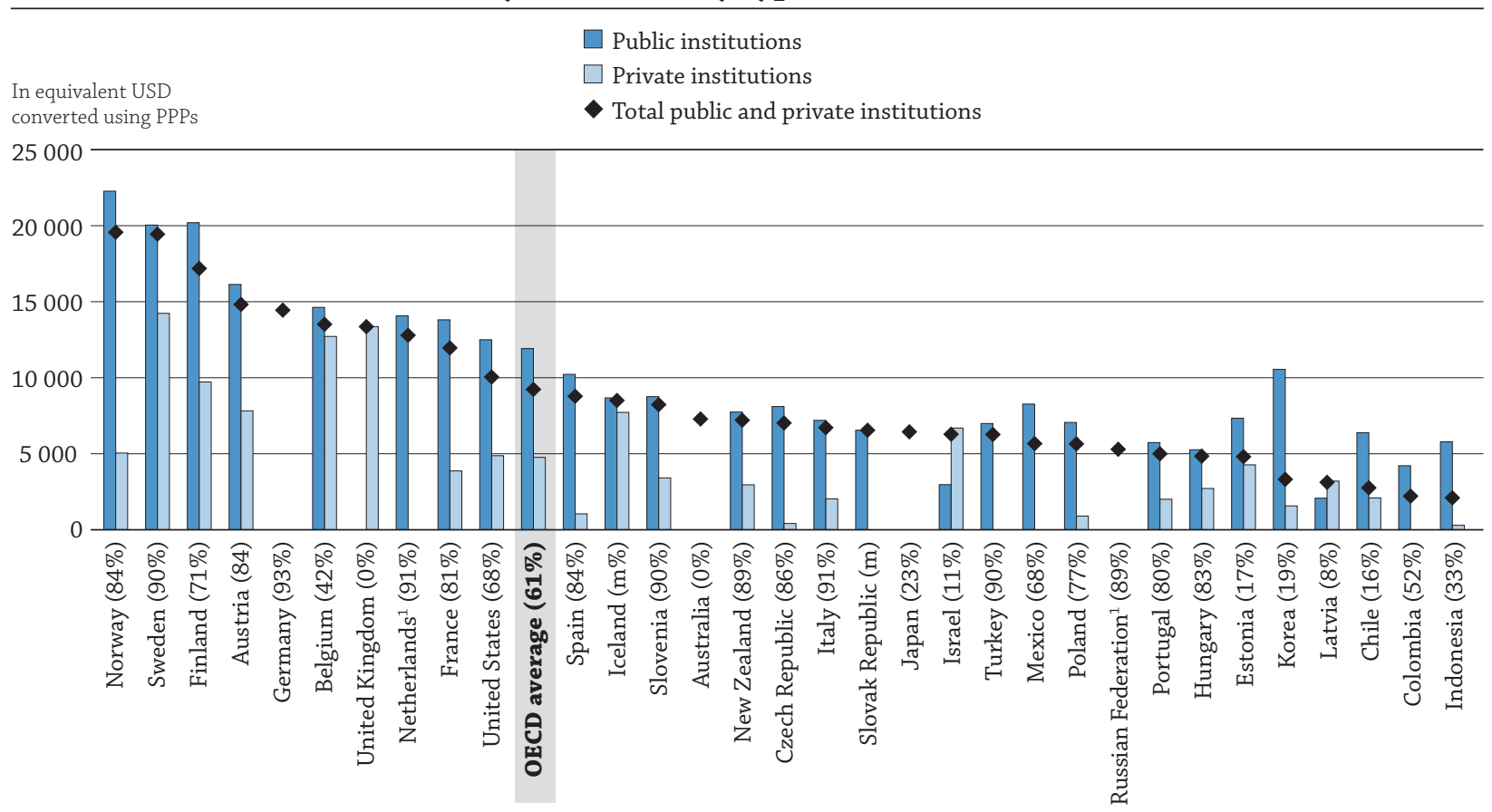

Note: The figures into brackets represent the percentage of students enrolled in public institutions in tertiary education, based on full-time equivalents. 1. Government-dependent institutions are included with public institutions.

Countries are ranked in descending order of public expenditure on public and private educational institutions per student.

Source: OECD. Table B3.3. See Annex 3 for notes (www.oecd.org/education/education-at-a-glance-19991487.htm).

StatLink 제개 http://dx.doi.org/10.1787/888933284014

\section{Definitions}

Other private entities include private businesses and non-profit organisations, e.g. religious organisations, charitable organisations and business and labour associations.

Private spending includes all direct expenditure on educational institutions, whether partially covered by public subsidies or not. Expenditure by private companies on the work-based element of school- and work-based training of apprentices and students is also taken into account. Public subsidies attributable to households, included in private spending, are shown separately.

The public and private proportions of expenditure on educational institutions are the percentages of total spending originating in, or generated by, the public and private sectors.

Public expenditure is related to all students at public and private institutions, whether these institutions receive public funding or not. 


\section{Methodology}

Data refer to the financial year 2012 and are based on the UOE data collection on education statistics administered by the OECD in 2014 (for details see Annex 3 at www.oecd.org/education/education-at-a-glance-19991487.htm).

Not all spending on instructional goods and services occurs within educational institutions. For example, families may purchase commercial textbooks and materials or seek private tutoring for their children outside educational institutions. At the tertiary level, students' living expenses and foregone earnings can also account for a significant proportion of the costs of education. All expenditure outside educational institutions, even if publicly subsidised, is excluded from this indicator. Public subsidies for educational expenditure outside institutions are discussed in Indicators B4 and B5.

A portion of the budgets of educational institutions is related to ancillary services offered to students, including student welfare services (student meals, housing and transport). Part of the cost of these services is covered by fees collected from students and is included in the indicator.

Expenditure on educational institutions is calculated on a cash-accounting basis and, as such, represents a snapshot of expenditure in the reference year. Many countries operate a loan payment/repayment system at the tertiary level. While public loan payments are taken into account, loan repayments from private individuals are not, and so the private contribution to education costs may be under-represented.

The data on expenditure for 2000, 2005, 2008, 2010 and 2011 were updated based on a survey in 2014, and expenditure for 2000 to 2011 were adjusted to the methods and definitions used in the current UOE data collection.

\section{Note regarding data from Israel}

The statistical data for Israel are supplied by and are under the responsibility of the relevant Israeli authorities. The use of such data by the OECD is without prejudice to the status of the Golan Heights, East Jerusalem and Israeli settlements in the West Bank under the terms of international law.

\section{Indicator B3 Tables}

StatLink 잠ㄴ http://dx.doi.org/10.1787/888933285464

Table B3.1 Relative proportions of public and private expenditure on educational institutions, by level of education (2012)

Table B3.2a Trends in the proportion of public expenditure on educational institutions and index of change in public and private expenditure, at primary, secondary, post-secondary non-tertiary level (2000, 2005, 2008, 2010 to 2012)

Table B3.2b Trends in the proportion of public expenditure on tertiary education institutions and index of change in public and private expenditure (2000, 2005, 2008, 2010 to 2012)

Table B3.3 Annual public expenditure on educational institutions per student, by type of institution (2012)

Cut-off date for the data: 23 October 2015. Updates can be found on line at http://dx.doi.org/10.1787/eag-data-en. 
Table B3.1. Relative proportions of public and private expenditure on educational institutions, by level of education (2012)

Distribution of public and private sources of funds for educational institutions after transfers from public sources

\begin{tabular}{|c|c|c|c|c|c|c|c|c|c|c|c|c|c|c|c|}
\hline & \multicolumn{5}{|c|}{$\begin{array}{l}\text { Primary, secondary } \\
\text { and post-secondary non-tertiary }\end{array}$} & \multicolumn{5}{|c|}{ Tertiary } & \multicolumn{5}{|c|}{$\begin{array}{c}\text { Primary to tertiary } \\
\text { (including undistributed programmes) }\end{array}$} \\
\hline & 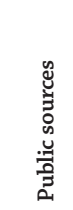 & \multicolumn{3}{|c|}{ Private sources } & 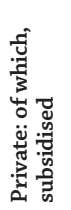 & 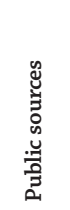 & \multicolumn{3}{|c|}{ Private sources } & 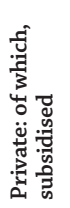 & 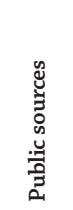 & \multicolumn{3}{|c|}{ Private sources } & 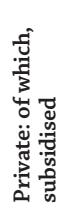 \\
\hline & $(1)$ & (2) & (3) & (4) & $(5)$ & (6) & $(7)$ & (8) & (9) & $(10)$ & (11) & (12) & (13) & $(14)$ & (15) \\
\hline Australia & 82.4 & 14.9 & 2.7 & 17.6 & 3.6 & 44.9 & 40.0 & 15.0 & 55.1 & 10.1 & 71.7 & 22.1 & 6.2 & 28.3 & 5.4 \\
\hline Austria & 96.0 & 3.1 & 1.0 & 4.0 & 4.5 & 95.3 & 1.9 & 2.8 & 4.7 & 9.4 & 95.7 & 2.7 & 1.6 & 4.3 & 6.2 \\
\hline Belgium & 96.3 & 3.6 & 0.1 & 3.7 & 3.0 & 89.9 & 4.9 & 5.2 & 10.1 & 15.7 & 94.9 & 3.8 & 1.3 & 5.1 & 5.9 \\
\hline Canada $^{2,3}$ & 91.0 & 4.0 & 5.0 & 9.0 & $\mathrm{~m}$ & 54.9 & 23.4 & 21.7 & 45.1 & 4.3 & 76.2 & 12.0 & 11.8 & 23.8 & 1.7 \\
\hline Chile $^{4}$ & 78.0 & 21.3 & 0.7 & 22.0 & 0.9 & 34.6 & 54.8 & 10.7 & 65.4 & 6.7 & 60.1 & 35.1 & 4.8 & 39.9 & 3.3 \\
\hline Czech Republic & 91.0 & 7.0 & 2.0 & 9.0 & 3.1 & 79.3 & 8.5 & 12.3 & 20.7 & 1.4 & 87.3 & 7.7 & 5.0 & 12.7 & 2.5 \\
\hline Denmark & 97.2 & 2.8 & 0.0 & 2.8 & 7.2 & $\mathbf{m}$ & $\mathrm{m}$ & $\mathrm{m}$ & $\mathbf{m}$ & $\mathrm{m}$ & $\mathbf{m}$ & $\mathrm{m}$ & $\mathrm{m}$ & $\mathbf{m}$ & $\mathrm{m}$ \\
\hline Finland & 99.3 & $\mathrm{x}(4)$ & $x(4)$ & 0.7 & 3.6 & 96.2 & $\mathrm{x}(9)$ & $\mathrm{x}(9)$ & 3.8 & 15.0 & 98.3 & $\mathrm{x}(14)$ & $\mathrm{x}(14)$ & 1.7 & 7.2 \\
\hline France & 91.0 & 7.5 & 1.5 & 9.0 & 3.3 & 79.8 & 10.6 & 9.6 & 20.2 & 7.3 & 88.0 & 8.3 & 3.7 & 12.0 & 4.4 \\
\hline Germany & 86.5 & $\mathrm{x}(4)$ & $\mathrm{x}(4)$ & 13.5 & $\mathrm{~m}$ & 85.9 & $\mathrm{x}(9)$ & $\mathrm{x}(9)$ & 14.1 & $\mathrm{~m}$ & 86.6 & $\mathrm{x}(14)$ & $\mathrm{x}(14)$ & 13.4 & $\mathrm{~m}$ \\
\hline Greece & $\mathbf{m}$ & $\mathrm{m}$ & $\mathrm{m}$ & $\mathbf{m}$ & $\mathrm{m}$ & $\mathbf{m}$ & $\mathrm{m}$ & $\mathrm{m}$ & $\mathbf{m}$ & $\mathrm{m}$ & $\mathbf{m}$ & $\mathrm{m}$ & $\mathrm{m}$ & $\mathbf{m}$ & $\mathrm{m}$ \\
\hline Hungary & 94.2 & $\mathrm{x}(4)$ & $\mathrm{x}(4)$ & 5.8 & 3.8 & 54.4 & $\mathrm{x}(9)$ & $\mathrm{x}(9)$ & 45.6 & 9.8 & 81.5 & $\mathrm{x}(14)$ & $x(14)$ & 18.5 & 5.5 \\
\hline Iceland & 96.0 & 3.7 & 0.4 & 4.0 & a & 90.6 & 8.7 & 0.7 & 9.4 & a & 92.1 & 7.1 & 0.8 & 7.9 & $\mathrm{a}$ \\
\hline Ireland & 95.7 & 4.3 & $\mathrm{~m}$ & 4.3 & 6.7 & 81.8 & 15.8 & 2.4 & 18.2 & 17.8 & 92.6 & 6.9 & 0.5 & 7.4 & 9.1 \\
\hline Israel & 88.9 & 7.5 & 3.6 & 11.1 & 1.1 & 52.4 & 29.6 & 18.0 & 47.6 & 5.9 & 77.1 & 13.9 & 9.0 & 22.9 & 2.4 \\
\hline Italy $^{5}$ & 95.5 & 4.4 & 0.1 & 4.5 & 2.1 & 66.0 & 26.5 & 7.5 & 34.0 & 19.6 & 88.4 & 9.7 & 1.9 & 11.6 & 6.3 \\
\hline Japan $^{3}$ & 92.9 & 5.1 & 2.1 & 7.1 & $\mathrm{~m}$ & 34.3 & 51.6 & 14.1 & 65.7 & $\mathrm{~m}$ & 70.1 & 19.7 & 10.2 & 29.9 & $\mathrm{~m}$ \\
\hline Korea & 83.9 & 14.4 & 1.7 & 16.1 & 1.6 & 29.3 & 42.1 & 28.6 & 70.7 & 6.5 & 66.5 & 22.6 & 11.0 & 33.5 & 3.8 \\
\hline Luxembourg & 97.8 & 2.0 & 0.2 & 2.2 & 1.7 & 94.8 & 2.8 & 2.4 & 5.2 & 11.8 & 97.4 & 2.1 & 0.5 & 2.6 & 2.9 \\
\hline Mexico & 82.8 & 17.1 & 0.1 & 17.2 & 5.5 & 69.7 & 30.0 & 0.3 & 30.3 & 3.7 & 80.3 & 19.5 & 0.2 & 19.7 & 5.0 \\
\hline Netherlands & 86.7 & 4.4 & 9.0 & 13.3 & 6.4 & 70.5 & 15.6 & 13.9 & 29.5 & 9.5 & 81.7 & 7.8 & 10.5 & 18.3 & 7.4 \\
\hline New Zealand & 82.5 & 12.8 & 4.7 & 17.5 & 1.9 & 52.4 & 33.1 & 14.5 & 47.6 & 11.4 & 74.2 & 18.4 & 7.4 & 25.8 & 4.5 \\
\hline Norway $^{3}$ & $\mathbf{m}$ & $\mathrm{m}$ & $\mathrm{m}$ & $\mathbf{m}$ & $\mathrm{m}$ & 96.1 & 3.3 & 0.6 & 3.9 & 18.0 & $\mathbf{m}$ & $\mathrm{m}$ & $\mathrm{m}$ & $\mathbf{m}$ & $\mathrm{m}$ \\
\hline Slovak Republic ${ }^{3}$ & 88.1 & 9.8 & 2.1 & 11.9 & 4.4 & 73.8 & 13.8 & 12.4 & 26.2 & 18.7 & 84.6 & 10.6 & 4.8 & 15.4 & 8.3 \\
\hline Slovenia & 91.0 & 8.6 & 0.3 & 9.0 & 2.9 & 86.1 & 11.4 & 2.5 & 13.9 & 15.9 & 89.8 & 9.3 & 0.9 & 10.2 & 6.0 \\
\hline Spain & 88.7 & 10.5 & 0.8 & 11.3 & 1.6 & 73.1 & 23.0 & 4.0 & 26.9 & 8.3 & 84.2 & 14.1 & 1.7 & 15.8 & 3.5 \\
\hline Sweden & 100.0 & 0.0 & 0.0 & 0.0 & 0.0 & 89.3 & 0.4 & 10.4 & 10.7 & 0.0 & 96.7 & 0.1 & 3.1 & 3.3 & 0.0 \\
\hline Switzerland & 88.5 & 0.0 & 11.5 & 11.5 & 1.4 & $\mathbf{m}$ & $\mathrm{m}$ & $\mathrm{m}$ & $\mathbf{m}$ & $\mathrm{m}$ & $\mathbf{m}$ & $\mathrm{m}$ & $\mathrm{m}$ & $\mathbf{m}$ & $\mathrm{m}$ \\
\hline Turkey & 85.4 & 14.6 & $\mathrm{~m}$ & 14.6 & 1.0 & 80.4 & 19.6 & $\mathrm{~m}$ & 19.6 & 5.1 & 83.8 & 16.2 & $\mathrm{~m}$ & 16.2 & 2.3 \\
\hline United Kingdom & 84.0 & 13.7 & 2.4 & 16.0 & 5.9 & 56.9 & 19.3 & 23.8 & 43.1 & 8.7 & 76.4 & 15.2 & 8.3 & 23.6 & 6.7 \\
\hline United States ${ }^{3}$ & 92.0 & 8.0 & $\mathrm{~m}$ & 8.0 & $\mathrm{~m}$ & 37.8 & 45.8 & 16.4 & 62.2 & 12.7 & 68.4 & 24.5 & 7.1 & 31.6 & 5.5 \\
\hline OECD average & 90.6 & 7.9 & 2.2 & 9.4 & 3.1 & 69.7 & 21.7 & 10.0 & 30.3 & 10.1 & 83.5 & 12.8 & 4.5 & 16.5 & 4.7 \\
\hline EU21 average & 92.8 & 6.2 & 1.4 & 7.2 & 3.5 & 78.1 & 14.0 & 8.0 & 21.9 & 11.0 & 88.6 & 8.3 & 3.0 & 11.4 & 5.2 \\
\hline $\begin{array}{l}\text { Argentina } \\
\text { Brazil }\end{array}$ & $\begin{array}{l}\mathbf{m} \\
\mathbf{m}\end{array}$ & $\begin{array}{l}\mathrm{m} \\
\mathrm{m}\end{array}$ & $\begin{array}{l}\mathrm{m} \\
\mathrm{m}\end{array}$ & $\begin{array}{l}\mathbf{m} \\
\mathbf{m}\end{array}$ & $\begin{array}{l}\mathrm{m} \\
\mathrm{m}\end{array}$ & $\begin{array}{l}\mathbf{m} \\
\mathbf{m}\end{array}$ & $\begin{array}{l}\mathrm{m} \\
\mathrm{m}\end{array}$ & $\begin{array}{l}\mathrm{m} \\
\mathrm{m}\end{array}$ & $\begin{array}{l}\mathbf{m} \\
\mathbf{m}\end{array}$ & $\begin{array}{l}\mathrm{m} \\
\mathrm{m}\end{array}$ & $\begin{array}{l}\mathbf{m} \\
\mathbf{m}\end{array}$ & $\begin{array}{l}\mathrm{m} \\
\mathrm{m}\end{array}$ & $\begin{array}{l}\mathrm{m} \\
\mathrm{m}\end{array}$ & $\begin{array}{l}\mathbf{m} \\
\mathbf{m}\end{array}$ & $\begin{array}{l}\mathrm{m} \\
\mathrm{m}\end{array}$ \\
\hline China & $\mathbf{m}$ & $\mathrm{m}$ & $\mathrm{m}$ & $\mathbf{m}$ & $\mathrm{m}$ & $\mathbf{m}$ & $\mathrm{m}$ & $\mathrm{m}$ & $\mathbf{m}$ & $\mathrm{m}$ & $\mathbf{m}$ & $\mathrm{m}$ & $\mathrm{m}$ & $\mathbf{m}$ & $\mathrm{m}$ \\
\hline Colombia $^{4}$ & 78.0 & 21.8 & 0.2 & 22.0 & 2.4 & 42.6 & 57.4 & 0.0 & 57.4 & 5.4 & 69.1 & 30.7 & 0.2 & 30.9 & 3.1 \\
\hline India & m & $\mathrm{m}$ & $\mathrm{m}$ & $\mathbf{m}$ & $\mathrm{m}$ & m & $\mathrm{m}$ & $\mathrm{m}$ & m & $\mathrm{m}$ & $\mathbf{m}$ & $\mathrm{m}$ & $\mathrm{m}$ & m & $\mathrm{m}$ \\
\hline Indonesia $^{4}$ & 90.6 & 9.4 & $\mathrm{~m}$ & 9.4 & 1.2 & 70.7 & 29.3 & $\mathrm{~m}$ & 29.3 & 2.2 & 87.7 & 12.3 & $\mathrm{~m}$ & 12.3 & 1.3 \\
\hline Latvia & 97.7 & 2.1 & 0.2 & 2.3 & 4.0 & 63.6 & 36.2 & 0.2 & 36.4 & 10.8 & 87.1 & 12.8 & 0.2 & 12.9 & 6.2 \\
\hline Russian Federation & 96.7 & 2.6 & 0.7 & 3.3 & $\mathrm{~m}$ & 63.5 & 23.8 & 12.7 & 36.5 & $\mathrm{~m}$ & 85.4 & 9.8 & 4.9 & 14.6 & $\mathrm{~m}$ \\
\hline Saudi Arabia & m & $\mathrm{m}$ & $\mathrm{m}$ & $\mathbf{m}$ & $\mathrm{m}$ & $\mathbf{m}$ & $\mathrm{m}$ & $\mathrm{m}$ & $\mathbf{m}$ & $\mathrm{m}$ & $\mathbf{m}$ & $\mathrm{m}$ & $\mathrm{m}$ & $\mathbf{m}$ & $\mathrm{m}$ \\
\hline South Africa & $\mathbf{m}$ & $\mathrm{m}$ & $\mathrm{m}$ & $\mathbf{m}$ & $\mathrm{m}$ & $\mathbf{m}$ & $\mathrm{m}$ & $\mathrm{m}$ & $\mathbf{m}$ & $\mathrm{m}$ & $\mathbf{m}$ & $\mathrm{m}$ & $\mathrm{m}$ & $\mathbf{m}$ & $\mathrm{m}$ \\
\hline
\end{tabular}

\begin{tabular}{l|l|l|l|l|l|l|l|l|l|l|l|l|l|l|l|l|l|} 
G20 average & $\mathbf{m}$ & $\mathrm{m}$ & $\mathrm{m}$ & $\mathbf{m}$ & $\mathrm{m}$ & $\mathbf{m}$ & $\mathrm{m}$ & $\mathrm{m}$ & $\mathbf{m}$ & $\mathrm{m}$ & $\mathbf{m}$ & $\mathrm{m}$ & $\mathrm{m}$ & $\mathbf{m}$ & $\mathrm{m}$
\end{tabular}

Note: Proportions of total expenditure from public sources in early childhood education is available in Indicator C2.

1. Including subsidies attributable to payments to educational institutions received from public sources.

2. Year of reference 2011 instead of 2012

3. Some levels of education are included with others. Refer to " $\mathrm{x}$ " code in Table B1.1a for details.

4. Year of reference 2013 instead of 2012.

5. Excludes short-cycle tertiary programmes.

Source: OECD. Argentina, China, Colombia, India, Indonesia, Saudi Arabia, South Africa: UNESCO Institute for Statistics. Latvia: Eurostat. See Annex 3 for notes (www.oecd.org/education/education-at-a-glance-19991487.htm).

Please refer to the Reader's Guide for information concerning symbols for missing data and abbreviations.

StatLink 需点 http://dx.doi.org/10.1787/888933285473 
Table B3.2a. Trends in the proportion of public expenditure ${ }^{1}$ on educational institutions and index of change in public and private expenditure, at primary, secondary, post-secondary non-tertiary level (2000, 2005, 2008, 2010 to 2012)

Index of change of public and private sources of funds for educational institutions after transfers from public sources, by year

\begin{tabular}{|c|c|c|c|c|c|c|c|c|c|c|c|c|c|c|c|c|}
\hline & \multirow{2}{*}{\multicolumn{6}{|c|}{$\begin{array}{l}\text { Share of public expenditure } \\
\text { on educational institutions } \\
\text { (\%) }\end{array}$}} & \multicolumn{10}{|c|}{$\begin{array}{l}\text { Index of change between } 2000 \text { and } 2012 \text { in expenditure } \\
\text { on educational institutions } \\
(2005=100 \text {, constant prices })\end{array}$} \\
\hline & & & & & & & \multicolumn{5}{|c|}{ Public sources } & \multicolumn{5}{|c|}{ Private sources $^{2}$} \\
\hline & 2000 & 2005 & 2008 & 2010 & 2011 & 2012 & 2000 & 2008 & 2010 & 2011 & 2012 & 2000 & 2008 & 2010 & 2011 & 2012 \\
\hline & (1) & (2) & (3) & (4) & (5) & (6) & (7) & (8) & (9) & (10) & (11) & $(12)$ & (13) & (14) & (15) & (16) \\
\hline Australia & 83.7 & 83.5 & 82.5 & 84.7 & 83.6 & 82.4 & 83 & 108 & 135 & 130 & 129 & 82 & 116 & 123 & 129 & 139 \\
\hline Austria & $\mathrm{m}$ & $\mathrm{m}$ & $\mathrm{m}$ & $\mathrm{m}$ & $\mathrm{m}$ & 96.0 & $\mathrm{~m}$ & $\mathrm{~m}$ & $\mathrm{~m}$ & $\mathrm{~m}$ & $\mathrm{~m}$ & $\mathrm{~m}$ & $\mathrm{~m}$ & $\mathrm{~m}$ & $\mathrm{~m}$ & $\mathrm{~m}$ \\
\hline Belgium & $\mathrm{m}$ & 94.9 & 95.2 & 96.0 & 96.2 & 96.3 & $\mathrm{~m}$ & 113 & 113 & 114 & 116 & $\mathrm{~m}$ & 106 & 88 & 83 & 83 \\
\hline Canada $^{3,4}$ & 92.4 & 89.9 & 88.6 & 89.3 & 89.7 & 91.0 & 86 & 104 & 116 & 117 & 116 & 63 & 118 & 123 & 120 & 102 \\
\hline Chile $^{5}$ & 68.4 & 69.8 & 78.4 & 78.6 & 78.3 & 78.0 & 94 & 145 & 141 & 165 & 156 & 100 & 92 & 89 & 105 & 101 \\
\hline Czech Republic & 91.7 & 89.9 & 90.4 & 90.8 & 90.9 & 91.0 & 78 & 106 & 111 & 114 & 115 & 63 & 101 & 100 & 102 & 102 \\
\hline Denmark $^{3}$ & 97.8 & 97.9 & 97.6 & 97.6 & 97.2 & 97.2 & 86 & 99 & 108 & 99 & 79 & 89 & 112 & 124 & 134 & $\mathrm{~m}$ \\
\hline Estonia & $\mathrm{m}$ & 98.9 & 99.0 & 98.7 & 98.9 & 99.1 & 81 & 124 & 109 & 103 & 105 & $\mathrm{~m}$ & 119 & 129 & 108 & 89 \\
\hline Finland & 99.3 & 99.2 & 99.0 & 99.2 & 99.3 & 99.3 & 83 & 107 & 112 & 113 & 112 & 66 & 126 & 104 & 97 & 99 \\
\hline France & 91.6 & 91.4 & 91.2 & 91.4 & 91.2 & 91.0 & 99 & 102 & 106 & 104 & 104 & 97 & 105 & 106 & 107 & 108 \\
\hline Germany & $\mathrm{m}$ & $\mathrm{m}$ & $\mathrm{m}$ & 87.1 & 87.2 & 86.5 & $\mathrm{~m}$ & $\mathrm{~m}$ & $\mathrm{~m}$ & $\mathrm{~m}$ & $\mathrm{~m}$ & $\mathrm{~m}$ & $\mathrm{~m}$ & $\mathrm{~m}$ & $\mathrm{~m}$ & $\mathrm{~m}$ \\
\hline Greece & 91.7 & 92.5 & $\mathrm{~m}$ & $\mathrm{~m}$ & $\mathrm{~m}$ & $\mathrm{~m}$ & $\mathrm{~m}$ & $\mathrm{~m}$ & $\mathrm{~m}$ & $\mathrm{~m}$ & $\mathrm{~m}$ & $\mathrm{~m}$ & $\mathrm{~m}$ & $\mathrm{~m}$ & $\mathrm{~m}$ & $\mathrm{~m}$ \\
\hline Hungary & 92.7 & 95.5 & 100.0 & 100.0 & 100.0 & 94.2 & 68 & 96 & 85 & 79 & 75 & $\mathrm{~m}$ & $\mathrm{~m}$ & $\mathrm{~m}$ & $\mathrm{~m}$ & $\mathrm{~m}$ \\
\hline Iceland & 96.4 & 96.2 & 96.4 & 96.2 & 96.3 & 96.0 & 73 & 108 & 96 & 99 & 98 & 70 & 103 & 96 & 97 & 105 \\
\hline Ireland & 96.0 & 96.8 & 97.7 & 95.9 & 95.8 & 95.7 & 68 & 132 & 138 & 136 & 137 & 87 & 97 & 179 & 183 & 187 \\
\hline Israel & 94.8 & 93.0 & 93.0 & 92.4 & 89.5 & 88.9 & 101 & 120 & 129 & 139 & 147 & 74 & 120 & 142 & 217 & 243 \\
\hline Italy & 97.8 & 96.3 & 97.1 & 96.6 & 96.2 & 95.5 & 95 & 105 & 98 & 93 & 88 & 55 & 81 & 89 & 96 & 109 \\
\hline Japan $^{3}$ & 89.8 & 90.1 & 90.0 & 93.0 & 93.0 & 92.9 & 98 & 102 & 108 & 108 & 110 & 101 & 103 & 73 & 74 & 76 \\
\hline Korea & 80.8 & 77.0 & 77.8 & 78.5 & 80.7 & 83.9 & 72 & 116 & 128 & 133 & 136 & 58 & 111 & 118 & 107 & 88 \\
\hline Luxembourg & $\mathrm{m}$ & $\mathrm{m}$ & $\mathrm{m}$ & $\mathrm{m}$ & $\mathrm{m}$ & 97.8 & $\mathrm{~m}$ & $\mathrm{~m}$ & $\mathrm{~m}$ & $\mathrm{~m}$ & 96 & $\mathrm{~m}$ & $\mathrm{~m}$ & $\mathrm{~m}$ & $\mathrm{~m}$ & $\mathrm{~m}$ \\
\hline Mexico & 86.1 & 82.9 & 82.9 & 82.7 & 82.6 & 82.8 & 84 & 103 & 111 & 115 & 119 & 65 & 103 & 112 & 118 & 119 \\
\hline Netherlands & 86.1 & 87.1 & 86.6 & 86.9 & 86.6 & 86.7 & 81 & 105 & 114 & 113 & 112 & 89 & 111 & 116 & 118 & 117 \\
\hline New Zealand & $\mathrm{m}$ & $\mathrm{m}$ & $\mathrm{m}$ & $\mathrm{m}$ & $\mathrm{m}$ & 82.5 & $\mathrm{~m}$ & $\mathrm{~m}$ & $\mathrm{~m}$ & $\mathrm{~m}$ & $\mathrm{~m}$ & $\mathrm{~m}$ & $\mathrm{~m}$ & $\mathrm{~m}$ & $\mathrm{~m}$ & $\mathrm{~m}$ \\
\hline Norway & 99.0 & $\mathrm{~m}$ & $\mathrm{~m}$ & $\mathrm{~m}$ & $\mathrm{~m}$ & $\mathrm{~m}$ & 87 & 107 & 113 & 112 & 112 & $\mathrm{~m}$ & $\mathrm{~m}$ & $\mathrm{~m}$ & $\mathrm{~m}$ & $\mathrm{~m}$ \\
\hline Poland & 95.4 & 98.2 & 94.7 & 93.8 & 93.9 & 92.0 & 87 & 110 & 116 & 115 & 115 & 226 & 337 & 417 & 402 & 538 \\
\hline Portugal & 99.9 & 99.9 & 99.9 & 100.0 & 99.9 & 85.2 & 100 & 95 & 108 & 101 & $\mathrm{~m}$ & 101 & 89 & 87 & 89 & $\mathrm{~m}$ \\
\hline Slovak Republic ${ }^{3}$ & 97.6 & 86.2 & 84.8 & 88.0 & 88.6 & 88.1 & 84 & 113 & 138 & 129 & 128 & 13 & 126 & 117 & 104 & 108 \\
\hline Slovenia & $\mathrm{m}$ & 91.9 & 91.7 & 91.3 & 91.1 & 91.0 & $\mathrm{~m}$ & 104 & 103 & 100 & 96 & $\mathrm{~m}$ & 107 & 110 & 110 & 108 \\
\hline Spain & 93.0 & 93.5 & 93.1 & 91.8 & 91.1 & 88.7 & 92 & 115 & 117 & 113 & 105 & 99 & 122 & 150 & 158 & 192 \\
\hline Sweden & 99.9 & 99.9 & 99.9 & 99.9 & 100.0 & 100.0 & 88 & 103 & 102 & 103 & 103 & a & a & a & a & a \\
\hline Switzerland & 88.9 & 86.9 & 86.3 & 88.1 & 88.3 & 88.5 & 89 & 102 & 108 & 111 & 112 & 74 & 108 & 98 & 98 & 97 \\
\hline Turkey & $\mathrm{m}$ & $\mathrm{m}$ & $\mathrm{m}$ & $\mathrm{m}$ & 86.8 & 85.4 & 71 & 121 & 147 & 149 & 165 & $\mathrm{~m}$ & $\mathrm{~m}$ & $\mathrm{~m}$ & $\mathrm{~m}$ & $\mathrm{~m}$ \\
\hline United Kingdom & $\mathrm{m}$ & $\mathrm{m}$ & $\mathrm{m}$ & $\mathrm{m}$ & $\mathrm{m}$ & 84.0 & 75 & 94 & 102 & 113 & 114 & $\mathrm{~m}$ & $\mathrm{~m}$ & $\mathrm{~m}$ & $\mathrm{~m}$ & $\mathrm{~m}$ \\
\hline United States & 91.7 & 91.8 & 91.8 & 92.3 & 91.9 & 92.0 & 86 & 111 & 110 & 107 & 104 & 87 & 111 & 102 & 105 & 101 \\
\hline OECD average & 92.1 & 91.5 & 91.7 & 91.9 & 91.6 & 90.6 & 85 & 109 & 114 & 115 & 114 & 84 & 118 & 125 & 128 & 137 \\
\hline $\begin{array}{l}\text { OECD average for } \\
19 \text { countries with } \\
\text { data available for } \\
\text { all reference years }\end{array}$ & 90.6 & 89.5 & 89.6 & 90.0 & 89.9 & 89.7 & 86 & 111 & 117 & 119 & 118 & 83 & 121 & 129 & 134 & 144 \\
\hline EU21 average & 95.1 & 94.7 & 94.9 & 94.4 & 94.4 & 92.8 & 84 & 107 & 110 & 108 & 106 & 90 & 124 & 137 & 135 & 153 \\
\hline
\end{tabular}

\begin{tabular}{|c|c|c|c|c|c|c|c|c|c|c|c|c|c|c|c|c|}
\hline Argentina & $\mathrm{m}$ & $\mathrm{m}$ & $\mathrm{m}$ & $\mathrm{m}$ & $\mathrm{m}$ & $\mathrm{m}$ & $\mathrm{m}$ & $\mathrm{m}$ & $\mathrm{m}$ & $\mathrm{m}$ & $m$ & $\mathrm{~m}$ & $\mathrm{~m}$ & $\mathrm{~m}$ & $\mathrm{~m}$ & $\mathrm{~m}$ \\
\hline Brazil & $\mathrm{m}$ & $\mathrm{m}$ & $\mathrm{m}$ & $\mathrm{m}$ & $\mathrm{m}$ & $\mathrm{m}$ & 66 & 146 & 170 & 175 & 182 & $\mathrm{~m}$ & $\mathrm{~m}$ & $\mathrm{~m}$ & $\mathrm{~m}$ & $\mathrm{~m}$ \\
\hline China & $\mathrm{m}$ & $\mathrm{m}$ & $\mathrm{m}$ & $\mathrm{m}$ & $\mathrm{m}$ & $\mathrm{m}$ & $\mathrm{m}$ & $\mathrm{m}$ & $\mathrm{m}$ & $\mathrm{m}$ & $\mathrm{m}$ & $\mathrm{m}$ & $\mathrm{m}$ & $\mathrm{m}$ & $\mathrm{m}$ & $\mathrm{m}$ \\
\hline Colombia $^{5}$ & $\mathrm{~m}$ & $\mathrm{~m}$ & $\mathrm{~m}$ & $\mathrm{~m}$ & $\mathrm{~m}$ & 78.0 & $\mathrm{~m}$ & $\mathrm{~m}$ & $\mathrm{~m}$ & $\mathrm{~m}$ & $\mathrm{~m}$ & $\mathrm{~m}$ & $\mathrm{~m}$ & $\mathrm{~m}$ & $\mathrm{~m}$ & $\mathrm{~m}$ \\
\hline India & $\mathrm{m}$ & $\mathrm{m}$ & $\mathrm{m}$ & $\mathrm{m}$ & $\mathrm{m}$ & $\mathrm{m}$ & $\mathrm{m}$ & $\mathrm{m}$ & $\mathrm{m}$ & $\mathrm{m}$ & $\mathrm{m}$ & $\mathrm{m}$ & $\mathrm{m}$ & $\mathrm{m}$ & $\mathrm{m}$ & $\mathrm{m}$ \\
\hline Indonesia $^{5}$ & $\mathrm{~m}$ & $\mathrm{~m}$ & $\mathrm{~m}$ & $\mathrm{~m}$ & $\mathrm{~m}$ & 90.6 & $\mathrm{~m}$ & $\mathrm{~m}$ & $\mathrm{~m}$ & $\mathrm{~m}$ & $\mathrm{~m}$ & $\mathrm{~m}$ & $\mathrm{~m}$ & $\mathrm{~m}$ & $\mathrm{~m}$ & $\mathrm{~m}$ \\
\hline Latvia & $\mathrm{m}$ & $\mathrm{m}$ & $\mathrm{m}$ & $\mathrm{m}$ & $\mathrm{m}$ & 97.7 & $\mathrm{~m}$ & $\mathrm{~m}$ & $\mathrm{~m}$ & $\mathrm{~m}$ & $\mathrm{~m}$ & $\mathrm{~m}$ & $\mathrm{~m}$ & $\mathrm{~m}$ & $\mathrm{~m}$ & $\mathrm{~m}$ \\
\hline Russian Federation & $\mathrm{m}$ & $\mathrm{m}$ & $\mathrm{m}$ & $\mathrm{m}$ & $\mathrm{m}$ & 96.7 & 66 & 132 & 126 & 129 & 151 & $\mathrm{~m}$ & $\mathrm{~m}$ & $\mathrm{~m}$ & $\mathrm{~m}$ & $\mathrm{~m}$ \\
\hline Saudi Arabia & $\mathrm{m}$ & $\mathrm{m}$ & $\mathrm{m}$ & $\mathrm{m}$ & $\mathrm{m}$ & $\mathrm{m}$ & $\mathrm{m}$ & $\mathrm{m}$ & $\mathrm{m}$ & $\mathrm{m}$ & $\mathrm{m}$ & $\mathrm{m}$ & $\mathrm{m}$ & $\mathrm{m}$ & $\mathrm{m}$ & $\mathrm{m}$ \\
\hline South Africa & $\mathrm{m}$ & $\mathrm{m}$ & $\mathrm{m}$ & $\mathrm{m}$ & $\mathrm{m}$ & $\mathrm{m}$ & $\mathrm{m}$ & $\mathrm{m}$ & $\mathrm{m}$ & $\mathrm{m}$ & $\mathrm{m}$ & $\mathrm{m}$ & $\mathrm{m}$ & $\mathrm{m}$ & $\mathrm{m}$ & $\mathrm{m}$ \\
\hline G20 average & $\mathrm{m}$ & $\mathrm{m}$ & $\mathrm{m}$ & $\mathrm{m}$ & $\mathrm{m}$ & $\mathrm{m}$ & $\mathrm{m}$ & $\mathrm{m}$ & $\mathrm{m}$ & $\mathrm{m}$ & $\mathrm{m}$ & $\mathrm{m}$ & $\mathrm{m}$ & $\mathrm{m}$ & $\mathrm{m}$ & $\mathrm{m}$ \\
\hline
\end{tabular}

1. Excluding international funds in public and total expenditure on educational institutions.

2. Including subsidies attribuable to payments to educational institutions received from public sources.

3. Some levels of education are included with others. Refer to " $\mathrm{x}$ " code in Table B1.1a for details.

4. Year of reference 2011 instead of 2012.

5. Year of reference 2013 instead of 2012.

Source: OECD. Argentina, China, Colombia, India, Indonesia, Saudi Arabia, South Africa: UNESCO Institute for Statistics. Latvia: Eurostat. See Annex 3 for notes (www.oecd.org/education/education-at-a-glance-19991487.htm).

Please refer to the Reader's Guide for information concerning symbols for missing data and abbreviations.

StatLink तnा SL http://dx.doi.org/10.1787/888933285480 
Table B3.2b. Trends in the proportion of public expenditure ${ }^{1}$ on tertiary education institutions and index of change in public and private expenditure (2000, 2005, 2008, 2010 to 2012)

Index of change of public and private sources of funds for educational institutions after transfers from public sources, by year

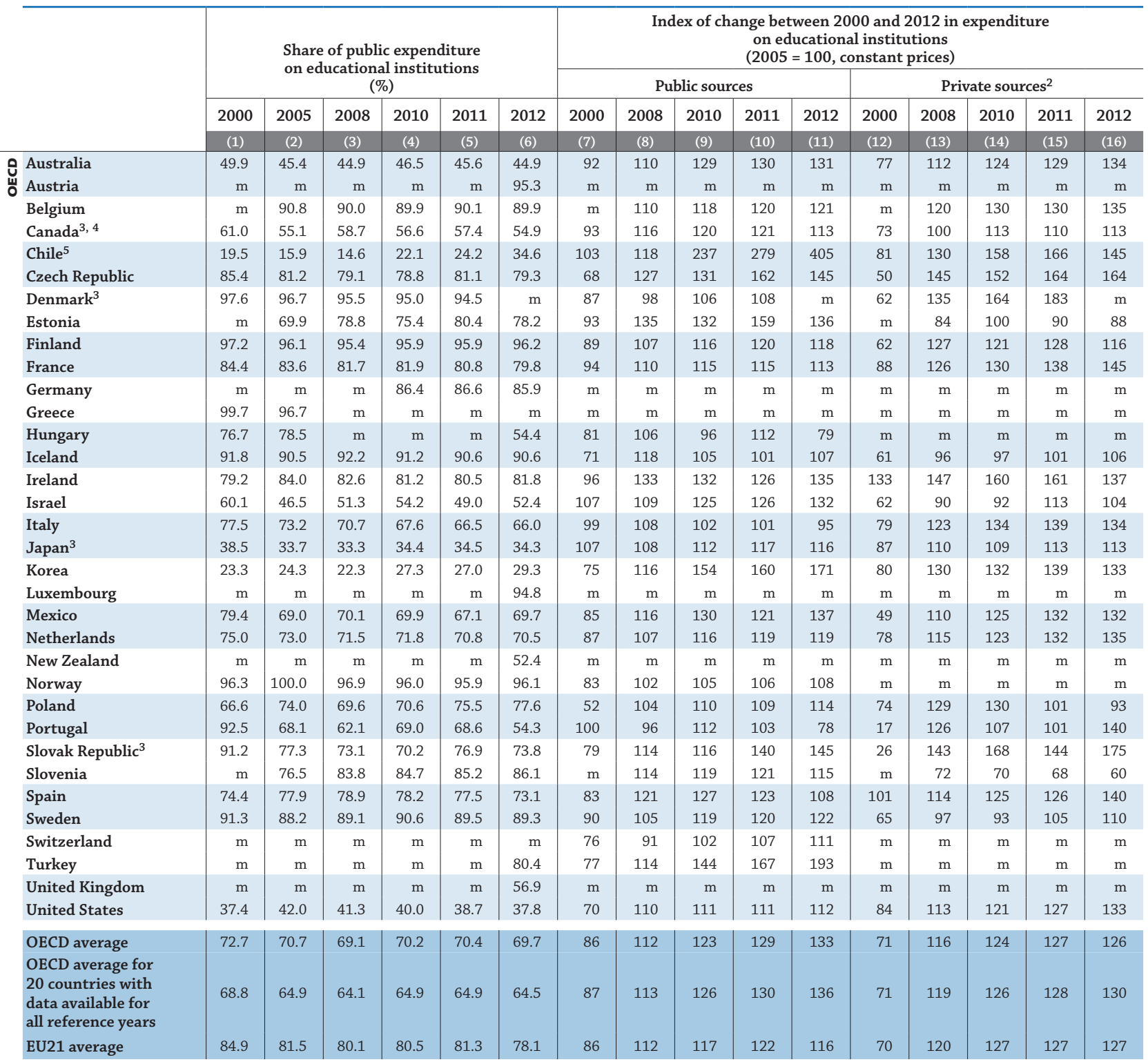

\begin{tabular}{|c|c|c|c|c|c|c|c|c|c|c|c|c|c|c|c|c|}
\hline $\begin{array}{l}\text { Argentina } \\
\text { Brazil }\end{array}$ & $\begin{array}{l}\mathrm{m} \\
\mathrm{m}\end{array}$ & $\begin{array}{l}\mathrm{m} \\
\mathrm{m}\end{array}$ & $\begin{array}{l}\mathrm{m} \\
\mathrm{m}\end{array}$ & $\begin{array}{l}\mathrm{m} \\
\mathrm{m}\end{array}$ & $\begin{array}{l}\mathrm{m} \\
\mathrm{m}\end{array}$ & $\begin{array}{l}\mathrm{m} \\
\mathrm{m}\end{array}$ & $\begin{array}{r}\mathrm{m} \\
79\end{array}$ & $\begin{array}{r}\mathrm{m} \\
119\end{array}$ & $\begin{array}{r}\mathrm{m} \\
148\end{array}$ & $\begin{array}{r}\mathrm{m} \\
155\end{array}$ & $\begin{array}{r}\mathrm{m} \\
149\end{array}$ & $\begin{array}{l}\mathrm{m} \\
\mathrm{m}\end{array}$ & $\begin{array}{l}\mathrm{m} \\
\mathrm{m}\end{array}$ & $\begin{array}{l}\mathrm{m} \\
\mathrm{m}\end{array}$ & $\begin{array}{l}\mathrm{m} \\
\mathrm{m}\end{array}$ & $\begin{array}{l}\mathrm{m} \\
\mathrm{m}\end{array}$ \\
\hline ¿ China & $\mathrm{m}$ & $\mathrm{m}$ & $\mathrm{m}$ & $\mathrm{m}$ & $\mathrm{m}$ & $\mathrm{m}$ & $\mathrm{m}$ & $\mathrm{m}$ & $\mathrm{m}$ & $\mathrm{m}$ & $\mathrm{m}$ & $\mathrm{m}$ & $\mathrm{m}$ & $\mathrm{m}$ & $\mathrm{m}$ & $\mathrm{m}$ \\
\hline Colombia $^{5}$ & $\mathrm{~m}$ & $\mathrm{~m}$ & $\mathrm{~m}$ & $\mathrm{~m}$ & $\mathrm{~m}$ & 42.6 & $\mathrm{~m}$ & $\mathrm{~m}$ & $\mathrm{~m}$ & $\mathrm{~m}$ & $\mathrm{~m}$ & $\mathrm{~m}$ & $\mathrm{~m}$ & $\mathrm{~m}$ & $\mathrm{~m}$ & $\mathrm{~m}$ \\
\hline Indonesia $^{5}$ & $\mathrm{~m}$ & $\mathrm{~m}$ & $\mathrm{~m}$ & $\mathrm{~m}$ & $\mathrm{~m}$ & 70.7 & $\mathrm{~m}$ & $\mathrm{~m}$ & $\mathrm{~m}$ & $\mathrm{~m}$ & $\mathrm{~m}$ & $\mathrm{~m}$ & $\mathrm{~m}$ & $\mathrm{~m}$ & $\mathrm{~m}$ & $\mathrm{~m}$ \\
\hline Latvia & $\mathrm{m}$ & $\mathrm{m}$ & $\mathrm{m}$ & $\mathrm{m}$ & $\mathrm{m}$ & 63.6 & $\mathrm{~m}$ & $\mathrm{~m}$ & $\mathrm{~m}$ & $\mathrm{~m}$ & $\mathrm{~m}$ & $\mathrm{~m}$ & $\mathrm{~m}$ & $\mathrm{~m}$ & $\mathrm{~m}$ & $\mathrm{~m}$ \\
\hline Russian Federation & $\mathrm{m}$ & $\mathrm{m}$ & $\mathrm{m}$ & $\mathrm{m}$ & $\mathrm{m}$ & 63.5 & 44 & 147 & 145 & 136 & 142 & $\mathrm{~m}$ & $\mathrm{~m}$ & $\mathrm{~m}$ & $\mathrm{~m}$ & $\mathrm{~m}$ \\
\hline South Africa & $\mathrm{m}$ & $\mathrm{m}$ & $\mathrm{m}$ & $\mathrm{m}$ & $\mathrm{m}$ & $\mathrm{m}$ & $\mathrm{m}$ & $\mathrm{m}$ & $\mathrm{m}$ & $\mathrm{m}$ & $\mathrm{m}$ & $\mathrm{m}$ & $\mathrm{m}$ & $\mathrm{m}$ & $\mathrm{m}$ & $\mathrm{m}$ \\
\hline G20 average & $\mathrm{m}$ & $\mathrm{m}$ & $\mathrm{m}$ & $\mathrm{m}$ & $\mathrm{m}$ & $\mathrm{m}$ & $\mathrm{m}$ & $\mathrm{m}$ & $\mathrm{m}$ & $\mathrm{m}$ & $\mathrm{m}$ & $\mathrm{m}$ & $\mathrm{m}$ & $\mathrm{m}$ & $\mathrm{m}$ & $\mathrm{m}$ \\
\hline
\end{tabular}

1. Excluding international funds in public and total expenditure on educational institutions.

2. Including subsidies attribuable to payments to educational institutions received from public sources.

3. Some levels of education are included with others. Refer to " $\mathrm{x}$ " code in Table B1.1a for details.

4. Year of reference 2011 instead of 2012.

5. Year of reference 2013 instead of 2012

Source: OECD. Argentina, China, Colombia, India, Indonesia, Saudi Arabia, South Africa: UNESCO Institute for Statistics. Latvia: Eurostat. See Annex 3 for notes (www.oecd.org/education/education-at-a-glance-19991487.htm).

Please refer to the Reader's Guide for information concerning symbols for missing data and abbreviations.

StatLink 젶ㄴ http://dx.doi.org/10.1787/888933285491 
Table B3.3. Annual public expenditure on educational institutions per student, by type of institution (2012)

In equivalent USD converted using PPPs for GDP, by level of education and type of institution, based on full-time equivalents

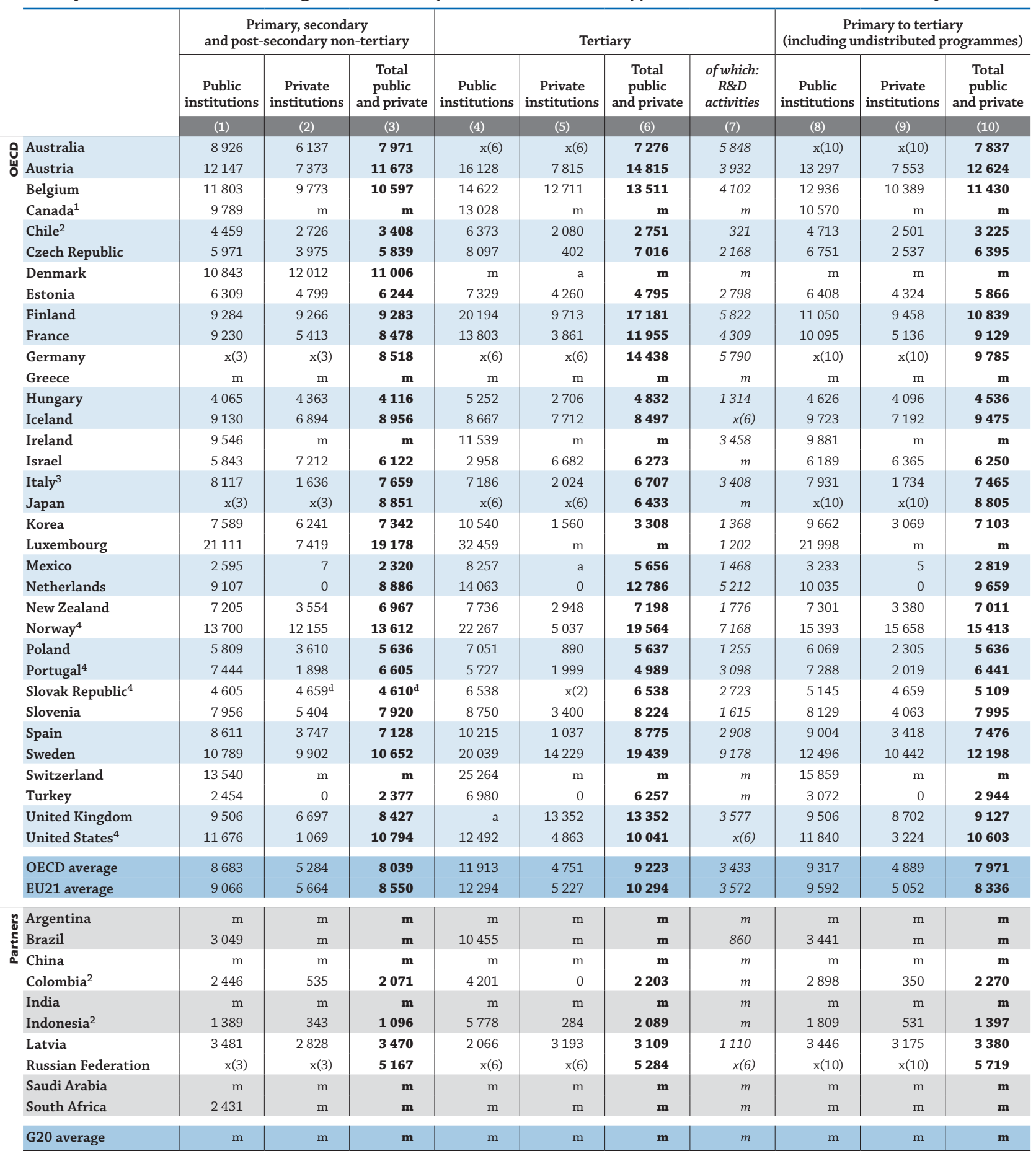

1. Year of reference 2011.

2. Year of reference 2013

3. Excluding post-secondary non-tertiary education and short-cycle tertiary programmes.

4. Some levels of education are included with others. Refer to " $x$ " code in Table B1.1a for details.

Source: OECD. Argentina, China, Colombia, India, Indonesia, Saudi Arabia, South Africa: UNESCO Institute for Statistics. Latvia: Eurostat. See Annex 3 for notes (www.oecd.org/education/education-at-a-glance-19991487.htm).

Please refer to the Reader's Guide for information concerning symbols for missing data and abbreviations.

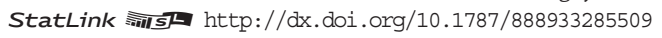




\section{WHAT IS THE TOTAL PUBLIC SPENDING ON EDUCATION}

- Primary to tertiary education accounts for $11.6 \%$ of total public spending, on average across OECD countries, ranging from $8 \%$ or less in Hungary, Italy and Spain, to more than $18 \%$ in Mexico and New Zealand.

- The proportion of public expenditure devoted to primary to tertiary education decreased between 2005 and 2012 in nearly two-thirds of the countries with available data for both years, and remained stable for most others, except, most notably, in Brazil and Israel, where it increased by 3 percentage points or more.

- During the shorter period 2008-12, the height of the economic crisis, the share of public expenditure devoted to primary to tertiary education decreased by $2 \%$ as public expenditure on education grew at a lower rate (or decreased at a faster rate) than public expenditure on all other services in 16 of the 26 OECD countries with available data.

\section{Chart B4.1. Total public expenditure on primary to tertiary education as a percentage of total public expenditure $(2005,2008,2012)$}

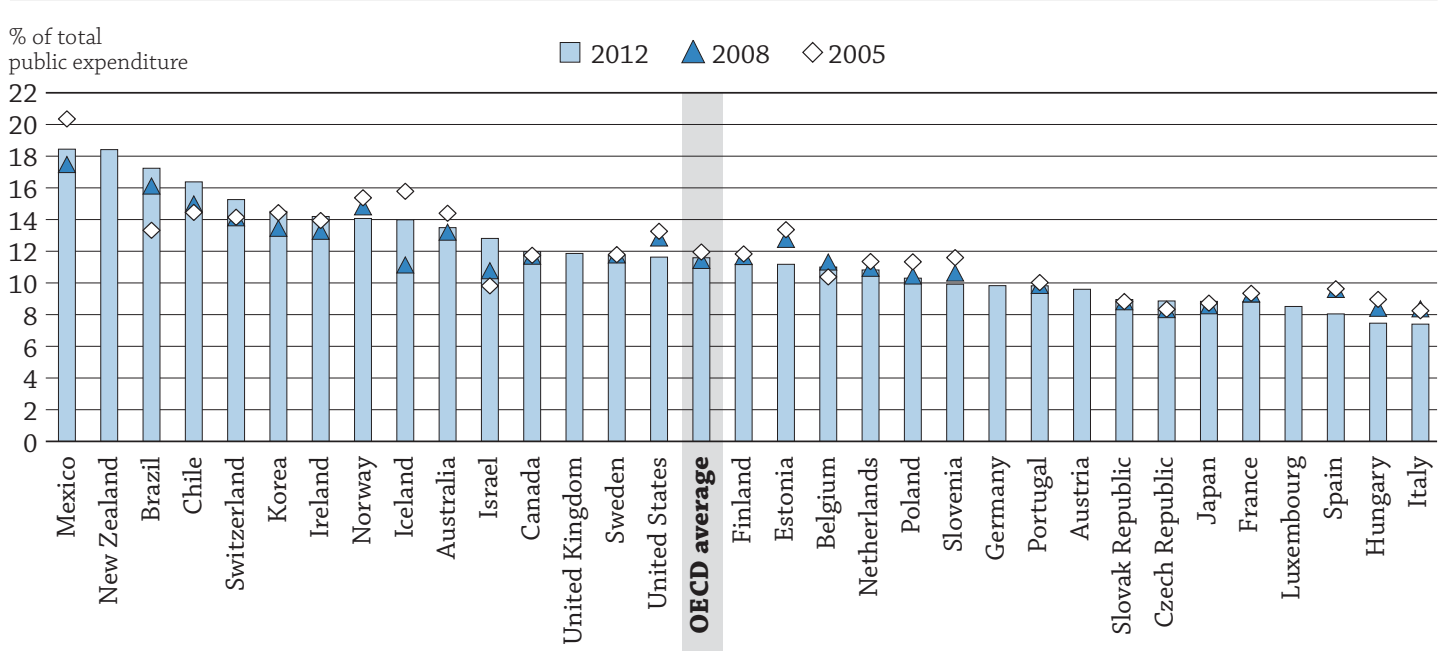

Countries are ranked in descending order of total public expenditure on primary to tertiary education as a percentage of total public expenditure in 2012.

Source: OECD. Table B4.2. See Annex 3 for notes (www.oecd.org/education/education-at-a-glance-19991487.htm).

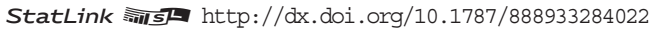

\section{Context}

Countries' decisions concerning budget allocations to various sectors, including education, health care, social security and defence, depend not only on the countries' priorities, but also on whether markets, alone, can provide those services adequately, especially at the tertiary level of education. Markets may fail to do so if the public benefits are greater than the private benefits; thus government funding can help to increase access to tertiary education.

However, the economic crisis has put pressure on public budgets to the extent that less public resources may be allocated to education. This, in turn, may affect access to or the outcomes and quality of education. At the same time, the demand for education and training from people who are not in work may increase, requiring more spending on education. Yet higher expenditure is not necessarily associated with better outcomes or the quality of education. In addition, levels of expenditure are affected by many factors (see Indicator B7) that need to be taken into account when comparing countries.

This indicator presents total public spending on education, relative to both a country's total public spending and to its gross domestic product, to account for the relative sizes of public budgets. In addition, it includes data on the different sources of public funding invested in education (central, regional and local government) and on the transfers of funds between these levels of government. 


\section{Other findings}

- While public expenditure on education as a percentage of total public expenditure on all services decreased, on average, across OECD countries between 2005 and 2012, public expenditure on education relative to GDP increased slightly during this period.

- Most OECD and partner countries (33 out of 38 countries with available data) spend more than twice as much on primary, secondary and post-secondary non-tertiary education combined as on tertiary education.

- At the primary, secondary and post-secondary non-tertiary levels of education, only New Zealand had an entirely centralised public funding system.

\section{Trends}

Between 2000 and 2012, the percentage of total public expenditure devoted to primary to tertiary education decreased slightly in half of the countries with available data (13 of 25 countries). The decrease was especially substantial (one percentage point or more) in eight countries and exceeded two percentage points in Estonia and Mexico. However, the share also increased substantially (by two percentage points or more) in some countries, most notably Brazil, Chile, Israel and the Slovak Republic (Table B4.2).

There is no clear pattern regarding public expenditure on education as a percentage of GDP between 2000 and 2012. The share of public expenditure devoted to education decreased in half of the countries between 2000 and 2012, as did public expenditure on education as a percentage of GDP in around one-third of countries (8 out of 27 countries) during this period. On average across OECD countries with available data for both years, expenditure on education as a percentage of GDP increased.

Between 2008 and 2012, in two-thirds of countries (18 of 27 countries with available data), both public expenditure on education and total public expenditure for all services increased. However, in 17 of 27 countries, public expenditure on all services grew faster or decreased slower than public expenditure on education (Table B4.2). 


\section{Analysis}

\section{Overall level of public resources invested in education}

In 2012, total public expenditure on primary to tertiary education as a percentage of total public expenditure for all services averaged $11.6 \%$ in OECD countries, ranging from $8.0 \%$ or less in Hungary (7.5\%), Italy (7.4\%) and Spain (8.0\%) to $18 \%$ or more in Mexico (18.4\%) and New Zealand (18.4\%) (Chart B4.1 and Table B4.1).

In most countries, and on average across OECD countries, more than two-thirds of total public expenditure on primary to tertiary education as a percentage of total public expenditure was devoted to primary, secondary and post-secondary non-tertiary education. This is primarily explained by the near-universal enrolment rates at these levels of education (see Indicator $\mathrm{C} 1$ ) and the demographic structure of the population.

Public expenditure devoted to tertiary education amounts to $25.9 \%$ of public expenditure from primary to tertiary education, on average across OECD countries. The percentages range from about $18 \%$ or less in Brazil (18.2\%), Israel (18.1\%), Korea (18.2\%), Luxembourg (12.0\%) and Portugal (18.3\%) to 30\% or more in Canada (34.6\%), Finland (33.6\%), Germany (30.5\%), the Netherlands (30.9\%), Norway (32.5\%), Sweden (33.0\%) and the United States (30.5\%), and exceeded 35\% in Austria (36.7\%) (Table B4.1).

When public expenditure on education is considered as a proportion of total public spending, the relative sizes of public budgets must be taken into account. Indeed, the picture is different when looking at public expenditure on education as a percentage of GDP, compared with public expenditure on education as a percentage of total public expenditure. In 2012, public expenditure on primary to tertiary education as a proportion of GDP was 3.5\% or less in Indonesia (3.3\%), the Russian Federation (3.4\%) and the Slovak Republic (3.5\%). At the other end of the spectrum, only Norway (7.7\%) spent more than $7 \%$ of its GDP on primary to tertiary education - well above the OECD average of $4.8 \%$ (Table B4.1).

\section{Chart B4.2. Total public expenditure as a percentage of GDP $(2005,2012)$}

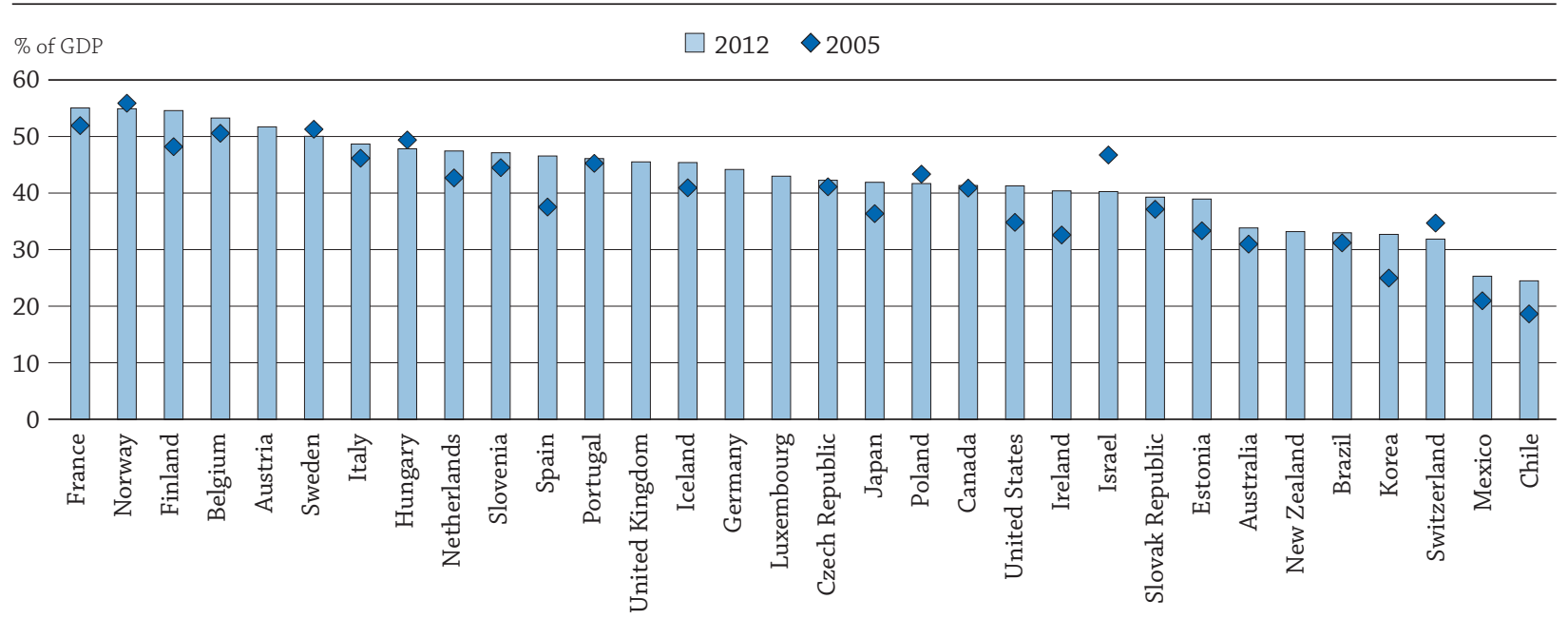

Note: This chart represents public expenditure on all services and not simply public expenditure on education.

Countries are ranked in descending order of total public expenditure as a percentage of GDP in 2012.

Source: OECD. Annex 2. See Annex 3 for notes (www.oecd.org/education/education-at-a-glance-19991487.htm).

StatLink त्ञाI J http://dx.doi.org/10.1787/888933284034

Contrary to expectations, the five countries with the highest total public expenditure on primary to tertiary education as a percentage of total public expenditure in 2012 - namely, Brazil, Chile, Mexico, New Zealand and Switzerland (Chart B4.1) - are at the bottom end of the spectrum in total public expenditure on all services as a percentage of GDP (Chart B4.2). Norway is the exception with high proportions on both counts.

When looking at total public expenditure on all services (including health, social security and the environment), and not simply public expenditure on education, as a proportion of GDP, rates differ greatly among countries. In 2012, almost one in five countries with available data reported that total public expenditure on all services was more than $50 \%$ of GDP, including France (55.1\%) where total public expenditure on all services was more than 55\% of GDP. At the other extreme, total public expenditure on all services accounted for about $25 \%$ or less in Chile and Mexico (24.5\% and 25.3\% of their GDP respectively) (Chart B4.2 and Annex 2). 


\section{Changes in total public expenditure on education as a percentage of total public expenditure between 2000 and 2012}

\section{An increase was observed between 2000 and 2005...}

Over a period of 5 years (2000-05), public expenditure on primary to tertiary education as a percentage of total public expenditure increased in two-thirds of countries (17 of 28 countries with available data for both 2000 and 2005), and by 0.2 percentage points on average across $\mathrm{OECD}$ countries. Among other countries, public expenditure on primary to tertiary education as a percentage of total public expenditure decreased most significantly (by 1 percentage point or more) in France and Portugal.

During this period, public expenditure on primary to tertiary education as a percentage of GDP also increased, by 0.2 percentage point, on average across OECD countries with data for both years. In two-thirds of countries (18 of 28 countries), public expenditure on education as a percentage of GDP grew more than public expenditure on education as a percentage of total public expenditure, reflecting slower growth in GDP than in public expenditure (Table B4.2).

\section{...but a drop was seen after 2005, coinciding with the 2008 financial crisis.}

Spending patterns changed considerably between 2005 and 2012. During this seven-year period, public expenditure on education as a percentage of total public expenditure decreased in nearly two-thirds of countries (16 of 27 countries with available data), and by an average of 0.5 percentage point among OECD countries with available data for both years.

The decrease was the largest in Estonia (-2.2 percentage points), Iceland (-1.8) and Mexico (-1.9) and was also substantial (-1 percentage point or more) in Hungary, Norway, Poland, Slovenia, Spain and the United States. Nevertheless, one-third of countries showed an increase in expenditure on education as a percentage of total public expenditure from 2005 to 2012. The increases were largest (1 percentage point or more) in Brazil (3.9 percentage points), Chile (1.9), Israel (3.0) and Switzerland (1.1).

Comparing data from 2005 and 2012 also shows a different pattern regarding the proportion of GDP spent on education, because GDP was also affected by the economic crisis. As a result, public expenditure on primary to tertiary education as a percentage of GDP increased or remained stable in two-thirds of countries (21 of 29 countries). On average among OECD countries with available data for all years, the increase was 0.1 percentage point. This share decreased the most (by 0.9 percentage point) in Hungary and Norway (Table B4.2 and Box B2.1 in Indicator B2).

\section{First effect of the economic crisis: In half of the countries, public expenditure on education varied at a slower rate than public expenditure for all services}

The variations observed between 2008 and 2012 are possibly linked to the effects of the global economic crisis, which began in 2008. The crisis put more pressure on overall public budgets, requiring governments to prioritise allocations among education and other key public sectors, such as health and social security (Table B4.2 and Chart B4.3).

Between 2008 and 2012, there was no clear global trend concerning the evolution of public expenditure on primary to tertiary education as a percentage of total public expenditure as was the case for the period 2000-05.

Nevertheless, in two-thirds of countries (18 of 27 countries with available data), public expenditure on education and total public expenditure for all services both increased between 2008 and 2012. In 10 of these 18 countries, public expenditure on all services grew faster than public expenditure on education (Table B4.2 and Chart B4.3). The differences were greatest in France, Korea and Norway. In the eight other countries, public expenditure on education grew faster than public expenditure for all services. In these 18 countries, growth in public expenditure for all services ranged from $5 \%$ in Sweden to 15\% or more in Australia, Brazil, Chile and Mexico, and exceeded 30\% in Korea.

Among the remaining third of countries (9 of 27 countries with available data), total public expenditure on all services and public expenditure on education both decreased in five (Estonia, Hungary, Iceland, Italy and Slovenia). In Iceland, public expenditure on all services shrank by as much as $22 \%$, and public expenditure on education also fell, but not as steeply (by 8\%). In Estonia, Hungary, Italy and Slovenia, public expenditure on education declined more steeply than public expenditure on all services (Table B4.2 and Chart B4.3).

In the last four countries (Ireland, Portugal, Spain and the United States), public expenditure on all services and public expenditure on education diverged between 2008 and 2012. Public expenditure on education decreased (as well as the share of public expenditure on education as a percentage of total public expenditure) in Portugal, Spain and the United States, and increased (as did the share of public expenditure on education as a percentage of total public expenditure) in Ireland. 


\section{Chart B4.3. Index of change between 2008 and 2012 in total public expenditure on education as a percentage of total public expenditure}

\section{Primary to tertiary education (2008 $=100,2012$ constant prices)}

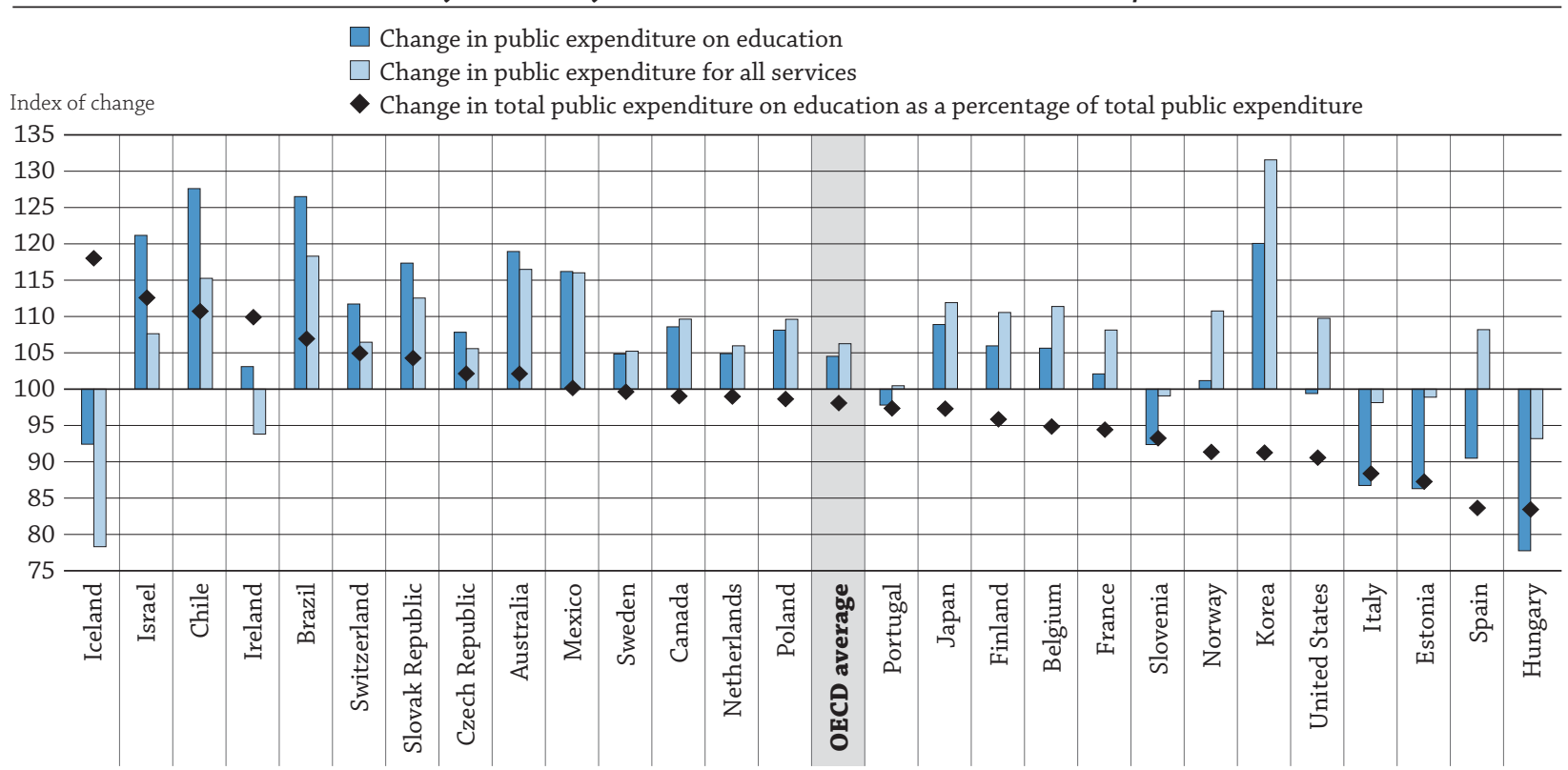

Countries are ranked in descending order of the change in total public expenditure on primary to tertiary education as a percentage of total public expenditure. Source: OECD. Table B4.2. See Annex 3 for notes (www.oecd.org/education/education-at-a-glance-19991487.htm).

StatLink 제매 http://dx.doi.org/10.1787/888933284040

\section{Sources of public funding invested in education}

All government sources of expenditure on education, apart from international sources, are classified into three different levels of government: central, regional and local. In some countries, the funding of education is centralised; in others, funding can become decentralised after transfers among the different levels of government.

In recent years, many schools have become more autonomous and decentralised organisations (see Indicator D6 in Education at a Glance 2012 [OECD, 2012]); they have also become more accountable to students, parents and the public at large for their outcomes. The results from the OECD Programme for International Student Assessment (PISA) suggest that when autonomy and accountability are intelligently combined, they tend to be associated with better student performance (OECD, 2013).

Public funding is more centralised at the tertiary level than at lower levels of education (see Tables B4.3 and B4.6 in Education at a Glance 2014). In 2012, on average across OECD countries, 52.6\% of public funds for primary, secondary and post-secondary non-tertiary education combined came from the central government, before transfers (Table B4.3).

For primary, secondary and post-secondary non-tertiary education combined, the share of initial public funds from the central government differed greatly among countries. Six countries reported a share of less than $10 \%$, namely Canada (3.6\%), Denmark (5.8\%), Germany (7.4\%), Norway (9.3\%), Poland (4.8\%) and Switzerland (3.6\%). In Canada, funding for primary and secondary education is provided at the provincial/territorial level, with the exception of a small amount of federal funding for some First Nations/Aboriginal schools. At the other extreme, public funds came nearly exclusively from the central government in Ireland, New Zealand and Turkey, and more than $90 \%$ of initial public funds came from the central government in Chile (94.9\%), the Netherlands (91.0\%), Portugal (90.6\%) and Slovenia (90.4\%).

Nevertheless, this picture changes when transfers among levels of government are taken into account. After these transfers, less than 5\% of public funds came from central sources in Australia (3.9\%), Canada (2.9\%), Japan (1.8\%), Korea (0.8\%), Poland (3.8\%), Switzerland (0.2\%) and the United States (0.5\%). Only New Zealand had an entirely centralised funding system even after taking transfers into account (Chart B4.4 and Table B4.3). On average across OECD countries, $40.6 \%$ of public funds for primary, secondary and post-secondary non-tertiary levels combined came from local sources, after transfers, compared with $26.7 \%$ before transfers. 


\section{Chart B4.4. Distribution of initial sources of public funds for education by level of government in primary, secondary and post-secondary non-tertiary education (2012)}

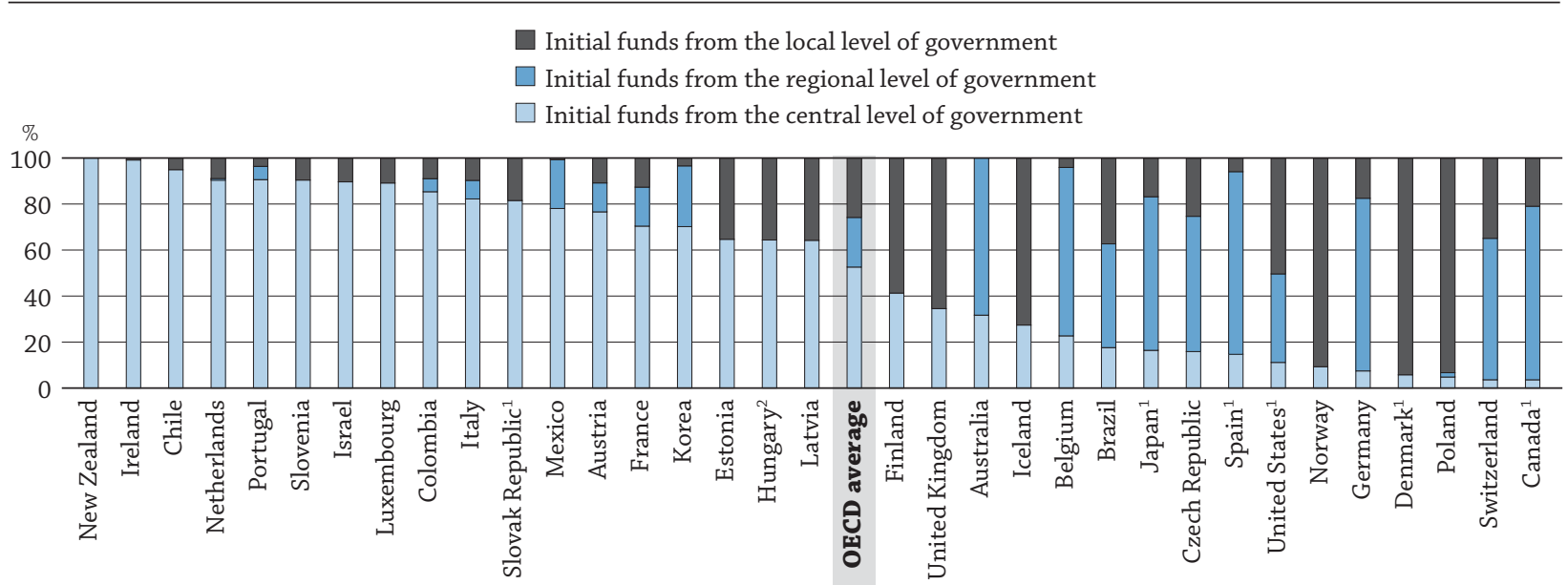

\section{Change in the proportion of educational funds received from the different levels of government between initial and final purchasers of educational resources (2012) In percentage points}

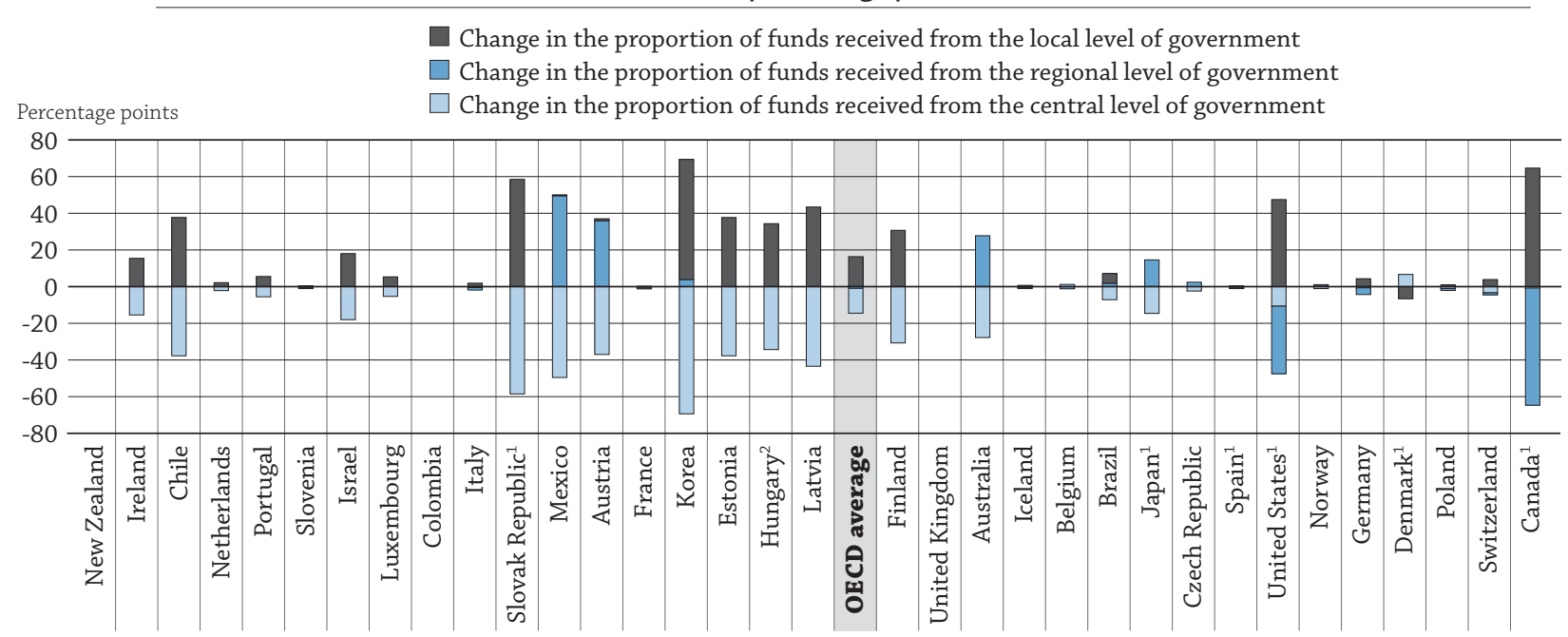

1. Some levels of education are included with others. Refer to "x" code in Table B1.1a for details.

2. Funds from local level include funds from regional level of government.

Countries are ranked in descending order of the share of initial sources of funds from the central level of government.

Source: OECD. Table B4.3. See Annex 3 for notes (www.oecd.org/education/education-at-a-glance-19991487.htm)

StatLink 게개 http://dx.doi.org/10.1787/888933284057

The extent of transfers of public funds from central to lower levels of government varies widely between countries. The difference after transfers from central to lower levels of government represents more than 30 percentage points in Austria, Chile, Estonia, Finland and Hungary, and more than 40 percentage points in Korea, Latvia, Mexico and the Slovak Republic. In Austria, Canada and the United States, the difference after transfers from regional to local sources of public funds exceeds 30 percentage points (Chart B4.4).

\section{Definitions}

Public expenditure on education covers expenditure on educational institutions and support for students' living costs and for other private expenditure outside institutions. It includes expenditure by all public entities, including ministries other than ministries of education, local and regional governments, and other public agencies. OECD countries differ in the ways in which they use public money for education. Public funds may flow directly to institutions or may be channelled to institutions via government programmes or via households. They may also be restricted to the purchase of educational services or be used to support students' living costs. 
All government sources of expenditure on education, apart from international sources, can be classified into three levels: central (national) government, regional government (province, state, Bundesland, etc.), and local government (municipality, district, commune, etc.). The terms "regional" and "local" apply to governments whose responsibilities are exercised within certain geographical subdivisions of a country. They do not apply to government bodies whose roles are not geographically circumscribed but are defined in terms of responsibility for particular services, functions or categories of students.

Total public expenditure, also referred to as total public spending, corresponds to the non-repayable current and capital expenditure of all levels of government: central, regional and local. It includes direct public expenditure on educational institutions as well as public support to households (e.g. scholarships and loans to students for tuition fees and student living costs) and to other private entities for education (e.g. subsidies to companies or labour organisations that operate apprenticeship programmes).

\section{Methodology}

Data refer to the financial year 2012 and are based on the UOE data collection on education statistics administered by the OECD in 2014 (for details see Annex 3 at www.oecd.org/education/education-at-a-glance-19991487.htm).

Figures for total public expenditure and GDP have been taken from the OECD National Accounts Database (see Annex 2) and use the System of National Accounts 1993.

Educational expenditure is expressed as a percentage of a country's total public sector expenditure and as a percentage of GDP.

Though expenditure on debt servicing (e.g. interest payments) is included in total public expenditure, it is excluded from public expenditure on education. The reason is that some countries cannot separate interest payments for education from those for other services. This means that public expenditure on education as a percentage of total public expenditure may be underestimated in countries in which interest payments represent a large proportion of total public expenditure on all services.

\section{Note regarding data from Israel}

The statistical data for Israel are supplied by and are under the responsibility of the relevant Israeli authorities. The use of such data by the OECD is without prejudice to the status of the Golan Heights, East Jerusalem and Israeli settlements in the West Bank under the terms of international law.

\section{References}

OECD (2014), Education at a Glance 2014: OECD Indicators, OECD Publishing, Paris, http://dx.doi.org/10.1787/eag-2014-en. OECD (2013), PISA 2012 Results: What Makes Schools Successful (Volume IV): Resources, Policies and Practices, PISA, OECD Publishing, Paris, http://dx.doi.org/10.1787/9789264201156-en.

OECD (2012), Education at a Glance 2012: OECD Indicators, OECD Publishing, Paris, http://dx.doi.org/10.1787/eag-2012-en.

\section{Indicator B4 Tables}

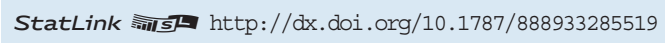

Table B4.1 Total public expenditure on education (2012)

Table B4.2 Trends in total public expenditure on primary to tertiary education $(2000,2005,2008,2010,2011$, and 2012)

Table B4.3 Sources of public funds for education, for primary, secondary and post-secondary non-tertiary education (2012)

\footnotetext{
Cut-off date for the data: 23 October 2015. Updates can be found on line at http://dx.doi.org/10.1787/eag-data-en.
} 
Table B4.1. Total public expenditure on education (2012)

Direct public expenditure on educational institutions plus public subsidies to households ${ }^{1}$ and other private entities, as a percentage of total public expenditure and as a percentage of GDP, by level of education

\begin{tabular}{|c|c|c|c|c|c|c|}
\hline & \multicolumn{3}{|c|}{$\begin{array}{l}\text { Public expenditure }{ }^{1} \text { on education as a percentage } \\
\text { of total public expenditure }\end{array}$} & \multicolumn{3}{|c|}{$\begin{array}{l}\text { Public expenditure }{ }^{1} \text { on education } \\
\text { as a percentage of GDP }\end{array}$} \\
\hline & $\begin{array}{l}\text { Primary, secondary } \\
\text { and post-secondary } \\
\text { non-tertiary }\end{array}$ & All tertiary & $\begin{array}{l}\text { Primary to tertiary } \\
\text { (including } \\
\text { undistributed } \\
\text { programmes) }\end{array}$ & $\begin{array}{l}\text { Primary, secondary } \\
\text { and post-secondary } \\
\text { non-tertiary }\end{array}$ & All tertiary & $\begin{array}{l}\text { Primary to tertiary } \\
\text { (including } \\
\text { undistributed } \\
\text { programmes) }\end{array}$ \\
\hline & (6) & (9) & (10) & (16) & (19) & (20) \\
\hline Australia & 10.1 & 3.4 & 13.5 & 3.4 & 1.1 & 4.6 \\
\hline Austria & 6.1 & 3.5 & 9.6 & 3.1 & 1.8 & 5.0 \\
\hline Belgium & 8.1 & 2.7 & 11.0 & 4.3 & 1.4 & 5.9 \\
\hline Canada $^{2,3}$ & 7.8 & 4.1 & 12.0 & 3.2 & 1.7 & 5.0 \\
\hline Chile $^{4}$ & 11.6 & 4.8 & 16.4 & 2.8 & 1.2 & 4.0 \\
\hline Czech Republic & 6.1 & 2.4 & 8.9 & 2.6 & 1.0 & 3.7 \\
\hline Denmark & 8.6 & $\mathrm{~m}$ & $\mathbf{m}$ & 5.0 & $\mathrm{~m}$ & $\mathbf{m}$ \\
\hline Estonia & 8.4 & 2.7 & 11.2 & 3.3 & 1.0 & 4.4 \\
\hline Finland & 7.4 & 3.8 & 11.2 & 4.1 & 2.1 & 6.1 \\
\hline France & 6.6 & 2.2 & 8.8 & 3.6 & 1.2 & 4.8 \\
\hline Germany & 6.7 & 3.0 & 9.8 & 2.9 & 1.3 & 4.3 \\
\hline Greece & $\mathrm{m}$ & $\mathrm{m}$ & $\mathbf{m}$ & $\mathrm{m}$ & $\mathrm{m}$ & $\mathbf{m}$ \\
\hline Hungary & 5.3 & 1.7 & 7.5 & 2.6 & 0.8 & 3.6 \\
\hline Iceland & 10.0 & 3.2 & 14.0 & 4.5 & 1.4 & 6.4 \\
\hline Ireland & 11.1 & 3.1 & 14.2 & 4.5 & 1.3 & 5.7 \\
\hline Israel & 9.8 & 2.3 & 12.8 & 4.0 & 0.9 & 5.2 \\
\hline Italy & 5.8 & 1.6 & 7.4 & 2.8 & 0.8 & 3.6 \\
\hline $\mathrm{Japan}^{3}$ & 6.5 & $1.8^{\mathrm{d}}$ & 8.8 & 2.7 & $0.8^{\mathrm{d}}$ & 3.7 \\
\hline Korea & 9.6 & 2.6 & 14.5 & 3.2 & 0.9 & 4.8 \\
\hline Luxembourg & 7.5 & 1.0 & 8.5 & 3.2 & 0.4 & 3.7 \\
\hline Mexico & 13.5 & 4.0 & 18.4 & 3.4 & 1.0 & 4.7 \\
\hline Netherlands & 7.5 & 3.3 & 10.8 & 3.5 & 1.6 & 5.1 \\
\hline New Zealand & 13.2 & 5.2 & 18.4 & 4.4 & 1.7 & 6.1 \\
\hline Norway $^{3}$ & $9.0^{\mathrm{d}}$ & 4.6 & 14.1 & $4.9^{\mathrm{d}}$ & 2.5 & 7.7 \\
\hline Poland & 7.6 & 2.7 & 10.3 & 3.2 & 1.1 & 4.3 \\
\hline Portugal $^{3}$ & 7.8 & $1.8^{\mathrm{d}}$ & 9.8 & 3.6 & $0.8^{\mathrm{d}}$ & 4.5 \\
\hline Slovak Republic ${ }^{3}$ & $6.3^{\mathrm{d}}$ & 2.4 & 9.0 & $2.5^{\mathrm{d}}$ & 0.9 & 3.5 \\
\hline Slovenia & 7.4 & 2.5 & 9.9 & 3.5 & 1.2 & 4.7 \\
\hline Spain & 5.9 & 2.1 & 8.0 & 2.8 & 1.0 & 3.7 \\
\hline Sweden & 7.9 & 3.9 & 11.7 & 3.9 & 1.9 & 5.9 \\
\hline Switzerland & 10.8 & 4.2 & 15.3 & 3.5 & 1.3 & 4.9 \\
\hline Turkey & $\mathrm{m}$ & $\mathrm{m}$ & $\mathbf{m}$ & 2.6 & 1.4 & 3.9 \\
\hline United Kingdom & 8.8 & 3.0 & 11.9 & 4.0 & 1.4 & 5.4 \\
\hline United States $^{3}$ & 8.1 & $3.5^{\mathrm{d}}$ & 11.6 & 3.3 & $1.5^{\mathrm{d}}$ & 4.8 \\
\hline OECD average & 8.3 & 3.0 & 11.6 & 3.5 & 1.3 & 4.8 \\
\hline EU21 average & 7.3 & 2.6 & 10.0 & 3.4 & 1.2 & 4.6 \\
\hline Argentina & $\mathrm{m}$ & $\mathrm{m}$ & $\mathbf{m}$ & $\mathrm{m}$ & $\mathrm{m}$ & $\mathbf{m}$ \\
\hline Brazil & 14.1 & 3.1 & 17.2 & 4.7 & 1.0 & 5.7 \\
\hline China & $\mathrm{m}$ & $\mathrm{m}$ & $\mathbf{m}$ & $\mathrm{m}$ & $\mathrm{m}$ & $\mathbf{m}$ \\
\hline Colombia $^{4}$ & $\mathrm{~m}$ & $\mathrm{~m}$ & $\mathbf{m}$ & 3.6 & 0.9 & 4.9 \\
\hline India & $\mathrm{m}$ & $\mathrm{m}$ & $\mathbf{m}$ & $\mathrm{m}$ & $\mathrm{m}$ & $\mathbf{m}$ \\
\hline Indonesia $^{4}$ & $\mathrm{~m}$ & $\mathrm{~m}$ & $\mathbf{m}$ & 2.3 & 0.6 & 3.3 \\
\hline Latvia & $\mathrm{m}$ & $\mathrm{m}$ & $\mathbf{m}$ & 2.9 & 1.0 & 3.8 \\
\hline Russian Federation & $\mathrm{m}$ & $\mathrm{m}$ & $\mathbf{m}$ & 2.2 & 0.9 & 3.4 \\
\hline Saudi Arabia & $\mathrm{m}$ & $\mathrm{m}$ & $\mathbf{m}$ & $\mathrm{m}$ & $\mathrm{m}$ & $\mathbf{m}$ \\
\hline South Africa & $\mathrm{m}$ & $\mathrm{m}$ & $\mathbf{m}$ & 4.8 & 0.8 & 6.1 \\
\hline G20 average & $\mathrm{m}$ & $\mathrm{m}$ & $\mathbf{m}$ & $\mathrm{m}$ & $\mathrm{m}$ & $\mathbf{m}$ \\
\hline
\end{tabular}

Note: Values for each level of education (columns 1 to 5, 7, 8, 11 to 15, 17 and 18) are available for consultation on line (see Statlink below).

1. Public expenditure presented in this table includes public subsidies to households for living costs (scholarships and grants to students/households and students loans), which are not spent on educational institutions. Therefore the figures presented here exceed those on public spending on institutions found in Table B2.3.

2. Year of reference 2011 instead of 2012.

3. Some levels of education are included with others. Refer to " $x$ " code in Table B1.1a for details.

4. Year of reference 2013 instead of 2012.

Source: OECD. Argentina, China, Colombia, India, Indonesia, Saudi Arabia, South Africa: UNESCO Institute for Statistics. Latvia: Eurostat. See annex 3 for notes (www.oecd.org/education/education-at-a-glance-19991487.htm).

Please refer to the Reader's Guide for information concerning symbols for missing data and abbreviations.

StatLink 제 SL http://dx.doi.org/10.1787/888933285522 
Table B4.2. Trends in total public expenditure on primary to tertiary education $(2000,2005,2008,2010,2011$ and 2012)

Direct public expenditure on educational institutions plus public subsidies to households ${ }^{1}$ and other private entities, as a percentage of total public expenditure and as a percentage of GDP, for primary to tertiary levels of education combined by year

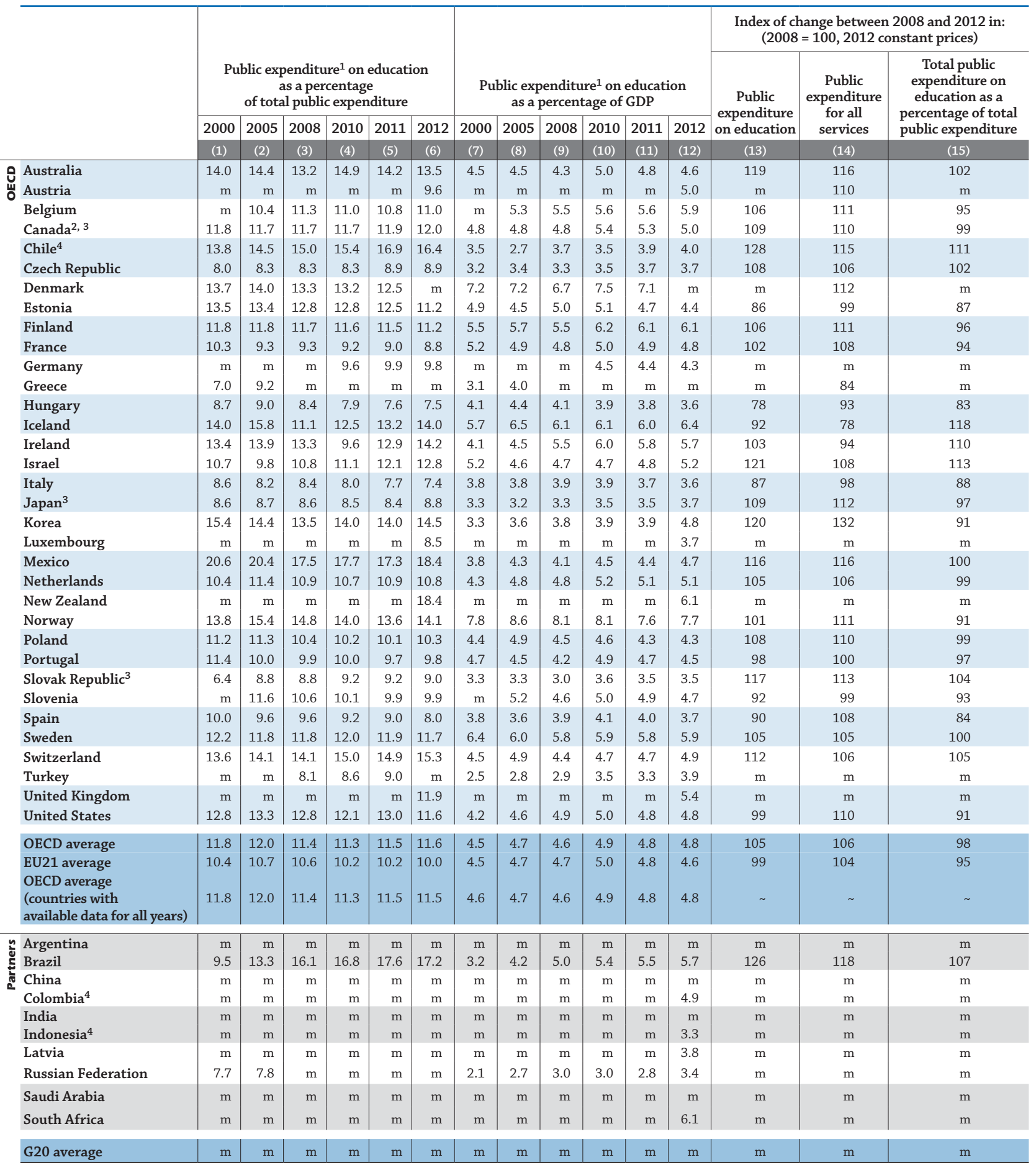

1. Public expenditure presented in this table includes public subsidies to households for living costs (scholarships and grants to students/households and students loans), which are not spent on educational institutions. Thus the figures presented here exceed those on public spending on institutions found in Table B2.3.

2. Year of reference 2011 instead of 2012

3. Some levels of education are included with others. Refer to " $\mathrm{x}$ " code in Table B1.1a for details.

4. Year of reference 2013 instead of 2012. Data refer to 2009-2012 instead of 2008-2011.

Source: OECD. Argentina, China, Colombia, India, Indonesia, Saudi Arabia, South Africa: UNESCO Institute for Statistics. Latvia: Eurostat. See Annex 3 for notes (www.oecd.org/education/education-at-a-glance-19991487.htm).

Please refer to the Reader's Guide for information concerning symbols for missing data and abbreviations.

StatLink 제 जी http://dx.doi.org/10.1787/888933285538 
Table B4.3 Sources of public funds for education, for primary, secondary and post-secondary non-tertiary education (2012)

Before and after transfers

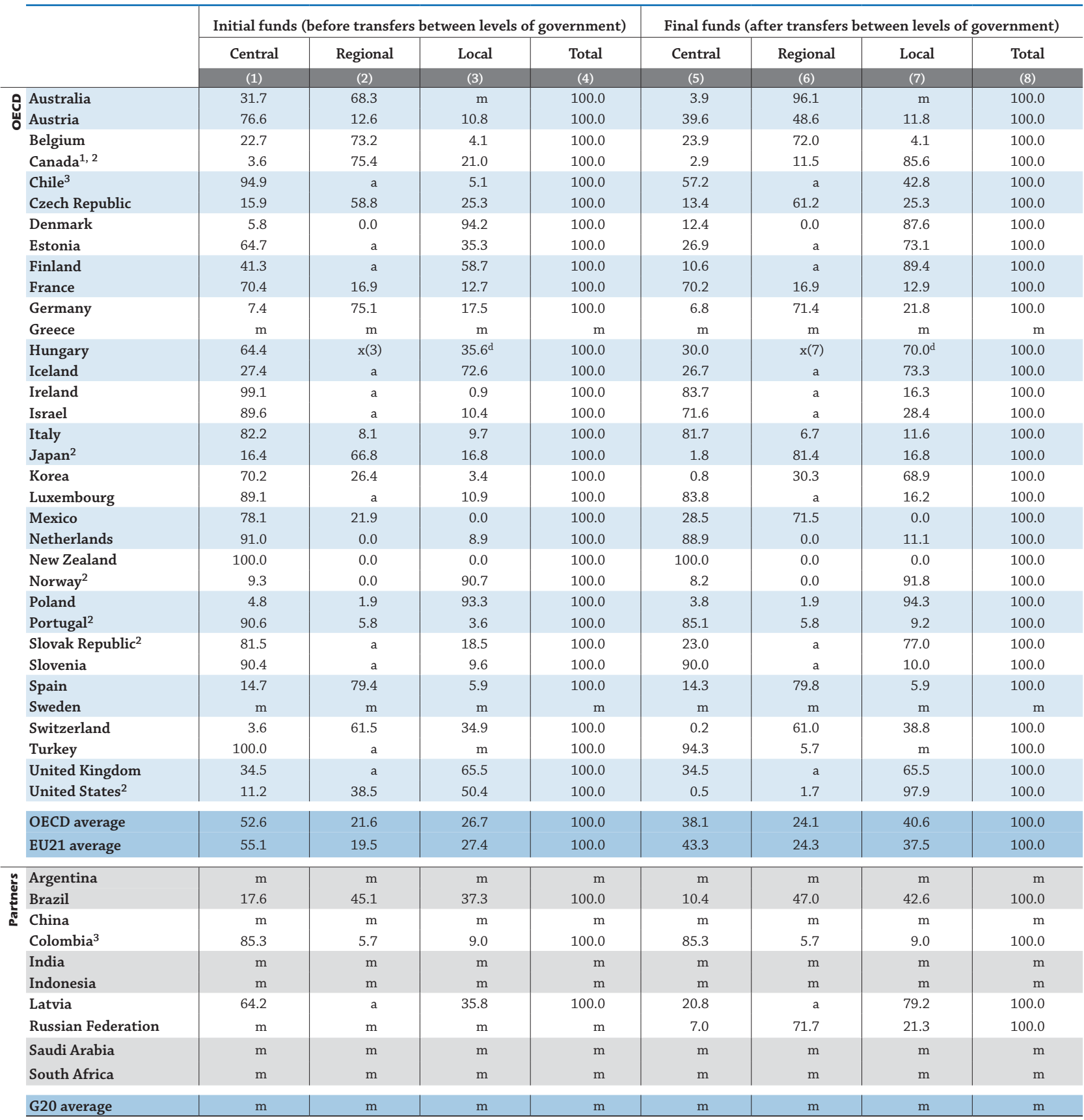

1.Year of reference 2011.

2. Some levels of education are included with others. Refer to " $x$ " code in Table B1.1a for details.

3. Year of reference 2013.

Source: OECD. Argentina, China, Colombia, India, Indonesia, Saudi Arabia, South Africa: UNESCO Institute for Statistics. Latvia: Eurostat. See Annex 3 for notes (www.oecd.org/education/education-at-a-glance-19991487.htm).

Please refer to the Reader's Guide for information concerning symbols for missing data and abbreviations.

StatLink 젝ㄴ http://dx.doi.org/10.1787/888933285547 


\section{HOW MUCH DO TERTIARY STUDENTS PAY AND WHAT PUBLIC SUPPORT DO THEY RECEIVE?}

- OECD countries differ significantly in the amount of tuition fees charged by their tertiary institutions. In eight OECD countries, public institutions charge no tuition fees for full-time students in bachelor or equivalent programmes. However, in more than half of the remaining countries with available data, public institutions charge annual tuition fees in excess of USD 2000 for national students.

- In all OECD countries, people with a master's, doctoral or equivalent degree have better labour market opportunities compared to those with only a bachelor's degree. However, in one-third of OECD countries, tuition fees charged by public institutions for master's and doctorate or equivalent programmes are not much higher than those charged for bachelor's degree programmes. The difference in tuition fees between bachelor's and master's degree programmes is more than USD 1400 only in Australia, Colombia, Korea and the United States.

- An increasing number of OECD countries charge higher tuition fees for international students than for national students, and many also differentiate tuition fees by field of education, largely because of the relevance of the different qualifications on the labour market.

\section{Chart B5.1. Average tuition fees charged by public institutions related to the proportion of students who benefit from public loans and/or scholarships/grants at bachelor's and equivalent level (2013-14)} For full-time national students, in USD converted using PPPs for GDP, academic year 2013/14

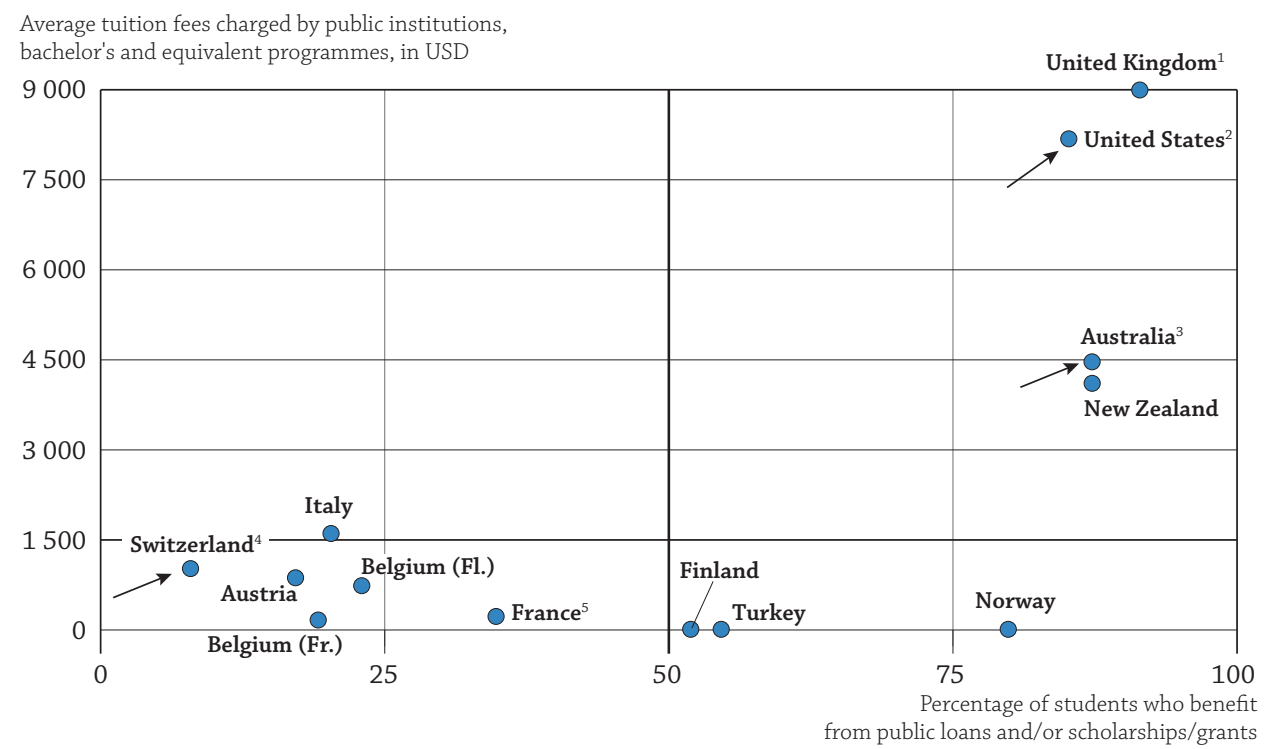

Note: Arrows show how the average tuition fees and the proportion of students who benefit from public support have changed since 1995 further to reforms.

1. Tuition fees refer to England only.

2. Reference year 2011-12

3. Only includes the major Australian Government scholarships programmes. It excludes all scholarships provided by education institutions and the private sector.

4. Swiss data refer to the financial year 2013 and the academic year 2012/2013.

5. Tuition fees range from USD 215 to USD 715 for university programmes depending from the Ministry of Higher Education. Sources: OECD. Tables B5.1a and B5.3. See Annex 3 for notes (www.oecd.org/education/education-at-a-glance-19991487.htm).

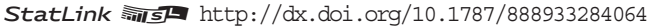

\section{Context}

Many countries have similar goals for tertiary education, such as strengthening the knowledge economy, increasing access for students, boosting completion rates, and ensuring the financial stability of their higher education systems. However, OECD countries differ in the way the spending on tertiary education is shared among governments, students and their families and other private entities, and in the financial support they provide to students. 
Policy decisions relating to tuition fees affect both the cost of tertiary education to students and the resources available to tertiary institutions. Tuition fees paid by students and their families can play a significant role in funding tertiary educational institutions (see Indicator B3) and also affect decisions to enrol in tertiary programmes within the country or abroad (see Indicator C4).

Public support to students and their families enables governments to encourage participation in education - particularly among low-income students - by covering part of the cost of education and related expenses. In this way, governments address access and equality of opportunity issues. The impact of such support should therefore be judged, at least partly, by examining participation and retention in, and completion of, tertiary education.

Public support to students also indirectly funds tertiary institutions. Channelling funding to institutions through students may also help to increase competition among institutions and to be more responsive to student needs. Since aid for students' living costs can serve as a substitute for income from work, public subsidies may enhance educational attainment by allowing students to work less. This support comes in many forms, including means-based subsidies, family allowances for students, tax allowances for students or their parents, or other household transfers. Governments will strive to strike the right balance among these different subsidies, especially in a period of financial crisis. Based on a given amount of subsidies, public support, such as tax reductions or family allowances, may provide less support for low-income students than means-tested subsidies, as the former are not targeted specifically to support low-income students. However, they may still help to reduce financial disparities among households with and without children in education.

\section{Other findings}

- Countries with high tuition fees tend to be those where private entities, such as enterprises, also contribute the most to funding tertiary institutions.

- The high entry rates into tertiary education in some countries that charge no tuition fees may also be related to these countries' highly developed financial support systems for students, and not just to the absence of tuitions fees.

- OECD data show no strong cross-country relationship between levels of tuition fees and participation in tertiary education. However, among countries with high tuition fees, student financial support systems that offer loans with income-contingent repayment combined with means-tested grants may help to promote access and equity while sharing the costs of higher education between the state and students.

\section{Trends}

As reported in Education at a Glance 2012, between 1995 and 2010, 14 of the 25 countries with available information implemented reforms to tuition fees. In all of these 14 countries except Iceland and the Slovak Republic, the reforms were combined with a change in the level of public support available to students.

Since 2010, ten countries have introduced reforms on tuition fees in tertiary education. These reforms related to all levels of tertiary education in Australia, Belgium (French Community), Estonia, Hungary, Italy, Korea, New Zealand, Turkey and the United Kingdom; in Sweden, they focused on bachelor's and master's or equivalent levels. In all these countries except Belgium (French Community), New Zealand and Turkey, these reforms were combined with changes in the support system of students. These reforms usually share two different objectives: on the one hand, the need to ensure stability of funding for tertiary institutions, and on the other hand, to ensure that all students have access to tertiary studies. For example, in the United Kingdom, tuition fees doubled - and nearly tripled in some universities - in 2012, as part of a government plan to stabilise university finances. In parallel, conditions for student loan repayment have been changed to adapt to the changes in tuition fees (see Box B5.2). 


\section{Analysis}

\section{Annual tuition fees charged by tertiary educational institutions for national bachelor's degree students}

The level of tuition fees charged by tertiary educational institutions - as well as the level and type of financial assistance countries provide through their student-support systems - are among the most hotly debated publicpolicy topics in education today. The different ways to combine tuition fees and financial support to students can greatly influence the access to and equity in tertiary education. Striking the right balance between providing sufficient support to institutions through tuition fees and maintaining access and equity is challenging.

Several factors influence the level of tuition fees, such as the salary of professors, in the competition to hire the best ones in a global academic market; the development of non-teaching services (employability services, relations with companies); the growth of digital learning; and investments to support internationalisation.

On the one hand, higher tuition fees increase the resources available to educational institutions, support their efforts to maintain quality academic programmes and develop new ones, and can help institutions accommodate increases in student enrolment. However, high tuition fees may also restrict access to tertiary education for students - particularly those from low-income backgrounds - in the absence of a strong system of public support to help them pay or reimburse the cost of their studies. In addition, high tuition fees may prevent some students from pursuing fields that require extended periods of study, especially when labour market opportunities are not sufficient in these fields.

On the other hand, lower tuition fees can help to promote student access and equity in higher education, particularly among disadvantaged populations. However, they may also constrain the ability of tertiary institutions to maintain an appropriate quality of education, especially in light of the massive expansion of tertiary education in all OECD countries in recent years. Moreover budgetary pressures stemming from the global economic crisis may make it more difficult for countries that have lower tuition fees to sustain this model in the future.

Differentiating tuition fees (by level of education, field of education, student background or mode of delivery) is a way for countries to adjust the level of tuition fees to take into account equity issues to access tertiary education, costs to provide education and labour market opportunities.

There are large differences among countries in the average tuition fees charged by tertiary educational institutions for national students in bachelor's degree or equivalent programmes. In the four Nordic countries with available data (Denmark, Finland, Norway and Sweden), and in Estonia, the Slovak Republic, Slovenia and Turkey, public institutions do not charge tuition fees for full-time students. However tuition fees can be charged in Estonia to part-time students; in the Slovak Republic to students enrolled in two or more programmes; and, in Turkey, to students enrolled in evening programmes and to those who have not graduated within the theoretical duration of a programme.

By contrast, tuition fees for public institutions are higher than USD 2000 in more than half of the countries with available data, and they exceed USD 4000 in Australia, Canada, Korea and New Zealand, USD 5000 in Japan, and USD 8000 in the United Kingdom (for government-dependent private institutions in England only) and the United States. Meanwhile, in Austria, Belgium (Flemish and French Communities), Colombia, Italy and Switzerland, students are charged lower tuition fees (under USD 2 000) for bachelor's programmes in public institutions. Among the EU21 countries for which data are available, only Italy, the Netherlands and the United Kingdom (in government-dependent private institutions) have annual tuition fees that exceed USD 1500 per full-time national student (Table B5.1a and Chart B5.2).

\section{Differentiation of tuition fees across tertiary programmes and between fields of education}

In all OECD countries with available data, people with a master's, doctorate or equivalent degrees have higher earnings advantages and better labour market opportunities compared to those with only a bachelor's degree (see Indicators A5 and A6). However, the tuition fees charged by public institutions for national students in master's and doctorate or equivalent programmes are generally not much higher than those charged for bachelor's programmes. In one-third of OECD countries, similar tuition fees are charged by public institutions to full-time students regardless of the level of the programme. No tuition fee is charged in Denmark, Estonia, Finland, Norway, the Slovak Republic, Sweden (for national students) and Turkey; and similar tuition fees are charged in the different levels of tertiary education in Austria (about USD 860), Canada (about USD 4760 to 4960 for bachelor's and master's programmes), Japan (about USD 5 150), the Netherlands (USD 2300 for bachelor's and master's programmes) and the United Kingdom (about USD 9 000, in government-dependent private institutions). 


\section{Chart B5.2. Average annual tuition fees charged by public institutions at bachelor's or equivalent level (2013-14) \\ Tuition fees charged to full-time national students, converted in USD using PPPs for GDP, academic year 2013/14}

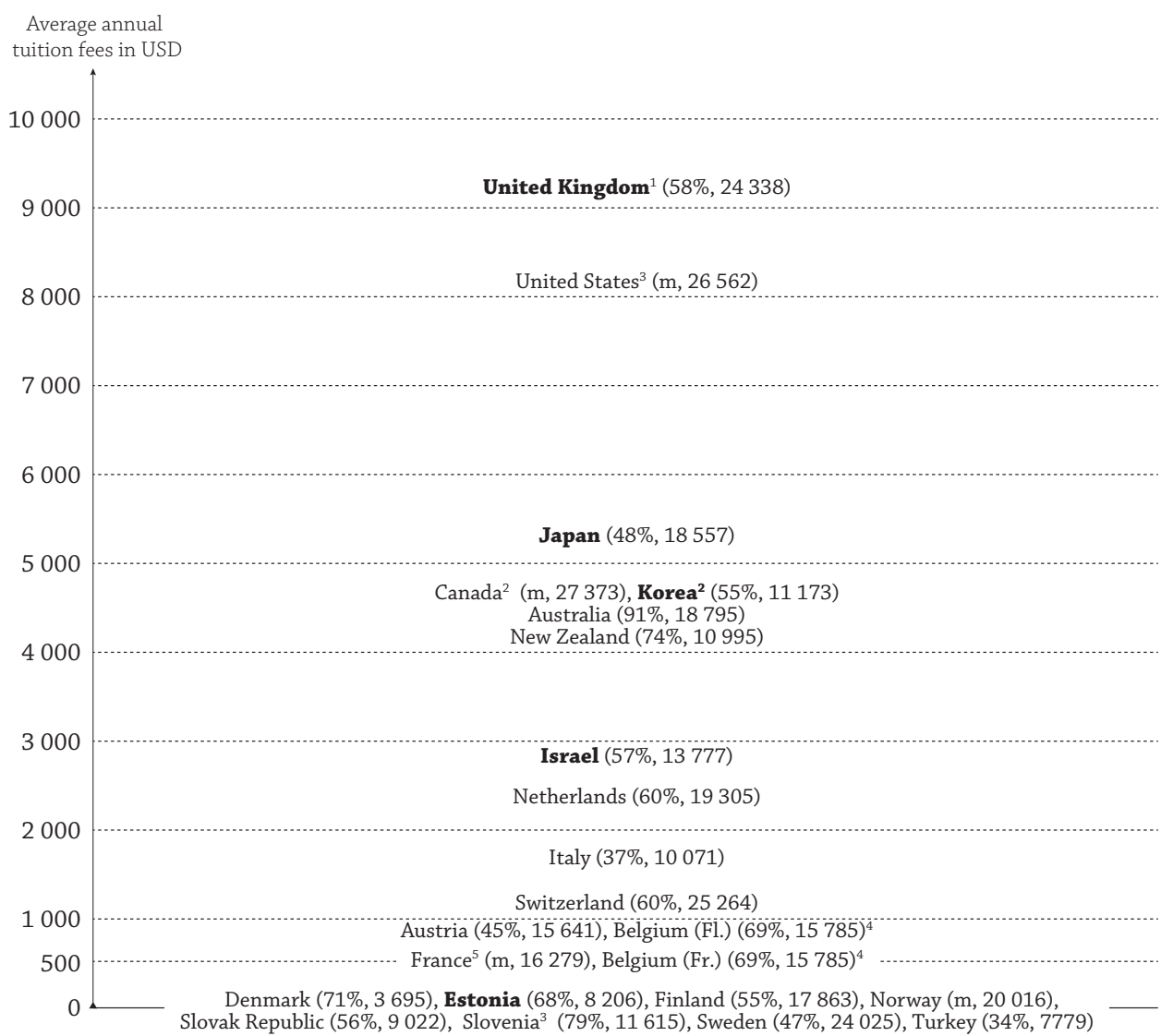

Notes: This chart shows the annual tuition fees charged in equivalent USD converted using PPPs. Countries in bold indicate that tuition fees refer to public institutions but more than two-thirds of students are enrolled in private institutions. Expenditure per student (in USD - all services, including R\&D) in bachelor's, master's, doctoral or equivalent programmes and the net entry rate in bachelor's or equivalent programmes are added next to country names (and refer to 2012-13 reference year).

This chart does not take into account grants, subsidies or loans that partially or fully offset the student's tuition fees.

1. Public institutions do not exist at this level of education and almost all students are enrolled in government-dependent private institutions. Tuition fees refer to England only.

2. Reference year 2014-15 for tuition fees (2014 in Korea).

3. Reference year 2011-12 for tuition fees.

4. Expenditure per student and entry rate refer bachelor, master, doctorate or equivalent programmes for the whole Belgium.

5. Tuition fees range from USD 215 to USD 715 for university programmes depending from the Ministry of Higher Education.

Sources: OECD. Tables B1.1a and B5.1a and Indicator C3. See Annex 3 for notes (www.oecd.org/education/education-at-a-glance-19991487.htm).

Please refer to the Reader's Guide for information concerning symbols for missing data and abbreviations.

StatLink הills $\mathrm{sttp} / / / \mathrm{dx}$.doi.org/10.1787/888933284079

However, the difference between bachelor's and master's programmes in tuition fees charged to national students is substantial in some countries. Tuition fees for master's programmes in public institutions are $30 \%$ higher than those for bachelor's programmes in Korea and the United States, $60 \%$ higher in Australia, and more than four times higher in Belgium (French Community) and Colombia (tuition fees for bachelor's programmes are less than USD 600 in these two countries). Expressed in USD, these differences range between USD 1500 and USD 2900 in Australia, Korea and the United States (Table B5.1a, and Table B5.1c, available on line).

Among countries with available data on tuition fees charged by public institutions for national students in doctoral programmes, tuition fees are much lower than those charged for bachelor's and master's programmes in a few countries, including Australia, Belgium (Flemish Community) and Switzerland. For example, in Australia, tuition fees in public institutions amounts to USD 314 for a doctorate, compared with USD 4473 for a bachelor's programme, as very few national doctoral students pay any fee in Australia (less than $5 \%$ of doctoral students in public institutions). 
However, in Colombia, Korea, Slovenia and the United States, tuition fees charged for doctoral programmes in public institutions are higher than those for bachelor's and master's programmes. This is also true in independent private institutions, except in Slovenia where data are not available (Table B5.1a, and Table B5.1d, available on line)

When tertiary institutions charge tuition fees for students, these fees are also differentiated by field of education in more than half of the countries with available data. The exceptions are Belgium (Flemish and French Communities), Italy, the Netherlands and Switzerland. Australia shows the widest spectrum of fees in public educational institutions, with highest fees being nearly three times the level of the lowest fees for master's programmes (from USD 3876 in education to USD 10231 in social sciences, business and law). A ratio of 1 to 3 between tuition fees by field of education also appears for bachelor's and master's programmes in independent private institutions in Australia, and for bachelor's programmes in public institutions in Colombia (Table B5.2, available on line, and Box B5.1).

\section{Box B5.1. Bases for differentiation of tuition fees by field of education}

Differentiating tuition fees by field of education is a way for countries to adjust the level of tuition fees to take into account equity issues to access tertiary education, costs to provide education and labour market opportunities. The table below shows that the main rationale for differentiating fees is the relevance of the different qualifications on the labour market. This is one of the bases for differentiating tuition fees in all countries with available data, except the United States, where differences in tuition fees between fields of education result from differences in tuition fees between institutions rather than differences within institutions. In Australia for example, tuition fee differentiation is linked to the level of salaries that graduates in certain disciplines can expect to receive.

However, the public cost of the field of education is also used to differentiate tuition fees in Australia, as well as in Hungary and New Zealand. In these countries, the higher the cost of the field of education, the higher the level of tuition fees charged by educational institutions.

\section{Chart B5.a. Differentiation of level of tuition fees by field of education, tertiary education (2013-14)}

\begin{tabular}{|c|c|c|c|c|c|}
\hline & & \multicolumn{4}{|r|}{ National students } \\
\hline & & \multirow[b]{2}{*}{$\begin{array}{l}\text { Differentiation } \\
\text { of level of } \\
\text { tuition fees } \\
\text { by field of } \\
\text { education }\end{array}$} & \multicolumn{3}{|c|}{ Reasons for differentiation of level of tuition fees by field of education } \\
\hline & & & $\begin{array}{c}\text { Relevance } \\
\text { of the different } \\
\text { qualifications on } \\
\text { the labour market }\end{array}$ & $\begin{array}{l}\text { Public } \\
\text { cost } \\
\text { of the } \\
\text { studies }\end{array}$ & Other \\
\hline & & $(1)$ & $(2)$ & $(3)$ & $(4)$ \\
\hline \multirow{13}{*}{$\begin{array}{l}\text { 己̛ } \\
\text { ô }\end{array}$} & Australia & Yes & Yes & Yes & No \\
\hline & Austria & Yes & No & No & No \\
\hline & France & Yes & $\mathrm{m}$ & $\mathrm{m}$ & \\
\hline & Canada & Yes & Yes & No & No \\
\hline & Hungary & Yes & Yes & Yes & No \\
\hline & Israel & Yes & Yes & No & No \\
\hline & Korea & Yes & Yes & No & No \\
\hline & New Zealand & Yes & Yes & Yes & No \\
\hline & Norway & Yes & Yes & Yes & No \\
\hline & Slovak Republic ${ }^{1}$ & Yes & Yes & No & No \\
\hline & Slovenia $^{2}$ & Yes & $\mathrm{m}$ & $\mathrm{m}$ & $\mathrm{m}$ \\
\hline & United Kingdom & Yes & Yes & No & No \\
\hline & United States & Yes & No & No & $\begin{array}{l}\text { Differences in tuition fees by field of education are a result of differences } \\
\text { in tuition charged at different institutions (not differences in tuition fees } \\
\text { charged within an institution for different fields of education). }\end{array}$ \\
\hline \multirow[t]{2}{*}{$\frac{n}{5}$} & Colombia & Yes & Yes & No & $\begin{array}{l}\text { Each higher education institution defines the level of tuition fees and } \\
\text { the methodology to determine the level of fees (they are usually associated } \\
\text { to the socioeconomic conditions of students and their families). }\end{array}$ \\
\hline & $\begin{array}{l}\text { Note: Countries wi } \\
\text { 1. Differentiation o } \\
\text { 2. Differentiation } \\
\text { tuition fees. } \\
\text { Source: OECD. See }\end{array}$ & $\begin{array}{l}\text { hout differentation } \\
\text { tuition fees in inde } \\
\text { Annex } 3 \text { for notes }\end{array}$ & $\begin{array}{l}\text { of tuition fees by fiel } \\
\text { pendent private insti } \\
\text { ctoral or equivalent } \\
\text { vww.oecd.org/educat }\end{array}$ & $\begin{array}{l}\text { I of educat } \\
\text { utions on } \\
\text { on/educat }\end{array}$ & $\begin{array}{l}\text { ion are not reported in this table. } \\
\text { y. } \\
\text { es only. In bachelor's, master's or equivalent level, full-time students do not pa } \\
\text { ion-at-a-glance-19991487.htm). }\end{array}$ \\
\hline
\end{tabular}




\section{Tuition fees for non-national students}

National policies regarding tuition fees and financial aid to students generally cover all students studying in the country's educational institutions. Countries' policies also take international students into account. Differences between national and international students, in the fees they are charged or the financial support they may receive from the country in which they study, can, along with other factors, such as the public support that these students may receive from the country they come from, have an impact on the flows of international students. These differences can attract students to study in some countries or discourage students from studying in others (see Indicator C4), especially in a context where an increasing number of OECD countries are charging higher tuition fees for international students.

In the majority of countries with available data (20 out of 38), the tuition fees charged by public educational institutions may differ for national and international students enrolled in the same programme. However, in countries from the European Union and the European Economic Area (EEA), the same tuition fees are charged for nationals and students from the EU and EEA countries. In Austria, for example, the average tuition fees charged by public institutions for students who are not citizens of EU or European Economic Area (EEA) countries are twice the fees charged for citizens of these countries (for bachelor, master and doctorate programmes in public institutions). Similar policies are found in Australia, Belgium (French and Flemish communities), Canada, Chile, the Czech Republic, Denmark (as of 2006-07), Estonia, Ireland, the Netherlands, New Zealand (except for foreign $\mathrm{PhD}$ students), Poland, Portugal, the Russian Federation, Sweden (as of 2011), Turkey, the United Kingdom and the United States. In these countries, tuition fees vary based on citizenship or on an individual's residence, and can also vary according to fields of education, as in Sweden (see Table B5.6, available on line, and Indicator C4 and Box C4.2).

\section{Grants and loans to students}

OECD research (OECD, 2008) suggests that having a robust financial support system is important for ensuring good outcomes for students in higher education, and that the type of aid is also critical. A key question in many OECD countries is whether financial support for students in tertiary education should be provided primarily in the form of grants or loans. Governments support students' living or educational costs through different combinations of these two types of support. Tax reductions and tax credits for education are not included in this indicator. Advocates of student loans argue that loans allow available resources to be spread further. If the amount spent on grants were used to guarantee or subsidise loans instead, more aid would be available to more students, and overall access to higher education would increase.

\section{Chart B5.3. Distribution of scholarships/grants and public loans to students in bachelor's or equivalent level (2013-14)}

\section{Percentage of students}

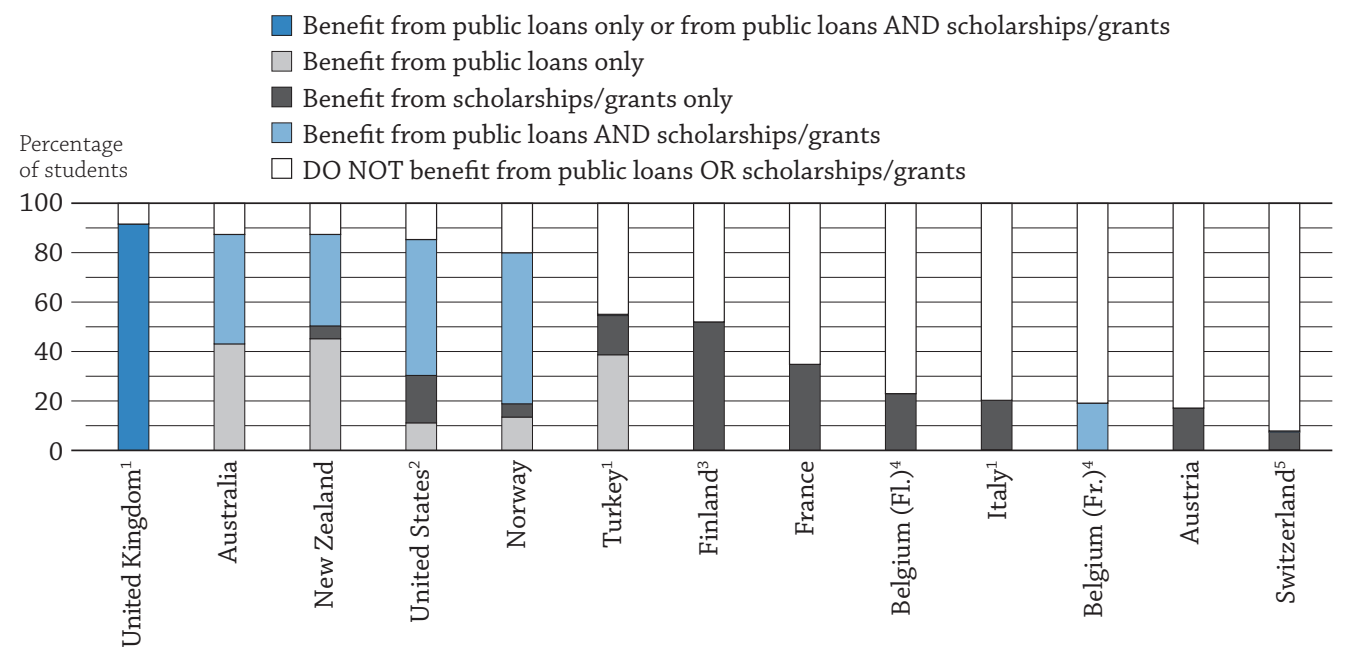

1. Reference year 2014-15.

2. Reference year 2011-12.

3. Includes master's, doctoral or equivalent levels.

4. Includes master's or equivalent level.

5. Bachelor's or equivalent level includes short-cycle tertiary programmes. Swiss data refer to the financial year 2013 and the academic year $2012 / 2013$.

Countries are ranked in descending order of the percentage of students receiving financial support for their studies.

Source: OECD. Table B5.3. See Annex 3 for notes (www.oecd.org/education/education-at-a-glance-19991487.htm).

StatLink 제에 $\mathrm{http}: / / \mathrm{dx}$.doi.org/10.1787/888933284082 
Loans also shift some of the cost of education to those who benefit most from higher education, namely, the individual student reflecting the high private returns of completing tertiary education (see Indicator A7). Opponents of loans argue that student loans are less effective than grants in encouraging low-income students to pursue their education. They also argue that loans may be less efficient than anticipated because of the various types of support provided to borrowers or lenders and the costs of administration and servicing. Finally, high level of student debt may have adverse effects both for students and for governments, if large numbers of students are unable to repay their loans (see Box B5.1 in Education at a Glance 2014).

Among the few countries with available data on the distribution of financial support to bachelor students in public institutions, $75 \%$ or more students in Australia, New Zealand, Norway, the United Kingdom and the United States benefit from public loans or scholarships/grants. Excluding Norway, these countries are also among countries with the highest tuitions fees in OECD countries. In Austria, Belgium (Flemish and French Communities), France, Italy and Switzerland, tuition fees are moderate, and most students in these countries do not benefit from financial support, but those who do usually receive such support in the form of scholarships and grants. Only bachelor's degree students in Belgium (French Community) benefit from a combination of both loans and scholarships/grants. In Finland and Turkey, public institutions do not charge tuition fees, and most students benefit from scholarships (Finland) or from scholarships/grants or loans (Turkey) (Table B5.3 and Chart B5.3).

\section{Country approaches to funding tertiary education}

Although many countries have similar goals for tertiary education, such as strengthening the knowledge economy, increasing access for students, encouraging high completion rates, and ensuring the financial stability of their higher education systems, countries differ dramatically in the way the cost of higher education is shared among governments, students and their families, and other private entities - and in the financial support they provide to students.

Whereas the cost of tertiary education, and the level of support available to students, varies markedly across OECD countries, some patterns can be identified to draw a classification of approaches to funding tertiary education. Countries can be grouped into four models, according to two factors: the level of tuition fees, and the financial support available through the country's student financial aid system for tertiary education.

There is no single model for financing tertiary education. Countries in which tertiary institutions charge similar tuition fees may vary in the proportion of students benefiting from public support and/or in the average amount of these subsidies (Tables B5.1a and B5.3, and Chart B5.1). However, arrangements regarding the tuition fees charged by tertiary educational institutions and financial support to students have been the subject of reforms in many OECD countries in recent years, to ensure that tertiary institutions have the necessary financial and human resources to face the increasing student population, and to ensure equity in access to tertiary studies, so that some countries have moved from one model to another over this period (Chart B5.1 and Box B5.2 on changes in tuition fees and financial support to student).

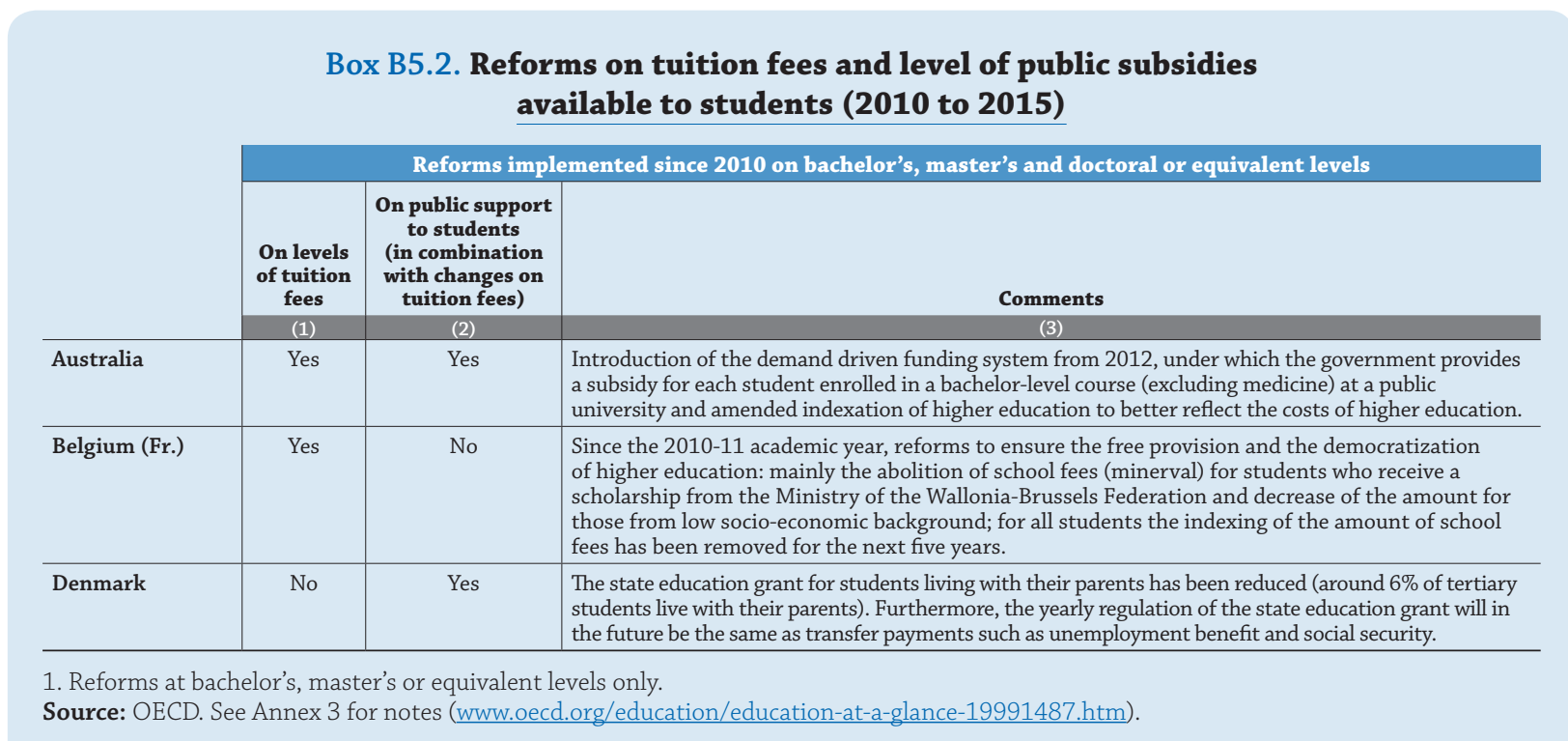




\section{Box B5.2. (continued) Reforms on tuition fees and level of public subsidies available to students (2010 to 2015)}

\begin{tabular}{|c|c|c|c|}
\hline & \multicolumn{3}{|c|}{ Reforms implemented since 2010 on bachelor's, master's and doctoral or equivalent levels } \\
\hline & $\begin{array}{l}\text { On levels } \\
\text { of tuition } \\
\text { fees }\end{array}$ & $\begin{array}{l}\text { On public support } \\
\text { to students } \\
\text { (in combination } \\
\text { with changes on } \\
\text { tuition fees) }\end{array}$ & Comments \\
\hline & (1) & (2) & (3) \\
\hline Estonia & Yes & Yes & $\begin{array}{l}\text { To enhance the accessability of tertiary level education and to increase the efficiency of studies, } \\
\text { conditions for a university to demand the reimbursement of study costs from a student is based } \\
\text { on condition relating to full-time enrolment; Estonian being the language of instruction of the } \\
\text { programme, and completion of the studyload by the student. } \\
\text { New needs-based student support system introduced in } 2013 / 2014 \text {. Students from less privileged } \\
\text { families can apply for study allowance (EUR } 75-220 \text { per month) when studying full time and in } \\
\text { Estonian language. In addition, starting from } 2015 \text {, need-based special allowance was introduced if } \\
\text { the application for a need-based study allowance of a student has been declined and the economic } \\
\text { situation of the family has been changed since. The possibility to apply for special study loans from } \\
\text { banks has remained. }\end{array}$ \\
\hline Finland & No & No & $\begin{array}{l}\text { The new Finnish government from } 2015 \text { plans to introduce tuition fees for students coming from } \\
\text { outside the EU and European Economic Area to study in Finland. Between } 2010 \text { and } 2014 \text { there } \\
\text { was a tuition fee trial period when it was possible for higher education institutions to charge fees } \\
\text { to foreign students coming from outside the EU or the European Economic Area and studying in } \\
\text { university and polytechnic programmes at master's level given in a foreign language. }\end{array}$ \\
\hline France & No & No & $\begin{array}{l}\text { Changes in } 2013 \text { and } 2014 \text { to increase the financial support to tertiary students (increase in the } \\
\text { amount of scholarships; in the number of scholarships to students and conditions to benefit from } \\
\text { scholarship extended). }\end{array}$ \\
\hline Hungary & Yes & Yes & $\begin{array}{l}\text { There are fully state-financed, partially state-financed, as well as full-paid tuition places in the } \\
\text { Hungarian higher education. In } 2012-13 \text { academic year, the number of fully financed places in } \\
\text { tertiary insitutions has been decreased (by } 27 \% \text { ) and the number of places that are } 50 \% \text { financed } \\
\text { by the state have been increased (by a lower rate). This reduction has mainly affected fields of study } \\
\text { such as law and economics, with science and technology being better supported.) } \\
\text { In } 2012-13 \text { academic year, a new student loan form (namely Diákhitel2) was launched for all } \\
\text { students who pay the cost of studies ("cost-refunding" or "tuition fee"), besides Diákhitel1. } \\
\text { Diákhitel2 can be used only for the cost of studies. }\end{array}$ \\
\hline Italy & Yes & Yes & $\begin{array}{l}\text { Following the adoption of a general university reform in } 2010 \text {, the students' support system } \\
\text { is currently undergoing significant change. The main aims of the reform are to strengthen the } \\
\text { opportunities for students coming from a low socio-economic background and to promote merit } \\
\text { amongst all students. The main measures are the definition of minimum standards of student } \\
\text { services, to be guaranteed to all students coming from a low socio-economic background, and } \\
\text { the creation of a national fund to support the most successful students. In this context, an } \\
\text { Observatory on Students' Welfare (Osservatorio per il Diritto allo Studio) was created in } 2013 \text { to } \\
\text { collect information on the student population, to monitor and report on students' support services, } \\
\text { and to advise the Ministry on standards for the student support system. While the required } \\
\text { legislation to implement these reforms has already been approved, administrative procedures and } \\
\text { implementation measures are currently being developed. }\end{array}$ \\
\hline Korea & Yes & Yes & $\begin{array}{l}\text { Reforms in } 2012 \text { to increase the level of public support for higher education, with the goal of } \\
\text { expanding access to and improving equity in tertiary education. National scholarships to students } \\
\text { since } 2012 \text { by combining and expanding the existing scholarships for low-income students. }\end{array}$ \\
\hline Netherlands & No & No & No reform, but tuition fees are corrected each year for inflation. \\
\hline New Zealand & Yes & No & $\begin{array}{l}\text { Control increases in tuition fees : limits on how much a provider may increase all fees and course } \\
\text { costs are defined by the Ministry. This level is set each year and since } 2011 \text { has been } 4 \% \text {. A level of } \\
3 \% \text { is proposed for the } 2016 \text { calendar year. }\end{array}$ \\
\hline Slovak Republic & No & No & $\begin{array}{l}\text { The conditions of determining the maximum amount of tuition fees have been amended; specific } \\
\text { charges are determined by each school separately in its internal regulation. }\end{array}$ \\
\hline Sweden $^{1}$ & Yes & Yes & $\begin{array}{l}\text { Tuition fees were introduced for non-EEA students } 2011 \text { in higher education institutions, except at } \\
\text { doctoral level, and at the same time public stipend programmes were introduced. These stipends are } \\
\text { distributed via other state agencies than CSN. }\end{array}$ \\
\hline Turkey & Yes & No & $\begin{array}{l}\text { As of the academic year 2012-13, students in first education (regular morning programmes) and } \\
\text { open education programmes are not charged tuition fees over the course of the theoretical duration } \\
\text { of the programmes. Tuition fees are paid only by students in public institutions who are enrolled in } \\
\text { evening programmes and those who have not graduated from a programme within the theoretical } \\
\text { duration. }\end{array}$ \\
\hline United Kingdom & Yes & Yes & $\begin{array}{l}\text { For new students starting courses in England from } 2012-13 \text { the maximum tuition fee cap increased } \\
\text { to GBP } 9000 \text { per year (from GBP } 3 \text { 290). Tuition fee loans available to students also increased to } \\
\text { GBP } 9000 \text { per year, with repayment terms also changing (earnings threshold at which repayments } \\
\text { start increased; a real interest rate to be charged when income is above the earnings threshold; } \\
\text { earnings thresholds will be increased annually in line with earnings; the length of time before all } \\
\text { debts are written off is extended from } 25 \text { to } 30 \text { years; extension of free loans to part-time students). }\end{array}$ \\
\hline United States & No & No & $\begin{array}{l}\text { Prior to } 2010 \text { the federal government guaranteed student loans provided by banks and non-profit } \\
\text { lenders. In 2010, the guaranteed loan program was eliminated and all U.S. federal student loans } \\
\text { became direct loans (originated and funded directly by the U.S. Department of Education). }\end{array}$ \\
\hline
\end{tabular}

1. Reforms at bachelor's, master's or equivalent levels only.

Source: OECD. See Annex 3 for notes (www.oecd.org/education/education-at-a-glance-19991487.htm). 


\section{Model 1: Countries with no or low tuition fees and generous student-support systems}

This group is composed of the Nordic countries: Denmark, Finland, Iceland, Norway and Sweden. Students pay no tuition fee and benefit from generous public support for higher education. In these countries, more than 55\% of students benefit from public grants, public loans, or a combination of the two (Table B5.3, and Table B5.4 in Education at a Glance 2014). These countries have more progressive tax structures (OECD, 2011) and individuals face high income tax rates. The average entry rate into bachelor programmes for this group - $59 \%$ - is above the OECD average of 56\% (see Indicator C3, Table C3.1). These entry rates may also reflect the attractiveness of these countries' highly-developed student financial support systems, not just the absence of tuition fees.

The approach to funding tertiary education in this model reflects these countries' deeply rooted social values, such as equality of opportunity and social equity. The notion that government should provide its citizens with tertiary education at no charge to the individual is a salient feature of the culture of education in these countries: the funding of both institutions and students is based on the principle that access to tertiary education is a right, rather than a privilege.

However, during the past decade, Denmark and Sweden (as of 2011) decided to introduce tuition fees for international students to increase the resources available for their tertiary institutions and/or to improve the quality of the programmes they offer (Sweden). Iceland also considered doing so, and between 2010 and 2014, Finland implemented a tuition fee trial period for higher education institutions to charge fees to some foreign students coming from outside EU or the European Economic Area. Such a change may discourage international students from studying in these countries. Sweden has seen a reduction in the number of international students in the country since this reform. Between autumn 2010 and autumn 2011 the number of students who were not part of an exchange programme and came from outside the European Economic Area and Switzerland decreased by almost 80\% (Swedish Higher Education Authority, 2013).

\section{Model 2: Countries with high tuition fees and well-developed student-support systems}

The second group includes Australia, Canada, the Netherlands, New Zealand, the United Kingdom and the United States. These countries have potentially high financial obstacles to entry into tertiary education, but they also offer significant public support to students. The average entry rate to bachelor programmes for this group of countries is $71 \%$, significantly above the OECD average (56\%) and higher than most countries with low tuition fees.

Since 1995, the United Kingdom has moved from Model 4 (countries with lower tuition fees and less-developed student-support systems) to Model 2 (Chart B5.1), and the Netherlands moved from Model 1 to Model 2 as tuition fees increased and the student-support system is well-developed (see Chart B5.1 in Education at a Glance 2014). Countries in Model 2 tend to be those where private entities (e.g. private businesses and non-profit organisations) contribute the most to financing tertiary institutions. In other words, in Model 2 countries, the cost of education is shared among government, households and private companies (see Chart B3.2 and Table B3.1).

Tuition fees charged by public institutions for bachelor's programmes (government-dependent private institutions in the United Kingdom) exceed USD 4000 in all these countries (except the Netherlands, where they reach USD 2 300). At least $85 \%$ of tertiary students receive support from public loans or scholarships/grants in Australia, New Zealand, the United Kingdom and the United States, the four countries with data available (Tables B5.1a and B5.3). Studentsupport systems are well-developed and mostly accommodate the needs of the entire student population (Table B5.3 and Table B5.3 in Education at a Glance 2014).

In this group of countries, access to tertiary education is above the OECD average. For example, Australia and New Zealand have among the highest entry rates into bachelor's programmes ( $91 \%$ and $74 \%$, respectively), although these rates also reflect the high proportion of international students enrolled (entry rates excluding international students are still above the average). Entry rates were also above the OECD average (56\%) in the Netherlands (60\%) and the United Kingdom (58\%) in 2013. These countries spend more on core services (services directly related to instruction) per tertiary student than the OECD average and have a relatively high level of revenue from income tax as a percentage of GDP, compared to the OECD average. The Netherlands is an outlier, as its level of income taxation is below the OECD average (see Table B1.1b, available on line, and Table C3.1).

OECD research (OECD, 2008) suggests that, in general, this model can be an effective way for countries to increase access to higher education. However, during periods of economic crisis, high tuition fees impose a considerable financial burden on students and their families and can discourage some of them from entering tertiary education, even when relatively high levels of student support are available. 


\section{Model 3: Countries with high tuition fees and less-developed student-support systems}

In Chile, Japan and Korea, most students are charged high tuition fees (more than USD 4700 for bachelor's programme in public institutions in Japan and Korea in 2013-14; more than USD 5800 for Chile, as based on data from Education at a Glance 2014), but student-support systems are somewhat less developed than those in Models 1 and 2. This approach can impose a heavy financial burden on students and their families. Entry rates into bachelor's programmes are slightly above or below the OECD average (58\% in Chile, $48 \%$ in Japan and 55\% in Korea). In Japan and Korea, some students who excel academically but have difficulty financing their studies can benefit from reduced tuition and/or admission fees or receive total exemptions.

Japan and Korea are among the countries with the lowest levels of public expenditure allocated to tertiary education as a percentage of GDP (see Chart B2.2). This partially explains the small proportion of students who benefit from public loans. However, both countries have recently implemented reforms to improve their student-support systems. In Korea, reforms in 2012 aimed to expand access to and improve equity in tertiary education by offering national scholarships to students and by combining and expanding existing scholarships for low-income students.

\section{Model 4: Countries with low tuition fees and less-developed student-support systems}

The fourth group includes all other European countries for which data are available (Austria, Belgium, France, Italy, and Switzerland in this edition, but also the Czech Republic, Ireland, Poland, Portugal and Spain as based on data from Education at a Glance 2014). All of these countries charge moderate tuition fees compared to those in Models 2 and 3, although since 1995, reforms were implemented in some of these countries - particularly Austria and Italy - to increase tuition fees in public institutions (Chart B5.1 and Box B5.1 in Education at a Glance 2012). Model 4 countries have relatively low financial barriers to entry into bachelor's programmes, combined with relatively low levels of support for students, which are mainly targeted to specific groups. Tuition fees charged by public institutions in this group never exceed USD 1600 , and in countries for which data are available, most students do not benefit from public support (Tables B5.1a and B5.3). Turkey is moving from Model 4 to Model 1. As of academic year 2012/13, tuition fees are no longer charged in public institutions for students in first education (regular morning programmes) and open education programmes, and most students benefit from student loans or scholarship/grants. These reforms aim to facilitate access to tertiary education for all.

In Model 4 countries, tertiary institutions usually depend heavily on the state for funding, and participation in tertiary education is typically below the OECD average. The average entry rate into bachelor's programmes in this group of countries - 52\% - is relatively low; but in some countries, such as Austria and Spain this is complemented by aboveaverage entry rates into short-cycle tertiary programmes. Similarly, expenditure per student in bachelor's, master's or doctoral programmes is also comparatively low (see Chart B5.2 and Indicator B1). While high tuition fees can raise potential barriers to student participation, Model 4 suggests that lower tuition fees, which are assumed to ease access to education, do not necessarily guarantee greater access to tertiary education.

In these countries, students and their families can benefit from support provided by sources other than the ministry of education (e.g. housing allowances, tax reductions and/or tax credits for education), but these are not covered in this analysis. In France, for example, of total state funding, housing allowances represent about $90 \%$ of scholarships/ grants, and about one-third of students benefit from them. Poland is notable in that most students enrolled in public institutions have their studies fully subsidised by the state, while students enrolled in part-time studies pay the full costs of tuition.

In Model 4 countries, loan systems, such as public loans or loans guaranteed by the state, are not available or are only available to a small proportion of students in these countries (Table B5.3). At the same time, the level of public spending and the tax revenue from income as a percentage of GDP vary significantly more among this group of countries than in the other groups.

\section{Implementation of public loan systems and amount of public loans}

According to available data, public loan systems (see Box B5.1 on types of student loans in Education at a Glance 2014) are particularly well-developed in countries with high tuition fees, including Australia, the United Kingdom and the United States, where some $62 \%$ or more of students benefit from a public loan during their tertiary studies in bachelor's, master's, doctoral or equivalent programmes (only 32\% of doctoral students in the United States). Public loan systems are also well-developed in countries where tertiary institutions do not charge tuition fees for national students, such as Denmark (35\%), Norway (68\%) and Sweden (52\%). 
The financial support that students receive from public loans during their studies cannot be solely analysed in light of the proportion of students who have loans. The support for students also depends on the amount they can receive in public loans. Among the 20 countries with available data, the average annual gross amount of public loan available to students exceeds USD 4000 in all countries (with available data) where the majority of student benefit from a public loan. It also exceeds this amount in countries where a small or even marginal proportion of students benefit from a public loan (for example in Japan) (Table B5.4).

Interestingly, the larger the proportion of students who have a loan, the higher the average annual gross amount of loans available to each student. On the one hand, in Belgium (French Community), Estonia and Finland, 9\% to 22\% of students benefit from a loan, and the average annual gross amount of loan in these countries is no more than USD 3 500. On the other hand, in Australia, Norway, Sweden, the United Kingdom and the United States, most students take out a loan (52\% to $92 \%$ of students) and the average annual gross amount of loan exceeds USD 4000 (Table B5.4).

The comparison of average tuition fees and average amounts of loans should be interpreted with caution since, in a given education programme, the amount of a loan can vary widely among students, even if the programme's tuition fees are the same. Nevertheless, such a comparison provides some insight into whether students take a loan to cover tuition fees and living expenses. In the OECD countries for which data on annual gross amounts of loans are available (in public and private institutions combined), the average amount of public loan exceeds the average tuition fee charged by public institutions, except in Australia, Canada (for bachelor's degree students), the United Kingdom and the United States (at bachelor's and doctoral levels) - four countries with among the highest tuitions fees for bachelor's, master's and doctoral programmes. This suggests that public loans may help support students' living expenses during their studies, but not necessarily in countries where tuition fees charged to students are the highest. In Canada, the average amount of public loan does not exceed the average tuition fee charged by public institution, but data refer only to the federal portion of the loan; students typically receive another portion provided by the province or territory, often raising the total so that some living expenses are covered.

Among the countries where public institutions charge average tuition fees above USD 2000 for bachelor's programmes and for which data on annual gross amounts of loans are available, the average amount of a student loan exceeds the amount of fees only in Japan, the Netherlands and New Zealand. By contrast, in the United Kingdom (for governmentdependent private institutions) and the United States, the average tuition fee is much higher than the average student loan (in the United States, many students have both grants and loans). The largest differences between average tuition fees and the average amount of loans are observed in the Nordic countries (Denmark, Finland, Norway and Sweden), Estonia and Turkey, where no tuition fee is charged by institutions and a large proportion of students benefit from a public loan (or a loan guaranteed by the state). The average loan in these countries ranges from about USD 2700 in Finland (private loan guaranteed by the state) to USD 10000 in Norway (Table B5.1a and Table B5.4).

Public loan systems also offer some financial aid to students through the interest rate that these students may have to pay, the repayment system or even remission/forgiveness mechanisms (Table B5.3).

\section{Financial support through interest rates}

The financial benefits from reducing interest rates on public or private loans is twofold, as the interest rates supported by students during and after their studies may differ. Comparing interest rates among countries is difficult, as the structure of interest rates, both public and private, is not known and can vary significantly among countries, such that a given interest rate may be considered high in one country and low in another. However, differences in rates during and after tertiary studies seem intended to reduce the financial burden on students during their studies.

In Canada, Japan, New Zealand and the Slovak Republic, there is no nominal interest rate on a public loan during the period of studies; but after this period, students/graduates may incur an interest charge related to the cost of government borrowing or even higher. For example, New Zealand, which made loans interest-free for borrowers while they reside in New Zealand, charges an interest rate on loans to borrowers who are overseas. Australia, Brazil, Colombia, Estonia, Hungary, Korea, the Netherlands and Sweden do not differentiate between the interest rate borne by student during and after their studies. In Australia, a real interest rate is not charged on loans; instead, the part of a loan that has remained unpaid for 11 months or more is indexed to ensure that the real value of the loan is maintained (Table B5.4).

\section{Repayment of loans}

The current reporting of household expenditure on education as part of private expenditure (see Indicator B3) does not take into account the repayment of public loans by previous recipients. The repayment period varies among 
countries, ranging from ten years or less in Australia, Canada, Estonia, New Zealand, the Slovak Republic and Turkey, to twenty years or more in Norway, Sweden and in the United States (for income based repayments).

Among the 16 countries with available data on repayment systems, four English-speaking countries (Australia, New Zealand and the United Kingdom, and the United States for part of the student's loans) as well as Hungary, Korea (for part of the student's loans) and the Netherlands make the repayment of loans dependent on graduates' level of income. Among countries with income-contingent repayment systems, the minimum annual income threshold above which borrowers have to reimburse the loan varies largely between countries. While it is about USD 13000 in New Zealand, it varies among other countries from USD 20000 in the Netherlands to more than USD 30000 in Australia and the United Kingdom (Table B5.5).

Besides repayment, schemes for remission (delaying the repayment) and/or forgiveness of student loans exist in nearly all countries with a student-loan system. These systems may benefit to significant proportions of students who took a loan during their studies. Among countries with available information, the proportion of students benefiting from remission and/or forgiveness varies from $2 \%$ or less in Australia, Finland, Hungary, Japan, New Zealand and Sweden, to $10 \%$ in the Netherlands. This can translate into significant proportions of loans that are not repaid. In Australia, Canada and the Netherlands, it is estimated that $10 \%$ or more of the loans are expected not to be repaid.

The conditions to benefit from such mechanisms vary between countries. Death, disability or poor financial situation of the graduate who took the loan are commonly accepted reasons for obtaining forgiveness or a remission. Furthermore, conditions for remission and/or forgiveness are linked in some countries to the labour market situation or to students' results. For example, in the United States, teachers and individuals in public service may apply to loan-forgiveness programmes; in Australia, graduates of specific fields (and employed in a related occupation) and graduates who take up related occupations or work in specified locations benefit from remission through a reduction of their repayments. In Colombia and Japan, some graduates with particularly outstanding results may also expect forgiveness of all or part of their student loan.

\section{Debt at graduation}

During economic crises, when young graduates may have difficulties in finding a job, the level of debt at graduation becomes a concern. When labour market opportunities are scarce, many graduates may to go back to school - and risk assuming even more debt.

In several countries, most students are in debt at graduation. Countries whose tertiary institutions charge high tuition fees are also those whose students have the highest levels of debt at graduation. In countries with a relatively small proportion of graduates in debt, the debt burden is also lighter. For example, in Finland, fewer than one in two students is in debt at graduation for an average of about USD 8300 , while in the United Kingdom (England only), nine out of ten graduates have debt from loans of an average of more than USD 30000 (Table B5.4).

However, and contrary to what could be expected, graduates in countries with no tuition fee can also have a high level of debt at graduation. This is the case of students in Norway and Sweden, where the average annual gross amount of loan available to each students reaches around USD 10000 and USD 6 800, respectively, and also covers student living expenses, as there is no tuition fee for tertiary studies. In addition, compared to countries with higher tuition fees, income is generally lower after graduation and taxes are higher in these countries (see Model 1).

\section{Definitions}

Average tuition fees charged in public and private tertiary institutions distinguishes tuition fees between shortcycle, bachelor, master, doctorate or equivalent programmes. This indicator gives an overview of tuition fees at each level by type of institution and shows the proportions of students who do or do not receive scholarships/grants that fully or partially cover tuition fees. Levels of tuition fees and associated proportions of students should be interpreted with caution as they are derived from the weighted average of the main programmes.

Student loans refer to the full range of student loans in order to provide information on the level of support received by students. The gross amount of loans provides an appropriate measure of the financial aid to current participants in education. Interest payments and repayments of principal by borrowers should be taken into account when assessing the net cost of student loans to public and private lenders. In most countries, loan repayments do not flow to education authorities, and the money is not available to them to cover other expenditures on education. 
OECD indicators take the full amount of scholarships and loans (gross) into account when discussing financial aid to current students. Some OECD countries also have difficulty quantifying the amount of loans to students. Therefore, data on student loans should be treated with some caution.

\section{Methodology}

Data refer to the financial year 2013 or school year 2013-14 and are based on a special survey administered by the OECD and undertaken in 2015 (for details see Annex 3 at www.oecd.org/education/education-at-a-glance-19991487. htm).

Amounts of tuition fees and amounts of loans in national currency are converted into equivalent USD by dividing the national currency by the purchasing power parity (PPP) index for GDP. Amounts of tuition fees and associated proportions of students should be interpreted with caution as they represent the weighted average of the main tertiary programmes and do not cover all educational institutions.

\section{Note regarding data from Israel}

The statistical data for Israel are supplied by and are under the responsibility of the relevant Israeli authorities. The use of such data by the OECD is without prejudice to the status of the Golan Heights, East Jerusalem and Israeli settlements in the West Bank under the terms of international law.

\section{References}

OECD (2011), Revenue Statistics 2011, OECD Publishing, Paris, http://dx.doi.org/10.1787/ctpa-rev-data-en.

OECD (2008), Tertiary Education for the Knowledge Society: Volume 1 and Volume 2, OECD Publishing, Paris, http://dx.doi.org/ 10.1787/9789264046535-en.

Swedish Higher Education Authority (2013), "Fewer Students from Asia after the Tuition Reform”, Statistical Analysis, Stockholm.

\section{Indicator B5 Tables}

StatLink त्माज्ञा http://dx.doi.org/10.1787/888933285558

Table B5.1a Estimated annual average tuition fees charged by educational institutions (bachelor's, master's, doctoral or equivalent level) (2013-14)

WEB Table B5.1b Estimated annual average tuition fees charged by educational institutions for students in bachelor's or equivalent programmes (2013-14)

WEB Table B5.1c Estimated annual average tuition fees charged by educational institutions for students in master's or equivalent programmes (2013-14)

WEB Table B5.1d Estimated annual average tuition fees charged by educational institutions for students in doctoral or equivalent programmes (2013-14)

WEB Table B5.1e Estimated annual average tuition fees charged by educational institutions for students in short-cycle tertiary programmes (2013-14)

WEB Table B5.2 Average tuition fees charged by tertiary institutions for bachelor's, master's, doctoral or equivalent programmes, by field of education (2013-14)

Table B5.3 Financial support to students and tuition fees charged by educational institutions (2013-14)

Table B5.4 Public loans to students in bachelor's, master's, doctoral or equivalent programmes (2013-14)

Table B5.5 Repayment and remission of public loans to students in bachelor's, master's, doctoral or equivalent programmes (academic year 2013/14)

WEB Table B5.6 Estimated annual average tuition fees charged by educational institutions for international students (2013-14) 
Table B5.1a. [1/2] Estimated annual average tuition fees charged by educational institutions (bachelor's, master's, doctoral or equivalent level) ${ }^{1}$ (2013-14)

National students, in equivalent USD converted using PPPs, by type of institutions and degree structure, based on full-time students, academic year 2013-14

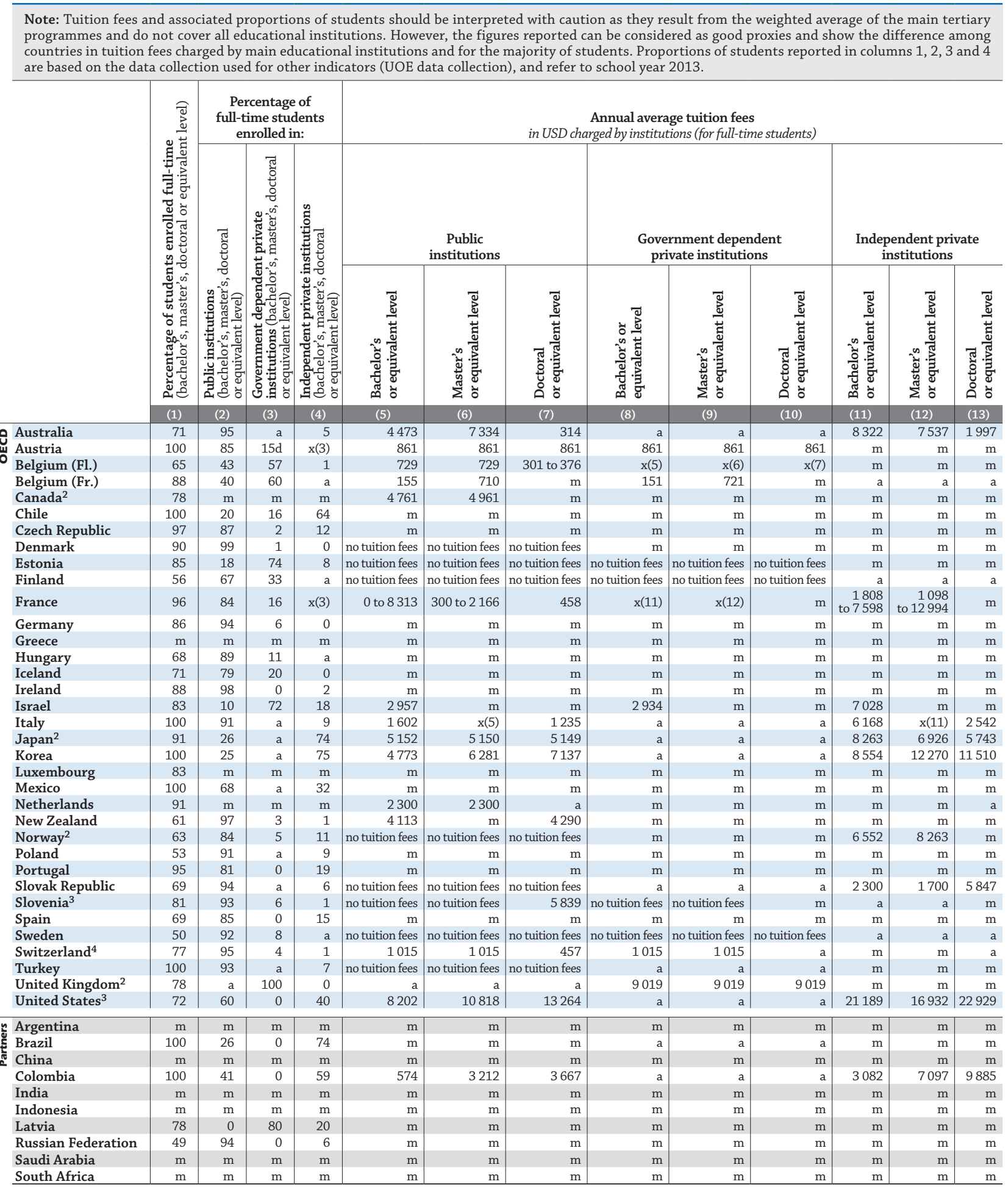

1. Scholarships/grants that the student may receive are not taken into account.

2. Reference year 2014-15 for tuition fees (in Japan, for public institutions only).

3. Reference year 2011-12 for tuition fees.

4. Financial reference year 2013 and academic reference year 2012-13.

Source: OECD. See Annex 3 for notes (www.oecd.org/education/education-at-a-glance-19991487.htm).

Please refer to the Reader's Guide for information concerning symbols for missing data and abbreviations.

StatLink 제개 http://dx.doi.org/10.1787/888933285566 
Table B5.1a. [2/2] Estimated annual average tuition fees charged by educational institutions (bachelor's, master's, doctoral or equivalent level) ${ }^{1}$ (2013-14)

National students, in equivalent USD converted using PPPs, by type of institutions and degree structure, based on full-time students, academic year 2013-14

\begin{abstract}
Note: Tuition fees and associated proportions of students should be interpreted with caution as they result from the weighted average of the main tertiary programmes and do not cover all educational institutions. However, the figures reported can be considered as good proxies and show the difference among countries in tuition fees charged by main educational institutions and for the majority of students. Proportions of students reported in columns 1 , 2, 3 and 4 are based on the data collection used for other indicators (UOE data collection), and refer to school year 2013.
\end{abstract}

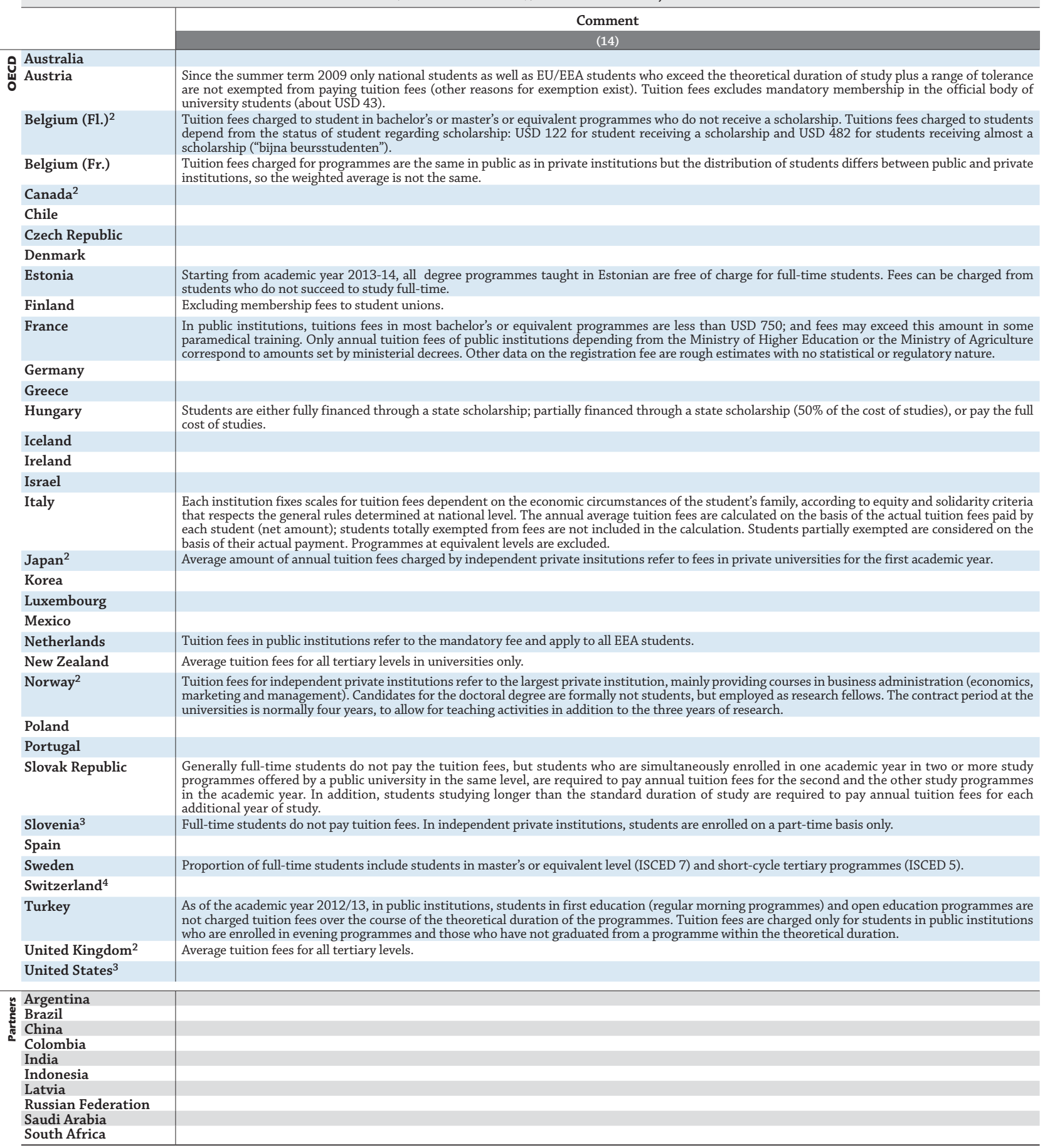

1. Scholarships/grants that the student may receive are not taken into account.

2. Reference year 2014-15 for tuition fees (in Japan, for public institutions only).

3. Reference year 2011-12 for tuition fees.

4. Financial reference year 2013 and academic reference year 2012-13.

Source: OECD. See Annex 3 for notes (www.oecd.org/education/education-at-a-glance-19991487.htm).

Please refer to the Reader's Guide for information concerning symbols for missing data and abbreviations.

StatLink 제그 http://dx.doi.org/10.1787/888933285566 
Table B5.3. Financial support to students and tuition fees charged by educational institutions (2013-14) National students, based on full-time students, academic year 2013-14

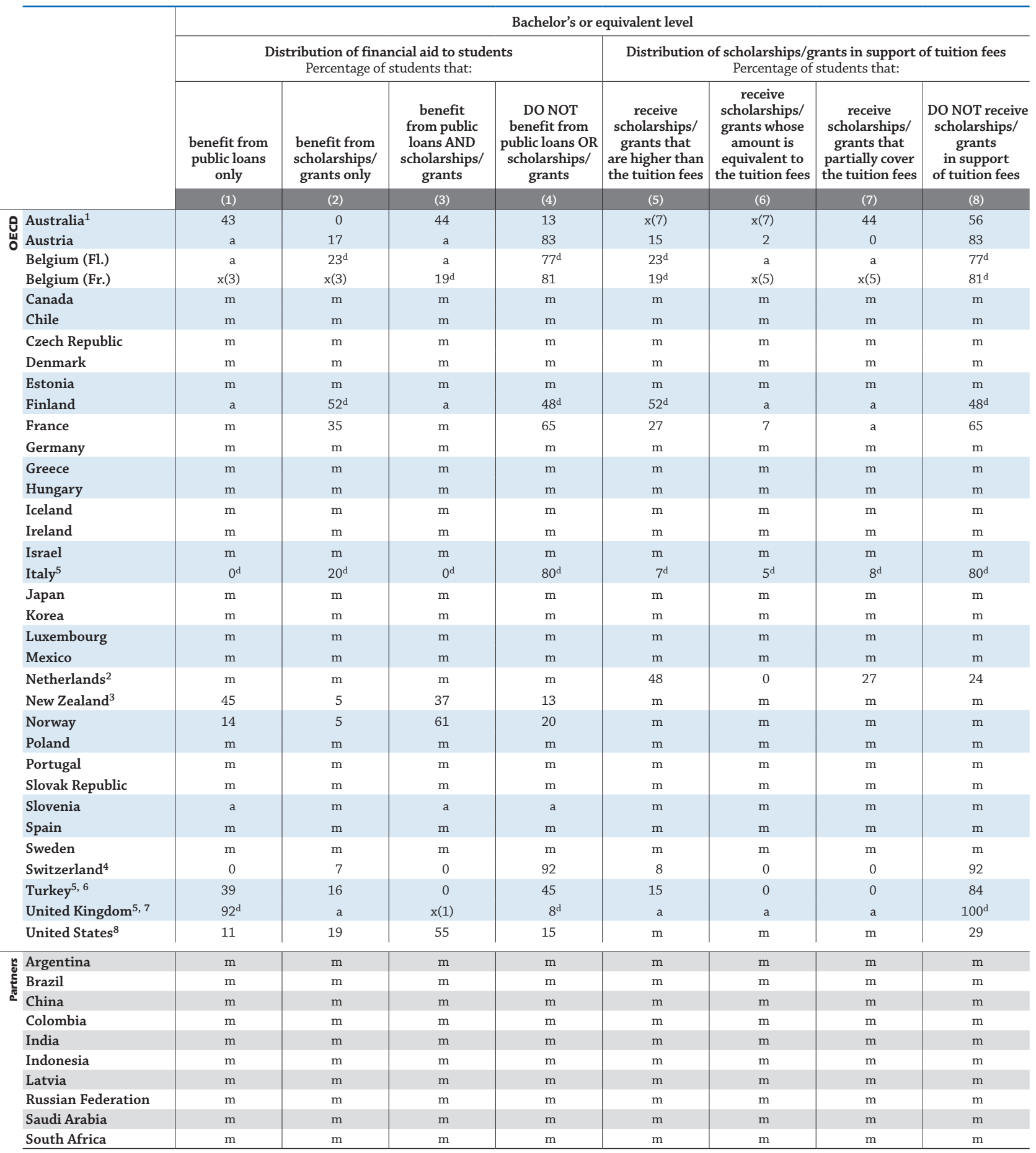

Note: Distribution of financial aid to students and scholarships/grants in support of tuition fees at master's and doctoral or equivalent levels are available on line.

1. Only includes the major Australian Government scholarships programmes. It excludes all scholarships provided by education institutions and the private sector.

2. Public institutions only.

3. Average values for full-time students across all levels of tertiary education.

4. Bachelor's or equivalent level includes short-cycle tertiary programmes. Swiss data refer to the financial year 2013 and the academic year $2012 / 2013$.

5. Reference year 2014-15.

6. Students who benefit from scholarships/grants only includes those receiving public scholarships/grants.

7. Excludes independent private institutions.

8. Reference year 2011-12.

Source: OECD. See Annex 3 for notes (www.oecd.org/education/education-at-a-glance-19991487.htm).

Please refer to the Reader's Guide for information concerning symbols for missing data and abbreviations.

StatLink 젶ㄴ http://dx.doi.org/10.1787/888933285571 
Table B5.4. [1/2] Public loans to students in bachelor's, master's, doctoral or equivalent programmes (2013-14)

National students, in USD converted using PPPs

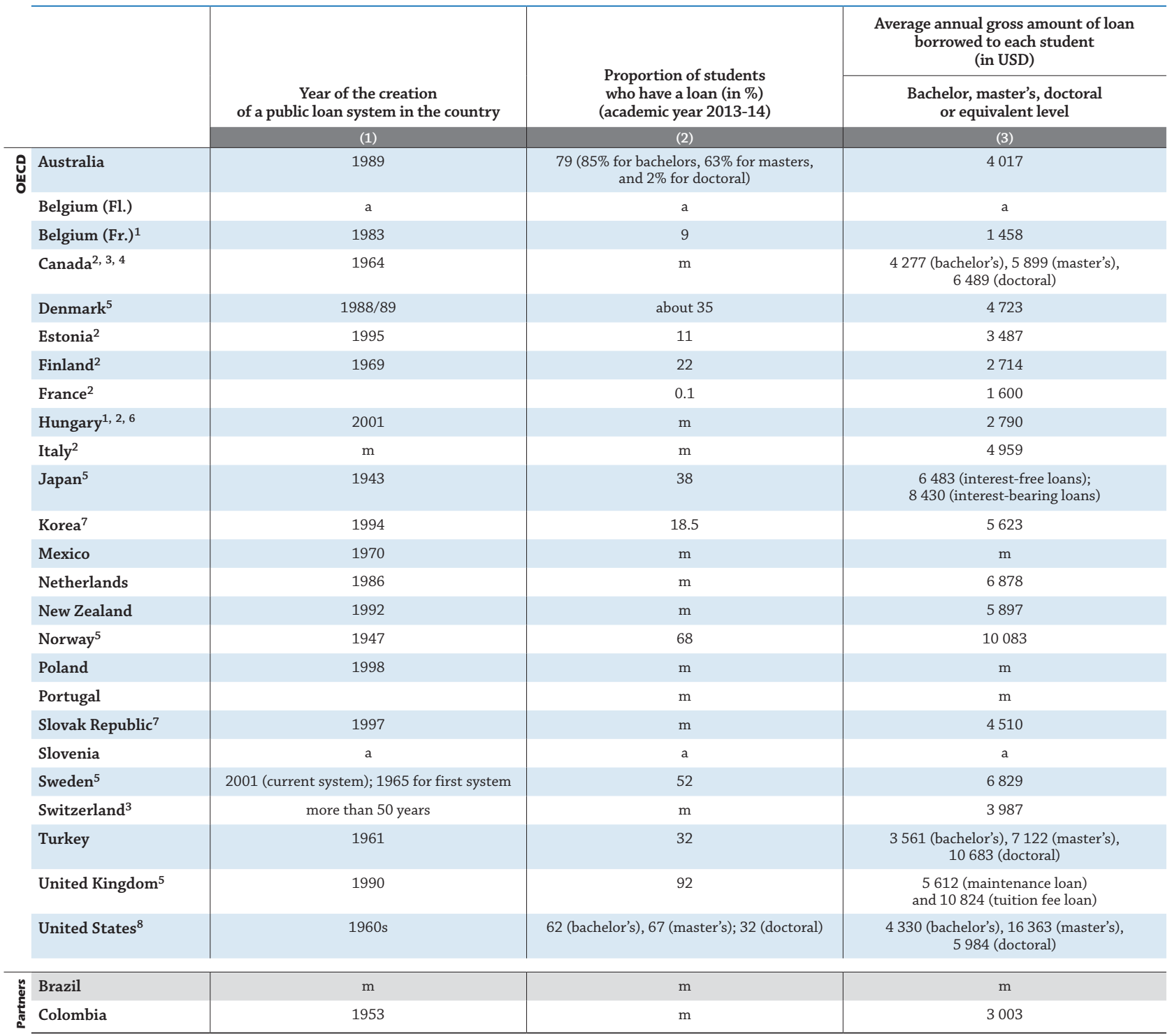

Note: Columns 4, 5 and 6 available on line display the average annual gross amount of loan at bachelor's, master's and doctoral or equivalent levels.

1. All students in bachelor's, master's, doctoral or equivalent programmes.

2. Private loan guaranteed by the state rather than public loan (in Italy, for the majority of student loans).

3. Reference year 2012-13.

4. Only includes information on the federal portion of student financial assistance, that is to say $60 \%$ of student loans provided in the provinces participating in the Canada Student Loans Program (CSLP). Excludes the province of Quebec (about 25\% of the Canadian population) that does not participate in the CSLP.

5. Reference year 2014-15 (for Japan, 2013-14 reference year for debt at graduation).

6. Data refer to Diákhitel1 only. In the academic year 2012-13 a new student loan form (Diákhitel2) was launched, besides Diákhitel1. Diákhitel2 can be used only for the cost of studies ("cost-refunding" or "tuition fee"), while Diákhitel1 can be used for any purpose (e.g. student living expenses).

7. Includes short-cycle tertiary programmes.

8. Reference year 2011-12 for the proportion of students with student loans; reference year 2014-15 for information on interest rates.

Source: OECD. See Annex 3 for notes (www.oecd.org/education/education-at-a-glance-19991487.htm).

Please refer to the Reader's Guide for information concerning symbols for missing data and abbreviations.

StatLink : 
Table B5.4. [2/2] Public loans to students in bachelor's, master's, doctoral or equivalent programmes (2013-14)

National students, in USD converted using PPPs

\begin{tabular}{|c|c|c|c|c|c|}
\hline & & \multicolumn{2}{|c|}{ Subsidy through reduced interest rate } & \multicolumn{2}{|r|}{ Debt at graduation } \\
\hline & & $\begin{array}{l}\text { Interest rate } \\
\text { during studies }\end{array}$ & $\begin{array}{l}\text { Interest rate } \\
\text { after studies }\end{array}$ & $\begin{array}{c}\text { Percentage } \\
\text { of graduates with debt } \\
\text { (in \%) }\end{array}$ & $\begin{array}{l}\text { Average debt at graduation } \\
\text { (in USD) }\end{array}$ \\
\hline & & $(7)$ & $(8)$ & (9) & (10) \\
\hline \multirow{23}{*}{ : } & Australia & $2 \%$ (no real interest rate) & $2 \%$ (no real interest rate) & $74 \%$ & $\mathrm{~m}$ \\
\hline & Belgium (Fl.) & a & $\mathrm{a}$ & $\mathrm{a}$ & a \\
\hline & Canada $^{2,3,4}$ & No nominal interest rate & $5.4 \%$ & $\mathrm{~m}$ & 12422 \\
\hline & Denmark $^{5}$ & $\begin{array}{l}4.0 \% \text { (interest rate higher than } \\
\text { cost of government borrowing) }\end{array}$ & $\begin{array}{l}1.0 \% \text { (interest rate higher equal } \\
\text { to cost of government borrowing) }\end{array}$ & $57 \%$ & 14856 \\
\hline & Estonia $^{2}$ & $5.0 \%$ & $5.0 \%$ & $\mathrm{~m}$ & $\mathrm{~m}$ \\
\hline & Finland $^{2}$ & $1.0 \%$ & $\begin{array}{l}\text { Full interest rate agreed } \\
\text { with the private bank }\end{array}$ & $43.5 \%$ & 8291 \\
\hline & France $^{2}$ & $\mathrm{~m}$ & $\mathrm{~m}$ & $\mathrm{~m}$ & $\mathrm{~m}$ \\
\hline & Hungary $^{1,}$ 2, 6 & $\begin{array}{l}\text { Variable ( } 7.5 \% \text { to } 6.5 \%) \\
\text { for Diákhitel1 }\end{array}$ & $\begin{array}{l}\text { Variable ( } 7.5 \% \text { to } 6.5 \%) \\
\text { for Diákhitel1 }\end{array}$ & $\mathrm{m}$ & $\mathrm{m}$ \\
\hline & Italy $^{2}$ & $\mathrm{~m}$ & $\mathrm{~m}$ & $\mathrm{~m}$ & $\mathrm{~m}$ \\
\hline & Japan $^{5}$ & No nominal nor real interest rate & $\begin{array}{l}\text { Maximum of } 3 \% \text {, } \\
\text { rest paid by government }\end{array}$ & $\mathrm{m}$ & 29942 \\
\hline & Korea $^{7}$ & $2.9 \%$ & $2.9 \%$ & $\mathrm{~m}$ & $\mathrm{~m}$ \\
\hline & Mexico & $\mathrm{m}$ & $\mathrm{m}$ & $\mathrm{m}$ & $\mathrm{m}$ \\
\hline & Netherlands & $\begin{array}{l}\text { Cost of government borrowing } \\
(0.12 \%)\end{array}$ & $\begin{array}{l}\text { Cost of government borrowing } \\
(0.12 \%)\end{array}$ & $67 \%$ & 18100 \\
\hline & New Zealand & No nominal nor real interest rate & $\begin{array}{c}\text { No nominal interest rate } \\
\text { if New Zealand based, } \\
5.9 \% \text { otherwise }\end{array}$ & $\mathrm{m}$ & $\begin{array}{l}\text { USD } 13437 \text { (2014 average for } \\
\text { both graduates and non-graduate borrowers } \\
\text { who have left study, and regardless } \\
\text { of what level they studied at) }\end{array}$ \\
\hline & Norway $^{5}$ & $\begin{array}{l}\text { a (repayment of the loan } \\
\text { starts after graduation) }\end{array}$ & $\begin{array}{l}2.52 \% \text { (cost of government } \\
\text { borrowing, }+1.25 \% \text { to cover } \\
\text { defaulting costs) }\end{array}$ & $\mathrm{m}$ & 26826 \\
\hline & Portugal & $\mathrm{m}$ & $\mathrm{m}$ & $\mathrm{m}$ & $\mathrm{m}$ \\
\hline & Slovak Republic ${ }^{7}$ & No nominal nor real interest rate & $3.19 \%$ & $0.81 \%$ & 3247 \\
\hline & Slovenia & a & a & a & a \\
\hline & Sweden ${ }^{5}$ & $1 \%$ & $1 \%$ & $77 \%$ & 22789 \\
\hline & Switzerland ${ }^{3}$ & $\mathrm{~m}$ & $\mathrm{~m}$ & $\mathrm{~m}$ & $\mathrm{~m}$ \\
\hline & Turkey & $\begin{array}{l}\text { a (repayment of the loan } \\
\text { starts after graduation) }\end{array}$ & $\begin{array}{l}\text { Based on the domestic producer } \\
\text { price index }\end{array}$ & $\mathrm{m}$ & $\mathrm{m}$ \\
\hline & United Kingdom 5 & $\begin{array}{l}\text { Retail price index, plus } 3 \% \\
\quad(5.5 \% \text { for } 2014-15)\end{array}$ & $\begin{array}{c}\text { from retail price index } \\
\text { (2.5\% for 2014-15) to retail price } \\
\text { index, plus 3\% (5.5\% for 2014-15), } \\
\text { based on earnings }\end{array}$ & $91.6 \%$ & 30349 \\
\hline & United States $^{8}$ & $\begin{array}{l}0 \text { to } 7.21 \% \\
\text { (cost of government borrowing) }\end{array}$ & $\begin{array}{c}4.66 \text { to } 7.21 \% \\
\text { (cost of government borrowing) }\end{array}$ & $\mathrm{m}$ & $\mathrm{m}$ \\
\hline \multirow{2}{*}{ 峁 } & Brazil & $3.4 \%$ & $3.4 \%$ & $\mathrm{~m}$ & $\mathrm{~m}$ \\
\hline & Colombia & $\begin{array}{c}\text { Consumer price index to consumer } \\
\text { proce index }+8 \%\end{array}$ & $\begin{array}{l}\text { Consumer price index to consumer } \\
\text { proce index }+8 \%\end{array}$ & $\mathrm{~m}$ & 7298 \\
\hline
\end{tabular}

Note: Columns 4, 5 and 6 available on line display the average annual gross amount of loan at bachelor's, master's and doctoral or equivalent levels.

1. All students in bachelor's, master's, doctoral or equivalent programmes.

2. Private loan guaranteed by the state rather than public loan (in Italy, for the majority of student loans).

3. Reference year 2012-13.

4. Only includes information on the federal portion of student financial assistance, that is to say $60 \%$ of student loans provided in the provinces participating in the Canada Student Loans Program (CSLP). Excludes the province of Quebec (about 25\% of the Canadian population) that does not participate in the CSLP.

5. Reference year 2014-15 (for Japan, 2013-14 reference year for debt at graduation).

6. Data refer to Diákhitel1 only. In the academic year 2012-13 a new student loan form (Diákhitel2) was launched, besides Diákhitel1. Diákhitel2 can be used only for the cost of studies ("cost-refunding" or "tuition fee"), while Diákhitel1 can be used for any purpose (e.g. student living expenses).

7. Includes short-cycle tertiary programmes.

8. Reference years 2011-12 for the proportion of students with student loans; reference year 2014-15 for information on interest rates.

Source: OECD. See Annex 3 for notes (www.oecd.org/education/education-at-a-glance-19991487.htm).

Please refer to the Reader's Guide for information concerning symbols for missing data and abbreviations.

StatLink त्ञाज http://dx.doi.org/10.1787/888933285585 
Table B5.5. [1/2] Repayment and remission of public loans to students in bachelor's, master's, doctoral or equivalent programmes (academic year 2013-14) National students, in USD converted using PPPs

\begin{tabular}{|c|c|c|c|c|c|}
\hline & \multicolumn{5}{|c|}{ Repayment } \\
\hline & Repayment system & $\begin{array}{l}\text { Annual minimum } \\
\text { income threshold } \\
\text { (in USD) }\end{array}$ & $\begin{array}{l}\text { Duration of typical } \\
\text { amortisation period } \\
\text { (in years) }\end{array}$ & $\begin{array}{l}\text { Estimated annual } \\
\text { income of recent } \\
\text { graduates (in USD) }\end{array}$ & $\begin{array}{l}\text { Average annual amount } \\
\text { of repayment (in USD) }\end{array}$ \\
\hline & (1) & (2) & (3) & $(4)$ & $(5)$ \\
\hline Australia & Income contingent & 33709 & 8,5 & 34492 & 2424 \\
\hline Belgium (Fl.) & a & a & a & $\mathrm{m}$ & a \\
\hline Belgium (Fr.) & $\mathrm{m}$ & $\mathrm{m}$ & $\mathrm{m}$ & $\mathrm{m}$ & $\mathrm{m}$ \\
\hline Canada $^{1,2,3}$ & $\mathrm{~m}$ & $\mathrm{~m}$ & 9.5 & $\mathrm{~m}$ & $\mathrm{~m}$ \\
\hline Denmark $^{4}$ & Mortgage style & a & 7 to 15 & $\mathrm{~m}$ & $\mathrm{~m}$ \\
\hline Estonia & Mortgage style & a & 8 to 10 & $\begin{array}{c}21556 \\
\text { (gross salary in 2012) }\end{array}$ & $\mathrm{m}$ \\
\hline Finland & Mortgage style & a & 5 to 15 & 37574 & 1530 \\
\hline France & $\mathrm{m}$ & $\mathrm{m}$ & $\mathrm{m}$ & $\mathrm{m}$ & $\mathrm{m}$ \\
\hline Hungary 5 & $\begin{array}{l}\text { Income } \\
\text { contingent }\end{array}$ & None & 10 to 15 & $\mathrm{~m}$ & $\begin{array}{l}1259 \text { (Diákhitel1); } \\
664 \text { ((Diákhitel2) }\end{array}$ \\
\hline Italy & $\mathrm{m}$ & $\mathrm{m}$ & $\mathrm{m}$ & $\mathrm{m}$ & $\mathrm{m}$ \\
\hline Japan $^{4}$ & Mortgage style & a & 15 & $\mathrm{~m}$ & 2178 (from 1064 to 10024 ) \\
\hline Korea $^{6}$ & $\begin{array}{l}\text { Income contingent } \\
\text { and mortgage style }\end{array}$ & $\begin{array}{l}\text { About } 21755 \text { (income } \\
\quad \text { contingent loan); } \\
\text { a (mortgage style loan) }\end{array}$ & $\begin{array}{c}\text { m (income contingent loan); } \\
\text { up to } 10 \text { years (mortgage style loan) }\end{array}$ & $\mathrm{m}$ & $\mathrm{m}$ \\
\hline Netherlands & $\begin{array}{l}\text { Income } \\
\text { contingent }\end{array}$ & 19516 & 15 & $\mathrm{~m}$ & 1086 \\
\hline New Zealand & $\begin{array}{l}\text { Income } \\
\text { contingent }\end{array}$ & 12996 & 7 & $\mathrm{~m}$ & $\begin{array}{l}1907 \text { (12\% of income amount } \\
\text { above income threshold, } \\
\text { plus any voluntary repayments) }\end{array}$ \\
\hline Norway & Mortgage style & a & 20 & $\mathrm{~m}$ & 1609 \\
\hline Slovak Republic ${ }^{7}$ & Mortgage style & a & 7.1 (from 5 to 10 ) & $\mathrm{m}$ & 780 (from 86 to 2300 ) \\
\hline Slovenia & a & a & a & a & a \\
\hline Sweden ${ }^{4}$ & Mortgage style & a & 25 & $\mathrm{~m}$ & Typically 756 \\
\hline United Kingdom ${ }^{4}$ & $\begin{array}{l}\text { Income } \\
\text { contingent }\end{array}$ & 30062 & $\mathrm{~m}$ & 30778 & $\begin{array}{l}616 \text { (1st year of repayment } \\
\text { for } 2012 \text { cohort) to } 1560 \text { ( } 8 \text { th year } \\
\text { of repayment for } 2005 \text { cohort) }\end{array}$ \\
\hline United States & $\begin{array}{c}\text { Mortgage style } \\
\text { and income contingent }\end{array}$ & $\mathrm{m}$ & $\mid \begin{array}{c}10 \text { (mortgage style repayment); } \\
20 \text { to } 25 \text { (income based repayment; } \\
\text { predicted period) }\end{array}$ & 24448 & $\mathrm{~m}$ \\
\hline Brazil & $\mathrm{m}$ & $\mathrm{m}$ & $\mathrm{m}$ & $\mathrm{m}$ & $\mathrm{m}$ \\
\hline Colombia & Mortgage style & a & $\begin{array}{l}\text { From same to twice the time of the } \\
\text { study period }\end{array}$ & 18982 & $\mathrm{~m}$ \\
\hline
\end{tabular}

1. Private loan guaranteed by the state rather than public loan (in Italy, for the majority of student loans).

2. Reference year 2012-13.

3. Only includes information on the federal portion of student financial assistance, that is to say $60 \%$ of student loans provided in the provinces participating in the

Canada Student Loans Program (CSLP). Excludes the province of Quebec (about 25\% of the Canadian population) that does not participate in the CSLP.

4. Reference year 2014-15.

5. In the academic year 2012-13, a new student loan form (namely Diákhitel2) was launched, besides Diákhitel1. Diákhitel2 can be used only for the cost of studies ("cost-refunding" or "tuition fee"), while Diákhitel1 can be used for any purpose (e.g. student living expenses).

6. Eligibility rule: Income Contingent Student Loans, if 35 years old or younger, 7 th income decile or below, took 12 credits or more and gained 70 points or higher (maximum 100 points). General Installment Student Loans, if 55 years old or younger, 8th income decile or above, undergraduate and graduate students, took 12 credits or more, and gained 70 points or higher (maximum 100 points).

7. Includes short-cycle tertiary programmes.

Source: OECD. See Annex 3 for notes (www.oecd.org/education/education-at-a-glance-19991487.htm).

Please refer to the Reader's Guide for information concerning symbols for missing data and abbreviations.

StatLink 젬 http://dx.doi.org/10.1787/888933285591 
Table B5.5. [2/2] Repayment and remission of public loans to students in bachelor's, master's, doctoral or equivalent programmes (academic year 2013-14) National students, in USD converted using PPPs

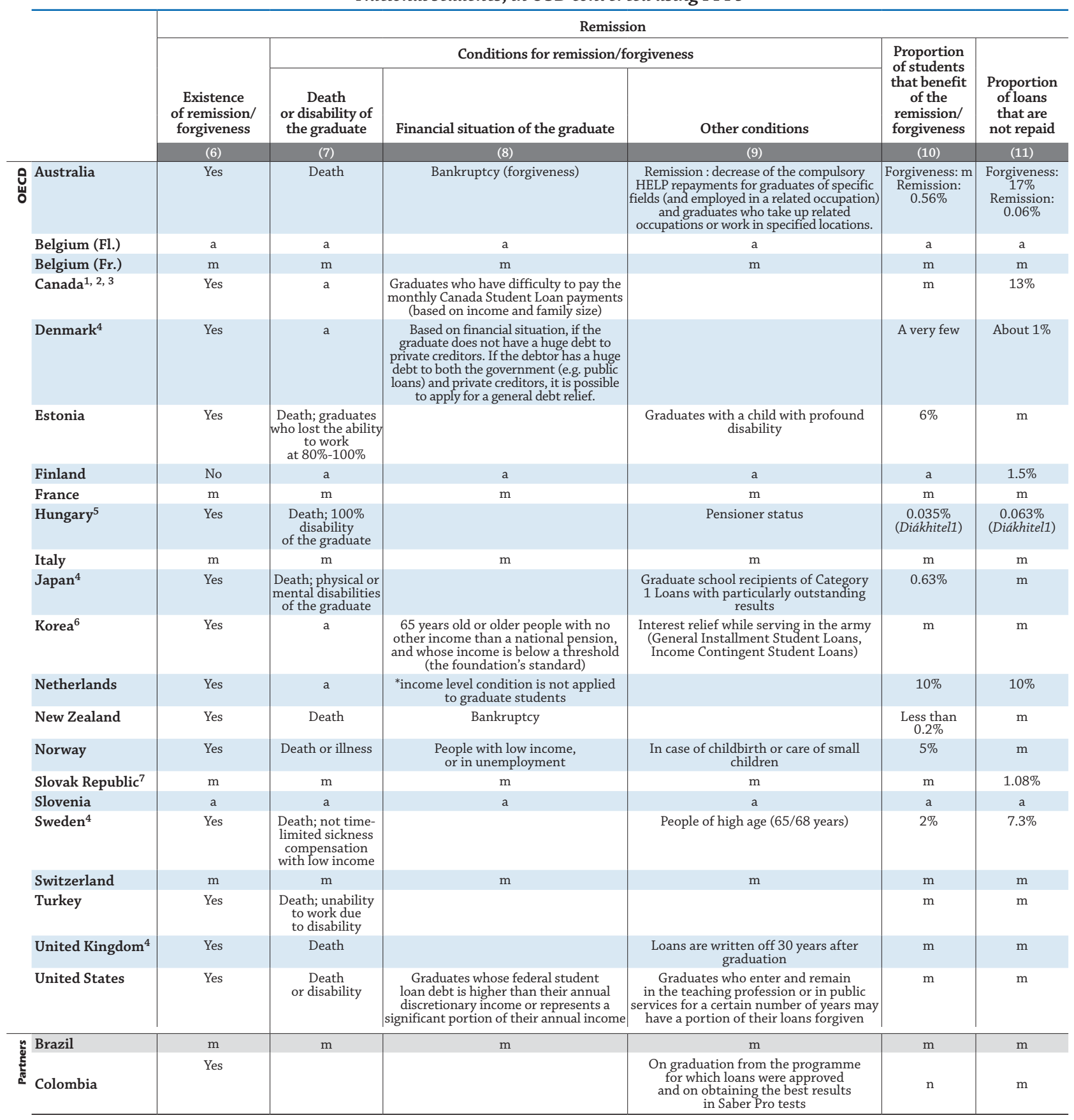

1. Private loan guaranteed by the state rather than public loan (in Italy, for the majority of student loans).

2. Reference year 2012-13.

3. Only includes information on the federal portion of student financial assistance, that is to say $60 \%$ of student loans provided in the provinces participating in the

Canada Student Loans Program (CSLP). Excludes the province of Quebec (about 25\% of the Canadian population) that does not participate in the CSLP.

4. Reference year 2014-15.

5. In the academic year 2012/2013, a new student loan form (namely Diákhitel2) was launched, besides Diákhitel1. Diákhitel2 can be used only for the cost of studies ("cost-refunding" or "tuition fee"), while Diákhitel1 can be used for any purpose (e.g. student living expenses).

6. Eligibility rule: Income Contingent Student Loans, if 35 years old or younger, 7th income decile or below, took 12 credits or more and gained 70 points or higher (maximum 100 points). General Installment Student Loans, if 55 years old or younger, 8th income decile or above, undergraduate and graduate students, took 12 credits or more, and gained 70 points or higher (maximum 100 points).

7. Includes short-cycle tertiary programmes.

8. Reference years 2011-12 for the proportion of students with student loans; 2012-13 for debt at graduation, 2014-15 otherwise.

Source: OECD. See Annex 3 for notes (www.oecd.org/education/education-at-a-glance-19991487.htm).

Please refer to the Reader's Guide for information concerning symbols for missing data and abbreviations.

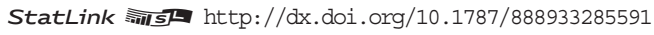




\section{ON WHAT RESOURCES AND SERVICES IS EDUCATION FUNDING SPENT?}

- About $90 \%$ or more of total expenditure on education is devoted to current expenditure, on average across OECD countries, and in most OECD countries, at both primary, secondary, post-secondary non-tertiary levels combined and at tertiary level.

- In two-thirds (24 of 33) of OECD and partner countries with available data, the share of total expenditure devoted to capital expenditure is larger at the tertiary level than at primary, secondary and post-secondary non-tertiary levels combined. This may be because new buildings have had to be constructed in recent years to accommodate the expansion of tertiary education.

- In OECD and partner countries with available data, most current expenditure goes to compensating education staff (teachers and others).

\section{Chart B6.1. Distribution of current expenditure on educational institutions for primary, secondary and post-secondary non-tertiary education (2012)}

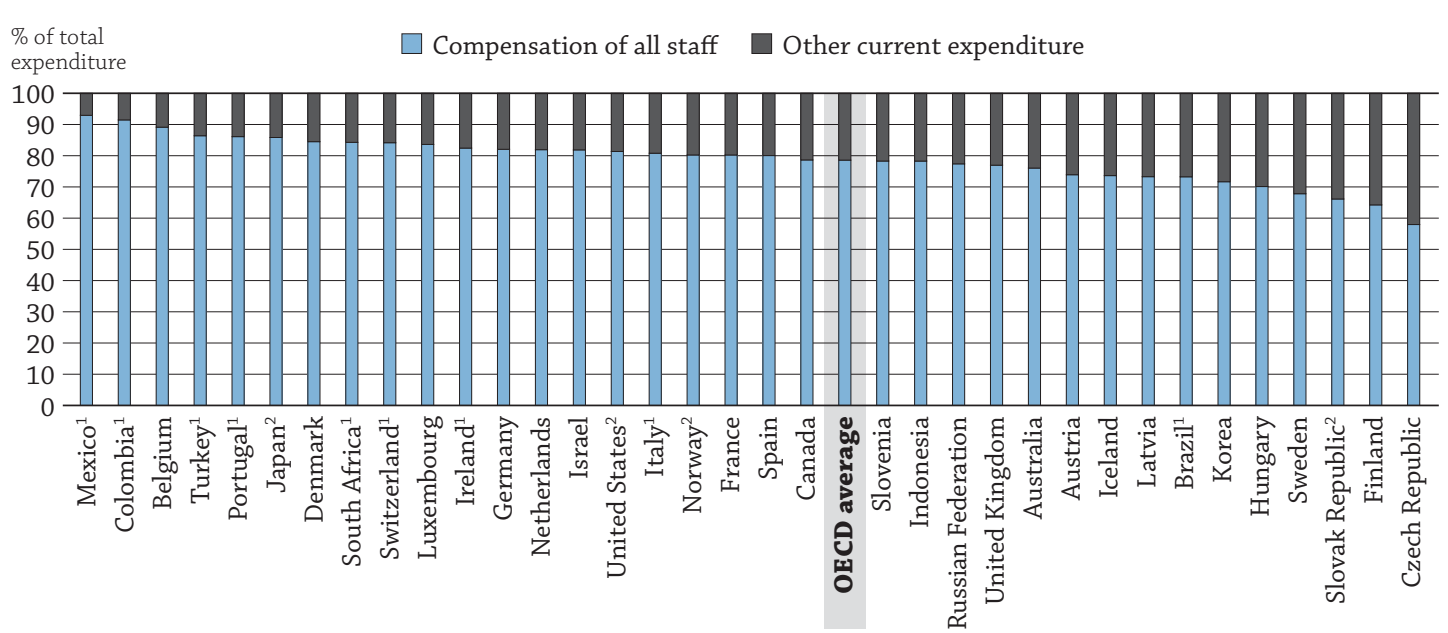

1. Public institutions only.

2. Some levels of education are included with others. Refer to "x" code in Table B1.1a for details.

Countries are ranked in descending order of the share of compensation of all staff in primary, secondary and post-secondary non-tertiary education.

Source: OECD. Table B6.2. See Annex 3 for notes (www.oecd.org/education/education-at-a-glance-19991487.htm).

StatLink 제내 http://dx.doi.org/10.1787/888933284092

\section{Context}

Decisions about how resources are allocated affect the material conditions under which instruction takes place and can also influence the nature of instruction.

While savings can be made by cutting capital expenditure (such as not building new schools) and some current expenditure (not purchasing certain teaching materials), when pressures on education budgets increase, changes in spending on staff have the greatest impact on overall spending. Still, saving money by reducing salaries and benefits or cutting the number of teachers and other staff is unpopular politically and possibly counterproductive, in that it discourages good teachers from wanting to enter or remain in the profession. In fact, in addition to managing material resources more efficiently, human resources must also be well-managed in order to improve the quality of education systems. Deferring expenditures, such as hiring new teachers or salary increases, are temporary measures in response to pressures on public budgets.

This indicator describes the resources and services on which money for education is spent. It shows the difference between current and capital expenditure. Capital expenditure can be affected by expanding enrolments, which often require the construction of new buildings. This indicator also presents details on where current expenditure is spent, either on compensation of education staff or elsewhere. Current expenditure is mainly affected by teachers' salaries (see Indicator D3), but also by 
pension systems, the age distribution of teachers, and the size of the non-teaching staff employed in education. In addition, educational institutions offer not only instruction but other services, such as meals, transport, housing services and/or research activities. All these expenditures are addressed in this indicator.

\section{INDICATOR B6}

\section{Other findings}

- Current expenditure devoted to purposes other than compensating staff is largest at the tertiary level in almost all countries except Brazil and Iceland; and it reaches 33\% of all current expenditure, on average across OECD countries. In five OECD and partner countries, this proportion is $40 \%$ or larger. These large proportions could be explained by the higher costs of facilities and equipment in tertiary education compared to other levels of education.

- At the primary, secondary and post-secondary non-tertiary levels of education, OECD countries spend an average of $21 \%$ of current expenditure for purposes other than compensating education personnel. In most countries, there is little difference between primary and secondary education in the proportion of current expenditure used for purposes other than compensation. However, the difference exceeds 5 percentage points in the Czech Republic, Denmark, South Africa and Turkey, and 10 percentage points in Indonesia, Ireland and Luxembourg.

- Most current expenditure is related to compensation of staff in nearly all countries except, at the tertiary level, the Czech Republic and Indonesia. Only Brazil and Iceland devote $80 \%$ or more of current expenditure to staff compensation at the tertiary level; 19 countries devote similar shares of current expenditure to staff compensation at the primary, secondary and post-secondary nontertiary levels of education combined. 


\section{Analysis}

\section{Current and capital expenditure by educational institutions}

Education expenditure includes both current and capital expenditure. Current expenditure by educational institutions takes account of the spending on school resources used each year to operate schools. It includes, for instance, the compensation of teachers and other staff, maintenance of school buildings, students' meals or the rental of school buildings and other facilities. Capital expenditure by educational institutions refers to spending on assets that last longer than one year. It includes, for instance, spending on the construction, renovation and major repair of school buildings.

The largest share of expenditure is current expenditure, given the labour-intensive nature of instruction. In 2012, about $90 \%$ or more of total expenditure was devoted to current expenditure at the primary, secondary and postsecondary non-tertiary levels of education combined and at the tertiary level, on average among OECD countries. Current expenditure amounts to more than $78 \%$ of total expenditure at each level of education in every OECD and partner country. The share varies from $86 \%$ (Indonesia) to nearly $98 \%$ (Austria) in primary education; from nearly $86 \%$ (Portugal) to more than $98 \%$ (Austria and South Africa) in secondary education; and from $78 \%$ (Turkey) to $97 \%$ (Belgium) in tertiary education (Tables B6.1 and B6.2 and Chart B6.2).

The OECD average share of current expenditure differs by three percentage points between primary, secondary and post-secondary non-tertiary education combined (93.0\%) and tertiary education (90.0\%). Nevertheless, differences among countries between the share of current expenditure on primary, secondary and post-secondary non-tertiary education combined and on tertiary education can be relatively large. In most countries, the share of current expenditure on the former levels of education is larger than on the latter level. The main exceptions are Finland, Israel, the Netherlands, Norway, Portugal and Sweden where the share of current expenditure on tertiary education exceeds the share in primary, secondary and post-secondary non-tertiary education combined by more than one to four percentage points. In contrast, the share of current expenditure at primary, secondary and postsecondary non-tertiary education combined exceeds the share in tertiary education by five percentage points or more in Australia, Hungary, Indonesia, Italy, Latvia, Poland, the Slovak Republic, Spain and Turkey.

The differences among countries are likely to reflect how the different levels of education are organised in each country, as well as the degree to which the expansion in enrolments requires the construction of new buildings, especially at the tertiary level. Capital expenditure on tertiary education exceeds $15 \%$ in Indonesia (20.2\%), Latvia (18.4\%), Poland (18.4\%), the Slovak Republic (18.8\%) and Turkey (22.0\%). The ways countries report expenditure related to university buildings may also explain differences in the share of current and capital expenditure at the tertiary level. For example, the buildings and lands used for education can be owned, used free of charge, or rented by the institutions; and the amount of current and capital expenditure partly depends on the type of real estate management used in the country (see Box B6.1 in Education at a Glance 2012 [OECD, 2012]).

\section{Distribution of current expenditure}

Current expenditure by educational institutions can be subdivided further into three broad functional categories: compensation of teachers, compensation of other staff, and other current expenditures. Other current expenditures include, for instance, teaching materials and supplies, maintenance of school buildings, students' meals and rental of school facilities. The amount allocated to each of these categories depends partly on current and projected changes in enrolments, on the salaries of education personnel, and on the costs of maintenance and construction of education facilities. Despite the fact that the shares of these categories do not change much from year to year, countries' decisions might affect not only the amounts allocated, but also these shares.

At the primary, secondary and post-secondary non-tertiary levels, more than $62 \%$ of current expenditure is devoted to compensating teachers, more than $15 \%$ to compensating other staff, and more than $21 \%$ to expenditure other than compensation, on average across OECD countries. For tertiary education, nearly $40 \%$ of current expenditure is devoted to the compensation of teachers, on average across OECD countries, as larger shares are devoted to compensating other staff (more than 26\%) and other current expenditure (about 33\%).

There are relatively large differences in how current expenditure is allocated between the primary, secondary, and post-secondary non-tertiary levels combined and tertiary education. For instance, in all countries, the share devoted to compensation of teachers is larger at the combined primary, secondary and post-secondary non-tertiary levels of education than at the tertiary level. 


\section{Chart B6.2. Distribution of current and capital expenditure on educational institutions (2012)}

By resource category and level of education

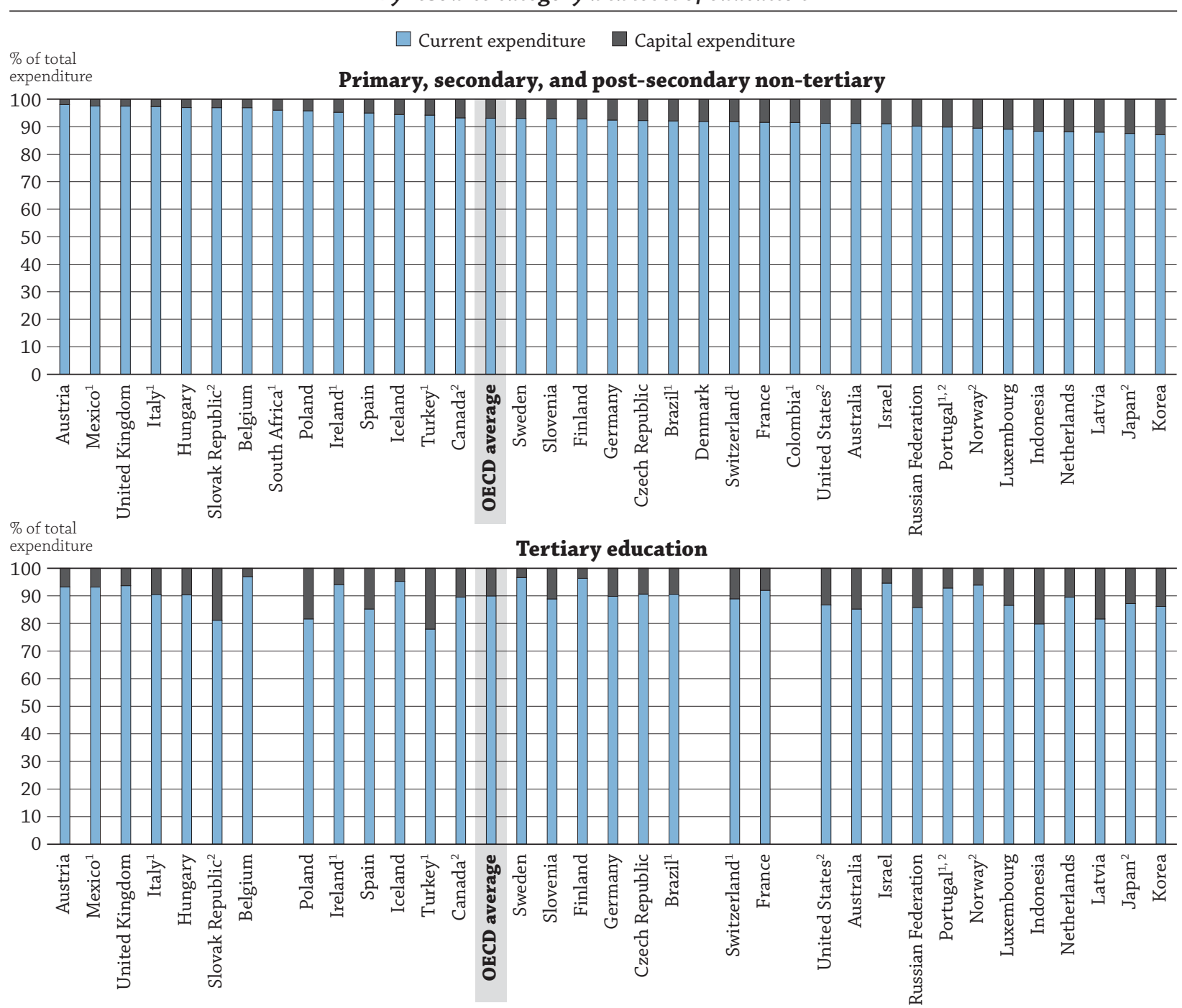

1. Public institutions only (for Italy, except in tertiary education).

2. Some levels of education are included with others. Refer to " $\mathrm{x}$ " code in Table B1.1a for details.

Countries are ranked in descending order of the share of current expenditure on primary, secondary and post-secondary non-tertiary education.

Source: OECD. Table B6.2. See Annex 3 for notes (www.oecd.org/education/education-at-a-glance-19991487.htm).

StatLink 제개 http://dx.doi.org/10.1787/888933284103

The share of other current expenditure is more than $30 \%$ in primary, secondary and post-secondary non-tertiary education combined in only four countries, namely the Czech Republic (42.0\%), Finland (35.7\%), the Slovak Republic (33.9\%) and Sweden (32.2\%). In contrast, at the tertiary level, this share is more than $30 \%$ in more than half of OECD countries with available data and less than $20 \%$ in only two countries: Brazil (18.9\%) and Iceland (17.4\%).

The variation in current expenditure not devoted to compensation of staff between levels of education reflects differences in the size of administrative systems (for instance, the number of employees or the equipment available to administrative staff) across these levels. The cost of facilities and equipment is expected to be higher in tertiary education than in other levels of education. Meanwhile, the differences among countries in compensation of other staff likely reflect the degree to which education personnel, such as principals, guidance counsellors, bus drivers, school nurses, janitors and maintenance workers, are included in the category "non-teaching staff" (see Indicator D2). Compensation of staff involved in research and development at the tertiary level may also explain part of the differences, between countries and between levels of education, in the share of current expenditure devoted to compensation of other staff. 


\section{Definitions}

Capital expenditure refers to spending on assets that last longer than one year, including construction, renovation or major repair of buildings and new or replacement equipment. The capital expenditure reported here represents the value of educational capital acquired or created during the year in question - that is, the amount of capital formation - regardless of whether the capital expenditure was financed from current revenue or through borrowing. Neither current nor capital expenditure includes debt servicing.

Current expenditure refers to spending on goods and services consumed within the current year and requiring recurrent production in order to sustain educational services. Current expenditure by educational institutions other than on compensation of personnel includes expenditure on sub-contracted services such as support services (e.g. maintenance of school buildings), ancillary services (e.g. preparation of meals for students) and rental of school buildings and other facilities. These services are obtained from outside providers, unlike the services provided by the education authorities or by the educational institutions using their own personnel.

\section{Methodology}

Data refer to the financial year 2012 and are based on the UOE data collection on education statistics administered by the OECD in 2014 (for details see Annex 3 at www.oecd.org/education/education-at-a-glance-19991487.htm).

Calculations cover expenditure by public institutions or, where available, by both public and private institutions.

\section{Note regarding data from Israel}

The statistical data for Israel are supplied by and are under the responsibility of the relevant Israeli authorities. The use of such data by the OECD is without prejudice to the status of the Golan Heights, East Jerusalem and Israeli settlements in the West Bank under the terms of international law.

\section{References}

OECD (2012), Education at a Glance 2012: OECD Indicators, OECD Publishing, Paris, http://dx.doi.org/10.1787/eag-2012-en.

\section{Indicator B6 Tables}

StatLink 젶ㄴ http://dx.doi.org/10.1787/888933285627

Table B6.1 Expenditure by primary and secondary educational institutions, by resource category (2012)

Table B6.2 Expenditure by educational institutions, by resource category and level of education (2012) 
Table B6.1. Expenditure by primary and secondary educational institutions, by resource category (2012)

Distribution of total and current expenditure by educational institutions from public and private sources

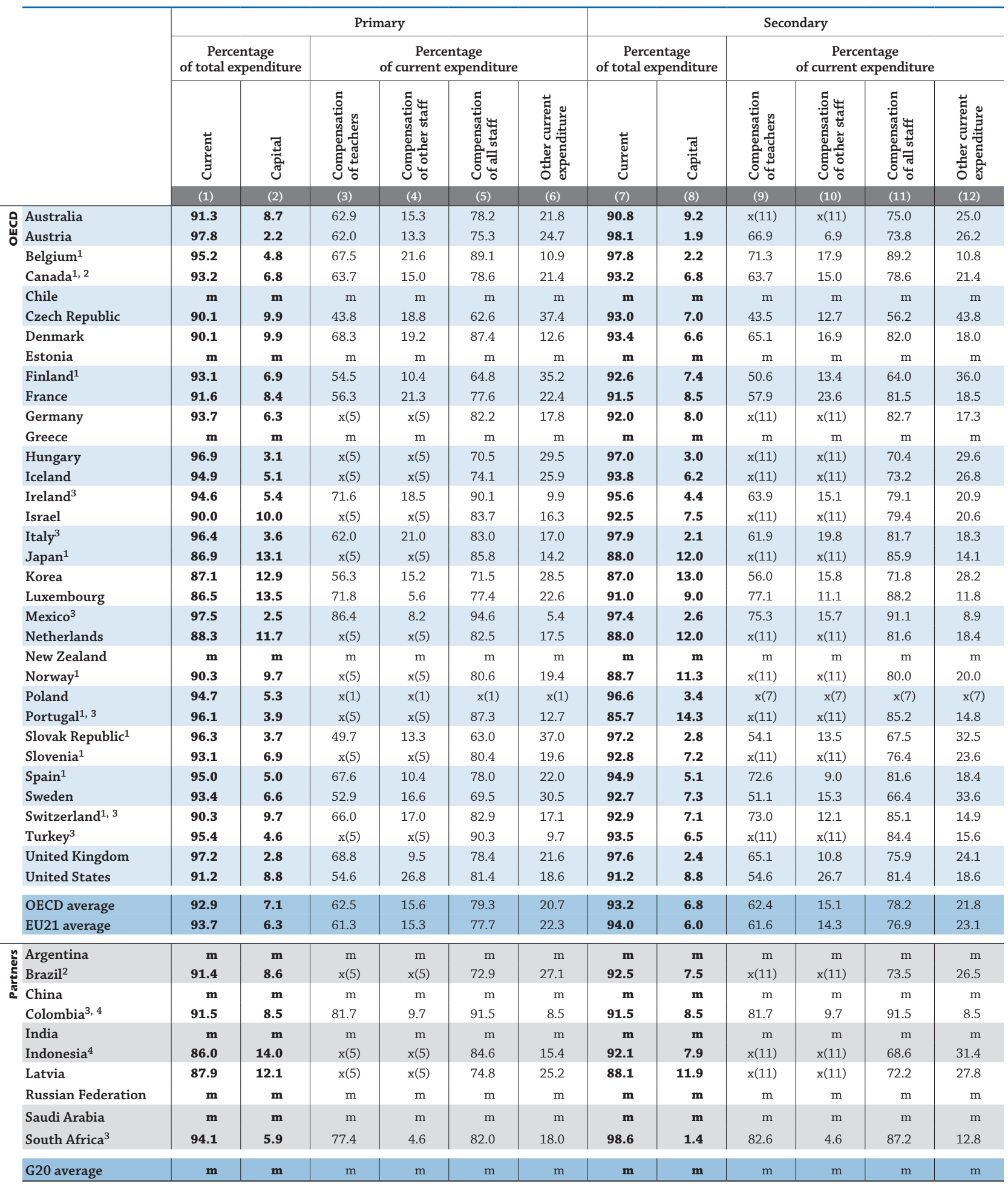

1. Some levels of education are included with others. Refer to " $\mathrm{x}$ " code in Table B1.1a for details.

2. Year of reference 2011.

3. Public institutions only.

4. Year of reference 2013.

Source: OECD. Argentina, China, Colombia, India, Indonesia, Saudi Arabia, South Africa: UNESCO Institute for Statistics. Latvia: Eurostat. See Annex 3 for notes (www.oecd.org/education/education-at-a-glance-19991487.htm).

Please refer to the Reader's Guide for information concerning symbols for missing data and abbreviations.

StatLink त्ञाज http://dx.doi.org/10.1787/888933285635 
Table B6.2. Expenditure by educational institutions, by resource category and level of education (2012) Distribution of total and current expenditure by educational institutions from public and private sources

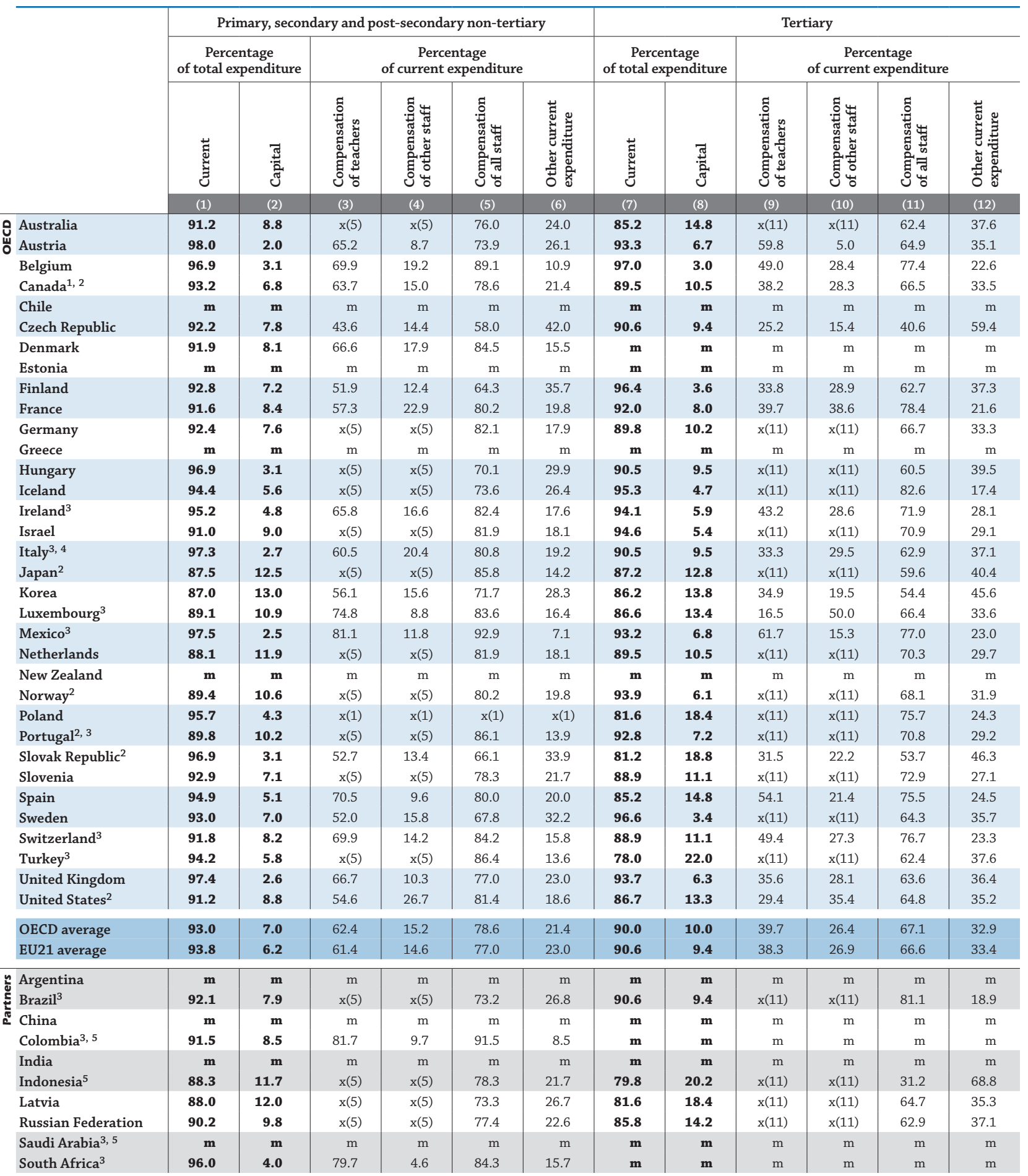

\begin{tabular}{l|l|l|l|l|l|l|l|l|l|l|l|l|l|l} 
G20 average & $\mathbf{m}$ & $\mathbf{m}$ & $\mathrm{m}$ & $\mathrm{m}$ & $\mathrm{m}$ & $\mathrm{m}$ & $\mathbf{m}$ & $\mathbf{m}$ & $\mathrm{m}$ & $\mathrm{m}$ & $\mathrm{m}$ & $\mathrm{m}$
\end{tabular}

1. Year of reference 2011

2. Some levels of education are included with others. Refer to "X" code in Table B1.1a for details.

3. Public institutions only (For Italy and the United Kingdom, except in tertiary education. For Luxembourg, in tertiary education only).

4. Excludes short-cycle tertiary programmes.

5. Year of reference 2013

Source: OECD. Argentina, China, Colombia, India, Indonesia, Saudi Arabia, South Africa: UNESCO Institute for Statistics. Latvia: Eurostat. See Annex 3 for notes (www.oecd.org/education/education-at-a-glance-19991487.htm).

Please refer to the Reader's Guide for information concerning symbols for missing data and abbreviations.

StatLink sils http://dx.doi.org/10.1787/888933285646 



\section{WHICH FACTORS INFLUENCE THE LEVEL OF EXPENDITURE ON EDUCATION?}

- Four factors influence expenditure on education related to the salary cost of teachers per student: instruction time of students, teaching time of teachers, teachers' salaries and estimated class size. Consequently, a given level of the salary cost of teachers per student may result from different combinations of these four factors.

- In most countries, the salary cost of teachers per student increases with the level of education.

- Between 2010 and 2013, the salary cost of teachers per student increased in a majority of countries at the primary and lower secondary levels of education. On average, it increased by $2.6 \%$ (from USD 2550 to USD 2 616) at the primary level and by 1.0\% (from USD 3185 to USD 3 215) at the lower secondary level.

\section{Chart B7.1. Salary cost of teachers per student, by level of education (2013)} In USD

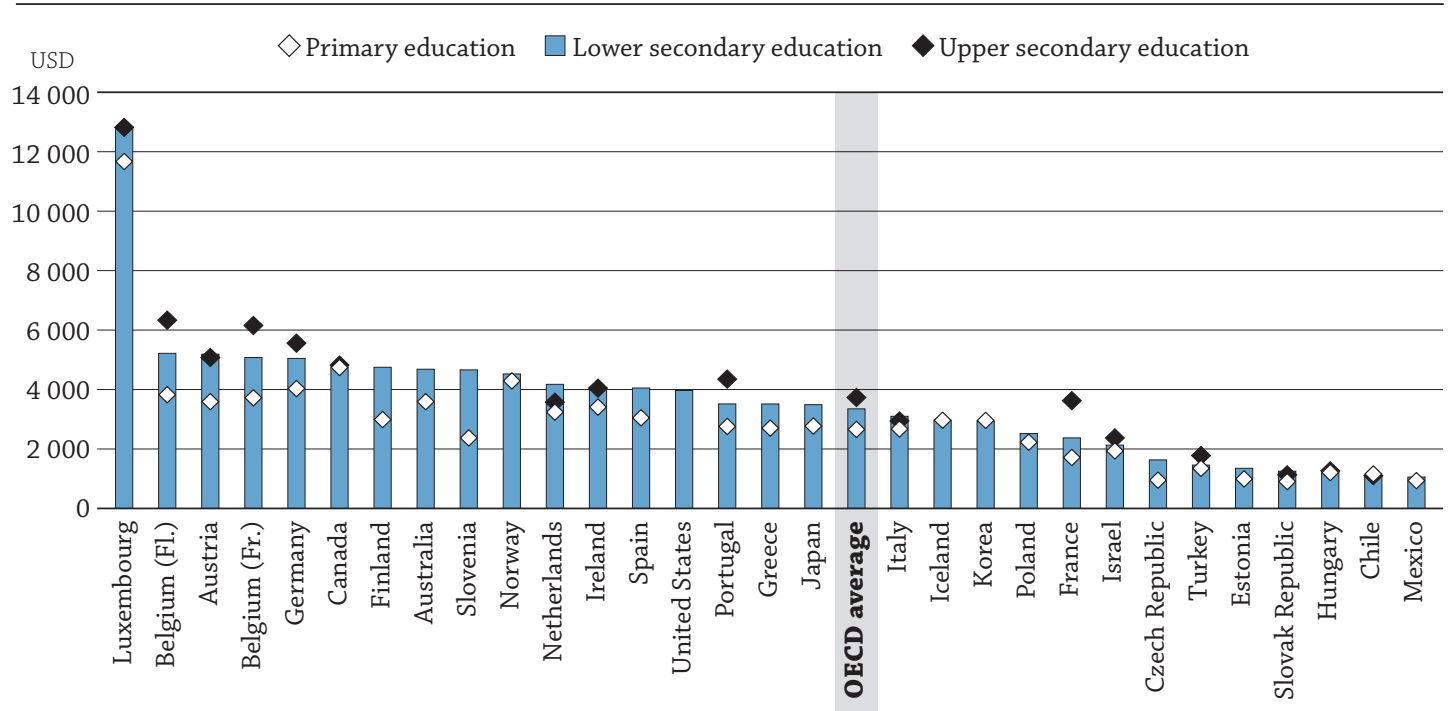

Countries are ranked in descending order of the salary cost of teachers per student in lower secondary education.

Source: OECD. Table B7.1. See Annex 3 for notes (www.oecd.org/education/education-at-a-glance-19991487.htm).

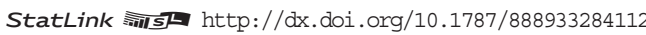

\section{Context}

Governments have become increasingly interested in the relationship between the amount of resources devoted to education and student learning outcomes. Governments seek to provide more and better education for their populations while, at the same time, ensuring that public funding is used efficiently, particularly when public budgets are being tightened. Teachers' compensation is usually the largest part of expenditure on education and thus of expenditure per student (see Indicator B6). The salary cost of teachers is a function of the instruction time of students, the teaching time of teachers, teachers' salaries and the number of teachers needed to teach students, which depends on estimated class size (Box B7.1).

Differences among countries in these four factors may explain differences in the level of expenditure per student. Similarly, a given level of expenditure may be associated with different combinations of these factors. This indicator examines the choices countries make when investing their resources in primary and secondary education, and explores how changing policy choices between 2005, 2010 and 2013 relating to these four factors affected the salary cost of teachers. However, some of these choices do not necessary reflect policy decisions but, rather, demographic changes, such as shrinking numbers of students. Thus, for example, in countries where enrolments have been declining in recent years, class size would also shrink (assuming all other factors remain constant), unless there was a simultaneous drop in the number of teachers as well. 


\section{Other findings}

- Similar levels of expenditure among countries can mask a variety of contrasting policy choices. This helps to explain why there is no simple relationship between overall spending on education and the level of student performance. For example, at the upper secondary level of education, Austria and Canada had similar levels of salary costs of teachers per student in 2013, above the average. In Austria, this was the result of the combination of above-average teachers' salaries and instruction time, and below-average teaching time and estimated class size. In Canada, it was mostly the result of above-average teachers' salaries and, to a lesser extent, of below-average estimated class size, whose effects were slightly counterbalanced by above-average teaching time and below-average instruction time.

- Teachers' salaries are most often the primary factor influencing the difference from the average salary cost of teachers per student at each level of education; estimated class size is the second factor.

- When differences in countries' wealth are taken into account, teachers' salaries are less often the primary factor influencing the difference from the average salary cost of teachers per student.

\section{Trends}

The increase in the salary cost of teachers per student between 2010 and 2013 was mostly influenced by changes in two factors: teachers' salaries and estimated class size. During this period, among countries with available data for both years, teachers' salaries decreased by an average of less than $1 \%$ at the primary and lower secondary levels, while estimated class size decreased by $1 \%$ at the primary level and increased by more than $10 \%$ at the lower secondary level. Variations in the other two factors, instruction time and teaching time, are usually smaller in most countries, but the average is influenced by large variations in some countries. Teaching time at the lower secondary level varied the most during the period, and increased, on average, by $2.9 \%$ among countries with available data for both years.

At the primary and lower secondary levels of education, a similar number of countries increased and decreased teachers' salaries and/or estimated class size between 2010 and 2013. These changes resulted in an increase in the salary cost per student in the majority of countries and on average across countries with available data for both years. However, the salary cost per student decreased in some countries during this period, most significantly (by around $15 \%$ or more) in Portugal and Spain at both primary and lower secondary levels. In both of these countries, decreases in teachers' salaries combined with increases in estimated class size are the main reasons for decreases in the salary cost of teachers per student. Some other countries also introduced reforms since 2005 that affected the salary cost of teachers per student. For instance, in Hungary, teaching time was increased at the secondary level in 2006, decreasing the number of teachers required at this level. That, in turn, reduced expenditure on teachers' salaries. Italy implemented reforms on class size to increase slightly the number of students per class. This resulted in a decrease in the salary cost of teachers per student (see Table B7.5 in Education at a Glance 2012 [OECD, 2012]). 


\section{Analysis}

\section{Variation in the salary cost of teachers per student, by level of education}

Per-student expenditure reflects the structural and institutional factors that relate to the organisation of schools and curricula. Expenditure can be broken down into the compensation of teachers and other expenditure (defined as expenditure for all purposes other than teacher compensation). Teacher compensation usually constitutes the largest part of expenditure on education. As a result, the level of teacher compensation divided by the number of students (referred to here as "salary cost of teachers per student") is the main proportion of expenditure per student.

\section{Box B7.1. Relationship between salary cost of teachers per student and instruction time of students, teaching time of teachers, teachers' salaries and class size}

One way to analyse the factors that have an impact on expenditure per student and to measure the extent of their effects is to compare the differences between national figures and the OECD average. This analysis computes the differences in expenditure per student among countries and the OECD average, and then calculates the contribution of these different factors to the variation from the OECD average.

This exercise is based on a mathematical relationship between the different factors and follows the method presented in the Canadian publication Education Statistics Bulletin (2005) (see explanations in Annex 3). Educational expenditure is mathematically linked to factors related to a country's school context (number of hours of instruction time for students, number of teaching hours for teachers, estimated class size) and one factor relating to teachers (statutory salary).

Expenditure is broken down into compensation of teachers and other expenditure (defined as all expenditure other than compensation of teachers). Compensation of teachers divided by the number of students, or "the salary cost of teachers per student" (CCS), is estimated through the following calculation:

$$
C C S=S A L \times \text { inst } T \times \frac{1}{\text { teach } T} \times \frac{1}{\text { ClassSize }}=\frac{S A L}{\text { Ratiostud/teacher }}
$$

SAL: teachers' salaries (estimated by statutory salary after 15 years of experience)

inst T: instruction time of students (estimated as the annual intended instruction time, in hours, for students)

teachT: teaching time of teachers (estimated as the annual number of teaching hours for teachers)

ClassSize: a proxy for class size

Ratiostud/teacher: the ratio of students to teaching staff

With the exception of class size (which is not computed at the upper secondary level, as class size is difficult to define and compare because students at this level may attend several classes depending on the subject area), values for the different variables can be obtained from the indicators published in Education at a Glance (Chapter D). However, for the purpose of the analysis, an "estimated" class size or proxy class size is computed based on the ratio of students to teaching staff and the number of teaching hours and instruction hours (see Box D2.2). As a proxy, this estimated class size should be interpreted with caution.

Using this mathematical relationship and comparing a country's values for the four factors to the OECD averages makes it possible to measure both the direct and indirect contribution of each of these four factors to the variation in salary cost per student between that country and the OECD average (for more details, see Annex 3). For example, in the case where only two factors interact, if a worker receives a $10 \%$ increase in the hourly wage and increases the number of hours of work by $20 \%$, his/her earnings will increase by $32 \%$ as a result of the direct contribution of each of these variations $(0.1+0.2)$ and the indirect contribution of these variations due to the combination of the two factors $\left(0.1^{*} 0.2\right)$. To account for differences in countries' level of wealth when comparing salary costs per student, salary cost per student, as well as teachers' salaries, can be divided by GDP per capita (on the assumption that GDP per capita is an estimate of countries' level of wealth). This makes it possible to compare countries' "relative" salary cost per student (see Education at a Glance 2015 tables, available on line).

The salary cost of teachers per student is estimated based on theoretical values: statutory salaries at a specific point in the salary scale, theoretical instruction time of student and statutory teaching time of teachers; and estimated class size. As a consequence, this measure may differ from the actual salary cost of teachers resulting from the combination of actual average values for these four factors. This also explains part of the differences between this indicator and Indicators B1, B2, B3 and B6 that are based on actual expenditure and student population at each level of education. 
The salary cost of teachers per student is based on the instruction time of students, the teaching time of teachers, teachers' salaries and the number of teachers needed to teach students, which depends on estimated class size (Box B7.1). As a consequence, differences among countries in these four factors may explain differences in the level of expenditure. In the same way, a given level of expenditure may result from a different combination of these factors.

Salary costs of teachers per student show a common pattern across OECD countries: they usually rise with the level of education taught. However, in some countries they are lower at the upper secondary level than at the lower secondary level. Overall, among OECD countries with available data for each of these different levels in 2013, the average salary cost of teachers per student is USD 2677 per primary student, USD 3350 per lower secondary student and USD 3749 per upper secondary student (Chart B7.1).

\section{Disparities in salary cost of teachers among OECD countries}

The variation in salary cost of teachers per student between levels of education is significant among countries. In 2013, there was a difference of less than USD 100 in Canada, Chile, Hungary, Iceland and Mexico among these three levels of education, but the difference was over USD 1900 in France and exceeded USD 2000 in Belgium (Flemish and French Communities) and Slovenia (Table B7.1 and Chart B7.1).

This increase in the salary cost of teachers per student with the level of education taught is partly the result of increases in teachers' salaries and in the instruction time of students at higher education levels. In 2013, the OECD average salary varies from USD 41864 at the primary level to USD 43634 at the lower secondary level and USD 45701 at the upper secondary level. Meanwhile, the OECD average annual instruction time varies from 794 hours at the primary level, to 905 hours at the lower secondary level and 929 hours at the upper secondary level. The increase is also related to the fact that teaching time generally decreases as the level of education increases, implying that more teachers are necessary to teach a given number of students (the OECD average annual teaching time in 2013 decreases from 772 hours at the primary level, to 694 hours at the lower secondary level to 643 hours at the upper secondary level). However, larger classes at higher levels of education tend to reduce the salary cost per student (the OECD average estimated class size increases between primary, lower secondary and upper secondary levels from 15.2 students to 17.3 students to 17.6 students, respectively) (Tables B7.2a and b, and Table B7.2c, available on line).

\section{Variations in salary costs of teachers per student between 2010 and 2013}

The salary cost of teachers per student also varies over time in a given level of education. These changes are only analysed at the primary and lower secondary levels of education because trend data are not available at the upper secondary level. This analysis is also limited to countries with data for both 2010 and 2013 (24 and 23 countries, respectively, for the primary and lower secondary levels), as comparable data over a larger period (for 2005, 2010 and 2013) are available for fewer countries.

The salary cost of teachers per student increased slightly (by less than $3 \%$ ) at both the primary and lower secondary levels between 2010 and 2013 (from USD 2550 to USD 2616 at the primary level and from USD 3185 to USD 3215 at the lower secondary level), on average across the countries with available data for both years (Chart B7.2).

In most countries, the salary cost of teachers per student at both of these levels of education increased between 2010 and 2013. The increase reached 25\% or more in Korea at the primary level and exceeded 35\% in Israel at the primary level and in Poland at the lower secondary level (Chart B7.3).

However, the salary cost of teachers per student also fell between 2010 and 2013 in a significant number of countries, most notably in Portugal (by 29\% at the primary level and 34\% at the lower secondary level) and Spain (by around $15 \%$ at the primary level and $24 \%$ at the lower secondary level). Decreases of more than $10 \%$ and up to $15 \%$ in the salary cost of teachers per student were also observed at the primary level in Hungary and Italy, and at the lower secondary level in Belgium (French Community), Hungary and Slovenia (Chart B7.2).

\section{Impact of teachers' salaries and class size on salary cost of teachers per student}

Of the four factors that determine the level of the salary cost of teachers, two are largely responsible for the wide variations in this cost: teachers' salaries and class size. Between 2010 and 2013, among countries with available data for this period, average teachers' salaries (expressed in constant prices) decreased by less than $1 \%$ at the primary and lower secondary levels, while estimated class size decreased by $1 \%$ at the primary level and increased by more than $10 \%$ at the lower secondary level (Tables B7.2a and b). 


\section{Chart B7.2. Change in the salary cost of teachers per student, primary and lower secondary education (2005, 2010 and 2013)}

In USD

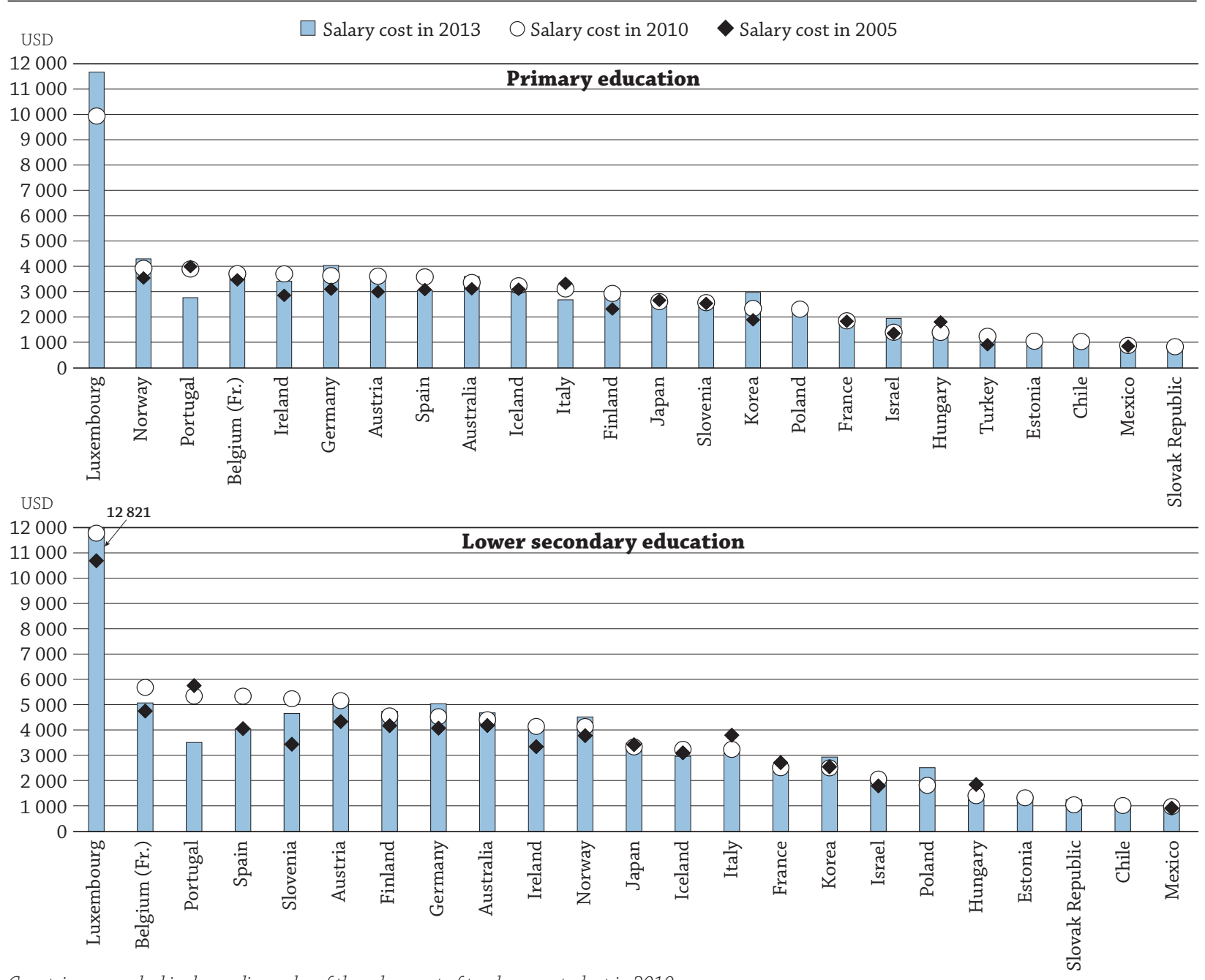

Countries are ranked in descending order of the salary cost of teacher per student in 2010.

Source: OECD. Tables B7.3 and B7.4. See Annex 3 for notes (www.oecd.org/education/education-at-a-glance-19991487.htm).

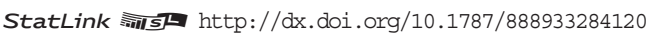

At both the primary and lower secondary levels, the small decrease in average teachers' salaries (in real terms) across OECD countries with comparable data for 2010 and 2013 resulted from decreases and increases in a similar number of countries. Teachers' salaries decreased most notably (by 10\% or more) in Hungary, Portugal and Spain at both these levels, and this contributed (with the increase in estimated class size) to the decrease in the salary cost of teachers per student in these countries (Chart B7.3).

Among countries with data for both 2010 and 2013, the small decrease in average estimated class size at the primary level and the large increase in average estimated class size at the lower secondary level also resulted from decreases and increases in a similar number of countries. At the primary and lower secondary levels, the largest reductions were observed in countries that had relatively large estimated classes in 2010 (Chile and Israel at the primary level, and Poland at the lower secondary level) and also in countries with below-average estimated class size in 2010 (Luxembourg at both primary and lower secondary levels). In all four of these countries, this resulted in an increase of the salary cost per student. Nevertheless, estimated class size also increased significantly in some countries, contributing to a decrease in salary cost per student. This was the case most notably in Portugal (from 10.6 to 14.3 at the primary level and from 9.5 to 15.3 at the lower secondary level), in Poland at the primary level (from 9.3 to 11.2 students), and in the French Community of Belgium (from 11.6 to 13.7 students) and Spain (from 14.9 to 17.2 students) at the lower secondary level. 


\section{Chart B7.3. Change in the salary cost of teachers per student, teachers' salaries and estimated class size, primary and lower secondary education (2010, 2013) Change in percentage, between 2010 and 2013}

Change in teachers' salary $\square$ Change in estimated class size

Change in salary cost of teachers per student

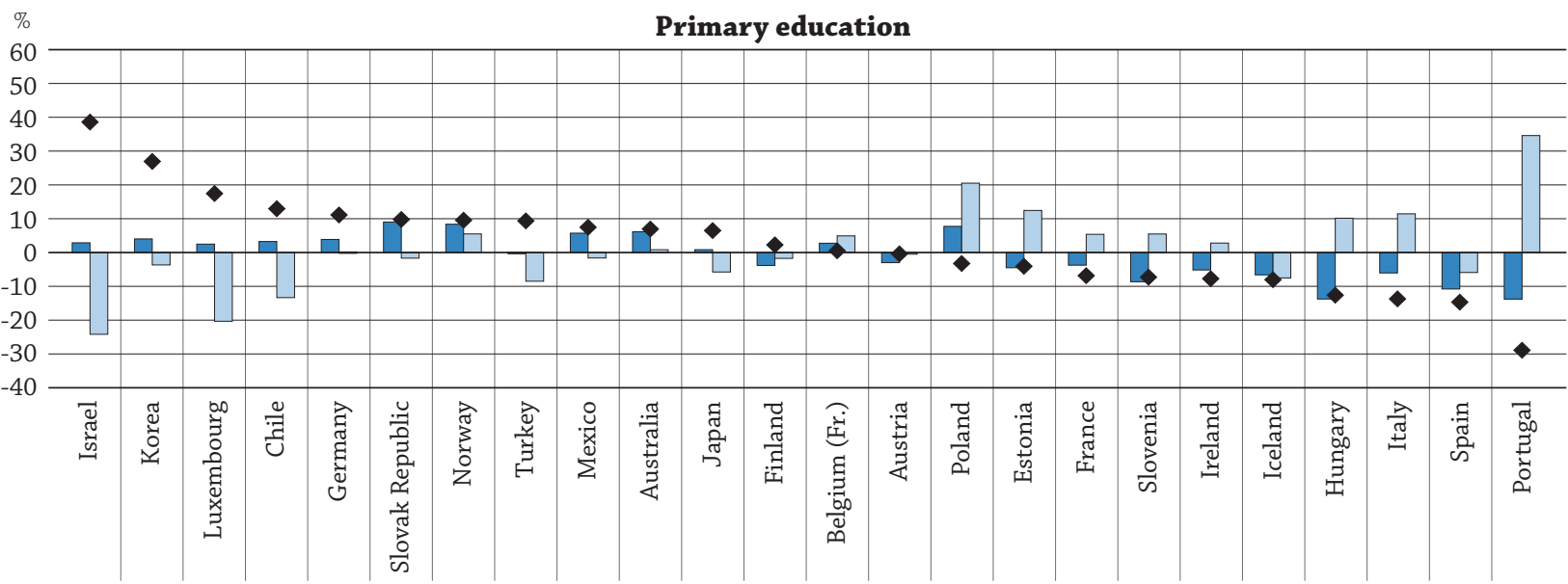

Lower secondary education

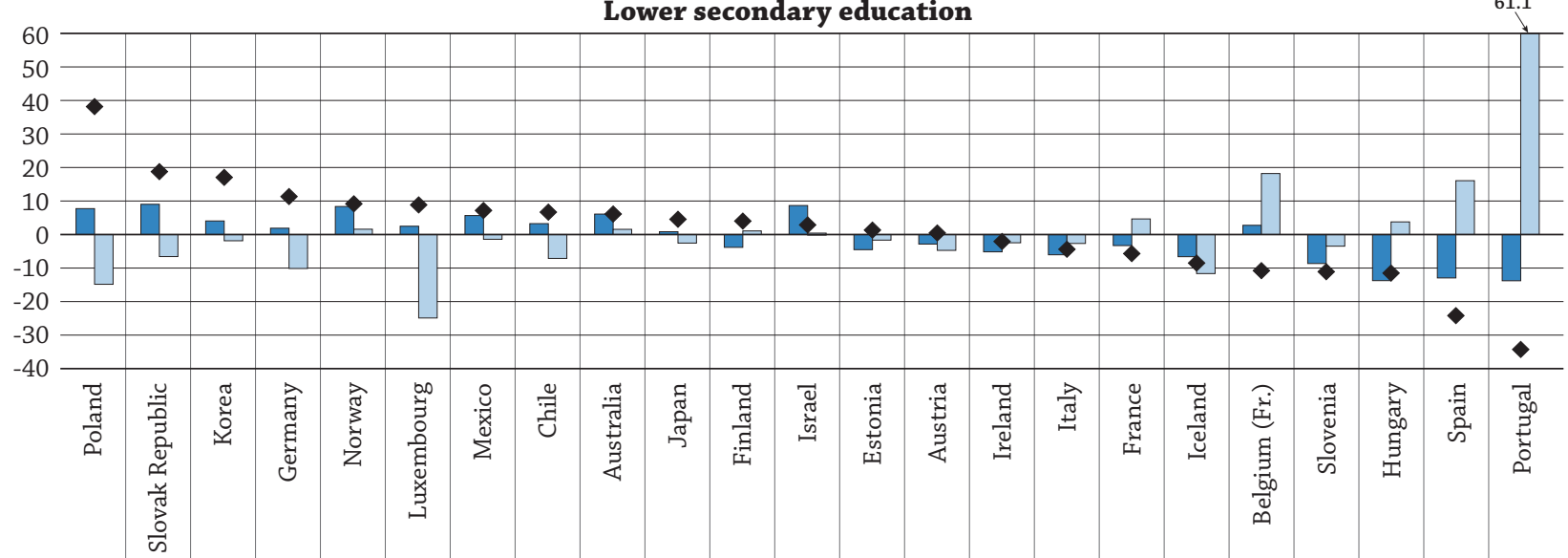

Countries are ranked in descending order of the change in the salary cost of teachers per student between 2010 and 2013

Source: OECD. Tables B7.2a, B7.2b, B7.3 and B7.4. See Annex 3 for notes (www.oecd.org/education/education-at-a-glance-19991487.htm). StatLink त्ञाज $h$ htp://dx.doi.org/10.1787/888933284134

Changes in instruction time and teaching time, the two other factors influencing the salary cost of teachers, averaged from $-0.5 \%$ to $2.9 \%$ at both primary and lower secondary levels (among countries with available data for both years) during the same period (Tables B7.2a and B7.2b). This may reflect the political sensitivity of implementing reforms in these areas (see Table B7.5 in Education at a Glance 2012).

Nevertheless, in a small number of countries, instruction time and/or teaching time did change significantly. For example in Norway, Poland and Portugal, reforms were recently introduced to increase instruction time in reading and mathematics. Between 2010 and 2013, teaching time changed most significantly (by more than 100 hours) in Korea (a decrease from 807 to 667 hours at the primary level) and Luxembourg (an increase from 634 to 739 hours at the lower secondary level). Instruction time changed also significantly (by more than 100 hours) between 2010 and 2013 in some countries as well. At the lower secondary level, instruction time decreased by more than 100 hours in Iceland and increased by more than 100 hours in Portugal.

\section{Relationship between expenditure on education and policy choices}

Higher levels of expenditure on education cannot automatically be equated with better performance by education systems. This is not surprising, as countries spending similar amounts on education do not necessarily have similar education policies and practices. For example, at the upper secondary level of education, Austria and Canada had similar levels of salary cost of teachers per student in 2013 (USD 5093 and USD 4 839, respectively) both higher 
than the OECD average. In Canada, this was largely because teachers' salaries were higher than the average, whereas in Austria, it was the result of the combination of below-average estimated class size and teaching time and, to a lesser extent, of above-average instruction time and teachers' salaries. Hungary and the Slovak Republic also had similar salary cost of teachers per student (below the average). Both countries had similar teachers' salaries and teaching time, but the two other factors (instruction time and estimated class size) influenced the salary cost of teachers in different ways in the two countries (Table B7.5 and Chart B7.4).

In addition, even though countries may make similar policy choices, those choices can result in different levels of salary cost of teachers per student. For example, in Canada and Ireland, the salary cost of teachers per student at the upper secondary level is the result of balancing two opposing effects: above-average teaching time reduces the salary cost of teachers per student relative to the OECD average, and relatively high teachers' salaries (and small estimated class size in Canada) increase the salary cost of teachers per student relative to the OECD average.

The salary cost of teachers per student resulting from this combination is above the OECD average in these two countries, but the difference from the OECD average varies from USD 315 more in Ireland to USD 1091 more in Canada (Table B7.5 and Chart B7.4).

\section{Chart B7.4. Contribution of various factors to salary cost of teachers per student, upper secondary education (2013)

$$
\text { In USD }
$$

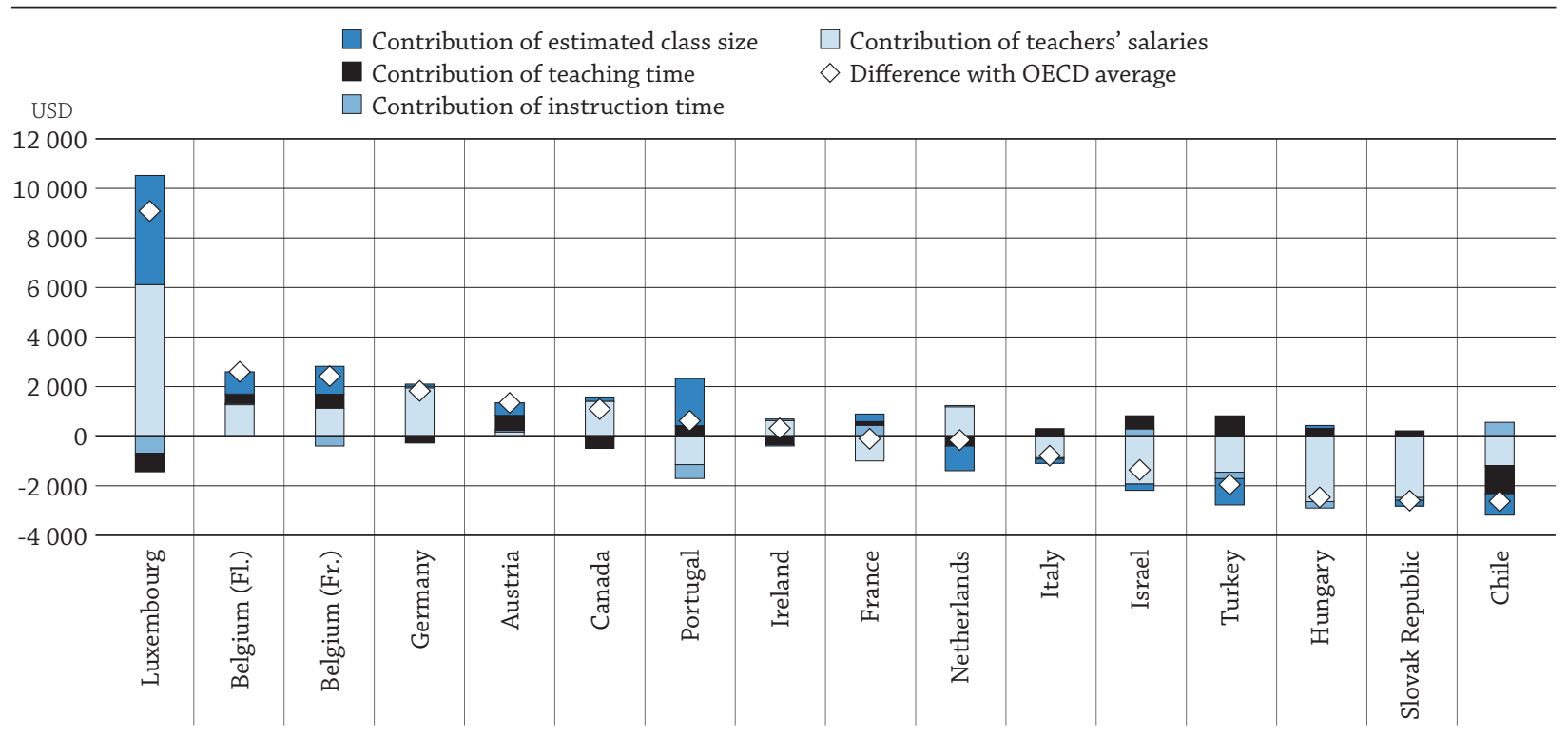

\section{How to read this chart}

This chart shows the contribution (in USD) of the factors influencing the difference between salary cost of teachers per student in the country and the OECD average. For example, in Chile, the salary cost of teachers per student is USD 2624 lower than the OECD average. This is because Chile has lower teachers' salaries (- USD 1 183) than the OECD average, above-average instruction time for students (+ USD 557), above-average teaching time for teachers (- USD 1 136), and above-average estimated class size (- USD 863).

Note: Contributions expressed in percentage of GDP per capita are also available in a similar chart on line. Countries are ranked in descending order of the difference between the salary cost of teachers per student and the OECD average. Sources: OECD. Table B7.5. See Annex 3 for notes (www.oecd.org/education/education-at-a-glance-19991487.htm).

StatLink 제이 http://dx.doi.org/10.1787/888933284144

\section{Main factors influencing the salary cost of teachers per student, by level of education}

Comparing the salary cost of teachers per student to the OECD average and how the four factors contribute to this difference allows for an analysis of the extent of each factor's impact on the differences in salary cost of teachers per student. At each level of education, teachers' salaries are most often the primary factor - that is, the factor with the largest impact - influencing the difference in the average salary cost of teachers per student. Among countries with available data in 2013, teachers' salaries were the primary factor in 22 of 29 countries at the primary level, in 17 of 30 countries at the lower secondary level, and in 14 of 16 countries at the upper secondary level. This is true in countries with the highest and lowest levels of salary cost of teachers per student. 
For example, at the lower secondary level, the above-average salaries of teachers is the main factor influencing the difference from the average salary cost of teachers per student in the country with the highest salary cost (Luxembourg), as well as in seven of the ten countries with the lowest salary cost of teachers per student (Chile, the Czech Republic, Estonia, Hungary, Israel, Poland and the Slovak Republic) (Table B7.4).

Estimated class size is the second most influential factor responsible for the difference in salary cost of teachers per student at each level of education (for 4 countries at the primary level, 11 countries at the lower secondary level, and 1 country at the upper secondary level). At the lower secondary level, below-average estimated class size is the primary factor in 3 of the 6 countries with the highest salary cost of teachers per student, namely Austria and Belgium (Flemish and French Communities) (Box B7.2 and Table B7.4).

\section{Box B7.2. Main factors influencing salary cost of teacher per student, by level of education (2013)}

\begin{tabular}{|c|c|c|c|}
\hline & Primary education & Lower secondary education & Upper secondary education \\
\hline Salary & $\begin{array}{c}22 \text { countries } \\
\text { AUS (+), BFL (+), BFR (+), CAN (+), } \\
\text { CHL (-), CZE (-), EST (-), DEU (+), } \\
\text { GRC (-), HUN (-), ISL (-), IRL (+), } \\
\text { ISR (-), ITA (-), JPN (+), KOR (+), } \\
\text { LUX (+), NLD (+), POL (-), PRT(-), } \\
\text { SVK (-), TUR (-) }\end{array}$ & $\begin{array}{c}17 \text { countries } \\
\text { AUS }(+), \text { CAN }(+), \text { CHL (-), CZE (-), } \\
\text { EST (-), DEU (+), GRC (-), } \\
\text { HUN (-), ISL (-), IRL (+), ISR (-), } \\
\text { ITA (-), LUX (+), NLD (+), POL (-), } \\
\text { PRT (-), SVK(-) }\end{array}$ & $\begin{array}{c}14 \text { countries } \\
\operatorname{BFL}(+), \operatorname{BFR}(+), \text { CAN }(+), \\
\text { CHL (-), FRA (-), DEU (+), } \\
\text { HUN (-), IRL (+), ISR (-), } \\
\text { ITA (-), LUX (+), NLD (+), } \\
\text { SVK (-), TUR (-) }\end{array}$ \\
\hline $\begin{array}{l}\text { Instruction } \\
\text { time }\end{array}$ & $\begin{array}{l}1 \text { country } \\
\text { FIN (-) }\end{array}$ & $\begin{array}{l}1 \text { country } \\
\text { ESP }(+)\end{array}$ & 0 country \\
\hline Teaching time & $\begin{array}{c}2 \text { countries } \\
\text { FRA (-), SVN (+) }\end{array}$ & $\begin{array}{l}1 \text { country } \\
\text { USA (-) }\end{array}$ & $\begin{array}{l}1 \text { country } \\
\text { AUT }(+)\end{array}$ \\
\hline $\begin{array}{l}\text { Estimated } \\
\text { class size }\end{array}$ & $\begin{array}{c}4 \text { countries } \\
\operatorname{AUT}(+), \operatorname{MEX}(-), \\
\operatorname{NOR}(+), \operatorname{ESP}(+)\end{array}$ & $\begin{array}{c}11 \text { countries } \\
\text { AUT (+), BFL (+), BFR (+), FIN (+), } \\
\text { FRA (-), JPN }(-), \operatorname{KOR}(-), \operatorname{MEX}(-), \\
\text { NOR }(+), \operatorname{SVN}(+), \operatorname{TUR}(-)\end{array}$ & $\begin{array}{l}1 \text { country } \\
\text { PRT (+) }\end{array}$ \\
\hline
\end{tabular}

Note: For each level of education, countries are included in the cell corresponding to the factor which has the largest impact (measured in USD) on the salary cost of teachers' per student. The positive or negative signs show whether the factor increases or decreases the salary cost of teacher per student. Sources: OECD. Tables B7.3, B7.4 and B7.5. See Annex 3 for notes (www.oecd.org/education/education-at-a-glance-19991487.htm).

Please refer to the Reader's Guide for information concerning symbols for missing data and abbreviations.

StatLink 제의 http://dx.doi.org/10.1787/888933285727

\section{Main factors influencing the salary cost of teachers per student, after accounting for countries' wealth}

The level of teachers' salaries and, in turn, the level of the salary cost of teachers per student, depend on a country's relative wealth. To control for differences in wealth among countries, the levels of teachers' salaries (and salary cost per student) relative to GDP per capita were analysed. Comparing the relative salary cost of teachers per student using this analysis affects the ranking of countries (Chart B7.4 continued, available on line). However, the position of only a few countries changes significantly compared to the analysis in USD. At the upper secondary level, Luxembourg has the highest salary cost of teachers per student in USD, mainly as a result of the high USD salaries, but not as a proportion of per capita GDP, even if the salary cost of teachers per student is still above the OECD average because of below-average estimated class size. As a result, teachers' salaries, as a percentage of per capita GDP, do not raise the salary cost of teachers per student (Tables B7.3 continued, B7.4 continued, B7.5 continued, and Chart B7.4 continued, available on line).

Even after accounting for differences in countries' wealth, teachers' salaries, as a percentage of GDP per capita, and estimated class size are the main factors influencing variations from the average salary cost of teachers per student at each level of education (Box B7.2 continued, available on line). 


\section{Methodology}

Data referring to the 2013 school year, as well as 2005 and 2010 data relating to salaries of teachers and teaching time are based on the UOE data collection on education statistics and on the Survey on Teachers and the Curriculum, which were both administered by the OECD in 2014. Other data referring to the 2005 and 2010 school year are based on the UOE data collection on education statistics, and on the Survey on Teachers and the Curriculum, which were both administered by the OECD and published in the 2007 and 2012 editions of Education at a Glance (data on ratio of student to teaching staff and instruction time). Data for 2013 instruction time refer to 2014 data from the 2014 edition of Education at a Glance. The consistency of 2005, 2010 and 2013 data has been validated (for details, see Annex 3 at www.oecd.org/education/education-at-a-glance-19991487.htm).

Salary cost of teachers per student is calculated based on teachers' salaries, the number of hours of instruction for students, the number of hours of teaching for teachers, and the estimated class size (a proxy of the class size; see Box D2.2). In most cases, the values for these variables are derived from Education at a Glance (see above). At upper secondary level, teachers' salaries and teaching time refer to general programmes. Teachers' salaries in national currencies are converted into equivalent USD by dividing the national currency figure by the purchasing power parity (PPP) index for private consumption, following the methodology used in Indicator D3 on teachers' salaries, which results in the salary cost per student expressed in equivalent USD. Further details on the analysis of these factors are available in Annex 3 at www.oecd.org/education/education-at-a-glance-19991487.htm.

Note regarding data from Israel

The statistical data for Israel are supplied by and are under the responsibility of the relevant Israeli authorities. The use of such data by the OECD is without prejudice to the status of the Golan Heights, East Jerusalem and Israeli settlements in the West Bank under the terms of international law.

\section{Reference}

OECD (2012), Education at a Glance 2012: OECD Indicators, OECD Publishing, Paris, http://dx.doi.org/10.1787/eag-2012-en.

\section{Indicator B7 Tables}

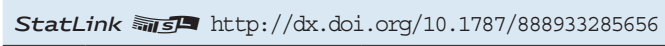

\begin{tabular}{|c|c|c|}
\hline & Table B7.1 & Salary cost of teachers per student, by level of education (2013) \\
\hline & Table B7.2a & $\begin{array}{l}\text { Factors used to compute the salary cost of teachers per student, in primary education } \\
(2005,2010 \text { and } 2013)\end{array}$ \\
\hline & Table B7.2b & $\begin{array}{l}\text { Factors used to compute the salary cost of teachers per student, in lower secondary education } \\
(2005,2010 \text { and } 2013)\end{array}$ \\
\hline WEB & Table B7.2c & $\begin{array}{l}\text { Factors used to compute the salary cost of teachers per student, in upper secondary education } \\
\text { (2013) }\end{array}$ \\
\hline & Table B7.3 & $\begin{array}{l}\text { Contribution of various factors to salary cost of teachers per student in primary education } \\
(2005,2010 \text { and } 2013)\end{array}$ \\
\hline WEB & Table B7.3 (continued) & Contribution of various factors to salary cost of teachers per student in primary education (2013) \\
\hline & Table B7.4 & $\begin{array}{l}\text { Contribution of various factors to salary cost of teachers per student } \\
\text { in lower secondary education }(2005,2010 \text { and 2013) }\end{array}$ \\
\hline$W E B$ & Table B7.4 (continued) & $\begin{array}{l}\text { Contribution of various factors to salary cost of teachers per student } \\
\text { in lower secondary education (2013) }\end{array}$ \\
\hline & Table B7.5 & $\begin{array}{l}\text { Contribution of various factors to salary cost of teachers per student } \\
\text { in upper secondary education (2013) }\end{array}$ \\
\hline WEB & Table B7.5 (continued) & $\begin{array}{l}\text { Contribution of various factors to salary cost of teachers per student } \\
\text { in upper secondary education (2013) }\end{array}$ \\
\hline
\end{tabular}

\section{Additional material available on line only}

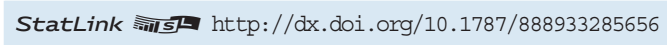

WEB Chart B7.4 (continued) Contribution of various factors to salary cost of teachers per student, upper secondary education (2012)

WEB Box B7.2 (continued) Main factors influencing salary cost of teachers per student as a percentage of per capita GDP, by level of education (2013) 
Table B7.1. Salary cost of teachers per student, by level of education (2013) In equivalent USD, converted using PPPs for private consumption, and in percentage of per capita GDP

\begin{tabular}{|c|c|c|c|c|c|c|}
\hline & \multicolumn{3}{|c|}{$\begin{array}{l}\text { Salary cost of teachers per student } \\
\text { (in USD) }\end{array}$} & \multicolumn{3}{|c|}{$\begin{array}{l}\text { Salary cost of teachers per student } \\
\text { (in percentage of GDP per capita) }\end{array}$} \\
\hline & Primary & Lower secondary & Upper secondary & Primary & Lower secondary & Upper secondary \\
\hline & (1) & (2) & (3) & (4) & (5) & (6) \\
\hline Australia & 3608 & 4684 & $\mathrm{~m}$ & 8.1 & 10.6 & $\mathrm{~m}$ \\
\hline Austria & 3609 & 5191 & 5093 & 8.2 & 11.8 & 11.6 \\
\hline Belgium (Fl.) & 3842 & 5218 & 6344 & 9.8 & 13.4 & 16.2 \\
\hline Belgium (Fr.) & 3739 & 5078 & 6167 & 9.6 & 13.0 & 15.8 \\
\hline Canada & 4755 & 4755 & 4839 & 11.4 & 11.4 & 11.6 \\
\hline Chile & 1181 & 1095 & 1124 & 6.0 & 5.5 & 5.7 \\
\hline Czech Republic & 973 & 1633 & $\mathrm{~m}$ & 3.7 & 6.2 & $\mathrm{~m}$ \\
\hline Denmark & $\mathrm{m}$ & $\mathrm{m}$ & $\mathrm{m}$ & $\mathrm{m}$ & $\mathrm{m}$ & $\mathrm{m}$ \\
\hline England & $\mathrm{m}$ & $\mathrm{m}$ & $\mathrm{m}$ & $\mathrm{m}$ & $\mathrm{m}$ & $\mathrm{m}$ \\
\hline Estonia & 1015 & 1350 & $\mathrm{~m}$ & 4.5 & 6.0 & $\mathrm{~m}$ \\
\hline Finland & 3008 & 4749 & $\mathrm{~m}$ & 8.0 & 12.7 & $\mathrm{~m}$ \\
\hline France & 1735 & 2374 & 3643 & 4.8 & 6.6 & 10.1 \\
\hline Germany & 4047 & 5047 & 5573 & 9.7 & 12.1 & 13.4 \\
\hline Greece & 2720 & 3515 & $\mathrm{~m}$ & 12.1 & 15.7 & $\mathrm{~m}$ \\
\hline Hungary & 1229 & 1252 & 1287 & 5.9 & 6.0 & 6.1 \\
\hline Iceland & 2985 & 2970 & $\mathrm{~m}$ & 7.4 & 7.4 & $\mathrm{~m}$ \\
\hline Ireland & 3426 & 4063 & 4063 & 8.7 & 10.4 & 10.4 \\
\hline Israel & 1956 & 2131 & 2391 & 6.5 & 7.0 & 7.9 \\
\hline Italy & 2692 & 3100 & 2963 & 8.5 & 9.8 & 9.4 \\
\hline Japan & 2790 & 3491 & $\mathrm{~m}$ & 8.3 & 10.4 & $\mathrm{~m}$ \\
\hline Korea & 2981 & 2941 & $\mathrm{~m}$ & 9.6 & 9.4 & $\mathrm{~m}$ \\
\hline Luxembourg & 11674 & 12821 & 12821 & 13.7 & 15.0 & 15.0 \\
\hline Mexico & 958 & 1057 & $\mathrm{~m}$ & 6.5 & 7.2 & $\mathrm{~m}$ \\
\hline Netherlands & 3258 & 4176 & 3593 & 7.6 & 9.7 & 8.4 \\
\hline New Zealand & $\mathrm{m}$ & $\mathrm{m}$ & $\mathrm{m}$ & $\mathrm{m}$ & $\mathrm{m}$ & $\mathrm{m}$ \\
\hline Norway & 4307 & 4525 & $\mathrm{~m}$ & 7.0 & 7.4 & $\mathrm{~m}$ \\
\hline Poland & 2247 & 2519 & $\mathrm{~m}$ & 9.9 & 11.1 & $\mathrm{~m}$ \\
\hline Portugal & 2777 & 3516 & 4366 & 11.4 & 14.4 & 17.9 \\
\hline Scotland & $\mathrm{m}$ & $\mathrm{m}$ & $\mathrm{m}$ & $\mathrm{m}$ & $\mathrm{m}$ & $\mathrm{m}$ \\
\hline Slovak Republic & 924 & 1254 & 1152 & 3.9 & 5.2 & 4.8 \\
\hline Slovenia & 2392 & 4661 & $\mathrm{~m}$ & 9.2 & 17.9 & $\mathrm{~m}$ \\
\hline Spain & 3067 & 4052 & $\mathrm{~m}$ & 10.4 & 13.8 & $\mathrm{~m}$ \\
\hline Sweden & $\mathrm{m}$ & $\mathrm{m}$ & $\mathrm{m}$ & $\mathrm{m}$ & $\mathrm{m}$ & $\mathrm{m}$ \\
\hline Switzerland & $\mathrm{m}$ & $\mathrm{m}$ & $\mathrm{m}$ & $\mathrm{m}$ & $\mathrm{m}$ & $\mathrm{m}$ \\
\hline Turkey & 1368 & 1459 & 1800 & 8.5 & 9.1 & 11.2 \\
\hline United States & $\mathrm{m}$ & 3967 & $\mathrm{~m}$ & $\mathrm{~m}$ & 7.5 & $\mathrm{~m}$ \\
\hline OECD average & 2677 & 3350 & 3749 & 7.9 & 9.4 & 10.4 \\
\hline
\end{tabular}

Source: OECD. See Annex 3 for notes (www.oecd.org/education/education-at-a-glance-19991487.htm).

Please refer to the Reader's Guide for information concerning symbols for missing data and abbreviations.

StatLink 세대 http://dx.doi.org/10.1787/888933285662 
Table B7.2a. [1/2] Factors used to compute the salary cost of teachers per student, in primary education $(2005,2010$ and 2013)

\begin{tabular}{|c|c|c|c|c|c|c|c|c|c|c|c|c|}
\hline & \multicolumn{4}{|c|}{$\begin{array}{l}\text { Teachers' salary } \\
\text { (annual, in USD. } 2013 \text { constant prices) }\end{array}$} & \multicolumn{4}{|c|}{$\begin{array}{c}\text { Instruction time } \\
\text { (for students, hours per year) }\end{array}$} & \multicolumn{4}{|c|}{$\begin{array}{c}\text { Teaching time } \\
\text { (for teachers, hours per year) }\end{array}$} \\
\hline & 2005 & 2010 & 2013 & $\begin{array}{c}\text { Variation } \\
\text { 2010-2013 } \\
\text { (\%) }\end{array}$ & 2005 & 2010 & 2013 & $\begin{array}{c}\text { Variation } \\
2010-2013 \\
(\%)\end{array}$ & 2005 & 2010 & 2013 & $\begin{array}{c}\text { Variation } \\
2010-2013 \\
(\%)\end{array}$ \\
\hline & (1) & (2) & (3) & (4) & (5) & (6) & (7) & (8) & (9) & (10) & (11) & (12) \\
\hline Australia $^{1}$ & 50684 & 53076 & 56335 & 6.1 & 952 & 982 & 1010 & 2.9 & 888 & 868 & 879 & 1.3 \\
\hline Austria $^{1}$ & 42514 & 44344 & 43015 & -3.0 & 690 & 690 & 705 & 2.2 & 774 & 779 & 779 & 0.0 \\
\hline Belgium (Fl.) & 47103 & 47821 & 48690 & 1.8 & $\mathrm{~m}$ & $\mathrm{~m}$ & 821 & $\mathbf{m}$ & 752 & 752 & 752 & 0.0 \\
\hline Belgium (Fr.) ${ }^{1}$ & 44457 & 46111 & 47381 & 2.8 & 840 & 840 & 849 & 1.1 & 722 & 732 & 721 & -1.5 \\
\hline Canada & $\mathrm{m}$ & $\mathrm{m}$ & 66702 & $\mathbf{m}$ & $\mathrm{m}$ & 917 & 919 & 0.3 & $\mathrm{~m}$ & 799 & 796 & -0.4 \\
\hline Chile $^{1}$ & $\mathrm{~m}$ & 25771 & 26610 & 3.3 & $\mathrm{~m}$ & 1083 & 1049 & -3.2 & 1128 & 1105 & 1129 & 2.2 \\
\hline Czech Republic ${ }^{2}$ & $\mathrm{~m}$ & $\mathrm{~m}$ & 18273 & $\mathbf{m}$ & 661 & 588 & 676 & 15.0 & 813 & 862 & 827 & -4.1 \\
\hline Denmark & 51700 & 54558 & 52672 & -3.5 & 671 & 701 & 754 & 7.6 & 640 & 650 & 662 & 1.8 \\
\hline England & 54792 & 50317 & 47279 & -6.0 & 880 & 893 & 861 & -3.5 & $\mathrm{~m}$ & 684 & 722 & 5.6 \\
\hline Estonia $^{1}$ & 10070 & 13857 & 13233 & -4.5 & 752 & 595 & 661 & 11.0 & 630 & 630 & 619 & -1.7 \\
\hline Finland $^{1}$ & 37024 & 41276 & 39701 & -3.8 & 530 & 608 & 632 & 3.9 & 677 & 680 & 677 & -0.5 \\
\hline France $^{1}$ & 35709 & 34804 & 33500 & -3.7 & 918 & 847 & 864 & 2.0 & 924 & 924 & 924 & 0.0 \\
\hline Germany $^{1}$ & 58592 & 60865 & 63221 & 3.9 & 627 & 641 & 683 & 6.5 & 808 & 805 & 800 & -0.6 \\
\hline Greece & 34859 & 35333 & 25826 & -26.9 & 864 & 720 & 783 & 8.8 & 604 & 589 & 569 & -3.4 \\
\hline Hungary $^{1}$ & 19280 & 15143 & 13061 & -13.8 & 555 & 555 & 616 & 11.0 & 583 & 604 & 601 & -0.5 \\
\hline Iceland $^{1}$ & 35173 & 33350 & 31145 & -6.6 & 720 & 800 & 729 & -8.9 & 671 & 624 & 624 & 0.0 \\
\hline Ireland $^{1}$ & 51371 & 59108 & 56057 & -5.2 & 941 & 915 & 915 & 0.0 & 915 & 915 & 915 & 0.0 \\
\hline Israel $^{1}$ & 23621 & 29035 & 29869 & 2.9 & 666 & 914 & 957 & 4.7 & 731 & 820 & 840 & 2.5 \\
\hline Italy $^{1}$ & 35402 & 35367 & 33230 & -6.0 & 990 & 891 & 891 & 0.0 & 739 & 770 & 752 & -2.3 \\
\hline Japan $^{1}$ & 51724 & 48139 & 48546 & 0.8 & 707 & 735 & 762 & 3.7 & 578 & 707 & 736 & 4.2 \\
\hline Korea $^{1}$ & 53256 & 49598 & 51594 & 4.0 & 667 & 667 & 648 & -2.9 & 883 & 807 & 667 & -17.4 \\
\hline Luxembourg $^{1}$ & 73586 & 100460 & 102956 & 2.5 & 847 & 924 & 924 & 0.0 & 774 & 739 & 810 & 9.5 \\
\hline Mexico $^{1}$ & 24353 & 25097 & 26533 & 5.7 & 800 & 800 & 800 & 0.0 & 800 & 800 & 800 & 0.0 \\
\hline Netherlands & $\mathrm{m}$ & $\mathrm{m}$ & 54001 & $\mathbf{m}$ & 940 & 940 & 940 & 0.0 & 930 & 930 & 930 & 0.0 \\
\hline New Zealand & $\mathrm{m}$ & $\mathrm{m}$ & 43292 & $\mathbf{m}$ & $\mathrm{m}$ & $\mathrm{m}$ & $\mathrm{m}$ & $\mathbf{m}$ & $\mathrm{m}$ & $\mathrm{m}$ & 922 & $\mathbf{m}$ \\
\hline Norway $^{1}$ & 38749 & 41099 & 44538 & 8.4 & 599 & 701 & 748 & 6.7 & 741 & 741 & 741 & 0.0 \\
\hline Poland ${ }^{1}$ & 20453 & 23132 & 24921 & 7.7 & $\mathrm{~m}$ & 600 & 635 & 5.8 & $\mathrm{~m}$ & 644 & 629 & -2.3 \\
\hline Portugal $^{1}$ & 43221 & 42528 & 36663 & -13.8 & 855 & 757 & 806 & 6.5 & 765 & 779 & 747 & -4.0 \\
\hline Scotland & 48098 & 47148 & 43991 & -6.7 & a & $\mathrm{a}$ & $\mathrm{a}$ & $\mathbf{m}$ & 893 & 855 & 855 & 0.0 \\
\hline Slovak Republic ${ }^{1}$ & $\mathrm{~m}$ & 14354 & 15650 & 9.0 & $\mathrm{~m}$ & 695 & 680 & -2.0 & $\mathrm{~m}$ & 841 & 832 & -1.1 \\
\hline Slovenia $^{1}$ & 38258 & 41882 & 38261 & -8.6 & 621 & 621 & 664 & 7.0 & 627 & 627 & 627 & 0.0 \\
\hline Spain $^{1}$ & 44294 & 47288 & 42187 & -10.8 & 793 & 875 & 787 & -10.0 & 880 & 880 & 880 & 0.0 \\
\hline Sweden & 34971 & $\mathrm{~m}$ & 38175 & $\mathbf{m}$ & 741 & 741 & 754 & 1.8 & $\mathrm{~m}$ & $\mathrm{~m}$ & a & $\mathbf{m}$ \\
\hline Switzerland & 60689 & 61677 & 63504 & 3.0 & $\mathrm{~m}$ & $\mathrm{~m}$ & $\mathrm{~m}$ & $\mathbf{m}$ & $\mathrm{m}$ & $\mathrm{m}$ & $\mathrm{m}$ & m \\
\hline Turkey $^{1}$ & 23762 & 27122 & 27139 & 0.1 & 720 & 720 & 720 & 0.0 & 720 & 720 & 720 & 0.0 \\
\hline United States & 60284 & 55802 & 59339 & 6.3 & $\mathrm{~m}$ & $\mathrm{~m}$ & 967 & $\mathbf{m}$ & $\mathrm{m}$ & $\mathrm{m}$ & $\mathrm{m}$ & $\mathbf{m}$ \\
\hline OECD average & 41602 & 42112 & 41864 & -1.8 & 761 & 773 & 794 & 2.5 & 771 & 771 & 772 & -0.4 \\
\hline $\begin{array}{l}\text { Average for } 24 \text { countries } \\
\text { with all data available } \\
\text { for } 2010 \text { and } 2013\end{array}$ & 〜 & $\mid 39700$ & 39389 & -0.8 & & 769 & 781 & 1.5 & $\sim$ & 773 & 769 & -0.5 \\
\hline
\end{tabular}

Note: Data in this table come either from chapter D (for 2005, 2010 and 2013 data relating to salaries of teachers and teaching time, 2013 data on ratio of student to teaching staff) or from 2007 and 2012 editions of Education at a Glance (data on ratio of student to teaching staff and instruction time). Data for 2013 instruction time refer to 2014 data from the 2014 edition of Education at a Glance. Please see notes on these data in these tables.

1. Countries with all data available for both 2010 and 2013.

2. Current instruction time for 2005 and 2010, minimum instruction time for 2013.

Source: OECD. See Annex 3 for notes (www.oecd.org/education/education-at-a-glance-19991487.htm).

Please refer to the Reader's Guide for information concerning symbols for missing data and abbreviations.

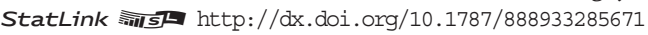


Table B7.2a. [2/2] Factors used to compute the salary cost of teachers per student, in primary education $(2005,2010$ and 2013)

\begin{tabular}{|c|c|c|c|c|c|c|c|c|}
\hline & \multicolumn{4}{|c|}{$\begin{array}{l}\text { Ratio of students to teaching staff } \\
\text { (number of students per teacher) }\end{array}$} & \multicolumn{4}{|c|}{$\begin{array}{c}\text { Estimated class size } \\
\text { (number of students per classroom) }\end{array}$} \\
\hline & 2005 & 2010 & 2013 & $\begin{array}{c}\text { Variation } \\
2010-2013 \\
(\%)\end{array}$ & 2005 & 2010 & 2013 & $\begin{array}{c}\text { Variation } \\
\text { 2010-2013 } \\
\text { (\%) }\end{array}$ \\
\hline & (13) & (14) & (15) & (16) & $(17)=(13)^{*}(5) /(9)$ & $(18)=(14)^{*}(6) /(10)$ & $(19)=(15)^{*}(7) /(11)$ & (20) \\
\hline Australia ${ }^{1}$ & 16.2 & 15.7 & 15.6 & -0.8 & 17.4 & 17.8 & 17.9 & 0.8 \\
\hline Austria ${ }^{1}$ & 14.1 & 12.2 & 11.9 & -2.6 & 12.6 & 10.8 & 10.8 & -0.5 \\
\hline Belgium (Fl.) & 12.8 & 12.4 & 12.7 & 2.2 & $\mathrm{~m}$ & $\mathrm{~m}$ & 13.8 & $\mathbf{m}$ \\
\hline Belgium (Fr.) $)^{1}$ & 12.8 & 12.4 & 12.7 & 2.2 & 14.9 & 14.2 & 14.9 & 4.9 \\
\hline Canada & $\mathrm{m}$ & 17.9 & 14.0 & -21.7 & $\mathrm{~m}$ & 20.6 & 16.2 & -21.2 \\
\hline Chile $^{1}$ & 25.9 & 24.6 & 22.5 & -8.6 & $\mathrm{~m}$ & 24.2 & 20.9 & -13.3 \\
\hline Czech Republic ${ }^{2}$ & 17.5 & 18.7 & 18.8 & 0.6 & 14.3 & 12.7 & 15.4 & 20.6 \\
\hline Denmark & 11.9 & 11.5 & $\mathrm{~m}$ & $\mathbf{m}$ & 12.5 & 12.4 & $\mathrm{~m}$ & $\mathbf{m}$ \\
\hline England & 14.9 & 19.8 & $\mathrm{~m}$ & $\mathbf{m}$ & $\mathrm{m}$ & 25.9 & $\mathrm{~m}$ & $\mathbf{m}$ \\
\hline Estonia $^{1}$ & $\mathrm{~m}$ & 13.1 & 13.0 & -0.5 & $\mathrm{~m}$ & 12.4 & 13.9 & 12.4 \\
\hline Finland $^{1}$ & 15.9 & 14.0 & 13.2 & -5.9 & 12.5 & 12.5 & 12.3 & -1.7 \\
\hline France $^{1}$ & 19.4 & 18.7 & 19.3 & 3.3 & 19.2 & 17.1 & 18.1 & 5.4 \\
\hline Germany $^{1}$ & 18.8 & 16.7 & 15.6 & -6.5 & 14.6 & 13.3 & 13.3 & 0.2 \\
\hline Greece & 11.1 & $\mathrm{~m}$ & 9.5 & $\mathbf{m}$ & 15.8 & $\mathrm{~m}$ & 13.1 & $\mathbf{m}$ \\
\hline Hungary $^{1}$ & 10.6 & 10.8 & 10.6 & -1.3 & 10.1 & 9.9 & 10.9 & 10.1 \\
\hline Iceland $^{1}$ & 11.3 & 10.3 & 10.4 & 1.5 & $\mathrm{~m}$ & 13.2 & 12.2 & -7.5 \\
\hline Ireland $^{1}$ & 17.9 & 15.9 & 16.4 & 2.8 & 18.4 & 15.9 & 16.4 & 2.8 \\
\hline Israel $^{1}$ & 17.3 & 20.6 & 15.3 & -25.8 & 15.7 & 22.9 & 17.4 & -24.2 \\
\hline Italy $^{1}$ & 10.6 & 11.3 & 12.3 & 8.9 & 14.2 & 13.1 & 14.6 & 11.4 \\
\hline $\mathrm{Japan}^{1}$ & 19.4 & 18.4 & 17.4 & -5.3 & 23.7 & 19.1 & 18.0 & -5.8 \\
\hline Korea $^{1}$ & 28.0 & 21.1 & 17.3 & -18.1 & 21.2 & 17.4 & 16.8 & -3.7 \\
\hline Luxembourg $^{1}$ & $\mathrm{~m}$ & 10.1 & 8.8 & -12.7 & $\mathrm{~m}$ & 12.6 & 10.1 & -20.3 \\
\hline Mexico $^{1}$ & 28.3 & 28.1 & 27.7 & -1.6 & 28.3 & 28.1 & 27.7 & -1.6 \\
\hline Netherlands & 15.9 & 15.7 & 16.6 & 5.3 & 16.1 & 15.9 & 16.8 & 5.3 \\
\hline New Zealand & 18.1 & 16.2 & 16.4 & 1.0 & $\mathrm{~m}$ & $\mathrm{~m}$ & $\mathrm{~m}$ & $\mathbf{m}$ \\
\hline Norway $^{1}$ & 10.9 & 10.5 & 10.3 & -1.1 & 8.8 & 9.9 & 10.4 & 5.5 \\
\hline Poland ${ }^{1}$ & 11.7 & 10.0 & 11.1 & 11.4 & $\mathrm{~m}$ & 9.3 & 11.2 & 20.5 \\
\hline Portugal $^{1}$ & 10.8 & 10.9 & 13.2 & 21.3 & 12.1 & 10.6 & 14.3 & 34.6 \\
\hline Scotland & 14.9 & 19.8 & $\mathrm{~m}$ & $\mathbf{m}$ & $\mathrm{m}$ & $\mathrm{m}$ & $\mathrm{m}$ & $\mathbf{m}$ \\
\hline Slovak Republic ${ }^{1}$ & 18.9 & 17.1 & 16.9 & -0.7 & $\mathrm{~m}$ & 14.1 & 13.9 & -1.7 \\
\hline Slovenia $^{1}$ & 15.0 & 16.2 & 16.0 & -1.5 & 14.9 & 16.1 & 16.9 & 5.5 \\
\hline Spain $^{1}$ & 14.3 & 13.2 & 13.8 & 4.6 & 12.9 & 13.1 & 12.3 & -5.9 \\
\hline Sweden & 12.2 & 11.7 & 12.7 & 8.6 & $\mathrm{~m}$ & $\mathrm{~m}$ & $\mathrm{~m}$ & $\mathbf{m}$ \\
\hline Switzerland & 14.6 & 14.9 & 14.8 & -1.0 & $\mathrm{~m}$ & $\mathrm{~m}$ & $\mathrm{~m}$ & $\mathbf{m}$ \\
\hline Turkey $^{1}$ & 25.8 & 21.7 & 19.8 & -8.5 & 25.8 & 21.7 & 19.8 & -8.5 \\
\hline United States & 14.9 & 14.5 & 15.3 & 5.6 & $\mathrm{~m}$ & $\mathrm{~m}$ & $\mathrm{~m}$ & $\mathbf{m}$ \\
\hline OECD average & 16.1 & 15.6 & 14.9 & -1.4 & 16.2 & 15.8 & 15.2 & 0.9 \\
\hline $\begin{array}{l}\text { Average for } 24 \text { countries } \\
\text { with all data available } \\
\text { for } 2010 \text { and } 2013\end{array}$ & $\sim$ & 15.6 & 17.1 & 9.6 & $\sim$ & 15.5 & 15.3 & -1.3 \\
\hline
\end{tabular}

Note: Data in this table come either from chapter D (for 2005, 2010 and 2013 data relating to salaries of teachers and teaching time, 2013 data on ratio of student to teaching staff) or from 2007 and 2012 editions of Education at a Glance (data on ratio of student to teaching staff and instruction time). Data for 2013 instruction time refer to 2014 data from the 2014 edition of Education at a Glance. Please see notes on these data in these tables.

1. Countries with all data available for both 2010 and 2013.

2. Current instruction time for 2005 and 2010, minimum instruction time for 2013

Source: OECD. See Annex 3 for notes (www.oecd.org/education/education-at-a-glance-19991487.htm).

Please refer to the Reader's Guide for information concerning symbols for missing data and abbreviations.

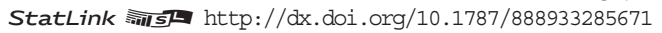


Table B7.2b. [1/2] Factors used to compute the salary cost of teachers per student, in lower secondary education $(2005,2010$ and 2013)

\begin{tabular}{|c|c|c|c|c|c|c|c|c|c|c|c|c|}
\hline & \multicolumn{4}{|c|}{$\begin{array}{l}\text { Teachers' salary } \\
\text { (annual in USD, } 2013 \text { constant prices) }\end{array}$} & \multicolumn{4}{|c|}{$\begin{array}{c}\text { Instruction time } \\
\text { (for students, hours per year) }\end{array}$} & \multicolumn{4}{|c|}{$\begin{array}{c}\text { Teaching time } \\
\text { (for teachers, hours per year) }\end{array}$} \\
\hline & 2005 & 2010 & 2013 & $\begin{array}{c}\text { Variation } \\
\text { 2010-2013 } \\
\text { (\%) }\end{array}$ & 2005 & 2010 & 2013 & $\begin{array}{c}\text { Variation } \\
2010-2013 \\
(\%)\end{array}$ & 2005 & 2010 & 2013 & $\begin{array}{c}\text { Variation } \\
2010-2013 \\
(\%)\end{array}$ \\
\hline & (1) & (2) & (3) & (4) & (5) & (6) & (7) & (8) & (9) & (10) & (11) & (12) \\
\hline Australia $^{1}$ & 50801 & 53076 & 56315 & 6.1 & 970 & 997 & 1015 & 1.8 & 810 & 819 & 821 & 0.3 \\
\hline Austria $^{1}$ & 46053 & 47996 & 46631 & -2.8 & 913 & 914 & 900 & -1.5 & 607 & 607 & 607 & 0.0 \\
\hline Belgium (Fl.) & 47103 & 47821 & 48690 & 1.8 & $\mathrm{~m}$ & $\mathrm{~m}$ & 928 & $\mathbf{m}$ & 684 & 669 & 669 & 0.0 \\
\hline Belgium (Fr.) ${ }^{1}$ & 44956 & 46111 & 47381 & 2.8 & 960 & 960 & 971 & 1.1 & 662 & 671 & 661 & -1.5 \\
\hline Canada & $\mathrm{m}$ & $\mathrm{m}$ & 66702 & $\mathbf{m}$ & $\mathrm{m}$ & 922 & 921 & -0.1 & $\mathrm{~m}$ & 740 & 743 & 0.4 \\
\hline Chile $^{1}$ & $\mathrm{~m}$ & 25771 & 26610 & 3.3 & $\mathrm{~m}$ & 1083 & 1062 & -2.0 & 1128 & 1105 & 1129 & 2.2 \\
\hline Czech Republic ${ }^{2}$ & $\mathrm{~m}$ & $\mathrm{~m}$ & 18273 & $\mathbf{m}$ & 902 & 862 & 874 & 1.3 & 647 & 647 & 620 & -4.1 \\
\hline Denmark & 51700 & 55344 & 53431 & -3.5 & 880 & 900 & 930 & 3.3 & 640 & 650 & 662 & 1.8 \\
\hline England & 54792 & 50317 & 47279 & -6.0 & 900 & 925 & 911 & -1.5 & $\mathrm{~m}$ & 703 & 745 & 5.9 \\
\hline Estonia $^{1}$ & 10070 & 13857 & 13233 & -4.5 & 1073 & 802 & 823 & 2.5 & 630 & 630 & 619 & -1.7 \\
\hline Finland $^{1}$ & 41697 & 44578 & 42877 & -3.8 & 796 & 777 & 844 & 8.7 & 592 & 595 & 592 & -0.5 \\
\hline France $^{1}$ & 38567 & 37834 & 36589 & -3.3 & 959 & 971 & 991 & 2.1 & 648 & 648 & 648 & 0.0 \\
\hline Germany $^{1}$ & 63357 & 67426 & 68698 & 1.9 & 872 & 887 & 866 & -2.3 & 758 & 756 & 752 & -0.5 \\
\hline Greece & 34859 & 35333 & 25826 & -26.9 & 998 & 796 & 785 & -1.3 & 434 & 415 & 415 & 0.0 \\
\hline Hungary $^{1}$ & 19280 & 15143 & 13061 & -13.8 & 717 & 671 & 710 & 5.9 & 555 & 604 & 601 & -0.5 \\
\hline Iceland $^{1}$ & 35173 & 33350 & 31145 & -6.6 & 872 & 969 & 839 & -13.4 & 671 & 624 & 624 & 0.0 \\
\hline Ireland $^{1}$ & 51924 & 59749 & 56667 & -5.2 & 848 & 929 & 935 & 0.7 & 735 & 735 & 735 & 0.0 \\
\hline Israel $^{1}$ & 24071 & 26428 & 28715 & 8.7 & 971 & 981 & 1004 & 2.3 & 579 & 598 & 644 & 7.6 \\
\hline Italy $^{1}$ & 38563 & 38534 & 36207 & -6.0 & 1016 & 1023 & 990 & -3.2 & 605 & 630 & 616 & -2.3 \\
\hline Japan $^{1}$ & 51724 & 48139 & 48546 & 0.8 & 869 & 877 & 895 & 2.1 & 505 & 602 & 608 & 1.1 \\
\hline Korea $^{1}$ & 53127 & 49485 & 51489 & 4.1 & 867 & 859 & 842 & -2.0 & 621 & 627 & 557 & -11.2 \\
\hline Luxembourg $^{1}$ & 96227 & 107575 & 110243 & 2.5 & 782 & 908 & 845 & -6.9 & 642 & 634 & 739 & 16.7 \\
\hline Mexico $^{1}$ & 31129 & 32257 & 34083 & 5.7 & 1167 & 1167 & 1167 & 0.0 & 1047 & 1047 & 1047 & 0.0 \\
\hline Netherlands & $\mathrm{m}$ & $\mathrm{m}$ & 66831 & $\mathbf{m}$ & 1067 & 1000 & 1000 & 0.0 & 750 & 750 & 750 & 0.0 \\
\hline New Zealand & $\mathrm{m}$ & $\mathrm{m}$ & 44509 & $\mathbf{m}$ & $\mathrm{m}$ & $\mathrm{m}$ & $\mathrm{m}$ & $\mathbf{m}$ & $\mathrm{m}$ & $\mathrm{m}$ & 841 & $\mathbf{m}$ \\
\hline Norway $^{1}$ & 38749 & 41099 & 44538 & 8.4 & 827 & 836 & 868 & 3.8 & 656 & 654 & 663 & 1.5 \\
\hline Poland ${ }^{1}$ & 20453 & 23132 & 24921 & 7.7 & $\mathrm{~m}$ & 765 & 810 & 5.9 & $\mathrm{~m}$ & 572 & 555 & -3.0 \\
\hline Portugal $^{1}$ & 43221 & 42528 & 36663 & -13.8 & 880 & 757 & 892 & 17.8 & 623 & 634 & 609 & -4.0 \\
\hline Scotland & 48098 & 47148 & 43991 & -6.7 & a & $\mathrm{a}$ & $\mathrm{a}$ & $\mathbf{a}$ & 893 & 855 & 855 & 0.0 \\
\hline Slovak Republic ${ }^{1}$ & $\mathrm{~m}$ & 14354 & 15650 & 9.0 & $\mathrm{~m}$ & 822 & 828 & 0.7 & $\mathrm{~m}$ & 652 & 645 & -1.1 \\
\hline Slovenia $^{1}$ & 38258 & 41882 & 38261 & -8.6 & 791 & 817 & 767 & -6.1 & 627 & 627 & 627 & 0.0 \\
\hline Spain $^{1}$ & 50864 & 53880 & 46907 & -12.9 & 956 & 1050 & 1061 & 1.1 & 713 & 713 & 713 & 0.0 \\
\hline Sweden & 35860 & $\mathrm{~m}$ & 38852 & $\mathbf{m}$ & 741 & 741 & 754 & 1.8 & $\mathrm{~m}$ & $\mathrm{~m}$ & $\mathrm{a}$ & $\mathbf{m}$ \\
\hline Switzerland & 69260 & 70052 & 71929 & 2.7 & $\mathrm{~m}$ & $\mathrm{~m}$ & $\mathrm{~m}$ & m & $\mathrm{m}$ & $\mathrm{m}$ & $\mathrm{m}$ & m \\
\hline Turkey & 25116 & 28279 & 28110 & -0.6 & 791 & 768 & 840 & 9.4 & 504 & 504 & 504 & 0.0 \\
\hline United States & 55361 & 59163 & 60965 & 3.0 & $\mathrm{~m}$ & $\mathrm{~m}$ & 1011 & $\mathbf{m}$ & $\mathrm{m}$ & $\mathrm{m}$ & 981 & $\mathbf{m}$ \\
\hline OECD average & 43680 & 43795 & 43634 & -1.8 & 903 & 895 & 905 & 1.0 & 677 & 679 & 694 & 0.2 \\
\hline $\begin{array}{l}\text { Average for } 23 \text { countries } \\
\text { with all data available } \\
\text { for } 2010 \text { and } 2013\end{array}$ & $\sim$ & $\mid 41921$ & 41540 & -0.9 & & 905 & 910 & 0.5 & $\sim$ & 686 & 706 & 2.9 \\
\hline
\end{tabular}

Note: Data in this table come either from chapter D (for 2005, 2010 and 2013 data relating to salaries of teachers and teaching time, 2013 data on ratio of student to teaching staff) or from 2007 and 2012 editions of Education at a Glance (data on ratio of student to teaching staff and instruction time). Data for 2013 instruction time refer to 2014 data from the 2014 edition of Education at a Glance. Please see notes on these data in these tables.

1. Countries with all data available for both 2010 and 2013.

2. Current instruction time for 2000 and 2005, minimum instruction time for 2013.

Source: OECD. See Annex 3 for notes (www.oecd.org/education/education-at-a-glance-19991487.htm).

Please refer to the Reader's Guide for information concerning symbols for missing data and abbreviations.

StatLink त्ताजा http://dx.doi.org/10.1787/888933285680 
Table B7.2b. [2/2] Factors used to compute the salary cost of teachers per student, in lower secondary education $(2005,2010$ and 2013)

\begin{tabular}{|c|c|c|c|c|c|c|c|c|}
\hline & \multicolumn{4}{|c|}{$\begin{array}{l}\text { Ratio of students to teaching staff } \\
\text { (number of students per teacher) }\end{array}$} & \multicolumn{4}{|c|}{$\begin{array}{c}\text { Estimated class size } \\
\text { (number of students per classroom) }\end{array}$} \\
\hline & 2005 & 2010 & 2013 & $\begin{array}{c}\text { Variation } \\
2010-2013 \\
(\%)\end{array}$ & 2005 & 2010 & 2013 & $\begin{array}{c}\text { Variation } \\
2010-2013 \\
\text { (\%) }\end{array}$ \\
\hline & (13) & (14) & (15) & (16) & $(17)=(13)^{*}(5) /(9)$ & $(18)=(14)^{*}(6) /(10)$ & $(19)=(15)^{*}(7) /(11)$ & (20) \\
\hline Australia $^{1}$ & 12.1 & 12.0 & 12.0 & 0.0 & 14.5 & 14.6 & 14.9 & 1.6 \\
\hline Austria $^{1}$ & 10.6 & 9.3 & 9.0 & -3.3 & 16.0 & 14.0 & 13.3 & -4.7 \\
\hline Belgium (Fl.) & 9.4 & 8.1 & 9.3 & 15.2 & $\mathrm{~m}$ & $\mathrm{~m}$ & 12.9 & $\mathbf{m}$ \\
\hline Belgium (Fr.) ${ }^{1}$ & 9.4 & 8.1 & 9.3 & 15.2 & 13.7 & 11.6 & 13.7 & 18.2 \\
\hline Canada & $\mathrm{m}$ & 17.9 & 14.0 & -21.7 & $\mathrm{~m}$ & 22.3 & 17.4 & -22.1 \\
\hline Chile $^{1}$ & 25.9 & 25.1 & 24.3 & -3.2 & $\mathrm{~m}$ & 24.6 & 22.9 & -7.1 \\
\hline Czech Republic ${ }^{2}$ & 13.5 & 11.2 & 11.2 & -0.2 & 18.8 & 14.9 & 15.8 & 5.4 \\
\hline Denmark & 11.9 & 11.5 & $\mathrm{~m}$ & $\mathbf{m}$ & 16.4 & 15.9 & $\mathrm{~m}$ & $\mathbf{m}$ \\
\hline England & 15.1 & 17.1 & $\mathrm{~m}$ & $\mathbf{m}$ & $\mathrm{m}$ & 22.5 & $\mathrm{~m}$ & $\mathbf{m}$ \\
\hline Estonia $^{1}$ & $\mathrm{~m}$ & 10.4 & 9.8 & -5.8 & $\mathrm{~m}$ & 13.2 & 13.0 & -1.7 \\
\hline Finland $^{1}$ & 10.0 & 9.8 & 9.0 & -7.5 & 13.4 & 12.7 & 12.9 & 1.1 \\
\hline France $^{1}$ & 14.2 & 15.0 & 15.4 & 2.5 & 21.0 & 22.5 & 23.6 & 4.7 \\
\hline Germany $^{1}$ & 15.5 & 14.9 & 13.6 & -8.5 & 17.9 & 17.4 & 15.7 & -10.1 \\
\hline Greece & 7.9 & $\mathrm{~m}$ & 7.3 & $\mathbf{m}$ & 18.1 & $\mathrm{~m}$ & 13.9 & m \\
\hline Hungary $^{1}$ & 10.4 & 10.7 & 10.4 & -2.5 & 13.4 & 11.9 & 12.3 & 3.8 \\
\hline Iceland $^{1}$ & 11.3 & 10.3 & 10.5 & 2.1 & 14.7 & 16.0 & 14.1 & -11.6 \\
\hline Ireland $^{1}$ & 15.5 & 14.4 & 13.9 & -3.1 & 17.9 & 18.2 & 17.8 & -2.5 \\
\hline Israel $^{1}$ & 13.4 & 12.8 & 13.5 & 5.6 & 22.4 & 20.9 & 21.0 & 0.4 \\
\hline Italy $^{1}$ & 10.1 & 11.9 & 11.7 & -1.7 & 17.0 & 19.3 & 18.8 & -2.7 \\
\hline $\mathrm{Japan}^{1}$ & 15.1 & 14.4 & 13.9 & -3.5 & 26.0 & 21.0 & 20.5 & -2.6 \\
\hline Korea $^{1}$ & 20.8 & 19.7 & 17.5 & -11.1 & 29.0 & 27.0 & 26.5 & -1.9 \\
\hline Luxembourg ${ }^{1}$ & 9.0 & 9.1 & 8.6 & -5.8 & 11.0 & 13.1 & 9.8 & -24.9 \\
\hline Mexico $^{1}$ & 33.7 & 32.7 & 32.2 & -1.4 & 37.6 & 36.5 & 35.9 & -1.4 \\
\hline Netherlands & 16.2 & 16.5 & 16.0 & -3.0 & 23.1 & 22.0 & $\mathrm{~m}$ & $\mathbf{m}$ \\
\hline New Zealand & 16.8 & 16.3 & 16.4 & 0.5 & $\mathrm{~m}$ & $\mathrm{~m}$ & $\mathrm{~m}$ & $\mathbf{m}$ \\
\hline Norway $^{1}$ & 10.2 & 9.9 & 9.8 & -0.7 & 12.9 & 12.7 & 12.9 & 1.6 \\
\hline Poland $^{1}$ & 12.7 & 12.7 & 9.9 & -22.0 & $\mathrm{~m}$ & 17.0 & 14.4 & -14.8 \\
\hline Portugal $^{1}$ & 7.5 & 7.9 & 10.4 & 31.2 & 10.6 & 9.5 & 15.3 & 61.1 \\
\hline Scotland & 15.1 & 17.1 & $\mathrm{~m}$ & $\mathbf{m}$ & $\mathrm{m}$ & $\mathrm{m}$ & $\mathrm{m}$ & m \\
\hline Slovak Republic ${ }^{1}$ & 14.1 & 13.6 & 12.5 & -8.2 & $\mathrm{~m}$ & 17.1 & 16.0 & -6.6 \\
\hline Slovenia $^{1}$ & 11.1 & 8.0 & 8.2 & 2.8 & 14.0 & 10.4 & 10.0 & -3.5 \\
\hline Spain $^{1}$ & 12.5 & 10.1 & 11.6 & 14.8 & 16.8 & 14.9 & 17.2 & 16.1 \\
\hline Sweden & 12.0 & 11.4 & 12.0 & 5.2 & $\mathrm{~m}$ & $\mathrm{~m}$ & $\mathrm{~m}$ & $\mathbf{m}$ \\
\hline Switzerland & 11.7 & 11.8 & 11.8 & -0.1 & $\mathrm{~m}$ & $\mathrm{~m}$ & $\mathrm{~m}$ & $\mathbf{m}$ \\
\hline Turkey & $\mathrm{m}$ & $\mathrm{m}$ & 19.3 & $\mathbf{m}$ & $\mathrm{m}$ & $\mathrm{m}$ & 32.1 & $\mathbf{m}$ \\
\hline United States & 15.1 & 14.0 & 15.4 & 9.9 & $\mathrm{~m}$ & $\mathrm{~m}$ & 15.8 & $\mathbf{m}$ \\
\hline OECD average & 13.6 & 13.4 & 13.0 & -0.3 & 18.1 & 17.6 & 17.3 & -0.2 \\
\hline $\begin{array}{l}\text { Average for } 23 \text { countries } \\
\text { with all data available } \\
\text { for } 2010 \text { and } 2013\end{array}$ & $\sim$ & 13.2 & 12.9 & -1.8 & $\sim$ & 15.5 & 17.1 & 10.4 \\
\hline
\end{tabular}

Note: Data in this table come either from chapter D (for 2005, 2010 and 2013 data relating to salaries of teachers and teaching time, 2013 data on ratio of student to teaching staff) or from 2007 and 2012 editions of Education at a Glance (data on ratio of student to teaching staff and instruction time).

Data for 2013 instruction time refer to 2014 data from the 2014 edition of Education at a Glance. Please see notes on these data in these tables.

1. Countries with all data available for both 2010 and 2013.

2. Current instruction time for 2000 and 2005, minimum instruction time for 2013

Source: OECD. See Annex 3 for notes (www.oecd.org/education/education-at-a-glance-19991487.htm).

Please refer to the Reader's Guide for information concerning symbols for missing data and abbreviations.

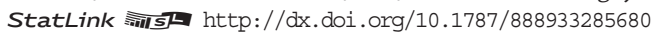


Table B7.3. Contribution of various factors to salary cost of teachers per student in primary education (2005, 2010 and 2013)

In equivalent USD, converted using PPPs for private consumption

\begin{tabular}{|c|c|c|c|c|c|c|c|c|}
\hline & \multirow{2}{*}{\multicolumn{3}{|c|}{$\begin{array}{l}\text { Salary cost of teachers } \\
\text { per student }\end{array}$}} & \multirow{3}{*}{$\begin{array}{c}\text { Difference } \\
\text { (in USD) from } \\
\text { the } 2013 \\
\text { OECD average } \\
\text { of } \\
\text { USD } 2 \mathbf{6 7 7} \\
2013\end{array}$} & \multicolumn{4}{|c|}{$\begin{array}{l}\text { Contribution of the underlying factors to the difference } \\
\text { from the OECD average }\end{array}$} \\
\hline & & & & & \multirow[t]{2}{*}{$\begin{array}{c}\text { Effect (in USD) } \\
\text { of teachers' salary } \\
\text { below/above } \\
\text { the } 2013 \\
\text { OECD average } \\
\text { of } \\
\text { USD } 39960 \\
2013\end{array}$} & \multirow[t]{2}{*}{$\begin{array}{c}\begin{array}{c}\text { Effect (in USD) } \\
\text { of instruction time } \\
\text { (for students) } \\
\text { below/above } \\
\text { the } 2013 \\
\text { OECD average } \\
\text { of } \\
789 \text { hours }\end{array} \\
2013\end{array}$} & \multirow[t]{2}{*}{$\begin{array}{c}\text { Effect (in USD) } \\
\text { of teaching time } \\
\text { (for teachers) } \\
\text { below/above } \\
\text { the } 2013 \\
\begin{array}{c}\text { OECD average } \\
\text { of }\end{array} \\
770 \text { hours } \\
2013\end{array}$} & \multirow[t]{2}{*}{ 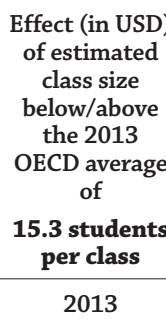 } \\
\hline & 2005 & 2010 & 2013 & & & & & \\
\hline & (1) & (2) & (3) & $(4)=(5)+(6)+(7)+(8)$ & (5) & (6) & (7) & (8) \\
\hline Australia & 3125 & 3373 & 3608 & 931 & 1082 & 781 & -424 & -508 \\
\hline Austria & 3007 & 3623 & 3609 & 932 & 232 & -355 & -39 & 1094 \\
\hline Belgium (Fl.) & $\mathrm{m}$ & $\mathrm{m}$ & 3842 & 1165 & 636 & 131 & 75 & 324 \\
\hline Belgium (Fr.) & 3477 & 3720 & 3739 & 1062 & 541 & 235 & 208 & 78 \\
\hline Canada & $\mathrm{m}$ & $\mathrm{m}$ & 4755 & 2078 & 1853 & 563 & -124 & -214 \\
\hline Chile & $\mathrm{m}$ & 1046 & 1181 & -1496 & -753 & 553 & -710 & -585 \\
\hline Czech Republic & $\mathrm{m}$ & $\mathrm{m}$ & 973 & -1704 & -1301 & -270 & -127 & -7 \\
\hline Denmark & 4343 & 4738 & $\mathrm{~m}$ & $\mathbf{m}$ & $\mathrm{m}$ & $\mathrm{m}$ & $\mathrm{m}$ & $\mathrm{m}$ \\
\hline England & $\mathrm{m}$ & 2537 & $\mathrm{~m}$ & $\mathbf{m}$ & $\mathrm{m}$ & $\mathrm{m}$ & $\mathrm{m}$ & $\mathrm{m}$ \\
\hline Estonia & $\mathrm{m}$ & 1058 & 1015 & -1662 & -1931 & -333 & 420 & 181 \\
\hline Finland & 2329 & 2942 & 3008 & 331 & -19 & -635 & 368 & 616 \\
\hline France & 1845 & 1862 & 1735 & -943 & -384 & 200 & -397 & -362 \\
\hline Germany & 3110 & 3643 & 4047 & 1370 & 1529 & -490 & -130 & 462 \\
\hline Greece & 3150 & $\mathrm{~m}$ & 2720 & 43 & -1209 & -20 & 834 & 438 \\
\hline Hungary & 1817 & 1406 & 1229 & -1448 & -2188 & -511 & 527 & 723 \\
\hline Iceland & 3108 & 3246 & 2985 & 308 & -716 & -228 & 601 & 651 \\
\hline Ireland & 2866 & 3714 & 3426 & 749 & 1035 & 456 & -534 & -208 \\
\hline Israel & 1368 & 1412 & 1956 & -721 & -672 & 452 & -202 & -298 \\
\hline Italy & 3339 & 3120 & 2692 & 15 & -497 & 328 & 62 & 122 \\
\hline Japan & 2670 & 2620 & 2790 & 113 & 535 & -94 & 122 & -450 \\
\hline Korea & 1902 & 2349 & 2981 & 304 & 729 & -565 & 409 & -269 \\
\hline Luxembourg & $\mathrm{m}$ & 9940 & 11674 & 8997 & 5648 & 1033 & -337 & 2653 \\
\hline Mexico & 860 & 892 & 958 & -1719 & -691 & 25 & -67 & -986 \\
\hline Netherlands & $\mathrm{m}$ & $\mathrm{m}$ & 3258 & 581 & 899 & 524 & -569 & -274 \\
\hline New Zealand & $\mathrm{m}$ & $\mathrm{m}$ & $\mathrm{m}$ & $\mathbf{m}$ & $\mathrm{m}$ & $\mathrm{m}$ & $\mathrm{m}$ & $\mathrm{m}$ \\
\hline Norway & 3551 & 3931 & 4307 & 1630 & 375 & -186 & 132 & 1309 \\
\hline Poland & $\mathrm{m}$ & 2322 & 2247 & -430 & -1191 & -551 & 515 & 797 \\
\hline Portugal & 4003 & 3905 & 2777 & 99 & -235 & 60 & 82 & 193 \\
\hline Scotland & $\mathrm{m}$ & $\mathrm{m}$ & $\mathrm{m}$ & $\mathbf{m}$ & $\mathrm{m}$ & $\mathrm{m}$ & $\mathrm{m}$ & $\mathrm{m}$ \\
\hline Slovak Republic & $\mathrm{m}$ & 842 & 924 & -1753 & -1535 & -259 & -137 & 177 \\
\hline Slovenia & 2549 & 2580 & 2392 & -285 & -111 & -437 & 524 & -261 \\
\hline Spain & 3094 & 3594 & 3067 & 390 & 156 & -5 & -386 & 625 \\
\hline Sweden & $\mathrm{m}$ & $\mathrm{m}$ & $\mathrm{m}$ & $\mathbf{m}$ & $\mathrm{m}$ & $\mathrm{m}$ & $\mathrm{m}$ & $\mathrm{m}$ \\
\hline Switzerland & $\mathrm{m}$ & $\mathrm{m}$ & $\mathrm{m}$ & $\mathbf{m}$ & $\mathrm{m}$ & $\mathrm{m}$ & $\mathrm{m}$ & $\mathrm{m}$ \\
\hline Turkey & 921 & 1251 & 1368 & -1309 & -753 & -180 & 133 & -509 \\
\hline United States & $\mathrm{m}$ & $\mathrm{m}$ & $\mathrm{m}$ & $\mathbf{m}$ & $\mathrm{m}$ & $\mathrm{m}$ & $\mathrm{m}$ & $\mathrm{m}$ \\
\hline $\begin{array}{l}\text { OECD average } \\
\text { (countries with } \\
\text { available data for both } \\
2010 \text { and 2013) }\end{array}$ & $\sim$ & 2550 & 2616 & $\sim$ & $\sim$ & $\sim$ & $\sim$ & $\sim$ \\
\hline
\end{tabular}

Source: OECD. See Annex 3 for notes (www.oecd.org/education/education-at-a-glance-19991487.htm). Please refer to the Reader's Guide for information concerning symbols for missing data and abbreviations.

StatLink iㅔ S1 http://dx.doi.org/10.1787/888933285697 
Table B7.4. Contribution of various factors to salary cost of teachers per student in lower secondary education (2005, 2010 and 2013)

In equivalent USD, converted using PPPs for private consumption

\begin{tabular}{|c|c|c|c|c|c|c|c|c|}
\hline & \multirow{2}{*}{\multicolumn{3}{|c|}{$\begin{array}{l}\text { Salary cost of teacher } \\
\text { per student }\end{array}$}} & \multirow[b]{2}{*}{\begin{tabular}{|} 
Difference (in USD) \\
from the 2013 \\
OECD average \\
of \\
USD $3 \mathbf{3 5 0}$
\end{tabular}} & \multicolumn{4}{|c|}{$\begin{array}{l}\text { Contribution of the underlying factors to the difference } \\
\text { from the OECD average }\end{array}$} \\
\hline & & & & & $\begin{array}{c}\text { Effect (in USD) } \\
\text { of teachers' salary } \\
\text { below/above } \\
\text { the } 2013 \\
\text { OECD average } \\
\text { of } \\
\text { USD } 43 \mathbf{6 3 4}\end{array}$ & $\begin{array}{c}\text { Effect (in USD) } \\
\text { of instruction time } \\
\text { (for students) } \\
\text { below/above } \\
\text { the } 2013 \\
\text { OECD average } \\
\text { of } \\
905 \text { hours }\end{array}$ & $\begin{array}{c}\text { Effect (in USD) } \\
\text { of teaching time } \\
\text { (for teachers) } \\
\text { below/above } \\
\text { the } 2013 \\
\text { OECD average } \\
\text { of } \\
694 \text { hours }\end{array}$ & $\begin{array}{c}\text { Effect (in USD) } \\
\text { of estimated } \\
\text { class size } \\
\text { below/above } \\
\text { the } 2013 \\
\text { OECD average } \\
\text { of } \\
17 \text { students } \\
\text { per class }\end{array}$ \\
\hline & 2005 & 2010 & 2013 & 2013 & 2013 & 2013 & 2013 & 2013 \\
\hline & (1) & (2) & (3) & $(4)=(5)+(6)+(7)+(8)$ & (5) & (6) & (7) & (8) \\
\hline Australia & 4185 & 4414 & 4684 & 1335 & 1019 & 458 & -681 & 538 \\
\hline Austria & 4341 & 5167 & 5191 & 1841 & 280 & -25 & 567 & 1019 \\
\hline Belgium (Fl.) & $\mathrm{m}$ & $\mathrm{m}$ & 5218 & 1869 & 464 & 105 & 154 & 1146 \\
\hline Belgium (Fr.) & 4758 & 5691 & 5078 & 1728 & 343 & 290 & 203 & 893 \\
\hline Canada & $\mathrm{m}$ & $\mathrm{m}$ & 4755 & 1406 & 1708 & 71 & -280 & -93 \\
\hline Chile & $\mathrm{m}$ & 1027 & 1095 & -2254 & -1001 & 342 & -985 & -610 \\
\hline Czech Republic & $\mathrm{m}$ & $\mathrm{m}$ & 1633 & -1716 & -2106 & -90 & 287 & 193 \\
\hline Denmark & 4343 & 4806 & $\mathrm{~m}$ & $\mathbf{m}$ & $\mathrm{m}$ & $\mathrm{m}$ & $\mathrm{m}$ & $\mathrm{m}$ \\
\hline England & $\mathrm{m}$ & 2937 & $\mathrm{~m}$ & $\mathbf{m}$ & $\mathrm{m}$ & $\mathrm{m}$ & $\mathrm{m}$ & $\mathrm{m}$ \\
\hline Estonia & $\mathrm{m}$ & 1332 & 1350 & -2000 & -2721 & -237 & 285 & 673 \\
\hline Finland & 4179 & 4566 & 4749 & 1400 & -71 & -283 & 638 & 1115 \\
\hline France & 2722 & 2518 & 2374 & -975 & -503 & 260 & 197 & -929 \\
\hline Germany & 4081 & 4534 & 5047 & 1698 & 1885 & -187 & -339 & 339 \\
\hline Greece & 4426 & $\mathrm{~m}$ & 3515 & 166 & -1895 & -513 & 1849 & 724 \\
\hline Hungary & 1857 & 1415 & 1252 & -2098 & -2669 & -579 & 354 & 796 \\
\hline Iceland & 3108 & 3246 & 2970 & -380 & -1075 & -245 & 341 & 599 \\
\hline Ireland & 3350 & 4149 & 4063 & 713 & 968 & 120 & -213 & -162 \\
\hline Israel & 1800 & 2072 & 2131 & -1218 & -1132 & 284 & 208 & -578 \\
\hline Italy & 3802 & 3243 & 3100 & -250 & -604 & 290 & 389 & -324 \\
\hline Japan & 3430 & 3339 & 3491 & 141 & 367 & -39 & 455 & -642 \\
\hline Korea & 2554 & 2513 & 2941 & -408 & 533 & -235 & 710 & -1416 \\
\hline Luxembourg & 10692 & 11780 & 12821 & 9471 & 6502 & -537 & -491 & 3998 \\
\hline Mexico & 923 & 986 & 1057 & -2293 & -514 & 552 & -842 & -1488 \\
\hline Netherlands & $\mathrm{m}$ & $\mathrm{m}$ & 4176 & 826 & 1619 & 380 & -297 & -875 \\
\hline New Zealand & $\mathrm{m}$ & $\mathrm{m}$ & $\mathrm{m}$ & $\mathbf{m}$ & $\mathrm{m}$ & $\mathrm{m}$ & $\mathrm{m}$ & $\mathrm{m}$ \\
\hline Norway & 3785 & 4145 & 4525 & 1176 & 81 & -166 & 179 & 1082 \\
\hline Poland & $\mathrm{m}$ & 1823 & 2519 & -830 & -1666 & -335 & 677 & 493 \\
\hline Portugal & 5763 & 5350 & 3516 & 166 & -601 & -51 & 452 & 366 \\
\hline Scotland & $\mathrm{m}$ & $\mathrm{m}$ & $\mathrm{m}$ & $\mathbf{m}$ & $\mathrm{m}$ & $\mathrm{m}$ & $\mathrm{m}$ & $\mathrm{m}$ \\
\hline Slovak Republic & $\mathrm{m}$ & 1056 & 1254 & -2096 & -2198 & -206 & 170 & 138 \\
\hline Slovenia & 3447 & 5243 & 4661 & 1312 & -538 & -678 & 413 & 2114 \\
\hline Spain & 4062 & 5345 & 4052 & 702 & 267 & 586 & -99 & -52 \\
\hline Sweden & $\mathrm{m}$ & $\mathrm{m}$ & $\mathrm{m}$ & $\mathbf{m}$ & $\mathrm{m}$ & $\mathrm{m}$ & $\mathrm{m}$ & $\mathrm{m}$ \\
\hline Switzerland & $\mathrm{m}$ & $\mathrm{m}$ & $\mathrm{m}$ & $\mathbf{m}$ & $\mathrm{m}$ & $\mathrm{m}$ & $\mathrm{m}$ & $\mathrm{m}$ \\
\hline Turkey & $\mathrm{m}$ & $\mathrm{m}$ & 1459 & -1891 & -1029 & -180 & 787 & -1470 \\
\hline United States & $\mathrm{m}$ & $\mathrm{m}$ & 3967 & 617 & 1240 & 410 & -1296 & 262 \\
\hline $\begin{array}{l}\text { OECD average } \\
\text { (countries with } \\
\text { available data for both } \\
2010 \text { and 2013) }\end{array}$ & $\sim$ & 3185 & 3215 & $\sim$ & $\sim$ & $\sim$ & $\sim$ & \\
\hline
\end{tabular}

Source: OECD. See Annex 3 for notes (www.oecd.org/education/education-at-a-glance-19991487.htm).

Please refer to the Reader's Guide for information concerning symbols for missing data and abbreviations.

StatLink 제일 http://dx.doi.org/10.1787/888933285709 
Table B7.5. Contribution of various factors to salary cost of teachers per student in upper secondary education (2013)

In equivalent USD, converted using PPPs for private consumption

\begin{tabular}{|c|c|c|c|c|c|c|}
\hline & \multirow[b]{2}{*}{$\begin{array}{l}\text { Salary cost } \\
\text { of teacher } \\
\text { per student }\end{array}$} & \multirow[b]{2}{*}{$\begin{array}{l}\text { Difference from } \\
\text { the OECD average } \\
\text { of } \\
\text { USD } 3749\end{array}$} & \multicolumn{4}{|c|}{ Contribution of the underlying factors to the difference from the OECD average } \\
\hline & & & $\begin{array}{l}\text { Effect (in USD) } \\
\text { of teachers' salary } \\
\text { below/above } \\
\text { the OECD average } \\
\text { of } \\
\text { USD } 48248\end{array}$ & $\begin{array}{l}\text { Effect (in USD) } \\
\text { of instruction time } \\
\text { (for students) } \\
\text { below/above } \\
\text { the OECD average } \\
\text { of } \\
921 \text { hours }\end{array}$ & $\begin{array}{l}\text { Effect (in USD) } \\
\text { of teaching time } \\
\text { (for teachers) } \\
\text { below/above } \\
\text { the OECD average } \\
\text { of } \\
674 \text { hours }\end{array}$ & $\begin{array}{l}\text { Effect (in USD) } \\
\text { of estimated class } \\
\text { size below/above } \\
\text { the OECD average } \\
\text { of } \\
17.6 \text { students } \\
\text { per class }\end{array}$ \\
\hline & (1) & $(2)=(3)+(4)+(5)+(6)$ & (3) & (4) & (5) & (6) \\
\hline \multirow{2}{*}{$\begin{array}{l}\text { Australia } \\
\text { Austria }\end{array}$} & $\mathrm{m}$ & $\mathbf{m}$ & $\mathrm{m}$ & $\mathrm{m}$ & $\mathrm{m}$ & $\mathrm{m}$ \\
\hline & 5093 & 1344 & 173 & 73 & 595 & 503 \\
\hline Belgium (Fl.) & 6344 & 2596 & 1283 & 40 & 378 & 895 \\
\hline Belgium (Fr.) & 6167 & 2419 & 1130 & -397 & 562 & 1124 \\
\hline Canada & 4839 & 1091 & 1408 & -61 & -430 & 173 \\
\hline Chile & 1124 & -2624 & -1183 & 557 & -1136 & -863 \\
\hline Denmark & $\mathrm{m}$ & $\mathbf{m}$ & $\mathrm{m}$ & $\mathrm{m}$ & $\mathrm{m}$ & $\mathrm{m}$ \\
\hline England & $\mathrm{m}$ & m & $\mathrm{m}$ & $\mathrm{m}$ & $\mathrm{m}$ & $\mathrm{m}$ \\
\hline Estonia & $\mathrm{m}$ & $\mathbf{m}$ & $\mathrm{m}$ & $\mathrm{m}$ & $\mathrm{m}$ & $\mathrm{m}$ \\
\hline Finland & $\mathrm{m}$ & $\mathbf{m}$ & $\mathrm{m}$ & $\mathrm{m}$ & $\mathrm{m}$ & $\mathrm{m}$ \\
\hline France & 3643 & -105 & -998 & 440 & 147 & 305 \\
\hline Germany & 5573 & 1824 & 1948 & 63 & -274 & 88 \\
\hline Greece & m & $\mathbf{m}$ & m & $\mathrm{m}$ & $\mathrm{m}$ & $\mathrm{m}$ \\
\hline Hungary & 1287 & -2462 & -2638 & -256 & 312 & 120 \\
\hline Iceland & $\mathrm{m}$ & m & $\mathrm{m}$ & $\mathrm{m}$ & $\mathrm{m}$ & $\mathrm{m}$ \\
\hline Ireland & 4063 & 315 & 629 & 62 & -337 & -39 \\
\hline Israel & 2391 & -1358 & -1929 & 294 & 530 & -253 \\
\hline Italy & 2963 & -786 & -868 & -60 & 306 & -164 \\
\hline Japan & $\mathrm{m}$ & m & m & $\mathrm{m}$ & $\mathrm{m}$ & $\mathrm{m}$ \\
\hline Mexico & $\mathrm{m}$ & m & $\mathrm{m}$ & $\mathrm{m}$ & $\mathrm{m}$ & $\mathrm{m}$ \\
\hline Netherlands & 3593 & -156 & 1216 & 19 & -397 & -994 \\
\hline New Zealand & $\mathrm{m}$ & m & $\mathrm{m}$ & $\mathrm{m}$ & $\mathrm{m}$ & $\mathrm{m}$ \\
\hline Norway & $\mathrm{m}$ & $\mathbf{m}$ & $\mathrm{m}$ & $\mathrm{m}$ & $\mathrm{m}$ & $\mathrm{m}$ \\
\hline Poland & $\mathrm{m}$ & $\mathbf{m}$ & $\mathrm{m}$ & $\mathrm{m}$ & $\mathrm{m}$ & $\mathrm{m}$ \\
\hline Portugal & 4366 & 618 & -1145 & -560 & 424 & 1899 \\
\hline Scotland & $\mathrm{m}$ & $\mathbf{m}$ & $\mathrm{m}$ & $\mathrm{m}$ & $\mathrm{m}$ & $\mathrm{m}$ \\
\hline Slovak Republic & 1152 & -2596 & -2470 & -112 & 217 & -231 \\
\hline Slovenia & $\mathrm{m}$ & $\mathbf{m}$ & $\mathrm{m}$ & $\mathrm{m}$ & $\mathrm{m}$ & $\mathrm{m}$ \\
\hline Spain & $\mathrm{m}$ & $\mathbf{m}$ & $\mathrm{m}$ & $\mathrm{m}$ & $\mathrm{m}$ & $\mathrm{m}$ \\
\hline Sweden & $\mathrm{m}$ & $\mathbf{m}$ & $\mathrm{m}$ & $\mathrm{m}$ & $\mathrm{m}$ & $\mathrm{m}$ \\
\hline Switzerland & $\mathrm{m}$ & $\mathbf{m}$ & $\mathrm{m}$ & $\mathrm{m}$ & $\mathrm{m}$ & $\mathrm{m}$ \\
\hline Turkey & 1800 & -1949 & -1452 & -259 & 821 & -1059 \\
\hline United States & $\mathrm{m}$ & $\mathbf{m}$ & $\mathrm{m}$ & $\mathrm{m}$ & $\mathrm{m}$ & $\mathrm{m}$ \\
\hline
\end{tabular}

Source: OECD. See Annex 3 for notes (www.oecd.org/education/education-at-a-glance-19991487.htm).

Please refer to the Reader's Guide for information concerning symbols for missing data and abbreviations.

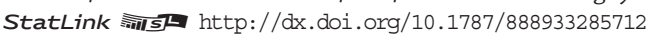




\section{Chapter}

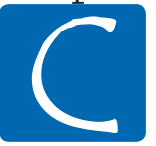

\section{ACCESS TO EDUCATION, PARTICIPATION AND PROGRESSION}

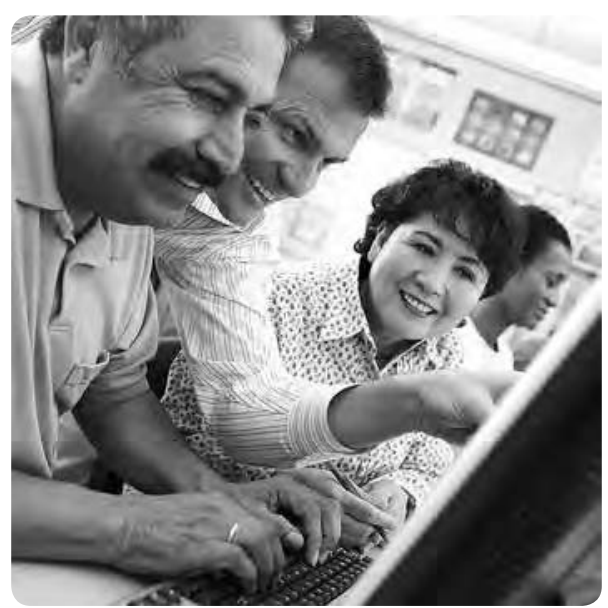

Indicator C1 Who participates in education?

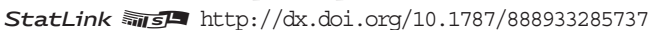

Indicator C2 How do early childhood education systems differ around the world? StatLink 部 SD http://dx.doi.org/10.1787/888933285801

Indicator C3 How many students are expected to enter tertiary education?

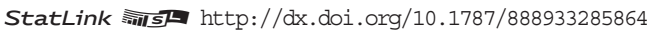

Indicator C4 Who studies abroad and where?

StatLink 需s http://dx.doi.org/10.1787/888933285911

Indicator C5 Transition from school to work: Where are the 15-29 year-olds? StatLink 게대 http://dx.doi.org/10.1787/888933285971

Indicator C6 How many adults participate in education and learning?

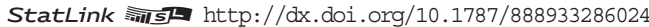




\section{WHO PARTICIPATES IN EDUCATION?}

- Access to education for 5-14 year-olds is universal in all OECD and most partner countries with available data.

INDICATOR C1

- In 2013, enrolment rates among 15-19 year-olds were greater than $70 \%$ in 32 of the 37 OECD and partner countries with available data.

- In almost all OECD countries, more than one in five 20-29 year-olds participated in education in 2013.

Chart C1.1. Enrolment rates of 15-19 and 20-29 year-olds (2013)

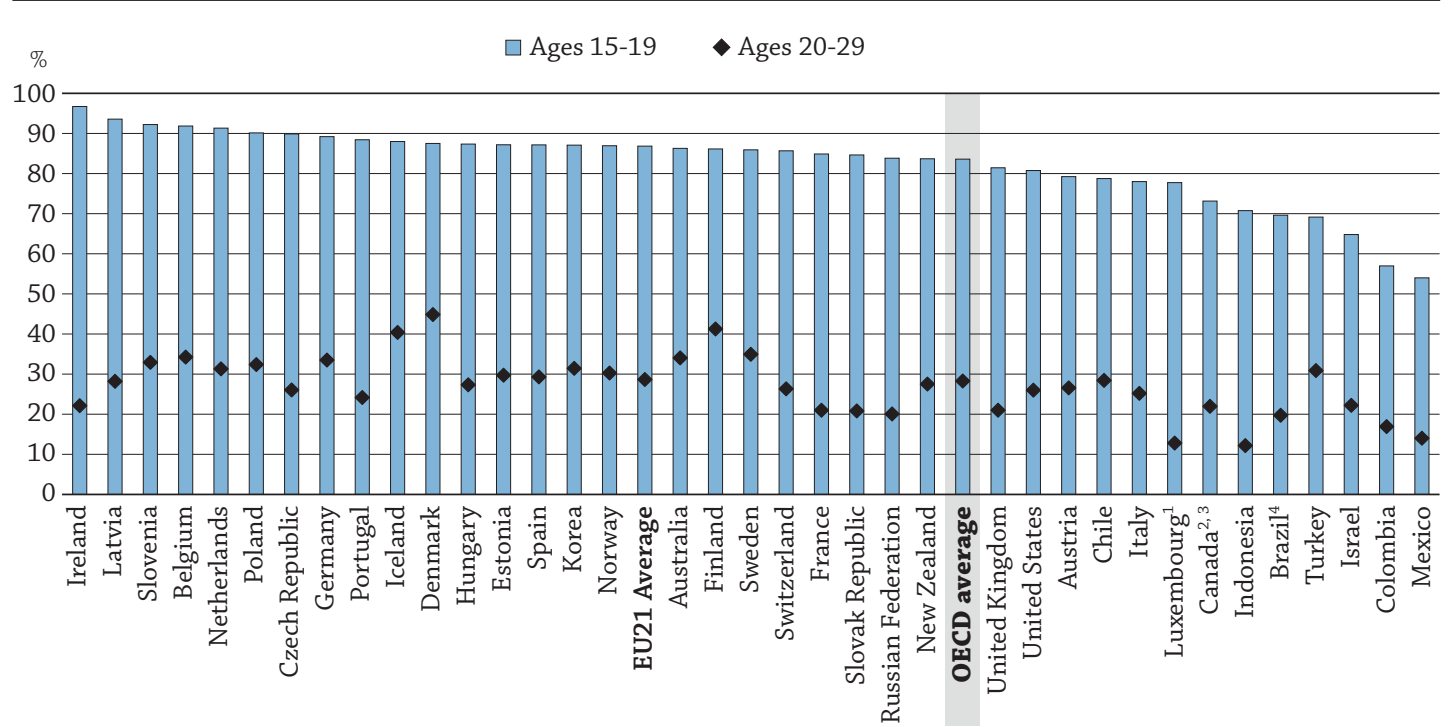

1. Underestimated because many resident students go to school in the neighbouring countries.

2. Excludes post-secondary non-tertiary education.

3. Year of reference 2012.

4. Excludes enrolments in ISCED 7 and 8.

Countries are ranked in descending order of the enrolment rates of 15-19 year-olds.

Source: OECD. Table C1.1a. See Annex 3 for notes (www.oecd.org/education/education-at-a-glance- 19991487.htm).

StatLink 제개 http://dx.doi.org/10.1787/888933284155

\section{Context}

In times of economic hardship, the advantage of education for labour-market prospects becomes even clearer. Education systems in OECD and most G20 countries now provide universal access to basic education, such that both pre-primary and upper secondary education are becoming universal in most countries (see also Indicator C2). The expansion of upper secondary education is both a response to increasing demand and a result of various policy changes, ranging from more flexible curricula and a reshaping of vocational studies, to efforts to expand access to education to the entire population. While the same changes have been made to tertiary education, participation rates at the higher level of education are significantly lower.

Upper secondary education has become the minimum qualification for a smooth and successful transition into the labour market; attainment of this level of education reduces the risk of unemployment (see Indicator A5). Successful completion of upper secondary programmes is vital for addressing equity issues (OECD, 2010a; OECD, 2011), but graduation rates vary widely among OECD countries (see Indicator A2). To help ensure good returns for individuals, education systems must be able to help students acquire the skills they need both to make them employable in the short term, and to enable them to pursue learning throughout their working lives (OECD, 2010b). The deep structural changes that have occurred in the global labour market over the past decades suggest that bettereducated individuals will continue to have an advantage as the labour market becomes increasingly more knowledge-based. 


\section{Other findings}

- Based on 2013 enrolment patterns, a 5-year-old in an OECD country can expect to participate in more than 17 years of full-time and part-time education, on average, before reaching the age of 40 . The expected duration of education ranged from 13 years in Saudi Arabia to 19 years or more in Australia, Belgium, Denmark, Finland, Iceland and Sweden.

- Across OECD countries in 2013 , at least $90 \%$ of the school-age population participated in an average of 13 years of formal education. Twenty-eight out of the 40 countries with available data were equal or above this OECD average, while 12 countries were below the average.

\section{Trends}

Between 2005 and 2013, enrolment rates for 15-19 year-olds in OECD countries increased, on average, from $82 \%$ to $84 \%$. While the rates increased by nearly 30 percentage points during this period in Turkey, they remained virtually unchanged in France, Hungary, Israel and the Slovak Republic (Table C1.6, available on line). In 2013, enrolment rates for 15-19 year-olds were still 70\% or lower in Brazil, Colombia, Israel, Mexico and Turkey. Among 20-29 year-olds, enrolment rates in OECD countries increased, on average, 4 percentage points, from $24 \%$ in 2005 to $28 \%$ in 2013. 


\section{Analysis}

In 20 of the 40 OECD and partner countries with available data in 2013, full enrolment in education (defined here as enrolment rates exceeding $90 \%$ of the population of the age range covering a certain level of studies) begins between the ages of 3 and 4 (age 2 in Denmark, Iceland and Norway); in the other 17 countries, full enrolment starts between the ages of 5 and 6, except in the Russian Federation and Saudi Arabia, where it starts at 7. In most OECD and partner countries, at least $75 \%$ of 3-4 year-olds were enrolled in either pre-primary or primary programmes (Table C1.1a and Indicator C2). In Belgium, Denmark, France, Iceland, Israel, Italy, New Zealand, Norway, Spain and the United Kingdom, enrolment rates among 3-4 year-olds reached at least 96\% in 2013.

\section{Box C1.1. Expected years in education}

The estimation of expected years in education includes enrolment in all forms of formal education, including non-continuous and incomplete participation. Thus, based on 2013 enrolment patterns, a 5-year-old in an OECD country can expect to participate in education for more than 17 years, on average, before reaching the age of 40. More specifically, this person can expect to be enrolled for around one year in early childhood education, nearly 6 years in primary education, 3 years in lower secondary education, 4 years in upper secondary education, 0.3 years in post-secondary non-tertiary education, and 3 years in tertiary education. This same student can expect to participate in full-time studies for 16 years and in part-time studies for around one year. Women can expect to be enrolled in full-time education for about 17 years while men can expect to be enrolled for 16 years, on average.

Among countries with available data, the expected number of years in education ranges from 15 years or less in Colombia, Indonesia, Japan, Luxembourg, Mexico and Saudi Arabia, to 19 years or more in Australia, Belgium, Denmark, Finland, Iceland and Sweden. (Table C1.5).

Enrolment in an education programme is not limited to a particular age range. Based on 2013 data, Australia, Belgium, Finland, Iceland, New Zealand and Sweden show significant shares of their adult populations particularly adults who are between 40 and 64 - participating in education. This is explained by larger parttime enrolments and/or by lifelong learning programmes in these countries. For instance, credit-based systems in Sweden allow adults to study selected parts of a programme in formal education as a way to upgrade their skills in a specific area.

Expected years in education is only an estimate of the potential number of years an individual may expect to be in education. This estimation is not comparable to educational attainment, and may also differ from projections of future attainment, because the time spent in a given programme may change within the population.

\section{Participation in compulsory education}

Compulsory education varies across countries. In 2013, the starting age of compulsory education ranged from age 4 in Brazil, Luxembourg and Mexico, to age 7 in Estonia, Finland, Indonesia, the Russian Federation, South Africa and Sweden. In the United Kingdom and the United States, the starting age ranged between ages 4 and 5 and ages 4 and 6, respectively; in Switzerland, the age range was from 5 to 7 (as the reference date for the data collection is 1 January, children could be a year younger when they enter compulsory education).

Compulsory education comprises primary and lower secondary programmes in all OECD countries, and upper secondary education in most of them, according to the theoretical age ranges associated with the different levels of education in each country. Enrolment rates among 5-14 year-olds are higher than $90 \%$, i.e. there is universal coverage of basic education, in nearly all OECD and partner countries with available data. In 2013, enrolment rates in 34 out of the 39 countries with available data for this age range were around 95\% or higher (Table C1.1a and Table X1.3).

\section{Participation in upper secondary education}

In recent years, countries have increased the diversity of their upper secondary programmes. This diversification is both a response to the growing demand for upper secondary education and a result of changes in curricula. Curricula have gradually evolved from separating general and vocational programmes to offering more comprehensive programmes that include both types of learning, leading to more flexible pathways into further education or the labour market. 
Based on 2013 data, enrolment rates among 15-19 year-olds, i.e. those typically in upper secondary programmes or in transition to upper levels of education, reached at least $80 \%$ in 26 of the 37 OECD and partner countries with available data. In Belgium, Ireland, Latvia, the Netherlands, Poland, and Slovenia, these rates were higher than $90 \%$ (Table C1.1a). By contrast, the proportion of people of this age group who were not enrolled in education exceeded $20 \%$ in Austria, Canada, Chile, Italy and Luxembourg, and exceeded $30 \%$ in Brazil and Turkey. In Israel, more than $35 \%$ of the age group were not enrolled in education, largely due to conscription, while in Colombia and Mexico this proportion exceeded 40\% (Table C1.1a and Chart C1.2).

\section{Chart C1.2. Distribution of 15-19 year-olds enrolled in upper secondary education, by programme orientation (2013)}

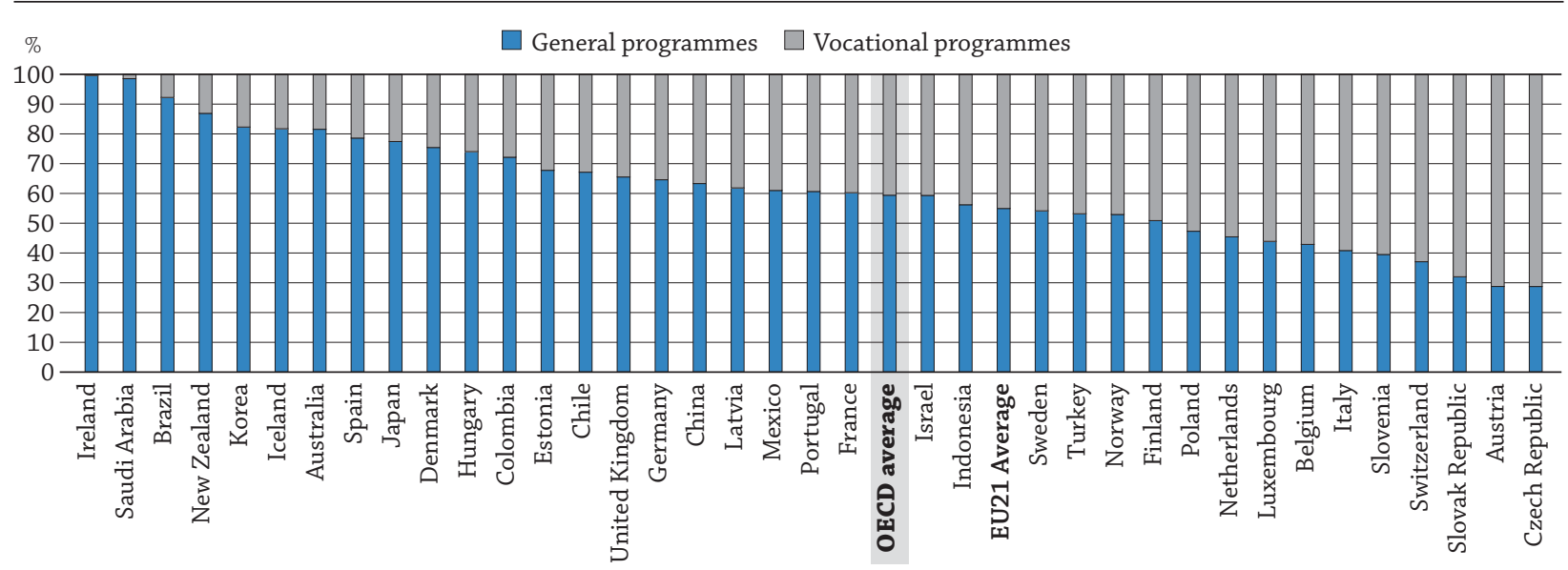

Countries are ranked in descending order of the share of students in general programmes.

Source: OECD. Table C1.2. See Annex 3 for notes (www.oecd.org/education/education-at-a-glance- 19991487.htm).

StatLink त्ताs http://dx.doi.org/10.1787/888933284162

In 2013 , at least $85 \%$ of 15 -year-olds in 36 of the 38 countries with available data were enrolled in secondary education (Table C1.1b, available on line).

The variation in upper secondary enrolment rates reflects different completion requirements or age limits. For example, in the Netherlands, students aged 20 years and older represent $42 \%$ of total enrolments in upper secondary vocational programmes (Table C1.2). Education policies, combined with other factors, such as longer programmes, grade repetition, and late entry into the labour market or participation in education while employed, among others, have resulted in larger numbers of older students participating in upper secondary education (see Indicator A2). Consequently, in some OECD countries, around one in five to one in three 20-year-olds is still enrolled in upper secondary education. This is the case in Denmark (33\%), Germany (23\%), Luxembourg (25\%), the Netherlands (27\%) and Switzerland (25\%) (Table C1.1b, available on line).

\section{Vocational education and training (VET) programmes}

Many countries have recently renewed their interest in vocational education and training (VET) programmes, as these programmes are seen as effective in developing skills among those who would otherwise lack qualifications to ensure a smooth and successful transition into the labour market (OECD, 2010a). Countries with well-established vocational and apprenticeship programmes have been more effective in holding the line on youth unemployment (see Indicator (5). At the same time, some consider vocational education a less-attractive option than academic education; and some research suggests that participation in vocational education increases the risk of unemployment at later ages (Hanushek et al., 2011).

In most countries, a student who successfully completes an apprenticeship programme is usually awarded an upper secondary or post-secondary qualification. In some countries, it is possible to earn higher qualifications, like the Advanced Diploma awarded in Australia. Vocational programmes in OECD countries offer different combinations of vocational studies along with apprenticeship programmes. Upper secondary students in many education systems can enrol in vocational programmes, but some OECD countries delay vocational training until students graduate from upper secondary education. For instance, while vocational programmes are offered as upper secondary education in Austria, Hungary and Spain, similar programmes are typically offered as post-secondary education in Canada (see Indicator A2). 
In more than one-third of the countries for which 2013 data are available, the percentage of students who participated in vocational programmes exceeded $50 \%$ of all students enrolled in upper secondary education - and this proportion was at least 70\% in Austria, the Czech Republic and Finland. In the other two-thirds of countries, more than 50\% of upper secondary students were enrolled in general programmes rather than in VET. This proportion was larger than 80\% in Brazil, Ireland, Korea, Saudi Arabia and South Africa (Table C1.2).

In most countries, vocational education at the upper secondary level is school-based only. However, some countries offer programmes that combine both school and work. Some $60 \%$ of all upper secondary students in Switzerland are enrolled in these combined vocational programmes as are more than $30 \%$ of all upper secondary students in Austria, China, Denmark, Germany and Latvia (Table C1.2).

\section{Participation of young adults in education}

In 2013, an average of 28\% of 20-29 year-olds in OECD countries were enrolled in some type of education. The largest proportions of this age group enrolled in education (more than 40\%) were found in Denmark and Finland. Meanwhile, in Indonesia, Luxembourg and Mexico less than 15\% of young adults in this age group were enrolled (Table C1.1a and Chart C1.1).

In most of the countries analysed, 20-year-olds are typically enrolled in tertiary education. In 2013 , nearly $37 \%$ of 20 -year-olds in OECD countries were enrolled in tertiary education, on average. In Korea, seven in ten 20-year-olds were enrolled in this level of education, whereas in Belgium, Ireland and Slovenia, at least one in two people of this age were enrolled. By contrast, fewer than one in four 20-year-olds in Brazil, China, Colombia, Denmark, Germany, Iceland, Israel, Luxembourg, Mexico, Sweden and Switzerland were enrolled in tertiary education (Table C1.1b, available on line).

Returning to or continuing studies is an option for adults who want to improve and diversify their skills and make themselves more adaptable to the changing demands of the labour market. In the current context of high unemployment and changing skills needs in the labour market, some countries, such as Chile, have established specific policies to encourage adults to pursue vocational studies.

\section{Gender differences}

Recent studies have emphasised the importance of having a more balanced approach to gender, given that half of the economic growth in OECD countries over the past 50 years can be attributed to higher educational attainment, which, in turn, has been achieved mainly because more girls and women are participating in all levels of education (OECD, 2012).

The gender gap in enrolment is relatively narrow for people younger than 20 , but it increases among older individuals. On average across OECD countries, $30 \%$ of $20-29$ year-old women and $27 \%$ of men the same age participated in education in 2013. There were important differences within countries. In Poland, for instance, 38\% of women were enrolled while only $27 \%$ of men were. In Poland, Slovenia and Sweden, the enrolment rate for women was at least 11 percentage points higher than that for men. In Korea, the 14 percentage-point difference in favour of men's enrolment rates compared with women's enrolment rates in 2013 is linked to delayed graduation among men completing their mandatory military service.

In most countries, enrolment rates among 30-39 year-olds were also higher among women than men. Australia, Finland, Iceland, New Zealand and Sweden had the highest rates of women of this age participating in education, with Sweden showing the widest gender gap, of almost eight percentage points (Table C1.1a).

\section{Part-time studies}

The new International Standard Classification of Education 2011 (ISCED 2011), used for the first time in this edition of Education at a Glance, allows for more disaggregated data for the tertiary level of education, among others. Instead of the ISCED-97 tertiary type A and type B programmes discussed in previous editions, tertiary education is now broken down into short-cycle tertiary programmes, bachelor's, master's and doctoral or equivalent programmes.

Students in tertiary education are more likely to enrol full time rather than part time, regardless of their choice of programme. In 2013, more than $77 \%$ of tertiary students, on average across OECD countries with available data, were enrolled full time, and in Austria, Colombia, the Czech Republic, Mexico, Portugal and Turkey, more than 95\% of students were. Students are more likely to be enrolled full time in bachelor's or equivalent programmes (OECD average of $80 \%)$. Although most of the students enrolled in short-cycle tertiary programmes are also enrolled full time (OECD average of $73 \%$ ), the proportion of these students is the smallest among all tertiary students. 
In $2013,27 \%$ of students in short-cycle tertiary programmes were enrolled part time, on average across OECD countries. In Australia and the United States, more than $50 \%$ of enrolments in these programmes were part time, in New Zealand, more than $60 \%$ were, in Belgium, $72 \%$ were, and in the United Kingdom, $89 \%$ of enrolments in short-cycle tertiary programmes were part time. There are, however, significant gender differences in some countries. In the Netherlands, for example, $37 \%$ of women were enrolled part time compared to $27 \%$ of men. In Germany, Ireland and New Zealand, the gender difference in these proportions is around eight percentage points (Table C1.3).

Part-time enrolments in bachelor's or equivalent programmes did not exceed full-time enrolments in any country except Sweden, where 55\% of students in these programmes study part time. In Poland, $47 \%$ of students at this level of education are enrolled part time. In 17 of the 31 countries with available data, more than $20 \%$ of enrolments in bachelor's or equivalent programmes were part time (Table C1.3).

Twenty-nine of the 33 OECD and partner countries with available data offered part-time enrolments in master's or equivalent programmes in 2013. More than $50 \%$ of students in master's or equivalent programmes in Finland, New Zealand and the Russian Federation chose to enrol part time - far more than the OECD average of 26\%. In Australia, Ireland, Luxembourg, Poland, the United Kingdom and the United States, more than $40 \%$ of students at this level of education also chose part-time enrolment (Table C1.3).

\section{The relative size of the public and private sectors}

In most OECD and partner countries, most students, from primary through tertiary education, are enrolled in public institutions. On average across OECD countries in 2013, around $90 \%$ of primary students, $86 \%$ of lower secondary students and $81 \%$ of upper secondary students were enrolled in public schools. At least $90 \%$ of all lower secondary students in 35 of the 39 countries with available data attended either public or government-dependent private institutions; and the same percentage was observed among upper secondary students in 31 of the 39 countries with data available. (Table C1.4a)

Based on the new ISCED 2011 classification, 69\% of tertiary students were enrolled in public institutions in 2013, on average across OECD countries. At least $90 \%$ of students in tertiary education were enrolled in public institutions in Australia, Denmark, Germany, Ireland, Italy, Saudi Arabia, South Africa, Sweden and Turkey. In contrast, less than $20 \%$ of tertiary students were enrolled in public institutions in Chile, Estonia, Israel, Korea, Latvia and the United Kingdom (where $100 \%$ of students were enrolled in government-dependent private institutions) (Table C1.4b and Chart C1.3).

\section{Chart C1.3. Distribution of students in tertiary education, by type of institutions (2013)}

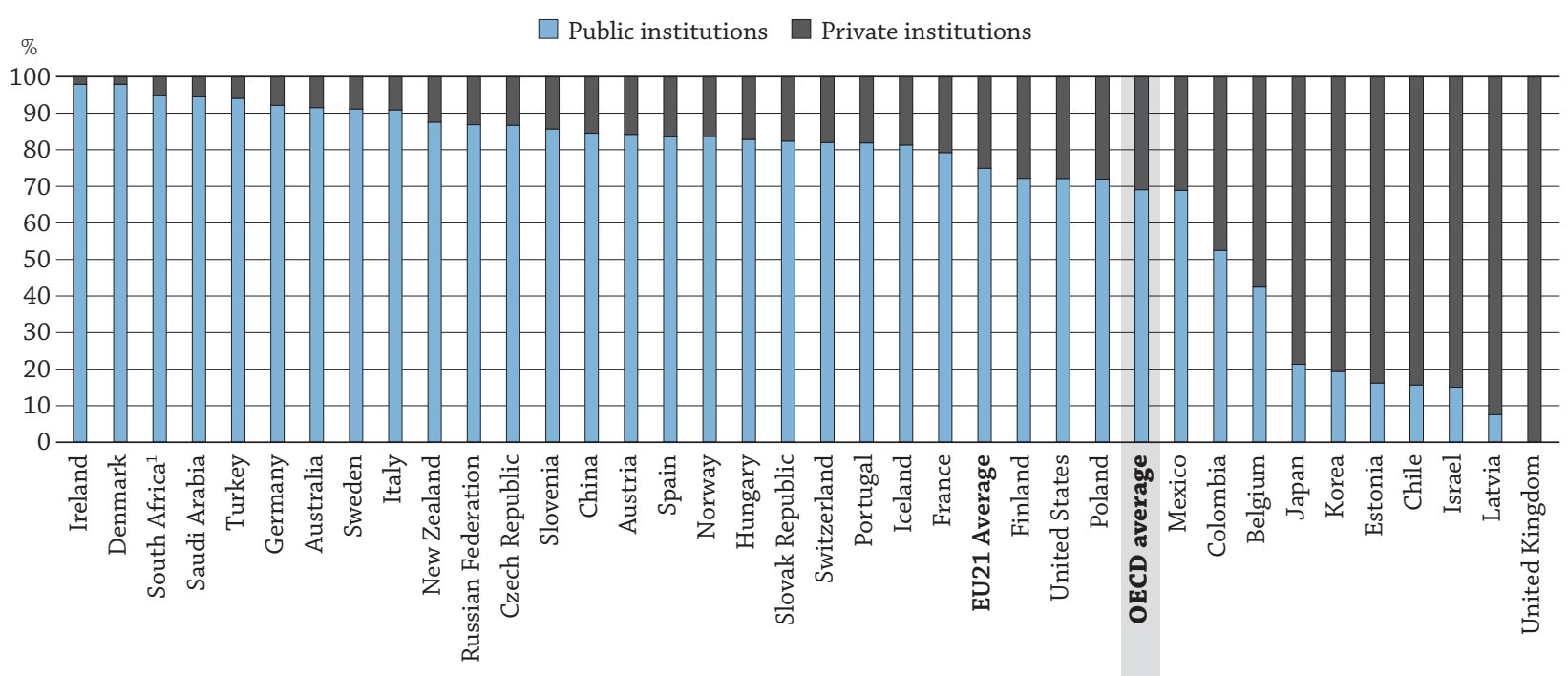

1. Year of reference 2012.

Countries are ranked in descending order of the share of students in public institutions.

Source: OECD. Table C1.4b. See Annex 3 for notes (www.oecd.org/education/education-at-a-glance- 19991487.htm).

StatLink 제대 http://dx.doi.org/10.1787/888933284178 
Public institutions provide $100 \%$ of short-cycle tertiary education in Finland, Luxembourg and Saudi Arabia, while in Denmark, Mexico, the Russian Federation, South Africa, Turkey and the United States, at least $90 \%$ of enrolments in these programmes are in public institutions (see Indicator C3 for details on the different fields of education that students choose for this level of education in these countries). Similarly, 95\% or more of enrolments in master's or equivalent programmes in Australia, China, Denmark, Germany, Ireland, New Zealand, Norway, Saudi Arabia, South Africa and Switzerland are in public institutions. All enrolments in doctoral or equivalent programmes in China, the Czech Republic, Denmark, Finland, Germany, the Netherlands, New Zealand, the Russian Federation, Saudi Arabia, South Africa and Switzerland are in public institutions (Table C1.4b).

\section{Definitions}

Intensity of participation refers to students' commitment of time to their studies. A full-time student is one whose commitment to study (both inside and outside of an educational institution) represents $75 \%$ or more of the school week, as it applies locally at that level of education, and if the student would normally be expected to be in the programme for the entire school academic year. A part-time student is one whose commitment is less that $75 \%$ of the school week, or one who is expected to be in the programme for less that the full school year.

Secondary programmes can be subdivided into three categories, based on the degree to which they are oriented towards a specific class of occupations or trades and lead to a qualification that is relevant to the labour market:

In combined school- and work-based programmes, less than $75 \%$ of the curriculum is presented in the school environment or through distance education. These programmes can be organised in conjunction with education authorities or institutions and include apprenticeship programmes that involve concurrent school-based and workbased training, and programmes that involve alternating periods of attendance at educational institutions and participation in work-based training (sometimes referred to as "sandwich" programmes).

In school-based programmes, instruction takes place (either partially or exclusively) in educational institutions. These include special training centres run by public or private authorities or enterprise-based special training centres if these qualify as educational institutions. These programmes can have an on-the-job training component involving some practical experience at the workplace. Programmes are classified as school-based if at least $75 \%$ of the programme curriculum is presented in the school environment. This may include distance education.

General education programmes are not explicitly designed to prepare participants for specific occupations or trades, or for entry into further vocational or technical education programmes (less than $25 \%$ of programme content is vocational or technical).

The degree to which a programme has a vocational or general orientation does not necessarily determine whether participants have access to tertiary education. In several OECD countries, vocationally oriented programmes are designed to prepare students for further study at the tertiary level, and in some countries general programmes do not always provide direct access to further education.

Vocational or technical education programmes prepare participants for direct entry into specific occupations without further training. Successful completion of such programmes leads to a vocational or technical qualification that is relevant to the labour market. Vocational programmes are further divided into two categories (school-based and combined school- and work-based programmes), based on the amount of training provided in school as opposed to the workplace.

\section{Methodology}

Data on enrolments are for the school year 2012/13 and are based on the UOE data collection on education systems administered annually by the OECD. Except where otherwise noted, figures are based on head counts; that is, they do not distinguish between full-time and part-time study because the concept of part-time study is not recognised by some countries. In some OECD countries, part-time education is only partially covered in the reported data. Net enrolment rates, expressed as percentages in Table $\mathrm{C} 1.1 \mathrm{a}$, are calculated by dividing the number of students of a particular age group enrolled in all levels of education by the size of the population of that age group.

In Table C1.6 (available on line), data on trends in enrolment rates for the years 2005 and 2013 are based on a special survey carried out in March 2015 among OECD countries, Brazil and the Russian Federation.

Expected years in education are calculated as the proportion of the population enrolled at specific ages over an age range. The main assumption is that every year of full enrolment would correspond to a full year of expected education for an individual below that age. 


\section{Note regarding data from Israel}

The statistical data for Israel are supplied by and are under the responsibility of the relevant Israeli authorities. The use of such data by the OECD is without prejudice to the status of the Golan Heights, East Jerusalem and Israeli settlements in the West Bank under the terms of international law.

\section{References}

Hanushek, E., L. Woessmann and L. Zhang (2011), "General Education, Vocational Education, and Labor-Market Outcomes over the Life-Cycle”, IZA Discussion Paper, No. 6083, Institute for the Study of Labor (IZA), Bonn, October 2011.

OECD (2012), Closing the Gender Gap: Act Now, OECD Publishing, Paris, http://dx.doi.org/10.1787/9789264179370-en.

OECD (2011), "Access to education, participation and progression", in Equity and Quality in Education: Supporting Disadvantaged Students and Schools, OECD Publishing, Paris, http://dx.doi.org/10.1787/9789264130852-en.

OECD (2010a), PISA 2009 Results: Overcoming Social Background: Equity in Learning Opportunities and Outcomes (Volume II), PISA, OECD Publishing, Paris, http://dx.doi.org/10.1787/9789264091504-en.

OECD (2010b), Learning for Jobs, OECD Reviews of Vocational Education and Training, OECD Publishing, Paris, http://dx.doi. org/10.1787/9789264087460-en.

\section{Indicator C1 Tables}

\begin{tabular}{|c|c|c|}
\hline & Table C1.1a & Enrolment rates, by age group (2013) \\
\hline WEB & Table C1.1b & Percentage of students between the ages of 15 and 20 enrolled by level of education (2013) \\
\hline & Table C1.2 & $\begin{array}{l}\text { Percentage of students in upper secondary and post-secondary non-tertiary education, } \\
\text { by programme orientation and age group (2013) }\end{array}$ \\
\hline & Table C1.3 & $\begin{array}{l}\text { Percentage of students in tertiary ISCED levels and total tertiary, by intensity of study } \\
\text { and gender (2013) }\end{array}$ \\
\hline & Table C1.4a & Percentage of students in primary and secondary education, by type of institution (2013) \\
\hline & Table C1.4b & Percentage of students in tertiary ISCED level and total tertiary, by type of institution (2013) \\
\hline & Table C1.5 & $\begin{array}{l}\text { Expected years in education from age } 5 \text { through } 39 \text {, by level of education, intensity of study } \\
\text { and gender (2013) }\end{array}$ \\
\hline WEB & Table C1.6 & Trends in enrolment rates of $15-19$ and 20-29 year-olds (2005-2013) \\
\hline
\end{tabular}

Cut-off date for the data: 23 October 2015. Updates can be found on line at http://dx.doi.org/10.1787/eag-data-en. 
Table C1.1a. Enrolment rates, by age group (2013)

Students in full-time and part-time programmes, in both public and private institutions

\begin{tabular}{|c|c|c|c|c|c|c|c|c|c|c|c|c|c|c|}
\hline & $\begin{array}{l}\text { population } \\
\text { of school age } \\
\text { are enrolled }\end{array}$ & $\begin{array}{l}\text { population } \\
\text { of school age } \\
\text { are enrolled }\end{array}$ & $\begin{array}{c}\text { Age } 2 \\
\text { and } \\
\text { under }^{1}\end{array}$ & $\begin{array}{c}\text { Ages } \\
3-4\end{array}$ & $\begin{array}{l}\text { Ages } \\
5-14\end{array}$ & $\begin{array}{c}\text { Ages } \\
15-19\end{array}$ & $\mathrm{M}+\mathrm{W}$ & Men & Women & $\mathrm{M}+\mathrm{W}$ & Men & Women & \begin{tabular}{|c|} 
Ages \\
$40-64$
\end{tabular} & $\begin{array}{c}\text { Enrolment } \\
\text { rate of total } \\
\text { population }\end{array}$ \\
\hline & (1) & (2) & (3) & (4) & (5) & (6) & (7) & $(8)$ & (9) & (10) & (11) & (12) & (13) & (14) \\
\hline \& Australia & 13 & $5-17$ & 36 & 72 & 100 & 86 & 34 & 33 & 35 & 13 & 13 & 14 & 7 & 30 \\
\hline ठّ Austria & 13 & $4-16$ & 15 & 81 & 98 & 79 & 27 & 25 & 28 & 6 & 6 & 6 & 1 & 21 \\
\hline Belgium & 15 & $3-17$ & 17 & 98 & 98 & 92 & 34 & 30 & 39 & 10 & 8 & 11 & 5 & 27 \\
\hline Canada $^{2,3}$ & 12 & $5-16$ & $\mathrm{~m}$ & $\mathrm{~m}$ & 100 & 73 & 22 & 20 & 24 & 5 & 4 & 5 & 1 & 18 \\
\hline Chile & 13 & $5-17$ & 18 & 67 & 97 & 79 & 28 & 27 & 29 & 6 & 6 & 6 & 1 & 28 \\
\hline Czech Republic & 13 & $6-18$ & 6 & 71 & 98 & 90 & 26 & 23 & 29 & 3 & 3 & 4 & 1 & 20 \\
\hline Denmark & 16 & $2-17$ & 61 & 97 & 99 & 88 & 45 & 42 & 48 & 9 & 8 & 10 & 2 & 29 \\
\hline Estonia & 14 & $4-17$ & 30 & 89 & 96 & 87 & 30 & 26 & 34 & 7 & 5 & 9 & 1 & 23 \\
\hline Finland & 13 & $6-18$ & 28 & 71 & 97 & 86 & 41 & 38 & 44 & 16 & 15 & 18 & 5 & 27 \\
\hline France & 15 & $3-17$ & 4 & 100 & 99 & 85 & 21 & 19 & 23 & 1 & 1 & 1 & 1 & 23 \\
\hline Germany & 15 & $3-17$ & 33 & 94 & 99 & 89 & 34 & 34 & 33 & 4 & 5 & 4 & 0 & 20 \\
\hline Greece & $\mathrm{m}$ & $\mathrm{m}$ & $\mathrm{m}$ & $\mathrm{m}$ & $\mathrm{m}$ & $\mathrm{m}$ & $\mathrm{m}$ & $\mathrm{m}$ & $\mathrm{m}$ & $\mathrm{m}$ & $\mathrm{m}$ & $\mathrm{m}$ & $\mathrm{m}$ & $\mathrm{m}$ \\
\hline Hungary & 14 & $4-17$ & 3 & 84 & 97 & 87 & 27 & 26 & 29 & 4 & 3 & 5 & 1 & 21 \\
\hline Iceland & 16 & $2-17$ & 44 & 96 & 99 & 88 & 40 & 37 & 44 & 14 & 11 & 17 & 5 & 33 \\
\hline Ireland & 15 & $4-18$ & 0 & 70 & 100 & 97 & 22 & 22 & 22 & 4 & 4 & 4 & 1 & 26 \\
\hline Israel & 15 & $3-17$ & 31 & 100 & 98 & 65 & 22 & 19 & 25 & 6 & 7 & 6 & 2 & 33 \\
\hline Italy & 15 & $3-17$ & 5 & 96 & 100 & 78 & 25 & 22 & 28 & 2 & 2 & 3 & 0 & 18 \\
\hline $\mathrm{Japan}^{4}$ & 14 & $4-17$ & 0 & 88 & 100 & $\mathrm{~m}$ & $\mathrm{~m}$ & $\mathrm{~m}$ & $\mathrm{~m}$ & $\mathrm{~m}$ & $\mathrm{~m}$ & $\mathrm{~m}$ & $\mathrm{~m}$ & 16 \\
\hline Korea & 14 & $3-17$ & 63 & 92 & 98 & 87 & 31 & 38 & 24 & 2 & 2 & 2 & 1 & 24 \\
\hline Luxembourg & 12 & $4-15$ & 2 & 85 & 97 & 78 & 13 & 13 & 12 & 2 & 2 & 1 & 0 & 20 \\
\hline Mexico & 9 & $5-13$ & 2 & 66 & 100 & 54 & 14 & 15 & 13 & 3 & 3 & 3 & 2 & 30 \\
\hline Netherlands & 14 & $4-17$ & 0 & 91 & 100 & 91 & 31 & 32 & 31 & 3 & 3 & 3 & 1 & 24 \\
\hline New Zealand & 15 & $3-17$ & 41 & 98 & 100 & 84 & 28 & 25 & 30 & 10 & 8 & 12 & 5 & 30 \\
\hline Norway & 17 & $2-18$ & 55 & 96 & 100 & 87 & 30 & 27 & 33 & 7 & 6 & 9 & 2 & 28 \\
\hline Poland & 13 & $5-18$ & 2 & 60 & 96 & 90 & 32 & 27 & 38 & 3 & 2 & 4 & 2 & 22 \\
\hline Portugal & 14 & $4-17$ & 0 & 84 & 100 & 88 & 24 & 24 & 24 & 4 & 4 & 4 & 1 & 20 \\
\hline Slovak Republic & 12 & $6-17$ & 4 & 68 & 94 & 85 & 21 & 17 & 24 & 3 & 2 & 4 & 1 & 20 \\
\hline Slovenia & 15 & $4-18$ & 37 & 87 & 97 & 92 & 33 & 27 & 39 & 3 & 3 & 4 & 1 & 21 \\
\hline Spain & 15 & $3-17$ & 32 & 96 & 97 & 87 & 29 & 28 & 31 & 5 & 5 & 5 & 2 & 22 \\
\hline Sweden & 16 & $3-18$ & 46 & 94 & 98 & 86 & 35 & 29 & 41 & 14 & 10 & 18 & 4 & 27 \\
\hline Switzerland & 13 & $5-17$ & 0 & 22 & 100 & 86 & 26 & 27 & 26 & 4 & 5 & 4 & 1 & 19 \\
\hline Turkey & 9 & $6-14$ & $\mathrm{~m}$ & 22 & 96 & 69 & 31 & 33 & 29 & 8 & 8 & 7 & 1 & 29 \\
\hline United Kingdom & 15 & $3-17$ & 11 & 96 & 98 & 81 & 21 & 20 & 22 & 6 & 5 & 8 & 3 & 24 \\
\hline United States & 12 & $5-16$ & $\mathrm{~m}$ & 54 & 97 & 81 & 26 & 23 & 29 & 8 & 6 & 9 & 2 & 26 \\
\hline OECD average & 13 & $4-16$ & 21 & 81 & 98 & 84 & 28 & 27 & 30 & 6 & 5 & 7 & 2 & 24 \\
\hline EU21 average & 14 & $4-16$ & 17 & 86 & 98 & 87 & 29 & 26 & 31 & 6 & 5 & 6 & 2 & 23 \\
\hline Argentina ${ }^{2}$ & 12 & $5-16$ & $\mathrm{~m}$ & $\mathrm{~m}$ & $\mathrm{~m}$ & $\mathrm{~m}$ & $\mathrm{~m}$ & $\mathrm{~m}$ & $\mathrm{~m}$ & $\mathrm{~m}$ & $\mathrm{~m}$ & $\mathrm{~m}$ & $\mathrm{~m}$ & $\mathrm{~m}$ \\
\hline Brazil $^{5}$ & 10 & $6-15$ & 16 & 65 & 95 & 70 & 20 & 18 & 21 & 7 & 6 & 8 & 2 & 28 \\
\hline ¿ China & $\mathrm{m}$ & $\mathrm{m}$ & $\mathrm{m}$ & $\mathrm{m}$ & $\mathrm{m}$ & $\mathrm{m}$ & $\mathrm{m}$ & $\mathrm{m}$ & $\mathrm{m}$ & $\mathrm{m}$ & $\mathrm{m}$ & $\mathrm{m}$ & $\mathrm{m}$ & $\mathrm{m}$ \\
\hline Colombia & 7 & $5-13$ & 19 & 62 & 93 & 57 & 17 & 16 & 18 & 6 & 6 & 7 & 2 & 30 \\
\hline India & $\mathrm{m}$ & $\mathrm{m}$ & $\mathrm{m}$ & $\mathrm{m}$ & $\mathrm{m}$ & $\mathrm{m}$ & $\mathrm{m}$ & $\mathrm{m}$ & $\mathrm{m}$ & $\mathrm{m}$ & $\mathrm{m}$ & $\mathrm{m}$ & $\mathrm{m}$ & $\mathrm{m}$ \\
\hline Indonesia & 8 & $6-15$ & 2 & 27 & 90 & 71 & 12 & 12 & 13 & 0 & 0 & 0 & 0 & 27 \\
\hline Latvia & 15 & $4-18$ & 0 & 86 & 98 & 94 & 28 & 25 & 32 & 5 & 4 & 6 & 1 & 21 \\
\hline Russian Federation & 11 & $7-17$ & 18 & 76 & 93 & 84 & 20 & 18 & 22 & 3 & 3 & 4 & 0 & 20 \\
\hline Saudi Arabia & 5 & $7-11$ & 0 & 17 & 88 & $\mathrm{~m}$ & $\mathrm{~m}$ & $\mathrm{~m}$ & $\mathrm{~m}$ & 1 & 1 & 1 & 1 & 30 \\
\hline South Africa ${ }^{2}$ & $\mathrm{~m}$ & $\mathrm{~m}$ & $\mathrm{~m}$ & $\mathrm{~m}$ & $\mathrm{~m}$ & $\mathrm{~m}$ & $\mathrm{~m}$ & $\mathrm{~m}$ & $\mathrm{~m}$ & 0 & 0 & 0 & 0 & 27 \\
\hline
\end{tabular}

G20 average

Notes: Mismatches between the coverage of the population data and the enrolment data mean that the participation rates may be underestimated for countries such as Luxembourg that are net exporters of students and may be overestimated for those that are net importers. Rates above $100 \%$ in the calculation are shown in italics.

1. Includes only institution-based early childhood programmes that have an intentional education component. These are not the only form of effective education available below the age of 3 , therefore inferences about access to and quality of early childhood education and care should be made with caution. In countries where an integrated system of pre-primary and care exists enrolment rate is noted as not applicable for children aged 2 and under.

2. Year of reference 2012

3. Excludes post-secondary non-tertiary education.

4. Excludes students aged 17 and older enrolled in tertiary education.

5. Excludes enrolments in ISCED 7 and 8.

Source: OECD. Argentina, China, Colombia, India, Indonesia, Saudi Arabia and South Africa: UNESCO Institute for Statistics. Latvia: Eurostat. See Annex 3 for notes (www.oecd.org/education/education-at-a-glance-19991487.htm).

Please refer to the Reader's Guide for information concerning symbols for missing data and abbreviations.

StatLink 제에 http://dx.doi.org/10.1787/888933285743 
Table C1.2. Percentage of students in upper secondary and post-secondary non-tertiary education, by programme orientation and age group (2013)

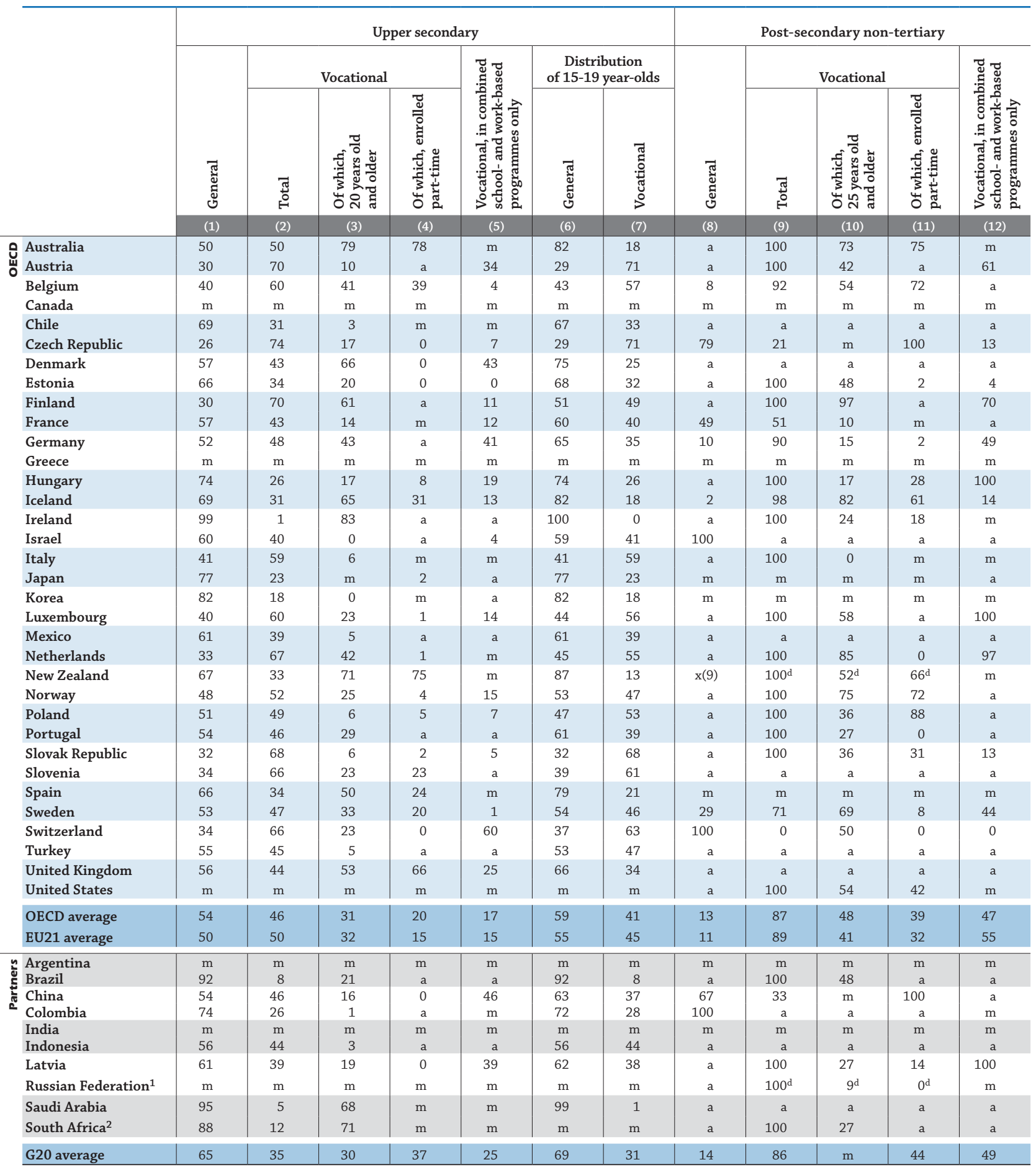

1. Upper secondary vocational programmes are partially included in post-secondary non-tertiary and tertiary programmes.

2. Year of reference 2012.

Source: OECD. Argentina, China, Colombia, India, Indonesia, Saudi Arabia and South Africa: UNESCO Institute for Statistics. Latvia: Eurostat. See Annex 3 for notes (www.oecd.org/education/education-at-a-glance-19991487.htm).

Please refer to the Reader's Guide for information concerning symbols for missing data and abbreviations.

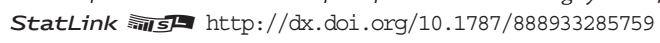


Table C1.3. Percentage of students in tertiary ISCED levels and total tertiary, by intensity of study and gender (2013)

\begin{tabular}{|c|c|c|c|c|c|c|c|c|c|c|c|c|c|c|c|c|}
\hline & \multicolumn{4}{|c|}{ Short-cycle tertiary } & \multicolumn{4}{|c|}{ Bachelor's or equivalent } & \multicolumn{4}{|c|}{ Master's or equivalent } & \multicolumn{4}{|c|}{ Total tertiary } \\
\hline & \multirow[b]{2}{*}{ 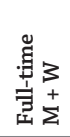 } & \multicolumn{3}{|c|}{ Part-time } & \multirow[b]{2}{*}{ 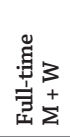 } & \multicolumn{3}{|c|}{ Part-time } & \multirow[b]{2}{*}{ 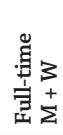 } & \multicolumn{3}{|c|}{ Part-time } & \multirow[b]{2}{*}{ 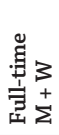 } & \multicolumn{3}{|c|}{ Part-time } \\
\hline & & $\begin{array}{l}3 \\
+ \\
\Sigma\end{array}$ & $\sum_{\Sigma}^{\tilde{J}}$ & 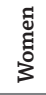 & & $\begin{array}{l}3 \\
+ \\
\sum\end{array}$ & $\stackrel{\tilde{\Xi}}{\Sigma}$ & $\begin{array}{l}\text { घี } \\
\text { घूँ } \\
\text { ふे }\end{array}$ & & $\begin{array}{l}3 \\
+ \\
\Sigma\end{array}$ & $\tilde{\Xi}_{\Sigma}^{\tilde{I}}$ & 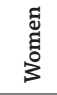 & & $\begin{array}{l}3 \\
+ \\
\Sigma\end{array}$ & $\sum_{\Sigma}^{\tilde{J}}$ & $\begin{array}{l}\text { घี } \\
\text { घू } \\
\text { ふे }\end{array}$ \\
\hline & (1) & (2) & (3) & (4) & (5) & (6) & (7) & (8) & (9) & $(10)$ & (11) & (12) & (13) & (14) & (15) & (16) \\
\hline Australia & 46 & 54 & 51 & 57 & 75 & 25 & 23 & 27 & 54 & 46 & 44 & 48 & 67 & 33 & 31 & 35 \\
\hline Austria & 100 & $\mathrm{a}$ & $\mathrm{a}$ & $a$ & 100 & a & $\mathrm{a}$ & $\mathrm{a}$ & 100 & $\mathrm{a}$ & a & a & 100 & $\mathrm{a}$ & $\mathrm{a}$ & $\mathrm{a}$ \\
\hline Belgium & 28 & 72 & 90 & 60 & 73 & 27 & 28 & 26 & 64 & 36 & 41 & 32 & 70 & 30 & 32 & 29 \\
\hline Canada $^{1}$ & 88 & 12 & 11 & 13 & 79 & 21 & 20 & 22 & 69 & 31 & 28 & 34 & 80 & 20 & 18 & 21 \\
\hline Chile & $\mathrm{m}$ & $\mathrm{m}$ & $\mathrm{m}$ & $\mathrm{m}$ & $\mathrm{m}$ & $\mathrm{m}$ & $\mathrm{m}$ & $\mathrm{m}$ & $\mathrm{m}$ & $\mathrm{m}$ & $\mathrm{m}$ & $\mathrm{m}$ & $\mathrm{m}$ & $\mathrm{m}$ & $\mathrm{m}$ & $\mathrm{m}$ \\
\hline Czech Republic & 100 & 0 & 0 & 0 & 99 & 1 & 1 & 2 & 94 & 6 & 4 & 7 & 97 & 3 & 2 & 3 \\
\hline Denmark & 71 & 29 & 26 & 33 & 89 & 11 & 10 & 12 & 94 & 6 & 7 & 6 & $\mathrm{~m}$ & $\mathrm{~m}$ & $\mathrm{~m}$ & $\mathrm{~m}$ \\
\hline Estonia & $\mathrm{a}$ & $\mathrm{a}$ & $\mathrm{a}$ & a & 85 & 15 & 18 & 13 & 85 & 15 & 17 & 14 & 85 & 15 & 17 & 13 \\
\hline Finland & 100 & a & a & a & 65 & 35 & 41 & 29 & 41 & 59 & 59 & 59 & 56 & 44 & 48 & 40 \\
\hline France & $\mathrm{m}$ & $\mathrm{m}$ & $\mathrm{m}$ & $\mathrm{m}$ & $\mathrm{m}$ & $\mathrm{m}$ & $\mathrm{m}$ & $\mathrm{m}$ & $\mathrm{m}$ & $\mathrm{m}$ & $\mathrm{m}$ & $\mathrm{m}$ & $\mathrm{m}$ & $\mathrm{m}$ & $\mathrm{m}$ & $\mathrm{m}$ \\
\hline Germany & 68 & 32 & 25 & 33 & 90 & 10 & 10 & 9 & 95 & 5 & 6 & 3 & 86 & 14 & 16 & 12 \\
\hline Greece & $\mathrm{m}$ & $\mathrm{m}$ & $\mathrm{m}$ & $\mathrm{m}$ & $\mathrm{m}$ & $\mathrm{m}$ & $\mathrm{m}$ & $\mathrm{m}$ & $\mathrm{m}$ & $\mathrm{m}$ & $\mathrm{m}$ & $\mathrm{m}$ & $\mathrm{m}$ & $\mathrm{m}$ & $\mathrm{m}$ & $\mathrm{m}$ \\
\hline Hungary & 71 & 29 & 26 & 30 & 67 & 33 & 28 & 37 & 71 & 29 & 25 & 31 & 69 & 31 & 28 & 34 \\
\hline Iceland & 52 & 48 & 55 & 39 & 75 & 25 & 23 & 26 & 60 & 40 & 37 & 41 & 71 & 29 & 27 & 30 \\
\hline Ireland & 72 & 28 & 24 & 32 & 95 & 5 & 5 & 4 & 59 & 41 & 42 & 40 & 85 & 15 & 15 & 15 \\
\hline Israel & 100 & $\mathrm{a}$ & a & a & 80 & 20 & 19 & 21 & 94 & 6 & 7 & 5 & 86 & 14 & 13 & 15 \\
\hline Italy & $\mathrm{m}$ & $\mathrm{m}$ & $\mathrm{m}$ & $\mathrm{m}$ & $\mathrm{m}$ & $\mathrm{m}$ & $\mathrm{m}$ & $\mathrm{m}$ & $\mathrm{m}$ & $\mathrm{m}$ & $\mathrm{m}$ & $\mathrm{m}$ & $\mathrm{m}$ & $\mathrm{m}$ & $\mathrm{m}$ & $\mathrm{m}$ \\
\hline Japan & 97 & 3 & 2 & 3 & 90 & 10 & 8 & 12 & 98 & 2 & 2 & 3 & 92 & 8 & 6 & 9 \\
\hline Korea & $\mathrm{m}$ & $\mathrm{m}$ & $\mathrm{m}$ & $\mathrm{m}$ & $\mathrm{m}$ & $\mathrm{m}$ & $\mathrm{m}$ & $\mathrm{m}$ & $\mathrm{m}$ & $\mathrm{m}$ & $\mathrm{m}$ & $\mathrm{m}$ & $\mathrm{m}$ & $\mathrm{m}$ & $\mathrm{m}$ & $\mathrm{m}$ \\
\hline Luxembourg & 100 & 0 & 0 & 0 & 99 & 1 & 0 & 1 & 57 & 43 & 49 & 38 & 83 & 17 & 18 & 15 \\
\hline Mexico & 100 & $\mathrm{a}$ & a & a & 100 & a & a & a & 100 & a & a & a & 100 & a & $\mathrm{a}$ & a \\
\hline Netherlands & 69 & 31 & 27 & 37 & 92 & 8 & 8 & 9 & 86 & 14 & 11 & 17 & 91 & 9 & 8 & 10 \\
\hline New Zealand & 38 & 62 & 57 & 66 & 63 & 37 & 34 & 39 & 36 & 64 & 63 & 65 & 56 & 44 & 42 & 46 \\
\hline Norway & 60 & 40 & 46 & 24 & 63 & 37 & 32 & 40 & 71 & 29 & 25 & 31 & 63 & 37 & 34 & 40 \\
\hline Poland & 67 & 33 & 32 & 33 & 53 & 47 & 45 & 49 & 52 & 48 & 45 & 50 & 53 & 47 & 45 & 48 \\
\hline Portugal & a & $\mathrm{a}$ & a & a & 95 & 5 & 6 & 4 & 96 & 4 & 5 & 3 & 95 & 5 & 6 & 4 \\
\hline Slovak Republic & 82 & 18 & 15 & 19 & $69^{\mathrm{d}}$ & $31^{\mathrm{d}}$ & $26^{\mathrm{d}}$ & $34^{\mathrm{d}}$ & $\mathrm{x}(5)$ & $\mathrm{x}(6)$ & $\mathrm{x}(7)$ & $\mathrm{x}(8)$ & 69 & 31 & 27 & 34 \\
\hline Slovenia & 54 & 46 & 46 & 45 & 81 & 19 & 17 & 20 & 89 & 11 & 10 & 12 & 77 & 23 & 23 & 22 \\
\hline Spain & 92 & 8 & 5 & 11 & 71 & 29 & 31 & 27 & 64 & 36 & 39 & 34 & 73 & 27 & 28 & 26 \\
\hline Sweden & 91 & 9 & 11 & 7 & 45 & 55 & 55 & 55 & 61 & 39 & 34 & 43 & 53 & 47 & 44 & 49 \\
\hline Switzerland & 73 & 27 & 32 & 23 & 71 & 29 & 35 & 23 & 86 & 14 & 16 & 12 & 76 & 24 & 28 & 19 \\
\hline Turkey & 100 & a & a & a & 100 & $\mathrm{a}$ & $\mathrm{a}$ & $\mathrm{a}$ & 100 & a & $\mathrm{a}$ & a & 100 & $\mathrm{a}$ & a & a \\
\hline United Kingdom & 11 & 89 & 87 & 91 & 86 & 14 & 13 & 15 & 51 & 49 & 47 & 50 & 69 & 31 & 28 & 33 \\
\hline United States & 46 & 54 & 52 & 55 & 77 & 23 & 22 & 24 & 54 & 46 & 42 & 48 & 62 & 38 & 35 & 39 \\
\hline OECD average & 73 & 27 & 27 & 26 & 80 & 20 & 19 & 20 & 74 & 26 & 25 & 26 & 77 & 23 & 22 & 23 \\
\hline EU21 average & 74 & 26 & 26 & 27 & 81 & 19 & 19 & 19 & 74 & 26 & 26 & 26 & 77 & 23 & 23 & 23 \\
\hline
\end{tabular}

\begin{tabular}{|c|c|c|c|c|c|c|c|c|c|c|c|c|c|c|c|c|}
\hline $\begin{array}{l}\text { Argentina } \\
\text { Brazil }\end{array}$ & $\begin{array}{r}\mathrm{m} \\
100\end{array}$ & $\begin{array}{r}\mathrm{m} \\
\mathrm{a}\end{array}$ & $\begin{array}{r}\mathrm{m} \\
\mathrm{a}\end{array}$ & $\begin{array}{r}\mathrm{m} \\
\mathrm{a}\end{array}$ & $\begin{array}{r}\mathrm{m} \\
100\end{array}$ & $\begin{array}{c}\mathrm{m} \\
\mathrm{a}\end{array}$ & $\begin{array}{c}\mathrm{m} \\
\mathrm{a}\end{array}$ & $\begin{array}{c}\mathrm{m} \\
\mathrm{a}\end{array}$ & $\begin{array}{l}\mathrm{m} \\
\mathrm{m}\end{array}$ & $\begin{array}{l}\mathrm{m} \\
\mathrm{m}\end{array}$ & $\begin{array}{l}\mathrm{m} \\
\mathrm{m}\end{array}$ & $\begin{array}{l}\mathrm{m} \\
\mathrm{m}\end{array}$ & $\begin{array}{l}\mathrm{m} \\
\mathrm{m}\end{array}$ & $\begin{array}{l}\mathrm{m} \\
\mathrm{m}\end{array}$ & $\begin{array}{l}\mathrm{m} \\
\mathrm{m}\end{array}$ & $\begin{array}{l}\mathrm{m} \\
\mathrm{m}\end{array}$ \\
\hline China & 69 & 31 & 32 & 30 & 80 & 20 & 20 & 20 & 72 & 28 & 33 & 21 & 75 & 25 & 26 & 24 \\
\hline Colombia & 100 & $\mathrm{a}$ & a & a & 100 & a & a & a & 100 & a & a & a & 100 & a & a & a \\
\hline India & $\mathrm{m}$ & $\mathrm{m}$ & $\mathrm{m}$ & $\mathrm{m}$ & $\mathrm{m}$ & $\mathrm{m}$ & $\mathrm{m}$ & $\mathrm{m}$ & $\mathrm{m}$ & $\mathrm{m}$ & $\mathrm{m}$ & $\mathrm{m}$ & $\mathrm{m}$ & $\mathrm{m}$ & $\mathrm{m}$ & $\mathrm{m}$ \\
\hline Indonesia & $\mathrm{m}$ & a & a & a & 100 & a & a & a & 100 & a & a & a & $\mathrm{m}$ & $\mathrm{m}$ & $\mathrm{m}$ & $\mathrm{m}$ \\
\hline Latvia & 51 & 49 & 51 & 48 & 76 & 24 & 24 & 25 & 84 & 16 & 15 & 16 & 73 & 27 & 27 & 27 \\
\hline Russian Federation ${ }^{2}$ & $79^{\mathrm{d}}$ & $21^{\mathrm{d}}$ & $22^{\mathrm{d}}$ & $19^{\mathrm{d}}$ & $\mathrm{x}(9)$ & $\mathrm{x}(10)$ & $\mathrm{x}(11)$ & $\mathrm{x}(12)$ & $49^{d}$ & $51^{\mathrm{d}}$ & $51^{\mathrm{d}}$ & $52^{\mathrm{d}}$ & $57^{\mathrm{d}}$ & $43^{\mathrm{d}}$ & $42^{\mathrm{d}}$ & $44^{\mathrm{d}}$ \\
\hline Saudi Arabia & $\mathrm{m}$ & $\mathrm{m}$ & $\mathrm{m}$ & $\mathrm{m}$ & $\mathrm{m}$ & $\mathrm{m}$ & $\mathrm{m}$ & $\mathrm{m}$ & $\mathrm{m}$ & $\mathrm{m}$ & $\mathrm{m}$ & $\mathrm{m}$ & $\mathrm{m}$ & $\mathrm{m}$ & $\mathrm{m}$ & $\mathrm{m}$ \\
\hline South Africa ${ }^{1}$ & $\mathrm{~m}$ & $\mathrm{~m}$ & $\mathrm{~m}$ & $\mathrm{~m}$ & $\mathrm{~m}$ & $\mathrm{~m}$ & $\mathrm{~m}$ & $\mathrm{~m}$ & $\mathrm{~m}$ & $\mathrm{~m}$ & $\mathrm{~m}$ & $\mathrm{~m}$ & $\mathrm{~m}$ & $\mathrm{~m}$ & $\mathrm{~m}$ & $\mathrm{~m}$ \\
\hline
\end{tabular}

1. Year of reference 2012.

2. Upper secondary vocational programmes are partially included in post-secondary non-tertiary and tertiary programmes.

Source: OECD. Argentina, China, Colombia, India, Indonesia, Saudi Arabia and South Africa: UNESCO Institute for Statistics. Latvia: Eurostat. See Annex 3 for notes (www.oecd.org/education/education-at-a-glance-19991487.htm).

Please refer to the Reader's Guide for information concerning symbols for missing data and abbreviations.

StatLink 젶ㄴ http://dx.doi.org/10.1787/888933285765 
Table C1.4a. Percentage of students in primary and secondary education, by type of institution (2013)

\begin{tabular}{|c|c|c|c|c|c|c|c|c|c|c|c|c|}
\hline & \multicolumn{4}{|c|}{ Primary } & \multicolumn{4}{|c|}{ Lower Secondary } & \multicolumn{4}{|c|}{ Upper Secondary } \\
\hline & \multirow[b]{2}{*}{ 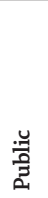 } & \multicolumn{3}{|c|}{ Private, of which } & \multirow[b]{2}{*}{ 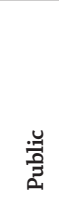 } & \multicolumn{3}{|c|}{ Private, of which } & \multirow[b]{2}{*}{ 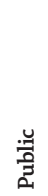 } & \multicolumn{3}{|c|}{ Private, of which } \\
\hline & & 今ే & 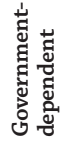 & 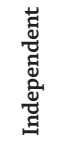 & & సేّ & 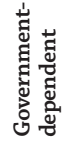 & 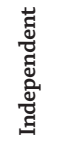 & & $\begin{array}{l}\text { సే } \\
\stackrel{0}{0}\end{array}$ & 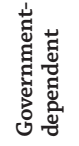 & 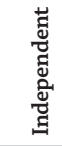 \\
\hline & (1) & (2) & (3) & (4) & (5) & (6) & (7) & (8) & (9) & (10) & (11) & (12) \\
\hline Australia & 69 & 31 & 31 & $\mathrm{a}$ & 63 & 37 & 37 & $\mathrm{~m}$ & 63 & 37 & 37 & $\mathrm{~m}$ \\
\hline Austria & 94 & 6 & $6^{\mathrm{d}}$ & $x(3)$ & 91 & 9 & $9^{d}$ & $\mathrm{x}(7)$ & 90 & 10 & $10^{\mathrm{d}}$ & $\mathrm{x}(11)$ \\
\hline Belgium & 46 & 54 & 54 & $\mathrm{~m}$ & 42 & 58 & 58 & $\mathrm{~m}$ & 41 & 59 & 59 & $\mathrm{~m}$ \\
\hline Canada $^{1}$ & 94 & 6 & $6^{\mathrm{d}}$ & $\mathrm{x}(3)$ & 91 & 9 & $9^{d}$ & $x(7)$ & 94 & 6 & $6^{\mathrm{d}}$ & $\mathrm{x}(11)$ \\
\hline Chile & $\mathrm{m}$ & $\mathrm{m}$ & $\mathrm{m}$ & $\mathrm{m}$ & $\mathrm{m}$ & $\mathrm{m}$ & $\mathrm{m}$ & $\mathrm{m}$ & $\mathrm{m}$ & $\mathrm{m}$ & $\mathrm{m}$ & $\mathrm{m}$ \\
\hline Czech Republic & 98 & 2 & 2 & a & 97 & 3 & 3 & a & 85 & 15 & 15 & a \\
\hline Denmark & 85 & 15 & 15 & 0 & 73 & 27 & 26 & 1 & 98 & 2 & 2 & 0 \\
\hline Estonia & 95 & 5 & a & 5 & 96 & 4 & a & 4 & 97 & 3 & a & 3 \\
\hline Finland & 98 & 2 & 2 & $\mathrm{a}$ & 95 & 5 & 5 & a & 81 & 19 & 19 & a \\
\hline France & 85 & 15 & 14 & 0 & 78 & 22 & 22 & 0 & 68 & 32 & 31 & 1 \\
\hline Germany & 95 & 5 & $5^{d}$ & $\mathrm{x}(3)$ & 90 & 10 & $10^{\mathrm{d}}$ & $x(7)$ & 92 & 8 & $8^{\mathrm{d}}$ & $\mathrm{x}(11)$ \\
\hline Greece & $\mathrm{m}$ & $\mathrm{m}$ & $\mathrm{m}$ & $\mathrm{m}$ & $\mathrm{m}$ & $\mathrm{m}$ & $\mathrm{m}$ & $\mathrm{m}$ & $\mathrm{m}$ & $\mathrm{m}$ & $\mathrm{m}$ & $\mathrm{m}$ \\
\hline Hungary & 86 & 14 & 14 & a & 85 & 15 & 15 & a & 73 & 27 & 27 & a \\
\hline Iceland & 97 & 3 & 3 & 0 & 99 & 1 & 1 & 0 & 80 & 20 & 19 & 1 \\
\hline Ireland & 99 & 1 & 0 & 1 & 100 & 0 & 0 & 0 & 98 & 2 & 0 & 2 \\
\hline Israel & 77 & 23 & 23 & $\mathrm{a}$ & 84 & 16 & 16 & a & 94 & 6 & 6 & a \\
\hline Italy & 93 & 7 & a & 7 & 96 & 4 & $\mathrm{a}$ & 4 & 91 & 9 & 5 & 4 \\
\hline Japan & 99 & 1 & $\mathrm{a}$ & 1 & 93 & 7 & a & 7 & 68 & 32 & a & 32 \\
\hline Korea & 98 & 2 & a & 2 & 82 & 18 & 18 & a & 56 & 44 & 44 & $\mathrm{a}$ \\
\hline Luxembourg & 90 & 10 & 0 & 9 & 81 & 19 & 10 & 9 & 83 & 17 & 7 & 10 \\
\hline Mexico & 91 & 9 & a & 9 & 89 & 11 & a & 11 & 83 & 17 & a & 17 \\
\hline Netherlands & $\mathrm{m}$ & $\mathrm{m}$ & $\mathrm{m}$ & $\mathrm{m}$ & $\mathrm{m}$ & $\mathrm{m}$ & $\mathrm{m}$ & $\mathrm{m}$ & $\mathrm{m}$ & $\mathrm{m}$ & $\mathrm{m}$ & $\mathrm{m}$ \\
\hline New Zealand & 98 & 2 & 0 & 2 & 95 & 5 & 0 & 5 & 85 & 15 & 10 & 5 \\
\hline Norway & 97 & 3 & 2 & 0 & 97 & 3 & 3 & 0 & 89 & 11 & 11 & 0 \\
\hline Poland & 96 & 4 & 1 & 3 & 94 & 6 & 2 & 4 & 84 & 16 & 1 & 15 \\
\hline Portugal & 88 & 12 & 4 & 8 & 87 & 13 & 7 & 6 & 79 & 21 & 5 & 16 \\
\hline Slovak Republic & 94 & 6 & 6 & a & 93 & 7 & 7 & a & 85 & 15 & 15 & a \\
\hline Slovenia & 99 & 1 & 1 & 0 & 100 & 0 & 0 & 0 & 96 & 4 & 2 & 2 \\
\hline Spain & 68 & 32 & 28 & 4 & 71 & 29 & 27 & 3 & 75 & 25 & 17 & 8 \\
\hline Sweden & 90 & 10 & 10 & 0 & 85 & 15 & 15 & 0 & 82 & 18 & 18 & 0 \\
\hline Switzerland & 94 & 6 & 1 & 4 & 91 & 9 & 3 & 6 & 85 & 15 & 9 & 5 \\
\hline Turkey & 97 & 3 & $\mathrm{a}$ & 3 & 97 & 3 & a & 3 & 97 & 3 & $\mathrm{a}$ & 3 \\
\hline United Kingdom & 89 & 11 & 6 & 4 & 48 & 52 & 47 & 6 & 26 & 74 & 69 & 5 \\
\hline United States & 92 & 8 & a & 8 & 92 & 8 & a & 8 & 92 & 8 & a & 8 \\
\hline OECD average & 90 & 10 & $\sim$ & $\sim$ & 86 & 14 & $\sim$ & $\sim$ & 81 & 19 & $\sim$ & $\sim$ \\
\hline EU21 average & 89 & 11 & $\sim$ & & 84 & 16 & $\sim$ & $\sim$ & 80 & 20 & $\sim$ & $\sim$ \\
\hline Argentina & $\mathrm{m}$ & $\mathrm{m}$ & $\mathrm{m}$ & $\mathrm{m}$ & $\mathrm{m}$ & $\mathrm{m}$ & $\mathrm{m}$ & $\mathrm{m}$ & $\mathrm{m}$ & $\mathrm{m}$ & $\mathrm{m}$ & $\mathrm{m}$ \\
\hline Brazil & 84 & 16 & $\mathrm{a}$ & 16 & 88 & 12 & $\mathrm{a}$ & 12 & 86 & 14 & $\mathrm{a}$ & 14 \\
\hline China & 94 & 6 & $6^{\mathrm{d}}$ & $\mathrm{x}(3)$ & 91 & 9 & $9^{d}$ & $x(7)$ & 90 & 10 & $10^{\mathrm{d}}$ & $\mathrm{x}(11)$ \\
\hline Colombia & 82 & 18 & 0 & 18 & 81 & 19 & 0 & 19 & 75 & 25 & 0 & 25 \\
\hline India & $\mathrm{m}$ & $\mathrm{m}$ & $\mathrm{m}$ & $\mathrm{m}$ & $\mathrm{m}$ & $\mathrm{m}$ & $\mathrm{m}$ & $\mathrm{m}$ & $\mathrm{m}$ & $\mathrm{m}$ & $\mathrm{m}$ & $\mathrm{m}$ \\
\hline Indonesia & 82 & 18 & a & 18 & 64 & 36 & $\mathrm{a}$ & 36 & 50 & 50 & a & 50 \\
\hline Latvia & 99 & 1 & $\mathrm{a}$ & 1 & 99 & 1 & a & 1 & 98 & 2 & a & 2 \\
\hline Russian Federation & 99 & 1 & a & 1 & 99 & 1 & $\mathrm{a}$ & 1 & 98 & 2 & $\mathrm{a}$ & 2 \\
\hline Saudi Arabia & 90 & 10 & $\mathrm{x}(2)$ & $x(2)$ & 92 & 8 & $\mathrm{x}(6)$ & $\mathrm{x}(6)$ & 80 & 20 & $\mathrm{x}(10)$ & $\mathrm{x}(10)$ \\
\hline South Africa ${ }^{1}$ & 96 & 4 & $x(2)$ & $x(2)$ & 96 & 4 & $\mathrm{x}(6)$ & $\mathrm{x}(6)$ & 94 & 6 & $x(10)$ & $\mathrm{x}(10)$ \\
\hline G20 average & 91 & 9 & $\sim$ & $\sim$ & 85 & 15 & $\sim$ & $\sim$ & 78 & 22 & $\sim$ & $\sim$ \\
\hline
\end{tabular}

1. Year of reference 2012.

Source: OECD. Argentina, China, Colombia, India, Indonesia, Saudi Arabia and South Africa: UNESCO Institute for Statistics. Latvia: Eurostat. See Annex 3 for notes (www.oecd.org/education/education-at-a-glance-19991487.htm).

Please refer to the Reader's Guide for information concerning symbols for missing data and abbreviations.

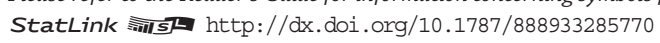


Table C1.4b. Percentage of students in tertiary ISCED level and total tertiary, by type of institution (2013)

\begin{tabular}{|c|c|c|c|c|c|c|c|c|c|c|c|c|c|c|c|c|c|c|c|}
\hline \multicolumn{4}{|c|}{ Total Tertiary } & \multicolumn{4}{|c|}{ Short-cycle tertiary } & \multicolumn{4}{|c|}{ Bachelor's or equivalent } & \multicolumn{4}{|c|}{ Master's or equivalent } & \multicolumn{4}{|c|}{ Doctoral or equivalent } \\
\hline \multirow[b]{2}{*}{ 駦 } & \multicolumn{3}{|c|}{ Private, of which } & \multirow[b]{2}{*}{ 总 } & \multicolumn{3}{|c|}{ Private, of which } & \multirow[b]{2}{*}{ 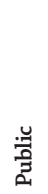 } & \multicolumn{3}{|c|}{ Private, of which } & \multirow[b]{2}{*}{ 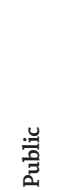 } & \multicolumn{3}{|c|}{ Private, of which } & & \multicolumn{3}{|c|}{ f which } \\
\hline & $\begin{array}{l}\text { ]ే } \\
\stackrel{0}{\circ}\end{array}$ & 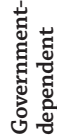 & 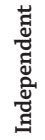 & & స్ & 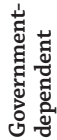 & 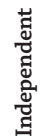 & & 胥 & 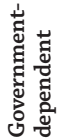 & 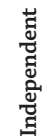 & & 胥 & 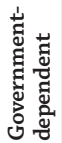 & 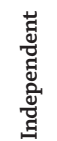 & 总 & 胥 & 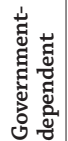 & 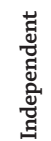 \\
\hline () & (2) & (3) & (4) & (5) & (6) & $(7)$ & (8) & (9) & (10) & (11) & (12) & (13) & (14) & (15) & (16) & (17) & (18) & (19) & (20) \\
\hline
\end{tabular}

\begin{tabular}{|c|c|c|c|c|c|c|c|c|c|c|c|c|c|c|c|c|c|c|c|c|}
\hline & & & & & & & & & & & & & & & & & & & & \\
\hline Q Australia & 92 & 8 & 3 & 6 & 74 & 26 & 16 & 10 & 95 & 5 & 0 & 5 & 95 & 5 & a & 5 & 99 & 1 & a & 1 \\
\hline む̈ Austria & 84 & 16 & $16^{\mathrm{d}}$ & $x(3)$ & 82 & 18 & $18^{d}$ & $x(7)$ & 79 & 21 & $21^{\mathrm{d}}$ & $\mathrm{x}(11)$ & 89 & 11 & $11^{\mathrm{d}}$ & $\mathrm{x}(15)$ & 99 & 1 & $1^{\mathrm{d}}$ & $\mathrm{x}(19)$ \\
\hline Belgium & 42 & 58 & 57 & $\mathrm{~m}$ & 37 & 63 & 63 & $\mathrm{~m}$ & 43 & 57 & 57 & 0 & 40 & 60 & 60 & 1 & 58 & 42 & 42 & 0 \\
\hline Canada & $\mathrm{m}$ & $\mathrm{m}$ & $\mathrm{m}$ & $\mathrm{m}$ & $\mathrm{m}$ & $\mathrm{m}$ & $\mathrm{m}$ & $\mathrm{m}$ & $\mathrm{m}$ & $\mathrm{m}$ & $\mathrm{m}$ & $\mathrm{m}$ & $\mathrm{m}$ & $\mathrm{m}$ & $\mathrm{m}$ & $\mathrm{m}$ & $\mathrm{m}$ & $\mathrm{m}$ & $\mathrm{m}$ & $\mathrm{m}$ \\
\hline Chile & 16 & 84 & 12 & 72 & 3 & 97 & 2 & 94 & 20 & 80 & 15 & 65 & 26 & 74 & 16 & 58 & 44 & 56 & 44 & 11 \\
\hline Czech Republic & 87 & 13 & 2 & 11 & 86 & 14 & 14 & a & 84 & 16 & 3 & 13 & 90 & 10 & a & 10 & 100 & 0 & a & 0 \\
\hline Denmark & 98 & 2 & 2 & 0 & 97 & 3 & 3 & 0 & 97 & 3 & 3 & 0 & 100 & 0 & 0 & 0 & 100 & 0 & 0 & 0 \\
\hline Estonia & 16 & 84 & 74 & 10 & $\mathrm{a}$ & a & a & a & 23 & 77 & 64 & 13 & 1 & 99 & 95 & 4 & 0 & 100 & 99 & 1 \\
\hline Finland & 72 & 28 & 28 & a & 100 & 0 & 0 & a & 65 & 35 & 35 & a & 92 & 8 & 8 & a & 100 & 0 & 0 & a \\
\hline France & 79 & 21 & 3 & 18 & 67 & 33 & 12 & 21 & 86 & 14 & 2 & 13 & 78 & 22 & 0 & 22 & 99 & 1 & 0 & 1 \\
\hline Germany & 92 & 8 & $8^{\mathrm{d}}$ & $\mathrm{x}(3)$ & 80 & 20 & $20^{\mathrm{d}}$ & $\mathrm{x}(7)$ & 88 & 12 & $12^{\mathrm{d}}$ & $\mathrm{x}(11)$ & 97 & 3 & $3^{\mathrm{d}}$ & $\mathrm{x}(15)$ & 100 & 0 & 0 & 0 \\
\hline Greece & $\mathrm{m}$ & $\mathrm{m}$ & $\mathrm{m}$ & $\mathrm{m}$ & $\mathrm{m}$ & $\mathrm{m}$ & $\mathrm{m}$ & $\mathrm{m}$ & $\mathrm{m}$ & $\mathrm{m}$ & $\mathrm{m}$ & $\mathrm{m}$ & $\mathrm{m}$ & $\mathrm{m}$ & $\mathrm{m}$ & $\mathrm{m}$ & $\mathrm{m}$ & $\mathrm{m}$ & $\mathrm{m}$ & $\mathrm{m}$ \\
\hline Hungary & 83 & 17 & 17 & $\mathrm{a}$ & 44 & 56 & 56 & $\mathrm{a}$ & 87 & 13 & 13 & a & 89 & 11 & 11 & a & 94 & 6 & 6 & a \\
\hline Iceland & 81 & 19 & 19 & 0 & 45 & 55 & 55 & 0 & 81 & 19 & 19 & 0 & 83 & 17 & 17 & 0 & 95 & 5 & 5 & 0 \\
\hline Ireland & 98 & 2 & $\mathrm{~m}$ & $\mathrm{~m}$ & $\mathrm{~m}$ & $\mathrm{~m}$ & $\mathrm{~m}$ & $\mathrm{~m}$ & 98 & 2 & 0 & 2 & 97 & 3 & 0 & 3 & 99 & 1 & 0 & 1 \\
\hline Israel & 15 & 85 & 73 & 12 & 38 & 62 & 62 & 0 & 12 & 88 & 73 & 16 & 7 & 93 & 79 & 14 & 0 & 100 & 100 & 0 \\
\hline Italy & 91 & 9 & a & 9 & 0 & 100 & 0 & 100 & 90 & 10 & a & 10 & 92 & 8 & a & 8 & 95 & 5 & 0 & 5 \\
\hline Japan & 21 & 79 & a & 79 & 8 & 92 & a & 92 & 20 & 80 & a & 80 & 47 & 53 & a & 53 & 75 & 25 & a & 25 \\
\hline Korea & 19 & 81 & a & 81 & 2 & 98 & a & 98 & 24 & 76 & a & 76 & 31 & 69 & a & 69 & 36 & 64 & a & 64 \\
\hline Luxembourg & $\mathrm{m}$ & $\mathrm{m}$ & $\mathrm{m}$ & $\mathrm{m}$ & 100 & 0 & 0 & 0 & $\mathrm{~m}$ & $\mathrm{~m}$ & $\mathrm{~m}$ & $\mathrm{~m}$ & $\mathrm{~m}$ & $\mathrm{~m}$ & $\mathrm{~m}$ & $\mathrm{~m}$ & $\mathrm{~m}$ & $\mathrm{~m}$ & $\mathrm{~m}$ & $\mathrm{~m}$ \\
\hline Mexico & 69 & 31 & a & 31 & 96 & 4 & a & 4 & 69 & 31 & a & 31 & 47 & 53 & a & 53 & 67 & 33 & $\mathrm{a}$ & 33 \\
\hline Netherlands & $\mathrm{m}$ & $\mathrm{m}$ & $\mathrm{m}$ & $\mathrm{m}$ & $\mathrm{m}$ & $\mathrm{m}$ & $\mathrm{m}$ & $\mathrm{m}$ & $\mathrm{m}$ & $\mathrm{m}$ & $\mathrm{m}$ & $\mathrm{m}$ & $\mathrm{m}$ & $\mathrm{m}$ & $\mathrm{m}$ & $\mathrm{m}$ & 100 & a & a & a \\
\hline New Zealand & 88 & 12 & 11 & 1 & 57 & 43 & 40 & 4 & 96 & 4 & 4 & 0 & 97 & 3 & 3 & 0 & 100 & 0 & 0 & 0 \\
\hline Norway & 83 & 17 & 7 & 9 & 60 & 40 & 40 & 0 & 81 & 19 & 7 & 12 & 95 & 5 & 2 & 3 & 98 & 2 & 1 & 1 \\
\hline Poland & 72 & 28 & $\mathrm{a}$ & 28 & 89 & 11 & $\mathrm{a}$ & 11 & 70 & 30 & a & 30 & 74 & 26 & a & 26 & 93 & 7 & a & 7 \\
\hline Portugal & 82 & 18 & 0 & 18 & a & $\mathrm{a}$ & a & a & 79 & 21 & 0 & 21 & 85 & 15 & 0 & 15 & 93 & 7 & 0 & 7 \\
\hline Slovak Republic & 82 & 18 & 0 & 17 & 74 & 26 & 26 & a & 81 & 19 & 0 & 19 & 83 & 17 & 0 & 17 & 94 & 6 & 0 & 6 \\
\hline Slovenia & 86 & 14 & 6 & 8 & 76 & 24 & 2 & 21 & 85 & 15 & 7 & 8 & 92 & 8 & 5 & 2 & 87 & 13 & 4 & 10 \\
\hline Spain & 84 & 16 & 2 & 14 & 80 & 20 & 13 & 7 & 84 & 16 & 0 & 16 & 85 & 15 & 0 & 15 & 92 & 8 & 0 & 8 \\
\hline Sweden & 91 & 9 & 9 & 0 & 51 & 49 & 49 & 0 & 95 & 5 & 5 & 0 & 92 & 8 & 8 & 0 & 93 & 7 & 7 & 0 \\
\hline Switzerland & 82 & 18 & 9 & 9 & 8 & 92 & 19 & 73 & 80 & 20 & 11 & 9 & 95 & 5 & 3 & 2 & 100 & 0 & 0 & 0 \\
\hline Turkey & 94 & 6 & a & 6 & 97 & 3 & a & 3 & 94 & 6 & a & 6 & 85 & 15 & $\mathrm{a}$ & 15 & 94 & 6 & a & 6 \\
\hline United Kingdom & a & 100 & 100 & $\mathrm{a}$ & a & 100 & 100 & a & a & 100 & 100 & a & a & 100 & 100 & $\mathrm{a}$ & a & 100 & 100 & a \\
\hline United States & 72 & 28 & a & 28 & 90 & 10 & a & 10 & 66 & 34 & a & 34 & 46 & 54 & a & 54 & 62 & 38 & a & 38 \\
\hline OECD average & 69 & 31 & $\sim$ & & 59 & 41 & & & 69 & 31 & $\sim$ & $\sim$ & 71 & 29 & $\sim$ & $\sim$ & 80 & 20 & $\sim$ & $\sim$ \\
\hline EU21 average & 74 & 26 & $\sim$ & $\sim$ & 66 & 34 & $\sim$ & & 74 & 26 & $\sim$ & $\sim$ & 76 & 24 & $\sim$ & $\sim$ & 84 & 16 & $\sim$ & \\
\hline
\end{tabular}

\begin{tabular}{|c|c|c|c|c|c|c|c|c|c|c|c|c|c|c|c|c|c|c|c|c|}
\hline $\begin{array}{l}\text { Argentina } \\
\text { Brazil }\end{array}$ & $\begin{array}{l}\mathrm{m} \\
\mathrm{m}\end{array}$ & $\begin{array}{l}\mathrm{m} \\
\mathrm{m}\end{array}$ & $\begin{array}{l}\mathrm{m} \\
\mathrm{m}\end{array}$ & $\begin{array}{l}\mathrm{m} \\
\mathrm{m}\end{array}$ & $\begin{array}{c}\mathrm{m} \\
58\end{array}$ & $\begin{array}{l}\mathrm{m} \\
42\end{array}$ & $\begin{array}{c}\mathrm{m} \\
\mathrm{a}\end{array}$ & $\begin{array}{l}m \\
42\end{array}$ & $\begin{array}{l}\mathrm{m} \\
\mathrm{m}\end{array}$ & $\begin{array}{l}\mathrm{m} \\
\mathrm{m}\end{array}$ & $\begin{array}{l}\mathrm{m} \\
\mathrm{m}\end{array}$ & $\begin{array}{l}\mathrm{m} \\
\mathrm{m}\end{array}$ & $\begin{array}{l}\mathrm{m} \\
\mathrm{m}\end{array}$ & $\begin{array}{l}\mathrm{m} \\
\mathrm{m}\end{array}$ & $\begin{array}{l}\mathrm{m} \\
\mathrm{m}\end{array}$ & $\begin{array}{l}\mathrm{m} \\
\mathrm{m}\end{array}$ & $\begin{array}{l}\mathrm{m} \\
\mathrm{m}\end{array}$ & $\begin{array}{l}\mathrm{m} \\
\mathrm{m}\end{array}$ & $\begin{array}{l}\mathrm{m} \\
\mathrm{m}\end{array}$ & $\begin{array}{l}\mathrm{m} \\
\mathrm{m}\end{array}$ \\
\hline China & 85 & 15 & 15 & a & 87 & 13 & 13 & $\mathrm{a}$ & 81 & 19 & 19 & a & 100 & a & $\mathrm{a}$ & $\mathrm{a}$ & 100 & a & a & a \\
\hline Colombia & 52 & 48 & 0 & 48 & 76 & 24 & 0 & 24 & 42 & 58 & 0 & 58 & 28 & 72 & 0 & 72 & 68 & 32 & 0 & 32 \\
\hline India & $\mathrm{m}$ & $\mathrm{m}$ & $\mathrm{m}$ & $\mathrm{m}$ & $\mathrm{m}$ & $\mathrm{m}$ & $\mathrm{m}$ & $\mathrm{m}$ & $\mathrm{m}$ & $\mathrm{m}$ & $\mathrm{m}$ & $\mathrm{m}$ & $\mathrm{m}$ & $\mathrm{m}$ & $\mathrm{m}$ & $\mathrm{m}$ & $\mathrm{m}$ & $\mathrm{m}$ & $\mathrm{m}$ & $\mathrm{m}$ \\
\hline Indonesia & $\mathrm{m}$ & $\mathrm{m}$ & $\mathrm{m}$ & $\mathrm{m}$ & $\mathrm{m}$ & $\mathrm{m}$ & $\mathrm{m}$ & $\mathrm{m}$ & 32 & 68 & a & 68 & 50 & 50 & $\mathrm{a}$ & 50 & 79 & 21 & a & 21 \\
\hline Latvia & 8 & 92 & 65 & 27 & 41 & 59 & 18 & 41 & $\mathrm{a}$ & 100 & 72 & 28 & $\mathrm{a}$ & 100 & 92 & 8 & a & 100 & 93 & 7 \\
\hline Russian Federation & 87 & 13 & $\mathrm{a}$ & 13 & 95 & 5 & $\mathrm{a}$ & 5 & 83 & 17 & $\mathrm{a}$ & 17 & 86 & 14 & $\mathrm{a}$ & 14 & 100 & 0 & a & 0 \\
\hline Saudi Arabia & 94 & 6 & $x(2)$ & $x(2)$ & 100 & 0 & $x(6)$ & $x(6)$ & 94 & 6 & $\mathrm{x}(10)$ & $x(10)$ & 82 & 18 & $\mathrm{x}(14)$ & $x(14)$ & 100 & 0 & 0 & 0 \\
\hline South Africa ${ }^{1}$ & 95 & 5 & $\mathrm{x}(2)$ & $\mathrm{x}(2)$ & 90 & 10 & $\mathrm{x}(6)$ & $\mathrm{x}(6)$ & 97 & 3 & $\mathrm{x}(10)$ & $x(10)$ & 95 & 5 & $\mathrm{x}(14)$ & $\mathrm{x}(14)$ & 100 & 0 & 0 & 0 \\
\hline G20 average & 71 & 29 & $\sim$ & $\sim$ & 63 & 37 & $\sim$ & $\sim$ & 68 & 32 & $\sim$ & & 69 & 31 & $\sim$ & $\sim$ & 80 & 20 & $\sim$ & \\
\hline
\end{tabular}

1. Year of reference 2012.

Source: OECD. Argentina, China, Colombia, India, Indonesia, Saudi Arabia and South Africa: UNESCO Institute for Statistics. Latvia: Eurostat. See Annex 3 for notes (www.oecd.org/education/education-at-a-glance-19991487.htm).

Please refer to the Reader's Guide for information concerning symbols for missing data and abbreviations.

StatLink 部 
Table C1.5. Expected years in education from age 5 through 39, by level of education, intensity of study and gender (2013)

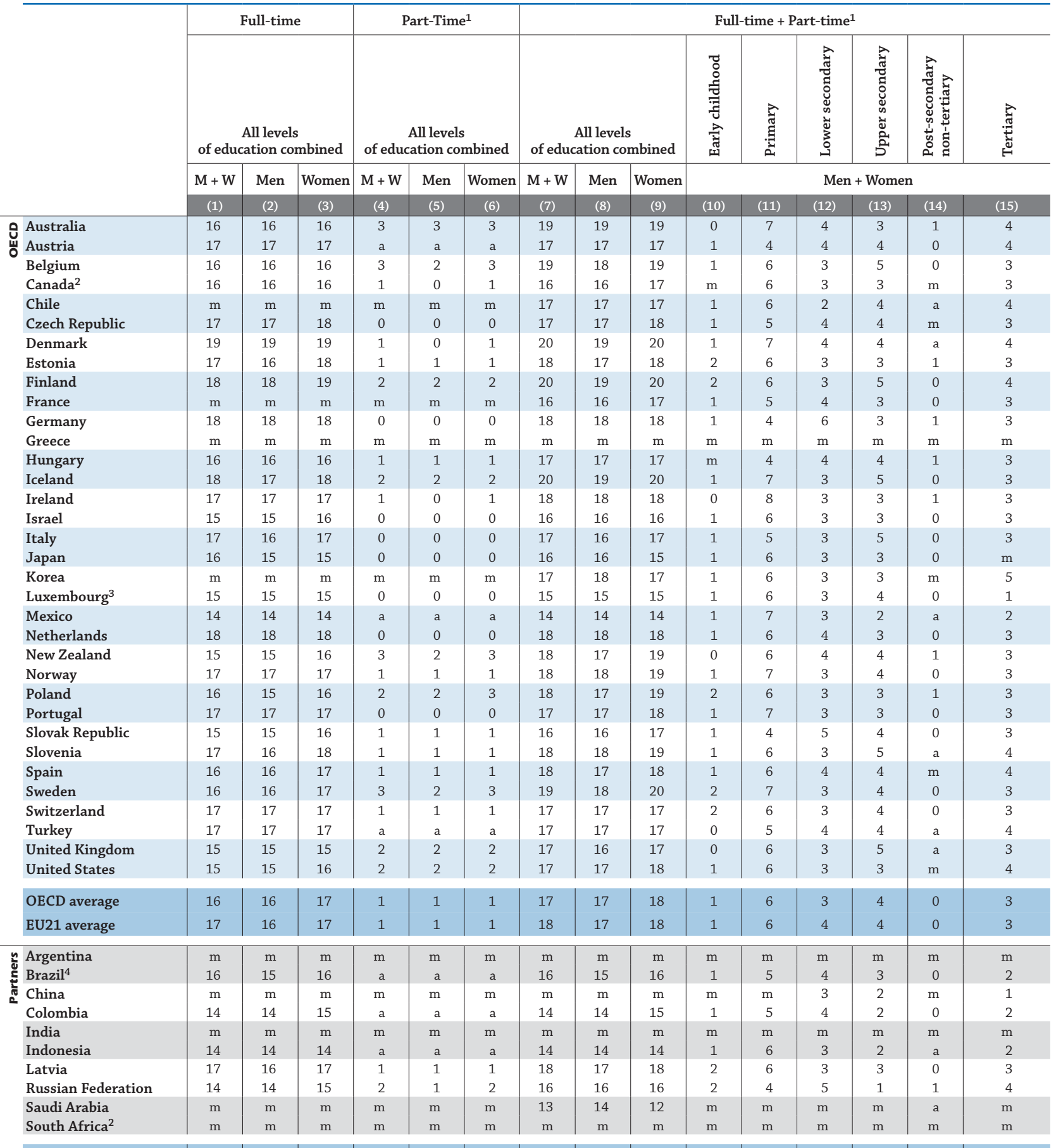
G20 average

1. Expected years in part-time education must be interpreted with caution since they may reflect variations due to different intensities of participation among countries, levels and individuals of different ages.

2. Year of reference 2012.

3. High levels of enrolment abroad and immigration may affect expected years in education.

4. Excludes enrolments in ISCED 7 and 8.

Source: OECD. Argentina, China, Colombia, India, Indonesia, Saudi Arabia and South Africa: UNESCO Institute for Statistics. Latvia: EUROSTAT. See Annex 3 for notes (www.oecd.org/education/education-at-a-glance-19991487.htm).

Please refer to the Reader's Guide for information concerning symbols for missing data and abbreviations.

StatLink 세대 http://dx.doi.org/10.1787/888933285796 


\section{HOW DO EARLY CHILDHOOD EDUCATION SYSTEMS DIFFER AROUND THE WORLD?}

- Fifteen-year-old students who had attended at least one year of pre-primary education perform better on the OECD Programme for International Student Assessment (PISA) test than those who did not, even after accounting for their socio-economic backgrounds.

- Early childhood education is particularly beneficial for students with an immigrant background. Among 15-year-old immigrant students who arrived in their OECD host country before the age of 6 , the gap in performance between those who had attended pre-primary education and those who had not is equivalent to around two years of schooling.

- In a majority of OECD countries, education now begins for most children well before they are 5 years old. Some $74 \%$ of 3-year-olds are enrolled in early childhood education across OECD countries; among OECD countries that are part of the European Union, $80 \%$ of 3-year-olds are enrolled.

\section{Chart C2.1. Enrolment rates at age 3 and 4 in early childhood education (2013)}

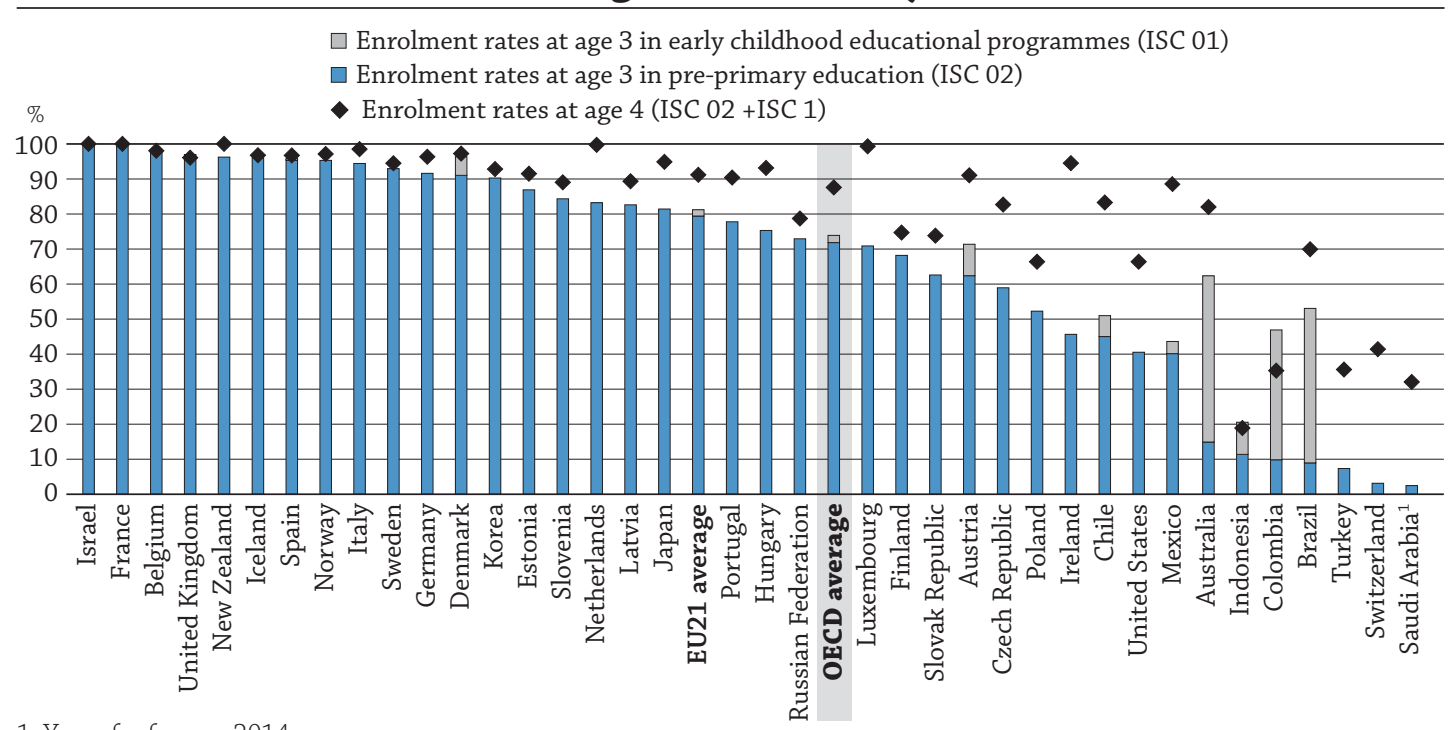

1. Year of reference 2014.

Countries are ranked in descending order of the enrolment rates of 3 year-olds in pre-primary programmes.

Source: OECD. Table C2.1. See Annex 3 for notes (www.oecd.org/education/education-at-a-glance- 19991487.htm).

StatLink 제이 $h t t p: / / d x . d o i . o r g / 10.1787 / 888933284184$

\section{Context}

As family structures change, so do the relative ages of parents. More women and men are waiting until later in life to begin their families. They do so for a number of reasons, including planning for greater financial security and emotional maturity, taking more time to find a stable relationship, and committing to their careers before turning their attention to having children. As younger and older parents are also more likely to be in the workforce today, there is a growing need for early childhood education. In addition, there is increasing awareness of the key role that early childhood education plays in the cognitive and emotional development of the young. As a result, ensuring the quality of early childhood education and care $(E C E C)$ has become a policy priority in many countries.

Enrolling children in early childhood education can also mitigate social inequalities and promote better student outcomes overall. Many of the inequalities found in education systems are already evident when children enter formal schooling and persist as they progress through the school system. Because inequalities tend to grow when school is not compulsory, earlier entrance into the school system may reduce these inequalities. In addition, pre-primary education helps to prepare children to enter and succeed in formal schooling.

There are many different ECEC systems and structures within OECD countries. Consequently, there is also a range of different approaches to identifying the boundary between early childhood education and childcare (Box C2.1 and see the Definitions section). These differences should be taken into account when drawing conclusions from international comparisons. 


\section{Other findings}

- Almost nine out of ten 4-year-olds (88\%) are enrolled in early childhood or primary education across OECD countries.

- Some $84 \%$ of funding of pre-primary educational institutions in European OECD countries come from public sources compared to $80 \%$ on average across OECD countries.

- Expenditure on pre-primary education accounts for an average of $0.6 \%$ of GDP while expenditure on early childhood education development accounts for an average of $0.4 \%$ of GDP.

- In most countries, the proportions of children enrolled in private early childhood education are considerably larger than those enrolled in private primary and secondary educational institutions. Thus, more than $50 \%$ of children enrolled in early childhood development programmes attend private institutions, on average. This can result in heavy financial burdens for parents, even when government subsidies are provided.

- The ratio of children to teaching staff is an indicator of the resources devoted to early childhood education. The child-teacher ratio at the pre-primarylevel, excluding non-teachingstaff (e.g.teachers' aides), ranges from more than 20 children per teacher in Chile, China, Colombia, France, Indonesia and Mexico, to fewer than 10 in Estonia, Iceland, New Zealand, the Russian Federation, Slovenia, Sweden and the United Kingdom.

- Some countries make extensive use of teachers' aides in pre-primary education. Twelve countries reported smaller ratios of children to contact staff than of children to teaching staff. As a result, the ratios of children to contact staff in pre-primary education are substantially lower than the ratios of children to teaching staff (more than two fewer children) in Austria, Chile, China, France, Norway and the Russian Federation.

\section{Trends}

Over the past decade, many countries have expanded early childhood education. This increased focus has resulted in the extension of compulsory education to lower ages in some countries, free early childhood education, universal provision of early childhood education, and the creation of programmes that integrate care with formal pre-primary education.

On average among those OECD countries with 2005 and 2013 data, enrolments in pre-primary education rose from 52\% of 3-year-olds in 2005 to $72 \%$ in 2013, and from $69 \%$ of 4 -year-olds in 2005 to $85 \%$ in 2013. The enrolment rates of 4-year-olds in pre-primary education increased by 20 percentage points or more in Australia, Chile, Korea, Mexico, Poland, Russian Federation and Turkey between 2005 and 2013.

\section{Note}

ISCED level 0 refers to early childhood programmes that have an intentional education component. ISCED level 0 programmes target children below the age of entry into primary education (ISCED level 1). These programmes aim to develop cognitive, physical and socio-emotional skills necessary for participation and well-being in school and society.

Thanks to the new ISCED classification, level 0 covers now early childhood education for all ages, including very young children. Programmes are sub-classified into two categories, depending on the level of complexity of the educational content: early childhood educational development (code 01) and pre-primary education (code 02). Early childhood educational development programmes (code 01) are generally designed for children younger than 3. They are introduced as a new category in ISCED 2011 and were not covered by ISCED-97. Pre-primary education (code 02) corresponds exactly to level 0 in ISCED-97. Early childhood educational development programmes are not provided in Belgium (except in the Flemish Community), the Czech Republic, France, Ireland, Italy, Japan, Luxembourg, Poland, Portugal, the Slovak Republic and Switzerland. In these countries, other structures exist, but the programmes providing ECEC are outside the scope of ISCED 2011 or outside the scope of the UOE data collection (see more details in Box C2.1 and in the Definitions section). 


\section{Analysis}

In a majority of OECD countries, ECEC policy has paralleled the evolution of women's participation in the labour force. More and more women have become salaried employees since the 1970s, as the service- and knowledge-based economies expanded. Because economic prosperity depends on maintaining a high employment-to-population ratio, encouraging more women to enter the labour market has prompted greater government interest in expanding ECEC services. In the 1970s and 1980s, European governments, in particular, put family and childcare policies into place to encourage couples to have children and ensure that it is feasible for women to combine work and family responsibilities (OECD, 2013a; 2011a).

There is a growing body of evidence that children who start strong in their learning and well-being will have better outcomes when they grow older. Such evidence has prompted policy makers to design early interventions and re-think their education spending patterns to gain "value for money".

\section{Enrolment in early childhood education}

Early childhood education, as defined in the ISCED 2011 classification, is the initial stage of organised instruction for many children and can play a significant role in their development. While primary and lower secondary enrolment patterns are fairly similar throughout OECD countries, there is significant variation in early childhood education programmes among OECD and other G20 countries. This includes the overall level of participation in programmes, the typical starting age for children, financing and the duration of the programme (Table C2.5).

In most OECD countries, education now begins for most children well before they are 5 years old. Almost nine out of ten 4-year-olds (88\%) are enrolled in pre-primary and primary education across OECD countries. In the OECD countries that are part of the European Union, $91 \%$ of 4-year-olds are enrolled. Enrolment rates for pre-primary and primary education at this age vary from $95 \%$ or more in Belgium, Denmark, France, Germany, Iceland, Ireland, Israel, Italy, Japan, Luxembourg, the Netherlands, New Zealand, Norway, Spain and the United Kingdom, to less than $60 \%$ in Colombia, Indonesia, Saudi Arabia and Turkey. Switzerland also falls into this group, but because enrolment in integrated programmes is not reported for those countries (see the Definitions section), the true enrolment rate cannot be calculated and is likely to be higher than that reported here. In Switzerland, the enrolment rate in early childhood education is highest among 5-year-old children (Table C2.1).

\section{Enrolment in early childhood education and PISA performance at age 15}

On average across OECD countries, $74 \%$ of the 15-year-old students assessed by the OECD Programme for International Student Assessment (PISA) reported that they had attended more than one year of pre-primary education. According to students' responses, enrolment in more than one year of pre-primary education was nearly universal about ten years ago in Belgium, France, Hungary, Iceland, Japan and the Netherlands, where over $90 \%$ of 15 -year-olds reported that they had attended pre-primary education for more than one year. Pre-primary education is rare in Turkey, where fewer than $30 \%$ of 15 -year-olds had attended pre-primary education for any period of time. More than one year of pre-primary education is uncommon in Australia, Chile, Ireland and Poland, where fewer than $52 \%$ of students reported that they had attended pre-primary education for that length of time (see OECD, 2013b, Table IV.3.33).

\section{Box C2.1. Coverage of early childhood education programmes (ISCED 01 and ISCED 02) in Indicator C2}

In ISCED 2011 (and now for the first time in Education at a Glance 2015), level 0 covers early childhood education for all ages, including very young children. Programmes are sub-classified into two categories depending on age and the level of complexity of the educational content: early childhood educational development (code 010) and pre-primary education (code 020). Early childhood educational development programmes (code 010) are generally designed for children younger than 3 . They are introduced as a new category in ISCED 2011 and were not covered by ISCED 1997. Pre-primary education (code 020) corresponds exactly to level 0 in ISCED 1997.

Data from age-integrated programmes designed to include children both younger and older than 3 should be allocated to 010 and 020 , according to the age of the children, as indicated above. This may involve estimation of expenditures and personnel at levels 010 and 020, respectively. 
However, some countries internally define early childhood education more broadly than others. Thus, the comparability of international statistics on programmes at ISCED level 0 depends on each country's willingness and ability to report data for this level according to a standard international definition, even if that definition diverges from the one that the country uses in compiling its own national statistics. In this regard, the data reported in Education at a Glance as ISCED level 0 programmes may differ from national reporting of early childhood education.

To ensure international comparability of data, several criteria need to be met to determine whether or not a programme should be classified as ISCED level 0 and included in reporting. For a programme to be reported as ISCED level 0, it must:

- Have adequate intentional educational properties; and

- Be institutionalised; and

- Be targeted at children within the age range starting from age 0 up to the age of entry into ISCED level 1 education; and also

- Meet the minimum intensity/duration (an intensity of at least 2 hours per day; and a duration of at least 100 days a year).

Programmes should wherever possible also:

- Have a regulatory framework recognised by the relevant national authorities; $\underline{\text { and }}$

- Have trained or accredited staff as per the appropriate regulatory framework.Programmes that provide childcare only (i.e. supervision, nutrition and health) are excluded from this Indicator. Where both educational and non-educational programmes exist and it is possible to enrol in each independently, only the educational programmes are reported in Indicator C2. For example, in an institution that offers a daytime educational programme as well as extended afternoon or evening childcare programmes, and where parents may choose to enrol their child in either or both programmes, only the daytime educational programme is reported in Indicator $\mathrm{C} 2$. Integrated programmes in which the non-educational portion is greater than the educational portion may be included as long as the educational portion meets certain criteria.

ISCED level 0 also excludes purely family-based arrangements that may be purposeful but do not meet the UOE definition of a "programme" (e.g. informal learning by children from their parents, other relatives or friends is not included under ISCED level 0). Also excluded are learning activities delivered from private homes or other institutionalised centres that are outside the jurisdiction of an appropriate national early childhood education authority or regulatory body, regardless of whether the activities are organised in the style of an approved early childhood education programme. An example of this would be a private citizen who, of his or her own volition, provides learning opportunities for young children that nominally meet the ISCED level criteria around intentional education, intensity/duration and staff qualification requirements, but who is not recognised by an authorising body.

Examples of programmes to be excluded from reporting include:

- Programmes where attendance can be ad-hoc or of a drop-in style where individual children will not experience a continuity of structured learning opportunities.

- Short-duration programmes, such as vacation care, which may have an educational curriculum but not a sustained period of instruction or learning opportunities.

- Programmes with intentional educational properties but with no minimum level of attendance, such as when parents are free to choose an intensity and duration of their child's attendance that does not meet the ISCED level 0 criteria.

- Early childhood services that are open for extended hours and provide intentional educational activities during these hours, but do not require a minimum intensity/duration of attendance or enrolment.

OECD countries will continue working together to improve methods of reporting statistics on early childhood education. Country-specific information can be found in Annex 3 of this publication and in Table C2.5. 
PISA analyses find that in most countries, students who had attended at least one year of pre-primary education tend to perform better than those who had not, even after accounting for students' socio-economic background. PISA research also shows that the relationship between pre-primary attendance and performance tends to be stronger in school systems with longer duration pre-primary education, smaller child-to-teacher ratios in pre-primary education, and higher public expenditure per child at the pre-primary level (OECD 2013b, Table IV.3.33).

Participation in early childhood education is particularly beneficial for children with an immigrant background. Across OECD countries, an average of 69\% of 3-6 year-old immigrant children were enrolled in pre-primary education in 2012 - an attendance rate that was 7 percentage points lower than among their native-born peers. In the European Union, immigrant children are only marginally less likely than native-born children to attend pre-primary education. There are, however, some exceptions: in the Czech Republic, Italy and Norway, for example, attendance rates are 10 percentage points lower among immigrant children (see Figure 13.5 in Indicators of Immigrant Integration 2015: Settling In [OECD/European Union, 2015]).

Among immigrant children of comparable socio-economic backgrounds who had arrived in their OECD host country before the age of 6 , those who had attended pre-primary education scored better in the PISA reading assessment, when they were 15 years old, than those who had not attended pre-primary education in their OECD host country. The performance gap between the two groups is 75 score points, equivalent to around two years of schooling (the performance gap related to pre-school attendance is narrower, but still considerable, among non-immigrant 15-year-old students). The benefits of early childhood education for immigrant children are particularly significant in France, Finland, Israel and the United States. This finding has special resonance in the United States, where proportion of immigrant children who are enrolled in early childhood education is relatively small (Chart C2.2).

In addition, early arrival in the host country (e.g. before the age of six) contributes to better results among immigrant children. Thus, those who arrived in their OECD host country between the ages of 6 and 10 earn PISA reading scores that are, on average, 19 points lower compared to those who had arrived in their host country before the age of 6 . The gap exceeds 39 score points (the equivalent of around one year of schooling) in Belgium, Finland, France, Germany, Iceland and Israel (see Table 13.A1.6 in Indicators of Immigrant Integration 2015: Settling In [OECD/European Union, 2015]).

\section{Chart C2.2. Mean PISA scores of 15-year-old students who did or did not attend preschool in the host country (2012)}

Differences in PISA reading scores after accounting for socio-economic background

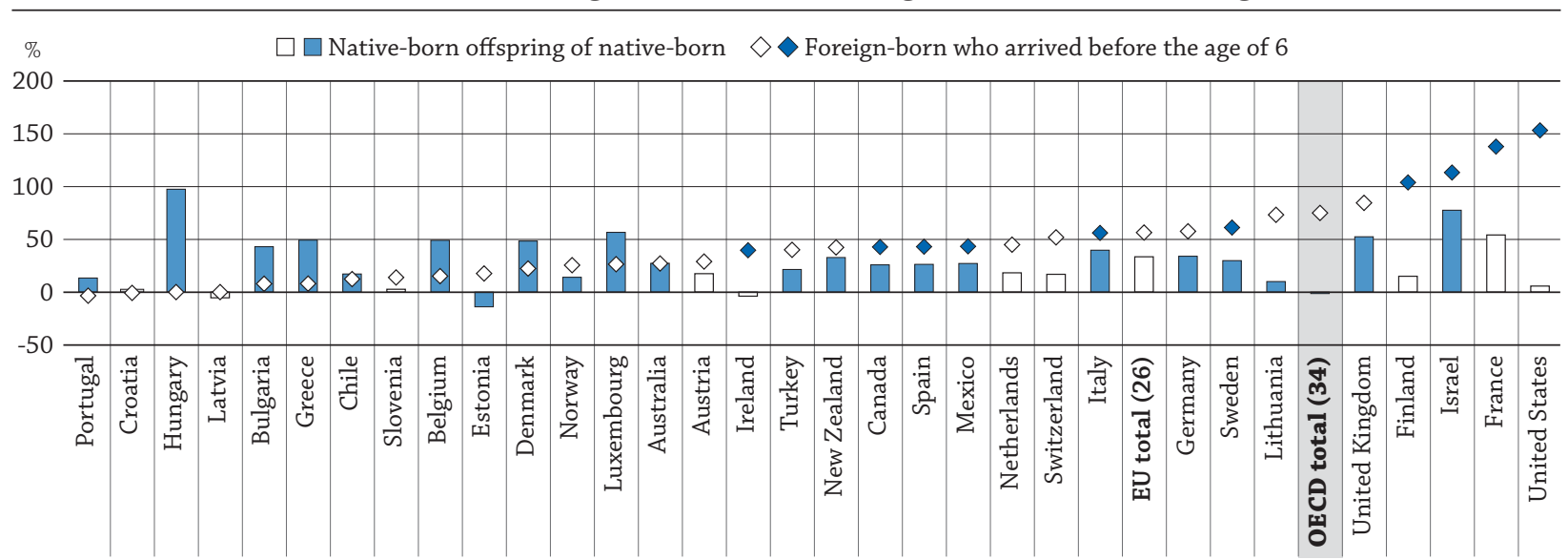

Note: White bars and markers indicate differences which are not statistically significant (with a probability of 0.05). Positive figures means that those who had attended preschool scored better in the PISA reading assessment than those who had not attended preschool.

Countries are ranked in ascending order of the difference in PISA reading scores between the immigrants who arrived in their OECD host country before the age of 6 and who had attended pre-primary education and those who had not attended pre-primary education.

Source: OECD Programme of International Student Assessment (PISA). Indicators of Immigrant Integration 2015: Settling In (http://dx.doi.org/ 10.1787/9789264234024-en), Figure 13.6. See Annex 3 for notes (www.oecd.org/education/education-at-a-glance- 19991487.htm).

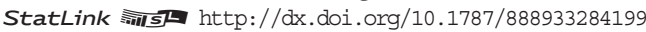

Early childhood education programmes for even younger children are not as extensive. In some countries, demand for early childhood education for children aged 3 and under far outstrips supply, even in countries that provide for long parental leave. Four out of ten 2-year-olds are enrolled in early childhood education across OECD countries, as a whole, growing to almost three out of four (74\%) for 3-year-olds. The highest enrolment rates of 3-year-olds in early 
childhood education are found in Belgium, Denmark, France, Iceland, Italy, New Zealand, Norway, Spain and the United Kingdom. In countries where public funding for parental leave is limited, many working parents must either look to the private market, where parents' ability to pay significantly influences access to quality services, or else rely on informal arrangements with family, friends and neighbours (Table C2.1, Chart C2.1 and Starting Strong III [OECD, 2011b]).

Some countries have made access to pre-primary education almost universal for children by the time they are three. The availability of early childhood education is growing quickly in most countries. On average among OECD countries with 2005 and 2013 data, enrolments in pre-primary education rose from 52\% of 3-year-olds in 2005 to $72 \%$ in 2013, and from 69\% of 4-year-olds in 2005 to 85\% in 2013 (Table C2.1).

\section{Early childhood education, by type of institutions}

As countries continue to expand their early childhood education programmes, it will be important to consider parents' needs and expectations regarding accessibility, cost, programme and staff quality and accountability. When parents' needs for quality, accessibility or accountability are not met in public institutions, some parents may be more inclined to send their children to private pre-primary institutions, childcare programmes, or extracurricular activities. (Shin et al., 2009).

\section{Chart C2.3. Percentage of pupils enrolled in public institutions in early childhood education (2013)}

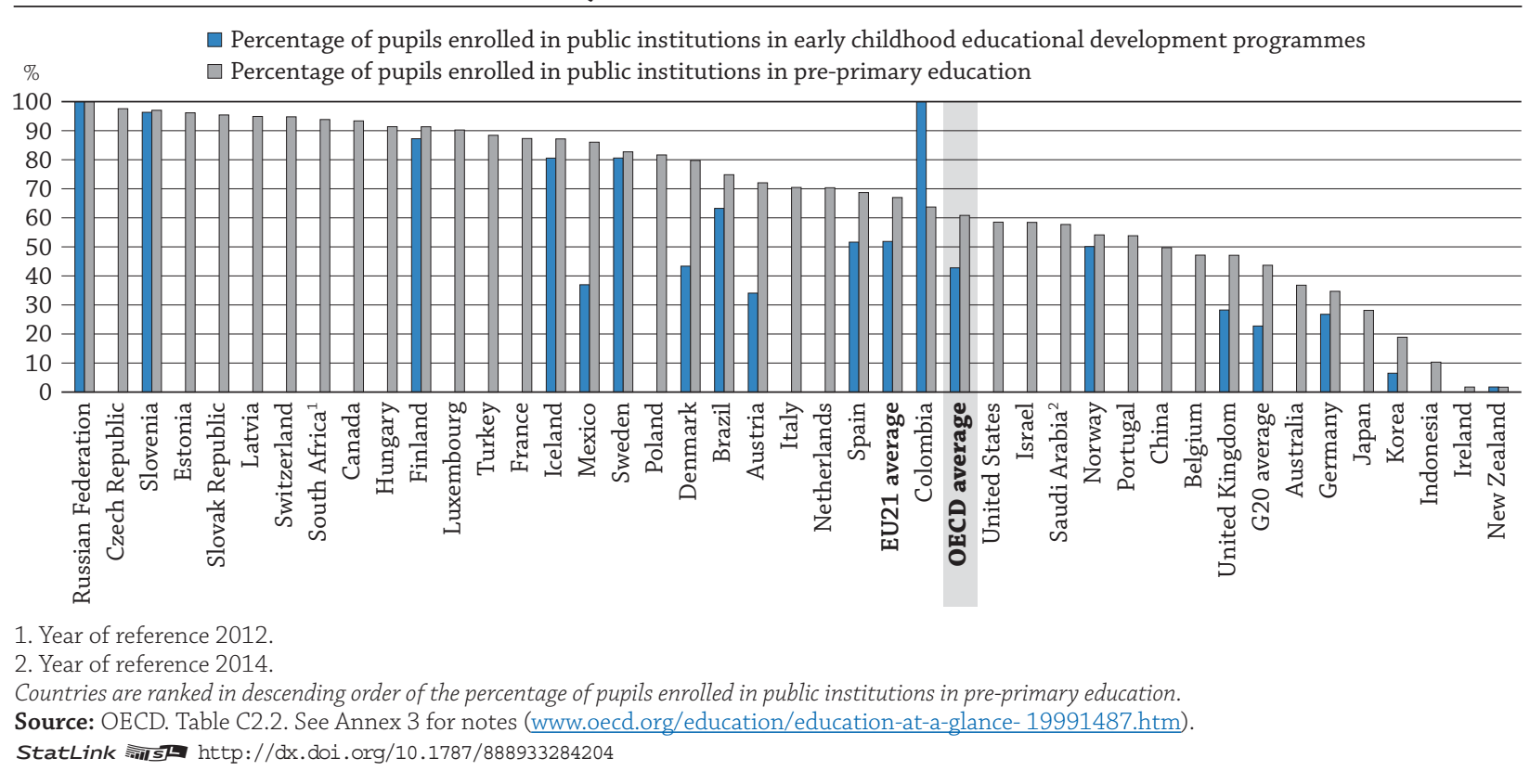

In most countries, a minority of students attends private schools at the primary through upper secondary levels. However, the proportions of children enrolled in private pre-primary schools are considerably larger: some $15 \%$ of children in pre-primary education are enrolled in independent private schools on average across OECD countries. When considering pre-primary independent private and government-dependent private schools together, 39\% of children are enrolled in private pre-primary programmes. This proportion exceeds $50 \%$ in Australia, Belgium, Germany, Indonesia, Ireland, Japan, Korea, New Zealand and the United Kingdom (Table C2.2 and Chart C2.3). Moreover, in 12 out of 13 OECD countries with available data, except New Zealand, the share of children enrolled in private (both independent and government-dependent) early childhood education development programmes (ISCED 01) is larger than the share enrolled in private pre-primary (ISCED 02) schools. In New Zealand, almost all early childhood education institutions are private government-dependent, and these cover $98 \%$ of enrolled children at both ISCED 01 and ISCED 02 levels. On average, about 58\% of all children enrolled in early childhood development programmes are in independent or government-dependent private schools while only 39\% of preprimary children are. In most of these countries, the proportion of private funding is also larger for early childhood education development programmes (ISCED 01) than for pre-primary programmes (ISCED 02). This can result in heavy financial burdens for parents, even when government subsidies are provided (Table C2.2 and Chart C2.3). 


\section{Financing early childhood education}

Sustained public funding is critical for supporting the growth and quality of early childhood education programmes. Appropriate funding helps to recruit professional staff who are qualified to support children's cognitive, social and emotional development. Investment in early childhood facilities and materials also helps support the development of child-centred environments for learning. In countries that do not channel sufficient public funding to cover both quantity and quality, some parents may be more inclined to send their children to private ECEC services, which implies heavy financial burdens (OECD, 2011b); others may prefer to stay home, which can hinder a parent's participation in the labour force (OECD, 2011a).

Public expenditure on pre-primary education is mainly used to support public institutions, but in some countries it also funds private institutions, to varying degrees. At the pre-primary level, annual expenditure, from both public and private sources, per child for both public and private institutions averages USD 8008 in OECD countries. However, expenditure varies from USD 4000 or less in Israel, Latvia and South Africa, to more than USD 10000 in Australia, Iceland, Luxembourg, Sweden, the United Kingdom and the United States (Table C2.3, and see Table B1.1a in Indicator B1).

At the early childhood educational development level, annual expenditure, from both public and private sources, per child, for both public and private institutions averages USD 12324 in OECD countries with available data.

\section{Chart C2.4. Expenditure on pre-primary educational institutions (2012)} As a percentage of GDP, by funding source

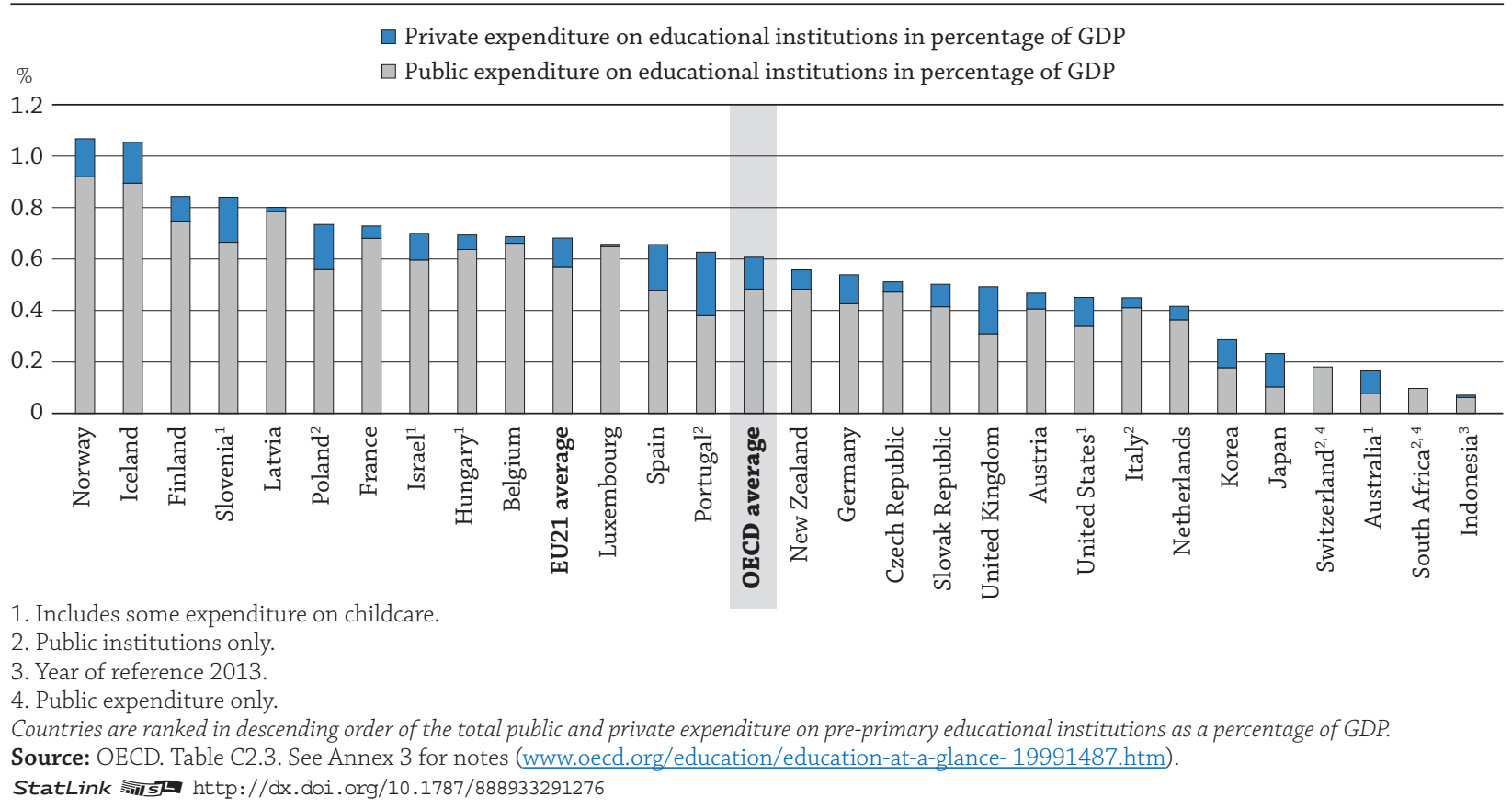

Expenditure on pre-primary education accounts for an average of $0.6 \%$ of the collective GDP. Differences between countries are significant. For example, while $0.1 \%$ or less of GDP is spent on pre-primary education in Indonesia and South Africa, $0.8 \%$ or more is spent in Finland, Iceland, Latvia, Norway, Slovenia, and Sweden (Table C2.3 and Chart C2.4). These differences are largely explained by enrolment rates, legal entitlements and costs, and the different starting age for primary education; they are also influenced by the extent to which this indicator covers private early childhood education. In Switzerland, the absence of data on integrated programmes at this level is also likely to understate the true level of expenditure and enrolments in early childhood education programmes (see more details in Box C2.1), and may affect the comparability of the data to that of other countries. Inferences on access to and quality of ECEC should therefore be made with caution (Table C2.3 and Box C2.1). Differences in expenditure as a percentage of GDP could be influenced by the duration of programmes. In some countries, like New Zealand, pre-primary education spans two years, while in other countries it spans three or even four years (see Table C2.5). The duration of a programme has an impact on the level of expenditure devoted to early childhood education. 
Publicly funded pre-primary education tends to be more strongly developed in the European than the non-European countries of the OECD. In Europe, the concept of universal access to education for 3-6 year-olds is generally accepted. Most countries in this region provide all children with at least two years of free, publicly funded pre-primary education in schools before they begin primary education. With the exception of Ireland and the Netherlands, such access is generally a statutory right from the age of 3 , and in some countries, even before then. Compared to primary, secondary and post-secondary non-tertiary education, pre-primary institutions receive the largest proportion of funds (20\%) from private sources. However, this proportion varies widely, ranging from $5 \%$ or less in Belgium, Latvia and Luxembourg, to 50\% or more in Australia and Japan (Table C2.3 and Starting Strong II [OECD, 2006]).

\section{The child-teacher ratio varies considerably across OECD countries}

Research demonstrates that enriched, stimulating environments and high-quality pedagogy are fostered by betterqualified practitioners, and that better-quality staff-child interactions facilitate better learning outcomes. While qualifications are one of the strongest predictors of staff quality, the level of qualification tells only part of the story. Qualifications indicate how much specialised and practical training is included in initial staff education, what types of professional development and education are available to, and taken up by, staff, and how many years of experience staff have accumulated. In addition, working conditions can influence professional satisfaction, which is likely to affect the ability and willingness of professionals to build relationships and interact attentively with children (Shin et al., 2009). High turnover disrupts the continuity of care, undermines professional development efforts, lowers overall quality, and adversely affects child outcomes.

The ratio of children to teaching staff is also an important indicator of the resources devoted to education. That ratio is obtained by dividing the number of full-time equivalent children at a given level of education by the number of full-time equivalent teachers at that level and in similar types of institutions. However, this ratio does not take into account instruction time for students, nor how much time teachers spend teaching. Therefore, it cannot be interpreted in terms of class size. The number of children per class summarises different factors, but distinguishing between these factors helps to identify differences in the quality of education systems (see Indicator D2).

Table C2.2 shows the ratio of children to teaching staff and also the ratio of children to contact staff (e.g. teachers and non-professional staff [teachers' aides]) in early childhood education. Some countries make extensive use of teachers' aides at the pre-primary level. Twelve out of $24 \mathrm{OECD}$ and G20 countries reported smaller ratios of children to contact staff (Column 10 of Table C2.2) than of children to teaching staff. The ratios of children to contact staff are substantially lower in Austria, Chile, China, France, Norway and the Russia Federation. On average across OECD countries, there are 14 children for every teacher in pre-primary education. The child-teacher ratio, excluding teachers' aides, ranges from more than 20 children per teacher in Chile, China, Colombia, France, Indonesia and Mexico, to fewer than 10 in Estonia, Iceland, New Zealand, the Russian Federation, Slovenia, Sweden and the United Kingdom (Table C2.2 and Chart C2.5).

\section{Chart C2.5. Ratio of pupils to teaching staff in early childhood education (2013)} Public and private institutions, calculation based on full-time equivalents

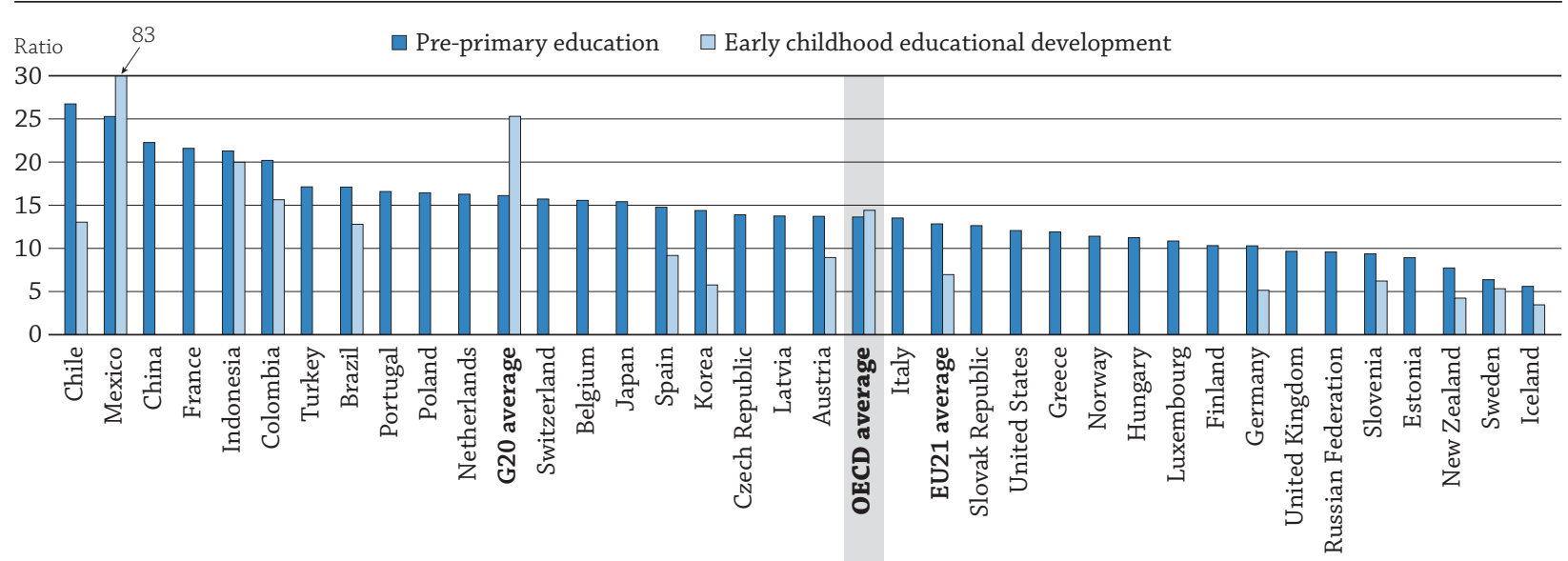

Note: The figures should be interpreted with some caution because the indicator compares the teacher/student ratios in countries with "education-only" and "integrated education and daycare" programmes. In some countries, the staff requirements in these two types of provision are very different. Countries are ranked in descending order of students to teaching staff ratios in pre-primary education.

Source: OECD. Table C2.2. See Annex 3 for notes (www.oecd.org/education/education-at-a-glance- 19991487.htm).

StatLink 제그 http://dx.doi.org/10.1787/888933291280 


\section{Definitions}

Education-only programmes in early childhood education are those that primarily offer education services for a short period of the day. Working parents usually have to use additional care services in the morning and/or afternoon.

Integrated programmes in early childhood education are those that provide both early childhood education and care in the same programme.

Some variations at the national level cannot be presented, and information on the "characteristics of programmes" has been simplified in some cases. For example, in some countries, the starting age of early childhood education programmes differs among jurisdictions or regions. In these instances, the information that is the most common or typical is reported.

ISCED level 0 refers to early childhood programmes that have an intentional education component. ISCED level 0 programmes target children below the age of entry into primary education (ISCED level 1).

Programmes at ISCED level 0 are typically designed with a holistic approach to support children's early cognitive, language, physical, social and emotional development and to introduce young children to organised instruction in an institutionalised setting. At this level, programmes are not necessarily highly structured, but they are designed to provide an organised and purposeful set of learning activities in a safe environment. They allow children to learn through interaction with other children under the guidance of staff/educators, typically through creative and play-based activities.

ISCED level 0 refers to those early childhood programmes that have an intentional education component. These programmes aim to develop the socio-emotional skills necessary for participation in school and society, to develop some of the skills needed for academic readiness, and to prepare children for entry into primary education.

Along with an intentional child-development and education focus, a key defining factor of ISCED level 0 programmes is the sustained intensity and duration of delivery of intentional educational activities. These are what differentiate ISCED level 0 from other programmes, such as childcare and occasional, after hours or vacation care.

ISCED level 0 programmes target children below the age of entry into ISCED level 1 . There are two categories of ISCED level 0 programmes: ISCED 010 - early childhood educational development, and ISCED 020 - pre-primary education. ISCED 010 has intentional educational content designed for younger children (typically in the age range of 0 to 2 years), while ISCED 020 is typically designed for children from age 3 to the start of primary education (ISCED level 1).

Programmes classified at ISCED level 0 may be referred to in many ways nationally, for example: early childhood education and development, play school, reception, pre-primary, pre-school, Kindergarten, Kita, Krippe, or educación inicial. For programmes provided in crèches, daycare centres, private homes, nurseries, Tagespflege or guarderías, it is important to ensure that they meet the ISCED level 0 classification criteria specified below. For international comparability purposes, the term "early childhood education" is used to label ISCED level 0 (see also Box C2.1 and ISCED 2011 Operational Manual: Guidelines for Classifying National Education Programmes and Related Qualifications (OECD/Eurostat/UNESCO Institute for Statistics [2015]).

In addition to the above, the educational properties of ISCED level 0 programmes can be further described as follows:

- ISCED 010 - Early childhood educational development

The learning environment is visually stimulating and language rich, and fosters self-expression with an emphasis on language acquisition and the use of language for meaningful communication. There are opportunities for active play so that children can exercise their co-ordination and motor skills under supervision and in interaction with staff. Typically aimed at very young children aged 0-2.

\section{- ISCED 020 - Pre-primary education}

Through interaction with peers and educators, children improve their use of language and their social skills, start to develop logical and reasoning skills, and talk through their thought processes. They are also introduced to alphabetical and mathematical concepts, understanding and use of language, and are encouraged to explore their surrounding world and environment. Supervised gross motor activities (i.e. physical exercise through games and other activities) and play-based activities can be used as learning opportunities to promote social interactions with peers and to develop skills, autonomy and school readiness. Aimed at children in the years immediately prior to starting compulsory schooling, typically aged 3-5. 


\section{Methodology}

ISCED level 0 programmes are usually school-based or otherwise institutionalised for a group of children. As the institutions authorised to provide ISCED level 0 programmes vary between jurisdictions (e.g. centre-based, community-based, home-based), to be reported in the UOE collection both the programme and the mode or institution of delivery should be recognised within the respective early childhood education system. Particular care is given to programmes delivered from home-based settings: if the programme meets the criteria as set out above and is recognised under the respective regulations, it is included in reporting.

To further ensure international comparability of data, once a programme has been identified as an ISCED level 0 early childhood education programme by meeting the criteria above, the following rules apply when collecting data on the programmes for UOE purposes. These rules are applied to programmes in their entirety (not just to the intentional education component):

\section{Full-time equivalents for enrolments}

The concepts used to define full-time and part-time participation at other ISCED levels, such as study load, student participation, and the academic value or progress that the study represents, are not easily applicable to ISCED level 0. In addition, the daily or weekly hours that represent a typical full-time enrolment in an education programme at ISCED level 0 varies widely between countries. Because of this, full-time-equivalents cannot be calculated for ISCED level 0 programmes in the same way as for other ISCED levels.

A consensus has not been reached on a methodology for calculating FTE for enrolments at ISCED 0 level but it is recommended in UOE reporting to estimate children enrolled in full-time equivalents by ISCED 0 enrolment headcount (i.e. all enrolments counted as full-time). Headcount is not a satisfying criterion for full-time equivalent for indicators, such as expenditure per student (even if it is accepted for enrolment comparisons), but most countries are in favour of this solution; the same guarantee was not offered by other estimation methods.

\section{Institutions that provide both education programmes and childcare programmes}

In some countries, institutions providing early childhood education also provide extended day or evening childcare programmes. Education programmes traditionally provided during the day may now be provided outside these hours to offer further flexibility to parents and carers of children. These are given special consideration in reporting.

Where the childcare components are distinctly separate from early childhood education components (for example, the two components are offered as individual programmes in which children must enrol separately), the childcare components are excluded from reporting. If the programme is in the form of extended day or evening programmes that meet all of the criteria listed above, they are included in reporting as educational programmes.

Where both education and noneducation programmes exist and it is possible to enrol in each independently, only the education programmes are reported. Integrated programmes are included when the non-education portion is greater than the education portion, only when the education portion meets the criteria listed above. For example, in an institution that offers a daytime education programme as well as extended afternoon or evening childcare programmes, and parents may choose to enrol their child in either or both programmes, only the daytime educational programme is reported in the UOE data collection.

\section{Note regarding data from Israel}

The statistical data for Israel are supplied by and are under the responsibility of the relevant Israeli authorities. The use of such data by the OECD is without prejudice to the status of the Golan Heights, East Jerusalem and Israeli settlements in the West Bank under the terms of international law.

\section{References}

OECD (2013a), "How Do Early Childhood Education and Care (ECEC) Policies, Systems and Quality Vary Across OECD Countries?", Education Indicators in Focus, No. 11, OECD Publishing, Paris, http://dx.doi.org/10.1787/5k49czkz4bq2-en.

OECD (2013b), PISA 2012 Results: What Makes Schools Successful (Volume IV): Resources, Policies and Practices, PISA, OECD Publishing, Paris, http://dx.doi.org/10.1787/9789264201156-en.

OECD (2011a), How's Life?: Measuring Well-being, OECD Publishing, Paris, http://dx.doi.org/10.1787/9789264121164-en. 
OECD (2011b), Starting Strong III: A Quality Toolbox for Early Childhood Education and Care, OECD Publishing, Paris, http://dx.doi. org/10.1787/9789264123564-en.

OECD (2006), Starting Strong II: Early Childhood Education and Care, OECD Publishing, Paris, http://dx.doi.org/10.1787/ 9789264035461-en.

OECD/European Union (2015), Indicators of Immigrant Integration 2015: Settling In, OECD Publishing, Paris, http://dx.doi.org/ 10.1787/9789264234024-en.

OECD/Eurostat/UNESCO Institute for Statistics (2015), ISCED 2011 Operational Manual: Guidelines for Classifying National Education Programmes and Related Qualifications, OECD Publishing, Paris, http://dx.doi.org/10.1787/9789264228368-en.

Shin, E., M. Jung and E. Park (2009), “A Survey on the Development of the Pre-school Free Service Model”, Research Report of the Korean Educational Development Institute, Seoul.

\section{Indicator C2 Tables}

StatLink त्माज्ञा http://dx.doi.org/10.1787/888933285801

Table C2.1 Enrolment rates in early childhood education and primary education, by age $(2005,2013)$

Table C2.2 Profile of early childhood educational development programmes and pre-primary education (2013)

Table C2.3 Expenditure on early childhood educational institutions (2012)

Table C2.4 Profile of education-only and integrated pre-primary programmes (2013)

Table C2.5 Coverage of early childhood education programmes in OECD and partner countries

Cut-off date for the data: 23 October 2015. Updates can be found on line at http://dx.doi.org/10.1787/eag-data-en. 
Table C2.1. Enrolment rates in early childhood education and primary education, by age $(2005,2013)$

\begin{tabular}{|c|c|c|c|c|c|c|c|c|c|c|c|c|c|c|c|c|c|c|c|}
\hline & \multicolumn{15}{|c|}{ Enrolment rates (2013) } & \multicolumn{4}{|c|}{ Enrolment rates (2005) } \\
\hline & \multicolumn{3}{|c|}{ Age 2} & \multicolumn{3}{|c|}{ Age 3} & \multicolumn{3}{|c|}{ Age 4} & \multicolumn{3}{|c|}{ Age 5} & \multicolumn{3}{|c|}{ Age 6} & \multirow{2}{*}{ 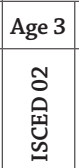 } & \multicolumn{3}{|c|}{ Age 4} \\
\hline & 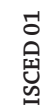 & 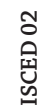 & సేّ & 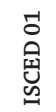 & 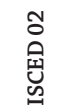 & 予 & 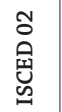 & 至 & 胥 & 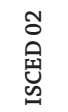 & 䓢 & సేّ & 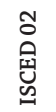 & 跑 & సేّ & & 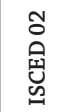 & 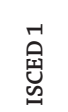 & సేّ \\
\hline & (1) & (2) & (3) & (4) & (5) & $(6)$ & (7) & (8) & (9) & (10) & (11) & (12) & (13) & (14) & (15) & (16) & (17) & (18) & (19) \\
\hline Australia & 55 & 0 & 55 & 47 & 15 & 62 & 80 & 2 & 82 & 16 & 85 & 100 & 0 & 100 & 100 & 17 & 51 & 2 & 53 \\
\hline Austria & 27 & 7 & 34 & 9 & 62 & 71 & 91 & 0 & 91 & 96 & 0 & 96 & 40 & 58 & 98 & $\mathrm{~m}$ & $\mathrm{~m}$ & $\mathrm{~m}$ & $\mathrm{~m}$ \\
\hline Belgium & a & 51 & 51 & $\mathrm{a}$ & 98 & 98 & 98 & 0 & 98 & 97 & 1 & 98 & 4 & 93 & 98 & $\mathrm{~m}$ & $\mathrm{~m}$ & 0 & $\mathrm{~m}$ \\
\hline Canada & $\mathrm{m}$ & $\mathrm{m}$ & $\mathrm{m}$ & $\mathrm{m}$ & $\mathrm{m}$ & $\mathrm{m}$ & $\mathrm{m}$ & 0 & $\mathrm{~m}$ & 92 & 0 & 92 & $\mathrm{~m}$ & 99 & $\mathrm{~m}$ & $\mathrm{~m}$ & $\mathrm{~m}$ & $\mathrm{~m}$ & $\mathrm{~m}$ \\
\hline Chile & 27 & 2 & 29 & 6 & 45 & 51 & 83 & 0 & 83 & 93 & 0 & 93 & 13 & 84 & 97 & 23 & 30 & 12 & 42 \\
\hline Czech Republic & $\mathrm{a}$ & 18 & 18 & a & 59 & 59 & 83 & 0 & 83 & 89 & 0 & 89 & 48 & 48 & 97 & 66 & 91 & 0 & 91 \\
\hline Denmark & 91 & 1 & 92 & 5 & 91 & 96 & 97 & 0 & 97 & 97 & 2 & 99 & 8 & 92 & 99 & $\mathrm{~m}$ & $\mathrm{~m}$ & $\mathrm{~m}$ & $\mathrm{~m}$ \\
\hline Estonia & $x(2)$ & $67^{\mathrm{d}}$ & 67 & $\mathrm{x}(5)$ & $87^{d}$ & 87 & $91^{\mathrm{d}}$ & 0 & 91 & $90^{\mathrm{d}}$ & 0 & 90 & $79^{\mathrm{d}}$ & 14 & 92 & 80 & 84 & 0 & 84 \\
\hline Finland & 52 & 0 & 52 & 0 & 68 & 68 & 75 & 0 & 75 & 80 & 0 & 80 & 97 & 0 & 98 & 62 & 69 & 0 & 69 \\
\hline France & a & 11 & 11 & a & 100 & 100 & 100 & 0 & 100 & 100 & 1 & 100 & 2 & 99 & 100 & 101 & 101 & 0 & 101 \\
\hline Germany & 59 & 0 & 59 & 0 & 92 & 92 & 96 & 0 & 96 & 97 & 0 & 98 & 34 & 62 & 96 & 80 & 89 & 0 & 89 \\
\hline Greece & $\mathrm{m}$ & $\mathrm{m}$ & $\mathrm{m}$ & $\mathrm{m}$ & $\mathrm{m}$ & $\mathrm{m}$ & $\mathrm{m}$ & $\mathrm{m}$ & $\mathrm{m}$ & $\mathrm{m}$ & $\mathrm{m}$ & $\mathrm{m}$ & $\mathrm{m}$ & $\mathrm{m}$ & $\mathrm{m}$ & a & 56 & 0 & 56 \\
\hline Hungary & $\mathrm{m}$ & 9 & $\mathrm{~m}$ & $\mathrm{~m}$ & 75 & $\mathrm{~m}$ & 93 & 0 & 93 & 96 & 0 & 96 & 69 & 23 & 92 & 73 & 91 & 0 & 91 \\
\hline Iceland & 95 & 0 & 95 & 0 & 96 & 96 & 97 & 0 & 97 & 95 & 0 & 96 & 0 & 99 & 99 & $\mathrm{~m}$ & $\mathrm{~m}$ & $\mathrm{~m}$ & $\mathrm{~m}$ \\
\hline Ireland & a & 0 & 0 & a & 46 & 46 & 57 & 37 & 95 & 3 & 99 & 100 & 0 & 100 & 100 & $\mathrm{~m}$ & $\mathrm{~m}$ & 44 & $\mathrm{~m}$ \\
\hline Israel & 45 & 0 & 45 & 0 & 100 & 100 & 100 & 0 & 100 & 99 & 0 & 99 & 15 & 83 & 98 & 66 & 84 & 0 & 84 \\
\hline Italy & $\mathrm{a}$ & 16 & 16 & a & 94 & 94 & 98 & a & 98 & 90 & 9 & 99 & 1 & 99 & 100 & 99 & 102 & 0 & 102 \\
\hline Japan & a & a & a & a & 81 & 81 & 95 & a & 95 & 97 & a & 97 & a & 100 & 100 & 69 & 95 & a & 95 \\
\hline Korea & 83 & 0 & 83 & 0 & 90 & 90 & 93 & 0 & 93 & 91 & 1 & 91 & 0 & 93 & 93 & 14 & 30 & 0 & 30 \\
\hline Luxembourg & $\mathrm{a}$ & 5 & 5 & a & 71 & 71 & 99 & 0 & 99 & 95 & 5 & 100 & 5 & 91 & 96 & 62 & 95 & a & 95 \\
\hline Mexico & 5 & 0 & 5 & 3 & 40 & 44 & 89 & 0 & 89 & 84 & 28 & 100 & 1 & 100 & 100 & 23 & 69 & 0 & 69 \\
\hline Netherlands & $\mathrm{a}$ & 0 & 0 & $\mathrm{a}$ & 83 & 83 & 100 & 0 & 100 & 99 & 0 & 99 & 0 & 100 & 100 & $\mathrm{~m}$ & 98 & 0 & 98 \\
\hline New Zealand & 65 & 0 & 65 & 0 & 96 & 96 & 100 & 0 & 100 & 4 & 97 & 100 & 0 & 100 & 100 & $\mathrm{~m}$ & $\mathrm{~m}$ & 0 & $\mathrm{~m}$ \\
\hline Norway & 90 & 0 & 90 & 0 & 95 & 95 & 97 & 0 & 97 & 98 & 0 & 98 & 1 & 100 & 100 & $\mathrm{~m}$ & $\mathrm{~m}$ & 0 & $\mathrm{~m}$ \\
\hline Poland & $\mathrm{a}$ & 6 & 6 & a & 52 & 52 & 66 & a & 66 & 92 & 8 & 100 & 78 & 9 & 86 & 28 & 38 & 0 & 38 \\
\hline Portugal & a & 0 & 0 & a & 78 & 78 & 90 & 0 & 90 & 97 & 0 & 98 & 6 & 96 & 100 & 61 & 84 & 0 & 84 \\
\hline Slovak Republic & a & 12 & 12 & a & 63 & 63 & 74 & 0 & 74 & 81 & 0 & 81 & 41 & 50 & 90 & $\mathrm{~m}$ & $\mathrm{~m}$ & 0 & $\mathrm{~m}$ \\
\hline Slovenia & 68 & 0 & 68 & 0 & 84 & 84 & 89 & 0 & 89 & 91 & 0 & 91 & 5 & 92 & 98 & 67 & 76 & 0 & 76 \\
\hline Spain & 52 & 0 & 52 & 0 & 96 & 96 & 97 & 0 & 97 & 97 & 0 & 97 & 1 & 96 & 97 & 94 & 99 & 0 & 99 \\
\hline Sweden & 88 & 0 & 88 & 0 & 93 & 93 & 94 & 0 & 94 & 95 & 0 & 95 & 96 & 1 & 97 & $\mathrm{~m}$ & $\mathrm{~m}$ & $\mathrm{~m}$ & $\mathrm{~m}$ \\
\hline Switzerland & $\mathrm{a}$ & 0 & 0 & a & 3 & 3 & 41 & 0 & 41 & 96 & 0 & 97 & 58 & 42 & 100 & 9 & 39 & 0 & 39 \\
\hline Turkey & $\mathrm{m}$ & $\mathrm{m}$ & $\mathrm{m}$ & a & 7 & 7 & 36 & a & 36 & 41 & 32 & 74 & $\mathrm{a}$ & 99 & 99 & 2 & 5 & 0 & 5 \\
\hline United Kingdom & 32 & 0 & 32 & 0 & 97 & 97 & 62 & 34 & 96 & 0 & 97 & 97 & 0 & 97 & 97 & $\mathrm{~m}$ & $\mathrm{~m}$ & 32 & $\mathrm{~m}$ \\
\hline United States & $\mathrm{m}$ & $\mathrm{m}$ & $\mathrm{m}$ & a & 41 & 41 & 66 & 0 & 66 & 85 & 5 & 90 & 21 & 76 & 97 & 39 & 68 & 0 & 68 \\
\hline OECD average & 33 & 6 & 39 & 2 & 72 & 74 & 85 & 2 & 88 & 81 & 14 & 95 & 22 & 75 & 97 & 52 & 69 & 3 & 72 \\
\hline EU21 average & 26 & 10 & 35 & 1 & 79 & 80 & 88 & 4 & 91 & 84 & 11 & 95 & 31 & 66 & 97 & 67 & 84 & 4 & 84 \\
\hline Argentina & $\mathrm{m}$ & $\mathrm{m}$ & $\mathrm{m}$ & $\mathrm{m}$ & $\mathrm{m}$ & $\mathrm{m}$ & $\mathrm{m}$ & $\mathrm{m}$ & $\mathrm{m}$ & $\mathrm{m}$ & $\mathrm{m}$ & $\mathrm{m}$ & $\mathrm{m}$ & $\mathrm{m}$ & $\mathrm{m}$ & $\mathrm{m}$ & $\mathrm{m}$ & $\mathrm{m}$ & $\mathrm{m}$ \\
\hline Brazil & 31 & 1 & 32 & 44 & 9 & 53 & 70 & 0 & 70 & 81 & 7 & 88 & 10 & 85 & 95 & $\mathrm{~m}$ & $\mathrm{~m}$ & $\mathrm{~m}$ & $\mathrm{~m}$ \\
\hline China & $\mathrm{a}$ & $\mathrm{m}$ & $\mathrm{m}$ & $\mathrm{a}$ & $\mathrm{m}$ & $\mathrm{m}$ & $\mathrm{m}$ & $\mathrm{m}$ & $\mathrm{m}$ & $\mathrm{m}$ & $\mathrm{m}$ & $\mathrm{m}$ & $\mathrm{m}$ & $\mathrm{m}$ & $\mathrm{m}$ & $\mathrm{m}$ & $\mathrm{m}$ & $\mathrm{m}$ & $\mathrm{m}$ \\
\hline Colombia & 31 & 2 & 33 & 37 & 10 & 47 & 34 & 1 & 35 & 60 & 19 & 79 & 8 & 78 & 86 & $\mathrm{~m}$ & $\mathrm{~m}$ & $\mathrm{~m}$ & $\mathrm{~m}$ \\
\hline India & $\mathrm{m}$ & $\mathrm{m}$ & $\mathrm{m}$ & $\mathrm{m}$ & $\mathrm{m}$ & $\mathrm{m}$ & $\mathrm{m}$ & $\mathrm{m}$ & $\mathrm{m}$ & $\mathrm{m}$ & $\mathrm{m}$ & $\mathrm{m}$ & $\mathrm{m}$ & $\mathrm{m}$ & $\mathrm{m}$ & $\mathrm{m}$ & $\mathrm{m}$ & $\mathrm{m}$ & $\mathrm{m}$ \\
\hline Indonesia & 7 & 0 & 7 & 9 & 11 & 21 & 19 & 0 & 19 & 23 & 4 & 27 & 50 & 70 & 100 & $\mathrm{~m}$ & $\mathrm{~m}$ & $\mathrm{~m}$ & $\mathrm{~m}$ \\
\hline Latvia & $\mathrm{a}$ & 0 & 0 & a & 83 & 83 & 89 & 0 & 89 & 96 & 0 & 96 & 93 & 4 & 97 & 66 & 73 & 0 & 73 \\
\hline Russian Federation & 51 & 0 & 51 & 0 & 73 & 73 & 79 & $\mathrm{a}$ & 79 & 80 & 1 & 81 & 75 & 13 & 88 & 55 & 55 & 0 & 55 \\
\hline Saudi Arabia ${ }^{1}$ & $a$ & $\mathrm{~m}$ & $\mathrm{~m}$ & a & 2 & 2 & 32 & 0 & 32 & 15 & 0 & 15 & 0 & 83 & 83 & $\mathrm{~m}$ & $\mathrm{~m}$ & $\mathrm{~m}$ & $\mathrm{~m}$ \\
\hline South Africa ${ }^{2}$ & a & $\mathrm{m}$ & $\mathrm{m}$ & a & $\mathrm{m}$ & $\mathrm{m}$ & $\mathrm{m}$ & $\mathrm{m}$ & $\mathrm{m}$ & $\mathrm{m}$ & $\mathrm{m}$ & $\mathrm{m}$ & $\mathrm{m}$ & $\mathrm{m}$ & $\mathrm{m}$ & $\mathrm{m}$ & $\mathrm{m}$ & $\mathrm{m}$ & $\mathrm{m}$ \\
\hline
\end{tabular}

\begin{tabular}{l|c|c|c|c|c|c|c|c|c|c|c|c|c|c|c|c|c|c|c|} 
G20 average & $\mathrm{m}$ & $\mathrm{m}$ & $\mathrm{m}$ & $\mathrm{m}$ & $\mathrm{m}$ & $\mathrm{m}$ & $\mathrm{m}$ & $\mathrm{m}$ & $\mathrm{m}$ & $\mathrm{m}$ & $\mathrm{m}$ & $\mathrm{m}$ & $\mathrm{m}$ & $\mathrm{m}$ & $\mathrm{m}$ & $\mathrm{m}$ & $\mathrm{m}$ & $\mathrm{m}$ & $\mathrm{m}$
\end{tabular}

Note: In cases where a category is not applicable (code "a") in a country, the value zero has been imputed for the purpose of calculating the OECD and EU21 averages. Early childhood education target children aged below the age of entry into ISCED level 1. There are two categories of ISCED level 0 programmes: early childhood educational development (ISCED 01) and pre-primary education (ISCED 02). Enrolment rates at young ages should be interpreted with care; mismatches between the coverage of the population data and the enrolment data mean that the participation rates may be underestimated for countries such as Luxembourg that are net exporters of students and may be overestimated for those that are net importers.

1. Year of reference 2014.

2. Year of reference 2012.

Source: OECD. Argentina, China, Colombia, Indonesia: UNESCO Institute for Statistics. Latvia: Eurostat. See Annex 3 for notes (www.oecd.org/education/educationat-a-glance-19991487.htm).

Please refer to the Reader's Guide for information concerning symbols for missing data and abbreviations.

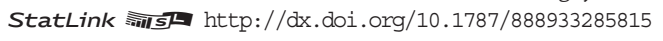


Table C2.2. Profile of early childhood educational development programmes and pre-primary education (2013)

Early childhood educational development programmes=ISCED 01, pre-primary education=ISCED 02

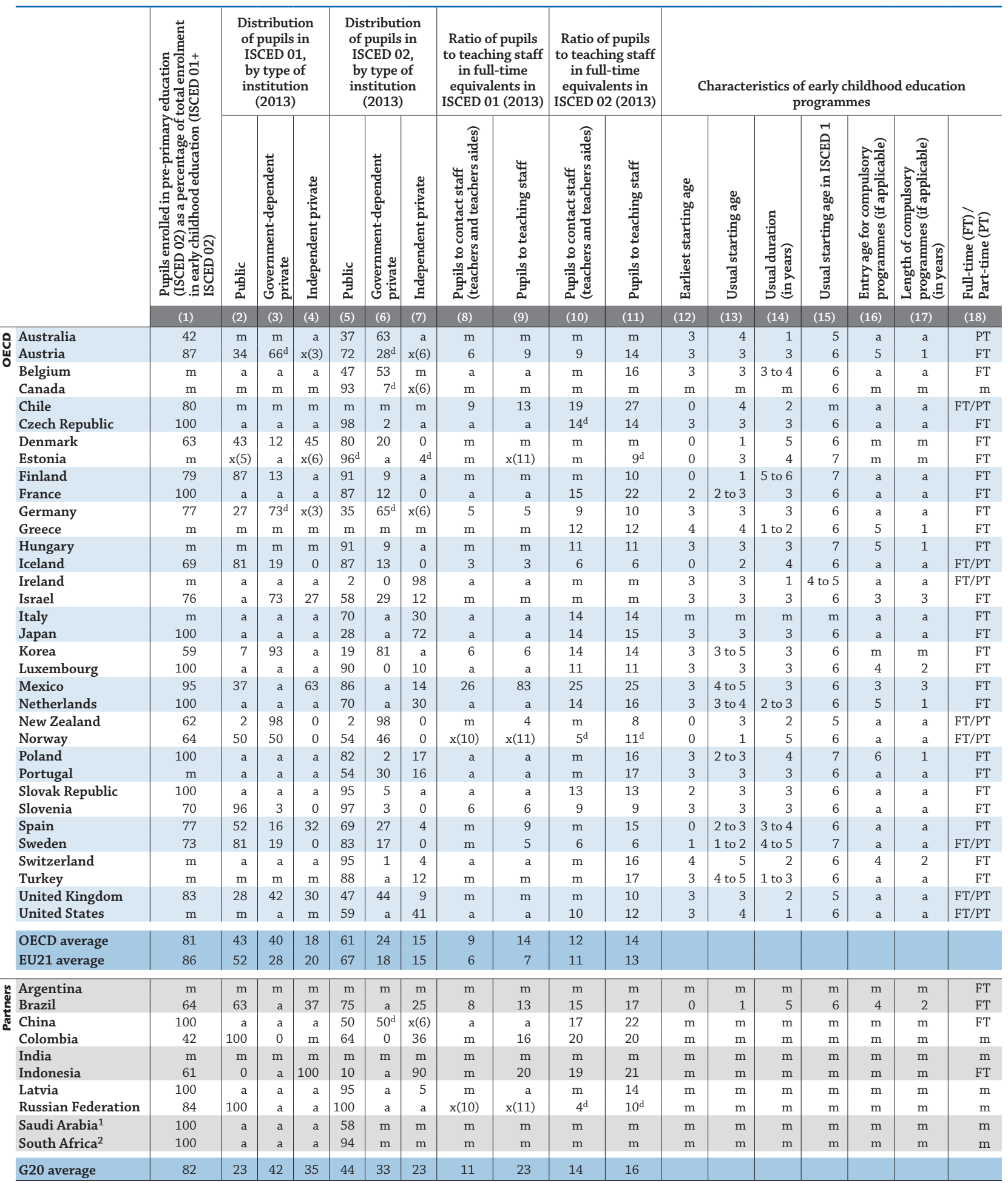

1. Year of reference 2014.

2. Year of reference 2012

Source: OECD. Argentina, China, Colombia, Indonesia, Saudi Arabia, South Africa: UNESCO Institute for Statistics. Latvia: Eurostat. See Annex 3 for notes (www.oecd.org/education/education-at-a-glance-19991487.htm).

Please refer to the Reader's Guide for information concerning symbols for missing data and abbreviations.

StatLink 제그 http://dx.doi.org/10.1787/888933285821 
Table C2.3. Expenditure on early childhood educational institutions (2012)

\begin{tabular}{|c|c|c|c|c|c|c|c|c|c|}
\hline & \multicolumn{3}{|c|}{$\begin{array}{l}\text { Expenditure on educational institutions } \\
\text { as a percentage of GDP }\end{array}$} & \multicolumn{3}{|c|}{$\begin{array}{l}\text { Proportions of total expenditure } \\
\text { from public sources }\end{array}$} & \multicolumn{3}{|c|}{$\begin{array}{l}\text { Annual expenditure per student } \\
\text { by educational institutions for all services }\end{array}$} \\
\hline & $\begin{array}{c}\text { Early } \\
\text { childhood } \\
\text { educational } \\
\text { development }\end{array}$ & Pre-primary & $\begin{array}{l}\text { All early } \\
\text { childhood } \\
\text { education }\end{array}$ & $\begin{array}{c}\text { Early } \\
\text { childhood } \\
\text { educational } \\
\text { development }\end{array}$ & Pre-primary & $\begin{array}{l}\text { All early } \\
\text { childhood } \\
\text { education }\end{array}$ & $\begin{array}{c}\text { Early } \\
\text { childhood } \\
\text { educational } \\
\text { development }\end{array}$ & Pre-primary & $\begin{array}{l}\text { All early } \\
\text { childhood } \\
\text { education }\end{array}$ \\
\hline & (1) & (2) & (3) & (4) & (5) & (6) & (7) & (8) & (9) \\
\hline Australia $^{1}$ & 0.3 & 0.2 & 0.4 & 4 & 47 & 21 & 10054 & 10298 & 10146 \\
\hline Austria & 0.1 & 0.5 & 0.6 & 69 & 87 & 84 & 9434 & 7716 & 7954 \\
\hline Belgium & $\mathrm{m}$ & 0.7 & $\mathrm{~m}$ & $\mathrm{~m}$ & 96 & $\mathrm{~m}$ & $\mathrm{~m}$ & 6975 & $\mathrm{~m}$ \\
\hline Canada & $\mathrm{m}$ & $\mathrm{m}$ & $\mathrm{m}$ & $\mathrm{m}$ & $\mathrm{m}$ & $\mathrm{m}$ & $\mathrm{m}$ & $\mathrm{m}$ & $\mathrm{m}$ \\
\hline Chile $^{2}$ & $\mathrm{x}(3)$ & $\mathrm{x}(3)$ & 0.9 & $\mathrm{x}(6)$ & $\mathrm{x}(6)$ & 82 & $\mathrm{x}(9)$ & $\mathrm{x}(9)$ & 4599 \\
\hline Czech Republic & a & 0.5 & 0.5 & a & 92 & 92 & a & 4447 & 4447 \\
\hline Denmark $^{1}$ & $\mathrm{x}(3)$ & $x(3)$ & 1.4 & $\mathrm{x}(6)$ & $\mathrm{x}(6)$ & 81 & $\mathrm{x}(9)$ & $\mathrm{x}(9)$ & 10911 \\
\hline Estonia & $\mathrm{x}(3)$ & $x(3)$ & 0.4 & $\mathrm{x}(6)$ & $\mathrm{x}(6)$ & 99 & $\mathrm{x}(9)$ & $\mathrm{x}(9)$ & 2193 \\
\hline Finland & 0.4 & 0.8 & 1.2 & 90 & 89 & 89 & 17860 & 9998 & 11559 \\
\hline France & a & 0.7 & 0.7 & $\mathrm{a}$ & 93 & 93 & $\mathrm{a}$ & 6969 & 6969 \\
\hline Germany & 0.3 & 0.5 & 0.8 & 70 & 79 & 76 & 13720 & 8568 & 9744 \\
\hline Greece & $\mathrm{m}$ & $\mathrm{m}$ & $\mathrm{m}$ & $\mathrm{m}$ & $\mathrm{m}$ & $\mathrm{m}$ & $\mathrm{m}$ & $\mathrm{m}$ & $\mathrm{m}$ \\
\hline Hungary $^{1}$ & $\mathrm{~m}$ & 0.7 & $\mathrm{~m}$ & $\mathrm{~m}$ & 92 & $\mathrm{~m}$ & $\mathrm{~m}$ & 4539 & $\mathrm{~m}$ \\
\hline Iceland & 0.6 & 1.1 & 1.7 & 88 & 85 & 86 & 12969 & 10250 & 11096 \\
\hline Ireland & $\mathrm{m}$ & $\mathrm{m}$ & $\mathrm{m}$ & $\mathrm{m}$ & $\mathrm{m}$ & $\mathrm{m}$ & $\mathrm{m}$ & $\mathrm{m}$ & $\mathrm{m}$ \\
\hline Israel $^{1}$ & $\mathrm{~m}$ & 0.7 & $\mathrm{~m}$ & $\mathrm{~m}$ & 85 & $\mathrm{~m}$ & $\mathrm{~m}$ & 3416 & $\mathrm{~m}$ \\
\hline Italy ${ }^{3}$ & $\mathrm{a}$ & 0.4 & 0.4 & $\mathrm{a}$ & 91 & 91 & $\mathrm{a}$ & 7892 & 7892 \\
\hline Japan & a & 0.2 & 0.2 & $\mathrm{a}$ & 44 & 44 & $\mathrm{a}$ & 5872 & 5872 \\
\hline Korea & $\mathrm{m}$ & 0.3 & $\mathrm{~m}$ & $\mathrm{~m}$ & 62 & $\mathrm{~m}$ & $\mathrm{~m}$ & 5674 & $\mathrm{~m}$ \\
\hline Luxembourg & $\mathrm{m}$ & 0.7 & $\mathrm{~m}$ & $\mathrm{~m}$ & 99 & $\mathrm{~m}$ & $\mathrm{~m}$ & 19719 & $\mathrm{~m}$ \\
\hline Mexico & $\mathrm{x}(3)$ & $\mathrm{x}(3)$ & 0.6 & $\mathrm{x}(6)$ & $\mathrm{x}(6)$ & 83 & $\mathrm{x}(9)$ & $\mathrm{x}(9)$ & 2445 \\
\hline Netherlands & a & 0.4 & 0.4 & a & 87 & 87 & a & 8176 & 8176 \\
\hline New Zealand & 0.4 & 0.6 & 1.0 & 72 & 87 & 80 & 12656 & 9670 & 10726 \\
\hline Norway & 1.0 & 1.1 & 2.1 & 86 & 86 & 86 & 15604 & 9050 & 11383 \\
\hline Poland ${ }^{3}$ & a & 0.7 & 0.7 & $\mathrm{a}$ & 76 & 76 & $\mathrm{a}$ & 6505 & 6505 \\
\hline Portugal $^{3}$ & $\mathrm{~m}$ & 0.6 & $\mathrm{~m}$ & $\mathrm{~m}^{\mathrm{d}}$ & 61 & $\mathrm{~m}$ & $\mathrm{~m}^{\mathrm{d}}$ & 5713 & $\mathrm{~m}$ \\
\hline Slovak Republic & $\mathrm{a}$ & 0.5 & 0.5 & $\mathrm{a}$ & 83 & 83 & $\mathrm{a}$ & 4694 & 4694 \\
\hline Slovenia $^{1}$ & 0.4 & 0.8 & 1.3 & 75 & 79 & 78 & 11665 & 7472 & 8726 \\
\hline Spain & 0.2 & 0.7 & 0.9 & 62 & 73 & 70 & 7924 & 6182 & 6588 \\
\hline Sweden & 0.5 & 1.2 & 1.8 & $\mathrm{~m}$ & $\mathrm{~m}$ & $\mathrm{~m}$ & 14180 & 12212 & 12752 \\
\hline Switzerland ${ }^{3}$ & $\mathrm{a}$ & 0.2 & 0.2 & $\mathrm{a}$ & $\mathrm{m}$ & $\mathrm{m}$ & a & 5457 & 5457 \\
\hline Turkey & $\mathrm{m}$ & $\mathrm{m}$ & $\mathrm{m}$ & $\mathrm{m}$ & $\mathrm{m}$ & $\mathrm{m}$ & $\mathrm{m}$ & $\mathrm{m}$ & $\mathrm{m}$ \\
\hline United Kingdom & 0.1 & 0.5 & 0.6 & 64 & 63 & 63 & 9495 & 10699 & 10548 \\
\hline United States ${ }^{1}$ & $\mathrm{~m}$ & 0.5 & $\mathrm{~m}$ & $\mathrm{~m}$ & 75 & $\mathrm{~m}$ & $\mathrm{~m}$ & 10042 & $\mathrm{~m}$ \\
\hline OECD average & 0.4 & 0.6 & 0.8 & 68 & 80 & 78 & 12324 & 8008 & 7886 \\
\hline EU21 average & 0.3 & 0.7 & 0.8 & 72 & 84 & 83 & 12040 & 8146 & 7977 \\
\hline Argentina & $\mathrm{m}$ & $\mathrm{m}$ & $\mathrm{m}$ & $\mathrm{m}$ & $\mathrm{m}$ & $\mathrm{m}$ & $\mathrm{m}$ & $\mathrm{m}$ & $\mathrm{m}$ \\
\hline Brazil 1,3 & $\mathrm{x}(3)$ & $\mathrm{x}(3)$ & 0.6 & $\mathrm{~m}$ & $\mathrm{~m}$ & $\mathrm{~m}$ & $\mathrm{x}(9)$ & $\mathrm{x}(9)$ & 2939 \\
\hline China & $\mathrm{m}$ & $\mathrm{m}$ & $\mathrm{m}$ & $\mathrm{m}$ & $\mathrm{m}$ & $\mathrm{m}$ & $\mathrm{m}$ & $\mathrm{m}$ & $\mathrm{m}$ \\
\hline Colombia $^{2}$ & $x(3)$ & $x(3)$ & 0.5 & $x(6)$ & $\mathrm{x}(6)$ & 57 & $x(9)$ & $x(9)$ & 1236 \\
\hline India & $\mathrm{m}$ & $\mathrm{m}$ & $\mathrm{m}$ & $\mathrm{m}$ & $\mathrm{m}$ & $\mathrm{m}$ & $\mathrm{m}$ & $\mathrm{m}$ & $\mathrm{m}$ \\
\hline Indonesia $^{2}$ & $\mathrm{~m}$ & 0.1 & $\mathrm{~m}$ & $\mathrm{~m}$ & 88 & $\mathrm{~m}$ & $\mathrm{~m}$ & $\mathrm{~m}$ & $\mathrm{~m}$ \\
\hline Latvia & $\mathrm{a}$ & 0.8 & 0.8 & $\mathrm{a}$ & 98 & 98 & $\mathrm{a}$ & 3067 & 3067 \\
\hline Russian Federation & $x(3)$ & $x(3)$ & 0.8 & $\mathrm{x}(6)$ & $x(6)$ & 89 & $x(9)$ & $\mathrm{x}(9)$ & 4887 \\
\hline Saudi Arabia & $\mathrm{m}$ & $\mathrm{m}$ & $\mathrm{m}$ & $\mathrm{m}$ & $\mathrm{m}$ & $\mathrm{m}$ & $\mathrm{m}$ & $\mathrm{m}$ & $\mathrm{m}$ \\
\hline South Africa ${ }^{3}$ & a & 0.1 & 0.1 & $\mathrm{a}$ & $\mathrm{m}$ & $\mathrm{m}$ & $\mathrm{a}$ & 806 & 806 \\
\hline G20 average & $\mathrm{m}$ & $\mathrm{m}$ & $\mathrm{m}$ & $\mathrm{m}$ & $\mathrm{m}$ & $\mathrm{m}$ & $\mathrm{m}$ & $\mathrm{m}$ & $\mathrm{m}$ \\
\hline
\end{tabular}

1. Includes some expenditure on childcare.

2. Year of reference 2013.

3. Public institutions only.

Source: OECD. Argentina, China, Colombia, India, Indonesia, Saudi Arabia, South Africa: UNESCO Institute for Statistics. Latvia: Eurostat. See Annex 3 for notes (www.oecd.org/education/education-at-a-glance-19991487.htm).

Please refer to the Reader's Guide for information concerning symbols for missing data and abbreviations.

StatLink त्नाज् http://dx.doi.org/10.1787/888933285830 
Table C2.4. Profile of education-only and integrated pre-primary programmes (2013)

\begin{tabular}{|c|c|c|c|c|c|c|c|c|c|}
\hline & \multicolumn{3}{|c|}{ Education-only programmes } & \multicolumn{3}{|c|}{$\begin{array}{l}\text { Integrated programmes } \\
\text { (includes education and childcare services) }\end{array}$} & \multicolumn{3}{|c|}{$\begin{array}{l}\text { Relative proportion of enrolments } \\
\text { reported in Education at a Glance (\%) }\end{array}$} \\
\hline & $\underset{\text { nationally }}{\text { Exist }}$ & $\begin{array}{l}\text { Delivered } \\
\text { by qualified } \\
\text { teacher }\end{array}$ & $\begin{array}{c}\text { Have a formal } \\
\text { curriculum }\end{array}$ & $\begin{array}{c}\text { Exist } \\
\text { nationally }\end{array}$ & $\begin{array}{l}\text { Delivered } \\
\text { by qualified } \\
\text { teacher }\end{array}$ & $\begin{array}{l}\text { Have a formal } \\
\text { curriculum }\end{array}$ & $\begin{array}{c}\text { Education-only } \\
\text { programmes }\end{array}$ & $\begin{array}{c}\text { Integrated } \\
\text { programmes }\end{array}$ & Total \\
\hline & (1) & (2) & (3) & (4) & (5) & (6) & (7) & (8) & (9) \\
\hline Australia & Yes & Yes & Yes & Yes & Yes & Yes & $\mathrm{x}(9)$ & $\mathrm{x}(9)$ & 100 \\
\hline ปै Austria & Yes & Yes & Yes & Yes & Yes & No & 3 & 97 & 100 \\
\hline Belgium & Yes & Yes & Yes & No & a & a & 100 & $\mathrm{a}$ & 100 \\
\hline Canada & Yes & Yes & Yes & Yes & Yes & Yes & $\mathrm{m}$ & $\mathrm{m}$ & $\mathrm{m}$ \\
\hline Chile & Yes & Yes & Yes & Yes & Yes & Yes & $\mathrm{x}(9)$ & $\mathrm{x}(9)$ & 100 \\
\hline Czech Republic & Yes & Yes & Yes & No & $\mathrm{a}$ & $\mathrm{a}$ & 100 & $\mathrm{a}$ & 100 \\
\hline Denmark & No & $\mathrm{a}$ & $\mathrm{a}$ & Yes & Yes & Yes & $\mathrm{a}$ & 100 & 100 \\
\hline Estonia & No & $\mathrm{a}$ & $\mathrm{a}$ & Yes & Yes & Yes & $\mathrm{a}$ & 100 & 100 \\
\hline Finland & Yes & Yes & Yes & Yes & Yes & Yes & 31 & 69 & 100 \\
\hline France & Yes & Yes & Yes & No & a & a & 100 & a & 100 \\
\hline Germany & Yes & Yes & Yes & No & a & $\mathrm{a}$ & 100 & $\mathrm{a}$ & 100 \\
\hline Greece & Yes & Yes & Yes & Yes & $\mathrm{m}$ & $\mathrm{m}$ & 100 & $\mathrm{~m}$ & 100 \\
\hline Hungary & No & $\mathrm{a}$ & $\mathrm{a}$ & Yes & Yes & Yes & $\mathrm{a}$ & 100 & 100 \\
\hline Iceland & Yes & Yes & Yes & Yes & Yes & Yes & 1 & 99 & 100 \\
\hline Ireland & No & $\mathrm{a}$ & $\mathrm{a}$ & Yes & $\mathrm{a}$ & $\mathrm{a}$ & $\mathrm{a}$ & 100 & 100 \\
\hline Israel & Yes & Yes & Yes & Yes & Yes & Yes & 98 & 2 & 100 \\
\hline Italy & No & $\mathrm{a}$ & $\mathrm{a}$ & Yes & $\mathrm{m}$ & $\mathrm{m}$ & $\mathrm{a}$ & 100 & $\mathrm{~m}$ \\
\hline Japan & Yes & Yes & Yes & Yes & Varies & Varies & $\mathrm{x}(9)$ & $\mathrm{x}(9)$ & 100 \\
\hline Korea & Yes & Yes & Yes & Yes & Yes & Yes & $\mathrm{x}(9)$ & $\mathrm{x}(9)$ & 100 \\
\hline Luxembourg & Yes & Yes & Yes & No & $\mathrm{a}$ & $\mathrm{a}$ & 100 & $\mathrm{a}$ & 100 \\
\hline Mexico & Yes & Yes & Yes & Yes & Yes & Yes & 99 & 1 & 100 \\
\hline Netherlands & Yes & Yes & Yes & Yes & No & Varies & 70 & 30 & 100 \\
\hline New Zealand & No & $\mathrm{a}$ & $\mathrm{a}$ & Yes & Yes & Yes & $\mathrm{a}$ & 100 & 100 \\
\hline Norway & No & a & a & Yes & Yes & Yes & a & 100 & 100 \\
\hline Poland & Yes & Yes & Yes & No & a & a & 100 & a & 100 \\
\hline Portugal & No & $\mathrm{a}$ & a & Yes & Yes & Yes & a & 100 & 100 \\
\hline Slovak Republic & Yes & Yes & Yes & No & a & $\mathrm{a}$ & 100 & $\mathrm{a}$ & 100 \\
\hline Slovenia & No & $\mathrm{a}$ & $\mathrm{a}$ & Yes & Yes & Yes & $\mathrm{a}$ & 100 & 100 \\
\hline Spain & Yes & Yes & Yes & No & a & a & 100 & a & 100 \\
\hline Sweden & Yes & Yes & Yes & Yes & Yes & Yes & 17 & 83 & 100 \\
\hline Switzerland & Yes & Yes & Yes & Yes & Yes & $\mathrm{m}$ & 100 & $\mathrm{~m}$ & 100 \\
\hline Turkey & Yes & Yes & Yes & No & a & a & 100 & $\mathrm{a}$ & 100 \\
\hline United Kingdom & Yes & Yes & Yes & Yes & Varies & Yes & $\mathrm{x}(9)$ & $\mathrm{x}(9)$ & 100 \\
\hline United States & Yes & Varies & Varies & Yes & Varies & Varies & $\mathrm{x}(9)$ & $\mathrm{x}(9)$ & 100 \\
\hline Argentina & $\mathrm{m}$ & $\mathrm{m}$ & $\mathrm{m}$ & $\mathrm{m}$ & $\mathrm{m}$ & $\mathrm{m}$ & $\mathrm{m}$ & $\mathrm{m}$ & $\mathrm{m}$ \\
\hline Brazil & Yes & Yes & No & Yes & Yes & No & $x(9)$ & $\mathrm{x}(9)$ & 100 \\
\hline China & $\mathrm{m}$ & $\mathrm{m}$ & $\mathrm{m}$ & $\mathrm{m}$ & $\mathrm{m}$ & $\mathrm{m}$ & $\mathrm{m}$ & $\mathrm{m}$ & $\mathrm{m}$ \\
\hline Colombia & $\mathrm{m}$ & $\mathrm{m}$ & $\mathrm{m}$ & $\mathrm{m}$ & $\mathrm{m}$ & $\mathrm{m}$ & $\mathrm{m}$ & $\mathrm{m}$ & $\mathrm{m}$ \\
\hline India & $\mathrm{m}$ & $\mathrm{m}$ & $\mathrm{m}$ & $\mathrm{m}$ & $\mathrm{m}$ & $\mathrm{m}$ & $\mathrm{m}$ & $\mathrm{m}$ & $\mathrm{m}$ \\
\hline Indonesia & $\mathrm{m}$ & $\mathrm{m}$ & $\mathrm{m}$ & $\mathrm{m}$ & $\mathrm{m}$ & $\mathrm{m}$ & $\mathrm{m}$ & $\mathrm{m}$ & $\mathrm{m}$ \\
\hline Latvia & $\mathrm{m}$ & $\mathrm{m}$ & $\mathrm{m}$ & $\mathrm{m}$ & $\mathrm{m}$ & $\mathrm{m}$ & $\mathrm{m}$ & $\mathrm{m}$ & $\mathrm{m}$ \\
\hline Russian Federation & $\mathrm{m}$ & $\mathrm{m}$ & $\mathrm{m}$ & $\mathrm{m}$ & $\mathrm{m}$ & $\mathrm{m}$ & $\mathrm{m}$ & $\mathrm{m}$ & $\mathrm{m}$ \\
\hline Saudi Arabia & $\mathrm{m}$ & $\mathrm{m}$ & $\mathrm{m}$ & $\mathrm{m}$ & $\mathrm{m}$ & $\mathrm{m}$ & $\mathrm{m}$ & $\mathrm{m}$ & $\mathrm{m}$ \\
\hline South Africa & $\mathrm{m}$ & $\mathrm{m}$ & $\mathrm{m}$ & $\mathrm{m}$ & $\mathrm{m}$ & $\mathrm{m}$ & $\mathrm{m}$ & $\mathrm{m}$ & $\mathrm{m}$ \\
\hline
\end{tabular}

Source: OECD, INES Working Party special data collection on early childhood education programmes. See Annex 3 for notes (www.oecd.org/education/education-ata-glance-19991487.htm).

Please refer to the Reader's Guide for information concerning symbols for missing data and abbreviations.

StatLink त्नाजम http://dx.doi.org/10.1787/888933285840 
Table C2.5. [1/3] Coverage of early childhood education programmes in OECD and partner countries ISCED 01 and ISCED 02, based on ISCED 2011 classifications

\begin{tabular}{|c|c|c|c|c|c|c|c|c|}
\hline & \multicolumn{4}{|c|}{ ISCED 010 - Early childhood development programmes } & \multicolumn{4}{|c|}{020 - Pre-primary education } \\
\hline & 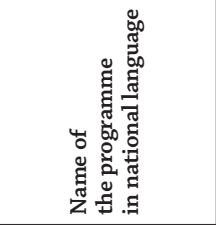 & 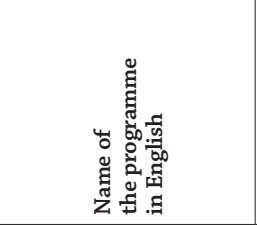 & 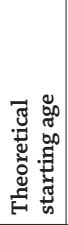 & 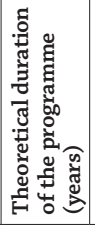 & 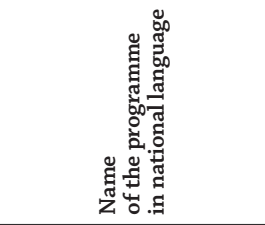 & 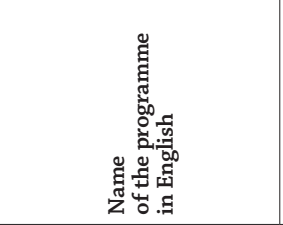 & 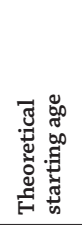 & 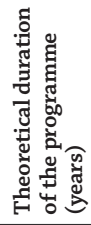 \\
\hline Australia & $\begin{array}{l}\text { Early childhood } \\
\text { education }\end{array}$ & $\begin{array}{l}\text { Early childhood } \\
\text { education }\end{array}$ & 0 & $2-4$ & Pre-primary, preschool & $\begin{array}{l}\text { Preschool programs } \\
\text { delivered in educational } \\
\text { institution settings or } \\
\text { educational } \\
\text { long-day care settings. }\end{array}$ & 4 & 1 \\
\hline \multirow[t]{2}{*}{ Austria } & \multirow[t]{2}{*}{ Kinderkrippe } & \multirow[t]{2}{*}{ Crèche } & \multirow[t]{2}{*}{0} & \multirow[t]{2}{*}{3} & Kindergarten & Kindergarten & 3 & 3 \\
\hline & & & & & Vorschulstufe & $\begin{array}{l}\text { Pre-primary stage } \\
\text { (of primary school) }\end{array}$ & 6 & 1 \\
\hline Belgium (Fl.) & Kinderopvang & Childcare & 0 & $2.5-3$ & $\begin{array}{l}\text { Buitengewoon } \\
\text { kleuteronderwijs }\end{array}$ & Special nursery education & $2.5-3$ & 3 \\
\hline \multirow[t]{2}{*}{ Belgium (Fr.) } & \multirow{2}{*}{\multicolumn{4}{|c|}{$\mathrm{a}$}} & $\begin{array}{l}\text { Enseignement maternel } \\
\text { ordinaire }\end{array}$ & $\begin{array}{l}\text { Regular pre-primary } \\
\text { education }\end{array}$ & $2.5-3$ & 3 \\
\hline & & & & & $\begin{array}{l}\text { Enseignement maternel } \\
\text { spécialisé }\end{array}$ & $\begin{array}{l}\text { Special pre-primary } \\
\text { education }\end{array}$ & $2.5-3$ & 3 \\
\hline Canada & $\begin{array}{l}\text { Early childhood } \\
\text { development or } \\
\text { equivalent }\end{array}$ & $\begin{array}{l}\text { Pre-elementary education } \\
\text { or equivalent - Early } \\
\text { childhood development }\end{array}$ & $3-4$ & $1-2$ & Kindergarten & $\begin{array}{l}\text { Pre-elementary education } \\
\text { or equivalent - } \\
\text { Kindergarten }\end{array}$ & $4-5$ & 1 \\
\hline Chile & $\begin{array}{l}\text { Educación Parvularia } \\
\text { (Sala Cuna y Nivel } \\
\text { Medio Menor) }\end{array}$ & $\begin{array}{l}\text { Pre-primary education } \\
\text { (Day care and Lower } \\
\text { Middle Level) }\end{array}$ & $0-2$ & 3 & $\begin{array}{l}\text { Educación Parvularia } \\
\text { (Nivel Medio Mayor, } \\
\text { Nivel de Transición } 1 \\
\text { y Nivel de Transición 2) }\end{array}$ & $\begin{array}{l}\text { Pre-primary education } \\
\text { (Upper middle level, } \\
\text { 1st transition level } \\
\text { and 2nd transition level) }\end{array}$ & $3-5$ & 3 \\
\hline \multirow[t]{2}{*}{ Czech Republic } & \multirow{2}{*}{\multicolumn{4}{|c|}{ a }} & Mateřská škola & Kindergarten & 3 & 3 \\
\hline & & & & & $\begin{array}{l}\text { Přípravné třídy pro děti se } \\
\text { sociálním znevýhodněním }\end{array}$ & $\begin{array}{l}\text { Preparatory classes for } \\
\text { socially disadvantaged } \\
\text { children }\end{array}$ & 6 & 1 \\
\hline Denmark & Vuggestue & Nursery school & $0-2$ & 3 & Børnehave & Kindergarten & $3-5$ & 2 \\
\hline Estonia & \multicolumn{4}{|c|}{ Included with ISCED-02 } & $\begin{array}{c}\text { Alusharidus } \\
\text { (alushariduse } \\
\text { raamõppekava) }\end{array}$ & \begin{tabular}{|c|} 
Pre-primary education \\
(general study programme of \\
pre-primary education)
\end{tabular} & 0 & 6 \\
\hline \multirow[t]{3}{*}{ Finland } & $\begin{array}{l}\text { 0-2-v. lapset } \\
\text { päiväkodeissa }\end{array}$ & \begin{tabular}{|c|} 
Kindergartens \\
(0 to 2 -year-old children), \\
including special \\
education programmes
\end{tabular} & $0-2$ & $1-3$ & 3-5-v. lapset päiväkodeissa & $\begin{array}{c}\text { Kindergartens } \\
\text { ( } 3 \text { to } 5 \text {-year-old children), } \\
\text { including special education } \\
\text { programmes }\end{array}$ & $3-5$ & $1-3$ \\
\hline & \multirow[t]{2}{*}{$\begin{array}{l}\text { 0-2-v. lapset } \\
\text { perhepäivähoidossa }\end{array}$} & \multirow[t]{2}{*}{$\begin{array}{c}\text { Family day care } \\
\text { (0 to 2-year-old children), } \\
\text { including special } \\
\text { education programmes }\end{array}$} & \multirow[t]{2}{*}{$0-2$} & \multirow[t]{2}{*}{$1-3$} & 6-v. lasten esiopetus & $\begin{array}{c}\text { Pre-primary education } \\
\text { for 6-year-old children } \\
\text { in kindergartens and } \\
\text { comprehensive schools, } \\
\text { including special education } \\
\text { programmes }\end{array}$ & 6 & 1 \\
\hline & & & & & $\begin{array}{l}\text { 3-5-v. lapset } \\
\text { perhepäivähoidossa }\end{array}$ & $\begin{array}{c}\text { Family day care } \\
\text { (3 to 5-year-old children), } \\
\text { including special education } \\
\text { programmes }\end{array}$ & $3-5$ & $1-3$ \\
\hline France & \multicolumn{4}{|c|}{ a } & $\begin{array}{l}\text { Enseignement } \\
\text { préélémentaire }\end{array}$ & Pre-elementary education & $2-3$ & 3 \\
\hline \multirow[t]{3}{*}{ Germany } & \multirow[t]{3}{*}{ Krippen } & \multirow[t]{3}{*}{ Crèche, Day nursery } & \multirow[t]{3}{*}{0} & \multirow[t]{3}{*}{$2-3$} & 01 Kindergärten & Kindergarten & 3 & 3 \\
\hline & & & & & 02 Schulkindergärten & School kindergarten & 6 & 1 \\
\hline & & & & & 03 Vorklassen & Pre-school classes & 5 & 1 \\
\hline Greece & $\begin{array}{l}\text { Vrefonipiakos } \\
\text { stathmos }\end{array}$ & $\begin{array}{l}\text { Kindergarten } \\
\text { Early childhood }\end{array}$ & 0 & $1-3$ & Nipiagogio & Pre-primary & $4-5$ & $1-2$ \\
\hline Hungary & $\begin{array}{l}\text { Bölcsőde } \\
\text { (2 évestől) }\end{array}$ & Créche & 2 & 1 & Óvoda & \begin{tabular}{|l|} 
Kindergarten (of which one \\
year pre-school education)
\end{tabular} & 3 & 3 \\
\hline \multirow[t]{2}{*}{ Iceland } & \multirow[t]{2}{*}{ Leikskóli I } & \multirow[t]{2}{*}{ Pre-primary schools I } & 0 & $1-3$ & Leikskóli II & Pre-primary schools II & 3 & $\begin{array}{c}0 \text { to } \\
3 \text { years, } \\
\text { variable }\end{array}$ \\
\hline & & & & & 5 ára bekkur & 0 grade for 5 -year-olds & 5 & 1 \\
\hline
\end{tabular}

Source: ISCED 2011 mappings. For more details, see Annex 3 for notes (www.oecd.org/education/education-at-a-glance-19991487.htm).

Please refer to the Reader's Guide for information concerning symbols for missing data and abbreviations.

StatLink 체개 http://dx.doi.org/10.1787/888933285850 
Table C2.5. [2/3] Coverage of early childhood education programmes in OECD and partner countries ISCED 01 and ISCED 02, based on ISCED 2011 classifications

\begin{tabular}{|c|c|c|c|c|c|c|c|c|}
\hline & \multicolumn{4}{|c|}{ ISCED 010 - Early childhood development programmes } & \multicolumn{4}{|c|}{020 - Pre-primary education } \\
\hline & 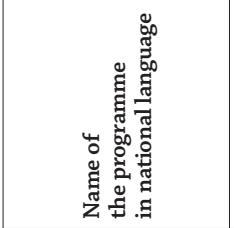 & 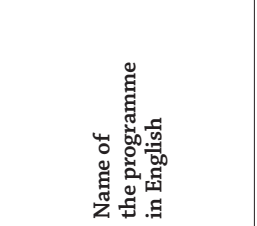 & 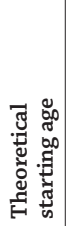 & 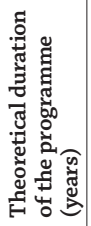 & 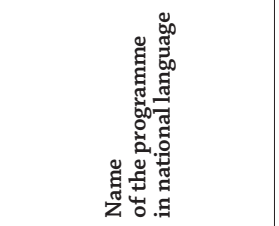 & 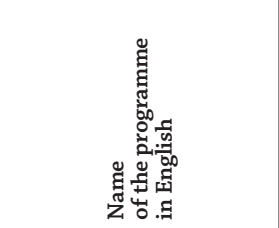 & 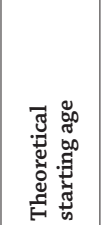 & 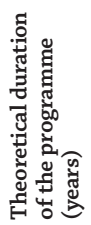 \\
\hline \multirow{3}{*}{ Ireland } & \multirow{3}{*}{\multicolumn{4}{|c|}{ a }} & Early start & Early start & $3-4$ & 1 \\
\hline & & & & & $\begin{array}{l}\text { Traveller Pre-School } \\
\text { Programmes }\end{array}$ & $\begin{array}{l}\text { Traveller Pre-School } \\
\text { Programmes }\end{array}$ & $3-4$ & 1 \\
\hline & & & & & $\begin{array}{l}\text { Privately provided } \\
\text { Pre-Primary education - } \\
\text { Early Childhood Care and } \\
\text { Education (ECCE) Scheme } \\
\text { and the Community } \\
\text { Childcare Subvention } \\
\text { (CCS) Programme }\end{array}$ & $\begin{array}{l}\text { Privately provided } \\
\text { Pre-Primary education - } \\
\text { Early Childhood Care and } \\
\text { Education (ECCE) Scheme } \\
\text { and the Community } \\
\text { Childcare Subvention } \\
\text { (CCS) Programme }\end{array}$ & $\begin{array}{c}3 \text { years } \\
2 \text { months } \\
\text { to } \\
4 \text { years } \\
6 \text { months }\end{array}$ & 1 \\
\hline \multirow[t]{2}{*}{ Israel } & \multirow[t]{2}{*}{$\begin{array}{l}\text { Hinuh be ganey } \\
\text { misrad ha kalkala } \\
\text { or harevacha }\end{array}$} & \multirow[t]{2}{*}{$\begin{array}{l}\text { Early childhood education } \\
\text { supervised by Ministry } \\
\text { of Economy or by } \\
\text { Ministry of Welfare }\end{array}$} & \multirow[t]{2}{*}{0} & \multirow[t]{2}{*}{3} & $\begin{array}{c}\text { Hinuh kdam yesody-ganey } \\
\text { yeladim-ziburi } \\
\text { (misrad ha kalkala, } \\
\text { misrad ha revacha } \\
\text { ve misrad ha hinuh) }\end{array}$ & $\begin{array}{c}\text { Pre-primary } \\
\text { education-public } \\
\text { (supervised by Ministry } \\
\text { of Economy, Ministry } \\
\text { of Welfare or by MoE) }\end{array}$ & 3 & 3 \\
\hline & & & & & $\begin{array}{c}\text { Hinuh kdam yesody-ganey } \\
\text { yeladim-prati }\end{array}$ & $\begin{array}{c}\text { Pre-primary } \\
\text { education-independent } \\
\text { private }\end{array}$ & 3 & 3 \\
\hline Italy & \multicolumn{4}{|c|}{$\mathrm{a}$} & Scuola dell'infanzia & Pre-primary school & 3 & 3 \\
\hline \multirow[t]{3}{*}{ Japan } & \multirow{3}{*}{\multicolumn{4}{|c|}{$\mathrm{a}$}} & Yochien & Kindergarte & $3-5$ & $1-3$ \\
\hline & & & & & $\begin{array}{c}\text { Tokubetsu-shien-gakko } \\
\text { Yochi-bu }\end{array}$ & $\begin{array}{l}\text { School for special needs } \\
\text { education, kindergarten } \\
\text { department }\end{array}$ & $3-5$ & $1-3$ \\
\hline & & & & & Hoikusho & Day nursery & $3-5$ & $1-3$ \\
\hline Korea & $\begin{array}{c}\text { 어린이집 }(0 \text { - } 2 \text { 세) } \\
\text { (Eorinyijip, age } 0 \text { - 2) }\end{array}$ & $\begin{array}{l}\text { Infant course, } \\
\text { Childcare centre }\end{array}$ & $0-2$ & $1-3$ & $\begin{array}{c}\text { 어린이집 (3 - 5세) } \\
\text { (Eorinyijip, age } 3 \text { - 5) }\end{array}$ & $\begin{array}{l}\text { Kindergarten course, } \\
\text { Childcare centre }\end{array}$ & $3-5$ & $1-3$ \\
\hline \multirow[t]{4}{*}{ Luxembourg } & \multirow{4}{*}{\multicolumn{4}{|c|}{$\mathrm{a}$}} & $\begin{array}{l}\text { Enseignement fondamental/ } \\
\text { cycle 1-éducation précoce }\end{array}$ & Early maturity education & 3 & 1 \\
\hline & & & & & Éducation précoce & $\begin{array}{l}\text { Early maturity education } \\
\text { (independent private } \\
\text { institutions) }\end{array}$ & $<4$ & 1 \\
\hline & & & & & $\begin{array}{c}\text { Enseignement } \\
\text { fondamental/cycle } 1 \text { - } \\
\text { éducation préscolaire } \\
\text { (Spillschoul) }\end{array}$ & Pre-primary education & 4 & 2 \\
\hline & & & & & Éducation préscolaire & $\begin{array}{c}\text { Pre-primary education } \\
\text { (independent private } \\
\text { institutions) }\end{array}$ & 4 & 2 \\
\hline Mexico & Educación Inicial & $\begin{array}{l}\text { Early Childhood } \\
\text { Education }\end{array}$ & 0 & 3 & Educación Preescolar & Pre-primary Education & 3 & $2-3$ \\
\hline \multirow[t]{2}{*}{ Netherlands } & $\begin{array}{c}\text { Early childhood } \\
\text { education }\end{array}$ & $\begin{array}{l}\text { Early childhood } \\
\text { education }\end{array}$ & 0 & $<=3$ & Voorschools onderwijs & $\begin{array}{l}\text { Pre-school education in day } \\
\text { care centres and play groups }\end{array}$ & 3 & 1 \\
\hline & $\begin{array}{l}\text { Barnehage, } \\
0 \text { - } 2 \text { åringer }\end{array}$ & Kindergarten & 0 & 2 & $\begin{array}{c}\text { Basisonderwijs en speciaal } \\
\text { basisonderwijs, groep } \\
1 \text { en } 2\end{array}$ & $\begin{array}{l}\text { Pre-primary education in } \\
\text { school settings, including } \\
\text { pre-primary special needs } \\
\text { education group } \\
\text { (class) } 1 \text { and } 2\end{array}$ & 4 & 2 \\
\hline New Zealand & $\begin{array}{l}\text { Early childhood } \\
\text { education }\end{array}$ & $\begin{array}{l}\text { Early childhood } \\
\text { education }\end{array}$ & 0 & $<=3$ & Early childhood education & Early childhood education & 3 & 2 \\
\hline Norway & Barnehage, 0 - 2 åringer & Kindergarten & 0 & 2 & Barnehage, 3 - 5 åringer & Kindergarten & 3 & 3 \\
\hline \multirow[t]{2}{*}{ Poland } & \multirow{2}{*}{\multicolumn{4}{|c|}{$\mathrm{a}$}} & Wychowanie przedszkolne & Pre-school education & 3 & 4 \\
\hline & & & & & $\begin{array}{c}\text { Wychowanie przedszkolne } \\
\text { specjalne }\end{array}$ & Special pre-school education & 3 & 4 \\
\hline Portugal & & $\mathrm{a}$ & & & Educação pré-escolar & Pre-primary education & $3-5$ & 3 \\
\hline
\end{tabular}

Source: ISCED-2011 mappings. For more details, see Annex 3 for notes (www.oecd.org/education/education-at-a-glance-19991487.htm).

Please refer to the Reader's Guide for information concerning symbols for missing data and abbreviations.

StatLink त्ताज् http://dx.doi.org/10.1787/888933285850 
Table C2.5. [3/3] Coverage of early childhood education programmes in OECD and partner countries ISCED 01 and ISCED 02, based on ISCED 2011 classifications

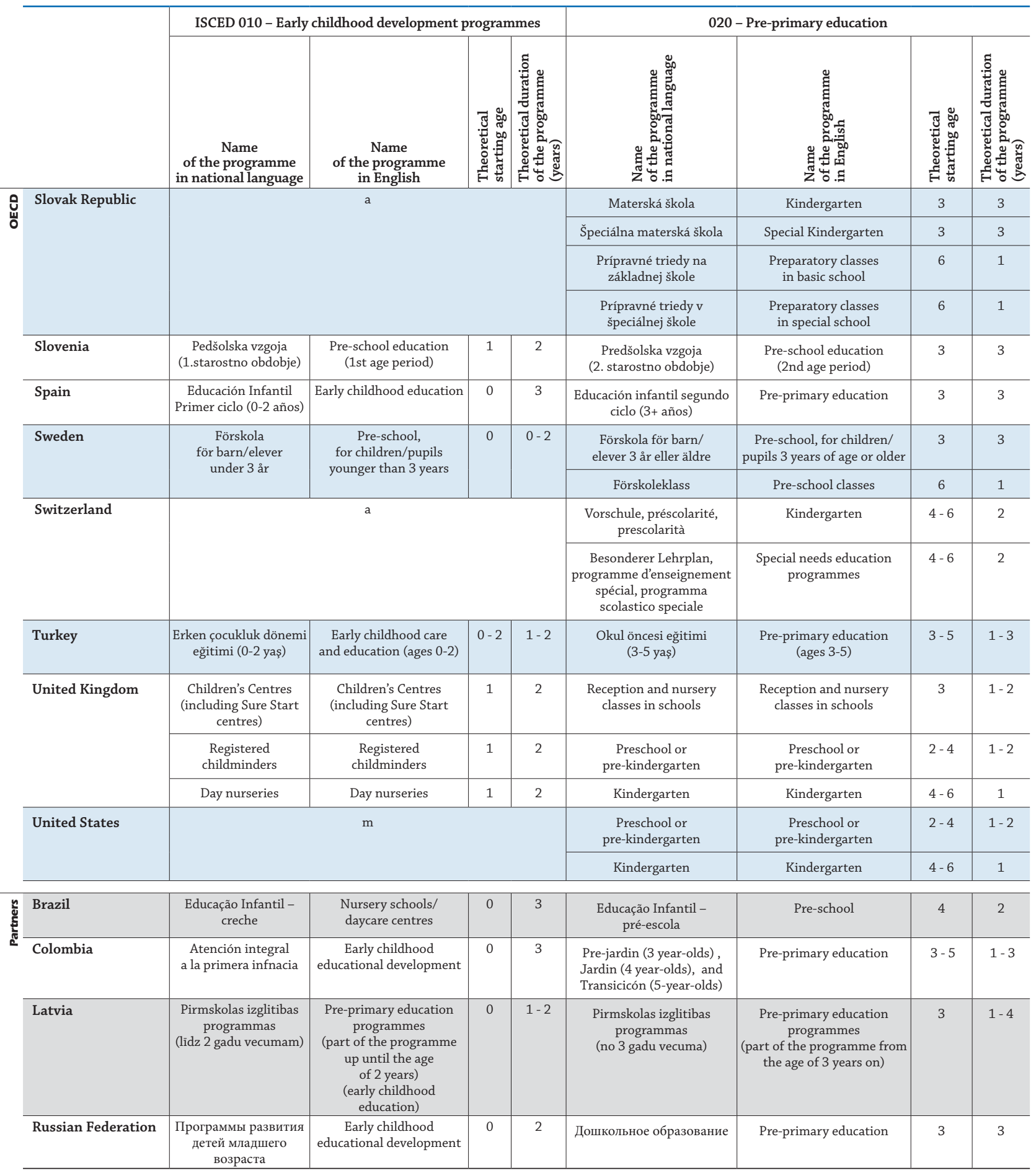

Source: ISCED-2011 mappings. For more details, see Annex 3 for notes (www.oecd.org/education/education-at-a-glance-19991487.htm).

Please refer to the Reader's Guide for information concerning symbols for missing data and abbreviations.

StatLink त्नाज http://dx.doi.org/10.1787/888933285850 


\section{HOW MANY STUDENTS ARE EXPECTED TO ENTER TERTIARY EDUCATION?}

- Some $57 \%$ of young adults in OECD countries are expected to enter a bachelor's degree or equivalent programme over their lifetime, and $22 \%$ are expected to enter a master's degree or equivalent programme over their lifetime.

- In all OECD countries except Korea, the most popular fields of education chosen by new entrants into tertiary programmes are social sciences, business and law.

- On average across OECD countries, $54 \%$ of new entrants into tertiary education are women, and $82 \%$ are under the age of 25 . Some $13 \%$ of all entrants are international students.

\section{Chart C3.1. First-time tertiary entry rates (2013)}

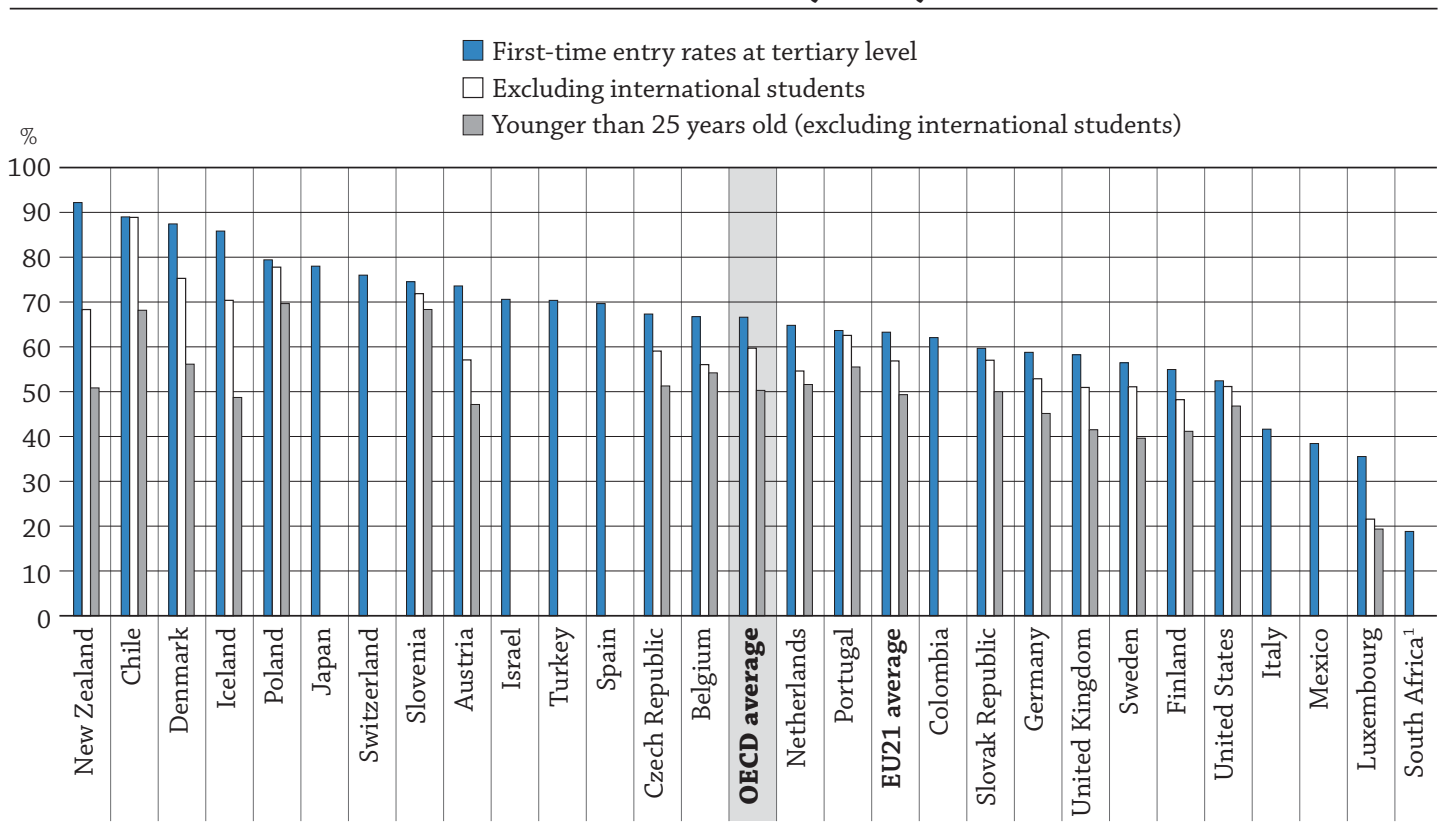

Note: Mismatches between the coverage of the population data and the new-entrants data mean that the entry rates for those countries that are net exporters of students may be underestimated and those that are net importers may be overestimated. The adjusted entry rates seek to compensate for that. Please refer to Annex 3 for further specific information by country. 1. Year of reference 2012

Countries are ranked in descending order of entry rate at tertiary level.

Source: OECD. Table C3.1. See Annex 3 for notes (www.oecd.org/education/education-at-a-glance- 19991487.htm).

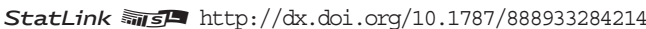

\section{Context}

Entry rates estimate the proportion of people who are expected to enter a specific type of tertiary education programme during their lifetime. They provide some indication of the accessibility of tertiary education, the perceived value of attending tertiary programmes, and the degree to which a population is acquiring the high-level skills and knowledge that can create and fuel knowledge-based economies. High entry and enrolment rates in tertiary education imply that a highly educated labour force is being developed and maintained.

In OECD countries, the belief that skills acquired through higher education are valued more than those held by people with lower educational attainment stems from the perception, both real and feared, that "routine" jobs can be mechanised or performed in low-wage countries. There is also a common understanding that knowledge and innovation are key to sustaining economic growth. Tertiary institutions not only have to meet growing demand by expanding the number of places they offer, they also have to adapt their programmes and teaching methods to match the diverse needs of a new generation of students. 


\section{Other findings}

- At least one in 25 students in Austria, Germany, Switzerland and the United Kingdom is expected to enter a doctoral programme over their lifetime, but fewer than one in 200 students in Chile, China, Colombia, Indonesia, Mexico and Saudi Arabia is expected to do so.

- Based on current patterns, it is estimated that an average of $18 \%$ of today's young adults in OECD countries will enter a short tertiary programme over their lifetime, and $57 \%$ will enter a bachelor's degree or equivalent programme.

- In Austria, Luxembourg and New Zealand, more than one in five entrants into a bachelor's programme are international students, well above the OECD average of $9 \%$.

- On average, $23 \%$ of students entering master's-level tertiary education do so as part of a long firstdegree programme; in Sweden, more than $90 \%$ of these students do.

\section{Note}

Entry rates represent the percentage of an age cohort that is expected to enter a tertiary programme over a lifetime. This estimate is based on the number of new entrants in 2013 and the age distribution of this group. Therefore, the entry rates are based on a "synthetic cohort" assumption, according to which the current pattern of entry constitutes the best estimate of the behaviour of today's young adults over their lifetime.

Entry rates are sensitive to changes in the education system, such as the introduction of new programmes. For example, during the implementation of the Bologna Process, some students in European countries stayed for longer than expected in tertiary education, while others postponed their entrance to be given a degree adaptable to the new classification. Entry rates can be very high, and even greater than $100 \%$ (thus clearly indicating that the synthetic cohort assumption is implausible), during a period when there is an unexpectedly high number of entrants.

In some countries, high entry rates may reflect a temporary phenomenon - namely the effects of economic cycles and crises, when prospective students align their expectations to the realities of the job market, or government incentives. Second-chance programmes, through which the government encourages older students to re-join education, can also boost entry rates.

A surge in the number of international students can temporarily inflate entry rates. The percentage of expected new entrants into tertiary programmes changes dramatically when international students are excluded from the calculation. Together with older students, international students are a significant share of the total student population in some countries, and their numbers can artificially inflate the proportion of today's young adults who are expected to enter a tertiary programme. When international and older students are not counted, some countries are notable for their high tertiary entry rates. 


\section{Analysis}

\section{Overall access to tertiary education}

The transition to ISCED 2011 helps to distinguish between the various levels of tertiary education - including short-cycle tertiary, bachelor's degrees, master's degrees and doctoral programmes - in Education at a Glance 2015.

It is estimated that $67 \%$ of young adults in OECD countries will enter tertiary education at least once during their lifetime if current patterns of entry continue. This average drops to $60 \%$ when international students are excluded and to $51 \%$ if only domestic students younger than 25 are considered (Chart C3.1). Some countries have very high tertiary entry rates largely because of popular short-cycle programmes. In Chile, for example, around 89\% of young people are expected to enter tertiary education at least once in their lifetime - with $45 \%$ of them entering shortcycle programmes (Tables C3.1 and C3.2).

Some $18 \%$ of tertiary students across OECD countries enter short-cycle programmes as do $12 \%$ of tertiary students in the 21 members of the European Union that are also part of the OECD. In some countries, such as Chile, more than $40 \%$ of students are expected to enter short-cycle tertiary education, while in 6 out of 30 countries, $1 \%$ or less are. These programmes do not exist in Estonia, Finland, Greece and Portugal (Tables C3.1 and C3.2).

In most countries, the largest proportion of tertiary students enters bachelor's degree programmes (ISCED 6). Across OECD countries, 57\% of young people will enter one of these programmes during their lifetime, although this rate varies widely across countries. In Luxembourg, for example, given the large proportion of its citizens who study abroad, first-time entry rates stand at only $22 \%$ at the bachelor's level. Conversely, Australia, which has a large population of international tertiary students, has a first-time entry rate of $91 \%$. When international entrants are excluded from the calculation, Australia's entry rate falls to $76 \%$.

Many OECD countries invest heavily to provide education beyond the bachelor's level. Some countries have entry rates as high as $46 \%$ for master's programmes (Poland) and around 5\% for doctoral programmes (Germany and Switzerland).

Around 22\% of students across OECD countries are expected to enter a master's programme over their lifetime, and $14 \%$ of domestic students are expected to enter those programmes before the age of 30 . After excluding international students from the calculation, entry rates into master's programmes vary from $35 \%$ and $37 \%$ in Iceland and the Slovak Republic, respectively, to 3\% in China.

Only $2 \%$ of young people will enter a doctoral programme over their lifetime, and only $1 \%$ of all domestic students are expected to do so before the age of 30 .

\section{International students}

As previously discussed, international students are of great relevance in understanding how entry rates describe a country's education system. Many of those entering a certain level of education may come from abroad or may have attained the previous level of their education in a foreign country, which substantially alters the indicators. For example, when international students are excluded, the entry rates for bachelor's degree programmes decrease by an average of 2 percentage points.

At the master's and doctoral levels, the change in rates is also relevant after accounting for international students. The first-time entry rate for master's programmes, calculated only for domestic students, is 3 percentage points lower than that for all students, on average. First-time entry rates at the doctoral level decreases from $2.5 \%$ to $1.8 \%$, which is also a relatively large difference. Indicator C4 discusses in greater detail students' motivation for pursuing higher education, particularly master's and doctoral programmes, in other countries.

\section{Students above the typical age}

The "typical age" is the age at which most students enter a given education level. After excluding students above the typical age at entry, there are substantial differences in the estimates for first-time tertiary entry rates for domestic students, ranging from $60 \%$ to $50 \%$, on average. This means that half of all young people across OECD countries are expected to enter a tertiary-level programme before the age of 25 (Table C3.1). But in some countries, students first entering this level of education are older. In Iceland, Israel and Switzerland, for example, at least 30\% of those entering tertiary education are older than 25 (Table C3.2).

Doctoral entry rates are also affected by this adjustment in the calculations. Although $1.8 \%$ of all domestic youth are expected to enter a $\mathrm{PhD}$ programme, only $1.1 \%$ will do so before they turn 30 . 


\section{Profile of first-time entrants into tertiary education}

\section{By level of education}

Knowing the level at which students enter tertiary education helps to determine the depth and length of the studies in which they engage. Most education systems begin tertiary education at the bachelor's-degree level.

Chart C3.2. shows that across OECD countries, $72 \%$ of new entrants at the tertiary level start at the bachelor's level and about $10 \%$ begin at the master's level or equivalent, essentially corresponding to long first degrees. Some $18 \%$ of new entrants, on average, enter short tertiary programmes, although in Turkey, $50 \%$ or more of new entrants do. In Luxembourg, Portugal, Sweden and Switzerland, more than one out of five new entrants enter master's programmes (Table C3.2).

\section{Chart C3.2. Distribution of first-time new entrants, by level of education (2013)}

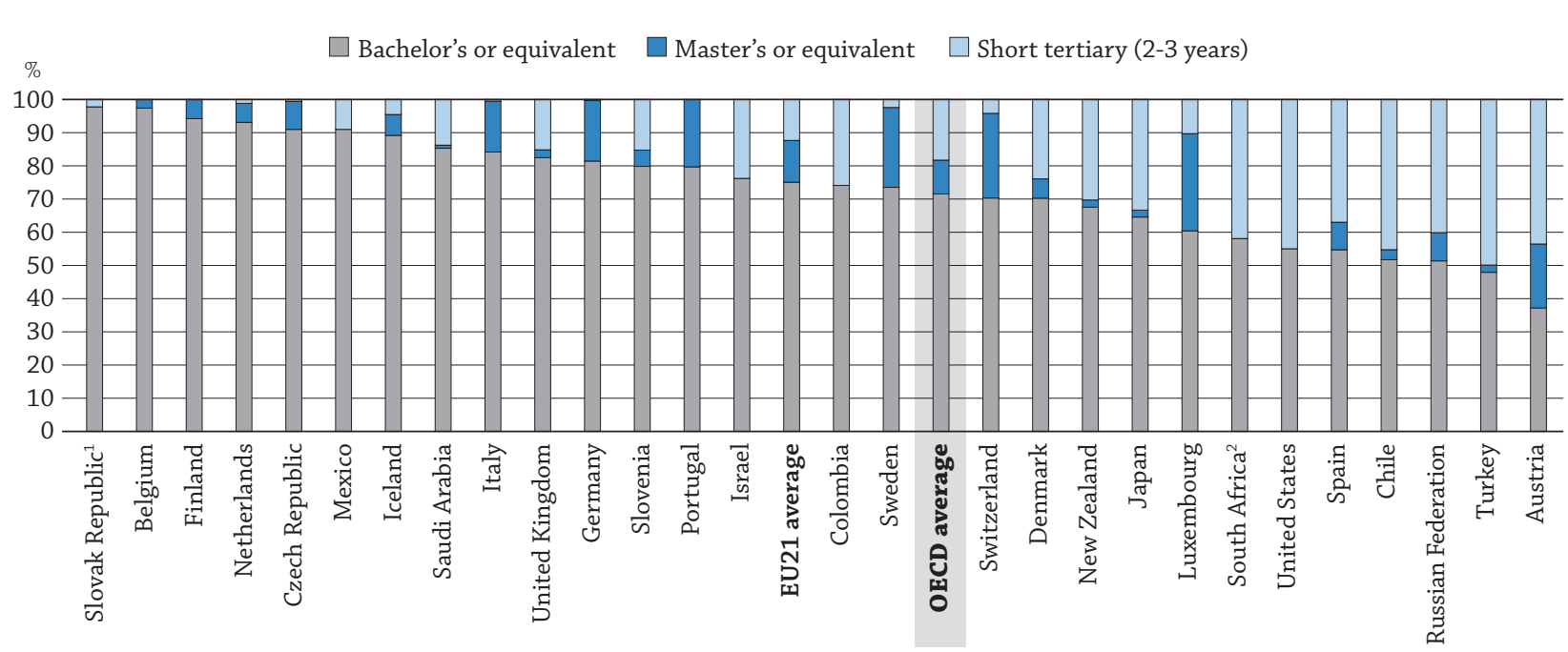

1. Master's degrees are included with Bachelor's degrees.

2. Year of reference 2012.

Countries are ranked in decreasing order of the percentage of first-time entrants in bachelor's degrees or equivalent.

Source: OECD. Table C3.2. See Annex 3 for notes (www.oecd.org/education/education-at-a-glance- 19991487.htm).

StatLink 젬ㄴ http://dx.doi.org/10.1787/888933284224

\section{Women's participation in tertiary education}

Women make up the majority of entrants into tertiary education in all countries except Mexico, Saudi Arabia, Switzerland and Turkey. On average across OECD countries, 54\% of new entrants are women. The largest shares of female new entrants (58\%) are found in the Czech Republic, Iceland and Sweden. Nevertheless, equal participation of men and women at a given education level does not imply evenly balanced distribution across fields of study. Women are over-represented in programmes that will lead to relatively lower-paying jobs, namely teaching and nursing, while men are over-represented in science, technology, engineering and mathematics (STEM) fields. Table C3.3. shows the gender gaps in fields of study at the bachelor's level (see section below).

\section{Proportion of new entrants above the typical age}

The age of new entrants into tertiary education varies across OECD countries because of differences in the typical age at which students graduate from upper secondary education, the intake capacity of institutions (admissions with numerus clausus, one of many methods used to limit the number of students who may study at a tertiary institution), the opportunity cost of entering the labour market before enrolling in tertiary education, and cultural expectations.

During the recent economic crisis, some young people postponed entry into the labour market and remained in education. Some governments have also developed second-chance programmes, aimed at people who left school early, to raise the level of skills available in the workforce and increase opportunities for people to acquire practical education and competencies. Nevertheless, entering tertiary education at a later stage is more costly from both public and personal perspectives. It means that for a period of time, the productive potential of individuals is untapped. 
As a result, tax revenues are lower and public expenditures may be higher (see Indicator B7). Older students may face more difficulties combining work and study and thus may be unable to complete the programmes on time. Understanding that delays in completing education are costly to the education system, governments are introducing measures to foster timely completion.

The proportion of older first-time entrants into tertiary programmes may reflect the flexibility of the programmes and their suitability to students outside the typical age group. It may also reveal the value placed on work experience before entering higher education, which is a characteristic of countries with small proportions of entrants below the typical age (less than 75\%), namely Colombia, Denmark, Iceland, Israel, Luxembourg and Switzerland. Older entrants can also reflect a response to policies aimed at expanding lifelong learning and more flexible access to tertiary education. The reasons differ substantially from one country to another. For instance, in Australia, taking a gap year before entering tertiary education has become a trend. In 2009/10, almost one in four students took a gap year, and $51 \%$ of them declared "work" as their main reason for taking the year off from education (Lumsden and Stanwick, 2012).

\section{Share of international students}

In most countries, all international students enrolling for the first time in a country are counted as new entrants, regardless of their previous education in other countries. To highlight the impact of international students on entry rates, Chart C3.1 shows both unadjusted and adjusted entry rates (i.e. the entry rate when international students are excluded from consideration).

The total share of international students entering a tertiary programme for the first time ranges from close to zero in Chile to over $40 \%$ in Luxembourg. It is also high (around 25\%) in Austria and New Zealand. On average, however, $13 \%$ of all new entrants in OECD countries come from abroad (Table C3.2).

\section{Share of new entrants, by field of education}

In all countries with available data, except Korea, more students pursue tertiary programmes in the fields of social sciences, business and law than in any other subject. In Korea, $25 \%$ of new entrants pursue their studies in engineering, manufacturing and construction.

Science-related fields, which include science and engineering, manufacturing and construction, are comparatively less popular. On average, the least popular fields are agriculture (2\%) and services (6\%) (Table C3.2).

\section{Bachelor's programmes}

Bachelor's degrees are the most popular diplomas of tertiary education in all countries, meaning that students are more likely to enter this level of education than any other level of tertiary education. Almost three out of four people who enter tertiary education for the first time will enrol in a bachelor's degree programme (Table C3.2). Some 57\% of young people across OECD countries are expected to enter a bachelor's degree programme at some point in their lifetime (Table C3.1).

In 31 of the 35 countries for which data are available, women are more likely than men to enter a bachelor's programme. In Sweden, $61 \%$ of all entrants at that level are women as are $44 \%$ of all entrants into bachelor's programmes in Japan (Table C3.3).

Traditionally, students enter a bachelor's programme immediately after having completed upper secondary education, and this remains true in many countries. On average, $83 \%$ of new entrants into a bachelor's programme are younger than 25. In 6 of the 26 countries for which these proportions are calculated, $90 \%$ or more of new entrants are below the typical age of 25 . However, in some countries, the transition from upper secondary to tertiary education may occur at a later age because of time spent in the labour force or the military. The fact that some countries require young people to serve in the armed forces postpones their entry into tertiary education. For example, Israel and Switzerland, where $68 \%$ and $67 \%$, respectively, of new entrants to bachelor's programmes are under the age of 25 , both have mandatory conscription.

Over half (54\%) of those who enter a bachelor's programme do so in education, humanities or social sciences and $27 \%$, on average, enter an engineering or science programme. Some $12 \%$ of new entrants choose to pursue health and welfare studies. The largest proportions of entrants into a bachelor's programme in education, humanities or social sciences (70\% or more) are observed in Israel and Luxembourg, while in Germany, $40 \%$ of all entrants into bachelor's programmes choose the field of science and engineering (Table C3.3). 
Although more women than men choose education, humanities or social sciences to study at the bachelor's level (except in Japan, where only 15\% of new entrants into these fields are women), a larger share of men than women chooses engineering and science. Meanwhile, $78 \%$ of all entrants into bachelor's-level health and welfare studies are women, on average (Table C3.3).

The share of international entrants at the bachelor's level varies widely across OECD countries, from 25\% in Luxembourg and 24\% in Austria to less than 1\% in Chile and China (Table C3.3). The countries with the largest shares of international students see a steep drop in their entry rates when international students are excluded from the calculations. In Australia, first-time entry rates into bachelor's programmes drop from $91 \%$ to $76 \%$ when international students are excluded from the calculation (Table C3.1).

\section{Short-cycle programmes}

Compared to other education levels, short tertiary programmes have the most diverse profile of entrants. Although $54 \%$ of new entrants into short tertiary programmes are women, on average, this proportion varies from less than $25 \%$ in Italy and Saudi Arabia to $81 \%$ in Poland.

On average across OECD countries, $68 \%$ of those entering a short-cycle programme (ISCED 5) are younger than 25; in seven countries, more than $80 \%$ are. By contrast, in Denmark, Iceland, Sweden and the United Kingdom, fewer than one in two new entrants is under 25.

A small proportion of international students enters short-cycle tertiary programmes, although around $20 \%$ of international students in New Zealand, the United Kingdom and 30\% in Iceland do.

\section{Master's programmes}

This year, thanks to the new data collection, Education at a Glance can provide analysis on bachelor's and master's programmes separately. Both programmes are increasingly popular, particularly in OECD countries.

Master's programmes attract a larger share of international students than bachelor's programmes (see Indicator C4). On average, $21 \%$ of all first-time entrants at the master's level are international students.

Of all new entrants, $27 \%$ are older than 30 , the typical age for earning a master's degree. The percentage range from more than 50\% in Chile and Colombia to less than 5\% in Belgium and Indonesia.

\section{Chart C3.3. Percentage of entrants below typical age (2013)}

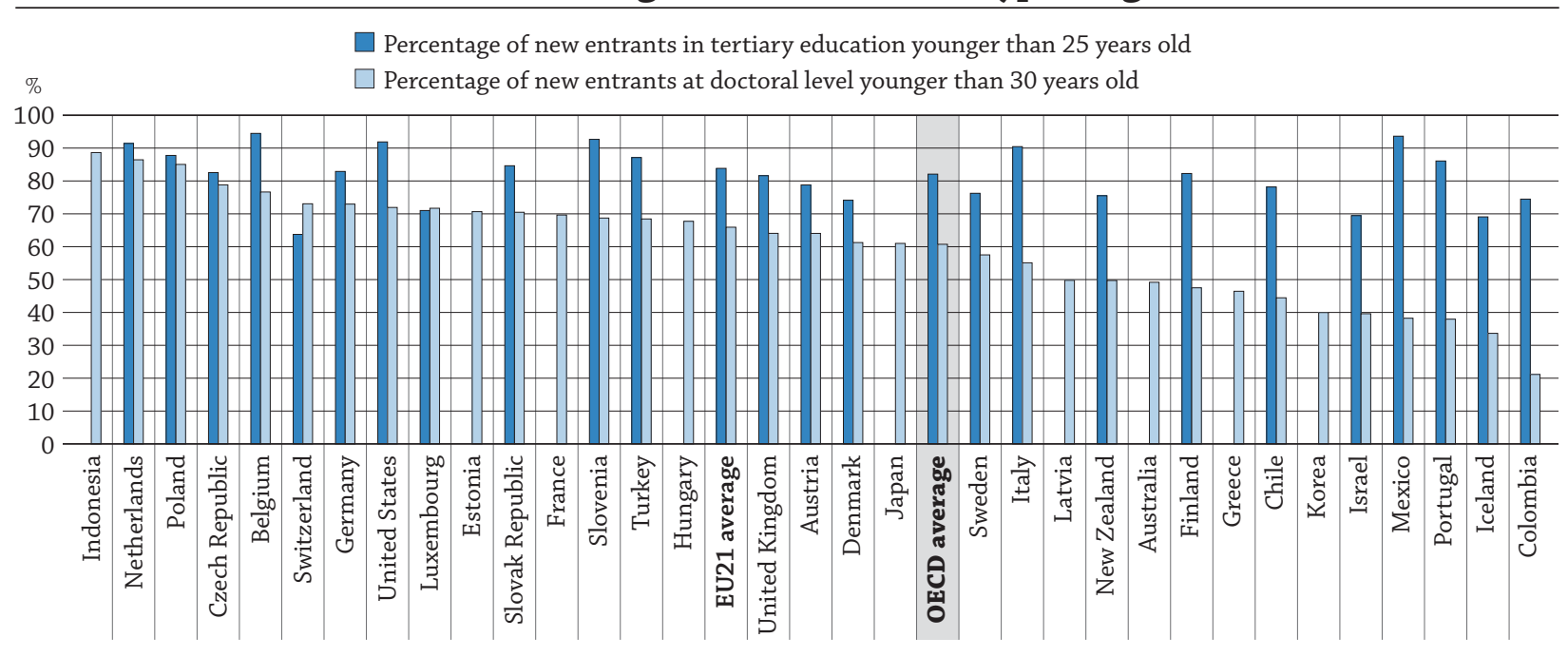

Countries are ranked in descending order of the percentage of new entrants at doctoral level younger than 30 years old.

Source: OECD. Tables C3.2 and C3.4. See Annex 3 for notes (www.oecd.org/education/education-at-a-glance- 19991487.htm).

StatLink 젶ㄴ http://dx.doi.org/10.1787/888933284233

The proportion of students entering a long first-degree programme out of the total of those entering a master's degree or equivalent programme varies across countries. In Sweden, over $94 \%$ of entrants into a master's programme (ISCED 7) do so as part of a long first degree. By contrast, in Denmark, Mexico and Switzerland, long first-degree programmes are rare; and in Indonesia, Korea and the Netherlands there is no such programme. 
On average across OECD countries, 56\% of those entering master's programmes are women. Below-average proportions of women entering master's programmes are observed in China (47\%), Indonesia (48\%), Japan (33\%), Saudi Arabia (43\%), Switzerland (48\%) and Turkey (44\%).

\section{Doctoral programmes}

Graduate-level research, particularly at the doctoral level, plays a crucial role in innovation and economic growth, and contributes significantly to the national and international knowledge base. Businesses are attracted to countries that make this level of research readily available (Halse and Mowbray, 2011; Smith, 2010), while individuals who attain this level of education benefit from higher wages and higher employment rates (see Indicators A5 and A6).

Several countries are developing doctoral programmes or changing the funding policy to attract international students. Attracting the best students from around the world helps to ensure that a country plays a leading role in research and innovation (Smith, 2010). Not surprisingly, in 8 of the 22 countries for which data are available, more than $40 \%$ of students entering doctoral programmes are international students - as are more than $90 \%$ of students entering these programmes in Luxembourg.

On average across OECD countries, $61 \%$ of entrants at the doctoral level are younger than 30 (Table C3.4). A larger share of younger entrants may reflect lower dropout rates and greater emphasis on acquiring specialised skills with a first degree in tertiary education. Some countries offer incentives, such as grants, scholarships, international mobility programmes, part-time jobs and distance learning, to encourage students to pursue advanced studies straight after completion of their first degree in tertiary education. By contrast, tuition fees, availability of scholarships, and/or cultural expectations, such as being expected to enter the labour force by a certain age or to gain professional experience prior to entering advanced education may explain why some new entrants are older.

\section{Definitions}

Entry rate is the sum of age-specific entry rates, calculated by dividing the number of entrants of a certain age into a certain education level by the total population of that age.

Entry rate adjusted for international students is the entry rate when calculated excluding international students in the numerator of each age-specific entry rate.

Entry rate below typical age is the sum of age-specific entry rates for age groups below the typical age.

International students are those students who left their country of origin and moved to another country for the purpose of study. International students enrolling for the first time in a programme are considered first-time entrants.

New entrants are students who enrol at the relevant level of education for the first time.

Tertiary-level entry rate is an estimated probability, based on current entry patterns, that a young adult will enter tertiary education during his or her lifetime.

\section{Methodology}

Data refer to the academic year 2012/13 and are based on the UOE data collection on education statistics administered by the OECD in 2014 (for details, see Annex 3 at www.oecd.org/education/education-at-a-glance-19991487.htm). The fields of education used in the UOE data collection instruments follow the revised ISCED 11 classification by field of education. The same classification is used for all levels of education.

Table C3.1, and Table C3.5, available on line, show the sum of net entry rates for all ages. Tables C3.2, C3.3 and C3.4 present the share of entrants with different profiles.

The net entry rate for a specific age is obtained by dividing the number of first-time entrants of that age for each type of tertiary education by the total population in the corresponding age group. The sum of net entry rates is calculated by adding the rates for each year of age. The result represents an estimate of the probability that a young person will enter tertiary education in his/her lifetime if current age-specific entry rates continue.

Note regarding data from Israel

The statistical data for Israel are supplied by and are under the responsibility of the relevant Israeli authorities. The use of such data by the OECD is without prejudice to the status of the Golan Heights, East Jerusalem and Israeli settlements in the West Bank under the terms of international law. 


\section{References}

Halse C. and S. Mowbray (2011), “The Impact of the Doctorate”, Studies in Higher Education, 36:5, pp. 513-525, http://dx.doi.org/ 10.1080/03075079.2011.594590.

Lumsden, M. and J. Stanwick (2012), “Who Takes A Gap Year And Why?” Longitudinal Surveys of Australian Youth, Briefing Paper 28. National Centre for Vocational Education Research (NCVER), Adelaide, Australia.

OECD (2013), Education at a Glance 2013: OECD Indicators, OECD Publishing, Paris, http://dx.doi.org/10.1787/eag-2013-en.

Smith, A. (2010), One Step Beyond: Making the Most of Postgraduate Education, Report for the UK Department for Business, Innovation and Skills.

\section{Indicator C3 Tables}

StatLink 젶ㄴ http://dx.doi.org/10.1787/888933285864

Table C3.1 First-time entry rates, by tertiary ISCED level (2013)

Table C3.2 Profile of first-time new entrants into tertiary education (2013)

Table C3.3 Profile of first-time new entrants into bachelor's programmes (2013)

Table C3.4 Profile of first-time new entrants, by level of education (2013)

WEB Table C3.5 Tertiary entry rates, by ISCED level and year $(2005,2013)$

Cut-off date for the data: 23 October 2015. Updates can be found on line at http://dx.doi.org/10.1787/eag-data-en. 
Table C3.1. First-time entry rates, by tertiary ISCED level (2013) Sum of age-specific entry rates, by demographic group

\begin{tabular}{|c|c|c|c|c|c|c|c|c|c|c|c|c|c|c|c|}
\hline & \multicolumn{3}{|c|}{$\begin{array}{c}\text { Short tertiary ( } 2-3 \text { years }) \\
\text { ISCED } 5\end{array}$} & \multicolumn{3}{|c|}{$\begin{array}{l}\text { Bachelor's or equivalent } \\
\text { ISCED } 6\end{array}$} & \multicolumn{3}{|c|}{$\begin{array}{l}\text { Master's or equivalent } \\
\text { ISCED } 7\end{array}$} & \multicolumn{3}{|c|}{$\begin{array}{l}\text { Doctoral or equivalent } \\
\text { ISCED } 8\end{array}$} & \multicolumn{3}{|c|}{ First-time tertiary } \\
\hline & \multirow[b]{2}{*}{ Total } & \multicolumn{2}{|c|}{$\begin{array}{c}\text { Excluding } \\
\text { international } \\
\text { students }\end{array}$} & \multirow[b]{2}{*}{ Total } & \multicolumn{2}{|c|}{$\begin{array}{c}\text { Excluding } \\
\text { international } \\
\text { students }\end{array}$} & \multirow[b]{2}{*}{ Total } & \multicolumn{2}{|c|}{$\begin{array}{c}\text { Excluding } \\
\text { international } \\
\text { students }\end{array}$} & \multirow[b]{2}{*}{ Total } & \multicolumn{2}{|c|}{$\begin{array}{c}\text { Excluding } \\
\text { international } \\
\text { students }\end{array}$} & \multirow[b]{2}{*}{ Total } & \multicolumn{2}{|c|}{$\begin{array}{c}\text { Excluding } \\
\text { international } \\
\text { students }\end{array}$} \\
\hline & & Total & $\left|\begin{array}{l}\text { Younger } \\
\text { than } 25\end{array}\right|$ & & Total & $\begin{array}{l}\text { Younger } \\
\text { than } 25\end{array}$ & & Total & $\begin{array}{l}\text { Younger } \\
\text { than } 30\end{array}$ & & Total & $\begin{array}{l}\text { Younger } \\
\text { than } 30\end{array}$ & & Total & $\begin{array}{l}\text { Younger } \\
\text { than } 25\end{array}$ \\
\hline & (1) & (2) & (3) & (4) & (5) & (6) & (7) & (8) & (9) & (10) & (11) & (12) & (13) & (14) & (15) \\
\hline Australia & $\mathrm{m}$ & $\mathrm{m}$ & $\mathrm{m}$ & 91 & 76 & 60 & 28 & 15 & 7 & 3.6 & 2.2 & 0.9 & $\mathrm{~m}$ & $\mathrm{~m}$ & $\mathrm{~m}$ \\
\hline Austria & 35 & 35 & 29 & 45 & 34 & 26 & 28 & 20 & 17 & 4.0 & 2.6 & 1.7 & 74 & 57 & 47 \\
\hline Belgium & $\mathrm{m}$ & $\mathrm{m}$ & $\mathrm{m}$ & 69 & 62 & 60 & 26 & 21 & 20 & 1.0 & 0.5 & 0.5 & 67 & 56 & 54 \\
\hline Canada & $\mathrm{m}$ & $\mathrm{m}$ & $\mathrm{m}$ & $\mathrm{m}$ & $\mathrm{m}$ & $\mathrm{m}$ & $\mathrm{m}$ & $\mathrm{m}$ & $\mathrm{m}$ & $\mathrm{m}$ & $\mathrm{m}$ & $\mathrm{m}$ & $\mathrm{m}$ & $\mathrm{m}$ & $\mathrm{m}$ \\
\hline Chile & 49 & 49 & 34 & 58 & 58 & 45 & 13 & 12 & 6 & 0.4 & 0.4 & 0.2 & 89 & 89 & 68 \\
\hline Czech Republic & 0 & 0 & 0 & 64 & 58 & 49 & 31 & 27 & 24 & 3.5 & 3.0 & 2.4 & 67 & 59 & 51 \\
\hline Denmark & 32 & 29 & 11 & 71 & 66 & 50 & 32 & 25 & 21 & 3.7 & 2.5 & 1.4 & 87 & 75 & 56 \\
\hline Estonia & $\mathrm{a}$ & a & $\mathrm{a}$ & 70 & 68 & 55 & 25 & 24 & 19 & 2.0 & 1.8 & 1.2 & $\mathrm{~m}$ & $\mathrm{~m}$ & $\mathrm{~m}$ \\
\hline Finland & $a$ & a & $\mathrm{a}$ & 55 & 51 & 41 & 11 & 8 & 4 & 2.6 & 1.9 & 0.9 & 55 & 48 & 41 \\
\hline France & $\mathrm{m}$ & $\mathrm{m}$ & $\mathrm{m}$ & $\mathrm{m}$ & $\mathrm{m}$ & $\mathrm{m}$ & $\mathrm{m}$ & $\mathrm{m}$ & $\mathrm{m}$ & 2.5 & $\mathrm{~m}$ & $\mathrm{~m}$ & $\mathrm{~m}$ & $\mathrm{~m}$ & $\mathrm{~m}$ \\
\hline Germany & 0 & 0 & 0 & 48 & 46 & 38 & 25 & 18 & 17 & 5.4 & 3.9 & 4.0 & 59 & 53 & 45 \\
\hline Greece & a & $\mathrm{a}$ & $\mathrm{a}$ & 66 & $\mathrm{~m}$ & $\mathrm{~m}$ & 11 & $\mathrm{~m}$ & $\mathrm{~m}$ & 2.1 & $\mathrm{~m}$ & $\mathrm{~m}$ & $\mathrm{~m}$ & $\mathrm{~m}$ & $\mathrm{~m}$ \\
\hline Hungary & 13 & $\mathrm{~m}$ & $\mathrm{~m}$ & 41 & $\mathrm{~m}$ & $\mathrm{~m}$ & 14 & $\mathrm{~m}$ & $\mathrm{~m}$ & 1.7 & $\mathrm{~m}$ & $\mathrm{~m}$ & $\mathrm{~m}$ & $\mathrm{~m}$ & $\mathrm{~m}$ \\
\hline Iceland & 6 & 4 & 1 & 80 & 68 & 48 & 39 & 35 & 17 & 2.5 & 1.8 & 0.5 & 86 & 70 & 49 \\
\hline Ireland & 20 & 20 & 17 & 59 & 57 & 53 & $\mathrm{~m}$ & $\mathrm{~m}$ & $\mathrm{~m}$ & $\mathrm{~m}$ & $\mathrm{~m}$ & $\mathrm{~m}$ & $\mathrm{~m}$ & $\mathrm{~m}$ & $\mathrm{~m}$ \\
\hline Israel & 23 & $\mathrm{~m}$ & $\mathrm{~m}$ & 57 & 55 & 37 & 21 & 20 & 9 & 1.8 & 1.7 & 0.6 & 71 & $\mathrm{~m}$ & $\mathrm{~m}$ \\
\hline Italy & 0 & $\mathrm{~m}$ & $\mathrm{~m}$ & 37 & $\mathrm{~m}$ & $\mathrm{~m}$ & 23 & $\mathrm{~m}$ & $\mathrm{~m}$ & 1.7 & $\mathrm{~m}$ & $\mathrm{~m}$ & 42 & $\mathrm{~m}$ & $\mathrm{~m}$ \\
\hline Japan & 28 & $\mathrm{~m}$ & $\mathrm{~m}$ & 48 & $\mathrm{~m}$ & $\mathrm{~m}$ & 9 & 8 & 7 & 1.2 & 1.0 & $\mathrm{~m}$ & 78 & $\mathrm{~m}$ & $\mathrm{~m}$ \\
\hline Korea & 34 & $\mathrm{~m}$ & $\mathrm{~m}$ & 55 & $\mathrm{~m}$ & $\mathrm{~m}$ & 14 & $\mathrm{~m}$ & $\mathrm{~m}$ & 3.3 & $\mathrm{~m}$ & $\mathrm{~m}$ & $\mathrm{~m}$ & $\mathrm{~m}$ & $\mathrm{~m}$ \\
\hline Luxembourg & 4 & 3 & 3 & 22 & 17 & 16 & 30 & 10 & 7 & 0.7 & 0.1 & 0.0 & 36 & 22 & 19 \\
\hline Mexico & 3 & $\mathrm{~m}$ & $\mathrm{~m}$ & 35 & $\mathrm{~m}$ & $\mathrm{~m}$ & 4 & $\mathrm{~m}$ & $\mathrm{~m}$ & 0.4 & $\mathrm{~m}$ & $\mathrm{~m}$ & 38 & $\mathrm{~m}$ & $\mathrm{~m}$ \\
\hline Netherlands & 1 & 1 & 0 & 60 & 54 & 51 & 17 & 13 & 12 & 1.2 & 0.7 & 0.7 & 65 & 55 & 52 \\
\hline New Zealand & 38 & 30 & 13 & 74 & 58 & 43 & 9 & 7 & 3 & 2.7 & 1.3 & 0.5 & 92 & 68 & 51 \\
\hline Norway & $\mathrm{m}$ & $\mathrm{m}$ & $\mathrm{m}$ & $\mathrm{m}$ & $\mathrm{m}$ & $\mathrm{m}$ & $\mathrm{m}$ & $\mathrm{m}$ & $\mathrm{m}$ & $\mathrm{m}$ & $\mathrm{m}$ & $\mathrm{m}$ & $\mathrm{m}$ & $\mathrm{m}$ & $\mathrm{m}$ \\
\hline Poland & 1 & 1 & 1 & 73 & $\mathrm{~m}$ & $\mathrm{~m}$ & 46 & $\mathrm{~m}$ & $\mathrm{~m}$ & 3.0 & $\mathrm{~m}$ & $\mathrm{~m}$ & 79 & 78 & 70 \\
\hline Portugal & $\mathrm{a}$ & $\mathrm{a}$ & a & 52 & 51 & 45 & 36 & 34 & 27 & 3.3 & 2.7 & 1.2 & 64 & 63 & 56 \\
\hline Slovak Republic & 1 & 1 & 1 & 56 & 54 & $\mathrm{~m}$ & 39 & 37 & $\mathrm{~m}$ & 2.9 & 2.7 & 2.0 & 60 & 57 & 50 \\
\hline Slovenia & 28 & 28 & 19 & 79 & 77 & 71 & 28 & 27 & 23 & 2.7 & 2.5 & 1.8 & 75 & 72 & 68 \\
\hline Spain & 26 & $\mathrm{~m}$ & $\mathrm{~m}$ & 46 & 46 & 42 & 10 & 8 & 7 & $\mathrm{~m}$ & $\mathrm{~m}$ & $\mathrm{~m}$ & 70 & $\mathrm{~m}$ & $\mathrm{~m}$ \\
\hline Sweden & 9 & 9 & 4 & 47 & 45 & 33 & 29 & 24 & 18 & 2.7 & 1.6 & 0.7 & 56 & 51 & 40 \\
\hline Switzerland & 5 & $\mathrm{~m}$ & $\mathrm{~m}$ & 60 & $\mathrm{~m}$ & $\mathrm{~m}$ & 21 & $\mathrm{~m}$ & $\mathrm{~m}$ & 4.9 & $\mathrm{~m}$ & $\mathrm{~m}$ & 76 & $\mathrm{~m}$ & $\mathrm{~m}$ \\
\hline Turkey & 35 & $\mathrm{~m}$ & $\mathrm{~m}$ & 34 & $\mathrm{~m}$ & $\mathrm{~m}$ & 8 & $\mathrm{~m}$ & $\mathrm{~m}$ & 1.5 & $\mathrm{~m}$ & $\mathrm{~m}$ & 70 & $\mathrm{~m}$ & $\mathrm{~m}$ \\
\hline United Kingdom & 9 & 7 & 3 & 58 & 48 & 40 & 28 & 15 & 8 & 4.0 & 2.2 & 1.4 & 58 & 51 & 42 \\
\hline United States & 39 & 38 & 27 & $\mathrm{~m}$ & $\mathrm{~m}$ & $\mathrm{~m}$ & 13 & 12 & 7 & 1.2 & 0.7 & 0.4 & 52 & 51 & 47 \\
\hline OECD average & 18 & $\mathrm{~m}$ & $\mathrm{~m}$ & 57 & 55 & 45 & 22 & 20 & 14 & 2.5 & 1.8 & 1.1 & 67 & 60 & 50 \\
\hline EU21 average & 12 & $\mathrm{~m}$ & $\mathrm{~m}$ & 56 & 52 & 45 & 26 & 21 & 16 & 2.7 & 2.0 & 1.4 & 63 & 57 & 49 \\
\hline Argentina & $\mathrm{m}$ & $\mathrm{m}$ & $\mathrm{m}$ & $\mathrm{m}$ & $\mathrm{m}$ & $\mathrm{m}$ & $\mathrm{m}$ & $\mathrm{m}$ & $\mathrm{m}$ & $\mathrm{m}$ & $\mathrm{m}$ & $\mathrm{m}$ & $\mathrm{m}$ & $\mathrm{m}$ & $\mathrm{m}$ \\
\hline Brazil & $\mathrm{m}$ & $\mathrm{m}$ & $\mathrm{m}$ & $\mathrm{m}$ & $\mathrm{m}$ & $\mathrm{m}$ & $\mathrm{m}$ & $\mathrm{m}$ & $\mathrm{m}$ & $\mathrm{m}$ & $\mathrm{m}$ & $\mathrm{m}$ & $\mathrm{m}$ & $\mathrm{m}$ & $\mathrm{m}$ \\
\hline China & 25 & 25 & $\mathrm{~m}$ & 25 & 25 & $\mathrm{~m}$ & 3 & 3 & $\mathrm{~m}$ & 0.3 & 0.3 & $\mathrm{~m}$ & $\mathrm{~m}$ & $\mathrm{~m}$ & $\mathrm{~m}$ \\
\hline Colombia & 16 & $\mathrm{~m}$ & $\mathrm{~m}$ & 46 & $\mathrm{~m}$ & $\mathrm{~m}$ & 11 & $\mathrm{~m}$ & $\mathrm{~m}$ & 0.1 & $\mathrm{~m}$ & $\mathrm{~m}$ & 62 & $\mathrm{~m}$ & $\mathrm{~m}$ \\
\hline India & $\mathrm{m}$ & $\mathrm{m}$ & $\mathrm{m}$ & $\mathrm{m}$ & $\mathrm{m}$ & $\mathrm{m}$ & $\mathrm{m}$ & $\mathrm{m}$ & $\mathrm{m}$ & & $\mathrm{m}$ & $\mathrm{m}$ & $\mathrm{m}$ & $\mathrm{m}$ & $\mathrm{m}$ \\
\hline Indonesia & $\mathrm{m}$ & $\mathrm{m}$ & $\mathrm{m}$ & 29 & $\mathrm{~m}$ & $\mathrm{~m}$ & 2 & $\mathrm{~m}$ & $\mathrm{~m}$ & 0.1 & $\mathrm{~m}$ & $\mathrm{~m}$ & $\mathrm{~m}$ & $\mathrm{~m}$ & $\mathrm{~m}$ \\
\hline Latvia & 26 & $\mathrm{~m}$ & $\mathrm{~m}$ & 77 & $\mathrm{~m}$ & $\mathrm{~m}$ & 18 & $\mathrm{~m}$ & $\mathrm{~m}$ & 2.3 & $\mathrm{~m}$ & $\mathrm{~m}$ & $\mathrm{~m}$ & $\mathrm{~m}$ & $\mathrm{~m}$ \\
\hline Russian Federation & 38 & $\mathrm{~m}$ & $\mathrm{~m}$ & 72 & $\mathrm{~m}$ & $\mathrm{~m}$ & 11 & $\mathrm{~m}$ & $\mathrm{~m}$ & 2.0 & $\mathrm{~m}$ & $\mathrm{~m}$ & $\mathrm{~m}$ & $\mathrm{~m}$ & $\mathrm{~m}$ \\
\hline Saudi Arabia & 12 & $\mathrm{~m}$ & $\mathrm{~m}$ & 72 & $\mathrm{~m}$ & $\mathrm{~m}$ & 3 & $\mathrm{~m}$ & $\mathrm{~m}$ & 0.2 & $\mathrm{~m}$ & $\mathrm{~m}$ & $\mathrm{~m}$ & $\mathrm{~m}$ & $\mathrm{~m}$ \\
\hline South Africa ${ }^{1}$ & $\mathrm{~m}$ & $\mathrm{~m}$ & $\mathrm{~m}$ & $\mathrm{~m}$ & $\mathrm{~m}$ & $\mathrm{~m}$ & $\mathrm{~m}$ & $\mathrm{~m}$ & $\mathrm{~m}$ & $\mathrm{~m}$ & $\mathrm{~m}$ & $\mathrm{~m}$ & 19 & $\mathrm{~m}$ & $\mathrm{~m}$ \\
\hline
\end{tabular}

\section{G20 average}

Note: Mismatches between the coverage of the population data and the new-entrants data mean that the entry rates for those countries that are net exporters of students may be underestimated and those that are net importers may be overestimated. The adjusted entry rates seek to compensate for that. Please refer to Annex 3 for further specific information by country.

1. Year of reference 2012.

Source: OECD. Argentina, China, Colombia, India, Indonesia, Saudi Arabia, South Africa: UNESCO Institute for Statistics. Latvia: Eurostat. See Annex 3 for notes (www.oecd.org/education/education-at-a-glance-19991487.htm).

Please refer to the Reader's Guide for information concerning symbols for missing data and abbreviations.

StatLink त्ञाज http://dx.doi.org/10.1787/888933285873 
Table C3.2. Profile of first-time new entrants into tertiary education (2013)

\begin{tabular}{|c|c|c|c|c|c|c|c|c|c|c|c|c|c|c|}
\hline & \multirow{2}{*}{ 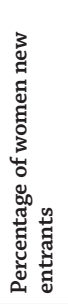 } & \multirow{2}{*}{ 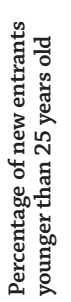 } & \multirow{2}{*}{ 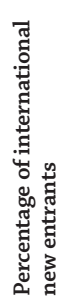 } & \multicolumn{3}{|c|}{$\begin{array}{l}\text { Percentage of first-time new } \\
\text { entrants by level } \\
\text { of education }\end{array}$} & \multicolumn{8}{|c|}{$\begin{array}{l}\text { Percentage of new entrants by field of education } \\
\text { (for all tertiary levels) }\end{array}$} \\
\hline & & & & 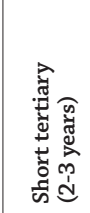 & 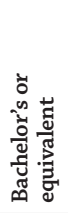 & 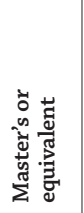 & 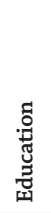 & 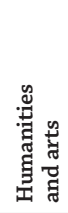 & 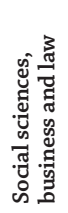 & 芯 & 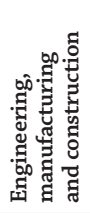 & 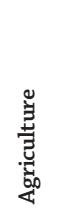 & 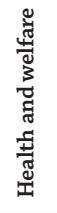 & 岸 \\
\hline & (1) & (2) & (3) & (4) & (5) & (6) & (7) & (8) & (9) & (10) & (11) & (12) & (13) & (14) \\
\hline Australia & $\mathrm{m}$ & $\mathrm{m}$ & $\mathrm{m}$ & $\mathrm{m}$ & $\mathrm{m}$ & $\mathrm{m}$ & $\mathrm{m}$ & $\mathrm{m}$ & $\mathrm{m}$ & $\mathrm{m}$ & $\mathrm{m}$ & $\mathrm{m}$ & $\mathrm{m}$ & $\mathrm{m}$ \\
\hline Austria & 54 & 79 & 23 & 44 & 37 & 19 & 13 & 10 & 33 & 11 & 19 & 2 & 6 & 7 \\
\hline Belgium & 56 & 94 & 17 & $\mathrm{~m}$ & 97 & 3 & $\mathrm{~m}$ & $\mathrm{~m}$ & $\mathrm{~m}$ & $\mathrm{~m}$ & $\mathrm{~m}$ & $\mathrm{~m}$ & $\mathrm{~m}$ & $\mathrm{~m}$ \\
\hline Canada & $\mathrm{m}$ & $\mathrm{m}$ & $\mathrm{m}$ & $\mathrm{m}$ & $\mathrm{m}$ & $\mathrm{m}$ & $\mathrm{m}$ & $\mathrm{m}$ & $\mathrm{m}$ & $\mathrm{m}$ & $\mathrm{m}$ & $\mathrm{m}$ & $\mathrm{m}$ & $\mathrm{m}$ \\
\hline Chile & 52 & 78 & 0 & 45 & 52 & 3 & 10 & 4 & 25 & 6 & 20 & 2 & 19 & 14 \\
\hline Czech Republic & 58 & 83 & 12 & 1 & 91 & 8 & 9 & 9 & 32 & 13 & 15 & 5 & 12 & 6 \\
\hline Denmark & 54 & 74 & 14 & 24 & 70 & 6 & 7 & 11 & 42 & 9 & 11 & 1 & 17 & 2 \\
\hline Estonia & $\mathrm{m}$ & $\mathrm{m}$ & $\mathrm{m}$ & $\mathrm{m}$ & $\mathrm{m}$ & $\mathrm{m}$ & 7 & 13 & 29 & 15 & 16 & 2 & 10 & 8 \\
\hline Finland & 55 & 82 & 12 & a & 94 & 6 & 4 & 10 & 23 & 9 & 23 & 2 & 20 & 8 \\
\hline France & $\mathrm{m}$ & $\mathrm{m}$ & $\mathrm{m}$ & $\mathrm{m}$ & $\mathrm{m}$ & $\mathrm{m}$ & $\mathrm{m}$ & $\mathrm{m}$ & $\mathrm{m}$ & $\mathrm{m}$ & $\mathrm{m}$ & $\mathrm{m}$ & $\mathrm{m}$ & $\mathrm{m}$ \\
\hline Germany & 50 & 83 & 10 & 0 & 81 & 18 & 10 & 11 & 30 & 16 & 22 & 2 & 6 & 3 \\
\hline Greece & $\mathrm{m}$ & $\mathrm{m}$ & $\mathrm{m}$ & $\mathrm{m}$ & $\mathrm{m}$ & $\mathrm{m}$ & 8 & 14 & 29 & 14 & 19 & 4 & 11 & 2 \\
\hline Hungary & $\mathrm{m}$ & $\mathrm{m}$ & $\mathrm{m}$ & $\mathrm{m}$ & $\mathrm{m}$ & $\mathrm{m}$ & 6 & 11 & 37 & 10 & 14 & 3 & 9 & 11 \\
\hline Iceland & 58 & 69 & 18 & 5 & 89 & 6 & 11 & 16 & 36 & 12 & 10 & 1 & 12 & 3 \\
\hline Ireland & $\mathrm{m}$ & $\mathrm{m}$ & $\mathrm{m}$ & $\mathrm{m}$ & $\mathrm{m}$ & $\mathrm{m}$ & $\mathrm{m}$ & $\mathrm{m}$ & $\mathrm{m}$ & $\mathrm{m}$ & $\mathrm{m}$ & $\mathrm{m}$ & $\mathrm{m}$ & $\mathrm{m}$ \\
\hline Israel & 56 & 69 & $\mathrm{~m}$ & 24 & 76 & $\mathrm{a}$ & 18 & 9 & 35 & 9 & 20 & 0 & 8 & 0 \\
\hline Italy & 56 & 90 & $\mathrm{~m}$ & 1 & 84 & 15 & 4 & 17 & 33 & 11 & 19 & 3 & 10 & 3 \\
\hline Japan & 50 & $\mathrm{~m}$ & $\mathrm{~m}$ & 36 & 62 & 2 & 9 & 15 & 27 & 3 & 17 & 3 & 16 & 9 \\
\hline Korea & $\mathrm{m}$ & $\mathrm{m}$ & $\mathrm{m}$ & $\mathrm{m}$ & $\mathrm{m}$ & $\mathrm{m}$ & 7 & 18 & 20 & 7 & 25 & 1 & 14 & 7 \\
\hline Luxembourg & 53 & 71 & 41 & 10 & 60 & 29 & 16 & 11 & 49 & 10 & 5 & 1 & 9 & 0 \\
\hline Mexico & 49 & 94 & $\mathrm{~m}$ & 9 & 91 & $\mathrm{~m}$ & 10 & 5 & 41 & 5 & 26 & 2 & 10 & 1 \\
\hline Netherlands & 52 & 91 & 16 & 1 & 93 & 6 & 9 & 8 & 40 & 8 & 9 & 1 & 18 & 6 \\
\hline New Zealand & 55 & 76 & 26 & 30 & 68 & 2 & 8 & 16 & 34 & 17 & 8 & 1 & 12 & 5 \\
\hline Norway & $\mathrm{m}$ & $\mathrm{m}$ & $\mathrm{m}$ & $\mathrm{m}$ & $\mathrm{m}$ & $\mathrm{m}$ & $\mathrm{m}$ & $\mathrm{m}$ & $\mathrm{m}$ & $\mathrm{m}$ & $\mathrm{m}$ & $\mathrm{m}$ & $\mathrm{m}$ & $\mathrm{m}$ \\
\hline Poland & 56 & 88 & 2 & a & $\mathrm{m}$ & $\mathrm{m}$ & 10 & 10 & 34 & 9 & 17 & 2 & 8 & 9 \\
\hline Portugal & 56 & 86 & 2 & a & 80 & 20 & 8 & 11 & 34 & 8 & 18 & 2 & 13 & 7 \\
\hline Slovak Republic & 57 & 85 & 5 & 2 & $98^{\mathrm{d}}$ & $\mathrm{x}(6)$ & 13 & 7 & 31 & 10 & 16 & 2 & 14 & 7 \\
\hline Slovenia & 53 & 93 & 4 & 15 & 80 & 5 & 6 & 8 & 33 & 11 & 21 & 4 & 7 & 11 \\
\hline Spain & 53 & 84 & $\mathrm{~m}$ & 37 & 55 & 8 & $\mathrm{~m}$ & $\mathrm{~m}$ & $\mathrm{~m}$ & $\mathrm{~m}$ & $\mathrm{~m}$ & $\mathrm{~m}$ & $\mathrm{~m}$ & $\mathrm{~m}$ \\
\hline Sweden & 58 & 76 & 9 & 2 & 74 & 24 & 11 & 14 & 28 & 10 & 18 & 1 & 16 & 3 \\
\hline Switzerland & 49 & 64 & $\mathrm{~m}$ & 4 & 70 & 26 & 8 & 9 & 37 & 9 & 16 & 1 & 12 & 6 \\
\hline Turkey & 49 & 87 & $\mathrm{~m}$ & 50 & 48 & 2 & 7 & 13 & 39 & 7 & 16 & 3 & 9 & 5 \\
\hline United Kingdom & 55 & 82 & 13 & 15 & 82 & 2 & 10 & 16 & 29 & 18 & 9 & 1 & 15 & 1 \\
\hline United States & 53 & 92 & 2 & 45 & 55 & a & $\mathrm{m}$ & $\mathrm{m}$ & $\mathrm{m}$ & $\mathrm{m}$ & $\mathrm{m}$ & $\mathrm{m}$ & $\mathrm{m}$ & $\mathrm{m}$ \\
\hline OECD average & 54 & 82 & 13 & 18 & 72 & 10 & 9 & 11 & 33 & 10 & 17 & 2 & 12 & 6 \\
\hline EU21 average & 55 & 84 & 13 & 12 & 75 & 13 & 9 & 11 & 33 & 11 & 16 & 2 & 12 & 5 \\
\hline
\end{tabular}

\begin{tabular}{l|c|c|c|c|c|c|c|c|c|c|c|c|c|c}
\hline Argentina & $\mathrm{m}$ & $\mathrm{m}$ & $\mathrm{m}$ & $\mathrm{m}$ & $\mathrm{m}$ & $\mathrm{m}$ & $\mathrm{m}$ & $\mathrm{m}$ & $\mathrm{m}$ & $\mathrm{m}$ & $\mathrm{m}$ & $\mathrm{m}$ & $\mathrm{m}$ & $\mathrm{m}$ \\
Brazil & $\mathrm{m}$ & $\mathrm{m}$ & $\mathrm{m}$ & $\mathrm{m}$ & $\mathrm{m}$ & $\mathrm{m}$ & $\mathrm{m}$ & $\mathrm{m}$ & $\mathrm{m}$ & $\mathrm{m}$ & $\mathrm{m}$ & $\mathrm{m}$ & $\mathrm{m}$ & $\mathrm{m}$ \\
B & $\mathrm{m}$ & $\mathrm{m}$ & $\mathrm{m}$ & $\mathrm{m}$ & $\mathrm{m}$ & $\mathrm{m}$ & $\mathrm{m}$ & $\mathrm{m}$ & $\mathrm{m}$ & $\mathrm{m}$ & $\mathrm{m}$ & $\mathrm{m}$ & $\mathrm{m}$ & $\mathrm{m}$ \\
China & 51 & 74 & $\mathrm{~m}$ & 26 & 74 & $\mathrm{a}$ & 9 & 4 & 48 & 4 & 24 & 2 & 7 & 3 \\
Colombia & $\mathrm{m}$ & $\mathrm{m}$ & $\mathrm{m}$ & $\mathrm{m}$ & $\mathrm{m}$ & $\mathrm{m}$ & $\mathrm{m}$ & $\mathrm{m}$ & $\mathrm{m}$ & $\mathrm{m}$ & $\mathrm{m}$ & $\mathrm{m}$ & $\mathrm{m}$ & $\mathrm{m}$ \\
India & $\mathrm{m}$ & $\mathrm{m}$ & $\mathrm{m}$ & $\mathrm{m}$ & $\mathrm{m}$ & $\mathrm{m}$ & $\mathrm{m}$ & $\mathrm{m}$ & $\mathrm{m}$ & $\mathrm{m}$ & $\mathrm{m}$ & $\mathrm{m}$ & $\mathrm{m}$ & $\mathrm{m}$ \\
Indonesia & $\mathrm{m}$ & $\mathrm{m}$ & $\mathrm{m}$ & $\mathrm{m}$ & $\mathrm{m}$ & $\mathrm{m}$ & 6 & 9 & 40 & 8 & 16 & 2 & 12 & 7 \\
Latvia & $\mathrm{m}$ & $\mathrm{m}$ & $\mathrm{m}$ & 40 & 51 & 8 & 8 & 4 & 38 & 8 & 27 & 2 & 7 & 7 \\
Russian Federation & 45 & $\mathrm{~m}$ & $\mathrm{~m}$ & 14 & 85 & 1 & $\mathrm{~m}$ & $\mathrm{~m}$ & $\mathrm{~m}$ & $\mathrm{~m}$ & $\mathrm{~m}$ & $\mathrm{~m}$ & $\mathrm{~m}$ & $\mathrm{~m}$ \\
Saudi Arabia & 57 & 78 & $\mathrm{~m}$ & 42 & 58 & $\mathrm{a}$ & $\mathrm{m}$ & $\mathrm{m}$ & $\mathrm{m}$ & $\mathrm{m}$ & $\mathrm{m}$ & $\mathrm{m}$ & $\mathrm{m}$ & $\mathrm{m}$
\end{tabular}

\begin{tabular}{l|c|c|c|c|c|c|c|c|c|c|c|c|c|c} 
G20 average & $\mathrm{m}$ & $\mathrm{m}$ & $\mathrm{m}$ & $\mathrm{m}$ & $\mathrm{m}$ & $\mathrm{m}$ & $\mathrm{m}$ & $\mathrm{m}$ & $\mathrm{m}$ & $\mathrm{m}$ & $\mathrm{m}$ & $\mathrm{m}$ & $\mathrm{m}$ & $\mathrm{m}$ \\
\hline
\end{tabular}

Note: Columns 1 to 6 refer to students entering tertiary education for the first time, while columns 7 to 14 refer to the sum of all students entering a given tertiary level for the first time.

1. Year of reference 2012

Source: OECD. Argentina, China, Colombia, India, Indonesia, Saudi Arabia, South Africa: UNESCO Institute for Statistics. Latvia: Eurostat. See Annex 3 for notes (www.oecd.org/education/education-at-a-glance-19991487.htm).

Please refer to the Reader's Guide for information concerning symbols for missing data and abbreviations.

StatLink 세대 http://dx.doi.org/10.1787/888933285889 
Table C3.3. Profile of first-time new entrants into bachelor's programmes (2013)

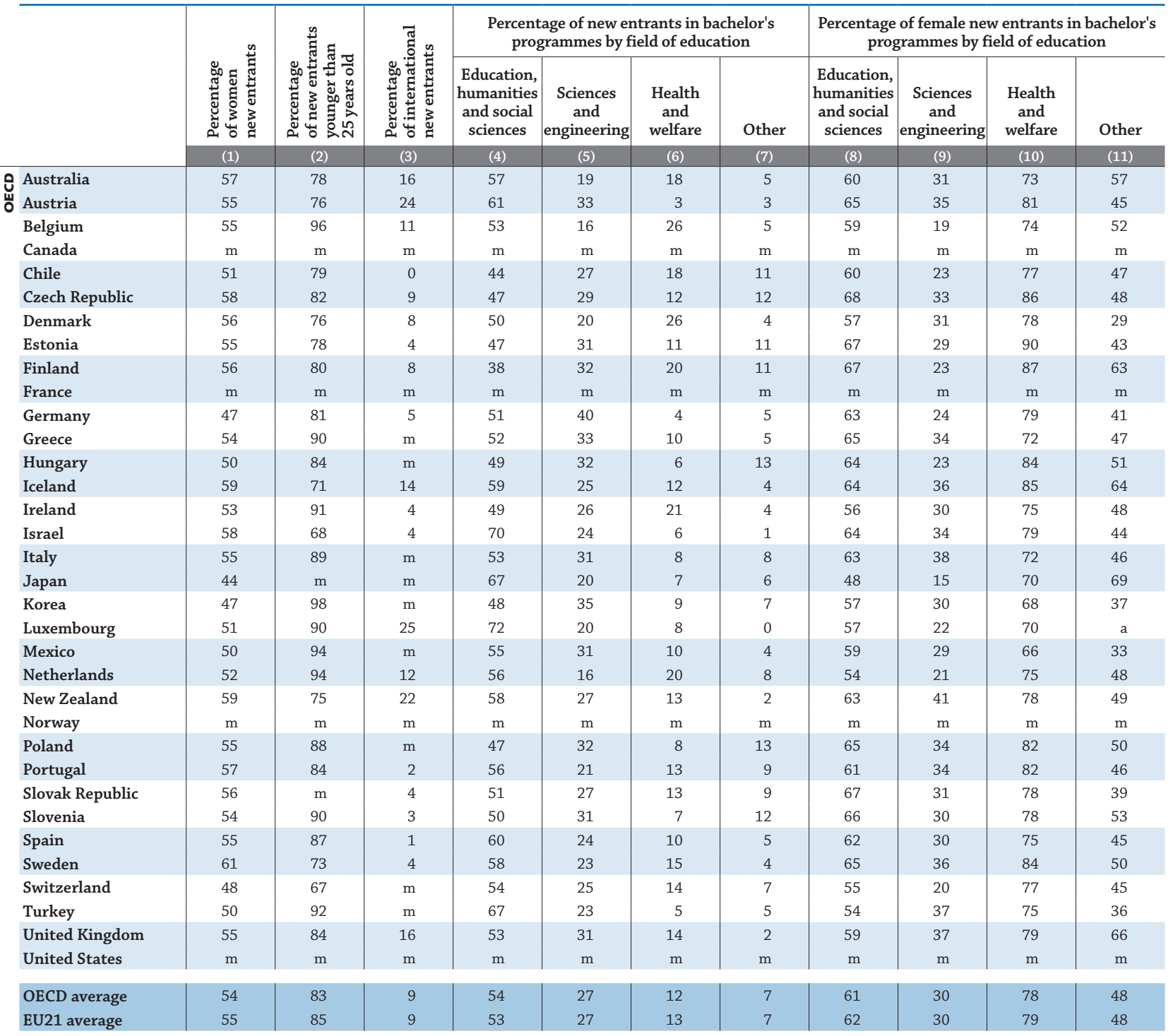

\begin{tabular}{|c|c|c|c|c|c|c|c|c|c|c|c|}
\hline $\begin{array}{l}\text { Argentina } \\
\text { Brazil }\end{array}$ & $\begin{array}{l}\mathrm{m} \\
\mathrm{m}\end{array}$ & $\begin{array}{l}\mathrm{m} \\
\mathrm{m}\end{array}$ & $\begin{array}{l}\mathrm{m} \\
\mathrm{m}\end{array}$ & $\begin{array}{l}\mathrm{m} \\
\mathrm{m}\end{array}$ & $\begin{array}{l}\mathrm{m} \\
\mathrm{m}\end{array}$ & $\begin{array}{l}\mathrm{m} \\
\mathrm{m}\end{array}$ & $\begin{array}{l}\mathrm{m} \\
\mathrm{m}\end{array}$ & $\begin{array}{l}\mathrm{m} \\
\mathrm{m}\end{array}$ & $\begin{array}{l}\mathrm{m} \\
\mathrm{m}\end{array}$ & $\begin{array}{l}\mathrm{m} \\
\mathrm{m}\end{array}$ & $\begin{array}{l}\mathrm{m} \\
\mathrm{m}\end{array}$ \\
\hline China & 54 & $\mathrm{~m}$ & 0 & $\mathrm{~m}$ & $\mathrm{~m}$ & $\mathrm{~m}$ & $\mathrm{~m}$ & $\mathrm{~m}$ & $\mathrm{~m}$ & $\mathrm{~m}$ & $\mathrm{~m}$ \\
\hline Colombia & 54 & 75 & $\mathrm{~m}$ & 60 & 29 & 8 & 2 & 61 & 34 & 74 & 43 \\
\hline India & $\mathrm{m}$ & $\mathrm{m}$ & $\mathrm{m}$ & $\mathrm{m}$ & $\mathrm{m}$ & $\mathrm{m}$ & $\mathrm{m}$ & $\mathrm{m}$ & $\mathrm{m}$ & $\mathrm{m}$ & $\mathrm{m}$ \\
\hline Indonesia & 51 & 100 & $\mathrm{~m}$ & 61 & 21 & 12 & 6 & 54 & 36 & 71 & 36 \\
\hline Latvia & 55 & 73 & $\mathrm{~m}$ & 56 & 25 & 11 & 9 & 65 & 23 & 78 & 54 \\
\hline Russian Federation & $\mathrm{m}$ & $\mathrm{m}$ & $\mathrm{m}$ & 63 & 29 & 1 & 7 & $\mathrm{~m}$ & $\mathrm{~m}$ & $\mathrm{~m}$ & $\mathrm{~m}$ \\
\hline Saudi Arabia & 48 & $\mathrm{~m}$ & $\mathrm{~m}$ & $\mathrm{~m}$ & $\mathrm{~m}$ & $\mathrm{~m}$ & $\mathrm{~m}$ & $\mathrm{~m}$ & $\mathrm{~m}$ & $\mathrm{~m}$ & $\mathrm{~m}$ \\
\hline South Africa & $\mathrm{m}$ & $\mathrm{m}$ & $\mathrm{m}$ & $\mathrm{m}$ & $\mathrm{m}$ & $\mathrm{m}$ & $\mathrm{m}$ & $\mathrm{m}$ & $\mathrm{m}$ & $\mathrm{m}$ & $\mathrm{m}$ \\
\hline G20 average & 51 & $\mathrm{~m}$ & $\mathrm{~m}$ & $\mathrm{~m}$ & $\mathrm{~m}$ & $\mathrm{~m}$ & $\mathrm{~m}$ & $\mathrm{~m}$ & $\mathrm{~m}$ & $\mathrm{~m}$ & $\mathrm{~m}$ \\
\hline
\end{tabular}

Source: OECD. Argentina, China, Colombia, India, Indonesia, Saudi Arabia, South Africa: UNESCO Institute for Statistics. Latvia: Eurostat. See Annex 3 for notes (www.oecd.org/education/education-at-a-glance-19991487.htm).

Please refer to the Reader's Guide for information concerning symbols for missing data and abbreviations.

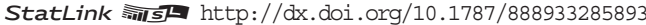


Table C3.4. Profile of first-time new entrants, by level of education (2013) Descriptive indicators for first-time entrants into ISCED 5, 7 and 8

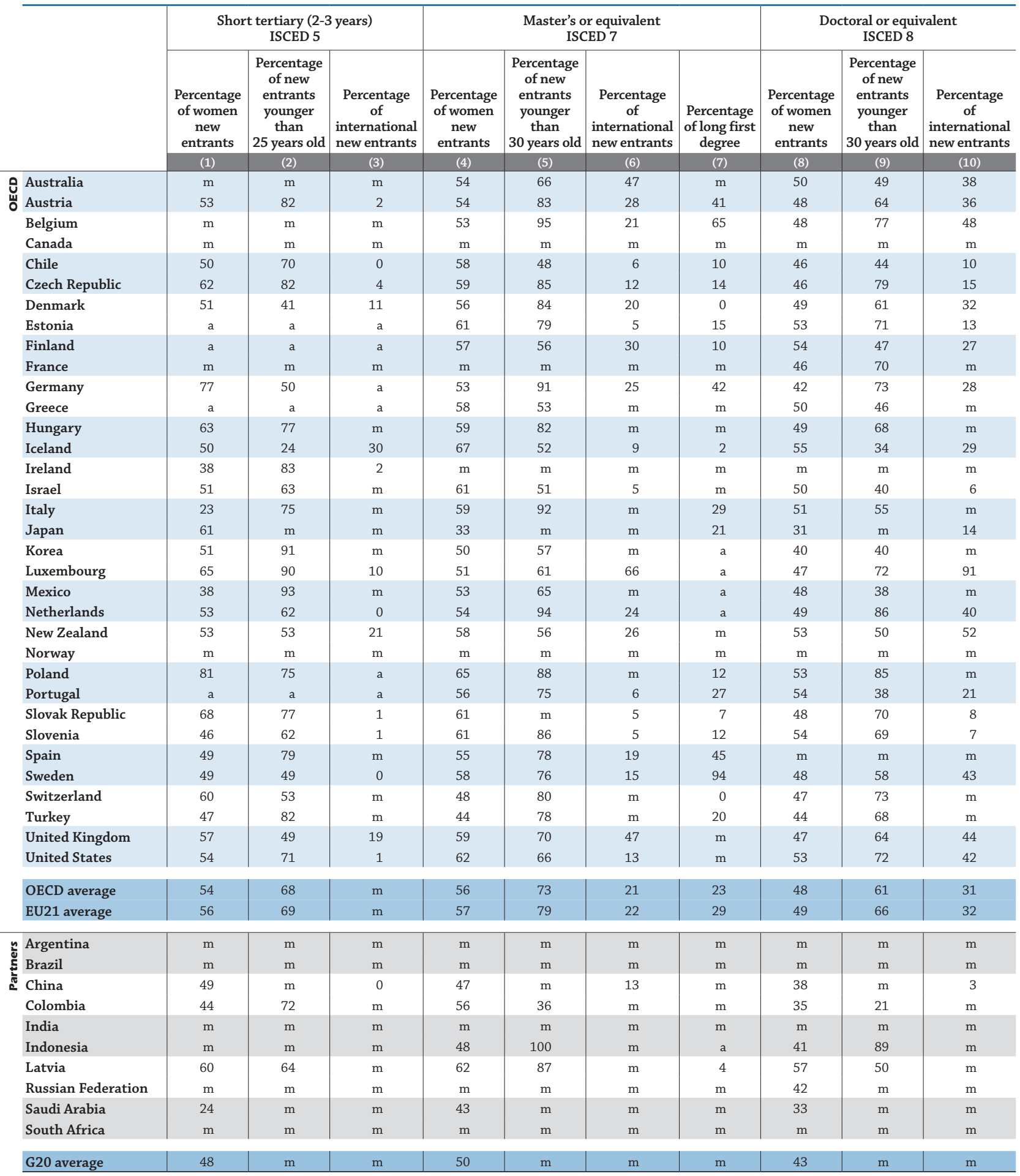

Source: OECD. Argentina, China, Colombia, India, Indonesia, Saudi Arabia, South Africa: UNESCO Institute for Statistics. Latvia: Eurostat. See Annex 3 for notes (www.oecd.org/education/education-at-a-glance-19991487.htm).

Please refer to the Reader's Guide for information concerning symbols for missing data and abbreviations.

StatLink 젶ㄴ http://dx.doi.org/10.1787/888933285907 


\section{WHO STUDIES ABROAD AND WHERE?}

- In 2013, more than 4 million students were enrolled in tertiary education outside their country of citizenship. Australia, Austria, Luxembourg, New Zealand, Switzerland and the United Kingdom have the largest proportion of international students as a percentage of their total tertiary enrolments.

- Students from Asia represent 53\% of international students enrolled worldwide. China is the country with the largest numbers of citizens enrolled abroad, followed by India and Germany.

- The proportion of international students among total enrolments tends to be much larger at the most advanced levels of tertiary education. On average across OECD countries $24 \%$ of students enrolled in doctoral or equivalent programmes are international students, against an average of $9 \%$ in all levels of tertiary education.

Chart C4.1. Student mobility in tertiary education (2013) International or foreign student enrolments as a percentage of total tertiary education

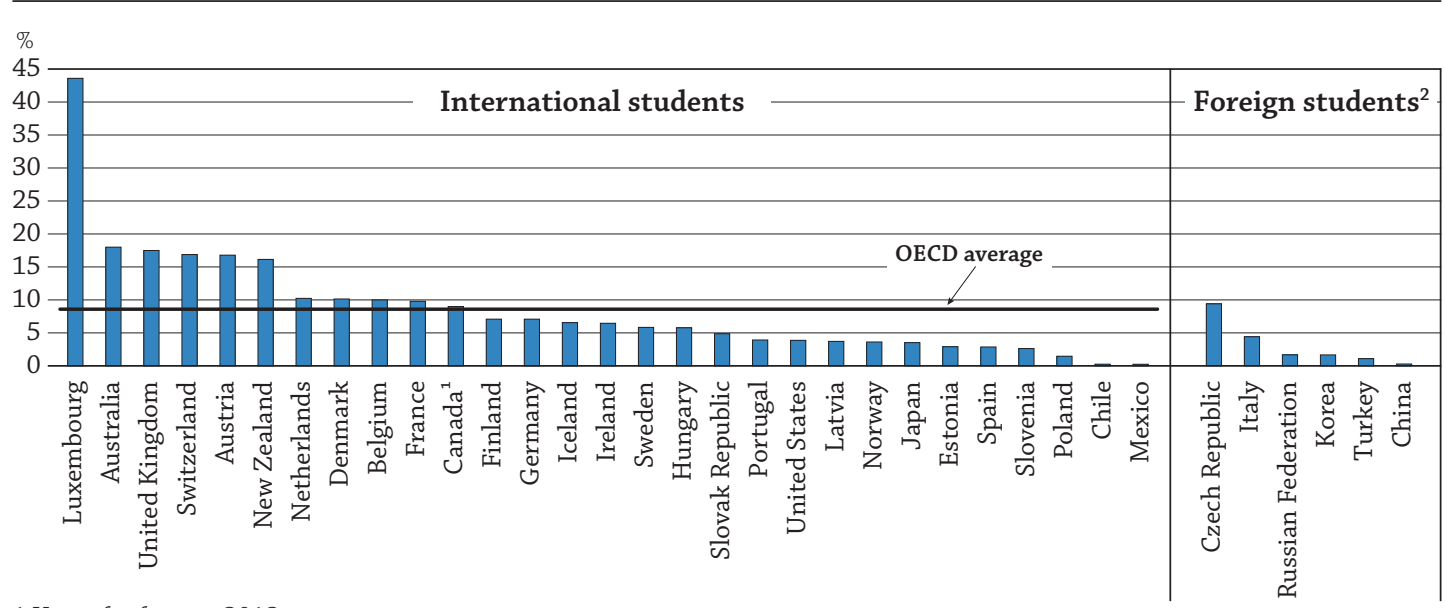

1.Year of reference 2012.

2. Foreign students are defined on the basis of their country of citizenship, these data are not comparable with data on international students and are therefore presented separately in the chart.

Countries are ranked in descending order of the percentage of international or foreign students in total tertiary education.

Source: OECD. Table C4.1. See Annex 3 for notes (www.oecd.org/education/education-at-a-glance- 19991487.htm).

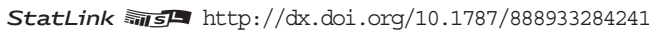

\section{Context}

As national economies become more interconnected and participation in education expands, tertiary education emerges as a means to broaden students' horizons and help them to better understand the world's languages, cultures and business methods. Tertiary education is becoming more international through a number of means, for example distance education, international education-related internships and training experiences, crossborder delivery of academic programmes and offshore satellite campuses. Among the phenomena related to the internationalisation of tertiary education, enrolling in a study programme abroad is receiving considerable attention from students and policy-makers. By providing an opportunity to expand knowledge of other societies and languages, studying abroad is an important cultural and personal experience for students as well as a way to improve their employability in the globalised sectors of the labour market.

Student mobility has increased dramatically over the recent past, due to a range of factors. The exploding demand for tertiary education worldwide and the perceived value of studying at prestigious postsecondary institutions abroad contribute to an increasing and diversified flow of international students, ranging from those who cannot find a place to study in post-secondary education at home to students of high academic achievement studying at high-quality programmes and institutions. In addition, the educational value associated with a diverse student body, the substantial revenues that can be earned by expanding education for international students, and economic and political considerations prompted some governments and institutions make major efforts to attract students from outside their national borders (Altbach and Knight, 2007; Knight, 2008). 
One key economic consideration for countries hosting international students is that these students can later become high-skilled workers. Countries that "export" students to other countries for studying purposes risk losing, permanently, many of their talented citizens (what is commonly known as "brain drain"); but the fact that many developing countries sponsor a number of international students suggests that at least some of these students will return to their home country or establish social and business links between their home and host countries, developing what some authors (e.g. Solimano, 2002) call "brain circulation".

In the current economic climate, shrinking support for scholarships and grants, as well as tighter budgets for individuals, may slow the pace of student mobility. But limited labour market opportunities in students' countries of origin may increase the attractiveness of studying abroad as a way to gain a competitive edge, and thus boost student mobility.

Throughout this indicator, the terms "international students" refers to students who have moved from their country of origin with the purpose of studying (according to the criterion of country of prior education or the criterion of usual residence, see the Definitions section within this Indicator). The term "foreign students" refers to students who are not citizens of the countries in which they are enrolled, but may be long-term residents or were born in that country. In general, international students are a subset of foreign students (see the Definitions section at the end of this indicator).

\section{Other findings}

- Australia, Canada, France, Germany, Japan, the United Kingdom and the United States together receive more than $50 \%$ of all international students worldwide.

- In 2013, the number of international students enrolled in tertiary education in OECD countries was, on average, three times the number of students from OECD countries studying abroad.

- In some countries, international students are concentrated in some particular fields of study. For example, $53 \%$ of the international students in the Slovak Republic study health and welfare, $40 \%$ of those in Iceland are enrolled in the humanities and arts, and 23\% of those in Chile study education.

\section{Trends}

Estimates for 2013 of the number of individuals worldwide who moved abroad with the purpose of study (i.e. international students) are not comparable with previous years, because too few countries were providing this information in the past. However, time series of the total number of students enrolled abroad (foreign students) can be constructed until 2012 (Box C4.3). These time series show that during 2005-12 the number of foreign tertiary students enrolled worldwide increased by $50 \%$. 


\section{Analysis}

\section{Extent of international student mobility in tertiary education}

Among countries for which data on international students are available, Australia, Austria, Luxembourg, New Zealand, Switzerland and the United Kingdom show the highest levels of incoming student mobility, measured as the proportion of international students among total tertiary enrolment. In Luxembourg, $44 \%$ of students enrolled in tertiary education are from another country. Similarly, international students represent $16 \%$ or more of total tertiary enrolments in Australia, Austria, New Zealand, Switzerland, and the United Kingdom. In contrast, international students account for 3\% or less of total tertiary enrolments in Chile, Estonia, Mexico, Poland, Slovenia and Spain (Table C4.1 and Chart C4.1).

Among countries using the definition of international students based on country of citizenship, the Czech Republic had the largest proportion of foreign students (9\%) of the total enrolled at the tertiary level. In contrast, foreign enrolments represented less than $2 \%$ of total tertiary enrolments in China, Korea, the Russian Federation and Turkey (Table C4.1 and Chart C4.1).

\section{Proportion of international students at different levels of tertiary education}

The proportion of international students is different at different levels of tertiary education. It is highest for the most advanced tertiary education programmes, at the master's or doctoral level, or equivalent. This could be because capacity constraints in the countries of origin may be particularly severe at these levels of education; the returns to study abroad, and in more prestigious institutions, may be higher for master's or doctoral programmes than at lower levels of tertiary education; and students in these programmes may be a particular subgroup of the population that is more likely to travel and live abroad, independently from their educational choices. Attracting international students in doctoral or equivalent programmes is particularly appealing to host countries because of their potential contribution to research and development, either as students or later as highly qualified immigrants.

Comparing the distribution of international and foreign students across countries by level of tertiary education gives a fair indication of which programmes are relatively more attractive in each country.

\section{Chart C4.2. Student mobility in tertiary education, by ISCED level (2013)}

International or foreign student enrolments as a percentage of total tertiary education

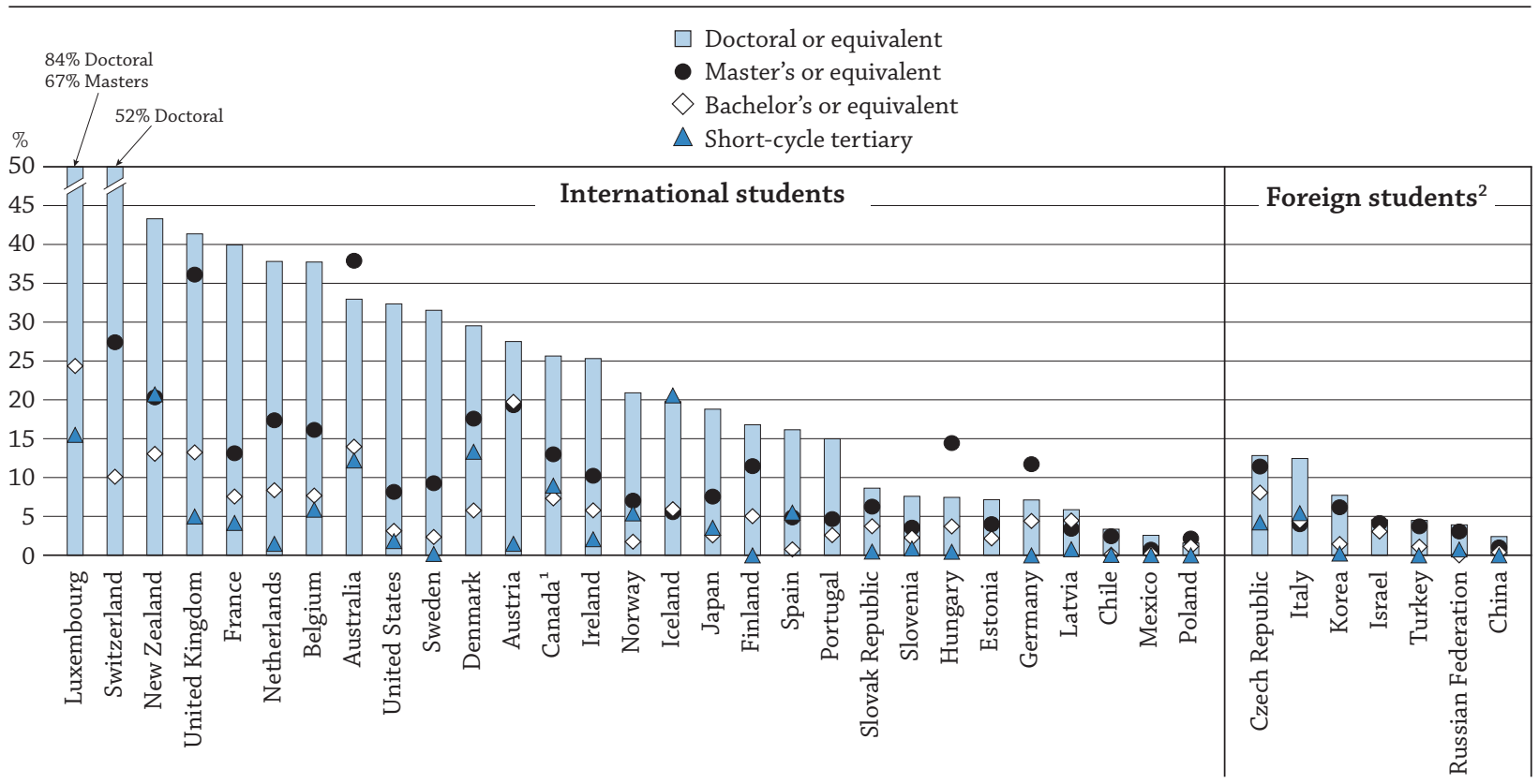

1.Year of reference 2012 .

2. Foreign students are defined on the basis of their country of citizenship, these data are not comparable with data on international students and are therefore presented separately in the chart.

Countries are ranked in descending order of the percentage of international or foreign students in doctoral or equivalent programmes.

Source: OECD. Table C4.1. See Annex 3 for notes (www.oecd.org/education/education-at-a-glance- 19991487.htm).

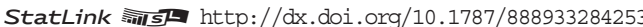


In 2013, on average across OECD countries, the share of international students in short-cycle (typically vocational) tertiary programmes (5\%) was smaller than at any other level of tertiary education. However, in some countries, international students were more represented in short-cycle programmes than at the bachelor's or master's (or equivalent) level. This is the case in Iceland and New Zealand, in which international students represent $21 \%$ of the total number of students enrolled in these programmes, Spain (6\%) and, among countries with data on foreign students, Italy (5\%) (Table C4.1 and Chart C4.2).

International enrolments at the bachelor's level were also relatively low $(6 \%)$ on average across OECD countries. Among the countries for which data are available, they exceeded enrolments at the master's level only in Austria and Latvia, where international students represented $20 \%$ and $4 \%$ of total enrolments at the bachelor's or equivalent level, respectively.

The proportion of international students was much higher at the most advanced levels of education: on average across OECD countries, $14 \%$ of students in master's programmes or the equivalent were international students as were $24 \%$ of students at the doctoral level. Luxembourg had the largest proportion of international students at the master's or equivalent level (67\%) followed by Australia (38\%), the United Kingdom (36\%) and Switzerland (27\%) (Table C4.1 and Chart C4.2).

For all reporting countries, except Australia, Germany, Hungary and Poland, the largest proportion of international students is found in doctoral or equivalent programmes. In Luxembourg and Switzerland, the majority of the students enrolled at this level are international. The proportion of international students enrolled in programmes at the doctoral or equivalent level is also large (exceeding 35\%) in Belgium, France, the Netherlands, New Zealand, Switzerland and the United Kingdom. In contrast, this proportion is 5\% or smaller in Chile, Mexico, Poland and, among the countries that reported data based on the criteria of citizenship, China, Israel, the Russian Federation and Turkey.

\section{Proportion of international students in different fields of study}

On average across the OECD countries with data on international students, more than one-third of international students enroll in the field of social sciences, business and law. Other popular fields are engineering, manufacturing and construction (14\%), health and welfare (13\%), the humanities and arts (13\%) and the sciences (11\%). The fields enrolling the smallest proportions of international students, on average, are agriculture (2\%), education and services (both 4\%) (Table C4.2).

However, there are appreciable differences between countries. In Australia, Estonia and Luxembourg, for example, the majority of international students is enrolled in the field of social sciences, business and law, whereas in the Slovak Republic the majority of international students study health and welfare. Other countries have a particularly large share of international students concentrated in one of the other fields: in Chile, $23 \%$ of international students are enrolled in education; in Iceland, $40 \%$ are enrolled in the humanities and arts; in Sweden, $20 \%$ are enrolled in the sciences; and in Finland, 31\% are enrolled in engineering. A relatively large proportion of international students studying a particular subject in a particular country may indicate a comparative advantage of this country in this field of education, good work opportunities in related fields of work, or that admission policies for this field of education are less restrictive in this country than in others.

Indicator A3 extends this analysis by showing the number of international students in each field of study relative to the total number of students in that field of study, by level of tertiary education.

\section{Major destinations of international students}

OECD countries attract $73 \%$ of all students enrolled abroad in countries reporting data to the OECD or the UNESCO Institute for Statistics. Within the OECD area, EU21 countries host the largest proportion (35\%) of international students. Some $71 \%$ of international students enrolled in EU21 countries come from another EU21 country, which may be partly an effect of EU mobility policies. North America is also an attractive region for international students, as the United States and Canada combined account for $23 \%$ of the total. The profile of international students in this region is more diverse than that observed in the European Union. For instance, although 55\% of Canadians studying abroad are in the United States, they account for only $3 \%$ of the international students studying in the United States. Similarly, $11 \%$ of Americans studying abroad chose Canada, but they account for only $6 \%$ of all international students enrolled in tertiary education in Canada (Tables C4.3 and C4.4, and Table C4.6, available on line).

Among the countries providing data on international students, Australia, Canada, France, Germany, Japan, the United Kingdom and the United States enrolled more than one in two international students in 2013. In absolute 
terms, the United States hosted the largest number of all international students (19\% of the total), followed by the United Kingdom (10\%), Australia and France (6\%), Germany (5\%), Canada and Japan (both 3\%) and, among the countries with data on foreign students only, the Russian Federation (3\%). Although these destinations account for more than half of all tertiary students pursuing their studies abroad, other countries play a substantial role in the international education market (Chart C4.3, and Table C4.6, available on line). Besides the eight major destinations, significant numbers of students from abroad were enrolled in Austria, China, Italy, the Netherlands and Saudi Arabia (2\%) in 2013 (Table C4.6). Notice that, since the statistics displayed in Chart C4.3, and in Table C4.6 (available on line) relate to international students, they are not directly comparable with the statistics on the distribution of foreign students by country of destination presented in the previous edition of Education at a Glance (OECD, 2014a).

\section{Chart C4.3. Distribution of foreign and international students in tertiary education, by country of destination (2013) \\ Percentage of foreign and international tertiary students reported to the OECD who are enrolled in each country of destination}

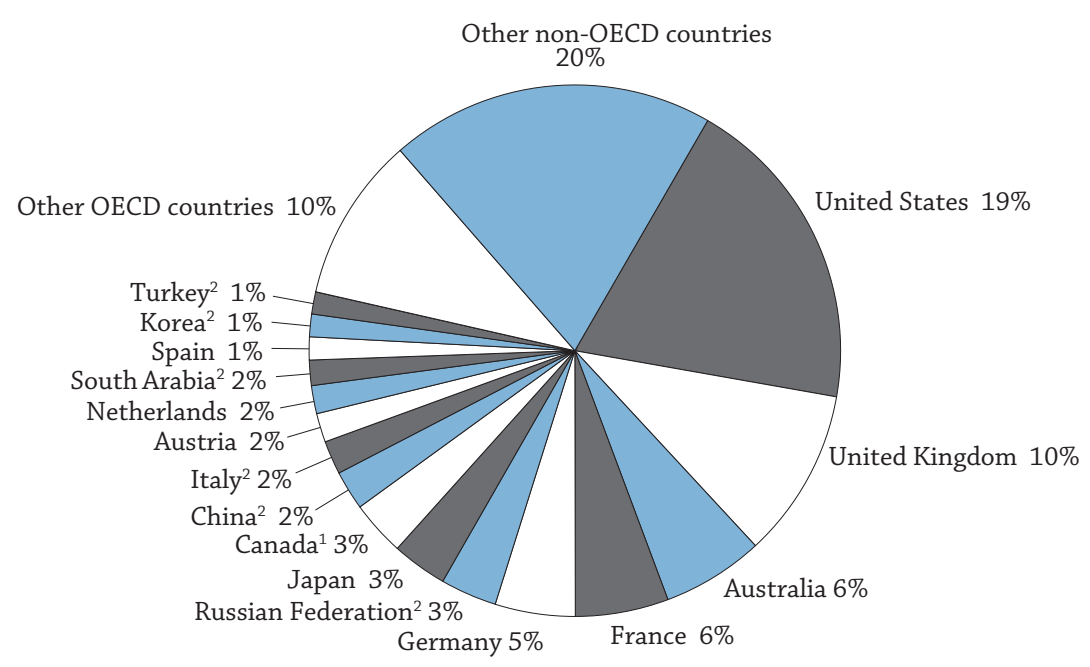

1.Year of reference 2012.

2. Data refer to foreign instead of international students.

Source: OECD. Table C4.4, and Table C4.6, available on line. See Annex 3 for notes (www.oecd.org/education/education-at-a-glance- 19991487.htm). StatLink 젶ㄴ http://dx.doi.org/10.1787/888933284267

\section{Underlying factors in students' choice of a country of study}

\section{Language of instruction}

The language spoken and used in instruction is likely to affect international students' choices over their potential destination countries. Countries whose language of instruction is widely spoken and read, such as English, French, German, Russian and Spanish, can be particularly attractive to international students, both in absolute and relative terms. Japan is a notable exception: despite a language of instruction that is not widely used around the world, it enrols large numbers of international students, $93 \%$ of whom are from Asia (Table C4.3).

The prevalence of predominantly English-speaking destinations, such as Australia, Canada, New Zealand, the United Kingdom and the United States, in part reflects the progressive adoption of English as a global language. It may also reflect the fact that students intending to study abroad are likely to have learned English in their home country or wish to improve their English-language skills through immersion in a native English-speaking context.

English-taught tertiary education programmes are offered in an increasing number of institutions in non-Englishspeaking countries. The diffusion of English as a medium of instruction is especially noticeable in the Nordic countries (Box C4.1). 


\section{Box C4.1. European countries offering tertiary education programmes in English}

The proportion of tertiary education institutions offering English-Taught Programmes (ETPs) at ISCED level 5 or 6 in 2013/2014 varied greatly across European countries. Within the sample for which the Academic Cooperation Association (ACA) collected data (Wächter and Maiworm, 2014), the three countries in which a majority of insitutions offer at least one programme entirely taught in English are Finland (83\%), Sweden (81\%) and the Netherlands (65\%). These countries also score well in terms of international student enrolment (Chart C4.3). In general, the proportion of institutions offering ETPs tends to be above average in the the groups of countries that Wächter and Maiworm (2014) define as "Nordic" and "Central-West Europe", and below average in Southern and East European countries, with Slovenia (9\%) and Croatia (7\%) showing the lowest values.

The proportion of institutions offering ETPs may not be the best indicator for measuring the diffusion of ETPs across tertiary education systems. When this indicator is compared with an alternative, such as the proportion of students enrolled in ETPs, it turns out that the two measures are far from perfectly correlated $(r=0.54)$. However, international data on the proportion of institutions offering ETPs are, for the moment, more reliable (Wächter and Maiworm, 2014, p. 36).

\section{Chart C4.a. Percentage of tertiary education education institutions offering ETPs at ISCED level 5 or 6, academic year 2013/2014}

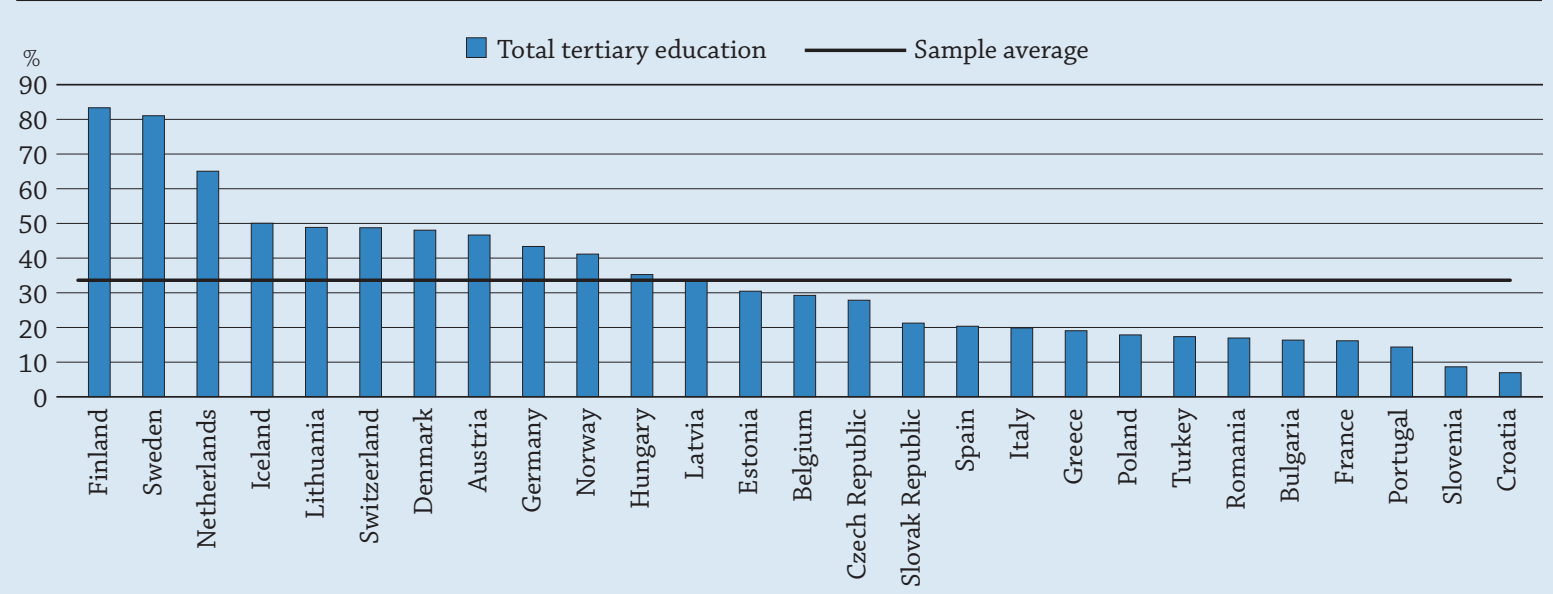

Countries are ranked in descending order of the percentage of tertiary education education institutions offering ETPs at ISCED level 5 or 6. Source: Wächter and Maiworm (2014), Table 1.4, www.aca-secretariat.be/index.php?id=792.

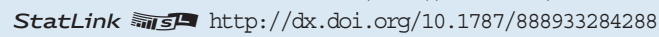

\section{Quality of programmes}

International students select their study destination based, at least in part, on the quality of education offered, as perceived from a wide array of information on, and rankings of, higher education programmes now available, both in print and on line. The large proportion of top-ranked higher education institutions in the principal destination countries, and the growing number of institutions in these rankings that are based in fast-growing student destinations draw attention to the increasing importance of quality in attracting students. There seems to be a correlation between the position of universities in international university rankings and their attractiveness to international students (e.g. Marconi, 2013). Besides rankings, other sources of information and the overall academic reputation of particular institutions or programmes are likely to play a large role.

\section{Tuition fees}

Tuition fees make up a substantial part of the cost of studying (see Indicator A7), and it is reasonable to expect that students take them into consideration when deciding where to study abroad. The cost of education differs substantially across countries, as well as the level of public subsidies and support (see Indicators B3 and B5). 
Furthermore, in some countries public subsidies and support can be mostly directed towards national students, so that tuition fees are differentiated for national and international students (Box C4.2). In other cases, the same tuition fees apply to students coming from a specific sub-group of countries as to national students. For example, among EU countries, international students from other EU countries are treated as domestic students with respect to tuition fee charges (European Commission, 2010). Finally, some countries make no difference between national and international students from any country of origin in terms of tuition fees. All these factors create a large variation in the tuition fees that international students have to pay (Box C4.2).

\section{Box C4.2. Tuition fees for international students}

The tuition fees that international students have to pay to enrol in tertiary education in different countries can be very different. For example, in 2013 international students paid no tuition fees for enrolling in Finland, Iceland, Norway, the Slovak Republic or Slovenia at the bachelor's or equivalent level in a public institution. Conversely, at this type of institution and this level of education the average annual tuition fees for international students were on average around 15000 USD PPP or higher in Australia, Canada, Estonia (for some educational programmes only), New Zealand and the United States (Indicator B5). In many countries, the tuition fees paid by international students are higher than for nationals (Table C.4a).

\section{Table C4.a. Differentiation in tuition fees between domestic and international students}

\begin{tabular}{l|l}
\hline Tuition fee structure & OECD and other G20 countries \\
\hline $\begin{array}{l}\text { Different tuition fees for international } \\
\text { students than for domestic students }\end{array}$ & $\begin{array}{l}\text { Australia, }{ }^{1} \text { Austria, }{ }^{2} \text { Belgium, }{ }^{2,3} \text { Canada, Chile, the Czech Republic, }{ }^{2} \\
\text { Denmark, }{ }^{2} \text { Estonia, }{ }^{2} \text { Greece, Ireland, Luxembourg, the Netherlands, }{ }^{2} \\
\text { New Zealand, }{ }^{4} \text { Poland, }{ }^{2} \text { the Russian Federation, Sweden, }{ }^{5} \text { Turkey, the } \\
\text { United Kingdom, }{ }^{2} \text { the United States. }{ }^{6}\end{array}$ \\
\hline $\begin{array}{l}\text { Same tuition fees for international } \\
\text { and domestic students }\end{array}$ & $\begin{array}{l}\text { Brazil, Colombia, France, Germany, Hungary, Israel, Italy, Japan, }{ }^{7} \text { Korea, } \\
\text { Mexico, }{ }^{8} \text { Portugal, Spain, Switzerland. }\end{array}$ \\
\hline
\end{tabular}

\begin{tabular}{l|l}
\hline No tuition fees neither for international & Finland, Iceland, Norway, Slovak Republic, Slovenia. ${ }^{9}$
\end{tabular}

nor domestic students

1. International students (excepting students from New Zealand) are not eligible for government-subsidised places in Australia and therefore pay the full fee. While this typically results in international students having higher tuition fees than domestic students, who are usually given subsidised places, some domestic students in public universities and all students in independent-private universities are full-fee paying and pay the same tuition fees as international students.

2. For non-European Union or non-European Economic Area students. For the other EU countries for which information is missing, the EU regulation according to which international students from other EU countries are treated as domestic students with respect to tuition fee charges (European Commission, 2010) is relevant.

3. In Belgium (Flemish Community), for non-EEA students, the institutions have the autonomy to fix the amount of the tuition fee, except for some categories of students (for example, refugees, asylum seekers).

4. Except for students in advanced research programmes, or for students from Australia.

5. There are no tuition fees for national students in Sweden. Fees apply only for students from outside the EEA and Switzerland.

6. In public institutions, international students pay the same fees as domestic out-of-state students. However, since most domestic students are enrolled in-state, international students pay higher tuition fees than most domestic students, in practice. In private universities, the fees are the same for national and international students.

7. This information applies only to public institutions.

8. Some institutions charge higher tuition fees for international students.

9. No tuition fees in bachelor's and master's programmes for EU students and for citizens of countries outside the EU that signed bilateral or multilateral agreements on educational co-operation with Slovenia, and for students who are themselves, or their parents, residents of the Republic of Slovenia (residence for tax purposes); others pay the same tuition fees as part-time students. International students in doctoral programmes pay similar amounts of tuition fees as other students.

Source: OECD. Indicator B5. See Annex 3 for notes (www.oecd.org/education/education-at-a-glance-19991487.htm).

Countries that charge international students the full cost of education reap significant economic benefits. Several countries in the Asia-Pacific region have actually made international education an explicit part of their socio-economic development strategy and have initiated policies to attract international students on a revenue-generating or at least a cost-recovery basis. New Zealand has successfully adopted differentiated tuition fees for international students (except those enrolling in $\mathrm{PhDs}$ ), and it continues to attract a large number of international students (Table C4.1). 
This suggests that tuition fees do not necessarily discourage prospective international students, as long as the quality of education provided is high and its potential returns make the investment worthwhile.

\section{Immigration policy}

In recent years, several OECD countries have eased their immigration policies to encourage the temporary or permanent immigration of international students (OECD, 2014b). This makes these countries more attractive to students by improving their job prospects and increases the pool of talent from which their economies can draw. For example, Canada and Australia allow international students to stay in the country after their studies to look for a job for a maximum of three and four years, respectively. Most other OECD countries issue similar job-search permits for international students for a shorter duration. Students are issued a work permit only if, within the duration of their job-search permit, they find a job matching their qualifications according to some criteria. Some countries in which these criteria were particularly strict, such as France, have recently relaxed them (OECD, 2014b), which will presumably help them to attract and retain international students.

\section{Other factors}

The decision if and where to study abroad is often a complex one, and students base it on a number of other factors such as: recognition of foreign degrees and of workload carried out abroad, including government policies to facilitate the transfer of credits between home and host institutions; the quality and admission policies of tertiary education in the home country; future opportunities to come back to work in the home country; and cultural aspirations. In addition, geographical, trade or migration links between countries can play a large role. This is true both for current geopolitical areas such as the European Union and the North American Free Trade Agreement area, and those related to historical links, such as the former Soviet Union, the Commonwealth or the Francophonie.

\section{Profile of international student intake in different destinations Global balance of student mobility in OECD countries}

OECD countries receive more international students than they send to study abroad for tertiary education. In 2013, OECD countries hosted three international students for every citizen who was studying outside his or her country of origin. In absolute terms, this represents 2.9 million international students in OECD countries, compared to less than 1 million students studying outside their OECD country of citizenship. As $89 \%$ of OECD citizens studying abroad study in another OECD country, about seven out of ten international students in the OECD area come from a country that is not an OECD member (Tables C4.4 and C4.5).

At the country level, the balance varies greatly. While in Australia there are more than 20 international students for each Australian student abroad, the ratio is less than 1 in Chile, Estonia, Iceland, Ireland, Latvia, Luxembourg, Mexico, Norway, the Slovak Republic, Slovenia and, among countries with data on foreign students, Brazil, Israel, Korea and Saudi Arabia. In addition to Australia, all the other countries among the five with the highest ratio of incoming international students per national student abroad (equal or above 6:1) have English as an official language (either legally or de facto): New Zealand, South Africa, the United Kingdom and the United States (Table C4.5).

\section{Main regions of origin}

Students from Asia form the largest group of international students enrolled in countries reporting data to the OECD or the UNESCO Institute for Statistics: 53\% of the total in all reporting destinations (Chart C4.4). In particular, students from China account for $22 \%$ of all international students enrolled in tertiary education in the OECD area, the highest share among all reporting countries (Table C4.3). Some $31 \%$ of all Chinese students studying abroad are enrolled in the United States, while more than $45 \%$ choose either Australia, Canada, Japan, Korea, or the United Kingdom (Table C4.4). The second-largest proportion of international students within the OECD comes from India (6\%), almost half of which goes to the United States.

Within OECD countries, the largest sender is Germany, accounting for $3.9 \%$ of all international students in tertiary education in the OECD area, followed by Korea (3.6\%), France (2.4\%) and the United States (1.7\%).

Across OECD countries in 2013, an average of 19\% of all international students came from neighbouring countries that share land or maritime borders with the host country (Table C4.5, and Table C4.6, available on line). Among OECD and partner countries, more than $60 \%$ of international or foreign students came from neighbouring countries in the Czech Republic, Estonia, Japan, Korea, Luxembourg, Poland, the Slovak Republic and the Russian Federation. 


\section{Chart C4.4. Distribution of foreign and international students in tertiary education, by region of origin (2013) \\ Percentage of foreign and international tertiary students enrolled worldwide}

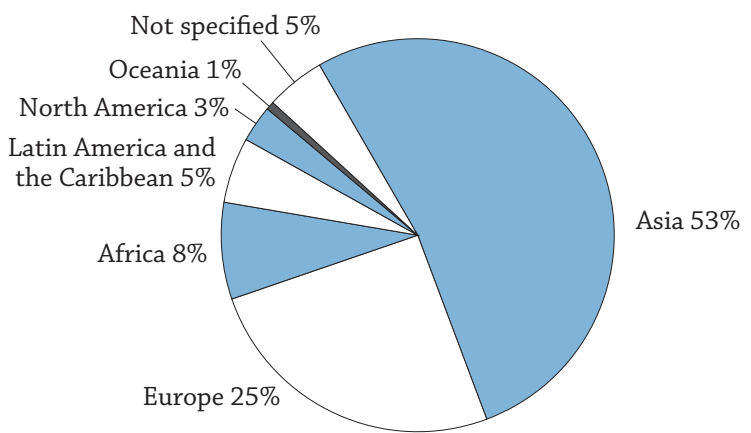

Source: OECD. Table C4.3. See Annex 3 for notes (www.oecd.org/education/education-at-a-glance- 19991487.htm).

StatLink 제개 http://dx.doi.org/10.1787/888933284277

In some cases, mobility from neighbouring countries reflects local patterns of mobility - students in border regions studying abroad but also relatively close to home. For example, although data are not available, many Belgian, French and German students in Luxembourg could have family living within a few hundred kilometres from the location where they study. In some other cases, mobility from neighbouring countries could reflect historic patterns of mobility developed within a formerly unified country which divided into two or more countries. For example, $74 \%$ of foreign students in the Czech Republic come from the Slovak Republic (Table C4.4).

\section{Box C4.3. Long-term trends in the global number of foreign students}

Comparable data on the global number of foreign students are available only until 2012, because since this year many countries started supplying data on international students only. As a result, trends in the global number of foreign students are based on the data until 2012 published in the previous edition of this publication (OECD, 2014a). These data show that, over the past three decades, the number of students enrolled outside their country of citizenship has risen dramatically, from 0.8 million worldwide in 1975 to 4.5 million in 2012 , a more than fivefold increase (Chart C4.b). The increase in global demand for tertiary education, reduced transportation and communication costs, and the internationalisation of labour markets for highly skilled people has given students stronger incentives to study abroad as part of their tertiary education. In addition, many governments and supra-national institutions have shown interest in promoting academic, cultural, social and political ties among countries. This is most evident in the European Union, which, in 2011, set the ambitious goal that, by 2020, 20\% of its graduates from higher education would have experience of tertiarylevel study or training abroad (Council of the European Union, 2011).

\section{Chart C4.b. Long-term growth in the number of students enrolled outside their country of citizenship (1975-2012) \\ Growth in internationalisation of tertiary education (in millions)}
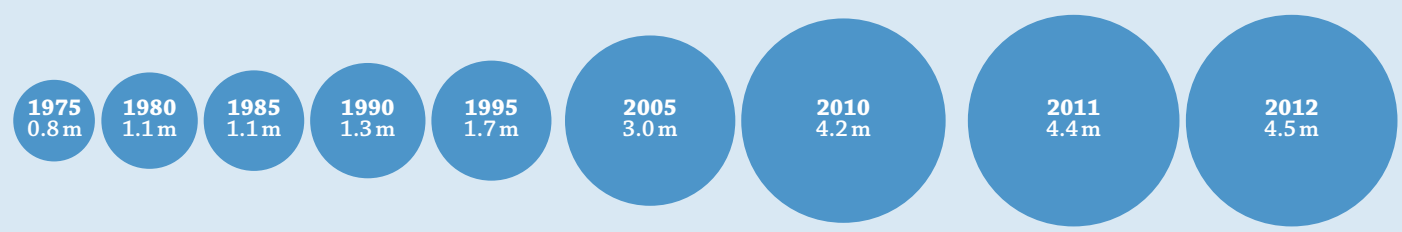

Data on foreign enrolment worldwide comes from both the OECD and the UNESCO Institute for Statistics (UIS). UIS provided the data on all countries for 1975-95 and most of the non-OECD countries for 2000, 2005, 2010, 2011 and 2012. The OECD provided the data on OECD countries and other non-OECD economies in 2000 and 2012. Both sources use similar definitions, thus making their combination possible. Missing data were imputed with the closest data reports to ensure that breaks in data coverage do not result in breaks in time series. 


\section{Definitions}

The country of prior education is the country in which students obtained the qualification required to enrol in their current level of education. Country-specific operational definitions of international students are indicated in the tables as well as in Annex 3 (www.oecd.org/education/education-at-a-glance-19991487.htm).

Foreign students are those who are not citizens of the country in which the data are collected. While pragmatic and operational, this classification is inappropriate for capturing student mobility because of differing national policies regarding the naturalisation of immigrants. For instance, Australia has a greater propensity to grant permanent residence to its immigrant populations than Switzerland. This implies that even when the proportion of foreign students in tertiary enrolment is similar for both countries, the proportion of international students in tertiary education is smaller in Switzerland than in Australia. Therefore, for student mobility and bilateral comparisons, interpretations of data based on the concept of foreign students should be made with caution.

International students are those who left their country of origin and moved to another country for the purpose of study. Depending on country-specific immigration legislation, mobility arrangements, such as the free mobility of individuals within the EU and the EEA, and data availability, international students may be defined as students who are not permanent or usual residents of their country of study or alternatively as students who obtained their prior education in a different country.

Permanent or usual residence in the reporting country is defined according to national legislation. In practice, this means holding a student visa or permit, or electing a foreign country of domicile in the year prior to entering the education system of the country reporting the data.

\section{Methodology}

Data on international and foreign students refer to the academic year 2012/13 unless otherwise indicated and are based on the UOE data collection on education statistics administered by the OECD in 2013.

The fields of education used in the UOE data collection instruments follow the revised ISCED classification by field of education. The same classification is used for all levels of education (for details see Annex 3 at www.oecd.org/education/ education-at-a-glance-19991487.htm). Additional data from the UNESCO Institute for Statistics are also included.

Data on international and foreign students are obtained from enrolments in their countries of destination. The method used for obtaining data on international and foreign students is therefore the same as that used for collecting data on total enrolments, i.e. records of regularly enrolled students in an education programme.

Domestic and international students are usually counted on a specific day or period of the year. This procedure makes it possible to measure the proportion of international enrolments in an education system, but the actual number of individuals involved may be much higher since many students study abroad for less than a full academic year, or participate in exchange programmes that do not require enrolment, such as inter-university exchanges or short-term advanced research programmes.

Trends are based on data until 2012, because since this year a considerable number of countries supply data only on international students, and not on foreign students. This enhances the cross-country comparisons, but it introduces a break in the time series. The data do not include students enrolled in countries that did not report international or foreign students to the OECD or to the UNESCO Institute for Statistics. All statements on students enrolled abroad may therefore underestimate the actual number of citizens studying abroad (Table C4.3), especially in cases where many citizens study in countries that did not report their foreign students to the OECD or UNESCO Institute for Statistics, such as India.

Data on the total number of students enrolled abroad are based on the number of international students counts and, for the countries for which these are not available, on foreign students counts. The data do not include students enrolled in countries that did not report international or foreign students to the OECD or to the UNESCO Institute for Statistics. Aggregates, market shares and proportions of international students coming from particular countries rely on this estimate of the total (Tables C4.4, C4.5, and Table C4.6, available on line, Charts C4.3 and C4.4).

\section{Note regarding data from Israel}

The statistical data for Israel are supplied by and are under the responsibility of the relevant Israeli authorities. The use of such data by the OECD is without prejudice to the status of the Golan Heights, East Jerusalem and Israeli settlements in the West Bank under the terms of international law. 


\section{References}

Altbach, P.G. and J. Knight (2007), “The Internationalization of Higher Education: Motivations and Realities”, Journal of Studies in International Education, Vol. 11, pp. 290-305.

Council of the European Union (2011), "Council Conclusions on the Modernisation of Higher Education", 3128th Education, Youth, Culture and Sport Council Meeting, Brussels, 28 and 29 November 2011, Council of the European Union, Brussels.

European Commission (2010), A Guide to the Rights of Mobile Students in the European Union, European Commission, Brussels.

Knight, J. (2008), Higher Education in Turmoil: The Changing World of Internationalization, Sense Publishers, Rotterdam.

Marconi, G. (2013), "Rankings, Accreditations and International Exchange Students", IZA Journal of European Labor Studies, Vol. 2, No. 5, http://www.izajoels.com/content/2/1/5.

OECD (2014a), Education at a Glance 2014: OECD Indicators, OECD Publishing, Paris, http://dx.doi.org/10.1787/eag-2014-en.

OECD (2014b), International Migration Outlook 2014, OECD Publishing, Paris, http://dx.doi.org/10.1787/migr outlook-2014-en.

Solimano, A. (2002), Globalizing Talent and Human Capital: Implications for Developing Countries, United Nations, Santiago.

Wächter, B. and F. Maiworm (eds.) (2014), English-Taught Programmes in European Higher Education: The State of Play in 2014, ACA Papers on International Cooperation in Education, Lemmens, Bonn.

\section{Indicator C4 Tables}

StatLink 제은 http://dx.doi.org/10.1787/888933285911

\section{Table C4.1 International student mobility and foreign students in tertiary education (2013)}

Table C4.2 Distribution of international and foreign students enrolled in tertiary programmes, by field of education (2013)

Table C4.3 Distribution of international and foreign students in tertiary education, by country of origin (2013)

Table C4.4 Students abroad in tertiary education, by country of destination (2013)

Table C4.5 Mobility patterns of foreign and international students (2013)

WEB Table C4.6 Number of foreign and international students in tertiary education, by country of origin and destination, and market shares in international education (2013)

Cut-off date for the data: 23 October 2015. Updates can be found on line at http://dx.doi.org/10.1787/eag-data-en. 
Table C4.1. International student mobility and foreign students in tertiary education (2013) International and foreign students enrolled as a percentage of all students (international plus domestic)

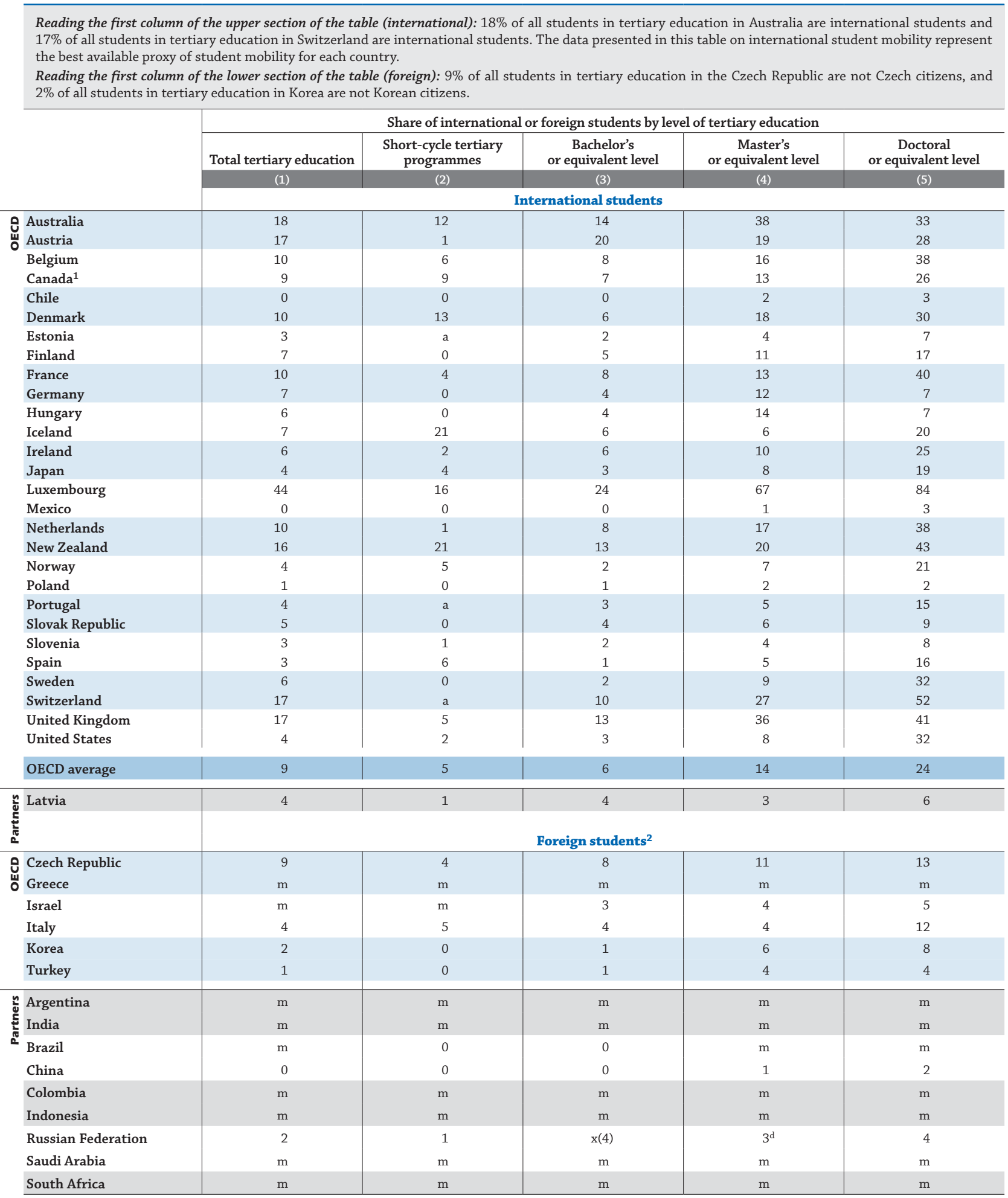

Note: Countries using the "foreign students" definition are not taken into account in the OECD average.

1. Year of reference 2012 .

2. Foreign students are defined on the basis of their country of citizenship, these data are not comparable with data on international students and are therefore presented separately in the table.

Source: OECD. Argentina, China, Colombia, India, Indonesia, Saudi Arabia and South Africa: UNESCO Institute for Statistics. Latvia: Eurostat. See Annex 3 for notes (www.oecd.org/education/education-at-a-glance-19991487.htm).

Please refer to the Reader's Guide for information concerning symbols for missing data and abbreviations.

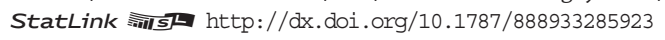


Table C4.2. Distribution of international and foreign students enrolled in tertiary programmes, by field of education (2013)

\begin{tabular}{|c|c|c|c|c|c|c|c|c|c|c|c|c|c|}
\hline \multirow[b]{2}{*}{ 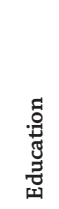 } & \multirow[b]{2}{*}{ 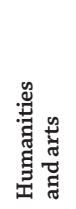 } & \multirow[b]{2}{*}{ 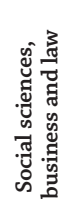 } & \multicolumn{5}{|c|}{ Sciences } & \multirow[b]{2}{*}{ 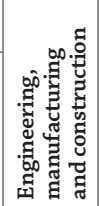 } & \multirow[b]{2}{*}{ 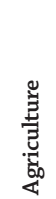 } & \multirow[b]{2}{*}{ 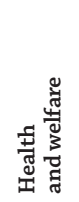 } & \multirow[b]{2}{*}{ 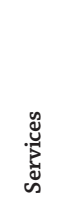 } & \multirow[b]{2}{*}{ 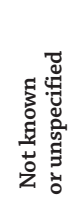 } & \multirow[b]{2}{*}{ 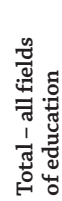 } \\
\hline & & & 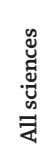 & 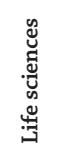 & 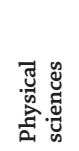 & 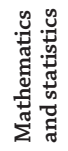 & 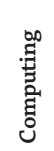 & & & & & & \\
\hline (1) & (2) & (3) & (4) & (5) & (6) & (7) & (8) & (9) & $(10)$ & (11) & (12) & (13) & (14) \\
\hline
\end{tabular}

\begin{tabular}{|c|c|c|c|c|c|c|c|c|c|c|c|c|c|c|}
\hline & \multicolumn{14}{|c|}{ International students } \\
\hline oิ Australia & 2 & 6 & 52 & 12 & 3 & 2 & 1 & 7 & 13 & 1 & 10 & 2 & 0 & 100 \\
\hline む̈ Austria & 6 & 17 & 38 & 12 & 4 & 3 & 1 & 4 & 15 & 2 & 8 & 2 & 0 & 100 \\
\hline Belgium & 4 & 14 & 23 & 7 & 2 & 3 & 1 & 1 & 11 & 5 & 34 & 2 & 1 & 100 \\
\hline Canada $^{1}$ & 1 & 6 & 41 & 15 & 4 & 3 & 3 & 5 & 18 & 1 & 5 & 2 & 10 & 100 \\
\hline Chile & 23 & 6 & 32 & 4 & 1 & 1 & 0 & 1 & 14 & 2 & 7 & 13 & 0 & 100 \\
\hline Denmark & 3 & 10 & 41 & 11 & 1 & 1 & 2 & 7 & 20 & 3 & 11 & 1 & 0 & 100 \\
\hline Estonia & 0 & 16 & 55 & 9 & 1 & 1 & 0 & 6 & 7 & 8 & 5 & 1 & 0 & 100 \\
\hline Finland & 2 & 11 & 27 & 11 & 2 & 2 & 0 & 6 & 31 & 2 & 10 & 6 & 0 & 100 \\
\hline France & 1 & 17 & 38 & 18 & 3 & 5 & 3 & 6 & 14 & 0 & 7 & 2 & 2 & 100 \\
\hline Germany ${ }^{2}$ & 5 & 19 & 26 & 15 & $\mathrm{~m}$ & $\mathrm{~m}$ & $\mathrm{~m}$ & $\mathrm{~m}$ & 25 & 2 & 6 & 2 & 1 & 100 \\
\hline Hungary & 3 & 11 & 21 & 4 & 1 & 1 & 0 & 2 & 9 & 8 & 40 & 4 & 0 & 100 \\
\hline Iceland & 7 & 40 & 25 & 14 & 5 & 7 & 0 & 2 & 6 & 2 & 5 & 1 & 0 & 100 \\
\hline Ireland & $\mathrm{m}$ & $\mathrm{m}$ & $\mathrm{m}$ & $\mathrm{m}$ & $\mathrm{m}$ & $\mathrm{m}$ & $\mathrm{m}$ & $\mathrm{m}$ & $\mathrm{m}$ & $\mathrm{m}$ & $\mathrm{m}$ & $\mathrm{m}$ & $\mathrm{m}$ & $\mathrm{m}$ \\
\hline Japan & 2 & 21 & 38 & 2 & $\mathrm{~m}$ & $\mathrm{~m}$ & $\mathrm{~m}$ & $\mathrm{~m}$ & 17 & 2 & 2 & 2 & 13 & 100 \\
\hline Luxembourg & 6 & 9 & 62 & 12 & 4 & 2 & 1 & 5 & 5 & 1 & 3 & 0 & 3 & 100 \\
\hline Mexico & $\mathrm{m}$ & $\mathrm{m}$ & $\mathrm{m}$ & $\mathrm{m}$ & $\mathrm{m}$ & $\mathrm{m}$ & $\mathrm{m}$ & $\mathrm{m}$ & $\mathrm{m}$ & $\mathrm{m}$ & $\mathrm{m}$ & $\mathrm{m}$ & $\mathrm{m}$ & $\mathrm{m}$ \\
\hline Netherlands & 3 & 12 & 43 & 7 & $\mathrm{~m}$ & $\mathrm{~m}$ & $\mathrm{~m}$ & $\mathrm{~m}$ & 11 & 2 & 13 & 8 & 2 & 100 \\
\hline New Zealand & 4 & 9 & 39 & 18 & 4 & 3 & 3 & 9 & 9 & 1 & 7 & 7 & 6 & 100 \\
\hline Norway & 5 & 17 & 26 & 14 & 3 & 4 & 1 & 6 & 12 & 2 & 10 & 5 & 10 & 100 \\
\hline Poland & 2 & 11 & 42 & 7 & 1 & 1 & 0 & 5 & 7 & 1 & 22 & 8 & 0 & 100 \\
\hline Portugal & 6 & 13 & 35 & 11 & 4 & 4 & 1 & 2 & 18 & 1 & 10 & 6 & 0 & 100 \\
\hline Slovak Republic & 11 & 5 & 18 & 2 & 1 & 0 & 0 & 1 & 6 & 2 & 53 & 2 & 0 & 100 \\
\hline Slovenia & 5 & 13 & 39 & 13 & 3 & 2 & 1 & 6 & 15 & 3 & 7 & 5 & 0 & 100 \\
\hline Spain & 4 & 9 & 30 & 8 & 1 & 1 & 0 & 5 & 15 & 1 & 26 & 7 & 0 & 100 \\
\hline Sweden & 3 & 11 & 25 & 20 & 6 & 6 & 2 & 6 & 27 & 1 & 11 & 2 & 0 & 100 \\
\hline Switzerland & 5 & 16 & 33 & 18 & 5 & 7 & 2 & 3 & 17 & 1 & 7 & 2 & 1 & 100 \\
\hline United Kingdom & 2 & 13 & 45 & 15 & 5 & 3 & 2 & 5 & 15 & 1 & 8 & 2 & 0 & 100 \\
\hline United States & 2 & 13 & 33 & 18 & 6 & 4 & 2 & 6 & 16 & 1 & 9 & 3 & 4 & 100 \\
\hline OECD average & 4 & 13 & 36 & 11 & 3 & 3 & 1 & 5 & 14 & 2 & 13 & 4 & 2 & 100 \\
\hline
\end{tabular}

\begin{tabular}{|c|c|c|c|c|c|c|c|c|c|c|c|c|c|c|}
\hline \multirow[t]{2}{*}{ Latvia } & 1 & 8 & 42 & 4 & 0 & 0 & 0 & 4 & 5 & 0 & 27 & 12 & 0 & 100 \\
\hline & \multicolumn{14}{|c|}{ Foreign students ${ }^{2}$} \\
\hline \& Czech Republic & 2 & 10 & 38 & 16 & 3 & 3 & 1 & 9 & 11 & 3 & 17 & 4 & 0 & 100 \\
\hline ప̈ Greece & $\mathrm{m}$ & $\mathrm{m}$ & $\mathrm{m}$ & $\mathrm{m}$ & $\mathrm{m}$ & $\mathrm{m}$ & $\mathrm{m}$ & $\mathrm{m}$ & $\mathrm{m}$ & $\mathrm{m}$ & $\mathrm{m}$ & $\mathrm{m}$ & $\mathrm{m}$ & $\mathrm{m}$ \\
\hline Israel & $\mathrm{m}$ & $\mathrm{m}$ & $\mathrm{m}$ & $\mathrm{m}$ & $\mathrm{m}$ & $\mathrm{m}$ & $\mathrm{m}$ & $\mathrm{m}$ & $\mathrm{m}$ & $\mathrm{m}$ & $\mathrm{m}$ & $\mathrm{m}$ & $\mathrm{m}$ & $\mathrm{m}$ \\
\hline Italy & 2 & 21 & 31 & 7 & 2 & 2 & 1 & 2 & 21 & 2 & 15 & 2 & 0 & 100 \\
\hline Korea & 2 & 22 & 45 & 5 & 2 & 1 & 0 & 2 & 16 & 1 & 4 & 4 & 0 & 100 \\
\hline Turkey & 6 & 13 & 38 & 9 & 2 & 3 & 1 & 3 & 16 & 2 & 12 & 5 & 0 & 100 \\
\hline
\end{tabular}

\begin{tabular}{|c|c|c|c|c|c|c|c|c|c|c|c|c|c|c|}
\hline Argentina $^{1}$ & $\mathrm{~m}$ & $\mathrm{~m}$ & $\mathrm{~m}$ & $\mathrm{~m}$ & $\mathrm{~m}$ & $\mathrm{~m}$ & $\mathrm{~m}$ & $\mathrm{~m}$ & $\mathrm{~m}$ & $\mathrm{~m}$ & $\mathrm{~m}$ & $\mathrm{~m}$ & $\mathrm{~m}$ & $\mathrm{~m}$ \\
\hline India & $\mathrm{m}$ & $\mathrm{m}$ & $\mathrm{m}$ & $\mathrm{m}$ & $\mathrm{m}$ & $\mathrm{m}$ & $\mathrm{m}$ & $\mathrm{m}$ & $\mathrm{m}$ & $\mathrm{m}$ & $\mathrm{m}$ & $\mathrm{m}$ & $\mathrm{m}$ & $\mathrm{m}$ \\
\hline Brazil & $\mathrm{m}$ & $\mathrm{m}$ & $\mathrm{m}$ & $\mathrm{m}$ & $\mathrm{m}$ & $\mathrm{m}$ & $\mathrm{m}$ & $\mathrm{m}$ & $\mathrm{m}$ & $\mathrm{m}$ & $\mathrm{m}$ & $\mathrm{m}$ & $\mathrm{m}$ & $\mathrm{m}$ \\
\hline China & $\mathrm{m}$ & $\mathrm{m}$ & $\mathrm{m}$ & $\mathrm{m}$ & $\mathrm{m}$ & $\mathrm{m}$ & $\mathrm{m}$ & $\mathrm{m}$ & $\mathrm{m}$ & $\mathrm{m}$ & $\mathrm{m}$ & $\mathrm{m}$ & $\mathrm{m}$ & $\mathrm{m}$ \\
\hline Colombia & $\mathrm{m}$ & $\mathrm{m}$ & $\mathrm{m}$ & $\mathrm{m}$ & $\mathrm{m}$ & $\mathrm{m}$ & $\mathrm{m}$ & $\mathrm{m}$ & $\mathrm{m}$ & $\mathrm{m}$ & $\mathrm{m}$ & $\mathrm{m}$ & $\mathrm{m}$ & $\mathrm{m}$ \\
\hline Indonesia & $\mathrm{m}$ & $\mathrm{m}$ & $\mathrm{m}$ & $\mathrm{m}$ & $\mathrm{m}$ & $\mathrm{m}$ & $\mathrm{m}$ & $\mathrm{m}$ & $\mathrm{m}$ & $\mathrm{m}$ & $\mathrm{m}$ & $\mathrm{m}$ & $\mathrm{m}$ & $\mathrm{m}$ \\
\hline Russian Federation & $\mathrm{m}$ & $\mathrm{m}$ & $\mathrm{m}$ & $\mathrm{m}$ & $\mathrm{m}$ & $\mathrm{m}$ & $\mathrm{m}$ & $\mathrm{m}$ & $\mathrm{m}$ & $\mathrm{m}$ & $\mathrm{m}$ & $\mathrm{m}$ & $\mathrm{m}$ & $\mathrm{m}$ \\
\hline Saudi Arabia & $\mathrm{m}$ & $\mathrm{m}$ & $\mathrm{m}$ & $\mathrm{m}$ & $\mathrm{m}$ & $\mathrm{m}$ & $\mathrm{m}$ & $\mathrm{m}$ & $\mathrm{m}$ & $\mathrm{m}$ & $\mathrm{m}$ & $\mathrm{m}$ & $\mathrm{m}$ & $\mathrm{m}$ \\
\hline South Africa & $\mathrm{m}$ & $\mathrm{m}$ & $\mathrm{m}$ & $\mathrm{m}$ & $\mathrm{m}$ & $\mathrm{m}$ & $\mathrm{m}$ & $\mathrm{m}$ & $\mathrm{m}$ & $\mathrm{m}$ & $\mathrm{m}$ & $\mathrm{m}$ & $\mathrm{m}$ & $\mathrm{m}$ \\
\hline
\end{tabular}

Note: Countries using the "foreign students" definition are not taken into account in the OECD average.

1. Year of reference 2012.

2. Foreign students are defined on the basis of their country of citizenship; these data are not comparable with data on international students and are therefore presented separately in the table and chart.

Source: OECD. Argentina, China, Colombia, India, Indonesia, Saudi Arabia and South Africa: UNESCO Institute for Statistics. Latvia: Eurostat. See Annex 3 for notes (www.oecd.org/education/education-at-a-glance-19991487.htm).

Please refer to the Reader's Guide for information concerning symbols for missing data and abbreviations.

StatLink त्गार् http://dx.doi.org/10.1787/888933285934 


\section{Table C4.3. [1/2] Distribution of international and foreign students in tertiary education, by country of origin (2013)}

International and foreign students enrolled in tertiary education from a given country of origin as a percentage of all international or foreign students in the country of destination, based on head counts

The table shows for each country the proportion of international students in tertiary education who are residents of or had their prior education in a given country of origin.
When data on student mobility are not available, the table shows the proportion of foreign students in tertiary education that have citizenship of a given country of origin.
Reading the first column: $2.7 \%$ of international tertiary students in Australia come from Korea, $1.2 \%$ of international tertiary students in Australia come from the United States, etc.
Reading the seventh column: $49.3 \%$ of international tertiary students in Estonia come from Finland, $3.0 \%$ of international tertiary students in Estonia come from Turkey, etc.
Reading column 29: $60.5 \%$ of foreign tertiary students in the Czech Republic are Slovak citizens, $0.8 \%$ of foreign tertiary students in the Czech Republic are Norwegian citizens, etc.

OECD destination countries

International students

Countries of origin

Australia

Belgium

Canada

Canada

Czech Republic

Denmark

Estonia

Finland

France

Germany

Greece

Hungary

Iceland

Ireland

Israel

Italy

Japan

Korea

Luxembourg

Mexico

Netherlands

New Zealand

Norway

Poland

Plovak Republic

Slovenia

Spain

Sweden

Switzerland

Turkey

United Kingdom

United States

Total from OECD

Argentina

Brazil

China

India

Indonesia

Latvia

Russian Federation

Saudi Arabia

South Africa

Total from other G20 and

partner countries

Main geographic regions

Total from Africa

Total from Asia

Total from Europ

of which, from EU21 countries

Total from North America

Total from Oceania

Total from Latin America

and the Caribbean

Not specified

Total from all countries

2. Excludes doctoral or equivalent programmes (for Germany, these programmes are included only in main geographic regions).

3. Foreign students are defined on the basis of their country of citizenship; these data are not comparable with data on international students and are therefore presented separately in the table.

4. Excludes students in short-cycle tertiary programmes.

Source: OECD. Argentina, China, Colombia, India, Indonesia, Saudi Arabia and South Africa: UNESCO Institute for Statistics. Latvia: Eurostat. See Annex 3 for notes (www.oecd.org/education/education-at-a-glance-19991487.htm).

Please refer to the Reader's Guide for information concerning symbols for missing data and abbreviations.

StatLink 젝ㄴ http://dx.doi.org/10.1787/888933285949

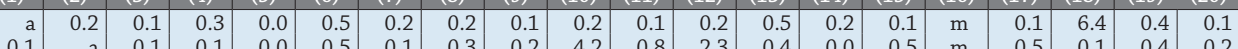

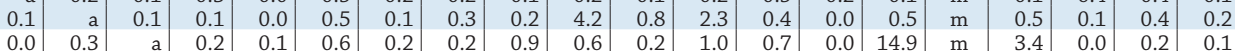

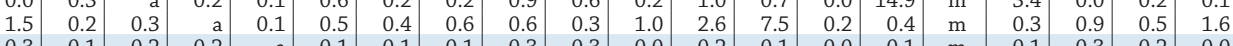
\begin{tabular}{|l|l|l|l|l|l|l|l|l|l|l|l|l|l|l|l|l|l|l|l|l|}
0.3 & 0.1 & 0.2 & 0.2 & $\mathrm{a}$ & 0.1 & 0.1 & 0.1 & 0.3 & 0.3 & 0.0 & 0.2 & 0.1 & 0.0 & 0.1 & $\mathrm{~m}$ & 0.1 & 0.3 & 0.2 & 0.0 \\
\hline
\end{tabular}

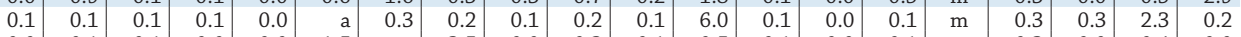

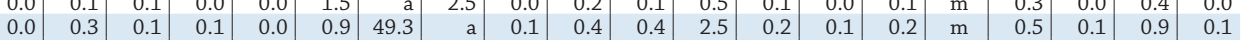

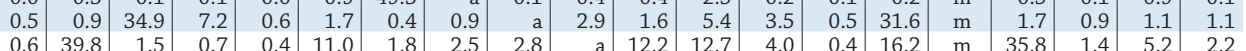

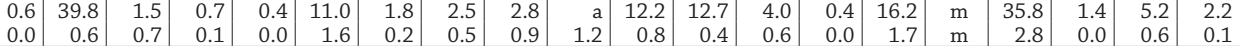
\begin{tabular}{l|l|l|l|l|l|l|l|l|l|l|l|l|l|l|l|l|l|l|l}
0.0 & 2.3 & 0.2 & 0.0 & 0.0 & 1.9 & 0.4 & 0.8 & 0.2 & 0.8 & $\mathrm{a}$ & 1.2 & 0.2 & 0.1 & 0.5 & $\mathrm{~m}$ & 0.7 & 0.0 & 0.4 & 0.2 \\
\hline & 0.0 & 0.0 & 0.0 & 0.0 & 4.0 & 0.2 & 0.1 & 0.0 & 0.0 & 0.6 & $\mathrm{a}$ & 0.0 & 0.0 & 0.0 & $\mathrm{~m}$ & 0.2 & 0.0 & 1.4 & 0.0
\end{tabular} \begin{tabular}{|l|l|l|l|l|l|l|l|l|l|l|l|l|l|l|l|l|l|l|l|l|l|l|l|l|l}
0.1 & 0.1 & 0.1 & 0.1 & 0.0 & 0.3 & 0.2 & 0.2 & 0.2 & 0.2 & 1.1 & 0.4 & $\mathrm{a}$ & 0.0 & 0.1 & $\mathrm{~m}$ & 0.3 & 0.1 & 0.1 & 0.3 \\
\hline
\end{tabular} \begin{tabular}{l|l|l|l|l|l|l|l|l|l|l|l|l|l|l|l|l|l|l|l}
0.1 & 0.2 & 0.1 & 0.7 & 0.0 & 0.2 & 0.1 & 0.1 & 0.1 & 0.8 & 3.6 & 0.2 & 0.1 & 0.0 & 0.1 & $\mathrm{~m}$ & 0.1 & 0.0 & 0.1 & 0.2
\end{tabular} \begin{tabular}{|r|r|r|r|r|r|r|r|r|r|r|r|r|r|r|r|r|r|r|r|r|}
0.2 & 11.0 & 1.7 & 0.3 & 0.2 & 2.7 & 1.1 & 1.2 & 2.7 & 2.2 & 1.2 & 3.8 & 1.8 & 0.1 & 2.7 & $\mathrm{~m}$ & 2.2 & 0.2 & 1.4 & 1.0 \\
\hline
\end{tabular}

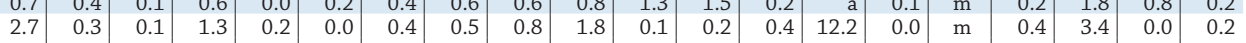

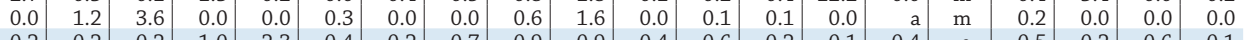

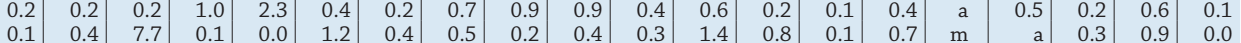

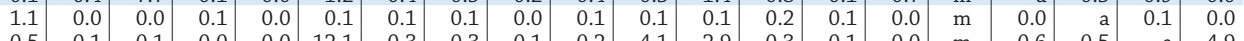

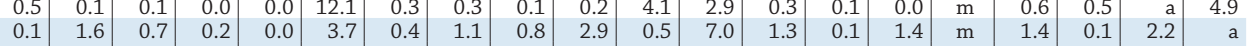

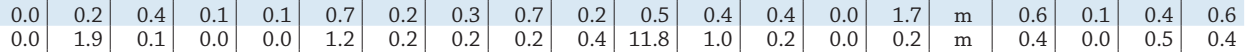
\begin{tabular}{|l|l|l|l|l|l|l|l|l|r|r|r|r|r|r|r|r|r|r|r|}
0.0 & 1.9 & 0.1 & 0.0 & 0.0 & 1.2 & 0.2 & 0.2 & 0.2 & 0.4 & 11.8 & 1.0 & 0.2 & 0.0 & 0.2 & $\mathrm{~m}$ & 0.4 & 0.0 & 0.5 & 0.4 \\
0.0 & 0.9 & 0.0 & 0.0 & 0.0 & 0.2 & 0.1 & 0.1 & 0.0 & 0.1 & 0.1 & 0.6 & 0.1 & 0.0 & 0.1 & $\mathrm{~m}$ & 0.2 & 0.0 & 0.1 & 0.1
\end{tabular} \begin{tabular}{|l|l|l|l|l|l|l|l|l|l|l|l|l|l|l|l|l|l|l|l}
0.1 & 0.9 & 1.0 & 0.1 & 0.4 & 2.3 & 0.8 & 1.0 & 1.9 & 2.7 & 2.2 & 4.6 & 1.4 & 0.1 & 1.0 & $\mathrm{~m}$ & 1.4 & 0.1 & 1.6 & 3.5 \\
\hline
\end{tabular} \begin{tabular}{l|l|l|l|l|l|l|l|l|l|l|l|l|l|l|l|l|l|l|l}
0.3 & 0.3 & 0.1 & 0.1 & 0.1 & 8.5 & 0.5 & 2.0 & 0.2 & 0.3 & 2.2 & 5.4 & 0.2 & 0.2 & 0.2 & $\mathrm{~m}$ & 0.4 & 0.3 & 8.2 & 3.8 \\
0.1 & 1.1 & 0.3 & 0.2 & 0.0 & 0.4 & 0.1 & 0.1 & 0.5 & 1.2 & 0.2 & 0.6 & 0.3 & 0.0 & 0.3 & $\mathrm{~m}$ & 0.4 & 0.1 & 0.4 & 0.1
\end{tabular} \begin{tabular}{r|r|r|r|r|r|r|r|r|r|r|r|r|r|r|r|r|r|r|r|r|r|}
0.2 & 4.4 & 0.4 & 0.5 & 0.0 & 0.5 & 3.0 & 0.8 & 0.8 & 2.8 & 2.8 & 0.3 & 0.2 & 0.1 & 0.6 & $\mathrm{~m}$ & 0.8 & 0.1 & 0.6 & 2.0 \\
\hline & 0.9 & 0.5 & 0.8 & 0.0 & 2.2 & 0.6 & 1.0 & 0.9 & 0.8 & 1.6 & 4.0 & 16.4 & 0.3 & 0.7 & $\mathrm{~m}$ & 1.9 & 1.3 & 1.1 & 0.7
\end{tabular} \begin{tabular}{l|l|l|l|l|l|l|l|l|l|l|l|l|l|l|l|l|l|l|l|l|l|l|l|l|l}
1.2 & 1.1 & 0.5 & 5.5 & 0.5 & 2.1 & 1.8 & 1.5 & 1.4 & 2.0 & 2.2 & 6.5 & 9.0 & 1.5 & 1.2 & $\mathrm{~m}$ & 0.9 & 5.4 & 2.2 & 3.1
\end{tabular} \begin{tabular}{l|l|l|l|l|l|l|l|l|l|l|l|l|l|l|l|} 
& & &
\end{tabular} \begin{tabular}{ll|l|l|l|l|l|l|l|l|l|l|l|l|l|l|l|l|r|r|}
0.1 & 0.1 & 0.1 & 0.1 & 2.2 & 0.1 & 0.0 & 0.1 & 0.3 & 0.2 & 0.0 & 0.2 & 0.0 & 0.0 & 0.2 & $\mathrm{~m}$ & 0.0 & 0.1 & 0.2 & 0.0 \\
0.4 & 0.3 & 0.3 & 0.5 & 9.5 & 0.5 & 0.4 & 0.4 & 1.7 & 1.3 & 0.1 & 0.2 & 0.3 & 0.2 & 0.2 & $\mathrm{~m}$ & 0.3 & 0.3 & 1.0 & 0.1 \\
\hline
\end{tabular}

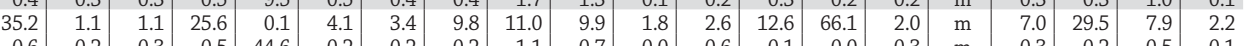
\begin{tabular}{r|r|r|r|r|r|r|r|r|r|r|r|r|r|r|r|r|r|r|r|r|r|r|}
0.6 & 0.2 & 0.3 & 0.5 & 44.6 & 0.2 & 0.2 & 0.2 & 1.1 & 0.7 & 0.0 & 0.6 & 0.1 & 0.0 & 0.3 & $\mathrm{~m}$ & 0.3 & 0.2 & 0.5 & 0.1 \\
\hline 6.5 & 0.5 & 0.5 & 7.1 & 0.1 & 0.9 & 1.0 & 2.9 & 0.8 & 2.9 & 0.4 & 1.3 & 4.2 & 0.4 & 0.9 & $\mathrm{~m}$ & 1.3 & 16.6 & 2.6 & 0.8
\end{tabular}

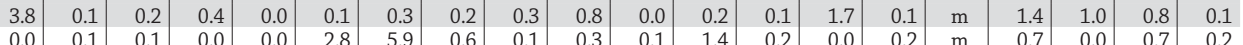
\begin{tabular}{|l|l|l|l|l|l|l|l|l|l|l|l|l|l|l|l|l|l|l|l}
0.4 & 1.4 & 0.6 & 0.4 & 0.0 & 0.6 & 9.0 & 10.1 & 1.6 & 4.8 & 1.0 & 2.2 & 0.5 & 0.2 & 1.3 & $\mathrm{~m}$ & 0.9 & 0.8 & 6.8 & 2.1 \\
\hline
\end{tabular} \begin{tabular}{|l|l|l|l|l|l|l|l|l|l|l|l|l|l|l|l|l|l|l|l|l|l|l|l|l|}
0.3 & 0.1 & 0.1 & 0.2 & 0.0 & 0.2 & 0.1 & 0.1 & 0.0 & 0.1 & 0.0 & 0.1 & 0.4 & 0.0 & 0.1 & $\mathrm{~m}$ & 0.2 & 0.2 & 0.3 & 0.1 \\
\hline
\end{tabular} \begin{tabular}{|l|l|l|l|l|l|l|l|l|l|l|l|l|l|l|l|l|l|l|l|}
49.1 & 3.9 & 3.4 & 38.1 & 56.5 & 9.5 & 20.3 & 24.3 & 17.1 & 21.1 & 4.6 & 8.7 & 22.3 & 68.9 & 5.2 & $\mathrm{~m}$ & 12.2 & 51.1 & 20.9 & 7.5 \\
\hline
\end{tabular}

\begin{tabular}{l|l|l|l|l|l|l|l|l|l|l|l|l|l|l|l|l|l|l|l|}
2.9 & 1.3 & 12.0 & 9.5 & 0.2 & 2.5 & 1.4 & 11.9 & 40.9 & 8.3 & 4.6 & 1.7 & 4.6 & 0.8 & 8.0 & $\mathrm{~m}$ & 1.6 & 1.0 & 12.6 & 2.2 \\
\end{tabular} \begin{tabular}{|r|r|r|r|r|r|r|r|r|r|r|r|r|r|r|r|r|r|r|r|r|r|r|}
\hline 85.2 & 10.8 & 5.6 & 56.7 & 0.5 & 11.2 & 15.5 & 35.2 & 23.2 & 32.7 & 22.0 & 10.7 & 41.2 & 93.5 & 6.9 & $\mathrm{~m}$ & 14.5 & 69.5 & 34.7 & 16.2 \\
\hline 4.3 & 82.0 & 5.4 & 11.9 & 2.0 & 81.4 & 79.1 & 3.0 & 20.1 & 43.6 & 69.5 & 75.2 & 3.7 & 2.9 & 82.1 & $\mathrm{~m}$ & 64.2 & 7.0 & 46.1 & 76.0 \\
\hline
\end{tabular}

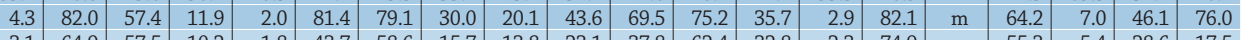

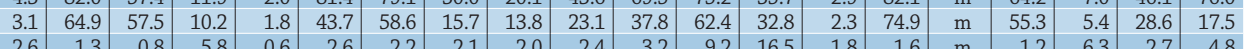
\begin{tabular}{|l|l|l|l|l|l|l|l|l|l|l|l|l|l|l|l|l|l|r|r|}
2.6 & 1.3 & 0.8 & 5.8 & 0.6 & 2.6 & 2.2 & 2.1 & 2.0 & 2.4 & 3.2 & 9.2 & 16.5 & 1.8 & 1.6 & $\mathrm{~m}$ & 1.2 & 6.3 & 2.7 & 4.8 \\
\hline 2.0 & 0.2 & 0.1 & 0.5 & 0.0 & 0.6 & 0.3 & 0.3 & 0.2 & 0.3 & 0.2 & 0.3 & 0.7 & 0.4 & 0.1 & $\mathrm{~m}$ & 0.1 & 9.2 & 0.4 & 0.1 \\
\hline
\end{tabular} \begin{tabular}{|l|l|l|l|l|l|l|l|l|l|l|l|l|l|l|l|l|l|l|l|}
\hline 2.0 & 1.2 & 2.0 & 6.0 & 95.4 & 1.8 & 1.4 & 2.1 & 6.0 & 5.1 & 0.6 & 2.8 & 1.3 & 0.7 & 1.3 & $\mathrm{~m}$ & 2.5 & 1.2 & 3.4 & 0.6 \\
\hline
\end{tabular} \begin{tabular}{|l|l|l|l|l|l|l|l|l|l|l|l|l|l|l|l|l|r|r|r|}
0.8 & 3.2 & 22.2 & 9.6 & 1.3 & 0.0 & 0.1 & 18.4 & 7.6 & 7.6 & 0.0 & 0.1 & 0.0 & 0.0 & 0.0 & $\mathrm{~m}$ & 15.7 & 5.9 & 0.0 & 0.1 \\
\hline
\end{tabular} \begin{tabular}{|l|l|l|l|l|l|l|l|l|l|l|l|l|l|l|l|l|l|l|l|}
100.0 & 100.0 & 100.0 & 100.0 & 100.0 & 100.0 & 100.0 & 100.0 & 100.0 & 100.0 & 100.0 & 100.0 & 100.0 & 100.0 & 100.0 & $\mathrm{~m}$ & 100.0 & 100.0 & 100.0 & 100.0 \\
\hline
\end{tabular}

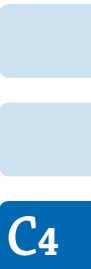

(4) 
Table C4.3. [2/2] Distribution of international and foreign students in tertiary education, by country of origin (2013)

International and foreign students enrolled in tertiary education from a given country of origin as a percentage of all international or foreign students in the country of destination, based on head counts

The table shows for each country the proportion of international students in tertiary education who are residents of or had their prior education in a given country of origin. When data on student mobility are not available, the table shows the proportion of foreign students in tertiary education that have citizenship of a given country of origin. Reading the first column: $2.7 \%$ of international tertiary students in Australia come from Korea, $1.2 \%$ of international tertiary students in Australia come from the United States, etc. Reading the seventh column: $49.3 \%$ of international tertiary students in Estonia come from Finland, $3.0 \%$ of international tertiary students in Estonia come from Turkey, etc.

Reading column 29: $60.5 \%$ of foreign tertiary students in the Czech Republic are Slovak citizens, $0.8 \%$ of foreign tertiary students in the Czech Republic are Norwegian citizens, etc.

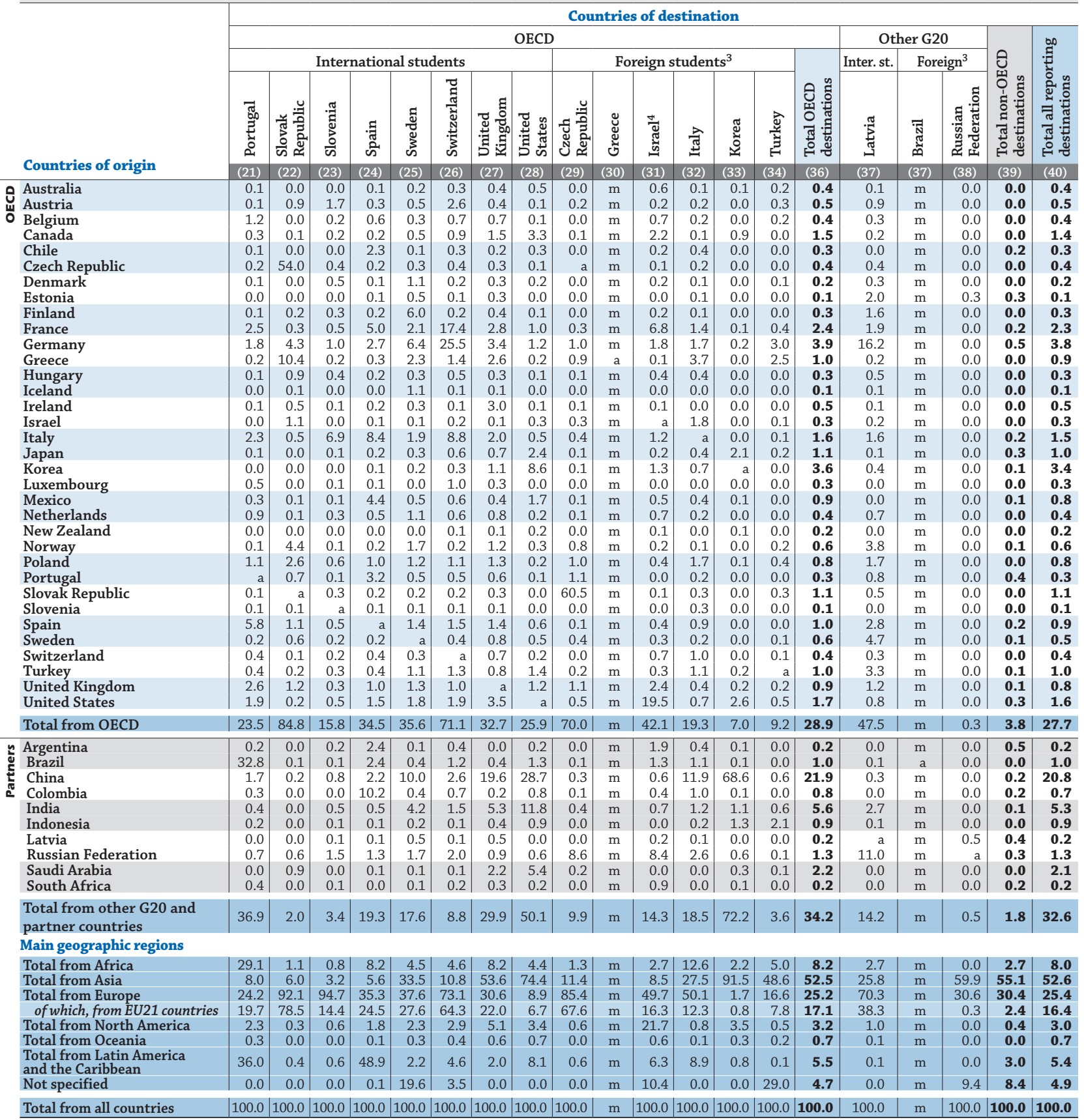

1. Year of reference 2012

2. Excludes doctoral or equivalent programmes (for Germany, these programmes are included only in main geographic regions).

3. Foreign students are defined on the basis of their country of citizenship; these data are not comparable with data on international students and are therefore presented separately in the table.

4. Excludes students in short-cycle tertiary programmes.

Source: OECD. Argentina, China, Colombia, India, Indonesia, Saudi Arabia and South Africa: UNESCO Institute for Statistics. Latvia: Eurostat. See Annex 3 for notes (www.oecd.org/education/education-at-a-glance-19991487.htm).

Please refer to the Reader's Guide for information concerning symbols for missing data and abbreviations.

StatLink त्ञात http://dx.doi.org/10.1787/888933285949 
Table C4.4. [1/2] Students abroad in tertiary education, by country of destination (2013)

Number of foreign and international students enrolled in tertiary education in a given country of destination as a percentage of all students enrolled abroad in reporting destinations, based on head counts

\begin{tabular}{|c|c|c|c|c|c|c|c|c|c|c|c|c|c|c|c|c|c|c|c|c|c|c|}
\hline \multicolumn{23}{|c|}{$\begin{array}{l}\text { The table shows for each country the proportion of students studying abroad in tertiary education in a given country of destination. } \\
\text { Reading the second column: } 4.9 \% \text { of Czech citizens enrolled in tertiary education abroad study in Austria, } 15.9 \% \text { of Italian citizens enrolled in tertiary education abroad study } \\
\text { in Austria, etc. } \\
\text { Reading the first row: } 2.4 \% \text { of Australian citizens enrolled in tertiary education abroad study in France, } 21.8 \% \text { of Australian citizens enrolled in tertiary education abroad } \\
\text { study in New Zealand, etc. }\end{array}$} \\
\hline \multirow[b]{4}{*}{ Country of origin } & \multicolumn{22}{|c|}{ Countries of destination } \\
\hline & \multicolumn{22}{|c|}{ OECD } \\
\hline & 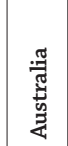 & 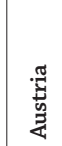 & 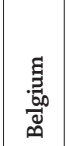 & 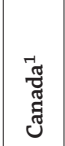 & चี & ن & 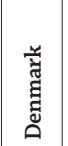 & 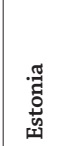 & 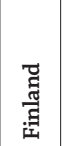 & 苟 & 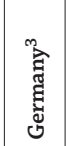 & 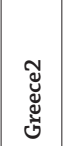 & 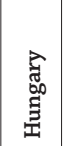 & 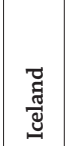 & 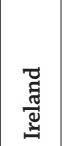 & 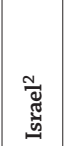 & 誉 & 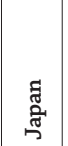 & 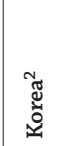 & 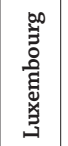 & 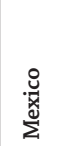 & 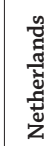 \\
\hline & (1) & (2) & (3) & (4) & (5) & (6) & (7) & (8) & (9) & (10) & (11) & (12) & (13) & (14) & (15) & (16) & (17) & (18) & (19) & (20) & $(21)$ & (22) \\
\hline Australia & $\mathrm{a}$ & 1.1 & 0.3 & 2.8 & 0.0 & 0.1 & 1.2 & 0.0 & 0.3 & 2.4 & 3.8 & $\mathrm{~m}$ & 0.1 & 0.0 & 0.6 & 0.5 & 0.5 & 2.8 & 0.6 & 0.0 & $\mathrm{~m}$ & 0.6 \\
\hline Austria & 1.4 & $\mathrm{a}$ & 0.2 & 0.7 & 0.0 & 0.5 & 0.9 & 0.0 & 0.5 & 2.6 & 51.8 & $\mathrm{~m}$ & 1.1 & 0.2 & 0.3 & 0.1 & 1.2 & 0.4 & 0.1 & 0.1 & $\mathrm{~m}$ & 2.3 \\
\hline Belgium & 0.8 & 1.4 & $\mathrm{a}$ & 1.7 & 0.0 & 0.1 & 1.5 & 0.0 & 0.4 & 17.0 & 8.6 & $\mathrm{~m}$ & 0.3 & 0.1 & 0.7 & 0.6 & 1.4 & 0.4 & 0.1 & 3.4 & $\mathrm{~m}$ & 18.7 \\
\hline Canada & 7.6 & 0.3 & 0.2 & $\mathrm{a}$ & 0.0 & 0.1 & 0.3 & 0.0 & 0.3 & 2.8 & 1.3 & $\mathrm{~m}$ & 0.4 & 0.1 & 2.0 & 0.5 & 0.2 & 0.6 & 1.1 & 0.0 & $\mathrm{~m}$ & 0.5 \\
\hline Chile & 6.4 & 0.6 & 0.8 & 2.4 & $\mathrm{a}$ & 0.1 & 0.4 & 0.0 & 0.3 & 7.6 & 6.2 & $\mathrm{~m}$ & 0.1 & 0.0 & 0.1 & 0.2 & 3.5 & 0.3 & 0.1 & 0.0 & $\mathrm{~m}$ & 0.6 \\
\hline Czech Republic & 0.9 & 4.9 & 0.3 & 0.6 & 0.0 & $\mathrm{a}$ & 1.4 & 0.2 & 0.5 & 5.2 & 10.9 & $\mathrm{~m}$ & 0.3 & 0.2 & 0.1 & 0.1 & 1.0 & 0.4 & 0.1 & 0.1 & $\mathrm{~m}$ & 1.9 \\
\hline Denmark & 3.3 & 1.5 & 0.4 & 1.4 & 0.0 & 0.1 & $\mathrm{a}$ & 0.1 & 0.8 & 3.0 & 8.4 & $\mathrm{~m}$ & 0.3 & 1.4 & 0.3 & 0.4 & 0.8 & 1.0 & 0.1 & 0.1 & $\mathrm{~m}$ & 3.7 \\
\hline Estonia & 0.8 & 1.7 & 0.5 & 0.2 & 0.0 & 0.2 & 10.1 & $\mathrm{a}$ & 12.1 & 2.4 & 11.0 & $\mathrm{~m}$ & 0.3 & 0.1 & 0.3 & 0.1 & 1.3 & 0.5 & 0.0 & 0.0 & $\mathrm{~m}$ & 4.2 \\
\hline Finland & 1.3 & 2.6 & 0.5 & 0.9 & 0.0 & 0.1 & 3.2 & 10.9 & a & 3.3 & 9.0 & $\mathrm{~m}$ & 0.9 & 0.4 & 0.3 & 0.2 & 0.9 & 1.6 & 0.1 & 0.1 & $\mathrm{~m}$ & 3.9 \\
\hline France & 1.5 & 0.9 & 20.7 & 12.9 & 0.0 & 0.2 & 0.7 & 0.0 & 0.3 & $\mathrm{a}$ & 7.5 & $\mathrm{~m}$ & 0.4 & 0.1 & 0.6 & 0.9 & 1.6 & 1.0 & 0.1 & 1.2 & $\mathrm{~m}$ & 1.5 \\
\hline Germany & 1.2 & 23.4 & 0.6 & 0.8 & 0.0 & 0.3 & 2.7 & 0.0 & 0.5 & 5.3 & $\mathrm{a}$ & $\mathrm{m}$ & 2.1 & 0.1 & 0.4 & 0.2 & 1.2 & 0.5 & 0.1 & 0.4 & $\mathrm{~m}$ & 20.5 \\
\hline Greece & 0.3 & 1.2 & 0.8 & 0.3 & 0.0 & 1.0 & 1.3 & 0.0 & 0.3 & 5.4 & 6.4 & $\mathrm{a}$ & 0.5 & 0.0 & 0.2 & 0.0 & 8.2 & 0.1 & 0.0 & 0.1 & $\mathrm{a}$ & 5.3 \\
\hline Hungary & 1.2 & 18.6 & 1.2 & 0.6 & 0.0 & 0.6 & 6.3 & 0.1 & 1.9 & 5.7 & 18.1 & $\mathrm{~m}$ & $\mathrm{a}$ & 0.2 & 0.3 & 0.4 & 4.0 & 1.1 & 0.1 & 0.1 & $\mathrm{~m}$ & 5.1 \\
\hline Iceland & 1.1 & 0.8 & 0.3 & 1.9 & 0.0 & 0.2 & 41.6 & 0.1 & 0.6 & 1.5 & 3.3 & $\mathrm{~m}$ & 4.4 & $\mathrm{a}$ & 0.2 & 0.0 & 0.9 & 0.7 & 0.0 & 0.0 & $\mathrm{~m}$ & 4.1 \\
\hline Ireland & 1.6 & 0.6 & 0.2 & 0.7 & 0.0 & 0.2 & 0.4 & 0.0 & 0.2 & 2.5 & 2.1 & $\mathrm{~m}$ & 1.4 & 0.0 & $\mathrm{a}$ & 0.0 & 0.2 & 0.1 & 0.1 & 0.0 & $\mathrm{~m}$ & 1.2 \\
\hline Israel & 1.8 & 0.6 & 0.2 & 5.6 & 0.0 & 0.7 & 0.3 & 0.0 & 0.1 & 1.1 & 9.1 & $\mathrm{~m}$ & 4.3 & 0.0 & 0.1 & $\mathrm{a}$ & 8.7 & 0.2 & 0.0 & 0.0 & $\mathrm{~m}$ & 0.6 \\
\hline Italy & 1.2 & 15.9 & 1.5 & 0.8 & 0.0 & 0.3 & 1.6 & 0.0 & 0.5 & 12.4 & 8.8 & $\mathrm{~m}$ & 0.5 & 0.1 & 0.5 & 0.2 & $\mathrm{a}$ & 0.4 & 0.0 & 0.2 & $\mathrm{~m}$ & 3.0 \\
\hline Japan & 5.2 & 0.8 & 0.2 & 2.5 & 0.0 & 0.2 & 0.2 & 0.0 & 0.4 & 4.1 & 5.0 & $\mathrm{~m}$ & 0.8 & 0.1 & 0.1 & 0.1 & 1.0 & $\mathrm{a}$ & 3.5 & 0.0 & $\mathrm{~m}$ & 0.4 \\
\hline Korea & 6.1 & 0.2 & 0.0 & 1.6 & 0.0 & 0.0 & 0.0 & 0.0 & 0.1 & 1.7 & 3.1 & $\mathrm{~m}$ & 0.0 & 0.0 & 0.0 & 0.1 & 0.5 & 14.8 & $\mathrm{a}$ & 0.0 & $\mathrm{~m}$ & 0.2 \\
\hline Luxembc & 0.1 & 9.3 & 17.7 & 0.3 & 0.0 & 0.0 & 0.9 & 0.0 & 0.1 & 14.5 & 35.2 & $\mathrm{~m}$ & 0.0 & 0.0 & 0.2 & 0.0 & 0.1 & 0.0 & 0.0 & $\mathrm{a}$ & $\mathrm{m}$ & 1.5 \\
\hline Mexico & 2.1 & 0.5 & 0.4 & 4.8 & 0.2 & 0.1 & 0.4 & 0.0 & 0.5 & 7.4 & 6.6 & $\mathrm{~m}$ & 0.3 & 0.0 & 0.1 & 0.2 & 1.3 & 0.6 & 0.2 & 0.0 & $\mathrm{a}$ & 1.2 \\
\hline Netherlands & 1.8 & 2.1 & 24.4 & 0.7 & 0.0 & 0.2 & 2.6 & 0.0 & 0.7 & 3.5 & 5.6 & $\mathrm{~m}$ & 0.5 & 0.1 & 0.8 & 0.5 & 1.2 & 0.9 & 0.1 & 0.1 & $\mathrm{~m}$ & $\mathrm{a}$ \\
\hline New Zealand & 50.6 & 0.6 & 0.1 & 2.0 & 0.0 & 0.1 & 0.7 & 0.0 & 0.3 & 1.1 & 2.0 & $\mathrm{~m}$ & 0.3 & 0.0 & 0.4 & 0.1 & 0.2 & 1.5 & 1.2 & 0.0 & $\mathrm{~m}$ & 0.4 \\
\hline Norway & 6.4 & 0.4 & 0.2 & 0.2 & 0.0 & 1.8 & 19.8 & 0.0 & 0.3 & 1.8 & 2.0 & $\mathrm{~m}$ & 4.7 & 0.2 & 0.2 & 0.1 & 0.6 & .5 & 0.0 & .0 & $\mathrm{~m}$ & 2.1 \\
\hline Poland & 0.8 & 4.9 & 1.3 & 1.2 & 0.0 & 1.6 & 4.6 & 0.0 & 1.0 & 7.7 & 24.3 & $\mathrm{~m}$ & 0.5 & 0.4 & 0.7 & 0.2 & 5.9 & 0.4 & 0.1 & 0.2 & $\mathrm{~m}$ & 4.0 \\
\hline Portugal & 1.1 & 1.3 & 1.7 & 1.5 & 0.0 & 3.9 & 1.7 & 0.0 & 0.5 & 13.4 & 4.1 & $\mathrm{~m}$ & 0.9 & 0.0 & 0.4 & 0.0 & 1.7 & 0.3 & 0.0 & 0.4 & $\mathrm{~m}$ & 3.7 \\
\hline Slovak Republic & 0.3 & 4.0 & 0.2 & 0.1 & 0.0 & 73.8 & 1.1 & 0.0 & 0.1 & 1.1 & 2.6 & $\mathrm{~m}$ & 7.4 & 0.0 & 0.1 & 0.0 & 0.6 & 0.1 & 0.0 & 0.0 & $\mathrm{~m}$ & 0.9 \\
\hline Slovenia & 1.0 & 24.9 & 0.7 & 1.0 & 0.0 & 0.7 & 2.3 & 0.0 & 0.7 & 2.8 & 9.9 & $\mathrm{~m}$ & 0.8 & 0.3 & 0.4 & 0.1 & 10.0 & 0.5 & 0.1 & 0.1 & $\mathrm{~m}$ & 4.9 \\
\hline Spain & 0.8 & 2.2 & 1.5 & 0.4 & 0.0 & 0.2 & 2.3 & 0.0 & 0.8 & 14.4 & 17.6 & $\mathrm{~m}$ & 1.5 & 0.2 & 0.6 & 0.1 & 2.5 & .5 & 0.1 & 1 & $\mathrm{~m}$ & 3.2 \\
\hline Sweden & 3.9 & 1.1 & 0.4 & 0.8 & 0.0 & 1.0 & 14.0 & 0.1 & 2.4 & 2.3 & 3.1 & $\mathrm{~m}$ & 2.5 & 0.4 & 0.2 & 0.2 & 0.7 & 1.3 & 0.1 & 0.0 & $\mathrm{~m}$ & 1.7 \\
\hline Switzerla & 3.1 & 6.6 & 1.3 & 1.9 & 0.0 & 0.1 & 0.9 & 0.0 & 0.3 & 9.3 & 18.8 & $\mathrm{~m}$ & 0.3 & 0.1 & 0.4 & 0.6 & 7.1 & 0.5 & 0.1 & 0.1 & $\mathrm{~m}$ & 2.2 \\
\hline Turkey & 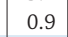 & 6.1 & 0.4 & 1.4 & 0.0 & 0.2 & 0.3 & 0.1 & 0.3 & 3.5 & 10.5 & $\mathrm{~m}$ & 1.1 & 0.0 & 0.1 & 0.1 & 1.8 & 0.3 & 0.2 & 0.0 & $\mathrm{~m}$ & 1.1 \\
\hline United Kingdom & 5.7 & 2.1 & 0.8 & 3.6 & 0.0 & 1.5 & 2.2 & 0.0 & 0.8 & 6.9 & 5.1 & $\mathrm{~m}$ & 1.1 & 0.2 & 7.2 & 0.9 & 1.0 & 1.4 & 0.3 & 0.1 & $\mathrm{~m}$ & 4.5 \\
\hline United States & 4.3 & 1.2 & 0.3 & $\mid 11.3$ & 0.0 & 0.3 & 0.9 & 0.1 & 0.5 & 4.8 & 5.9 & $\mathrm{~m}$ & 0.7 & 0.1 & 1.7 & 3.0 & 0.8 & 3.2 & 2.2 & 0.1 & $\mathrm{~m}$ & 0.9 \\
\hline Total from $\mathrm{OE}$ & 3.1 & 5.4 & 2.6 & 2.9 & 0.0 & 2.9 & 2.0 & 0.1 & 0.5 & 4.8 & 7.1 & $\mathrm{~m}$ & 1.2 & 0.1 & 0.7 & 0.5 & 1.7 & 2.4 & 0.4 & 0.2 & $\mathrm{~m}$ & 4.3 \\
\hline of which, fr & 1.4 & 8.5 & 4.5 & 2.6 & 0.0 & 5.0 & 2.4 & 0.2 & 0.6 & 5.9 & 8.4 & $\mathrm{~m}$ & 1.5 & 0.1 & 0.8 & 0.3 & 1.9 & 0.6 & 0.1 & 0.4 & $\mathrm{~m}$ & 7.1 \\
\hline Argentina & 1.6 & 0.5 & 0.3 & 1. & $0 . \varepsilon$ & 0.0 & 0.4 & 0.0 & 0.2 & 8.2 & 4.2 & $\mathrm{~m}$ & 0.0 & 0.0 & 0.0 & 2.5 & 4.1 & 0.7 & 0.4 & 0.1 & $\mathrm{~m}$ & 0.4 \\
\hline Brazil & 2.8 & 0.6 & 0.5 & 2.0 & 0.9 & 0.1 & 0.5 & 0.0 & 0.3 & 11.7 & 7.7 & $\mathrm{~m}$ & 0.0 & 0.0 & 0.1 & 0.4 & 2.7 & 0.8 & 0.1 & 0.0 & $\mathrm{~m}$ & 0.6 \\
\hline China & 12.1 & 0.1 & 0.1 & 4.7 & 0.0 & 0.0 & 0.2 & 0.0 & 0.3 & 3.5 & 2.7 & $\mathrm{~m}$ & 0.1 & 0.0 & 0.2 & 0.0 & 1.3 & 12.3 & 5.2 & 0.0 & $\mathrm{~m}$ & 0.7 \\
\hline Colombia & 5.3 & 0.5 & 0.5 & 2.5 & 5.0 & 0.1 & 0.2 & 0.0 & 0.2 & 9.3 & 5.2 & $\mathrm{~m}$ & 0.0 & 0.0 & 0.0 & 0.1 & 3.2 & 0.2 & 0.1 & 0.0 & $\mathrm{~m}$ & 0.9 \\
\hline India & 8.4 & 0.2 & 0.1 & 5.0 & 0.0 & 0.1 & 0.1 & 0.0 & 0.3 & 1.0 & 2.9 & $\mathrm{~m}$ & 0.0 & 0.0 & 0.3 & 0.0 & 0.5 & 0.3 & 0.3 & 0.0 & $\mathrm{~m}$ & 0.5 \\
\hline Indonesia & 21.7 & 0.2 & 0.2 & 1.4 & 0.0 & 0.0 & 0.1 & 0.0 & 0.1 & 1.5 & 3.6 & $\mathrm{~m}$ & 0.0 & 0.0 & 0.0 & 0.0 & 0.3 & 5.1 & 1.7 & 0.0 & $\mathrm{~m}$ & 2.2 \\
\hline Latvia & 0.4 & 1.4 & 0.6 & \begin{tabular}{|l|} 
\\
0.3
\end{tabular} & 0.0 & 0.1 & 12.1 & 1.6 & 1.8 & 2.5 & 9.9 & $\mathrm{~m}$ & 0.3 & 0.3 & 0.4 & 0.2 & 1.8 & 0.4 & 0.0 & 0.1 & $\mathrm{~m}$ & 7.3 \\
\hline Russian Federation & 1.7 & 1.8 & 0.5 & 0.9 & 0.0 & 6.2 & 0.3 & 0.3 & 4.0 & 6.5 & 17.0 & $\mathrm{~m}$ & 0.4 & 0.0 & 0.1 & 1.6 & 3.8 & 0.6 & 0.6 & 0.1 & $\mathrm{~m}$ & 1.1 \\
\hline Saudi Arabia & 6.4 & 0.1 & 0.0 & 5.9 & 0.0 & 0.1 & 0.0 & 0.0 & 0.0 & 0.6 & 0.3 & $\mathrm{~m}$ & 0.3 & 0.0 & 0.6 & 0.0 & 0.0 & 0.4 & 0.2 & 0.0 & $\mathrm{~m}$ & 0.1 \\
\hline South Africa & 10.4 & 0.7 & 0.8 & 2.7 & 0.0 & 0.2 & 0.7 & 0.0 & 0.3 & 1.3 & 2.8 & $\mathrm{~m}$ & 0.1 & 0.0 & 0.7 & 1.2 & 0.3 & 0.2 & 0.7 & 0.0 & $\mathrm{~m}$ & 1.5 \\
\hline $\begin{array}{l}\text { Total from other G20 } \\
\text { countries }\end{array}$ & 10.7 & 0.2 & 0.1 & 4.5 & 0.1 & 0.3 & 0.2 & 0.0 & 0.5 & 3.4 & 3.6 & $\mathrm{~m}$ & 0.1 & 0.0 & 0.2 & 0.1 & 1.3 & 8.2 & 3.5 & 0.0 & $\mathrm{~m}$ & 0.7 \\
\hline Total from all countrie & 6.2 & 1.8 & 1.1 & 3.4 & 0.1 & 1.0 & 0.7 & 0.0 & 0.5 & 5.7 & 4.9 & 0.7 & 0.5 & 0.0 & 0.3 & 0.3 & 2.0 & 3.4 & 1.4 & 0.1 & 0.2 & 1.7 \\
\hline
\end{tabular}

Note: The proportion of students abroad is based only on the total of students enrolled in countries reporting data to the OECD and UNESCO Institute for Statistics.

1. Year of reference 2012.

2. Data refer to foreign instead of international students.

3. Excludes doctoral or equivalent programmes.

Source: OECD. Argentina, China, Colombia, India, Indonesia, Saudi Arabia and South Africa: UNESCO Institute for Statistics. Latvia: Eurostat. See Annex 3 for notes (www.oecd.org/education/education-at-a-glance-19991487.htm).

Please refer to the Reader's Guide for information concerning symbols for missing data and abbreviations.

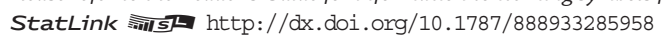


Table C4.4. [2/2] Students abroad in tertiary education, by country of destination (2013)

Number of foreign and international students enrolled in tertiary education in a given country of destination as a percentage of all students enrolled abroad in reporting destinations, based on head counts

\begin{tabular}{|c|c|c|c|c|c|c|c|c|c|c|c|c|c|c|c|c|c|c|c|}
\hline \multirow[b]{3}{*}{ Country of origin } & \multicolumn{12}{|c|}{ OECD } & \multirow[b]{2}{*}{ 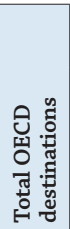 } & \multirow[b]{2}{*}{ 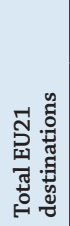 } & \multicolumn{3}{|c|}{$\begin{array}{l}\text { Other G20 and } \\
\text { partner countries }\end{array}$} & \multirow[b]{2}{*}{ 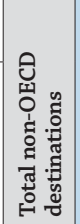 } & \multirow{2}{*}{ 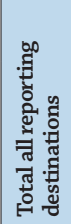 } \\
\hline & 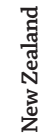 & 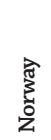 & $\begin{array}{l}\text { ్̆ㅁ } \\
\text { 융 }\end{array}$ & $\begin{array}{l}\text { ]ే } \\
\text { है } \\
\text { है }\end{array}$ & 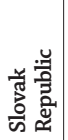 & 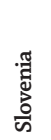 & $\begin{array}{l}\text { मूँ } \\
\text { की }\end{array}$ & 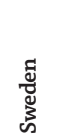 & 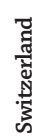 & 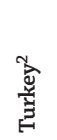 & 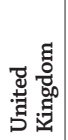 & 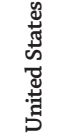 & & & 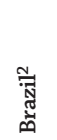 & 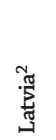 & 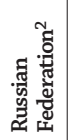 & & \\
\hline & (23) & (24) & (25) & (26) & (27) & (28) & (29) & (30) & (31) & (32) & (33) & $(34)$ & (35) & (36) & (37) & (38) & (39) & $(40)$ & (41) \\
\hline Australia & 21.8 & 0.3 & 0.1 & 0.1 & 0.0 & 0.0 & 0.4 & 0.5 & 1.1 & 0.7 & 15.3 & 32.6 & 90.8 & 27.6 & $\mathrm{~m}$ & 0.0 & 0.0 & 9.2 & 100.0 \\
\hline Austri & 0.4 & 0.2 & 0.3 & & 0.6 & 0.3 & 1.0 & 0.8 & 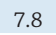 & 1.2 & 11.2 & 6.2 & 94.4 & 75.8 & $\mathrm{~m}$ & 0.2 & 0.0 & 5.6 & 100.0 \\
\hline Belgium & 0.1 & 0.1 & 0.2 & 1.4 & 0.0 & 0.0 & 2.6 & 0.6 & 2.5 & 0.8 & 22.8 & 6.8 & 95.2 & 81.2 & $\mathrm{~m}$ & 0.1 & .0 & 4.8 & 100.0 \\
\hline Canada & 0.8 & 0.1 & 1.0 & 0.1 & 0.0 & 0.0 & 0.3 & 0.3 & 0.9 & 0.1 & 12.9 & 54.9 & 89.7 & 23.0 & $\mathrm{~m}$ & 0.0 & .0 & 10.3 & 100.0 \\
\hline Chile & 1.1 & 0.2 & 0.0 & 0.1 & 0.0 & 0.0 & 13.4 & 0.2 & 1.2 & 0.0 & 6.7 & 22.9 & 75.7 & 40.8 & $\mathrm{~m}$ & 0.0 & .0 & 24.3 & 100.0 \\
\hline Czech 1 & 0.1 & 0.4 & 6.4 & 0.2 & 43.8 & 0.1 & 0.9 & 0.5 & 1.3 & 0.1 & 9.8 & 5.9 & 98.5 & 88.4 & $\mathrm{~m}$ & 0.1 & .0 & 1.5 & 100.0 \\
\hline Denm & 2.6 & 4.0 & 0.9 & 0.1 & 0.1 & 0.2 & 1.2 & 5.2 & 1.4 & 0.7 & 26.0 & 24.3 & 93.9 & 53.4 & $\mathrm{~m}$ & 0.2 & U & 6.1 & 100.0 \\
\hline Estonia & 0.0 & 0.8 & 0.2 & 0.1 & 0.0 & 0.0 & 0.9 & 3.0 & 1.0 & 0.1 & 25.9 & 4.7 & 82.7 & 74.4 & $\mathrm{~m}$ & 1.6 & 7 & 17.3 & 100.0 \\
\hline Finland & 0.4 & 1.0 & 0.4 & 0.1 & 0.2 & 0.1 & 1.4 & 18.0 & 1.1 & 0.1 & 21.4 & 7.8 & 92.2 & 77.4 & $\mathrm{~m}$ & 0.7 & 0 & 7.8 & 100.0 \\
\hline France & 0. & 0.1 & 0.4 & 0 & 0.0 & 0.0 & 3.7 & 0.7 & 10.9 & 0.3 & 15.2 & 10.5 & 95.0 & 56.2 & $\mathrm{~m}$ & 0.1 & & 5.0 & 100.0 \\
\hline Germany & 0.5 & 0.4 & 0.5 & 0.2 & 0.4 & 0.0 & 1.3 & 1.3 & 10.0 & 1.3 & 11.8 & 7.8 & 95.6 & 72.8 & $\mathrm{~m}$ & 0.5 & 0 & 4.4 & 100.0 \\
\hline Greece & 0.0 & 0.2 & 0.1 & 0.1 & 2.9 & 0.0 & 0.5 & 1.6 & 1.8 & 3.8 & 29.5 & 5.3 & 77.2 & 65.4 & $\mathrm{~m}$ & 0.0 & 0 & 22.8 & 100.0 \\
\hline Hungar & 0.2 & 0.4 & 0.7 & 0.1 & 1.0 & 0.1 & 1.3 & 0.9 & 2.5 & 0.2 & 13.6 & 7.4 & 94.2 & 79.8 & $\mathrm{~m}$ & 0.2 & & 5.8 & 100.0 \\
\hline Icelan & 0. & 4.5 & 0.2 & 0.1 & 0.3 & 0.0 & 0.8 & 9.5 & 1.3 & 0.1 & 7.5 & 12.9 & 9.0 & 76.5 & $\mathrm{~m}$ & 0.1 & 0 & 1.0 & 100.0 \\
\hline Ireland & 0.3 & 0.0 & 0.4 & 0.1 & 0.3 & 0.0 & 0.6 & 0.4 & 0.3 & 0.1 & 76.4 & 6.7 & 7.5 & 87.6 & $\mathrm{~m}$ & 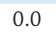 & & 2.5 & 100.0 \\
\hline Israel & 0 & 0. & 0.4 & 0. & 0.6 & 0. & 0. & 0.1 & 0. & 0.2 & 2.9 & 13.6 & 52.2 & 30. & $\mathrm{~m}$ & 0 & 0 & \begin{tabular}{|l|} 
\\
\end{tabular} & 100.0 \\
\hline Italy & 0 & 0. & 0.5 & 0. & 0.1 & 0. & 9. & 1.0 & 8. & 0.1 & 16.8 & 8.3 & 4.5 & 74. & $\mathrm{~m}$ & 0.1 & & 5.5 & 100.0 \\
\hline Japan & 2. & 0.2 & 0.1 & 0.0 & 0.0 & 0.0 & 0.3 & 0.3 & 0. & 0.4 & 9.3 & 56.4 & 4.4 & 23. & $\mathrm{~m}$ & 0 & & 5.6 & 100.0 \\
\hline Kore & 1. & 0.0 & 0.1 & 0.0 & 0.0 & 0. & 0.1 & 0.0 & 0. & 0.0 & 4.1 & 60.8 & 5.1 & 10. & $\mathrm{~m}$ & 0.0 & & 4.9 & 100.0 \\
\hline $\mathrm{Lu}$ & 0 & 0. & 0. & 0.8 & 0. & 0. & 0. & 0.1 & 5. & 0.0 & 11.7 & 0.9 & 9.5 & 92. & $\mathrm{~m}$ & 0.0 & & 0.5 & 100.0 \\
\hline Mex & & 0.2 & 0. & 0. & 0. & 0. & 8. & 0 & 1. & 0.0 & 5.6 & 49.0 & 92.8 & 34. & $\mathrm{~m}$ & 0 & & 7.2 & 100.0 \\
\hline Neth & 0 & 0.6 & 0.1 & 0. & 0.0 & 0. & $2 .($ & 1.9 & 2.1 & 0.0 & 23.3 & 13.2 & 91.1 & 70.3 & $\mathrm{~m}$ & 0.2 & & 8.9 & 100.0 \\
\hline New & 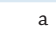 & 0.1 & 0.1 & 0.1 & 0.0 & 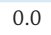 & 0.1 & 02 & 09 & 0.0 & 8.9 & 22.9 & 94.9 & 15.5 & $\mathrm{~m}$ & مि & & 5.1 & 100.0 \\
\hline Nory & $\perp$ & $\mathrm{a}$ & 7.6 & 0. & 2.5 & 0. & 0. & 2 & 0.5 & 0.7 & 26.8 & 11.1 & 4.8 & 73. & $\mathrm{~m}$ & 7 & & 5.2 & 100.0 \\
\hline Polan & 0.1 & 0.9 & 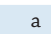 & 0.6 & 1.1 & 0.1 & 2. & 1.3 & 2.1 & 1.0 & 22.0 & (1) & 7.7 & 84.1 & $\mathrm{~m}$ & 2 & & 2.3 & 100.0 \\
\hline Portug & & & 1. & $\mathrm{a}$ & 0.7 & & 15. & & & & 20.7 & & 6.6 & 72.9 & $\mathrm{~m}$ & & & 13.4 & 100.0 \\
\hline Slovak & 0.0 & 0. & 0. & 0.0 & 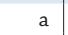 & 0.0 & 0.3 & 0.1 & 0. & 0.5 & 4.2 & 1. & 9.6 & 97. & $\mathrm{~m}$ & 0.1 & & 0.4 & 100.0 \\
\hline Slove & & & 0.9 & 4 & 0.3 & 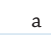 & 1.2 & & 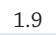 & 0.4 & 12.7 & & 8.4 & 74. & $\mathrm{~m}$ & م & & 11.6 & 100.0 \\
\hline Spa & & 0. & 3. & 2 & 0.4 & . & 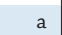 & 1.2 & 2. & 0.0 & 19.7 & 16.0 & 5.5 & 74. & $\mathrm{~m}$ & 0.3 & & 4.5 & 100.0 \\
\hline & & 4.2 & 5.9 & 0.2 & 0.4 & 0.0 & 0.8 & a & 1.2 & 0.2 & 18.3 & 22.9 & 90.6 & 54.9 & $\mathrm{~m}$ & 0.9 & 0 & 9.4 & 100.0 \\
\hline $\mathrm{Su}$ & & & & & & & 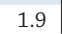 & & 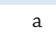 & 0.4 & 23.5 & 113 & 92.8 & 74. & $\mathrm{~m}$ & & & 7.2 & 100.0 \\
\hline Turke & & 0. & 1. & 0 & & 0. & 0. & 0.5 & 1.2 & $\mathrm{a}$ & 6.4 & 21.0 & 9.6 & 34.3 & $\mathrm{~m}$ & & & 40.4 & 100.0 \\
\hline Unit & 1. & 0.3 & 0. & 1. & 0.4 & 0. & 1. & 1. & 1.6 & 0.4 & a & 31.0 & 86.0 & 38.8 & $\mathrm{~m}$ & 0.1 & 0 & 14.0 & 100.0 \\
\hline United States & 3.4 & 0.3 & 1.3 & 0.4 & 0.0 & 0.0 & 1.3 & 0.7 & 1.4 & 0.4 & 22.1 & a & 73.6 & 44.0 & $\mathrm{~m}$ & 0.0 & 0.0 & 26.4 & 100.0 \\
\hline Total $\mathrm{f}$ & 1.1 & 0. & 0. & 0.4 & 0. & $0 .($ & 2. & 0. & 3.5 & 0.5 & 14.3 & 21.3 & 3 & 53. & $\mathrm{~m}$ & 0.2 & 0 & 10.7 & 100.0 \\
\hline of which, from EU21 & 0.4 & 0.5 & 0.9 & 0.5 & 1.5 & 0.1 & 2.6 & 1.3 & 5.6 & 0.8 & 17.1 & 9.8 & 93.4 & 71.1 & $\mathrm{~m}$ & 0.2 & 0.1 & 6.6 & 100.0 \\
\hline Arg & & & 0.1 & 0.4 & 0.0 & & 17 & & 2.1 & 0.0 & 2. & & 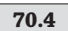 & & $\mathrm{m}$ & & & 29.6 & 100.0 \\
\hline Braz & & & 01 & 14.6 & & & - & & 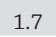 & 0.0 & 4.8 & & 0.2 & 18 & a & & & 9.8 & 100.0 \\
\hline China & 1.7 & 0. & 0.1 & 0.0 & 0. & 0.0 & 0.2 & 0.3 & 0.2 & 0.0 & 11.2 & 30.9 & 88.2 & 20.9 & $\mathrm{~m}$ & 0.0 & 0 & 11.8 & 100.0 \\
\hline Colomb & 0.2 & 0.2 & 0.1 & 0. & 0. & 0.0 & 21.5 & 0.4 & 1.2 & 0.0 & 3.5 & 23.4 & 84.1 & 45.7 & $\mathrm{~m}$ & 0.0 & 0 & 15.9 & 100.0 \\
\hline Indi & & 0. & 0. & & & 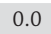 & 0 & 0 & 0. & 0.2 & 11.5 & & 84.9 & 18. & $\mathrm{~m}$ & 0.0 & & $\mid 15.1$ & 100.0 \\
\hline Indonesi & 1.0 & 0.2 & 0.1 & 0.1 & $0 . c$ & 0.0 & 0.1 & 0.1 & 0.1 & 2.6 & 3.8 & 16.8 & 63.0 & 12.4 & $\mathrm{~m}$ & 0.0 & 0.0 & 37.0 & 100.0 \\
\hline Latvia & 0.0 & 1.0 & 1.0 & 0.1 & 0.1 & 0.0 & 0.6 & 1.8 & 0.7 & 0.0 & 30.6 & 4.1 & 81.6 & 74.1 & $\mathrm{~m}$ & a & 9.7 & 18.4 & 100.0 \\
\hline Russ & & 1.1 & 1.0 & 0. & & 0. & 1. & 0.8 & 1.7 & 0.1 & 6.5 & 8.4 & 69.3 & 52. & $\mathrm{~m}$ & .7 & a & 30.7 & 100.0 \\
\hline & 1 & 0. & 0.6 & 0. & & 0. & 0. & 0.0 & 0.0 & 0.1 & 12.0 & 54.8 & 4.0 & 15.0 & $\mathrm{~m}$ & 0.0 & 0.0 & 16.0 & 100.0 \\
\hline South Africa & 1.3 & 0.3 & 0.2 & 0.8 & 0.0 & 0.0 & 0.3 & 0.4 & 1.3 & 0.3 & 16.3 & 23.2 & 9.0 & 27.4 & $\mathrm{~m}$ & 0.0 & 0 & 1.0 & 100.0 \\
\hline $\begin{array}{l}\text { Total from other G20 } \\
\text { countries }\end{array}$ & 1.8 & 0.2 & 0.2 & 0.5 & 0.0 & 0.0 & 0.9 & 0.4 & 0.4 & 0.2 & 10.9 & 34.3 & 3 & 8 & $\mathrm{~m}$ & 0.0 & 0.1 & 14.7 & 100.0 \\
\hline Total from all countrie & 1.0 & 0.2 & 0.7 & 0.4 & 0.3 & 0.1 & 1.4 & 0.6 & 1.2 & 1.3 & 10.3 & 19.4 & 72.9 & 34.8 & 0.4 & 0.1 & 3.4 & 27.1 & 100.0 \\
\hline
\end{tabular}

Note: The proportion of students abroad is based only on the total of students enrolled in countries reporting data to the OECD and UNESCO Institute for Statistics.

1. Year of reference 2012.

2. Data refer to foreign instead of international (mobile) students.

3. Excludes doctoral or equivalent programmes.

Source: OECD. Argentina, China, Colombia, India, Indonesia, Saudi Arabia and South Africa: UNESCO Institute for Statistics. Latvia: Eurostat. See Annex 3 for notes (www.oecd.org/education/education-at-a-glance-19991487.htm).

Please refer to the Reader's Guide for information concerning symbols for missing data and abbreviations.

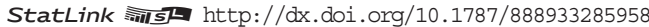


Table C4.5. Mobility patterns of foreign and international students (2013)

Regional and cross-border mobility, balance on mobility and use of the official language of the host country in countries of origin

\begin{tabular}{|c|c|c|c|c|}
\hline & $\begin{array}{l}\text { Percentage of national tertiary } \\
\text { students enrolled abroad }\end{array}$ & $\begin{array}{c}\text { Number } \\
\text { of international students } \\
\text { per national student abroad }\end{array}$ & $\begin{array}{c}\text { Percentage } \\
\text { of international students } \\
\text { from neighbouring countries }{ }^{1}\end{array}$ & $\begin{array}{l}\text { Percentage of students } \\
\text { from countries with } \\
\text { the same official language }\end{array}$ \\
\hline & (1) & (2) & (3) & (4) \\
\hline ○ Austria & 4 & 4 & 58 & 54 \\
\hline Belgium & 3 & 3 & 48 & 58 \\
\hline Canada $^{2}$ & 3 & 3 & 6 & 32 \\
\hline Chile & 1 & 0 & 18 & 84 \\
\hline Czech Republic ${ }^{3}$ & 3 & 3 & 63 & 0 \\
\hline Denmark & 2 & 6 & 43 & 0 \\
\hline Estonia & 7 & 0 & 65 & 0 \\
\hline Finland & 3 & 3 & 15 & 2 \\
\hline France & 3 & 3 & 15 & 27 \\
\hline Germany & 4 & 2 & 16 & 10 \\
\hline Hungary & 3 & 2 & 38 & 0 \\
\hline Iceland & 14 & 0 & 9 & 0 \\
\hline Ireland & 8 & 1 & 16 & 45 \\
\hline Israel $^{3}$ & 4 & 1 & 1 & 0 \\
\hline Italy $^{3}$ & 3 & 2 & 25 & 4 \\
\hline Japan & 1 & 4 & 79 & 0 \\
\hline Korea $^{3}$ & 3 & 0 & 71 & 0 \\
\hline Luxembourg & 68 & 0 & 63 & 35 \\
\hline Mexico & 1 & 0 & $\mathrm{~m}$ & $\mathrm{~m}$ \\
\hline Netherlands & 2 & 5 & 41 & 4 \\
\hline New Zealand & 2 & 8 & 9 & 36 \\
\hline Norway & 7 & 1 & 21 & 0 \\
\hline Poland & 1 & 1 & 62 & 0 \\
\hline Portugal & 3 & 1 & 6 & 62 \\
\hline Spain & 2 & 2 & 24 & 43 \\
\hline Sweden & 4 & 1 & 20 & 6 \\
\hline Switzerland & 5 & 4 & 56 & 61 \\
\hline Turkey $^{3}$ & 1 & 1 & 20 & 11 \\
\hline United Kingdom & 1 & 14 & 12 & 25 \\
\hline United States $^{4}$ & 0 & 12 & 6 & 22 \\
\hline OECD total & 2 & 3 & 19 & 21 \\
\hline EU21 total & 3 & 3 & 23 & 21 \\
\hline 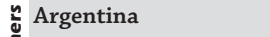 & $\mathrm{m}$ & $\mathrm{m}$ & $\mathrm{m}$ & $\mathrm{m}$ \\
\hline $\mathrm{Brazil}^{3}$ & 0 & 0 & $\mathrm{~m}$ & $\mathrm{~m}$ \\
\hline China ${ }^{3}$ & 2 & $\mathrm{~m}$ & $\mathrm{~m}$ & $\mathrm{~m}$ \\
\hline Colombia $^{3}$ & 1 & $\mathrm{~m}$ & $\mathrm{~m}$ & $\mathrm{~m}$ \\
\hline India & $\mathrm{m}$ & $\mathrm{m}$ & $\mathrm{m}$ & $\mathrm{m}$ \\
\hline Indonesia $^{3}$ & 1 & $\mathrm{~m}$ & $\mathrm{~m}$ & $\mathrm{~m}$ \\
\hline Latvia & 7 & 1 & $\mathrm{~m}$ & $\mathrm{~m}$ \\
\hline Russian Federation ${ }^{3,5}$ & 1 & 3 & 87 & 46 \\
\hline Saudi Arabia ${ }^{3}$ & 6 & 1 & 32 & 44 \\
\hline South Africa ${ }^{2,3}$ & 1 & 6 & 48 & 70 \\
\hline
\end{tabular}

1. The neighbouring countries considered have land or maritime borders with the host country.

2. Year of reference 2012.

3. Data refer to foreign instead of international students.

4. Even though there is no official language in the United States, English has been used for Column (4).

5. The percentage of foreign students coming from neighbouring countries includes those from former Soviet Union countries, mostly of central Asia.

Source: OECD and UNESCO Institute for Statistics for most data on non-OECD countries. Latvia: Eurostat. CIA World Factbook 2014 for worldwide official languages. See Annex 3 for notes (www.oecd.org/education/education-at-a-glance-19991487.htm).

Please refer to the Reader's Guide for information concerning symbols for missing data and abbreviations.

StatLink 제으 http://dx.doi.org/10.1787/888933285960 


\section{TRANSITION FROM SCHOOL TO WORK: WHERE ARE THE 15-29 YEAR-OLDS?}

- The percentage of 20-24 year-olds not in education ranges from less than $40 \%$ in Denmark and Slovenia to over $70 \%$ in Brazil, Colombia, Israel and Mexico.

- On average across OECD countries, $18 \%$ of 20-24 year-olds are neither employed nor in education or training (NEET).

- Countries in which a large share of 15-29 year-olds are employed and study at the same time usually show small proportions of students who work 35 hours or more per week. In the Czech Republic, Denmark, Iceland, the Netherlands and Norway, more than $25 \%$ of adults study and work the same time, but less than $30 \%$ of them work 35 or more hours per week.

\section{Chart C5.1. NEET population among 20-24 year-olds, by gender (2014)}

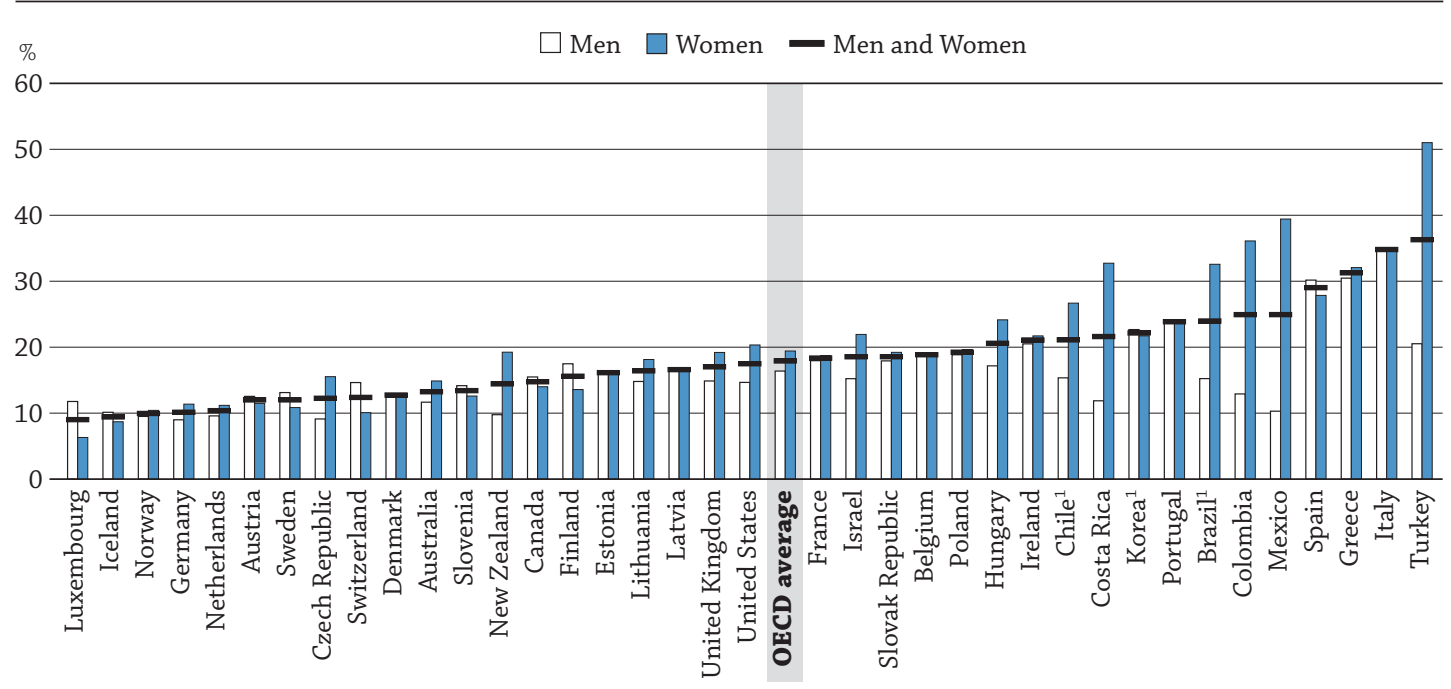

Note: NEET refer to young people neither in employment nor in education or training

1. Brazil, Chile, Korea: Year of reference 2013.

Countries are ranked in ascending order of the percentage of the 20-24 year-old NEET population of men and women.

Source: OECD. Table C5.2a. See Annex 3 for notes (www.oecd.org/education/education-at-a-glance- 19991487.htm).

StatLink 젶ㄴ http://dx.doi.org/10.1787/888933284290

\section{Context}

The length and the quality of the schooling that individuals receive have an impact on students' transition from education to work, as do labour market conditions, the economic environment and demographics. For example, in some countries, young people traditionally complete schooling before they look for work; in others, education and employment are concurrent. In some countries, there is little difference between how young women and men experience their transitions from school to work, while in other countries, significant proportions of young women raise families full time after leaving the education system and do not enter the labour force.

The ageing of the population in OECD countries should favour employment among young people as, theoretically, when older people leave the labour market their jobs are made available to the young. However, during recessionary periods, fewer job vacancies make the transition from school to work substantially more difficult for young people, as those with more work experience are favoured over new entrants into the labour market. When labour market conditions are unfavourable, young people often tend to stay in education longer, because high unemployment rates drive down the opportunity costs of education. At the same time, most countries are adopting policies that raise the age of retirement. Delaying retirement slows job rotation, which tends to lead to a decrease in job vacancies. This may account for differences in the number of young people (entrants) and older people (leavers) in the labour market. 
To improve the transition from school to work, regardless of the economic climate, education systems should aim to ensure that individuals have the skills that are needed in the labour market. During recessions, public investment in education could be a sensible way to counterbalance unemployment and invest in future economic growth by building the needed skills. In addition, public investment could be directed towards potential employers in the form of incentives to hire young people.

\section{Other findings}

- On average across OECD countries, $48 \%$ of 15-29 year-olds were in education in 2014. Of the remaining 52\%, 36\% held a job, $7 \%$ were unemployed, and $9 \%$ were outside of the labour force.

- Women are more often neither in employment nor in education or training (NEET) than men. Among 20-24 year-olds, $19.4 \%$ of women and $16.4 \%$ of men were NEET in 2014, on average across OECD countries. In Mexico and Turkey, the gender difference in the shares of 20-24 year-olds who were NEET was around 30 percentage points.

- Across OECD countries, and among all young people who are employed and no longer in school, men are more likely to find a full-time job than women. On average, $82 \%$ of young men work full time compared with $67 \%$ of young women.

\section{Trends}

Most countries have encouraged students to continue their studies beyond compulsory schooling. As a result, the average number of years of formal education expected after compulsory schooling has increased considerably. On average across OECD countries, since 2000, about one year has been added to the duration of formal education; in the Czech Republic, the Netherlands, the Slovak Republic and Turkey, two years or more have been added (Table C5.1b, available on line).

Governments' efforts to improve educational attainment among their populations have resulted in significant changes in participation in education over the years. In 2000, an average of $35 \%$ of 20-24 year-olds in OECD countries were in education; by 2014, that proportion had grown to $46 \%$ (Table C5.2b, available on line).

During the same period, the proportion of 20-24 year-olds not in education but employed fell from $42 \%$ to $36 \%$. While the percentage of individuals in education increased steadily between 2000 and 2014 , trends in the proportion of 20-24 year-olds who were neither in employment nor in education or training (NEET) remained stable at around 17-19\% between 2000 and 2014 (Table C5.2b, available on line). 


\section{Analysis}

\section{Young people in education or not, and their labour market status}

Young people tend to make the transition from education to the labour market between the ages of 15 and 29 . As expected, the older individuals in this age band are less likely to be enrolled in educational institutions than the younger individuals. In 2014, on average across OECD countries, $86 \%$ of $15-19$ year-olds, $46 \%$ of $20-24$ year-olds and $17 \%$ of 25-29 year-olds were in education. In some countries, relatively large shares young people study and work at the same time. For example, in Australia, Denmark, Germany, Iceland, the Netherlands, Norway and Switzerland, more than one in four 20-24 year-olds study and work concurrently (Table C5.2a).

Among those who were not in education in 2014 (i.e. $14 \%$ of $15-19$ year-olds, $54 \%$ of $20-24$ year-olds and $83 \%$ of 25-29 year-olds), what was their status in the labour market? Chart C5.2 shows that, on average, among all 20-24 year-olds, 36\% were not in education and employed, about $9 \%$ were not in education and unemployed, and $9 \%$ were not in education and inactive (i.e. not employed and not looking for a job). The percentage of 20-24 year-olds not in education ranged from less than $40 \%$ in Denmark and Slovenia to over $70 \%$ in Brazil, Colombia, Israel and Mexico (Table C5.2a).

\section{Chart C5.2. Percentage of 20-24 year-olds in education/not in education, by work status (2014)}

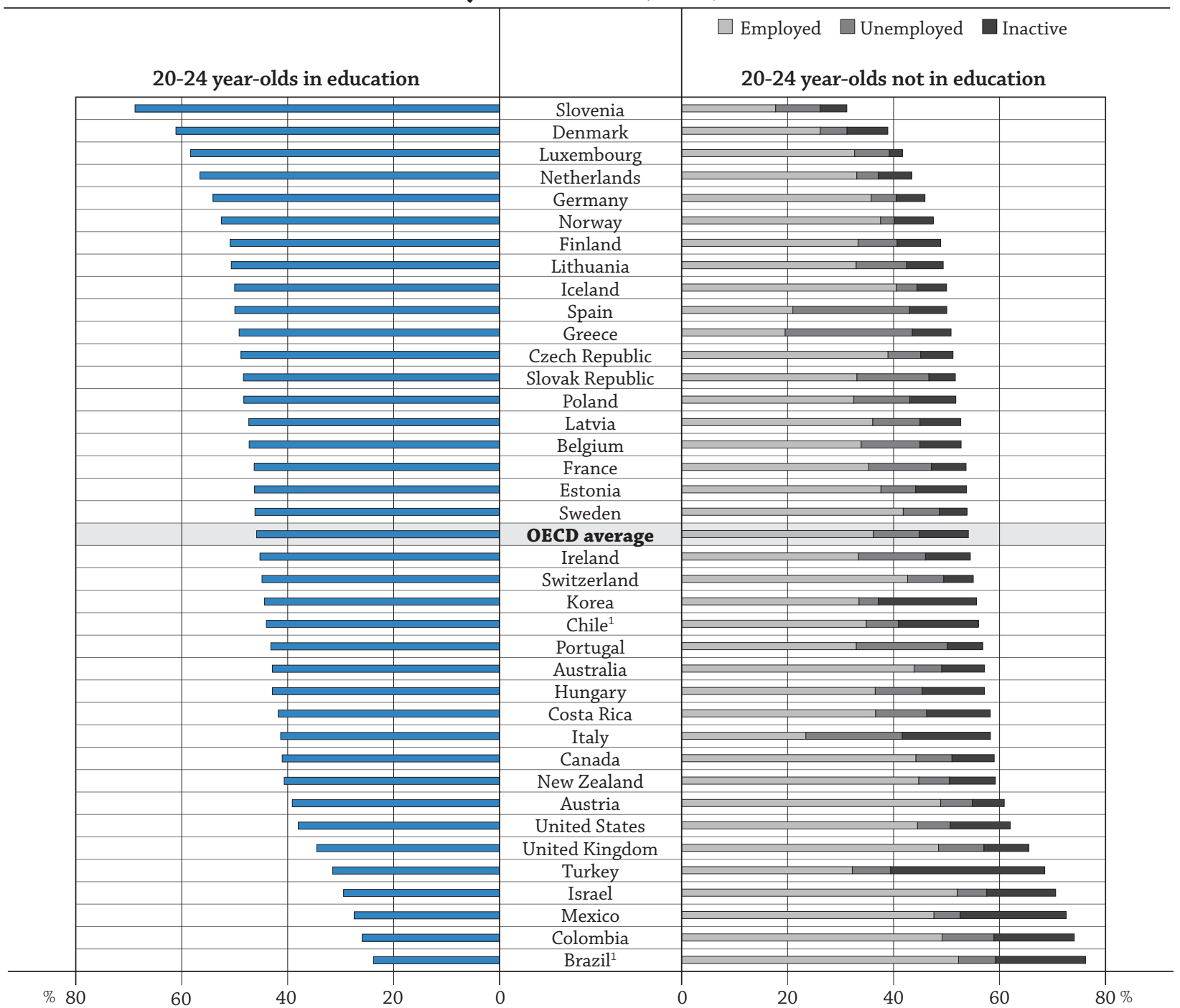

1. Brazil, Chile: Year of reference 2013.

Countries are ranked in ascending order of the percentage of 20-24 year-olds not in education.

Source: OECD. Table C5.2a. See Annex 3 for notes (www.oecd.org/education/education-at-a-glance- 19991487.htm).

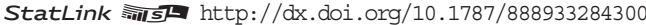


Participation in education and the labour market also differs according to educational attainment. Those who have completed tertiary education (which represents the highest level of education) and are no longer in education are usually employed, whereas individuals who are not in education and who did not complete upper secondary education are distributed almost evenly among employment, unemployment or inactivity. In Mexico and Turkey, less than $40 \%$ of $15-29$ year-olds with only lower secondary education who did not complete upper secondary education are still in school. In the Czech Republic, Denmark, Finland, Lithuania, Luxembourg, Poland, the Slovak Republic, Slovenia, Sweden and Switzerland, $80 \%$ or more of young people who did not finish upper secondary education are still in school (Table C5.3a).

\section{Young people who are neither in employment nor in education or training (NEET)}

Unemployment and employment rates are useful indicators of how people engage in the labour market. Young individuals are particularly likely to delay their entry into the labour market or drop out of the labour force and become inactive. While increasing numbers of young people tend to stay in education beyond the age of compulsory schooling without being active in the labour market, it would be inappropriate to consider them as a high-risk group. Consequently, the proportion of young people who are neither in employment nor in education or training (NEET) is a better measure of the difficulties young people face when they are searching for a job, as it includes not only those who do not manage to find a job (unemployed) but also those who do not actively seek employment (inactive).

On average across OECD countries in $2014,15.5 \%$ of $15-29$ year-olds, $7.2 \%$ of $15-19$ year-olds, $17.9 \%$ of 20-24 year-olds and $20.5 \%$ of 25-29 year-olds were NEET (Table C5.2a).

The most important ages to study when analysing the NEET population are 20-24 year-olds. At this age, compulsory education does not affect the proportion of inactive or unemployed. However, when analysing the proportion of NEET, it is important to remember that a significant proportion of 20-24 year-olds are continuing their studies after compulsory education.

In 2014, Greece, Italy and Turkey were the only countries where more than $30 \%$ of $20-24$ year-olds were NEET. Turkey has the highest proportion of NEET, but it is also the only country among these three to show a decrease in the percentage of NEET between 2005 and 2014, from 49.7\% in 2005 to 36.3\% in 2014 (Table C5.2b, available on line).

Germany's share of 20-24 year-old NEET (18.7\%) was above the OECD average (17.4\%) in 2005, but by 2014, that share fell back to $10.1 \%$, well below the OECD average of $17.9 \%$. In fact, the proportion of 20-24 year-old NEET in Germany is one of the smallest among OECD countries along with those in Iceland (9.4\%), Luxembourg (9.0\%), the Netherlands (10.4\%) and Norway (10.0\%) (Table C5.2a).

Women are more often NEET than men. Some 16.4\% of 20-24 year-old men were NEET in 2014 compared with $19.4 \%$ of women that age, on average across OECD countries. In Mexico and Turkey, the gender difference in the shares of 20-24 year-olds who were NEET in 2014 is around 30 percentage points. The greatest difference in favour of women is observed in Luxembourg, where $6.3 \%$ of women but $11.8 \%$ of men were NEET. Chart C5.1 shows that there is no direct association between a country's overall proportion of NEET and the gender gap in shares of NEET. In Italy and Turkey, more than $30 \%$ of 20-24 year-olds were NEET; but while the gender gap among NEET of those ages is large in Turkey, it is almost non-existent in Italy (Table C5.2a).

\section{Working hours}

The extent to which 15-29 year-olds participate in the labour market not only varies in terms of employment, but it also varies in intensity of work. As the transition between school and work will most likely occur during this period in a young person's life, work intensity should be analysed separately between students and non-students. The latter are largely young people who recently finished their education and are available to work full time, while students have to divide their time between work and school. The participation levels between these two groups are therefore very different. Students show lower levels of employment and fewer hours worked, while non-students show higher employment rates and dedicate more hours to work.

\section{Working and studying at the same time: Finding a good balance}

The varying levels of employment among 15-29 year-old students can be explained by cultural, economic or social differences across countries. For instance, in some countries, students may wait until they finish their studies before looking for a job, while in other countries, young people may be more inclined to work during their studies to gain some experience on the labour market or to finance their studies (and/or other expenses). In the latter case, when work and education/learning are pursued simultaneously, it becomes important to consider how much time students are devoting to work to determine whether those working hours are becoming an obstacle to education. 
While there is no clear international recommendation for the appropriate number of hours a student should work, studies have shown that the skills and the work experience students gain in the labour market can be beneficial for both the academic and the professional spheres. The combination of work and study can provide students with the opportunity to try different jobs before fully entering the world of work. Employment can help students to gain financial independence from their parents, develop a sense of responsibility, enhance self-accomplishment and social integration, and develop knowledge and skills that will help them find work after their studies (Dundes and Marx, 2006; Murier, 2006; OECD, 2010).

Countries in which a large share of 15-29 year-olds are employed and study at the same time usually show small proportions of students working 35 hours or more per week. In the Czech Republic, Denmark, the Netherlands, Norway and Sweden, more than $25 \%$ of young adults study and work the same time, but less than $30 \%$ of them work 35 or more hours per week. The picture in Austria and Germany is different because of the prevalence of work-study programmes. In these two countries, about one in five young adults studied and worked at the same time in 2014, and about one in two of them worked 35 hours per week or more. Thus, in a number of countries, the large proportion of students working long hours is part of an arrangement between school and future employers. By contrast, in countries where less than $10 \%$ of young adults study and work simultaneously, about $50 \%$ of students work full time, on average (Table C5.4b).

Chart C5.3. Percentage of 15-29 not in education, by number of hours worked in a reference week (2014)

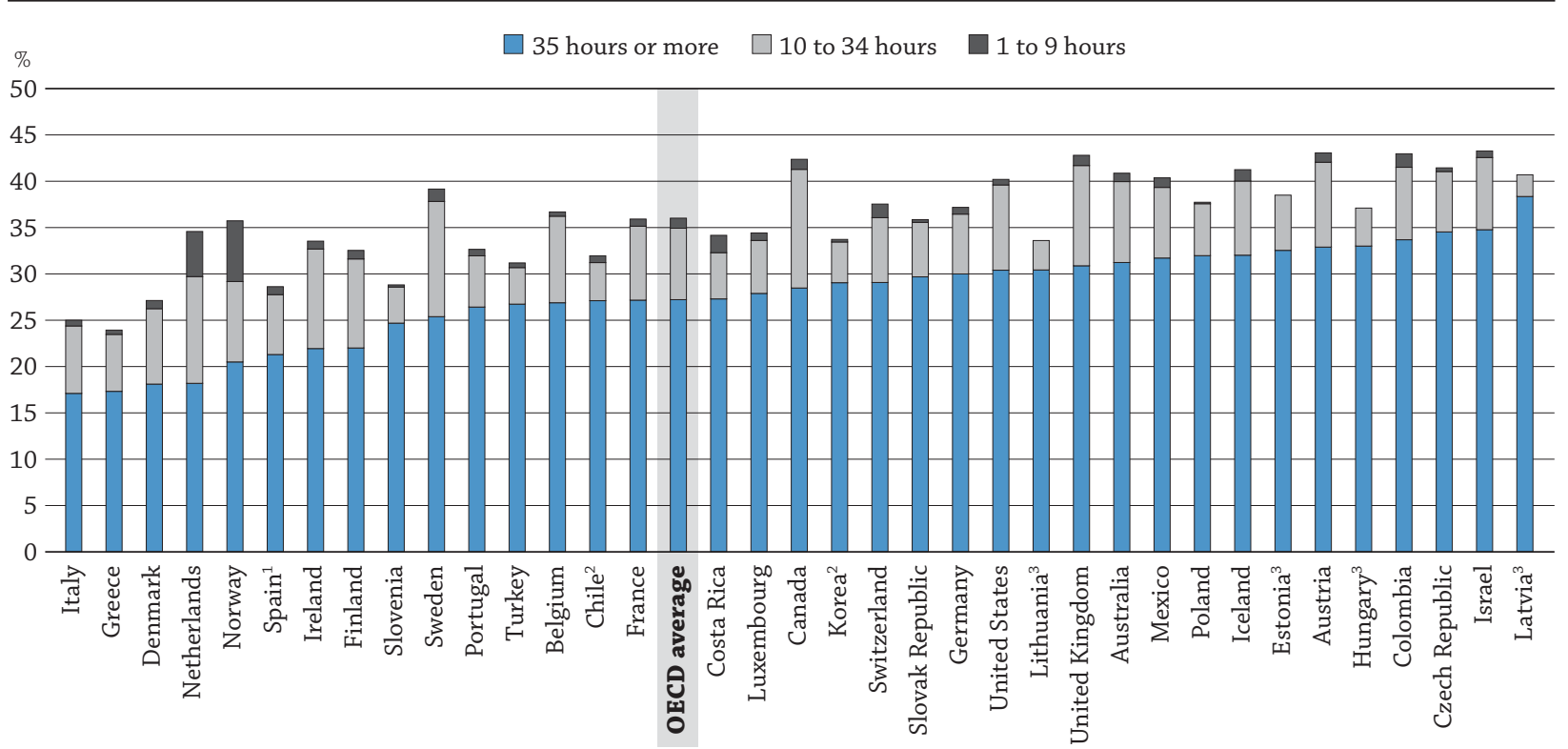

Note: Hours worked represent the actual number of hours worked per week, including overtime. When actual hours worked per week were equal to zero, the usual hours worked were used instead. When a country could not provide the actual number of hours worked per week, the usual hours worked were used.

1. Spain: Data refer to 16-29 year-olds.

2. Chile, Korea: Year of reference 2013

3. Estonia, Hungary, Latvia and Lithuania: Category " 1 to 9 hours" is not shown because there are too few observations to provide reliable estimates. Countries are ranked in ascending order of the percentage of 15-29 year-olds not in education and working 35 hours or more in a reference week.

Source: OECD. Tables C5.4b. See Annex 3 for notes (www.oecd.org/education/education-at-a-glance- 19991487.htm).

StatLink 쳎ㄴ $h$ ttp://dx.doi.org/10.1787/888933284314

\section{Seeking a full-time job when leaving school}

Across OECD countries, one in three 15-29 year-olds is no longer in education, but is employed. Chart C5.3 shows that most young people who are working take up a full-time job after finishing their studies. In some countries, such as the Netherlands and Norway, there are significant proportions of 15-29 year-olds who are not in education but are employed and working less than 35 hours per week ( $47 \%$ and $43 \%$, respectively). Young people who work part time without being in education do so for different reasons. Some may decide to work part time to accommodate family-related tasks, such as taking care of children or other family members; others may want to work full time but are unable to find a position (Table C5.4b). 
Chart C5.4 shows that across OECD countries, among all young people who are employed and no longer in education, men are more likely to find a full-time job than women. On average, $82 \%$ of young men work full time compared with $67 \%$ of young women. The largest gender gaps - more than 20 percentage points - are found in Belgium, Italy, Luxembourg and the Netherlands. For example, in the Netherlands, among all employed people who are no longer in education, $67 \%$ of men work full time compared to $37 \%$ of women. The smallest gender gap, less than 5 percentage points, is observed in the Czech Republic, Hungary, Korea, Latvia, Lithuania and the Slovak Republic (Table C5.4b).

\section{Chart C5.4. Percentage of 15-29 year-olds who work 35 hours or more per week among all employed young adults not in education, by gender (2014)}

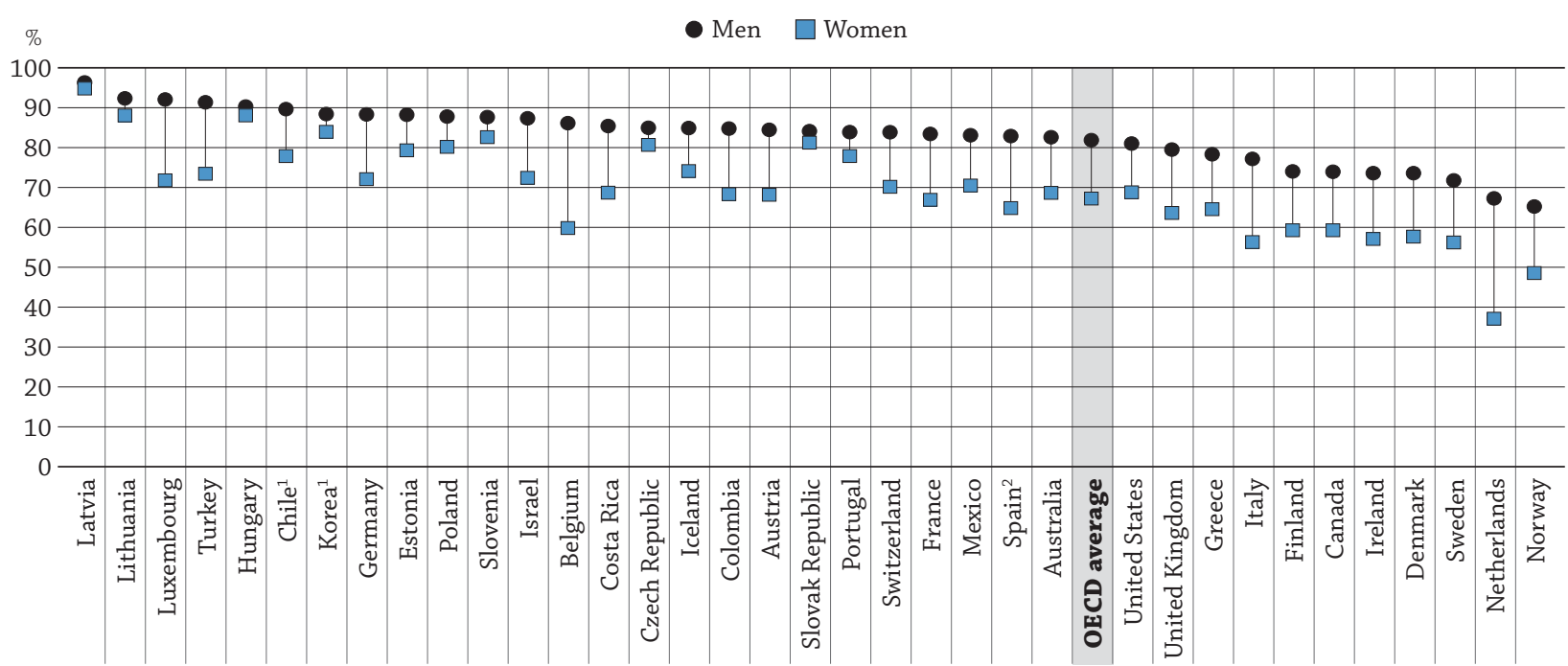

Note: Hours worked represent the actual number of hours worked per week, including overtime. When actual hours worked per week were equal to zero, the usual hours worked were used instead. When a country could not provide the actual number of hours worked per week, the usual hours worked were used.

1. Chile, Korea: Year of reference 2013.

2. Spain: Data refer to 16-29 year-olds.

Countries are ranked in descending order of the percentage of 15-29 year-old men not in education and working 35 hours or more in a reference week.

Source: OECD. Tables C5.4b. See Annex 3 for notes (www.oecd.org/education/education-at-a-glance- 19991487.htm).

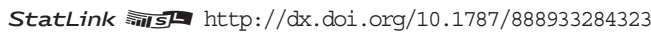

\section{Expected years in education}

In 2014, a typical 15-year-old in an OECD country could expect to spend about 7 additional years in formal education during the subsequent 15 years of his or her life. During these seven years in education, he/she could expect to hold a job for two years (including work-study programmes) and be unemployed or inactive for 5 years. Then, almost eight years will be spent not in education, during which he/she could expect to be employed for roughly five and a half years, to be unemployed for just over one year, and to be out of the labour force - that is, neither in education nor seeking work - for just over one year. There are large differences among countries: in Brazil, Colombia, Mexico and Turkey, a 15-year-old student could expect to spend an average of about five more years in education, while in Denmark and Slovenia, he or she could expect to spend an average of nine more years in education (Table C5.1a, available on line).

In most countries, years spent in education are normally not combined with work; the only exceptions are Denmark, Iceland, the Netherlands and Switzerland, where young people spend an average of four years or more working (including work-study programmes) while studying. On average across OECD countries, students spend nearly two out of seven years in education working while studying (Table C5.1a, available on line).

There are no large gender differences in expected years in education, but women tend to spend more time in education than men, except in Austria, Chile, Colombia, France, Germany, Israel, Japan, Luxembourg, the Netherlands, New Zealand, Norway, Portugal, Switzerland and the United Kingdom where the number of expected years in education for men and women are similar. In Korea and Turkey, the number of expected years in education is higher for men than for women. In some other countries, gender differences in employment among those who have left education are large. For example, in Mexico and Turkey, men work over three years more than women (Table C5.1a, available on line). 


\section{Definitions}

Employed individuals are those who, during the survey reference week: work for pay (employees) or profit (self-employed and unpaid family workers) for at least one hour; or have a job but are temporarily not at work (through injury, illness, holiday, strike or lock-out, educational or training leave, maternity or parental leave, etc.).

Hours worked are the number of hours actually worked, defined as the sum of all periods spent on direct and ancillary activities to produce goods and services. The number of hours actually worked covers all hours including extra hours regardless of whether they were paid or not. The reference period for the actual work would be the week of reference. Data on usual number of hours worked were used in the cases where the number of actual hours worked could not be collected.

Inactive individuals are those who are, during the survey's reference week, neither employed nor unemployed, i.e. individuals who are not looking for a job. The number of inactive individuals is calculated by subtracting the number of active people (labour force) from the number of all working-age people.

Levels of education: In this Indicator two ISCED (International Standard Classification of Education) classifications are used: ISCED 2011 and ISCED-97.

When it is specified that ISCED 2011 is used, the levels of education are defined as: below upper secondary corresponds to ISCED 2011 Levels 0, 1 and 2, and includes recognised qualifications from ISCED 2011 Level 3 programmes, which are not considered as sufficient for ISCED 2011 Level 3 completion, and without direct access to post-secondary non-tertiary education or tertiary education; upper secondary or post-secondary non-tertiary corresponds to ISCED 2011 Levels 3 and 4; and tertiary corresponds to ISCED 2011 Levels 5, 6, 7 and 8 (UNESCO Institute for Statistics, 2012).

When it is specified that ISCED-97 is used, the levels of education are defined as: below upper secondary corresponds to ISCED-97 Levels 0, 1, 2 and 3C short programmes; upper secondary or post-secondary non-tertiary corresponds to ISCED-97 Levels 3A, 3B, 3C long programmes, and Level 4; and tertiary corresponds to ISCED-97 Levels 5A, 5B and 6.

See in the section About the new ISCED 2011 classification at the beginning of this publication for a presentation of all ISCED 2011 levels and Annex 3 for a presentation of all ISCED-97 levels.

NEET: Neither in employment nor in education or training.

Unemployed individuals are those who are, during the survey reference week, without work (i.e. neither had a job nor were at work for one hour or more in paid employment or self-employment), actively seeking employment (i.e. had taken specific steps during the four weeks prior to the reference week to seek paid employment or self-employment), and currently available to start work (i.e. were available for paid employment or self-employment before the end of the two weeks following the reference week).

\section{Methodology}

Data on population, educational attainment and labour market status for most countries are taken from OECD and Eurostat databases, which are compiled from National Labour Force Surveys by the OECD LSO (Labour Market, Economic and Social Outcomes of Learning) Network, and usually refer to the first quarter, or the average of the first three months of the calendar year. Some discrepancies may exist in the data collected. For example some countries may refer to all jobs instead of main job. See Annex 3 (www.oecd.org/education/education-at-a-glance-19991487. $\mathrm{htm}$ ) for additional information.

For Israel, the proportion of NEETs in 2013 is not comparable with data from 2011 and previous years. Conscripts into the army are considered to be employed in 2013, as opposed to 2011 and the previous year, when they were counted as not in the labour force.

Note regarding data from Israel

The statistical data for Israel are supplied by and are under the responsibility of the relevant Israeli authorities. The use of such data by the OECD is without prejudice to the status of the Golan Heights, East Jerusalem and Israeli settlements in the West Bank under the terms of international law. 


\section{References}

Dundes, L. and J. Marx (2006), "Balancing Work and Academics in College: Why Do Students Working 10 to 19 Hours per Week Excel?”, Journal of College Student Retention: Research, Theory and Practice, Vol. 8, No. 1.

Murier, T. (2006), “L’activité professionnelle des élèves et des étudiants : Une étude basée sur les résultats de l’enquête suisse sur la population active 1996-2005”, Actualité OFS, No. 3, Neuchâtel.

OECD (2010), "How good is part-time work?", in OECD Employment Outlook 2010: Moving beyond the Jobs Crisis, OECD Publishing, Paris, http://dx.doi.org/10.1787/empl_outlook-2010-5-en.

UNESCO Institute for Statistics (2012), International Standard Classification of Education: ISCED 2011, UNESCO-UIS, Montreal, Canada, www.uis.unesco.org/Education/Documents/isced-2011-en.pdf.

\section{Indicator C5 Tables}

StatLink 젶ㄴ http://dx.doi.org/10.1787/888933285971

WEB Table C5.1a Expected years in education/not in education from age 15 through 29, by work status and gender (2014)

WEB Table C5.1b Trends in expected years in education/not in education from age 15 through 29, by gender $(2000,2005,2010$ and 2014)

Table C5.2a Percentage of 15-29 year-olds in education/not in education, by work status, age group and gender (2014)

WEB Table C5.2b Trends in the percentage of 15-29 year-olds in education/not in education, employed or not, by age group and gender (2000, 2005, 2010 and 2014)

Table C5.3a Percentage of 15-29 year-olds in education/not in education, by educational attainment, work status and gender (2014)

WEB Table C5.3b Trends in the percentage of 15-29 year-olds in education/not in education, employed or not, by educational attainment and gender (2006, 2010 and 2014)

Table C5.4a Percentage of 15-29 year-olds in education/not in education, by number of hours worked and age group (2014)

Table C5.4b Percentage of 15-29 year-olds in education/not in education, by number of hours worked and gender (2014) 
Table C5.2a. [1/2] Percentage of 15-29 year-olds in education/not in education, by work status, age group and gender (2014)

\begin{tabular}{|c|c|c|c|c|c|c|c|c|c|c|c|c|}
\hline & \multicolumn{12}{|c|}{ 20-24 year-olds } \\
\hline & \multicolumn{12}{|c|}{ Young men } \\
\hline & \multicolumn{6}{|c|}{ In education } & \multicolumn{5}{|c|}{ Not in education } & \multirow[b]{3}{*}{ 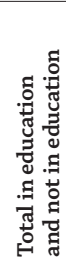 } \\
\hline & \multicolumn{3}{|c|}{ Employed } & \multirow[b]{2}{*}{ 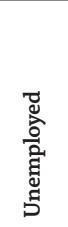 } & \multirow[b]{2}{*}{ 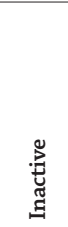 } & \multirow[b]{2}{*}{ 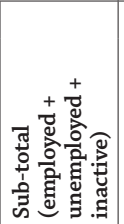 } & \multirow[b]{2}{*}{ 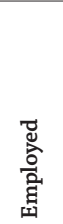 } & \multirow[b]{2}{*}{$\begin{array}{l}\text { 島 } \\
\text { 至 }\end{array}$} & \multicolumn{2}{|c|}{ NEET } & \multirow[b]{2}{*}{ 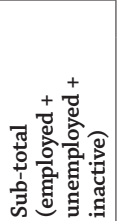 } & \\
\hline & 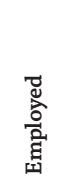 & 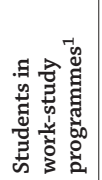 & 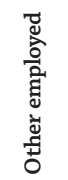 & & & & & & 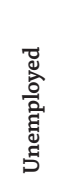 & 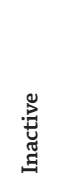 & & \\
\hline & (85) & (86) & $(87)$ & (88) & (89) & (90) & (91) & (92) & (93) & (94) & (95) & $(96)$ \\
\hline Australia & 24.2 & 7.2 & 17.0 & 2.8 & 15.0 & 41.9 & 46.4 & 11.7 & 6.2 & 5.4 & 58.1 & 100 \\
\hline Austria & 15.9 & 3.5 & 12.4 & 1.8 & 19.3 & 37.0 & 50.4 & 12.5 & 7.1 & 5.4 & 63.0 & 100 \\
\hline Belgium & 3.8 & c & 2.6 & c & 39.4 & 43.9 & 37.3 & 18.8 & 12.9 & 5.9 & 56.1 & 100 \\
\hline Canada & 15.7 & $\mathrm{x}(87)$ & 15.7 & 1.5 & 19.4 & 36.6 & 47.9 & 15.5 & 8.6 & 6.8 & 63.4 & 100 \\
\hline Chile $^{2}$ & 10.7 & $\mathrm{x}(87)$ & 10.7 & 2.2 & 29.2 & 42.1 & 42.6 & 15.4 & 6.7 & 8.6 & 57.9 & 100 \\
\hline Czech Republic & 10.3 & 6.7 & 3.6 & c & 31.8 & 42.6 & 48.3 & 9.1 & 6.9 & 2.2 & 57.4 & 100 \\
\hline Denmark & 31.8 & $\mathrm{x}(87)$ & 31.8 & 4.9 & 21.3 & 58.0 & 29.2 & 12.8 & 5.6 & 7.2 & 42.0 & 100 \\
\hline Estonia & 12.6 & $\mathrm{x}(87)$ & 12.6 & c & 26.6 & 40.8 & 43.3 & 16.0 & 9.0 & 7.0 & 59.2 & 100 \\
\hline Finland & 14.2 & $\mathrm{x}(87)$ & 14.2 & 4.9 & 25.5 & 44.6 & 37.8 & 17.5 & 10.5 & 7.0 & 55.3 & 100 \\
\hline France & 12.4 & $\mathrm{x}(87)$ & 12.4 & 1.0 & 31.1 & 44.5 & 37.5 & 18.0 & 13.5 & 4.5 & 55.5 & 100 \\
\hline Germany & 26.6 & 15.3 & 11.4 & 0.9 & 25.7 & 53.2 & 37.8 & 9.0 & 5.4 & 3.6 & 46.8 & 100 \\
\hline Greece & 2.6 & a & 2.6 & 2.4 & 41.1 & 46.1 & 23.4 & 30.5 & 24.1 & 6.3 & 53.9 & 100 \\
\hline Hungary & 2.2 & a & 2.2 & c & 37.1 & 39.8 & 43.1 & 17.2 & 9.1 & 8.1 & 60.2 & 100 \\
\hline Iceland & 30.9 & a & 30.9 & c & 13.3 & 47.2 & 42.7 & 10.1 & 5.5 & 4.6 & 52.8 & 100 \\
\hline Ireland & 12.5 & a & 12.5 & $2.0^{x}$ & 31.5 & 46.0 & 33.2 & 20.5 & 15.4 & 5.1 & 53.7 & 100 \\
\hline Israel & 8.4 & $\mathrm{x}(87)$ & 8.4 & 1.2 & 15.8 & 25.4 & 59.4 & 15.2 & 5.7 & 9.5 & 74.6 & 100 \\
\hline Italy & 1.7 & a & 1.7 & 1.3 & 34.0 & 37.0 & 28.0 & 34.6 & 20.0 & 14.6 & 62.5 & 100 \\
\hline Japan & $\mathrm{m}$ & $\mathrm{m}$ & $\mathrm{m}$ & $\mathrm{m}$ & $\mathrm{m}$ & $\mathrm{m}$ & $\mathrm{m}$ & $\mathrm{m}$ & $\mathrm{m}$ & $\mathrm{m}$ & $\mathrm{m}$ & $\mathrm{m}$ \\
\hline Korea $^{2}$ & 9.4 & a & 9.4 & 0.7 & 38.5 & 48.6 & 28.7 & 22.7 & 3.3 & 19.3 & 51.4 & 100 \\
\hline Luxembourg & $3.7^{x}$ & a & $3.7^{\mathrm{r}}$ & c & 53.3 & 57.7 & 30.6 & 11.8 & 11.0 & c & 42.3 & 100 \\
\hline Mexico & 9.2 & a & 9.2 & 0.8 & 18.1 & 28.1 & 61.6 & 10.3 & 6.1 & 4.2 & 71.9 & 100 \\
\hline Netherlands & 34.9 & $\mathrm{x}(87)$ & 34.9 & 3.6 & 18.4 & 56.8 & 33.6 & 9.6 & 3.9 & 5.6 & 43.2 & 100 \\
\hline New Zealand & 20.2 & $\mathrm{a}$ & 20.2 & 1.4 & 19.6 & 41.2 & 48.8 & 9.8 & 5.5 & 4.2 & 58.5 & 100 \\
\hline Norway & 30.5 & 19.5 & 10.9 & 1.6 & 19.3 & 51.4 & 39.1 & 9.5 & 3.3 & 6.2 & 48.6 & 100 \\
\hline Poland & 9.4 & a & 9.4 & 1.7 & 29.0 & 40.1 & 41.0 & 18.9 & 12.1 & 6.7 & 59.9 & 100 \\
\hline Portugal & 4.3 & a & 4.3 & 3.1 & 34.8 & 42.2 & 34.1 & 23.7 & 17.4 & 6.3 & 57.8 & 100 \\
\hline Slovak Republic & 1.9 & c & c & c & 37.1 & 39.8 & 42.3 & 17.9 & 16.7 & $1.2^{\mathrm{r}}$ & 60.2 & 100 \\
\hline Slovenia & 13.5 & $\mathrm{x}(87)$ & 13.5 & $3.2^{r}$ & 43.8 & 60.5 & 25.3 & 14.2 & 9.3 & $4.8^{\mathrm{r}}$ & 39.5 & 100 \\
\hline Spain & 4.8 & $\mathrm{x}(87)$ & 4.8 & 8.0 & 34.2 & 46.9 & 22.9 & 30.2 & 24.1 & 6.1 & 53.1 & 100 \\
\hline Sweden & 9.9 & a & 9.9 & 7.4 & 23.7 & 41.0 & 45.8 & 13.1 & 8.4 & 4.7 & 59.0 & 100 \\
\hline Switzerland & 24.1 & 10.2 & 13.9 & c & 16.7 & 42.0 & 43.1 & 14.6 & 8.6 & 6.0 & 57.7 & 100 \\
\hline Turkey & 17.1 & a & 17.1 & 2.9 & 16.8 & 36.8 & 42.7 & 20.5 & 9.5 & 11.1 & 63.2 & 100 \\
\hline United Kingdom & 14.1 & 3.8 & 10.3 & 2.0 & 18.5 & 34.6 & 50.5 & 14.9 & 10.6 & 4.2 & 65.4 & 100 \\
\hline United States & 16.7 & $\mathrm{x}(87)$ & 16.7 & 1.1 & 18.8 & 36.6 & 48.7 & 14.7 & 6.2 & 8.4 & 63.4 & 100 \\
\hline OECD average (excluding Japan) & 13.9 & $\mathrm{~m}$ & 12.2 & 2.6 & 27.2 & 43.4 & 40.2 & 16.3 & 10.0 & 6.6 & 56.5 & 100 \\
\hline EU21 average & 11.6 & $\mathrm{~m}$ & 10.5 & 3.2 & 31.3 & 45.4 & 36.9 & 17.6 & 12.0 & 5.8 & 54.6 & 100 \\
\hline
\end{tabular}

\begin{tabular}{|c|c|c|c|c|c|c|c|c|c|c|c|c|}
\hline Argentina & $\mathrm{m}$ & $\mathrm{m}$ & $\mathrm{m}$ & $\mathrm{m}$ & $\mathrm{m}$ & $\mathrm{m}$ & $\mathrm{m}$ & $\mathrm{m}$ & $\mathrm{m}$ & $\mathrm{m}$ & $\mathrm{m}$ & $\mathrm{m}$ \\
\hline Erazil $^{2}$ & 13.6 & a & 13.6 & 1.7 & 6.2 & 21.6 & 63.2 & 15.2 & 6.3 & 9.0 & 78.4 & 100 \\
\hline China & $\mathrm{m}$ & $\mathrm{m}$ & $\mathrm{m}$ & $\mathrm{m}$ & $\mathrm{m}$ & $\mathrm{m}$ & $\mathrm{m}$ & $\mathrm{m}$ & $\mathrm{m}$ & $\mathrm{m}$ & $\mathrm{m}$ & $\mathrm{m}$ \\
\hline Colombia & 12.1 & a & 12.1 & 2.7 & 10.0 & 24.7 & 62.4 & 12.9 & 8.6 & 4.3 & 75.3 & 100 \\
\hline Costa Rica & 21.8 & a & 21.8 & 6.1 & 11.1 & 39.0 & 49.1 & 11.9 & 9.2 & 2.7 & 61.0 & 100 \\
\hline India & $\mathrm{m}$ & $\mathrm{m}$ & $\mathrm{m}$ & $\mathrm{m}$ & $\mathrm{m}$ & $\mathrm{m}$ & $\mathrm{m}$ & $\mathrm{m}$ & $\mathrm{m}$ & $\mathrm{m}$ & $\mathrm{m}$ & $\mathrm{m}$ \\
\hline Indonesia & $\mathrm{m}$ & $\mathrm{m}$ & $\mathrm{m}$ & $\mathrm{m}$ & $\mathrm{m}$ & $\mathrm{m}$ & $\mathrm{m}$ & $\mathrm{m}$ & $\mathrm{m}$ & $\mathrm{m}$ & $\mathrm{m}$ & $\mathrm{m}$ \\
\hline Latvia & 17.0 & $\mathrm{a}$ & 17.0 & $3.0^{x}$ & 22.4 & 42.4 & 40.8 & 16.9 & 10.7 & 6.1 & 57.6 & 100 \\
\hline Lithuania & 12.1 & a & c & c & 32.0 & 46.0 & 39.2 & 14.8 & 10.1 & c & 54.0 & 100 \\
\hline Russian Federation & $\mathrm{m}$ & $\mathrm{m}$ & $\mathrm{m}$ & $\mathrm{m}$ & $\mathrm{m}$ & $\mathrm{m}$ & $\mathrm{m}$ & $\mathrm{m}$ & $\mathrm{m}$ & $\mathrm{m}$ & $\mathrm{m}$ & $\mathrm{m}$ \\
\hline Saudi Arabia & $\mathrm{m}$ & $\mathrm{m}$ & $\mathrm{m}$ & $\mathrm{m}$ & $\mathrm{m}$ & $\mathrm{m}$ & $\mathrm{m}$ & $\mathrm{m}$ & $\mathrm{m}$ & $\mathrm{m}$ & $\mathrm{m}$ & $\mathrm{m}$ \\
\hline South Africa & $\mathrm{m}$ & $\mathrm{m}$ & $\mathrm{m}$ & $\mathrm{m}$ & $\mathrm{m}$ & $\mathrm{m}$ & $\mathrm{m}$ & $\mathrm{m}$ & $\mathrm{m}$ & $\mathrm{m}$ & $\mathrm{m}$ & $\mathrm{m}$ \\
\hline G20 average & $\mathrm{m}$ & $\mathrm{m}$ & $\mathrm{m}$ & $\mathrm{m}$ & $\mathrm{m}$ & $\mathrm{m}$ & $\mathrm{m}$ & $\mathrm{m}$ & $\mathrm{m}$ & $\mathrm{m}$ & $\mathrm{m}$ & $\mathrm{m}$ \\
\hline
\end{tabular}

Notes: NEET refer to young people neither in employment nor in education or training. Columns showing data for total population and/or other age groups are available for consultation on line (see StatLink below).

1. Students in work-study programmes are considered to be both in education and employed, irrespective of their labour market status according to the ILO definition. 2. Brazil, Chile, Korea: Data for 2014 refer to year 2013.

Source: OECD. Latvia, Lithuania: Eurostat. See Annex 3 for notes (www.oecd.org/education/education-at-a-glance-19991487.htm).

Please refer to the Reader's Guide for information concerning symbols for missing data and abbreviations.

StatLink 젶ㄴ http://dx.doi.org/10.1787/888933285989 
Table C5.2a. [2/2] Percentage of 15-29 year-olds in education/not in education, by work status, age group and gender (2014)

\begin{tabular}{|c|c|c|c|c|c|c|c|c|c|c|c|c|}
\hline & \multicolumn{12}{|c|}{$20-24$ year-olds } \\
\hline & \multicolumn{12}{|c|}{ Young women } \\
\hline & \multicolumn{6}{|c|}{ In education } & \multicolumn{5}{|c|}{ Not in education } & \multirow{3}{*}{ 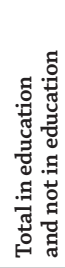 } \\
\hline & & \multicolumn{2}{|c|}{ Employed } & \multirow[b]{2}{*}{ 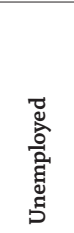 } & \multirow[b]{2}{*}{ 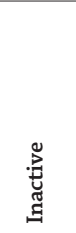 } & \multirow[b]{2}{*}{ 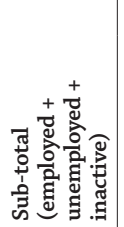 } & \multirow[b]{2}{*}{ 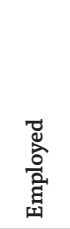 } & \multirow[b]{2}{*}{$\begin{array}{l}\text { 䛼 } \\
\text { 穵 }\end{array}$} & \multicolumn{2}{|c|}{ NEET } & \multirow[b]{2}{*}{ 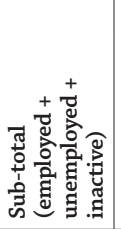 } & \\
\hline & 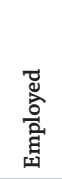 & 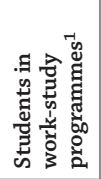 & 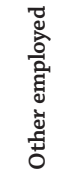 & & & & & & 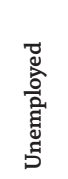 & 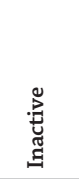 & & \\
\hline & (97) & (98) & $(99)$ & (100) & (101) & (102) & (103) & (104) & (105) & (106) & (107) & (108) \\
\hline Australia & 27.5 & $0.5 \mathrm{r}$ & 27.0 & 2.9 & 13.6 & 43.9 & 41.2 & 14.9 & 4.1 & 10.7 & 56.1 & 100 \\
\hline Austria & 16.8 & 2.9 & 13.9 & 2.5 & 21.9 & 41.3 & 47.3 & 11.5 & 4.8 & 6.7 & 58.7 & 100 \\
\hline Belgium & 4.9 & c & 3.8 & 1.5 & 44.4 & 50.8 & 30.4 & 18.9 & 9.2 & 9.6 & 49.2 & 100 \\
\hline Canada & 24.4 & $\mathrm{x}(99)$ & 24.4 & 1.9 & 19.4 & 45.7 & 40.3 & 14.0 & 4.9 & 9.1 & 54.3 & 100 \\
\hline Chile $^{2}$ & 9.2 & $x(99)$ & 9.2 & 3.4 & 33.3 & 46.0 & 27.4 & 26.7 & 5.4 & 21.2 & 54.0 & 100 \\
\hline Czech Republic & 10.1 & 4.9 & 5.2 & c & 44.6 & 55.3 & 29.2 & 15.5 & 5.4 & 10.1 & 44.7 & 100 \\
\hline Denmark & 35.0 & $x(99)$ & 35.0 & 4.0 & 25.3 & 64.3 & 23.1 & 12.7 & 4.5 & 8.2 & 35.7 & 100 \\
\hline Estonia & 20.0 & $x(99)$ & 20.0 & c & 31.0 & 52.2 & 31.5 & 16.3 & 3.9 & 12.4 & 47.8 & 100 \\
\hline Finland & 25.6 & $x(99)$ & 25.6 & 4.9 & 27.1 & 57.6 & 28.4 & 13.6 & 4.1 & 9.5 & 42.0 & 100 \\
\hline France & 10.3 & $\mathrm{x}(99)$ & 10.3 & 1.2 & 36.7 & 48.2 & 33.1 & 18.7 & 10.1 & 8.6 & 51.8 & 100 \\
\hline Germany & 28.5 & 13.3 & 15.2 & 0.7 & 25.9 & 55.0 & 33.6 & 11.4 & 4.0 & 7.3 & 45.0 & 100 \\
\hline Greece & 2.4 & $\mathrm{a}$ & 2.4 & 2.6 & 47.2 & 52.2 & 15.7 & 32.1 & 23.8 & 8.2 & 47.8 & 100 \\
\hline Hungary & 2.6 & a & 2.6 & c & 43.0 & 46.2 & 29.7 & 24.1 & 8.6 & 15.5 & 53.8 & 100 \\
\hline Iceland & 39.4 & a & 39.4 & c & 11.8 & 53.0 & 38.4 & 8.7 & c & 6.6 & 47.0 & 100 \\
\hline Ireland & 12.0 & a & 12.0 & $1.6^{r}$ & 31.0 & 44.5 & 33.5 & 21.7 & 9.7 & 12.0 & 55.2 & 100 \\
\hline Israel & 16.6 & $\mathrm{x}(99)$ & 16.6 & 1.4 & 15.6 & 33.6 & 44.5 & 21.9 & 5.4 & 16.6 & 66.4 & 100 \\
\hline Italy & 2.5 & a & 2.5 & 1.4 & 42.0 & 45.9 & 18.7 & 35.1 & 16.3 & 18.8 & 53.7 & 100 \\
\hline Japan & $\mathrm{m}$ & $\mathrm{m}$ & $\mathrm{m}$ & $\mathrm{m}$ & $\mathrm{m}$ & $\mathrm{m}$ & $\mathrm{m}$ & $\mathrm{m}$ & $\mathrm{m}$ & $\mathrm{m}$ & $\mathrm{m}$ & $\mathrm{m}$ \\
\hline Korea $^{2}$ & 10.1 & $\mathrm{a}$ & 10.1 & 0.8 & 29.9 & 40.8 & 37.5 & 21.7 & 3.9 & 17.8 & 59.2 & 100 \\
\hline Luxembourg & 11.4 & $\mathrm{a}$ & 11.4 & $3.9^{r}$ & 43.7 & 59.0 & 34.7 & $6.3^{\mathrm{r}}$ & c & $4.1^{r}$ & 41.0 & 100 \\
\hline Mexico & 6.6 & a & 6.6 & 0.9 & 19.4 & 26.8 & 33.8 & 39.4 & 3.8 & 35.7 & 73.2 & 100 \\
\hline Netherlands & 33.8 & $\mathrm{x}(99)$ & 33.8 & 4.1 & 18.5 & 56.4 & 32.5 & 11.2 & 4.2 & 7.0 & 43.6 & 100 \\
\hline New Zealand & 20.1 & a & 20.1 & 2.9 & 17.2 & 40.1 & 40.6 & 19.2 & 6.0 & 13.2 & 59.8 & 100 \\
\hline Norway & 31.1 & 8.2 & 22.9 & 1.7 & 21.0 & 53.7 & 35.8 & 10.4 & 1.8 & 8.6 & 46.3 & 100 \\
\hline Poland & 12.9 & a & 12.9 & 3.0 & 41.1 & 57.0 & 23.4 & 19.6 & 8.8 & 10.8 & 43.0 & 100 \\
\hline Portugal & 4.6 & a & 4.6 & 4.2 & 35.3 & 44.2 & 31.8 & 24.1 & 16.9 & 7.2 & 55.8 & 100 \\
\hline Slovak Republic & 3.1 & c & c & c & 53.5 & 57.4 & 23.4 & 19.2 & 10.3 & 8.9 & 42.6 & 100 \\
\hline Slovenia & 22.5 & $\mathrm{x}(99)$ & 22.5 & $2.7^{x}$ & 52.7 & 77.9 & 9.5 & 12.6 & $7.3^{\mathrm{r}}$ & $5.3^{\mathrm{r}}$ & 22.1 & 100 \\
\hline Spain & 7.4 & $\mathrm{x}(99)$ & 7.4 & 8.7 & 37.0 & 53.1 & 19.0 & 27.9 & 19.9 & 7.9 & 46.9 & 100 \\
\hline Sweden & 16.6 & a & 16.6 & 7.1 & 27.8 & 51.6 & 37.6 & 10.8 & 5.2 & 5.6 & 48.4 & 100 \\
\hline Switzerland & 27.5 & 7.4 & 20.0 & 2.7 & 17.7 & 47.8 & 42.1 & 10.1 & 5.0 & 5.1 & 52.2 & 100 \\
\hline Turkey & 7.0 & a & 7.0 & 2.1 & 17.5 & 26.6 & 22.4 & 51.0 & 5.1 & 45.9 & 73.4 & 100 \\
\hline United Kingdom & 15.1 & 1.9 & 13.2 & 1.6 & 17.8 & 34.5 & 46.3 & 19.2 & 6.5 & 12.7 & 65.5 & 100 \\
\hline United States & 21.7 & $x(99)$ & 21.7 & 1.2 & 16.6 & 39.4 & 40.2 & 20.3 & 6.2 & 14.2 & 60.6 & 100 \\
\hline OECD average (excluding Japan) & 16.3 & $\mathrm{~m}$ & 15.5 & 2.8 & 29.7 & 48.5 & 32.0 & 19.4 & 7.6 & 12.2 & 51.4 & 100 \\
\hline EU21 average & 14.2 & $\mathrm{~m}$ & 13.5 & 3.3 & 35.6 & 52.6 & 29.2 & 18.2 & 9.2 & 9.4 & 47.4 & 100 \\
\hline Argentina & $\mathrm{m}$ & $\mathrm{m}$ & $\mathrm{m}$ & $\mathrm{m}$ & $\mathrm{m}$ & $\mathrm{m}$ & $\mathrm{m}$ & $\mathrm{m}$ & $\mathrm{m}$ & $\mathrm{m}$ & $\mathrm{m}$ & $\mathrm{m}$ \\
\hline Brazil $^{2}$ & 14.0 & a & $\mathrm{m}$ & 2.2 & 9.8 & 26.0 & 41.4 & 32.6 & 7.7 & 24.9 & 74.0 & 100 \\
\hline China & $\mathrm{m}$ & $\mathrm{m}$ & $\mathrm{m}$ & $\mathrm{m}$ & $\mathrm{m}$ & $\mathrm{m}$ & $\mathrm{m}$ & $\mathrm{m}$ & $\mathrm{m}$ & $\mathrm{m}$ & $\mathrm{m}$ & $\mathrm{m}$ \\
\hline Colombia & 11.8 & $\mathrm{a}$ & $\mathrm{m}$ & 3.2 & 12.0 & 27.1 & 36.8 & 36.1 & 10.9 & 25.2 & 72.9 & 100 \\
\hline Costa Rica & 16.7 & a & $\mathrm{m}$ & 8.4 & 19.9 & 45.0 & 22.3 & 32.7 & 10.1 & 22.6 & 55.0 & 100 \\
\hline India & $\mathrm{m}$ & $\mathrm{m}$ & $\mathrm{m}$ & $\mathrm{m}$ & $\mathrm{m}$ & $\mathrm{m}$ & $\mathrm{m}$ & $\mathrm{m}$ & $\mathrm{m}$ & $\mathrm{m}$ & $\mathrm{m}$ & $\mathrm{m}$ \\
\hline Indonesia & $\mathrm{m}$ & $\mathrm{m}$ & $\mathrm{m}$ & $\mathrm{m}$ & $\mathrm{m}$ & $\mathrm{m}$ & $\mathrm{m}$ & $\mathrm{m}$ & $\mathrm{m}$ & $\mathrm{m}$ & $\mathrm{m}$ & $\mathrm{m}$ \\
\hline Latvia & 14.6 & a & $\mathrm{m}$ & c & 36.7 & 52.5 & 31.2 & 16.3 & 7.0 & 9.3 & 47.5 & 100 \\
\hline Lithuania & 13.3 & $\mathrm{a}$ & c & c & 40.1 & 55.7 & 26.2 & 18.1 & 8.9 & 9.2 & 44.3 & 100 \\
\hline Russian Federation & $\mathrm{m}$ & $\mathrm{m}$ & $\mathrm{m}$ & $\mathrm{m}$ & $\mathrm{m}$ & $\mathrm{m}$ & $\mathrm{m}$ & $\mathrm{m}$ & $\mathrm{m}$ & $\mathrm{m}$ & $\mathrm{m}$ & $\mathrm{m}$ \\
\hline Saudi Arabia & $\mathrm{m}$ & $\mathrm{m}$ & $\mathrm{m}$ & $\mathrm{m}$ & $\mathrm{m}$ & $\mathrm{m}$ & $\mathrm{m}$ & $\mathrm{m}$ & $\mathrm{m}$ & $\mathrm{m}$ & $\mathrm{m}$ & $\mathrm{m}$ \\
\hline South Africa & $\mathrm{m}$ & $\mathrm{m}$ & $\mathrm{m}$ & $\mathrm{m}$ & $\mathrm{m}$ & $\mathrm{m}$ & $\mathrm{m}$ & $\mathrm{m}$ & $\mathrm{m}$ & $\mathrm{m}$ & $\mathrm{m}$ & $\mathrm{m}$ \\
\hline G20 average & $\mathrm{m}$ & $\mathrm{m}$ & $\mathrm{m}$ & $\mathrm{m}$ & $\mathrm{m}$ & $\mathrm{m}$ & $\mathrm{m}$ & $\mathrm{m}$ & $\mathrm{m}$ & $\mathrm{m}$ & $\mathrm{m}$ & $\mathrm{m}$ \\
\hline
\end{tabular}

Notes: NEET refer to young people neither in employment nor in education or training. Columns showing data for total population and/or other age groups are available for consultation on line (see StatLink below).

1. Students in work-study programmes are considered to be both in education and employed, irrespective of their labour market status according to the ILO definition. 2. Brazil, Chile, Korea: Data for 2014 refer to year 2013.

Source: OECD. Latvia, Lithuania: Eurostat. See Annex 3 for notes (www.oecd.org/education/education-at-a-glance-19991487.htm).

Please refer to the Reader's Guide for information concerning symbols for missing data and abbreviations.

StatLink त्तारा http://dx.doi.org/10.1787/888933285989 
Table C5.3a. Percentage of 15-29 year-olds in education/not in education, by educational attainment, work status and gender (2014)

\begin{tabular}{|c|c|c|c|c|c|c|c|c|c|c|c|c|}
\hline & \multicolumn{12}{|c|}{ Tertiary } \\
\hline & \multicolumn{12}{|c|}{ Total (young men + young women) } \\
\hline & \multicolumn{6}{|c|}{ In education } & \multicolumn{5}{|c|}{ Not in education } & \multirow{3}{*}{ 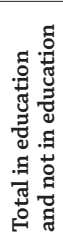 } \\
\hline & & \multicolumn{2}{|c|}{ Employed } & \multirow[b]{2}{*}{ 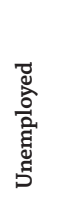 } & \multirow[b]{2}{*}{ 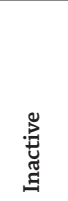 } & \multirow[b]{2}{*}{ 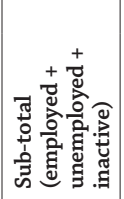 } & \multirow[b]{2}{*}{ 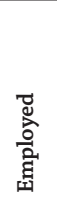 } & \multirow[b]{2}{*}{$\begin{array}{l}\text { 旨 } \\
\text { 昰 }\end{array}$} & \multicolumn{2}{|c|}{ NEET } & \multirow[b]{2}{*}{ 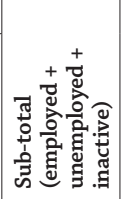 } & \\
\hline & 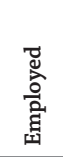 & 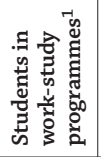 & 离 & & & & & & 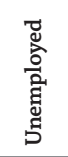 & 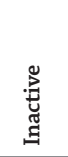 & & \\
\hline & (73) & (74) & (75) & (76) & (77) & (78) & (79) & (80) & $(81)$ & $(82)$ & (83) & (84) \\
\hline Australia & 15.2 & c & 15.0 & 1.4 & 8.7 & 25.3 & 66.1 & 8.6 & 2.5 & 6.1 & 74.7 & 100 \\
\hline Austria & 20.8 & c & 20.5 & 2.4 & 15.0 & 38.2 & 55.9 & 5.9 & 3.1 & 2.9 & 61.8 & 100 \\
\hline Belgium & 5.5 & c & 5.2 & c & 16.0 & 22.2 & 68.3 & 9.5 & 6.0 & 3.5 & 77.8 & 100 \\
\hline Canada & 13.4 & $\mathrm{x}(75)$ & 13.4 & 1.0 & 9.8 & 24.2 & 65.0 & 10.8 & 5.2 & 5.6 & 75.8 & 100 \\
\hline Chile $^{2}$ & 5.8 & $\mathrm{a}$ & 5.8 & 1.0 & 7.0 & 13.8 & 68.2 & 18.0 & 8.6 & 9.4 & 86.2 & 100 \\
\hline Czech Republic & 11.0 & $\mathrm{~m}$ & 10.9 & c & 20.0 & 31.8 & 56.4 & 11.8 & 4.4 & 7.4 & 68.2 & 100 \\
\hline Denmark & 27.4 & $x(75)$ & 27.4 & $2.4^{\mathrm{r}}$ & 9.6 & 39.4 & 49.3 & 11.3 & 7.6 & 3.6 & 60.6 & 100 \\
\hline Estonia & 16.6 & $\mathrm{x}(75)$ & 16.6 & c & 8.2 & 26.3 & 60.0 & 13.7 & 4.8 & 8.9 & 73.7 & 100 \\
\hline Finland & 17.7 & $\mathrm{x}(75)$ & 17.7 & $1.8^{\mathrm{r}}$ & 6.9 & 26.4 & 60.4 & 13.2 & 5.6 & 7.5 & 73.6 & 100 \\
\hline France & 10.6 & $x(75)$ & 10.6 & 0.5 & 16.2 & 27.4 & 60.9 & 11.7 & 8.1 & 3.6 & 72.6 & 100 \\
\hline Germany & 14.5 & 1.1 & 13.3 & 1.3 & 9.7 & 25.5 & 68.9 & 5.6 & 2.3 & 3.2 & 74.5 & 100 \\
\hline Greece & 2.2 & $\mathrm{a}$ & 2.2 & $1.5^{\mathrm{r}}$ & 5.4 & 9.1 & 50.6 & 40.3 & 35.8 & 4.5 & 90.9 & 100 \\
\hline Hungary & 3.6 & a & 3.6 & c & 9.8 & 13.7 & 71.8 & 14.5 & 5.7 & 8.8 & 86.3 & 100 \\
\hline Iceland & 14.8 & a & 14.8 & c & c & 21.6 & 73.1 & c & c & c & 78.4 & 100 \\
\hline Ireland & 7.7 & a & 7.7 & $1.2^{\mathrm{r}}$ & 11.7 & 20.7 & 67.4 & 11.9 & 7.4 & 4.5 & 79.3 & 100 \\
\hline Israel & 15.2 & $x(75)$ & 15.2 & 0.7 & 4.2 & 20.0 & 67.6 & 12.3 & 5.3 & 7.1 & 80.0 & 100 \\
\hline Italy & 4.5 & a & 4.5 & 1.7 & 29.4 & 35.5 & 34.5 & 29.2 & 14.0 & 15.2 & 63.7 & 100 \\
\hline Japan & $\mathrm{m}$ & $\mathrm{m}$ & $\mathrm{m}$ & $\mathrm{m}$ & $\mathrm{m}$ & $\mathrm{m}$ & $\mathrm{m}$ & $\mathrm{m}$ & $\mathrm{m}$ & $\mathrm{m}$ & $\mathrm{m}$ & $\mathrm{m}$ \\
\hline Korea $^{2}$ & 1.4 & $\mathrm{a}$ & 1.4 & 0.0 & 1.6 & 3.0 & 72.2 & 24.8 & 5.8 & 18.9 & 97.0 & 100 \\
\hline Luxembourg & 11.5 & a & 11.5 & $\mathrm{~m}$ & 19.9 & 31.3 & 59.5 & 9.1 & 7.3 & c & 68.7 & 100 \\
\hline Mexico & 8.0 & a & 8.0 & 0.7 & 12.1 & 20.7 & 63.0 & 16.2 & 7.4 & 8.8 & 79.3 & 100 \\
\hline Netherlands & 21.4 & $x(75)$ & 21.4 & 1.6 & 8.7 & 31.7 & 62.4 & 5.9 & 3.1 & 2.9 & 68.3 & 100 \\
\hline New Zealand & 12.8 & a & 12.8 & 0.7 & 6.9 & 20.4 & 70.9 & 8.7 & 3.8 & 4.9 & 79.6 & 100 \\
\hline Norway & 12.3 & $\mathrm{~m}$ & 12.3 & 0.8 & 13.0 & 26.2 & 68.5 & 5.2 & 2.4 & 2.8 & 73.8 & 100 \\
\hline Poland & 10.6 & a & 10.6 & 1.6 & 12.2 & 24.5 & 63.0 & 12.5 & 7.1 & 5.4 & 75.5 & 100 \\
\hline Portugal & 8.8 & $\mathrm{a}$ & 8.8 & 3.1 & 18.1 & 30.0 & 51.8 & 18.1 & 13.9 & 4.2 & 70.0 & 100 \\
\hline Slovak Republic & 4.5 & c & c & c & 26.8 & 31.6 & 52.3 & 16.1 & 9.1 & 7.0 & 68.4 & 100 \\
\hline Slovenia & 17.2 & $\mathrm{x}(75)$ & 17.2 & $4.0^{\mathrm{r}}$ & 12.4 & 33.6 & 50.5 & 15.9 & 11.6 & $4.3^{r}$ & 66.4 & 100 \\
\hline Spain $^{3}$ & 10.9 & $\mathrm{x}(75)$ & 10.9 & 7.4 & 14.3 & 32.6 & 46.7 & 20.7 & 16.7 & 4.0 & 67.4 & 100 \\
\hline Sweden & 17.7 & a & 17.7 & 4.4 & 17.3 & 39.5 & 55.0 & 5.5 & 3.0 & 2.5 & 60.5 & 100 \\
\hline Switzerland & 15.8 & c & 15.3 & 1.2 & 7.6 & 24.7 & 67.8 & 7.2 & 4.3 & 3.0 & 75.1 & 100 \\
\hline Turkey & 18.9 & a & 18.9 & 3.1 & 6.2 & 28.2 & 46.3 & 25.6 & 10.2 & 15.4 & 71.8 & 100 \\
\hline United Kingdom & 11.8 & 0.9 & 10.8 & 1.3 & 8.9 & 21.9 & 69.8 & 8.3 & 4.1 & 4.2 & 78.1 & 100 \\
\hline United States & 13.6 & $\mathrm{x}(75)$ & 13.6 & 0.4 & 8.4 & 22.4 & 66.5 & 11.1 & 3.7 & 7.4 & 77.6 & 100 \\
\hline OECD average (excluding Japan) & 12.2 & $\mathrm{~m}$ & 12.4 & 1.8 & 11.9 & 25.6 & 60.9 & 13.7 & 7.5 & 6.4 & 74.4 & 100 \\
\hline EU21 average & 12.2 & $\mathrm{~m}$ & 12.5 & 2.4 & 14.1 & 28.2 & 57.9 & 13.8 & 8.6 & 5.4 & 71.7 & 100 \\
\hline
\end{tabular}

\begin{tabular}{|c|c|c|c|c|c|c|c|c|c|c|c|c|}
\hline Argentina & $\mathrm{m}$ & $\mathrm{m}$ & $\mathrm{m}$ & $\mathrm{m}$ & $\mathrm{m}$ & $\mathrm{m}$ & $\mathrm{m}$ & $\mathrm{m}$ & $\mathrm{m}$ & $\mathrm{m}$ & $\mathrm{m}$ & $\mathrm{m}$ \\
\hline Brazil $^{2}$ & 3.1 & $\mathrm{a}$ & 3.1 & 0.3 & 1.2 & 4.7 & 82.1 & 13.3 & 5.6 & 7.7 & 95.3 & 100 \\
\hline China & $\mathrm{m}$ & $\mathrm{m}$ & $\mathrm{m}$ & $\mathrm{m}$ & $\mathrm{m}$ & $\mathrm{m}$ & $\mathrm{m}$ & $\mathrm{m}$ & $\mathrm{m}$ & $\mathrm{m}$ & $\mathrm{m}$ & $\mathrm{m}$ \\
\hline Colombia & 15.5 & $\mathrm{a}$ & 15.5 & 2.5 & 4.2 & 22.3 & 61.2 & 16.5 & 10.7 & 5.9 & 77.7 & 100 \\
\hline Costa Rica & 28.7 & $\mathrm{a}$ & 29.0 & 7.0 & 6.7 & 42.4 & 47.8 & 9.8 & 7.5 & 2.3 & 57.6 & 100 \\
\hline India & $\mathrm{m}$ & $\mathrm{m}$ & $\mathrm{m}$ & $\mathrm{m}$ & $\mathrm{m}$ & $\mathrm{m}$ & $\mathrm{m}$ & $\mathrm{m}$ & $\mathrm{m}$ & $\mathrm{m}$ & $\mathrm{m}$ & $\mathrm{m}$ \\
\hline Indonesia & $\mathrm{m}$ & $\mathrm{m}$ & $\mathrm{m}$ & $\mathrm{m}$ & $\mathrm{m}$ & $\mathrm{m}$ & $\mathrm{m}$ & $\mathrm{m}$ & $\mathrm{m}$ & $\mathrm{m}$ & $\mathrm{m}$ & $\mathrm{m}$ \\
\hline Latvia & 12.3 & $\mathrm{a}$ & 12.3 & c & 4.7 & 17.8 & 69.8 & 12.4 & $3.4^{r}$ & 9.0 & 82.2 & 100 \\
\hline Lithuania & c & $\mathrm{a}$ & c & c & c & 9.1 & 78.9 & 12.0 & 7.0 & c & 90.9 & 100 \\
\hline Russian Federation & $\mathrm{m}$ & $\mathrm{m}$ & $\mathrm{m}$ & $\mathrm{m}$ & $\mathrm{m}$ & $\mathrm{m}$ & $\mathrm{m}$ & $\mathrm{m}$ & $\mathrm{m}$ & $\mathrm{m}$ & $\mathrm{m}$ & $\mathrm{m}$ \\
\hline Saudi Arabia & $\mathrm{m}$ & $\mathrm{m}$ & $\mathrm{m}$ & $\mathrm{m}$ & $\mathrm{m}$ & $\mathrm{m}$ & $\mathrm{m}$ & $\mathrm{m}$ & $\mathrm{m}$ & $\mathrm{m}$ & $\mathrm{m}$ & $\mathrm{m}$ \\
\hline South Africa & $\mathrm{m}$ & $\mathrm{m}$ & $\mathrm{m}$ & $\mathrm{m}$ & $\mathrm{m}$ & $\mathrm{m}$ & $\mathrm{m}$ & $\mathrm{m}$ & $\mathrm{m}$ & $\mathrm{m}$ & $\mathrm{m}$ & $\mathrm{m}$ \\
\hline G20 average & $\mathrm{m}$ & $\mathrm{m}$ & $\mathrm{m}$ & $\mathrm{m}$ & $\mathrm{m}$ & $\mathrm{m}$ & $\mathrm{m}$ & $\mathrm{m}$ & $\mathrm{m}$ & $\mathrm{m}$ & $\mathrm{m}$ & $\mathrm{m}$ \\
\hline
\end{tabular}

Notes: NEET refer to young people neither in employment nor in education or training. Data refer to ISCED 2011. In Brazil data refer to ISCED-97. See the description of the levels of education in the Definitions section. Columns showing data broken down by gender and/or other educational attainment groups are available for consultation on line (see StatLink below).

1. Students in work-study programmes are considered to be both in education and employed, irrespective of their labour market status according to the ILO definition.

2. Brazil, Chile, Korea: Data for 2014 refer to year 2013.

3. Spain: Data refer to $16-29$ year-olds.

Source: OECD. Latvia, Lithuania: Eurostat. See Annex 3 for notes (www.oecd.org/education/education-at-a-glance-19991487.htm).

Please refer to the Reader's Guide for information concerning symbols for missing data and abbreviations.

StatLink त्ञाs http://dx.doi.org/10.1787/888933285997 
Table C5.4a. Percentage of 15-29 year-olds in education/not in education, by number of hours worked and age group (2014)

\begin{tabular}{|c|c|c|c|c|c|c|c|c|c|c|c|c|c|c|}
\hline & \multicolumn{14}{|c|}{ 20-24 year-olds } \\
\hline & \multicolumn{7}{|c|}{ In education } & \multicolumn{7}{|c|}{ Not in education } \\
\hline & \multicolumn{5}{|c|}{$\begin{array}{l}\text { Employed - Number of hours } \\
\text { worked per week }\end{array}$} & \multirow[b]{2}{*}{ 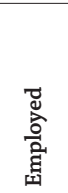 } & \multirow[b]{2}{*}{ 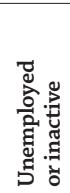 } & \multicolumn{5}{|c|}{$\begin{array}{l}\text { Employed - Number of hours } \\
\text { worked per week }\end{array}$} & \multirow[b]{2}{*}{ 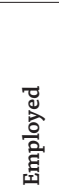 } & \multirow[b]{2}{*}{ 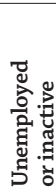 } \\
\hline & $1-9$ & $10-19$ & $20-34$ & $35+$ & $\begin{array}{l}\text { క్ } \\
\text { छ్ } \\
\text { है }\end{array}$ & & & $1-9$ & $10-19$ & $20-34$ & $35+$ & $\begin{array}{l}\text { ई } \\
0 \\
\text { छ } \\
\text { है }\end{array}$ & & \\
\hline & (15) & (16) & (17) & (18) & (19) & (20) & (21) & $(22)$ & (23) & (24) & $(25)$ & $(26)$ & (27) & (28) \\
\hline Australia & 3.6 & $\mathrm{x}(17)$ & $14.0^{\mathrm{d}}$ & 8.2 & 0.0 & 25.8 & 17.1 & 1.2 & $\mathrm{x}(24)$ & $10.7^{\mathrm{d}}$ & 31.9 & 0.0 & 43.8 & 13.2 \\
\hline Austria & 3.9 & 4.2 & 2.7 & 5.5 & 16.4 & 16.4 & 22.8 & 0.8 & 1.2 & 7.6 & 39.3 & 0.0 & 48.8 & 12.0 \\
\hline Belgium & 1.3 & c & 0.8 & 1.2 & c & 4.3 & 43.0 & c & 2.2 & 7.5 & 23.8 & c & 33.9 & 18.9 \\
\hline Canada & 4.4 & 8.3 & 5.2 & 2.2 & 0.0 & 20.0 & 21.0 & 1.2 & 3.1 & 12.5 & 27.4 & 0.0 & 44.2 & 14.8 \\
\hline Chile $^{1}$ & 1.1 & 1.1 & 1.9 & 5.6 & 0.3 & 9.9 & 34.1 & 0.7 & 1.3 & 3.1 & 29.1 & 0.7 & 34.9 & 21.1 \\
\hline Czech Republic & c & 0.8 & 1.8 & 1.9 & 5.3 & 10.2 & 38.6 & c & 0.9 & 5.0 & 32.6 & c & 38.9 & 12.3 \\
\hline Denmark & 12.4 & 8.9 & 3.3 & 8.6 & c & 33.4 & 27.7 & 1.5 & 2.5 & 6.4 & 15.7 & 0.0 & 26.1 & 12.7 \\
\hline Estonia & c & 1.5 & 7.1 & 5.9 & c & 16.2 & 30.1 & c & c & 4.2 & 29.8 & 2.4 & 37.6 & 16.1 \\
\hline Finland & 5.5 & 5.7 & 4.7 & 3.4 & c & 19.7 & 31.2 & 1.3 & 2.2 & 8.5 & 21.1 & c & 33.3 & 15.6 \\
\hline France & 1.4 & 2.0 & 1.5 & 6.4 & 0.1 & 11.3 & 35.0 & 0.9 & 1.9 & 6.6 & 25.8 & 0.1 & 35.3 & 18.3 \\
\hline Germany & 4.4 & 3.8 & 2.1 & 17.2 & 0.0 & 27.5 & 26.6 & 0.6 & 1.7 & 3.9 & 29.6 & 0.0 & 35.8 & 10.1 \\
\hline Greece & c & $0.5^{r}$ & $0.9^{r}$ & 1.1 & 0.0 & 2.5 & 46.7 & $0.4^{x}$ & 1.1 & 4.7 & 13.4 & 0.0 & 19.5 & 31.3 \\
\hline Hungary & c & c & 1.0 & 1.0 & c & 2.4 & 40.5 & c & c & 3.6 & 32.1 & c & 36.5 & 20.6 \\
\hline Iceland & 7.4 & 9.2 & 7.0 & 11.4 & 0.0 & 35.1 & 14.9 & c & c & 5.2 & 32.8 & 0.0 & 40.5 & 9.4 \\
\hline Ireland & 1.9 & 4.9 & 2.8 & 2.6 & c & 12.2 & 33.0 & $0.9^{r}$ & 2.3 & 9.2 & 20.7 & c & 33.4 & 21.1 \\
\hline Israel & 1.9 & 2.7 & 4.6 & 3.3 & 0.0 & 12.4 & 17.0 & 0.9 & 2.3 & 7.7 & 41.1 & 0.0 & 52.0 & 18.5 \\
\hline Italy & 0.4 & 0.4 & 0.7 & 0.5 & c & 2.1 & 39.3 & 0.6 & 1.2 & 6.1 & 15.3 & 0.3 & 23.4 & 34.8 \\
\hline Japan & $\mathrm{m}$ & $\mathrm{m}$ & $\mathrm{m}$ & $\mathrm{m}$ & $\mathrm{m}$ & $\mathrm{m}$ & $\mathrm{m}$ & $\mathrm{m}$ & $\mathrm{m}$ & $\mathrm{m}$ & $\mathrm{m}$ & $\mathrm{m}$ & $\mathrm{m}$ & $\mathrm{m}$ \\
\hline Korea $^{1}$ & 1.6 & $\mathrm{x}(17)$ & $5.2^{\mathrm{d}}$ & 2.9 & 0.0 & 9.8 & 34.6 & 0.4 & $\mathrm{x}(24)$ & $4.8^{\mathrm{d}}$ & 28.0 & 0.3 & 33.5 & 22.2 \\
\hline Luxembourg & $c$ & c & $2.2^{r}$ & 3.7 & c & 7.6 & 50.8 & c & $3.3^{r}$ & 4.1 & 21.9 & $1.9^{\mathrm{r}}$ & 32.6 & 9.0 \\
\hline Mexico & 0.7 & 1.1 & 2.0 & 3.9 & 0.2 & 7.9 & 19.6 & 1.0 & 2.5 & 5.8 & 37.2 & 1.0 & 47.6 & 24.9 \\
\hline Netherlands & 14.3 & 8.0 & 6.3 & 4.8 & 0.9 & 34.3 & 22.3 & 4.4 & 2.6 & 9.1 & 15.8 & 1.1 & 33.0 & 10.4 \\
\hline New Zealand & $\mathrm{x}(19)$ & $\mathrm{x}(19)$ & $\mathrm{x}(19)$ & $\mathrm{x}(19)$ & $20.1^{\mathrm{d}}$ & 20.1 & 20.5 & $x(26)$ & $\mathrm{x}(26)$ & $\mathrm{x}(26)$ & $x(26)$ & $44.7^{\mathrm{d}}$ & 44.7 & 14.4 \\
\hline Norway & 9.9 & 5.8 & 5.0 & 7.9 & 0.0 & 30.8 & 21.7 & 6.2 & 3.0 & 7.4 & 21.0 & 0.0 & 37.5 & 10.0 \\
\hline Poland & 0.3 & 0.7 & 2.9 & 7.3 & 0.0 & 11.1 & 37.2 & 0.0 & 0.7 & 4.1 & 27.6 & 0.0 & 32.5 & 19.2 \\
\hline Portugal & c & c & 0.8 & 1.8 & c & 4.4 & 38.8 & c & 1.8 & 4.6 & 24.3 & 1.5 & 32.9 & 23.9 \\
\hline Slovak Republic & 0.0 & c & $0.7^{\mathrm{r}}$ & 1.2 & 0.0 & 2.5 & 45.9 & c & 1.2 & 4.2 & 27.2 & c & 33.1 & 18.6 \\
\hline Slovenia & $2.3^{\mathrm{r}}$ & $3.2^{\mathrm{r}}$ & 7.2 & 5.0 & 0.0 & 17.8 & 51.1 & c & $1.2^{\mathrm{r}}$ & $1.5^{\mathrm{r}}$ & 15.0 & 0.0 & 17.7 & 13.4 \\
\hline Spain & 1.2 & $\mathrm{x}(17)$ & $3.1^{\mathrm{d}}$ & 1.7 & 0.0 & 6.1 & 43.9 & 0.9 & $\mathrm{x}(24)$ & $5.7^{\mathrm{d}}$ & 14.4 & 0.0 & 21.0 & 29.0 \\
\hline Sweden & 4.8 & 3.5 & 2.9 & 1.9 & c & 13.2 & 33.0 & 1.7 & 3.3 & $11.7^{\mathrm{d}}$ & 25.0 & c & 41.8 & 12.0 \\
\hline Switzerland & 4.6 & 3.1 & 3.4 & 4.2 & 10.5 & 25.7 & 19.1 & 0.9 & 0.9 & 5.5 & 30.7 & 4.5 & 42.6 & 12.4 \\
\hline Turkey & 0.2 & 0.6 & 1.0 & 10.0 & 0.0 & 11.8 & 19.7 & 0.4 & 1.6 & 2.5 & 27.7 & 0.0 & 32.2 & 36.3 \\
\hline United Kingdom & 2.2 & 3.9 & 2.7 & 4.7 & 1.2 & 14.6 & 19.9 & 1.2 & 4.2 & 8.1 & 33.4 & 1.5 & 48.4 & 17.0 \\
\hline United States & 1.6 & 4.3 & 7.9 & 5.3 & 0.1 & 19.2 & 18.9 & 0.7 & 2.0 & 9.2 & 32.6 & 0.0 & 44.5 & 17.5 \\
\hline OECD average (excluding Japan) & 3.6 & 3.7 & 3.6 & 4.8 & 2.4 & 15.1 & 30.8 & 1.3 & 2.0 & 6.3 & 26.4 & 2.3 & 36.2 & 17.9 \\
\hline EU21 average & 3.8 & 3.5 & 2.8 & 4.2 & 2.2 & 12.9 & 36.1 & 1.2 & 2.0 & 6.0 & 24.0 & 0.6 & 33.1 & 17.9 \\
\hline
\end{tabular}

\begin{tabular}{|c|c|c|c|c|c|c|c|c|c|c|c|c|c|c|}
\hline $\begin{array}{l}\text { Argentina } \\
\text { Brazil }^{1}\end{array}$ & $\begin{array}{c}\mathrm{m} \\
\mathrm{x}(19)\end{array}$ & $\begin{array}{c}\mathrm{m} \\
\mathrm{x}(19)\end{array}$ & $\begin{array}{c}\mathrm{m} \\
\mathrm{x}(19)\end{array}$ & $\begin{array}{r}\mathrm{m} \\
\mathrm{x}(19)\end{array}$ & $\begin{array}{c}\mathrm{m} \\
13.8^{\mathrm{d}}\end{array}$ & $\begin{array}{r}\mathrm{m} \\
13.8\end{array}$ & $\begin{array}{r}\mathrm{m} \\
10.0\end{array}$ & $\begin{array}{c}\mathrm{m} \\
\mathrm{x}(26)\end{array}$ & $\begin{array}{r}m \\
x(26)\end{array}$ & $\begin{array}{c}\mathrm{m} \\
\mathrm{x}(26)\end{array}$ & $\begin{array}{c}\mathrm{m} \\
\mathrm{x}(26)\end{array}$ & $\begin{array}{c}\mathrm{m} \\
52.2^{\mathrm{d}}\end{array}$ & $\begin{array}{r}\mathrm{m} \\
52.2\end{array}$ & $\begin{array}{r}\mathrm{m} \\
24.0\end{array}$ \\
\hline China & $\mathrm{m}$ & $\mathrm{m}$ & $\mathrm{m}$ & $\mathrm{m}$ & $\mathrm{m}$ & $\mathrm{m}$ & $\mathrm{m}$ & $\mathrm{m}$ & $\mathrm{m}$ & $\mathrm{m}$ & $\mathrm{m}$ & $\mathrm{m}$ & $\mathrm{m}$ & $\mathrm{m}$ \\
\hline Colombia & 1.1 & 1.3 & 2.3 & 7.3 & 0.0 & 11.9 & 14.0 & 1.6 & 2.5 & 6.2 & 38.8 & 0.0 & 49.1 & 24.9 \\
\hline Costa Rica & 1.4 & 1.5 & 2.1 & 14.4 & 0.0 & 19.4 & 22.4 & 1.9 & 2.0 & 2.9 & 29.8 & 0.0 & 36.6 & 21.6 \\
\hline India & $\mathrm{m}$ & $\mathrm{m}$ & $\mathrm{m}$ & $\mathrm{m}$ & $\mathrm{m}$ & $\mathrm{m}$ & $\mathrm{m}$ & $\mathrm{m}$ & $\mathrm{m}$ & $\mathrm{m}$ & $\mathrm{m}$ & $\mathrm{m}$ & $\mathrm{m}$ & $\mathrm{m}$ \\
\hline Indonesia & $\mathrm{m}$ & $\mathrm{m}$ & $\mathrm{m}$ & $\mathrm{m}$ & $\mathrm{m}$ & $\mathrm{m}$ & $\mathrm{m}$ & $\mathrm{m}$ & $\mathrm{m}$ & $\mathrm{m}$ & $\mathrm{m}$ & $\mathrm{m}$ & $\mathrm{m}$ & $\mathrm{m}$ \\
\hline Latvia & c & c & 5.2 & 10.0 & 0.0 & 15.8 & 31.5 & 0.0 & c & $1.3^{r}$ & 33.2 & c & 36.1 & 16.6 \\
\hline Lithuania & c & c & 4.1 & 8.1 & c & 12.7 & 38.0 & c & c & c & 28.9 & c & 32.9 & 16.4 \\
\hline Russian Federation & $\mathrm{m}$ & $\mathrm{m}$ & $\mathrm{m}$ & $\mathrm{m}$ & $\mathrm{m}$ & $\mathrm{m}$ & $\mathrm{m}$ & $\mathrm{m}$ & $\mathrm{m}$ & $\mathrm{m}$ & $\mathrm{m}$ & $\mathrm{m}$ & $\mathrm{m}$ & $\mathrm{m}$ \\
\hline Saudi Arabia & $\mathrm{m}$ & $\mathrm{m}$ & $\mathrm{m}$ & $\mathrm{m}$ & $\mathrm{m}$ & $\mathrm{m}$ & $\mathrm{m}$ & $\mathrm{m}$ & $\mathrm{m}$ & $\mathrm{m}$ & $\mathrm{m}$ & $\mathrm{m}$ & $\mathrm{m}$ & $\mathrm{m}$ \\
\hline South Africa & $\mathrm{m}$ & $\mathrm{m}$ & $\mathrm{m}$ & $\mathrm{m}$ & $\mathrm{m}$ & $\mathrm{m}$ & $\mathrm{m}$ & $\mathrm{m}$ & $\mathrm{m}$ & $\mathrm{m}$ & $\mathrm{m}$ & $\mathrm{m}$ & $\mathrm{m}$ & $\mathrm{m}$ \\
\hline
\end{tabular}

\begin{tabular}{l|l|l|l|l|l|l|l|l|l|l|l|l|l|l|} 
G20 average & $\mathrm{m}$ & $\mathrm{m}$ & $\mathrm{m}$ & $\mathrm{m}$ & $\mathrm{m}$ & $\mathrm{m}$ & $\mathrm{m}$ & $\mathrm{m}$ & $\mathrm{m}$ & $\mathrm{m}$ & $\mathrm{m}$ & $\mathrm{m}$ & $\mathrm{m}$ & $\mathrm{m}$ \\
\hline
\end{tabular}

Notes: Hours worked represent the actual number of hours worked per week, including overtime. When actual hours worked per week were equal to zero, the usual hours worked were used instead. When a country could not provide the actual number of hours worked per week, the usual hours worked were used. Columns showing data for other age groups are available for consultation on line (see StatLink below).

1. Brazil, Chile, Korea: Data for 2014 refer to year 2013.

Source: OECD. Latvia, Lithuania: Eurostat. See Annex 3 for notes (www.oecd.org/education/education-at-a-glance-19991487.htm).

Please refer to the Reader's Guide for information concerning symbols for missing data and abbreviations.

StatLink 제이 http://dx.doi.org/10.1787/888933286000 
Table C5.4b. Percentage of 15-29 year-olds in education/not in education, by number of hours worked and gender (2014)

\begin{tabular}{|c|c|c|c|c|c|c|c|c|c|c|c|c|c|c|}
\hline & \multicolumn{14}{|c|}{ Total (young men + young women) } \\
\hline & \multicolumn{7}{|c|}{ In education } & \multicolumn{7}{|c|}{ Not in education } \\
\hline & \multicolumn{5}{|c|}{$\begin{array}{l}\text { Employed - Number of hours } \\
\text { worked per week }\end{array}$} & \multirow[b]{2}{*}{ 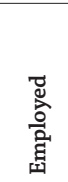 } & \multirow[b]{2}{*}{ 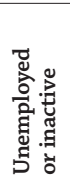 } & \multicolumn{5}{|c|}{$\begin{array}{l}\text { Employed - Number of hours } \\
\text { worked per week }\end{array}$} & \multirow[b]{2}{*}{ 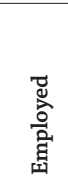 } & \multirow[b]{2}{*}{ 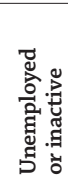 } \\
\hline & $1-9$ & $10-19$ & $20-34$ & $35+$ & $\begin{array}{l}\text { ईु } \\
\text { है } \\
\text { है }\end{array}$ & & & $1-9$ & $10-19$ & $20-34$ & $35+$ & 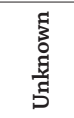 & & \\
\hline & (1) & (2) & (3) & (4) & (5) & (6) & (7) & (8) & (9) & (10) & (11) & (12) & (13) & (14) \\
\hline Australia & 5.5 & $\mathrm{x}(3)$ & $10.6^{\mathrm{d}}$ & 7.3 & 0.0 & 23.5 & 23.0 & 0.9 & $\mathrm{x}(10)$ & $8.7^{\mathrm{d}}$ & 31.2 & 0.0 & 40.9 & 12.6 \\
\hline Austria & 3.3 & 2.6 & 2.7 & 9.2 & 0.0 & 17.9 & 27.5 & 1.0 & 1.8 & 7.4 & 32.9 & 0.0 & 43.1 & 11.6 \\
\hline Belgium & 0.9 & c & 0.8 & 1.3 & 0.5 & 3.8 & 44.3 & 0.5 & 2.1 & 7.2 & 26.9 & c & 36.9 & 15.0 \\
\hline Canada & 5.1 & 7.1 & 3.6 & 1.8 & 0.0 & 17.6 & 26.6 & 1.1 & 2.6 & 10.2 & 28.5 & 0.0 & 42.4 & 13.4 \\
\hline Chile $^{1}$ & 0.7 & 0.8 & 1.4 & 4.6 & 0.2 & 7.6 & 41.0 & 0.7 & 1.1 & 3.0 & 27.1 & 0.7 & 32.7 & 18.8 \\
\hline Czech Republic & 0.2 & 0.6 & 1.4 & 2.0 & 21.3 & 25.5 & 20.5 & 0.4 & 0.9 & 5.6 & 34.5 & c & 41.5 & 12.5 \\
\hline Denmark & 13.5 & 8.1 & 2.2 & 6.2 & c & 30.0 & 32.1 & 0.9 & 1.9 & 6.2 & 18.1 & 0.0 & 27.1 & 10.7 \\
\hline Estonia & 0.7 & 1.2 & 3.7 & 5.3 & c & 11.1 & 34.2 & c & 1.3 & 4.7 & 32.5 & 1.2 & 40.1 & 14.6 \\
\hline Finland & 4.7 & 3.7 & 3.1 & 3.9 & $0.3^{r}$ & 15.7 & 38.6 & 0.9 & 1.9 & 7.7 & 22.0 & c & 32.6 & 12.9 \\
\hline France & 0.8 & 1.0 & 0.9 & 4.5 & 0.0 & 7.2 & 40.5 & 0.8 & 1.7 & 6.2 & 27.2 & 0.0 & 36.0 & 16.3 \\
\hline Germany & 3.5 & 2.6 & 1.8 & 12.4 & 0.0 & 20.3 & 33.4 & 0.7 & 1.7 & 4.7 & 30.0 & 0.0 & 37.2 & 9.2 \\
\hline Greece & c & $0.3^{x}$ & 0.6 & 1.0 & 0.0 & 1.8 & 45.9 & 0.5 & 1.2 & 4.9 & 17.3 & 0.0 & 23.9 & 28.3 \\
\hline Hungary & c & c & 0.5 & 1.0 & c & 1.7 & 43.4 & c & 0.5 & 3.6 & 33.0 & c & 37.4 & 17.5 \\
\hline Iceland & 7.7 & 7.7 & 6.3 & 9.9 & 0.0 & 31.6 & 18.4 & 1.2 & 2.3 & 5.7 & 32.0 & 0.0 & 41.2 & 8.8 \\
\hline Ireland & 1.5 & 2.5 & 1.7 & 1.7 & c & 7.5 & 40.4 & 0.8 & 2.4 & 8.3 & 21.9 & 0.4 & 34.0 & 18.0 \\
\hline Israel & 2.2 & 2.5 & 4.1 & 3.9 & 0.0 & 12.6 & 30.2 & 0.7 & 1.9 & 5.9 & 34.8 & 0.0 & 43.3 & 13.8 \\
\hline Italy & 0.2 & 0.3 & 0.6 & 0.6 & 0.0 & 1.8 & 44.9 & 0.6 & 1.3 & 5.9 & 17.1 & 0.3 & 25.3 & 27.6 \\
\hline $\mathrm{Japan}^{2}$ & $\mathrm{x}(5)$ & $\mathrm{x}(5)$ & $\mathrm{x}(5)$ & $\mathrm{x}(5)$ & $10.8^{\mathrm{d}}$ & 10.8 & 53.5 & $\mathrm{x}(12)$ & $x(12)$ & $\mathrm{x}(12)$ & $\mathrm{x}(12)$ & $29.2^{\mathrm{d}}$ & 29.2 & 6.6 \\
\hline Korea $^{1}$ & 0.7 & $\mathrm{x}(3)$ & $2.9^{\mathrm{d}}$ & 1.8 & 0.0 & 5.5 & 42.2 & 0.3 & $\mathrm{x}(10)$ & $4.4^{\mathrm{d}}$ & 29.0 & 0.5 & 34.3 & 18.0 \\
\hline Luxembourg & 1.5 & $0.7^{\mathrm{r}}$ & 1.9 & 2.9 & $0.7^{\mathrm{r}}$ & 7.6 & 48.0 & $0.8^{\mathrm{r}}$ & 1.2 & 4.5 & 27.9 & 1.8 & 36.2 & 8.2 \\
\hline Mexico & 1.0 & 1.4 & 1.9 & 3.1 & 0.2 & 7.5 & 28.7 & 1.0 & 2.2 & 5.4 & 31.7 & 1.0 & 41.4 & 22.4 \\
\hline Netherlands & 14.4 & 6.5 & 4.7 & 3.9 & 1.2 & 30.7 & 24.5 & 4.9 & 2.4 & 9.1 & 18.2 & 1.0 & 35.5 & 9.2 \\
\hline New Zealand & $\mathrm{x}(5)$ & $\mathrm{x}(5)$ & $\mathrm{x}(5)$ & $\mathrm{x}(5)$ & $16.6^{\mathrm{d}}$ & 16.6 & 28.5 & $\mathrm{x}(12)$ & $\mathrm{x}(12)$ & $\mathrm{x}(12)$ & $\mathrm{x}(12)$ & $41.8^{\mathrm{d}}$ & 41.8 & 12.9 \\
\hline Norway & 10.0 & 4.4 & 3.6 & 6.4 & 0.0 & 25.8 & 30.0 & 6.6 & 2.4 & 6.3 & 20.5 & 0.0 & 35.7 & 8.5 \\
\hline Poland & 0.1 & 0.5 & 1.5 & 4.3 & 0.0 & 6.5 & 39.9 & 0.2 & 0.8 & 4.8 & 32.0 & 0.0 & 37.7 & 15.8 \\
\hline Portugal & 0.5 & 0.3 & 0.7 & 1.7 & 0.3 & 3.6 & 44.4 & 0.7 & 1.3 & 4.2 & 26.4 & 1.7 & 34.3 & 17.7 \\
\hline Slovak Republic & 0.0 & 0.3 & 0.5 & 1.1 & 0.0 & 2.0 & 43.1 & 0.3 & 1.3 & 4.6 & 29.7 & 0.3 & 36.2 & 18.7 \\
\hline Slovenia & 1.5 & 1.8 & 3.5 & 5.6 & 0.0 & 12.3 & 45.2 & $0.2^{r}$ & $0.9^{r}$ & 3.0 & 24.7 & 0.0 & 28.8 & 13.7 \\
\hline Spain $^{3}$ & 0.8 & $\mathrm{x}(3)$ & $2.2^{\mathrm{d}}$ & 2.2 & 0.0 & 5.1 & 40.4 & 0.9 & $\mathrm{x}(10)$ & $6.5^{\mathrm{d}}$ & 21.3 & 0.0 & 28.6 & 25.8 \\
\hline Sweden & 4.6 & 2.7 & 2.3 & 2.2 & c & 11.9 & 39.4 & 1.3 & 2.6 & 9.8 & 25.4 & $0.1^{r}$ & 39.3 & 9.4 \\
\hline Switzerland & 3.3 & 1.9 & 2.4 & 3.4 & 16.2 & 27.2 & 21.7 & 1.5 & 1.8 & 5.2 & 29.1 & 3.6 & 41.2 & 9.9 \\
\hline Turkey & 0.2 & 0.6 & 1.4 & 7.1 & 0.0 & 9.2 & 28.0 & 0.5 & 1.3 & 2.6 & 26.7 & 0.0 & 31.2 & 31.6 \\
\hline United Kingdom & 3.0 & 3.1 & 2.1 & 4.1 & 1.1 & 13.3 & 28.2 & 1.1 & 3.6 & 7.2 & 30.9 & 1.3 & 44.1 & 14.4 \\
\hline United States & 1.7 & 3.7 & 5.4 & 3.8 & 0.1 & 14.6 & 30.1 & 0.6 & 1.6 & 7.6 & 30.4 & 0.0 & 40.3 & 15.0 \\
\hline OECD average (excluding Japan) & 3.1 & 2.5 & 2.6 & 4.1 & 2.1 & 13.2 & 34.8 & 1.1 & 1.7 & 6.0 & 27.2 & 1.9 & 36.4 & 15.5 \\
\hline EU21 average & 2.9 & 2.1 & 1.9 & 3.7 & 1.6 & 11.3 & 38.1 & 0.9 & 1.6 & 6.0 & 26.2 & 0.5 & 35.0 & 15.6 \\
\hline
\end{tabular}

\begin{tabular}{|c|c|c|c|c|c|c|c|c|c|c|c|c|c|c|}
\hline $\begin{array}{l}\text { Argentina } \\
\text { Brazil }^{1}\end{array}$ & $\begin{array}{c}\mathrm{m} \\
\mathrm{x}(5)\end{array}$ & $\begin{array}{c}\mathrm{m} \\
\mathrm{x}(5)\end{array}$ & $\begin{array}{c}\mathrm{m} \\
\mathrm{x}(5)\end{array}$ & $\begin{array}{c}\mathrm{m} \\
\mathrm{x}(5)\end{array}$ & $\begin{array}{c}\mathrm{m} \\
13.0^{\mathrm{d}}\end{array}$ & $\begin{array}{r}\mathrm{m} \\
13.0\end{array}$ & $\begin{array}{r}\mathrm{m} \\
22.7\end{array}$ & $\begin{array}{c}\mathrm{m} \\
\mathrm{x}(12)\end{array}$ & $\begin{array}{c}\mathrm{m} \\
\mathrm{x}(12)\end{array}$ & $\begin{array}{c}\mathrm{m} \\
\mathrm{x}(12)\end{array}$ & $\begin{array}{c}\mathrm{m} \\
\mathrm{x}(12)\end{array}$ & $\begin{array}{c}\mathrm{m} \\
44.0^{\mathrm{d}}\end{array}$ & $\begin{array}{r}\mathrm{m} \\
44.0\end{array}$ & $\begin{array}{r}\mathrm{m} \\
20.3\end{array}$ \\
\hline है China & $\mathrm{m}$ & $\mathrm{m}$ & $\mathrm{m}$ & $\mathrm{m}$ & $\mathrm{m}$ & $\mathrm{m}$ & $\mathrm{m}$ & $\mathrm{m}$ & $\mathrm{m}$ & $\mathrm{m}$ & $\mathrm{m}$ & $\mathrm{m}$ & $\mathrm{m}$ & $\mathrm{m}$ \\
\hline Colombia & 1.3 & 1.6 & 2.1 & 5.8 & 0.0 & 10.9 & 24.7 & 1.5 & 2.3 & 5.5 & 33.7 & 0.0 & 43.0 & 21.4 \\
\hline Costa Rica & 1.1 & 1.4 & 2.0 & 10.8 & 0.0 & 15.3 & 31.7 & 1.9 & 1.7 & 3.3 & 27.3 & 0.0 & 34.2 & 18.8 \\
\hline India & $\mathrm{m}$ & $\mathrm{m}$ & $\mathrm{m}$ & $\mathrm{m}$ & $\mathrm{m}$ & $\mathrm{m}$ & $\mathrm{m}$ & $\mathrm{m}$ & $\mathrm{m}$ & $\mathrm{m}$ & $\mathrm{m}$ & $\mathrm{m}$ & $\mathrm{m}$ & $\mathrm{m}$ \\
\hline Indonesia & $\mathrm{m}$ & $\mathrm{m}$ & $\mathrm{m}$ & $\mathrm{m}$ & $\mathrm{m}$ & $\mathrm{m}$ & $\mathrm{m}$ & $\mathrm{m}$ & $\mathrm{m}$ & $\mathrm{m}$ & $\mathrm{m}$ & $\mathrm{m}$ & $\mathrm{m}$ & $\mathrm{m}$ \\
\hline Latvia & c & c & 2.0 & 6.5 & 0.0 & 8.9 & 35.2 & 0.0 & $0.6^{r}$ & 1.7 & 38.4 & $0.6^{r}$ & 41.3 & 14.5 \\
\hline Lithuania & c & c & 2.0 & 4.7 & c & 7.0 & 44.0 & c & c & 3.2 & 30.4 & c & 34.8 & 14.2 \\
\hline Russian Federation & $\mathrm{m}$ & $\mathrm{m}$ & $\mathrm{m}$ & $\mathrm{m}$ & $\mathrm{m}$ & $\mathrm{m}$ & $\mathrm{m}$ & $\mathrm{m}$ & $\mathrm{m}$ & $\mathrm{m}$ & $\mathrm{m}$ & $\mathrm{m}$ & $\mathrm{m}$ & $\mathrm{m}$ \\
\hline Saudi Arabia & $\mathrm{m}$ & $\mathrm{m}$ & $\mathrm{m}$ & $\mathrm{m}$ & $\mathrm{m}$ & $\mathrm{m}$ & $\mathrm{m}$ & $\mathrm{m}$ & $\mathrm{m}$ & $\mathrm{m}$ & $\mathrm{m}$ & $\mathrm{m}$ & $\mathrm{m}$ & $\mathrm{m}$ \\
\hline South Africa & $\mathrm{m}$ & $\mathrm{m}$ & $\mathrm{m}$ & $\mathrm{m}$ & $\mathrm{m}$ & $\mathrm{m}$ & $\mathrm{m}$ & $\mathrm{m}$ & $\mathrm{m}$ & $\mathrm{m}$ & $\mathrm{m}$ & $\mathrm{m}$ & $\mathrm{m}$ & $\mathrm{m}$ \\
\hline G20 average & $\mathrm{m}$ & $\mathrm{m}$ & $\mathrm{m}$ & $\mathrm{m}$ & $\mathrm{m}$ & $\mathrm{m}$ & $\mathrm{m}$ & $\mathrm{m}$ & $\mathrm{m}$ & $\mathrm{m}$ & $\mathrm{m}$ & $\mathrm{m}$ & $\mathrm{m}$ & $\mathrm{m}$ \\
\hline
\end{tabular}

Notes: Hours worked represent the actual number of hours worked per week, including overtime. When actual hours worked per week were equal to zero, the usual hours worked were used instead. When a country could not provide the actual number of hours worked per week, the usual hours worked were used. Columns showing data broken down by gender are available for consultation on line (see StatLink below).

1. Brazil, Chile, Korea: Data for 2014 refer to year 2013.

2. Japan: Data for 15-29 year-olds refer to 15-24 year-olds.

3. Spain: Data for 15-29 year-olds refer to 16-19 year-olds.

Source: OECD. Latvia, Lithuania: Eurostat. See Annex 3 for notes (www.oecd.org/education/education-at-a-glance-19991487.htm).

Please refer to the Reader's Guide for information concerning symbols for missing data and abbreviations.

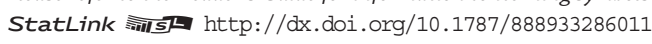





\section{HOW MANY ADULTS PARTICIPATE IN EDUCATION AND LEARNING?}

- Across OECD countries and sub-national entities that participated in the Survey of Adult Skills (PIAAC) in 2012, about 50\% of all employed adults participate in employer-sponsored formal and/or non-formal education in a given year. The proportion ranges from more than $60 \%$ in Denmark, Finland, the Netherlands and Norway, to less than 40\% in France, Italy, Poland, the Russian Federation and the Slovak Republic.

- About $60 \%$ of employed adults with good skills in information and communication technology (ICT) and in problem solving participate in employer-sponsored formal and/or non-formal education, while only $18 \%$ of adults who have no computer experience do.

- About $60 \%$ of workers in the most skilled occupations participate in employer-sponsored formal and/or non-formal education, while about $25 \%$ of workers in elementary occupations do.

\section{Chart C6.1. Participation in employer-sponsored formal and/or non-formal education, by skills and readiness to use information and communication technologies for problem solving (2012)}

Survey of Adult Skills, employed 25-64 year-olds
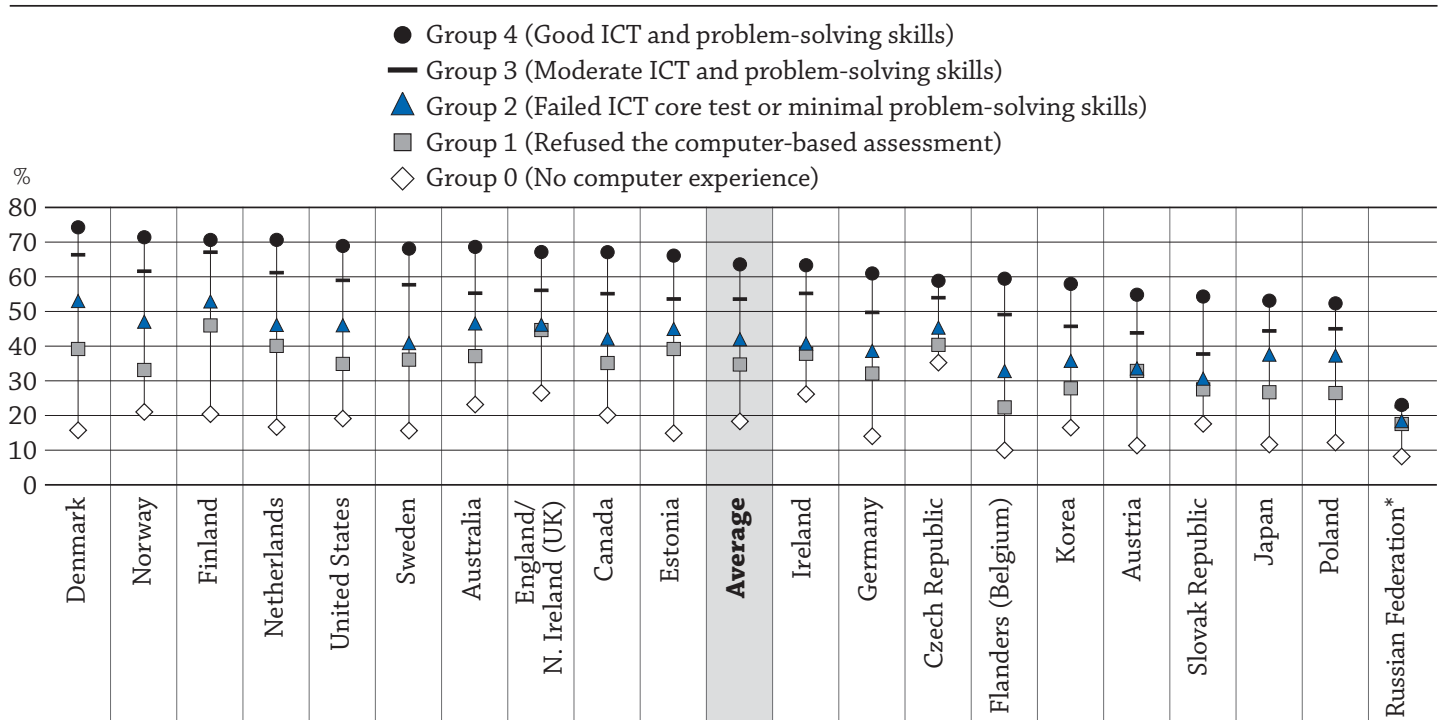

* See note on data for the Russian Federation in the Methodology section.

Countries are ranked in descending order of participation in employer-sponsored formal and/or non-formal education among people in Group 4 (Good ICT and problem-solving skills).

Source: OECD. Table C6.1 (P). See Annex 3 for notes (www.oecd.org/education/education-at-a-glance- 19991487.htm).

StatLink : तils $\mathrm{http}: / / \mathrm{dx} . \mathrm{doi}$. org $/ 10.1787 / 888933284339$

\section{Context}

Adult learning can play an important role in helping adults to develop and maintain key informationprocessing skills, and acquire other knowledge and skills, throughout life. It is crucial to provide, and ensure access to, organised learning opportunities for adults beyond initial formal education, especially for workers who need to adapt to changes throughout their careers. The relevance of continued learning opportunities now extends to workers in both high- and low-skilled occupations. In high-technology sectors, workers need to update their competencies and keep pace with rapidly changing techniques. Workers in low-technology sectors and those performing low-skilled tasks must learn to be adaptable, since they are at higher risk of losing their job, as routine tasks are increasingly performed by machines, and companies may relocate to countries with lower labour costs (OECD, 2013). In general, the higher the productivity of a worker, the more interested an employer might be in investing in his or her human capital. 


\section{Other findings}

- Participation in employer-sponsored formal and/or non-formal education in all countries is strongly related to proficiency levels in key skills such as literacy and numeracy as well as to educational attainment. These factors combine to create a virtuous circle for persons with high skills proficiency and educational attainment who tend to acquire yet more skills through attending adult education activities. The factors also combine to establish a vicious circle of low educational attainment, low skills proficiency, and lack of support for institutionalised learning to redress skills deficiencies.

- Participation rates in employer-sponsored formal and/or non-formal education are highest among workers who most frequently use reading, writing and numeracy skills at work.

- Across OECD countries and sub-national entities that participated in the Survey of Adult Skills, $57 \%$ of full-time workers with indefinite contracts participate in employer-sponsored formal and/or non-formal education while $33 \%$ of part-time workers with limited contracts do.

- In all countries, 25-34 year-old workers are more likely to participate in employer-sponsored formal and/or non-formal education than 55-64 year-old workers. 


\section{Analysis}

\section{Employer-sponsored formal and/or non-formal education}

Employers support their employees' participation in education and training activities when they have an interest in investing in their employees' human capital. Employer support can take the form of time, i.e. providing educational activities that take place fully or partly during paid working hours, or financial support, i.e. giving grants to employees to participate in educational activities.

Across OECD countries and sub-national entities that participated in the Survey of Adult Skills, about 50\% of all employed adults participate in employer-sponsored formal and/or non-formal education in a given year. The proportion ranges from more than $60 \%$ in Denmark, Finland, the Netherlands and Norway, to less than $40 \%$ in France, Italy, Poland, the Russian Federation and the Slovak Republic (Table C6.1 [P]).

The large variation in participation in adult learning activities suggests that there are significant differences in learning cultures, learning opportunities at work, and adult education structures. Results from the Survey of Adult Skills, a product of the OECD Programme for the International Assessment of Adult Competencies (PIAAC), show a clear relationship between the extent of participation in organised adult learning activities and the average level of key information-processing skills in a given country.

\section{Participation as related to ICT skills and educational attainment}

The 2012 Survey of Adult Skills measured adults' proficiency in using ICT to solve problems. Chart C6.1 shows that, across OECD countries and sub-national entities that participated in the Survey of Adult Skills, $63 \%$ of adults with good ICT and problem-solving skills participate in employer-sponsored formal and/or non-formal education, while only $18 \%$ of those who have no computer experience do. The most highly skilled adults are thus about three times more likely to participate in employer-sponsored formal and/or non-formal education than are the least skilled adults (Table C6.1 [P]).

All participating OECD countries and sub-national entities show increasing participation as the level of proficiency increases. The most skilled adults are more than three times more likely to participate in employer-sponsored formal and/or non-formal education than those with no skills, except in Australia, the Czech Republic, England (UK), Ireland, Northern Ireland (UK) and the Russian Federation, where the most-skilled adults are two or less than three times more likely to participate in such activities than adults with no skills. In the Czech Republic, England (UK), Ireland and Northern Ireland (UK) at least 25\% of those with no computer experience participate in employer-sponsored formal and/or non-formal education. In Australia, Denmark, England (UK), Finland, the Netherlands, Norway, Sweden and the United States, about two out of three (67\% or more) workers with good ICT and problem-solving skills receive support for further learning from their employers (Table C6.1 [P]).

The frequency with which adults use ICT and problem-solving skills at work is also related to participation in employer-sponsored formal and/or non-formal education. The ICT activities surveyed include: using e-mail, Internet, spreadsheets, word processors, programming languages; conducting transactions on line; and participating in online discussions (conferences, chats). The respondents indicated how often they engage in each activity: never; less than once a month; less than once a week, but at least once a month; at least once a week but not every day; or every day. The index created to measure frequency of use compares the frequency of the respondent's activities to the frequency of activities of all adults assessed.

Across OECD countries and sub-national entities that participated in the Survey of Adult Skills, the rate of participation in employer-sponsored formal and/or non-formal education increases from $36 \%$ among those who never engage in the ICT activities cited, to $67 \%$ among those who engage in these activities most often, on average. Meanwhile, at least $60 \%$ of adults who engage in these activities moderately or often (i.e. less than once a week but at least once a month, at least once a week but not every day, or every day) participate in employer-sponsored formal and/or non-formal education programmes, which suggests that there might be a ceiling effect (Table C6.3e, available on line).

Workers' educational attainment is also closely related to participation in employer-sponsored formal and/or non-formal education. Across OECD countries and sub-national entities that participated in the Survey of Adult Skills, workers with high educational attainment are about twice as likely to participate than are workers with low education (62\% among those with tertiary education compared with $29 \%$ among those with below upper secondary education as their highest level of attainment). This difference is larger in countries with lower participation rates, in general (correlation coefficient $=0.86)($ Table C6.2a). 


\section{Participation as related to the work environment}

The demands of the job also affect workers' need and wish for education and training. One indication of the level of skills demanded in a job is the occupation of the worker. The Survey of Adult Skills distinguishes among four groups of occupations: skilled occupations (managers, professionals, technicians and associate professionals); semi-skilled white-collar occupations (clerical support workers, service and sales workers); semi-skilled blue-collar occupations (skilled manual workers); and elementary occupations.

Chart C6.2 includes both information from the Survey of Adult Skills and information from the Adult Education Survey (AES) for countries that did not participate in the Survey of Adult Skills. The results are presented in separated sections as they are not directly comparable. On the left side of the chart, data from the Survey of Adult Skills are presenting data for year 2012 on formal and/or non-formal education while on the right side of the chart data from the AES are for year 2011 and refer to employer-sponsored, job-related, non-formal education and training.

On average, across OECD countries and sub-national entities that participated in the Survey of Adult Skills, $62 \%$ of workers in the most skilled occupations participate in employer-sponsored formal and/or non-formal education, while $26 \%$ of workers in elementary occupations do. The former group is thus more than twice as likely to participate than the latter group (Table 6.2c). The results support the thesis that participation in employer-sponsored formal and/or non-formal education is more likely among adults in occupations that require more skills. Workers' occupations have been identified as the single most important factor in determining the frequency with which adults use their skills in the workplace (OECD, 2013, p. 181).

\section{Chart C6.2. Participation in employer-sponsored education, by occupation (2011, 2012)} Survey of Adult Skills and Adult Education Survey, employed 25-64 year-olds

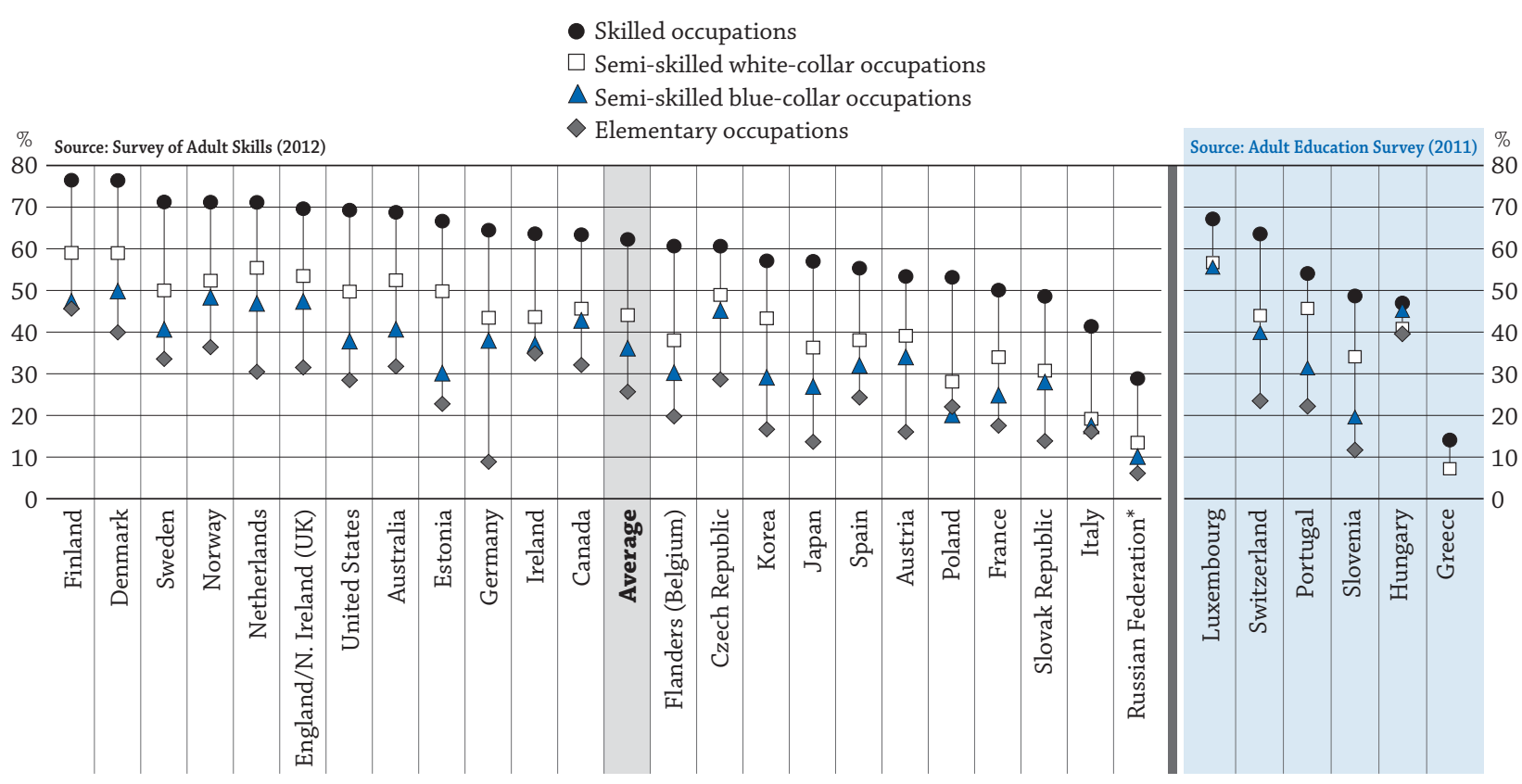

Notes: The data for the countries having participated in the Survey of Adult Skills refer to "employer-sponsored formal and/or non-formal education". The data for the countries having participated in the Eurostat Adult Education Survey (AES) refer to "employer-sponsored", job-related, non-formal education and training".

* See note on data for the Russian Federation in the Methodology section.

Countries are ranked in descending order of participation in employer-sponsored formal and/or non-formal education among people in skilled occupations. Source: OECD. Table C6.2c. See Annex 3 for notes (www.oecd.org/education/education-at-a-glance- 19991487.htm).

StatLink त्गाज http://dx.doi.org/10.1787/888933284342

Workers in skilled occupations participate most often in employer-sponsored formal and/or non-formal education; workers in elementary occupations participate the least. For countries and sub-national entities that participated in the Survey of Adult Skills, in Italy and Poland, all workers who are not in skilled occupations participate at the same, relatively low, rate. Austria, Canada, the Czech Republic, Germany, Norway and the Slovak Republic show small differences between semi-skilled blue-collar and white-collar workers, while in most other countries 
white-collar workers participate more in employer-sponsored formal and/or non-formal education. In Denmark, Finland and Norway, more than $35 \%$ of workers in elementary occupations participate in employer-sponsored formal and/or non-formal education, while more than $70 \%$ workers in skilled occupations do. Countries with the lowest participation for workers in skilled occupations are Austria, France, Italy, Japan, Korea, Poland, the Russian Federation, the Slovak Republic and Spain, where less than $60 \%$ of workers in skilled occupations participate in such learning activities. For countries that participated in the AES, the highest participation from workers in elementary occupation is found in Hungary, where $40 \%$ of workers in elementary occupations participate in employer-sponsored, job-related, non-formal education and training; for workers in skilled occupations, the highest proportion is found in Luxembourg, where about $67 \%$ of workers in skilled occupations participate in such learning activities (Table C6.2c).

\section{Participation as related to type of employment contract and industry}

Perhaps not surprising, part-time workers, those who work up to 30 hours per week, are less likely to participate in formal and/or non-formal education, especially employer-sponsored participation, than full-time workers. Across OECD countries and sub-national entities that participated in the Survey of Adult Skills, 52\% of full-time workers, those who work 30 hours per week or more, participate in such education, while $35 \%$ of part-time workers do. Workers on limited contracts also tend to participate less (43\%) than workers with unlimited contracts (55\%) (Table C6.2d).

\section{Chart C6.3. Participation in employer-sponsored formal and/or non-formal education, by working hours and contract type (2012) \\ Survey of Adult Skills, employed 25-64 year-olds}

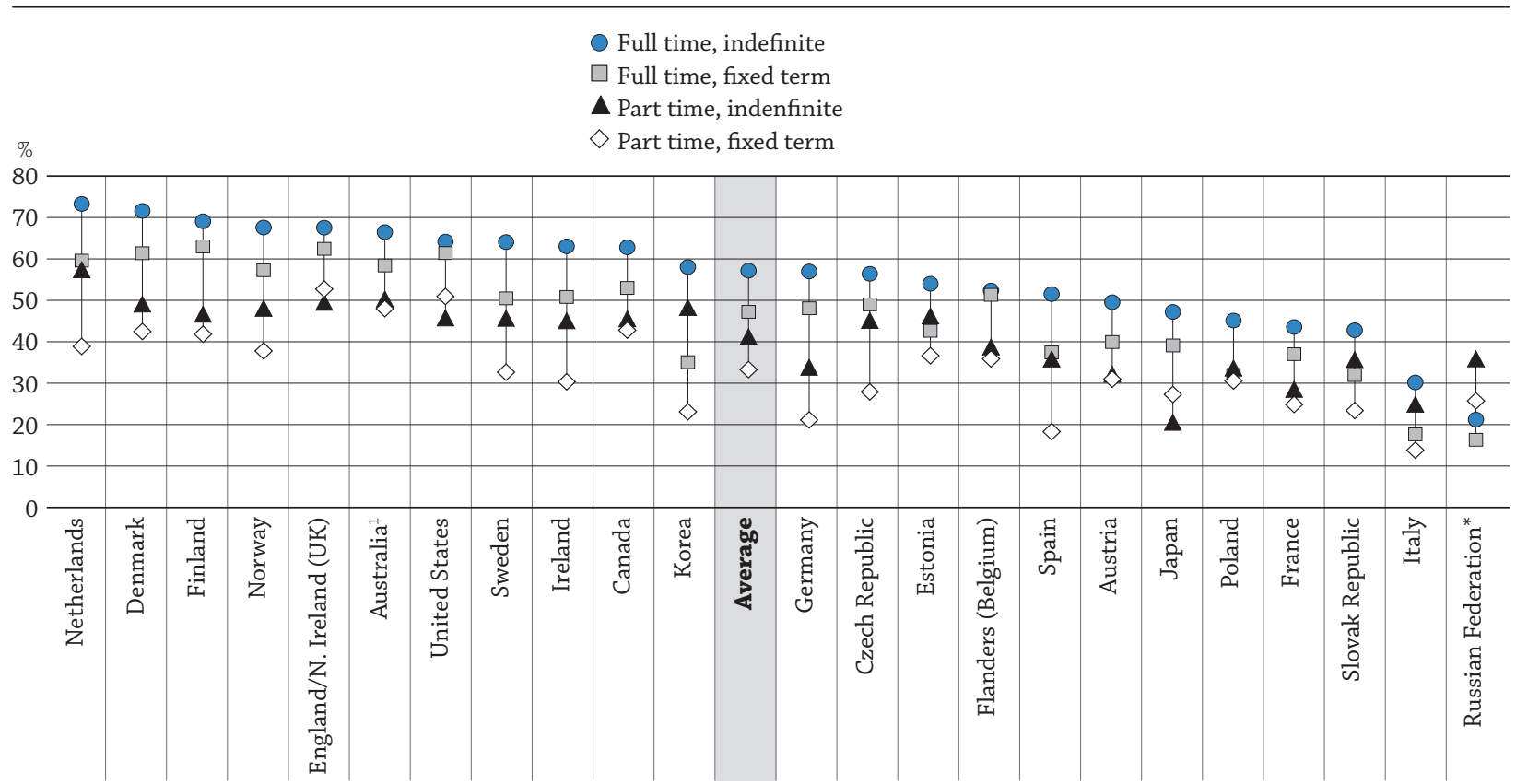

1. For Australia, data based on full time/part time are using a variable which is topped at 60 hours per week while there is no upper limit for other countries.

* See note on data for the Russian Federation in the Methodology section.

Countries are ranked in descending order of participation in employer-sponsored formal and/or non-formal education among people who work full time and have an indefinite contract.

Source: OECD. Table C6.2d. See Annex 3 for notes (www.oecd.org/education/education-at-a-glance- 19991487.htm).

StatLink 䨛项 http://dx.doi.org/10.1787/888933284355

Chart C6.3 shows the effect on participation of the two variables above combined. Employers see less of a risk investing in full-time workers with unlimited contracts, since they will probably stay longer in the firm and repay the investment with greater productivity. The opposite is true for part-time workers with limited contracts. The results confirm this: across OECD countries and sub-national entities that participated in the Survey of Adult Skills, $57 \%$ of full-time workers with unlimited contracts participate in employer-sponsored formal and/or non-formal education, while only $33 \%$ of part-time workers with limited contracts do (Table 6.2d). 
The classification of industries used in the Survey of Adult Skills (i.e. resource industries, goods-producing industries, lower-tier services and upper-tier services) reflects the skills demands of the broad categories. As may be expected, workers in upper-tier service industries have the highest participation rates in all countries, while employees in resource industries have the lowest participation rates in most countries. Across participating OECD countries and sub-national entities, $59 \%$ of workers in upper-tier services participate in such education, while $36 \%$ of workers in resource industries do. Participation rates in the goods-producing industries and the lower-tier services vary, presumably reflecting the skills demands of the goods-producing industries in the countries (Table C6.2e, available on line). See Annex 3 for more details on the type of industries (www.oecd.org/education/education-at-aglance-19991487.htm).

\section{Participation as related to other types of learning at work}

A further indication of the degree in which the working environment is also a learning environment is the availability of other learning activities at the workplace. The index that measures this includes learning new things from supervisors or co-workers; learning-by-doing; and keeping up-to-date with new products or services. Chart C6.4 shows that workers who learn more at work through other activities participate most in employer-sponsored education. It also shows that there is a ceiling effect: while the participation rate rises steeply between those who never learn at work to those who occationally learn at work, it tends to level off among those who frequently learn at work through other activities. This ceiling effect can also be observed in the three single variables that constitute the index, i.e. it is not due to the construction of the index (Table C6.3a).

\section{Chart C6.4. Participation in employer-sponsored formal and/or non-formal education, by use of learning at work (2012) \\ Survey of Adult Skills, employed 25-64 year-olds}

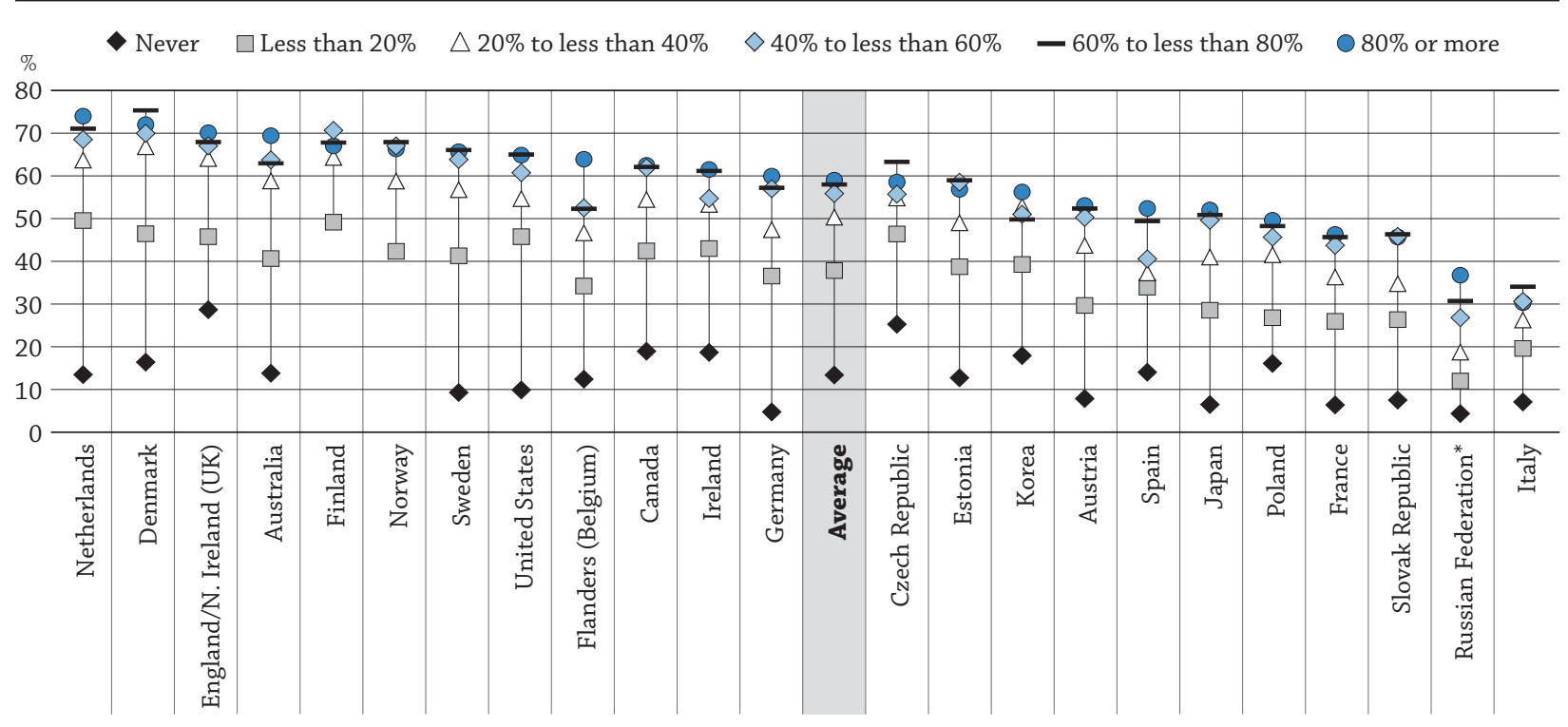

* See note on data for the Russian Federation in the Methodology section.

Countries are ranked in descending order of participation in employer-sponsored formal and/or non-formal education among people who have an index of learning at work of $80 \%$ or more.

Source: OECD. Table C6.3a. See Annex 3 for notes (www.oecd.org/education/education-at-a-glance- 19991487.htm).

StatLink 제내 http://dx.doi.org/10.1787/888933284364

Across OECD countries and sub-national entities that participated in the Survey of Adult Skills, $13 \%$ of the workers who indicated that they never engage in any of the learning activities participate in employer-sponsored formal and/or non-formal education, while $59 \%$ of workers who engage in other learning activities the most often do. The latter group is four times more likely to participate than the former group. In France, Germany, Japan, the Russian Federation and Sweden, workers' participation in employer-sponsored formal and/or non-formal education differs by a magnitude of more than seven times, depending on their engagement in other learning activities at work. These countries show low rates of participation among workers who never engage in such learning activities. Conversely, many countries with relatively high rates of participation in employer-sponsored 
formal and/or non-formal education (over 16\%) among workers who never engage in other learning activities at work show relatively small differences (less than four times) in participation in other learning activities at work. These countries and sub-national entities are Canada, the Czech Republic, England (UK), Ireland, Korea, Northern Ireland (UK) and Poland (Table C6.3a).

\section{Participation as related to the use of information-processing skills at work}

The Survey of Adult Skills asked adults to indicate the frequence with which they use various skills at work. These skills were then grouped into indicies as information-processing skills (including reading, writing, numeracy, ICT and problem-solving skills) and other generic skills (including task discretion, learning at work, influencing skills, co-operative skills, self-organising skills, dexterity, and physical skills) (OECD, 2013).

All of the information-processing skills measured show a fairly strong relationship with participation in employersponsored formal and/or non-formal education. The results confirm the general hypothesis of higher participation in employer-sponsored formal and/or non-formal education of adults with higher skills use (Tables C6.3b to C6.3e, available on line).

The index of reading at work that was created to measure that activity includes eight different tasks. Chart C6.5 shows that across OECD countries and sub-national entities that participated in the Survey of Adult Skills, $67 \%$ of adults who read most frequently at work participate in employer-sponsored formal and/or non-formal education. By contrast, only $14 \%$ of those who reported that they never read at work participate. Participation in employer-sponsored formal and/or non-formal education rises steadily the more frequently people read at work (Table C6.3b, available on line).

\section{Chart C6.5. Participation in employer-sponsored formal and/or non-formal education, by use of reading skills at work (2012) \\ Survey of Adult Skills, employed 25-64 year-olds}

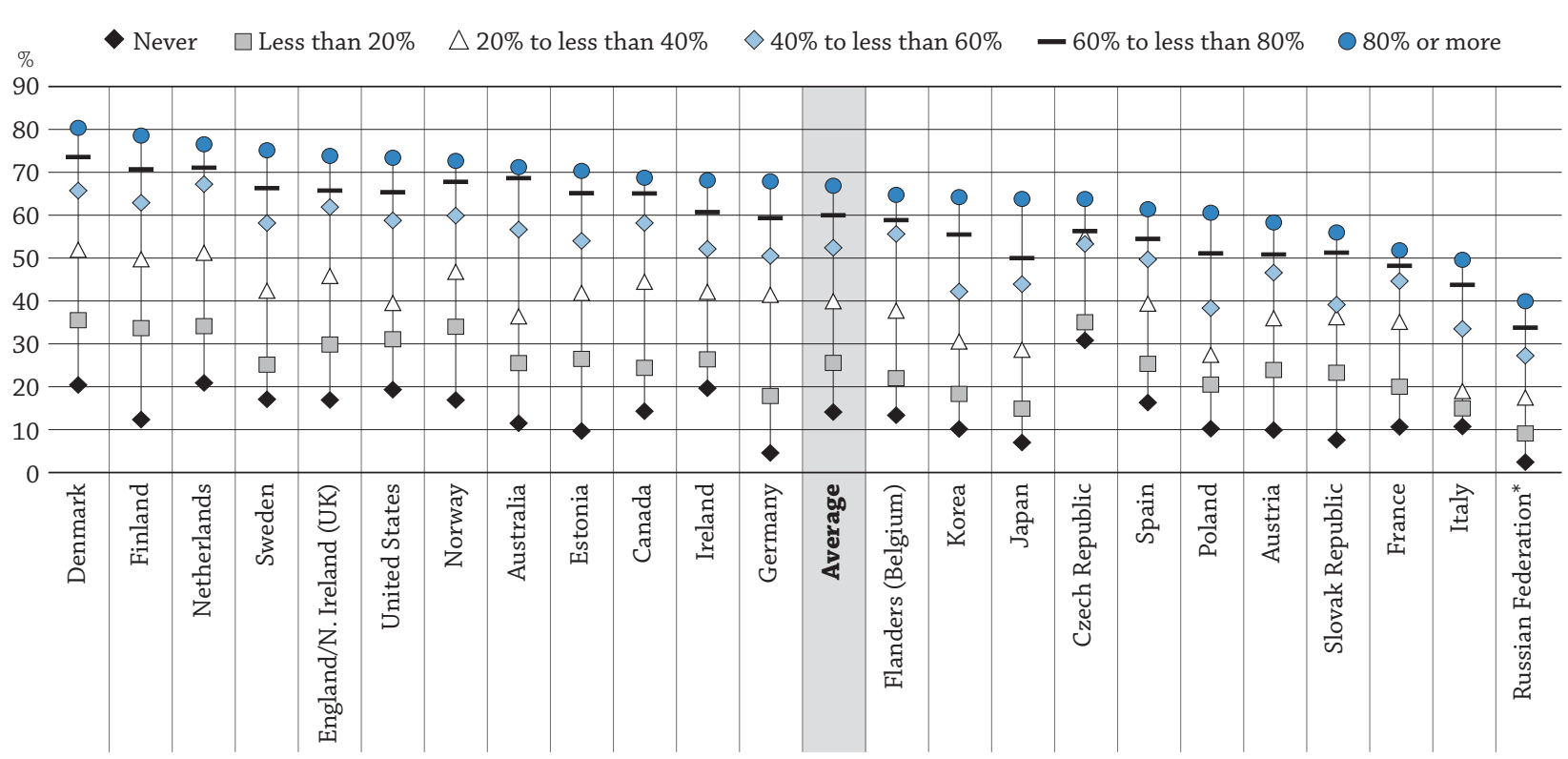

* See note on data for the Russian Federation in the Methodology section.

Countries are ranked in descending order of participation in employer-sponsored formal and/or non-formal education among people who have an index of use of reading skills at work of $80 \%$ or more.

Source: OECD. Table C6.3b, available on line. See Annex 3 for notes (www.oecd.org/education/education-at-a-glance- 19991487.htm).

StatLink 세에 $h t t p: / / d x . d o i . o r g / 10.1787 / 888933284377$

In Denmark, Finland, the Netherlands and Sweden more than three out of four workers who read frequently at work participate in employer-sponsored formal and/or non-formal education (75\% or more). At the other extreme, in Austria, Estonia, Germany, Japan, the Russian Federation and the Slovak Republic, fewer than one in ten (less than 10\%) of the workers who never read at the workplace participate in such learning activities (Table C6.3b, available on line). 
Across OECD countries and sub-national entities that participated in the Survey of Adult Skills, 19\% of the workers who never write at work participate in employer-sponsored formal and/or non-formal education, compared to $67 \%$ of those who write the mostly frequently at work. Participation rates tend to rise as adults write more at work (Table C6.3c, available on line).

Similar results are found when considering the use of numeracy skills at work. On average across OECD countries and sub-national entities that participated in the Survey of Adult Skills, 63\% of workers who use their numeracy skills most frequently at work participate in employer-sponsored formal and/or non-formal education, compared to $33 \%$ of those who never use such skills (Table C6.3d, available on line).

\section{Participation as related to the use of certain generic skills at work}

Chart C6.6 shows that using influencing skills at work are strongly related to participation in employer-sponsored formal and/or non-formal education. Participation rates rise steadily as the use of these skills at work increases from $33 \%$ among workers who never use influencing skills at work to $63 \%$ among workers who use these skills most frequently. The latter are thus twice as likely to participate in employer-sponsored education as the former (Table C6.3f, available on line).

\section{Chart C6.6. Participation in employer-sponsored formal and/or non-formal education, by use of influencing skills at work (2012)}

Survey of Adult Skills, employed 25-64 year-olds

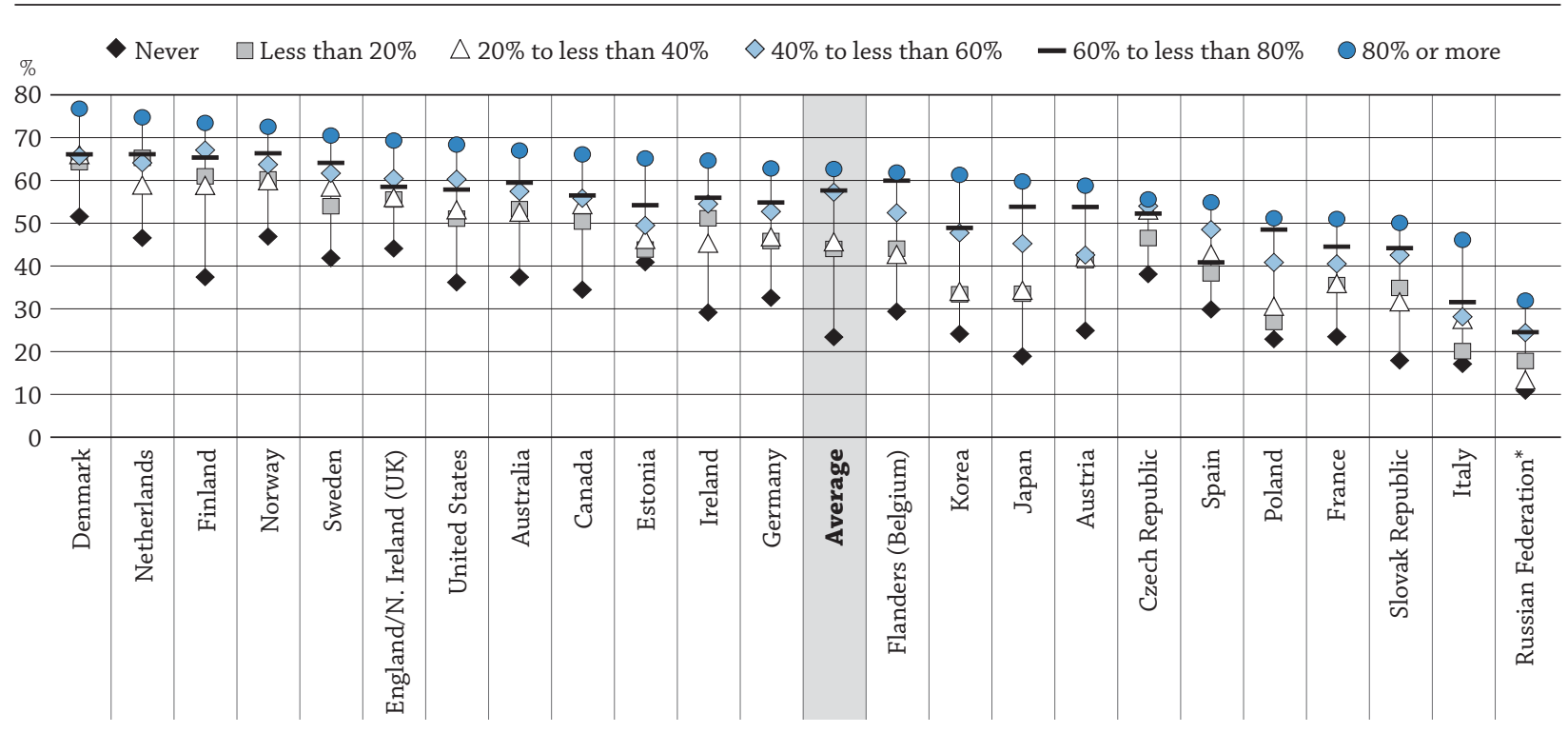

* See note on data for the Russian Federation in the Methodology section.

Countries are ranked in descending order of participation in employer-sponsored formal and/or non-formal education among people who have an index of use of influencing skills at work of $80 \%$ or more.

Source: OECD. Table C6.3f, available on line. See Annex 3 for notes (www.oecd.org/education/education-at-a-glance-19991487.htm).

StatLink 제개 http://dx.doi.org/10.1787/888933284386

For most countries participation in employer-sponsored formal and/or non-formal education rises steadily with the frequency of the workers' use of influencing skills at work. In Austria, the Czech Republic, Flanders (Belgium), Germany and Poland the participation rates of the two groups with the highest frequency of use of influencing skills are close together (5 percentage-point difference or less), suggesting a mild ceiling effect (Table C6.3f, available on line).

Workers who never use task-discretion skills (choosing or changing the sequence of job tasks, the speed of work, working hours; and deciding how to do the job) at work participate least in employer-sponsored formal and/or non-formal education. Across OECD countries and sub-national entities that participated in the Survey of Adult Skills, only $29 \%$ of such workers participate, on average. In most countries, the participation rate rises steeply between those who never use task-discretion skills at work and those who use those skills occationally at work, but falls for those who use those skills the most (Table C6.3g, available on line). 


\section{Definitions}

Adults refers to 25-64 year-olds.

Education and training: Formal education is planned education provided in the system of schools, colleges, universities and other formal educational institutions that normally constitutes a continuous "ladder" of full-time education for children and young people. The providers may be public or private. Non-formal education is sustained educational activity that does not correspond exactly to the definition of formal education. Non-formal education may take place both within and outside educational institutions and cater to individuals of all ages. Depending on country contexts, it may cover education programmes in adult literacy, basic education for out-of-school children, life skills, work skills, and general culture. The Survey of Adult Skills uses a list of possible non-formal education activities, including open or distance-learning courses, private lessons, organised sessions for on-the-job training, and workshops or seminars to prompt respondents to list all of their learning activities during the previous 12 months. Some of these learning activities might be of short duration.

Employer-sponsored formal and/or non-formal education: Employer support can be offered in the form of time (i.e. educational activities that take place fully or partly during paid working hours), or financial support (giving grants to employees to participate in educational activities).

Generic skills at work: Learning at work corresponds to learning new things from supervisors or co-workers; learning-by-doing; and keeping up-to-date with new products or services; influencing skills corresponds to instructing, teaching or training people; making speeches or presentations; selling products or services; advising people; planning others' activities; persuading or influencing others; and negotiating; task discretion corresponds to choosing or changing the sequence of job tasks, the speed of work, working hours; and deciding how to do the job; and co-operative skills corresponds to co-operating or collaborating with co-workers.

Index of: use of learning at work, use of reading skills at work, use of writing skills at work, use of numeracy skills at work, use of ICT skills at work, use of influencing skills at work, use of task discretion at work, and use of co-operative skills at work: The indices are categorised as Warm's mean weighted likelihood estimation (WLE). It is derived from variables that are based on a Likert scale from "Never" to "Every day". For these skills-use variables, numerical comparisons between the use of different skills are possible: a value of 0 indicates that the skill is never used; a value of 1 indicates that it is used less than once a month; a value of 2 indicates that the skill is used less than once a week but at least once a month; a value of 3 indicates that it is used at least once a week but not every day; and a value of 4 indicates that it is used every day. The categories should therefore be interpreted based on the frequency of the activity, with "Never" being the least frequent and "80\% or more" being the most frequent. For more details on the index, see page 143 of OECD Skills Outlook 2013: First Results from the Survey of Adult Skills (OECD, 2013).

Industry type: resource industries; goods-producing industries; lower-tier services; and upper-tier services are defined by the variable ISIC2C. See Annex 3 for the detailed list of industries included in each group.

Information-processing skills: Reading corresponds to reading documents (directions, instructions, letters, memos, e-mails, articles, books, manuals, bills, invoices, diagrams, maps); writing corresponds to writing documents (letters, memos, e-mails, articles, reports, forms); numeracy corresponds to calculating prices, costs or budgets; use of fractions, decimals or percentages; use of calculators; preparing graphs or tables; algebra or formulas; use of advanced mathematics or statistics (calculus, trigonometry, regressions); and ICT skills corresponds to using e-mail, Internet, spreadsheets, word processors, programming languages; conducting transactions on line; and participating in online discussions (conferences, chats).

Levels of education: Below upper secondary corresponds to ISCED-97 Levels 0, 1, 2 and 3C short programmes; upper secondary or post-secondary non-tertiary corresponds to ISCED-97 Levels 3A, 3B, 3C long programmes, and Level 4; and tertiary corresponds to ISCED-97 Levels 5A, 5B and 6.

Occupation: Skilled occupations include legislators, senior officials and managers (ISCO 1), professionals (ISCO 2), technicians and associate professionals (ISCO 3); semi-skilled white-collar occupations include clerks (ISCO 4), service workers, and shop and market sales workers (ISCO 5); semi-skilled blue-collar occupations include skilled agricultural and fishery workers (ISCO 6), craft and related trades workers (ISCO 7), and plant and machine operators and assemblers (ISCO 8), and elementary occupations include low-skilled occupations (ISCO 9).

Skill groups refer to skills and readiness to use information and communication technologies (ICT) for problem solving in technology-rich environments. Each group is described in terms of the characteristics of the types of 
tasks that can be successfully completed by adults and the related scores in the assessment of problem solving in technology-rich environments in the Survey of Adult Skills.

- Group 0 (no computer experience)

- Group 1 (refused the computer-based assessment)

- Group 2 (failed ICT core test or minimal problem-solving skills - scored below Level 1 in the problem solving in technology-rich environments assessment)

- Group 3 (moderate ICT and problem-solving skills - scored at Level 1 in the problem solving in technology-rich environments assessment)

- Group 4 (good ICT and problem-solving skills - scored at Level 2 or Level 3 in the problem solving in technologyrich environments assessment)

Working hours and contract type: Full time refers to more than 30 hours per week and fixed-term contract includes fixed-term contract, temporary employment agency contract, and an apprenticeship or other training scheme.

\section{Methodology}

All data are based on the Survey of Adult Skills (PIAAC) (2012). PIAAC is the OECD Programme for the International Assessment of Adult Competencies.

A number of skills-use variables are taken directly from questions asked in the background questionnaire of the Survey of Adult Skills. Other variables have been derived based on more than one question from the background questionnaire. These variables have been transformed so that they have a mean of 2 and a standard deviation of 1 across the pooled sample of all participating countries, thus allowing for meaningful comparisons across countries (OECD, 2013, p. 143). For more detailed information, see the Technical Report of the Survey of Adult Skills (OECD, 2014, Chapter 20) and see Annex 3 for notes (www.oecd.org/education/education-at-a-glance-19991487.htm).

Note regarding data from the Russian Federation in the Survey of Adult Skills (PIAAC)

Readers should note that the sample for the Russian Federation does not include the population of the Moscow municipal area. The data published, therefore, do not represent the entire resident population aged 16-65 in Russia but rather the population of Russia excluding the population residing in the Moscow municipal area. More detailed information regarding the data from the Russian Federation as well as that of other countries can be found in the Technical Report of the Survey of Adult Skills (OECD, 2014).

\section{References}

OECD (2014), Technical Report of the Survey of Adult Skills, www.oecd.org/site/piaac/ Technical\%20Report 17OCT13.pdf, pre-publication copy.

OECD (2013), OECD Skills Outlook 2013: First Results from the Survey of Adult Skills, OECD Publishing, Paris, http://dx.doi. org/10.1787/9789264204256-en.

\section{Indicator C6 Tables}

StatLink त्माज् http://dx.doi.org/10.1787/888933286024

WEB Table C6.1 (L) Participation in employer-sponsored formal and/or non-formal education, by literacy proficiency level (2012)

WEB Table C6.1 (N) Participation in employer-sponsored formal and/or non-formal education, by numeracy proficiency level (2012)

Table C6.1 (P) Participation in employer-sponsored formal and/or non-formal education, by skills and readiness to use information and communication technologies for problem solving (2012)

Table C6.2a Participation in employer-sponsored education, by educational attainment $(2011,2012)$

WEB Table C6.2b

Participation in employer-sponsored education, by gender and age group $(2011,2012)$

Table C6.2c

Participation in employer-sponsored education, by occupation $(2011,2012)$

Table C6.2d

Participation in employer-sponsored formal and/or non-formal education, by working hours and contract type (2012)

WEB Table C6.2e Participation in employer-sponsored formal and/or non-formal education, by industry (2012) 
Table C6.3a Participation in employer-sponsored formal and/or non-formal education, by use of learning at work (2012)

WEB Table C6.3b Participation in employer-sponsored formal and/or non-formal education, by use of reading skills at work (2012)

WEB Table C6.3c

Participation in employer-sponsored formal and/or non-formal education, by use of writing skills at work (2012) Participation in employer-sponsored formal and/or non-formal education, by use of numeracy skills at work (2012)

WEB Table C6.3e Participation in employer-sponsored formal and/or non-formal education, by use of ICT skills at work (2012)

WEB Table C6.3f Participation in employer-sponsored formal and/or non-formal education, by use of influencing skills at work (2012)

WEB Table C6.3g Participation in employer-sponsored formal and/or non-formal education, by use of task discretion at work (2012) 
Table C6.1 (P). Participation in employer-sponsored formal and/or non-formal education, by skills and readiness to use information and communication technologies

for problem solving (2012)

Survey of Adult Skills, employed 25-64 year-olds

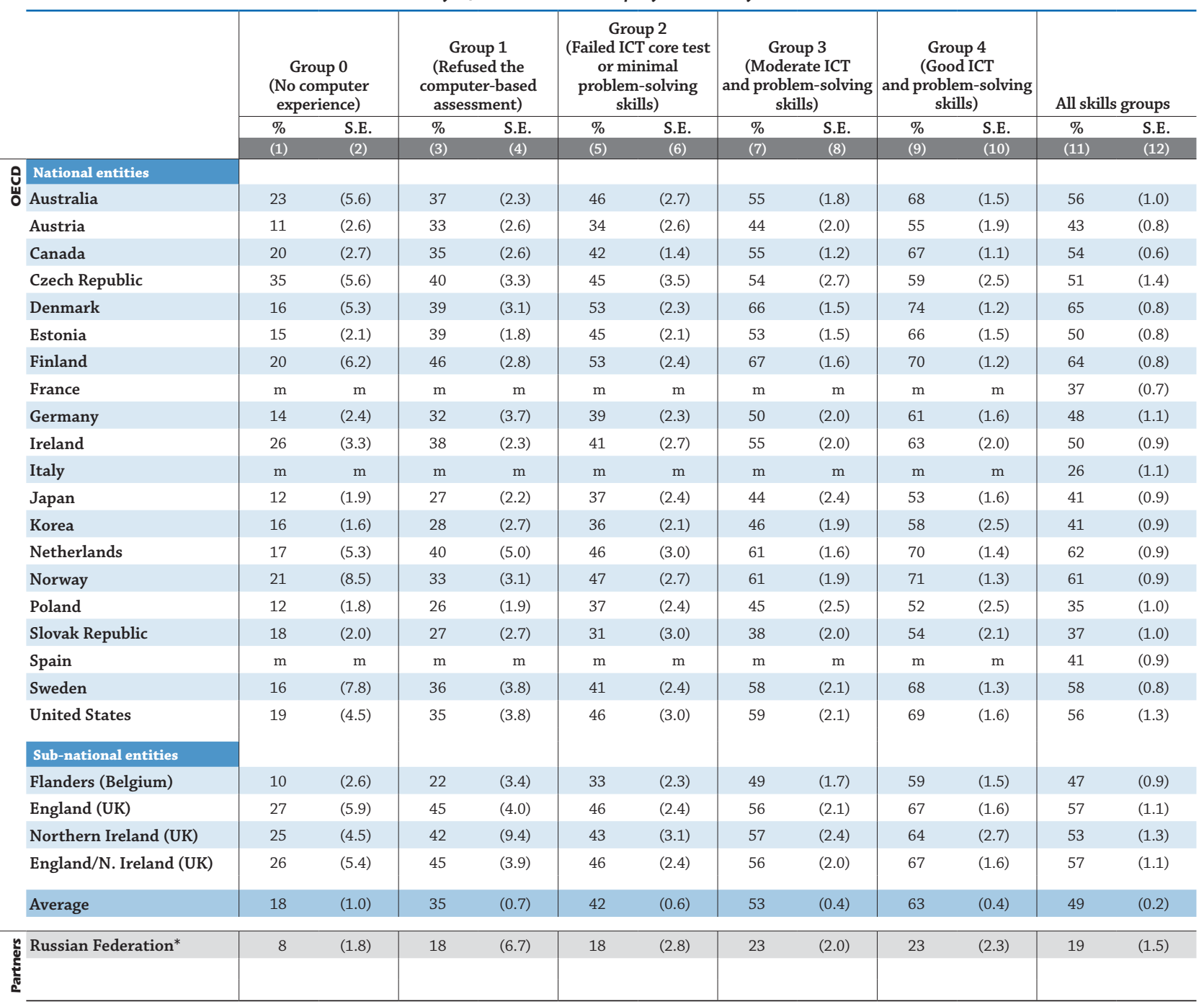

Notes: Participation in education or training during previous 12 months. The category "All skills groups" corresponds to the average participation in employersponsored formal and/or non-formal education for the adults across all skills groups.

* See note on data for the Russian Federation in the Methodology section.

Source: OECD. Survey of Adult Skills (PIAAC) (2012). See Annex 3 for notes (www.oecd.org/education/education-at-a-glance-19991487.htm).

Please refer to the Reader's Guide for information concerning symbols for missing data and abbreviations.

StatLink 젶ㄴ http://dx.doi.org/10.1787/888933286032 
Table C6.2a. Participation in employer-sponsored education, by educational attainment $(2011,2012)$

Employed 25-64 year-olds

\begin{tabular}{|c|c|c|c|c|c|c|c|c|}
\hline & \multicolumn{8}{|c|}{ Formal and/or non-formal education, Survey of Adult Skills (2012) } \\
\hline & \multicolumn{2}{|c|}{ Below upper secondary } & \multicolumn{2}{|c|}{$\begin{array}{c}\text { Upper secondary } \\
\text { or post-secondary non-tertiary }\end{array}$} & \multicolumn{2}{|c|}{ Tertiary } & \multicolumn{2}{|c|}{ All levels of education } \\
\hline & $\%$ & S.E. & $\%$ & S.E. & $\%$ & S.E. & $\%$ & S.E. \\
\hline & (1) & (2) & (3) & (4) & (5) & (6) & (7) & (8) \\
\hline \multicolumn{9}{|l|}{ National entities } \\
\hline Australia & 37 & (1.9) & 52 & (1.4) & 70 & $(1.2)$ & 56 & (1.0) \\
\hline Austria & 25 & $(2.1)$ & 42 & (1.1) & 57 & (2.1) & 43 & $(0.8)$ \\
\hline Canada & 30 & (1.9) & 48 & (1.0) & 62 & $(0.8)$ & 54 & (0.6) \\
\hline Czech Republic & 33 & (3.8) & 50 & (1.6) & 59 & (3.2) & 51 & (1.4) \\
\hline Denmark & 46 & $(2.2)$ & 61 & (1.3) & 76 & (1.0) & 65 & $(0.8)$ \\
\hline Estonia & 30 & $(2.2)$ & 41 & (1.2) & 63 & (1.0) & 50 & $(0.8)$ \\
\hline Finland & 39 & (3.0) & 57 & (1.3) & 74 & (1.1) & 64 & $(0.8)$ \\
\hline France & 21 & (1.4) & 34 & (1.1) & 51 & $(1.2)$ & 37 & $(0.7)$ \\
\hline Germany & 20 & $(2.9)$ & 43 & (1.4) & 61 & (1.6) & 48 & (1.1) \\
\hline Ireland & 32 & $(2.2)$ & 43 & (1.4) & 64 & (1.5) & 50 & $(0.9)$ \\
\hline Italy & 15 & (1.8) & 28 & (1.4) & 48 & (2.4) & 26 & (1.1) \\
\hline Japan & 22 & $(2.5)$ & 32 & (1.4) & 52 & $(1.2)$ & 41 & $(0.9)$ \\
\hline Korea & 18 & (1.4) & 33 & (1.3) & 58 & (1.4) & 41 & (0.9) \\
\hline Netherlands & 44 & (1.7) & 60 & (1.5) & 74 & $(1.2)$ & 62 & $(0.9)$ \\
\hline Norway & 43 & $(2.2)$ & 58 & (1.7) & 72 & (1.1) & 61 & $(0.9)$ \\
\hline Poland & 18 & $(3.2)$ & 25 & (1.2) & 53 & (1.8) & 35 & (1.0) \\
\hline Slovak Republic & 12 & $(2.1)$ & 33 & (1.4) & 55 & (1.7) & 37 & (1.0) \\
\hline Spain & 25 & (1.4) & 40 & (2.4) & 56 & (1.3) & 41 & (0.9) \\
\hline Sweden & 38 & $(2.9)$ & 56 & (1.2) & 69 & $(1.2)$ & 58 & $(0.8)$ \\
\hline United States & 25 & $(3.2)$ & 49 & (1.7) & 70 & (1.3) & 56 & (1.3) \\
\hline \multicolumn{9}{|l|}{ Sub-national entities } \\
\hline Flanders (Belgium) & 24 & $(2.5)$ & 38 & (1.5) & 61 & (1.5) & 47 & $(0.9)$ \\
\hline England (UK) & 41 & $(2.6)$ & 55 & (1.5) & 67 & (1.6) & 57 & (1.1) \\
\hline Northern Ireland (UK) & 31 & $(2.5)$ & 54 & (2.4) & 67 & (1.8) & 53 & (1.3) \\
\hline England/N. Ireland (UK) & 41 & $(2.5)$ & 55 & (1.5) & 67 & (1.5) & 57 & (1.1) \\
\hline Average & 29 & $(0.5)$ & 44 & $(0.3)$ & 62 & $(0.3)$ & 49 & $(0.2)$ \\
\hline Russian Federation* & 11 & (7.6) & 9 & (1.6) & 23 & (1.5) & 19 & (1.5) \\
\hline
\end{tabular}

\begin{tabular}{|c|c|c|c|c|c|c|c|c|}
\hline & \multicolumn{8}{|c|}{ Job-related non-formal education, Adult Education Survey (2011) } \\
\hline & \multicolumn{2}{|c|}{ Below upper secondary } & \multicolumn{2}{|c|}{$\begin{array}{c}\text { Upper secondary } \\
\text { or post-secondary non-tertiary }\end{array}$} & \multicolumn{2}{|c|}{ Tertiary } & \multicolumn{2}{|c|}{ All levels of education } \\
\hline & $\%$ & S.E. & $\%$ & S.E. & $\%$ & S.E. & $\%$ & S.E. \\
\hline & (1) & (2) & (3) & (4) & (5) & (6) & (7) & (8) \\
\hline Q Greece & 0 & $\mathrm{~m}$ & 3 & $\mathrm{~m}$ & 12 & $\mathrm{~m}$ & 5 & $\mathrm{~m}$ \\
\hline ڤ̈ Hungary & 17 & $\mathrm{~m}$ & 29 & $\mathrm{~m}$ & 40 & $\mathrm{~m}$ & 29 & $\mathrm{~m}$ \\
\hline Luxembourg & 36 & $\mathrm{~m}$ & 49 & $\mathrm{~m}$ & 55 & $\mathrm{~m}$ & 48 & $\mathrm{~m}$ \\
\hline Portugal & 21 & $\mathrm{~m}$ & 41 & $\mathrm{~m}$ & 50 & $\mathrm{~m}$ & 29 & $\mathrm{~m}$ \\
\hline Slovenia & 6 & $\mathrm{~m}$ & 20 & $\mathrm{~m}$ & 46 & $\mathrm{~m}$ & 23 & $\mathrm{~m}$ \\
\hline Switzerland & 17 & $\mathrm{~m}$ & 42 & $\mathrm{~m}$ & 60 & $\mathrm{~m}$ & 45 & $\mathrm{~m}$ \\
\hline
\end{tabular}

Notes: Participation in education or training during previous 12 months. The category "All levels of education" corresponds to the average participation in employersponsored formal and/or non-formal education for the adults across all education levels.

* See note on data for the Russian Federation in the Methodology section.

Source: OECD. Survey of Adult Skills (PIAAC) (2012) and Adult Education Survey (AES) (2011). See Annex 3 for notes (www.oecd.org/education/education-ata-glance-19991487.htm).

Please refer to the Reader's Guide for information concerning symbols for missing data and abbreviations.

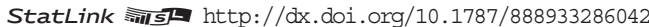


Table C6.2c. Participation in employer-sponsored education, by occupation $(2011,2012)$ Survey of Adult Skills, employed 25-64 year-olds

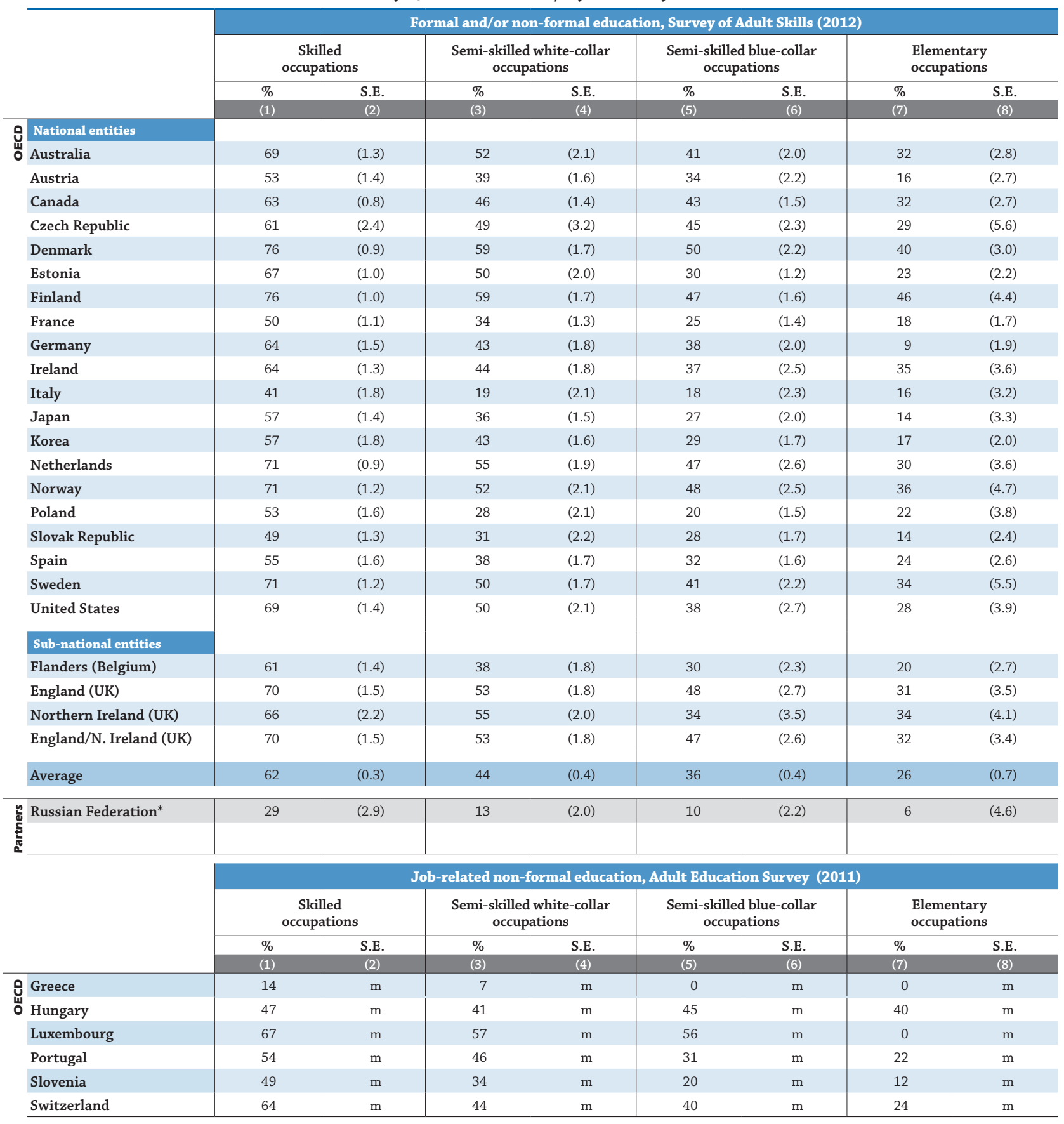

Note: Participation in education or training during previous 12 months.

* See note on data for the Russian Federation in the Methodology section.

Source: OECD. Survey of Adult Skills (PIAAC) (2012) and Adult Education Survey (AES) (2011). See Annex 3 for notes (www.oecd.org/education/education-ata-glance-19991487.htm).

Please refer to the Reader's Guide for information concerning symbols for missing data and abbreviations.

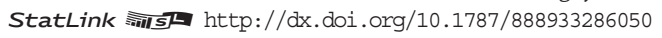


Table C6.2d. Participation in employer-sponsored formal and/or non-formal education, by working hours and contract type (2012)

Survey of Adult Skills, employed 25-64 year-olds

\begin{tabular}{|c|c|c|c|c|c|c|c|c|}
\hline & \multicolumn{8}{|c|}{ Working hours by contract type } \\
\hline & \multicolumn{2}{|c|}{ Full time, indefinite } & \multicolumn{2}{|c|}{ Full time, fixed term } & \multicolumn{2}{|c|}{ Part time, indefinite } & \multicolumn{2}{|c|}{ Part time, fixed term } \\
\hline & $\%$ & S.E. & $\%$ & S.E. & $\%$ & S.E. & $\%$ & S.E. \\
\hline \multicolumn{9}{|l|}{ National entities } \\
\hline Australia $^{1}$ & 67 & $(1.3)$ & 58 & $(1.9)$ & 50 & $(2.5)$ & 48 & (3.0) \\
\hline Austria & 50 & $(1.1)$ & 40 & $(3.8)$ & 32 & $(2.4)$ & 31 & $(5.4)$ \\
\hline Czech Republic & 56 & (1.8) & 49 & $(4.7)$ & 45 & $(7.7)$ & 28 & (7.3) \\
\hline Denmark & 72 & $(0.9)$ & 61 & $(2.9)$ & 49 & $(2.8)$ & 42 & $(6.8)$ \\
\hline Estonia & 54 & $(1.0)$ & 43 & $(2.4)$ & 46 & $(3.2)$ & 37 & $(4.4)$ \\
\hline Finland & 69 & $(0.8)$ & 63 & $(2.6)$ & 47 & (3.7) & 42 & $(5.8)$ \\
\hline France & 44 & $(0.9)$ & 37 & (3.1) & 29 & $(2.0)$ & 25 & $(4.0)$ \\
\hline Germany & 57 & (1.3) & 48 & $(3.7)$ & 34 & $(2.5)$ & 21 & (3.3) \\
\hline Italy & 30 & $(1.5)$ & 18 & $(3.0)$ & 25 & (3.4) & 14 & $(4.9)$ \\
\hline Japan & 47 & $(1.2)$ & 39 & $(3.2)$ & 21 & $(2.1)$ & 27 & (3.0) \\
\hline Korea & 58 & (1.4) & 35 & (1.6) & 48 & $(5.2)$ & 23 & (3.3) \\
\hline Netherlands & 73 & $(1.2)$ & 60 & (3.5) & 57 & (1.8) & 39 & (3.8) \\
\hline Norway & 68 & (1.1) & 57 & $(3.7)$ & 48 & $(3.0)$ & 38 & (4.9) \\
\hline Poland & 45 & $(1.5)$ & 32 & $(2.0)$ & 34 & $(4.7)$ & 31 & $(4.7)$ \\
\hline Slovak Republic & 43 & $(1.2)$ & 32 & (2.9) & 36 & $(6.7)$ & 23 & (7.5) \\
\hline Spain & 52 & (1.1) & 37 & $(2.5)$ & 36 & $(4.0)$ & 18 & (3.5) \\
\hline Sweden & 64 & $(0.9)$ & 51 & $(3.8)$ & 46 & $(4.1)$ & 33 & $(5.1)$ \\
\hline United States & 64 & (1.8) & 61 & $(1.8)$ & 46 & $(6.6)$ & 51 & (3.7) \\
\hline \multicolumn{9}{|l|}{ Sub-national entities } \\
\hline Flanders (Belgium) & 52 & (1.3) & 51 & $(5.4)$ & 39 & $(2.3)$ & 36 & (8.9) \\
\hline England (UK) & 68 & (1.4) & 63 & (3.4) & 50 & $(2.7)$ & 53 & $(5.2)$ \\
\hline Average & 57 & $(0.3)$ & 47 & $(0.7)$ & 41 & $(0.8)$ & 33 & (1.1) \\
\hline Russian Federation* & 21 & (1.8) & 16 & (2.7) & 36 & $(5.2)$ & 26 & (2.8) \\
\hline
\end{tabular}

Notes: Participation in education or training during previous 12 months. Additional columns on working hours and contract type are available for consultation on line (see StatLink below).

1. For Australia, data based on full time/part time are using a variable which is topped at 60 hours per week while there is no upper limit for other countries.

* See note on data for the Russian Federation in the Methodology section.

Source: OECD. Survey of Adult Skills (PIAAC) (2012). See Annex 3 for notes (www.oecd.org/education/education-at-a-glance-19991487.htm).

Please refer to the Reader's Guide for information concerning symbols for missing data and abbreviations.

StatLink silst http://dx.doi.org/10.1787/888933286060 
Table C6.3a. Participation in employer-sponsored formal and/or non-formal education, by use of learning at work (2012)

Survey of Adult Skills, employed 25-64 year-olds

\begin{tabular}{|c|c|c|c|c|c|c|c|c|c|c|c|c|}
\hline & \multicolumn{12}{|c|}{ Index of learning at work ${ }^{1}$} \\
\hline & \multicolumn{2}{|c|}{ Never } & \multicolumn{2}{|c|}{ Less than $20 \%$} & \multicolumn{2}{|c|}{$\begin{array}{c}20 \% \text { to } \\
\text { less than } 40 \%\end{array}$} & \multicolumn{2}{|c|}{$\begin{array}{c}40 \% \text { to } \\
\text { less than } 60 \%\end{array}$} & \multicolumn{2}{|c|}{$\begin{array}{c}60 \% \text { to } \\
\text { less than } 80 \%\end{array}$} & \multicolumn{2}{|c|}{$80 \%$ or more } \\
\hline & $\%$ & S.E. & $\%$ & S.E. & $\%$ & S.E. & $\%$ & S.E. & $\%$ & S.E. & $\%$ & S.E. \\
\hline & (1) & (2) & (3) & (4) & (5) & (6) & (7) & (8) & (9) & (10) & (11) & (12) \\
\hline \multicolumn{13}{|l|}{ National entities } \\
\hline Australia & 14 & $(4.3)$ & 41 & $(2.6)$ & 59 & (2.5) & 64 & $(1.8)$ & 63 & $(2.2)$ & 69 & $(2.1)$ \\
\hline Austria & 8 & $(4.4)$ & 30 & (1.9) & 44 & (1.8) & 50 & (1.9) & 52 & (2.3) & 53 & (3.1) \\
\hline Canada & 19 & (3.3) & 42 & (1.9) & 54 & (1.5) & 62 & $(1.2)$ & 62 & $(1.3)$ & 62 & $(1.4)$ \\
\hline Czech Republic & 25 & $(7.0)$ & 46 & $(2.8)$ & 55 & (3.2) & 56 & (2.9) & 63 & (3.6) & 59 & (4.3) \\
\hline Denmark & 16 & $(6.1)$ & 46 & $(2.2)$ & 67 & (1.6) & 70 & (1.7) & 75 & (1.6) & 72 & (1.8) \\
\hline Estonia & 13 & $(3.0)$ & 39 & (1.8) & 49 & (1.7) & 59 & $(1.5)$ & 59 & $(2.1)$ & 57 & (1.8) \\
\hline Finland & c & c & 49 & $(2.4)$ & 64 & (2.1) & 71 & $(1.6)$ & 68 & (1.9) & 67 & (2.0) \\
\hline France & 6 & (1.9) & 26 & $(1.7)$ & 36 & (1.7) & 44 & $(1.8)$ & 46 & $(1.7)$ & 46 & (1.6) \\
\hline Germany & 5 & $(2.8)$ & 37 & $(2.1)$ & 47 & (2.0) & 57 & (1.9) & 57 & $(2.6)$ & 60 & (3.1) \\
\hline Ireland & 19 & $(4.6)$ & 43 & $(2.1)$ & 53 & (2.5) & 55 & $(2.5)$ & 61 & $(2.2)$ & 62 & (2.1) \\
\hline Italy & 7 & (3.1) & 20 & $(2.2)$ & 26 & (2.2) & 31 & $(2.6)$ & 34 & $(3.0)$ & 30 & (2.9) \\
\hline Japan & 6 & $(2.2)$ & 29 & (1.7) & 41 & (2.0) & 50 & (1.7) & 51 & $(2.1)$ & 52 & (3.5) \\
\hline Korea & 18 & $(2.1)$ & 39 & (1.6) & 53 & (2.0) & 51 & $(2.4)$ & 50 & $(2.9)$ & 56 & (4.1) \\
\hline Netherlands & 13 & $(4.0)$ & 50 & $(2.1)$ & 64 & (2.0) & 69 & (1.9) & 71 & $(2.0)$ & 74 & (2.5) \\
\hline Norway & c & c & 42 & $(3.0)$ & 59 & (2.0) & 67 & (1.4) & 68 & $(1.7)$ & 66 & (1.9) \\
\hline Poland & 16 & (3.6) & 27 & (1.7) & 42 & (2.4) & 46 & (3.3) & 48 & $(2.4)$ & 50 & $(2.8)$ \\
\hline Slovak Republic & 8 & $(2.8)$ & 26 & (1.9) & 35 & (2.4) & 46 & $(2.2)$ & 46 & $(2.4)$ & 46 & (2.6) \\
\hline Spain & 14 & (3.4) & 34 & $(2.7)$ & 37 & (2.6) & 41 & $(2.8)$ & 49 & $(2.5)$ & 52 & (1.5) \\
\hline Sweden & 9 & $(5.8)$ & 41 & (2.6) & 57 & $(2.2)$ & 64 & (1.7) & 66 & (1.9) & 66 & $(2.1)$ \\
\hline United States & 10 & $(4.4)$ & 46 & $(3.1)$ & 55 & (2.7) & 61 & $(2.2)$ & 65 & $(2.1)$ & 65 & (1.7) \\
\hline \multicolumn{13}{|l|}{ Sub-national entities } \\
\hline Flanders (Belgium) & 12 & $(4.1)$ & 34 & $(2.1)$ & 47 & (1.8) & 53 & $(1.7)$ & 52 & $(2.6)$ & 64 & $(2.4)$ \\
\hline England (UK) & 29 & $(5.9)$ & 46 & $(2.4)$ & 64 & (2.6) & 67 & $(2.1)$ & 68 & $(2.3)$ & 70 & $(2.5)$ \\
\hline Northern Ireland (UK) & 20 & $(5.5)$ & 44 & (2.8) & 60 & (2.9) & 65 & (3.1) & 66 & (3.5) & 62 & (3.9) \\
\hline England/N. Ireland (UK) & 29 & $(5.6)$ & 46 & $(2.4)$ & 64 & (2.5) & 67 & $(2.0)$ & 68 & $(2.2)$ & 70 & $(2.4)$ \\
\hline Average & 13 & $(0.9)$ & 38 & $(0.5)$ & 50 & $(0.5)$ & 56 & $(0.4)$ & 58 & $(0.5)$ & 59 & $(0.5)$ \\
\hline Russian Federation* & 4 & $(2.0)$ & 12 & (2.3) & 19 & (2.2) & 27 & (3.3) & 31 & (3.4) & 37 & $(4.1)$ \\
\hline
\end{tabular}

Note: Participation in education or training during previous 12 months.

1. The index of learning at work is categorised as Warm's mean weighted likelihood estimation (WLE). It is derived from variables that are based on a Likert scale from "Never" to "Every day". The categories should therefore be interpreted based on the frequency of the activity, with "Never" being the least frequent and " $80 \%$ or more" being the most frequent. For more details on the index, see page 143 of the OECD Skills Outlook 2013: First Results from the Survey of Adult Skills. (http://dx.doi. org/10.1787/9789264204256-en).

* See note on data for the Russian Federation in the Methodology section.

Source: OECD. Survey of Adult Skills (PIAAC) (2012). See Annex 3 for notes (www.oecd.org/education/education-at-a-glance-19991487.htm).

Please refer to the Reader's Guide for information concerning symbols for missing data and abbreviations.

StatLink 제개 http://dx.doi.org/10.1787/888933286070 



\section{Chapter}

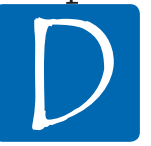

\section{THE LEARNING ENVIRONMENT AND ORGANISATION OF SCHOOLS}

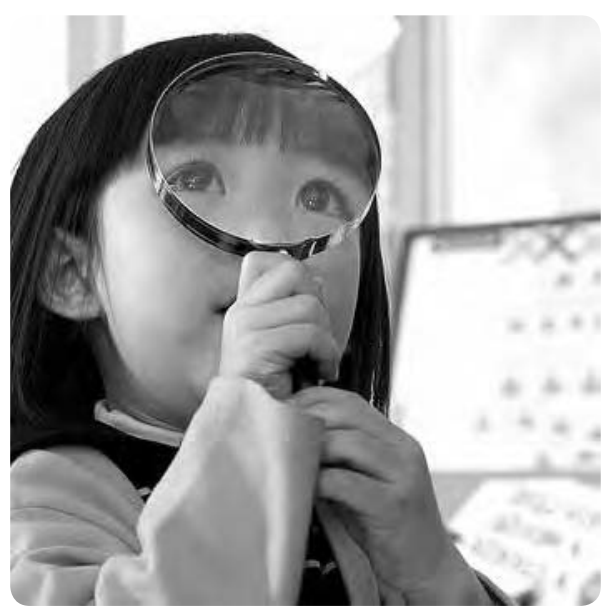

Indicator D1 How much time do students spend in the classroom? StatLink ज्ञा5 http://dx.doi.org/10.1787/888933286089

Indicator D2 What is the student-teacher ratio and how big are classes? StatLink Aताs http://dx.doi.org/10.1787/888933286136

Indicator D3 How much are teachers paid?

StatLink नills http://dx.doi.org/10.1787/888933286177

Indicator D4 How much time do teachers spend teaching? StatLink ज्ञाइ http://dx.doi.org/10.1787/888933286246

Indicator D5 Who are the teachers?

StatLink 需政 http://dx.doi.org/10.1787/888933286275

Indicator D6 What evaluation and assessment mechanisms are in place? StatLink 形 S http://dx.doi.org/10.1787/888933286339

Indicator D7 What teacher and school leader appraisal systems are in place?

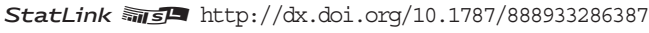

Indicator D8 To what extent is information and communication technology used in teaching and learning?

StatLink ज्ञाता http://dx.doi.org/10.1787/888933286444 


\section{HOW MUCH TIME DO STUDENTS SPEND IN THE CLASSROOM?}

- Students in OECD countries receive an average of 7570 hours of compulsory instruction during their primary and lower secondary education.

- On average across OECD countries, instruction in reading, writing and literature, mathematics, and the arts represents $46 \%$ of compulsory instruction time for primary school students; instruction in reading, writing and literature, second and other languages, and mathematics represents $38 \%$ of

INDICATOR D1 compulsory instruction time for lower secondary school students.

\section{Chart D1.1. Compulsory instruction time in general education (2015) In primary and lower secondary education}

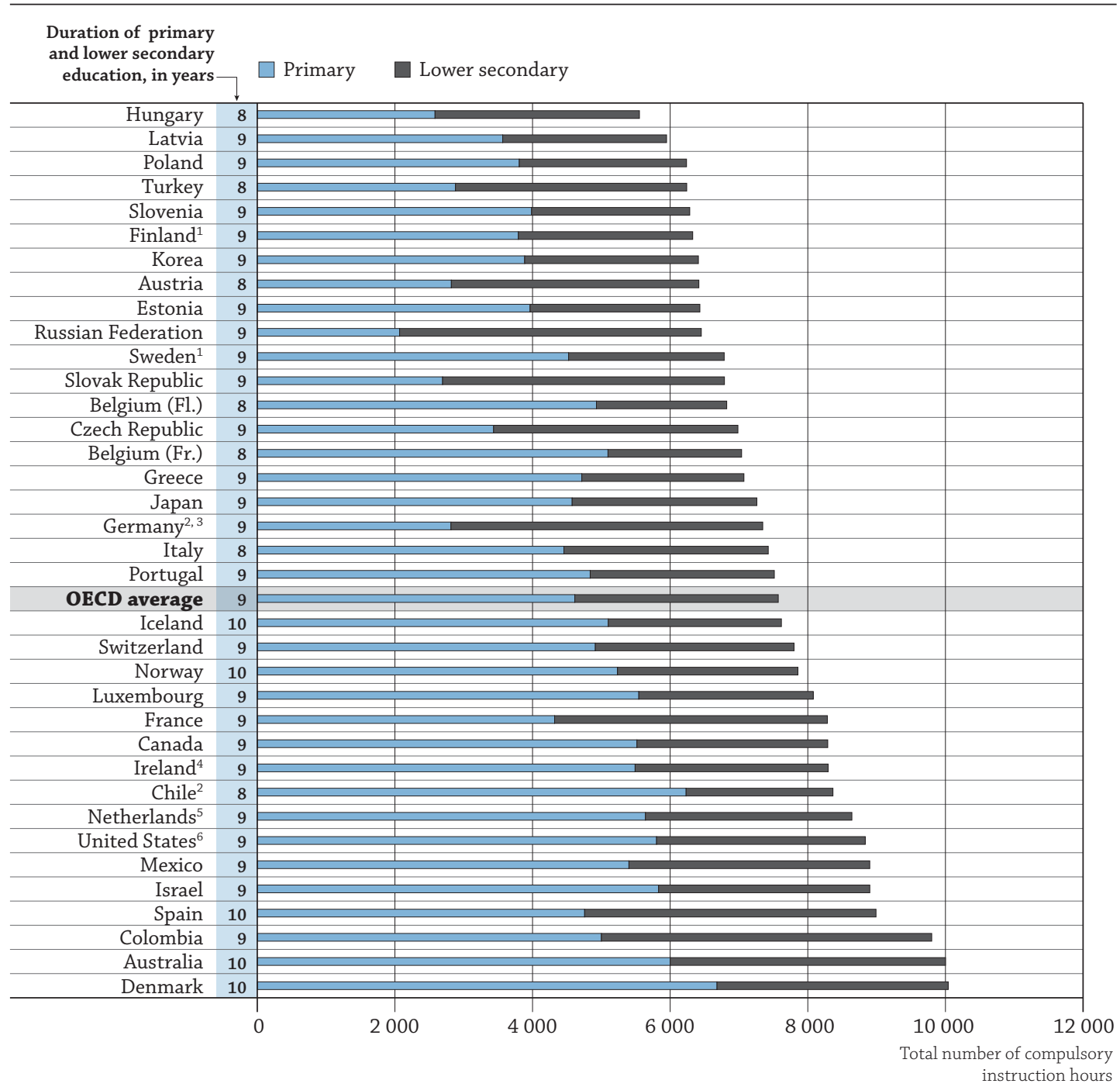

1. Estimated number of hours by level of education based on the average number of hours per year, as the allocation of instruction time across multiple grades is flexible.

2. Year of reference 2014

3. Excludes the last year of compulsory education, which can be classified at either the lower secondary or the upper secondary level.

4. Actual instruction time for lower secondary education.

5. The number of grades in lower secondary education is three or four, depending on the track. The 4th year of pre-vocational secondary education (VMBO) was excluded from the calculation.

6. Year of reference 2012.

Countries are ranked in ascending order of the total number of compulsory instruction hours.

Source: OECD. Table D1.1. See Annex 3 for notes (www.oecd.org/education/education-at-a-glance-19991487.htm).

StatLink त्ताजी http://dx.doi.org/10.1787/888933284394 


\section{Context}

Providing instruction in formal classroom settings accounts for a large portion of public investment in education. Countries make various choices concerning the overall amount of time devoted to instruction and which subjects are compulsory. These choices reflect national and/or regional priorities and preferences concerning what material students should be taught and at what age. Countries usually have statutory or regulatory requirements regarding hours of instruction. These are most often stipulated as the minimum number of hours of instruction a school must offer, and are based on the understanding that sufficient time is required for good learning outcomes. Matching resources with students' needs and making optimal use of time are central to education policy. Teachers' salaries, institutional maintenance and provision of other educational resources constitute the main costs of education. The length of time during which these resources are made available to students (as partly shown in this Indicator) is an important factor in determining how funds for education are allocated (see Indicator B7, which shows the factors influencing the salary cost of teachers per student).

\section{Other findings}

- In OECD countries, compulsory instruction time for primary students averages 804 hours per year; lower secondary students receive an average of 112 more hours of compulsory education per year than primary students do.

- The proportion of the compulsory curriculum for primary students that is devoted to reading, writing and literature ranges from 18\% in Poland to $37 \%$ in France; for lower secondary students, it ranges from $12 \%$ in Australia, the Czech Republic, Finland, Ireland and Japan to 33\% in Italy.

- The proportion of the compulsory curriculum devoted to mathematics at primary level ranges from $13 \%$ in Denmark and Greece to $27 \%$ in Portugal; at the lower secondary level it ranges from $11 \%$ in Greece to $20 \%$ in Italy.

- In OECD countries, an average of $12 \%$ (for primary students) and 6\% (for lower secondary students) of compulsory instruction time is devoted to compulsory subjects with a flexible timetable. An average of $5 \%$ of compulsory instruction time for both groups of students is devoted to compulsory flexible subjects chosen by schools.

- In about one-third of countries with available data, the allocation of instruction time across grades is flexible, i.e. instruction time for a specific subject is defined for a certain number of grades, or even the whole of compulsory education, without specifying the time to be allocated to each grade. 


\section{Analysis}

\section{Compulsory general education}

Annual instruction time should be examined together with the length of compulsory education. In some countries, the duration of compulsory education is shorter and students bear a heavier workload; in other countries, the workload is distributed evenly over more years, which ultimately means a larger number of total instruction hours for all.

D1 In three out of four countries with available data, students start primary education at the age of 6 . However, in Estonia, Finland, Latvia, the Russian Federation and Sweden, students do not start until age 7. In Poland, primary education is compulsory for 7-year-olds and for 6-year-old children born in the first semester of 2008. Only in Australia, England, New Zealand and Scotland does primary education start at age 5. There is also substantial variation in the duration of primary education. On average, primary education lasts six years, but ranges from four years in Austria, Germany, Hungary, the Slovak Republic and Turkey, to seven years in Denmark, Iceland, Norway and Scotland. Lower secondary education averages three years but ranges from two years in Belgium (Flemish and French Communities) and Chile to five years in the Russian Federation and the Slovak Republic. In around two out of three countries with available data, at least one year of upper secondary education is part of compulsory full-time education (Table D1.2).

Countries also allocate annual instruction time differently over the year. On average across OECD countries, primary and lower secondary students attend 185 and 183 instruction days per year, respectively. However, students in France (primary and lower secondary), Greece (lower secondary), Iceland (primary and lower secondary), Ireland (lower secondary), Latvia (primary), Luxembourg (lower secondary) and the Russian Federation (primary) attend 170 instruction days, or fewer, per year. In contrast, primary and lower secondary school students in Brazil, Colombia, Israel, Italy, Japan and Mexico attend at least 200 instruction days per year (Table D1.2).

\section{Compulsory instruction time}

Compulsory instruction time refers to the amount and allocation of instruction time that has to be provided in almost every public school and must be attended by almost all public sector students, as per public regulations.

Students in OECD countries attend an average of 4614 hours of instruction during primary school and an average of 2957 hours during lower secondary education. While the average total compulsory instruction time for primary and lower secondary students in OECD countries is 7570 hours, formal instruction-time requirements range from 5553 hours in Hungary to 10040 hours in Denmark (Table D1.1).

In England and Scotland regulations do not prescribe compulsory instruction time in schools. However, schools are required to allow sufficient instruction time to deliver a broad and balanced curriculum that includes all statutory requirements.

Compulsory instruction time can differ from actual instruction time, as it only captures the time spent by students in formal classroom settings. This is only a part of the total time students spend receiving instruction. Instruction also occurs outside the classroom and/or school. In some countries, secondary school students are encouraged to take after-school classes in subjects already taught in school to help them improve their performance. Students can participate in after-school lessons in the form of remedial "catch-up" classes or enrichment courses, with individual tutors or in group lessons provided by school teachers, or in other independent courses. These lessons can be financed through public funds or by students and their families (see Box D1.1 in OECD, 2011).

This indicator captures intended instruction time, as established in public regulations, as a measure of learning in formal classroom settings. It does not show the actual number of hours of instruction that students receive and does not cover learning outside of the formal classroom setting.

\section{Intended instruction time}

Total intended instruction time is the estimated number of hours during which schools are obliged to offer instruction in compulsory, and if applicable, non-compulsory subjects.

Intended and compulsory instruction time are of the same length (i.e. intended instruction time is fully compulsory), for primary and lower secondary students in about three out of four countries with available data. However in Denmark, Finland, France (lower secondary), Greece (Primary), Poland, Portugal and Slovenia, the intended instruction time is at least $4 \%$ longer than the compulsory instruction time. 


\section{Instruction time per subject}

Primary students spend an average of $46 \%$ of the compulsory instruction time on three subjects: reading, writing and literature (22\%), mathematics (15\%) and the arts (9\%). Together with physical education and health (8\%), natural sciences (7\%) and social studies (6\%), these six study areas form the major part of the curriculum in all OECD countries where instruction time per subject is specified. Second and other languages, religion, information and communication technologies (ICT), technology, practical and vocational skills, and other subjects make up the remainder (15\%) of the non-flexible compulsory curriculum at the primary level (Table D1.3a and Chart D1.2a).

\section{Chart D1.2a. Instruction time per subject in primary education (2015)} As a percentage of total compulsory instruction time

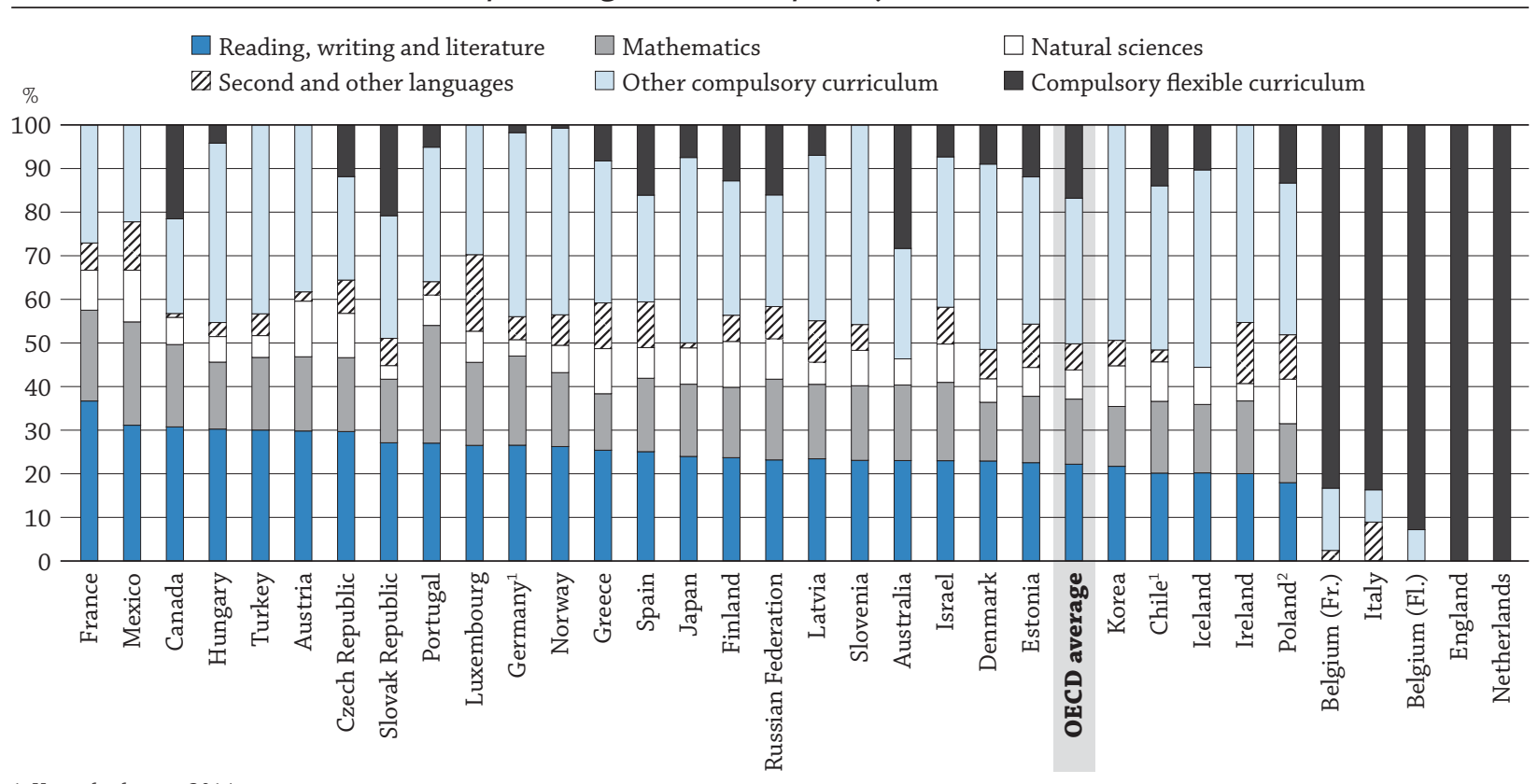

1. Year of reference 2014

2. Excludes the first three years of primary education for which a large proportion of the time allocated to compulsory subjects is flexible. Countries are ranked in descending order of the proportion of instruction hours devoted to reading, writing and literature.

Source: OECD. Table D1.3a. See Annex 3 for notes (www.oecd.org/education/education-at-a-glance-19991487.htm).

StatLink 제그 http://dx.doi.org/10.1787/888933284408

At the lower secondary level, an average of $38 \%$ of the compulsory curriculum is composed of three subjects: reading, writing and literature (14\%), second and other languages (13\%) and mathematics (12\%). On average, an additional $11 \%$ of the compulsory curriculum is devoted to natural sciences and $10 \%$ to social studies. Together with physical education and health (7\%) and the arts (6\%), these seven study areas form the major part of the curriculum for this level of education in all OECD countries where instruction time per subject is specified. Religion, ICT, technology, practical and vocational skills, and other subjects make up the remainder (11\%) of the non-flexible compulsory curriculum for students at this level of education (Table D1.3b and Chart D1.2b).

This is a significant shift in the allocation of time from primary schooling. Instruction in reading, writing and literature drops from $22 \%$ of the compulsory instruction time to $14 \%$. Instruction in mathematics drops from $15 \%$ to $12 \%$ of compulsory instruction time. Conversely, instruction in both natural science and social studies climbs from $7 \%$ and $6 \%$ of the compulsory curriculum to $11 \%$ and $10 \%$, respectively, while instruction in other languages (second and others) climbs from 6\% to 13\%. At the national level, instruction in second and other languages accounts for the largest share of the compulsory core curriculum at the lower secondary level in Finland (together with natural sciences), France, Germany, Israel, Japan, Latvia, Luxembourg, Norway and Poland (together with reading, writing and literature) (Tables D1.3a and b).

At the lower secondary level, there is substantial variation in how countries allocate time among the different subjects within the compulsory curriculum. For example, reading, writing and literature accounts for $12 \%$ of compulsory instruction time in Australia, the Czech Republic, Finland and Japan, while it accounts for more than $25 \%$ of compulsory instruction time in Greece and Italy. In Ireland, reading, writing and literature is taught in two national languages and 
therefore the actual estimation of the combined percentage can reach about $24 \%$ of the total compulsory instruction time. In Canada and Greece, instruction in second language accounts for $7 \%$ or less of compulsory instruction time while it accounts for $17 \%$ in Luxembourg. In addition, in slightly less than half of countries with available data, studying other language in addition to a second language is compulsory for lower secondary students.

\section{Chart D1.2b. Instruction time per subject in general lower secondary education (2015)}

As a percentage of total compulsory instruction time
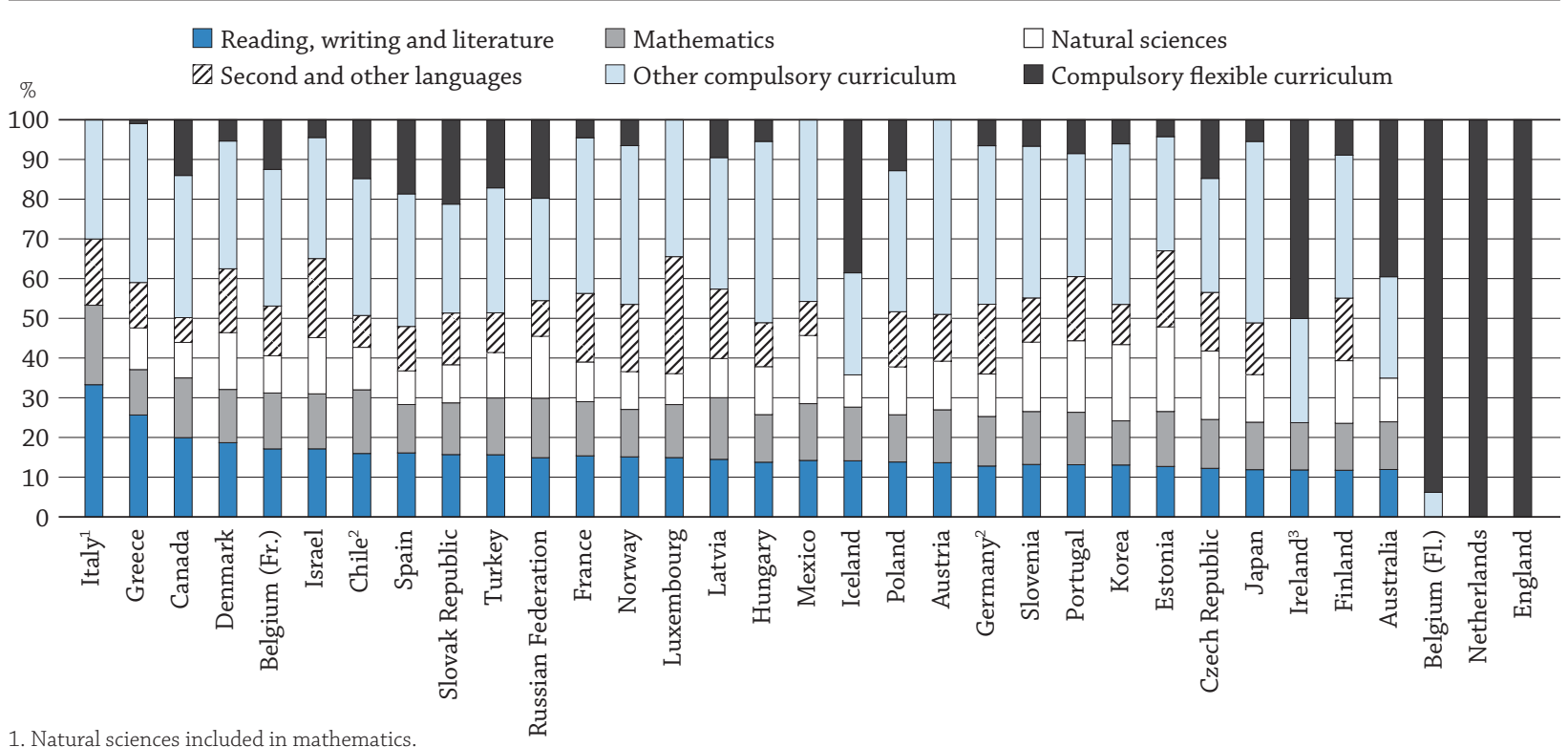

1. Natural sciences included in mathematics.

2. Year of reference 2014.

3. Actual instruction time.

Countries are ranked in descending order of the proportion of instruction hours devoted to reading, writing and literature.

Source: OECD. Table D1.3b. See Annex 3 for notes (www.oecd.org/education/education-at-a-glance-19991487.htm).

StatLink 게피 http://dx.doi.org/10.1787/888933284418

As seen at the primary and lower secondary levels, there are significant differences in how time is allocated to school subjects as students grow older. On average across OECD countries, $25 \%$ of instruction time for 7 -year-olds is devoted to reading, writing and literature; for 11 -year-olds, $17 \%$ of instruction time is devoted to those subjects, while $12 \%$ of instruction time for 15-year-olds is devoted to those subjects. By contrast, whereas an average of $3 \%$ of instruction time for 7-year-olds is devoted to the teaching of second language, $9 \%$ of instruction time for 11-year-olds is spent studying a second language and $2 \%$ studying other languages, and $9 \%$ and $4 \%$ of instruction time for 15 -year-olds is devoted to the second and other languages, respectively. The share of instruction time dedicated to natural sciences increases from $6 \%$ (for 7 -year-olds) to $8 \%$ (for 11 -year-olds) to $11 \%$ (for 15 -year-olds), while instruction time in social studies increases from $5 \%$ (for 7-year-olds) to $8 \%$ (for 11-year-olds) to $9 \%$ (for 15-year-olds). The portion of instruction time dedicated to arts slips from $9 \%$ and $8 \%$ for 7 - and 11 -year-old students, respectively, to $4 \%$ for 15 -year-olds, while time dedicated to physical education remains constant at $9 \%$ and $8 \%$ for 7 - and 11-year-old students, respectively, and drops to $6 \%$ for 15 -year-old students (Tables D1.5b, $\mathrm{f}$ and $\mathrm{j}$, available on line).

\section{Box D1.1. Recess and breaks during the school day}

Learning in the classroom demands that students be focused and concentrated for long periods of time. Research has found that some time spent outside the classroom during the school day, in activities other than instruction itself can help improve students' performance in the classroom. In primary education, breaks in instruction allow pupils to play, rest and freely interact with their peers to further develop cognitive, emotional and social skills. Research suggests that students may then apply those skills in the classroom, thus improving their learning (Pellegrini and Bohn, 2005; Pellegrini et al., 2002). OECD countries increasingly consider recess and breaks as an important component of the school day. 
How breaks are organised in OECD countries depends on how education systems are governed and the degree of autonomy that individual schools enjoy. Decisions on the length of breaks, or even on the moment of the day in which they should occur, are generally taken by the decision making body responsible for the entire instruction time. In countries where responsibility is largely left to the schools or schools' governing bodies, as in Australia, Canada, England (UK), Italy, the Netherlands and Switzerland, schools have the liberty to take decisions about the organisation of breaks too. In other countries, such as Colombia, the decision is left to school principals and head teachers.

In some countries, although regulations on breaks have been established centrally, their implementation is left to schools and/or their governing bodies. In the Flemish Community of Belgium, for instance, legislation stipulates the amount of time that students should devote to breaks at primary (at least a one-hour lunch break) and secondary (at least a 50-minute lunch break) levels. However, schools and school associations or networks are granted the authority to take the final decision and determine the length of the breaks as they see fit.

In most OECD countries, breaks are generally organised in accordance with daily compulsory instruction time. The time students spend in school is most often defined by the amount of instruction they are supposed to receive daily and weekly. In most countries, the school day is divided into lessons that last 45 to 50 minutes, allowing for short breaks between them to make up an entire hour. Across OECD countries, 10- to 15-minute breaks are generally long enough to allow students to change classrooms and visit the restroom. These short breaks are different in length and purpose to longer breaks also observed in the majority of countries. During longer breaks, students can have breakfast or lunch and are commonly supervised by a teacher or group of teachers.

In primary education, long breaks are common, and in some cases are even compulsory. In Spain, for example, breaks in primary school are considered as part of compulsory instruction time. Primary students in Spain have a half-hour break every day in the middle of the morning session that is considered part of the five daily instruction hours. In several countries, a lunch break is set as part of the learning process, where students learn about hygiene, healthy eating habits and/or recycling waste.

In several countries, long breaks can be found at all levels of education. In Australia, schools at all levels of education tend to have one morning recess that lasts about 20 minutes, then a longer lunch break. In Canada, there is a midday break for lunch in primary through upper secondary education. In both countries, long breaks can last around 40 to 60 minutes. Breaks can also occur throughout the day. In Switzerland, for example, schools usually organise two long breaks, one in the morning and a second in the afternoon. In Chile, schools with a large number of students may divide students up into two or more groups, by grade or age, for their breaks.

Schools can use recess and breaks for different purposes. They can use breaks as a way of helping students who have to commute a long distance to school or to harmonise the end of classes when the duration of lesson periods are different across grades, as in the Czech Republic, where 10-minute breaks can be shortened to five minutes. In Denmark, municipalities often propose to use breaks and recess as an integrated part of daily exercise and physical activities for students, at all grade levels. This is also the case in Slovenia, where schools sometimes organise a long break intended for students to practice sports in the gym and on school's outdoor playing fields.

\section{Flexibility in the curriculum}

In most countries, central and state authorities establish regulations or recommendations regarding instruction time and the curriculum. However, local authorities, schools, teachers and/or students also have varying degrees of freedom in organising instruction time or in choosing subjects.

In about one in three countries with available data, the allocation of instruction time across grades is flexible, i.e. instruction time for a specific subject is defined for a certain number of grades, or even the whole of compulsory education, without specifying the time to be allocated to each grade. In such cases, schools/local authorities are free to decide how much time should be allocated for each grade (Table D1.2).

Setting compulsory subjects within a flexible timetable is more common at the primary level where, on average across $\mathrm{OECD}$ countries, it accounts for $12 \%$ of the compulsory instruction time. In this case, compulsory subjects and total instruction time are specified, but not the time to be allocated to each subject. Local authorities, schools and/ or teachers are free to decide how much time should be allocated to each compulsory subject. In Belgium (Flemish and French Communities) and Italy, compulsory subjects within a flexible timetable account for $80 \%$ or more of instruction time at the primary level. At the primary and lower secondary levels, England and the Netherlands 
allow complete flexibility in allocating instruction time across compulsory subjects. In Scotland, at both primary and lower secondary levels, some compulsory subjects are specified, but there is no regulation on total instruction time, which is the responsibility of local authorities and schools themselves.

Flexibility in the choice of subjects is less common across OECD countries. On average, $5 \%$ of compulsory instruction time is allocated to subjects chosen by schools at the primary level. At the lower secondary level, $5 \%$ of compulsory instruction time is allocated to subjects chosen by schools and another $5 \%$ to subjects chosen by the students. However, some countries allocate a substantial part of the compulsory instruction time to flexible subjects. For example, in Australia (primary level), Belgium (French and Flemish communities, lower secondary), Canada (lower secondary), Chile, the Czech Republic, Estonia (primary level), and Poland, at least 10\% of compulsory instruction time is allocated to subjects chosen by schools; up to $21 \%$ of compulsory instruction time is so allocated in the Slovak Republic. In Australia, Iceland, Ireland, Spain and Turkey, at least 17\% of compulsory instruction time is allocated to subjects chosen by lower secondary students (Tables D1.3a and b).

\section{Non-compulsory instruction time}

Non-compulsory instruction time is rare across OECD countries. Only about six countries devote some time to noncompulsory instruction at primary level and the number rises to seven at the lower secondary level. Across these countries, non-compulsory instruction time is equivalent to an average of $3 \%$ of the total compulsory instruction time for primary students, and $2 \%$ for lower secondary students. Nevertheless, a considerable amount of additional non-compulsory instruction time is provided in some countries. At the primary level, additional non-compulsory time accounts for 35\% in Greece and 23\% in Portugal. At the lower secondary level, non-compulsory instruction time accounts for $19 \%$ of the total compulsory instruction time in Slovenia and 10\% in France (Tables D1.3a and b).

\section{Box D1.2. Extracurricular activities at school}

In addition to formal instruction time, students may participate in extracurricular activities before and/or after the school day or during school holidays, on school premises.

In OECD and partner countries, extracurricular activities are more commonly offered during the school year (before and/or after classes) than during school holidays. Although schools often have the autonomy to decide whether they provide these activities or not, it is sometimes compulsory for all schools to offer extracurricular activities. This is the case, for example, in Poland and Slovenia. In Hungary, not only do primary and lower secondary schools have to organise extracurricular activities until $4 \mathrm{pm}$, but students are required to attend them.

These activities can be organised by schools, as in Brazil and Hungary, by municipalities, as in Israel, or by volunteer school staff, as in Ireland. External public partners are also often involved in organising extracurricular activities on school premises as are private stakeholders, though less commonly so. For example, in Portugal, these activities can be organised by parent associations and non-governmental organisations. In the Czech Republic, Estonia, Iceland, Italy, Japan and Slovenia, occasional additional payments are offered to teachers in primary to upper secondary education, to participate in these extracurricular activities (see Indicator D3).

Before- and/or after-school activities typically include childcare (at the primary level), tutoring or remedial courses, sports and/or artistic and cultural activities. In Hungary (upper secondary level) and Turkey, these activities also include community service; in Spain, classes in foreign languages, ICT and reading and writing workshops are offered.

\section{Definitions}

Compulsory curriculum refers to the amount and allocation of instruction time that has to be provided in almost every public school and must be attended by almost all public sector students. The compulsory curriculum may be flexible as local authorities, schools, teachers and/or pupils may have varying degrees of freedom to choose the subjects and/or the allocation of compulsory instruction time.

Compulsory flexible subjects chosen by schools refers to the total amount of compulsory instruction time indicated by the central authorities, which regional authorities, local authorities, schools or teachers allocate to subjects of their choice (or subjects they chose from a list defined by central education authorities). It is compulsory for the school to offer one of these subjects and students must attend it. 
Compulsory options chosen by the students refers to the total amount of instruction time in one or more subjects that pupils have to select (from a set of subjects that are compulsory for schools to offer) in order to cover part of their compulsory instruction time.

Compulsory subjects with a flexible timetable refers to the total amount of instruction time indicated by the central authorities for a given group of subjects, which regional authorities, local authorities, schools or teachers allocate to individual subjects. There is flexibility in the time spent on a subject, but not in the subjects to be taught.

Flexible allocation of instruction time across multiple grades refers to the case when the curriculum only indicates the total instruction time for a specific subject for a certain number of grades, or even the whole of compulsory education, without specifying the time to be allocated to each grade. In such cases, schools/local authorities are free to decide how much time should be assigned for each grade.

Instruction time refers to the time a public school is expected to provide instruction to students on all the subjects integrated into the compulsory and non-compulsory curriculum, on school premises or in before-/after-school activities, that are formal parts of the compulsory programme. Instruction time excludes breaks between classes or other types of interruptions, non-compulsory time outside the school day, time dedicated to homework activities, and individual tutoring or private study.

Intended instruction time refers to the number of hours per year of the compulsory and non-compulsory part of the curriculum that students are entitled to receive in public schools. The intended curriculum can based on regulations or standards of the central (or top level) education authorities or may be established as a set of recommendations at the regional level.

The non-compulsory part of the curriculum refers to the total amount of instruction time to which students are entitled beyond the compulsory hours of instruction and that almost every public school is expected to provide. Subjects can vary from school to school or from region to region and take the form of elective subjects. Students are not required to choose one of the elective subjects, but all public schools are expected to offer this possibility.

\section{Methodology}

Data on instruction time are from the 2014 Joint Eurydice-OECD Instruction time data collection and refer to instruction time during compulsory primary and full-time (lower and upper) secondary general education for the school year 2014/15.

In editions of Education at a Glance prior to 2014, data on instruction time was collected through another survey using a different scope, methodology and definitions than the 2013 Joint Eurydice-OECD instruction time data collection first published in Education at a Glance 2014. As a result, data on instruction time are not comparable with those published in previous editions of Education at a Glance.

This indicator captures intended instruction time, as established in public regulations, as a measure of learning in formal classroom settings. It does not show the actual number of hours of instruction that students receive and does not cover learning outside of the formal classroom setting. Differences may exist across countries between the regulatory minimum hours of instruction and the actual hours of instruction received by students. A study conducted by Regioplan Beleidsonderzoek in the Netherlands showed that, given such factors as school timetables, lesson cancellations and teacher absenteeism, schools may not consistently attain the regulatory minimum instruction time (see Box D1.1 in OECD, 2007).

The indicator also illustrates how minimum instruction hours are allocated across different curricular areas. It shows the intended net hours of instruction for those grades that are part of compulsory full-time general education. Although the data are difficult to compare among countries because of different curricular policies, they nevertheless provide an indication of how much formal instruction time is considered necessary for students to achieve the desired educational goals.

When the allocation of instruction time across grades is flexible, i.e. instruction time for a specific subject is defined for a certain number of grades, or even the whole of compulsory education, without specifying the time to be allocated to each grade, instruction time per age or level of education was estimated by dividing the total number of instruction hours per the number of grades.

Notes on definitions and methodologies for each country related to this indicator are provided in Annex 3, available at www.oecd.org/education/education-at-a-glance-19991487.htm. 


\section{Note regarding data from Israel}

The statistical data for Israel are supplied by and are under the responsibility of the relevant Israeli authorities. The use of such data by the OECD is without prejudice to the status of the Golan Heights, East Jerusalem and Israeli settlements in the West Bank under the terms of international law.

\section{References}

D1 OECD (2011), Education at a Glance 2011: OECD Indicators, OECD Publishing, Paris, http://dx.doi.org/10.1787/eag-2011-en. OECD (2007), Education at a Glance 2007: OECD Indicators, OECD Publishing, Paris, http://dx.doi.org/10.1787/eag-2007-en.

Pellegrini, A.D. and C. Bohn (2005), “The role of recess in children's cognitive performance and school adjustment”, Educational Researcher, Vol. 34/1, pp. 13-19.

Pellegrini, A.D., K. Kato, P. Blatchford and E. Baines (2002), "A short-term longitudinal study of children's playground games across the first year of school: Implications for social competence and adjustment to school”, American Educational Research Journal, Vol. 39/4, pp. 991-1015.

\section{Indicator D1 Tables}

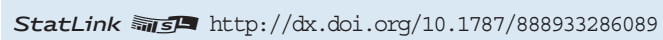

\begin{tabular}{rll}
\hline Table D1.1 & Instruction time in compulsory general education (2015) \\
\hline Table D1.2 & Organisation of compulsory general education (2015) \\
\hline Table D1.3a & Instruction time per subject in primary education (2015) \\
Table D1.3b & Instruction time per subject in general lower secondary education (2015) \\
\hline WEB & Table D1.4 & Instruction time in compulsory general education, by age (2015) \\
\hline WEB & Table D1.5a & Instruction time per subject for 6-year-olds (2015) \\
\hline WEB & Table D1.5b & Instruction time per subject for 7-year-olds (2015) \\
\hline WEB & Table D1.5c & Instruction time per subject for 8-year-olds (2015) \\
\hline WEB & Table D1.5d & Instruction time per subject for 9-year-olds (2015) \\
\hline WEB & Table D1.5e & Instruction time per subject for 10-year-olds (2015) \\
WEB & Table D1.5f & Instruction time per subject for 11-year-olds (2015) \\
\hline WEB & Table D1.5g & Instruction time per subject for 12-year-olds (2015) \\
\hline WEB & Table D1.5h & Instruction time per subject for 13-year-olds (2015) \\
\hline WEB & Table D1.5i & Instruction time per subject for 14-year-olds (2015) \\
\hline WEB & Table D1.5j & Instruction time per subject for 15-year-olds (2015) \\
WEB Table D1.5k & Instruction time per subject for 16-year-olds (2015) \\
\hline WEB & Table D1.51 & Instruction time per subject for 17-year-olds (2015) \\
\hline
\end{tabular}


Table D1.1. [1/2] Instruction time in compulsory general education ${ }^{1}$ (2015) By level of education, in public institutions

\begin{tabular}{|c|c|c|c|c|c|c|c|c|c|c|c|}
\hline & \multicolumn{7}{|c|}{ Primary } & \multicolumn{4}{|c|}{ Lower secondary } \\
\hline & \multirow[b]{2}{*}{$\begin{array}{c}\text { Number } \\
\text { of grades } \\
\text { that are part } \\
\text { of compulsory } \\
\text { education }\end{array}$} & \multicolumn{3}{|c|}{ Average hours per year } & \multicolumn{3}{|c|}{ Total number of hours } & \multirow[b]{2}{*}{$\begin{array}{c}\text { Number } \\
\text { of grades } \\
\text { that are part } \\
\text { of compulsory } \\
\text { education }\end{array}$} & \multicolumn{3}{|c|}{ Average hours per year } \\
\hline & & 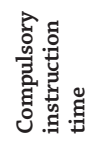 & 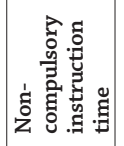 & 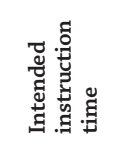 & 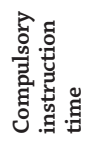 & 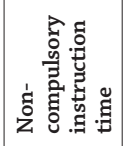 & 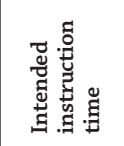 & & 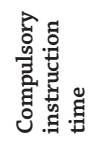 & 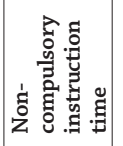 & 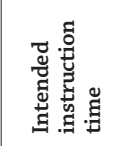 \\
\hline & (1) & (2) & (3) & $(4)=(2)+(3)$ & (5) & (6) & $(7)=(5)+(6)$ & (8) & (9) & (10) & $(11)=(9)+(10)$ \\
\hline Australia & 6 & 1000 & $\mathrm{~m}$ & $\mathrm{~m}$ & 6000 & $\mathrm{~m}$ & $\mathrm{~m}$ & 4 & 1000 & $\mathrm{~m}$ & $\mathrm{~m}$ \\
\hline $\begin{array}{l}\text { Austria } \\
\text { Belgium (FI) }\end{array}$ & 4 & 705 & $\mathrm{~m}$ & $\mathrm{~m}$ & 2820 & $\mathrm{~m}$ & $\mathrm{~m}$ & 4 & 899 & $\mathrm{~m}$ & $\mathrm{~m}$ \\
\hline & 6 & 821 & $\mathrm{a}$ & 821 & 4928 & $\mathrm{a}$ & 4928 & 2 & 947 & a & 947 \\
\hline Belgium (Fr. $)^{2}$ & 6 & 849 & $\mathrm{~m}$ & $\mathrm{~m}$ & 5096 & $\mathrm{~m}$ & $\mathrm{~m}$ & 2 & 971 & $\mathrm{~m}$ & $\mathrm{~m}$ \\
\hline Canada & 6 & 919 & $\mathrm{a}$ & 919 & 5516 & $\mathrm{a}$ & 5516 & 3 & 924 & 6 & 930 \\
\hline Chile $^{3}$ & 6 & 1039 & a & 1039 & 6231 & $\mathrm{a}$ & 6231 & 2 & 1067 & a & 1067 \\
\hline Czech Republic & 5 & 687 & $\mathrm{~m}$ & $\mathrm{~m}$ & 3434 & $\mathrm{~m}$ & $\mathrm{~m}$ & 4 & 888 & $\mathrm{~m}$ & $\mathrm{~m}$ \\
\hline Denmark & 7 & 954 & 97 & 1051 & 6680 & 680 & 7360 & 3 & 1120 & 80 & 1200 \\
\hline England $^{4}$ & 6 & $\mathrm{a}$ & $\mathrm{a}$ & $\mathrm{a}$ & $\mathrm{a}$ & $\mathrm{a}$ & $\mathrm{a}$ & 3 & $\mathrm{a}$ & a & a \\
\hline Estonia & 6 & 661 & a & 661 & 3964 & a & 3964 & 3 & 823 & a & 823 \\
\hline Finland $^{5}$ & 6 & 632 & 29 & 661 & 3794 & 171 & 3965 & 3 & 844 & 57 & 901 \\
\hline France & 5 & 864 & $\mathrm{a}$ & 864 & 4320 & $\mathrm{a}$ & 4320 & 4 & 991 & 99 & 1090 \\
\hline Germany ${ }^{3,6}$ & 4 & 703 & a & 703 & 2814 & a & 2814 & 5 & 906 & a & 906 \\
\hline Greece & 6 & 786 & 279 & 1065 & 4715 & 1672 & 6387 & 3 & 785 & $\mathrm{n}$ & 785 \\
\hline Hungary & 4 & 646 & a & 646 & 2583 & $\mathrm{a}$ & 2583 & 4 & 743 & a & 743 \\
\hline Iceland & 7 & 729 & a & 729 & 5100 & a & 5100 & 3 & 839 & a & 839 \\
\hline Ireland $^{7}$ & 6 & 915 & a & 915 & 5490 & $\mathrm{a}$ & 5490 & 3 & 935 & a & 935 \\
\hline Israel & 6 & 972 & $\mathrm{n}$ & 972 & 5831 & $\mathrm{n}$ & 5831 & 3 & 1023 & $\mathrm{n}$ & 1023 \\
\hline Italy & 5 & 891 & a & 891 & 4455 & $\mathrm{a}$ & 4455 & 3 & 990 & a & 990 \\
\hline Japan & 6 & 763 & a & 763 & 4575 & a & 4575 & 3 & 895 & $\mathrm{a}$ & 895 \\
\hline Korea & 6 & 648 & a & 648 & 3885 & a & 3885 & 3 & 842 & a & 842 \\
\hline Luxembourg & 6 & 924 & a & 924 & 5544 & a & 5544 & 3 & 845 & a & 845 \\
\hline Mexico & 6 & 900 & $\mathrm{a}$ & 900 & 5400 & a & 5400 & 3 & 1167 & a & 1167 \\
\hline Netherlands ${ }^{8}$ & 6 & 940 & $\mathrm{~m}$ & $\mathrm{~m}$ & 5640 & $\mathrm{~m}$ & $\mathrm{~m}$ & 3 & 1000 & $\mathrm{~m}$ & $\mathrm{~m}$ \\
\hline New Zealand & 6 & $\mathrm{~m}$ & $\mathrm{~m}$ & $\mathrm{~m}$ & $\mathrm{~m}$ & $\mathrm{~m}$ & $\mathrm{~m}$ & 4 & $\mathrm{~m}$ & $\mathrm{~m}$ & $\mathrm{~m}$ \\
\hline Norway & 7 & 748 & $\mathrm{a}$ & 748 & 5234 & a & 5234 & 3 & 874 & a & 874 \\
\hline Poland & 6 & 635 & 58 & 693 & 3807 & 349 & 4156 & 3 & 810 & 65 & 875 \\
\hline Portugal & 6 & 806 & 189 & 995 & 4838 & 1133 & 5971 & 3 & 892 & 27 & 919 \\
\hline Scotland ${ }^{4}$ & 7 & $\mathrm{a}$ & a & a & a & $\mathrm{a}$ & a & 3 & a & a & a \\
\hline Slovak Republic & 4 & 673 & a & 673 & 2693 & a & 2693 & 5 & 819 & a & 819 \\
\hline Slovenia & 6 & 664 & 114 & 778 & 3986 & 683 & 4669 & 3 & 766 & 145 & 911 \\
\hline Spain & 6 & 793 & $\mathrm{a}$ & 793 & 4757 & $\mathrm{a}$ & 4757 & 4 & 1059 & $\mathrm{a}$ & 1059 \\
\hline Sweden 5 & 6 & 754 & $\mathrm{~m}$ & $\mathrm{~m}$ & 4523 & $\mathrm{~m}$ & $\mathrm{~m}$ & 3 & 754 & $\mathrm{~m}$ & $\mathrm{~m}$ \\
\hline Switzerland & 6 & 819 & $\mathrm{~m}$ & $\mathrm{~m}$ & 4912 & $\mathrm{~m}$ & $\mathrm{~m}$ & 3 & 963 & $\mathrm{~m}$ & $\mathrm{~m}$ \\
\hline Turkey & 4 & 720 & $\mathrm{a}$ & 720 & 2880 & $\mathrm{a}$ & 2880 & 4 & 840 & a & 840 \\
\hline United States $^{9}$ & 6 & 967 & $\mathrm{~m}$ & $\mathrm{~m}$ & 5802 & $\mathrm{~m}$ & $\mathrm{~m}$ & 3 & 1011 & $\mathrm{~m}$ & $\mathrm{~m}$ \\
\hline OECD average $^{4}$ & 6 & 804 & 31 & $\sim$ & 4614 & 188 & $\sim$ & 3 & 916 & 19 & $\sim$ \\
\hline EU21 average ${ }^{4}$ & 6 & 776 & 48 & 〜 & 4328 & 293 & $\sim$ & 3 & 895 & 30 & $\sim$ \\
\hline Argentina & $\mathrm{m}$ & $\mathrm{m}$ & $\mathrm{m}$ & $\mathrm{m}$ & $\mathrm{m}$ & $\mathrm{m}$ & $\mathrm{m}$ & $\mathrm{m}$ & $\mathrm{m}$ & $\mathrm{m}$ & $\mathrm{m}$ \\
\hline Brazil & 5 & $\mathrm{~m}$ & $\mathrm{~m}$ & $\mathrm{~m}$ & $\mathrm{~m}$ & $\mathrm{~m}$ & $\mathrm{~m}$ & 4 & $\mathrm{~m}$ & $\mathrm{~m}$ & $\mathrm{~m}$ \\
\hline China & $\mathrm{m}$ & $\mathrm{m}$ & $\mathrm{m}$ & $\mathrm{m}$ & $\mathrm{m}$ & $\mathrm{m}$ & $\mathrm{m}$ & $\mathrm{m}$ & $\mathrm{m}$ & $\mathrm{m}$ & $\mathrm{m}$ \\
\hline Colombia & 5 & 1000 & $\mathrm{~m}$ & $\mathrm{~m}$ & 5000 & $\mathrm{~m}$ & $\mathrm{~m}$ & 4 & 1200 & $\mathrm{~m}$ & $\mathrm{~m}$ \\
\hline India & $\mathrm{m}$ & $\mathrm{m}$ & $\mathrm{m}$ & $\mathrm{m}$ & $\mathrm{m}$ & $\mathrm{m}$ & $\mathrm{m}$ & $\mathrm{m}$ & $\mathrm{m}$ & $\mathrm{m}$ & $\mathrm{m}$ \\
\hline Indonesia & $\mathrm{m}$ & $\mathrm{m}$ & $\mathrm{m}$ & $\mathrm{m}$ & $\mathrm{m}$ & $\mathrm{m}$ & $\mathrm{m}$ & $\mathrm{m}$ & $\mathrm{m}$ & $\mathrm{m}$ & $\mathrm{m}$ \\
\hline Latvia & 6 & 594 & $\mathrm{~m}$ & $\mathrm{~m}$ & 3566 & $\mathrm{~m}$ & $\mathrm{~m}$ & 3 & 794 & $\mathrm{~m}$ & $\mathrm{~m}$ \\
\hline Russian Federation & 4 & 517 & $\mathrm{~m}$ & $\mathrm{~m}$ & 2068 & $\mathrm{~m}$ & $\mathrm{~m}$ & 5 & 877 & $\mathrm{~m}$ & $\mathrm{~m}$ \\
\hline Saudi Arabia & $\mathrm{m}$ & $\mathrm{m}$ & $\mathrm{m}$ & $\mathrm{m}$ & $\mathrm{m}$ & $\mathrm{m}$ & $\mathrm{m}$ & $\mathrm{m}$ & $\mathrm{m}$ & $\mathrm{m}$ & $\mathrm{m}$ \\
\hline South Africa & $\mathrm{m}$ & $\mathrm{m}$ & $\mathrm{m}$ & $\mathrm{m}$ & $\mathrm{m}$ & $\mathrm{m}$ & $\mathrm{m}$ & $\mathrm{m}$ & $\mathrm{m}$ & $\mathrm{m}$ & $\mathrm{m}$ \\
\hline G20 average & $\mathrm{m}$ & $\mathrm{m}$ & $\mathrm{m}$ & $\mathrm{m}$ & $\mathrm{m}$ & $\mathrm{m}$ & $\mathrm{m}$ & $\mathrm{m}$ & $\mathrm{m}$ & $\mathrm{m}$ & $\mathrm{m}$ \\
\hline
\end{tabular}

Note: Columns showing instruction time in compulsory upper secondary education (i.e. columns 19-25) are available for consultation on line (see StatLink below).

1. Refers to full-time compulsory education and excludes pre-primary education, even if compulsory.

2. It is compulsory for students to attend full-time education up to the age of 15 or 16 . However, those in general education must continue until they are 18.

3. Year of reference 2014.

4. England and Scotland are not included in the averages

5. Estimated number of hours by level of education based on the average number of hours per year, as the allocation of instruction time across multiple grades is flexible.

6. Excludes the last year of compulsory education, which can be classified at either the lower secondary or the upper secondary level.

7. Actual instruction time for lower and upper secondary education.

8. The number of grades in lower secondary education is three or four, depending on the track. The 4th year of pre-vocational secondary education (VMBO) was excluded from the calculation.

9. Year of reference 2012.

Source: OECD. See Annex 3 for notes (www.oecd.org/education/education-at-a-glance-19991487.htm).

Please refer to the Reader's Guide for information concerning symbols for missing data and abbreviations.

StatLink 需政 http://dx.doi.org/10.1787/888933286093 
Table D1.1. [2/2] Instruction time in compulsory general education ${ }^{\mathbf{1}}$ (2015) By level of education, in public institutions

\begin{tabular}{|c|c|c|c|c|c|c|}
\hline \multirow{2}{*}{\multicolumn{3}{|c|}{$\begin{array}{c}\text { Lower secondary } \\
\text { Total number of hours }\end{array}$}} & \multicolumn{4}{|c|}{ Primary and lower secondary } \\
\hline & & & \multirow[b]{2}{*}{$\begin{array}{c}\text { Theoretical } \\
\text { duration in years }\end{array}$} & \multicolumn{3}{|c|}{ Total number of hours } \\
\hline 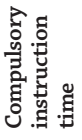 & 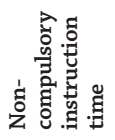 & 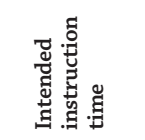 & & 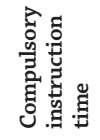 & 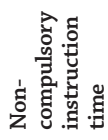 & 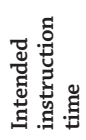 \\
\hline (12) & (13) & $(14)=(12)+(13)$ & (15) & (16) & (17) & (18) \\
\hline
\end{tabular}

\begin{tabular}{|c|c|c|c|c|c|c|c|}
\hline & (12) & (13) & $(14)=(12)+(13)$ & (15) & $(16)$ & (17) & (18) \\
\hline $\begin{array}{l}\text { Australia } \\
\text { Austria }\end{array}$ & $\begin{array}{l}4000 \\
3597\end{array}$ & $\begin{array}{l}\mathrm{m} \\
\mathrm{m}\end{array}$ & $\begin{array}{l}\mathrm{m} \\
\mathrm{m}\end{array}$ & $\begin{array}{r}10 \\
8\end{array}$ & $\begin{array}{r}10000 \\
6417\end{array}$ & $\begin{array}{l}\mathbf{m} \\
\mathbf{m}\end{array}$ & $\begin{array}{l}\mathbf{m} \\
\mathbf{m}\end{array}$ \\
\hline Belgium (Fl.) $)^{2}$ & 1893 & $\mathrm{a}$ & 1893 & 8 & 6821 & $\mathbf{a}$ & 6821 \\
\hline Belgium (Fr.) ${ }^{2}$ & 1941 & $\mathrm{~m}$ & $\mathrm{~m}$ & 8 & 7037 & $\mathbf{m}$ & $\mathbf{m}$ \\
\hline Chile $^{3}$ & 2134 & $\mathrm{a}$ & 2134 & 8 & 8365 & $\mathbf{a}$ & 8365 \\
\hline $\begin{array}{l}\text { Czech Republic } \\
\text { Denmark }\end{array}$ & $\begin{array}{l}3550 \\
3360\end{array}$ & $\begin{array}{r}m \\
240\end{array}$ & $\begin{array}{r}\mathrm{m} \\
3600\end{array}$ & $\begin{array}{r}9 \\
10\end{array}$ & $\begin{array}{r}6984 \\
10040\end{array}$ & $\begin{array}{r}m \\
920\end{array}$ & $\begin{array}{r}m \\
10960\end{array}$ \\
\hline $\begin{array}{l}\text { England }^{4} \\
\text { Estonia }\end{array}$ & $\begin{array}{r}a \\
2468\end{array}$ & $\begin{array}{l}\mathrm{a} \\
\mathrm{a}\end{array}$ & $\begin{array}{r}a \\
2468\end{array}$ & $\begin{array}{l}9 \\
9\end{array}$ & $\begin{array}{r}a \\
6431\end{array}$ & $\mathbf{a}$ & $\begin{array}{r}a \\
6431\end{array}$ \\
\hline Finland ${ }^{5}$ & 2533 & 171 & 2704 & 9 & 6327 & 342 & 6669 \\
\hline France & 3964 & 396 & 4360 & 9 & 8284 & 396 & 8680 \\
\hline Germany $^{3,6}$ & 4531 & $\mathrm{a}$ & 4531 & 9 & 7345 & $\mathbf{a}$ & 7345 \\
\hline Greece & 2356 & $\mathrm{n}$ & 2356 & 9 & 7071 & 1672 & 8744 \\
\hline Hungary & 2970 & a & 2970 & 8 & 5553 & $\mathbf{a}$ & 5553 \\
\hline Iceland & 2516 & $\mathrm{a}$ & 2516 & 10 & 7616 & $\mathbf{a}$ & 7616 \\
\hline Ireland ${ }^{7}$ & 2806 & a & 2806 & 9 & 8296 & $\mathbf{a}$ & 8296 \\
\hline Israel & 3070 & $\mathrm{n}$ & 3070 & 9 & 8901 & $\mathbf{n}$ & 8901 \\
\hline Japan & 2684 & a & 2684 & 9 & 7260 & $\mathbf{a}$ & 7260 \\
\hline Korea & 2525 & $\mathrm{a}$ & 2525 & 9 & 6410 & $\mathbf{a}$ & 6410 \\
\hline Luxembourg & 2535 & $\mathrm{a}$ & 2535 & 9 & 8079 & $\mathbf{a}$ & 8079 \\
\hline Mexico & 3500 & a & 3500 & 9 & 8900 & $\mathbf{a}$ & 8900 \\
\hline Netherlands ${ }^{8}$ & 3000 & $\mathrm{~m}$ & $\mathrm{~m}$ & 9 & 8640 & m & $\mathbf{m}$ \\
\hline New Zealand & $\mathrm{m}$ & $\mathrm{m}$ & $\mathrm{m}$ & 10 & $\mathbf{m}$ & $\mathbf{m}$ & $\mathbf{m}$ \\
\hline Norway & 2622 & $\mathrm{a}$ & 2622 & 10 & 7856 & $\mathbf{a}$ & 7856 \\
\hline Poland & 2430 & 194 & 2624 & 9 & 6237 & 542 & 6779 \\
\hline Portugal & 2675 & 80 & 2756 & 9 & 7513 & 1214 & 8726 \\
\hline Scotland $^{4}$ & a & a & a & 10 & a & $\mathbf{a}$ & a \\
\hline Slovak Republic & 4095 & a & 4095 & 9 & 6788 & a & 6788 \\
\hline Slovenia & 2298 & 435 & 2733 & 9 & 6284 & 1118 & 7401 \\
\hline Spain & 4234 & $\mathrm{a}$ & 4234 & 10 & 8991 & $\mathbf{a}$ & 8991 \\
\hline Sweden $^{5}$ & 2262 & $\mathrm{~m}$ & $\mathrm{~m}$ & 9 & 6785 & m & m \\
\hline Switzerland & 2888 & $\mathrm{~m}$ & $\mathrm{~m}$ & 9 & 7800 & $\mathbf{m}$ & m \\
\hline Turkey & 3360 & $\mathrm{a}$ & 3360 & 8 & 6240 & $\mathbf{a}$ & 6240 \\
\hline United States $^{9}$ & 3033 & $\mathrm{~m}$ & $\mathrm{~m}$ & 9 & 8835 & $\mathbf{m}$ & m \\
\hline
\end{tabular}

\begin{tabular}{|c|c|c|c|c|c|c|c|}
\hline $\begin{array}{l}\text { Argentina } \\
\text { 힌 Brazil }\end{array}$ & $\begin{array}{l}\mathrm{m} \\
\mathrm{m}\end{array}$ & $\begin{array}{l}\mathrm{m} \\
\mathrm{m}\end{array}$ & $\begin{array}{l}\mathrm{m} \\
\mathrm{m}\end{array}$ & $\begin{array}{r}\text { m } \\
9\end{array}$ & $\begin{array}{l}\mathbf{m} \\
\mathbf{m}\end{array}$ & $\begin{array}{l}\mathbf{m} \\
\mathbf{m}\end{array}$ & $\begin{array}{l}\mathbf{m} \\
\mathbf{m}\end{array}$ \\
\hline $\begin{array}{l}\text { China } \\
\text { Colombia }\end{array}$ & $\begin{array}{r}m \\
4800\end{array}$ & $\begin{array}{l}\mathrm{m} \\
\mathrm{m}\end{array}$ & $\begin{array}{l}\mathrm{m} \\
\mathrm{m}\end{array}$ & $\begin{array}{r}\mathbf{m} \\
\mathbf{9}\end{array}$ & $\begin{array}{r}m \\
9800\end{array}$ & $\begin{array}{l}\mathbf{m} \\
\mathbf{m}\end{array}$ & $\begin{array}{l}\mathbf{m} \\
\mathbf{m}\end{array}$ \\
\hline $\begin{array}{l}\text { India } \\
\text { Indonesia }\end{array}$ & $\begin{array}{l}\mathrm{m} \\
\mathrm{m}\end{array}$ & $\begin{array}{l}\mathrm{m} \\
\mathrm{m}\end{array}$ & $\begin{array}{l}\mathrm{m} \\
\mathrm{m}\end{array}$ & $\begin{array}{l}\mathbf{m} \\
\mathbf{m}\end{array}$ & $\begin{array}{l}\mathbf{m} \\
\mathbf{m}\end{array}$ & $\begin{array}{l}\mathbf{m} \\
\mathbf{m}\end{array}$ & $\begin{array}{l}\mathbf{m} \\
\mathbf{m}\end{array}$ \\
\hline $\begin{array}{l}\text { Latvia } \\
\text { Russian Federation }\end{array}$ & $\begin{array}{l}2381 \\
4384\end{array}$ & $\begin{array}{l}\mathrm{m} \\
\mathrm{m}\end{array}$ & $\begin{array}{l}\mathrm{m} \\
\mathrm{m}\end{array}$ & $\begin{array}{l}9 \\
9\end{array}$ & $\begin{array}{l}5947 \\
6452\end{array}$ & $\begin{array}{l}\mathbf{m} \\
\mathbf{m}\end{array}$ & $\begin{array}{l}\mathbf{m} \\
\mathbf{m}\end{array}$ \\
\hline $\begin{array}{l}\text { Saudi Arabia } \\
\text { South Africa }\end{array}$ & $\begin{array}{l}\mathrm{m} \\
\mathrm{m}\end{array}$ & $\begin{array}{l}\mathrm{m} \\
\mathrm{m}\end{array}$ & $\begin{array}{l}\mathrm{m} \\
\mathrm{m}\end{array}$ & $\begin{array}{l}\mathbf{m} \\
\mathbf{m}\end{array}$ & $\begin{array}{l}\mathbf{m} \\
\mathbf{m}\end{array}$ & $\begin{array}{l}\mathbf{m} \\
\mathbf{m}\end{array}$ & $\begin{array}{l}\mathbf{m} \\
\mathbf{m}\end{array}$ \\
\hline G20 average & $\mathrm{m}$ & $\mathrm{m}$ & $\mathrm{m}$ & $\mathbf{m}$ & m & $\mathbf{m}$ & m \\
\hline
\end{tabular}

Note: Columns showing instruction time in compulsory upper secondary education (i.e. columns 19-25) are available for consultation on line (see StatLink below). 1. Refers to full-time compulsory education and excludes pre-primary education, even if compulsory.

2. It is compulsory for students to attend full-time education up to the age of 15 or 16 . However, those in general education must continue until they are 18 .

3. Year of reference 2014.

4. England and Scotland are not included in the averages.

5. Estimated number of hours by level of education based on the average number of hours per year, as the allocation of instruction time across multiple grades is flexible.

6. Excludes the last year of compulsory education, which can be classified at either the lower secondary or the upper secondary level.

7. Actual instruction time for lower and upper secondary education.

8. The number of grades in lower secondary education is three or four, depending on the track. The 4th year of pre-vocational secondary education (VMBO) was excluded from the calculation.

9. Year of reference 2012.

Source: OECD. See Annex 3 for notes (www.oecd.org/education/education-at-a-glance-19991487.htm).

Please refer to the Reader's Guide for information concerning symbols for missing data and abbreviations.

StatLink 케느 http://dx.doi.org/10.1787/888933286093 
Table D1.2. Organisation of compulsory general education ${ }^{1}$ (2015) By level of education, in public institutions

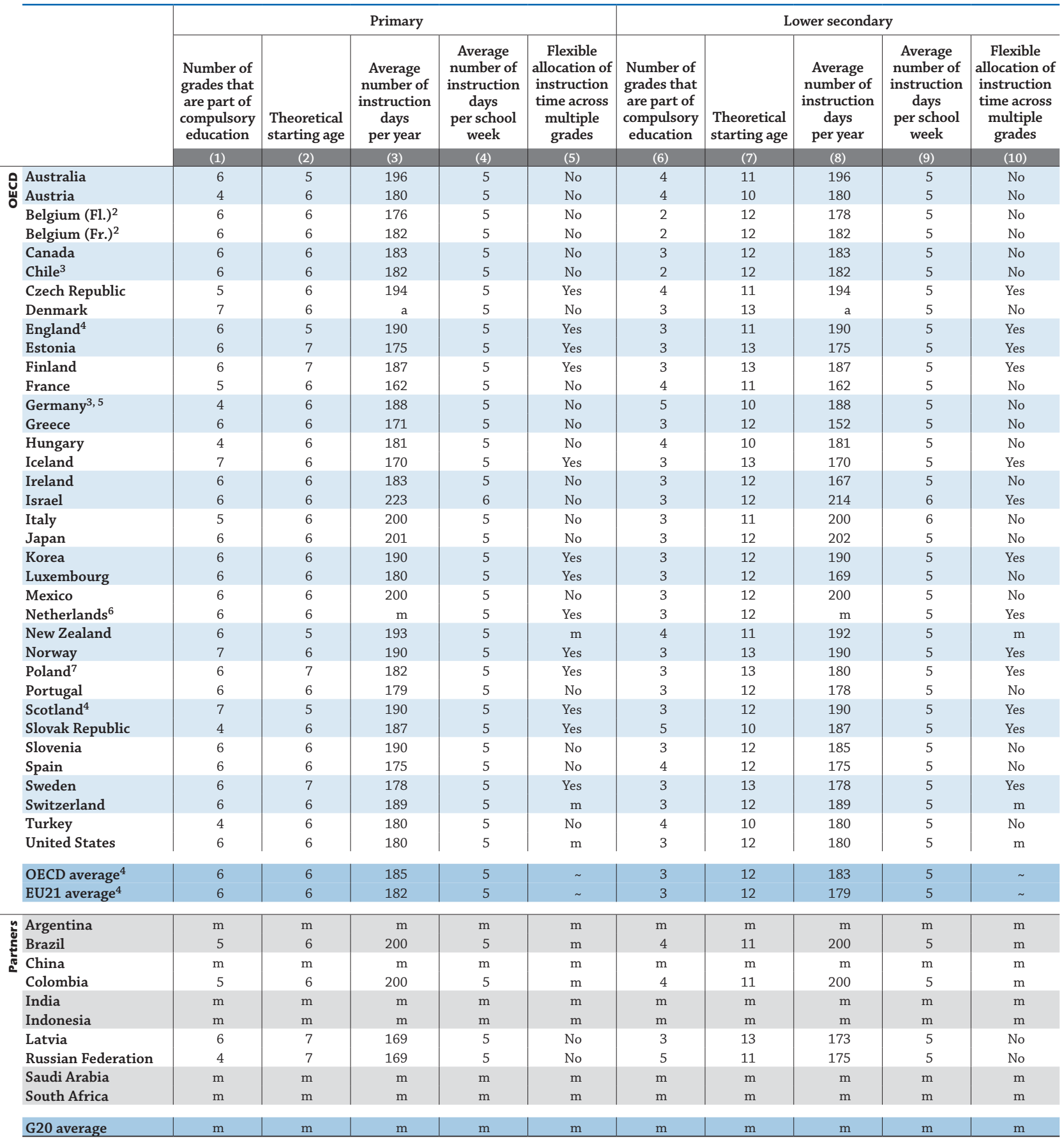

Note: Columns showing the organisation of compulsory upper secondary education (i.e. columns 11-15) are available for consultation on line (see StatLink below).

1. Refers to full-time compulsory education and excludes pre-primary education, even if compulsory.

2. It is compulsory for students to attend full-time education up to the age of 15 or 16 . However, those in general education must continue until they are 18 .

3. Year of reference 2014

4. England and Scotland are not included in the averages.

5. Excludes the last year of compulsory education, which can be classified at either the lower secondary or the upper secondary level.

6. The number of grades in lower secondary education is three or four, depending on the track. The 4th year of pre-vocational secondary education (VMBO) was excluded from the calculation.

7. In the 2014/15 school year, primary education was compulsory for 6-year-old children born in the first semester of 2008 .

Source: OECD. See Annex 3 for notes (www.oecd.org/education/education-at-a-glance-19991487.htm).

Please refer to the Reader's Guide for information concerning symbols for missing data and abbreviations.

StatLink 겍ㄴ http://dx.doi.org/10.1787/888933286104 
Table D1.3a. Instruction time per subject in primary education (2015) As a percentage of total compulsory instruction time

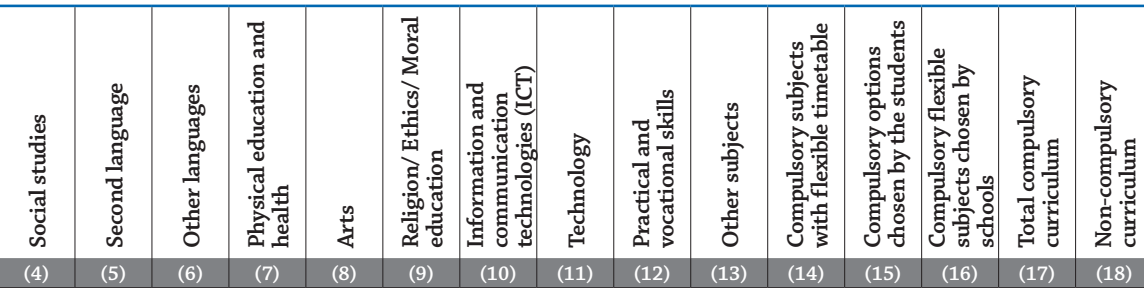

\begin{tabular}{|c|c|c|c|c|c|c|c|c|c|c|c|c|c|c|c|c|c|c|}
\hline Australia & 23 & 17 & 6 & 9 & $x(16)$ & $\mathrm{x}(16)$ & 8 & 5 & $\mathrm{x}(4)$ & $\mathrm{x}(11)$ & 4 & $\mathrm{x}(11)$ & $\mathrm{x}(16)$ & $\mathrm{x}(16)$ & $\mathrm{m}$ & 28 & 100 & $\mathrm{~m}$ \\
\hline Austria & 30 & 17 & 13 & $x(3)$ & 2 & $\mathrm{n}$ & 11 & 9 & 9 & $\mathrm{x}(17)$ & $\mathrm{x}(3)$ & 6 & 4 & a & $\mathrm{n}$ & a & 100 & $\mathrm{~m}$ \\
\hline Belgium (Fl. $)^{1}$ & $\mathrm{x}(14)$ & $\mathrm{x}(14)$ & $\mathrm{x}(14)$ & $\mathrm{x}(3)$ & $\mathrm{n}$ & a & $\mathrm{x}(14)$ & $\mathrm{x}(14)$ & 7 & $\mathrm{a}$ & $\mathrm{x}(3)$ & a & $\mathrm{a}$ & 93 & $\mathrm{n}$ & $\mathrm{x}(14)$ & 100 & a \\
\hline Belgium (Fr.) ${ }^{1}$ & $\mathrm{x}(14)$ & $\mathrm{x}(14)$ & $\mathrm{x}(14)$ & $\mathrm{x}(14)$ & 2 & a & 7 & $\mathrm{x}(14)$ & 7 & a & $\mathrm{x}(14)$ & a & a & 83 & a & $\mathrm{n}$ & 100 & $\mathrm{~m}$ \\
\hline Chile $^{2}$ & 20 & 16 & 9 & 9 & 3 & $\mathrm{x}(16)$ & 9 & 10 & 5 & $\mathrm{x}(16)$ & 3 & $\mathrm{x}(16)$ & 2 & a & a & 14 & 100 & a \\
\hline Czech Republic & 30 & 17 & 10 & $\mathrm{x}(3)$ & 8 & a & 8 & 10 & $\mathrm{x}(13)$ & 1 & 4 & $\mathrm{x}(11)$ & $\mathrm{x}(16)$ & a & $\mathrm{x}(16)$ & 12 & 100 & $\mathrm{~m}$ \\
\hline Denmark & 23 & 13 & 5 & 3 & 5 & 1 & 7 & 9 & 4 & $\mathrm{a}$ & a & 5 & 16 & 9 & $\mathrm{n}$ & $\mathrm{n}$ & 100 & 10 \\
\hline England $^{3}$ & $\mathrm{x}(14)$ & $\mathrm{x}(14)$ & $\mathrm{x}(14)$ & $x(14)$ & $x(14)$ & a & $\mathrm{x}(14)$ & $x(14)$ & $\mathrm{x}(14)$ & $\mathrm{x}(14)$ & $\mathrm{x}(14)$ & a & a & 100 & a & a & $\mathbf{a}$ & a \\
\hline Finland & 24 & 16 & 11 & 2 & 6 & $\mathrm{n}$ & 10 & 13 & 5 & $\mathrm{a}$ & $\mathrm{a}$ & a & $\mathrm{n}$ & 6 & a & 7 & 100 & 5 \\
\hline France & 37 & 21 & 9 & 5 & 6 & a & 13 & 9 & $\mathrm{x}(17)$ & $\mathrm{x}(3)$ & $\mathrm{n}$ & a & a & $\mathrm{n}$ & a & $\mathrm{n}$ & 100 & a \\
\hline Germany $^{2}$ & 26 & 20 & 4 & 6 & 5 & $\mathrm{n}$ & 11 & 14 & 7 & $\mathrm{n}$ & 1 & $\mathrm{n}$ & 3 & a & 2 & a & 100 & a \\
\hline Greece & 25 & 13 & 10 & 7 & 8 & 2 & 8 & 10 & 4 & 3 & a & a & a & a & a & 8 & 100 & 35 \\
\hline Hungary & 30 & 15 & 6 & a & 3 & a & 19 & 15 & 2 & 1 & 4 & a & a & a & a & 4 & 100 & a \\
\hline Iceland & 20 & 16 & 8 & 13 & $x(14)$ & $\mathrm{x}(14)$ & 9 & 19 & $\mathrm{x}(4)$ & 3 & a & $\mathrm{x}(8)$ & $\mathrm{x}(15)$ & 5 & 5 & a & 100 & a \\
\hline Ireland ${ }^{4}$ & 20 & 17 & 4 & 8 & 14 & $a$ & 4 & 12 & 10 & $\mathrm{x}(17)$ & $x(3)$ & a & 11 & a & a & $\mathrm{m}$ & 100 & $\mathrm{a}$ \\
\hline Israel & 23 & 18 & 9 & 8 & 6 & 2 & 6 & 5 & 11 & a & $\mathrm{x}(3)$ & 4 & $\mathrm{n}$ & $\mathrm{n}$ & $\mathrm{n}$ & 7 & 100 & $\mathrm{n}$ \\
\hline Japan & 24 & 17 & 8 & 8 & 1 & a & 10 & 12 & 3 & a & a & a & 10 & 7 & a & a & 100 & a \\
\hline Korea & 22 & 14 & 9 & 9 & 6 & a & 7 & 9 & $\mathrm{x}(4)$ & $\mathrm{x}(13)$ & $\mathrm{x}(12)$ & $\mathrm{x}(3)$ & 24 & a & a & a & 100 & a \\
\hline Luxembourg $^{4}$ & 26 & 19 & 7 & 2 & $\mathrm{x}(1)$ & 18 & 10 & 11 & 7 & a & a & a & a & a & a & a & 100 & a \\
\hline Mexico & 31 & 24 & 12 & 9 & 11 & a & 4 & 4 & 4 & a & a & a & a & $\mathrm{a}$ & $\mathrm{a}$ & a & 100 & a \\
\hline Netherlands & $\mathrm{x}(14)$ & $\mathrm{x}(14)$ & $\mathrm{x}(14)$ & $x(14)$ & $x(14)$ & a & $\mathrm{x}(14)$ & $x(14)$ & $x(14)$ & $\mathrm{x}(14)$ & $\mathrm{x}(14)$ & $\mathrm{x}(14)$ & $\mathrm{a}$ & 100 & a & $\mathrm{a}$ & 100 & $\mathrm{~m}$ \\
\hline New Zealand & $\mathrm{m}$ & $\mathrm{m}$ & $\mathrm{m}$ & $\mathrm{m}$ & $\mathrm{m}$ & $\mathrm{m}$ & $\mathrm{m}$ & $\mathrm{m}$ & $\mathrm{m}$ & $\mathrm{m}$ & $\mathrm{m}$ & $\mathrm{m}$ & $\mathrm{m}$ & $\mathrm{m}$ & $\mathrm{m}$ & $\mathrm{m}$ & $\mathbf{m}$ & $\mathrm{m}$ \\
\hline Norway & 26 & 17 & 6 & 7 & 7 & a & 11 & 15 & 8 & a & a & 2 & a & a & a & 1 & 100 & a \\
\hline Poland ${ }^{5}$ & 18 & 14 & 10 & 5 & 10 & a & 14 & 7 & $\mathrm{x}(18)$ & 3 & 3 & a & 3 & $\mathrm{n}$ & $\mathrm{a}$ & 13 & 100 & 9 \\
\hline Portugal & 27 & 27 & 7 & 8 & 3 & a & 8 & 9 & $x(18)$ & $\mathrm{x}(18)$ & 2 & a & 4 & a & $\mathrm{a}$ & 5 & 100 & 23 \\
\hline Scotland ${ }^{3}$ & $\mathrm{x}(14)$ & $\mathrm{x}(14)$ & $\mathrm{x}(14)$ & $\mathrm{x}(14)$ & $\mathrm{x}(14)$ & $\mathrm{x}(14)$ & $\mathrm{x}(14)$ & $\mathrm{x}(14)$ & $\mathrm{x}(14)$ & $\mathrm{x}(14)$ & $\mathrm{x}(14)$ & $\mathrm{x}(14)$ & a & a & a & a & $\mathbf{a}$ & a \\
\hline Slovak Republic & 27 & 15 & 3 & 3 & 6 & $\mathrm{x}(16)$ & 8 & 8 & 4 & 3 & a & 1 & $x(16)$ & a & $\mathrm{x}(16)$ & 21 & 100 & a \\
\hline Slovenia & 23 & 17 & 8 & 7 & 6 & $\mathrm{x}(18)$ & 15 & 16 & $\mathrm{x}(4)$ & $\mathrm{x}(17)$ & 6 & 2 & 1 & a & a & a & 100 & 17 \\
\hline Spain & 25 & 17 & 7 & 7 & 10 & $\mathrm{n}$ & 9 & 9 & $\mathrm{x}(15)$ & $\mathrm{a}$ & a & a & $\mathrm{n}$ & a & 7 & 9 & 100 & a \\
\hline Sweden & $\mathrm{m}$ & $\mathrm{m}$ & $\mathrm{m}$ & $\mathrm{m}$ & $\mathrm{m}$ & $\mathrm{m}$ & $\mathrm{m}$ & $\mathrm{m}$ & $\mathrm{m}$ & $\mathrm{m}$ & $\mathrm{m}$ & $\mathrm{m}$ & $\mathrm{m}$ & $\mathrm{m}$ & $\mathrm{m}$ & $\mathrm{m}$ & $\mathbf{m}$ & $\mathrm{m}$ \\
\hline Switzerland & $\mathrm{m}$ & $\mathrm{m}$ & $\mathrm{m}$ & $\mathrm{m}$ & $\mathrm{m}$ & $\mathrm{m}$ & $\mathrm{m}$ & $\mathrm{m}$ & $\mathrm{m}$ & $\mathrm{m}$ & $\mathrm{m}$ & $\mathrm{m}$ & $\mathrm{m}$ & $\mathrm{m}$ & $\mathrm{m}$ & $\mathrm{m}$ & $\mathbf{m}$ & $\mathrm{m}$ \\
\hline Turkey & 30 & 17 & 5 & 13 & 5 & a & 14 & 7 & 2 & a & a & 1 & 7 & a & $\mathrm{a}$ & a & 100 & a \\
\hline United States & $\mathrm{m}$ & $\mathrm{m}$ & $\mathrm{m}$ & $\mathrm{m}$ & $\mathrm{m}$ & $\mathrm{m}$ & $\mathrm{m}$ & $\mathrm{m}$ & $\mathrm{m}$ & $\mathrm{m}$ & $\mathrm{m}$ & $\mathrm{m}$ & $\mathrm{m}$ & $\mathrm{m}$ & $\mathrm{m}$ & $\mathrm{m}$ & $\mathbf{m}$ & $\mathrm{m}$ \\
\hline Argentina & $\mathrm{m}$ & $\mathrm{m}$ & $\mathrm{m}$ & $\mathrm{m}$ & $\mathrm{m}$ & $\mathrm{m}$ & $\mathrm{m}$ & $\mathrm{m}$ & $\mathrm{m}$ & $\mathrm{m}$ & $\mathrm{m}$ & $\mathrm{m}$ & $\mathrm{m}$ & $\mathrm{m}$ & $\mathrm{m}$ & $\mathrm{m}$ & $\mathbf{m}$ & $\mathrm{m}$ \\
\hline Erazil & $\mathrm{m}$ & $\mathrm{m}$ & $\mathrm{m}$ & $\mathrm{m}$ & $\mathrm{m}$ & a & $\mathrm{m}$ & $\mathrm{m}$ & $\mathrm{m}$ & $\mathrm{m}$ & a & a & $\mathrm{m}$ & $\mathrm{m}$ & $\mathrm{m}$ & $\mathrm{m}$ & $\mathbf{m}$ & $\mathrm{m}$ \\
\hline China & $\mathrm{m}$ & $\mathrm{m}$ & $\mathrm{m}$ & $\mathrm{m}$ & $\mathrm{m}$ & $\mathrm{m}$ & $\mathrm{m}$ & $\mathrm{m}$ & $\mathrm{m}$ & $\mathrm{m}$ & $\mathrm{m}$ & $\mathrm{m}$ & $\mathrm{m}$ & $\mathrm{m}$ & $\mathrm{m}$ & $\mathrm{m}$ & $\mathbf{m}$ & $\mathrm{m}$ \\
\hline Colombia & $\mathrm{m}$ & $\mathrm{m}$ & $\mathrm{m}$ & $\mathrm{m}$ & $\mathrm{m}$ & $\mathrm{m}$ & $\mathrm{m}$ & $\mathrm{m}$ & $\mathrm{m}$ & $\mathrm{m}$ & $\mathrm{m}$ & $\mathrm{m}$ & $\mathrm{m}$ & $\mathrm{m}$ & $\mathrm{m}$ & $\mathrm{m}$ & $\mathbf{m}$ & $\mathrm{m}$ \\
\hline India & $\mathrm{m}$ & $\mathrm{m}$ & $\mathrm{m}$ & $\mathrm{m}$ & $\mathrm{m}$ & $\mathrm{m}$ & $\mathrm{m}$ & $\mathrm{m}$ & $\mathrm{m}$ & $\mathrm{m}$ & $\mathrm{m}$ & $\mathrm{m}$ & $\mathrm{m}$ & $\mathrm{m}$ & $\mathrm{m}$ & $\mathrm{m}$ & $\mathbf{m}$ & $\mathrm{m}$ \\
\hline Indonesia & $\mathrm{m}$ & $\mathrm{m}$ & $\mathrm{m}$ & $\mathrm{m}$ & $\mathrm{m}$ & $\mathrm{m}$ & $\mathrm{m}$ & $\mathrm{m}$ & $\mathrm{m}$ & $\mathrm{m}$ & $\mathrm{m}$ & $\mathrm{m}$ & $\mathrm{m}$ & $\mathrm{m}$ & $\mathrm{m}$ & $\mathrm{m}$ & $\mathbf{m}$ & $\mathrm{m}$ \\
\hline Latvia & 23 & 17 & 5 & 6 & 8 & 1 & 8 & 13 & 2 & 1 & a & 4 & 4 & a & a & 7 & 100 & $\mathrm{~m}$ \\
\hline Russian Federation & 23 & 19 & 9 & a & 7 & a & 9 & 9 & a & a & 7 & a & a & 16 & a & $\mathrm{m}$ & 100 & $\mathrm{~m}$ \\
\hline Saudi Arabia & $\mathrm{m}$ & $\mathrm{m}$ & $\mathrm{m}$ & $\mathrm{m}$ & $\mathrm{m}$ & $\mathrm{m}$ & $\mathrm{m}$ & $\mathrm{m}$ & $\mathrm{m}$ & $\mathrm{m}$ & $\mathrm{m}$ & $\mathrm{m}$ & $\mathrm{m}$ & $\mathrm{m}$ & $\mathrm{m}$ & $\mathrm{m}$ & $\mathbf{m}$ & $\mathrm{m}$ \\
\hline South Africa & $\mathrm{m}$ & $\mathrm{m}$ & $\mathrm{m}$ & $\mathrm{m}$ & $\mathrm{m}$ & $\mathrm{m}$ & $\mathrm{m}$ & $\mathrm{m}$ & $\mathrm{m}$ & $\mathrm{m}$ & $\mathrm{m}$ & $\mathrm{m}$ & $\mathrm{m}$ & $\mathrm{m}$ & $\mathrm{m}$ & $\mathrm{m}$ & $\mathbf{m}$ & $\mathrm{m}$ \\
\hline G20 average & $\mathrm{m}$ & $\mathrm{m}$ & $\mathrm{m}$ & $\mathrm{m}$ & $\mathrm{m}$ & $\mathrm{m}$ & $\mathrm{m}$ & $\mathrm{m}$ & $\mathrm{m}$ & $\mathrm{m}$ & $\mathrm{m}$ & $\mathrm{m}$ & $\mathrm{m}$ & $\mathrm{m}$ & $\mathrm{m}$ & $\mathrm{m}$ & m & $\mathrm{m}$ \\
\hline
\end{tabular}

Notes: Please refer to Tables D1.5a to D1.5l, available on line, for instruction time per subject for each age (see StatLink below).

The averages were adjusted to $100 \%$ and do not correspond exactly to the average of each column.

1. It is compulsory for students to attend full-time education up to the age of 15 or 16 . However, those in general education must continue until they are 18 .

2. Year of reference 2014

3 England and Scotland are not included in the averages.

4. The second language of instruction includes other national languages taught.

5. Excludes the first three years of primary education for which a large proportion of the time allocated to compulsory subjects is flexible.

Source: OECD. See Annex 3 for notes (www.oecd.org/education/education-at-a-glance-19991487.htm).

Please refer to the Reader's Guide for information concerning symbols for missing data and abbreviations.

StatLink 제이 http://dx.doi.org/10.1787/888933286110 
Table D1.3b. Instruction time per subject in general lower secondary education (2015) As a percentage of total compulsory instruction time

\begin{tabular}{|c|c|c|c|c|c|c|c|c|c|c|c|c|c|c|c|c|c|c|}
\hline & 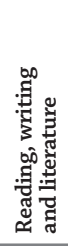 & 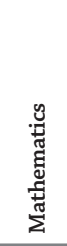 & 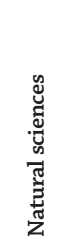 & 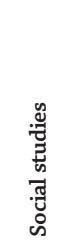 & 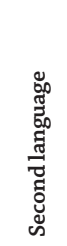 & 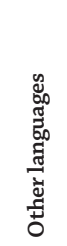 & 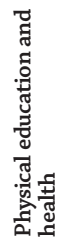 & $\frac{n}{4}$ & 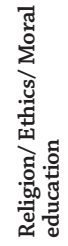 & 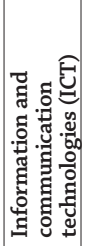 & 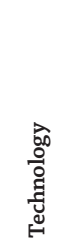 & 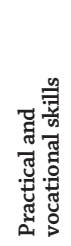 & 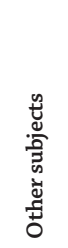 & 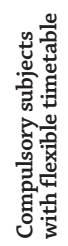 & 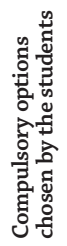 & 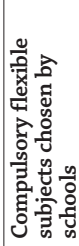 & 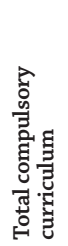 & 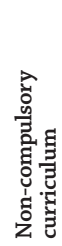 \\
\hline & (1) & (2) & (3) & (4) & (5) & (6) & (7) & (8) & (9) & (10) & $(11)$ & (12) & $(13)$ & (14) & $(15)$ & (16) & (17) & (18) \\
\hline Australia $^{1}$ & 12 & 12 & 11 & 10 & $\mathrm{x}(16)$ & $\mathrm{x}(16)$ & 8 & 4 & $\mathrm{x}(4)$ & $\mathrm{x}(11)$ & 4 & $\mathrm{x}(11)$ & $\mathrm{x}(16)$ & $\mathrm{x}(16)$ & 18 & 22 & 100 & $\mathrm{~m}$ \\
\hline Austria & 14 & 13 & 12 & 11 & 12 & $\mathrm{n}$ & 11 & 12 & 7 & $\mathrm{x}(17)$ & $\mathrm{n}$ & 8 & $\mathrm{n}$ & $\mathrm{a}$ & $\mathrm{n}$ & $\mathrm{a}$ & 100 & $\mathrm{~m}$ \\
\hline Belgium (Fl. $)^{2}$ & $\mathrm{x}(14)$ & $\mathrm{x}(14)$ & $\mathrm{x}(14)$ & $\mathrm{x}(14)$ & $\mathrm{x}(14)$ & $\mathrm{x}(14)$ & $\mathrm{x}(14)$ & $\mathrm{x}(14)$ & 6 & $\mathrm{a}$ & $\mathrm{x}(14)$ & $\mathrm{a}$ & $\mathrm{a}$ & 73 & $\mathrm{n}$ & 20 & 100 & $\mathrm{a}$ \\
\hline Belgium (Fr.) ${ }^{2}$ & 17 & 14 & 9 & 13 & 13 & a & 9 & 3 & 6 & $\mathrm{~m}$ & 3 & $\mathrm{~m}$ & $\mathrm{x}(15)$ & $\mathrm{n}$ & $x(16)$ & 13 & 100 & $\mathrm{~m}$ \\
\hline Canada & 20 & 15 & 9 & 13 & 6 & $\mathrm{a}$ & 10 & 6 & 2 & $\mathrm{a}$ & 3 & 1 & 1 & 2 & 1 & 11 & 100 & 1 \\
\hline Chile $^{3}$ & 16 & 16 & 11 & 11 & 8 & $\mathrm{x}(16)$ & 5 & 8 & 5 & $\mathrm{x}(16)$ & 3 & $\mathrm{x}(16)$ & 3 & $\mathrm{a}$ & $\mathrm{a}$ & 15 & 100 & a \\
\hline Czech Republic & 12 & 12 & 17 & 9 & 10 & 5 & 8 & 8 & $x(13)$ & 1 & 2 & $\mathrm{x}(11)$ & $\mathrm{x}(16)$ & a & $x(16)$ & 15 & 100 & $\mathrm{~m}$ \\
\hline Denmark & 19 & 13 & 14 & 8 & 8 & 8 & 5 & $\mathrm{x}(15)$ & 2 & $\mathrm{x}(15)$ & $\mathrm{x}(15)$ & 2 & 15 & $\mathrm{a}$ & 5 & $\mathrm{n}$ & 100 & 7 \\
\hline England $^{4}$ & $\mathrm{x}(14)$ & $\mathrm{x}(14)$ & $\mathrm{x}(14)$ & $\mathrm{x}(14)$ & $\mathrm{x}(14)$ & a & $\mathrm{x}(14)$ & $\mathrm{x}(14)$ & $x(14)$ & $\mathrm{x}(14)$ & $\mathrm{x}(14)$ & $\mathrm{x}(15)$ & a & 100 & $\mathrm{x}(14)$ & a & a & a \\
\hline Estonia & 13 & 14 & 21 & 11 & 10 & 10 & 6 & 6 & $x(16)$ & $x(16)$ & 5 & $\mathrm{a}$ & a & a & $\mathrm{a}$ & 4 & 100 & $\mathrm{a}$ \\
\hline Finland & 12 & 12 & 16 & 8 & 9 & 7 & 9 & 9 & 4 & a & a & a & 6 & 4 & $\mathrm{a}$ & 5 & 100 & 7 \\
\hline France & 15 & 14 & 10 & 11 & 12 & 5 & 12 & 7 & $\mathrm{x}(4)$ & $\mathrm{x}(11)$ & 6 & $\mathrm{x}(15)$ & 3 & $\mathrm{n}$ & 4 & 1 & 100 & 10 \\
\hline Germany $^{3}$ & 13 & 12 & 11 & 11 & 12 & 6 & 9 & 9 & 5 & 1 & 2 & 2 & 1 & a & 7 & a & 100 & a \\
\hline Greece & 26 & 11 & 10 & 12 & 6 & 6 & 7 & 6 & 6 & 3 & 2 & 5 & a & $\mathrm{a}$ & $\mathrm{a}$ & 1 & 100 & $\mathrm{n}$ \\
\hline Hungary & 14 & 12 & 12 & 11 & 11 & a & 16 & 7 & 3 & 3 & 3 & a & 3 & a & $\mathrm{a}$ & 5 & 100 & a \\
\hline Iceland & 14 & 14 & 8 & 8 & $\mathrm{x}(14)$ & $\mathrm{x}(14)$ & 8 & 8 & $\mathrm{x}(4)$ & 2 & $\mathrm{a}$ & $x(8)$ & $\mathrm{x}(15)$ & 19 & 20 & $\mathrm{a}$ & 100 & a \\
\hline Ireland $d^{5,6}$ & 12 & 12 & $\mathrm{x}(15)$ & 17 & $\mathrm{x}(15)$ & $\mathrm{x}(15)$ & 7 & $\mathrm{x}(15)$ & 2 & $\mathrm{x}(15)$ & $\mathrm{x}(15)$ & $\mathrm{x}(15)$ & $\mathrm{x}(14)$ & 10 & 40 & $\mathrm{~m}$ & 100 & a \\
\hline Israel & 17 & 14 & 14 & 15 & 11 & 9 & 5 & $\mathrm{x}(16)$ & 9 & $\mathrm{x}(3)$ & $\mathrm{x}(3)$ & 1 & $\mathrm{n}$ & 3 & $\mathrm{n}$ & 2 & 100 & $\mathrm{n}$ \\
\hline Italy & 33 & 20 & $\mathrm{x}(2)$ & $\mathrm{x}(1)$ & 10 & 7 & 7 & 13 & 3 & a & 7 & $\mathrm{a}$ & $\mathrm{a}$ & $\mathrm{n}$ & $\mathrm{a}$ & $\mathrm{x}(17)$ & 100 & a \\
\hline Japan & 12 & 12 & 12 & 11 & 13 & $\mathrm{a}$ & 10 & 7 & 3 & a & 3 & a & 12 & 5 & $\mathrm{a}$ & $\mathrm{a}$ & 100 & a \\
\hline Korea & 13 & 11 & 19 & 15 & 10 & a & 8 & 8 & $\mathrm{x}(4)$ & $\mathrm{x}(12)$ & $\mathrm{x}(12)$ & $\mathrm{x}(3)$ & 9 & a & $\mathrm{x}(16)$ & 6 & 100 & a \\
\hline Luxembourg 6 & 15 & 13 & 8 & 11 & 17 & 13 & 8 & 9 & 7 & a & $\mathrm{a}$ & $\mathrm{a}$ & a & a & $\mathrm{a}$ & a & 100 & a \\
\hline Mexico & 14 & 14 & 17 & 12 & 9 & a & 6 & 6 & 8 & $\mathrm{a}$ & 11 & $\mathrm{a}$ & 3 & a & $\mathrm{a}$ & a & 100 & a \\
\hline Netherlands & $\mathrm{x}(14)$ & $\mathrm{x}(14)$ & $\mathrm{x}(14)$ & $\mathrm{x}(14)$ & $\mathrm{x}(14)$ & $\mathrm{x}(14)$ & $\mathrm{x}(14)$ & $\mathrm{x}(14)$ & $\mathrm{x}(14)$ & $\mathrm{x}(14)$ & $\mathrm{x}(14)$ & $\mathrm{x}(14)$ & a & 100 & $\mathrm{a}$ & a & 100 & $\mathrm{~m}$ \\
\hline New Zealand & $\mathrm{m}$ & $\mathrm{m}$ & $\mathrm{m}$ & $\mathrm{m}$ & $\mathrm{m}$ & $\mathrm{m}$ & $\mathrm{m}$ & $\mathrm{m}$ & $\mathrm{m}$ & $\mathrm{m}$ & $\mathrm{m}$ & $\mathrm{m}$ & $\mathrm{m}$ & $\mathrm{m}$ & $\mathrm{m}$ & $\mathrm{m}$ & $\mathbf{m}$ & $\mathrm{m}$ \\
\hline Norway & 15 & 12 & 9 & 9 & 8 & 8 & 9 & 9 & 6 & a & $\mathrm{a}$ & 7 & $\mathrm{a}$ & $\mathrm{a}$ & 7 & a & 100 & a \\
\hline Poland & 14 & 12 & 12 & 12 & 14 & $\mathrm{x}(5)$ & 12 & 4 & $\mathrm{x}(18)$ & 2 & 2 & $\mathrm{a}$ & 4 & a & $\mathrm{a}$ & 13 & 100 & 8 \\
\hline Portugal & 13 & 13 & 18 & 14 & 8 & 8 & 7 & 7 & $\mathrm{x}(18)$ & 2 & $\mathrm{n}$ & $\mathrm{a}$ & $\mathrm{n}$ & a & $\mathrm{a}$ & 9 & 100 & 3 \\
\hline Scotland ${ }^{4}$ & $\mathrm{x}(14)$ & $\mathrm{x}(14)$ & $\mathrm{x}(14)$ & $\mathrm{x}(14)$ & $\mathrm{x}(14)$ & $\mathrm{x}(14)$ & $\mathrm{x}(14)$ & $\mathrm{x}(14)$ & $\mathrm{x}(14)$ & $\mathrm{x}(14)$ & $\mathrm{x}(14)$ & $\mathrm{x}(14)$ & $\mathrm{a}$ & a & $\mathrm{a}$ & a & $\mathbf{a}$ & a \\
\hline Slovak Republic & 16 & 13 & 10 & 10 & 10 & 3 & 7 & 5 & 3 & 1 & $\mathrm{x}(16)$ & 1 & $\mathrm{x}(16)$ & $\mathrm{a}$ & $\mathrm{x}(16)$ & 21 & 100 & a \\
\hline Slovenia & 13 & 13 & 17 & 15 & 11 & $\mathrm{x}(15)$ & 9 & 8 & $\mathrm{x}(4)$ & $\mathrm{x}(17)$ & 4 & $\mathrm{a}$ & 2 & a & 7 & $\mathrm{a}$ & 100 & 19 \\
\hline Spain & 16 & 12 & 8 & 11 & 11 & $\mathrm{n}$ & 7 & 7 & $x(15)$ & $\mathrm{x}(11)$ & 5 & $\mathrm{a}$ & 3 & a & 18 & $\mathrm{a}$ & 100 & a \\
\hline Sweden & $\mathrm{m}$ & $\mathrm{m}$ & $\mathrm{m}$ & $\mathrm{m}$ & $\mathrm{m}$ & $\mathrm{m}$ & $\mathrm{m}$ & $\mathrm{m}$ & $\mathrm{m}$ & $\mathrm{m}$ & $\mathrm{m}$ & $\mathrm{m}$ & $\mathrm{m}$ & $\mathrm{m}$ & $\mathrm{m}$ & $\mathrm{m}$ & $\mathbf{m}$ & $\mathrm{m}$ \\
\hline Switzerland & $\mathrm{m}$ & $\mathrm{m}$ & $\mathrm{m}$ & $\mathrm{m}$ & $\mathrm{m}$ & $\mathrm{m}$ & $\mathrm{m}$ & $\mathrm{m}$ & $\mathrm{m}$ & $\mathrm{m}$ & $\mathrm{m}$ & $\mathrm{m}$ & $\mathrm{m}$ & $\mathrm{m}$ & $\mathrm{m}$ & $\mathrm{m}$ & $\mathbf{m}$ & $\mathrm{m}$ \\
\hline Turkey & 16 & 14 & 11 & 8 & 10 & $\mathrm{a}$ & 6 & 6 & 6 & 3 & 3 & 1 & $\mathrm{a}$ & $\mathrm{a}$ & 17 & $\mathrm{a}$ & 100 & a \\
\hline United States & $\mathrm{m}$ & $\mathrm{m}$ & $\mathrm{m}$ & $\mathrm{m}$ & $\mathrm{m}$ & $\mathrm{m}$ & $\mathrm{m}$ & $\mathrm{m}$ & $\mathrm{m}$ & $\mathrm{m}$ & $\mathrm{m}$ & $\mathrm{m}$ & $\mathrm{m}$ & $\mathrm{m}$ & $\mathrm{m}$ & $\mathrm{m}$ & $\mathbf{m}$ & $\mathrm{m}$ \\
\hline OECD average ${ }^{4}$ & 14 & 12 & 11 & 10 & 9 & 4 & 7 & 6 & 4 & 1 & 3 & 1 & 2 & 6 & 5 & 5 & 100 & 2 \\
\hline EU21 average ${ }^{4}$ & 14 & 11 & 11 & 10 & 9 & 4 & 7 & 6 & 4 & 1 & 2 & 1 & 2 & 8 & 4 & 5 & 100 & 3 \\
\hline Argentina & $\mathrm{m}$ & $\mathrm{m}$ & $\mathrm{m}$ & $\mathrm{m}$ & $\mathrm{m}$ & $\mathrm{m}$ & $\mathrm{m}$ & $\mathrm{m}$ & $\mathrm{m}$ & $\mathrm{m}$ & $\mathrm{m}$ & $\mathrm{m}$ & $\mathrm{m}$ & $\mathrm{m}$ & $\mathrm{m}$ & $\mathrm{m}$ & $\mathbf{m}$ & $\mathrm{m}$ \\
\hline Brazi & $\mathrm{m}$ & $\mathrm{m}$ & $\mathrm{m}$ & $\mathrm{m}$ & $\mathrm{m}$ & a & $\mathrm{m}$ & $\mathrm{m}$ & $\mathrm{m}$ & $\mathrm{m}$ & $\mathrm{a}$ & $\mathrm{m}$ & $\mathrm{m}$ & $\mathrm{m}$ & $\mathrm{m}$ & $\mathrm{m}$ & $\mathbf{m}$ & $\mathrm{m}$ \\
\hline China & $\mathrm{m}$ & $\mathrm{m}$ & $\mathrm{m}$ & $\mathrm{m}$ & $\mathrm{m}$ & $\mathrm{m}$ & $\mathrm{m}$ & $\mathrm{m}$ & $\mathrm{m}$ & $\mathrm{m}$ & $\mathrm{m}$ & $\mathrm{m}$ & $\mathrm{m}$ & $\mathrm{m}$ & $\mathrm{m}$ & $\mathrm{m}$ & $\mathbf{m}$ & $\mathrm{m}$ \\
\hline Colombia & $\mathrm{m}$ & $\mathrm{m}$ & $\mathrm{m}$ & $\mathrm{m}$ & $\mathrm{m}$ & $\mathrm{m}$ & $\mathrm{m}$ & $\mathrm{m}$ & $\mathrm{m}$ & $\mathrm{m}$ & $\mathrm{m}$ & $\mathrm{m}$ & $\mathrm{m}$ & $\mathrm{m}$ & $\mathrm{m}$ & $\mathrm{m}$ & $\mathbf{m}$ & $\mathrm{m}$ \\
\hline India & $\mathrm{m}$ & $\mathrm{m}$ & $\mathrm{m}$ & $\mathrm{m}$ & $\mathrm{m}$ & $\mathrm{m}$ & $\mathrm{m}$ & $\mathrm{m}$ & $\mathrm{m}$ & $\mathrm{m}$ & $\mathrm{m}$ & $\mathrm{m}$ & $\mathrm{m}$ & $\mathrm{m}$ & $\mathrm{m}$ & $\mathrm{m}$ & $\mathbf{m}$ & $\mathrm{m}$ \\
\hline Indonesia & $\mathrm{m}$ & $\mathrm{m}$ & $\mathrm{m}$ & $\mathrm{m}$ & $\mathrm{m}$ & $\mathrm{m}$ & $\mathrm{m}$ & $\mathrm{m}$ & $\mathrm{m}$ & $\mathrm{m}$ & $\mathrm{m}$ & $\mathrm{m}$ & $\mathrm{m}$ & $\mathrm{m}$ & $\mathrm{m}$ & $\mathrm{m}$ & $\mathbf{m}$ & $\mathrm{m}$ \\
\hline Latvia & 15 & 16 & 10 & 14 & 9 & 9 & 6 & 6 & a & 1 & $\mathrm{a}$ & 4 & 3 & $\mathrm{a}$ & $\mathrm{a}$ & 10 & 100 & $\mathrm{~m}$ \\
\hline Russian Federation & 15 & 15 & 16 & 8 & 9 & $\mathrm{a}$ & 6 & 5 & $\mathrm{a}$ & 2 & 4 & 1 & a & 20 & $\mathrm{~m}$ & $\mathrm{~m}$ & 100 & $\mathrm{~m}$ \\
\hline Saudi Arabia & $\mathrm{m}$ & $\mathrm{m}$ & $\mathrm{m}$ & $\mathrm{m}$ & $\mathrm{m}$ & $\mathrm{m}$ & $\mathrm{m}$ & $\mathrm{m}$ & $\mathrm{m}$ & $\mathrm{m}$ & $\mathrm{m}$ & $\mathrm{m}$ & $\mathrm{m}$ & $\mathrm{m}$ & $\mathrm{m}$ & $\mathrm{m}$ & $\mathbf{m}$ & $\mathrm{m}$ \\
\hline South Africa & $\mathrm{m}$ & $\mathrm{m}$ & $\mathrm{m}$ & $\mathrm{m}$ & $\mathrm{m}$ & $\mathrm{m}$ & $\mathrm{m}$ & $\mathrm{m}$ & $\mathrm{m}$ & $\mathrm{m}$ & $\mathrm{m}$ & $\mathrm{m}$ & $\mathrm{m}$ & $\mathrm{m}$ & $\mathrm{m}$ & $\mathrm{m}$ & $\mathbf{m}$ & $\mathrm{m}$ \\
\hline G20 average & $\mathrm{m}$ & $\mathrm{m}$ & $\mathrm{m}$ & $\mathrm{m}$ & $\mathrm{m}$ & $\mathrm{m}$ & $\mathrm{m}$ & $\mathrm{m}$ & $\mathrm{m}$ & $\mathrm{m}$ & $\mathrm{m}$ & $\mathrm{m}$ & $\mathrm{m}$ & $\mathrm{m}$ & $\mathrm{m}$ & $\mathrm{m}$ & m & $\mathrm{m}$ \\
\hline
\end{tabular}

Notes: Please refer to Tables D1.5a to D1.5l, available on line, for instruction time per subject for each age (see StatLink below)

The averages were adjusted to $100 \%$ and do not correspond exactly to the average of each column.

1. The intended instruction times derived from the Australian Curriculum assumes that certain subjects, which may be considered compulsory in years 7 and 8 , could be delivered to students as electives in years 9 and 10 .

2. It is compulsory for students to attend full-time education up to the age of 15 or 16 . However, those in general education must continue until they are 18 .

3. Year of reference 2014.

4. England and Scotland are not included in the averages.

5. Actual instruction time.

6. The second language of instruction includes other national languages taught.

Source: OECD. See Annex 3 for notes (www.oecd.org/education/education-at-a-glance-19991487.htm).

Please refer to the Reader's Guide for information concerning symbols for missing data and abbreviations.

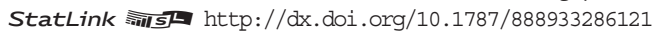




\section{WHAT IS THE STUDENT-TEACHER RATIO AND HOW BIG ARE CLASSES?}

- The average primary school class in OECD countries has 21 students, and this average increases to 24 in lower secondary education.

- The difference in average class size between public and private institutions in primary education varies substantially across OECD countries, but is considerably larger in partner countries.

INDICATOR D2 - There are 15 students per teacher in primary education, on average across OECD countries.

\section{Chart D2.1. Average class size in educational institutions, by level of education (2013)}

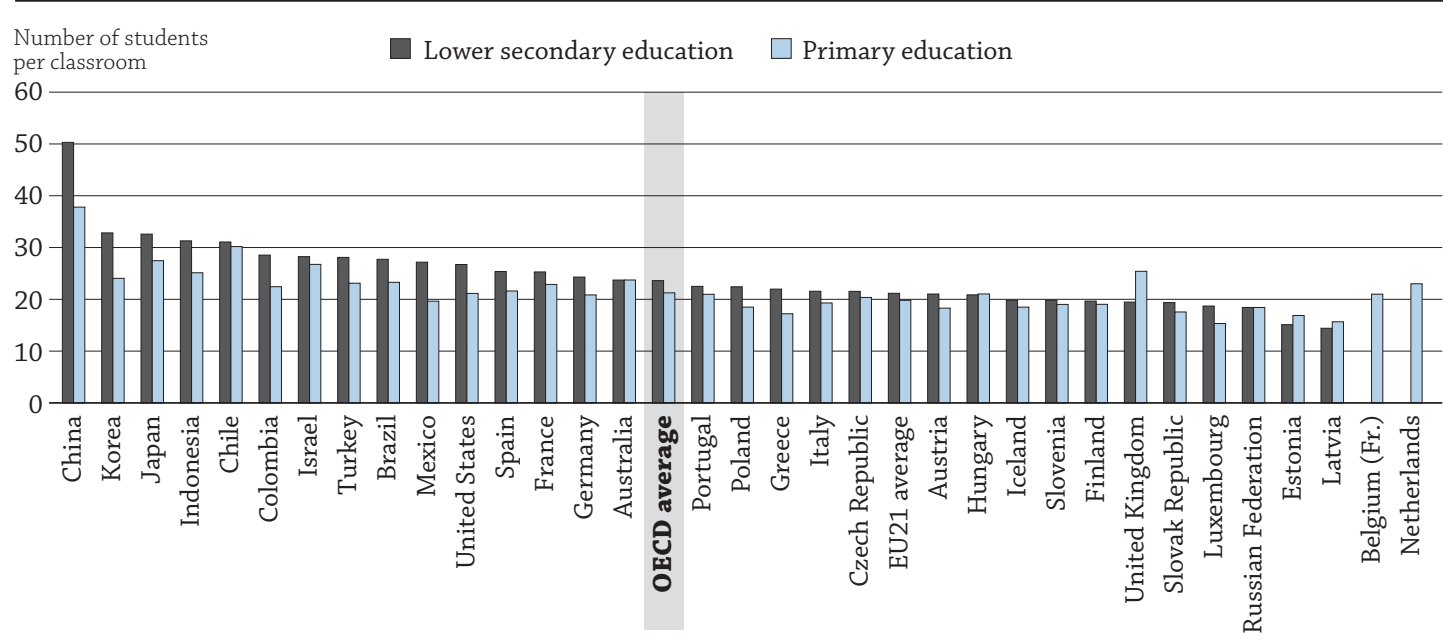

Countries are ranked in descending order of average class size in lower secondary education.

Source: OECD. Table D2.1. See Annex 3 for notes (www.oecd.org/education/education-at-a-glance-19991487.htm).

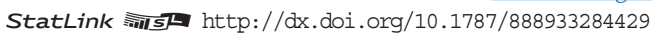

\section{Context}

Class size and student-teacher ratios are much-discussed aspects of education and, along with students' instruction time (see Indicator D1), teachers' working time (see Indicator D4), and the division of teachers' time between teaching and other duties, are among the determinants of the demand for teachers. Together with teachers' salaries (see Indicator D3) and the age distribution of teachers (see Indicator D5), class size and student-teacher ratios also have a considerable impact on the level of current expenditure on education (see Indicators B6 and B7).

Smaller classes are often seen as beneficial because they allow teachers to focus more on the needs of individual students and reduce the amount of class time needed to deal with disruptions. Yet, while there is some evidence that smaller classes may benefit specific groups of students, such as those from disadvantaged backgrounds (Finn, 1998; Krueger, 2002; Piketty and Valdenaire, 2006), overall, evidence of the effect of differences in class size on student performance is weak. Given recent findings from the 2013 OECD Teaching and Learning International Survey (TALIS), however, larger classes seem to be associated with a higher percentage of students with behavioural problems and with more class time spent keeping order as opposed to teaching and learning (see Box D2.1).

The ratio of students to teaching staff indicates how resources for education are allocated. Smaller student-teacher ratios often have to be weighed against higher salaries for teachers, investing in their professional development, greater investment in teaching technology, or more widespread use of assistant teachers and other paraprofessionals whose salaries are often considerably lower than those of qualified teachers. As larger numbers of children with special needs are integrated into mainstream classes, more use of specialised personnel and support services may limit the resources available for reducing student-teacher ratios. 


\section{Other findings}

- With the exceptions of Chile, Colombia, Korea, Luxembourg and Mexico, the student-teacher ratio decreases or stays the same in all countries with available data between primary and lower secondary level, despite a general increase in class size between these levels.

- On average across OECD countries, the student-teacher ratio in secondary education is slightly lower in private than in public institutions. This is most striking in Mexico where, at the secondary level, there are at least 17 students more per teacher in public than in private institutions.

- Class size varies significantly across countries. The biggest classes in primary education are observed in Chile and China, with 30 and 38 students per classroom, respectively, whereas in Latvia and Luxembourg classes have fewer than 17 students, on average. 


\section{Analysis}

\section{Average class size in primary and lower secondary education}

The average primary class in OECD countries had more than 21 pupils in 2013. There are fewer than 26 pupils per primary classroom in nearly all of the countries with available data, with the exception of Chile, China, Israel and Japan.

At the lower secondary level, the average class in OECD countries has 24 students. Among all countries with available data on this level of education, that number varies from fewer than 20 students in Estonia, Latvia, Luxembourg, the Russian Federation, the Slovak Republic and the United Kingdom, to around 33 students per class in Japan and Korea and 50 students in China (Table D2.1).

The number of students per class tends to increase between primary and lower secondary education. In China and Korea, the increase in average class size exceeds seven students. Meanwhile, the United Kingdom and, to a lesser extent, Estonia and Latvia show a drop in the number of students per class between these two levels of education (Chart D2.1).

The Indicator on class size is limited to primary and lower secondary education because class size is difficult to define and compare at higher levels, where students often attend several different classes, depending on the subject area.

\section{Box D2.1. Relationship between average class size and classroom environment}

Despite the extensive literature on the effects of class size on education, evidence of the impact of class size is mixed in research. The often-cited relationship between class size and student performance, for example, has been found to be beneficial only to specific groups and in certain contexts, such as in schools considered at-risk, or for struggling students. Overall, there is little evidence that class size, by itself, determines achievement. Indeed, results from the OECD Programme for International Student Assessment (PISA) do not provide evidence of a relationship between class size and the performance of 15-year-olds. Instead, PISA finds that countries should prioritise policies to improve teacher quality - e.g. by raising salaries to attract good candidates and retain effective teachers - even if the trade-off is larger classes. The 2013 OECD Teaching and Learning International Survey (TALIS) also reports that class size is not a strong determinant of teachers' job satisfaction, or even of whether they use pedagogies involving small groups, project-based tasks or information and communication technologies. Nevertheless, one relationship merits further investigation: class size and classroom environment.

Generally speaking, smaller class sizes are perceived as allowing teachers to spend less time managing the class and more time with each student (OECD, 2012). Evidence from TALIS can help shed light on this relationship. After asking teachers how much class time they devote to teaching and learning activities versus administrative tasks and keeping order (or behaviour management), TALIS found that teachers spend $79 \%$ of their time, on average, on teaching and learning. But that proportion varies widely - from $87 \%$ in Bulgaria to $67 \%$ in Brazil and class size could explain part of that difference.

Chart D2.a shows that larger classes are correlated with less time spent on actual teaching and learning and with more time spent on keeping order in the classroom (although not shown, larger classes are also correlated with more class time spent on administrative tasks). Specifically, one additional student added to an averagesize class is associated with a 0.5 percentage-point decrease in time spent on teaching and learning activities.

The charts also highlight an important mechanism through which class size may influence the percentage of time devoted to teaching and learning. The size of each bubble represents the proportion of lower secondary teachers who reported having more than $10 \%$ of students with behaviour problems in their classes (OECD, 2014). So the larger the bubble, the higher the share of teachers who reported that more than $10 \%$ of their students have behaviour problems. There is a positive correlation between average class size and the reported proportion of students with behaviour problems. This correlation is important because teachers who teach classes where more than one in ten students have behaviour problems spend almost twice as much time keeping order in the classroom as their peers with less than $10 \%$ of such students in their class (OECD, 2015). In other words, larger classes are associated with a higher proportion of students with behavioural problems, which, in turn, is associated with less time spent on teaching and learning activities. 
Time spent on teaching and learning is an essential component of effective education environments. That is especially true given that time spent keeping order in the classroom and on administrative tasks are both associated with lower levels of teacher self-efficacy and job satisfaction. Therefore, these findings suggest that teachers in countries and schools with larger classes and more challenging classroom compositions may be in greater need of interventions to help them use class time more effectively.

\section{Chart D2.a. Relationship between average class size and time spent teaching/learning and time spent keeping order in the classroom in lower secondary education (2013)}
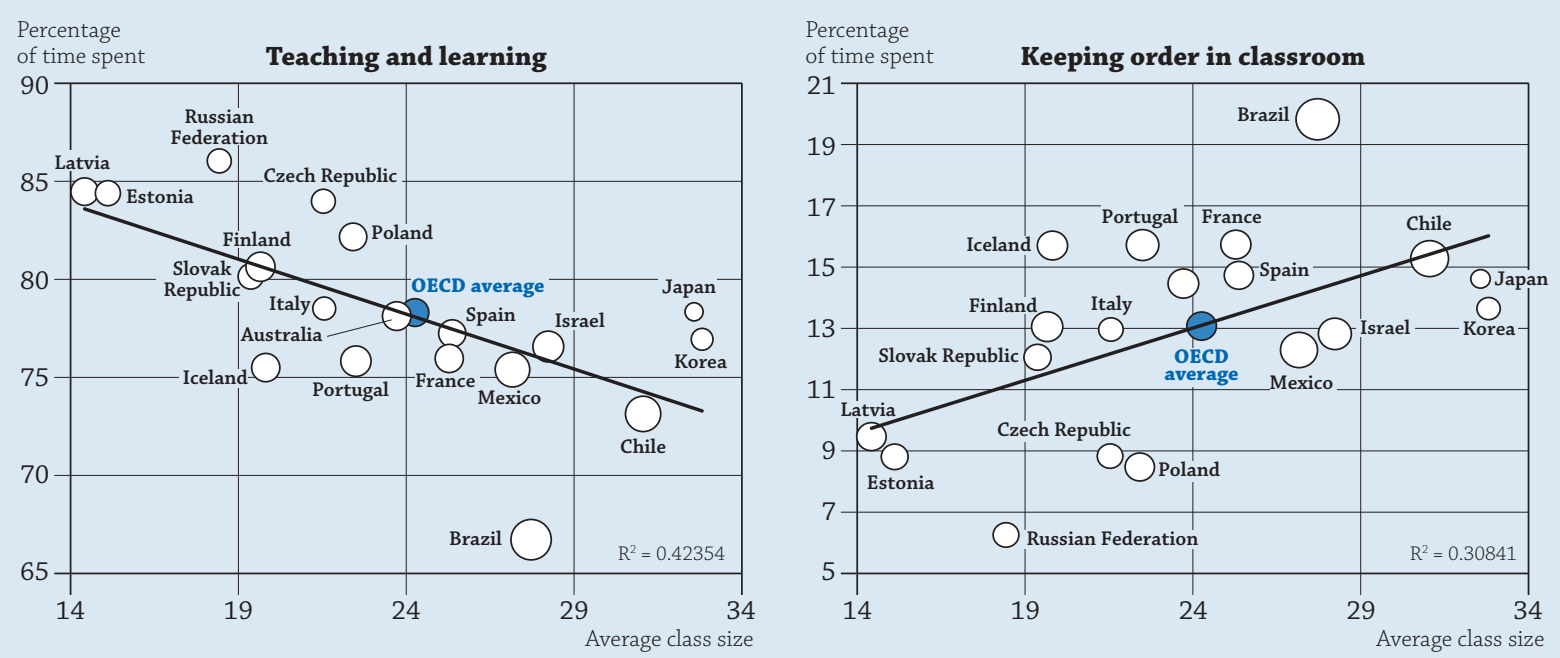

Note: The size of each bubble represents the proportion of lower secondary teachers who reported having more than $10 \%$ of students with behaviour problems in their classes (OECD, 2014).

Source: OECD. Data on average class size: Table D2.1. Data on use of class time: (OECD, 2014), TALIS 2013 Results: An International Perspective on Teaching and Learning, TALIS, http://dx.doi.org/10.1787/9789264196261-en.

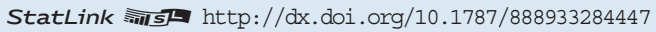

\section{Class size in public and private institutions}

Class size is one factor that parents may consider when deciding on a school for their children; and the difference in average class size between public and private schools (and between different types of private institutions) could influence enrolment.

In most OECD countries, average class size does not differ between public and private institutions by more than two students per class in both primary and lower secondary education (Table D2.1). However, there are marked differences among countries. For example, in Brazil, Colombia, the Czech Republic, Latvia, Poland, the Russian Federation and the United Kingdom, the average primary school class in public institutions is larger than the average class in a private school by more than four students. However, with the exception of Brazil, Colombia and the United Kingdom, the private sector is relatively small in all of these countries, representing $5 \%$ of students, at most, at the primary level (see Table C1.4a). In contrast, in China and Luxembourg, the average class in private institutions is larger than that in public institutions by four or more students.

The comparison of class size between public and private institutions shows a mixed picture at the lower secondary level, where private institutions are more prevalent. The average class in lower secondary private institutions is larger than in public institutions in 8 countries, smaller in 18 countries and the same in 4 countries. The differences, however, tend to be smaller than in primary education.

In countries where private (including both government-dependent and independent) institutions are more prevalent at the primary level (i.e. countries where more than $15 \%$ of students are enrolled in these institutions), such as Australia, Brazil, Colombia, Indonesia, Israel and Spain, there may be large differences in class size between public and private institutions. In Australia and Spain private institutions tend to have more students per class than public schools (see Tables C1.4a and D2.1). This suggests that in some countries, where a substantial proportion of students and families choose private schools, class size is not a determining factor in their decision. 


\section{Student-teacher ratios}

The ratio of students to teaching staff compares the number of students (full-time equivalent) to the number of teachers (full-time equivalent) at a given level of education and in similar types of institutions. However, this ratio does not take into account the amount of instruction time for students compared to the length of a teacher's working day, nor how much time teachers spend teaching. Therefore, it cannot be interpreted in terms of class size (Box D2.2).

At the primary level, there are 15 students for every teacher, on average across OECD countries. The studentteacher ratio ranges from 28 students per teacher in Mexico to 10 or fewer in Greece, Iceland, Luxembourg and Norway (Chart D2.2).

Student-teacher ratios also vary, and to a larger extent, at the secondary school level, ranging from 30 students per full-time equivalent teacher in Mexico to fewer than 10 in Austria, Greece, Latvia, Luxembourg, Portugal and the Russian Federation. On average across OECD countries, there are about 13 students per teacher at the secondary level (Table D2.2).

As the differences in student-teacher ratios indicate, there are fewer full-time equivalent students per full-time equivalent teacher at the secondary level than at the primary level of education. In most countries, the studentteacher ratio decreases between primary and lower secondary school despite an increase in class size. This is true in all but five countries: Chile, Colombia, Korea, Luxembourg and Mexico. However, the student-teacher ratio in Luxembourg is very low in both levels of education.

\section{Chart D2.2. Ratio of students to teaching staff in educational institutions, by level of education (2013)}

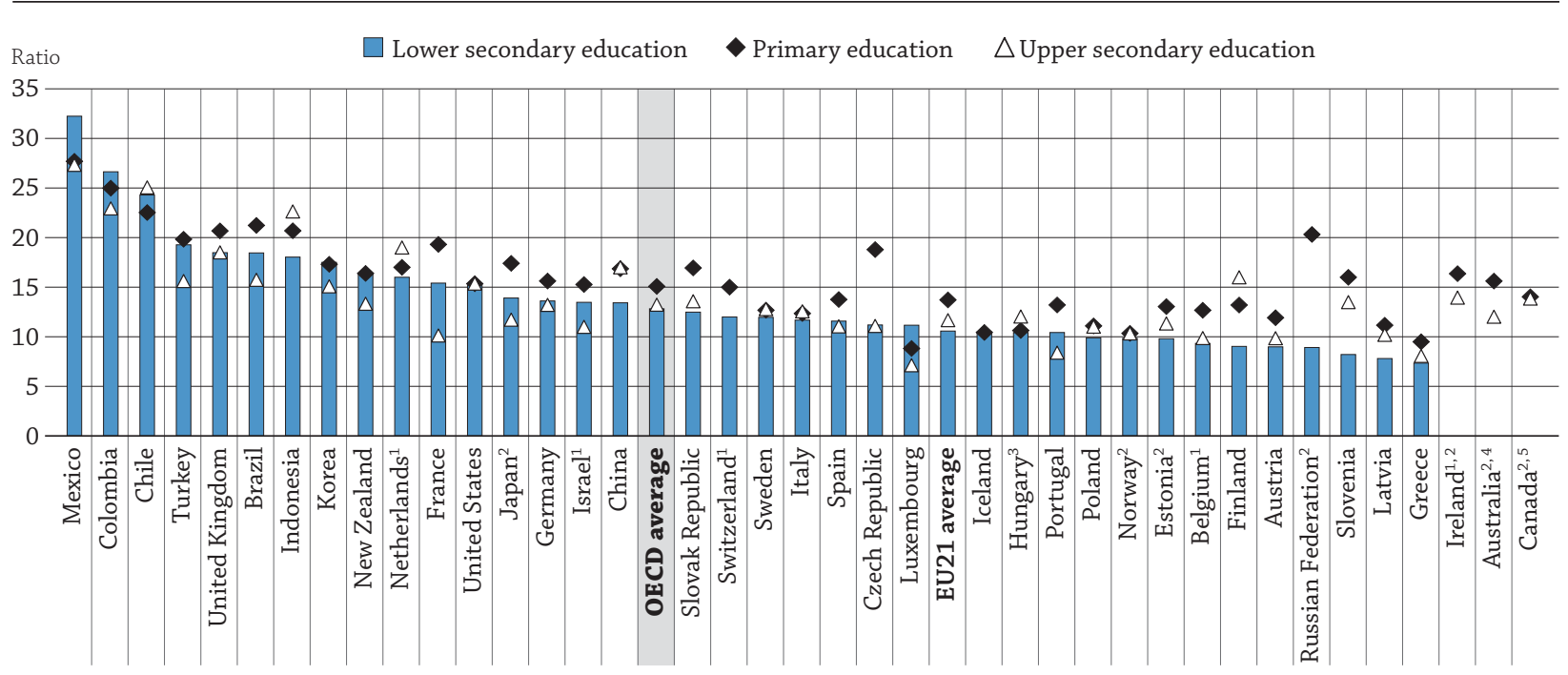

1. Public institutions only. For Israel, public institutions only for upper secondary education. For Belgium, data does not include independent private institutions.

2. Some levels of education are included with others. Please refer to " $x$ " code in Table D2.2 for details.

3. Includes data on management personnel.

4. Includes only general programmes in upper secondary education.

5. Year of reference 2012.

Countries are ranked in descending order of ratio of students to teaching staff in lower secondary education in 2013.

Source: OECD. Table D2.2. See Annex 3 for notes (www.oecd.org/education/education-at-a-glance-19991487.htm).

StatLink 젬ㄴ http://dx.doi.org/10.1787/888933284432

This reduction in the student-teacher ratio reflects differences in annual instruction time: since annual instruction time tends to increase with the level of education (see Indicator D1), so does the number of teachers. It may also result from delays in matching the teaching force to demographic changes, or from differences in teaching hours for teachers at different levels of education (the number of teaching hours tends to decrease with the level of education, as teacher specialisation increases). The general trend is consistent among countries, but evidence is mixed as to whether smaller student-teacher ratios are more desirable, from an education perspective, at higher levels of education. 
At the tertiary level, the student-teacher ratio ranges from over 20 students per teacher in Belgium, Brazil, Colombia, the Czech Republic, Indonesia and Turkey to 10 in Norway (Table D2.2). However, comparisons at this level should be made with caution since it is difficult to calculate full-time equivalent students and teachers on a comparable basis. In 6 of the 18 countries with comparable data at the tertiary level, the ratio of students to teaching staff is lower in short-cycle tertiary education than in bachelor's, master's, doctoral or equivalent levels. Among countries in which the ratio of students to teaching staff is higher in short-cycle tertiary education than in bachelor's, master's, doctoral or equivalent levels, Turkey displays the largest difference: 55 to 1 in short-cycle tertiary education and 17 to 1 in bachelor's, master's, doctoral or equivalent levels (Table D2.2).

Differences between public and private institutions in student-teacher ratios are similar to those observed in class size. On average among the countries for which data are available, the ratios of students to teaching staff are slightly lower in private institutions than in public institutions at the lower and upper secondary levels (Table D2.3). The largest differences between public and private institutions are in Brazil, Colombia, Mexico, Turkey and the United Kingdom, where, at the lower secondary level, there are at least seven more students per teacher in public institutions than in private institutions. At the upper secondary level in Mexico, the difference in student-teacher ratios between public and private institutions (a difference of 18 students per teacher) is even larger than that at the lower secondary level (16 students per teacher).

However, in some countries, the student-teacher ratio is lower in public institutions than in private institutions. At the lower secondary level, this difference is most pronounced in Luxembourg, which has some 24 students per teacher in private institutions, compared to 10 students per teacher in public institutions.

\section{Box D2.2. What is the relationship between class size and the student-teacher ratio?}

The student-teacher ratio is calculated by dividing the number of full-time equivalent students by the number of full-time equivalent teachers at a given level of education and type of institution. Class size, on the other hand, takes into account a number of different elements: the ratio of students to teaching staff, the number of classes or students for which a teacher is responsible, the amount of instruction time compared to the length of teachers' working days, the proportion of time teachers spend teaching, how students are grouped within classes, and team-teaching arrangements.

For example, in a school of 100 full-time students and 10 full-time teachers, the student-teacher ratio is 10 to 1 . If teachers' work week is estimated to include 20 hours of teaching, and if instruction time for each student is 30 hours per week, then regardless of how students are grouped in the school, one way to estimate average class size is as follows:

Estimated class size $=10$ students per teacher $*$ ( 30 hours of instruction time per student $/ 20$ hours of teaching per teacher) $=15$ students.

Using a different approach, the class size presented in Table D2.1 is defined as the number of students who are following a common course of study, based on the highest number of common courses (usually compulsory studies), and excluding teaching in subgroups. The estimated class size will be close to the average class size in Table D2.1 where teaching in subgroups is less frequent, such as in primary and lower secondary education.

Given the difference between student-teacher ratio and average class size, it is possible for countries with similar student-teacher ratios to have different class sizes. For example, at the primary level, Israel and the United States have similar ratios of student to teaching staff (15 students per teacher - Table D2.2), but the average class size differs substantially (21 students in the United States and 27 in Israel - Table D2.1).

\section{Definitions}

Instructional personnel (teaching staff) includes two categories:

- Teachers' aides and teaching/research assistants include non-professional personnel or students who support teachers in providing instruction to students.

- Teaching staff refers to professional personnel directly involved in teaching students. The classification includes classroom teachers, special-education teachers and other teachers who work with a whole class of students in a classroom, in small groups in a resource room, or in one-to-one teaching situations inside or outside a regular class. Teaching staff also includes department chairpersons whose duties include some teaching, but excludes non-professional personnel who support teachers in providing instruction to students, such as teachers' aides and other paraprofessional personnel. 


\section{Methodology}

Data refer to the academic year 2012/13 and are based on the UOE data collection on education statistics administered by the OECD in 2013 (for details see Annex 3 at www.oecd.org/education/education-at-a-glance-19991487.htm).

Class size is calculated by dividing the number of students enrolled by the number of classes. In order to ensure comparability among countries, special-needs programmes are excluded. Data include only regular programmes at primary and lower secondary levels of education, and exclude teaching in sub-groups outside the regular classroom setting.

The ratio of students to teaching staff is obtained by dividing the number of full-time equivalent students at a given level of education by the number of full-time equivalent teachers at that level and in similar types of institutions.

Notes on definitions and methodologies regarding this indicator for each country are presented in Annex 3 at www.oecd.org/education/education-at-a-glance-19991487.htm.

\section{Note regarding data from Israel}

The statistical data for Israel are supplied by and are under the responsibility of the relevant Israeli authorities. The use of such data by the OECD is without prejudice to the status of the Golan Heights, East Jerusalem and Israeli settlements in the West Bank under the terms of international law.

\section{References}

Finn, J. (1998), Class Size and Students at Risk: What is Known? What is Next?, US Department of Education, Office of Educational Research and Improvement, National Institute on the Education of At-Risk Students, Washington, DC.

Krueger, A.B. (2002), "Economic Considerations and Class Size”, National Bureau of Economic Research Working Paper, No. 8875.

OECD (2015), “Improving School Climate and Students' Opportunities to Learn”, Teaching in Focus, No. 9, OECD Publishing, Paris, http://dx.doi.org/10.1787/5js7sf14gd7b-en.

OECD (2014), TALIS 2013 Results: An International Perspective on Teaching and Learning, OECD Publishing, Paris, http://dx.doi. org/10.1787/9789264196261-en.

OECD (2012), “How Does Class Size Vary Around the World?”, Education Indicators in Focus, No. 9, OECD Publishing, Paris, http://dx.doi.org/10.1787/5k8x7gvpr9jc-en.

Piketty, T. and M. Valdenaire (2006), L'Impact de la taille des classes sur la réussite scolaire dans les écoles, collèges et lycées français : Estimations à partir du panel primaire 1997 et du panel secondaire 1995, Ministère de l'Éducation nationale, de l'Enseignement supérieur et de la Recherche, Direction de l'évaluation et de la prospective, Paris.

\section{Indicator D2 Tables}

StatLink 세댄 http://dx.doi.org/10.1787/888933286136

Table D2.1 Average class size, by type of institution and level of education (2013)

Table D2.2 Ratio of students to teaching staff in educational institutions (2013)

Table D2.3 Ratio of students to teaching staff, by type of institution (2013)

Cut-off date for the data: 23 October 2015. Updates can be found on line at http://dx.doi.org/10.1787/eag-data-en. 
Table D2.1. Average class size, by type of institution and level of education (2013) Calculations based on number of students and number of classes

\begin{tabular}{|c|c|c|c|c|c|c|c|c|c|c|}
\hline & \multicolumn{5}{|c|}{ Primary education } & \multicolumn{5}{|c|}{$\begin{array}{l}\text { Lower secondary education } \\
\text { (general programmes) }\end{array}$} \\
\hline & \multirow{2}{*}{ 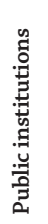 } & \multicolumn{3}{|c|}{ Private institutions } & \multirow{2}{*}{ 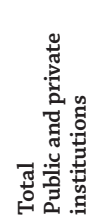 } & \multirow{2}{*}{ 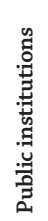 } & \multicolumn{3}{|c|}{ Private institutions } & \multirow{2}{*}{ 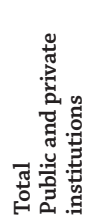 } \\
\hline & & r. & 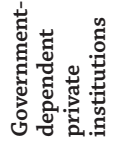 & 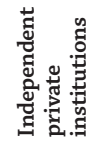 & & & r. & 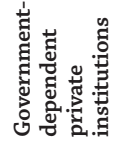 & 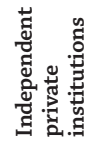 & \\
\hline & (1) & (2) & (3) & (4) & (5) & (6) & (7) & (8) & (9) & (10) \\
\hline Australia & 23 & 25 & 25 & $\mathrm{a}$ & 24 & 23 & 25 & 25 & $\mathrm{a}$ & 24 \\
\hline Austria & 18 & 19 & $19^{\mathrm{d}}$ & $\mathrm{x}(3)$ & 18 & 21 & 22 & $22^{\mathrm{d}}$ & $\mathrm{x}(8)$ & 21 \\
\hline Belgium (Fr.) & 20 & 22 & 22 & a & 21 & $\mathrm{~m}$ & $\mathrm{~m}$ & $\mathrm{~m}$ & $\mathrm{~m}$ & $\mathbf{m}$ \\
\hline Canada & $\mathrm{m}$ & $\mathrm{m}$ & $\mathrm{m}$ & $\mathrm{m}$ & $\mathbf{m}$ & $\mathrm{m}$ & $\mathrm{m}$ & $\mathrm{m}$ & $\mathrm{m}$ & $\mathbf{m}$ \\
\hline Chile & 29 & 31 & 32 & 24 & 30 & 31 & 31 & 33 & 25 & 31 \\
\hline Czech Republic & 20 & 15 & 15 & a & 20 & 22 & 19 & 19 & a & 22 \\
\hline Denmark & 21 & $\mathrm{~m}$ & 19 & $\mathrm{~m}$ & $\mathbf{m}$ & 21 & $\mathrm{~m}$ & 20 & $\mathrm{~m}$ & $\mathbf{m}$ \\
\hline Estonia & 17 & 16 & $\mathrm{x}(4)$ & $16^{\mathrm{d}}$ & 17 & 15 & 12 & $\mathrm{x}(9)$ & $12^{\mathrm{d}}$ & 15 \\
\hline Finland & 19 & 17 & 17 & $\mathrm{a}$ & 19 & 20 & 20 & 20 & $\mathrm{a}$ & 20 \\
\hline France & 23 & 23 & $23^{\mathrm{d}}$ & $\mathrm{x}(3)$ & 23 & 25 & 26 & 26 & 14 & 25 \\
\hline Germany & 21 & 21 & $x(2)$ & $x(2)$ & 21 & 24 & 24 & $\mathrm{x}(7)$ & $\mathrm{x}(7)$ & 24 \\
\hline Greece & 17 & 19 & a & 19 & 17 & 22 & 23 & $\mathrm{a}$ & 23 & 22 \\
\hline Hungary & 21 & 20 & 20 & $\mathrm{a}$ & 21 & 21 & 20 & 20 & $\mathrm{a}$ & 21 \\
\hline Iceland & 19 & 16 & 16 & $\mathrm{a}$ & 18 & 20 & 13 & 13 & a & 20 \\
\hline Ireland & 25 & $\mathrm{~m}$ & $\mathrm{a}$ & $\mathrm{m}$ & $\mathbf{m}$ & $\mathrm{m}$ & $\mathrm{m}$ & a & $\mathrm{m}$ & $\mathbf{m}$ \\
\hline Israel & 28 & 24 & 24 & $\mathrm{a}$ & 27 & 29 & 24 & 24 & a & 28 \\
\hline Italy & 19 & 20 & $\mathrm{a}$ & 20 & 19 & 22 & 22 & $\mathrm{a}$ & 22 & 22 \\
\hline Japan & 27 & 30 & a & 30 & 27 & 32 & 34 & a & 34 & 33 \\
\hline Korea & 24 & 29 & a & 29 & 24 & 33 & 32 & 32 & a & 33 \\
\hline Luxembourg & 15 & 19 & 16 & 19 & 15 & 19 & 18 & 20 & 17 & 19 \\
\hline Mexico & 20 & 19 & $\mathrm{a}$ & 19 & 20 & 28 & 24 & a & 24 & 27 \\
\hline Netherlands & $23^{\mathrm{d}}$ & $\mathrm{m}$ & $\mathrm{x}(1)$ & $\mathrm{m}$ & 23 & $\mathrm{~m}$ & $\mathrm{~m}$ & a & $\mathrm{m}$ & $\mathbf{m}$ \\
\hline New Zealand & $\mathrm{m}$ & $\mathrm{m}$ & $\mathrm{m}$ & $\mathrm{m}$ & $\mathbf{m}$ & $\mathrm{m}$ & $\mathrm{m}$ & $\mathrm{m}$ & $\mathrm{m}$ & $\mathbf{m}$ \\
\hline Norway $^{1}$ & $\mathrm{~m}$ & $\mathrm{~m}$ & $\mathrm{~m}$ & $\mathrm{~m}$ & $\mathbf{m}$ & $\mathrm{m}$ & $\mathrm{m}$ & $\mathrm{m}$ & $\mathrm{m}$ & $\mathbf{m}$ \\
\hline Poland & 19 & 11 & 10 & 12 & 18 & 23 & 17 & 23 & 16 & 22 \\
\hline Portugal & 21 & 21 & 23 & 20 & 21 & 22 & 23 & 25 & 22 & 22 \\
\hline Slovak Republic & 18 & 17 & 17 & a & 18 & 19 & 18 & 18 & a & 19 \\
\hline Slovenia & 19 & 22 & 22 & a & 19 & 20 & 19 & 19 & a & 20 \\
\hline Spain & 21 & 24 & 25 & 22 & 22 & 25 & 26 & 27 & 22 & 25 \\
\hline Sweden & $\mathrm{m}$ & $\mathrm{m}$ & $\mathrm{m}$ & $\mathrm{m}$ & m & $\mathrm{m}$ & $\mathrm{m}$ & $\mathrm{m}$ & $\mathrm{m}$ & $\mathbf{m}$ \\
\hline Switzerland & $\mathrm{m}$ & $\mathrm{m}$ & $\mathrm{m}$ & $\mathrm{m}$ & $\mathbf{m}$ & $\mathrm{m}$ & $\mathrm{m}$ & $\mathrm{m}$ & $\mathrm{m}$ & $\mathbf{m}$ \\
\hline Turkey & 23 & 20 & $\mathrm{a}$ & 20 & 23 & 28 & 20 & $\mathrm{a}$ & 20 & 28 \\
\hline United Kingdom & 27 & 18 & 27 & 12 & 25 & 20 & 19 & 21 & 12 & 19 \\
\hline United States & 22 & 18 & a & 18 & 21 & 28 & 20 & a & 20 & 27 \\
\hline OECD average & 21 & 21 & 21 & 20 & 21 & 24 & 22 & 23 & 20 & 24 \\
\hline EU21 average & 20 & 19 & 20 & 17 & 20 & 21 & 21 & 21 & 18 & 21 \\
\hline Argentina & $\mathrm{m}$ & $\mathrm{m}$ & $\mathrm{m}$ & $\mathrm{m}$ & $\mathbf{m}$ & $\mathrm{m}$ & $\mathrm{m}$ & $\mathrm{m}$ & $\mathrm{m}$ & $\mathbf{m}$ \\
\hline Brazil & 25 & 18 & $\mathrm{a}$ & 18 & 23 & 28 & 24 & $\mathrm{a}$ & 24 & 28 \\
\hline China & 37 & 44 & $44^{\mathrm{d}}$ & $\mathrm{x}(3)$ & 38 & 50 & 52 & $52^{\mathrm{d}}$ & $\mathrm{x}(8)$ & 50 \\
\hline Colombia & 24 & 19 & $\mathrm{a}$ & 19 & 22 & 30 & 25 & a & 25 & 29 \\
\hline India & $\mathrm{m}$ & $\mathrm{m}$ & $\mathrm{m}$ & $\mathrm{m}$ & $\mathbf{m}$ & $\mathrm{m}$ & $\mathrm{m}$ & $\mathrm{m}$ & $\mathrm{m}$ & $\mathbf{m}$ \\
\hline Indonesia & 26 & 22 & a & 22 & 25 & 31 & 31 & a & 31 & 31 \\
\hline Latvia & 16 & 8 & $\mathrm{a}$ & 8 & 16 & 15 & 9 & a & 9 & 14 \\
\hline Russian Federation & 18 & 13 & $\mathrm{a}$ & 13 & 18 & 19 & 11 & $\mathrm{a}$ & 11 & 18 \\
\hline Saudi Arabia & $\mathrm{m}$ & $\mathrm{m}$ & $\mathrm{m}$ & $\mathrm{m}$ & $\mathbf{m}$ & $\mathrm{m}$ & $\mathrm{m}$ & $\mathrm{m}$ & $\mathrm{m}$ & $\mathbf{m}$ \\
\hline South Africa & $\mathrm{m}$ & $\mathrm{m}$ & $\mathrm{m}$ & $\mathrm{m}$ & $\mathbf{m}$ & $\mathrm{m}$ & $\mathrm{m}$ & $\mathrm{m}$ & $\mathrm{m}$ & $\mathbf{m}$ \\
\hline G20 average & 24 & 23 & 30 & 20 & 24 & 28 & 26 & 31 & 21 & 28 \\
\hline
\end{tabular}

1. Students are organised in groups that vary in size during the school day.

Sources: OECD. Argentina, China, Colombia, India, Indonesia, Saudi Arabia, South Africa: UNESCO Institute for Statistics. Latvia: Eurostat. See Annex 3 for notes (www.oecd.org/education/education-at-a-glance-19991487.htm).

Please refer to the Reader's Guide for information concerning symbols for missing data and abbreviations.

StatLink 제근 http://dx.doi.org/10.1787/888933286142 
Table D2.2. Ratio of students to teaching staff in educational institutions (2013) By level of education, calculations based on full-time equivalents

\section{D2}

\begin{tabular}{|c|c|c|c|c|c|c|c|c|}
\hline & \multirow[b]{2}{*}{$\begin{array}{l}\text { Primary } \\
\text { education }\end{array}$} & \multicolumn{3}{|c|}{ Secondary education } & \multirow[b]{2}{*}{$\begin{array}{l}\text { Post-secondary } \\
\text { non-tertiary } \\
\text { education }\end{array}$} & \multicolumn{3}{|c|}{ Tertiary education } \\
\hline & & $\begin{array}{c}\text { Lower } \\
\text { secondary } \\
\text { education }\end{array}$ & $\begin{array}{c}\text { Upper } \\
\text { secondary } \\
\text { education }\end{array}$ & $\begin{array}{l}\text { All secondary } \\
\text { education }\end{array}$ & & $\begin{array}{l}\text { Short-cycle } \\
\text { tertiary } \\
\text { education }\end{array}$ & \begin{tabular}{|c|} 
Bachelor's, \\
master's, \\
doctoral or \\
equivalent level
\end{tabular} & $\begin{array}{c}\text { All tertiary } \\
\text { education }\end{array}$ \\
\hline & (1) & (2) & (3) & (4) & (5) & (6) & (7) & (8) \\
\hline Australia $^{1}$ & 16 & $\mathrm{x}(3)$ & $12^{\mathrm{d}}$ & 12 & $\mathrm{~m}$ & $\mathrm{~m}$ & 14 & 14 \\
\hline Austria & 12 & 9 & 10 & 9 & 10 & 9 & 17 & 15 \\
\hline Belgium $^{2}$ & 13 & 9 & 10 & 10 & 15 & $\mathrm{x}(7)$ & $21^{\mathrm{d}}$ & 21 \\
\hline Canada $^{3,4}$ & $14^{\mathrm{d}}$ & $\mathrm{x}(1)$ & 14 & 14 & $\mathrm{~m}$ & $\mathrm{~m}$ & $\mathrm{~m}$ & $\mathrm{~m}$ \\
\hline Chile & 23 & 24 & 25 & 25 & $\mathrm{a}$ & $\mathrm{m}$ & $\mathrm{m}$ & $\mathrm{m}$ \\
\hline Czech Republic & 19 & 11 & 11 & 11 & 31 & 13 & 22 & 22 \\
\hline Denmark & $\mathrm{m}$ & $\mathrm{m}$ & $\mathrm{m}$ & $\mathrm{m}$ & $\mathrm{m}$ & $\mathrm{m}$ & $\mathrm{m}$ & $\mathrm{m}$ \\
\hline Estonia & 13 & 10 & $11^{\mathrm{d}}$ & $11^{\mathrm{d}}$ & $\mathrm{x}(3)$ & $\mathrm{m}$ & $\mathrm{m}$ & $\mathrm{m}$ \\
\hline Finland & 13 & 9 & 16 & 13 & 17 & $\mathrm{a}$ & 14 & 14 \\
\hline France & 19 & 15 & 10 & 13 & $x(6,7)$ & $17^{\mathrm{d}}$ & $17^{\mathrm{d}}$ & $17^{\mathrm{d}}$ \\
\hline Germany & 16 & 14 & 13 & 13 & 13 & 15 & 12 & 12 \\
\hline Greece & 9 & 7 & 8 & 8 & $\mathrm{~m}$ & $\mathrm{a}$ & $\mathrm{m}$ & $\mathrm{m}$ \\
\hline Hungary ${ }^{5}$ & 11 & 10 & 12 & 11 & 13 & 19 & 14 & 15 \\
\hline Iceland & 10 & 10 & $\mathrm{~m}$ & $\mathrm{~m}$ & $\mathrm{~m}$ & $\mathrm{~m}$ & $\mathrm{~m}$ & $\mathrm{~m}$ \\
\hline Ireland $^{2}$ & 16 & $\mathrm{x}(3)$ & $14^{\mathrm{d}}$ & 14 & $\mathrm{~m}$ & $\mathrm{x}(8)$ & $20^{\mathrm{d}}$ & 20 \\
\hline Israel $^{2}$ & 15 & 13 & 11 & 12 & $\mathrm{~m}$ & $\mathrm{~m}$ & $\mathrm{~m}$ & $\mathrm{~m}$ \\
\hline Italy & 12 & 12 & 13 & 12 & $\mathrm{~m}$ & a & 19 & 19 \\
\hline Japan $^{6}$ & 17 & 14 & $12^{\mathrm{d}}$ & $13^{\mathrm{d}}$ & $\mathrm{m}$ & $\mathrm{m}$ & $\mathrm{m}$ & $\mathrm{m}$ \\
\hline Korea & 17 & 18 & 15 & 16 & $\mathrm{~m}$ & $\mathrm{~m}$ & $\mathrm{~m}$ & $\mathrm{~m}$ \\
\hline Luxembourg & 9 & 11 & 7 & 9 & $\mathrm{~m}$ & $\mathrm{~m}$ & $\mathrm{~m}$ & $\mathrm{~m}$ \\
\hline Mexico & 28 & 32 & 27 & 30 & $\mathrm{a}$ & 17 & 14 & 14 \\
\hline Netherlands ${ }^{2}$ & 17 & 16 & 19 & 17 & 20 & 15 & 15 & 15 \\
\hline New Zealand & 16 & 16 & 13 & 15 & 21 & 16 & 17 & 17 \\
\hline Norway & 10 & 10 & $10^{\mathrm{d}}$ & $10^{\mathrm{d}}$ & $\mathrm{x}(3)$ & $\mathrm{x}(3)$ & 10 & 10 \\
\hline Poland & 11 & 10 & 11 & 10 & 16 & 8 & 15 & 15 \\
\hline Portugal & 13 & 10 & $8^{\mathrm{d}}$ & $9^{d}$ & $\mathrm{x}(3,7)$ & a & $14^{\mathrm{d}}$ & $14^{\mathrm{d}}$ \\
\hline Slovak Republic & 17 & 12 & 14 & 13 & 13 & 9 & 14 & 14 \\
\hline Slovenia & 16 & 8 & 13 & 11 & a & 21 & 18 & 18 \\
\hline Spain & 14 & 12 & 11 & 11 & $\mathrm{~m}$ & 12 & 12 & 12 \\
\hline Sweden & 13 & 12 & 13 & 12 & 11 & 10 & 11 & 11 \\
\hline Switzerland ${ }^{2}$ & 15 & 12 & $\mathrm{~m}$ & $\mathrm{~m}$ & $\mathrm{~m}$ & $\mathrm{~m}$ & $\mathrm{~m}$ & $\mathrm{~m}$ \\
\hline Turkey & 20 & 19 & 16 & 17 & a & 55 & 17 & 22 \\
\hline United Kingdom & 21 & 18 & 19 & 18 & $\mathrm{a}$ & 20 & 18 & 18 \\
\hline United States & 15 & 15 & 15 & 15 & $x(8)$ & $\mathrm{x}(8)$ & $\mathrm{x}(8)$ & $15^{\mathrm{d}}$ \\
\hline OECD average & 15 & 13 & 13 & 13 & 16 & 17 & 16 & 16 \\
\hline EU21 average & 14 & 11 & 12 & 12 & 16 & 14 & 16 & 16 \\
\hline
\end{tabular}

\begin{tabular}{|c|c|c|c|c|c|c|c|c|}
\hline $\begin{array}{l}\text { Argentina } \\
\text { 닣 Brazil }\end{array}$ & $\begin{array}{l}\mathrm{m} \\
21\end{array}$ & $\begin{array}{c}\mathrm{m} \\
18\end{array}$ & $\begin{array}{c}\mathrm{m} \\
16\end{array}$ & $\begin{array}{r}\mathrm{m} \\
17\end{array}$ & $\begin{array}{r}\mathrm{m} \\
17\end{array}$ & $\begin{array}{c}\mathrm{m} \\
57\end{array}$ & $\begin{array}{r}\mathrm{m} \\
27\end{array}$ & $\begin{array}{r}\mathrm{m} \\
27\end{array}$ \\
\hline China & 17 & 13 & 17 & 15 & $\mathrm{~m}$ & $\mathrm{~m}$ & $\mathrm{~m}$ & $\mathrm{~m}$ \\
\hline Colombia & 25 & 27 & 23 & 25 & 12 & $\mathrm{x}(7)$ & $24^{\mathrm{d}}$ & 35 \\
\hline India & $\mathrm{m}$ & $\mathrm{m}$ & $\mathrm{m}$ & $\mathrm{m}$ & $\mathrm{m}$ & $\mathrm{m}$ & $\mathrm{m}$ & $\mathrm{m}$ \\
\hline Indonesia & 21 & 18 & 23 & 20 & a & $\mathrm{x}(7)$ & $31^{\mathrm{d}}$ & 31 \\
\hline Latvia & 11 & 8 & 10 & 9 & 16 & 25 & 19 & 20 \\
\hline Russian Federation & 20 & $9^{d}$ & $x(2)$ & 9 & 17 & 11 & 11 & 11 \\
\hline Saudi Arabia & $\mathrm{m}$ & $\mathrm{m}$ & $\mathrm{m}$ & $\mathrm{m}$ & $\mathrm{m}$ & $\mathrm{m}$ & $\mathrm{m}$ & $\mathrm{m}$ \\
\hline South Africa & $\mathrm{m}$ & $\mathrm{m}$ & $\mathrm{m}$ & $\mathrm{m}$ & $\mathrm{m}$ & $\mathrm{m}$ & $\mathrm{m}$ & $\mathrm{m}$ \\
\hline G20 average & 18 & 17 & 16 & 16 & $\mathrm{~m}$ & $\mathrm{~m}$ & $\mathrm{~m}$ & $\mathrm{~m}$ \\
\hline
\end{tabular}

1. Includes only general programmes in lower and upper secondary education.

2. Public institutions only. For Israel, public institutions only for upper secondary education. For Belgium, data does not include independent private institutions.

3. Year of reference 2012.

4. Primary includes pre-primary.

5. Includes data on management personnel.

6. Upper secondary includes programmes from post-secondary non-tertiary education.

Source: OECD. Argentina, China, Colombia, India, Indonesia, Saudi Arabia, South Africa: UNESCO Institute for Statistics. Latvia: Eurostat. See Annex 3 for notes (www.oecd.org/education/education-at-a-glance-19991487.htm).

Please refer to the Reader's Guide for information concerning symbols for missing data and abbreviations.

StatLink 젶ㄴ http://dx.doi.org/10.1787/888933286152 
Table D2.3. Ratio of students to teaching staff, by type of institution (2013) By level of education,calculations based on full-time equivalents

\begin{tabular}{|c|c|c|c|c|c|c|c|c|c|c|c|c|}
\hline & \multicolumn{4}{|c|}{ Lower secondary education } & \multicolumn{4}{|c|}{ Upper secondary education } & \multicolumn{4}{|c|}{ All secondary programmes } \\
\hline & \multirow{2}{*}{ 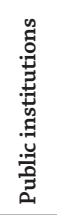 } & \multicolumn{3}{|c|}{ Private institutions } & \multirow{2}{*}{ 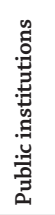 } & \multicolumn{3}{|c|}{ Private institutions } & \multirow{2}{*}{ 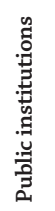 } & \multicolumn{3}{|c|}{ Private institutions } \\
\hline & & 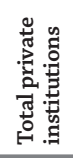 & 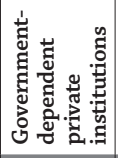 & 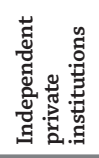 & & 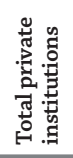 & 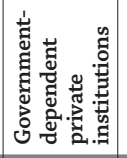 & 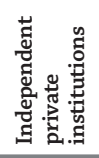 & & 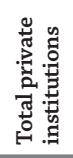 & 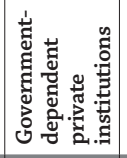 & 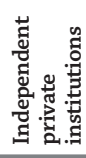 \\
\hline Australia $^{1}$ & $\mathrm{x}(5)$ & $\mathrm{x}(6)$ & $\mathrm{x}(7)$ & a & $12^{\mathrm{d}}$ & $12^{\mathrm{d}}$ & $12^{\mathrm{d}}$ & a & 12 & 12 & 12 & $\mathrm{a}$ \\
\hline Austria & 9 & 11 & $11^{\mathrm{d}}$ & $x(3)$ & 10 & 9 & $9^{d}$ & $\mathrm{x}(7)$ & 9 & 10 & $10^{\mathrm{d}}$ & $\mathrm{x}(11)$ \\
\hline Belgium & 9 & $\mathrm{~m}$ & 9 & $\mathrm{~m}$ & 10 & $\mathrm{~m}$ & 10 & $\mathrm{~m}$ & 10 & $\mathrm{~m}$ & 10 & $\mathrm{~m}$ \\
\hline Canada $^{2}$ & $\mathrm{~m}$ & $\mathrm{~m}$ & $\mathrm{~m}$ & $\mathrm{~m}$ & 14 & 12 & $12^{\mathrm{d}}$ & $\mathrm{x}(7)$ & 14 & 12 & $12^{\mathrm{d}}$ & $\mathrm{x}(11)$ \\
\hline Chile & 21 & 27 & 29 & 22 & 24 & 26 & 28 & 16 & 23 & 26 & 28 & 18 \\
\hline Czech Republic & 11 & 10 & 10 & a & 11 & 12 & 12 & a & 11 & 12 & 12 & a \\
\hline Denmark & $\mathrm{m}$ & $\mathrm{m}$ & $\mathrm{m}$ & $\mathrm{m}$ & $\mathrm{m}$ & $\mathrm{m}$ & $\mathrm{m}$ & $\mathrm{m}$ & $\mathrm{m}$ & $\mathrm{m}$ & $\mathrm{m}$ & $\mathrm{m}$ \\
\hline Finland & 9 & 9 & 9 & $\mathrm{a}$ & 16 & 16 & 16 & $\mathrm{a}$ & 12 & 15 & 15 & a \\
\hline France & 15 & $\mathrm{~m}$ & 18 & $\mathrm{~m}$ & 10 & $\mathrm{~m}$ & 11 & $\mathrm{~m}$ & 12 & $\mathrm{~m}$ & 14 & $\mathrm{~m}$ \\
\hline Germany & 14 & 13 & $\mathrm{x}(2)$ & $\mathrm{x}(2)$ & 13 & 12 & $\mathrm{x}(6)$ & $\mathrm{x}(6)$ & 14 & 13 & $\mathrm{x}(11)$ & $\mathrm{x}(11)$ \\
\hline Greece & 7 & 7 & $\mathrm{~m}$ & 7 & 8 & 8 & $\mathrm{~m}$ & 8 & 8 & 8 & $\mathrm{~m}$ & 8 \\
\hline Hungary ${ }^{4}$ & 10 & 10 & 10 & a & 12 & 12 & 12 & $\mathrm{a}$ & 11 & 11 & 11 & $\mathrm{a}$ \\
\hline Iceland & 11 & 3 & 3 & a & $\mathrm{m}$ & $\mathrm{m}$ & $\mathrm{m}$ & $\mathrm{m}$ & $\mathrm{m}$ & $\mathrm{m}$ & $\mathrm{m}$ & $\mathrm{m}$ \\
\hline Ireland & $\mathrm{x}(5)$ & $\mathrm{m}$ & $\mathrm{m}$ & $\mathrm{m}$ & $14^{\mathrm{d}}$ & $\mathrm{m}$ & $\mathrm{a}$ & $\mathrm{m}$ & 14 & $\mathrm{~m}$ & $\mathrm{~m}$ & $\mathrm{~m}$ \\
\hline Israel & 14 & 7 & 7 & a & 11 & $\mathrm{~m}$ & $\mathrm{~m}$ & a & 12 & $\mathrm{~m}$ & $\mathrm{~m}$ & a \\
\hline Italy & 12 & 11 & a & 11 & 13 & 7 & $\mathrm{a}$ & 7 & 12 & 8 & a & 8 \\
\hline Japan $^{3}$ & 14 & 12 & a & 12 & $11^{\mathrm{d}}$ & $13^{d}$ & a & $13^{d}$ & $13^{\mathrm{d}}$ & $13^{\mathrm{d}}$ & a & $13^{\mathrm{d}}$ \\
\hline Korea & 17 & 18 & 18 & $\mathrm{a}$ & 14 & 16 & 16 & a & 16 & 17 & 17 & $\mathrm{a}$ \\
\hline Luxembourg & 10 & 24 & 12 & a & 9 & 4 & 12 & 2 & 9 & 6 & 12 & 4 \\
\hline Mexico & 35 & 19 & a & 19 & 33 & 15 & a & 15 & 34 & 17 & a & 17 \\
\hline Netherlands & 16 & $\mathrm{~m}$ & a & $\mathrm{m}$ & 19 & $\mathrm{~m}$ & a & $\mathrm{m}$ & 17 & $\mathrm{~m}$ & $\mathrm{a}$ & $\mathrm{m}$ \\
\hline New Zealand & 17 & 13 & a & 13 & 14 & 12 & 13 & 10 & 15 & 12 & 13 & 11 \\
\hline Norway $^{5}$ & 10 & 9 & $9^{d}$ & $x(3)$ & $10^{\mathrm{d}}$ & $16^{\mathrm{d}}$ & $16^{\mathrm{d}}$ & $\mathrm{x}(7)$ & $10^{\mathrm{d}}$ & $14^{\mathrm{d}}$ & $14^{\mathrm{d}}$ & $\mathrm{x}(11)$ \\
\hline Slovak Republic & 13 & 12 & 12 & a & 14 & 12 & 12 & $\mathrm{a}$ & 13 & 12 & 12 & $\mathrm{a}$ \\
\hline Slovenia & 8 & 8 & 8 & a & 14 & 13 & 10 & 32 & 11 & 13 & 10 & 32 \\
\hline Spain & 10 & 15 & 15 & 14 & 10 & 14 & 15 & 13 & 10 & 14 & 15 & 13 \\
\hline Sweden & 11 & 17 & 17 & $\mathrm{a}$ & 12 & 14 & 14 & $\mathrm{a}$ & 12 & 15 & 15 & a \\
\hline Switzerland & 12 & $\mathrm{~m}$ & $\mathrm{~m}$ & $\mathrm{~m}$ & $\mathrm{~m}$ & $\mathrm{~m}$ & $\mathrm{~m}$ & $\mathrm{~m}$ & $\mathrm{~m}$ & $\mathrm{~m}$ & $\mathrm{~m}$ & $\mathrm{~m}$ \\
\hline Turkey & 20 & 9 & $\mathrm{a}$ & 9 & 16 & 7 & $\mathrm{a}$ & 7 & 18 & 8 & $\mathrm{a}$ & 8 \\
\hline United Kingdom & 27 & 14 & 16 & 7 & 22 & 18 & 19 & 9 & 24 & 16 & 18 & 8 \\
\hline United States & 16 & 11 & $\mathrm{a}$ & 11 & 16 & 11 & $\mathrm{a}$ & 11 & 16 & 11 & $\mathrm{a}$ & 11 \\
\hline OECD average & 14 & 12 & 13 & 12 & 14 & 12 & 14 & 11 & 14 & 12 & 14 & 12 \\
\hline EU21 average & 12 & 12 & 12 & 9 & 12 & 11 & 13 & 11 & 12 & 11 & 13 & 11 \\
\hline
\end{tabular}

\begin{tabular}{|c|c|c|c|c|c|c|c|c|c|c|c|c|}
\hline $\begin{array}{l}\text { Argentina } \\
\text { Brazil }\end{array}$ & $\begin{array}{r}\mathrm{m} \\
20\end{array}$ & $\begin{array}{c}\mathrm{m} \\
12\end{array}$ & $\begin{array}{c}\mathrm{m} \\
\mathrm{a}\end{array}$ & $\begin{array}{c}\mathrm{m} \\
12\end{array}$ & $\begin{array}{r}\mathrm{m} \\
17\end{array}$ & $\begin{array}{c}\mathrm{m} \\
10\end{array}$ & $\begin{array}{r}\mathrm{m} \\
\mathrm{a}\end{array}$ & $\begin{array}{r}\mathrm{m} \\
10\end{array}$ & $\begin{array}{r}\mathrm{m} \\
19\end{array}$ & $\begin{array}{c}\mathrm{m} \\
11\end{array}$ & $\begin{array}{c}\mathrm{m} \\
\mathrm{a}\end{array}$ & $\begin{array}{r}\mathrm{m} \\
11\end{array}$ \\
\hline$e^{\pi}$ China & 13 & 18 & $18^{\mathrm{d}}$ & $\mathrm{x}(3)$ & 18 & 10 & $10^{\mathrm{d}}$ & $x(3)$ & 15 & 13 & $13^{\mathrm{d}}$ & $\mathrm{x}(11)$ \\
\hline Colombia & 31 & 17 & $\mathrm{a}$ & 17 & 27 & 16 & a & 16 & 30 & 17 & $\mathrm{a}$ & 17 \\
\hline India & $\mathrm{m}$ & $\mathrm{m}$ & $\mathrm{m}$ & $\mathrm{m}$ & $\mathrm{m}$ & $\mathrm{m}$ & $\mathrm{m}$ & $\mathrm{m}$ & $\mathrm{m}$ & $\mathrm{m}$ & $\mathrm{m}$ & $\mathrm{m}$ \\
\hline Indonesia & 20 & 16 & a & 16 & 18 & 28 & a & 28 & 19 & 21 & a & 21 \\
\hline Latvia & 8 & 4 & a & 4 & 10 & 7 & a & 7 & 9 & 6 & a & 6 \\
\hline Russian Federation & $9^{d}$ & $4^{\mathrm{d}}$ & a & $4^{\mathrm{d}}$ & $\mathrm{x}(1)$ & $x(2)$ & $\mathrm{a}$ & $\mathrm{x}(4)$ & 9 & 4 & $\mathrm{a}$ & 4 \\
\hline South Africa & $\mathrm{m}$ & $\mathrm{m}$ & $\mathrm{m}$ & $\mathrm{m}$ & $\mathrm{m}$ & $\mathrm{m}$ & $\mathrm{m}$ & $\mathrm{m}$ & $\mathrm{m}$ & $\mathrm{m}$ & $\mathrm{m}$ & $\mathrm{m}$ \\
\hline
\end{tabular}

South Africa

\begin{tabular}{l|l|l|l|l|}
18 & 13 & 18 & 11 & 16 \\
\hline
\end{tabular}

1. Includes only general programmes in lower and upper secondary education.

2. Year of reference 2012.

3. Upper secondary includes programmes from post-secondary education.

4. Includes data on management personnel.

5. Upper secondary includes programmes from post-secondary non-tertiary and short-cycle tertiary education.

Source: OECD. Argentina, China, Colombia, India, Indonesia, Saudi Arabia, South Africa: UNESCO Institute for Statistics. Latvia: Eurostat. See Annex 3 for notes (www.oecd.org/education/education-at-a-glance-19991487.htm).

Please refer to the Reader's Guide for information concerning symbols for missing data and abbreviations.

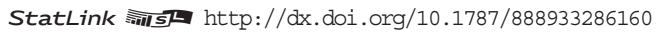




\section{HOW MUCH ARE TEACHERS PAID?}

- On average across OECD countries, pre-primary and primary teachers earn $78 \%$ of the salary of a similarly-educated, 25-64 year-old full-time, full-year worker, lower secondary teachers are paid $80 \%$, and upper secondary teachers are paid $82 \%$ of that benchmark salary.

- The statutory salaries of teachers with 15 years of experience and typical qualifications average USD 38653 at the pre-primary level, USD 41245 at the primary level, USD 42825 at the lower secondary level, and USD 44600 at the upper secondary level.

\section{Chart D3.1. Teachers' salaries relative to earnings for similarly educated workers (2013)}

Salaries of lower secondary teachers teaching general programmes in public institutions

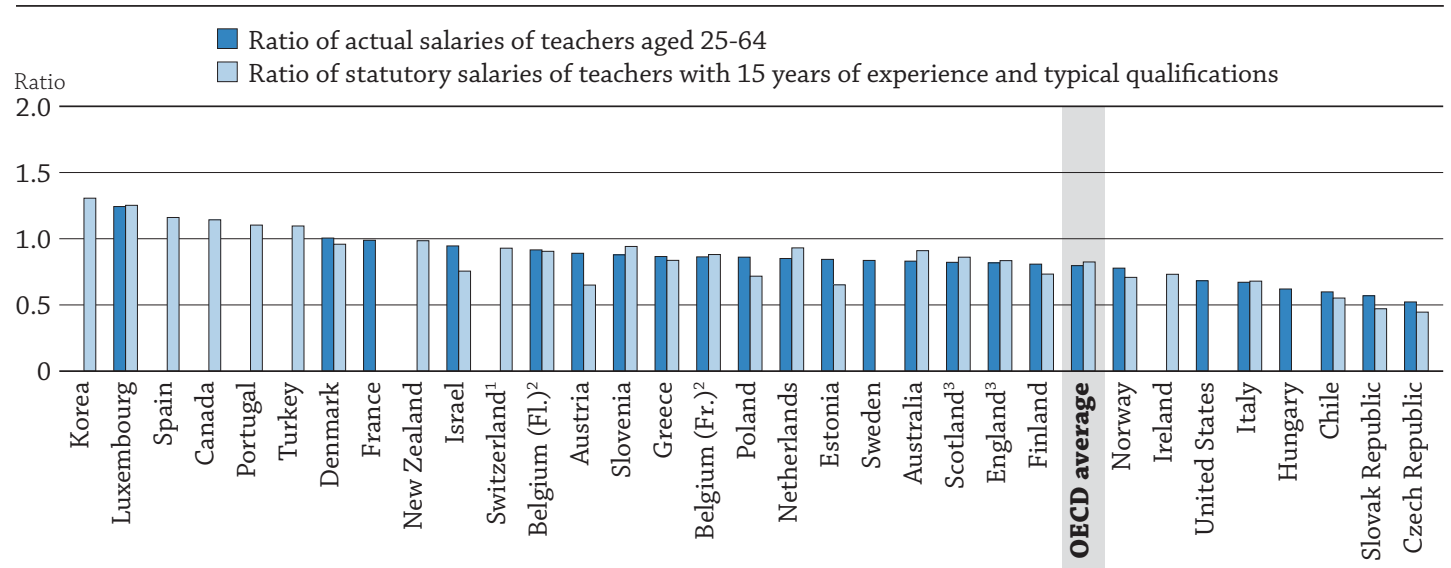

Notes: The definition of teachers' typical qualification is based on a broad concept including the typical ISCED level of attainment and other criteria, as discussed in Box D3.2. For further details on the different metrics used to calculate these ratios, please refer to the Methodology section.

1. Statutory salaries of teachers with 11 years of experience and minimum qualification instead of 15 years of experience and typical qualifications.

2. Data on earnings for full-time, full-year workers with tertiary education refer to Belgium.

3. Data on earnings for full-time, full-year workers with tertiary education refer to the United Kingdom.

Countries are ranked in descending order of the ratio of teachers' salaries to earnings for full-time, full-year workers with tertiary education aged 25-64.

Source: OECD. Table D3.2a, and Table D3.2b, available on line. See Annex 3 for notes (www.oecd.org/education/education-at-a-glance-19991487.htm).

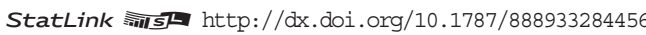

\section{Context}

Teachers' salaries represent the largest single cost in formal education and have a direct impact on the attractiveness of the teaching profession. They influence decisions to enrol in teacher education, become a teacher after graduation (as graduates' career choices are associated with relative earnings in teaching and non-teaching occupations, and their likely growth over time), return to the teaching profession after a career interruption, and/or remain a teacher (as, in general, the higher the salaries, the fewer the people who choose to leave the profession) (OECD, 2005). Burgeoning national debt, spurred by governments' responses to the financial crisis of late 2008, have put pressure on policy makers to reduce government expenditure - particularly on public payrolls. Since compensation and working conditions are important for attracting, developing and retaining skilled and high-quality teachers, policy makers should carefully consider teachers' salaries as they try to ensure both quality teaching and sustainable education budgets (see Indicators B6 and B7). 


\section{Other findings}

- In most OECD countries, teachers' salaries increase with the level of education they teach. For example, the salary of an upper secondary school teacher with 15 years of experience and typical qualification in Belgium, Finland, Hungary and the Slovak Republic is at least $25 \%$ higher than that of a pre-primary school teacher with the same experience and typical qualification.

- Salaries at the top of the scale for teachers with typical qualifications are, on average, $64 \%, 66 \%$, $65 \%$ and $66 \%$ higher, respectively, than starting salaries in pre-primary, primary, lower secondary and upper secondary education. The difference tends to be greatest when it takes many years to progress through the scale. In countries where it takes 30 years or more to reach the top of the salary scale, salaries at that level can be more than $90 \%$ higher, on average, than starting salaries.

- Teachers with maximum qualifications at the top of their salary scales are paid, on average, USD 49176 at the pre-primary level, USD 51177 at the primary level, USD 53786 at the lower secondary level, and USD 54666 at the upper secondary level.

- In 11 out of 28 countries with available data, the average annual salaries of upper secondary teachers, including bonuses and allowances, are at least 10\% higher than statutory salaries for upper secondary teachers with 15 years of experience and typical qualifications.

- In the 17 countries with available data, the average annual salary for full-time equivalent tertiary academic instructional faculty members varies across countries, ranging from USD 13348 in Latvia to USD 74305 in Germany. For the 12 countries with available data, the average salaries for men are consistently higher than those for women.

\section{Trends}

Between 2000 and 2013, teachers' salaries rose, in real terms, in all countries with available data, except Denmark (upper secondary), England, France and Italy. However, in most countries, salaries increased less since 2005 than between 2000 and 2005. The economic downturn in 2008 also had a direct impact on teachers' salaries, which were either frozen or cut in some countries. As a consequence, the number of countries showing an increase in salaries, in real terms, between 2008 and 2013 shrank to about one in two OECD countries. 


\section{Analysis}

\section{Statutory teachers' salaries}

Teachers' salaries are one component of teachers' total compensation. Other benefits, such as regional allowances for teaching in remote areas, family allowances, reduced rates on public transport and tax allowances on the purchase of instructional materials, may also form part of teachers' total remuneration. There are also large differences in taxation and social-benefits systems in OECD countries. All this should be borne in mind when comparing statutory salaries across countries.

Teachers' salaries vary widely across countries. The salaries of lower secondary school teachers with 15 years of experience and typical qualification range from less than USD 15000 in Estonia and Hungary, to more than USD 60000 in Canada, Germany, the Netherlands and the United States and exceed USD 100000 in Luxembourg (Table D3.1a and Chart D3.2).

In most countries, teachers' salaries increase with the level of education taught. In Belgium, Denmark, Hungary, the Netherlands, Norway and the Slovak Republic, upper secondary teachers with 15 years of experience and typical qualification earn between $20 \%$ and $40 \%$ more than pre-primary teachers with the same experience; they earn around 55\% more in Finland. In Finland and the Slovak Republic, the difference is mainly explained by the gap between pre-primary and primary teachers' salaries. In Belgium, teachers' salaries at the upper secondary level are significantly higher than at the other levels of education. In Hungary, the main differences are found between upper secondary and lower secondary teachers' salaries, while there is no difference between the salaries of lower secondary and primary teachers. The differences between salaries at each level of education should be interpreted in light of the requirements to enter the teaching profession (see Education at a Glance 2014 [OECD, 2014], Indicator D6).

In Australia, Korea, Slovenia, Turkey and the United States, there is less than a $5 \%$ difference between salaries for upper secondary and pre-primary teachers with 15 years of experience and typical qualification; in England, Greece, Poland, Portugal, Scotland, teachers receive the same salary irrespective of the level of education taught. This is also true in the Czech Republic, Estonia, Japan, Slovenia and the Slovak Republic at the primary, lower secondary and upper secondary levels. In Israel, there is a $17 \%$ difference between the salaries of an upper secondary teacher and a pre-primary teacher in favour of the latter. This difference is the result of the "New Horizon" reform, begun in 2008 and partially implemented by 2013, that increased salaries for pre-primary, primary and lower secondary teachers. Another reform, launched in 2012, aims to raise salaries for upper secondary teachers. In Luxembourg, primary teachers with 15 years of experience earned around 50\% less than secondary teachers with the same amount of experience prior to a reform in 2009. Now, the difference between primary and secondary school teachers' salaries is less than $10 \%$.

Differences in teachers' salaries at different education levels may influence how schools and school systems attract and retain teachers and may also influence the extent to which teachers move among education levels.

\section{Box D3.1. Tertiary faculty salaries}

There have been substantial increases in enrolment rates in tertiary education programmes over the past two decades. These have been accompanied by growth in spending on tertiary education and an expansion of the facilities and staffing to meet the needs of these new students. Policy makers and the public across $\mathrm{OECD}$ countries have become increasingly concerned about the capacity for tertiary education institutions to continue to recruit sufficient numbers of high-quality instructors.

One way to attract and maintain high-quality instructional staff is to offer competitive salaries. In a 2015 survey, preliminary data were gathered about the structures for determining faculty salaries and the average salaries offered by public and government-dependent private tertiary institutions (details available in Annex 3). The survey found that the structures for determining tertiary faculty salaries were markedly different in many countries compared to those structures for determining primary and secondary teachers' salaries. Most countries use national salary schedules as a basis for determining tertiary faculty salaries, as they do for determining primary and secondary teachers' salaries. However, in most countries, individual institutions have discretion in modifying these payment levels, which primary and secondary schools do not have. The criteria used for setting faculty salaries include educational attainment and length of experience, which are also used for teachers at the primary and secondary levels, but in addition they included academic rank, field(s) of instruction and research experience, which are not commonly considered for primary and secondary teachers. For example, in 25 out of 32 reporting countries, research experience was cited as a relevant factor in determining faculty salaries. 
The 2015 survey also obtained estimates of the actual average annual salary for all full-time equivalent (FTE) tertiary academic instructors for a recent year (2012/13 for most reporting countries). Though preliminary, these results reveal some interesting findings. Seventeen OECD and partner countries reported salary averages for FTE tertiary faculty, ranging from USD 13348 in Latvia to USD 74305 in Germany (tertiary faculty salaries were converted using PPPs for private consumption from the OECD National Accounts database). Among the 12 countries that provided separate data on men and women, the average salaries for men were consistently higher than the average salaries for women. Twelve countries also provided actual average annual salaries for full-time full professors. Average salaries for these senior faculty were consistently higher among OECD and partner countries, averaging 18\% (Norway) to 105\% (the Czech Republic) higher than the averages for all FTE tertiary faculty in their countries. Salaries for full-time full professors were highest in Germany (USD 107 889), the United States (USD 101685 ) and the United Kingdom (USD 97 079) among all reporting countries. Similar to the pattern observed for faculty salaries overall, salaries for male professors were higher than salaries for female professors in all reporting countries except Portugal. However, the salary differential between male and female professors was smaller than those reported for all faculty, with Iceland and Norway reporting a differential of less than $2 \%$.

\section{Minimum and typical qualifications}

Differences in statutory salaries across countries should be interpreted in light of the concepts of typical and minimum qualifications. The minimum qualifications required to teach at a given education level refers to the typical duration and type of training required to enter the profession (see Education at a Glance 2014 [OECD, 2014], Indicator D6) and does not include other requirements to become a licensed teacher in the public school system, such as probation years.

The definition of teachers' typical qualifications varies by country (Box D3.1). The typical level of qualifications refers to the level of qualifications and training teachers typically have, and may include certificates and qualifications obtained while in the teaching profession. The qualifications of teachers are "typical" in that they represent the qualifications held by the largest proportion of teachers in the system, in a given year.

However, differences between the minimum and typical qualifications of teachers are by no means the general rule. In 17 of the 36 countries with available data, there are no differences between minimum and typical qualifications throughout a teacher's career. In the remaining 19 countries, differences in teachers' statutory salaries may reflect differences in whether teachers hold the typical or the minimum qualifications, at least in one education level and at least at one point in their career; at starting salary, after ten years of experience, after 15 years of experience, or at the top of the salary scale. Yet the salary difference related to having qualifications other than the minimum varies across countries.

In Chile, England, Iceland, Israel and Mexico, starting salaries are the same among all teachers. It is only after teachers have spent some time in the school system that the salaries of teachers with minimum and typical qualifications start to diverge. In Belgium (French Community), Colombia, Canada, the Czech Republic, Ireland and the United States, teachers with typical qualifications have higher statutory salaries than teachers with minimum qualifications at all points of a teacher's career, including starting salaries, at all levels of education for which information is available. This is true in Australia as well, except at the top of the salary scale, where salaries do not generally depend on teachers' qualifications. In Norway, statutory salaries are higher for teachers with typical qualifications at all stages of their career and all education levels except pre-primary, as there is no difference between minimum and typical qualifications at that level of education. Conversely, in Poland, the statutory salaries of teachers with typical qualifications are higher than those of teachers with minimum qualifications at all levels of education except upper secondary, since most teachers in Poland have a master's degree or the equivalent (ISCED 7), which is the qualification required to teach upper secondary but not other levels of education (Table D3.1a, and Table D3.1b, available on line).

In Poland, the difference in statutory salaries can be substantial between teachers with the minimum and typical qualifications and 15 years of experience, ranging from about 13\% among lower secondary teachers, to about $26 \%$ among pre-primary and primary teachers. In Mexico, the differences are also large - about 24\% or more at all levels of education for which information is available. In Belgium (French Community), the differences among teachers with 15 years of experience are about $5 \%$ among teachers in pre-primary through lower secondary education, yet the difference is about $30 \%$ among upper secondary teachers. Conversely, in the Czech Republic, the difference in statutory salaries among teachers with 15 years of experience but who have different qualifications is about $4 \%$ from primary through upper secondary education, but $24 \%$ at the pre-primary level (Table D3.1a, and Table D3.1b, available on line). 


\section{Box D3.2. Typical qualifications of teachers}

In most OECD countries, teachers are required to have a specific level of attainment or type of diploma to enter the teaching profession, or even a combination of qualifications. Typical qualifications generally involve the completion of requirements beyond teachers' typical educational attainment (Tables D3.2a, and Table D3.2b, available on line). Very often, teachers have to undergo training, gain practical experience and/or demonstrate their skills over probation periods to become fully qualified teachers. Sometimes they have to satisfy additional criteria, such as passing competitive examinations, to be able to teach or to reach higher levels in pay scales and degrees of responsibility in the school system. Criteria may also change depending on the level of education at which they teach (for further information see Education at a Glance 2014 [OECD, 2014], Indicator D6).

As a result, the minimum qualifications required to enter the teaching profession may not be the most commonly held qualifications in the teaching force. In several education systems, the "typical" teacher has most likely undergone certification and qualification processes beyond the minimum requirements, and has reached a given position in a salary scale. This is what is referred to as the typical qualification of teachers, and it varies depending on the country and the school system.

Variations between the minimum and the typical qualifications of teachers currently teaching are often seen in countries where policy or legislation has recently changed and the requirements for entering the teaching profession have been raised or lowered. In some countries, differences between the minimum and typical qualifications of teachers are not seen at the starting point of teachers' careers but among teachers who have spent a few years in the education system.

In Chile, Iceland and Israel, teachers' professional development activities have an effect on the definition of teachers' qualifications and on their salaries. In Iceland, for example, even if most practicing teachers hold a master's degree or equivalent (ISCED 7), they are required to spend 150 hours of each school year in professional development activities, which may include formal education with equivalences in the European Credits Transfer System (ECTS). Therefore, the typical qualification of a teacher with 15 years of experience in Iceland includes all additional ECTS attained through professional development activities during the course of his or her years as a teacher.

Difference between the minimum and typical qualifications can also arise in systems where several types of qualifications (types of diploma and/or ISCED levels of attainment) are accepted for entrance into the teaching profession or where there are alternative pathways. In Australia, for example, starting in 2013, the minimum qualifications for new teachers are four years (or equivalent) of tertiary education (ISCED 6). Graduate teachers must have completed a qualification that meets the requirements of a nationally accredited programme of initial teacher education. Yet, the level of initial teacher education courses varies across a series of four-year or longer full-time equivalent higher education qualifications (at either ISCED 6 or ISCED 7), that enable alternative or flexible pathways into the teaching profession and that meet the requirements of the Australian Qualifications Framework (AQF). The differences in qualifications may entail differences in salaries even at the starting point of a teaching career, as the number of years of training may have an effect on the teacher's entry salary step.

By contrast, in the French Community of Belgium, minimum and typical qualifications are generally associated with the diploma awarded to a teacher at the end of initial teacher education, which is required for teaching at a given ISCED level. Yet teacher shortages at some education levels have led to the reallocation of teachers with different qualifications. For example, lower secondary teachers have been reallocated to make up for shortages of upper secondary teachers, resulting in a modification of the typical qualifications at the upper secondary level.

Differences between minimum and typical qualifications can be indicators of teachers' progression throughout their careers or of the changes in an education system over time. These examples show that across OECD countries the teaching force is in constant development, and that the criteria for teachers to enter the teaching profession and to move forward within it are far from static.

Across OECD countries with available information, in almost two-thirds of countries, the typical attainment of teachers is a bachelor's degree or the equivalent (ISCED 6), and in over a third of countries, it is a master's degree or the equivalent (ISCED 7). However, in a small handful of countries, the typical attainment of pre-primary teachers is lower than a bachelor's degree (ISCED 6). In Australia, Chile, England, France, Greece, Iceland, Israel, Korea, Mexico 
and Scotland, the typical attainment of teachers is the same across education levels from pre-primary through upper secondary education. Yet in some countries, the typical attainment of teachers varies for one level only, such as in pre-primary education in the Czech Republic, Finland, Germany, Japan and the Slovak Republic, or in upper secondary education in Belgium, Denmark, Hungary and Norway (Table D3.2a, and Table D3.2b, available on line).

\section{Starting and maximum teachers' salaries}

Education systems compete with other sectors of the economy to attract high-quality graduates as teachers. Research shows that salaries and alternative employment opportunities are important influences on the attractiveness of teaching (Santiago, 2004). Teachers' starting salaries relative to other non-teaching occupations and the likely growth in earnings have a huge influence over a graduate's decision to become a teacher. Countries that are looking to increase the supply of teachers, especially those with an ageing teacher workforce and/or a growing school-age population, might consider offering more attractive starting wages and career prospects. However, to ensure a well-qualified teaching workforce, efforts must be made not only to recruit and select only the most competent and qualified teachers, but also to retain effective teachers.

At the lower secondary level, new teachers entering the profession with the typical qualifications earn, on average, USD 31 013. This starting salary ranges from below USD 15000 in Estonia, Hungary and the Slovak Republic, to more than USD 40000 in Denmark, Germany, Luxembourg, Norway, Spain and the United States. For teachers at the top of the salary scale and with the maximum qualifications, salaries average USD 53 786. This maximum salary ranges from less than USD 20000 in Estonia, Hungary and the Slovak Republic, to USD 75000 or more in Austria and Korea, and more than USD 130000 in Luxembourg.

Most countries with starting salaries below the OECD average also show lower maximum salaries. At the lower secondary level, some exceptions are Japan, Korea, and Mexico, where starting salaries are at least $6 \%$ lower than the OECD average, but maximum salaries are significantly higher. In Scotland, although starting salaries are at least $10 \%$ below the OECD average, maximum salaries are very close to the OECD average. The opposite is true for Denmark, where starting salaries are more than 30\% higher than the OECD average while maximum salaries are closer to the OECD average. In Sweden, the starting salaries are slightly above the OECD average, but the maximum salaries are more than $20 \%$ lower than the OECD average. (Chart D3.2, and Table D3.6a, available on line).

\section{Chart D3.2. Lower secondary teachers' salaries at different points in teachers' careers (2013)} Annual statutory salaries of teachers in public institutions, in equivalent USD converted using PPPs

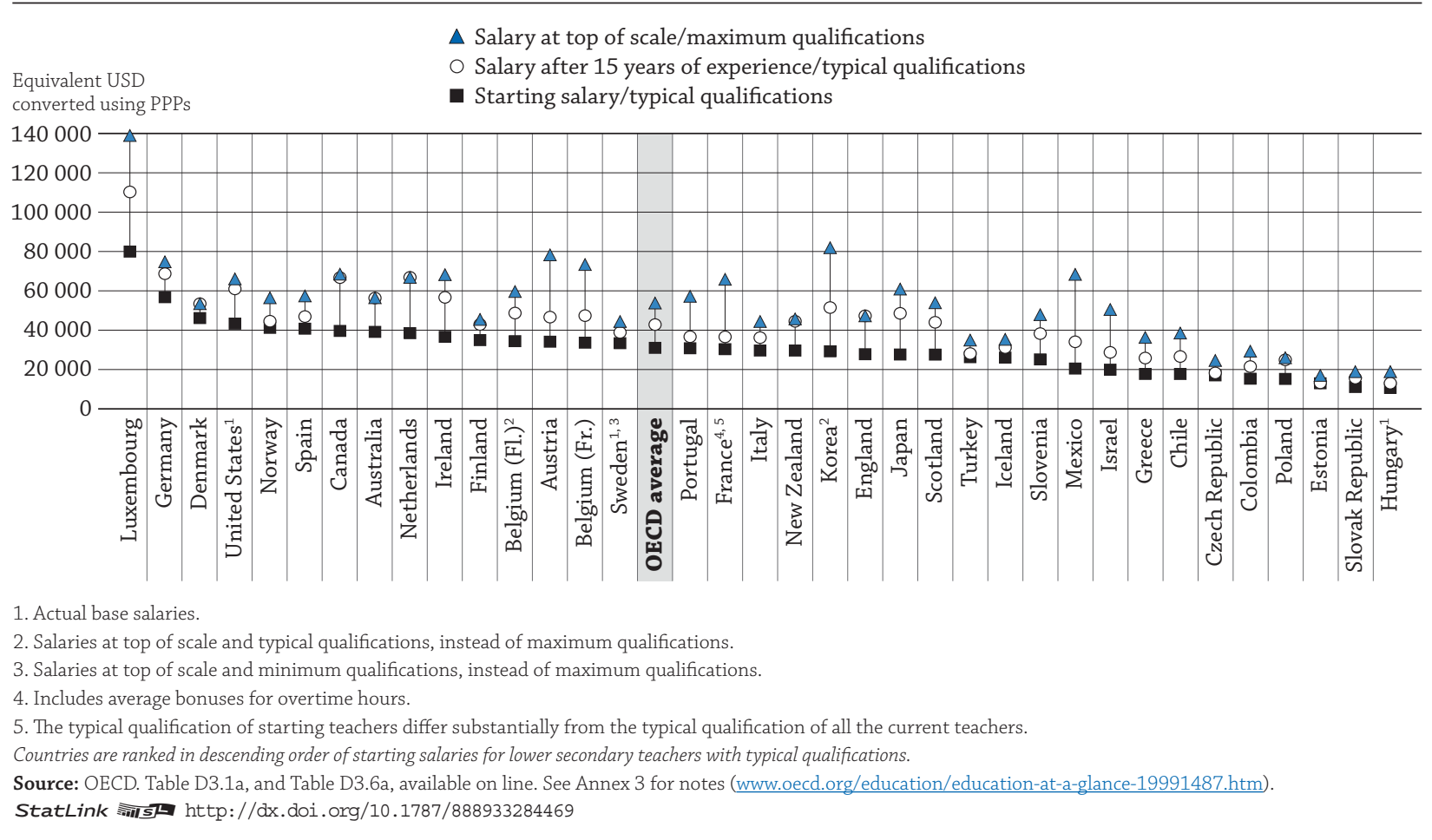


A number of countries have relatively flat/compressed salary scales. The difference between starting and maximum salaries is less than $30 \%$ in the Czech Republic (pre-primary), Denmark, Finland (pre-primary), Norway (pre-primary and upper secondary) and in Sweden (pre-primary).

Weak financial incentives may make it more difficult to retain teachers as teachers approach the peak of their earnings. However, there may be some benefits to compressed pay scales. It is often argued, for example, that organisations in which there are smaller differences in salaries among employees enjoy more trust, freer flows of information and more collegiality among co-workers.

By contrast, maximum salaries are at least double the starting salaries in Austria, Chile, Greece, Israel and Korea at all levels of education, in the French community of Belgium at pre-primary, primary and lower secondary levels, in France at lower and upper secondary levels, in Hungary at the upper secondary level, and in Japan at primary and secondary levels. Maximum salaries are more than three times higher than starting salaries in Mexico at pre-primary, primary and lower secondary levels (Chart D3.2, and Table D3.6a, available on line).

When analysing starting and maximum salaries, it is important to bear in mind that "typical" qualifications refer to the most widely held qualifications across the teaching force in a given year; not all teachers in the system hold this qualification. Thus, the typical qualification of starting teachers and teachers at the top of the salary scale (and therefore their salaries) may differ substantially from the typical qualification of all teachers in a school system.

The salary premium for higher qualifications, at the top of the salary scale, also varies across countries. At the lower secondary level, while there is no difference between salaries at the top of the scale for teachers with minimum and maximum qualifications in 13 of 36 countries with data for both, teachers at the top of the scale holding the maximum qualifications in the French community of Belgium, Colombia, the Czech Republic, France, Israel, Norway and the Slovak Republic earn at least $25 \%$ more than teachers with the same experience, but with minimum training. This salary gap is widest in Mexico, where teachers at the top of the scale holding the maximum qualifications earn more than twice of those with the same experience, but with minimum qualification. A similar picture is seen at the upper secondary level (Tables D3.1b and D3.6b, available on line).

When considering the salary structure for teachers, it is important to remember that not all teachers reach the top of the salary scale, and that only few of them hold the maximum qualifications. For example, in Greece and Italy, less than 5\% of all teachers were at the top of the salary scale in 2013; and in France, only 5\% of all teachers were lower secondary teachers with the maximum qualifications.

\section{Teaching experience and salary scales}

Salary structures usually define the salaries paid to teachers at different points in their careers. Deferred compensation, which rewards employees for staying in organisations or professions and for meeting established performance criteria, is also used in teachers' salary structures. OECD data on teachers' salaries are limited to information on statutory salaries at four points of the salary scale: starting salaries, salaries after ten years of service, salaries after 15 years of experience, and salaries at the top of the scale. As mentioned above, further qualifications can influence differences in starting and maximum salary and lead to wage increases in some countries.

In OECD countries, teachers' salaries rise during the course of a career, although the rate of change differs across countries. Statutory salaries for lower secondary school teachers with typical qualifications and ten and 15 years of experience are, respectively, $26 \%$ and $39 \%$ higher, on average, than starting salaries. In addition, salaries at the top of the scale, which is reached after an average of 24 years of experience, are $65 \%$ higher, on average, than starting salaries. In Hungary, Israel, Italy, Korea and Spain, lower secondary school teachers reach the top of the salary scale only after 35 years of service or more; in Greece, the top of the scale is reached after 45 years of service. By contrast, lower secondary teachers in Australia, Colombia, Estonia, New Zealand and Scotland reach the highest step on the salary scale within six to nine years (Tables D3.1a and D3.3a).

\section{Statutory salaries per hour of net teaching time}

The average statutory salary per teaching hour after 15 years of experience and with typical qualifications is USD 53 for primary teachers, USD 63 for lower secondary teachers, and USD 71 for upper secondary teachers in general education. Chile, the Czech Republic (primary and lower secondary levels), Estonia, Hungary and the Slovak Republic show the lowest salaries per teaching hour: less than USD 30. By contrast, salaries per teaching hour are USD 90 or more in Canada, Germany and Korea at the lower and upper secondary levels, and in Belgium, Denmark, Japan and Norway at the upper secondary level. They exceed USD 115 in Luxembourg at all levels. For pre-primary teachers with typical qualifications, the average statutory salary per teaching hour after 15 years of experience is USD 43. However, in about a third of the countries, pre-primary teachers with 15 years of experience and typical qualifications earn less than USD 30 per teaching hour (Table D3.3a). 
Because secondary teachers are required to teach fewer hours than primary teachers, their salaries per teaching hour are usually higher than those of teachers at lower levels of education, even in countries where statutory salaries are similar (see Indicator D4). On average among OECD countries, upper secondary teachers' salaries per teaching hour exceed those of primary teachers by about 34\%. In Scotland, there is no difference, while in Denmark upper secondary teachers earn $87 \%$ of the salary of primary teachers per teaching hour (Table D3.3a).

However, the difference in salaries between primary and secondary teachers may disappear when comparing salaries per hour of working time. In Portugal, for example, there is a $23 \%$ difference in salaries per teaching hour between primary and upper secondary teachers, even though statutory salaries and total working time are actually the same at these levels. The difference is explained by the fact that primary teachers spend more time in teaching activities than upper secondary teachers do (see Table D4.1).

\section{Trends since 2000}

Comparing salaries of teachers with typical qualifications in 2000 and 2013, teachers' salaries increased overall in real terms in most countries with available data. Notable exceptions are Denmark (upper secondary), England and France, where there was a decline of about $8 \%$ to $10 \%$; and Italy (secondary education), where a slight decline in teachers' salaries in real terms occurred. In Estonia (primary to upper secondary), Finland (primary), Ireland, Israel (pre-primary to lower secondary), Mexico (pre-primary to lower secondary) and Scotland (pre-primary), salaries increased by at least $20 \%$ over this period (Table D3.5a).

However, between 2005 and 2013, only slightly more than half of OECD countries with available data showed an increase in their salaries in real terms. In the French Community of Belgium, Estonia (primary to upper secondary), Israel and Turkey, most of the increase in teachers' salaries occurred after 2005. In Poland, salaries increased by at least $20 \%$ since 2005 at all levels of education - the result of a 2007 government programme that aimed to increase teachers' salaries successively between 2008 and 2013. The government reform was implemented to improve the quality of education by providing financial incentives to attract high-quality teachers.

By contrast, in Greece and Hungary, salaries decreased by at least 26\% since 2005 (Chart D3.3). However, these decreases occurred largely between 2008 and 2013. This reflects the impact of the economic downturn in 2008 on teachers' salaries, which were either frozen or cut in these countries between 2008 and 2013 (Box D3.3). Half of OECD countries with available data showed an increase in salaries, in real terms, between 2008 and 2013. In England, Iceland, Portugal, Scotland and Spain, salaries fell by at least 5\% between 2008 and 2013 (Table D3.5a).

\section{Chart D3.3. Change in lower secondary teachers' salaries $(2000,2005,2013)$ Index of change between 2000 and 2013 (2005 = 100, constant prices), for teachers with 15 years of experience and typical qualifications}

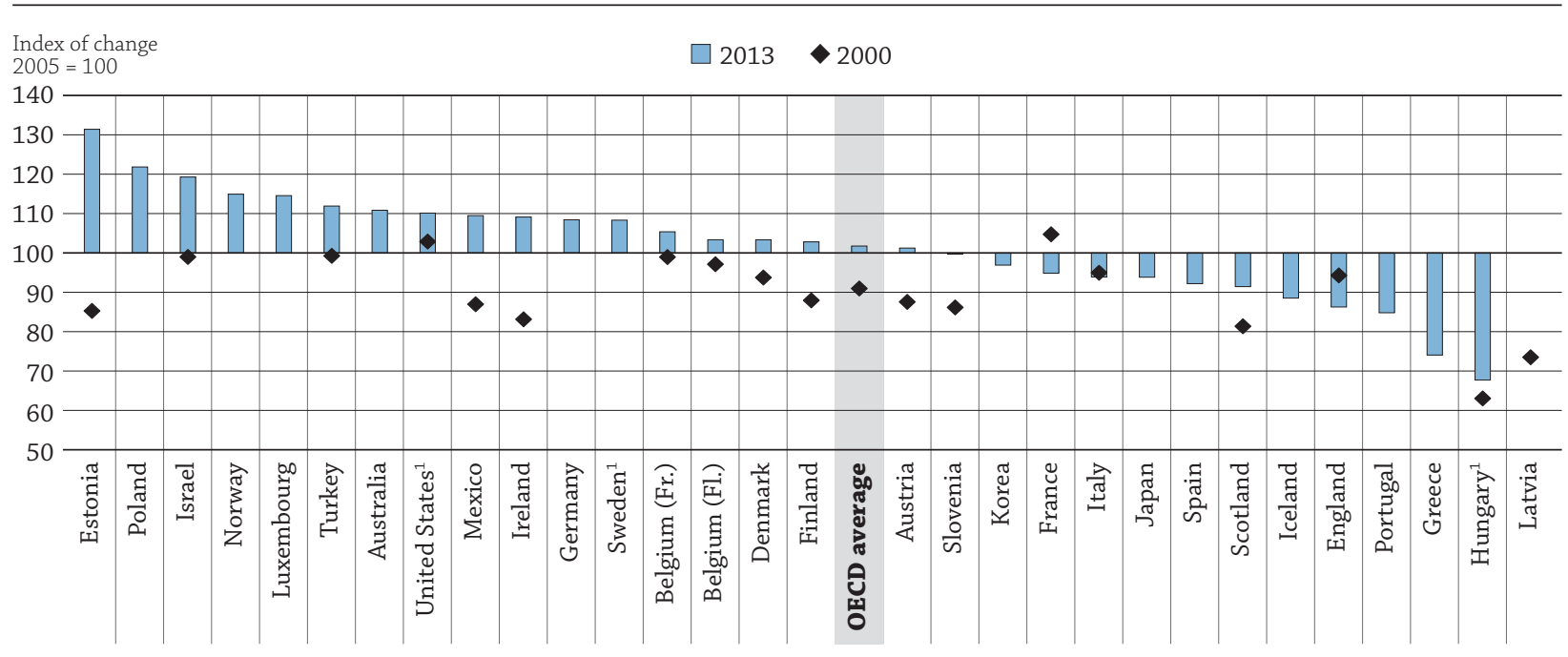

1. Actual base salaries.

Countries are ranked in descending order of the index of change, between 2005 and 2013, in the salaries of lower secondary teachers with 15 years of experience.

Source: OECD. Table D3.5a. See Annex 3 for notes (www.oecd.org/education/education-at-a-glance-19991487.htm).

StatLink 젝ㄴ http://dx.doi.org/10.1787/888933284478 
The above analysis on trends in salaries is based on teachers with 15 years of experience and typical qualifications (a proxy for mid-career teachers). But teachers at certain stages of their career may experience more rapid pay increases than teachers at another stage of their career. For example, some countries that have been experiencing teacher shortages may implement targeted policies to improve the attractiveness of the profession by increasing the salaries of beginning teachers (OECD, 2005). In France, for example, starting teachers received an increase in pay in 2010 and 2011.

In most countries, similar increases and decreases in teachers' salaries were seen at the primary, lower secondary and upper secondary levels. However, in Israel and Luxembourg, they increased significantly more at the primary than at the secondary level between 2005 and 2013. In both Israel and Luxembourg, the difference in the index of change between primary and secondary teachers' salaries is due to reforms that aimed to increase primary teachers' salaries. In Israel, this is largely the result of the gradual implementation of the "New Horizon" reform in primary and lower secondary schools, begun in 2008, following an agreement between the education authorities and the Israeli Teachers Union (for primary and lower secondary education). This reform includes higher teacher pay in exchange for more working hours (see Indicator D4). In the academic year 2012/2013 for example, 77\% of full-time equivalent teachers in pre-primary education, $91 \%$ in primary education and $49 \%$ in lower secondary education were included in the reform. In this same year, a similar reform ("Oz Letmura") was introduced at the upper secondary level, affecting $25 \%$ of full-time equivalent teachers.

\section{Box D3.3. Effect of the economic crisis}

The financial and economic crisis that hit the world economy in the last months of 2008 significantly affected the salaries for civil servants and public sector workers in general. On average across OECD countries with available data, teachers' salaries decreased, for the first time since 2000, by about $5 \%$ at all levels of education between 2009 and 2013.

\section{Chart D3.a. Change in teachers' salaries in OECD countries (2005-13)}

$O E C D$ average index of change, among countries with data for all reference years, for teachers with 15 years of experience and minimum qualifications ( $2005=100$, constant prices)

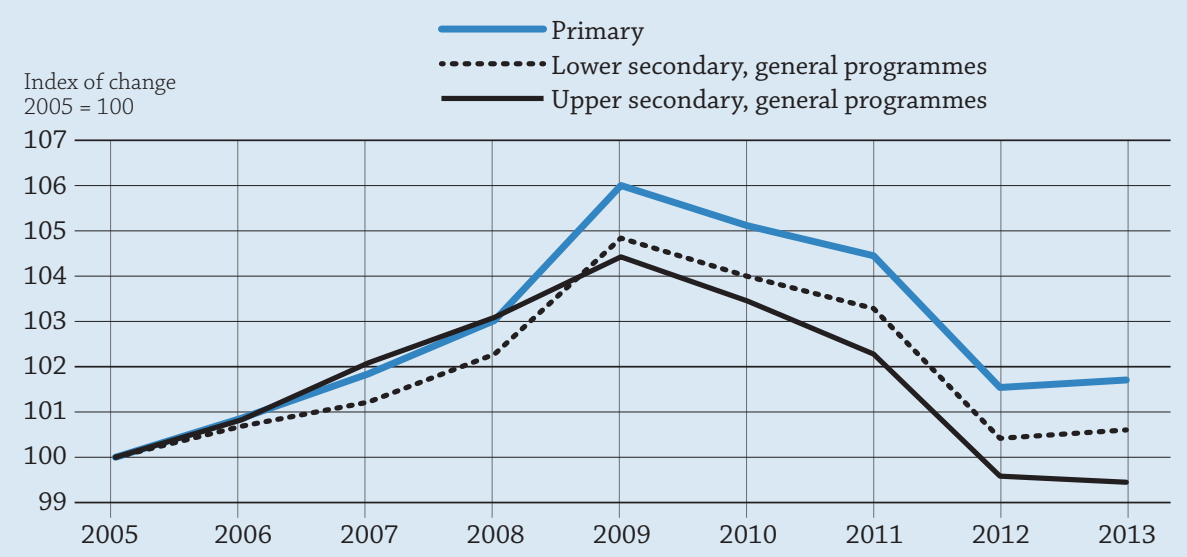

Source: OECD. Table D3.5b, available on line. See Annex 3 for notes (www.oecd.org/education/education-at-a-glance-19991487.htm). StatLink त्ञात http://dx.doi.org/10.1787/888933284483

In England, Estonia, Greece, Hungary, Ireland, Italy, Portugal, Scotland and Spain, teachers' salaries were significantly affected by the crisis. In Estonia, minimum teachers' salaries were cut back to their 2008 levels in 2010 and were frozen at that level. In Greece, various reductions in teachers' benefits and allowances have affected teachers' salaries since 2010 . As a result, gross salaries fell by more than $25 \%$, in real terms, between 2009 and 2013. In addition, Greek teachers also saw their net salaries shrink as a tax for solidarity was created. This tax increased the level of taxation on teachers' already reduced gross salary; and the insurance coverage paid by teachers is still calculated based on their earlier, higher salaries. In Hungary, the 13th month of salary 
(a supplemental bonus that was paid to all employees) was suspended in 2009. Although a compensatory bonus was paid to all public-sector employees whose wages where under a certain threshold, the base salary of teachers was still considerably affected. The continued decrease in teachers' salaries is due to a reduction in additional payments, such as for extra teaching lessons. These additional payments were a significant component of teachers' total compensation, paid above base salaries.

In Spain, all civil servants saw their salaries reduced in July 2010. The extent of the decrease depended on the annual amount earned but it affected both the base salary and bonuses. In Ireland, teachers' salaries were reduced as of 1 January 2010 as part of a public service-wide reduction in pay. In addition, teachers who entered the profession after 1 January 2011 are paid according to a new salary scale that is 10\% lower than the salary scale that applied to those previously recruited. In Portugal in 2011, using a method defined in a new law and as part of a reform package, salaries higher than EUR 1500 were reduced. They fell again in 2012 as civil servants were paid salaries covering 12 months, not 14 months, as had previously been the case. In England, teachers' salaries were frozen between 2011 and 2012 at all levels of education, followed by a below-inflation increase of $1 \%$ in the following year for the public sector as a whole, all due to the financial crisis. As teachers were in a three-year pay settlement, the pay freeze was applied later for teachers than for other public-sector workers. Similarly, the Scottish Negotiating Committee for Teachers (SNCT) agreed to freeze teachers' pay from April 2011 until March 2013. While teachers in Scotland are not classified as civil servants, this agreement mirrored the freezes applied to the pay of civil servants in Scotland. In Italy, teachers' salaries were frozen from 2011. This salary freeze affected all civil servants, including teachers, and was introduced in response to the international economic situation and in order to meet the public finance targets set by the EU.

The economic downturn may also have an influence on the supply of teachers. In general, when the general economy is weak, and there is high unemployment among graduates and low graduate earnings, teaching might seem to be a more attractive job choice than other occupations (OECD, 2005).

\section{Actual average salaries}

Statutory salaries as reported by most of the countries in this indicator must be distinguished from actual expenditures on wages by governments and from teachers' actual average salaries, which are influenced by factors such as the level of experience of the teaching force and the prevalence of bonuses and allowances in the compensation system. Unlike statutory salaries, actual salaries may include work-related payments, such as annual bonuses, results-related bonuses, extra pay for holidays, sick-leave pay and other additional payments. This distinction is important, as bonuses and allowances can represent a significant addition to base salaries.

In the Slovak Republic, for example, most teachers receive bonuses, such as personal valuations/appraisals, on a monthly basis. Depending on the financial resources of the school and the evaluation of individual teachers, teachers' average salaries in that country, including these bonuses, can be double the base statutory salary.

The comparison of actual annual salaries of all teachers aged 25-64 with statutory salaries for teachers with 15 years of experience and typical qualifications shows that in Austria, the Czech Republic (upper secondary level), Estonia (primary and secondary levels), France (upper secondary level), Hungary, Iceland (upper secondary level), Israel (primary and secondary levels), Poland (lower secondary level) and the Slovak Republic, average actual salaries, including bonuses and allowances, are at least 20\% higher than statutory salaries for teachers with 15 years of experience. By contrast, in Australia, the Netherlands and the United States at all education levels, in Slovenia at pre-primary, primary and lower secondary levels, and in England and Luxembourg at pre-primary and primary levels, actual salaries of teachers aged 25-64 are at least 5\% lower than statutory salaries for teachers with 15 years of experience and typical qualifications (Tables D3.1a and D3.4).

In some countries, average actual teachers' salaries vary more across education levels than do statutory salaries for teachers with 15 years of experience and typical qualifications. In the Czech Republic, Denmark, Finland, France and Israel, the gap between average actual salaries of upper secondary teachers and average actual salaries of pre-primary teachers is at least 15 percentage points greater than the difference in their statutory salaries. In Poland, there is a difference of around 15\% between average actual salaries at the pre-primary and primary levels, despite similar statutory salaries at these levels. The variety of bonuses available for different levels of education partly explains these differences (see Annex 3, available on line). 


\section{Box D3.4. Actual average salaries, by age group and gender}

The actual salaries of older teachers (those aged 55-64) are, on average, $40 \%$ (pre-primary level), $41 \%$ (primary level), $41 \%$ (lower secondary level) and $43 \%$ (upper secondary) higher than actual salaries of younger teachers (those aged 25-34).

When teachers' salaries compared to similarly-educated, full-time, full-year 25-64 year-old workers are disaggregated by age, the ratio differs among age groups. Relative teachers' salaries are about ten percentage points higher among the youngest adults (25-34 year-olds) than among the older age groups (55-64 year-olds). The higher ratio among the youngest adults compared to other age groups indicates the attractiveness of entering the teaching profession. However, this ratio shrinks as teachers age, indicating that teachers' salaries may evolve at a slower rate than for other workers, and that the salaries of other similarly educated professionals are more attractive as the work force ages.

A comparison of the actual salaries of male and female teachers shows that differences in actual salaries are very small - less than $3 \%$, on average. Female teachers earn, on average only slightly more than male teachers at the pre-primary level and slightly less at the primary, lower secondary and upper secondary levels.

Larger gender differences are shown in the ratio of teachers' salaries to earnings for similarly-educated workers aged 25-64. On average across all levels of education, male teachers aged 25-64 earn less than 75\% of the salary of a tertiary-educated, 25-64 year-old full time, full-year male worker. Female teachers aged 25-64 are paid more than $90 \%$ to up to $93 \%$ (secondary level) of that benchmark salary. This higher ratio among female teachers reflects the persistent gender gap in earnings in the labour market. It also suggests that the teaching profession may be more attractive to women than men compared to other professions (Tables D3.2a and D3.4).

\section{Formation of base salary and additional payments: Incentives and allowances}

Statutory salaries provide valuable information about the base salaries that are paid to teachers. However, teachers' statutory salaries, based on pay scales, are only one component of teachers' total compensation. In addition to base pay scales, school systems use schemes that offer additional payments, such as allowances, bonuses or other rewards to teachers. These may take the form of financial remuneration and/or reduction in the number of teaching hours. Criteria for additional payments vary across countries. These additional payments may explain certain differences between statutory scheduled salaries and actual average salaries.

New data provides some insights into the conditions and criteria on which teachers' salaries are based (Table D3.7c, and Tables D3.7a, b and d, and D3.8a through D3.8d, available on line). These data suggest that in the large majority of countries, core tasks like teaching, planning or preparing lessons, marking student's work, participating in general administrative work, communicating with parents, supervising students, and working with colleagues are mandatory for teachers (implicitly or explicitly) and rarely considered as meriting bonuses or additional payments.

Shouldering other responsibilities, however, often entails having some sort of extra compensation. In about half of the countries with information available for lower secondary teachers, teachers who participate in school management activities in addition to fulfilling their teaching duties receive some sort of compensation, either reduced teaching time, as in Denmark, Finland, Luxembourg, Portugal and the Slovak Republic, or an annual additional payment, as in England, France, Hungary, Ireland, Italy, Japan, Korea, Norway and Spain. It is also common to see additional payments, either annual or occasional, when teachers teach more classes or hours than required by their full-time contract, for being a class or form teacher, or for performing special tasks, like training student teachers (Table D3.7c).

Occasional additional payments are also awarded when teachers show outstanding performance, as is the case for lower secondary teachers in the Czech Republic, Estonia, Israel, Japan, Korea, Poland, the Slovak Republic, Slovenia and Turkey. Additional payments can also include bonuses for special teaching conditions; for teaching students with special needs in regular schools, and for teaching in disadvantaged, remote or high-cost areas. 
Compensation for teachers' tasks can be established in several ways. In most countries, central or state authorities determine whether teachers' tasks, particularly core tasks like teaching, planning and preparing lessons or marking students' work, are mandatory. These authorities generally also decide whether completion of these core tasks is considered when establishing base salaries or additional payments. In countries where schools have high levels of autonomy, like the Netherlands, Sweden and the United States, some decisions on core tasks, like teaching, are taken at the school level, either by the school principal, head teacher or the school board (Table D3.8c, available on line).

Decisions about other teacher responsibilities - like teaching more classes or hours than required by full-time contract, participating in extracurricular activities, or acting as class or form teacher - are often taken at the school level. In many countries, school principals, head teachers or school boards determine if these sorts of tasks are mandatory, and who performs them. Decisions on whether these tasks should be compensated for through base salary or additional payments, and even decisions on the amount to be paid to teachers who perform such tasks, are often taken by other authorities. For example, in more than half of the countries with available information, decisions on teaching more hours, at the lower secondary level, than required by full-time contract are taken at the school level; but in only seven countries are decisions about whether compensation for completing these tasks should come from the base salary and/or additional payments decided at the school level, and in only five countries is the amount to be paid to teachers for performing the task decided at that level. In the remaining countries, these decisions are taken by central, state, provincial or local authorities.

\section{Teachers' salaries relative to earnings for tertiary-educated workers}

Young people's decision to undertake teacher training, and graduates' decision to enter or stay in the profession, are influenced by the salaries of teachers relative to those of other occupations requiring similar qualifications and by likely salary increases. In most OECD countries, a tertiary degree is required to become a teacher at all levels of education (Table D3.2a, and Table D3.2b, available on line), so the likely alternative to teacher education is a similar tertiary education programme. Thus, to interpret salary levels in different countries and reflect comparative labour-market conditions, teachers' salaries are compared to those of other similarly educated professionals: 25-64 year-old full-time, full-year workers with a similar tertiary education (Table D3.2a).

A comparison of the average annual salaries (including bonuses and allowances) of teachers in public institutions with the wages of similarly educated workers, based on teachers' typical attainment level shows that, on average, pre-primary and primary teachers' salaries amount to $78 \%$ of full-time, full-year earnings for 25-64 year-olds with similar educational attainment, lower secondary teachers are paid $80 \%$, and upper secondary teachers earn $82 \%$ of that benchmark salary.

A second benchmark is based on the actual salaries of all teachers, relative to earnings for full-time, full-year workers with tertiary education (ISCED 5 to 8). Against this benchmark, pre-primary teachers' salaries amount to $73 \%$ of full-time, full-year earnings, on average, among 25-64 year-olds with tertiary education, primary teachers earn $80 \%$, lower secondary teachers are paid $86 \%$, and upper secondary teachers earn $91 \%$ of the benchmark salary (Table D3.2a and Chart D3.1).

Upper secondary teachers in only five of the 25 countries with available data earn as much as or more than workers with a similar educational attainment. In almost all countries with available information, and at almost all levels of education, teachers earn less than similarly educated workers. Relative salaries for teachers are highest in Belgium (upper secondary), Luxembourg, Switzerland (upper secondary), where teachers' salaries are at least 8\% higher than those of comparably educated workers. The lowest relative teachers' salaries, compared to the salaries of other professionals with comparable education, are found in the Czech Republic at the primary and lower secondary levels and in Hungary at the upper secondary level, where teachers' salaries are less than $55 \%$ of what a full-time, full-year worker with a similar educational attainment earns (Table D3.2a and Chart D3.1).

\section{Definitions}

Actual salaries for teachers aged 25-64 refer to the annual average earnings received by full-time teachers aged 25-64, before taxes. It includes work-related payments, such as annual bonuses, results-related bonuses, extra pay for holidays and sick-leave pay. Income from other sources, such as government social transfers, investment income, and any other income that is not directly related to their profession, are not included.

An adjustment to base salary is defined as any difference in salary between what a particular teacher actually receives for work performed at school and the amount that he or she would expect to receive on the basis of experience (i.e. number of years in the teaching profession). Adjustments may be temporary or permanent, and they can effectively move a teacher off the scale and to a different salary scale or to a higher step on the same salary scale. 
Earnings for workers with tertiary education are average earnings for full-time, full-year workers aged 25-64 with an education at ISCED 5/6/7 or 8 level. The relative salary indicator is calculated for the latest year with available earnings data. For countries in which teachers' salaries and workers' earnings information are not available for the same year (e.g. Australia, Canada, Chile, Finland, France, Italy, the Netherlands, Poland, Spain and Sweden), the indicator is adjusted for inflation using the deflators for private consumption. Reference statistics for earnings for workers with tertiary education are provided in Annex 3.

Salaries after 15 years of experience refer to the scheduled annual salary of a full-time classroom teacher. Statutory salaries may refer to the salaries of teachers with the minimum training necessary to be fully qualified, or salaries of teachers with the typical qualifications, plus 15 years of experience.

Starting salaries refer to the average scheduled gross salary per year for a full-time classroom teacher with the minimum training necessary to be fully qualified at the beginning of the teaching career; maximum salaries refer to the maximum annual salary (top of the salary scale) for a full-time classroom teacher with the maximum qualifications recognised for compensation.

Statutory salaries refer to scheduled salaries according to official pay scales. The salaries reported are gross (total sum paid by the employer) less the employer's contribution to social security and pension, according to existing salary scales. Salaries are "before tax", i.e. before deductions for income tax. In Table D3.3a, and Table D3.3b (available on line), salary per hour of net contact time divides a teacher's annual statutory salary by the annual net teaching time in hours (see Table D4.1).

Typical educational attainment refers to teachers' typical diploma according to the following ISCED 2011Attainment Codes:

- ISCED-A 343/353/344/354: Upper secondary

- ISCED-A 443/453/444/454: Post-secondary non-tertiary

- ISCED-A 540/550: Short cycle tertiary

- ISCED-A 660: Bachelor's or equivalent

- ISCED-A 760: Master's or equivalent

\section{Methodology}

Data on statutory teachers' salaries and bonuses are derived from the 2014 OECD-INES Survey on Teachers and the Curriculum. Data refer to the school year 2012/13 and are reported in accordance with formal policies for public institutions.

Data on teachers' salary at upper secondary level refer only to general programmes.

Measuring the statutory salary of a full-time teacher relative to the number of hours per year that a teacher is required to spend teaching does not adjust salaries for the amount of time that teachers spend in various other teachingrelated activities. Since the proportion of teachers' working time spent teaching varies across OECD countries, statutory salaries per hour of net teaching time must be interpreted with caution (see Indicator D4). However, it can provide an estimate of the cost of the actual time teachers spend in the classroom.

Gross teachers' salaries were converted using PPPs for private consumption from the OECD National Accounts database. Prior to the 2012 edition of Education at a Glance (OECD, 2012), salaries used to be converted using PPPs for GDP. As a consequence, teachers' salaries in USD (Tables D3.1a, and Table D3.1b, available on line) are not directly comparable with the figures published prior to the 2012 edition of Education at a Glance. Information on trends in teachers' salaries can be found in Table D3.5a, and Table D3.5b, available on line. As a complement to Table D 3.1a, and Table D3.1b, available on line, which presents teachers' salaries in equivalent USD, converted using PPPs, tables with teachers' salaries in national currency are included in Annex 2. The period of reference for teachers' salaries is from 1 July 2012 to 30 June 2013. The reference date for PPPs is 2012-13 except for some Southern Hemisphere countries (e.g. Australia and New Zealand) where the academic year runs from January to December. In these countries the reference year is the calendar year (i.e. 2013).

For calculation of changes in teachers' salaries (Table D3.5a, and Table D3.5b, available on line), the deflator for private consumption is used to convert salaries to 2005 prices.

In most countries, the criteria to determine the typical qualification and typical attainment of teachers are based on a principle of absolute majority, i.e. the level of education attained by more than half of all current teachers in the system.

In Table D3.2a, the ratios of teachers' salaries to earnings for full-time, full-year workers with tertiary education aged 25-64 are calculated using the annual average salaries (including bonuses and allowances) for teachers 
aged 25-64, for countries with available data (see Table D3.4). The information on teachers' typical attainment level is used to define the denominator for the ratios if data on the wages of workers by ISCED level of attainment is available (i.e. the earnings for full-time, full-year workers). For countries whose data on the wages of workers by ISCED level of attainment is not available, information on all tertiary-educated workers was used. The same procedure was used in Table D3.2b, available on line, but the ratios are calculated using the statutory salaries of teachers with 15 years of experience instead of their actual salaries. That table shows information for countries with available information on statutory salaries. Information on the typical attainment level used as a reference for the calculation of relative teachers' salaries is indicated in Table D3.2a, and Table D3.2b, available on line. A description of the ISCED levels can be found in the Definitions section.

Notes on definitions and methodologies for each country are provided in Annex 3 at www.oecd.org/education/ education-at-a-glance-19991487.htm.

\section{Note regarding data from Israel}

The statistical data for Israel are supplied by and are under the responsibility of the relevant Israeli authorities. The use of such data by the OECD is without prejudice to the status of the Golan Heights, East Jerusalem and Israeli settlements in the West Bank under the terms of international law.

\section{References}

OECD (2014), Education at a Glance 2014: OECD Indicators, OECD Publishing, Paris, http://dx.doi.org/10.1787/eag-2014-en. OECD (2012), Education at a Glance 2012: OECD Indicators, OECD Publishing, Paris, http://dx.doi.org/10.1787/eag-2012-en. OECD (2005), Teachers Matter: Attracting, Developing and Retaining Effective Teachers, OECD Publishing, Paris, http://dx.doi. org/10.1787/9789264018044-en.

Santiago, P. (2004), “The Labour Market for Teachers”, in G. Johnes and J. Johnes (eds), International Handbook on the Economics of Education, Edward Elgar, Cheltenham.

\section{Indicator D3 Tables}

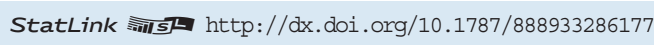

Table D3.1a Teachers' statutory salaries, based on typical qualifications, at different points in teachers' careers (2013)

WEB Table D3.1b Teachers' statutory salaries, based on minimum qualifications, at different points in teachers' careers (2013)

Table D3.2a Teachers' actual salaries relative to wages of similarly educated workers (2013)

WEB Table D3.2b Teachers' statutory salaries relative to wages of similarly educated workers (2013)

Table D3.3a Comparison of teachers' statutory salaries, based on typical qualifications (2013)

WEB Table D3.3b Comparison of teachers' statutory salaries, based on minimum qualifications (2013)

Table D3.4 Average actual teachers' salaries (2013)

Table D3.5a Trends in teachers' salaries, based on typical qualifications, between 2000 and 2013

WEB Table D3.5b Trends in teachers' salaries, based on minimum qualifications, between 2000 and 2013

WEB Table D3.6a Starting/Maximum teachers' statutory salaries, based on typical/maximum qualifications (2013)

WEB Table D3.6b Starting/Maximum teachers' statutory salaries, based on minimum/maximum qualifications (2013)

WEB Table D3.7a Teachers' tasks and other criteria related to teachers' base salaries and additional payments, pre-primary level (2013)

WEB Table D3.7b Teachers' tasks and other criteria related to teachers' base salaries and additional payments, primary level (2013)

Table D3.7c Teachers' tasks and other criteria related to teachers' base salaries and additional payments, lower secondary level (2013)

WEB Table D3.7d Teachers' tasks and other criteria related to teachers' base salaries and additional payments, upper secondary (2013)

WEB Table D3.8a Determining teachers' base salaries and additional payments, pre-primary level (2013)

WEB Table D3.8b Determining teachers' base salaries and additional payments, primary level (2013)

WEB Table D3.8c Determining teachers' base salaries and additional payments, lower secondary level (2013)

WEB Table D3.8d Determining teachers' base salaries and additional payments, upper secondary level (2013) 
Table D3.1a. [1/2] Teachers' statutory salaries, based on typical qualifications, at different points in teachers' careers (2013)

Annual teachers' salaries, in public institutions, in equivalent USD converted using PPPs for private consumption

\begin{tabular}{|c|c|c|c|c|c|c|c|c|}
\hline & \multicolumn{4}{|c|}{ Pre-primary } & \multicolumn{4}{|c|}{ Primary } \\
\hline & $\begin{array}{c}\text { Starting } \\
\text { salary }\end{array}$ & $\begin{array}{c}\text { Salary } \\
\text { after } 10 \text { years } \\
\text { of experience }\end{array}$ & $\begin{array}{c}\text { Salary } \\
\text { after } 15 \text { years } \\
\text { of experience }\end{array}$ & $\begin{array}{c}\text { Salary at top } \\
\text { of scale }\end{array}$ & $\begin{array}{c}\text { Starting } \\
\text { salary }\end{array}$ & $\begin{array}{c}\text { Salary } \\
\text { after } 10 \text { years } \\
\text { of experience }\end{array}$ & $\begin{array}{c}\text { Salary } \\
\text { after } 15 \text { years } \\
\text { of experience }\end{array}$ & $\begin{array}{l}\text { Salary at top } \\
\text { of scale }\end{array}$ \\
\hline & (1) & (2) & (3) & (4) & (5) & (6) & (7) & (8) \\
\hline Australia $^{1}$ & 39776 & 56782 & 56782 & 57025 & 39177 & 56335 & 56335 & 56521 \\
\hline Austria $^{2}$ & 32610 & 38376 & 43015 & 64014 & 32610 & 38376 & 43015 & 64014 \\
\hline Belgium (Fl.) & 34411 & 43219 & 48690 & 59633 & 34411 & 43219 & 48690 & 59633 \\
\hline Canada & $\mathrm{m}$ & $\mathrm{m}$ & $\mathrm{m}$ & $\mathrm{m}$ & 39608 & 63557 & 66702 & 66702 \\
\hline Chile $^{1,3}$ & 17733 & 23736 & 26610 & 37110 & 17733 & 23736 & 26610 & 37110 \\
\hline Czech Republic & 16537 & 16743 & 17099 & 18232 & 17033 & 17529 & 18273 & 20795 \\
\hline Denmark $^{3}$ & 40284 & 45724 & 45724 & 45724 & 45860 & 50958 & 52672 & 52672 \\
\hline England & 27768 & 45595 & 47279 & 47279 & 27768 & 45595 & 47279 & 47279 \\
\hline Estonia & $\mathrm{m}$ & $\mathrm{m}$ & $\mathrm{m}$ & $\mathrm{m}$ & 13004 & 13233 & 13233 & 17015 \\
\hline Finland $^{4}$ & 27645 & 29857 & 29857 & 29857 & 32356 & 37453 & 39701 & 42083 \\
\hline Greece & 17760 & 22460 & 25826 & 34901 & 17760 & 22460 & 25826 & 34901 \\
\hline Hungary $^{7}$ & 10295 & 11498 & 12305 & 16306 & 10647 & 12177 & 13061 & 17362 \\
\hline Iceland $^{3}$ & 24906 & 24906 & 28459 & 28459 & 26046 & 29165 & 31145 & 31145 \\
\hline Ireland & $\mathrm{m}$ & $\mathrm{m}$ & $\mathrm{m}$ & $\mathrm{m}$ & 34899 & 50248 & 56057 & 63165 \\
\hline Israel & 22368 & 27298 & 30960 & 57513 & 19806 & 25732 & 29869 & 51855 \\
\hline Italy & 27509 & 30262 & 33230 & 40437 & 27509 & 30262 & 33230 & 40437 \\
\hline Japan $^{3}$ & $\mathrm{~m}$ & $\mathrm{~m}$ & $\mathrm{~m}$ & $\mathrm{~m}$ & 27627 & 41036 & 48546 & 60878 \\
\hline Korea & 29357 & 44193 & 51594 & 82002 & 29357 & 44193 & 51594 & 82002 \\
\hline Luxembourg & 68873 & 91203 & 102956 & 123406 & 68873 & 91203 & 102956 & 123406 \\
\hline Mexico & 15944 & 20779 & 26533 & 34048 & 15944 & 20779 & 26533 & 34048 \\
\hline Netherlands & 36456 & 45228 & 54001 & 54001 & 36456 & 45228 & 54001 & 54001 \\
\hline New Zealand & $\mathrm{m}$ & $\mathrm{m}$ & $\mathrm{m}$ & $\mathrm{m}$ & 29124 & 43292 & 43292 & 43292 \\
\hline Norway & 35685 & 40882 & 40882 & 40882 & 41177 & 44538 & 44538 & 48662 \\
\hline Poland & 15220 & 20402 & 24921 & 25980 & 15220 & 20402 & 24921 & 25980 \\
\hline Portugal & 30806 & 33740 & 36663 & 57201 & 30806 & 33740 & 36663 & 57201 \\
\hline Slovenia & 25134 & 29905 & 36818 & 42333 & 25134 & 31077 & 38261 & 45764 \\
\hline Spain & 36422 & 39468 & 42187 & 51265 & 36422 & 39468 & 42187 & 51265 \\
\hline Sweden ${ }^{7}$ & 33383 & 35822 & 36885 & 38714 & 32991 & 36817 & 38175 & 43595 \\
\hline Switzerland & $\mathrm{m}$ & $\mathrm{m}$ & $\mathrm{m}$ & $\mathrm{m}$ & $\mathrm{m}$ & $\mathrm{m}$ & $\mathrm{m}$ & $\mathrm{m}$ \\
\hline Turkey & 25295 & 26107 & 27139 & 29342 & 25295 & 26107 & 27139 & 29342 \\
\hline United States $^{7}$ & 42590 & 51275 & 58202 & 70978 & 41606 & 53799 & 59339 & 66938 \\
\hline OECD average & 28730 & 35300 & 38653 & 46564 & 29807 & 37795 & 41245 & 48706 \\
\hline EU21 average & 28976 & 35387 & 38688 & 45649 & 30032 & 36980 & 40519 & 47662 \\
\hline Argentina & $\mathrm{m}$ & $\mathrm{m}$ & $\mathrm{m}$ & $\mathrm{m}$ & $\mathrm{m}$ & $\mathrm{m}$ & $\mathrm{m}$ & $\mathrm{m}$ \\
\hline Brazil & $\mathrm{m}$ & $\mathrm{m}$ & $\mathrm{m}$ & $\mathrm{m}$ & $\mathrm{m}$ & $\mathrm{m}$ & $\mathrm{m}$ & $\mathrm{m}$ \\
\hline China & $\mathrm{m}$ & $\mathrm{m}$ & $\mathrm{m}$ & $\mathrm{m}$ & $\mathrm{m}$ & $\mathrm{m}$ & $\mathrm{m}$ & $\mathrm{m}$ \\
\hline Colombia & 15324 & 19661 & 19661 & 19661 & 15416 & 20807 & 20807 & 20807 \\
\hline India & $\mathrm{m}$ & $\mathrm{m}$ & $\mathrm{m}$ & $\mathrm{m}$ & $\mathrm{m}$ & $\mathrm{m}$ & $\mathrm{m}$ & $\mathrm{m}$ \\
\hline Indonesia & $\mathrm{m}$ & $\mathrm{m}$ & $\mathrm{m}$ & $\mathrm{m}$ & $\mathrm{m}$ & $\mathrm{m}$ & $\mathrm{m}$ & $\mathrm{m}$ \\
\hline Latvia & $\mathrm{a}$ & $\mathrm{m}$ & $\mathrm{m}$ & $\mathrm{a}$ & $\mathrm{a}$ & $\mathrm{m}$ & $\mathrm{m}$ & $\mathrm{a}$ \\
\hline Russian Federation & $\mathrm{m}$ & $\mathrm{m}$ & $\mathrm{m}$ & $\mathrm{m}$ & $\mathrm{m}$ & $\mathrm{m}$ & $\mathrm{m}$ & $\mathrm{m}$ \\
\hline Saudi Arabia & $\mathrm{m}$ & $\mathrm{m}$ & $\mathrm{m}$ & $\mathrm{m}$ & $\mathrm{m}$ & $\mathrm{m}$ & $\mathrm{m}$ & $\mathrm{m}$ \\
\hline South Africa & $\mathrm{m}$ & $\mathrm{m}$ & $\mathrm{m}$ & $\mathrm{m}$ & $\mathrm{m}$ & $\mathrm{m}$ & $\mathrm{m}$ & $\mathrm{m}$ \\
\hline G20 average & $\mathrm{m}$ & $\mathrm{m}$ & $\mathrm{m}$ & $\mathrm{m}$ & $\mathrm{m}$ & $\mathrm{m}$ & $\mathrm{m}$ & $\mathrm{m}$ \\
\hline
\end{tabular}

Note: The definition of teachers' typical qualification is based on a broad concept, including the typical ISCED level of attainment and other criteria. Please see Box D3.2 for further details.

1. Statutory salaries do not include the the part of social security contributions and pension-scheme contributions paid by the employees.

2. Figures for the pre-primary level refer to primary teachers (in primary schools only) teaching pre-primary classes.

3. Statutory salaries include the part of social security contributions and pension-scheme contributions paid by the employers.

4. Includes data on the majority, i.e. kindergarten teachers only for pre-primary education.

5. Includes average bonuses for overtime hours for lower and upper secondary teachers.

6. The typical qualification of starting teachers differ substantially from the typical qualification of all the current teachers.

7. Actual base salaries.

Source: OECD. See Annex 3 for notes (www.oecd.org/education/education-at-a-glance-19991487.htm).

Please refer to the Reader's Guide for information concerning symbols for missing data and abbreviations.

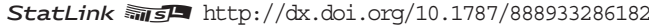


Table D3.1a. [2/2] Teachers' statutory salaries, based on typical qualifications, at different points in teachers' careers (2013)

Annual teachers' salaries, in public institutions, in equivalent USD converted using PPPs for private consumption

\begin{tabular}{|c|c|c|c|c|c|c|c|c|}
\hline & \multicolumn{4}{|c|}{ Lower secondary, general programmes } & \multicolumn{4}{|c|}{ Upper secondary, general programmes } \\
\hline & $\begin{array}{l}\text { Starting } \\
\text { salary }\end{array}$ & $\begin{array}{c}\text { Salary } \\
\text { after } 10 \text { years } \\
\text { of experience }\end{array}$ & $\begin{array}{c}\text { Salary } \\
\text { after } 15 \text { years } \\
\text { of experience }\end{array}$ & $\begin{array}{c}\text { Salary at top } \\
\text { of scale }\end{array}$ & $\begin{array}{l}\text { Starting } \\
\text { salary }\end{array}$ & $\begin{array}{c}\text { Salary } \\
\text { after } 10 \text { years } \\
\text { of experience }\end{array}$ & $\begin{array}{c}\text { Salary } \\
\text { after } 15 \text { years } \\
\text { of experience }\end{array}$ & $\begin{array}{c}\text { Salary at top } \\
\text { of scale }\end{array}$ \\
\hline & (9) & $(10)$ & (11) & $(12)$ & $(13)$ & $(14)$ & $(15)$ & $(16)$ \\
\hline Australia ${ }^{1}$ & 39125 & 56315 & 56315 & 56474 & 39125 & 56315 & 56315 & 56474 \\
\hline 。̆ Austria ${ }^{2}$ & 34143 & 41509 & 46631 & 66378 & 35794 & 44029 & 50183 & 74195 \\
\hline Belgium (Fl.) & 34411 & 43219 & 48690 & 59633 & 42996 & 54874 & 62614 & 75514 \\
\hline Canada & 39608 & 63557 & 66702 & 66702 & 39775 & 63878 & 67022 & 67022 \\
\hline Chile $^{1,3}$ & 17733 & 23736 & 26610 & 37110 & 18838 & 25155 & 28179 & 39229 \\
\hline Czech Republic & 17033 & 17529 & 18273 & 20795 & 17033 & 17529 & 18273 & 20795 \\
\hline Denmark $^{3}$ & 46144 & 51640 & 53431 & 53431 & 46218 & 54979 & 54979 & 54979 \\
\hline England & 27768 & 45595 & 47279 & 47279 & 27768 & 45595 & 47279 & 47279 \\
\hline Estonia & 13004 & 13233 & 13233 & 17015 & 13004 & 13233 & 13233 & 17015 \\
\hline Finland $^{4}$ & 34945 & 40450 & 42877 & 45449 & 37056 & 44504 & 46284 & 49061 \\
\hline Greece & 17760 & 22460 & 25826 & 34901 & 17760 & 22460 & 25826 & 34901 \\
\hline Hungary $^{7}$ & 10647 & 12177 & 13061 & 17362 & 11617 & 13978 & 15491 & 21738 \\
\hline Iceland $^{3}$ & 26046 & 29165 & 31145 & 31145 & 25181 & 28864 & 31425 & 32840 \\
\hline Ireland & 36612 & 52257 & 56667 & 63774 & 36612 & 52257 & 56667 & 63774 \\
\hline Israel & 19917 & 25481 & 28715 & 44106 & 19302 & 22861 & 25681 & 40241 \\
\hline Italy & 29655 & 32851 & 36207 & 44408 & 29655 & 33649 & 37221 & 46425 \\
\hline Japan $^{3}$ & 27627 & 41036 & 48546 & 60878 & 27627 & 41036 & 48546 & 62542 \\
\hline Korea & 29252 & 44088 & 51489 & 81897 & 29252 & 44088 & 51489 & 81897 \\
\hline Luxembourg & 79920 & 99900 & 110243 & 138920 & 79920 & 99900 & 110243 & 138920 \\
\hline Mexico & 20492 & 26581 & 34083 & 43506 & $\mathrm{~m}$ & $\mathrm{~m}$ & $\mathrm{~m}$ & $\mathrm{~m}$ \\
\hline Netherlands & 38473 & 55697 & 66831 & 66831 & 38473 & 55697 & 66831 & 66831 \\
\hline New Zealand & 29635 & 44509 & 44509 & 44509 & 30145 & 45726 & 45726 & 45726 \\
\hline Norway & 41177 & 44538 & 44538 & 48662 & 45601 & 50289 & 50289 & 56452 \\
\hline Poland & 15220 & 20402 & 24921 & 25980 & 15220 & 20402 & 24921 & 25980 \\
\hline Portugal & 30806 & 33740 & 36663 & 57201 & 30806 & 33740 & 36663 & 57201 \\
\hline Slovenia & 25134 & 31077 & 38261 & 45764 & 25134 & 31077 & 38261 & 45764 \\
\hline Spain & 40752 & 44124 & 46907 & 57398 & 40752 & 44124 & 46907 & 57398 \\
\hline Sweden ${ }^{7}$ & 33383 & 37442 & 38852 & 44398 & 34692 & 38997 & 40733 & 46566 \\
\hline Switzerland & $\mathrm{m}$ & $\mathrm{m}$ & $\mathrm{m}$ & $\mathrm{m}$ & $\mathrm{m}$ & $\mathrm{m}$ & $\mathrm{m}$ & $\mathrm{m}$ \\
\hline Turkey & 26266 & 26730 & 28110 & 30313 & 26266 & 26730 & 28110 & 30313 \\
\hline United States ${ }^{7}$ & 43324 & 53758 & 60965 & 66022 & 42695 & 54843 & 59948 & 67016 \\
\hline OECD average & 31013 & 39268 & 42825 & 50414 & 32260 & 41077 & 44600 & 52822 \\
\hline EU21 average & 31533 & 38908 & 42485 & 50138 & 32741 & 40732 & 44507 & 52879 \\
\hline n Argentina & $\mathrm{m}$ & $\mathrm{m}$ & $\mathrm{m}$ & $\mathrm{m}$ & $\mathrm{m}$ & $\mathrm{m}$ & $\mathrm{m}$ & $\mathrm{m}$ \\
\hline Erazil & $\mathrm{m}$ & $\mathrm{m}$ & $\mathrm{m}$ & $\mathrm{m}$ & $\mathrm{m}$ & $\mathrm{m}$ & $\mathrm{m}$ & $\mathrm{m}$ \\
\hline China & $\mathrm{m}$ & $\mathrm{m}$ & $\mathrm{m}$ & $\mathrm{m}$ & $\mathrm{m}$ & $\mathrm{m}$ & $\mathrm{m}$ & $\mathrm{m}$ \\
\hline Colombia & 15331 & 21499 & 21499 & 21499 & 15331 & 21499 & 21499 & 21499 \\
\hline India & $\mathrm{m}$ & $\mathrm{m}$ & $\mathrm{m}$ & $\mathrm{m}$ & $\mathrm{m}$ & $\mathrm{m}$ & $\mathrm{m}$ & $\mathrm{m}$ \\
\hline Indonesia & $\mathrm{m}$ & $\mathrm{m}$ & $\mathrm{m}$ & $\mathrm{m}$ & $\mathrm{m}$ & $\mathrm{m}$ & $\mathrm{m}$ & $\mathrm{m}$ \\
\hline Latvia & a & $\mathrm{m}$ & $\mathrm{m}$ & $\mathrm{a}$ & $\mathrm{a}$ & $\mathrm{m}$ & $\mathrm{m}$ & a \\
\hline Russian Federation & $\mathrm{m}$ & $\mathrm{m}$ & $\mathrm{m}$ & $\mathrm{m}$ & $\mathrm{m}$ & $\mathrm{m}$ & $\mathrm{m}$ & $\mathrm{m}$ \\
\hline Saudi Arabia & $\mathrm{m}$ & $\mathrm{m}$ & $\mathrm{m}$ & $\mathrm{m}$ & $\mathrm{m}$ & $\mathrm{m}$ & $\mathrm{m}$ & $\mathrm{m}$ \\
\hline South Africa & $\mathrm{m}$ & $\mathrm{m}$ & $\mathrm{m}$ & $\mathrm{m}$ & $\mathrm{m}$ & $\mathrm{m}$ & $\mathrm{m}$ & $\mathrm{m}$ \\
\hline G20 average & $\mathrm{m}$ & $\mathrm{m}$ & $\mathrm{m}$ & $\mathrm{m}$ & $\mathrm{m}$ & $\mathrm{m}$ & $\mathrm{m}$ & $\mathrm{m}$ \\
\hline
\end{tabular}

Note: The definition of teachers' typical qualification is based on a broad concept, including the typical ISCED level of attainment and other criteria. Please see Box D3.2 for further details.

1. Statutory salaries do not include the the part of social security contributions and pension-scheme contributions paid by the employees.

2. Figures for the pre-primary level refer to primary teachers (in primary schools only) teaching pre-primary classes.

3. Statutory salaries include the part of social security contributions and pension-scheme contributions paid by the employers.

4. Includes data on the majority, i.e. kindergarten teachers only for pre-primary education.

5. Includes average bonuses for overtime hours for lower and upper secondary teachers.

6. The typical qualification of starting teachers differ substantially from the typical qualification of all the current teachers.

7. Actual base salaries.

Source: OECD. See Annex 3 for notes (www.oecd.org/education/education-at-a-glance-19991487.htm).

Please refer to the Reader's Guide for information concerning symbols for missing data and abbreviations.

StatLink 젝ㄴ http://dx.doi.org/10.1787/888933286182 
Table D3.2a. Teachers' actual salaries relative to wages of similarly educated workers (2013) Ratio of salary, by age group and gender, using annual average salaries (including bonuses and allowances) of teachers in public institutions relative to the wages of workers with similar educational attainment

\begin{tabular}{|c|c|c|c|c|c|c|c|c|c|}
\hline & \multirow[b]{2}{*}{$\begin{array}{l}\text { Year of } \\
\text { reference }\end{array}$} & \multicolumn{4}{|c|}{ Typical educational attainment (ISCED 2011-A) } & \multicolumn{4}{|c|}{ 25-64 year-olds } \\
\hline & & Pre-primary & Primary & $\begin{array}{l}\text { Lower } \\
\text { secondary, } \\
\text { general } \\
\text { programmes }\end{array}$ & $\begin{array}{l}\text { Upper } \\
\text { secondary, } \\
\text { general } \\
\text { programmes }\end{array}$ & Pre-primary & Primary & $\begin{array}{l}\text { Lower } \\
\text { secondary, } \\
\text { general } \\
\text { programmes }\end{array}$ & $\begin{array}{c}\text { Upper } \\
\text { secondary, } \\
\text { general } \\
\text { programmes }\end{array}$ \\
\hline & (1) & (2) & (3) & (4) & (5) & (6) & $(7)$ & $(8)$ & (9) \\
\hline & \multicolumn{9}{|c|}{$\begin{array}{l}\text { Actual salaries of all teachers, relative to earnings for full-time, full-year similarly-educated workers } \\
\text { based on teachers' attainment level (ISCED 2011-A) }\end{array}$} \\
\hline Belgium (Fl. $)^{1}$ & 2013 & 660 & 660 & 660 & 760 & 0.92 & 0.93 & 0.92 & 1.20 \\
\hline Belgium (Fr.) ${ }^{1}$ & 2013 & 660 & 660 & 660 & 760 & 0.87 & 0.87 & 0.86 & 1.10 \\
\hline Canada & $\mathrm{m}$ & $\mathrm{m}$ & 660 & 660 & 660 & $\mathrm{~m}$ & $\mathrm{~m}$ & $\mathrm{~m}$ & $\mathrm{~m}$ \\
\hline Chile & 2013 & 660 & 660 & 660 & 660 & 0.55 & 0.59 & 0.60 & 0.63 \\
\hline Czech Republic & 2013 & 354 & 760 & 760 & 760 & $\mathrm{~m}$ & 0.52 & 0.52 & 0.56 \\
\hline Denmark $^{2}$ & 2013 & 660 & 660 & 660 & 760 & 0.82 & 0.99 & 1.01 & 0.83 \\
\hline England $^{3}$ & 2013 & 760 & 760 & 760 & 760 & 0.75 & 0.75 & 0.82 & 0.82 \\
\hline Finland ${ }^{4}$ & 2012 & 660 & 760 & 760 & 760 & 0.73 & 0.74 & 0.81 & 0.91 \\
\hline France $^{5}$ & 2013 & 660 & 660 & 660 & 660 & 0.84 & 0.84 & 0.99 & 1.08 \\
\hline Greece & 2013 & 660 & 660 & 660 & 660 & 0.86 & 0.86 & 0.87 & 0.87 \\
\hline Hungary & 2013 & 660 & 660 & 660 & 760 & 0.57 & 0.62 & 0.62 & 0.48 \\
\hline Iceland & $\mathrm{m}$ & 760 & 760 & 760 & 760 & $\mathrm{~m}$ & $\mathrm{~m}$ & $\mathrm{~m}$ & $\mathrm{~m}$ \\
\hline Ireland & $\mathrm{m}$ & $\mathrm{m}$ & 665 & 667 & 667 & $\mathrm{~m}$ & $\mathrm{~m}$ & $\mathrm{~m}$ & $\mathrm{~m}$ \\
\hline Israel & 2013 & 660 & 660 & 660 & 660 & 0.91 & 0.96 & 0.95 & 0.91 \\
\hline Japan & $\mathrm{m}$ & $540 / 660$ & 660 & 660 & 660 & $\mathrm{~m}$ & $\mathrm{~m}$ & $\mathrm{~m}$ & $\mathrm{~m}$ \\
\hline Korea & $\mathrm{m}$ & 660 & 660 & 660 & 660 & $\mathrm{~m}$ & $\mathrm{~m}$ & $\mathrm{~m}$ & $\mathrm{~m}$ \\
\hline Mexico & $\mathrm{m}$ & 665 & 665 & 665 & 665 & $\mathrm{~m}$ & $\mathrm{~m}$ & $\mathrm{~m}$ & $\mathrm{~m}$ \\
\hline New Zealand & $\mathrm{m}$ & $\mathrm{m}$ & $\mathrm{m}$ & $\mathrm{m}$ & $\mathrm{m}$ & $\mathrm{m}$ & $\mathrm{m}$ & $\mathrm{m}$ & $\mathrm{m}$ \\
\hline Norway & 2013 & 660 & 660 & 660 & 760 & 0.69 & 0.78 & 0.78 & 0.65 \\
\hline Scotland ${ }^{3,6}$ & 2013 & 660 & 660 & 660 & 660 & 0.82 & 0.82 & 0.82 & 0.82 \\
\hline Slovak Republic ${ }^{7}$ & 2013 & $354 / 660$ & 760 & 760 & 760 & 0.75 & 0.57 & 0.57 & 0.57 \\
\hline OECD average & $\sim$ & $\sim$ & $\sim$ & $\sim$ & $\sim$ & 0.78 & 0.78 & 0.80 & 0.82 \\
\hline
\end{tabular}

\begin{tabular}{|c|c|c|c|c|c|c|c|c|c|}
\hline $\begin{array}{l}\text { Austria }{ }^{8,9} \\
\text { Estonia }\end{array}$ & $\begin{array}{l}2013 \\
2013\end{array}$ & $\tilde{\sim}$ & $\tilde{\sim}$ & $\tilde{\sim}$ & $\tilde{\sim}$ & $\begin{array}{l}0.77 \\
0.59\end{array}$ & $\begin{array}{l}0.77 \\
0.84\end{array}$ & $\begin{array}{l}0.89 \\
0.84\end{array}$ & $\begin{array}{l}0.97 \\
0.84\end{array}$ \\
\hline$\overline{0}$ Italy & 2013 & $\sim$ & $\sim$ & $\sim$ & $\sim$ & 0.63 & 0.63 & 0.67 & 0.73 \\
\hline Luxembourg & 2013 & $\sim$ & $\sim$ & $\sim$ & $\sim$ & 1.09 & 1.09 & 1.24 & 1.24 \\
\hline Netherlands & 2013 & $\sim$ & $\sim$ & $\sim$ & $\sim$ & 0.69 & 0.69 & 0.85 & 0.85 \\
\hline Poland & 2013 & $\sim$ & $\sim$ & $\sim$ & $\sim$ & 0.74 & 0.85 & 0.86 & 0.84 \\
\hline Portugal & $\mathrm{m}$ & $\sim$ & $\sim$ & $\sim$ & $\sim$ & $\mathrm{m}$ & $\mathrm{m}$ & $\mathrm{m}$ & $\mathrm{m}$ \\
\hline Slovenia $^{10}$ & 2013 & $\sim$ & $\sim$ & $\sim$ & $\sim$ & 0.65 & 0.86 & 0.88 & 0.94 \\
\hline Spain & $\mathrm{m}$ & $\sim$ & $\sim$ & $\sim$ & $\sim$ & $\mathrm{m}$ & $\mathrm{m}$ & $\mathrm{m}$ & $\mathrm{m}$ \\
\hline Switzerland & 2013 & $\sim$ & $\sim$ & $\sim$ & $\sim$ & $\mathrm{m}$ & $\mathrm{m}$ & $\mathrm{m}$ & 1.08 \\
\hline Turkey & $\mathrm{m}$ & $\sim$ & $\sim$ & & $\sim$ & $\mathrm{m}$ & $\mathrm{m}$ & $\mathrm{m}$ & $\mathrm{m}$ \\
\hline United States & 2013 & $\sim$ & $\sim$ & $\sim$ & $\sim$ & 0.65 & 0.67 & 0.68 & 0.71 \\
\hline OECD average & $\sim$ & $\sim$ & $\sim$ & $\sim$ & $\sim$ & 0.73 & 0.80 & 0.86 & 0.91 \\
\hline n Argentina & $\mathrm{m}$ & $\tilde{\sim}$ & $\tilde{\sim}$ & $\tilde{\sim}$ & $\begin{array}{l}\sim \sim \\
\sim\end{array}$ & $\mathrm{m}$ & $\mathrm{m}$ & $\mathrm{m}$ & $\mathrm{m}$ \\
\hline schazin & $\mathrm{m}$ & $\sim$ & $\sim$ & $\sim$ & $\sim$ & $\mathrm{m}$ & $\mathrm{m}$ & $\mathrm{m}$ & $\mathrm{m}$ \\
\hline Colombia & $\mathrm{m}$ & $\sim$ & $\sim$ & $\sim$ & $\sim$ & $\mathrm{m}$ & $\mathrm{m}$ & $\mathrm{m}$ & $\mathrm{m}$ \\
\hline Indonesia & $\mathrm{m}$ & $\sim$ & $\sim$ & $\sim$ & $\sim$ & $\mathrm{m}$ & $\mathrm{m}$ & $\mathrm{m}$ & $\mathrm{m}$ \\
\hline Latvia & $\mathrm{m}$ & $\sim$ & $\sim$ & $\sim$ & $\sim$ & $\mathrm{m}$ & $\mathrm{m}$ & $\mathrm{m}$ & $\mathrm{m}$ \\
\hline Russian Federation & $\mathrm{m}$ & $\sim$ & $\sim$ & $\sim$ & $\sim$ & $\mathrm{m}$ & $\mathrm{m}$ & $\mathrm{m}$ & $\mathrm{m}$ \\
\hline Saudi Arabia & $\mathrm{m}$ & $\sim$ & $\sim$ & $\sim$ & $\sim$ & $\mathrm{m}$ & $\mathrm{m}$ & $\mathrm{m}$ & $\mathrm{m}$ \\
\hline South Africa & $\mathrm{m}$ & $\sim$ & $\sim$ & $\sim$ & $\sim$ & $\mathrm{m}$ & $\mathrm{m}$ & $\mathrm{m}$ & $\mathrm{m}$ \\
\hline G20 average & $\sim$ & $\sim$ & $\sim$ & $\sim$ & $\sim$ & $\mathrm{m}$ & $\mathrm{m}$ & $\mathrm{m}$ & $\mathrm{m}$ \\
\hline
\end{tabular}

Notes: Columns showing actual salaries of all teachers relative to wages of similarly educated workers, broken down by age groups and gender (i.e. columns 10-33) are available for consultation on line (see StatLink below). Columns on teachers' typical educational attainment (i.e. columns 2-5) refer to teachers typical diploma, according to the ISCED 2011 attainment level (descriptions available in the Definitions section).

1. Data on earnings for full-time, full-year workers with tertiary education refer to Belgium.

2. Also includes data on actual salaries of teachers in early childhood educational development programmes for pre-primary education.

3. Data on earnings for full-time, full-year workers with tertiary education refer to the United Kingdom.

4. Includes data on the majority, i.e. kindergarten teachers only for pre-primary education.

5. Ratios are overestimated as a proportion of teachers teaching at the secondary level have a higher attainment (i.e. ISCED 7).

6. Includes all teachers, irrespective of their age.

7. Data refers to the ratio of teachers with the typical ISCED 354 qualification for pre-primary education.

8. Figures for the pre-primary level refer to primary teachers (in primary schools only) teaching pre-primary classes.

9. Also includes data on actual salaries of headmasters, deputies and assistants.

10. Also includes data on actual salaries of pre-school teaching assistants for pre-primary education.

Source: OECD. See Annex 3 for notes (www.oecd.org/education/education-at-a-glance-19991487.htm).

Please refer to the Reader's Guide for information concerning symbols for missing data and abbreviations.

StatLink 제개 http://dx.doi.org/10.1787/888933286198 
Table D3.3a. Comparison of teachers' statutory salaries, based on typical qualifications (2013)

Ratio of salaries at different points in teachers' careers, and salary per hour in USD converted using PPPs for private consumption

\begin{tabular}{|c|c|c|c|c|c|c|c|c|c|c|}
\hline & \multicolumn{4}{|c|}{ Ratio of salary at top of scale to starting salary } & \multirow[b]{2}{*}{\begin{tabular}{|c} 
Years from \\
starting to top \\
salary (lower \\
secondary)
\end{tabular}} & \multicolumn{4}{|c|}{$\begin{array}{l}\text { Salary per hour of net contact (teaching) } \\
\text { time after } 15 \text { years of experience }\end{array}$} & \multirow{2}{*}{$\begin{array}{c}\text { Ratio of salary } \\
\text { per teaching } \\
\text { hour of upper } \\
\text { secondary } \\
\text { teachers } \\
\text { to primary } \\
\text { teachers (after } \\
15 \text { years of } \\
\text { experience) }\end{array}$} \\
\hline & $\begin{array}{c}\text { Pre- } \\
\text { primary }\end{array}$ & Primary & $\begin{array}{c}\text { Lower } \\
\text { secondary, } \\
\text { general } \\
\text { programmes }\end{array}$ & $\begin{array}{c}\text { Upper } \\
\text { secondary, } \\
\text { general } \\
\text { programmes }\end{array}$ & & $\begin{array}{c}\text { Pre- } \\
\text { primary }\end{array}$ & Primary & $\begin{array}{c}\text { Lower } \\
\text { secondary, } \\
\text { general } \\
\text { programmes }\end{array}$ & $\begin{array}{c}\text { Upper } \\
\text { secondary, } \\
\text { general } \\
\text { programmes }\end{array}$ & \\
\hline & (1) & (2) & (3) & (4) & (5) & (6) & (7) & (8) & (9) & (10) \\
\hline Australia & 1.43 & 1.44 & 1.44 & 1.44 & 8 & 63 & 64 & 69 & 69 & 1.08 \\
\hline Austria $^{1}$ & 1.96 & 1.96 & 1.94 & 2.07 & 34 & 55 & 55 & 77 & 85 & 1.54 \\
\hline Belgium (Fl.) & 1.73 & 1.73 & 1.73 & 1.76 & 27 & 66 & 65 & 73 & 100 & 1.55 \\
\hline Belgium (Fr.) & 1.72 & 1.72 & 1.72 & 1.75 & 27 & 60 & 66 & 72 & 101 & 1.54 \\
\hline Canada & $\mathrm{m}$ & 1.68 & 1.68 & 1.69 & 11 & $\mathrm{~m}$ & 84 & 90 & 90 & 1.07 \\
\hline Chile & 2.09 & 2.09 & 2.09 & 2.08 & 30 & 24 & 24 & 24 & 25 & 1.06 \\
\hline Czech Republic & 1.10 & 1.22 & 1.22 & 1.22 & 27 & 15 & 22 & 29 & 31 & 1.40 \\
\hline Denmark & 1.14 & 1.15 & 1.16 & 1.19 & 12 & $\mathrm{~m}$ & 80 & 81 & 149 & 1.87 \\
\hline England & 1.70 & 1.70 & 1.70 & 1.70 & 12 & 65 & 65 & 63 & 63 & 0.97 \\
\hline Estonia & $\mathrm{m}$ & 1.31 & 1.31 & 1.31 & 7 & $\mathrm{~m}$ & 21 & 21 & 23 & 1.09 \\
\hline Finland $^{2}$ & 1.08 & 1.30 & 1.30 & 1.32 & 20 & $\mathrm{~m}$ & 59 & 72 & 84 & 1.43 \\
\hline France $^{3}$ & 1.81 & 1.81 & 1.74 & 1.73 & 29 & 36 & 36 & 56 & 57 & 1.57 \\
\hline Germany & $\mathrm{m}$ & 1.31 & 1.32 & 1.38 & 28 & $\mathrm{~m}$ & 79 & 91 & 103 & 1.30 \\
\hline Greece & 1.97 & 1.97 & 1.97 & 1.97 & 45 & 38 & 45 & 62 & 62 & 1.37 \\
\hline Hungary ${ }^{4}$ & 1.58 & 1.63 & 1.63 & 1.87 & 40 & 11 & 22 & 22 & 26 & 1.19 \\
\hline Iceland & 1.34 & 1.20 & 1.20 & 1.30 & 18 & 19 & 50 & 50 & 58 & 1.16 \\
\hline Ireland & $\mathrm{m}$ & 1.81 & 1.74 & 1.74 & 22 & $\mathrm{~m}$ & 61 & 77 & 77 & 1.26 \\
\hline Israel & 2.57 & 2.62 & 2.21 & 2.08 & 36 & 30 & 36 & 45 & 45 & 1.27 \\
\hline Italy & 1.47 & 1.47 & 1.50 & 1.57 & 35 & 36 & 44 & 59 & 60 & 1.37 \\
\hline Japan & $\mathrm{m}$ & 2.20 & 2.20 & 2.26 & 34 & $\mathrm{~m}$ & 66 & 80 & 95 & 1.43 \\
\hline Korea & 2.79 & 2.79 & 2.80 & 2.80 & 37 & 90 & 77 & 92 & 94 & 1.21 \\
\hline Luxembourg & 1.79 & 1.79 & 1.74 & 1.74 & 30 & 117 & 127 & 149 & 149 & 1.17 \\
\hline Mexico & 2.14 & 2.14 & 2.12 & $\mathrm{~m}$ & 14 & 50 & 33 & 33 & $\mathrm{~m}$ & $\mathrm{~m}$ \\
\hline Netherlands & 1.48 & 1.48 & 1.74 & 1.74 & 13 & 58 & 58 & 89 & 89 & 1.53 \\
\hline New Zealand & $\mathrm{m}$ & 1.49 & 1.50 & 1.52 & 7 & $\mathrm{~m}$ & 47 & 53 & 60 & 1.28 \\
\hline Norway & 1.15 & 1.18 & 1.18 & 1.24 & 16 & 27 & 60 & 67 & 96 & 1.60 \\
\hline Poland & 1.71 & 1.71 & 1.71 & 1.71 & 20 & 23 & 43 & 51 & 52 & 1.20 \\
\hline Portugal & 1.86 & 1.86 & 1.86 & 1.86 & 34 & 38 & 49 & 60 & 60 & 1.23 \\
\hline Scotland & 1.60 & 1.60 & 1.60 & 1.60 & 6 & 51 & 51 & 51 & 51 & 1.00 \\
\hline Slovak Republic & 1.24 & 1.52 & 1.52 & 1.52 & 32 & 10 & 19 & 24 & 25 & 1.35 \\
\hline Slovenia & 1.68 & 1.82 & 1.82 & 1.82 & 25 & 28 & 61 & 61 & 67 & 1.10 \\
\hline Spain & 1.41 & 1.41 & 1.41 & 1.41 & 38 & 48 & 48 & 66 & 68 & 1.41 \\
\hline Sweden ${ }^{4}$ & 1.16 & 1.32 & 1.33 & 1.34 & $\mathrm{a}$ & $\mathrm{m}$ & $\mathrm{m}$ & $\mathrm{m}$ & $\mathrm{m}$ & $\mathrm{m}$ \\
\hline Switzerland & $\mathrm{m}$ & $\mathrm{m}$ & $\mathrm{m}$ & $\mathrm{m}$ & 26 & $\mathrm{~m}$ & $\mathrm{~m}$ & $\mathrm{~m}$ & $\mathrm{~m}$ & $\mathrm{~m}$ \\
\hline Turkey & 1.16 & 1.16 & 1.15 & 1.15 & 27 & 25 & 38 & 56 & 56 & 1.48 \\
\hline United States ${ }^{4}$ & 1.67 & 1.61 & 1.52 & 1.57 & $\mathrm{~m}$ & $\mathrm{~m}$ & $\mathrm{~m}$ & 62 & $\mathrm{~m}$ & $\mathrm{~m}$ \\
\hline OECD average & 1.64 & 1.66 & 1.65 & 1.66 & 24 & 43 & 53 & 63 & 71 & 1.32 \\
\hline EU21 average & 1.56 & 1.59 & 1.60 & 1.62 & 26 & 44 & 54 & 64 & 72 & 1.34 \\
\hline Argentina & $\mathrm{m}$ & $\mathrm{m}$ & $\mathrm{m}$ & $\mathrm{m}$ & $\mathrm{m}$ & $\mathrm{m}$ & $\mathrm{m}$ & $\mathrm{m}$ & $\mathrm{m}$ & $\mathrm{m}$ \\
\hline Brazil & $\mathrm{m}$ & $\mathrm{m}$ & $\mathrm{m}$ & $\mathrm{m}$ & $\mathrm{m}$ & $\mathrm{m}$ & $\mathrm{m}$ & $\mathrm{m}$ & $\mathrm{m}$ & $\mathrm{m}$ \\
\hline China & $\mathrm{m}$ & $\mathrm{m}$ & $\mathrm{m}$ & $\mathrm{m}$ & $\mathrm{m}$ & $\mathrm{m}$ & $\mathrm{m}$ & $\mathrm{m}$ & $\mathrm{m}$ & $\mathrm{m}$ \\
\hline Colombia & 1.28 & 1.35 & 1.40 & 1.40 & 9 & $\mathrm{~m}$ & $\mathrm{~m}$ & $\mathrm{~m}$ & $\mathrm{~m}$ & $\mathrm{~m}$ \\
\hline India & $\mathrm{m}$ & $\mathrm{m}$ & $\mathrm{m}$ & $\mathrm{m}$ & $\mathrm{m}$ & $\mathrm{m}$ & $\mathrm{m}$ & $\mathrm{m}$ & $\mathrm{m}$ & $\mathrm{m}$ \\
\hline Indonesia & $\mathrm{m}$ & $\mathrm{m}$ & $\mathrm{m}$ & $\mathrm{m}$ & $\mathrm{m}$ & $\mathrm{m}$ & $\mathrm{m}$ & $\mathrm{m}$ & $\mathrm{m}$ & $\mathrm{m}$ \\
\hline Latvia & a & $\mathrm{a}$ & $\mathrm{a}$ & a & 10 & $\mathrm{~m}$ & $\mathrm{~m}$ & $\mathrm{~m}$ & $\mathrm{~m}$ & $\mathrm{~m}$ \\
\hline Russian Federation & $\mathrm{m}$ & $\mathrm{m}$ & $\mathrm{m}$ & $\mathrm{m}$ & $\mathrm{m}$ & $\mathrm{m}$ & $\mathrm{m}$ & $\mathrm{m}$ & $\mathrm{m}$ & $\mathrm{m}$ \\
\hline Saudi Arabia & $\mathrm{m}$ & $\mathrm{m}$ & $\mathrm{m}$ & $\mathrm{m}$ & $\mathrm{m}$ & $\mathrm{m}$ & $\mathrm{m}$ & $\mathrm{m}$ & $\mathrm{m}$ & $\mathrm{m}$ \\
\hline South Africa & $\mathrm{m}$ & $\mathrm{m}$ & $\mathrm{m}$ & $\mathrm{m}$ & $\mathrm{m}$ & $\mathrm{m}$ & $\mathrm{m}$ & $\mathrm{m}$ & $\mathrm{m}$ & $\mathrm{m}$ \\
\hline G20 average & $\mathrm{m}$ & $\mathrm{m}$ & $\mathrm{m}$ & $\mathrm{m}$ & $\mathrm{m}$ & $\mathrm{m}$ & $\mathrm{m}$ & $\mathrm{m}$ & $\mathrm{m}$ & $\mathrm{m}$ \\
\hline
\end{tabular}

Note: The definition of teachers' typical qualification is based on a broad concept, including the typical ISCED level of attainment and other criteria. Please see Box D3.2 for further details.

1. Figures for the pre-primary level refer to primary teachers (in primary schools only) teaching pre-primary classes.

2. Includes data on the majority, i.e. kindergarten teachers only for pre-primary education.

3. The typical qualification of starting teachers differ substantially from the typical qualification of all the current teachers.

4. Actual base salaries.

Source: OECD. See Annex 3 for notes (www.oecd.org/education/education-at-a-glance-19991487.htm).

Please refer to the Reader's Guide for information concerning symbols for missing data and abbreviations.

StatLink 게개 http://dx.doi.org/10.1787/888933286208 
Table D3.4. Average actual teachers' salaries (2013)

Annual average salaries (including bonuses and allowances) of teachers in public institutions, in equivalent USD converted using PPPs for private consumption, by age group and gender

\begin{tabular}{|c|c|c|c|c|}
\hline & (1) & (2) & (3) & (4) \\
\hline Q Australia & 49535 & 50737 & 51417 & 51457 \\
\hline แ̆ Austria ${ }^{1,2}$ & 55346 & 55346 & 63863 & 69632 \\
\hline Belgium (Fl.) & 49573 & 50162 & 49268 & 64471 \\
\hline Belgium (Fr.) & 46924 & 46881 & 46429 & 59375 \\
\hline Canada & $\mathrm{m}$ & $\mathrm{m}$ & $\mathrm{m}$ & $\mathrm{m}$ \\
\hline Chile & 26520 & 28573 & 28849 & 30512 \\
\hline Czech Republic & 18557 & 21479 & 21426 & 23032 \\
\hline Denmark $^{3}$ & 45867 & 55305 & 56025 & 62390 \\
\hline England & 42399 & 42399 & 46327 & 46327 \\
\hline Estonia & 12009 & 17141 & 17141 & 17141 \\
\hline Finland $^{4}$ & 31907 & 43538 & 47898 & 54128 \\
\hline France & 36440 & 36120 & 42702 & 46864 \\
\hline Germany & $\mathrm{m}$ & 60618 & 66510 & 72521 \\
\hline Greece & 26466 & 26466 & 26717 & 26717 \\
\hline Hungary & 15674 & 17062 & 17062 & 19327 \\
\hline Iceland & $\mathrm{m}$ & $\mathrm{m}$ & $\mathrm{m}$ & 41283 \\
\hline Ireland & $\mathrm{m}$ & $\mathrm{m}$ & $\mathrm{m}$ & $\mathrm{m}$ \\
\hline Israel & 34581 & 36394 & 35949 & 34527 \\
\hline Italy & 33379 & 33379 & 35757 & 38675 \\
\hline Japan & $\mathrm{m}$ & $\mathrm{m}$ & $\mathrm{m}$ & $\mathrm{m}$ \\
\hline Korea & $\mathrm{m}$ & $\mathrm{m}$ & $\mathrm{m}$ & $\mathrm{m}$ \\
\hline Luxembourg & 96140 & 96140 & 109420 & 109420 \\
\hline Mexico & $\mathrm{m}$ & $\mathrm{m}$ & $\mathrm{m}$ & $\mathrm{m}$ \\
\hline Netherlands & 49533 & 49533 & 61078 & 61078 \\
\hline New Zealand & $\mathrm{m}$ & $\mathrm{m}$ & $\mathrm{m}$ & $\mathrm{m}$ \\
\hline Norway & 43082 & 48923 & 48923 & 52072 \\
\hline Poland & 25681 & 29434 & 29912 & 29252 \\
\hline Portugal & $\mathrm{m}$ & $\mathrm{m}$ & $\mathrm{m}$ & $\mathrm{m}$ \\
\hline Scotland ${ }^{5}$ & 42012 & 42012 & 42012 & 42012 \\
\hline Slovak Republic & 14063 & 18918 & 18918 & 18859 \\
\hline Slovenia $^{6}$ & 26385 & 35044 & 35688 & 38378 \\
\hline Spain & $\mathrm{m}$ & $\mathrm{m}$ & $\mathrm{m}$ & $\mathrm{m}$ \\
\hline Sweden ${ }^{7}$ & 35290 & 38258 & 39026 & 41013 \\
\hline Switzerland & $\mathrm{m}$ & $\mathrm{m}$ & $\mathrm{m}$ & 83412 \\
\hline Turkey & $\mathrm{m}$ & $\mathrm{m}$ & $\mathrm{m}$ & $\mathrm{m}$ \\
\hline United States & 49800 & 51334 & 52343 & 54083 \\
\hline Average & 37798 & 41248 & 43626 & 47702 \\
\hline Argentina & $\mathrm{m}$ & $\mathrm{m}$ & $\mathrm{m}$ & $\mathrm{m}$ \\
\hline Brazil & $\mathrm{m}$ & $\mathrm{m}$ & $\mathrm{m}$ & $\mathrm{m}$ \\
\hline China & $\mathrm{m}$ & $\mathrm{m}$ & $\mathrm{m}$ & $\mathrm{m}$ \\
\hline Colombia & $\mathrm{m}$ & $\mathrm{m}$ & $\mathrm{m}$ & $\mathrm{m}$ \\
\hline India & $\mathrm{m}$ & $\mathrm{m}$ & $\mathrm{m}$ & $\mathrm{m}$ \\
\hline Indonesia & $\mathrm{m}$ & $\mathrm{m}$ & $\mathrm{m}$ & $\mathrm{m}$ \\
\hline Latvia & $\mathrm{m}$ & $\mathrm{m}$ & $\mathrm{m}$ & $\mathrm{m}$ \\
\hline Russian Federation 8 & 24995 & 25648 & 25648 & 25648 \\
\hline Saudi Arabia & $\mathrm{m}$ & $\mathrm{m}$ & $\mathrm{m}$ & $\mathrm{m}$ \\
\hline South Africa & $\mathrm{m}$ & $\mathrm{m}$ & $\mathrm{m}$ & $\mathrm{m}$ \\
\hline Average & $\mathrm{m}$ & $\mathrm{m}$ & $\mathrm{m}$ & $\mathrm{m}$ \\
\hline
\end{tabular}

Note: Columns showing average actual teachers' salaries, broken down by age groups and gender (i.e. columns 5-28), are available for consultation on line (see StatLink below).

1. Figures for the pre-primary level refer to primary teachers (in primary schools only) teaching pre-primary classes.

2. Also includes data on actual salaries of headmasters, deputies and assistants.

3. Also includes also data on actual salaries of teachers in early childhood educational development programmes for pre-primary education.

4. Includes data on the majority, i.e. kindergarten teachers only for pre-primary education.

5. Includes all teachers, irrespective of their age.

6. Also includes data on actual salaries of pre-school teaching assistants for pre-primary education.

7. Average actual teachers' salaries, not including bonuses and allowances.

8. Average actual teachers' salaries for all teachers, irrespective of the level of education they teach.

Source: OECD. See Annex 3 for notes (www.oecd.org/education/education-at-a-glance-19991487.htm).

Please refer to the Reader's Guide for information concerning symbols for missing data and abbreviations.

StatLink त्ञाज ht htp://dx.doi.org/10.1787/888933286213 
Table D3.5a. Trends in teachers' salaries, based on typical qualifications, between 2000 and 2013

Index of change between 2000 and 2013 in teachers' statutory salaries after 15 years of experience $(2005=100)$, by level of education, converted to constant prices using deflators for private consumption

\begin{tabular}{|c|c|c|c|c|c|c|c|c|c|c|c|c|c|c|c|c|c|c|c|c|}
\hline & \multicolumn{5}{|c|}{ Pre-primary } & \multicolumn{5}{|c|}{ Primary } & \multicolumn{5}{|c|}{$\begin{array}{l}\text { Lower secondary, } \\
\text { general programmes }\end{array}$} & \multicolumn{5}{|c|}{$\begin{array}{l}\text { Upper secondary, } \\
\text { general programmes }\end{array}$} \\
\hline & 2000 & 2005 & 2011 & 2012 & 2013 & 2000 & 2005 & 2011 & 2012 & 2013 & 2000 & 2005 & 2011 & 2012 & 2013 & 2000 & 2005 & 2011 & 2012 & 2013 \\
\hline & (1) & (2) & (8) & (9) & (10) & (11) & (12) & (18) & (19) & (20) & (21) & (22) & (28) & (29) & (30) & (31) & (32) & (38) & (39) & $(40)$ \\
\hline Australia & $\mathrm{m}$ & 100 & 105 & 106 & 112 & $\mathrm{~m}$ & 100 & 106 & 107 & 111 & $\mathrm{~m}$ & 100 & 107 & 107 & 111 & $\mathrm{~m}$ & 100 & 107 & 107 & 111 \\
\hline Austria $^{1,2}$ & $\mathrm{~m}$ & 100 & 103 & 102 & 101 & 91 & 100 & 103 & 102 & 101 & 88 & 100 & 103 & 102 & 101 & 95 & 100 & 109 & 108 & 107 \\
\hline Belgium (Fl.) & $\mathrm{m}$ & 100 & 102 & 101 & 103 & 92 & 100 & 102 & 101 & 103 & 97 & 100 & 102 & 101 & 103 & 97 & 100 & 102 & 102 & 104 \\
\hline Belgium (Fr.) & 94 & 100 & 105 & 104 & 107 & 94 & 100 & 105 & 104 & 107 & 99 & 100 & 103 & 103 & 105 & 99 & 100 & 103 & 103 & 105 \\
\hline Canada & $\mathrm{m}$ & $\mathrm{m}$ & $\mathrm{m}$ & $\mathrm{m}$ & $\mathrm{m}$ & $\mathrm{m}$ & $\mathrm{m}$ & $\mathrm{m}$ & $\mathrm{m}$ & $\mathrm{m}$ & $\mathrm{m}$ & $\mathrm{m}$ & $\mathrm{m}$ & $\mathrm{m}$ & $\mathrm{m}$ & $\mathrm{m}$ & $\mathrm{m}$ & $\mathrm{m}$ & $\mathrm{m}$ & $\mathrm{m}$ \\
\hline Chile & $\mathrm{m}$ & $\mathrm{m}$ & $\mathrm{m}$ & $\mathrm{m}$ & $\mathrm{m}$ & $\mathrm{m}$ & $\mathrm{m}$ & $\mathrm{m}$ & $\mathrm{m}$ & $\mathrm{m}$ & $\mathrm{m}$ & $\mathrm{m}$ & $\mathrm{m}$ & $\mathrm{m}$ & $\mathrm{m}$ & $\mathrm{m}$ & $\mathrm{m}$ & $\mathrm{m}$ & $\mathrm{m}$ & $\mathrm{m}$ \\
\hline Czech Republic & $\mathrm{m}$ & $\mathrm{m}$ & $\mathrm{m}$ & $\mathrm{m}$ & $\mathrm{m}$ & $\mathrm{m}$ & $\mathrm{m}$ & $\mathrm{m}$ & $\mathrm{m}$ & $\mathrm{m}$ & $\mathrm{m}$ & $\mathrm{m}$ & $\mathrm{m}$ & $\mathrm{m}$ & $\mathrm{m}$ & $\mathrm{m}$ & $\mathrm{m}$ & $\mathrm{m}$ & $\mathrm{m}$ & $\mathrm{m}$ \\
\hline Denmark & 88 & 100 & 99 & 98 & 97 & 94 & 100 & 103 & 100 & 102 & 94 & 100 & 104 & 102 & 103 & 107 & 100 & 101 & 98 & 97 \\
\hline England & 94 & 100 & 90 & 88 & 86 & 94 & 100 & 90 & 88 & 86 & 94 & 100 & 90 & 88 & 86 & 94 & 100 & 90 & 88 & 86 \\
\hline Estonia & $\mathrm{m}$ & $\mathrm{m}$ & $\mathrm{m}$ & $\mathrm{m}$ & $\mathrm{m}$ & 85 & 100 & 132 & 126 & 131 & 85 & 100 & 132 & 126 & 131 & 85 & 100 & 132 & 126 & 131 \\
\hline Finland & 92 & 100 & 109 & 108 & 106 & 87 & 100 & 110 & 109 & 107 & 88 & 100 & 106 & 104 & 103 & 92 & 100 & 106 & 107 & 105 \\
\hline France & 105 & 100 & 97 & 95 & 94 & 105 & 100 & 97 & 95 & 94 & 105 & 100 & 98 & 96 & 95 & 104 & 100 & 97 & 96 & 95 \\
\hline Germany & $\mathrm{m}$ & $\mathrm{m}$ & $\mathrm{m}$ & $\mathrm{m}$ & $\mathrm{m}$ & $\mathrm{m}$ & 100 & 106 & 107 & 108 & $\mathrm{~m}$ & 100 & 108 & 108 & 108 & $\mathrm{~m}$ & 100 & 103 & 102 & 103 \\
\hline Greece & $\mathrm{m}$ & 100 & 86 & 78 & 74 & $\mathrm{~m}$ & 100 & 86 & 78 & 74 & $\mathrm{~m}$ & 100 & 86 & 78 & 74 & $\mathrm{~m}$ & 100 & 86 & 78 & 74 \\
\hline Hungary ${ }^{3}$ & 59 & 100 & 79 & 75 & 71 & 63 & 100 & 76 & 71 & 68 & 63 & 100 & 76 & 71 & 68 & 63 & 100 & 71 & 66 & 64 \\
\hline Iceland & $\mathrm{m}$ & 100 & 93 & 97 & 89 & $\mathrm{~m}$ & 100 & 92 & 89 & 89 & $\mathrm{~m}$ & 100 & 92 & 89 & 89 & $\mathrm{~m}$ & 100 & 86 & 88 & 87 \\
\hline Ireland & $\mathrm{m}$ & $\mathrm{m}$ & $\mathrm{m}$ & $\mathrm{m}$ & $\mathrm{m}$ & 83 & 100 & 113 & 111 & 109 & 83 & 100 & 113 & 111 & 109 & 83 & 100 & 113 & 111 & 109 \\
\hline Israel & 95 & 100 & 122 & 131 & 131 & 99 & 100 & 130 & 130 & 126 & 99 & 100 & 116 & 115 & 119 & 100 & 100 & 101 & 112 & 110 \\
\hline Italy & $\mathrm{m}$ & 100 & 98 & 96 & 94 & 94 & 100 & 98 & 96 & 94 & 95 & 100 & 99 & 96 & 94 & 95 & 100 & 99 & 96 & 94 \\
\hline Japan & $\mathrm{m}$ & $\mathrm{m}$ & $\mathrm{m}$ & $\mathrm{m}$ & $\mathrm{m}$ & $\mathrm{m}$ & 100 & 93 & 93 & 94 & $\mathrm{~m}$ & 100 & 93 & 93 & 94 & $\mathrm{~m}$ & 100 & 93 & 93 & 94 \\
\hline Korea & $\mathrm{m}$ & 100 & 98 & 98 & 100 & $\mathrm{~m}$ & 100 & 95 & 96 & 97 & $\mathrm{~m}$ & 100 & 95 & 96 & 97 & $\mathrm{~m}$ & 100 & 95 & 96 & 97 \\
\hline Luxembourg & $\mathrm{m}$ & 100 & 134 & 138 & 140 & $\mathrm{~m}$ & 100 & 134 & 138 & 140 & $\mathrm{~m}$ & 100 & 110 & 113 & 115 & $\mathrm{~m}$ & 100 & 110 & 113 & 115 \\
\hline Mexico & 87 & 100 & 107 & 108 & 109 & 87 & 100 & 107 & 108 & 109 & 87 & 100 & 107 & 109 & 109 & $\mathrm{~m}$ & $\mathrm{~m}$ & $\mathrm{~m}$ & $\mathrm{~m}$ & $\mathrm{~m}$ \\
\hline Netherlands & $\mathrm{m}$ & $\mathrm{m}$ & $\mathrm{m}$ & $\mathrm{m}$ & $\mathrm{m}$ & $\mathrm{m}$ & $\mathrm{m}$ & $\mathrm{m}$ & $\mathrm{m}$ & $\mathrm{m}$ & $\mathrm{m}$ & $\mathrm{m}$ & $\mathrm{m}$ & $\mathrm{m}$ & $\mathrm{m}$ & $\mathrm{m}$ & $\mathrm{m}$ & $\mathrm{m}$ & $\mathrm{m}$ & $\mathrm{m}$ \\
\hline New Zealand & $\mathrm{m}$ & $\mathrm{m}$ & $\mathrm{m}$ & $\mathrm{m}$ & $\mathrm{m}$ & $\mathrm{m}$ & $\mathrm{m}$ & $\mathrm{m}$ & $\mathrm{m}$ & $\mathrm{m}$ & $\mathrm{m}$ & $\mathrm{m}$ & $\mathrm{m}$ & $\mathrm{m}$ & $\mathrm{m}$ & $\mathrm{m}$ & $\mathrm{m}$ & $\mathrm{m}$ & $\mathrm{m}$ & $\mathrm{m}$ \\
\hline Norway & $\mathrm{m}$ & 100 & 116 & 116 & 120 & $\mathrm{~m}$ & 100 & 110 & 111 & 115 & $\mathrm{~m}$ & 100 & 110 & 111 & 115 & $\mathrm{~m}$ & 100 & 112 & 112 & 117 \\
\hline Poland & $\mathrm{m}$ & 100 & 117 & 120 & 122 & $\mathrm{~m}$ & 100 & 117 & 120 & 122 & $\mathrm{~m}$ & 100 & 117 & 120 & 122 & $\mathrm{~m}$ & 100 & 117 & 120 & 122 \\
\hline Portugal & $\mathrm{m}$ & 100 & 100 & 86 & 85 & $\mathrm{~m}$ & 100 & 100 & 86 & 85 & $\mathrm{~m}$ & 100 & 100 & 86 & 85 & $\mathrm{~m}$ & 100 & 100 & 86 & 85 \\
\hline Scotland & 50 & 100 & 96 & 93 & 91 & 81 & 100 & 96 & 93 & 91 & 81 & 100 & 96 & 93 & 91 & 81 & 100 & 96 & 93 & 91 \\
\hline Slovak Republic & $\mathrm{m}$ & $\mathrm{m}$ & $\mathrm{m}$ & $\mathrm{m}$ & $\mathrm{m}$ & $\mathrm{m}$ & $\mathrm{m}$ & $\mathrm{m}$ & $\mathrm{m}$ & $\mathrm{m}$ & $\mathrm{m}$ & $\mathrm{m}$ & $\mathrm{m}$ & $\mathrm{m}$ & $\mathrm{m}$ & $\mathrm{m}$ & $\mathrm{m}$ & $\mathrm{m}$ & $\mathrm{m}$ & $\mathrm{m}$ \\
\hline Slovenia & $\mathrm{m}$ & $\mathrm{m}$ & $\mathrm{m}$ & $\mathrm{m}$ & $\mathrm{m}$ & 86 & 100 & 109 & 105 & 100 & 86 & 100 & 109 & 105 & 100 & 86 & 100 & 109 & 105 & 100 \\
\hline Spain & $\mathrm{m}$ & 100 & 102 & 98 & 95 & $\mathrm{~m}$ & 100 & 102 & 98 & 95 & $\mathrm{~m}$ & 100 & 100 & 95 & 92 & $\mathrm{~m}$ & 100 & 100 & 95 & 92 \\
\hline Sweden ${ }^{3}$ & $\mathrm{~m}$ & 100 & 109 & $\mathrm{~m}$ & 114 & $\mathrm{~m}$ & 100 & 102 & $\mathrm{~m}$ & 109 & $\mathrm{~m}$ & 100 & 103 & $\mathrm{~m}$ & 108 & $\mathrm{~m}$ & 100 & 101 & $\mathrm{~m}$ & 105 \\
\hline Switzerland & $\mathrm{m}$ & $\mathrm{m}$ & $\mathrm{m}$ & $\mathrm{m}$ & $\mathrm{m}$ & $\mathrm{m}$ & $\mathrm{m}$ & $\mathrm{m}$ & $\mathrm{m}$ & $\mathrm{m}$ & $\mathrm{m}$ & $\mathrm{m}$ & $\mathrm{m}$ & $\mathrm{m}$ & $\mathrm{m}$ & $\mathrm{m}$ & $\mathrm{m}$ & $\mathrm{m}$ & $\mathrm{m}$ & $\mathrm{m}$ \\
\hline Turkey & 99 & 100 & 112 & 112 & 114 & 99 & 100 & 112 & 112 & 114 & 99 & 100 & 109 & 110 & 112 & 99 & 100 & 109 & 110 & 112 \\
\hline United States $^{3}$ & 98 & 100 & $\mathrm{~m}$ & 119 & 120 & 82 & 100 & 93 & 98 & 98 & 103 & 100 & 107 & 110 & 110 & 98 & 100 & 102 & 103 & 103 \\
\hline OECD average & 87 & 100 & 103 & 103 & 103 & 89 & 100 & 104 & 103 & 103 & 91 & 100 & 103 & 101 & 102 & 93 & 100 & 102 & 100 & 101 \\
\hline $\begin{array}{l}\text { OECD average for } \\
\text { countries with data } \\
\text { available for all } \\
\text { reference years }\end{array}$ & 86 & 100 & 101 & 101 & 101 & 89 & 100 & 104 & 103 & 102 & 91 & 100 & 104 & 103 & 102 & 87 & 94 & 96 & 96 & 95 \\
\hline $\begin{array}{l}\text { EU21 average for } \\
\text { countries with data } \\
\text { available for all } \\
\text { reference years }\end{array}$ & 83 & 100 & 96 & 95 & 93 & 89 & 100 & 102 & 100 & 100 & 89 & 100 & 102 & 100 & 99 & 91 & 100 & 102 & 100 & 99 \\
\hline
\end{tabular}

\begin{tabular}{|c|c|c|c|c|c|c|c|c|c|c|c|c|c|c|c|c|c|c|c|c|}
\hline $\begin{array}{l}\text { Argentina } \\
\text { Brazil }\end{array}$ & $\begin{array}{l}\mathrm{m} \\
\mathrm{m}\end{array}$ & $\begin{array}{l}\mathrm{m} \\
\mathrm{m}\end{array}$ & $\begin{array}{l}\mathrm{m} \\
\mathrm{m}\end{array}$ & $\begin{array}{l}\mathrm{m} \\
\mathrm{m}\end{array}$ & $\begin{array}{l}\mathrm{m} \\
\mathrm{m}\end{array}$ & $\begin{array}{l}\mathrm{m} \\
\mathrm{m}\end{array}$ & $\begin{array}{l}\mathrm{m} \\
\mathrm{m}\end{array}$ & $\begin{array}{l}\mathrm{m} \\
\mathrm{m}\end{array}$ & $\begin{array}{l}\mathrm{m} \\
\mathrm{m}\end{array}$ & $\begin{array}{l}\mathrm{m} \\
\mathrm{m}\end{array}$ & $\begin{array}{l}\mathrm{m} \\
\mathrm{m}\end{array}$ & $\begin{array}{l}\mathrm{m} \\
\mathrm{m}\end{array}$ & $\begin{array}{l}\mathrm{m} \\
\mathrm{m}\end{array}$ & $\begin{array}{l}\mathrm{m} \\
\mathrm{m}\end{array}$ & $\begin{array}{l}\mathrm{m} \\
\mathrm{m}\end{array}$ & $\begin{array}{l}\mathrm{m} \\
\mathrm{m}\end{array}$ & $\begin{array}{l}\mathrm{m} \\
\mathrm{m}\end{array}$ & $\begin{array}{l}\mathrm{m} \\
\mathrm{m}\end{array}$ & $\begin{array}{l}\mathrm{m} \\
\mathrm{m}\end{array}$ & $\begin{array}{l}\mathrm{m} \\
\mathrm{m}\end{array}$ \\
\hline China & $\mathrm{m}$ & $\mathrm{m}$ & $\mathrm{m}$ & $\mathrm{m}$ & $\mathrm{m}$ & $\mathrm{m}$ & $\mathrm{m}$ & $\mathrm{m}$ & $\mathrm{m}$ & $\mathrm{m}$ & $\mathrm{m}$ & $\mathrm{m}$ & $\mathrm{m}$ & $\mathrm{m}$ & $\mathrm{m}$ & $\mathrm{m}$ & $\mathrm{m}$ & $\mathrm{m}$ & $\mathrm{m}$ & $\mathrm{m}$ \\
\hline Colombia & $\mathrm{m}$ & $\mathrm{m}$ & $\mathrm{m}$ & $\mathrm{m}$ & $\mathrm{m}$ & $\mathrm{m}$ & $\mathrm{m}$ & $\mathrm{m}$ & $\mathrm{m}$ & $\mathrm{m}$ & $\mathrm{m}$ & $\mathrm{m}$ & $\mathrm{m}$ & $\mathrm{m}$ & $\mathrm{m}$ & $\mathrm{m}$ & $\mathrm{m}$ & $\mathrm{m}$ & $\mathrm{m}$ & $\mathrm{m}$ \\
\hline $\begin{array}{l}\text { India } \\
\text { Indonesia }\end{array}$ & $\begin{array}{l}\mathrm{m} \\
\mathrm{m}\end{array}$ & $\begin{array}{l}\mathrm{m} \\
\mathrm{m}\end{array}$ & $\begin{array}{l}\mathrm{m} \\
\mathrm{m}\end{array}$ & $\begin{array}{l}\mathrm{m} \\
\mathrm{m}\end{array}$ & $\begin{array}{l}\mathrm{m} \\
\mathrm{m}\end{array}$ & $\begin{array}{l}\mathrm{m} \\
\mathrm{m}\end{array}$ & $\begin{array}{l}\mathrm{m} \\
\mathrm{m}\end{array}$ & $\begin{array}{l}\mathrm{m} \\
\mathrm{m}\end{array}$ & $\begin{array}{l}\mathrm{m} \\
\mathrm{m}\end{array}$ & $\begin{array}{l}\mathrm{m} \\
\mathrm{m}\end{array}$ & $\begin{array}{l}\mathrm{m} \\
\mathrm{m}\end{array}$ & $\begin{array}{l}\mathrm{m} \\
\mathrm{m}\end{array}$ & $\begin{array}{l}\mathrm{m} \\
\mathrm{m}\end{array}$ & $\begin{array}{l}\mathrm{m} \\
\mathrm{m}\end{array}$ & $\begin{array}{l}\mathrm{m} \\
\mathrm{m}\end{array}$ & $\begin{array}{l}\mathrm{m} \\
\mathrm{m}\end{array}$ & $\begin{array}{l}\mathrm{m} \\
\mathrm{m}\end{array}$ & $\begin{array}{l}\mathrm{m} \\
\mathrm{m}\end{array}$ & $\begin{array}{l}\mathrm{m} \\
\mathrm{m}\end{array}$ & $\begin{array}{l}\mathrm{m} \\
\mathrm{m}\end{array}$ \\
\hline Latvia & 74 & 100 & 125 & 128 & $\mathrm{~m}$ & 74 & 100 & 125 & 128 & $\mathrm{~m}$ & 74 & 100 & 125 & 128 & $\mathrm{~m}$ & 74 & 100 & 125 & 128 & $\mathrm{~m}$ \\
\hline Russian Federation & $\mathrm{m}$ & $\mathrm{m}$ & $\mathrm{m}$ & $\mathrm{m}$ & $\mathrm{m}$ & $\mathrm{m}$ & $\mathrm{m}$ & $\mathrm{m}$ & $\mathrm{m}$ & $\mathrm{m}$ & $\mathrm{m}$ & $\mathrm{m}$ & $\mathrm{m}$ & $\mathrm{m}$ & $\mathrm{m}$ & $\mathrm{m}$ & $\mathrm{m}$ & $\mathrm{m}$ & $\mathrm{m}$ & $\mathrm{m}$ \\
\hline Saudi Arabia & $\mathrm{m}$ & $\mathrm{m}$ & $\mathrm{m}$ & $\mathrm{m}$ & $\mathrm{m}$ & $\mathrm{m}$ & $\mathrm{m}$ & $\mathrm{m}$ & $\mathrm{m}$ & $\mathrm{m}$ & $\mathrm{m}$ & $\mathrm{m}$ & $\mathrm{m}$ & $\mathrm{m}$ & $\mathrm{m}$ & $\mathrm{m}$ & $\mathrm{m}$ & $\mathrm{m}$ & $\mathrm{m}$ & $\mathrm{m}$ \\
\hline South Africa & $\mathrm{m}$ & $\mathrm{m}$ & $\mathrm{m}$ & $\mathrm{m}$ & $\mathrm{m}$ & $\mathrm{m}$ & $\mathrm{m}$ & $\mathrm{m}$ & $\mathrm{m}$ & $\mathrm{m}$ & $\mathrm{m}$ & $\mathrm{m}$ & $\mathrm{m}$ & $\mathrm{m}$ & $\mathrm{m}$ & $\mathrm{m}$ & $\mathrm{m}$ & $\mathrm{m}$ & $\mathrm{m}$ & $\mathrm{m}$ \\
\hline G20 average & $\mathrm{m}$ & $\mathrm{m}$ & $\mathrm{m}$ & $\mathrm{m}$ & $\mathrm{m}$ & $\mathrm{m}$ & $\mathrm{m}$ & $\mathrm{m}$ & $\mathrm{m}$ & $\mathrm{m}$ & $\mathrm{m}$ & $\mathrm{m}$ & $\mathrm{m}$ & $\mathrm{m}$ & $\mathrm{m}$ & $\mathrm{m}$ & $\mathrm{m}$ & $\mathrm{m}$ & $\mathrm{m}$ & $\mathrm{m}$ \\
\hline
\end{tabular}

Notes: Years 2006, 2007, 2008, 2009, 2010 (i.e. columns 3-7; 13-17; 23-27; 33-37) are available for consultation on line (see StatLink below). The definition of teachers' typical qualification is based on a broad concept, including the typical ISCED level of attainment and other criteria. Please see Box D3.2 for further details.

1. Break in time series following methodological changes in 2007 for upper secondary education.

2. Figures for the pre-primary level refer to primary teachers (in primary schools only) teaching pre-primary classes.

3. Actual base salaries.

Source: OECD. See Annex 3 for notes (www.oecd.org/education/education-at-a-glance-19991487.htm).

Please refer to the Reader's Guide for information concerning symbols for missing data and abbreviations.

StatLink 젝ㄴ http://dx.doi.org/10.1787/888933286220 


\section{Table D3.7c. [1/4] Teachers' tasks and other criteria related to teachers' base salaries and additional payments, lower secondary level (2013)}

Criteria for base salary and additional payments awarded to teachers in public institutions

\begin{tabular}{|c|c|c|c|c|c|c|}
\hline \multicolumn{7}{|c|}{ Tasks } \\
\hline Teaching & $\begin{array}{l}\text { Individual } \\
\text { planning } \\
\text { or preparation } \\
\text { of lessons } \\
\text { either at school } \\
\text { or elsewhere }\end{array}$ & $\begin{array}{c}\text { Marking/ } \\
\text { correcting } \\
\text { of student work }\end{array}$ & $\begin{array}{c}\text { General } \\
\text { administrative } \\
\text { work (including } \\
\text { communication, } \\
\text { paperwork and } \\
\text { other clerical } \\
\text { duties undertaken } \\
\text { as part of the job) }\end{array}$ & $\begin{array}{l}\text { Communication } \\
\text { and co-operation } \\
\text { with parents } \\
\text { or guardians }\end{array}$ & $\begin{array}{l}\text { Supervision } \\
\text { of students } \\
\text { during breaks }\end{array}$ & $\begin{array}{c}\text { Team work } \\
\text { and dialogue } \\
\text { with colleagues } \\
\text { at school or } \\
\text { elsewhere }\end{array}$ \\
\hline
\end{tabular}

\begin{tabular}{|c|c|c|c|c|c|c|c|c|c|c|c|c|c|c|c|c|c|c|c|c|c|}
\hline & & (2) & (v) & $(x)$ & (1) & $(8)$ & (7) & (8) & (9) & (10) & (11) & (12) & (13) & (14) & (15) & (16) & (17) & (18) & (19) & (20) & \\
\hline Australia & $\mathrm{m}$ & $\mathrm{m}$ & $\mathrm{m}$ & $\mathrm{m}$ & $\mathrm{m}$ & $\mathrm{m}$ & $\mathrm{m}$ & $\mathrm{m}$ & $\mathrm{m}$ & $\mathrm{m}$ & $\mathrm{m}$ & $\mathrm{m}$ & $\mathrm{m}$ & $\mathrm{m}$ & $\mathrm{m}$ & $\mathrm{m}$ & $\mathrm{m}$ & $\mathrm{m}$ & $\mathrm{m}$ & $\mathrm{m}$ & $\mathrm{m}$ \\
\hline Austria & $\mathrm{m}$ & $\mathrm{m}$ & $\mathrm{m}$ & $\mathrm{m}$ & $\mathrm{m}$ & $\mathrm{m}$ & $\mathrm{m}$ & $\mathrm{m}$ & $\mathrm{m}$ & $\mathrm{m}$ & $\mathrm{m}$ & $\mathrm{m}$ & $\mathrm{m}$ & $\mathrm{m}$ & $\mathrm{m}$ & $\mathrm{m}$ & $\mathrm{m}$ & $\mathrm{m}$ & $\mathrm{m}$ & $\mathrm{m}$ & $\mathrm{m}$ \\
\hline Belgium (Fl.) & Yes & Yes & 1 & Yes & No & $\mathrm{a}$ & DoS & No & $\mathrm{a}$ & DoS & No & a & DoS & No & $\mathrm{a}$ & Dos & No & $\mathrm{a}$ & Dos & No & a \\
\hline Belgium (Fr.) & Yes & No & $a$ & Yes & No & $\mathrm{a}$ & Yes & No & $\mathrm{a}$ & Yes & No & a & Yes & No & $\mathrm{a}$ & No & No & $\mathrm{a}$ & No & No & a \\
\hline Canada & $\mathrm{m}$ & $\mathrm{m}$ & $\mathrm{m}$ & $\mathrm{m}$ & $\mathrm{m}$ & $\mathrm{m}$ & $\mathrm{m}$ & $\mathrm{m}$ & $\mathrm{m}$ & $\mathrm{m}$ & $\mathrm{m}$ & $\mathrm{m}$ & $\mathrm{m}$ & $\mathrm{m}$ & $\mathrm{m}$ & $\mathrm{m}$ & $\mathrm{m}$ & $\mathrm{m}$ & $\mathrm{m}$ & $\mathrm{m}$ & $\mathrm{m}$ \\
\hline Chile & $\mathrm{m}$ & $\mathrm{m}$ & $\mathrm{m}$ & $\mathrm{m}$ & $\mathrm{m}$ & $\mathrm{m}$ & $\mathrm{m}$ & $\mathrm{m}$ & $\mathrm{m}$ & $\mathrm{m}$ & $\mathrm{m}$ & $\mathrm{m}$ & $\mathrm{m}$ & $\mathrm{m}$ & $\mathrm{m}$ & $\mathrm{m}$ & $\mathrm{m}$ & $\mathrm{m}$ & $\mathrm{m}$ & $\mathrm{m}$ & $\mathrm{m}$ \\
\hline Czech Republic & Yes & Yes & 1 & No & No & $\mathrm{a}$ & No & No & $\mathrm{a}$ & DoS & Yes & 5 & No & No & $\mathrm{a}$ & Dos & No & $\mathrm{a}$ & Dos & No & $\mathrm{a}$ \\
\hline Denmark & Yes & No & $a$ & Yes & No & $\mathrm{a}$ & Yes & No & $\mathrm{a}$ & Yes & No & a & Yes & No & a & $\mathrm{m}$ & No & $\mathrm{a}$ & Yes & No & $\mathrm{a}$ \\
\hline England & Yes & Yes & 1 & Yes & Yes & 1 & Yes & Yes & 1 & DoS & DoS & 1 & Yes & Yes & 1 & Dos & DoS & 1 & Yes & Yes & 1 \\
\hline Estonia & Yes & Yes & 1 & Yes & Yes & 1 & Yes & Yes & 1 & Yes & Yes & 1 & Yes & Yes & 1 & Dos & Yes & 3 & Yes & Yes & 1 \\
\hline Finland & Yes & Yes & 1 & Yes & Yes & 1 & Yes & Yes & 1 & Yes & Yes & 1 & Yes & Yes & 1 & Dos & Yes & 1 & Yes & Yes & 1 \\
\hline France & Yes & Yes & 1 & No & Yes & 1 & Yes & Yes & 1 & Yes & Yes & 1 & Yes & Yes & 1 & $a$ & $\mathrm{a}$ & a & No & No & a \\
\hline Germany & Yes & Yes & 1 & Yes & Yes & 1 & Yes & Yes & 1 & DoS & Yes & 1 & Yes & Yes & 1 & DoS & Yes & 1 & No & Yes & 1 \\
\hline Greece & Yes & Yes & 1 & Yes & Yes & 1 & Yes & Yes & 1 & Yes & Yes & 1 & Yes & Yes & 1 & $\mathrm{~m}$ & $\mathrm{~m}$ & 1 & Yes & Yes & 1 \\
\hline Hungary & Yes & No & $a$ & Yes & No & a & DoS & No & a & DoS & No & a & Yes & No & $\mathrm{a}$ & No & No & $\mathrm{a}$ & Yes & No & $a$ \\
\hline Iceland & Yes & Yes & 1 & Yes & Yes & 1 & Yes & Yes & 1 & DoS & DoS & 5 & DoS & No & $\mathrm{m}$ & No & No & $\mathrm{m}$ & Dos & Yes & 2 \\
\hline Ireland & Yes & Yes & 1 & Yes & Yes & 1 & Yes & Yes & 1 & Yes & Yes & 1 & Yes & Yes & 1 & Yes & Yes & 1 & Yes & Yes & 1 \\
\hline Israel & Yes & Yes & 1 & Yes & Yes & 1 & Yes & Yes & 1 & Yes & Yes & 1 & Yes & Yes & 1 & Yes & Yes & 1 & Yes & Yes & 1 \\
\hline Italy & Yes & $\mathrm{a}$ & $\mathrm{a}$ & Yes & $\mathrm{a}$ & a & Yes & $\mathrm{a}$ & $\mathrm{a}$ & Yes & $a$ & a & Yes & a & a & Yes & a & $\mathrm{a}$ & Yes & a & a \\
\hline Japan & Yes & Yes & 1 & Yes & Yes & 1 & Yes & Yes & 1 & Yes & Yes & 1 & Yes & Yes & 1 & Yes & Yes & 1 & Yes & Yes & 1 \\
\hline Korea & Yes & Yes & 1 & Yes & Yes & 1 & Yes & Yes & 1 & Yes & Yes & 1 & Yes & Yes & 1 & Yes & Yes & 1 & Yes & Yes & 1 \\
\hline Luxembourg & Yes & Yes & 1 & Yes & Yes & 1 & Yes & Yes & 1 & Yes & Yes & 1 & Yes & Yes & 1 & Yes & Yes & 1 & Yes & Yes & 1 \\
\hline Mexico & $\mathrm{m}$ & $\mathrm{m}$ & $\mathrm{m}$ & $\mathrm{m}$ & $\mathrm{m}$ & $\mathrm{m}$ & $\mathrm{m}$ & $\mathrm{m}$ & $\mathrm{m}$ & $\mathrm{m}$ & $\mathrm{m}$ & $\mathrm{m}$ & $\mathrm{m}$ & $\mathrm{m}$ & $\mathrm{m}$ & $\mathrm{m}$ & $\mathrm{m}$ & $\mathrm{m}$ & $\mathrm{m}$ & $\mathrm{m}$ & $\mathrm{m}$ \\
\hline Netherlands & Dos & Yes & 1 & Dos & Yes & 1 & DoS & Yes & 1 & DoS & Yes & 1 & DoS & Yes & 1 & Dos & Yes & 1 & Dos & Yes & 1 \\
\hline New Zealand & $\mathrm{m}$ & $\mathrm{m}$ & $\mathrm{m}$ & $\mathrm{m}$ & $\mathrm{m}$ & $\mathrm{m}$ & $\mathrm{m}$ & $\mathrm{m}$ & $\mathrm{m}$ & $\mathrm{m}$ & $\mathrm{m}$ & $\mathrm{m}$ & $\mathrm{m}$ & $\mathrm{m}$ & $\mathrm{m}$ & $\mathrm{m}$ & $\mathrm{m}$ & $\mathrm{m}$ & $\mathrm{m}$ & $\mathrm{m}$ & $\mathrm{m}$ \\
\hline Norway & Yes & No & $\mathrm{a}$ & Yes & No & a & Yes & No & a & Yes & No & $\mathrm{a}$ & Yes & No & a & Dos & No & $\mathrm{a}$ & Dos & No & a \\
\hline Poland & Yes & Yes & 1 & Yes & No & a & Yes & No & a & Yes & No & a & Yes & No & a & Yes & No & $\mathrm{a}$ & Yes & No & a \\
\hline Portugal & Yes & No & $\mathrm{a}$ & Yes & No & $\mathrm{a}$ & Yes & No & a & Yes & No & a & Yes & No & a & No & No & $\mathrm{a}$ & Yes & No & a \\
\hline Scotland & Yes & Yes & 1 & Yes & Yes & 1 & Yes & Yes & 1 & No & No & a & Yes & Yes & 1 & No & No & $\mathrm{a}$ & Yes & Yes & 1 \\
\hline Slovak Rep & Yes & Yes & 1 & Yes & No & $\mathrm{a}$ & Yes & No & a & Yes & No & a & Yes & No & a & Yes & No & $a$ & Yes & No & a \\
\hline Slovenia & Yes & Yes & 1 & Yes & Yes & 1 & Yes & Yes & 1 & Yes & Yes & 1 & Yes & Yes & 1 & DoS & Yes & 1 & Yes & Yes & 1 \\
\hline Spain & Yes & $\mathrm{a}$ & a & Yes & $\mathrm{a}$ & a & Yes & a & a & Yes & $\mathrm{a}$ & a & Yes & $\mathrm{a}$ & a & a & $\mathrm{a}$ & $\mathrm{a}$ & Yes & a & a \\
\hline Sweden & Yes & DoS & $\mathrm{a}$ & Yes & DoS & a & Yes & Dos & $\mathrm{a}$ & Yes & DoS & $\mathrm{a}$ & Yes & DoS & a & Dos & DoS & $\mathrm{a}$ & Yes & DoS & a \\
\hline Switzerland & Yes & $\mathrm{m}$ & $\mathrm{m}$ & $\mathrm{m}$ & $\mathrm{m}$ & $\mathrm{m}$ & $\mathrm{m}$ & $\mathrm{m}$ & $\mathrm{m}$ & $\mathrm{m}$ & $\mathrm{m}$ & $\mathrm{m}$ & $\mathrm{m}$ & $\mathrm{m}$ & $\mathrm{m}$ & $\mathrm{m}$ & $\mathrm{m}$ & $\mathrm{m}$ & $\mathrm{m}$ & $\mathrm{m}$ & $\mathrm{m}$ \\
\hline Turkey & Yes & Yes & 1 & Yes & Yes & 4 & Yes & No & $\mathrm{a}$ & Yes & No & $\mathrm{a}$ & Yes & No & $\mathrm{a}$ & Yes & No & $\mathrm{a}$ & Yes & No & $\mathrm{a}$ \\
\hline
\end{tabular}

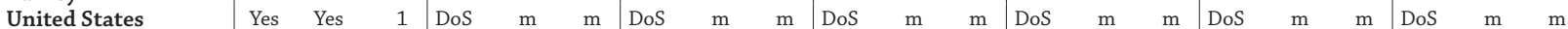

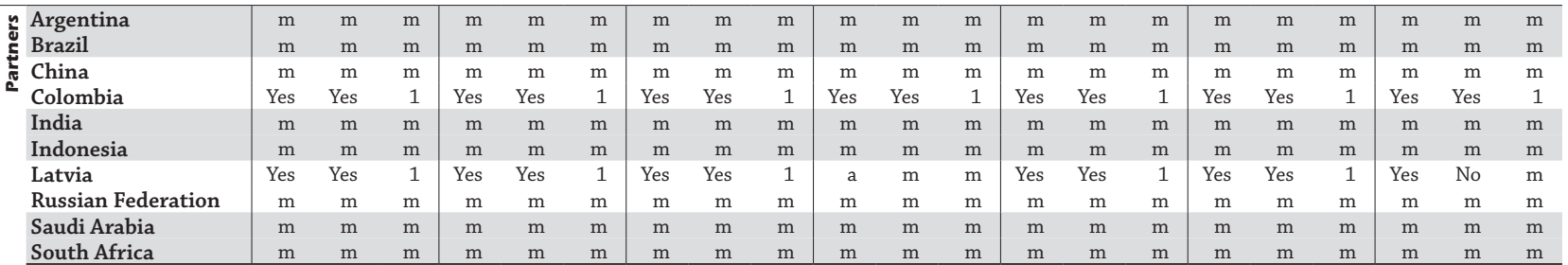

Is the performance mandatory for teachers? Yes: Yes, it is mandatory

DoS: Yes, at the discretion of individual schools

DoS: At the discretion of individual schools

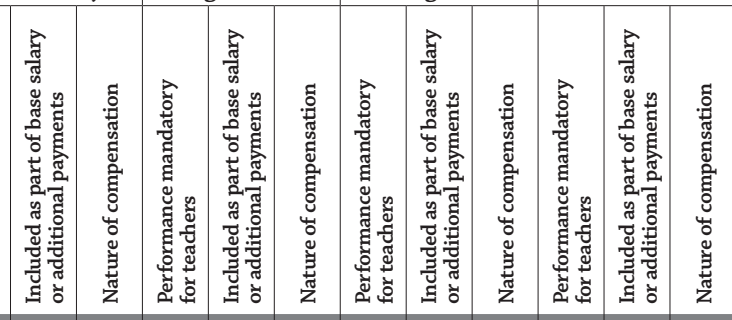


Table D3.7c. [2/4] Teachers' tasks and other criteria related to teachers' base salaries and additional payments, lower secondary level (2013)

Criteria for base salary and additional payments awarded to teachers in public institutions

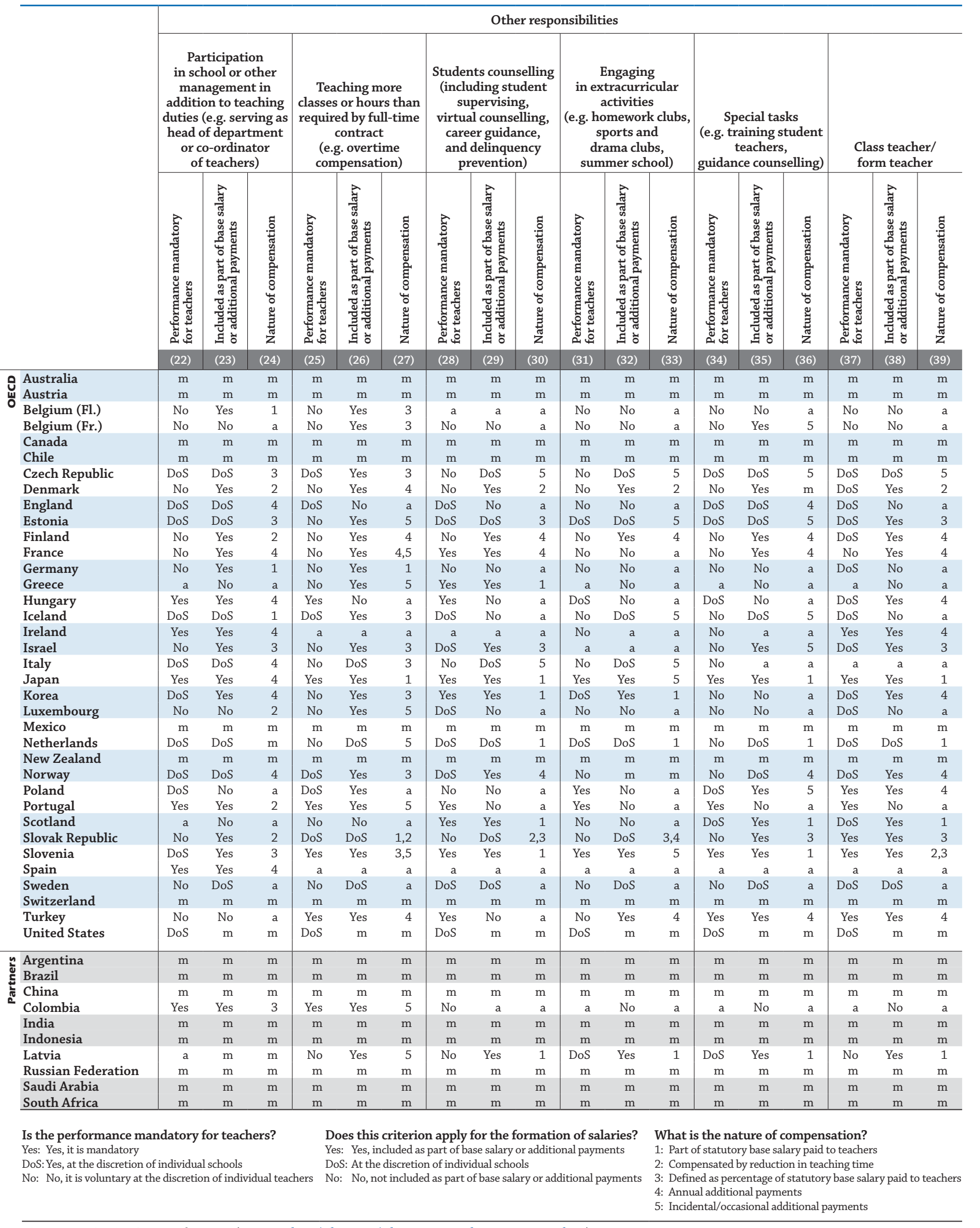

Source: OECD. See Annex 3 for notes (www.oecd.org/education/education-at-a-glance-19991487.htm)

Please refer to the Reader's Guide for information concerning symbols for missing data and abbreviations.

StatLink 젶ㄴ http://dx.doi.org/10.1787/888933286236 


\section{Table D3.7c. [3/4] Teachers' tasks and other criteria related to teachers' base salaries and additional payments, lower secondary level (2013)}

Criteria for base salary and additional payments awarded to teachers in public institutions

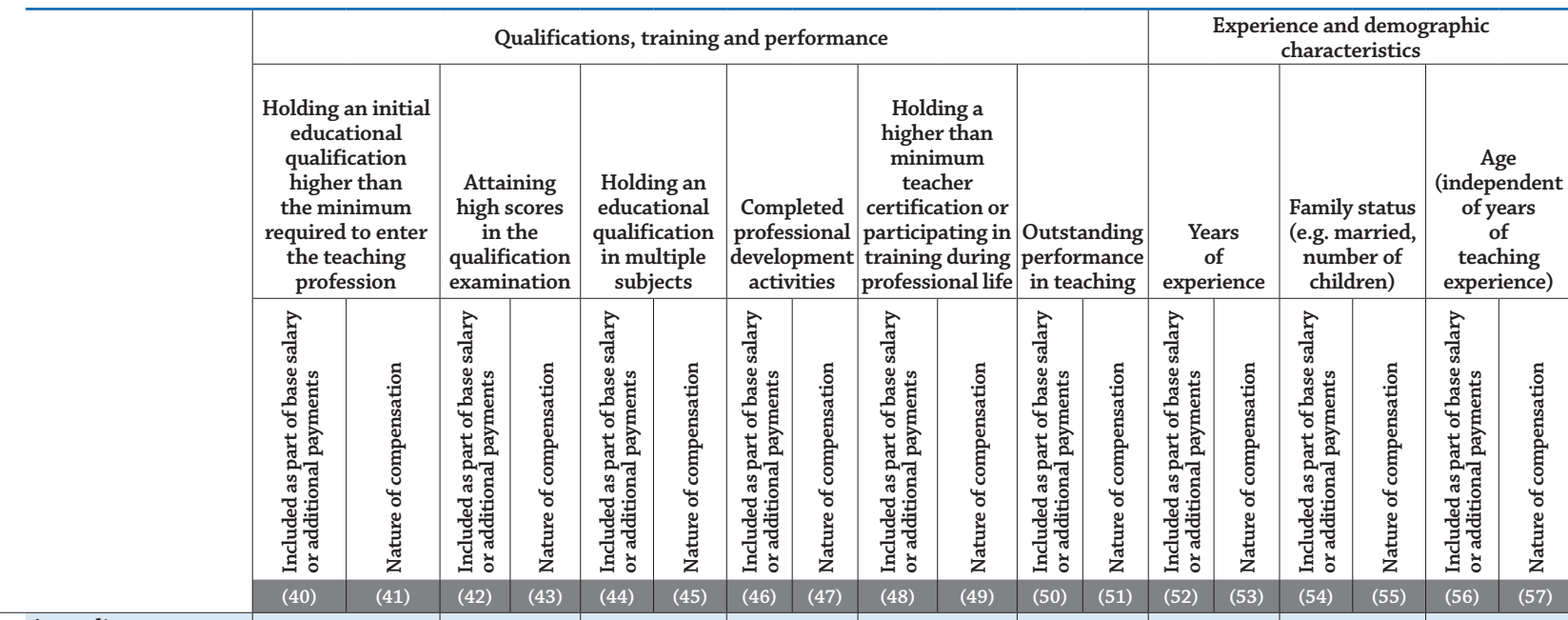

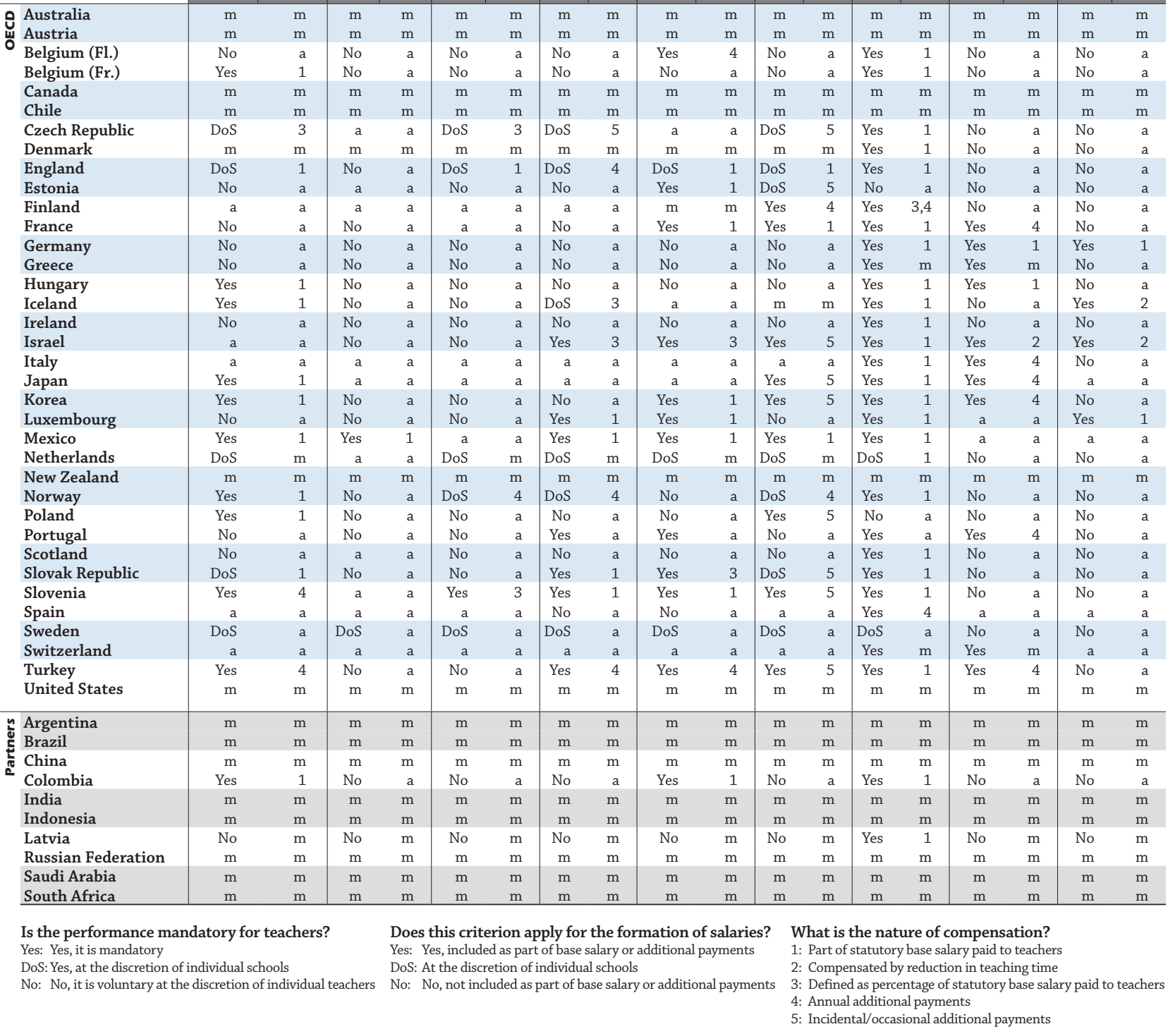

Source: OECD. See Annex 3 for notes (www.oecd.org/education/education-at-a-glance-19991487.htm).

Please refer to the Reader's Guide for information concerning symbols for missing data and abbreviations.

StatLink 젶ㄴ http://dx.doi.org/10.1787/888933286236 
Table D3.7c. [4/4] Teachers' tasks and other criteria related to teachers' base salaries and additional payments, lower secondary level (2013)

Criteria for base salary and additional payments awarded to teachers in public institutions

\begin{tabular}{|c|c|c|c|c|c|c|c|c|c|c|}
\hline & \multicolumn{6}{|c|}{ Teaching conditions } & \multicolumn{4}{|c|}{ Benefits } \\
\hline & \multicolumn{2}{|c|}{$\begin{array}{l}\text { Teaching courses } \\
\text { ina particular field } \\
\text { (e.g. mathematics or } \\
\text { science) }\end{array}$} & \multicolumn{2}{|c|}{$\begin{array}{l}\text { Teaching students with } \\
\text { special educational needs } \\
\text { (in regular schools) }\end{array}$} & \multicolumn{2}{|c|}{$\begin{array}{c}\text { Teaching in a } \\
\text { disadvantaged, } \\
\text { remote or high cost area } \\
\text { (location allowance) }\end{array}$} & \multicolumn{2}{|c|}{$\begin{array}{c}\text { Holiday benefits } \\
\begin{array}{c}\text { (e.g. for religious and/or } \\
\text { official holidays) }\end{array}\end{array}$} & \multicolumn{2}{|c|}{ Thirteen month benefits } \\
\hline & 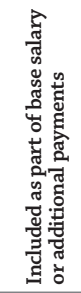 & 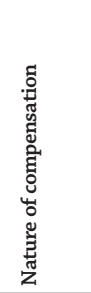 & 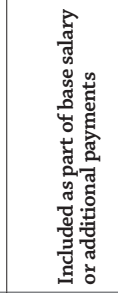 & 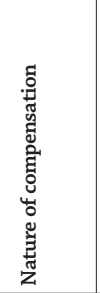 & 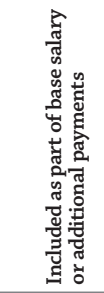 & 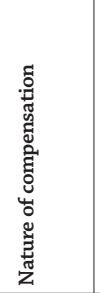 & 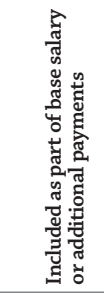 & 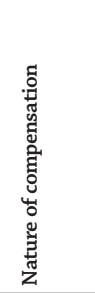 & 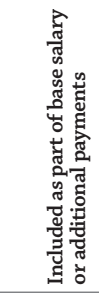 & 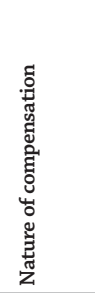 \\
\hline & (58) & $(59)$ & $(60)$ & $(61)$ & $(62)$ & (63) & $(64)$ & $(65)$ & $(66)$ & $(67)$ \\
\hline $\begin{array}{l}\text { Australia } \\
\text { Austria }\end{array}$ & $\begin{array}{l}\mathrm{m} \\
\mathrm{m}\end{array}$ & $\begin{array}{l}\mathrm{m} \\
\mathrm{m}\end{array}$ & $\begin{array}{l}\mathrm{m} \\
\mathrm{m}\end{array}$ & $\begin{array}{l}\mathrm{m} \\
\mathrm{m}\end{array}$ & $\begin{array}{l}\mathrm{m} \\
\mathrm{m}\end{array}$ & $\begin{array}{l}\mathrm{m} \\
\mathrm{m}\end{array}$ & $\begin{array}{l}\mathrm{m} \\
\mathrm{m}\end{array}$ & $\begin{array}{l}\mathrm{m} \\
\mathrm{m}\end{array}$ & $\begin{array}{l}\mathrm{m} \\
\mathrm{m}\end{array}$ & $\begin{array}{l}\mathrm{m} \\
\mathrm{m}\end{array}$ \\
\hline Belgium (Fl.) & No & $\mathrm{a}$ & No & $\mathrm{a}$ & No & $\mathrm{a}$ & No & $\mathrm{a}$ & No & $\mathrm{a}$ \\
\hline Belgium (Fr.) & No & $\mathrm{a}$ & No & a & No & a & Yes & 4 & No & $\mathrm{a}$ \\
\hline $\begin{array}{l}\text { Canada } \\
\text { Chile }\end{array}$ & $\mathrm{m}$ & $\begin{array}{l}\mathrm{m} \\
\mathrm{m}\end{array}$ & $\begin{array}{l}\mathrm{m} \\
\mathrm{m}\end{array}$ & $\begin{array}{l}\mathrm{m} \\
\mathrm{m}\end{array}$ & $\begin{array}{l}\mathrm{m} \\
\mathrm{m}\end{array}$ & $\begin{array}{l}\mathrm{m} \\
\mathrm{m}\end{array}$ & $\begin{array}{l}\mathrm{m} \\
\mathrm{m}\end{array}$ & $\begin{array}{l}\mathrm{m} \\
\mathrm{m}\end{array}$ & $\begin{array}{l}\mathrm{m} \\
\mathrm{m}\end{array}$ & $\begin{array}{l}\mathrm{m} \\
\mathrm{m}\end{array}$ \\
\hline $\begin{array}{l}\text { Czech Republic } \\
\text { Denmark }\end{array}$ & $\begin{array}{c}\text { No } \\
m\end{array}$ & $\mathrm{a}$ & $\begin{array}{l}\text { Yes } \\
\text { Yes }\end{array}$ & $\begin{array}{l}1 \\
2\end{array}$ & $\begin{array}{c}\text { No } \\
m\end{array}$ & $\mathrm{a}$ & $\begin{array}{l}\text { No } \\
m\end{array}$ & $\mathrm{a}$ & $\begin{array}{l}\text { No } \\
m\end{array}$ & $\begin{array}{l}\mathrm{a} \\
\mathrm{m}\end{array}$ \\
\hline England & Yes & 4 & DoS & 4 & Yes & 4 & No & $\mathrm{a}$ & a & $\mathrm{a}$ \\
\hline Estonia & No & a & Yes & 2 & No & a & No & a & Dos & 5 \\
\hline Finland & No & a & No & a & Yes & 1 & Yes & 4 & $\mathrm{~m}$ & $\mathrm{~m}$ \\
\hline France & $\mathrm{a}$ & $\mathrm{a}$ & Yes & 4 & Yes & 4 & No & $\mathrm{a}$ & No & $\mathrm{a}$ \\
\hline Germany & No & a & No & a & No & a & No & a & Yes & 4 \\
\hline Greece & a & $\mathrm{a}$ & a & a & Yes & $\mathrm{m}$ & No & $\mathrm{a}$ & No & $\mathrm{a}$ \\
\hline Hungary & No & 3 & No & 3 & Yes & 4 & No & a & No & $\mathrm{a}$ \\
\hline Iceland & No & $\mathrm{a}$ & Yes & 1 & No & $\mathrm{a}$ & Yes & 4 & No & $\mathrm{a}$ \\
\hline Ireland & No & $\mathrm{a}$ & No & $\mathrm{a}$ & No & $\mathrm{a}$ & No & $\mathrm{a}$ & No & $\mathrm{a}$ \\
\hline Israel & No & $\mathrm{a}$ & Yes & 4 & Yes & 4 & No & a & No & a \\
\hline Italy & $\mathrm{a}$ & a & $\mathrm{a}$ & $\mathrm{a}$ & No & $\mathrm{a}$ & $\mathrm{a}$ & a & Yes & 4 \\
\hline Japan & $\mathrm{a}$ & $\mathrm{a}$ & Yes & 4 & Yes & 4 & No & a & No & $\mathrm{a}$ \\
\hline Korea & Yes & 4 & Yes & 4 & Yes & 4 & Yes & 3 & $\mathrm{a}$ & a \\
\hline Luxembourg & No & $\mathrm{a}$ & No & $\mathrm{a}$ & $a$ & $a$ & No & $\mathrm{a}$ & Yes & 4 \\
\hline Mexico & $\mathrm{a}$ & $\mathrm{a}$ & $\mathrm{a}$ & $\mathrm{a}$ & Yes & 1 & $\mathrm{a}$ & $\mathrm{a}$ & a & $\mathrm{a}$ \\
\hline Netherlands & DoS & $\mathrm{m}$ & Dos & $\mathrm{m}$ & DoS & $\mathrm{m}$ & Yes & 1 & Yes & 1 \\
\hline New Zealand & $\mathrm{m}$ & $\mathrm{m}$ & $\mathrm{m}$ & $\mathrm{m}$ & $\mathrm{m}$ & $\mathrm{m}$ & $\mathrm{m}$ & $\mathrm{m}$ & $\mathrm{m}$ & $\mathrm{m}$ \\
\hline Norway & Dos & 4 & Dos & 4 & Dos & 4 & No & $\mathrm{a}$ & No & $a$ \\
\hline Poland & No & $\mathrm{a}$ & Yes & 4 & Yes & 3 & Yes & 1 & Yes & 1 \\
\hline Portugal & No & $\mathrm{a}$ & No & $\mathrm{a}$ & $\mathrm{a}$ & a & No & $\mathrm{a}$ & No & a \\
\hline Scotland & No & a & No & $\mathrm{a}$ & Yes & 4 & a & $\mathrm{a}$ & No & a \\
\hline Slovak Republic & Yes & 1 & Yes & 1 & a & a & No & $\mathrm{a}$ & No & $\mathrm{a}$ \\
\hline Slovenia & No & $\mathrm{a}$ & Yes & 1 & No & a & Yes & 3 & No & $\mathrm{a}$ \\
\hline Spain & $\mathrm{a}$ & a & $\mathrm{a}$ & a & Yes & 4 & $\mathrm{a}$ & a & $\mathrm{a}$ & $\mathrm{a}$ \\
\hline Sweden & DoS & a & DoS & a & DoS & a & No & a & a & a \\
\hline Switzerland & $\mathrm{a}$ & a & Yes & $\mathrm{m}$ & a & a & $\mathrm{m}$ & $\mathrm{m}$ & $\mathrm{m}$ & $\mathrm{m}$ \\
\hline Turkey & No & $\mathrm{a}$ & No & $\mathrm{a}$ & Yes & 1 & No & $\mathrm{a}$ & No & $\mathrm{a}$ \\
\hline United States & $\mathrm{m}$ & $\mathrm{m}$ & $\mathrm{m}$ & $\mathrm{m}$ & $\mathrm{m}$ & $\mathrm{m}$ & $\mathrm{m}$ & $\mathrm{m}$ & $\mathrm{a}$ & $\mathrm{a}$ \\
\hline \multirow{10}{*}{$\begin{array}{l}\text { Indonesia } \\
\text { Latvia } \\
\text { Russian Feder } \\
\text { Saudi Arabia } \\
\text { South Africa } \\
\end{array}$} & $\mathrm{m}$ & $\mathrm{m}$ & $\mathrm{m}$ & $\mathrm{m}$ & $\mathrm{m}$ & $\mathrm{m}$ & $\mathrm{m}$ & $\mathrm{m}$ & $\mathrm{m}$ & $\mathrm{m}$ \\
\hline & $\mathrm{m}$ & $\mathrm{m}$ & $\mathrm{m}$ & $\mathrm{m}$ & $\mathrm{m}$ & $\mathrm{m}$ & $\mathrm{m}$ & $\mathrm{m}$ & $\mathrm{m}$ & $\mathrm{m}$ \\
\hline & $\mathrm{m}$ & $\mathrm{m}$ & $\mathrm{m}$ & $\mathrm{m}$ & $\mathrm{m}$ & $\mathrm{m}$ & $\mathrm{m}$ & $\mathrm{m}$ & $\mathrm{m}$ & $\mathrm{m}$ \\
\hline & No & $\mathrm{a}$ & No & $\mathrm{a}$ & Yes & 3 & No & $a$ & No & $a$ \\
\hline & $\mathrm{m}$ & $\mathrm{m}$ & $\mathrm{m}$ & $\mathrm{m}$ & $\mathrm{m}$ & $\mathrm{m}$ & $\mathrm{m}$ & $\mathrm{m}$ & $\mathrm{m}$ & $\mathrm{m}$ \\
\hline & $\mathrm{m}$ & $\mathrm{m}$ & $\mathrm{m}$ & $\mathrm{m}$ & $\mathrm{m}$ & $\mathrm{m}$ & $\mathrm{m}$ & $\mathrm{m}$ & $\mathrm{m}$ & $\mathrm{m}$ \\
\hline & No & $\mathrm{m}$ & Yes & 3 & Yes & 3 & Yes & $\mathrm{m}$ & No & $\mathrm{m}$ \\
\hline & $\mathrm{m}$ & $\mathrm{m}$ & $\mathrm{m}$ & $\mathrm{m}$ & $\mathrm{m}$ & $\mathrm{m}$ & $\mathrm{m}$ & $\mathrm{m}$ & $\mathrm{m}$ & $\mathrm{m}$ \\
\hline & $\mathrm{m}$ & $\mathrm{m}$ & $\mathrm{m}$ & $\mathrm{m}$ & $\mathrm{m}$ & $\mathrm{m}$ & $\mathrm{m}$ & $\mathrm{m}$ & $\mathrm{m}$ & $\mathrm{m}$ \\
\hline & $\mathrm{m}$ & $\mathrm{m}$ & $\mathrm{m}$ & $\mathrm{m}$ & $\mathrm{m}$ & $\mathrm{m}$ & $\mathrm{m}$ & $\mathrm{m}$ & $\mathrm{m}$ & $\mathrm{m}$ \\
\hline \multicolumn{3}{|c|}{$\begin{array}{l}\text { Is the performance mandatory for teachers? } \\
\text { Yes: Yes, it is mandatory } \\
\text { DoS: Yes, at the discretion of individual schools } \\
\text { No: No, it is voluntary at the discretion of individual teachers }\end{array}$} & $\begin{array}{l}\text { Does this c } \\
\text { Yes: Yes, incl } \\
\text { DoS: At the d } \\
\text { No: No, not }\end{array}$ & $\begin{array}{l}\text { apply for } \\
\text { part of base } \\
\text { of individu. } \\
\text { as part of b. }\end{array}$ & $\begin{array}{l}\text { the forma } \\
\text { salary or add } \\
\text { al schools } \\
\text { ase salary or }\end{array}$ & $\begin{array}{l}\text { salaries? } \\
\text { lyments } \\
\text { payments }\end{array}$ & $\begin{array}{l}\text { What is t } \\
\text { 1: Part of s } \\
\text { 2: Compen } \\
\text { 3: Defined } \\
\text { 4: Annual } \\
\text { 5: Incident }\end{array}$ & $\begin{array}{l}\text { ure of com } \\
\text { base salary } \\
\text { reduction i } \\
\text { ntage of sta } \\
\text { al payments } \\
\text { ional additi }\end{array}$ & $\begin{array}{l}\text { pensation } \\
\text { paid to teach } \\
\text { teaching tir } \\
\text { utory base s } \\
\text { nal payment }\end{array}$ & to teachers \\
\hline
\end{tabular}

Source: OECD. See Annex 3 for notes (www.oecd.org/education/education-at-a-glance-19991487.htm). Please refer to the Reader's Guide for information concerning symbols for missing data and abbreviations.

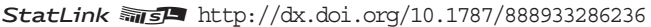




\section{HOW MUCH TIME DO TEACHERS SPEND TEACHING?}

- Public school teachers teach an average of 1005 hours per year at the pre-primary level, 772 hours at the primary level, 694 hours at the lower secondary level, and 643 hours at the upper secondary level of education.

- In the majority of countries with available data, the amount of teaching time in primary, lower secondary and upper secondary education remained largely unchanged between 2000 and 2013.

\section{Chart D4.1. Number of teaching hours per year in general lower secondary education, in 2000, 2005 and 2013}

Net statutory contact time in public institutions

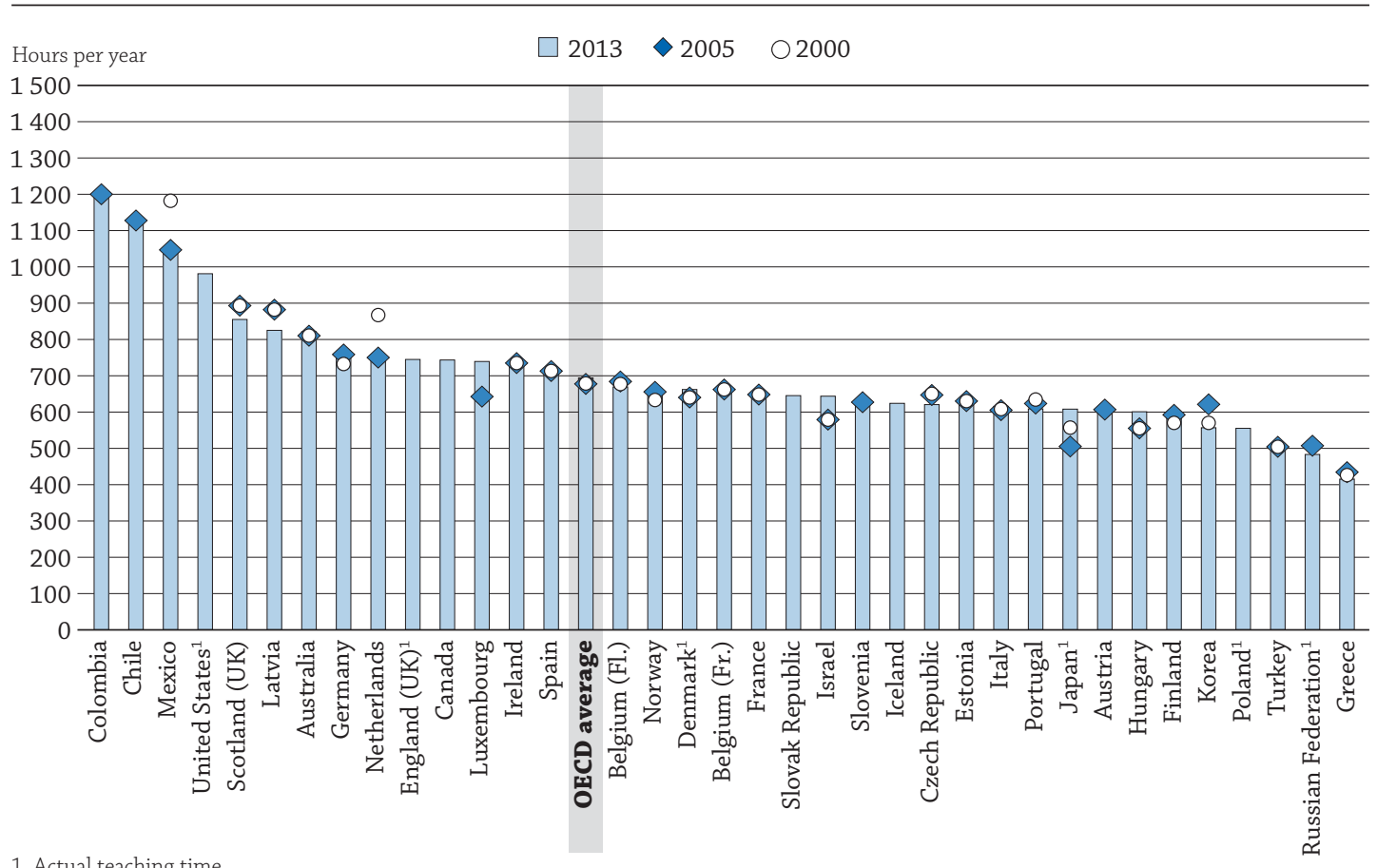

1. Actual teaching time.

Countries are ranked in descending order of the number of teaching hours per year in lower secondary education in 2013.

Source: OECD. Table D4.2. See Annex 3 for notes (www.oecd.org/education/education-at-a-glance-19991487.htm).

StatLink 部西 http://dx.doi.org/10.1787/888933284491

\section{Context}

Although statutory working hours and teaching hours only partly determine teachers' actual workload, they do offer valuable insight into the demands placed on teachers in different countries. Teaching hours and the extent of non-teaching duties may also affect the attractiveness of the teaching profession. Together with teachers' salaries (see Indicator D3) and average class size (see Indicator D2), this indicator presents some key measures regarding the working lives of teachers.

The proportion of statutory working time spent teaching provides information on the amount of time available for non-teaching activities such as lesson preparation, correction, in-service training and staff meetings. A large proportion of statutory working time spent teaching may indicate that less time is devoted to tasks such as assessing students and preparing lessons. It also could indicate that teachers have to perform these tasks on their own time and to work more hours than required by statutory working time.

In addition to class size and the ratio of students to teaching staff (see Indicator D2), students' hours of instruction (see Indicator D1) and teachers' salaries (see Indicator D3), the amount of time teachers spend teaching also affects the financial resources countries need to allocate to education (see Indicator B7). 


\section{Other findings}

- At all levels of education, the number of teaching hours per year required of the average public school teacher varies considerably across countries and tends to decrease as the level of education increases.

- On average, pre-primary teachers are required to teach about 30\% more hours than primary school teachers, but the time during which teachers are required to be working at school, or their total working time, is often more similar for these two levels of education.

- Required teaching time at the pre-primary level in public schools varies more across countries than it does at any other level.

- The average number of teaching hours in public pre-primary schools is 1005 hours per year, but it ranges from 532 hours per school year in Mexico to 1792 hours per school year in Sweden.

- Public primary school teachers teach an average of 772 hours per year, but teaching time ranges from less than 570 hours in Greece and the Russian Federation to over 1000 hours in Chile.

- The number of teaching hours in public lower secondary schools averages 694 hours per year, but ranges from 415 hours in Greece to over 1000 hours in Chile, Colombia and Mexico.

- Teachers in public upper secondary schools teach an average of 643 hours per year, but ranges from 369 hours in Denmark to over 1000 hours in Chile and Colombia.

- Most countries regulate the number of working hours per year that teachers are formally required to work, including teaching and non-teaching activities. Some of these countries regulated the specific number of hours required at school; others set the overall working time, including hours at school and elsewhere.

\section{Trends}

While there has been little change in average teaching hours over the past decade, some countries with available data reported an increase or decrease of $10 \%$ or more in teaching time in pre-primary, primary, lower secondary and/or upper secondary education between 2000 and 2013. In Korea, however, net teaching time at the primary level dropped dramatically by more than $20 \%$ between 2000 and 2013. 


\section{Analysis}

\section{Teaching time}

At all levels of education, countries vary considerably in the number of teaching hours per year required of the average public school teacher.

Required teaching time at the pre-primary level in public schools varies more across countries than it does at any other level. The number of teaching days ranges from 144 days in France to 227 in Iceland; annual teaching hours range from less than 700 hours in Greece, Korea and Mexico to more than 1500 in Norway and Sweden. On average across OECD countries, teachers at this level of education are required to teach 1005 hours per year, spread over 40 weeks or 191 days of teaching (Table D4.1 and Chart D4.2).

Primary school teachers are required to teach an average of 772 hours per year. In most countries with available data, teachers are required to teach between 3 and 6 hours a day. The exceptions are Chile and France, where teachers teach slightly more than 6 hours per day ( 5 days per week in Chile and 4.5 in France). There is no set rule on how teaching time is distributed throughout the year. In Spain, for example, primary school teachers must teach 880 hours per year, about 100 hours more than the OECD average. However, those teaching hours are spread over fewer days of instruction than the OECD average because primary school teachers in Spain teach an average of five hours per day compared to the OECD average of 4.22 hours.

Lower secondary school teachers in general programmes teach an average of 694 hours per year. The teaching time at the lower secondary level ranges from less than 600 hours in Finland, Greece, Korea, Poland, the Russian Federation and Turkey to more than 1000 hours in Chile, Colombia and Mexico.

A teacher of general subjects in upper secondary education has an average teaching load of 643 hours per year. Teaching time exceeds 800 hours in only six countries: Australia, Chile, Colombia, Latvia, Mexico and Scotland. However, in Chile and Scotland, the reported hours refer to the maximum time teachers can be required to teach and not to their typical teaching load. In contrast, teachers are required to teach less than 500 hours per year in Denmark, Greece and the Russian Federation. Teachers in Finland, Greece, Japan, Korea, Norway, the Russian Federation, Slovenia and Turkey teach for three hours or less per day, on average, compared to more than five hours in Chile, Colombia and Latvia.

\section{Chart D4.2. Number of teaching hours per year, by level of education (2013)} Net statutory contact time in public institutions

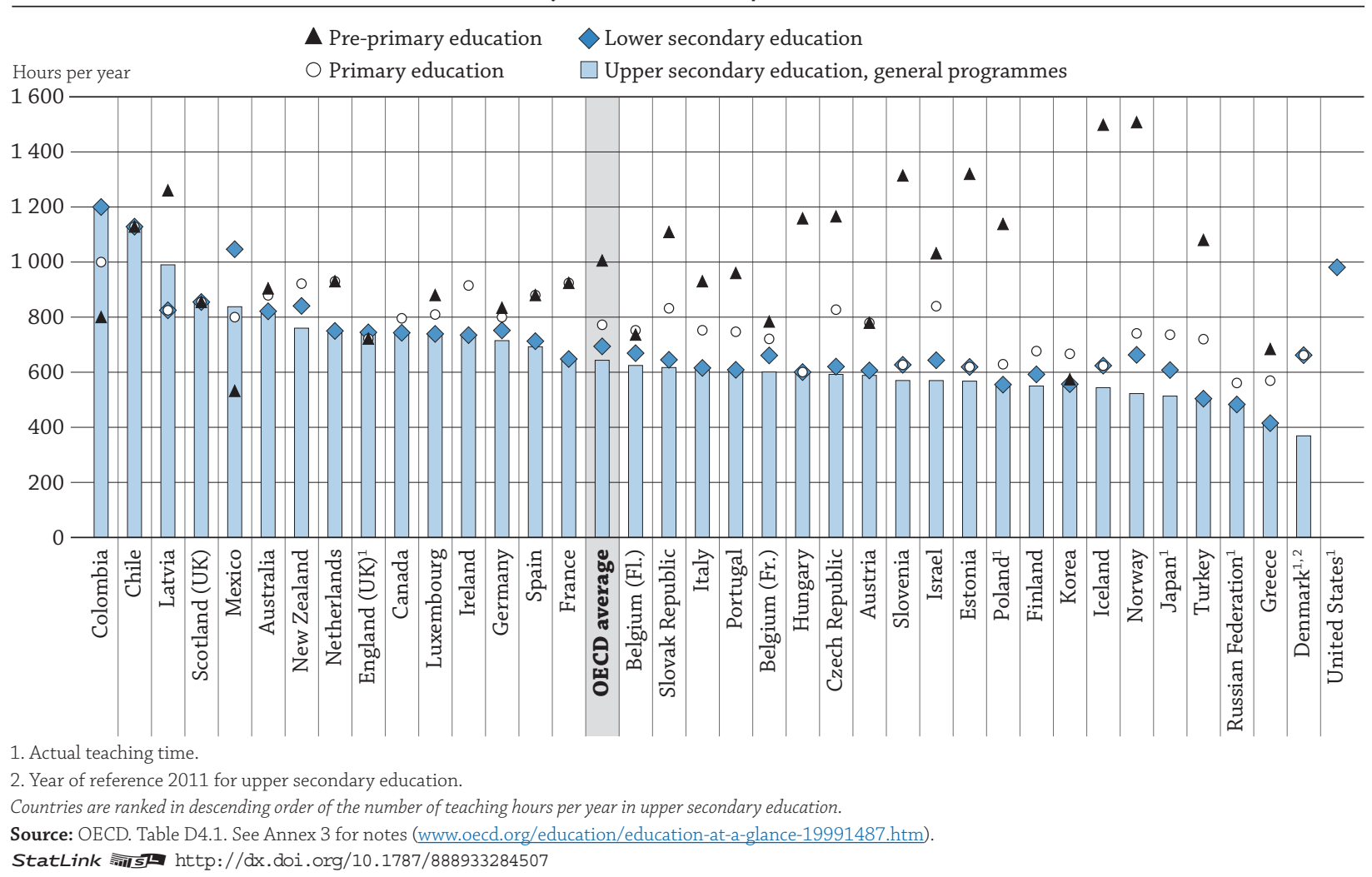


Reported teaching time refers to net contact time, excluding periods of time formally allowed for breaks between lessons or groups of lesson and preparation time. The exclusion of these breaks in some countries, but not in others, may explain some of these differences. Variations in how teaching time is reported (minimum, typical or maximum) across countries, may also explain some of these differences.

\section{Differences in teaching time between levels of education}

In most countries, teaching time at the upper secondary level is less than at the pre-primary level. The exceptions are Chile and Scotland, where the maximum time teachers can be required to teach is the same, irrespective of the level of education taught, and Colombia, England and Mexico, where secondary school teachers are required to teach more hours than pre-primary school teachers (Table D4.1 and Chart D4.2).

Teaching time requirements vary the most between the pre-primary and primary levels of education. On average, pre-primary school teachers are required to spend almost 30\% more time in the classroom than primary school teachers. In Estonia, Iceland, Norway and Slovenia, pre-primary school teachers are required to teach at least twice the amount of time per year as primary school teachers.

In the Czech Republic, France, Greece, Israel and Turkey, primary school teachers have at least $11 \%$ more annual teaching time than lower secondary school teachers. In contrast, there is no difference in Chile, Denmark, Estonia, Hungary, Iceland, Latvia, Scotland and Slovenia. Colombia, England and Mexico are the only countries in which the teaching load for primary school teachers is lighter than for lower secondary school teachers.

Teaching time at the lower and upper secondary levels is similar across most countries. However, in Mexico and Norway, the annual required teaching time at the lower secondary level is at least $20 \%$ more than at the upper secondary level. This difference amounts to almost $80 \%$ in Denmark. In Latvia, the annual required teaching time for upper secondary teachers is about $15 \%$ higher than it is for lower secondary teachers.

\section{Actual teaching time}

Statutory teaching time, as reported by most of the countries in this indicator, must be distinguished from actual teaching time. Actual teaching time is the annual average number of hours that full-time teachers teach a group or a class of students, including overtime, and thus provides a picture of teachers' actual teaching load.

Only a few countries could report both statutory and actual teaching time, but these data suggest that actual teaching time can sometimes differ from statutory requirements. In Slovenia, for example, lower secondary teachers work around 5\% more than the statutory benchmark time, while in Poland, actual teaching time is up to $13 \%$ more than statutory requirements. By contrast, in Estonia, actual teaching time is about $5 \%$ less than statutory teaching time at the lower secondary level (Chart D4.4, available on line).

It is difficult to determine why there are differences between statutory and actual teaching time. Some of these variations can be the result of overtime due to teacher absenteeism or shortage of teachers. Some may be explained by the nature of the data, as figures on statutory teaching time refer to official requirements and agreements, whereas actual teaching time is based on administrative registers, statistical databases, representative sample surveys or other representative sources.

\section{Trends in teaching time}

While there has been little change in average teaching hours over the past decade, some countries with available data reported an increase or decrease of $10 \%$ or more in teaching time in pre-primary, primary, lower secondary and/or upper secondary education between 2000 and 2013 (Table D4.2 and Chart D4.1).

At the pre-primary level, there was a 11\% increase in teaching time between 2000 and 2013 in Germany, while pre-primary teaching time decreased by $10 \%$ in Scotland over the same period.

In Israel and Japan, there was an increase of about 15\% in teaching time at the primary level between 2000 and 2013. In Israel, this increase in teaching and working time is part of the "New Horizon" reform that has been gradually implemented since 2008. One of the key measures of this reform was to lengthen teachers' workweek to accommodate small-group teaching in exchange for more generous compensation. Teachers' working time was increased from 30 to 36 hours per week and now includes five hours of small-group teaching in primary schools. To compensate, salaries have been raised substantially (see Indicator D3).

Upper secondary school teachers in Iceland were required to teach 20\% more in 2005 than in 2000, then, between 2005 and 2013, their teaching time was reduced slightly from 560 to 544 hours per year. Secondary school teachers in Luxembourg were required to teach 15\% more hours in 2013 than in 2005; and teaching time for secondary teachers in Japan increased by around $20 \%$ during the same period. 
D4 In Turkey, the reduction in teaching and working time for upper secondary teachers in 2013 is related to shorter classes in ISCED 3. General upper secondary classes were cut from 45 to 40 minutes in 2013. As a result, teachers' total annual teaching time was reduced as compared to previous years.

\section{Teachers' working time}

In most countries, teachers are formally required to work a specified total number of hours per week, including teaching and non-teaching time, as stipulated in collective agreements or other contractual arrangements, to earn their full-time salary. Some countries also regulate the time a teacher has to be present in the school. Within this framework, however, countries differ in how they allocate time for each activity (Chart D4.3).

More than half of OECD countries specify the time during which teachers are required to be available at school, for both teaching and non-teaching activities, at one or various levels of education. In more than half of these countries, the difference between the time upper secondary school teachers and pre-primary school teachers are required to be available at school is less than $10 \%$. In Israel, Norway, Sweden and Turkey, pre-primary teachers are required to be available at school at least $30 \%$ more hours than upper secondary school teachers (Table D4.1).

In Austria (pre-primary, primary and lower secondary education), the French Community of Belgium (pre-primary and primary education), the Czech Republic, Denmark, France (lower and upper secondary education), Germany, Japan (primary, lower and upper secondary education), Latvia, the Netherlands and Slovenia, teachers' total annual statutory working time, at school and elsewhere, is specified, but the allocation of time spent at school and time spent elsewhere is not.

In Sweden, although the total working time per year is decided through collective agreements, the school leader decides on the number of working hours per week and on the use of teachers' time (teaching or non-teaching activities).

In addition, workload and teaching load requirements may evolve throughout the career. While in some countries, such as in Germany, some beginning teachers might have a reduced teaching load as part of their induction programmes, some countries also encourage older teachers to stay in the teaching profession by diversifying their duties and reducing their teaching hours. For example, Greece reduces teaching hours according to how many years a teacher has served. At the secondary level, teachers are required to teach 21 class sessions per week. After 6 years, this drops to 19 sessions, and after 12 years to 18 sessions. After 20 years of service, teachers are required to teach 16 class sessions a week - more than $25 \%$ less than teachers who have just started their careers. However, the remaining hours of teachers' working time must be spent at school.

\section{Non-teaching time}

Although teaching time is a substantial component of teachers' workloads, assessing students, preparing lessons, correcting students' work, in-service training and staff meetings should also be taken into account when analysing the demands placed on teachers in different countries. The amount of time available for these non-teaching activities varies across countries, and a large proportion of statutory working time spent teaching may indicate that less time is devoted to activities such as assessing students and preparing lessons.

In the majority of countries, teachers' working time is determined by the statutory teaching time specified in working regulations. In addition, in most countries, teachers are formally required to work a specific number of hours per year. This may be specified either as the number of hours teachers must be available at school for teaching and non-teaching activities, or as the number of total working hours. Both correspond to official working hours as specified in contractual agreements. In Israel, for example, recent reforms take into account further working hours at school beyond teaching time. Regulations now specify the working time required at school, including teaching and non-teaching time, rather than overall working time. All non-teaching tasks that teachers previously performed at home are now required to be completed at school. 
Chart D4.3. Percentage of lower secondary teachers' working time spent teaching (2013) Net teaching time as a percentage of total statutory working time and working time required at school

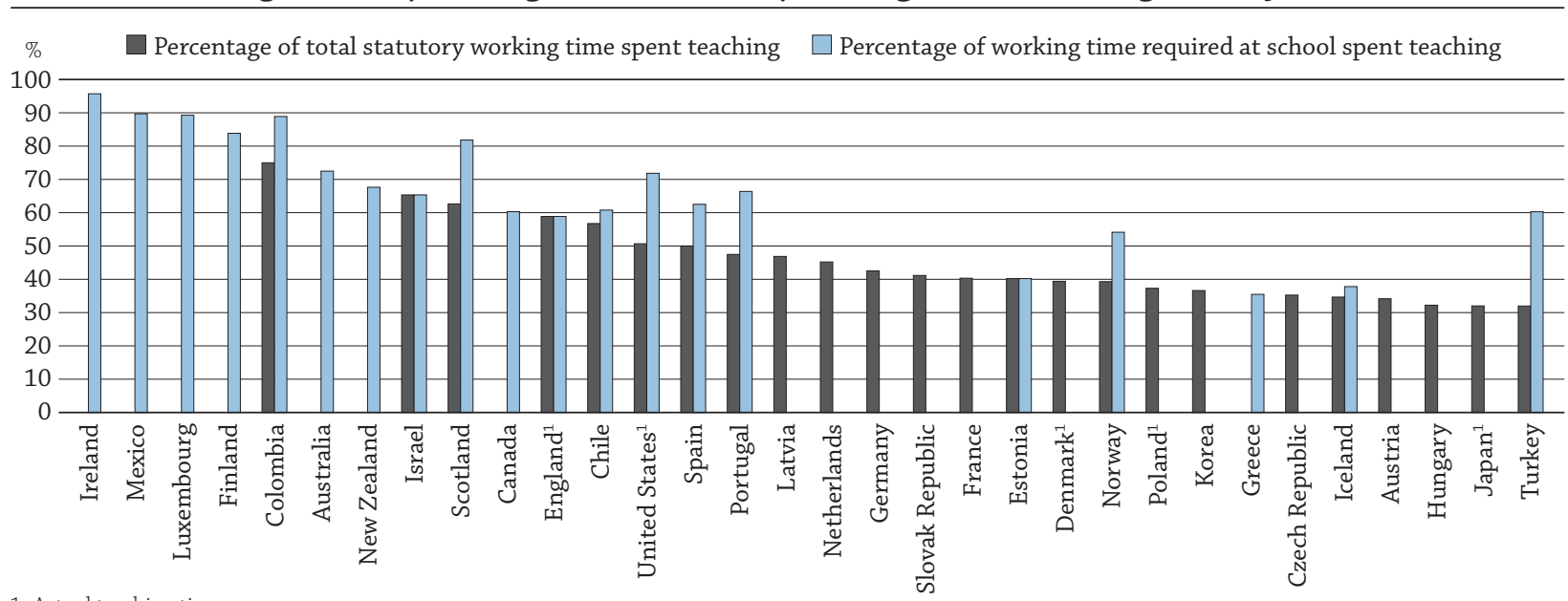

1. Actual teaching time.

Countries are ranked in descending order of the percentage of lower secondary teachers' total working time spent teaching. Source: OECD. Table D4.1. See Annex 3 for notes (www.oecd.org/education/education-at-a-glance-19991487.htm). StatLink 젶ㄴ http://dx.doi.org/10.1787/888933284511

In the 25 countries with data for both teaching and total working time for lower secondary teachers, the percentage of teachers' working time spent teaching ranges from less than 35\% in Austria, Hungary, Iceland, Japan and Turkey, to $65 \%$ in Israel (Chart D4.3).

In the 20 countries that specify both teaching time and the amount of time that lower secondary teachers are required to be available at school, the percentage of teachers' timetabled or legislated working time at school spent teaching ranges from less than $40 \%$ in Estonia, Greece and Iceland to more than $90 \%$ in Ireland.

In Austria (upper secondary level), Belgium (Flemish and French Community, secondary level), Italy and Japan (pre-primary level), there are no formal requirements regarding time spent on non-teaching activities. However, this does not mean that teachers are given total freedom to carry out other tasks. In the Flemish Community of Belgium, although there are no regulations regarding the time devoted to preparing lessons, correcting tests, marking students' papers, etc., additional non-teaching hours at school are set at the school level. In Italy, there is a requirement of up to 80 hours of scheduled non-teaching collegial work at school per year. Of these 80 hours, up to 40 hours of compulsory working time per year are dedicated to meetings of the teachers' assembly, staff planning meetings and meetings with parents; the remaining compulsory 40 hours are dedicated to class councils (Table D4.1).

\section{Box D4.1. Non-teaching tasks required of teachers in lower secondary education (2013)}

Non-teaching tasks are a part of teachers' workload and working conditions. These non-teaching activities required by legislation, regulations or agreements between stakeholders (e.g. teachers' unions, local authorities, school boards, etc.) do not necessarily reflect the actual participation of teachers in non-teaching activities, but provide an insight into the breadth and complexity of teachers' roles.

First findings in Education at a Glance 2014 (OECD, 2014) show that, according to regulations, individual planning or preparing lessons, teamwork and dialogue with colleagues and communicating and co-operating with parents are the most common non-teaching tasks required of lower secondary teachers during their statutory working time at school or statutory total working time. These tasks were required in at least 20 of the 34 countries with available data. Marking/correcting student work, general administrative communication and paperwork and professional development activities were also required in around half of the countries with available data. Lower secondary teachers are required to supervise students during breaks, provide counselling and guidance to students, or and/or participate in school management in around one-third of the countries, and only 8 countries require that lower secondary teachers engage in extracurricular activities after school. 
In a recent ad-hoc survey, countries were asked whether completing the required tasks and additional responsibilities are mandatory or voluntarily for the teacher. Results show that teachers often perform such tasks as engaging in extracurricular activities, training student teachers, guidance counselling, and participating in school or other management activities voluntarily. In almost half of the countries, individual teachers decided whether or not to perform these tasks. Responsibilities such as being class/form teacher or supervising students during breaks are largely distributed at the school level. In more than half of the countries, teamwork and dialogue with colleagues as well as general administrative work are mandatory for teachers. In almost all countries, it is mandatory for teachers to teach, plan lessons, mark and correct students' work and communicate with other teachers. Most countries require teachers to perform these tasks through central or state mandate; four countries reported that school principals determine which tasks are required of teachers.

\section{Chart D4.a. Tasks and responsibilities lower secondary teachers are required to perform (2013) \\ For lower secondary teachers teaching general programmes}

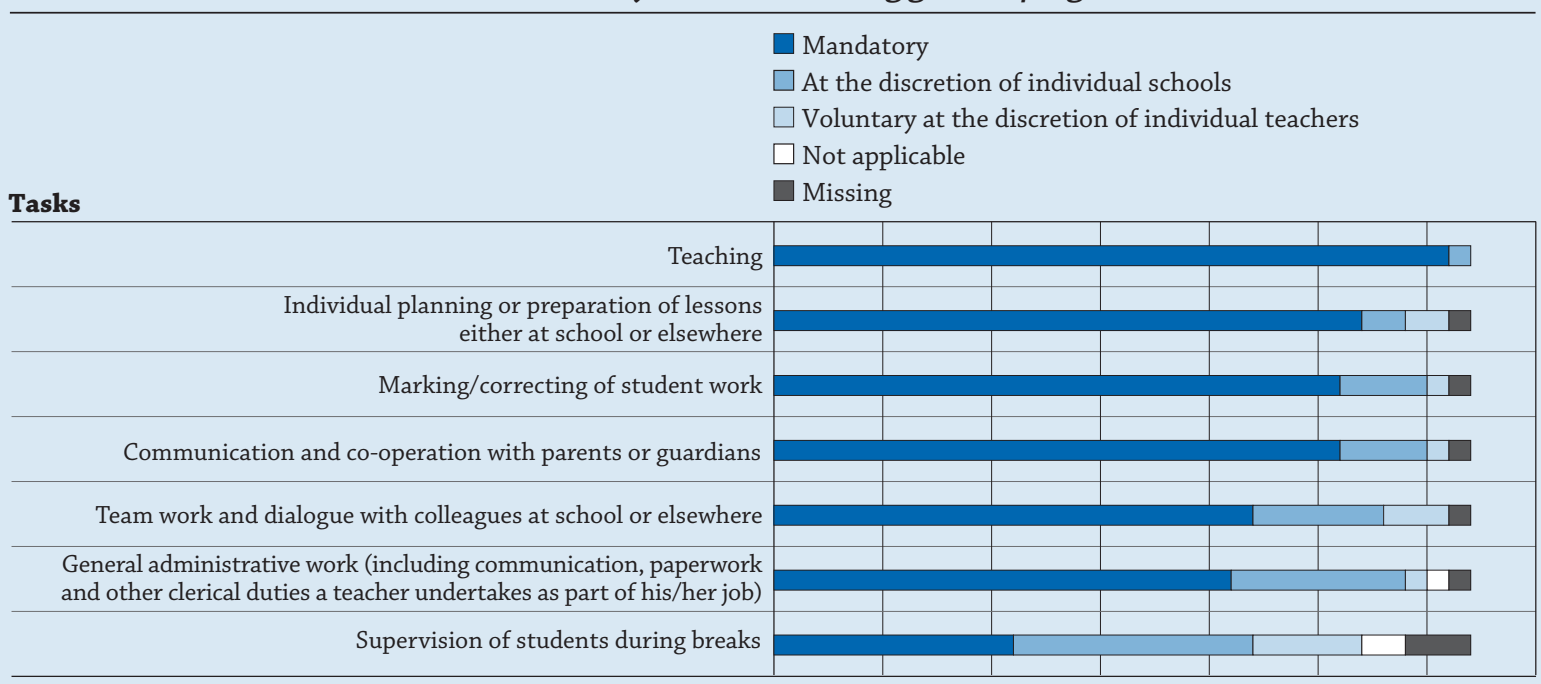

\section{Other responsibilities}

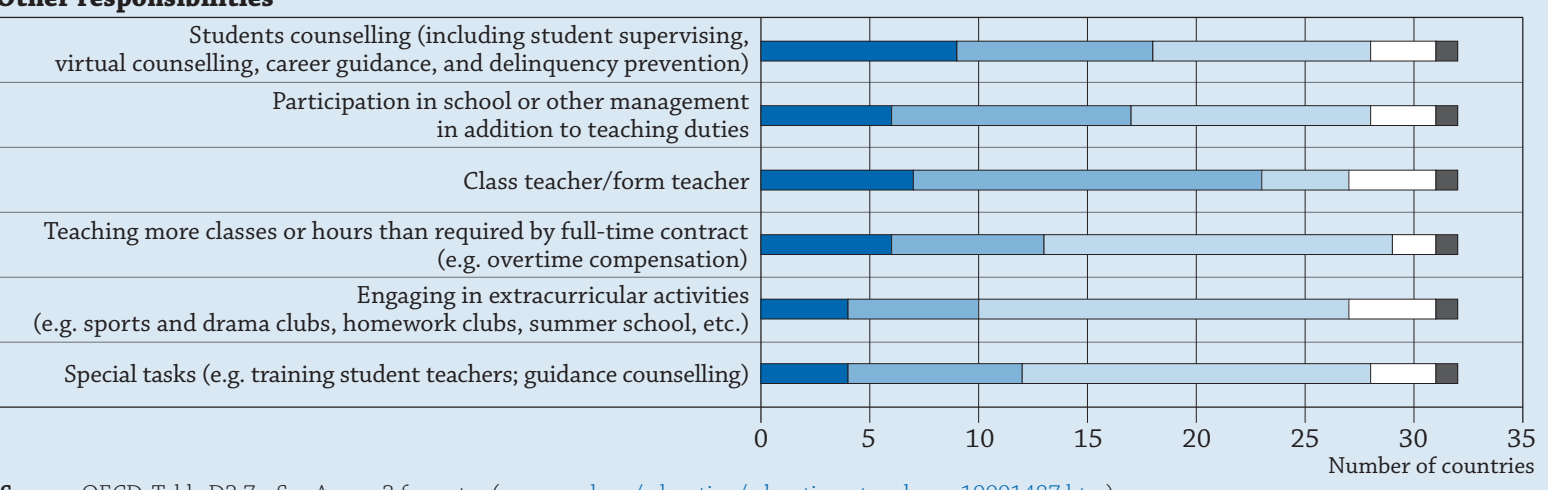

Source: OECD. Table D3.7c. See Annex 3 for notes (www.oecd.org/education/education-at-a-glance-19991487.htm).

StatLink 제기 http://dx.doi.org/10.1787/888933284521

\section{Definitions}

Actual teaching time is the annual average number of hours that full-time teachers teach a group or class of students including all extra hours such as overtime. The data can be from administrative registers, statistical databases, representative sample surveys or other representative sources.

The number of teaching days is the number of teaching weeks multiplied by the number of days per week a teacher teaches, less the number of days on which the school is closed for holidays.

The number of teaching weeks refers to the number of weeks of instruction excluding holiday weeks. 
Statutory teaching time is defined as the scheduled number of 60-minute hours per year that a full-time teacher teaches a group or class of students as set by policy, teachers' contracts of employment or other official documents. Teaching time can be defined on a weekly or annual basis. Annual teaching time is normally calculated as the number of teaching days per year multiplied by the number of hours a teacher teaches per day (excluding preparation time and periods of time formally allowed for breaks between lessons or groups of lessons). At the primary level, short breaks between lessons are included if the classroom teacher is responsible for the class during these breaks.

Total statutory working time refers to the number of hours that a full-time teacher is expected to work as set by policy. It can be defined on a weekly or annual basis. It does not include paid overtime. According to a country's formal policy, working time can refer to:

- the time directly associated with teaching and other curricular activities for students, such as assignments and tests; and

- the time directly associated with teaching and hours devoted to other activities related to teaching, such as preparing lessons, counselling students, correcting assignments and tests, professional development, meetings with parents, staff meetings, and general school tasks.

Working time required at school refers to the time teachers are required to spend working at school, including teaching and non-teaching time.

\section{Methodology}

Data are from the 2014 OECD-INES Survey on Teachers and the Curriculum and refer to the school year 2012/13.

In interpreting differences in teaching hours among countries, net contact time, as used here, does not necessarily correspond to the teaching load. Although contact time is a substantial component of teachers' workloads, preparing for classes and necessary follow-up, including correcting students' work, also need to be included when making comparisons. Other relevant elements, such as the number of subjects taught, the number of students taught, and the number of years a teacher teaches the same students, should also be taken into account.

Notes on definitions and methodologies for each country are provided in Annex 3 at www.oecd.org/education/ education-at-a-glance-19991487.htm.

\section{Note regarding data from Israel}

The statistical data for Israel are supplied by and are under the responsibility of the relevant Israeli authorities. The use of such data by the OECD is without prejudice to the status of the Golan Heights, East Jerusalem and Israeli settlements in the West Bank under the terms of international law.

\section{Reference}

OECD (2014), Education at a Glance 2014: OECD Indicators, Indicator D4, OECD Publishing, Paris, http://dx.doi.org/10.1787/ eag-2014-en.

\section{Indicator D4 Tables}

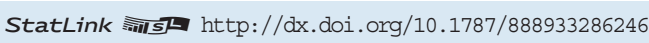

Table D4.1 Organisation of teachers' working time (2013)

Table D4.2 Number of teaching hours per year (2000, 2005, 2010 and 2013) 
Table D4.1. Organisation of teachers' working time (2013)

Number of statutory teaching weeks, teaching days, net teaching hours, and teachers' working time in public institutions over the school year

\begin{tabular}{|c|c|c|c|c|c|c|c|c|c|c|c|c|c|c|c|c|c|c|c|c|}
\hline & \multicolumn{4}{|c|}{$\begin{array}{c}\text { Number of weeks } \\
\text { of teaching }\end{array}$} & \multicolumn{4}{|c|}{$\begin{array}{c}\text { Number of days } \\
\text { of teaching }\end{array}$} & \multicolumn{4}{|c|}{$\begin{array}{l}\text { Net teaching time, } \\
\text { in hours }\end{array}$} & \multicolumn{4}{|c|}{$\begin{array}{l}\text { Working time required } \\
\text { at school, in hours }\end{array}$} & \multicolumn{4}{|c|}{$\begin{array}{c}\text { Total statutory } \\
\text { working time, in hours }\end{array}$} \\
\hline & 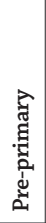 & 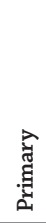 & 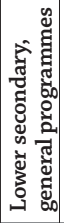 & 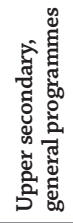 & 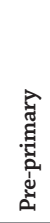 & 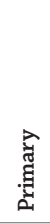 & 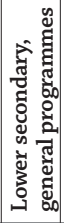 & 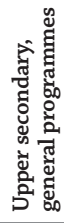 & 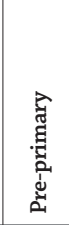 & 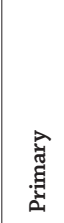 & 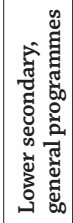 & 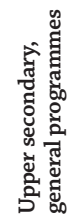 & 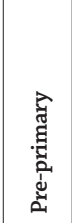 & 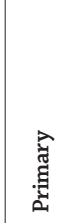 & 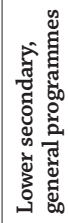 & 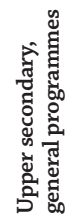 & 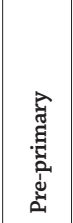 & 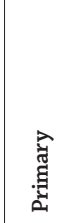 & 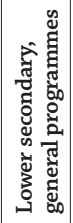 & 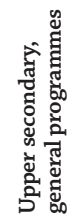 \\
\hline & (1) & $(2)$ & (3) & (4) & (5) & (6) & (7) & $(8)$ & (9) & (10) & (11) & (12) & (13) & $(14)$ & (15) & (16) & (17) & (18) & (19) & (20) \\
\hline Austr & 40 & 40 & 40 & 40 & 196 & 196 & 196 & 194 & 904 & 879 & 821 & 812 & 1227 & 1117 & 1133 & 1133 & $\mathrm{a}$ & a & & \\
\hline Aust & 38 & 38 & 38 & 38 & 180 & 180 & 180 & 180 & 779 & 779 & 607 & 589 & $\mathrm{a}$ & $a$ & $a$ & a & 1776 & 1776 & 1776 & 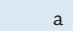 \\
\hline Belgium (Fl. $)^{1}$ & 37 & 37 & 37 & 37 & 177 & 177 & 179 & 179 & 736 & 752 & 669 & 625 & 920 & 920 & a & $\mathrm{a}$ & $\mathrm{a}$ & $a$ & $\mathrm{a}$ & a \\
\hline Belg & 37 & 37 & 37 & 37 & 181 & 181 & 181 & 181 & 784 & 721 & 661 & 601 & $\mathrm{a}$ & a & a & $\mathrm{a}$ & 962 & 962 & $\mathrm{a}$ & $\mathrm{a}$ \\
\hline Canada $^{1}$ & $\mathrm{~m}$ & 37 & 37 & 37 & $\mathrm{~m}$ & 183 & 183 & 183 & $\mathrm{~m}$ & 796 & 743 & 745 & $\mathrm{~m}$ & 1227 & 1232 & 1236 & $\mathrm{~m}$ & a & $\mathrm{a}$ & . \\
\hline Chi & 38 & 38 & 38 & 38 & 181 & 181 & 181 & 181 & 1129 & 1129 & 1129 & 1129 & 1857 & 1857 & 1857 & 1857 & 1989 & 1989 & 1989 & 1989 \\
\hline Czech Rep & 39 & 39 & 39 & 39 & 188 & 188 & 188 & 188 & 1166 & 827 & 620 & 592 & $\mathrm{a}$ & a & $\mathrm{a}$ & $\mathrm{a}$ & 1760 & 1760 & 1760 & 1760 \\
\hline & a & a & $\mathrm{a}$ & $\mathrm{a}$ & a & a & a & a & a & 662 & 662 & 369 & $a$ & a & a & $\mathrm{a}$ & 1680 & 1680 & 1680 & 1680 \\
\hline Eng & 38 & 38 & 38 & 38 & 190 & 190 & 190 & 190 & 722 & 722 & 745 & 745 & 1265 & 1265 & 1265 & 1265 & 1265 & 1265 & 1265 & 1265 \\
\hline Esto & 46 & 35 & 35 & 35 & 220 & 172 & 172 & 172 & 1320 & 619 & 619 & 568 & 1610 & 1540 & 1540 & 1540 & 1610 & 1540 & 1540 & 1540 \\
\hline Finland $^{5}$ & $\mathrm{~m}$ & 38 & 38 & 38 & $\mathrm{~m}$ & 188 & 188 & 188 & $\mathrm{~m}$ & 677 & 592 & 550 & $\mathrm{~m}$ & 791 & 706 & 645 & $\mathrm{a}$ & $a$ & $\mathrm{a}$ & 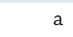 \\
\hline France $^{1}$ & 36 & 36 & 36 & 36 & 144 & 144 & $\mathrm{a}$ & $\mathrm{a}$ & 924 & 924 & 648 & 648 & 972 & 972 & $\mathrm{a}$ & $\mathrm{a}$ & 1607 & 1607 & 1607 & 1607 \\
\hline Germany & 40 & 40 & 40 & 40 & 193 & 193 & 193 & 193 & 834 & 800 & & 715 & a & a & a & $\mathrm{a}$ & 1768 & 1768 & 1768 & 1768 \\
\hline Greece $^{1}$ & 35 & 35 & 31 & 31 & 171 & 171 & 152 & 152 & 684 & 569 & 415 & 415 & 1140 & 1140 & 1170 & 1170 & a & $\mathrm{a}$ & $\mathrm{a}$ & a \\
\hline Hung & 36 & 36 & 36 & 36 & 182 & 182 & 182 & 181 & 1158 & 601 & 601 & 597 & $\mathrm{~m}$ & $\mathrm{~m}$ & $\mathrm{~m}$ & $\mathrm{~m}$ & 1864 & 1864 & 1864 & 1864 \\
\hline Iceland $^{1}$ & 48 & 37 & 37 & 35 & 227 & 180 & 180 & 170 & 1498 & 624 & 624 & 544 & 1800 & 1650 & 1650 & 1720 & 1800 & 1800 & 1800 & 1800 \\
\hline Ireland $^{1}$ & $\mathrm{~m}$ & 37 & 33 & 33 & $\mathrm{~m}$ & 183 & 167 & 167 & $\mathrm{~m}$ & 915 & 735 & 735 & $\mathrm{~m}$ & 1073 & 768 & 768 & a & a & a & a \\
\hline Israel $^{1}$ & 38 & 38 & 36 & 36 & 182 & 182 & 175 & 175 & 1032 & 840 & 644 & 570 & 1054 & 1225 & 985 & 811 & 1054 & 1225 & 985 & 811 \\
\hline Italy $^{1}$ & 42 & 39 & 39 & 39 & 186 & 171 & 171 & 171 & 930 & 752 & 616 & 616 & a & a & a & $\mathrm{a}$ & $\mathrm{m}$ & $\mathrm{m}$ & $\mathrm{m}$ & $\mathrm{m}$ \\
\hline Japan $^{4}$ & 39 & 40 & 40 & 39 & $\mathrm{~m}$ & 201 & 202 & 197 & $\mathrm{~m}$ & 736 & 608 & 513 & a & a & $\mathrm{a}$ & $\mathrm{a}$ & $\mathrm{a}$ & 1899 & 1899 & 1899 \\
\hline Korea $^{5}$ & 36 & 38 & 38 & 38 & 180 & 190 & 190 & 190 & 574 & 667 & 557 & 549 & $\mathrm{a}$ & a & a & $\mathrm{a}$ & 1520 & 1520 & 1520 & 1520 \\
\hline Luxembourg $^{1}$ & 36 & 36 & 36 & 36 & 176 & 176 & 176 & 176 & 880 & 810 & 739 & 739 & 1060 & 990 & 828 & 828 & a & a & a & a \\
\hline Mexico $^{1}$ & 42 & 42 & 42 & 36 & 200 & 200 & 200 & 171 & 532 & 800 & 1047 & 838 & 772 & 800 & 1167 & 971 & $\mathrm{a}$ & a & $\mathrm{a}$ & a \\
\hline Netherlands ${ }^{3}$ & 40 & 40 & $\mathrm{~m}$ & $\mathrm{~m}$ & 195 & 195 & $\mathrm{~m}$ & $\mathrm{~m}$ & 930 & 930 & 750 & 750 & $\mathrm{a}$ & a & a & $\mathrm{a}$ & 1659 & 1659 & 1659 & 1659 \\
\hline New Zealand ${ }^{1}$ & $\mathrm{~m}$ & 38 & 38 & 38 & $\mathrm{~m}$ & 192 & 191 & 190 & $\mathrm{~m}$ & 922 & 841 & 760 & $\mathrm{~m}$ & 1536 & 1243 & 950 & $\mathrm{a}$ & a & $\mathrm{a}$ & a \\
\hline Norway $^{3}$ & 45 & 38 & 38 & 38 & 225 & 190 & 190 & 190 & 1508 & 741 & 663 & 523 & 1508 & 1300 & 1225 & 1150 & a & 1688 & 1688 & 1688 \\
\hline Poland ${ }^{4}$ & 45 & 38 & 37 & 37 & 216 & 184 & 182 & 179 & 1138 & 629 & 555 & 551 & $\mathrm{~m}$ & $\mathrm{~m}$ & $\mathrm{~m}$ & $\mathrm{~m}$ & 1800 & 1504 & 1488 & 1472 \\
\hline Portugal $^{3}$ & 41 & 36 & 36 & 36 & 192 & 166 & 166 & 166 & 960 & 747 & 609 & 609 & 1105 & 1016 & 917 & 917 & 1412 & 1282 & 1282 & 1282 \\
\hline Scotland ${ }^{3}$ & 38 & 38 & 38 & 38 & 190 & 190 & 190 & 190 & 855 & 855 & 855 & 855 & 1045 & 1045 & 1045 & 1045 & 1365 & 1365 & 1365 & 1365 \\
\hline Slovak Republic ${ }^{1}$ & 42 & 38 & 38 & 38 & 198 & 187 & 187 & 187 & 1109 & 832 & 645 & 617 & $\mathrm{~m}$ & $\mathrm{~m}$ & $\mathrm{~m}$ & $\mathrm{~m}$ & 1568 & 1568 & 1568 & 1568 \\
\hline Slovenia $^{1}$ & 46 & 40 & 40 & 40 & 219 & 190 & 190 & 190 & 1314 & 627 & 627 & 570 & $\mathrm{a}$ & a & a & $\mathrm{a}$ & $\mathrm{m}$ & $\mathrm{m}$ & $\mathrm{m}$ & $\mathrm{m}$ \\
\hline Spain $^{1}$ & 37 & 37 & 37 & 36 & 176 & 176 & 176 & 171 & 880 & 880 & 713 & 693 & 1140 & 1140 & 1140 & 1140 & 1425 & 1425 & 1425 & 1425 \\
\hline Sweden ${ }^{1}$ & 47 & $a$ & a & a & 224 & a & $\mathrm{a}$ & a & 1792 & a & $a$ & $a$ & 1792 & 1360 & 1360 & 1360 & a & 1767 & 1767 & 1767 \\
\hline Switzerland & $\mathrm{m}$ & $\mathrm{m}$ & $\mathrm{m}$ & $\mathrm{m}$ & $\mathrm{m}$ & $\mathrm{m}$ & $\mathrm{m}$ & $\mathrm{m}$ & $\mathrm{m}$ & $\mathrm{m}$ & $\mathrm{m}$ & $\mathrm{m}$ & $\mathrm{m}$ & $\mathrm{m}$ & $\mathrm{m}$ & $\mathrm{m}$ & $\mathrm{m}$ & $\mathrm{m}$ & $\mathrm{m}$ & $\mathrm{m}$ \\
\hline Turkey $^{1}$ & 38 & 38 & 38 & 38 & 180 & 180 & 180 & 180 & 1080 & 720 & 504 & 504 & 1160 & 980 & 836 & 836 & 1576 & 1576 & 1576 & 1576 \\
\hline
\end{tabular}

United States ${ }^{4}$

\begin{tabular}{l|l|l|l|l|l|l|l|l|l|l|l|l|l|l|l|l|l|l|l|l|l|l|l|l|l|l|}
36 & 36 & 36 & 36 & 180 & 180 & 180 & 180 & $\mathrm{~m}$ & $\mathrm{~m}$ & 981 & $\mathrm{~m}$ & 1365 & 1362 & 1366 & 1365 & 1890 & 1922 & 1936 & 1960
\end{tabular}

\begin{tabular}{l|l|l|l|l|l|l|l|l|l|l|l|l|l|l|l|l|l|l|l|l|}
\hline OECD average & 40 & 38 & 37 & 37 & 191 & 183 & 182 & 181 & 1005 & 772 & 694 & 643 & 1266 & 1196 & 1172 & 1135 & 1588 & 1600 & 1618 & 1603
\end{tabular} \begin{tabular}{l|l|l|l|l|l|l|l|l|l|l|l|l|l|l|l|l|l|l|l|l|l|} 
EU21 average & 40 & 38 & 37 & 37 & 190 & 180 & 179 & 179 & 995 & 756 & 656 & 625 & 1205 & 1104 & 1074 & 1068 & 1568 & 1549 & 1588 & 1573
\end{tabular}

\begin{tabular}{|c|c|c|c|c|c|c|c|c|c|c|c|c|c|c|c|c|c|c|c|c|}
\hline $\begin{array}{l}\text { Argentina } \\
\text { Brazil }{ }^{1}\end{array}$ & $\begin{array}{r}\mathrm{m} \\
42\end{array}$ & $\begin{array}{r}\mathrm{m} \\
42\end{array}$ & $\begin{array}{l}m \\
42\end{array}$ & $\begin{array}{r}m \\
42\end{array}$ & \begin{tabular}{r|}
$m$ \\
203
\end{tabular} & $\begin{array}{r}m \\
203\end{array}$ & $\begin{array}{r}m \\
203\end{array}$ & $\begin{array}{r}\mathrm{m} \\
203\end{array}$ & $\begin{array}{l}\mathrm{m} \\
\mathrm{m}\end{array}$ & $\begin{array}{l}\mathrm{m} \\
\mathrm{m}\end{array}$ & \begin{tabular}{l|}
$\mathrm{m}$ \\
$\mathrm{m}$
\end{tabular} & $\begin{array}{l}\mathrm{m} \\
\mathrm{m}\end{array}$ & $\begin{array}{r}\mathrm{m} \\
\mathrm{a}\end{array}$ & $\begin{array}{r}\mathrm{m} \\
\mathrm{a}\end{array}$ & $\begin{array}{r}\mathrm{m} \\
\mathrm{a}\end{array}$ & $\begin{array}{r}\mathrm{m} \\
\mathrm{a}\end{array}$ & $\begin{array}{l}\mathrm{m} \\
\mathrm{m}\end{array}$ & $\begin{array}{l}\mathrm{m} \\
\mathrm{m}\end{array}$ & \begin{tabular}{l|}
$\mathrm{m}$ \\
$\mathrm{m}$
\end{tabular} & $\begin{array}{l}\mathrm{m} \\
\mathrm{m}\end{array}$ \\
\hline $\begin{array}{l}\text { China } \\
\text { Colombia }{ }^{1}\end{array}$ & $\begin{array}{r}\mathrm{m} \\
40\end{array}$ & $\begin{array}{r}\mathrm{m} \\
40\end{array}$ & $\begin{array}{r}\mathrm{m} \\
40\end{array}$ & $\begin{array}{r}\mathrm{m} \\
40\end{array}$ & $\begin{array}{r}m \\
200\end{array}$ & $\begin{array}{r}\mathrm{m} \\
200\end{array}$ & $\begin{array}{r}m \\
200\end{array}$ & $\begin{array}{r}m \\
200\end{array}$ & $\begin{array}{r}\mathrm{m} \\
800\end{array}$ & $\begin{array}{r}\mathrm{m} \\
1000\end{array}$ & $\begin{array}{r}\mathrm{m} \\
1200\end{array}$ & $\begin{array}{r}\text { m } \\
1200\end{array}$ & $\begin{array}{r}\mathrm{m} \\
1350\end{array}$ & $\begin{array}{r}\mathrm{m} \\
1350\end{array}$ & $\begin{array}{r}\mathrm{m} \\
1350\end{array}$ & $\begin{array}{r}\mathrm{m} \\
1350\end{array}$ & $\begin{array}{r}\mathrm{m} \\
1600\end{array}$ & $\begin{array}{r}\mathrm{m} \\
1600\end{array}$ & $\begin{array}{r}\mathrm{m} \\
1600\end{array}$ & $\begin{array}{r}\mathrm{m} \\
1600\end{array}$ \\
\hline $\begin{array}{l}\text { India } \\
\text { Indonesia }\end{array}$ & $\begin{array}{l}\mathrm{m} \\
\mathrm{m}\end{array}$ & $\begin{array}{l}\mathrm{m} \\
\mathrm{m}\end{array}$ & $\begin{array}{l}\mathrm{m} \\
\mathrm{m}\end{array}$ & $\begin{array}{l}\mathrm{m} \\
\mathrm{m}\end{array}$ & $\begin{array}{l}\mathrm{m} \\
\mathrm{m}\end{array}$ & $\begin{array}{l}\mathrm{m} \\
\mathrm{m}\end{array}$ & $\begin{array}{l}\mathrm{m} \\
\mathrm{m}\end{array}$ & $\begin{array}{l}\mathrm{m} \\
\mathrm{m}\end{array}$ & $\begin{array}{l}\mathrm{m} \\
\mathrm{m}\end{array}$ & $\begin{array}{l}\mathrm{m} \\
\mathrm{m}\end{array}$ & $\begin{array}{l}\mathrm{m} \\
\mathrm{m}\end{array}$ & $\begin{array}{l}\mathrm{m} \\
\mathrm{m}\end{array}$ & $\begin{array}{l}\mathrm{m} \\
\mathrm{m}\end{array}$ & $\begin{array}{l}\mathrm{m} \\
\mathrm{m}\end{array}$ & $\begin{array}{l}\mathrm{m} \\
\mathrm{m}\end{array}$ & $\begin{array}{l}\mathrm{m} \\
\mathrm{m}\end{array}$ & $\begin{array}{l}\mathrm{m} \\
\mathrm{m}\end{array}$ & $\begin{array}{l}\mathrm{m} \\
\mathrm{m}\end{array}$ & $\begin{array}{l}\mathrm{m} \\
\mathrm{m}\end{array}$ & $\begin{array}{l}\mathrm{m} \\
\mathrm{m}\end{array}$ \\
\hline Latvia & 44 & 35 & 35 & 35 & 210 & 165 & 165 & 165 & 1260 & 825 & 825 & 990 & a & a & a & $\mathrm{a}$ & 1760 & 1760 & 1760 & 1760 \\
\hline Russian Federation ${ }^{4}$ & $\mathrm{~m}$ & 34 & 35 & 35 & $\mathrm{~m}$ & 170 & 210 & 210 & $\mathrm{~m}$ & 561 & 483 & 483 & $\mathrm{~m}$ & $\mathrm{~m}$ & $\mathrm{~m}$ & $\mathrm{~m}$ & $\mathrm{~m}$ & $\mathrm{~m}$ & $\mathrm{~m}$ & $\mathrm{~m}$ \\
\hline $\begin{array}{l}\text { Saudi Arabia } \\
\text { South Africa }\end{array}$ & $\begin{array}{l}\mathrm{m} \\
\mathrm{m}\end{array}$ & $\begin{array}{l}\mathrm{m} \\
\mathrm{m}\end{array}$ & $\begin{array}{l}\mathrm{m} \\
\mathrm{m}\end{array}$ & $\begin{array}{l}\mathrm{m} \\
\mathrm{m}\end{array}$ & $\begin{array}{l}\mathrm{m} \\
\mathrm{m}\end{array}$ & $\begin{array}{l}\mathrm{m} \\
\mathrm{m}\end{array}$ & $\begin{array}{l}\mathrm{m} \\
\mathrm{m}\end{array}$ & $\begin{array}{l}\mathrm{m} \\
\mathrm{m}\end{array}$ & $\begin{array}{l}\mathrm{m} \\
\mathrm{m}\end{array}$ & $\begin{array}{l}\mathrm{m} \\
\mathrm{m}\end{array}$ & $\begin{array}{l}\mathrm{m} \\
\mathrm{m}\end{array}$ & $\begin{array}{l}\mathrm{m} \\
\mathrm{m}\end{array}$ & $\begin{array}{l}\mathrm{m} \\
\mathrm{m}\end{array}$ & $\begin{array}{l}\mathrm{m} \\
\mathrm{m}\end{array}$ & $\begin{array}{l}\mathrm{m} \\
\mathrm{m}\end{array}$ & $\begin{array}{l}\mathrm{m} \\
\mathrm{m}\end{array}$ & $\begin{array}{l}\mathrm{m} \\
\mathrm{m}\end{array}$ & $\begin{array}{l}\mathrm{m} \\
\mathrm{m}\end{array}$ & $\begin{array}{l}\mathrm{m} \\
\mathrm{m}\end{array}$ & $\begin{array}{l}\mathrm{m} \\
\mathrm{m}\end{array}$ \\
\hline G20 average & $\mathrm{m}$ & $\mathrm{m}$ & $\mathrm{m}$ & $\mathrm{m}$ & $\mathrm{m}$ & $\mathrm{m}$ & $\mathrm{m}$ & $\mathrm{m}$ & $\mathrm{m}$ & $\mathrm{m}$ & $\mathrm{m}$ & $\mathrm{m}$ & $\mathrm{m}$ & $\mathrm{m}$ & $\mathrm{m}$ & $\mathrm{m}$ & $\mathrm{m}$ & $\mathrm{m}$ & $\mathrm{m}$ & $\mathrm{m}$ \\
\hline
\end{tabular}

1. Typical teaching time.

2. Figures for the pre-primary level refer to primary teachers (in primary schools only) teaching pre-primary classes.

3. Maximum teaching time.

4. Actual teaching time.

5. Minimum teaching time.

6. Year of reference 2011 for upper secondary education.

Source: OECD. See Annex 3 for notes (www.oecd.org/education/education-at-a-glance-19991487.htm).

Please refer to the Reader's Guide for information concerning symbols for missing data and abbreviations.

StatLink 제며 http://dx.doi.org/10.1787/888933286256 
Table D4.2. Number of teaching hours per year (2000, 2005, 2010 and 2013) Net statutory contact time in public institutions, by level of education

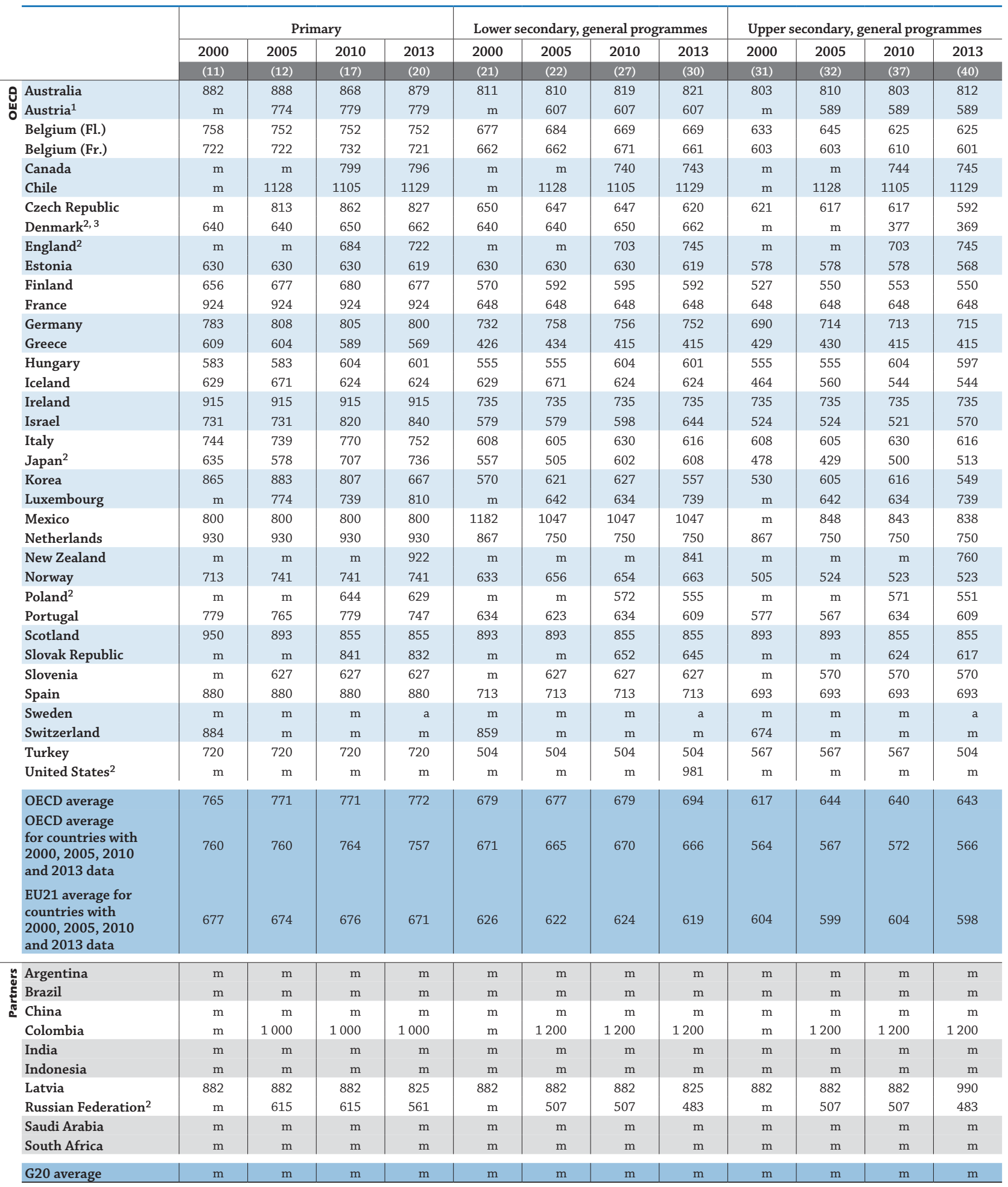

Notes: Data on years 2000 to 2013 for pre-primary education (i.e. columns 1-10) are available for consultation on line. Data on years 2006, 2007, 2008, 2009, 2011 and 2012 for primary education, lower secondary education and upper secondary education (i.e. columns 13-16; 18-19; 23-26; 28-29; 33-36; 38-39) are available for consultation on line (see StatLink below).

1. Figures for the pre-primary level refer to primary teachers (in primary schools only) teaching pre-primary classes.

2. Actual teaching time.

3. Year of reference 2011 instead of 2012 and 2013 for upper secondary education.

Source: OECD. See Annex 3 for notes (www.oecd.org/education/education-at-a-glance-19991487.htm).

Please refer to the Reader's Guide for information concerning symbols for missing data and abbreviations.

StatLink 케개 http://dx.doi.org/10.1787/888933286263 


\section{WHO ARE THE TEACHERS?}

- On average across OECD countries, 30\% of primary school teachers were at least 50 years old in 2013. The average increases to $34 \%$ at the lower secondary level and to $38 \%$ at the upper secondary level.

- More than two out of three teachers and academic staff are women, on average across OECD countries; but the percentage of female teachers decreases as the level of education increases: $96 \%$ at the pre-primary level, $82 \%$ at the primary level, $68 \%$ at the lower secondary level, $58 \%$ at the upper secondary level, and $42 \%$ at the tertiary level.

- On average, $83 \%$ of teachers have moderate or good skills in using information and communication technologies (ICT) to solve problems.

\section{Chart D5.1. Age distribution of teachers in primary education (2013) Distribution of teachers in educational institutions, by age group}

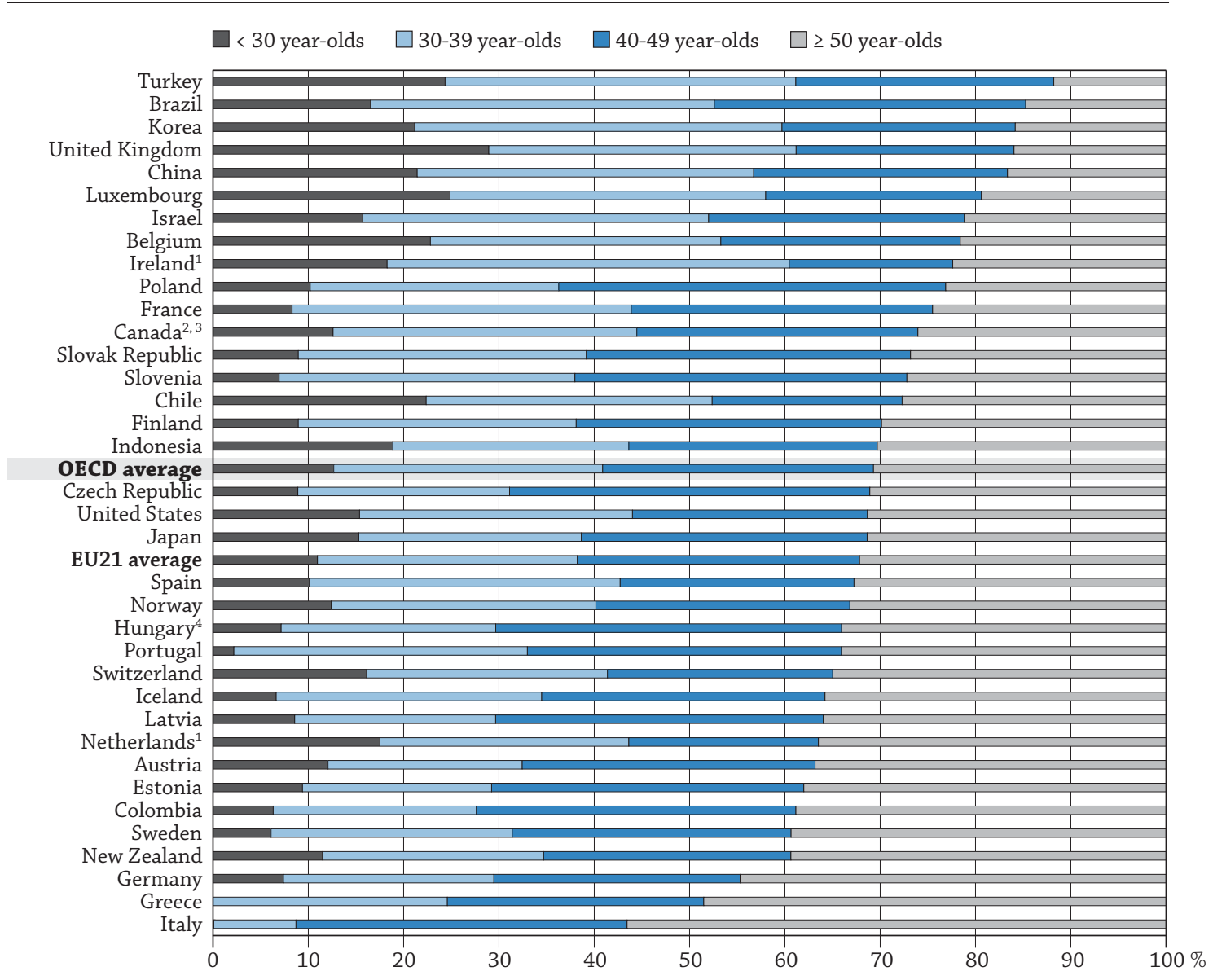

1. Public institutions only.

2. Year of reference 2012 .

3. Primary includes pre-primary and lower secondary.

4. Includes data on management personnel.

Countries are ranked in ascending order of the percentage of teachers aged 50 years or older at the primary level.

Source: OECD. Table D5.1. See Annex 3 for notes (www.oecd.org/education/education-at-a-glance-19991487.htm).

StatLink 需正 http://dx.doi.org/10.1787/888933284530

\section{Context}

The demand for teachers depends on a range of factors, including the age structure of the school-age population, average class size, the required instruction time for students, the use of teaching assistants and other "non-classroom" staff in schools, enrolment rates at the different levels of education, and starting and ending age of compulsory education. With large proportions of teachers in several OECD countries set to reach retirement age in the next decade, and/or the projected increase in the size of 
the school-age population, governments will be under pressure to recruit and train new teachers. Given compelling evidence that the calibre of teachers is the most significant in-school determinant of student achievement, concerted efforts must be made to attract top talent to the teaching profession and to provide high-quality training (Hiebert and Stigler, 1999; OECD, 2005).

Teacher-retention policies need to promote work environments that encourage effective teachers to continue teaching. In addition, as teaching at the pre-primary, primary and lower secondary levels remains largely dominated by women, this gender imbalance in the teaching profession and its impact on student learning warrant detailed study.

\section{INDICATOR D5}

\section{Other findings}

- In nearly all countries except Finland, Latvia and the Russian Federation, most teachers at the tertiary level are men.

- The United Kingdom has the largest proportion (29\%) of primary teachers under the age of 30 of all countries with available data. By contrast, Greece and Italy have almost no primary teachers in that age group.

- Teachers in Korea use their ICT skills at work more than adults in any other country or subnational entity that participated in the Survey of Adult Skills, a product of the OECD Programme for the International Assessment of Adult Competencies (PIAAC). Korea also has one of the largest proportions of teachers with good skills in using ICT to solve problems.

\section{Trends}

Between 2005 and 2013, the proportion of secondary teachers aged 50 or older climbed by 3 percentage points, on average among countries with comparable data. The increase is 10 percentage points or more in Greece, Korea, Portugal and Slovenia; Austria saw a 19 percentage-point increase in this proportion during the period. In countries that stand to lose a significant number of teachers through retirement and whose school-age population is expected to remain the same or grow, governments will have to boost the appeal of teaching to upper secondary and tertiary students, expand teacher-training programmes and, if necessary, provide alternate routes to certification for mid-career professionals intent on changing careers. Fiscal constraints - particularly those driven by pension obligations and health-care costs for retirees - are likely to result in greater pressure on governments to reduce academic offerings, increase class size, integrate more self-paced, online learning, or implement some combination of these measures (Abrams, 2011; Peterson, 2010). 


\section{Analysis}

\section{Age distribution of teachers}

The age distribution of teachers varies considerably across countries and can be affected by a variety of factors, such as the size and age distribution of the population, the duration of tertiary education, or teachers' salaries and working conditions. Declining birth rates, for example, may drive down the demand for new teachers; longer tertiary education can delay the entrance of teachers to the labour market. Competitive salaries and good working conditions may, in some countries, attract young people to teaching and, in others, help to retain effective teachers.

The age distribution of teachers is similar for the primary and secondary levels of education: about $82 \%$ of teachers are aged between 30 and 59. At the primary level, $30 \%$ of school teachers are at least 50 years old, on average across OECD countries. The proportion exceeds $40 \%$ in Germany, Greece and Italy. At the other end of the spectrum, in most countries with available data, only $15 \%$ or less of primary teachers are under the age of 30 . Only in Belgium, Chile, China, Korea, Luxembourg, Turkey and the United Kingdom does the proportion of primary teachers under the age of 30 equal or exceed 20\% (Chart D5.1).

\section{Chart D5.2. Age distribution of teachers in upper secondary education (2013) Distribution of teachers in educational institutions, by age group}

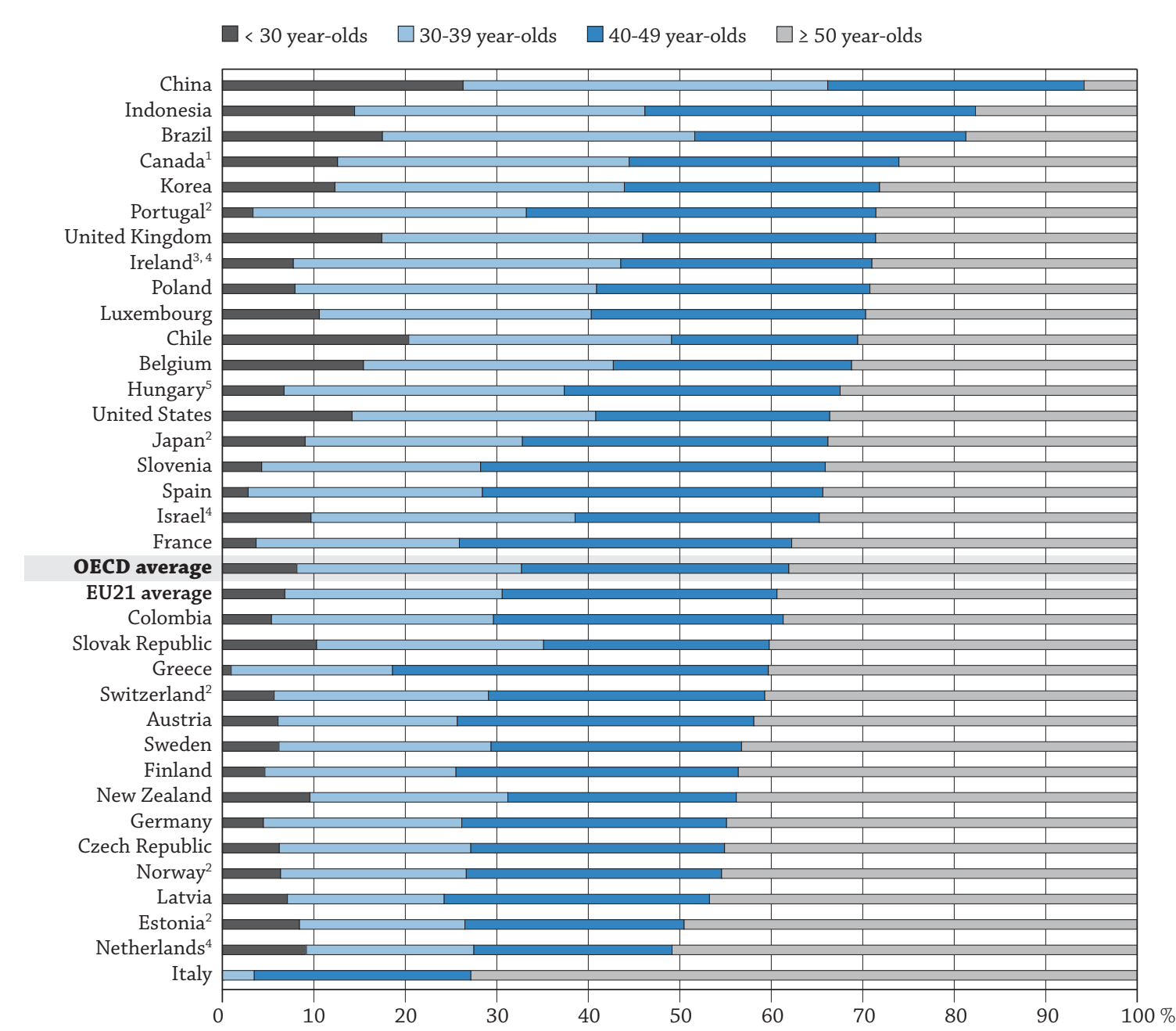

1. Year of reference 2012.

2. Upper secondary includes programmes from post-secondary non-tertiary.

3. Upper secondary includes lower secondary.

4. Public institutions only.

5. Includes data on management personnel.

Countries are ranked in ascending order of the percentage of teachers aged 50 years or older at the secondary level.

Source: OECD. Table D5.1. See Annex 3 for notes (www.oecd.org/education/education-at-a-glance-19991487.htm).

StatLink त्राजम http://dx.doi.org/10.1787/888933284546 
At the lower secondary level, on average across OECD countries, $34 \%$ of teachers are at least 50 years old, of which $7 \%$ are 60 or older. The proportion of lower secondary teachers aged 60 or older varies from $1 \%$ or less in China, Indonesia, Japan, Korea and Turkey, to 19\% in Estonia and Italy. At the upper secondary level, the proportion of teachers aged 50 or older is 4 percentage points larger than it is in lower secondary education, on average across OECD countries. Only in Brazil (52\%) and China (66\%) are most upper secondary teachers below the age of 40.

The ageing of the teaching force has a number of implications for countries' education systems. In addition to prompting recruitment and training efforts to replace retiring teachers, it may also affect budgetary decisions. In most school systems, there is a positive link between teachers' salaries and years of teaching experience. Thus, the ageing of teachers increases school costs, which, in turn, can limit the resources available to implement other initiatives (see Indicator D3).

\section{Gender profile of teachers}

On average across OECD countries, more than two out of three teachers in all levels of education combined are women. The highest proportions of female teachers, however, are concentrated in the earlier years of schooling and shrink at each successive level of education. Indeed, women represent only $42 \%$ of the teaching staff at the tertiary level, on average across OECD countries.

In 33 of the 36 OECD and G20 countries with available data, 93\% or more of pre-primary teachers are women. The exceptions are France, the Netherlands and the United Kingdom where 83\%, 87\% and 90\% of pre-primary teachers, respectively, are women. In primary education, women occupy $82 \%$ of teaching positions, on average across OECD countries. This percentage varies widely, however, from 58\% in Turkey to $99 \%$ in the Russian Federation (Chart D5.3).

Even though female teachers are still the majority in lower and upper secondary education, the proportion of male teachers is larger at these levels than at the pre-primary and primary levels. On average across OECD countries, $68 \%$ of teachers are women in lower secondary education. Indeed, female teachers comprise over half of the teaching staff at that level in all but one country with available data, Japan, where women represent $42 \%$ of the teaching staff. At the upper secondary level, the OECD average drops to $58 \%$, and the proportion of female teachers varies considerably from $28 \%$ in Japan to $81 \%$ in Latvia.

Chart D5.3. Gender distribution of teachers (2013)

Percentage of women among teaching staff in public and private institutions, by level of education

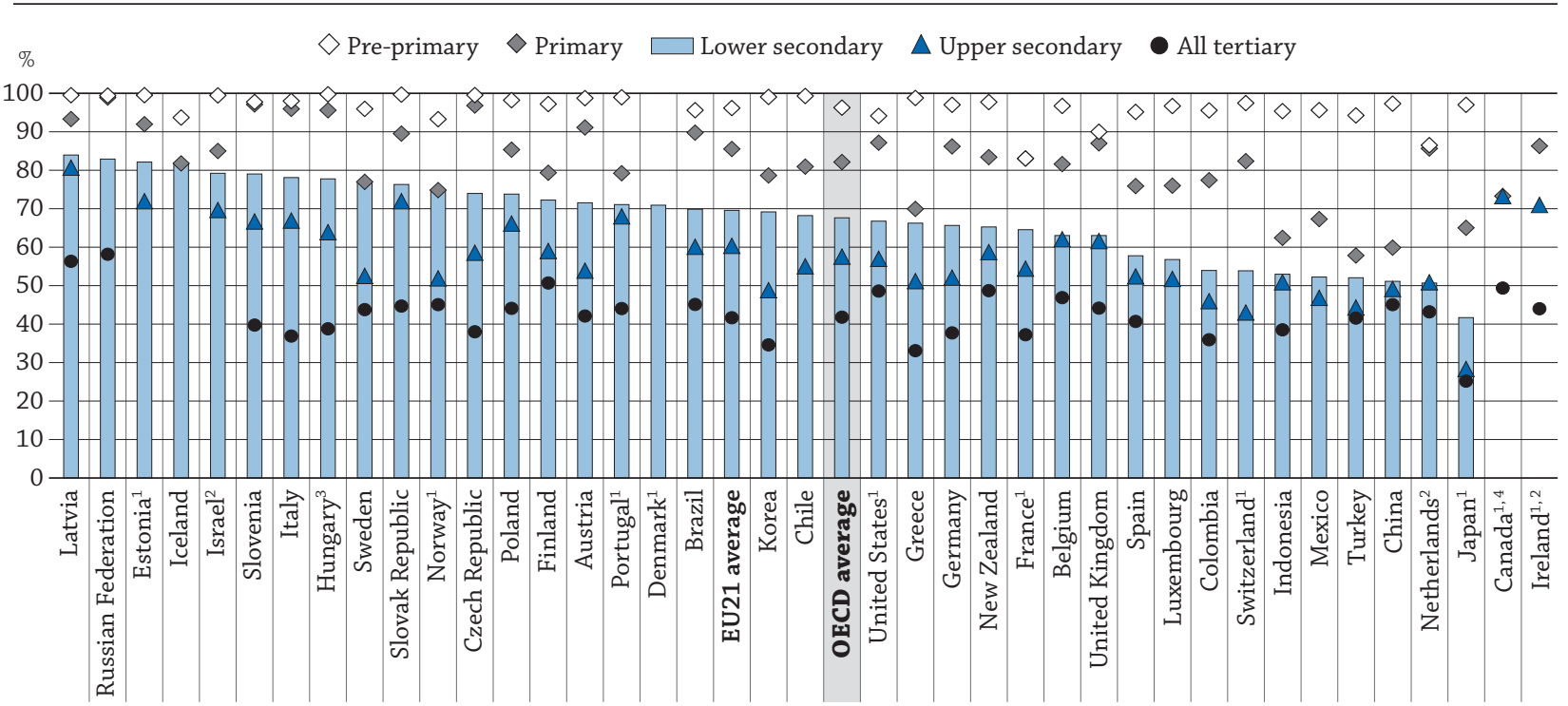

1. Some levels of education are included with others. Please refer to " $x$ " code in Table D5.3 for details.

2. Public institutions only. For the Netherlands, private data are available and included for pre-primary education. For Israel, public institutions only for pre-primary and upper secondary education.

3. Includes data on management personnel.

4. Year of reference 2012.

Countries are ranked in descending order of the percentage of female teachers at the lower secondary level.

Source: OECD. Table D5.3. See Annex 3 for notes (www.oecd.org/education/education-at-a-glance-19991487.htm).

StatLink 젶ㄴ http://dx.doi.org/10.1787/888933284558 
At the tertiary level, the gender profile of teachers is reversed. Male teachers represent $58 \%$ of the teaching staff at that level, on average across OECD countries. As at the lower and upper secondary levels, Japan has the smallest share of female teachers at the tertiary level $-25 \%$. Of the 26 OECD countries with available data, only one Finland - has a larger share of female teachers (51\%) than male teachers at this level.

The potential impact of gender imbalance in the teaching profession on student achievement, student motivation and teacher retention is worthy of study, especially in countries where few men are attracted to the profession (Drudy, 2008; OECD, 2005; 2009). There is little evidence that a teacher's gender has an impact on student performance (e.g. Antecol, Eren and Ozbeklik, 2012; Holmlund and Sund, 2008), but some research has shown that female teachers' attitudes towards school subjects, such as mathematics, can influence their female students' achievement (Beilock et al., 2009; OECD, 2014a).

In addition, school leadership does not reflect the gender balance among teachers (OECD, 2014a). While the proportion of male teachers in primary schools is relatively small in many countries, there is an over-representation of male principals, relative to male teachers, especially at that level of education. This suggests that male teachers tend to be promoted to principal positions more often than female teachers - which is surprising, given that most principals are former teachers, and most teachers are female.

\section{Change in the age distribution of teachers between 2005 and 2013}

The average annual growth rate between 2005 and 2013 in the proportion of secondary teachers aged 50 or older varied considerably among countries. In Austria, Korea, Portugal and Slovenia, it was more than $4 \%$. The proportion of secondary teachers aged 50 or older increased the most in Korea, by an average of $8.5 \%$ per year. In France, Germany, Ireland, Luxembourg and the United Kingdom, the proportion of secondary teachers aged 50 or older decreased by an average of $1 \%$ or more per year during the period (Table D5.2).

In all countries, changes in the number of teachers should be balanced against changes in the school-age population. In countries with an increase in the school-age population over the period (see Indicator $\mathrm{C} 1$ ), new teachers will be needed to compensate for the significant number of staff hired in the late 1960s and 1970s who will reach retirement age over the next decade. Teacher-training programmes will likely have to grow in these countries, and incentives for students to enter the teaching profession may have to increase (see Indicator D6 in Education at a Glance [OECD, 2014b]).

\section{Skills and readiness to use information and communication technologies for problem solving}

The 2012 Survey of Adult Skills, a product of the OECD Programme for the International Assessment of Adult Competencies (PIAAC), measured problem-solving skills in technology-rich environments and estimated the frequency with which adults use those skills at work and at home. Greater proficiency in problem solving in technology-rich environments reflects both better problem-solving skills and better skills in using digital technology, communication tools and networks to acquire and evaluate information, communicate with others and perform practical tasks (PIAAC Expert Group in Problem Solving in Technology-Rich Environments, 2009).

The information gathered through the Survey of Adult Skills allows for the creation of an indicator that measures skills and readiness to use ICT for problem solving. This indicator combines data about performance in the problemsolving assessment and information about why some adults did not participate in the computer-based assessment and thus do not have a score in problem solving (see the Definitions section at the end of this chapter).

\section{Teachers' skills}

Based on data drawn from the Survey of Adult Skills, Chart D5.4 shows that, on average, 47\% of teachers (defined as those who teach both pre-primary and primary school, primary teachers and secondary teachers) have good ICT and problem-solving skills (Group 4). This proportion ranges from $64 \%$ in Korea and $62 \%$ in England/ Northern Ireland (UK) to less than 30\% in the Russian Federation (29\%), Estonia (27\%) and Poland (25\%). On average across participating countries and sub-national entities, $83 \%$ of teachers have moderate or good ICT and problem-solving skills (Table D5.4a).

\section{Teachers' use of information and communication technologies at work}

In the Survey of Adult Skills, respondents were asked if they had the computer skills needed to do their job. In all national and sub-national entities that participated in the survey, $87 \%$ of teachers replied that they did. In the Czech Republic (99\%) and Korea (97\%) more than 95\% of teachers replied affirmatively. In general, the use of ICT skills at work is around the average (index value of 1.9) in the Czech Republic, while it is the highest recorded (index value of 2.5) in Korea. By contrast, much lower percentages of teachers in Japan (63\%) and Norway (72\%) reported that they had the computer skills needed to do their job (Table D5.4b). 
Chart D5.4. Teachers' skills and readiness to use information and communication technologies for problem solving (2012)

Survey of Adult Skills, teachers who teach both pre-primary and primary school, primary teachers and secondary teachers, 25-64 year-olds

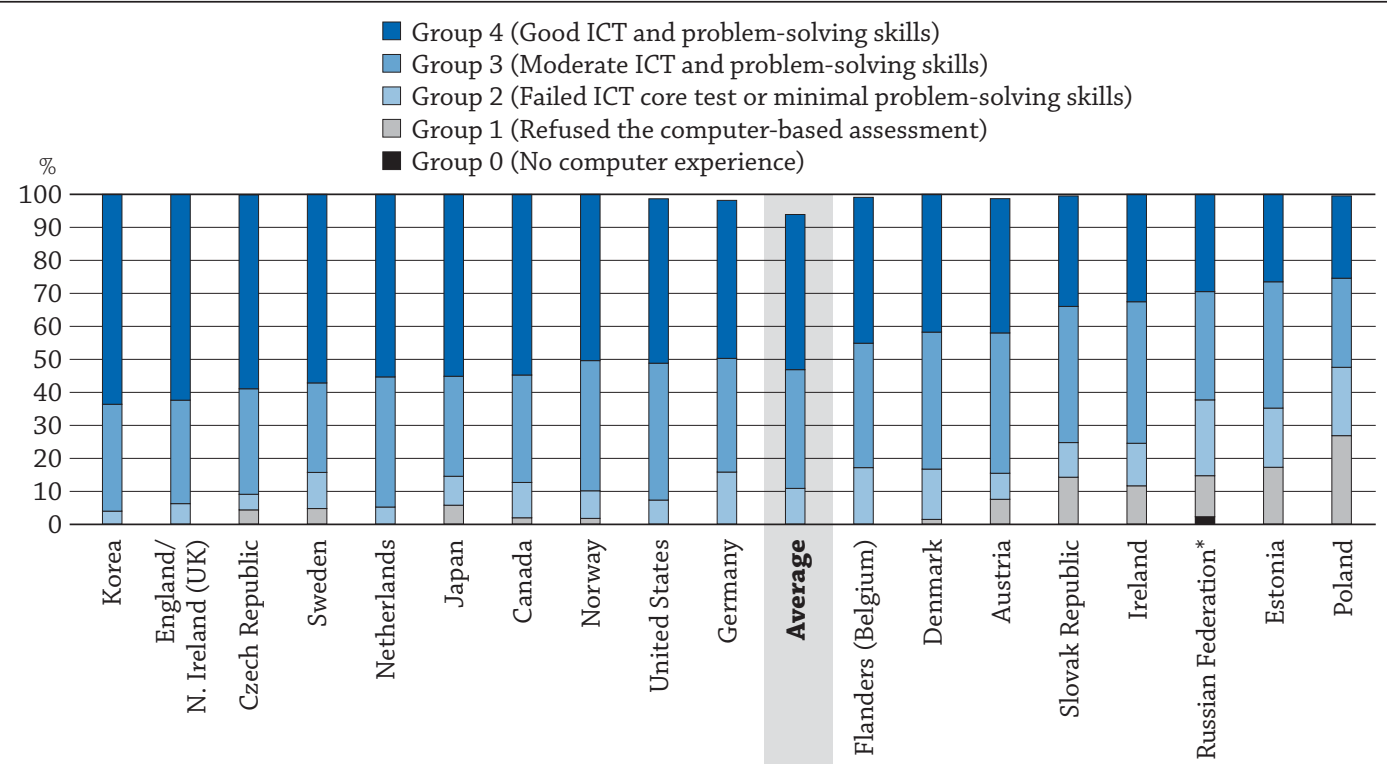

Notes: Teachers who teach both pre-primary and primary school, primary teachers and secondary teachers refer to teachers who were currently working as teachers at the moment of the survey. The bars may not add up to $100 \%$ because of the presence categories for which there are too few observations to provide reliable estimates.

* See note on data for the Russian Federation in the Methodology section.

Countries are ranked in descending order of the percentage of teachers with good ICT and problem-solving skills (Group 4).

Source: OECD. Table D5.4a. See Annex 3 for notes (www.oecd.org/education/education-at-a-glance-19991487.htm).

StatLink त्ञात http://dx.doi.org/10.1787/888933284562

Chart D5.5. Relationship between teachers' use of information and communication technologies skills at work and proficiency in those skills (2012)

Survey of Adult Skills, teachers who teach both pre-primary and primary school, primary teachers and secondary teachers, 25-64 year-olds

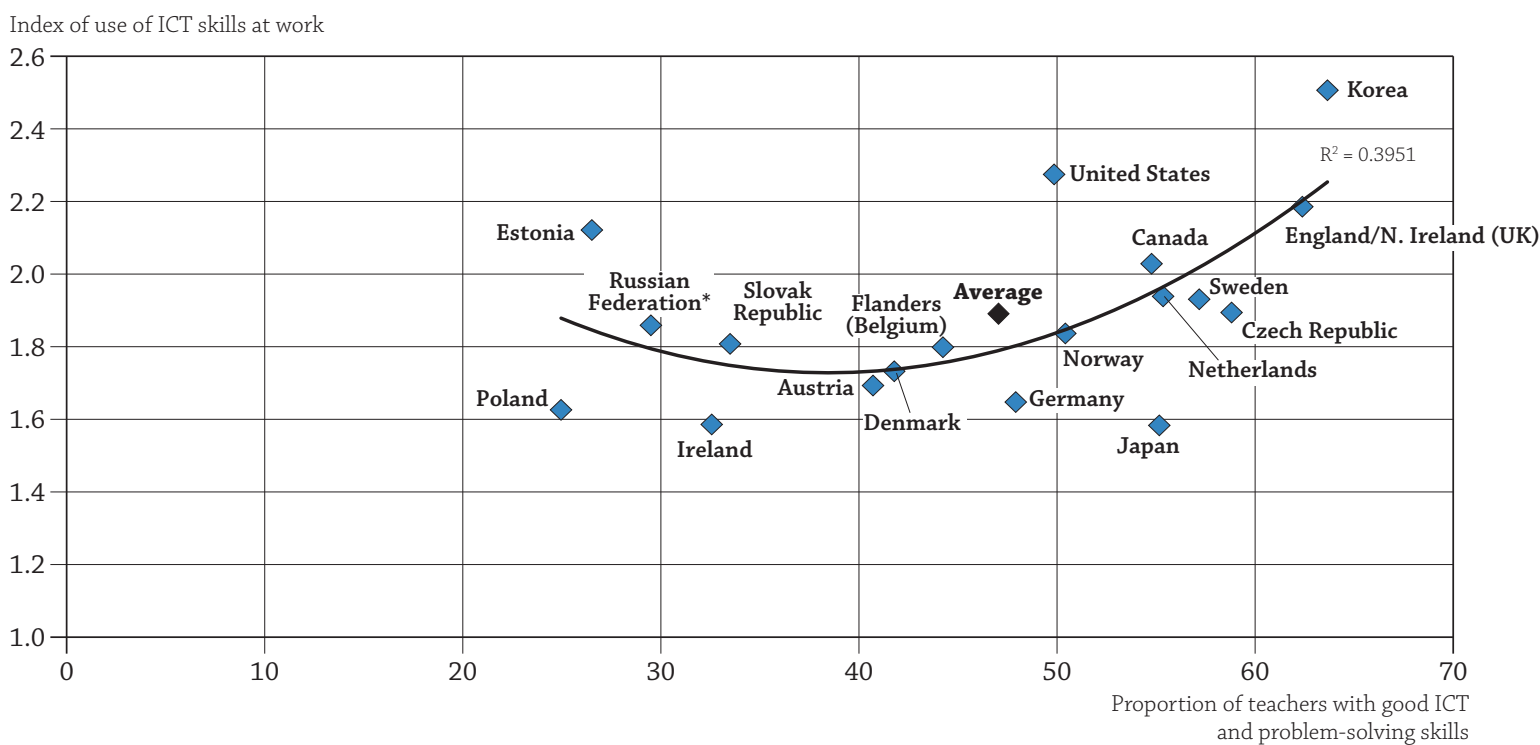

Notes: Teachers who teach both pre-primary and primary school, primary teachers and secondary teachers refer to teachers who were currently working as teachers at the moment of the survey. The index of use of ICT indicates the frequency of use of ICT skills at work. The higher the index, the more frequent the use of ICT skills at work. See the Definitions section.

* See note on data for the Russian Federation in the Methodology section.

Source: OECD. Tables D5.4a and D5.4b. See Annex 3 for notes (www.oecd.org/education/education-at-a-glance-19991487.htm).

StatLink 제그 http://dx.doi.org/10.1787/888933284572 
Respondents were also asked about the level of ICT skills required at work. On average, $73 \%$ reported that moderate or complex ICT skills were required. These percentages ranged from below $55 \%$ in Poland (50\%) and the Russian Federation (51\%) to $85 \%$ or more in Korea (85\%), the Netherlands (87\%), Japan (88\%) and Estonia (89\%). Only $1 \%$ to $7 \%$ of teachers reported that complex ICT skills are required at work; the vast majority of teachers reported that moderate ICT skills are required (Table D5.4b).

Chart D5.5 shows the positive relationship between teachers' use of ICT skills at work and the percentage of teachers with good ICT and problem-solving skills (Group 4). The proportion of teachers with good ICT and problem-solving skills (Group 4) tends to increase as teachers use those skills more at work. For example, in Poland 21\% of teachers have good ICT and problem-solving skills (Group 4) and they use those skills at work less than the average (1.6 on the index of skills use at work, while the average is 1.9). By contrast, in Korea, $64 \%$ of teachers have good ICT and problem-solving skills (Group 4) and use their skills at work far more than the average (index value of 2.5) - the highest values among all countries and sub-national entities that participated in the survey. Estonia and Japan are the two outliers in this chart. Teachers in Estonia use ICT skills frequently at work (index value of 2.1), yet a relatively small proportion of teachers (27\%) have good ICT and problem-solving skills, while the reverse is observed in Japan, where teachers use of ICT skills at work is below average (index value of 1.6) while a majority of teachers (55\%) has good ICT and problem-solving skills (Tables D5.4a and D5.4b).

\section{Definitions}

ICT Skills required at work refers to the use of computers needed at work. Four levels of use are identified: "ICT skills not required at work" corresponds to individuals who reported they do not use a computer in their job; "Straightforward" indicates using a computer for routine tasks, such as data entry or sending and receiving e-mails; "Moderate" indicates using a computer for word-processing, spreadsheets or database management; and "Complex" indicates developing software or modifying computer games, programming using languages like java, sql, php or perl, or maintaining a computer network.

Index of use of ICT skills at work indicates the frequency of use of ICT skills at work. The higher the value on the index, the more frequent the use of ICT skills at work. The variable was derived from several questions from the background questionnaire of the Survey of Adult Skills, and has been transformed so that it has a mean of 2 and a standard deviation of 1 across the pooled sample of all participating countries. For more details, see page 143 of the OECD Skills Outlook 2013: First Results from the Survey of Adult Skills (OECD, 2013).

ISCED type of final qualification refers to the type of education qualification that a new teacher would be required to have to teach primary, lower secondary or upper secondary school (general programmes) in the public sector.

Skill groups refer to skills and readiness to use information and communication technologies (ICT) for problem solving in technology-rich environments. Each group is described in terms of the characteristics of the types of tasks that can be successfully completed by adults and the related scores in the assessment of problem solving in technology-rich environments in the Survey of Adult Skills.

- Group 0 (no computer experience)

- Group 1 (refused the computer-based assessment)

- Group 2 (failed ICT core stage 1 or minimal problem-solving skills - scored below Level 1 in the problem solving in technology-rich environments assessment)

- Group 3 (moderate ICT and problem-solving skills - scored at Level 1 in the problem solving in technology-rich environments assessment)

- Group 4 (good ICT and problem-solving skills - scored at Level 2 or Level 3 in the problem solving in technologyrich environments assessment)

\section{Methodology}

Data refer to the academic year 2012/13 and are based on the UOE data collection on education statistics administered by the OECD in 2013 (for details, see Annex 3 at www.oecd.org/education/education-at-a-glance-19991487.htm). Data on teachers by age for 2005 may have been revised in 2015 to ensure consistency with 2013 data.

Data on skills and readiness to use ICT for problem solving are based on data from the Survey of Adult Skills (PIAAC) (2012), which was not specifically designed for teachers. The sample is smaller than in other indicators that use the whole population, explaining why standard errors are slightly higher than usual. Data should, therefore, be interpreted with caution. PIAAC is the OECD Programme for the International Assessment of Adult Competencies. 


\section{Note regarding data from Israel}

The statistical data for Israel are supplied by and are under the responsibility of the relevant Israeli authorities. The use of such data by the OECD is without prejudice to the status of the Golan Heights, East Jerusalem and Israeli settlements in the West Bank under the terms of international law.

\section{Note regarding data from the Russian Federation in the Survey of Adult Skills (PIAAC)}

Readers should note that the sample for the Russian Federation does not include the population of the Moscow municipal area. The data published, therefore, do not represent the entire resident population aged 16-65 in Russia but rather the population of Russia excluding the population residing in the Moscow municipal area. More detailed information regarding the data from the Russian Federation as well as that of other countries can be found in the Technical Report of the Survey of Adult Skills (OECD, 2014c).

\section{References}

Abrams, S.E. (2011), “Technology in Moderation”, The Teachers College Record, www.tcrecord.org/content.asp? contentid=16584. Antecol, H., O. Eren and S. Ozbeklik (2012), “The Effect of Teacher Gender on Student Achievement in Primary School: Evidence from a Randomized Experiment”, IZA Discussion Paper, No. 6453, http://ftp.iza.org/dp6453.pdf.

Beilock, S.L. et al. (2009), "Female Teachers' Math Anxiety Affects Girls' Math Achievement”, Proceedings of the National Academy of Science of the United States of America-PNAS, Vol. 107/5, pp .1860-1863.

Drudy, S. (2008), "Gender Balance/Gender Bias: The Teaching Profession and the Impact of Feminisation”, Gender and Education, Vol. 20/4, pp. 309-323.

Hiebert, J. and J. Stigler (1999), The Teaching Gap: Best Ideas from the World's Teachers for Improving Education in the Classroom, Free Press, New York.

Holmlund, H. and K. Sund (2008), "Is the Gender Gap in School Performance Affected by the Sex of the Teacher?", Labour Economics, Vol. 15, pp. 37-53.

OECD (2014a), TALIS 2013 Results: An International Perspective on Teaching and Learning, OECD Publishing, Paris, http://dx.doi. org/10.1787/9789264196261-en.

OECD (2014b), Education at a Glance 2014: OECD Indicators, OECD Publishing, Paris, http://dx.doi.org/10.1787/eag-2014-en.

OECD (2014c), Technical Report of the Survey of Adult Skills, www.oecd.org/site/piaac/ Technical\%20Report 17OCT13.pdf, pre-publication copy.

OECD (2013), OECD Skills Outlook 2013: First Results from the Survey of Adult Skills, OECD Publishing, Paris, http://dx.doi. org/10.1787/9789264204256-en.

OECD (2009), Creating Effective Teaching and Learning Environments: First Results from TALIS, OECD Publishing, Paris, http:// dx.doi.org/10.1787/9789264068780-en.

OECD (2005), Teachers Matter: Attracting, Developing and Retaining Effective Teachers, Education and Training Policy, OECD Publishing, Paris, http://dx.doi.org/10.1787/9789264018044-en.

Peterson, P. (2010), Saving Schools: From Horace Mann to Virtual Learning, Harvard University Press, Cambridge.

PIAAC Expert Group in Problem Solving in Technology-Rich Environments (2009), "PIAAC problem solving in technologyrich environments: a conceptual framework", OECD Education Working Papers, No. 36, OECD Publishing, Paris, http://dx.doi. org/10.1787/220262483674.

\section{Indicator D5 Tables}

StatLink : ints http://dx.doi.org/10.1787/888933286275

Table D5.1 Age distribution of teachers (2013)

Table D5.2 Age distribution of teachers (2005, 2013)

Table D5.3 Gender distribution of teachers (2013)

Table D5.4a Teachers' skills and readiness to use information and communication technologies for problem solving (2012)

Table D5.4b Teachers' use of information and communication technologies at work, ICT skills required at work, and teachers' confidence in their computer skills (2012) 
Table D5.1. Age distribution of teachers (2013)

Percentage of teachers in public and private institutions, by level of education and age group, based on head counts

\begin{tabular}{|c|c|c|c|c|c|c|c|c|c|c|c|c|c|c|}
\hline \multicolumn{5}{|c|}{ Primary } & \multicolumn{5}{|c|}{ Lower secondary } & \multicolumn{5}{|c|}{ Upper secondary } \\
\hline $\begin{array}{l}<30 \\
\text { years }\end{array}$ & $\begin{array}{l}30-39 \\
\text { years }\end{array}$ & $\begin{array}{l}40-49 \\
\text { years }\end{array}$ & $\begin{array}{l}50-59 \\
\text { years }\end{array}$ & $\begin{array}{l}>=60 \\
\text { years }\end{array}$ & $\begin{array}{l}<30 \\
\text { years }\end{array}$ & $\begin{array}{l}\text { 30-39 } \\
\text { years }\end{array}$ & $\begin{array}{l}40-49 \\
\text { years }\end{array}$ & $\begin{array}{l}50-59 \\
\text { years }\end{array}$ & $\begin{array}{l}>=60 \\
\text { years }\end{array}$ & $\begin{array}{l}<30 \\
\text { years }\end{array}$ & $\begin{array}{l}30-39 \\
\text { years }\end{array}$ & $\begin{array}{l}40-49 \\
\text { years }\end{array}$ & $\begin{array}{l}50-59 \\
\text { years }\end{array}$ & $\begin{array}{l}>=60 \\
\text { years }\end{array}$ \\
\hline (1) & (2) & (3) & (4) & (5) & (6) & (7) & (8) & (9) & (10) & (11) & (12) & (13) & (14) & (15) \\
\hline
\end{tabular}

Q Australia

ठै Austria

Belgium
Canada $^{1,2}$

Chile

Czech Republic

Denmark

Estonia $^{3}$

Finland

France

Germany

Greece

Hungary ${ }^{4}$

Iceland

Ireland $^{5}$

Israel $^{5}$

Italy

$\mathrm{Japan}^{3}$

\begin{tabular}{c|c|c|c|c|c|c|c|c|c|c|c|c|c|c|c|}
$(1)$ & $(2)$ & $(3)$ & $(4)$ & $(5)$ & $(6)$ & $(7)$ & $(8)$ & $(9)$ & $(10)$ & $(11)$ & $(12)$ & $(13)$ & $(14)$ & $(15)$ \\
\hline $\mathrm{m}$ & $\mathrm{m}$ & $\mathrm{m}$ & $\mathrm{m}$ & $\mathrm{m}$ & $\mathrm{m}$ & $\mathrm{m}$ & $\mathrm{m}$ & $\mathrm{m}$ & $\mathrm{m}$ & $\mathrm{m}$ & $\mathrm{m}$ & $\mathrm{m}$ & $\mathrm{m}$ & $\mathrm{m}$
\end{tabular}

Korea

Luxembourg

Mexico

Netherlands ${ }^{5}$

New Zealand

Norway $^{3}$

Poland

Portugal $^{3}$

Slovak Republic

Slovenia

Spain

Sweden

Switzerland ${ }^{3}$

Turkey

United Kingdom

United States

\begin{tabular}{|c|c|c|c|}
\hline $\mathrm{m}$ & $\mathrm{m}$ & $\mathrm{m}$ & $\mathrm{m}$ \\
\hline 31 & 34 & 3 & 7 \\
\hline 25 & 21 & 1 & 18 \\
\hline $29^{d}$ & $22^{\mathrm{d}}$ & $5^{\mathrm{d}}$ & $\mathrm{x}(1)$ \\
\hline 20 & 20 & 7 & 21 \\
\hline 38 & 27 & 4 & 9 \\
\hline $\mathrm{x}(8)$ & $\mathrm{x}(9)$ & $\mathrm{x}(10)$ & $5^{\mathrm{d}}$ \\
\hline 33 & 27 & 11 & 8 \\
\hline 32 & 26 & 4 & 9 \\
\hline 32 & 23 & 1 & 9 \\
\hline 26 & 31 & 14 & 7 \\
\hline 27 & 46 & 3 & 1 \\
\hline 36 & 33 & 1 & 6 \\
\hline 30 & 24 & 12 & 7 \\
\hline 17 & 19 & 3 & $\mathrm{x}(11)$ \\
\hline 27 & 18 & 3 & 11 \\
\hline 35 & 43 & 13 & 0 \\
\hline 30 & 30 & 1 & 13 \\
\hline 24 & 13 & 2 & 13 \\
\hline 23 & 18 & 1 & 22 \\
\hline $\mathrm{m}$ & $\mathrm{m}$ & $\mathrm{m}$ & $\mathrm{m}$ \\
\hline 20 & 29 & 8 & 14 \\
\hline 26 & 27 & 13 & 11 \\
\hline 27 & 21 & 12 & 12 \\
\hline 41 & 22 & 2 & 10 \\
\hline 33 & 31 & 3 & 1 \\
\hline 34 & 23 & 4 & 14 \\
\hline 35 & 27 & 1 & 6 \\
\hline 25 & 28 & 5 & 3 \\
\hline 29 & 24 & 15 & 6 \\
\hline 24 & 29 & 6 & 11 \\
\hline 27 & 11 & 1 & 35 \\
\hline 23 & 13 & 3 & 22 \\
\hline 25 & 24 & 8 & 17 \\
\hline
\end{tabular}

\begin{tabular}{|c|c|c|c|c|c|c|c|c|}
\hline $\mathrm{m}$ & $\mathrm{m}$ & $\mathrm{m}$ & $\mathrm{m}$ & $\mathrm{m}$ & $\mathrm{m}$ & $\mathrm{m}$ & $\mathrm{m}$ & $\mathrm{m}$ \\
\hline 17 & 27 & 45 & 4 & 6 & 20 & 32 & 37 & 5 \\
\hline 28 & 25 & 26 & 3 & 15 & 27 & 26 & 28 & 3 \\
\hline$x(2)$ & $x(3)$ & $\mathrm{x}(4)$ & $x(5)$ & 13 & 32 & 29 & 22 & 5 \\
\hline 28 & 19 & 21 & 9 & 20 & 29 & 20 & 22 & 9 \\
\hline 25 & 35 & 27 & 5 & 6 & 21 & 28 & 35 & 10 \\
\hline $30^{\mathrm{d}}$ & $29^{d}$ & $25^{\mathrm{d}}$ & $11^{\mathrm{d}}$ & $\mathrm{m}$ & $\mathrm{m}$ & $\mathrm{m}$ & $\mathrm{m}$ & $\mathrm{m}$ \\
\hline 17 & 26 & 31 & 19 & $8^{\mathrm{d}}$ & $18^{\mathrm{d}}$ & $24^{\mathrm{d}}$ & $31^{\mathrm{d}}$ & $19^{\mathrm{d}}$ \\
\hline 31 & 31 & 25 & 5 & 5 & 21 & 31 & 31 & 12 \\
\hline 33 & 31 & 23 & 5 & 4 & 22 & 36 & 29 & 8 \\
\hline 19 & 23 & 35 & 15 & 4 & 22 & 29 & 32 & 13 \\
\hline 20 & 41 & 34 & 3 & 1 & 18 & 41 & 36 & 4 \\
\hline 23 & 33 & 36 & 2 & 7 & 31 & 30 & 28 & 5 \\
\hline 28 & 30 & 24 & 12 & $\mathrm{~m}$ & $\mathrm{~m}$ & $\mathrm{~m}$ & $\mathrm{~m}$ & $\mathrm{~m}$ \\
\hline (12) & $\mathrm{x}(13)$ & $x(14)$ & $x(15)$ & $8^{\mathrm{d}}$ & $36^{\mathrm{d}}$ & $27^{\mathrm{d}}$ & $24^{\mathrm{d}}$ & $5^{\mathrm{d}}$ \\
\hline 31 & 31 & 22 & 6 & 10 & 29 & 27 & 23 & 12 \\
\hline 8 & 29 & 44 & 19 & 0 & 3 & 24 & 57 & 16 \\
\hline 25 & 34 & 26 & 1 & $9^{d}$ & $24^{\mathrm{d}}$ & $33^{\mathrm{d}}$ & $30^{\mathrm{d}}$ & $4^{\mathrm{d}}$ \\
\hline 33 & 32 & 22 & 1 & 12 & 32 & 28 & 27 & 1 \\
\hline 39 & 22 & 15 & 2 & 11 & 30 & 30 & 25 & 4 \\
\hline $\mathrm{m}$ & $\mathrm{m}$ & $\mathrm{m}$ & $\mathrm{m}$ & $\mathrm{m}$ & $\mathrm{m}$ & $\mathrm{m}$ & $\mathrm{m}$ & $\mathrm{m}$ \\
\hline 23 & 21 & 31 & 11 & 9 & 18 & 22 & 37 & 14 \\
\hline 23 & 24 & 28 & 14 & 10 & 22 & 25 & 29 & 15 \\
\hline 28 & 27 & 21 & 12 & $6^{\mathrm{d}}$ & $20^{\mathrm{d}}$ & $28^{\mathrm{d}}$ & $27^{\mathrm{d}}$ & $18^{\mathrm{d}}$ \\
\hline 36 & 33 & 20 & 2 & 8 & 33 & 30 & 23 & 7 \\
\hline 25 & 41 & 30 & 3 & $3^{\mathrm{d}}$ & $30^{\mathrm{d}}$ & $38^{\mathrm{d}}$ & $25^{\mathrm{d}}$ & $3^{\mathrm{d}}$ \\
\hline 28 & 22 & 29 & 7 & 10 & 25 & 25 & 32 & 9 \\
\hline 34 & 28 & 30 & 2 & 4 & 24 & 38 & 28 & 6 \\
\hline 26 & 37 & 29 & 5 & 3 & 26 & 37 & 30 & 5 \\
\hline 25 & 29 & 24 & 16 & 6 & 23 & 27 & 27 & 17 \\
\hline 28 & 25 & 28 & 8 & $6^{\mathrm{d}}$ & $23^{\mathrm{d}}$ & $30^{\mathrm{d}}$ & $31^{\mathrm{d}}$ & $10^{\mathrm{d}}$ \\
\hline 41 & 16 & 7 & 0 & $\mathrm{~m}$ & $\mathrm{~m}$ & $\mathrm{~m}$ & $\mathrm{~m}$ & $\mathrm{~m}$ \\
\hline 33 & 24 & 18 & 4 & 17 & 29 & 25 & 21 & 7 \\
\hline 29 & 25 & 22 & 8 & 14 & 27 & 26 & 23 & 10 \\
\hline
\end{tabular}

\begin{tabular}{l|l|l|l|l|l|l|l|l|l|l|l|l|l|l|l|} 
OECD average & 13 & 28 & 28 & 25 & 5 & 11 & 27 & 28 & 27 & 7 & 8 & 25 & 29 & 29 & 9 \\
EU21 average & 11 & 27 & 30 & 27 & 5 & 9 & 26 & 29 & 29 & 7 & 7 & 24 & 30 & 31 & 9
\end{tabular}

\begin{tabular}{|c|c|c|c|c|c|c|c|c|c|c|c|c|c|c|c|}
\hline Argentina & $\mathrm{m}$ & $\mathrm{m}$ & $\mathrm{m}$ & $\mathrm{m}$ & $\mathrm{m}$ & $\mathrm{m}$ & $\mathrm{m}$ & $\mathrm{m}$ & $\mathrm{m}$ & $\mathrm{m}$ & $\mathrm{m}$ & $\mathrm{m}$ & $\mathrm{m}$ & $\mathrm{m}$ & $\mathrm{m}$ \\
\hline Brazil & 17 & 36 & 33 & 13 & 2 & 18 & 35 & 30 & 15 & 3 & 18 & 34 & 30 & 16 & 3 \\
\hline China & 21 & 35 & 27 & 17 & 0 & 22 & 42 & 28 & 8 & 0 & 26 & 40 & 28 & 6 & 0 \\
\hline Colombia & 6 & 21 & 34 & 30 & 9 & 5 & 24 & 32 & 30 & 9 & 5 & 24 & 32 & 29 & 9 \\
\hline India & $\mathrm{m}$ & $\mathrm{m}$ & $\mathrm{m}$ & $\mathrm{m}$ & $\mathrm{m}$ & $\mathrm{m}$ & $\mathrm{m}$ & $\mathrm{m}$ & $\mathrm{m}$ & $\mathrm{m}$ & $\mathrm{m}$ & $\mathrm{m}$ & $\mathrm{m}$ & $\mathrm{m}$ & $\mathrm{m}$ \\
\hline Indonesia & 19 & 25 & 26 & 30 & 0 & 14 & 26 & 41 & 19 & 0 & 14 & 32 & 36 & 18 & 0 \\
\hline Latvia & 9 & 21 & 34 & 28 & 8 & 6 & 18 & 32 & 34 & 10 & 7 & 17 & 29 & 33 & 13 \\
\hline Russian Federation & $\mathrm{m}$ & $\mathrm{m}$ & $\mathrm{m}$ & $\mathrm{m}$ & $\mathrm{m}$ & $\mathrm{m}$ & $\mathrm{m}$ & $\mathrm{m}$ & $\mathrm{m}$ & $\mathrm{m}$ & $\mathrm{m}$ & $\mathrm{m}$ & $\mathrm{m}$ & $\mathrm{m}$ & $\mathrm{m}$ \\
\hline Saudi Arabia & $\mathrm{m}$ & $\mathrm{m}$ & $\mathrm{m}$ & $\mathrm{m}$ & $\mathrm{m}$ & $\mathrm{m}$ & $\mathrm{m}$ & $\mathrm{m}$ & $\mathrm{m}$ & $\mathrm{m}$ & $\mathrm{m}$ & $\mathrm{m}$ & $\mathrm{m}$ & $\mathrm{m}$ & $\mathrm{m}$ \\
\hline South Africa & $\mathrm{m}$ & $\mathrm{m}$ & $\mathrm{m}$ & $\mathrm{m}$ & $\mathrm{m}$ & $\mathrm{m}$ & $\mathrm{m}$ & $\mathrm{m}$ & $\mathrm{m}$ & $\mathrm{m}$ & $\mathrm{m}$ & $\mathrm{m}$ & $\mathrm{m}$ & $\mathrm{m}$ & $\mathrm{m}$ \\
\hline
\end{tabular}

G20 average

1. Year of reference 2012

2. Primary includes pre-primary.

3. Upper secondary includes programmes from post-secondary non-tertiary.

4. Includes data on management personnel.

5. Public institutions only. For Israel, public institutions only for upper secondary education.

Source: OECD. Argentina, China, Colombia, India, Indonesia, Saudi Arabia, South Africa: UNESCO Institute for Statistics. Latvia: Eurostat. See Annex 3 for notes (www.oecd.org/education/education-at-a-glance-19991487.htm).

Please refer to the Reader's Guide for information concerning symbols for missing data and abbreviations.

StatLink 겍ㄴ http://dx.doi.org/10.1787/888933286280 
Table D5.2. Age distribution of teachers $(2005,2013)$

Percentage of teachers in public and private institutions, by level of education and age group, based on head counts

\begin{tabular}{|c|c|c|c|c|c|c|c|c|c|c|c|}
\hline & \multicolumn{5}{|c|}{ Secondary (2013) } & \multicolumn{5}{|c|}{ Secondary (2005) } & \multirow{2}{*}{$\begin{array}{c}\begin{array}{c}\text { Percentage of teachers } \\
\text { aged } 50 \text { years or older }\end{array} \\
\begin{array}{c}\text { Average annual growth rate } \\
(2005-2013)\end{array}\end{array}$} \\
\hline & $\begin{array}{l}<30 \\
\text { years }\end{array}$ & $\begin{array}{l}30-39 \\
\text { years }\end{array}$ & $\begin{array}{l}40-49 \\
\text { years }\end{array}$ & $\begin{array}{l}50-59 \\
\text { years }\end{array}$ & $\begin{array}{l}>=60 \\
\text { years }\end{array}$ & $\begin{array}{c}<30 \\
\text { years }\end{array}$ & $\begin{array}{l}30-39 \\
\text { years }\end{array}$ & $\begin{array}{l}40-49 \\
\text { years }\end{array}$ & $\begin{array}{l}50-59 \\
\text { years }\end{array}$ & $\begin{array}{l}>=60 \\
\text { years }\end{array}$ & \\
\hline & (1) & (2) & (3) & (4) & (5) & (6) & (7) & (8) & (9) & (10) & (11) \\
\hline Australia & $\mathrm{m}$ & $\mathrm{m}$ & $\mathrm{m}$ & $\mathrm{m}$ & $\mathrm{m}$ & $\mathrm{m}$ & $\mathrm{m}$ & $\mathrm{m}$ & $\mathrm{m}$ & $\mathrm{m}$ & $\mathrm{m}$ \\
\hline Austria & 7 & 18 & 30 & 41 & 4 & 7 & 22 & 45 & 25 & 1 & 7.2 \\
\hline Belgium & 17 & 28 & 26 & 28 & 3 & 17 & 23 & 31 & 27 & 2 & 0.2 \\
\hline Canada $^{1}$ & 13 & 32 & 29 & 22 & 5 & $\mathrm{~m}$ & $\mathrm{~m}$ & $\mathrm{~m}$ & $\mathrm{~m}$ & $\mathrm{~m}$ & $\mathrm{~m}$ \\
\hline Chile & 21 & 29 & 20 & 22 & 9 & 12 & 25 & 30 & 25 & 7 & -0.8 \\
\hline Czech Republic & 7 & 23 & 31 & 31 & 8 & $\mathrm{~m}$ & $\mathrm{~m}$ & $\mathrm{~m}$ & $\mathrm{~m}$ & $\mathrm{~m}$ & $\mathrm{~m}$ \\
\hline Denmark & $\mathrm{m}$ & $\mathrm{m}$ & $\mathrm{m}$ & $\mathrm{m}$ & $\mathrm{m}$ & $\mathrm{m}$ & $\mathrm{m}$ & $\mathrm{m}$ & $\mathrm{m}$ & $\mathrm{m}$ & $\mathrm{m}$ \\
\hline Estonia $^{2}$ & $8^{d}$ & $17^{\mathrm{d}}$ & $25^{\mathrm{d}}$ & $31^{\mathrm{d}}$ & $19^{d}$ & $\mathrm{~m}$ & $\mathrm{~m}$ & $\mathrm{~m}$ & $\mathrm{~m}$ & $\mathrm{~m}$ & $\mathrm{~m}$ \\
\hline Finland & 7 & 25 & 31 & 28 & 9 & 8 & 25 & 30 & 32 & 5 & 0.1 \\
\hline France & 6 & 28 & 34 & 26 & 7 & 12 & 29 & 24 & 34 & 1 & -1.0 \\
\hline Germany $^{3}$ & 6 & 20 & 25 & 34 & 14 & 3 & 18 & 26 & 44 & 9 & -1.0 \\
\hline Greece & 1 & 19 & 41 & 35 & 4 & 6 & 24 & 41 & 27 & 2 & 3.8 \\
\hline Hungary ${ }^{4}$ & 6 & 27 & 31 & 32 & 3 & 15 & 26 & 30 & 24 & 4 & 2.6 \\
\hline Iceland $^{5}$ & $\mathrm{~m}$ & $\mathrm{~m}$ & $\mathrm{~m}$ & $\mathrm{~m}$ & $\mathrm{~m}$ & $11^{\mathrm{d}}$ & $27^{\mathrm{d}}$ & $30^{\mathrm{d}}$ & $25^{d}$ & $8^{d}$ & $\mathrm{~m}$ \\
\hline Ireland $^{6}$ & 8 & 36 & 27 & 24 & 5 & 11 & 25 & 27 & 29 & 7 & -2.8 \\
\hline Israel $^{6}$ & 10 & 30 & 28 & 23 & 10 & 10 & 29 & 30 & 26 & 5 & 0.6 \\
\hline Italy & 0 & 5 & 26 & 51 & 18 & 0 & 6 & 32 & 55 & 8 & 1.3 \\
\hline $\mathrm{Japan}^{2,7}$ & $11^{\mathrm{d}}$ & $24^{\mathrm{d}}$ & $34^{\mathrm{d}}$ & $28^{\mathrm{d}}$ & $3^{d}$ & 9 & 28 & 40 & 21 & 2 & 3.7 \\
\hline Korea & 13 & 32 & 30 & 24 & 1 & 17 & 30 & 40 & 12 & 1 & 8.5 \\
\hline Luxembourg $^{8}$ & 15 & 33 & 27 & 22 & 3 & 18 & 25 & 26 & 29 & 2 & -2.6 \\
\hline Mexico & $\mathrm{m}$ & $\mathrm{m}$ & $\mathrm{m}$ & $\mathrm{m}$ & $\mathrm{m}$ & $\mathrm{m}$ & $\mathrm{m}$ & $\mathrm{m}$ & $\mathrm{m}$ & $\mathrm{m}$ & $\mathrm{m}$ \\
\hline Netherlands ${ }^{6,8}$ & 12 & 21 & 21 & 34 & 12 & 10 & 17 & 31 & 37 & 5 & 1.1 \\
\hline New Zealand & 10 & 22 & 25 & 28 & 15 & 14 & 21 & 29 & 29 & 8 & 2.0 \\
\hline Norway $^{2}$ & $9^{d}$ & $24^{\mathrm{d}}$ & $27^{\mathrm{d}}$ & $25^{\mathrm{d}}$ & $15^{\mathrm{d}}$ & $\mathrm{m}$ & $\mathrm{m}$ & $\mathrm{m}$ & $\mathrm{m}$ & $\mathrm{m}$ & $\mathrm{m}$ \\
\hline Poland & 9 & 34 & 31 & 21 & 5 & 16 & 33 & 29 & 19 & 3 & 2.1 \\
\hline Portugal $^{2}$ & $2^{d}$ & $28^{\mathrm{d}}$ & $39^{d}$ & $27^{\mathrm{d}}$ & $3^{d}$ & 16 & 35 & 31 & 16 & 2 & 6.2 \\
\hline Slovak Republic & 12 & 27 & 23 & 30 & 8 & 16 & 21 & 25 & 30 & 7 & 0.1 \\
\hline Slovenia & 5 & 29 & 33 & 29 & 4 & 11 & 33 & 34 & 20 & 2 & 5.0 \\
\hline Spain & 3 & 26 & 37 & 29 & 5 & 8 & 32 & 35 & 21 & 4 & 3.8 \\
\hline Sweden & 6 & 24 & 28 & 26 & 16 & 10 & 24 & 24 & 30 & 13 & -0.3 \\
\hline Switzerland $^{2}$ & $9^{d}$ & $26^{\mathrm{d}}$ & $27^{\mathrm{d}}$ & $29^{d}$ & $9^{d}$ & 13 & 24 & 30 & 28 & 5 & 1.7 \\
\hline Turkey & 35 & 41 & 16 & 7 & 0 & $\mathrm{~m}$ & $\mathrm{~m}$ & $\mathrm{~m}$ & $\mathrm{~m}$ & $\mathrm{~m}$ & $\mathrm{~m}$ \\
\hline United Kingdom & 19 & 30 & 25 & 20 & 6 & 15 & 24 & 28 & 31 & 2 & -2.9 \\
\hline United States & 16 & 28 & 25 & 23 & 9 & 17 & 26 & 23 & 26 & 8 & -0.8 \\
\hline
\end{tabular}

\begin{tabular}{|c|c|c|c|c|c|c|c|c|c|c|c|}
\hline OECD average & 10 & 26 & 28 & 28 & 8 & 12 & 25 & 31 & 27 & 5 & $\sim$ \\
\hline $\begin{array}{l}\text { Average for countries } \\
\text { with available data for } \\
\text { both reference years }\end{array}$ & 9 & 26 & 29 & 28 & 7 & 12 & 25 & 30 & 28 & 5 & 1.5 \\
\hline EU21 average & 8 & 25 & 30 & 30 & 8 & 11 & 25 & 30 & 29 & 4 & $\sim$ \\
\hline
\end{tabular}

\begin{tabular}{|c|c|c|c|c|c|c|c|c|c|c|c|}
\hline Argentina & $\mathrm{m}$ & $\mathrm{m}$ & $\mathrm{m}$ & $\mathrm{m}$ & $\mathrm{m}$ & $\mathrm{m}$ & $\mathrm{m}$ & $\mathrm{m}$ & $\mathrm{m}$ & $\mathrm{m}$ & $\mathrm{m}$ \\
\hline Brazil & 18 & 34 & 30 & 15 & 3 & $\mathrm{~m}$ & $\mathrm{~m}$ & $\mathrm{~m}$ & $\mathrm{~m}$ & $\mathrm{~m}$ & $\mathrm{~m}$ \\
\hline China & 24 & 41 & 28 & 7 & 0 & $\mathrm{~m}$ & $\mathrm{~m}$ & $\mathrm{~m}$ & $\mathrm{~m}$ & $\mathrm{~m}$ & $\mathrm{~m}$ \\
\hline Colombia & 5 & 24 & 32 & 30 & 9 & $\mathrm{~m}$ & $\mathrm{~m}$ & $\mathrm{~m}$ & $\mathrm{~m}$ & $\mathrm{~m}$ & $\mathrm{~m}$ \\
\hline India & $\mathrm{m}$ & $\mathrm{m}$ & $\mathrm{m}$ & $\mathrm{m}$ & $\mathrm{m}$ & $\mathrm{m}$ & $\mathrm{m}$ & $\mathrm{m}$ & $\mathrm{m}$ & $\mathrm{m}$ & $\mathrm{m}$ \\
\hline Indonesia & 14 & 29 & 39 & 18 & 0 & $\mathrm{~m}$ & $\mathrm{~m}$ & $\mathrm{~m}$ & $\mathrm{~m}$ & $\mathrm{~m}$ & $\mathrm{~m}$ \\
\hline Latvia & 7 & 18 & 30 & 34 & 12 & $\mathrm{~m}$ & $\mathrm{~m}$ & $\mathrm{~m}$ & $\mathrm{~m}$ & $\mathrm{~m}$ & $\mathrm{~m}$ \\
\hline Russian Federation & $\mathrm{m}$ & $\mathrm{m}$ & $\mathrm{m}$ & $\mathrm{m}$ & $\mathrm{m}$ & $\mathrm{m}$ & $\mathrm{m}$ & $\mathrm{m}$ & $\mathrm{m}$ & $\mathrm{m}$ & $\mathrm{m}$ \\
\hline Saudi Arabia & $\mathrm{m}$ & $\mathrm{m}$ & $\mathrm{m}$ & $\mathrm{m}$ & $\mathrm{m}$ & $\mathrm{m}$ & $\mathrm{m}$ & $\mathrm{m}$ & $\mathrm{m}$ & $\mathrm{m}$ & $\mathrm{m}$ \\
\hline South Africa & $\mathrm{m}$ & $\mathrm{m}$ & $\mathrm{m}$ & $\mathrm{m}$ & $\mathrm{m}$ & $\mathrm{m}$ & $\mathrm{m}$ & $\mathrm{m}$ & $\mathrm{m}$ & $\mathrm{m}$ & $\mathrm{m}$ \\
\hline G20 average & 15 & 29 & 28 & 23 & 5 & $\mathrm{~m}$ & $\mathrm{~m}$ & $\mathrm{~m}$ & $\mathrm{~m}$ & $\mathrm{~m}$ & $\mathrm{~m}$ \\
\hline
\end{tabular}

1. Year of reference 2012.

2. Upper secondary includes programmes from post-secondary non-tertiary.

3. Year of reference 2006 instead of 2005.

4. Includes data on management personnel.

5. Secondary includes primary education.

6. Public institutions only. For Israel, public institutions only for upper secondary education.

7. Year of reference 2004 instead of 2005.

8. Secondary in 2005 only includes upper secondary education.

Source: OECD. Argentina, China, Colombia, India, Indonesia, Saudi Arabia, South Africa: UNESCO Institute for Statistics. Latvia: Eurostat. See Annex 3 for notes (www.oecd.org/education/education-at-a-glance-19991487.htm).

Please refer to the Reader's Guide for information concerning symbols for missing data and abbreviations.

StatLink 제피 http://dx.doi.org/10.1787/888933286291 
Table D5.3. Gender distribution of teachers (2013)

Percentage of women among teaching staff in public and private institutions by level of education, based on head counts

\begin{tabular}{|c|c|c|c|c|c|c|c|c|c|c|c|}
\hline & \multirow[b]{2}{*}{$\begin{array}{c}\text { Pre- } \\
\text { primary } \\
\text { education }\end{array}$} & \multirow[b]{2}{*}{ Primary } & \multirow[b]{2}{*}{$\begin{array}{c}\text { Lower } \\
\text { secondary }\end{array}$} & \multicolumn{3}{|c|}{ Upper secondary } & \multirow[b]{2}{*}{$\begin{array}{c}\text { Post- } \\
\text { secondary } \\
\text { non- } \\
\text { tertiary }\end{array}$} & \multicolumn{3}{|c|}{ Tertiary } & \multirow[b]{2}{*}{$\begin{array}{l}\text { All levels } \\
\text { of } \\
\text { education }\end{array}$} \\
\hline & & & & 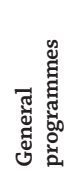 & 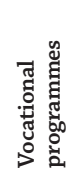 & 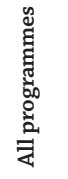 & & 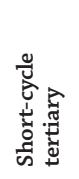 & 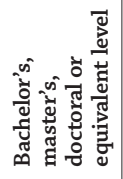 & 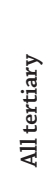 & \\
\hline & (1) & (2) & (3) & (4) & (5) & (6) & (7) & (8) & (9) & (10) & (11) \\
\hline Australia & $\mathrm{m}$ & $\mathrm{m}$ & $\mathrm{m}$ & $\mathrm{m}$ & $\mathrm{m}$ & $\mathrm{m}$ & $\mathrm{m}$ & $\mathrm{m}$ & 44 & 44 & $\mathrm{~m}$ \\
\hline Austria & 99 & 91 & 72 & 62 & 49 & 54 & 68 & 53 & 40 & 42 & 65 \\
\hline Belgium & 97 & 82 & 63 & 62 & 62 & 62 & 46 & $\mathrm{x}(10)$ & $\mathrm{x}(10)$ & 47 & 70 \\
\hline Canada $^{1}$ & $x(2)$ & $73^{\mathrm{d}}$ & $\mathrm{x}(2)$ & $73^{\mathrm{d}}$ & $\mathrm{x}(4)$ & 73 & $\mathrm{~m}$ & 54 & 43 & 49 & $\mathrm{~m}$ \\
\hline Chile & 99 & 81 & 68 & 57 & 50 & 55 & a & $\mathrm{m}$ & $\mathrm{m}$ & $\mathrm{m}$ & $\mathrm{m}$ \\
\hline Czech Republic & 100 & 97 & 74 & 59 & 59 & 59 & 96 & 81 & 38 & 38 & 75 \\
\hline Denmark & $\mathrm{m}$ & $\mathrm{x}(3)$ & $71^{\mathrm{d}}$ & $\mathrm{m}$ & $\mathrm{m}$ & $\mathrm{m}$ & $\mathrm{m}$ & $\mathrm{m}$ & $\mathrm{m}$ & $\mathrm{m}$ & $\mathrm{m}$ \\
\hline Estonia $^{2}$ & $100^{\mathrm{d}}$ & 92 & 82 & 78 & $64^{\mathrm{d}}$ & $72^{\mathrm{d}}$ & $\mathrm{x}(5)$ & $\mathrm{m}$ & $\mathrm{m}$ & $\mathrm{m}$ & $\mathrm{m}$ \\
\hline Finland & 97 & 79 & 72 & 69 & 54 & 59 & 53 & $\mathrm{a}$ & 51 & 51 & 71 \\
\hline France & 83 & 83 & 65 & 56 & 52 & 54 & $\mathrm{x}(10)$ & $39^{d}$ & $37^{\mathrm{d}}$ & $37^{\mathrm{d}}$ & 66 \\
\hline Germany & 97 & 86 & 66 & 54 & 47 & 52 & 56 & 43 & 38 & 38 & 65 \\
\hline Greece & 99 & 70 & 66 & 54 & 47 & 51 & 56 & a & 33 & 33 & 63 \\
\hline Hungary ${ }^{3}$ & 100 & 96 & 78 & 68 & 49 & 64 & 53 & 50 & 37 & 39 & 76 \\
\hline Iceland & 94 & 82 & 82 & $\mathrm{~m}$ & $\mathrm{~m}$ & $\mathrm{~m}$ & $\mathrm{~m}$ & $\mathrm{~m}$ & $\mathrm{~m}$ & $\mathrm{~m}$ & $\mathrm{~m}$ \\
\hline Ireland $^{4}$ & $\mathrm{~m}$ & 86 & $\mathrm{x}(4)$ & $71^{\mathrm{d}}$ & $\mathrm{m}$ & $71^{\mathrm{d}}$ & $\mathrm{m}$ & $\mathrm{x}(9)$ & $44^{\mathrm{d}}$ & 44 & $\mathrm{~m}$ \\
\hline Israel $^{4}$ & 99 & 85 & 79 & $70^{\mathrm{d}}$ & $x(4)$ & 70 & $\mathrm{~m}$ & $\mathrm{~m}$ & $\mathrm{~m}$ & $\mathrm{~m}$ & $\mathrm{~m}$ \\
\hline Italy & 98 & 96 & 78 & 74 & 62 & 67 & $\mathrm{~m}$ & $\mathrm{a}$ & 37 & 37 & $\mathrm{~m}$ \\
\hline Japan $^{5}$ & 97 & 65 & 42 & $\mathrm{~m}$ & $\mathrm{~m}$ & $28^{\mathrm{d}}$ & $\mathrm{x}(6,10)$ & $47^{d}$ & $19^{\mathrm{d}}$ & $25^{d}$ & 48 \\
\hline Korea & 99 & 79 & 69 & 50 & 43 & 49 & $\mathrm{~m}$ & 43 & 32 & 35 & 60 \\
\hline Luxembourg & 97 & 76 & 57 & 57 & 45 & 52 & $\mathrm{~m}$ & $\mathrm{~m}$ & $\mathrm{~m}$ & $\mathrm{~m}$ & $\mathrm{~m}$ \\
\hline Mexico & 96 & 67 & 52 & 48 & 45 & 47 & a & $\mathrm{m}$ & $\mathrm{m}$ & $\mathrm{m}$ & $\mathrm{m}$ \\
\hline Netherlands ${ }^{4}$ & 87 & 86 & 51 & 51 & 51 & 51 & 51 & (9) & $43^{d}$ & 43 & 66 \\
\hline New Zealand & 98 & 83 & 65 & 60 & 54 & 59 & 54 & 49 & 49 & 49 & 70 \\
\hline Norway $^{2}$ & $93^{\mathrm{d}}$ & 75 & 75 & $\mathrm{x}(6)$ & $\mathrm{x}(6)$ & $52^{\mathrm{d}}$ & $\mathrm{x}(6)$ & $\mathrm{x}(6)$ & 45 & 45 & 69 \\
\hline Poland & 98 & 85 & 74 & 71 & 62 & 66 & 65 & 69 & 44 & 44 & 74 \\
\hline Portugal & 99 & 79 & 71 & $68^{\mathrm{d}}$ & $\mathrm{x}(4)$ & 68 & $x(4,9)$ & $\mathrm{a}$ & $44^{\mathrm{d}}$ & $44^{\mathrm{d}}$ & 70 \\
\hline Slovak Republic & 100 & 90 & 76 & 74 & 71 & 72 & 67 & 64 & 44 & 45 & 76 \\
\hline Slovenia & 98 & 97 & 79 & 70 & 64 & 67 & a & 47 & 38 & 40 & 75 \\
\hline Spain & 95 & 76 & 58 & 55 & 47 & 52 & $\mathrm{~m}$ & 45 & 40 & 41 & $\mathrm{~m}$ \\
\hline Sweden & 96 & 77 & 77 & 52 & 54 & 53 & 43 & 43 & 44 & 44 & 74 \\
\hline Switzerland & 97 & 82 & 54 & 45 & $42^{\mathrm{d}}$ & $43^{\mathrm{d}}$ & $\mathrm{x}(5)$ & $\mathrm{m}$ & 33 & 33 & 60 \\
\hline Turkey & 94 & 58 & 52 & 45 & 44 & 44 & a & 34 & 43 & 42 & 53 \\
\hline United Kingdom & 90 & 87 & 63 & 63 & 59 & 62 & a & 48 & 44 & 44 & 69 \\
\hline United States & 94 & 87 & 67 & $57^{d}$ & $\mathrm{x}(4)$ & 57 & $x(10)$ & $\mathrm{x}(10)$ & $\mathrm{x}(10)$ & $49^{d}$ & 70 \\
\hline OECD average & 96 & 82 & 68 & 61 & 53 & 58 & 59 & 51 & 40 & 42 & 67 \\
\hline EU21 average & 96 & 86 & 70 & 63 & 55 & 60 & 60 & 53 & 41 & 42 & 70 \\
\hline
\end{tabular}

\begin{tabular}{|c|c|c|c|c|c|c|c|c|c|c|c|}
\hline $\begin{array}{l}\text { Argentina } \\
\text { Brazil }\end{array}$ & $\begin{array}{l}\mathrm{m} \\
96\end{array}$ & $\begin{array}{r}\mathrm{m} \\
90\end{array}$ & $\begin{array}{r}\mathrm{m} \\
70\end{array}$ & $\begin{array}{l}\mathrm{m} \\
62\end{array}$ & $\begin{array}{c}\mathrm{m} \\
51\end{array}$ & $\begin{array}{l}\mathrm{m} \\
60\end{array}$ & $\begin{array}{l}\mathrm{m} \\
46\end{array}$ & $\begin{array}{r}m \\
43\end{array}$ & $\begin{array}{l}\mathrm{m} \\
45\end{array}$ & $\begin{array}{l}\mathrm{m} \\
45\end{array}$ & $\begin{array}{l}\mathrm{m} \\
72\end{array}$ \\
\hline china & 97 & 60 & 51 & 49 & 49 & 49 & $\mathrm{x}(10)$ & $47^{\mathrm{d}}$ & $44^{\mathrm{d}}$ & $45^{\mathrm{d}}$ & 58 \\
\hline Colombia & 96 & 77 & 54 & $46^{\mathrm{d}}$ & $\mathrm{x}(4)$ & 46 & 64 & $\mathrm{x}(9)$ & $36^{\mathrm{d}}$ & 36 & 61 \\
\hline India & $\mathrm{m}$ & $\mathrm{m}$ & $\mathrm{m}$ & $\mathrm{m}$ & $\mathrm{m}$ & $\mathrm{m}$ & $\mathrm{m}$ & $\mathrm{m}$ & $\mathrm{m}$ & $\mathrm{m}$ & $\mathrm{m}$ \\
\hline Indonesia & 95 & 62 & 53 & 52 & 49 & 51 & a & (9) & $39^{d}$ & 39 & 60 \\
\hline Latvia & 99 & 93 & 84 & 85 & 70 & 81 & 70 & 68 & 54 & 56 & 83 \\
\hline Russian Federation & 99 & 99 & $83^{\mathrm{d}}$ & $\mathrm{x}(3)$ & $\mathrm{x}(7,8)$ & $x(3,7,8)$ & $63^{\mathrm{d}}$ & $73^{\mathrm{d}}$ & 52 & $58^{\mathrm{d}}$ & 82 \\
\hline South Africa & $\mathrm{m}$ & $\mathrm{m}$ & $\mathrm{m}$ & $\mathrm{m}$ & $\mathrm{m}$ & $\mathrm{m}$ & $\mathrm{m}$ & $\mathrm{m}$ & $\mathrm{m}$ & $\mathrm{m}$ & $\mathrm{m}$ \\
\hline G20 average & 95 & 78 & 62 & 57 & 50 & 53 & 55 & 47 & 40 & 42 & 64 \\
\hline
\end{tabular}

Note: The data in "All levels of education" does not include early childhood educational development (ISCED 01).

1. Year of reference 2012

2. Pre-primary includes early childhood education

3. Includes data on management personnel.

4. Public institutions only. For the Netherlands, private data are available and included for pre-primary education. For Israel, public institutions only for pre-primary and upper secondary education.

5. Upper secondary includes programmes from post-secondary non-tertiary.

Source: OECD. Argentina, China, Colombia, India, Indonesia, Saudi Arabia, South Africa: UNESCO Institute for Statistics. Latvia: Eurostat. See Annex 3 for notes (www.oecd.org/education/education-at-a-glance-19991487.htm).

Please refer to the Reader's Guide for information concerning symbols for missing data and abbreviations.

StatLink 제의 http://dx.doi.org/10.1787/888933286309 
Table D5.4a. Teachers' skills and readiness to use information and communication technologies for problem solving (2012)

Teachers who teach both pre-primary and primary school, primary teachers and secondary teachers, 25-64 year-olds

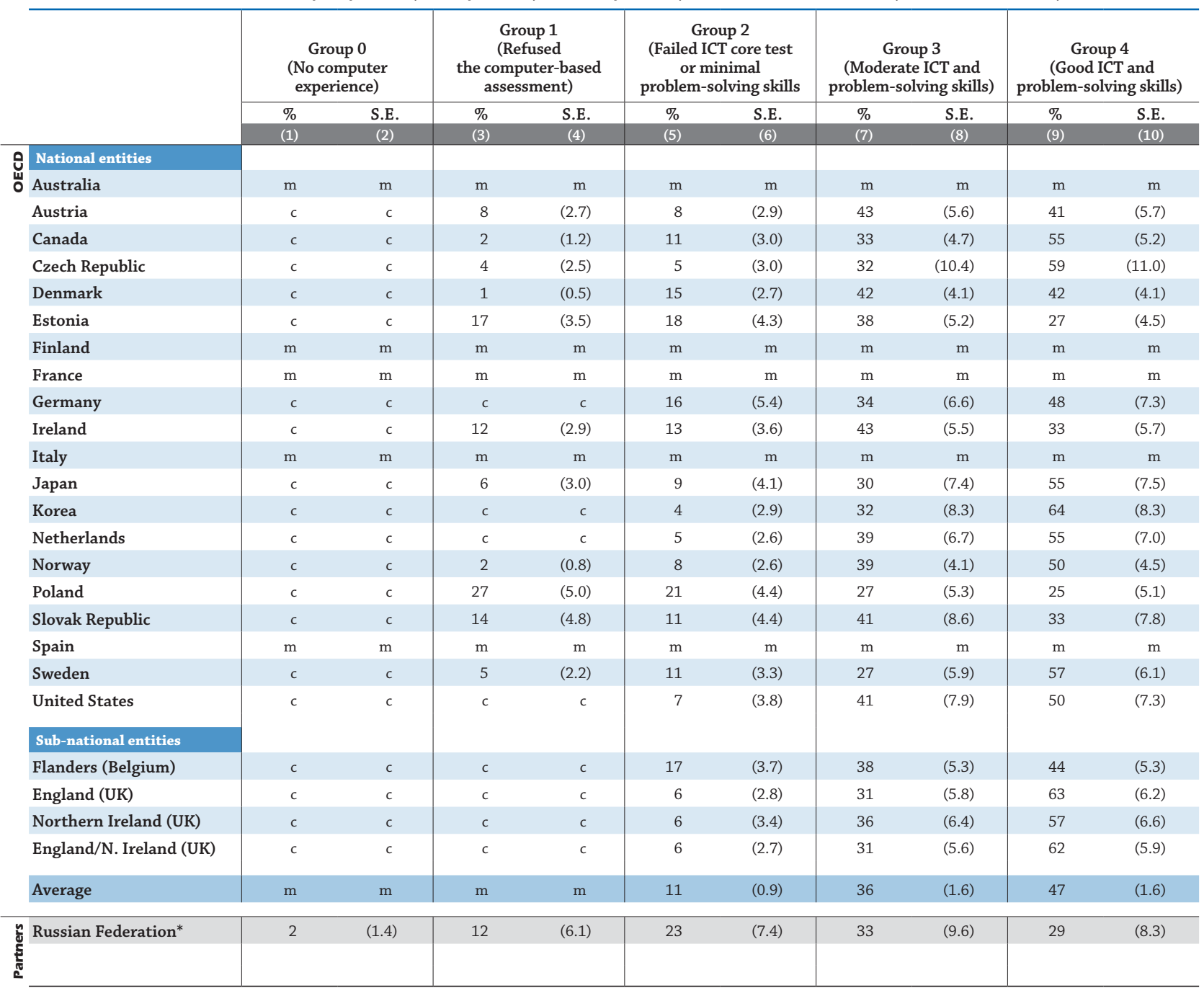

Note: Teachers who teach both pre-primary and primary school, primary teachers and secondary teachers refer to teachers who were currently working as teachers at the moment of the survey.

* See note on data for the Russian Federation in the Methodology section.

Source: OECD. Survey of Adult Skills (PIAAC) (2012). See Annex 3 for notes (www.oecd.org/education/education-at-a-glance-19991487.htm).

Please refer to the Reader's Guide for information concerning symbols for missing data and abbreviations.

StatLink 제그 http://dx.doi.org/10.1787/888933286310 
Table D5.4b. Teachers' use of information and communication technologies at work, ICT skills required at work, and teachers' confidence in their computer skills (2012) Teachers who teach both pre-primary and primary school, primary teachers and secondary teachers, 25-64 year-olds

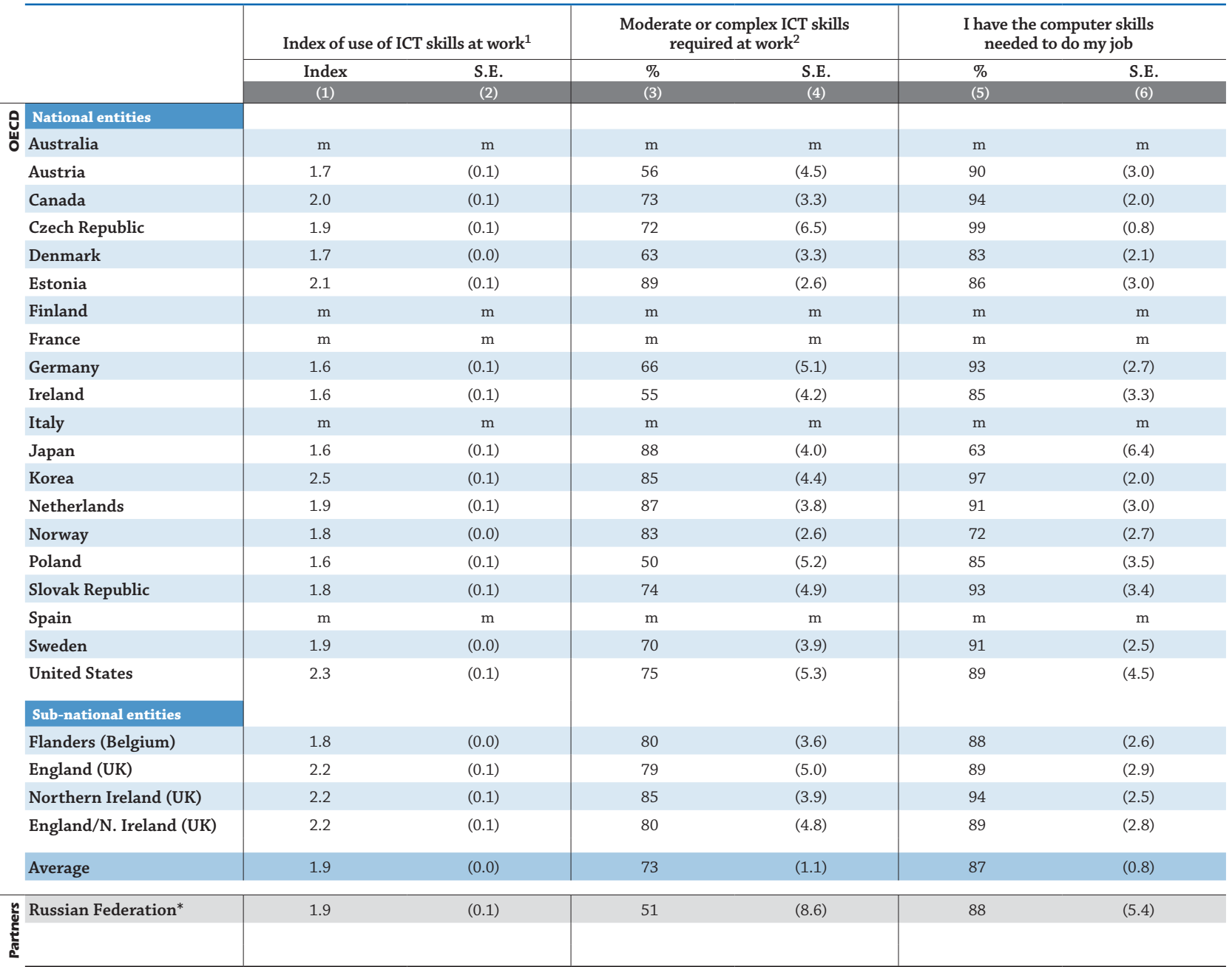

Note: Teachers who teach both pre-primary and primary school, primary teachers and secondary teachers refer to teachers who were currently working as teachers at the moment of the survey.

* See note on data for the Russian Federation in the Methodology section.

1. The index of use of ICT indicates the frequency of use of ICT skills at work. The higher the index, the more frequent the use of ICT skills at work. See the Definitions section.

2. Other categories are: "ICT skills not required at work" and "Straightforward ICT skills required at work".

Source: OECD. Survey of Adult Skills (PIAAC) (2012). See Annex 3 for notes (www.oecd.org/education/education-at-a-glance-19991487.htm).

Please refer to the Reader's Guide for information concerning symbols for missing data and abbreviations.

StatLink 제그 http://dx.doi.org/10.1787/888933286328 



\section{WHAT EVALUATION AND ASSESSMENT MECHANISMS ARE IN PLACE?}

- National examinations are most prevalent at the upper secondary level (31 countries), while national assessments are more common at the primary (32 countries) and lower secondary (28 countries) levels.

- Evaluation of schools is the area most frequently reported to be highly influenced by school inspection, in spite of the fact that school inspection practices vary considerably across countries.

- Although teacher appraisals are legislated in 30 countries, and school leader appraisals are legislated in 22 countries, some countries with no such legislation use similar evaluation practices.

\section{Chart D6.1. Evaluation and assessment mechanisms in public schools (2015)} In general programmes

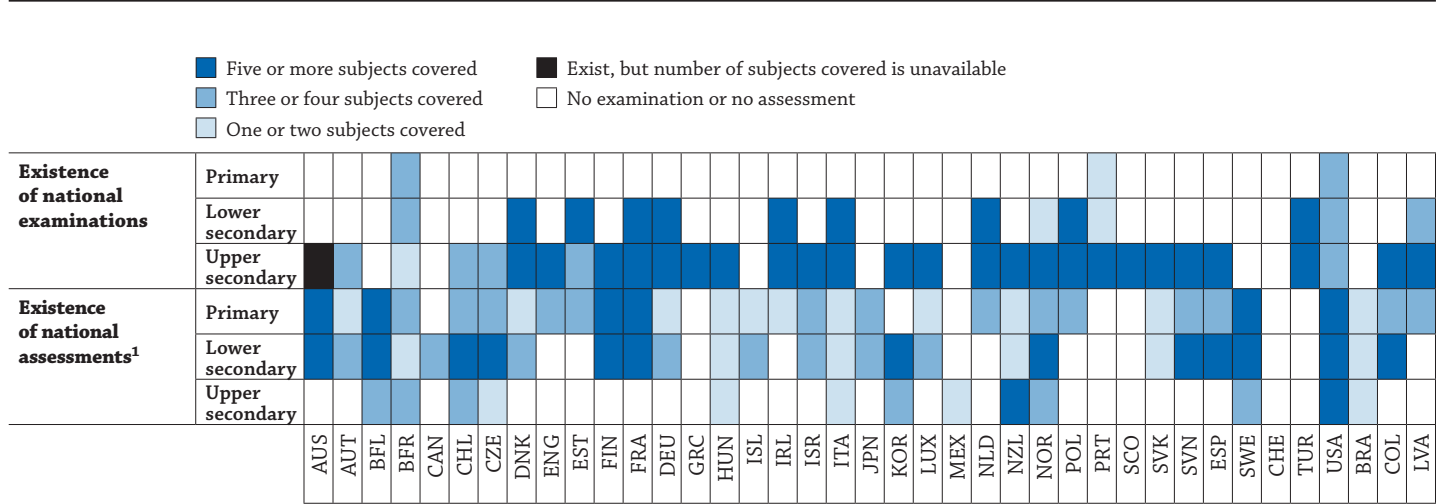

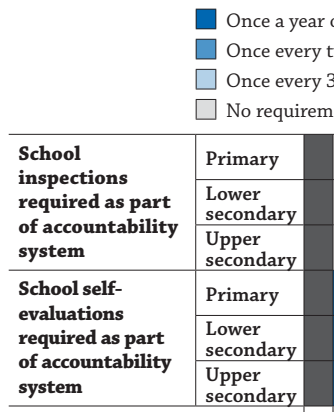
$\mathbb{Z}$ Have self-evaluations that are not part of accountability system $\square$ No required inspections or self-evaluations $\square$ Missing
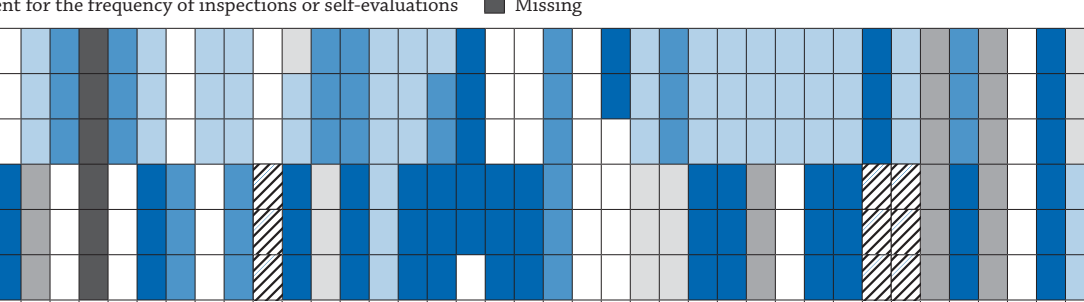

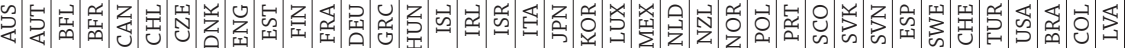

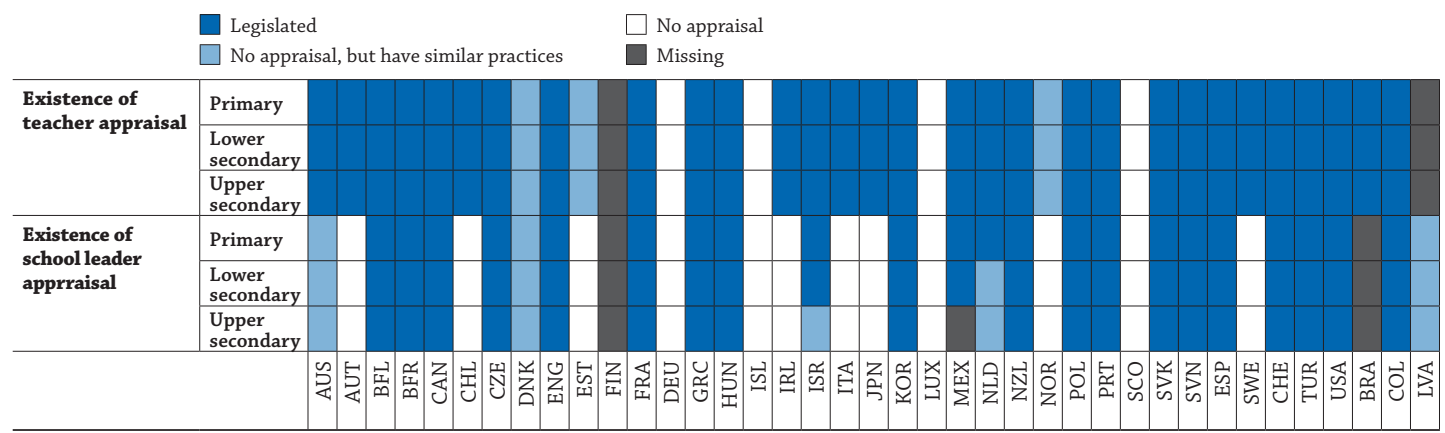

1. Number of subjects covered in the assessment framework (subjects may be tested on a rotation basis).

Source: OECD. Tables D6.2a, b, and c, D6.6a, b, and c, D6.10a, b, and c, D6.12a, b, and c, D7.2 and D7.7, available on line. See Annex 3 for notes (www.oecd.org/education/education-at-a-glance-19991487.htm).

StatLink 需正 http://dx.doi.org/10.1787/888933284582 


\section{Context}

Initially, evaluations in the education sector focused on specific programmes. As the field of evaluation developed, more and more components of the education system became targets for evaluation. Today, evaluation in education, based on data that is systematically and regularly collected, encompasses assessing the quality and effectiveness of programmes or projects, educators, including teachers and school leaders, and schools, school districts and school systems.

In education systems, data for evaluations are collected in a variety of ways: through student examinations and assessments, school inspections, school self-evaluations and reports of schools' compliance with regional or national rules and regulations. Most countries use a combination of these mechanisms, sometimes as part of a larger system of accountability.

\section{Other findings}

- Three countries reported conducting national examinations at the primary level, 14 countries reported conducting national examinations at the lower secondary level, and 31 countries reported conducting such examinations at the upper secondary level.

- The two most common purposes for national examinations at the upper secondary level are to determine student entry into tertiary education (27 countries) and for student certification, graduation or grade completion (24 countries).

- Twenty-eight countries reported having national or central-level assessments at the lower secondary level, while 11 countries reported that they had no national assessment at this level. National assessments are more common at the primary level (32 countries), although the number of subjects covered is larger at the lower secondary level.

- The two subjects most commonly covered in national assessments are reading, writing and literature, and mathematics.

- The three main uses of national assessments at the lower secondary level are to provide teachers with student diagnostic information (17 countries), to evaluate school performance (16 countries), and to provide parents with formative feedback (14 countries).

- In 30 countries, school inspections are a component of lower secondary school accountability systems.

- School self-evaluation is reported to be a component of accountability systems in 27 countries. Seven countries reported that their schools engage in self-evaluations which are not part of the formal accountability system.

- While it is common for countries to report that examinations, assessments, school inspections and school self-evaluations play a significant role in evaluating school performance, these activities are less commonly used to evaluate teachers and school administrators.

\section{Trends}

Between 2009 and 2015, there were only modest changes in the proportion of countries that reported having national examinations at the primary and lower secondary levels, while more countries reported using national examinations at the upper secondary level in 2015 than in 2009.

During the same period, there was a small increase in the number of countries that reported having national assessments at the lower secondary level and only minor changes in the proportion of schools that had national assessments at the primary and upper secondary levels.

The proportion of countries reporting that they require school inspections has not changed in recent years, although there was a slight increase in the proportion of countries that reported that school self-evaluations had become mandatory. 


\section{Analysis}

This Indicator presents data on the various mechanisms that countries may use for the evaluation and assessment of their education system. It builds upon earlier work in Education at a Glance 2011 (OECD, 2011), Indicator D5, which focused on accountability mechanisms. The scope of this Indicator was guided by the conceptual framework elaborated in the OECD Reviews of Evaluation and Assessment in Education (OECD, 2013) and by the INES Network for the Collection and Adjudication of System-Level Descriptive Information on Educational Structures, Policies and Practices (NESLI), part of the OECD Indicators of Education Systems (INES) programme. Teacher appraisal and school leader appraisal, which are discussed in Indicator D7, are also seen as activities that fit into the OECD framework for evaluation and assessment.

\section{National examinations}

National/Central examinations, which apply to nearly all students, are standardised tests of what students are expected to know or be able to do that have a formal consequence for students, such as an impact on a student's eligibility to progress to a higher level of education or to complete an officially recognised degree. National examinations are most prevalent at the upper secondary level, while national assessments are most prevalent at the primary level (see Chart D6.1). While the data collected for this Indicator covers primary, lower secondary and upper secondary levels, the following discussion focuses on examinations at the upper secondary level.

Three out of 39 countries with available data reported having national examinations at the primary level, 14 countries reported having national examinations at the lower secondary level, and 31 countries reported having national examinations at the upper secondary level. Only eight countries reported that they had no national examination at the upper secondary level.

Twenty-three countries reported that it is compulsory for public schools to participate in national examinations at the upper secondary level, and 17 countries reported that government-dependent private schools are required to participate in the examinations at that level. Twenty-four countries reported that $100 \%$ of all public schools participate in national examinations, and five other countries reported that between $76 \%$ and $99 \%$ of schools participate. Seventeen countries reported that all government-dependent private schools participate in national examinations and another three countries reported that between $76 \%$ and $99 \%$ of their government-dependent private schools participate. Even if it is not mandatory for schools and/or students to participate in national examinations - for example in England, Finland and Poland - the vast majority of schools and students tend to do so (Table D6.1c).

National examinations are standardised at various levels, although most (26 countries) are standardised at the central level. In five countries examinations are standardised at the state level.

Most countries develop their examinations at the national level or, in the case of federations, at the state or provincial level. England reported that private companies are involved in developing national examinations. By contrast, the responsibility for grading examinations usually includes or lies at the intermediate or local level.

National examinations cover a wide array of subjects or subject areas. At the upper secondary level, the most common exam subjects are reading, writing, and literature (all 30 countries with available data reported that they test this subject); mathematics (29 countries; Belgium [French Community] is the only subnational entity that does not test this subject); other languages (27 countries); natural sciences (26 countries); and social studies (26 countries). Arts (17 countries), information and communication technology (ICT) and technology (14 countries each) are also commonly covered. Religion (10 countries), physical education, and practical and vocational skills (9 countries each), and other subjects ( 6 countries) are less common.

The number of subjects covered by national examinations ranges widely, from 9 to 12 subjects in Denmark, England, France, Germany, Greece, Ireland, Israel, Italy, Luxembourg, New Zealand, Scotland, the Slovak Republic and Slovenia, to two or three in Austria, Belgium (French Community), the Czech Republic, Estonia and the United States (Chart D6.1, Table D6.1c, and Table D6.2c, available on line).

Although all students are tested in reading, writing and literature in 22 of the 30 countries with available data, in six of the remaining countries students can choose to be tested in these subjects, while in Norway, only a selection of students are tested in these subjects. By contrast, 16 of the 26 countries that cover natural science in the examination allow students to decide whether or not to take the test. In six countries, all students are tested, while in Denmark, Italy and Norway, only selected students are tested. Twelve of 29 countries test all students in mathematics, while 12 countries give students a choice, and four countries test only selected students (Table D6.2c, available on line). 


\section{Chart D6.2. Main purposes and uses of national/central examinations and assessments (2015)} In general programmes

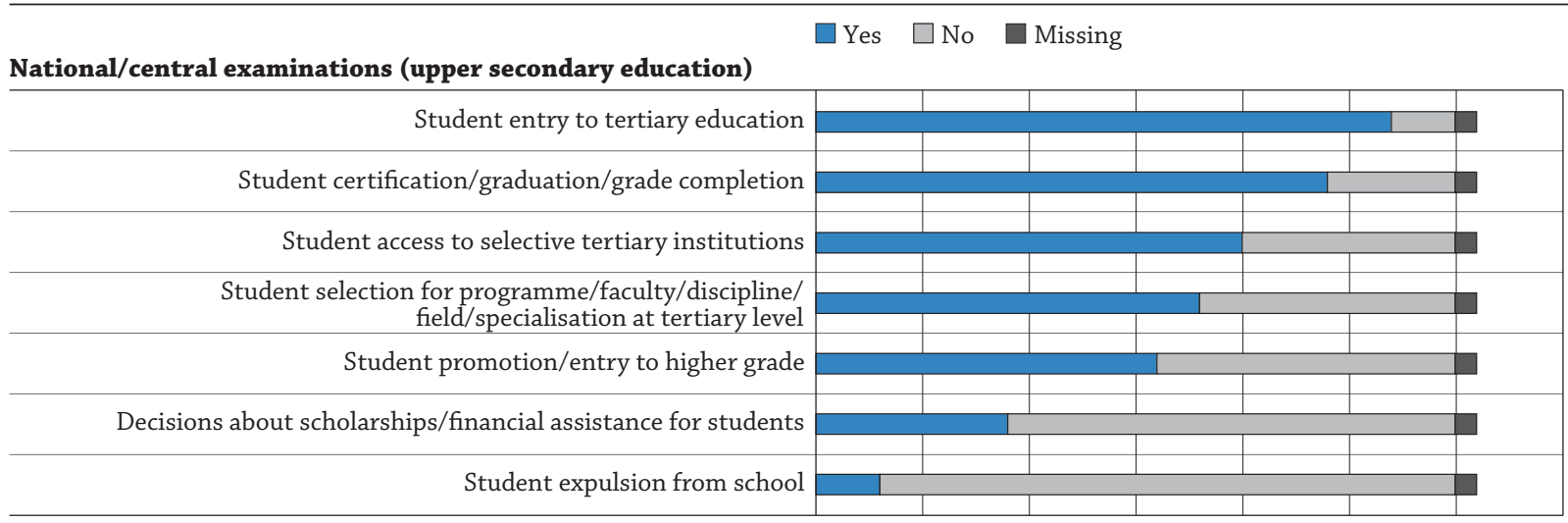

National/central assessments (lower secondary education)

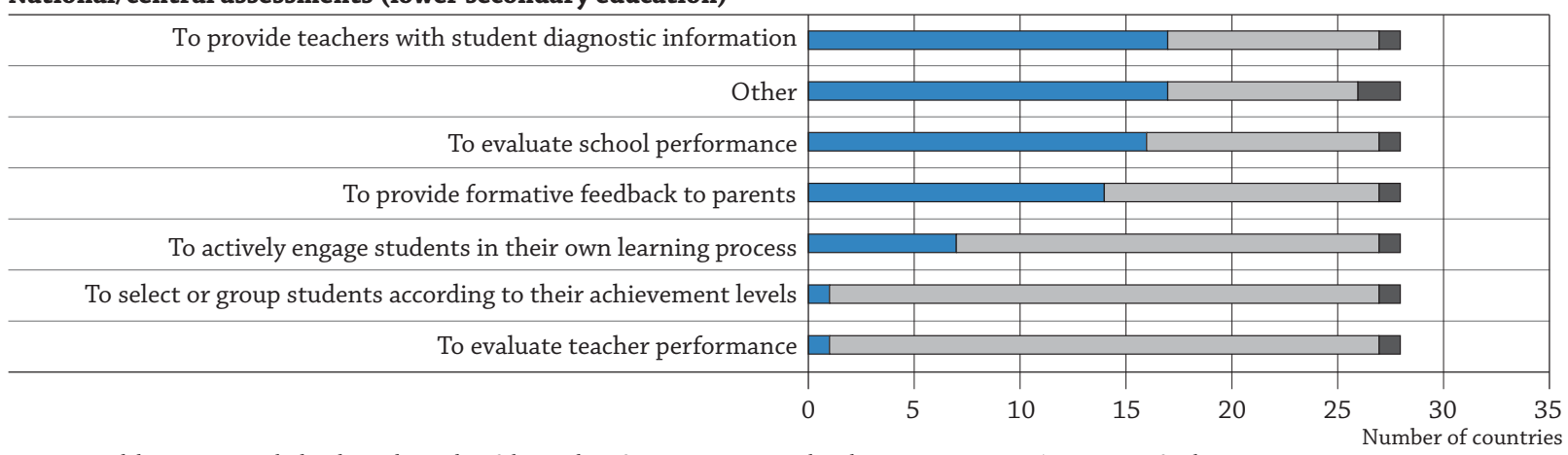

Purposes and decisions are ranked in descending order of the number of countries reporting that they use examinations/assessments for these. Source: OECD. Tables D6.1c and D6.5b. See Annex 3 for notes (www.oecd.org/education/education-at-a-glance-19991487.htm). StatLink : Ails $h$ htp://dx.doi.org/10.1787/888933284598

National examinations at the upper secondary level are most often used to determine entry into tertiary education (27 countries) and student certification, graduation or grade completion (24 countries) (Chart D6.2 and Table D6.1c).

Countries report results of national examinations in different ways. In many countries, results are not reported in isolation. They often include some type of contextual information since school performance outcomes and the characteristics of the student population are often correlated (OECD, 2013). This allows for comparisons at aggregate levels or by the characteristics of schools and of the student population. Specifically, ten countries indicate that results are context sensitive; in 27 countries, the results show the level of performance attained in the most recent year; 16 countries compare results with other groups or populations of students. No country, with the exception of Finland and the Slovak Republic (upper secondary level), indicated reporting the ranking of schools when communicating results. In fact, in a few countries steps are taken by the government/education authorities to prohibit or prevent the ranking of schools. However, 18 of 29 countries indicated that the media or other groups report upper secondary school rankings (Table D6.1c, and Table D6.4, available on line).

Thirty countries reported that results from national examinations are shared directly with others (i.e. information is received without having requested it) in addition to education authorities. "Others" include school administrators (28 countries), classroom teachers ( 22 countries), parents (20 countries) and students ( 29 countries). Twenty-five countries reported that they share results directly with the media and general public (Table D6.3, available on line).

Between 2009 and 2015, there were no significant changes in the number of countries that reported conducting national examinations at the primary level (data from 2009 were reported in Education at a Glance 2011 [OECD, 2011]). The French Community of Belgium reported that it established examinations at the lower secondary level in 2011, but they did not become compulsory until 2013.

More countries reported that they conduct national examinations at the upper secondary level in 2015 than what was reported for 2009 in Education at a Glance 2011 (OECD, 2011). National examinations were introduced at this level in Austria in 2014/15 for general programmes, and in 2015/16, Austria will introduce examinations for vocational 
programmes. The French Community of Belgium and the Czech Republic introduced examinations at this level in 2011. Spain and Turkey reported changes in their national examinations either because the examinations are now considered compulsory for all schools or because the purpose of the examinations had changed (see Annex 3 for more details).

\section{National assessments}

Like national examinations, national assessments are based on standardised student achievement tests. However, results from assessments do not have an impact on students' progression through schooling or on certification.

Twenty-eight countries reported having national or central-level assessments at the lower secondary level, while 11 countries reported that they have no national assessments at this level. National assessments are more common at the primary level (32 countries), although the number of subjects covered appears to be larger at the lower secondary level. National assessments are less common in upper secondary school (13 countries) where national examinations are more prevalent. The findings in this section focus on assessments at the lower secondary level unless otherwise indicated.

In most countries that conduct national assessments, all schools are required to participate. Seventeen countries reported that it is compulsory for all public schools to participate in national assessments; 14 countries reported that all government-dependent private schools are required to participate. In a handful of countries, only a sampling of schools was required to participate. Seven countries sample public schools and five countries sample governmentdependent private schools. In Finland and the United States, national sample-based assessments are used to allow central authorities to evaluate the performance of the school system more broadly. The National Assessment of Educational Progress in the United States is administered by the federal government and is based on a sample of schools, allowing policy makers to monitor the relative performance of the school system and conduct extensive research (Chart D6.1, Table D6.5b, and Tables D6.5a and c, available on line).

National assessments are commonly standardised at the central level (22 countries), although in four countries the assessments are standardised at the state level, and in Canada the assessments are standardised at the level of provinces and territories. In most (23) countries, national assessments are developed at the national level or, in the case of federations, at the state or provincial level (three countries). Twenty-one countries reported that assessments are graded at the central or state level, while four countries reported that assessments are graded locally, often within the school itself. Two countries reported that the responsibility for grading includes both the central and school level.

National assessments cover a wide array of subjects, but the two most commonly covered at all levels of education are reading, writing and literature, and mathematics. All countries which have national assessments cover these two subjects at the primary level, while they are covered in nine out of ten countries at the lower and upper secondary levels. Other subjects commonly covered in assessments are natural sciences (by two out of three countries at the lower secondary level) and other languages (by three out of five countries at the lower, and half of the countries at upper secondary levels) (Table D6.5b, and Tables D6.5a and c, D6.6a, b and c, available on line).

At the lower secondary level, while around three-quarters of countries with national assessments test all students in reading, writing and literature (19 of 25 countries) and mathematics (18 of 25 countries), the other quarter of the countries test only a selection/sample of students. The opposite is seen in natural sciences, where around a third of countries test all students (7 of 19 countries) (Table D6.6b, available on line).

At the lower secondary level, many countries reported that reading, writing and literature (16 of 25 countries) and mathematics (15 of 25 countries) are tested annually, while eight in 25 countries reported that these subjects are tested on a rotation basis. In nine of 16 countries, other languages are tested annually; in four of those 16 countries, they are tested on a rotation basis and in three countries they are tested on some other basis. The remaining subjects are mostly tested in rotation.

The three most commonly reported main purposes of national assessments at the lower secondary level are to provide teachers with student diagnostic information (17 countries), to evaluate school performance (16 countries), and to provide parents with formative feedback (14 countries) (Chart D6.2 and Table D6.5b).

Countries report results of lower secondary assessments in different ways. In twenty-five countries, the results show the level of performance for the most recent year; 26 countries compare the results with other groups or populations of students; and 11 countries report the results with other indicators of school quality (Table D6.5b). 
In 26 of 27 countries, results from national assessments are shared with external audiences in addition to education authorities. In all 26 countries, the results are shared directly with school administrators, and in 22 countries results are shared directly with classroom teachers. School administrators and teachers receive aggregated results; in a third of the countries, they also receive the results of individual students. In 23 countries, results from national assessments are shared directly with parents and/or students. In 24 countries, only aggregated results are shared directly with the media (Table D6.7, available on line).

Although education authorities do not report school rankings, 10 of 25 countries with available data indicated that the media or other groups prepare and publicise rankings of schools based on mean student performance in the national assessment (Table D6.8, available on line).

Only a few countries reported changes in their national assessments between 2011 and 2015. Austria introduced a national assessment at the primary and lower secondary levels in 2013, and the Czech Republic established an assessment in 2011/12 for their primary and lower secondary schools. Germany also expanded its national assessment (Ländervergleich) at the primary and lower secondary levels. The Slovak Republic reported that a national assessment was established in 2012 at the primary level, but only a small proportion of schools participate. Korea reported a number of changes in its primary school assessments over the years, including broadening the assessment to include all students in 1993, then opting for only sampling in 1998, and then reverting to all students in 2008. Since 2013, Korea has stopped administering an assessment at the primary level. In Mexico, the primary and lower secondary assessments were discontinued for the school year 2013/14, and resumed in the school year 2014/15 under a new scheme called PLANEA, which is currently underway. See Annex 3 for more details about recent changes in national assessments.

\section{School inspection}

A school inspection is a mandated, formal process of external evaluation that aims to hold schools accountable. It involves one or more trained inspectors who evaluate quality based on a standard procedure. The results of a school inspection are given to the school in a formal report and are used to identify strengths and weaknesses. The reports are often also shared with higher-level education authorities who use this information to evaluate schools and hold them accountable for the public resources they receive. In some countries, the inspectors' reports are also made available to parents and the public.

School inspection practices vary considerably in how they are conducted, who controls the process and the areas that are inspected (see Tables D6.9, and Tables D6.10a, b, and c, available on line). Inspections may focus on such areas as compliance with rules and regulations, student achievement, staff, administration, curriculum and the school environment. Schools may be rewarded or sanctioned based on results from these inspections.

In 30 countries, lower secondary school inspections are a component of the school-accountability system (Chart D6.1). In nine countries, school inspections are a component of the school-accreditation process through which schools are granted recognition or credentials if they meet or exceed minimum standards. While school inspections commonly involve all schools, in 13 countries school inspections are targeted at low-performing schools. Hungary is developing a school-inspection system to be put in place in 2014/15.

Twelve countries reported that public lower secondary schools are inspected at least once every three years. Six countries reported the same frequency of inspections in government-dependent private schools. Eight countries reported that public schools are inspected every two or three years; and four countries reported that they occur more frequently. Fifteen countries reported that public schools are inspected once every three of more years. In the Netherlands, although school inspections take place every four years, annual risk analysis can trigger more frequent inspections.

In 20 countries, school inspections are reported to be highly structured, while in six countries inspections are partially structured, and in two countries inspections are unstructured. Sixteen countries reported that inspections are organised exclusively at the national level and six countries reported that they are organised only at the state level. Most school inspections are conducted by teams of inspectors (19 countries), but in six countries, inspections are conducted by a single person. School inspections cover a wide array of topics, most commonly compliance with rules and regulations, and quality of instruction, and least frequently, financial management (Chart D6.3, and Table D6.10b, available on line).

Results from school inspections are most commonly used to evaluate school performance, although they are also used to evaluate school administration and to determine whether or not to close schools or evaluate individual teachers. School inspections rarely affect decisions about remuneration and bonuses for teachers or school budgets (Chart D6.4, and Table D6.16, available on line). 


\section{Chart D6.3. Distribution of areas addressed during school inspections and school self-evaluations (2015) \\ In general programmes, lower secondary education}

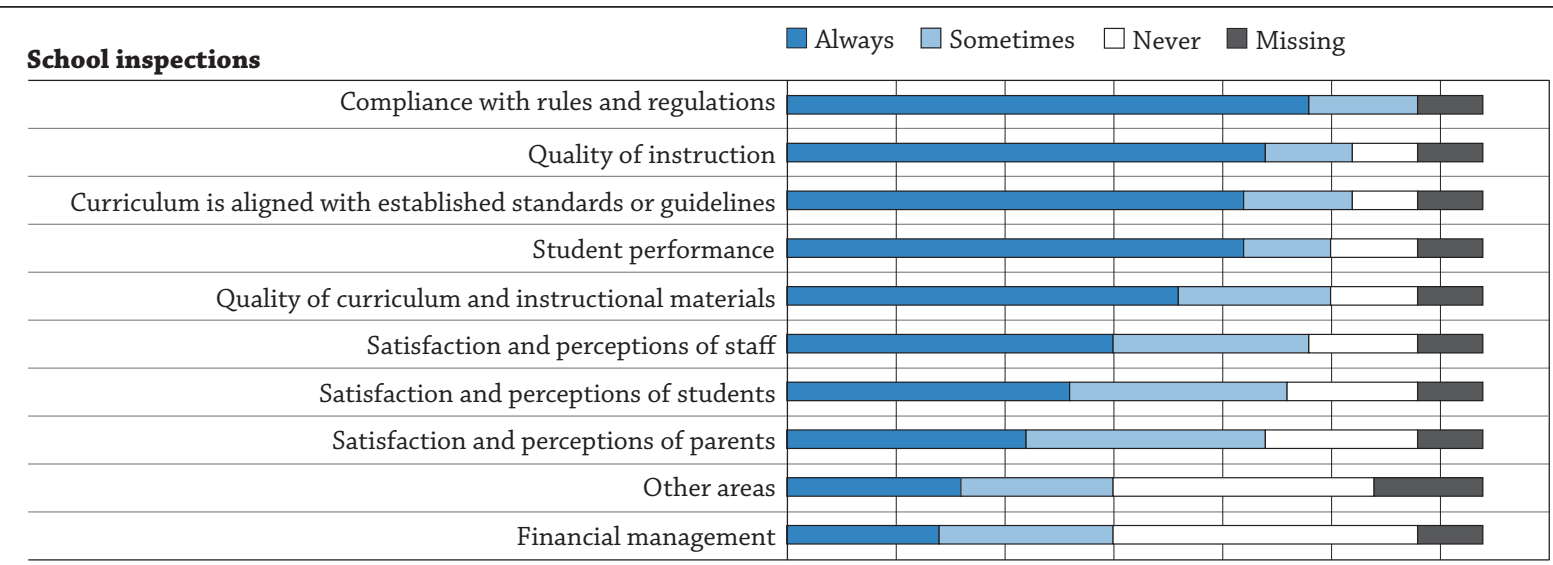

School self-evaluations

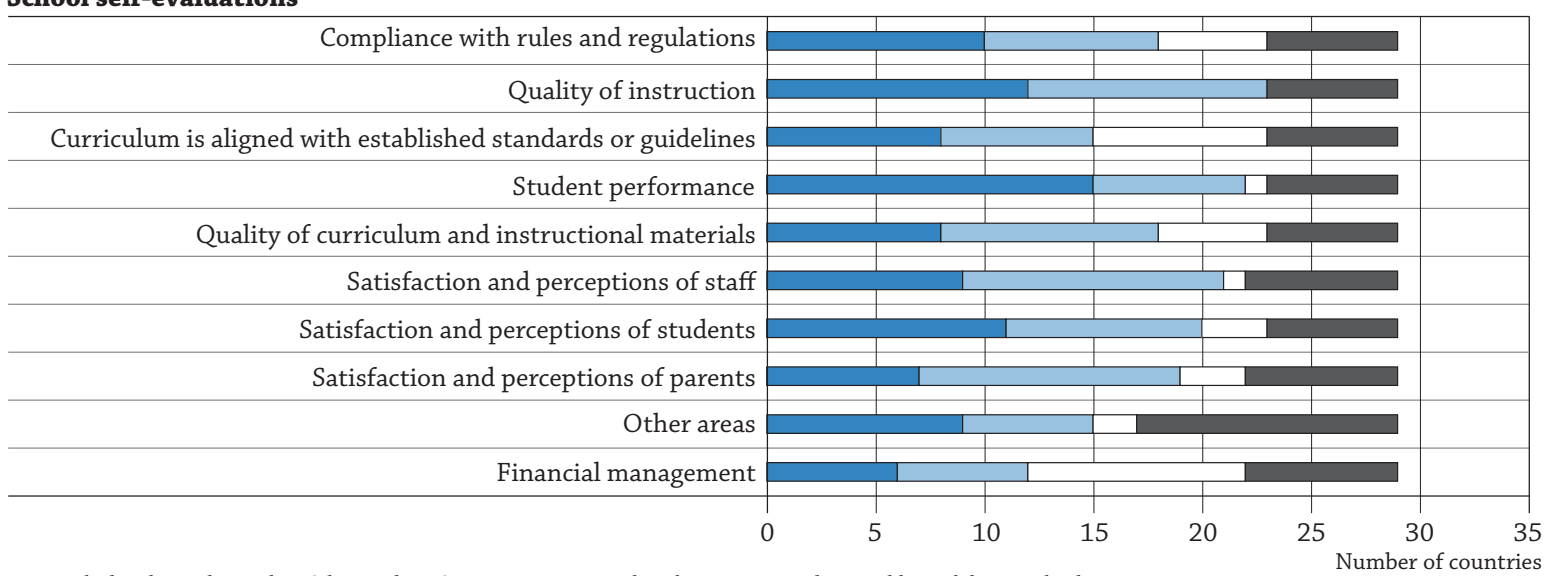

Areas are ranked in descending order of the number of countries reporting that these areas are always addressed during school inspections.

Source: OECD. Tables D6.10b and D6.12b, available on line. See Annex 3 for notes (www.oecd.org/education/education-at-a-glance-19991487.htm)

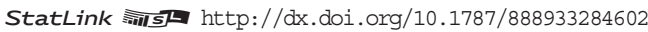

Twenty-six countries reported that results of school inspections at the lower secondary level are shared with external audiences; only two countries reported that these results are not shared. Table D6.10b, available on line, shows the external audiences that have direct or indirect access to school-inspection findings.

\section{School self-evaluation}

In a self-evaluation, a school systematically reviews the quality of the instruction and education services provided and school outcomes. Formal self-evaluation activities are mandated by higher-level authorities. When schools are required to conduct self-evaluations, a set of questionnaires or tools usually structures the activity. Results from self-evaluations can be used to inform internal audiences, or they could be used to inform school inspectors or accreditation teams. In fact, self-evaluations are often designed in connection with an external evaluation activity, such as a school inspection or a school accreditation visit. The results are more suitable for use as the basis for improvement. Some of the advantages of self-evaluations are that they are less costly and results can be more easily interpreted in light of the local context. The main disadvantage is that results are often seen by external groups as less credible and less suitable for accountability purposes.

Twenty-seven countries reported that school self-evaluation at the lower secondary level is a component of their accountability systems, and three countries reported that their schools engage in self-evaluation practices which are not part of the formal accountability system. Four countries reported practicing school self-evaluation both as part of the accountability system and outside of it. Because school self-evaluations are less frequently overseen by central authorities, some countries were not able to report on how frequently schools conduct self-evaluations (Chart D6.1, Table D6.9, and Table D6.12b, available on line). 
School self-evaluations are required or regulated more often in public schools than in government-dependent private schools. Fifteen countries reported that their public schools conduct self-evaluations at least once a year, while eight countries reported that government-dependent private schools conduct these activities annually. While most schools use self-evaluations as the basis for improvement activities, 14 countries reported that school selfevaluations are a component of the school-inspection process, and two countries reported that they are a component of an accreditation process. Chart D6.3 shows that the most common areas covered by school self-evaluations are student performance, quality of instruction, student satisfaction, and compliance with rules and regulations (Table D6.12b, available on line).

The number of countries reporting that school self-evaluation is a required part of the accountability system increased slightly in recent years. Self-evaluations are required in schools in the Flemish Community of Belgium since 2009; since 2013/14, self-evaluations have been conducted in lower secondary schools in Austria and Greece. They became mandatory in Ireland in 2012 and in Italy in 2014/15. In England, all schools are encouraged to conduct self-evaluations, although the statutory requirement to complete a standardised self-evaluation form was removed in 2010. In Scotland, local authorities are obliged by law to ensure continuous improvement in their schools. All schools are expected to evaluate their own work, but these procedures are not considered to be formal school self-evaluations. In Sweden, as in many other countries, school self-evaluations are expected or are required to be conducted regularly, although results are not systematically collected or analysed centrally.

In Finland, the municipalities have a statutory duty to participate in national evaluations as well as evaluate the education they provide. Forms and procedures of local evaluation can be decided locally. Self-evaluation of schools and education providers as well as national sample-based evaluations of learning outcomes play a key role in the Finnish evaluation system (or quality assurance system).

Nineteen of 22 countries reported that results from school self-evaluations are shared with external audiences, such as higher-level education authorities, school inspectors, parents or the general public. In 12 countries the results are shared directly with higher-level authorities, but 7 countries reported that results from self-evaluations are not shared with these authorities (Table D6.12b, available on line).

\section{Compliance reporting}

A large part of accountability involves schools submitting data and information to higher levels of authority. To a lesser extent, parents and students, as well as the general public, also need to know about the extent to which their schools comply with established laws and regulations. Compliance reporting aims to confirm that schools are adhering to these laws and regulations.

Given the nature of internal reporting, a considerable portion of regulatory accountability is not available for public scrutiny, although some of the information that schools submit may appear in reports released to parents, students or the general public.

Countries were asked whether they report data on eight specific domains to education authorities (Table D6.13, and Tables D6.13a and b, available on line). Nearly all (34) countries indicated that public schools report data on student populations to regional or national authorities. Public schools also report data to regional or national authorities on facilities and grounds (27 countries), teacher qualifications/credentials (25 countries), curriculum (24 countries), closing budget or financial audit from previous year (23 countries), safety issues (23 countries), issues related to governance (20 countries), and proposed budget for the subsequent year (19 countries).

The local school board is another common recipient of the data and information schools provide as part of reporting; parents, students and the general public are least likely to receive this information. This is not surprising, given that compliance reporting is all about demonstrating accountability to higher-level authorities. While national and regional education authorities typically receive data on students and teachers, they are less likely to request data on school governance and future budgets, which are more important for local authorities or school boards. Data concerning safety issues is more commonly reported to lower-level education authorities than to regional or national authorities.

Government-dependent private schools are more likely to report compliance data to their school boards compared with public schools, which also report to local, regional and national education authorities.

Although a number of countries still file "hard copy" compliance reports on paper, most countries now use Internetbased forms to submit compliance-related data. Thirty-one of 32 countries indicated that they use Internet-based reporting for at least some student data, and 20 countries reported that they use Internet reporting to submit data on teacher qualifications, curriculum, and facilities and grounds in public schools (Tables D6.14a and b, available on line). 


\section{School influence or control over evaluation and assessment activities}

Generally, schools have little or no influence on national examinations and, in some countries, only marginal influence on national assessments. Schools have little or no influence in designing or controlling school inspections. These inspections are devised by higher-level authorities and are conducted by external inspectors. By definition, school self-evaluations are largely controlled by individual schools. The influence of local schools on teacher and school leader appraisals varies considerably from country to country (see Indicator D7) (Table D6.17, available on line).

\section{Influence and use of evaluation and assessment mechanisms}

Chart D6.4 shows the relative influence of four key evaluation activities and how these activities affect five general decisions. Country responses on how evaluation activities are related to decisions concerning school budgets and teachers' salaries are shown in Table D6.16 (available on line).

While it is common for countries to report that examinations, assessments, school inspections and school selfevaluation have considerable influence on the evaluation of school performance, these mechanisms and activities are less frequently conducted to evaluate administrators and teachers.

School inspection is the activity most directly linked to evaluation of school performance: 13 countries reported that school inspections have a high influence on the evaluation of schools. Examinations and assessment results were each reported to have no influence in 10 countries. While seven countries reported that results from school inspections have a high influence on decisions about school closure, the other evaluation activities seem to have little or no influence on such decisions. Evaluation of individual teachers is not highly influenced by any of the mechanisms.

\section{Chart D6.4. Level of influence of various evaluation and assessment mechanisms (2015)} In general programmes, all levels of education

\begin{tabular}{l} 
National/central examinations \\
\hline Evaluation of school performance \\
\hline Evaluation of school administration \\
\hline Provision of assistance to teachers to improve their teaching skills \\
\hline Likelihood of school closure \\
\hline Evaluation of individual teachers
\end{tabular}

\section{National/central assessments}

$\begin{array}{r}\text { Evaluation of school performance } \\ \hline \text { Evaluation of school administration } \\ \hline \text { Provision of assistance to teachers to improve their teaching skills } \\ \hline \text { Likelihood of school closure } \\ \hline \text { Evaluation of individual teachers }\end{array}$

School inspections

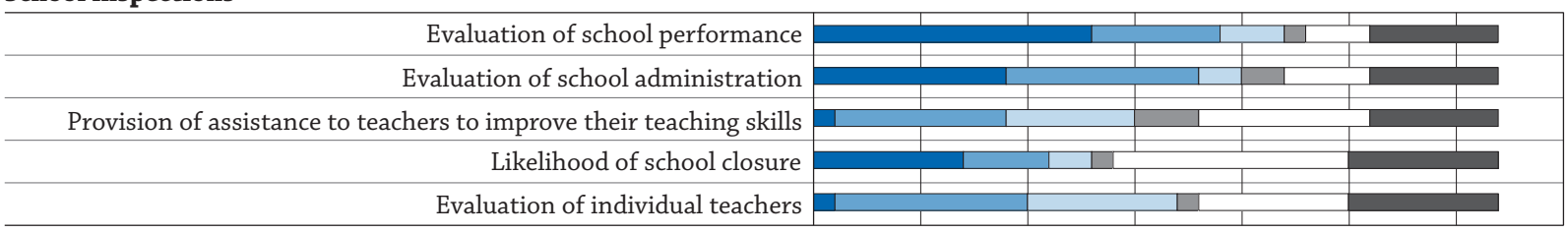

School self-evaluations

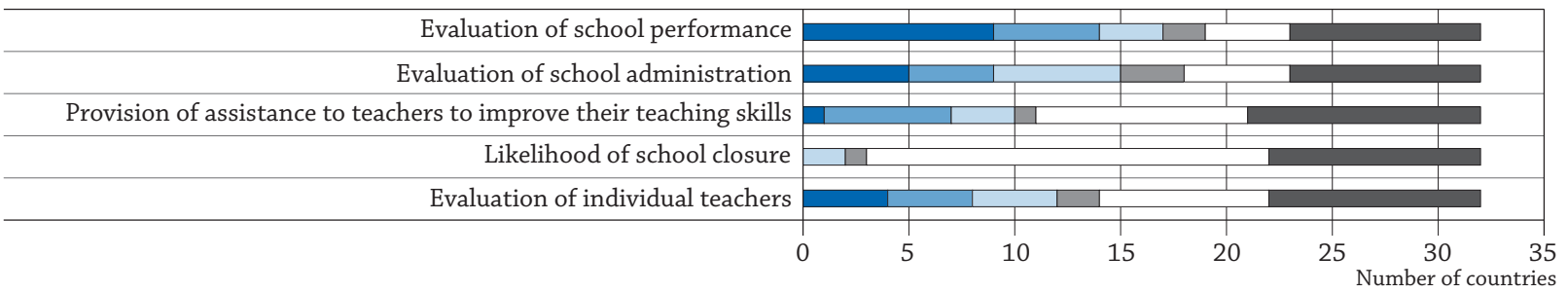




\section{Summative and/or formative}

Chart D6.5 contrasts the extent to which countries see four general evaluation activities as either formative or summative in nature. The purpose of formative evaluation is to "improve", while the purpose of summative evaluation is to "prove". Formative evaluations examine implementation and aim to obtain information that can be used to help improve the effectiveness of a teacher, a programme or a school. They are more relevant for internal audiences. Summative evaluations are often conducted at the end of a programme or at least at a time when long-term outcomes are available. Summative evaluations are closely linked to accountability to higher-level education authorities and audiences outside the school.

National examinations are predominantly seen as summative, in that they inform decisions about student matriculation, advancement or placement. National assessments are seen as both formative and summative. School inspections are frequently used for both formative (18 countries) and summative (14 countries) purposes. Meanwhile, 20 countries indicated that they use school self-evaluations for formative purposes; only 7 countries reported using school self-evaluations for summative purposes (Table D6.18, available on line).

\section{Chart D6.5. Extent to which evaluation and assessment activities are used for formative and/or summative purposes (2015)}

In general programmes, all levels of education

\begin{tabular}{ll}
\hline & $\square \square$ High level of use \\
& $\square \square$ Moderate level of use \\
$\begin{array}{l}\text { Number } \\
\text { of countries }\end{array}$ & $\square \square$ Low level of use \\
$30 \longrightarrow$ Not used \\
\hline
\end{tabular}

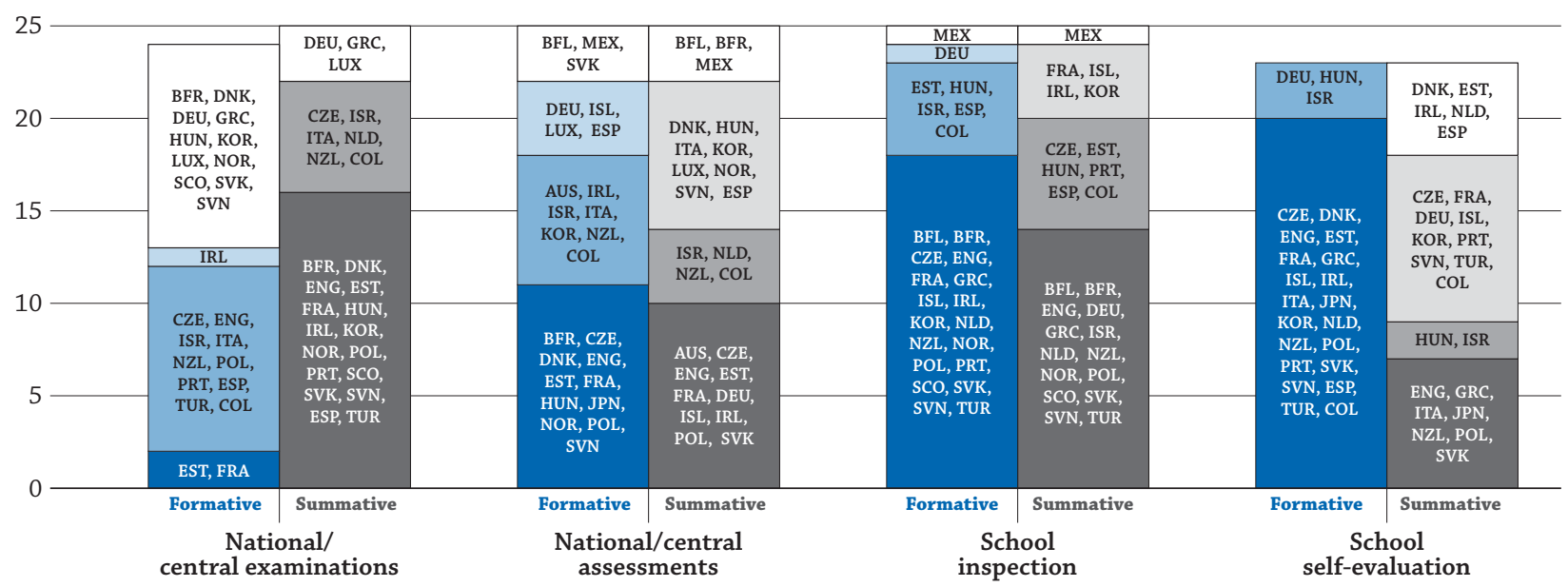

Source: OECD. Table D6.18, available on line. See Annex 3 for notes (www.oecd.org/education/education-at-a-glance-19991487.htm). StatLink त्राजा http://dx.doi.org/10.1787/888933284624

\section{Definitions}

Accountability means "to take account of". It refers to the interaction, in a hierarchical relationship, between those who have power and those who are delegated authority. Those who are delegated authority have to report on what they are doing with this authority or responsibility. In its simplest sense, accountability can refer to information and transparency in operations and outcomes. Regulatory (compliance) accountability concerns adherence to relevant laws and regulations.

Evaluation refers to the process of determining the merit, worth, value or performance of something.

National assessments are based on standardised student achievement tests. However, national assessments do not affect students' progression through school or certification.

National examinations are standardised student tests that have a formal consequence for students, such as an impact on a student's eligibility to progress to a higher level of education or to complete an officially-recognised degree. 
School inspection is defined as a mandated, formal process of external evaluation with the aim of holding schools accountable. Formal school inspection is not conducted by internal staff, parents, the community or the media.

School self-evaluation refers to an activity in which schools systematically review the quality of the instruction and education services provided, as well as school outcomes. This activity involves internal evaluation that is formative in nature.

\section{Methodology}

D6 Data are from the 2014 OECD-INES Survey on Evaluation and Assessment and refer to the school year 2014/15.

Notes on definitions and methodologies for each country are provided in Annex 3 at www.oecd.org/education/ education-at-a-glance-19991487.htm.

\section{Note regarding data from Israel}

The statistical data for Israel are supplied by and are under the responsibility of the relevant Israeli authorities. The use of such data by the OECD is without prejudice to the status of the Golan Heights, East Jerusalem and Israeli settlements in the West Bank under the terms of international law.

\section{References}

OECD (2013), Synergies for Better Learning: An International Perspective on Evaluation and Assessment, OECD Reviews of Evaluation and Assessment in Education, OECD Publishing, Paris, http://dx.doi.org/10.1787/9789264190658-en.

OECD (2011), Education at a Glance 2011: OECD Indicators, OECD Publishing, Paris, http://dx.doi.org/10.1787/eag-2011-en.

Scriven, M. (1991), Evaluation Thesaurus, 4th edition, Sage Publications, Inc., Newbury Park, California.

Worthen, B.R., J. Sanders and J. Fitzpatrick (1997), Program Evaluation: Alternative Approaches and Practical Guidelines, Longman Publishers, White Plains, New York.

\section{Indicator D6 Tables}

\section{StatLink 제기 http://dx.doi.org/10.1787/888933286339}

WEB Table D6.1a National/central examinations at the primary level (2015)

WEB Table D6.1b National/central examinations at the lower secondary level (2015)

Table D6.1c National/central examinations at the upper secondary level (2015)

WEB Table D6.2a Subjects tested in national/central examinations in primary education (2015)

WEB Table D6.2b Subjects tested in national/central examinations in lower secondary education (2015)

WEB Table D6.2c Subjects tested in national/central examinations in upper secondary education (2015)

WEB Table D6.3 Sharing results from national/central examinations (2015)

WEB Table D6.4 Ranking of schools based on results from national/central examinations (2015)

WEB Table D6.5a National/central assessments at the primary level (2015)

Table D6.5b National/central assessments at the lower secondary level (2015)

WEB Table D6.5c National/central assessments at the upper secondary level (2015)

WEB Table D6.6a Subjects tested in national/central assessments in primary education (2015)

WEB Table D6.6b Subjects tested in national/central assessments in lower secondary education (2015)

WEB Table D6.6c Subjects tested in national/central assessments in upper secondary education (2015)

WEB Table D6.7 Sharing results from national/central assessments (2015)

WEB Table D6.8 Ranking of schools based on results from national/central assessments (2015)

Table D6.9 School inspection and school self-evaluation at the lower secondary level (2015)

WEB Table D6.10a School inspection at the primary level (2015)

WEB Table D6.10b School inspection at the lower secondary level (2015)

WEB Table D6.10c School inspection at the upper secondary level (2015)

WEB Table D6.11 Diverse models of school inspection (2015) 
WEB Table D6.12a School self-evaluation at the primary level (2015)

WEB Table D6.12b School self-evaluation at the lower secondary level (2015)

WEB Table D6.12c School self-evaluation at the upper secondary level (2015)

Table D6.13 Public school compliance-oriented reports, by domains and receiving groups (2015)

WEB Table D6.13a Public school compliance-oriented reports, by domains and receiving groups (2015)

WEB Table D6.13b Government-dependent private school compliance-oriented reports, by domains and receiving groups (2015)

WEB Table D6.14a Means and methods for collecting and reporting regulatory accountability data, public schools (2015)

WEB Table D6.14b Means and methods for collecting and reporting regulatory accountability data, government-dependent private schools (2015)

WEB Table D6.15 Existence and use of other forms or measures of evaluation and assessment mechanisms (2015)

WEB Table D6.16 Level of influence of various evaluation and assessment mechanisms (2015)

WEB Table D6.17 Extent of school influence or control over various evaluation and assessment mechanisms (2015)

WEB Table D6.18 Extent to which evaluation and assessment activities are used for formative and/or summative purposes (2015) 
Table D6.1c. National/central examinations at the upper secondary level (2015) In general programmes

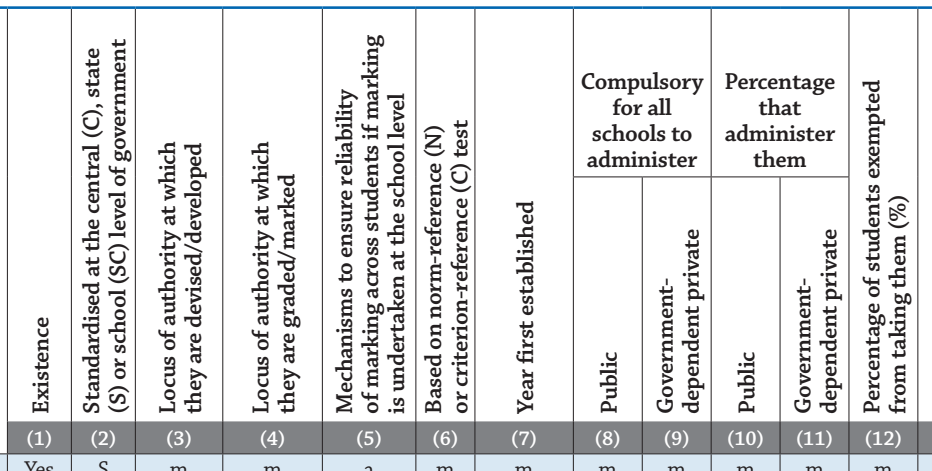

\author{
Subjects covered:
}

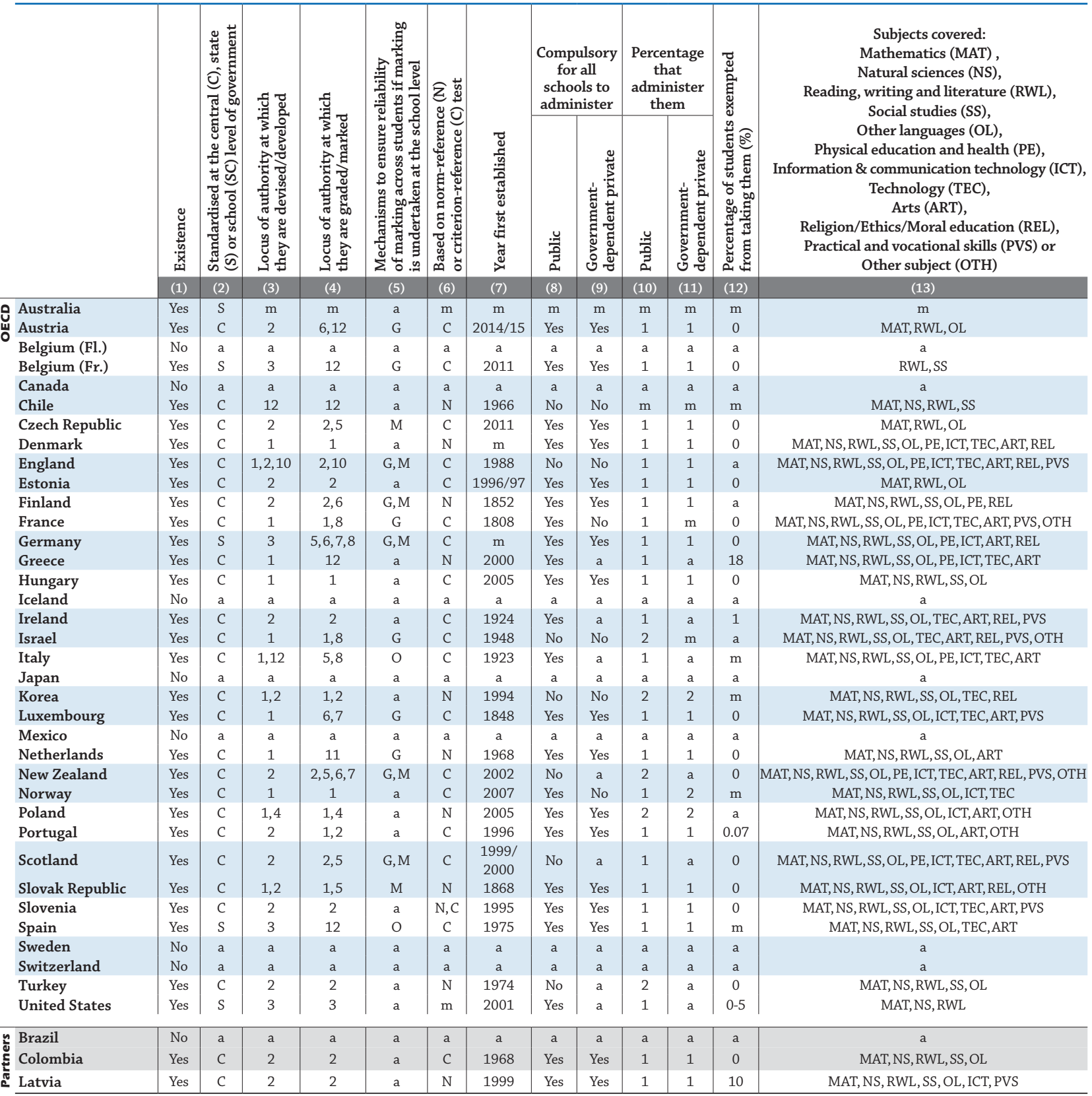

Locus of authority for developing/marking/grading examinations

Mechanisms to ensure reliability of marking across students
1: Central authority or government
2: Central agency responsible for assessment or certification
3: State education authorities or governments
4: Provincial/regional education authorities or governments
5: School, school board or committee
6: The students' own teacher
7: Another teacher from within the school
8: A teacher from another school
9: Subject/discipline association
10: Private company
11: Depends on the subject

12: Other

G: Availability of national guidance materials for marking student performance
on the examination

on the examination

O: Other

Percentage of schools administering examinations

1: All schools

2: Between $76 \%$ and $99 \%$ of schools

3: Between $51 \%$ and $75 \%$ of schools

4: Between $26 \%$ and $50 \%$ of schools

5: Between $11 \%$ and $25 \%$ of schools

6: $10 \%$ or less of schools

Notes: Federal states or countries with highly decentralised school systems may have different regulations in states, provinces or regions. Please refer to Annex 3 for additional information.

Columns showing the main purposes and uses of national/central examinations (i.e. columns 14-21), as well as the features used when reporting results (i.e. columns 22-28) are available for consultation on line (see StatLink below).

Source: OECD. See Annex 3 for notes (www.oecd.org/education/education-at-a-glance-19991487.htm).

Please refer to the Reader's Guide for information concerning symbols for missing data and abbreviations.

StatLink 제개 http://dx.doi.org/10.1787/888933286347 
Table D6.5b. National/central assessments at the lower secondary level (2015) In general programmes

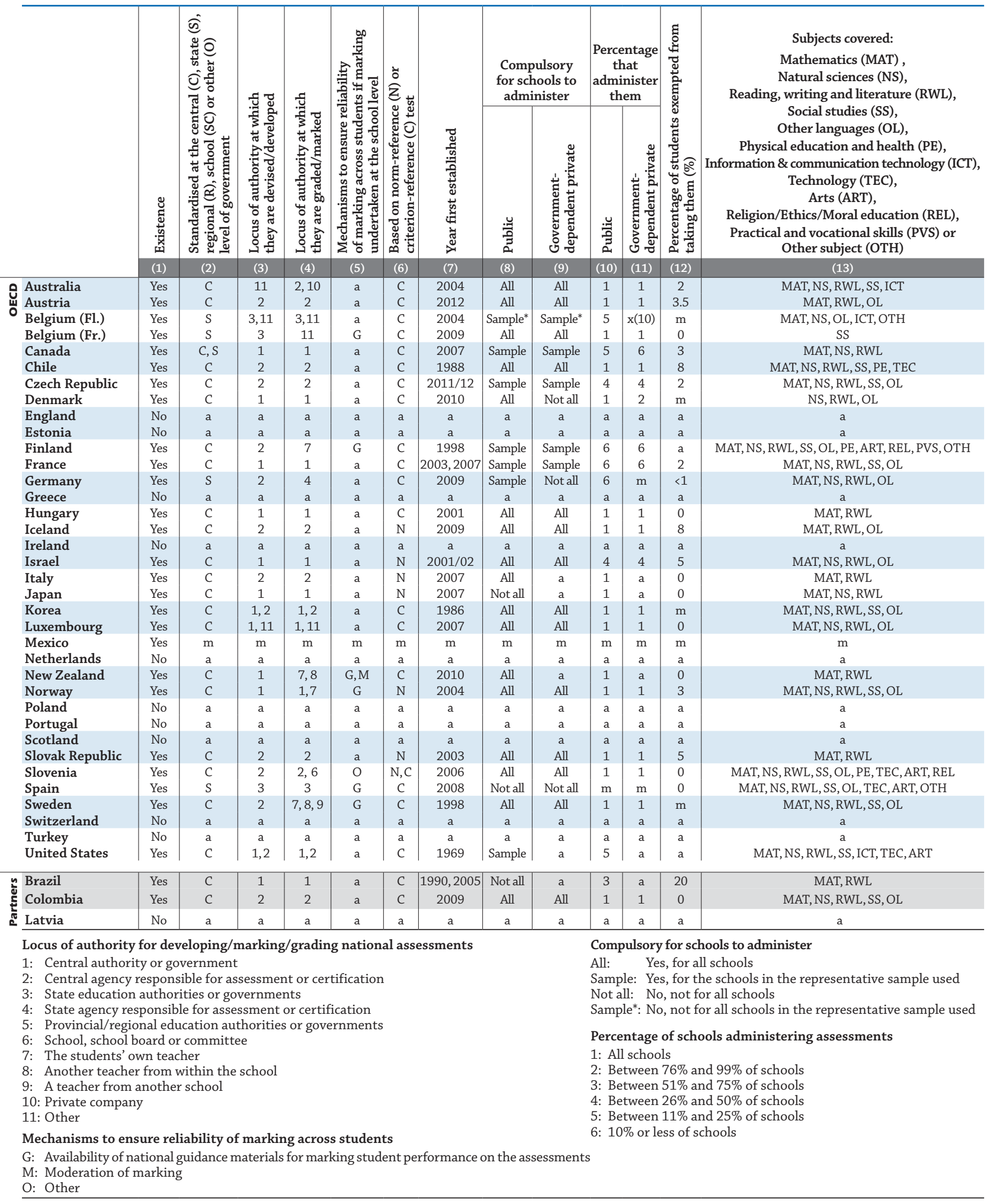

Notes: Federal states or countries with highly decentralised school systems may have different regulations in states, provinces or regions. Please refer to Annex 3 for additional information.

Columns showing the main purposes and uses of national/central assessments (i.e. columns 14-20), as well as the features used when reporting results (i.e. columns 21-27) are available for consultation on line (see StatLink below).

Source: OECD. See Annex 3 for notes (www.oecd.org/education/education-at-a-glance-19991487.htm).

Please refer to the Reader's Guide for information concerning symbols for missing data and abbreviations.

StatLink 젝ㄴ http://dx.doi.org/10.1787/888933286352 
Table D6.9. [1/2] School inspection and school self-evaluation at the lower secondary level (2015) In general programmes

\begin{tabular}{|c|c|c|c|c|c|c|c|c|c|c|}
\hline & \multicolumn{10}{|c|}{ School inspection } \\
\hline & \multirow{2}{*}{ 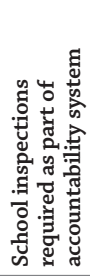 } & \multicolumn{2}{|c|}{$\begin{array}{c}\text { Frequency } \\
\text { of school inspections }\end{array}$} & \multicolumn{2}{|c|}{$\begin{array}{l}\text { Percentage of schools } \\
\text { that have inspections } \\
\text { conducted each year (\%) }\end{array}$} & \multirow{2}{*}{ 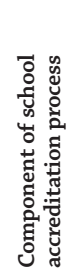 } & \multirow{2}{*}{ 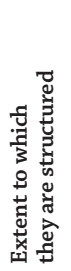 } & \multirow{2}{*}{ 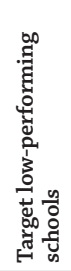 } & \multirow{2}{*}{ 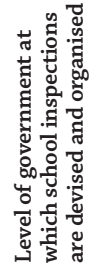 } & \multirow{2}{*}{ 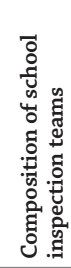 } \\
\hline & & Public & $\begin{array}{l}\text { Government- } \\
\text { dependent } \\
\text { private }\end{array}$ & Public & $\begin{array}{c}\text { Government- } \\
\text { dependent } \\
\text { private }\end{array}$ & & & & & \\
\hline & (1) & (2) & (3) & (4) & (5) & (6) & (7) & (8) & (9) & (10) \\
\hline $\begin{array}{l}\text { Australia } \\
\text { Austria }\end{array}$ & $\begin{array}{l}\mathrm{m} \\
\text { No }\end{array}$ & $\begin{array}{c}\mathrm{m} \\
\mathrm{a}\end{array}$ & $\begin{array}{c}\mathrm{m} \\
\mathrm{a}\end{array}$ & $\begin{array}{c}\mathrm{m} \\
\mathrm{a}\end{array}$ & $\begin{array}{c}\mathrm{m} \\
\mathrm{a}\end{array}$ & $\begin{array}{l}\mathrm{m} \\
\mathrm{a}\end{array}$ & $\begin{array}{l}\mathrm{m} \\
\mathrm{a}\end{array}$ & $\begin{array}{l}\mathrm{m} \\
\mathrm{a}\end{array}$ & $\begin{array}{c}\mathrm{m} \\
\mathrm{a}\end{array}$ & $\begin{array}{c}\mathrm{m} \\
\mathrm{a}\end{array}$ \\
\hline Belgium (Fl.) & Yes & 6 & 6 & 12.5 & 12.5 & Yes & $\mathrm{H}$ & Yes & 2 & Team \\
\hline Belgium (Fr.) & Yes & 5 & 5 & 33 & 33 & Yes & $\mathrm{H}$ & No & 2 & Single \\
\hline $\begin{array}{l}\text { Canada } \\
\text { Chile }\end{array}$ & $\begin{array}{l}\mathrm{m} \\
\text { Yes }\end{array}$ & $\begin{array}{l}\mathrm{m} \\
4\end{array}$ & $\begin{array}{l}\mathrm{m} \\
4\end{array}$ & $\begin{array}{l}\mathrm{m} \\
\mathrm{m}\end{array}$ & $\begin{array}{l}\mathrm{m} \\
\mathrm{m}\end{array}$ & $\begin{array}{l}\mathrm{m} \\
\text { No }\end{array}$ & $\begin{array}{l}\mathrm{m} \\
\mathrm{H}\end{array}$ & $\begin{array}{l}\mathrm{m} \\
\text { Yes }\end{array}$ & $\begin{array}{l}\mathrm{m} \\
1\end{array}$ & $\begin{array}{c}\mathrm{m} \\
\text { Team }\end{array}$ \\
\hline Czech Republic & Yes & 6 & 6 & 25 & 25 & Yes & $\mathrm{H}$ & Yes & 1 & Team \\
\hline Denmark & No & a & a & $\mathrm{a}$ & a & a & a & a & a & a \\
\hline England & Yes & 6 & 6 & 25 & 25 & No & $\mathrm{H}$ & Yes & 1 & Team \\
\hline Estonia & Yes & 6 & 6 & $\mathrm{~m}$ & $\mathrm{~m}$ & No & $\mathrm{H}$ & No & 1 & Single \\
\hline Finland & No & a & a & a & a & a & a & a & a & a \\
\hline France & Yes & 6 & 6 & $\mathrm{~m}$ & $\mathrm{~m}$ & No & $\mathrm{P}$ & No & 1 & Single \\
\hline Germany & Yes & 4 & 1 & 50 & $\mathrm{~m}$ & No & $\mathrm{H}$ & No & 2 & Team \\
\hline Greece & Yes & 4 & a & $\mathrm{m}$ & a & No & $\mathrm{U}$ & Yes & 3 & Team \\
\hline Hungary & Yes & 6 & 6 & $\mathrm{~m}$ & $\mathrm{~m}$ & No & $\mathrm{H}$ & No & 1 & Team \\
\hline Iceland & Yes & 6 & 6 & 7 & $\mathrm{~m}$ & No & $\mathrm{H}$ & No & 5 & Team \\
\hline Ireland & Yes & 4 & a & 81 & a & Yes & $\mathrm{H}$ & Yes & 1 & Mixed \\
\hline Israel & Yes & 2 & 3 & 100 & 100 & Yes & $\mathrm{H}$ & Yes & 1 & Single \\
\hline Italy & No & a & a & $\mathrm{a}$ & $\mathrm{a}$ & $\mathrm{a}$ & a & $\mathrm{a}$ & a & a \\
\hline Japan & No & a & a & a & $\mathrm{a}$ & a & a & a & a & a \\
\hline Korea & Yes & 5 & 5 & 33 & 33 & No & $\mathrm{P}$ & No & 1,3 & Team \\
\hline Luxembourg & No & a & a & a & a & a & a & a & a & a \\
\hline Mexico & Yes & 2 & a & 50 & a & Yes & $\mathrm{U}$ & No & 2 & Single \\
\hline Netherlands & Yes & 6 & 6 & 100 & 100 & No & $\mathrm{P}$ & Yes & 1 & Team \\
\hline New Zealand & Yes & 5 & a & 33 & $a$ & No & $\mathrm{P}$ & Yes & 1 & Team \\
\hline Norway & Yes & 6 & 6 & $15-20$ & 5 & No & $P$ & No & 1 & Team \\
\hline Poland & Yes & 6 & 6 & 20 & 20 & No & $\mathrm{H}$ & Yes & 1,3 & Team \\
\hline Portugal & Yes & 6 & 1 & 25 & a & No & $\mathrm{H}$ & No & 1 & Team \\
\hline Scotland & Yes & 6 & a & 10 & a & No & $\mathrm{H}$ & No & 1 & Team \\
\hline Slovak Republic & Yes & 6 & 6 & 20 & 20 & No & $\mathrm{H}$ & No & 1 & Team \\
\hline Slovenia & Yes & 6 & 6 & 35 & $\mathrm{~m}$ & No & $\mathrm{H}$ & No & 1 & Mixed \\
\hline Spain & Yes & 2 & 2 & 100 & 100 & No & $\mathrm{H}$ & No & 2 & Single \\
\hline Sweden $^{1}$ & Yes & 6 & 6 & 33 & 33 & No & $\mathrm{P}$ & Yes & 1 & Mixed \\
\hline Switzerland & Yes & $\mathrm{m}$ & $\mathrm{m}$ & $\mathrm{m}$ & $\mathrm{m}$ & $\mathrm{m}$ & $\mathrm{m}$ & $\mathrm{m}$ & $\mathrm{m}$ & $\mathrm{m}$ \\
\hline Turkey & Yes & 5 & a & 33 & a & No & $\mathrm{H}$ & No & 3 & Team \\
\hline United States & Yes & $\mathrm{m}$ & a & $\mathrm{m}$ & a & Yes & $\mathrm{m}$ & Yes & $2,5,6$ & Team \\
\hline Brazil & No & $\mathrm{a}$ & a & & & $\mathrm{a}$ & & $\mathrm{a}$ & a & $\mathrm{a}$ \\
\hline Colombia & Yes & 3 & 3 & 25 & 100 & Yes & $\mathrm{H}$ & Yes & 3 & Team \\
\hline Latvia & Yes & 1 & 1 & a & $\mathrm{a}$ & Yes & $\mathrm{H}$ & No & 2 & Mixed \\
\hline
\end{tabular}

Frequency of school inspections/self-evaluations

1: There is no requirement for school inspections/self-evaluations

2: More often than once a year

3: Once a year

4: Once every two years

5: Once every three years

6: Once every three or more years
Levels of government

1: Central authority or government

2: State authorities or governments

3: Provincial/regional authorities or government

4: Sub-regional or inter-municipal authorities or governments

5: Local authorities or governments
Extent to which structured

H: Highly structured

P: Partially structured

U: Unstructured

Note: Federal states or countries with highly decentralised school systems may have different regulations in states, provinces or regions. Please refer to Annex 3 for additional information.

1. Year of reference for school inspection is 2014

Source: OECD. See Annex 3 for notes (www.oecd.org/education/education-at-a-glance-19991487.htm).

Please refer to the Reader's Guide for information concerning symbols for missing data and abbreviations.

StatLink 첸 http://dx.doi.org/10.1787/888933286369 
Table D6.9. [2/2] School inspection and school self-evaluation at the lower secondary level (2015) In general programmes

\begin{tabular}{|c|c|c|c|c|c|c|c|c|c|c|c|}
\hline & \multicolumn{11}{|c|}{ School self-evaluation } \\
\hline & \multirow{2}{*}{ 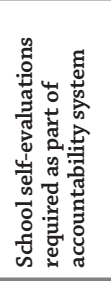 } & \multirow{2}{*}{ 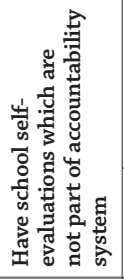 } & \multicolumn{2}{|c|}{$\begin{array}{l}\text { Frequency of school } \\
\text { self-evaluations }\end{array}$} & \multicolumn{2}{|c|}{$\begin{array}{c}\text { Percentage of schools } \\
\text { required to conduct } \\
\text { self-evaluation each year (\%) }\end{array}$} & \multirow{2}{*}{ 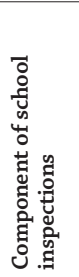 } & \multirow{2}{*}{ 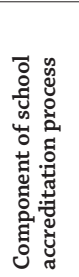 } & \multirow{2}{*}{ 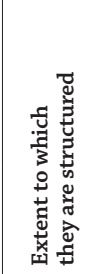 } & \multirow{2}{*}{ 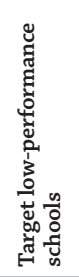 } & \multirow{2}{*}{ 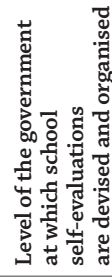 } \\
\hline & & & Public & $\begin{array}{c}\text { Government- } \\
\text { dependent } \\
\text { private }\end{array}$ & Public & $\begin{array}{c}\text { Government- } \\
\text { dependent } \\
\text { private }\end{array}$ & & & & & \\
\hline & (11) & (12) & (13) & (14) & (15) & (16) & (17) & (18) & (19) & (20) & (21) \\
\hline Australia & $\mathrm{m}$ & $\mathrm{m}$ & $\mathrm{m}$ & $\mathrm{m}$ & $\mathrm{m}$ & $\mathrm{m}$ & $\mathrm{m}$ & $\mathrm{m}$ & $\mathrm{m}$ & $\mathrm{m}$ & $\mathrm{m}$ \\
\hline Austria & Yes & No & 3 & 3 & 100 & 100 & No & No & $\mathrm{P}$ & No & 1 \\
\hline Belgium (Fl.) & Yes & Yes & $\mathrm{m}$ & $\mathrm{m}$ & $\mathrm{m}$ & $\mathrm{m}$ & Yes & No & $\mathrm{U}$ & No & 6 \\
\hline Belgium (Fr.) & No & No & $\mathrm{a}$ & a & $\mathrm{a}$ & a & a & a & a & a & a \\
\hline Canada & $\mathrm{m}$ & $\mathrm{m}$ & $\mathrm{m}$ & $\mathrm{m}$ & $\mathrm{m}$ & $\mathrm{m}$ & $\mathrm{m}$ & $\mathrm{m}$ & $\mathrm{m}$ & $\mathrm{m}$ & $\mathrm{m}$ \\
\hline Chile & No & No & a & a & $\mathrm{a}$ & $\mathrm{a}$ & a & a & $\mathrm{a}$ & a & a \\
\hline Czech Republic & Yes & No & 3 & 3 & 100 & 100 & No & No & $\mathrm{P}$ & No & 6 \\
\hline Denmark & Yes & No & 5 & 5 & 33 & 33 & No & No & $\mathrm{P}$ & No & 5 \\
\hline England & No & No & a & a & a & a & a & a & a & a & a \\
\hline Estonia & Yes & No & 5 & 5 & 33 & 33 & No & No & $\mathrm{U}$ & No & 6 \\
\hline Finland & No & Yes & a & $\mathrm{a}$ & $\mathrm{a}$ & a & a & a & a & a & a \\
\hline France & Yes & No & 3 & 6 & 100 & $\mathrm{~m}$ & Yes & No & $\mathrm{P}$ & No & 1 \\
\hline Germany & Yes & $\mathrm{m}$ & 1 & 1 & $\mathrm{~m}$ & $\mathrm{~m}$ & No & No & $\mathrm{m}$ & No & $\mathrm{m}$ \\
\hline Greece & Yes & No & 3 & 3 & 100 & 100 & Yes & No & $\mathrm{H}$ & No & 1 \\
\hline Hungary & Yes & No & 6 & 4 & 0 & 0 & Yes & No & $\mathrm{H}$ & No & 6 \\
\hline Iceland & Yes & No & 3 & 3 & 100 & 100 & Yes & No & $\mathrm{U}$ & No & 6 \\
\hline Ireland & Yes & Yes & 3 & a & 100 & a & Yes & No & $\mathrm{P}$ & No & 1 \\
\hline Israel & Yes & No & 3 & 3 & 100 & 100 & Yes & No & $\mathrm{P}$ & No & $1,4,6$ \\
\hline Italy & Yes & No & 3 & $\mathrm{a}$ & 100 & $\mathrm{a}$ & No & No & $\mathrm{P}$ & No & 1 \\
\hline Japan & Yes & No & 2 & $\mathrm{a}$ & 100 & a & No & No & $\mathrm{m}$ & No & 6 \\
\hline Korea & Yes & No & 5 & 5 & 33 & 33 & No & No & $\mathrm{P}$ & No & 1,3 \\
\hline Luxembourg & No & No & a & a & a & a & a & a & a & a & a \\
\hline Mexico & No & No & a & $\mathrm{a}$ & a & a & a & a & a & a & a \\
\hline Netherlands & Yes & $\mathrm{m}$ & 1 & 1 & a & a & Yes & No & $\mathrm{U}$ & No & 6 \\
\hline New Zealand & Yes & No & 1 & a & 100 & a & No & No & U & No & 6 \\
\hline Norway & Yes & No & 3 & 3 & 100 & 100 & Yes & No & $\mathrm{P}$ & No & $1,5,6$ \\
\hline Poland & Yes & No & 3 & 3 & 100 & 100 & Yes & No & $\mathrm{U}$ & Yes & 6 \\
\hline Portugal & Yes & Yes & $\mathrm{m}$ & $\mathrm{m}$ & $\mathrm{m}$ & $\mathrm{m}$ & Yes & No & $\mathrm{P}$ & No & 6 \\
\hline Scotland & No & No & a & a & $\mathrm{a}$ & $\mathrm{a}$ & $\mathrm{a}$ & a & a & a & a \\
\hline Slovak Republic & Yes & No & 3 & 3 & 100 & 100 & Yes & No & $\mathrm{P}$ & No & 1 \\
\hline Slovenia & Yes & No & 3 & $\mathrm{~m}$ & 100 & $\mathrm{~m}$ & Yes & No & U & No & 6 \\
\hline Spain & No & Yes & a & $\mathrm{a}$ & $\mathrm{a}$ & a & a & a & a & a & a \\
\hline Sweden $^{1}$ & No & Yes & a & a & a & a & a & a & a & a & a \\
\hline Switzerland & Yes & No & $\mathrm{m}$ & $\mathrm{m}$ & $\mathrm{m}$ & $\mathrm{m}$ & $\mathrm{m}$ & $\mathrm{m}$ & $\mathrm{m}$ & $\mathrm{m}$ & $\mathrm{m}$ \\
\hline Turkey & Yes & No & 3 & $\mathrm{a}$ & 100 & a & No & No & $\mathrm{H}$ & No & 1 \\
\hline United States & Yes & Yes & $\mathrm{m}$ & a & $\mathrm{m}$ & a & $\mathrm{m}$ & Yes & $\mathrm{m}$ & $\mathrm{m}$ & $2,5,6$ \\
\hline Brazil & No & $\mathrm{m}$ & $a$ & $\mathrm{a}$ & $\mathrm{a}$ & $\mathrm{a}$ & a & a & a & a & a \\
\hline Colombia & Yes & No & 3 & $\mathrm{~m}$ & 100 & $\mathrm{~m}$ & Yes & No & H & No & 6 \\
\hline Latvia & Yes & No & 6 & 6 & 100 & 100 & No & Yes & $\mathrm{H}$ & No & 6 \\
\hline \multicolumn{4}{|c|}{ Frequency of school inspections/self-evaluations } & \multicolumn{5}{|c|}{ Levels of government } & \multicolumn{3}{|c|}{ Extent to which structured } \\
\hline \multicolumn{4}{|c|}{$\begin{array}{l}\text { 1: There is no requirement for school inspections/self-evaluations } \\
\text { 2: More often than once a year } \\
\text { 3: Once a year } \\
\text { 4: Once every two years } \\
\text { 5: Once every three years } \\
\text { 6: Once every three or more years }\end{array}$} & \multicolumn{5}{|c|}{$\begin{array}{l}\text { 1: Central authority or government } \\
\text { 2: State authorities or governments } \\
\text { 3: Provincial/regional authorities or go } \\
\text { 4: Sub-regional or inter-municipal auth } \\
\text { 5: Local authorities or governments } \\
\text { 6: School, school board or committee }\end{array}$} & \multicolumn{3}{|c|}{$\begin{array}{l}\text { H: Highly structured } \\
\text { P: Partially structured } \\
\text { U: Unstructured }\end{array}$} \\
\hline
\end{tabular}

Note: Federal states or countries with highly decentralised school systems may have different regulations in states, provinces or regions. Please refer to Annex 3 for additional information.

1. Year of reference for school inspection is 2014.

Source: OECD. See Annex 3 for notes (www.oecd.org/education/education-at-a-glance-19991487.htm).

Please refer to the Reader's Guide for information concerning symbols for missing data and abbreviations.

StatLink त्ञाज http://dx.doi.org/10.1787/888933286369 
Table D6.13. Public school compliance-oriented reports, by domains and receiving groups (2015) In general programmes

\begin{abstract}
The table shows for each country the group(s) to which public schools are expected to submit compliance-oriented reports on a given domain.
Reading columns 1, 9 and 17: In Israel, public schools are expected to submit student data reports to the school board (S), to the municipal or local government/education authority $(\mathrm{L})$, to the regional and national government/education authority $(\mathrm{R}, \mathrm{N})$ as well as parents, students $(\mathrm{P})$ and the general public $(\mathrm{G})$.
\end{abstract}

\begin{tabular}{|c|c|c|c|c|c|c|c|c|c|c|c|c|c|c|c|c|c|c|c|c|c|c|c|}
\hline & Mun & & & & & & & & & $\begin{array}{l}\text { ucati } \\
\text { Natio } \\
\text { ducat }\end{array}$ & 10018 & a & 20 & & & & & & & & & & \\
\hline 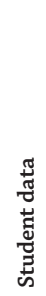 & 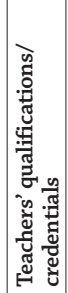 & 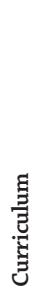 & 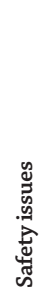 & 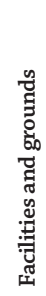 & 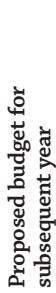 & 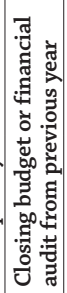 & 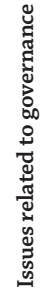 & 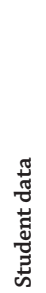 & 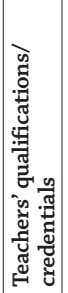 & 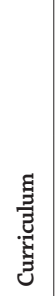 & 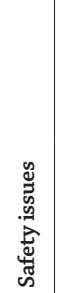 & 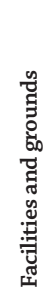 & 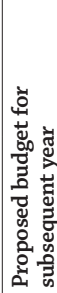 & 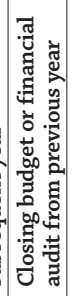 & 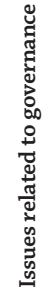 & 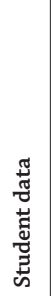 & 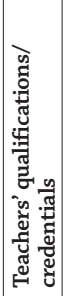 & 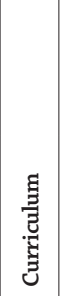 & 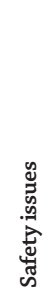 & 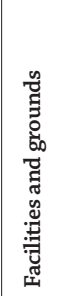 & 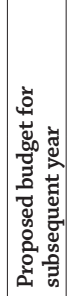 & 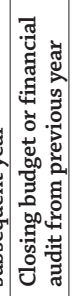 & 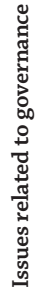 \\
\hline
\end{tabular}

\begin{tabular}{|c|c|c|c|c|c|c|c|c|c|c|c|c|c|c|c|c|c|c|c|c|c|c|c|c|}
\hline $\begin{array}{l}\text { Australia } \\
\text { ous Austria }\end{array}$ & $\begin{array}{l}\mathrm{m} \\
\mathrm{L}\end{array}$ & $\begin{array}{c}\mathrm{m} \\
\mathrm{L}\end{array}$ & $\begin{array}{c}\mathrm{m} \\
\mathrm{S}, \mathrm{L}\end{array}$ & $\begin{array}{l}\mathrm{m} \\
\text { No }\end{array}$ & $\begin{array}{l}\mathrm{m} \\
\mathrm{L}\end{array}$ & $\begin{array}{l}\mathrm{m} \\
\mathrm{L}\end{array}$ & $\begin{array}{l}\mathrm{m} \\
\mathrm{L}\end{array}$ & $\begin{array}{l}\mathrm{m} \\
\text { No }\end{array}$ & $\begin{array}{c}\mathrm{m} \\
\mathrm{R}, \mathrm{N}\end{array}$ & $\begin{array}{c}\mathrm{m} \\
\mathrm{R}, \mathrm{N}\end{array}$ & $\begin{array}{c}\mathrm{m} \\
\mathrm{R}, \mathrm{N}\end{array}$ & $\begin{array}{l}\mathrm{m} \\
\text { No }\end{array}$ & $\begin{array}{c}\mathrm{m} \\
\mathrm{R}, \mathrm{N}\end{array}$ & $\begin{array}{c}\mathrm{m} \\
\mathrm{R}, \mathrm{N}\end{array}$ & $\begin{array}{c}\mathrm{m} \\
\mathrm{R}, \mathrm{N}\end{array}$ & $\begin{array}{l}\mathrm{m} \\
\text { No }\end{array}$ & $\begin{array}{l}\mathrm{m} \\
\mathrm{m}\end{array}$ & $\begin{array}{l}\mathrm{m} \\
\mathrm{m}\end{array}$ & $\begin{array}{l}\mathrm{m} \\
\mathrm{m}\end{array}$ & $\begin{array}{l}\mathrm{m} \\
\text { No }\end{array}$ & $\begin{array}{l}\mathrm{m} \\
\mathrm{m}\end{array}$ & $\begin{array}{l}\mathrm{m} \\
\mathrm{m}\end{array}$ & $\begin{array}{l}\mathrm{m} \\
\mathrm{m}\end{array}$ & $\begin{array}{l}\mathrm{m} \\
\text { No }\end{array}$ \\
\hline Belgium (Fl.) & s & s & $S$ & S,L & S,L & s & s & S & $\mathrm{N}$ & $\mathrm{N}$ & $\mathrm{N}$ & $\mathrm{N}$ & $\mathrm{N}$ & No & $\mathrm{N}$ & $\mathrm{N}$ & No & No & P & No & No & $\mathrm{P}$ & No & $P, G$ \\
\hline Belgium (Fr.) & S,L & $\mathrm{L}$ & No & S,L & $S, L$ & S,L & S, L & No & $\mathrm{R}, \mathrm{N}$ & $\mathrm{R}, \mathrm{N}$ & No & $\mathrm{R}, \mathrm{N}$ & $R, N$ & $R, N$ & $R, N$ & No & No & No & No & No & No & No & No & No \\
\hline Canada & $\mathrm{m}$ & $\mathrm{m}$ & $\mathrm{m}$ & $\mathrm{m}$ & $\mathrm{m}$ & $\mathrm{m}$ & $\mathrm{m}$ & $\mathrm{m}$ & $\mathrm{m}$ & $\mathrm{m}$ & $\mathrm{m}$ & $\mathrm{m}$ & $\mathrm{m}$ & $\mathrm{m}$ & $\mathrm{m}$ & $\mathrm{m}$ & $\mathrm{m}$ & $\mathrm{m}$ & $\mathrm{m}$ & $\mathrm{m}$ & $\mathrm{m}$ & $\mathrm{m}$ & $\mathrm{m}$ & $\mathrm{m}$ \\
\hline Chile & L & $\mathrm{L}$ & $\mathrm{L}$ & $\mathrm{L}$ & $\mathrm{L}$ & $\mathrm{L}$ & $\mathrm{L}$ & $\mathrm{L}$ & $\mathrm{N}$ & $\mathrm{N}$ & $\mathrm{N}$ & $\mathrm{N}$ & $\mathrm{N}$ & No & No & No & No & No & No & No & No & No & No & No \\
\hline Czech Republic & S,L & $\mathrm{S}, \mathrm{L}$ & $\mathrm{S}, \mathrm{L}$ & $\mathrm{S}, \mathrm{L}$ & $\mathrm{S}, \mathrm{L}$ & $\mathrm{S}, \mathrm{L}$ & S, L & S,L & $\mathrm{R}$ & $\mathrm{R}$ & $\mathrm{R}$ & $\mathrm{R}$ & $\mathrm{R}$ & $\mathrm{R}$ & $\mathrm{R}$ & $\mathrm{R}$ & $\mathrm{P}, \mathrm{G}$ & No & $P, G$ & No & No & $\mathrm{P}$ & $\mathrm{P}$ & No \\
\hline Denmark & S,L & $\mathrm{L}$ & $\mathrm{S}, \mathrm{L}$ & $\mathrm{m}$ & $\mathrm{m}$ & $\mathrm{S}, \mathrm{L}$ & S,L & $\mathrm{S}, \mathrm{L}$ & $\mathrm{R}, \mathrm{N}$ & No & $\mathrm{N}$ & No & $\mathrm{N}$ & $\mathrm{N}$ & $\mathrm{N}$ & $\mathrm{N}$ & G & No & $\mathrm{P}, \mathrm{G}$ & No & No & No & No & No \\
\hline England & S,L & $s$ & s & S,L & S,L & $\mathrm{S}, \mathrm{L}$ & S, L & S,L & $\mathrm{N}$ & No & No & No & No & No & No & No & No & No & No & No & No & No & No & No \\
\hline Estonia & $\mathrm{L}$ & S & S & s & S & $\mathrm{S}, \mathrm{L}$ & s & S & $\mathrm{N}$ & No & $\mathrm{N}$ & No & No & No & No & No & G & G & No & No & No & No & No & No \\
\hline Finland & L & L & $\mathrm{m}$ & $\mathrm{m}$ & $\mathrm{m}$ & $\mathrm{m}$ & $\mathrm{m}$ & $\mathrm{m}$ & No & No & No & No & No & No & No & No & $\mathrm{m}$ & $\mathrm{m}$ & $\mathrm{m}$ & $\mathrm{m}$ & $\mathrm{m}$ & $\mathrm{m}$ & $\mathrm{m}$ & $\mathrm{m}$ \\
\hline France & No & No & $\mathrm{L}$ & $\mathrm{S}, \mathrm{L}$ & No & No & No & No & $\mathrm{R}, \mathrm{N}$ & $\mathrm{R}, \mathrm{N}$ & $R, N$ & $\mathrm{R}$ & $\mathrm{R}, \mathrm{N}$ & $R, N$ & $\mathrm{R}, \mathrm{N}$ & $\mathrm{R}, \mathrm{N}$ & No & No & $\mathrm{P}, \mathrm{G}$ & No & No & No & No & No \\
\hline Germany & S,L & $\mathrm{S}, \mathrm{L}$ & $\mathrm{S}, \mathrm{L}$ & S, L & $S, L$ & $\mathrm{~S}, \mathrm{~L}$ & S, L & $\mathrm{m}$ & $\mathrm{R}$ & R & $\mathrm{R}$ & $\mathrm{R}$ & $\mathrm{R}$ & $\mathrm{R}$ & No & $\mathrm{m}$ & $\mathrm{P}, \mathrm{G}$ & No & $\mathrm{P}, \mathrm{G}$ & $\mathrm{P}, \mathrm{G}$ & No & No & No & $\mathrm{m}$ \\
\hline Greece & $\mathrm{L}$ & $\mathrm{S}, \mathrm{L}$ & $\mathrm{S}, \mathrm{L}$ & S, L & $\mathrm{S}, \mathrm{L}$ & No & S, L & $\mathrm{S}, \mathrm{L}$ & $\mathrm{R}, \mathrm{N}$ & $\mathrm{R}, \mathrm{N}$ & $R, N$ & $\mathrm{R}, \mathrm{N}$ & $\mathrm{R}, \mathrm{N}$ & No & $\mathrm{R}, \mathrm{N}$ & $\mathrm{R}, \mathrm{N}$ & No & No & P & P, G & $\mathrm{P}, \mathrm{G}$ & No & No & No \\
\hline Hungary & No & No & No & No & No & No & No & No & $\mathrm{N}$ & No & No & No & No & No & No & No & No & No & No & No & No & No & No & No \\
\hline Iceland & S,L & $\mathrm{S}, \mathrm{L}$ & $\mathrm{S}, \mathrm{L}$ & $\mathrm{S}, \mathrm{L}$ & $\mathrm{S}, \mathrm{L}$ & $\mathrm{S}, \mathrm{L}$ & S, L & S,L & $\mathrm{N}$ & $\mathrm{N}$ & $\mathrm{N}$ & No & No & No & No & $\mathrm{N}$ & No & No & $\mathrm{P}, \mathrm{G}$ & No & No & No & No & No \\
\hline Ireland & S & S & S & S & S & S & S & S & $\mathrm{R}, \mathrm{N}$ & $\mathrm{R}, \mathrm{N}$ & R,N & $\mathrm{R}$ & $\mathrm{R}$ & $\mathrm{R}$ & $\mathrm{R}, \mathrm{N}$ & $\mathrm{R}, \mathrm{N}$ & No & No & No & No & No & No & No & $\mathrm{P}$ \\
\hline Israel & S,L & $\mathrm{S}, \mathrm{L}$ & $\mathrm{S}, \mathrm{L}$ & L & $S, L$ & $\mathrm{~S}, \mathrm{~L}$ & S, L & No & $\mathrm{R}, \mathrm{N}$ & $\mathrm{R}, \mathrm{N}$ & $R, N$ & $\mathrm{R}, \mathrm{N}$ & $\mathrm{R}, \mathrm{N}$ & $R, N$ & $\mathrm{R}, \mathrm{N}$ & No & $\mathrm{P}, \mathrm{G}$ & No & $\mathrm{P}$ & P & P & No & $\mathrm{P}$ & No \\
\hline Italy & L & No & S & S,L & L & - & S & No & $\mathrm{R}, \mathrm{N}$ & $\mathrm{R}, \mathrm{N}$ & R,N & $\mathrm{N}$ & $\mathrm{R}$ & No & No & No & No & No & No & No & No & No & No & No \\
\hline Japan & $\mathrm{m}$ & $\mathrm{m}$ & $\mathrm{m}$ & $\mathrm{m}$ & $\mathrm{m}$ & $\mathrm{m}$ & $\mathrm{m}$ & $\mathrm{m}$ & $\mathrm{N}$ & No & No & $\mathrm{N}$ & $\mathrm{N}$ & No & No & No & $\mathrm{m}$ & $\mathrm{m}$ & $\mathrm{m}$ & $\mathrm{m}$ & $\mathrm{m}$ & $\mathrm{m}$ & $\mathrm{m}$ & $\mathrm{m}$ \\
\hline Korea & L & L & $\mathrm{S}, \mathrm{L}$ & $\mathrm{S}, \mathrm{L}$ & L & $\mathrm{S}, \mathrm{L}$ & S, L & S,L & $\mathrm{R}, \mathrm{N}$ & $\mathrm{R}, \mathrm{N}$ & $R, N$ & $\mathrm{R}, \mathrm{N}$ & $\mathrm{R}, \mathrm{N}$ & $R, N$ & $R, N$ & $\mathrm{R}, \mathrm{N}$ & G & G & G & G & G & G & G & G \\
\hline Luxembourg & No & No & No & No & No & No & No & No & $\mathrm{N}$ & $\mathrm{N}$ & $\mathrm{N}$ & $\mathrm{N}$ & $\mathrm{N}$ & $\mathrm{N}$ & No & $\mathrm{N}$ & No & No & No & No & No & No & No & No \\
\hline Mexico & S & s & No & No & $s$ & No & No & No & $\mathrm{R}, \mathrm{N}$ & $\mathrm{R}, \mathrm{N}$ & No & No & $\mathrm{R}, \mathrm{N}$ & No & No & No & G & No & No & No & No & No & No & No \\
\hline Netherlands & s & $S$ & $S$ & s & $S$ & $S$ & s & $s$ & $\mathrm{~N}$ & No & No & $\mathrm{N}$ & $\mathrm{N}$ & No & $\mathrm{N}$ & $\mathrm{N}$ & No & No & P & P & No & No & No & No \\
\hline New Zealand & s & S & 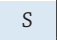 & S & S & No & S & S & $\mathrm{N}$ & $\mathrm{N}$ & No & No & No & No & $\mathrm{N}$ & No & No & No & No & No & No & No & No & No \\
\hline Norway & $\mathrm{m}$ & $\mathrm{m}$ & $\mathrm{m}$ & $\mathrm{m}$ & $\mathrm{m}$ & $\mathrm{m}$ & $\mathrm{m}$ & $\mathrm{m}$ & $\mathrm{m}$ & $\mathrm{m}$ & $\mathrm{m}$ & $\mathrm{m}$ & $\mathrm{m}$ & $\mathrm{m}$ & $\mathrm{m}$ & $\mathrm{m}$ & $\mathrm{m}$ & $\mathrm{m}$ & $\mathrm{m}$ & $\mathrm{m}$ & $\mathrm{m}$ & $\mathrm{m}$ & $\mathrm{m}$ & $\mathrm{m}$ \\
\hline Poland & L & L & No & L & $\mathrm{L}$ & $\mathrm{L}$ & L & $\mathrm{L}$ & $\mathrm{N}$ & $\mathrm{N}$ & No & $\mathrm{N}$ & $\mathrm{N}$ & No & $\mathrm{N}$ & $\mathrm{N}$ & $\mathrm{m}$ & $\mathrm{m}$ & $\mathrm{m}$ & $\mathrm{m}$ & $\mathrm{m}$ & $\mathrm{m}$ & $\mathrm{m}$ & $\mathrm{m}$ \\
\hline Portugal & $s$ & No & $\mathrm{S}, \mathrm{L}$ & $S, L$ & s & $s$ & $s$ & S,L & $\mathrm{R}, \mathrm{N}$ & $\mathrm{R}, \mathrm{N}$ & $R, N$ & $\mathrm{R}, \mathrm{N}$ & $\mathrm{R}, \mathrm{N}$ & $\mathrm{R}, \mathrm{N}$ & $\mathrm{R}, \mathrm{N}$ & $\mathrm{R}, \mathrm{N}$ & No & No & $\mathrm{P}$ & $\mathrm{P}$ & No & No & No & $\mathrm{P}$ \\
\hline Scotland & $\mathrm{L}$ & No & L & $\mathrm{L}$ & No & No & L & No & $\mathrm{N}$ & No & No & No & No & No & No & No & G & No & $P, G$ & No & No & No & No & No \\
\hline Slovak Republic & S,L & No & $\mathrm{S}, \mathrm{L}$ & S,L & $\mathrm{S}, \mathrm{L}$ & $\mathrm{S}, \mathrm{L}$ & S, L & $\mathrm{S}, \mathrm{L}$ & $\mathrm{R}, \mathrm{N}$ & $\mathrm{N}$ & $\mathrm{R}, \mathrm{N}$ & $\mathrm{R}, \mathrm{N}$ & $\mathrm{R}, \mathrm{N}$ & $\mathrm{R}, \mathrm{N}$ & $\mathrm{R}, \mathrm{N}$ & $\mathrm{R}, \mathrm{N}$ & No & No & No & No & No & No & No & No \\
\hline Slovenia & S,L & S, L & $\mathrm{S}, \mathrm{L}$ & s & S,L & $\mathrm{S}, \mathrm{L}$ & s & S,L & $\mathrm{N}$ & $\mathrm{N}$ & $\mathrm{N}$ & No & $\mathrm{N}$ & $\mathrm{N}$ & $\mathrm{N}$ & $\mathrm{N}$ & $P, G$ & No & $P, G$ & $\mathrm{P}$ & $P, G$ & No & G & G \\
\hline Spain & S,L & S, L & $\mathrm{S}, \mathrm{L}$ & S,L & S,L & $\mathrm{S}, \mathrm{L}$ & S, L & S,L & $\mathrm{R}, \mathrm{N}$ & $\mathrm{R}, \mathrm{N}$ & R,N & $\mathrm{R}, \mathrm{N}$ & $R, N$ & $R, N$ & $\mathrm{R}, \mathrm{N}$ & $\mathrm{R}, \mathrm{N}$ & $\mathrm{P}$ & $\mathrm{P}$ & $\mathrm{P}$ & $\mathrm{P}$ & $\mathrm{P}$ & $\mathrm{P}$ & $\mathrm{P}$ & $\mathrm{P}$ \\
\hline $\begin{array}{l}\text { Sweden } \\
\text { Switzerland }\end{array}$ & $\begin{array}{l}\mathrm{m} \\
\mathrm{m}\end{array}$ & $\begin{array}{l}\mathrm{m} \\
\mathrm{m}\end{array}$ & $\begin{array}{l}\mathrm{m} \\
\mathrm{m}\end{array}$ & $\begin{array}{l}\mathrm{m} \\
\mathrm{m}\end{array}$ & $\begin{array}{l}\mathrm{m} \\
\mathrm{m}\end{array}$ & $\begin{array}{l}\mathrm{m} \\
\mathrm{m}\end{array}$ & $\begin{array}{l}\mathrm{m} \\
\mathrm{m}\end{array}$ & $\begin{array}{l}\mathrm{m} \\
\mathrm{m}\end{array}$ & $\begin{array}{l}N \\
\mathrm{~m}\end{array}$ & $\begin{array}{c}\text { No } \\
\mathrm{m}\end{array}$ & $\begin{array}{l}\mathrm{m} \\
\mathrm{m}\end{array}$ & $\begin{array}{c}\text { No } \\
\mathrm{m}\end{array}$ & $\begin{array}{c}\text { No } \\
\mathrm{m}\end{array}$ & $\begin{array}{c}\text { No } \\
\mathrm{m}\end{array}$ & & $\begin{array}{c}\text { No } \\
\mathrm{m}\end{array}$ & $\begin{array}{l}\mathrm{m} \\
\mathrm{m}\end{array}$ & $\begin{array}{l}\mathrm{m} \\
\mathrm{m}\end{array}$ & & $\begin{array}{l}\mathrm{m} \\
\mathrm{m}\end{array}$ & $\begin{array}{l}\mathrm{m} \\
\mathrm{m}\end{array}$ & $\begin{array}{l}\mathrm{m} \\
\mathrm{m}\end{array}$ & $\begin{array}{l}\mathrm{m} \\
\mathrm{m}\end{array}$ & $\begin{array}{l}\mathrm{m} \\
\mathrm{m}\end{array}$ \\
\hline Turkey & $\mathrm{L}$ & No & $\mathrm{L}$ & $\mathrm{L}$ & $\mathrm{L}$ & $\mathrm{L}$ & $\mathrm{L}$ & $\mathrm{L}$ & $\mathrm{R}, \mathrm{N}$ & No & $R, N$ & $\mathrm{R}, \mathrm{N}$ & $\mathrm{R}, \mathrm{N}$ & $R, N$ & $\mathrm{R}, \mathrm{N}$ & $\mathrm{R}, \mathrm{N}$ & No & No & No & No & No & No & No & No \\
\hline United States & $\mathrm{S}, \mathrm{L}$ & $S, L$ & $\mathrm{~S}, \mathrm{~L}$ & $\mathrm{~S}, \mathrm{~L}$ & S,L & S,L & S, L & $S, L$ & $\mathrm{R}, \mathrm{N}$ & $\mathrm{R}$ & $\mathrm{R}, \mathrm{N}$ & $\mathrm{R}, \mathrm{N}$ & $\mathrm{R}$ & $\mathrm{R}$ & $\mathrm{R}$ & $\mathrm{R}$ & $\mathrm{P}, \mathrm{G}$ & No & No & P & No & $P, G$ & $P, G$ & No \\
\hline Brazil & $\mathrm{L}$ & $\mathrm{L}$ & $\mathrm{L}$ & $\mathrm{L}$ & $\mathrm{L}$ & 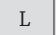 & $\mathrm{L}$ & $\mathrm{L}$ & $\mathrm{R}, \mathrm{N}$ & $\mathrm{R}, \mathrm{N}$ & $\mathrm{R}$ & $\mathrm{R}$ & $\mathrm{R}, \mathrm{N}$ & $\mathrm{R}$ & $\mathrm{R}$ & $\mathrm{R}$ & No & No & No & No & No & $\mathrm{P}$ & $\mathrm{P}$ & No \\
\hline Colombia & S,L & $S, L$ & $\mathrm{~S}, \mathrm{~L}$ & S, L & S,L & S,L & S, L & S,L & $\mathrm{R}, \mathrm{N}$ & $\mathrm{R}, \mathrm{N}$ & R,N & $\mathrm{R}, \mathrm{N}$ & $\mathrm{R}, \mathrm{N}$ & $\mathrm{R}, \mathrm{N}$ & $R, N$ & $\mathrm{R}, \mathrm{N}$ & No & P & $\mathrm{P}, \mathrm{G}$ & $\mathrm{P}, \mathrm{G}$ & $P$ & No & $\mathrm{P}, \mathrm{G}$ & No \\
\hline Latvia & S,L & $\mathrm{S}, \mathrm{L}$ & $\mathrm{S}, \mathrm{L}$ & $\mathrm{S}, \mathrm{L}$ & $\mathrm{S}, \mathrm{L}$ & $\mathrm{S}, \mathrm{L}$ & S, L & $\mathrm{S}, \mathrm{L}$ & $\mathrm{R}, \mathrm{N}$ & $\mathrm{R}, \mathrm{N}$ & $R, N$ & $\mathrm{R}, \mathrm{N}$ & $\mathrm{R}, \mathrm{N}$ & $\mathrm{R}, \mathrm{N}$ & $\mathrm{R}, \mathrm{N}$ & $\mathrm{R}, \mathrm{N}$ & No & No & $\mathrm{P}, \mathrm{G}$ & $\mathrm{P}, \mathrm{G}$ & No & No & No & No \\
\hline
\end{tabular}

Note: Federal states or countries with highly decentralised school systems may have different regulations in states, provinces or regions. Please refer to Annex 3 for additional information.

Source: OECD. See Annex 3 for notes (www.oecd.org/education/education-at-a-glance-19991487.htm).

Please refer to the Reader's Guide for information concerning symbols for missing data and abbreviations.

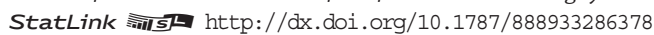





\section{WHAT TEACHER AND SCHOOL LEADER APPRAISAL SYSTEMS ARE IN PLACE?}

- Teacher appraisal is legislated/required by policy or regulation in 30 of 37 OECD and partner countries with available data.

- School leader appraisal at the lower secondary level is legislated and covered by a policy framework in 21 of 37 countries. School leader appraisal is mandatory in all countries for which data are available except Poland, where it is voluntary.

\section{Chart D7.1. Frequency of various types of teacher and school leader appraisals covered by policy framework (2015) \\ In general programmes, lower secondary education}

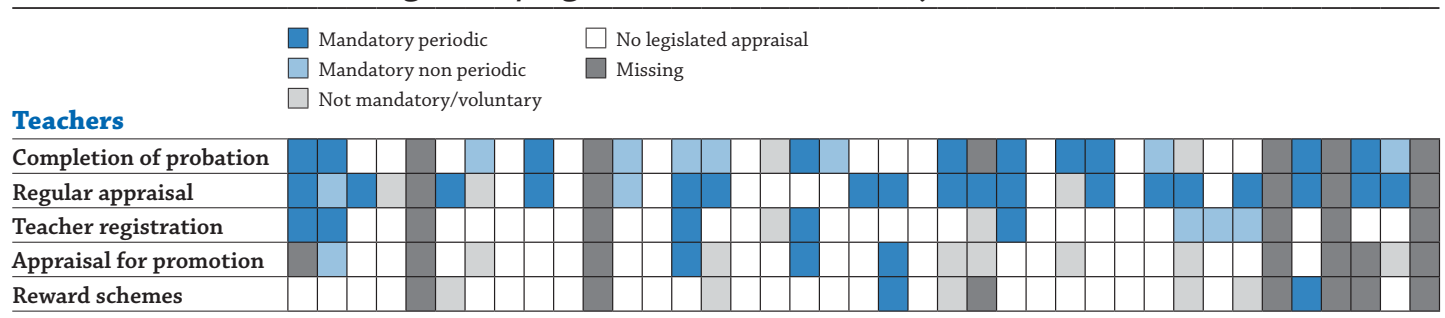

School leaders

School leader appraisal

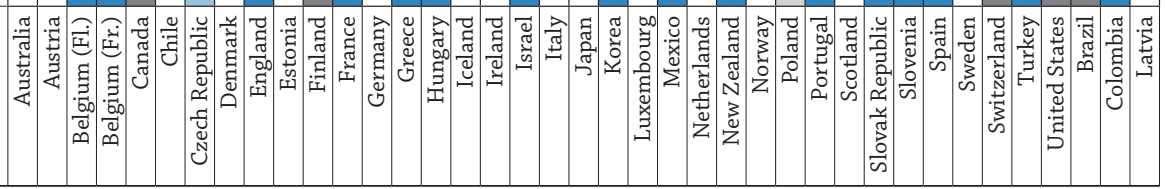

Source: OECD. Tables D7.3b and D7.8b. See Annex 3 for notes (www.oecd.org/education/education-at-a-glance-19991487.htm).

StatLink त्राज http://dx.doi.org/10.1787/888933284638

\section{Context}

Monitoring and appraising teachers is central to improving schools and learning environments. If well designed, teacher appraisal and feedback systems can be used as tools to increase teacher effectiveness and achieve better learning outcomes. Appraisal can help to increase the focus on instruction and teachers' professional learning. Appraisal and feedback systems can also help to create better school organisation by allowing teachers to progress in their career and to take on new roles and responsibilities based on a solid evaluation of their performance. They also offer an opportunity to recognise and reward effective teaching (OECD, 2013).

Backed by a growing research base, policy makers have become increasingly aware of the significance of school leadership for effective teaching and learning. A growing number of countries have developed initiatives to strengthen the leadership capacity of their schools. While research on the effects of different appraisal schemes is limited, some evidence suggests potential benefits of the appraisal of individual school leaders as a means of communicating a vision of effective leadership and improving school leaders' practices and behaviours. 


\section{Other findings}

- While regular appraisal, appraisal for the completion of probation, and appraisal for teacher registration is mandatory in the majority of countries for which data are available, appraisal for promotion and reward schemes are usually voluntary.

- Most countries use two or more different types of teacher appraisal, depending on the purpose of the appraisal.

- Lower secondary teachers eligible and included in the policy framework for teacher appraisal include public school teachers (in at least 9 of 10 countries) and teachers in government-dependent private schools (in at least 7 of 10 countries with available data) and independent private schools (in around half of countries with available data).

- Teachers are usually appraised in relation to decisions on employment status. In the case of regular appraisal and appraisal upon completion of probation, the next most common purpose for teacher appraisal is in relation to concerns about performance.

- In 24 of 28 countries, central education authorities (19 countries) or state education authorities (7 countries) determine the procedures for regular teacher appraisal.

- A combination of actors is responsible for evaluating teachers, but the most common evaluator is the school principal or director.

- Teacher appraisals generally focus on planning and preparation, instruction and classroom environment.

- In at least four out of five countries, classroom observations and interviews with teachers are used in regular teacher appraisals and in teacher appraisal at the completion of probation. Although student outcomes are used in 8 of 19 countries for regular appraisals, they are infrequently used for teacher registration and at the completion of probation.

- Nineteen of the 37 countries with available data reported that school leader appraisal is required by government policy or regulation and is implemented across the country.

- In all but two countries with available data, central or state education authorities determine the procedures for school leader appraisal.

- All countries with available data reported that school leaders are appraised on general leadership and pedagogical/instructional leadership, and all but one country also appraise school leaders on organisation of development and resource management. At least four in five countries also focus on school climate, community relations, interpersonal skills, and evaluation and accountability.

- Eleven out of 18 countries with available data reported that school leader appraisal is used for decisions about career advancement and 11 out of 19 countries indicated that school leader appraisal is used to inform decisions on school leaders' professional development activities.

- Rewards or incentives for good performance by school leaders was reported by 11 of 15 countries with available data.

- All but a few countries reported that they use teacher and school leader appraisal for both formative and summative purposes. 


\section{Analysis}

\section{Teacher appraisal}

Teacher appraisal is the evaluation of individual teachers resulting in judgements about their competencies and performance. Traditionally, teacher appraisal focused on formative feedback. In recent years, a number of countries have been working to reform teacher-appraisal systems and are considering summative judgements that can inform decisions about tenure, advancement and pay incentives. In the United States, several models that aim to incorporate improvements in students' scores on standardised assessments as a source of evidence of teacher effectiveness are under consideration. Data systems and current practices, however, do not easily lend themselves to such ends. The following discussion focuses on teacher appraisal at the lower secondary level.

\section{Prevalence and nature of teacher appraisal}

Some 30 of the 37 countries with available data reported having policy frameworks (national or state laws or regulations) in place to regulate one or more types of teacher appraisal. While 26 of those 30 countries implement these policy frameworks countrywide, they are implemented at the provincial/territorial level in Canada and only in some states in the United States. While teacher appraisal is required only in public institutions in England, it is also widely practiced in government-dependent and independent private institutions. Although there is no government policy for teacher appraisal in Denmark, Estonia and Norway, these countries have similar practices. Only Germany, Iceland, Luxembourg and Scotland reported that teachers are not appraised (see Chart D6.1). Eight countries reported that all teachers (100\%) are appraised, and four additional countries reported that between $80 \%$ and $99 \%$ of teachers are appraised. In the Netherlands and Spain, around 70\% of teachers are appraised, while in Belgium (French Community), Hungary and Israel, less than 30\% of teachers are appraised each year (Table D7.1, and Table D7.2, available on line).

Countries use a range of different approaches for appraisal, depending on the purpose of the appraisal. Countries were asked to describe and respond to questions related to each of five different types of teacher appraisal:

i) Teachers on probation: This involves a teacher's entry into the profession.

ii) Regular appraisal: This typically involves a process internal to the school regulated by general labour-law provisions requiring the employers of teachers to regularly appraise the performance and results of their employees.

iii) Teacher registration: This is the process designed to determine and officially confirm a teacher as competent for teaching.

iv) Appraisal for promotion: This is often voluntary and takes place in relation to decisions on employment status (most countries integrate this activity with regular appraisal).

v) Appraisal for rewards: This involves teacher appraisal explicitly designed to identify a select number of highperforming teachers to reward and acknowledge.

In order to have a comprehensive and coherent framework of teacher appraisal that will support continuous learning for individual teachers throughout their career and for the profession as a whole (Darling-Hammond, 2012), countries often combine two or more types of appraisal in their policy framework. Although the regular appraisal category is most commonly cited, most countries reported the existence of two or most types of teacher appraisal.

The most common type of teacher appraisal regulated by existing policy frameworks is regular teacher appraisal (24 of 28 countries). Here, 20 countries reported that regular appraisal is mandatory; 18 countries conduct this appraisal at regular intervals/on a set cycle, most commonly, every year. Although regular appraisal is legislated in Belgium (French Community), the Czech Republic and Poland, it is voluntary and needs to be requested by teachers themselves (Table D7.3b).

Two other types of appraisal used for performance management, appraisal for teacher registration and appraisal for promotion, are less frequently used (used in 11 and 12 countries, respectively). While in 8 of 10 countries with available data it is mandatory for teachers to be appraised for teacher registration, in only 4 of 11 countries with available data are it mandatory for teachers to be appraised for promotion.

Another common approach to teacher appraisal is the appraisal of individual teachers upon completion of a probationary period (21 of 28 countries). Of the 19 countries with available data, 17 reported that this type of appraisal is mandatory and 2 reported that it is voluntary. Of those countries where it is mandatory, 10 reported that appraisals are carried out periodically at regular intervals, usually annually. Only 9 of 27 countries with available data had policy frameworks for teacher reward schemes. 


\section{Governance and responsibilities for teacher appraisal}

The lower secondary teachers eligible and included in the policy framework for teacher appraisal include public school teachers (in at least 9 of 10 countries) and teachers in government-dependent schools (in at least 7 of 10 countries with available data) and independent private schools (in around half of countries with available data). The type of teachers included in the policy framework depends on the type of appraisal undertaken. For regular appraisal, the framework most commonly includes public school teachers (all 24 countries), permanent teachers (22 of 23 countries), teachers on fixed-term contracts (18 of 22 countries) and all teachers regardless of contract status (16 of 21 countries). By definition, appraisal for the completion of probation focuses on teachers on probation (18 of 20 countries). Appraisal for teacher registration focuses on teachers on fixed-term contracts (8 of 10 countries), permanent teachers ( 6 of 10 countries) and provisionally registered teachers (6 of 10 countries). Permanent teachers are the focus of appraisal for promotion in 10 of 11 countries, and of appraisal for reward schemes in all 8 countries for which data are available (Table D7.3b).

The circumstances under which teachers are appraised vary considerably among countries. Most commonly, teachers are appraised in relation to decisions on employment status. In the case of regular appraisal and appraisal upon completion of probation, the next most common reasons for appraisal are as a result of performance problems, at the discretion of the school principal, the teacher volunteers or requests to be appraised and in response to a complaint. Appraisals for teacher registration are usually conducted in relation to decisions on employment, at the initiative of the teacher, or in response to a complaint or performance problems. Appraisals for promotion are most frequently related to decisions on employment status and are usually conducted at the initiative of the teacher. Similarly, most often teachers volunteer for reward schemes appraisals.

The obligation for teachers to undertake appraisals varies according to the type of appraisal. While regular appraisal, appraisal for the completion of probation, and appraisal for teacher registration are mandatory in the majority of countries, appraisals for promotion and reward schemes are usually voluntary. Although regular appraisal is mandatory in 20 countries, it is voluntary in 3 countries. Some 18 of 20 countries reported that regular appraisal is carried out periodically at regular intervals. Appraisal for the completion of probation is mandatory in 17 countries (it is periodic in 10 of these countries), and voluntary in 2 countries. Similarly, appraisal for teacher registration is mandatory in 8 countries (it is periodic in 5 countries) and voluntary in 2 countries. By contrast, appraisal for promotion is voluntary in 7 countries and mandatory in 4 countries, and appraisal for reward schemes is voluntary in 5 countries and mandatory in 2 countries. Although the frequency of appraisal varies, most countries with periodic appraisals reported that they are conducted annually.

In most countries (24 of 28 countries), central education authorities (19 countries) and/or state education authorities ( 7 countries) determine the procedures for teacher appraisal. The central and state education authorities also share this responsibility with regional (Korea), sub-regional (2 countries) and local (4 countries) education authorities. In Japan, regional and local education authorities are in charge of determining the procedures. Together with education authorities, the school principal (7 countries), the school board (7 countries), school organising bodies ( 4 countries), teachers' professional organisations ( 3 countries) and the deputy school principal ( 2 countries) also play a role in determining the procedures. The procedures for determining teacher appraisal are determined primarily by teachers' professional organisations in Ireland and by school boards in the Netherlands.

In teacher appraisal, a combination of actors is responsible for evaluating teachers. For all the different types of appraisal, the most common evaluator is the school principal and education authorities from various levels. Another common evaluator for the completion of probation and for teacher registration is the supervisor (10 of 20 countries and 4 of 10 countries, respectively). For regular appraisal, other school leaders (8 of 23 countries) and supervisors (6 of 22 countries) are also common evaluators. External accredited evaluators and peer evaluators at the same school are also involved in appraisals in a few countries.

\section{Common procedures used and sources of information}

In at least $75 \%$ of countries, the main standards against which teachers are appraised are national or state teaching standards. A description of the general and professional duties of teachers is also frequently used for all types of appraisal, except appraisal for teacher registration (reported to be used by $20 \%$ of countries). Other common references/standards used by a handful of countries are the teacher code of conduct, school development plan or school project, and the school's internal regulations (Table D7.4b).

The key domains and aspects of the job covered in teacher appraisal are planning and preparation, instruction and classroom environment. This is followed by professional development, contribution to school development and links to the community. All five types of teacher appraisal cover these aspects. 
A range of methods, instruments and information sources is used to appraise teachers; countries draw from a mix of these to appraise different aspects of a teacher's performance. The most frequently used methods in regular appraisal, appraisal at the completion of probation and teacher registration are classroom observation and interviews of teachers (at least 4 in 5 countries). Teacher portfolio and teacher self-appraisal are also often used, while parent and/or student surveys are less often used as sources of information for regular appraisal, appraisal at the completion of probation and teacher registration. Although student outcomes are used in 8 of 19 countries in regular appraisals, they are less often used in appraisals for teacher registration and at completion of probation. The most frequently used sources of information in appraisal for promotion and reward schemes are teacher portfolio and classroom observation, followed by teacher self-appraisal and interviews of teachers.

Teacher appraisal results in a rating, i.e. a category on a scale of teacher performance, in 15 of 18 countries for appraisal at the completion of probation, in 9 of 11 countries for appraisal for promotion, in 13 of 20 countries for regular appraisal, in 6 of 10 countries for appraisal for teacher registration, and in 3 of 6 countries for appraisal for reward schemes. All countries reported having mechanisms to appeal teacher-registration appraisal results, while 4 in 5 countries reported having these mechanisms for appraisal at the completion of probation and for appraisal for promotion. Appeal mechanisms for regular appraisal are in place in 2 out of 3 countries.

\section{Chart D7.2. Use of results from regular teacher appraisals (2015)}

For lower secondary teachers teaching general programmes

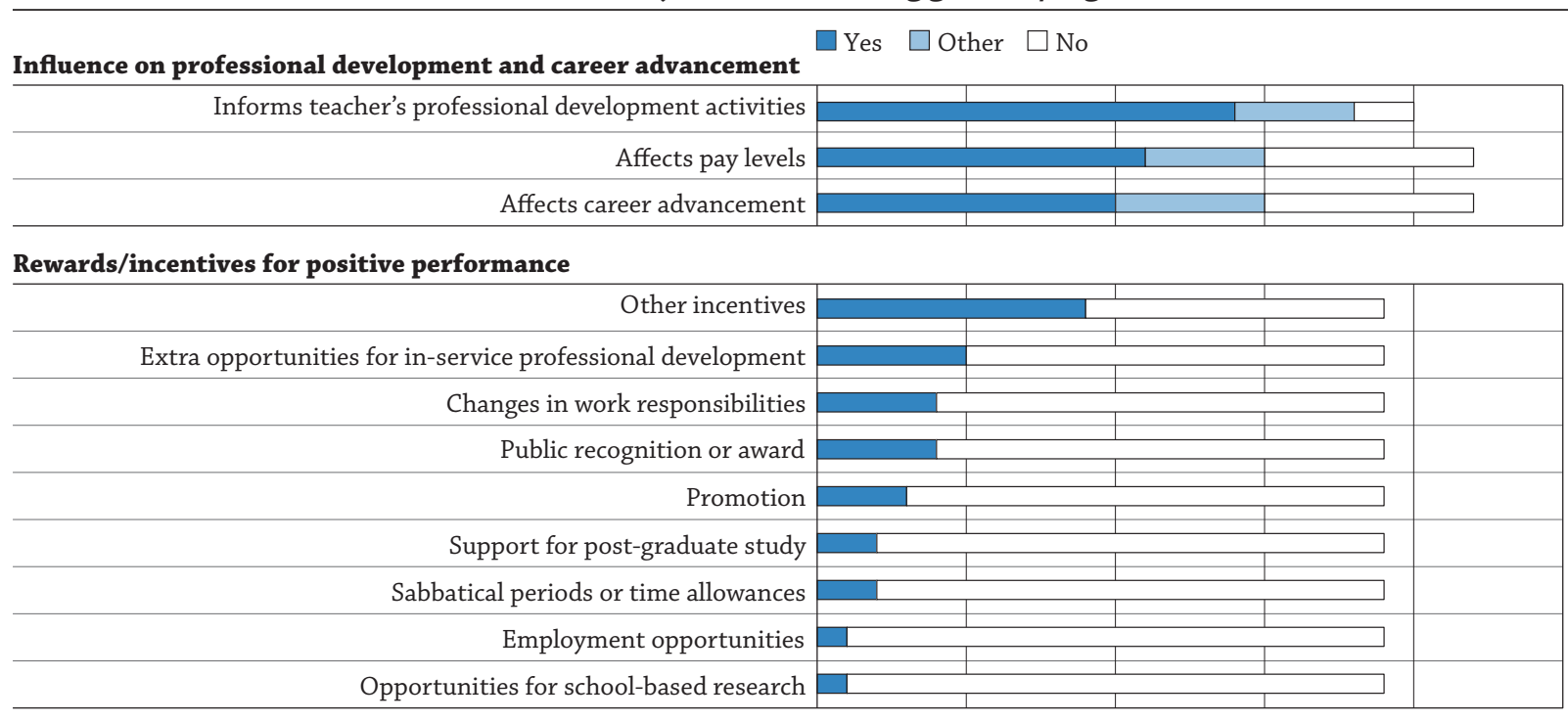

\section{Responses/disincentives to underperformance}

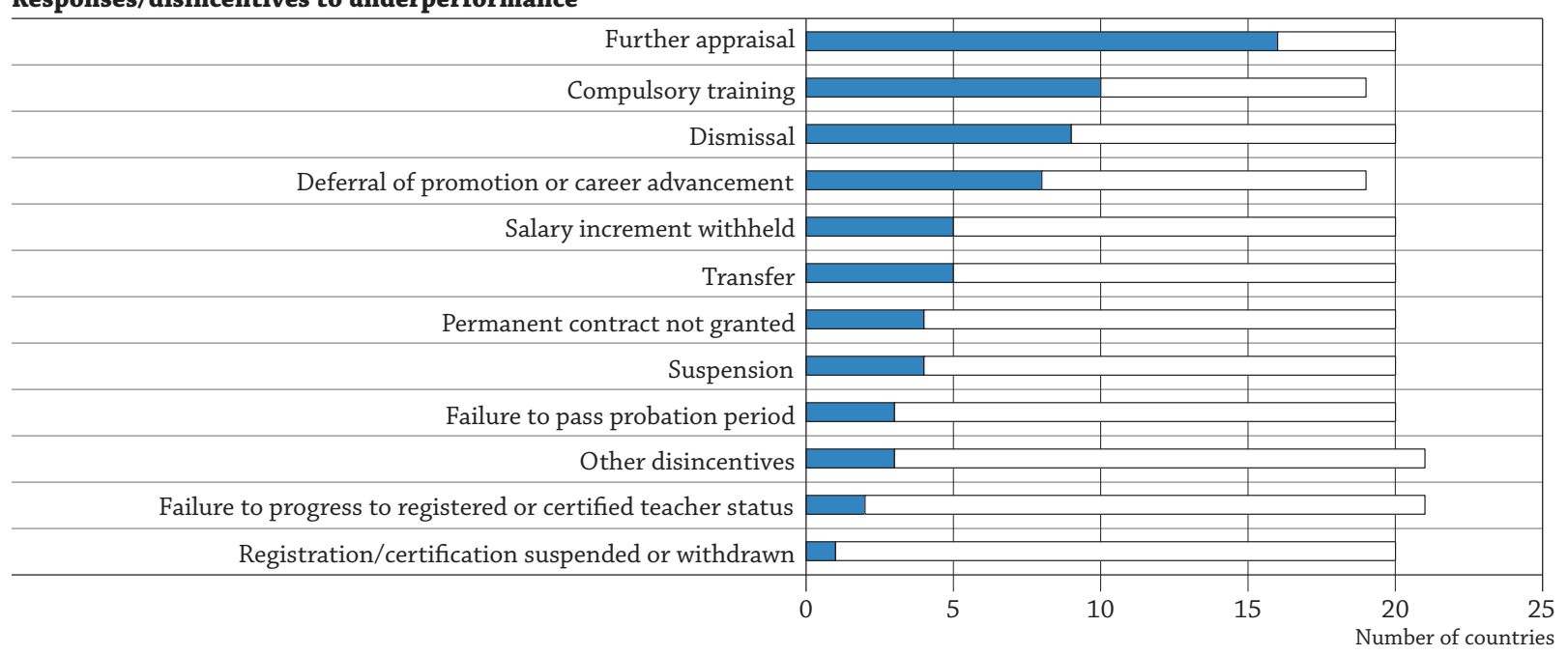




\section{Use of results from teacher appraisal}

In 14 of 20 countries, results from regular teacher appraisal inform decisions about teachers' professional development activities. These results can also affect teachers' pay (11 countries) and career advancement (10 countries). In 9 of 19 countries, positive performance in a regular appraisal may not result in any reward, but 10 countries reported that results could be used for rewards or incentives. The most common rewards include more opportunities for in-service professional development, public recognition and changes in work responsibility. Underperformance in regular appraisal has consequences for teachers, the most common being the need for further appraisal (16 countries) and compulsory training (10 countries). Other responses to underperformance include dismissal (9 countries), deferral of promotion or career advancement (8 countries), withholding of pay raise ( 5 countries), transfer to another school (5 countries) and suspension (4 countries) (Chart D7.2, and Table D7.5b, available on line).

In 12 of 20 countries, appraisals for teachers in probation influence decisions on access to contracts. While these results also affect teachers' pay in 9 countries, they have no effect in 9 other countries. In 11 countries, results are also used to inform teachers' professional development activities. While the successful completion of the probation period may lead to rewards or incentives (e.g. employment opportunities) in 9 countries, it does not in 10 other countries. Not surprisingly, in all 19 countries, underperformance in this appraisal will result in a failure to pass the probation period. Other common sanctions or responses to underperformance include dismissal (12 countries), permanent contract not granted (11 countries), further appraisal (11 countries), failure to progress to registered or certified teacher status ( 7 countries) and compulsory training (6 countries).

Results of appraisal for promotion are used to determine teachers' professional development activities in 5 of 9 countries. These results may also affect teachers' career advancement ( 8 of 11 countries) and pay ( 7 of 11 countries). Outstanding performance may lead to a promotion (8 of 10 countries), while underperformance can cause deferral of promotion or career advancement (7 of 10 countries) or withholding of a pay raise (4 of 10 countries). In 6 of 8 countries, results of appraisal for rewards schemes affect teachers' pay.

Results of appraisals for teacher registration influence decisions on access to contracts, renewal of fixed-term contracts or access to a permanent position (5 of 10 countries). While outstanding performance can also lead to promotions in 2 of 9 countries, it leads to no such reward in the other 7 countries. However, underperformance can lead to a failure to progress to registered or certified teacher status (8 of 9 countries), a permanent contract not being granted ( 6 of 9 countries), further appraisal ( 4 of 8 countries), registration or certification suspended or withheld and even dismissal ( 4 of 9 countries).

Although teacher appraisals may play a role in influencing salaries and career progression, other factors or criteria are also used. Chart D7.6 and Table D7.6 (available on line) explore the relative level of influence of these other factors.

\section{School leader appraisal}

School leader appraisal is not as well developed and practiced as teacher appraisal. Even though the importance of effective school leadership is widely recognised, many countries lack the tools and mechanisms for appraising their school leaders. However, since school leadership is critical to ensure effective teaching and learning, an increasing number of countries are developing initiatives to strengthen the leadership capacity of their schools.

School leaders are primarily responsible for school management and administration. The school leader holds the highest leadership position within the school, such as the headmaster, head teacher or principal, or a middle-level position in a leadership capacity, such as deputy school directors or department heads. Generally, there is no more than one director per school.

The following discussion focuses on school leader appraisal at the lower secondary level.

\section{Prevalence and nature of school leader appraisal}

Some 19 of 37 countries with available data reported that school leader appraisal is required by government policy or regulation and is implemented across the country. Although school leader appraisal is required only in public institutions in England, it is widely practiced in private institutions as well. In Canada, the requirement for school leader appraisal varies by province or territory, while in the United States, school leader appraisal is only implemented in some states. In Israel, it is required only at the primary and lower secondary levels, but it is also widely practiced at the upper secondary level. Similarly, in the Netherlands, it is only required at the primary level, although it is widely practiced at the secondary level. 
Australia, Denmark and Latvia do not require school leader appraisals, but they do have similar practices. Finland grants extensive local autonomy in the evaluation of education, including school leader appraisal. In Latvia, school leaders are appraised in the context of accreditation of schools and programmes. In the remaining countries with available data, appraisal of school leaders is not required (see Chart D6.1, and Table D7.7, available on line). Nine countries reported that all school leaders $(100 \%)$ are appraised. The Slovak Republic reported that at least $99 \%$ of its school leaders are appraised, while England reported that all public school leaders and a large majority of private school leaders are appraised. In Spain, $70 \%$ of school leaders are appraised, whereas in Colombia, only around 20\% of school leaders are appraised.

\section{Governance and responsibilities for school leader appraisal}

In all 19 countries with available data, school principals are eligible and included in the policy framework for school leader appraisal. In 7 of these countries, only school principals are eligible/included. In 11 of the 19 countries, deputy school principals and, in England, assistant head teachers are also included. In Mexico, New Zealand and Portugal school principals, deputy principals, middle leaders and department heads are included in the policy framework.

The circumstances in which school leaders are appraised vary considerably. The most common circumstance under which school leaders are appraised is in relation to decisions on employment status (13 countries). School leaders are also appraised at the discretion of the school board ( 6 countries) or school director (5 countries). In 4 countries, school leaders can also be appraised as a result of a complaint, and in 6 countries, school leader appraisal is voluntary (Table D7.8b).

In all countries with available data, school leader appraisal is mandatory except in Poland where it is voluntary, i.e. the school leader initiates the appraisal him/herself. With the exception of the Czech Republic and France (at the primary level), all countries that reported having mandatory school leader appraisal indicated that the appraisal is carried out periodically at regular intervals. The frequency with which school leaders are appraised varies greatly across countries. Among the 16 countries that require periodic appraisals of their school leaders, these appraisals take place annually in Colombia, England, Korea, New Zealand, the Slovak Republic, Slovenia and Spain; in France (secondary level) and Israel (primary and lower secondary levels), these appraisals take place once every three years. The interval between appraisals ranges from once every four years in Belgium (Flemish Community), Greece, Mexico, Portugal and Turkey (primary and lower secondary levels), to up to every five years in Belgium (French Community) and Hungary (Table D7.8b, and Tables D7.8a and c, available on line).

The procedures for school leader appraisal are determined at various levels of government, or by an education authority, alone or together with other education bodies. In all countries with available data except the Czech Republic and the Slovak Republic, central or state education authorities determine the procedures for school leader appraisals. In the Czech Republic, regional education authorities determine the procedures for appraisal; in the United States, state and local education authorities do. In Korea, there are different kinds of school leader appraisal and central, regional and local education authorities all play a role in determining the procedures for all or some kinds of appraisal. In addition to education authorities, school organising bodies ( 5 countries), school board (3 countries), and central agencies (3 countries) also play a role in determining the procedures. In the Slovak Republic, the entity that established the school determines the procedures for appraising the school principal (rather than the central education authority), and the school principal determines the procedures for appraising the deputy principal (Table D7.8b).

A combination of actors is responsible for evaluating school leaders. The most common scenario is a combination of evaluators, which includes education authorities from various levels, the school principal, an external accredited evaluator, members of the school organising body, the school board and/or intermediate agencies.

\section{Common procedures used and sources of information}

All countries appraise school leaders against one or multiple reference standards that state clearly what school leaders are expected to know and do. All countries with available data, except Belgium (Flemish Community), the Czech Republic and Portugal appraise school leaders against national or state and/or regional standards for school leadership. The second most common standard used is a description of the general and professional duties of school leaders (12 countries), which is used by both Belgium (Flemish Community) and the Czech Republic. Although less common, codes of conduct (5 countries), school development plans or school projects (5 countries) and school internal regulations ( 4 countries) are also used by some countries (Table D7.9b).

School leader appraisals focus on a variety of areas, competencies and responsibilities. All countries with available data reported that school leaders are appraised on general leadership and pedagogical/instructional leadership. 
These countries (except Hungary) also appraise school leaders on organisation/planning of development and resource management. In at least 4 of 5 countries, school leader appraisals also focus on school climate, community relations, interpersonal skills, and evaluation and accountability.

To get an accurate, fair and reliable picture of school leaders' performance, countries use a mixture/range of instruments and information sources. Among the 18 countries with available data, the most common instruments or information sources are interviews of the school leader by an evaluator (15 countries), school leader portfolio (13 countries), and school leader self-appraisal (12 countries). Other common sources include school visits (10 countries) and student outcomes (9 countries). Around one in three countries also considers the views of stakeholders through student, parent and teacher surveys.

In 2 out of 3 countries, the appraisal of a school leader results in a rating, i.e. a category on a scale of school-leader performance. Four out of 5 countries have mechanisms or procedures in place through which school leaders can appeal their appraisal results.

\section{Use of results from school leader appraisal}

Eleven out of 18 countries with available data reported that school leader appraisal is used for decisions about career advancement. Half (9 out of 18) of the countries with available data use school leader appraisals to inform school leaders' professional development activities, while 6 countries reported that they do not do so (Chart D7.3, and Table D7.10b, available on line).

Seven countries reported that school leader appraisal results influence decisions about promotion, and 3 countries reported that these results influence the speed with which a school leader progresses in the career structure or salary scale. In Colombia, results influence both. Of the remaining 7 countries with available data, 4 reported that results influence other career advancement, while 3 reported that results have no influence at all.

\section{Chart D7.3. Use of results from school leader appraisals (2015)}

In general programmes, lower secondary education

\begin{tabular}{l} 
Influence on professional development and career advancement \\
\hline Affects career advancement \\
\hline Informs school leader's professional development activities \\
Rewards/incentives for positive performance \\
\hline Permanent salary increment \\
\hline One-off financial bonus \\
\hline Extra professional development opportunities
\end{tabular}

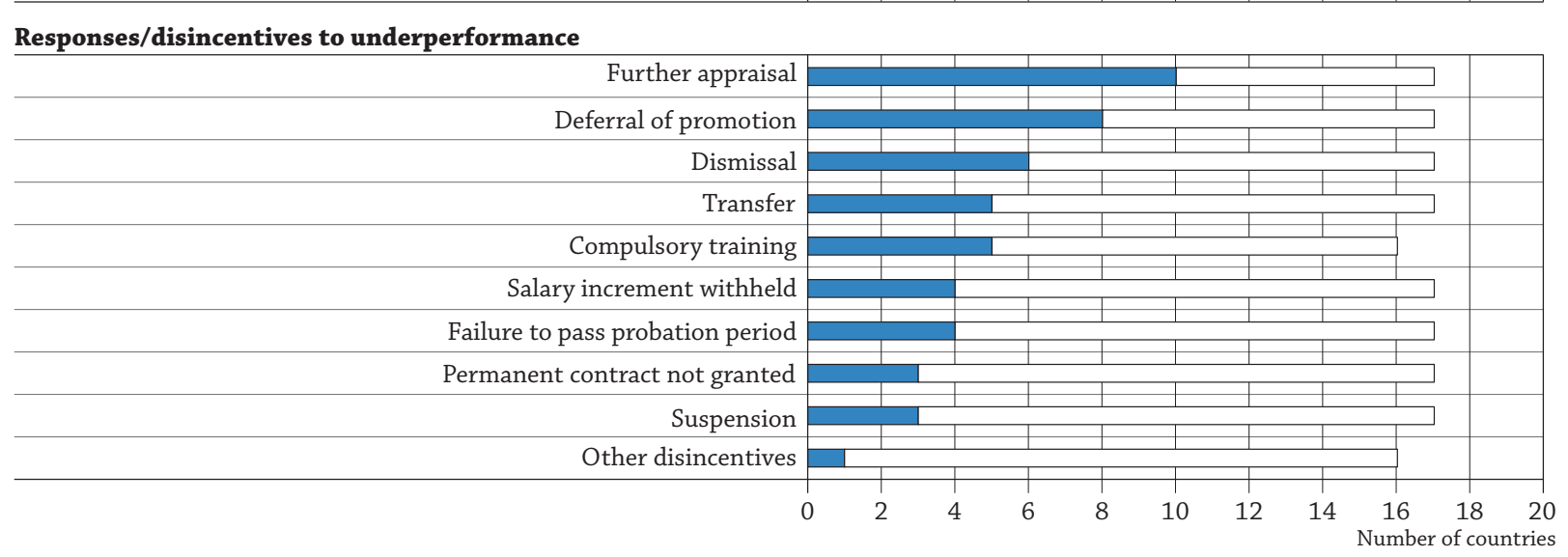

Actions/Rewards/Sanctions are ranked in descending order of the number of countries reporting that they use school leader appraisal results for these purposes and decisions. Source: OECD. Table D7.10b, available on line. See Annex 3 for notes (www.oecd.org/education/education-at-a-glance-19991487.htm).

StatLink 젶ㄴ http://dx.doi.org/10.1787/888933284655 
While 11 of the 15 countries with available data may reward lower secondary school leaders for positive performance in their appraisal, 4 countries do not. These rewards may take the form of one or a combination of financial rewards/incentives for the school leaders. School leaders may be rewarded with a permanent pay raise (8 countries), a one-off financial bonus ( 5 countries), and/or a pay raise for a fixed period of time ( 1 country). In Korea and Mexico, school leaders may be rewarded with additional professional development opportunities. In the Czech Republic, the school and organising bodies determine the reward offered.

The most common response to school leaders' underperformance is further appraisal (10 of 17 countries), followed by deferral of promotion (8 countries). In some countries, underperformance may lead to dismissal (6 countries), transfer to another school (5 countries), failure to pass a probation period (4 countries) or suspension (3 countries). While England, the Slovak Republic and Spain may reward outstanding school leaders financially, they may also sanction underperforming school leaders financially by withholding pay raises. Colombia, Greece, Korea, Mexico and Portugal require school leaders to participate in training.

Although school leader appraisals may play a role in influencing salaries and career progression, other factors or criteria are also used. Chart D7.6 and Table D7.11 (available on line) explore the relative level of influence of these other factors.

\section{School influence or control over teacher and school leader appraisal activities}

The influence of local schools on teacher and school leader appraisal varies considerably from country to country. At the lower secondary level, schools have a high or moderate level of influence or control over teacher appraisal in 16 of the 22 countries with available data, and little or no influence in the remaining 6 countries. By contrast, 10 of the 18 countries with available data reported schools as having little or no influence over school leader appraisal, while 5 countries reported schools as having a high level of influence and another 3 countries reported that schools have a moderate level of influence (Table D7.13, available on line).

\section{Influence of teacher and school leader appraisal over various decisions}

As expected, teacher appraisal has a high-to-moderate level of influence over the evaluation of individual teachers (15 of 24 countries) and on providing assistance to teachers to improve their teaching skills ( 14 of 23 countries). By contrast, it has no influence over the likelihood of school closure (22 of 23 countries) and little to no influence over the size of the school budget ( 21 of 22 countries), the evaluation of the school administration ( 21 of 25 countries) or the evaluation of the school's performance (19 of 25 countries). In most countries, teacher appraisal has little or no influence on teachers' salaries or bonuses. However, in a few countries, outstanding performance can lead to a pay raise and/or one-time bonus (Chart D7.4, and Table D7.12, available on line).

While school leader appraisal has high influence over the evaluation of school administration in seven of the 19 countries with available data, it has no influence in nine countries. Its influence on the evaluation of the school or individual teachers is also non-existent or low, except in Colombia, Czech Republic, Mexico, Poland, Portugal, the Slovak Republic and Turkey. In most countries with available data, school leader appraisal has no influence on the school budget, the likelihood of school closure, or teachers' salaries or bonuses; in a handful of countries, it has a low influence on these outcomes.

\section{Use of teacher and school leader appraisal for formative and summative purposes}

Teacher and school leader appraisal are useful in themselves and as part of a broader system of evaluation within schools. The overall purpose of teacher and school leader appraisals is to provide formative feedback to teachers and school leaders. For example, teachers need feedback on their performance to help them identify how to improve their teaching practice and, with the support of effective school leadership, to create professional learning communities within schools. The summative purpose of teacher and school leader appraisal is to judge their effectiveness so that a variety of employment decisions and actions (like career advancement or salary progression, and the allocation of financial rewards or sanctions) can be made.

Sixteen countries reported using teacher appraisal for both formative and summative purposes. Eight of these countries reported this activity as having a high level of use, 3 reported a moderate level of use, and 2 reported a low level of use for both formative and summative purposes. Korea and Turkey reported that this activity has a moderate level of use for formative purposes, but a low level of use for summative purposes. By contrast, the Czech Republic reported a high level of use for summative purposes and a low level of use for formative purposes. Three countries reported using teacher appraisal for formative purposes only: Belgium (French Community) and Italy where it is highly used, and Australia where it is moderately used (Chart D7.5, and Table D7.14, available on line). 


\section{Chart D7.4. Level of influence of teacher and school leader appraisals over various decisions (2015) \\ In general programmes}

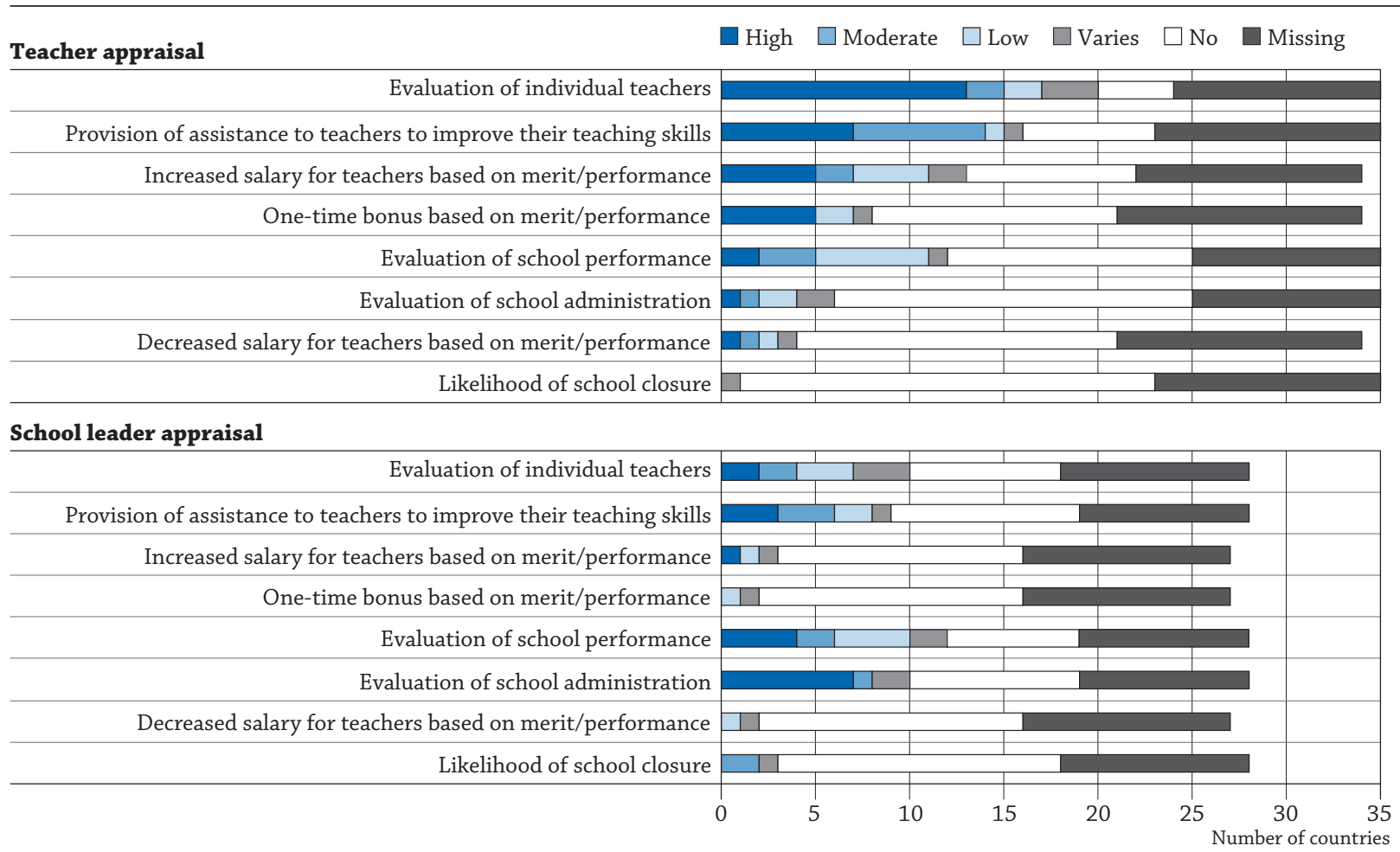

Actions/Rewards/Sanctions are ranked in descending order of the number of countries reporting that teacher appraisal has a high level of influence on these decisions. Source: OECD. Table D7.12, available on line. See Annex 3 for notes (www.oecd.org/education/education-at-a-glance-19991487.htm).

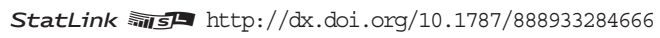

\section{Chart D7.5. Extent to which teacher and school leader appraisals are used for formative and/or summative purposes (2015)}

In general programmes

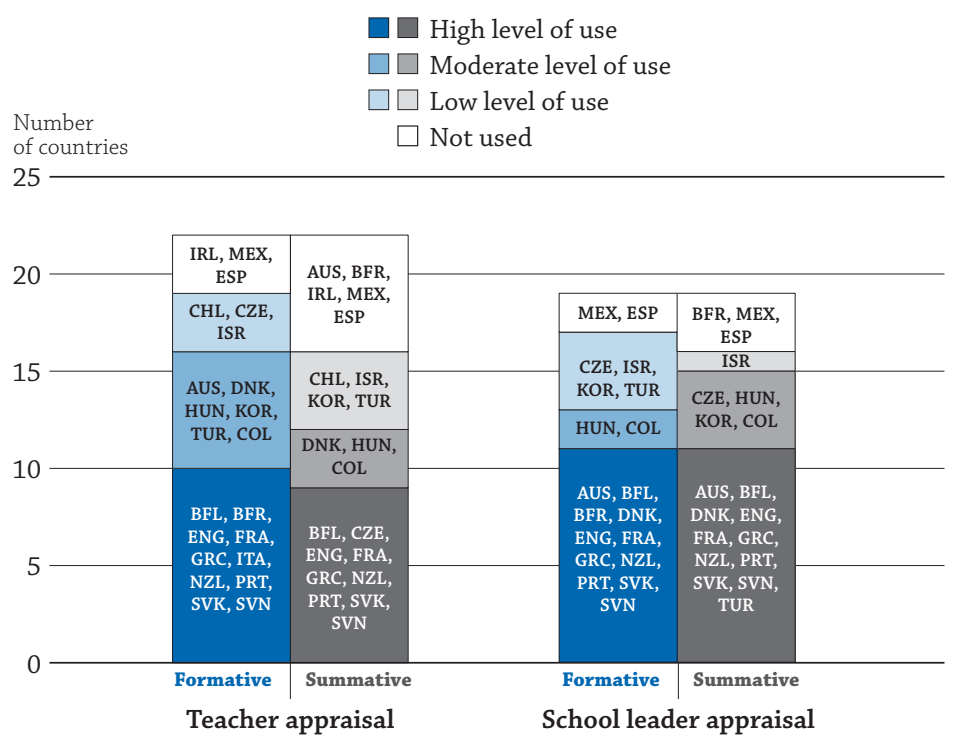

Source: OECD. Table D7.14, available on line. See Annex 3 for notes (www.oecd.org/education/education-at-a-glance-19991487.htm). StatLink 젝ㄴ http://dx.doi.org/10.1787/888933284674 


\section{D7 Definitions}

Professional development refers to in-service training that aims to update, develop and broaden the knowledge of teachers and school leaders. This includes any activity that develops an individual's skills, knowledge, expertise and other characteristics, through personal study and reflection as well as through formal courses.

References/standards refers to documents that provide the basis for appraisal criteria by defining what teachers and school leaders should know and be able to do, the tasks they should perform, and the regulations with which they should comply.

School leader/administrator appraisal (i.e. evaluation) refers to the external evaluation of individual school leaders to make a judgement about their work and performance using objective criteria. Results of school leadership evaluations may be used to inform professional development activities, career advancement, and rewards.

Teacher appraisal, also referred to as teacher evaluation, refers to the evaluation of individual teachers to make a judgement about their work and performance using objective criteria. Results of teacher appraisals may be used to inform professional development activities, certification, career advancement and rewards. The term "teachers" covers all professional personnel directly involved in teaching students, including classroom teachers and other teachers who work with students as a whole class in a classroom, in small groups in a resource room, or in one-to-one teaching inside or outside a regular classroom.

\section{Methodology}

Data are from the 2014 OECD-INES Survey on Evaluation and Assessment and refer to the school year 2014/15.

Notes on definitions and methodologies for each country are provided in Annex 3 at www.oecd.org/education/ education-at-a-glance-19991487.htm.

\section{Note regarding data from Israel}

The statistical data for Israel are supplied by and are under the responsibility of the relevant Israeli authorities. The use of such data by the OECD is without prejudice to the status of the Golan Heights, East Jerusalem and Israeli settlements in the West Bank under the terms of international law.

\section{References}

Darling-Hammond, L. (2012), "The right start: Creating a strong foundation for teaching career", Phi Delta Kappan, Vol. 94, No. 3, pp. 8-13.

OECD (2013), Synergies for Better Learning: An International Perspective on Evaluation and Assessment, OECD Reviews of Evaluation and Assessment in Education, OECD Publishing, Paris, http://dx.doi.org/10.1787/9789264190658-en.

\section{Indicator D7 Tables}

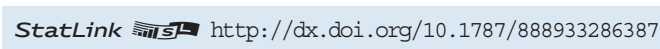

Table D7.1 Teacher appraisal and school leader appraisal at the lower secondary level (2015)

WEB Table D7.2 Teacher appraisal (2015)

WEB Table D7.3a Teacher appraisal at the primary level: Eligibility, governance and responsibilities (2015)

Table D7.3b Teacher appraisal at the lower secondary level: Eligibility, governance and responsibilities (2015)

WEB Table D7.3c Teacher appraisal at the upper secondary level: Eligibility, governance and responsibilities (2015)

WEB Table D7.4a Features of teacher appraisal at the primary level (2015) 
Table D7.4b Features of teacher appraisal at the lower secondary level (2015)

WEB Table D7.4c Features of teacher appraisal at the upper secondary level (2015)

WEB Table D7.5a Use of results from teacher appraisal at the primary level (2015)

WEB Table D7.5b Use of results from teacher appraisal at the lower secondary level (2015)

WEB Table D7.5c Use of results from teacher appraisal at the upper secondary level (2015)

WEB Table D7.6 Factors that influence teachers' salaries and career progression (2015)

WEB Table D7.7 School leader appraisal (2015)

WEB Table D7.8a School leader appraisal at the primary level: Eligibility, governance and responsibilities (2015)

Table D7.8b School leader appraisal at the lower secondary level: Eligibility, governance and responsibilities (2015)

WEB Table D7.8c School leader appraisal at the upper secondary level: Eligibility, governance and responsibilities (2015)

WEB Table D7.9a Features of school leader appraisal at the primary level (2015)

Table D7.9b Features of school leader appraisal at the lower secondary level (2015)

WEB Table D7.9c Features of school leader appraisal at the upper secondary level (2015)

WEB Table D7.10a Use of results from school leader appraisal at the primary level (2015)

WEB Table D7.10b Use of results from school leader appraisal at the lower secondary level (2015)

WEB Table D7.10c Use of results from school leader appraisal at the upper secondary level (2015)

WEB Table D7.11 Factors that influence school leaders' salaries and career progression (2015)

WEB Table D7.12 Level of influence of teacher and school leader appraisal over various decisions (2015)

WEB Table D7.13 Extent of school influence or control over teacher and school leader appraisal activities (2015)

WEB Table D7.14 Extent to which teacher and school leader appraisal are used for formative and/or summative purposes (2015) 
Table D7.1. Teacher appraisal and school leader appraisal at the lower secondary level (2015) In general programmes

\begin{tabular}{|c|c|c|c|c|c|c|c|c|c|c|c|}
\hline & \multicolumn{8}{|c|}{ Teacher appraisal } & \multicolumn{3}{|c|}{ School leader appraisal } \\
\hline & \multirow[b]{2}{*}{\begin{tabular}{|c|} 
Existence \\
of teacher \\
appraisal
\end{tabular}} & \multicolumn{2}{|c|}{$\begin{array}{l}\text { Policy implementation } \\
\text { or practice } \\
\text { (if not legislated) }\end{array}$} & \multicolumn{5}{|c|}{$\begin{array}{l}\text { Types of teacher appraisal } \\
\text { covered by policy framework }\end{array}$} & \multirow[b]{2}{*}{$\begin{array}{c}\text { Existence } \\
\text { of school } \\
\text { leader } \\
\text { appraisal }\end{array}$} & \multicolumn{2}{|c|}{$\begin{array}{c}\text { Policy implementation } \\
\text { or practice } \\
\text { (if not legislated) }\end{array}$} \\
\hline & & 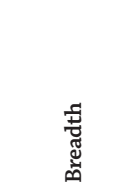 & 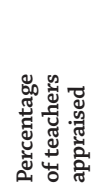 & 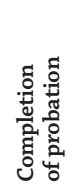 & 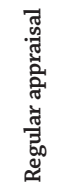 & పี & 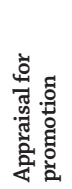 & 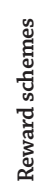 & & 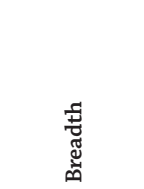 & 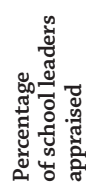 \\
\hline & (1) & (2) & (3) & (4) & (5) & (6) & (7) & $(8)$ & (9) & (10) & (11) \\
\hline Australia & $\mathrm{L}$ & Countrywide & $\mathrm{m}$ & Yes & Yes & Yes & $\mathrm{m}$ & No & $\mathrm{P}$ & Countrywide & $\mathrm{m}$ \\
\hline Austria & $\mathrm{L}$ & Countrywide & $\mathrm{m}$ & Yes & Yes & Yes & Yes & No & $\mathrm{N}$ & a & $\mathrm{a}$ \\
\hline Belgium (Fl.) & $\mathrm{L}$ & Countrywide & $\mathrm{m}$ & No & Yes & No & No & No & $\mathrm{L}$ & Countrywide & $\mathrm{m}$ \\
\hline Belgium (Fr.) & $\mathrm{L}$ & Countrywide & 5 & No & Yes & No & No & No & $\mathrm{L}$ & Countrywide & 100 \\
\hline Canada & $\mathrm{L}$ & Some states & $\mathrm{m}$ & $\mathrm{m}$ & $\mathrm{m}$ & $\mathrm{m}$ & $\mathrm{m}$ & $\mathrm{m}$ & $\mathrm{L}$ & Some states & $\mathrm{m}$ \\
\hline Czech Republic & $\mathrm{L}$ & Countrywide & 100 & Yes & Yes & No & Yes & No & $\mathrm{L}$ & Countrywide & 100 \\
\hline Denmark & $\mathrm{P}$ & Countrywide & 95 & a & a & $\mathrm{a}$ & a & $\mathrm{a}$ & $\mathrm{P}$ & Countrywide & 100 \\
\hline England $^{1}$ & $\mathrm{~L}$ & Some schools & 90 & Yes & Yes & No & No & No & $\mathrm{L}$ & Some schools & 90 \\
\hline Estonia & $\mathrm{P}$ & Countrywide & 80 & a & a & a & $\mathrm{a}$ & a & $\mathrm{N}$ & a & $\mathrm{a}$ \\
\hline Finland & $\mathrm{m}$ & $\mathrm{m}$ & $\mathrm{m}$ & $\mathrm{m}$ & $\mathrm{m}$ & $\mathrm{m}$ & $\mathrm{m}$ & $\mathrm{m}$ & $\mathrm{m}$ & $\mathrm{m}$ & $\mathrm{m}$ \\
\hline France & $\mathrm{L}$ & Countrywide & 100 & Yes & Yes & No & No & No & $\mathrm{L}$ & Countrywide & $\mathrm{m}$ \\
\hline Germany & $\mathrm{N}$ & a & a & a & a & a & a & $\mathrm{a}$ & $\mathrm{N}$ & a & a \\
\hline Greece & $\mathrm{L}$ & Countrywide & 100 & Yes & Yes & Yes & Yes & No & $\mathrm{L}$ & Countrywide & 100 \\
\hline Hungary & $\mathrm{L}$ & Countrywide & 15 & Yes & Yes & No & Yes & Yes & $\mathrm{L}$ & Countrywide & $\mathrm{m}$ \\
\hline Iceland & $\mathrm{N}$ & a & a & $\mathrm{a}$ & $\mathrm{a}$ & $\mathrm{a}$ & $\mathrm{a}$ & $\mathrm{a}$ & $\mathrm{N}$ & a & $\mathrm{a}$ \\
\hline Ireland & $\mathrm{L}$ & Countrywide & $\mathrm{m}$ & Yes & No & Yes & No & No & $\mathrm{N}$ & a & a \\
\hline Israel & $\mathrm{L}$ & Countrywide & 25 & Yes & No & Yes & Yes & No & $\mathrm{L}$ & Countrywide & 100 \\
\hline Italy & $\mathrm{L}$ & Countrywide & 5 & Yes & No & No & No & No & $\mathrm{N}$ & $\mathrm{a}$ & a \\
\hline Japan & $\mathrm{L}$ & Countrywide & $\mathrm{m}$ & No & Yes & No & No & No & $\mathrm{N}$ & $\mathrm{a}$ & $\mathrm{a}$ \\
\hline Korea & $\mathrm{L}$ & Countrywide & $\mathrm{m}$ & No & Yes & No & Yes & Yes & $\mathrm{L}$ & Countrywide & $\mathrm{m}$ \\
\hline Netherlands & $\mathrm{L}$ & Countrywide & 68 & Yes & Yes & Yes & Yes & Yes & $\mathrm{P}$ & $\mathrm{m}$ & $\mathrm{m}$ \\
\hline New Zealand & $\mathrm{L}$ & Countrywide & 100 & Yes & Yes & Yes & No & No & $\mathrm{L}$ & Countrywide & 100 \\
\hline Norway & $\mathrm{P}$ & $\mathrm{m}$ & $\mathrm{m}$ & a & $\mathrm{a}$ & a & a & $\mathrm{a}$ & $\mathrm{N}$ & a & a \\
\hline Poland & $\mathrm{L}$ & Countrywide & $\mathrm{m}$ & Yes & Yes & No & Yes & No & $\mathrm{L}$ & Countrywide & $\mathrm{m}$ \\
\hline Portugal & $\mathrm{L}$ & Countrywide & $\mathrm{m}$ & Yes & Yes & No & No & No & $\mathrm{L}$ & Countrywide & 100 \\
\hline Scotland & $\mathrm{N}$ & $\mathrm{a}$ & a & a & $\mathrm{a}$ & a & a & $\mathrm{a}$ & $\mathrm{N}$ & a & a \\
\hline Slovak Republic & $\mathrm{L}$ & Countrywide & 100 & Yes & Yes & No & No & No & $\mathrm{L}$ & Countrywide & 99 \\
\hline Slovenia & $\mathrm{L}$ & Countrywide & 100 & Yes & Yes & Yes & Yes & Yes & $\mathrm{L}$ & Countrywide & 100 \\
\hline Spain & $\mathrm{L}$ & Countrywide & 74 & No & No & Yes & No & No & $\mathrm{L}$ & Countrywide & 70 \\
\hline Sweden & $\mathrm{L}$ & Countrywide & 100 & No & Yes & Yes & No & Yes & $\mathrm{N}$ & a & $\mathrm{a}$ \\
\hline Switzerland & $\mathrm{L}$ & Countrywide & $\mathrm{m}$ & $\mathrm{m}$ & $\mathrm{m}$ & $\mathrm{m}$ & $\mathrm{m}$ & $\mathrm{m}$ & $\mathrm{L}$ & Countrywide & $\mathrm{m}$ \\
\hline Turkey & $\mathrm{L}$ & Countrywide & 100 & Yes & Yes & No & No & Yes & $\mathrm{L}$ & Countrywide & 100 \\
\hline United States & $\mathrm{L}$ & Some states & $\mathrm{m}$ & Yes & Yes & Yes & Yes & Yes & $\mathrm{L}$ & Some states & $\mathrm{m}$ \\
\hline Brazil & $\mathrm{L}$ & $\mathrm{m}$ & $\mathrm{m}$ & Yes & Yes & No & $\mathrm{m}$ & $\mathrm{m}$ & $\mathrm{m}$ & $\mathrm{m}$ & $\mathrm{m}$ \\
\hline Colombia & $\mathrm{L}$ & Countrywide & 48 & Yes & Yes & No & Yes & No & $\mathrm{L}$ & Countrywide & 20 \\
\hline Latvia & $\mathrm{m}$ & $\mathrm{m}$ & $\mathrm{m}$ & $\mathrm{m}$ & $\mathrm{m}$ & $\mathrm{m}$ & $\mathrm{m}$ & $\mathrm{m}$ & $\mathrm{P}$ & Countrywide & $\mathrm{m}$ \\
\hline
\end{tabular}

Existence of teacher/school leader appraisal

L: Legislated

P: No teacher/school leader appraisal, but have similar practices

N: No teacher/school leader appraisal or similar practices

Note: Federal states or countries with highly decentralised school systems may have different regulations in states, provinces or regions. Please refer to Annex 3 for additional information.

1. Teacher appraisal is legislated in public institutions, and not legislated (but widely practised) in private institutions.

Source: OECD. See Annex 3 for notes (www.oecd.org/education/education-at-a-glance-19991487.htm).

Please refer to the Reader's Guide for information concerning symbols for missing data and abbreviations.

StatLink 제이 http://dx.doi.org/10.1787/888933286394 
Table D7.3b. [1/4] Teacher appraisal at the lower secondary level: Eligibility, governance and responsibilities (2015)

For teachers teaching general programmes

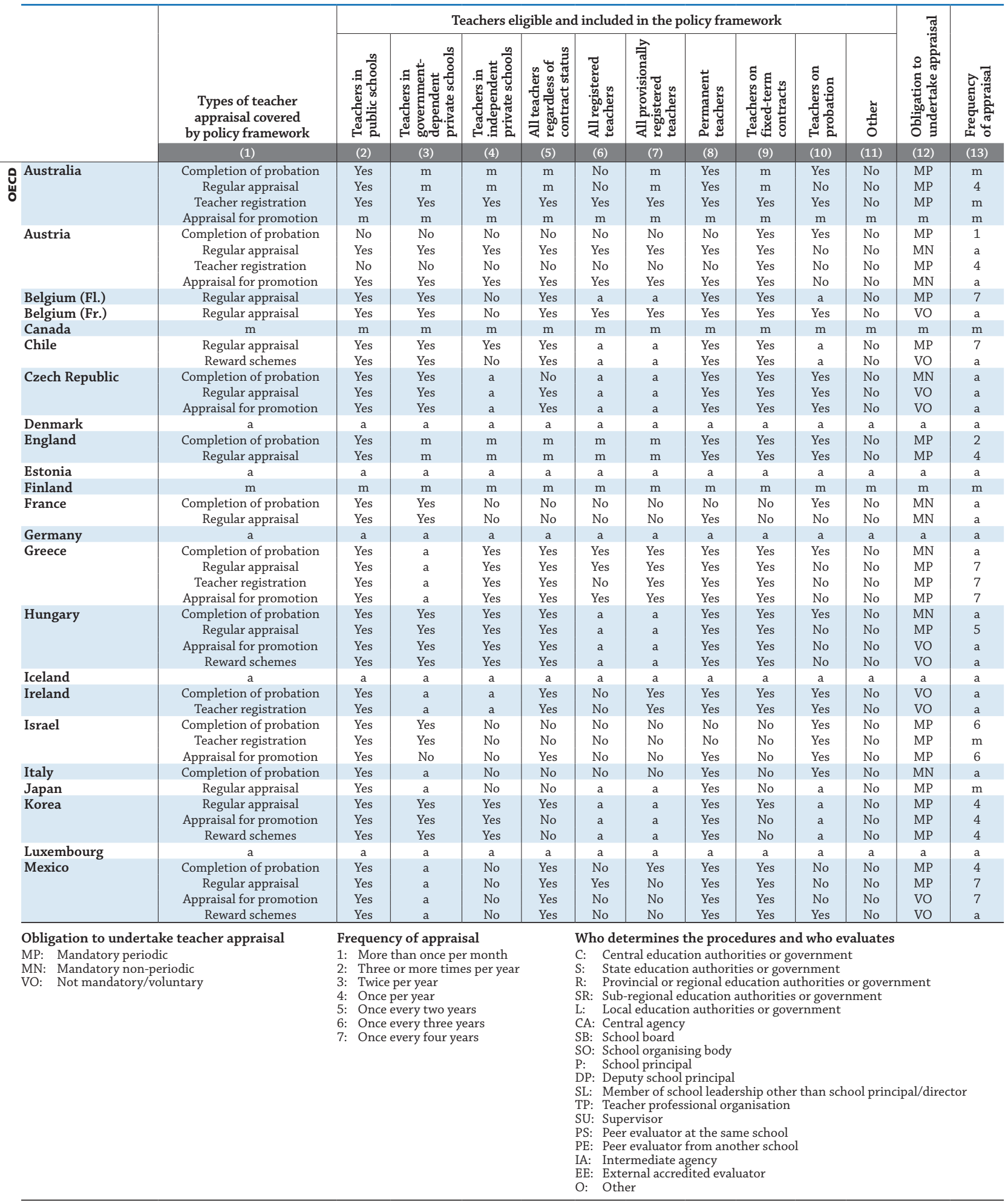

Notes: Federal states or countries with highly decentralised school systems may have different regulations in states, provinces or regions. Please refer to Annex 3 for additional information.

Individual columns for who determines the procedures for appraisal (i.e. columns 22-33) and who evaluates (i.e. columns 35-50) are available for consultation on line (see StatLink below).

Source: OECD. See Annex 3 for notes (www.oecd.org/education/education-at-a-glance-19991487.htm).

Please refer to the Reader's Guide for information concerning symbols for missing data and abbreviations.

StatLink 제매 http://dx.doi.org/10.1787/888933286404 
Table D7.3b. [2/4] Teacher appraisal at the lower secondary level: Eligibility, governance and responsibilities (2015) For teachers teaching general programmes

\begin{tabular}{|c|c|c|c|c|c|c|c|c|c|c|}
\hline & & & Circur & mstances & under whi & ich apprais & & & & \\
\hline & $\begin{array}{c}\text { Types of teacher } \\
\text { appraisal covered } \\
\text { by policy framework }\end{array}$ & 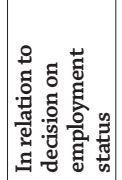 & 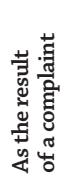 & 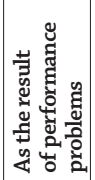 & 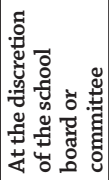 & 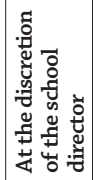 & 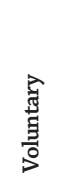 & 节 & $\begin{array}{l}\text { Who determines } \\
\text { the procedures } \\
\text { for appraisal }\end{array}$ & $\begin{array}{c}\text { Who are } \\
\text { the evaluators }\end{array}$ \\
\hline & (1) & (14) & (15) & (16) & (17) & (18) & (19) & (20) & (21) & (34) \\
\hline Australia & $\begin{array}{l}\text { Completion of probation } \\
\text { Regular appraisal } \\
\text { Teacher registration } \\
\text { Appraisal for promotion }\end{array}$ & $\begin{array}{c}\mathrm{m} \\
\mathrm{m} \\
\text { Yes } \\
\mathrm{m}\end{array}$ & $\begin{array}{l}\mathrm{m} \\
\mathrm{m} \\
\mathrm{m} \\
\mathrm{m}\end{array}$ & $\begin{array}{l}\mathrm{m} \\
\text { Yes } \\
\text { No } \\
\mathrm{m}\end{array}$ & $\begin{array}{c}\mathrm{m} \\
\mathrm{m} \\
\mathrm{No} \\
\mathrm{m}\end{array}$ & $\begin{array}{l}\mathrm{m} \\
\text { Yes } \\
\text { No } \\
\mathrm{m}\end{array}$ & $\begin{array}{c}\mathrm{m} \\
\mathrm{m} \\
\mathrm{No} \\
\mathrm{m}\end{array}$ & $\begin{array}{l}\mathrm{m} \\
\text { Yes } \\
\text { No } \\
\mathrm{m}\end{array}$ & $\begin{array}{l}\text { S, SR, SB, P } \\
\text { S, SR, SB, P } \\
\text { S } \\
\text { m }\end{array}$ & $\begin{array}{l}\text { S, P, SL, SU } \\
\text { P, SL, SU } \\
\text { S } \\
\text { m }\end{array}$ \\
\hline Austria & $\begin{array}{l}\text { Completion of probation } \\
\text { Regular appraisal } \\
\text { Teacher registration } \\
\text { Appraisal for promotion }\end{array}$ & $\begin{array}{l}\text { Yes } \\
\text { Yes } \\
\text { Yes } \\
\text { Yes }\end{array}$ & $\begin{array}{l}\text { No } \\
\text { Yes } \\
\text { No } \\
\text { No }\end{array}$ & $\begin{array}{l}\text { No } \\
\text { Yes } \\
\text { No } \\
\text { No }\end{array}$ & $\begin{array}{l}\text { No } \\
\text { No } \\
\text { No } \\
\text { No }\end{array}$ & $\begin{array}{l}\text { No } \\
\text { Yes } \\
\text { No } \\
\text { No }\end{array}$ & $\begin{array}{l}\text { No } \\
\text { Yes } \\
\text { No } \\
\text { Yes }\end{array}$ & $\begin{array}{l}\text { No } \\
\text { No } \\
\text { No } \\
\text { No }\end{array}$ & $\begin{array}{l}C \\
C \\
C \\
C\end{array}$ & $\begin{array}{c}\text { S, R, P } \\
\text { S, R, P, IA } \\
\text { S, R, P, IA } \\
\text { S, R, IA }\end{array}$ \\
\hline Belgium (Fl.) & Regular appraisal & Yes & No & No & Yes & Yes & No & No & S, SO, P & SO, P, SL \\
\hline Belgium (Fr.) & Regular appraisal & Yes & Yes & Yes & Yes & Yes & No & No & S & S \\
\hline Canada & $\mathrm{m}$ & $\mathrm{m}$ & $\mathrm{m}$ & $\mathrm{m}$ & $\mathrm{m}$ & $\mathrm{m}$ & $\mathrm{m}$ & $\mathrm{m}$ & $\mathrm{m}$ & $\mathrm{m}$ \\
\hline Czech Republic & $\begin{array}{l}\text { Completion of probation } \\
\text { Regular appraisal } \\
\text { Appraisal for promotion }\end{array}$ & $\begin{array}{l}\text { Yes } \\
\text { Yes } \\
\text { Yes }\end{array}$ & $\begin{array}{l}\text { No } \\
\text { No } \\
\text { No }\end{array}$ & $\begin{array}{l}\text { No } \\
\text { No } \\
\text { No }\end{array}$ & $\begin{array}{l}\text { No } \\
\text { No } \\
\text { No }\end{array}$ & $\begin{array}{l}\text { No } \\
\text { Yes } \\
\text { Yes }\end{array}$ & $\begin{array}{l}\text { No } \\
\text { No } \\
\text { No }\end{array}$ & $\begin{array}{l}\text { No } \\
\text { No } \\
\text { No }\end{array}$ & $\begin{array}{l}\mathrm{P} \\
\mathrm{C}, \mathrm{P} \\
\mathrm{C}, \mathrm{P}\end{array}$ & $\begin{array}{l}\text { P, SU } \\
\text { P, O } \\
\text { P, O }\end{array}$ \\
\hline Denmark & a & a & a & a & a & a & a & $\mathrm{a}$ & a & a \\
\hline England & $\begin{array}{l}\text { Completion of probation } \\
\text { Regular appraisal }\end{array}$ & $\begin{array}{l}\text { No } \\
\text { No }\end{array}$ & $\begin{array}{l}\text { No } \\
\text { No }\end{array}$ & $\begin{array}{l}\text { Yes } \\
\text { Yes }\end{array}$ & $\begin{array}{l}\text { No } \\
\text { No }\end{array}$ & $\begin{array}{l}\text { No } \\
\text { No }\end{array}$ & $\begin{array}{l}\text { Yes } \\
\text { Yes }\end{array}$ & $\begin{array}{l}\text { No } \\
\text { No }\end{array}$ & $\begin{array}{c}\text { C, S } \\
\text { C }\end{array}$ & $\begin{array}{l}\text { L, P } \\
\text { L, P }\end{array}$ \\
\hline Estonia & $\mathrm{a}$ & $\mathrm{a}$ & $\mathrm{a}$ & $\mathrm{a}$ & $\mathrm{a}$ & $\mathrm{a}$ & $\mathrm{a}$ & a & $\mathrm{a}$ & $\mathrm{a}$ \\
\hline Finland & $\mathrm{m}$ & $\mathrm{m}$ & $\mathrm{m}$ & $\mathrm{m}$ & $\mathrm{m}$ & $\mathrm{m}$ & $\mathrm{m}$ & $\mathrm{m}$ & $\mathrm{m}$ & $\mathrm{m}$ \\
\hline France & $\begin{array}{l}\text { Completion of probation } \\
\text { Regular appraisal }\end{array}$ & $\begin{array}{l}\text { Yes } \\
\text { Yes }\end{array}$ & $\begin{array}{l}\text { Yes } \\
\text { Yes }\end{array}$ & $\begin{array}{l}\text { Yes } \\
\text { Yes }\end{array}$ & $\begin{array}{l}\text { No } \\
\text { No }\end{array}$ & $\begin{array}{l}\text { No } \\
\text { No }\end{array}$ & $\begin{array}{l}\text { No } \\
\text { Yes }\end{array}$ & $\begin{array}{l}\text { No } \\
\text { No }\end{array}$ & $\begin{array}{l}\mathrm{C} \\
\mathrm{C}\end{array}$ & $\begin{array}{l}\text { C, P, SU, IA } \\
\text { C, P, IA }\end{array}$ \\
\hline Germany & a & $\mathrm{a}$ & $\mathrm{a}$ & $\mathrm{a}$ & $\mathrm{a}$ & $\mathrm{a}$ & $\mathrm{a}$ & $\mathrm{a}$ & a & a \\
\hline Greece & $\begin{array}{l}\text { Completion of probation } \\
\text { Regular appraisal } \\
\text { Teacher registration } \\
\text { Appraisal for promotion }\end{array}$ & $\begin{array}{l}\text { Yes } \\
\text { Yes } \\
\text { Yes } \\
\text { Yes }\end{array}$ & $\begin{array}{l}\text { No } \\
\text { No } \\
\text { No } \\
\text { No }\end{array}$ & $\begin{array}{l}\text { No } \\
\text { No } \\
\text { No } \\
\text { No }\end{array}$ & $\begin{array}{l}\text { No } \\
\text { No } \\
\text { No } \\
\text { No }\end{array}$ & $\begin{array}{l}\text { No } \\
\text { No } \\
\text { No } \\
\text { No }\end{array}$ & $\begin{array}{l}\text { No } \\
\text { Yes } \\
\text { Yes } \\
\text { Yes }\end{array}$ & $\begin{array}{l}\text { No } \\
\text { No } \\
\text { No } \\
\text { No }\end{array}$ & $\begin{array}{c}\text { C } \\
\text { C, CA, SB, SO } \\
\text { C, CA, SB, SO } \\
\text { C, CA, SB, SO }\end{array}$ & $\begin{array}{c}\text { C, P, EE } \\
\text { C, SB, P, EE } \\
\text { C, SB, P, EE } \\
\text { C, SB, P, EE }\end{array}$ \\
\hline Hungary & $\begin{array}{l}\text { Completion of probation } \\
\text { Regular appraisal } \\
\text { Appraisal for promotion } \\
\text { Reward schemes }\end{array}$ & $\begin{array}{l}\text { Yes } \\
\text { Yes } \\
\text { Yes } \\
\text { Yes }\end{array}$ & $\begin{array}{l}\text { No } \\
\text { Yes } \\
\text { No } \\
\text { No }\end{array}$ & $\begin{array}{l}\text { No } \\
\text { Yes } \\
\text { No } \\
\text { No }\end{array}$ & $\begin{array}{l}\text { No } \\
\text { No } \\
\text { No } \\
\text { No }\end{array}$ & $\begin{array}{l}\text { No } \\
\text { No } \\
\text { No } \\
\text { No }\end{array}$ & $\begin{array}{l}\text { No } \\
\text { Yes } \\
\text { Yes } \\
\text { Yes }\end{array}$ & $\begin{array}{l}\text { No } \\
\text { No } \\
\text { No } \\
\text { No }\end{array}$ & $\begin{array}{l}\text { C, } \mathrm{CA} \\
\text { C, CA } \\
\text { C, CA } \\
\text { C, CA }\end{array}$ & $\begin{array}{l}\mathrm{P}, \mathrm{O} \\
\mathrm{R}, \mathrm{P} \\
\mathrm{R}, \mathrm{P} \\
\mathrm{R}, \mathrm{P}\end{array}$ \\
\hline Israel & $\begin{array}{l}\text { Completion of probation } \\
\text { Teacher registration } \\
\text { Appraisal for promotion }\end{array}$ & $\begin{array}{l}\text { Yes } \\
\text { Yes } \\
\text { Yes }\end{array}$ & $\begin{array}{l}\text { No } \\
\text { No } \\
\text { No }\end{array}$ & $\begin{array}{l}\text { Yes } \\
\text { No } \\
\text { No }\end{array}$ & $\begin{array}{l}\text { No } \\
\text { No } \\
\text { No }\end{array}$ & $\begin{array}{l}\text { Yes } \\
\text { No } \\
\text { No }\end{array}$ & $\begin{array}{l}\text { No } \\
\text { No } \\
\text { No }\end{array}$ & $\begin{array}{l}\text { No } \\
\text { No } \\
\text { No }\end{array}$ & $\begin{array}{l}C \\
C \\
C\end{array}$ & $\begin{array}{l}\text { C, P } \\
\text { C, P, O } \\
\text { C, } \mathrm{P}, \mathrm{O}\end{array}$ \\
\hline Italy & Completion of probation & Yes & No & No & No & No & No & No & SB & $\mathrm{O}$ \\
\hline Korea & $\begin{array}{l}\text { Regular appraisal } \\
\text { Appraisal for promotion } \\
\text { Reward schemes }\end{array}$ & $\begin{array}{l}\text { No } \\
\text { No } \\
\text { No }\end{array}$ & $\begin{array}{l}\text { No } \\
\text { No } \\
\text { No }\end{array}$ & $\begin{array}{l}\text { No } \\
\text { No } \\
\text { No }\end{array}$ & $\begin{array}{l}\text { No } \\
\text { No } \\
\text { No }\end{array}$ & $\begin{array}{l}\text { No } \\
\text { No } \\
\text { No }\end{array}$ & $\begin{array}{l}\text { No } \\
\text { No } \\
\text { No }\end{array}$ & $\begin{array}{l}\text { Yes } \\
\text { Yes } \\
\text { Yes }\end{array}$ & $\begin{array}{c}\text { C, R, L, SB } \\
\text { C, R } \\
\text { C, R, L, SB }\end{array}$ & $\begin{array}{l}\text { P, SL, PS, O } \\
\text { P, PS } \\
\text { P, PS }\end{array}$ \\
\hline $\begin{array}{l}\text { Luxembourg } \\
\text { Mexico }\end{array}$ & $\begin{array}{l}\text { a } \\
\text { Completion of probation } \\
\text { Regular appraisal } \\
\text { Appraisal for promotion } \\
\text { Reward schemes }\end{array}$ & $\begin{array}{l}\text { a } \\
\text { Yes } \\
\text { Yes } \\
\text { Yes } \\
\text { Yes } \\
\end{array}$ & $\begin{array}{l}\text { a } \\
\text { No } \\
\text { No } \\
\text { No } \\
\text { No }\end{array}$ & $\begin{array}{l}\text { a } \\
\text { No } \\
\text { No } \\
\text { No } \\
\text { No }\end{array}$ & $\begin{array}{l}\text { a } \\
\text { No } \\
\text { No } \\
\text { No } \\
\text { No }\end{array}$ & $\begin{array}{l}\text { a } \\
\text { No } \\
\text { No } \\
\text { No } \\
\text { No }\end{array}$ & $\begin{array}{l}\text { a } \\
\text { No } \\
\text { No } \\
\text { Yes } \\
\text { Yes }\end{array}$ & $\begin{array}{l}\text { a } \\
\text { No } \\
\text { No } \\
\text { No } \\
\text { No }\end{array}$ & $\begin{array}{l}a \\
C \\
c \\
C \\
c\end{array}$ & $\begin{array}{l}\text { a } \\
C \\
C \\
C, P, P S \\
C \\
\end{array}$ \\
\hline Obligation to ur & teacher appraisal & Frequenc) & app & sal & & Who de & $\operatorname{rmin}$ & p & lures and who $\epsilon$ & \\
\hline $\begin{array}{ll}\text { MP: } & \text { Mandatory } \\
\text { MN: } & \text { Mandatory } \\
\text { VO: } & \text { Not mandat }\end{array}$ & & $\begin{array}{l}\text { 1: More tha } \\
\text { 2: Three or } \\
\text { 3: Twice pe } \\
\text { 4: Once per } \\
\text { 5: Once eve } \\
\text { 6: Once eve } \\
\text { 7: Once eve }\end{array}$ & $\begin{array}{l}\text { n once p } \\
\text { more tin } \\
\text { ryear } \\
\text { year } \\
\text { ry two y } \\
\text { ry three } \\
\text { ry four y }\end{array}$ & $\begin{array}{l}\text { er month } \\
\text { mes per ye } \\
\text { ears } \\
\text { years } \\
\text { years }\end{array}$ & & $\begin{array}{ll}\text { C: } & \text { Centr } \\
\text { S: } & \text { State } \\
\text { R: } & \text { Provi } \\
\text { SR: Sub-r } \\
\text { L: } & \text { Local } \\
\text { CA: Centr } \\
\text { SB: Schoc } \\
\text { SO: Schoc } \\
\text { P: Schoc } \\
\text { DP: Depu } \\
\text { SL: Mem } \\
\text { TP: Teach } \\
\text { SU: Super } \\
\text { PS: Peer } \\
\text { PE: Peer } \\
\text { IA: Inter } \\
\text { EE: Exter } \\
\text { O: } & \text { Othe }\end{array}$ & $\begin{array}{l}\text { al educ } \\
\text { educat } \\
\text { ncial or } \\
\text { egional } \\
\text { educat } \\
\text { al agen } \\
\text { l boarc } \\
\text { l orgar } \\
\text { l princ } \\
\text { ty scho } \\
\text { per of s } \\
\text { er prof } \\
\text { visor } \\
\text { valuat } \\
\text { valuat } \\
\text { nediat } \\
\text { nal acc } \\
\text {. }\end{array}$ & $\begin{array}{l}\text { on aut } \\
\text { auth } \\
\text { gional } \\
\text { lucatio } \\
\text { auth } \\
\text { aug boc } \\
1 \text { princi } \\
\text { bol lea } \\
\text { ional } \\
\text { at the } \\
\text { rom a } \\
\text { gency } \\
\text { ited ex }\end{array}$ & $\begin{array}{l}\text { rities or government } \\
\text { ies or government } \\
\text { ucation authorities o } \\
\text { uuthorities or govern } \\
\text { ies or government } \\
\text { ship other than scho } \\
\text { anisation } \\
\text { ne school } \\
\text { ther school } \\
\text { uator }\end{array}$ & ernment \\
\hline
\end{tabular}

Notes: Federal states or countries with highly decentralised school systems may have different regulations in states, provinces or regions. Please refer to Annex 3 for additional information.

Individual columns for who determines the procedures for appraisal (i.e. columns 22-33) and who evaluates (i.e. columns 35-50) are available for consultation on line (see StatLink below).

Source: OECD. See Annex 3 for notes (www.oecd.org/education/education-at-a-glance-19991487.htm).

Please refer to the Reader's Guide for information concerning symbols for missing data and abbreviations.

StatLink 제개 http://dx.doi.org/10.1787/888933286404 
Table D7.3b. [3/4] Teacher appraisal at the lower secondary level: Eligibility, governance and responsibilities (2015)

For teachers teaching general programmes

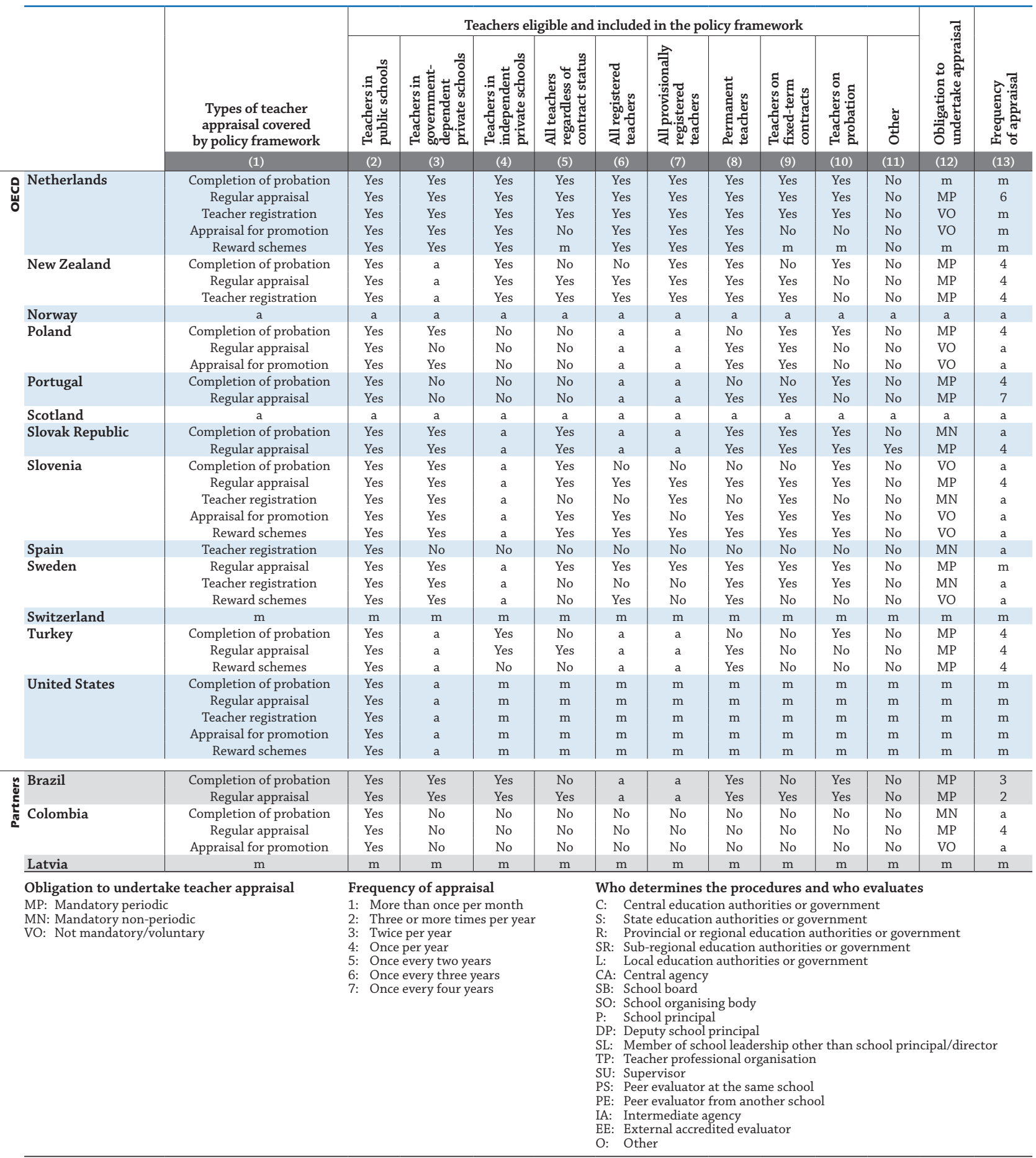

Notes: Federal states or countries with highly decentralised school systems may have different regulations in states, provinces or regions. Please refer to Annex 3 for additional information.

Individual columns for who determines the procedures for appraisal (i.e. columns 22-33) and who evaluates (i.e. columns 35-50) are available for consultation on line (see StatLink below).

Source: OECD. See Annex 3 for notes (www.oecd.org/education/education-at-a-glance-19991487.htm).

Please refer to the Reader's Guide for information concerning symbols for missing data and abbreviations.

StatLink 제그 http://dx.doi.org/10.1787/888933286404 
Table D7.3b. [4/4] Teacher appraisal at the lower secondary level: Eligibility, governance and responsibilities (2015)

For teachers teaching general programmes

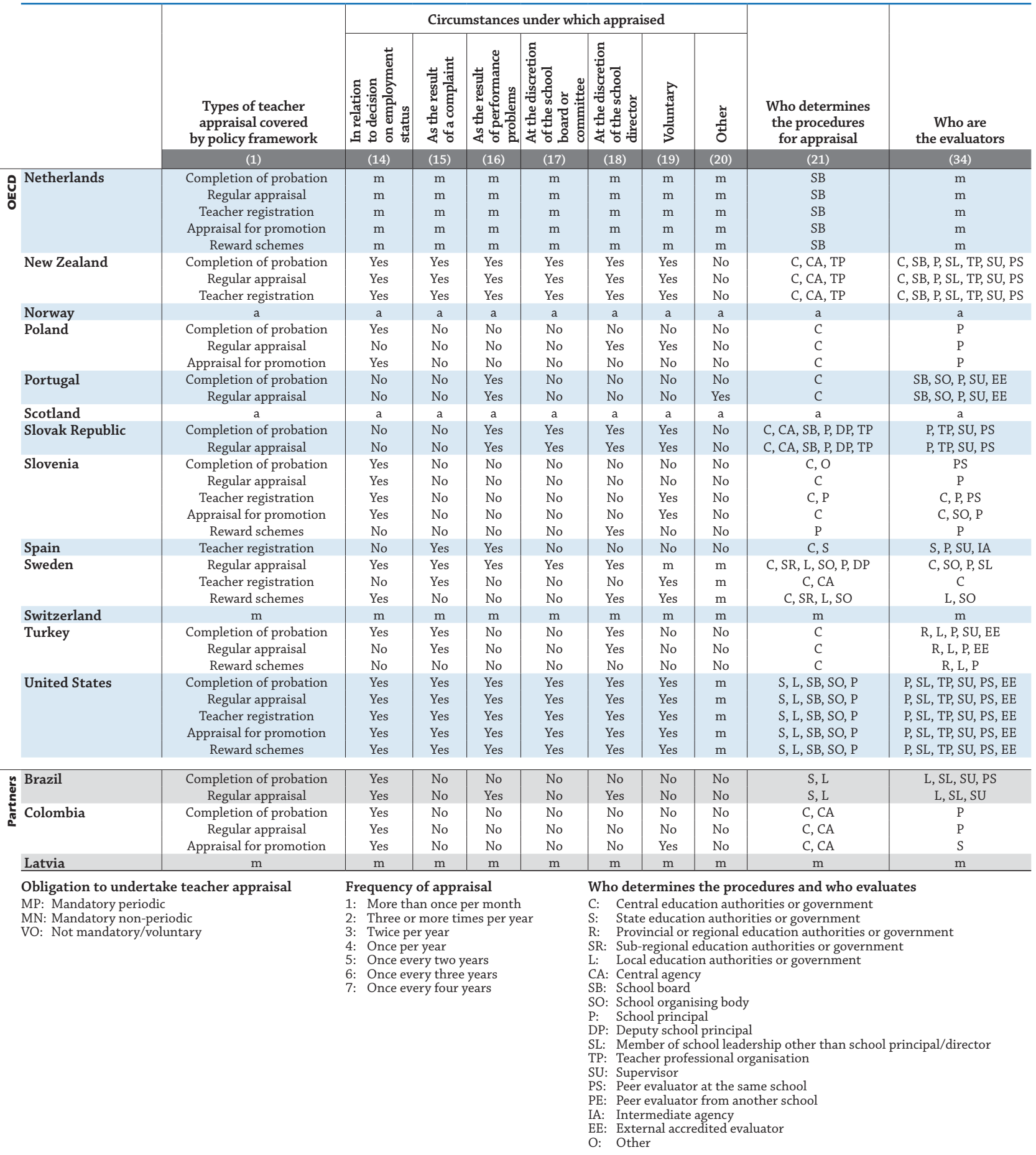

Notes: Federal states or countries with highly decentralised school systems may have different regulations in states, provinces or regions. Please refer to Annex 3 for additional information.

Individual columns for who determines the procedures for appraisal (i.e. columns 22-33) and who evaluates (i.e. columns 35-50) are available for consultation on line (see StatLink below).

Source: OECD. See Annex 3 for notes (www.oecd.org/education/education-at-a-glance-19991487.htm).

Please refer to the Reader's Guide for information concerning symbols for missing data and abbreviations.

StatLink 섹ㄴ http://dx.doi.org/10.1787/888933286404 
Table D7.4b. [1/2] Features of teacher appraisal at the lower secondary level (2015) For teachers teaching general programmes

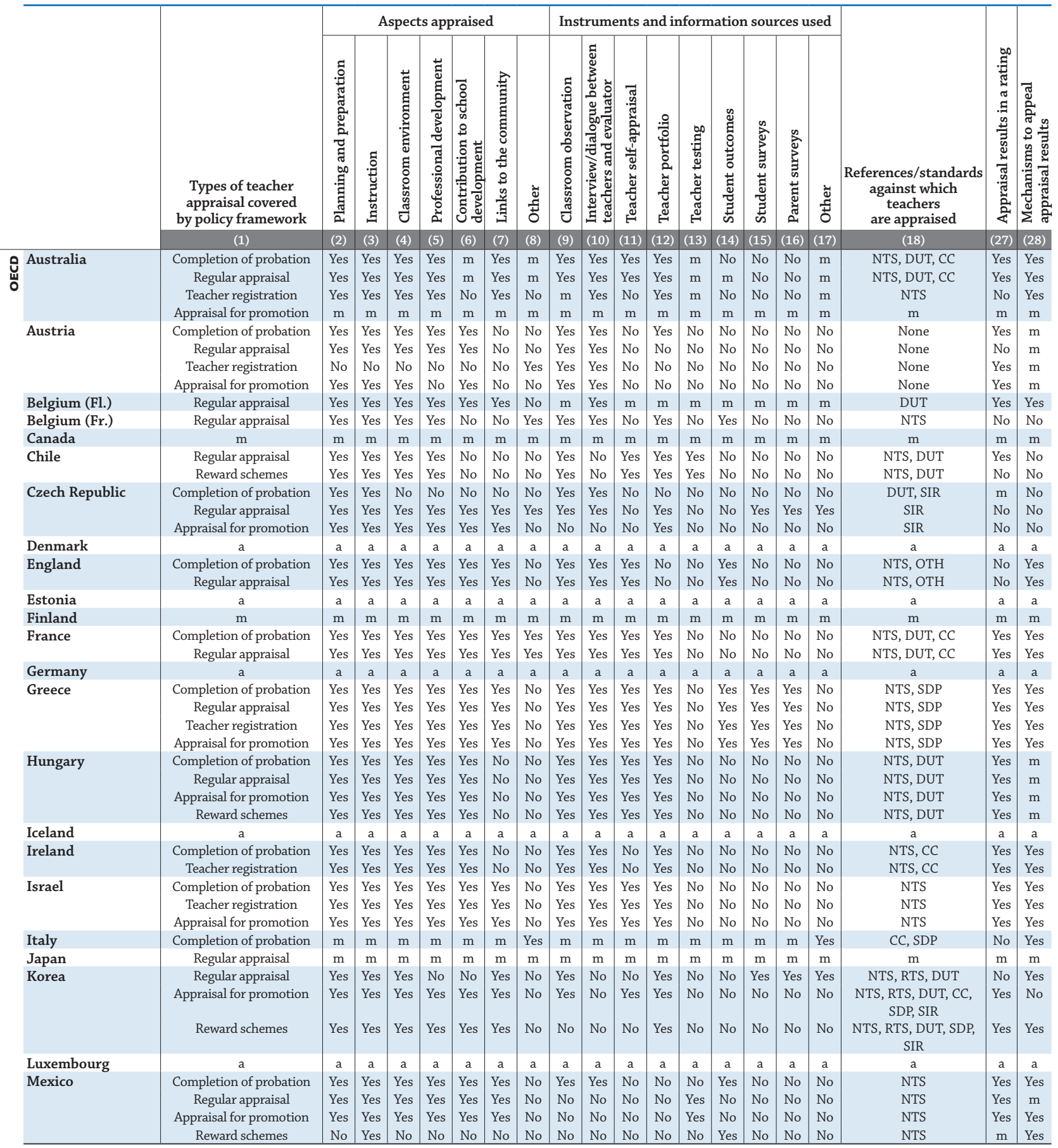

References/standards against which teachers are appraised

None: None

NTS: National or state teaching standards

RTS: Regional or intermediate teaching standards

DUT: A description of the general and professional duties of teachers

CC: Code of conduct

SDP: School development plan or school project

SIR: School internal regulations

OTH: Other

Notes: Federal states or countries with highly decentralised school systems may have different regulations in states, provinces or regions. Please refer to Annex 3 for additional information.

Individual columns for the references against which teachers are appraised (i.e. columns 19-26) are available for consultation on line (see StatLink below).

Source: OECD. See Annex 3 for notes (www.oecd.org/education/education-at-a-glance-19991487.htm).

Please refer to the Reader's Guide for information concerning symbols for missing data and abbreviations.

StatLink त्गाज http://dx.doi.org/10.1787/888933286413 
Table D7.4b. [2/2] Features of teacher appraisal at the lower secondary level (2015) For teachers teaching general programmes

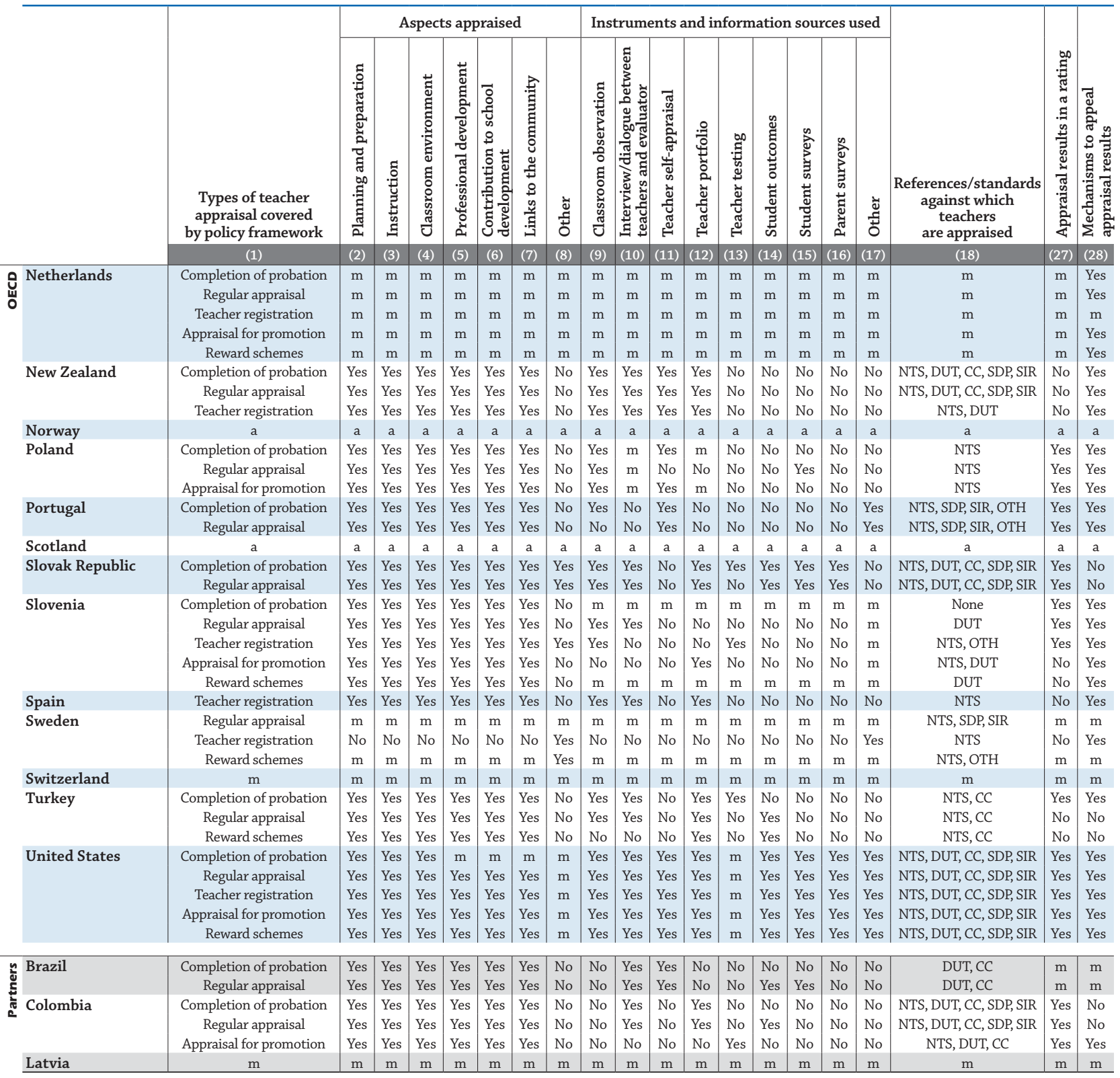

References/standards against which teachers are appraised

None: None

NTS: National or state teaching standards

RTS: Regional or intermediate teaching standards

DUT: A description of the general and professional duties of teachers

CC: Code of conduct

SDP: School development plan or school project

SIR: School internal regulations

OTH: Other

Notes: Federal states or countries with highly decentralised school systems may have different regulations in states, provinces or regions. Please refer to Annex 3 for additional information.

Individual columns for the references against which teachers are appraised (i.e. columns 19-26) are available for consultation on line (see StatLink below).

Source: OECD. See Annex 3 for notes (www.oecd.org/education/education-at-a-glance-19991487.htm).

Please refer to the Reader's Guide for information concerning symbols for missing data and abbreviations.

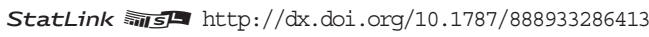


Table D7.8b. School leader appraisal at the lower secondary level: Eligibility, governance and responsibilities (2015)

In general programmes

\begin{tabular}{|c|c|c|c|c|c|c|c|c|c|c|c|c|c|c|c|c|c|}
\hline & & & $\begin{array}{l}\text { nool lea } \\
\text { the poli }\end{array}$ & $\begin{array}{l}\text { ders i } \\
\text { cy fra }\end{array}$ & $\begin{array}{l}\text { nclud } \\
\text { mewc }\end{array}$ & & & & & Circum & tances under & which & apprais & & & & \\
\hline & 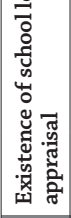 & 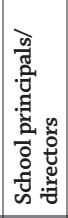 & 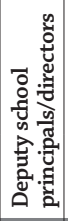 & 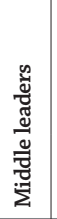 & 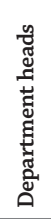 & ఫ్ّ̆ & 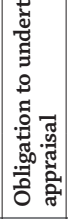 & 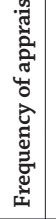 & 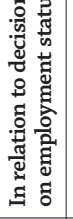 & 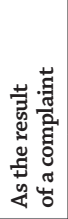 & 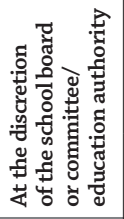 & 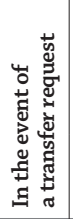 & 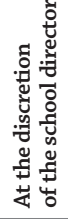 & 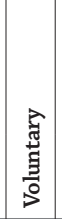 & 毠 & $\begin{array}{l}\text { Who determines } \\
\text { the procedures } \\
\text { for appraisal }\end{array}$ & $\begin{array}{l}\text { Who are } \\
\text { the evaluators }\end{array}$ \\
\hline & (1) & (2) & (3) & (4) & (5) & (6) & (7) & (8) & (9) & (10) & (11) & (12) & (13) & (14) & (15) & (16) & (30) \\
\hline $\begin{array}{l}\text { Australia } \\
\text { Austria }\end{array}$ & $\begin{array}{l}\mathrm{P} \\
\mathrm{N}\end{array}$ & $\begin{array}{l}\mathrm{a} \\
\mathrm{a}\end{array}$ & $\begin{array}{l}\mathrm{a} \\
\mathrm{a}\end{array}$ & $\begin{array}{l}\mathrm{a} \\
\mathrm{a}\end{array}$ & $\begin{array}{l}\mathrm{a} \\
\mathrm{a}\end{array}$ & $\begin{array}{l}\mathrm{a} \\
\mathrm{a}\end{array}$ & $\begin{array}{l}\mathrm{a} \\
\mathrm{a}\end{array}$ & $\begin{array}{l}\mathrm{a} \\
\mathrm{a}\end{array}$ & $\begin{array}{l}\mathrm{a} \\
\mathrm{a}\end{array}$ & $\begin{array}{l}\mathrm{a} \\
\mathrm{a}\end{array}$ & $\begin{array}{l}\mathrm{a} \\
\mathrm{a}\end{array}$ & $\begin{array}{l}\mathrm{a} \\
\mathrm{a}\end{array}$ & $\begin{array}{l}\mathrm{a} \\
\mathrm{a}\end{array}$ & $\begin{array}{l}\mathrm{a} \\
\mathrm{a}\end{array}$ & $\begin{array}{l}\mathrm{a} \\
\mathrm{a}\end{array}$ & $\begin{array}{l}\mathrm{a} \\
\mathrm{a}\end{array}$ & $\begin{array}{l}\mathrm{a} \\
\mathrm{a}\end{array}$ \\
\hline Belgium (Fl.) & L & Yes & No & Yes & No & No & MP & 3 & Yes & No & Yes & No & Yes & No & No & S, SO & P, SO \\
\hline Belgium (Fr.) & $\mathrm{L}$ & Yes & No & $\mathrm{a}$ & $\mathrm{a}$ & $\mathrm{a}$ & MP & 4 & Yes & No & Yes & No & No & No & No & S, SO & $\mathrm{S}, \mathrm{SO}, \mathrm{O}$ \\
\hline Canada & $\mathrm{L}$ & $\mathrm{m}$ & $\mathrm{m}$ & $\mathrm{m}$ & $\mathrm{m}$ & $\mathrm{m}$ & $\mathrm{m}$ & $\mathrm{m}$ & $\mathrm{m}$ & $\mathrm{m}$ & $\mathrm{m}$ & $\mathrm{m}$ & $\mathrm{m}$ & $\mathrm{m}$ & $\mathrm{m}$ & $\mathrm{m}$ & $\mathrm{m}$ \\
\hline Chile & $\mathrm{N}$ & a & a & $\mathrm{a}$ & $a$ & $\mathrm{a}$ & a & $a$ & a & a & a & a & a & $\mathrm{a}$ & a & a & a \\
\hline Czech Republic & $\mathrm{L}$ & Yes & No & No & No & No & MN & $a$ & Yes & No & No & No & No & No & No & $\mathrm{R}, \mathrm{SO}$ & SR, SO \\
\hline Denmark & $\mathrm{P}$ & $\mathrm{a}$ & $\mathrm{a}$ & $\mathrm{a}$ & $\mathrm{a}$ & $\mathrm{a}$ & $\mathrm{a}$ & $\mathrm{a}$ & $\mathrm{a}$ & a & a & a & $\mathrm{a}$ & a & $\mathrm{a}$ & $\mathrm{a}$ & a \\
\hline England & $\mathrm{L}$ & Yes & Yes & No & No & Yes & MP & 1 & No & No & No & No & No & Yes & $\mathrm{m}$ & c & L, SB, EE \\
\hline Estonia & $\mathrm{N}$ & a & a & $\mathrm{a}$ & $\mathrm{a}$ & $\mathrm{a}$ & a & $\mathrm{a}$ & a & $\mathrm{a}$ & a & a & a & $\mathrm{a}$ & a & a & $\mathrm{a}$ \\
\hline Finland & $\mathrm{m}$ & $\mathrm{m}$ & $\mathrm{m}$ & $\mathrm{m}$ & $\mathrm{m}$ & $\mathrm{m}$ & $\mathrm{m}$ & $\mathrm{m}$ & $\mathrm{m}$ & $\mathrm{m}$ & $\mathrm{m}$ & $\mathrm{m}$ & $\mathrm{m}$ & $\mathrm{m}$ & $\mathrm{m}$ & $\mathrm{m}$ & $\mathrm{m}$ \\
\hline France & $\mathrm{L}$ & Yes & Yes & No & No & No & MP & 2 & No & Yes & No & Yes & No & No & No & c & c \\
\hline Germany & $\mathrm{N}$ & $\mathrm{a}$ & $\mathrm{a}$ & $\mathrm{a}$ & $\mathrm{a}$ & $\mathrm{a}$ & a & $\mathrm{a}$ & $\mathrm{a}$ & $\mathrm{a}$ & $\mathrm{a}$ & a & a & $\mathrm{a}$ & $a$ & a & a \\
\hline Greece & L & Yes & No & No & No & No & MP & 3 & Yes & No & No & No & No & Yes & No & C & C, IA \\
\hline Hungary & $\mathrm{L}$ & Yes & No & No & No & No & MP & 4 & Yes & No & No & No & No & No & Yes & C, CA & P, EE, O \\
\hline Iceland & $\mathrm{N}$ & $\mathrm{a}$ & $\mathrm{a}$ & $\mathrm{a}$ & $a$ & $\mathrm{a}$ & $\mathrm{a}$ & a & $\mathrm{a}$ & a & $\mathrm{a}$ & a & $\mathrm{a}$ & $\mathrm{a}$ & $\mathrm{a}$ & $\mathrm{a}$ & $\mathrm{a}$ \\
\hline Ireland & $\mathrm{N}$ & a & a & $\mathrm{a}$ & $\mathrm{a}$ & $\mathrm{a}$ & a & $a$ & a & a & a & a & $\mathrm{a}$ & a & $\mathrm{a}$ & a & a \\
\hline Israel & $\mathrm{L}$ & Yes & Yes & No & No & No & MP & 2 & Yes & No & No & No & Yes & Yes & No & C, SA & $\mathrm{R}$ \\
\hline Italy & $\mathrm{N}$ & $\mathrm{a}$ & $\mathrm{a}$ & $\mathrm{a}$ & $\mathrm{a}$ & $\mathrm{a}$ & a & $a$ & a & a & a & a & a & a & $a$ & a & a \\
\hline Japan & $\mathrm{N}$ & $\mathrm{a}$ & $\mathrm{a}$ & $\mathrm{a}$ & a & $\mathrm{a}$ & a & $a$ & $\mathrm{a}$ & $\mathrm{a}$ & a & a & $\mathrm{a}$ & $\mathrm{a}$ & $\mathrm{a}$ & $\mathrm{a}$ & $\mathrm{a}$ \\
\hline Korea & $\mathrm{L}$ & Yes & Yes & $\mathrm{a}$ & $\mathrm{a}$ & No & MP & 1 & No & No & Yes & No & No & No & No & $C, R, L, S B$ & P, DP, EE, O \\
\hline Luxembourg & $\mathrm{N}$ & $\mathrm{a}$ & $\mathrm{a}$ & $a$ & $\mathrm{a}$ & $a$ & a & $\mathrm{a}$ & a & a & $\mathrm{a}$ & $\mathrm{a}$ & $\mathrm{a}$ & $\mathrm{a}$ & $a$ & a & a \\
\hline Mexico & $\mathrm{L}$ & Yes & Yes & Yes & Yes & No & MP & 3 & Yes & No & No & No & No & Yes & No & C & C \\
\hline Netherlands & $\mathrm{P}$ & $\mathrm{a}$ & $\mathrm{a}$ & $\mathrm{a}$ & $\mathrm{a}$ & $\mathrm{a}$ & a & a & $\mathrm{a}$ & $\mathrm{a}$ & a & $\mathrm{a}$ & a & $\mathrm{a}$ & $a$ & $\mathrm{a}$ & $\mathrm{a}$ \\
\hline New Zealand & $\mathrm{L}$ & Yes & Yes & Yes & Yes & No & MP & 1 & Yes & No & Yes & No & No & No & No & C, CA, SB, P, DP, SP & C, SB, P, SL, PE \\
\hline Norway & $\mathrm{N}$ & $\mathrm{a}$ & $\mathrm{a}$ & $\mathrm{a}$ & $\mathrm{a}$ & $\mathrm{a}$ & $\mathrm{a}$ & $\mathrm{a}$ & $\mathrm{a}$ & $\mathrm{a}$ & a & a & $\mathrm{a}$ & a & $a$ & a & a \\
\hline Poland & $\mathrm{L}$ & Yes & No & No & No & No & VO & $a$ & No & No & Yes & No & No & Yes & No & c & $\mathrm{L}$ \\
\hline Portugal & $\mathrm{L}$ & Yes & Yes & Yes & Yes & No & MP & 3 & No & No & No & No & No & No & Yes & c & SB, IA \\
\hline Scotland & $\mathrm{N}$ & $\mathrm{a}$ & $\mathrm{a}$ & $\mathrm{a}$ & $a$ & $\mathrm{a}$ & a & $\mathrm{a}$ & a & $\mathrm{a}$ & a & a & $\mathrm{a}$ & $\mathrm{a}$ & $a$ & a & a \\
\hline Slovak Republic & $\mathrm{L}$ & Yes & Yes & No & No & No & MP & 1 & No & Yes & No & No & No & No & No & SO, P, O & $\mathrm{P}, \mathrm{SO}$ \\
\hline Slovenia & $\mathrm{L}$ & Yes & Yes & $\mathrm{a}$ & $\mathrm{a}$ & No & MP & 1 & Yes & No & No & No & No & No & No & c & C, SB, P, SO, IA \\
\hline Spain & $\mathrm{L}$ & Yes & No & No & No & No & MP & 1 & Yes & No & No & No & No & No & No & s & S, IA \\
\hline Sweden & $\mathrm{N}$ & $\mathrm{a}$ & $\mathrm{a}$ & $a$ & $\mathrm{a}$ & $\mathrm{a}$ & $\mathrm{a}$ & $\mathrm{a}$ & a & a & a & a & a & a & $\mathrm{a}$ & a & a \\
\hline Switzerland & $\mathrm{L}$ & $\mathrm{m}$ & $\mathrm{m}$ & $\mathrm{m}$ & $\mathrm{m}$ & $\mathrm{m}$ & $\mathrm{m}$ & $\mathrm{m}$ & $\mathrm{m}$ & $\mathrm{m}$ & $\mathrm{m}$ & $\mathrm{m}$ & $\mathrm{m}$ & $\mathrm{m}$ & $\mathrm{m}$ & $\mathrm{m}$ & $\mathrm{m}$ \\
\hline Turkey & $\mathrm{L}$ & Yes & No & No & No & No & MP & 3 & Yes & Yes & No & No & Yes & No & No & c & R, L, EE, O \\
\hline United States & $\mathrm{L}$ & Yes & Yes & $\mathrm{m}$ & $\mathrm{m}$ & $\mathrm{m}$ & $\mathrm{m}$ & $\mathrm{m}$ & Yes & Yes & Yes & Yes & Yes & Yes & $\mid$ Yes $\mid$ & $S, L, S B, S O, S P$ & S, L, SB, PS, EE, PE \\
\hline Brazil & $\mathrm{m}$ & $\mathrm{m}$ & $\mathrm{m}$ & $\mathrm{m}$ & $\mathrm{m}$ & $\mathrm{m}$ & $\mathrm{m}$ & $\mathrm{m}$ & $\mathrm{m}$ & $\mathrm{m}$ & $\mathrm{m}$ & $\mathrm{m}$ & $\mathrm{m}$ & $\mathrm{m}$ & $\mathrm{m}$ & $\mathrm{m}$ & $\mathrm{m}$ \\
\hline Colombia & $\mathrm{L}$ & Yes & Yes & No & No & No & MP & 1 & Yes & No & No & No & Yes & No & No & C, CA & $S / L$ \\
\hline Latvia & $\mathrm{P}$ & $\mathrm{a}$ & $\mathrm{a}$ & $\mathrm{a}$ & $\mathrm{a}$ & $\mathrm{a}$ & $\mathrm{a}$ & $\mathrm{a}$ & $\mathrm{a}$ & $\mathrm{a}$ & $\mathrm{a}$ & $\mathrm{a}$ & $\mathrm{a}$ & $\mathrm{a}$ & $\mathrm{a}$ & $\mathrm{a}$ & $\mathrm{a}$ \\
\hline Existence of scho & eader a & pprais & & & & & equen & $y$ of a & apprais & & Who de & mines $t$ & he pro & cedur & res an & d who evaluates & \\
\hline L: Legislated & & & & & & & Once p & er year & & & C: Cer & educatic & on auth & orities & s or go & hent & \\
\hline P: No school leader & aisal & ut h & im & $\ln ^{2}$ & & & Once e & very th & hree yea & & CA: Central & $\begin{array}{l}\text { agency } \\
\text { and }\end{array}$ & & & & & \\
\hline N: No school leader & praisal o & $\operatorname{sim}$ & ar pra & & & & Once e & very fo & our year & & S: State ec & lucation & author & ities or & r gove & ernment & \\
\hline Obligation to un & take an & pnraisa & Jnroce & dure & & & Once & & ive $y$ & & SA: State ag & ency & lo & & & & \\
\hline MP. & take ap & ppralsa & 11 proce & & & & & & & & $\begin{array}{l}\text { R: } \text { Provinc } \\
\text { SP. }\end{array}$ & ial or reg & gional e & dux & $\mathrm{t}=\mathrm{d}$ & & \\
\hline $\begin{array}{l}\text { MP: Mandatory pe } \\
\text { MN. Mandatory }\end{array}$ & $\begin{array}{l}\text { ic } \\
\text { riodic }\end{array}$ & & & & & & & & & & $\begin{array}{l}\text { SR: Sub-reg } \\
\text { L: Local ec }\end{array}$ & $\begin{array}{l}\text { ional int } \\
\text { lucation }\end{array}$ & $\begin{array}{l}\text { ter-mun } \\
\text { author }\end{array}$ & $\begin{array}{l}\text { nicipal } \\
\text { ities ol }\end{array}$ & educa & $\begin{array}{l}\text { ation authoritie } \\
\text { ernment }\end{array}$ & rnment \\
\hline VO: Not mandato & oluntar & & & & & & & & & & SB: School & ooard or & commi & ittee & & & \\
\hline & & & & & & & & & & & $\begin{array}{l}\text { SO: School } \\
\text { P: School }\end{array}$ & $\begin{array}{l}\text { organisir } \\
\text { orincipal }\end{array}$ & $\begin{array}{l}\text { ng body } \\
\text { 1/direct }\end{array}$ & & & & \\
\hline & & & & & & & & & & & DP: Deputy & school p & rincipa & 1/direc & ctor & & \\
\hline & & & & & & & & & & & SP: Profess & onal org & janisati & ion for & schoo & ol leaders & \\
\hline & & & & & & & & & & & SL: Membe & rof sche & pol leade & ership & other & than school prin & /director \\
\hline & & & & & & & & & & & TP: Teacher & profess & ional or & rganisa & ation & & \\
\hline & & & & & & & & & & & SU: Supervi & sor & & & & & \\
\hline & & & & & & & & & & & PS: Peer ev & luator a & it the sa & ame sch & hool & & \\
\hline & & & & & & & & & & & PE: Peer ev & luator $\mathrm{f}$ & rom an & other s & school & & \\
\hline & & & & & & & & & & & IA: Int & & & & & & \\
\hline & & & & & & & & & & & $\begin{array}{l}\text { EE: Externa } \\
\text { O: Other }\end{array}$ & & & & & & \\
\hline
\end{tabular}

Notes: Federal states or countries with highly decentralised school systems may have different regulations in states, provinces or regions. Please refer to Annex 3 for additional information.

Individual columns for who determines the procedures for appraisal (i.e. columns 17-29) and who evaluates (i.e. columns 31-45) are available for consultation on line (see StatLink below).

Source: OECD. See Annex 3 for notes (www.oecd.org/education/education-at-a-glance-19991487.htm)

Please refer to the Reader's Guide for information concerning symbols for missing data and abbreviations.

StatLink 部 St http://dx.doi.org/10.1787/888933286426 
Table D7.9b. Features of school leader appraisal at the lower secondary level (2015) In general programmes

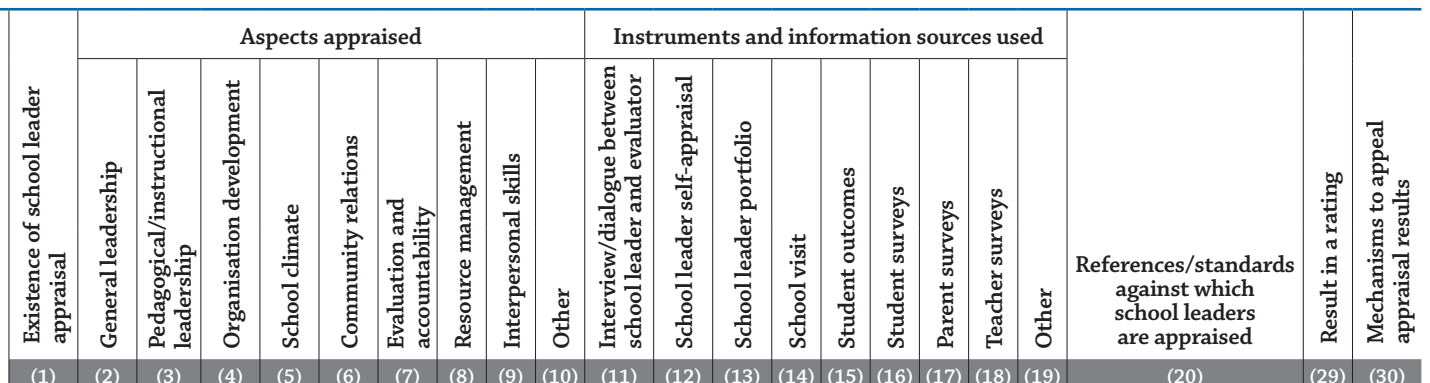

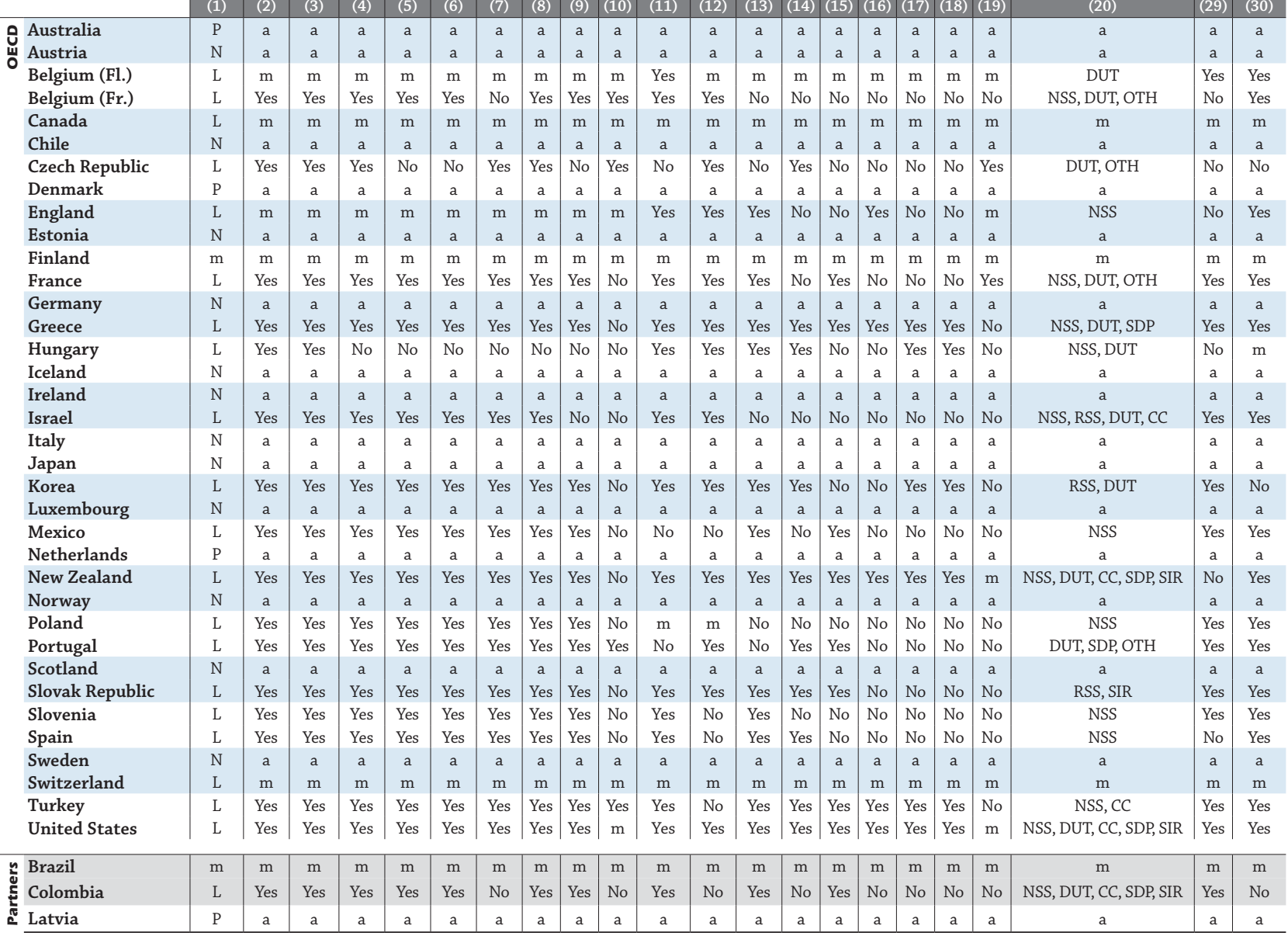

Existence of school leader appraisal

L: Legislated

P: No school leader appraisal, but have similar practices

$\mathrm{N}$ : No school leader appraisal or similar practices
References/standards against which school leaders are appraised

None: None

NSS: National or state standards for school leadership

RSS: Regional or intermediate standards for school leadership

DUT: A description of the general and professional duties of school leaders

CC: Code of conduct

SDP: School development plan or school project

SIR: School internal regulations

OTH: Other

Notes: Federal states or countries with highly decentralised school systems may have different regulations in states, provinces or regions. Please refer to Annex 3 for additional information.

Individual columns for the references against which school leaders are appraised (i.e. columns 21-28) are available for consultation on line (see StatLink below).

Source: OECD. See Annex 3 for notes (www.oecd.org/education/education-at-a-glance-19991487.htm).

Please refer to the Reader's Guide for information concerning symbols for missing data and abbreviations.

StatLink 젶ㄴ http://dx.doi.org/10.1787/888933286432 



\section{TO WHAT EXTENT IS INFORMATION AND COMMUNICATION TECHNOLOGY USED IN TEACHING AND LEARNING?}

- Virtually all 15-year-old students in OECD countries are in schools that make at least one computer available to them; but there is considerable variation in the ratio of students to computers, from less than 1 student per computer in Australia to 45 students per computer in Turkey.

- On average across OECD countries, around $15 \%$ of students reported that they first accessed the Internet at age 6 or younger.

INDICATOR D8

- Only $17 \%$ of students spend one hour or more at school using the Internet during a typical school day, on average across OECD countries, while more than $36 \%$ of them do not spend any time using the Internet at school.

\section{Chart D8.1. How old were 15 year-old students when they first accessed the Internet? (PISA 2012)}

Age distribution based on results from students' self-reports

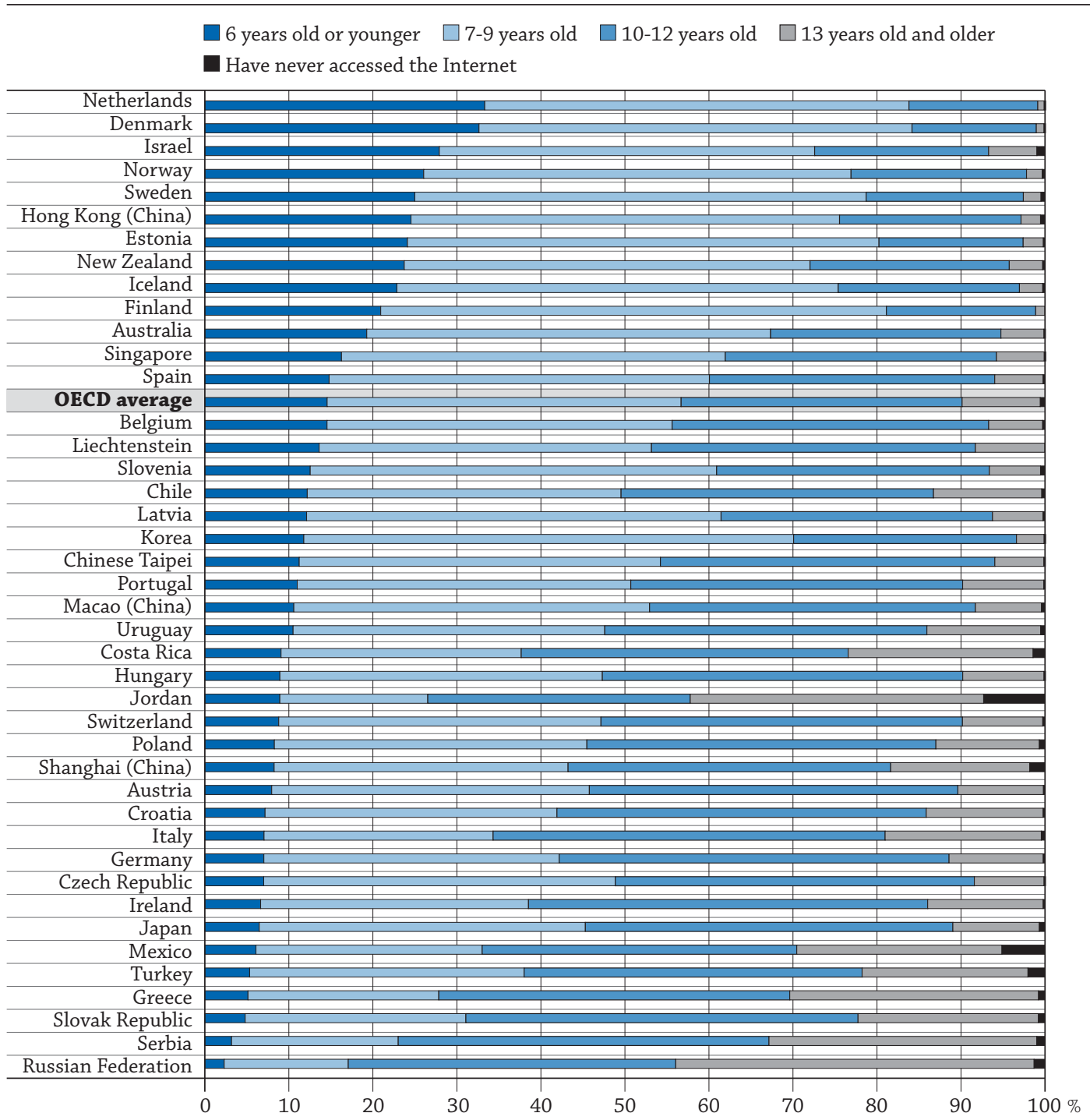

Countries and economies are ranked in descending order of the percentage of students who reported that they had first accessed the Internet when they were 6 or younger.

Source: OECD. Table D8.1. See Annex 3 for notes (www.oecd.org/education/education-at-a-glance-19991487.htm).

StatLink 제그 http://dx.doi.org/10.1787/888933284683 


\section{Context}

Information and communication technology (ICT) is a major component of economic growth in all OECD countries. Given that young people today need to be skilled in using these technologies as students, job-seekers or workers, consumers and responsible citizens, those who have no access to or experience in using ICT will find it increasingly difficult to participate fully in economic, social and civic life. However, basic ICT skills may not add value unless they are well paired with cognitive skills and other skills, such as creativity, communication skills, team work and perseverance.

Schools need sufficient ICT resources to help students both to learn how to use and benefit from these technologies and to acquire new knowledge and skills, in other subjects, through using them. ICT can also help teachers and school administrators to work more efficiently. The distribution of resources across and within education systems has long been an important issue for both equity and excellence in education. Given the rapid advances in technology, and the central role ICT now plays in all aspects of life, education policy makers need to consider how to ensure that ICT resources and students' access to those resources are provided equitably within education systems.

\section{Other findings}

- On average, 15-year-old boys score 4 points higher on the computer-based PISA reading test than on the paper-based reading test. By contrast, 15-year-old girls perform 8 points lower in digital reading than in paper-based reading test, on average.

- In all countries and economies that participated in the OECD Programme for International Student Assessment (PISA) in 2012, the gender gap in reading performance is narrower in digital reading than in print reading. Girls outperform boys in digital reading by an average of 26 score points, compared to an average of 38 score points - the equivalent of nearly one year of schooling - in print reading.

- Teachers who participated in the 2013 OECD Teaching and Learning International Survey (TALIS) (OECD, 2014a) reported that the areas in which they most need professional development are in teaching students with special needs and developing ICT skills for teaching.

- An average of only $40 \%$ of lower secondary teachers who participated in TALIS reported that students frequently use ICT for projects or class work. This suggests that, despite large investments in ICT across school systems, in many countries, teachers are still not systematically inclined and prepared to use these tools in their teaching.

- Given teachers' self-reported need for training in how to use ICT in their teaching, TALIS finds no correlation between the ICT-related professional development activities offered and teachers' participation rates in those programmes. That lack of coherence could be costly if the teachers who feel they need further training do not have access to it, or if the training is not well-targeted.

\section{Trends}

- Students in 2012 were less likely than their counterparts were in 2003 to attend schools whose principal reported that the school's capacity to provide instruction is hindered by a lack of computers and computer software.

- According to principals' reports, the number of 15-year-old students per school computer did not change significantly across OECD countries, on average. In 2012 as in 2009 there were between four and five students to every school computer, on average across OECD countries. 


\section{Analysis}

\section{ICT resources in schools}

\section{Quality of schools' educational resources}

In 2012, the OECD Programme for International Student Assessment (PISA) asked school principals to report whether their school's capacity to provide instruction was hindered by a shortage or inadequacy of: science laboratory equipment, instructional materials (e.g. textbooks), computers for instruction, computer software for instruction, and library materials. The responses were combined to create an index of quality of schools' educational resources that has a mean of zero and a standard deviation of 1 in OECD countries. Positive values reflect principals' perceptions that a shortage of educational resources hinders learning to a lesser extent than the OECD average, and negative values indicate that school principals believe the shortage hinders learning to a greater extent.

In 2012, an average of less than $10 \%$ of 15 -year-old students across OECD countries attended schools whose principals reported that the school's capacity to provide instruction was hindered a lot by a shortage or inadequacy of educational resources (e.g. traditional textbooks, library materials, a science laboratory, computers and computer software). Thus, for example, only $9 \%$ of students were in schools whose principals reported that instruction was hindered a lot by a shortage of computers for instruction, and only $5 \%$ were in schools whose principals reported that instruction was hindered by a shortage of computer software. More globally, a shortage of computers for instruction hinders learning to a greater extent in Brazil, Greece, Iceland, Indonesia, Mexico, Sweden, Tunisia and Turkey: at least 15\% of students attended schools whose principals reported that the school's capacity to provide instruction was hindered a lot by a shortage of computers. By contrast, principals are the most positive in Australia, the Czech Republic, France, Hong-Kong (China), Hungary, Italy, Korea, Macao (China) and the Slovak Republic and reported for more than $96 \%$ of them that instruction in their school is not hindered by a shortage of computers (Table D8.2).

\section{Chart D8.2. Change between 2003 and 2012 in the index of quality of schools' educational resources (e.g. textbooks, computers for instruction, computer software)}

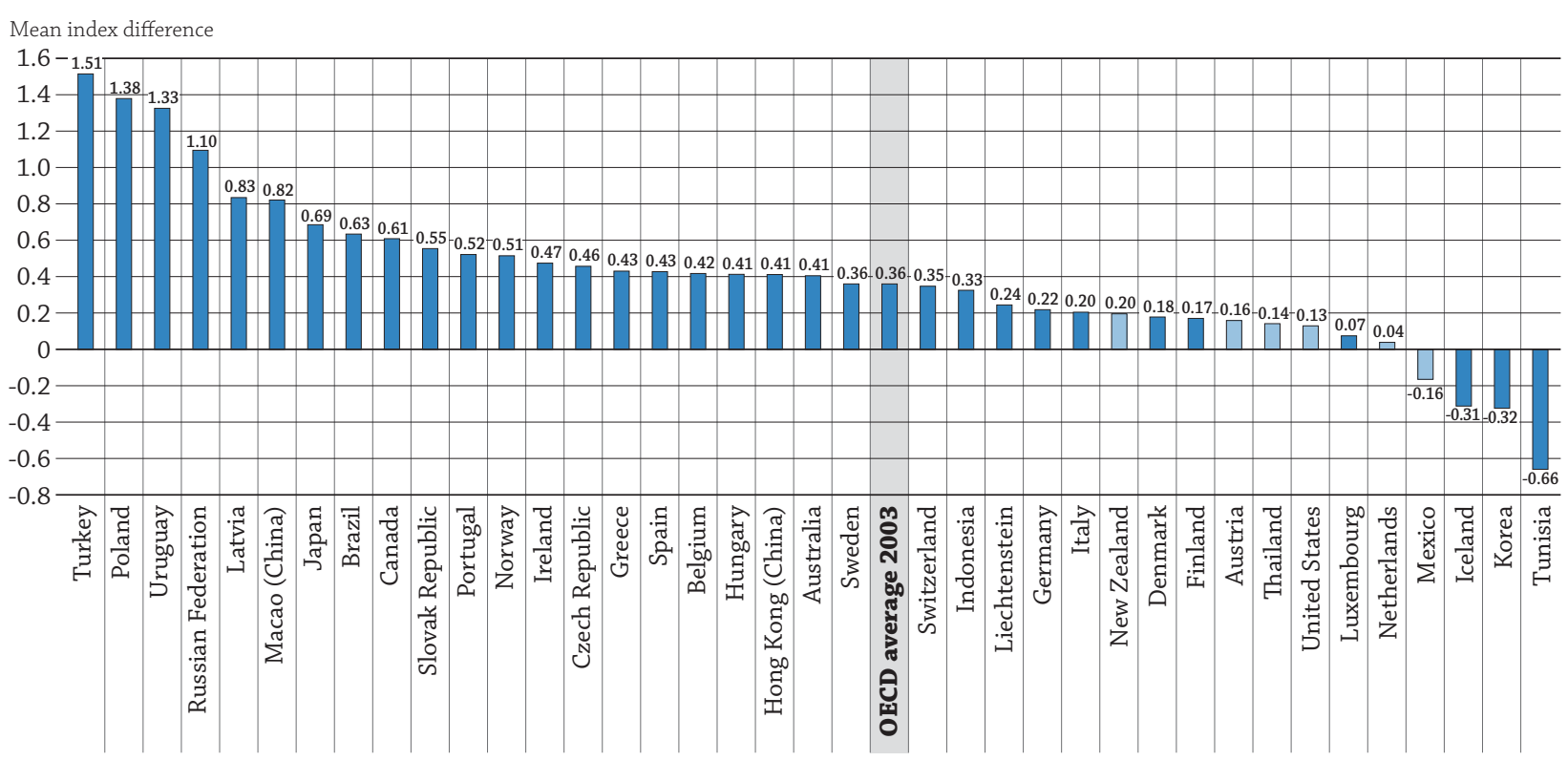

Notes: The index of quality of school educational resources was derived from the items measuring school principals' perceptions of potential factors hindering instruction at their school (SC14, from the PISA 2012 school questionnaire). Higher values on this index indicate better quality of educational resources in 2012 . Dark blue bars indicate differences that are statistically significant. For comparability over time, PISA 2003 values on the index of schools' educational resources have been rescaled to the PISA 2012 scale of the index.

Countries and economies are ranked in descending order of the change between 2003 and 2012 in the index of quality of schools' educational resources.

Source: OECD. Table D8.2. See Annex 3 for notes (www.oecd.org/education/education-at-a-glance-19991487.htm).

StatLink 젝ㄴ http://dx.doi.org/10.1787/888933284698

Moreover, the schools seem to be better equipped in new technologies in 2012 than in 2003. Thus, students in 2012 were less likely than their counterparts in 2003 to attend schools whose principal reported that the school's capacity to provide instruction is hindered by a lack of computers and computer software. Thus, for example, in 26 of the 38 countries and economies with comparable data, there were fewer school principals in 2012 than in 2003 who reported that their school's capacity to provide instruction was hindered by a shortage of computers. 
The largest improvements between 2003 and 2012 were observed in Australia, Belgium, Brazil, Germany, Ireland, the Netherlands, the Russian Federation and Uruguay. By contrast, the shortage of computers for instruction was greater in 2012 than in 2003 - signalling a greater likelihood that students attend schools where a lack of computers hinders the school's capacity to provide instruction - in Iceland, Indonesia, Mexico and Tunisia (Table D8.2).

The overall trend among OECD countries shows that the shortage of educational resources (e.g. traditional textbooks, library materials, a science laboratory, computers and computer software) hindered the school's capacity to provide instruction to a lesser extent in 2012 than in 2003. This trend was observed across all school types, advantaged and disadvantaged schools, private and public schools, lower and upper secondary programmes, and urban and rural schools). (OECD [2013], Table IV.3.45).

\section{Number of students per computer}

Given that students' use of ICT for learning partly depends on the extent to which they can have access to a computer, a key indication of access to ICT resources is the number of students per school computer. Across OECD countries, virtually all students attend schools with at least one computer. The number of students per computer is based on principals' reports about the number of students in the national modal grade for 15-year-olds and on the number of computers available for these students. On average across OECD countries in 2012, there were five students for every school computer. Brazil, Costa Rica, Indonesia, Mexico and Turkey had the largest number (at least 15) of students per computer, while Australia, the Czech Republic, Macao (China), New Zealand, Norway, the Slovak Republic, the United Kingdom and the United States had the smallest number (fewer than two) of students per school computer (Table D8.1).

According to principals' reports, the number of 15-year-old students per school computer did not change significantly across OECD countries, on average. In 2012 as in 2009 there were between four and five students to every school computer, on average across OECD countries. Globally, the number of students per school computer decreased significantly in 12 of the 49 countries/economies with comparable data, and increased only in five - most notably in Turkey (from 12 to 45). The change in Turkey may have been partly the result of an increase in the student population during this period rather than a reduction in the number of computers available to them (Table D8.1).

\section{First Internet access and intensity of Internet use at school}

\section{Number of students who have never used a computer}

The most basic measure of students' access to and familiarity with ICT is whether or not they have used a computer. PISA 2012 found that virtually all 15-year-old boys and girls in all participating countries and economies had accessed the Internet by the time they took the PISA test. In 2012, on average across OECD countries, less than $1 \%$ of students reported that they had never used a computer or accessed the Internet. In Mexico, $5 \%$ of students so reported, and Jordan showed the highest levels of non-use, with $7 \%$ of students reporting that they had never accessed the Internet (OECD [2015a]).

On average across OECD countries, around $15 \%$ of students reported that they first accessed the Internet before they set foot in a classroom (i.e. at the age of 6 or earlier), and around $40 \%$ of students reported that they were between 7 and 9 years old when they first used the Internet. On average, boys were 4 percentage points more likely than girls to have used the Internet before the age of 6 (Table D8.1, Chart D8.1 and OECD [2015b]).

PISA 2012 asked students how much time they spend using the computer in classroom lessons during a typical school day. Interpretation of ICT use in classroom lessons, measured by minutes and hours, is one way researchers can determine the extent to which ICT is included in classroom activities. On average across OECD countries, only $17 \%$ of students reported that they spend one hour or more using the Internet at school during a school day, while more than $36 \%$ reported that they do not spend any time at school using the Internet (Table D8.1).

According to their own reports, students in OECD countries spend an average of 25 minutes per day on line at school. In Australia, students spend an average of 58 minutes per day on line at school; in Denmark students spend an average of 46 minutes per day on line at school; in Greece they spend 42 minutes per day on line at school; and in Sweden students spend an average of 39 minutes per day on line at school. By contrast, in Germany, Italy, Japan, Jordan, Korea, Macao (China), Poland, Shanghai (China), Singapore, Turkey and Uruguay, at least 50\% of students reported that they spend no time at school using the Internet (Table D8.1). However, the association between the intensity of Internet use at school and PISA performance in reading is not linear. Thus, while PISA results suggest that limited use of computers at school may be better than not using computers at all, using computers more intensively than the OECD average tends to be associated with significantly poorer student performance. 
The use of ICT is linked to better student performance only in certain contexts, such as when computer software and Internet connections help to increase study time and practice (OECD [2015b]).

Although computers are becoming familiar pieces of hardware in many classrooms, most 15-year-olds who use computers regularly do so outside of school, on weekends, during their leisure time, and generally not for school work. On average across OECD countries, boys reported using the Internet for 144 minutes and girls for 130 minutes on typical weekdays. Perhaps surprisingly, boys also reported using the Internet at school more than girls: in 26 countries, boys spend more time using the Internet at school on a typical weekday than girls do (OECD [2015b]).

\section{Gender differences in digital- and print-reading performance}

But being familiar with smartphones and computers does not necessarily mean that a student can use those devices competently or know how to critically assess the information he or she collects through them. The learning outcomes that are associated with digital technologies depend, to a great extent, on how - and how frequently - students use them.

PISA 2012 evaluated not only how skilled 15-year-olds are in gathering and processing information that they acquire when reading printed texts, but also how proficient they are in reading digital material. PISA found that some countries have been far more successful than others in helping students to equip themselves to participate fully in the digital age. For example, 15-year-old boys and girls in Australia, Brazil, Korea, Singapore, Sweden and the United States perform better in digital reading than in print reading, while the opposite is observed in Germany, Hungary, Israel, Poland, Shanghai (China), Spain and the United Arab Emirates. Among those countries where students perform better in digital reading, Korea recently developed a "Smart Education" policy that includes building or improving school infrastructure so that it accommodates new technologies, and training teachers in the use of these technologies (Table D8.3 and Chart D8.3).

The assessment also revealed some interesting differences between girls and boys in their skills in the digital domain. On average, 15-year-old boys score 4 points higher on the computer-based PISA reading test than on the paper-based reading test. By contrast, 15-year-old girls perform 8 points lower in digital reading than in paperbased reading test, on average. As a result, while girls outperform boys in both print and digital reading, the gender gap tends to be narrower in digital reading. On average among the countries that took part in both assessments, girls outperformed boys by 38 points - the equivalent of one year of formal schooling - in print reading, but by only 26 points in digital reading. There is still a marked difference in performance in favour of girls in digital reading, but it is less extreme than the disparity between boys' and girls' performance in print reading. In all participating countries the gender gap in performance was wider in print than in digital reading, and the difference exceeds 15 score points in France, Israel, Italy, Korea, Macao (China), Portugal, the Russian Federation, the Slovak Republic, Slovenia, Sweden, and Chinese Taipei (Table D8.3 and Chart D8.3).

The variations in the size of the gender gap among countries do not seem to be associated with the absolute levels of performance. For example, among countries performing below the OECD average in digital and print reading, Austria has a substantially narrower gap between boys and girls in digital reading (27 points) than in print reading (37 points), while the gaps between Spanish boys and girls in digital (24 points) and print (29 points) reading are almost the same. Of the 32 countries that participated in the digital reading assessment in 2012, those with the widest gender gaps in digital reading, namely Estonia, Hungary, Norway, Poland, Slovenia, Sweden and the United Arab Emirates, tend to have a comparatively wide gender gap in print reading as well. In these countries, whatever factors might explain the performance differences between boys and girls in the digital medium seem to be the same, or at least have a similar effect, as those that account for performance differences in the print medium (Table D8.3 and Chart D8.3).

Results from the PISA report, The ABC of Gender Equality in Education: Aptitude, Behaviour, Confidence (OECD, 2015b), suggest that boys tend to do better in reading when they take a computer-based test largely because of their greater familiarity computers, which, in turn, is linked to the greater amount of time they spend playing video games. The more frequently students play one-player video games and collaborative online games, which boys tend to play more than girls, the worse their relative performance on paper-based tests. Frequent video gaming appears to "crowd out" other activities, such as doing homework regularly, that help students to acquire reading and mathematics skills. In computer-based tests, the negative effects of video gaming may be counterbalanced by its positive effects on students' ability to navigate through digital texts. And students who frequently play video games will, necessarily, be more at ease - and may even prefer - taking a test using a computer. 


\title{
Chart D8.3. Mean score-point difference between paper-and-pencil and computer-delivered reading test ${ }^{1}$ (PISA 2012)
}

\author{
15-year-old students, by gender
}

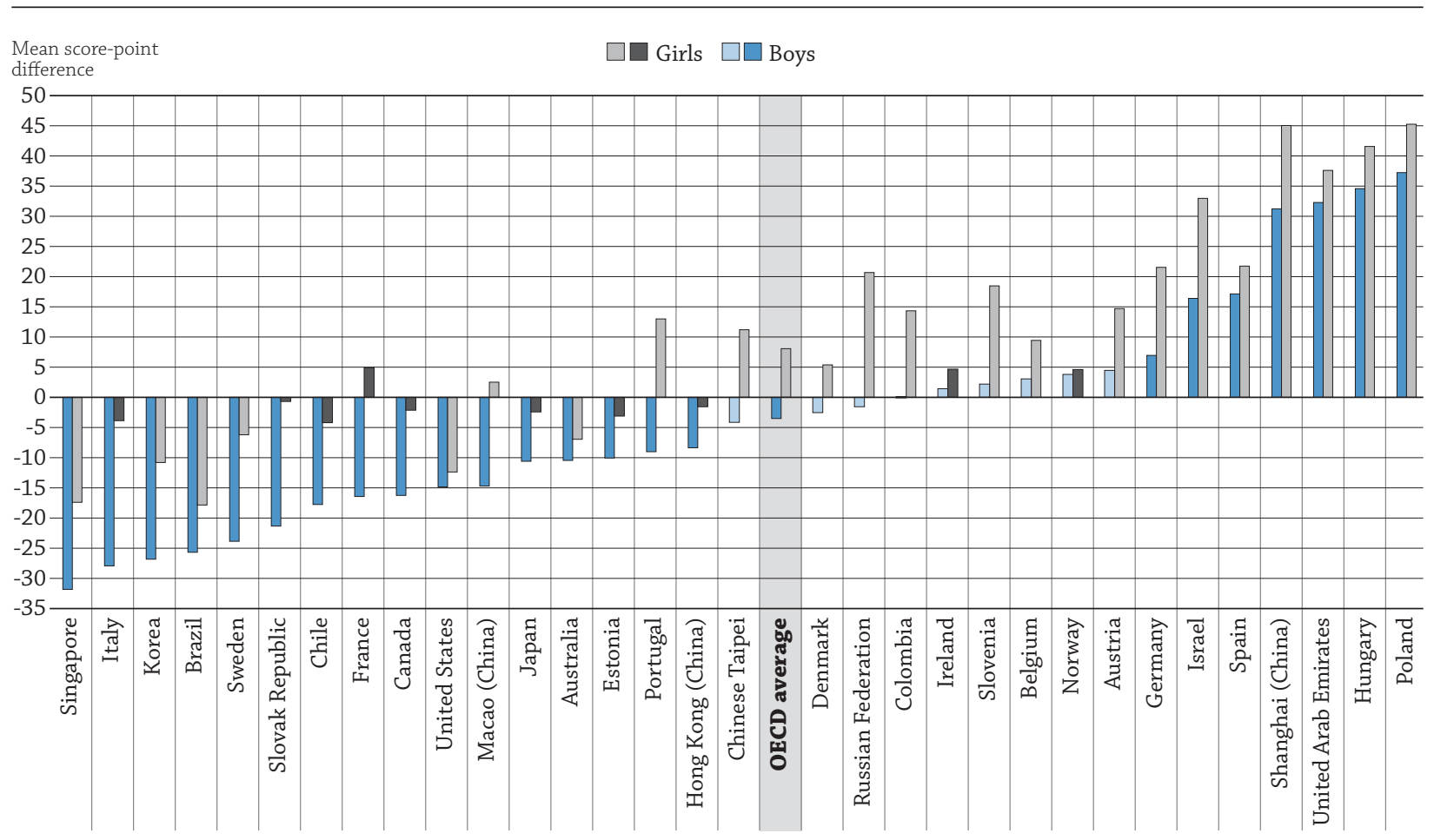

Note: Differences that are statistically significant are marked in grey and dark blue.

1. Negative figures mean that 15 -year-old students have obtained better performances on computer-delivered reading test.

Countries and economies are ranked in ascending order of the mean score-point difference between paper-and-pencil and computer-delivered reading test of boys.

Source: OECD. Table D8.3. See Annex 3 for notes (www.oecd.org/education/education-at-a-glance-19991487.htm).

StatLink 제그 http://dx.doi.org/10.1787/888933284709

\section{Teachers and ICT}

Teaching practices deployed by teachers can play a significant role in the degree to which students learn. The OECD Teaching and Learning International Survey (TALIS) conducted in 2013 asked lower secondary school teachers to choose a particular class from their teaching schedule and then respond to a series of questions about the frequency with which they used a number of teaching practices in this class. Of the eight practices examined, the two that teachers reported using most frequently, on average across countries, are presenting a summary of recently learned content and checking students' exercise books or homework (around 80\% of teachers, on average, reported using these practices). (See Table 6.1 in the TALIS survey [OECD, 2014a]).

In contrast, $40 \%$ of lower secondary teachers reported that students use ICT for projects or class work "frequently" or "in all or nearly all lessons". However, this average masks large disparities among countries. For example, in Australia, Chile, Denmark, Mexico, New Zealand, Norway and Abu Dhabi (the United Arab Emirates), more than one in two teachers reported that students use ICT "frequently" or "in all or nearly all lessons", while fewer than one in four teachers in Croatia, Finland, France, Israel, Japan, Malaysia, Serbia and Shanghai (China) reported so (Table D8.4 and Chart D8.4).

Despite an increasing number of new initiatives to develop ICT skills for teaching and greater investments in new technologies (OECD [2015c]), these figures show that teachers are still not systematically using these tools in their teaching. This may be because, among other things, teachers feel they are not sufficiently skilled in using ICT themselves. The PISA study showed that, among all teachers, those who are more inclined and better prepared for student-oriented teaching practices, such as group work, individualised learning and project work, are more likely to use digital resources, according to students' reports (OECD [2015b]). In addition, when asked to rank their professional development needs, teachers across all countries and economies that participated in TALIS in 2013 cited teaching students with special needs first, followed by teaching with ICT (18\% of teachers, on average) and using new technologies in the workplace ( $16 \%$ of teachers, on average). Even larger proportions 
of teachers cited the need for professional development in teaching with ICT and using new technologies in the workplace in Brazil (27\% and 37\%, respectively), Georgia (31\% and 39\%, respectively), Italy (36\% and 32\%, respectively) and Malaysia (38\% and 31\%, respectively) (Table D8.4).

Providing further support, either through professional development or initial teacher training, to encourage teachers to use ICT tools in their teaching should be a priority. In addition, teachers should be encouraged and given the time to collaborate with their colleagues. TALIS finds that teachers who reported that they participate in professional development activities involving collaborative research, observation visits to other schools, or a network of teachers are more likely to have reported that they use teaching practices that involve small groups of students and ICT.

\section{Chart D8.4. Information and communications technology: Teaching practices, teachers' needs for professional development and participation in professional development activities (TALIS 2013)}

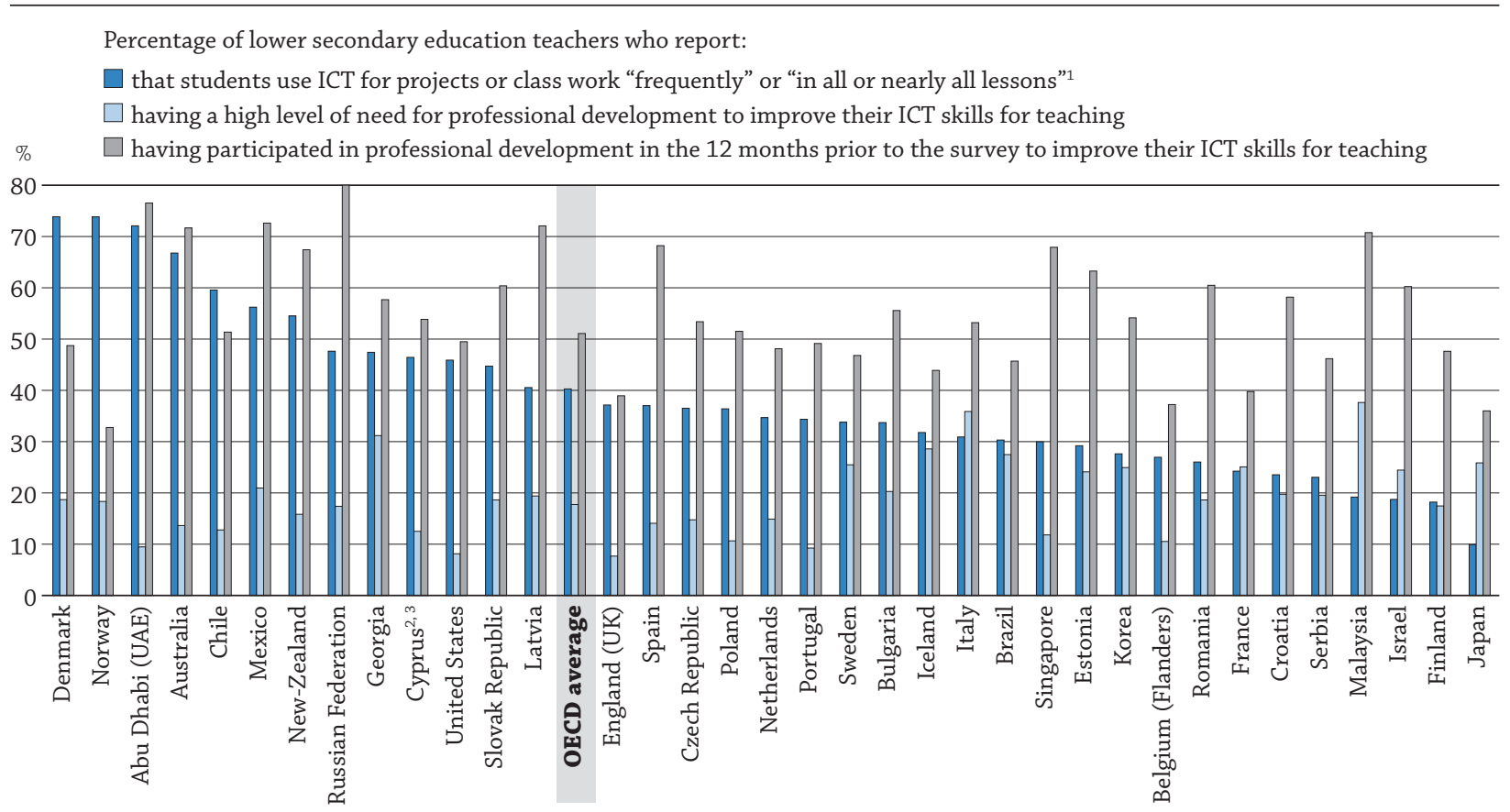

1. These data are reported by teachers and refer to a randomly chosen class they currently teach from their weekly timetable.

2. Note by Turkey: The information in this document with reference to "Cyprus" relates to the southern part of the Island. There is no single authority representing both Turkish and Greek Cypriot people on the Island. Turkey recognises the Turkish Republic of Northern Cyprus (TRNC). Until a lasting and equitable solution is found within the context of the United Nations, Turkey shall preserve its position concerning the "Cyprus issue".

3. Note by all the European Union Member States of the OECD and the European Union: The Republic of Cyprus is recognised by all members of the United Nations with the exception of Turkey. The information in this document relates to the area under the effective control of the Government of the Republic of Cyprus.

Countries and economies are ranked in descending order, based on the overall percentage of teachers who report that students use ICT for projects or class work "frequently" or "in all or nearly all lessons".

Source: OECD. Table D8.4. See Annex 3 for notes (www.oecd.org/education/education-at-a-glance-19991487.htm).

StatLink 제피 http://dx.doi.org/10.1787/888933284717

\section{Professional development}

No matter how good initial teacher education is, it cannot be expected to prepare teachers for all the challenges they will face during their first job as a teacher. Therefore, professional development, at all points in a teacher's career, is necessary to keep up with changing research, tools, practices and students' needs.

One in two teachers reported that they had participated in at least one professional development activity to improve their ICT skills for teaching during the 12 months prior to the TALIS survey. Although the reported participation rates in professional development activities related to ICT vary widely across countries (from $33 \%$ in Norway to $81 \%$ in the Russian Federation), teachers generally indicated that their professional development activities have a moderate or large positive impact on their teaching. Thus, between $64 \%$ of teachers in England and more than $90 \%$ of teachers in Portugal, Romania and the Slovak Republic, on average, reported that professional development activities to improve their ICT skills for teaching had a positive impact (Table D8.4). 
Given what teachers reported about their professional development needs, one would expect to see strong correlations between the reported need and participation rates in relevant professional development activities. But the data reported in Table D8.4 show otherwise. In many countries, the supply of professional development activities does not match the demand. That lack of coherence could be costly if the teachers who feel they need further training do not have access to it, or if the training is not well-targeted.

For example, in Italy, 36 \% of lower secondary school teachers indicated that they have a high need for professional development to improve their ICT skills for teaching (the second highest percentage of teachers among all participating countries/economies), but an average of 53\% of lower secondary teachers reported that they had participated in professional development activities in this area in the 12 months prior to the survey. Similarly, only $12 \%$ of lower secondary teachers indicated that they have a high need for professional development in ICT in Singapore while $68 \%$ of them participated in professional development activities in this area in the 12 months prior to the survey (Table D8.4 and Chart D8.4).

\section{Methodology}

All the data published in this Indicator came from the TALIS and PISA surveys.

The number of students per school computer was derived from dividing the number of students in the modal grade for 15-year-olds by the number of computers for educational purposes available to students in the modal grade for 15-year-olds.

The index of quality of school educational resources was derived from six items measuring school principals' perceptions of potential factors hindering instruction at their school (SC14, from the PISA 2012 school questionnaire). These factors are: shortage or inadequacy of science laboratory equipment; shortage or inadequacy of instructional materials; shortage or inadequacy of computers for instruction; lack or inadequacy of Internet connectivity; shortage or inadequacy of computer software for instruction; and shortage or inadequacy of library materials. As all items were inverted for scaling, higher values on this index indicate better quality of educational resources. For trends analyses, the PISA 2003 values of the index of quality of educational resources were rescaled to be comparable to those in PISA 2012. As a result, values for the index of quality educational resources for PISA 2003 reported in this Indicator may differ from those reported in Learning for Tomorrow's World: First Results from PISA 2003. One of the questions included to compute the index of quality of educational resources in PISA 2012 ("lack or inadequacy of Internet connection") was not included in the PISA 2003 questionnaire. Estimation of the PISA 2003 index treats this question as missing and, under the assumption that the relationship between the items remains unchanged with the inclusion of the new questions, the PISA 2003 and PISA 2012 values on the index of quality of educational resources are comparable after the rescaling. For more information regarding the indices, please refer to the PISA 2012 Technical Report (OECD [2014b]).

Thirty-two countries participated in the digital reading assessment in PISA 2012. When a country participated in the digital reading assessment option, it was expected that student sampling of the digital reading assessment would occur in every school that participated in the paper-based PISA survey. The overall sample size requirement for the digital reading assessment was 1200 assessed students within each country. The recommended Target Cluster Size (TCS) for the digital reading assessment was 14 students per sampled school. While 14 students for each of 150 schools (the typical number of PISA schools) would potentially yield 2100 students, the large TCS was chosen to account for the fact that some schools would not have adequate computer resources. The TCS of 14 also accounted for the loss in the digital reading assessment sample that would accrue from prior losses in the paper-based PISA sample. It was a requirement that all students who participated in the digital reading assessment also took part in the paper-based PISA assessment. The student sample for the digital reading assessment was selected at the same time that the paper-based PISA student sample was selected in each school by a student-sampling software. Therefore, any student sampled for both assessments who did not provide responses to the paper-based PISA assessment was an automatic loss to the digital reading assessment.

\section{Note regarding data from Israel}

The statistical data for Israel are supplied by and are under the responsibility of the relevant Israeli authorities. The use of such data by the OECD is without prejudice to the status of the Golan Heights, East Jerusalem and Israeli settlements in the West Bank under the terms of international law. 


\section{References}

OECD (2015a), Students, Computers and Learning: Making the Connection, PISA, OECD Publishing, Paris, http://dx.doi.org/10.1787/ 9789264239555-en.

OECD (2015b), The ABC of Gender Equality in Education: Aptitude, Behaviour, Confidence, PISA, OECD Publishing, Paris, http:// dx.doi.org/10.1787/9789264229945-en.

OECD (2015c), Education Policy Outlook 2015: Making Reforms Happen, OECD Publishing, Paris, http://dx.doi.org/10.1787/ 9789264225442-en.

D8 OECD (2014a), TALIS 2013 Results: An International Perspective on Teaching and Learning, OECD Publishing, Paris, http://dx.doi. org/10.1787/9789264196261-en.

OECD (2014b), PISA 2012 Technical Report, PISA, OECD, Paris, www.oecd.org/pisa/pisaproducts/pisa2012technicalreport.htm. OECD (2013), PISA 2012 Results: What Makes Schools Successful? (Volume IV): Resources, Policies and Practices, PISA, OECD Publishing, Paris, http://dx.doi.org/10.1787/9789264201156-en.

\section{Indicator D8 Tables}

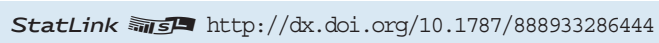

Table D8.1 Computer availability, first access to Internet and intensity of Internet use at school (PISA 2012)

Table D8.2 Change between 2003 and 2012 in the quality of schools' educational resources

Table D8.3 PISA score in reading for 15-year-olds and mean score-point difference between paper-and-pencil and computer-delivered reading test, by gender (PISA 2012)

Table D8.4 Teachers and information and communication technology 
Table D8.1. [1/2] Computer availability, first access to Internet and intensity of Internet use at school (PISA 2012)

\begin{tabular}{|c|c|c|c|c|c|c|c|c|c|c|c|c|c|c|}
\hline & \multicolumn{4}{|c|}{$\begin{array}{l}\text { Ratio of } 15 \text {-year-old students } \\
\text { to computers available to them } \\
\text { (results based on principals' reports) }\end{array}$} & \multicolumn{10}{|c|}{$\begin{array}{l}\text { How old were 15-year-old students when they first accessed the Internet? } \\
\text { (results based on students' self-reports) }\end{array}$} \\
\hline & \multicolumn{2}{|c|}{2009} & \multicolumn{2}{|c|}{2012} & \multicolumn{2}{|c|}{$\begin{array}{l}6 \text { years old } \\
\text { or younger }\end{array}$} & \multicolumn{2}{|c|}{$7-9$ years old } & \multicolumn{2}{|c|}{$10-12$ years old } & \multicolumn{2}{|c|}{$\begin{array}{l}13 \text { years old } \\
\text { or older }\end{array}$} & \multicolumn{2}{|c|}{$\begin{array}{l}\text { Never accessed } \\
\text { the Internet }\end{array}$} \\
\hline & Mean & S.E. & Mean & S.E. & $\%$ & S.E. & $\%$ & S.E. & $\%$ & S.E. & $\%$ & S.E. & $\%$ & S.E. \\
\hline & (1) & (2) & (3) & (4) & (5) & (6) & (7) & (8) & (9) & (10) & (11) & (12) & (13) & (14) \\
\hline Australia & 2.4 & (1.1) & 0.9 & $(0.0)$ & 19.3 & $(0.4)$ & 48.1 & $(0.4)$ & 27.4 & $(0.4)$ & 5.1 & $(0.2)$ & 0.1 & $(0.0)$ \\
\hline Щّ Austria & 2.4 & $(0.4)$ & 2.9 & $(0.5)$ & 8.0 & $(0.4)$ & 37.8 & $(0.7)$ & 43.9 & $(0.7)$ & 10.2 & $(0.6)$ & 0.2 & $(0.1)$ \\
\hline Belgium & 3.0 & $(0.2)$ & 2.8 & $(0.3)$ & 14.5 & $(0.5)$ & 41.1 & $(0.6)$ & 37.7 & $(0.7)$ & 6.4 & $(0.3)$ & 0.3 & $(0.1)$ \\
\hline Canada & 2.0 & $(0.1)$ & 2.8 & (1.0) & $\mathrm{m}$ & $\mathrm{m}$ & $\mathrm{m}$ & $\mathrm{m}$ & $\mathrm{m}$ & $\mathrm{m}$ & $\mathrm{m}$ & $\mathrm{m}$ & $\mathrm{m}$ & $\mathrm{m}$ \\
\hline Chile & 6.2 & $(0.5)$ & 4.7 & $(0.9)$ & 12.2 & $(0.4)$ & 37.4 & $(0.8)$ & 37.2 & $(0.9)$ & 12.9 & $(0.6)$ & 0.4 & $(0.1)$ \\
\hline Czech Republic & 2.2 & $(0.1)$ & 1.6 & (0.1) & 7.0 & $(0.4)$ & 41.9 & $(0.9)$ & 42.7 & (1.0) & 8.3 & $(0.5)$ & 0.1 & $(0.1)$ \\
\hline Denmark & 1.8 & $(0.1)$ & 2.4 & $(0.3)$ & 32.6 & $(0.8)$ & 51.5 & $(0.7)$ & 14.8 & $(0.6)$ & 0.9 & $(0.1)$ & 0.1 & $(0.0)$ \\
\hline Estonia & 2.5 & $(0.1)$ & 2.1 & $(0.1)$ & 24.1 & $(0.8)$ & 56.1 & $(0.9)$ & 17.2 & $(0.7)$ & 2.4 & $(0.3)$ & 0.2 & $(0.1)$ \\
\hline Finland & 3.0 & $(0.1)$ & 3.1 & $(0.1)$ & 20.9 & $(0.6)$ & 60.2 & $(0.6)$ & 17.8 & $(0.5)$ & 1.1 & $(0.2)$ & 0.0 & c \\
\hline France & $\mathrm{m}$ & $\mathrm{m}$ & 2.9 & $(0.2)$ & $\mathrm{m}$ & $\mathrm{m}$ & $\mathrm{m}$ & $\mathrm{m}$ & $\mathrm{m}$ & $\mathrm{m}$ & $\mathrm{m}$ & $\mathrm{m}$ & $\mathrm{m}$ & $\mathrm{m}$ \\
\hline Germany & 3.0 & $(0.2)$ & 4.2 & (1.3) & 7.0 & $(0.4)$ & 35.2 & $(0.8)$ & 46.4 & $(0.7)$ & 11.2 & $(0.5)$ & 0.2 & $(0.1)$ \\
\hline Greece & 7.8 & $(1.2)$ & 8.2 & (1.1) & 5.1 & $(0.3)$ & 22.7 & $(0.6)$ & 41.8 & $(0.7)$ & 29.6 & $(0.7)$ & 0.8 & $(0.1)$ \\
\hline Hungary & 2.4 & $(0.1)$ & 2.2 & $(0.1)$ & 8.9 & $(0.5)$ & 38.4 & (1.0) & 42.9 & $(0.9)$ & 9.7 & $(0.6)$ & 0.1 & $(0.1)$ \\
\hline Iceland & 2.2 & $(0.0)$ & 4.1 & $(0.0)$ & 22.9 & $(0.7)$ & 52.5 & $(0.8)$ & 21.6 & $(0.7)$ & 2.8 & $(0.3)$ & 0.3 & $(0.1)$ \\
\hline Ireland & 3.6 & $(1.2)$ & 2.6 & $(0.2)$ & 6.6 & $(0.4)$ & 31.9 & $(0.8)$ & 47.5 & $(0.8)$ & 13.7 & $(0.5)$ & 0.2 & $(0.1)$ \\
\hline Israel & 5.2 & $(0.6)$ & 4.7 & $(0.6)$ & 27.9 & $(0.8)$ & 44.7 & $(0.8)$ & 20.7 & $(0.7)$ & 5.7 & $(0.5)$ & 0.9 & $(0.2)$ \\
\hline Italy & 3.7 & $(0.1)$ & 4.1 & $(0.5)$ & 7.0 & $(0.2)$ & 27.3 & $(0.4)$ & 46.7 & $(0.5)$ & 18.6 & $(0.4)$ & 0.4 & $(0.1)$ \\
\hline Japan & 3.7 & $(0.1)$ & 3.6 & $(0.1)$ & 6.5 & $(0.4)$ & 38.8 & $(0.6)$ & 43.8 & $(0.7)$ & 10.3 & $(0.5)$ & 0.7 & $(0.1)$ \\
\hline Korea & 4.6 & $(0.2)$ & 5.3 & $(0.2)$ & 11.8 & $(0.5)$ & 58.3 & $(0.7)$ & 26.5 & $(0.8)$ & 3.3 & $(0.3)$ & 0.1 & $(0.0)$ \\
\hline Luxembourg & 12.1 & $(0.0)$ & 2.2 & $(0.0)$ & $\mathrm{m}$ & $\mathrm{m}$ & $\mathrm{m}$ & $\mathrm{m}$ & $\mathrm{m}$ & $\mathrm{m}$ & $\mathrm{m}$ & $\mathrm{m}$ & $\mathrm{m}$ & $\mathrm{m}$ \\
\hline Mexico & 20.8 & (7.6) & 15.5 & (2.0) & 6.1 & $(0.3)$ & 26.9 & $(0.5)$ & 37.5 & $(0.4)$ & 24.4 & $(0.5)$ & 5.1 & $(0.4)$ \\
\hline Netherlands & 2.6 & $(0.2)$ & 2.6 & $(0.2)$ & 33.3 & $(0.9)$ & 50.5 & $(0.9)$ & 15.3 & $(0.6)$ & 0.8 & $(0.1)$ & 0.1 & $(0.0)$ \\
\hline New Zealand & 1.4 & $(0.0)$ & 1.2 & $(0.1)$ & 23.7 & $(0.8)$ & 48.3 & $(0.9)$ & 23.7 & $(0.7)$ & 4.0 & $(0.3)$ & 0.3 & $(0.1)$ \\
\hline Norway & 1.8 & $(0.1)$ & 1.7 & $(0.1)$ & 26.1 & $(0.7)$ & 50.9 & $(0.9)$ & 20.9 & $(0.6)$ & 1.9 & $(0.2)$ & 0.3 & $(0.1)$ \\
\hline Poland & 4.9 & $(0.2)$ & 4.0 & $(0.1)$ & 8.3 & $(0.5)$ & 37.2 & $(0.8)$ & 41.6 & $(0.9)$ & 12.3 & $(0.6)$ & 0.7 & $(0.1)$ \\
\hline Portugal & 2.2 & $(0.1)$ & 3.7 & $(0.3)$ & 11.0 & $(0.6)$ & 39.7 & $(0.7)$ & 39.5 & $(0.8)$ & 9.7 & $(0.5)$ & 0.1 & $(0.0)$ \\
\hline Slovak Republic & 3.1 & $(0.3)$ & 2.0 & $(0.2)$ & 4.8 & $(0.4)$ & 26.3 & $(0.8)$ & 46.7 & $(0.9)$ & 21.5 & $(0.8)$ & 0.8 & $(0.2)$ \\
\hline Slovenia & 4.8 & $(0.0)$ & 3.3 & $(0.0)$ & 12.5 & $(0.5)$ & 48.4 & $(0.8)$ & 32.5 & $(0.7)$ & 6.1 & $(0.4)$ & 0.5 & $(0.1)$ \\
\hline Spain & 2.2 & $(0.1)$ & 2.2 & $(0.1)$ & 14.8 & $(0.4)$ & 45.3 & $(0.6)$ & 34.0 & $(0.5)$ & 5.8 & $(0.3)$ & 0.2 & $(0.1)$ \\
\hline Sweden & 3.6 & $(0.2)$ & 3.7 & $(0.8)$ & 25.0 & $(0.7)$ & 53.7 & $(0.8)$ & 18.7 & $(0.6)$ & 2.1 & $(0.2)$ & 0.5 & $(0.1)$ \\
\hline Switzerland & 2.7 & $(0.1)$ & 2.7 & $(0.2)$ & 8.8 & $(0.4)$ & 38.4 & $(0.7)$ & 43.0 & $(0.7)$ & 9.6 & $(0.4)$ & 0.3 & $(0.1)$ \\
\hline Turkey & 12.1 & $(2.0)$ & 44.9 & (9.7) & 5.3 & $(0.4)$ & 32.7 & $(0.8)$ & 40.2 & $(0.8)$ & 19.7 & $(0.7)$ & 2.0 & $(0.3)$ \\
\hline United Kingdom & 1.4 & $(0.1)$ & 1.4 & $(0.1)$ & $\mathrm{m}$ & $\mathrm{m}$ & $\mathrm{m}$ & $\mathrm{m}$ & $\mathrm{m}$ & $\mathrm{m}$ & $\mathrm{m}$ & $\mathrm{m}$ & $\mathrm{m}$ & $\mathrm{m}$ \\
\hline United States & 2.5 & $(0.4)$ & 1.8 & $(0.2)$ & $\mathrm{m}$ & $\mathrm{m}$ & $\mathrm{m}$ & $\mathrm{m}$ & $\mathrm{m}$ & $\mathrm{m}$ & $\mathrm{m}$ & $\mathrm{m}$ & $\mathrm{m}$ & $\mathrm{m}$ \\
\hline OECD average & 4.2 & $(0.2)$ & 4.7 & $(0.3)$ & 14.6 & $(0.1)$ & 42.1 & $(0.1)$ & 33.5 & $(0.1)$ & 9.3 & $(0.1)$ & 0.5 & $(0.0)$ \\
\hline Brazil & 34.0 & $(4.6)$ & 22.1 & (2.7) & $\mathrm{m}$ & $\mathrm{m}$ & $\mathrm{m}$ & $\mathrm{m}$ & $\mathrm{m}$ & $\mathrm{m}$ & $\mathrm{m}$ & $\mathrm{m}$ & $\mathrm{m}$ & $\mathrm{m}$ \\
\hline Colombia & 6.1 & $(0.9)$ & 3.7 & $(0.2)$ & $\mathrm{m}$ & $\mathrm{m}$ & $\mathrm{m}$ & $\mathrm{m}$ & $\mathrm{m}$ & $\mathrm{m}$ & $\mathrm{m}$ & $\mathrm{m}$ & $\mathrm{m}$ & $\mathrm{m}$ \\
\hline ¿ Costa Rica & 19.5 & (3.3) & 17.7 & (3.1) & 9.1 & $(0.5)$ & 28.6 & $(0.9)$ & 38.9 & $(0.8)$ & 22.0 & (1.1) & 1.4 & $(0.3)$ \\
\hline Croatia & 4.2 & $(0.2)$ & 5.0 & $(0.2)$ & 7.2 & $(0.4)$ & 34.7 & $(0.8)$ & 44.0 & $(0.8)$ & 13.9 & $(0.6)$ & 0.2 & $(0.1)$ \\
\hline Hong Kong (China) & 1.9 & $(0.1)$ & 2.2 & $(0.3)$ & 24.5 & $(1.0)$ & 51.0 & $(1.0)$ & 21.6 & $(0.7)$ & 2.3 & $(0.3)$ & 0.5 & $(0.1)$ \\
\hline Indonesia & 22.8 & $(2.8)$ & 16.4 & (2.2) & $\mathrm{m}$ & $\mathrm{m}$ & $\mathrm{m}$ & $\mathrm{m}$ & $\mathrm{m}$ & $\mathrm{m}$ & $\mathrm{m}$ & $\mathrm{m}$ & $\mathrm{m}$ & $\mathrm{m}$ \\
\hline Jordan & 4.3 & $(0.3)$ & 5.0 & $(0.5)$ & 8.9 & $(0.5)$ & 17.6 & $(0.6)$ & 31.2 & $(0.7)$ & 34.9 & $(0.7)$ & 7.3 & $(0.5)$ \\
\hline Latvia & 2.1 & $(0.1)$ & 2.2 & $(0.6)$ & 12.1 & $(0.7)$ & 49.3 & (1.0) & 32.3 & (1.0) & 6.0 & $(0.4)$ & 0.2 & $(0.1)$ \\
\hline Liechtenstein & 2.4 & $(0.0)$ & 2.1 & $(0.0)$ & 13.6 & $(2.1)$ & 39.6 & (3.0) & 38.6 & (2.9) & 8.3 & (1.5) & 0.0 & c \\
\hline Macao (China) & 2.5 & $(0.0)$ & 1.3 & $(0.0)$ & 10.6 & $(0.4)$ & 42.3 & $(0.7)$ & 38.8 & $(0.7)$ & 7.9 & $(0.3)$ & 0.4 & $(0.1)$ \\
\hline Russian Federation & 4.0 & $(0.6)$ & 3.0 & $(0.1)$ & 2.3 & $(0.2)$ & 14.8 & $(0.6)$ & 39.0 & $(0.9)$ & 42.7 & $(1.2)$ & 1.3 & $(0.2)$ \\
\hline Serbia & 7.1 & $(0.9)$ & 8.8 & (2.4) & 3.2 & $(0.3)$ & 19.8 & $(0.7)$ & 44.1 & $(0.8)$ & 31.9 & $(0.8)$ & 1.0 & $(0.1)$ \\
\hline Shanghai (China) & 4.8 & $(2.2)$ & 2.9 & $(0.2)$ & 8.3 & $(0.5)$ & 35.0 & $(0.9)$ & 38.4 & $(0.6)$ & 16.5 & $(0.8)$ & 1.8 & $(0.3)$ \\
\hline Singapore & 2.0 & $(0.0)$ & 2.0 & $(0.0)$ & 16.3 & $(0.5)$ & 45.7 & $(0.6)$ & 32.3 & $(0.7)$ & 5.7 & $(0.3)$ & 0.1 & $(0.0)$ \\
\hline Chinese Taipei & 5.0 & $(0.3)$ & 5.8 & (1.1) & 11.2 & $(0.5)$ & 43.0 & $(0.8)$ & 39.8 & $(0.7)$ & 5.8 & $(0.4)$ & 0.1 & $(0.0)$ \\
\hline Uruguay & 13.1 & (1.8) & 8.7 & $(0.6)$ & 10.5 & $(0.5)$ & 37.1 & $(0.8)$ & 38.4 & $(0.7)$ & 13.5 & $(0.6)$ & 0.5 & $(0.1)$ \\
\hline G20 average & $\mathrm{m}$ & $\mathrm{m}$ & $\mathrm{m}$ & $\mathrm{m}$ & $\mathrm{m}$ & $\mathrm{m}$ & $\mathrm{m}$ & $\mathrm{m}$ & $\mathrm{m}$ & $\mathrm{m}$ & $\mathrm{m}$ & $\mathrm{m}$ & $\mathrm{m}$ & $\mathrm{m}$ \\
\hline
\end{tabular}

1. The number of students per computer is based on principals' reports about the number of students in the national modal grade for 15-year-olds and on the number of computers available for these students. In schools where no computer is available, the number of students per computer is set equal to $1+$ the number of students reported by the principal.

Source: OECD. PISA 2012 Database.

Please refer to the Reader's Guide for information concerning symbols for missing data and abbreviations.

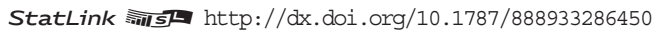


Table D8.1. [2/2] Computer availability, first access to Internet and intensity of Internet use at school (PISA 2012)

\begin{tabular}{|c|c|c|c|c|c|c|c|c|c|c|c|c|c|c|}
\hline & \multicolumn{14}{|c|}{ During a typical weekday, for how long do 15-year-old students use the Internet at school? (results based on students' self-reports) } \\
\hline & \multicolumn{2}{|c|}{ No time } & \multicolumn{2}{|c|}{ 1-60 minutes } & \multicolumn{2}{|c|}{$\begin{array}{c}\text { Between } \\
1 \text { and } 2 \text { hours }\end{array}$} & \multicolumn{2}{|c|}{$\begin{array}{c}\text { Between } \\
2 \text { and } 4 \text { hours }\end{array}$} & \multicolumn{2}{|c|}{$\begin{array}{c}\text { Between } \\
4 \text { and } 6 \text { hours }\end{array}$} & \multicolumn{2}{|c|}{$\begin{array}{l}\text { More than } \\
6 \text { hours }\end{array}$} & \multicolumn{2}{|c|}{$\begin{array}{l}\text { Average daily time } \\
\text { spent using the } \\
\text { Internet at school } \\
\text { (lower bound) }\end{array}$} \\
\hline & $\%$ & S.E. & $\%$ & S.E. & $\%$ & S.E. & $\%$ & S.E. & $\%$ & S.E. & $\%$ & S.E. & Minutes & S.E. \\
\hline & (15) & $(16)$ & (17) & $(18)$ & (19) & $(20)$ & (21) & (22) & (23) & (24) & $(25)$ & $(26)$ & (27) & $(28)$ \\
\hline Australia & 6.7 & $(0.3)$ & 48.6 & $(0.7)$ & 23.4 & $(0.6)$ & 14.7 & $(0.5)$ & 4.9 & $(0.3)$ & 1.7 & $(0.1)$ & 58 & (1.1) \\
\hline Austria & 25.3 & (1.0) & 53.0 & (1.0) & 13.3 & $(0.6)$ & 5.0 & $(0.4)$ & 2.0 & $(0.3)$ & 1.3 & $(0.2)$ & 29 & (1.3) \\
\hline Belgium & 47.8 & $(0.9)$ & 36.8 & $(0.7)$ & 8.6 & $(0.3)$ & 4.4 & $(0.3)$ & 1.3 & $(0.1)$ & 1.1 & $(0.1)$ & 22 & $(0.8)$ \\
\hline Canada & $\mathrm{m}$ & $\mathrm{m}$ & $\mathrm{m}$ & $\mathrm{m}$ & $\mathrm{m}$ & $\mathrm{m}$ & $\mathrm{m}$ & $\mathrm{m}$ & $\mathrm{m}$ & $\mathrm{m}$ & $\mathrm{m}$ & $\mathrm{m}$ & $\mathrm{m}$ & $\mathrm{m}$ \\
\hline Chile & 40.1 & (1.4) & 38.6 & (1.1) & 12.6 & $(0.8)$ & 4.8 & $(0.3)$ & 1.8 & $(0.2)$ & 2.1 & $(0.2)$ & 30 & (1.1) \\
\hline Czech Republic & 36.3 & (1.3) & 51.6 & (1.2) & 7.4 & $(0.6)$ & 2.5 & $(0.3)$ & 1.2 & $(0.2)$ & 1.0 & $(0.2)$ & 18 & (1.0) \\
\hline Denmark & 6.7 & $(0.4)$ & 61.4 & $(1.2)$ & 16.2 & $(0.6)$ & 9.7 & $(0.8)$ & 4.3 & $(0.4)$ & 1.7 & $(0.3)$ & 46 & (2.1) \\
\hline Estonia & 34.0 & (1.0) & 52.2 & (1.0) & 6.3 & $(0.4)$ & 4.3 & $(0.3)$ & 1.8 & $(0.2)$ & 1.4 & $(0.2)$ & 23 & $(1.0)$ \\
\hline Finland & 32.8 & $(0.9)$ & 55.7 & $(0.9)$ & 6.9 & $(0.4)$ & 2.8 & $(0.3)$ & 1.0 & $(0.2)$ & 0.7 & $(0.1)$ & 18 & $(0.8)$ \\
\hline France & $\mathrm{m}$ & $\mathrm{m}$ & $\mathrm{m}$ & $\mathrm{m}$ & $\mathrm{m}$ & $\mathrm{m}$ & $\mathrm{m}$ & $\mathrm{m}$ & $\mathrm{m}$ & $\mathrm{m}$ & $\mathrm{m}$ & $\mathrm{m}$ & $\mathrm{m}$ & $\mathrm{m}$ \\
\hline Germany & 51.4 & (1.1) & 40.2 & (1.1) & 4.5 & $(0.4)$ & 2.2 & $(0.2)$ & 0.8 & $(0.2)$ & 0.9 & $(0.2)$ & 14 & $(0.9)$ \\
\hline Greece & 36.5 & (1.3) & 35.4 & (1.1) & 13.2 & $(0.6)$ & 8.2 & $(0.7)$ & 3.4 & $(0.3)$ & 3.2 & $(0.3)$ & 42 & (1.6) \\
\hline Hungary & 34.5 & (1.2) & 47.1 & (1.2) & 9.3 & $(0.5)$ & 4.7 & $(0.4)$ & 2.2 & $(0.2)$ & 2.2 & $(0.2)$ & 30 & (1.3) \\
\hline Iceland & 35.7 & $(0.8)$ & 52.7 & $(0.8)$ & 4.7 & $(0.4)$ & 3.7 & $(0.4)$ & 1.8 & $(0.2)$ & 1.4 & $(0.2)$ & 20 & $(1.0)$ \\
\hline Ireland & 45.5 & $(1.3)$ & 44.4 & (1.2) & 6.1 & $(0.4)$ & 2.6 & (0.3) & 0.7 & (0.1) & 0.7 & $(0.1)$ & 16 & $(0.7)$ \\
\hline Israel & 45.6 & (1.2) & 39.2 & (1.2) & 7.2 & $(0.4)$ & 3.6 & (0.3) & 1.8 & $(0.2)$ & 2.5 & $(0.3)$ & 25 & (1.5) \\
\hline Italy & 56.9 & $(0.7)$ & 29.0 & $(0.6)$ & 9.2 & $(0.3)$ & 2.8 & $(0.1)$ & 1.0 & $(0.1)$ & 1.1 & $(0.1)$ & 19 & $(0.5)$ \\
\hline Japan & 62.0 & (1.2) & 30.5 & (1.2) & 5.7 & $(0.5)$ & 1.3 & $(0.2)$ & 0.3 & (0.1) & 0.3 & $(0.1)$ & 13 & $(0.5)$ \\
\hline Korea & 68.3 & (1.6) & 24.7 & (1.5) & 4.4 & $(0.4)$ & 2.3 & (0.3) & 0.2 & (0.1) & 0.1 & $(0.0)$ & 9 & $(0.6)$ \\
\hline Luxembourg & $\mathrm{m}$ & $\mathrm{m}$ & $\mathrm{m}$ & $\mathrm{m}$ & $\mathrm{m}$ & $\mathrm{m}$ & $\mathrm{m}$ & $\mathrm{m}$ & $\mathrm{m}$ & $\mathrm{m}$ & $\mathrm{m}$ & $\mathrm{m}$ & $\mathrm{m}$ & $\mathrm{m}$ \\
\hline Mexico & 42.6 & $(0.8)$ & 38.5 & $(0.6)$ & 12.1 & $(0.5)$ & 4.0 & $(0.2)$ & 1.4 & $(0.1)$ & 1.5 & $(0.1)$ & 26 & $(0.6)$ \\
\hline Netherlands & 17.8 & (1.1) & 67.3 & (1.1) & 8.2 & $(0.5)$ & 3.5 & (0.3) & 1.3 & $(0.2)$ & 1.9 & $(0.3)$ & 26 & (1.3) \\
\hline New Zealand & 21.8 & (1.0) & 62.6 & (1.0) & 9.1 & $(0.6)$ & 4.1 & $(0.4)$ & 1.3 & $(0.2)$ & 1.1 & $(0.2)$ & 25 & (1.1) \\
\hline Norway & 14.8 & (1.1) & 70.1 & (1.1) & 9.7 & $(0.7)$ & 3.3 & $(0.4)$ & 1.4 & $(0.2)$ & 0.7 & $(0.1)$ & 24 & (1.4) \\
\hline Poland & 50.2 & (1.5) & 42.8 & (1.4) & 3.7 & $(0.3)$ & 1.7 & $(0.2)$ & 0.7 & $(0.1)$ & 0.9 & $(0.2)$ & 13 & $(0.9)$ \\
\hline Portugal & 40.9 & (1.3) & 43.0 & (1.2) & 8.3 & $(0.5)$ & 3.9 & $(0.4)$ & 2.2 & $(0.3)$ & 1.5 & $(0.3)$ & 24 & (2.0) \\
\hline Slovak Republic & 25.0 & $(1.2)$ & 56.0 & $(1.2)$ & 9.9 & $(0.6)$ & 4.7 & $(0.3)$ & 1.9 & $(0.2)$ & 2.5 & $(0.2)$ & 32 & (1.3) \\
\hline Slovenia & 26.7 & $(0.8)$ & 53.3 & $(0.7)$ & 12.2 & $(0.5)$ & 4.5 & $(0.2)$ & 1.7 & $(0.2)$ & 1.7 & $(0.2)$ & 28 & $(0.9)$ \\
\hline Spain & 32.7 & (1.0) & 45.1 & $(0.8)$ & 12.0 & $(0.3)$ & 5.7 & $(0.3)$ & 2.3 & $(0.3)$ & 2.1 & $(0.3)$ & 34 & (1.4) \\
\hline Sweden & 16.3 & (1.0) & 60.1 & (1.6) & 11.2 & $(0.8)$ & 6.4 & $(0.9)$ & 3.5 & $(0.5)$ & 2.5 & $(0.4)$ & 39 & (2.9) \\
\hline Switzerland & 32.3 & (1.0) & 56.9 & (1.0) & 6.9 & $(0.4)$ & 2.6 & $(0.3)$ & 0.7 & $(0.2)$ & 0.6 & $(0.1)$ & 16 & $(0.9)$ \\
\hline Turkey & 63.4 & (1.4) & 25.7 & (1.1) & 6.3 & $(0.4)$ & 2.5 & $(0.3)$ & 1.1 & $(0.2)$ & 1.1 & $(0.2)$ & 15 & $(0.9)$ \\
\hline United Kingdom & $\mathrm{m}$ & $\mathrm{m}$ & $\mathrm{m}$ & $\mathrm{m}$ & $\mathrm{m}$ & $\mathrm{m}$ & $\mathrm{m}$ & $\mathrm{m}$ & $\mathrm{m}$ & $\mathrm{m}$ & $\mathrm{m}$ & $\mathrm{m}$ & $\mathrm{m}$ & $\mathrm{m}$ \\
\hline United States & $\mathrm{m}$ & $\mathrm{m}$ & $\mathrm{m}$ & $\mathrm{m}$ & $\mathrm{m}$ & $\mathrm{m}$ & $\mathrm{m}$ & $\mathrm{m}$ & $\mathrm{m}$ & $\mathrm{m}$ & $\mathrm{m}$ & $\mathrm{m}$ & $\mathrm{m}$ & $\mathrm{m}$ \\
\hline OECD average & 36.2 & $(0.2)$ & 47.0 & $(0.2)$ & 9.3 & $(0.1)$ & 4.4 & $(0.1)$ & 1.7 & $(0.0)$ & 1.4 & $(0.0)$ & 25 & $(0.2)$ \\
\hline
\end{tabular}

\begin{tabular}{|c|c|c|c|c|c|c|c|c|c|c|c|c|c|c|}
\hline$\frac{n}{g}$ Brazil & $\mathrm{m}$ & $\mathrm{m}$ & $\mathrm{m}$ & $\mathrm{m}$ & $\mathrm{m}$ & $\mathrm{m}$ & $\mathrm{m}$ & $\mathrm{m}$ & $\mathrm{m}$ & $\mathrm{m}$ & $\mathrm{m}$ & $\mathrm{m}$ & $\mathrm{m}$ & $\mathrm{m}$ \\
\hline Colombia & $\mathrm{m}$ & $\mathrm{m}$ & $\mathrm{m}$ & $\mathrm{m}$ & $\mathrm{m}$ & $\mathrm{m}$ & $\mathrm{m}$ & $\mathrm{m}$ & $\mathrm{m}$ & $\mathrm{m}$ & $\mathrm{m}$ & $\mathrm{m}$ & $\mathrm{m}$ & $\mathrm{m}$ \\
\hline${ }^{\pi}$ Costa Rica & 45.5 & (1.3) & 35.3 & (1.1) & 10.3 & $(0.5)$ & 4.5 & $(0.4)$ & 2.2 & $(0.2)$ & 2.2 & $(0.3)$ & 29 & (1.3) \\
\hline Croatia & 39.3 & (1.0) & 45.4 & (1.1) & 8.2 & $(0.5)$ & 3.6 & $(0.3)$ & 1.5 & $(0.2)$ & 1.9 & $(0.2)$ & 23 & (1.1) \\
\hline Hong Kong (China) & 49.6 & $(1.2)$ & 43.3 & $(1.2)$ & 4.0 & $(0.3)$ & 1.7 & $(0.2)$ & 1.0 & $(0.2)$ & 0.4 & $(0.1)$ & 11 & $(0.9)$ \\
\hline Indonesia & $\mathrm{m}$ & $\mathrm{m}$ & $\mathrm{m}$ & $\mathrm{m}$ & $\mathrm{m}$ & $\mathrm{m}$ & $\mathrm{m}$ & $\mathrm{m}$ & $\mathrm{m}$ & $\mathrm{m}$ & $\mathrm{m}$ & $\mathrm{m}$ & $\mathrm{m}$ & $\mathrm{m}$ \\
\hline Jordan & 50.1 & (1.5) & 35.5 & (1.3) & 8.2 & $(0.5)$ & 2.8 & $(0.2)$ & 1.2 & $(0.2)$ & 2.2 & $(0.2)$ & 23 & $(0.9)$ \\
\hline Latvia & 47.6 & (1.1) & 41.5 & $(0.9)$ & 5.3 & $(0.5)$ & 3.2 & $(0.3)$ & 1.5 & $(0.2)$ & 0.8 & $(0.2)$ & 17 & (1.0) \\
\hline Liechtenstein & 22.1 & (2.6) & 66.8 & (3.1) & 6.4 & (1.4) & 3.2 & (1.1) & 1.1 & $(0.6)$ & 0.3 & $(0.3)$ & 18 & (2.3) \\
\hline Macao (China) & 56.3 & $(0.6)$ & 34.4 & $(0.7)$ & 5.3 & $(0.3)$ & 2.6 & $(0.2)$ & 0.7 & $(0.1)$ & 0.7 & $(0.1)$ & 14 & $(0.5)$ \\
\hline Russian Federation & 38.5 & $(0.9)$ & 41.2 & $(1.0)$ & 8.6 & $(0.4)$ & 6.0 & (0.3) & 2.4 & $(0.2)$ & 3.3 & $(0.3)$ & 34 & (1.2) \\
\hline Serbia & 46.4 & $(1.7)$ & 40.8 & $(1.5)$ & 7.6 & $(0.5)$ & 2.6 & $(0.3)$ & 1.0 & $(0.2)$ & 1.6 & $(0.2)$ & 20 & (1.1) \\
\hline Shanghai (China) & 75.0 & $(1.2)$ & 18.0 & $(1.0)$ & 4.0 & $(0.3)$ & 1.7 & $(0.2)$ & 0.6 & $(0.1)$ & 0.7 & $(0.1)$ & 10 & $(0.8)$ \\
\hline Singapore & 52.0 & $(0.7)$ & 33.4 & $(0.6)$ & 9.2 & $(0.4)$ & 3.5 & $(0.4)$ & 1.0 & $(0.1)$ & 1.0 & $(0.2)$ & 20 & (1.0) \\
\hline Chinese Taipei & 49.0 & $(1.6)$ & 34.2 & $(1.5)$ & 10.8 & $(0.7)$ & 3.5 & $(0.3)$ & 1.4 & $(0.2)$ & 1.1 & $(0.1)$ & 23 & (1.0) \\
\hline Uruguay & 50.6 & $(1.3)$ & 30.1 & $(1.1)$ & 9.3 & (0.5) & 5.1 & $(0.4)$ & 2.4 & $(0.2)$ & 2.5 & $(0.2)$ & 30 & (1.2) \\
\hline G20 average & $\mathrm{m}$ & $\mathrm{m}$ & $\mathrm{m}$ & $\mathrm{m}$ & $\mathrm{m}$ & $\mathrm{m}$ & $\mathrm{m}$ & $\mathrm{m}$ & $\mathrm{m}$ & $\mathrm{m}$ & $\mathrm{m}$ & $\mathrm{m}$ & $\mathrm{m}$ & $\mathrm{m}$ \\
\hline
\end{tabular}

1. The number of students per computer is based on principals' reports about the number of students in the national modal grade for 15-year-olds and on the number of computers available for these students. In schools where no computer is available, the number of students per computer is set equal to $1+$ the number of students reported by the principal.

Source: OECD. PISA 2012 Database.

Please refer to the Reader's Guide for information concerning symbols for missing data and abbreviations.

StatLink 젶ㄴ http://dx.doi.org/10.1787/888933286450 
Table D8.2. [1/2] Change between 2003 and 2012 in the quality of schools' educational resources Results based on PISA school principals' reports

\begin{tabular}{|c|c|c|c|c|c|c|c|c|c|c|c|c|}
\hline & \multicolumn{12}{|c|}{ PISA 2003} \\
\hline & \multicolumn{2}{|c|}{$\begin{array}{c}\text { Index of quality } \\
\text { of schools' educational } \\
\text { resources }^{1}\end{array}$} & \multicolumn{10}{|c|}{$\begin{array}{l}\text { Percentage of students in schools whose principal reported that the school's capacity to provide instruction } \\
\text { is hindered a lot by a shortage or inadequacy of the following: }\end{array}$} \\
\hline & Mean index & S.E. & $\%$ & S.E. & $\%$ & S.E. & $\%$ & S.E. & $\%$ & S.E. & $\%$ & S.E. \\
\hline Australia & 0.27 & $(0.07)$ & 9.5 & (1.7) & 2.2 & $(0.9)$ & 13.1 & (1.8) & 0.7 & $(0.5)$ & 3.1 & $(0.9)$ \\
\hline Austria & 0.06 & $(0.08)$ & 1.4 & $(0.9)$ & 0.9 & $(0.7)$ & 11.6 & $(2.7)$ & 2.9 & (1.4) & 6.5 & (2.1) \\
\hline Belgium & -0.12 & $(0.06)$ & 8.2 & (1.9) & 11.2 & $(2.2)$ & 25.0 & $(3.0)$ & 4.0 & (1.3) & 10.5 & $(2.1)$ \\
\hline Canada & -0.34 & $(0.05)$ & 8.0 & (1.1) & 2.5 & $(0.8)$ & 14.6 & (1.5) & 4.5 & (1.1) & 10.8 & (1.3) \\
\hline Czech Republic & -0.41 & $(0.06)$ & 19.8 & $(2.0)$ & 0.6 & $(0.6)$ & 5.0 & (1.4) & 3.8 & (1.2) & 22.9 & $(3.0)$ \\
\hline Denmark & -0.32 & $(0.07)$ & 0.9 & $(0.7)$ & 1.4 & $(1.0)$ & 5.0 & (1.7) & 2.7 & (1.2) & 4.0 & (1.6) \\
\hline Finland & -0.37 & $(0.06)$ & 0.7 & $(0.7)$ & 0.0 & $(0.0)$ & 7.9 & $(2.0)$ & 0.8 & $(0.7)$ & 4.6 & (1.7) \\
\hline France & $\mathrm{m}$ & $\mathrm{m}$ & $\mathrm{m}$ & $\mathrm{m}$ & $\mathrm{m}$ & $\mathrm{m}$ & $\mathrm{m}$ & $\mathrm{m}$ & $\mathrm{m}$ & $\mathrm{m}$ & $\mathrm{m}$ & $\mathrm{m}$ \\
\hline Greece & -0.78 & $(0.13)$ & 11.0 & $(3.2)$ & 21.5 & $(5.0)$ & 10.7 & (3.9) & 23.3 & $(4.5)$ & 21.2 & $(4.2)$ \\
\hline Hungary & -0.24 & $(0.08)$ & 1.0 & $(0.6)$ & 0.0 & c & 9.4 & $(2.4)$ & 1.5 & (1.1) & 28.5 & (3.6) \\
\hline Iceland & -0.03 & $(0.00)$ & 2.2 & $(0.1)$ & 0.6 & $(0.1)$ & 7.5 & $(0.1)$ & 3.4 & $(0.0)$ & 1.9 & $(0.0)$ \\
\hline Ireland & -0.36 & $(0.08)$ & 1.3 & $(0.9)$ & 2.6 & $(0.9)$ & 50.5 & $(4.6)$ & 0.8 & $(0.8)$ & 21.7 & (3.7) \\
\hline Italy & -0.16 & $(0.07)$ & 4.1 & $(1.5)$ & 4.5 & $(1.3)$ & 10.3 & $(2.2)$ & 6.5 & (1.9) & 6.8 & (2.1) \\
\hline Japan & -0.25 & $(0.10)$ & 8.2 & $(2.3)$ & 5.5 & (1.9) & 0.0 & c & 8.9 & (2.4) & 9.6 & $(2.5)$ \\
\hline Korea & 0.38 & $(0.06)$ & 3.8 & (1.6) & 2.0 & $(1.2)$ & 2.4 & $(1.2)$ & 0.6 & $(0.7)$ & 0.6 & $(0.7)$ \\
\hline Luxembourg & -0.04 & $(0.00)$ & 13.1 & $(0.0)$ & 10.9 & $(0.0)$ & 15.3 & $(0.0)$ & 0.0 & c & 4.3 & $(0.0)$ \\
\hline Mexico & -0.69 & $(0.09)$ & 8.6 & (1.9) & 9.3 & $(2.2)$ & 20.8 & $(2.8)$ & 11.3 & $(2.1)$ & 15.4 & $(2.4)$ \\
\hline Netherlands & 0.15 & $(0.06)$ & 5.6 & $(2.1)$ & 8.0 & $(2.5)$ & 27.1 & (3.7) & 1.0 & $(0.7)$ & 2.8 & (1.9) \\
\hline New Zealand & 0.00 & $(0.06)$ & 6.2 & $(1.4)$ & 7.8 & $(1.5)$ & 8.2 & (1.6) & 2.7 & (1.4) & 5.7 & (1.8) \\
\hline Norway & -0.70 & $(0.05)$ & 3.1 & (1.3) & 0.7 & $(0.7)$ & 4.9 & (1.7) & 2.7 & (1.3) & 5.5 & (1.6) \\
\hline Poland & -1.02 & $(0.07)$ & 19.0 & (3.3) & 5.3 & $(1.8)$ & 8.5 & (2.1) & 18.4 & $(2.8)$ & 16.5 & $(2.8)$ \\
\hline Portugal & -0.35 & $(0.07)$ & 1.2 & $(0.8)$ & 5.2 & (1.9) & 5.4 & (1.9) & 1.1 & $(0.9)$ & 3.8 & (1.6) \\
\hline Slovak Republic & -1.10 & $(0.05)$ & 11.4 & (1.9) & 0.8 & $(0.6)$ & 5.1 & (1.5) & 19.9 & $(2.7)$ & 53.9 & (3.3) \\
\hline Spain & -0.41 & $(0.07)$ & 5.6 & (1.8) & 6.4 & $(2.1)$ & 16.8 & $(2.5)$ & 6.3 & (1.8) & 7.5 & (1.5) \\
\hline Sweden & -0.31 & $(0.07)$ & 8.9 & $(2.2)$ & 3.9 & $(1.4)$ & 8.2 & $(2.1)$ & 4.9 & (1.7) & 3.9 & (1.5) \\
\hline United States & 0.25 & $(0.09)$ & 2.8 & $(1.0)$ & 2.3 & $(1.2)$ & 8.2 & (1.5) & 2.0 & $(0.9)$ & 6.9 & $(2.1)$ \\
\hline OECD average & -0.31 & $(0.01)$ & 7.9 & $(0.4)$ & 6.3 & $(0.4)$ & 13.4 & $(0.5)$ & 7.0 & $(0.4)$ & 11.8 & $(0.4)$ \\
\hline Brazil & -1.17 & $(0.10)$ & 17.9 & (3.3) & 11.4 & $(2.4)$ & 31.9 & (3.5) & 20.3 & $(2.7)$ & 29.5 & (3.1) \\
\hline Hong Kong (China) & 0.03 & $(0.08)$ & 2.2 & $(2.2)$ & 1.4 & $(1.0)$ & 3.4 & (1.5) & 0.8 & $(0.8)$ & 1.5 & $(1.0)$ \\
\hline Indonesia & -1.08 & $(0.09)$ & 36.2 & (3.8) & 43.0 & $(4.0)$ & 13.2 & $(2.3)$ & 47.9 & (3.9) & 38.9 & (3.7) \\
\hline Latvia & -0.80 & $(0.07)$ & 4.3 & $(1.7)$ & 1.0 & $(1.0)$ & 9.9 & $(2.7)$ & 9.4 & $(2.3)$ & 16.1 & $(2.8)$ \\
\hline Liechtenstein & 0.52 & $(0.01)$ & 0.0 & c & 0.0 & c & 9.5 & $(0.1)$ & 0.0 & c & 1.2 & $(0.0)$ \\
\hline Macao (China) & -0.46 & $(0.00)$ & 2.4 & $(0.0)$ & 13.0 & $(0.2)$ & 3.2 & $(0.0)$ & 0.3 & $(0.0)$ & 0.0 & c \\
\hline Russian Federation & -1.58 & $(0.08)$ & 16.3 & $(2.7)$ & 10.3 & $(2.8)$ & 24.3 & (3.9) & 27.6 & $(3.6)$ & 27.0 & $(3.2)$ \\
\hline Thailand & -0.82 & $(0.10)$ & 11.7 & $(2.7)$ & 3.0 & $(1.4)$ & 16.4 & (2.9) & 15.8 & $(3.0)$ & 13.5 & $(2.9)$ \\
\hline Tunisia & -0.68 & $(0.07)$ & 6.8 & $(2.1)$ & 6.3 & $(1.9)$ & 24.5 & $(3.0)$ & 5.1 & $(1.8)$ & 3.1 & $(1.4)$ \\
\hline Uruguay & -1.21 & $(0.09)$ & 18.5 & $(3.4)$ & 14.3 & $(3.2)$ & 29.7 & $(4.5)$ & 31.8 & (3.8) & 46.2 & $(4.0)$ \\
\hline G20 average & $\mathrm{m}$ & $\mathrm{m}$ & $\mathrm{m}$ & $\mathrm{m}$ & $\mathrm{m}$ & $\mathrm{m}$ & $\mathrm{m}$ & $\mathrm{m}$ & $\mathrm{m}$ & $\mathrm{m}$ & $\mathrm{m}$ & $\mathrm{m}$ \\
\hline
\end{tabular}

Notes: Values for Change between 2003 and 2012 (PISA 2012 - PISA 2003) in the index of quality of schools' educational resources which are bolded indicate a statistical significance between the 2003 and 2012 index for that country.

Only countries and economies with comparable data from PISA 2003 and PISA 2012 are shown.

For comparability over time, PISA 2003 values on the index of quality of schools' educational resources have been rescaled to the PISA 2012 scale of the index. PISA 2003 results reported in this table may thus differ from those presented in Learning for Tomorrow's World: First Results from PISA 2003 (OECD, 2004 ) (see Annex A5 for more details).

1.The index of quality of school educational resources was derived from the items included in this table measuring school principals' perceptions of potential factors hindering instruction at their school (SC14, from the PISA 2012 school questionnaire). Higher values on this index indicate better quality of educational resources.

Source: OECD. PISA 2012 Results (Volume IV): What Makes Schools Successful? Resources, Policies and Practices, Table IV.3.43.

Please refer to the Reader's Guide for information concerning symbols for missing data and abbreviations.

StatLink 제그 http://dx.doi.org/10.1787/888933286465 
Table D8.2. [2/2] Change between 2003 and 2012 in the quality of schools' educational resources Results based on PISA school principals' reports

\section{PISA 2012}

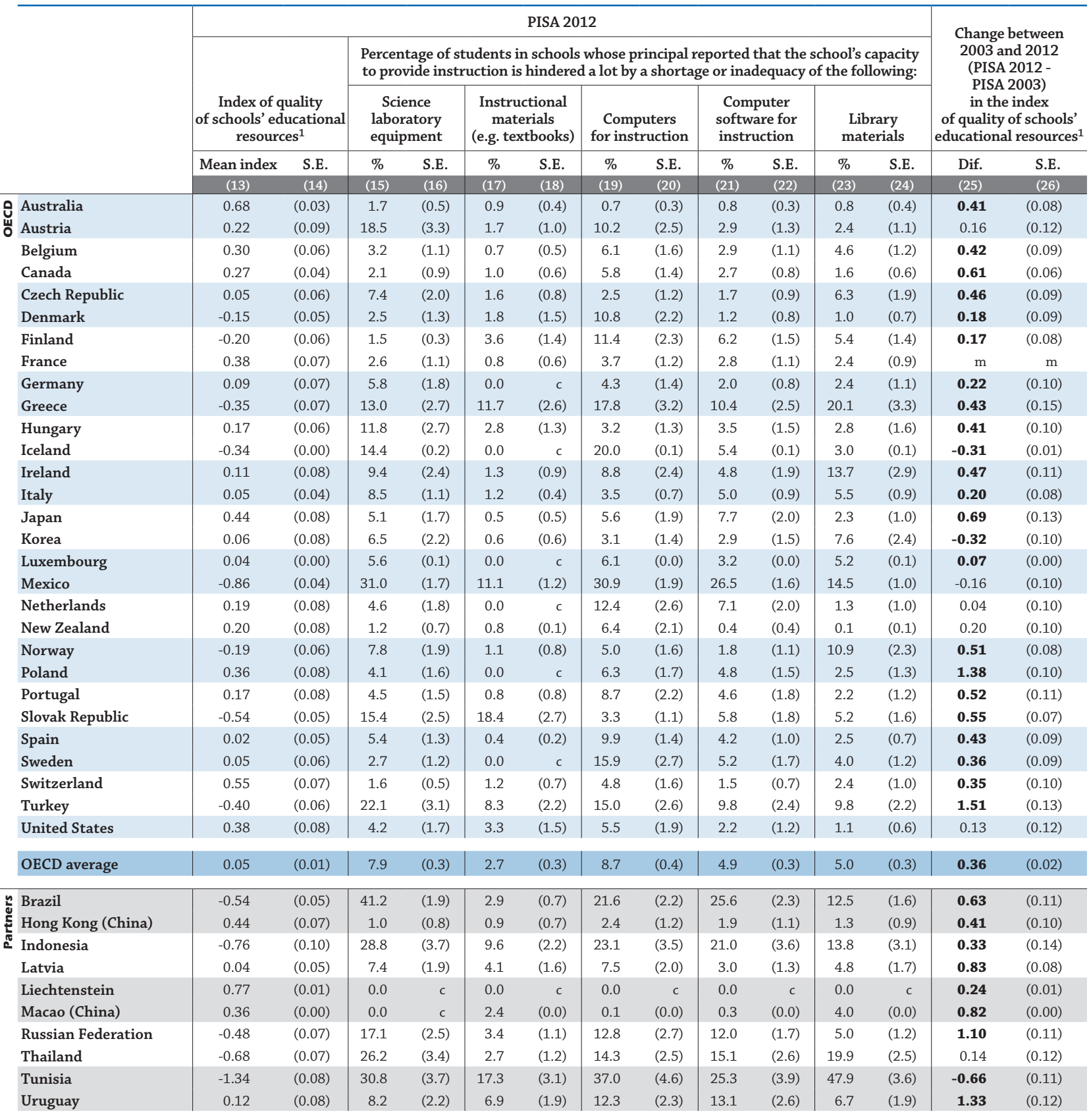

\section{G20 average}

\begin{tabular}{l|l}
$\mathrm{m}$ & $\mathrm{m}$
\end{tabular} $\mathrm{m} \quad \mathrm{m}$

$$
\mathrm{m} \quad \mathrm{m} \quad \mathrm{m}
$$

Notes: Values for Change between 2003 and 2012 (PISA 2012 - PISA 2003) in the index of quality of schools' educational resources which are bolded indicate a statistical significance between the 2003 and 2012 index for that country.

Only countries and economies with comparable data from PISA 2003 and PISA 2012 are shown.

For comparability over time, PISA 2003 values on the index of quality of schools' educational resources have been rescaled to the PISA 2012 scale of the index. PISA 2003 results reported in this table may thus differ from those presented in Learning for Tomorrow's World: First Results from PISA 2003 (OECD, 2004 ) (see Annex A5 for more details).

1.The index of quality of school educational resources was derived from the items included in this table measuring school principals' perceptions of potential factors hindering instruction at their school (SC14, from the PISA 2012 school questionnaire). Higher values on this index indicate better quality of educational resources. Source: OECD. PISA 2012 Results (Volume IV): What Makes Schools Successful? Resources, Policies and Practices, Table IV.3.43.

Please refer to the Reader's Guide for information concerning symbols for missing data and abbreviations.

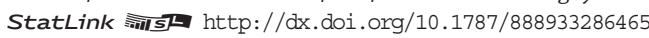


Table D8.3. PISA score in reading for 15-year-olds and mean score-point difference between paper-and-pencil and computer-delivered reading test, by gender (PISA 2012)

Mean score and variation

\begin{tabular}{|c|c|c|c|c|c|c|c|c|c|c|c|c|c|c|}
\hline & \multicolumn{8}{|c|}{$\begin{array}{l}\text { Mean score in reading and gender differences } \\
\text { (based on paper-based assessment) }\end{array}$} & \multicolumn{6}{|c|}{$\begin{array}{l}\text { Mean score-point difference between paper-and-pencil } \\
\text { and computer-delivered reading test }{ }^{1}\end{array}$} \\
\hline & \multicolumn{2}{|c|}{ Boys and girls } & \multicolumn{2}{|c|}{ Boys } & \multicolumn{2}{|c|}{ Girls } & \multicolumn{2}{|c|}{$\begin{array}{l}\text { Difference } \\
\quad(B-G)\end{array}$} & \multicolumn{2}{|c|}{ Boys } & \multicolumn{2}{|c|}{ Girls } & \multicolumn{2}{|c|}{$\begin{array}{l}\text { Difference } \\
\quad(B-G)\end{array}$} \\
\hline & Mean & S.E. & $\begin{array}{l}\text { Mean } \\
\text { score }\end{array}$ & S.E. & $\begin{array}{l}\text { Mean } \\
\text { score }\end{array}$ & S.E. & $\begin{array}{l}\text { Score } \\
\text { dif. }\end{array}$ & S.E. & $\begin{array}{l}\text { Score } \\
\text { dif. }\end{array}$ & S.E. & $\begin{array}{l}\text { Score } \\
\text { dif. }\end{array}$ & S.E. & $\begin{array}{l}\text { Score } \\
\text { dif. }\end{array}$ & S.E. \\
\hline & (1) & (2) & (3) & (4) & (5) & (6) & (7) & (8) & (9) & (10) & (11) & (12) & (13) & (14) \\
\hline Australia & 512 & (1.6) & 495 & $(2.3)$ & 530 & $(2.0)$ & -34 & (2.9) & -10 & (1.7) & -7 & (1.5) & -4 & (1.6) \\
\hline Austria & 490 & $(2.8)$ & 471 & $(4.0)$ & 508 & (3.4) & -37 & $(5.0)$ & 4 & (3.7) & 15 & (3.3) & -10 & (3.7) \\
\hline Belgium & 509 & (2.3) & 493 & $(3.0)$ & 525 & $(2.7)$ & -32 & (3.5) & 3 & (2.6) & 9 & (2.4) & -6 & $(2.5)$ \\
\hline Canada & 523 & (1.9) & 506 & $(2.3)$ & 541 & (2.1) & -35 & (2.1) & -16 & (2.4) & -2 & (2.4) & -14 & (1.3) \\
\hline Chile & 441 & (2.9) & 430 & $(3.8)$ & 452 & $(2.9)$ & -23 & (3.3) & -18 & (2.9) & -4 & (2.9) & -14 & $(2.5)$ \\
\hline Denmark & 496 & (2.6) & 481 & (3.3) & 512 & $(2.6)$ & -31 & $(2.8)$ & -3 & (2.8) & 5 & (2.5) & -8 & (1.9) \\
\hline Estonia & 516 & $(2.0)$ & 494 & $(2.4)$ & 538 & $(2.3)$ & -44 & $(2.4)$ & -10 & (2.5) & -3 & (2.4) & -7 & (1.6) \\
\hline France & 505 & $(2.8)$ & 483 & $(3.8)$ & 527 & $(3.0)$ & -44 & $(4.2)$ & -16 & (3.2) & 5 & (3.4) & -21 & $(2.4)$ \\
\hline Germany & 508 & $(2.8)$ & 486 & $(2.9)$ & 530 & (3.1) & -44 & (2.5) & 7 & (3.2) & 22 & (2.8) & -15 & (2.0) \\
\hline Hungary & 488 & (3.2) & 468 & (3.9) & 508 & (3.3) & -40 & (3.6) & 35 & (3.7) & 42 & (3.5) & -7 & (3.0) \\
\hline Ireland & 523 & $(2.6)$ & 509 & (3.5) & 538 & (3.0) & -29 & $(4.2)$ & 1 & $(4.0)$ & 5 & (2.8) & -3 & (3.9) \\
\hline Israel & 486 & $(5.0)$ & 463 & $(8.2)$ & 507 & (3.9) & -44 & (7.9) & 16 & (4.2) & 33 & (3.5) & -17 & $(4.3)$ \\
\hline Italy & 490 & $(2.0)$ & 471 & $(2.5)$ & 510 & $(2.3)$ & -39 & $(2.6)$ & -28 & (4.6) & -4 & (3.2) & -24 & $(4.3)$ \\
\hline Japan & 538 & $(3.7)$ & 527 & $(4.7)$ & 551 & (3.6) & -24 & $(4.1)$ & -11 & (2.7) & -2 & (2.4) & -8 & $(2.7)$ \\
\hline Korea & 536 & (3.9) & 525 & $(5.0)$ & 548 & $(4.5)$ & -23 & $(5.4)$ & -27 & (3.3) & -11 & (3.1) & -16 & (3.6) \\
\hline Norway & 504 & $(3.2)$ & 481 & (3.3) & 528 & (3.9) & -46 & (3.3) & 4 & (3.7) & 5 & (3.9) & -1 & $(2.2)$ \\
\hline Poland & 518 & (3.1) & 497 & (3.7) & 539 & (3.1) & -42 & $(2.9)$ & 37 & (3.4) & 45 & (3.4) & -8 & $(2.0)$ \\
\hline Portugal & 488 & (3.8) & 468 & $(4.2)$ & 508 & (3.7) & -39 & $(2.7)$ & -9 & (3.1) & 13 & (2.7) & -22 & (1.9) \\
\hline Slovak Republic & 463 & $(4.2)$ & 444 & $(4.6)$ & 483 & $(5.1)$ & -39 & $(4.6)$ & -21 & (2.7) & -1 & (2.6) & -21 & (2.6) \\
\hline Slovenia & 481 & $(1.2)$ & 454 & $(1.7)$ & 510 & $(1.8)$ & -56 & $(2.7)$ & 2 & (1.4) & 18 & (1.4) & -16 & (1.5) \\
\hline Spain & 488 & (1.9) & 474 & $(2.3)$ & 503 & (1.9) & -29 & $(2.0)$ & 17 & $(4.2)$ & 22 & (3.8) & -5 & (2.4) \\
\hline Sweden & 483 & (3.0) & 458 & $(4.0)$ & 509 & $(2.8)$ & -51 & (3.6) & -24 & (3.2) & -6 & (2.6) & -18 & (2.1) \\
\hline United States & 498 & $(3.7)$ & 482 & $(4.1)$ & 513 & $(3.8)$ & -31 & $(2.6)$ & -15 & (3.0) & -12 & (2.7) & -2 & (1.6) \\
\hline OECD average & 496 & $(0.5)$ & 478 & $(0.6)$ & 515 & $(0.5)$ & -38 & $(0.6)$ & -4 & $(0.7)$ & 8 & (0.6) & -12 & $(0.6)$ \\
\hline Brazil & 410 & $(2.1)$ & 394 & $(2.4)$ & 425 & $(2.2)$ & -31 & (1.9) & -26 & (3.4) & -18 & (3.5) & -8 & $(2.1)$ \\
\hline Colombia & 403 & (3.4) & 394 & (3.9) & 412 & (3.8) & -19 & (3.5) & 0 & (3.3) & 14 & (3.4) & -14 & (2.7) \\
\hline Hong Kong (China) & 545 & $(2.8)$ & 533 & $(3.8)$ & 558 & (3.3) & -25 & $(4.7)$ & -8 & (3.3) & -2 & (3.3) & -7 & $(2.8)$ \\
\hline Macao (China) & 509 & $(0.9)$ & 492 & (1.4) & 527 & (1.1) & -36 & (1.7) & -15 & (1.5) & 3 & (1.1) & -17 & (1.9) \\
\hline Russian Federation & 475 & $(3.0)$ & 455 & $(3.5)$ & 495 & $(3.2)$ & -40 & $(3.0)$ & -2 & (3.6) & 21 & (3.1) & -22 & (2.3) \\
\hline Shanghai (China) & 570 & $(2.9)$ & 557 & (3.3) & 581 & $(2.8)$ & -24 & (2.5) & 31 & (2.8) & 45 & (2.3) & -14 & $(2.0)$ \\
\hline Singapore & 542 & (1.4) & 527 & (1.9) & 559 & (1.9) & -32 & $(2.6)$ & -32 & (1.0) & -17 & (1.2) & -14 & (1.5) \\
\hline Chinese Taipei & 523 & $(3.0)$ & 507 & $(4.3)$ & 539 & $(4.3)$ & -32 & (6.4) & -4 & (2.3) & 11 & (2.2) & -15 & $(2.1)$ \\
\hline United Arab Emirates & 442 & $(2.5)$ & 413 & (3.9) & 469 & $(3.2)$ & -55 & $(4.8)$ & 32 & (3.5) & 38 & (3.4) & -5 & $(4.9)$ \\
\hline G20 average & $\mathrm{m}$ & $\mathrm{m}$ & $\mathrm{m}$ & $\mathrm{m}$ & $\mathrm{m}$ & $\mathrm{m}$ & $\mathrm{m}$ & $\mathrm{m}$ & $\mathrm{m}$ & $\mathrm{m}$ & $\mathrm{m}$ & $\mathrm{m}$ & $\mathrm{m}$ & $\mathrm{m}$ \\
\hline
\end{tabular}

Note: Differences that are statistically significant are indicated in bold. Only countries that have participated in the optional digital reading assessment in PISA 2012 are shown.

1. Negative figures (in columns 9, 11 and 13) mean that 15-year-old students have obtained better performances on computer-delivered reading test.

Source: OECD. PISA 2012 Database.

Please refer to the Reader's Guide for information concerning symbols for missing data and abbreviations.

StatLink 제그 http://dx.doi.org/10.1787/888933286476 
Table D8.4. Teachers and information and communication technology Results from TALIS 2013, percentage of lower secondary education teachers

\begin{tabular}{|c|c|c|c|c|c|c|c|c|c|c|c|c|c|}
\hline \multirow{3}{*}{\multicolumn{2}{|c|}{$\begin{array}{c}\text { Percentage } \\
\text { of lower secondary } \\
\text { education teachers } \\
\text { indicating students } \\
\text { use ICT for projects } \\
\text { or class work } \\
\text { "frequently" or } \\
\text { "in all or nearly } \\
\text { all lessons"1 }\end{array}$}} & \multicolumn{4}{|c|}{$\begin{array}{l}\text { Percentage of lower secondary } \\
\text { education teachers indicating } \\
\text { they have a high level of need } \\
\text { for professional development } \\
\text { in the following areas: }\end{array}$} & \multicolumn{8}{|c|}{$\begin{array}{l}\text { Percentage of lower secondary education teachers who report } \\
\text { having participated in professional development with the following } \\
\text { content in the } 12 \text { months prior to the survey and percentage } \\
\text { of participating teachers who report a moderate or large positive impact } \\
\text { of this professional development on their teaching }\end{array}$} \\
\hline & & \multirow{2}{*}{\multicolumn{2}{|c|}{$\begin{array}{c}\text { ICT skills } \\
\text { for teaching }\end{array}$}} & \multirow{2}{*}{\multicolumn{2}{|c|}{$\begin{array}{c}\text { New } \\
\text { technologies } \\
\text { in the workplace }\end{array}$}} & \multicolumn{4}{|c|}{ ICT skills for teaching } & \multicolumn{4}{|c|}{ New technologies in the workplace } \\
\hline & & & & & & \multicolumn{2}{|c|}{ Participation } & \multicolumn{2}{|c|}{$\begin{array}{c}\text { Moderate or large } \\
\text { positive impact }\end{array}$} & \multicolumn{2}{|c|}{ Participation } & \multicolumn{2}{|c|}{$\begin{array}{c}\text { Moderate or large } \\
\text { positive impact }\end{array}$} \\
\hline$\%$ & S.E. & $\%$ & S.E. & $\%$ & S.E. & $\%$ & S.E. & $\%$ & S.E. & $\%$ & S.E. & $\%$ & S.E. \\
\hline$(1)$ & $(2)$ & (3) & $(4)$ & (5) & $(6)$ & (7) & $(8)$ & (9) & $(10)$ & (11) & $(12)$ & (13) & $(14)$ \\
\hline 67 & (1.9) & 14 & $(0.9)$ & 12 & $(0.8)$ & 72 & $(1.7)$ & 70 & $(1.8)$ & 57 & $(1.8)$ & 68 & $(2.0)$ \\
\hline 27 & $(1.1)$ & 11 & $(0.7)$ & 5 & $(0.5)$ & 37 & $(1.8)$ & 80 & (1.5) & 13 & $(0.8)$ & 82 & $(2.4)$ \\
\hline 60 & (2.3) & 13 & $(0.9)$ & 17 & (1.1) & 51 & (2.2) & 87 & (1.7) & 38 & (1.8) & 86 & (2.3) \\
\hline 37 & (1.1) & 15 & $(0.7)$ & 10 & $(0.7)$ & 53 & (1.6) & 83 & (1.3) & 42 & (1.4) & 82 & (1.5) \\
\hline 74 & (1.9) & 19 & $(1.2)$ & 14 & (1.1) & 49 & (1.9) & 81 & (1.6) & 29 & $(2.0)$ & 78 & (2.3) \\
\hline 37 & (1.4) & 8 & $(0.7)$ & 8 & $(0.6)$ & 39 & (1.7) & 64 & (1.5) & 32 & (1.7) & 64 & (2.1) \\
\hline 29 & (1.3) & 24 & $(0.9)$ & 21 & (1.0) & 63 & (1.3) & 84 & (1.1) & 47 & (1.7) & 84 & (1.3) \\
\hline 18 & $(0.9)$ & 17 & (1.0) & 14 & $(0.8)$ & 48 & (1.9) & 68 & (1.9) & 42 & (1.7) & 63 & (2.3) \\
\hline 24 & (1.0) & 25 & $(0.9)$ & 17 & $(0.7)$ & 40 & (1.4) & 77 & (1.7) & 11 & $(0.8)$ & 74 & (3.1) \\
\hline 32 & (1.4) & 29 & (1.5) & 19 & (1.2) & 44 & (1.4) & 78 & (1.9) & 34 & (1.5) & 80 & $(2.4)$ \\
\hline 19 & (1.3) & 24 & (1.2) & 23 & (0.9) & 60 & (1.6) & 79 & (1.5) & 48 & (1.4) & 78 & (1.4) \\
\hline 31 & (1.4) & 36 & $(0.8)$ & 32 & $(0.9)$ & 53 & (1.3) & 82 & (1.4) & 45 & (1.4) & 80 & (1.6) \\
\hline 10 & $(0.6)$ & 26 & $(0.9)$ & 16 & $(0.7)$ & 36 & (1.4) & 69 & (1.9) & 15 & $(0.9)$ & 69 & $(2.5)$ \\
\hline 28 & (1.2) & 25 & (1.1) & 19 & (1.0) & 54 & (1.3) & 90 & (0.8) & 37 & (1.0) & 91 & (0.8) \\
\hline 56 & $(1.2)$ & 21 & (1.0) & 28 & (1.1) & 73 & $(1.0)$ & 84 & $(1.0)$ & 55 & (1.4) & 81 & (1.1) \\
\hline 55 & (1.6) & 16 & $(0.9)$ & 14 & $(0.7)$ & 67 & (1.3) & 70 & (1.5) & 49 & (1.4) & 69 & (1.4) \\
\hline 35 & (2.1) & 15 & (1.1) & 12 & (1.2) & 48 & (1.9) & 73 & (1.9) & 30 & $(2.1)$ & 71 & $(2.6)$ \\
\hline 74 & (1.7) & 18 & (1.4) & 9 & $(0.5)$ & 33 & (2.1) & 78 & (2.3) & 7 & (1.0) & 77 & $(4.8)$ \\
\hline 36 & (1.5) & 11 & $(0.8)$ & 13 & $(0.8)$ & 52 & (1.5) & 85 & (1.1) & 41 & (1.5) & 84 & $(1.4)$ \\
\hline 34 & $(0.9)$ & 9 & $(0.5)$ & 9 & $(0.6)$ & 49 & (1.6) & 92 & $(0.9)$ & 36 & (1.4) & 92 & (1.1) \\
\hline 45 & (1.3) & 19 & $(0.9)$ & 15 & $(0.7)$ & 60 & (1.3) & 92 & $(0.8)$ & 33 & (1.4) & 90 & $(1.2)$ \\
\hline 37 & (1.3) & 14 & $(0.7)$ & 14 & $(0.7)$ & 68 & (1.6) & 87 & $(0.9)$ & 56 & (1.5) & 86 & (1.1) \\
\hline 34 & (1.7) & 25 & $(0.8)$ & 18 & $(0.8)$ & 47 & (1.6) & 66 & (1.9) & 37 & (1.7) & 65 & $(2.2)$ \\
\hline 46 & (1.8) & 8 & $(0.8)$ & 15 & $(1.0)$ & 49 & $(2.0)$ & 73 & (1.8) & 57 & $(2.2)$ & 73 & (1.6) \\
\hline 40 & (1.4) & 18 & $(0.9)$ & 15 & (0.8) & 51 & (1.6) & 80 & (1.5) & 36 & (1.5) & 79 & (2.0) \\
\hline 72 & (1.7) & 9 & $(0.8)$ & 18 & (1.3) & 77 & (1.4) & 90 & $(1.0)$ & 69 & (1.7) & 88 & (1.0) \\
\hline 30 & (1.1) & 27 & $(0.7)$ & 37 & $(0.9)$ & 46 & (1.0) & 79 & (1.0) & 53 & $(1.2)$ & 79 & (1.0) \\
\hline 34 & (1.3) & 20 & $(0.9)$ & 23 & (1.3) & 56 & (1.8) & 85 & (1.5) & 53 & (1.7) & 82 & (1.5) \\
\hline 24 & $(0.9)$ & 20 & $(0.9)$ & 24 & (0.9) & 58 & (1.5) & 73 & (1.1) & 41 & (1.3) & 74 & (1.3) \\
\hline 46 & (1.4) & 13 & $(0.7)$ & 20 & (1.0) & 54 & (1.6) & 81 & (1.9) & 48 & (1.4) & 78 & (2.1) \\
\hline 47 & (1.8) & 31 & (1.4) & 39 & (1.1) & 58 & (1.9) & 89 & (1.5) & 33 & (1.8) & 85 & (1.9) \\
\hline 41 & (1.5) & 19 & (1.1) & 24 & (1.0) & 72 & (1.5) & 87 & $(1.2)$ & 59 & (1.6) & 86 & (1.3) \\
\hline 19 & (1.3) & 38 & $(1.2)$ & 31 & (1.0) & 71 & (1.3) & 88 & $(0.8)$ & 56 & (1.3) & 83 & (1.1) \\
\hline 26 & $(1.2)$ & 19 & $(0.9)$ & 22 & $(0.9)$ & 60 & (1.4) & 91 & (1.0) & 30 & $(1.2)$ & 88 & (1.4) \\
\hline 48 & (1.5) & 17 & (1.0) & 21 & $(0.9)$ & 81 & (1.1) & 87 & (1.1) & 89 & $(0.9)$ & 89 & (0.9) \\
\hline 23 & $(0.9)$ & 20 & $(0.8)$ & 21 & $(0.8)$ & 46 & $(1.2)$ & 84 & $(1.2)$ & 33 & (1.3) & 83 & (1.3) \\
\hline 30 & $(0.8)$ & 12 & $(0.6)$ & 10 & $(0.6)$ & 68 & $(0.8)$ & 73 & $(1.0)$ & 40 & $(0.9)$ & 69 & (1.5) \\
\hline 15 & $(0.9)$ & 25 & $(0.9)$ & 16 & $(0.8)$ & 64 & (1.1) & 83 & $(1.0)$ & 26 & (1.1) & 82 & (1.3) \\
\hline $\mathrm{m}$ & $\mathrm{m}$ & $\mathrm{m}$ & $\mathrm{m}$ & $\mathrm{m}$ & $\mathrm{m}$ & $\mathrm{m}$ & $\mathrm{m}$ & $\mathrm{m}$ & $\mathrm{m}$ & $\mathrm{m}$ & $\mathrm{m}$ & $\mathrm{m}$ & $\mathrm{m}$ \\
\hline
\end{tabular}

1. These data are reported by teachers and refer to a randomly chosen class they currently teach from their weekly timetable.

2. Footnote by Turkey: The information in this document with reference to "Cyprus" relates to the southern part of the Island. There is no single authority representing both Turkish and Greek Cypriot people on the Island. Turkey recognises the Turkish Republic of Northern Cyprus (TRNC). Until a lasting and equitable solution is found within the context of the United Nations, Turkey shall preserve its position concerning the "Cyprus issue".

3. Note by all the European Union Member States of the OECD and the European Union: The Republic of Cyprus is recognised by all members of the United Nations with the exception of Turkey. The information in this document relates to the area under the effective control of the Government of the Republic of Cyprus. Source: OECD. TALIS 2013 Results: An International Perspective on Teaching and Learning, Tables 4.10, 4.12 and 6.1.

Please refer to the Reader's Guide for information concerning symbols for missing data and abbreviations.

StatLink 젶ㄴ http://dx.doi.org/10.1787/888933286483 


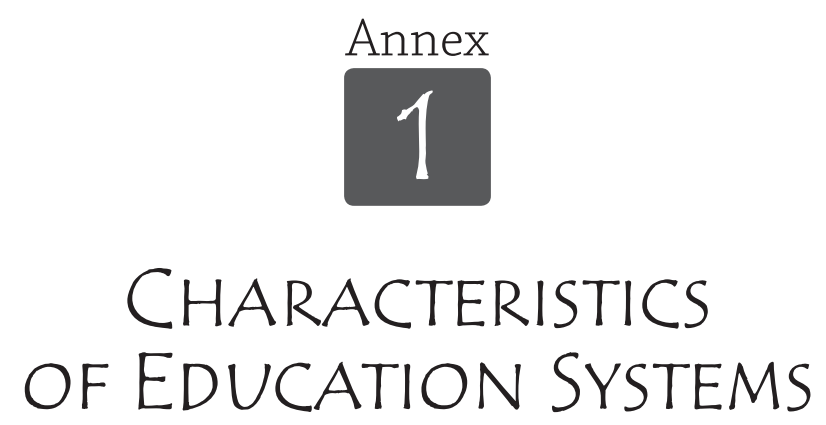

All tables in Annex 1 are available on line at:

StatLink : inls http://dx.doi.org/10.1787/888933286494

\section{Note regarding data from Israel}

The statistical data for Israel are supplied by and are under the responsibility of the relevant Israeli authorities. The use of such data by the OECD is without prejudice to the status of the Golan Heights, East Jerusalem and Israeli settlements in the West Bank under the terms of international law. 
Table X1.1a. [1/2] Typical graduation ages, by level of education (2013)

The typical age refers to the age of the students at the beginning of the school year; students will generally be one year older than the age indicated when they graduate at the end of the school year. The typical age is used for the gross graduation rate calculation.

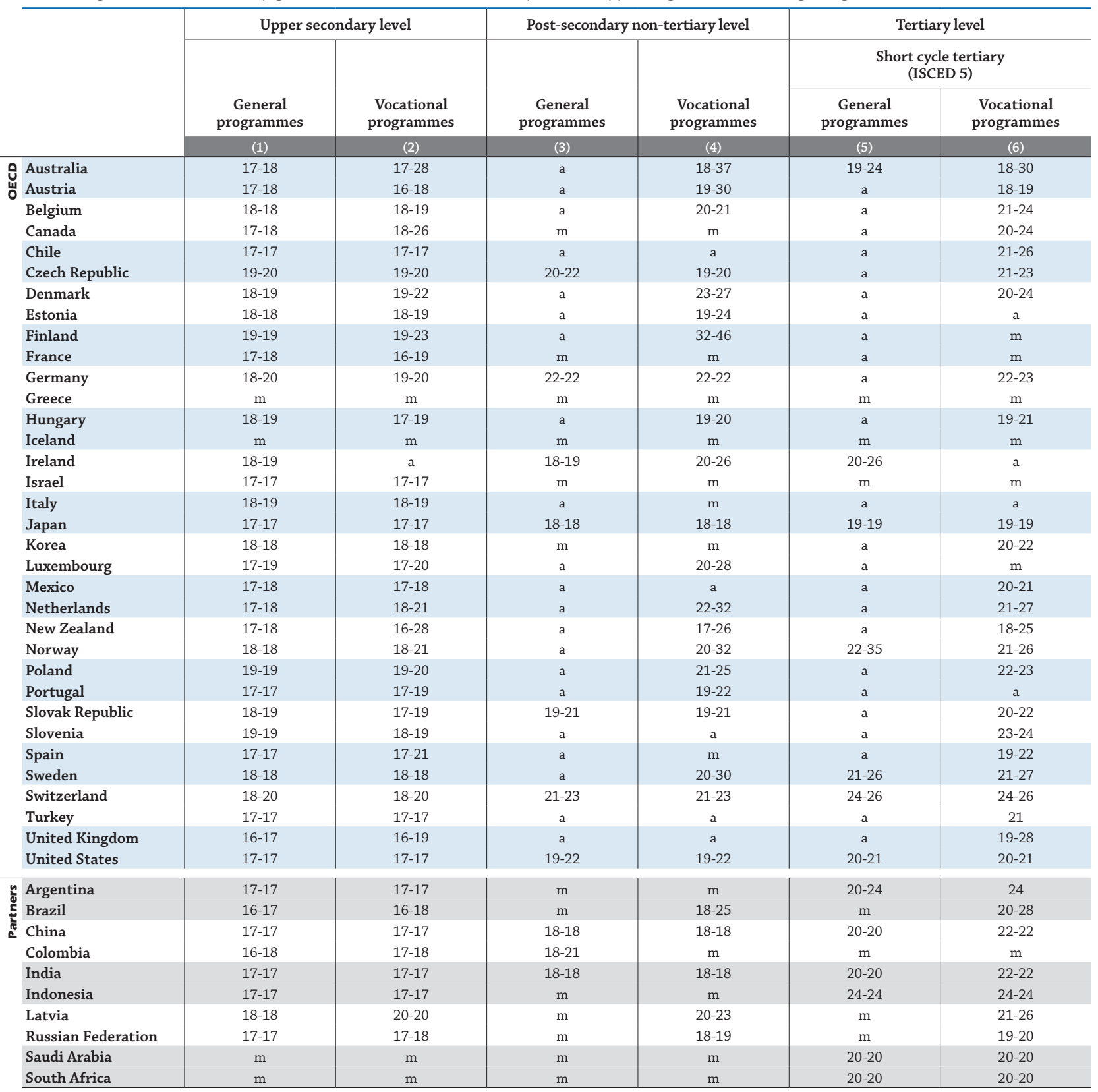

Sources: OECD. Argentina, China, Colombia, India, Indonesia, Saudi Arabia, South Africa: UNESCO Institute for Statistics. Latvia: Eurostat. See Annex 3 for notes (www.oecd.org/education/education-at-a-glance-19991487.htm).

Please refer to the Reader's Guide for information concerning symbols for missing data and abbreviations.

StatLink 켚ㄴ http://dx.doi.org/10.1787/888933286506 
Table X1.1a. [2/2] Typical graduation ages, by level of education (2013)

The typical age refers to the age of the students at the beginning of the school year; students will generally be one year older than the age indicated when they graduate at the end of the school year. The typical age is used for the gross graduation rate calculation.

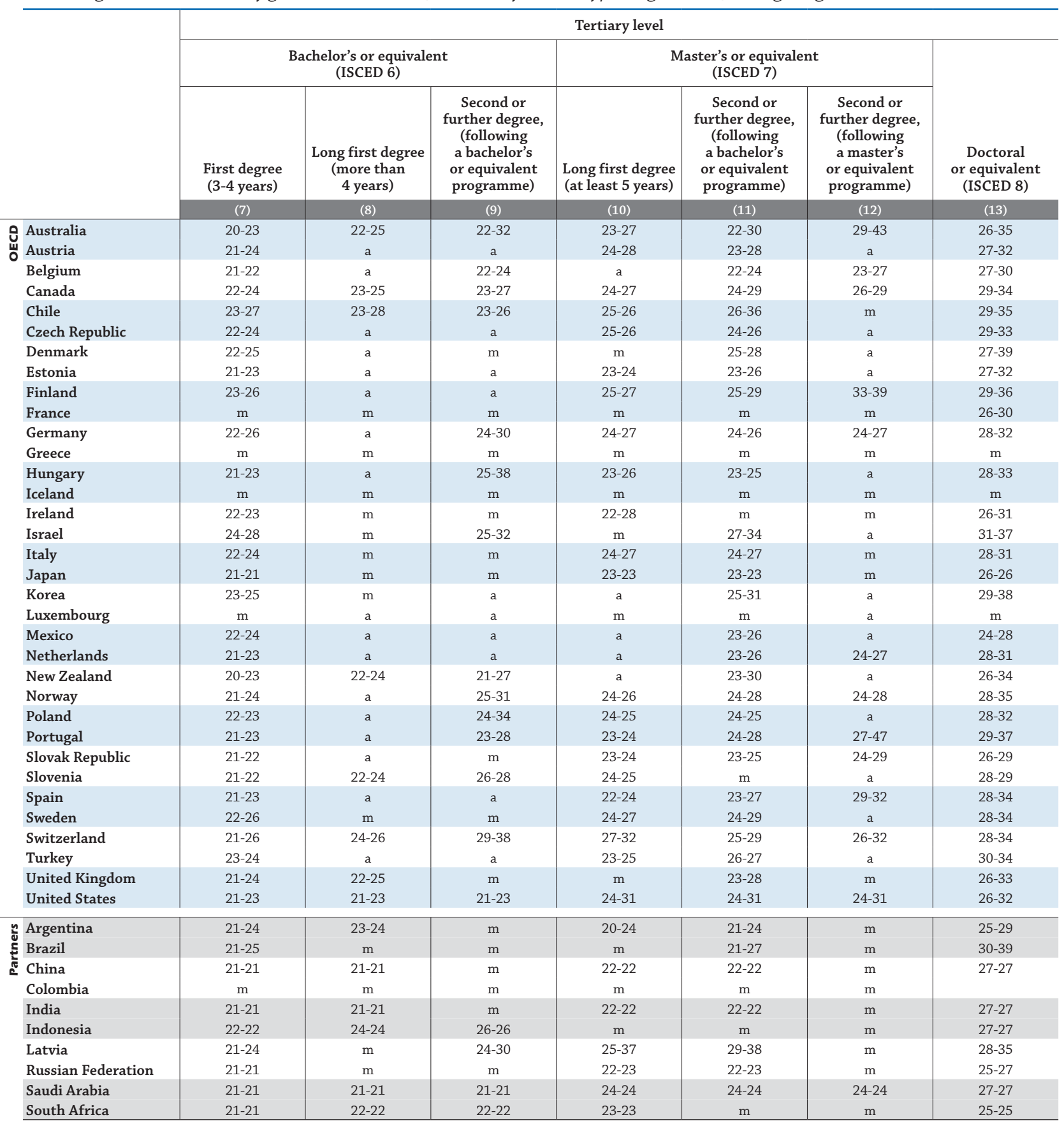

Sources: OECD. Argentina, China, Colombia, India, Indonesia, Saudi Arabia, South Africa: UNESCO Institute for Statistics. Latvia: Eurostat. See Annex 3 for notes (www.oecd.org/education/education-at-a-glance-19991487.htm).

Please refer to the Reader's Guide for information concerning symbols for missing data and abbreviations.

StatLink 게그 http://dx.doi.org/10.1787/888933286506 
Table X1.1b. Typical age of entry by level of education (2013)

The typical age refers to the age of the students at the beginning of the school year; students will generally be one year older than the age indicated when they graduate at the end of the school year. The typical age is used for the gross graduation rate calculation.

\begin{tabular}{|c|c|c|c|c|c|c|}
\hline & $\begin{array}{l}\text { Upper secondary } \\
\text { (ISCED 3) }\end{array}$ & $\begin{array}{l}\text { Post-secondary } \\
\text { non-tertiary } \\
\text { (ISCED 4) }\end{array}$ & $\begin{array}{l}\text { Short cycle tertiary } \\
\text { (ISCED 5) }\end{array}$ & $\begin{array}{c}\text { Bachelor's } \\
\text { or equivalent } \\
\text { (ISCED 6) }\end{array}$ & $\begin{array}{l}\text { Master's } \\
\text { or equivalent } \\
\text { (ISCED 7) }\end{array}$ & $\begin{array}{l}\text { Doctoral } \\
\text { or equivalent } \\
\text { (ISCED 8) }\end{array}$ \\
\hline & (1) & (2) & (3) & (4) & (5) & (6) \\
\hline Australia & $\mathrm{m}$ & $\mathrm{m}$ & $\mathrm{m}$ & $18-20$ & $21-26$ & $22-30$ \\
\hline Austria & $14-15$ & $17-22$ & $17-18$ & $19-21$ & $19-24$ & $24-28$ \\
\hline Belgium & $14-16$ & $18-22$ & $18-19$ & $18-19$ & $21-23$ & $23-26$ \\
\hline Canada & 15 & 18 & $18-19$ & $18-19$ & $21-27$ & $25-30$ \\
\hline Chile & $14-14$ & a & $18-21$ & $18-18$ & $24-33$ & $24-33$ \\
\hline Czech Republic & $15-16$ & $20-29$ & $19-21$ & $19-20$ & $22-24$ & $24-26$ \\
\hline Denmark & $16-17$ & $\mathrm{a}$ & $19-27$ & $20-22$ & $23-25$ & $25-29$ \\
\hline Estonia & $16-19$ & $19-24$ & a & $19-22$ & $22-26$ & $24-28$ \\
\hline Finland & $16-16$ & $31-43$ & $33-36$ & $19-20$ & $22-29$ & $25-30$ \\
\hline France & $15-17$ & $\mathrm{~m}$ & $\mathrm{~m}$ & $\mathrm{~m}$ & $18-19$ & $23-26$ \\
\hline Germany & $15-18$ & $19-21$ & $21-25$ & $19-21$ & $19-24$ & $25-29$ \\
\hline Greece & $\mathrm{m}$ & $\mathrm{m}$ & $\mathrm{m}$ & $\mathrm{m}$ & $\mathrm{m}$ & $\mathrm{m}$ \\
\hline Hungary & $15-15$ & $19-20$ & $19-21$ & $19-20$ & $19-24$ & $24-27$ \\
\hline Iceland & $\mathrm{m}$ & $\mathrm{m}$ & $\mathrm{m}$ & $\mathrm{m}$ & $\mathrm{m}$ & $\mathrm{m}$ \\
\hline Ireland & $15-16$ & $18-20$ & $18-19$ & $18-19$ & $20-21$ & $20-23$ \\
\hline Israel & $15-15$ & $20-25$ & $18-23$ & $21-25$ & $24-31$ & $26-32$ \\
\hline Italy & $14-14$ & $17-18$ & $20-21$ & $20-20$ & $20-20$ & $26-29$ \\
\hline Japan & $15-15$ & $18-18$ & $18-18$ & $18-18$ & $22-22$ & $24-24$ \\
\hline Korea & $15-15$ & $\mathrm{~m}$ & $18-18$ & $18-18$ & $22-27$ & $23-32$ \\
\hline Luxembourg & $15-19$ & $\mathrm{a}$ & $22-22$ & $18-18$ & $20-20$ & $24-24$ \\
\hline Mexico & $15-15$ & $\mathrm{a}$ & $18-19$ & $18-19$ & $24-28$ & $24-34$ \\
\hline Netherlands & $16-19$ & $22-36$ & $19-26$ & $18-20$ & $22-24$ & $24-26$ \\
\hline New Zealand & $15-16$ & $17-24$ & $17-25$ & $18-20$ & $21-28$ & $22-30$ \\
\hline Norway & $16-16$ & $19-31$ & $19-23$ & $19-20$ & $19-20$ & $25-29$ \\
\hline Poland & $16-16$ & $19-23$ & $19-20$ & $19-20$ & $22-24$ & $24-26$ \\
\hline Portugal & $15-15$ & $18-20$ & a & $18-20$ & $18-23$ & $23-31$ \\
\hline Slovak Republic & $15-18$ & $18-20$ & $19-20$ & $19-21$ & $22-23$ & $24-26$ \\
\hline Slovenia & $15-15$ & $\mathrm{a}$ & $19-25$ & $19-20$ & $22-24$ & $24-26$ \\
\hline Spain & $15-15$ & $\mathrm{~m}$ & $18-20$ & $18-18$ & $18-23$ & $\mathrm{~m}$ \\
\hline Sweden & $16-16$ & $19-25$ & $19-25$ & $19-21$ & $19-24$ & $26-33$ \\
\hline Switzerland & $15-17$ & $18-24$ & $18-23$ & $19-22$ & $22-25$ & $25-28$ \\
\hline Turkey & $14-14$ & $\mathrm{a}$ & $18-19$ & $18-19$ & $23-25$ & $26-27$ \\
\hline United Kingdom & $16-18$ & a & $18-27$ & $18-21$ & $21-30$ & $22-27$ \\
\hline United States & $15-15$ & $18-25$ & $18-22$ & $18-19$ & $22-28$ & $22-27$ \\
\hline Argentina & $15-15$ & $\mathrm{~m}$ & $18-18$ & 18 & $\mathrm{~m}$ & $25-25$ \\
\hline Brazil & $\mathrm{m}$ & $\mathrm{m}$ & $\mathrm{m}$ & $\mathrm{m}$ & $\mathrm{m}$ & $\mathrm{m}$ \\
\hline China & $15-15$ & $\mathrm{~m}$ & $17-17$ & 17 & $\mathrm{~m}$ & $21-21$ \\
\hline Colombia & $\mathrm{m}$ & $\mathrm{m}$ & $\mathrm{m}$ & $\mathrm{m}$ & $\mathrm{m}$ & $\mathrm{m}$ \\
\hline India & $\mathrm{m}$ & $\mathrm{m}$ & $\mathrm{m}$ & $\mathrm{m}$ & $\mathrm{m}$ & $\mathrm{m}$ \\
\hline Indonesia & $16-16$ & a & $19-19$ & 19 & $23-25$ & $25-25$ \\
\hline Latvia & $15-16$ & $19-21$ & $19-22$ & $19-20$ & $21-23$ & $24-31$ \\
\hline Russian Federation & $15-16$ & $17-18$ & $17-18$ & $17-18$ & $21-22$ & $23-24$ \\
\hline Saudi Arabia & $\mathrm{m}$ & $\mathrm{m}$ & $\mathrm{m}$ & $\mathrm{m}$ & $\mathrm{m}$ & $\mathrm{m}$ \\
\hline South Africa & $\mathrm{m}$ & $\mathrm{m}$ & $\mathrm{m}$ & $\mathrm{m}$ & $\mathrm{m}$ & $\mathrm{m}$ \\
\hline
\end{tabular}

Sources: OECD. Argentina, China, Colombia, India, Indonesia, Saudi Arabia, South Africa: UNESCO Institute for Statistics. Latvia: Eurostat. See Annex 3 for notes (www.oecd.org/education/education-at-a-glance-19991487.htm).

Please refer to the Reader's Guide for information concerning symbols for missing data and abbreviations.

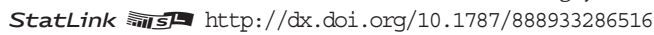


Table X1.2a. School year and financial year used for the calculation of indicators, OECD countries

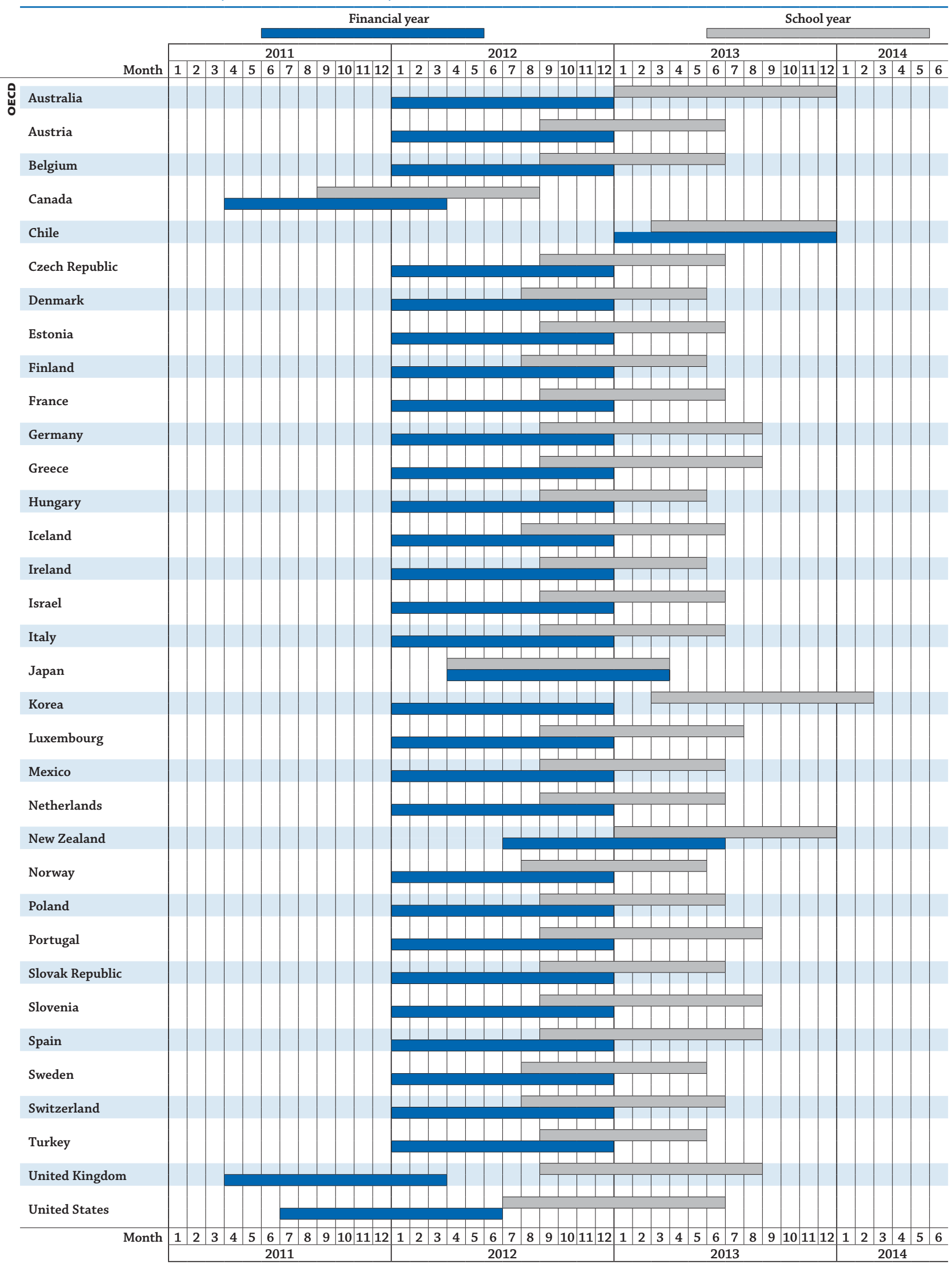

Sources: OECD. See Annex 3 for notes (www.oecd.org/education/education-at-a-glance-19991487.htm).

StatLink त्ताड़ा http://dx.doi.org/10.1787/888933286528 
Table X1.2b. School year and financial year used for the calculation of indicators, partner countries

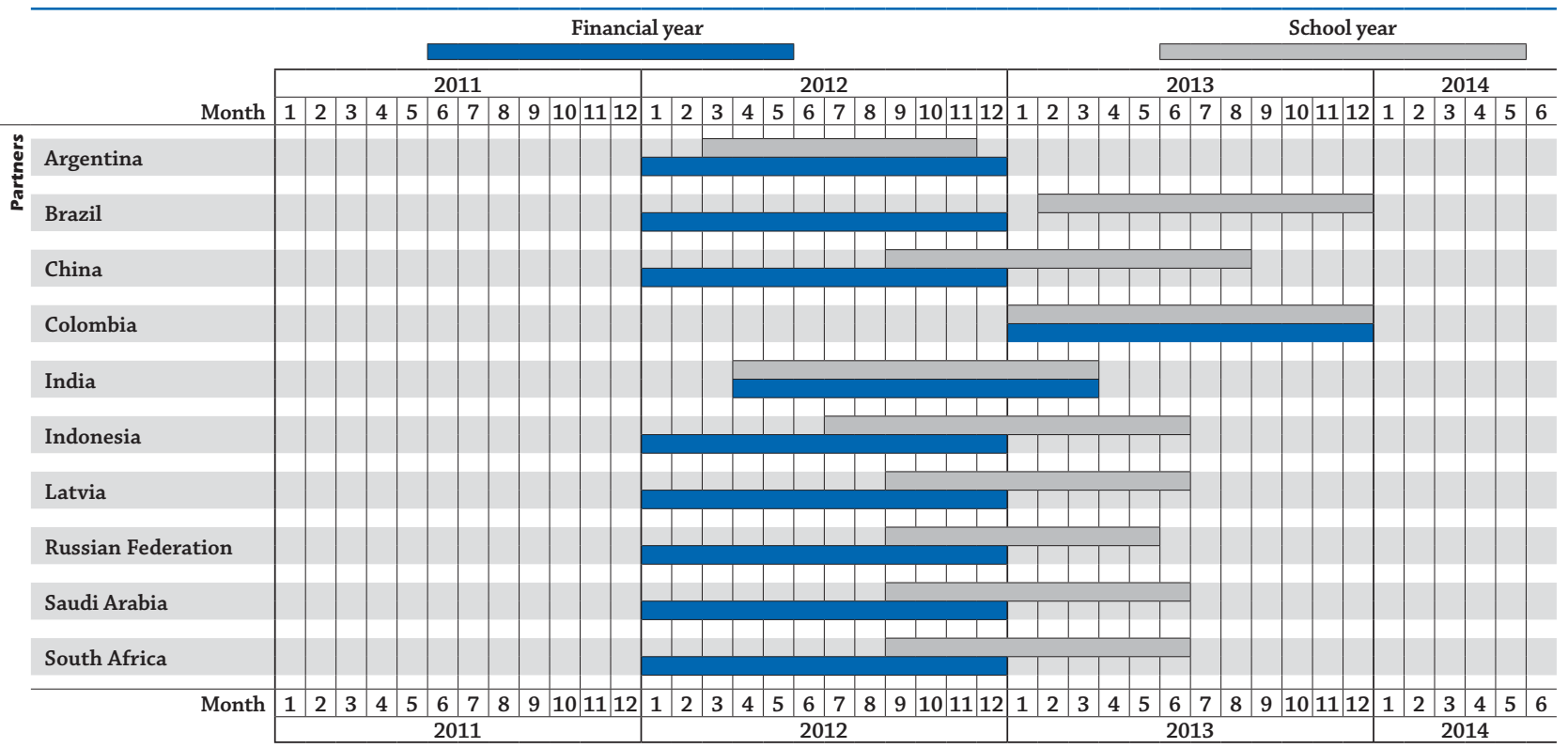

Sources: OECD. Argentina, China, Colombia, India, Indonesia, Saudi Arabia, South Africa: UNESCO Institute for Statistics. Latvia: Eurostat. See Annex 3 for notes (www.oecd.org/education/education-at-a-glance-19991487.htm).

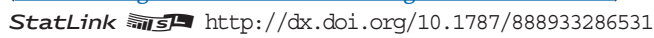


Table X1.3 Starting and ending age for students in compulsory education (2013)

\begin{tabular}{|c|c|c|}
\hline & \multicolumn{2}{|c|}{ Compulsory education } \\
\hline & Starting age & Ending age \\
\hline & (1) & (2) \\
\hline Australia & 6 & 17 \\
\hline Austria & 6 & 15 \\
\hline Belgium & 6 & 18 \\
\hline Canada $^{1}$ & 6 & $16-18$ \\
\hline Chile & 6 & 18 \\
\hline Czech Republic & 6 & 15 \\
\hline Denmark & 6 & 16 \\
\hline Estonia & 7 & 16 \\
\hline Finland & 7 & 16 \\
\hline France & 6 & 16 \\
\hline Germany & 6 & 18 \\
\hline Greece & 5 & $14-15$ \\
\hline Hungary & 5 & 16 \\
\hline Iceland & 6 & 16 \\
\hline Ireland & 6 & 16 \\
\hline Israel & 5 & 17 \\
\hline Italy & 6 & 16 \\
\hline Japan & 6 & 15 \\
\hline Korea & 6 & 14 \\
\hline Luxembourg & 4 & 16 \\
\hline Mexico & 4 & 15 \\
\hline Netherlands & 5 & 18 \\
\hline New Zealand & 5 & 16 \\
\hline Norway & 6 & 16 \\
\hline Poland & 5 & 16 \\
\hline Portugal & 6 & 18 \\
\hline Slovak Republic & 6 & 16 \\
\hline Slovenia & 6 & 14 \\
\hline Spain & 6 & 16 \\
\hline Sweden & 7 & 16 \\
\hline Switzerland & $5-7$ & 15 \\
\hline Turkey & $5-6$ & 17 \\
\hline United Kingdom & $4-5$ & 16 \\
\hline United States & $4-6$ & 17 \\
\hline OECD average & 6 & 16 \\
\hline EU21 average & 6 & 16 \\
\hline Argentina $^{1}$ & 5 & 17 \\
\hline Brazil & 4 & 17 \\
\hline China & $\mathrm{m}$ & $\mathrm{m}$ \\
\hline Colombia & 5 & 15 \\
\hline India & $\mathrm{m}$ & $\mathrm{m}$ \\
\hline Indonesia & 7 & 15 \\
\hline Latvia & 5 & 16 \\
\hline Russian Federation & 7 & 17 \\
\hline Saudi Arabia & 6 & 11 \\
\hline South Africa ${ }^{1}$ & 7 & 15 \\
\hline G20 average & $\mathrm{m}$ & $\mathrm{m}$ \\
\hline
\end{tabular}

Note: Ending age of compulsory education is the age at which compulsory schooling ends. For example, an ending age of 18 indicates that all students under 18 are legally obliged to participate in education.

1. Year of reference 2012

Sources: OECD. Argentina, China, Colombia, India, Indonesia, Saudi Arabia, South Africa: UNESCO Institute for Statistics. Latvia: Eurostat. See Annex 3 for notes (www.oecd.org/education/education-at-a-glance-19991487.htm).

Please refer to the Reader's Guide for information concerning symbols for missing data and abbreviations.

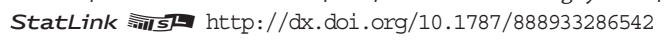





\section{Annex}

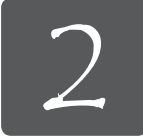

\section{REFERENCE STATISTICS}

All tables in Annex 2 are available on line at:

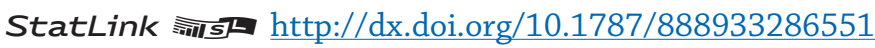

\section{Note regarding data from Israel}

The statistical data for Israel are supplied by and are under the responsibility of the relevant Israeli authorities. The use of such data by the OECD is without prejudice to the status of the Golan Heights, East Jerusalem and Israeli settlements in the West Bank under the terms of international law. 
Table X2.1. Overview of the economic context using basic variables (reference period: calendar year 2012, 2012 current prices)

\begin{tabular}{|c|c|c|c|c|c|}
\hline & $\begin{array}{l}\text { Total public expenditure } \\
\text { as a percentage of GDP }\end{array}$ & $\begin{array}{c}\text { GDP per capita } \\
\text { (in equivalent USD } \\
\text { converted using PPPs) }\end{array}$ & $\begin{array}{l}\text { GDP deflator } \\
(2008=100)\end{array}$ & $\begin{array}{l}\text { GDP deflator } \\
(2005=100)\end{array}$ & $\begin{array}{l}\text { GDP deflator } \\
(2000=100)\end{array}$ \\
\hline & (1) & (2) & (3) & (4) & (5) \\
\hline Australia & 33.9 & 43158 & 109.0 & 125.5 & 149.8 \\
\hline Austria & 51.7 & 44892 & 106.6 & 113.1 & 123.3 \\
\hline Belgium & 53.3 & 41684 & 107.7 & 115.1 & 127.6 \\
\hline Canada $^{1}$ & 41.4 & 42585 & 107.9 & 118.1 & 129.6 \\
\hline Chile $^{2}$ & 24.5 & 21260 & 115.9 & 126.8 & 179.4 \\
\hline Czech Republic & 42.3 & 28679 & 102.4 & 108.9 & 123.4 \\
\hline Denmark & 58.1 & 43564 & 107.1 & 116.8 & 130.7 \\
\hline Estonia & 39.0 & 24689 & 107.9 & 140.3 & 181.4 \\
\hline Finland & 54.6 & 40209 & 108.0 & 115.4 & 122.5 \\
\hline France & 55.1 & 37347 & 103.4 & 110.9 & 121.9 \\
\hline Germany & 43.3 & 42730 & 105.3 & 108.3 & 114.4 \\
\hline Greece & 53.5 & 25462 & 104.2 & 116.2 & 135.1 \\
\hline Hungary & 47.8 & 22494 & 112.1 & 128.4 & 175.5 \\
\hline Iceland & 45.4 & 40464 & 120.5 & 152.4 & 186.6 \\
\hline Ireland & 40.4 & 45210 & 96.6 & 99.1 & 122.6 \\
\hline Israel & 40.3 & 31296 & 111.7 & 116.3 & 124.1 \\
\hline Italy & 48.7 & 35334 & 105.5 & 112.9 & 129.5 \\
\hline Japan & 41.9 & 35695 & 94.7 & 91.6 & 85.3 \\
\hline Korea & 32.7 & 32022 & 109.6 & 115.4 & 132.6 \\
\hline Luxembourg & 43.0 & 91754 & 114.5 & 128.6 & 142.2 \\
\hline Mexico & 25.3 & 16767 & 117.4 & 139.0 & 194.7 \\
\hline Netherlands & 47.5 & 46062 & 103.0 & 109.7 & 124.6 \\
\hline New Zealand & 33.2 & 32165 & 105.6 & 117.2 & 132.1 \\
\hline Norway $^{3}$ & 54.9 & 51368 & 111.8 & 126.8 & 145.9 \\
\hline Poland & 41.7 & 22869 & 111.5 & 122.2 & 139.4 \\
\hline Portugal & 46.1 & 27204 & 101.1 & 109.3 & 129.3 \\
\hline Slovak Republic & 39.3 & 25725 & 102.2 & 109.4 & 136.5 \\
\hline Slovenia & 47.1 & 28455 & 103.8 & 115.5 & 149.6 \\
\hline Spain & 46.6 & 32775 & 100.7 & 110.5 & 134.7 \\
\hline Sweden & 50.1 & 43869 & 105.7 & 114.4 & 122.8 \\
\hline Switzerland & 31.9 & 55623 & 100.8 & 107.1 & 110.0 \\
\hline Turkey & $\mathrm{m}$ & 18002 & 129.2 & 168.0 & 523.5 \\
\hline United Kingdom & 45.5 & 37170 & 109.2 & 118.7 & 133.2 \\
\hline United States & 41.3 & 49895 & 106.0 & 114.3 & 128.4 \\
\hline Argentina & $\mathrm{m}$ & 14680 & $\mathrm{~m}$ & $\mathrm{~m}$ & $\mathrm{~m}$ \\
\hline Brazil & 33.0 & 12583 & 130.7 & 159.1 & 252.5 \\
\hline China & $\mathrm{m}$ & 10917 & $\mathrm{~m}$ & $\mathrm{~m}$ & $\mathrm{~m}$ \\
\hline Colombia $^{2}$ & $\mathrm{~m}$ & 12125 & $\mathrm{~m}$ & $\mathrm{~m}$ & $\mathrm{~m}$ \\
\hline India & $\mathrm{m}$ & $\mathrm{m}$ & $\mathrm{m}$ & $\mathrm{m}$ & $\mathrm{m}$ \\
\hline Indonesia $^{2}$ & $\mathrm{~m}$ & 10023 & $\mathrm{~m}$ & $\mathrm{~m}$ & $\mathrm{~m}$ \\
\hline Latvia & $\mathrm{m}$ & 15004 & $\mathrm{~m}$ & $\mathrm{~m}$ & $\mathrm{~m}$ \\
\hline Russian Federation & $\mathrm{m}$ & 24085 & 145.1 & 224.3 & 493.2 \\
\hline Saudi Arabia & $\mathrm{m}$ & $\mathrm{m}$ & $\mathrm{m}$ & $\mathrm{m}$ & $\mathrm{m}$ \\
\hline South Africa & $\mathrm{m}$ & 12555 & $\mathrm{~m}$ & $\mathrm{~m}$ & $\mathrm{~m}$ \\
\hline
\end{tabular}

1. Year of reference 2011

2. Year of reference 2013 instead of 2012. GDP deflators refer to 2001-13 instead of 2000-12, and to 2006-13 instead of 2005-12.

3. The GDP Mainland market value is used for Norway.

Source: OECD. See Annex 3 for notes (www.oecd.org/education/education-at-a-glance-19991487.htm)

Please refer to the Reader's Guide for information concerning symbols for missing data and abbreviations.

StatLink 게게 http://dx.doi.org/10.1787/888933286560 
Table X2.2. Basic reference statistics (reference period: calendar year 2012, 2012 current prices) ${ }^{1}$

\begin{tabular}{|c|c|c|c|c|c|c|c|}
\hline & $\begin{array}{l}\text { Gross domestic } \\
\text { product } \\
\text { (in millions of } \\
\text { local currency) }{ }^{2}\end{array}$ & $\begin{array}{c}\text { Gross } \\
\text { domestic product } \\
\text { (adjusted to } \\
\text { financial year) }\end{array}$ & $\begin{array}{l}\text { Total public } \\
\text { expenditure } \\
\text { (in millions of } \\
\text { local currency) }\end{array}$ & $\begin{array}{l}\text { Total population } \\
\text { in thousand } \\
\text { (mid-year } \\
\text { estimates) }\end{array}$ & $\begin{array}{c}\text { Purchasing power } \\
\text { parity for GDP } \\
\text { (PPP) } \\
(\mathrm{USD}=1)\end{array}$ & $\begin{array}{c}\text { Purchasing power } \\
\text { parity for GDP } \\
(\text { PPP) } \\
(\text { Euro area }=1)\end{array}$ & $\begin{array}{l}\text { Purchasing power } \\
\text { parity for private } \\
\text { consumption } \\
(\text { PPP) } \\
(\mathrm{USD}=1)\end{array}$ \\
\hline & (1) & (2) & (3) & (4) & (5) & (6) & (7) \\
\hline Australia & 1520944 & a & 515094 & 23152 & 1.5222 & 1.9515 & 1.5805 \\
\hline Austria & 317213 & a & 164052 & 8426 & 0.8386 & 1.0751 & 0.8574 \\
\hline Belgium & 388254 & a & 206852 & 11054 & 0.8426 & 1.0803 & 0.9056 \\
\hline Canada $^{4}$ & 1770014 & 1785318 & 738481 & 34343 & 1.2207 & 1.5651 & 1.2999 \\
\hline Chile $^{5}$ & 129600791 & a & 31782047 & 17403 & 350.2946 & 449.0957 & 370.1642 \\
\hline Czech Republic & 4047675 & a & 1711712 & 10509 & 13.4300 & 17.2179 & 15.5650 \\
\hline Denmark & 1866779 & $\mathrm{a}$ & 1084834 & 5591 & 7.6643 & 9.8260 & 8.5705 \\
\hline Estonia & 17637 & a & 6873 & 1325 & 0.5391 & 0.6911 & 0.6214 \\
\hline Finland & 199793 & a & 109071 & 5414 & 0.9178 & 1.1766 & 1.0019 \\
\hline France & 2091059 & a & 1151257 & 65609 & 0.8534 & 1.0941 & 0.9019 \\
\hline Greece & 194204 & $\mathrm{a}$ & 103822 & 11093 & 0.6876 & 0.8815 & 0.7793 \\
\hline Hungary & 28548800 & a & 13658622 & 9920 & 127.9363 & 164.0209 & 145.0074 \\
\hline Iceland & 1774001 & a & 805617 & 321 & 136.6984 & 175.2543 & 145.9209 \\
\hline Ireland & 172755 & a & 69811 & 4590 & 0.8325 & 1.0673 & 0.9621 \\
\hline Israel & 991762 & a & 399528 & 7911 & 4.0060 & 5.1359 & 4.4700 \\
\hline Italy & 1628004 & a & 792583 & 60339 & 0.7636 & 0.9790 & 0.8385 \\
\hline Japan $^{6}$ & 475110400 & 476364800 & 199725200 & 127552 & 104.6281 & 134.1386 & 121.3658 \\
\hline Korea & 1377456700 & a & 450811900 & 50004 & 860.2495 & 1102.8839 & 910.4712 \\
\hline Luxembourg & 43812 & a & 18843 & 532 & 0.8984 & 1.1518 & 0.9938 \\
\hline Mexico & 15561472 & a & 3942261 & 117054 & 7.9290 & 10.1654 & 8.9529 \\
\hline Netherlands & 640644 & a & 304035 & 16752 & 0.8303 & 1.0644 & 0.8822 \\
\hline New Zealand & 211632 & a & 70306 & 4444 & 1.4806 & 1.8982 & 1.6071 \\
\hline Norway $^{7}$ & 2295395 & a & 1260543 & 5019 & 8.9032 & 11.4144 & 9.8063 \\
\hline Poland & 1615895 & a & 673930 & 38534 & 1.8337 & 2.3509 & 1.9923 \\
\hline Slovenia & 36006 & a & 16975 & 2057 & 0.6152 & 0.7887 & 0.7078 \\
\hline Spain & 1055158 & a & 491414 & 46766 & 0.6884 & 0.8826 & 0.7898 \\
\hline Sweden & 3684800 & a & 1844276 & 9519 & 8.8236 & 11.3123 & 9.4636 \\
\hline Switzerland & 624592 & a & 199208 & 8039 & 1.3968 & 1.7908 & 1.6538 \\
\hline Turkey & 1416798 & a & $\mathrm{m}$ & 74899 & 1.0508 & 1.3472 & 1.1474 \\
\hline United Kingdom & 1655384 & 1645957 & 749427 & 63705 & 0.6951 & 0.8912 & 0.7046 \\
\hline United States & 16163150 & 15679235 & 6474354 & 314246 & 1.0000 & 1.2821 & 1.0000 \\
\hline \multicolumn{2}{|l|}{ Euro area } & & & & 0.78 & & \\
\hline Argentina & 2744829 & a & $\mathrm{m}$ & 41087 & 4.5508 & 5.8344 & $\mathrm{~m}$ \\
\hline Brazil & 4402537 & a & 1453357 & 196877 & 1.7771 & 2.2783 & $\mathrm{~m}$ \\
\hline China & 51947010 & a & $\mathrm{m}$ & 1354040 & 3.5140 & 4.5052 & $\mathrm{~m}$ \\
\hline Colombia $^{5}$ & 665441000 & a & $\mathrm{m}$ & 46582 & 1178.1469 & 1510.4448 & $\mathrm{~m}$ \\
\hline India & $\mathrm{m}$ & $\mathrm{a}$ & $\mathrm{m}$ & 1227193 & 15.9149 & 20.4038 & $\mathrm{~m}$ \\
\hline Indonesia ${ }^{5}$ & 9524736500 & a & $\mathrm{m}$ & 249866 & 3803.3510 & 4876.0911 & $\mathrm{~m}$ \\
\hline Latvia & 15492 & a & $\mathrm{m}$ & 2034 & 0.5077 & 0.6509 & $\mathrm{~m}$ \\
\hline Russian Federation & 62218378 & a & $\mathrm{m}$ & 143170 & 18.0435 & 23.1327 & 17.4149 \\
\hline Saudi Arabia & $\mathrm{m}$ & a & $\mathrm{m}$ & $\mathrm{m}$ & $\mathrm{m}$ & $\mathrm{m}$ & $\mathrm{m}$ \\
\hline South Africa & 3138980 & a & $\mathrm{m}$ & 51012 & 4.9010 & 6.2833 & $\mathrm{~m}$ \\
\hline
\end{tabular}

1. Data on GDP, PPPs and total public expenditure in countries in the Euro area are provided in Euros.

2. GDP calculated for the fiscal year in Australia and GDP and total public expenditure calculated for the fiscal year in New Zealand.

3. For countries where GDP is not reported for the same reference period as data on educational finance, GDP is estimated as: wt-1 (GDPt - 1) + wt (GDPt), where wt and wt 1 are the weights for the respective portions of the two reference periods for GDP which fall within the educational financial year. Adjustments were made in Chapter B for Canada, Japan, the United Kingdom and the United States.

4. Year of reference 2011.

5. Year of reference 2013

6. Total public expenditure adjusted to financial year.

7. The GDP Mainland market value is used for Norway.

Source: OECD. See Annex 3 for notes (www.oecd.org/education/education-at-a-glance-19991487.htm).

Please refer to the Reader's Guide for information concerning symbols for missing data and abbreviations.

StatLink 제의 http://dx.doi.org/10.1787/888933286577 
Table X2.3. [1/2] Basic reference statistics (reference period: calendar year 2000, 2005, 2008, 2010, 2011) ${ }^{1}$

\begin{tabular}{|c|c|c|c|c|c|c|c|c|c|c|}
\hline & \multicolumn{5}{|c|}{$\begin{array}{l}\text { Gross domestic product } \\
\text { (in millions of local currency, current prices) }\end{array}$} & \multicolumn{5}{|c|}{$\begin{array}{c}\text { Total public expenditure } \\
\text { (in millions of local currency, current prices) }\end{array}$} \\
\hline & 2000 & 2005 & 2008 & 2010 & 2011 & 2000 & 2005 & 2008 & 2010 & 2011 \\
\hline & $(1)$ & $(2)$ & (3) & $(4)$ & (5) & (6) & (7) & $(8)$ & $(9)$ & $(10)$ \\
\hline Australia & 705275 & 997968 & 1258074 & 1407865 & 1488028 & 225913 & 309431 & 405784 & 473579 & 498406 \\
\hline Austria & 213196 & 253009 & 291930 & 294208 & 308675 & 108287 & 122585 & 139494 & 150593 & 151994 \\
\hline Belgium & 257605 & 311150 & 355066 & 365747 & 379991 & 123943 & 157399 & 172484 & 187026 & 197422 \\
\hline Chile $^{2}$ & 42094989 & 82018171 & 96443761 & 121402822 & 129600791 & 10559689 & 15327440 & 23797395 & 27847954 & 30050204 \\
\hline Czech Republic & 2372630 & 3257972 & 4015346 & 3953651 & 4022410 & 945255 & 1340123 & 1583527 & 1661774 & 1653244 \\
\hline Denmark & 1326912 & 1586537 & 1797547 & 1798649 & 1833404 & 694479 & 815717 & 903263 & 1016158 & 1034208 \\
\hline Estonia & 6171 & 11260 & 16511 & 14709 & 16404 & 2225 & 3757 & 6441 & 5828 & 6109 \\
\hline Finland & 136261 & 164387 & 193711 & 187100 & 196869 & 63903 & 79262 & 91372 & 99707 & 104259 \\
\hline France & 1485303 & 1771978 & 1995850 & 1998481 & 2059284 & 744119 & 920351 & 1030025 & 1095602 & 1118728 \\
\hline Greece & 141732 & 199153 & 242096 & 226210 & 207752 & 63693 & 86097 & 117992 & 114302 & 108003 \\
\hline Hungary & 13293578 & 22331300 & 26949316 & 26946030 & 28035033 & 6251647 & 11032047 & 13070489 & 13252926 & 13834811 \\
\hline Iceland & 703445 & 1057998 & 1547817 & 1621053 & 1700558 & 286259 & 433346 & 853725 & 791880 & 771800 \\
\hline Ireland & 107799 & 169153 & 186870 & 164931 & 171042 & 33010 & 55177 & 77009 & 103427 & 76536 \\
\hline Israel & 535966 & 633762 & 767547 & 870843 & 924618 & 261087 & 296289 & 332256 & 367301 & 365561 \\
\hline Italy & 1239759 & 1490409 & 1632933 & 1605694 & 1638857 & 549577 & 688251 & 765537 & 782101 & 788137 \\
\hline Japan & 508780800 & 504599000 & 493691650 & 479616000 & 472260700 & 193917400 & 183640900 & 188561300 & 195879800 & 199103100 \\
\hline Korea & 635184600 & 919797300 & 1104492200 & 1265308000 & 1332681000 & 135324800 & 230062600 & 312548300 & 353006600 & 373227400 \\
\hline Luxembourg & 23122 & 29771 & 37523 & 39371 & 42410 & 8270 & 12573 & 14624 & 17098 & 17882 \\
\hline Mexico & 6132389 & 9424602 & 12256864 & 13266858 & 14508784 & 1139998 & 1979808 & 2894807 & 3355288 & 3655757 \\
\hline Netherlands & 448701 & 540656 & 635794 & 631512 & 642929 & 187162 & 230884 & 278455 & 304447 & 302269 \\
\hline New Zealand & 118446 & 161615 & 185608 & 199108 & 207392 & 36559 & 49320 & 64002 & 70450 & 69076 \\
\hline Norway $^{3}$ & 1113894 & 1464974 & 1862873 & 1987362 & 2157835 & 626569 & 818805 & 1018107 & 1149163 & 1207768 \\
\hline Poland & 746255 & 984919 & 1277322 & 1437357 & 1553582 & 294012 & 427147 & 551403 & 643465 & 663757 \\
\hline Slovenia & 18902 & 29227 & 37951 & 36220 & 36868 & 8636 & 13011 & 16511 & 17894 & 18350 \\
\hline Spain & 646250 & 930566 & 1116207 & 1080913 & 1075147 & 246890 & 349501 & 450948 & 485467 & 480111 \\
\hline Sweden & 2380358 & 2907352 & 3387599 & 3519994 & 3656577 & 1248029 & 1491382 & 1657889 & 1746603 & 1792006 \\
\hline Switzerland & 458779 & 507463 & 597381 & 606146 & 618325 & 151837 & 176236 & 187914 & 189561 & 196889 \\
\hline Turkey & 166658 & 648932 & 950534 & 1098799 & 1297713 & $\mathrm{~m}$ & $\mathrm{~m}$ & 345392 & 442178 & 485001 \\
\hline United Kingdom & 1023512 & 1326660 & 1518675 & 1558365 & 1617677 & 358902 & 553033 & 686738 & 738598 & 736445 \\
\hline United States & 10284780 & 13093720 & 14718590 & 14964380 & 15517930 & 3353547 & 4563353 & 5567081 & 6153839 & 5754000 \\
\hline Brazil & 1179482 & 2147240 & 3032204 & 3770085 & 4143015 & 394349 & 670514 & 939831 & 1211373 & 1308035 \\
\hline Russian Federation & 7298009 & 21609766 & 41276849 & 46308541 & 55967227 & 2016630 & 7380575 & $\mathrm{~m}$ & $\mathrm{~m}$ & $\mathrm{~m}$ \\
\hline
\end{tabular}

1. Data on GDP, and total public expenditure in countries in the Euro area are provided in Euros.

2. Years of reference 2001, 2006, 2009, 2011 and 2012 instead of 2000, 2005, 2008, 2010 and 2011.

3. The GDP Mainland market value is used for Norway.

Source: OECD. See Annex 3 for notes (www.oecd.org/education/education-at-a-glance-19991487.htm)

Please refer to the Reader's Guide for information concerning symbols for missing data and abbreviations.

StatLink 제으 http://dx.doi.org/10.1787/888933286586 
Table X2.3. [2/2] Basic reference statistics (reference period: calendar year 2000, 2005, 2008, 2010, 2011) ${ }^{1}$

\begin{tabular}{|c|c|c|c|c|c|c|}
\hline & \multicolumn{3}{|c|}{$\begin{array}{l}\text { Gross Domestic Product } \\
\text { (in millions of local currency, } 2012 \text { constant prices) }\end{array}$} & \multicolumn{3}{|c|}{$\begin{array}{c}\text { Total Public Expenditure } \\
\text { (in millions of local currency, } 2012 \text { constant prices) }\end{array}$} \\
\hline & 2008 & 2010 & 2011 & 2008 & 2010 & 2011 \\
\hline & $(11)$ & $(12)$ & (13) & (14) & (15) & (16) \\
\hline Australia & 1371034 & 1430317 & 1483654 & 442219 & 481131 & 496940 \\
\hline Austria & 311246 & 305049 & 314429 & 148723 & 156142 & 154827 \\
\hline Belgium & 382327 & 381623 & 387873 & 185727 & 195144 & 201517 \\
\hline Chile $^{2}$ & 111759872 & 125027571 & 131134365 & 27576629 & 28679416 & 30405790 \\
\hline Czech Republic & 4111210 & 4001777 & 4080472 & 1621333 & 1682002 & 1677108 \\
\hline Denmark & 1925955 & 1857798 & 1879078 & 967788 & 1049575 & 1059972 \\
\hline Estonia & 17815 & 15567 & 16853 & 6950 & 6168 & 6276 \\
\hline Finland & 209169 & 197612 & 202681 & 98664 & 105309 & 107337 \\
\hline France & 2063017 & 2041566 & 2084095 & 1064689 & 1119222 & 1132207 \\
\hline Greece & 252327 & 228095 & 207877 & 122978 & 115255 & 108068 \\
\hline Hungary & 30221464 & 28464834 & 28978563 & 14657489 & 13999923 & 14300427 \\
\hline Iceland & 1865164 & 1717284 & 1753959 & 1028763 & 838889 & 796036 \\
\hline Ireland & 180580 & 168626 & 173299 & 74417 & 105744 & 77546 \\
\hline Israel & 857573 & 924158 & 962951 & 371227 & 389788 & 380716 \\
\hline Italy & 1722693 & 1656179 & 1665871 & 807618 & 806691 & 801128 \\
\hline Japan & 467302218 & 466371161 & 467865676 & 178482082 & 190470480 & 197250176 \\
\hline Korea & 1210975823 & 1298839506 & 1346680975 & 342680949 & 362361510 & 377148199 \\
\hline Luxembourg & 42967 & 42769 & 43884 & 16746 & 18574 & 18503 \\
\hline Mexico & 14388718 & 14418513 & 14972048 & 3398305 & 3646550 & 3772485 \\
\hline Netherlands & 655138 & 640324 & 650937 & 286927 & 308695 & 306034 \\
\hline New Zealand & 196080 & 201724 & 206423 & 67613 & 71376 & 68753 \\
\hline Norway $^{3}$ & 2082486 & 2083187 & 2211899 & 1138131 & 1204572 & 1238028 \\
\hline Poland & 1424257 & 1515860 & 1588011 & 614833 & 678609 & 678466 \\
\hline Slovenia & 39384 & 36758 & 36981 & 17134 & 18160 & 18406 \\
\hline Spain & 1124344 & 1084346 & 1077682 & 454235 & 487009 & 481243 \\
\hline Sweden & 3581641 & 3599250 & 3695326 & 1752853 & 1785930 & 1810996 \\
\hline Switzerland & 602215 & 606825 & 617748 & 189435 & 189774 & 196705 \\
\hline Turkey & 1227670 & 1275403 & 1387322 & 446094 & 513247 & 518491 \\
\hline United Kingdom & 1659099 & 1617909 & 1644546 & 750237 & 766819 & 748677 \\
\hline United States & 15597149 & 15547779 & 15797034 & 5899382 & 6393751 & 5857491 \\
\hline \multirow{2}{*}{ 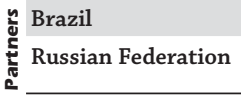 } & 3963739 & 4341660 & 4364448 & 1228560 & 1395027 & 1377946 \\
\hline & 59887606 & 57691503 & 60150289 & $\mathrm{~m}$ & $\mathrm{~m}$ & $\mathrm{~m}$ \\
\hline
\end{tabular}

1. Data on GDP, and total public expenditure in countries in the Euro area are provided in Euros.

2. Years of reference 2001, 2006, 2009, 2011 and 2012 instead of 2000, 2005, 2008, 2010 and 2011.

3. The GDP Mainland market value is used for Norway.

Source: OECD. See Annex 3 for notes (www.oecd.org/education/education-at-a-glance-19991487.htm).

Please refer to the Reader's Guide for information concerning symbols for missing data and abbreviations.

StatLink 젣ㄴ $\mathrm{http}$ ://dx.doi.org/10.1787/888933286586 
Table X2.4a. [1/2] 'Teachers' statutory salaries at different points in their careers, for teachers with typical qualification (2013)

Annual salaries in public institutions for teachers with typical qualification, in national currency

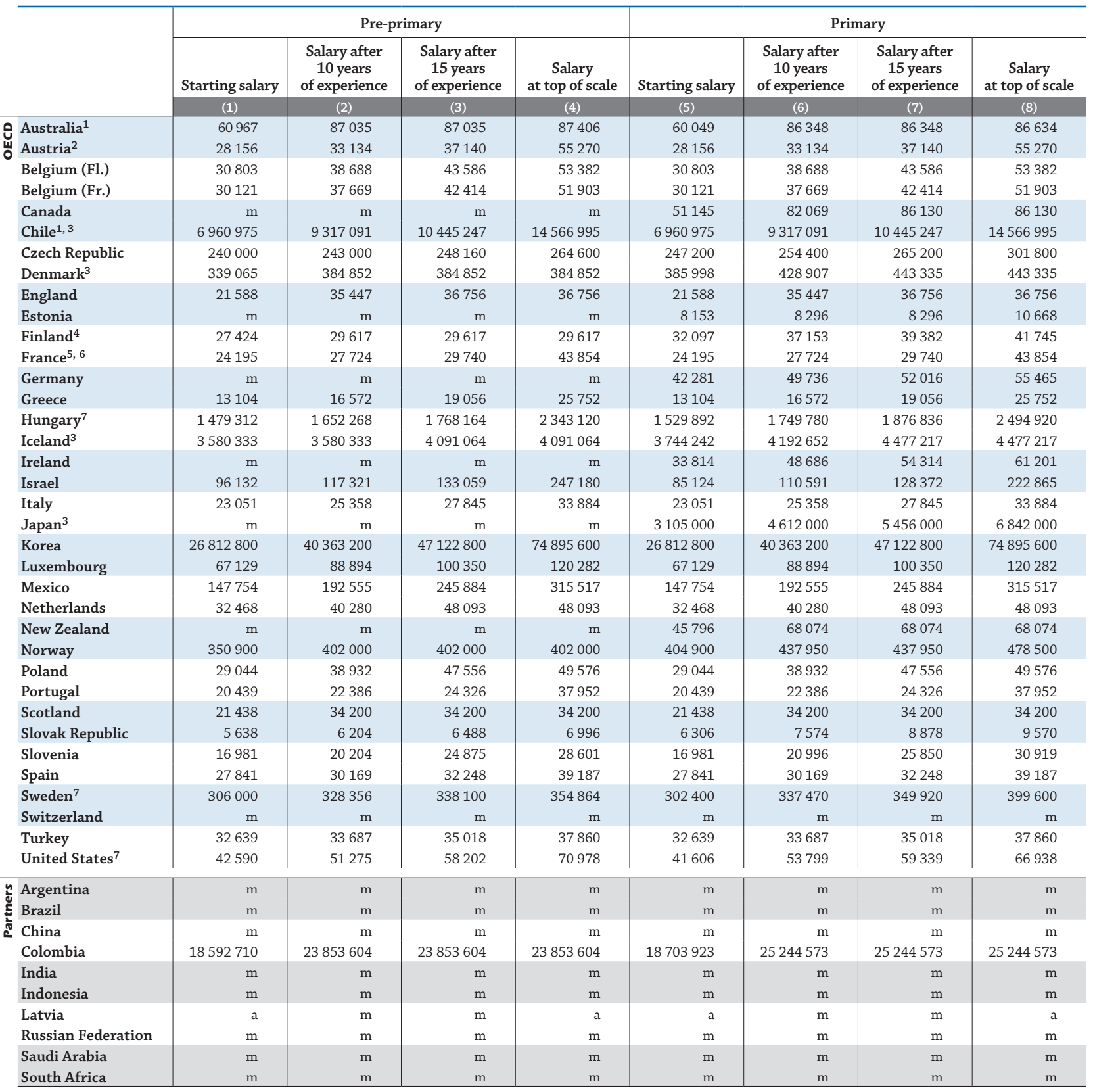

1. Statutory salaries do not include the part of social security contributions and pension scheme contributions paid by the employees.

2. Figures for the pre-primary level refer to primary teachers (in primary schools only) teaching pre-primary classes.

3. Statutory salaries include the part of social security contributions and pension scheme contributions paid by the employers.

4. Includes data on the majority, i.e. kindergarten teachers only for pre-primary education.

5. Includes average bonuses for overtime hours for lower and upper secondary teachers.

6. The typical qualification of starting teachers differ substantially from the typical qualification of all the current teachers.

7. Actual base salaries.

Source: OECD. See Annex 3 for notes (www.oecd.org/education/education-at-a-glance-19991487.htm).

Please refer to the Reader's Guide for information concerning symbols for missing data and abbreviations.

StatLink Nilst http://dx.doi.org/10.1787/888933286596 
Table X2.4a. [2/2] Teachers' statutory salaries at different points in their careers, for teachers with typical qualification (2013)

Annual salaries in public institutions for teachers with typical qualification, in national currency

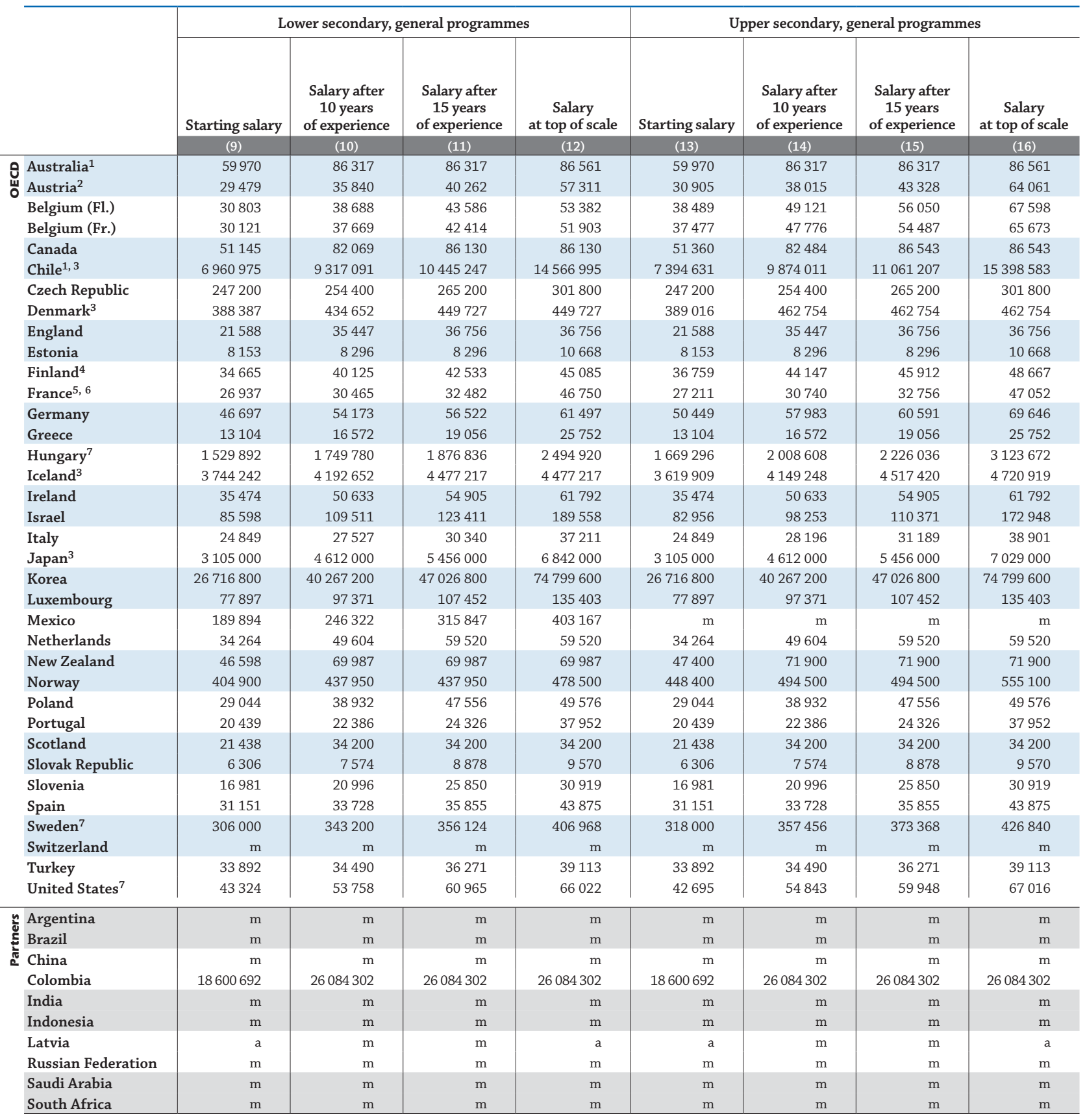

1. Statutory salaries do not include the part of social security contributions and pension scheme contributions paid by the employees.

2. Figures for the pre-primary level refer to primary teachers (in primary schools only) teaching pre-primary classes.

3. Statutory salaries include the part of social security contributions and pension scheme contributions paid by the employers.

4. Includes data on the majority, i.e. kindergarten teachers only for pre-primary education.

5. Includes average bonuses for overtime hours for lower and upper secondary teachers.

6. The typical qualification of starting teachers differ substantially from the typical qualification of all the current teachers.

7. Actual base salaries.

Source: OECD. See Annex 3 for notes (www.oecd.org/education/education-at-a-glance-19991487.htm).

Please refer to the Reader's Guide for information concerning symbols for missing data and abbreviations.

StatLink 제그 http://dx.doi.org/10.1787/888933286596 
Table X2.4b. [1/2] Teachers' statutory salaries at different points in their careers, for teachers with minimum qualification (2013)

Annual salaries in public institutions for teachers with minimum qualification, in national currency

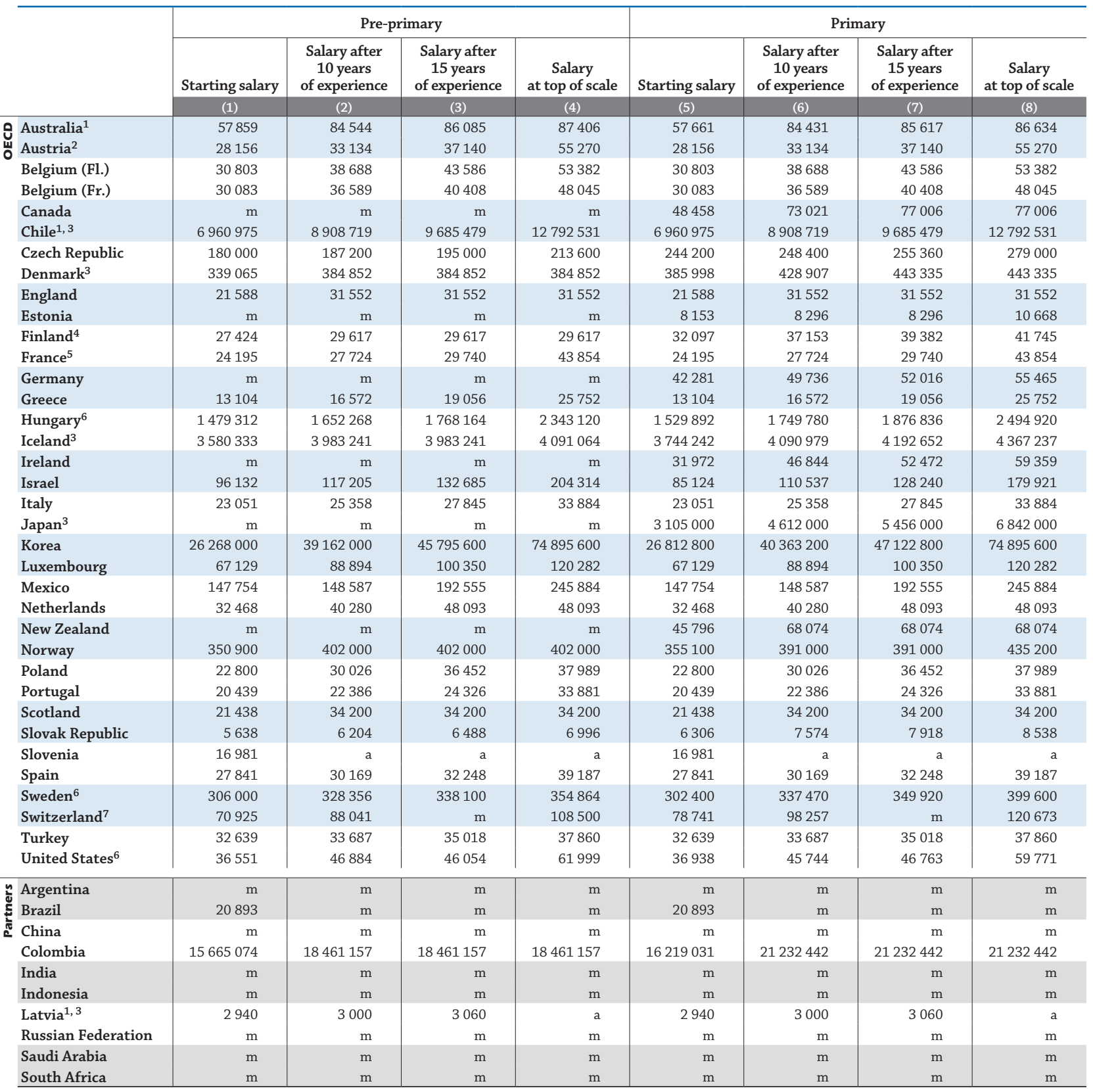

1. Statutory salaries do not include the part of social security contributions and pension scheme contributions paid by the employees.

2. Figures for the pre-primary level refer to primary teachers (in primary schools only) teaching pre-primary classes.

3. Statutory salaries include the part of social security contributions and pension scheme contributions paid by the employers.

4. Includes data on the majority, i.e. kindergarten teachers only for pre-primary education.

5. Includes average bonuses for overtime hours for lower and upper secondary teachers.

6. Actual base salaries.

7. Salaries after 11 years of experience for columns 2, 6, 10 and 14

Source: OECD. See Annex 3 for notes (www.oecd.org/education/education-at-a-glance-19991487.htm).

Please refer to the Reader's Guide for information concerning symbols for missing data and abbreviations.

StatLink 部实 http://dx.doi.org/10.1787/888933286604 
Table X2.4b. [2/2] Teachers' statutory salaries at different points in their careers, for teachers with minimum qualification (2013)

Annual salaries in public institutions for teachers with minimum qualification, in national currency

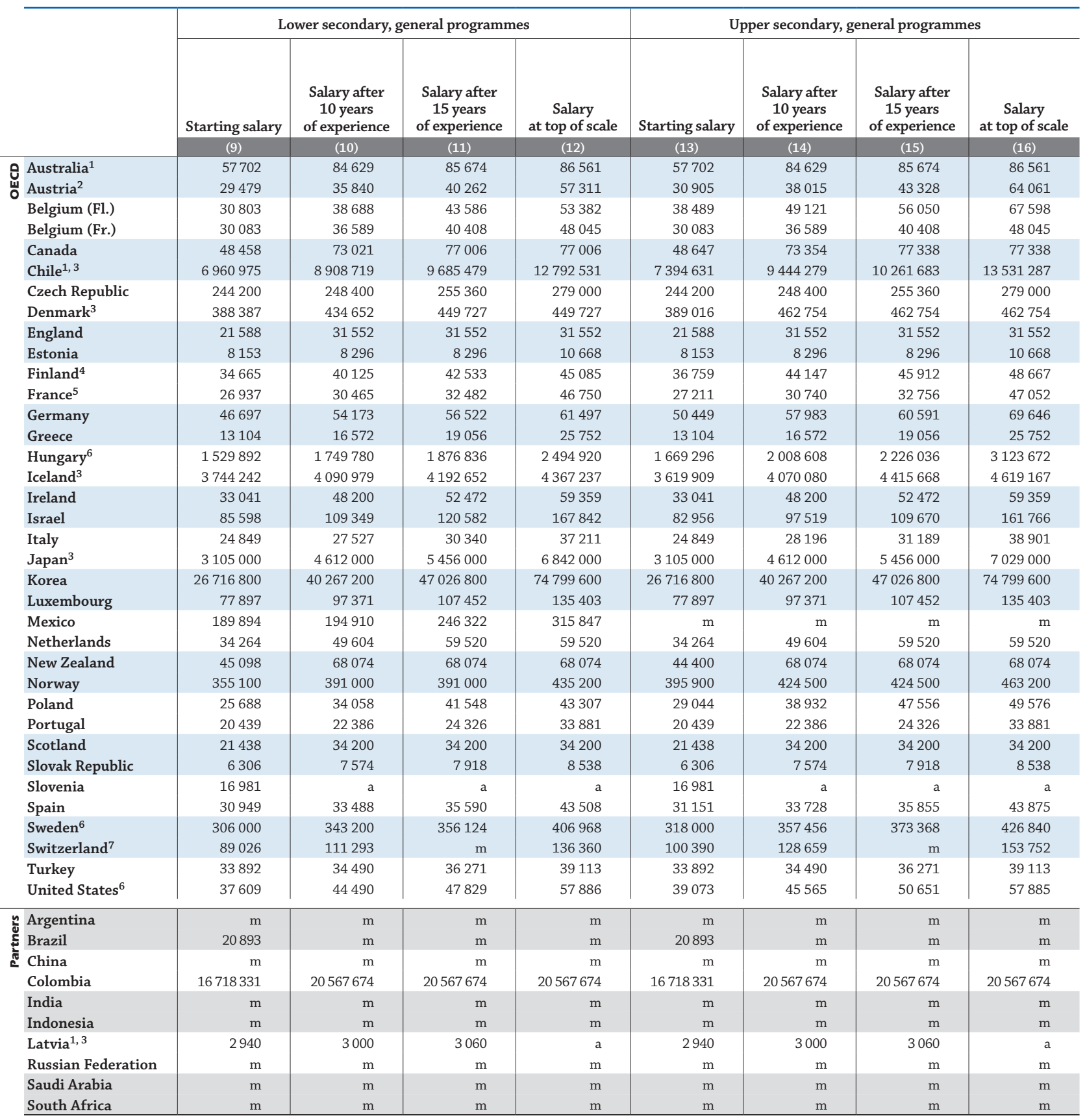

1. Statutory salaries do not include the part of social security contributions and pension scheme contributions paid by the employees.

2. Figures for the pre-primary level refer to primary teachers (in primary schools only) teaching pre-primary classes.

3. Statutory salaries include the part of social security contributions and pension scheme contributions paid by the employers.

4. Includes data on the majority, i.e. kindergarten teachers only for pre-primary education.

5. Includes average bonuses for overtime hours for lower and upper secondary teachers.

6. Actual base salaries.

7. Salaries after 11 years of experience for columns 2, 6, 10 and 14 .

Source: OECD. See Annex 3 for notes (www.oecd.org/education/education-at-a-glance-19991487.htm).

Please refer to the Reader's Guide for information concerning symbols for missing data and abbreviations.

StatLink 제그 http://dx.doi.org/10.1787/888933286604 
Table X2.4c. [1/2] Trends in teachers' salaries between 2000 and 2013, for teachers with typical qualification ${ }^{\mathbf{1}}$

Annual statutory teachers' salaries in public institutions for teachers with 15 years of experience and typical qualification, by level of education, in national currency

\begin{tabular}{|c|c|c|c|c|c|c|c|c|c|c|}
\hline & \multicolumn{5}{|c|}{ Pre-primary } & \multicolumn{5}{|c|}{ Primary } \\
\hline & 2000 & 2005 & 2011 & 2012 & 2013 & 2000 & 2005 & 2011 & 2012 & 2013 \\
\hline & (1) & (2) & (8) & (9) & (10) & (11) & (12) & (18) & (19) & $(20)$ \\
\hline Austriaa $^{2,3}$ & $\mathrm{~m}$ & 31050 & 35889 & 36653 & 37140 & 25826181 & 31050 & 35889 & 36653 & 37140 \\
\hline Belgium (Fl.) & $\mathrm{m}$ & 35417 & 41094 & 41968 & 43586 & 29579 & 35417 & 41094 & 41968 & 43586 \\
\hline Belgium (Fr.) & 28485 & 33427 & 39905 & 40785 & 42414 & 28485 & 33427 & 39905 & 40785 & 42414 \\
\hline Canada & $\mathrm{m}$ & $\mathrm{m}$ & $\mathrm{m}$ & $\mathrm{m}$ & $\mathrm{m}$ & $\mathrm{m}$ & $\mathrm{m}$ & $\mathrm{m}$ & $\mathrm{m}$ & 86130 \\
\hline Chile & $\mathrm{m}$ & $\mathrm{m}$ & 9474152 & 9947847 & 10445247 & $\mathrm{~m}$ & $\mathrm{~m}$ & 9474152 & 9947847 & 10445247 \\
\hline Czech Republic & $\mathrm{m}$ & $\mathrm{m}$ & $\mathrm{m}$ & $\mathrm{m}$ & 248160 & $\mathrm{~m}$ & $\mathrm{~m}$ & $\mathrm{~m}$ & $\mathrm{~m}$ & 265200 \\
\hline Denmark & 269948 & 334577 & 375122 & 382384 & 384852 & 315530 & 367323 & 428628 & 429083 & 443335 \\
\hline England & 30018 & 33978 & 36756 & 36756 & 36756 & 30018 & 33978 & 36756 & 36756 & 36756 \\
\hline Estonia & $\mathrm{m}$ & $\mathrm{m}$ & $\mathrm{m}$ & $\mathrm{m}$ & $\mathrm{m}$ & 3068 & 4379 & 7728 & 7728 & 8296 \\
\hline Finland & 19956 & 23333 & 28671 & 29191 & 29617 & 24961 & 30791 & 38222 & 38850 & 39382 \\
\hline France & 27288 & 28395 & 29831 & 29888 & 29740 & 27288 & 28395 & 29831 & 29888 & 29740 \\
\hline Germany & $\mathrm{m}$ & $\mathrm{m}$ & $\mathrm{m}$ & $\mathrm{m}$ & $\mathrm{m}$ & $\mathrm{m}$ & 43320 & 49587 & 50991 & 52016 \\
\hline Hungary ${ }^{4}$ & 751668 & 1739076 & 1779564 & 1778004 & 1768164 & 897168 & 1944576 & 1911204 & 1890288 & 1876836 \\
\hline Iceland & $\mathrm{m}$ & 2821586 & 3901395 & 4258019 & 4091064 & $\mathrm{~m}$ & 3100440 & 4264973 & 4321578 & 4477217 \\
\hline Ireland & $\mathrm{m}$ & $\mathrm{m}$ & $\mathrm{m}$ & $\mathrm{m}$ & $\mathrm{m}$ & 33370 & 48206 & 54314 & 54314 & 54314 \\
\hline Israel & 72174 & 82076 & 117644 & 129950 & 133059 & 75912 & 82179 & 125440 & 129562 & 128372 \\
\hline Italy & $\mathrm{m}$ & 25234 & 27845 & 27845 & 27845 & 20849 & 25234 & 27845 & 27845 & 27845 \\
\hline Japan & $\mathrm{m}$ & $\mathrm{m}$ & $\mathrm{m}$ & $\mathrm{m}$ & $\mathrm{m}$ & $\mathrm{m}$ & 6236000 & 5456000 & 5456000 & 5456000 \\
\hline Korea & $\mathrm{m}$ & 38608000 & 44222400 & 45800400 & 47122800 & $\mathrm{~m}$ & 39712000 & 44222400 & 45800400 & 47122800 \\
\hline Luxembourg & $\mathrm{m}$ & 62139 & 93182 & 97902 & 100350 & $\mathrm{~m}$ & 62139 & 93182 & 97902 & 100350 \\
\hline Mexico & 110833 & 159128 & 225605 & 235139 & 245884 & 110833 & 159128 & 225605 & 235139 & 245884 \\
\hline Netherlands & $\mathrm{m}$ & $\mathrm{m}$ & $\mathrm{m}$ & $\mathrm{m}$ & 48093 & $\mathrm{~m}$ & $\mathrm{~m}$ & $\mathrm{~m}$ & $\mathrm{~m}$ & 48093 \\
\hline New Zealand & $\mathrm{m}$ & $\mathrm{m}$ & $\mathrm{m}$ & $\mathrm{m}$ & $\mathrm{m}$ & $\mathrm{m}$ & $\mathrm{m}$ & $\mathrm{m}$ & $\mathrm{m}$ & 68074 \\
\hline Norway & $\mathrm{m}$ & 287000 & 374500 & 381500 & 402000 & $\mathrm{~m}$ & 327500 & 408500 & 415650 & 437950 \\
\hline Poland & $\mathrm{m}$ & 31216 & 42860 & 45785 & 47556 & $\mathrm{~m}$ & 31216 & 42860 & 45785 & 47556 \\
\hline Portugal & $\mathrm{m}$ & 24759 & 28069 & 24326 & 24326 & $\mathrm{~m}$ & 24759 & 28069 & 24326 & 24326 \\
\hline Scotland & 14022 & 29827 & 34200 & 34200 & 34200 & 22743 & 29827 & 34200 & 34200 & 34200 \\
\hline Slovak Republic & $\mathrm{m}$ & $\mathrm{m}$ & 6156 & 6236 & 6488 & $\mathrm{~m}$ & $\mathrm{~m}$ & 7518 & 7614 & 8878 \\
\hline Sweden $^{4}$ & $\mathrm{~m}$ & 261000 & 318000 & $\mathrm{~m}$ & 338100 & $\mathrm{~m}$ & 283200 & 322600 & $\mathrm{~m}$ & 349920 \\
\hline Switzerland & $\mathrm{m}$ & $\mathrm{m}$ & $\mathrm{m}$ & $\mathrm{m}$ & $\mathrm{m}$ & $\mathrm{m}$ & $\mathrm{m}$ & $\mathrm{m}$ & $\mathrm{m}$ & $\mathrm{m}$ \\
\hline Turkey & 4560 & 16464 & 29426 & 32049 & 35018 & 4560 & 16464 & 29426 & 32049 & 35018 \\
\hline United States ${ }^{4}$ & 36758 & 41501 & $\mathrm{~m}$ & 57249 & 58202 & 38046 & 51413 & 53801 & 58367 & 59339 \\
\hline Argentina & $\mathrm{m}$ & $\mathrm{m}$ & $\mathrm{m}$ & $\mathrm{m}$ & $\mathrm{m}$ & $\mathrm{m}$ & $\mathrm{m}$ & $\mathrm{m}$ & $\mathrm{m}$ & $\mathrm{m}$ \\
\hline Brazil & $\mathrm{m}$ & $\mathrm{m}$ & $\mathrm{m}$ & $\mathrm{m}$ & $\mathrm{m}$ & $\mathrm{m}$ & $\mathrm{m}$ & $\mathrm{m}$ & $\mathrm{m}$ & $\mathrm{m}$ \\
\hline China & $\mathrm{m}$ & $\mathrm{m}$ & $\mathrm{m}$ & $\mathrm{m}$ & $\mathrm{m}$ & $\mathrm{m}$ & $\mathrm{m}$ & $\mathrm{m}$ & $\mathrm{m}$ & $\mathrm{m}$ \\
\hline Colombia & $\mathrm{m}$ & $\mathrm{m}$ & $\mathrm{m}$ & $\mathrm{m}$ & 23853604 & $\mathrm{~m}$ & $\mathrm{~m}$ & $\mathrm{~m}$ & $\mathrm{~m}$ & 25244573 \\
\hline India & $\mathrm{m}$ & $\mathrm{m}$ & $\mathrm{m}$ & $\mathrm{m}$ & $\mathrm{m}$ & $\mathrm{m}$ & $\mathrm{m}$ & $\mathrm{m}$ & $\mathrm{m}$ & $\mathrm{m}$ \\
\hline Indonesia & $\mathrm{m}$ & $\mathrm{m}$ & $\mathrm{m}$ & $\mathrm{m}$ & $\mathrm{m}$ & $\mathrm{m}$ & $\mathrm{m}$ & $\mathrm{m}$ & $\mathrm{m}$ & $\mathrm{m}$ \\
\hline Latvia & 1321 & 2321 & 4071 & 4341 & $\mathrm{~m}$ & 1321 & 2321 & 4071 & 4341 & $\mathrm{~m}$ \\
\hline Russian Federation & $\mathrm{m}$ & $\mathrm{m}$ & $\mathrm{m}$ & $\mathrm{m}$ & $\mathrm{m}$ & $\mathrm{m}$ & $\mathrm{m}$ & $\mathrm{m}$ & $\mathrm{m}$ & $\mathrm{m}$ \\
\hline Saudi Arabia & $\mathrm{m}$ & $\mathrm{m}$ & $\mathrm{m}$ & $\mathrm{m}$ & $\mathrm{m}$ & $\mathrm{m}$ & $\mathrm{m}$ & $\mathrm{m}$ & $\mathrm{m}$ & $\mathrm{m}$ \\
\hline South Africa & $\mathrm{m}$ & $\mathrm{m}$ & $\mathrm{m}$ & $\mathrm{m}$ & $\mathrm{m}$ & $\mathrm{m}$ & $\mathrm{m}$ & $\mathrm{m}$ & $\mathrm{m}$ & $\mathrm{m}$ \\
\hline
\end{tabular}

Note: Years 2006, 2007, 2008, 2009, 2010 (i.e. columns 3-7; 13-17; 23-27; 33-37) are available for consultation on line (see StatLink below).

1. Data on salaries for countries now in the Euro area are shown in euros.

2. Break in time series following methodological changes in 2007 for upper secondary education.

3. Figures for the pre-primary level refer to primary teachers (in primary schools only) teaching pre-primary classes.

4. Actual base salaries.

Source: OECD. See Annex 3 for notes (www.oecd.org/education/education-at-a-glance-19991487.htm).

Please refer to the Reader's Guide for information concerning symbols for missing data and abbreviations.

StatLink 츔느 http://dx.doi.org/10.1787/888933286619 
Table X2.4c. [2/2] Trends in teachers' salaries between 2000 and 2013, for teachers with typical qualification ${ }^{1}$

Annual statutory teachers' salaries in public institutions for teachers with 15 years of experience and typical qualification, by level of education, in national currency

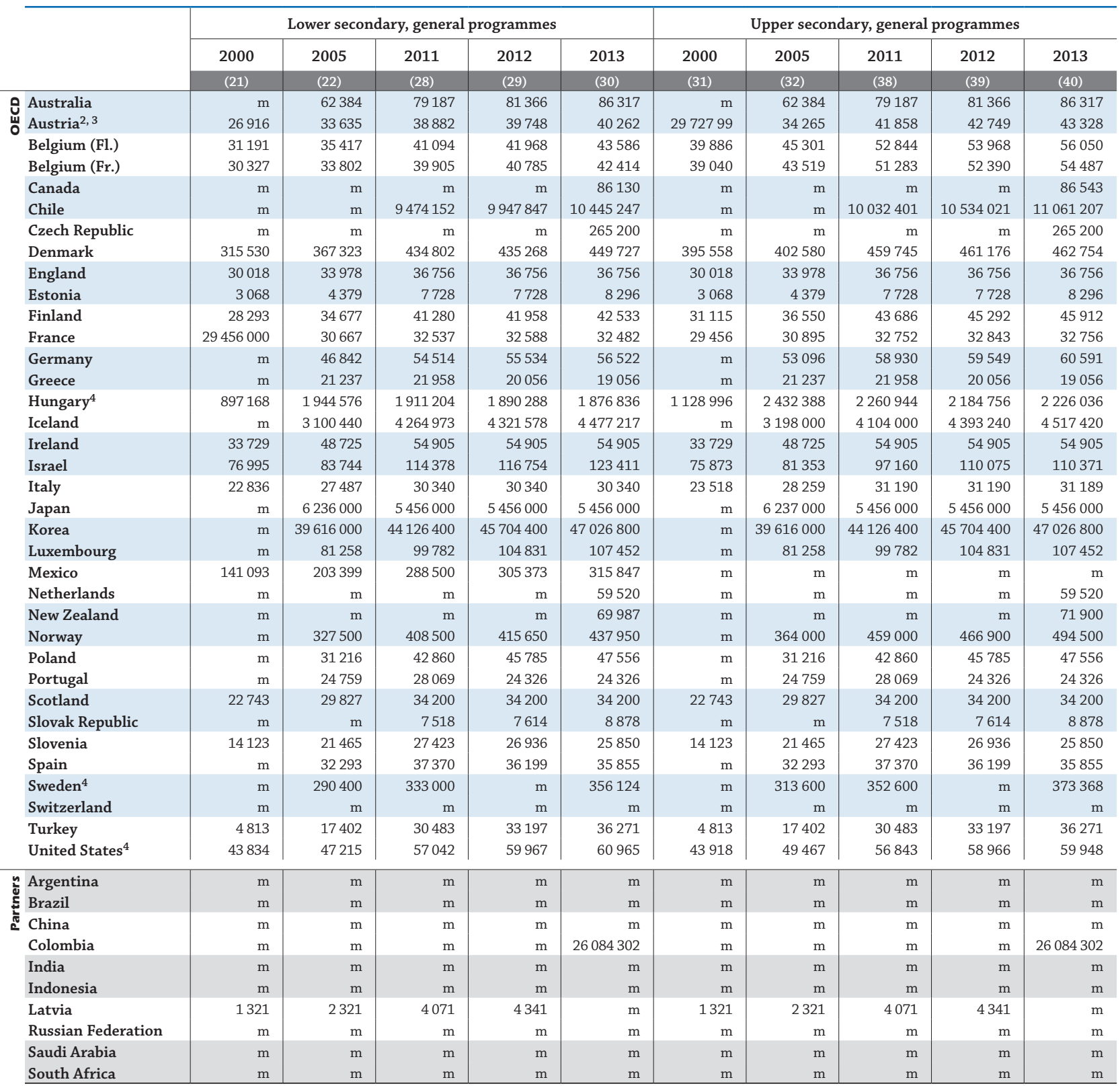

Note: Years 2006, 2007, 2008, 2009, 2010 (i.e. columns 3-7; 13-17; 23-27; 33-37) are available for consultation on line (see StatLink below).

1. Data on salaries for countries now in the Euro area are shown in euros.

2. Break in time series following methodological changes in 2007 for upper secondary education.

3. Figures for the pre-primary level refer to primary teachers (in primary schools only) teaching pre-primary classes.

4. Actual base salaries.

Source: OECD. See Annex 3 for notes (www.oecd.org/education/education-at-a-glance-19991487.htm).

Please refer to the Reader's Guide for information concerning symbols for missing data and abbreviations.

StatLink 제 जी http://dx.doi.org/10.1787/888933286619 
Table X2.4d. [1/2] Trends in teachers' salaries between 2000 and 2013, for teachers with minimum qualification ${ }^{1}$

Annual statutory teachers' salaries in public institutions for teachers with 15 years of experience and minimum qualification, by level of education, in national currency

\begin{tabular}{|c|c|c|c|c|c|c|c|c|c|c|}
\hline & \multicolumn{5}{|c|}{ Pre-primary } & \multicolumn{5}{|c|}{ Primary } \\
\hline & 2000 & 2005 & 2011 & 2012 & 2013 & 2000 & 2005 & 2011 & 2012 & 2013 \\
\hline & $(1)$ & (2) & (8) & (9) & $(10)$ & $(11)$ & $(12)$ & (18) & (19) & $(20)$ \\
\hline Austria $^{2,3}$ & $\mathrm{~m}$ & 31050 & 35889 & 36653 & 37140 & 2582618 & 31050 & 35889 & 36653 & 37140 \\
\hline Belgium (Fl.) & $\mathrm{m}$ & 35417 & 41094 & 41968 & 43586 & 29579 & 35417 & 41094 & 41968 & 43586 \\
\hline Belgium (Fr.) & 28485 & 32188 & 38015 & 38857 & 40408 & 28485 & 32188 & 38015 & 38857 & 40408 \\
\hline Canada & $\mathrm{m}$ & $\mathrm{m}$ & $\mathrm{m}$ & $\mathrm{m}$ & $\mathrm{m}$ & $\mathrm{m}$ & $\mathrm{m}$ & 73154 & 74981 & 77006 \\
\hline Chile & $\mathrm{m}$ & $\mathrm{m}$ & 8785016 & 9224259 & 9685479 & $\mathrm{~m}$ & $\mathrm{~m}$ & 8785016 & 9224259 & 9685479 \\
\hline Czech Republic ${ }^{4}$ & $\mathrm{~m}$ & 279001 & 274829 & 195000 & 195000 & 125501 & 250559 & 311793 & 255360 & 255360 \\
\hline Denmark & 269948 & 334577 & 375122 & 382384 & 384852 & 315530 & 367323 & 428628 & 429083 & 443335 \\
\hline England & 23958 & 27123 & 31552 & 31552 & 31552 & 23958 & 27123 & 31552 & 31552 & 31552 \\
\hline Estonia & $\mathrm{m}$ & $\mathrm{m}$ & $\mathrm{m}$ & $\mathrm{m}$ & $\mathrm{m}$ & 3068 & 4379 & 7728 & 7728 & 8296 \\
\hline Finland & 19956 & 23333 & 28671 & 29191 & 29617 & 24961 & 30791 & 38222 & 38850 & 39382 \\
\hline France & 27288 & 28395 & 29831 & 29888 & 29740 & 27288 & 28395 & 29831 & 29888 & 29740 \\
\hline Germany & $\mathrm{m}$ & $\mathrm{m}$ & $\mathrm{m}$ & $\mathrm{m}$ & $\mathrm{m}$ & $\mathrm{m}$ & 43320 & 49587 & 50991 & 52016 \\
\hline Hungary ${ }^{5}$ & 751668 & 1739076 & 1779564 & 1778004 & 1768164 & 897168 & 1944576 & 1911204 & 1890288 & 1876836 \\
\hline Iceland & $\mathrm{m}$ & 2257836 & 3409863 & 3721409 & 3983241 & 1884000 & 2573556 & 3987224 & 4047201 & 4192652 \\
\hline Ireland & $\mathrm{m}$ & $\mathrm{m}$ & $\mathrm{m}$ & $\mathrm{m}$ & $\mathrm{m}$ & 32251 & 46591 & 52472 & 52472 & 52472 \\
\hline Israel & 68894 & 74610 & 115884 & 126521 & 132685 & 68421 & 73496 & 121858 & 125606 & 128240 \\
\hline Italy & $\mathrm{m}$ & 25234 & 27845 & 27845 & 27845 & 20849 & 25234 & 27845 & 27845 & 27845 \\
\hline Japan & $\mathrm{m}$ & $\mathrm{m}$ & $\mathrm{m}$ & $\mathrm{m}$ & $\mathrm{m}$ & 6645000 & 6236000 & 5456000 & 5456000 & 5456000 \\
\hline Korea & $\mathrm{m}$ & 38608000 & 42987600 & 44515200 & 45795600 & 26757000 & 39712000 & 44222400 & 45800400 & 47122800 \\
\hline Luxembourg & $\mathrm{m}$ & 62139 & 93182 & 97902 & 100350 & $\mathrm{~m}$ & 62139 & 93182 & 97902 & 100350 \\
\hline Mexico & 86748 & 124082 & 176627 & 183981 & 192555 & 86748 & 124082 & 176627 & 183981 & 192555 \\
\hline Netherlands & $\mathrm{m}$ & $\mathrm{m}$ & $\mathrm{m}$ & $\mathrm{m}$ & 48093 & $\mathrm{~m}$ & $\mathrm{~m}$ & $\mathrm{~m}$ & $\mathrm{~m}$ & 48093 \\
\hline New Zealand & $\mathrm{m}$ & $\mathrm{m}$ & $\mathrm{m}$ & $\mathrm{m}$ & $\mathrm{m}$ & $\mathrm{m}$ & $\mathrm{m}$ & $\mathrm{m}$ & $\mathrm{m}$ & 68074 \\
\hline Norway & $\mathrm{m}$ & 298812 & 374500 & 381500 & 402000 & $\mathrm{~m}$ & 302000 & 370000 & 377000 & 391000 \\
\hline Poland & $\mathrm{m}$ & 23328 & 32878 & 35101 & 36452 & $\mathrm{~m}$ & 23328 & 32878 & 35101 & 36452 \\
\hline Portugal & $\mathrm{m}$ & 22775 & 28069 & 24326 & 24326 & 17180 & 22775 & 28069 & 24326 & 24326 \\
\hline Scotland & 14022 & 29827 & 34200 & 34200 & 34200 & 22743 & 29827 & 34200 & 34200 & 34200 \\
\hline Slovak Republic & $\mathrm{m}$ & $\mathrm{m}$ & 6156 & 6236 & 6488 & $\mathrm{~m}$ & $\mathrm{~m}$ & 7518 & 7614 & 7918 \\
\hline Sweden $^{5}$ & $\mathrm{~m}$ & 261000 & 318000 & $\mathrm{~m}$ & 338100 & 248300 & 283200 & 322600 & $\mathrm{~m}$ & 349920 \\
\hline Switzerland $^{6}$ & $\mathrm{~m}$ & 77925 & 85904 & 87198 & 88041 & 85513 & 90341 & 96798 & 97436 & 98257 \\
\hline Turkey & 4560 & 16464 & 29426 & 32049 & 35018 & 4560 & 16464 & 29426 & 32049 & 35018 \\
\hline United States $^{5}$ & 36758 & 41500 & $\mathrm{~m}$ & 45300 & 46054 & 38040 & 41114 & 46122 & 45998 & 46763 \\
\hline Argentina & $\mathrm{m}$ & $\mathrm{m}$ & $\mathrm{m}$ & $\mathrm{m}$ & $\mathrm{m}$ & $\mathrm{m}$ & $\mathrm{m}$ & $\mathrm{m}$ & $\mathrm{m}$ & $\mathrm{m}$ \\
\hline Brazil & $\mathrm{m}$ & $\mathrm{m}$ & $\mathrm{m}$ & $\mathrm{m}$ & $\mathrm{m}$ & $\mathrm{m}$ & $\mathrm{m}$ & $\mathrm{m}$ & $\mathrm{m}$ & $\mathrm{m}$ \\
\hline China & $\mathrm{m}$ & $\mathrm{m}$ & $\mathrm{m}$ & $\mathrm{m}$ & $\mathrm{m}$ & $\mathrm{m}$ & $\mathrm{m}$ & $\mathrm{m}$ & $\mathrm{m}$ & $\mathrm{m}$ \\
\hline Colombia & $\mathrm{m}$ & $\mathrm{m}$ & $\mathrm{m}$ & $\mathrm{m}$ & 18461157 & $\mathrm{~m}$ & $\mathrm{~m}$ & $\mathrm{~m}$ & $\mathrm{~m}$ & 21232442 \\
\hline India & $\mathrm{m}$ & $\mathrm{m}$ & $\mathrm{m}$ & $\mathrm{m}$ & $\mathrm{m}$ & $\mathrm{m}$ & $\mathrm{m}$ & $\mathrm{m}$ & $\mathrm{m}$ & $\mathrm{m}$ \\
\hline Indonesia & $\mathrm{m}$ & $\mathrm{m}$ & $\mathrm{m}$ & $\mathrm{m}$ & $\mathrm{m}$ & $\mathrm{m}$ & $\mathrm{m}$ & $\mathrm{m}$ & $\mathrm{m}$ & $\mathrm{m}$ \\
\hline Latvia & 1321 & 2321 & 4071 & 4341 & 3060 & 1321 & 2321 & 4071 & 4341 & 3060 \\
\hline Russian Federation & $\mathrm{m}$ & $\mathrm{m}$ & $\mathrm{m}$ & $\mathrm{m}$ & $\mathrm{m}$ & $\mathrm{m}$ & $\mathrm{m}$ & $\mathrm{m}$ & $\mathrm{m}$ & $\mathrm{m}$ \\
\hline Saudi Arabia & $\mathrm{m}$ & $\mathrm{m}$ & $\mathrm{m}$ & $\mathrm{m}$ & $\mathrm{m}$ & $\mathrm{m}$ & $\mathrm{m}$ & $\mathrm{m}$ & $\mathrm{m}$ & $\mathrm{m}$ \\
\hline South Africa & $\mathrm{m}$ & $\mathrm{m}$ & $\mathrm{m}$ & $\mathrm{m}$ & $\mathrm{m}$ & $\mathrm{m}$ & $\mathrm{m}$ & $\mathrm{m}$ & $\mathrm{m}$ & $\mathrm{m}$ \\
\hline
\end{tabular}

Note: Years 2006, 2007, 2008, 2009, 2010 (i.e. columns 3-7; 13-17; 23-27; 33-37) are available for consultation on line (see StatLink below).

1. Data on salaries for countries now in the Euro area are shown in euros.

2. Break in time series following methodological changes in 2007 for upper secondary education.

3. Figures for the pre-primary level refer to primary teachers (in primary schools only) teaching pre-primary classes.

4. Break in time series following methodological changes in 2012

5. Actual base salaries.

6. Salaries after 11 years of experience.

Source: OECD. See Annex 3 for notes (www.oecd.org/education/education-at-a-glance-19991487.htm).

Please refer to the Reader's Guide for information concerning symbols for missing data and abbreviations.

StatLink 제으 http://dx.doi.org/10.1787/888933286624 
Table X2.4d. [2/2] Trends in teachers' salaries between 2000 and 2013, for teachers with minimum qualification ${ }^{1}$

Annual statutory teachers' salaries in public institutions for teachers with 15 years of experience and minimum qualification, by level of education, in national currency

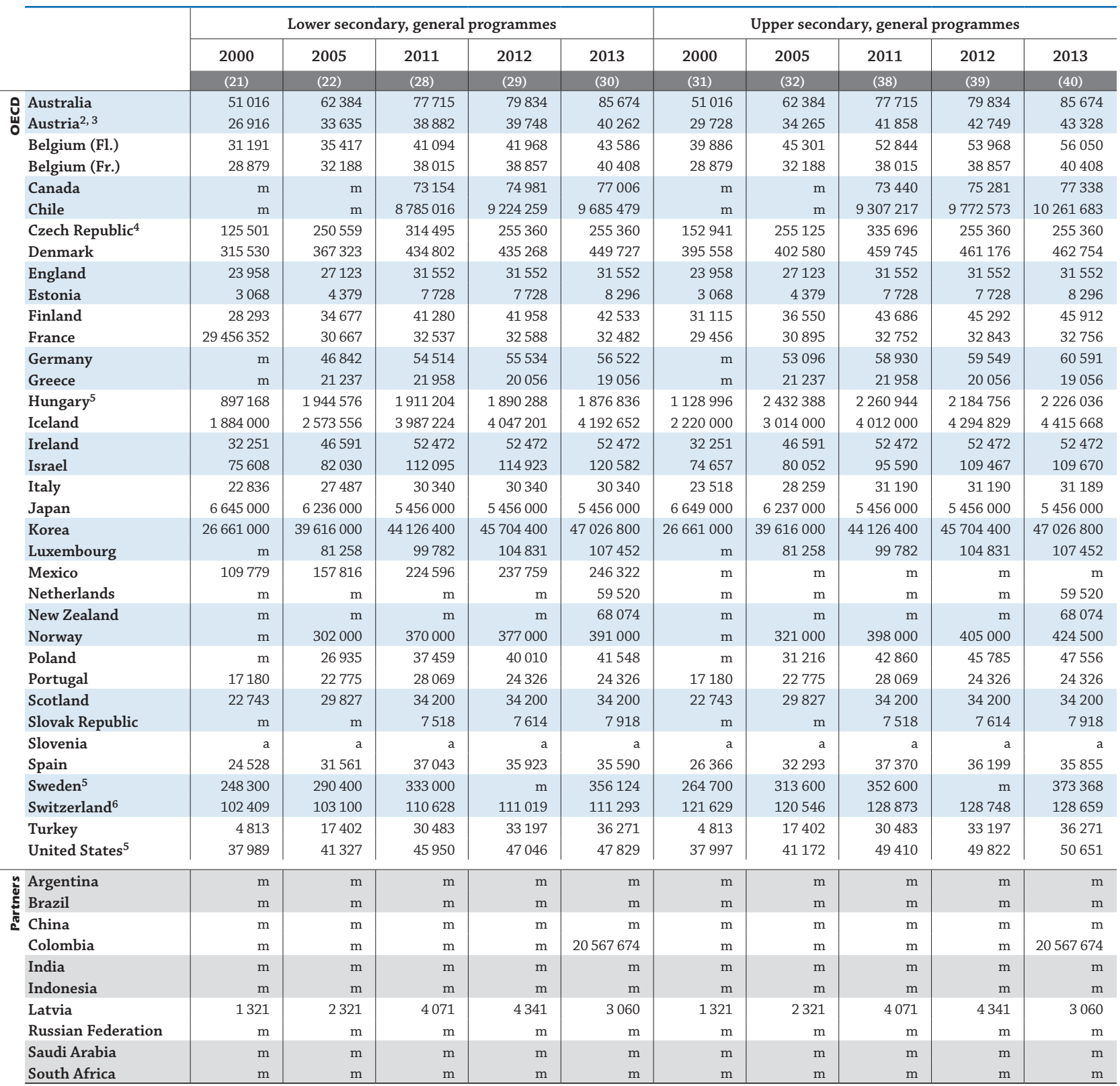

Note: Years 2006, 2007, 2008, 2009, 2010 (i.e. columns 3-7; 13-17; 23-27; 33-37) are available for consultation on line (see StatLink below).

1. Data on salaries for countries now in the Euro area are shown in euros.

2. Break in time series following methodological changes in 2007 for upper secondary education.

3. Figures for the pre-primary level refer to primary teachers (in primary schools only) teaching pre-primary classes.

4. Break in time series following methodological changes in 2012.

5. Actual base salaries.

6. Salaries after 11 years of experience.

Source: OECD. See Annex 3 for notes (www.oecd.org/education/education-at-a-glance-19991487.htm).

Please refer to the Reader's Guide for information concerning symbols for missing data and abbreviations.

StatLink 제근 http://dx.doi.org/10.1787/888933286624 
Table X2.4e. Reference statistics used in calculating teachers' salaries $(2000,2005-13)$

\begin{tabular}{|c|c|c|c|c|c|c|c|c|c|c|c|c|c|c|}
\hline & \multicolumn{3}{|c|}{$\begin{array}{l}\text { Purchasing power parity for } \\
\text { private consumption (PPP) }\end{array}$} & \multicolumn{10}{|c|}{ Private consumption deflators $(2005=100)$} & \multirow{2}{*}{$\begin{array}{c}\text { Reference } \\
\text { year for } 2013 \\
\text { salary data }\end{array}$} \\
\hline & 2012 & 2013 & Jan 2013 & $\begin{array}{c}\text { Jan } \\
2000\end{array}$ & $\begin{array}{c}\text { Jan } \\
2005\end{array}$ & $\begin{array}{c}\text { Jan } \\
2006\end{array}$ & $\begin{array}{c}\text { Jan } \\
2007\end{array}$ & $\begin{array}{c}\text { Jan } \\
2008\end{array}$ & $\begin{array}{c}\text { Jan } \\
2009\end{array}$ & $\begin{array}{c}\text { Jan } \\
2010\end{array}$ & $\begin{array}{c}\text { Jan } \\
2011\end{array}$ & $\begin{array}{c}\text { Jan } \\
2012\end{array}$ & $\begin{array}{c}\text { Jan } \\
2013\end{array}$ & \\
\hline & (1) & (2) & (3) & (4) & (5) & $(6)$ & (7) & $(8)$ & (9) & (10) & (11) & (12) & (13) & (14) \\
\hline Australia & 1.53 & 1.54 & 1.53 & 88 & 100 & 103 & 106 & 110 & 113 & 116 & 118 & 122 & 125 & 2013 \\
\hline Austria & 0.86 & 0.87 & 0.86 & 91 & 100 & 102 & 104 & 107 & 108 & 110 & 113 & 116 & 118 & 2013 \\
\hline Belgium (Fl.) ${ }^{2}$ & 0.89 & 0.90 & 0.90 & 91 & 100 & 103 & 106 & 109 & 111 & 111 & 114 & 117 & 119 & 2013 \\
\hline Canada & 1.29 & 1.29 & 1.29 & 91 & 100 & 102 & 103 & 105 & 106 & 106 & 108 & 110 & 112 & $2012 / 2013$ \\
\hline Chile & 391.92 & 393.15 & 392.53 & 86 & 100 & 104 & 107 & 113 & 118 & 121 & 125 & 130 & 134 & 2013 \\
\hline Czech Republic & 14.56 & 14.47 & 14.51 & 90 & 100 & 101 & 104 & 108 & 111 & 112 & 113 & 115 & 117 & $2012 / 2013$ \\
\hline Denmark & 8.41 & 8.42 & 8.42 & 92 & 100 & 102 & 104 & 106 & 109 & 111 & 113 & 116 & 118 & $2012 / 2013$ \\
\hline England $^{3}$ & 0.77 & 0.79 & 0.78 & 94 & 100 & 103 & 105 & 109 & 112 & 115 & 120 & 123 & 125 & $2012 / 2013$ \\
\hline Estonia & 0.62 & 0.63 & 0.63 & 82 & 100 & 105 & 112 & 121 & 126 & 128 & 134 & 140 & 144 & $2012 / 2013$ \\
\hline Finland & 0.99 & 1.00 & 0.99 & 93 & 100 & 101 & 103 & 106 & 108 & 110 & 113 & 116 & 119 & 2013 \\
\hline Greece & 0.75 & 0.72 & 0.74 & 87 & 100 & 103 & 107 & 111 & 114 & 116 & 120 & 122 & 121 & 2013 \\
\hline Hungary & 143.79 & 143.60 & 143.70 & 73 & 100 & 103 & 109 & 115 & 121 & 125 & 130 & 137 & 142 & 2013 \\
\hline Iceland & 142.56 & 144.95 & 143.75 & 81 & 100 & 105 & 112 & 122 & 137 & 145 & 149 & 156 & 163 & $2012 / 2013$ \\
\hline Ireland & 0.97 & 0.97 & 0.97 & 83 & 100 & 102 & 105 & 107 & 105 & 100 & 100 & 102 & 103 & $2012 / 2013$ \\
\hline Israel & 4.26 & 4.34 & 4.30 & 93 & 100 & 102 & 104 & 108 & 111 & 114 & 118 & 121 & 124 & $2012 / 2013$ \\
\hline Italy & 0.84 & 0.84 & 0.84 & 87 & 100 & 102 & 105 & 108 & 109 & 110 & 112 & 115 & 118 & $2012 / 2013$ \\
\hline Japan & 112.69 & 112.08 & 112.39 & 105 & 100 & 100 & 99 & 99 & 98 & 96 & 95 & 94 & 93 & $2012 / 2013$ \\
\hline Korea & 911.96 & 914.72 & 913.34 & 84 & 100 & 102 & 104 & 107 & 111 & 114 & 117 & 121 & 122 & 2013 \\
\hline Luxembourg & 0.97 & 0.98 & 0.97 & 90 & 100 & 103 & 105 & 107 & 109 & 110 & 112 & 114 & 115 & $2012 / 2013$ \\
\hline Mexico & 9.18 & 9.36 & 9.27 & 80 & 100 & 104 & 109 & 115 & 121 & 127 & 132 & 137 & 142 & $2012 / 2013$ \\
\hline Netherlands & 0.88 & 0.90 & 0.89 & 88 & 100 & 102 & 104 & 107 & 107 & 107 & 109 & 111 & 112 & $2012 / 2013$ \\
\hline New Zealand & 1.58 & 1.57 & 1.57 & 92 & 100 & 102 & 105 & 107 & 111 & 113 & 115 & 117 & 117 & 2013 \\
\hline Norway & 9.73 & 9.94 & 9.83 & 91 & 100 & 101 & 103 & 106 & 109 & 111 & 113 & 114 & 116 & $2012 / 2013$ \\
\hline Poland & 1.92 & 1.90 & 1.91 & 85 & 100 & 102 & 104 & 107 & 111 & 114 & 118 & 123 & 125 & $2012 / 2013$ \\
\hline Portugal & 0.67 & 0.66 & 0.66 & 85 & 100 & 104 & 107 & 111 & 111 & 111 & 113 & 115 & 116 & $2012 / 2013$ \\
\hline Scotland ${ }^{3}$ & 0.77 & 0.79 & 0.78 & 94 & 100 & 103 & 105 & 109 & 112 & 115 & 120 & 123 & 125 & $2012 / 2013$ \\
\hline Spain & 0.77 & 0.76 & 0.76 & 85 & 100 & 104 & 107 & 111 & 112 & 113 & 115 & 118 & 120 & $2012 / 2013$ \\
\hline Sweden & 9.11 & 9.22 & 9.17 & 93 & 100 & 101 & 103 & 105 & 108 & 110 & 111 & 113 & 113 & 2013 \\
\hline Switzerland & 1.55 & 1.54 & 1.55 & 97 & 100 & 101 & 103 & 104 & 105 & 105 & 105 & 105 & 104 & $2012 / 2013$ \\
\hline Turkey & 1.25 & 1.33 & 1.29 & 28 & 100 & 109 & 118 & 128 & 138 & 147 & 160 & 174 & 186 & 2013 \\
\hline United States & 1.00 & 1.00 & 1.00 & 90 & 100 & 103 & 105 & 108 & 110 & 111 & 113 & 115 & 117 & $2012 / 2013$ \\
\hline Argentina & $\mathrm{m}$ & $\mathrm{m}$ & $\mathrm{m}$ & $\mathrm{m}$ & $\mathrm{m}$ & $\mathrm{m}$ & $\mathrm{m}$ & $\mathrm{m}$ & $\mathrm{m}$ & $\mathrm{m}$ & $\mathrm{m}$ & $\mathrm{m}$ & $\mathrm{m}$ & $\mathrm{m}$ \\
\hline Brazil & 1.71 & 1.79 & 1.75 & 65 & 100 & 106 & 111 & 117 & 125 & 132 & 141 & 150 & 161 & 2013 \\
\hline China & $\mathrm{m}$ & $\mathrm{m}$ & $\mathrm{m}$ & $\mathrm{m}$ & $\mathrm{m}$ & $\mathrm{m}$ & $\mathrm{m}$ & $\mathrm{m}$ & $\mathrm{m}$ & $\mathrm{m}$ & $\mathrm{m}$ & $\mathrm{m}$ & $\mathrm{m}$ & $\mathrm{m}$ \\
\hline Colombia & 1209.94 & 1216.60 & 1213.27 & 72 & 100 & 104 & 109 & 115 & 120 & 124 & 128 & 133 & 136 & 2013 \\
\hline India & $\mathrm{m}$ & $\mathrm{m}$ & $\mathrm{m}$ & $\mathrm{m}$ & $\mathrm{m}$ & $\mathrm{m}$ & $\mathrm{m}$ & $\mathrm{m}$ & $\mathrm{m}$ & $\mathrm{m}$ & $\mathrm{m}$ & $\mathrm{m}$ & $\mathrm{m}$ & $\mathrm{m}$ \\
\hline Indonesia & $\mathrm{m}$ & $\mathrm{m}$ & $\mathrm{m}$ & $\mathrm{m}$ & $\mathrm{m}$ & $\mathrm{m}$ & $\mathrm{m}$ & $\mathrm{m}$ & $\mathrm{m}$ & $\mathrm{m}$ & $\mathrm{m}$ & $\mathrm{m}$ & $\mathrm{m}$ & $\mathrm{m}$ \\
\hline Latvia & 0.58 & 0.58 & 0.58 & 77 & 100 & 110 & 122 & 137 & 143 & 138 & 140 & 147 & 149 & $2012 / 2013$ \\
\hline Russian Federation & 16.74 & 16.72 & 16.73 & $\mathrm{~m}$ & $\mathrm{~m}$ & $\mathrm{~m}$ & $\mathrm{~m}$ & $\mathrm{~m}$ & $\mathrm{~m}$ & $\mathrm{~m}$ & $\mathrm{~m}$ & $\mathrm{~m}$ & $\mathrm{~m}$ & $2012 / 2013$ \\
\hline Saudi Arabia & $\mathrm{m}$ & $\mathrm{m}$ & $\mathrm{m}$ & $\mathrm{m}$ & $\mathrm{m}$ & $\mathrm{m}$ & $\mathrm{m}$ & $\mathrm{m}$ & $\mathrm{m}$ & $\mathrm{m}$ & $\mathrm{m}$ & $\mathrm{m}$ & $\mathrm{m}$ & $\mathrm{m}$ \\
\hline South Africa & $\mathrm{m}$ & $\mathrm{m}$ & $\mathrm{m}$ & $\mathrm{m}$ & $\mathrm{m}$ & $\mathrm{m}$ & $\mathrm{m}$ & $\mathrm{m}$ & $\mathrm{m}$ & $\mathrm{m}$ & $\mathrm{m}$ & $\mathrm{m}$ & $\mathrm{m}$ & $\mathrm{m}$ \\
\hline
\end{tabular}

1. Data on PPPs and GDP for countries now in the Euro area are shown in euros.

2. Data on PPPs and deflators refer to Belgium.

3. Data on PPPs and deflators refer to the United Kingdom.

Source: OECD. See Annex 3 for notes (www.oecd.org/education/education-at-a-glance-19991487.htm).

Please refer to the Reader's Guide for information concerning symbols for missing data and abbreviations.

StatLink 젝ㄴ http://dx.doi.org/10.1787/888933286638 
Table X2.4f. Average actual teachers' salaries in national currency (2013)

Average gross annual salary of teachers aged 25-64

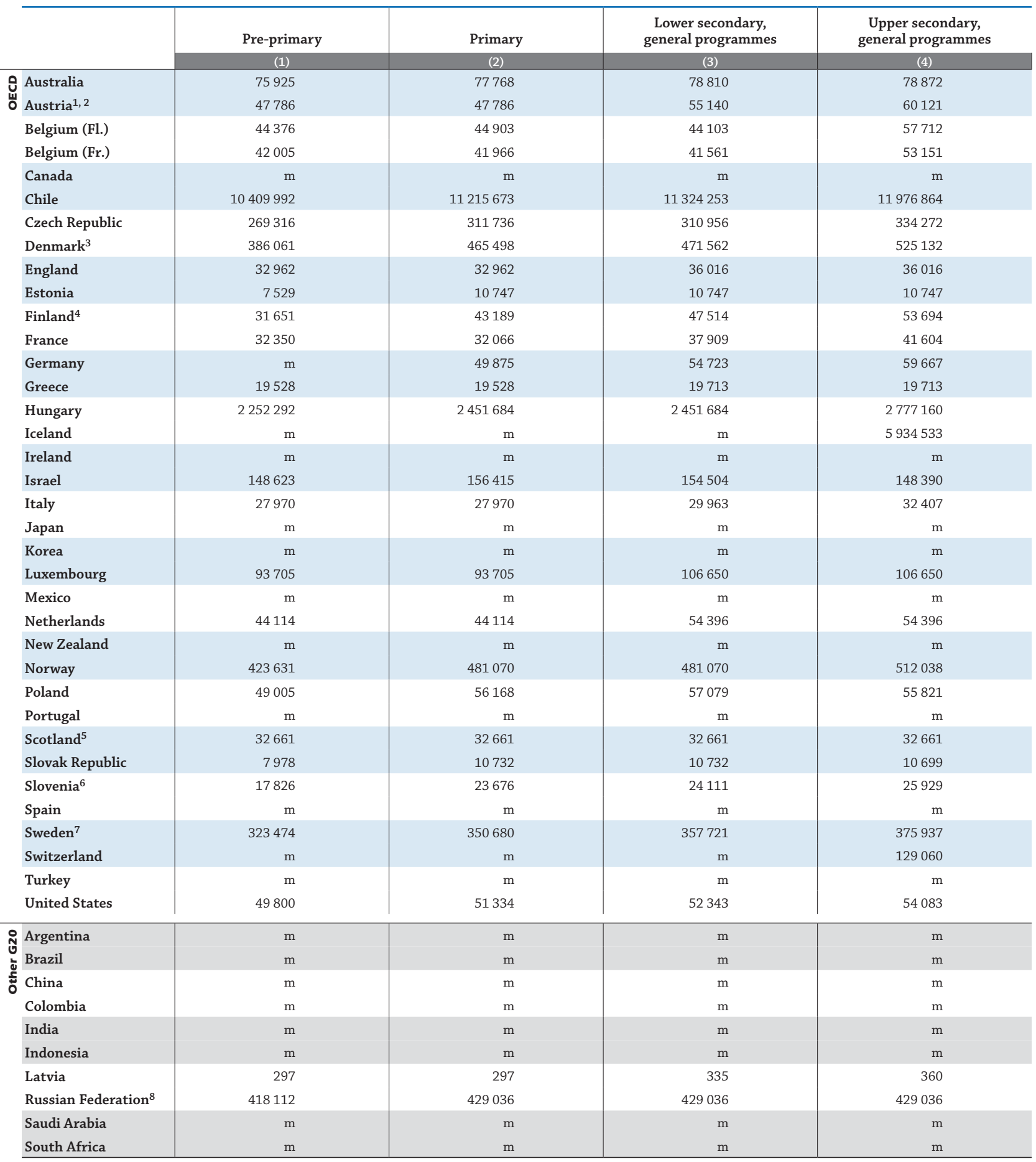

1. Figures for the pre-primary level refer to primary teachers (in primary schools only) teaching pre-primary classes.

2. Includes also data on actual salaries of headmasters, deputies and assistants.

3. Includes also data on actual salaries of teachers in early childhood educational development programmes for pre-primary education

4. Includes data on the majority, i.e. kindergarten teachers only for pre-primary education.

5. Includes all teachers, irrespective of their age.

6. Includes also data on actual salaries of pre-school teacher assistants for pre-primary education.

7. Average actual teachers' salaries, not including bonuses and allowances.

8. Average actual teachers' salaries for all teachers, irrespective of the level of education they teach.

Source: OECD. See Annex 3 for notes (www.oecd.org/education/education-at-a-glance-19991487.htm).

Please refer to the Reader's Guide for information concerning symbols for missing data and abbreviations.

StatLink 제이 $\mathrm{http}: / / \mathrm{dx}$.doi.org/10.1787/888933286640 


\section{General notes}

\section{Definitions}

Gross domestic product (GDP) refers to the producers' value of the gross outputs of resident producers, including distributive trades and transport, less the value of purchasers' intermediate consumption plus import duties. GDP is expressed in local money (in millions). For countries which provide this information for a reference year that is different from the calendar year (such as Australia and New Zealand), adjustments are made by linearly weighting their GDP between two adjacent national reference years to match the calendar year.

The GDP deflator is obtained by dividing the GDP expressed at current prices by the GDP expressed at constant prices. This provides an indication of the relative price level in a country.

GDP per capita is the gross domestic product (in equivalent USD converted using PPPs) divided by the population.

Purchasing power parity exchange rates (PPP) are the currency exchange rates that equalise the purchasing power of different currencies. This means that a given sum of money when converted into different currencies at the PPP rates will buy the same basket of goods and services in all countries. In other words, PPPs are the rates of currency conversion which eliminate the differences in price levels among countries. Thus, when expenditure on GDP for different countries is converted into a common currency by means of PPPs, it is, in effect, expressed at the same set of international prices so that comparisons between countries reflect only differences in the volume of goods and services purchased.

Total public expenditure, as used for the calculation of the education indicators, corresponds to the non-repayable current and capital expenditure of all levels of government. Current expenditure includes final consumption expenditure (e.g. compensation of employees, consumption of intermediate goods and services, consumption of fixed capital, and military expenditure), property income paid, subsidies, and other current transfers paid (e.g. social security, social assistance, pensions and other welfare benefits). Capital expenditure is spending to acquire and/or improve fixed capital assets, land, intangible assets, government stocks, and non-military, non-financial assets, and spending to finance net capital transfers.

\section{Sources}

The 2015 edition of the National Accounts of OECD Countries: Detailed Tables, Volume II.

OECD Analytical Database, February 2015. 


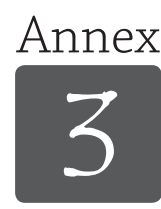

\section{SOURCES, METHODS AND TECHNICAL NOTES}

Annex 3 on sources and methods is available in electronic form only. It can be found at:

www.oecd.org/education/education-at-a-glance-19991487.htm 



\title{
CONTRIBUTORS TO THIS PUBLICATION
}

\begin{abstract}
Many people have contributed to the development of this publication.
The following lists the names of the country representatives who have taken part to the INES meetings and to the preparatory work leading to the publication of Education at a Glance 2015: OECD Indicators.
\end{abstract}

The OECD wishes to thank them all for their valuable efforts.

\author{
INES Working Party \\ Ms Maria Laura ALONSO (Argentina) \\ Mr Julián FALCONE (Argentina) \\ Ms Marcela JÁUREGUI (Argentina) \\ Ms Cheryl HOPKINS (Australia) \\ Mr Stuart FAUNT (Australia) \\ Mr Karl BAIGENT (Australia) \\ Mr Paul CMIEL (Australia) \\ Ms Rebecca SMEDLEY (Australia) \\ Mr Andreas GRIMM (Austria) \\ Ms Sabine MARTINSCHITZ (Austria) \\ Mr Mark NÉMET (Austria) \\ Mr Wolfgang PAULI (Austria) \\ Ms Helga POSSET (Austria) \\ Ms Natascha RIHA (Austria) \\ Mr Philippe DIEU (Belgium) \\ Ms Isabelle ERAUW (Belgium) \\ Ms Nathalie JAUNIAUX (Belgium) \\ Mr Guy STOFFELEN (Belgium) \\ Mr Raymond VAN DE SIJPE (Belgium) \\ Ms Ann VAN DRIESSCHE (Belgium) \\ Mr Daniel Jaime CAPISTRANO DE OLIVEIRA (Brazil) \\ Ms Carla D'Lourdes DO NASCIMENTO (Brazil) \\ Ms Juliana MARQUES DA SILVA (Brazil) \\ Ms Ana Carolina SILVA CIROTTO (Brazil) \\ Mr Patric BLOUIN (Canada) \\ Mr Patrice DE BROUCKER (Canada) \\ Mr Tomasz GLUSZYNSKI (Canada) \\ Ms Amanda HODGKINSON (Canada) \\ Mr Michael MARTIN (Canada) \\ Mr Enzo PIZZOFERRATO (Canada) \\ Mr Janusz ZIEMINSKI (Canada) \\ Ms María Paz DONOSO (Chile) \\ Ms Paola LEIVA (Chile) \\ Mr Francisco MENESES (Chile) \\ Mr Fabián RAMÍREZ (Chile) \\ Mr Roberto SCHURCH (Chile) \\ Ms María José SEPÚLVEDA (Chile) \\ Mr Juan Carlos BOLIVAR (Colombia) \\ Ms Jennifer DIAZ (Colombia) \\ Mr Javier Andrés RUBIO (Colombia) \\ Ms Azucena VALLEJO (Colombia) \\ Ms Elsa Nelly VELASCO (Colombia)

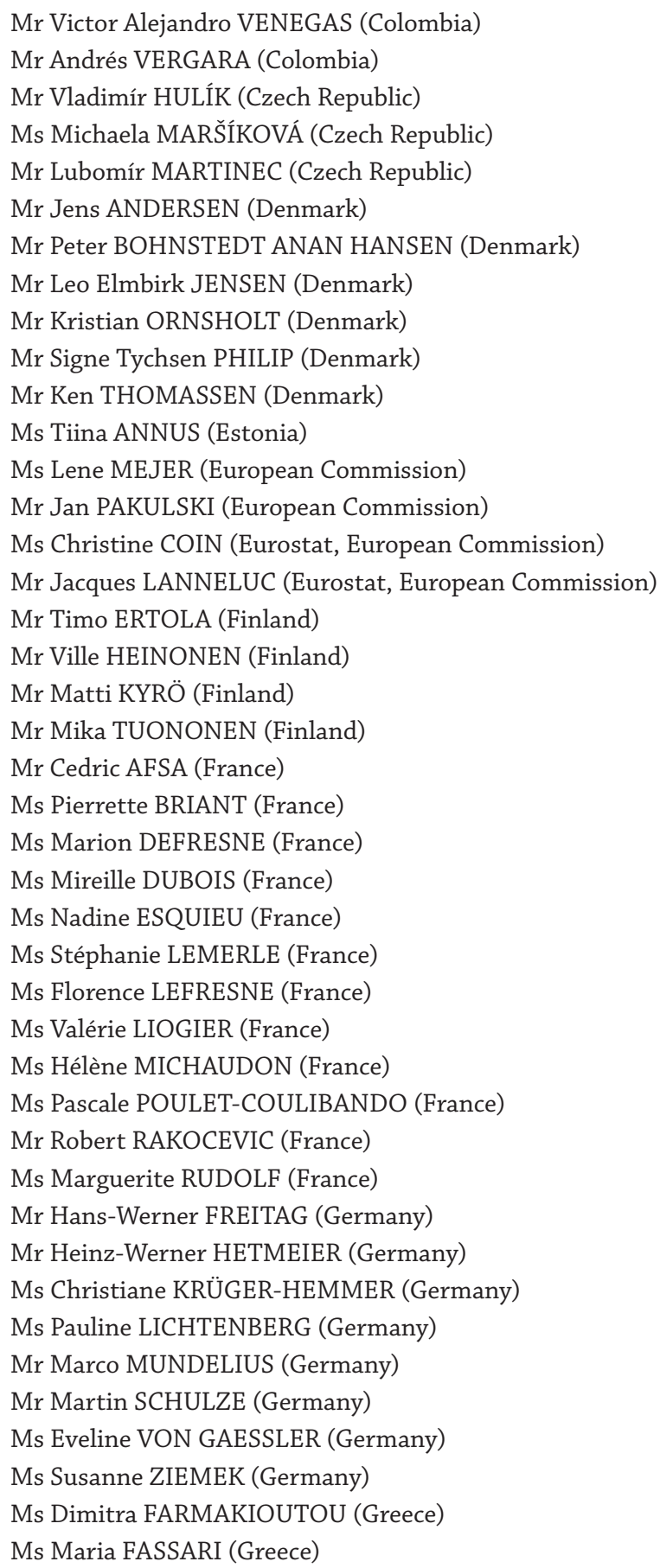


Mr Konstantinos KAMPANAKIS (Greece) Ms Akrivi NIKOLAKOPOULOU (Greece) Ms Athena PLESSA-PAPADAKI (Greece) Ms Tünde HAGYMÁSY (Hungary) Mr Tibor KÖNYVESI (Hungary) Mr László LIMBACHER (Hungary) Mr Kristián SZÉLL (Hungary) Mr Gunnar J. ÁRNASON (Iceland) Mr Julius BJORNSSON (Iceland) Ms Ásta URBANCIC (Iceland) Ms Ida KINTAMANI (Indonesia) Mr Yul Yunazwin NAZARUDDIN (Indonesia) Ms Siti SOFIA (Indonesia) Mr Gary Ó DONNCHADHA (Ireland) Mr Diarmuid REIDY (Ireland) Ms Nicola TICKNER (Ireland) Ms Sophie ARTSEV (Israel) Ms Yael ATIYAH (Israel) Mr Yoav AZULAY (Israel) Mr Yonatan BARON (Israel) Ms Lilach BITON (Israel) Ms Nava BRENNER (Israel) Ms Livnat GAVRIELOV (Israel) Mr Yosef GIDANIAN (Israel) Mr Pinhas KLEIN (Israel) Mr Aviel KRENTZLER (Israel) Mr Daniel LEVI-MAZLOUM (Israel) Ms Iris Avigail MATATYAHU (Israel) Mr Haim PORTNOY (Israel) Ms Naama STEINBERG (Israel) Ms Francesca BROTTO (Italy) Mr Massimiliano CICCIA (Italy) Ms Daniela DI ASCENZO (Italy) Ms Paola DI GIROLAMO (Italy) Ms Maria Teresa MORANA (Italy) Ms Claudia PIZZELLA (Italy) Mr Paolo SESTITO (Italy) Mr Paolo TURCHETTI (Italy) Mr Yuki MATSUO (Japan) Mr Takashi MURAO (Japan) Mr Yutaro NAGANO (Japan) Mr Hiromi SASAI (Japan) Mr Kenichiro TAKAHASHI (Japan) Ms Kumiko TANSHO-HIRABAYASHI (Japan) Ms Natsue SAITO (Japan)

Ms Hiroe HINO (Japan)

Mr Koji YANAGISAWA (Japan)

Ms Sujin CHOI (Korea)

Ms Jeongwon HWANG (Korea)

Ms Yoon Hee IM (Korea)

Ms Young Ok KIM (Korea)

Ms Hae Suk LEE (Korea)

Ms Hyun Mi LEE (Korea)

Ms Won Hee NA (Korea)

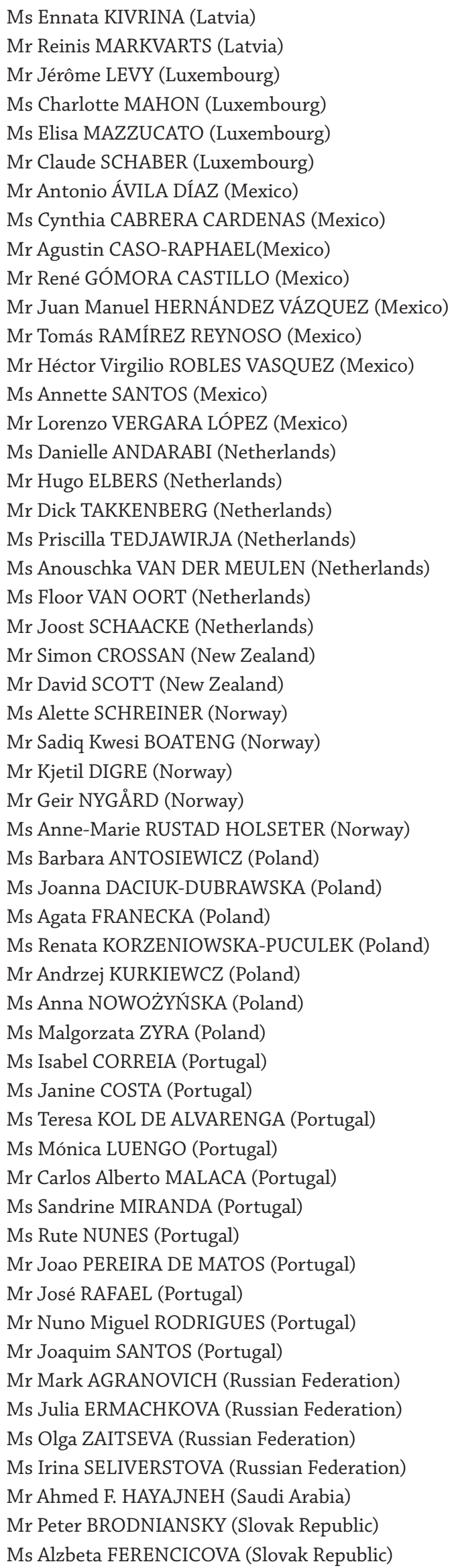


Ms Gabriela SLODICKOVA (Slovak Republic)

Mr Frantisek ZAJICEK (Slovak Republic)

Ms Barbara KRESAL-STERNIŠA (Slovenia)

Ms Breda LOŽAR (Slovenia)

Ms Tatjana ŠKRBEC (Slovenia)

Ms Andreja KOZMELJ (Slovenia)

Ms Darja VIDMAR (Slovenia)

Ms Bheki MPANZA (South Africa)

Mr Jacques APPELGRYN (South Africa)

Mr Nyokong MOSIUOA (South Africa)

Ms Hersheela NARSEE (South Africa)

Mr Vicente ALCAÑIZ MIÑANO (Spain)

Ms Laura ALONSO CARMONA (Spain)

Mr Eduardo DE LA FUENTE FUENTE (Spain)

Mr Jesús IBAÑEZ MILLA (Spain)

Mr Joaquín MARTÍN MUÑOZ (Spain)

Ms Cristina MONEO OCAÑA (Spain)

Mr Ismael SANZ LABRADOR (Spain)

Ms Carmen UREÑA UREÑA (Spain)

Ms Anna ERIKSSON (Sweden)

Mr Andreas FRODELL (Sweden)
Ms Maria GÖTHERSTRÖM (Sweden)

Ms Marie KAHLROTH (Sweden)

Ms Eva-Marie LARSSON (Sweden)

Mr Torbjörn LINDQVIST (Sweden)

Mr Kenny PETERSSON (Sweden)

Mr Hans-Åke ÖSTRÖM (Sweden)

Mr Alexander GERLINGS (Switzerland)

Ms Katrin HOLENSTEIN (Switzerland)

Mr Emanuel VON ERLACH (Switzerland)

Ms Hümeyra ALTUNTAŞ (Turkey)

Ms Nihan ERDAL (Turkey)

Ms Dilek GÜLEÇYÜZ (Turkey)

Ms Nur SALMANOĞLU (Turkey)

Ms Anuja SINGH (UNESCO Institute for Statistics)

Mr Said OULD AHMEDOU (UNESCO Institute for Statistics)

Ms Elisabeth BOYLING (United Kingdom)

Ms Emily KNOWLES (United Kingdom)

Ms Rachel DINKES (United States)

Ms Jana KEMP (United States)

Ms Ashley ROBERTS (United States)

Mr Thomas SNYDER (Chair INES Working Party, United States)

Network on Labour Market, Economic and Social Outcomes of Learning (LSO)

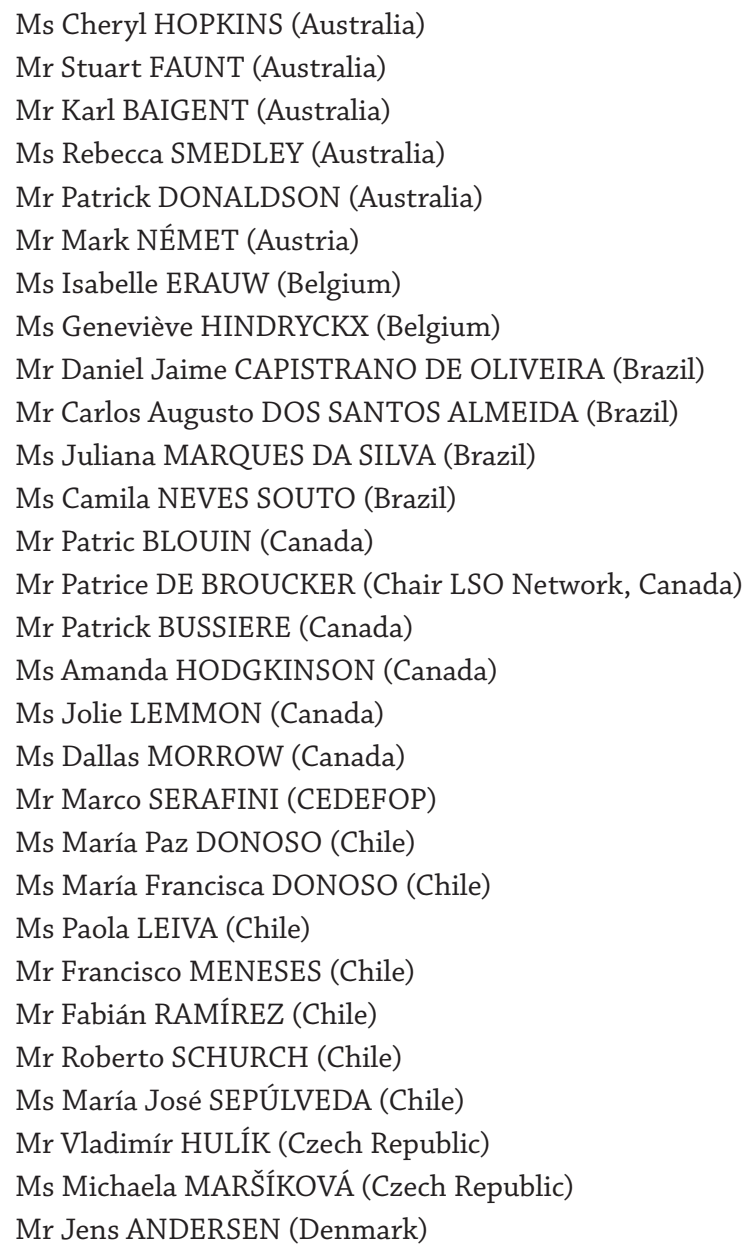

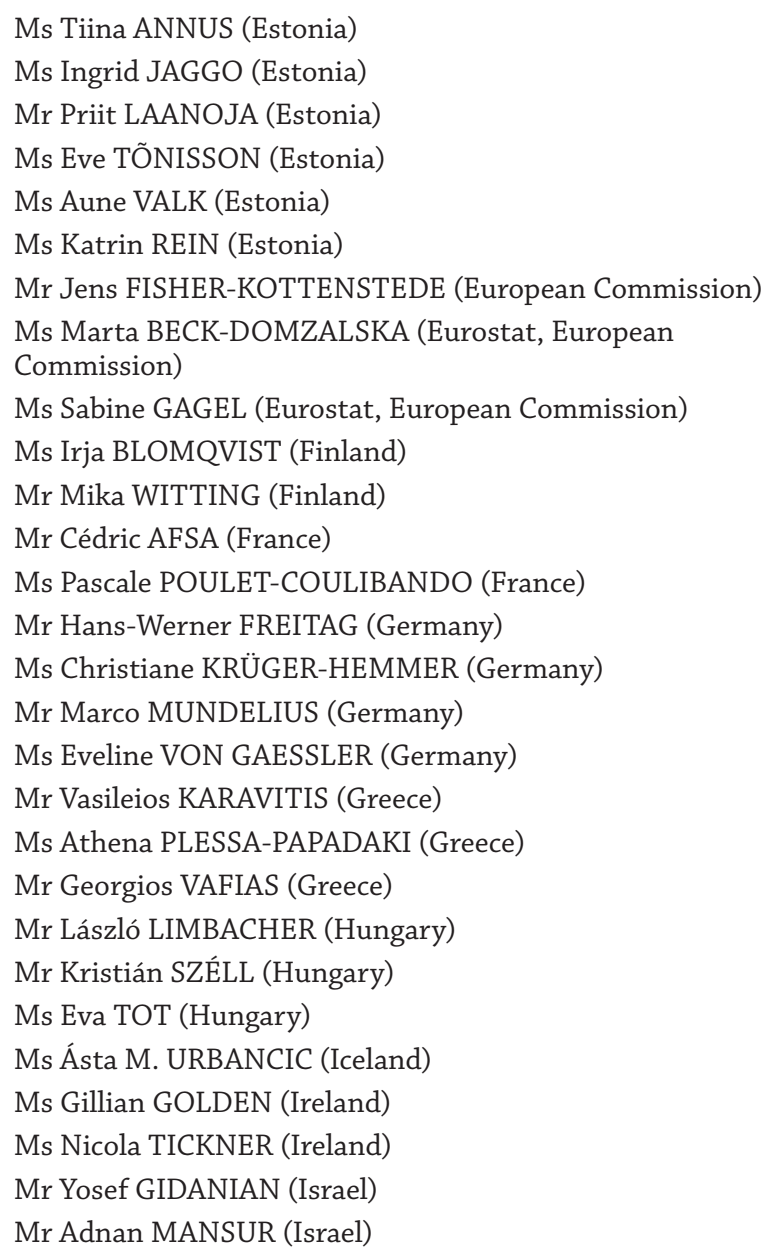


Mr Haim PORTNOY (Israel)

Mr Dan SHEINBERG (Israel)

Ms Raffaella CASCIOLI (Italy)

Mr Gaetano PROTO (Italy)

Ms Liana VERZICCO (Italy)

Ms Kaori MOCHIZUKI (Japan)

Ms Kumiko TANSHO-HIRABAYASHI (Japan)

Mr Chang Kyun CHAE (Korea)

Ms Jeongwon HWANG (Korea)

Ms Sung Bin MOON (Korea)

Mr Kirak RYU (Korea)

Mr Jung-seung Thomas YANG (Korea)

Mr Jérôme LEVY (Luxembourg)

Ms Karin MEYER (Luxembourg)

Mr Juan Manuel HERNÁNDEZ VÁZQUEZ (Mexico)

Mr Héctor ROBLES (Mexico)

Mr Gerardo TERRAZAS (Mexico)

Mr Ted REININGA (Netherlands)

Ms Tanja TRAAG (Netherlands)

Mr Francis VAN DER MOOREN (Netherlands)

Mr Bernard VERLAAN (Netherlands)

Mr Simon CROSSAN (New Zealand)

Mr David SCOTT (New Zealand)

Mr Sadiq-Kwesi BOATENG (Norway)

Ms Hild Marte BJØRNSEN (Norway)

Mr Geir NYGÅRD (Norway)

Ms Anne-Marie RUSTAD HOLSETER (Norway)

Mr Jacek MASLANKOWSKI (Poland)

Ms Anna NOWOŻYŃSKA (Poland)
Mr Carlos Alberto MALACA (Portugal)

Mr Joaquim SANTOS (Portugal)

Mr Mark AGRANOVICH (Russian Federation)

Ms Natalia KOVALEVA (Russian Federation)

Ms Elena SABELNIKOVA (Russian Federation)

Ms Olga ZAITSEVA (Russian Federation)

Mr Frantisek BLANAR (Slovak Republic)

Ms Gabriela JAKUBOVÁ (Slovak Republic)

Ms Tatjana SKRBEC (Slovenia)

Ms Irena SVETIN (Slovenia)

Mr Matej DIVJAK (Slovenia)

Ms Raquel HIDALGO (Spain)

Mr Raúl SAN SEGUNDO (Spain)

Ms Carmen UREÑA UREÑA (Spain)

Mr Torbjorn LINDQVIST (Sweden)

Mr Kenny PETERSSON (Sweden)

Mr Russell SCHMIEDER (Sweden)

Ms Wayra CABALLERO LIARDET (Switzerland)

Mr Emanuel VON ERLACH (Switzerland)

Mr Mutlu ALBAYRAK (Turkey)

Ms Hümeyra ALTUNTAŞ (Turkey)

Ms Dilek GÜLEÇYÜZ (Turkey)

Mr Cengiz SARAÇOĞLU (Turkey)

Mr Friedrich HUEBLER (UNESCO Institute for Statistics)

Ms Alison KENNEDY (UNESCO Institute for Statistics)

Mr Anthony CLARKE (United Kingdom)

Ms Rachel DINKES (United States)

Ms Ashley ROBERTS (United States)

Mr Thomas SNYDER (United States)

Network for the Collection and Adjudication of System-Level Descriptive Information on Educational Structures, Policies and Practices (NESLI)

Mr Karl BAIGENT (Australia)

Mr Stuart FAUNT (Australia)

Ms Cheryl HOPKINS (Australia)

Ms Rebecca SMEDLEY (Australia)

Mr Andreas GRIMM (Austria)

Mr Philippe DIEU (Belgium)

Ms Nathalie JAUNIAUX (Belgium)

Ms Bernadette SCHREUER (Belgium)

Mr Raymond VAN DE SIJPE (Belgium)

Ms Ann VAN DRIESSCHE (Belgium)

Mr Daniel Jaime CAPISTRANO DE OLIVEIRA (Brazil)

Ms Juliana MARQUES DA SILVA (Brazil)

Mr Richard FRANZ (Canada)

Ms Jolie LEMMON (Canada)

Ms María Paz DONOSO (Chile)

Ms Paola LEIVA (Chile)

Mr Francisco MENESES (Chile)

Mr Fabián RAMÍREZ (Chile)

Mr Roberto SCHURCH (Chile)

Ms María José SEPÚLVEDA (Chile)

Mr Vladimír HULÍK (Czech Republic)
Ms Michaela MARŠÍKOVÁ (Czech Republic)

Mr Lubomír MARTINEC (Czech Republic)

Mr Jorgen Balling RASMUSSEN (Denmark)

Ms Tiina ANNUS (Estonia)

Ms Hanna KANEP (Estonia)

Ms Kristel VAHER (Estonia)

Ms Lene MEJER (European Commission)

Ms Nathalie BAIDAK (Eurydice)

Ms Arlette DELHAXHE (Eurydice)

Ms Petra PACKALEN (Finland)

Mr Mika VÄISÄNEN (Finland)

Ms Kristiina VOLMARI (Finland)

Ms Florence LEFRESNE (France)

Mr Robert RAKOCEVIC (France)

Ms Pia BRUGGER (Germany)

Mr Marco MUNDELIUS (Germany)

Ms Dimitra FARMAKIOTOU (Greece)

Ms Maria FASSARI (Greece)

Ms Eudokia KARDAMITSI (Greece)

Mr Georgios MALLIOS (Greece)

Mr Stylianos MERKOURIS (Greece) 
Mr Konstantinos PAPACHRISTOS (Greece)

Ms Athena PLESSA-PAPADAKI (Greece)

Ms Anna IMRE (Hungary)

Mr Gunnar J. ÁRNASON (Iceland)

Ms Asta URBANCIC (Iceland)

Mr Gary Ó DONNCHADHA (Ireland)

Ms Nicola TICKNER (Ireland)

Mr Yoav AZULAY (Israel)

Ms Livnat GAVRIELOV (Israel)

Mr Yosef GIDANIAN (Israel)

Mr Pinhas KLEIN (Israel)

Mr Daniel LEVI-MAZLOUM (Israel)

Mr David MAAGAN (Israel)

Mr Haim PORTNOY (Israel)

Ms Gianna BARBIERI (Italy)

Ms Lucia DE FABRIZIO (Italy)

Mr Naruhiko KAWASE (Japan)

Ms Kayo KIRIHARA (Japan)

Ms Kumiko TANSHO-HIRABAYASHI (Japan)

Ms Sujin CHOI (Korea)

Ms Jeongwon HWANG (Korea)

Ms Sung Bim MOON (Korea)

Mr Gilles HIRT (Luxembourg)

Mr Jérôme LEVY (Luxembourg)

Ms Ana Maria ACEVES ESTRADA (Mexico)

Mr Antonio ÁVILA DÍAZ (Mexico)

Ms Cynthia CABRERA CARDENAS (Mexico)

Mr Juan Martín SOCA DE IÑIGO (Mexico)

Ms Linda DE PAEPE (Netherlands)

Mr Dick VAN VLIET (Netherlands)

Mr Hans RUESINK (Netherlands)

Ms Marian HULSHOF (Netherlands)

Mr Simon CROSSAN (New Zealand)

Mr Cyril MAKO (New Zealand)

Mr David SCOTT (New Zealand)
Mr Kjetil HELGELAND (Norway)

Mr Kjetil DIGRE (Norway)

Ms Renata KARNAS (Poland)

Ms Renata KORZENIOWSKA-PUCULEK (Poland)

Ms Anna NOWOŻYŃSKA (Poland)

Mr Joaquim SANTOS (Portugal)

Mr Mark AGRANOVICH (Russian Federation)

Ms Alzbeta FERENCICOVA (Slovak Republic)

Ms Gabriela SLODICKOVA (Slovak Republic)

Ms Andreja BARLE LAKOTA (Slovenia)

Ms Ksenija BREGAR GOLOBIČ (Slovenia)

Ms Barbara KRESAL-STERNIŠA (Slovenia)

Ms Tanja TAŠTANOSKA (Slovenia)

Ms Duša MARJETIČ (Slovenia)

Ms Nataša HAFNER-VOJČIĆ (Slovenia)

Mr Inmaculada CABEZALÍ MONTERO (Spain)

Ms Laura ALONSO CARMONA (Spain)

Ms María CÓRDOBA HITA (Spain)

Mr Joaquin MARTIN MUÑOZ (Spain)

Ms Camilla THINSZ FJELLSTROM (Sweden)

Ms Helena WINTGREN (Sweden)

Ms Rejane DEPPIERRAZ (Switzerland)

Ms Katrin MÜHLEMANN (Switzerland)

Ms Hümeyra ALTUNTAŞ (Turkey)

Ms Nihan ERDAL (Turkey)

Ms Dilek GÜLEÇYÜZ (Turkey)

Mr Olivier Labé (UNESCO Institute for Statistics)

Ms Elisabeth BOYLING (United Kingdom)

Ms Louise CUTHBERTSON (United Kingdom)

Mr Adrian HIGGINBOTHAM (United Kingdom)

Mr Christopher MORRISS (United Kingdom)

Ms Rachel DINKES (United States)

Ms Jana KEMP (United States)

Mr Thomas SNYDER (Chair NESLI Network, United States)

Other contributors to this publication

Ms Anna BORKOWSKY (LSO consultant)

BRANTRA SPRL (French translation)

Gavan CONLON (LSO consultant)

Marguerita LANE (LSO consultant)
Mr Gary MIRON (NESLI consultant)

Mr Dan SHERMAN (LSO consultant)

Ms Fung-Kwan TAM (layout) 



\section{EDUCATION INDICATORS IN FOCUS}

Education Indicators in Focus is a series of OECD briefs that highlight specific indicators in Education at a Glance that are of particular interest to policy makers and practitioners. These briefs provide a detailed look into current issues in pre-primary, primary and secondary education, higher education, and adult outcomes from a global perspective. They contain an engaging mix of text, tables and charts that describe the international context of the most pressing questions in education policy and practice.

The complete series is available at:

English: http://dx.doi.org/10.1787/22267077

French: http://dx.doi.org/10.1787/22267093

"How do differences in social and cultural background influence access to higher education and the completion of studies?", Education Indicators in Focus, No. 35 (2015)

http://dx.doi.org/10.1787/5jrs703c47s1-en

"What are the advantages today of having an upper secondary qualification?", Education Indicators in Focus, No. 34 (2015)

http://dx.doi.org/10.1787/5jrw5p4jn426-en

“Focus on vocational education and training (VET) programmes", Education Indicators in Focus, No. 33 (2015) http://dx.doi.org/10.1787/5jrxtk4cg7wg-en

“Are education and skills being distributed more inclusively?", Education Indicators in Focus, No. 32 (2015)

http://dx.doi.org/10.1787/5js0bsgdtr28-en

“How is the global talent pool changing (2013, 2030)?", Education Indicators in Focus, No. 31 (2015)

http://dx.doi.org/10.1787/5js33lf9jk41-en

“Education and employment: What are the gender differences?”, Education Indicators in Focus, No. 30 (2015) http://dx.doi.org/10.1787/5js4q17gg540-en

"How much time do teachers spend on teaching and non-teaching activities?", Education Indicators in Focus, No. 29 (2015)

http://dx.doi.org/10.1787/5js64kndz1f3-en

"Are young people attaining higher levels of education than their parents?", Education Indicators in Focus, No. 28 (2015)

http://dx.doi.org/10.1787/5js7lx8zx90r-en

“What are the earnings advantages from education?", Education Indicators in Focus, No. 27 (2014)

http://dx.doi.org/10.1787/5jxrcllj8pwl-en

“Learning Begets Learning: Adult Participation in Lifelong Education”, Education Indicators in Focus, No. 26 (2014)

http://dx.doi.org/10.1787/5jxsvvmr9z8n-en

"Who are the doctorate holders and where do their qualifications lead them?", Education Indicators in Focus, No. 25 (2014)

http://dx.doi.org/10.1787/5jxv8xsvp1g2-en

"How innovative is the education sector?", Education Indicators in Focus, No. 24 (2014)

http://dx.doi.org/10.1787/5jz1157b915d-en 
"At what age do university students earn their first degree?", Education Indicators in Focus, No. 23 (2014) http://dx.doi.org/10.1787/5jz3wl5rvjtk-en

"How much time do primary and lower secondary students spend in the classroom?", Education Indicators in Focus, No. 22 (2014) http://dx.doi.org/10.1787/5jz44fnl1t6k-en

"How much are teachers paid and how much does it matter?", Education Indicators in Focus, No. 21 (2014) http://dx.doi.org/10.1787/5jz6wn8xjvvh-en

"How old are the teachers?", Education Indicators in Focus, No. 20 (2014)

http://dx.doi.org/10.1787/5jz76b5dhsnx-en

“What are tertiary students choosing to study?", Education Indicators in Focus, No. 19 (2014)

http://dx.doi.org/10.1787/5jz8ssmzg5q4-en

"What is the impact of the economic crisis on public education spending?", Education Indicators in Focus, No. 18 (2013)

http://dx.doi.org/10.1787/5jzbb2sprz20-en

“Does upper secondary vocational education and training improve the prospects of young adults?", Education Indicators in Focus, No. 17 (2013)

http://dx.doi.org/10.1787/5jzbb2st8851-en

"How can countries best produce a highly-qualified young labour force?", Education Indicators in Focus, No. 16 (2013)

http://dx.doi.org/10.1787/5k3wb8khp3zn-en

“How are university students changing?", Education Indicators in Focus, No. 15 (2013)

http://dx.doi.org/10.1787/5k3z04ch3d5c-en

"How is international student mobility shaping up?", Education Indicators in Focus, No. 14 (2013)

http://dx.doi.org/10.1787/5k43k8r4k821-en

"How difficult is it to move from school to work?", Education Indicators in Focus, No. 13 (2013)

http://dx.doi.org/10.1787/5k44zcplv70q-en

"Which factors determine the level of expenditure on teaching staff?", Education Indicators in Focus, No. 12 (2013)

http://dx.doi.org/10.1787/5k4818h31242-en

"How do early childhood education and care (ECEC) policies, systems and quality vary across OECD countries?", Education Indicators in Focus, No. 11 (2013)

http://dx.doi.org/10.1787/5k49czkz4bq2-en

"What are the social benefits of education?", Education Indicators in Focus, No. 10 (2013)

http://dx.doi.org/10.1787/5k4ddxnl39vk-en

“How does class size vary around the world?", Education Indicators in Focus, No. 9 (2012)

http://dx.doi.org/10.1787/5k8x7gvpr9jc-en

"Is increasing private expenditure, especially in tertiary education, associated with less public funding and less equitable access?", Education Indicators in Focus, No. 8 (2012)

http://dx.doi.org/10.1787/5k8zs43nlm42-en

"How well are countries educating young people to the level needed for a job and a living wage?", Education Indicators in Focus, No. 7 (2012)

http://dx.doi.org/10.1787/5k91d4fsqj0w-en

"What are the returns on higher education for individuals and countries?", Education Indicators in Focus, No. 6 (2012)

http://dx.doi.org/10.1787/5k961169d8tg-en 
“How is the global talent pool changing?", Education Indicators in Focus, No. 5 (2012)

http://dx.doi.org/10.1787/5k97krns40d4-en

"How pronounced is income inequality around the world - and how can education help reduce it?", Education Indicators in Focus, No. 4 (2012)

http://dx.doi.org/10.1787/5k97krntvqtf-en

"How are girls doing in school - and women doing in employment - around the world?", Education Indicators in Focus, No. 3 (2012)

http://dx.doi.org/10.1787/5k9csf9bxzs7-en

"How are countries around the world supporting students in higher education?", Education Indicators in Focus, No. 2 (2012)

http://dx.doi.org/10.1787/5k9fd0kd59f4-en

"How has the global economic crisis affect people with different levels of education?", Education Indicators in Focus, No. 1 (2012)

http://dx.doi.org/10.1787/5k9fgpwlc6s0-en 



\section{ORGANISATION FOR ECONOMIC CO-OPERATION AND DEVELOPMENT}

The OECD is a unique forum where governments work together to address the economic, social and environmental challenges of globalisation. The OECD is also at the forefront of efforts to understand and to help governments respond to new developments and concerns, such as corporate governance, the information economy and the challenges of an ageing population. The Organisation provides a setting where governments can compare policy experiences, seek answers to common problems, identify good practice and work to co-ordinate domestic and international policies.

The OECD member countries are: Australia, Austria, Belgium, Canada, Chile, the Czech Republic, Denmark, Estonia, Finland, France, Germany, Greece, Hungary, Iceland, Ireland, Israel, Italy, Japan, Korea, Luxembourg, Mexico, the Netherlands, New Zealand, Norway, Poland, Portugal, the Slovak Republic, Slovenia, Spain, Sweden, Switzerland, Turkey, the United Kingdom and the United States. The European Union takes part in the work of the OECD.

OECD Publishing disseminates widely the results of the Organisation's statistics gathering and research on economic, social and environmental issues, as well as the conventions, guidelines and standards agreed by its members. 


\title{
Education at a Glance 2015 OECD INDICATORS
}

Education at a Glance: OECD Indicators is the authoritative source for information on the state of education around the world. It provides data on the structure, finances and performance of education systems in the 34 OECD countries and a number of partner countries.

With more than 100 charts, 150 tables and links to another 150 tables on line, Education at a Glance 2015 provides key information on the output of educational institutions; the impact of learning across countries; the financial and human resources invested in education; access, participation and progression in education; and the learning environment and organisation of schools.

New material in the 2015 edition includes:

- 2014 data on educational attainment and participation in the labour market and 2015 data on instruction time, and on appraisal, evaluation and assessment mechanisms;

- more detailed analysis of participation in early childhood and tertiary levels of education;

- indicators on the impact of skills on employment and earnings, gender differences in education and employment, and teacher and school leader appraisal systems;

- analysis of first generation tertiary-educated adults and their educational and social mobility, labour market outcomes for recent graduates, and participation in employer-sponsored formal and/or non-formal education;

- data and analysis on the skills and readiness to use information and communication technology for problem solving in teaching and learning;

- information on recess and breaks during the school day, teachers' typical qualifications, criteria for determining teachers' base salaries and additional payments for teachers, and salaries

for tertiary faculty; and

- for the first time, data from Costa Rica and Lithuania.

The Excel ${ }^{\mathrm{TM}}$ spreadsheets used to create the tables and charts in Education at a Glance are available via the StatLinks provided throughout the publication. Tables and charts, as well as the complete OECD education database, are available via the OECD education website at www.oecd.org/education/education-at-aglance-19991487.htm. Updated data can be found on line at http://dx.doi.org/10.1787/eag-data-en.

\author{
Contents \\ Chapter A. The output of educational institutions and the impact of learning \\ Chapter B. Financial and human resources invested in education \\ Chapter C. Access to education, participation and progression \\ Chapter D. The learning environment and organisation of schools
}

Please cite this publication as:

OECD (2015), Education at a Glance 2015: OECD Indicators, OECD Publishing.

http://dx.doi.org/10.1787/eag-2015-en

This work is published on the OECD iLibrary, which gathers all OECD books, periodicals and statistical databases. Visit www.oecd-ilibrary.org and do not hesitate to contact us for more information.

\section{5}

